\title{
SUPPORTING DOCUMENT FOR THE HISTORICAL TANK CONTENT ESTIMATE FOR
}

\section{S TANK FARM}

\section{WORK ORDER ER4945}

\author{
Prepared for
}

\author{
Westinghouse Hanford Company \\ June 1994
}

Prepared by

ICF Kaiser Hanford Company

Richland, Washington 


\section{DISCLAIMER}

Portions of this document may be illegible in electronic image products. Images are produced from the best available original document. 


\section{DISTRIBUTION SHEET}

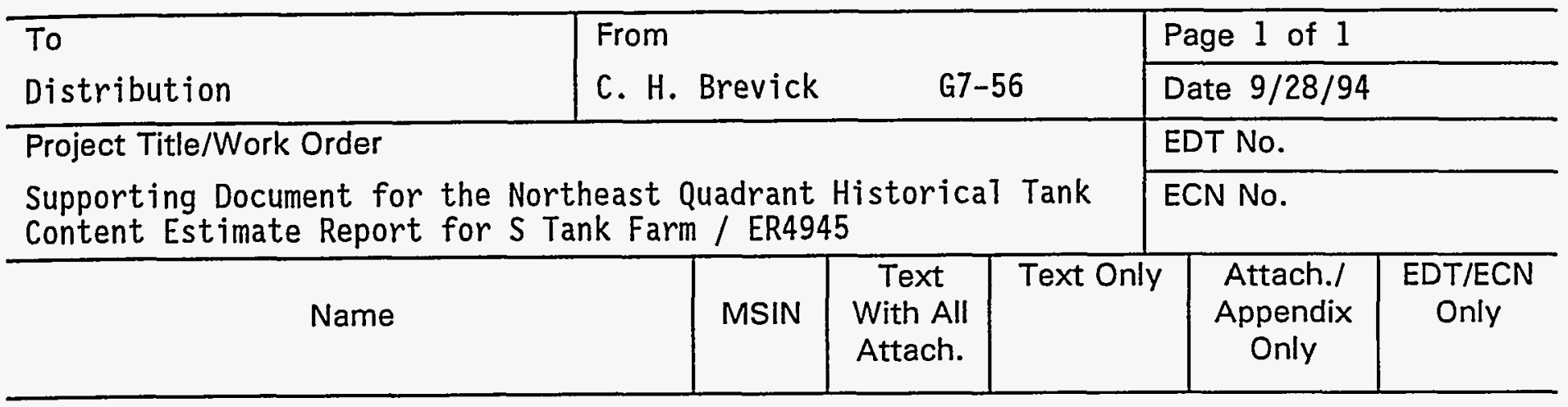

Westinghouse Hanford Company

T. M. Brown

G. Jansen

P. Sathyanarayana

B. C. Simpson -

TFIC (Tank Farm Information Center)

Central Files

OSTI

$\begin{array}{ll}\text { R2-12 } & X \\ H 6-33 & X \\ \text { R2-12 } & X(2) \\ \text { R2-12 } & X \\ \text { RI-20 } & X \\ \text { L8-04 } & X(2) \\ \text { L8-07 } & X(2)\end{array}$

ICF-Kaiser Hanford Company

C. H. Brevick

$\begin{array}{ll}G 7-56 & X \\ G 7-57 & X \\ S 2-54 & X \\ G 7-57 & X \\ E 6-46 & X \\ G 7-57 & X \\ G 7-57 & X \\ E 6-63 & 0 \\ G 3-11 & X\end{array}$

L. A. Gaddis

M. W. Hoffmann

E. D. Johnson

R. L. Newe 11

W. W. Pickett

A. C. Walsh

ICF-KH Publications

ICF-KH Doc Control

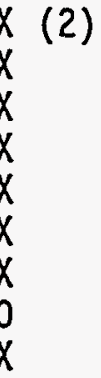

Department of Energy - Richland Operations

J. M. Clark

S7-54

$x$

Los Alamos National Laboratory

S. F. Agnew

Los Alamos National Laboratory

$X(2)$

MS-J586 - CST-14

P.0. Box 1663

Los Alamos, NM 87545

Los Alamos Technical Associates

D. S. DeLorenzo

Los A7amos Technical Associates

750 Swift, Suite 4

Richland, WA 99352 $x$ 


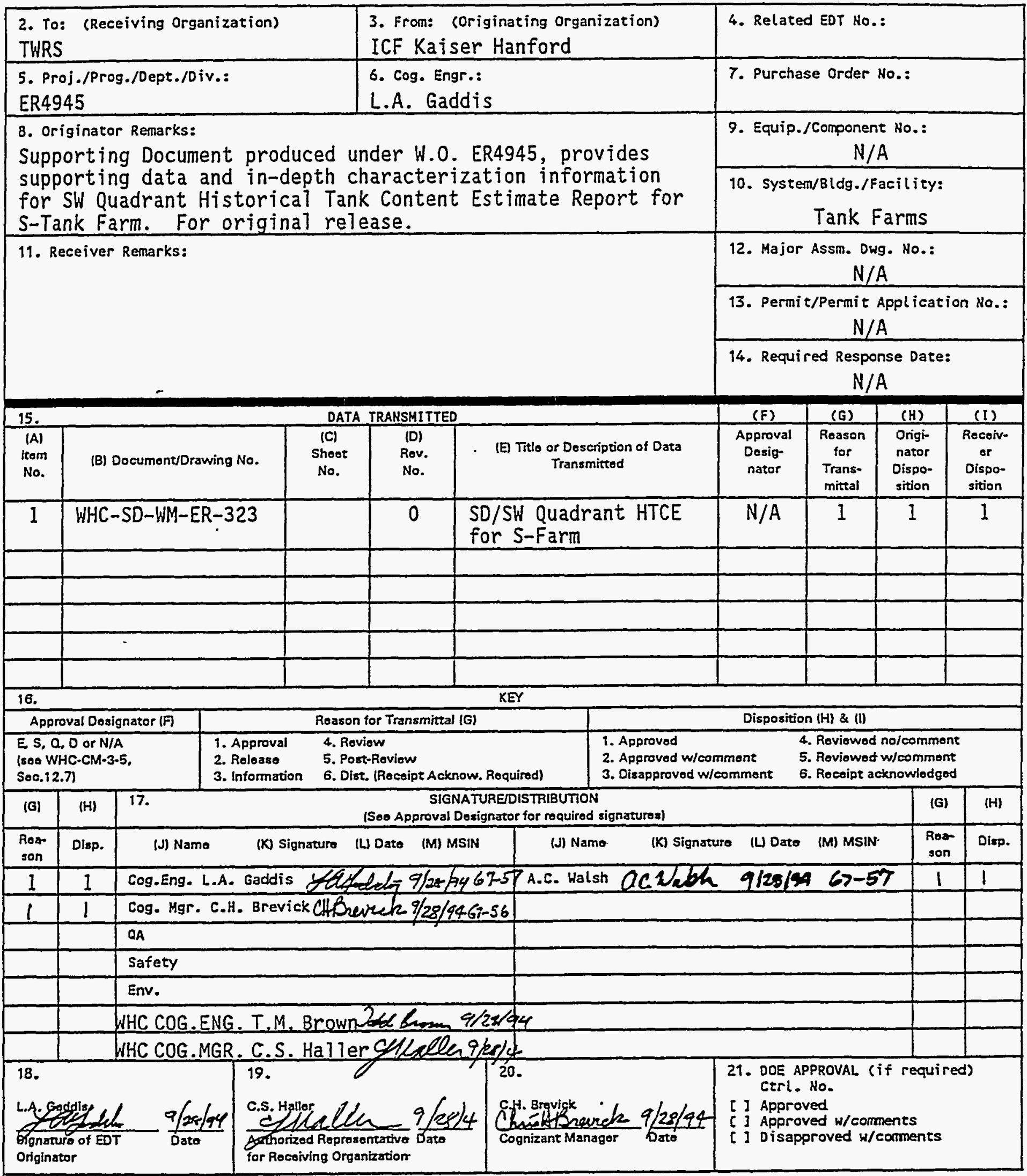




\section{RELEASE AUTHORIZATION}

Document Number: HHC-SD-WM-ER-323, REV.0

Document Title: Supporting Document for the SW Quadrant Historical

- Tank Content Estimate Report for S-Tank Farm

Release Date: $\quad$ September 28, 1994

This document was reviewed following the procedures described in WHC-CM-3-4 and is:

APPROVED FOR PUBLIC RELEASE

WHC Information Release Administration Specialist:

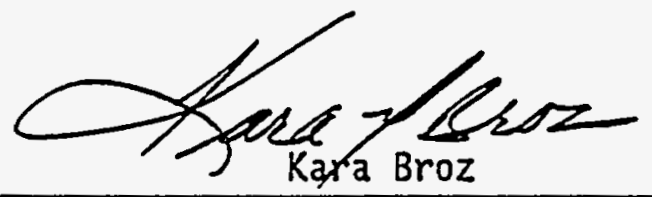

(Signature) 
2. Title

Supporting Document for the SW Quadrant Historical Tank Content Estimate for S-Tank Farm

\section{Key Hords}

S-Tank Farm, Tank Waste Characterization, Historical Tank Content Estimate, Historical Waste Transfer, tank waste level data, tank physical information, waste temperature, dry well data, liquid observation well, analytical data, waste sampling.
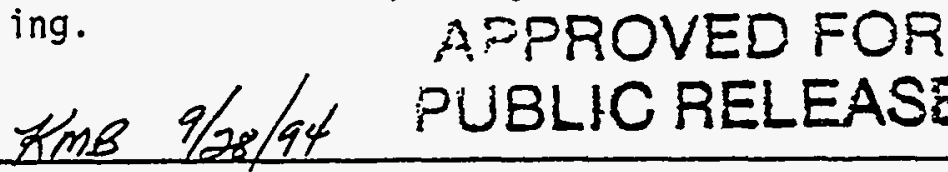

3. Number

WHC-SD-WM-ER-323
4. Rev No.

0

\section{Author}

Name: C.H. Brevick,

L.A. Gaddis, A.C. Walsh

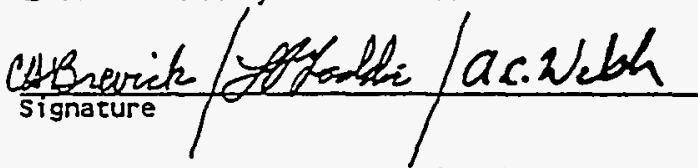

organization/charge code $58250 /$

ER4945/Tank Farm Technical Support

\section{Abstract}

This Supporting Document provides historical characterization information gathered on S-Tank Farm, such as historical waste transfer and level data, tank physical information, temperature data, sampling data, and dry well and liquid observation well data for Historical Tank Content Estimate of the SW Quadrant at the Hanford 200 West Area.
8. PUREOSE AND USE OF DOCUMENT - This document was prepared for use with in the U.S. Department of Energy and its contractors $1 t$ is to be used onty to perform, direct, or integrate work under U.S. Department of foergy contracts. This docynent is not approved for public release untit ceviewed.
PATENT STATUS - This document since it is transmitted in advance of patent clearance is made avaitable in confidence solely for use in performane of work under eantracts with the U.S:- Department of Energy. This document is not to de-aubl ished nor its contents pherwise disseminated or used for purposes orker than specifies above before patent approval for such release or use thas been secured, upon request, from the Patent Counsel, U.S. Department of Energy field Office, Richland, WA.

DISCLAIMER - This report was prepared as an account of work sponsored by an agency of the United States Government. Neither the United States Government nor any agency thereof, nor any of their employees, nor any of their contractors, subcontractors or their employees, makes any warranty, express or implied, or assumes any legal liability or responsibility for the accuracy, completeness, or any third party's use or the results of such use of any information, apparatus, product, or process disclosed, or represents that its use would not infringe privately owned rights. Reference herein to any specific cormercial product, process, or service by trade name, trademark, manufacturer, or otherwise, does not necessarily constiture or imply its endorsenent, recomendation, or favoring by the United States Goverment or any agency thereof or its contractors or subcontractors. The views and opinions of authors expressed herein do not necessarily state or reflect those of the United States Government or any agency thereof.

9. Impact Level $N / A$
10. RELEASE STAMP

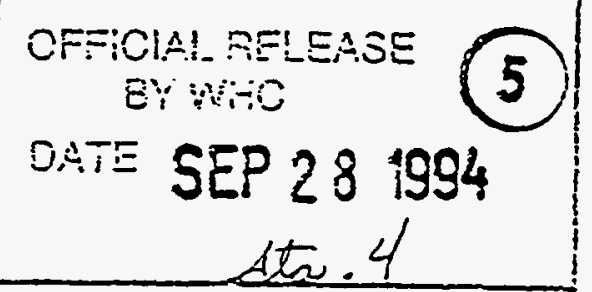




\section{SUPPORTING DOCUMENT FOR THE \\ HISTORICAL TANK CONTENT \\ ESTIMATE FOR \\ S TANK FARM}

WORK ORDER ER4945

Prepared by

ICF Kaiser Hanford Company

Richland, Washington

for

Westinghouse Hanford Company

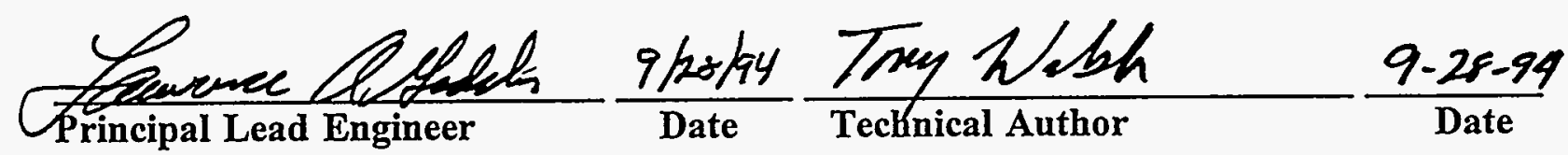

$\frac{\text { Chus HAFrevick }}{\text { Project Manager }} \frac{9 / 28 / 94}{\text { Date }} \frac{\text { Amomanauless }}{\text { Technical Docundents }} \frac{9 / 28 / 94}{\text { Date }}$

Westinghouse Hanford Company

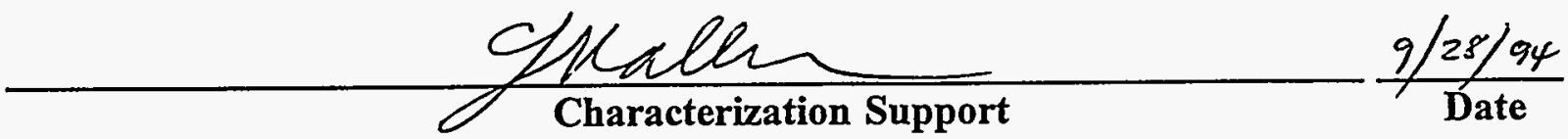


1.0 Introduction . . . . . . . . . . . . . . . . . . 1

1.1 Purpose . . . . . . . . . . . . . . . 1

1.2 Scope . . . . . . . . . . . . . . . . . . . . 1

1.3 Approach . . . . . . . . . . . . . . . . 1

1.3.1 Surveillance Techniques for the Hanford

Radioactive Mixed Waste Storage Tanks . 3

1.3.2 .. Approach and Development of Supporting Information Contained in the Historical

Tank Content Estimate and Supporting

1.3.3 Approach and Development of the Process-

Based Historical Tank Content Estimate - 8

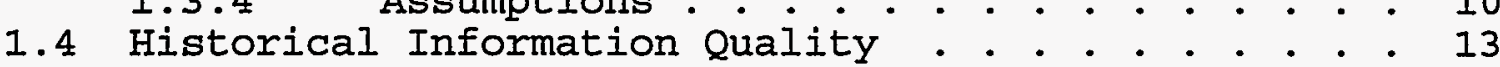

1.5 Background SW Quadrant . . . . . . . . . . . 16

1.5.1 Waste Generating Processes . . . . . . 17

1.5.2 Waste Management Operations . . . . . 21

1.5.3 Miscellaneous Waste Sources and

Equipment . . . . . . . . . . . 23

1.6 Safety Issues

1.6.1 Watch List Safety Issues . . . . . . . 27

1.6.2 Non-Watch List Safety Issues . . . . . 28

2.0 S Tank Farm . . . . . . . . . . . . . . . . 31

2.0.1 S Tank Farm History . . . . . . . . . 31

2.0.1.1 S Tank Farm Waste History . . . . 31

2.0.1.2 S Tank Farm Temperature History . . 32

2.0.1.3 S Tank Farm Integrity . . . . . . 32

2.0.1.4 S Tank Farm Historical Sample

Analysis Data ........ . . . 33

2.0.2 Current Status of $S$ Tank Farm . . . . . . 34

2.0.2.1 S Tank Farm In-Tank Photographs . . 34

2.1 Tank 241-S-10I . . . . . . . . . . . . . . . 35

2.1.1 241-S-101 Tank History . . . . . . . 35

2.1.1.1 Waste History Tank 241-S-101... . 35

2.1.1.2 Temperature History 241-S-101 . . . 35

2.1.1.3 Integrity of Tank 241-S-101 . . . . 36

2.1.1.4 Historical Sample Analysis Data for

Tank 241-S-101........ . 36

2.1.2 Current Status of Tank 241-S-101 . . . 36

$\begin{array}{ll}2.1 .2 .1 & \text { Waste Layer Volume Approximation } \\ & \text { for Tank 241-S-101 . . } 37\end{array}$

2.1.2.2 In-Tank Photograph 241-S-101... 37

2.1.3 Synopsis Tank 241-S-101........ . 37

2.2 Tank 241-S-102 . . . . . . . . . . . . . . . . 38

2.2.1 241-S-102 Tank History . . . . . . . 38

2.2.1.1 Waste History Tank 241-S-102. . . . 38

2.2.1.2 Temperature History 241-S-102 •. . 38

2.2.1.3 Integrity of Tank 241-S-102 . . . 39

2.2.1.4 Historical Sample Analysis Data for 
WHC-SD-WM-ER-323， Rev. 0

Tank 241-S-102 . . . . . . . . . . 40

2.2.2 . Current Status of Tank 241-S-102 . . . 40

2.2.2.1 Waste Layer Volume Approximation

for Tank 241-S-102 ....... 40

2.2.2.2 In-Tank Photograph 24I-S-I02... . 40

2.2.3 Synopsis Tank 241-S-102 . . . . . . . 41

2.3 Tank 241-S-103 . . . . . . . . . . . . . 42

2.3.1 241-S-103 Tank History . . . . . . 42

2.3.1.1 Waste History Tank 241-S-103. . . . 42

2.3.1.2 Temperature History 241-S-103 . . . 42

2.3.1.3 Integrity of Tank 241-S-103 . . . . 43

2.3.1.4 Historical Sample Analysis Data for

Tank 241-S-103........ . 43

2.3.2 Current Status of Tank 241-S-103 . . . 43

2.3.2.1 Waste Layer Volume Approximation

for Tank 241-S-103....... . 44

2.3.2.2 In-Tank Photograph 241-S-103... . 44

2.3 .3 Synopsis Tank 241-S-103 ........ 44

2.4 Tank 241-S-104 . . . . . . . . . . . . . . . 45

2.4.1 241-S-104 Tank History . . . . . . . 45

2.4.1.1 Waste History Tank 241-S-104.. . . 45

2.4.1.2 Temperature History 241-S-104 . . . 45

2.4.1.3 Integrity of Tank 241-S-104 . . . 46

2.4.1.4 Historical Sample Analysis Data for

Tank 241-S-104........ . 46

2.4.2 Current Status of Tank 241-S-104 . . . . 46

2.4.2.1 Waste Layer Volume Approximation

for Tank 241-S-104...... . 47

2.4.2.2 In-Tank Photograph 241-S-104... . 47

2.4.3 Synopsis Tank 241-S-104 . . . . . . . 47

2.5 Tank 241-S-105 . . . . . . . . . . . . . . 48

$2.5 .1241-S-105$ Tank History . . . . . 48

2.5.1.1 Waste History Tank 241-S-105. . . 48

2.5.1.2 Temperature History 241-S-105 . . . 48

2.5.1.3 Integrity of Tank 241-S-105 . . . . 49

2.5.1.4 Historical Sample Analysis Data for

Tank 241-S-105........ . 49

2.5.2 Current Status of Tank 241-S-105 . . . . 49

2.5.2.1 Waste Layer Volume Approximation

for Tank 241-S-105 ...... . 50

2.5.2.2 In-Tank Photograph 241-S-105... . 50

2.5.3 Synopsis Tank 241-S-105........ . 50

2.6 Tank 241-S-106 . . . . . . . . . . . . . . . . . . 51

2.6.1 241-S-106 Tank History . . . . . . . . . 51

2.6.1.1 Waste History Tank 241-S-106. . . 51

2.6.1.2 Temperature History 241-S-106 . . . 51

2.6.1.3 Integrity of Tank 241-S-106 . . . 52

2.6.1.4 Historical Sample Analysis Data for

Tank 241-S-106......... . 52

2.6.2 Current Status of Tank 241-S-106 . . . . 53

2.6.2.1 Waste Layer Volume Approximation

for Tank 241-S-106 .. . . . . . 53

2.6.2.2 In-Tank Photograph 241-S-106... 53

2.6.3 Synopsis Tank 241-S-106....... 53

- iii - $\quad 9 / 94$ 
2.7 Tank 241-S-107 . . . . . . . . . . . . . . . 54

2.7.1 - 241-S-107 Tank History . . . . . . . . 54

2.7.1.1 Waste History Tank 241-S-107... . 54

2.7.1.2 Temperature History 241-S-107 . . . 55

2.7.1.3 Integrity of Tank 241-S-107 . . . . 55

2.7.1.4 Historical Sample Analysis Data for Tank 241-S-107......... 55

2.7.2 Current Status of Tank 241-S-107 . . . 56

2.7.2.1 Waste Layer Volume Approximation

for Tank 241-S-107 .. . . . . . 56

2.7.3 Synopsis Tank 241-S-1......... 56

2.8 Tank 241-S-108 . . . . . . . . . . . . . . . . 57

2.8.1 241-S-108 Tank History . . . . . . . 57

2.8.1.1 Waste History Tank 241-S-108. . . 57

2.8.1.2 Temperature History 241-S-108 . . 57

2.8.1.3 Integrity of Tank 241-S-108.... 58

2.8.1.4 Historical Sample Analysis Data for Tank 241-S-108. . . . . . . . . 58

2.8.2 Current Status of Tank 241-S-108 . . . 59

$\begin{array}{ll}\text { 2.8.2.1 Waste Layer Volume Approximation } & \\ & \text { for Tank 24I-S-108 . . . } 59\end{array}$

2.8.2.2 In-Tank Photograph 241-S-108... . 59

2.8.3 Synopsis Tank 241-S-108 . . . . . . . . 59

2.9 Tank 24I-S-109 .. . . . . . . . . . . . . . 60

2.9.1 24I-S-109 Tank History . . . . . . . . 60

2.9.1.1 Waste History Tank 241-S-109. . . 60

2.9.1.2 Temperature History 241-S-109 . . . 60

2.9.1.3 Integrity of Tank 241-S-109 . . . . 61

2.9.1.4 Historical Sample Analysis Data for

Tank 241-S-109........ 61

2.9.2 Current Status of Tank 241-S-109 . . . . 61

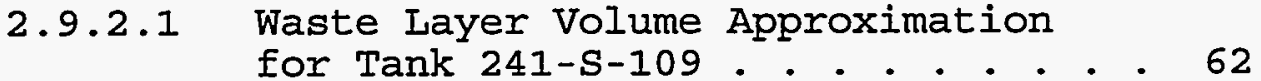

2.9.2.2 In-Tank Photograph 241-S-109:.: 62

2.9.3 Synopsis Tank 241-S-109 . . . . . . . 62

2.10 Tank 241-S-110 . . . . . . . . . . . . . 63

2.10.1 241-S-110 Tank History . . . . . . 63

2.10.1.1 Waste History Tank 241-S-110 . . . 63

2.10.1.2 Temperature History 241-S-110 . . . 63

2.10.1.3 Integrity of Tank 241-S-110 .... 64

2.10.1.4 Historical Sample Analysis Data for

Tank 241-S-110........ . 64

2.10.2 Current Status of Tank 241-S-110 . . . . 64

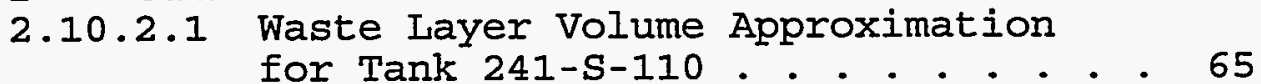

2.10.2.2 In-Tank Photograph 241-S-110.. . 65

2.10.3 Synopsis Tank 241-S-110 . . . . . . . . 65

2.11 Tank 241-S-111 . . . . . . . . . . . . . . . 66

2.11.1 241-S-111 Tank History . . . . . . . . 66

2.11.1.1 Waste History Tank 241-S-111.. . . 66

2.11.1.2 Temperature History 241-S-111 . . . 66

2.11.1.3 Integrity of Tank 241-S-111 . . . 67

2.11.1.4 Historical Sample Analysis Data for 
Tank 241-S-111... . . . . . . . 67

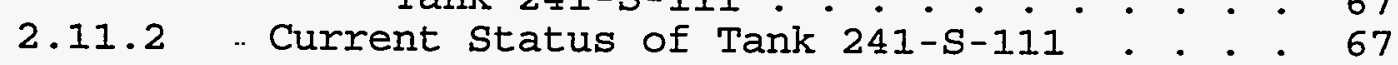

2.11.2.1 Waste Layer Volume Approximation

for Tank 241-S-111... . . . . . 68

2.11.2.2 In-Tank Photograph 241-S-111... . 68

2.11.3 Synopsis Tank 241-S-111. . . . . . . . 68

2.12 Tank 241-S-112 . . . . . . . . . . . . . . . . . . 69

2.12.1 241-S-112 Tank History . . . . . . . . . 69

2.12.1.1 Waste History Tank 241-S-112 . . . . 69

2.12.1.2 Temperature History 241-S-112 . . . 69

2.12.1.3 Integrity of Tank 241-S-112 . . . . 70

2.12.1.4 Historical Sample Analysis Data for

Tank 241-S-112. . . . . . . . . . . 70

2.12.2 Current Status of Tank $241-S-112$. . . . 70

2.12.2.1 Waste Layer Volume Approximation

for Tank 241-S-112 .. . . . . . . . 71

2.12.2.2 In-Tank Photograph 241-S-112... . 71

2.12.3 Synopsis Tank 241-S-112. . . . . . . . . 71 
WHC-SD-WM-ER-323, Rev. 0

APPENDICES

Appendix A. Glossary

Appendix B. References

Appendix C. Tank Level Histories and Data

Appendix D. Temperature Graphs and Data

Appendix E. Surface Level Graphs and Data

Appendix F. Drywell Graphs and Data

Appendix G. Riser Configuration and Tank Cross Section

Appendix H. Sampling Data

Appendix I. Tank Photographs

Appendix J. Unknown Tank Transfers

Appendix K. Tank Layering Comparison and Data 


\section{. INFORMATION FEEDBACK CARD}

\section{SUPPORTING DOCUMENT FOR THE HISTORICAL TANK CONTENT ESTIMATE FOR S TANK FARM ER4945}

\section{COMMENTS AND CONTRIBUTIONS}

The reader is requested to utilize this card to comment on this working document, report any discrepancies, or contribute new information to improve the accuracy and content of the report. Please use the space provided below, add additional pages if necessary, and return to the addressee on the reverse side. 
WHC-SD-WM-ER-323， Rev. 0
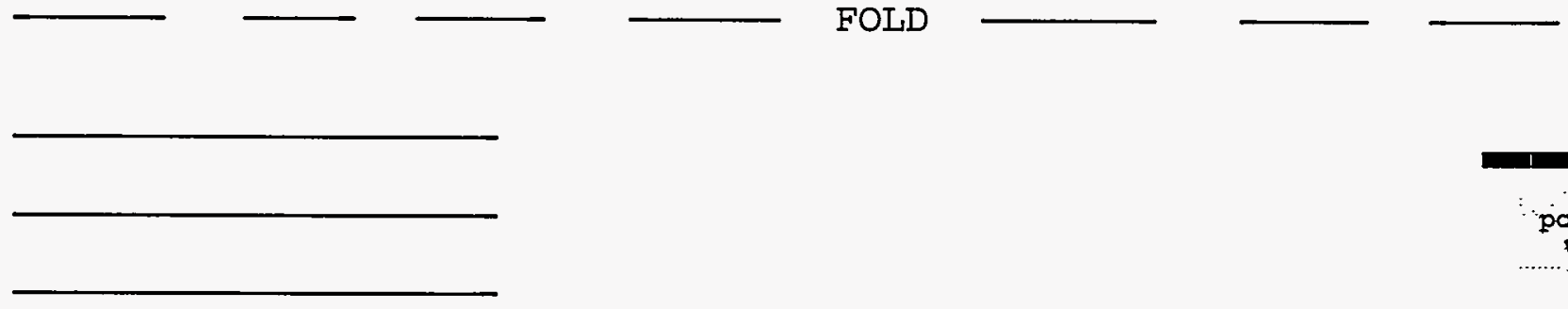

past affice

will not deliver without proper postage

Mr. Carl S. Haller

Manager, Characterization Support

Westinghouse Hanford Company

P.O. Box 1970

Richland, WA 99352

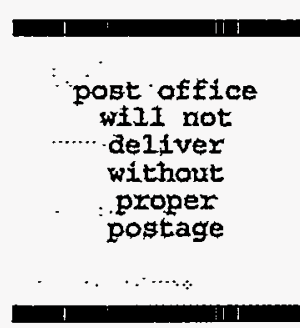




\subsection{Introduction}

\subsection{Purpose}

This document provides historical evaluations of the radioactive mixed wastes stored in the Hanford Site 200-East Area underground single-shell tanks (SSTs). A Historical Tank Content Estimate has been developed by reviewing the process histories, waste transfer data, and available physical and chemical characterization data from various Department of Energy (DOE) and Department of Defense (DOD) contractors.

The historical data will supplement information gathered from in-tank core sampling activities that are currently underway. A tank history review that is accompanied by current characterization data creates a complete and reliable inventory estimate. Additionally, historical review of the tanks may reveal anomalies or unusual contents that are critical to characterization and post characterization activities. Complete and accurate tank waste characterizations are critical first steps for DOE and Westinghouse Hanford Company safety programs, waste pretreatment, and waste retrieval activities.

\subsection{Scope}

The scope of this document is limited to all the SSTs in the $S$ Tank Farm of the southwest quadrant of the 200 West Area. For summarized characterization data on all SST's in the southwest quadrant of the 200 West area, refer to the Historical Tank Content Estimate Report (Brevick 1994). The northwest and southwest quadrants represent an approximate geographical division of the 200-West Area tank farms. The division was made as follows:

NW Quadrant: T, TX, TY

SW Quadrant: S, SX, U

The following map depicts the 200-East Area.

\subsection{Approach}

This report is a compilation of work performed by ICF Kaiser Hanford Company, Los Alamos National Laboratories, and Westinghouse Hanford Company.

Westinghouse Hanford Company requested that ICF Kaiser Hanford Company review the history of the tanks in each of the four quadrants and incorporate the tank layering models and inventory estimates being developed by Los Alamos National Laboratories into the report. Historical waste transfer and level data, tank physical information, temperature data, sampling data, and drywell and liquid observation well data have been compiled for this report. 


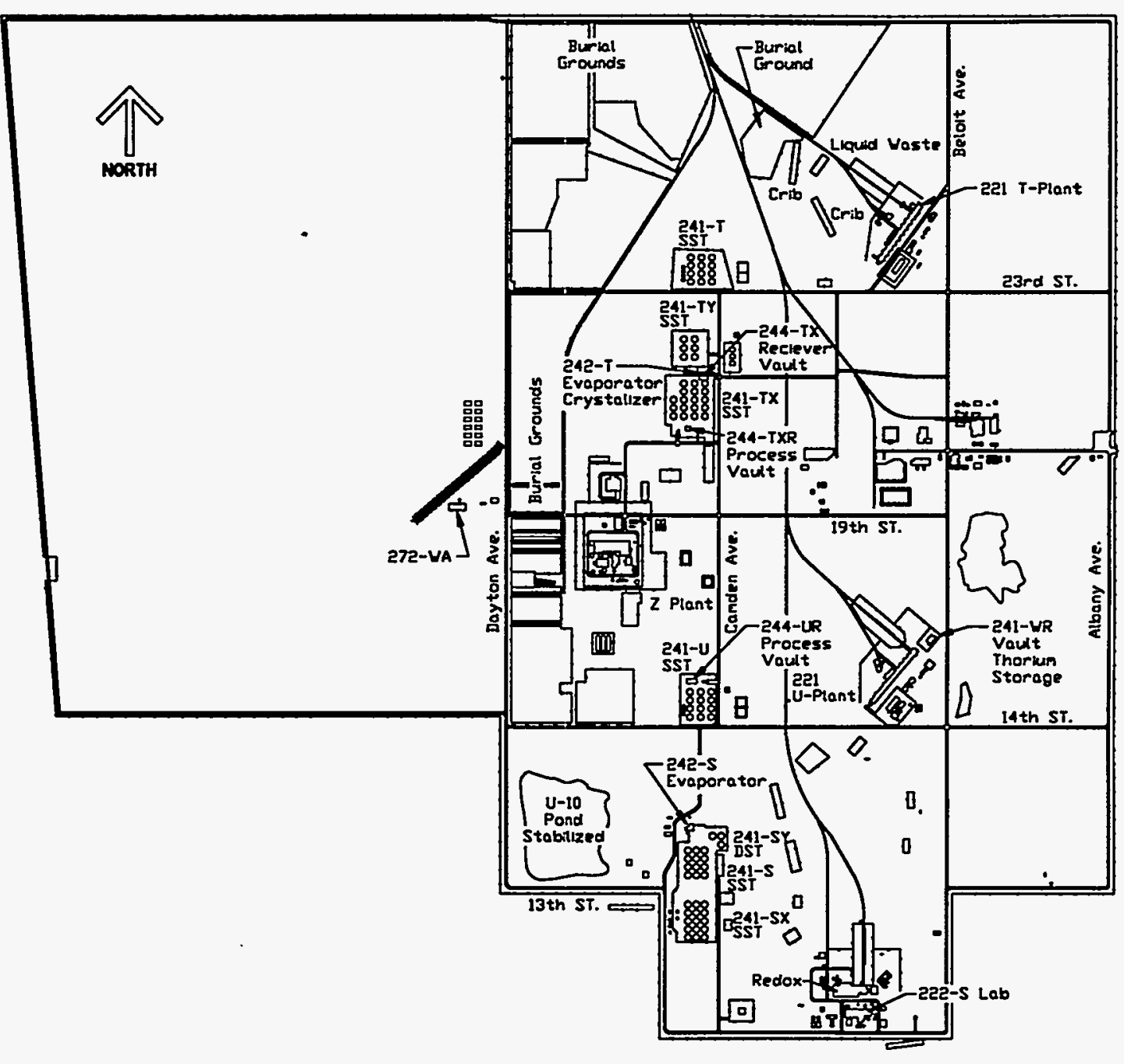

200 West Area

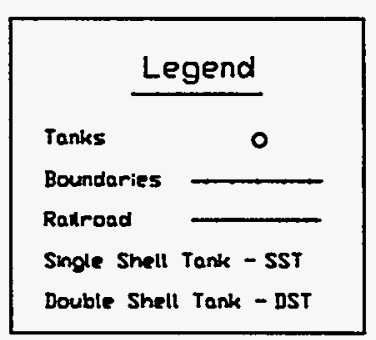


1.3.1 Surveillance Techniques for the Hanford Radioactive Mixed Waste Storage Tanks

Surveillance information is used for tank characterization. Tank integrity (i.e., sound, assumed leaker, assumed re-leaker) is investigated by comparing the changing liquid levels to liquid observation well data and drywell activity. Waste temperatures can be used to correlate the inventories of heat-generating radionuclides $\left({ }^{137} \mathrm{Cs}\right.$ and $\left.{ }^{91} \mathrm{Sr}\right)$ in the tanks. The instrumentation and methods used for tank surveillance are described in the following sections.

\section{In-Tank Surface Levels}

Surface levels within the SSTs are monitored by instruments consisting of calibrated steel tapes with an electrode plummet on the end that will complete a circuit when it contacts a conducting surface (i.e., liquid). The surface level measurements are usually taken with Food Instrument Corporation gauges and manual tapes. The tank monitoring device, manufactured by the Food Instrument Corporation, automatically moves up and down and contacts the waste. The gauge is often set to the intrusion mode by placing the plummet a short distance above the waste. If an intrusion occurs in the tank, the Food Instrument Corporation gauge will record the event when the surface level rises to meet the plummet. The manual tapes are similar to the Food Instrument Corporation gauge, except that manual operation of the tape and manual recording of the readings are required. Some tanks contain both types of level monitoring devices. The measurements are recorded on a computer automated surveillance system (CASS).

Interstitial Iiquid Level (Liquid Observation Wells)

Food Instrument Corporation gauges and manual tapes are limited to surface level measurement; therefore, liquid observation wells in many tanks have been installed specifically to monitor the interstitial liquid level within the SSTs. The interstitial liquid is the liquid trapped within and underneath the solid waste. Fiberglass or TEFZEL-reinforced epoxy-polyester resin liquid observation well tubes (by E.I. du Pont de Numours and Company) are sealed on the bottom and vertically installed approximately 1 in. from the tank bottom. Gamma, neutron, and acoustic probes may be lowered into the liquid observation wells to determine the interstitial liquid levels within the tank. A gamma probe detects the difference between saltcake and saltcake containing liquid, based on the principle that the liquid is the primary source of gamma emissions. A neutron probe distinguishes between the existing dry saltcake, wet saltcake, and liquid phases. Distinct differences exist in the neutron count rates of each material type. An acoustic probe emits a pulse that is reflected off the interface between the outer wall of the liquid observation well and the waste. A fiberglass/liquid interface produces a minimal reflected pulse and a fiberglass/air interface produces a maximum pulse reflection. Wet saltcake will fall between the limits appropriately; therefore, interpretation of the records will determine the liquid level. Each probe scan is fully automatic and 


$$
\text { WHC-SD-WM-ER-323, Rev. } 0
$$

successful scans are input to the computer automated surveillance system.

Internal Tank Temperatures (Thermocouples)

Temperatures usually are recorded automatically in the Computer Automated Surveillance System (CASS). Temperatures are monitored with thermocouple trees that are comprised of stainless steel pipe with 4 to 18 thermocouples. Usually, the lowest sensor is located approximately $4 \mathrm{in.}$ from the tank bottom and the other sensors progress up the .tree at 2-ft intervals. More frequent temperature readings are available for a few select tanks due to an upgrade in the surveillance data acquisition system to the tank monitor and control system (TMACS) in 1991.

Generally, a single thermocouple sensor is sent down the liquid observation well to take semiannual readings in tanks that do not contain a thermocouple tree. The temperatures are recorded manually in degrees Fahrenheit and are entered into the surveillance analysis computer system (SACS) that is currentIy using the ORACLE database.

\section{- External Leak Detection (Drywells)}

Drywells are located around the circumference of each SST and throughout the tank farms to determine if tank wastes have leaked. The drywells are generally 6-in. diameter carbon steel pipe, between 75- and 250-ft deep. The wells are termed dry because they are not deep enough to reach ground water. Scan profiles of radiation or moisture in the soil as a function of well depth are obtained with gamma radiation or neutron sensors. Scintillation monitors, Geiger-Mueller instruments, and neutron sensors are used to monitor the drywells. Scintillation monitors are radiation detection instruments based on the principle that light pulses are produced in some materials when they are exposed to radiation. Geiger-Mueller instruments detect low-level beta and gamma radiation. Neutron probes are equipped with a neutron source and detector that determine if moisture is present in the soil. The frequency of and probe used for drywell monitoring are specified in a schedule from the "Waste storage Tank Status and Leak Detection Criteria" (Welty 1989).

Drywell readings are recorded at 1 -ft intervals. The peak readings and the depth of the readings are recorded on a summary sheet. If a drywell indicates a new or unusual increase in activity, a rerun is requested and the drywell is retested. The wells are sealed when they are not being tested.

The drywells described in this report have a 6-digit computer identification number: the first two digits denote the tank farm (i.e., $\mathrm{A}=10, \mathrm{AX}=11, \mathrm{~B}=20, \mathrm{BX}=21, \mathrm{BY}=22$, and $\mathrm{C}=30$ ), the third and fourth are the last two digits of the tank number (i.e., 241-A-105 would be 10-05 and 00 indicates a non-specific tank), and the fifth and sixth describe the clockwise location of the well from due north. For example, the identification number of the first drywell for Tank 241-BY-102 would be 22-02-01. 
WHC-SD-WM-ER-323, Rev. 0

Laterals (i.e., horizontal drywells) are positioned under some SSTs to detect radionuclides in the soil which could be indicative of tank leakage. The drywells are monitored by radiation detection probes and occasionally by a temperature probe. Laterals have 4in. inside diameter steel pipes located 8 to $10 \mathrm{ft}$ below the concrete base of the tank, and each tank has three laterals. Laterals are located only in the A Tank Farm and a portion of the SX Tank Farm.

\section{In-Tank . Photography}

In-tank photography is another waste volume determination method used to resolve in-tank measurement anomalies and determine tank integrity. Photographs can help determine sludge and liquid levels, provide a degree of corroboration with other waste management processes, and are a source of information on foreign objects contained in the tanks. The photos can be compared to see if tank wastes with similar histories have similar characteristics.

1.3.2 Approach and Development of Supporting Information Contained in the Historical Tank Content Estimate and Supporting Documents

Extensive information was gathered for each tank. This document contains a comprehensive data set on each tank, that are summarized in the Historical Tank Characterization Estimates.

\section{Tank Level Histories}

The tank level histories are graphical representations of waste levels over the lifetime of a tank. They include information such as pH, in-tank photo dates, waste types, transfer data, and physical data. Information from the mid 1940 s to 1980 was compiled from two Westinghouse Hanford Company documents (Anderson 1990 and Welty 1988). Data from 1977 to the present were obtained from the monthly waste status summary reports. The following Hanford site operating contractors prepared summaries: Atlantic Richfield Hanford Company from 1973 to 1977, Rockwell Hanford Operations from 1977 to 1987, and Westinghouse Hanford Company from 1987 to present. Script files were imported from a spreadsheet to AutocAD 12 to graph the surface level data. Liquid observation well data were obtained from the Westinghouse Hanford Company Tank Farm Surveillance group in a spreadsheet format. Additional information was gathered from various documents and comments were added directly to each drawing.

The liquid, solid, and interstitial liquid levels (liquid observation wells data) are shown on the tank level history graphs as volumes and tank elevations. The following formulas were used to relate the volumes to the respective elevation for each tank farm design: 
FORMULAS

$\frac{\text { Total Gallons }}{2750 \frac{\text { Gallons }}{\text { Inch }}}=$ Total Inches

Total Gallons - 12,500 Gallons +12 Inches = Total Inches $2750 \frac{\text { Gallons }}{\text { Inch }}$

$\underline{\text { Total Gallons - 12,500 Gallons }}=$ Total Inches $2750 \frac{\text { Gallons }}{\text { Inch }}$

$\underline{\text { Total Gallons }-18,500 \text { Gallons }}+12$ Inches = Total Inches $2750 \frac{\text { Gallons }}{\text { Inch }}$

$\frac{\text { Total Gallons }-590 \text { Gallons }}{196 \frac{\text { Gallons }}{\text { Inch }}}+6$ Inches $=$ Total Inches

\begin{tabular}{|c|l|c|}
\hline Equation No. & Applicable Tank Farms: & Tank Diameter \\
\hline$I$ & A, AN, AP, AW, AX, AY, AZ, SY & $75 \mathrm{ft}$ \\
\hline 2 & BY, S, TX, TY & $75 \mathrm{ft}$ \\
\hline 3 & B, BX, C, T, U & $75 \mathrm{ft}$ \\
\hline 4 & SX & $75 \mathrm{ft}$ \\
\hline 5 & B, C, T, U (200 Series) & $20 \mathrm{ft}$ \\
\hline
\end{tabular}


WHC-SD-WM-ER-323, Rev . 0

Temperature Plots

There are two categories of temperature data, one or few temperature readings per day and thermocouple temperature data. The documentation used for the first category includes Waste status Summary Reports (Roberts, R.E. 2/58 - 10/58, Thress, M.A. 11/58 2/59, Lentz, J.E. 3/59-12/60, and Roberts, R.E. 6/61 - 6/62), RHO$C D-1172$, and HW-83906. The thermocouple temperature data were obtained from the Westinghouse Hanford Company ORACLE database commonly known as the Surveillance Analysis Computer System (SACS) and various retrieved hand-entered temperature data sheets. Temperature versus time plots were developed for both categories of temperature data. Historical temperature plots cover the time span between January 1953 and January 1973. The common temperature span used for these plots is 350 degrees. There are no tanks that have temperature data outside the sliding 350 degree temperature range.

No thermocouple information has been located prior to 1973 . The thermocouple temperature information was parsed in an EXCEL spreadsheet and graphed to show each individual thermocouple probe. January of 1973 is then the starting date for all thermocouple plots. The temperature range for all tank thermocouple plots in all tank farms was kept to a 120 degree $F$ span, except where the data exceeded this range. In the few tanks where the temperature span exceeds this sliding range the data are shown using the smallest temperature range possible. A consistent time frame and temperature range is essential in comparing temperature information from one tank to another. If documentation was available, thermocouple elevations were included in the plots for tanks currently containing thermocouple trees. Thermocouples are ordered from the bottom to the top of the tank. Therefore, a plot of thermocouple 1 over time would indicate the temperature near the bottom of the tank. In-tank temperature information is available in Appendix D.

\section{- Surface Level Plots}

The surface level data from 1991 to 1993 were obtained from the Westinghouse Hanford Company ORACLE database. The information was parsed in an EXCEL spreadsheet and graphed to show the waste level versus time. Current in-tank surface level information is available in Appendix E.

\section{Drywell Plots}

The drywell information was obtained from the manual records of the Westinghouse Hanford Company Tank Farm Surveillance Group. Data from 1990 to the present were input into an EXCEL spreadsheet and graphed to show the peaks in counts/second (c/s) and the peak depths in feet (ft) versus the date on the same chart. Tank drywell information is available in Appendix F.

\section{Tank Profiles and Other Drawings}

The drawings included in this report have been compiled from several sources including as-built drawings, the Waste storage Tank Status and Leak Detection Criteria (Welty 1988), and the Riser Configuration Document for Single-Shell Waste Tanks (Alstad 1991). 
The risers and penetrations throughout the tank dome are used for many purposes. . Some risers were installed during the original tank construction and others were added later.

Through the years, sleeves or smaller risers were installed in many of the larger risers. For the riser configurations in this report, a tank may have a riser number followed by a letter which means that a larger riser was fitted with smaller sleeves. Depending on the intended use, the riser sleeves can be as large as 12 in. or as small as 4 in. For example, a saltwell pump and screen require a 12-in. riser but a thermocouple tree requires only a 4-in. riser. The tank profile drawings are in Appendix $\mathrm{K}$.

\section{Photographs}

The tank farm photos were labeled to indicate the relative position of each tank in the farm and arrows were added to show the cascade series of each tank farm.

The latest in-tank and tank farm photos were obtained from Pacific Northwest Laboratory. In-tank photos have been arranged to show the contents of each tank. The auxiliary equipment within each photo collage was identified if possible and labeled appropriately. The latest in-tank and tank farm photos are available in Appendix I.

\section{Historical Sample Analysis Data}

The sampling data were obtained from the WHC Tank Waste and Retrieval Group. Tables were made for the samples that were taken prior to september 1989. The information was compiled in EXCEL to present the data in as similar a manner as possible. The tables include physical data, chemical analysis data by component, and radiological analysis data by component. Any other data included with the sample references are summarized as text. Tank historical sample analysis data are available in Appendix $\mathrm{H}$.

1.3.3 Approach and Development of the Process-Based Historical Tank Content Estimate

The process-based historical tank content estimates presented in this report were taken from work being performed by Los Alamos National Laboratories. The tank content estimates are the primary source of tank characterization data until the tank characterization reports are completed. The tank characterization reports will incorporate current core sampling and analysis results with historical-based tank content estimates to present the most current evaluation of the tank waste constituents.

Generating historical tank content estimates involves management and evaluation of large quantities of data. Several smaller components of the data management effort make up the system of generating historical tank estimates. The components are described in the following sections. 


$$
\text { WHC-SD-WM-ER-323， Rev. } 0
$$

\section{Waste Status and Transaction Record Summary Database}

The waste status and transaction record summary database (i.e., transaction summary) is a database of all known waste transfer records. Historically, when waste was pumped from a process plant to tank or tank to tank, a record of the pumped waste volume and type was kept. The transaction summary is the recorded waste level of the solids and supernatant.

The waste status and transaction record summary database is being partitioned into four separate sections (i.e., quadrants). The waste status and transaction record summary database for the northeast quadrant is available in WHC-SD-WM-TI-615 Rev. 0 (Agnew 1994).

\section{Tank Layering Model}

The tank layering model is derived from the waste status and transaction record summary database. The purpose of the tank layering model is to predict the waste types and volumes in each tank. The transaction summary database records the types of waste that enter each tank and the corresponding increase in the solids volume from each waste entry. Each waste type that increases the solids volume in the tank is recorded in the tank layering model. Therefore, the completeness and accuracy of the information in the tank layering model is directly related to the completeness and accuracy of the transaction summary database.

The tank layering model was created to develop historical tank content estimates. The results of the tank layering model are reported in this document. The tank layering model is presented graphically with the inventory estimate described below in Appendix $\mathrm{K}$.

\section{Defined Waste Types}

Early in the development of the historical tank content estimates, a need to compile the chemical compositions of all known waste types into one source document was recognized. This compilation was performed by Los Alamos National Laboratories. The Hanford defined waste document was developed independently of the waste status and transaction record summaries database and the tank layering model. However, the Hanford defined waste list will be used with the tank layering model to obtain the actual tank waste inventory estimates as described below.

The Hanford defined waste document gives the compositions for all known Hanford waste types that went into the double-shell and single-shell tanks. For more details of the methods used to develop these waste types, refer to the Hanford Defined Wastes Document (Agnew 1994).

\section{Inventory Estimates}

The end result of combining the tank layer model and the Hanford defined waste types is the tank inventory estimate. When the volumes and compositions of each waste type in a particular tank are known from the tank layering model and the Hanford defined 
wastes document, a calculation of the total chemical inventory in the tank is performed. The individual tank results are presented in tabular form in Appendix $\mathrm{K}$.

The inventory estimates developed by Los Alamos National Laboratory are quality checked against existing data from current sample analyses, thermal and physical models, and historical records. The checking constitutes a large portion of the time required to develop the estimates. When estimates are checked against existing data, the models used may be reevaluated. Thus, the process of generating estimates will iterate and the associated errors will be reduced over time as the historical knowledge of the tank waste operations increases.

\subsubsection{Assumptions}

The quality of the historical information available for the tanks often requires that assumptions and judgement calls be made on the applicability and validity of the data. Some assumptions were used to interpret the data.

\section{Temperature Data}

The lines joining the data points on the temperature plots were detached to show gaps in data of more than three years.

The single thermocouple tree in some tanks was removed and replaced with two trees. The original single thermocouple tree and tree 1 data were plotted on the same graph, and the tree 2 data were graphed separately.

No conclusion has been made on the status of thermocouples with less than 5 data points. In general, when there is thermocouple data with less than 5 data points, the data are retained on the temperature data sheets, but have not been plotted. Some possible explanations for the limited data are that the thermocouple failed, it was inoperable when readings were taken, or the operator misrepresented the number of thermocouples and the temperature data in the tank.

The thermocouples used in the trees were installed as purchased. There were no field calibrations before 1991. In 1991, a survey of Ferrocyanide Watch List thermocouple trees was conducted. The thermocouple tree probe temperatures were tested against probes inserted in liquid observation wells or by other methods as specified in Engineering Evaluation of Thermocouples in FeCN Watchlist Tanks, (Bussell 1992). Thermocouples, with equipment that could be repaired outside the tank, were made operable at this time.

It is assumed that the sinusoidal variances in the data can be attributed to seasonal and diurnal trends which are clear after 1991 due to an upgrade in thermocouple surveillance to a computerized data acquisition system, i.e., Tank Monitor and 
Control system (TMACS). Also, in general, the temperatures decrease from the bottom to the top of the tanks.

All the temperature data received by ICF Kaiser Hanford Company was reviewed for suspect data points. Data that obviously deviate from the temperature trend for a any tank are marked as suspect and designated in the comment column as either "suspect data (low)" or "suspect data (high)". Typically, suspect data sharply deviates from the data trend 15 degrees $F$ or more (See historical and thermocouple temperature data in Appendix D Temperature Graphs and Data).

After review of the data, it was determined that some data was recorded in degrees Celsius rather than in degrees Fahrenheit as it was electronically received. The data are converted to Fahrenheit and evaluated against the rest of the data set. Where the converted data are found to be consistent with the temperature trend, they are designated in the comment column as "raw data assumed degree C". Other delineations used to flag suspect data or substantiate the degree Celsius conversions are found from the Tank Waste Information Network System (TWINS) database, property of Battelle - Pacific Northwest Laboratory. Data recorded in degree $C$ or input incorrectly and confirmed with TWINS are delineated in the comments column as: "degree C/TWINS confirm" and "wrong input/TWINS confirm", respectively (See historical and thermocouple temperature data in Appendix D - Temperature Graphs and Data).

In addition, some of the lines of data were flagged in the comment column by Westinghouse Hanford Company (WHC) before transmission of the data. Some of the WHC comment flags include "cass" or "SET\#2 FROM CASS". It is assumed that "cass", an acronym for Computer Automated Surveillance System, applies to all the data taken on that particular day, and that "SET\#2 FROM CASS" applies to the contiguous data designated under the TREE SET \# column as 2 (See historical and thermocouple temperature data in Appendix D Temperature Graphs and Data).

Sources of temperature data are located in the footnotes of both the temperature plots and data. Some Historical Temperature plots use data from Waste status Summary Reports (Roberts, R.E. 2/58 - 10/58, Thress, M.A. 11/58 - 2/59, Lentz, J.E. 3/59-12/60, and Roberts, R.E. 6/61 - 6/62). If these reports are used, footnotes on the temperature plots indicate by including the source of data concisely as from "Waste Status Summary Reports" (See historical temperature plots in Appendix D - Temperature Graphs and Data).

\section{Drywell Data}

The drywell data did not appear to have had a quality review; therefore, the following assumptions were made about the data:

-A data point is considered suspect if an apparent rerun has been performed. 
-A data point is considered to be a rerun if the preceding data are erratic and the rerun is performed on the same day if monitored daily, within one or two days if monitored weekly, or within several days if monitored monthly or yearly.

-If suspect data were indeed relevant, the appropriate surveillance organizations would have followed the appropriate procedures to identify any problems.

\section{Sampling Data}

The data obtained from the historical analytical information are understood to be widely variable in type, quality, and scope. The data range from process control data obtained from a grab sample on a few selected analytes to a total characterization suite performed on a core sample. There are no specific quantitative data quality criteria for the information incorporated into the historical tank content estimate. A qualitative assessment of the information sources is done during construction of the historical tank content estimate. The available data are assessed for suitability and consistency in the study and each data source is weighted appropriately for its contribution to the overall estimates.

When the sampling data were compiled, a sample was considered a solids sample if it was greater than $30 \%$ solids. Sludge/slurry samples were included.

\section{Inventory Estimates}

Solid waste formations vary by type and are determined individually by examination of the historical fill records. Further refinement and bounding of the solids formation are provided by conformation to physically attainable systems (e.g., waste volumes contained in the tanks do not exceed the tank capacity and negative void fractions are not possible).

Waste stream compositions were based on process flow sheet information and previous analytical data.

Solids were evenly distributed throughout tank. The solids were assumed to be evenly distributed throughout the slurry during their transfer and their settling rate was assumed to be uniform throughout the tank.

Complete settling of the sludge occurred and the solids settled in a pseudo-pancake formation (i.e., flat layers conforming to the boundaries of the tank) which was a simplifying assumption. Localized mounding and pocketing in various areas of the tank may have occurred due to inlet/outlet phenomena (e.g., cascades and transfers from the floating suction pumps that remove or deposit sludge). This phenomenon would have affected only a small fraction of the tank area and does not impact the overall results of this study. However, spatial variability within physical configuration and process history is acknowledged. No method is currently available that would adequately model this discrepancy. An effort 
is underway to develop this concept and incorporate it into the tank characterization process, but it is incomplete at this time.

Solids from a specifically designated waste type are homogeneous, which is a simplifying assumption. Variation and layering of the sludge due to component concentration differences between batches, precipitate particle size, and particle density were quite likely. The effect of these factors on the distribution of analytes within the solids is unknown; however, the effect was probably small and not detectable with the present sample collection and analysis process.

Solids compaction did not occur and was not accounted for in the study which is a simplifying assumption. Although compaction probably occurred in the history of the tanks because of the formation of additional solids layers on top of each other, the degree of compression was not known and, therefore, was not incorporated.

Density and other physical characteristics of the solids were determined by individual waste type using available information or were based on approximate physical analogues.

The capacity of the tanks was never exceeded during transfers. Tank capacity is dependent on fill status, fill order, tank location in a cascade, and tank type.

Leaking of the tanks was not incorporated during the time of the study but was accounted for at the end.

Cascades, tank transfers, and crib line effluents were normally free of particulate; therefore, the majority of solids were confined to their initial receiver tanks and were not cribbed or transferred extensively from tank to tank. This assumption based on cases of plugged cascade lines when appreciable amounts of solids tried to cascade, and the pumping equipment typically was not designed to transfer of substantial quantities of solids (except when sluicing or specifically transferring solids). Monitors on the crib discharge lines should have indicated significant solids contamination, and the tank farm operating procedures would have prevented solids discharges to the ground.

\subsection{Historical Information Quality}

The quality of historical data compiled for this report varied greatly. Some data came from published documentation. Other data were retrieved electronically from various Westinghouse Hanford Company databases. The quality of data used in the estimates is described in the following section.

Level Histories

The level history narrative is designed to give a general description of the waste types contained in a specific tank from startup to the present. The history presents physical

$$
-13-\quad 9 / 94
$$


characteristics of the waste such as waste $\mathrm{pH}$ values and the latest maximum temperature of the waste. It also presents information on when the tank was removed from service, if it was stabilized, the method of stabilization, if it ever leaked, and the current stabilization status. It is not designed to be a detailed summary of every waste type and transaction for that tank. The waste types are general and usually are given only for large transactions (i.e., if a waste is not of sufficient volume to significantly impact the total volume of the tank then it was not reported in the narrative). For a more complete record of transactions and types of wastes involved, see the waste status and transaction record summary (Agnew 1994).

The accuracy of the tank level histories relies on the completeness of the Westinghouse Hanford Company document "A History of the 200 Area Tank Farms" (Anderson 1990); the monthly Westinghouse Hanford Company, Atlantic Richfield Hanford Company, and Rockwell Hanford operations waste summary reports; and the waste status and transaction record summary (Agnew 1994).

Surface level readings may be affected by several factors: plummet error, flushing water accumulation, and waste surface irregularities. Crystalline wastes (i.e., saltcake) can build up on the end of the plummet gradually and contact the waste which indicates a false surface level increase. Significant level discrepancies occur when the buildup breaks off or when the measuring instrument plummet is flushed to remove the saltcake buildups. Flushing the Food Instrument Corporation Gauge, manual tapes, or any other equipment may cause accumulated wash water to collect under the plummet which indicates a false increase in the overall volume of waste within the tank. Surface level readings often are difficult to obtain from tanks that have a relatively dry saltcake surface. Some tanks have crystalline buildup on in-tank equipment (i.e., pumps, thermocouples, liquid observation wells, and other protruding equipment). As the supernatant is pumped from the tanks, the crystalline structure may remain attached to the equipment and be suspended above the liquid. Therefore, an accurate surface level measurement would be difficult if the breakup of the crystalline structure was inconsistent and a nonuniform waste surface was created. Steel tapes that are bent or warped from operation or those discarded on the waste surface are other sources of altered surface level readings.

Routine measurements of solids and sludges in most SSTs were not reported in the Anderson document until 1965. The solids measurements are taken with a steel donut on a string and operators interpret the sludge level. The interpretation could introduce a wide range of variability in the recorded solids level. Sometimes estimates taken from photographic data and saltwell pumping activities are the only indications of the solids levels.

Liquid Observation Well Data

The liquid observation well data were obtained electronically

from the Westinghouse Hanford Company Surveillance Group. Some 
discrepancies exist in the liquid observation well readings for tanks with liquid waste surfaces. The discrepancies were supposed to be addressed in a December 1993 rebaselining of the liquid observation well data. Agreement between the liquid observation well plots and the data obtained from the surveillance group has been verified; however, errors in the data prior to the exchange of information could still exist. The liquid observation well data were understood to be unverified prior to the exchange. Therefore, the data should be reviewed before being used for any design purposes or safety evaluations.

\section{Temperature Readings}

The single-shell tank temperature profiles are provided by electronic data from Westinghouse Hanford Company. Agreement between the thermocouple plots and the data obtained from the surveillance group has been verified; however, errors in the data prior to the exchange of information could still exist. Thermocouples used in the thermocouple trees were purchased and installed according to the American Society of Testing and Materials (ASTM) standard. There were no field calibrations before 1991 when a survey of Ferrocyanide Watch List thermocouple trees was conducted and a few of the thermocouple trees were compared against a thermocouple probe inserted in a Iiquid observation well.

During a review of the plots, some thermocouples were found to have outlying data points and many of them have only one or two readings. The unusual readings may be the result of the thermocouples functioning on only one occasion, the operator misrepresenting the number of thermocouples in the tank, unnecessary thermocouples readings, or possible thermocouple failure.

Occasionally the operator would read the temperature in degrees celsius instead of degrees fahrenheit and would not indicate this on the data sheets. The thermocouple data were understood to be unverified prior to the exchange. An attempt has been made by ICF Kaiser Hanford to validate and verify the data, however, the data should be checked before being used for any design purposes or safety evaluations.

\section{Surface Level Data}

The surveillance automated computer system data were obtained electronically from the Westinghouse Hanford Company Surveillance Group and were plotted. The data are actual surface levels recorded from the surveillance equipment. If the surveillance equipment in a particular tank riser was removed from service, the readings may show a level change when a new instrument and/or riser is used, especially if the waste surface shows severe heterogeneity.

Agreement between the plots and the data obtained from the surveillance group has been verified; however, errors in the data prior to the exchange of information could still exist. The surveillance automated computer system data were understood to be 
WHC-SD-WM-ER-323, Rev . 0

unverified prior to the exchange. Therefore, the data should be reviewed before being used for any design purposes or safety evaluations.

\section{Drywell Data}

The drywell information is received on data sheets for each drywell. If the readings are questionable, Westinghouse Hanford Company often will request a rerun of the drywell scan. Because the reruns are not indicated on the data sheets, it can be difficult to determine if the data sheet has been. superseded. In some wells, several scans occurred on the same day with no explanation; therefore, the scan with the accepted results is not identifiable.

\section{Riser Configuration}

The riser configuration was determined from the WHC Riser Configuration Document for Single-Shell Waste Tanks (Alstad 1991). The information was current as of June 1991; therefore, any changes made after that date would not be included.

\section{Photographs}

The photo collages were made from the latest available in-tank photographs. In some tanks, photos have not been taken since the early 1970s. Some tanks had fogging problems in the vapor space which prevented use of the latest photos. Sometimes a review of the older photos was useful to determine the waste changes. Collages made from older photos may not be indicative of current tank contents, especially if the tank has been pumped since the last photo date.

\section{Historical Sample Analysis Data}

The historical sample results only refer to sample and analysis information obtained before August 1989. The historical sample and analysis results have not been validated by the characterization program. When validation occurs, they will be reissued in a later revision of the Historical Tank Content Estimate report and supporting documentation. The sample data often were retrieved from memos that indicated the results of the sample analysis. The analytical methods, holding times, and quality control information are unavailable. The location at which the sample was taken was not provided which leads to integration of some uncertainty into the sampling results. The analytical scope performed in many cases was quite narrow which limited the available data set. However, the data set is the only available historical chemical characterization information.

\subsection{Background sw Quadrant}

The SSTs located on the Hanford Site near Richland, Washington were built between 1943 and 1964 to provide interim storage for high-level nuclear wastes. Processes such as plutonium recovery from spent nuclear fuel rods and uranium metal recovery generated the millions of gallons of mixed radioactive hazardous waste solutions stored within these tanks. 
The southwest quadrant located in the 200-West exclusion area consists of 43 tanks grouped into three tank farms. The tank farms are: S Farm (twelve 758,000 gal tanks), SX Farm (fifteen 1,000,000 gal tanks), and U Farm (twelve 500,000 gal tanks and four 55,000 gal tanks). Single-shell tank construction consists of mild steel cylinders and bases inside reinforced concrete, and covered by a reinforced concrete dome. Depending upon the farm, the top of the tanks are buried between 6 and 11 ft underground.

Several. waste tanks are on watch lists. The definition of a watch list tank is explained in a later section of this report. Wastes stored in the $\mathrm{NE}$ quadrant were generated mostly from Plutonium Uranium Extraction (PUREX) and a Bismuth Phosphate Plant (B Plant); however, some tanks in the quadrant received wastes from the other onsite separation processes. The separation processes went through many changes as new technologies became available. Iiquid waste volumes were reduced by evaporators, in-tank solidification units, or chemical precipitation of radionuclides. The resulting supernatant was disposed of in the ground. Vaults were used for temporary storage or treatment of waste and they were the intermediate storage point for tank waste or waste awaiting further processing. A special rail car facility in the NE quadrant was used to unload onsite waste that was distributed to an evaporator, tanks, or processing facilities. The facilities were sources of the waste that exists in the SSTs of the NE quadrant.

\subsubsection{Waste Generating Processes}

Although not all of the processes listed below contributed waste directly to tanks in the NE quadrant, the waste they generated could have been indirectly transferred to the quadrant through tank to tank transfers. The plants and processes that generated waste now contained in the SSTs and the DSTs are presented in chronological order.

T Plant

T Plant was the first-full scale separations plant at Hanford. It was constructed in 1944 and was used as a separation facility for irradiated production reactor fuel until 1956. The bismuth phosphate separation process used at $T$ Plant was identical to the one used at B Plant. Since 1957, $T$ Plant has been used as a decontamination and repair facility. The facility was modified in 1978 for storage of pressurized water reactor (PWR) core II fuel assemblies. $T$ Plant provided facilities for decontamination, decomposition, repair, testing, shipping, and burial of contaminated tools and equipment from onsite and offsite locations.

The following wastes were sent to SSTs: waste solution from equipment decontamination efforts at $\mathrm{T}$ Plant, decontamination waste (DW), metal waste (MW) from the bismuth phosphate process, firstcycle (1C) decontamination waste from the bismuth phosphate process, and second-cycle (2C) decontamination waste from the bismuth phosphate process. 


\section{B Plant}

B Plant was constructed between August 1943 and February 1945. The bismuth phosphate process ran until 1952. One of the first plants built along with $T$ Plant, $B$ Plant was designed to separate plutonium from uranium and the bulk of fission products in irradiated fuel via the bismuth phosphate separation process. In 1968, B Plant became a waste fractionation plant and was retrofitted to remove cesium 137 and strontium 90 from PUREX acid waste, high-level supernatant liquids, and sludge from self-boiling liquid wastes.

B Plant contained 40 processing cells that served several functions (e.g. equipment storage, radionuclide purification, Iiquid waste concentration, and solvent extraction). Cells 5, 23, 24 and 25 were used as Iiquid waste concentrators for the B Plant processing. Cell 23 of B Plant was used as a waste concentrator from July 28, 1967 to February 2, 1968. Because B Plant was being upgraded to a waste fractionation plant, the Cell 23 concentrator was running specifically to concentrate intermediate level waste in the single-shell tanks.

In 1974, the Waste Encapsulation and Storage Facility (WESF) was added to B plant to encapsulate strontium and cesium that was recovered from the B Plant process. The cesium capsules were leased commercially for irradiation of food, medical supplies, and lumber. The strontium and cesium capsules are stored in water basins for future retrieval. Currently most of $B$ Plant is going through closure except for the plant sections that are needed to support the WESF mission. The closures result from a 1991 evaluation of the future B Plant mission.

The following B Plant waste streams were sent to SSTs: B Plant high-level waste (B), B Plant flush (BFSH), low-level waste from the waste fractionation plant (BL), complex concentrate (CC) or (CCPI), complexed waste (CPLX), fission products waste (FP), metal waste (MW), REDOX ion exchange waste processed at B Plant (RIX), ion exchange (IX), strontium sludge (SRS), cell 5 tank 6 waste (56), first-cycle waste (1C), and second-cycle waste (2C).

\section{Bismuth Phosphate Process}

The $B$ and $T$ Plants used a bismuth phosphate extraction to remove plutonium from uranium fuel elements. The extraction waste that resulted was a metal waste that still contained $90 \%$ of the fission products and $99 \%$ of the original uranium. The metal waste was sent to specific tank cascades in the 200-East and 200-West Areas. First-cycle decontamination waste (IC), which contained approximately $10 \%$ of the fission products, was also stored in other underground waste tanks in the 200 Areas.

\section{Plutonium Finishing Plant}

The Plutonium Finishing Plant (PFP or $\mathrm{Z}$ Plant), previously called Plutonium Recovery and Finishing Operations, began operation in late 1949 to process plutonium and prepare plutonium products. 
PFP operations included plutonium handling, plutonium reclamation, plutonium conversion, and decontamination and decommissioning.

Waste from this plant contained minor amounts of fission products and low concentrations of plutonium and other transuranic elements, but was high in metallic nitrates. Initially, the waste was discharged via cribs to soil columns, which absorbed the transuranic elements and retained them close to the point of discharge. Later waste from $\mathrm{z}$ plant was stored along with other waste in underground tanks.

The following waste was sent to tanks: waste from the Plutonium Reclamation Facility and the remote mechanical $C$ line (z).

\section{Uranium Oxide Plant}

The 224-U Building was completed in 1944 as part of the $U$ Plant complex. The building was converted to a $\mathrm{UO}_{3}$ plant in 1951 . The 224-UA building was constructed in 1957 with six installed calciners. The $\mathrm{UO}_{3}$ plant was able to handle the uranyl nitrate hexahydrate (UNH) stream from REDOX, $U$ Plant, and PUREX. The $\mathrm{UO}_{3}$ plant shut down in 1972 and restarted in 1984 with the feed Iines from REDOX and the $U$ Plant canyon disconnected. Since 1984, there have been 17 campaigns at the plant that averaged 8 days each. The plant can calcine UNH much faster than the PUREX plant can produce it. Final deactivation was ordered for the plants in 1992. In April 1993, the $\mathrm{UO}_{3}$ plant resumed operations to convert 200,000 gal of remaining UNH to uranium oxide powder. A final deactivation plan for the facility was prepared in the summer of 1993 and will be carried out in the next few years.

In the basic $\mathrm{UO}_{3}$ process, calcining consisted of concentrating and heating liquid UNH until it was converted to a stable orangeyellow powder. The nitric acid in the UNH solution was also recovered in the same process. The $\mathrm{UO}_{3}$ powder was the base material needed to manufacture uranium hexaflouride (UF 6 ) which is the primary feed material for gaseous diffusion plants in the United States.

\section{REDOX}

The Reduction and Oxidation extraction (REDOX) plant was built between May 1950 and August 1951 and operated until July 1967. Uranium and plutonium were extracted from solid uranium metal slugs that were irradiated in the Hanford reactors or piles into a methyl isobutyl ketone (hexone) solvent by a continuous solvent extraction process. The REDOX process was the first at Hanford to recover both plutonium and uranium.

The waste stream which was slightly acidic contained fission products and large quantities of aluminum nitrate that were used to promote the extraction of plutonium and uranium. The waste was then neutralized and stored in SSTs. 
The following wastes were sent to SSTs: REDOX process waste resulting from the dissolution of aluminum and zircaloy fuel element cladding, REDOX coating waste (CWR), REDOX high-level waste (R), and the supernatant liquid portion of waste generated by the REDOX process, REDOX supernatant (RSN). RSN is found above the sludge in the underground storage tanks.

U Plant

U Plant (221-U) was built as one of three original bismuth phosphate process - facilities although it was not used for that purpose. U Plant was modified extensively and used for the uranium recovery process. U Plant operated from 1952 to 1958. The main canyon at $U$ Plant currently stores failed equipment.

Uranium in waste from the $\mathrm{BiPO}_{4}$ process initially was stored in the SSTs. Isater it was mined by sluicing, dissolved in nitric acid, and processed through a solvent extraction process. For every gallon of metal waste originally stored, the uranium extraction process yielded approximately 1.4 gal of UR waste. The waste volume increase made development of a technology that would reduce the volume of the stored waste a priority. This need resulted in the development/implementation of the ferrocyanide scavenging process.

The waste sent to the SSTs from U Plant was called tributyl phosphate (TBP). In reality it contained very little TBP; therefore, to eliminate confusion, it will be referred to as uranium recovery (UR) waste in this report.

\section{Strontium Semiworks}

The strontium or hot semiworks processing facility (i.e., C Plant) was built in 1949 as a hot pilot plant for the REDOX process, but was later converted to a pilot plant for the PUREX process. In 1960, the plant was reactivated and used later as a process demonstration for the conversion of $B$ Plant to a waste fractionation facility and was used to recover strontium 90, promethium 147, and cesium 144 from PUREX waste. The building and building site have been decontaminated and decommissioned.

The strontium semiworks waste sent to SSTs was known as hot semiworks (HS), strontium semiworks (SSW), and fission products waste (FP).

\section{PUREX}

The Plutonium Uranium Extraction (PUREX) plant (i.e., A Plant) was an advanced solvent extraction process that used tributyl phosphate in a paraffin hydrocarbon solvent to recover uranium and plutonium from nitric acid solutions of irradiated uranium. PUREX was built between April 1953 and April 1955, it operated until 1972 when it was closed for 11 years. Two thorium campaigns were conducted in the PUREX plant between 1966 and 1971. The irradiated waste was similar to the PUREX waste but it contained thorium and uranium 233 rather than uranium and plutonium which was extracted from typical PUREX waste. It began operating again in November 
1983 and was shut down in 1988 after a safety violation. The PUREX Plant was shut down several times between 1988 and 1990 for various reasons. In October 1990, the PUREX Plant was put in standby mode and was eventually identified for closure in December 1992 by Secretary of Energy James Watkins.

The following PUREX waste streams were sent to SSTs: cladding waste (CW) or (CWP), organic solvent wash waste (OWW), neutralized PUREX plant acid waste (P), low-level waste from PUREX (PL), PUREX sludge supernatant Iiquid (PSS), organic wash waste using sodium carbonate (CARB), cesium feed (CF), and concentrated neutralized high-level waste (IWW) .

\subsubsection{Waste Management Operations}

This section describes the different waste concentrating methods used in the 200 Areas. Evaporating, concentrating, and scavenging are all methods used to reduce liquid volumes or precipitate solids. out of the supernatant solutions. The operations are presented in chronological order.

\section{2-T Evaporator}

The 242-T Evaporator was built in the early 1950s to reclaim nonboiling waste storage capacity in existing tanks. The evaporator was shut down in the summer of 1955 and modified for tributyl phosphate scavenging, although scavenging was never performed in this evaporator. The 242-T Evaporator restarted in 1965 and operated until 1976. Tank 241-TX-118 was the feed tank for the evaporator.

The following wastes were sent to tanks from the $242 \mathrm{~T}$ Evaporator: Slurry product from the evaporator bottoms (EB), evaporator feed $(E F)$, and terminal liquor (TL).

\section{2-B Evaporator}

The 242-B Evaporator was built south of the B Tank Farm and started operating in December 1951. It received feed waste until November 1954 and was shut down in September 1955. The evaporator was never reactivated. Tanks $241-B-105$ and -106 were used as the bottoms and feed tank respectively. The evaporator operated at atmospheric pressure.

\section{Ferrocyanide Scavenging}

Ferrocyanide scavenging was developed to reduce waste volumes. The ferrocyanide flow sheet was first tested in U Plant in October 1953. In-farm scavenging was completed in March 1956 and December 1957 for the 200-West and 200-East Areas respectively. The ferrocyanide scavenging program was designed to remove fission products such as ${ }^{137} \mathrm{Cs}$ (half life: $28.6 \mathrm{Yr}$ ) and ${ }^{90} \mathrm{Sr}$ (half life: $30.2 \mathrm{yx}$ ) from the UR and $1 \mathrm{C}$ wastes as a precipitate. Because the ${ }^{90} \mathrm{Sr}$ was essentially insoluble in the neutralized UR waste, the strontium compounds would precipitate as phosphates, sulfates, or hydrated oxides when the acidic waste was neutralized. However, common cesium compounds are quite soluble even at alkaline pHs. 
Development of the nickel ferrocyanide scavenging process provided a relatively simple process to precipitate ${ }^{137} \mathrm{Cs}$ and enhance the precipitation of ${ }^{90} \mathrm{Sr}$. The precipitating chemicals were added in stainless steel process vessels with agitation (i.e. U Plant, T Plant, or the $C R$ vault) and the waste slurry was routed to specific SSTs for settling. After the ${ }^{137} \mathrm{Cs}$ and ${ }^{90} \mathrm{Sr}$ precipitates settled, the clarified supernatant was sampled and disposed of in cribs. After the ${ }^{137} \mathrm{Cs}$ and ${ }^{90} \mathrm{Sr}$ precipitates settled, the clarified supernatant was sampled and disposed of in cribs.

- In-Tank Solidification

The primary function of the in-tank solidification systems was to concentrate the nonboiling waste directly inside of specially designed tanks that would produced a saltcake slurry. Each in-tank solidification system included a heat exchanger for water evaporation and a series of bottoms tanks. The first in-tank solidification unit (ITS \#1) operated with an airlift circulator through Tank 241-BY-102 and the second in-tank solidification unit (ITS \#2) operated with a similar circulator through Tank 241-BY112. Tank 241-BY-112 contained a 4,000 kW electric immersion heater. Tank 241-BY-101 contained an in-tank solidification prototype that was used only for demonstration purposes. In-tank solidification units 1 and 2 began operating in 1965 and 1968, respectively. In 1971 in-tank solidification unit 1 became the cooler for in-tank solidification unit 2. Both units were shut down in 1974.

\section{REDOX Concentrator}

The REDOX concentrator was used for volume reduction of dilute 200-West Area tank farm wastes by removing water. The concentrator received tank farm wastes from July 28, 1967 until June 30, 1972. Cell $D$ in the REDOX process was the concentration and neutralization section of the plant.

\section{2-S Evaporator/Crystallizer}

The 242-S Evaporator/Crystallizer began operating in November 1973 and shut down in 1981. It was designed as a reduced pressure (i.e., partial vacuum) evaporator/crystallizer. Aqueous salt wastes were converted in the evaporator to salt crystals for storage in underground tanks in the $\mathrm{S}$ and SX Tank Farms.

The following waste types were sent to SSTs from the 242-S Evaporator: non-complexed waste concentrated until the solution was nearly saturated with sodium aluminate known as double-shell slurry feed (DSSF), slurry product from the evaporator bottoms (EB), evaporator feed (EF), Hanford defense residual liquor (HDRL), partial neutralized feed waste (PNF), and terminal liquor (TI).

- 242-A Evaporator/Crystallizer

Construction of the 242-A Evaporator/Crystallizer was completed in 1976. The evaporator began operation in March 1977 with a design life of 10 years. In 1987, design changes were developed to extend the facilities operating life by 10 years. The 


$$
\text { WHC-SD-WM-ER-323， Rev. } 0
$$

evaporator was shut down in April 1989 because of regulatory issues and restarted in May 1994 after extensive modifications.

The following evaporated wastes were sent to SSTs: evaporator bottoms from B Plant low-level waste feed (BLEB), non-complexed waste concentrated in evaporators known as double-shell slurry feed (DSSF), evaporator feed (EF) or (EVAP), evaporator bottoms (EB), and terminal liquor (TL).

\subsubsection{Miscellaneous Waste Sources and Equipment}

There are various other sources of waste on the Hanford site, as well as other material added to the tanks. Some wastes are from the 300 Area, 100 Area production reactors, various laboratories, and catch tanks. Unique contents added to SSTs included laboratory wastes, diatomaceous earth, Portland cement, shroud tubes, ceramic balls, experimental fuel elements, and relatively small amounts of enriched uranium, plutonium, cobalt, and natural uranium.

The following wastes contribute to SST waste: diatomaceous earth (DE), Hanford defense residual liquor (HDRL), Hanford laboratory operations (HLO), filtered Hanford water $\left(\mathrm{H}_{2} \mathrm{O}\right)$, phosphate decontamination waste from $\mathrm{N}$ reactor $(\mathrm{N})$, and non-complexed waste (NCPL) .

\section{CriticaI Mass Lab}

The critical mass lab was used to develop data on the factors that are necessary to make a criticality or self-sustained nuclear reaction occur. Because plutonium is the key radioactive element on the Hanford site, the research was based on plutonium criticality safety in various fuel cycles. This facility was located next to the strontium semiworks and ran from the early 1950s to the early 1980s. The plutonium used in the lab was reprocessed in PUREX.

\section{4-AR, -BXR, and-CR Process Vaults}

There are three process vaults in the $N E$ quadrant: the $A R$ Vault, the BXR Vault, and the CR Vault. The vaults were composed of several process vessels or tanks used to prepare the waste for treatment or storage. Specific wastes from tanks can be pumped temporarily to the vaults and the wastes can be sent directly to desired tanks or processing facilities later.

The AR Vault was designed and constructed between 1964 and 1968 and is located next to the A and AX Tank Farms. The AR Vault has been in standby mode since 1978 .

The 244-BXR Vault is located south of the 241-BX Tank Farm and was constructed between 1950 and 1951. The BXR Vault began operating in 1952 and became inactive in 1956. The waste in the vault was difficult to handle, so the vault was high-pressure steam jetted in 1976. The vault was interim isolated after 1976 and interim stabilized in March 1985. Tank BXR-004 was removed in 1963 . 
The CR Vault was constructed in 1952 and is located next to the C Tank Farm. Saltwell waste from the C Tank Farm is interim stored in the CR Vault. The 244-BXR and -CR Vaults were constructed to aid in the recovery of uranium from bismuth phosphate metal waste. The $\mathrm{CR}$ Vault received waste. from the Strontium Semiworks Plant en route to the 241-C Tank Farm.

\section{4-AR and 204-S Railroad Car Facilities}

The 204-AR rail car unloading facility was built in 1981 and replaced the $204-\mathrm{S}$ rail car unloading facility. The facilities were built for unloading radioactive liquid waste tank cars.

Liquids or slurries from the 1314-N Building in the 100 Area and the 340 Building in the 300 Area are unloaded at the 204-AR unloading facility.

1.5.4 Timeline

The following timeline represents the times and spans of different events that occurred during the lifetime of major Hanford plants in the 200-East and 200-West Areas. The plants were the main contributors of waste currently contained in SSTs. The following list of abbreviations and corresponding meanings are used on the timeline:

PUREX: Plutonium uranium extraction process

$\mathrm{UO}_{3}: \quad$ Uranium trioxide

PFP : $\quad$ Plutonium finishing plant

RG-RB: Rubber glove - remote button line

RMA: Remote mechanical "A" line

RSE : Recuplex solvent extraction

RMC: Remote mechanical "C" line

PRF : $\quad$ Plutonium reclamation facility

PCB : Polychlorinated biphynels

WESF : Waste encapsulation and storage facility

ITS: In-tank solidification (units 1 and 2)

PWR: $\quad$ Pressurized water reactor

REDOX: Reduction oxidation process 


\section{PROCESSES PRODUCING WASTE CONTAIN}

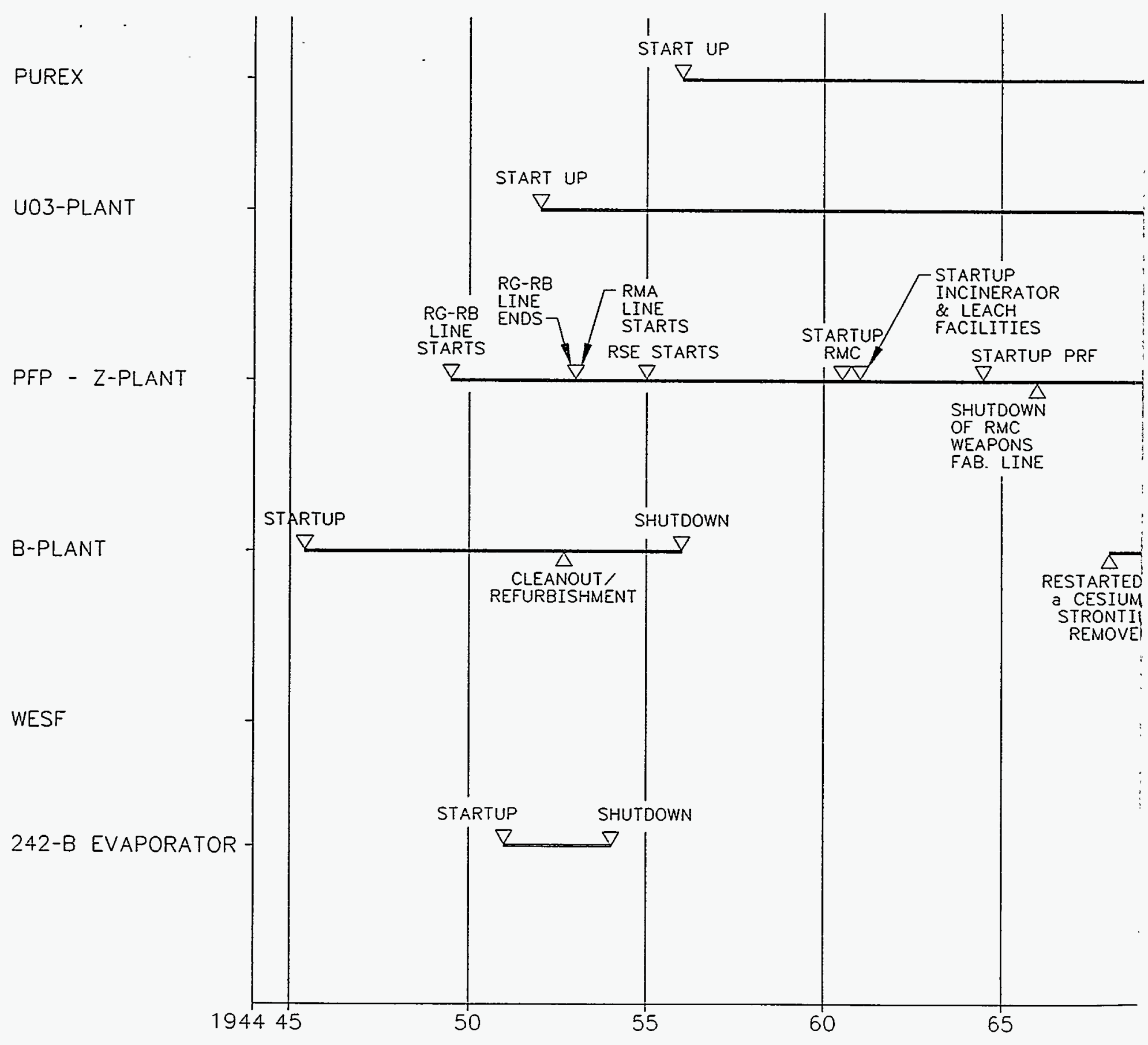




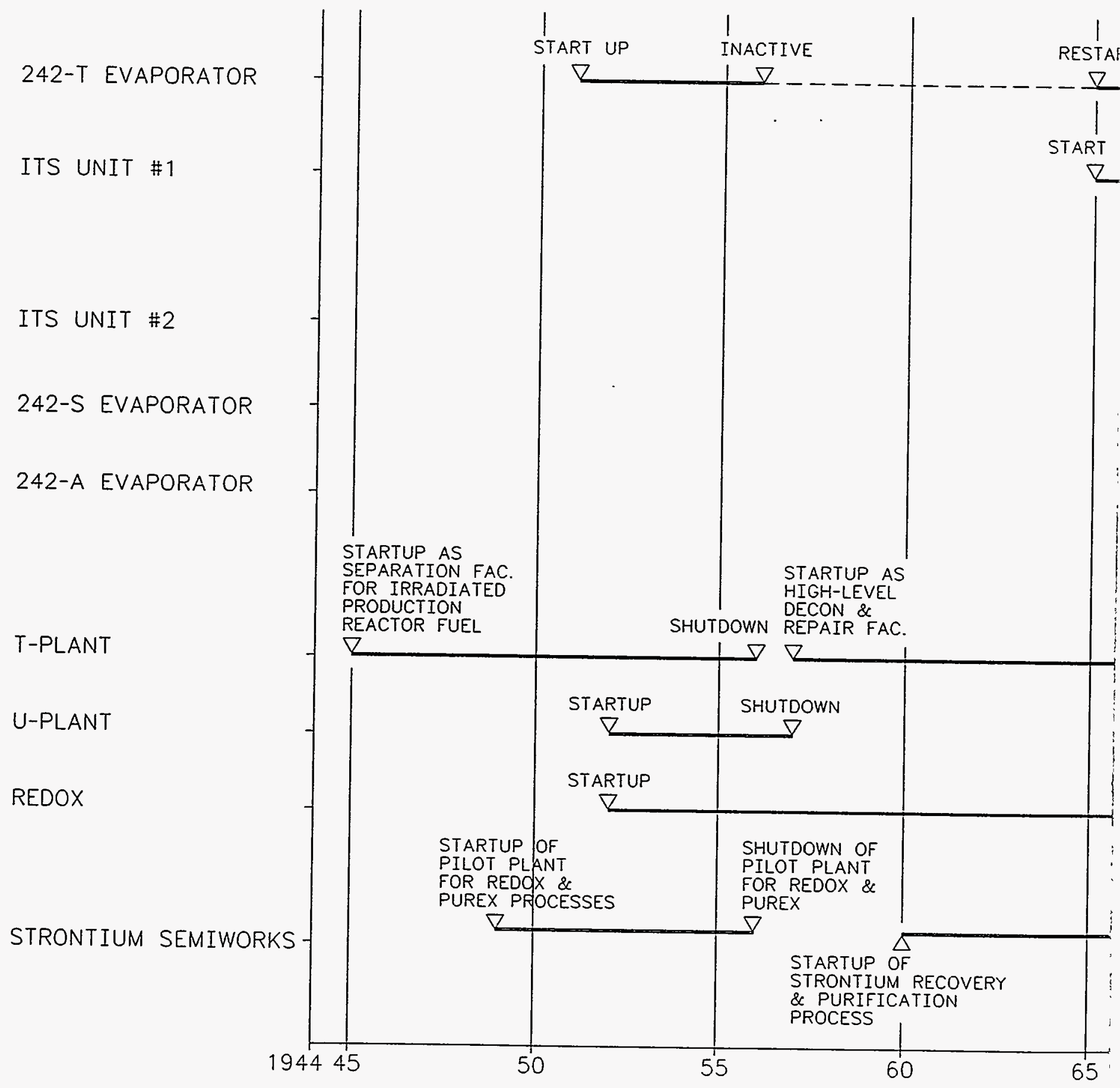


WHC-SD-WM-ER-323, Rev. O

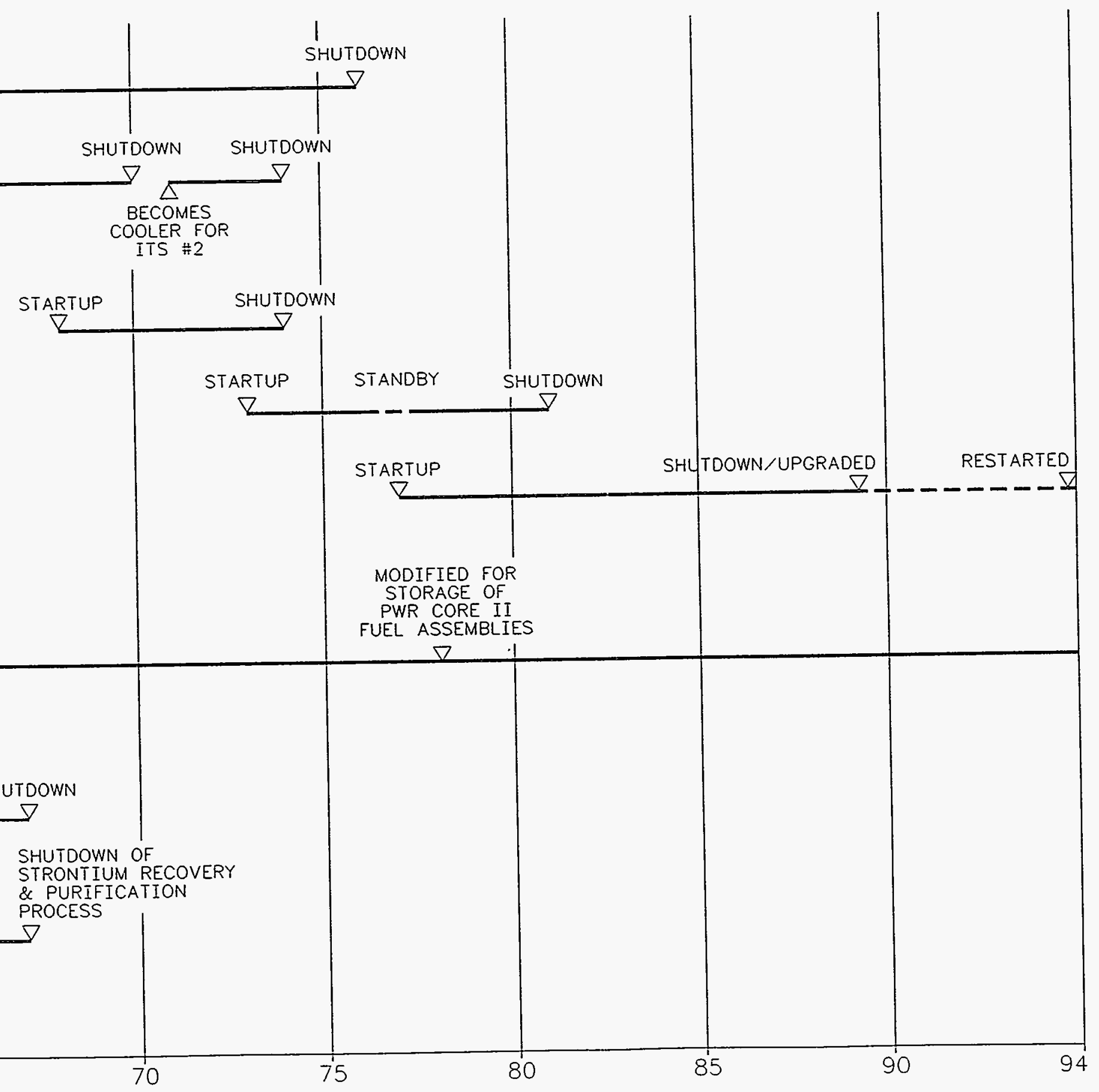




$$
\text { WHC-SD-WM-ER-323, Rev. } 0
$$

\subsection{Safety Issues}

The safety issues that effect the tanks can be divided into two groups: watch list and non-watch list. The watch lists are listings of the tanks believed to pose potential safety hazards to the environment and the public. Non-watch list issues are of concern because they may impact the environment.

\subsubsection{Watch List Safety Issues}

Issues in these tanks were identified as "issues/situations that contain most necessary conditions that could lead to worker (onsite) or offsite radiation exposure through an uncontrolled release of fission products" under Public Law 101-510, Section 3137, of the National Defense Authorization Act of Fiscal Year 1991 (i.e., the Wyden Amendment). As of October 1993, 45 SSTs and 6 double-shell tanks are on a watch list. In the NE quadrant, 21 of the 66 SSTs are on a watch list. The four watch list designations described in this report are: ferrocyanide, hydrogen/flammable gas, organic salts, and high-heat load. The following sections provide a general description and criteria of the different watch lists. For a more complete explanation, refer to the Hanford Site Tank Farm Facilities Interim Safety Basis (WHC 1993).

\section{Ferrocyanide}

A tank containing or believed to contain greater than 1,000 gram moles of ferrocyanide (dry basis) is on the Ferrocyanide Watch List.

\section{Hydrogen and Flammable Gas}

Most tanks were placed on the Hydrogen/Flammable Gas Watch List because of the potential to contain flammable gases rather than the verified presence of hazardous concentrations. Hydrogen/Flammable Gas Watch List tanks have been identified as unreviewed safety questions due to the concern of a flammable gas burn resulting in a radiological release.

\section{Organic Salts}

Tanks containing or believed to contain more than the equivalent of $10 \%$ by weight of sodium acetate or 3 wt\% total organic carbon (TOC) on a dry basis received a watch list designation as in the Tank Farm Surveillance and Waste status Summary Report (Hanlon 1993).

\section{High-Heat Loads}

Tanks with a heat load greater than 40,000 Btu/hr are designated as high-heat load tanks. Tank 241-C-106 is the only high-heat load tank on the High-Heat Load Watch List.

A tank that does not appear on a watch list but has a heat load less than 40,000 Btu/hr is considered a low-heat load tank by default. 
WHC-SD-WM-ER-323， Rev. 0

The following table lists the tanks in the SW quadrant that appeared on a watch..list as of November 1993. A current listing is published monthly in the Tank Farm Surveillance and Waste status Summary Report (Hanlon 1993).

SW Quadrant SST Watch List Tanks

\begin{tabular}{|c|c|c|c|}
\hline SST Tank & eategony & SST Tank & Category \\
\hline $241-S-102$ & $\begin{array}{c}\text { Hydrogen \& Organic } \\
\text { Salts }\end{array}$ & $241-S X-109$ & $\begin{array}{c}\text { Hydrogen, other tanks } \\
\text { vent through it }\end{array}$ \\
\hline$-S-111$ & Hydrogen & $-\mathrm{U}-103$ & Hydrogen \\
\hline$-S-112$ & Hydrogen & $-U-105$ & Hydrogen \\
\hline$-S X-101$ & Hydrogen & $-\mathrm{U}-106$ & Organic Salts \\
\hline$-S X-102$ & Hydrogen & $-U-107$ & $\begin{array}{c}\text { Hydrogen \& Organic } \\
\text { Salts }\end{array}$ \\
\hline$-S X-103$ & Hydrogen & $-\mathrm{U}-108$ & Hydrogen \\
\hline$-S X-104$ & Hydrogen & $-\mathrm{U}-109$ & Hydrogen \\
\hline$-S X-105$ & Hydrogen & $-U-111$ & Organic Salts \\
\hline$-S X-106$ & $\begin{array}{c}\text { Hydrogen \& Organic } \\
\text { Salts }\end{array}$ & & \\
\hline
\end{tabular}

1.6.2 Non-Watch List Safety Issues

Tank leaks are a safety hazard because of the potential chemical and radioactive liquid releases to the ground. Corrosion is the main cause of tank leaks and stabilization, integrity, and intrusion prevention are discussed in the corrosion section of this report. Criticality, tank bumps, and toxic vapor releases are other safety issues that do not require a watch list designation and/or continual monitoring under the wyden Amendment.

\section{Corrosion}

Corrosion is believed to be caused by localized or general reduction in SST mild carbon liner thickness. Localized liner thickness reduction is caused by three types of corrosion: pitting corrosion, stress corrosion cracking, and crevice cracking. General liner thickness reduction may be caused by uniform corrosion.

The SSTs were removed from service on or before November 21, 1980 and no longer accept wastes. When the SSTs are deactivated, an effort is made to minimize potential leaks into the earth by reducing the liquid waste contained in the sSTs. Primary stabilization is the first deactivation process of removing the supernatant or free liquid above the solid wastes within the SSTs. supernatant is removed by turbine or jet pumps in a saltwell system, low-volume submersible pumps, or in situ drying. In 
saltwell systems, the liquid is pumped from the waste through a pipe screen in a manner similar to ground water pumping. Tanks with less than 50,000 gal of drainable interstitial liquid and less than 5,000 gal of supernatant liquid are known as interim stabilized tanks. Iiquid waste is sent to the evaporator/crystallizer concentrating facilities and the concentrated waste is deposited into double-shell tanks (DSTs).

Eventually, sSTs will be interim stabilized and prepared for intrusion prevention.. (formerly. known as interim isolation). Stabilization efforts will be completed prior to final closure of the SSTs. Partial isolation of the tanks includes sealing the piping and risers that are not required for pumping or other stabilization methods. Intrusion prevention is the total physical and administrative effort involved in sealing inactive storage tank accesses against liquid additions while still allowing for longterm surveillance.

There are 67 assumed/confirmed leaking SSTs in the 200 East and West Areas with 11 in the SW quadrant. The remaining 33 tanks in the SW quadrant are sound. There are 11 interim stabilized tanks in the SW quadrant and 33 noninterim stabilized tanks. Intrusion prevention is in place for 18 SSTs in the sw quadrant, and 25 tanks are at the partial interim isolation stage.

Criticality

Criticality is an inadvertent self-sustained nuclear chain reaction. Studies have concluded that a nuclear criticality accident in the tank farms is probably not an imminent risk. However, definitive knowledge of the fissile material inventory and distribution within the tanks is lacking. Therefore, criticality remains a safety issue. The key criticality radionuclide on the Hanford site is plutonium. A fissile material criticality prevention specification of $125 \mathrm{~kg}$ has been placed on the tanks. An unusual occurrence report involving nuclear criticality was filed in June 1991 for Tank 241-C-104. An inventory of $56 \mathrm{~kg}$ was calculated later which indicated a low probability for criticality. Criticality is considered an unreviewed safety question but is not affected by the Wyden Amendment according to the Hanford Site Tank Farm Facilities Interim Safety Basis (WHC 1993).

\section{Tank Bumps}

Tank bumps have been an issue at Hanford since 1953 with the last bump occurring in 1968. The A Tank Farm is known to have had tank bumps. Tank bumps occur when heat from the hotter solid waste in the lower portion of the tank is suddenly transferred to a near boiling supernatant. Mixing can be achieved by "roll over" or sudden restart of airlift circulators which results in rapid fluid vaporization and a sudden internal tank pressurization that causes a bump. The three documented bumps that have occurred were due to airlift circulator failure followed by rapid startup of the airlift circulators which causes rapid mixing. Two key factors have been identified to control bumping: Iiquid and solid temperatures must be maintained as far away from the local boiling point as possible 


$$
\text { WHC-SD-WM-ER-323, Rev. } 0
$$

and airlift circulators and ventilation systems must be operated as designed. A tank bump is extremely unlikely if airlift circulators and vent flow rates are maintained at 500 to $1,000 \mathrm{ft}^{3} / \mathrm{min}$ per tank and $50 \mathrm{ft}^{3} / \mathrm{min}$ to the airlift circulators, according to the Hanford Site Tank Farm Facilities Interim Safety Basis (WHC 1993).

\section{Toxic Vapor Releases}

The safety concern of toxic vapor releases have been analyzed recently at Hanford. Odors have been released from the $C$ Tank Farm in the NE quadrant and from Tank 241-SY-101. Tank 241-C-105 has experienced releases that have resulted in worker exposure. If toxic vapors are determined to exist in a tank, three safety recommendations should be followed: determine a release rate, determine offsite and onsite exposure concentrations, and determine if the release is a spray effect. The entire issue of toxic gas releases at the tank farms has just begun to be understood, Hanford Site Tank Farm Facilities Interim Safety Basis (WHC 1993). 
WHC-SD-WM-ER-323, Rev. 0

\subsection{S Tank Farm \\ 2.0.1 S Tank Farm History}

The S Tank Farm was constructed between 1950 and 1951 under specification HW-3937. It is located in the 200-West Area north of 13th Street and the SX Tank Farm, south of 16th street, and west of Beloit Avenue. The $s$ Tank Farm contains 12 type III tanks constructed to the second-generation tank design. The design is similar to the original 75-ft diameter tanks but with an increased operating depth. The tanks have a 12-in. dish bottom, 4-ft knuckle radius, 75-ft diameter, 758,000-gal volume, and 23-ft operating level. The tanks were designed with a primary steel liner and $a$ concrete dome with various risers. The tanks are covered with approximately $8 \mathrm{ft}$ of overburden. Various coatings and materials were used to seal the inside and outside of the tank liners, domes, risers, and manholes. Cascade overflows are connected from Tank 241-S-101 to -102 to -103 , Tank 241-S-104 to -105 to -106 , Tank 241-S-107 to -108 to -109 , and Tank 241-S-110 to -111 to -112 . Tanks 241-S-103, $-106,-109$, and -112 were designed to overflow to cribs. All tanks overflow at a height of 285.5 in. or approximately $23.8 \mathrm{ft}$. The tanks were designed to withstand pH values of 8 to 10 , and fluid temperatures up to $220^{\circ} \mathrm{F}$. The tanks were not designed with a boiling period.

Ten tanks contain non-complexed waste and two tanks contain double-shell slurry feed. Two tanks are on the Hydrogen Watch List only, one tank is on the Hydrogen Watch List and the Organic Salts Watch Iist, and nine tanks are on the Low-Heat Load Nonwatch List. The $S$ Tank Farm received several types of REDOX plant waste in all four cascades. In 1953, surface condensers were installed that self-concentrated the wastes in the first two cascades by disposing of vapor condensate through cribbing. Further concentration in most $S$ Tank Farm tanks was achieved with the reduced pressure 242-S Evaporator/Crystallizer during the 1970s.

The 1993 aerial photograph shows tank and valve pit locations for the S Tank Farm. Arrows between the tanks show the direction of cascade overflows.

\subsubsection{S Tank Farm Waste History}

After startup of the 12 tanks in the $S$ Tank Farm in 1951, several types of REDOX Plant wastes were received. The tanks were almost filled with liquids by 1953; however, the wastes started to self-boil in the summer of 1952 which caused them to concentrate. To provide more tank space in 1953, surface condensers that concentrated the wastes in the first two cascades by disposing of vapor condensate to cribs were installed. Iiquid levels in the tanks fluctuated for the next 20 years, then the tanks filled rapidly with solids. The change can be attributed to the startup of the 242-s Evaporator/Concentrator because the tanks were used as receivers for evaporator waste products. The waste materials are concentrated solutions and the difference between solid and liquid 
may reflect only a small change in temperature or concentration. When the tanks were filled with solids, little could be done to increase their capacity with the technology that had been developed. The tanks were removed from service in the late 1970s or early 1980s.

\subsubsection{S Tank Farm Temperature History}

Each of the 12 tanks within the $S$ Tank Farm contain one thermocouple. tree designed to hold.6 to 14 thermocouple probes. Tanks 241-S-102, -111 , and -112 are on the Hydrogen Watch List and Tank 241-S-102 is on the Organic Salts Watch List. The watch list tanks have weekly temperature monitoring requirements and the remaining tanks have semiannual temperature monitoring requirements. Historical temperature information is also available between 1952 and 1973. The original historical temperature data, for some of the tanks, specified either sludge or supernatant temperatures. To integrate unusual delineations of the temperature monitoring location, the data has been evaluated as the highest daily temperature, or the average temperature if there are multiple daily readings.

\subsubsection{S Tank Farm Integrity}

The twelve 750,000-gal tanks in the $S$ Tank Farm are out of service. As of November 1993, only Tank 241-S-104 was categorized as an assumed leaker. Tank integrity surveillance methods include liquid observation wells, surface level measurements, and leak detection drywells. If a discrepancy is observed as a result of the surveillance activities; notification, reporting, documentation, and investigation of the surveillance anomaly (data outside the criteria limits, changing trend, or departure from steady state) is conducted in accordance with the appropriate procedure. The first plan of action is to determine if the discrepancy is caused by something other than a tank leak or intrusion.

All tanks in the S Tank Farm, except for Tanks 241-S-104 and 107, have liquid observation wells. The wells measure the interstitial liquid levels and are monitored weekly with a neutron probe and on request with a gamma probe. The maximum deviations from the established baseline values are a $0.3-\mathrm{ft}$ increase and a 0.4 -ft decrease.

Surface level monitoring for manual measurement devices in the $S$ Tank Farm is conducted daily or quarterly depending on the tank. The maximum allowable decrease from an established baseline is 0.5 , $1.0,2.0$, or 10.0 in. or no criterion depending on the tank. The maximum allowable increase from an established baseline is 2 or 3 in. depending on the tank. The no criterion designation was established for waste tanks containing sludge with little or no solution. For these tanks, a surface level decrease is not a valid means of leak detection because the level may decrease due to sludge slumpage (especially during saltwell pumping), tape flushes, 
WHC-SD-WM-ER-323， Rev . 0

and perforation of the exposed sludge at the point of probe contact. Tanks that have a saltcake surface and are equipped with an automatic Food Instrument Corporation gauge may have a 10-in. decrease criteria that was established to alert operating personnel if the gauge unwinds in search of a conductive liquid surface.

The S Tank Farm has 72 leak detection wells (i.e., drywells) that were drilled from 1944 to 1976; three are not tank specific. Drywell 40-00-06 had readings greater than the background radiation of $50 \mathrm{c} / \mathrm{s}$. The drywells in the s Tank Farm are monitored monthly or on request with scintillation or Geiger-Mueller probes. Increased activity action criteria for monitoring conducted with scintillation probes are as follows: if the normal readings are less than $200 \mathrm{c} / \mathrm{s}$, the readings must double and exceed $200 \mathrm{c} / \mathrm{s}$; if the normal readings are greater than $200 \mathrm{c} / \mathrm{s}$, the readings must double. Increased activity action criteria for monitoring conducted with Geiger-Mueller probes are as follows: if the normal readings are between 16 and $1,000 \mathrm{c} / \mathrm{s}$, the readings must triple; if the normal readings are greater than $1,000 \mathrm{c} / \mathrm{s}$, the readings must double. Drywells associated with assumed leaking tanks are monitored for contamination migration and the criteria limits apply only where new radiation peaks occur. If increasing drywell activity has not exceeded the action criteria stated above, appropriate personnel will determine if increased monitoring is required.

The S Tank Farm layout includes the peak drywell information, tank integrity information, and tank status information. The drywell information is based on summarized data prior to 1987 and data collected from January 1990 to the present.

\subsubsection{S Tank Farm Historical Sample Analysis Data}

The major waste products from the REDOX process are sodium nitrate, sodium aluminate, sodium silicate, chromium hydroxide, iron hydroxide, and zirconium oxide. The major evaporator product is sodium nitrate with minor amounts of phosphates, carbonates, and nitrites.

Test results from supernatant, sludge, and saltcake waste samples have been compiled to make up a historical collection or library. The data were selected from the collection for use in this report. In most cases, one sample was obtained and analyzed at a time which means that measurement variability cannot be assessed in the analytical procedures or in the sampling techniques. The presence or lack of homogeneity in the tanks cannot be evaluated from the data available.

Wastes were added to and removed from the tanks continuously until the late 1970s or early 1980s when the tanks were removed from service. Therefore, samples taken after activity ceased are probably more representative of the tank contents than earlier samples, although that may not always be true. The activities 
WHC-SD-WM-ER-323, Rev. 0

involved have to be considered to determine the value of each sample.

Samples were usually analyzed for a specific purpose such as to discover if the wastes in the tank could be sluiced, pumped, or concentrated further, or if supernatant could be sent to a crib. The specific nature of the samples often resulted in a limited look at the chemicals that may be in the tank. For these reasons, the data provide less information than desired in most cases. However, when used with caution, the data provides some useful information for characterization of tank contents.

\subsubsection{Current Status of $\mathrm{S}$ Tank Farm}

Tanks 241-S-102, -111, and -112 in the S Tank Farm were added to the watch list officially in January 1991. The three tanks are unreviewed safety questions due to the potential consequence of a radiological release resulting from a flammable gas burn. Previous safety studies did not investigate this potential. Tank 241-5-102 is identified on the Organic salts Watch List and is a safety concern because high organic concentration in the tank could support an exothermic reaction at elevated temperatures. Therefore, releases of high-level waste would be possible. Organic Salts watch List tanks must be monitored for temperature on a weekly basis. Non-watch list low- heat load tanks $(<40,000$ Btu/hr) include Tanks 241-s-101, -103, -104, -105, -106, -107, 108, -109, and -110. Low heat load tanks are monitored for temperature on a semiannual basis. The waste volume totals $5,688,000 \mathrm{gal}$ which is comprised of $54,000 \mathrm{gal}$ of supernatant; 549,000 gal of saltslurry; $4,046,000$ gal of saltcake; 175,000 gal of unknown waste; and 864,000 gal of sludge.

\subsubsection{S Tank Farm In-Tank Photographs}

The S Tank Farm photographs usually were taken from a side riser with the camera aimed toward the other side. Pictures of the entire inside surface area were taken and the photos were assembled into a collage portraying the interior of each tank. Most photographic collages provide a good representation of present tank contents because, except where noted, no activity has occurred to affect the tank since the photographs were taken. 
WHC-SD-WM-ER-323，Rev. 0

2.1 Tank 241-S-101

2.1.1 241-S-101 Tank History

2.1.1.1 Waste History Tank 241-S-101

The waste history narrative in the following paragraph is from "A History of the 200 Area Tank Farms" (Anderson 1990). Tank 241S-101 was filled with waste from the REDOX facility which it contained from the third quarter of 1953 until the third quarter of 1973. From the fourth quarter of 1973 until the third quarter of 1974, the tank received numerous types of waste from the $U$, $S$, and SX Tank Farms. The tank received bottoms and recycle streams from the 242-s Evaporator/Crystallizer from the fourth quarter 1974 until the second quarter of 1976. The tank contained various types of evaporator waste until the addition of partial neutralized feed in the second quarter of 1978. In the first quarter of 1980, the waste was classified as double-shell slurry feed. Presently, the waste is classified as non-complexed.

The waste history narrative of the primary additions that follow in this paragraph are from the "Waste status and Transaction Record Summary" (Agnew 1994). Tank 241-S-101 received REDOX waste from the third quarter of 1953 until the second quarter of 1954. From the first quarter of 1954 until the third quarter of 1955, the tank received REDOX cladding waste. The tank received wastewater from the third quarter of 1955 until the first quarter of 1974 .

Tank 241-S-101 was removed from service in 1980 and was partially isolated in December 1982. The tank level was adjusted in september 1980. In-tank photographs were taken on the following dates: June 1971, several in 1976, January 1977, two in 1978, July 1979, two in 1980, April 1983, and March 1988. The latest maximum temperature reading from July 1993 is $115^{\circ} \mathrm{F}$. Sketch ES-TKS-E67, which shows a graphical representation of the Tank 241-S-101 level history, and the historical waste level data used to make it are in Appendix C. Three pH readings of 14.0 in september $1974,13.6$ in November 1975, and 13.4 in December 1991 are shown on the sketch. Currently, the tank is categorized as sound and is non-stabilized.

\subsubsection{Temperature History 241-S-101}

There are six thermocouple probes on a single thermocouple tree in Tank 241-S-101 with elevations available for each individual probe in riser 14. Data for thermocouples 1, 3, and 5 span from January 1988 to March 1989 and thermocouples 2,4, and 6 span from January 1988 to July 1993. All thermocouples show similar trends with thermocouple 6 showing an overall cooler temperature of approximately $20^{\circ} \mathrm{F}$. Review of the Tank 241-S-101 level history readings indicates that probes 1 through 5 were within or near the solid level and probe 6 was in the vapor space.

The mean temperature of the first electronically available data set taken from all six thermocouples is $120^{\circ} \mathrm{F}$. A sum of the 
WHC-SD-WM-ER-323, Rev. 0

squares regression line fit for thermocouple 1, starting in 1988, shows a positive slope of $0.098 \pm 14^{\circ} \mathrm{F}$. A sum of the squares regression line fit for probes 2 through 6 , starting in 1988, shows a near zero slope $\pm 7^{\circ} \mathrm{F}$. From January 1988 to July 1993 , the median temperature was $124^{\circ} \mathrm{F}$ with a minimum of $90^{\circ} \mathrm{F}$ and a maximum of $169^{\circ} \mathrm{F}$. As of November 1993, thermocouples, 1,3,5, and 6 were out of service and a work order was issued. Tank 24I-S-10I has a semiannual temperature monitoring requirement.

Plots of thermocouple readings for Tank 241-s-101 are in Appendix D.

\subsubsection{Integrity of Tank 241-S-101}

Tank 241-S-101 is categorized as sound and is partially isolated. The Tank 241-S-101 surface level is monitored with a Food Instrument corporation gauge. The gauge is set in the intrusion mode for a 1-in. increase. If the Food Instrument Corporation gauge fails, manual field measurements will be conducted quarterly. The maximum allowable increase from the 162.3-in. baseline is 3 in. There is no criterion for a decrease. Due to the Food Instrument Corporation gauge setting, a limited number of surface level measurements are available. A figure in Appendix E graphically represents the surface level measurements taken from May 1992 through June 1993. The readings range from 162 to 160 in.

The Tank 241-S-101 liquid observation well is located in riser 2. The tank is monitored weekly with a neutron probe and on request with a gamma probe. The maximum deviations from the established baseline are a 0.3-ft increase and a 0.4-ft decrease. Sketch ES-TKS-E67 in Appendix $C$ is a graphical representation of the liquid observation well data.

Of the five drywells identified for Tank 241-S-101, none are considered active.

\subsubsection{Historical Sample Analysis Data for Tank 241-S-101}

The tank was used as a receiver for fresh feed en route to the 242-S Evaporator system. Data are included in this report for four sets of samples from Tank 241-S-101 that were analyzed between September 21, 1971 and December 5, 1975. The July 1975 sample was dark brown sludge which had the consistency of soft mud. The analysis indicated that the material sampled was primarily a nitrate and an aluminum compound. The two other samples analyzed were primarily liquid and most liquid was pumped from the tank in 1980. The results of these analyses will be found in Appendix $\mathrm{H}$.

\subsubsection{Current status of Tank 241-S-101}

Tank 241-S-101 entered service in July 1953 and currently stores 427,000 gal of waste. The waste is comprised of 12,000 gal of supernatant; $83,000 \mathrm{gal}$ of saltslurry; 121,000 gal of saltcake; 
WHC-SD-WM-ER-323, Rev. 0

and 211,000 gal of sludge with 90,000 gal of pumpable liquid remaining. The tank is identified as a low-heat load tank, is passively ventilated, and is categorized as sound, with partial interim isolation completed. Tank 241-s-101 is equipped to cascade to Tank 241-S-102 and is first in the three-tank cascade series. A plan view and tank cross-section are in Appendix $G$ that depict the approximate waste level and riser configuration. Tank 241-S101 has 12 risers and three are available for use: one 4-in. riser (no. 4) and two.12-in. risers (no. 7 and no. 8).

\subsubsection{Waste Layer Volume Approximation for Tank 241-S-101}

A comparative analysis of all layer approximations for the entire tank farm and the data used to derive the tank layer approximations are located in Appendix $\mathrm{K}$.

\subsubsection{In-Tank Photograph 241-S-101}

In the Tank 241-S-101 picture, most of the waste appears to be solidified (see Appendix I). A few small pools of liquid remain on the surface. The waste in the center of the tank looks like the dark brown sludgy material which was sampled in 1975. The crust that formed around the outside of the tank looks like saltcake. A manual tape, Food Instrument Corporation level probe, temperature probe, and liquid observation well are all visible and labelled. A discarded level measurement tape is lying on the waste surface at the base of the liquid observation well. Recent measurements indicate that this tank holds about 430,000 gal of waste and the waste measures about $14 \mathrm{ft}$ deep. There have been no changes in the tank after the pictures were taken in 1988 according to the waste level history diagram.

2.1.3 Syñopsis Tank 241-S-101

(To be completed) 
WHC-SD-WM-ER-323, Rev . 0

2.2 Tank 241-S-102

2.2.1 241-S-102 Tank History

2.2.1.1 Waste History Tank 241-S-102

The waste history narrative in the following paragraph is from "A History of the 200 Area Tank Farms" (Anderson 1990). Tank 241S-102 was filled with waste from the REDOX facility from the third quarter of 1953 to .the second quarter of 1965. From the third quarter of 1973 until the first quarter of 1976, the tank was used for feed from the 242-S Evaporator/Crystallizer and contained evaporator bottoms waste. In the second and third quarters of 1976, the tank received bottoms and recycle streams from the 242-S Evaporator/Crystallizer. From the fourth quarter of 1976 until the first quarter of 1978, the tank served as a low-heat evaporator feed tank. In the second quarter of 1978, the tank was designated as a future solids receiver and contained non-complexed waste. During the first, second, and third quarters of 1979, the tank contained partial neutralized feed. The waste was classified as double-shell slurry feed in the fourth quarter of 1979. Presently, the waste is classified as double-sheli slurry feed.

The waste history narrative of the primary additions that follow in this paragraph are from the "Waste status and Transaction Record Summary" (Agnew 1994). Tank 241-S-102 received wastewater from the third quarter of 1973 until the second quarter of 1976. During the third and fourth quarters of 1979, the tank received a $\mathrm{HNO}_{3} / \mathrm{KMnO}_{4}$ solution.

The tank was removed from service and labeled inactive in 1980. The tank was partially isolated in December 1982. The tank level was adjusted in October 1979 and August 1980. In-tank photographs were taken on the following dates: June 1971, October 1974, July 1975, several in 1976, November 1977, April 1979, several in 1980, two in 1983, and March 1988. Sketch ES-TKS-E68, which shows a graphical representation of the Tank 241-s-102 level history, and the historical waste level data used to make it are in Appendix C. Nineteen pH readings are listed on the sketch: $>14.0$ in November 1973, 11.9 in December 1973, 11.4 in December 1973, 12.4 in May 1974, 12.6 in June 1974, 13.2 in September 1974, >12.8 in October 1974, 14.0 in December 1974, 13.3 in February 1975, 13.2 in September 1975, 12.7 in July 1975, 12.2 in September 1975, 12.7 in October 1975, 14.0 in November $1975,13.7$ in November 1975, 12.8 in November 1975, 13.0 in January 1976, 13.2 in February 1976, and $>14.0$ in March 1980. The latest maximum temperature reading from October 1993 is $106^{\circ} \mathrm{F}$. Currently, the tank is categorized as sound and is non-stabilized.

\subsubsection{Temperature History 241-S-102}

There are six thermocouple probes on a single thermocouple tree in Tank 241-S-102 with elevations available for each probe. The data for all thermocouples span from January 1988 to December 
1993. The thermocouples show similar trends with thermocouple 6 showing an overall cooler temperature of approximately $20^{\circ} \mathrm{F}$. Review of the Tank 241-S-102 level history readings indicates that thermocouple probes 1 through 5 were in or near the solids level and probe 6 was in the vapor space.

The mean temperature of the first electronically available data set taken for the six thermocouples is $111^{\circ} \mathrm{F}$. A sum of the squares regression line fit for the six thermocouples, starting in 1988, shows a slightly negative, near zero slope $\pm 2.75^{\circ} \mathrm{F}$. A common outlying point is apparent for thermocouples 1 through 5 in February 1988. On the plot for thermocouple 6, minor seasonal temperature undulations begin to appear in January 1991. From January 1988 to December 1993, the median temperature was $105.85^{\circ} \mathrm{F}$ with a minimum of $71.2^{\circ} \mathrm{F}$ and a maximum of $122^{\circ} \mathrm{F}$. Tank $241-\mathrm{S}-101$ has weekly temperature readings because it is on the Hydrogen Watch List and the Organic Salts Watch List.

Plots of thermocouple readings for Tank 241-S-102 are in Appendix D.

\subsubsection{Integrity of Tank 241-S-102}

Tank 241-S-102 is categorized as sound, and is partially isolated. The Tank 241-S-102 surface level is monitored with a Food Instrument Corporation gauge. If the Food Instrument Corporation gauge fails, manual field measurements will be conducted quarterly. The maximum allowable deviation from the 202.3-in. baseline is a 3-in. increase or a 10-in. decrease. Appendix E contains a figure that graphically represents the surface level measurements from January 1991 to the present. The readings range from 207.5 to 202.2 in. and the plot indicates an increasing trend. The maximum allowable waste level is $205.3 \mathrm{in}$.

The Tank 241-S-102 liquid observation well is located in riser 5. The tank is monitored weekly with a neutron probe and on request with a gamma probe. The maximum deviations from the established baseline are a 0.3-ft increase or a 0.4 -ft decrease. Sketch ES-TKS-E68 in Appendix $C$ is a graphical representation of the liquid observation well data.

Eight drywells are identified for Tank 241-S-102. Drywells 40-02-03 (active prior to 1990, current readings $>200 \mathrm{c} / \mathrm{s}$ ) and 4002-08 (active prior to 1990, current readings < $200 \mathrm{c} / \mathrm{s}$ ) have or had readings greater than the $50 \mathrm{c} / \mathrm{s}$ background reading.

Graphical representations of the drywell data for the active drywells from January 1990 to the present are in Appendix F. The graphs include the peak c/s (some wells have multiple peaks), the depth at which the peak occurred, and the date of the reading. 
WHC-SD-WM-ER-323, Rev . 0

2.2.1.4 Historical Sample Analysis Data for Tank 241-S-102

Data have been tabulated for more than one hundred analytical evaluations of the tank contents that were performed from september 21, 1971 to March 28, 1980. The tank was a slurry receiver for the 242-S Evaporator. The earliest samples contained mostly nitrates but the others are difficult to generalize. Many samples were a yellow liquid but some were mixed liquids and solids or other colors such as green and amber. Later samples contained nitrates, nitrites, aluminates, and phosphates. Samples taken in 1979 and 1980 contained the same compounds as well as hydroxide, sulfate, and carbonate, which are all apparently sodium salts. Results from these analyses are found in Appendix $\mathrm{H}$.

\subsubsection{Current Status of Tank 24I-S-102}

Tank 241-S-102 currently stores 549,000 gal of waste. The waste is comprised of $341,000 \mathrm{gal}$ of saltslurry; $204,000 \mathrm{gal}$ of saltcake; and 4,000 gal of sludge with 208,000 gal of pumpable interstitial liquid remaining. The tank is on the Hydrogen Watch List and the Organic Salts Watch List, is passively ventilated, and is categorized as sound with partial interim isolation completed. Tank 241-S-102 is equipped to cascade to Tank 241-S-103 and is second in the three-tank cascade series. A plan view and tank cross-section in Appendix $G$ depict the approximate waste level and riser configuration. Tank 241-S-102 has 12 risers and two $12-i n$. risers, no. 6 and no. 7, are available for use.

\subsubsection{Waste Layer Volume Approximation for Tank 241-S-102}

A comparative analysis of all layer approximations for the entire tank farm and the data used to derive the tank layer approximations are located in Appendix $\mathrm{K}$.

\subsubsection{In-Tank Photograph 241-S-102}

The picture of the Tank 241-S-102 waste surface in Appendix I displays an interesting variety of different textures and colors. Most of the center of the tank appears to be filled with solids that are beginning to form a crust. The crust is forming on a liquid surface which indicates that liquid exists to an indeterminate depth in much of the tank. The crust has formed continuously around the perimeter of the tank. The material in the center of the tank appears to be comprised of a variety of different compounds or a least of different crystalline structures. Chemical analyses in 1979 and 1980 suggested that the tank contained sodium hydroxide, sodium sulfate, sodium carbonate, sodium phosphate, and possibly sodium aluminate. The manual tape, level gauge, temperature probe, and a saltwell screen are all visible and labelled. Manholes, risers, and nozzles are clearly seen in the picture. The tank was photographed in 1988 and there have been only slight changes in the tank since then according to the level history diagram. The tank contains approximately 550,000 gal of waste and it is filled to a depth of about $17 \mathrm{ft}$. 
WHC-SD-WM-ER-323，Rev. 0

2.2.3 Synopsis Tank 241-S-102

(To be completed) 
WHC-SD-WM-ER-323, Rev . 0

2.3 Tank 241-S-103

2.3.1 241-S-103 Tank History

2.3.1.1 Waste History Tank 241-S-103

The waste history narrative in the following paragraph is from "A History of the 200 Area Tank Farms" (Anderson 1990). Tank 241S-103 was filled with waste from the REDOX facility from the fourth quarter of 1953 until the fourth quarter of 1973. From the fourth quarter of 1973 until the second quarter of 1976, the tank received bottom and recycle streams from the 242-S Evaporator/Crystallizer. In the fourth quarter of 1976, the tank became a low-heat evaporator dump tank containing evaporator feed waste. Between the second quarter of 1978 and the fourth quarter of 1980, the tank was classified as having non-complexed, partial neutralized feed and double-shell slurry feed wastes. Presently, the tank contains double-shell slurry feed waste.

The waste history narrative of the primary additions that follow in this paragraph are from the "Waste status and Transaction Record Summary" (Agnew 1994). Tank 241-S-103 received wastewater during the second quarter of 1955. From the first quarter of 1978 until the fourth quarter of 1980 , the tank received a $\mathrm{HNO}_{3} / \mathrm{KMnO}_{4}$ solution.

The tank was removed from service in 1980 and was partially isolated in December 1982. The tank level was adjusted in November 1978, October 1979, and November 1980. In-tank photographs were taken on the following dates: July 1971, May 1978, April 1979, April 1981, January 1984, and June 1989. Sketch ES-TKS-E69, which shows a graphical representation of the Tank 241-S-103 level history, and the historical waste level data used to make it are in Appendix C. Four $\mathrm{pH}$ readings are shown on the sketch: 11.8 in January 1974, >14.5 in October 1974, 11.0 in October 1974, and 13.6 in October 1991. The latest maximum temperature reading from July 1993 is $85^{\circ} \mathrm{F}$. Presently, the tank is categorized as sound and is non-stabilized. The tank has what appears to be a saltcake buildup in the center of the tank that creates an uneven surface. The buildup is probably the reason for the liquid observation well (LOW) readings being higher than the waste level readings.

\subsubsection{Temperature History 241-S-103}

Seven thermocouple probes are on a single thermocouple tree in Tank 241-S-103 riser 4. Elevations are available for all thermocouple probes except probe 7. All thermocouple plots show similar trends with slight cooling of $10^{\circ} \mathrm{F}$ between thermocouples 1 and 6 . Temperature data from thermocouple probes $2,3,5$, and 6 span from January 1988 to July 1993. Thermocouple probe 1 temperature data span from January 1988 to February 1993 and thermocouple probe 4 temperature data span from January 1988 to January 1991. Thermocouple probe 7 contains only one temperature data point in November 1988. Review of the Tank 241-S-103 level history readings 
for the thermocouple tree indicate that probes 1 through 4 were within or near the solids level and probes 5 through 7 are in the tank vapor space.

The mean temperature of the first electronically available data set taken from thermocouples 1 through 6 is $87.8^{\circ} \mathrm{F}$. A sum of the squares regression line fit for the thermocouples, starting in 1988, shows a slightly negative near zero slope $\pm 7.5^{\circ} \mathrm{F}$. Outlying points are apparent for thermocouple 1 in July and August 1988 and thermocouple .2 in July 1988. From January 1988 to July 1993 , the median temperature was $87^{\circ} \mathrm{F}$ with a minimum of $69.5^{\circ} \mathrm{F}$ and a maximum of $130^{\circ} \mathrm{F}$. Tank $241-\mathrm{S}-103$ has a semiannual temperature measurement requirement.

Plots of thermocouple readings for Tank $241-5-103$ are found in Appendix D.

\subsubsection{Integrity of Tank 241-S-103}

Tank 241-S-103 is categorized as sound and is partially isolated. The Tank 241-U-103 surface level is monitored with a Food Instrument Corporation gauge. If the Food Instrument Corporation gauge fails, manual field measurements will be conducted daily. The maximum allowable deviation from the established 104.8-in. baseline is a 2-in. increase or a 2-in. decrease. A figure that graphically represents the surface level measurements from January 1991 to the present is in Appendix E. The surface level has an increasing trend with readings ranging from 105.1 to 102.2 in.

The Tank 241-S-103 liquid observation well is located in riser 2. The tank is monitored weekly with a neutron probe and on request with a gamma probe. The maximum deviation from the established baseline is a 0.3 -ft increase or a 0.4 -ft decrease. Sketch ES-TKS-E69 has a graphical representation of the liquid observation well data.

Seven drywells are identified for Tank 241-S-103. Drywell 40-03-09 was active prior to 1987 .

\subsubsection{Historical Sample Analysis Data for Tank 241-S-103}

Between September 21, 1971 and December 6 1974, four sets of samples from Tank 241-S-103 were analyzed. The tank was a slurry receiver for the 242-S Evaporator system. All samples analyzed were a clear yellow liquid. The level history diagram showed that the tank contained almost all supernatant at the time the samples were taken. The supernatant was pumped from the tank in 1980 . Results from the analyses are in Appendix $\mathrm{H}$.

\subsubsection{Current Status of Tank 241-S-103}

Tank 241-S-103 entered service in November 1953 and currently stores $248,000 \mathrm{gal}$ of waste. The waste is comprised of $17,000 \mathrm{gal}$ 
of supernatant; $103,000 \mathrm{gal}$ of saltslurry; 119,000 gal of saltcake; and 9,000 gal of unknown waste with 79,000 gal of pumpable liquid remaining. The tank is identified as a low-heat load tank, passively ventilated, and is categorized as sound with partial interim isolation completed. Tank 241-S-103 is third in the threetank cascade series. A plan view and tank cross-section that depict the approximate waste level and riser configuration are in Appendix G. Tank 241-S-103 has 12 risers, and three 12-in. risers (no. 6, no. 7 and no. 8) are available for use.

2.3.2.1 Waste Layer Volume Approximation for Tank 241-S-103

A comparative analysis of all layer approximations for the entire tank farm and the data used to derive the tank layer approximations are located in Appendix $\mathrm{K}$.

\subsubsection{In-Tank Photograph 241-S-103}

The photographic montage of the Tank 241-S-103 contents shows a light yellow fractured saltcake surface with several interspersed pools of supernatant and a large area of supernatant in the foreground (see Appendix I). A 42-in riser with several drain pipes can be seen protruding through the tank ceiling. A Food Instrument Corporation surface level probe and a manual tape measurement device are at the top center of the photo. The bright white dot at the center of the tank is the reflection of the camera strobe light. The tank contains approximately 250,000 gal of waste with a depth of approximately $8 \mathrm{ft}$. Data supplied indicate that there has been no activity in the tank since these photographs were taken; therefore, the picture should represent the current tank contents.

2.3.3 Synopsis Tank 241-S-103

(To be completed) 
WHC-SD-WM-ER-323, Rev. 0

2.4 Tank 241-S-104

2.4.1 241-S-104 Tank History

2.4.1.1 Waste History Tank 241-S-104

The waste history narrative in the following paragraph is from "A History of the 200 Area Tank Farms" (Anderson 1990). Tank 241S-104 was filled with waste from the REDOX facility from the first quarter of 1953 until the fourth quarter of 1974. In the fourth quarter of 1973, the tank was reported to be a suspect leaker and during the next two years, some of the waste was pumped to Tank 241-S-107. Presently, the waste is classified as non-complexed.

The waste history narrative of the primary additions that follow in this paragraph are from the "Waste status and Transaction Record Summary" (Agnew 1994). Tank 241-S-104 received REDOX cladding waste from the first quarter of 1953 until the third quarter of 1955. From the first quarter of 1953 until the second quarter of 1954, the tank received REDOX waste. During the second quarter of 1955, the tank received wastewater.

The tank was removed from service and was labeled an assumed leaker of $24,000 \mathrm{gal}$ in 1968. The tank was labeled inactive in 1977 and was primary stabilized in 1979. The tank was administratively interim stabilized in November 1984 and intrusion prevention was completed in september 1988. A saltwell pump and a jet pump were installed in 1974 and 1978 respectively. A total of $2,000 \mathrm{gal}$ of liquid was jet pumped between 1978 and 1980 . The tank level was adjusted in April 1982 and December 1984. In-tank photographs were taken on the following dates: June 1971, May 1976, May 1979, May 1980, April 1982, and December 1984. Sketch ES-TKS-E70, which shows a graphical representation of the Tank 241S-104 level history, and the historical waste level data used to make it are in Appendix C. A pH reading of $>12.6$ in December 1974 is listed on the sketch. The latest maximum temperature reading from July 1993 is $104^{\circ} \mathrm{F}$. Currently, the tank is categorized as an assumed leaker and is stabilized.

\subsubsection{Temperature History 241-S-104}

Tank 241-S-104 has 14 thermocouple probes on a single thermocouple tree in riser 4. Elevations are not available for any of the thermocouple probes. The temperature data span from November 1991 to July 1993. All thermocouple plots show similar trends throughout the data span, though thermocouple 5 shows an overall temperature decrease of approximately $20^{\circ} \mathrm{F}$. The same temperature decrease is apparent for thermocouples 6 through 14 .

The mean temperature of the first electronically available data set taken from all 14 thermocouples is $98.9^{\circ} \mathrm{F}$. A sum of the squares regression line fit for all 14 thermocouples, starting in 1991, shows a slightly negative slope $\pm 2.6^{\circ} \mathrm{F}$. From November 1991 to July 1993 , the median temperature was $89.75^{\circ} \mathrm{F}$ with a minimum of 
WHC-SD-WM-ER-323, Rev. 0

83.1 $1^{\circ} \mathrm{F}$ and a maximum of $112.7^{\circ} \mathrm{F}$. Tank $241-\mathrm{S}-104$ has semiannual temperature monitoring requirements.

Plots of thermocouple readings for Tank 241-S-104 are in Appendix D.

\subsubsection{Integrity of Tank 241-S-104}

Tank 241-S-104 is categorized as an assumed leaker and is interim stabilized with intrusion prevention completed. The Tank 241-S-104 surface level is monitored quarterly with a manual tape. The maximum allowable increase from the 114.5-in. baseline is 3 in. There is no criterion for a decrease. A figure that graphically represents the surface level measurements from January 1991 to the present is in Appendix $E$. The surface level has remained steady with the readings ranging from 114.5 to $112.5 \mathrm{in}$.

Tank 241-S-I04 does not have a liquid observation well. Four drywells are identified for Tank 241-S-104. Drywells 40-04-01 (active prior to 1990, no longer active), 40-04-05 (active prior to 1990, current readings $>200 \mathrm{c} / \mathrm{s}$ ), and 40-04-08 (active prior to 1990, current readings $<200 \mathrm{c} / \mathrm{s}$ ) have or had readings greater than the $50 \mathrm{c} / \mathrm{s}$ background radiation.

A graphical representation of the data for the active drywells from January 1990 to the present is in Appendix F. The graph includes the peak c/s (some wells have multiple peaks), the depth at which the peak occurred, and the date of the reading.

\subsubsection{Historical Sample Analysis.Data for Tank 24I-S-I04}

The results of samples taken from Tank 241-S-104 on August 12, 1974 and January 9, 1976 are included in this report. The 1976 sample was grey and had a consistency similar to stiff dough or putty with a tendency to be sticky. Sample data indicated that the tank contained various metal nitrates and a significant amount of an aluminum compound. The 1974 sample was $90 \%$ liquid and the tank contains only solids now. Data from the analyses of both samples are in Appendix $\mathrm{H}$.

\subsubsection{Current Status of Tank 241-S-104}

Tank 241-S-104 entered service in February 1953 and currently stores $294,000 \mathrm{gal}$ of waste. The waste is comprised of 1,000 gal of supernatant; $54,000 \mathrm{gal}$ of unknown waste; and 239,000 gal of sludge with 23,000 gal of pumpable liquid remaining. The tank is identified as a low-heat load tank, is passively ventilated, and is categorized as an assumed leaker with interim isolation and intrusion prevention completed. Tank 24I-S-104 is equipped to cascade to Tank 241-S-105 and is first in the three-tank cascade series. A plan view and tank cross-section that depict the approximate waste level and riser conifguration are in Appendix $G$. Tank 241-s-104 has 9 risers and four are available for use: two 4in. risers, no. 1 and no. 2 and two 12-in. risers, no. 7 and no. 8 . 
2.4.2.1 Waste Layer Volume Approximation for Tank 241-S-104

A comparative analysis of all layer approximations for the entire tank farm and the data used to derive the tank layer approximations are located in Appendix $\mathrm{K}$.

\subsubsection{In-Tank Photograph 241-S-104}

The photographic montage of Tank 241-S-104 shows solid material over the entire tank surface except for a few randomly dispersed small pools of yellow liquid (see appendix I) . A chemical sample in 1976 described the waste as grey with a consistency of dough or putty. In the photo, the waste appears brown or purple which may be due to lighting conditions in the tank. A saltwell screen penetrates what appears to be the deepest pool of liquid remaining in the tank. Behind the saltwell, the manual tape level measuring device is shown and there is a temperature probe further into the background. Because the photograph was taken after the tank was stabilized in 1984, it should represent the current tank conditions. The tank waste level has remained stable at almost $10 \mathrm{ft}$ which equates to approximately $290,000 \mathrm{gal}$.

\subsubsection{Synopsis Tank 241-S-104}

(To be completed) 
WHC-SD-WM-ER-323, Rev. 0

2.5 Tank 241-S-105

2.5.1 241-S-105 Tank History

2.5.1.1 Waste History Tank 24I-S-105

The waste history narrative in the following paragraph is from "A History of the 200 Area Tank Farms" (Anderson 1990). Tank 241S-105 was filled with waste from the REDOX facility from the second quarter of 1953 until the third quarter of 1973. In the first quarter of 1974, the tank received bottoms and recycle wastes from the 242-S Evaporator/Crystallizer until the tank was salt-filled in the fourth quarter of 1974. From the first quarter of 1978 until the third quarter of 1980, the tank received partial neutralized feed waste. In the fourth quarter of 1980 , the waste was classified as non-complexed.

Tank 241-S-105 did not receive any primary waste additions according to the "Waste status and Transaction Record Summary" (Agnew 1994) report.

The tank was removed from service in 1976, labeled inactive in 1978, primary stabilized in June 1979, partially isolated in December 1982, and jet pumped/interim stabilized in september 1988 . Intrusion prevention was completed in September 1990. The tank was salt-filled with a saltwell pump that was installed in 1974 . A jet pump was installed and initiated in 1978. A total of 114,300 gal of liquid was jet pumped between 1978 and 1980 . The tank level was adjusted in June 1979 and September 1988. In-tank photographs were taken on the following dates: July 1971, several in 1974, December 1978, May 1979, August 1980, October 1981, April 1987, and April 1989. Sketch ES-TKS-E71, which shows a graphical representation of the Tank 241-s-105 level history, and the historical waste level data used to make it are in Appendix $C$. Three $\mathrm{pH}$ readings are shown on the sketch: 11.6 in October 1973, 11.5 in January 1974, and $>14.0$ in March 1974. The latest maximum temperature reading from July 1993 is $73^{\circ} \mathrm{F}$. Presently, the tank is categorized as sound and is stabilized.

\subsubsection{Temperature History 241-S-105}

Fourteen thermocouple probes are on a single thermocouple tree in Tank 241-s-105 riser 4 with elevations available for all probes. All thermocouple temperature data span from January 1991 to July 1993. Review of the Tank 241-S-105 level history over the time span of readings for the thermocouple tree indicates that probes 1 through 7 were within the waste level and probes 8 through 14 were in the tank vapor space. The tank waste level has remained constant over the data span.

The mean temperature of the first electronically available data set taken from all 14 thermocouples is $69.5^{\circ} \mathrm{F}$. A sum of the squares regression line fit for all 14 thermocouples, starting in 1991, shows a slightly negative near zero slope of $\pm 2.7^{\circ} \mathrm{F}$. From 
January 1991 to July 1993, the median temperature was $70.8^{\circ} \mathrm{F}$ with a minimum of $63.7^{\circ} \mathrm{F}$ and a maximum of $79.1^{\circ} \mathrm{F}$. Tank $241-\mathrm{S}-105$ has a semiannual temperature reading requirement.

Plots of thermocouple readings for Tank 241-S-105 are in Appendix D.

\subsubsection{Integrity of Tank 241-S-105}

Tank 241-S-105 is categorized as sound and is interim stabilized with intrusion prevention completed. The Tank 241-S-105 surface level is monitored with a Food Instrument corporation gauge. The gauge is set in the intrusion mode for a 1-in. increase. If the Food Instrument Corporation gauge fails, manual field measurements will be conducted quarterly. The maximum allowable increase from the 158-in. baseline is 3 in. There is no criterion for a decrease. Data for a surface level plot are not available due to the Food Instrument Corporation gauge setting.

The Tank 241-S-105 liquid observation well is located in riser 14. The tank is monitored weekly with a neutron probe and on request with a gamma probe. The monitoring limits are a $0.3-\mathrm{ft}$ decrease and a 0.4-ft increase. Sketch ES-TKS-E71 is a graphical representation of the liquid observation well data.

Five drywells are identified for Tank 241-s-105. Drywell 4005-03 was active prior to 1987 .

\subsubsection{Historical Sample Analysis Data for Tank 241-S-105}

Tank 241-S-105 was used as a bottoms receiver for the 242-S Evaporator. Data from eight analytical investigations of Tank 241 S-105 contents were selected for inclusion in this report. The samples were analyzed between september 1971 and september 1974 . The level history diagram indicates that the 1974 samples are more representative of current tank contents than the earlier results. According to 1974 results, the tank contains terminal liquors that are composed primarily of sodium hydroxide, sodium nitrate, and sodium aluminate with lesser quantities of other sodium compounds. The tables that contain the results of these analytical investigations are in Appendix $\mathrm{H}$.

\subsubsection{Current Status of Tank 241-S-105}

Tank 241-S-105 entered service in 1953 and currently stores $456,000 \mathrm{gal}$ of waste. The waste is comprised of 454,000 gal of saltcake and $2,000 \mathrm{gal}$ of sludge with 13,000 gal of pumpable liquid remaining. The tank is categorized as sound with interim stabilization and intrusion prevention completed. Tank 241-S-105 is a low-heat, categorized as sound with interim stabilization and intrusion prevention completed, and is passively ventilated. Tank 241-S-105 is equipped to cascade to Tank 241-S-106 and is second in the three-tank cascade series. A plan view and tank cross-section that depict the approximate waste level and riser configuration are 
in Appendix G. Tank 241-S-105 has 12 risers and four are available for use: one 4-in.. riser (no. 2) and three 12-in. risers (no. 6, no. 7 , and no. 8).

\subsubsection{Waste Layer Volume Approximation for Tank 241-S-105}

A comparative analysis of all layer approximations for the entire tank farm and the data used to derive the tank layex approximations are located in Appendix $\mathrm{K}$.

\subsubsection{In-Tank Photograph 241-S-105}

The contents of Tank 24I-S-105 are completely dry, at least on the surface, according to the picture which was made from a series of 1989 photographs (see Appendix I). In 1974, the tank contained terminal liquors composed primarily of sodium hydroxide, sodium nitrate, and sodium aluminate. After 1974, the tank level dropped twice, probably indicating that the material in the tank was solidifying. Since these photos were made, the tank level has remained constant. A number of risers are clearly shown in this picture, as are a saltwell screen in the foreground and a temperature probe in the center of the tank. An old level measurement tape is clearly visible lying on the surface of the waste. The waste level in the tank is about $15 \mathrm{ft}$ which corresponds to a waste quantity of approximately 460,000 gal.

\subsubsection{Synopsis Tank 241-S-105}

(To be completed) 
2.6.1 241-S-106 Tank History

2.6.1.1 Waste History Tank 241-S-106

The waste history narrative in the following paragraph is from "A History of the 200 Area Tank Farms" (Anderson 1990). Tank 241S-106 was filled from the second quarter of 1953 with waste from the REDOX facility until the third quarter of 1973. From the fourth quarter of 1973 until the second quarter of 1976, the tank received bottoms and recycle streams from the 242-s Evaporator/Crystallizer. In the second quarter of 1976 , the tank was removed from service and was salt-filled. A saltwell was installed the following quarter. From the second quarter of 1976 until the second quarter of 1977, the tank received and/or contained evaporator feed. During the third and fourth quarters of 1977, the tank contained residual evaporator liquor. In the first quarter of 1978, the tank contained Hanford defense residual liquor. From the second quarter of 1978 until the third quarter of 1980, the tank received and/or contained partial neutralized feed. The waste has been classified as non-complexed since the fourth quarter of 1980 .

The waste history narrative of the primary additions that follow in this paragraph are from the "Waste status and Transaction Record Summary". (Agnew 1994). Tank 241-S-106 received wastewater from the third quarter of 1955 until the third quarter of 1973.

The tank was removed from service in 1976 and was partially isolated in 1982. A jet pump was installed and initiated in 1978 . A total of 99,800 gal of liquid was jet pumped between 1978 and 1980. A liquid observation well was installed in December 1980. The tank level was adjusted in September 1975 and June 1982. Intank photographs were taken on the following dates: July 1971, two in 1974, December 1976, May 1978, two in 1981, November 1982, October 1984, April 1987, and March 1989. Sketch ES-TKS-E72, which shows a graphical representation of the Tank 241-s-106 level history, and the historical waste level data used to make it are in Appendix C. Four pH readings are shown on the sketch: $>14.0$ in August 1974, 14.0 in December 1974, >14.0 in June 1975, and 13.2 in October 1991. The latest maximum temperature reading from July 1993 is $78^{\circ} \mathrm{F}$. Currently, the tank is categorized as sound and is non-stabilized.

\subsubsection{Temperature History 241-S-106}

There are 14 thermocouple probes on a single thermocouple tree in Tank 241-S-106 riser 2 with elevations available for each probe. Data for thermocouples 3 and 5 through 14 span from January 1991 to July 1993, and data for thermocouples 1,2 , and 4 span from November 1991 to July 1993. All thermocouples show similar trends with the exception of thermocouples 1 and 3 . The graph of the data from those thermocouples is more jagged and less flat than the 
other plots. Review of the Tank 241-S-106 level history readings for the thermocouple tree indicates that probes 1 through 8 were in or near the solids level and probes 9 through 14 appear to have been in the vapor space.

The mean temperature of the first electronically available data set was $71.7^{\circ} \mathrm{F}$. A sum of the squares regression line fit, starting in 1991 for thermocouples 2 through 14, shows a slightly negative near zero slope $\pm 3.4^{\circ} \mathrm{F}$. Thermocouple 1 has a slightly positive near zero slope \pm 7.2 .. From January 1991 to July 1993, the median temperature was $73^{\circ} \mathrm{F}$ with a minimum of $57.6^{\circ} \mathrm{F}$ and a maximum of $93.7^{\circ} \mathrm{F}$. Tank $241-\mathrm{S}-1.06$ has semiannual temperature monitoring requirements.

Plots of thermocouple readings for Tank 241-S-106 are in Appendix D.

\subsubsection{Integrity of Tank 241-S-106}

Tank 241-S-106 is categorized as sound and is partially isolated. The Tank 241-S-106 surface level is monitored with a Food Instrument Corporation gauge. If the Food Instrument Corporation gauge fails, manual field measurements will be conducted daily. The maximum allowable deviations from the established 176.8-in. baseline are a 3-in. increase and a 2-in. decrease. The figure that graphically represents the surface level measurements from January 1991 to the present is in Appendix E. The plot indicates an increasing trend in waste level. The readings range from 178.5 to 174.5 in.

The Tank 241-S-106 liquid observation well is located in riser 4. The tank is monitored weekly with a neutron probe and on request with a gamma probe. The monitoring limits are a 0.3 -ft decrease and a 0.4-ft increase. Sketch ES-TKS-E72 is a graphical representation of the liquid observation well data.

Six drywells are identified for Tank 241-S-106 and none are considered active.

\subsubsection{Historical Sample Analysis Data for Tank 241-S-106}

Data from seven analytical investigations that were conducted on Tank 241-S-106 waste contents from september 1971 to July 1975 were selected for inclusion in this report. Many of the studies were intended to determine whether or not tank contents were deliquescent because the tank was experiencing unexplained liquid level increases. The samples appeared different, but they all seemed to contain sodium nitrate, sodium hydroxide, sodium aluminate, and various other compounds in smaller quantities. Results from the analyses are in Appendix $\mathrm{H}$. 
Tank 241-S-106 entered service in 1953 and currently stores 543,000 gal of waste. The waste is comprised of $511,000 \mathrm{gal}$ of saltcake and $32,000 \mathrm{gal}$ of unknown waste with 162,000 gal of pumpable interstitial liquid remaining. The tank is identified as a low-heat load tank, is passively ventilated, and is categorized as sound with partial interim isolation completed. Tank 241-S-106 is third in the three-tank cascade series. A plan view and tank cross-section that depict the approximate waste level and riser configuration is in Appendix G. Tank 241-S-106 has 12 risers and four are available for use: two 12 -in. risers, no. 7 and no. 8, and two 4-in. risers, no. 11 and no. 14 .

\subsubsection{Waste Layer Volume Approximation for Tank 241-S-106}

A comparative analysis of all layer approximations for the entire tank farm and the data used to derive the tank layer approximations are located in Appendix $\mathrm{K}$.

\subsubsection{In-Tank Photograph 241-S-106}

The tank waste has formed a thick solid crust around the perimeter of the tank. A pool of liquid in the center of the tank extends down to an indeterminate depth. The crust appears to be composed of at least two or three different materials judging from color variations. Chemical samples from the tank were primarily sodium nitrate, sodium hydroxide, and sodium aluminate. The liquid observation well is in the foreground of the picture, a temperature probe is in the background, and the Food Instrument corporation level probe and a saltwell screen are in the middle (see Appendix I). The level history diagram indicates that tank contents have remained constant since the photographs were taken in 1989. The waste height in the tank is approximately $17 \mathrm{ft}$ which corresponds with a quantity of about $540,000 \mathrm{gal}$.

2.6.3 Synopsis Tank 241-S-106

(To be completed) 
WHC-SD-WM-ER-323, Rev . 0

\subsection{Tank 241-S-107}

2.7.1 241-S-107 Tank History

2.7.1.1 Waste History Tank 241-S-107

The waste history narrative in the following paragraph is from "A History of the 200 Area Tank Farms" (Anderson 1990). Tank 241S-107 was filled with waste from the REDOX facility from the fourth quarter of 1952 until the fourth quarter of 1956 . From the first quarter of 1957 until the third quarter of 1968, the tank received coating waste. The tank received evaporator bottoms waste from the REDOX evaporators from the fourth quarter of 1968. The tank began receiving evaporator bottoms waste from the REDOX evaporators from the second quarter of 1968 until the second quarter of 1971. The tank contained a mixture of REDOX and evaporator bottoms waste until the fourth quarter of 1973. From the first quarter of 1974 until the second quarter of 1980 , the tank received a wide variety of waste types. In the third quarter of 1980, the waste was classified as double-shell slurry feed. Presently, the tank waste is classified as non-complexed.

The waste history narrative of the primary additions that follow in this paragraph are from the "Waste status and Transaction Record Summary" (Agnew 1994). Tank 241-S-107 received REDOX waste from the third quarter of 1952 until the first quarter of 1972 . From the third quarter of 1952 until the first quarter of 1975, the tank received wastewater. The tank received REDOX cladding waste from the fourth quarter of 1954 until the first quarter of 1967. From the fourth quarter of 1968 until the second quarter of 1972, the tank received saltcake waste from the REDOX concentrator.

The tank was removed from service in 1980 and was partially isolated in December 1982. The tank level was adjusted in November 1978 and september 1980. In-tank photographs were taken on the following dates: August 1971, March 1974, February 1976, November 1977, May 1979, January 1983, and March 1987. Sketch ES-TKS-E73, which shows a graphical representation of the Tank 241-S-107 level history, and the historical waste level data used to make it are in Appendix $\mathrm{C}$. Nine $\mathrm{pH}$ readings are shown on the sketch: 11.9 in November 1973, 12.2 in November 1973, 14.0 in December 1974, >14.0 in March 1976, >14.0 in October 1978, >14.0 in April 1979, >14.0 in September 1980, 13.7 in October 1991, and 13.3 in October 1991. The latest maximum temperature reading from July 1993 is $107^{\circ} \mathrm{F}$. Currently, the tank is categorized as sound/non-stabilized. A figure that graphically represents the Tank 241-S-107 level history is in Appendix C. The increase in the tank liquid (supernatant) level in December 1993 is due to a comparison of 1983 and 1987 photo packages that indicate a level increase. Based on this influx, an elevation was extrapolated for 1993 which correlates with the current liquid level. 


\subsubsection{Temperature History 241-S-107}

There are six thermocouple probes on a single thermocouple tree in Tank 241-S-107 riser 4 with elevations available for each probe. The thermocouple probes have similar temperature trends that span from January 1988 to July 1993. Overall, the temperature readings decrease a maximum of $30^{\circ} \mathrm{F}$ from thermocouple 1 to thermocouple 6. Review of the Tank 241-S-107 level history readings for the thermocouple tree indicates that probes 1 through 5 were within or near the waste level and probe 6 appears to have been in the tank vapor space.

The mean temperature of the first electronically available data set for all six thermocouples is $108.5^{\circ} \mathrm{F}$. A sum of the squares regression line fit averaged for all six thermocouple probes, starting in 1988, shows a zero slope $\pm 6^{\circ} \mathrm{F}$. From January 1988 to July 1993, the median temperature was $108.9^{\circ} \mathrm{F}$ with a maximum of $139^{\circ} \mathrm{F}$ and a minimum of $78^{\circ} \mathrm{F}$. Tank 241-S-107 has a semiannual temperature monitoring requirement.

Plots of thermocouple readings for Tank 241-S-107 are in Appendix D.

\subsubsection{Integrity of Tank 241-S-107}

Tank 241-S-107 is categorized as sound and is partially isolated. The Tank 241-S-107 surface level is monitored with a Food Instrument Corporation gauge. If the Food Instrument Corporation gauge fails, manual field measurements will be conducted daily. The maximum allowable deviations from the 144.2-in. baseline established on May 27, 1993 are a 2-in. increase and a 1-in. decrease. A figure that graphically represents the surface level measurements from January 1991 to the present is in Appendix E. The surface level from May 27, 1993 to the present indicates an increasing trend and the readings range from 145.2 to 144.2 in. Occurrence Report no.76-81 was issued in July 1976 due to a liquid level decrease attributed to temperature and evaporative losses.

Tank 241-S-107 does not have a liquid observation well. Six drywells are identified for Tank 241-S-107 and none are considered active.

\subsubsection{Historical Sample Analysis Data for Tank 241-S-107}

Results from eight sets of samples taken from Tank 241-S-107 between November 1973 and September 1980 are included in this report. The samples taken prior to 1975 contained a large amount of liquid; therefore, the later samples are more representative of the current tank contents which are made up mostly solids. The most recent sample, analyzed on september 22, 1980, was made up of nitrates, nitrites, aluminates, hydroxides, phosphates, and carbonates and some organic material. The total organic carbon content of the tank is $4.0 \mathrm{~g} / 1$. Three samples analyzed in 1978 and 
WHC-SD-WM-ER-323， Rev. 0

1979 were similarly constituted. Viscosity and solids content were the main elements. of concern in many of the later samples. Results from these analyses are in Appendix $\mathrm{H}$.

\subsubsection{Current Status of Tank 241-S-107}

Tank 241-S-107 entered service in August 1952 and currently stores 376,000 gal of waste. The waste material is comprised of $14,000 \mathrm{gal}$ of supernatant; $22,000 \mathrm{gal}$ of saltslurry; $128,000 \mathrm{gal}$ of saltcake; and. 212,000 gal of sludge with 44,000 gal of pumpable liquid remaining. The tank is identified as a low-heat load tank, is passively ventilated, and is categorized as sound with partial interim isolation completed. Tank 241-s-107 is equipped to cascade to Tank 241-S-108 and is first in the three-tank cascade series. A plan view and tank cross-section that depict the approximate waste level and riser conifguration are in Appendix G. Tank 241-S107 has 12 risers and four are available for use: two 12-in risers, no. 6 and no. 7, and two 4-in. risers, no. 11 and no. 14.

\subsubsection{Waste Layer Volume Approximation for Tank 241-S-107}

A comparative analysis of all layer approximations for the entire tank farm and the data used to derive the tank layer approximations are located in Appendix $\mathrm{K}$.

\subsubsection{In-Tank Photograph 241-S-107}

The montage made from photographs inside Tank 241-S-107 shows reasonable detail around the perimeter but details are masked in the center which may be due to blurring from the flash (see Appendix I). A small amount of solid material appears to be clinging to the wall and the waste in the rest of the tank appears to be a liquid that has been described as sludgy. The waste history diagram indicates that the tank contains almost all solid material. The Food Instrument Corporation level probe and the temperature probe are clearly visible but blurring in the center photos obscures details. There is a strong image of a reflection from the strobe light in the center of the picture. The level gauge seems to be immersed in the liquid which seems to imply that tank contents are liquid rather than solid. Current measurements indicate that the tank holds about 376,000 gal of waste and the waste measures almost 12 ft deep.

2.7.3 Synopsis Tank 241-S-107

(To be completed) 
WHC-SD-WM-ER-323， Rev. 0

2.8 Tank $241-\mathrm{S}-108$

2.8.1 241-S-108 Tank History

2.8.1.1 Waste History Tank 241-S-108

The waste history narrative in the following paragraph is from "A History of the 200 Area Tank Farms" (Anderson 1990). Tank 241S-108 was filled with waste from the REDOX facility from the second quarter of 1952 until the fourth quarter of 1973. The tank received bottoms and recycle streams from the 242-s Evaporator/Crystallizer from the first quarter of 1974 until the third quarter of 1975. From the fourth quarter of 1975 until the first quarter of 1978, the tank received various types of evaporator wastes. In the second quarter of 1978, the tank received partial neutralized feed waste. The waste has been classified as non-complexed since the fourth quarter of 1980 .

Tank 241-S-108 did not receive any primary waste additions according to the "Waste Status and Transaction Record Summary" (Agnew 1994) report.

The tank was removed from service, labeled inactive, and primary stabilized in 1979. The tank was partially isolated in December 1982. A jet pump was installed and initiated in 1978. A total of $151,600 \mathrm{gal}$ of liquid was jet pumped between 1978 and 1980. The tank level was adjusted in June 1979 and April 1982 . In-tank photographs were taken on the following dates: July 1971, October 1974, January 1976, two in 1979, August 1980, November 1982, and March 1987. Sketch ES-TKS-E74, which shows a graphical representation of the Tank 241-S-108 level history, and the historical waste level data used to make it are in Appendix $C$. The sketch shows five pH readings of 12.0 in March 1965, 12.0 in July 1972, 11.5 in October 1973, $>14.0$ in June 1974, and 13.6 in November 1991. The latest maximum temperature reading from July 1993 is $85^{\circ} \mathrm{F}$. Presently, the tank is categorized as sound/nonstabilized.

\subsubsection{Temperature History 241-S-108}

There are 13 thermocouples on a single thermocouple tree in Tank 241-S-108 riser 4 with elevations available for each probe. No data are available for thermocouple 2. The data for all thermocouples span from January 1991 to July 1993 except for probes $4,11,12$, and 14 which span from November 1991 to July 1993. All thermocouple plots show similar trends with more irregularity present in probes 1 and 10 through 14 . Overall, the temperature readings decrease a maximum of $20^{\circ} \mathrm{F}$ from thermocouple 1 to thermocouple 13. Review of the Tank 241-s-108 level history readings for the thermocouple tree indicates that probes 1 through 9 were in or near the solids level and probes 10 through 14 appear to have been in the tank vapor space. 
The mean temperature of the first electronically available data set taken from all 13 thermocouples is $79^{\circ} \mathrm{F}$. A sum of the squares regression line fit averaged for the 13 thermocouple probes, starting in 1991, shows a zero slope $\pm 4^{\circ} \mathrm{F}$. From January 1991 to July 1993, the median temperature was $78^{\circ} \mathrm{F}$ with a maximum of $94^{\circ} \mathrm{F}$ and a minimum of $49.9^{\circ} \mathrm{F}$. Tank $241-\mathrm{S}-108$ has a semiannual temperature monitoring requirement.

Plots of the thermocouple readings for Tank 241-S-108 are in Appendix D.

\subsubsection{Integrity of Tank 241-S-108}

Tank 241-S-108 is categorized as sound and is partially isolated. The Tank 241-S-108 surface level is monitored with a Food Instrument Corporation gauge. The gauge is set in the intrusion mode for a 1-in. increase. If the Food Instrument Corporation gauge fails, manual field measurements will be conducted quarterly. The maximum allowable increase from the 172.8-in. baseline is 3 in. There is no criterion for a decrease. Due to the Food Instrument Corporation gauge setting, a limited number of surface level measurements is available. The figure that graphically represents the surface level measurements taken during the second quarter of 1993 is in Appendix E. The readings unexplainably range from 189.5 to 153.25 in.

The Tank 241-S-108 liquid observation well is located in riser 16. The tank is monitored weekly with a neutron probe and on request with a gamma probe. The monitoring limits are a $0.3-\mathrm{ft}$ decrease and a 0.4-ft increase. Sketch ES-TKS-E74 in Appendix C is a graphical representation of the liquid observation well data.

Five drywells are identified for Tank 241-S-108 and none are considered active.

\subsubsection{Historical Sample Analysis Data for Tank 241-S-108}

Data from four sets of samples were selected for this report; two samples of solids and one supernatant sample were analyzed on July 8, 1974. The tank was a slurry receiving tank for the $242-\mathrm{S}$ Evaporator/Crystallizer. The July samples were supposed to determine if tank contents were too concentrated or if they could be evaporated further. Although the constituents of the samples were basically the same, the percentages contained in each were quite different. The samples primarily contained sodium nitrate; and sodium hydroxide; and smaller amounts of sodium nitrite, sodium aluminate, and sodium carbonate. The solids in one of the samples came in two layers: the upper layer was a fine soft gray-colored solid and the lower layer was a coarse, yellow crystalline material. The other samples were from 1965, 1971, and 1973 when tank conditions were different. Results of the analyses are found in Appendix $\mathrm{H}$. 
2.8.2 Current Status of Tank 241-S-108

Tank 241-S-108 entered service in October 1952 and currently stores $604,000 \mathrm{gal}$ of waste. The waste is comprised of $599,000 \mathrm{gal}$ of saltcake and $5,000 \mathrm{gal}$ of sludge with 105,000 gal of pumpable liquid remaining. The tank is identified as a low-heat load tank, is passively ventilated, and is categorized as sound with partial interim isolation completed. Tank 241-S-108 is equipped to cascade to Tank 241-S-109 and is second in the three-tank cascade series. The plan view and tank cross-section that depict the approximate waste level and riser configuration are in Appendix G. Tank 241-S108 has 12 risers and two 12-in. risers, no. 6, and no. 7, are available for use.

\subsubsection{Waste Layer Volume Approximation for Tank 241-S-108}

A comparative analysis of all layer approximations for the entire tank farm and the data used to derive the tank layer approximations are located in Appendix $\mathrm{K}$.

\subsubsection{In-Tank Photograph 241-S-108}

From the Tank 241-S-108 picture the waste surface appears to be completely dry (see Appendix I). The waste looks well mixed except in the center and at about five. $o^{\prime}$ clock in the foreground where there may be some foreign material on the surface. A chemical analysis in 1974 indicated that the waste material was primarily sodium nitrate and sodium hydroxide. The manual tape, level gauge, temperature probe, and saltwell screen are all visible and labelled. Numerous manholes, risers, and nozzles are seen clearly in the picture. There is some debris, including a discarded manual tape, on the waste surface in the foreground at about five o'clock. Current measurements indicate that the tank holds about 600,000 gal of waste measuring about 19 ft deep. There have been no changes in the tank after the pictures were taken in 1987 according to the waste level history diagram.

2.8.3 Synopsis Tank 241-S-108

(To be completed) 
WHC-SD-WM-ER-323, Rev . 0

2.9 Tank 24I-S-109

2.9.1 241-S-109 Tank History

2.9.1.1 Waste History Tank 241-S-109

The waste history narrative in the following paragraph is from "A History of the 200 Area Tank Farms" (Anderson 1990). Tank 241S-109 was filled with waste from the REDOX facility from the fourth quarter of 1952 until the fourth quarter of 1973. From the first quarter of 1974 until the fourth quarter of 1975, the tank received bottoms and recycle streams from the 242-s Evaporator/Crystallizer. The tank was salt-filled in the fourth quarter of 1974. Between the first quarter of 1976 and the first quarter of 1978, the tank received various types of evaporator waste. The tank received partial neutralized feed waste from the second quarter of 1978 until the third quarter of 1980. The waste has been classified as non-complexed since the fourth quarter of 1980.

Tank 241-S-109 did not receive any primary waste additions according to the "Waste Status and Transaction Record Summary" (Agnew 1994) report.

The tank was primary stabilized and removed from service in 1979 and it was partially isolated in December 1982. A jet pump was installed and initiated in 1978. A total of 111,000 gal of liquid was jet pumped between 1978 and 1980. A liquid observation well was installed in December 1980. The tank level was adjusted in September 1975. In-tank photographs were taken on the following dates: July 1971, several in 1974, January 1976, May 1979, two in 1981, and August 1984. Sketch ES-TKS-E75, which shows a graphical representation of the Tank 241-S-109 level history, and the historical waste level data used to make it are in Appendix $C$. The sketch shows seven $\mathrm{pH}$ readings of 12.0 in July 1972, 11.5 in October 1973, >14.0 in August 1974, >13.1 in September 1974, 14.0 in November 1974, 13.0 in April 1975, and 13.2 in October 1991. The latest maximum temperature reading from January 1993 is $83^{\circ} \mathrm{F}$. As of July 1993, the temperature probe is out of service in this tank. Presently, the tank is categorized as sound/non-stabilized.

\subsubsection{Temperature History 241-S-109}

There are six thermocouple probes on a single thermocouple tree in Tank 241-S-109 riser 4 with elevations available for all probes. No data are available for probes two, three, four, five, six, and 8 and probes 1,9 , and 10 have only one data point. Probe 11 has two points and probe 12 has three points. The most data points were recorded for probe 7. Review of the Tank 241-5-109 level history readings for the thermocouple tree indicates that probes 1,7, and 9 were in or near the solids level and probes 10 through 12 appear to have been in the tank vapor space.

The mean temperature of the first electronically available data set was $68^{\circ} \mathrm{F}$. A sum of the squares regression line fit and 
variability analysis was not performed due to a limited number of data points. The median temperature is $66^{\circ} \mathrm{F}$ with a maximum of $82.9^{\circ} \mathrm{F}$ and a minimum of $55.3^{\circ} \mathrm{F}$. The thermocouples have been out of service since November 1993. Tank 241-S-109 has a semiannual temperature monitoring requirement.

Plots of thermocouple readings for Tank 241-S-109 are in Appendix C.

\subsubsection{Integrity of Tank 241-S-109}

Tank 241-S-109 is categorized as sound and is partially isolated. The Tank 241-S-109 surface level is monitored with a Food Instrument Corporation gauge. The gauge is set in the intrusion mode for a 1-in. increase. If the Food Instrument Corporation gauge fails, manual field measurements will be conducted quarterly. The maximum allowable increase from the 173.8-in. baseline is 3 in. There is no criterion for a decrease. Due to the Food Instrument Corporation gauge setting, a limited number of surface level measurements are available. The figure that graphically represents the surface level measurements taken during the first and second quarters of 1993 is in Appendix $E$. The readings range from 172.6 to 169.3 in.

The Tank 241-S-109 liquid observation well is located in riser 8. The tank is monitored weekly with a neutron probe and on request with a gamma probe. The maximum allowable deviations from the established baseline value are a $0.3-\mathrm{ft}$ increase and a $0.4-\mathrm{ft}$ decrease. Sketch ES-TKS-E75 in Appendix C has a graphical representation of the liquid observation well data.

Six drywells are identified for Tank 241-S-109 and none are considered active.

2.9.1.4 Historical Sample Analysis Data for Tank 241-S-109

Data are included in this report for five sets of samples from Tank 241-S-109 that were analyzed between December 18, 1973 and December 8, 1976. The 1976 sample consisted of coarse, yellowish granular crystals that were about 85 to 90 percent water soluble. In 1974, the sample consisted of yellow crystals and it was noted that the sample tended to take up water from the atmosphere. The analyses indicate that the tank contains mostly sodium nitrate and lesser amounts of various other sodium compounds. Tables presenting the results of the analyses are in Appendix $\mathrm{H}$.

\subsubsection{Current Status of Tank 241-S-109}

Tank 241-S-109 entered service in December 1952 and currently stores 568,000 gal of waste. The waste is comprised of 555,000 gal of saltcake and 13,000 gal of unknown waste with 119,000 gal of pumpable interstitial liquid remaining. The tank is identified as a low-heat load tank, is passively ventilated, and is categorized as sound with partial interim isolation completed. Tank 241-S-109 


$$
\text { WHC-SD-WM-ER-323, Rev. } 0
$$

is third in the three-tank cascade series. The plan view and tank cross-section that. depict the approximate waste level and riser conifguration are in Appendix G. Tank 241-S-109 has 12 risers, and five are available for use: two 12-in. risers (no. 6 and no. 7) and three 4-in. risers (no. no. 11, no. 14, and no. 16).

\subsubsection{Waste Layer Volume Approximation for Tank 241-S-109}

A comparative analysis of all layer approximations for the entire tank farm and the data used to derive the tank layer approximations are located in Appendix $\mathrm{K}$.

\subsubsection{In-Tank Photograph 241-S-109}

The Tank 241-S-109 picture shows the surface to be thoroughly dried out which corresponds with level history data (see Appendix I). Chemical samples from 1976 indicate that the tank primarily contained sodium nitrate and there has been no activity in the tank since then. Different colored areas on the surface show some lack of homogeneity in the waste material; however, such areas are not dominant in the picture. The level measurement device, temperature probe, and saltwell are in the background and some foreign contaminants are shown on the waste surface. The tank waste level has remained stable since the photographs were taken at approximately $18 \mathrm{ft}$ which corresponds with a waste content of about $570,000 \mathrm{gal}$.

\subsubsection{Synopsis Tank 24I-S-109}

(To be completed) 


\subsubsection{1-S-110 Tank History}

\subsubsection{Waste History Tank 241-S-110}

The waste history narrative in the following paragraph is from "A History of the 200 Area Tank Farms" (Anderson 1990). Tank 241S-110 was filled with waste from the REDOX facility from the second quarter of 1952 until the fourth quarter of 1973. From the first quarter of 1974 until the first quarter of 1978, the tank was filled with various wastes from the BX, S, SX, T, TX, and U Tank Farms and the 242-S Evaporator/Crystallizer. The tank was saltfilled during the third quarter of 1975. The tank received partial neutralized feed waste from the second quarter of 1978 until the third quarter of 1980. The waste has been classified as noncomplexed since the fourth quarter of 1980 .

The waste history narrative of the primary additions that follow in this paragraph are from the "Waste status and Transaction Record Summary" (Agnew 1994). Tank 241-S-110 received REDOX waste during the first, second, and third quarters of 1952. During the third and fourth quarters of 1953, the tank received REDOX cladding waste. From the first quarter of 1954 until the third quarter of 1974, the tank received wastewater.

The tank was removed from service and labeled inactive in 1976. It was primary stabilized in January 1979 and partially isolated in December 1982. A jet pump was installed and initiated in August 1978 and 185,900 gal were pumped from the tank. The tank level was adjusted in January 1979 and May 1992. In-tank photographs were taken on the following dates: July 1971, September 1973, several in 1975, and March 1987. Sketch ES-TKSE76, which shows a graphical representation of the Tank 241-S-110 level history, and the historical waste level data used to make it are in Appendix $\mathrm{C}$. The sketch shows two $\mathrm{pH}$ readings of $>14.0$ in July 1971 and 14.0 in December 1974. The latest maximum temperature reading from July 1993 is $116^{\circ} \mathrm{F}$. Presently, the tank is categorized as sound/non-stabilized.

\subsubsection{Temperature History 241-S-110}

There are six thermocouple probes on a single thermocouple tree in Tank 241-S-110 riser 4 with elevations available for each. The thermocouples show similar trends that span from January 1988 to July 1993. The temperature readings decrease a maximum of $45^{\circ} \mathrm{F}$ from thermocouple 1 to thermocouple 6 . Review of the tank level history readings for the thermocouple tree indicates that probes 1 through 5 were in or near the solids level with probe 6 in the vapor space.

The mean temperature of the first electronically available data set from all six thermocouples is $116^{\circ} \mathrm{F}$. A sum of the squares regression line fit averaged for the six thermocouple probes, 
WHC-SD-WM-ER-323, Rev. 0

starting in 1988 , shows a zero slope $\pm 6^{\circ} \mathrm{F}$. A common outlying point is apparent for all. the thermocouples in March 1989. From January 1988 to July 1993, the median temperature was $117^{\circ} \mathrm{F}$ with a maximum of $151^{\circ} \mathrm{F}$ and a minimum of $72.6^{\circ} \mathrm{F}$. Tank $241-\mathrm{S}-108$ has a semiannual temperature monitoring requirement.

Plots of the thermocouple readings for Tank $241-\mathrm{S}-110$ are in Appendix D.

\subsubsection{Integrity of Tank 241-S-110}

Tank 241-S-110 is categorized as sound and is partially isolated. The Tank 241-S-110 surface level is monitored with a Food Instrument Corporation gauge. The gauge is set in the intrusion mode for a 1 -in. increase. If the Food Instrument Corporation gauge fails, manual field measurements will be conducted quarterly. The maximum allowable increase from the 149.3-in. baseline is 3 in. There is no criterion for a decrease. Due to the Food Instrument Corporation gauge setting, a limited number of surface level measurements are available. The figure that graphically represents the surface level measurements taken during the first and second quarters of 1993 is in Appendix E. The readings range from 148.65 to 148.6 in.

The Tank 241-S-110 liquid observation well is located in riser 2. The tank is monitored weekly with a neutron probe and on request with a gamma probe. The maximum allowable deviation from the established baseline value is a 0.3 -ft increase and a $0.4-\mathrm{ft}$ decrease. Sketch ES-TKS-E76 in Appendix C has a graphical representation of the liquid observation well data.

Seven drywells are identified for Tank 241-S-110. Drywell 4010-01 was active prior to 1987.

\subsubsection{Historical Sample Analysis Data for Tank 241-S-110}

Results of five samples taken from Tank 241-S-110 between September 1971 and June 1975 are included in this report. Two samples are from 1975 and the most recent gave a typical mixture of sodium compounds. The 1974 samples may be more representative of a sludge layer lying below the saltcake layer. The sample from september 1974 was described as being composed of two layers; the top was dark brown and the bottom was gray with hard, large, white chunks distributed throughout. Appendix $\mathrm{H}$ contains tables that show the analyses results.

\subsubsection{Current Status of Tank 241-S-110}

Tank 241-S-110 entered service in 1952 and currently stores $390,000 \mathrm{gal}$ of waste. The waste is comprised of $277,000 \mathrm{gal}$ of saltcake and 113,000 gal of sludge with 103,000 gal of pumpable interstitial liquid remaining. The tank is identified as a lowheat load tank, is passively ventilated, and is categorized as sound with partial interim isolation completed. Tank 241-S-110 is 
equipped to cascade to Tank 241-S-111 and is first in the threetank cascade series. The plan view and tank cross-section that depict the approximate waste level and riser configuration are in Appendix G. Tank 241-S-110 has 12 risers and two 12-in. risers, no. 6 and no. 7, are readily available for use.

2.10.2.1 Waste Layer Volume Approximation for Tank 241-S-110

A comparative analysis of all layer approximations for the entire tank-farm-and. the data used to derive the tank layer approximations are located in Appendix $\mathrm{K}$.

\subsubsection{In-Tank Photograph 241-S-110}

There was a large level adjustment in the tank in 1992; therefore, the 1987 photographs in Appendix I probably do not accurately reflect the current appearance of the tank waste. The waste in the picture has formed a thick solid crust around the perimeter of the tank and there is a pool of liquid in the center of the tank which extends down to an indeterminate depth. The crust appears to be composed of several different materials judging from color variations. A temperature probe, manual tape, liquid observation well, and saltwell screen are visible in the picture. The current tank waste height is about 12 ft and the quantity of waste is approximately $390,000 \mathrm{gal}$. When the pictures were taken, the waste height was about $22 \mathrm{ft}$.

2.10.3 Synopsis Tank 241-S-110

(To be completed) 
WHC-SD-WM-ER-323， Rev. 0

2.11 Tank 241-S-111

2.11.1 241-S-111 Tank History

\subsubsection{Waste History Tank 241-S-111}

The waste history narrative in the following paragraph is from "A History of the 200 Area Tank Farms" (Anderson 1990). Tank 241S-111 was filled with waste from the REDOX facility from the third quarter of 1952 until the fourth quarter of 1973. The tank received bottoms and recycle streams from the 242-S Evaporator/Crystallizer from the first quarter of 1974 until the tank was salt-filled in the third quarter of 1975. The tank was removed from service in the second quarter of 1976. During the following quarter it was labeled inactive and salt-well pumped. The tank contained partial neutralized feed waste from the first quarter of 1978 until the third quarter of 1980 . The waste has been classified as non-complexed since the fourth quarter of 1980 .

Tank 241-s-111 did not receive any primary waste additions according to the "Waste Status and Transaction Record Summary" (Agnew 1994) report.

The tank was removed from service in 1972 and labeled inactive in 1978. It was primary stabilized in March 1978 and was partially isolated in December 1982. A pump was installed and initiated in 1975 and a jet pump was installed the following year. A total of $3,300 \mathrm{gal}$ of liquid was jet pumped between 1976 and 1980. The tank level was adjusted in September 1975 and April 1978. In-tank photographs were taken on the following dates: July 1971, two in 1974, September 1975, two in 1977, March 1978, April 1980, April 1983, May 1984, and October 1989. Sketch ES-TKS-E77, which shows a graphical representation of the Tank 241-S-111 level history and the historical waste level data used to make it are in Appendix $C$. The sketch shows five $\mathrm{pH}$ readings: $>14.0$ in July $1971,>14.0$ in August $1974,13.4$ in December 1974, >14.0 in May 1975, and $>14.0$ in June 1978. The latest maximum temperature reading from october 1993 is $92^{\circ} \mathrm{F}$. Currently, the tank is categorized as sound/nonstabilized.

\subsubsection{Temperature History 241-S-111}

There are 14 thermocouples on a single thermocouple tree in Tank 241-5-111 riser 4 with elevations available for each probe. All thermocouple plots show similar trends that span from January 1988 to December 1993. Review of the level history indicates that over the time span of readings, thermocouple probes 1 through 9 were in or near the solids level and probes 10 through 14 were in the vapor space.

The mean temperature of the first electronically available data set taken from all 14 thermocouples is $80.5^{\circ} \mathrm{F}$. A sum of the squares regression line fit for all 14 thermocouples, starting in 1988, shows a zero slope $\pm 2^{\circ} \mathrm{F}$. A low outlying temperature of 
WHC-SD-WM-ER-323, Rev. 0

63.6 occurred June 1993 for thermocouple 5. Annual temperature undulations or a seasonal trend is evident in thermocouples 5 through 13. From January 1988 to December 1993, the median temperature was $80^{\circ} \mathrm{F}$ with a maximum of $97^{\circ} \mathrm{F}$ and a minimum of $63.6^{\circ} \mathrm{F}$. Tank 241-S-111 has weekly temperature readings because it is on the Hydrogen Watch Iist.

Plots of thermocouple readings for Tank $241-S-111$ are in Appendix D.

\subsubsection{Integrity of Tank 241-S-111}

Tank 241-S-111 is categorized as sound and is partially isolated. The Tank 241-S-111 surface level is monitored with a Food Instrument Corporation gauge. If the Food Instrument Corporation gauge fails, manual field measurements will be conducted daily. The maximum allowable deviations from the 202.6in. baseline are a 3-in. increase and a 0.5-in. decrease. The figure that graphically represents the surface level measurements from January 1991 to the present is in Appendix E. The surface level for the past three years has remained steady with the readings ranging from 203.8 to 200.9 in.

The Tank 241-S-111 liquid observation well is located in riser 16. The tank is monitored weekly with a neutron probe and on request with a gamma probe. The maximum deviations from the established baseline are a 0.3-ft increase and a 0.4 -ft decrease. Sketch ES-TKS-E77 in Appendix $C$ has a graphical representation of the liquid observation well data.

Five drywells are identified for Tank 241-S-111 and none are considered active.

\subsubsection{Historical Sample Analysis Data for Tank 241-S-111}

Data from seven analytical investigations of Tank 241-S-111 contents were selected for inclusion in this report. The tank was a slurry receiving tank for the 242-S Evaporator. Five separate samples were analyzed on August 25, 1978. The salts, as received, were yellowish-green with variable crystal sizes. Samples taken in 1977 were dark green. The major constituent of all the samples was sodium nitrate. The samples contained lesser amounts of nitrite, carbonate, sulfate, and phosphate. Earlier samples from the tank yielded similar results. Twelve tables that contain the results of these investigations are in Appendix $\mathrm{H}$.

\subsubsection{Current Status of Tank 241-S-111}

Tank 241-S-111 entered service in 1952 and currently stores $596,000 \mathrm{gal}$ of waste. The waste is comprised of 10,000 gal of supernatant; $61,000 \mathrm{gal}$ of unknown waste; 447,000 gal of saltcake; and 139,000 gal of sludge with 134,000 gal of pumpable liquid remaining. The tank is identified as a Hydrogen watch List tank, is passively ventilated, and is categorized as sound with partial 
interim isolation completed. Tank 241-S-111 is equipped to cascade to Tank 241-s-112 and is second in the three-tank cascade series. The plan view and tank cross-section that depict the approximate waste level and riser configuration are in Appendix G. Tank 241-S111 has 12 risers and two 12-in. risers, no. 6 and no. 8, are available for use.

\subsubsection{Waste Layer Volume Approximation for Tank 241-S-111}

A comparative analysis of all layer approximations for the entire tank farm and the data used to derive the tank layer approximations are located in Appendix $\mathrm{K}$.

\subsubsection{In-Tank Photograph 241-S-111}

The montage of Tank 241-S-111 in Appendix I shows a surface that is one-third to one-half covered with solid material which appears to be floating on a liquid surface. The solid material in the tank was analyzed in 1978, The sample was reported to be yellowish green and the major constituent of the sample was sodium nitrate. The temperature probe, level gauge, liquid observation well, and saltwell are clearly visible in the picture along with three other risers and some inlet and outlet nozzles. There is some debris on the solid surface at about one o'clock near the temperature probe. The level history diagram shows about 600,000 gal of waste in the tank which has a depth of almost $19 \mathrm{ft}$. The level has not changed since the photographs were taken in 1989 .

2.11.3 Synopsis Tank 241-S-111

(To be completed) 
WHC-SD-WM-ER-323, Rev. 0

\subsection{Tank 241-S-112}

2.12.I 24I-S-112 Tank History

2.12.1.1 Waste History Tank 241-S-112

The waste history narrative in the following paragraph is from "A History of the 200 Area Tank Farms" (Anderson 1990). Tank 241S-112 was filled with waste from the REDOX facility from the third quarter of 1952 until the fourth quarter of 1973. The tank received bottoms and recycle streams from the 242-S Evaporator/Crystallizer from the first quarter of 1974 until the first quarter of 1975 when the tank was salt-filled. The tank continued to hold evaporator type waste until the first quarter of 1978. The tank contained partial neutralized feed waste from the second quarter of 1978 until it was reclassified as non-complexed waste in the fourth quarter of 1980. Presently, the waste is classified as non-complexed.

Tank 241-S-112 did not receive any primary waste additions according to the "Waste Status and Transaction Record Summary" (Agnew 1994) report.

The tank was removed from service in 1974 and labeled inactive in 1976. The tank was primary stabilized in June 1979 and was partially isolated in December 1982. A jet pump was installed and initiated in 1978. A total of $125,100 \mathrm{gal}$ of liquid was jet pumped between 1978 and 1980. The tank level was adjusted in July 1977, June 1979, and 1982. In-tank photographs were taken on the following dates: July 1971, September 1973, two in 1974, July 1977, May 1979, June 1981, and March 1987. Sketch ES-TKS-E78, which shows a graphical representation of the Tank 241-s-112 level history, and the historical waste level data used to make it are in Appendix C. The sketch shows four pH readings: $>14.0$ in July 1971, 12.2 in January 1974, >14.0 in May 1974, and 13.7 in December 1974. The latest maximum temperature reading from October 1993 is $84^{\circ} \mathrm{F}$. Presently, the tank is categorized as sound and is non-stabilized.

\subsubsection{Temperature History 241-S-112}

There are 14 thermocouple probes on a single thermocouple tree in Tank 241-S-112 riser 4 with thermocouple elevations unavailable. Data for thermocouples 3, 4, and 6 through 12 span from March 1991 to December 1993 and data for thermocouples $1,2,5,13$, and 14 span from February 1992 to December 1993. The temperature plots show similar trends with annual temperature undulations present in the higher thermocouples.

The mean temperature of the first electronically available data set taken from the thermocouples is $71^{\circ} \mathrm{F}$. A sum of the squares regression line fit for all 14 thermocouples, starting in 1991, shows a zero slope $\pm 5.3^{\circ} \mathrm{F}$. A common outlying point is apparent for thermocouples 1 through 8 in November 1993. From January 1991 to December 1993 , the median temperature was $77^{\circ} \mathrm{F}$ with 
WHC-SD-WM-ER-323， Rev. 0

a maximum of $92^{\circ} \mathrm{F}$ and a minimum of $47^{\circ} \mathrm{F}$. Tank $241-\mathrm{S}-112$ has weekly temperature readings because it is on the Hydrogen Watch List.

Plots of thermocouple readings for Tank 241-S-112 are in Appendix D.

\subsubsection{Integrity of Tank 241-S-112}

Tank 241-S-112 is categorized as sound and is partially isolated. The Tank 241-S-112 surface level is monitored with a Food Instrument Corporation gauge. The gauge is set in the intrusion mode for a 1-in. increase. If the Food Instrument Corporation gauge fails, manual field measurements will be conducted quarterly. The maximum allowable increase from the 196.4-in. baseline is 3 in. There is no criterion for a decrease. Due to the Food Instrument Corporation gauge setting, a limited number of surface level measurements are available. The figure that graphically represents the surface level measurements taken during the first and second quarters of 1993 is in Appendix E. The readings range from 195.9 to 195.7 in.

The Tank 241-S-112 liquid observation well is located in riser 8. The tank is monitored weekly with a neutron probe and on request with a gamma probe. The maximum deviations from the established baseline are a 0.3 -ft increase and a 0.4 -ft decrease. Sketch ES-TKS-E78 in Appendix C has a graphical representation of the liquid observation well data.

Five drywells are identified for Tank 241-S-112 and none are considered active.

\subsubsection{Historical Sample Analysis Data for Tank 241-S-112}

Data are included in this report for four samples from Tank 241-S-112 that were analyzed in 1974 and for one sample that was analyzed in 1971. Tank 241-S-112 was a 242-S Evaporator slurry receiving tank. The most recent analysis indicates that it contains mostly sodium nitrate and sodium carbonate with lesser amounts of various other sodium compounds. Sodium hydroxide content was high enough to cause the salt to pick up moisture from the atmosphere. Samples were about 50\% solids and were yellowishgreen. A principal concern of the sampling was to determine if the waste material had reached terminal concentration. Tables presenting the results of these analyses are in Appendix $\mathrm{H}$.

\subsubsection{Current Status of Tank 241-S-112}

Tank 241-S-112 entered service in July 1952 and currently stores 637,000 gal of waste. The waste is comprised of 631,000 gal of saltcake and $6,000 \mathrm{gal}$ of unknown waste with $112,000 \mathrm{gal}$ of pumpable interstitial liquid remaining. The tank is identified as a Hydrogen watch List tank, is passively ventilated, and is categorized as sound with partial interim isolation completed. Tank 241-S-112 is third in the three-tank cascade series. The plan 
view and tank cross-section that depict the approximate waste level and riser configuration are in Appendix G. Tank 241-S-112 has 12 risers, five are available for use: two 12-in. risers (no. 6 and no. 7) and three 4-in. risers (no. 2, no. 11, and no. 16).

\subsubsection{Waste Layer Volume Approximation for Tank 241-S-112}

A comparative analysis of all layer approximations for the entire tank farm and the data used to derive the tank layer approximations are- located in Appendix $\mathrm{K}$.

\subsubsection{In-Tank Photograph 241-S-112}

The picture of Tank 241-S-112 in Appendix I shows the waste in the tank is primarily a dry salt. Chemical analysis data from 1974 indicate that the tank contains mostly sodium nitrate. A plastic riser bag is visible near the center of photo as well as a metal pipe of unknown origin. A saltwell screen is also near the center of the photo. Various probes can be observed near the top of the photo including a liquid observation well, a temperature probe, a Food Instrument Corporation surface level probe, and other risers and nozzles. The tank contains approximately 640,000 gal of waste with a total depth of approximately $20 \mathrm{ft}$.

\subsubsection{Synopsis Tank 241-S-112}

(To be completed) 
WHC-SD-WM-ER-323, Rev. 0

\section{GLOSSARY}

0.17

0.33

1

IAYIN

IAZIN

IC

ICEB

$1 \mathrm{CS}$

Ist Generation Tank

222-B

$222-C$

$222-T$

$222-U$

224

224-F

$231 z$

242-A

$242-B$
Transaction flag key-monthly volumes derived from semiannual reports.

Transaction flag key-monthly volumes derived from quarterly reports.

Monthly report.

See Waste Abbreviations.

See Waste Abbreviations.

See Waste Abbreviations.

See Waste Abbreviations.

See Waste Abbreviations.

The original tank design encompassing Tank Farms B, C, T, U (excluding the 200 series tanks), and $B X$. These tanks have an operating capacity of 530,000 gal, a 75-ft diameter, a 12-in. dish bottom, and a 4-ft knuckle. Also see Type II tanks.

See Facilities.

See Facilities.

see Facilities.

See Facilities.

See Waste Abbreviations.

See Waste Abbreviations.

See Waste Abbreviations.

See Facilities.

See Facilities. 
$242-S$

242-T

2AYIN

2AZIN

$2 \mathrm{C}$

2nd Generation Tank

2SYIN

3

3AWIN

3rd Generation Tank

4th Generation Tank

5

$5-6 \#$

5AWIN

5th Generation Tank

6
See Facilities.

See Facilities.

See Waste Abbreviations.

See Waste Abbreviations.

See Waste Abbreviations.

Same as original tank design (1st generation, or type II) except the operating capacity was increased to 758,000 gal. Also see Type III tanks.

See Waste Abbreviations.

Quarterly report.

See Waste Abbreviations.

The first generation of the type IV tanks, contains the SX Tank Farm only. These tanks have a 1,000,000 gal operating capacity, a 75-ft diameter, a 14.875-in. dish bottom, and no knuckle. Also see Type IV tanks.

The second generation of the type IV tanks, contains the A Tank Farm only. These tanks are the same as the 3 rd generation except they have a flat bottom. Also see Type IV tanks.

See Waste Abbreviations.

Cells 5 and 6 from B-Plant.

See Waste Abbreviations.

The third generation of the type IV tanks, contains the AX Tank Farm only. These tanks are the same as the 4 th generation with the addition of grid drain slots beneath the steel liner bottom.

Semiannual report. 
WHC-SD-WM-ER-323, Rev. 0

6AWIN

A Plant (PUREX)

AI SLTCK

A2 SITSIRY

Active Tank

Active Drywell

$\mathrm{ADD}$

ADJ

AGE, AGING

Aging Waste

Airlift Circulator

Annulus

AR Vault

$\mathrm{AR}$
See Waste Abbreviations.

See Facilities.

See LANL Defined Waste List.

See LANL Defined Waste List.

A tank that contains more than 33,000 gal of waste and/or is still involved in waste management operations.

Drywell in which radiation readings of greater than 50 counts/second are detected. To be considered active, the readings must be consistent as to depth and radiation level for repeated readings.

Add primary waste from process.

Adjustment to waste amount-see CORR.

Waste Abbreviation.

High-level, first-cycle solvent extraction waste from the PUREX Plant (NCAW).

The airlift circulators are installed in tanks used to age wastes. The circulators promote mixing of the supernate and prevent agglomeration of radionuclides. By maintaining motion within the body of liquid, the circulators minimize superheat buildup and, consequently, minimize bumping.

A vessel space in the form of a ring; the space between concentric walls.

A structure containing tanks and chemical processing equipment that is used for waste processing or storage operations.

See IANL Defined Waste List. 
WHC-SD-WM-ER-323, Rev. 0

Assumed Ieaker

Assumed Re-leaker

Assumed Leaking Tank

B SLTCK

B

B Plant (222-B)

B860N

Background Radiation

Baseline

BFSH

BI

BLEB

BIIX

BLIXB

BNW

BNW
A waste storage tank for which surveillance data, in the past, has indicated a loss of liquid attributed to a breach of integrity.

A designation that exists after a tank has been declared an "assumed leaker" and then the surveillance data indicate a new loss of liquid attributed to a breach of integrity.

In 1984, the criteria designations of "suspect leaker," "questionable integrity," "confirmed leaker," "declared leaker," "borderline," and "dormant" were merged into one category now reported as "assumed leaker."

See LANL Defined Waste List.

See Waste Abbreviations.

See Facilities.

See Waste Abbreviations.

Radioactivity from naturally occurring sources; primarily radiation from cosmogenic and naturally occurring radionuclides.

A reference; for example, a specified liquid level or radiation level against which new information is compared.

See Waste Abbreviations.

See Waste Abbreviations.

See Waste Abbreviations.

See Waste Abbreviations.

See Waste Abbreviations.

Battelle Northwest Laboratory.

See Waste Abbreviations. 
BPDCC
BPDCS
BPDCV
BPFPS
BPLCS
BPLDC
BPLDN
Bumping, Tank Bump

BVCLN

BY SLTCK

C Plant (222-C)

C RLANT

Caisson

Calcine

CARB

CAS
See Waste Abbreviations.

See Waste Abbreviations.

See Waste Abbreviations.

See Waste Abbreviations.

See Waste Abbreviations.

See Waste Abbreviations.

See Waste Abbreviations.

A tank bump occurs when solids overheat in the lower portion of the tank. The hot solids rapidly transfer heat to the liquid, some of which quickly vaporizes. The sudden pressurization caused by vapor generation is called a "bump".

See Waste Abbreviations.

See LANL Defined Waste List.

See Facilities.

See SSW.

An underground structure used to store high-level waste; typical designs include corrugated metal or concrete cylinders, 55-gal drums welded end-toend, and vertical steel pipes below grade.

To heat a substance to a high temperature, but below its melting point, causing loss of volatile constituents such as moisture; refers also to the material produced by this process.

See Waste Abbreviations.

Cascade-see SET and END. This process filled three tanks with one pump using overflow siphoning. Normal use was with 
Cascade

CASS

Catch Tanks

CAW

$\mathrm{CC}$

CCGL

CCGR

CCPL

CCPIX

CCW

$\mathrm{CDF}$

CE

CELL 23 a sequence of tanks such as 101, 102, 103, or 110, 111, 112 .

Eleven of the single-shell tank farms (all except the AX Tank Farm) were equipped with overflow lines between tanks. The tanks were connected in series and were placed at different elevations creating a downhill gradient for liquids to flow from one tank to another.

Acronym for Computer Automated Surveillance system.

Small capacity single-shell tanks associated with diversion boxes and diverter stations. The tanks are designed to receive any transfer line cleanout, spills or leakage from the boxes, or leakage from the adjacent pipe encasement.

Current acid waste; this is PUREX acid HLW.

See Waste Abbreviations.

See Waste Abbreviations.

See Waste Abbreviations.

See Waste Abbreviations.

See Waste Abbreviations.

Concentrated customer waste.

Composition data file or transaction flag key-unit volume assumed to make stream active.

See Waste Abbreviations.

Waste from cell 23 at B-Plant. Cell 23 contained an evaporator.

See Waste Abbreviations.

$$
\text { A- } 6
$$


WHC-SD-WM-ER-323, Rev. 0

CF

CON

COND

Condensate

Conductivity Probe

Confirmed or

Storage Declared Leaker

COOL

CORR

CP

CPLX

CR Vault

Crib

Crust

CSED
See Waste Abbreviations.

See Waste Abbreviations.

Condensate-see EVAP, EB.

Iiquid formed from cooling vapors.

A device that completes an electrical circuit when contacted by a conductive material.

The designation of any underground waste tank where the data are considered sufficient to support a conclusion with 95 percent confidence that the tank has leaked.

Change in waste volume due to cooling. See CORR.

Correction to waste amount-see ADJ, LEAK, COOL.

See Waste Abbreviations.

See Waste Abbreviations.

Facility located adjacent to C Farm, used for scavenging campaign following uranium recovery. Ferrocyanide was added to tank supernatants in $C R$ Vault and the slurry was returned to C-Farm.

A linear excavation approximately $15 \mathrm{ft}$ in depth, with a perforated pipe in the bottom. The ditch is backfilled with broken rock or other loose material and then covered by soil and by a liquid impermeable membrane; the pipe is then used to distribute intermediate level liquid wastes along the crib.

A hard surface layer that has formed on top of the liquid in many waste tanks that contain concentrated solutions.

See Waste Abbreviations. 
CSR

CST

CSWLE

CSWLW

CTW

CW

CWP

CWP/ZR

CWP2

CWR

$\operatorname{CX70}$

D

DBP

DC

DCS

$D E$

$\mathrm{DE}$

Deliquescent

Desiccant

Diatomaceous Earth
See Waste Abbreviations.

See Waste Abbreviations.

See Waste Abbreviations.

See Waste Abbreviations.

Caustic waste for makeup.

See Waste Abbreviations

See Waste Abbreviations.

See Waste Abbreviations.

See LANL Defined Waste List.

See Waste Abbreviations.

See Waste Abbreviations.

Transaction Flag Key-Amount by difference.

Dibutyl phosphate.

See Waste Abbreviations.

Acroynm for dilute caustic solutions

Acronym for diatomaceous earth that was added to Tanks 241-BX-102, -SX-113, $-\mathrm{TX}-106$, and $-\mathrm{U}-104$ from 1970 to 1972.

See LANL Defined Waste List.

A solid that is capable of absorbing moisture from the air and becoming $a$ liquid.

A drying agent such as diatomaceous earth.

Diatomite, a light friable siliceous material, derived chiefly from diatom (algae) remains that is added to selected underground waste storage tanks 
DBP

DII

DILED

Ditch

Diversion Box

$\mathrm{DN} / \mathrm{PT}$

$\mathrm{DN} / \mathrm{PD}$

DN

Double-Shell Tank

Double-sheli slurry

Double-shell slurry Feed (DSSF)

Drywell to absorb and, thereby, immobilize residual liquids (i.e help to stabilize the tank).

Dibutyl phosphate.

Dilution

Dilute feed

A linearly oriented excavation often used for the temporary diversion or disposal of process waste streams.

A below grade concrete enclosure containing the remotely maintained jumpers and spare nozzles for the routing of waste solution to storage tank farms.

See Waste Abbreviations.

See Waste Abbreviations.

See Waste Abbreviations.

The newer underground waste storage tanks consisting of a concrete shell and two concentric carbon steel liners with an annular space between the liners.

Waste that exceeds the sodium aluminate saturation boundary in the evaporator without exceeding receiver tank composition limits. For reporting purposes, DSS is considered a solid.

Waste concentrated just before reaching the sodium aluminate saturation boundary in the evaporator without exceeding receiver tank composition limits. This form is not as concentrated as DSS.

A steel casing, generally 6 inches in diameter drilled into the ground to various depths, and used to insert monitoring instruments for measuring the presence of radioactivity or moisture 
WHC-SD-WM-ER-323, Rev. 0

content.

Drywell (in-tank)

DSS

DSSF

DUMM

DUMMY

DW

DWBIX

E

$\mathrm{EB}$

EDTA

$\mathrm{EF}$

EFD

END

Environs

EV

EVAP

EVAPF

Evaporator Feed
A sealed casing within a tank that is attached to a riser. It is used to insert experimental equipment, such as neutron or acoustical probes, to determine the level of drainable interstitial liquor.

See Waste Abbreviations.

See Waste Abbreviations.

See Waste Abbreviations.

See Waste Abbreviations.

See Waste Abbreviations.

See Waste Abbreviations.

Transaction flag key-waste transferred through evaporator.

See Waste Abbreviations.

Ethylenediaminetetraacetic acid.

See Waste Abbreviations.

See Waste Abbreviations.

Disconnect Cascaded Tanks, see CAS, Set.

Surrounding area, vicinity.

Evaporation.

See Waste Abbreviations.

See Waste Abbreviations.

Any waste liquid that can be concentrated to form saltcake; e.g., low heat waste, dilute interstitial liquor, aged waste, and other radioactive waste solutions. 
Evaporator-Crystallizer $242-A$ and $242-S$ waste concentration facilities that operate at a reduced pressure (vacuum) and are capable of producing a slurry containing about 30 volume percent solids at a specific gravity of greater than 1.6 .

EVS

Partial neutralization in the $242-\mathrm{S}$ Evaporator.

EVT

HEDTA destruction in the 242-B or 242-T Evaporators.

FD

See Waste Abbreviations.

FECN

Ferrocyanide wastes created during a scavenging campaign in 1953-57. See SCAV, POO, TOO.

Ferrocyanide

An ion composed of iron and cyanide with the chemical formula of $\mathrm{Fe}(\mathrm{CN})^{-4} 6$.

FIC

A Food Instrument Corporation automatic liquid level gauge based on a conductivity probe. At Hanford, they are electrically connected to a computer for data transmission, analysis, and reporting. Local readings may also be obtained from a dial.

First- And second-Cycle Decontamination Wastes

Waste contained 10 percent of the original fission product activity and 2 percent of the products. By-product cake solution was mixed with product waste and neutralized with 50 percent caustic. The waste contained a mixture of suspended solids, hydroxides, carbonate and phosphate, scavenger metals, chromium, iron, sodium, and silicofluoride.

FLSH

See Waste Abbreviations.

FP

Fission Product Waste. Waste produced at B Plant and Hot Semiworks in campaigns for $\mathrm{Cs}$ and $\mathrm{Sr}$ recovery during the 1960s. Cs was removed from PUREX supernatant and $\mathrm{S} r$ was removed from 
FP

GA

Gamma Ray

GAS

GM Instrument

GROUP

Gunite

$\mathrm{H}_{2} \mathrm{O}$

HDRL

HEAT

HEDTA

Heel

Hexone

High-Level Waste

Historical Information
PUREX sludge; both were removed from acidic waste.

See Waste Abbreviations.

Gain to tank.

Electromagnetic radiation sometimes emitted by the nuclei of radioactive substances during decay, similar in nature to $\mathrm{X}$-rays.

Slurry growth as a result of gas generation.

Instrument for detecting low-level beta and gamma radiation using a GeigerMueller tube.

A group of tanks where ITS averaged the supernatant phases-see ITS.

A building material consisting of a mixture of cement, sand, and water that is sprayed onto a mold.

Water.

See Waste Abbreviations.

A tank correction, see CORR, COOL.

$\mathrm{N}$ hydroxy-ethylenediaminetriacetic acid.

The amount remaining in a vessel or container after most of the contents have been removed.

Methyl isobutyl ketone, an organic solvent used in the REDOX solvent extraction process. Also known as Isobutyl methyl ketone, Methyl isobutyl ketone, 4-Methyl 2-pentanone, MIBK.

Waste from the fuel reprocessing operations in separations plants.

All information obtained from material 
HLO

HLO

HLW

HOT-SEMI

HS

HS

HWVP

$I \& S$

Inactive Tank

INST

Interim Isolated

Interim Stabilized referenced in published documentation and unpublished sources. Unpublished sources include electronically retrieved data (i.e. temperatures, liquid observation well, sampling, and surface level) and photographs.

See Waste Abbreviations.

Hanford Laboratory Operations.

See Waste Abbreviations.

See HS, SSW.

See Waste Abbreviations.

Hot Semi-Works. A pilot facility that had a variety of operations. C Plant.

Dilute, non-complexed waste from the vitrification plant.

Tank isolated and stabilized.

A tank that has been removed from liquid-processing service, has been pumped to less than $33,000 \mathrm{gal}$ of waste, and is waiting to be, or is in the process of being, stabilized and interim isolated. Includes all tanks not in active or active-restricted categories. Otherwise inactive contingency spares that would be used if an active tank failed are also included.

Change in tank level due to change in instrumentation.

An administrative designation reflecting the completion of the physical effort required to minimize the addition of liquids into an inactive storage tank, process vault, sump, catch tank, or diversion box.

A tank which contains less than 50,000 gallons of drainable interstitial liquid 
Interstitial Iiquor

Interstitial

Intrusion Mode

FIC Setting

Intrusion Prevention

ISO

ITS

IWW

IX

Jet Pump

Knuckle and has less than 5,000 gallons of supernatant liquid. If the tank was jet pumped to achieve interim stabilization, then the jet pump flow must also have been at or below 0.05 gallons per minute before interim stabilization is completed.

The liquid that fills the interstices or the void volume in the solid material in a waste tank.

The volume within a solid matrix made up of the crevices, cracks, and void spaces.

The FIC probe is positioned a short distance above the waste surface. If the surface level of the waste in the tank increases, thereby touching the probe tip, a positive indication is received.

This is an administrative designation reflecting the completion of the physical effort required to minimize the addition of liquid into an inactive storage tank, process vault, catch tank, sump, or diversion box.

Tank is interim-isolated.

The In-Tank Solidification Program was a program used to reduce waste volume directly by evaporating the wastes from inside certain tanks in the BX and $B Y$ Farms. The process used both hot air (ITS-1) and an electric heater (ITS-2).

See Waste Abbreviations.

See Waste Abbreviations.

A modified commercially available low capacity jet pump used as a salt well pump.

point where the side wall and the bottom 
L222S

L3A4A

LaF

Lateral

Leak Detection Pit

LEAK

LETE

Level Adjustment

Level History

Liquid Observation Well (LOW)

Iiquid Level Best Engineering Judgement Line curved surface of a tank meet.

See Waste Abbreviations.

See Waste Abbreviations.

See Waste Abbreviations.

Horizontal drywell under A Farm and certain SX Farm waste storage tanks.

Collection point for any leakage from $\mathrm{AX}$ Farm tanks. The pits are equipped with radiation and liquid detection instruments.

Tank leak volume, see CORR.

Liquid effluent treatment facility from $\mathrm{N}$-Reactor.

Any update in the waste inventory (or tank level) in a tank. The adjustments usually result from surveillance observations or historical investigations.

A diagram that shows the history of the waste level and waste level changes in a tank. The diagram also includes other related data.

Liquid observation wells are used to monitor the interstitial liquid level (ILL) in single-shell waste storage tanks. Three probes are used to monitor changes in the IIL: acoustic, gamma, and neutron. Each method can indicate intrusions or leakage by increases or decreases in the ILI.

During the initial fill of certain single-shell tanks, only the liquid level was reported. To adjust for the big increase in level height, which occurred when solids were added to the record, a sloped line was used to reflect solids volume between the 
LO

IUNC

LW

Metal Waste (MW)

Molarity

Mole

MW

MWF

$\mathrm{N}$

NCAW

NCPL

NCPLEX

NCPLX

NCRW

Neutralized PUREX Acid Waste initial fill and the time the solids data was recorded.

Loss from tank.

See Waste Abbreviations.

See Waste Abbreviations.

Waste from the extraction containing all the uranium, approximately 90 percent of the original fission product activity, and approximately 1 percent of the product. This waste was brought just to the neutral point with 50 percent caustic and then treated with an excess of sodium carbonate. This procedure yielded almost completely soluble waste at a minimum total volume. The exact composition of the carbonate compounds was not known but was assumed to be an uranium phosphate carbonate mixture.

Number of gram molecular weights (moles) per liter of solution.

The amount of a substance with weight equal to the molecular weight in grams.

See Waste Abbreviations.

See Waste Abbreviations.

See Waste Abbreviations.

See Waste Abbreviations.

See Waste Abbreviations.

See Waste Abbreviations.

See Waste Abbreviations.

See Waste Abbreviations.

The original plant in 1956 neutralized all the high-level waste and sent it to the 24I-A Tank Farm. As fission product 
Neutron Probe

NFAW

NHAW

NIT

Noise At The Bottom of Well--Drywell Probe

Non-Complexed (NCPLX)

NPH

NRAW

NRP82

NRPO4

NRSO4

NTA

Nuclear reactor

Open Hole salt Well recovery started, a portion of the waste was treated for strontium recovery and then neutralized. As of 1967, all of the high-level waste left PUREX as an acid solution for treatment at $B$ Plant.

Probe equipped with a neutron source and detector. They are used in dry well monitoring to determine the moisture content of the soil as one means for detecting leaks in underground waste storage tanks or pipelines.

See Waste Abbreviations.

See Waste Abbreviations.

$\mathrm{HNO}_{3} / \mathrm{KMNO}_{4}$ solution added during evaporator operation.

Erroneous reading caused by probe coming contact with foreign objects at the bottom of a well.

General waste term applied to all Hanford Site liquors not identified as complexed (containing organics).

Normal paraffin hydrocarbon.

See Waste Abbreviations.

See Waste Abbreviations.

See Waste Abbreviations.

See Waste Abbreviations.

Nitrilotriacetic acid.

A device for creating a controlled nuclear chain reaction using atomic fuel, as for the production of energy.

A well in which a pump is inserted in solid waste. Frequently used to remove the liquid from tanks containing less than 2 feet of sludge. 
WHC-SD-WM-ER-323, Rev. 0

Organic Wash Waste(OWW) The solvent used in PUREX was treated before reuse by washing with potassium permanganate and sodium carbonate, followed by dilute nitric acid and then a sodium carbonate wash.

Out-of-Service-Tank

OWW

$P$

P1 :

P2:

PADFG

PADWG

Partially Interim Isolated

PAS

PASF

PAW

PD

PDBNG

PDBSU

PDBTG

PDCSS
A tank that does not meet the definition of an in-service tank. Before september 1988, these tanks were defined as inactive. \{Note: all single-shell tanks (SSTs) are out of service.\}

See Waste Abbreviations.

See Waste Abbreviations.

See LANL Defined Waste List.

See LANL Defined Waste List.

See Waste Abbreviations.

See Waste Abbreviations.

The administrative designation reflecting the completion of the physical effort required for Interim Isolation except for the isolation of risers and piping that will be required for jet pumping or for other methods of stabilization.

See Waste Abbreviations.

See Waste Abbreviations.

See Waste Abbreviations.

See Waste Abbreviations.

See Waste Abbreviations.

See Waste Abbreviations.

See Waste Abbreviations.

See Waste Abbreviations. 
PDL87

PDL89

PDNSG

PDS87

PDS89

PDSLG

PDSUP

PFeCN

PFeCN1:

PFeCN2 :

PFMMS

PFP (Z-Plant)

PFP

PFPGR

PEPNT

PFPPT

PFPSL

$\mathrm{pH}$

Pile

PI

PML89

PMS89

PN
See Waste Abbreviations.

See Waste Abbreviation.

See Waste Abbreviations.

See Waste Abbreviations.

See Waste Abbreviations.

See Waste Abbreviations.

See Waste Abbreviations.

See Waste Abbreviations.

See IANL Defined Waste List.

See IANL Defined Waste List.

See Waste Abbreviations.

See facilities.

Plutonium finishing plant waste (see $Z$, 224, PRF) .

See Waste Abbreviations.

See Waste Abbreviations.

See Waste Abbreviations.

See Waste Abbreviations.

A measure of the hydrogen ion concentration in a solution.

An early term used to describe a nuclear reactor.

See Waste Abbreviations.

See Waste Abbreviations.

See Waste Abbreviations.

See Waste Abbreviations. 
WHC-SD-WM-ER-323, Rev. 0

PNF

POO-P\#\#

Portland Cement

PRF

Primary Addition

Primary Stabilization

Probe

PSI

PSS

PSSF

Psychrometry

PT
See Waste Abbreviations.

In-Plant scavenging with FeCN-see SCAV, TOO-T\#\#.

A hydraulic cement made by finely pulverizing the clinker produced by calcining to incipient fusion a mixture of clay and limestone or similar materials.

PIutonium Reclaimation Facility-Type of waste generated in Z-Plant for "Einishing wastes". Solvent based extraction process using $\mathrm{CCl}_{4} / \mathrm{TBP}$.

An addition of waste from a specific plant or process vault. These additions come from the "waste Status and Transaction Record Summary", WHC-SD-WMTI-614 \& -615 Rev. O DRAFT.

The condition of an inactive waste storage tank after all liquid above the solids, other than isolated surface pockets, has been removed. Isolated surface pockets of liquid are those not pumpable by conventional techniques.

A device used to get information about the environment. In this report, an instrument package designed to be inserted in drywells, risers, or ports to measure waste characteristics.

See Waste Abbreviations.

See Waste Abbreviations.

See Waste Abbreviations.

Determination of humidity or dew point from wet and dry bulb temperatures with the difference in the two used as a measure of dryness in the atmosphere.

See Waste Abbreviations. 


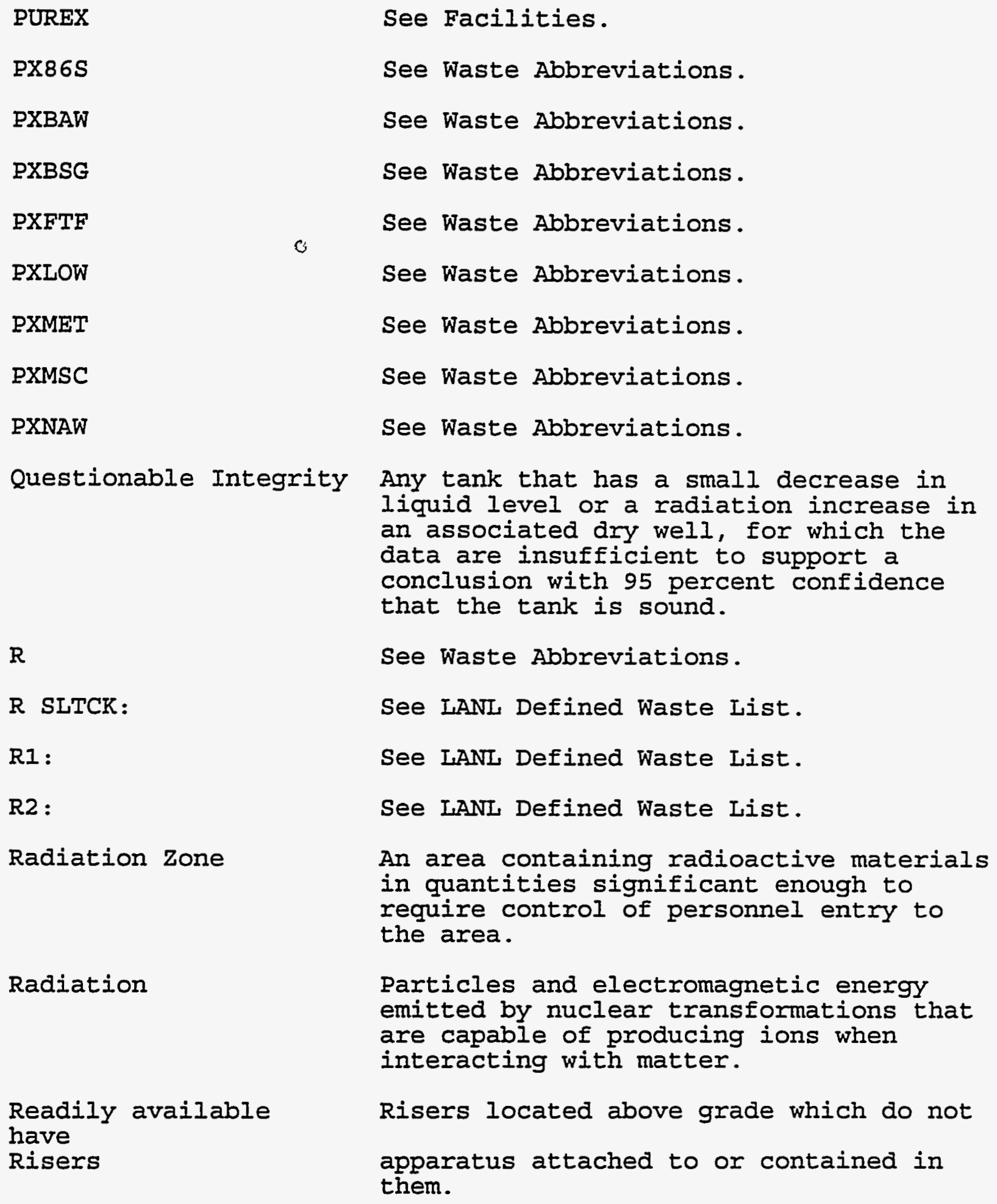
an associated dry well, for which the data are insufficient to support a conclusion with 95 percent confidence that the tank is sound.

$\mathbf{R}$

See Waste Abbreviations.

R SITCK:

See LANL Defined Waste List.

RI:

See IANL Defined Waste List.

R2 :

See IANL Defined Waste List.

Radiation Zone

An area containing radioactive materials in quantities significant enough to require control of personnel entry to the area.

Radiation

Particles and electromagnetic energy emitted by nuclear transformations that are capable of producing ions when interacting with matter.

Readily available have Risers

Risers located above grade which do not apparatus attached to or contained in them. 
REC

\section{REDOX}

Removed From Service

is not (Tanks)

Rerun Drywell Data

RESD

RIX

RMC

RSN

RSS

RTX

$S$

S-Plant (REDOX)

S1 SITCK:

S2 SLTSLRY:

Salt Cake

Salt Well

SCAV

Scavenged
Receive waste from another tank-see XFER.

See Facilities.

Any tank that is a confirmed leaker or intended for reuse.

Surveillance data which supersedes

"suspect data".

See Waste Abbreviations.

See Waste Abbreviations.

Remote Mechanical C-Line -Process used in Z-Plant.

See Waste Abbreviations.

See Waste Abbreviations.

See Waste Abbreviations.

Transaction Flag Key-Partial

neutralization (PNF).

See Facilities.

See LANL Defined Waste List.

See LANL Defined Waste List.

Crystallized nitrate and other salts deposited in waste tanks, usually after active measures are taken to remove moisture.

A hole drilled or sluiced into a salt cake and lined with a cylindrical screen to permit drainage and jet pumping of interstitial liquors.

Scavenging campaign with FECN on TBP, 1952-57. See TOO-T\#\#, POO-P\#\#.

Waste which has been treated with ferrocyanide to remove cesium from the

$$
\text { A- } 22
$$




$$
\text { WHC-SD-WM-ER-323, Rev. } 0
$$

Scintillation Monitor

Self Concentrating

Self-Evaporation

SET

SIX

SI

SL3SY

Sludge

Slugs

sluicing, or sluiced

Slurry

Sound Tank supernatant by precipitating it into the sludge.

A radiation detection instrument based on the principle that light pulses are produced in some materials when they are exposed to radiation.

High-level liquid radioactive waste whose constituent radionuclides contribute sufficient decay heat to cause the solution to boil and/or selfconcentrate.

A waste material in which moisture is being lost as the moisture changes to a vapor and diffuses into the atmosphere.

Connect cascaded tanks together-see CAS and END.

See Waste Abbreviations.

See Waste Abbreviations.

See Waste Abbreviations.

Primarily insoluble metal hydroxides and oxides precipitated from neutralized waste.

An early term for uranium fuel elements which had been machined or extruded into short cylinders which were then clad or encased in corrosion-resistant metals.

To wash with water. At Hanford, this has meant to dissolve or suspend in solution by action of a high pressure water stream.

Insoluble material suspended in water or aqueous solution.

The classification of a waste storage tank for which surveillance data indicates there has been no loss of liquid attributed to a breach of 
SPRG

$S R R$

SRS

SRS

SSW

STAB

Stabilization

Static Tank

Strontium Semi-Works

SU

Supernatant

(Supernatant Iiquid)

Surveillance

Suspect Data integrity.

Sparge-transfer of water or volume

See Waste Abbreviations.

See Waste Abbreviations.

See Waste Abbreviations.

See Waste Abbreviations.

Tank stabilized by removal of liquid. Both floating suction and salt-well jet pumps used to remove liquid.

The removal, or immobilization, as completely as possible, of the liquid contained in a radioactive waste storage tank by salt well pumping, open hole salt well pumping, diatomaceous earth addition, etc.

A tank with no significant change in liquid level or involvement in transfer operations during a stated period of time.

Called C-Plant or Hot Semi-Works, was a pilot plant for both Redox and Purex, then reconfigured for strontium recovery.

See Waste Abbreviations.

In waste storage tanks, the Iiquid quantity defined by the difference between the measured Iiquid level and the measured average solids level in a tank.

Regular or systematic watch kept over someone or something.

Surveillance data which does not fall within the expected range and is superseded by "reruns." 
SV

SW RCR

SWLIQ

SWLQW

$T$-Plant $(222-T)$

T1 SLTCK:

T2 SLTCK:

Tank Farm

TBP

TBP

TCO

Terminal Liquor

TFECN

$\mathrm{TH}$

Thermocouple Tree

Thermocouple,
Transaction Flag Key-Amount by

difference in solids.

Salt-well Receiver.

See Waste Abbreviations.

See Waste Abbreviation.

See Facilities.

See LANL Defined Waste List.

See IAANL Defined Waste List.

An area containing a number of storage tanks; i.e., a chemical tank farm for storage of chemicals used in a plant, or underground waste tank storage of radioactive waste.

Tributyl phosphate, a solvent used in the PUREX solvent extraction process.

See Waste Abbreviations.

See Waste Abbreviations.

The liquid product from the evaporationcrystallization process which, upon further concentration, forms an unacceptable solid for storage in single-shell tanks. Terminal liquor is characterized by a caustic concentration of approximately $5.5 \mathrm{M}$ (the caustic molarity will be lower if the aluminum salt saturation is reached first).

See Waste Abbreviations.

See Waste Abbreviations.

A group of thermocouples assembled in a pipe and inserted into a waste tank for measuring temperatures at regular

(normally 2 feet) vertical intervals.

A probe for measuring temperature, 
Thermocouple probe

Thermowell

THL

Thorium

TK

TL

TOO-T\#\#

TPLAL

TPLAN

TPLAS

TR

Trench

trFlag

Tributyl Phosphate (TBP) consisting of two dissimilar metal wires joined at one end (hot junction) with the free ends joined to a measuring instrument. Electrical potential changes due to temperature changes at the hot end are measured and calibrated to read out as temperature.

A well in a waste tank which contains thermocouples.

See Waste Abbreviations.

A chemical element which is also a fertile material. By fertile is meant that when subjected to radiation in a nuclear reactor it will be converted, in this case, to ${ }^{233}$ uranium, a potential fuel.

Tank. TK-17-2, however, was an early designation for B-Plant.

See Waste Abbreviations.

In-Tank scavenging with FECN, see SCAV, P\#\#.

See Waste Abbreviations.

See Waste Abbreviations.

See Waste Abbreviations.

Transfer from tank.

A deep furrow in the ground. At Hanford they are used for the disposal of solid waste.

Transaction Flag Keys-used by W-Trac-see $\mathrm{CDF}, \mathrm{D}, \mathrm{E}, \mathrm{S}, \mathrm{SV}, 1,3,6,0.17,0.33$.

A chemical compound, also an organic solvent used in the PUREX solvent extraction process. 
Type I Tank

Type II Tank

TYpe III Tank

Type IV Tank

Type V Tank

$U-P l a n t(222-U)$

U1U2

UNKAN

UNKNOWN :

UR

UR :

Watch List Tank
These are the 200 series $B, C, T$, and $U$ Farm tanks. They have an operating capacity of 55,000 gal, a $20-\mathrm{ft}$ diameter, a 6-in. dish bottom, and a 3-ft knuckle. There is no generation associated with type I tanks.

These are the original, 1st generation, tank designs, encompassing $B, C, T, U$ (excluding the 200 series tanks), and BX Tank Farms. Also see 1st Generation Tank.

These are the 2nd Generation tank designs, encompasing $B Y, S, T X$, and $T Y$ Tank Farms. Also see 2nd Generation Tank.

These include $3 \mathrm{rd}, 4 \mathrm{th}$, and 5 th generation tank designs, encompasing sx, $A$, and AX Tank Farms respectively. Also see $3 r d$ Generation Tank, 4th Generation Tank, and 5th Gneration Tank.

These are the first double shell tank designs, encompasing $A Y, A Z$, and SY Tank Farms.

See Facilities.

See Waste Abbreviations.

Unknown waste origin sink.

See LANL Defined Waste List.

Uranium recovery operation in 222-U, 1952-57. Created TBP (primary waste) and FECN (scavenging wastes). See TFECN, PFeCN, POO, TOO, FeCN.

See IANL Defined Waste List.

An underground storage tank containing waste that requires special safety precautions because it may have a serious potential for release of highlevel radioactive waste because of 
WATER

WTR

WVP

XFER

Z

Z-Plant

$Z-P L A N T$

ZAW

ZHIGH

ZLAB

ZLOW

ZPRFL

ZPRFS

ZRM

ZRMCL

ZRMCS

uncontrolled increases in temperature or pressure. Special restrictions have been placed on these tanks by "safety Measures for Waste Tanks at Hanford Nuclear Reservation," Section 3137 of the National Defense Authorization Act for Fiscal Year 1991, November 5, 1990, Public Law 101-501 (also known as the Wyden Amendment).

Flush water from various sources.

Water.

Waste volume projections

Transfer of waste out of tank.

See Waste Abbreviations

See Facilities

PFP. Plutonium Einishing Plant.

See Waste Abbreviations.

See Waste Abbreviations.

See Waste Abbreviations.

See Waste Abbreviations.

See Waste Abbreviations.

See Waste Abbreviations.

See Waste Abbreviations.

See Waste Abbreviations.

See Waste Abbreviations. 
WHC-SD-WM-ER-323， Rev . 0

\section{WASTE ABBREVIATIONS}

IAYIN

IAZIN

$1 \mathrm{C}$

ICEB

ICS

224

$224-F$

2312

2AYIN

2AZIN

$2 \mathrm{C}$

2SYIN

3AWIN

$5-6$

5AWIN

6AWIN

AGE, AGING

B

B860N

BFSH

$B I_{1}$
Concentrated complex waste from $101 \mathrm{AY}$ inventory

Pre 2-81 101AZ inventory

First cycle waste

Ist cycle evaporator bottoms

Ist cycle scavenging waste

224-U waste

224-U waste. LaF Pu finishing plant

Dilute, phosphate waste from $231 \mathrm{z}$

laboratories

Pre 2-81 102AY inventory

Pre 2-81 concentrated complex waste from 102Az inventory

Second cycle waste

Pre 2-81 102SY inventory

Pre 2-81 103AW inventory

B-Plant tank 5,6 waste

Pre 2-81 105AW inventory

Concentrated phosphate waste in 106AW inventory

Aging waste

B-Plant high- level waste

Dilute, non-complexed waste from B Plant cell drainage

B-Plant flush waste water

B-Plant low-level waste 
WHC-SD-WM-ER-323，Rev. 0

BLEB

BLIX

BLIXB

BNW

BPDCC

BPDCS

BPDCV

BPEPS

BPLCS

BPLDC

BPLDN

BVCLN

CARB

CC

CCGL

CCGR

CCPI

CCPIX
B-Plant low-level evaporator bottoms

B-Plant low level ion exchange?

B-Plant low level ion exchange bottoms?

Battelle Northwest Laboratory waste

Dilute, complexed waste from B Plant cesium processing

Dilute, complexed waste from B Plant strontium processing

Dilute, complexed waste from B Plant vessel clean-out

B Plant high Tru solids from retrieved PFP solids

Dilute, non-complexed waste from B plant strontium processing

Dilute, complexed waste from B Plant cesium processing

Dilute, non-complexed waste from B plant cesium processing

Dilute, non-complexed waste from B Plant vessel clean-out

PUREX organic wash waste

Complexant concentrate waste

B-Plant high Tru solids from retrieved complexed concentrate

Dilute, non-complexed waste from

retrieved

complexed

concentrate

Complexant concentrate

Complexant concentrate-see CPLX 


\begin{tabular}{|c|c|}
\hline $\mathrm{CE}$ & Evaporator concentrate \\
\hline CEM & Concrete-see CON \\
\hline CF & Cesium feed \\
\hline CON & Concrete-see CEM \\
\hline CP & $\begin{array}{l}\text { Concentrated phosphate waste from } \mathrm{N} \\
\text { Reactor decontamination }\end{array}$ \\
\hline CPLX & Complex waste \\
\hline CSFD & Cesium feed \\
\hline CSR & $\begin{array}{l}\text { Waste sent to B-Plant for cesium } \\
\text { recovery }\end{array}$ \\
\hline CST & Caustic solution \\
\hline CSWLE & Complexed salt well liquid east area \\
\hline CSWLW & Complexed salt well liquid west area \\
\hline CW & Coating waste \\
\hline CWP/ZR & $\begin{array}{l}\text { Coating waste (PUREX), Zirconium } \\
\text { cladding }\end{array}$ \\
\hline CWP & Coating waste (PUREX) \\
\hline CWR & Coating waste (REDOX) \\
\hline CX70 & $\begin{array}{l}\text { Dilute, complexed mixture hot-semiworks } \\
\text { Tru solids }\end{array}$ \\
\hline $\mathrm{DC}$ & Dilute complexed waste \\
\hline $\mathrm{DN} / \mathrm{PD}$ & DN with $P$ Tru solids \\
\hline DN & Dilute non-complexed waste \\
\hline $\mathrm{DN} / \mathrm{PT}$ & DN with PFP Tru solids \\
\hline DSS & Double-shell slurry \\
\hline DSSF & Double-shell slurry feed \\
\hline
\end{tabular}


WHC-SD-WM-ER-323， Rev. 0

\begin{tabular}{|c|c|}
\hline DUIMM & Dummy waste \\
\hline DUMMY & Dummy waste \\
\hline $\mathrm{DW}$ & Decontamination waste \\
\hline DWBIX & $\begin{array}{l}\text { Decontamination waste and B-Plant ion } \\
\text { exchange }\end{array}$ \\
\hline$E B$ & Evaporator bottoms \\
\hline$E F$ & Evaporator feed \\
\hline EFD & Evaporator feed dilute \\
\hline EVAP & Evaporator feed (post 1976) \\
\hline EVAPF & $\begin{array}{l}\text { Dilute, non-complexed waste from } \\
\text { evaporator pad flush }\end{array}$ \\
\hline FD & Feed dilute \\
\hline FLSH & Flush water \\
\hline FP & Fission product waste \\
\hline HDRL & Hanford defense residual liquor \\
\hline HLO & Hanford laboratory operations waste \\
\hline HLW & $\begin{array}{l}\text { High level waste-generic for all Hanford } \\
\text { tank wastes }\end{array}$ \\
\hline HS & Hot semiworks waste \\
\hline IWW & PUREX \#1 acid concentrator waste \\
\hline IX & Ion exchange waste \\
\hline I222S & $\begin{array}{l}2225 \text { laboratory, dilute non-complexed } \\
\text { waste }\end{array}$ \\
\hline I $3 A 4 A$ & $\begin{array}{l}\text { Dilute non-complexed laboratory wastes } \\
\text { from } 300 \& 400 \text { areas }\end{array}$ \\
\hline LaF & $\begin{array}{l}\text { Lanthanum fluoride waste generated in } \\
\text { PFP }\end{array}$ \\
\hline
\end{tabular}

$$
\text { A }-32
$$


LUNC

LW

MW

MWE

$\mathrm{N}$

NCAW

NCPLEX

NCPLX

NCPL

NCRW

NEAW

NHAW

NRAW

NRP82

NRPO4

NRSO4

OWW

P

RADFG

PADWG
Dilute, non-complexed waste from UNC fuels fabrication

Laboratory waste

Metal waste

Metal waste feed

$\mathrm{N}$-Reactor waste

Neutralized current acid waste, primary HLW stream from PUREX process

Non-complexed waste-see NCPLX

Non-complexed waste-see NCPLEX

Non-Complexed waste

Neutralized cladding removal waste-same as CWP.

Aging waste from PUREX/PFM high level waste

Aging waste from PUREX/PFM processing of NPR fuel

Aging waste from PUREX/PFM residue acid waste

Dilute, non-complexed waste from FY82 100-N area waste transfer

Dilute, phosphate waste from $100 \mathrm{~N}$ area

Dilute, non-complexed waste from $100 \mathrm{~N}$ area

Organic wash waste

PUREX waste

Purex ammonia destruction waste, from fuels grade fuel

Purex ammonia destruction waste, from 
WHC-SD-WM-ER-323, Rev. 0

weapons grade fuel

PAS

Purex acidified sludge

PASF

Purex ammonia scrubber feed

PAW

Purex acidified waste

PD

Purex decladding waste

PDBNG

Decladding sludge (non-Tru) from B Plant processing

PDBSU

Dilute, non-complexed waste from B Plant decladding waste

PDBTG

B Plant aging waste solids from Purex decladding waste

PDCSS

Dilute non-complexed Purex decladding waste, FY1986 only

PDI87

Purex decladding supernatant, 1987

PDI89

Purex decladding supernatant, non-Tru, spent metathesis removed

PDNSG

Non-Tru decladding sludge from Purex

PDS87

Purex decladding sludge

PDS89

Purex decladding sludge after FY89

PDSLG

PDSUP

PFeCN

Purex decladding sludge sol Purex

Dilute, non-complexed waste, Purex decladding waste

Ferrocyanide sludge produced by in-plant scavenging of waste from uranium recovery

PFMMS

Dilute, non-complexed waste from shear/leach processing of NPR fuel

PFPGR

Dilute, non-complexed waste from retrieved PFP solids 
WHC-SD-WM-ER-323， Rev. 0

\begin{tabular}{|c|c|}
\hline PFPNT & Non-tru sludge from the PFP sol $\mathrm{Z}$ Plant \\
\hline PFPPT & $\begin{array}{l}\text { Dilute, non-complexed waste from the PFP } \\
\text { (with TRUEX) }\end{array}$ \\
\hline PEPSL & High-tru sludge from the PFP sol $\mathrm{Z}$ Plant \\
\hline PL & PUREX low-level waste \\
\hline PML89 & Purex spent metathesis Iiquid after FY89 \\
\hline PMS89 & Purex spent metathesis solids after FY89 \\
\hline PN & Purex neutralized cladding waste \\
\hline PNE & Partial neutralized waste \\
\hline PSL & $\begin{array}{l}\text { Purex sludge sluiced during recovery of } \\
\text { strontium }\end{array}$ \\
\hline PSS & PUREX sludge supernatant \\
\hline PSSF & Purex sludge supernatant feed \\
\hline PT & Tru solids from $200 \mathrm{~W}$ \\
\hline 2X86S & $\begin{array}{l}\text { Dilute, non-complexed waste from Purex } \\
\text { miscellaneous streams (NPR fuel) FY86 }\end{array}$ \\
\hline PXBAW & $\begin{array}{l}\text { B Plant aging waste supernatant from } \\
\text { retrieved aging waste }\end{array}$ \\
\hline PXBSG & $\begin{array}{l}\text { B Plant aging waste solids from } \\
\text { retrieved aging waste }\end{array}$ \\
\hline PXFTF & $\begin{array}{l}\text { Dilute, non-complexed waste from Purex } \\
\text { miscelianeous streams (FFTF) }\end{array}$ \\
\hline PXLOW & Purex low level waste \\
\hline PXMET & $\begin{array}{l}\text { Purex dilute, non-complexed decladding: } \\
\text { spent metathesis }\end{array}$ \\
\hline PXMSC & $\begin{array}{l}\text { Dilute, non-complexed waste from Purex } \\
\text { miscellaneous streams (NPR fuel) }\end{array}$ \\
\hline PXNAW & Aging waste from Purex high level waste \\
\hline
\end{tabular}


WHC-SD-WM-ER-323, Rev. 0

R

RESD

RIX

RSN

RSS

RTX

SIX

SL

SL3SY

SRR

SRS

SRS

SSW

SU

SWIIQ

SWLQW

TBP

TCO

TFECN

TH

THL
REDOX waste

Residual evaporator liquor

REDOX ion exchange waste

REDOX supernatant

Redox sludge supernatant

Redox ion exchange

PUREX ion exchange waste

sludge

Double shell slurry from end of FY80, 103SY inventory

Waste sent to B-Plant for strontium recovery

strontium sludge

Strontium recovery supernatant

Strontium semiworks waste

Supernatant

Dilute, non-complexed waste from east area single shell tanks

Dilute, non-complexed waste from west area single shell tanks

Tri-Butyl Phosphate

Dilute non-complexed waste from terminal cleanout

Ferrocyanide sludge produced by in-tank or in-farm scavenging

Thoria HLW or cladding waste

Thoria low level waste 
WHC-SD-WM-ER-323, Rev. 0

TL

TPLAL

TPIAN

TPLAS

U1U2

Z

ZAW

ZHIGH

ZLAB

ZLOW

ZPREL

ZPRFS

ZRMCL

ZRMCS
Terminal Iiquor

Dilute, non-complexed waste from $T$ Plant

Dilute, non-complexed waste from $T$ Plant

Sludge from $T$ Plant operations

Dilute, non-complexed waste from U1/U2 groundwater pumping

Z-Plant waste

Purex waste stream from zirconium cladded fuel

Dilute, non-complexed waste from the PFP (without Truex)

Dilute, non-complexed waste from the PFP laboratories

Dilute, non-complexed waste from PreFY85 Z Plant operations

Dilute, non-complexed waste from PRF processing

PFP Tru solids from PRF processing

Dilute, non-complexed waste from PFP RMC processing

PFP Tru solids from PEP RMC processing 
WHC-SD-WM-ER-323, Rev. 0

A. LANL Defined Wastes

IANL DEFINED WASTE IIST

AI SLTCK

A2 SLTSIRY

AR

B SLTCK

BY SITCK

CWP2

DE

P1

P2

PFeCN1

PFeCN2

R SLTCK

RI

R2
Saltcake waste generated from the 242-A evaporator-crystallizer from 1977 until 1980.

Salt slurry waste generated from the 242-A evaporator-crystallizer from 1981 until 1994.

"Washed" PUREX sludge fromthe AR vault.

Saltcake waste generated from the 242-B evaporator from 1951 until 1955.

Saltcake waste generated from in-tank solidification units 1 and 2 between 1965 and 1974.

Cladding waste-PUREX 2???

Diatomaceous earth.

PUREX high-level waste generated between 1955 and 1962 .

PUREX high-level waste generated between 1963 and 1967.

Ferrocyanide sludge generated from inplant scavenging of waste from uranium recovery.

Same as PFeCN1, except used $0.0025 \mathrm{M}$ ferrocyanide.

Salt cake waste from the REDOX concentrator.

REDOX waste generated between 1952 and 1957 .

REDOX waste generated between 1958 and 1966. 
SI SLTCK

S2 SLTSLRY

TI SLTCK

T2 SLTCK

UNKNOWN

UR
Saltcake waste generated from the 242-S evaporator/crystallizer from 1973 until 1976.

Salt slurry waste generated from the 242-S evaporator-crystallizer from 1977 until 1980 .

Saltcake waste generated from the 242-T evaporator from 1951 until 1955.

Saltcake waste generated from the 242-T evaporator from 1965 until 1955.

Unkown waste type.

Uranium recovery waste (also known as tributyl phosphate (TBP) waste). 
WHC-SD-WM-ER-323， Rev. 0

\section{FACIIITIES}

A Plant (PUREX)

B Plant (222-B)

C Plant $(222-C)$

$S$ Plant (REDOX)

T Plant $(222-T)$

U Plant (222-U)

PFP ( $Z$ Plant)

$242-A$

$242-B$

$242-S$

$242-\mathrm{T}$
The facility at Hanford which contains the latest solvent extraction process for recovery of both plutonium and uranium.

On of the three original bismuthphosphate processing facilities. Later converted to a waste fractionation plant.

Initially a pilot plant for Redox, later a pilot plant for Purex and B-Plant waste partitioning.

The facility at Hanford which contains the original extraction process for recovery of both plutonium and uranium.

One of the three original bismuthphosphate processing facilities. Later converted to a decontamination facility.

One of the three original bismuthphosphate processing facilities. Later converted to a uranium recovery plant.

Plutonium Finishing Plant. The final operations for production of plutonium products are carried out in this facility.

A forced circulation vacuum evaporation system.

An evaporator that operates at atmospheric pressure. Also referred to as an open air type evaporator.

A forced circulation vacuum evaporation system.

An evaporator that operates at atmospheric pressure. Also referred to as an open air type evaporator. 
WHC-SD-WM-ER-323, Rev. 0

\section{REFERENCES}

Agnew, S.F., February 1994, Waste Status and Transaction Record Summary for the Northeast Quadrant, WHC-SD-WM-TI-615, REV. 0, Westinghouse Hanford Company, Richland, Washington.

Agnew, S.F., February 1994, Waste Status and Transaction Record Summary for the Southwest Quadrant, WHC-SD-WM-TI-614, REV. 0, Westinghouse Hanford Company, Richland, Washington.

Agnew, S.F., February 1994, Estimated Chemical and Radiochemical Inventories Spreadsheet: $N E$ Quadrant, $A, A X, B, B X, B Y, C$ Farms, WHC-SD-WM-TI-628, REV. 0, Westinghouse Hanford Company, Richland, Washington.

Agnew, S.F., February 1994, Estimated Chemical and Radiochemical Inventories Spreadsheet: $S W$ Quadrant, $S, S X, U$ Farms, WHC-SD-WMTI-631, REV. 0, Westinghouse Hanford Company, Richland, Washington.

Agnew, S.F. March 1994, Tank Layer Model (TLM) Spreadsheet: NE Quadrant, $A, A X, B, B X, B Y, C$ Farms, WHC-SD-WM-TI-627, REV. 0, Westinghouse Hanford Company, Richland, Washington.

Agnew, S.F. March 1994, Tank Layer Model (TLM) Spreadsheet: SW Quadrant, S, SX, U Farms, WHC-SD-WM-TI-630, REV. 0, Westinghouse Hanford Company, Richland, Washington.

Agnew, S.F. April 1994, Hanford Defined Wastes: Chemical and Radionuclide Compositions, WHC-SD-WM-TI-629, REV. 0, Westinghouse Hanford Company, Richland, Washington.

Allen, G.K., March 1976, Estimated Inventory of Chemicals Added to Underground Waste Tanks, 1944 through 1975, ARH-CD-610B, Atlantic Richfield Hanford Company, Richland, Washington.

Alstad, A.T., September 19, 1991, Riser Configuration Document for Single-Shell Waste Tanks, WHC-SD-RE-TI-053, Rev. 8, Westinghouse Hanford Company, Richland, Washington.

Anderson, J.D., January, 1972- December 1972, Waste Status Summary - Chemical Processing Division, ARH-2456, Atlantic Richfield Hanford Company, Richland, Washington.

Anderson, J.D., January, 1973- December 1973, Waste Status Summary - Chemical Processing Division, ARH-2794, Atlantic Richfield Hanford Company, Richland, Washington.

Anderson, J.D., January, 1974- December 1974, Waste Status Summary - Operations Division, ARH-CD-133, Atlantic Richfield Hanford Company, Richland, Washington. 
WHC-SD-WM-ER-323, Rev. 0

Anderson, J.D., January, 1974- December 1974, Waste Status Summary - Chemical Processing Division, ARH-CD-133, Atlantic Richfield Hanford Company, Richland, Washington.

Anderson, J.D., January, 1975- December 1975, Waste Status Summary - Production and Waste Management Division, ARH-CD-336, Atlantic Richfield Hanford Company, Richland, Washington.

Anderson, J.D., January, 1976- September 1976, Waste Status Summary - Production and Waste Management Division, ARH-CD-702, Atlantic Richfield Hanford Company, Richland, Washington.

Anderson, J.D., October, 1976- May 1977, Waste Status Summary Production and Waste Management Division, ARH-CD-822, Atlantic Richfield Hanford Company, Richland, Washington.

Anderson, J.D., June 1990, A History of the 200 Areas Tank Farms, WHC-MR-0132, Westinghouse Hanford Company, Richland, Washington.

ARCHO, October, 1967, Monthly Report, 200 Areas Operation, ARH-60 DEL, Atlantic Richfield Hanford Company, Richland, Washington.

ARHCO, June, 1969, SX Tank Farm Air Cooling Safety Analysis, ARH-1291, Atlantic Richfield Hanford Company, Richland, Washington.

ARHCO, July, 1972 through December, Chemical Processing Division Waste Status Summary, Quarterly Reports, ARH-2456 C and D, Atlantic Richfield Hanford Company, Richland, Washington.

ARHCO, January, 1973 through December 1973, Chemical Processing Division Waste Status Summary, Quarterly Reports, ARH-2694 A, B, $C$, and D, Atlantic Richfield Hanford Company, Richland, washington.

ARHCO, January, 1973 through September 1975, Operations Division Waste Status Summary, Quarterly Reports, ARH-CD-133 A, B, C, and D, Atlantic Richfield Hanford Company, Richland, Washington.

ARCHO, September, 1976, Production and Waste Management Division Waste Status Summary, Monthly Reports ARH-CD-702, Atlantic Richfield Hanford Company, Richland, Washington.

ARCHO, October, 1976 through June 1977, Production and Waste Management Division Waste Status Summary, Monthly Report, ARH-CD-822, Atlantic Richfield Hanford Company, Richland, Washington.

Babad, H., D. M. Camaioni, M. A. Lilga, W. D. Samuels, and D. M. Strachan, February 1993, Tank Waste Chemistry- $A$ New Understanding of Waste Aging, WHC-SA-1694-FP, Westinghouse Hanford Company, Richland, Washington. 
Boomer, R.D., June 1990, Functional Requirement Baseline for the Closure of Single-Shell Tanks, WHC-EP-0338, Westinghouse Hanford Company, Richland, Washington.

Borsheim, G.I., and B. C. Simpson, October 1991. An Assessment of the Inventories of the Ferrocyanide Watchlist Tanks, WHC-SD-WM-ER133 Rev. 0, Westinghouse Hanford Company, Richland, Washington.

Borsheim, G.I., August 2, 1989, Single-Shell Tank Isolation Safety Analysis Report, SD-WM-SAR-006-Rev 2, Westinghouse Hanford Company, Richland, Washington.

Borshiem, G. I. and N. W. Kirch, March 1991, Summary of Single-Shell Tank Waste Stability, WHC-EP-0347, Westinghouse Hanford Company, Richland, Washington.

Boyles, V.C., June 1981, Safety Analysis Report: Stabilization of Single-Shell Waste Storage Tanks by Saltwell Jet Pumping, RHO-SD-WM-SAR-034, Rev.0, Rockwell Hanford Operations, Richland, Washington.

Brevick, C.H., I. A. Gaddis, and W. W. Pickett, June, 1994, Historical Tank Content Estimate Report for the Northeast Quadrant of the Hanford 200 East Area, WHC-SD-WM-ER-349, Rev.0, ICF Kaiser Hanford Company, Richland, Washington.

Brevick, C.H., L. A. Gaddis, and W. W. Pickett, June, 1994, Historical Tank Content Estimate Report for the Southwest Quadrant of the Hanford 200 West Area, WHC-SD-WM-ER-352, Rev.0, ICF Kaiser Hanford Company, Richland, Washington.

Brown, W.G., April 26.1993, Hazard Identification and Evaluation for Non-Stabilized Single Shell Tanks, SD-WM-SAR-022, Rev. 0, Westinghouse Hanford Company, Richland, Washington.

Bussel1, J.H., February 3, 1992, Engineering Evaluation of Thermocouples in FeCN Watchlist Tanks, WHC-SD-WM-ER-134, Rev O-A, Westinghouse Hanford Company, Richland, Washington.

Cain, R.J., 1977, Isolation and Stabilization of Special Tanks, (Internal Letter to J.C. Womack) Rockwell Hanford Company, Richland, Washington.

Carpenter, G.K., April, 1953 - June 1953, Waste Status Summary Separations Section, HW-28043 etc., General Electric Company, Richland, Washington.

Carpenter, G.K., September, 1953 - May 1954, Waste Status Summary Separations Section, HW-29624 etc., General Electric Company, Richland, Washington. 
Carter, George J., April, 1986- September 1986, Waste Status Summary, RHO-RE-SR-14, Rockwell Hanford Operations, Richland, Washington.

Cooley, C.R., and G.L. Richardson; July 17, 1963, Hot Semiworks Strontium-90 Recovery Program, HW-72666, General Electric Company, Richland, Washington.

ERDA, December, 1975, Waste Management Operations, ERDA-1538, Energy Research and Development Administration, Richland, Washington.

Escobar, Glenn A., October, 1986- January 1987, Waste Status Summary, RHO-RE-SR-14, Rockwell Hanford Operations, Richland, Washington.

Farley, W.G., May 20, 1992, Safety Assessment for Thermocouple Tree System Installation and Operation in Nonleaking Ferrocyanide Tanks, WHC-SD-WM-SAD-014, Rev. I, Westinghouse Hanford Company, Richland, Washington.

GE Co., July 10, 1951, REDOX Technical Manual, HW-18700-Del, General Electric Company, Richland, Washington.

Gerber, M.S., September, 1992, Legend and Legacy: Fifty Years of Defense Production at the Hanford Site, WHC-MR-0293, Rev 2, Westinghouse Hanford Company, Richland, Washington

Gerber, M.S., November, 1993, A Brief History of the Purex and $\mathrm{UO}_{3}$ Facilities, WHC-MR-0437, Westinghouse Hanford Company, Richland, Washington.

Gerber, M.S., September,1993, Multiple Missions: The 300 Area in Hanford Site History, WHC-MR-0440, Westinghouse Hanford Company, Richland, Washington.

Hanlon, B.M., November, 1989- December, 1990, Tank Farm Surveillance and Waste Status Summary Report, WHC-EP-0182, Westinghouse Hanford Company, Richland, Washington.

Hanlon, B.M., January, 1991- December, 1991, Tank Farm Surveillance and Waste Status Summary Report, WHC-EP-0182, Westinghouse Hanford Company, Richland, Washington.

Hanlon, B.M., January, 1992- December, 1992, Tank Farm Surveillance and Waste Status Summary Report, WHC-EP-0182, Westinghouse Hanford Company, Richland, Washington.

Hanlon, B.M., July, 1993, Tank Farm Surveillance and Waste Status Summary Report for July 1993, WHC-EP-0182-64, Westinghouse Hanford Company, Richland, Washington.

Hanlon, B.M., January, 1993-October, 1993, Tank Farm Surveillance and Waste Status Summary Report, WHC-EP-0182, Westinghouse Hanford Company, Richland, Washington. 
WHC-SD-WM-ER-323, Rev . 0

Hatch, P., December 18, 1969, Limitations for use of Underground Waste Tanks, ARH-95I, Atlantic Richfield Hanford Company, Richland, Washington.

HEW, January 11, 1944, CVI, "Specifications for Construction of Composite Storage Tanks BIdg. No. 241," Project 9536, CVI 73550, Hanford Engineer Works, Richland, Washington.

Jenkins, C., M. Danielson, B. Rarig, R. Campbell, T. Ambalam, C. Kenoyer, and I. Hall, February, 1993, Engineering Evaluation of Alternatives Managing the Assumed Leak from SST 241-T-101, EEA-931, Kaiser Engineers Hanford, Richland, Washington.

Jungfleisch, F. M., March 1984, Preliminary Estimation of the Waste Inventories in Hanford Tanks through 1980, SD-WM-TI-057, Rev.0, Rockwell Hanford Operations, Richland, Washington.

KEH, February 1993, EEP Managing the Assumed Leak From SST, KEEA93-1, Kaiser Engineers Hanford, Richland, Washington.

Kirkman, M.J., G. L. Ritter, and P. W. Smith, March 6, 1969, BPlant Production Schedule and In-Tank Solidification Alternatives, ARH-900, Atlantic Richfield Hanford Company, Richland, Washington.

Kreig, S.A., W.W. Jenkins, K.J. Leist, K. G. Squires, and J. F. Thompson, June 1990, Single-Shell Tank Waste Retrieval Study, WHC-EP-0352, UC-721, Westinghouse Hanford Company, Richland, Washington.

Lentz, J.E., April 1959- December 1959, Waste Status Summary Chemical Processing Department, HW-58579 etc., General Electric Company, Richland, Washington.

Lentz, J.E., January 1960- December 1960, Waste Status Summary Chemical Processing Department, HW-63896 etc., General Electric Company, Richland, Washington.

Liverman, J.I., December 1975, Final Environmental Statement, Waste Management Operations Volume 1 and 2, ERDA-1538, Energy Research and Development Administration, Richland, Washington.

Iucas, G.E., June 1989, Waste Types in Hanford Single-Shell Tanks, WHC-SD-ER-TI-001, Rev. 0, Westinghouse Hanford Company, Richland, Washington.

McBride, J.P., July, 1953, Waste Status Summary - Separations Section, HW-29054, General Electric Company, Richland, Washington.

McCann, D.C., July, 1981-October 1981, Waste Status Summary, RHOCD-14, Rockwell Hanford Operations, Richland, Washington.

McCann, D.C., October, 1981- December 1982, Waste Status Summary, RHO-RE-SR-14, Rockwell Hanford Operations, Richland, Washington. 


$$
\text { WHC-SD-WM-ER-323, Rev . } 0
$$

McCann, D.C., January, 1983- December 1983, Waste Status. Summary, RHO-RE-SR-14, Rockwell Hanford Operations, Richland, Washington.

MCCann, D.C., January, 1984- February 1985, Waste Status Summary, RHO-RE-SR-14, Rockweli Hanford Operations, Richland, Washington.

Morganthaler, A.C., February, 1952 - March 1953, Waste Status Summary - Separations Section, HW-27897 etc., General Electric Company, Richland, Washington.

Mudd, O.C., January, 1979- December 1979, Waste Status Summary, RHO-CD-14, Rockwell Hanford Operations, Richland, Washington.

Mudd, O.C., January, 1980- December 1980, Waste Status Summary, RHO-CD-14, Rockwell Hanford Operations, Richland, Washington.

Mudd, O.C., January, 1981- June 1981, Waste Status Summary, RHO-CD14, Rockwell Hanford Operations, Richland, Washington.

Mulvey, C.A., June, 1977- December 1977, Waste Status Summary, RHOCD-14, Rockwell Hanford Operations, Richland, washington.

Mulvey, C.A., January, 1978- December 1978, Waste Status Summary, RHO-CD-14, Rockwell Hanford Operations, Richland, Washington.

Peterson, D.E., June, 1954- May 1955, Waste Status Summary Separations Section, HW-32389 etc., General Electric Company, Richland, Washington.

Peterson, D.E., June, 1955- May 1956, Waste Status Summary Separations Section, HW-38000 etc., General Electric Company, Richland, Washington.

Peterson, D.E., September, 1956- December 1956, Waste Status Summary - Chemical Processing Department, HW-45738 etc., General Electric Company, Richland, Washington.

Peterson, D.E., June, 1956- August 1956, Waste Status Summary Separations Section, HW-43895 etc., General Electric Company, Richland, Washington.

Pines, A.G., January 4, 1991, 244-AR Vault Safety Analysis Report, SD-WM-SAR 018 Rev. 0, Westinghouse Hanford Company, Richland, Washington.

Prosk, W., and D.A. Smith, January 1986, Tank Isolation Safety Analysis Report, SD-WM-SAR-006 Rev. 1, Rockwell Hanford Operations, Richland, Washington.

RHO, June 1977 through October 1981, Waste Status Summary, Monthly Reports RHO-CD-14, Rockwell Hanford Operations, Richland,

Washington. 
WHC-SD-WM-ER-323, Rev. 0

RHO, November 1981 through June 1987, Waste Status Summary, Monthly Reports RHO-RE-SR-014, Rockwell Hanford Operations, Richland, Washington.

Roberts, R.E., January, 1957- Decèmber 1957, Waste Status Summary Chemical Processing Department, HW-48144 etc., General Electric Company, Richland, Washington.

Roberts, R.E., January, 1958-October 1958, Waste Status Summary Chemical Processing Department, HW-54916 etc., General Electric Company, Richland, Washington.

Roberts, R.E., January, 1961- December 1961, Waste Status Summary Chemical Processing Department, HW-71610 etc., General Electric Company, Richland, Washington.

Roberts, R.E., January, 1962- December 1962, Waste Status Summary Chemical Processing Department, HW-74647 etc., General Electric Company, Richland, Washington.

Roberts, R.E., January, 1963- December 1963, Waste Status Summary Chemical Processing Department, HW-78279 etc., General Electric Company, Richland, Washington.

Roberts, R.E., January, 1964- December 1964, Waste Status Summary Chemical Processing Department, HW-83308 etc., General Electric Company, Richland, Washington.

Roberts, R.E., January, 1965- December 1965, Waste Status Summary Chemical Processing Department, HW-83906 etc., General Electric Company, Richland, Washington.

Roberts, R.E., October, 1966- December 1966, Waste Status Summary Chemical Processing Division, IsO-674, Isochem Inc., Richland, Washington.

Roberts, R.E., January, 1966- September 1966, Waste Status Summary - Chemical Processing Department, HW-83906 etc., General Electric Company, Richland, Washington.

Rodenhizer, D.G., September 30, 1987, Hanford Waste Tank Sluicing History, SD-WM-TI-302, Westinghouse Hanford Company, Richland, Washington.

Rutherford, M.J., August 31, 1948, Additional Waste Storage Facilities 200-East Area, Specification No. HW 3783, General Electric Company, Richland, Washington.

Scaief, C.C. III, October 19, 1993, TMACS I/O Termination Point Listing, WHC-8D-WM-TI-594, Rev. 0, Westinghouse Hanford Company, Richland, Washington. 
Smith, D.A., August 1986, SAR, Hazard Identification and-Evaluation for NonStabilized Single-Shell Tanks, SD-WM-SAR 022 Rev. 0, Rockwell Hanford Operations, Richland, Washington.

Stahl, S.M., November 22, 1993, Hanford Site Tank Farm Facilities Interim Safety Basis, Volume 1 and 2, WHC-SD-WM-ISB-001, Westinghouse Hanford Company, Richland, Washington.

Stong, F.S., September 29, 19860, Drywell Van In-Tank Liquid Observation Well Surveillance Data Interpretation, RHO-SD-WM-TI-237, Rockwell Hanford Operations, Richland, Washington.

ThanMai, T. T., April 27, 1993, Thermocouple Status Single-Shell and Double-Shel1 Waste Tanks, WHC-SD-WM-TI-553, Rev. 0, Westinghouse Hanford Company, Richland, Washington.

Thress, M A., November 1958- February 1959, Waste Status Summary Chemical Processing Department, HW-58579 etc., General Electric Company, Richland, Washington.

Thurman, Jack M., June, 1987 through December 1987, Tank Farm Surveillance and Waste Status Summary Report, Monthly Reports WHC-SP 0038, Westinghouse Hanford Company, Richland, Washington.

Thurman, Jack M., December, 1987 through June 1993, Tank Farm Surveillance and Waste Status Summary Report, Monthly Reports WHC-EP-0182-55, Westinghouse Hanford Company, Richland, Washington.

Thurman, Jack M., February, 1987- June 1987, Waste Status Summary, RHO-RE-SR-14, Rockwell Hanford Operations, Richland, Washington.

Thurman, Jack M., July, 1987-March, 1988, Waste Status Summary, WHC-SP-0038, Westinghouse Hanford Company, Richland, Washington.

Thurman, Jack M., April, 1988- December, 1988, Tank Farm Surveillance and Waste Status Summary Report, WHC-EP-0182, Westinghouse Hanford Company, Richland, Washington.

Thurman, Jack M., January, 1989- October, 1989, Tank Farm Surveillance and Waste Status Summary Report, WHC-EP-0182, Westinghouse Hanford Company, Richland, Washington.

U.S.DOE, December 1987, EIS, Disposal of Hanford Defense High Level, Transuranic, and Tank Wastes, DOE/EIS-0113 Volume 1-5, Department of Energy, Richland, Washington.

Uebelacker, D.L., October, 1968- December 1968, Waste Status Summary - Chemical Processing Division, ARH-1061, Atlantic Richfield Hanford Company, Richland, Washington.

Uebelacker, D.I., January, 1969- December 1969, Waste Status Summary - Chemical Processing Division, ARH-1200, Atlantic Richfield Hanford Company, Richland, Washington. 
Uebelacker, D.I., January, 1.970- December 1970, Waste Status Summary - Chemical Processing Division, ARH-1666, Atlantic Richfield Hanford Company, Richland, Washington.

Vail, Terry S., January, 1985- Maìch 1986, Waste Status Summary, RHO-RE-SR-14, Rockwell Hanford Operations; Richland, Washington.

Wagoner, J.D., March 18, 1993, Wyden Request, Document No. 9301156B R3, U.S. Department of Energy, Richland, Washington.

Waite, J.I., April 1991, Tank Wastes Discharged Directly to the Soil at the Hanford Site, WHC-MR-227, Westinghouse Hanford Company, Richland, Washington.

Welty, R. K., September 1988, Waste Storage Tank Status and Leak Detection Criteria, WHC-SD-WM-TI-356, Westinghouse Hanford Company, Vol. 1 and 2, Richland, Washington.

Welty, R.K. and Norma J. Vermeulen, September 12, 1989 with ECNs through November 08, 1993, Waste Storage Tank Status and Leak Detection Criteria, WHC-SD-WM-TI-357, Rev. 1K, Westinghouse Hanford Company, Richland, Washington.

WHC, September, 1991, Failure to Continuously Man Control Room During Transfer Per Procedure, Occurrence Report RL--WHC--Tank Farm-1991-1019, Westinghouse Hanford Company, Richland, Washington.

WHC, July, 1991, High Radiation Readings in 241-BY Drywells, Occurrence Report RL--WHC-Tank Farm-1991-1022, Westinghouse Hanford Company, Richland, Washington.

WHC, August 1993, Hanford Site Tank Farm Facilities Interim Safety Basis, WHC-SD-WM-ISB-001 Rev. 1, Volume 1 and Volume 2., Westinghouse Hanford Company,

WHC, March, 1993, Hanford Watchlist Tank Surveillance Trends, Letter to the Honorable Ron Wyden, Westinghouse Hanford Company, Richland, Washington.

WHC, July, 1993, Unauthorized Water Usage at 241-BY Tank Farm Results in Exceeding Raw Water Operational Limit, Occurrence Report RI--WHC-Tank Farm-1993-0050, Westinghouse Hanford Company, Richland, Washington.

Winters, W.I., I. Jensen, L.M. Sasaki, R.I. Weiss, J.F. Keller, A.J. Schmidt, and M.G. Woodruff, May 1989, Waste Characterization Plan for the Hanford Site Single-Shell Tanks, WHC-EP-0210, Westinghouse Hanford Company, Richland, Washington. 


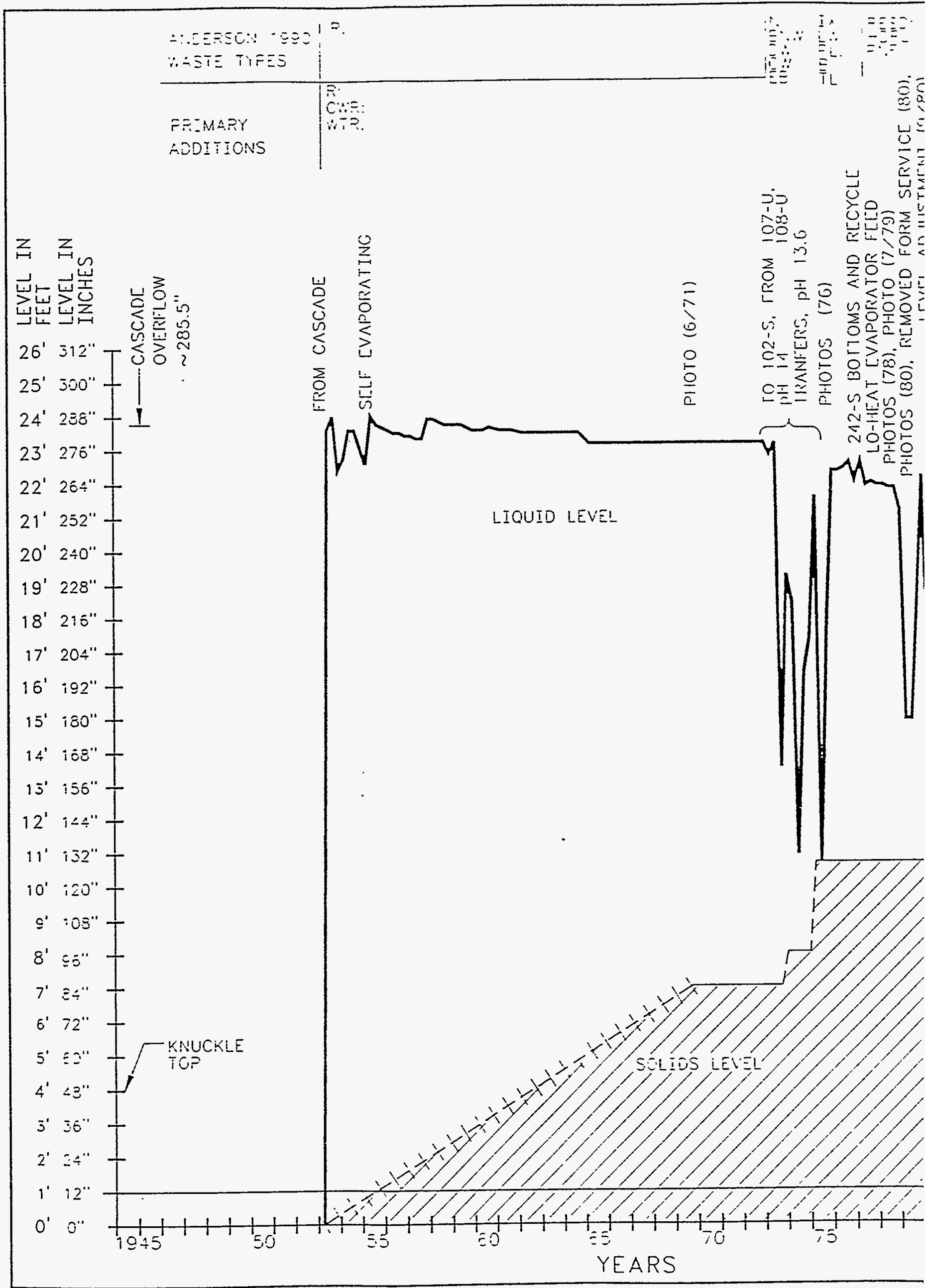




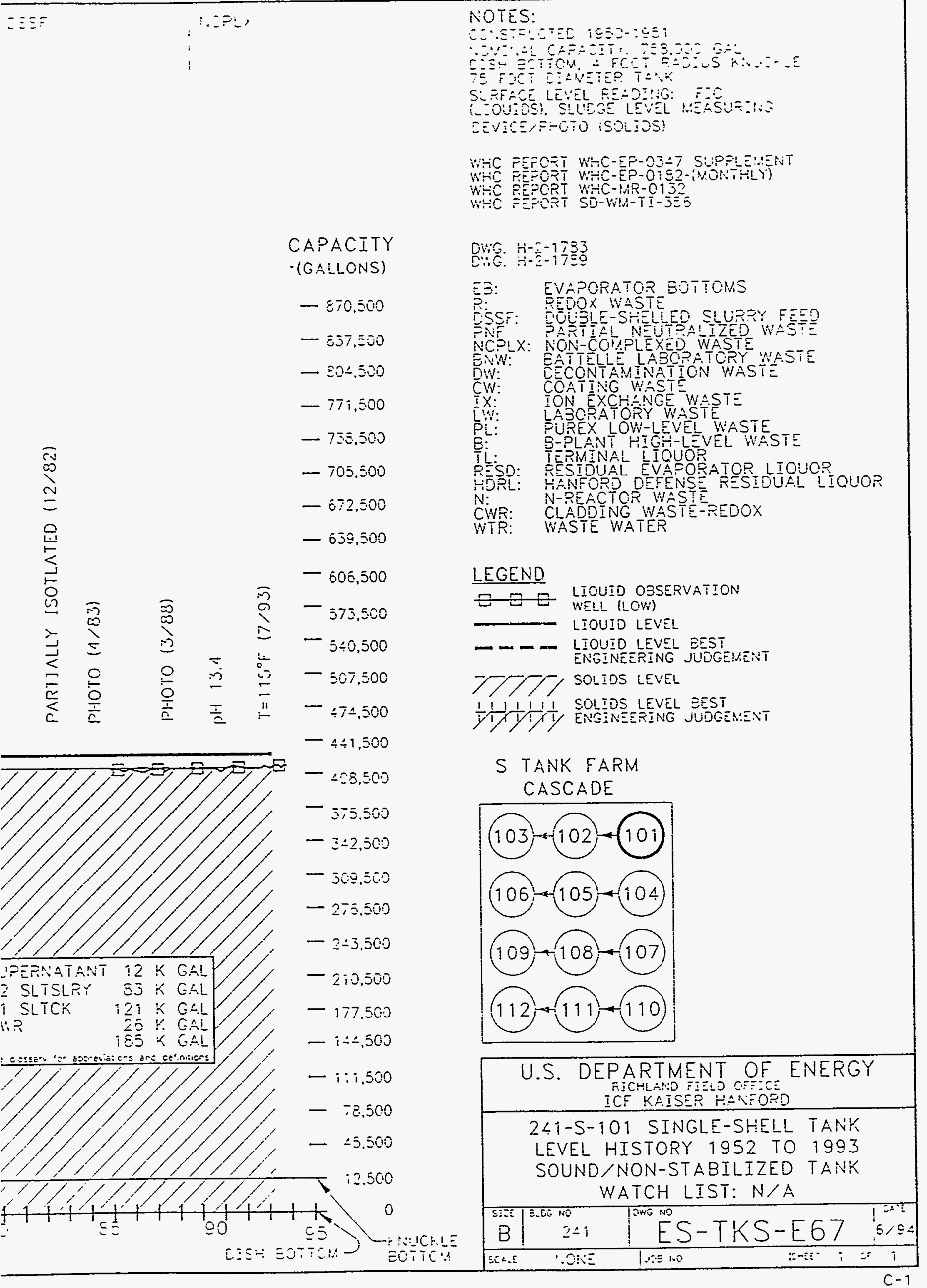




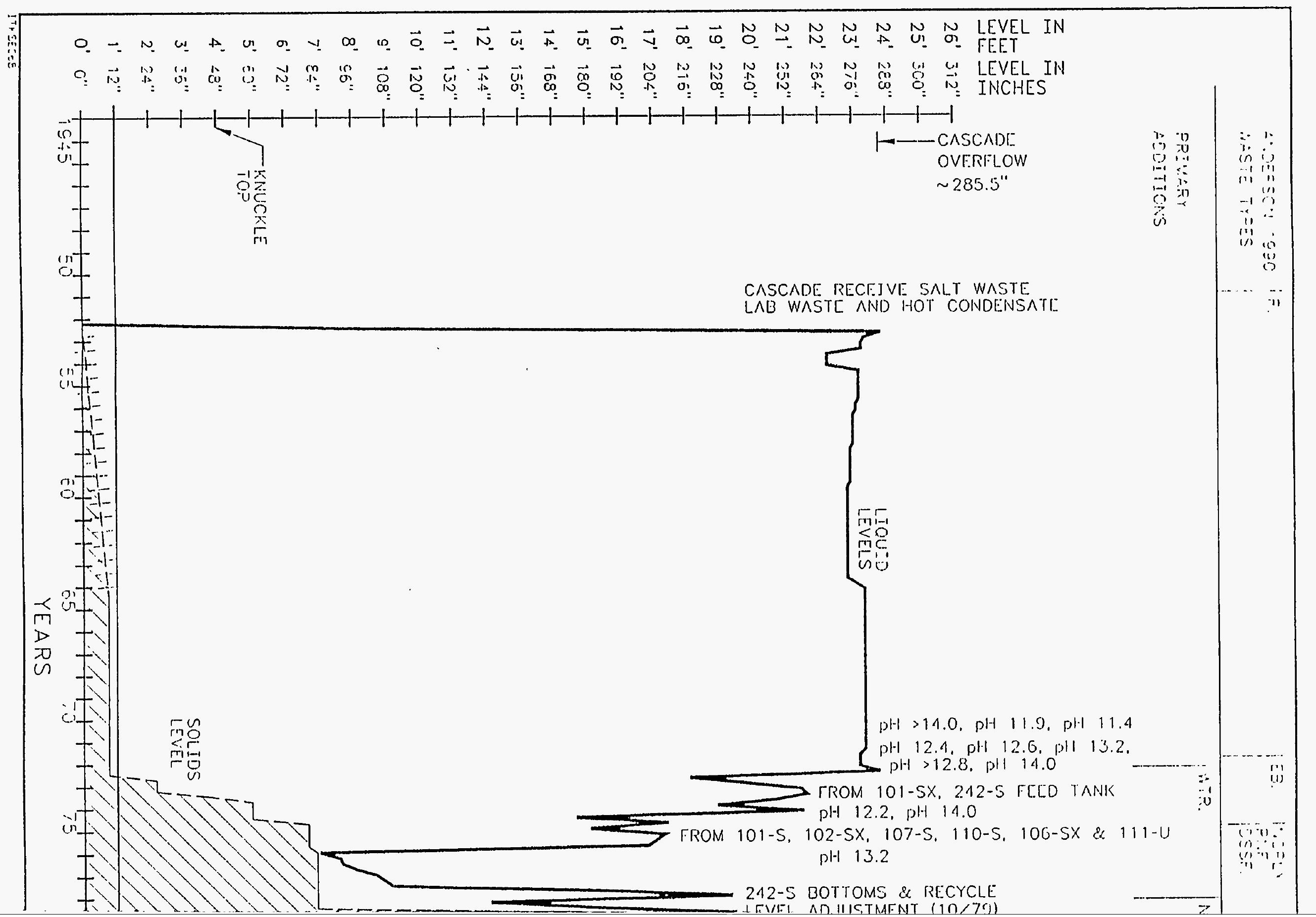




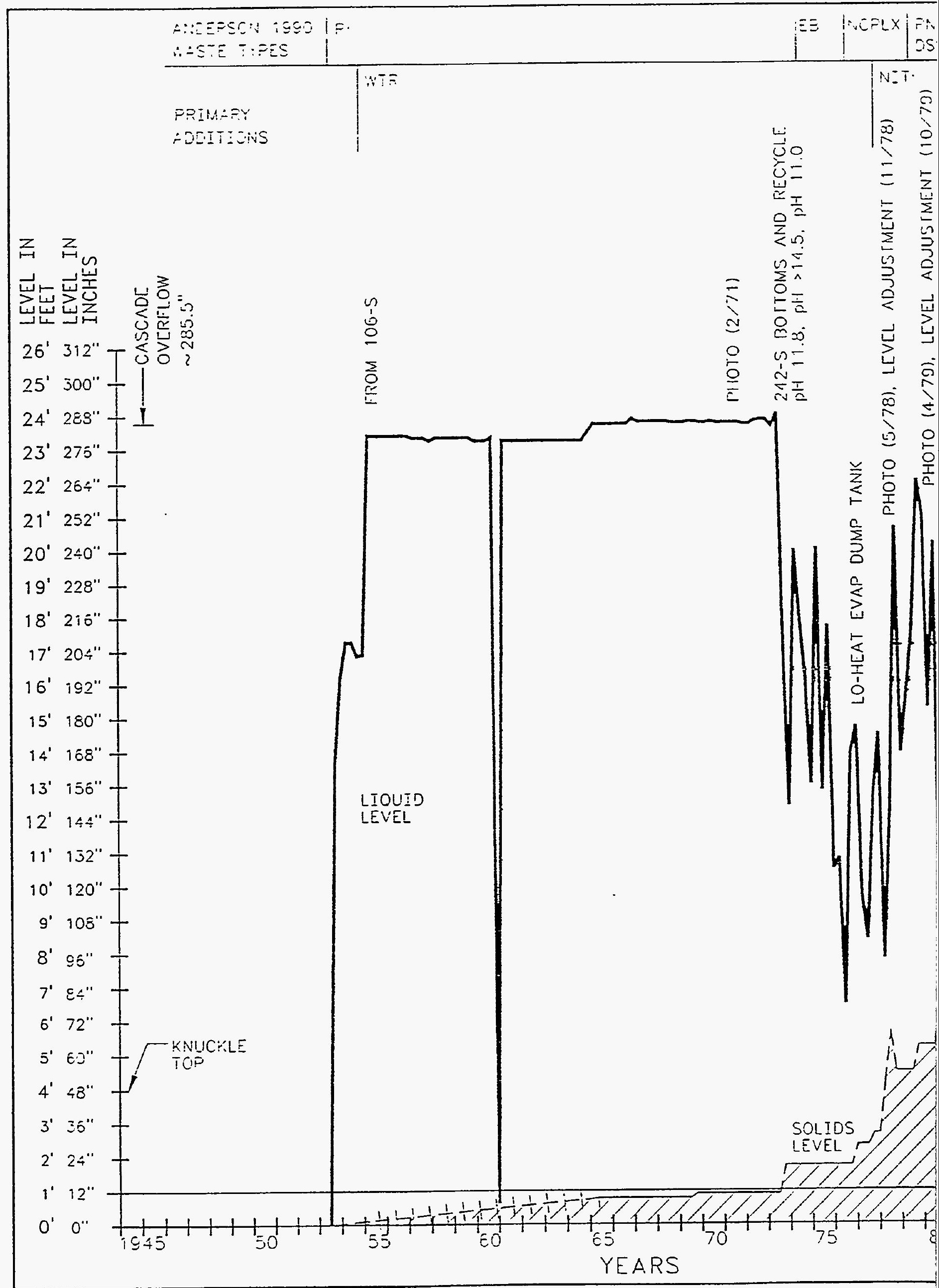




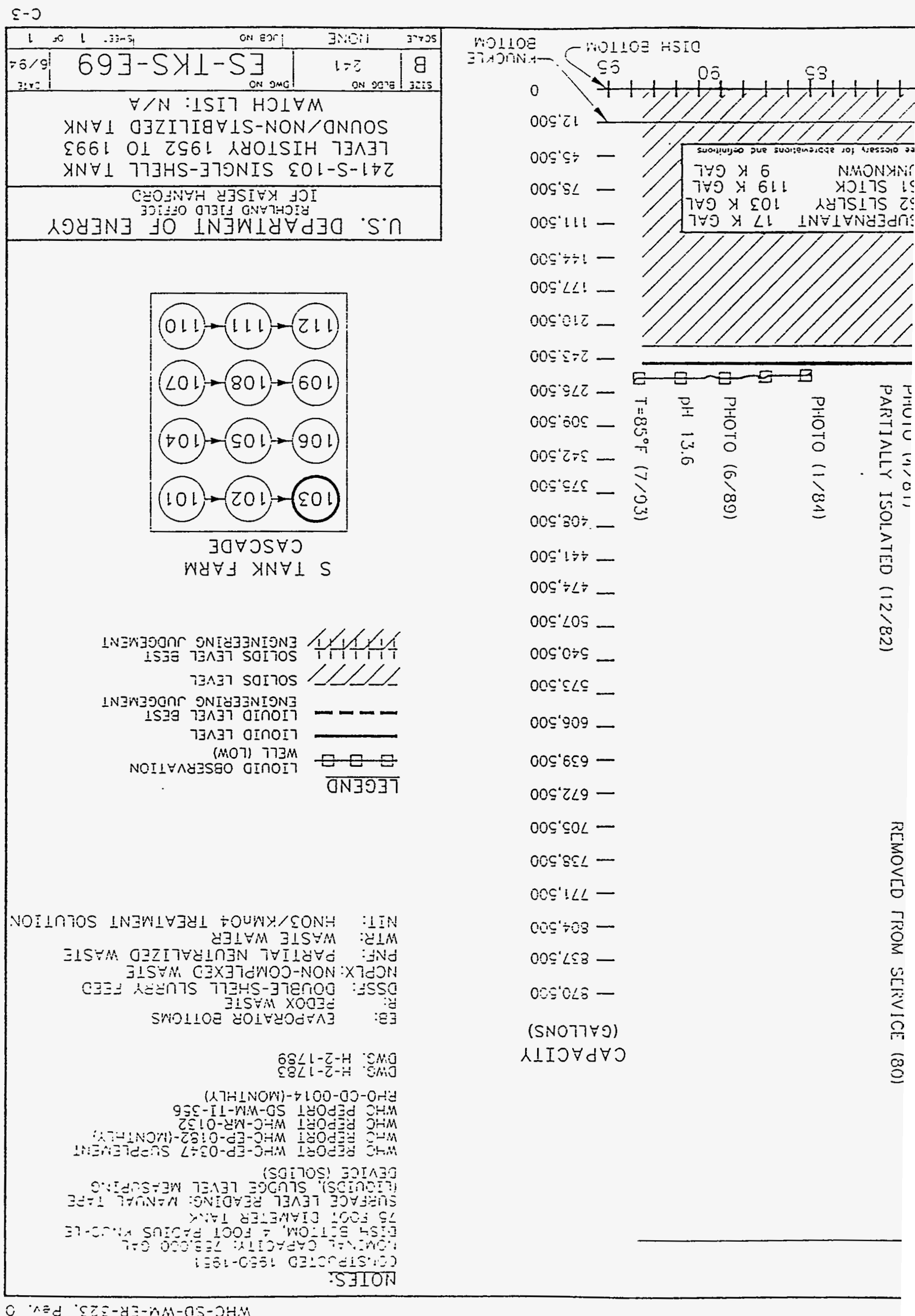




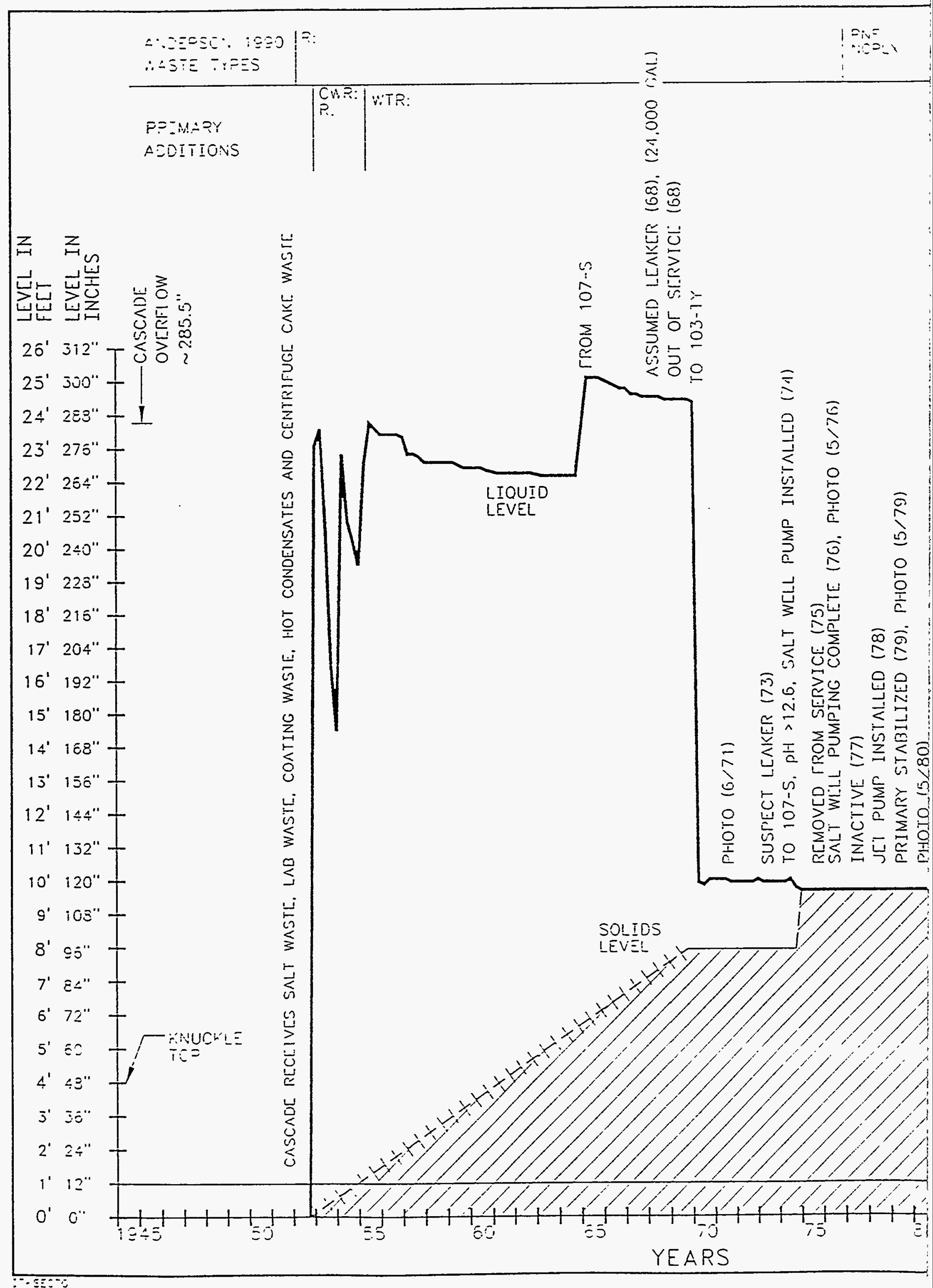


CAPACITY

E्ज

0

ㄴ.

$\stackrel{-}{\rightleftarrows}$

$\stackrel{c}{\leq}$

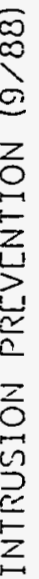

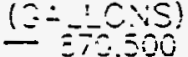

$-\overline{85}, 500$

- $\equiv 5-500$

$-77: 500$

$-75.500$

$-705,500$

- 072.500

$-05 \$ .500$

$-005.500$

- 575.500

$-5 \div 0.500$

$-507.500$

- $4 \div 500$

$\overline{0}-41.500$

$=-23.500$

$\stackrel{5}{\circ}-5: 5.500$

$11-3 \div 2,500$

$-308.500$

- 2?:500

$-245,550$

$-2: 2.500$

$-177.500$

$-i \div 500$

$-1 \cdot .5 x$

$-\quad-5.500$

- -5.500

$: 2.500$

GA

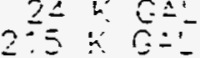

NOTES:

CONSTOUCTED :OSO-; EJ

MOA.

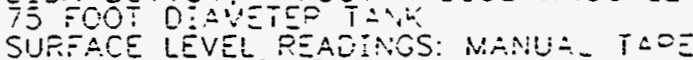

(IBUIDS ANO SOLIOS)

WHC PEPGRT WHC-EP-0jA7 SUPPLENENT

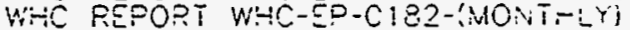

WHC REPORI W:IC-MR-O1J2

WHC PEPORT SD-WM-TI-350

P.HO-CD-0014-(MONTHLYY)

OWG $\mathrm{H}-2-1783$

OWG. $4-2-1789$

R: REDOX WASTE

PNF: PARTIAL NEUTRALIZED WASTE

NCPIX: NON-COMPLEXED WASTE

CWP: CLADDING WASTE-PEDOX

WTP: WASTE WATE?

\section{LEGEND}

C LEQUED OESERVATION

WELL LOW:

- - - LIQUDD LEVEL EESI ENGINEEPING JUDGENENT

77 SOLIOS LEVEL

I 1111 SOLIOS LEVEL EEST

Y $Y$ Y $T$ I ENGINEERING JUDGEMENT

$S$ TANK FARM

CASCADE

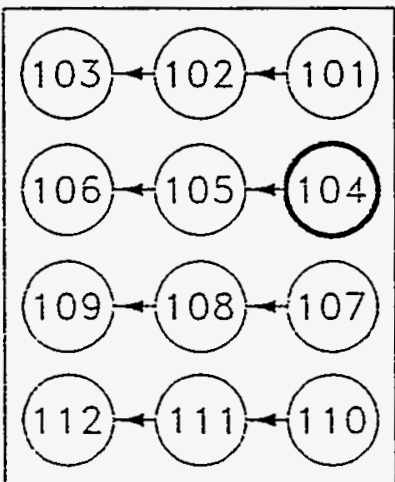

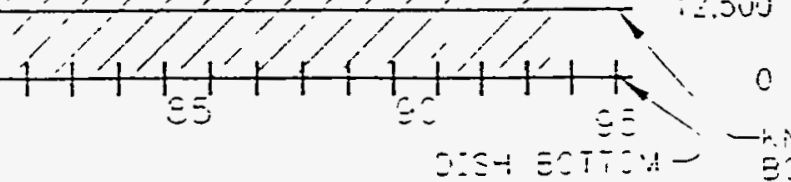

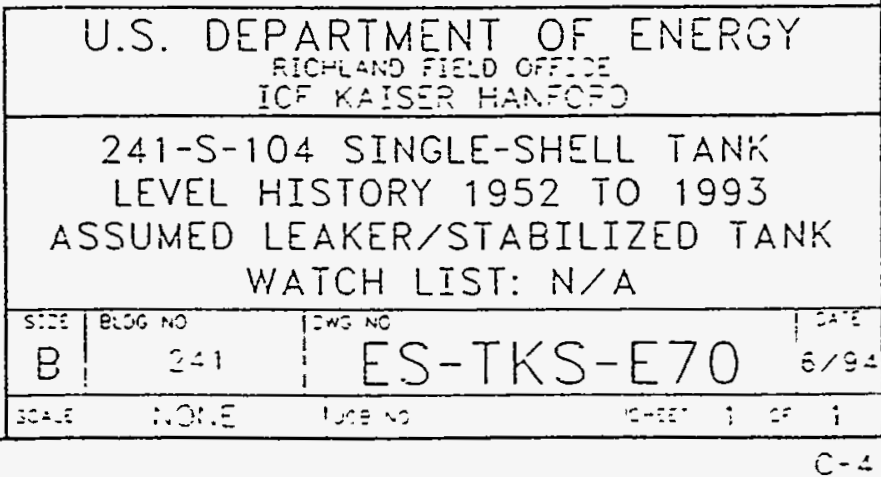




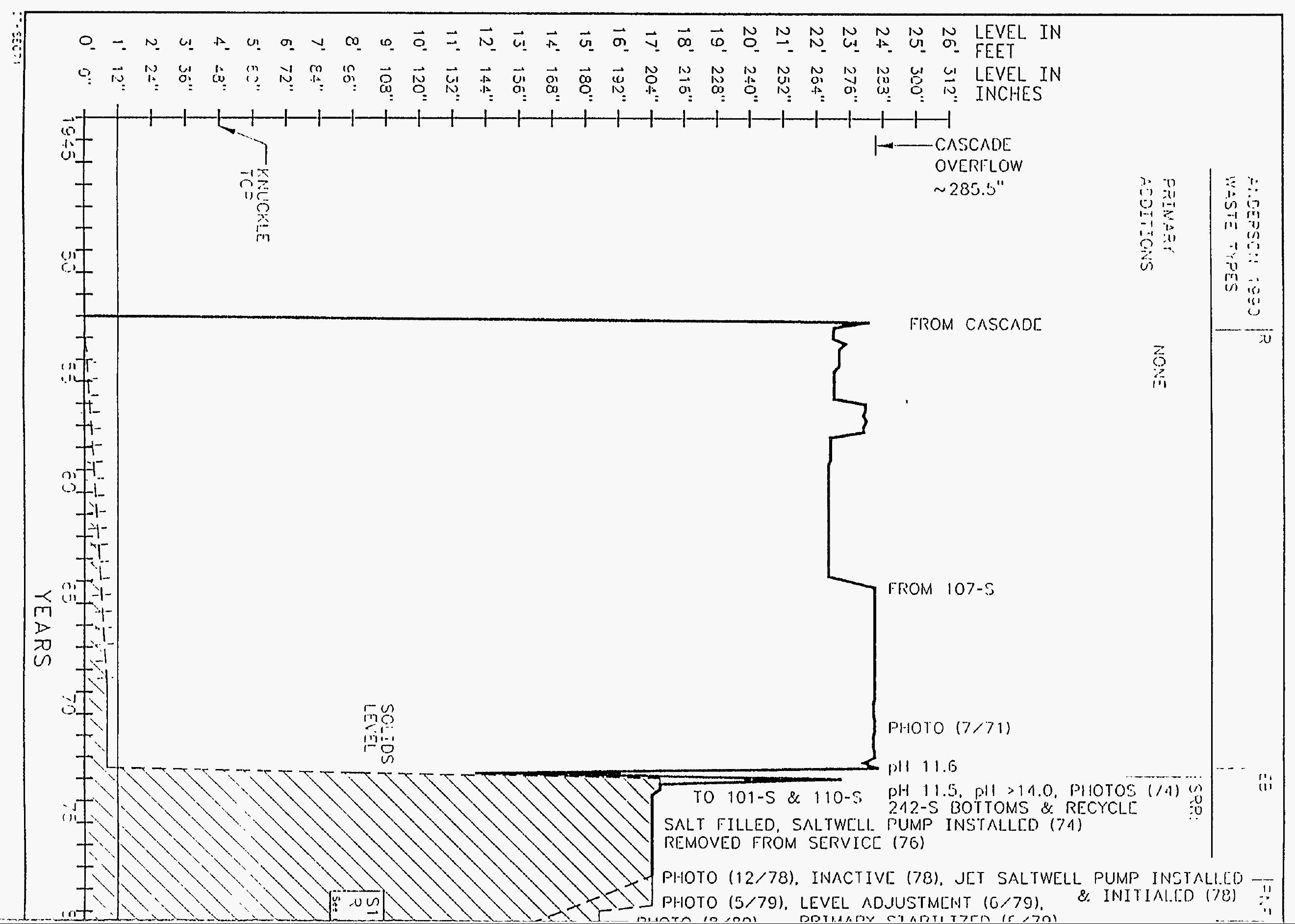




\begin{tabular}{|c|c|c|}
\hline$!$ & $\therefore \quad ! \quad \therefore=5$ & $\Xi \because \because$ \\
\hline $\begin{array}{ll}\therefore<\rho \\
\therefore=1\end{array}$ & $1 \angle 3-S H 1-S]_{x_{0}=1}$ & $==8$ \\
\hline & 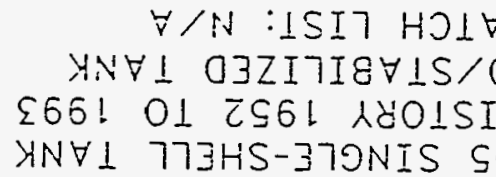 & $\begin{array}{l}705 \\
73 \wedge 37 \\
-S-127\end{array}$ \\
\hline & OdJNJ 30 INGNLUV & $0 . s^{\circ}$ \\
\hline
\end{tabular}

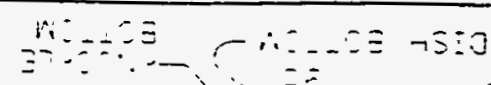

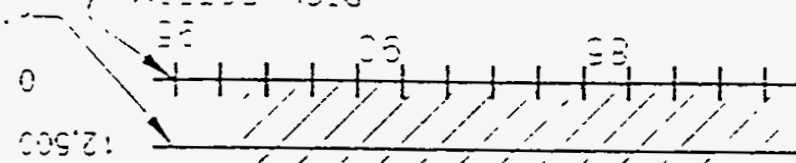

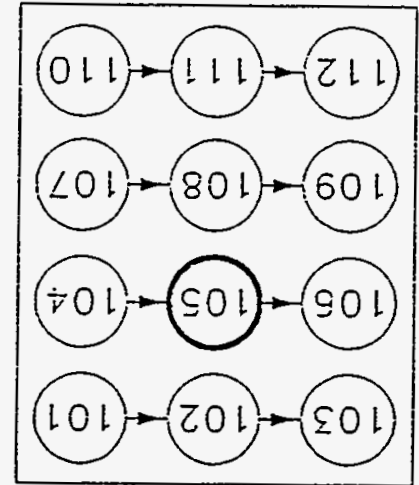

JOZOS $\forall 0$

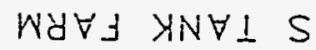

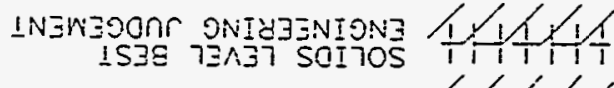

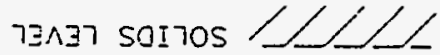
INENEOOR ONIDZZNIONZ

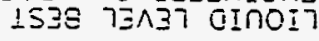

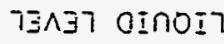
(MO) 7 Tin NC:LYAEZSEO CEกOIT

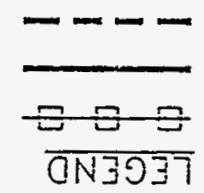

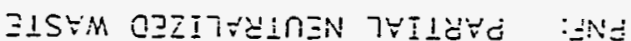

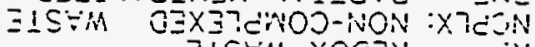

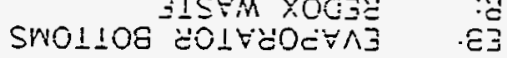

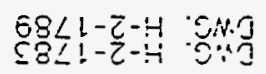

(MTHINON)--100-00-0H: SES-II-NM-OS ICOCJO OHM

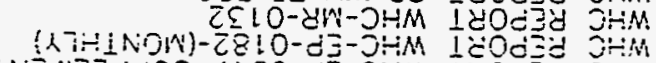

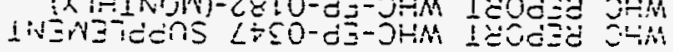
iscitosi

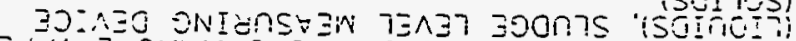

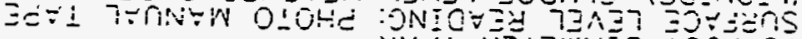
20 z-4an sn:

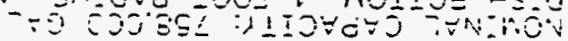

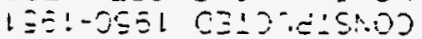
SIION ras:

osc $\subseteq=-$ ojs $\Xi-$

$\cos : 1:-$

[.:-

$\cos : 12:-$

ascoz-

แกะระ๐-

$0090: 2-$

cos'ลละ -

$\cos 6 \div 5$

ocs' $\subseteq:=$

$\cos 8: \div$

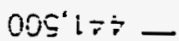

$00 S^{\prime} \bar{v} \angle \dot{r}$

OOS'LCS$0050 \div 5$

$009 \subseteq 1=$

$005 \cos$

oos'Es? -

$005210-$

oos's: -

OOS:SE -

$\cos ^{\circ}: 11-$

$005^{\circ} \div 5-2$

$00 \subseteq \angle \Sigma \Xi-$

on: $:=-$
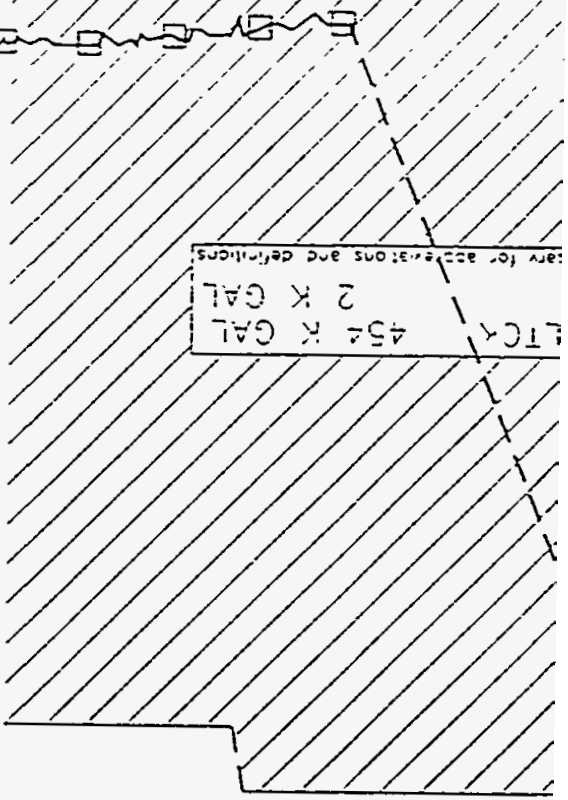

(SNOTIYO).

$\lambda \perp I O \forall d \forall O$

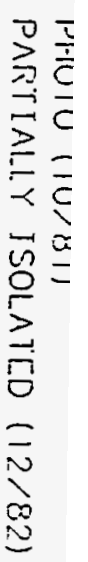

$\frac{\mathrm{D}}{\mathrm{S}}$

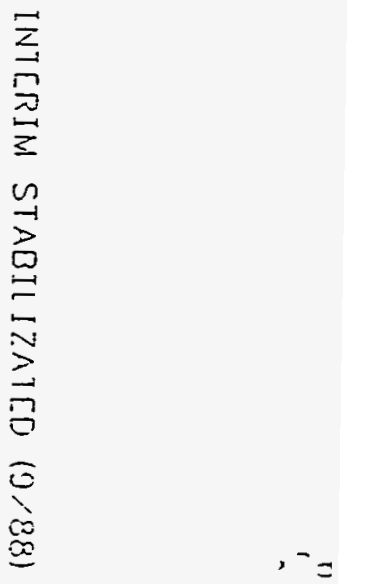




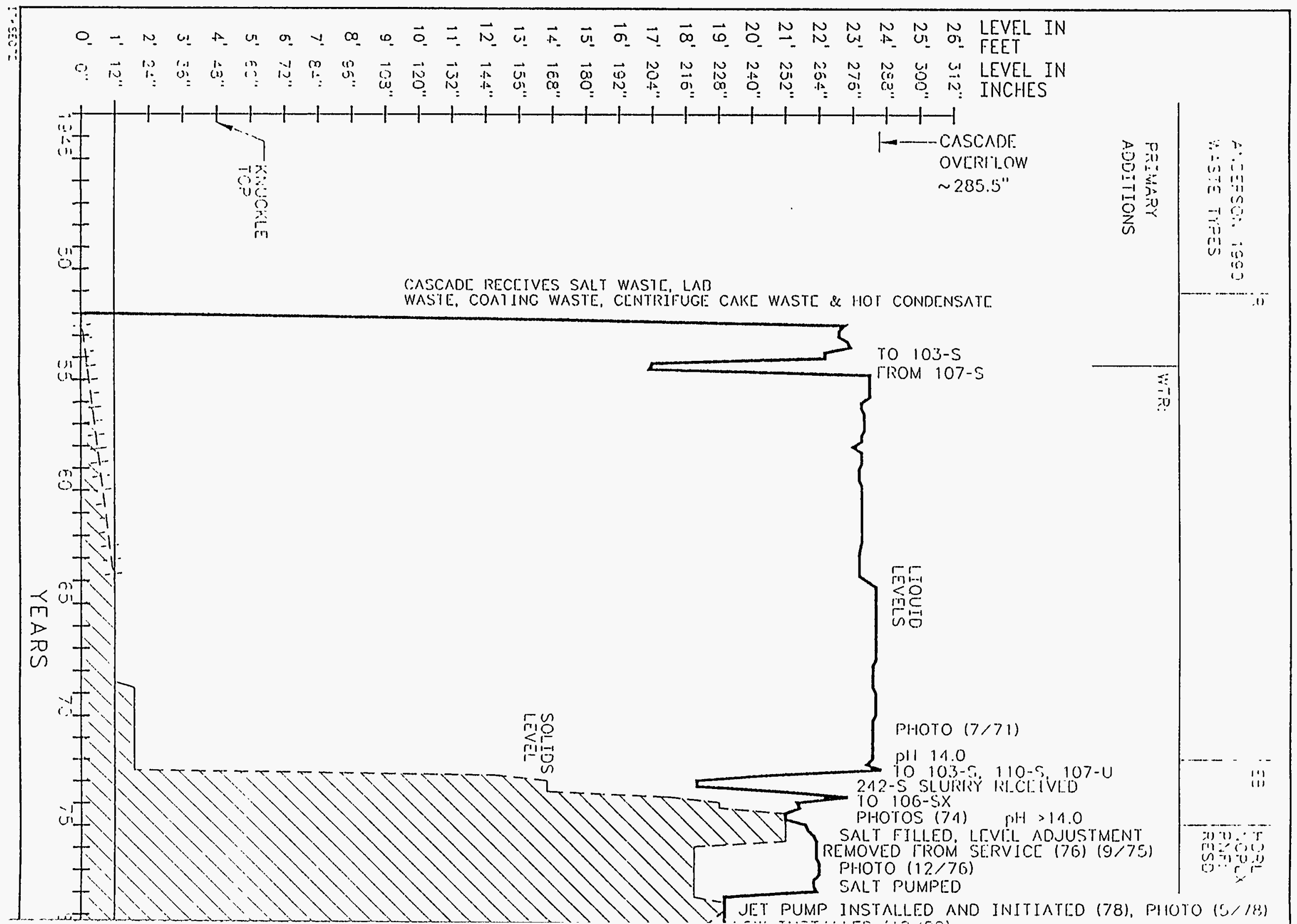




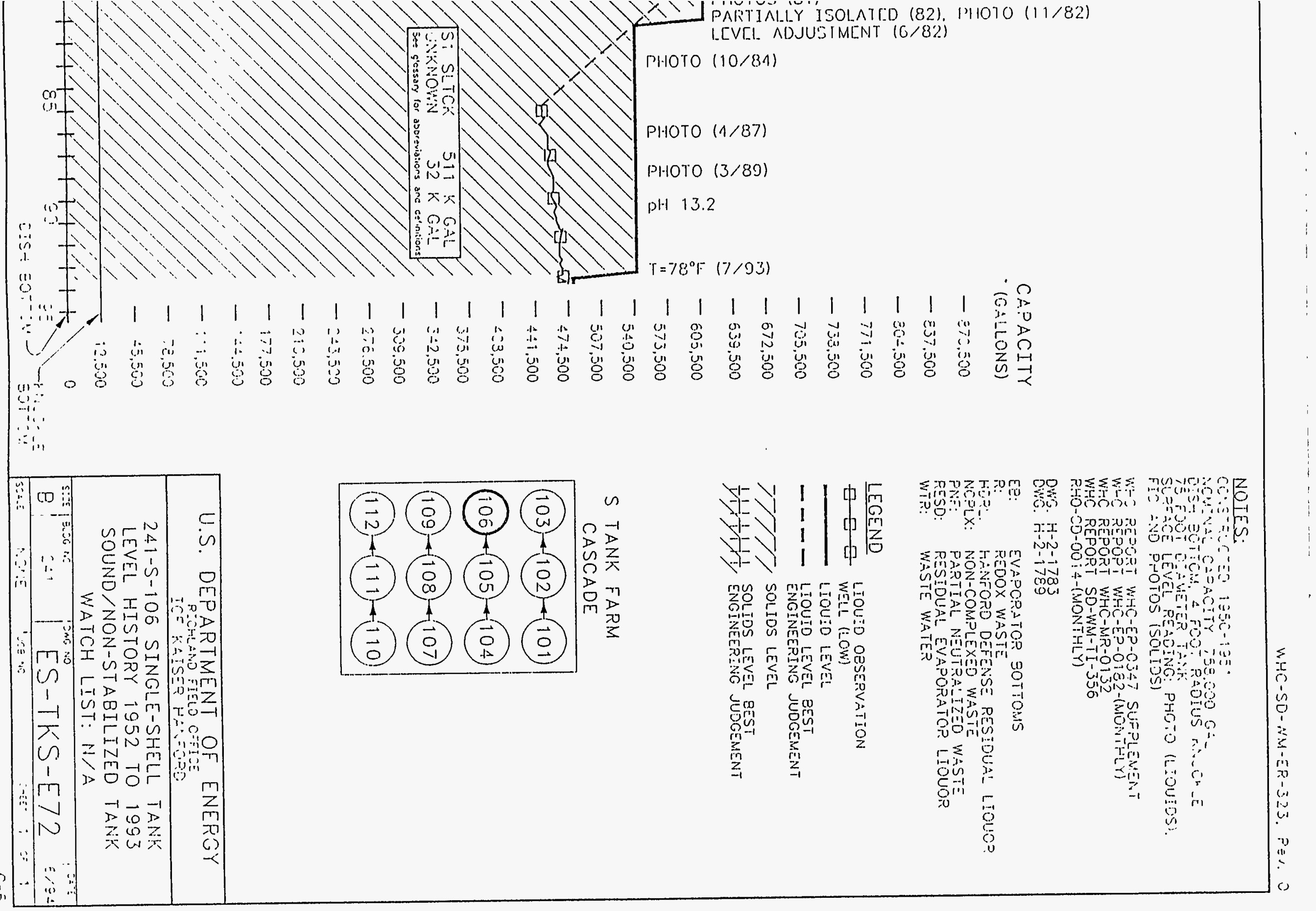




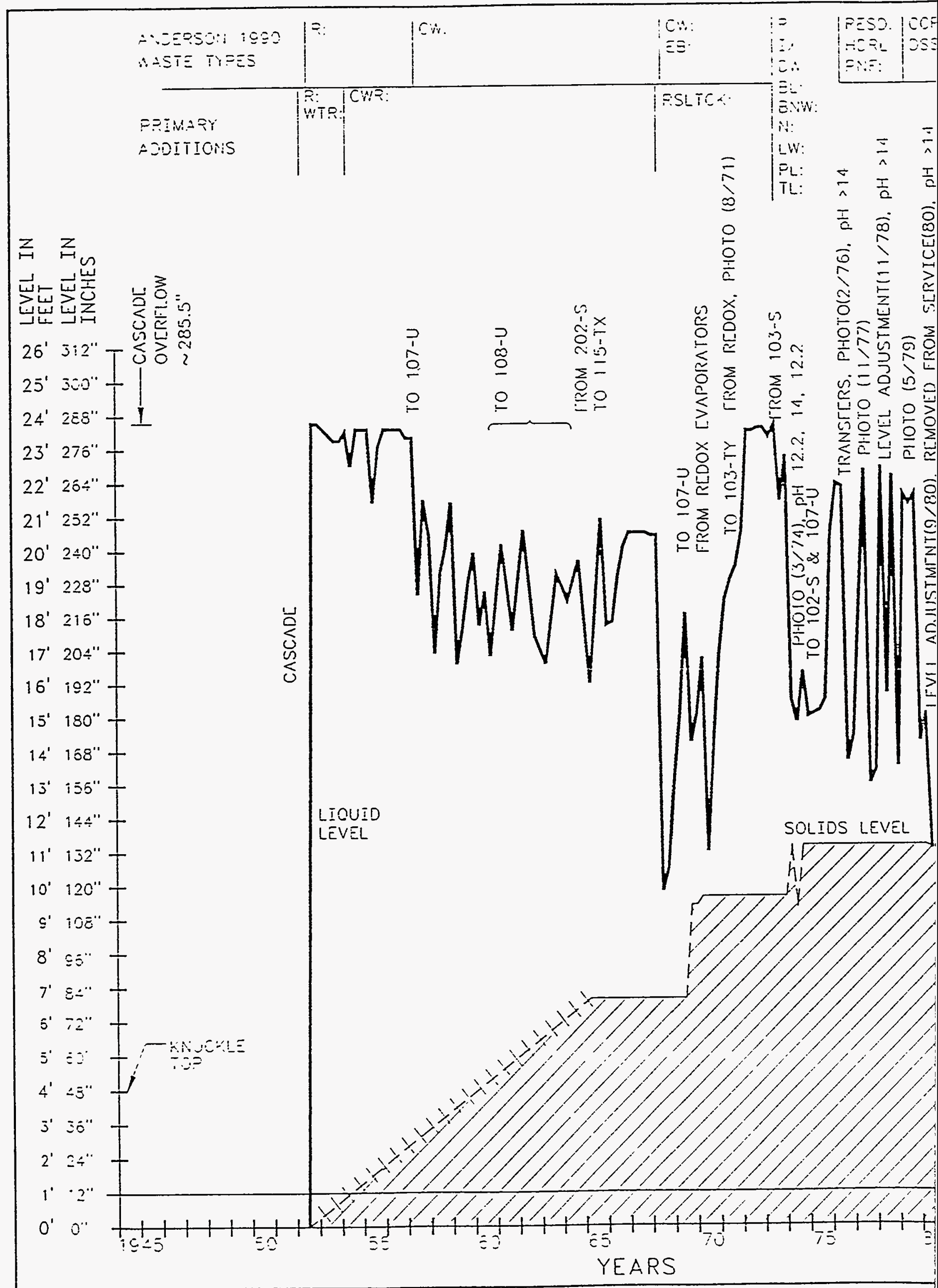




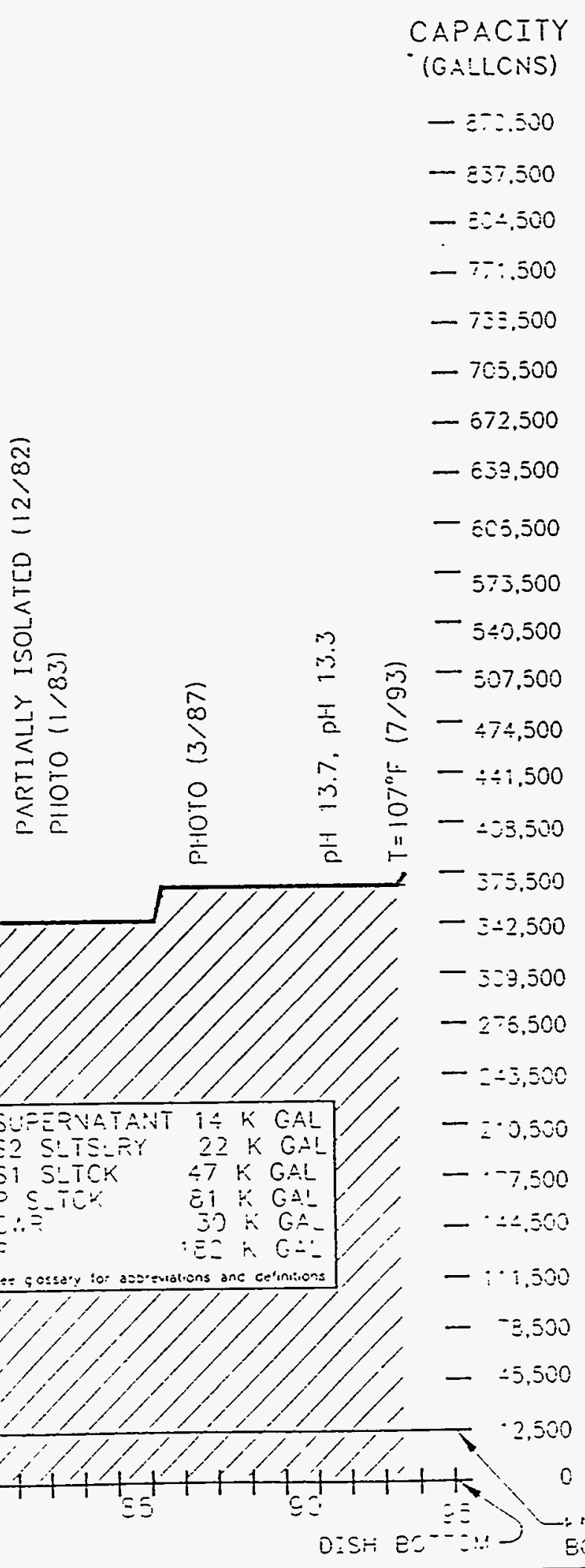

CAPACITY

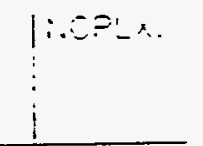

NOIES:

CCRSTSUCTED iO50- $i \leqq \equiv i$

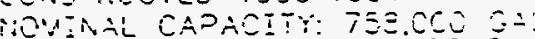

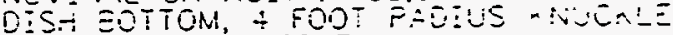

SUP FOCE LEVEL PEACING FIC AIOUIDS!.

SLLOGE LEVL MEASUPE OEVICE ANOD

bLiUTOS (SCLIOS)

WHAC FEPORT WHC-EP-OJA7 SUPPLEMENT

WHC REPORT WHC-EP-OIE2-(NONTHLY)

WHC REPORT WHC-MR-0132

WHC REPORT SO-WU-TI-J50

PHO-CD-CO I 4-(MONTHLY)

CWW.

OWG. $H-2-1789$

ES: EVAPORATOR EOTTOMS

R: REDOX WASTE

HOR!: LANEORD DEFENSE RESIOUAL LIOUOR

NCPLX: NON-COMPIEXED WIASTE

PIF: PARTIAL NEUTRALIZEO WASTE

RESO: RESIDUAL EVAPORATOR LIOUOR

CW: CORIING WASTE

IX: ION EXCHANGE WASTE

CW: CECONTAMINATION WASTE

?!: E-FIANT LOW-LEVEL WASTE

ENW: SATTELLE LAS WASTE

N: N-PEACTOR WASTE

IW: LAEORATCRY WASTE

PL: PUREX LOW-LEVEL WASTE

TL: TERMINA! LIQUOP

CCPLX: COMPIEXANT CONCENTRATE

DSSF: DOUBLE-SHELL SLURRY FEED

WIR: WASTE WATER

CWR: CLADDING WASTE-REDOX

RSLTCK: REDOX SALTCAKE WASTS

\section{LEGEND \\ $=$ LIQUID OBSERVATION \\ - WELL (LOW) \\ - - - LIOUIO LEVEL BEST \\ ENGINEERTiNO JUOGEMENT \\ $77 /$ SOLIOS LEVEL \\ LH H I SOLIDS LEVEL EEST \\ DTY T/, ENGINEERING JUDGEMENT}

S TANK FARM CASCADE

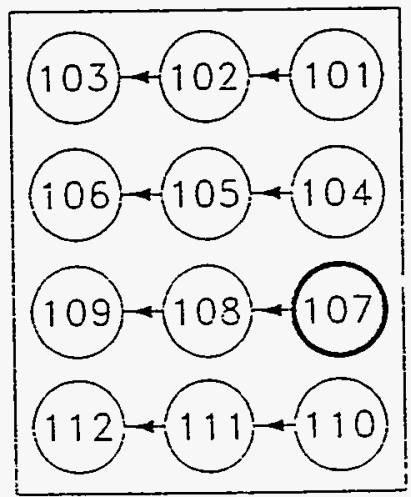

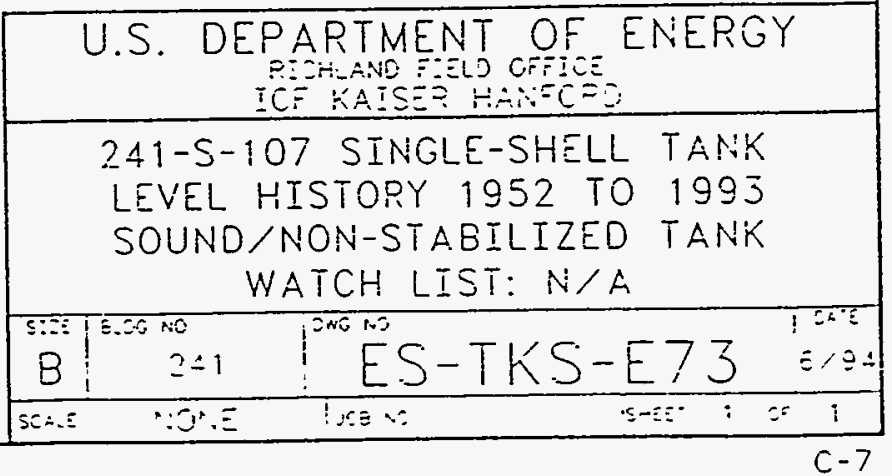




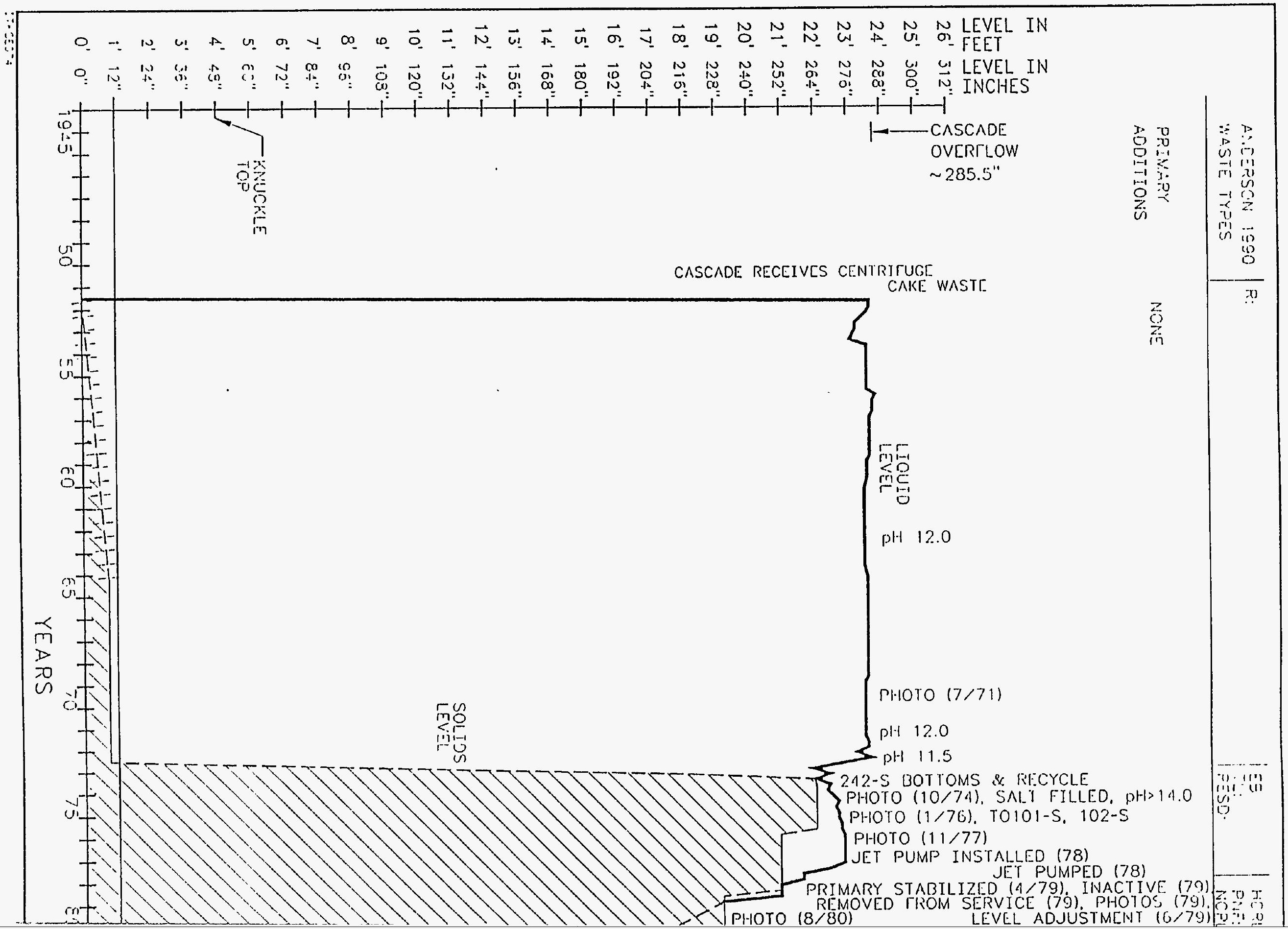




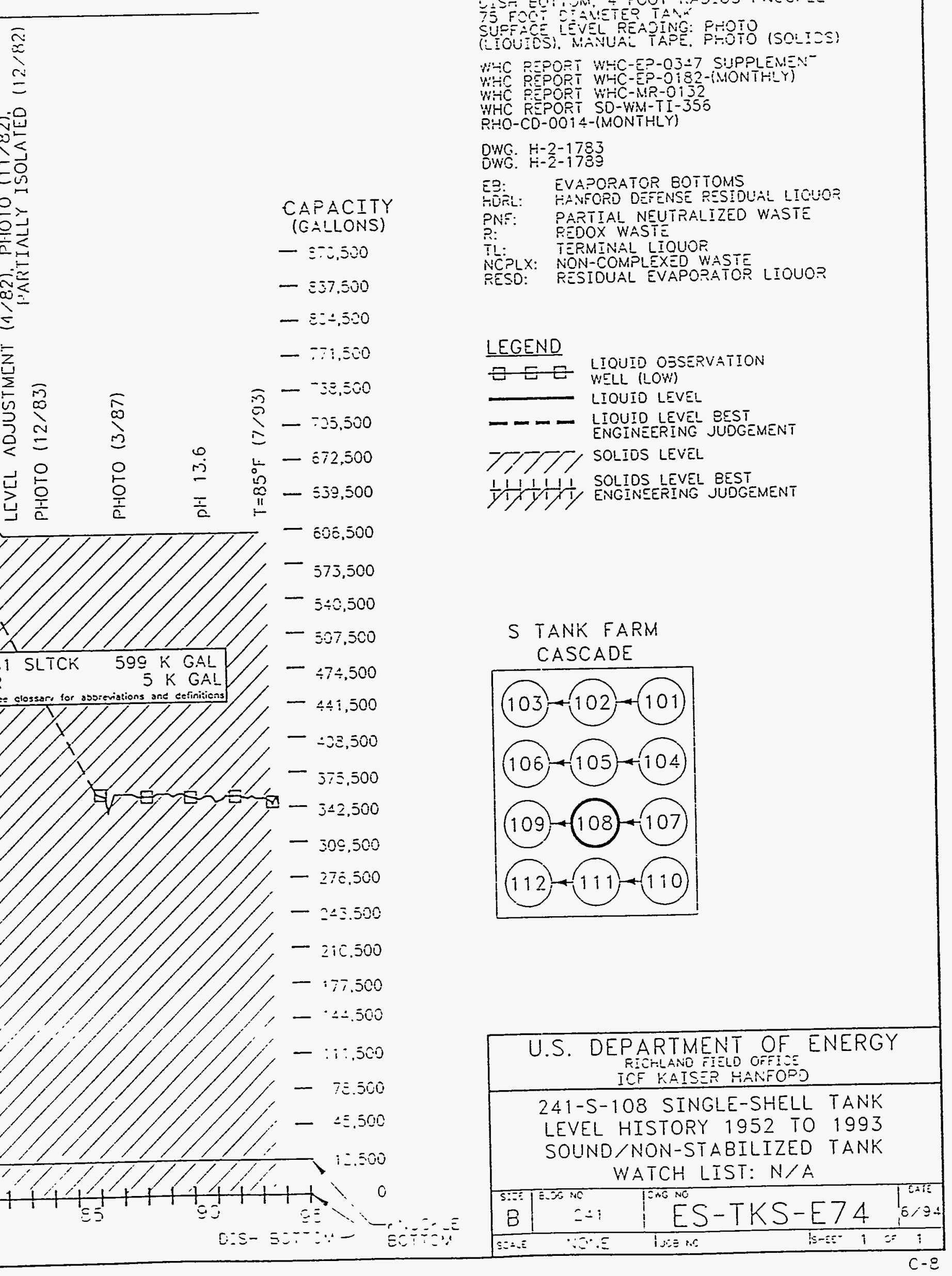

VOTES:

COI.STFUCTEO $1950-1951$

Or.

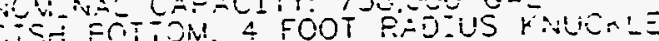

5 FOCI $T A M ! E T E O$ TANM

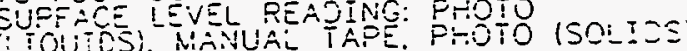

M-C EEPORT WLC-EP-OJ47 SUPPLENEN

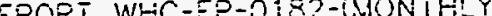

WHC PEPORT WHC-MR-O IJ2

RHO-CO-OOIA-IMONTHLY)

DWG $\mathrm{H}-2-1783$

OWG. H-2-1733

E3. EVAPORATGR EOTTOMS

HOF. HAVORO DEFENSE RESIDUAL LICUOR

PNF: PARTIAL NEUTRALIZED WASTE

REDOX WASTE

T. TERMINAI LIOUOR

NCPIX: NON-COMPLEXED WASTE

NCPLX: NON-COMPLEXEOPATOP LIOUOP

LEGEND

Z EE WEL (1OW)

- LIOUIO LEVEI

ENGINEERING JUDGEMEN

77 SOLIOS LEVEL

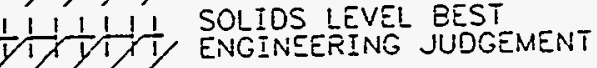

\section{S TANK FARM CASCADE}

U.S. DEPARTMENT OF ENERGY RICHLANO KATL HANFOP

241-S-108 SINGLE-SHELL TANK EVEL HISTORY 1952 TO 1993 SOUND/NON-STABILIZED TANK WATCH LIST: $N / A$

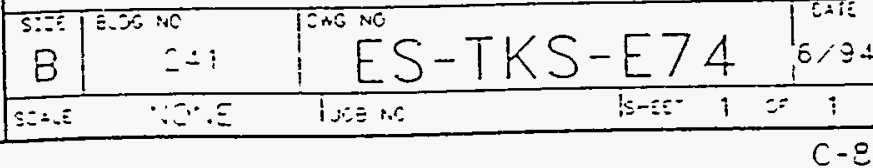




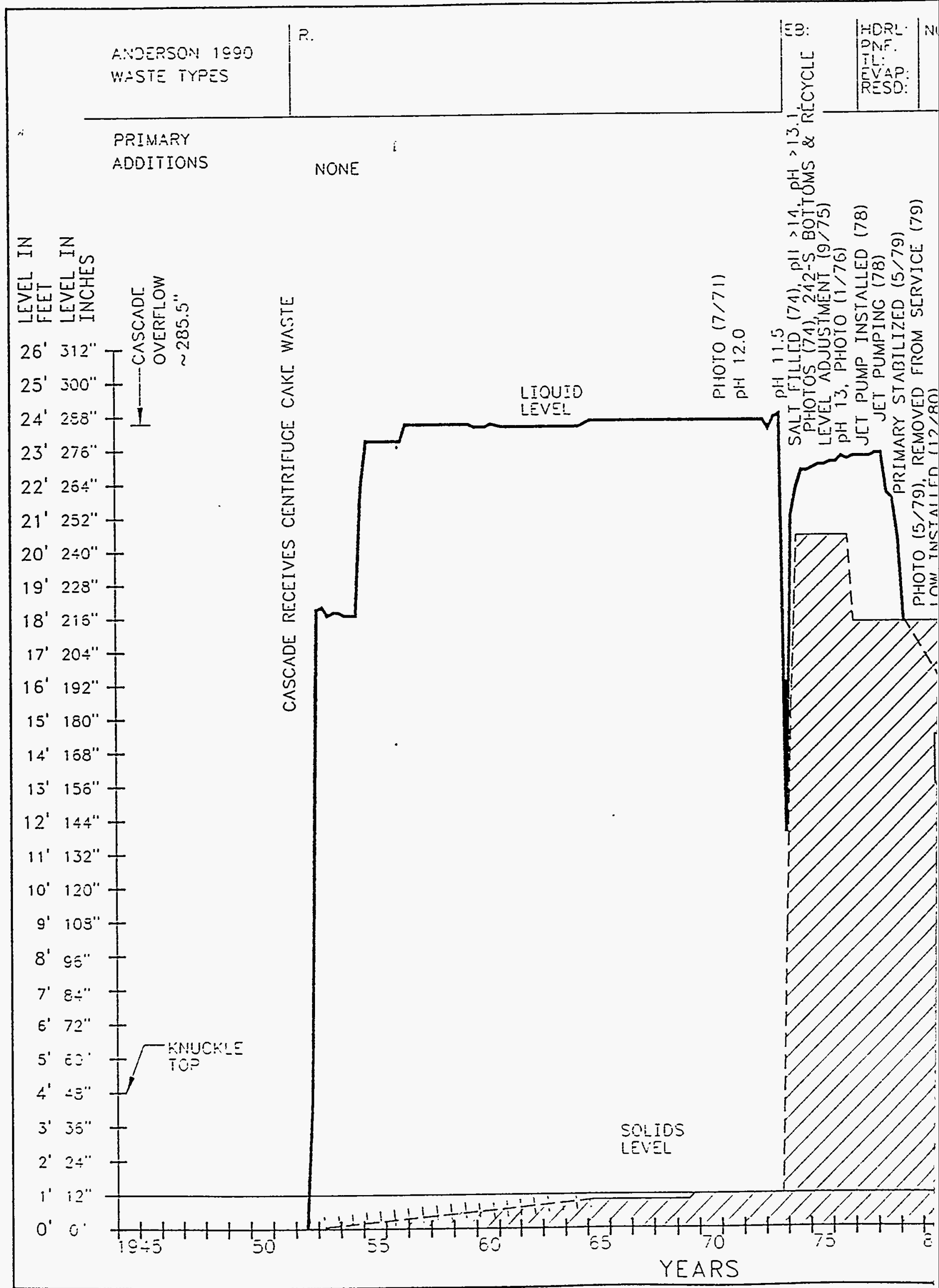




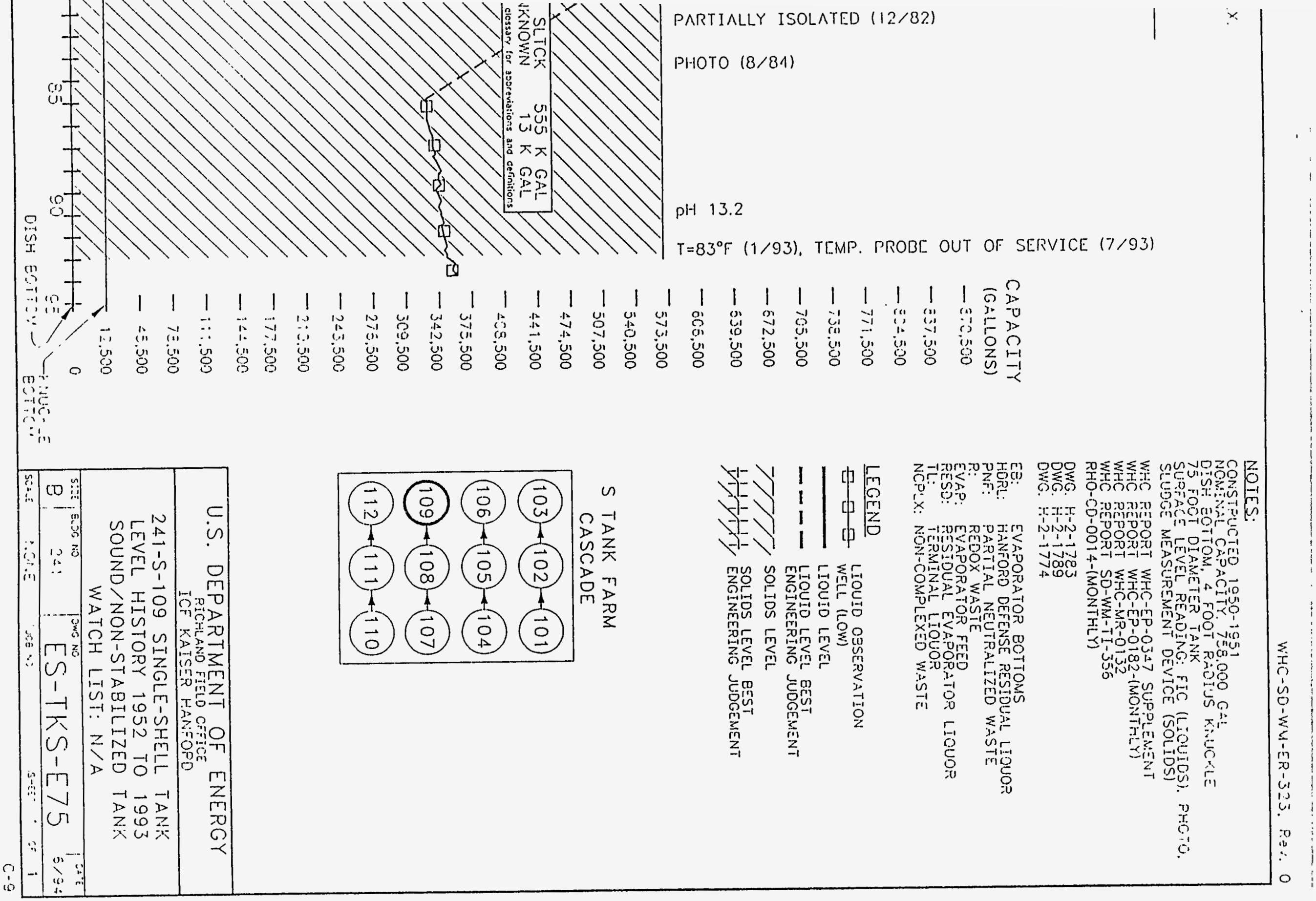




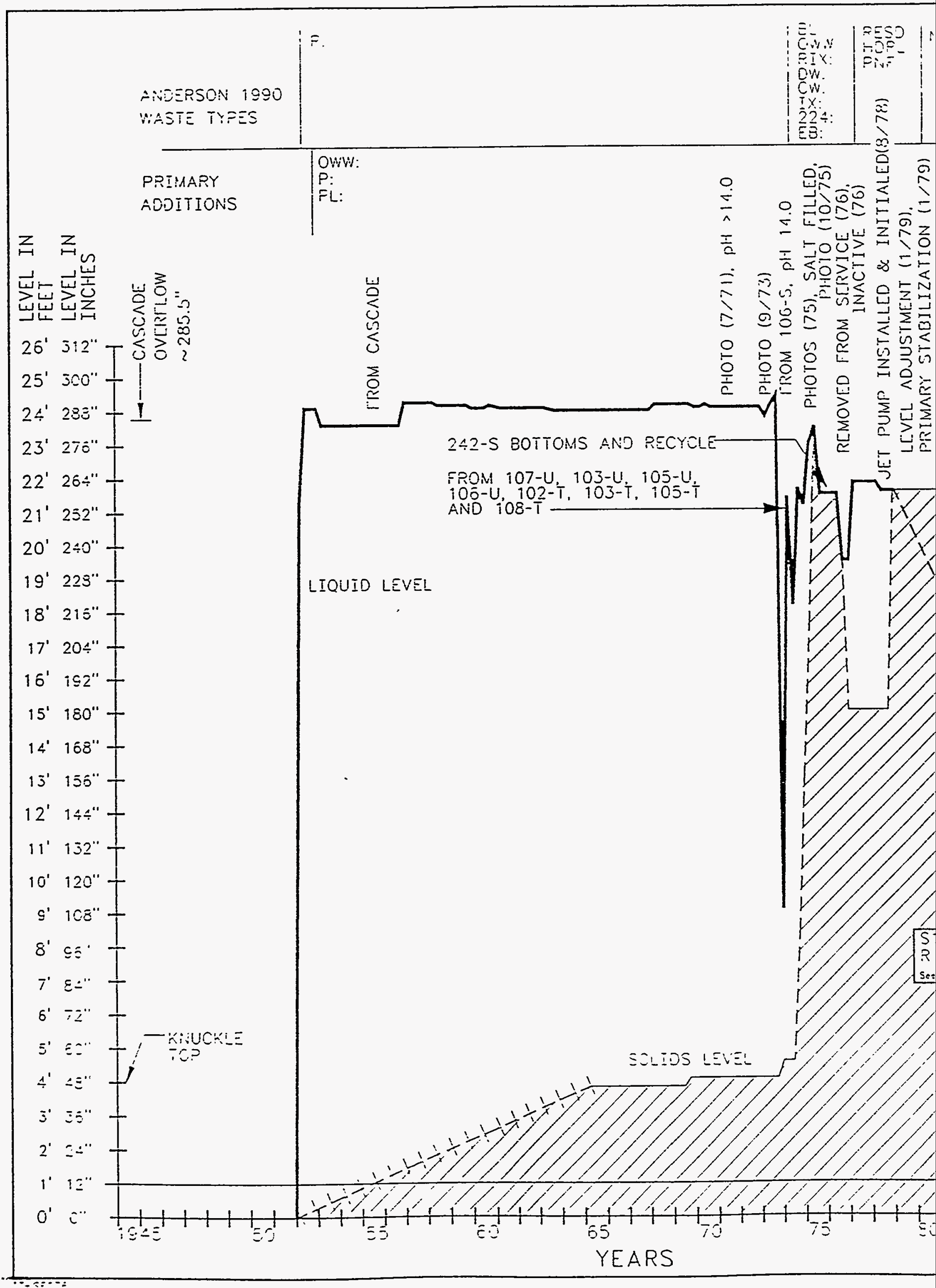




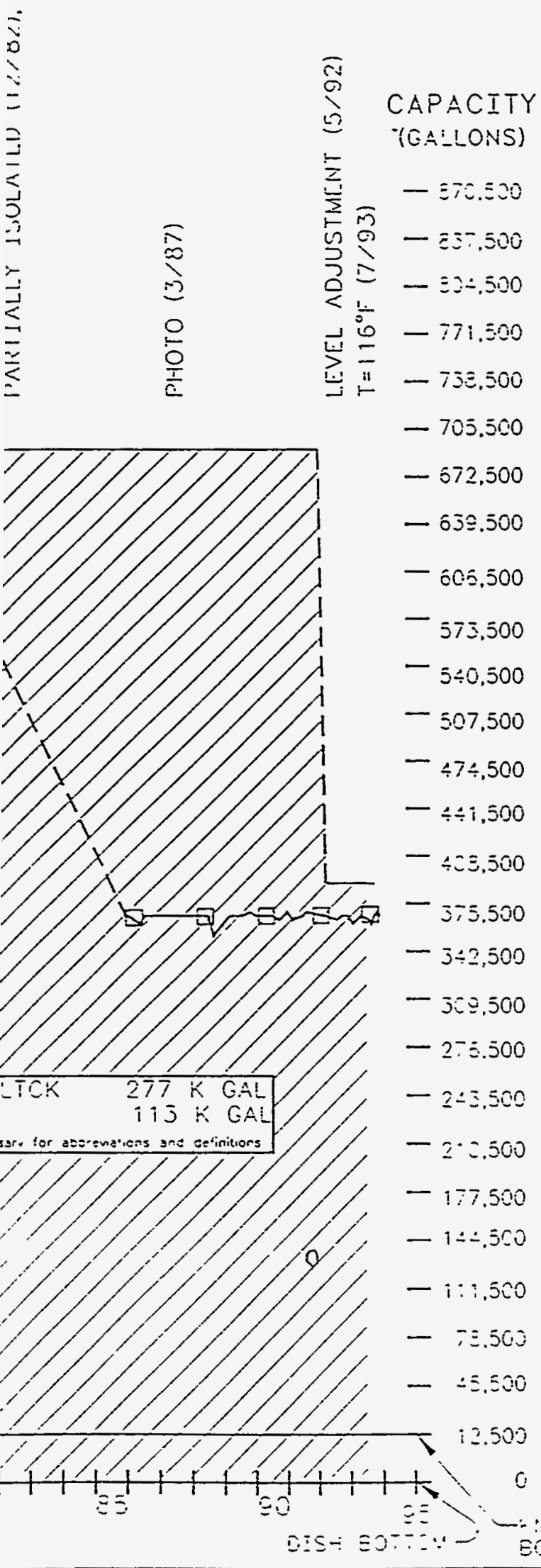

NOTES:

COAST:UC:EJ 1950-ic5i

NOA

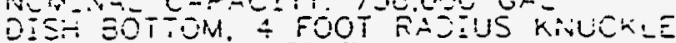
TSFOS DIALETER TAND

SUE=AOE LEVEL PEADING FIC (LIOUTSS!.

SLUOSE LEVEL MEASURING CEVICE ANO

FHOTES (SULIDS)

WHE FEFOET WHC-EP-OJ 77 SUPPLEMENT

WHE EEPOET WHC-EP-OIS2-\{́NONTHLYY

WHiC FEPPORT WHC-MR-0132

WHC PROORT SD-WM-TI-j50

RHO-CO-0O14-(MONTHLY)

DWG. $:-2-1733$

EZ: EVAPORATOR EOTTONS

P: REOOX WASTE

EVAT: EVAPORATOE FEED

HDO:- HANFORO DEEENSE RESIDUAL LICUOR

NCOLX: NON-COMPLEXED WASTE

PNE: PAETIAL NEUTRALIZEO WASTE

8L: B-FLANT LOW-LEVEL WASTE

OWW: CRGANIC WASH VIASTE

REX: REDOX ION EXCHANGE WASTE

DW: OECONTAMINATION WASTE

CW: COATING WASTE

IX: ION EXCHANGE WASTE

22:- 224-U WASTE

PESD: RESIDUAL EVAPORATOR LICUOR

CWR: CLADDING WASTE-REDOX

WTR: WASTE WATER

\section{LEGEND \\ E D LIOUTD OSSERVATION \\ 든 WELL (LOW) \\ LIOUID LEVEL \\ - - LIOUID LEVEL EEST \\ ENGINEERING JUDGEMENT \\ 777 SOLIOS LEVEI \\ 111 III SOLIOS LEVEL EEST \\ I 17 T $1 /$ ENGENEERING JUOGEMENT}

S TANK FARM CASCADE

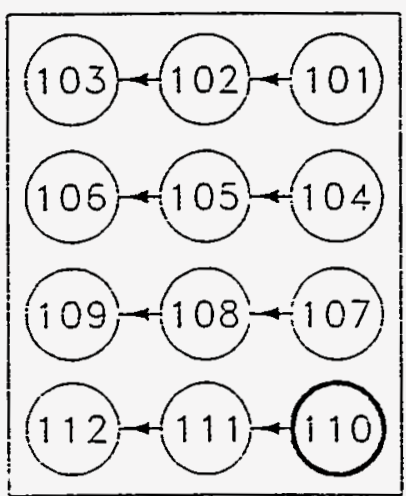

U.S. DEPARTMENT OF ENERGY

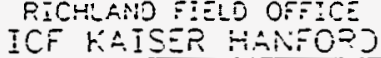

241-S-110 SINGLE-SHELL TANK LEVEL HISTORY 1952 TO 1993 SOUND/NON-STABILIZED TANK WATCH LIST: N/A

\begin{tabular}{|c|c|c|c|c|c|}
\hline S?:? & & \multicolumn{4}{|l|}{ 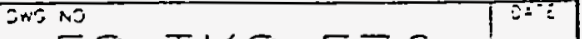 } \\
\hline$B$ & $2 \div i$ & ES & $E 7$ & & \\
\hline$\because \because=$ & $\because \equiv$ & 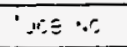 & $i_{s=?^{-}}$ & $i$ & 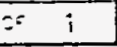 \\
\hline
\end{tabular}




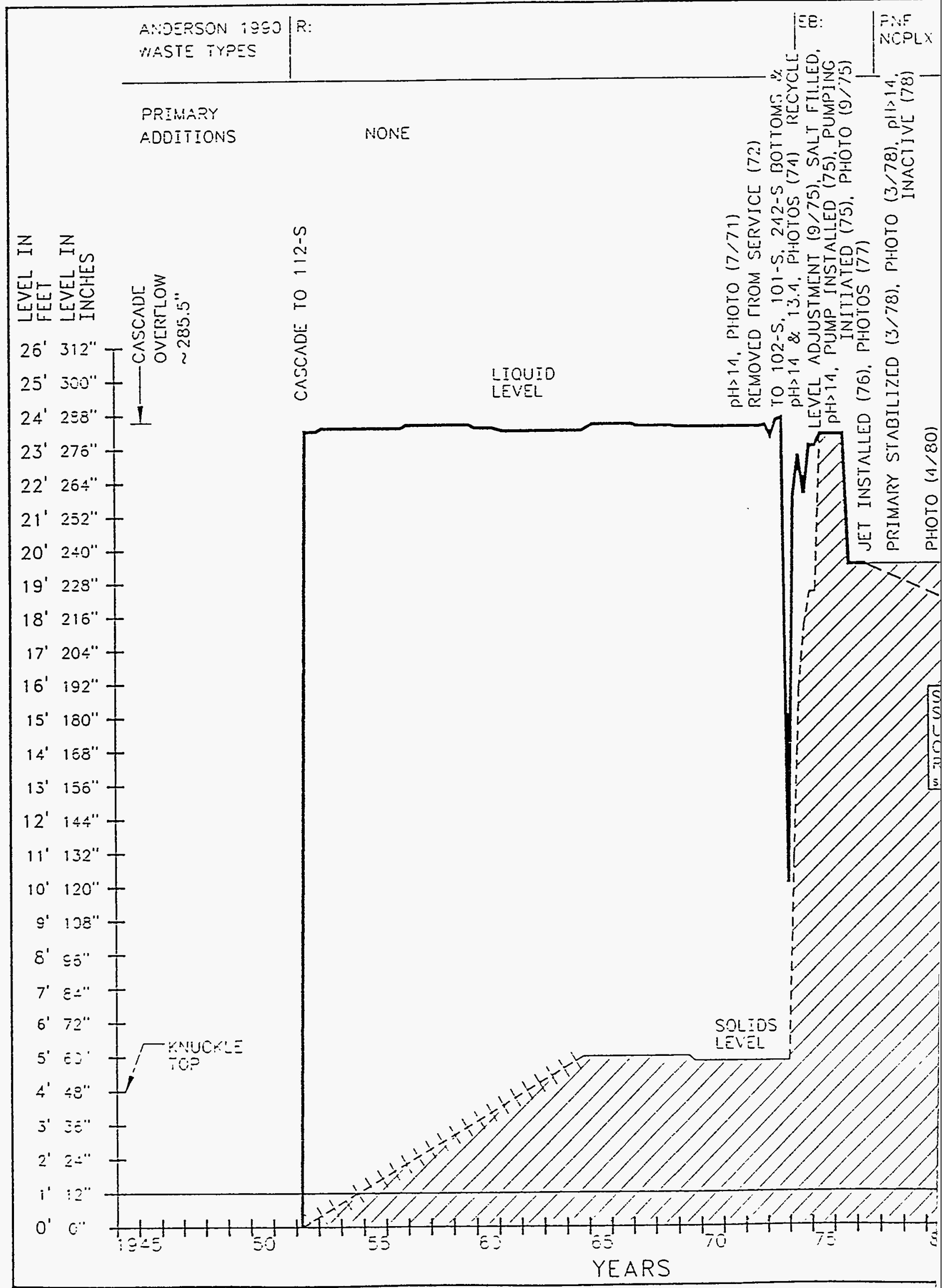




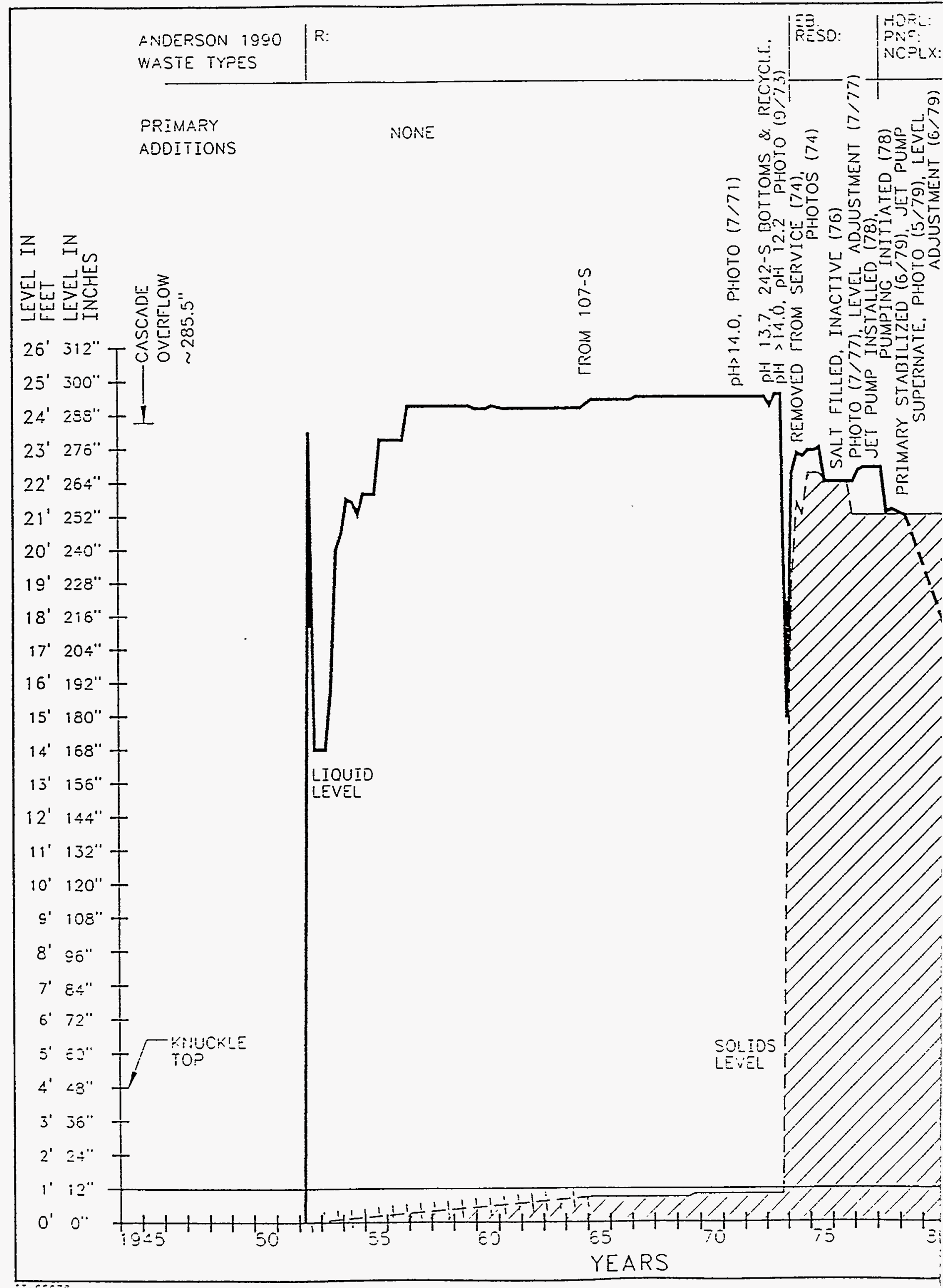




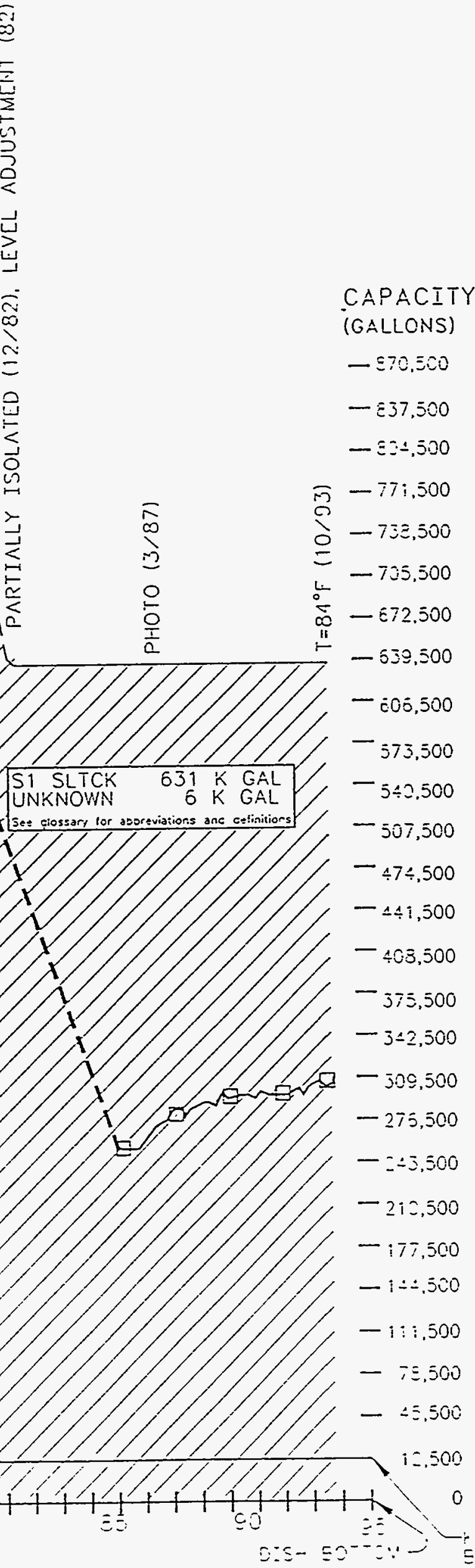

\section{NOTES:}

CONSTRUCTED iO50-i95!

NOA-NAL CAPACITY: $\$ 50.000$ 6AL

OIS: SOTTOM, A FOOT RADIUS KNUCK!E.

75 FOOT DIAMETER TANK

SUREACE LEVEL PEAOING: PHOTO (LIOUIO).

FIC AND FHOTO ISOLIDS)

WHC REPORT WHC-EP-03\%7 SUPPLEMENT

WHC REPORT WHC-EP-OI82-(MONTHLY)

WHC REPORT WHC-MR-0132

WHC REPORT SD-WM-TI-J56

RHO-CD-0014-(NONTHLY)

Dwi. $:-2-1783$

E8: EVAPORATOR ROTTOMS

HUE:- HANFORO DEFENSE RESIDUAL LIOUOR

PNF: PARTIAL NEUTRALIZED WASTE

P: REDOX WASTE

NCPLX: NON-COMPLEXED WASTE

PESO- RESIDUAL EVAPORATOR LIOUOR

\section{LEGEND}

OE LIQUID OESERVATION

WELL (LOW)

- LIOUID LEVEL

- - - LIOUID LEVEL BEST

ENGINEERING JUDGEMENT

77 SOLIOS LEVEL

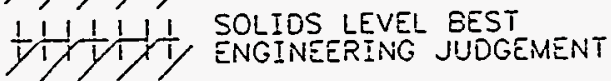

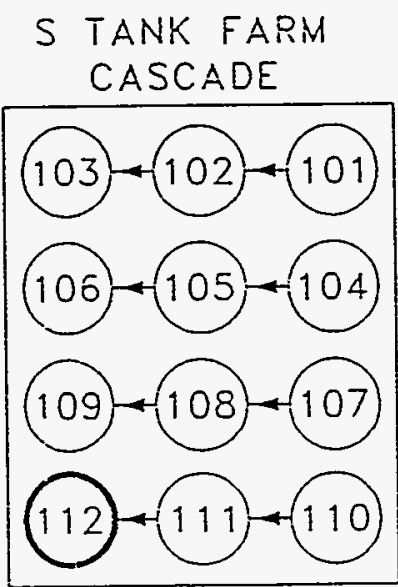

\begin{tabular}{|c|c|c|c|}
\hline \multicolumn{4}{|c|}{$\begin{array}{c}\text { U.S. DEPARTMENT OF ENERGY } \\
\text { RICHLAND FIELD OFFICE } \\
\text { ICF KAISER HANFORD }\end{array}$} \\
\hline \multicolumn{4}{|c|}{$\begin{array}{c}\text { 241-S-112 SINGLE-SHELL TANK } \\
\text { LEVEL HISTORY } 1952 \text { TO } 1993 \\
\text { SOUND/NON-STABILIZED TANK } \\
\text { WATCH LIST: HYDPOGEN }\end{array}$} \\
\hline $\begin{array}{l}5:=6 \\
8\end{array}$ & $\begin{array}{r}5: 06 \mathrm{Na} \\
2 \leq 1\end{array}$ & $E S-T K S-E 78$ & $\begin{array}{l}52: 6 \\
0 / 94 \\
0\end{array}$ \\
\hline$s=2=$ & 1.65 & 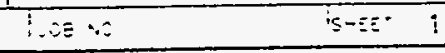 & $\because$ \\
\hline
\end{tabular}


241-S-101 SINGLE-SHELL WHC-SD-WM-ER-323, ReV. 0 TANK LEVEL HISTORY

\begin{tabular}{|c|c|c|c|c|c|}
\hline Year & Total & Total & Solids & Solids & LOW \\
\hline & ( $\mathrm{K}$ gal) & (in) & (K gal) & (in) & (in) \\
\hline \multicolumn{6}{|l|}{1.1952} \\
\hline \multicolumn{6}{|l|}{2} \\
\hline \multicolumn{6}{|l|}{3} \\
\hline \multicolumn{6}{|l|}{4} \\
\hline \multicolumn{6}{|l|}{$1-1953$} \\
\hline \multicolumn{6}{|l|}{2} \\
\hline 3 & 758 & 283 & 0 & 0 & \\
\hline 4 & 772 & 288 & 0 & 0 & \\
\hline $1-1954$ & 719 & 269 & 0 & 0 & \\
\hline 2 & 730 & 273 & 0 & 0 & \\
\hline 3 & 759 & 283 & 0 & 0 & \\
\hline 4 & 758 & 283 & 0 & 0 & \\
\hline 7.1955 & 741 & 277 & 0 & 0 & \\
\hline 2 & 725 & 271 & 0 & 0 & \\
\hline 3 & 772 & 288 & 0 & 0 & \\
\hline 4 & 763 & 285 & 0 & 0 & \\
\hline $1-1956$ & 761 & 284 & 0 & 0 & \\
\hline 2 & 757 & 283 & 0 & 0 & \\
\hline 3 & 756 & 282 & 0 & 0 & \\
\hline 4 & 756 & 282 & 0 & 0 & \\
\hline $1-1957$ & 752 & 281 & 0 & 0 & \\
\hline 2 & 752 & 281 & 0 & 0 & \\
\hline 3 & 750 & 280 & 0 & 0 & \\
\hline 4 & 750 & 280 & 0 & 0 & \\
\hline $1-1958$ & 769 & 287 & 0 & 0 & \\
\hline 2 & 769 & 287 & 0 & 0 & \\
\hline 3 & 766 & 286 & 0 & 0 & \\
\hline 4 & 763 & 285 & 0 & 0 & \\
\hline $1-1959$ & 763 & 285 & 0 & 0 & \\
\hline 2 & 763 & 285 & 0 & 0 & \\
\hline 3 & 762 & 285 & 0 & 0 & \\
\hline 4 & 761 & 284 & 0 & 0 & \\
\hline $7-1960$ & 757 & 283 & 0 & 0 & \\
\hline 2 & 757 & 283 & 0 & 0 & \\
\hline 3 & 757 & 283 & 0 & 0 & \\
\hline 4 & 761 & 284 & 0 & 0 & \\
\hline \multicolumn{6}{|l|}{$1-1961$} \\
\hline 2 & 758 & 283 & 0 & 0 & \\
\hline \multicolumn{6}{|l|}{3} \\
\hline 4 & 758 & 283 & 0 & 0 & \\
\hline \multicolumn{6}{|l|}{$1-1962$} \\
\hline 2 & 755 & 282 & 0 & 0 & \\
\hline \multicolumn{6}{|l|}{3} \\
\hline 4 & 755 & 282 & 0 & 0 & \\
\hline \multicolumn{6}{|l|}{$1-1963$} \\
\hline 2 & 755 & 282 & 0 & 0 & \\
\hline
\end{tabular}


241-S-101 SINGLE-SHELL WHC-SD-WM-ER-323， ReV. 0 TANK LEVEL HISTORY

\begin{tabular}{|c|c|c|c|c|c|}
\hline Year & Total & Total & Solids & Solids & LOW \\
\hline & (K gal) & (in) & (K gal) & (in) & (in) \\
\hline & & & & & \\
\hline \multicolumn{6}{|l|}{3} \\
\hline 4 & 755 & 282 & 0 & 0 & \\
\hline \multicolumn{6}{|l|}{$1-1964$} \\
\hline 2 & 755 & 282 & 0 & 0 & \\
\hline \multicolumn{6}{|l|}{3} \\
\hline 4 & 755 & 282 & 0 & 0 & \\
\hline \multicolumn{6}{|l|}{$9-1965$} \\
\hline 2 & 744 & 278 & 0 & 0 & \\
\hline 3 & 744 & 278 & 0 & 0 & \\
\hline 4 & 744 & 278 & 0 & 0 & \\
\hline 1-1966 & 744 & 278 & 0 & 0 & \\
\hline 2 & 744 & 278 & 0 & 0 & \\
\hline 3 & 744 & 278 & 0 & 0 & \\
\hline 4 & 744 & 278 & 0 & 0 & \\
\hline 7.1967 & 744 & 278 & 0 & 0 & \\
\hline 2 & 744 & 278 & 0 & 0 & \\
\hline 3 & 744 & 278 & 0 & 0 & \\
\hline 4 & 744 & 278 & 0 & 0 & \\
\hline $1-1968$ & 744 & 278 & 0 & 0 & \\
\hline 2 & 745 & 278 & 0 & 0 & \\
\hline 3 & 745 & 278 & 0 & 0 & \\
\hline 4 & 745 & 278 & 0 & 0 & \\
\hline $1-1969$ & 744 & 278 & 0 & 0 & \\
\hline 2 & 744 & 278 & 0 & 0 & \\
\hline 3 & 744 & 278 & 0 & 0 & \\
\hline 4 & 744 & 278 & 211 & 84 & \\
\hline $1-1970$ & 743 & 278 & 211 & 84 & \\
\hline 2 & 743 & 278 & 211 & 84 & \\
\hline 3 & 743 & 278 & 211 & 84 & \\
\hline 4 & 743 & 278 & 211 & 84 & \\
\hline $1-1971$ & 743 & 278 & 211 & 84 & \\
\hline 2 & 743 & 278 & 211 & 84 & \\
\hline 3 & 743 & 278 & 211 & 84 & \\
\hline 4 & 743 & 278 & 211 & 84 & \\
\hline $1-1972$ & 743 & 278 & 211 & 84 & \\
\hline 2 & 743 & 278 & 211 & 84 & \\
\hline 3 & 743 & 278 & 211 & 84 & \\
\hline 4 & 744 & 278 & 211 & 84 & \\
\hline $1-1973$ & 744 & 278 & 211 & 84 & - \\
\hline 2 & 734 & 274 & 211 & 84 & \\
\hline 3 & 744 & 278 & 211 & 84 & \\
\hline 4 & 426 & 162 & 211 & 84 & \\
\hline $1-1974$ & 616 & 231 & 244 & 96 & \\
\hline 2 & 588 & 221 & 244 & 96 & \\
\hline 3 & 339 & 131 & 244 & 96 & \\
\hline 4 & 519 & 196 & 244 & 96 & \\
\hline
\end{tabular}


241-S-101 SINGLE-SHELL WHC-SD-WM-ER-323， ReV.0 TANK LEVEL HISTORY

\begin{tabular}{|c|c|c|c|c|c|}
\hline Year & Total & Total & Solids & Solids & LOW \\
\hline & (K gal) & (in) & (K gal) & (in) & (in) \\
\hline & & & 210 & 96 & \\
\hline $1-1975$ & 552 & 208 & 244 & 90 & \\
\hline 2 & 692 & 259 & 332 & 128 & \\
\hline 3 & 332 & 128 & 332 & 128 & \\
\hline 4 & 560 & 211 & 332 & 128 & \\
\hline $1-1976$ & 717 & 268 & 332 & 128 & \\
\hline 2 & 717 & 268 & 332 & 128 & \\
\hline 3 & 719 & 269 & 332 & 128 & \\
\hline 4 & 725 & 271 & 332 & 128 & \\
\hline $1-1977$ & 708 & 265 & 332 & 128 & \\
\hline 2 & 725 & 271 & 332 & 128 & \\
\hline 3 & 703 & 263 & 332 & 128 & \\
\hline 4 & 706 & 264 & 332 & 128 & \\
\hline $1-1978$ & 703 & 263 & 332 & 128 & \\
\hline 2 & 703 & 263 & 332 & 128 & \\
\hline 3 & 700 & 262 & 332 & 128 & \\
\hline 4 & 700 & 262 & 332 & 128 & \\
\hline $1-1979$ & 678 & 254 & 332 & 128 & \\
\hline 2 & 472 & 179 & 332 & 128 & \\
\hline 3 & 472 & 179 & 332 & 128 & \\
\hline 4 & 585 & 220 & 332 & 128 & \\
\hline $1-1980$ & 711 & 266 & 332 & 128 & \\
\hline 2 & 520 & 197 & 332 & 128 & \\
\hline 3 & 427 & 163 & 415 & 158 & \\
\hline 4 & 427 & 163 & 415 & 158 & \\
\hline $1-1981$ & 427 & 163 & 415 & 158 & \\
\hline 2 & 427 & 163 & 415 & 158 & \\
\hline 3 & 427 & 163 & 415 & 158 & \\
\hline 4 & 427 & 163 & 415 & 158 & \\
\hline $1-1982$ & 427 & 163 & 415 & 158 & \\
\hline 2 & 427 & 163 & 415 & 158 & \\
\hline 3 & 427 & 163 & 415 & 158 & \\
\hline 4 & 427 & 163 & 415 & 158 & \\
\hline $1-1983$ & 427 & 163 & 415 & 158 & \\
\hline 2 & 427 & 163 & 415 & 158 & \\
\hline 3 & 427 & 163 & 415 & 158 & \\
\hline 4 & 427 & 163 & 415 & 158 & \\
\hline $1-1984$ & 427 & 163 & 415 & 158 & \\
\hline 2 & 427 & 163 & 415 & 158 & \\
\hline 3 & 427 & 163 & 415 & 158 & \\
\hline 4 & 427 & 163 & 415 & 158 & \\
\hline $1-1985$ & 427 & 163 & 415 & 158 & \\
\hline 2 & 427 & 163 & 415 & 158 & \\
\hline 3 & 427 & 163 & 415 & 158 & \\
\hline 4 & 427 & 163 & 415 & 158 & \\
\hline $1-1986$ & 427 & 163 & 415 & 158 & 160 \\
\hline 2 & 427 & 163 & 415 & 158 & 160 \\
\hline
\end{tabular}


241-S-101 SINGLE-SHELL WHC-SD-WM-ER-323, ReV. 0 TANK LEVEL HISTORY

\begin{tabular}{|c|c|c|c|c|c|}
\hline Year & Total & Total & Solids & Solids & LOW \\
\hline & ( $\mathrm{K}$ gal) & (in) & (K gal) & (in) & (in) \\
\hline & & & & & \\
\hline 3 & 427 & 163 & 415 & 158 & 160 \\
\hline 4 & 427 & 163 & 415 & 158 & 159 \\
\hline $1-1987$ & 427 & 163 & 415 & 158 & \\
\hline 2 & 427 & 163 & 415 & 158 & \\
\hline 3 & 427 & 163 & 415 & 158 & 160 \\
\hline 4 & 427 & 163 & 415 & 158 & 159 \\
\hline $1-1988$ & 427 & 163 & 415 & 158 & 160 \\
\hline 2 & 427 & 163 & 415 & 158 & 160 \\
\hline 3 & 427 & 163 & 415 & 158 & 159 \\
\hline 4 & 427 & 163 & 415 & 158 & 160 \\
\hline $1-1989$ & 427 & 163 & 415 & 158 & \\
\hline 2 & 427 & 163 & 415 & 158 & 160 \\
\hline 3 & 427 & 163 & 415 & 158 & 160 \\
\hline 4 & 427 & 163 & 415 & 158 & 160 \\
\hline $1-1990$ & 427 & 163 & 415 & 158 & 160 \\
\hline 2 & 427 & 163 & 415 & 158 & \\
\hline 3 & 427 & 163 & 415 & 158 & 160 \\
\hline 4 & 427 & 163 & 415 & 158 & 160 \\
\hline $1-1991$ & 427 & 163 & 415 & 158 & 160 \\
\hline 2 & 427 & 163 & 415 & 158 & 160 \\
\hline 3 & 427 & 163 & 415 & 158 & 161 \\
\hline 4 & 427 & 163 & 415 & 158 & 161 \\
\hline $7-1992$ & 427 & 163 & 415 & 158 & \\
\hline 2 & 427 & 163 & 415 & 158 & 160 \\
\hline 3 & 427 & 163 & 415 & 158 & \\
\hline 4 & 427 & 163 & 415 & 158 & 160 \\
\hline $1-1993$ & 427 & 163 & 415 & 158 & 161 \\
\hline 2 & 427 & 163 & 415 & 158 & 160 \\
\hline 3 & 427 & 163 & 415 & 158 & 161 \\
\hline 4 & 427 & 163 & 415 & 158 & 161 \\
\hline $1-1994$ & & & & & 160 \\
\hline
\end{tabular}


241-S-102 SINGLE-SHELL WHC-SD-WM-ER-323, REV. 0 TANK LEVEL HISTORY

\begin{tabular}{|c|c|c|c|c|c|}
\hline Year & Total & Total & Solids & Solids & LOW \\
\hline & (K gal) & (in) & (K gal) & (in) & (in) \\
\hline \multirow{2}{*}{\multicolumn{6}{|c|}{$1-1952$}} \\
\hline & & & & & \\
\hline \multicolumn{6}{|l|}{2} \\
\hline \multicolumn{6}{|l|}{3} \\
\hline \multicolumn{6}{|l|}{4} \\
\hline \multicolumn{6}{|l|}{$1-1953$} \\
\hline \multicolumn{6}{|l|}{2} \\
\hline 3 & 239 & 94 & 0 & 0 & \\
\hline 4 & 766 & 286 & 0 & 0 & \\
\hline $1-1954$ & 750 & 280 & 0 & 0 & \\
\hline 2 & 747 & 279 & 0 & 0 & \\
\hline 3 & 747 & 279 & 0 & 0 & \\
\hline 4 & 715 & 267 & 0 & 0 & \\
\hline $1-1955$ & 715 & 267 & 0 & 0 & \\
\hline 2 & 715 & 267 & 0 & 0 & \\
\hline 3 & 745 & 278 & 0 & 0 & \\
\hline 4 & 745 & 278 & 0 & 0 & \\
\hline $1-1956$ & 745 & 278 & 0 & 0 & \\
\hline 2 & 745 & 278 & 0 & 0 & \\
\hline 3 & 745 & 278 & 0 & 0 & \\
\hline 4 & 745 & 278 & 0 & 0 & \\
\hline $1-1957$ & 740 & 277 & 0 & 0 & \\
\hline 2 & 740 & 277 & 0 & 0 & \\
\hline 3 & 739 & 276 & 0 & 0 & \\
\hline 4 & 739 & 276 & 0 & 0 & \\
\hline $1-1958$ & 739 & 276 & 0 & 0 & \\
\hline 2 & 739 & 276 & 0 & 0 & \\
\hline 3 & 739 & 276 & 0 & 0 & \\
\hline 4 & 739 & 276 & 0 & 0 & \\
\hline $1-1959$ & 736 & 275 & 0 & 0 & \\
\hline 2 & 736 & 275 & 0 & 0 & \\
\hline 3 & 736 & 275 & 0 & 0 & \\
\hline 4 & 736 & 275 & 0 & 0 & \\
\hline $1-1960$ & 736 & 275 & 0 & 0 & \\
\hline 2 & 736 & 275 & 0 & 0 & \\
\hline 3 & 736 & 275 & 0 & 0 & \\
\hline 4 & 733 & 274 & 0 & 0 & \\
\hline \multicolumn{6}{|l|}{$1-1961$} \\
\hline 2 & 733 & 274 & 0 & 0 & \\
\hline 3 & & & & & - \\
\hline 4 & 733 & 274 & 0 & 0 & \\
\hline \multicolumn{6}{|l|}{$1-1962$} \\
\hline 2 & 733 & 274 & 0 & 0 & \\
\hline \multicolumn{6}{|l|}{3} \\
\hline 4 & 733 & 274 & 0 & 0 & \\
\hline \multicolumn{6}{|l|}{$1-1963$} \\
\hline 2 & 733 & 274 & 0 & 0 & \\
\hline
\end{tabular}


241-S-102 SINGLE-SHELL WHC-SD-WM-ER-323, REV. 0 TANK LEVEL HISTORY

\begin{tabular}{|c|c|c|c|c|c|}
\hline Year & Total & Total & Solids & Solids & LOW \\
\hline & (K gal) & (in) & (K gal) & (in) & (in) \\
\hline & & & & & \\
\hline 3 & & & & & \\
\hline 4 & 733 & 274 & 0 & 0 & \\
\hline $1-1964$ & & & & & \\
\hline 2 & 733 & 274 & 0 & 0 & \\
\hline 3 & & & & & \\
\hline 4 & 733 & 274 & 0 & 0 & \\
\hline $1-1965$ & & & & & \\
\hline 2 & 750 & 280 & 0 & 0 & \\
\hline 3 & 750 & 280 & 5 & 9 & \\
\hline 4 & 750 & 280 & 5 & 9 & \\
\hline $1-1966$ & 750 & 280 & 5 & 9 & \\
\hline 2 & 750 & 280 & 5 & 9 & \\
\hline 3 & 750 & 280 & 5 & 9 & \\
\hline 4 & 750 & 280 & 5 & 9 & \\
\hline $1-1967$ & 750 & 280 & 5 & 9 & \\
\hline 2 & 750 & 280 & 5 & 9 & \\
\hline 3 & 750 & 280 & 5 & 9 & \\
\hline 4 & 750 & 280 & 5 & 9 & \\
\hline $1-1968$ & 750 & 280 & 5 & 9 & \\
\hline 2 & 750 & 280 & 5 & 9 & \\
\hline 3 & 750 & 280 & 5 & 9 & \\
\hline 4 & 750 & 280 & 5 & 9 & \\
\hline $1-1969$ & 750 & 280 & 5 & 9 & \\
\hline 2 & 750 & 280 & 5 & 9 & \\
\hline 3 & 750 & 280 & 5 & 9 & \\
\hline 4 & 750 & 280 & 4 & 9 & \\
\hline $1-1970$ & 750 & 280 & 4 & 9 & \\
\hline 2 & 750 & 280 & 4 & 9 & \\
\hline 3 & 750 & 280 & 4 & 9 & \\
\hline 4 & 750 & 280 & 4 & 9 & \\
\hline $1-1971$ & 750 & 280 & 4 & 9 & \\
\hline 2 & 750 & 280 & 4 & 9 & \\
\hline 3 & 750 & 280 & 4 & 9 & \\
\hline 4 & 750 & 280 & 4 & 9 & \\
\hline $1-1972$ & 750 & 280 & 4 & 9 & \\
\hline 2 & 750 & 280 & 4 & 9 & \\
\hline 3 & 750 & 280 & 4 & 9 & \\
\hline 4 & 744 & 278 & 4 & 9 & \\
\hline $1-1973$ & 743 & 278 & 4 & 9 & \\
\hline 2 & 743 & 278 & 4 & 9 & \\
\hline 3 & 762 & 285 & 4 & 9 & \\
\hline 4 & 577 & 217 & 51 & 26 & \\
\hline $1-1974$ & 634 & 238 & 51 & 26 & \\
\hline 2 & 685 & 257 & 51 & 26 & \\
\hline 3 & 691 & 259 & 103 & 45 & \\
\hline 4 & 662 & 248 & 145 & 60 & \\
\hline
\end{tabular}


241-S-102 SINGLE-SHELL WHC-SD-WM-ER-323, REV. 0 TANK LEVEL HISTORY

\begin{tabular}{|c|c|c|c|c|c|}
\hline Year & Total & Total & Solids & Solids & LOW \\
\hline & ( $\mathrm{K}$ gal) & (in) & (K gal) & (in) & (in) \\
\hline & & & & & \\
\hline $1-1975$ & 604 & 227 & 145 & 60 & \\
\hline 2 & 689 & 258 & 145 & 60 & \\
\hline 3 & 464 & 176 & 145 & 60 & \\
\hline 4 & 554 & 209 & 200 & 80 & \\
\hline $1-1976$ & 477 & 181 & 200 & 80 & \\
\hline 2 & 552 & 208 & 200 & 80 & \\
\hline 3 & 543 & 205 & 200 & 80 & \\
\hline 4 & 535 & 202 & 200 & 80 & \\
\hline $1-1977$ & 211 & 84 & 208 & 83 & \\
\hline 2 & 230 & 91 & 208 & 83 & \\
\hline 3 & 233 & 92 & 208 & 83 & \\
\hline 4 & 246 & 97 & 208 & 83 & \\
\hline $1-1978$ & 266 & 104 & 208 & 83 & \\
\hline 2 & 274 & 107 & 208 & 83 & \\
\hline 3 & 282 & 110 & 208 & 83 & \\
\hline 4 & 483 & 183 & 208 & 83 & \\
\hline $1-1979$ & 618 & 232 & 208 & 83 & \\
\hline 2 & 378 & 145 & 208 & 83 & \\
\hline 3 & 480 & 182 & 208 & 83 & \\
\hline 4 & 618 & 232 & 510 & 193 & \\
\hline $1-1980$ & 609 & 229 & 510 & 193 & \\
\hline 2 & 561 & 211 & 510 & 193 & \\
\hline 3 & 555 & 209 & 555 & 209 & \\
\hline 4 & 555 & 209 & 555 & 209 & \\
\hline $1-1981$ & 555 & 209 & 555 & 209 & \\
\hline 2 & 555 & 209 & 555 & 209 & \\
\hline 3 & 555 & 209 & 555 & 209 & \\
\hline 4 & 555 & 209 & 555 & $209^{\circ}$ & \\
\hline $1-1982$ & 555 & 209 & 555 & 209 & \\
\hline 2 & 549 & 207 & 549 & 207 & \\
\hline 3 & 549 & 207 & 549 & 207 & \\
\hline 4 & 549 & 207 & 549 & 207 & \\
\hline $1-1983$ & 549 & 207 & 549 & 207 & \\
\hline 2 & 549 & 207 & 549 & 207 & \\
\hline 3 & 549 & 207 & 549 & 207 & \\
\hline 4 & 549 & 207 & 549 & 207 & \\
\hline $1-1984$ & 549 & 207 & 549 & 207 & \\
\hline 2 & 549 & 207 & 549 & 207 & \\
\hline 3 & 549 & 207 & 549 & 207 & \\
\hline 4 & 549 & 207 & 549 & 207 & \\
\hline $1-1985$ & 549 & 207 & 549 & 207 & \\
\hline 2 & 549 & 207 & 549 & 207 & \\
\hline 3 & 549 & 207 & 549 & 207 & \\
\hline 4 & 549 & 207 & 549 & 207 & \\
\hline $1-1986$ & 549 & 207 & 549 & 207 & 199 \\
\hline 2 & 549 & 207 & 549 & 207 & 199 \\
\hline
\end{tabular}


241-S-102 SINGLE-SHELL WHC-SD-WM-ER-323, ReV. 0 TANK LEVEL HISTORY

\begin{tabular}{|c|c|c|c|c|c|}
\hline Year & Total & Total & Solids & Solids & LOW \\
\hline & (K gal) & (in) & ( $\mathrm{K}$ gal) & (in) & (in) \\
\hline 3 & 549 & 207 & 549 & 207 & 199 \\
\hline 4 & 549 & 207 & 549 & 207 & 199 \\
\hline $1-1987$ & 549 & 207 & 549 & 207 & \\
\hline 2 & 549 & 207 & 549 & 207 & \\
\hline 3 & 549 & 207 & 549 & 207 & 199 \\
\hline 4 & 549 & 207 & 549 & 207 & 200 \\
\hline $1-1988$ & 549 & 207 & 549 & 207 & 199 \\
\hline 2 & 549 & 207 & 549 & 207 & 201 \\
\hline 3 & 549 & 207 & 549 & 207 & 197 \\
\hline 4 & 549 & 207 & 549 & 207 & 196 \\
\hline $1-1989$ & 549 & 207 & 549 & 207 & \\
\hline 2 & 549 & 207 & 549 & 207 & 201 \\
\hline 3 & 549 & 207 & 549 & 207 & 201 \\
\hline 4 & 549 & 207 & 549 & 207 & 200 \\
\hline $1-1990$ & 549 & 207 & 549 & 207 & 200 \\
\hline 2 & 549 & 207 & 549 & 207 & \\
\hline 3 & 549 & 207 & 549 & 207 & 201 \\
\hline 4 & 549 & 207 & 549 & 207 & 202 \\
\hline 1-1991 & 549 & 207 & 549 & 207 & 201 \\
\hline 2 & 549 & 207 & 549 & 207 & 201 \\
\hline 3 & 549 & 207 & 549 & 207 & 202 \\
\hline 4 & 549 & 207 & 549 & 207 & \\
\hline 1.1992 & 549 & 207 & 549 & 207 & \\
\hline 2 & 549 & 207 & 549 & 207 & 201 \\
\hline 3 & 549 & 207 & 549 & 207 & \\
\hline 4 & 549 & 207 & 549 & 207 & 201 \\
\hline $1-1993$ & 549 & 207 & 549 & 207 & 202 \\
\hline 2 & 549 & 207 & 549 & 207 & 203 \\
\hline 3 & & & & & 202 \\
\hline 4 & & & & & 203 \\
\hline
\end{tabular}


241-S-103 SINGLE-SHELL WHC-SD-WM-ER-323， ReV.0 TANK LEVEL HISTORY

\begin{tabular}{|c|c|c|c|c|c|}
\hline Year & Total & Total & Solids & Solids & LOW \\
\hline & (K gal) & (in) & (K gal) & (in) & (in) \\
\hline & & & & & \\
\hline \multicolumn{6}{|l|}{$1-1952$} \\
\hline \multicolumn{6}{|l|}{2} \\
\hline \multicolumn{6}{|l|}{3} \\
\hline \multicolumn{6}{|l|}{4} \\
\hline \multicolumn{6}{|l|}{$1-1953$} \\
\hline \multicolumn{6}{|l|}{2} \\
\hline 3 & 0 & 0 & 0 & 0 & \\
\hline $4 \quad \therefore$ & 430 & 164 & 0 & 0 & \\
\hline $1-1954$ & 514 & 194 & 0 & 0 & \\
\hline 2 & 549 & 207 & 0 & 0 & \\
\hline 3 & 549 & 207 & 0 & 0 & \\
\hline 4 & 536 & 202 & 0 & 0 & \\
\hline $1-1955$ & 537 & 203 & 0 & 0 & \\
\hline 2 & 752 & 281 & 0 & 0 & \\
\hline 3 & 752 & 281 & 0 & 0 & \\
\hline 4 & 752 & 281 & 0 & 0 & \\
\hline $1-1956$ & 752 & 281 & 0 & 0 & \\
\hline 2 & 752 & 281 & 0 & 0 & \\
\hline 3 & 752 & 281 & 0 & 0 & \\
\hline 4 & 752 & 281 & 0 & 0 & \\
\hline $1-1957$ & 752 & 281 & 0 & 0 & \\
\hline 2 & 750 & 280 & 0 & 0 & \\
\hline 3 & 750 & 280 & 0 & 0 & \\
\hline 4 & 750 & 280 & 0 & 0 & \\
\hline $1-1958$ & 747 & 279 & 0 & 0 & \\
\hline 2 & 750 & 280 & 0 & 0 & \\
\hline 3 & 750 & 280 & 0 & 0 & \\
\hline 4 & 750 & 280 & 0 & 0 & \\
\hline $1-1959$ & 750 & 280 & 0 & 0 & \\
\hline 2 & 750 & 280 & 0 & 0 & \\
\hline 3 & 750 & 280 & 0 & 0 & \\
\hline 4 & 750 & 280 & 0 & 0 & \\
\hline $1-1960$ & 747 & 279 & 0 & 0 & \\
\hline 2 & 747 & 279 & 0 & 0 & \\
\hline 3 & 747 & 279 & 0 & 0 & \\
\hline 4 & 750 & 280 & 0 & 0 & \\
\hline \multicolumn{6}{|l|}{$1-1961$} \\
\hline 2 & 747 & 279 & 0 & 0 & \\
\hline 3 & & & & & $\cdot$ \\
\hline 4 & 747 & 279 & 0 & 0 & \\
\hline \multicolumn{6}{|l|}{$1-1962$} \\
\hline 2 & 747 & 279 & 0 & 0 & \\
\hline \multicolumn{6}{|l|}{3} \\
\hline 4 & 747 & 279 & 0 & 0 & \\
\hline \multicolumn{6}{|l|}{$1-1963$} \\
\hline 2 & 747 & 279 & 0 & 0 & \\
\hline
\end{tabular}


241-S-103 SINGLE-SHELL WHC-SD-WM-ER-323, ReV. 0 TANK LEVEL HISTORY

\begin{tabular}{|c|c|c|c|c|c|}
\hline Year & Total & Total & Solids & Solids & LOW \\
\hline & (K gal) & (in) & (K gal) & (in) & (in) \\
\hline & & & & & \\
\hline \multicolumn{6}{|l|}{3} \\
\hline 4 & 747 & 279 & 0 & 0 & \\
\hline \multicolumn{6}{|l|}{$1-1964$} \\
\hline 2 & 747 & 279 & 0 & 0 & \\
\hline \multicolumn{6}{|l|}{3} \\
\hline 4 & 747 & 279 & 0 & 0 & \\
\hline \multicolumn{6}{|l|}{$1-1965$} \\
\hline 2 & 763 & 285 & 0 & 7 & \\
\hline 3 & 763 & 285 & 5 & 9 & \\
\hline 4 & 763 & 285 & 5 & 9 & \\
\hline $1-1966$ & 763 & 285 & 5 & 9 & \\
\hline 2 & 763 & 285 & 5 & 9 & \\
\hline 3 & 763 & 285 & 5 & 9 & \\
\hline 4 & 763 & 285 & 5 & 9 & \\
\hline $1-1967$ & 768 & 287 & 5 & 9 & \\
\hline 2 & 765 & 286 & 5 & 9 & \\
\hline 3 & 765 & 286 & 5 & 9 & \\
\hline 4 & 765 & 286 & 5 & 9 & \\
\hline $1-1968$ & 765 & 286 & 5 & 9 & \\
\hline 2 & 765 & 286 & 5 & 9 & \\
\hline 3 & 765 & 286 & 5 & 9 & \\
\hline 4 & 764 & 285 & 5 & 9 & \\
\hline $1-1969$ & 764 & 285 & 5 & 9 & \\
\hline 2 & 764 & 285 & 5 & 9 & \\
\hline 3 & 765 & 286 & 5 & 9 & \\
\hline 4 & 765 & 286 & 9 & 11 & \\
\hline $1-1970$ & 764 & 285 & 9 & 11 & \\
\hline 2 & 764 & 285 & 9 & 11 & \\
\hline 3 & 765 & 286 & 9 & 11 & \\
\hline 4 & 764 & 285 & 9 & 11 & \\
\hline $1-1971$ & 764 & 285 & 9 & 11 & \\
\hline 2 & 764 & 285 & 9 & 11 & \\
\hline 3 & 764 & 285 & 9 & 11 & \\
\hline 4 & 764 & 285 & 9 & 11 & \\
\hline $1-1972$ & 763 & 285 & 9 & 11 & \\
\hline 2 & 763 & 285 & 9 & 11 & \\
\hline 3 & 766 & 286 & 9 & 11 & \\
\hline 4 & 767 & 286 & 9 & 11 & \\
\hline $1-1973$ & 767 & 286 & 9 & 11 & \\
\hline 2 & 761 & 284 & 9 & 11 & \\
\hline 3 & 773 & 289 & 9 & 11 & \\
\hline 4 & 549 & 207 & 37 & 21 & \\
\hline $1-1974$ & 388 & 149 & 37 & 21 & \\
\hline 2 & 640 & 240 & 37 & 21 & \\
\hline 3 & 570 & 215 & 37 & 21 & \\
\hline 4 & 513 & 194 & 37 & 21 & \\
\hline
\end{tabular}


241-S-103 SINGLE-SHELL WHC-SD-WM-ER-323， ReV. 0 TANK LEVEL HISTORY

\begin{tabular}{|c|c|c|c|c|c|}
\hline Year & Total & Total & Solids & Solids & $i \quad$ LOW \\
\hline & (K gal) & (in) & (K gal) & (in) & (in) \\
\hline & & & & & $!$ \\
\hline $1-1975$ & 409 & 156 & 37 & 21 & \\
\hline 2 & 642 & 241 & 37 & 21 & 1 \\
\hline 3 & 403 & 154 & 37 & 21 & \\
\hline 4 & 565 & 213 & 37 & 21 & \\
\hline $1-1976$ & 326 & 126 & 37 & 21 & \\
\hline 2 & 337 & 130 & 37 & 21 & \\
\hline 3 & 194 & 78 & 37 & 21 & \\
\hline 4 & 439 & 167 & 37 & 21 & \\
\hline $1-1977$ & 466 & 177 & 57 & 28 & \\
\hline 2 & 301 & 117 & 57 & 28 & \\
\hline 3 & 257 & 101 & 57 & 28 & \\
\hline 4 & 395 & 151 & 68 & 32 & \\
\hline $1-1978$ & 459 & 174 & 68 & 32 & \\
\hline 2 & 238 & 94 & 120 & 51 & \\
\hline 3 & 387 & 148 & 167 & 68 & \\
\hline 4 & 662 & 248 & 128 & 54 & \\
\hline $1-1979$ & 440 & 167 & 128 & 54 & \\
\hline 2 & 499 & 189 & 128 & 54 & \\
\hline 3 & 560 & 211 & 128 & 54 & \\
\hline 4 & 708 & 265 & 153 & 63 & \\
\hline $1-1980$ & 675 & 253 & 153 & 63 & \\
\hline 2 & 484 & 183 & 153 & 63 & \\
\hline 3 & 647 & 243 & 153 & 63 & \\
\hline 4 & 248 & 98 & 231 & 91 & \\
\hline $1-1981$ & 248 & 98 & 231 & 91 & \\
\hline 2 & 248 & 98 & 231 & 91 & \\
\hline 3 & 248 & 98 & 231 & 91 & \\
\hline 4 & 248 & 98 & 231 & 91 & \\
\hline $1-1982$ & 248 & 98 & 231 & 91 & \\
\hline 2 & 248 & 98 & 231 & 91 & \\
\hline 3 & 248 & 98 & 231 & 91 & 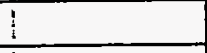 \\
\hline 4 & 248 & 98 & 231 & 91 & $!$ \\
\hline $1-1983$ & 248 & 98 & 231 & 91 & \\
\hline 2 & 248 & 98 & 231 & 91 & \\
\hline 3 & 248 & 98 & 231 & 91 & \\
\hline 4 & 248 & 98 & 231 & 91 & 1 \\
\hline $1-1984$ & 248 & 98 & 231 & 91 & 1 \\
\hline 2 & 248 & 98 & 231 & 91 & \\
\hline 3 & 248 & 98 & 231 & 91 & \\
\hline 4 & 248 & 98 & 231 & 91 & 1 \\
\hline $1-1985$ & 248 & 98 & 231 & 91 & 1 \\
\hline 2 & 248 & 98 & 231 & 91 & \\
\hline 3 & 248 & 98 & 231 & 91 & 1 \\
\hline 4 & 248 & 98 & 231 & 91 & 1 \\
\hline $1-1986$ & 248 & 98 & 231 & 91 & 101 \\
\hline 2 & 248 & 98 & 231 & 91 & 102 \\
\hline
\end{tabular}


241-S-103 SINGLE-SHELL WHC-SD-WM-ER-323, ReV. 0 TANK LEVEL HISTORY

\begin{tabular}{|c|c|c|c|c|c|}
\hline Year & Total & Total & Solids & Solids & LOW \\
\hline & (K gal) & (in) & (K gal) & (in) & (in) \\
\hline 3 & 248 & 98 & 231 & 91 & 107 \\
\hline 4 & 248 & 98 & 231 & 91 & 102 \\
\hline $1-1987$ & 248 & 98 & 231 & 91 & 102 \\
\hline 2 & 248 & 98 & 231 & 91 & \\
\hline 3 & 248 & 98 & 231 & 91 & 102 \\
\hline 4 & 248 & 98 & 231 & 91 & 102 \\
\hline $1-1988$ & 248 & 98 & 231 & 91 & 102 \\
\hline 2 & 248 & 98 & 231 & 91 & 103 \\
\hline 3 & 248 & 98 & 231 & 91 & 102 \\
\hline 4 & 248 & 98 & 231 & 91 & 102 \\
\hline $1-1989$ & 248 & 98 & 231 & 91 & \\
\hline 2 & 248 & 98 & 231 & 91 & 103 \\
\hline 3 & 248 & 98 & 231 & 91 & 103 \\
\hline 4 & 248 & 98 & 231 & 91 & 103 \\
\hline 1.1990 & 248 & 98 & 231 & 91 & 103 \\
\hline 2 & 248 & 98 & 231 & 91 & \\
\hline 3 & 248 & 98 & 231 & 91 & 102 \\
\hline 4 & 248 & 98 & 231 & 91 & 103 \\
\hline $1-1991$ & 248 & 98 & 231 & 91 & 103 \\
\hline 2 & 248 & 98 & 231 & 91 & 103 \\
\hline 3 & 248 & 98 & 231 & 91 & 103 \\
\hline 4 & 248 & 98 & 231 & 91 & 103 \\
\hline $1-1992$ & 248 & 98 & 231 & 91 & \\
\hline 2 & 248 & 98 & 231 & 91 & \\
\hline 3 & 248 & 98 & 231 & 91 & \\
\hline 4 & 248 & 98 & 231 & 91 & 103 \\
\hline $1-1993$ & 248 & 98 & 231 & 91 & 103 \\
\hline 2 & 248 & 98 & 231 & 91 & 103 \\
\hline 3 & 248 & 98 & 231 & 91 & 103 \\
\hline$\overline{4}$ & 248 & 98 & 231 & 91 & 103 \\
\hline $1-1994$ & & & & & 103 \\
\hline
\end{tabular}


241-S-104 SINGLE-SHELL

WHC-SD-WM-ER-323， Rev. 0 TANK LEVEL HISTORY

\begin{tabular}{|c|c|c|c|c|c|}
\hline Year & Total & Total & Solids & Solids & \\
\hline & (K gal) & (in) & (K gal) & (in) & \\
\hline & & & & & \\
\hline \multicolumn{6}{|l|}{$1-1952$} \\
\hline \multicolumn{6}{|l|}{2} \\
\hline \multicolumn{6}{|l|}{3} \\
\hline \multicolumn{6}{|l|}{4} \\
\hline $1-1953$ & 741 & 277 & 0 & 0 & \\
\hline 2 & 758 & 283 & 0 & 0 & \\
\hline 3 & 663 & 249 & 0 & 0 & \\
\hline 4 & 524 & 198 & 0 & 0 & \\
\hline $1-1954$ & 458 & 174 & 0 & 0 & \\
\hline 2 & 734 & 274 & 0 & 0 & \\
\hline 3 & 667 & 250 & 0 & 0 & \\
\hline 4 & 648 & 243 & 0 & 0 & \\
\hline $1-1955$ & 623 & 234 & 0 & 0 & \\
\hline 2 & 725 & 271 & 0 & 0 & \\
\hline 3 & 763 & 285 & 0 & 0 & \\
\hline 4 & 759 & 283 & 0 & 0 & \\
\hline $1-1956$ & 752 & 281 & 0 & 0 & \\
\hline 2 & 753 & 281 & 0 & 0 & \\
\hline 3 & 753 & 281 & 0 & 0 & \\
\hline 4 & 753 & 281 & 0 & 0 & \\
\hline $1-1957$ & 750 & 280 & 0 & 0 & \\
\hline 2 & 732 & 274 & 0 & 0 & \\
\hline 3 & 732 & 274 & 0 & 0 & \\
\hline 4 & 730 & 273 & 0 & 0 & \\
\hline $1-1958$ & 725 & 271 & 0 & 0 & \\
\hline 2 & 725 & 271 & 0 & 0 & \\
\hline 3 & 725 & 271 & 0 & 0 & \\
\hline 4 & 725 & 271 & 0 & 0 & \\
\hline $1-1959$ & 725 & 271 & 0 & 0 & \\
\hline 2 & 725 & 271 & 0 & 0 & \\
\hline 3 & 721 & 270 & 0 & 0 & \\
\hline 4 & 719 & 269 & 0 & 0 & \\
\hline $1-1960$ & 719 & 269 & 0 & 0 & \\
\hline 2 & 719 & 269 & 0 & 0 & \\
\hline 3 & 719 & 269 & 0 & 0 & \\
\hline 4 & 716 & 268 & 0 & 0 & \\
\hline \multicolumn{6}{|l|}{$1-1961$} \\
\hline 2 & 714 & 267 & 0 & 0 & \\
\hline 3 & & & & & - \\
\hline 4 & 714 & 267 & 0 & 0 & \\
\hline \multicolumn{6}{|l|}{$1-1962$} \\
\hline 2 & 714 & 267 & 0 & 0 & \\
\hline \multicolumn{6}{|l|}{3} \\
\hline 4 & 714 & 267 & 0 & 0 & \\
\hline \multicolumn{6}{|l|}{$1-1963$} \\
\hline 2 & 711 & 266 & 0 & 0 & \\
\hline
\end{tabular}


241-S-104 SINGLE-SHELL WHC-SD-WM-ER-323, ReV. 0 TANK LEVEL HISTORY

\begin{tabular}{|c|c|c|c|c|c|}
\hline Year & Total & Total & Solids & Solids & \\
\hline & (K gal) & (in) & (K gal) & (in) & \\
\hline \multirow{2}{*}{\multicolumn{6}{|c|}{3}} \\
\hline & & & & & \\
\hline 4 & 711 & 266 & 0 & 0 & \\
\hline \multicolumn{6}{|l|}{$1-1964$} \\
\hline 2 & 711 & 266 & 0 & 0 & \\
\hline \multicolumn{6}{|l|}{3} \\
\hline 4 & 711 & 266 & 0 & 0 & \\
\hline \multicolumn{6}{|l|}{$1-1965$} \\
\hline 2 & 807 & 301 & 0 & 0 & \\
\hline 3 & 807 & 301 & 0 & 0 & \\
\hline 4 & 807 & 301 & 0 & 0 & \\
\hline $1-1966$ & 805 & 300 & 0 & 0 & \\
\hline 2 & 802 & 299 & 0 & 0 & \\
\hline 3 & 799 & 298 & 0 & 0 & \\
\hline 4 & 796 & 297 & 0 & 0 & \\
\hline $1-1967$ & 796 & 297 & 0 & 0 & \\
\hline 2 & 791 & 295 & 0 & 0 & \\
\hline 3 & 790 & 295 & 0 & 0 & \\
\hline 4 & 789 & 294 & 0 & 0 & \\
\hline $1-1968$ & 787 & 294 & 0 & 0 & \\
\hline 2 & 787 & 294 & 0 & 0 & \\
\hline 3 & 787 & 294 & 0 & 0 & \\
\hline 4 & 785 & 293 & 0 & 0 & \\
\hline $1-1969$ & 785 & 293 & 0 & 0 & \\
\hline 2 & 784 & 293 & 0 & 0 & \\
\hline 3 & 784 & 293 & & & \\
\hline 4 & 784 & 293 & 241 & 95 & \\
\hline $1-1970$ & 783 & 292 & 241 & 95 & \\
\hline 2 & 307 & 119 & 241 & 95 & \\
\hline 3 & 304 & 118 & 241 & 95 & \\
\hline 4 & 310 & 120 & 241 & 95 & \\
\hline $1-1971$ & 310 & 120 & 241 & 95 & \\
\hline 2 & 309 & 120 & 241 & 95 & \\
\hline 3 & 309 & 120 & 241 & 95 & \\
\hline 4 & 308 & 119 & 241 & 95 & \\
\hline $1-1972$ & 308 & 119 & 241 & 95 & \\
\hline 2 & 308 & 119 & 241 & 95 & \\
\hline 3 & 308 & 119 & 241 & 95 & \\
\hline 4 & 308 & 119 & 241 & 95 & \\
\hline $1-1973$ & 309 & 120 & 241 & 95 & - \\
\hline 2 & 307 & 119 & 241 & 95 & \\
\hline 3 & 308 & 119 & 241 & 95 & \\
\hline 4 & 307 & 119 & 241 & 95 & \\
\hline $1-1974$ & 307 & 119 & 241 & 95 & \\
\hline 2 & 307 & 119 & 241 & 95 & \\
\hline 3 & 309 & 120 & 241 & 95 & \\
\hline 4 & 301 & 117 & 241 & 95 & \\
\hline
\end{tabular}


241-S-104 SINGLE-SHELL WHC-SD-WM-ER-323， Rev. 0 TANK LEVEL HISTORY

\begin{tabular}{|c|c|c|c|c|c|}
\hline Year & Total & Total & Solids & Solids & $i$ \\
\hline & (K gal) & (in) & ( $\mathrm{K}$ gal) & (in) & $i$ \\
\hline $1-1975$ & 299 & 116 & 299 & 116 & \\
\hline 2 & 299 & 116 & 299 & 116 & \\
\hline 3 & 299 & 116 & 299 & 116 & 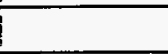 \\
\hline 4 & 299 & 116 & 299 & 116 & \\
\hline $1-1976$ & 299 & 116 & 299 & 116 & \\
\hline 2 & 299 & 116 & 299 & 116 & \\
\hline 3 & 299 & 116 & 299 & 116 & \\
\hline 4 & 299 & 116 & 299 & 116 & \\
\hline $1-1977$ & 299 & 116 & 299 & 116 & \\
\hline 2 & 299 & 116 & 299 & 116 & \\
\hline 3 & 299 & 116 & 299 & 116 & \\
\hline 4 & 299 & 116 & 299 & 116 & \\
\hline $1-1978$ & 299 & 116 & 299 & 116 & \\
\hline 2 & 299 & 116 & 299 & 116 & \\
\hline 3 & 299 & 116 & 299 & 116 & \\
\hline 4 & 299 & 116 & 299 & 116 & \\
\hline $1-1979$ & 299 & 116 & 299 & 116 & \\
\hline 2 & 299 & 116 & 299 & 116 & \\
\hline 3 & 299 & 116 & 299 & 116 & \\
\hline 4 & 299 & 116 & 299 & 116 & \\
\hline $1-1980$ & 299 & 116 & 299 & 116 & \\
\hline 2 & 299 & 116 & 299 & 116 & \\
\hline 3 & 299 & 116 & 299 & 116 & \\
\hline 4 & 299 & 116 & 299 & 116 & \\
\hline $1-1981$ & 299 & 116 & 299 & 116 & \\
\hline 2 & 299 & 116 & 299 & 116 & \\
\hline 3 & 299 & 116 & 299 & 116 & \\
\hline 4 & 299 & 116 & 299 & 116 & \\
\hline $1-1982$ & 299 & 116 & 299 & 116 & \\
\hline 2 & 294 & 114 & 293 & 114 & \\
\hline 3 & 294 & 114 & 293 & 114 & \\
\hline 4 & 294 & 114 & 293 & 114 & \\
\hline $1-1983$ & 294 & 114 & 293 & 114 & \\
\hline 2 & 294 & 114 & 293 & 114 & \\
\hline 3 & 294 & 114 & 293 & 114 & \\
\hline 4 & 294 & 114 & 293 & 114 & \\
\hline $1-1984$ & 294 & 114 & 293 & 114 & \\
\hline 2 & 294 & 114 & 293 & 114 & \\
\hline 3 & 294 & 114 & 293 & 114 & \\
\hline 4 & 294 & 114 & 293 & 114 & \\
\hline $1-1985$ & 294 & 114 & 293 & 114 & \\
\hline 2 & 294 & 114 & 293 & 114 & \\
\hline 3 & 294 & 114 & 293 & 114 & \\
\hline 4 & 294 & 114 & 293 & 114 & \\
\hline $1-1986$ & 294 & 114 & 293 & 114 & \\
\hline 2 & 294 & 114 & 293 & 114 & 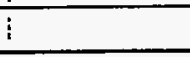 \\
\hline
\end{tabular}


241-S-104 SINGLE-SHELL WHC-SD-WM-ER-323， REV.0 TANK LEVEL HISTORY

\begin{tabular}{|c|c|c|c|c|}
\hline Year & Total & Total & Solids & Solids \\
\hline & ( $\mathrm{K}$ gal) & (in) & ( $\mathrm{K}$ gal) & (in) \\
\hline 3 & 294 & 114 & 202 & 99 \\
\hline 4 & 294 & 114 & $\frac{293}{293}$ & 114 \\
\hline $1-1987$ & 294 & 114 & 293 & 114 \\
\hline 2 & 294 & 114 & 293 & 114 \\
\hline 3 & 294 & 114 & 293 & 114 \\
\hline 4 & 294 & 114 & 293 & 114 \\
\hline $1-1988$ & 294 & 114 & 293 & 114 \\
\hline 2 & 294 & 114 & 293 & 114 \\
\hline 3 & 294 & 114 & 293 & 114 \\
\hline 4 & 294 & 114 & 293 & 114 \\
\hline $1-1989$ & 294 & 114 & 293 & 114 \\
\hline 2 & 294 & 114 & 293 & 114 \\
\hline 3 & 294 & 114 & 293 & 114 \\
\hline 4 & 294 & 114 & 293 & 114 \\
\hline $1-1990$ & 294 & 114 & 293 & 114 \\
\hline 2 & 294 & 114 & 293 & 114 \\
\hline 3 & 294 & 114 & 293 & 114 \\
\hline 4 & 294 & 114 & 293 & 114 \\
\hline $1-1991$ & 294 & 114 & 293 & 114 \\
\hline 2 & 294 & 114 & 293 & 114 \\
\hline 3 & 294 & 114 & 293 & 114 \\
\hline 4 & 294 & 114 & 293 & 114 \\
\hline $1-1992$ & 294 & 114 & 293 & 114 \\
\hline 2 & 294 & 114 & 293 & 114 \\
\hline 3 & 294 & 114 & 293 & 114 \\
\hline 4 & 294 & 114 & 293 & 114 \\
\hline $1-1993$ & 294 & 114 & 293 & 114 \\
\hline 2 & 294 & 114 & 293 & 114 \\
\hline \multicolumn{5}{|l|}{3} \\
\hline 4 & & & & \\
\hline
\end{tabular}


241-S-105 SINGLE-SHEL WHC-SD-WM-ER-323， ReV.0 L TANK LEVEL HISTORY

\begin{tabular}{|c|c|c|c|c|c|}
\hline Year & Total & Total & Solids & Solids & LOW \\
\hline & ( $\mathrm{K}$ gal) & (in) & (K gal) & (in) & (in) \\
\hline \multirow{2}{*}{\multicolumn{6}{|c|}{$1-1952$}} \\
\hline & & & & & \\
\hline \multicolumn{6}{|l|}{2} \\
\hline \multicolumn{6}{|l|}{3} \\
\hline \multicolumn{6}{|l|}{4} \\
\hline \multicolumn{6}{|l|}{$1-1953$} \\
\hline 2 & 758 & 283 & 0 & 0 & \\
\hline 3 & 723 & 270 & 0 & 0 & \\
\hline 4 & 722 & 270 & 0 & 0 & \\
\hline $1-1954$ & 722 & 270 & 0 & 0 & \\
\hline 2 & 734 & 274 & 0 & 0 & \\
\hline 3 & 728 & 272 & 0 & 0 & \\
\hline 4 & 728 & 272 & 0 & 0 & \\
\hline $1-1955$ & 728 & 272 & 0 & 0 & \\
\hline 2 & 728 & 272 & 0 & 0 & \\
\hline 3 & 723 & 270 & 0 & 0 & \\
\hline 4 & 723 & 270 & 0 & 0 & \\
\hline $1-1956$ & 723 & 270 & 0 & 0 & \\
\hline 2 & 723 & 270 & 0 & 0 & \\
\hline 3 & 723 & 270 & 0 & 0 & \\
\hline 4 & 723 & 270 & 0 & 0 & \\
\hline $1-1957$ & 754 & 282 & 0 & 0 & \\
\hline 2 & 754 & 282 & 0 & 0 & \\
\hline 3 & 752 & 281 & 0 & 0 & \\
\hline 4 & 755 & 282 & 0 & 0 & \\
\hline $1-1958$ & 752 & 281 & 0 & 0 & \\
\hline 2. & 752 & 281 & 0 & 0 & \\
\hline 3 & 719 & 269 & 0 & 0 & \\
\hline 4 & 719 & 269 & 0 & 0 & \\
\hline $1-1959$ & 719 & 269 & 0 & 0 & \\
\hline 2 & 719 & 269 & 0 & 0 & \\
\hline 3 & 719 & 269 & 0 & 0 & \\
\hline 4 & 717 & 268 & 0 & 0 & \\
\hline $1-1960$ & 717 & 268 & 0 & 0 & \\
\hline 2 & 717 & 268 & 0 & 0 & \\
\hline 3 & 717 & 268 & 0 & 0 & \\
\hline 4 & 717 & 268 & 0 & 0 & \\
\hline \multicolumn{6}{|l|}{$1-1961$} \\
\hline 2 & 717 & 268 & 0 & 0 & \\
\hline 3 & & & & & - \\
\hline 4 & 717 & 268 & 0 & 0 & \\
\hline \multicolumn{6}{|l|}{$1-1962$} \\
\hline 2 & 717 & 268 & 0 & 0 & \\
\hline \multicolumn{6}{|l|}{3} \\
\hline 4 & 717 & 268 & 0 & 0 & \\
\hline \multicolumn{6}{|l|}{$1-1963$} \\
\hline 2 & 717 & 268 & 0 & 0 & \\
\hline
\end{tabular}


241-S-105 SINGLE-SHEL WHC-SD-WM-ER-323, REV. 0

L TANK LEVEL HISTORY

\begin{tabular}{|c|c|c|c|c|c|}
\hline Year & Total & Total & Solids & Solids & LOW \\
\hline & (K gal) & (in) & (K gal) & (in) & (in) \\
\hline \multicolumn{6}{|l|}{3} \\
\hline 4 & 717 & 268 & 0 & 0 & \\
\hline \multicolumn{6}{|l|}{$1-1964$} \\
\hline 2 & 717 & 268 & 0 & 0 & \\
\hline \multicolumn{6}{|l|}{3} \\
\hline 4 & 717 & 268 & 0 & 0 & \\
\hline \multicolumn{6}{|l|}{$1-1965$} \\
\hline 2 & 763 & 285 & 0 & 0 & \\
\hline 3 & 763 & 285 & 0 & 0 & \\
\hline 4 & 763 & 285 & 0 & 0 & \\
\hline $1-1966$ & 763 & 285 & 0 & 0 & \\
\hline 2 & 763 & 285 & 0 & 0 & \\
\hline 3 & 763 & 285 & 0 & 0 & \\
\hline 4 & 763 & 285 & 0 & 0 & \\
\hline $1-1967$ & 763 & 285 & 0 & 0 & \\
\hline 2 & 763 & 285 & 0 & 0 & \\
\hline 3 & 763 & 285 & 0 & 0 & \\
\hline 4 & 763 & 285 & 0 & 0 & \\
\hline $1-1968$ & 763 & 285 & 0 & 0 & \\
\hline 2 & 763 & 285 & 0 & 0 & \\
\hline 3 & 763 & 285 & 0 & 0 & \\
\hline 4 & 763 & 285 & 0 & 0 & \\
\hline $1-1969$ & 763 & 285 & 0 & 0 & \\
\hline 2 & 763 & 285 & 0 & 0 & \\
\hline 3 & 763 & 285 & & & \\
\hline 4 & 763 & 285 & 2 & 8 & \\
\hline $1-1970$ & 763 & 285 & 2 & 8 & \\
\hline 2 & 763 & 285 & 2 & 8 & \\
\hline 3 & 762 & 285 & 2 & 8 & \\
\hline 4 & 762 & 285 & 2 & 8 & \\
\hline $1-1971$ & 762 & 285 & 2 & 8 & \\
\hline 2 & 763 & 285 & 2 & 8 & \\
\hline 3 & 763 & 285 & 2 & 8 & \\
\hline 4 & 763 & 285 & 2 & 8 & \\
\hline $1-1972$ & 762 & 285 & 2 & 8 & \\
\hline 2 & 762 & 285 & 2 & 8 & \\
\hline 3 & 762 & 285 & 2 & 8 & \\
\hline 4 & 763 & 285 & 2 & 8 & \\
\hline $1-1973$ & 763 & 285 & 2 & 8 & $\cdot$ \\
\hline 2 & 753 & 281 & 2 & 8 & \\
\hline 3 & 767 & 286 & 2 & 8 & \\
\hline 4 & 365 & 140 & 249 & 98 & \\
\hline $1-1974$ & 730 & 273 & 549 & 207 & \\
\hline 2 & 549 & 207 & 549 & 207 & \\
\hline 3 & 549 & 207 & 549 & 207 & \\
\hline 4 & 541 & 204 & 541 & 204 & \\
\hline
\end{tabular}


241-S-105 SINGLE-SHEL

WHC-SD-WM-ER-323， Rev. 0 L TANK LEVEL HISTORY

\begin{tabular}{|c|c|c|c|c|c|}
\hline Year & Total & Total & Solids & Solids & LOW \\
\hline & ( $\mathrm{K}$ gal) & (in) & (K gal) & (in) & (in) \\
\hline $1-1975$ & 541 & 204 & 541 & 204 & \\
\hline 2 & 541 & 204 & 541 & 204 & \\
\hline 3 & 541 & 204 & 541 & 204 & \\
\hline 4 & 541 & 204 & 541 & 204 & \\
\hline $1-1976$ & 541 & 204 & 541 & 204 & \\
\hline 2 & 541 & 204 & 541 & 204 & \\
\hline 3 & 541 & 204 & 541 & 204 & \\
\hline 4 & 541 & 204 & 541 & 204 & \\
\hline $1-1977$ & 541 & 204 & 541 & 204 & \\
\hline 2 & 541 & 204 & 541 & 204 & \\
\hline 3 & 541 & 204 & 541 & 204 & \\
\hline 4 & 541 & 204 & 541 & 204 & \\
\hline $1-1978$ & 541 & 204 & 541 & 204 & \\
\hline 2 & 541 & 204 & 541 & 204 & \\
\hline 3 & 541 & 204 & 541 & 204 & \\
\hline 4 & 541 & 204 & 541 & 204 & \\
\hline $1-1979$ & 541 & 204 & 541 & 204 & \\
\hline 2 & 541 & 204 & 541 & 204 & \\
\hline 3 & 541 & 204 & 541 & 204 & \\
\hline 4 & 541 & 204 & 541 & 204 & \\
\hline $1-1980$ & 488 & 185 & 488 & 185 & \\
\hline 2 & 488 & 185 & 488 & 185 & \\
\hline 3 & 488 & 185 & 488 & 185 & \\
\hline 4 & 488 & 185 & 488 & 185 & \\
\hline $1-1981$ & 488 & 185 & 488 & 185 & \\
\hline 2 & 488 & 185 & 488 & 185 & \\
\hline 3 & 488 & 185 & 488 & 185 & \\
\hline 4 & 488 & 185 & 488 & 185 & \\
\hline $1-1982$ & 488 & 185 & 488 & 185 & \\
\hline 2 & 488 & 185 & 488 & 185 & \\
\hline 3 & 488 & 185 & 488 & 185 & \\
\hline 4 & 488 & 185 & 488 & 185 & \\
\hline $1-1983$ & 488 & 185 & 488 & 185 & \\
\hline 2 & 488 & 185 & 488 & 185 & \\
\hline 3 & 488 & 185 & 488 & 185 & \\
\hline 4 & 488 & 185 & 488 & 185 & \\
\hline $1-1984$ & 488 & 185 & 488 & 185 & \\
\hline 2 & 488 & 185 & 488 & 185 & \\
\hline 3 & 488 & 185 & 488 & 185 & \\
\hline 4 & 488 & 185 & 488 & 185 & \\
\hline $1-1985$ & 488 & 185 & 488 & 185 & \\
\hline 2 & 488 & 185 & 488 & 185 & \\
\hline 3 & 488 & 185 & 488 & 185 & \\
\hline 4 & 488 & 185 & 488 & 185 & \\
\hline $1-1986$ & 488 & 185 & 488 & 185 & 49 \\
\hline 2 & 488 & 185 & 488 & 185 & 49 \\
\hline
\end{tabular}


241-S-105 SINGLE-SHEL WHC-SD-WM-ER-323, REV. 0 L TANK LEVEL HISTORY

\begin{tabular}{|c|c|c|c|c|c|}
\hline Year & Total & Total & Solids & Solids & LOW \\
\hline & (K gal) & (in) & (K gal) & (in) & (in) \\
\hline 3 & 488 & 185 & 488 & 185 & 49 \\
\hline 4 & 488 & 185 & 488 & 185 & 50 \\
\hline $1-1987$ & 488 & 185 & 488 & 185 & 47 \\
\hline 2 & 488 & 185 & 488 & 185 & \\
\hline 3 & 488 & 185 & 488 & 185 & 50 \\
\hline 4 & 488 & 185 & 488 & 185 & 49 \\
\hline 1.1988 & 488 & 185 & 488 & 185 & 50 \\
\hline 2 & 488 & 185 & 488 & 185 & 50 \\
\hline 3 & 456 & 173 & 456 & 173 & 49 \\
\hline 4 & 456 & 173 & 456 & 173 & 51 \\
\hline $1-1989$ & 456 & 173 & 456 & 173 & \\
\hline 2 & 456 & 173 & 456 & 173 & 51 \\
\hline 3 & 456 & 173 & 456 & 173 & 50 \\
\hline 4 & 456 & 173 & 456 & 173 & 52 \\
\hline $1-1990$ & 456 & 173 & 456 & 173 & 51 \\
\hline 2 & 456 & 173 & 456 & 173 & \\
\hline 3 & 456 & 173 & 456 & 173 & 52 \\
\hline 4 & 456 & 173 & 456 & 173 & 53 \\
\hline $1-1991$ & 456 & 173 & 456 & 173 & 52 \\
\hline 2 & 456 & 173 & 456 & 173 & 53 \\
\hline 3 & 456 & 173 & 456 & 173 & 51 \\
\hline 4 & 456 & 173 & 456 & 173 & 53 \\
\hline $1-1992$ & 456 & 173 & 456 & 173 & \\
\hline 2 & 456 & 173 & 456 & 173 & 53 \\
\hline 3 & 456 & 173 & 456 & 173 & \\
\hline 4 & 456 & 173 & 456 & 173 & 52 \\
\hline $1-1993$ & 456 & 173 & 456 & 173 & 52 \\
\hline 2 & 456 & 173 & 456 & 173 & 53 \\
\hline 3 & 456 & 173 & 456 & 173 & 52 \\
\hline 4 & 456 & 173 & 456 & 173 & 52 \\
\hline $1-1994$ & & & & & 53 \\
\hline
\end{tabular}


241-S-106 SINGLE-SHELL WHC-SD-WM-ER-323, ReV. 0 TANK LEVEL HISTORY

\begin{tabular}{|c|c|c|c|c|c|}
\hline Year & Total & Total & Solids & Solids & LOW \\
\hline & (K gal) & (in) & (K gal) & (in) & (in) \\
\hline \multicolumn{5}{|l|}{1.1952} & \\
\hline \multicolumn{6}{|l|}{2} \\
\hline \multicolumn{6}{|l|}{3} \\
\hline \multicolumn{6}{|l|}{4} \\
\hline \multicolumn{6}{|l|}{$1-1953$} \\
\hline 2 & 426 & 162 & 0 & 0 & \\
\hline 3 & 729 & 273 & 0 & 0 & \\
\hline 4 & 725 & 271 & 0 & 0 & \\
\hline $1-1954$ & 725 & 271 & 0 & 0 & \\
\hline 2 & 733 & 274 & 0 & 0 & \\
\hline 3 & 737 & 275 & 0 & 0 & \\
\hline 4 & 712 & 266 & 0 & 0 & \\
\hline $1-1955$ & 711 & 266 & 0 & 0 & \\
\hline 2 & 541 & 204 & 0 & 0 & \\
\hline 3 & 539 & 203 & 0 & 0 & \\
\hline 4 & 754 & 282 & 0 & 0 & \\
\hline $1-1956$ & 754 & 282 & 0 & 0 & \\
\hline 2 & 754 & 282 & 0 & 0 & \\
\hline 3 & 754 & 282 & 0 & 0 & \\
\hline 4 & 754 & 282 & 0 & 0 & \\
\hline $1-1957$ & 748 & 279 & 0 & 0 & \\
\hline 2 & 748 & 279 & 0 & 0 & \\
\hline 3 & 750 & 280 & 0 & 0 & \\
\hline 4 & 750 & 280 & 0 & 0 & \\
\hline $1-1958$ & 750 & 280 & 0 & 0 & \\
\hline 2 & 750 & 280 & 0 & 0 & \\
\hline 3 & 747 & 279 & 0 & 0 & \\
\hline 4 & 747 & 279 & 0 & 0 & \\
\hline $1-1959$ & 739 & 276 & 0 & 0 & \\
\hline 2 & 747 & 279 & 0 & 0 & \\
\hline 3 & 747 & 279 & 0 & 0 & \\
\hline 4 & 747 & 279 & 0 & 0 & \\
\hline $1-1960$ & 744 & 278 & 0 & 0 & \\
\hline 2 & 744 & 278 & 0 & 0 & \\
\hline 3 & 744 & 278 & 0 & 0 & \\
\hline 4 & 747 & 279 & 0 & 0 & \\
\hline \multicolumn{6}{|l|}{$1-1961$} \\
\hline 2 & 747 & 279 & 0 & 0 & \\
\hline \multicolumn{6}{|l|}{3} \\
\hline 4 & 747 & 279 & 0 & 0 & \\
\hline \multicolumn{6}{|l|}{$1-1962$} \\
\hline 2 & 747 & 279 & 0 & 0 & \\
\hline \multicolumn{6}{|l|}{3} \\
\hline 4 & 747 & 279 & 0 & 0 & \\
\hline \multicolumn{6}{|l|}{$1-1963$} \\
\hline 2 & 747 & 279 & 0 & 0 & \\
\hline
\end{tabular}


241-S-106 SINGLE-SHELL WHC-SD-WM-ER-323, ReV. 0 TANK LEVEL HISTORY

\begin{tabular}{|c|c|c|c|c|c|}
\hline Year & Total & Total & Solids & Solids & LOW \\
\hline & ( $\mathrm{K}$ gal) & (in) & (K gal) & (in) & (in) \\
\hline & & & & & \\
\hline \multicolumn{6}{|l|}{3} \\
\hline 4 & 744 & 278 & 0 & 0 & \\
\hline \multicolumn{6}{|l|}{$1-1964$} \\
\hline 2 & 744 & 278 & 0 & 0 & \\
\hline \multicolumn{6}{|l|}{3} \\
\hline 4 & 744 & 278 & 0 & 0 & \\
\hline \multicolumn{6}{|l|}{$1-1965$} \\
\hline 2 & 761 & 284 & 13 & 12 & \\
\hline 3 & 761 & 284 & 13 & 12 & \\
\hline 4 & 761 & 284 & 13 & 12 & \\
\hline $1-1966$ & 761 & 284 & 13 & 12 & \\
\hline 2 & 761 & 284 & 13 & 12 & \\
\hline 3 & 761 & 284 & 13 & 12 & \\
\hline 4 & 761 & 284 & 13 & 12 & \\
\hline $1-1967$ & 761 & 284 & 13 & 12 & \\
\hline 2 & 761 & 284 & 13 & 12 & \\
\hline 3 & 761 & 284 & 13 & 12 & \\
\hline 4 & 761 & 284 & 13 & 12 & \\
\hline $1-1968$ & 761 & 284 & 13 & 12 & \\
\hline 2 & 761 & 284 & 13 & 12 & \\
\hline 3 & 761 & 284 & 13 & 12 & \\
\hline 4 & 758 & 283 & 13 & 12 & \\
\hline $1-1969$ & 759 & 283 & 13 & 12 & \\
\hline 2 & 759 & 283 & 13 & 12 & \\
\hline 3 & 759 & 283 & 13 & 12 & \\
\hline 4 & 759 & 283 & 32 & 19 & \\
\hline $1-1970$ & 760 & 284 & 32 & 19 & \\
\hline 2 & 760 & 284 & 32 & 19 & \\
\hline 3 & 760 & 284 & 32 & 19 & \\
\hline 4 & 760 & 284 & 32 & 19 & \\
\hline $1-1971$ & 760 & 284 & 32 & 19 & \\
\hline 2 & 759 & 283 & 32 & 19 & \\
\hline 3 & 759 & 283 & 32 & 19 & \\
\hline 4 & 759 & 283 & 32 & 19 & \\
\hline $1-1972$ & 759 & 283 & 32 & 19 & \\
\hline 2 & 759 & 283 & 32 & 19 & \\
\hline 3 & 759 & 283 & 32 & 19 & \\
\hline 4 & 759 & 283 & 32 & 19 & \\
\hline $1-1973$ & 759 & 283 & 32 & 19 & - \\
\hline 2 & 753 & 281 & 32 & 19 & \\
\hline 3 & 766 & 286 & 32 & 19 & \\
\hline 4 & 657 & 246 & 384 & 147 & \\
\hline $1-1974$ & 585 & 220 & 436 & 166 & \\
\hline 2 & 584 & 220 & 436 & 166 & \\
\hline 3 & 669 & 251 & 436 & 166 & \\
\hline 4 & 733 & 274 & 557 & 210 & \\
\hline
\end{tabular}


241-S-106 SINGLE-SHELL WHC-SD-WM-ER-323， ReV.0 TANK LEVEL HISTORY

\begin{tabular}{|c|c|c|c|c|c|}
\hline Year & Total & Total & Solids & Solids & LOW \\
\hline & ( $\mathrm{K}$ gal) & (in) & ( $\mathrm{K}$ gal) & (in) & (in) \\
\hline & & & & & \\
\hline $1-1975$ & 684 & 256 & 607 & 228 & \\
\hline 2 & 686 & 257 & 607 & 228 & \\
\hline 3 & 673 & 252 & 673 & 252 & \\
\hline 4 & 673 & 252 & 673 & 252 & \\
\hline $1-1976$ & 692 & 259 & 673 & 252 & \\
\hline 2 & 695 & 260 & 673 & 252 & \\
\hline 3 & 700 & 262 & 673 & 252 & \\
\hline 4 & 703 & 263 & 673 & 252 & \\
\hline $1-1977$ & 703 & 263 & 582 & 219 & \\
\hline 2 & 703 & 263 & 582 & 219 & \\
\hline 3 & 703 & 263 & 582 & 219 & \\
\hline 4 & 706 & 264 & 582 & 219 & \\
\hline $1-1978$ & 706 & 264 & 582 & 219 & \\
\hline 2 & 706 & 264 & 582 & 219 & \\
\hline 3 & 700 & 262 & 582 & 219 & \\
\hline 4 & 700 & 262 & 582 & 219 & \\
\hline $1-1979$ & 703 & 263 & 582 & 219 & \\
\hline 2 & 612 & 230 & 582 & 219 & \\
\hline 3 & 612 & 230 & 612 & 230 & \\
\hline 4 & 612 & 230 & 612 & 230 & \\
\hline $1-1980$ & 612 & 230 & 612 & 230 & \\
\hline 2 & 612 & 230 & 612 & 230 & \\
\hline 3 & 612 & 230 & 612 & 230 & \\
\hline 4 & 612 & 230 & 612 & 230 & \\
\hline $1-1981$ & 612 & 230 & 612 & 230 & \\
\hline 2 & 612 & 230 & 612 & 230 & \\
\hline 3 & 612 & 230 & 612 & 230 & \\
\hline 4 & 612 & 230 & 612 & 230 & \\
\hline $1-1982$ & 612 & 230 & 612 & 230 & \\
\hline 2 & 612 & 230 & 611 & 230 & \\
\hline 3 & 543 & 205 & 543 & 205 & \\
\hline 4 & 543 & 205 & 543 & 205 & \\
\hline $1-1983$ & 543 & 205 & 543 & 205 & \\
\hline 2 & 543 & 205 & 543 & 205 & \\
\hline 3 & 543 & 205 & 543 & 205 & \\
\hline 4 & 543 & 205 & 543 & 205 & \\
\hline $1-1984$ & 543 & 205 & 543 & 205 & \\
\hline 2 & 543 & 205 & 543 & 205 & \\
\hline 3 & 543 & 205 & 543 & 205 & $=$ \\
\hline 4 & 543 & 205 & 543 & 205 & \\
\hline $1-1985$ & 543 & 205 & 543 & 205 & \\
\hline 2 & 543 & 205 & 543 & 205 & \\
\hline 3 & 543 & 205 & 543 & 205 & \\
\hline 4 & 543 & 205 & 543 & 205 & \\
\hline $1-1986$ & 543 & 205 & 543 & 205 & 171 \\
\hline 2 & 543 & 205 & 543 & 205 & 171 \\
\hline
\end{tabular}


241-S-106 SINGLE-SHELL WHC-SD-WM-ER-323，REV.0 TANK LEVEL HISTORY

\begin{tabular}{|c|c|c|c|c|c|}
\hline Year & Total & Total & Solids & Solids & LOW \\
\hline & (K gal) & (in) & (K gal) & (in) & (in) \\
\hline 3 & 543 & 205 & 543 & 205 & 171 \\
\hline 4 & 543 & 205 & 543 & 205 & 172 \\
\hline $1-1987$ & 543 & 205 & 543 & 205 & 170 \\
\hline 2 & 543 & 205 & 543 & 205 & \\
\hline 3 & 543 & 205 & 543 & 205 & 173 \\
\hline 4 & 543 & 205 & 543 & 205 & 173 \\
\hline $1-1988$ & 543 & 205 & 543 & 205 & 173 \\
\hline 2 & 543 & 205 & 543 & 205 & 174 \\
\hline 3 & 543 & 205 & 543 & 205 & 173 \\
\hline 4 & 543 & 205 & 543 & 205 & 173 \\
\hline $1-1989$ & 543 & 205 & 543 & 205 & \\
\hline 2 & 543 & 205 & 543 & 205 & 175 \\
\hline 3 & 543 & 205 & 543 & 205 & 175 \\
\hline 4 & 543 & 205 & 543 & 205 & 75 \\
\hline $1-1990$ & 543 & 205 & 543 & 205 & 174 \\
\hline 2 & 543 & 205 & 543 & 205 & \\
\hline 3 & 543 & 205 & 543 & 205 & 176 \\
\hline 4 & 543 & 205 & 543 & 205 & 175 \\
\hline $1-1991$ & 543 & 205 & 543 & 205 & 176 \\
\hline 2 & 543 & 205 & 543 & 205 & 176 \\
\hline 3 & 543 & 205 & 543 & 205 & 177 \\
\hline 4 & 543 & 205 & 543 & 205 & 178 \\
\hline $1-1992$ & 543 & 205 & 543 & 205 & \\
\hline 2 & 543 & 205 & 543 & 205 & 177 \\
\hline 3 & 543 & 205 & 543 & 205 & \\
\hline 4 & 543 & 205 & 543 & 205 & 177 \\
\hline $1-1993$ & 543 & 205 & 543 & 205 & 178 \\
\hline 2 & 543 & 205 & 543 & 205 & 177 \\
\hline 3 & 543 & 205 & 543 & 205 & 178 \\
\hline 4 & 479 & 182 & 475 & 180 & 177 \\
\hline $1-1994$ & 479 & 182 & 475 & 180 & 177 \\
\hline
\end{tabular}


241-S-107 SINGLE-SHELL WHC-SD-WM-ER-323, ReV. 0 TANK LEVEL HISTORY

\begin{tabular}{|c|c|c|c|c|c|}
\hline Year & Total & Total & Solids & Solids & \\
\hline & (K gal) & (in) & (K gal) & (in) & \\
\hline \multirow{2}{*}{\multicolumn{6}{|c|}{$1-1952$}} \\
\hline & & & & & \\
\hline \multicolumn{6}{|l|}{2} \\
\hline \multicolumn{6}{|l|}{3} \\
\hline 4 & 763 & 285 & 0 & 0 & \\
\hline $1-1953$ & 763 & 285 & 0 & 0 & \\
\hline 2 & 758 & 283 & 0 & 0 & \\
\hline 3 & 751 & 281 & 0 & 0 & \\
\hline 4 & 748 & 279 & 0 & 0 & \\
\hline $1-1954$ & 748 & 279 & 0 & 0 & \\
\hline 2 & 754 & 282 & 0 & 0 & \\
\hline 3 & 722 & 270 & 0 & 0 & \\
\hline 4 & 758 & 283 & 0 & 0 & \\
\hline $1-1955$ & 758 & 283 & 0 & 0 & \\
\hline 2 & 758 & 283 & 0 & 0 & \\
\hline 3 & 685 & 257 & 0 & 0 & \\
\hline 4 & 741 & 277 & 0 & 0 & \\
\hline $1-1956$ & 757 & 283 & 0 & 0 & \\
\hline 2 & 757 & 283 & 0 & 0 & \\
\hline 3 & 757 & 283 & 0 & 0 & \\
\hline 4 & 757 & 283 & 0 & 0 & \\
\hline 1.1957 & 750 & 280 & 0 & 0 & \\
\hline 2 & 750 & 280 & 0 & 0 & \\
\hline 3 & 596 & 224 & 0 & 0 & \\
\hline 4 & 689 & 258 & 0 & 0 & \\
\hline $1-1958$ & 653 & 245 & 0 & 0 & \\
\hline 2 & 538 & 203 & 0 & 0 & \\
\hline 3 & 618 & 232 & 0 & 0 & \\
\hline 4 & 642 & 241 & 0 & 0 & \\
\hline $1-1959$ & 686 & 257 & 0 & 0 & \\
\hline 2 & 528 & 199 & 0 & 0 & \\
\hline 3 & 562 & 212 & 0 & 0 & \\
\hline 4 & 607 & 228 & 0 & 0 & \\
\hline $1-1960$ & 637 & 239 & 0 & 0 & \\
\hline 2 & 564 & 213 & 0 & 0 & \\
\hline 3 & 598 & 225 & 0 & 0 & \\
\hline 4 & 536 & 202 & 0 & 0 & \\
\hline \multicolumn{6}{|l|}{$1-1961$} \\
\hline 2 & 645 & 242 & 0 & 0 & \\
\hline \multicolumn{6}{|l|}{3} \\
\hline 4 & 560 & 211 & 0 & 0 & \\
\hline \multicolumn{6}{|l|}{$1-1962$} \\
\hline 2 & 659 & 247 & 0 & 0 & \\
\hline \multicolumn{6}{|l|}{3} \\
\hline 4 & 555 & 209 & 0 & 0 & \\
\hline \multicolumn{6}{|l|}{$1-1963$} \\
\hline 2 & 527 & 199 & 0 & 0 & \\
\hline
\end{tabular}


241-S-107 SINGLE-SHELL WHC-SD-WM-ER-323, ReV. 0 TANK LEVEL HISTORY

\begin{tabular}{|c|c|c|c|c|c|}
\hline Year & Total & Total & Solids & Solids & \\
\hline & (K gal) & (in) & (K gal) & (in) & \\
\hline \multirow{2}{*}{\multicolumn{6}{|c|}{3}} \\
\hline & & & & & \\
\hline 4 & 615 & 231 & 0 & 0 & \\
\hline \multicolumn{6}{|l|}{$1-1964$} \\
\hline 2 & 590 & 222 & 0 & 0 & \\
\hline \multicolumn{6}{|l|}{3} \\
\hline 4 & 628 & 236 & 0 & 0 & \\
\hline \multicolumn{6}{|l|}{$1-1965$} \\
\hline 2 & 508 & 192 & 194 & 78 & \\
\hline 3 & 587 & 221 & 194 & 78 & \\
\hline 4 & 669 & 251 & 194 & 78 & \\
\hline $1-1966$ & 565 & 213 & 194 & 78 & \\
\hline 2 & 568 & 214 & 194 & 78 & \\
\hline 3 & 613 & 230 & 194 & 78 & \\
\hline 4 & 642 & 241 & 194 & 78 & \\
\hline $1-1967$ & 656 & 246 & 194 & 78 & \\
\hline 2 & 656 & 246 & 194 & 78 & \\
\hline 3 & 656 & 246 & 194 & 78 & \\
\hline 4 & 656 & 246 & 194 & 78 & \\
\hline $1-1968$ & 654 & 245 & 194 & 78 & \\
\hline 2 & 654 & 245 & 194 & 78 & \\
\hline 3 & 305 & 118 & 194 & 78 & \\
\hline 4 & 325 & 126 & 194 & 78 & \\
\hline $1-1969$ & 415 & 158 & 194 & 78 & \\
\hline 2 & 476 & 181 & 194 & 78 & \\
\hline 3 & 577 & 217 & 194 & 78 & \\
\hline 4 & 451 & 171 & 285 & 111 & \\
\hline $1-1970$ & 477 & 181 & 285 & 111 & \\
\hline 2 & 532 & 201 & 293 & 114 & \\
\hline 3 & 343 & 132 & 293 & 114 & \\
\hline 4 & 449 & 171 & 293 & 114 & \\
\hline 1.1971 & 536 & 202 & 293 & 114 & \\
\hline 2 & 589 & 222 & 293 & 114 & \\
\hline 3 & 609 & 229 & 293 & 114 & \\
\hline 4 & 622 & 234 & 293 & 114 & \\
\hline $1-1972$ & 655 & 246 & 293 & 114 & \\
\hline 2 & 756 & 282 & 293 & 114 & \\
\hline 3 & 756 & 282 & 293 & 114 & \\
\hline 4 & 757 & 283 & 293 & 114 & \\
\hline $1-1973$ & 757 & 283 & 293 & 114 & \\
\hline 2 & 749 & 280 & 293 & 114 & \\
\hline 3 & 761 & 284 & 293 & 114 & \\
\hline 4 & 686 & 257 & 293 & 114 & \\
\hline $1-1974$ & 731 & 273 & 293 & 114 & \\
\hline 2 & 492 & 186 & 343 & 132 & 1 \\
\hline 3 & 468 & 178 & 282 & 110 & \\
\hline 4 & 519 & 196 & 343 & 132 & \\
\hline
\end{tabular}


241-S-107 SINGLE-SHELL WHC-SD-WM-ER-323， Rev. 0 TANK LEVEL HISTORY

\begin{tabular}{|c|c|c|c|c|c|}
\hline Year & Total & Total & Solids & Solids & \\
\hline & (K gal) & (in) & (K gal) & (in) & \\
\hline $1-1975$ & 475 & 180 & 343 & 132 & \\
\hline 2 & 477 & 181 & 343 & 132 & \\
\hline 3 & 480 & 182 & 343 & 132 & \\
\hline 4 & 491 & 186 & 343 & 132 & \\
\hline $1-1976$ & 656 & 246 & 343 & 132 & \\
\hline 2 & 703 & 263 & 343 & 132 & \\
\hline 3 & 700 & 262 & 343 & 132 & \\
\hline 4 & 431 & 164 & 343 & 132 & \\
\hline $1-1977$ & 455 & 173 & 343 & 132 & \\
\hline 2 & 585 & 220 & 343 & 132 & \\
\hline 3 & 717 & 268 & 343 & 132 & \\
\hline 4 & 409 & 156 & 343 & 132 & \\
\hline $1-1978$ & 422 & 161 & 343 & 132 & \\
\hline 2 & 719 & 269 & 343 & 132 & \\
\hline 3 & 497 & 188 & 343 & 132 & \\
\hline 4 & 711 & 266 & 343 & 132 & \\
\hline $1-1979$ & 425 & 162 & 343 & 132 & \\
\hline 2 & 692 & 259 & 343 & 132 & \\
\hline 3 & 684 & 256 & 343 & 132 & \\
\hline 4 & 692 & 259 & 343 & 132 & \\
\hline $1-1980$ & 450 & 171 & 343 & 132 & \\
\hline 2 & 476 & 181 & 343 & 132 & \\
\hline 3 & 346 & 133 & 340 & 131 & \\
\hline 4 & 346 & 133 & 340 & 131 & \\
\hline 1.1981 & 346 & 133 & 340 & 131 & \\
\hline 2 & 346 & 133 & 340 & 131 & \\
\hline 3 & 346 & 133 & 340 & 131 & \\
\hline 4 & 346 & 133 & 340 & 131 & \\
\hline $1-1982$ & 346 & 133 & 340 & 131 & \\
\hline 2 & 346 & 133 & 340 & 131 & \\
\hline 3 & 346 & 133 & 340 & 131 & \\
\hline 4 & 346 & 133 & 340 & 131 & \\
\hline $1-1983$ & 346 & 133 & 340 & 131 & \\
\hline 2 & 346 & 133 & 340 & 131 & \\
\hline 3 & 346 & 133 & 340 & 131 & \\
\hline 4 & 346 & 133 & 340 & 131 & \\
\hline $1-1984$ & 346 & 133 & 340 & 131 & \\
\hline 2 & 346 & 133 & 340 & 131 & \\
\hline 3 & 346 & 133 & 340 & 131 & \\
\hline 4 & 346 & 133 & 340 & 131 & \\
\hline $1-1985$ & 346 & 133 & 340 & 131 & \\
\hline 2 & 346 & 133 & 340 & 131 & \\
\hline 3 & 346 & 133 & 340 & 131 & \\
\hline 4 & 346 & 133 & 340 & 131 & \\
\hline $1-1986$ & 346 & 133 & 340 & 131 & \\
\hline 2 & 346 & 133 & 340 & 131 & \\
\hline
\end{tabular}


241-S-107 SINGLE-SHELL WHC-SD-WM-ER-323, ReV. 0 TANK LEVEL HISTORY

\begin{tabular}{|c|c|c|c|c|}
\hline Year & Total & Total & Solids & Solids \\
\hline & (K gal) & (in) & (K gal) & (in) \\
\hline 3 & & 911 & & \\
\hline$\frac{3}{4}$ & $\frac{368}{200}$ & 141 & $\frac{362}{262}$ & 139 \\
\hline 4 & 368 & 741 & 362 & 139 \\
\hline $1-1987$ & 368 & 141 & 362 & 139 \\
\hline 2 & 368 & 141 & 362 & 139 \\
\hline 3 & 368 & 141 & 362 & 139 \\
\hline 4 & 368 & 141 & 362 & 139 \\
\hline $1-1988$ & 368 & 141 & 362 & 139 \\
\hline 2 & 368 & 141 & 362 & 139 \\
\hline 3 & 368 & 141 & 362 & 139 \\
\hline 4 & 368 & 141 & 362 & 139 \\
\hline $1-1989$ & 368 & 141 & 362 & 139 \\
\hline 2 & 368 & 141 & 362 & 139 \\
\hline 3 & 368 & 141 & 362 & 139 \\
\hline 4 & 368 & 141 & 362 & 139 \\
\hline $1-1990$ & 368 & 141 & 362 & 139 \\
\hline 2 & 368 & 141 & 362 & 139 \\
\hline 3 & 368 & 141 & 362 & 139 \\
\hline 4 & 368 & 141 & 362 & 139 \\
\hline 1-1991 & 368 & 141 & 362 & 139 \\
\hline 2 & 368 & 141 & 362 & 139 \\
\hline 3 & 368 & 141 & 362 & 139 \\
\hline 4 & 368 & 141 & 362 & 139 \\
\hline 1-1992 & 368 & 141 & 362 & 139 \\
\hline 2 & 368 & 141 & 362 & 139 \\
\hline 3 & 368 & 141 & 362 & 139 \\
\hline 4 & 368 & 141 & 362 & 139 \\
\hline $1-1993$ & 368 & 141 & 362 & 139 \\
\hline 2 & 368 & 141 & 362 & 139 \\
\hline 3 & 368 & 141 & 362 & 139 \\
\hline 4 & 376 & 144 & 362 & 139 \\
\hline
\end{tabular}


241-S-108 SINGLE-SHELL WHC-SD-WM-ER-323, ReV.0 TANK LEVEL HISTORY

\begin{tabular}{|c|c|c|c|c|c|}
\hline Year & Total & Total & Solids & Solids & LOW \\
\hline & (K gal) & (in) & (K gal) & (in) & (in) \\
\hline \multirow{2}{*}{\multicolumn{6}{|c|}{1.1952}} \\
\hline & & & & & \\
\hline \multicolumn{6}{|l|}{2} \\
\hline \multicolumn{6}{|l|}{3} \\
\hline 4 & 760 & 284 & 0 & 0 & \\
\hline $1-1953$ & 760 & 284 & 0 & 0 & \\
\hline 2 & 758 & 283 & 0 & 0 & \\
\hline 3 & 751 & 281 & 0 & 0 & \\
\hline 4 & 747 & 279 & 0 & 0 & \\
\hline $1-1954$ & 747 & 279 & 0 & 0 & \\
\hline 2 & 743 & 278 & 0 & 0 & \\
\hline 3 & 740 & 277 & 0 & 0 & \\
\hline 4 & 758 & 283 & 0 & 0 & \\
\hline $1-1955$ & 758 & 283 & 0 & 0 & \\
\hline 2 & 758 & 283 & 0 & 0 & \\
\hline 3 & 758 & 283 & 0 & 0 & \\
\hline 4 & 758 & 283 & 0 & 0 & \\
\hline $1-1956$ & 758 & 283 & 0 & 0 & \\
\hline 2 & 758 & 283 & 0 & 0 & \\
\hline 3 & 758 & 283 & 0 & 0 & \\
\hline 4 & 758 & 283 & 0 & 0 & \\
\hline $1-1957$ & 765 & 286 & 0 & 0 & \\
\hline 2 & 763 & 285 & 0 & $\overline{0}$ & \\
\hline 3 & 763 & 285 & 0 & 0 & \\
\hline 4 & 763 & 285 & 0 & 0 & \\
\hline $1-1958$ & 761 & 284 & 0 & 0 & \\
\hline 2 & 761 & 284 & 0 & 0 & \\
\hline 3 & 761 & 284 & 0 & 0 & \\
\hline 4 & 761 & 284 & 0 & 0 & \\
\hline $1-1959$ & 761 & 284 & 0 & 0 & \\
\hline 2 & 761 & 284 & 0 & 0 & \\
\hline 3 & 761 & 284 & 0 & 0 & \\
\hline 4 & 761 & 284 & 0 & 0 & \\
\hline $1-1960$ & 758 & 283 & 0 & 0 & \\
\hline 2 & 758 & 283 & 0 & 0 & \\
\hline 3 & 758 & 283 & 0 & 0 & \\
\hline 4 & 758 & 283 & 0 & 0 & \\
\hline \multicolumn{6}{|l|}{$1-1961$} \\
\hline 2 & 755 & 282 & 0 & 0 & \\
\hline \multicolumn{6}{|l|}{3} \\
\hline 4 & 755 & 282 & 0 & 0 & \\
\hline \multicolumn{6}{|l|}{$1-1962$} \\
\hline 2 & 755 & 282 & 0 & 0 & \\
\hline \multicolumn{6}{|l|}{3} \\
\hline 4 & 755 & 282 & 0 & 0 & \\
\hline \multicolumn{6}{|l|}{$1-1963$} \\
\hline 2 & 755 & 282 & 0 & 0 & \\
\hline
\end{tabular}


241-S-108 SINGLE-SHELL WHC-SD-WM-ER-323, ReV. 0 TANK LEVEL HISTORY

\begin{tabular}{|c|c|c|c|c|c|}
\hline Year & Total & Total & Solids & Solids & LOW \\
\hline & (K gal) & (in) & ( $\mathrm{K}$ gal) & (in) & (in) \\
\hline \multicolumn{6}{|l|}{3} \\
\hline 4 & 755 & 282 & 0 & 0 & \\
\hline \multicolumn{6}{|l|}{$1-1964$} \\
\hline 2 & 755 & 282 & 0 & 0 & \\
\hline \multicolumn{6}{|l|}{3} \\
\hline 4 & 755 & 282 & 0 & 0 & \\
\hline \multicolumn{6}{|l|}{$1-1965$} \\
\hline 2 & 758 & 283 & 4 & 9 & \\
\hline 3 & 758 & 283 & 4 & 9 & \\
\hline 4 & 758 & 283 & 4 & 9 & \\
\hline $1-1966$ & 758 & 283 & 4 & 9 & \\
\hline 2 & 758 & 283 & 4 & 9 & \\
\hline 3 & 758 & 283 & 4 & 9 & \\
\hline 4 & 758 & 283 & 4 & 9 & \\
\hline $1-1967$ & 758 & 283 & 4 & 9 & \\
\hline 2 & 758 & 283 & 4 & 9 & \\
\hline 3 & 758 & 283 & 4 & 9 & \\
\hline 4 & 758 & 283 & 4 & 9 & \\
\hline $1-1968$ & 758 & 283 & 4 & 9 & \\
\hline 2 & 757 & 283 & 4 & 9 & \\
\hline 3 & 757 & 283 & 4 & 9 & \\
\hline 4 & 757 & 283 & 4 & 9 & \\
\hline $1-1969$ & 757 & 283 & 4 & 9 & \\
\hline 2 & 757 & 283 & 4 & 9 & \\
\hline 3 & 757 & 283 & 4 & 9 & \\
\hline 4 & 757 & 283 & 5 & 9 & \\
\hline $1-1970$ & 756 & 282 & 5 & 9 & \\
\hline 2 & 756 & 282 & 5 & 9 & \\
\hline 3 & 756 & 282 & 5 & 9 & \\
\hline 4 & 756 & 282 & 5 & 9 & \\
\hline 1.1971 & 756 & 282 & 5 & 9 & \\
\hline 2 & 756 & 282 & 5 & 9 & \\
\hline 3 & 756 & 282 & 5 & 9 & \\
\hline 4 & 756 & 282 & 5 & 9 & \\
\hline $1-1972$ & 756 & 282 & 5 & 9 & \\
\hline 2 & 756 & 282 & 5 & 9 & \\
\hline 3 & 756 & 282 & 5 & 9 & \\
\hline 4 & 758 & 283 & 5 & 9 & \\
\hline $1-1973$ & 757 & 283 & 5 & 9 & \\
\hline 2 & 748 & 279 & 5 & 9 & \\
\hline 3 & 761 & 284 & 5 & 9 & \\
\hline 4 & & 7 & & 7 & \\
\hline $1-1974$ & 704 & 263 & 373 & 143 & \\
\hline 2 & 718 & 269 & 585 & 220 & \\
\hline 3 & 706 & 264 & 702 & 263 & \\
\hline 4 & 719 & 269 & 706 & 264 & \\
\hline
\end{tabular}


241-S-108 SINGLE-SHELL WHC-SD-WM-ER-323， ReV. 0 TANK LEVEL HISTORY

\begin{tabular}{|c|c|c|c|c|c|}
\hline Year & Total & Total & Solids & Solids & LOW \\
\hline & (K gal) & (in) & ( $\mathrm{K}$ gal) & (in) & (in) \\
\hline & & & & & \\
\hline $1-1975$ & 717 & 268 & 706 & 264 & \\
\hline 2 & 722 & 270 & 706 & 264 & \\
\hline 3 & 728 & 272 & 706 & 264 & \\
\hline 4 & 725 & 271 & 706 & 264 & \\
\hline $1-1976$ & 728 & 272 & 706 & 264 & \\
\hline 2 & 728 & 272 & 706 & 264 & \\
\hline 3 & 730 & 273 & 706 & 264 & \\
\hline 4 & 730 & 273 & 706 & 264 & \\
\hline $1-1977$ & 733 & 274 & 670 & 251 & \\
\hline 2 & 733 & 274 & 670 & 251 & \\
\hline 3 & 733 & 274 & 670 & 251 & \\
\hline 4 & 733 & 274 & 670 & 251 & \\
\hline $1-1978$ & 733 & 274 & 670 & 251 & \\
\hline 2 & 733 & 274 & 670 & 251 & \\
\hline 3 & 719 & 269 & 670 & 251 & \\
\hline 4 & 692 & 259 & 670 & 251 & \\
\hline $1-1979$ & 692 & 259 & 670 & 251 & \\
\hline 2 & 670 & 251 & 670 & 251 & \\
\hline 3 & 670 & 251 & 670 & 251 & \\
\hline 4 & 670 & 251 & 612 & 230 & \\
\hline $1-1980$ & 612 & 230 & 612 & 230 & \\
\hline 2 & 612 & 230 & 612 & 230 & \\
\hline 3 & 612 & 230 & 612 & 230 & \\
\hline 4 & 612 & 230 & 612 & 230 & \\
\hline $1-1981$ & 612 & 230 & 612 & 230 & \\
\hline 2 & 612 & 230 & 612 & 230 & \\
\hline 3 & 612 & 230 & 612 & 230 & \\
\hline 4 & 612 & 230 & 612 & 230 & \\
\hline $1-1982$ & 612 & 230 & 612 & 230 & \\
\hline 2 & 604 & 227 & 604 & 227 & \\
\hline 3 & 604 & 227 & 604 & 227 & \\
\hline 4 & 604 & 227 & 604 & 227 & \\
\hline $1-1983$ & 604 & 227 & 604 & 227 & \\
\hline 2 & 604 & 227 & 604 & 227 & \\
\hline 3 & 604 & 227 & 604 & 227 & \\
\hline 4 & 604 & 227 & 604 & 227 & \\
\hline $1-1984$ & 604 & 227 & 604 & 227 & \\
\hline 2 & 604 & 227 & 604 & 227 & \\
\hline 3 & 604 & 227 & 604 & 227 & \\
\hline 4 & 604 & 227 & 604 & 227 & \\
\hline $1-1985$ & 604 & 227 & 604 & 227 & \\
\hline 2 & 604 & 227 & 604 & 227 & \\
\hline 3 & 604 & 227 & 604 & 227 & \\
\hline 4 & 604 & 227 & 604 & 227 & \\
\hline $1-1986$ & 604 & 227 & 604 & 227 & 138 \\
\hline 2 & 604 & 227 & 604 & 227 & 138 \\
\hline
\end{tabular}


241-S-108 SINGLE-SHELL WHC-SD-WM-ER-323, ReV. 0 TANK LEVEL HISTORY

\begin{tabular}{|l|c|c|c|c|c|}
\hline Year & Total & Total & Solids & Solids & LOW \\
\hline & (K gal) & (in) & (K gal) & (in) & (in) \\
\hline 3 & & & & & \\
\hline 4 & 604 & 227 & 604 & 227 & 136 \\
\hline $1-1987$ & 604 & 227 & 604 & 227 & 138 \\
\hline 2 & 604 & 227 & 604 & 227 & 137 \\
\hline 3 & 604 & 227 & 604 & 227 & \\
\hline 4 & 604 & 227 & 604 & 227 & 137 \\
\hline $1-1988$ & 604 & 227 & 604 & 227 & 137 \\
\hline 2 & 604 & 227 & 604 & 227 & 136 \\
\hline 3 & 604 & 227 & 604 & 227 & 136 \\
\hline 4 & 604 & 227 & 604 & 227 & 136 \\
\hline $1-1989$ & 604 & 227 & 604 & 227 & 137 \\
\hline 2 & 604 & 227 & 604 & 227 & \\
\hline 3 & 604 & 227 & 604 & 227 & 137 \\
\hline 4 & 604 & 227 & 604 & 227 & 136 \\
\hline $1-1990$ & 604 & 227 & 604 & 227 & 137 \\
\hline 2 & 604 & 227 & 604 & 227 & 136 \\
\hline 3 & 604 & 227 & 604 & 227 & \\
\hline 4 & 604 & 227 & 604 & 227 & 135 \\
\hline $1-1991$ & 604 & 227 & 604 & 227 & 137 \\
\hline 2 & 604 & 227 & 604 & 227 & 136 \\
\hline 3 & 604 & 227 & 604 & 227 & 134 \\
\hline 4 & 604 & 227 & 604 & 227 & 135 \\
\hline $1-1992$ & 604 & 227 & 604 & 227 & 136 \\
\hline 2 & 604 & 227 & 604 & 227 & \\
\hline 3 & 604 & 227 & 604 & 227 & 136 \\
\hline 4 & 604 & 227 & 604 & 227 & \\
\hline $1-1993$ & 604 & 227 & 604 & 227 & 136 \\
\hline 2 & 604 & 227 & 604 & 227 & 135 \\
\hline 3 & 604 & 227 & 604 & 227 & 135 \\
\hline 4 & & & & & 135 \\
\hline & & & & & 135 \\
\hline
\end{tabular}


241-S-109 SINGLE-SHELL WHC-SD-WM-ER-323, Rev. 0 TANK LEVEL HISTORY

\begin{tabular}{|c|c|c|c|c|c|}
\hline Year & Total & Total & Solids & Solids & LOW \\
\hline & (K gal) & (in) & (K gal) & (in) & (in) \\
\hline \multirow{2}{*}{\multicolumn{6}{|c|}{$1-1952$}} \\
\hline & & & & & \\
\hline \multicolumn{6}{|l|}{2} \\
\hline \multicolumn{6}{|l|}{3} \\
\hline 4 & 103 & 45 & & & \\
\hline $1-1953$ & 582 & 219 & & & \\
\hline 2 & 585 & 220 & 0 & 0 & \\
\hline 3 & 575 & 217 & 0 & 0 & \\
\hline 4 & 579 & 218 & 0 & 0 & \\
\hline $1-1954$ & 579 & 218 & 0 & 0 & \\
\hline 2 & 576 & 217 & 0 & 0 & \\
\hline 3 & 576 & 217 & 0 & 0 & \\
\hline 4 & 576 & 217 & 0 & 0 & \\
\hline $1-1955$ & 704 & 263 & 0 & 0 & \\
\hline 2 & 747 & 279 & 0 & 0 & \\
\hline 3 & 747 & 279 & 0 & 0 & \\
\hline 4 & 747 & 279 & 0 & 0 & \\
\hline $1-1956$ & 747 & 279 & 0 & 0 & \\
\hline 2 & 747 & 279 & 0 & 0 & \\
\hline 3 & 747 & 279 & 0 & 0 & \\
\hline 4 & 747 & 279 & 0 & 0 & \\
\hline $1-1957$ & 764 & 285 & 0 & 0 & \\
\hline 2 & 764 & 285 & 0 & 0 & \\
\hline 3 & 763 & 285 & 0 & 0 & \\
\hline 4 & 763 & 285 & 0 & 0 & \\
\hline $1-1958$ & 763 & 285 & 0 & 0 & \\
\hline 2 & 763 & 285 & 0 & 0 & \\
\hline 3 & 763 & 285 & 0 & 0 & \\
\hline 4 & 763 & 285 & 0 & 0 & \\
\hline $1-1959$ & 763 & 285 & 0 & 0 & \\
\hline 2 & 763 & 285 & 0 & 0 & \\
\hline 3 & 763 & 285 & 0 & 0 & \\
\hline 4 & 763 & 285 & 0 & 0 & \\
\hline $1-1960$ & 761 & 284 & 0 & 0 & \\
\hline 2 & 761 & 284 & 0 & 0 & \\
\hline 3 & 761 & 284 & 0 & 0 & \\
\hline 4 & 763 & 285 & 0 & 0 & \\
\hline \multicolumn{6}{|l|}{$1-1961$} \\
\hline 2 & 761 & 284 & 0 & 0 & \\
\hline \multicolumn{6}{|l|}{3} \\
\hline 4 & 761 & 284 & 0 & 0 & \\
\hline \multicolumn{6}{|l|}{$1-1962$} \\
\hline 2 & 761 & 284 & 0 & 0 & \\
\hline \multicolumn{6}{|l|}{3} \\
\hline 4 & 761 & 284 & 0 & 0 & \\
\hline $1-1963$ & & & & & \\
\hline 2 & 761 & 284 & 0 & 0 & \\
\hline
\end{tabular}


241-S-109 SINGLE-SHELL WHC-SD-WM-ER-323, Rev. 0 TANK LEVEL HISTORY

\begin{tabular}{|c|c|c|c|c|c|}
\hline Year & Total & Total & Solids & Solids & LOW \\
\hline & (K gal) & (in) & (K gal) & (in) & (in) \\
\hline & & & & & i \\
\hline \multicolumn{6}{|l|}{3} \\
\hline 4 & 761 & 284 & 0 & 0 & 1 \\
\hline $1-1964$ & & & & & 1 \\
\hline 2 & 761 & 284 & 0 & 0 & $i$ \\
\hline \multicolumn{6}{|l|}{3} \\
\hline 4 & 761 & 284 & 0 & 0 & \\
\hline \multicolumn{6}{|l|}{$1-1965$} \\
\hline 2 & 766 & 286 & 7 & 10 & \\
\hline 3 & 766 & 286 & 7 & 10 & \\
\hline 4 & 766 & 286 & 7 & 10 & \\
\hline $1-1966$ & 766 & 286 & 7 & 10 & \\
\hline 2 & 766 & 286 & 7 & 10 & \\
\hline 3 & 766 & 286 & 7 & 10 & \\
\hline 4 & 766 & 286 & 7 & 10 & \\
\hline $1-1967$ & 766 & 286 & 7 & 10 & \\
\hline 2 & 766 & 286 & 7 & 10 & \\
\hline 3 & 766 & 286 & 7 & 10 & \\
\hline 4 & 766 & 286 & 7 & 10 & \\
\hline $1-1968$ & 766 & 286 & 7 & 10 & \\
\hline 2 & 766 & 286 & 7 & 10 & \\
\hline 3 & 766 & 286 & 7 & 10 & \\
\hline 4 & 765 & 286 & 7 & 10 & \\
\hline $1-1969$ & 765 & 286 & 7 & 10 & \\
\hline 2 & 765 & 286 & 7 & 10 & \\
\hline 3 & 765 & 286 & 7 & 10 & \\
\hline 4 & 765 & 286 & 13 & 12 & \\
\hline $1-1970$ & 765 & 286 & 13 & 12 & \\
\hline 2 & 765 & 286 & 13 & 12 & \\
\hline 3 & 765 & 286 & 13 & 12 & \\
\hline 4 & 765 & 286 & 13 & 12 & \\
\hline $9-1971$ & 765 & 286 & 13 & 12 & \\
\hline 2 & 765 & 286 & 13 & 12 & \\
\hline 3 & 765 & 286 & 13 & 12 & \\
\hline 4 & 765 & 286 & 13 & 12 & \\
\hline $1-1972$ & 765 & 286 & 13 & 12 & \\
\hline 2 & 765 & 286 & 13 & 12 & \\
\hline 3 & 765 & 286 & 13 & 12 & \\
\hline 4 & 765 & 286 & 13 & 12 & \\
\hline $1-1973$ & 765 & 286 & 13 & 12 & • \\
\hline 2 & 758 & 283 & 13 & 12 & \\
\hline 3 & 770 & 287 & 13 & 12 & 7 \\
\hline 4 & 771 & 288 & 13 & 12 & \\
\hline $1-1974$ & 361 & 139 & 263 & 103 & \\
\hline 2 & 672 & 252 & 488 & 185 & \\
\hline 3 & 700 & 262 & 653 & 245 & 1 \\
\hline 4 & 717 & 268 & 653 & 245 & $i$ \\
\hline
\end{tabular}


241-S-109 SINGLE-SHELL WHC-SD-WM-ER-323， ReV. 0 TANK LEVEL HISTORY

\begin{tabular}{|c|c|c|c|c|c|}
\hline Year & Total & Total & Solids & Solids & LOW \\
\hline & (K gal) & (in) & (K gal) & (in) & (in) \\
\hline $1-1975$ & 717 & 268 & 653 & 245 & \\
\hline 2 & 719 & 269 & 653 & 245 & \\
\hline 3 & 722 & 270 & 653 & 245 & \\
\hline 4 & 722 & 270 & 653 & 245 & \\
\hline $1-1976$ & 725 & 271 & 653 & 245 & \\
\hline 2 & 725 & 271 & 653 & 245 & \\
\hline 3 & 730 & 273 & 653 & 245 & \\
\hline 4 & 728 & 272 & 653 & 245 & \\
\hline $1-1977$ & 730 & 273 & 568 & 214 & \\
\hline 2 & 730 & 273 & 568 & 214 & \\
\hline 3 & 730 & 273 & 568 & 214 & \\
\hline 4 & 730 & 273 & 568 & 214 & \\
\hline $1-1978$ & 733 & 274 & 568 & 214 & \\
\hline 2 & 733 & 274 & 568 & 214 & \\
\hline 3 & 695 & 260 & 568 & 214 & \\
\hline 4 & 689 & 258 & 568 & 214 & \\
\hline $1-1979$ & 651 & 244 & 568 & 214 & \\
\hline 2 & 568 & 214 & 568 & 214 & \\
\hline 3 & 568 & 214 & 568 & 214 & \\
\hline 4 & 568 & 214 & 568 & 214 & \\
\hline $1-1980$ & 568 & 214 & 568 & 214 & \\
\hline 2 & 568 & 214 & 568 & 214 & \\
\hline 3 & 568 & 214 & 568 & 214 & \\
\hline 4 & 568 & 214 & 568 & 214 & \\
\hline $1-1981$ & 568 & 214 & 568 & 214 & \\
\hline 2 & 568 & 214 & 568 & 214 & \\
\hline 3 & 568 & 214 & 568 & 214 & \\
\hline 4 & 568 & 214 & 568 & 214 & \\
\hline $1-1982$ & 568 & 214 & 568 & 214 & \\
\hline 2 & 568 & 214 & 568 & 214 & \\
\hline 3 & 568 & 214 & 568 & 214 & \\
\hline 4 & 568 & 214 & 568 & 214 & \\
\hline $1-1983$ & 568 & 214 & 568 & 214 & \\
\hline 2 & 568 & 214 & 568 & 214 & \\
\hline 3 & 568 & 214 & 568 & 214 & \\
\hline 4 & 568 & 214 & 568 & 214 & \\
\hline $1-1984$ & 568 & 214 & 568 & 214 & \\
\hline 2 & 568 & 214 & 568 & 214 & . \\
\hline 3 & 568 & 214 & 568 & 214 & \\
\hline 4 & 568 & 214 & 568 & 214 & \\
\hline $1-1985$ & 568 & 214 & 568 & 214 & \\
\hline 2 & 568 & 214 & 568 & 214 & \\
\hline 3 & 568 & 214 & 568 & 214 & \\
\hline 4 & 568 & 214 & 568 & 214 & \\
\hline $1-1986$ & 568 & 214 & 568 & 214 & 127 \\
\hline 2 & 568 & 214 & 568 & 214 & 128 \\
\hline
\end{tabular}


241-S-109 SINGLE-SHELL WHC-SD-WM-ER-323， REV.0 TANK LEVEL HISTORY

\begin{tabular}{|l|c|c|c|c|c|}
\hline \multicolumn{1}{|c|}{ Year } & Total & Total & Solids & Solids & LOW \\
\hline & (K gal) & (in) & (K gal) & (in) & (in) \\
\hline 3 & & & & & \\
\hline 4 & 568 & 214 & 568 & 214 & 128 \\
\hline $1-1987$ & 568 & 214 & 568 & 214 & 128 \\
\hline 2 & 568 & 214 & 568 & 214 & 128 \\
\hline 3 & 568 & 214 & 568 & 214 & \\
\hline 4 & 568 & 214 & 568 & 214 & 129 \\
\hline $1-1988$ & 568 & 214 & 568 & 214 & 130 \\
\hline 2 & 568 & 214 & 568 & 214 & 130 \\
\hline 3 & 568 & 214 & 568 & 214 & 130 \\
\hline 4 & 568 & 214 & 568 & 214 & 130 \\
\hline $1-1989$ & 568 & 214 & 568 & 214 & 131 \\
\hline 2 & 568 & 214 & 568 & 214 & 133 \\
\hline 3 & 568 & 214 & 568 & 214 & 132 \\
\hline 4 & 568 & 214 & 568 & 214 & 133 \\
\hline $1-1990$ & 568 & 214 & 568 & 214 & 131 \\
\hline 2 & 568 & 214 & 568 & 214 & \\
\hline 3 & 568 & 214 & 568 & 214 & 133 \\
\hline 4 & 568 & 214 & 568 & 214 & 132 \\
\hline $1-1991$ & 568 & 214 & 568 & 214 & 133 \\
\hline 2 & 568 & 214 & 568 & 214 & 133 \\
\hline 3 & 568 & 214 & 568 & 214 & 134 \\
\hline 4 & 568 & 214 & 568 & 214 & 133 \\
\hline $1-1992$ & 568 & 214 & 568 & 214 & \\
\hline 2 & 568 & 214 & 568 & 214 & 134 \\
\hline 3 & 568 & 214 & 568 & 214 & \\
\hline 4 & 568 & 214 & 568 & 214 & 134 \\
\hline $1-1993$ & 568 & 214 & 568 & 214 & 135 \\
\hline 2 & 568 & 214 & 568 & 214 & 135 \\
\hline 3 & & & & & 137 \\
\hline 4 & & & & & 138 \\
\hline
\end{tabular}


241-S-110 SINGLE-SHELL WHC-SD-WM-ER-323, ReV. 0 TANK LEVEL HISTORY

\begin{tabular}{|c|c|c|c|c|c|}
\hline Year & Total & Total & Solids & Solids & LOW \\
\hline & ( $\mathrm{K}$ gal) & (in) & ( $\mathrm{K}$ gal) & (in) & (in) \\
\hline \multicolumn{6}{|l|}{$1-1952$} \\
\hline 2 & 681 & 255 & 0 & 0 & \\
\hline 3 & 774 & 289 & 0 & 0 & \\
\hline 4 & 774 & 289 & 0 & 0 & \\
\hline $1-1953$ & 774 & 289 & 0 & 0 & \\
\hline 2 & 758 & 283 & 0 & 0 & \\
\hline 3 & 758 & 283 & 0 & 0 & \\
\hline 4 & 758 & 283 & 0 & 0 & \\
\hline $1-1954$ & 758 & 283 & 0 & 0 & \\
\hline 2 & 758 & 283 & 0 & 0 & \\
\hline 3 & 758 & 283 & 0 & 0 & \\
\hline 4 & 758 & 283 & $\overline{0}$ & 0 & \\
\hline $1-1955$ & 758 & 283 & 0 & 0 & \\
\hline 2 & 758 & 283 & 0 & 0 & \\
\hline 3 & 758 & 283 & 0 & 0 & \\
\hline 4 & 758 & 283 & 0 & 0 & \\
\hline $1-1956$ & 758 & 283 & 0 & 0 & \\
\hline 2 & 758 & 283 & 0 & 0 & \\
\hline 3 & 758 & 283 & 0 & 0 & \\
\hline 4 & 758 & 283 & 0 & 0 & \\
\hline $1-1957$ & 781 & 291 & 0 & 0 & \\
\hline 2 & 781 & 291 & 0 & 0 & \\
\hline 3 & 780 & 291 & 0 & 0 & \\
\hline 4 & 780 & 291 & 0 & 0 & \\
\hline $1-1958$ & 780 & 291 & 0 & 0 & \\
\hline 2 & 780 & 291 & 0 & 0 & \\
\hline 3 & 777 & 290 & 0 & 0 & \\
\hline 4 & 777 & 290 & 0 & 0 & \\
\hline $1-1959$ & 777 & 290 & 0 & 0 & \\
\hline 2 & 777 & 290 & 0 & 0 & \\
\hline 3 & 777 & 290 & 0 & 0 & \\
\hline 4 & 777 & 290 & 0 & 0 & \\
\hline $1-1960$ & 774 & 289 & 0 & 0 & \\
\hline 2 & 774 & 289 & 0 & 0 & \\
\hline 3 & 774 & 289 & 0 & 0 & \\
\hline 4 & 777 & 290 & 0 & 0 & \\
\hline \multicolumn{6}{|l|}{$1-1961$} \\
\hline 2 & 774 & 289 & 0 & 0 & \\
\hline \multicolumn{6}{|l|}{3} \\
\hline 4 & 774 & 289 & 0 & 0 & \\
\hline \multicolumn{6}{|l|}{$1-1962$} \\
\hline 2 & 774 & 289 & 0 & 0 & \\
\hline \multicolumn{6}{|l|}{3} \\
\hline 4 & 774 & 289 & 0 & 0 & \\
\hline \multicolumn{6}{|l|}{$1-1963$} \\
\hline 2 & 774 & 289 & 0 & 0 & \\
\hline
\end{tabular}


241-S-110 SINGLE-SHELL WHC-SD-WM-ER-323, ReV.0 TANK LEVEL HISTORY

\begin{tabular}{|c|c|c|c|c|c|}
\hline Year & Total & Total & Solids & Solids & LOW \\
\hline & ( $\mathrm{K}$ gal) & (in) & ( $\mathrm{K}$ gal) & (in) & (in) \\
\hline \multicolumn{6}{|l|}{3} \\
\hline 4 & 772 & 288 & 0 & 0 & \\
\hline \multicolumn{6}{|l|}{$1-1964$} \\
\hline 2 & 772 & 288 & 0 & 0 & \\
\hline \multicolumn{6}{|l|}{3} \\
\hline 4 & 772 & 288 & 0 & 0 & \\
\hline \multicolumn{6}{|l|}{$1-1965$} \\
\hline 2 & 772 & 288 & 106 & 46 & \\
\hline 3 & 772 & 288 & 106 & 46 & \\
\hline 4 & 772 & 288 & 106 & 46 & \\
\hline $1-1966$ & 772 & 288 & 106 & 46 & \\
\hline 2 & 772 & 288 & 106 & 46 & \\
\hline 3 & 772 & 288 & 106 & 46 & \\
\hline 4 & 772 & 288 & 106 & 46 & \\
\hline $1-1967$ & 772 & 288 & 106 & 46 & \\
\hline 2 & 772 & 288 & 106 & 46 & \\
\hline 3 & 772 & 288 & 106 & 46 & \\
\hline 4 & 772 & 288 & 106 & 46 & \\
\hline $1-1968$ & 772 & 288 & 106 & 46 & \\
\hline 2 & 776 & 290 & 1.06 & 46 & \\
\hline 3 & 776 & 290 & 106 & 46 & \\
\hline 4 & 776 & 290 & 106 & 46 & \\
\hline $1-1969$ & 776 & 290 & 106 & 46 & \\
\hline 2 & 776 & 290 & 106 & 46 & \\
\hline 3 & 776 & 290 & 106 & 46 & \\
\hline 4 & 776 & 290 & 113 & 49 & \\
\hline $1-1970$ & 775 & 289 & 113 & 49 & \\
\hline 2 & 775 & 289 & 113 & 49 & \\
\hline 3 & 776 & 290 & 113 & 49 & \\
\hline 4 & 775 & 289 & 113 & 49 & \\
\hline $1-1971$ & 775 & 289 & 113 & 49 & \\
\hline 2 & 774 & 289 & 113 & 49 & \\
\hline 3 & 775 & 289 & 113 & 49 & \\
\hline 4 & 775 & 289 & 113 & 49 & \\
\hline $1-1972$ & 774 & 289 & 113 & 49 & \\
\hline 2 & 774 & 289 & 113 & 49 & \\
\hline 3 & 773 & 289 & 113 & 49 & \\
\hline 4 & 774 & 289 & 113 & 49 & \\
\hline $1-1973$ & 773 & 289 & 113 & 49 & $\cdot$ \\
\hline 2 & 766 & 286 & 113 & 49 & \\
\hline 3 & 778 & 290 & 113 & 49 & \\
\hline 4 & 786 & 293 & 113 & 49 & \\
\hline $1-1974$ & 280 & 109 & 131 & 55 & \\
\hline 2 & 686 & 257 & 131 & 55 & \\
\hline 3 & 580 & 218 & 131 & 55 & \\
\hline 4 & 695 & 260 & 255 & 100 & \\
\hline
\end{tabular}


241-S-110 SINGLE-SHELL WHC-SD-WM-ER-323, ReV. 0 TANK LEVEL HISTORY

\begin{tabular}{|c|c|c|c|c|c|}
\hline Year & Total & Total & Solids & Solids & LOW \\
\hline & (K gal) & (in) & ( $\mathrm{K}$ gal) & (in) & (in) \\
\hline & & & & & \\
\hline $1-1975$ & 678 & 254 & 414 & 158 & \\
\hline 2 & 739 & 276 & 560 & 211 & \\
\hline 3 & 755 & 282 & 755 & 282 & \\
\hline 4 & 689 & 258 & 689 & 258 & \\
\hline $1-1976$ & 689 & 258 & 689 & 258 & \\
\hline 2 & 689 & 258 & 689 & 258 & \\
\hline 3 & 689 & 258 & 689 & 258 & \\
\hline 4 & 623 & 234 & 623 & 234 & \\
\hline $1-1977$ & 623 & 234 & 475 & 180 & \\
\hline 2 & 700 & 262 & 475 & 180 & \\
\hline 3 & 700 & 262 & 475 & 180 & \\
\hline 4 & 700 & 262 & $47 \overline{5}$ & 180 & \\
\hline $1-1978$ & 700 & 262 & 475 & 180 & \\
\hline 2 & 700 & 262 & 475 & 180 & \\
\hline 3 & 692 & 259 & 475 & 180 & \\
\hline 4 & 692 & 259 & 475 & 180 & \\
\hline $1-1979$ & 692 & 259 & 692 & 259 & \\
\hline 2 & 692 & 259 & 692 & 259 & \\
\hline 3 & 692 & 259 & 692 & 259 & \\
\hline 4 & 692 & 259 & 692 & 259 & \\
\hline $1-1980$ & 692 & 259 & 692 & 259 & \\
\hline 2 & 692 & 259 & 692 & 259 & \\
\hline 3 & 692 & 259 & 692 & 259 & \\
\hline 4 & 692 & 259 & 692 & 259 & \\
\hline $1-1981$ & 692 & 259 & 692 & 259 & \\
\hline 2 & 692 & 259 & 692 & 259 & \\
\hline 3 & 692 & 259 & 692 & 259 & \\
\hline 4 & 692 & 259 & 692 & 259 & \\
\hline $1-1982$ & 692 & 259 & 692 & 259 & \\
\hline 2 & 692 & 259 & 692 & 259 & \\
\hline 3 & 692 & 259 & 692 & 259 & \\
\hline 4 & 692 & 259 & 692 & 259 & \\
\hline $1-1983$ & 692 & 259 & 692 & 259 & \\
\hline 2 & 692 & 259 & 692 & 259 & \\
\hline 3 & 692 & 259 & 692 & 259 & \\
\hline 4 & 692 & 259 & 692 & 259 & \\
\hline $1-1984$ & 692 & 259 & 692 & 259 & \\
\hline 2 & 692 & 259 & 692 & 259 & \\
\hline 3 & 692 & 259 & 692 & 259 & \\
\hline 4 & 692 & 259 & 692 & 259 & \\
\hline $1-1985$ & 692 & 259 & 692 & 259 & \\
\hline 2 & 692 & 259 & 692 & 259 & \\
\hline 3 & 692 & 259 & 692 & 259 & \\
\hline 4 & 692 & 259 & 692 & 259 & \\
\hline $1-1986$ & 692 & 259 & 692 & 259 & 141 \\
\hline 2 & 692 & 259 & 692 & 259 & 141 \\
\hline
\end{tabular}


241-S-110 SINGLE-SHELL WHC-SD-WM-ER-323, Rev.0 TANK LEVEL HISTORY

\begin{tabular}{|c|c|c|c|c|c|}
\hline Year & Total & Total & Solids & Solids & LOW \\
\hline & ( $\mathrm{K}$ gal) & (in) & (K gal) & (in) & (in) \\
\hline 3 & 692 & 259 & 692 & 259 & 139 \\
\hline 4 & 692 & 259 & 692 & 259 & 141 \\
\hline $1-1987$ & 692 & 259 & 692 & 259 & 141 \\
\hline 2 & 692 & 259 & 692 & 259 & \\
\hline 3 & 692 & 259 & 692 & 259 & 141 \\
\hline 4 & 692 & 259 & 692 & 259 & 141 \\
\hline $1-1988$ & 692 & 259 & 692 & 259 & 141 \\
\hline 2 & 692 & 259 & 692 & 259 & 141 \\
\hline 3 & 692 & 259 & 692 & 259 & 141 \\
\hline 4 & 692 & 259 & 692 & 259 & 142 \\
\hline $1-1989$ & 692 & 259 & 692 & 259 & \\
\hline 2 & 692 & 259 & 692 & 259 & 141 \\
\hline 3 & 692 & 259 & 692 & 259 & 141 \\
\hline 4 & 692 & 259 & 692 & 259 & 141 \\
\hline $1-1990$ & 692 & 259 & 692 & 259 & 141 \\
\hline 2 & 692 & 259 & 692 & 259 & \\
\hline 3 & 692 & 259 & 692 & 259 & 141 \\
\hline 4 & 692 & 259 & 692 & 259 & 140 \\
\hline $1-1991$ & 692 & 259 & 692 & 259 & 142 \\
\hline 2 & 692 & 259 & 692 & 259 & 140 \\
\hline 3 & 692 & 259 & 692 & 259 & 141 \\
\hline 4 & 692 & 259 & 692 & 259 & 142 \\
\hline $1-1992$ & 692 & 259 & 692 & 259 & \\
\hline 2 & 390 & 149 & 390 & 149 & 141 \\
\hline 3 & 390 & 149 & 390 & 149 & \\
\hline 4 & 390 & 149 & 390 & 149 & 141 \\
\hline $1-1993$ & 390 & 149 & 390 & 149 & 741 \\
\hline 2 & 390 & 149 & 390 & 149 & 139 \\
\hline 3 & 390 & 149 & 390 & 149 & 141 \\
\hline 4 & 390 & 149 & 390 & 149 & 141 \\
\hline $1-1994$ & & & & & 141 \\
\hline
\end{tabular}


241-S-111 SINGLE-SHELL WHC-SD-WM-ER-323, ReV.0 TANK LEVEL HISTORY

\begin{tabular}{|c|c|c|c|c|c|}
\hline YEAR & Liquid & Liquid & Solids & Solids & LOW \\
\hline & ( $\mathrm{K}$ gal) & (in) & (K gal) & (in) & (in) \\
\hline \multirow{2}{*}{\multicolumn{6}{|c|}{1.1952}} \\
\hline & & & & & \\
\hline \multicolumn{6}{|l|}{2} \\
\hline 3 & 755 & 282 & 0 & 0 & \\
\hline 4 & 755 & 282 & 0 & 0 & \\
\hline $1-1953$ & 755 & 282 & 0 & 0 & \\
\hline 2 & 758 & 283 & 0 & 0 & \\
\hline 3 & 758 & 283 & 0 & 0 & \\
\hline 4 & 758 & 283 & 0 & 0 & \\
\hline $1-1954$ & 758 & 283 & 0 & 0 & \\
\hline 2 & 758 & 283 & 0 & 0 & \\
\hline 3 & 758 & 283 & 0 & 0 & \\
\hline 4 & 758 & 283 & 0 & 0 & \\
\hline $1-1955$ & 758 & 283 & 0 & 0 & \\
\hline 2 & 758 & 283 & 0 & 0 & \\
\hline 3 & 758 & 283 & 0 & 0 & \\
\hline 4 & 758 & 283 & 0 & 0 & \\
\hline $1-1956$ & 758 & 283 & 0 & 0 & \\
\hline 2 & 758 & 283 & 0 & 0 & \\
\hline 3 & 758 & 283 & 0 & 0 & \\
\hline 4 & 758 & 283 & 0 & 0 & \\
\hline $1-1957$ & 761 & 284 & 0 & 0 & \\
\hline 2 & 761 & 284 & 0 & 0 & \\
\hline 3 & 761 & 284 & 0 & 0 & \\
\hline 4 & 761 & 284 & 0 & 0 & \\
\hline $1-1958$ & 761 & 284 & 0 & 0 & \\
\hline 2 & 761 & 284 & 0 & 0 & \\
\hline 3 & 761 & 284 & 0 & 0 & \\
\hline 4 & 761 & 284 & 0 & 0 & \\
\hline $1-1959$ & 761 & 284 & 0 & 0 & \\
\hline 2 & 761 & 284 & 0 & 0 & \\
\hline 3 & 761 & 284 & 0 & 0 & \\
\hline 4 & 761 & 284 & 0 & 0 & \\
\hline $1-1960$ & 758 & 283 & 0 & 0 & \\
\hline 2 & 758 & 283 & 0 & 0 & \\
\hline 3 & 758 & 283 & 0 & 0 & \\
\hline 4 & 758 & 283 & 0 & 0 & \\
\hline \multicolumn{6}{|l|}{$1-1961$} \\
\hline 2 & 755 & 282 & 0 & 0 & \\
\hline \multicolumn{6}{|l|}{3} \\
\hline 4 & 755 & 282 & 0 & 0 & \\
\hline \multicolumn{6}{|l|}{$1-1962$} \\
\hline 2 & 755 & 282 & 0 & 0 & \\
\hline \multicolumn{6}{|l|}{3} \\
\hline 4 & 755 & 282 & 0 & 0 & \\
\hline $1-1963$ & & & & & \\
\hline 2 & 755 & 282 & 0 & 0 & \\
\hline
\end{tabular}


241-S-111 SINGLE-SHELL WHC-SD-WM-ER-323， REV. 0 TANK LEVEL HISTORY

\begin{tabular}{|c|c|c|c|c|c|}
\hline YEAR & Liquid & Liquid & Solids & Solids & LOW \\
\hline & ( $\mathrm{K}$ gal) & (in) & ( $\mathrm{K}$ gal) & (in) & (in) \\
\hline \multirow{2}{*}{\multicolumn{5}{|c|}{3}} & \\
\hline 4 & & & & & \\
\hline \multicolumn{6}{|l|}{$1-1964$} \\
\hline 2 & 755 & 282 & 0 & 0 & \\
\hline \multicolumn{6}{|l|}{3} \\
\hline 4 & 755 & 282 & 0 & 0 & \\
\hline \multicolumn{6}{|l|}{1.1965} \\
\hline 2 & 761 & 284 & 144 & 60 & \\
\hline 3 & 761 & 284 & 144 & 60 & \\
\hline 4 & 761 & 284 & 144 & 60 & \\
\hline $1-1966$ & 761 & 284 & 144 & 60 & \\
\hline 2 & 761 & 284 & 144 & 60 & \\
\hline 3 & 761 & 284 & 144 & 60 & \\
\hline 4 & 761 & 284 & 144 & 60 & \\
\hline $1-1967$ & 761 & 284 & 144 & 60 & \\
\hline 2 & 759 & 283 & 144 & 60 & \\
\hline 3 & 759 & 283 & 144 & 60 & \\
\hline 4 & 759 & 283 & 144 & 60 & \\
\hline $1-1968$ & 759 & 283 & 144 & 60 & \\
\hline 2 & 759 & 283 & 144 & 60 & \\
\hline 3 & 759 & 283 & 144 & 60 & \\
\hline 4 & 759 & 283 & 144 & 60 & \\
\hline $1-1969$ & 758 & 283 & 144 & 60 & \\
\hline 2 & 758 & 283 & 144 & 60 & \\
\hline 3 & 758 & 283 & 144 & 60 & \\
\hline 4 & 758 & 283 & 139 & 58 & \\
\hline $1-1970$ & 758 & 283 & 139 & 58 & \\
\hline 2 & 758 & 283 & 139 & 58 & \\
\hline 3 & 758 & 283 & 139 & 58 & \\
\hline 4 & 758 & 283 & 139 & 58 & \\
\hline $1-1971$ & 758 & 283 & 139 & 58 & \\
\hline 2 & 758 & 283 & 139 & 58 & \\
\hline 3 & 758 & 283 & 139 & 58 & \\
\hline 4 & 758 & 283 & 139 & 58 & \\
\hline $1-1972$ & 758 & 283 & 139 & 58 & \\
\hline 2 & 758 & 283 & 139 & 58 & \\
\hline 3 & 758 & 283 & 139 & 58 & \\
\hline 4 & 758 & 283 & 139 & 58 & \\
\hline $1-1973$ & 759 & 283 & 139 & 58 & \\
\hline 2 & 749 & 280 & 139 & 58 & \\
\hline 3 & 764 & 285 & 139 & 58 & \\
\hline 4 & 766 & 286 & 139 & 58 & \\
\hline $1-1974$ & 311 & 121 & 139 & 58 & \\
\hline 2 & 689 & 258 & 334 & 129 & \\
\hline 3 & 730 & 273 & 502 & 190 & \\
\hline 4 & 692 & 259 & 563 & 212 & \\
\hline
\end{tabular}


241-S-111 SINGLE-SHELL WHC-SD-WM-ER-323, Rev. 0 TANK LEVEL HISTORY

\begin{tabular}{|c|c|c|c|c|c|}
\hline YEAR & Liquid & Liquid & Solids & Solids & LOW \\
\hline & (K gal) & (in) & (K gal) & (in) & (in) \\
\hline $1-1975$ & 739 & 276 & 596 & 224 & \\
\hline 2 & 739 & 276 & 596 & 224 & \\
\hline 3 & 750 & 280 & 750 & 280 & \\
\hline 4 & 750 & 280 & 750 & 280 & \\
\hline $1-1976$ & 750 & 280 & 750 & 280 & \\
\hline 2 & 750 & 280 & 750 & 280 & \\
\hline 3 & 750 & 280 & 750 & 280 & \\
\hline 4 & 623 & 234 & 623 & 234 & \\
\hline $1-1977$ & 623 & 234 & 623 & 234 & \\
\hline 2 & 623 & 234 & 623 & 234 & \\
\hline 3 & 623 & 234 & 623 & 234 & \\
\hline 4 & 623 & 234 & 623 & 234 & \\
\hline $1-1978$ & 623 & 234 & 623 & 234 & \\
\hline 2 & 623 & 234 & 623 & 234 & \\
\hline 3 & 623 & 234 & 623 & 234 & \\
\hline 4 & 623 & 234 & 623 & 234 & \\
\hline $1-1979$ & 623 & 234 & 623 & 234 & \\
\hline 2 & 623 & 234 & 623 & 234 & \\
\hline 3 & 623 & 234 & 623 & 234 & \\
\hline 4 & 623 & 234 & 623 & 234 & \\
\hline $1-1980$ & 623 & 234 & 623 & 234 & \\
\hline 2 & 623 & 234 & 623 & 234 & \\
\hline 3 & 623 & 234 & 623 & 234 & \\
\hline 4 & 623 & 234 & 623 & 234 & \\
\hline $1-1981$ & 623 & 234 & 623 & 234 & \\
\hline 2 & 623 & 234 & 623 & 234 & \\
\hline 3 & 623 & 234 & 623 & 234 & \\
\hline 4 & 623 & 234 & 623 & 234 & \\
\hline $1-1982$ & 623 & 234 & 623 & 234 & \\
\hline 2 & 596 & 224 & 586 & 221 & \\
\hline 3 & 596 & 224 & 586 & 221 & \\
\hline 4 & 596 & 224 & 586 & 221 & \\
\hline $1-1983$ & 596 & 224 & 586 & 221 & \\
\hline 2 & 596 & 224 & 586 & 221 & \\
\hline 3 & 596 & 224 & 586 & 221 & \\
\hline 4 & 596 & 224 & 586 & 221 & \\
\hline $1-1984$ & 596 & 224 & 586 & 221 & \\
\hline 2 & 596 & 224 & 586 & 221 & \\
\hline 3 & 596 & 224 & 586 & 221 & \\
\hline 4 & 596 & 224 & 586 & 221 & \\
\hline $1-1985$ & 596 & 224 & 586 & 221 & \\
\hline 2 & 596 & 224 & 586 & 221 & \\
\hline 3 & 596 & 224 & 586 & 221 & \\
\hline 4 & 596 & 224 & 586 & 221 & \\
\hline $1-1986$ & 596 & 224 & 586 & 221 & 203 \\
\hline 2 & 596 & 224 & 586 & 221 & 203 \\
\hline
\end{tabular}


241-S-111 SINGLE-SHELL WHC-SD-WM-ER-323, ReV. 0 TANK LEVEL HISTORY

\begin{tabular}{|c|c|c|c|c|c|}
\hline YEAR & Liquid & Liquid & Solids & Solids & LOW \\
\hline & ( $\mathrm{K}$ gal) & (in) & ( $\mathrm{K}$ gal) & (in) & (in) \\
\hline 3 & 596 & 224 & 586 & 221 & 204 \\
\hline 4 & 596 & 224 & 586 & 221 & 204 \\
\hline $1-1987$ & 596 & 224 & 586 & 221 & 203 \\
\hline 2 & 596 & 224 & 586 & 221 & \\
\hline 3 & 596 & 224 & 586 & 221 & 204 \\
\hline 4 & 596 & 224 & 586 & 221 & 203 \\
\hline $1-1988$ & 596 & 224 & 586 & 221 & 203 \\
\hline 2 & 596 & 224 & 586 & 221 & 204 \\
\hline 3 & 596 & 224 & 586 & 221 & 203 \\
\hline 4 & 596 & 224 & 586 & 221 & 203 \\
\hline $1-1989$ & 596 & 224 & 586 & 221 & \\
\hline 2 & 596 & 224 & 586 & 221 & 204 \\
\hline 3 & 596 & 224 & 586 & 221 & 204 \\
\hline 4 & 596 & 224 & 586 & 221 & 204 \\
\hline $1-1990$ & 596 & 224 & 586 & 221 & 202 \\
\hline 2 & 596 & 224 & 586 & 221 & \\
\hline 3 & 596 & 224 & 586 & 221 & 203 \\
\hline 4 & 596 & 224 & 586 & 221 & 203 \\
\hline $1-1991$ & 596 & 224 & 586 & 221 & 203 \\
\hline 2 & 596 & 224 & 586 & 221 & 204 \\
\hline 3 & 596 & 224 & 586 & 221 & 203 \\
\hline 4 & 596 & 224 & 586 & 221 & 203 \\
\hline \multicolumn{6}{|l|}{$1-1992$} \\
\hline 2 & 596 & 224 & 586 & 221 & 204 \\
\hline 3 & 596 & 224 & 586 & 221 & \\
\hline 4 & 596 & 224 & 586 & 221 & 204 \\
\hline $1-1993$ & 596 & 224 & 586 & 221 & 203 \\
\hline 2 & 596 & 224 & 586 & 221 & 204 \\
\hline 3 & & & & & 203 \\
\hline 4 & & & & & 203 \\
\hline
\end{tabular}


241-S-112 SINGLE-SHELL WHC-SD-WM-ER-323, REV. 0 TANK LEVEL HISTORY

\begin{tabular}{|c|c|c|c|c|c|}
\hline Year & Total & Total & Solids & Solids & LOW \\
\hline & (K gal) & (in) & ( $\mathrm{K}$ gal) & (in) & (in) \\
\hline \multirow{2}{*}{\multicolumn{6}{|c|}{$1-1952$}} \\
\hline & & & & & \\
\hline \multicolumn{6}{|l|}{2} \\
\hline 3 & 755 & 282 & 0 & 0 & \\
\hline 4 & 442 & 168 & 0 & 0 & \\
\hline $1-1953$ & 442 & 168 & 0 & 0 & \\
\hline 2 & 442 & 168 & 0 & 0 & \\
\hline 3 & 498 & 189 & 0 & 0 & \\
\hline 4 & 640 & 240 & 0 & 0 & \\
\hline $7-1954$ & 656 & 246 & 0 & 0 & \\
\hline 2 & 688 & 258 & 0 & 0 & \\
\hline 3 & 686 & 257 & 0 & 0 & \\
\hline 4 & 675 & 253 & 0 & 0 & \\
\hline $1-1955$ & 695 & 260 & 0 & 0 & \\
\hline 2 & 695 & 260 & 0 & 0 & \\
\hline 3 & 695 & 260 & 0 & 0 & \\
\hline 4 & 747 & 279 & 0 & 0 & \\
\hline $1-1956$ & 747 & 279 & 0 & 0 & \\
\hline 2 & 747 & 279 & 0 & 0 & \\
\hline 3 & 747 & 279 & 0 & 0 & \\
\hline 4 & 747 & 279 & 0 & 0 & \\
\hline $1-1957$ & 780 & 291 & 0 & 0 & \\
\hline 2 & 780 & 291 & 0 & 0 & \\
\hline 3 & 780 & 291 & 0 & 0 & \\
\hline 4 & 780 & 291 & 0 & 0 & \\
\hline $1-1958$ & 780 & 291 & 0 & 0 & \\
\hline 2 & 780 & 291 & 0 & 0 & \\
\hline 3 & 780 & 291 & 0 & 0 & \\
\hline 4 & 780 & 291 & 0 & 0 & \\
\hline $1-1959$ & 780 & 291 & 0 & 0 & \\
\hline 2 & 780 & 291 & 0 & 0 & \\
\hline 3 & 780 & 291 & 0 & 0 & \\
\hline 4 & 780 & 291 & 0 & 0 & \\
\hline $1-1960$ & 777 & 290 & 0 & 0 & \\
\hline 2 & 777 & 290 & 0 & 0 & \\
\hline 3 & 777 & 290 & 0 & 0 & \\
\hline 4 & 780 & 291 & 0 & 0 & \\
\hline \multicolumn{6}{|l|}{$1-1961$} \\
\hline 2 & 777 & 290 & 0 & 0 & \\
\hline 3 & & & & & - \\
\hline 4 & 777 & 290 & 0 & 0 & \\
\hline \multicolumn{6}{|l|}{$1-1962$} \\
\hline 2 & 777 & 290 & 0 & 0 & \\
\hline \multicolumn{6}{|l|}{3} \\
\hline 4 & 777 & 290 & 0 & 0 & \\
\hline \multicolumn{6}{|l|}{$1-1963$} \\
\hline 2 & 777 & 290 & 0 & 0 & \\
\hline
\end{tabular}


241-S-112 SINGLE-SHELL WHC-SD-WM-ER-323, REV. 0 TANK LEVEL HISTORY

\begin{tabular}{|c|c|c|c|c|c|}
\hline Year & Total & Total & Solids & Solids & LOW \\
\hline & (K gal) & (in) & (K gal) & (in) & (in) \\
\hline & & & & & \\
\hline \multicolumn{6}{|l|}{3} \\
\hline 4 & 777 & 290 & 0 & 0 & \\
\hline \multicolumn{6}{|l|}{$1-1964$} \\
\hline 2 & 777 & 290 & 0 & 0 & \\
\hline \multicolumn{6}{|l|}{3} \\
\hline 4 & 777 & 290 & 0 & 0 & \\
\hline \multicolumn{6}{|l|}{$1-1965$} \\
\hline 2 & 785 & 293 & 4 & 9 & \\
\hline 3 & 785 & 293 & 4 & 9 & \\
\hline 4 & 785 & 293 & 4 & 9 & \\
\hline $1-1966$ & 785 & 293 & 4 & 9 & \\
\hline 2 & 785 & 293 & 4 & 9 & \\
\hline 3 & 785 & 293 & 4 & 9 & \\
\hline 4 & 785 & 293 & 4 & 9 & \\
\hline $1-1967$ & 785 & 293 & 4 & 9 & \\
\hline 2 & 788 & 294 & 4 & 9 & \\
\hline 3 & 788 & 294 & 4 & 9 & \\
\hline 4 & 788 & 294 & 4 & 9 & \\
\hline $1-1968$ & 788 & 294 & 4 & 9 & \\
\hline 2 & 788 & 294 & 4 & 9 & \\
\hline 3 & 788 & 294 & 4 & 9 & \\
\hline 4 & 787 & 294 & 4 & 9 & \\
\hline $1-1969$ & 787 & 294 & 4 & 9 & \\
\hline 2 & 787 & 294 & 4 & 9 & \\
\hline 3 & 787 & 294 & 4 & 9 & \\
\hline 4 & 787 & 294 & 6 & 10 & \\
\hline $1-1970$ & 787 & 294 & 6 & 10 & \\
\hline 2 & 787 & 294 & 6 & 10 & \\
\hline 3 & 787 & 294 & 6 & 10 & \\
\hline 4 & 787 & 294 & 6 & 10 & \\
\hline $1-1971$ & 787 & 294 & 6 & 10 & \\
\hline 2 & 787 & 294 & 6 & 10 & \\
\hline 3 & 787 & 294 & 6 & 10 & \\
\hline 4 & 788 & 294 & 6 & 10 & \\
\hline $1-1972$ & 787 & 294 & 6 & 10 & \\
\hline 2 & 787 & 294 & 6 & 10 & \\
\hline 3 & 787 & 294 & 6 & 10 & \\
\hline 4 & 787 & 294 & 6 & 10 & \\
\hline $1-1973$ & 787 & 294 & 6 & 10 & \\
\hline 2 & 780 & 291 & 6 & 10 & \\
\hline 3 & 792 & 295 & 6 & 10 & \\
\hline 4 & 792 & 295 & 6 & 10 & \\
\hline $1-1974$ & 473 & 179 & 367 & 141 & \\
\hline 2 & 713 & 267 & 598 & 225 & \\
\hline 3 & 732 & 274 & 686 & 257 & \\
\hline 4 & 730 & 273 & 673 & 252 & \\
\hline
\end{tabular}


241-S-112 SINGLE-SHELL WHC-SD-WM-ER-323, REV. 0 TANK LEVEL HISTORY

\begin{tabular}{|c|c|c|c|c|c|}
\hline Year & Total & Total & Solids & Solids & LOW \\
\hline & (K gal) & (in) & (K gal) & (in) & (in) \\
\hline & & & & & \\
\hline $1-1975$ & 736 & 275 & 714 & 267 & \\
\hline 2 & 736 & 275 & 714 & 267 & \\
\hline 3 & 739 & 276 & 714 & 267 & \\
\hline 4 & 706 & 264 & 706 & 264 & \\
\hline $1-1976$ & 706 & 264 & 706 & 264 & \\
\hline 2 & 706 & 264 & 706 & 264 & \\
\hline 3 & 706 & 264 & 706 & 264 & \\
\hline 4 & 706 & 264 & 706 & 264 & \\
\hline $1-1977$ & 706 & 264 & 673 & 252 & \\
\hline 2 & 717 & 268 & 673 & 252 & \\
\hline 3 & 719 & 269 & 673 & 252 & \\
\hline 4 & 719 & 269 & 673 & 252 & \\
\hline $1-1978$ & 719 & 269 & 673 & 252 & \\
\hline 2 & 719 & 269 & 673 & 252 & \\
\hline 3 & 675 & 253 & 673 & 252 & \\
\hline 4 & 678 & 254 & 673 & 252 & \\
\hline $1-1979$ & 675 & 253 & 673 & 252 & \\
\hline 2 & 673 & 252 & 673 & 252 & \\
\hline 3 & 673 & 252 & 673 & 252 & \\
\hline 4 & 673 & 252 & 673 & 252 & \\
\hline $1-1980$ & 673 & 252 & 673 & 252 & \\
\hline 2 & 672 & 252 & 672 & 252 & \\
\hline 3 & 672 & 252 & 672 & 252 & \\
\hline 4 & 672 & 252 & 672 & 252 & \\
\hline $1-1981$ & 672 & 252 & 672 & 252 & \\
\hline 2 & 672 & 252 & 672 & 252 & \\
\hline 3 & 672 & 252 & 672 & 252 & \\
\hline 4 & 672 & 252 & 672 & 252 & \\
\hline $1-1982$ & 672 & 252 & 672 & 252 & \\
\hline 2 & 641 & 241 & 641 & 241 & \\
\hline 3 & 637 & 239 & 637 & 239 & \\
\hline 4 & 637 & 239 & 637 & 239 & \\
\hline $1-1983$ & 637 & 239 & 637 & 239 & \\
\hline 2 & 637 & 239 & 637 & 239 & \\
\hline 3 & 637 & 239 & 637 & 239 & \\
\hline 4 & 646 & 242 & 637 & 239 & \\
\hline $1-1984$ & 646 & 242 & 637 & 239 & \\
\hline 2 & 646 & 242 & 637 & 239 & \\
\hline 3 & 637 & 239 & 637 & 239 & 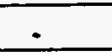 \\
\hline 4 & 637 & 239 & 637 & 239 & \\
\hline $1-1985$ & 637 & 239 & 637 & 239 & \\
\hline 2 & 637 & 239 & 637 & 239 & \\
\hline 3 & 637 & 239 & 637 & 239 & \\
\hline 4 & 637 & 239 & 637 & 239 & \\
\hline $1-1986$ & 637 & 239 & 637 & 239 & 101 \\
\hline 2 & 637 & 239 & 637 & 239 & 100 \\
\hline
\end{tabular}


241-S-112 SINGLE-SHELL WHC-SD-WM-ER-323, ReV. 0 TANK LEVEL HISTORY

\begin{tabular}{|l|c|c|c|c|c|}
\hline \multicolumn{1}{|c|}{ Year } & Total & Total & Solids & Solids & LOW \\
\hline & (K gal) & (in) & (K gal) & (in) & (in) \\
\hline 3 & & & & & \\
\hline 4 & 637 & 239 & 637 & 239 & \\
\hline $1-1987$ & 637 & 239 & 637 & 239 & \\
\hline 2 & 637 & 239 & 637 & 239 & 100 \\
\hline 3 & 637 & 239 & 637 & 239 & \\
\hline 4 & 637 & 239 & 637 & 239 & 106 \\
\hline $1-1988$ & 637 & 239 & 637 & 239 & 107 \\
\hline 2 & 637 & 239 & 637 & 239 & 108 \\
\hline 3 & 637 & 239 & 637 & 239 & 111 \\
\hline 4 & 637 & 239 & 637 & 239 & 109 \\
\hline $1-1989$ & 637 & 239 & 637 & 239 & 111 \\
\hline 2 & 637 & 239 & 637 & 239 & \\
\hline 3 & 637 & 239 & 637 & 239 & 113 \\
\hline 4 & 637 & 239 & 637 & 239 & 113 \\
\hline $1-1990$ & 637 & 239 & 637 & 239 & 115 \\
\hline 2 & 637 & 239 & 637 & 239 & 114 \\
\hline 3 & 637 & 239 & 637 & 239 & \\
\hline 4 & 637 & 239 & 637 & 239 & 115 \\
\hline $1-1991$ & 637 & 239 & 637 & 239 & 115 \\
\hline 2 & 637 & 239 & 637 & 239 & 115 \\
\hline 3 & 637 & 239 & 637 & 239 & 115 \\
\hline 4 & 637 & 239 & 637 & 239 & 115 \\
\hline $1-1992$ & 637 & 239 & 637 & 239 & \\
\hline 2 & 637 & 239 & 637 & 239 & 115 \\
\hline 3 & 637 & 239 & 637 & 239 & \\
\hline 4 & 637 & 239 & 637 & 239 & 115 \\
\hline $1-1993$ & 637 & 239 & 637 & 239 & 117 \\
\hline 2 & 637 & 239 & 637 & 239 & 118 \\
\hline 3 & 637 & 239 & 637 & 239 & 119 \\
\hline 4 & 523 & 198 & 523 & 198 & 118 \\
\hline
\end{tabular}


Tank 241-S-101

Historical Vapor Space Temperature

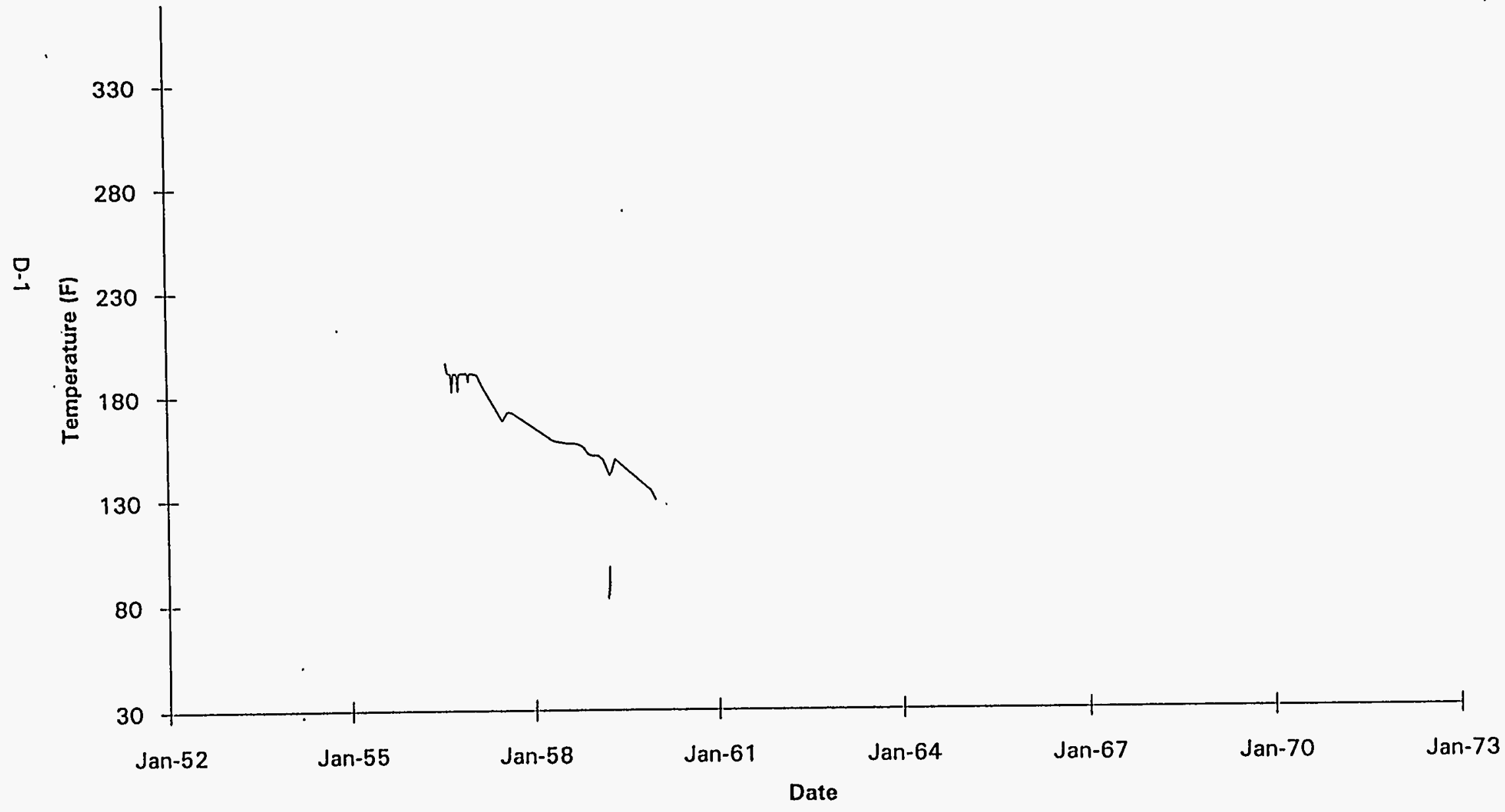


Tank 241-S-101

Historical Average Waste Temperature

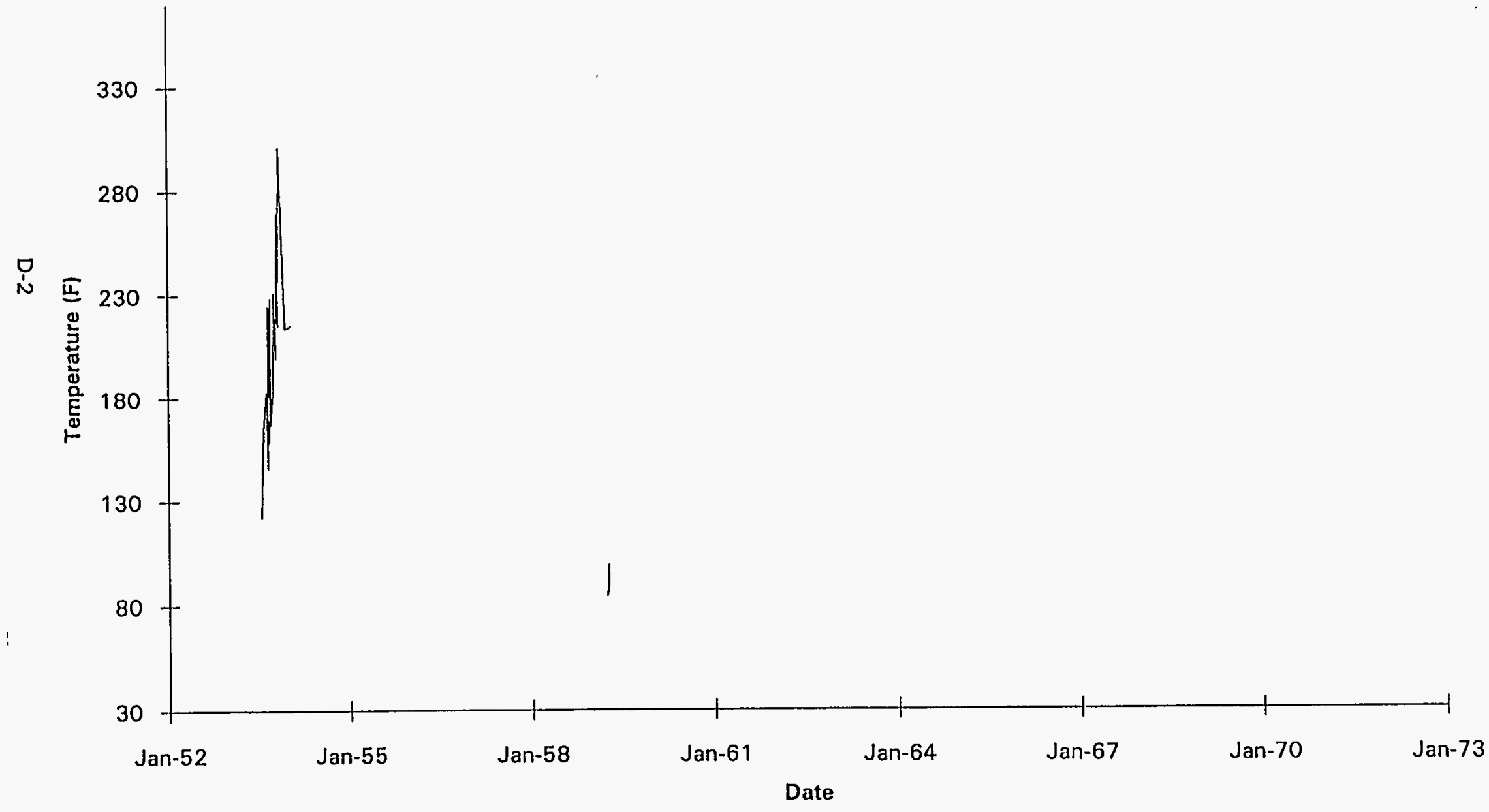


WHC-SD-WM-ER-323, Rev. 0
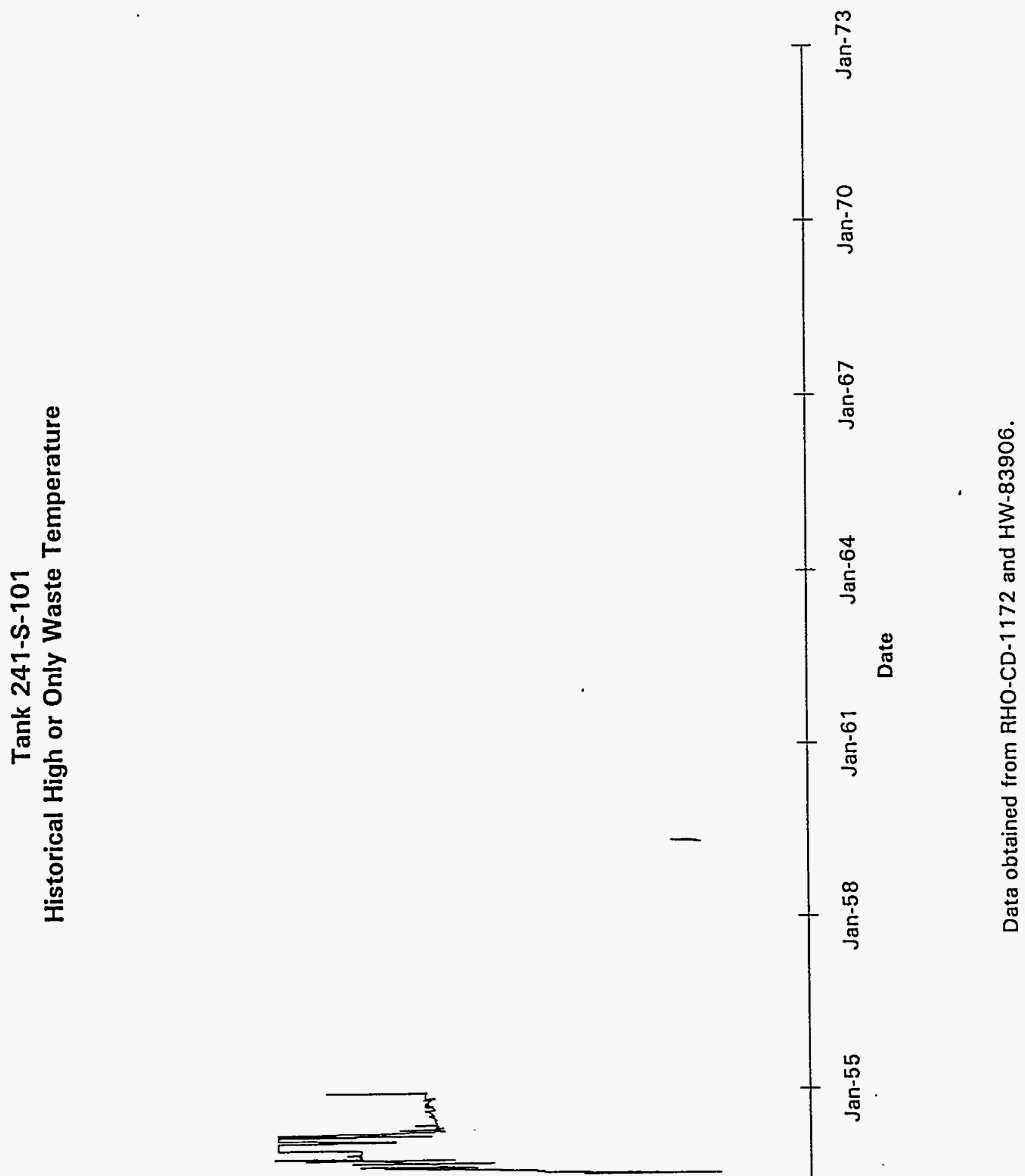

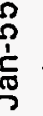

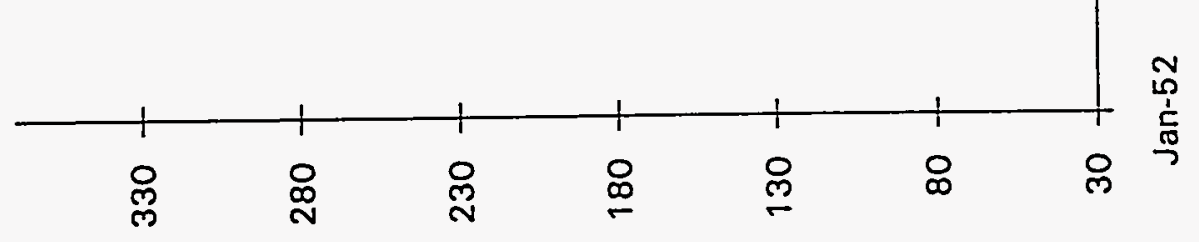

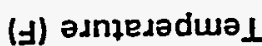

D-3 
WHC-SD-WM-ER-323, Rev. 0

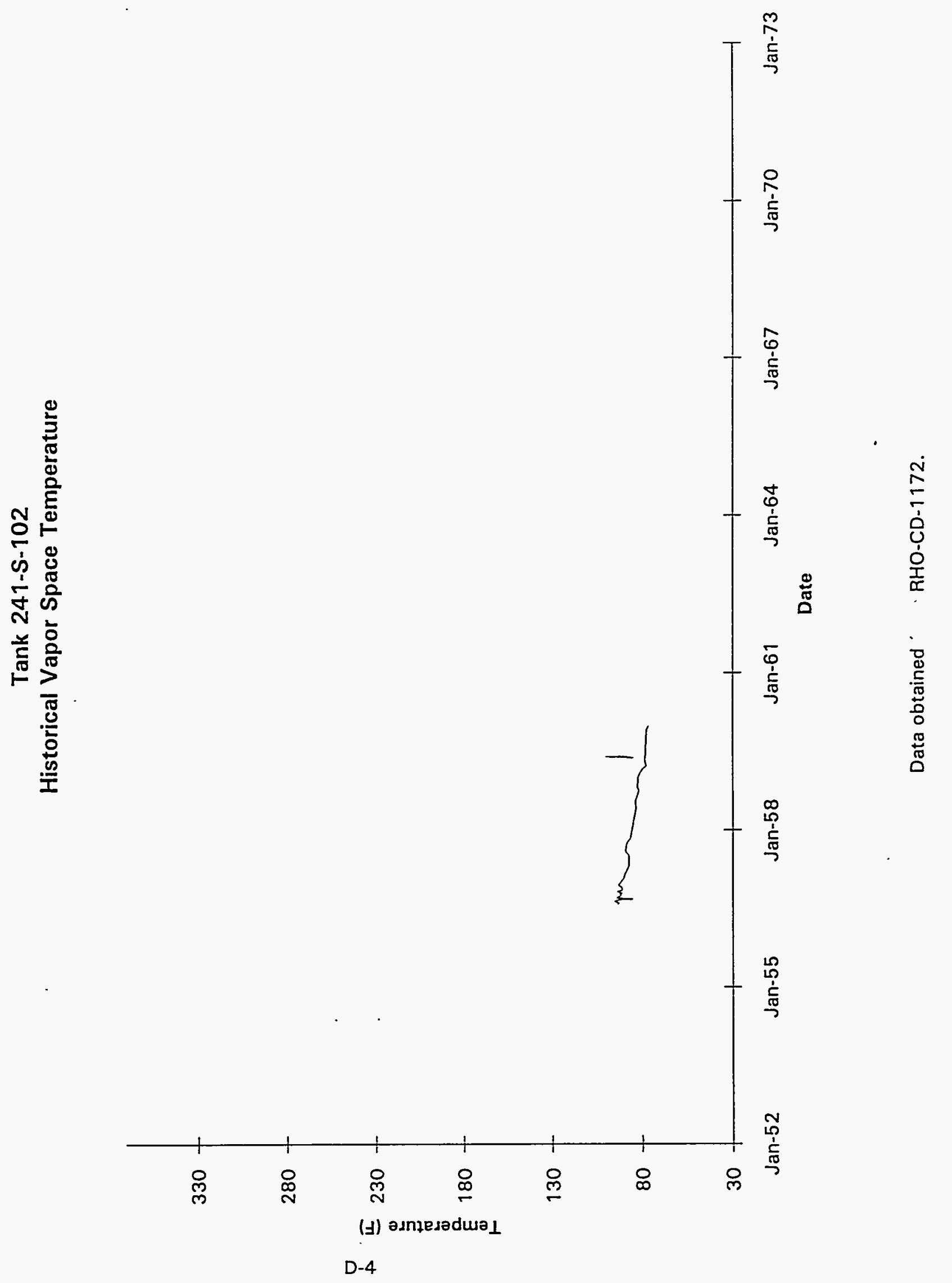


WHC-SD-WM-ER-323, Rev. 0
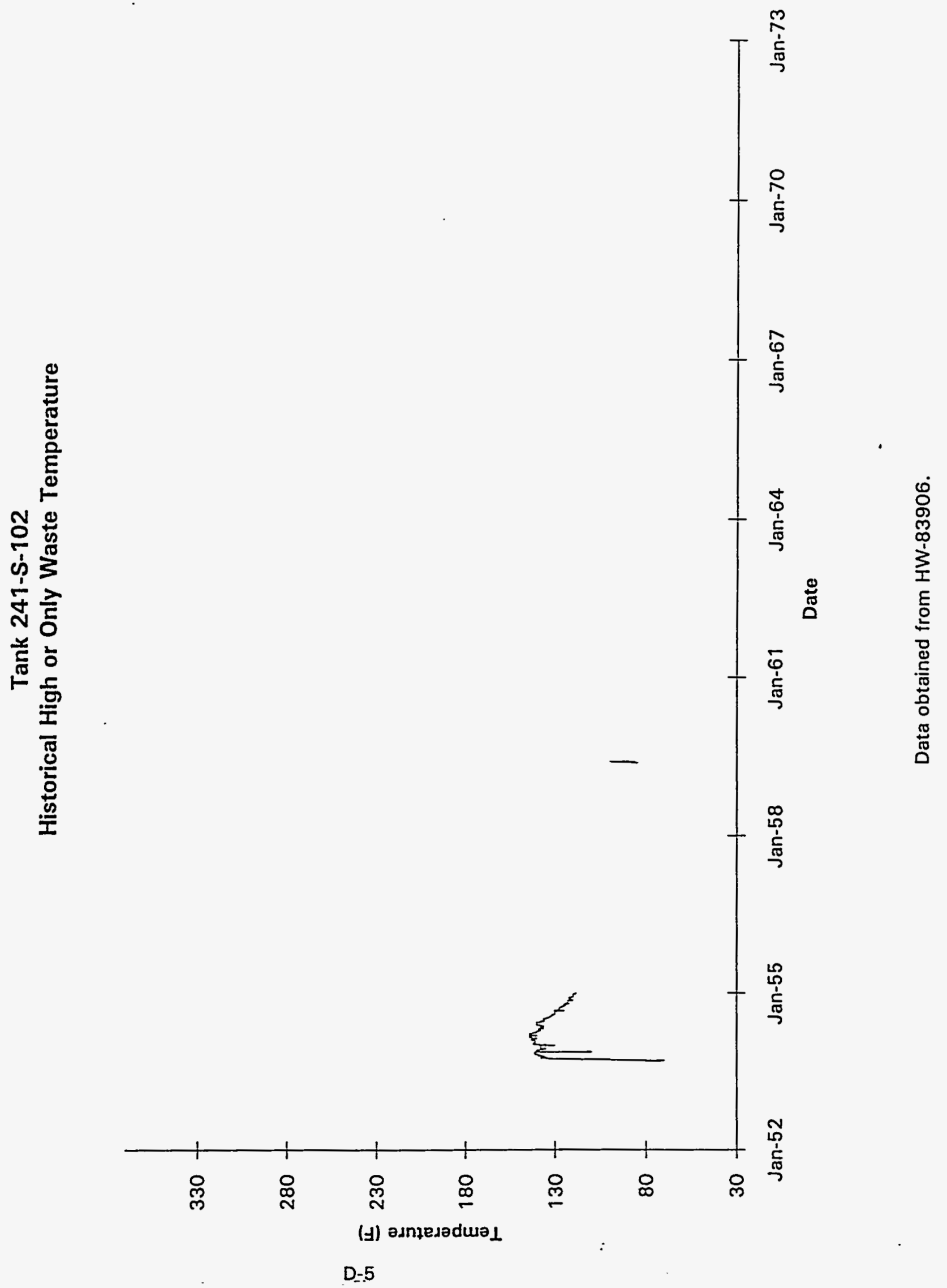
WHC-SD-WM-ER-323, Rev. 0
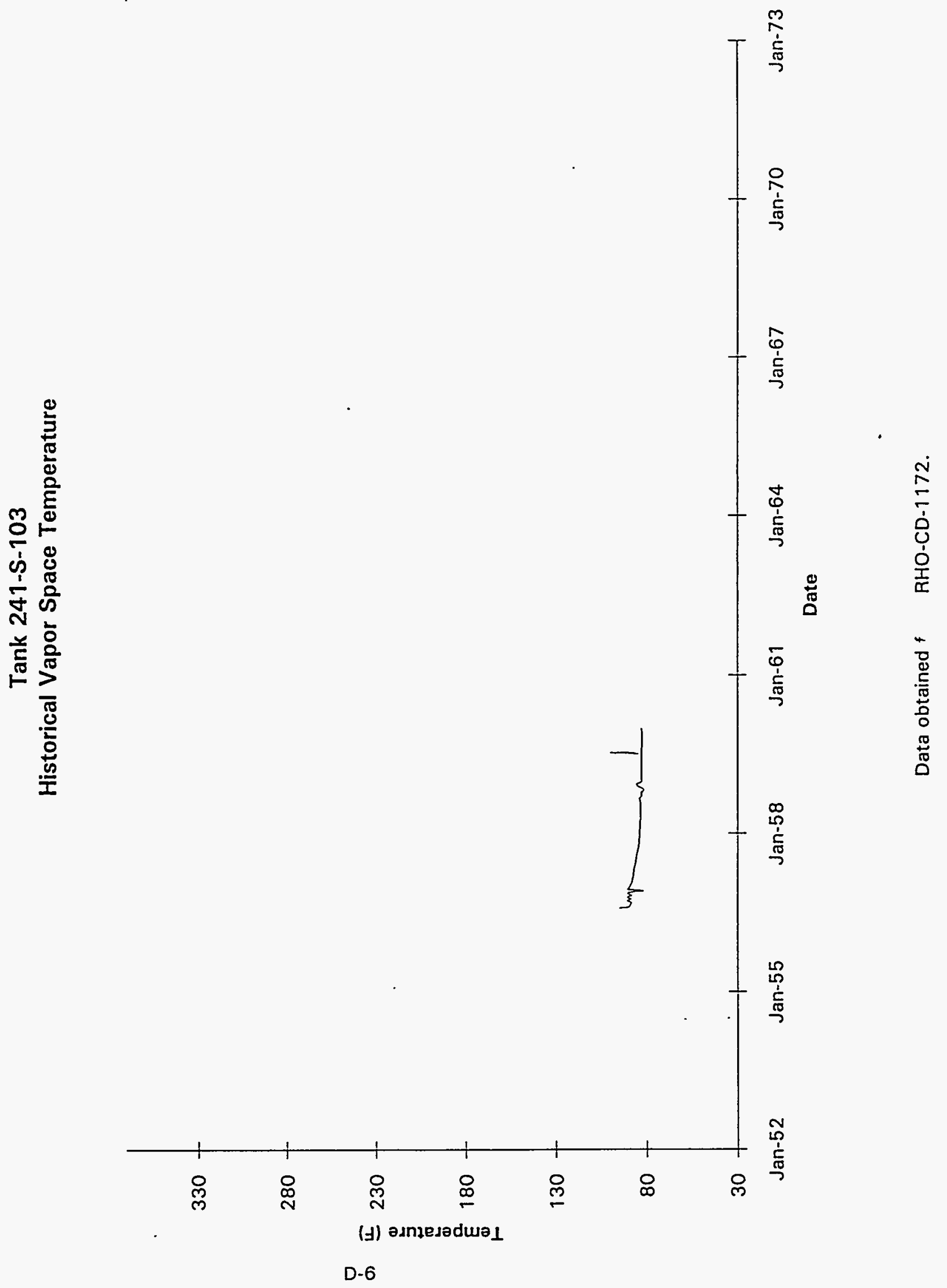
WHC-SD-WM-ER-323, Rev. 0
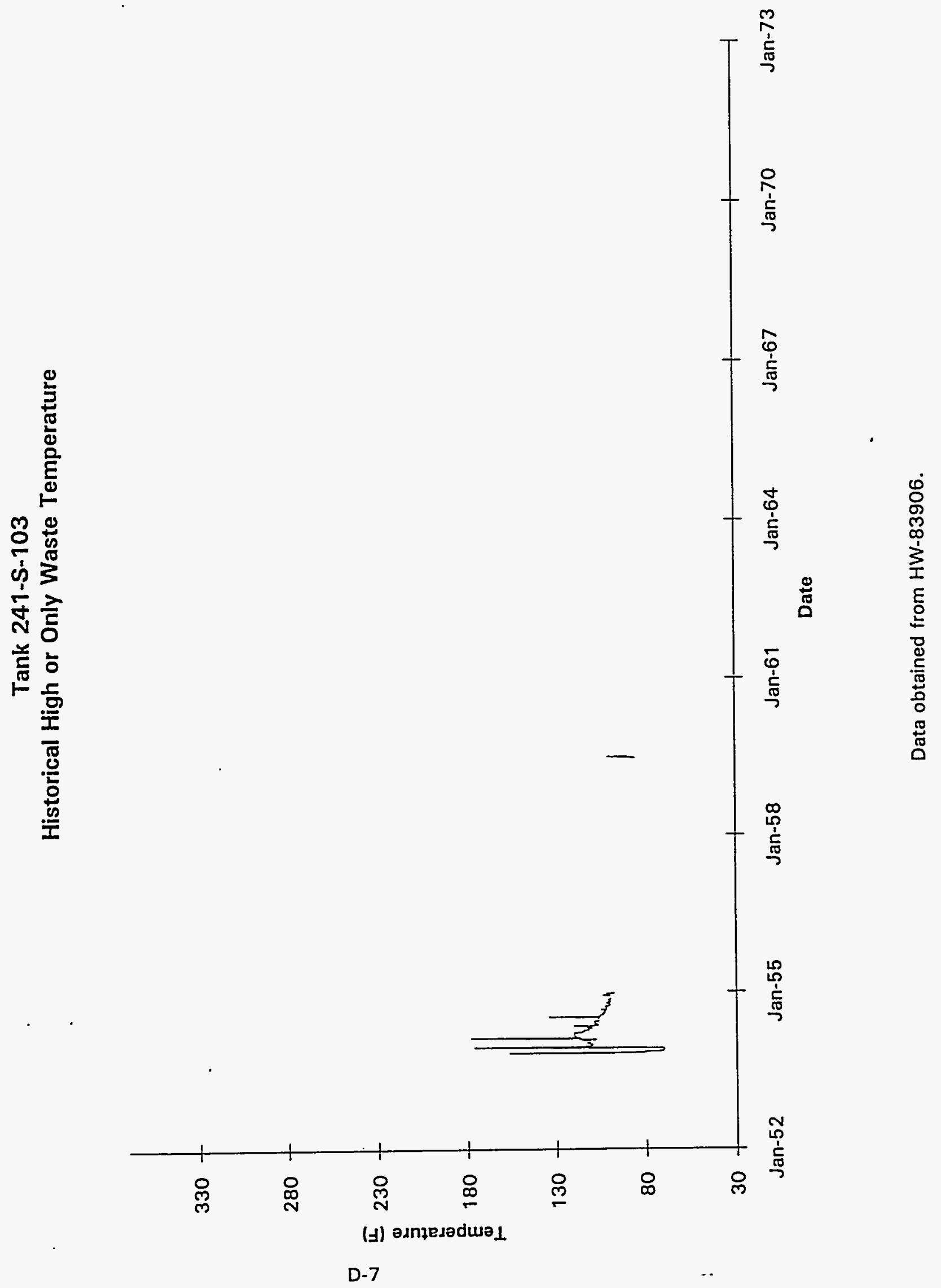
WHC-SD-WM-ER-323, Rev. 0
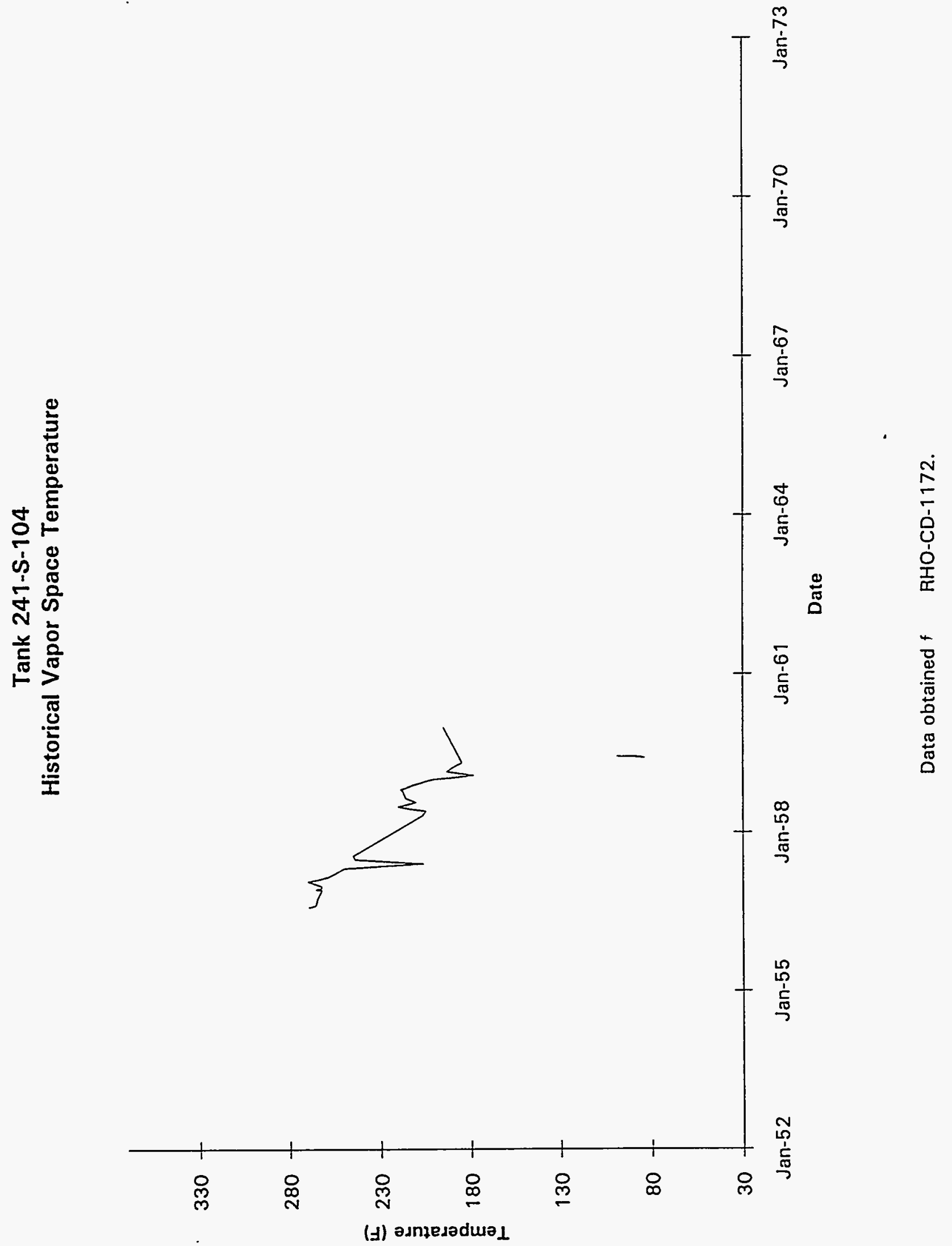

D-8 
WHC-SD-WM-ER-323, Rev. 0
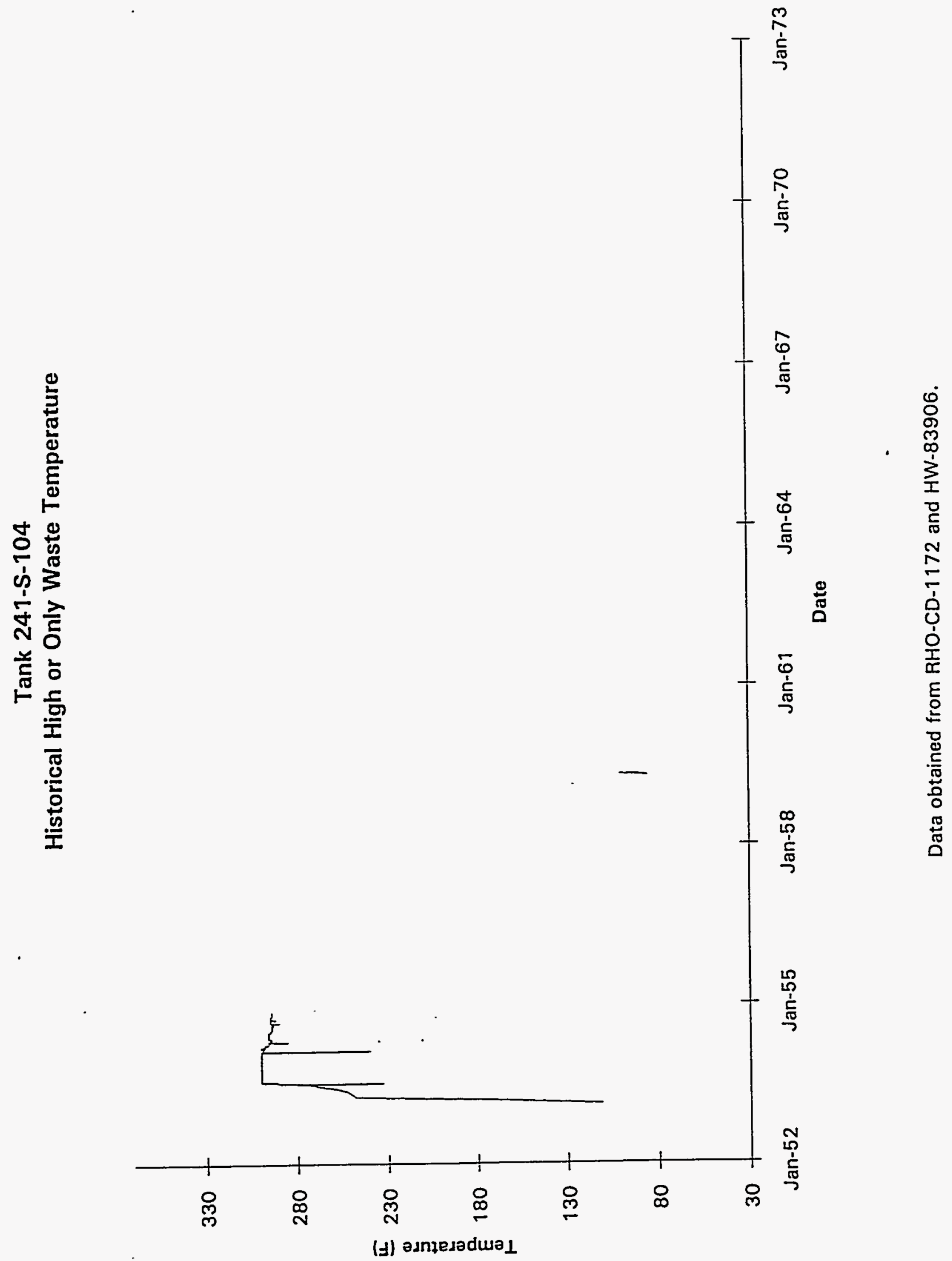

D-9 
Tank 241-S-105

Historical Vapor Space Temperature

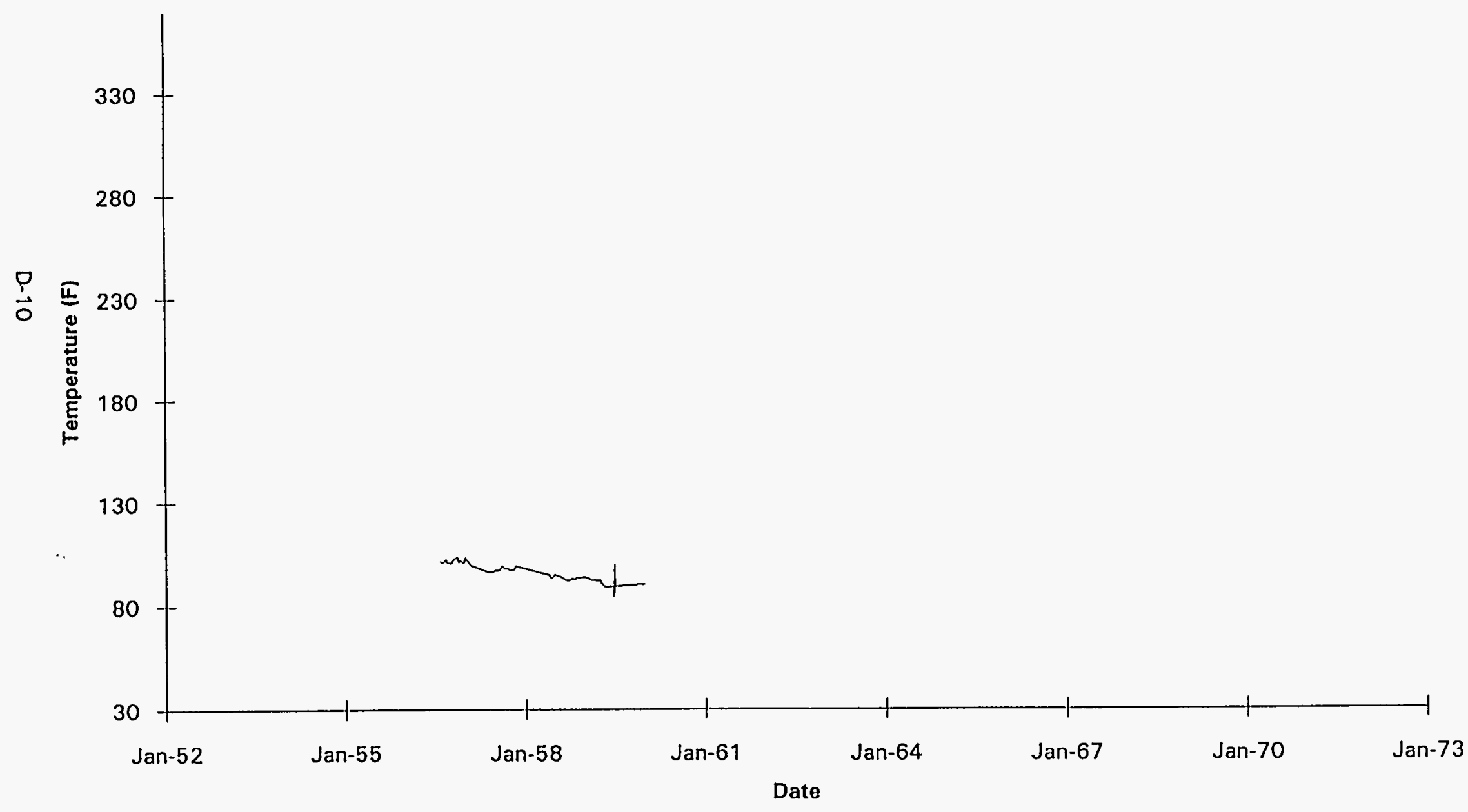


Tank 241-S-105

Historical High or Only Waste Temperature

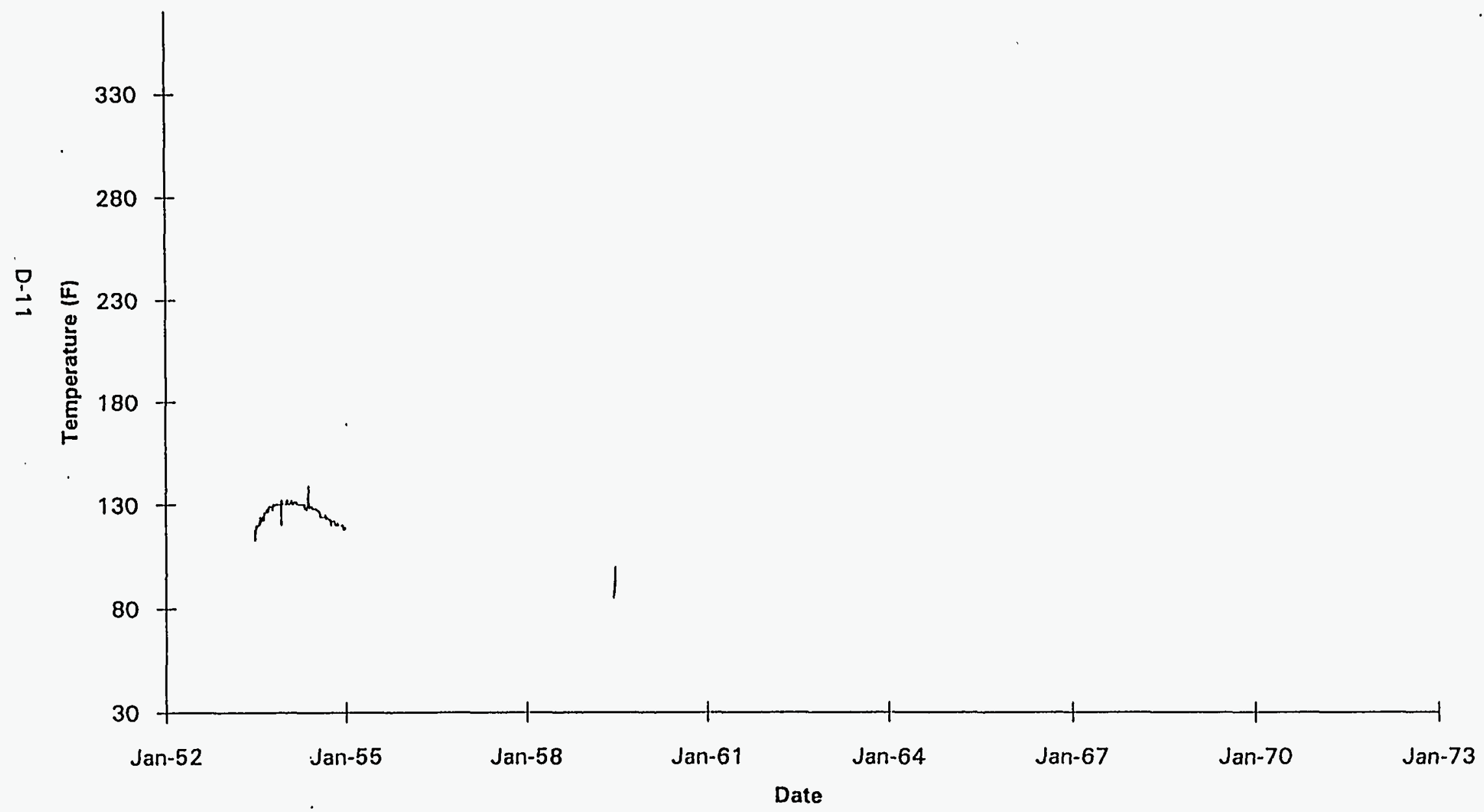

Data obtained from HW-83906. 
Tank 241-S-106

Historical Vapor Space Temperature

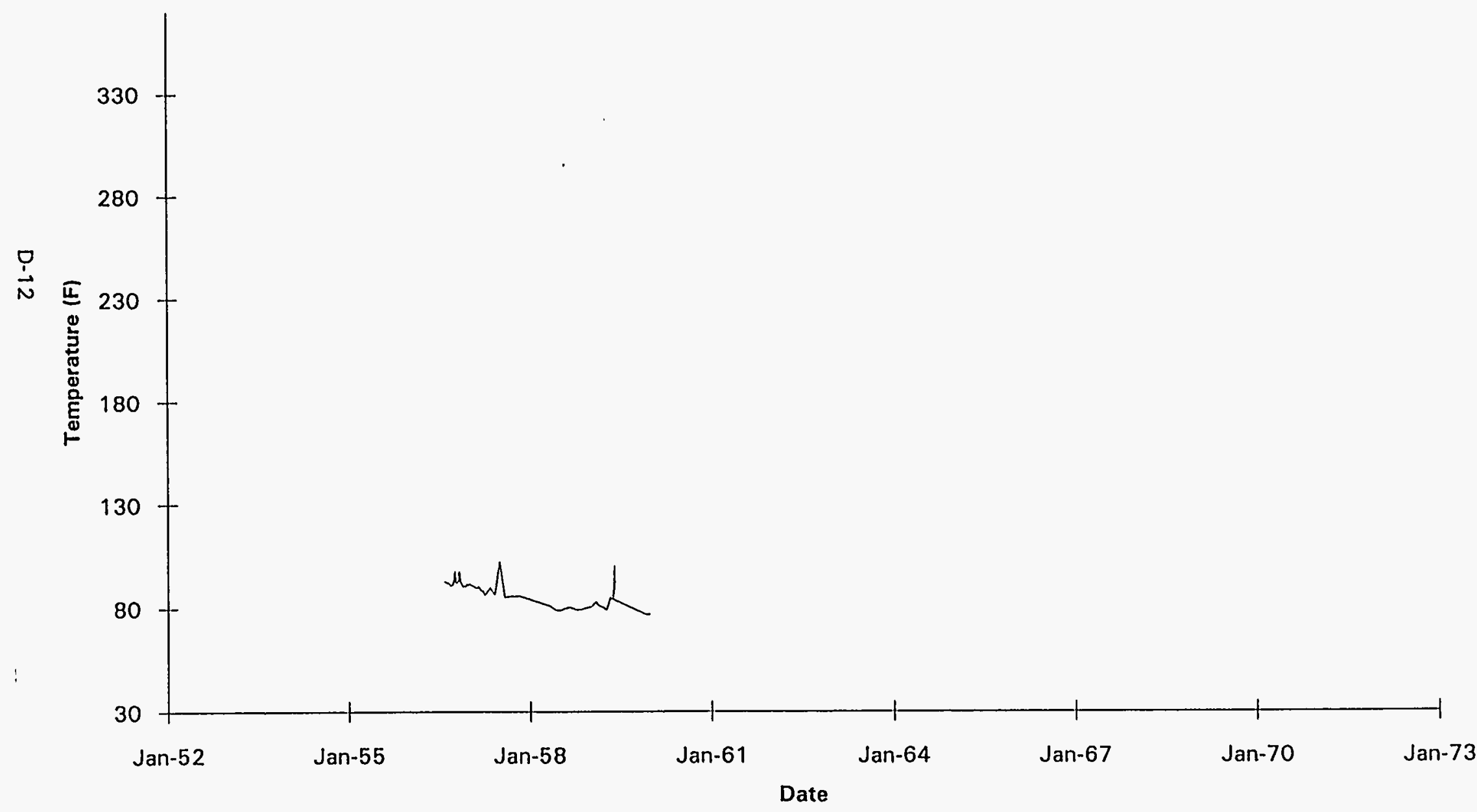

Data obtained ' 7 RHO-CD-1172. 
Tank 241-S-106

Historical High or Only Waste Temperature

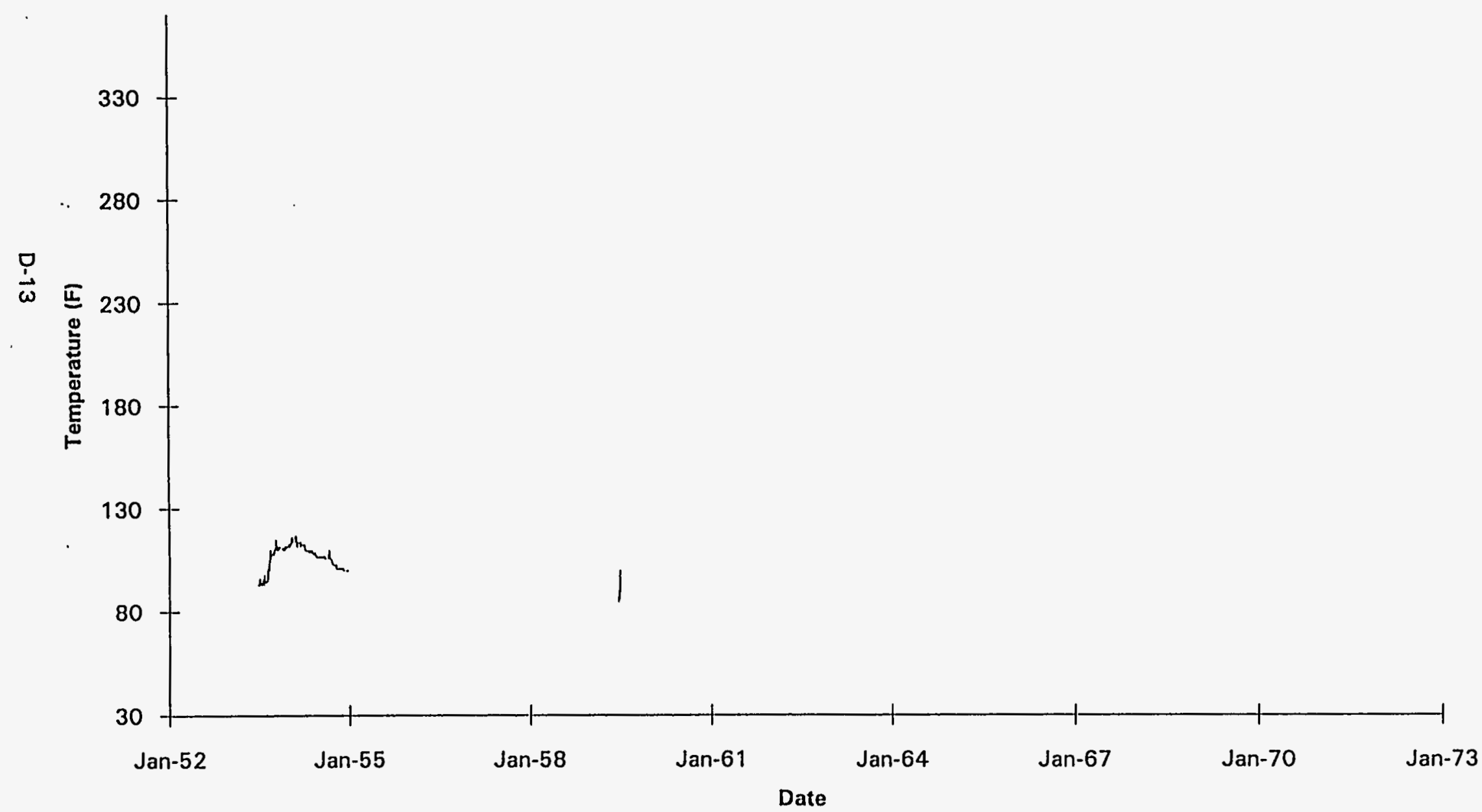

Data obtained from HW-83906. 
S107BULB.GPH

Tank 241-S-107

Historical Vapor Space Temperature

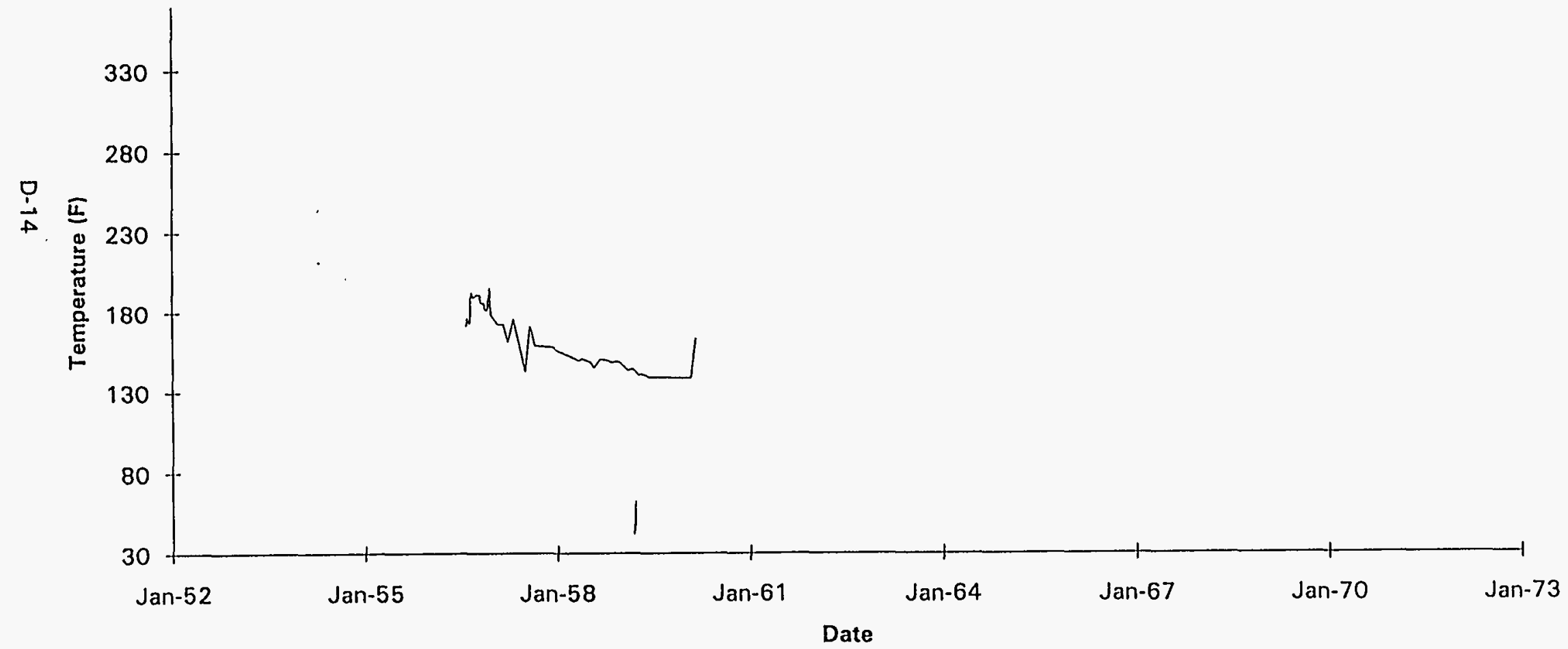


Tank 241-S-107

Historical Average Waste Temperature

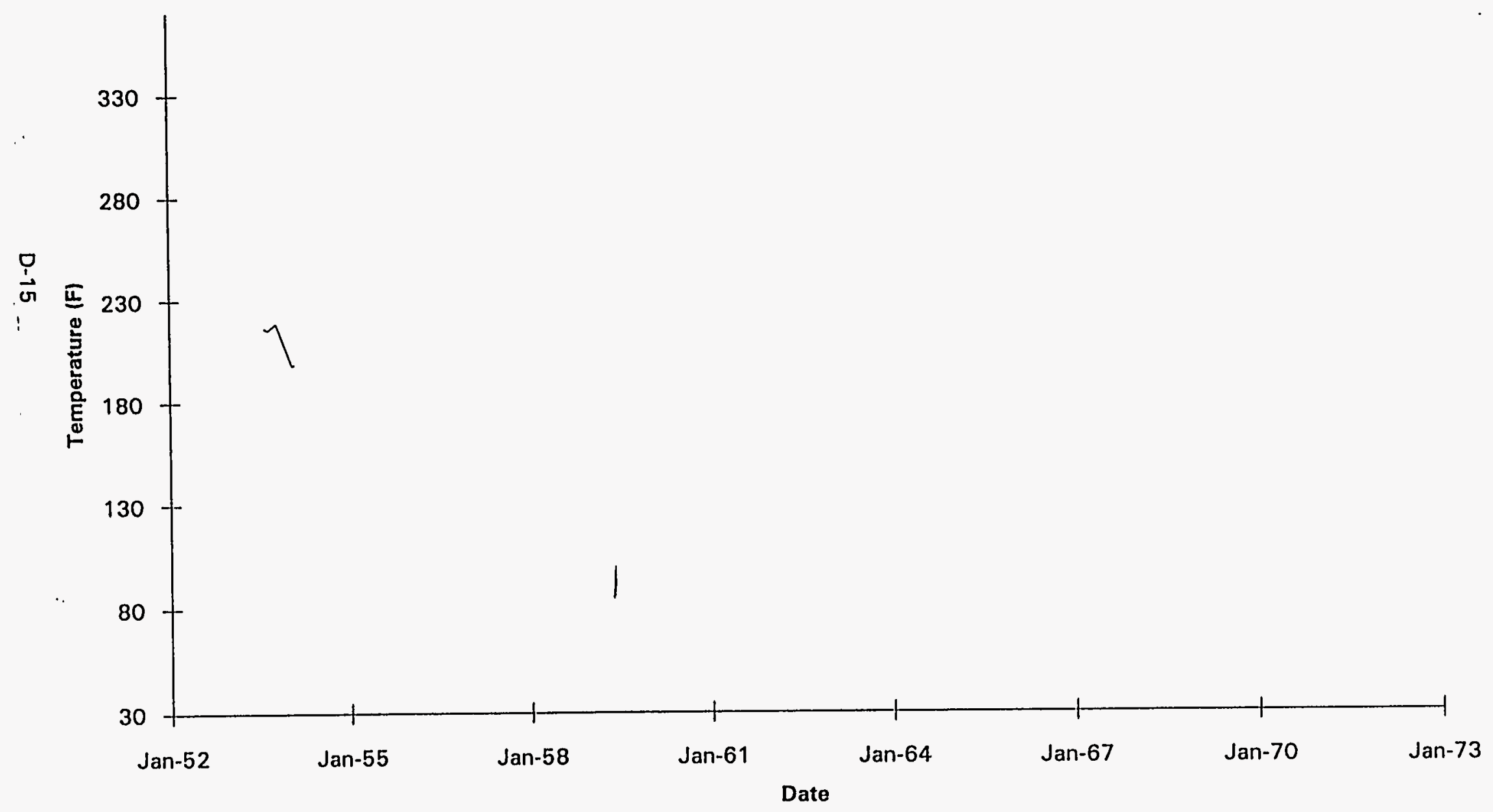


WHC-SD-WM-ER-323, Rev. O
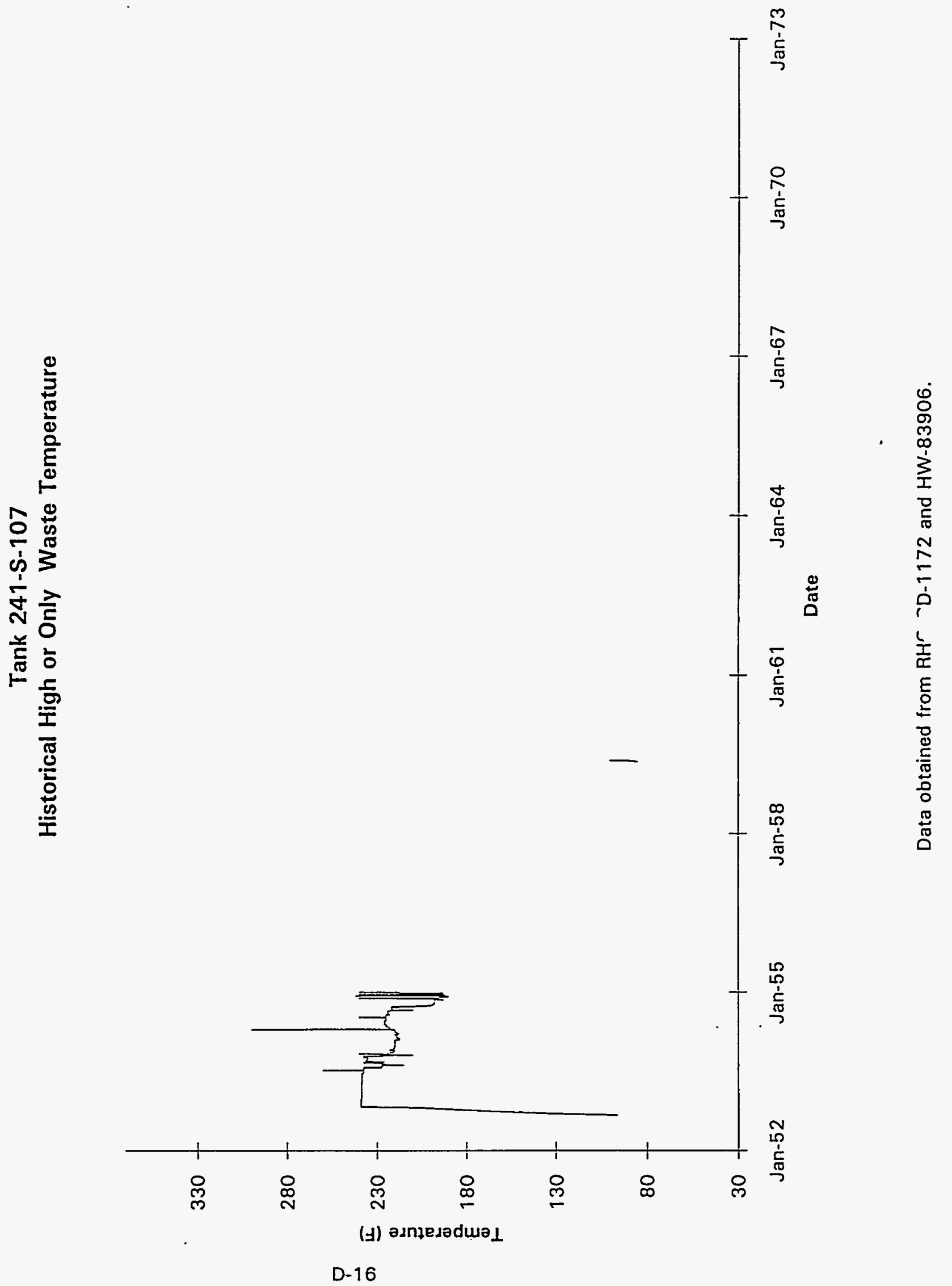
Tank 241-S-108

Historical Vapor Space Temperature

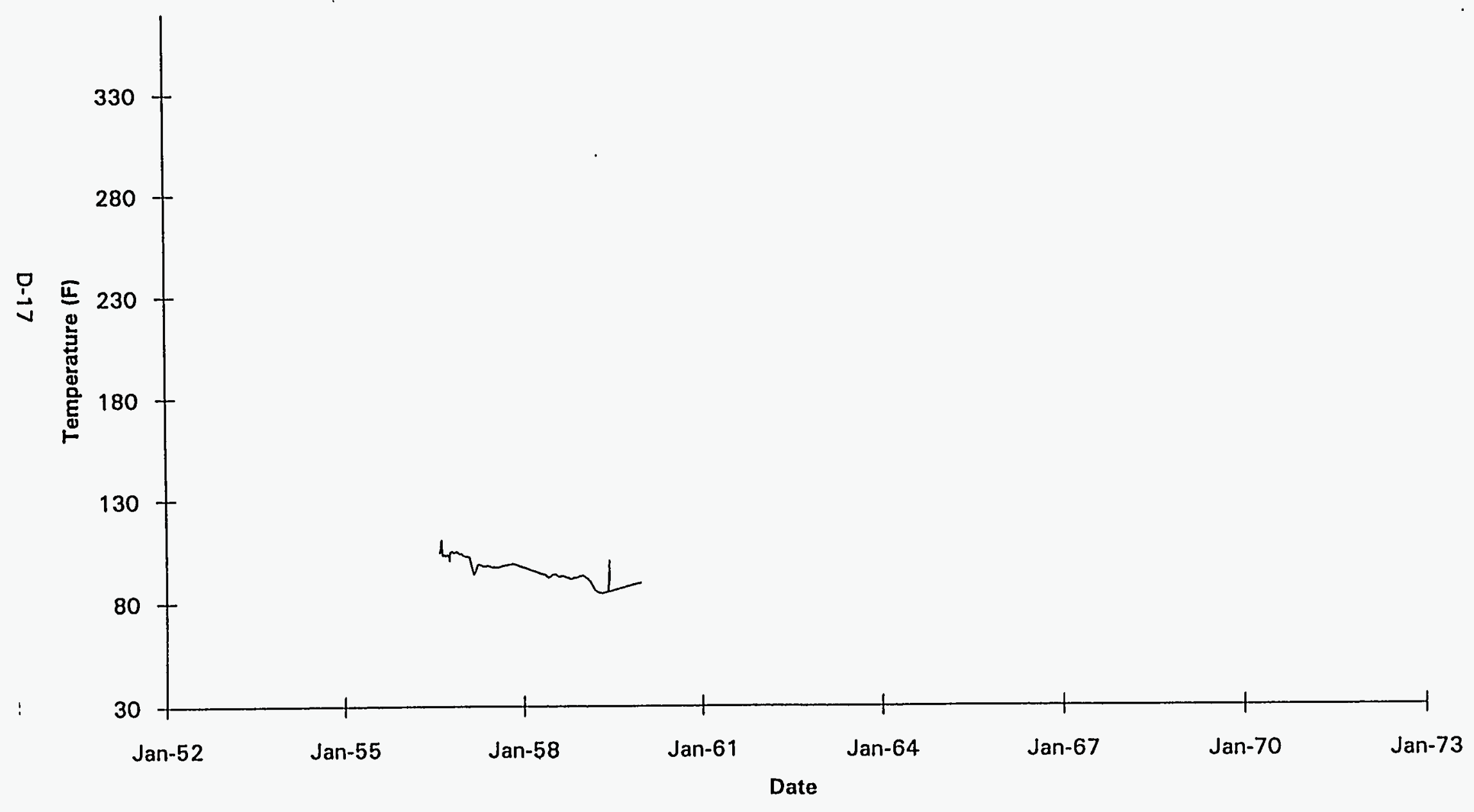

Data obtained from RHO-CD-1172. 
WHC-SD-WM-ER-323, Rev. 0
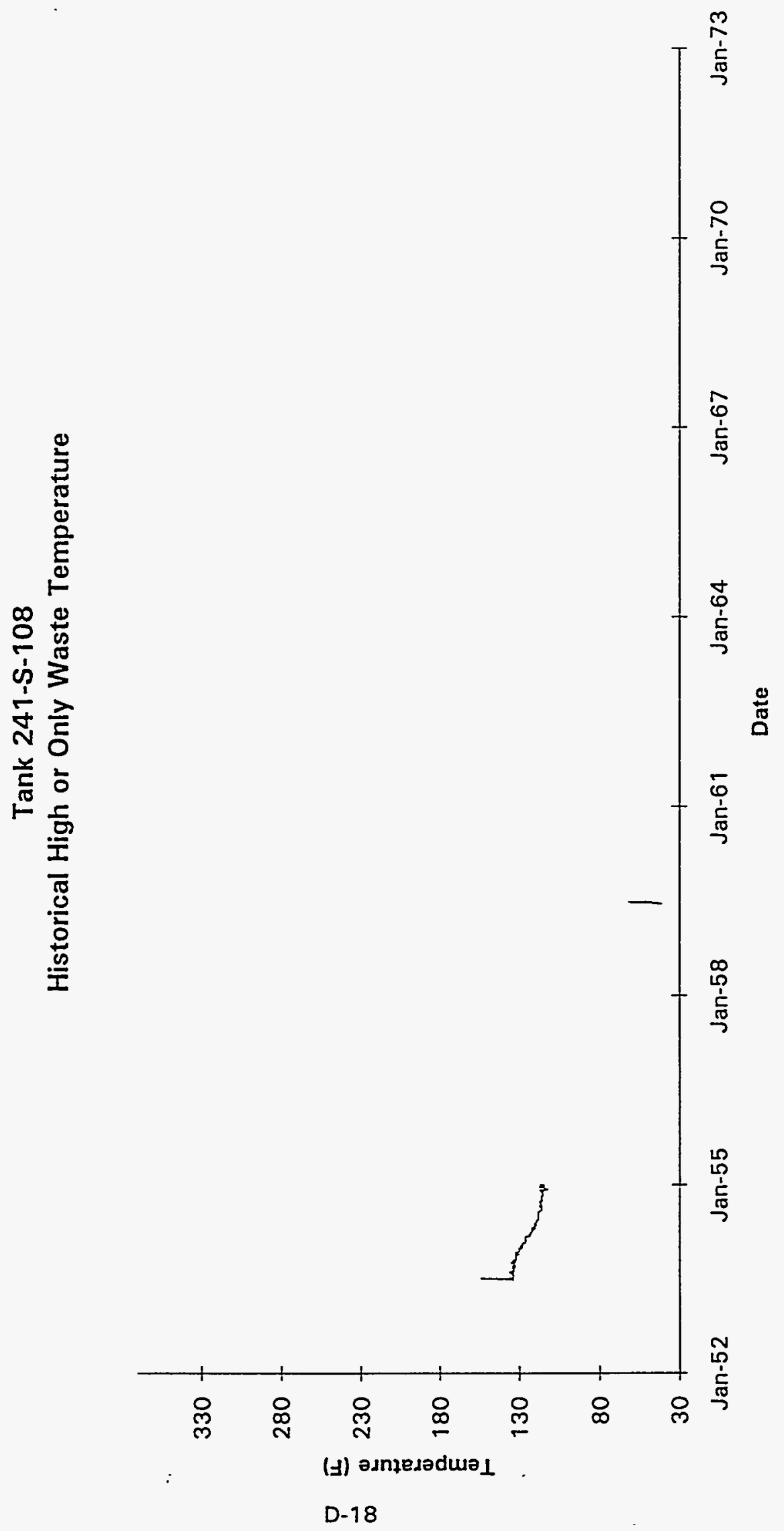

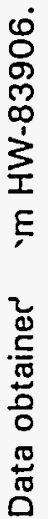


WHC-SD-WM-ER-323, Rev. 0

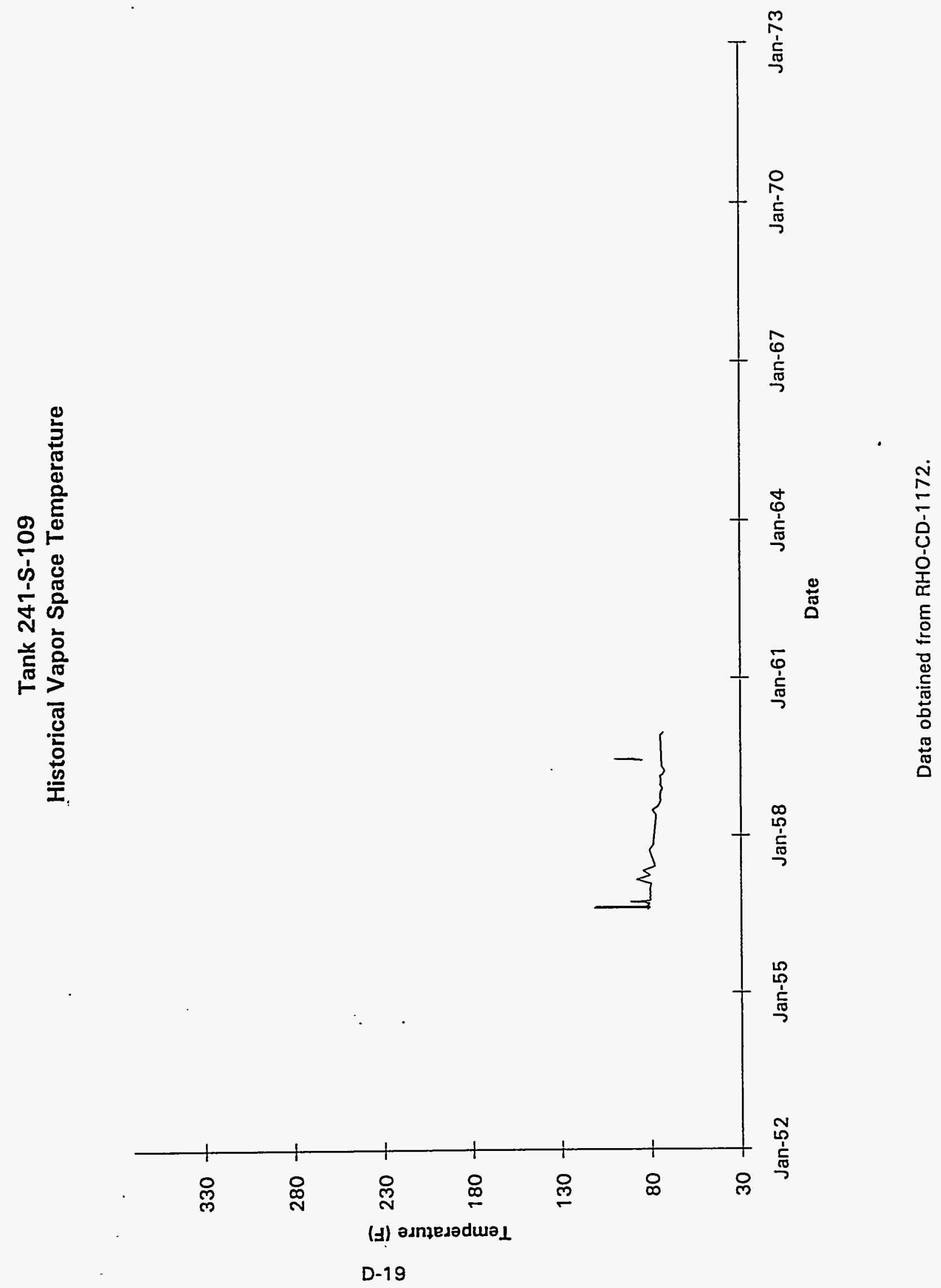


Tank 241-S-109

Historical High or Only Waste Temperature

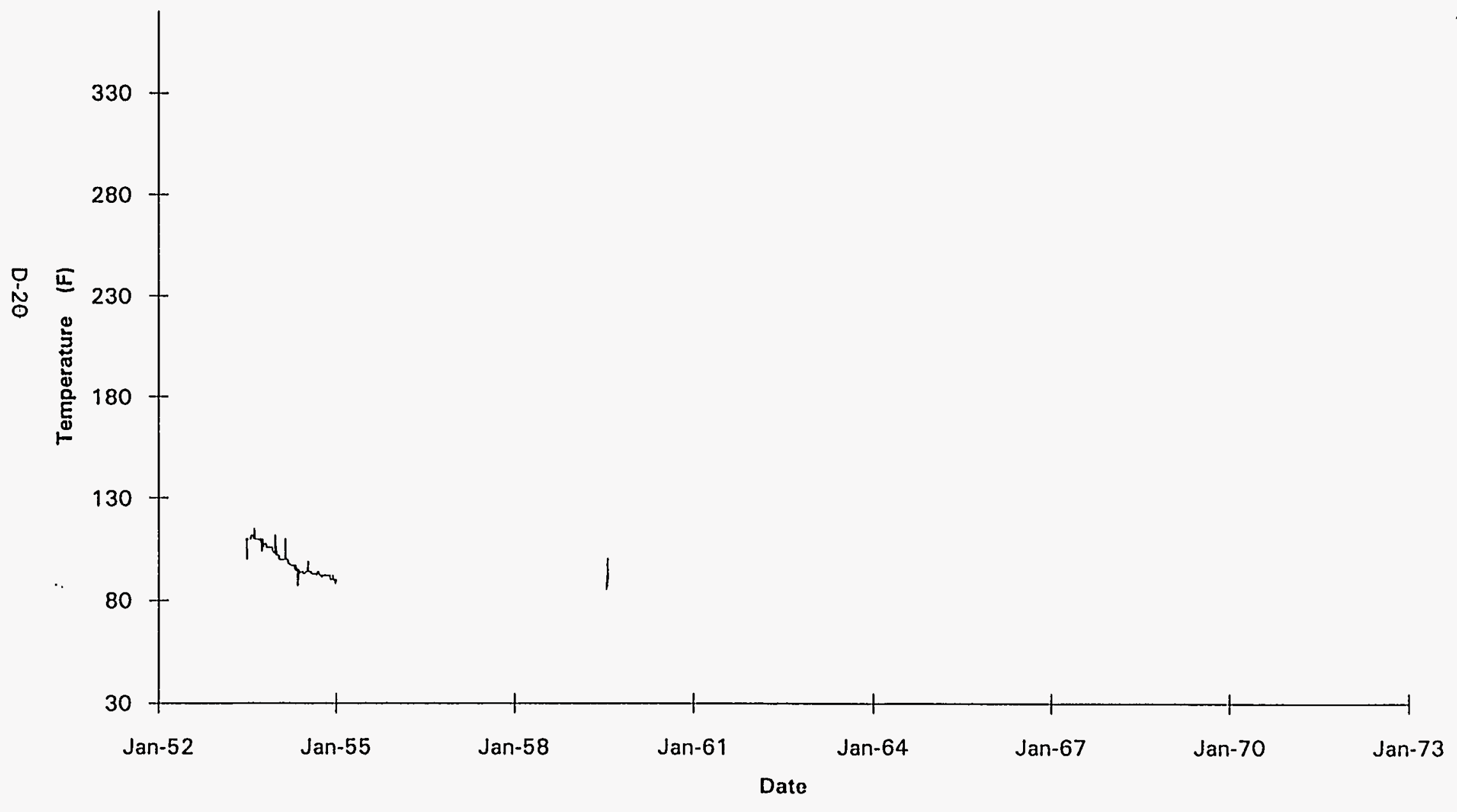


WHC-SD-WM-ER-323, Rev. 0
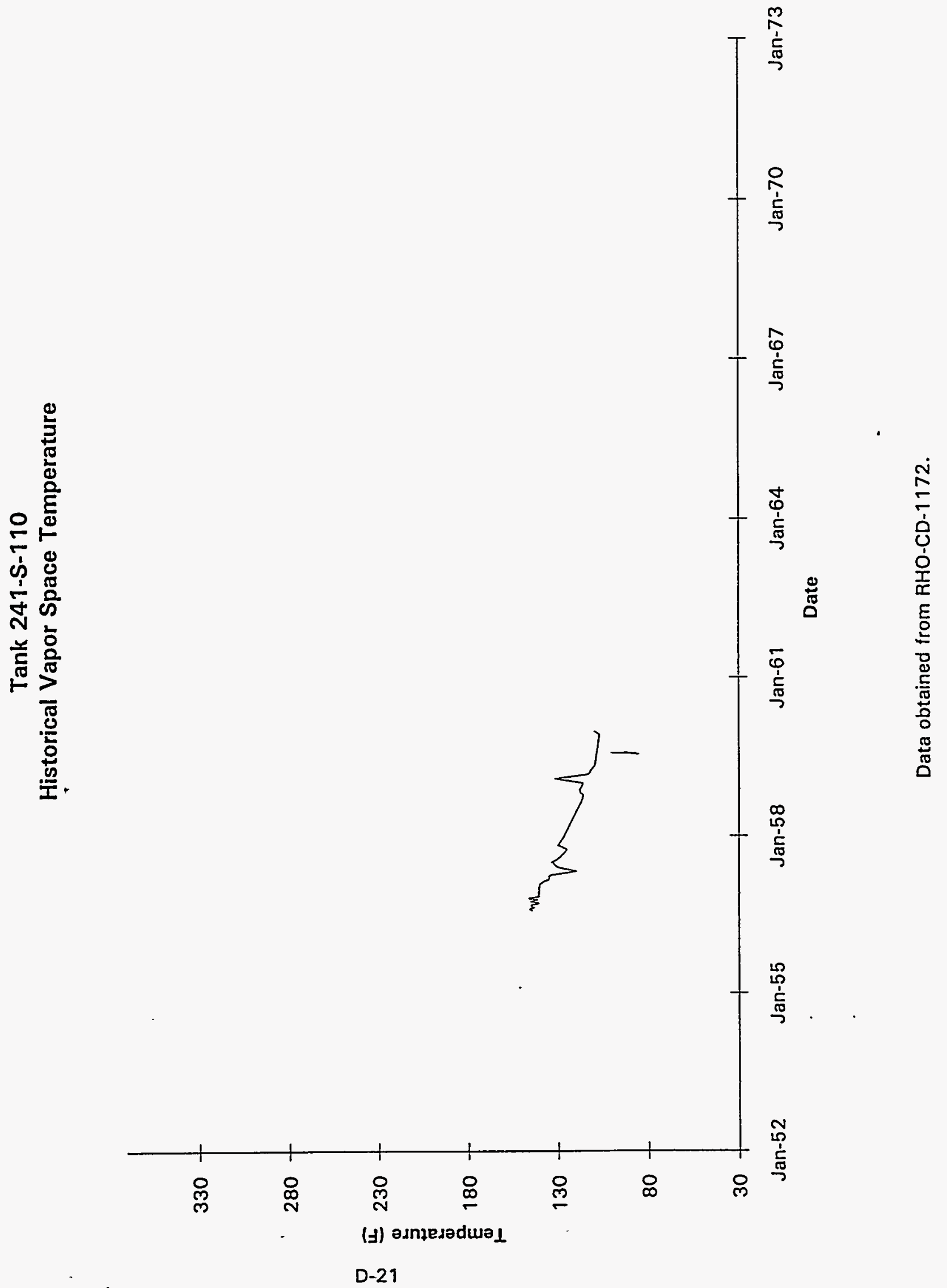
Tank 241-S-110

Historical High or Only Waste Temperature

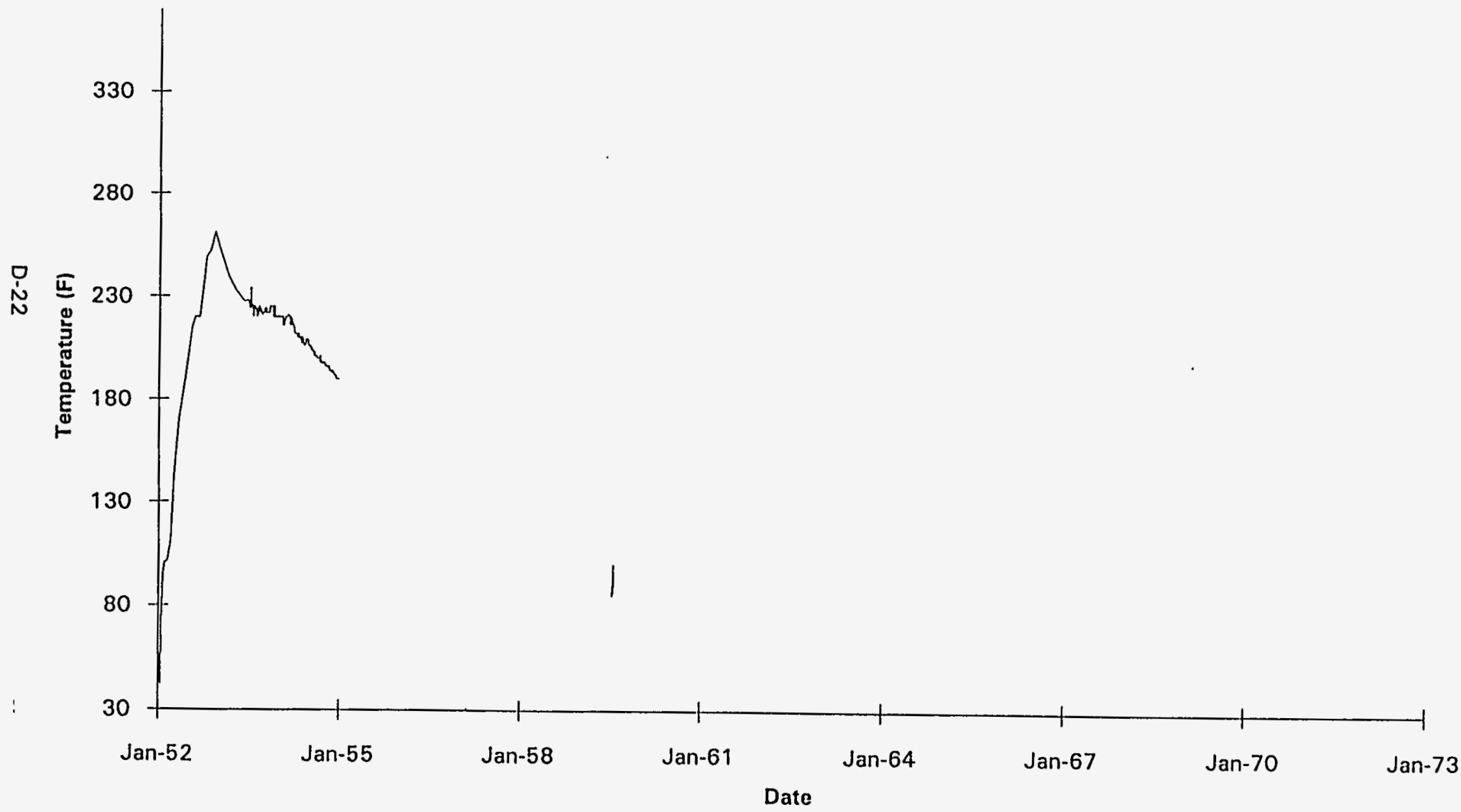

Data obtained from RHS $7-1172$ and HW-83906. 
Tank 241-S-111

Historical Vapor Space Temperature

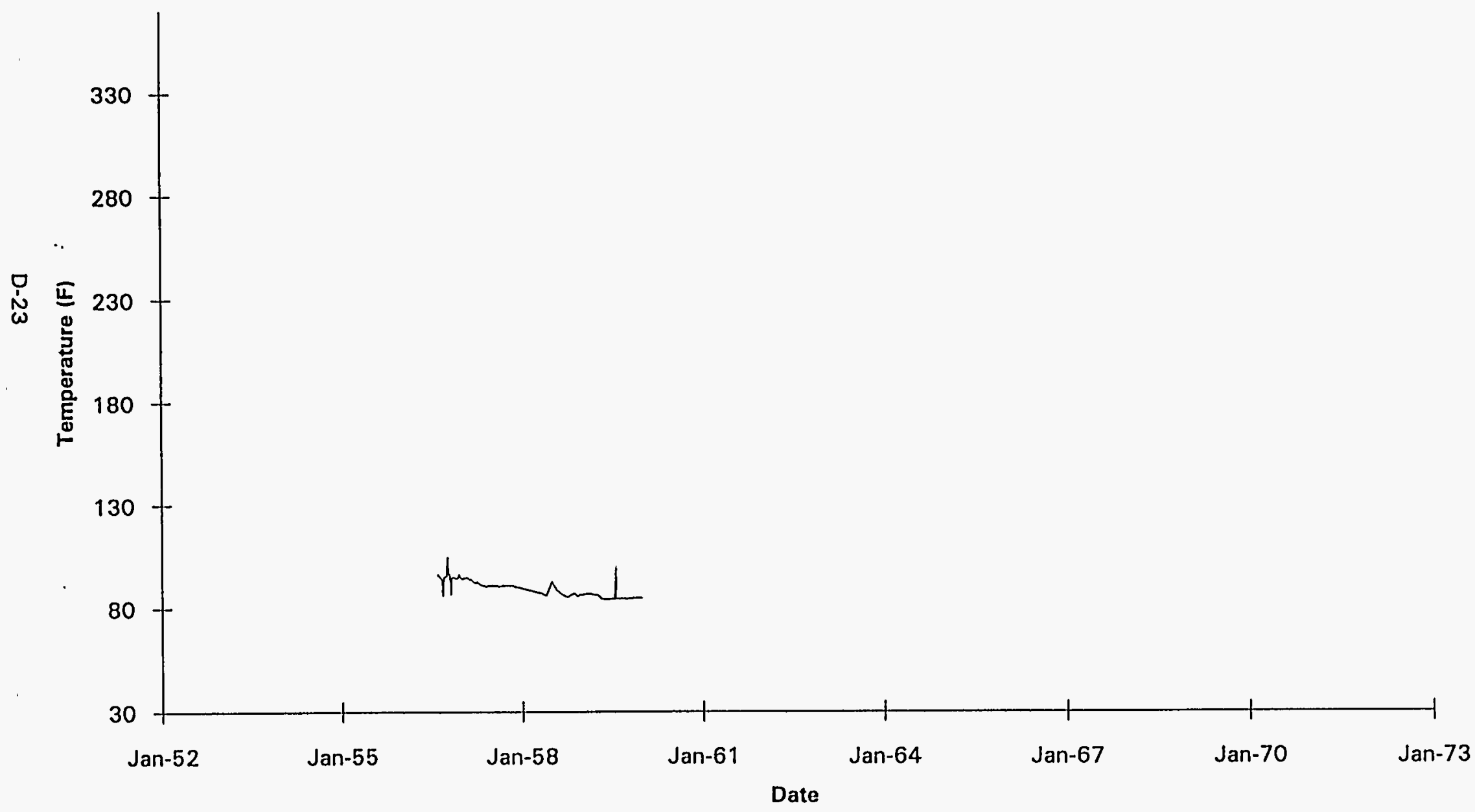


WHC-SD-WM-ER-323, Rev. 0
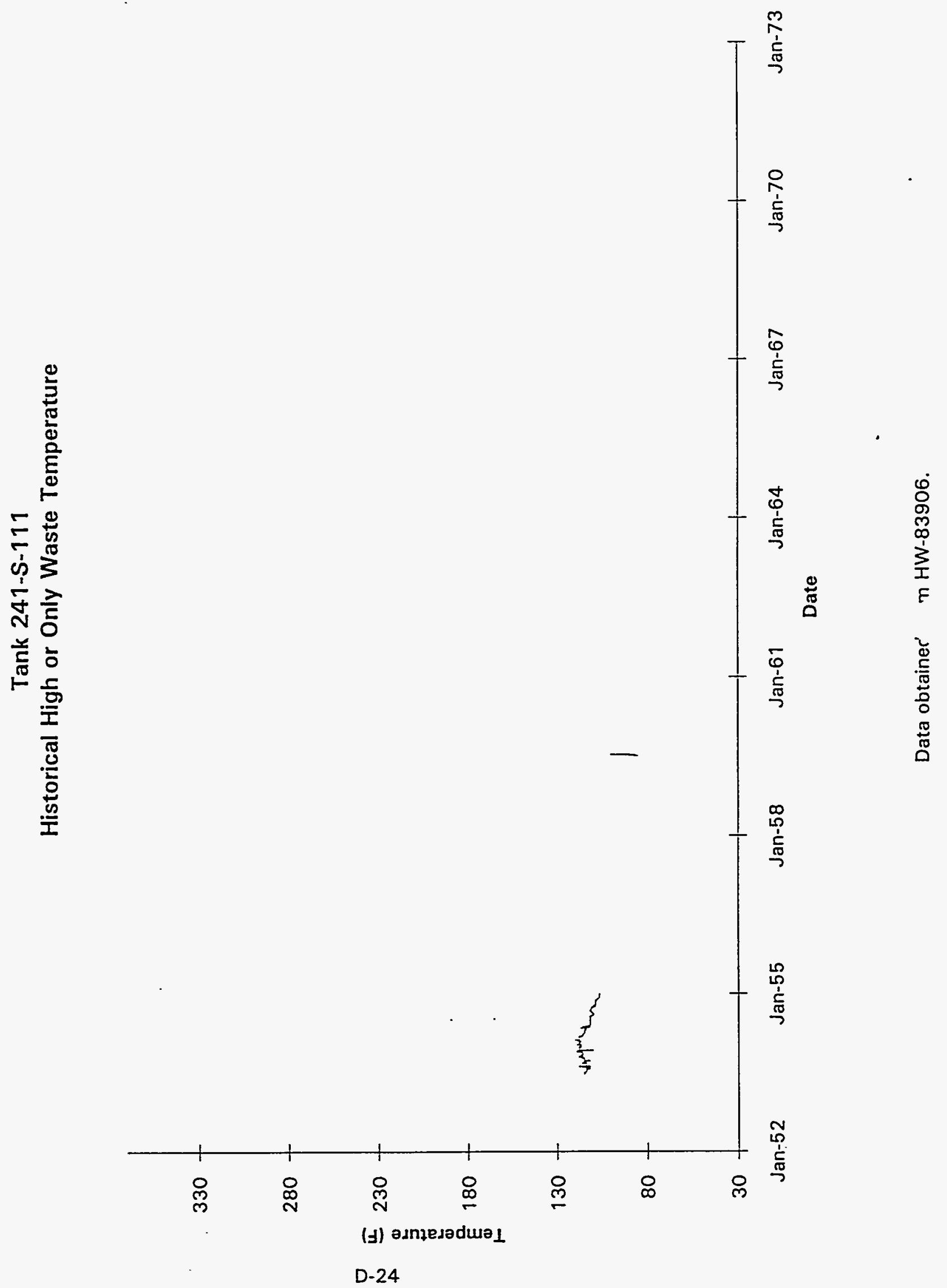
Tank 241-S-112

Historical Vapor Space Temperature

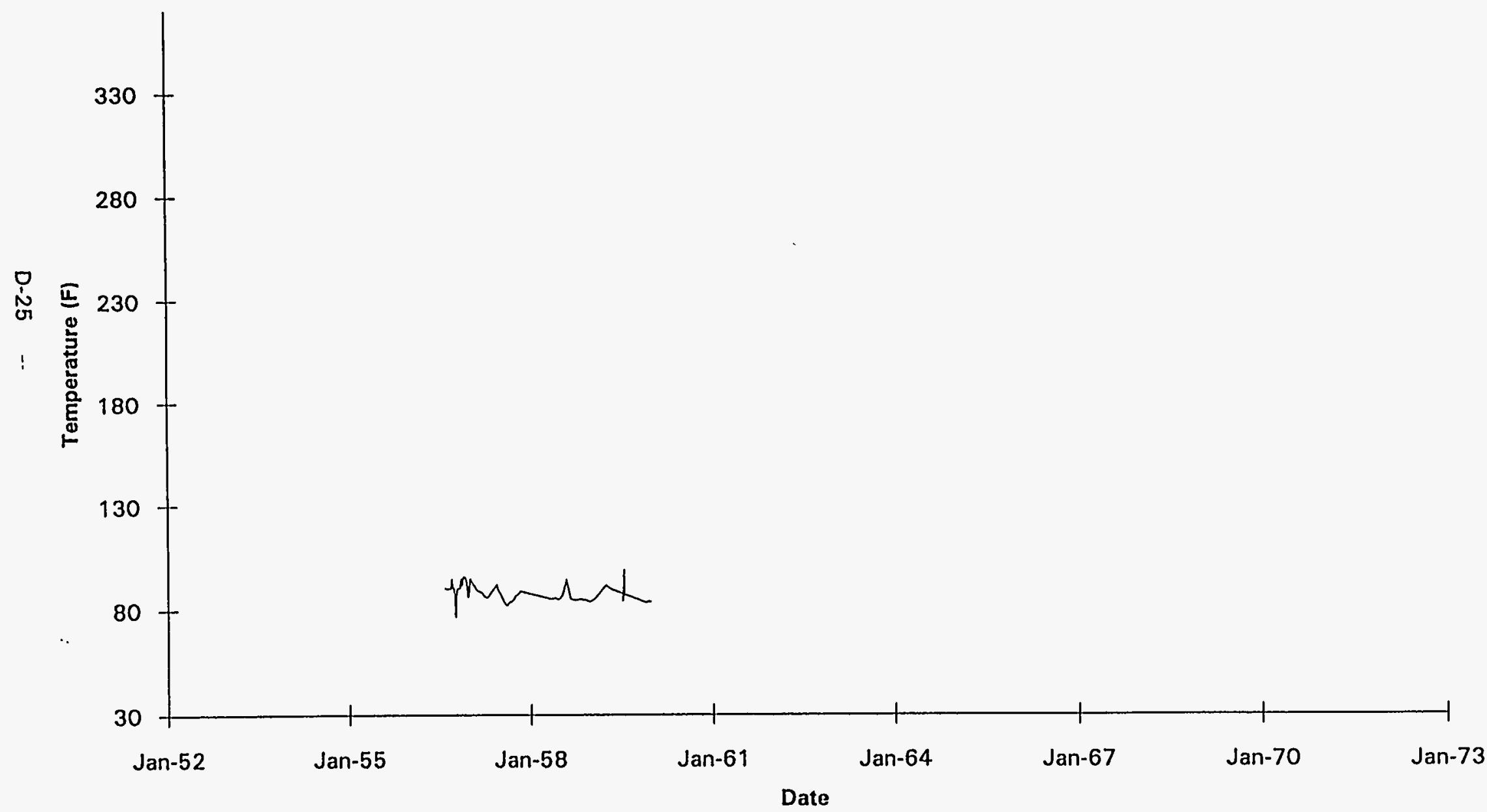


WHC-SD-WM-ER-323, Rev. O

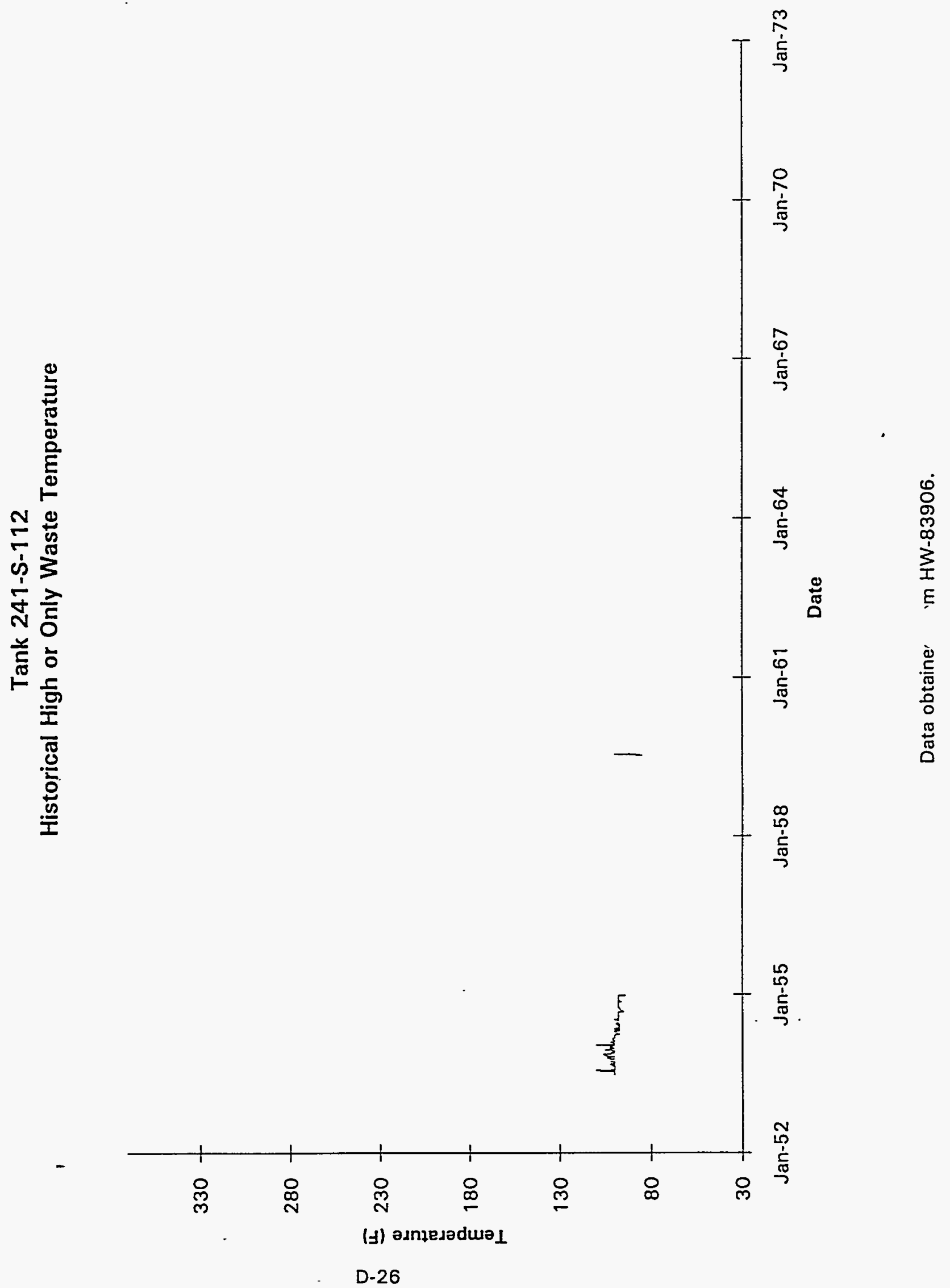




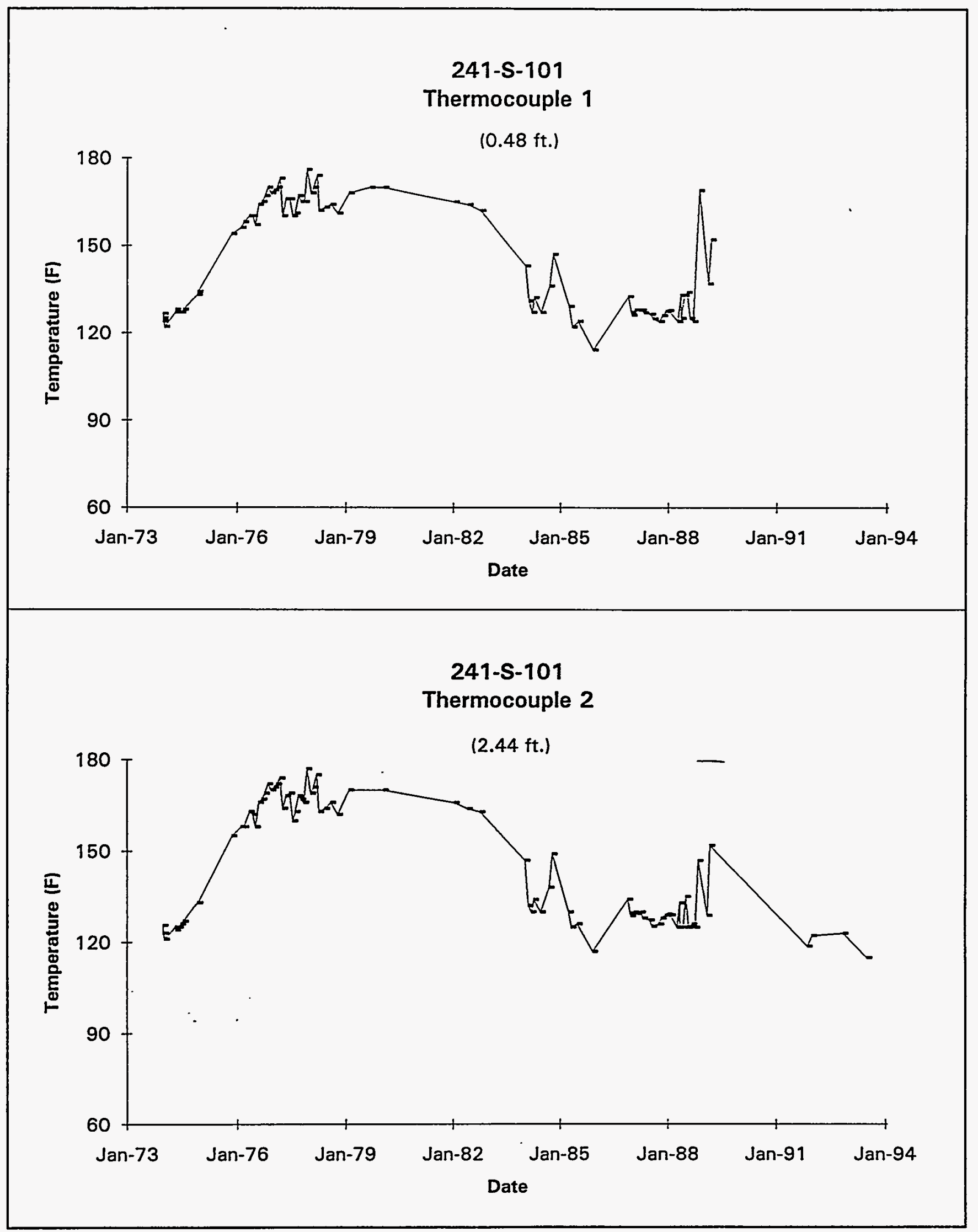

Data obtained from WHC Surveillance Analysis Computer System (SACS), February 24, 1994, Data Sheet TK-105-S to TK-101-S, and Archive box 1121869. 


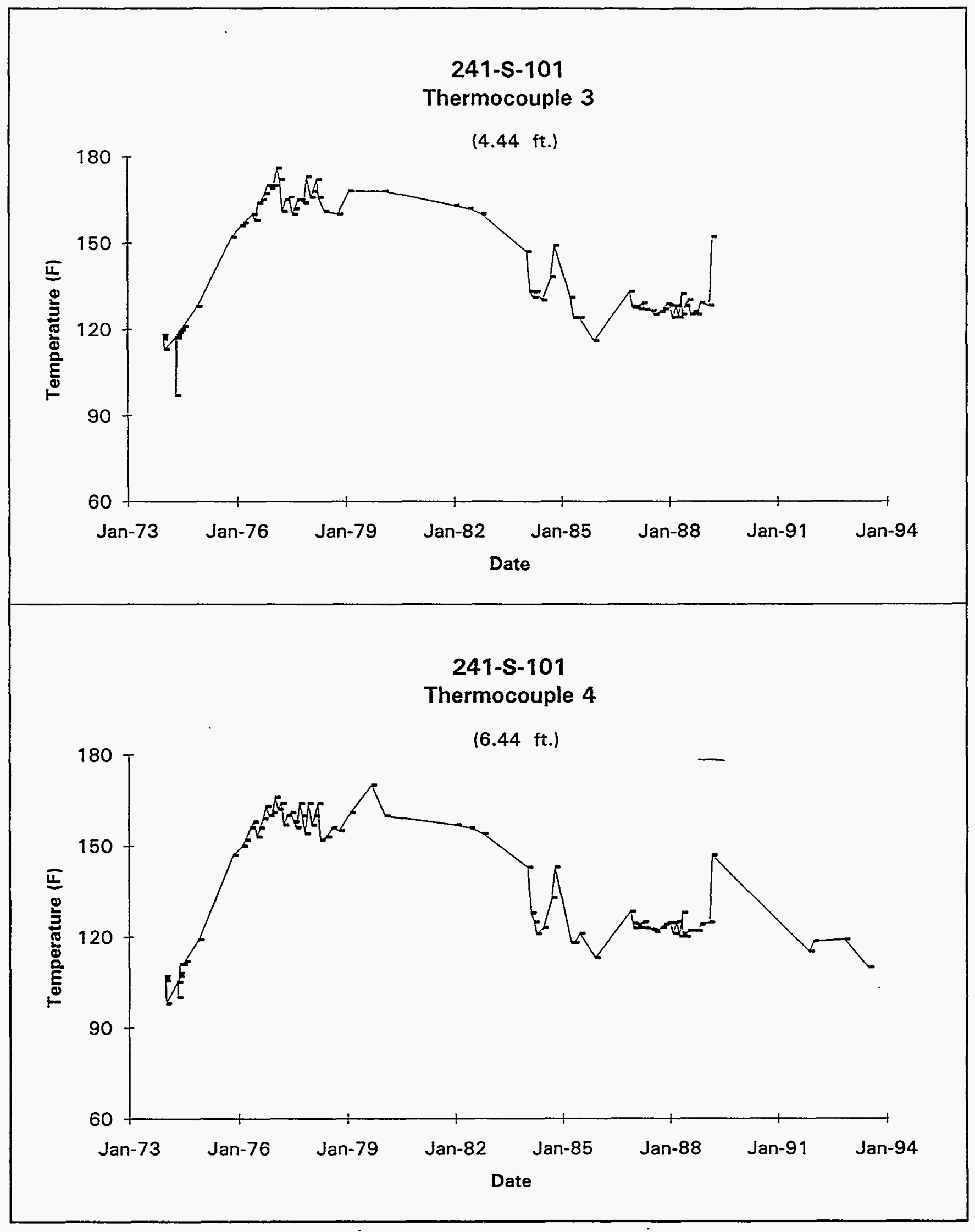

Data obtāined from WHC Surveillance Analysis Computer System (SACS), February 24, 1994, Data Sheet TK-105-S to TK-101-S, and Archive box 121869. 


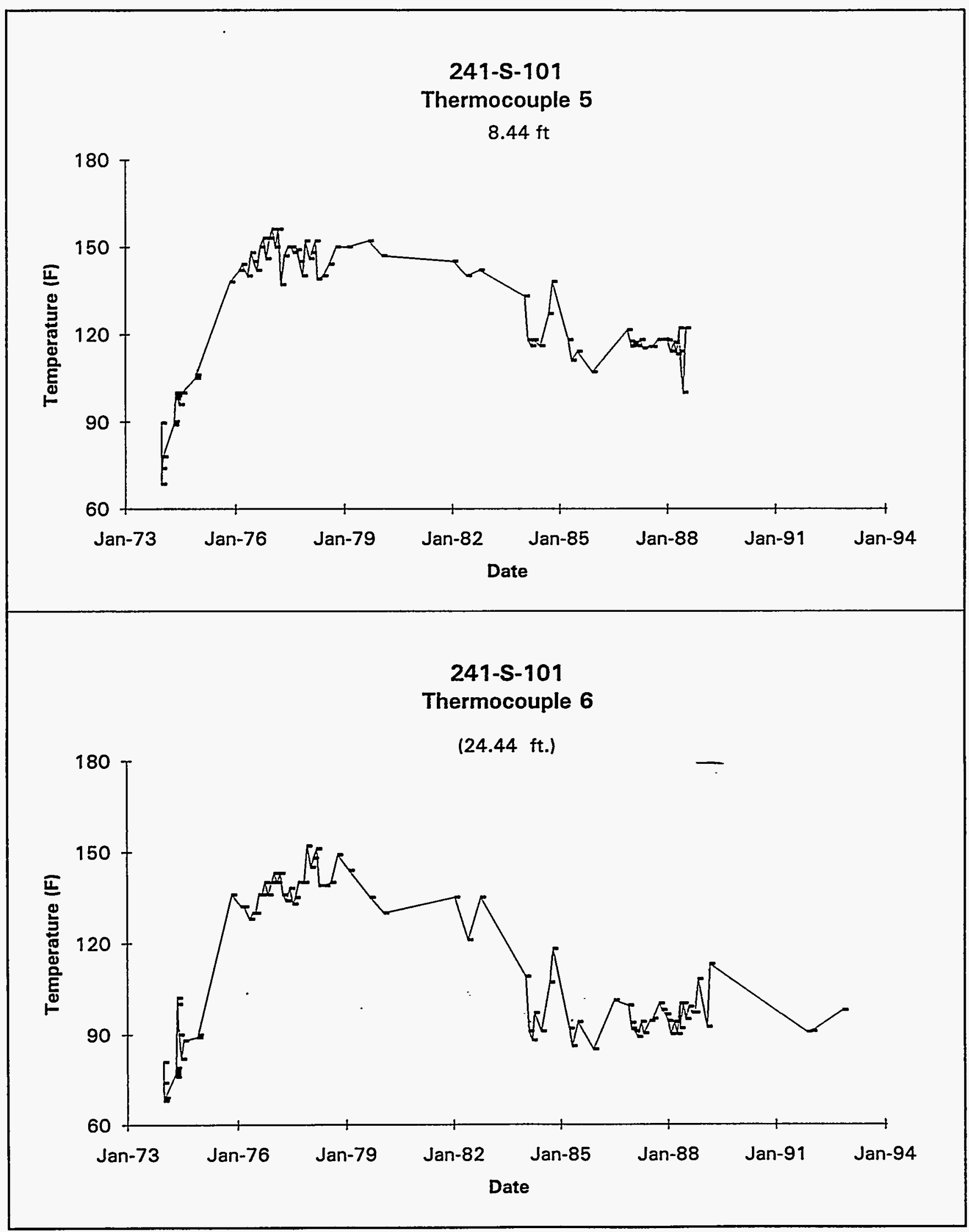

Dato obtained from WHC Surveillance Analysis Computer System (SACS), February 24, 1994, Data Sheet TK-105-S to TK-101-S, and Archive box 121869. 


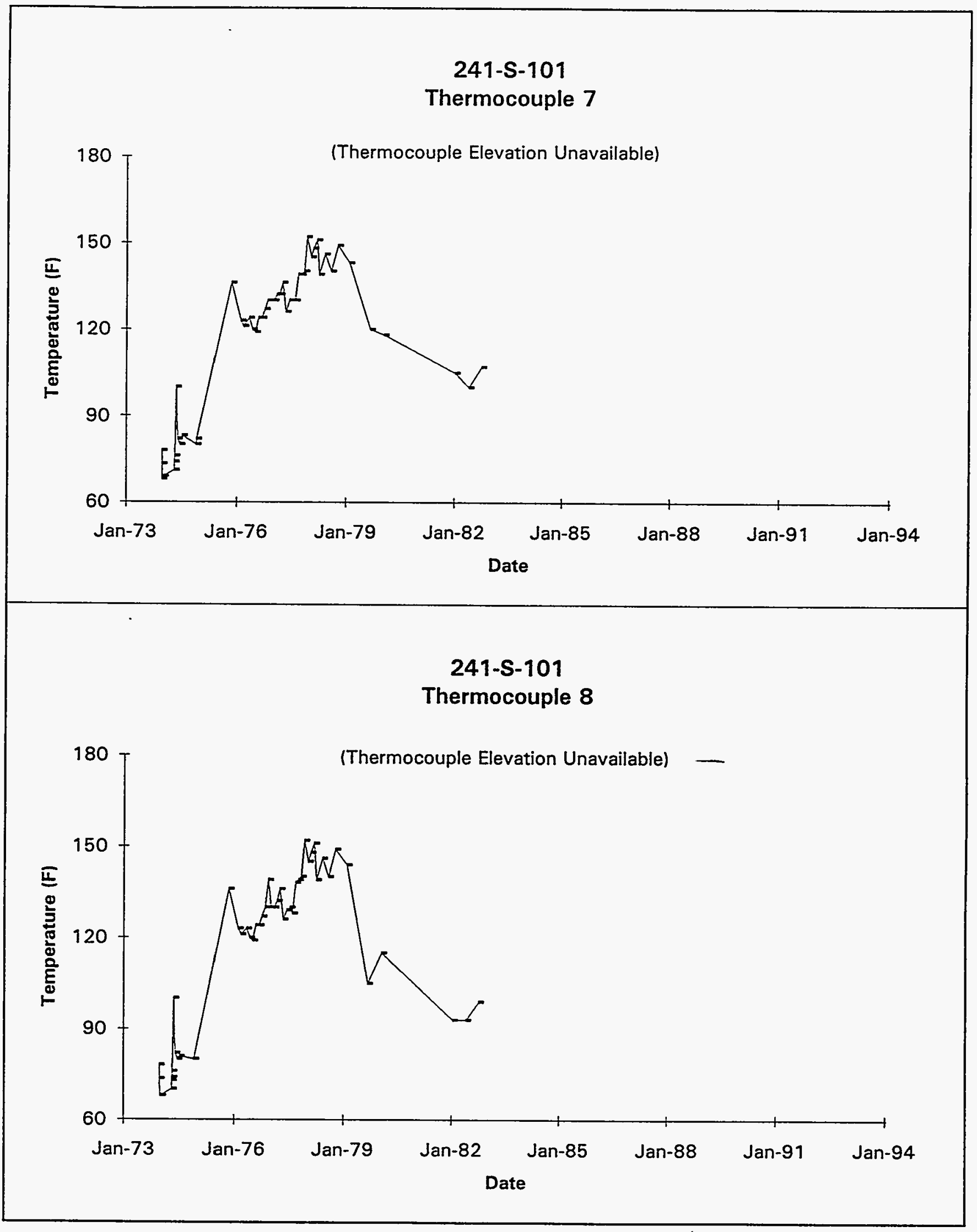

Data obtained from WHC Surveillance Analysis Computer System (SACS), February 24, 1994, Data Sheet TK-105-S to TK-101-S, and Archive bax 121869. 


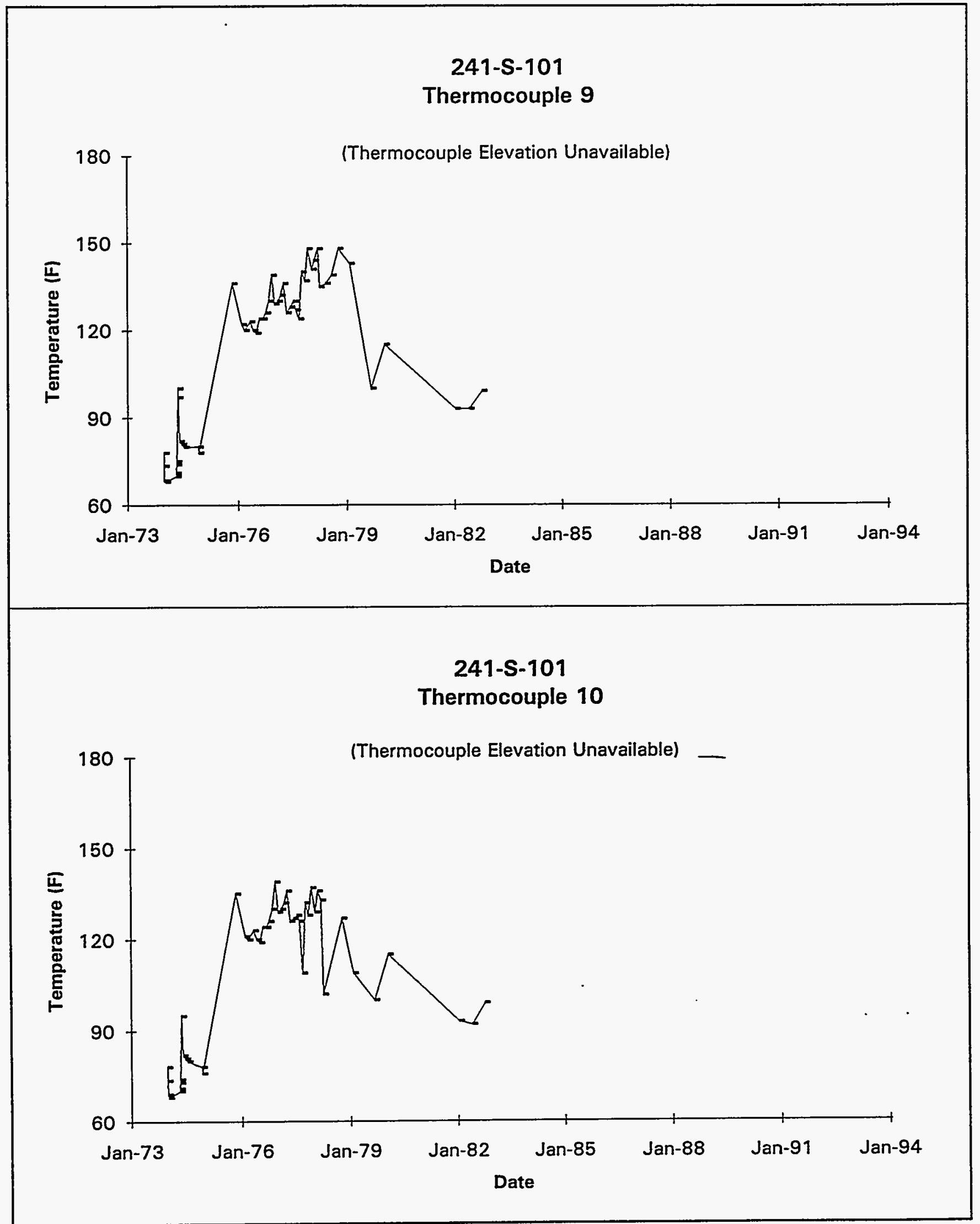

Data obtained from WHC Surveillance Analysis Computer System (SÄCS), February 24, 1994, Data Sheet TK-105-S to TK-101-S, and Archive box \$121869. 


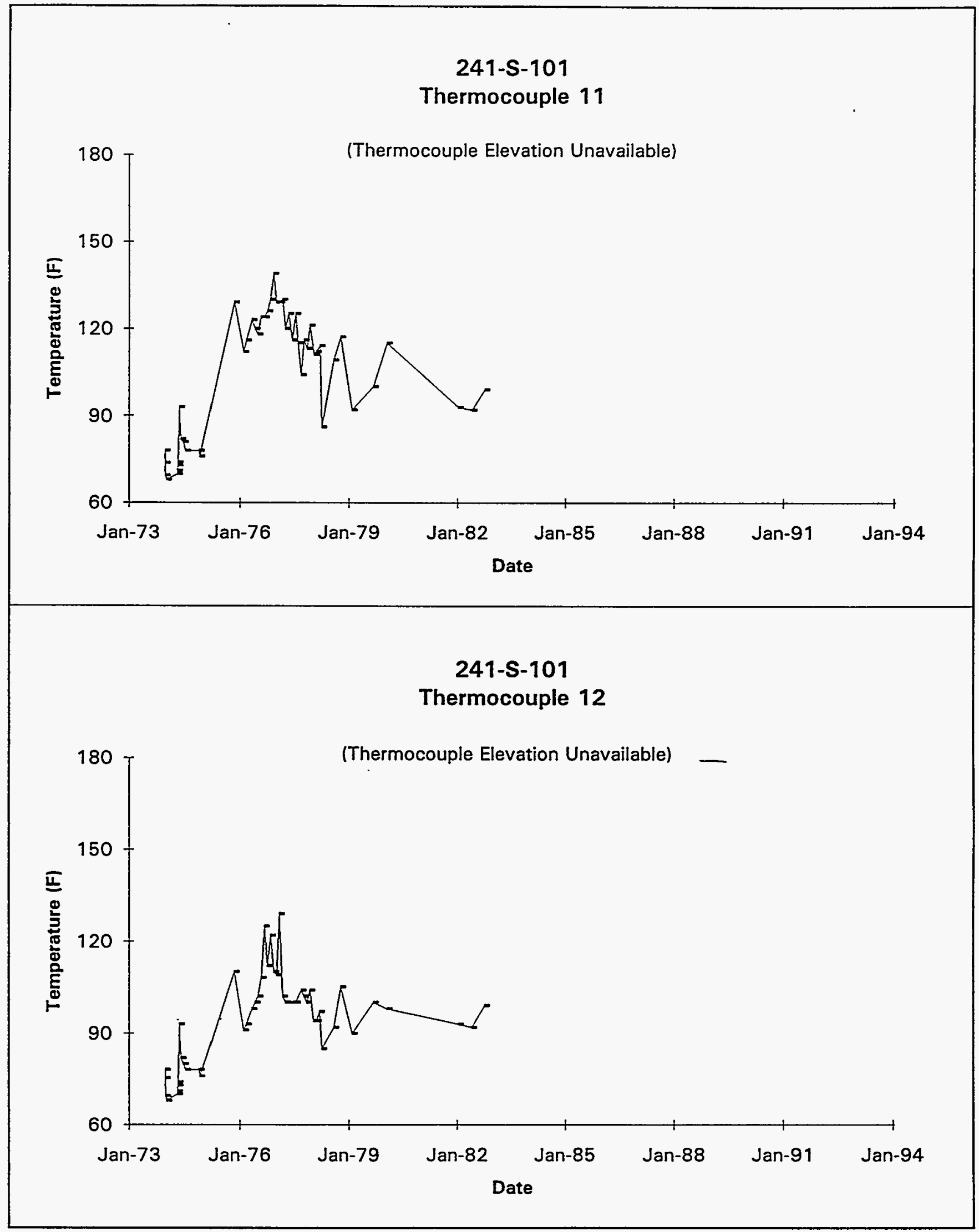

Data obteined from WHC Surveillance Anslysis Computer System (SACS), February 24, 1994, Data Sheet TK-105-S to TK-101-S, and Archive box $\# 121869$. 


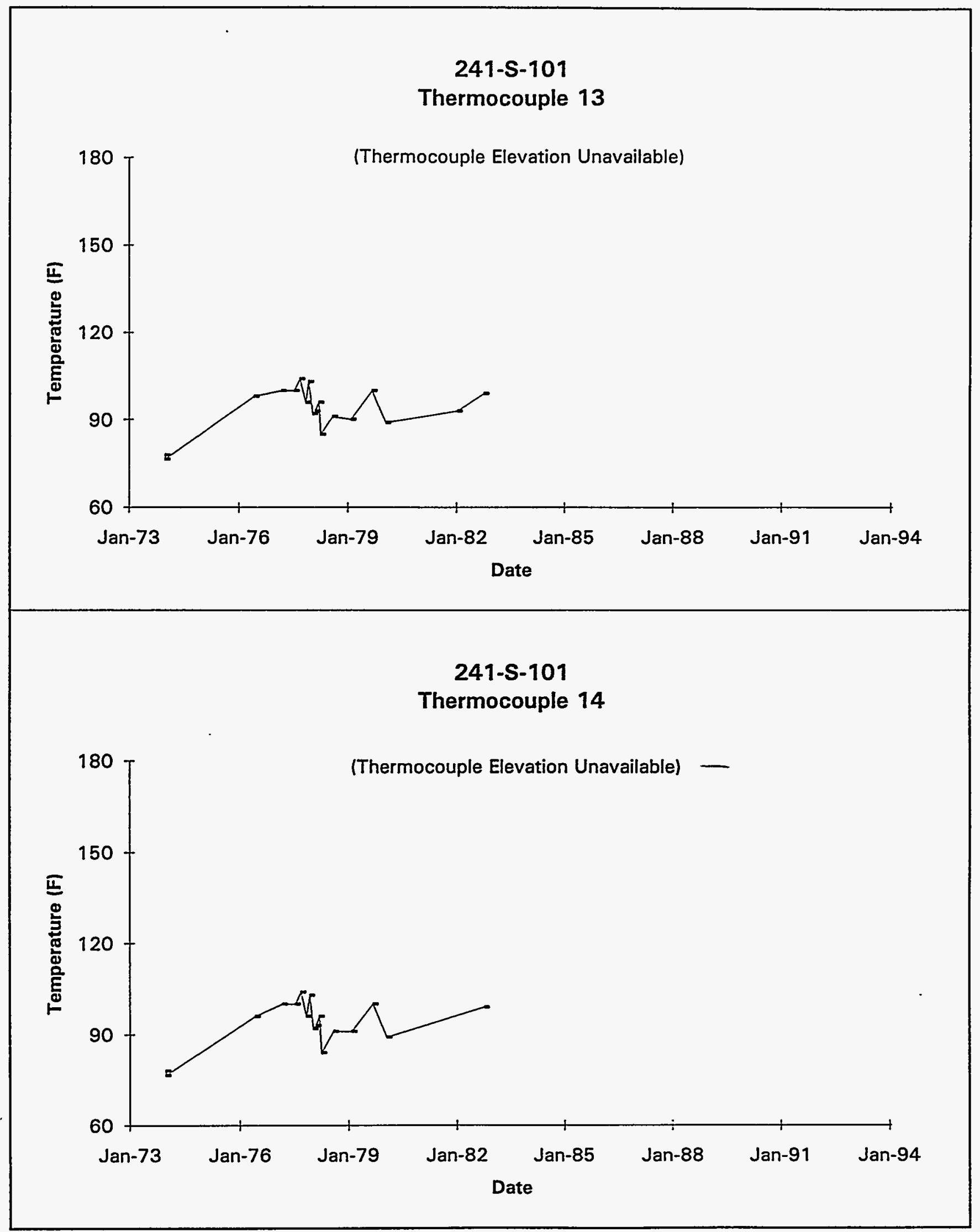

Data obiained frōm WHC Surveillance Analysis Computer System (SACS), February 24, 1994, Data Sheet TK-105-S to TK-101-S, and Archive box $\$ 121869$. 


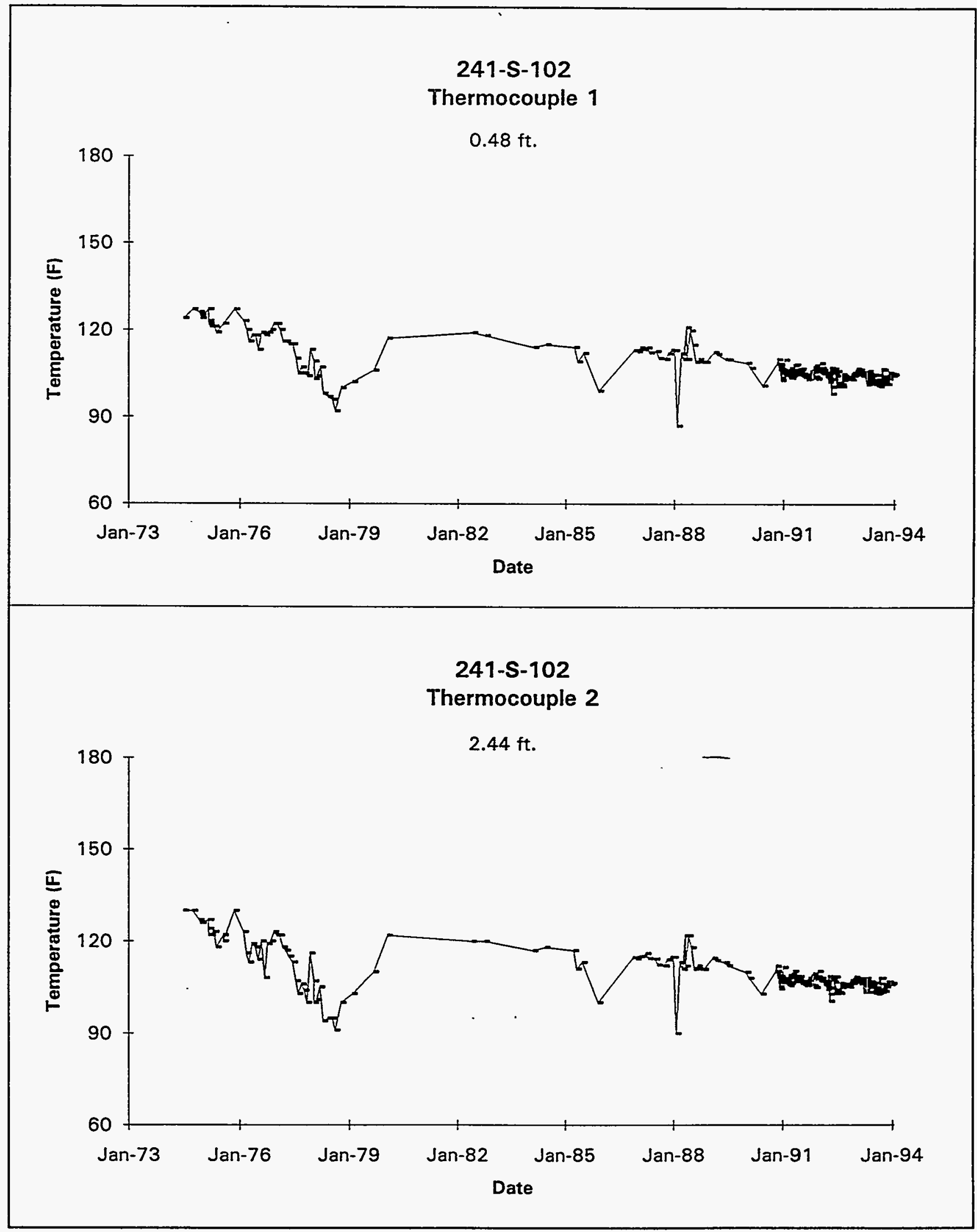

Data obtained from WHC Surveillance Analysis Computer System (SACS), February 24, 1994, 242-S Bottoms Tanks Transfer Record, Data Shaat TK-105-S to TK-101-S, and Archive box $\$ 121869$. 


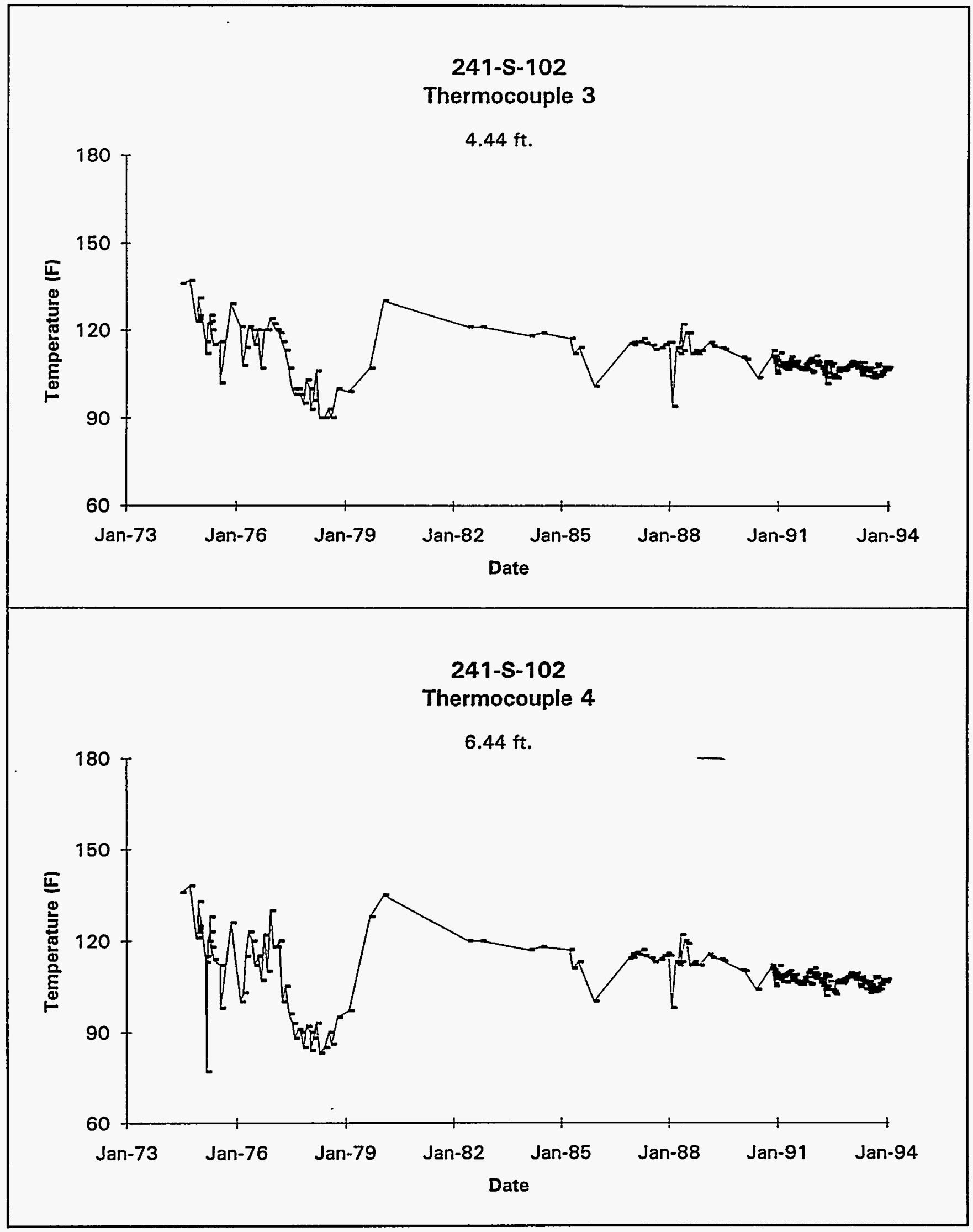

Data obtained from WHC Surveillance Analysis Computer System (SACS), February 24, 1994, 242-S Bottoms Tenks Transiet Record, Date Sheit TK-105-S to TK-101-S, and Archive box $\$ 121869$. 


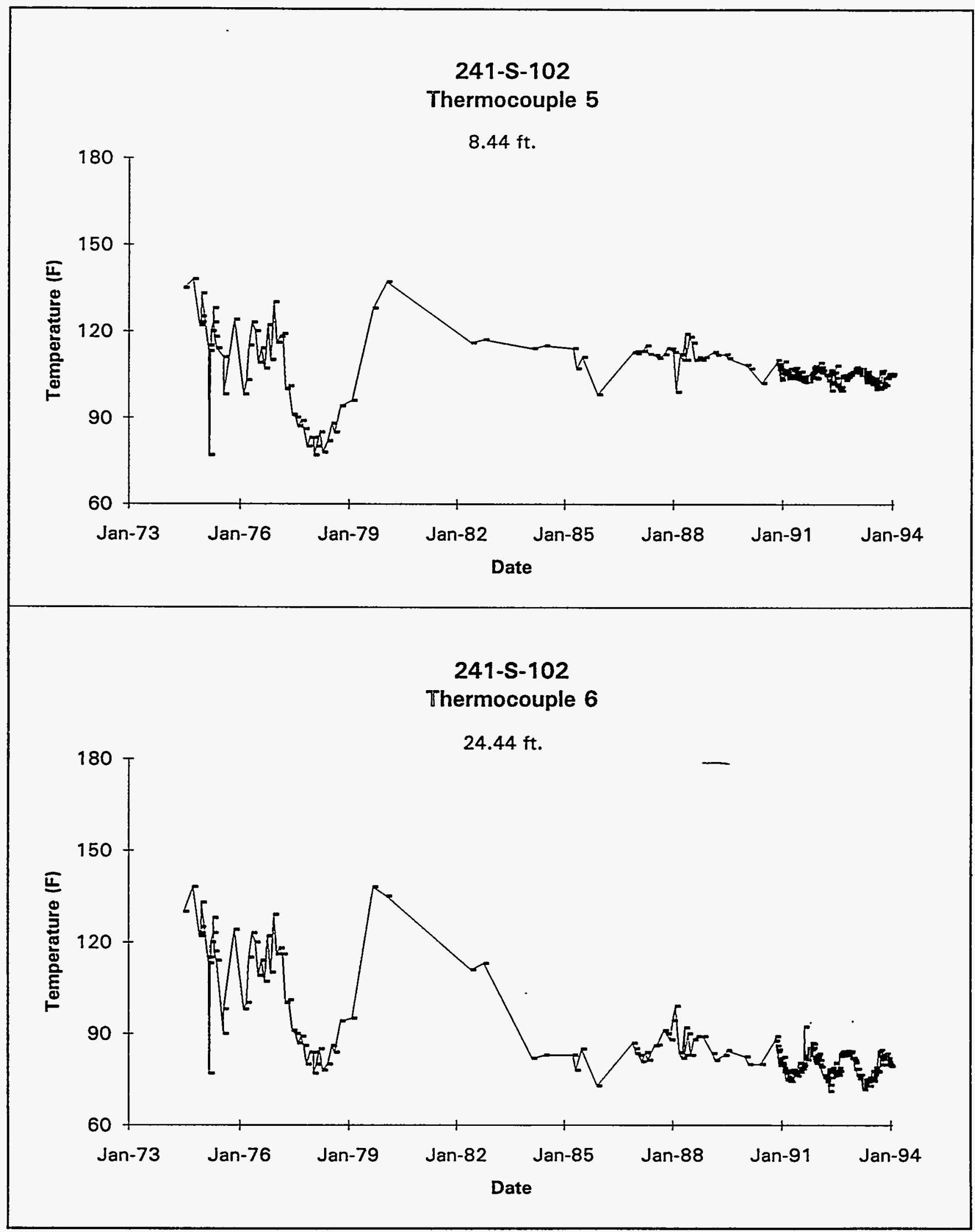

Data obtained from WHC Surveillance Analysis Computer System (SACS), February 24, 1994, 242-S Bottoms Tanks Transfer Record, Data Sheat TK-105-S to TK-101-S, and Aschive box $\$ 121869$ 


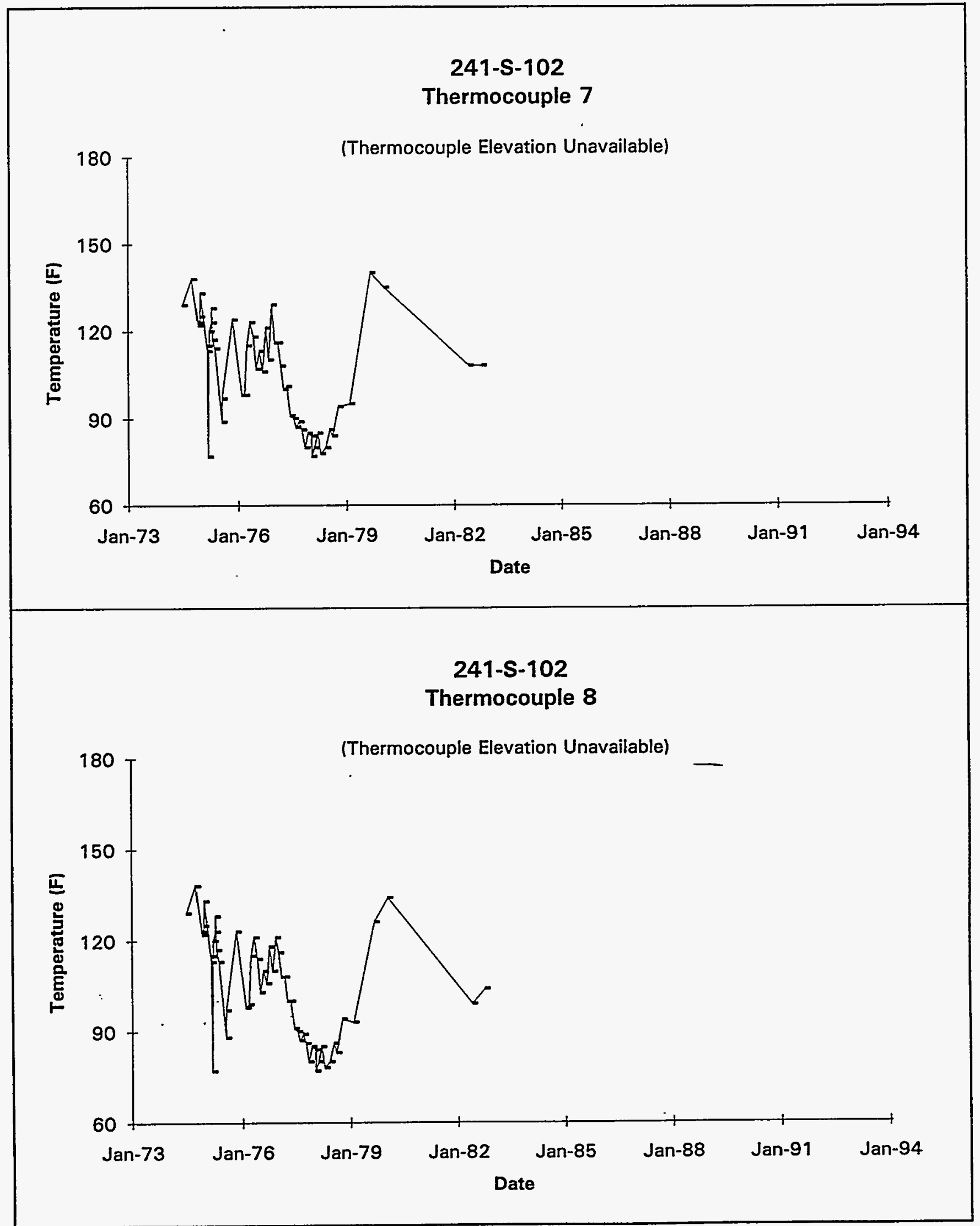

Data obtained Irom WHC Surveillance Analysis Computer System (SACS), February 24, 1994, 242-S Bottoms Tanks Transfer Record. Data Sheet TK-105-S to TK-101-S, and Archive box $\$ 121869$. 


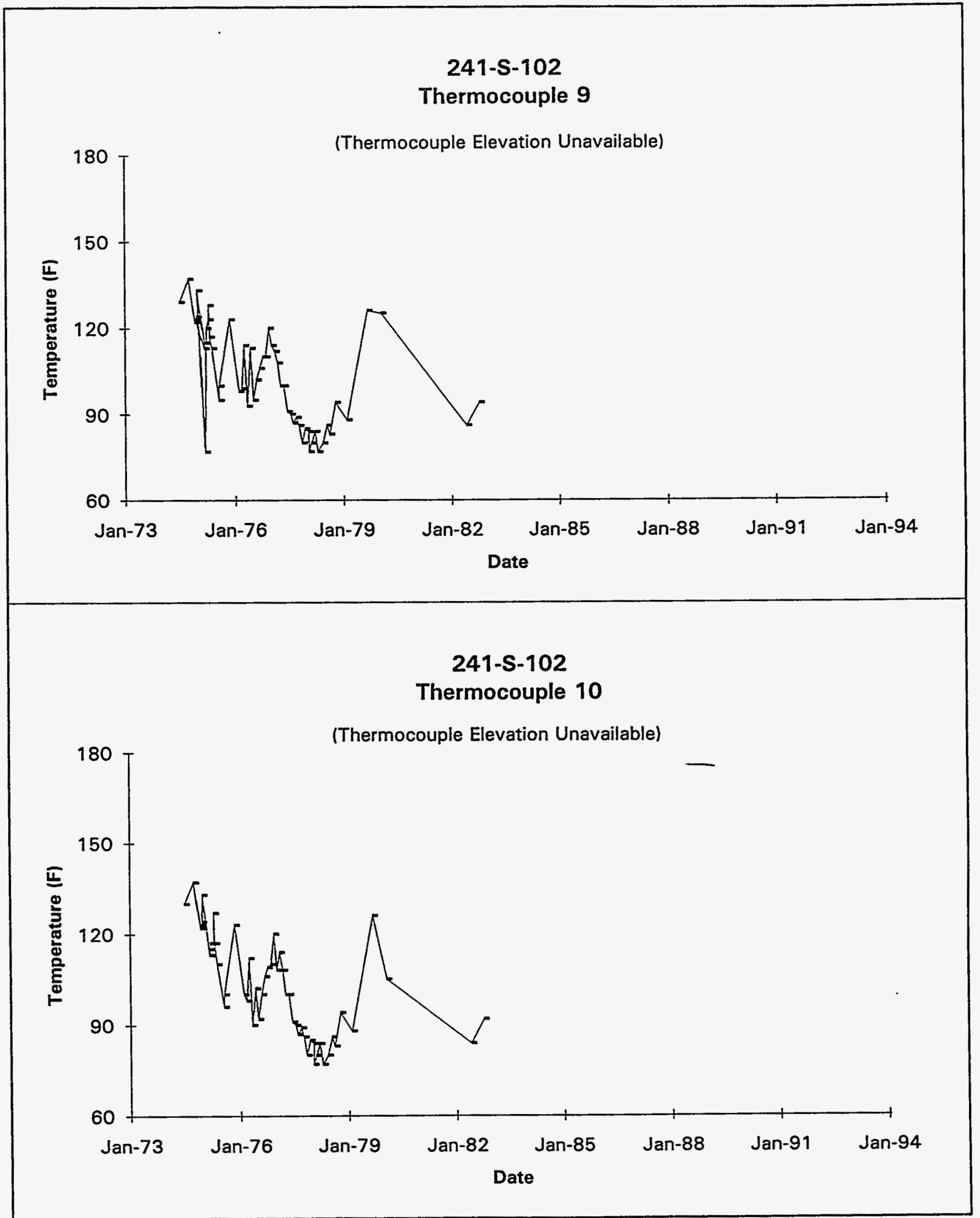

Data obtained from WhC Surveillance Analysis Computer System (SACS), February 24, 1994, 242-S Bottoms Tanks Trenster Record, Data Sheet TK-105-S to TK-101-S, and Archive box 121869 . 


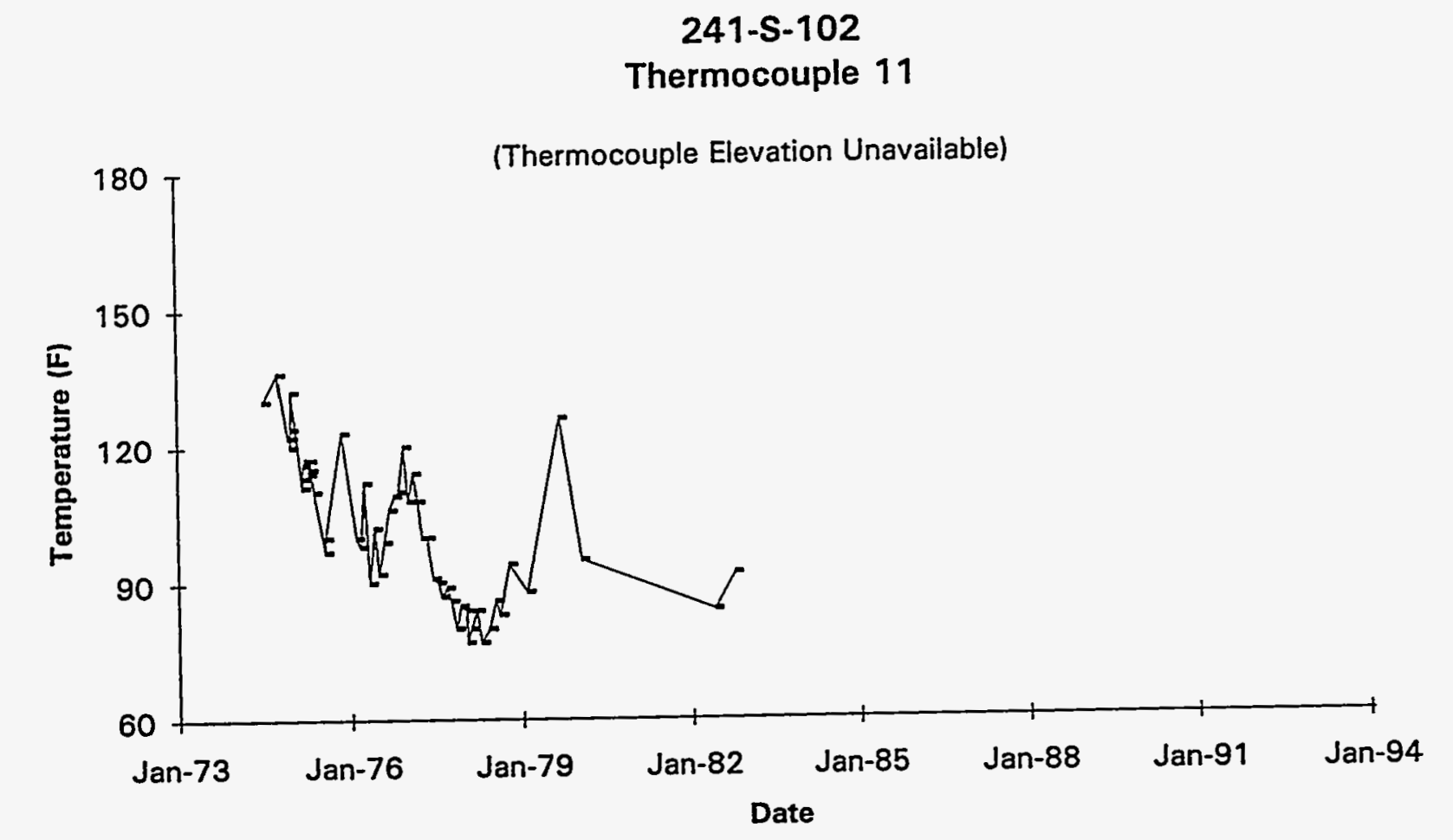

241-S-102

Thermocouple 12

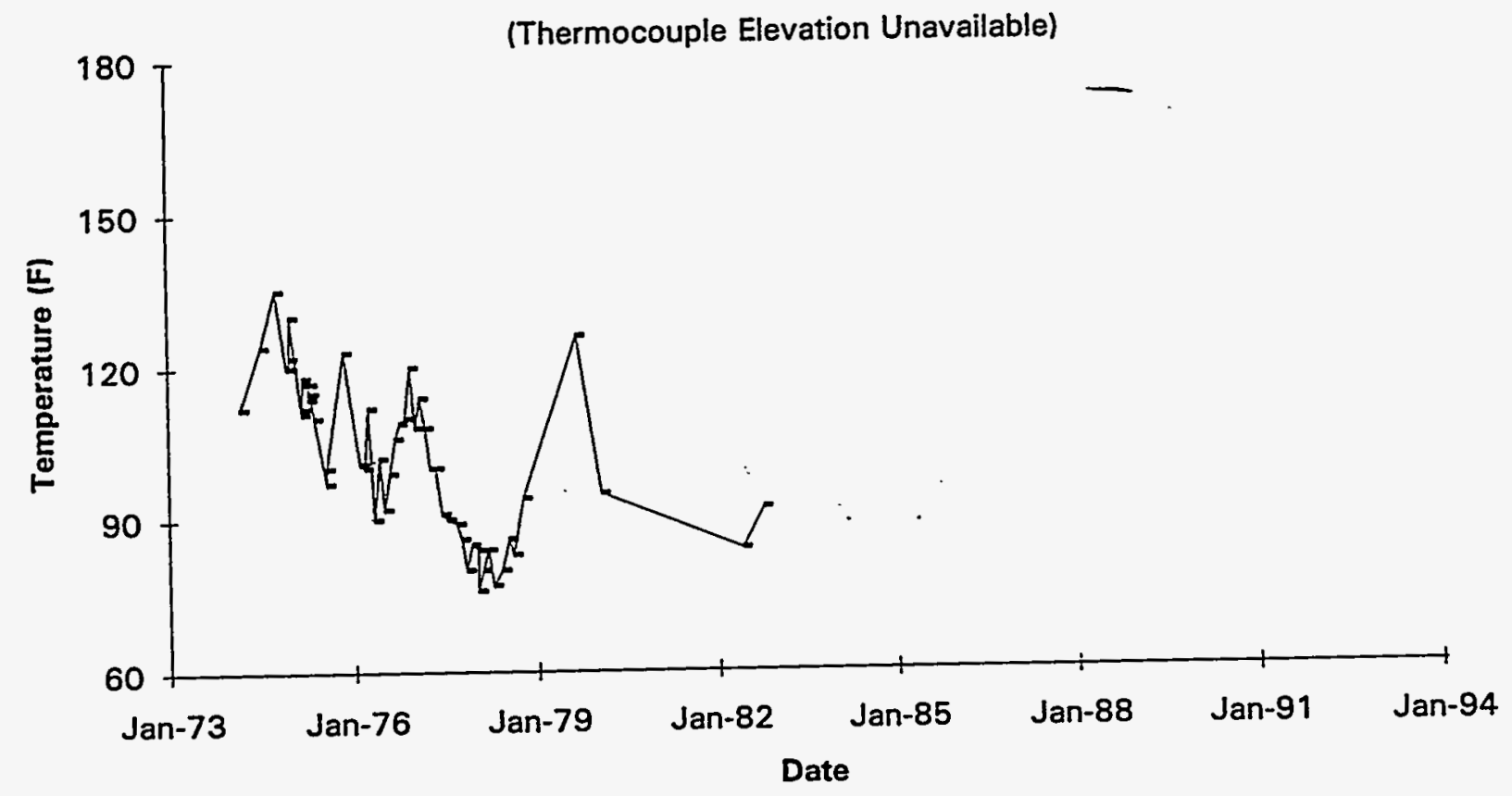

Dato obtained from WHC Surveillance Analysis Computar System (SACS). February 24, 1994, 242-S Bottoms Tanks Transter Record, Data Sheet TK-105-S to TK-101-S. and Archive box $\$ 121869$. 


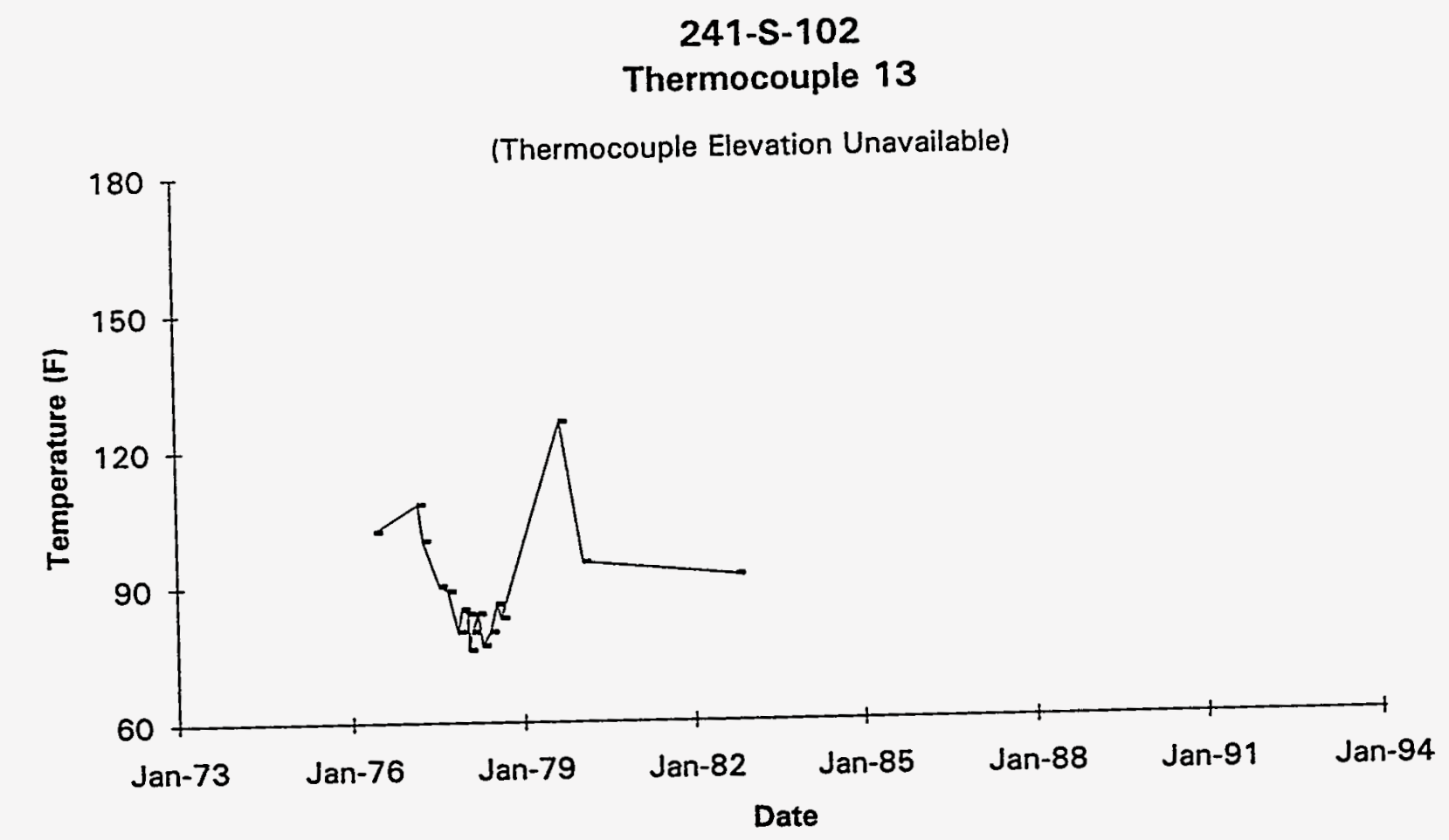

241-S-102

Thermocouple 14

(Thermocouple Elevation Unavailable)

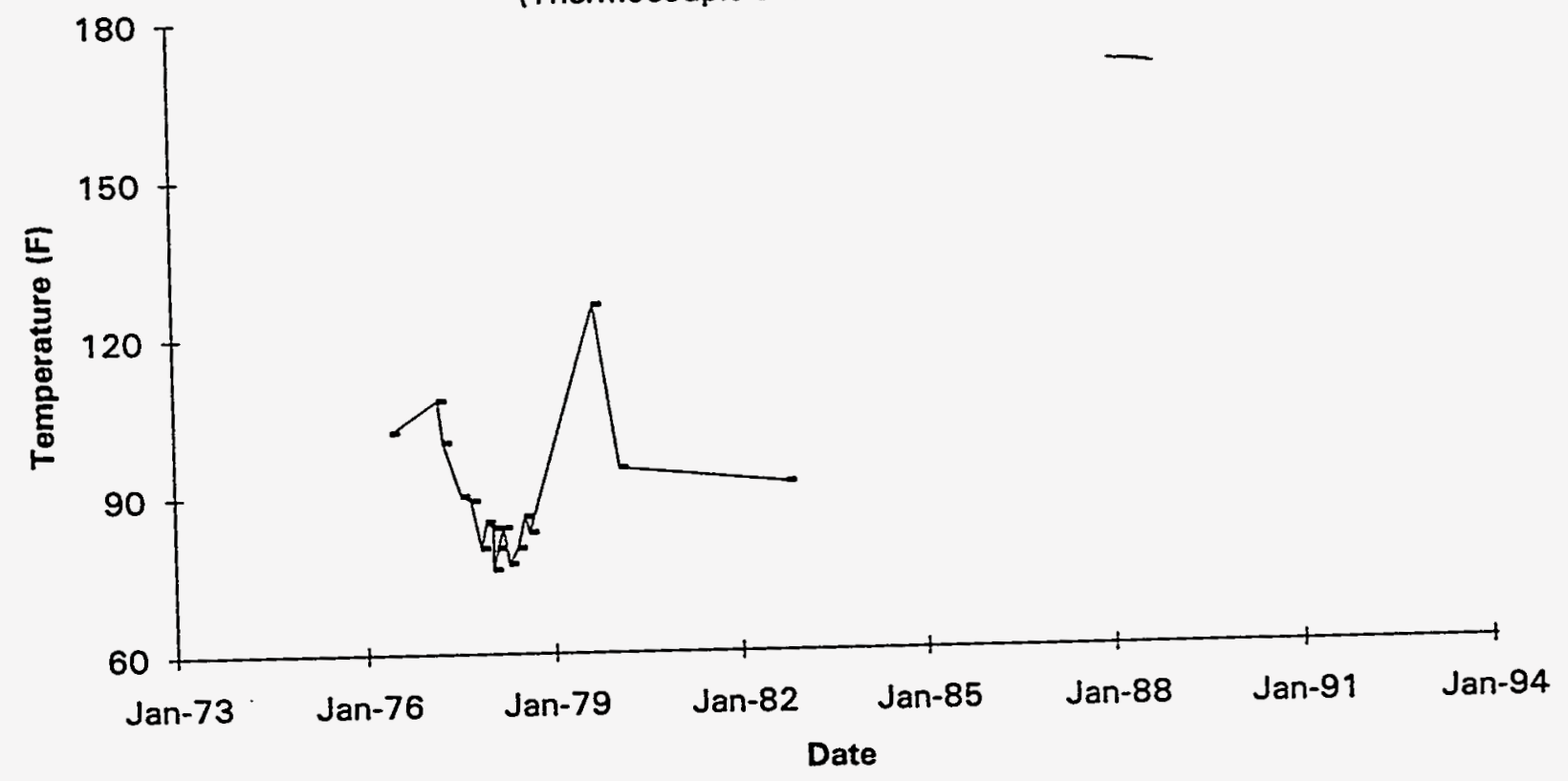

Whe Archive box $\$ 121869$.

$$
-0-40
$$




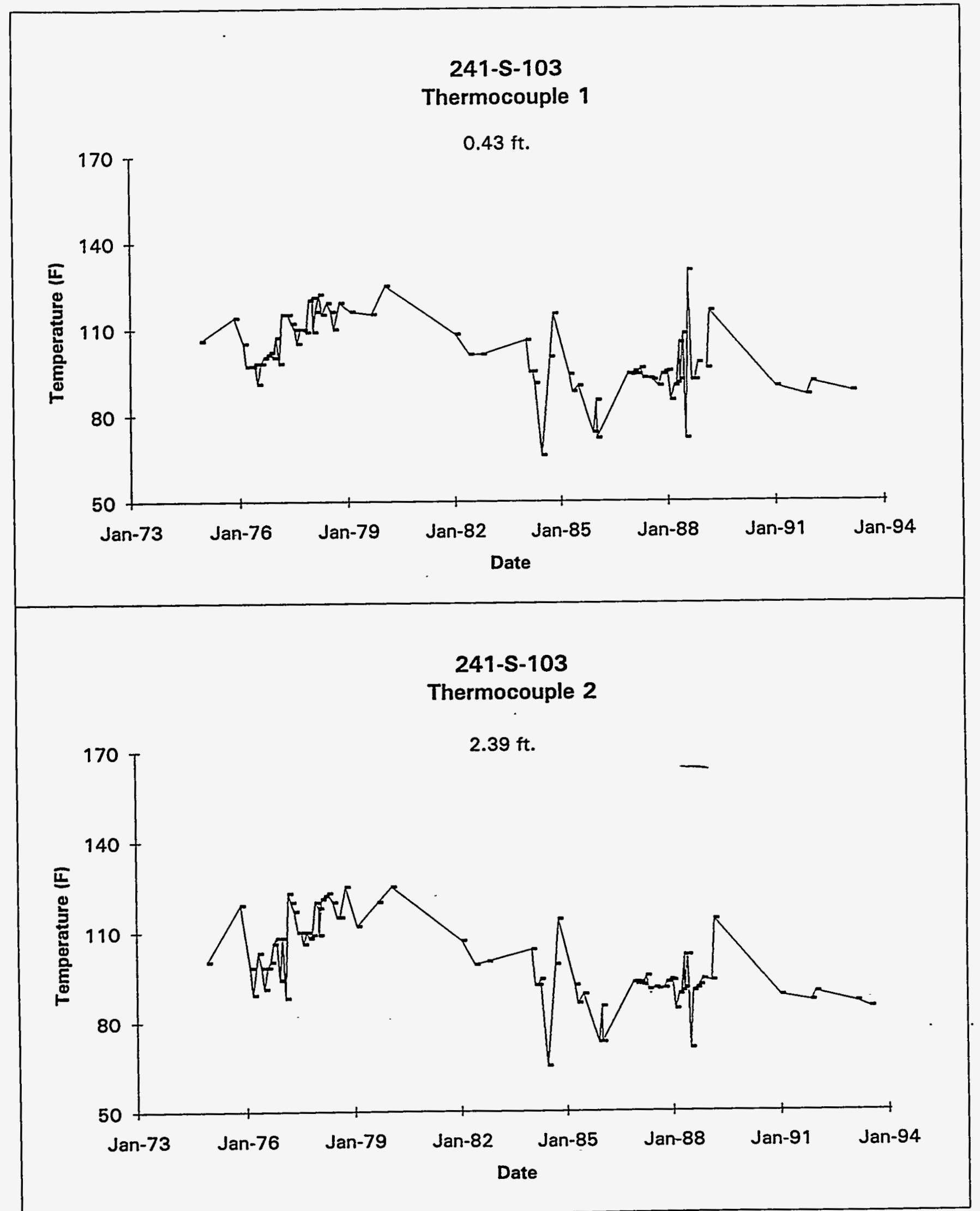

Data obtained from WHC Surveillance Analysis Computer System (SACS), February 24, 1994.

D-41 


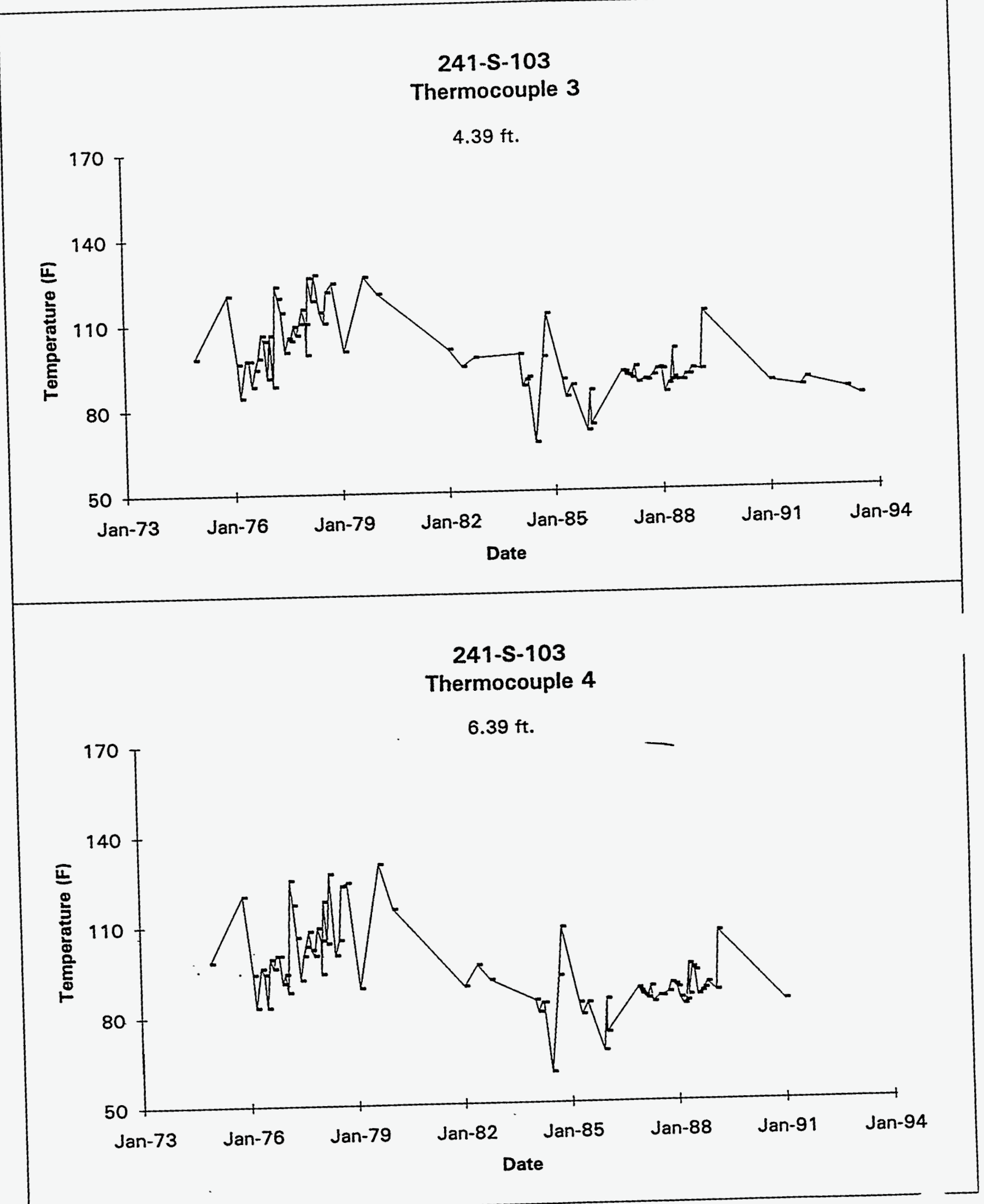

Data obtained from WHC Surveillance Analysis Computer System (SACS), February 24, 1994. 


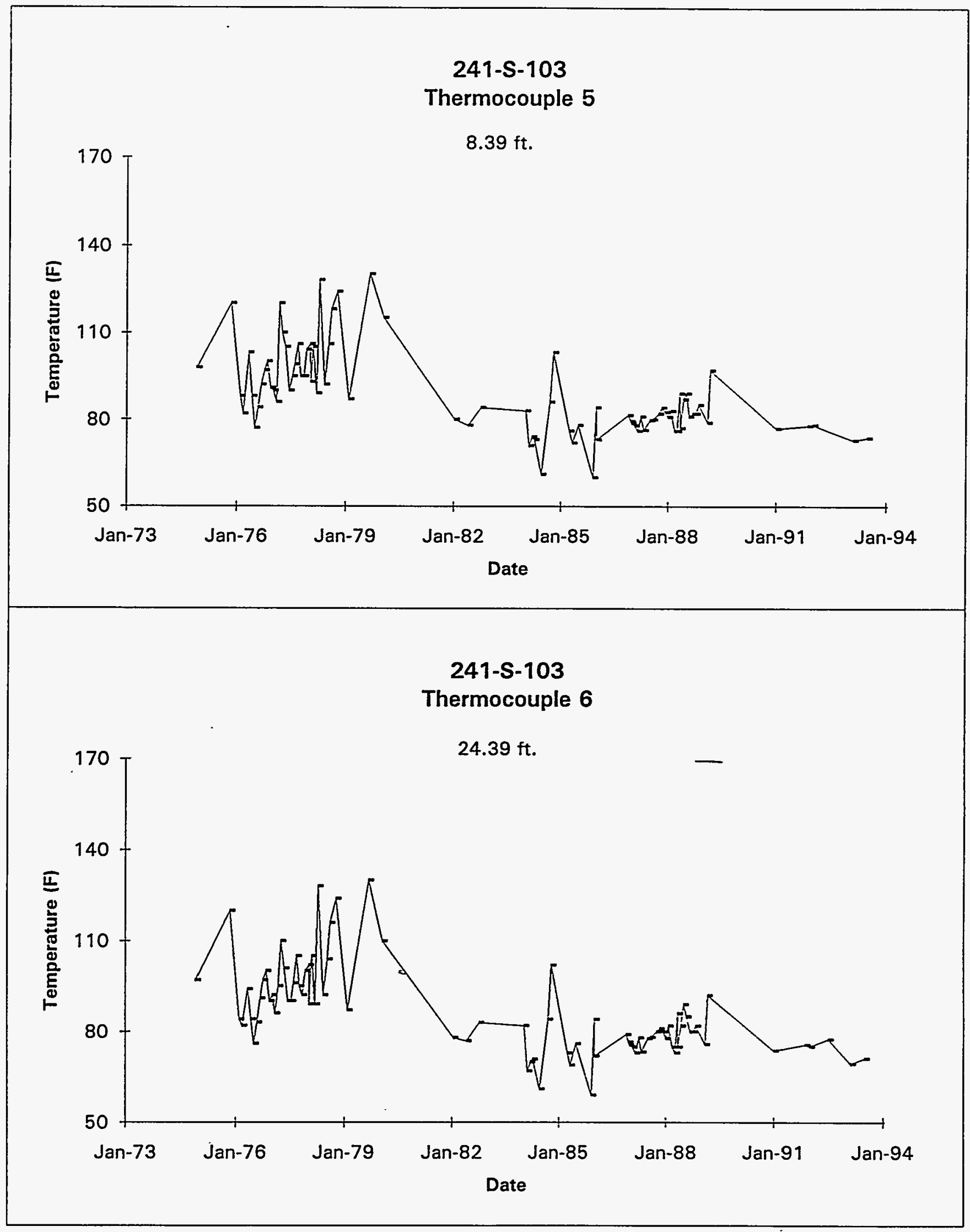

Data obtained from WHC Surveillance Analysis Computer System (SACS), February 24, 1994. 


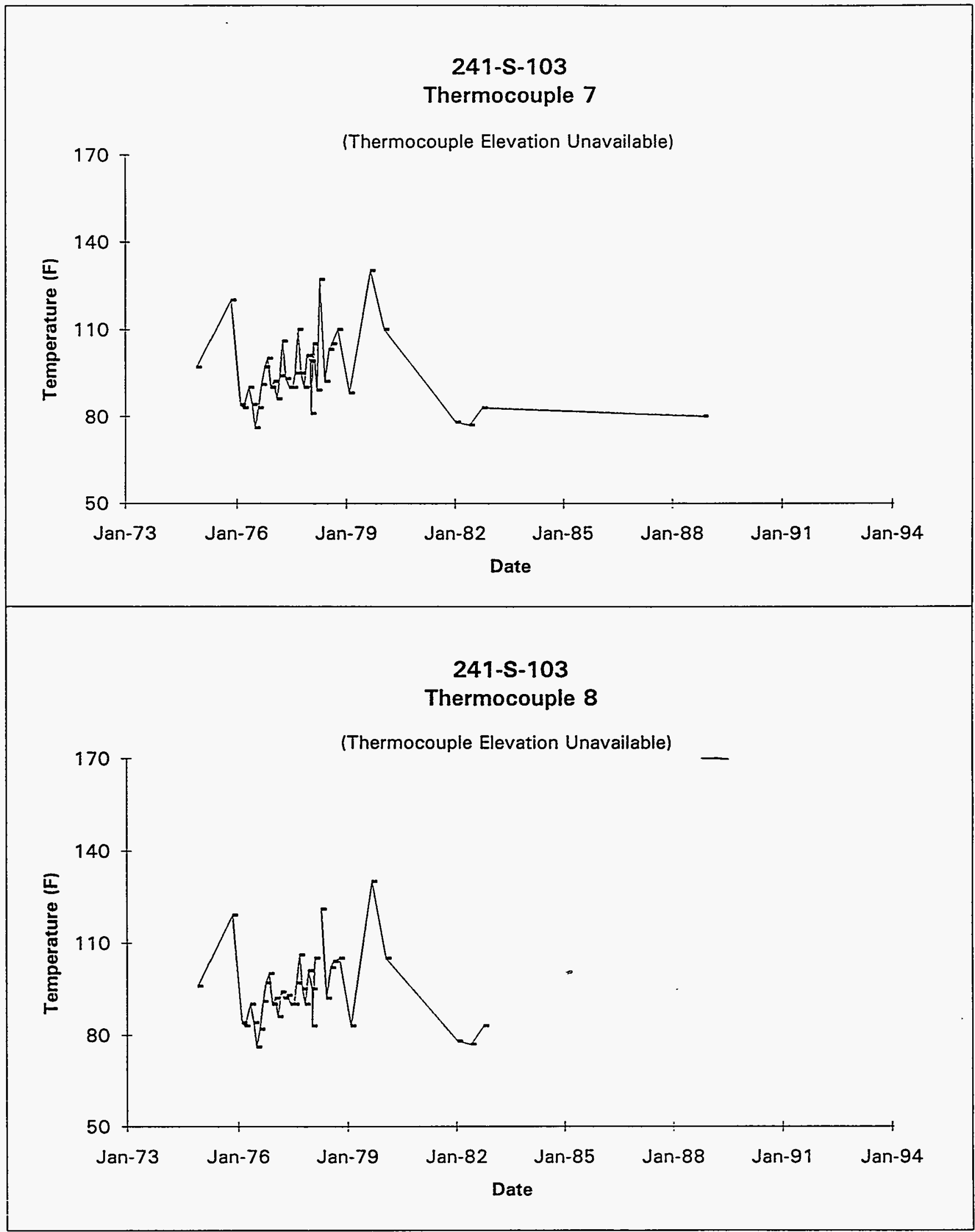

Data obtained from WHC Surveillance Analysis Computer System (SACS), February 24, 1994. 


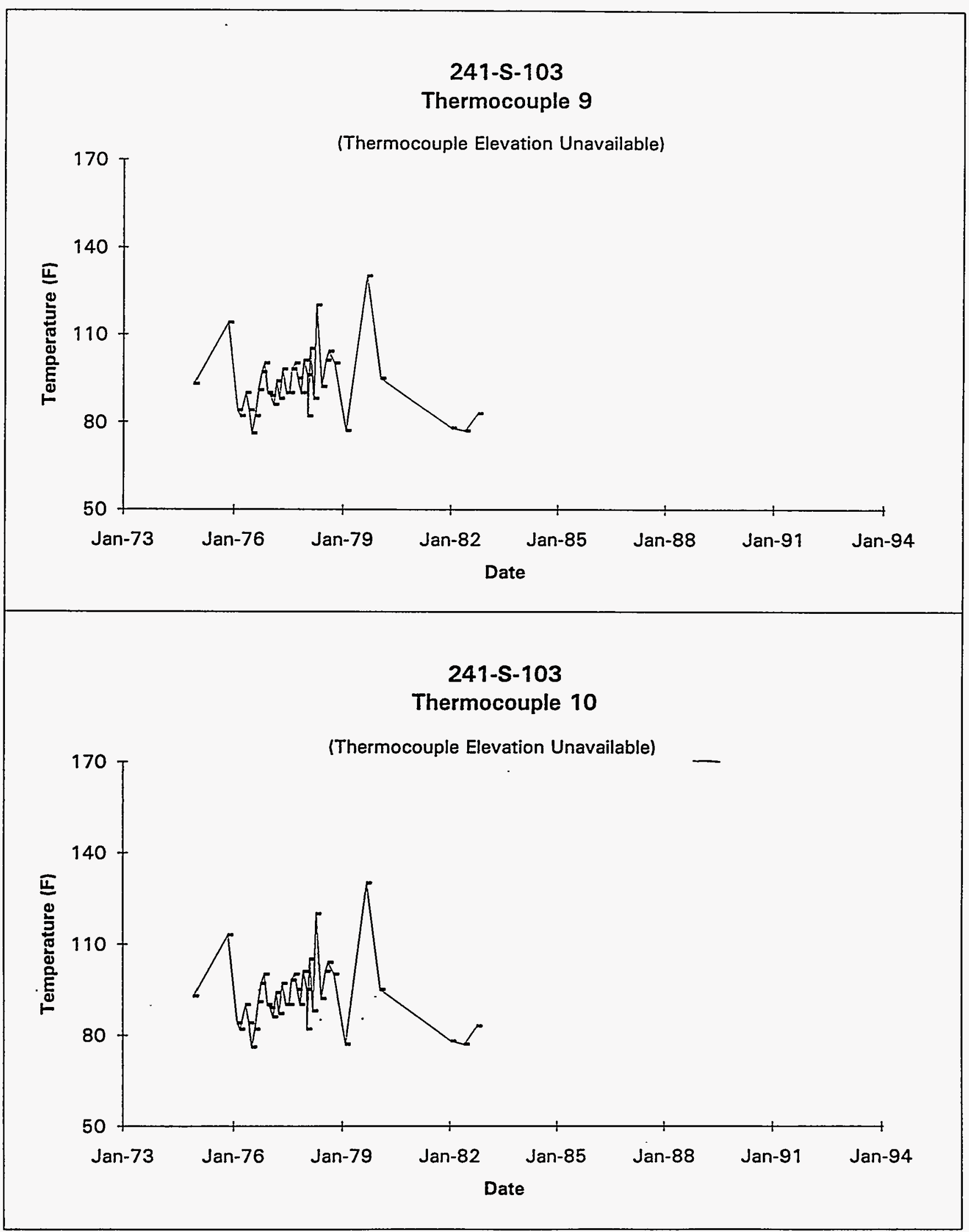

Data obtained from WHC Surveillance Analysis Computer System (SACS), February 24, 1994. 


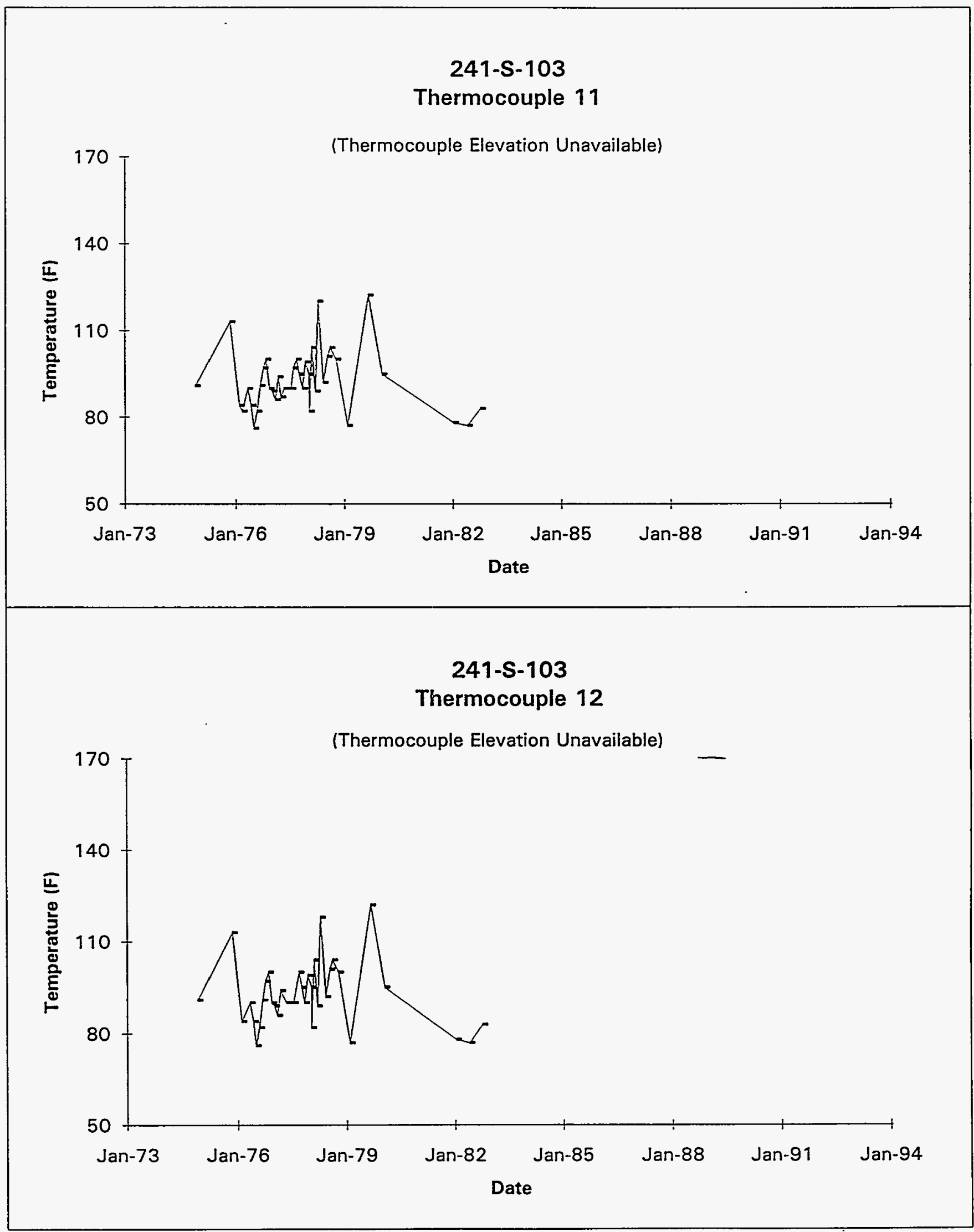

Data obtained from WHC Surveillance Analysis Computer System (SACS), February 24, 1994. 


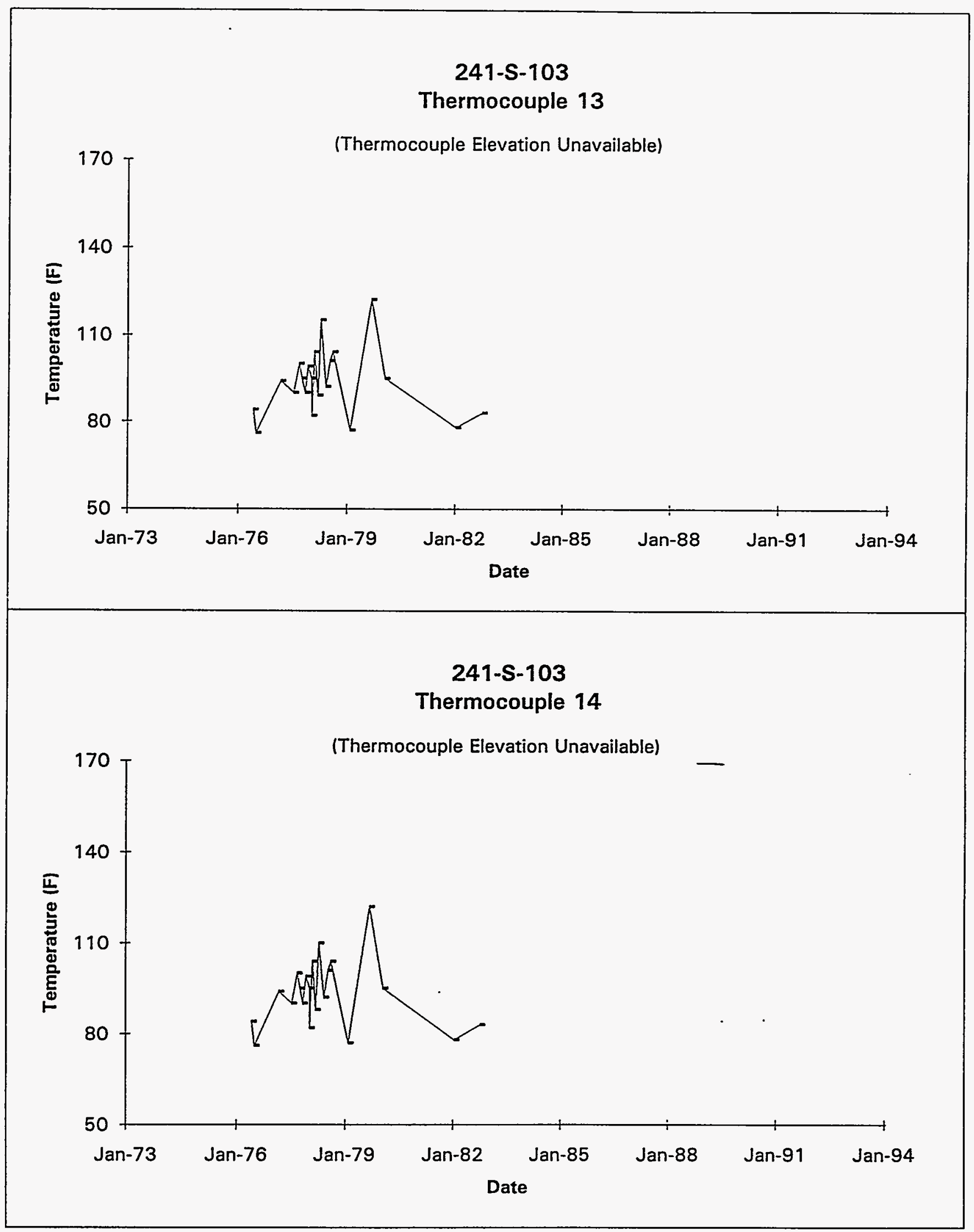

Data obtained from WHC Surveillance Analysis Computer System (SACS), February 24, 1994. 


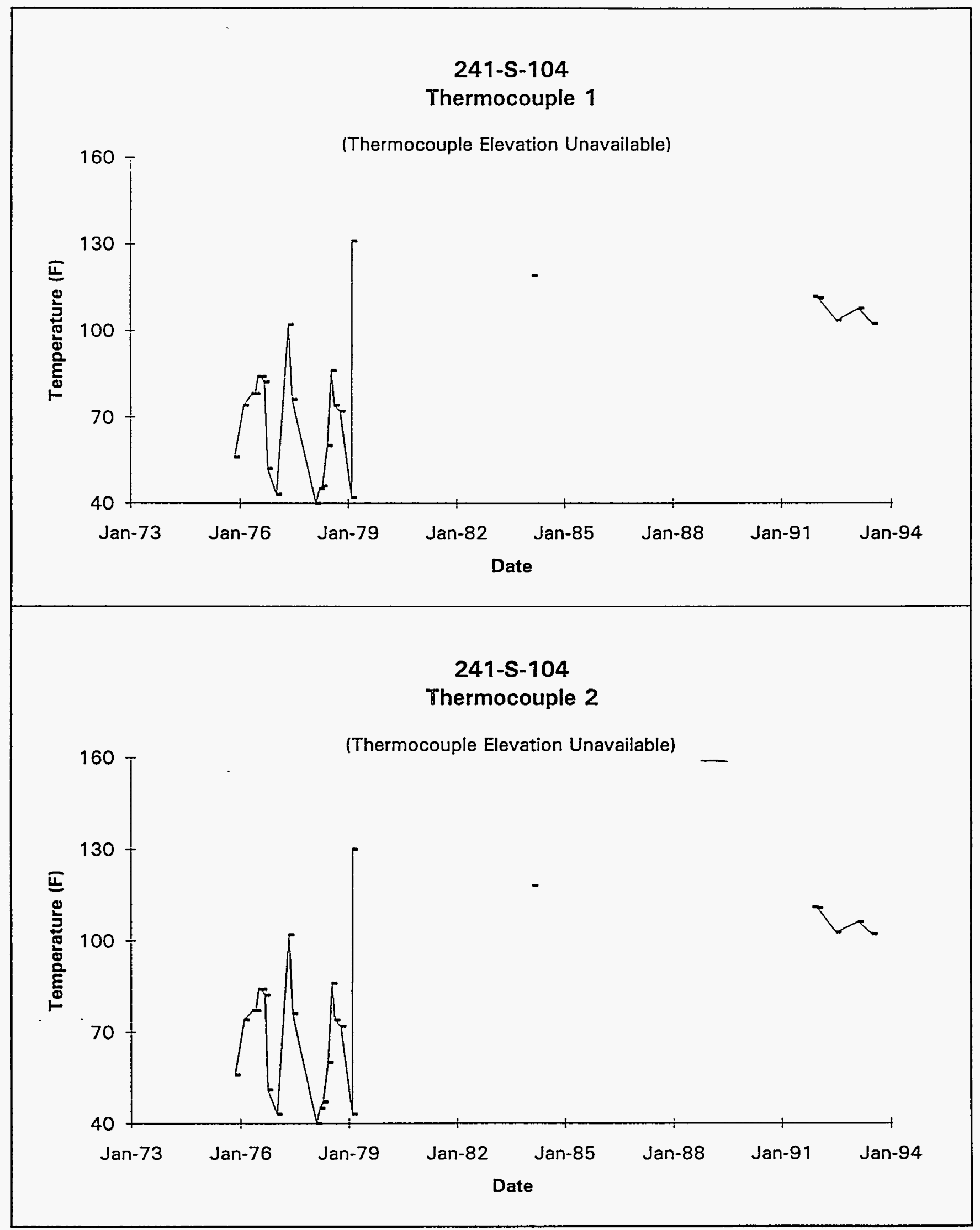

Data obtained from WHC Surveillance Analysis Computer System (SACS), February 24, 1994. 


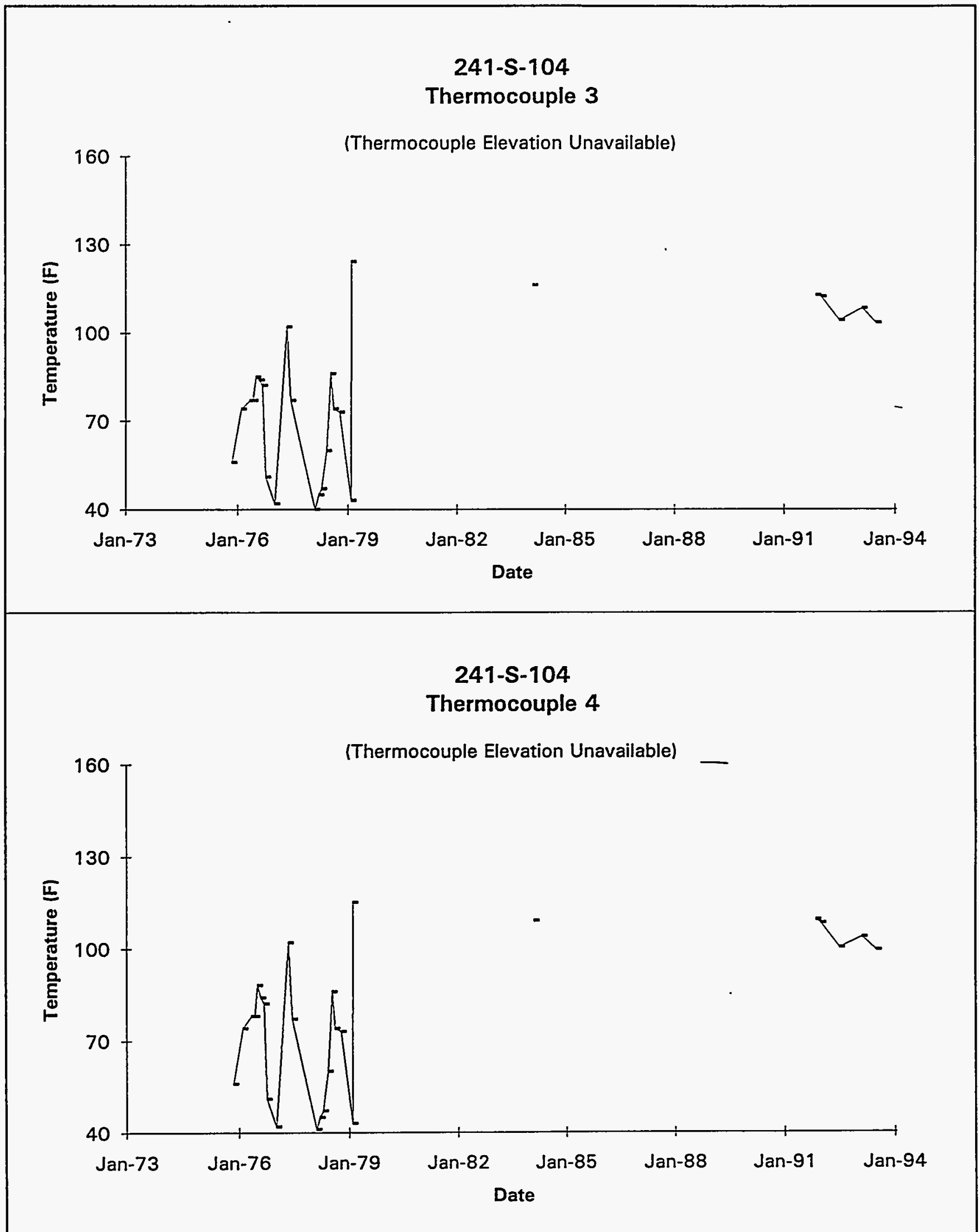

Data obtained from WHC Surveillance Analysis Computer System (SACS), February 24, 1994. 


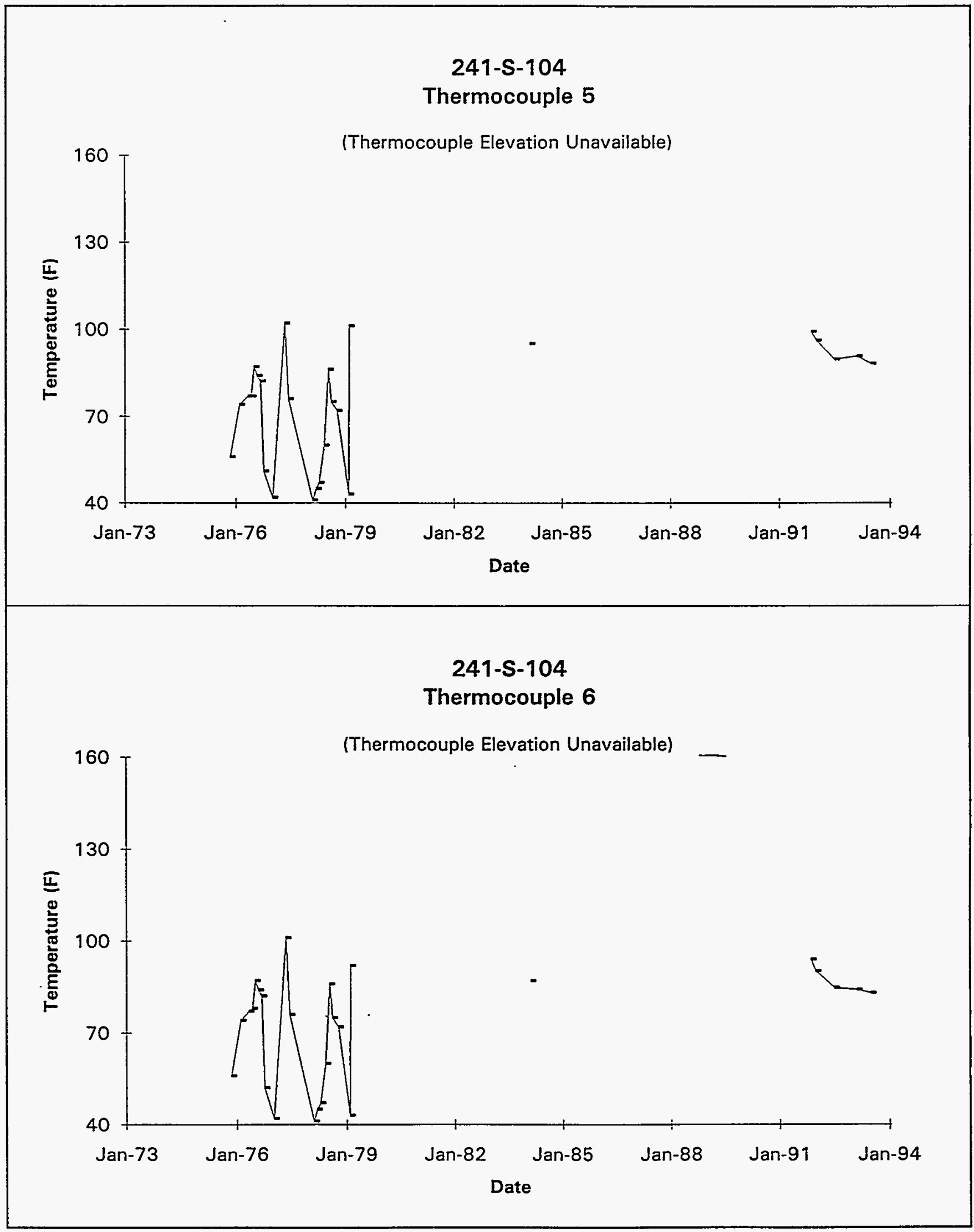

Data obtained from WHC Surveillance Analysis Computer System (SACS), February 24, 1994. 


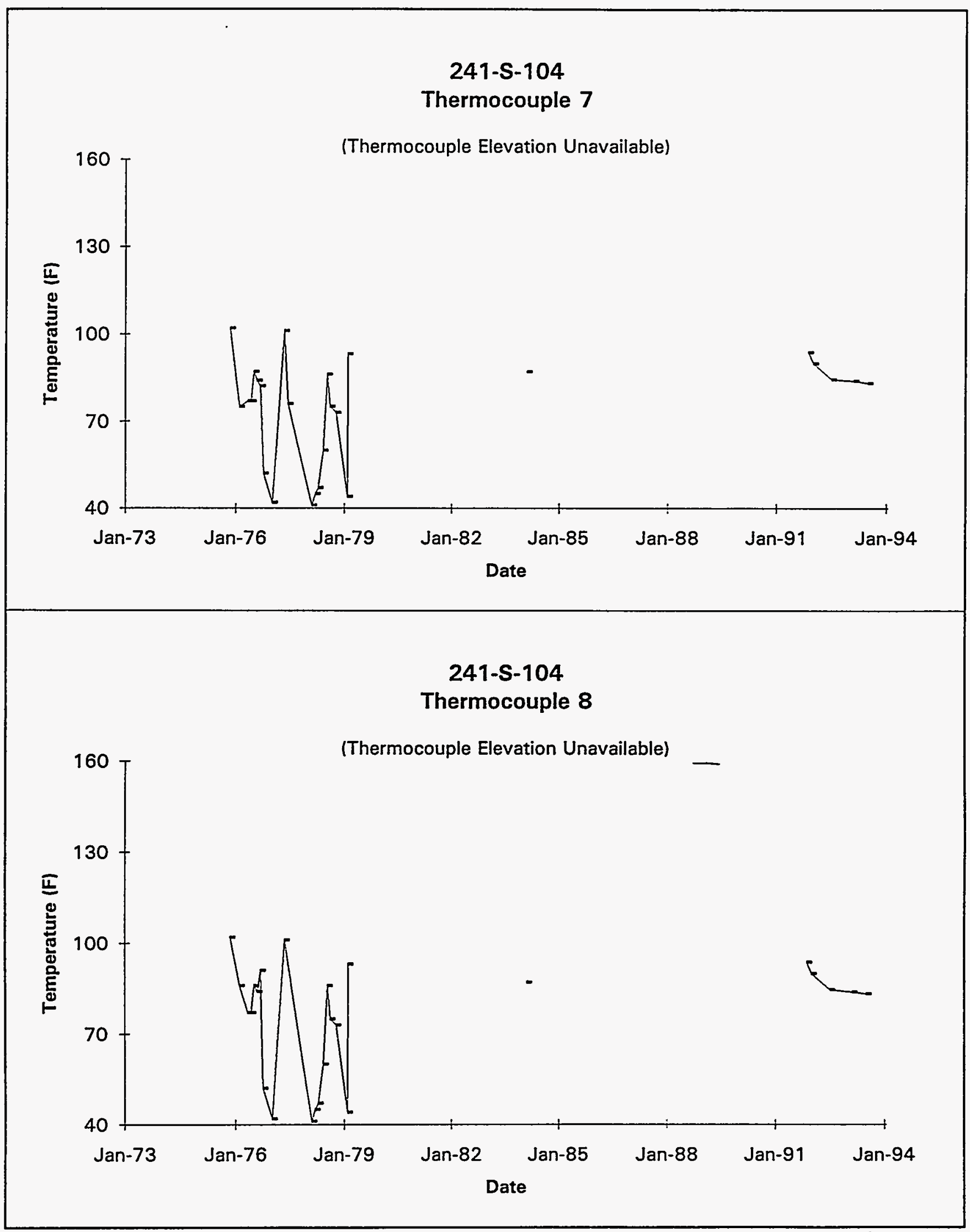

Data obtained from WHC Surveillance Analysis Computer System (SACS), February 24, 1994. 


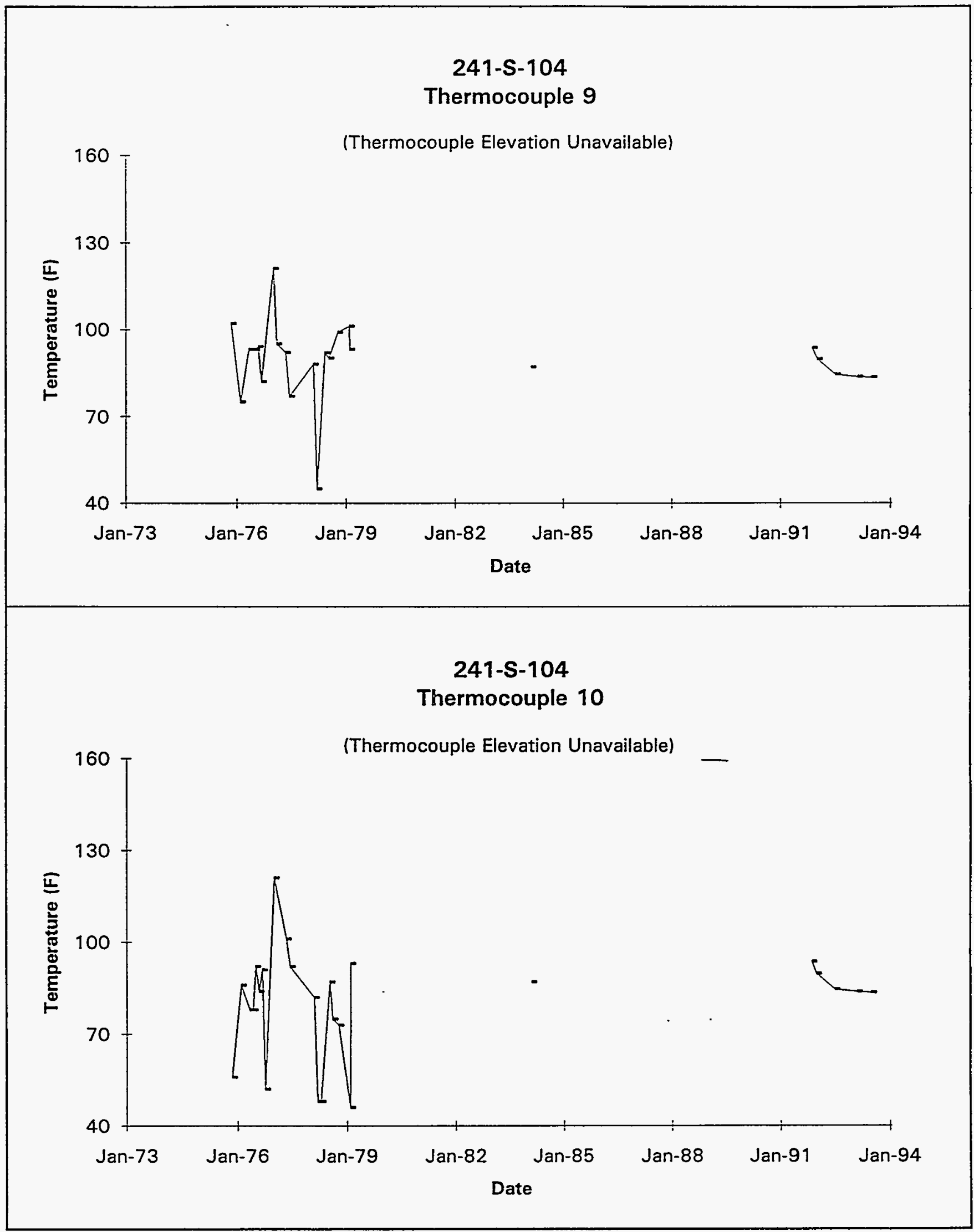

Data obtained from WHC Surveillance Analysis Computer System (SACS), February 24, 1994. 


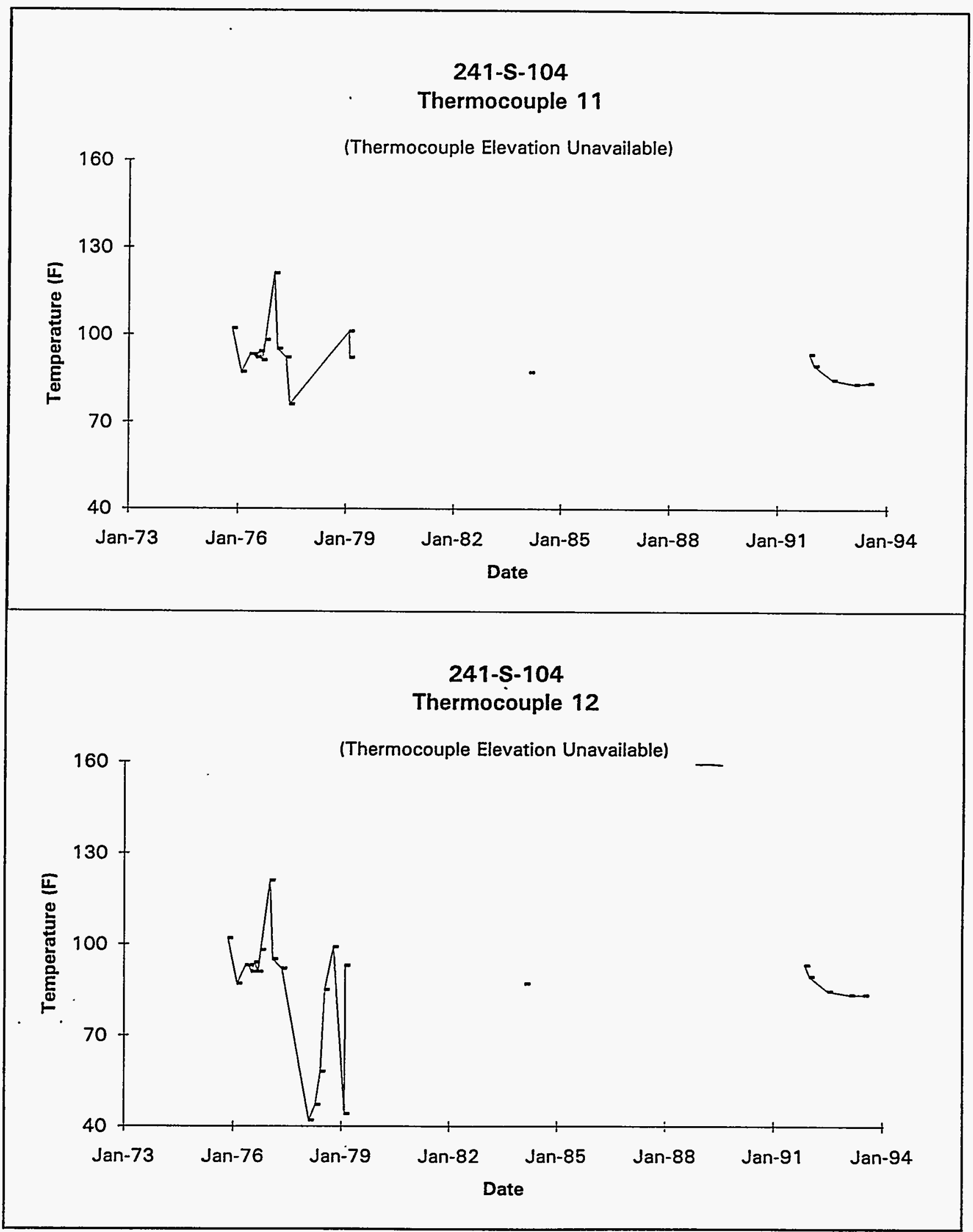

Data obtained from WHC Surveillance Analysis Computer System (SACS), February 24, 1994. 


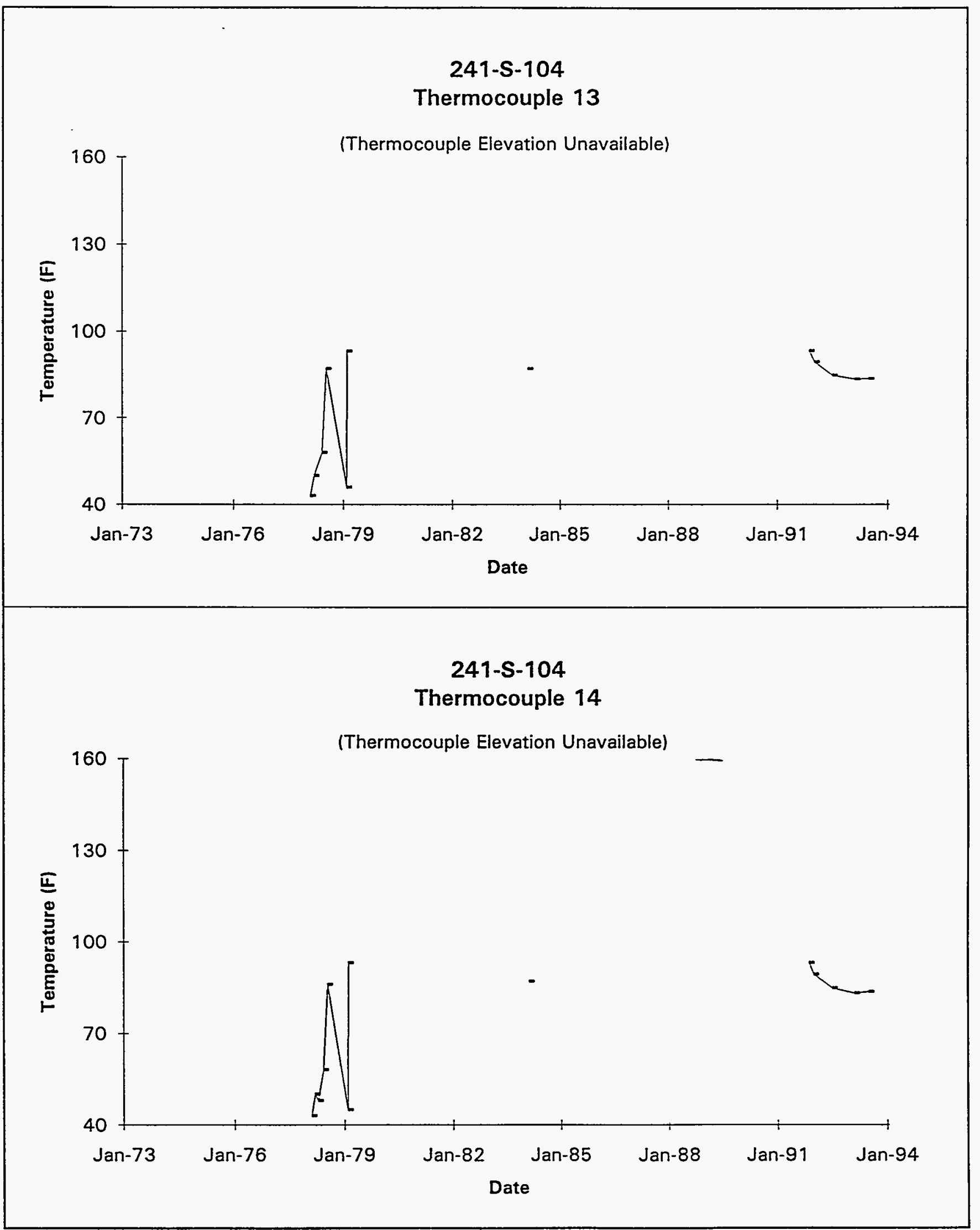

Data obtained from WHC Surveillance Analysis Computer System (SACS), February 24, 1994. 


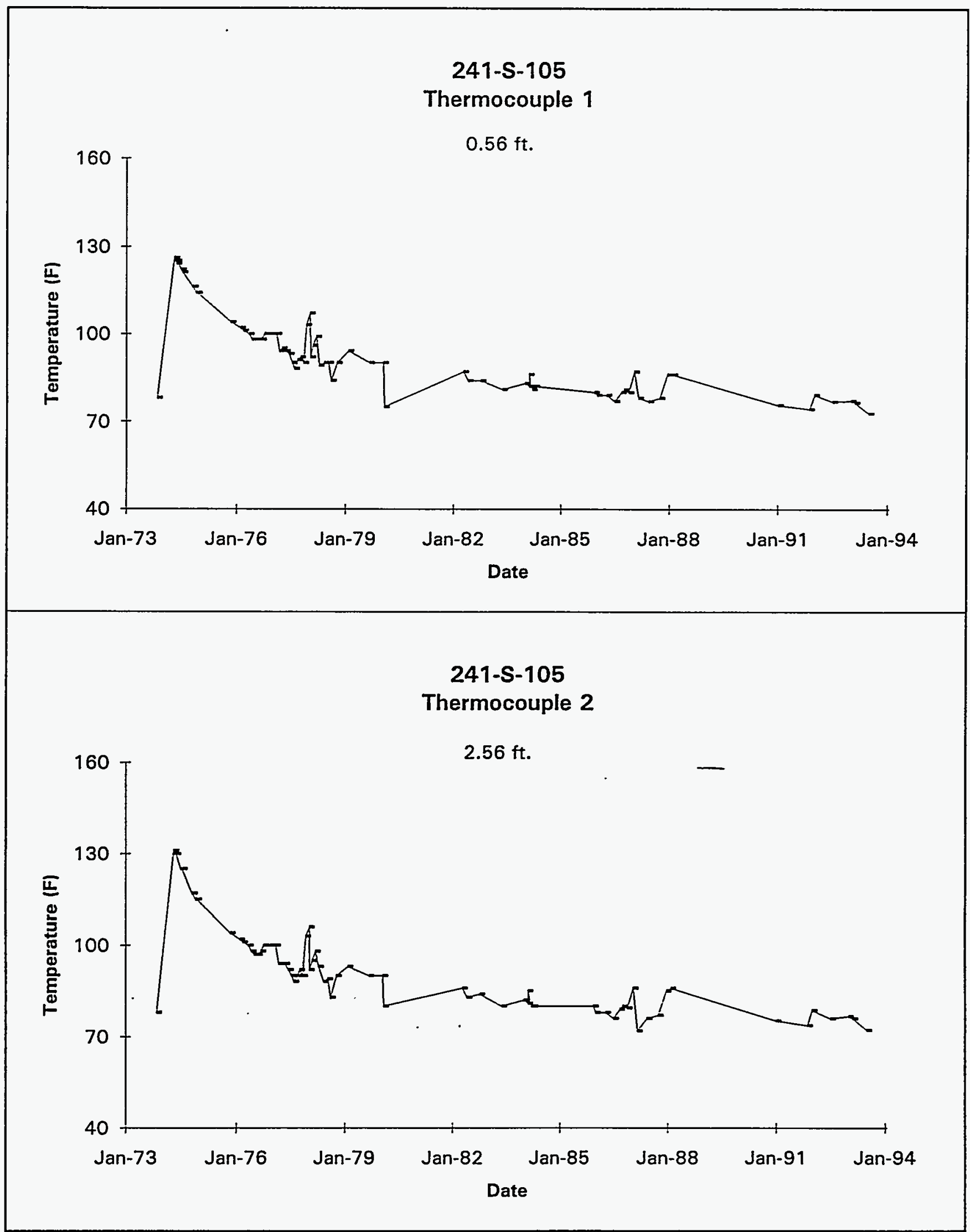

Data obtained from WHC Surveillance Analysis Computer System (SACS), February 24, 1994, Data Sheet TK-105-S to TK-101-S, and Archive box *121869. 


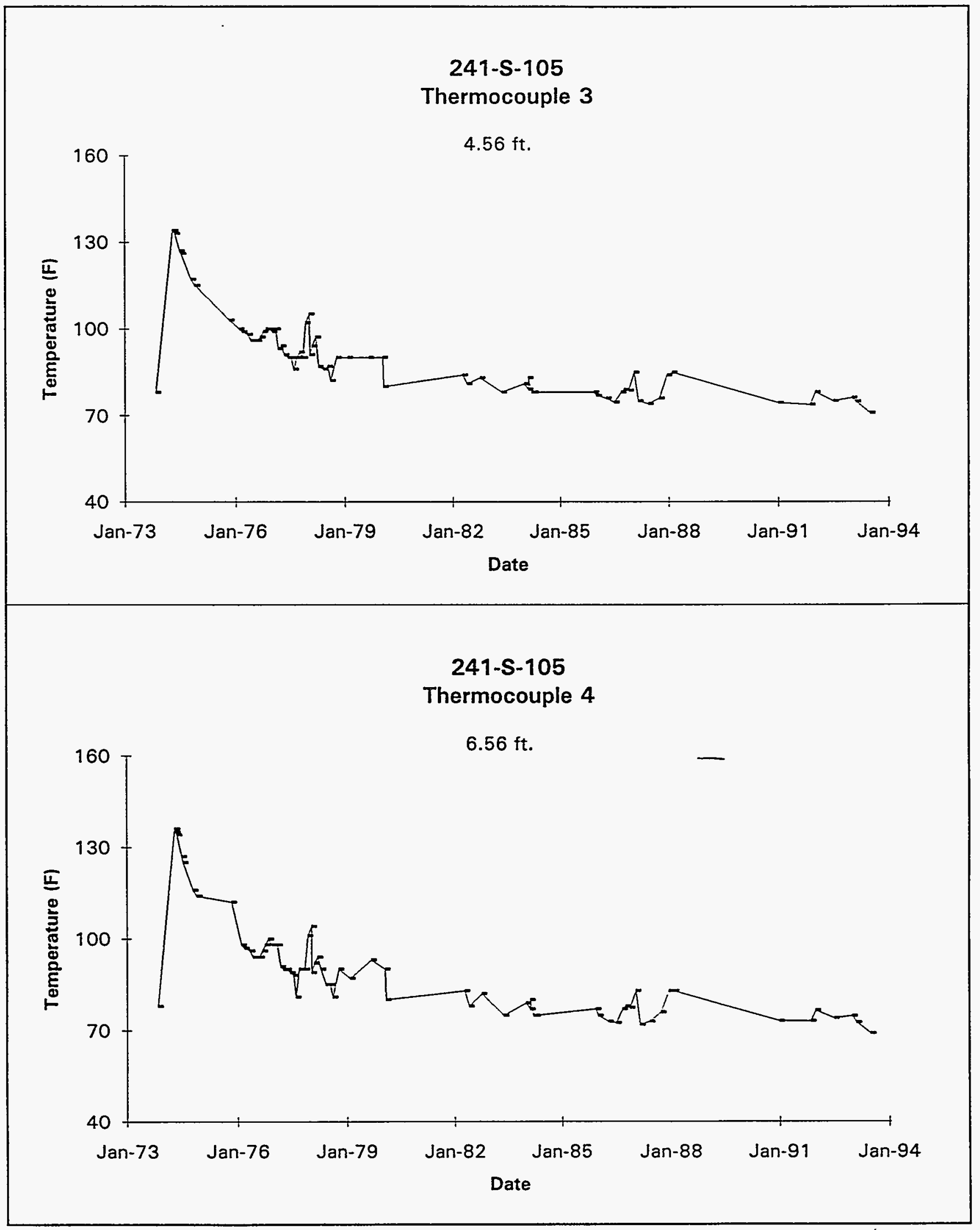

Data obtained from WHC Surveillance Analysis Computer System (SACS), February 24, 1994, Data Sheet TK-105-S to TK-101-S, and Archive box 121869. 


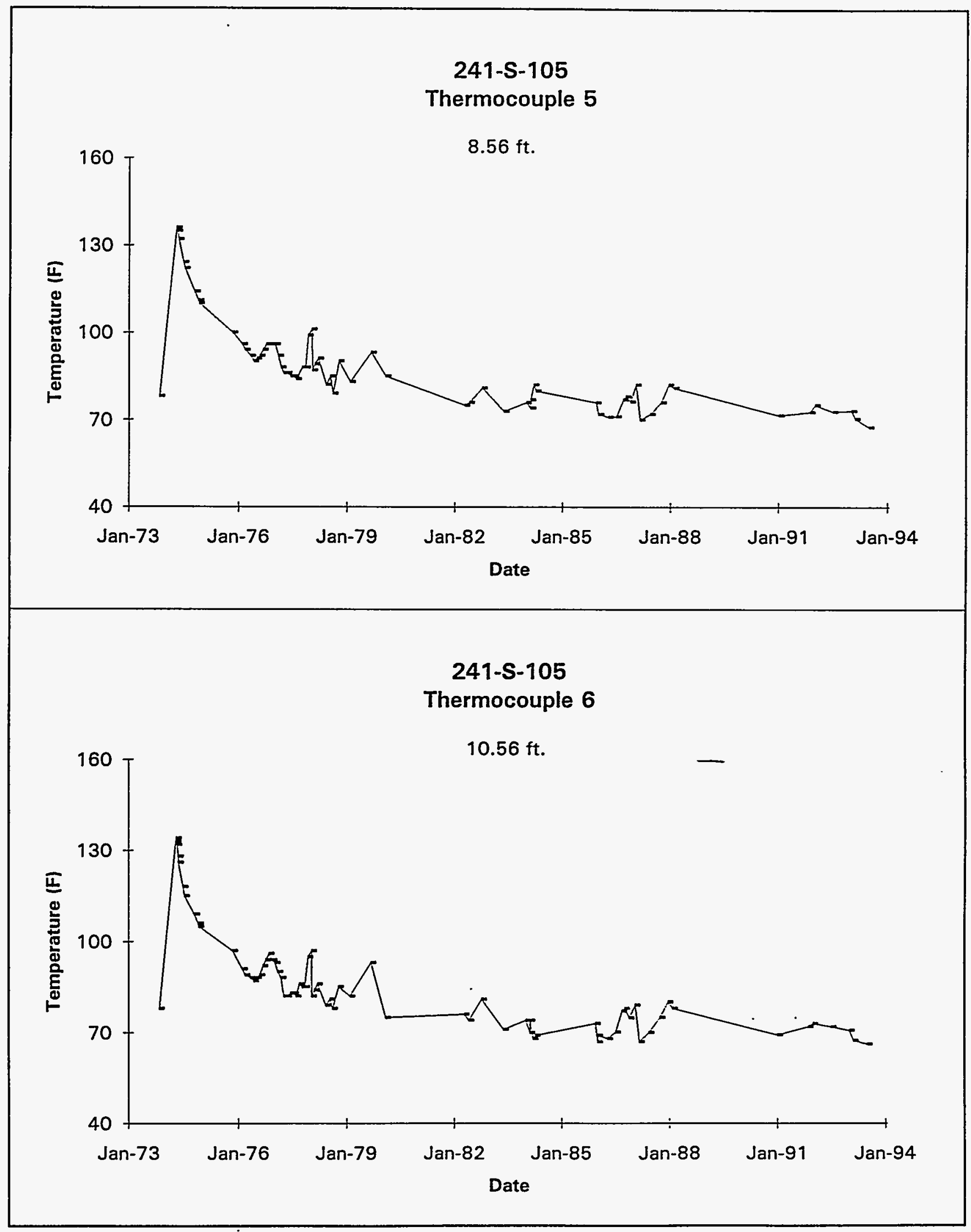

Data obtained from WHC Surveillance Analysis Computer System (SACS), February 24, 1994, Data Sheet TK-105-S to TK-101-S, and Archive box $\$ 121869$. 


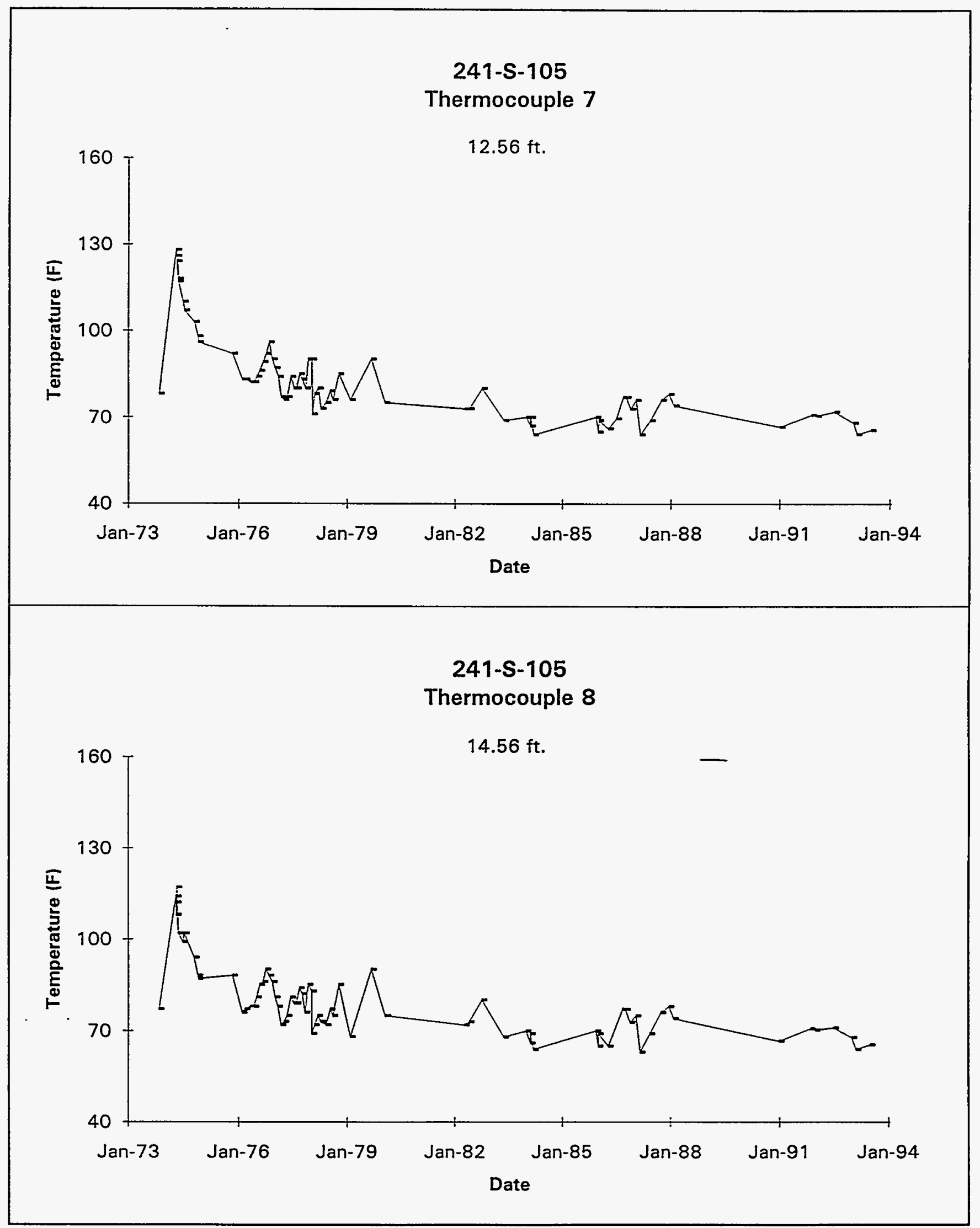




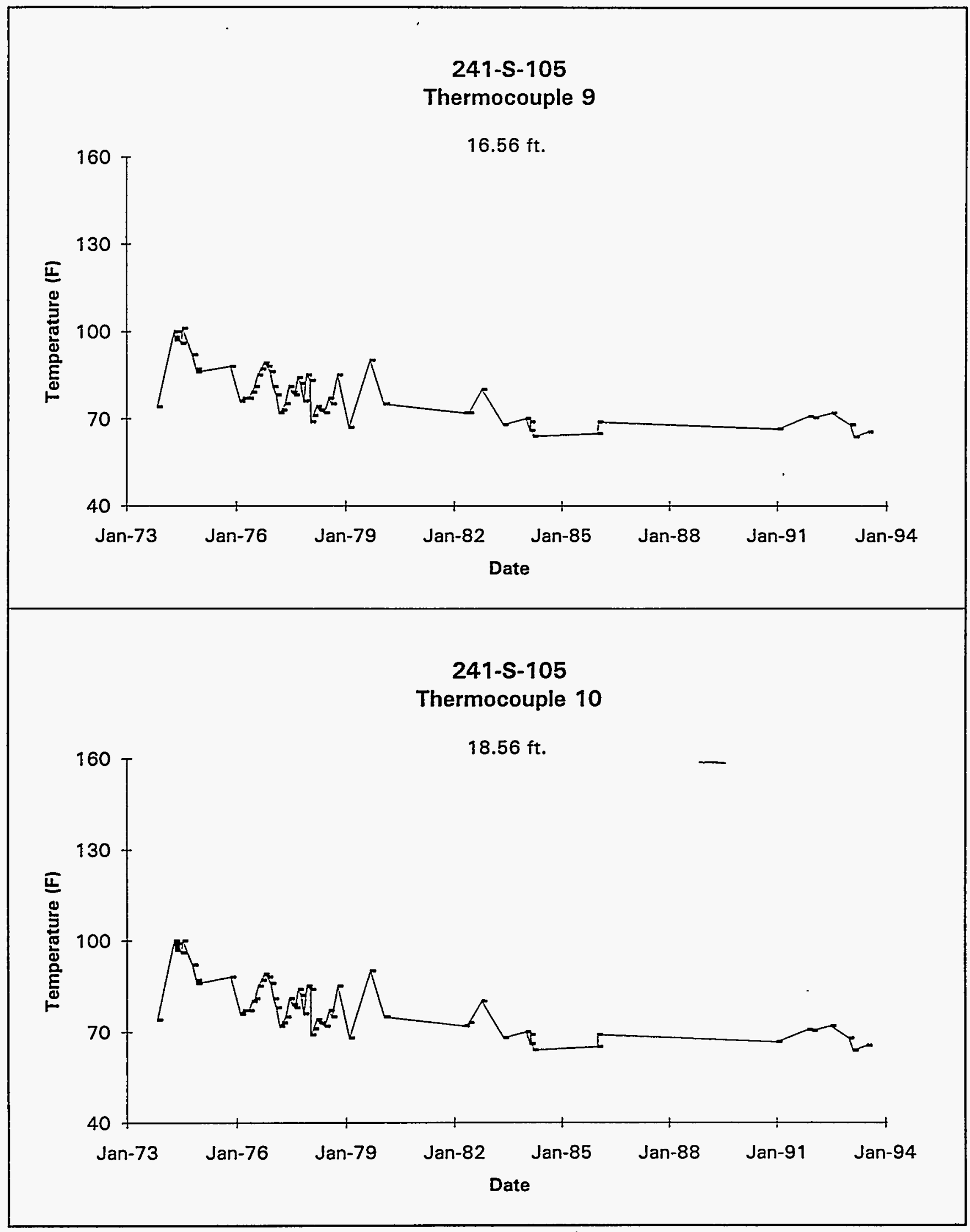

Data obtained from WHC Surveillance Analysis Computer System (SACS), February 24, 1994, Data Sheet TK-105-S to TK-101-S, and Archive box \$121869. 


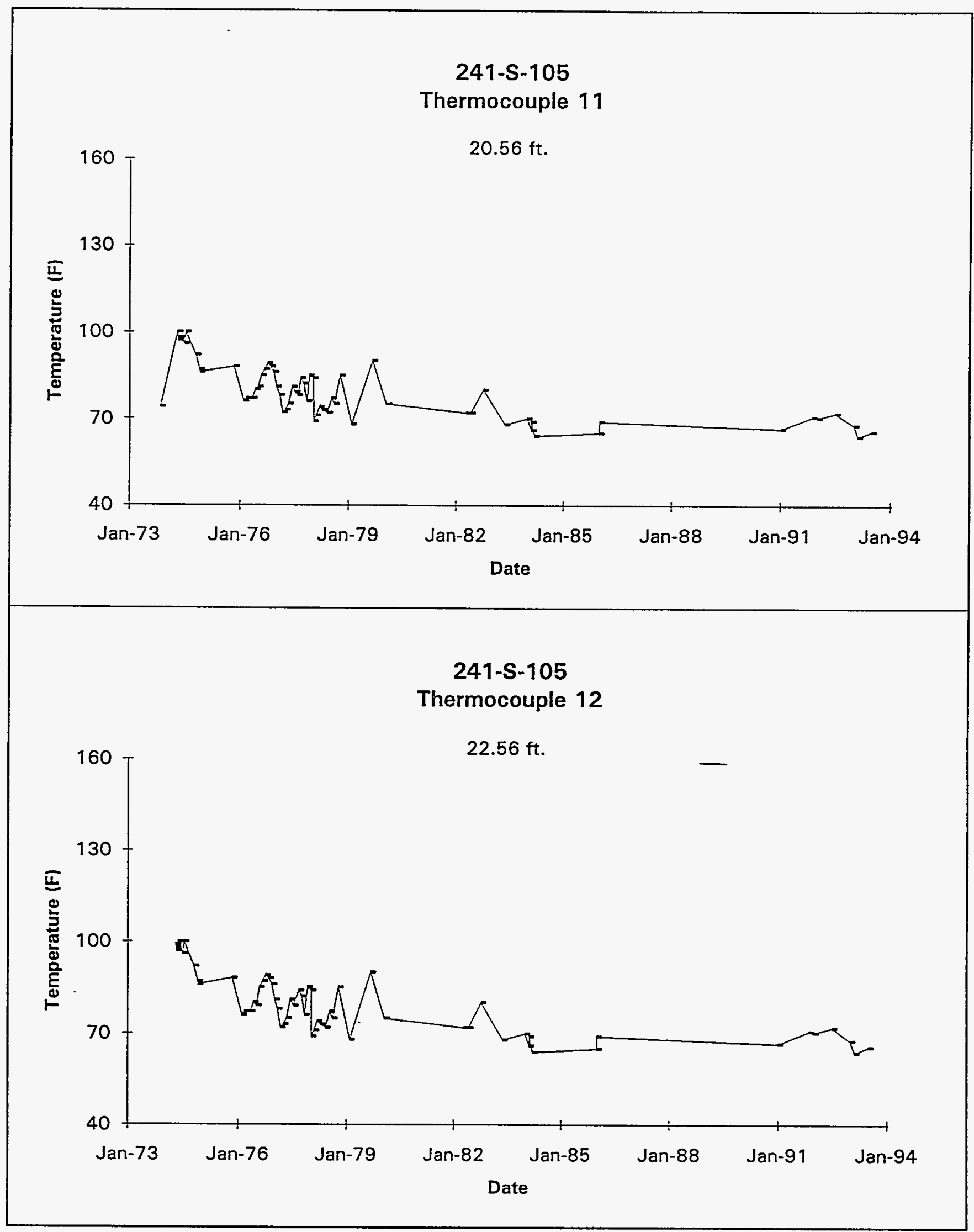




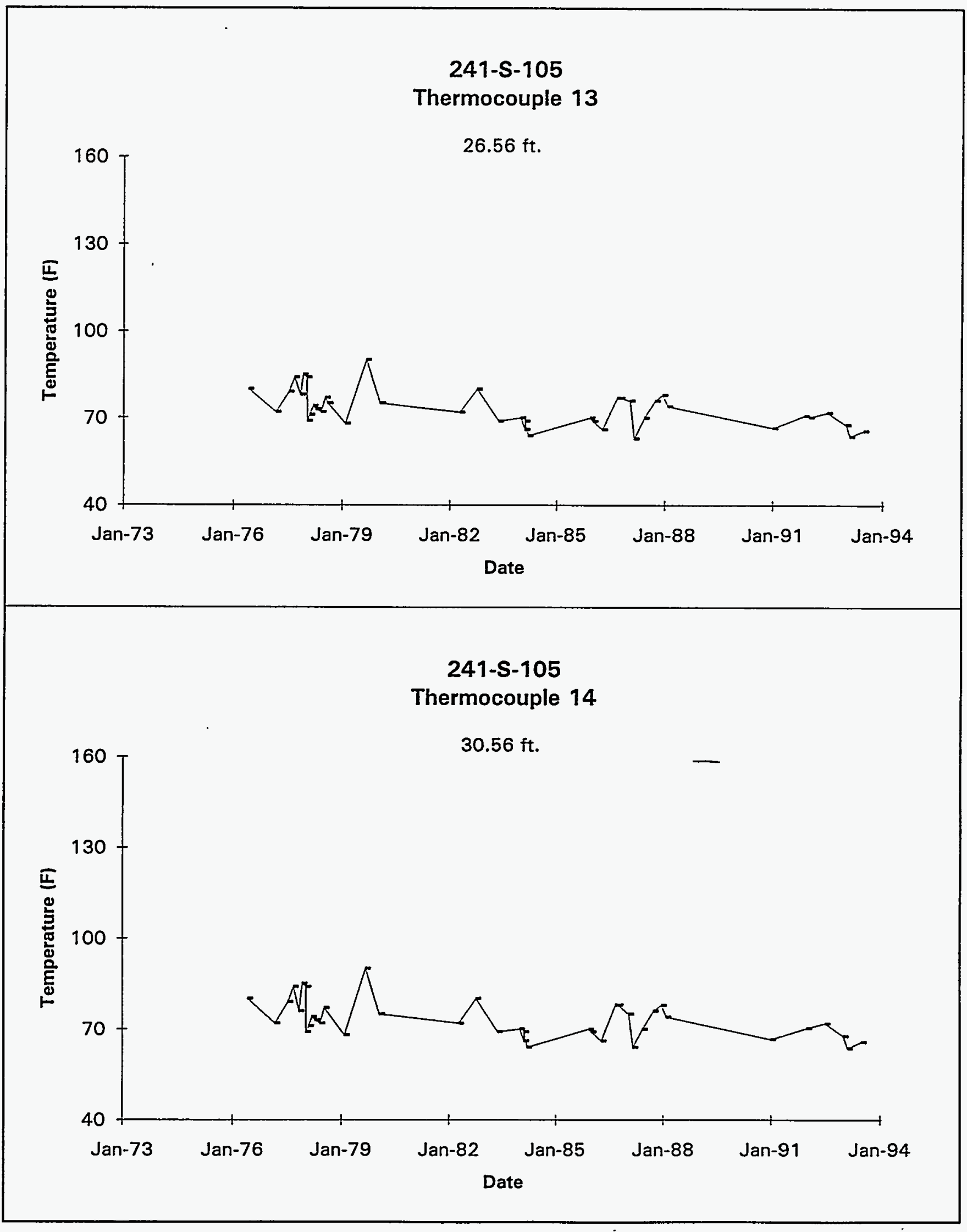

Data obtained from WHC Surveillance Analysis Computer S̄ystem (SACS). February 24, 1994, Data Sheet TK-105-S to TK-101-S, and Archive box $\$ 121869$. 


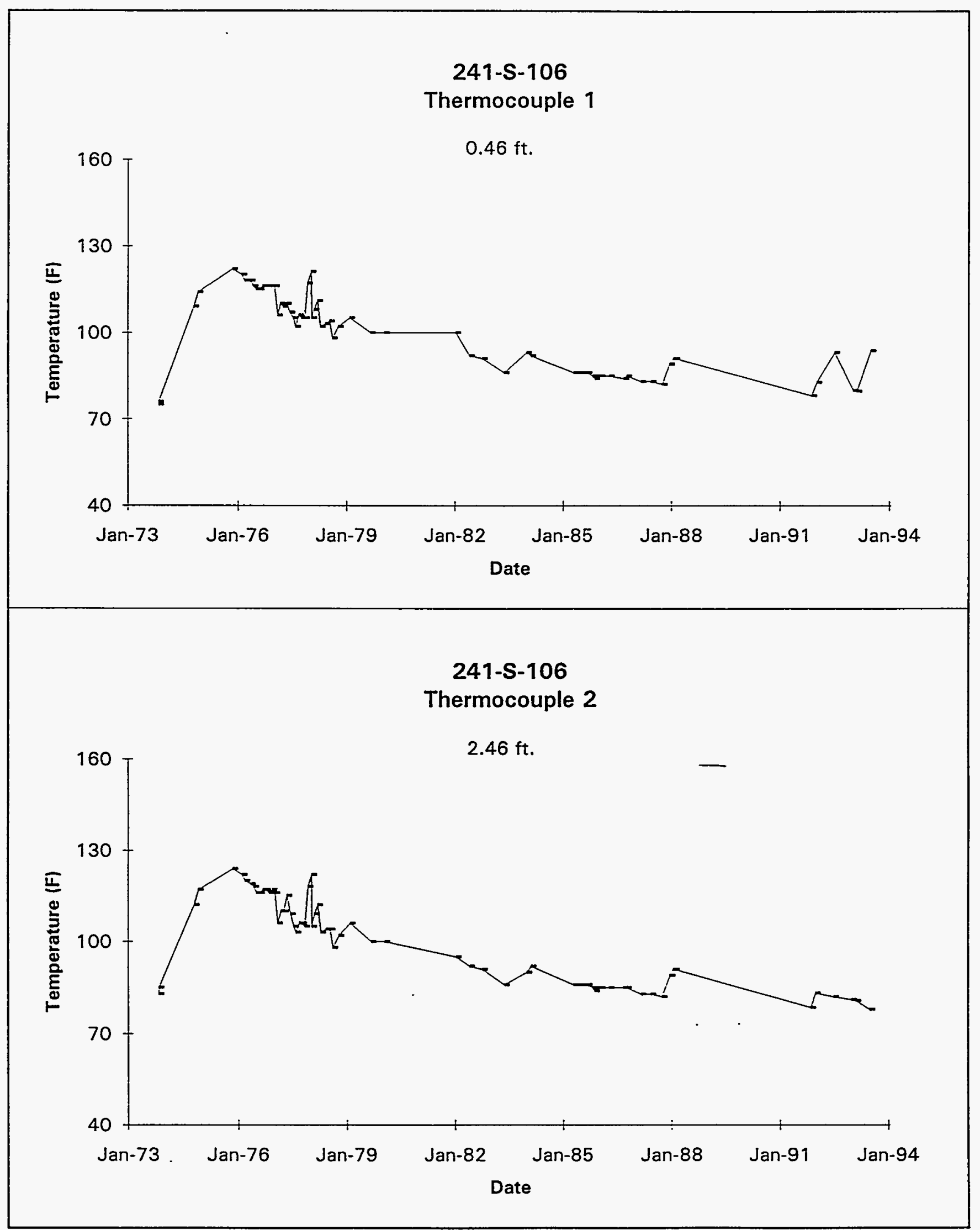




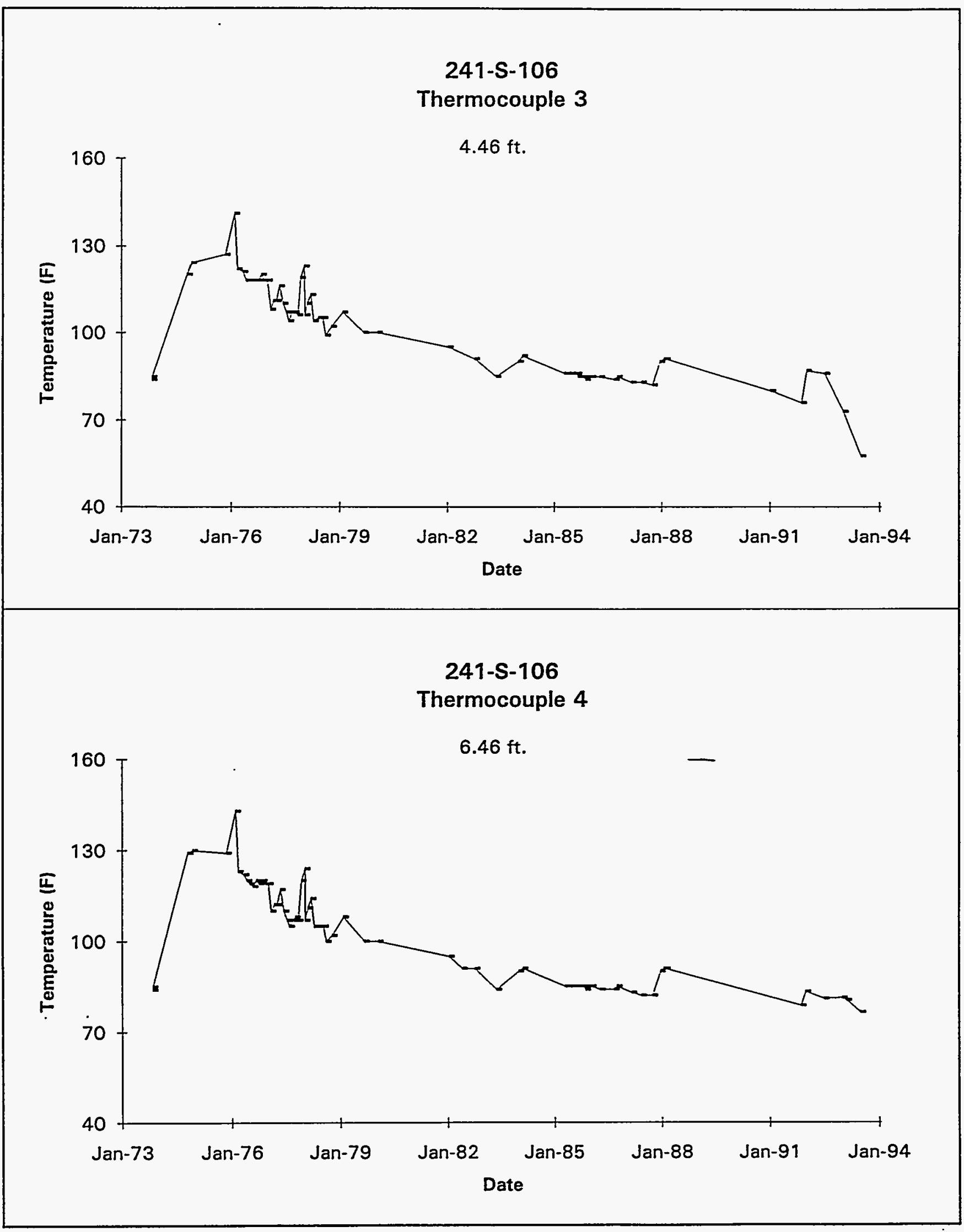

Data obtained from WHC Surveillance Analysis Computer System (SACS), February 24, 1994 and Archive box $\$ 121869$. 


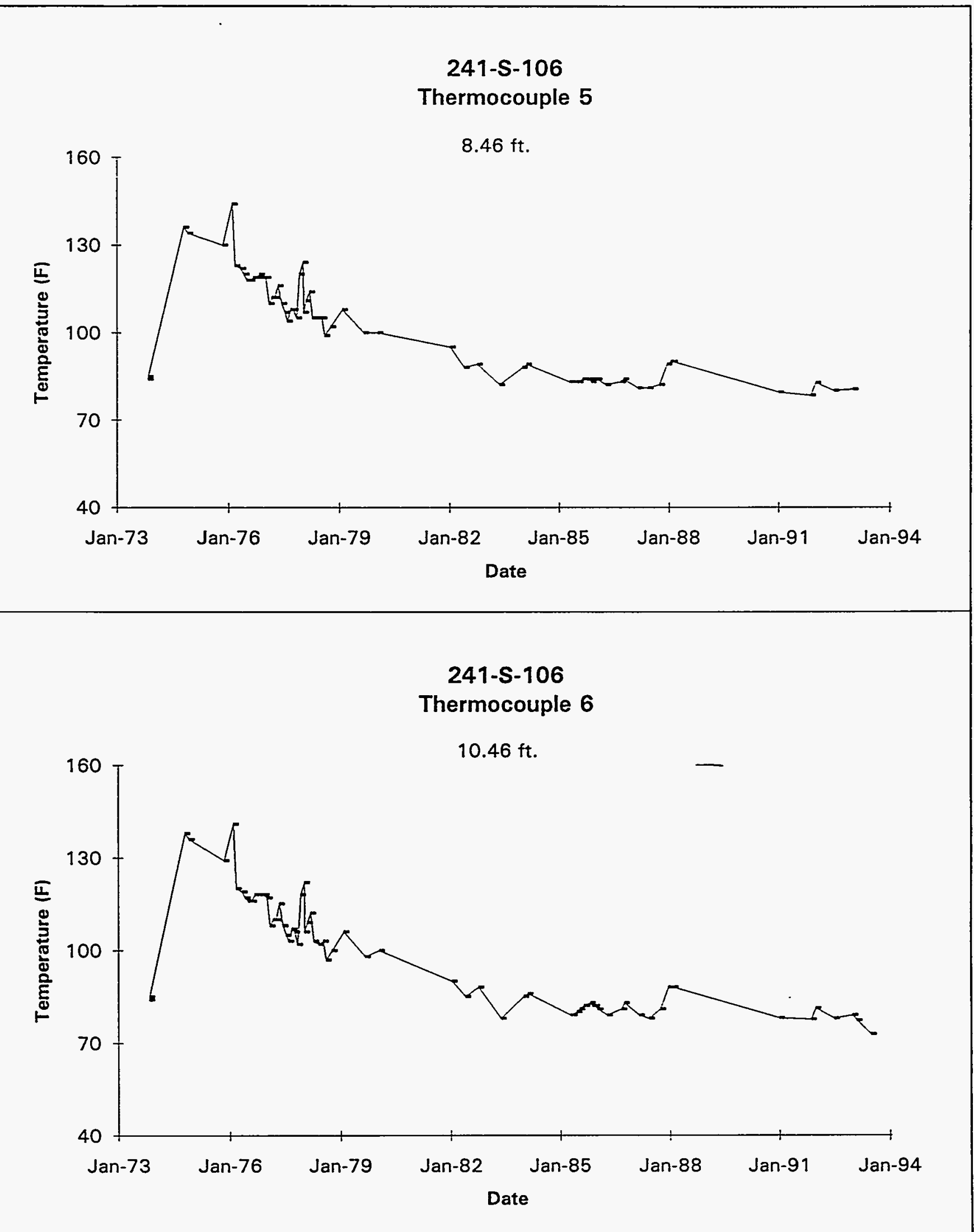

Data obtained from WHC Surveillance Analysis Computer System (SACS). February 24, 1994 and Archive box $\$ 121869$. 


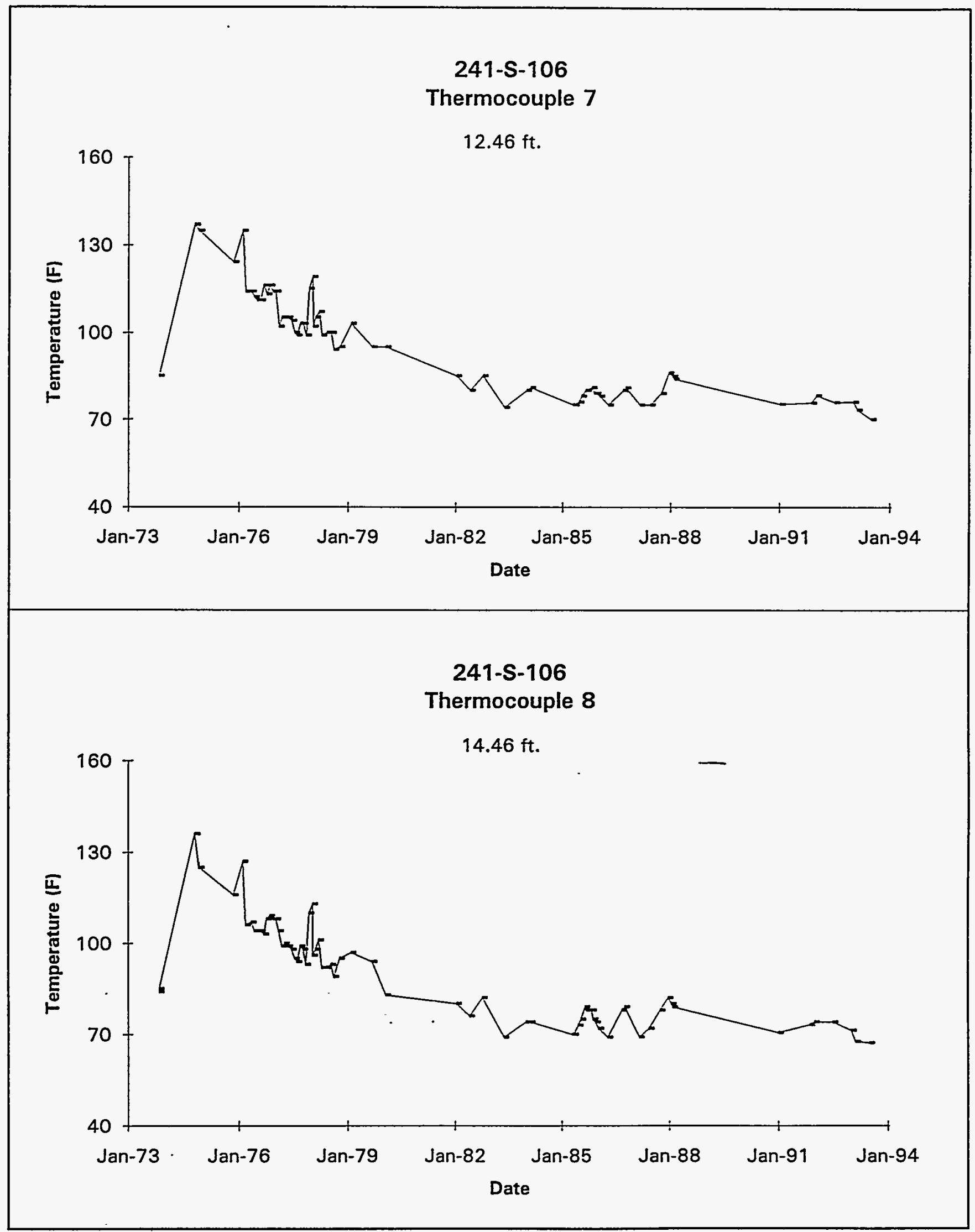




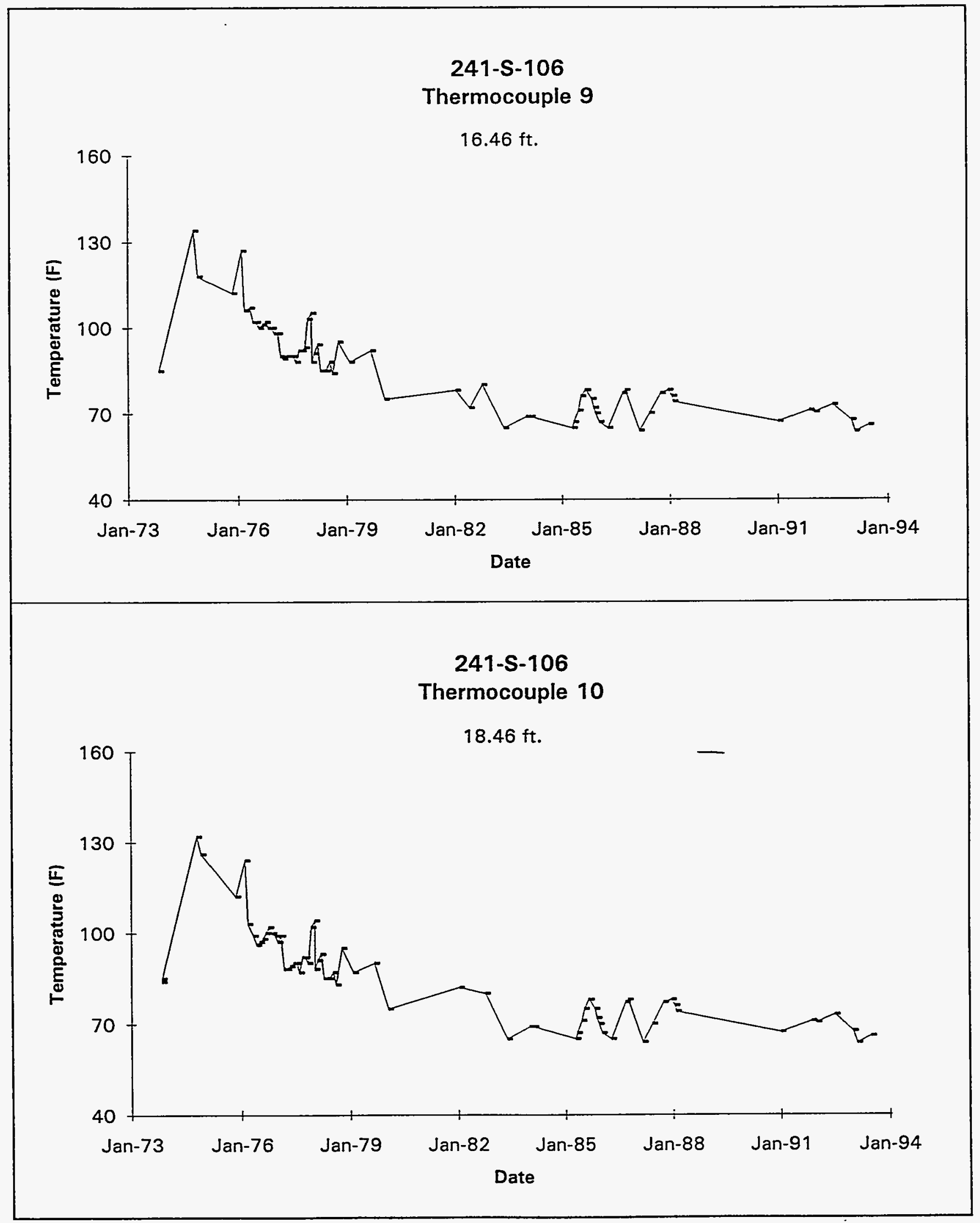

Data obtained from WHC Surveillance Analysis Computer System (SACS), February 24, 1994 and Archive box $\$ 121869$. 


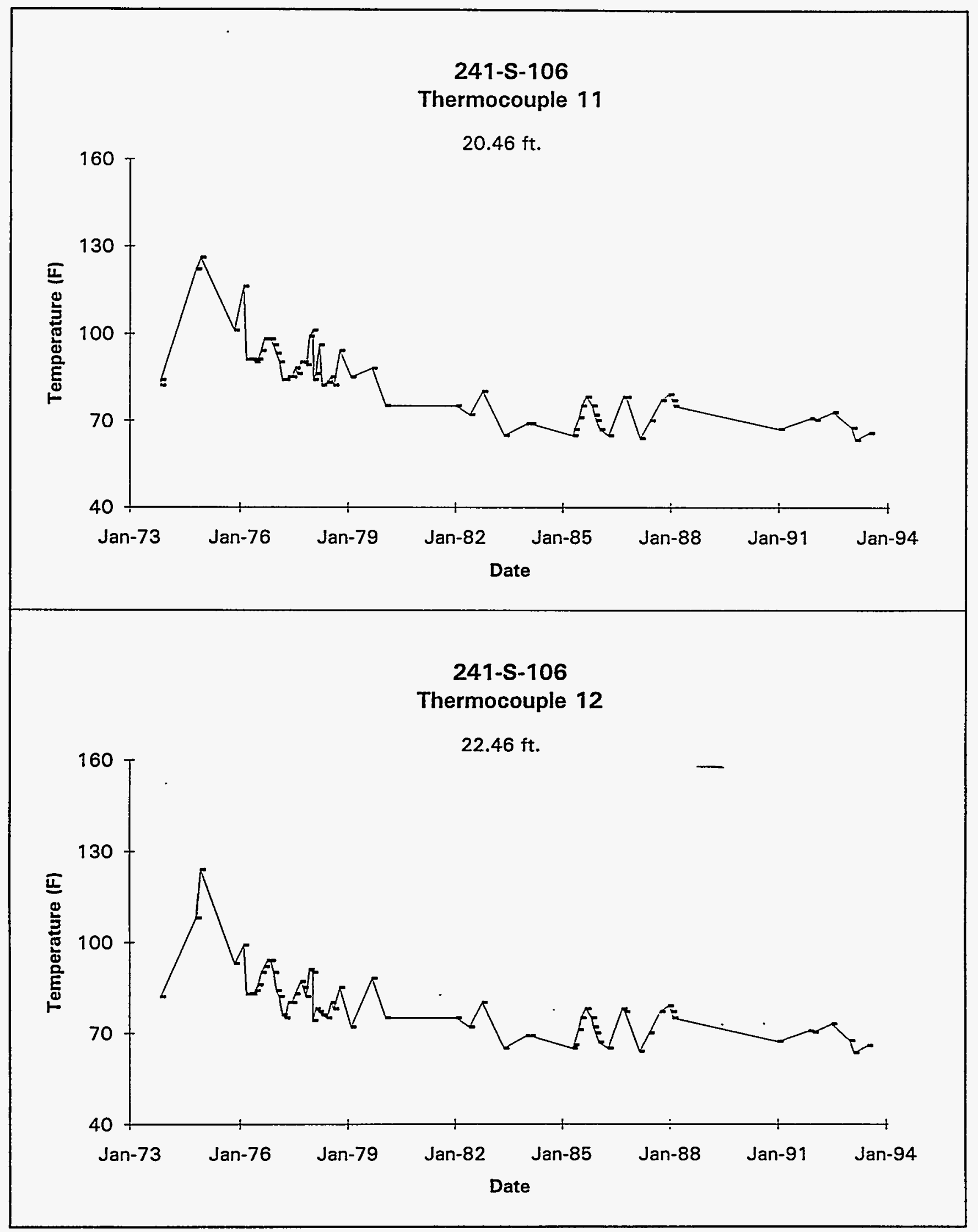

Data obtained from WHC Surveillance Analysis Compuner System (SACS), February 24, 1994 and Archive box $\$ 121869$. 


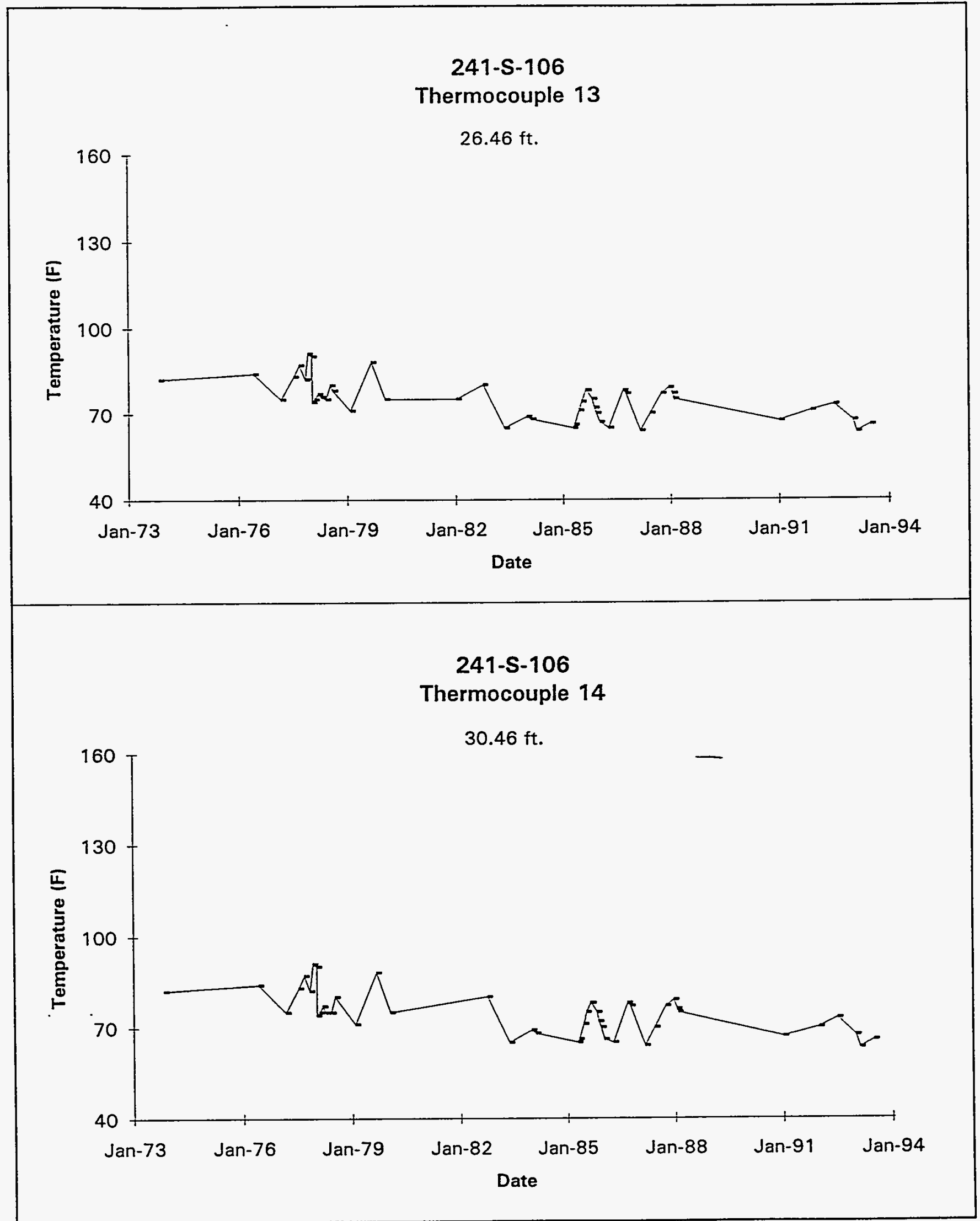

Data obtained from WHC Surveillance Analysis Computer System (SACS). February 24, 1994 and Archive box $\$ 121869$. 


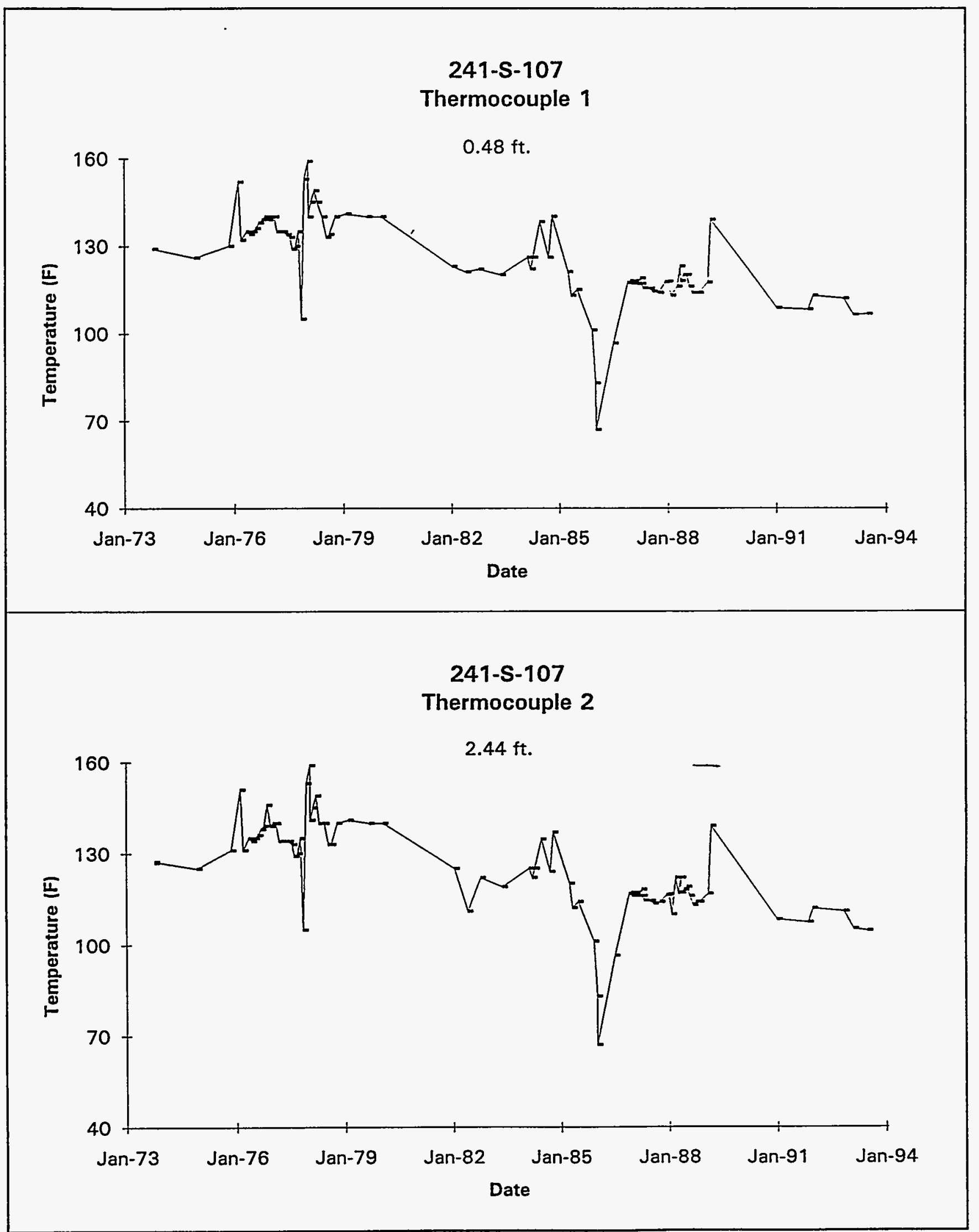

Data obtained from WHC Surveillance Analysis Computer System (SACS), February 24, 1994.

D-69 


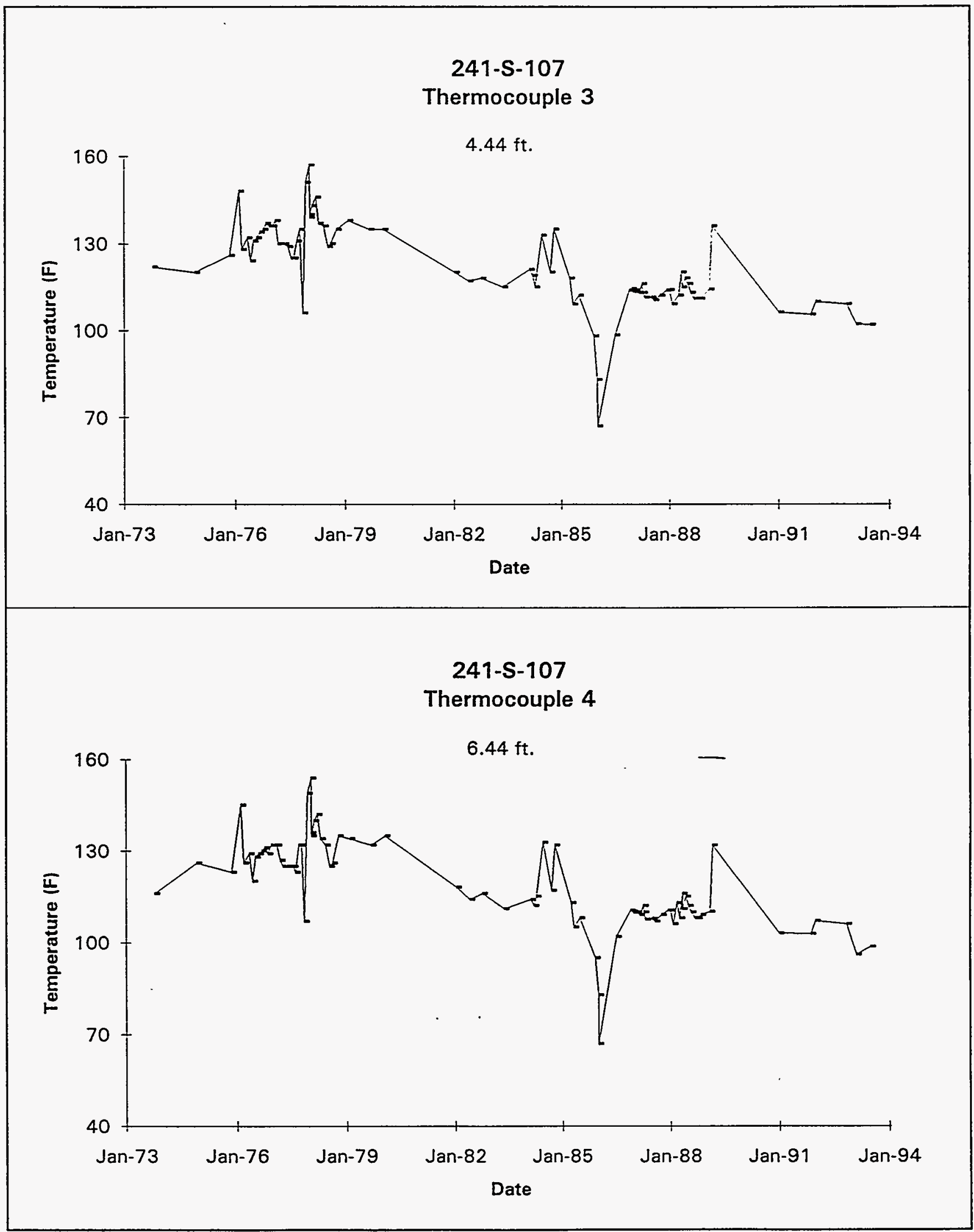

Data obtained from WHC Surveillance Analysis Computer System (SACS), February 24, 1994. 


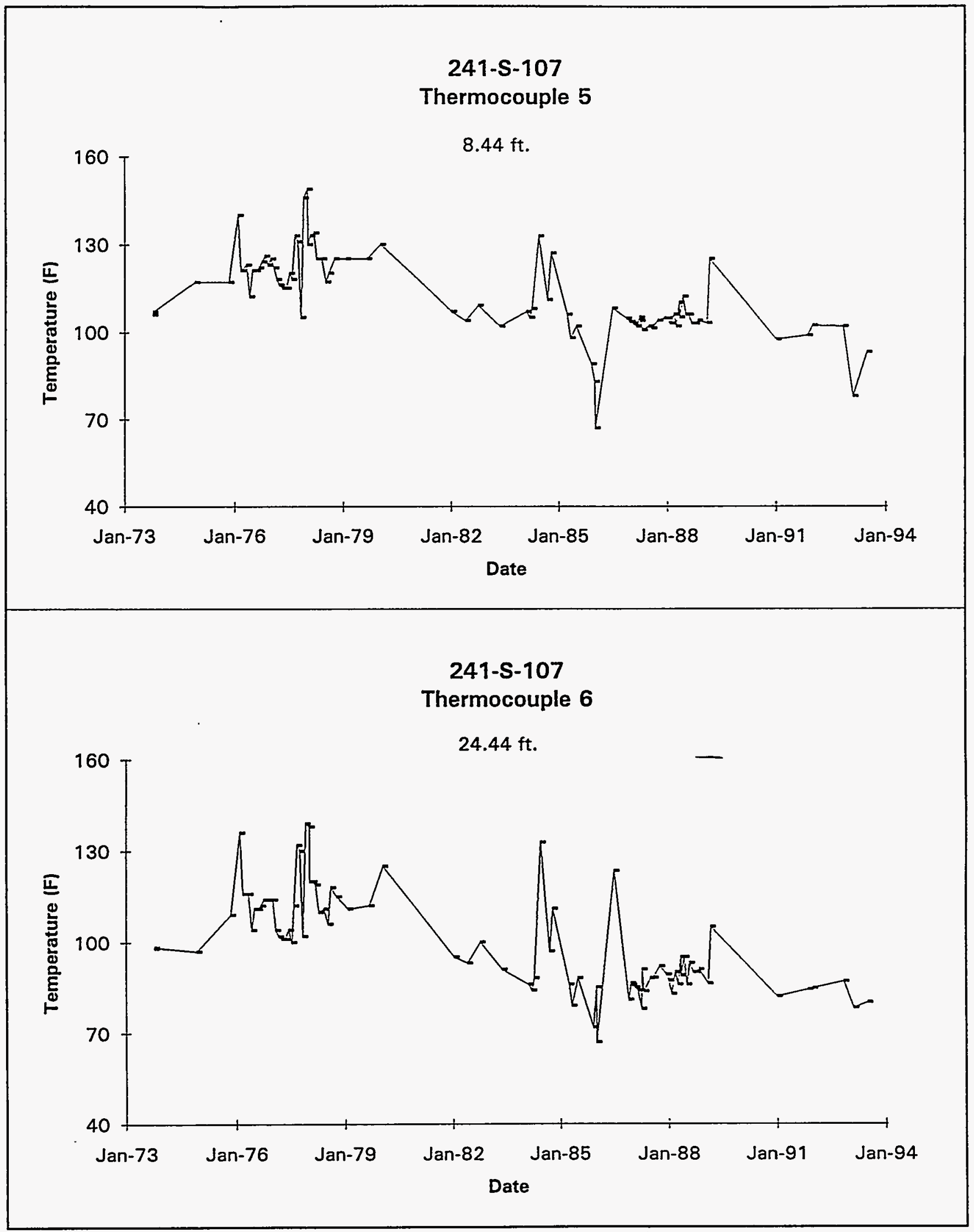

Data obtained from WHC Surveillance_Analysis Computer System (SACS), February 24, 1994. 


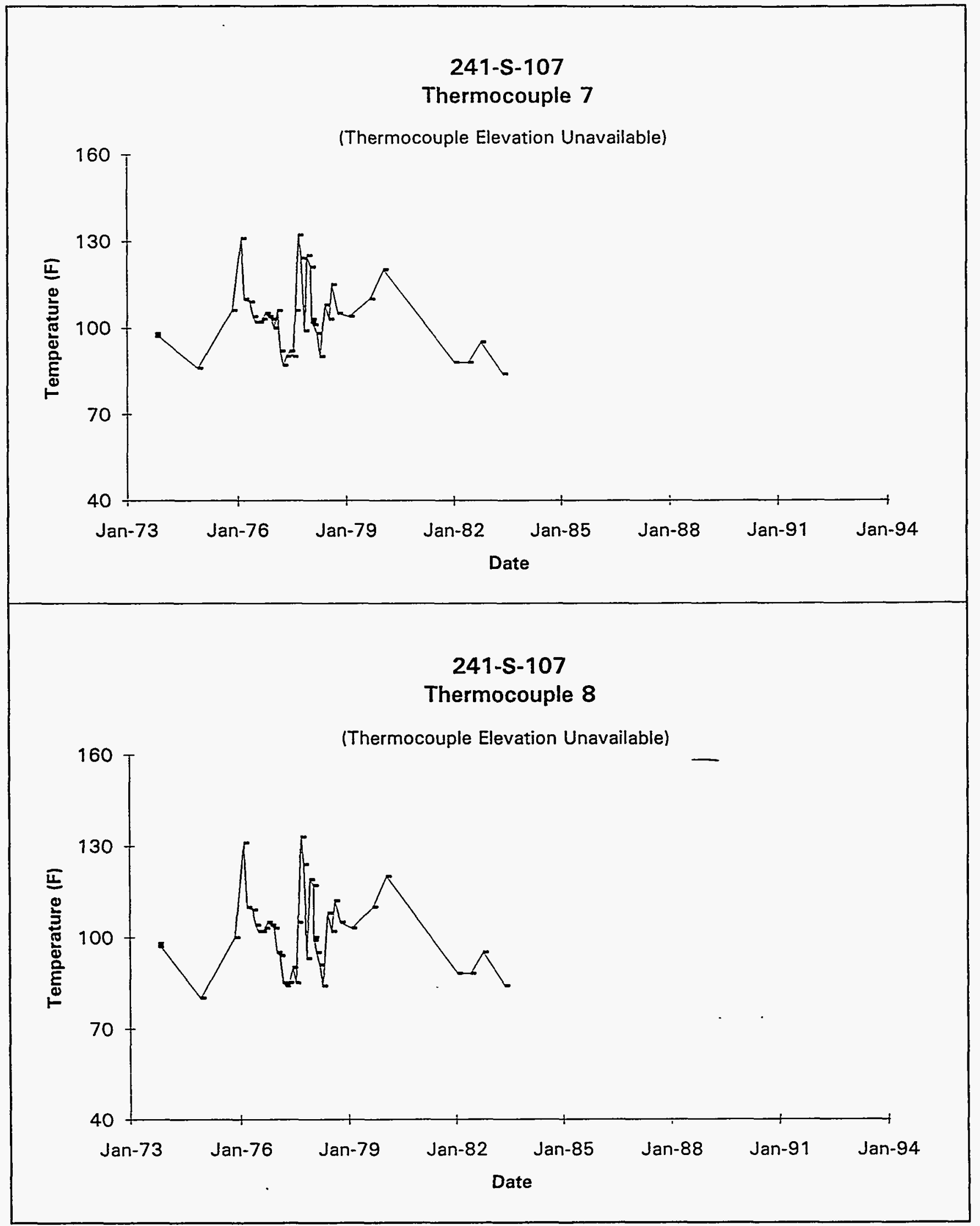

Data obtained from WHC Surveillance Analysis Computer System (SACS), February 24, 1994. 


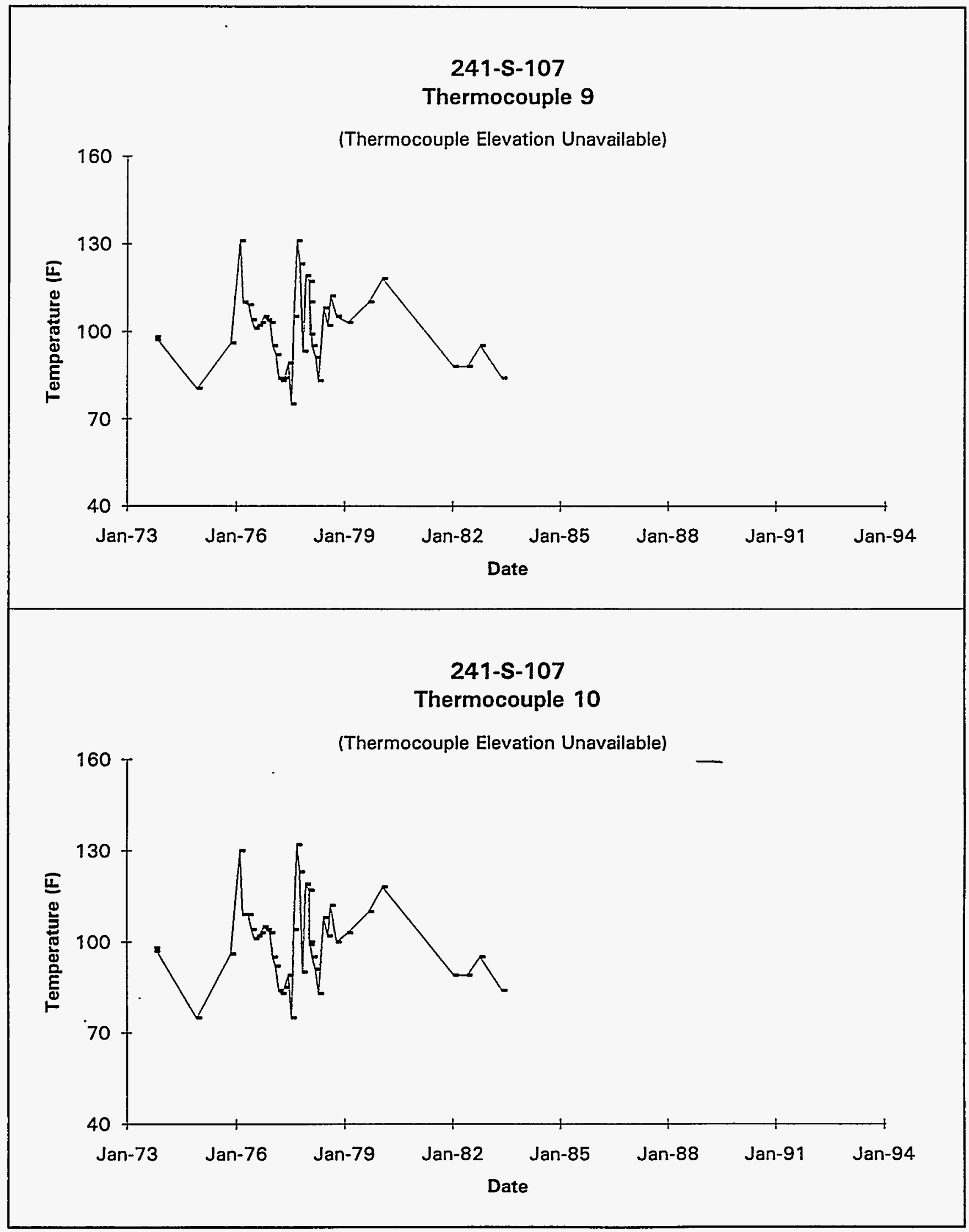

Data obtained from WHC Surveillance Analysis Computer System (SACS), February 24, 1994.

D-73 


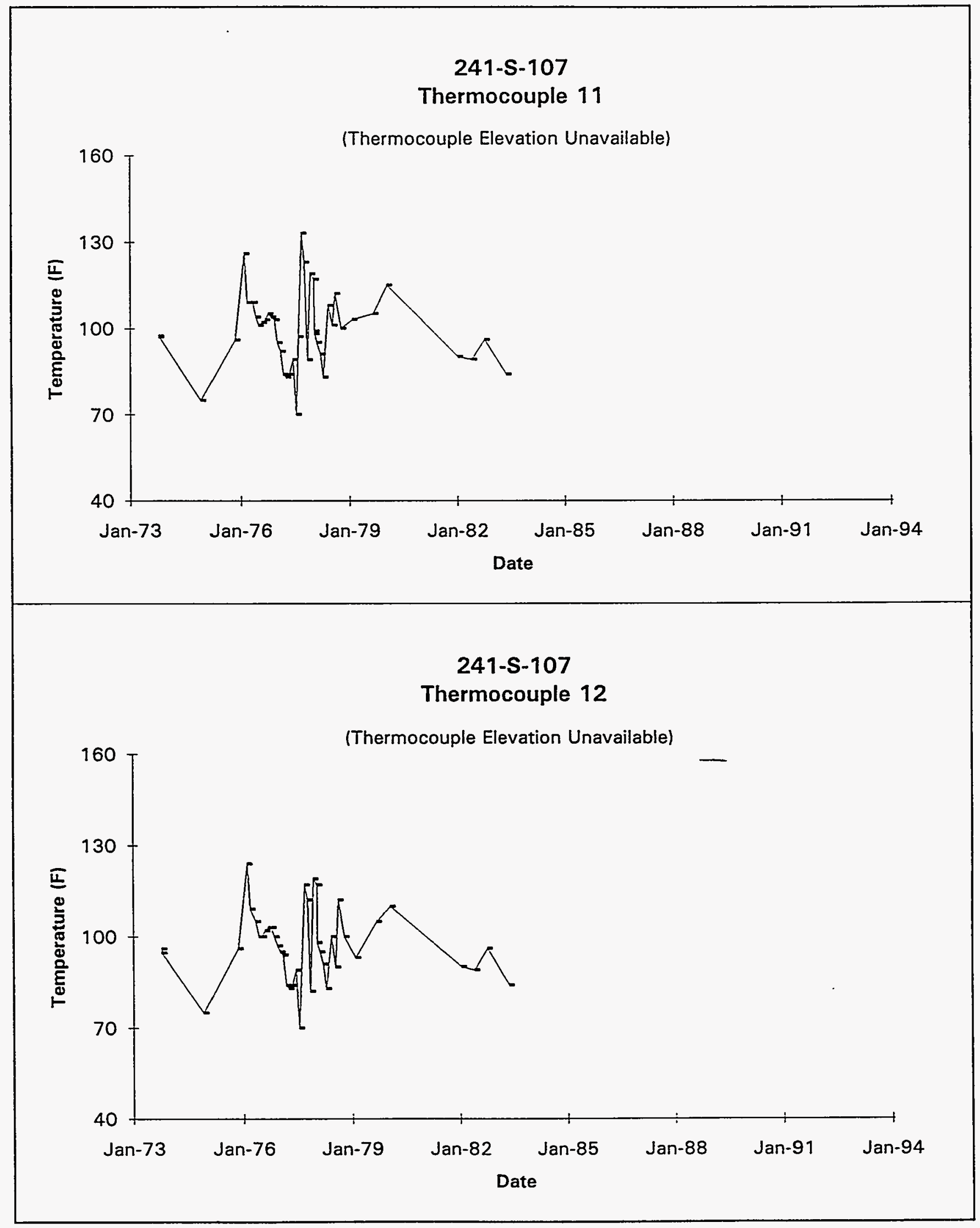

Data obtained from WHC Surveillance Analysis Computer System (SACS), February 24, 1994. 


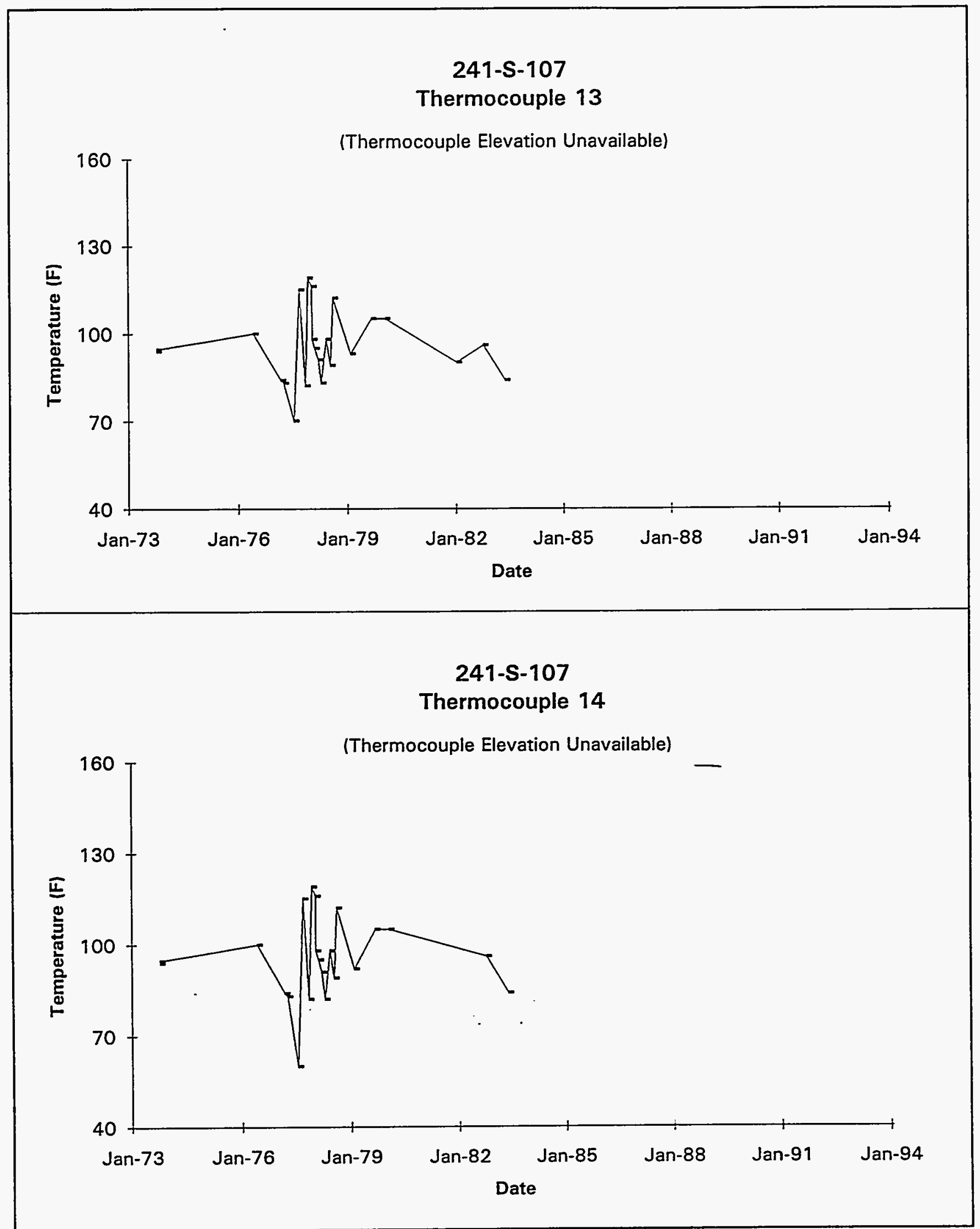

Data obtained from WHC Surveillance Analysis Computer System (SACS), February 24, 1994. 


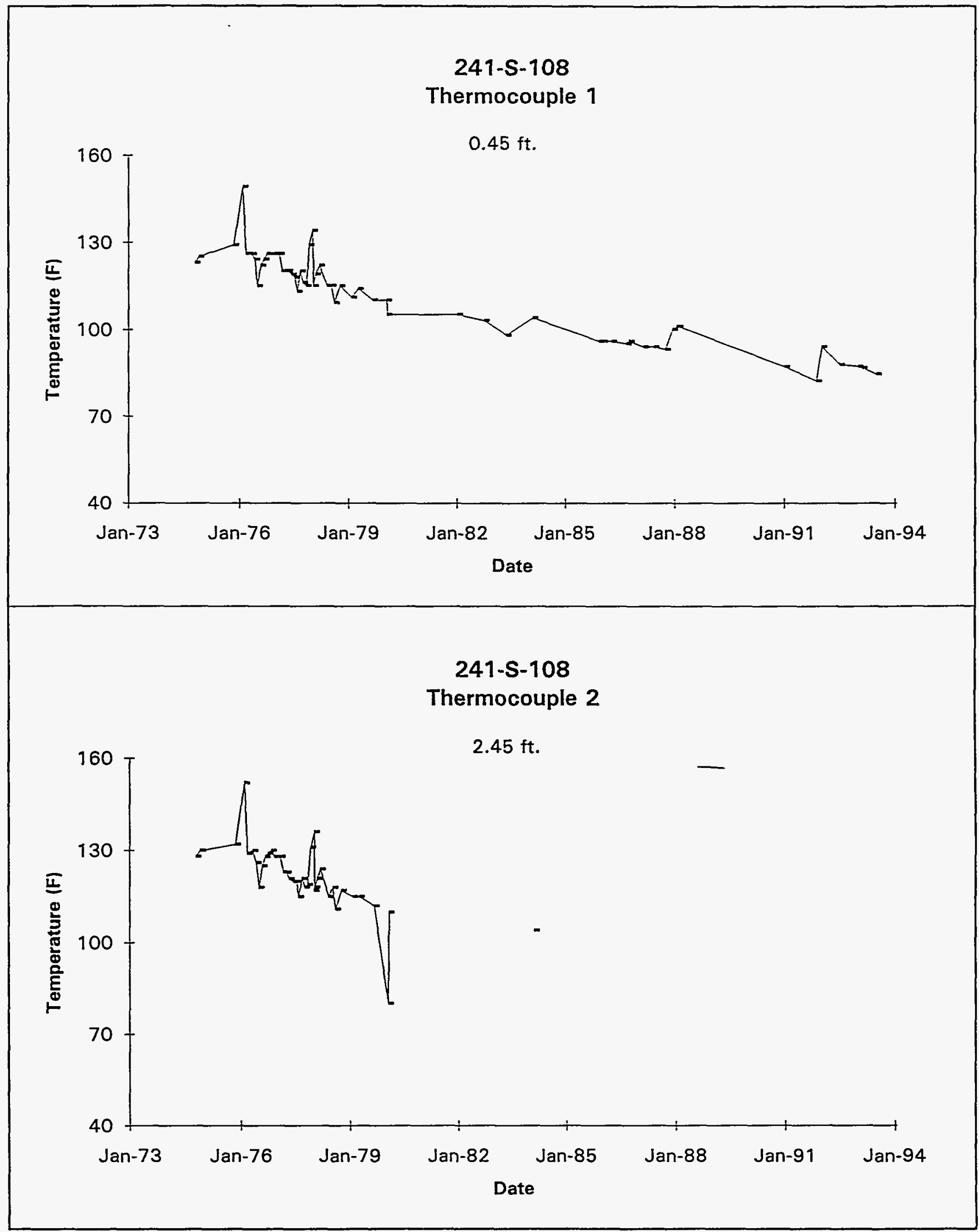

Data obtained from WHC Surveillance Analysis Computer System (SACS), February 24, 1994.

D-76 


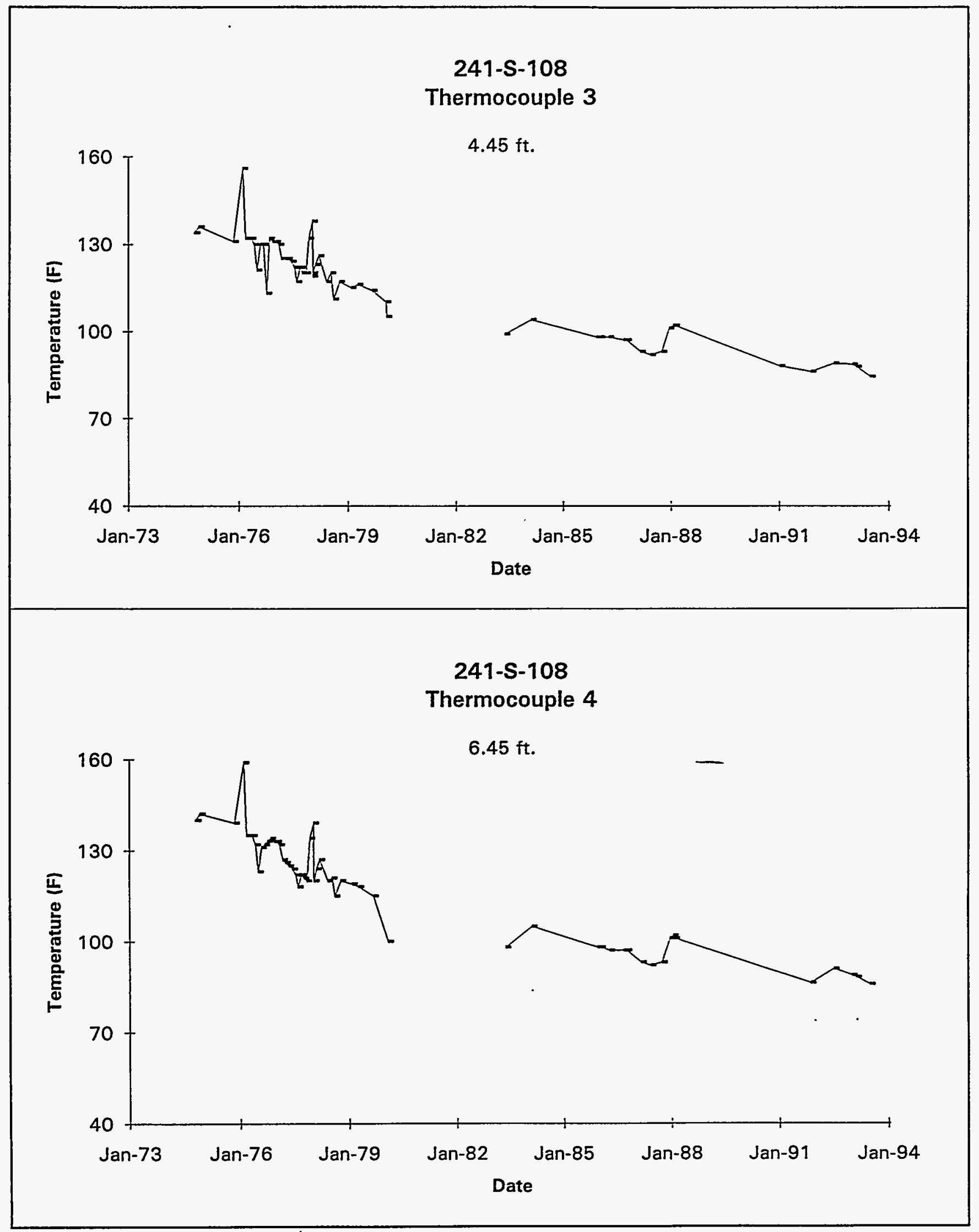

Data obtained from WHC Surveillance Analysis Computer System (SACS), February 24, 1994. 


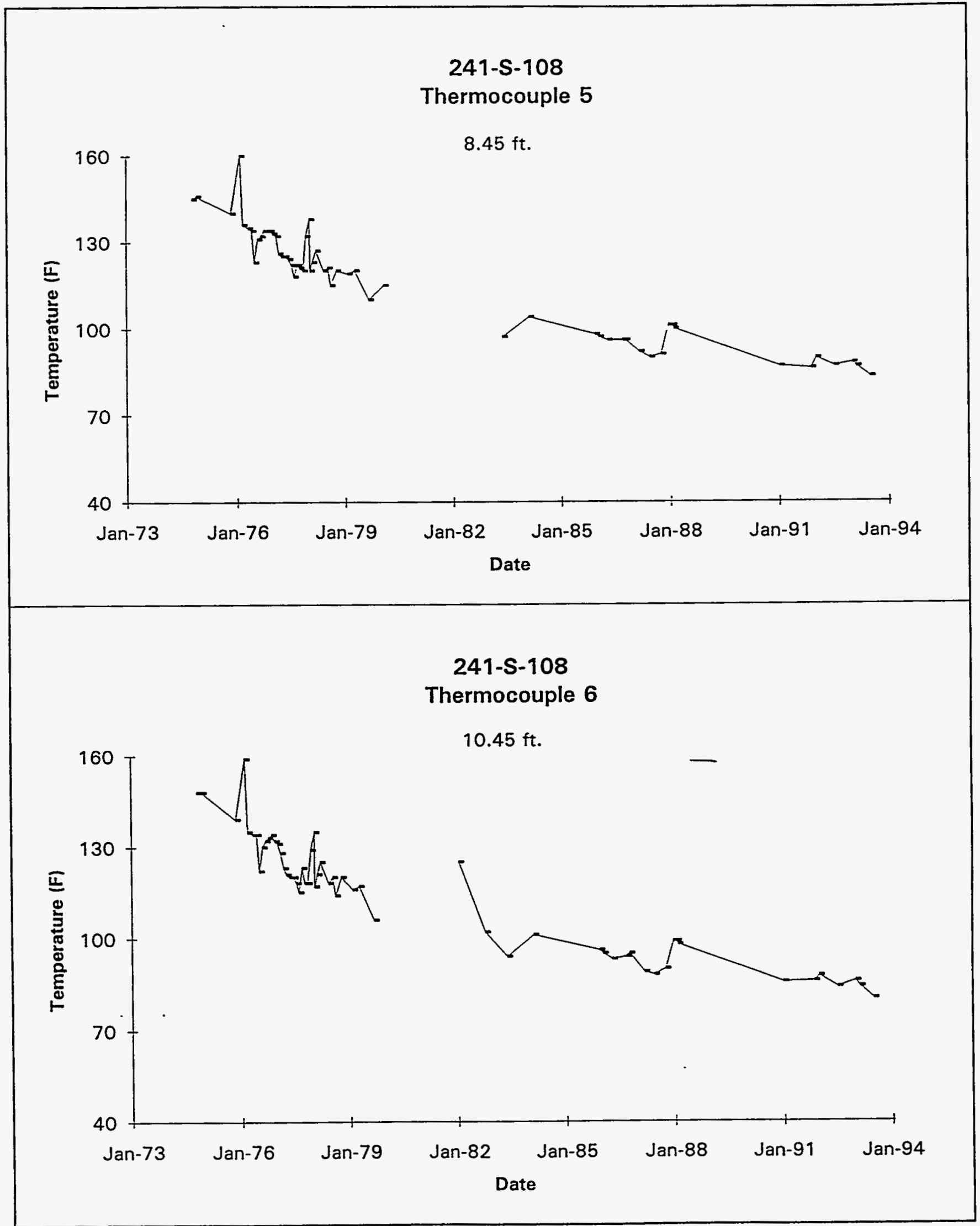

Data obtained from WHC Surveillance Analysis Computer System (SACS), February 24, 1994. 


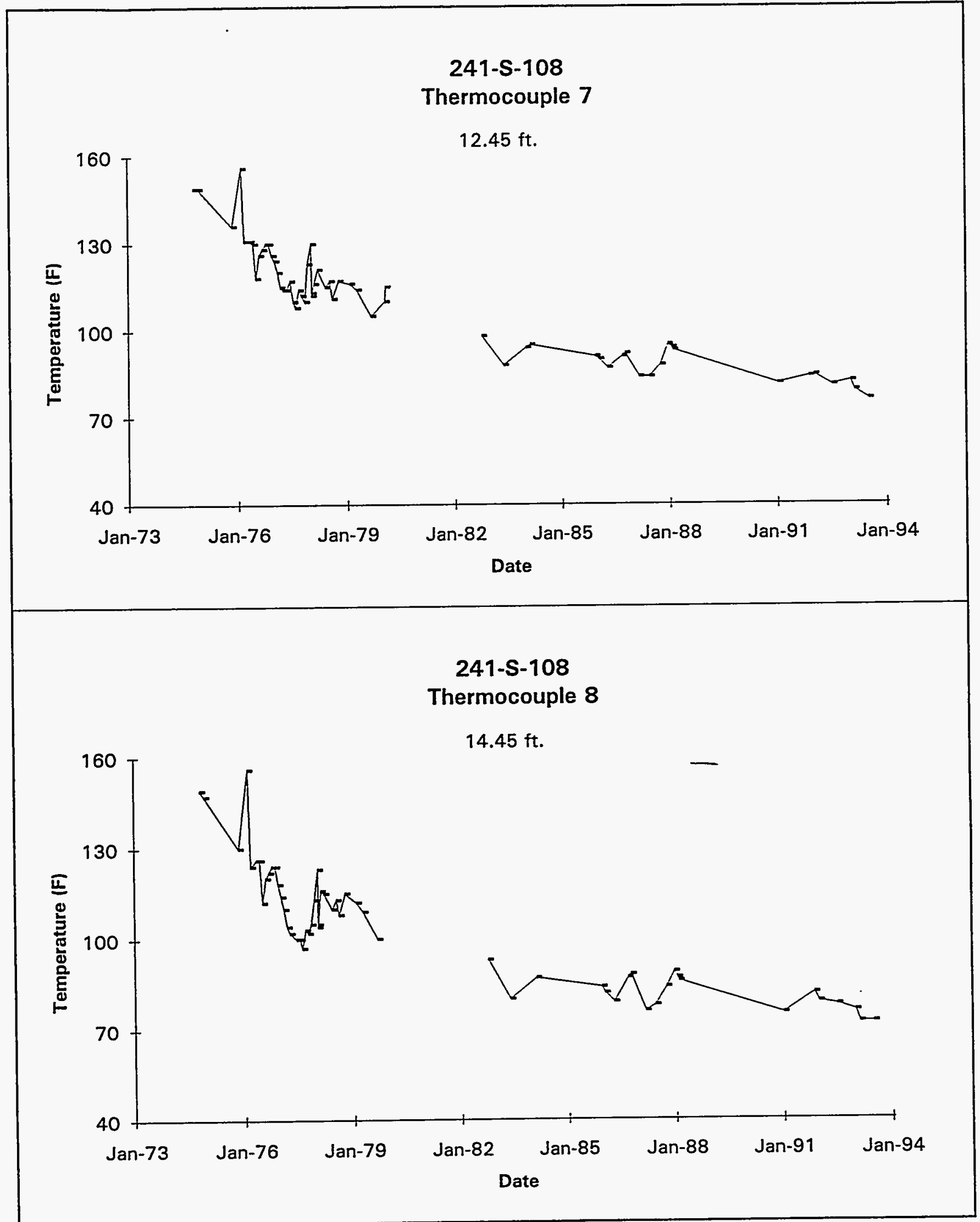

Data obtained from WHC Surveillance Analysis Computer System (SACS), February 24, 1994.

D-79 


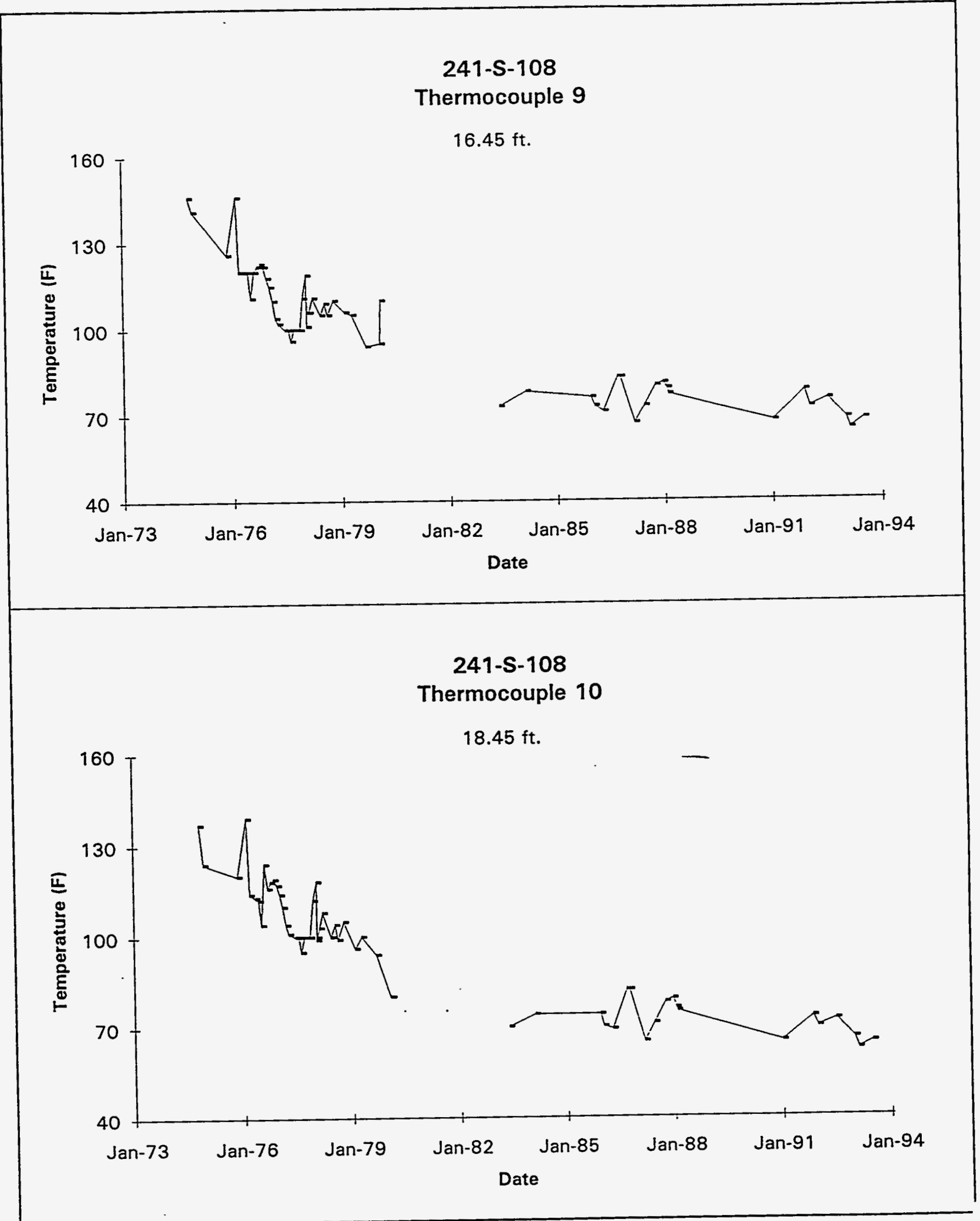

Data obtained from WHC Surveillance Analysis Computer System (SACS), February 24, 1994.

D-80 


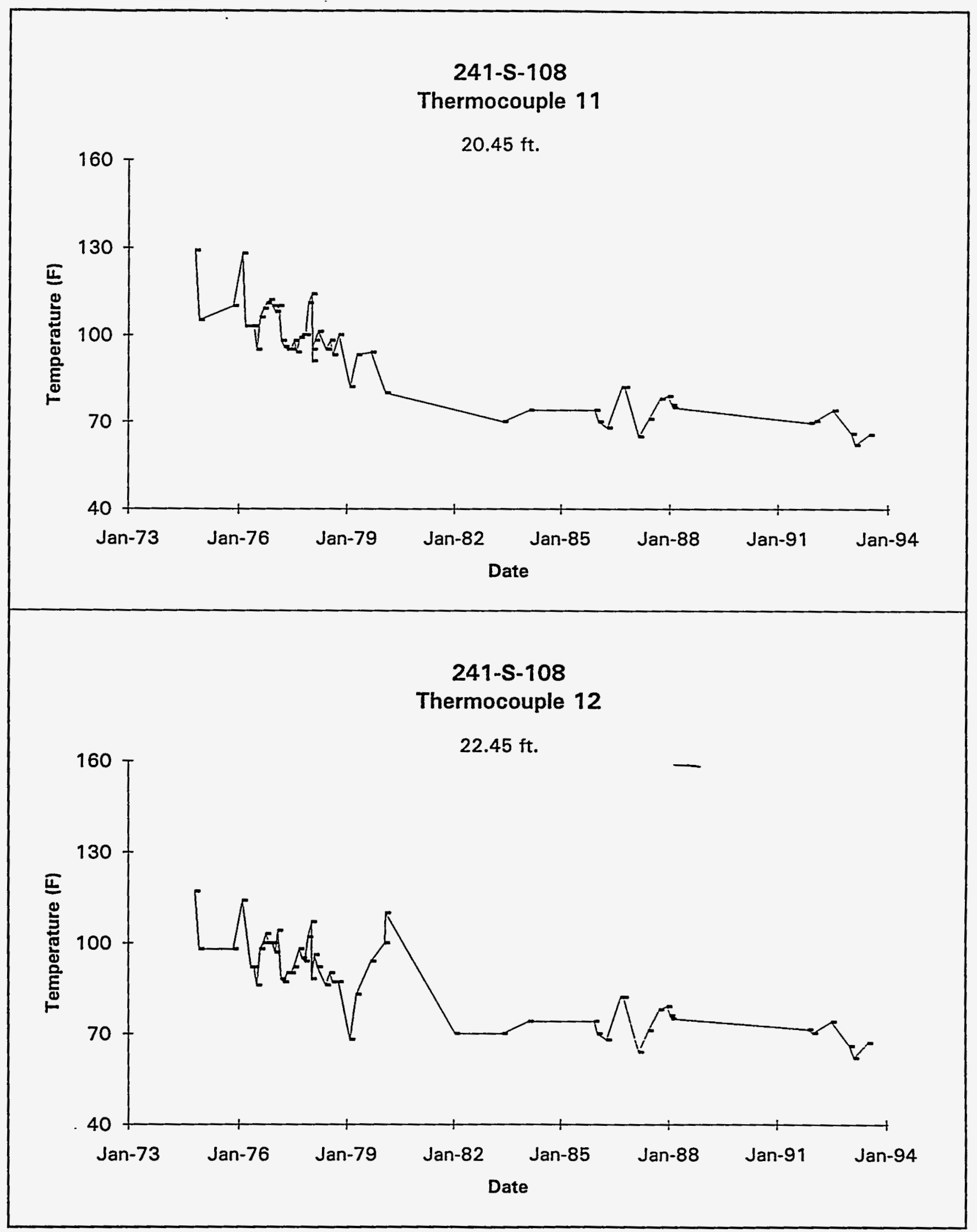

Data obtained from WHC Surveillance Analysis Computer System (SACS), February 24, 1994. 


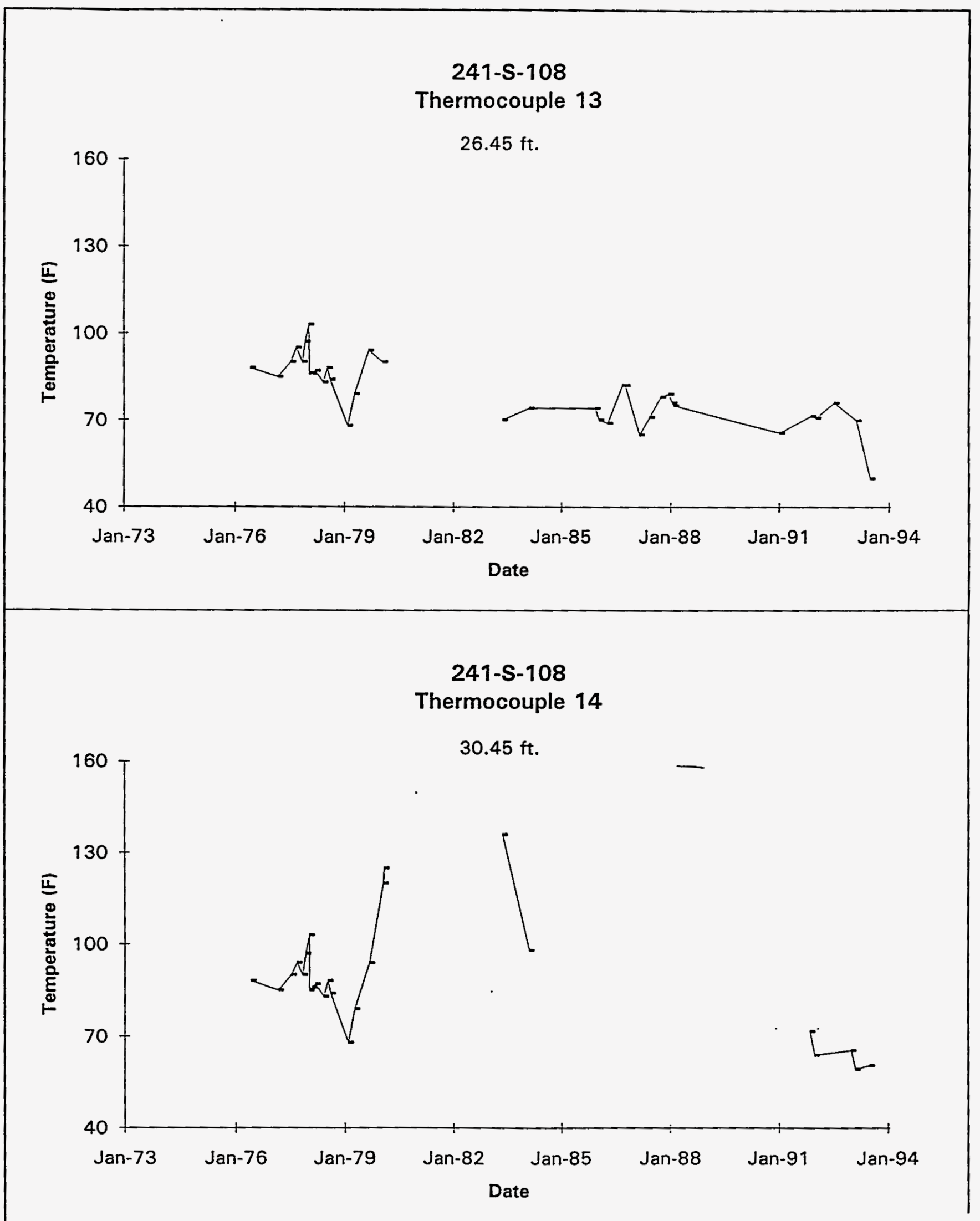

Data obtained from WHC Surveillance Analysis Computer System (SACS), February 24, 1994. 


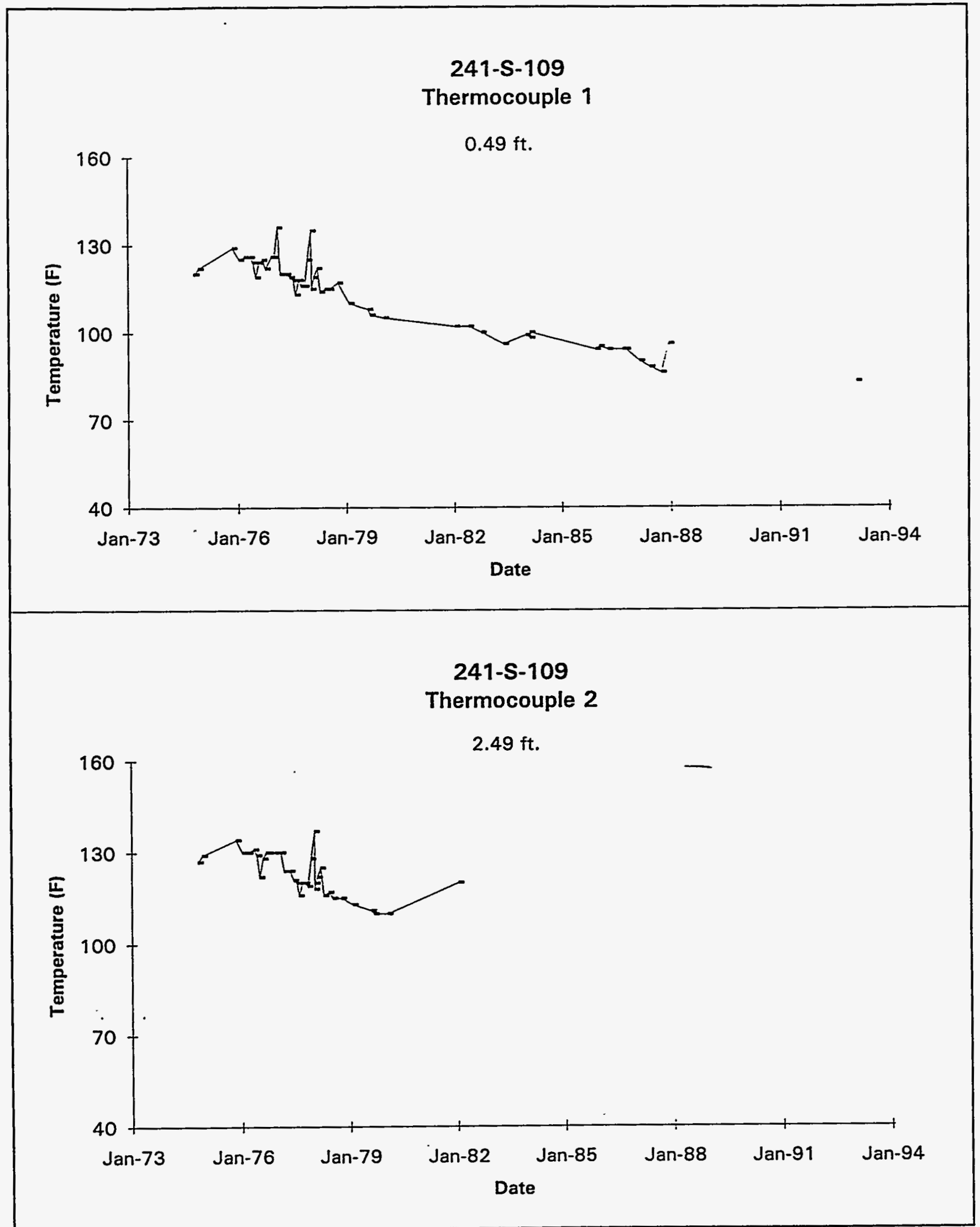

Data obtained from WHC Surveillance Analysis Computer System (SACS), February 24, 1994. D-83 


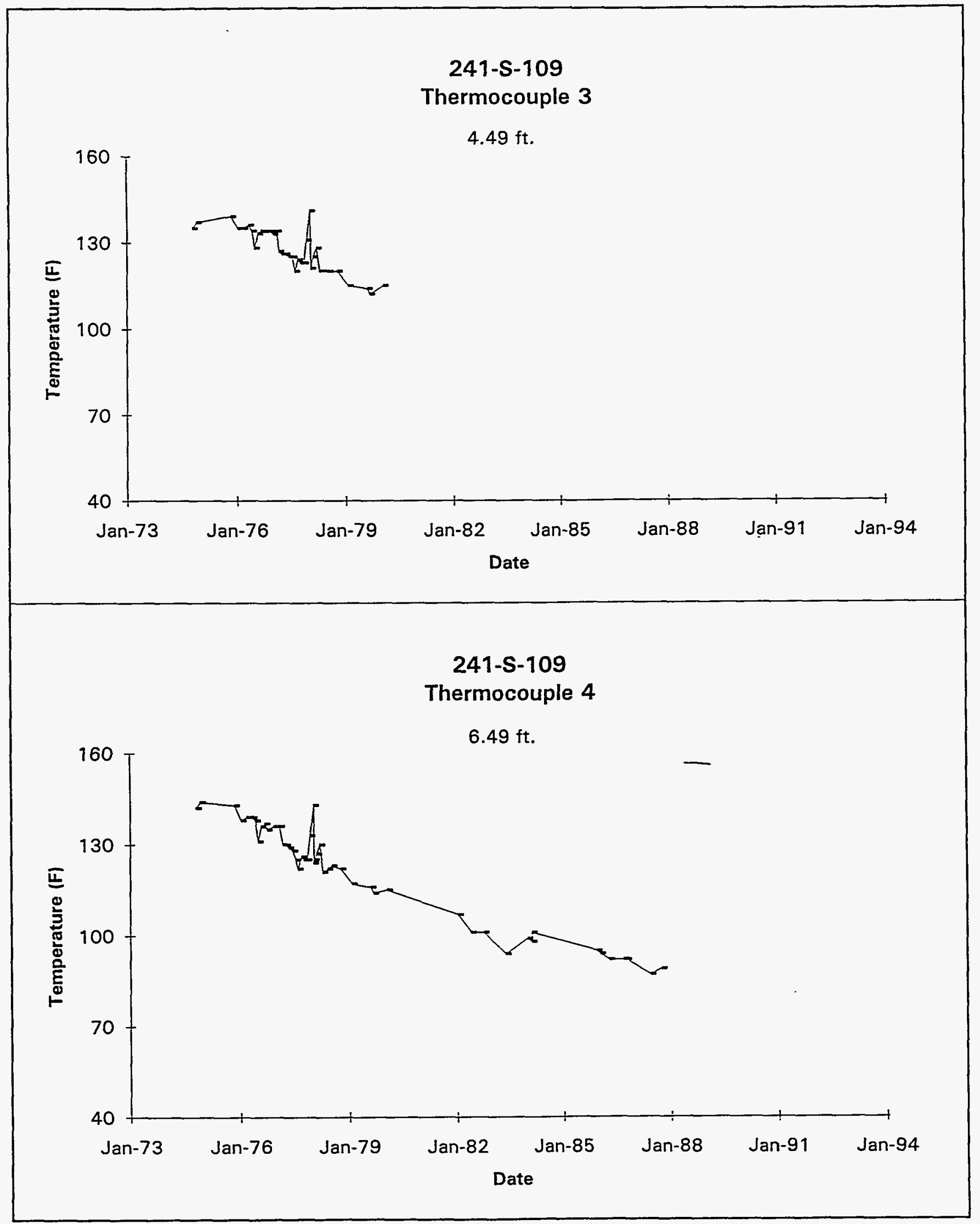

.. Data obtained from WHC Surveillance Analysis Computer System (SACS), February 24, 1994. 


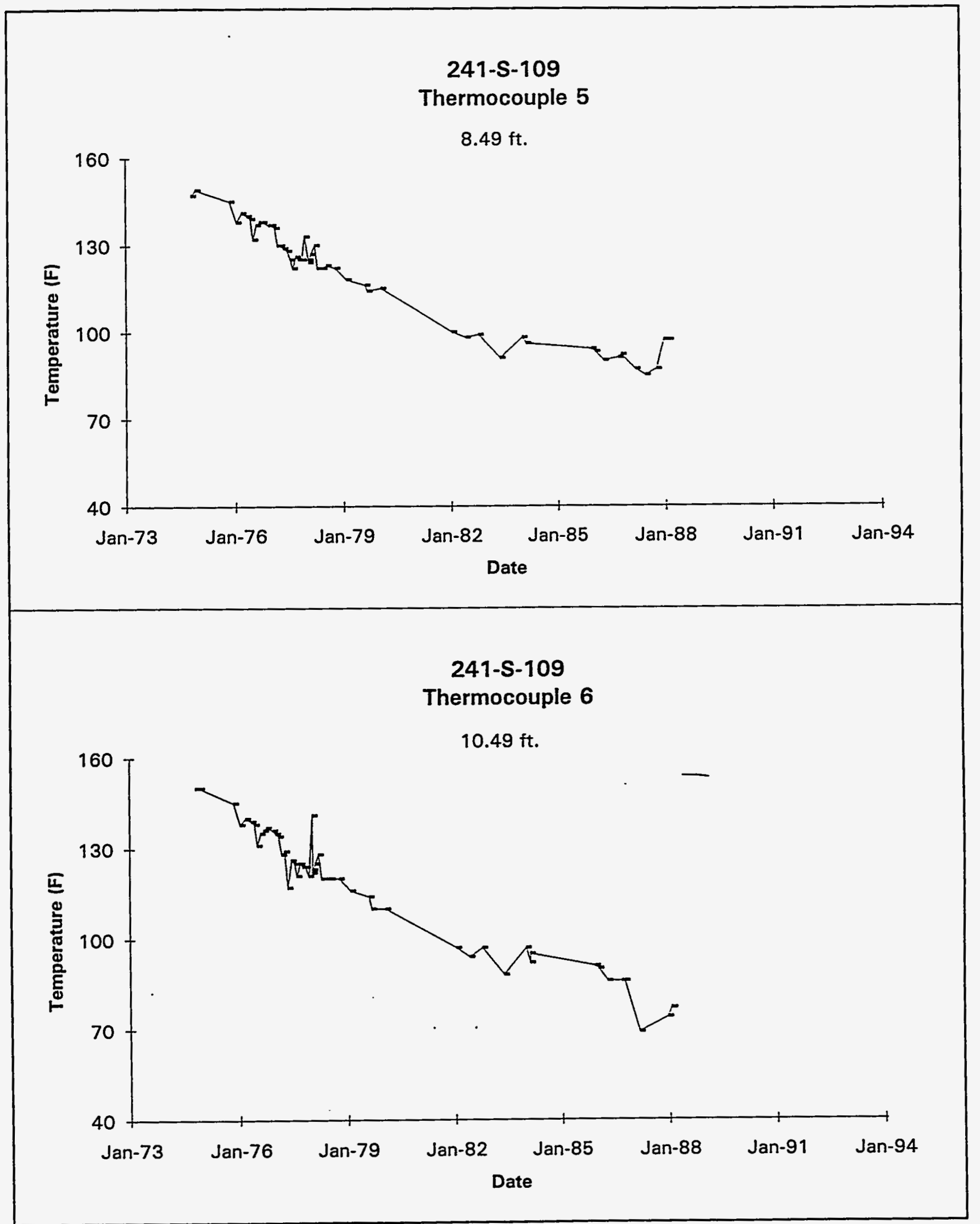

Data obtained from WHC Surveillance Analysis Computer System (SACS), February 24, 1994.

D-85 


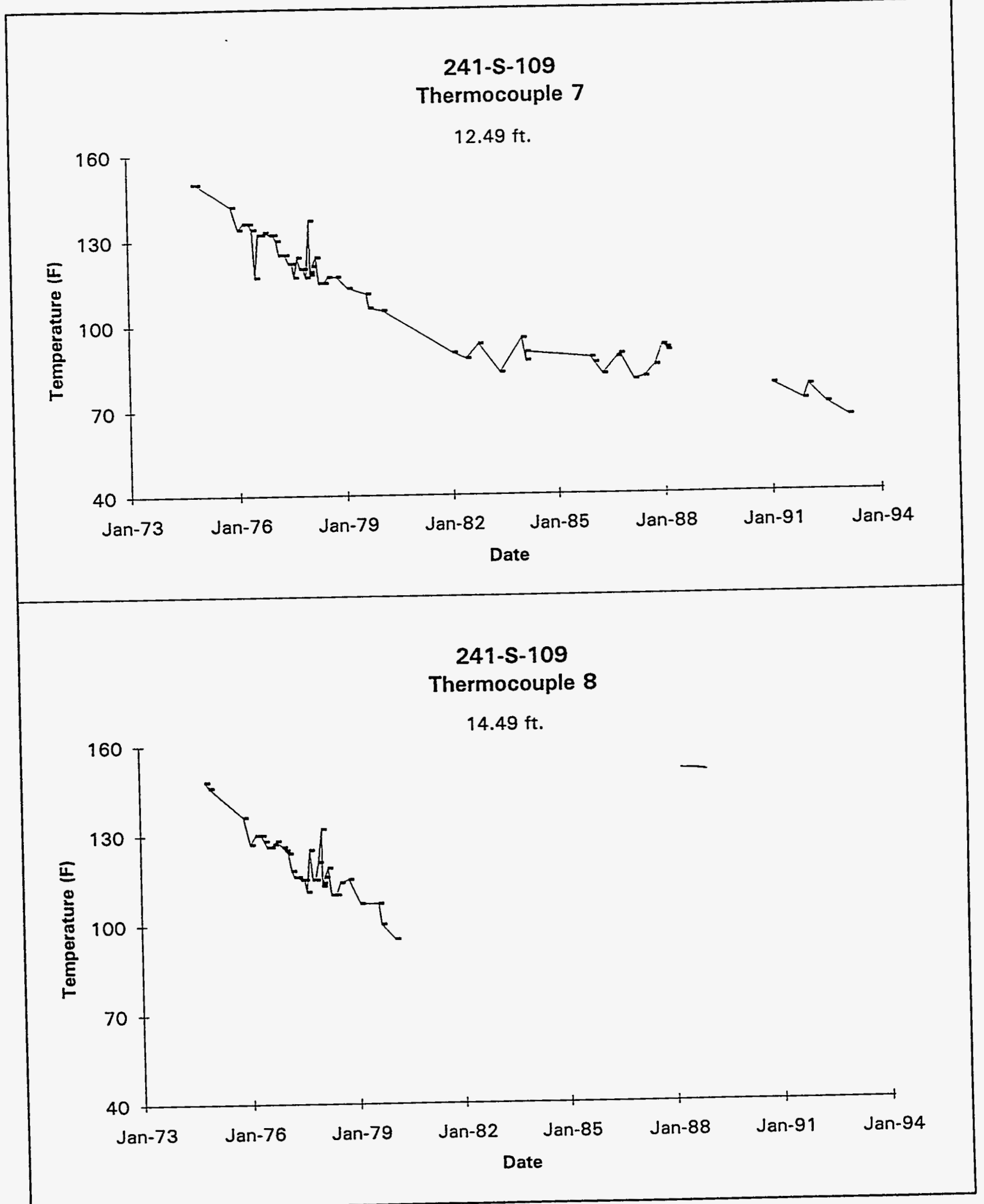

Data obtained from WHC Surveillance Analysis Computer System (SACS), February 24, 1994. 


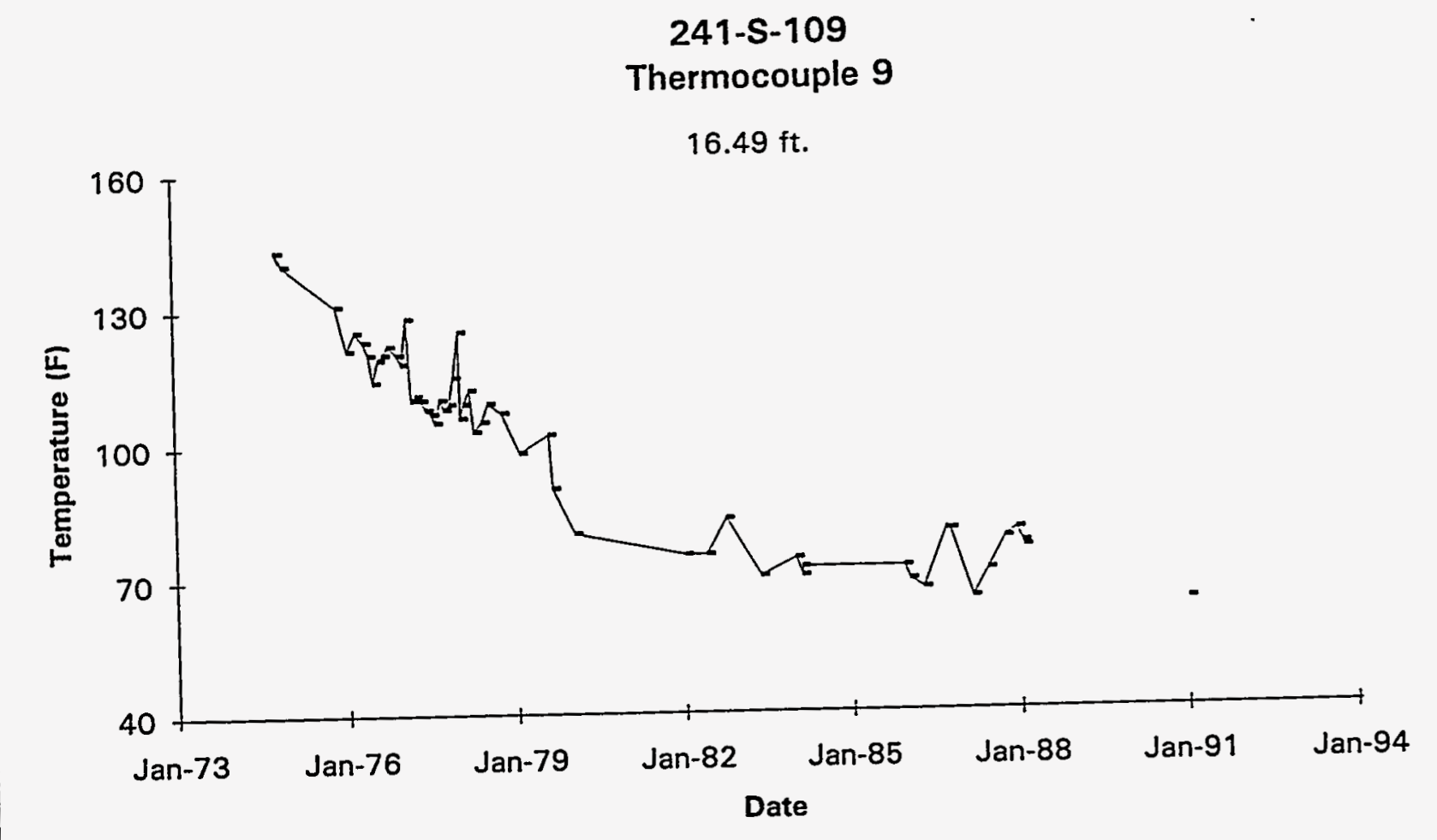

241-S-109

Thermocouple 10

$18.49 \mathrm{ft}$.

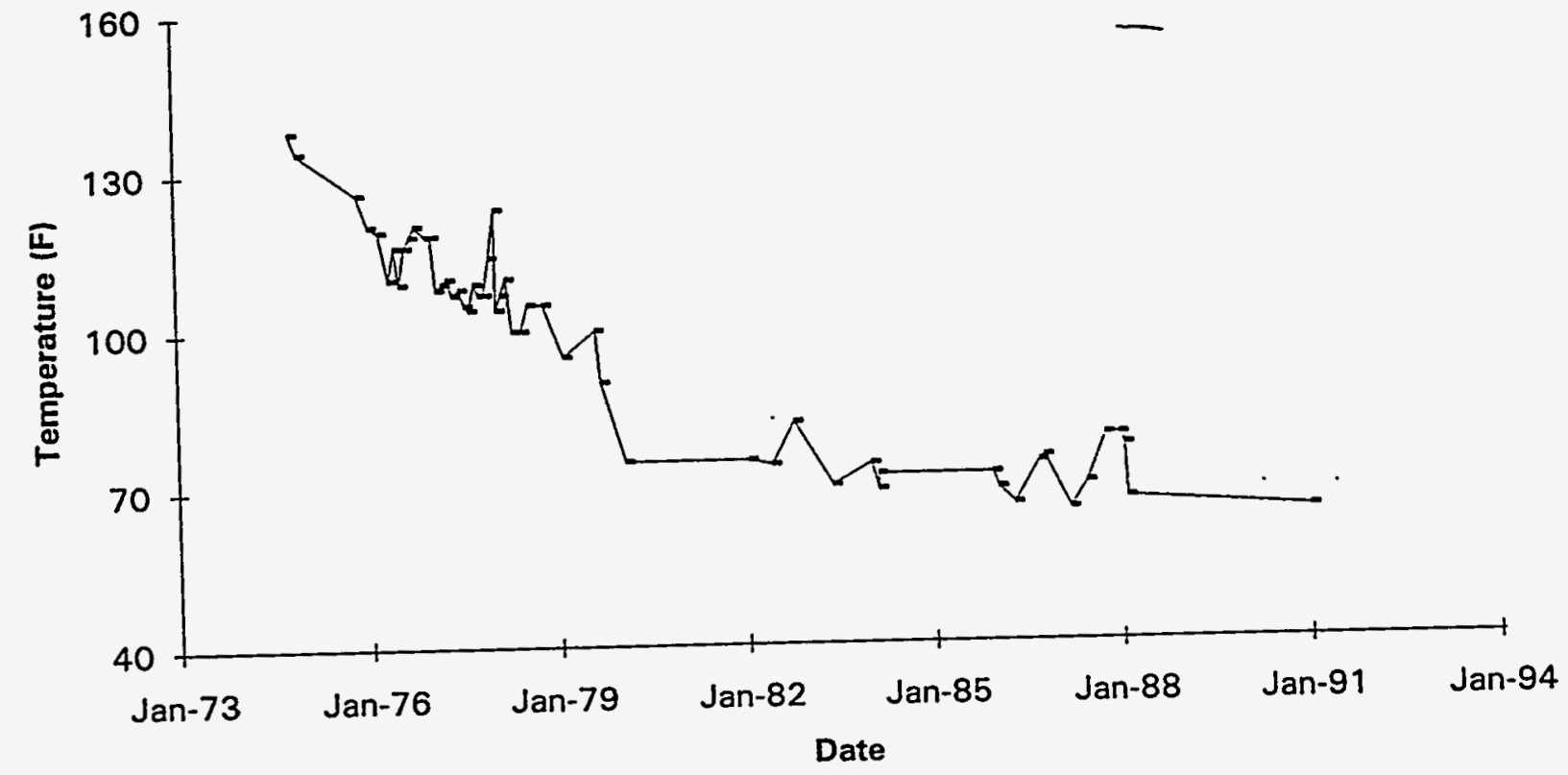

Data obtained from WHC Surveillance Analysis Computer System (SACS), February 24, 1994. 


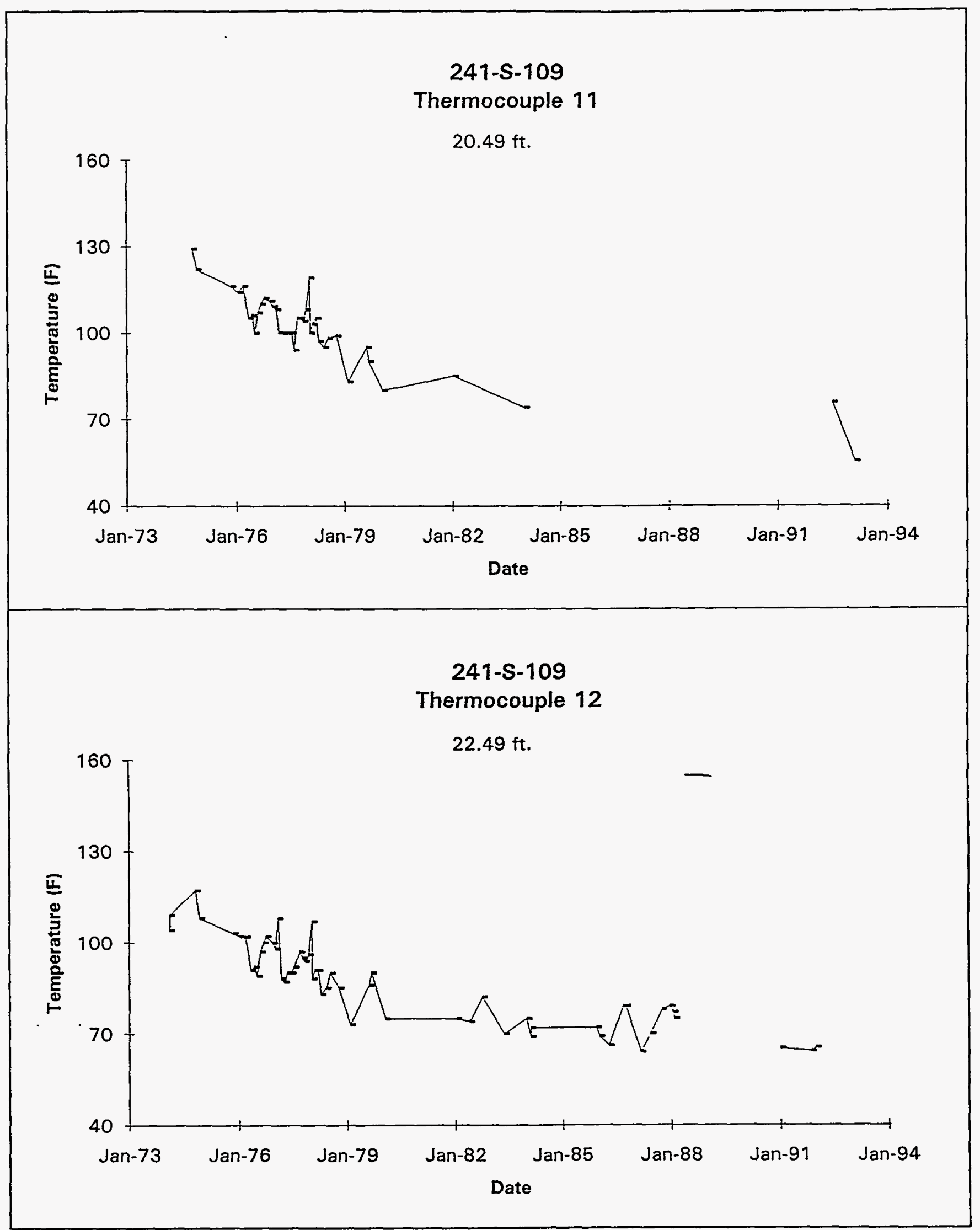

Data obtained from WHC Surveillance Analysis Computer System (SACS), February 24, 1994.

$$
\text { D-88 }
$$




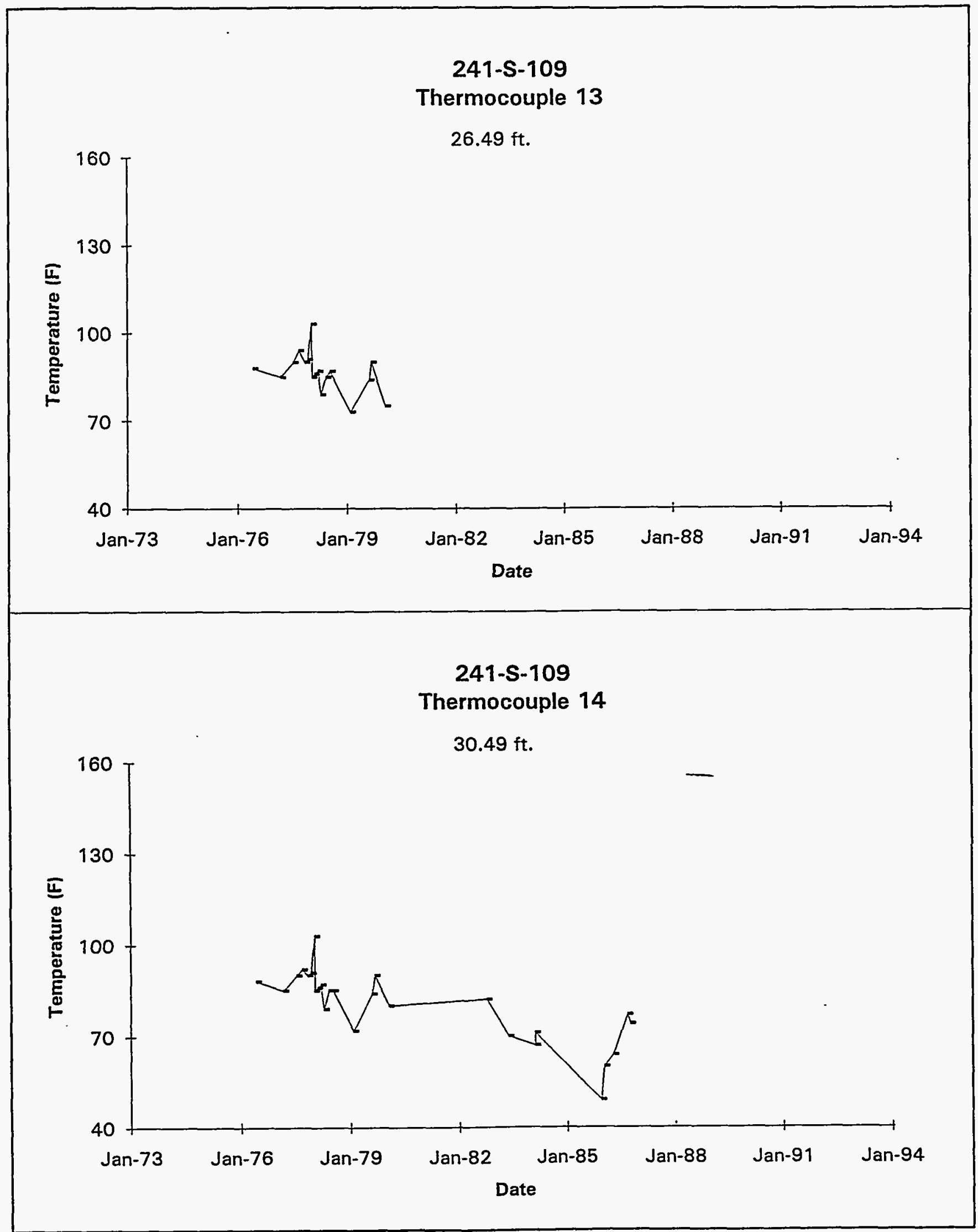

Data obtained from WHC Surveillance Analysis Computer System (SACS), February 24, 1994.

D-89 


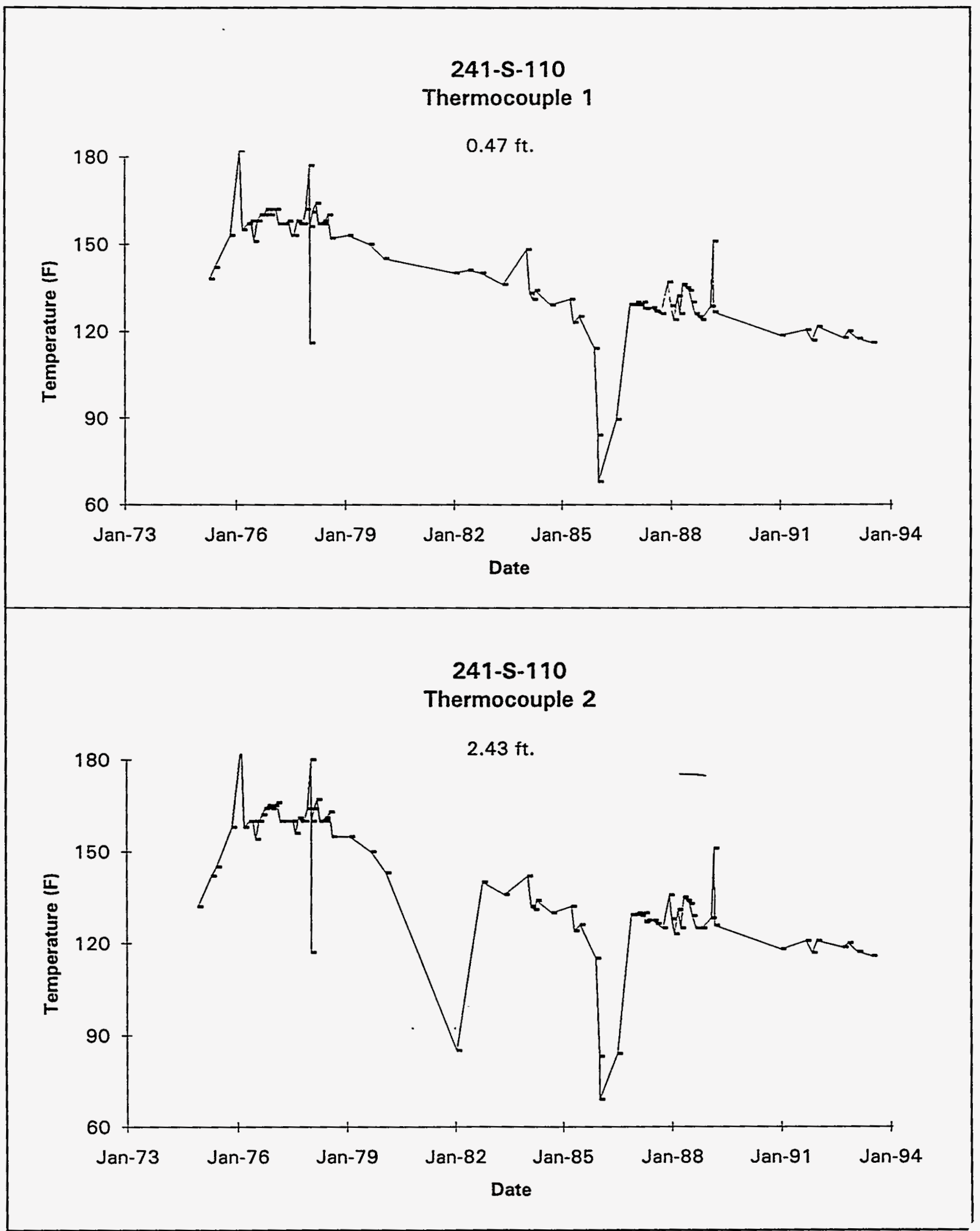

Data obtained from WHC Surveillance Analysis Computer System (SACS), February 24, 1994. 


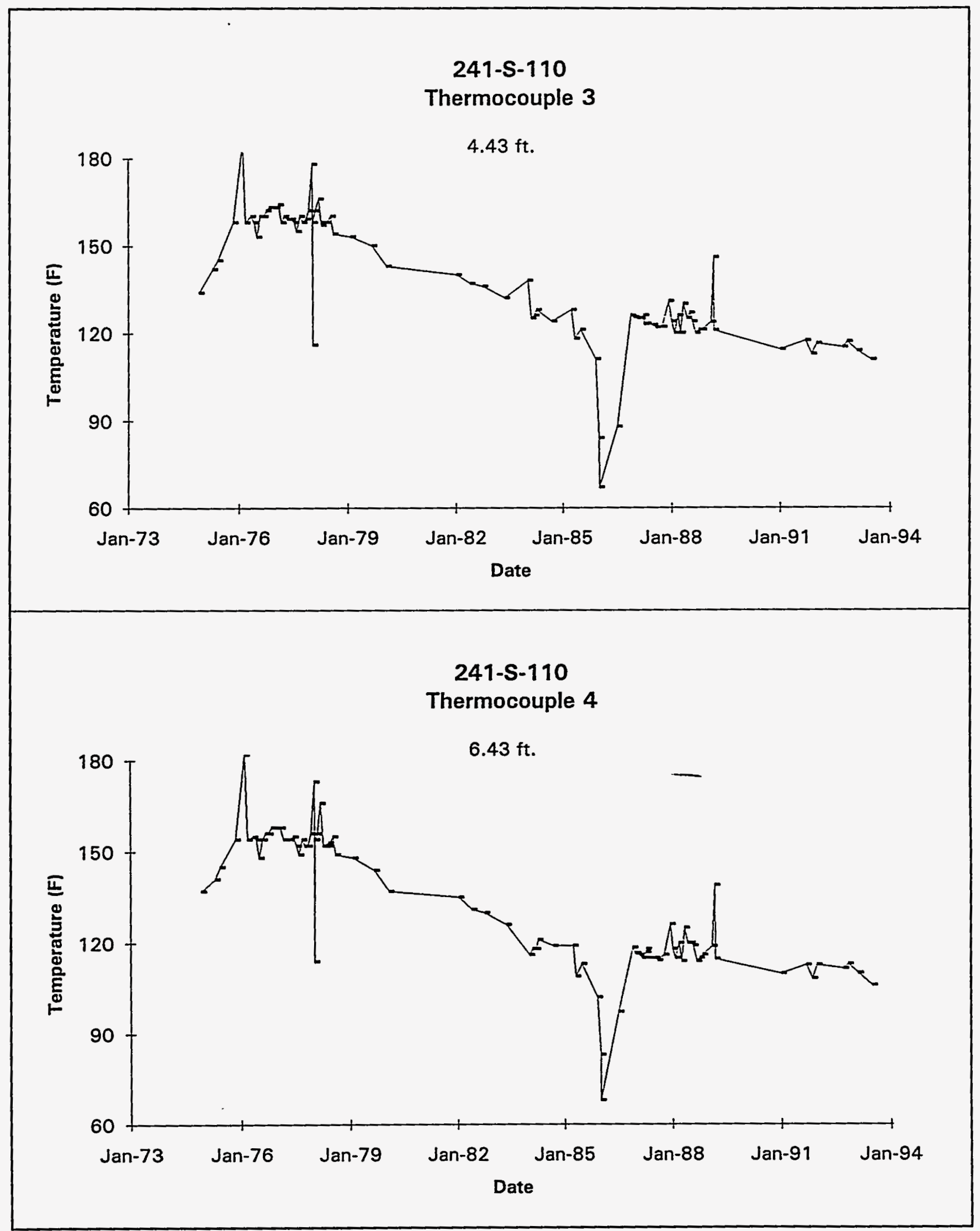

Data obtained from WHC Surveillance Analysis Computer System (SACS), February 24, 1994. 


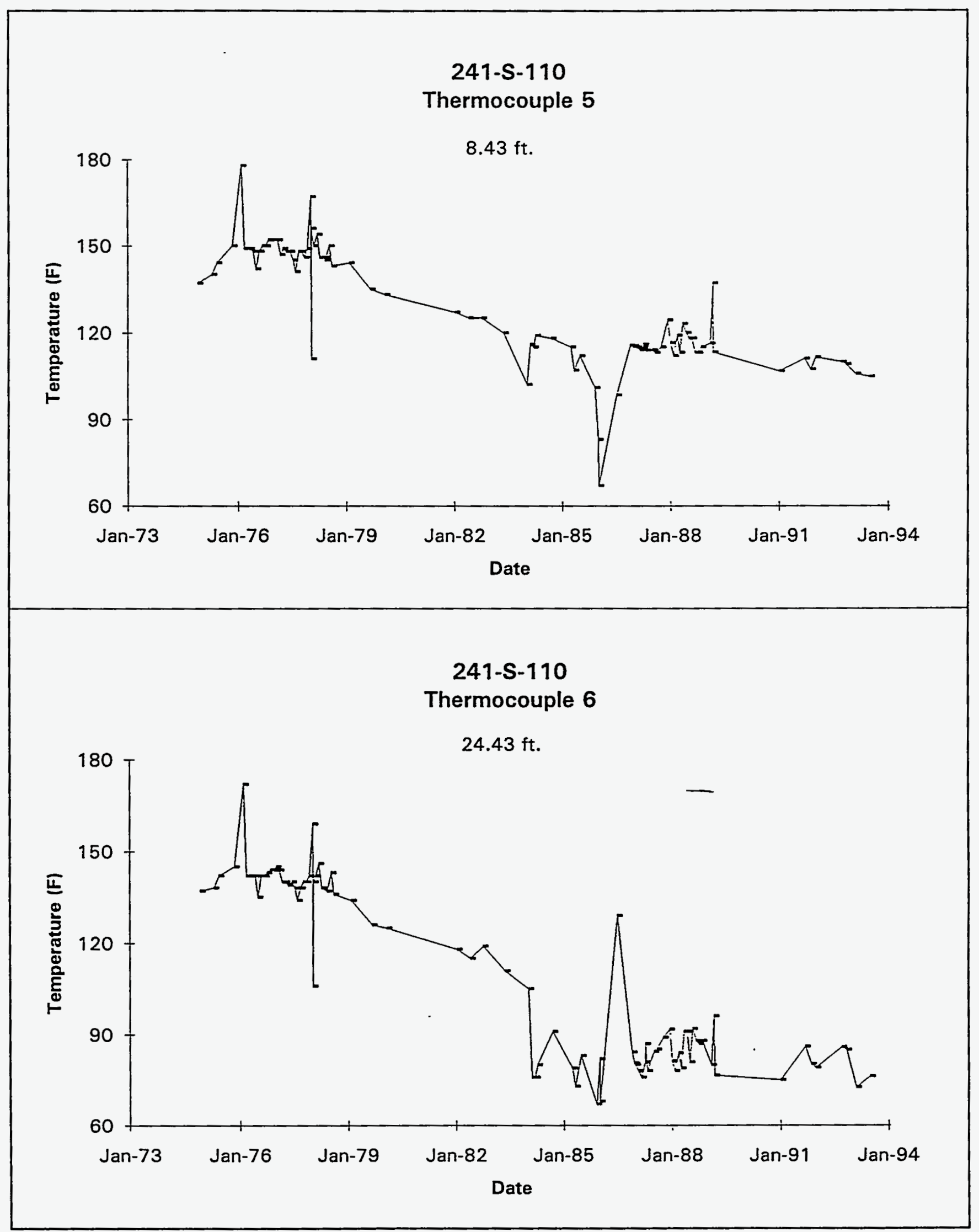

Data obtained from WHC Surveillance Analysis Computer System (SACS), February 24, 1994. 
241-S-110

Thermocouple 7

(Thermocouple Elevation Unavailable)

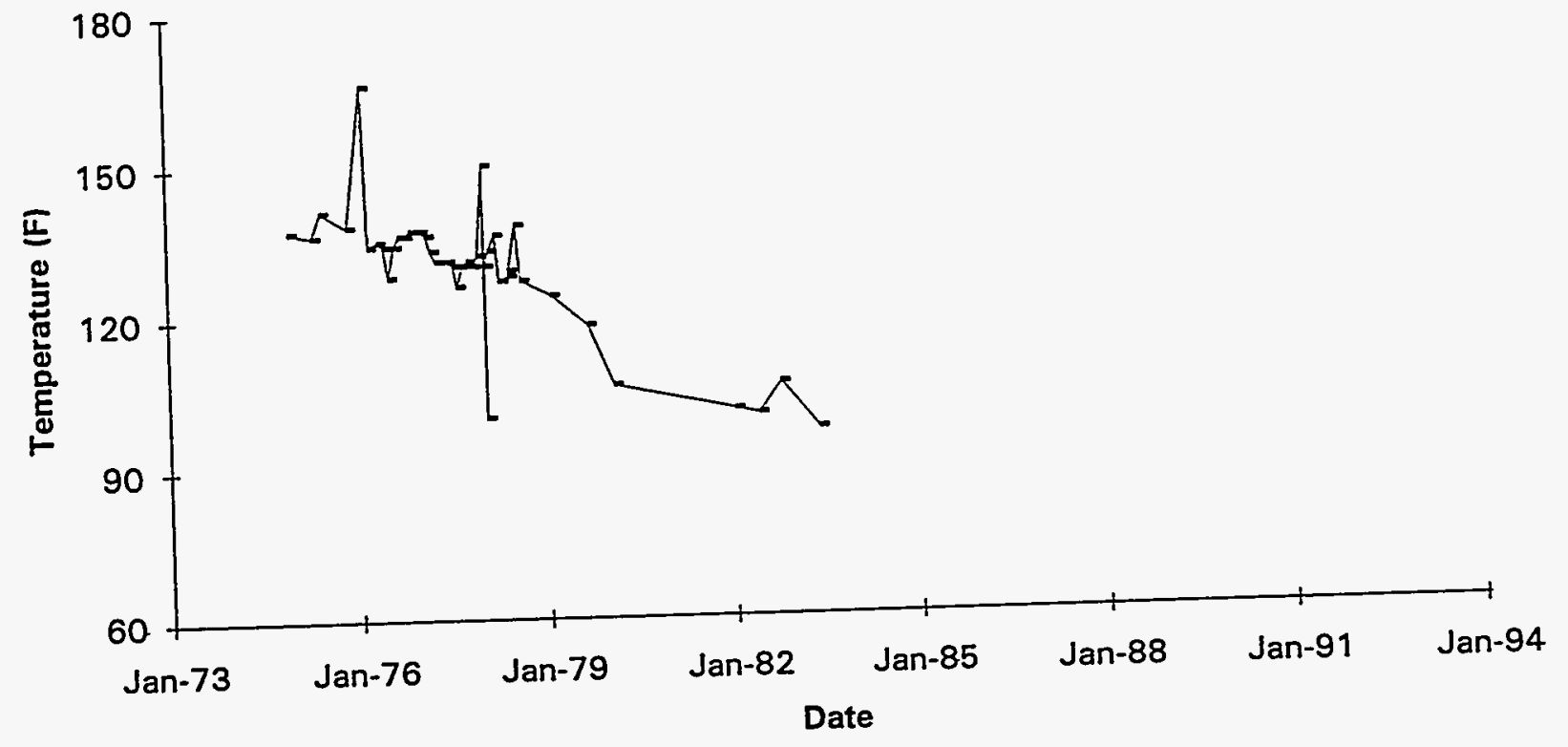

241-S-110

Thermocouple 8

(Thermocouple Elevation Unavailable)

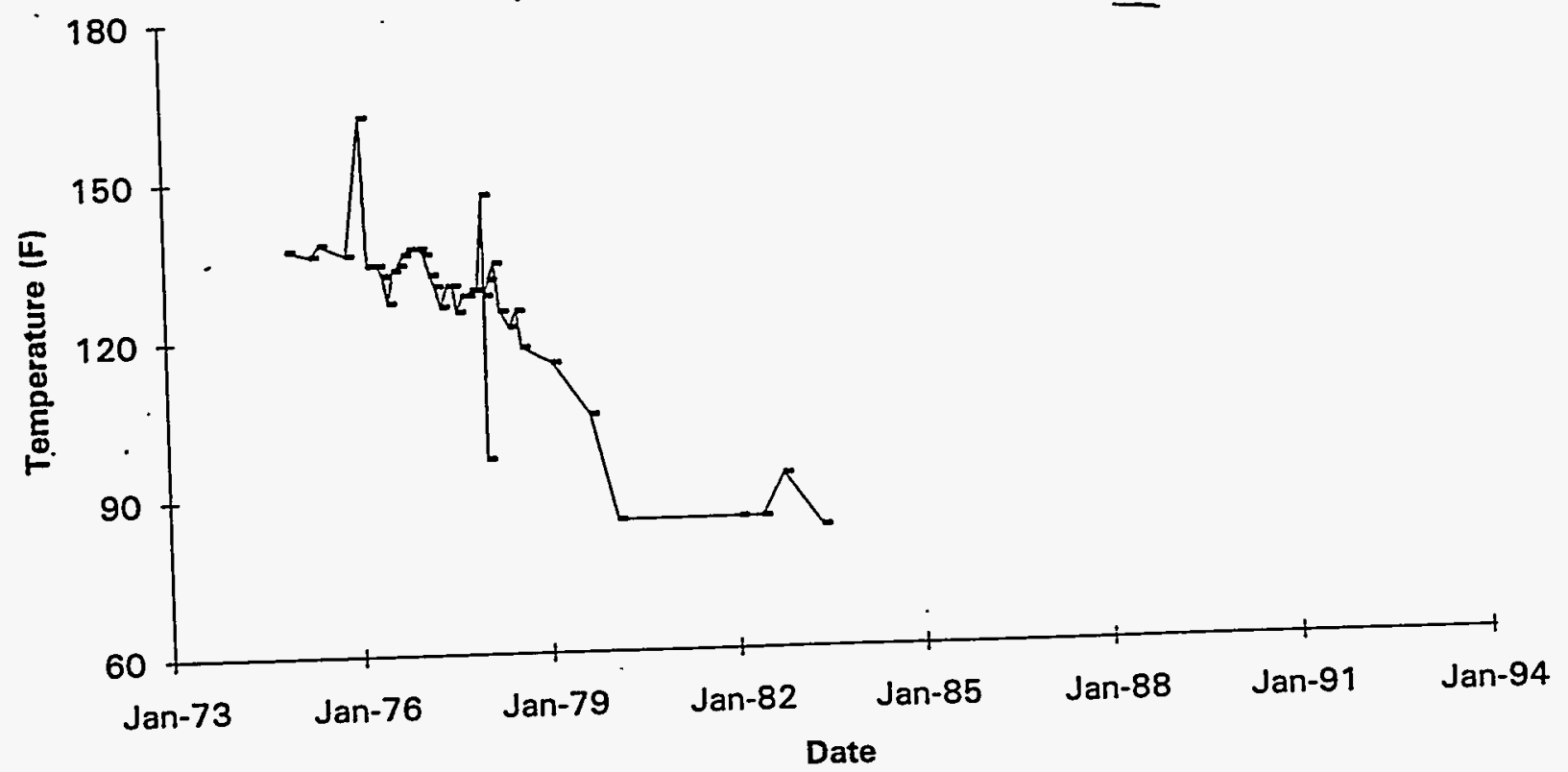

Data obtained from WHC Surveillance Analysis Computer System (SACS). February 24, 1994. 


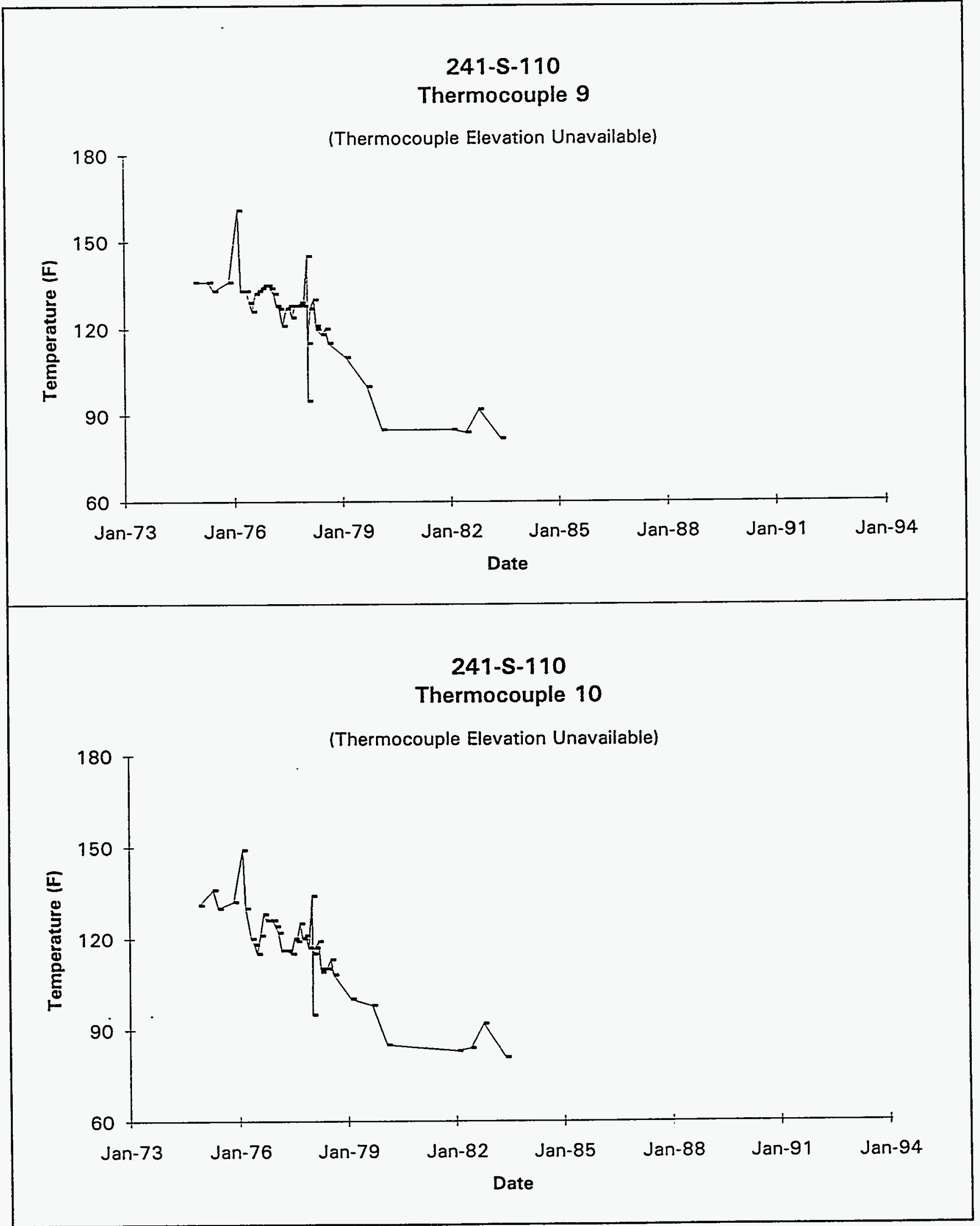

Data obtained from WHC Surveillance Analysis Computer System (SACS), February 24, 1994. 


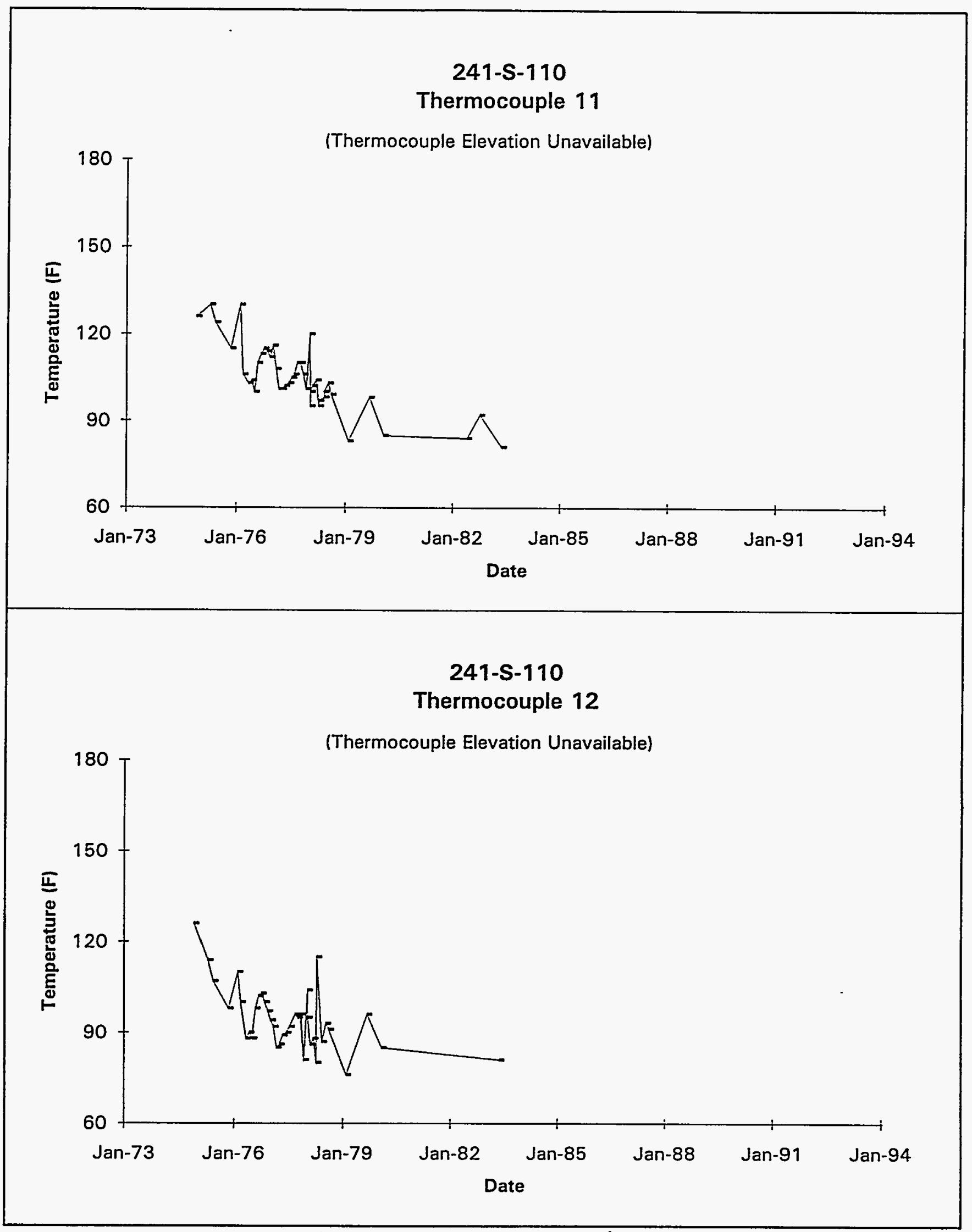

Data obtained from WHC Surveillance Analysis Computer System (SACS), February 24, 1994. 


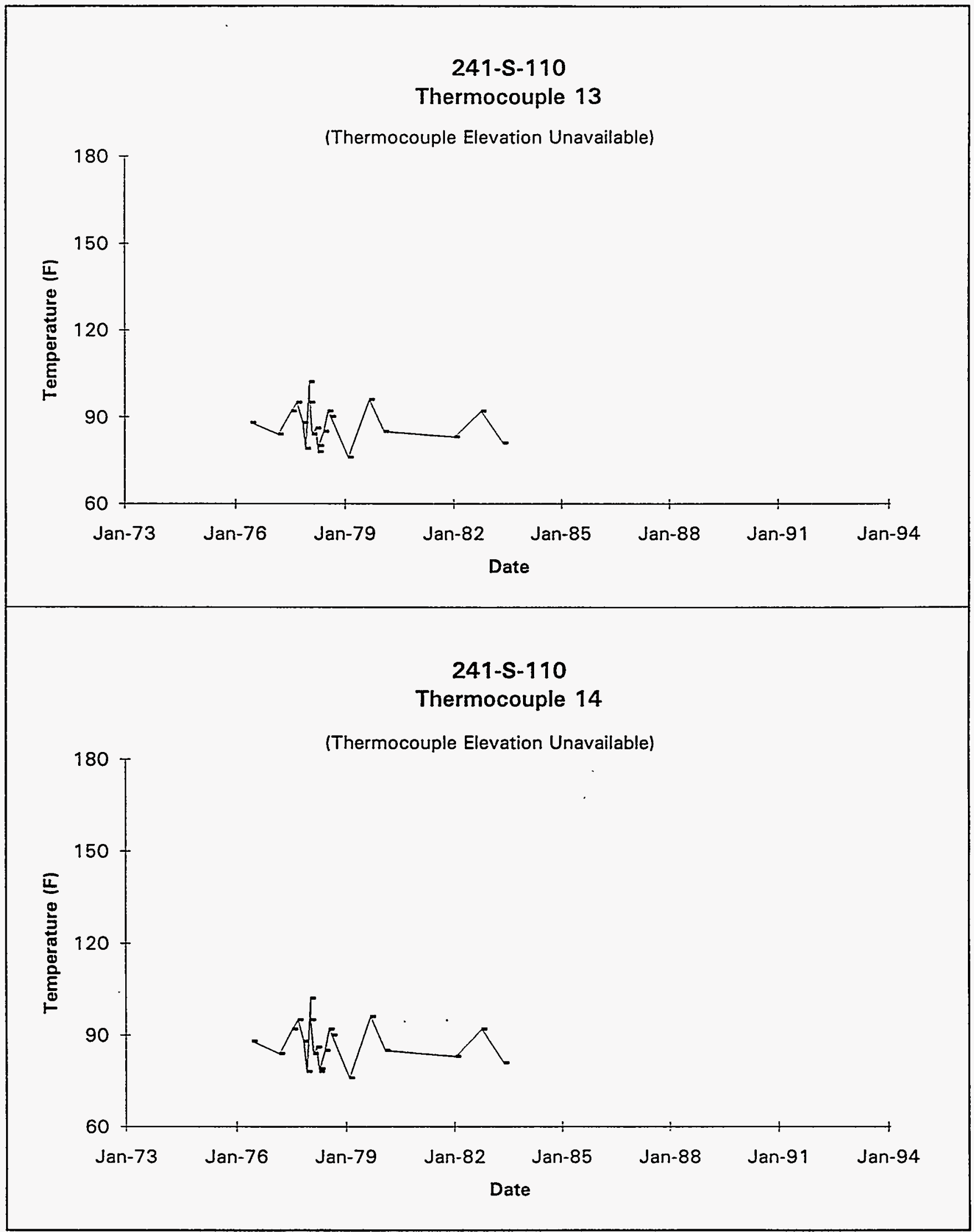

Data obtained from WHC Surveillance Analysis Computer System (SACS), February 24, 1994. 


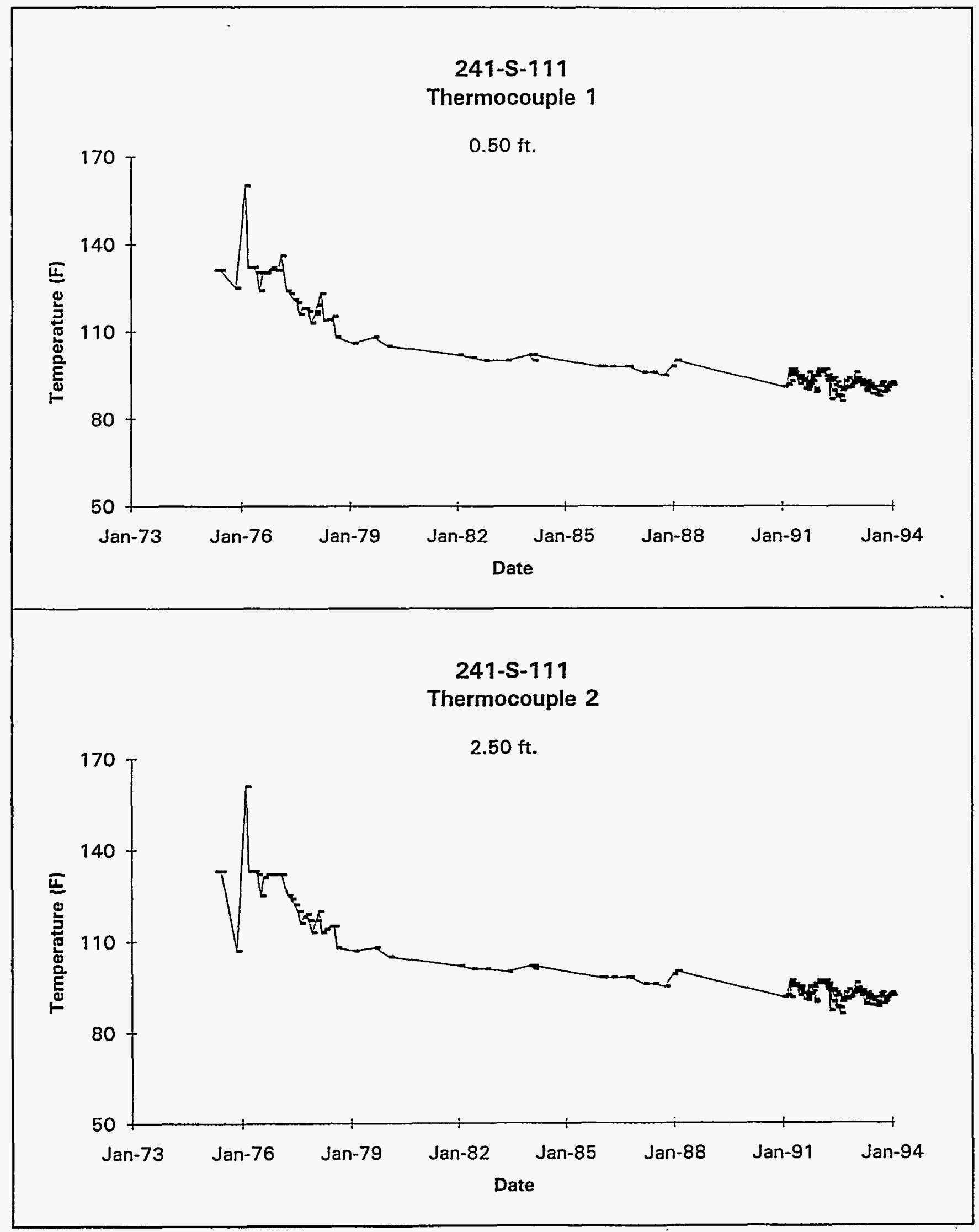

Data obtained from WHC Surveillance Analysis Computer System (SACS), February 24, 1994. 


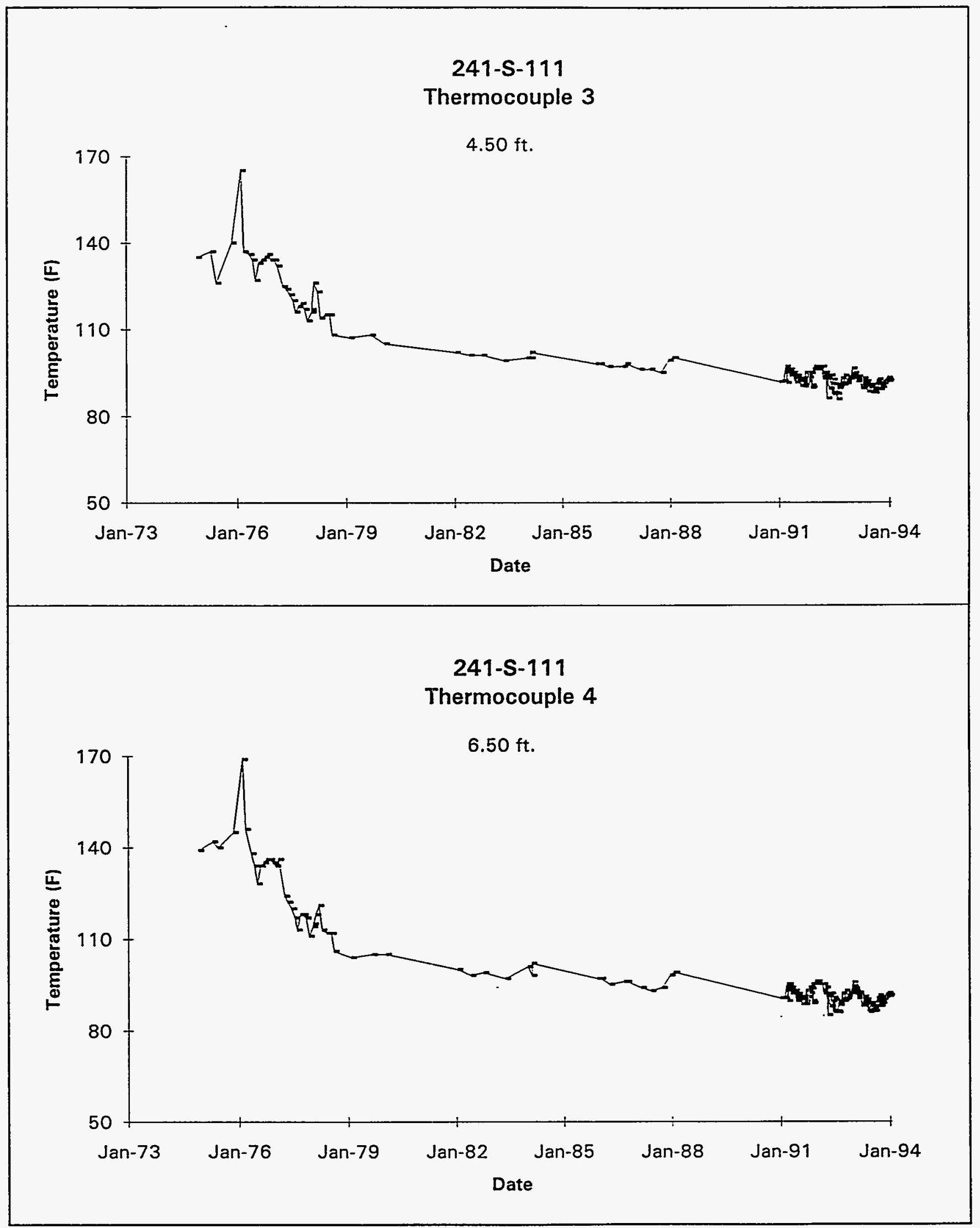

Data obtained from WHC Surveillance Analysis Computer System (SACS), February 24, 1994.

D-98 


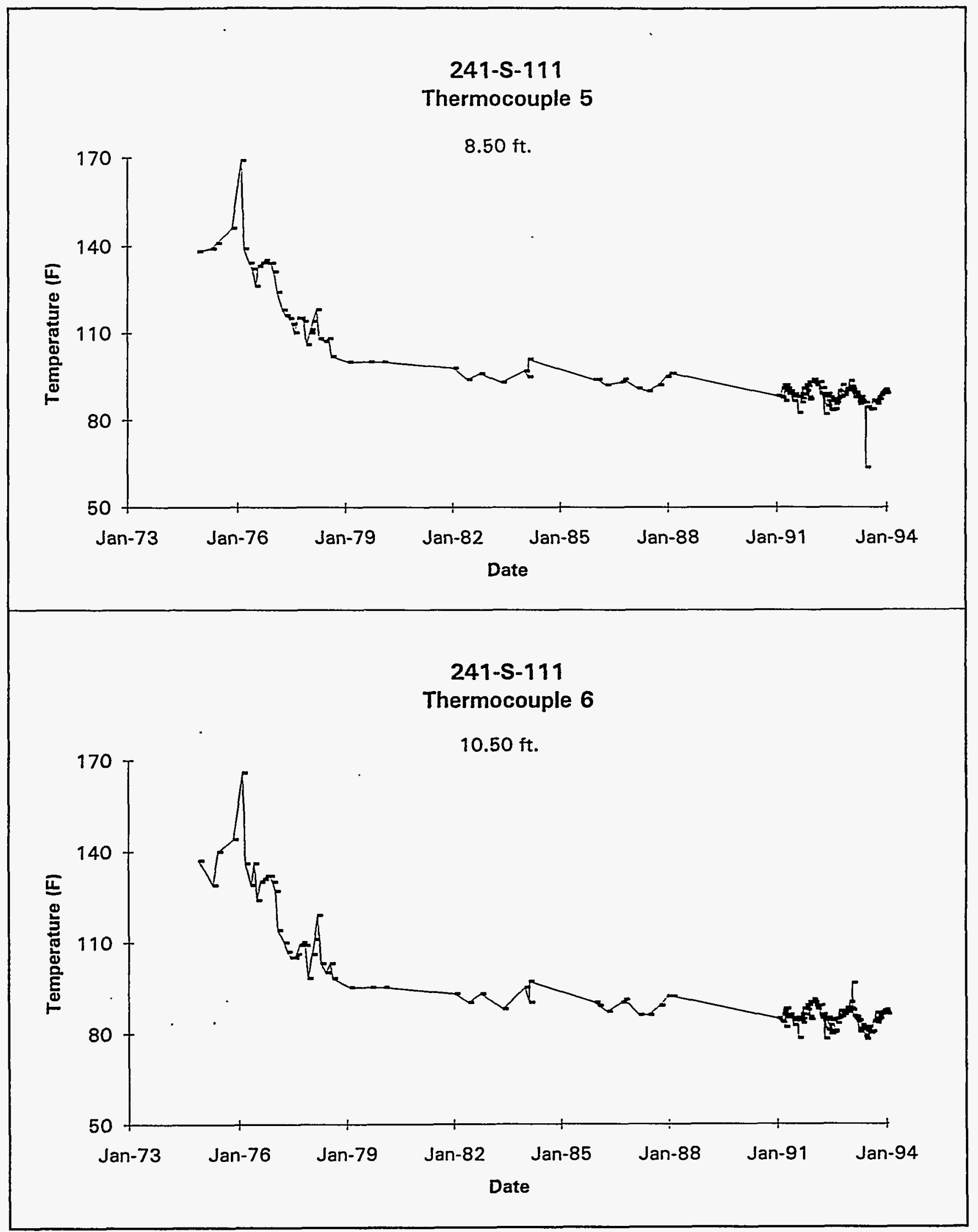

Data obtained from WHC Surveillance Analysis Computer System (SACS), February 24, 1994. 


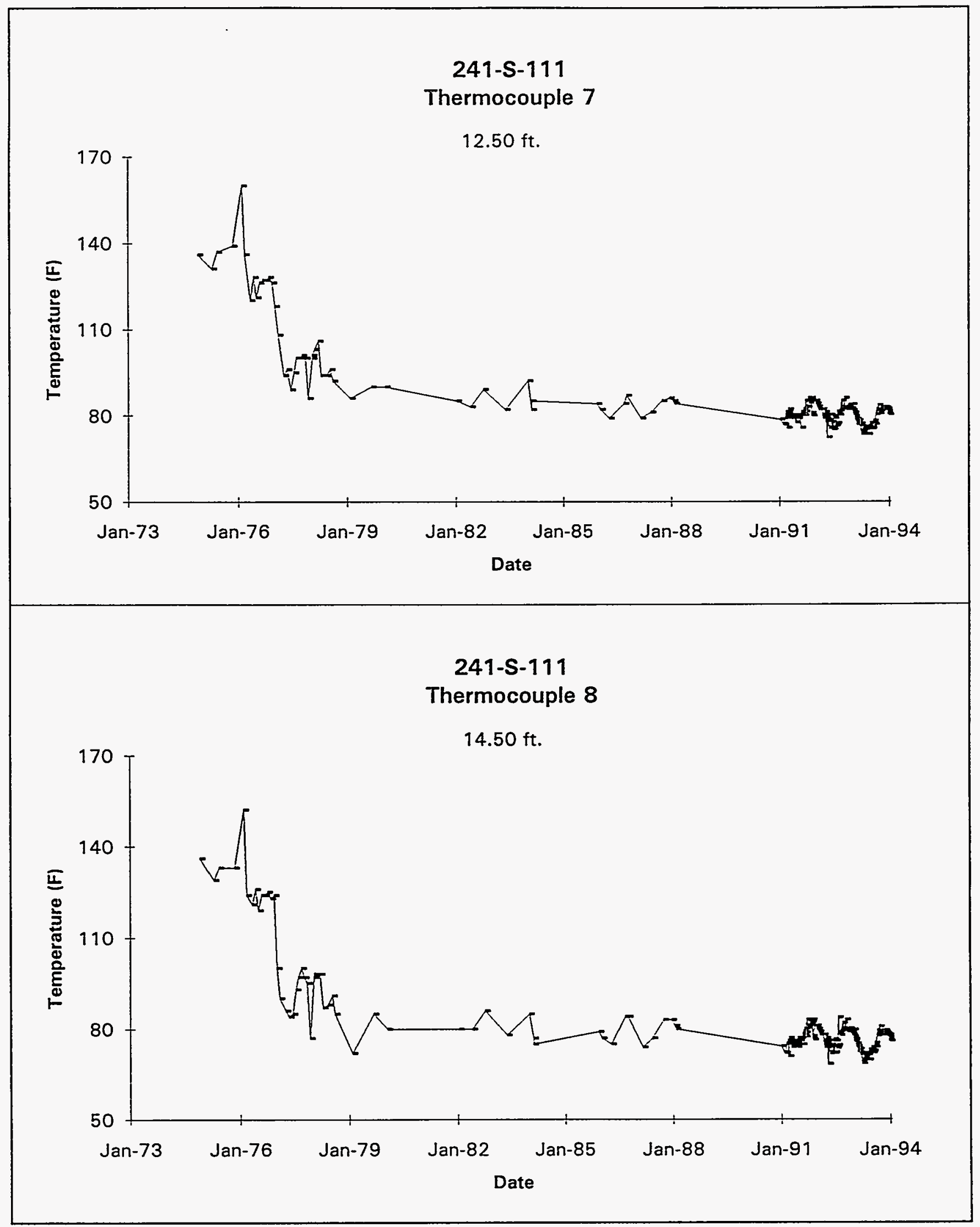

Data obtained from WHC Surveillance Analysis Computer System (SACS), February 24, 1994.

D-100 


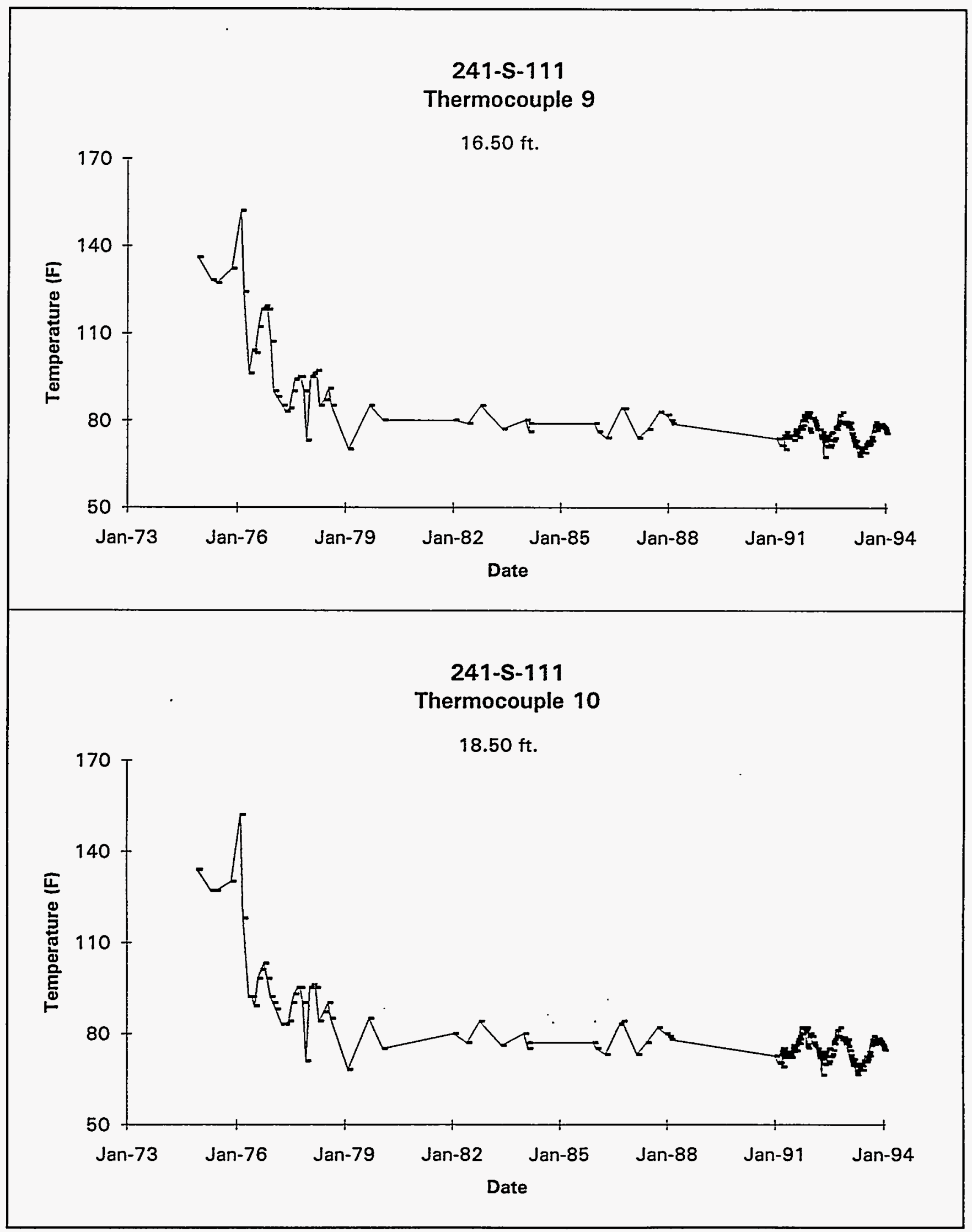

Data obtained from WHC Surveillance Analysis Computer System (SACS), February 24, 1994. 


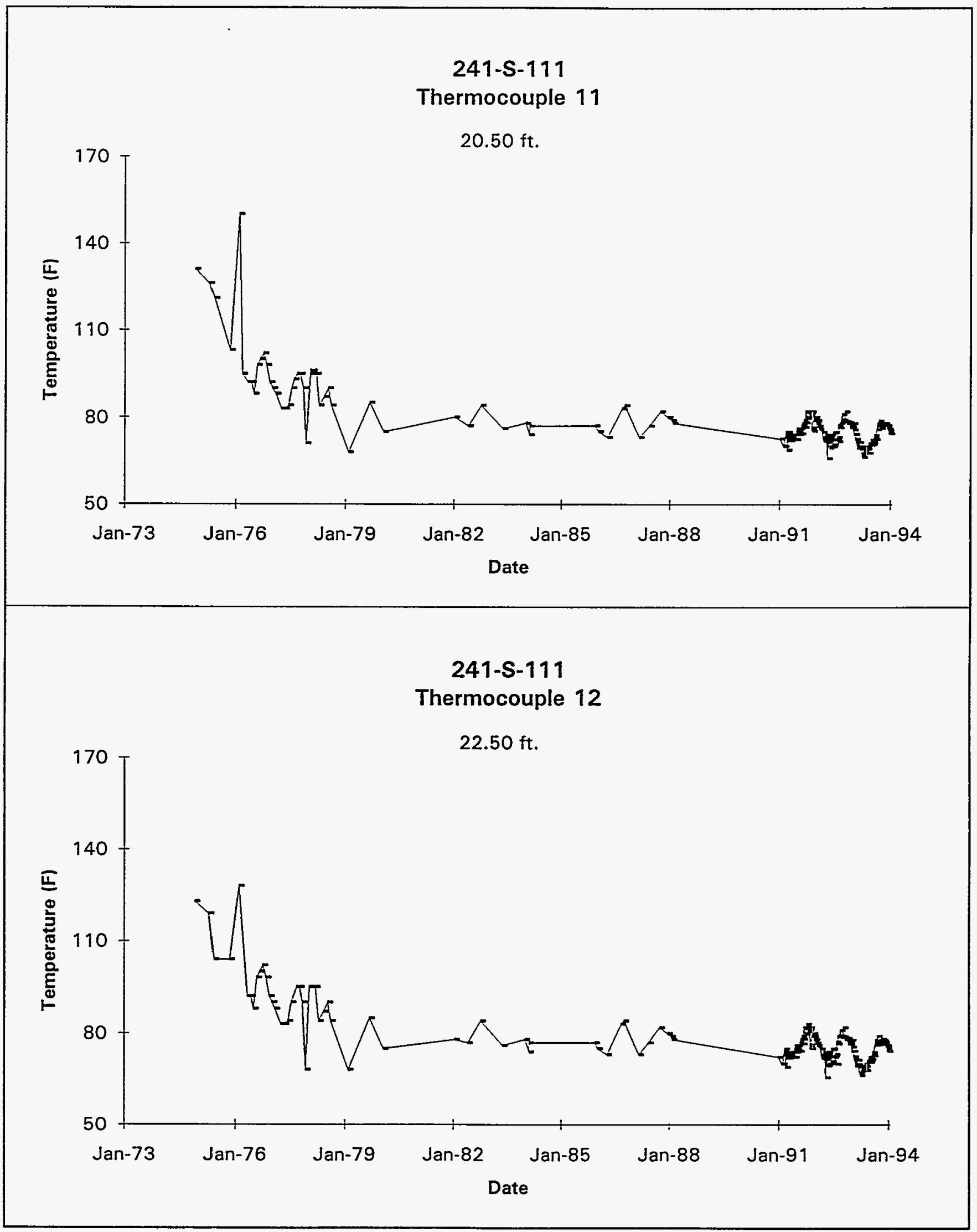

Data obtained from WHC Surveillance Analysis Computer System (SACS), February 24, 1994. 


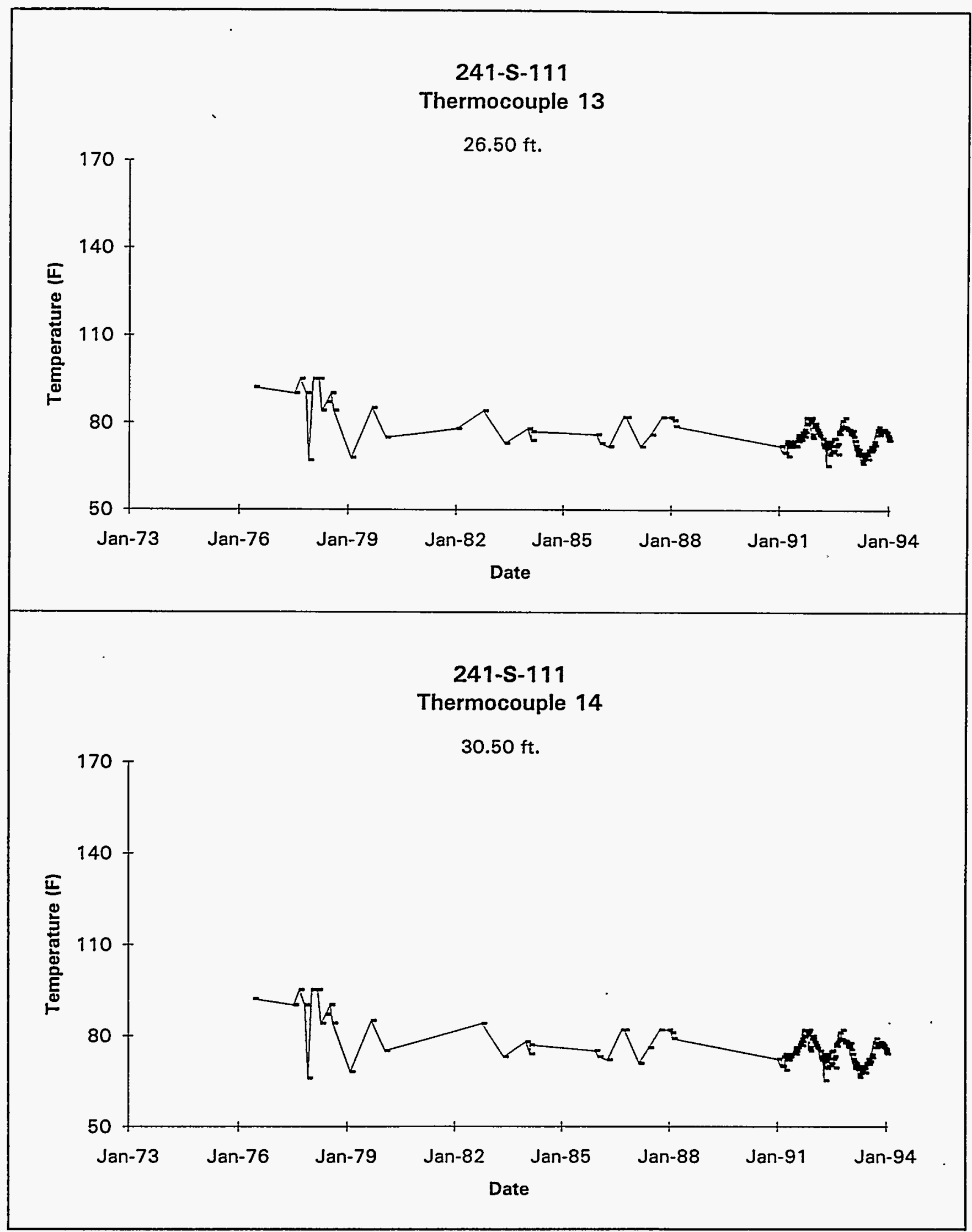

Data obtained from WHC Surveillance Analysis Computer System (SACS), February 24, 1994. 


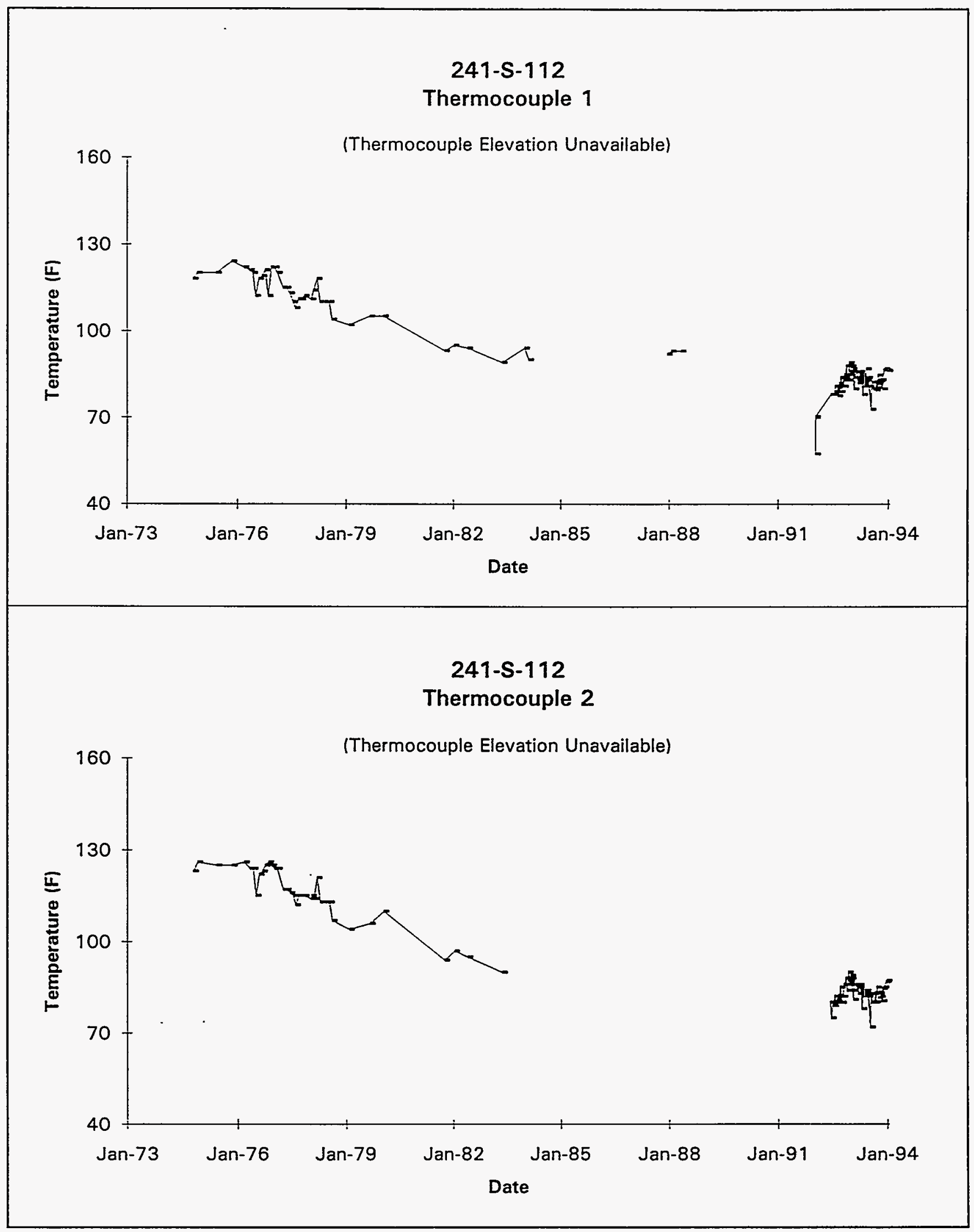

Data obtained from WHC Surveillance Analysis Computer System (SACS), February 24, 1994. 


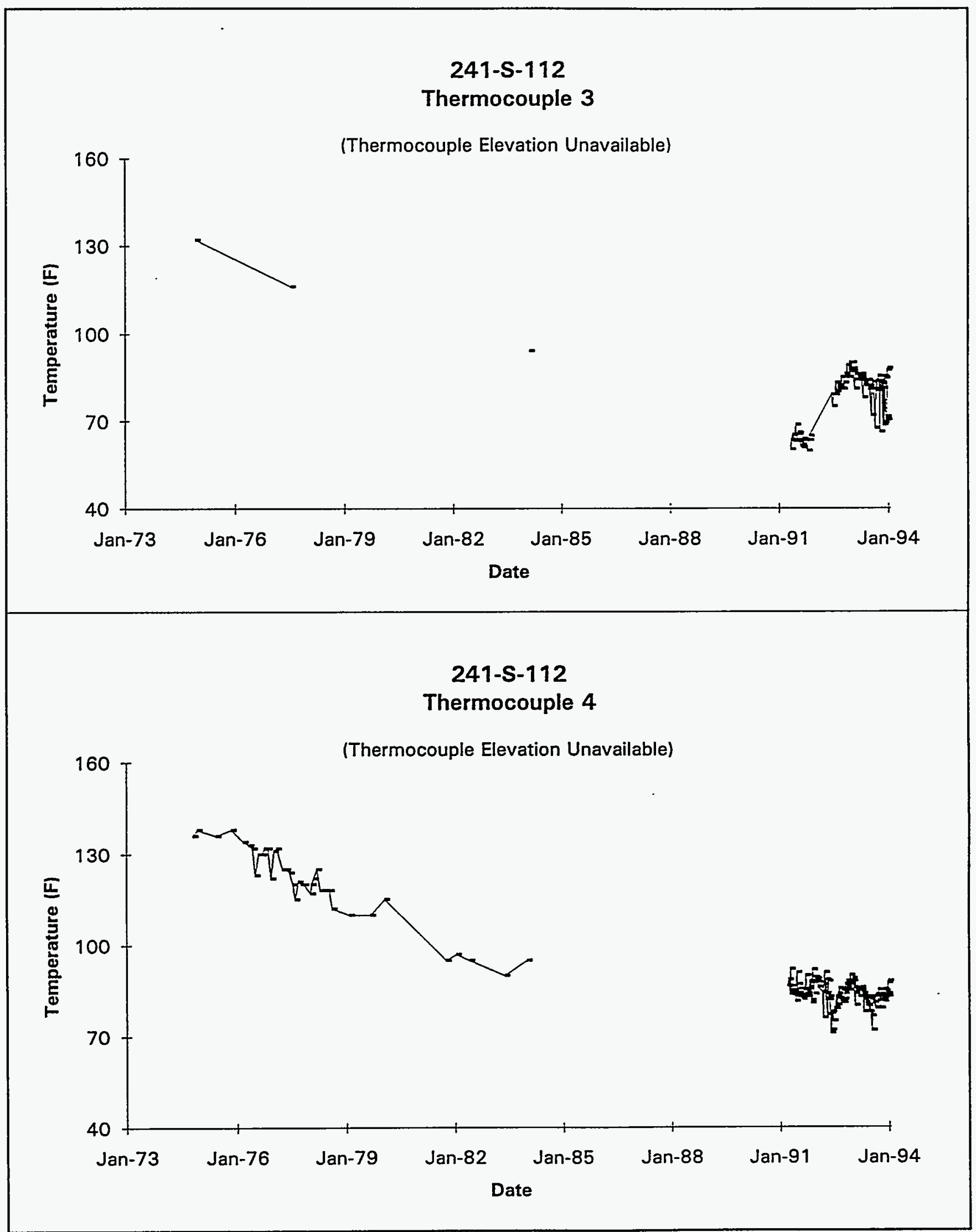

Data obtained from WHC Surveillance Analysis Computer System (SACS). February 24, 1994. 


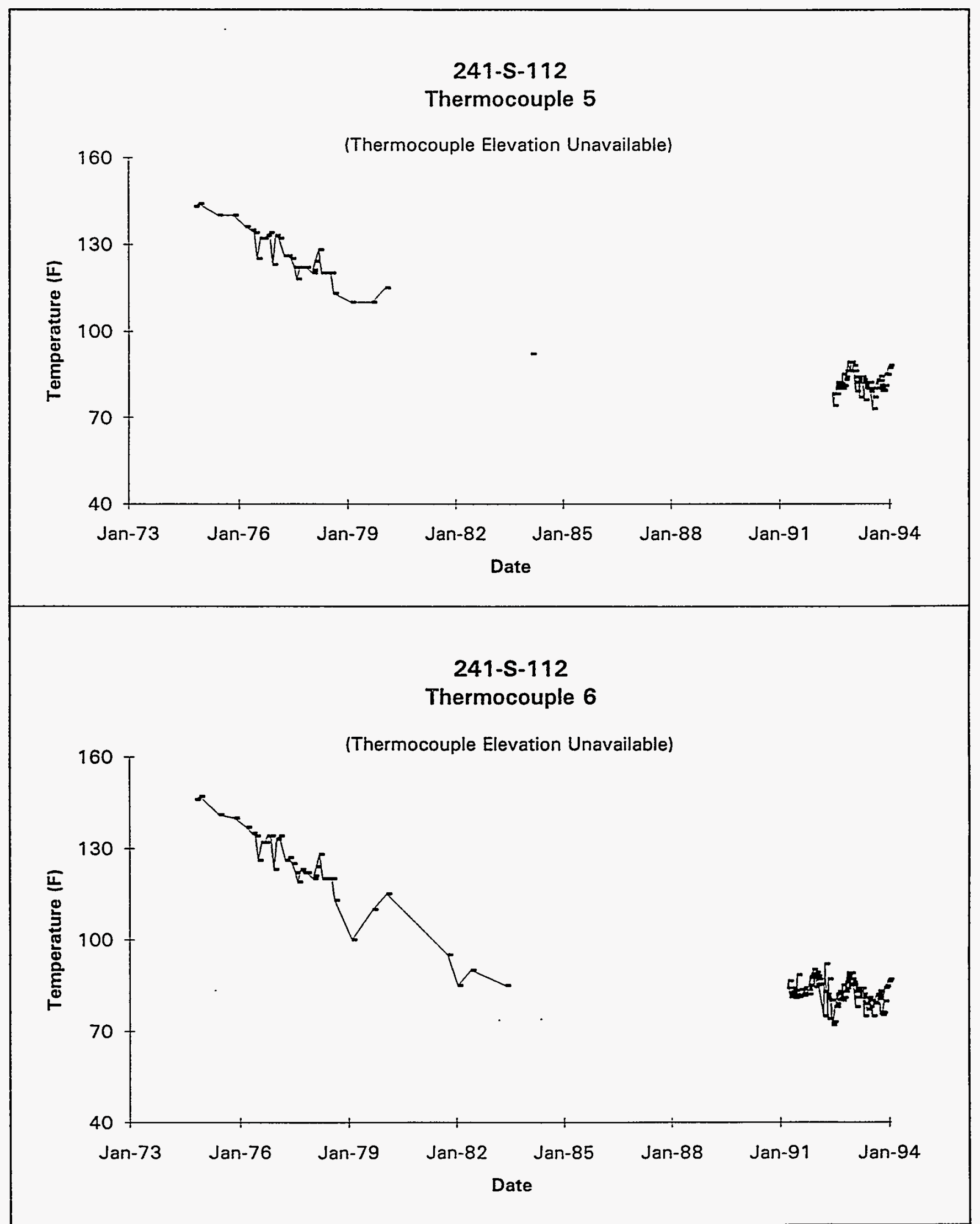

Data obtained from WHC Surveillance Analysis Computer System (SACS), February 24, 1994.

D-106 


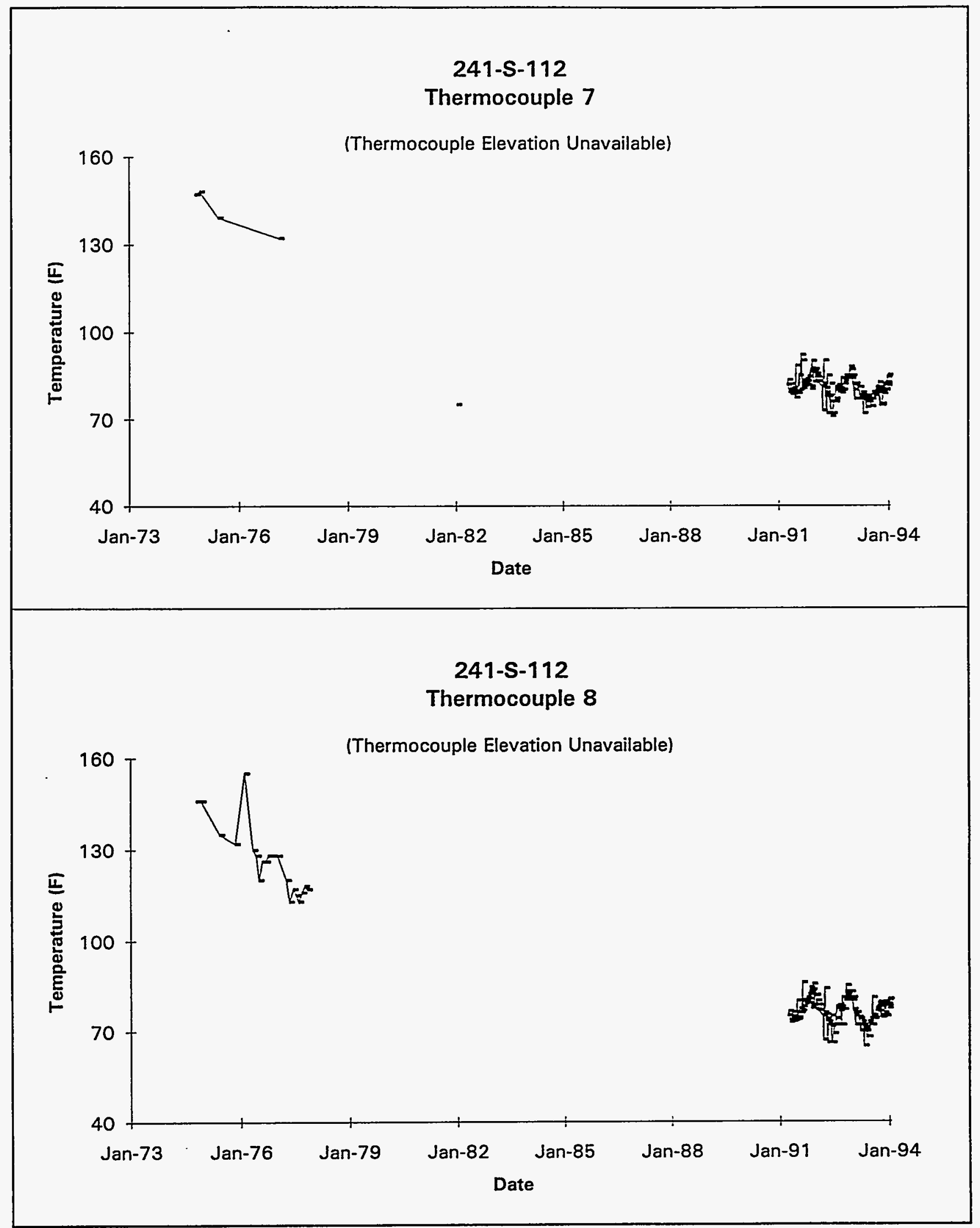

Data obtained from WHC Surveillance Analysis Computer System (SACS), February 24, 1994.

D-107 


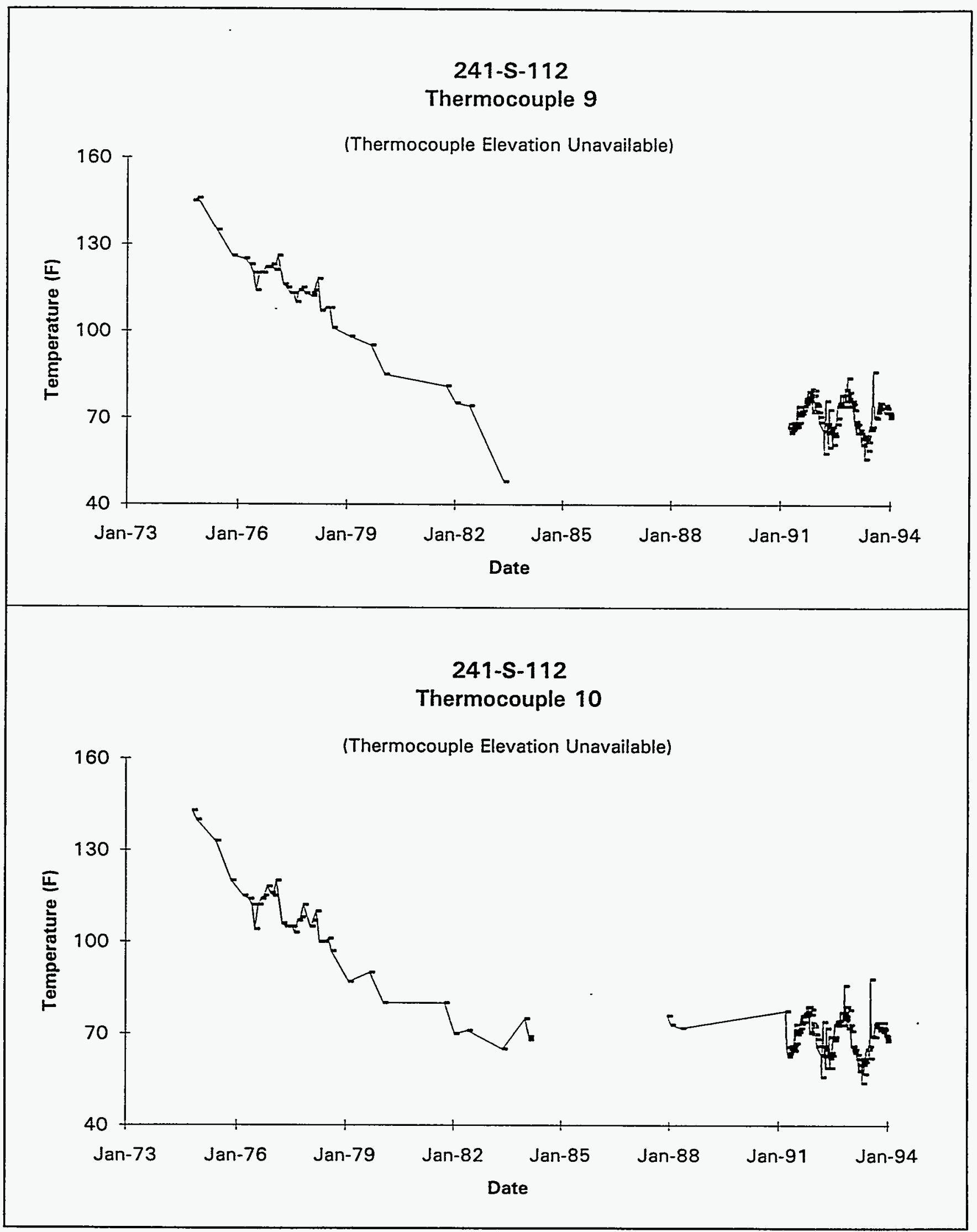

Data obtained from WHC Surveillance Analysis Computer System (SACS), February 24, 1994.

D-108 


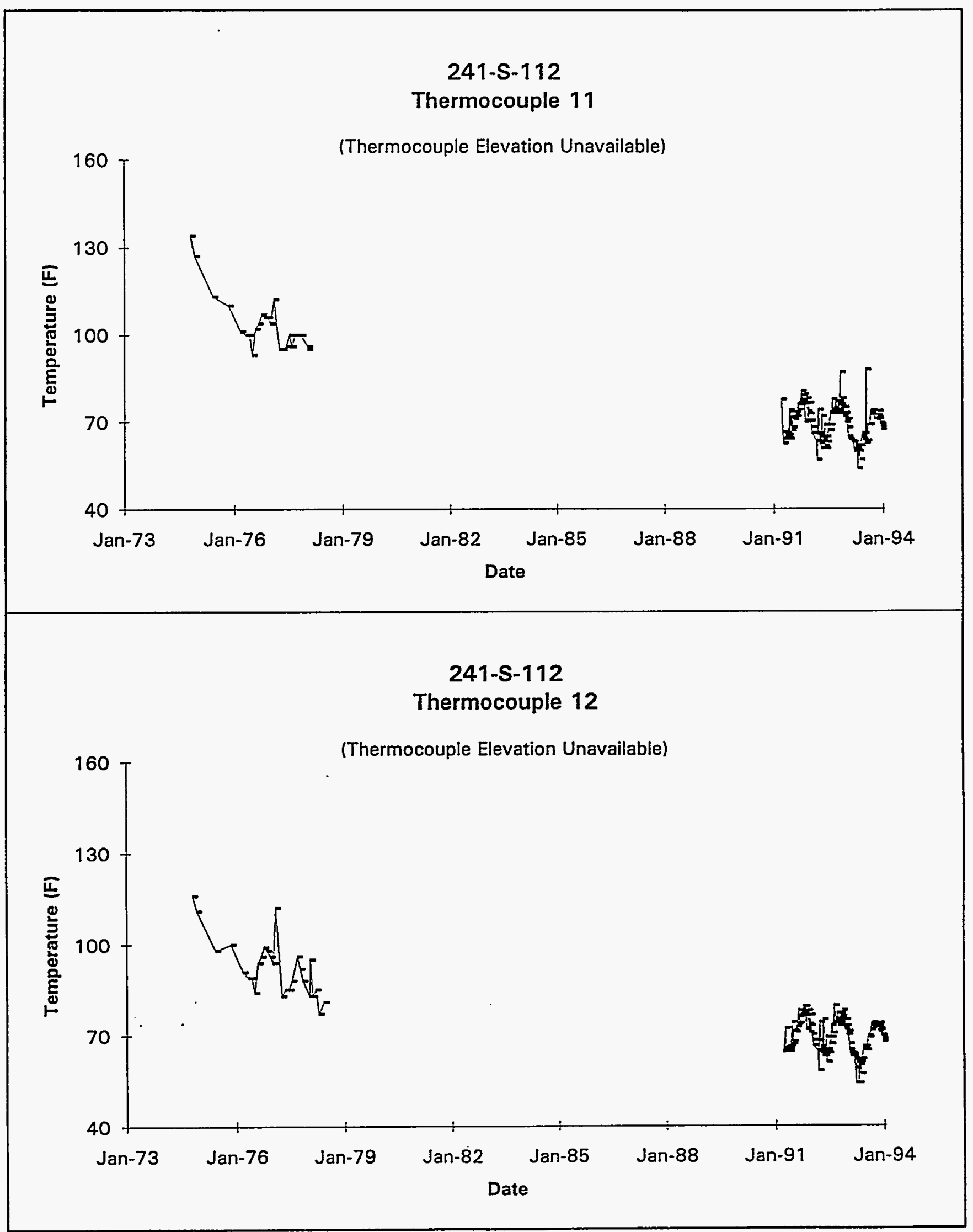

Data obtained from WHC Surveillance Analysis Computer System (SACS), February 24, 1994.

D-109 


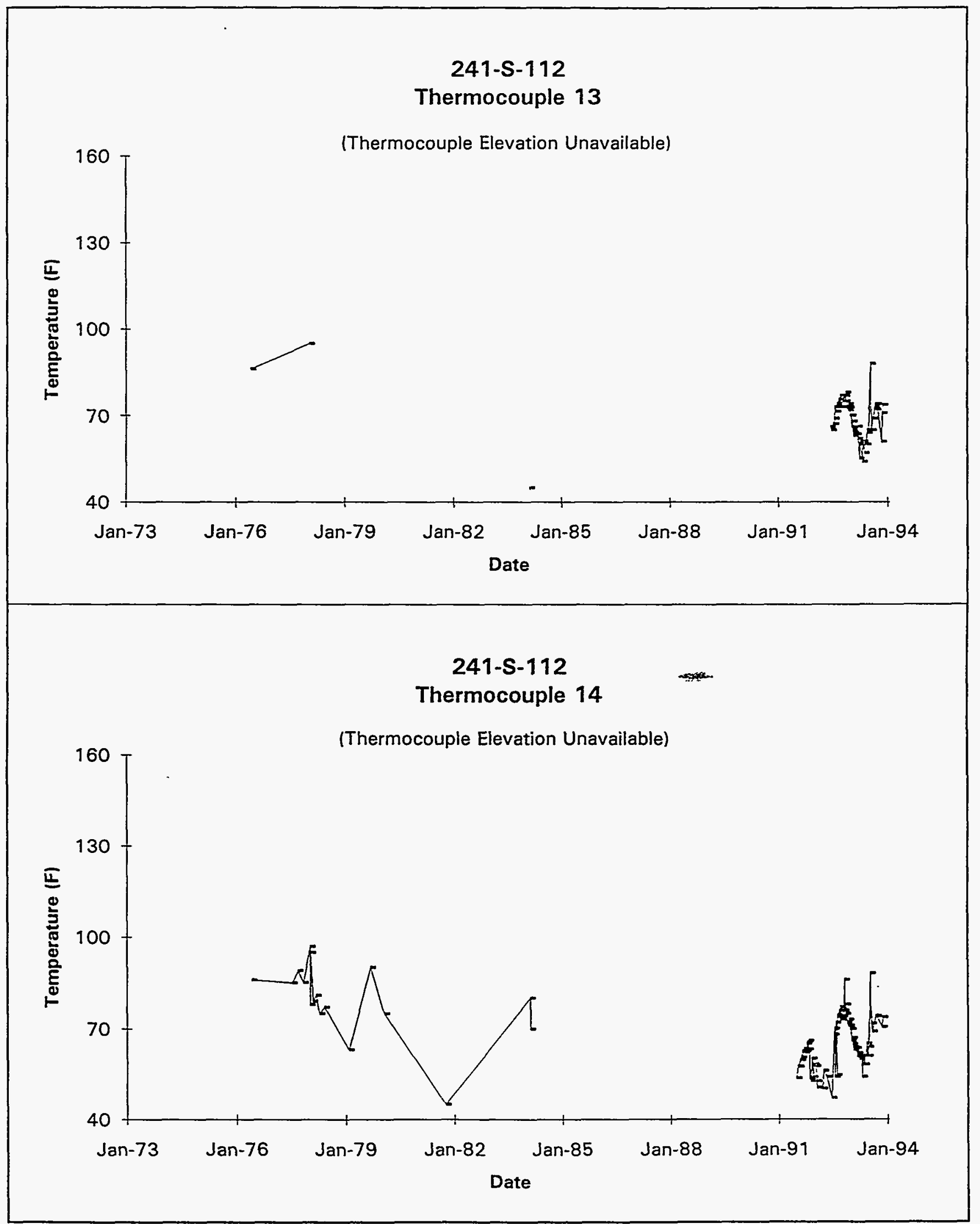

Data obtained from WHC Surveillance Analysis Computer System (SACS), February 24, 1994. 


\begin{tabular}{|c|c|c|c|c|c|c|c|}
\hline \multicolumn{8}{|c|}{ Historical Vapor Space Temperature Data } \\
\hline \multicolumn{8}{|c|}{ Tank 241-S-101 } \\
\hline & & & & & & & \\
\hline Date & Temp (C) & Temp (F) & Comments & Date & Temp (C) & Temp (F) & Comments \\
\hline $7 / 28 / 56$ & & 196 & & $11 / 13 / 56$ & & 191 & \\
\hline $8 / 6 / 56$ & & 191 & & $11 / 20 / 56$ & & 191 & \\
\hline $8 / 16 / 56$ & & 191 & & $11 / 25 / 56$ & & 191 & \\
\hline $8 / 22 / 56$ & & 191 & 8 & $11 / 29 / 56$ & & 191 & \\
\hline $8 / 26 / 56$ & & 191 & & $12 / 4 / 56$ & & 191 & \\
\hline $8 / 27 / 56$ & & 190 & 8 & $12 / 6 / 56$ & & 190 & \\
\hline $8 / 29 / 56$ & & 188 & & $12 / 7 / 56$ & & 189 & \\
\hline $8 / 30 / 56$ & & 187 & & $12 / 10 / 56$ & & 187 & \\
\hline $8 / 31 / 56$ & & 185 & & $12 / 11 / 56$ & & 187 & \\
\hline $9 / 1 / 56$ & & 184 & & $12 / 13 / 56$ & & 188 & \\
\hline $9 / 2 / 56$ & & 182 & & $12 / 14 / 56$ & & 191 & \\
\hline $9 / 3 / 56$ & & 183 & & $12 / 21 / 56$ & & 191 & \\
\hline $9 / 5 / 56$ & & 184 & & $12 / 28 / 56$ & & 191 & \\
\hline $9 / 6 / 56$ & & 185 & & $1 / 30 / 57$ & & 190 & \\
\hline $9 / 7 / 56$ & & 186 & & $2 / 28 / 57$ & & 185 & \\
\hline $9 / 8 / 56$ & & 188 & & $6 / 28 / 57$ & & 168 & \\
\hline $9 / 9 / 56$ & & 190 & & $8 / 1 / 57$ & & 172 & \\
\hline $9 / 11 / 56$ & & 191 & & $8 / 28 / 57$ & & 172 & \\
\hline $9 / 13 / 56$ & & 191 & 8 & $5 / 1 / 58$ & & 158 & \\
\hline $9 / 16 / 56$ & & 191 & & $7 / 28 / 58$ & & 157 & \\
\hline $9 / 25 / 56$ & & 191 & & $9 / 2 / 58$ & & 157 & \\
\hline $10 / 1 / 56$ & & 190 & 8 & $10 / 1 / 58$ & & 156 & \\
\hline $10 / 3 / 56$ & & 189 & 8 & $10 / 27 / 58$ & & 155 & \\
\hline $10 / 4 / 56$ & & 187 & & $11 / 26 / 58$ & & 151 & \\
\hline $10 / 6 / 56$ & & 185 & & $12 / 18 / 58$ & & 151 & \\
\hline $10 / 7 / 56$ & & 184 & & $1 / 15 / 59$ & & 151 & \\
\hline $10 / 8 / 56$ & & 182 & & $1 / 29 / 59$ & & 150 & \\
\hline $10 / 10 / 56$ & & 184 & & $2 / 20 / 59$ & & 149 & \\
\hline $10 / 11 / 56$ & & 186 & & $3 / 2 / 59$ & & 147 & \\
\hline $10 / 13 / 56$ & & 188 & & $3 / 18 / 59$ & & 143 & \\
\hline $10 / 14 / 56$ & & 190 & & $3 / 28 / 59$ & & 141 & \\
\hline $10 / 16 / 56$ & & 190 & & $4 / 11 / 59$ & & 143 & \\
\hline $10 / 18 / 56$ & & 191 & & $5 / 3 / 59$ & & 149 & \\
\hline $10 / 22 / 56$ & & 191 & & $12 / 2 / 59$ & & 134 & \\
\hline $10 / 29 / 56$ & & 191 & 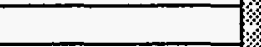 & $12 / 31 / 59$ & & 129 & \\
\hline $11 / 4 / 56$ & & 191 & & & & & \\
\hline
\end{tabular}


WHC-SD-WM-ER-323, Rev. 0

Historical Average Waste Temperature Data

\begin{tabular}{|c|c|c|c|c|c|c|c|c|}
\hline \multicolumn{9}{|c|}{ Historical Average Waste Temperature Data } \\
\hline \multicolumn{9}{|c|}{ Tank 241-S-101 } \\
\hline & & & & & & & & \\
\hline Date & Temp (C) & Temp (F) & Comments & & Date & Temp (C) & Temp (F) & Comments \\
\hline $7 / 18 / 53$ & & 122 & & & $9 / 20 / 53$ & & 175 & \\
\hline $7 / 31 / 53$ & & 164 & & & $9 / 27 / 53$ & & 183 & \\
\hline $8 / 14 / 53$ & & 183 & & & $9 / 30 / 53$ & & 231 & \\
\hline $8 / 23 / 53$ & & 145 & & & $10 / 11 / 53$ & & 199 & \\
\hline $8 / 25 / 53$ & & 224 & & & $10 / 20 / 53$ & & 269 & \\
\hline $9 / 2 / 53$ & & 223 & & & $10 / 25 / 53$ & & 215 & \\
\hline $9 / 4 / 53$ & & 158 & & & $10 / 31 / 53$ & & 301 & \\
\hline $9 / 9 / 53$ & & 228 & & & $12 / 6 / 53$ & & 214 & \\
\hline $9 / 12 / 53$ & & 166 & & m & $1 / 14 / 54$ & & 215 & \\
\hline
\end{tabular}

Data obtained from RHO-CD-1172 and HW-83906. 


\begin{tabular}{|c|c|c|c|c|c|c|c|}
\hline \multicolumn{8}{|c|}{ Historical High or Only Waste Temperature Data } \\
\hline \multicolumn{8}{|c|}{ Tank 241-S-101 } \\
\hline Date & Temp (C) & Temp (F) & Comments & Date & Temp (C) & Temo (F) & Comments \\
\hline $7 / 17 / 53$ & & & $\begin{array}{c}-60 \text { - suspect data, } \\
\text { low }\end{array}$ & $9 / 8 / 53$ & & 258 & \\
\hline $7 / 18 / 53$ & & 144 & & $9 / 9 / 53$ & & 246 & ASSUMPTION \\
\hline $7 / 19 / 53$ & & 75 & & 9/10/53 & & 213 & ASSUMPTION \\
\hline $7 / 20 / 53$ & & 100 & & $9 / 11 / 53$ & & 216 & ASSUMPTION \\
\hline $7 / 21 / 53$ & & 108 & & 9/13/53 & & 216 & ASSUMPTION \\
\hline $7 / 22 / 53$ & & 107 & & 9/14/53 & & 211 & ASSUMPTION \\
\hline $7 / 23 / 53$ & & 110 & & 9/15/53 & & 210 & ASSUMPTION \\
\hline $7 / 24 / 53$ & & 114 & & $9 / 16 / 53$ & & 218 & ASSUMPTION \\
\hline $7 / 25 / 53$ & & 116 & & $9 / 17 / 53$ & & 218 & ASSUMPTION \\
\hline $7 / 26 / 53$ & & 120 & & 9/18/53 & & 218 & ASSUMPTION \\
\hline $7 / 27 / 53$ & & 134 & & 9/19/53 & & 220 & ASSUMPTION \\
\hline $7 / 28 / 53$ & & 136 & & $9 / 20 / 53$ & & 220 & ASSUMPTION \\
\hline $7 / 29 / 53$ & & 145 & & $9 / 21 / 53$ & & 221 & ASSUMPTION \\
\hline $7 / 30 / 53$ & & 159 & & $9 / 22 / 53$ & & 223 & ASSUMPTION \\
\hline $7 / 31 / 53$ & & 164 & & $9 / 24 / 53$ & & 224 & ASSUMPTION \\
\hline $8 / 1 / 53$ & & 165 & & $9 / 25 / 53$ & & 224 & ASSUMPTION \\
\hline $8 / 2 / 53$ & & 168 & & $9 / 28 / 53$ & & 224 & ASSUMPTION \\
\hline $8 / 3 / 53$ & & 167 & & $9 / 29 / 53$ & & 190 & \\
\hline $8 / 4 / 53$ & & 167 & & $9 / 30 / 53$ & & 262 & ASSUMPTION \\
\hline $8 / 5 / 53$ & & 167 & & $10 / 1 / 53$ & & 200 & ASSUMPTION \\
\hline $8 / 6 / 53$ & & 165 & & $10 / 2 / 53$ & & 201 & ASSUMPTION \\
\hline $8 / 7 / 53$ & & 165 & & $10 / 3 / 53$ & & 232 & ASSUMPTION \\
\hline $8 / 8 / 53$ & & 164 & & $10 / 4 / 53$ & & 232 & ASSUMPTION \\
\hline $8 / 9 / 53$ & & 164 & & $10 / 5 / 53$ & & 233 & ASSUMPTION \\
\hline $8 / 10 / 53$ & & 166 & & $10 / 6 / 53$ & & 236 & ASSUMPTION \\
\hline $8 / 11 / 53$ & & 169 & & $10 / 7 / 53$ & & 237 & ASSUMPTION \\
\hline $8 / 12 / 53$ & & 172 & & $10 / 8 / 53$ & & 237 & ASSUMPTION \\
\hline $8 / 13 / 53$ & & 175 & & $10 / 9 / 53$ & & 237 & ASSUMPTION \\
\hline $8 / 14 / 53$ & & 188 & & $10 / 10 / 53$ & & 240 & ASSUMPTION \\
\hline $8 / 15 / 53$ & & 182 & & $10 / 11 / 53$ & & 242 & ASSUMPTION \\
\hline $8 / 16 / 53$ & & 186 & & $10 / 12 / 53$ & & 242 & ASSUMPTION \\
\hline $8 / 17 / 53$ & & 186 & & $10 / 14 / 53$ & & 246 & ASSUMPTION \\
\hline $8 / 18 / 53$ & & 992 & & $10 / 15 / 53$ & & 243 & ASSUMPTION \\
\hline $8 / 19 / 53$ & & 204 & & $10 / 16 / 53$ & & 246 & ASSUMPTION \\
\hline $8 / 20 / 53$ & & 206 & & $10 / 17 / 53$ & & 246 & ASSUMPTION \\
\hline $8 / 21 / 53$ & & 212 & & $10 / 18 / 53$ & & 250 & ASSUMPTION \\
\hline $8 / 22 / 53$ & & 219 & & $10 / 19 / 53$ & & 235 & ASSUMPTION \\
\hline $8 / 23 / 53$ & & 221 & & $10 / 20 / 53$ & & 286 & ASSUMPTION \\
\hline $8 / 24 / 53$ & & 228 & & $10 / 21 / 53$ & & 254 & ASSUMPTION \\
\hline $8 / 25 / 53$ & & 233 & & $10 / 22 / 53$ & & 210 & \\
\hline $8 / 26 / 53$ & & 238 & & $10 / 23 / 53$ & & 253 & ASSUMPTION \\
\hline $8 / 27 / 53$ & & 240 & & $10 / 24 / 53$ & & 252 & ASSUMPTION \\
\hline $8 / 28 / 53$ & & 242 & & $10 / 25 / 53$ & & 258 & ASSUMPTION \\
\hline $8 / 29 / 53$ & & 242 & & $10 / 26 / 53$ & & 257 & ASSUMPTION \\
\hline $8 / 30 / 53$ & & 246 & & $10 / 27 / 53$ & & 257 & ASSUMPTION \\
\hline $8 / 31 / 53$ & & 246 & & $10 / 28 / 53$ & & 257 & ASSUMPTION \\
\hline $9 / 1 / 53$ & & 199 & ASSUMPTION & $10 / 29 / 53$ & & 257 & ASSUMPTION \\
\hline $9 / 2 / 53$ & & 241 & ASSUMPTION & $10 / 30 / 53$ & & 257 & ASSUMPTION \\
\hline $9 / 3 / 53$ & & 205 & ASSUMPTION & $10 / 31 / 53$ & & 302 & \\
\hline $9 / 4 / 53$ & & 205 & ASSUMPTION & $11 / 2 / 53$ & & 257 & ASSUMPTION \\
\hline $9 / 5 / 53$ & & 206 & ASSUMPTION & $11 / 3 / 53$ & & 257 & ASSUMPTION \\
\hline $9 / 6 / 53$ & $\cdot$ & 200 & ASSUMPTION & $11 / 4 / 53$ & & 257 & ASSUMPTION \\
\hline $9 / 7 / 53$ & & 252 & & $11 / 5 / 53$ & & 258 & ASSUMPTION \\
\hline
\end{tabular}

Data obtained from RHO-CD-1172 and HW-83906. 


\begin{tabular}{|c|c|c|c|c|c|c|c|}
\hline \multicolumn{8}{|c|}{ Historical High or Only Waste Temperature Data } \\
\hline \multicolumn{8}{|c|}{ Tank 241-S-101 } \\
\hline & & & & & & & \\
\hline$\frac{\text { Date }}{11 / 6 / 53}$ & Temp (C) & $\frac{\text { Temp (F) }}{258}$ & $\frac{\text { Comments }}{\text { ASSUMPTION }}$ & $\frac{\text { Date }}{1 / 23 / 54}$ & Temp (c) & $\frac{1 \mathrm{emp}(\mathrm{r})}{300}$ & $\frac{\text { Comments }}{\text { NOT SPECIFIC }}$ \\
\hline $11 / 10 / 53$ & & 258 & ASSUMPTION & $1 / 24 / 54$ & & 300 & \\
\hline $11 / 11 / 53$ & & 259 & ASSUMPTION & $1 / 25 / 54$ & & 300 & NOT SPECIFIC \\
\hline $11 / 12 / 53$ & & 259 & ASSUMPTION & $1 / 26 / 54$ & & 300 & NOT SPECIFIC \\
\hline $11 / 13 / 53$ & & 257 & ASSUMPTION & $1 / 27 / 54$ & & 300 & NOT SPECIFIC \\
\hline $11 / 15 / 53$ & & 259 & ASSUMPTION & $1 / 28 / 54$ & & 300 & NOT SPECIFIC \\
\hline $11 / 16 / 53$ & & 259 & ASSUMPTION & $1 / 29 / 54$ & & 300 & NOT SPECIFIC \\
\hline $11 / 17 / 53$ & & 259 & ASSUMPTION & $2 / 1 / 54$ & & 300 & NOT SPECIFIC \\
\hline $11 / 18 / 53$ & & 259 & ASSUMPTION & $2 / 2 / 54$ & & 300 & NOT SPECIFIC \\
\hline $11 / 19 / 53$ & & 259 & ASSUMPTION & $2 / 3 / 54$ & & 300 & NOT SPECIFIC \\
\hline $11 / 20 / 53$ & & 258 & ASSUMPTION & $2 / 4 / 54$ & & 300 & NOT SPECIFIC \\
\hline $11 / 22 / 53$ & & 265 & ASSUMPTION & $2 / 5 / 54$ & & 300 & NOT SPECIFIC \\
\hline $11 / 23 / 53$ & & 258 & ASSUMPTION & $2 / 6 / 54$ & & 300 & NOT SPECIFIC \\
\hline $91 / 24 / 53$ & & 258 & ASSUMPTION & $2 / 7 / 54$ & & 300 & NOT SPECIFIC \\
\hline $11 / 25 / 53$ & & 258 & ASSUMPTION & $2 / 8 / 54$ & & 300 & NOT SPECIFIC \\
\hline $11 / 27 / 53$ & & 258 & ASSUMPTION & $2 / 9 / 54$ & & 300 & NOT SPECIFIC \\
\hline $11 / 29 / 53$ & & 263 & ASSUMPTION & $2 / 10 / 54$ & & 300 & NOT SPECIFIC \\
\hline $11 / 30 / 53$ & & 258 & ASSUMPTION & $2 / 11 / 54$ & & 300 & NOT SPECIFIC \\
\hline $12 / 1 / 53$ & & 258 & ASSUMPTION & $2 / 12 / 54$ & & 300 & \\
\hline $12 / 2 / 53$ & & 258 & ASSUMPTION & $2 / 13 / 54$ & & 240 & NOT SPECIFIC \\
\hline $12 / 3 / 53$ & & 258 & ASSUMPTION & $2 / 15 / 54$ & & 240 & NOT SPECIFIC \\
\hline $12 / 4 / 53$ & & 258 & ASSUMPTION & $2 / 16 / 54$ & & 240 & NOT SPECIFIC \\
\hline $12 / 5 / 53$ & & 258 & ASSUMPTION & $2 / 17 / 54$ & & 240 & NOT SPECIFIC \\
\hline $12 / 6 / 53$ & & 258 & ASSUMPTION & $2 / 18 / 54$ & & 240 & NOT SPECIFIC \\
\hline $12 / 7 / 53$ & & 258 & ASSUMPTION & $2 / 19 / 54$ & & 240 & NOT SPECIFIC \\
\hline $12 / 8 / 53$ & & 258 & ASSUMPTION & $2 / 20 / 54$ & & 240 & NOT SPECIFIC \\
\hline $12 / 14 / 53$ & & 258 & ASSUMPTION & $2 / 22 / 54$ & & 260 & ASSUMPTION \\
\hline $92 / 17 / 53$ & & 258 & ASSUMPTION & $2 / 23 / 54$ & & 260 & ASSUMPTION \\
\hline $12 / 18 / 53$ & & 258 & ASSUMPTION & $2 / 25 / 54$ & & 260 & ASSUMPTION \\
\hline $12 / 21 / 53$ & & 258 & ASSUMPTION & $2 / 26 / 54$ & & 300 & \\
\hline $12 / 22 / 53$ & & 258 & ASSUMPTION & $3 / 1 / 54$ & & 300 & \\
\hline $12 / 23 / 53$ & & 258 & ASSUMPTION & $3 / 2 / 54$ & & 300 & \\
\hline $12 / 24 / 53$ & & 270 & ASSUMPTION & $3 / 3 / 54$ & & 300 & \\
\hline $12 / 25 / 53$ & - & 260 & ASSUMPTION & $3 / 4 / 54$ & & 300 & NOT SPECIFIC \\
\hline $12 / 26 / 53$ & & 260 & ASSUMPTION & $3 / 5 / 54$ & & 300 & NOT SPECIFIC \\
\hline $12 / 28 / 53$ & & 300 & NOT SPECIFIC & $3 / 6 / 54$ & & 300 & NOT SPECIFIC \\
\hline $12 / 29 / 53$ & & 300 & NOT SPECIFIC & $3 / 7 / 54$ & & 300 & NOT SPECIFIC \\
\hline $12 / 30 / 53$ & & 300 & NOT SPECIFIC & $3 / 8 / 54$ & & 300 & NOT SPECIFIC \\
\hline $12 / 31 / 53$ & & 300 & NOT SPECIFIC & $3 / 9 / 54$ & & 300 & NOT SPECIFIC \\
\hline $1 / 4 / 54$ & & 300 & NOT SPECIFIC & $3 / 10 / 54$ & & 300 & NOT SPECIFIC \\
\hline $1 / 5 / 54$ & & 300 & NOT SPECIFIC & $3 / 12 / 54$ & & 300 & NOT SPECIFIC \\
\hline $1 / 6 / 54$ & & 300 & NOT SPECIFIC & $3 / 14 / 54$ & & 300 & \\
\hline $9 / 7 / 54$ & & 300 & NOT SPECIFIC & $3 / 15 / 54$ & & 300 & NOT SPECIFIC \\
\hline $9 / 8 / 54$ & & 300 & NOT SPECIFIC & $3 / 16 / 54$ & & 300 & NOT SPECIFIC \\
\hline $1 / 11 / 54$ & & 300 & NOT SPECIFIC & $3 / 17 / 54$ & & 300 & NOT SPECIFIC \\
\hline $1 / 12 / 54$ & & 300 & NOT SPECIFIC & $3 / 18 / 54$ & & 300 & NOT SPECIFIC \\
\hline $1 / 13 / 54$ & & 300 & NOT SPECIFIC & $3 / 19 / 54$ & & 300 & NOT SPECIFIC \\
\hline $1 / 14 / 54$ & & 300 & NOT SPECIFIC & $3 / 20 / 54$ & & 260 & ASSUMPTION \\
\hline $1 / 15 / 54$ & & 300 & NOT SPECIFIC & $3 / 21 / 54$ & & 260 & ASSUMPTION \\
\hline $1 / 18 / 54$ & & 300 & NOT SPECIFIC & $3 / 22 / 54$ & & 300 & NOT SPECIFIC \\
\hline $1 / 19 / 54$ & & 300 & NOT SPECIFIC & $3 / 24 / 54$ & & 222 & \\
\hline $1 / 20 / 54$ & & 300 & NOT SPECIFIC & $4 / 1 / 54$ & & 300 & \\
\hline $1 / 21 / 54$ & & 300 & NOT SPECIFIC & $4 / 5 / 54$ & & 300 & \\
\hline $1 / 22 / 54$ & & 300 & NOT SPECIFIC & $4 / 6 / 54$ & & 300 & \\
\hline
\end{tabular}

Data obtained from RHO-CD-1172 and HW-83906. 


\begin{tabular}{|c|c|c|c|c|c|c|c|}
\hline \multicolumn{8}{|c|}{ Historical High or Only Waste Temperature Data } \\
\hline & & & Tan & $-5-101$ & & & \\
\hline Date & Tomn ICI & Temn (F) & Comments & Date & Temo (C) & Temo (F) & Comments \\
\hline $4 / 7 / 54$ & & 300 & & $6 / 26 / 54$ & & 220 & \\
\hline $4 / 8 / 54$ & & 300 & & $6 / 28 / 54$ & & 220 & \\
\hline $4 / 9 / 54$ & & 300 & & $6 / 30 / 54$ & & 219 & \\
\hline $4 / 11 / 54$ & & 240 & & $7 / 2 / 54$ & & 220 & \\
\hline $4 / 12 / 54$ & & 250 & & $7 / 3 / 54$ & & 220 & \\
\hline $4 / 14 / 54$ & & 235 & ASSUMPTION & $7 / 4 / 54$ & & 220 & \\
\hline $4 / 15 / 54$ & & 235 & ASSUMPTION & $7 / 5 / 54$ & & 219 & \\
\hline $4 / 16 / 54$ & & 235 & ASSUMPTION & $7 / 6 / 54$ & & 220 & \\
\hline $4 / 17 / 54$ & & 220 & & $7 / 7 / 54$ & & 220 & \\
\hline $4 / 18 / 54$ & & 220 & & $7 / 10 / 54$ & & 220 & \\
\hline $4 / 19 / 54$ & & 220 & & $7 / 12 / 54$ & & 220 & \\
\hline $4 / 21 / 54$ & & 220 & & $7 / 13 / 54$ & & 221 & \\
\hline $4 / 23 / 54$ & & 220 & & $7 / 14 / 54$ & & 220 & \\
\hline $4 / 25 / 54$ & & 215 & & $7 / 16 / 54$ & & 220 & \\
\hline $4 / 26 / 54$ & & 218 & & $7 / 19 / 54$ & & 220 & \\
\hline $4 / 27 / 54$ & & 217 & & $7 / 20 / 54$ & & 222 & \\
\hline $4 / 28 / 54$ & & 217 & & $7 / 23 / 54$ & & 221 & \\
\hline $4 / 29 / 54$ & & 217 & & $7 / 26 / 54$ & & 221 & \\
\hline $4 / 30 / 54$ & & 217 & & $7 / 28 / 54$ & & 223 & \\
\hline $5 / 1 / 54$ & & 218 & & $7 / 29 / 54$ & & 223 & \\
\hline $5 / 2 / 54$ & & 238 & & $7 / 30 / 54$ & & 220 & \\
\hline $5 / 3 / 54$ & & 218 & & $7 / 31 / 54$ & & 220 & \\
\hline $5 / 4 / 54$ & & 218 & & $8 / 2 / 54$ & & 222 & \\
\hline $5 / 5 / 54$ & & 219 & & $8 / 4 / 54$ & & 222 & \\
\hline $5 / 7 / 54$ & & 219 & & $8 / 9 / 54$ & & 221 & \\
\hline $5 / 8 / 54$ & & 220 & & $8 / 10 / 54$ & & 222 & \\
\hline $5 / 10 / 54$ & & 219 & & $8 / 11 / 54$ & & 222 & \\
\hline $5 / 11 / 54$ & & 218 & & $8 / 13 / 54$ & & 222 & \\
\hline $5 / 12 / 54$ & & 218 & & $8 / 16 / 54$ & & 222 & \\
\hline $5 / 13 / 54$ & & 219 & & $8 / 17 / 54$ & & 222 & \\
\hline $5 / 14 / 54$ & & 216 & & $8 / 18 / 54$ & & 222 & \\
\hline $5 / 15 / 54$ & & 220 & & $8 / 19 / 54$ & & 222 & \\
\hline $5 / 16 / 54$ & & 220 & & $8 / 20 / 54$ & & 223 & \\
\hline $5 / 24 / 54$ & - & 218 & & $8 / 21 / 54$ & & 223 & \\
\hline $5 / 25 / 54$ & & 218 & & $8 / 22 / 54$ & & 223 & \\
\hline $5 / 26 / 54$ & & 218 & & $8 / 23 / 54$ & & 223 & \\
\hline $5 / 27 / 54$ & & 219 & & $8 / 26 / 54$ & & 223 & \\
\hline $5 / 28 / 54$ & & 219 & & $8 / 27 / 54$ & & 223 & \\
\hline $5 / 29 / 54$ & & 220 & & $8 / 28 / 54$ & & 225 & \\
\hline $5 / 30 / 54$ & & 225 & & $8 / 30 / 54$ & & 222 & \\
\hline $5 / 31 / 54$ & & 230 & & $8 / 31 / 54$ & & 222 & \\
\hline $6 / 1 / 54$ & & 220 & & $9 / 1 / 54$ & & 224 & \\
\hline $6 / 2 / 54$ & & 219 & & $9 / 2 / 54$ & & 220 & \\
\hline $6 / 3 / 54$ & & 219 & & $9 / 3 / 54$ & & 220 & \\
\hline $6 / 7 / 54$ & & 219 & & $9 / 4 / 54$ & & 220 & \\
\hline $6 / 8 / 54$ & & 219 & & $9 / 5 / 54$ & & 222 & \\
\hline $6 / 9 / 54$ & & 219 & & $9 / 7 / 54$ & & 220 & \\
\hline $6 / 14 / 54$ & & 219 & & $9 / 8 / 54$ & & 220 & \\
\hline $6 / 16 / 54$ & & 220 & & $9 / 9 / 54$ & & 220 & \\
\hline $6 / 18 / 54$ & & 220 & & $9 / 10 / 54$ & & 221 & \\
\hline $6 / 19 / 54$ & & 220 & & $9 / 11 / 54$ & & 220 & \\
\hline $6 / 21 / 54$ & & 220 & & $9 / 20 / 54$ & & 222 & \\
\hline $6 / 23 / 54$ & & 220 & & $9 / 21 / 54$ & & 224 & \\
\hline $6 / 25 / 54$ & & 220 & & $9 / 22 / 54$ & & 223 & \\
\hline
\end{tabular}

Data obtained from RHO-CD-1172 and HW-83906. 
WHC-SD-WM-ER-323, Rev.O I

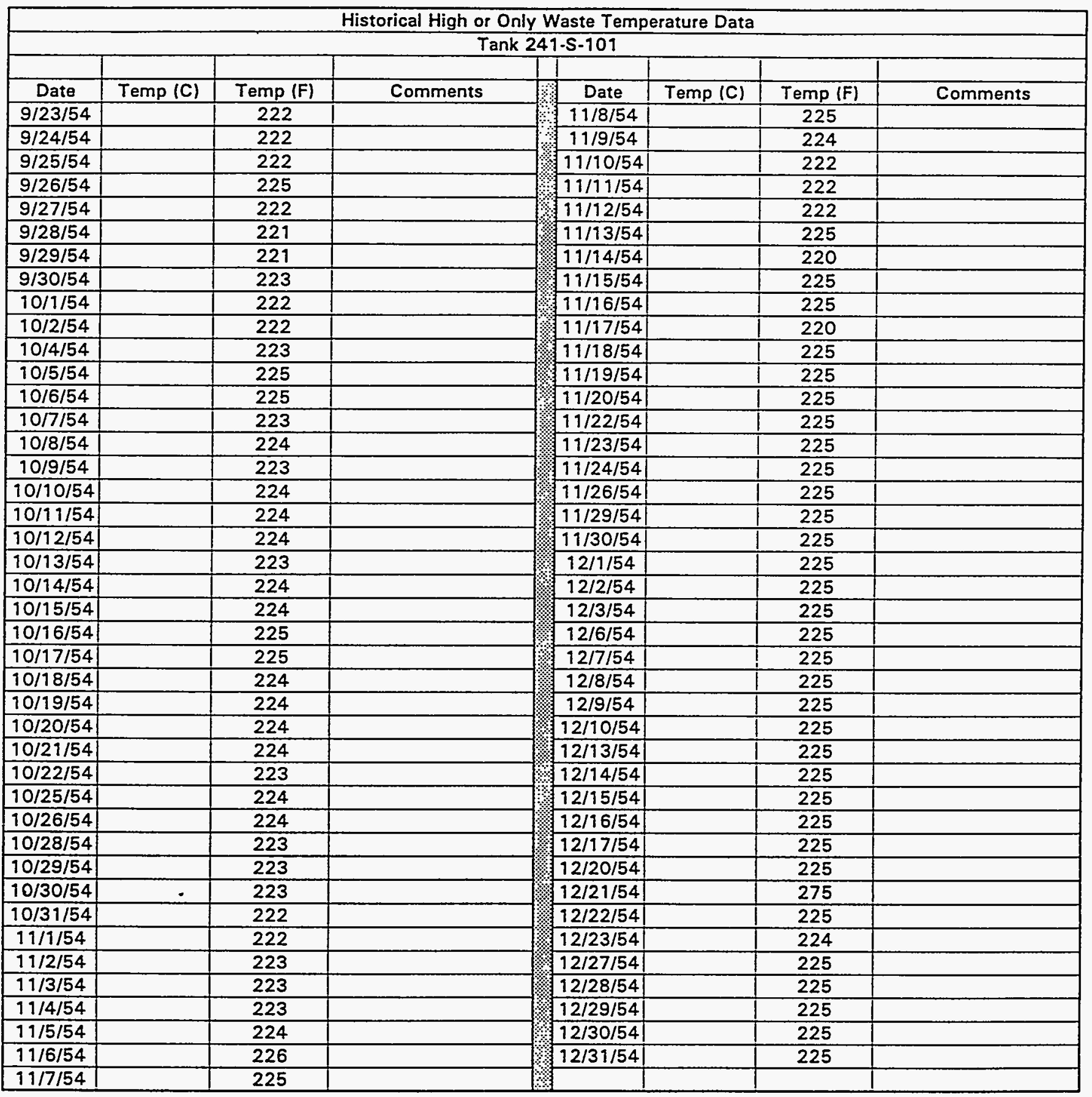

Data obtained from RHO-CD-1172 and HW-83906. 


\begin{tabular}{|c|c|c|c|c|c|c|c|}
\hline \multicolumn{8}{|c|}{ Historical Vapor Space Temperature Data } \\
\hline \multicolumn{8}{|c|}{ Tank 241.S-102 } \\
\hline & & & & & & & \\
\hline Date & Temp (C) & Temp (F) & Comments & Date & Temp (C) & Temp (F) & Comments \\
\hline $7 / 31 / 56$ & & 94 & & $11 / 11 / 56$ & & 91 & \\
\hline $8 / 8 / 56$ & & 93 & & $11 / 15 / 56$ & & 91 & \\
\hline $8 / 13 / 56$ & & 94 & & $11 / 18 / 56$ & & 91 & \\
\hline $8 / 21 / 56$ & & 95 & & $11 / 20 / 56$ & & 92 & \\
\hline $8 / 28 / 56$ & & 94 & & $11 / 26 / 56$ & & 92 & \\
\hline $8 / 30 / 56$ & & 93 & & $11 / 30 / 56$ & & 92 & \\
\hline $8 / 31 / 56$ & & 91 & & $12 / 3 / 56$ & & 92 & \\
\hline $9 / 2 / 56$ & & 89 & & $12 / 7 / 56$ & & 93 & \\
\hline $9 / 4 / 56$ & & 87 & & $12 / 10 / 56$ & & 93 & \\
\hline $9 / 5 / 56$ & & 85 & & $12 / 13 / 56$ & & 93 & \\
\hline $9 / 7 / 56$ & & 87 & & $9 / 30 / 57$ & & 90 & \\
\hline $9 / 9 / 56$ & & 89 & & $2 / 28 / 57$ & & 90 & \\
\hline $9 / 10 / 56$ & & 90 & & $3 / 16 / 57$ & & 89 & \\
\hline $9 / 11 / 56$ & & 92 & & $3 / 29 / 57$ & & 88 & \\
\hline $9 / 12 / 56$ & & 93 & & $4 / 30 / 57$ & & 87 & \\
\hline $9 / 14 / 56$ & & 94 & & $5 / 25 / 57$ & & 87 & \\
\hline $9 / 19 / 56$ & & 94 & & $6 / 13 / 57$ & & 87 & \\
\hline $9 / 21 / 56$ & & 93 & & $7 / 9 / 57$ & & 87 & \\
\hline $9 / 24 / 56$ & & 93 & & $7 / 27 / 57$ & & 89 & \\
\hline $9 / 27 / 56$ & & 92 & & $8 / 9 / 57$ & & 89 & \\
\hline $9 / 30 / 56$ & & 92 & & $9 / 6 / 57$ & & 89 & \\
\hline $10 / 1 / 56$ & & 92 & & $10 / 6 / 57$ & & 88 & \\
\hline $10 / 5 / 56$ & & 92 & & $10 / 31 / 57$ & & 87 & \\
\hline $10 / 8 / 56$ & & 92 & & $6 / 1 / 58$ & & 83 & \\
\hline $10 / 10 / 56$ & & 92 & & $7 / 28 / 58$ & & 84 & \\
\hline $10 / 11 / 56$ & & 93 & & $9 / 2 / 58$ & & 83 & \\
\hline $10 / 14 / 56$ & & 91 & & $10 / 3 / 58$ & & 82 & \\
\hline $10 / 16 / 56$ & & 91 & & $11 / 3 / 58$ & & 83 & \\
\hline $10 / 19 / 56$ & & 92 & & $12 / 4 / 58$ & & 82 & \\
\hline $10 / 23 / 56$ & & 93 & & $1 / 1 / 59$ & & 82 & \\
\hline $10 / 25 / 56$ & & 93 & & $1 / 31 / 59$ & & 81 & \\
\hline $10 / 27 / 56$ & & 94 & & $3 / 2 / 59$ & & 80 & \\
\hline $10 / 29 / 56$ & & 93 & & $3 / 25 / 59$ & & 78 & \\
\hline $111 / 1 / 56$ & $\cdot$ & 92 & & $4 / 3 / 59$ & & 78 & \\
\hline $11 / 2 / 56$ & & 92 & & $4 / 28 / 59$ & & 78 & \\
\hline $11 / 4 / 56$ & & 92 & & $11 / 30 / 59$ & & 78 & \\
\hline $11 / 7 / 56$ & & 91 & & $1 / 1 / 60$ & & 76 & \\
\hline
\end{tabular}

Data obtained from RHO-CD-1172. 


\begin{tabular}{|c|c|c|c|c|c|c|c|}
\hline \multicolumn{8}{|c|}{ Historical High or Only Waste Temperature Data } \\
\hline \multicolumn{8}{|c|}{ Tank 241-S-102 } \\
\hline Date & Temp (C) & Temo (F) & & & & & \\
\hline & & $\frac{\text { lemp (t) }}{72}$ & Comments & Date & Temp (C) & Temp (F) & Comments \\
\hline$\frac{9 / 10 / 53}{9 / 11 / 53}$ & & $\frac{72}{80}$ & & $11 / 5 / 53$ & & 141 & \\
\hline$\frac{9 / 11 / 53}{9 / 13 / 53}$ & & 80 & & $11 / 6 / 53$ & & 141 & \\
\hline $9 / 13 / 53$ & & 70 & & $11 / 10 / 53$ & & 141 & \\
\hline $9 / 14 / 53$ & & 70 & & $11 / 11 / 53$ & & 140 & \\
\hline $9 / 15 / 53$ & & 76 & & $11 / 11 / 53$ & & 140 & \\
\hline $9 / 16 / 53$ & & 70 & & $11 / 12 / 53$ & & 140 & \\
\hline $9 / 17 / 53$ & & 73 & & $11 / 13 / 53$ & & 140 & \\
\hline $9 / 18 / 53$ & & 90 & & $11 / 15 / 53$ & & 110 & \\
\hline $9 / 19 / 53$ & & 100 & & $11 / 16 / 53$ & & 140 & \\
\hline $9 / 20 / 53$ & & 100 & & $11 / 11 / 53$ & & 140 & \\
\hline $9 / 21 / 53$ & & 100 & & $11 / 12 / 53$ & & 140 & \\
\hline $9 / 22 / 53$ & & 100 & & $11 / 13 / 53$ & & 140 & \\
\hline $9 / 23 / 53$ & & 116 & & $11 / 15 / 53$ & & 110 & \\
\hline $9 / 24 / 53$ & & 116 & & $11 / 16 / 53$ & & 140 & \\
\hline $9 / 25 / 53$ & & 116 & & $11 / 17 / 53$ & & 140 & \\
\hline $9 / 26 / 53$ & & 132 & & $11 / 18 / 53$ & & 140 & \\
\hline $9 / 27 / 53$ & & 132 & & $11 / 19 / 53$ & & 140 & \\
\hline $9 / 28 / 53$ & & 132 & & $11 / 20 / 53$ & & 140 & \\
\hline $9 / 29 / 53$ & & 132 & & $11 / 22 / 53$ & & 140 & \\
\hline $9 / 30 / 53$ & & 132 & & $11 / 23 / 53$ & & 140 & \\
\hline $10 / 1 / 53$ & & 134 & & $11 / 24 / 53$ & & 140 & \\
\hline $10 / 2 / 53$ & & 135 & & $11 / 25 / 53$ & & 140 & \\
\hline $10 / 3 / 53$ & & 138 & & $11 / 27 / 53$ & & 138 & \\
\hline $10 / 4 / 53$ & & 138 & & $11 / 29 / 53$ & & 138 & \\
\hline $10 / 5 / 53$ & & 135 & & $11 / 30 / 53$ & & 138 & \\
\hline $10 / 6 / 53$ & & 135 & & $12 / 1 / 53$ & & 138 & \\
\hline $10 / 7 / 53$ & & 135 & & $12 / 2 / 53$ & & 136 & \\
\hline $10 / 8 / 53$ & & 135 & & $12 / 3 / 53$ & & 136 & \\
\hline $10 / 9 / 53$ & & 135 & & $12 / 4 / 53$ & & 136 & \\
\hline $10 / 10 / 53$ & & 136 & & $12 / 5 / 53$ & & 136 & \\
\hline $10 / 11 / 53$ & & 136 & & $12 / 6 / 53$ & & 135 & \\
\hline $10 / 12 / 53$ & & 136 & & $12 / 7 / 53$ & & 135 & \\
\hline $10 / 14 / 53$ & & 136 & & $12 / 8 / 53$ & & 138 & \\
\hline $1,0 / 15 / 53$ & & 136 & & $12 / 10 / 53$ & & 138 & \\
\hline $10 / 16 / 53$ & & 138 & & $12 / 11 / 53$ & & 138 & \\
\hline $10 / 17 / 53$ & & 139 & & $12 / 14 / 53$ & & 138 & \\
\hline $10 / 18 / 53$ & & 139 & & $12 / 15 / 53$ & & 138 & \\
\hline $10 / 19 / 53$ & & 139 & & $12 / 16 / 53$ & & 138 & \\
\hline $10 / 20 / 53$ & & 139 & & $12 / 17 / 53$ & & 138 & \\
\hline $10 / 21 / 53$ & & 139 & & $12 / 18 / 53$ & & 138 & \\
\hline $10 / 22 / 53$ & & 138 & & $12 / 21 / 53$ & & 138 & \\
\hline $10 / 23 / 53$ & & 139 & & $12 / 22 / 53$ & & 138 & \\
\hline $10 / 24 / 53$ & & 138 & & $1.2 / 23 / 53$ & & 138 & \\
\hline $10 / 25 / 53$ & & 140 & & $12 / 25 / 53$ & & 138 & \\
\hline $10 / 26 / 53$ & & 140 & & $12 / 26 / 53$ & & 138 & \\
\hline $10 / 27 / 53$ & & 140 & & $12 / 27 / 53$ & & 138 & \\
\hline $10 / 28 / 53$ & & 140 & & $12 / 28 / 53$ & & 138 & \\
\hline $10 / 29 / 53$ & & 140 & & $12 / 29 / 53$ & & 138 & \\
\hline $10 / 30 / 53$ & & 141 & & $12 / 30 / 53$ & & 138 & \\
\hline $10 / 31 / 53$ & & 941 & & $12 / 31 / 53$ & & 130 & \\
\hline $11 / 1 / 53$ & & 141 & & $1 / 4 / 54$ & & 138 & \\
\hline $111 / 2 / 53$ & & 141 & & $1 / 5 / 54$ & & 140 & \\
\hline $11 / 3 / 53$ & & 141 & & $1 / 6 / 54$ & & 142 & \\
\hline $111 / 4 / 53$ & & 141 & & $1 / 7 / 54$ & & 142 & \\
\hline
\end{tabular}

Data obtained from HW-83906. 


\begin{tabular}{|c|c|c|c|c|c|c|c|}
\hline \multicolumn{8}{|c|}{ Historical High or Only Waste Temperature Data } \\
\hline & & & & $S-102$ & & & \\
\hline Date & Temp (C) & Temp (F) & Comments & Date & Temp (C) & Temp (F) & \\
\hline $1 / 8 / 54$ & & 142 & & $4 / 5 / 54$ & ० & 140 & Comments \\
\hline $1 / 11 / 54$ & & 142 & & $4 / 6 / 54$ & & 140 & \\
\hline $1 / 12 / 54$ & & 142 & & $4 / 7 / 54$ & & 140 & \\
\hline $1 / 21 / 54$ & & 141 & & $4 / 16 / 54$ & & 138 & \\
\hline $1 / 22 / 54$ & & 141 & & $4 / 17 / 54$ & & 139 & \\
\hline $1 / 23 / 54$ & & 141 & & $4 / 18 / 54$ & & 139 & \\
\hline $1 / 24 / 54$ & & 141 & & $4 / 19 / 54$ & & 139 & \\
\hline $1 / 25 / 54$ & & 141 & & $4 / 21 / 54$ & & 139 & \\
\hline $1 / 26 / 54$ & & 141 & & $4 / 23 / 54$ & & 139 & \\
\hline $1 / 27 / 54$ & & 141 & & $4 / 24 / 54$ & & 136 & \\
\hline $1 / 28 / 54$ & & 141 & & $4 / 25 / 54$ & & 139 & \\
\hline $1 / 29 / 54$ & & 141 & & $4 / 26 / 54$ & & 139 & \\
\hline $2 / 1 / 54$ & & 141 & & $4 / 27 / 54$ & & 139 & \\
\hline $2 / 2 / 54$ & & 141 & & $4 / 28 / 54$ & & 137 & \\
\hline $2 / 3 / 54$ & & 142 & & $4 / 29 / 54$ & & 137 & \\
\hline $2 / 4 / 54$ & & 142 & & $4 / 30 / 54$ & & 137 & \\
\hline $2 / 5 / 54$ & & 142 & & $5 / 1 / 54$ & & 137 & \\
\hline $2 / 6 / 54$ & & 143 & & $5 / 3 / 54$ & & 137 & \\
\hline $2 / 7 / 54$ & & 143 & & $5 / 4 / 54$ & & 137 & \\
\hline $2 / 8 / 54$ & & 143 & & $5 / 5 / 54$ & & 136 & \\
\hline $2 / 9 / 54$ & & 143 & & $5 / 8 / 54$ & & 138 & \\
\hline $2 / 10 / 54$ & & 142 & & $5 / 9 / 54$ & & 138 & \\
\hline $2 / 11 / 54$ & & 142 & & $5 / 10 / 54$ & & 136 & \\
\hline $2 / 12 / 54$ & & 142 & & $5 / 12 / 54$ & & 136 & \\
\hline $2 / 13 / 54$ & & 140 & & $5 / 15 / 54$ & & 136 & \\
\hline $2 / 15 / 54$ & & 140 & & $5 / 16 / 54$ & & 137 & \\
\hline $2 / 16 / 54$ & & 140 & & $5 / 24 / 54$ & & 140 & \\
\hline $2 / 17 / 54$ & & 140 & & $5 / 26 / 54$ & & 140 & \\
\hline $2 / 18 / 54$ & & 143 & & $5 / 31 / 54$ & & 140 & \\
\hline $2 / 19 / 54$ & & 143 & & $6 / 1 / 54$ & & 140 & \\
\hline $2 / 22 / 54$ & & 143 & & $6 / 2 / 54$ & & 140 & \\
\hline $2 / 23 / 54$ & & 143 & & $6 / 3 / 54$ & & 140 & \\
\hline $2 / 25 / 54$ & & 144 & & $6 / 7 / 54$ & & 140 & \\
\hline $2 / 26 / 54$ & & 144 & & $6 / 8 / 54$ & & 140 & \\
\hline $3 / 1 / 54$ & & 144 & & $6 / 9 / 54$ & & 138 & \\
\hline $3 / 2 / 54$ & & 144 & & $6 / 14 / 54$ & & 138 & \\
\hline $3 / 3 / 54$ & & 143 & & $6 / 16 / 54$ & & 136 & \\
\hline $3 / 4 / 54$ & & 143 & & $6 / 18 / 54$ & & 136 & \\
\hline $3 / 5 / 54$ & & 143 & & $6 / 21 / 54$ & & 136 & \\
\hline $3 / 6 / 54$ & & 143 & & $6 / 23 / 54$ & & 136 & \\
\hline $3 / 7 / 54$ & & 140 & & $6 / 26 / 54$ & & 136 & \\
\hline $3 / 8 / 54$ & & 140 & & $6 / 28 / 54$ & & 136 & \\
\hline $3 / 9 / 54$ & & 144 & & $6 / 30 / 54$ & & 136 & \\
\hline $3 / 10 / 54$ & & 142 & & $7 / 5 / 54$ & & 136 & \\
\hline $3 / 12 / 54$ & & 142 & & $7 / 7 / 54$ & & 134 & \\
\hline $3 / 14 / 54$ & & 144 & & $7 / 12 / 54$ & & 134 & \\
\hline $3 / 15 / 54$ & & 144 & & $7 / 14 / 54$ & & 133 & \\
\hline $3 / 16 / 54$ & & 144 & & $7 / 19 / 54$ & & 133 & \\
\hline $3 / 17 / 54$ & & 144 & & $7 / 23 / 54$ & & 132 & \\
\hline $3 / 18 / 54$ & & 144 & & $7 / 26 / 54$ & & 132 & \\
\hline $3 / 19 / 54$ & & 144 & & $7 / 28 / 54$ & & 131 & \\
\hline $3 / 22 / 54$ & & 144 & & $8 / 2 / 54$ & & 131 & \\
\hline $3 / 24 / 54$ & & 142 & & $8 / 4 / 54$ & & 130 & \\
\hline $4 / 1 / 54$ & & 140 & & $8 / 9 / 54$ & & 130 & \\
\hline
\end{tabular}

Data obtained from HW-83906. 


\begin{tabular}{|c|c|c|c|c|c|c|c|}
\hline \multicolumn{8}{|c|}{ Historical High or Only Waste Temperature Data } \\
\hline \multicolumn{8}{|c|}{ Tank 241-S-102 } \\
\hline Date & Temp (C) & Temp (F) & Comments & Date & Temp (C) & Temp (F) & Comments \\
\hline $8 / 11 / 54$ & & 130 & & $11 / 4 / 54$ & & 123 & \\
\hline $8 / 16 / 54$ & & 130 & & $11 / 5 / 54$ & & 123 & \\
\hline $8 / 18 / 54$ & & 130 & & $11 / 6 / 54$ & & 120 & \\
\hline $8 / 23 / 54$ & & 130 & & $11 / 7 / 54$ & & 120 & \\
\hline $8 / 26 / 54$ & & 130 & & $11 / 8 / 54$ & & 120 & \\
\hline $8 / 28 / 54$ & & 125 & & $11 / 9 / 54$ & & 120 & \\
\hline $8 / 30 / 54$ & & 130 & & $11 / 10 / 54$ & & 122 & \\
\hline $8 / 31 / 54$ & & 130 & & $11 / 11 / 54$ & & 122 & \\
\hline $9 / 1 / 54$ & & 127 & & $11 / 12 / 54$ & & 122 & \\
\hline $9 / 2 / 54$ & & 128 & & $11 / 13 / 54$ & & 120 & \\
\hline $9 / 6 / 54$ & & 127 & & $11 / 14 / 54$ & & 120 & \\
\hline $9 / 8 / 54$ & & 128 & & $11 / 15 / 54$ & & 122 & \\
\hline $9 / 20 / 54$ & & 127 & & $11 / 16 / 54$ & & 122 & \\
\hline $9 / 21 / 54$ & & 126 & & $11 / 17 / 54$ & & 122 & \\
\hline $9 / 27 / 54$ & & 126 & & $11 / 18 / 54$ & & 122 & \\
\hline $9 / 30 / 54$ & & 124 & & $11 / 19 / 54$ & & 122 & \\
\hline $10 / 4 / 54$ & & 124 & & $11 / 20 / 54$ & & 122 & \\
\hline $10 / 5 / 54$ & & 125 & & $11 / 22 / 54$ & & 122 & \\
\hline $10 / 6 / 54$ & & 125 & & $11 / 23 / 54$ & & 121 & \\
\hline $10 / 8 / 54$ & & 125 & & $11 / 24 / 54$ & & 122 & \\
\hline $10 / 11 / 54$ & & 125 & & $11 / 26 / 54$ & & 122 & \\
\hline $10 / 12 / 54$ & & 124 & & $11 / 29 / 54$ & & 122 & \\
\hline $10 / 13 / 54$ & & 124 & & $11 / 30 / 54$ & & 122 & \\
\hline $10 / 14 / 54$ & & 124 & & $12 / 1 / 54$ & & 120 & \\
\hline $10 / 15 / 54$ & & 124 & & $12 / 2 / 54$ & & 120 & \\
\hline $10 / 17 / 54$ & & 122 & & $12 / 3 / 54$ & & 120 & \\
\hline $10 / 18 / 54$ & & 124 & & $12 / 6 / 54$ & & 120 & \\
\hline $10 / 19 / 54$ & & 124 & & $12 / 7 / 54$ & & 120 & \\
\hline $10 / 20 / 54$ & & 123 & & $12 / 8 / 54$ & & 120 & \\
\hline $10 / 22 / 54$ & & 123 & & $12 / 9 / 54$ & & 120 & \\
\hline $10 / 25 / 54$ & & 123 & & $12 / 10 / 54$ & & 120 & \\
\hline $10 / 26 / 54$ & & 123 & & $12 / 13 / 54$ & & -120 & \\
\hline $10 / 27 / 54$ & & 123 & & $12 / 14 / 54$ & & 120 & \\
\hline $10 / 28 / 54$ & & 123 & & $12 / 15 / 54$ & & 120 & \\
\hline $10 / 29 / 54$ & & 123 & & $12 / 17 / 54$ & & 120 & \\
\hline $10 / 30 / 54$ & & 123 & & $12 / 20 / 54$ & & 120 & \\
\hline $10 / 31 / 54$ & & 123 & & $12 / 22 / 54$ & & 119 & \\
\hline $11 / 1 / 54$ & & 123 & & $12 / 27 / 54$ & & 119 & \\
\hline $11 / 3 / 54$ & & 123 & & $12 / 29 / 54$ & & 118 & \\
\hline
\end{tabular}

Data obtained from HW-83906. 


\begin{tabular}{|c|c|c|c|c|c|c|c|}
\hline \multicolumn{8}{|c|}{ Historical Vapor Space Temperature Data } \\
\hline \multicolumn{8}{|c|}{ Tank 241-S-103 } \\
\hline & & & & & & & \\
\hline Date & $\operatorname{Temp}(\mathrm{C})$ & Temp (F) & Comments & Date & Temp (C) & Temp (F) & Comments \\
\hline $7 / 31 / 56$ & & 95 & & $11 / 26 / 56$ & & 82 & \\
\hline $8 / 2 / 56$ & & 93 & & $11 / 28 / 56$ & & 81 & \\
\hline $8 / 5 / 56$ & & 91 & & $11 / 30 / 56$ & & 83 & \\
\hline $8 / 6 / 56$ & & 90 & & $12 / 1 / 56$ & & 84 & \\
\hline $8 / 10 / 56$ & & 90 & & $12 / 2 / 56$ & & 85 & \\
\hline $8 / 13 / 56$ & & 89 & & $12 / 4 / 56$ & & 86 & \\
\hline $8 / 17 / 56$ & & 89 & & $12 / 5 / 56$ & & 87 & \\
\hline $8 / 22 / 56$ & & 89 & & $12 / 6 / 56$ & & 88 & \\
\hline $8 / 25 / 56$ & & 89 & & $12 / 7 / 56$ & & 89 & \\
\hline $8 / 29 / 56$ & & 89 & & $12 / 8 / 56$ & & 90 & \\
\hline $9 / 5 / 56$ & & 88 & & $12 / 10 / 56$ & & 90 & \\
\hline $9 / 9 / 56$ & & 88 & & $12 / 13 / 56$ & & 90 & \\
\hline $9 / 12 / 56$ & & 89 & & $12 / 16 / 56$ & & 90 & \\
\hline $9 / 15 / 56$ & & 89 & & $12 / 20 / 56$ & & 90 & \\
\hline $9 / 18 / 56$ & & 90 & & $12 / 24 / 56$ & & 89 & \\
\hline $9 / 22 / 56$ & & 90 & & $12 / 28 / 56$ & & 89 & \\
\hline $9 / 25 / 56$ & & 90 & & $12 / 31 / 56$ & & 89 & \\
\hline $9 / 29 / 56$ & & 90 & & $1 / 30 / 57$ & & 88 & \\
\hline $10 / 1 / 56$ & & 89 & & $3 / 16 / 57$ & & 87 & \\
\hline $10 / 3 / 56$ & & 88 & & $4 / 27 / 57$ & & 87 & \\
\hline $10 / 5 / 56$ & & 89 & & $5 / 31 / 57$ & & 86 & \\
\hline $10 / 8 / 56$ & & 90 & & $8 / 8 / 57$ & & 85 & \\
\hline $10 / 10 / 56$ & & 90 & & $10 / 4 / 57$ & & 84 & \\
\hline $10 / 13 / 56$ & & 90 & & $10 / 28 / 57$ & & 84 & \\
\hline $10 / 15 / 56$ & & 90 & & $5 / 4 / 58$ & & 83 & \\
\hline $10 / 18 / 56$ & & 90 & & $5 / 31 / 58$ & & 83 & \\
\hline $10 / 20 / 56$ & & 90 & & $6 / 30 / 58$ & & 83 & \\
\hline $10 / 25 / 56$ & & 88 & & $7 / 29 / 58$ & & 83 & \\
\hline $10 / 26 / 56$ & & 88 & & $9 / 1 / 58$ & & 84 & \\
\hline $10 / 30 / 56$ & & 88 & & $9 / 15 / 58$ & & 83 & \\
\hline $10 / 31 / 56$ & & 89 & & $10 / 12 / 58$ & & 83 & \\
\hline $11 / 3 / 56$ & & 90 & & $10 / 20 / 58$ & & 82 & \\
\hline $11 / 7 / 56$ & & 90 & & $10 / 30 / 58$ & & 81 & \\
\hline $11 / 9 / 56$ & & 90 & & $1117 / 58$ & & 82 & \\
\hline $11 / 13 / 56$ & & 90 & & $11 / 15 / 58$ & & 83 & \\
\hline $11 / 15 / 56$ & & 90 & & $11 / 24 / 58$ & & 85 & \\
\hline $11 / 18 / 56$ & & 89 & & $12 / 2 / 58$ & & 85 & \\
\hline $11 / 19 / 56$ & & 88 & & $12 / 10 / 58$ & & 85 & \\
\hline $11 / 21 / 56$ & & 87 & & $12 / 17 / 58$ & & 84 & \\
\hline $11 / 22 / 56$ & & 87 & & $12 / 24 / 58$ & & 83 & \\
\hline $11 / 24 / 56$ & & 86 & & $5 / 1 / 59$ & & 83 & \\
\hline $11 / 25 / 56$ & & 84 & & $11 / 30 / 59$ & & 82 & \\
\hline $11 / 26 / 56$ & & 83 & & $12 / 31 / 59$ & & 83 & \\
\hline
\end{tabular}

Data obtained from RHO-CD-1172. 


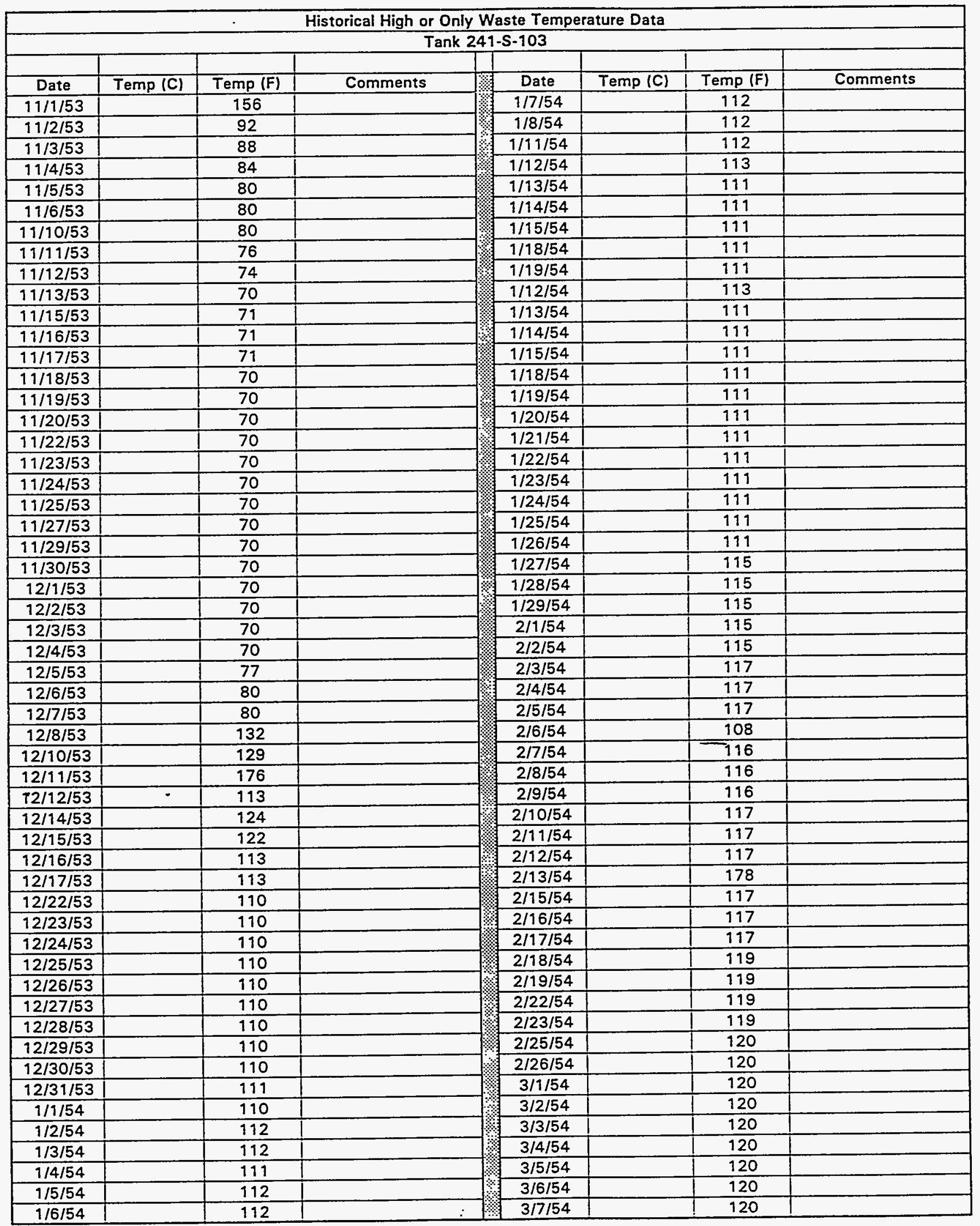

Data obtained from HW-83906. 


\begin{tabular}{|c|c|c|c|c|c|c|c|}
\hline \multicolumn{8}{|c|}{ Historical High or Only Waste Temperature Data } \\
\hline \multicolumn{8}{|c|}{ Tank 241-S-103 } \\
\hline & & & & & & & \\
\hline Date & Temp (C) & Temp (F) & Comments & Date & Temp (C) & Temp (F) & Comments \\
\hline $3 / 8 / 54$ & & 120 & & $6 / 28 / 54$ & & 107 & \\
\hline $3 / 9 / 54$ & & 120 & & $6 / 30 / 54$ & & 107 & \\
\hline $3 / 10 / 54$ & & 120 & & $7 / 5 / 54$ & & 107 & \\
\hline $3 / 19 / 54$ & & 120 & & $7 / 26 / 54$ & & 105 & \\
\hline $3 / 22 / 54$ & & 120 & & $7 / 28 / 54$ & & 104 & \\
\hline $3 / 24 / 54$ & & 116 & & $8 / 2 / 54$ & & 104 & \\
\hline $4 / 1 / 54$ & & 115 & & $8 / 4 / 54$ & & 104 & \\
\hline $4 / 5 / 54$ & & 115 & & $8 / 9 / 54$ & & 104 & \\
\hline $4 / 6 / 54$ & & 115 & & $8 / 11 / 54$ & & 103 & \\
\hline $4 / 7 / 54$ & & 115 & & $8 / 16 / 54$ & & 103 & \\
\hline $4 / 8 / 54$ & & 115 & & $8 / 18 / 54$ & & 103 & \\
\hline $4 / 9 / 54$ & & 115 & & $8 / 23 / 54$ & & 103 & \\
\hline $4 / 11 / 54$ & & 112 & & $8 / 26 / 54$ & & 103 & \\
\hline $4 / 12 / 54$ & & 112 & & $8 / 28 / 54$ & & 105 & \\
\hline $4 / 14 / 54$ & & 112 & & $8 / 30 / 54$ & & 103 & \\
\hline $4 / 15 / 54$ & & 112 & & $8 / 31 / 54$ & & 102 & \\
\hline $4 / 16 / 54$ & & 112 & & $9 / 1 / 54$ & & 102 & \\
\hline $4 / 17 / 54$ & & 112 & & $9 / 2 / 54$ & & 103 & \\
\hline $4 / 18 / 54$ & & 112 & & $9 / 6 / 54$ & & 102 & \\
\hline $4 / 19 / 54$ & & 112 & & $9 / 8 / 54$ & & 102 & \\
\hline $4 / 21 / 54$ & & 113 & & $9 / 20 / 54$ & & 103 & \\
\hline $4 / 23 / 54$ & & 113 & & $9 / 21 / 54$ & & 101 & \\
\hline $4 / 24 / 54$ & & 110 & & $9 / 27 / 54$ & & 101 & \\
\hline $4 / 25 / 54$ & & 112 & & $9 / 30 / 54$ & & 100 & \\
\hline $4 / 26 / 54$ & & 113 & & $10 / 4 / 54$ & & 100 & \\
\hline $4 / 27 / 54$ & & 113 & & $10 / 5 / 54$ & & 101 & \\
\hline $4 / 28 / 54$ & & 111 & & $10 / 6 / 54$ & & 101 & \\
\hline $4 / 29 / 54$ & & 111 & & $10 / 8 / 54$ & & 101 & \\
\hline $4 / 30 / 54$ & & 111 & & $10 / 11 / 54$ & & 101 & \\
\hline $5 / 1 / 54$ & & 112 & & $10 / 12 / 54$ & & 101 & \\
\hline $5 / 3 / 54$ & & 111 & & $10 / 13 / 54$ & & 101 & \\
\hline $5 / 4 / 54$ & & 111 & & $10 / 14 / 54$ & & 101 & \\
\hline $5 / 5 / 54$ & & 110 & & $10 / 15 / 54$ & & 102 & \\
\hline $.5 / 8 / 54$ & $\cdot$ & 110 & & $10 / 17 / 54$ & & 100 & \\
\hline $5 / 9 / 54$ & & 120 & & $10 / 18 / 54$ & & 101 & \\
\hline $5 / 10 / 54$ & & 110 & & $10 / 19 / 54$ & & 101 & \\
\hline $5 / 12 / 54$ & & 109 & & $10 / 20 / 54$ & & 100 & \\
\hline $5 / 15 / 54$ & & 107 & & $10 / 22 / 54$ & & 101 & \\
\hline $5 / 16 / 54$ & & 107 & & $10 / 25 / 54$ & & 100 & \\
\hline $5 / 24 / 54$ & & 108 & & $10 / 26 / 54$ & & 101 & \\
\hline $5 / 26 / 54$ & & 109 & & $10 / 27 / 54$ & & 100 & \\
\hline $5 / 31 / 54$ & & 109 & & $10 / 28 / 54$ & & 100 & \\
\hline $6 / 1 / 54$ & & 109 & & $10 / 29 / 54$ & & 100 & \\
\hline $6 / 2 / 54$ & & 109 & & $10 / 30 / 54$ & & 100 & \\
\hline $6 / 3 / 54$ & & 109 & & $10 / 31 / 54$ & & 100 & \\
\hline $6 / 7 / 54$ & & 109 & & $11 / 1 / 54$ & & 100 & \\
\hline $6 / 8 / 54$ & & 109 & & $11 / 3 / 54$ & & 100 & \\
\hline $6 / 9 / 54$ & & 107 & & $11 / 4 / 54$ & & 100 & \\
\hline $6 / 14 / 54$ & & 107 & & $11 / 5 / 54$ & & 101 & \\
\hline $6 / 16 / 54$ & & 107 & & $11 / 6 / 54$ & & 100 & \\
\hline $6 / 18 / 54$ & & 107 & & $11 / 7 / 54$ & & 100 & \\
\hline $6 / 21 / 54$ & & 107 & & $91 / 8 / 54$ & & 100 & \\
\hline $6 / 23 / 54$ & & 107 & & $11 / 9 / 54$ & & 100 & \\
\hline $6 / 26 / 54$ & & 107 & & $11 / 10 / 54$ & & 100 & \\
\hline
\end{tabular}

Data obtained from HW-83906. 
WHC-SD-WM-ER-323, Rev. O

\begin{tabular}{|c|c|c|c|c|c|c|c|}
\hline \multicolumn{8}{|c|}{ Historical High or Only Waste Temperature Data } \\
\hline \multicolumn{8}{|c|}{ Tank 241-S-103 } \\
\hline & & & & & & & \\
\hline Date & Temp (C) & Temp (F) & Comments & Date & Temp (C) & Temp (F) & Comments \\
\hline $11 / 11 / 54$ & & 100 & & $12 / 1 / 54$ & & 100 & \\
\hline $11 / 12 / 54$ & & 100 & & $12 / 2 / 54$ & & 100 & \\
\hline $11 / 13 / 54$ & & 100 & & $12 / 3 / 54$ & & 100 & \\
\hline $11 / 14 / 54$ & & 100 & & $12 / 6 / 54$ & & 104 & \\
\hline $11 / 15 / 54$ & & 100 & & $12 / 7 / 54$ & & 104 & \\
\hline $11 / 16 / 54$ & & 100 & & $12 / 8 / 54$ & & 100 & \\
\hline $11 / 17 / 54$ & & 100 & & $12 / 9 / 54$ & & 100 & \\
\hline $11 / 18 / 54$ & & 100 & & $12 / 10 / 54$ & & 100 & \\
\hline $11 / 19 / 54$ & & 100 & & $12 / 13 / 54$ & & 100 & \\
\hline $11 / 20 / 54$ & & 100 & & $12 / 14 / 54$ & & 100 & \\
\hline $11 / 22 / 54$ & & 100 & & $12 / 15 / 54$ & & 100 & \\
\hline $11 / 23 / 54$ & & 100 & & $12 / 17 / 54$ & & 102 & \\
\hline $11 / 24 / 54$ & & 100 & & $12 / 20 / 54$ & & 102 & \\
\hline $11 / 26 / 54$ & & 100 & & $12 / 22 / 54$ & & 98 & \\
\hline $11 / 29 / 54$ & & 100 & & $12 / 27 / 54$ & & 98 & \\
\hline $11 / 30 / 54$ & & 100 & & $12 / 29 / 54$ & & 98 & \\
\hline
\end{tabular}

Data obtained from HW-83906. 
WHC-SD-WM-ER-323, Rev. O 1

Historical Vapor Space Temperature Data

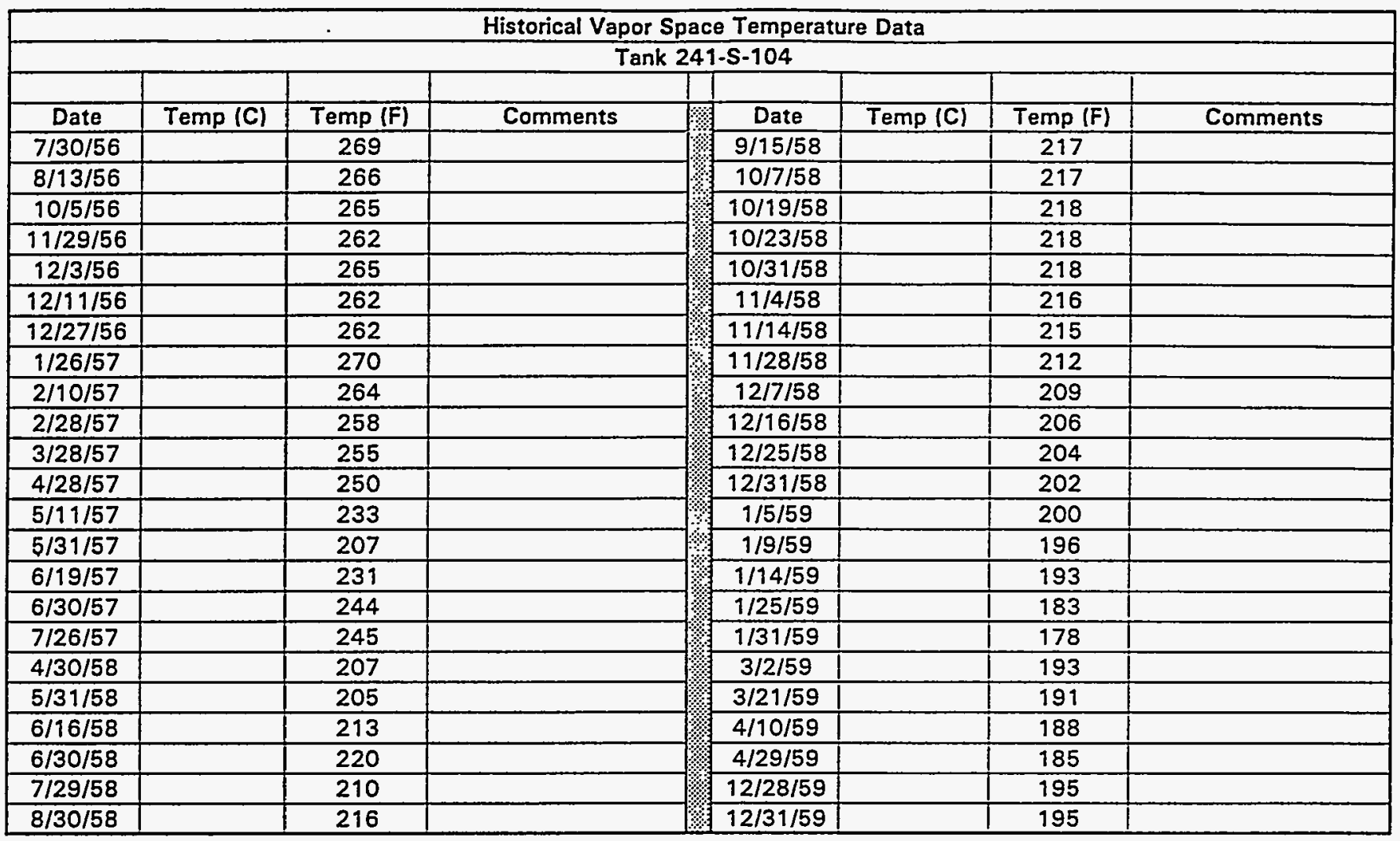




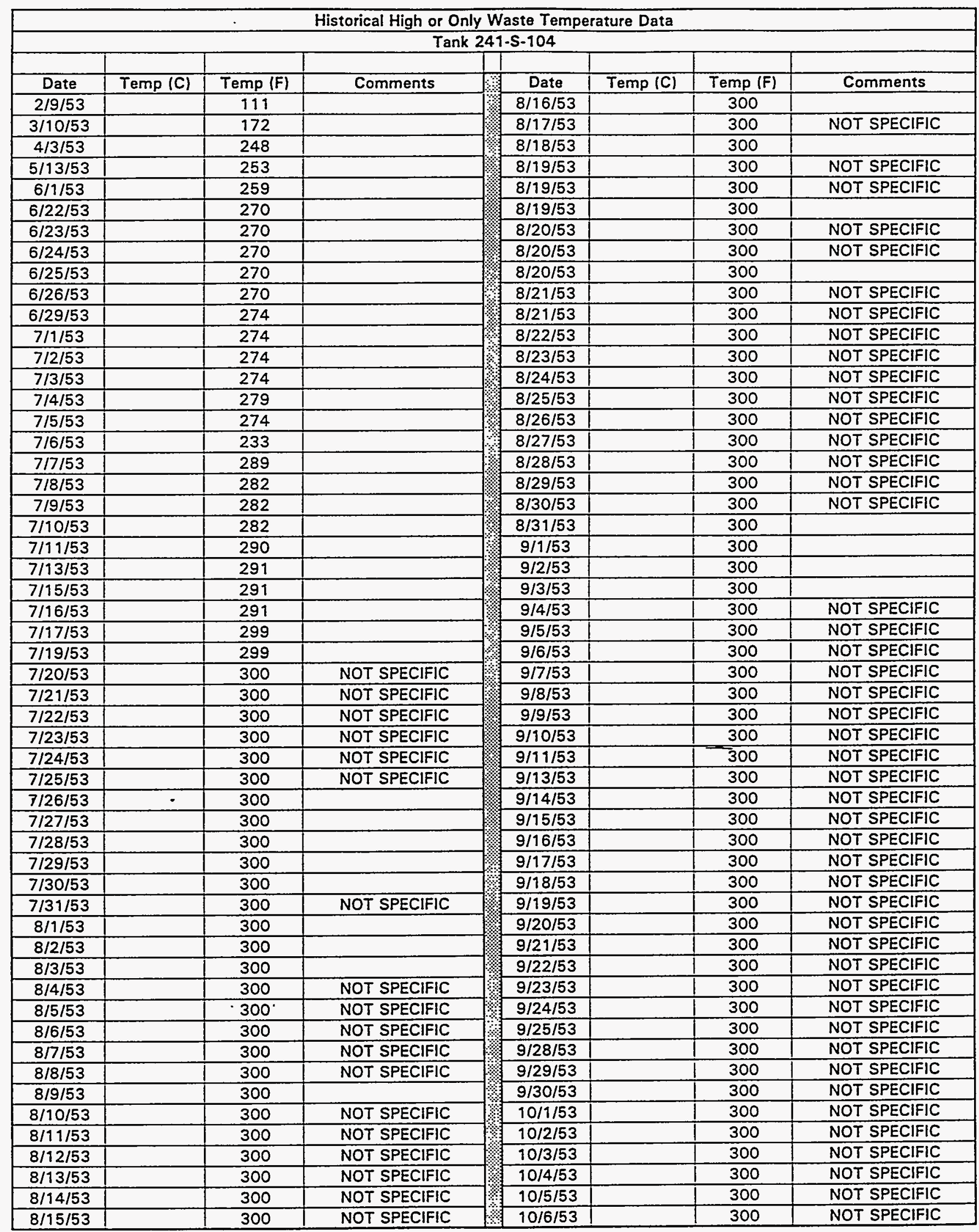

Data obtained from RHO-CD-1172 and HW-83906. 


\begin{tabular}{|c|c|c|c|c|c|c|c|}
\hline \multicolumn{8}{|c|}{ Historical High or Only Waste Temperature Data } \\
\hline \multicolumn{8}{|c|}{ Tank 241-S-104 } \\
\hline Date $_{4}$ & Temp (C) & Temp (F) & Comments & Date & Temp (C) & Temp (F) & \\
\hline $10 / 7 / 53$ & & 300 & NOT SPECIFIC & $12 / 24 / 53$ & & 300 & Comments \\
\hline $10 / 8 / 53$ & & 300 & NOT SPECIFIC & $12 / 30 / 53$ & & 300 & \\
\hline $10 / 9 / 53$ & & 300 & NOT SPECIFIC & $12 / 31 / 53$ & & 300 & \\
\hline $10 / 14 / 53$ & & 300 & NOT SPECIFIC & $1 / 4 / 54$ & & 300 & \\
\hline $10 / 15 / 53$ & & 300 & NOT SPECIFIC & $1 / 5 / 54$ & & 300 & \\
\hline $10 / 16 / 53$ & & 300 & NOT SPECIFIC & $1 / 6 / 54$ & & 300 & \\
\hline $10 / 17 / 53$ & & 300 & NOT SPECIFIC & $1 / 7 / 54$ & & 300 & \\
\hline $10 / 18 / 53$ & & 300 & NOT SPECIFIC & $1 / 8 / 54$ & & 300 & \\
\hline $10 / 19 / 53$ & & 300 & NOT SPECIFIC & $1 / 11 / 54$ & & 300 & \\
\hline $10 / 20 / 53$ & & 300 & & $1 / 12 / 54$ & & 300 & \\
\hline $10 / 21 / 53$ & & 300 & & $1 / 13 / 54$ & & 300 & \\
\hline $10 / 23 / 53$ & & 300 & & $1 / 14 / 54$ & & 300 & \\
\hline $10 / 24 / 53$ & & 300 & & $1 / 15 / 54$ & & 300 & \\
\hline $10 / 25 / 53$ & & 300 & & $1 / 18 / 54$ & & 300 & \\
\hline $10 / 26 / 53$ & & 300 & & $1 / 19 / 54$ & & 300 & \\
\hline $10 / 27 / 53$ & & 300 & & $1 / 20 / 54$ & & 300 & \\
\hline $10 / 28 / 53$ & & 300 & & $1 / 21 / 54$ & & 300 & \\
\hline $10 / 29 / 53$ & & 300 & & $1 / 22 / 54$ & & 300 & \\
\hline $10 / 30 / 53$ & & 300 & & $1 / 23 / 54$ & & 300 & \\
\hline $10 / 31 / 53$ & & 300 & & $1 / 24 / 54$ & & 300 & \\
\hline $11 / 2 / 53$ & & 300 & & $1 / 25 / 54$ & & 300 & \\
\hline $11 / 3 / 53$ & & 300 & & $1 / 26 / 54$ & & 300 & \\
\hline $11 / 4 / 53$ & & 300 & & $1 / 27 / 54$ & & 300 & \\
\hline $11 / 5 / 53$ & & 300 & & $1 / 28 / 54$ & & 300 & \\
\hline $11 / 6 / 53$ & & 300 & & $1 / 29 / 54$ & & 300 & \\
\hline $11 / 10 / 53$ & & 300 & & $2 / 1 / 54$ & & 300 & \\
\hline $11 / 11 / 53$ & & 300 & & $2 / 2 / 54$ & & 300 & \\
\hline $11 / 12 / 53$ & & 300 & & $2 / 3 / 54$ & & 300 & \\
\hline $11 / 13 / 53$ & & 300 & & $2 / 4 / 54$ & & 300 & \\
\hline $11 / 15 / 53$ & & 300 & & $2 / 5 / 54$ & & 300 & \\
\hline $11 / 16 / 53$ & & 300 & & $2 / 6 / 54$ & & 300 & \\
\hline $11 / 17 / 53$ & & 300 & & $2 / 7 / 54$ & & -300 & \\
\hline $11 / 18 / 53$ & & 300 & & $2 / 8 / 54$ & & 300 & \\
\hline $11 / 22 / 53$ & & 300 & & $2 / 9 / 54$ & & 300 & \\
\hline $11 / 23 / 53$ & & 300 & & $2 / 10 / 54$ & & 300 & \\
\hline $11 / 24 / 53$ & & 300 & & $2 / 11 / 54$ & & 299 & \\
\hline $11 / 25 / 53$ & & 300 & & $2 / 12 / 54$ & & 299 & \\
\hline $11 / 27 / 53$ & & 300 & & $2 / 13 / 54$ & & 240 & \\
\hline $11 / 30 / 53$ & & 300 & & $2 / 15 / 54$ & & 299 & \\
\hline $12 / 1 / 53$ & & 300 & & $2 / 16 / 54$ & & 299 & \\
\hline $12 / 2 / 53$ & & 300 & & $2 / 17 / 54$ & & 299 & \\
\hline $12 / 3 / 53$ & & 300 & & $2 / 18 / 54$ & & 299 & \\
\hline $12 / 4 / 53$ & & 300 & & $2 / 19 / 54$ & & 299 & \\
\hline $12 / 5 / 53$ & & 300 & & $2 / 22 / 54$ & & 299 & \\
\hline $12 / 6 / 53$ & & 300 & & $2 / 23 / 54$ & & 299 & \\
\hline $12 / 7 / 53$ & & 300 & & $2 / 25 / 54$ & & 299 & \\
\hline $12 / 8 / 53$ & & 300 & & $2 / 26 / 54$ & & 299 & \\
\hline $12 / 14 / 53$ & & 300 & & $3 / 1 / 54$ & & 298 & \\
\hline $12 / 15 / 53$ & & 300 & & $3 / 2 / 54$ & & 298 & \\
\hline $12 / 16 / 53$ & & 300 & & $3 / 4 / 54$ & & 298 & \\
\hline $12 / 17 / 53$ & & 300 & & $3 / 5 / 54$ & & 298 & \\
\hline $12 / 18 / 53$ & & 300 & & $3 / 6 / 54$ & & 300 & \\
\hline $12 / 21 / 53$ & & 300 & & $3 / 7 / 54$ & & 298 & \\
\hline $12 / 22 / 53$ & & 300 & & $3 / 8 / 54$ & & 298 & \\
\hline
\end{tabular}

Data obtained from RHO-CD-1172 and HW-83906. 


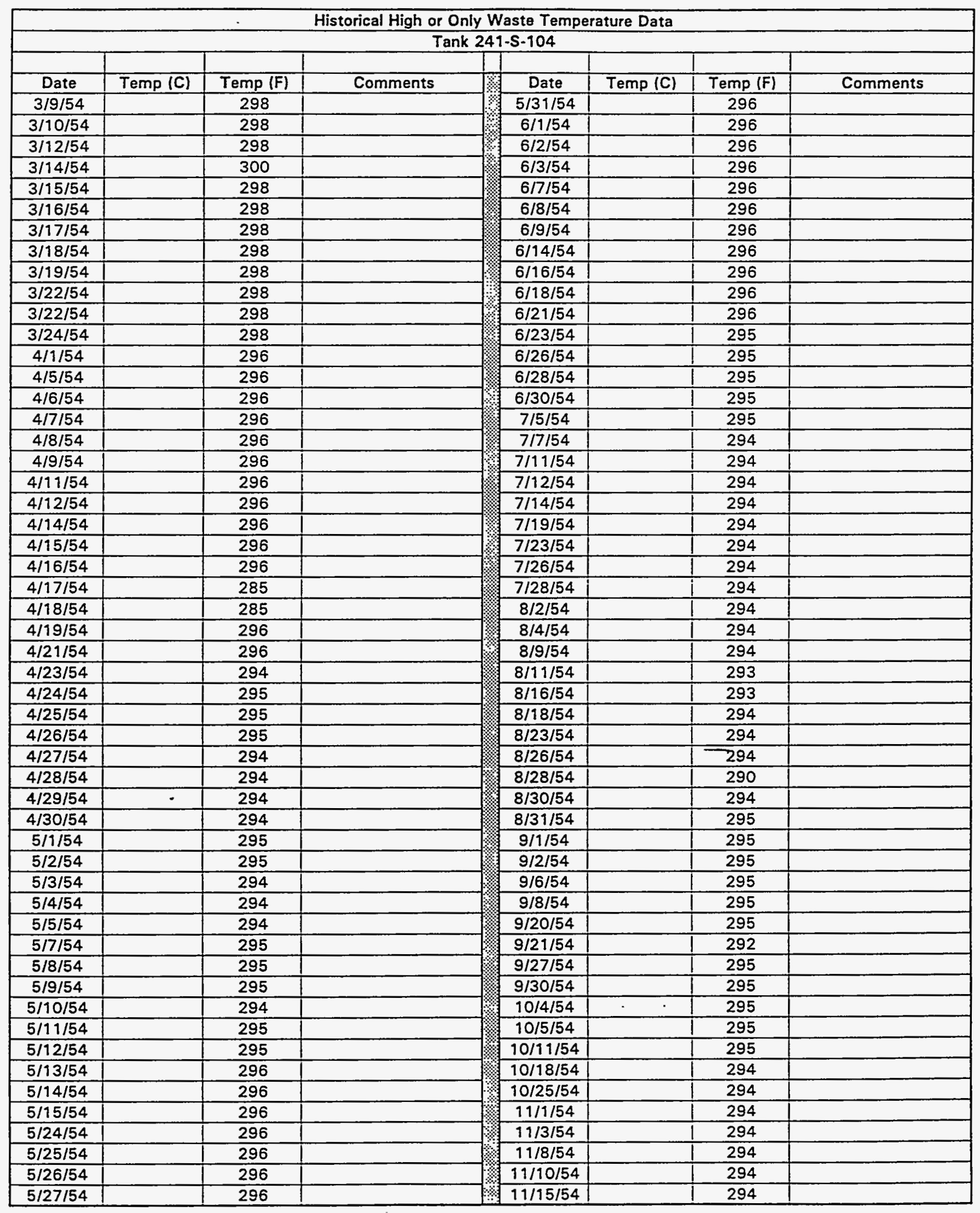

Data obtained from RHO-CD-1172 and HW-83906. 


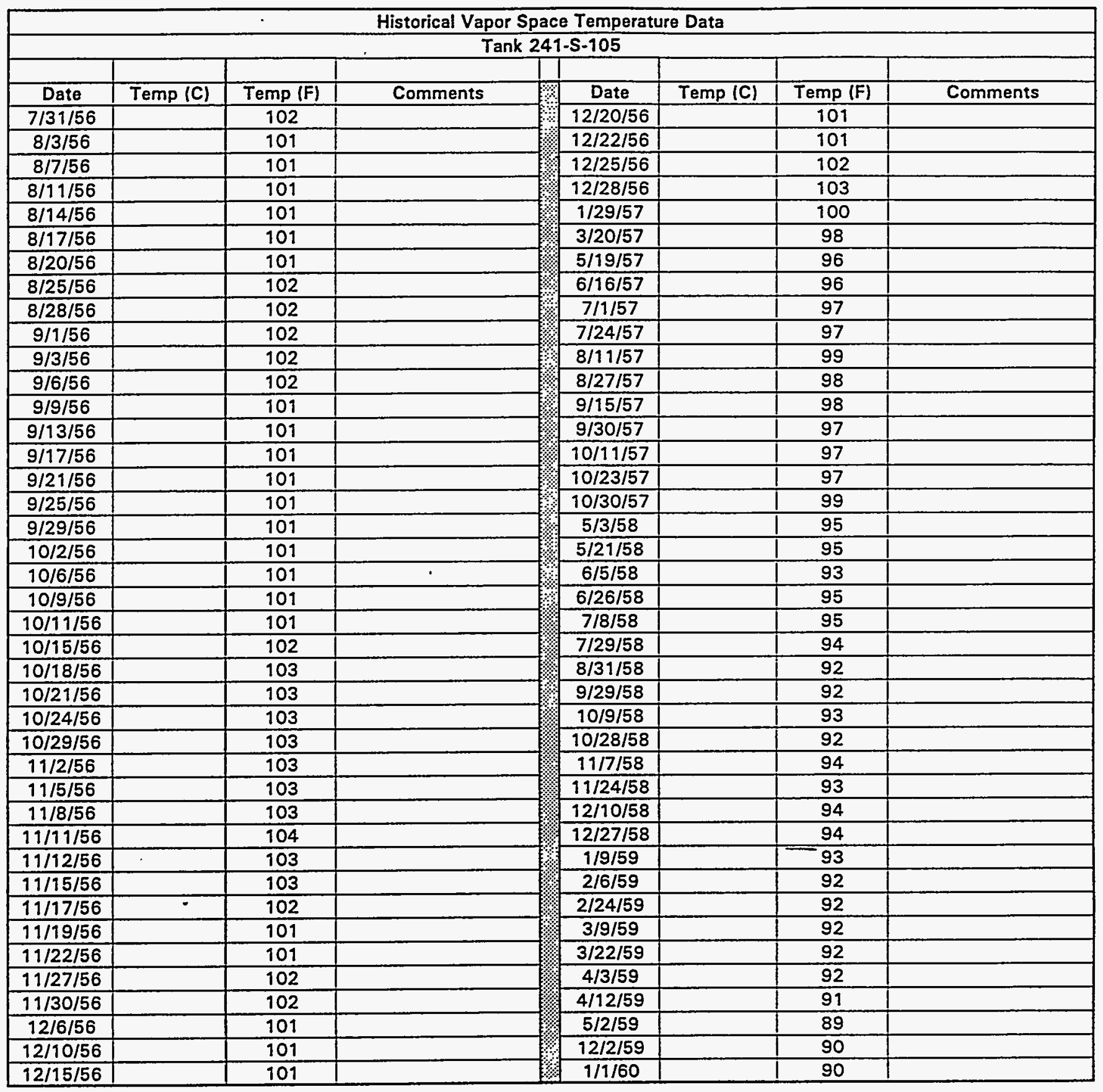

Data obtained from RHO-CD-1472. 


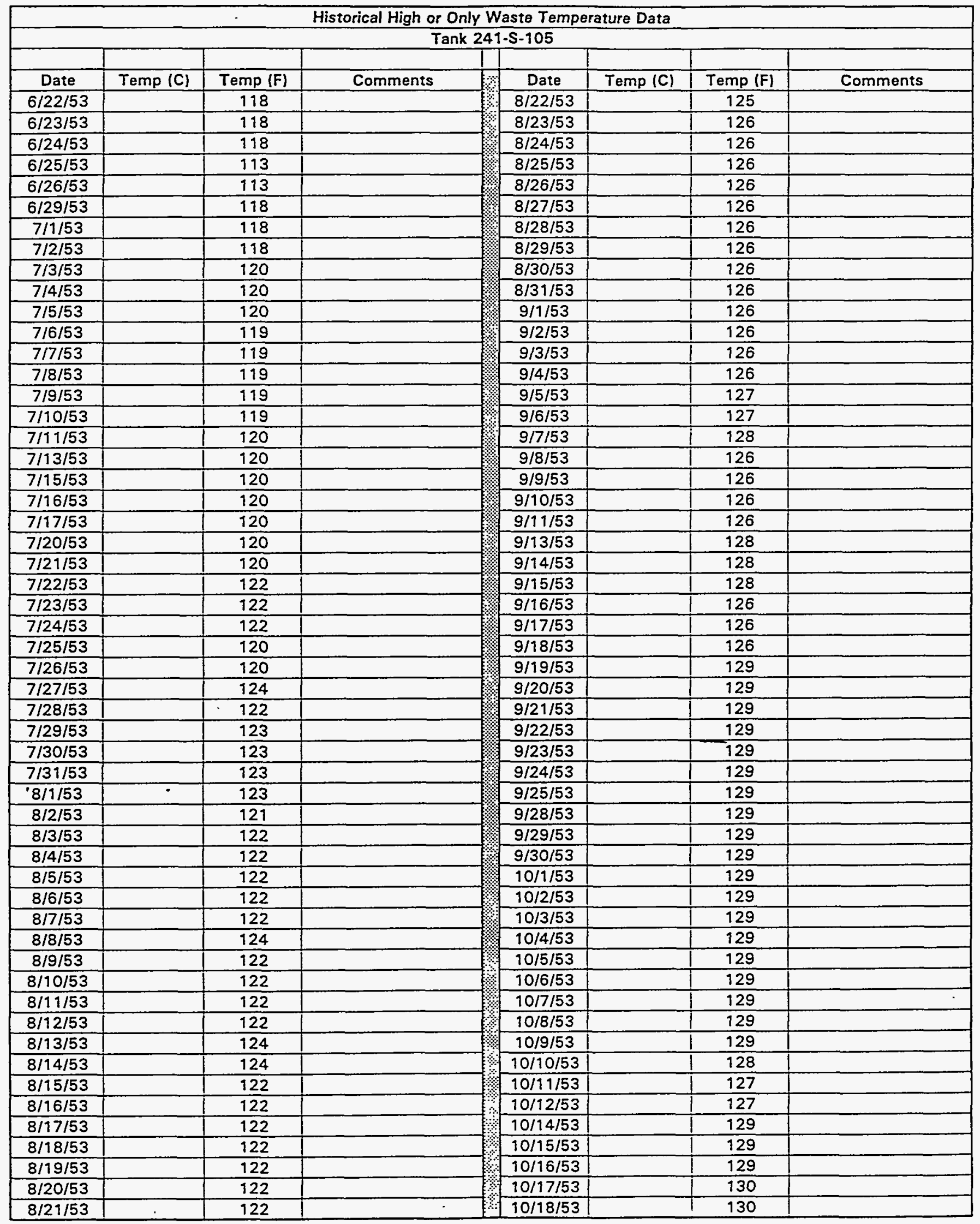

Data obtained from HW-83906. 


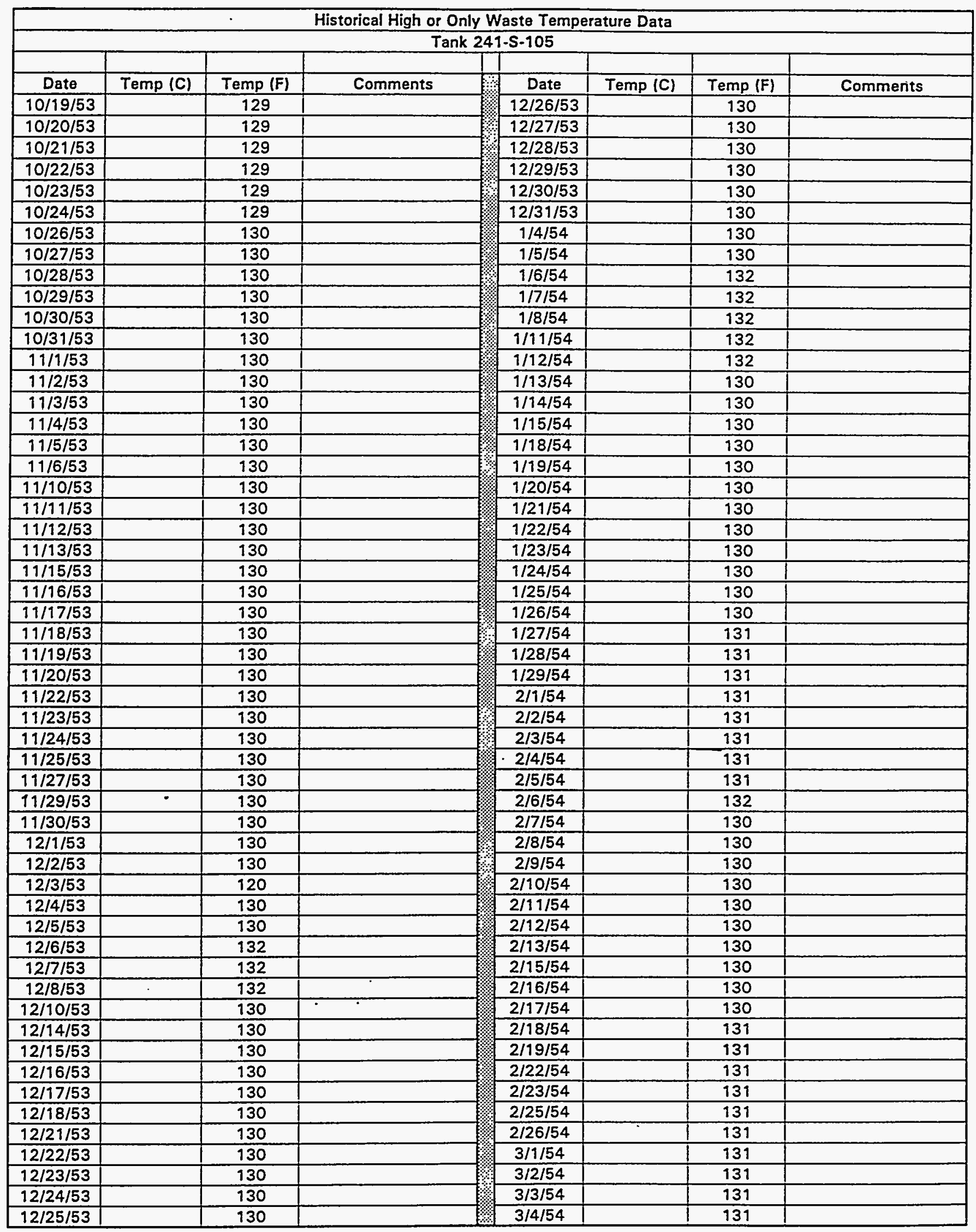

Data obtained from HW-83906. 


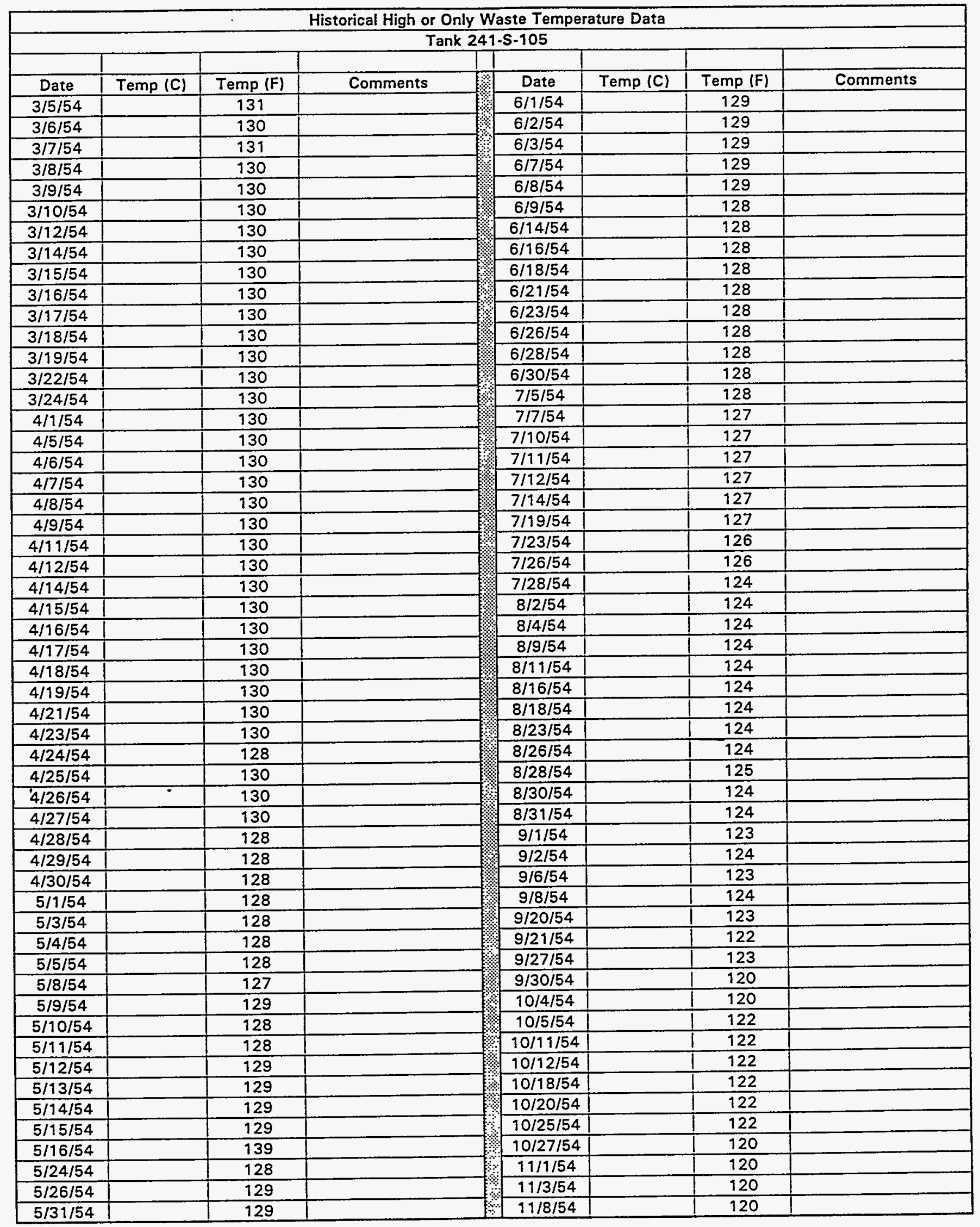

Data obtained from HW-83906. 
WHC-SD-WM-ER-323, Rev. O 1

Historical High or Only Waste Temperature Data

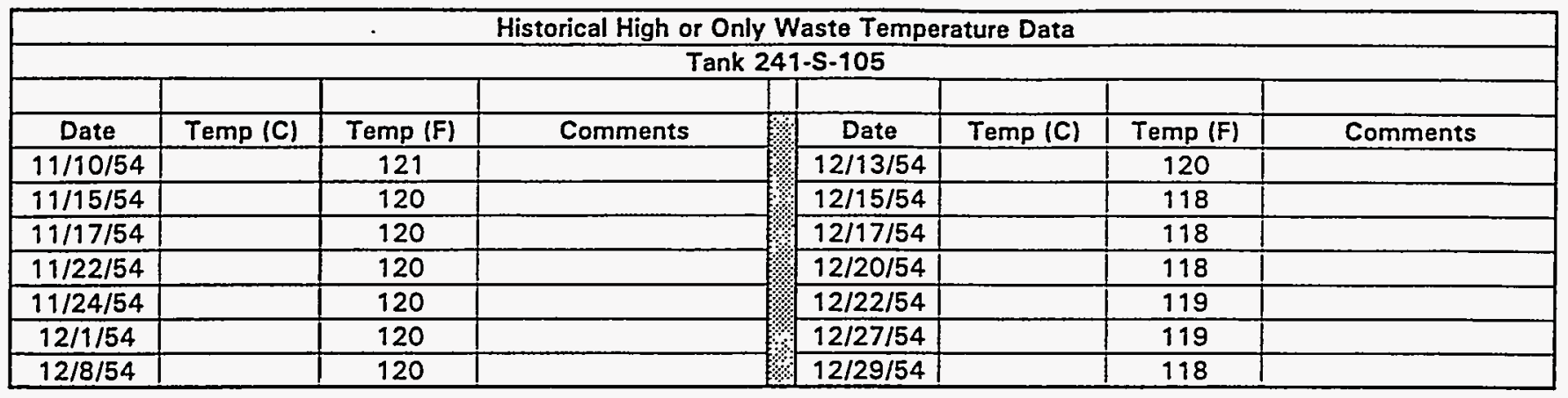




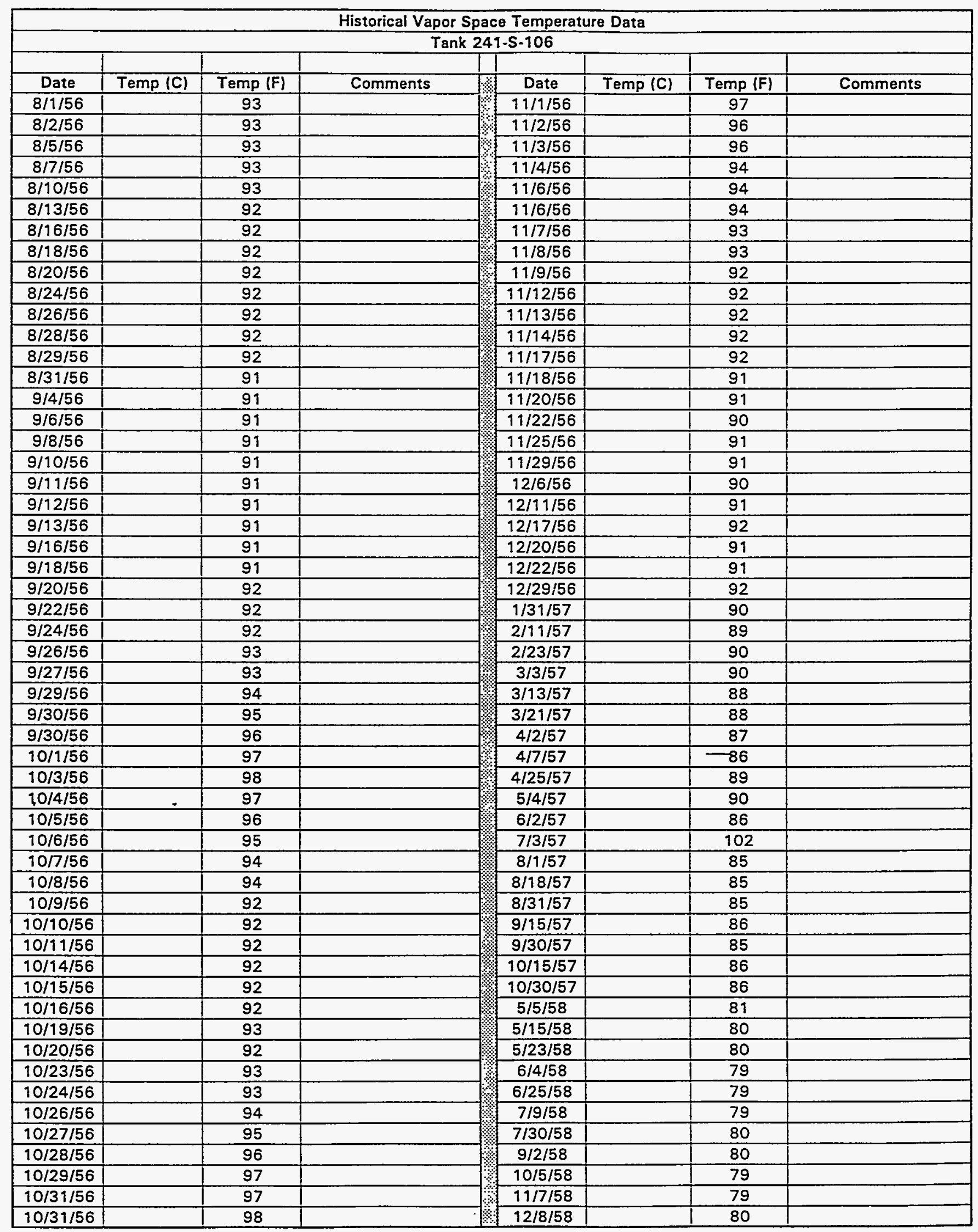

Data obtained from RHO-CD-1172. 
WHC-SD-WM-ER-323, Rev. 0 1

Historical Vapor Space Temperature Data

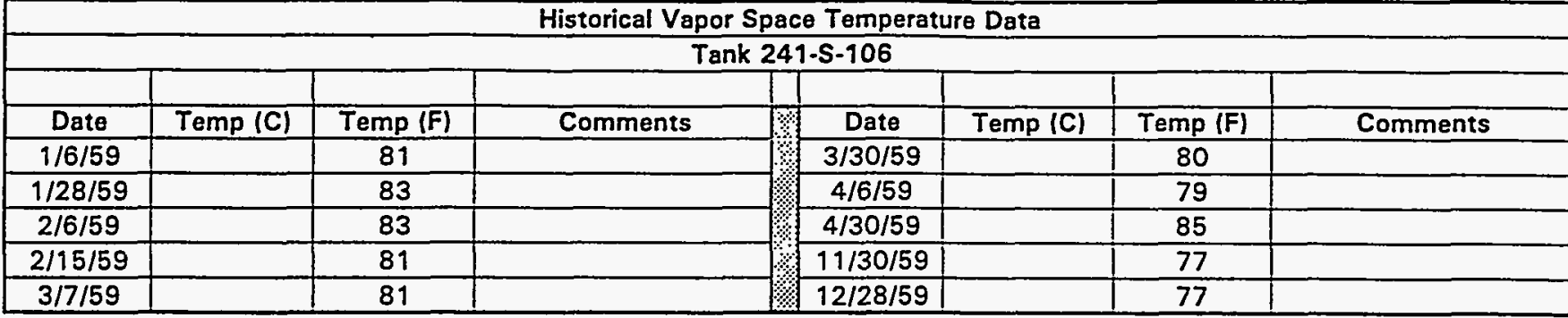




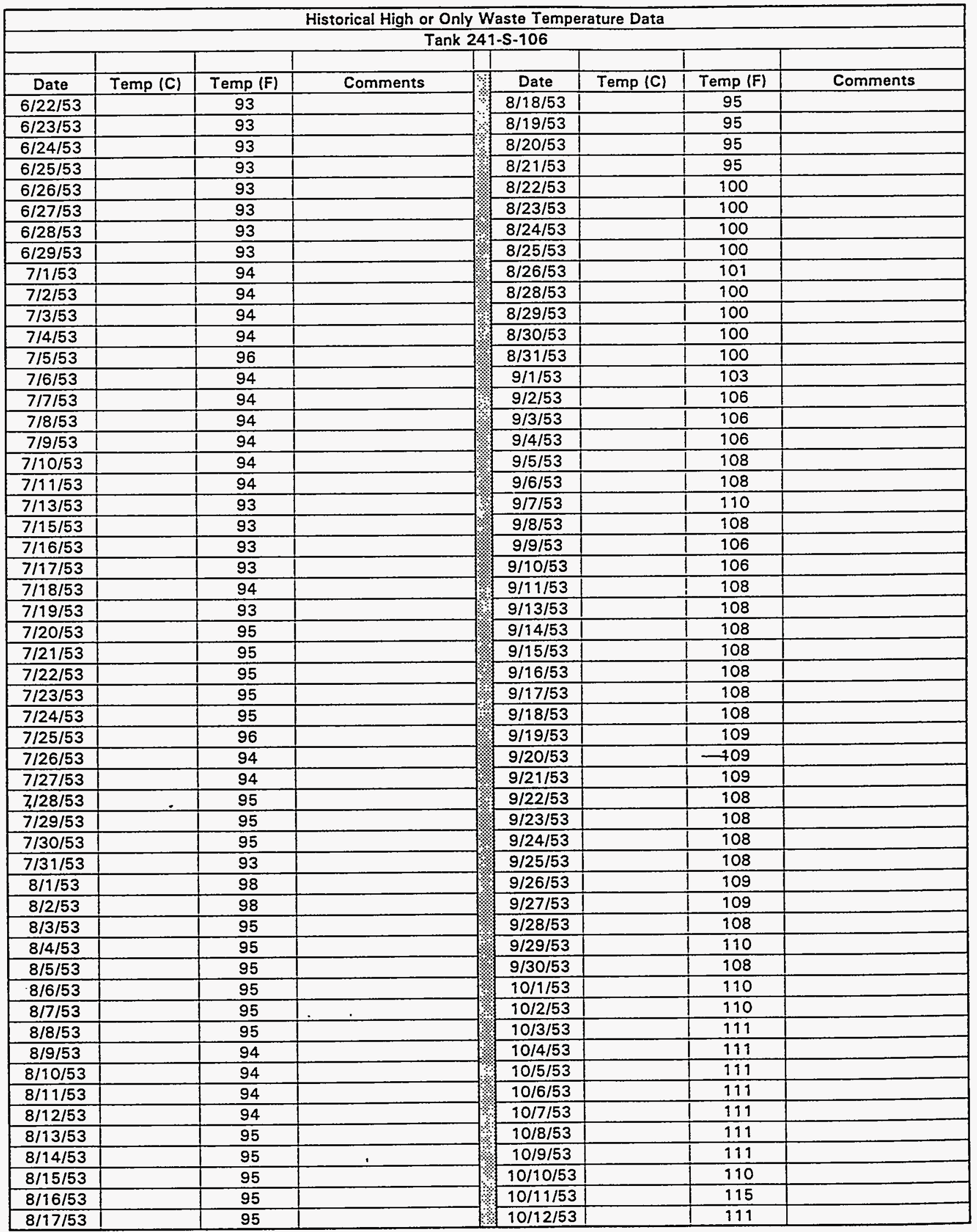

Data obtained from HW-83906. 


\begin{tabular}{|c|c|c|c|c|c|c|c|}
\hline \multicolumn{8}{|c|}{ Historical High or Only Waste Temperature Data } \\
\hline \multicolumn{8}{|c|}{ Tank 241-S-106 } \\
\hline & & & & & & & \\
\hline Date & Temp (C) & Temp (F) & Comments & Date & Temp (C) & Temp (F) & Comments \\
\hline $10 / 14 / 53$ & & 111 & & $12 / 17 / 53$ & & 112 & \\
\hline $10 / 15 / 53$ & & 111 & & $12 / 18 / 53$ & & 112 & \\
\hline $10 / 16 / 53$ & & 111 & & $12 / 21 / 53$ & & 112 & \\
\hline $10 / 17 / 53$ & & 110 & & $12 / 22 / 53$ & & 112 & \\
\hline $10 / 18 / 53$ & & 110 & & $12 / 23 / 53$ & & 112 & \\
\hline $10 / 19 / 53$ & & 110 & & $12 / 24 / 53$ & & 112 & \\
\hline $10 / 20 / 53$ & & 110 & & $12 / 25 / 53$ & & 112 & \\
\hline $10 / 21 / 53$ & & 110 & & $12 / 26 / 53$ & & 112 & \\
\hline $10 / 22 / 53$ & & 112 & & $12 / 27 / 53$ & & 113 & \\
\hline $10 / 23 / 53$ & & 111 & & $12 / 28 / 53$ & & 113 & \\
\hline $10 / 24 / 53$ & & 111 & & $12 / 29 / 53$ & & 113 & \\
\hline $10 / 25 / 53$ & & 110 & & $12 / 30 / 53$ & & 112 & \\
\hline $10 / 26 / 53$ & & 111 & & $12 / 31 / 53$ & & 112 & \\
\hline $10 / 27 / 53$ & & 111 & & $1 / 1 / 54$ & & 113 & \\
\hline $10 / 28 / 53$ & & 111 & & $1 / 2 / 54$ & & 113 & \\
\hline $10 / 29 / 53$ & & 111 & & $1 / 3 / 54$ & & 113 & \\
\hline $10 / 30 / 53$ & & 111 & & $1 / 4 / 54$ & & 112 & \\
\hline $10 / 31 / 53$ & & 112 & & $1 / 5 / 54$ & & 113 & \\
\hline $11 / 2 / 53$ & & 111 & & $1 / 6 / 54$ & & 113 & \\
\hline $11 / 3 / 53$ & & 111 & & $1 / 7 / 54$ & & 113 & \\
\hline $11 / 4 / 53$ & & 111 & & $1 / 8 / 54$ & & 113 & \\
\hline $11 / 5 / 53$ & & 111 & & $1 / 11 / 54$ & & 915 & \\
\hline $11 / 6 / 53$ & & 111 & & $1 / 12 / 54$ & & 115 & \\
\hline $11 / 10 / 53$ & & 111 & & $1 / 13 / 54$ & & 116 & \\
\hline $11 / 11 / 53$ & & 111 & & $1 / 14 / 54$ & & 116 & \\
\hline $11 / 12 / 53$ & & 111 & & $1 / 15 / 54$ & & 116 & \\
\hline $11 / 13 / 53$ & & 111 & & $1 / 18 / 54$ & & 116 & \\
\hline $11 / 15 / 53$ & & 111 & & $1 / 19 / 54$ & & 116 & \\
\hline $11 / 16 / 53$ & & 111 & & $1 / 20 / 54$ & & 114 & \\
\hline $11 / 17 / 53$ & & 111 & & $1 / 21 / 54$ & & 114 & \\
\hline $11 / 18 / 53$ & & 111 & & $1 / 22 / 54$ & & 114 & \\
\hline $11 / 19 / 53$ & & 111 & & $1 / 23 / 54$ & & 114 & \\
\hline $11 / 20 / 53$ & & 111 & & $1 / 24 / 54$ & & 114 & \\
\hline $11 / 22 / 53$ & & 110 & & $1 / 25 / 54$ & & 114 & \\
\hline $11 / 23 / 53$ & & 111 & & $1 / 26 / 54$ & & 114 & \\
\hline $11 / 24 / 53$ & & 119 & & $1 / 27 / 54$ & & 114 & \\
\hline $11 / 25 / 53$ & & 111 & & $1 / 28 / 54$ & & 114 & \\
\hline $19 / 27 / 53$ & & 111 & & $1 / 29 / 54$ & & 114 & \\
\hline $11 / 29 / 53$ & & 110 & & $2 / 1 / 54$ & & 114 & \\
\hline $11 / 30 / 53$ & & 110 & & $2 / 2 / 54$ & & 114 & \\
\hline $12 / 1 / 53$ & & 110 & & $2 / 3 / 54$ & & 114 & \\
\hline $12 / 2 / 53$ & & 110 & & $2 / 4 / 54$ & & 114 & \\
\hline $12 / 3 / 53$ & & 110 & & $2 / 5 / 54$ & & 114 & \\
\hline $12 / 4 / 53$ & & 110 & & $2 / 6 / 54$ & & 117 & \\
\hline $12 / 5 / 53$ & & 111 & & $2 / 7 / 54$ & & 117 & \\
\hline $12 / 6 / 53$ & & 112 & & $2 / 8 / 54$ & & 117 & \\
\hline $12 / 7 / 53$ & & 112 & & $2 / 9 / 54$ & & 117 & \\
\hline $12 / 8 / 53$ & & 112 & & $2 / 10 / 54$ & & 117 & \\
\hline $12 / 10 / 53$ & & 111 & & $2 / 11 / 54$ & & 117 & \\
\hline $12 / 11 / 53$ & & 111 & & $2 / 13 / 54$ & & 112 & \\
\hline $12 / 12 / 53$ & & 112 & & $2 / 15 / 54$ & & 112 & \\
\hline $12 / 14 / 53$ & & 112 & & $2 / 16 / 54$ & & 112 & \\
\hline $12 / 15 / 53$ & & 112 & & $2 / 17 / 54$ & & 112 & \\
\hline $12 / 16 / 53$ & & 112 & & $2 / 18 / 54$ & & 114 & \\
\hline
\end{tabular}

Data obtained from HW-83906.

D-137. 


\begin{tabular}{|c|c|c|c|c|c|c|c|}
\hline \multicolumn{8}{|c|}{ Historical High or Only Waste Temperature Data } \\
\hline \multicolumn{8}{|c|}{ Tank 241-S-106 } \\
\hline & & & & & & & \\
\hline Date & Temp (C) & Temp (F) & Comments & Date & Temp (C) & Temp (F) & Comments \\
\hline $2 / 19 / 54$ & & 114 & & $5 / 11 / 54$ & & 109 & \\
\hline $2 / 22 / 54$ & & 114 & & $5 / 12 / 54$ & & 109 & \\
\hline $2 / 23 / 54$ & & 114 & & $5 / 13 / 54$ & & 109 & \\
\hline $2 / 25 / 54$ & & 114 & & $5 / 14 / 54$ & & 109 & \\
\hline $2 / 26 / 54$ & & 114 & & $5 / 15 / 54$ & & 109 & \\
\hline $3 / 1 / 54$ & & 114 & & $5 / 16 / 54$ & & 110 & \\
\hline $3 / 2 / 54$ & & 114 & & $5 / 24 / 54$ & & 108 & \\
\hline $3 / 3 / 54$ & & 114 & & $5 / 26 / 54$ & & 108 & \\
\hline $3 / 4 / 54$ & & 114 & & $5 / 31 / 54$ & & 109 & \\
\hline $3 / 5 / 54$ & & 114 & & $6 / 1 / 54$ & & 109 & \\
\hline $3 / 6 / 54$ & & 114 & & $6 / 2 / 54$ & & 108 & \\
\hline $3 / 7 / 54$ & & 112 & & $6 / 3 / 54$ & & 108 & \\
\hline $3 / 8 / 54$ & & 114 & & $6 / 7 / 54$ & & 108 & \\
\hline $3 / 9 / 54$ & & 114 & & $6 / 8 / 54$ & & 108 & \\
\hline $3 / 10 / 54$ & & 113 & & $6 / 9 / 54$ & & 107 & \\
\hline $3 / 12 / 54$ & & 113 & & $6 / 14 / 54$ & & 107 & \\
\hline $3 / 14 / 54$ & & 112 & & $6 / 16 / 54$ & & 107 & \\
\hline $3 / 15 / 54$ & & 113 & & $6 / 18 / 54$ & & 107 & \\
\hline $3 / 16 / 54$ & & 113 & & $6 / 21 / 54$ & & 107 & \\
\hline $3 / 17 / 54$ & & 113 & & $6 / 23 / 54$ & & 107 & \\
\hline $3 / 18 / 54$ & & 113 & & $6 / 26 / 54$ & & 107 & \\
\hline $3 / 19 / 54$ & & 113 & & $6 / 28 / 54$ & & 107 & \\
\hline $3 / 22 / 54$ & & 113 & & $6 / 30 / 54$ & & 107 & \\
\hline $3 / 24 / 54$ & & 113 & & $7 / 5 / 54$ & & 107 & \\
\hline $4 / 1 / 54$ & & 113 & & $7 / 7 / 54$ & & 107 & \\
\hline $4 / 5 / 54$ & & 110 & & $7 / 10 / 54$ & & 107 & \\
\hline $4 / 6 / 54$ & & 110 & & $7 / 11 / 54$ & & 107 & \\
\hline $4 / 7 / 54$ & & 110 & & $7 / 12 / 54$ & & 107 & \\
\hline $4 / 8 / 54$ & & 110 & & $7 / 14 / 54$ & & 107 & \\
\hline $4 / 9 / 54$ & & 110 & & $7 / 19 / 54$ & & 107 & \\
\hline $4 / 11 / 54$ & & 110 & & $7 / 23 / 54$ & & 107 & \\
\hline $4 / 12 / 54$ & & 110 & & $7 / 26 / 54$ & & 707 & \\
\hline $4 / 14 / 54$ & & 110 & & $7 / 28 / 54$ & & 107 & \\
\hline $4 / 15 / 54$ & . & 910 & & $8 / 2 / 54$ & & 107 & \\
\hline $4 / 16 / 54$ & & 110 & & $8 / 4 / 54$ & & 106 & \\
\hline $4 / 17 / 54$ & & 110 & & $8 / 9 / 54$ & & 106 & \\
\hline $4 / 18 / 54$ & & 110 & & $8 / 11 / 54$ & & 106 & \\
\hline $4 / 19 / 54$ & & 110 & & $8 / 16 / 54$ & & 106 & \\
\hline $4 / 21 / 54$ & & 110 & & $8 / 18 / 54$ & & 106 & \\
\hline $4 / 23 / 54$ & & 110 & & $8 / 23 / 54$ & & 106 & \\
\hline $4 / 24 / 54$ & & 110 & & $8 / 26 / 54$ & & 106 & \\
\hline $4 / 25 / 54$ & & 910 & & $8 / 28 / 54$ & & 110 & \\
\hline $4 / 26 / 54$ & & 110 & & $8 / 30 / 54$ & & 106 & \\
\hline $4 / 27 / 54$ & & 110 & & $8 / 31 / 54$ & & 107 & \\
\hline $4 / 28 / 54$ & & 109 & & $9 / 1 / 54$ & & 106 & \\
\hline $4 / 29 / 54$ & & 109 & & $9 / 2 / 54$ & & 106 & \\
\hline $4 / 30 / 54$ & & 109 & & $9 / 6 / 54$ & & 106 & \\
\hline $5 / 1 / 54$ & & 110 & & $9 / 8 / 54$ & & 106 & \\
\hline $5 / 3 / 54$ & & 109 & & $9 / 20 / 54$ & & 103 & \\
\hline $5 / 4 / 54$ & & 109 & & $9 / 21 / 54$ & & 103 & \\
\hline $5 / 5 / 54$ & & 109 & & $9 / 27 / 54$ & & 103 & \\
\hline $5 / 8 / 54$ & & 110 & & $9 / 30 / 54$ & & 103 & \\
\hline $5 / 9 / 54$ & & 110 & & $10 / 4 / 54$ & & 103 & \\
\hline $5 / 10 / 54$ & & 109 & & $10 / 5 / 54$ & & 103 & \\
\hline
\end{tabular}

Data obtained from HW-83906. 
WHC-SD-WM-ER-323, Rev. O

\begin{tabular}{|c|c|c|c|c|c|c|c|}
\hline \multicolumn{8}{|c|}{ Historical High or Only Waste Temperature Data } \\
\hline \multicolumn{8}{|c|}{ Tank 241-S-106 } \\
\hline & & & & & & & \\
\hline Date & Temp (C) & Temp (F) & Comments & Date & Temp (C) & Temp (F) & Commentś \\
\hline $10 / 11 / 54$ & & 103 & & $11 / 22 / 54$ & & 101 & \\
\hline $10 / 12 / 54$ & & 101 & & $11 / 24 / 54$ & & 100 & \\
\hline $10 / 18 / 54$ & & 101 & & $12 / 1 / 54$ & & 100 & \\
\hline $10 / 20 / 54$ & & 101 & & $12 / 8 / 54$ & & 100 & \\
\hline $10 / 25 / 54$ & & 101 & & $12 / 13 / 54$ & & 100 & \\
\hline $10 / 27 / 54$ & & 101 & & $12 / 15 / 54$ & & 100 & \\
\hline $11 / 1 / 54$ & & 101 & & $12 / 17 / 54$ & & 100 & \\
\hline $11 / 3 / 54$ & & 101 & & $12 / 20 / 54$ & & 100 & \\
\hline $11 / 8 / 54$ & & 101 & & $12 / 22 / 54$ & & 100 & \\
\hline $11 / 10 / 54$ & & 101 & & $12 / 27 / 54$ & & 100 & \\
\hline $11 / 15 / 54$ & & 101 & & $12 / 29 / 54$ & & 100 & \\
\hline $11 / 17 / 54$ & & 101 & & $0-1-10$ & & & \\
\hline
\end{tabular}

Data obtained from HW-83906... 


\begin{tabular}{|c|c|c|c|c|c|c|c|}
\hline \multicolumn{8}{|c|}{ Historical Vapor Space Temperature Data } \\
\hline \multicolumn{8}{|c|}{ Tank 241-S-107 } \\
\hline & & & & & & & \\
\hline Date & Temp (C) & Temp (F) & Comments & Date & Temp (C) & Temp (F) & Comments \\
\hline $7 / 30 / 56$ & & 171 & & $12 / 3 / 56$ & & 184 & \\
\hline $8 / 1 / 56$ & & 172 & & $12 / 3 / 56$ & & 185 & \\
\hline $8 / 2 / 56$ & & 174 & & $12 / 4 / 56$ & & 187 & \\
\hline $8 / 5 / 56$ & & 175 & & $12 / 6 / 56$ & & 189 & \\
\hline $8 / 7 / 56$ & & 176 & & $12 / 8 / 56$ & & 191 & \\
\hline $8 / 9 / 56$ & & 175 & & $12 / 8 / 56$ & & 193 & \\
\hline $8 / 11 / 56$ & & 174 & & $12 / 10 / 56$ & & 194 & \\
\hline $8 / 13 / 56$ & & 173 & & $12 / 11 / 56$ & & 195 & \\
\hline $8 / 16 / 56$ & & 173 & & $12 / 12 / 56$ & & 193 & \\
\hline $8 / 19 / 56$ & & 173 & & $12 / 13 / 56$ & & 192 & \\
\hline $8 / 21 / 56$ & & 173 & & $12 / 14 / 56$ & & 191 & \\
\hline $8 / 22 / 56$ & & 173 & & $12 / 15 / 56$ & & 189 & \\
\hline $8 / 22 / 56$ & & 175 & & $12 / 15 / 56$ & & 187 & \\
\hline $8 / 23 / 56$ & & 176 & & $12 / 16 / 56$ & & 185 & \\
\hline $8 / 24 / 56$ & & 177 & & $12 / 17 / 56$ & & 184 & \\
\hline $8 / 24 / 56$ & & 179 & & $12 / 18 / 56$ & & 183 & \\
\hline $8 / 24 / 56$ & & 181 & & $12 / 18 / 56$ & & 181 & \\
\hline $8 / 24 / 56$ & & 183 & & $12 / 19 / 56$ & & 180 & \\
\hline $8 / 25 / 56$ & & 185 & & $12 / 20 / 56$ & & 179 & \\
\hline $8 / 27 / 56$ & & 188 & & $12 / 21 / 56$ & & 179 & \\
\hline $8 / 27 / 56$ & & 190 & & $12 / 23 / 56$ & & 178 & \\
\hline $8 / 27 / 56$ & & 192 & & $12 / 24 / 56$ & & 178 & \\
\hline $8 / 30 / 56$ & & 192 & & $12 / 26 / 56$ & & 178 & \\
\hline $9 / 2 / 56$ & & 191 & & $12 / 29 / 56$ & & 177 & \\
\hline $9 / 4 / 56$ & & 191 & & $1 / 29 / 57$ & & 172 & \\
\hline $9 / 6 / 56$ & & 190 & & $2 / 28 / 57$ & & 172 & \\
\hline $9 / 9 / 56$ & & 189 & & $3 / 29 / 57$ & & 161 & \\
\hline $9 / 13 / 56$ & & 189 & & $4 / 30 / 57$ & & 176 & \\
\hline $9 / 14 / 56$ & & 190 & & $7 / 3 / 57$ & & 142 & \\
\hline $9 / 18 / 56$ & & 191 & & $7 / 31 / 57$ & & 171 & \\
\hline $9 / 22 / 56$ & & 191 & & $8 / 6 / 57$ & & 169 & \\
\hline $9 / 25 / 56$ & & 191 & & $8 / 27 / 57$ & & 159 & \\
\hline $9 / 29 / 56$ & & 191 & & $12 / 12 / 57$ & & 758 & \\
\hline $10 / 2 / 56$ & & 191 & & $12 / 24 / 57$ & & 156 & \\
\hline $10 / 6 / 56$ & 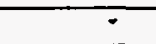 & 191 & & $2 / 28 / 58$ & & 153 & \\
\hline $10 / 9 / 56$ & & 191 & & $5 / 1 / 58$ & & 149 & \\
\hline $10 / 13 / 56$ & & 191 & & $5 / 26 / 58$ & & 150 & \\
\hline $10 / 16 / 56$ & & 199 & & $6 / 17 / 58$ & & 149 & \\
\hline $10 / 17 / 56$ & & 189 & & $7 / 10 / 58$ & & 148 & \\
\hline $10 / 20 / 56$ & & 189 & & $7 / 29 / 58$ & & 145 & \\
\hline $10 / 22 / 56$ & & 188 & & $9 / 2 / 58$ & & 150 & \\
\hline $10 / 24 / 56$ & & 186 & & $10 / 5 / 58$ & & 150 & \\
\hline $10 / 26 / 56$ & & 186 & & $11 / 9 / 58$ & & 148 & \\
\hline $10 / 31 / 56$ & & 186 & & $12 / 3 / 58$ & & 949 & \\
\hline $11 / 3 / 56$ & & 186 & & $12 / 23 / 58$ & & 148 & \\
\hline $11 / 7 / 56$ & & 186 & & $1 / 22 / 59$ & & 145 & \\
\hline $11 / 11 / 56$ & & 186 & & $2 / 8 / 59$ & & 143 & \\
\hline $11 / 13 / 56$ & & 185 & & $2 / 20 / 59$ & & 144 & \\
\hline $11 / 16 / 56$ & & 183 & & $3 / 7 / 59$ & & 144 & \\
\hline $11 / 16 / 56$ & & 182 & & $3 / 21 / 59$ & & 142 & \\
\hline $11 / 19 / 56$ & & 181 & & $4 / 8 / 59$ & & 140 & \\
\hline $11 / 23 / 56$ & & 182 & & $4 / 20 / 59$ & & 141 & \\
\hline $11 / 26 / 56$ & & 181 & & $5 / 2 / 59$ & & 141 & \\
\hline $11 / 29 / 56$ & & 182 & & $5 / 17 / 59$ & & 140 & \\
\hline $12 / 2 / 56$ & & 182 & & $6 / 6 / 59$ & & 139 & \\
\hline
\end{tabular}

Data obtained from RHO-CD-1172. 
WHC-SD-WM-ER-323, Rev. O

\begin{tabular}{|c|c|c|c|c|c|c|c|}
\hline \multicolumn{7}{|c|}{ Historical Vapor Space Temperature Data } \\
\hline & & & \multicolumn{3}{c|}{ Tank 241-5-107 } & & \\
\hline Date & Temp (C) & Temp (F) & Comments & Date & Temp (C) & Temp (F) & Comments \\
\hline $1 / 30 / 60$ & & 138 & & $3 / 1 / 60$ & & 163 & \\
\hline $2 / 8 / 60$ & & 145 & & & & & \\
\hline
\end{tabular}


WHC-SD-WM-ER-323, Rev. O 1

Historical Average Waste Temperature Data

\begin{tabular}{|c|c|c|c|c|c|c|c|}
\hline \multicolumn{8}{|c|}{ Tank 241-S-107 } \\
\hline & & & 5 & Dota & Tomn IC & Temp (F) & Comments \\
\hline $7 / 26 / 53$ & $\operatorname{ten}(\mathrm{N})$ & $\frac{1 \mathrm{emp}(1)}{216}$ & Cominerts & $1 / 8 / 54$ & & 198 & \\
\hline $8 / 18 / 53$ & & 216 & & $1 / 29 / 54$ & & 199 & \\
\hline $10 / 7 / 53$ & & 219 & & & & & \\
\hline
\end{tabular}




\begin{tabular}{|c|c|c|c|c|c|c|c|}
\hline \multirow{2}{*}{\multicolumn{8}{|c|}{ Historical High or Only Waste Temperature Data }} \\
\hline \multicolumn{7}{|c|}{ Tank 241-S-107 } & \\
\hline $9 / 2 / 52$ & & 96 & & $8 / 13 / 53$ & & 228 & ASSUMPTION \\
\hline $9 / 7 / 52$ & & 121 & & $8 / 14 / 53$ & & 228 & ASSUMPTION \\
\hline $9 / 11 / 52$ & & 131 & & $8 / 15 / 53$ & & 228 & ASSUMPTION \\
\hline $9 / 23 / 52$ & & 157 & & $8 / 16 / 53$ & & 215 & \\
\hline $10 / 3 / 52$ & & 170 & & $8 / 17 / 53$ & & 228 & ASSUMPTION \\
\hline $10 / 19 / 52$ & & 195 & & $8 / 18 / 53$ & & 228 & ASSUMPTION \\
\hline $10 / 24 / 52$ & & 206 & & $8 / 19 / 53$ & & 228 & ASSUMPTION \\
\hline $10 / 27 / 52$ & & 219 & & $8 / 20 / 53$ & & 228 & ASSUMPTION \\
\hline $10 / 30 / 52$ & & 239 & & $8 / 21 / 53$ & & 228 & ASSUMPTION \\
\hline $6 / 22 / 53$ & & 239 & ASSUMPTION & $8 / 22 / 53$ & & 227 & ASSUMPTION \\
\hline $6 / 23 / 53$ & & 238 & ASSUMPTION & $8 / 23 / 53$ & & 227 & ASSUMPTION \\
\hline $6 / 24 / 53$ & & 238 & ASSUMPTION & $8 / 24 / 53$ & & 227 & ASSUMPTION \\
\hline $6 / 25 / 53$ & & 238 & ASSUMPTION & $8 / 25 / 53$ & & 226 & ASSUMPTION \\
\hline $6 / 26 / 53$ & & 238 & ASSUMPTION & $8 / 26 / 53$ & & 227 & ASSUMPTION \\
\hline $6 / 26 / 53$ & & 238 & ASSUMPTION & $8 / 28 / 53$ & & 228 & \\
\hline $6 / 29 / 53$ & & 238 & ASSUMPTION & $8 / 29 / 53$ & & 228 & ASSUMPTION \\
\hline $7 / 1 / 53$ & & 238 & ASSUMPTION & $8 / 30 / 53$ & & 228 & ASSUMPTION \\
\hline $7 / 2 / 53$ & & 238 & ASSUMPTION & $8 / 31 / 53$ & & 238 & ASSUMPTION \\
\hline $7 / 3 / 53$ & & 238 & ASSUMPTION & $9 / 1 / 53$ & & 238 & ASSUMPTION \\
\hline $7 / 4 / 53$ & & 238 & ASSUMPTION & $9 / 2 / 53$ & & 236 & ASSUMPTION \\
\hline $7 / 6 / 53$ & & 238 & ASSUMPTION & $9 / 3 / 53$ & & 236 & ASSUMPTION \\
\hline $7 / 7 / 53$ & & 238 & ASSUMPTION & $9 / 4 / 53$ & & 236 & ASSUMPTION \\
\hline $7 / 8 / 53$ & & 238 & ASSUMPTION & $9 / 5 / 53$ & & 228 & ASSUMPTION \\
\hline $7 / 9 / 53$ & & 238 & ASSUMPTION & $9 / 6 / 53$ & & 226 & ASSUMPTION \\
\hline $7 / 10 / 53$ & & 238 & ASSUMPTION & $9 / 7 / 53$ & & 228 & ASSUMPTION \\
\hline $7 / 11 / 53$ & & 260 & NOT SPECIFIC & $9 / 8 / 53$ & & 228 & ASSUMPTION \\
\hline $7 / 13 / 53$ & & 238 & ASSUMPTION & $9 / 9 / 53$ & & 236 & ASSUMPTION \\
\hline $7 / 15 / 53$ & & 238 & ASSUMPTION & $9 / 10 / 53$ & & 236 & ASSUMPTION \\
\hline $7 / 16 / 53$ & & 238 & ASSUMPTION & $9 / 11 / 53$ & & 236 & ASSUMPTION \\
\hline $7 / 17 / 53$ & & 238 & ASSUMPTION & $9 / 13 / 53$ & & 236 & ASSUMPTION \\
\hline $7 / 20 / 53$ & & 238 & ASSUMPTION & $9 / 14 / 53$ & & 236 & ASSUMPTION \\
\hline $7 / 21 / 53$ & & 238 & ASSUMPTION & $9 / 15 / 53$ & & 237 & ASSUMPTION \\
\hline $7 / 22 / 53$ & & 238 & ASSUMPTION & $9 / 16 / 53$ & & 236 & ASSUMPTION \\
\hline $7 / 23 / 53$ & - & 238 & ASSUMPTION & $9 / 17 / 53$ & & 236 & ASSUMPTION \\
\hline $7 / 24 / 53$ & & 238 & ASSUMPTION & $9 / 18 / 53$ & & 236 & ASSUMPTION \\
\hline $7 / 25 / 53$ & & 238 & ASSUMPTION & $9 / 19 / 53$ & & 236 & ASSUMPTION \\
\hline $7 / 26 / 53$ & & 228 & ASSUMPTION & $9 / 20 / 53$ & & 236 & ASSUMPTION \\
\hline $7 / 27 / 53$ & & 238 & ASSUMPTION & $9 / 21 / 53$ & & 236 & ASSUMPTION \\
\hline $7 / 28 / 53$ & & 238 & ASSUMPTION & $9 / 22 / 53$ & & 236 & ASSUMPTION \\
\hline $7 / 29 / 53$ & & 238 & ASSUMPTION & $9 / 23 / 53$ & & 235 & ASSUMPTION \\
\hline $7 / 30 / 53$ & & 238 & ASSUMPTION & $9 / 24 / 53$ & & 235 & ASSUMPTION \\
\hline $7 / 31 / 53$ & & 238 & ASSUMPTION & $9 / 25 / 53$ & & 235 & ASSUMPTION \\
\hline $8 / 1 / 53$ & & 238 & ASSUMPTION & $9 / 28 / 53$ & & 235 & ASSUMPTION \\
\hline $8 / 2 / 53$ & & 238 & ASSUMPTION & $9 / 29 / 53$ & $\cdot$ & 235 & ASSUMPTION \\
\hline $8 / 3 / 53$ & & 228 & ASSUMPTION & $9 / 30 / 53$ & & 235 & ASSUMPTION \\
\hline $8 / 4 / 53$ & & 228 & ASSUMPTION & $10 / 1 / 53$ & & 235 & ASSUMPTION \\
\hline $8 / 5 / 53$ & & 228 & ASSUMPTION & $10 / 2 / 53$ & & 235 & ASSUMPTION \\
\hline $8 / 6 / 53$ & & 228 & ASSUMPTION & $10 / 3 / 53$ & & 235 & ASSUMPTION \\
\hline $8 / 7 / 53$ & & 228 & ASSUMPTION & $10 / 4 / 53$ & & 235 & ASSUMPTION \\
\hline $8 / 8 / 53$ & & 228 & ASSUMPTION & $10 / 5 / 53$ & & 235 & ASSUMPTION \\
\hline $8 / 9 / 53$ & & 227 & ASSUMPTION & $10 / 6 / 53$ & & 238 & ASSUMPTION \\
\hline $8 / 10 / 53$ & & 228 & ASSUMPTION & $10 / 7 / 53$ & & 238 & ASSUMPTION \\
\hline $8 / 11 / 53$ & & 228 & ASSUMPTION & $10 / 8 / 53$ & & 238 & ASSUMPTION \\
\hline $8 / 12 / 53$ & & 228 & ASSUMPTION & $10 / 9 / 53$ & & 238 & ASSUMPTION \\
\hline
\end{tabular}

Data obtained from HW-83906. 


\begin{tabular}{|c|c|c|c|c|c|c|c|}
\hline \multicolumn{8}{|c|}{ Historical High or Only Waste Temperature Data } \\
\hline \multicolumn{8}{|c|}{ Tank 241-S-107 } \\
\hline Date & Temp (C) & Temp (F) & Comments & Date & Temp (C) & Temp (F) & Comments \\
\hline $10 / 10 / 53$ & & 238 & ASSUMPTION & $12 / 14 / 53$ & & 220 & ASSUMPTION \\
\hline $10 / 11 / 53$ & & 238 & ASSUMPTION & $12 / 15 / 53$ & & 220 & ASSUMPTION \\
\hline $10 / 12 / 53$ & & 238 & ASSUMPTION & $12 / 16 / 53$ & & 220 & ASSUMPTION \\
\hline $10 / 14 / 53$ & & 238 & ASSUMPTION & $12 / 17 / 53$ & & 220 & ASSUMPTION \\
\hline $10 / 15 / 53$ & & 238 & ASSUMPTION & $12 / 18 / 53$ & & 220 & ASSUMPTION \\
\hline $10 / 16 / 53$ & & 238 & ASSUMPTION & $12 / 21 / 53$ & & 220 & ASSUMPTION \\
\hline $10 / 17 / 53$ & & 225 & ASSUMPTION & $12 / 22 / 53$ & & 220 & ASSUMPTION \\
\hline $10 / 18 / 53$ & & 225 & ASSUMPTION & $12 / 23 / 53$ & & 220 & ASSUMPTION \\
\hline $10 / 19 / 53$ & & 222 & ASSUMPTION & $12 / 24 / 53$ & & 220 & ASSUMPTION \\
\hline $10 / 20 / 53$ & & 222 & ASSUMPTION & $12 / 25 / 53$ & & 220 & ASSUMPTION \\
\hline $10 / 21 / 53$ & & 228 & ASSUMPTION & $12 / 26 / 53$ & & 220 & ASSUMPTION \\
\hline $10 / 22 / 53$ & & 225 & ASSUMPTION & $12 / 29 / 53$ & & 220 & ASSUMPTION \\
\hline $10 / 23 / 53$ & & 210 & & $12 / 30 / 53$ & & 220 & ASSUMPTION \\
\hline $10 / 24 / 53$ & & 210 & & $12 / 31 / 53$ & & 220 & ASSUMPTION \\
\hline $10 / 25 / 53$ & & 225 & ASSUMPTION & $1 / 4 / 54$ & & 220 & ASSUMPTION \\
\hline $10 / 26 / 53$ & & 225 & ASSUMPTION & $1 / 5 / 54$ & & 220 & ASSUMPTION \\
\hline $10 / 27 / 53$ & & 225 & ASSUMPTION & $1 / 6 / 54$ & & 220 & ASSUMPTION \\
\hline $10 / 28 / 53$ & & 225 & ASSUMPTION & $1 / 7 / 54$ & & 220 & ASSUMPTION \\
\hline $10 / 29 / 53$ & & 225 & ASSUMPTION & $1 / 8 / 54$ & & 220 & ASSUMPTION \\
\hline $10 / 30 / 53$ & & 225 & ASSUMPTION & $1 / 111 / 54$ & & 220 & ASSUMPTION \\
\hline $10 / 31 / 53$ & & 240 & NOT SPECIFIC & $1 / 12 / 54$ & & 220 & ASSUMPTION \\
\hline $11 / 2 / 53$ & & 223 & ASSUMPTION & $1 / 13 / 54$ & & 220 & ASSUMPTION \\
\hline $11 / 3 / 53$ & & 223 & ASSUMPTION & $1 / 14 / 54$ & & 220 & ASSUMPTION \\
\hline $11 / 4 / 53$ & & 223 & ASSUMPTION & $1 / 15 / 54$ & & 220 & ASSUMPTION \\
\hline $11 / 5 / 53$ & & 223 & ASSUMPTION & $1 / 18 / 54$ & & 220 & ASSUMPTION \\
\hline $11 / 6 / 53$ & & 223 & ASSUMPTION & $1 / 19 / 54$ & & 220 & ASSUMPTION \\
\hline $11 / 6 / 53$ & & 223 & ASSUMPTION & $1 / 20 / 54$ & & 220 & ASSUMPTION \\
\hline $11 / 10 / 53$ & & 223 & ASSUMPTION & $1 / 21 / 54$ & & 220 & ASSUMPTION \\
\hline $11 / 11 / 53$ & & 221 & ASSUMPTION & $1 / 22 / 54$ & & 220 & ASSUMPTION \\
\hline $11 / 11 / 53$ & & 221 & ASSUMPTION & $1 / 23 / 54$ & & 220 & ASSUMPTION \\
\hline $11 / 12 / 53$ & & 221 & ASSUMPTION & $1 / 24 / 54$ & & 220 & ASSUMPTION \\
\hline $11 / 13 / 53$ & & 221 & ASSUMPTION & $1 / 25 / 54$ & & 220 & ASSUMPTION \\
\hline $11 / 15 / 53$ & & 221 & ASSUMPTION & $1 / 26 / 54$ & & 220 & ASSUMPTION \\
\hline $11 / 16 / 53$ & 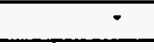 & 221 & ASSUMPTION & $1 / 27 / 54$ & & 220 & ASSUMPTION \\
\hline $11 / 17 / 53$ & & 221 & ASSUMPTION & $9 / 28 / 54$ & & 220 & ASSUMPTION \\
\hline $11 / 18 / 53$ & & 221 & ASSUMPTION & $1 / 29 / 54$ & & 220 & ASSUMPTION \\
\hline $11 / 19 / 53$ & & 221 & ASSUMPTION & $2 / 1 / 54$ & & 220 & ASSUMPTION \\
\hline $11 / 20 / 53$ & & 221 & ASSUMPTION & $2 / 2 / 54$ & & 220 & ASSUMPTION \\
\hline $11 / 22 / 53$ & & 220 & ASSUMPTION & $2 / 3 / 54$ & & 220 & ASSUMPTION \\
\hline $11 / 23 / 53$ & & 221 & ASSUMPTION & $2 / 4 / 54$ & & 220 & ASSUMPTION \\
\hline $11 / 24 / 53$ & & 221 & ASSUMPTION & $2 / 5 / 54$ & & 220 & ASSUMPTION \\
\hline $11 / 25 / 53$ & & 221 & ASSUMPTION & $2 / 6 / 54$ & & 218 & ASSUMPTION \\
\hline $11 / 27 / 53$ & & 223 & ASSUMPTION & $2 / 7 / 54$ & & 218 & ASSUMPTION \\
\hline $11 / 29 / 53$ & & 221 & ASSUMPTION & $2 / 8 / 54$ & & 218 & ASSUMPTION \\
\hline $11 / 30 / 53$ & & 221 & ASSUMPTION & $2 / 9 / 54$ & & 218 & ASSUMPTION \\
\hline $12 / 1 / 53$ & & 221 & ASSUMPTION & $2 / 10 / 54$ & & 218 & ASSUMPTION \\
\hline $12 / 2 / 53$ & & 221 & ASSUMPTION & $2 / 11 / 54$ & & 218 & ASSUMPTION \\
\hline $12 / 3 / 53$ & & 221 & ASSUMPTION & $2 / 12 / 54$ & & 218 & ASSUMPTION \\
\hline $12 / 4 / 53$ & & 221 & ASSUMPTION & $2 / 15 / 54$ & & 218 & ASSUMPTION \\
\hline $12 / 5 / 53$ & & 221 & ASSUMPTION & $2 / 16 / 54$ & & 218 & ASSUMPTION \\
\hline $12 / 6 / 53$ & & 221 & ASSUMPTION & $2 / 17 / 54$ & & 218 & ASSUMPTION \\
\hline $12 / 7 / 53$ & & 221 & ASSUMPTION & $2 / 18 / 54$ & & 219 & ASSUMPTION \\
\hline $12 / 8 / 53$ & & 221 & ASSUMPTION & $2 / 19 / 54$ & & 219 & ASSUMPTION \\
\hline $12 / 10 / 53$ & & 221 & ASSUMPTION & $2 / 22 / 54$ & & 219 & ASSUMPTION \\
\hline
\end{tabular}

Data obtained from HW- $\mathbf{8} 3906$. 


\begin{tabular}{|c|c|c|c|c|c|c|c|}
\hline \multicolumn{8}{|c|}{ Historical High or Only Waste Temperature Data } \\
\hline & & & & $-5-107$ & & & \\
\hline Date & Temp (C) & Temp (F) & Comments & Date & Temn (C) & Temn (F) & Comments \\
\hline $2 / 23 / 54$ & & 219 & ASSUMPTION & $5 / 16 / 54$ & & 225 & ASSUMPTION \\
\hline $2 / 25 / 54$ & & 219 & ASSUMPTION & $5 / 24 / 54$ & & 226 & ASSUMPTION \\
\hline $2 / 25 / 54$ & & 219 & ASSUMPTION & $5 / 26 / 54$ & & 226 & ASSUMPTION \\
\hline $2 / 26 / 54$ & & 219 & ASSUMPTION & $5 / 31 / 54$ & & 226 & ASSUMPTION \\
\hline $3 / 1 / 54$ & & 219 & ASSUMPTION & $6 / 1 / 54$ & & 226 & ASSUMPTION \\
\hline $3 / 2 / 54$ & & 219 & ASSUMPTION & $6 / 2 / 54$ & & 226 & ASSUMPTION \\
\hline $3 / 3 / 54$ & & 219 & ASSUMPTION & $6 / 3 / 54$ & & 226 & ASSUMPTION \\
\hline $3 / 4 / 54$ & & 219 & ASSUMPTION & $6 / 7 / 54$ & & 226 & ASSUMPTION \\
\hline $3 / 5 / 54$ & & 219 & ASSUMPTION & $6 / 8 / 54$ & & 226 & ASSUMPTION \\
\hline $3 / 6 / 54$ & & 219 & ASSUMPTION & $6 / 9 / 54$ & & 226 & ASSUMPTION \\
\hline $3 / 7 / 54$ & & 219 & ASSUMPTION & $6 / 14 / 54$ & & 226 & ASSUMPTION \\
\hline $3 / 8 / 54$ & & 219 & ASSUMPTION & $6 / 16 / 54$ & & 225 & ASSUMPTION \\
\hline $3 / 9 / 54$ & & 219 & ASSUMPTION & $6 / 18 / 54$ & & 225 & ASSUMPTION \\
\hline $3 / 10 / 54$ & & 219 & ASSUMPTION & $6 / 21 / 54$ & & 225 & ASSUMPTION \\
\hline $3 / 12 / 54$ & & 219 & ASSUMPTION & $6 / 22 / 54$ & & 225 & ASSUMPTION \\
\hline $3 / 14 / 54$ & & 220 & ASSUMPTION & $6 / 23 / 54$ & & 225 & ASSUMPTION \\
\hline $3 / 15 / 54$ & & 218 & ASSUMPTION & $6 / 26 / 54$ & & 225 & ASSUMPTION \\
\hline $3 / 16 / 54$ & & 218 & ASSUMPTION & $6 / 28 / 54$ & & 225 & ASSUMPTION \\
\hline $3 / 17 / 54$ & & 218 & ASSUMPTION & $6 / 30 / 54$ & & 225 & ASSUMPTION \\
\hline $3 / 18 / 54$ & & 218 & ASSUMPTION & $7 / 5 / 54$ & & 225 & ASSUMPTION \\
\hline $3 / 19 / 54$ & & 218 & ASSUMPTION & $7 / 7 / 54$ & & 225 & ASSUMPTION \\
\hline $3 / 22 / 54$ & & 218 & ASSUMPTION & $7 / 11 / 54$ & & 240 & NOT SPECIFIC \\
\hline $3 / 24 / 54$ & & 219 & ASSUMPTION & $7 / 12 / 54$ & & 225 & ASSUMPTION \\
\hline $4 / 1 / 54$ & & 220 & ASSUMPTION & $7 / 14 / 54$ & & 225 & ASSUMPTION \\
\hline $4 / 5 / 54$ & & 220 & ASSUMPTION & $7 / 19 / 54$ & & 225 & ASSUMPTION \\
\hline $4 / 6 / 54$ & & 220 & ASSUMPTION & $7 / 23 / 54$ & & 225 & ASSUMPTION \\
\hline $4 / 7 / 54$ & & 220 & ASSUMPTION & $7 / 26 / 54$ & & 225 & ASSUMPTION \\
\hline $4 / 8 / 54$ & & 220 & ASSUMPTION & $7 / 28 / 54$ & & 223 & ASSUMPTION \\
\hline $4 / 9 / 54$ & & 220 & ASSUMPTION & $8 / 2 / 54$ & & 224 & ASSUMPTION \\
\hline $4 / 11 / 54$ & & 220 & ASSUMPTION & $8 / 4 / 54$ & & 224 & ASSUMPTION \\
\hline $4 / 12 / 54$ & & 220 & ASSUMPTION & $8 / 9 / 54$ & & 224 & ASSUMPTION \\
\hline $4 / 14 / 54$ & & 220 & ASSUMPTION & $8 / 11 / 54$ & & 224 & ASSUMPTION \\
\hline $4 / 15 / 54$ & & 220 & ASSUMPTION & $8 / 16 / 54$ & & 224 & ASSUMPTION \\
\hline $4 / 16 / 54$ & & 220 & ASSUMPTION & $8 / 18 / 54$ & & 224 & ASSUMPTION \\
\hline $4 / 17 / 54$ & & 300 & NOT SPECIFIC & $8 / 23 / 54$ & & 224 & ASSUMPTION \\
\hline $4 / 18 / 54$ & & 300 & NOT SPECIFIC & $8 / 26 / 54$ & & 222 & ASSUMPTION \\
\hline $4 / 19 / 54$ & & 240 & NOT SPECIFIC & $8 / 28 / 54$ & & 210 & ASSUMPTION \\
\hline $4 / 21 / 54$ & & 223 & ASSUMPTION & $8 / 30 / 54$ & & 222 & ASSUMPTION \\
\hline $4 / 23 / 54$ & & 223 & ASSUMPTION & $8 / 31 / 54$ & & 222 & ASSUMPTION \\
\hline $4 / 25 / 54$ & & 223 & ASSUMPTION & $9 / 1 / 54$ & & 222 & ASSUMPTION \\
\hline $4 / 26 / 54$ & & 223 & ASSUMPTION & $9 / 2 / 54$ & & 222 & ASSUMPTION \\
\hline $4 / 27 / 54$ & & 223 & ASSUMPTION & $9 / 6 / 54$ & & 222 & ASSUMPTION \\
\hline $4 / 28 / 54$ & & 223 & ASSUMPTION & $9 / 8 / 54$ & & 222 & ASSUMPTION \\
\hline $4 / 29 / 54$ & & 223 & ASSUMPTION & $9 / 20 / 54$ & & 222 & ASSUMPTION \\
\hline $4 / 30 / 54$ & & 223 & ASSUMPTION & $9 / 21 / 54$ & & 222 & ASSUMPTION \\
\hline $5 / 1 / 54$ & & 223 & ASSUMPTION & $9 / 27 / 54$ & & 204 & \\
\hline $5 / 3 / 54$ & & 223 & ASSUMPTION & $9 / 30 / 54$ & & 199 & \\
\hline $5 / 4 / 54$ & & 223 & ASSUMPTION & $10 / 4 / 54$ & & 199 & \\
\hline $5 / 5 / 54$ & & 225 & ASSUMPTION & $10 / 5 / 54$ & & 199 & \\
\hline $5 / 8 / 54$ & & 225 & ASSUMPTION & $10 / 111 / 54$ & & 199 & \\
\hline $5 / 9 / 54$ & & 225 & ASSUMPTION & $10 / 12 / 54$ & & 198 & \\
\hline $5 / 10 / 54$ & & 225 & ASSUMPTION & $10 / 18 / 54$ & & 198 & \\
\hline $5 / 12 / 54$ & & 225 & ASSUMPTION & $10 / 20 / 54$ & & 198 & \\
\hline $5 / 15 / 54$ & & 225 & ASSUMPTION & $10 / 25 / 54$ & & 198 & \\
\hline
\end{tabular}

Data obtained from HW-83906. 
WHC-SD-WM-ER-323, Rev. O 1

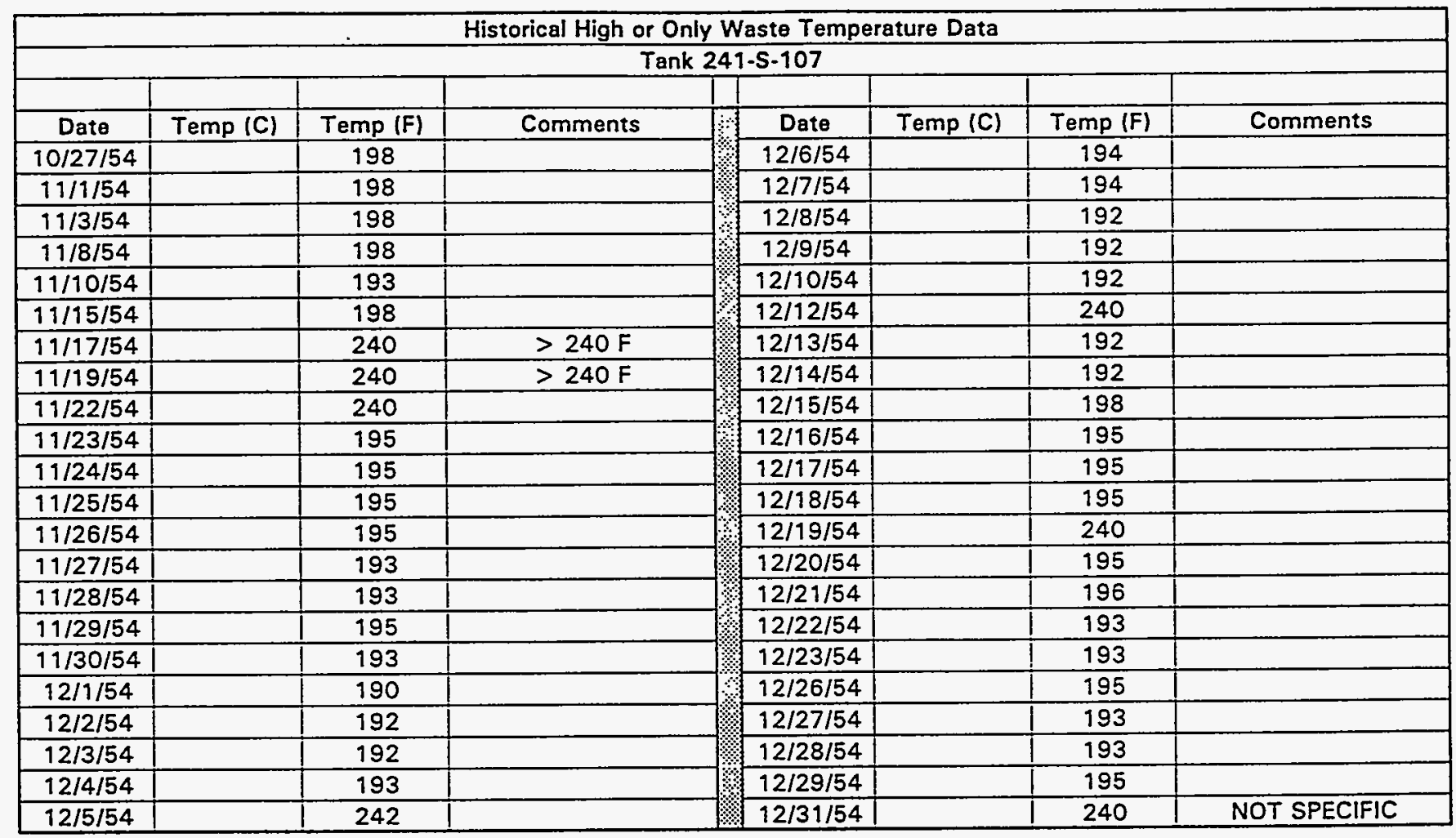

Data obtained from HW-83906. 
Historical Vapor Space Temperature Data

\begin{tabular}{|c|c|c|c|c|c|c|c|}
\hline \multicolumn{8}{|c|}{ Historical Vapor Space Temperature Data } \\
\hline \multicolumn{8}{|c|}{ Tank 241-S-108 } \\
\hline & & & & & & 1 & \\
\hline Date & Temp (C) & Temp (F) & Comments & Date & Temp (C) & Temp (F) & Comments \\
\hline $7 / 31 / 56$ & & 104 & & $12 / 6 / 56$ & & 104 & \\
\hline $8 / 1 / 56$ & & 104 & & $12 / 9 / 56$ & & 104 & \\
\hline $8 / 4 / 56$ & & 104 & & $12 / 12 / 56$ & & 104 & \\
\hline $8 / 5 / 56$ & & 104 & & $12 / 15 / 56$ & & 104 & \\
\hline $8 / 7 / 56$ & & 104 & & $12 / 18 / 56$ & & 104 & \\
\hline $8 / 8 / 56$ & & 105 & & $12 / 22 / 56$ & & 104 & \\
\hline $8 / 8 / 56$ & & 106 & & $12 / 24 / 56$ & & 103 & \\
\hline $8 / 9 / 56$ & & 106 & & $12 / 26 / 56$ & & 103 & \\
\hline $8 / 10 / 56$ & & 107 & & $2 / 1 / 57$ & & 102 & \\
\hline $8 / 11 / 56$ & & 107 & & $3 / 2 / 57$ & & 94 & \\
\hline $8 / 13 / 56$ & & 109 & & $3 / 10 / 57$ & & 95 & \\
\hline $8 / 14 / 56$ & & 110 & & $3 / 28 / 57$ & & 99 & \\
\hline $8 / 15 / 56$ & & 110 & & $4 / 17 / 57$ & & 98 & \\
\hline $8 / 16 / 56$ & & 109 & & $5 / 1 / 57$ & & 98 & \\
\hline $8 / 17 / 56$ & & 108 & & $5 / 25 / 57$ & & 98 & \\
\hline $8 / 19 / 56$ & & 107 & & $6 / 21 / 57$ & & 97 & \\
\hline $8 / 20 / 56$ & & 105 & & $7 / 11 / 57$ & & 97 & \\
\hline $8 / 21 / 56$ & & 104 & & $7 / 28 / 57$ & & 97 & \\
\hline $8 / 22 / 56$ & & 103 & & $8 / 15 / 57$ & & 98 & \\
\hline $8 / 23 / 56$ & & 103 & & $8 / 31 / 57$ & & 98 & \\
\hline $8 / 24 / 56$ & & 103 & & $9 / 24 / 57$ & & 98 & \\
\hline $8 / 26 / 56$ & & 103 & & $10 / 30 / 57$ & & 99 & \\
\hline $8 / 28 / 56$ & & 104 & & $5 / 5 / 58$ & & 94 & \\
\hline $8 / 31 / 56$ & & 103 & & $5 / 16 / 58$ & & 94 & \\
\hline $9 / 4 / 56$ & & 104 & & $5 / 27 / 58$ & & 93 & \\
\hline $9 / 6 / 56$ & & 104 & & $6 / 6 / 58$ & & 92 & \\
\hline $9 / 10 / 56$ & & 103 & & $6 / 17 / 58$ & & 93 & \\
\hline $9 / 12 / 56$ & & 102 & & $6 / 28 / 58$ & & 94 & \\
\hline $9 / 14 / 56$ & & 103 & & $7 / 11 / 58$ & & 94 & \\
\hline $9 / 17 / 56$ & & 104 & & $7 / 18 / 58$ & & 94 & \\
\hline $9 / 24 / 56$ & & 104 & & $7 / 25 / 58$ & & 93 & \\
\hline $9 / 27 / 56$ & & 103 & & $8 / 1 / 58$ & & 93 & \\
\hline $9 / 30 / 56$ & & 102 & & $9 / 2 / 58$ & & 93 & \\
\hline $10 / 3 / 56$ & & 100 & & $9 / 29 / 58$ & & 92 & \\
\hline $10 / 8 / 56$ & & 105 & & $10 / 19 / 58$ & & 91 & \\
\hline $10 / 13 / 56$ & & 105 & & $11 / 4 / 58$ & & 92 & \\
\hline $10 / 17 / 56$ & - & 105 & & $11 / 23 / 58$ & & 92 & \\
\hline $10 / 23 / 56$ & & 105 & & $12 / 13 / 58$ & & 93 & \\
\hline $10 / 24 / 56$ & & 104 & & $12 / 30 / 58$ & & 93 & \\
\hline $10 / 28 / 56$ & & 104 & & $1 / 14 / 59$ & & 92 & \\
\hline $11 / 1 / 56$ & & 105 & & $1 / 30 / 59$ & & 92 & \\
\hline $11 / 5 / 56$ & & 104 & & $2 / 13 / 59$ & & 90 & \\
\hline $11 / 13 / 56$ & & 105 & & $2 / 27 / 59$ & & 88 & \\
\hline $11 / 9 / 56$ & & 105 & & $3 / 12 / 59$ & & 86 & \\
\hline $11 / 18 / 56$ & & 105 & & $3 / 25 / 59$ & & 85 & \\
\hline $11 / 20 / 56$ & & 105 & & $4 / 14 / 59$ & & 85 & \\
\hline $11 / 25 / 56$ & & 104 & & $4 / 28 / 59$ & & 84 & \\
\hline $11 / 27 / 56$ & & 104 & & $12 / 1 / 59$ & & 89 & \\
\hline $12 / 1 / 56$ & & 104 & & $12 / 28 / 59$ & & 89 & \\
\hline $12 / 4 / 56$ & & 104 & & & & & \\
\hline
\end{tabular}

-. Data obtained from RHO-CD-1172. 
$8+l \cdot 0$

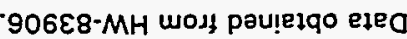

\begin{tabular}{|c|c|c|c|c|c|c|c|}
\hline & ZEL & & $\varepsilon G / \angle Z / O L$ & L & $\nabla \varepsilon !$ & & $\varepsilon \mathrm{G} / \mathrm{G} / \mathrm{B}$ \\
\hline & ZEL & & $\varepsilon g / 9 Z / O L$ & $\%$ & $\nabla \varepsilon l$ & & $\varepsilon \mathrm{g} / \triangleright \tau / 8$ \\
\hline & Zहl & & $\varepsilon S / \hbar z / O L$ & & $\nabla \varepsilon \downarrow$ & & $\varepsilon \mathrm{s} / \varepsilon 乙 / 8$ \\
\hline & ZEL & & ES/Ez/OL & 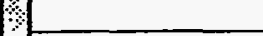 & $\varepsilon \varepsilon l$ & & $\varepsilon \varsigma / 2 Z / 8$ \\
\hline & Z८L & & $\varepsilon S / Z Z / O L$ & & $\varepsilon \varepsilon\llcorner$ & & $\varepsilon S / L Z / 8$ \\
\hline & $2 \varepsilon l$ & & $\varepsilon g / L 2 / O L$ & & $\varepsilon \varepsilon l$ & & $\varepsilon \mathrm{s} / 0 \mathrm{Z} / 8$ \\
\hline & $2 \varepsilon b$ & & $\varepsilon 9 / 0 Z / 01$ & & $\varepsilon \varepsilon\llcorner$ & & $\varepsilon S / 6 \mathrm{~L} / 8$ \\
\hline & टहL & & $\varepsilon \mathrm{E} / 6 \mathrm{l} / \mathrm{Ol}$ & & $t \varepsilon l$ & & $\varepsilon 9 / 81 / 8$ \\
\hline & $\nabla \varepsilon \downarrow$ & & $\varepsilon 9 / 8 \mathrm{l} / 0 \mathrm{l}$ & & $\nabla \varepsilon l$ & & $\varepsilon S / L L / 8$ \\
\hline & $2 \varepsilon !$ & & $\varepsilon g / \angle L / O L$ & & $\nabla \varepsilon b$ & & $\varepsilon g / 91 / 8$ \\
\hline & $z \varepsilon l$ & & $\varepsilon g / 9$ l/O & & $\nabla \varepsilon !$ & & $\varepsilon G / \mathcal{G} / / 8$ \\
\hline & ZEL & & $\varepsilon \mathrm{G} / \mathrm{SL} / O \mathrm{~L}$ & & $\mathrm{g \varepsilon l}$ & & $\varepsilon G / t h / 8$ \\
\hline & $2 \varepsilon l$ & & $\varepsilon g / t / / O L$ & $\cdot$ & gEl & & $\varepsilon \Phi / \varepsilon \downarrow / 8$ \\
\hline & $z \varepsilon \downarrow$ & & $\varepsilon g / 21 / 0 L$ & & SE! & & $\varepsilon S / 21 / 8$ \\
\hline & $\tau \varepsilon l$ & & $\varepsilon g / \downarrow b / O L$ & & $G \varepsilon \downarrow$ & & $\varepsilon G / L / / 8$ \\
\hline & $\nabla \varepsilon b$ & & $\varepsilon 9 / 01 / 06$ & \% & $9 \varepsilon \downarrow$ & & $\varepsilon 9 / 0 \mathrm{~L} / 8$ \\
\hline & $2 \varepsilon b$ & & $\varepsilon G / 6 / 0 \mathrm{~L}$ & 4 & $\varepsilon \varepsilon !$ & & $\varepsilon g / 6 / 8$ \\
\hline & $\tau \varepsilon \downarrow$ & & $\varepsilon 9 / 8 / 01$ & & $\nabla \varepsilon L$ & & $\varepsilon g / 8 / 8$ \\
\hline & $\tau \varepsilon \downarrow$ & & $\varepsilon S / L / O l$ & 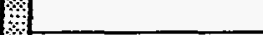 & $g \varepsilon !$ & & $\varepsilon g / L / 8$ \\
\hline & $\varepsilon \varepsilon \downarrow$ & & $\varepsilon \mathrm{G} / 9 / 0 \mathrm{~L}$ & & $\subseteq \varepsilon \downarrow$ & & $\varepsilon g / 9 / 8$ \\
\hline & $\underline{G \varepsilon l}$ & & $\varepsilon g / G / O L$ & & SEL & & $\varepsilon g / G / 8$ \\
\hline & $\varepsilon \varepsilon \downarrow$ & & $\varepsilon g / t / 0 l$ & & $\underline{9 !}$ & . & $\varepsilon g / t / 8$ \\
\hline & $\varepsilon \varepsilon l$ & & $\varepsilon g / \varepsilon / 01$ & & $g \varepsilon !$ & & $\varepsilon g / \varepsilon / 8$ \\
\hline & $\varepsilon \varepsilon !$ & & $\varepsilon 9 / 2 / 0 \mathrm{~L}$ & & $\nabla \varepsilon L$ & & $\varepsilon g / Z / 8$ \\
\hline & $\varepsilon \varepsilon 1$ & & $\varepsilon g / L / 0 !$ & & $\nabla \varepsilon l$ & & $\varepsilon g / \downarrow / 8$ \\
\hline & $\varepsilon \varepsilon !$ & & $\varepsilon q / 0 \varepsilon / 6$ & & $\nabla \varepsilon !$ & & $\varepsilon G / L \varepsilon / L$ \\
\hline & $\varepsilon \varepsilon l$ & & $\varepsilon g / 6 Z / 6$ & & $t \varepsilon l$ & & $\varepsilon S / 0 \varepsilon / L$ \\
\hline & $\varepsilon \varepsilon L$ & & $\varepsilon s / 8 Z / 6$ & & $t \varepsilon l$ & & $\varepsilon S / 6 Z / L$ \\
\hline & $\varepsilon \varepsilon \downarrow$ & & $\varepsilon S / S Z / 6$ & & $\nabla \varepsilon !$ & & $\varepsilon g / 8 Z / L$ \\
\hline & $\varepsilon \varepsilon !$ & & $\varepsilon g / t 2 / 6$ & & $\nabla \varepsilon !$ & & $\varepsilon g / L Z / L$ \\
\hline & $\varepsilon \varepsilon \downarrow$ & & $\varepsilon s / \varepsilon 己 / 6$ & & $\nabla \varepsilon b$ & & $\varepsilon G / 9 Z / L$ \\
\hline & $\varepsilon \varepsilon l$ & & $\varepsilon g / 2 z / 6$ & & $\nabla \varepsilon l$ & & $\varepsilon G / \subseteq Z / L$ \\
\hline & $\varepsilon \varepsilon \downarrow$ & & $\varepsilon g / L / / 6$ & & $t \varepsilon !$ & & $\varepsilon \subseteq / \downarrow Z / L$ \\
\hline & $\varepsilon \varepsilon l$ & & $\varepsilon s / 0 Z / 6$ & & $t \varepsilon L$ & & $\varepsilon g / \varepsilon \tau / L$ \\
\hline & $\nabla \varepsilon l$ & & $\varepsilon S / 6 L / 6$ & & $\nabla \varepsilon L$ & & $\varepsilon S / 2 Z / L$ \\
\hline & $Z \varepsilon L$ & & $\varepsilon 9 / 81 / 6$ & & $\nabla \varepsilon b$ & & $\varepsilon g /\lfloor z / L$ \\
\hline & $2 \varepsilon l$ & & $\varepsilon g / L ! / 6$ & & $\nabla \varepsilon l$ & & $\varepsilon \mathrm{g} / 0 \mathrm{Z} / L$ \\
\hline & Zहl & & $\varepsilon 9 / 91 / 6$ & & $\nabla \varepsilon l$ & & $\varepsilon g / L L / L$ \\
\hline & $\nabla \varepsilon l$ & & $\varepsilon \mathrm{G} / \mathrm{G} L / 6$ & & $\nabla \varepsilon l$ & & $\varepsilon S / 9 L / L$ \\
\hline & $\nabla \varepsilon !$ & & $\varepsilon G / b L / 6$ & & $\nabla \varepsilon l$ & & $\varepsilon g / G \downarrow / L$ \\
\hline & $\nabla \varepsilon l$ & & $\varepsilon \mathrm{g} / \varepsilon \downarrow / 6$ & & $\nabla \varepsilon l$ & & $\varepsilon G / \varepsilon \downarrow / L$ \\
\hline & $\nabla \varepsilon l$ & & $\varepsilon S / 1 / / 6$ & & $\nabla \varepsilon 1$ & & $\varepsilon g / L L / L$ \\
\hline & $\nabla \varepsilon !$ & & $\varepsilon g / 01 / 6$ & & $\nabla \varepsilon l$ & & $\varepsilon g / 0 \mathrm{~L} / L$ \\
\hline & $\nabla \varepsilon \downarrow$ & & $\varepsilon 9 / 6 / 6$ & & $\nabla \varepsilon l$ & & $\varepsilon g / 6 / L$ \\
\hline & $\nabla \varepsilon l$ & & $\varepsilon \mathrm{g} / 8 / 6$ & & $\nabla \varepsilon l$ & & $\varepsilon S / 8 / L$ \\
\hline & $\nabla \varepsilon l$ & & $\varepsilon g / L / 6$ & & $\nabla \varepsilon l$ & & $\varepsilon G / L / L$ \\
\hline & $Z \varepsilon I$ & & $\varepsilon g / 9 / 6$ & & $\nabla \varepsilon l$ & & $\varepsilon g / 9 / L$ \\
\hline & $\nabla \varepsilon l$ & & $\varepsilon g / G / 6$ & & $\nabla \varepsilon L$ & & $\varepsilon g / g / L$ \\
\hline & $\nabla \varepsilon l$ & & $\varepsilon g / t / 6$ & & $\nabla 91$ & & $\varepsilon g / \not / L$ \\
\hline & $\nabla \varepsilon \downarrow$ & & $\varepsilon s / \varepsilon / 6$ & & $\nabla \varepsilon l$ & & $\varepsilon G / \varepsilon / L$ \\
\hline & $\nabla \varepsilon L$ & & $\varepsilon g / Z / 6$ & & $\nabla \varepsilon l$ & & $\varepsilon S / Z / L$ \\
\hline & $\nabla \varepsilon l$ & & $\varepsilon g / L / 6$ & & $\nabla \varepsilon \downarrow$ & & $\varepsilon S / L / L$ \\
\hline & $\nabla \varepsilon l$ & & $\varepsilon \subseteq /\lfloor\varepsilon / 8$ & & $\nabla \varepsilon l$ & & $\varepsilon q / 6 Z / 9$ \\
\hline & $\nabla \varepsilon l$ & & $\varepsilon 9 / 0 \varepsilon / 8$ & & $\nabla \varepsilon !$ & & $\varepsilon q / 9 \tau / 9$ \\
\hline & $\nabla \varepsilon b$ & & $\varepsilon g / 62 / 8$ & 1 & $\nabla \varepsilon l$ & & $\varepsilon \Phi / \mathcal{G} / 9$ \\
\hline & $\nabla \varepsilon !$ & & $\varepsilon 9 / 82 / 8$ & & $\nabla \varepsilon l$ & & $\varepsilon G / t Z / 9$ \\
\hline & $\nabla \varepsilon \downarrow$ & & $\varepsilon g / \angle Z / 8$ & & $\nabla \varepsilon l$ & & $\varepsilon S / \varepsilon 乙 / 9$ \\
\hline & $\nabla \varepsilon l$ & & $\varepsilon \leqq / 9 Z / 8$ & & $\nabla \varepsilon l$ & & $\varepsilon S / 2 Z / 9$ \\
\hline s\}บow110 & (A) dwa 1 & (ग) dwal & opeg & sfuaumos & (A) dura 1 & (0) dwa 1 & काषव \\
\hline & & & & & & & \\
\hline \multicolumn{8}{|c|}{$80 L-S-L t Z \times 148$} \\
\hline \multicolumn{8}{|c|}{ 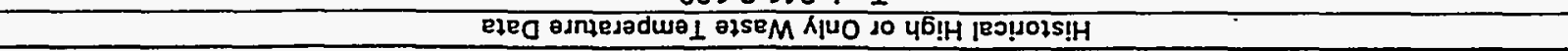 } \\
\hline
\end{tabular}

1

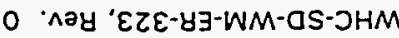




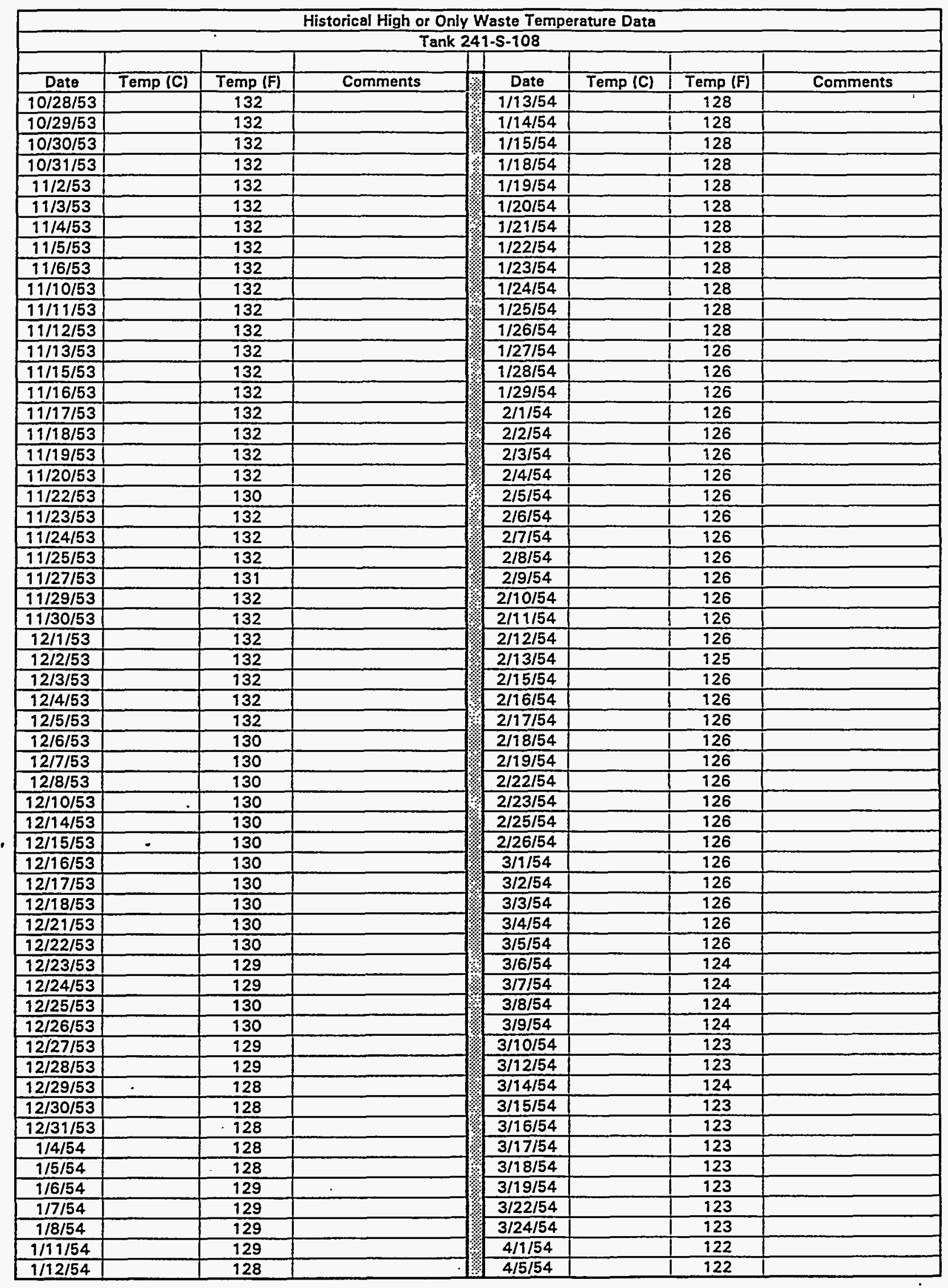

Data obtained from HW-83906. 


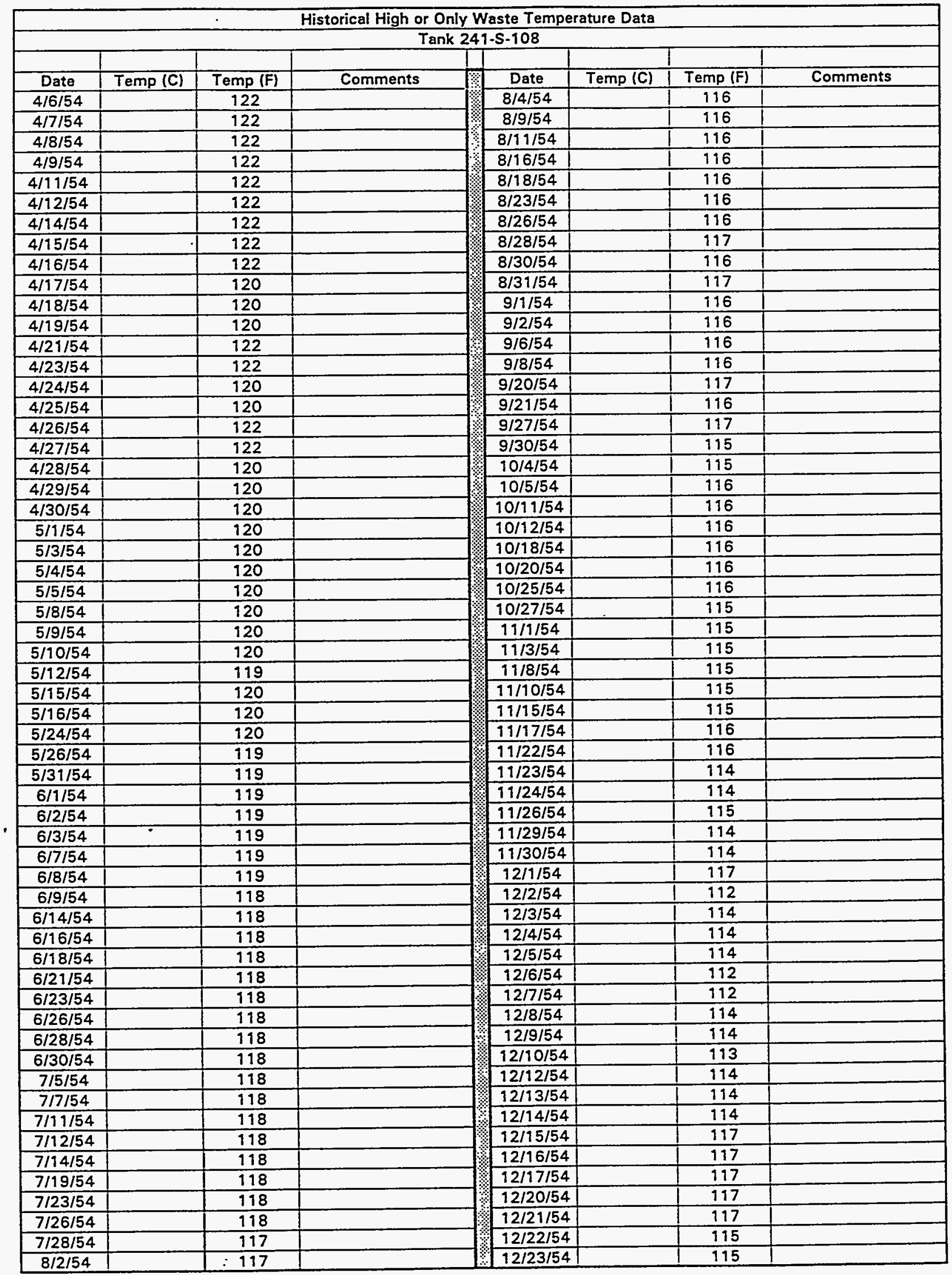

Data obtained from HW-83906. 
WHC-SD-WM-ERR-323, Rev. 0 1

Historical High or Only Waste Temperature Data

\begin{tabular}{|c|c|c|c|c|c|c|c|}
\hline \multicolumn{7}{|c|}{ Historical High or Only Waste Temperature Data } \\
\hline & & & & & & \\
\hline Date & Temp (C) & Temp (F) & Comments & Date & Temp (C) & Temp (F) & Comments \\
\hline $12 / 24 / 54$ & & 114 & & $12 / 29 / 54$ & & 114 & \\
\hline $12 / 25 / 54$ & & 114 & & $12 / 31 / 54$ & & 114 & \\
\hline $12 / 26 / 54$ & & 114 & & $1 / 1 / 55$ & & 116 & \\
\hline $12 / 27 / 54$ & & 115 & 115 & $1 / 2 / 55$ & & 117 & \\
\hline $12 / 28 / 54$ & & 115 & & & & \\
\hline
\end{tabular}




\begin{tabular}{|c|c|c|c|c|c|c|c|}
\hline \multicolumn{8}{|c|}{ Historical Vapor Space Temperature Data } \\
\hline \multicolumn{8}{|c|}{ Tank 241-S-109 } \\
\hline & & & & & & & \\
\hline Date & Temp (C) & Temp (F) & Comments & Date & Temp (C) & Temp (F) & Comments \\
\hline $8 / 1 / 56$ & & 82 & & $10 / 5 / 56$ & & 80 & \\
\hline $8 / 5 / 56$ & & 81 & & $10 / 11 / 56$ & & 80 & \\
\hline $8 / 8 / 56$ & & 81 & & $10 / 15 / 56$ & & 81 & \\
\hline $8 / 12 / 56$ & & 81 & & $10 / 19 / 56$ & & 81 & \\
\hline $8 / 14 / 56$ & & 112 & & $10 / 21 / 56$ & & 81 & \\
\hline $8 / 15 / 56$ & & 81 & & $10 / 24 / 56$ & & 81 & \\
\hline $8 / 19 / 56$ & & 81 & & $11 / 11 / 56$ & & 80 & \\
\hline $8 / 21 / 56$ & & 80 & & $11 / 20 / 56$ & & 81 & \\
\hline $8 / 23 / 56$ & & 111 & & $11 / 26 / 56$ & & 80 & \\
\hline $8 / 24 / 56$ & & 81 & & $12 / 5 / 56$ & & 81 & \\
\hline $8 / 27 / 56$ & & 81 & & $12 / 11 / 56$ & & 81 & \\
\hline $8 / 30 / 56$ & & 82 & & $12 / 20 / 56$ & & 81 & \\
\hline $8 / 31 / 56$ & & 82 & & $12 / 27 / 56$ & & 81 & \\
\hline $9 / 1 / 56$ & & 82 & & $12 / 30 / 56$ & & 81 & \\
\hline $9 / 4 / 56$ & & 89 & & $1 / 30 / 57$ & & 80 & \\
\hline $9 / 6 / 56$ & & 81 & & $3 / 1 / 57$ & & 88 & \\
\hline $9 / 9 / 56$ & & 81 & & $3 / 30 / 57$ & & 80 & \\
\hline $9 / 12 / 56$ & & 82 & & $5 / 2 / 57$ & & 84 & \\
\hline $9 / 12 / 56$ & & 82 & & $6 / 1 / 57$ & & 78 & \\
\hline $9 / 12 / 56$ & & 82 & & $7 / 9 / 57$ & & 79 & \\
\hline $9 / 16 / 56$ & & 81 & & $8 / 13 / 57$ & & 80 & \\
\hline $9 / 18 / 56$ & & 81 & & $9 / 24 / 57$ & & 81 & \\
\hline $9 / 19 / 56$ & & 82 & & $10 / 29 / 57$ & & 79 & \\
\hline $9 / 20 / 56$ & & 84 & & $5 / 2 / 58$ & & 77 & \\
\hline $9 / 21 / 56$ & & 85 & & $5 / 30 / 58$ & & 77 & \\
\hline $9 / 22 / 56$ & & 86 & & $7 / 1 / 58$ & & 79 & \\
\hline $9 / 23 / 56$ & & 88 & & $7 / 28 / 58$ & & 76 & \\
\hline $9 / 23 / 56$ & & 89 & & $9 / 2 / 58$ & & 74 & \\
\hline $9 / 24 / 56$ & & 90 & & $10 / 1 / 58$ & & 75 & \\
\hline $9 / 25 / 56$ & & 91 & & $11 / 3 / 58$ & & 74 & \\
\hline $9 / 27 / 56$ & & 91 & & $12 / 2 / 58$ & & 73 & \\
\hline $9 / 28 / 56$ & & 91 & & $12 / 26 / 58$ & & 75 & \\
\hline $9 / 28 / 56$ & & 89 & & $1 / 3 / 59$ & & 74 & \\
\hline $9 / 29 / 56$ & & 88 & & $2 / 1 / 59$ & & 74 & \\
\hline $9 / 29 / 56$ & & 87 & & $2 / 24 / 59$ & & 75 & \\
\hline $9 / 30 / 56$ & & 86 & & $3 / 3 / 59$ & & 73 & \\
\hline $10 / 1 / 56$ & & 85 & & $4 / 3 / 59$ & & 72 & \\
\hline $10 / 1 / 56$ & & 84 & & $4 / 30 / 59$ & & 74 & \\
\hline $10 / 1 / 56$ & & 84 & & $12 / 2 / 59$ & & 74 & \\
\hline $10 / 2 / 56$ & & 83 & & $12 / 15 / 59$ & & 73 & \\
\hline $10 / 3 / 56$ & & 81 & & $12 / 30 / 59$ & & 73 & \\
\hline $10 / 3 / 56$ & & 81 & & & & & \\
\hline
\end{tabular}




\begin{tabular}{|c|c|c|c|c|c|c|c|}
\hline \multicolumn{8}{|c|}{ Historical High or Only Waste Temperature Data } \\
\hline \multicolumn{8}{|c|}{ Tank 241-S.109 } \\
\hline Date & Temp (C) & Temp (F) & Comments & Date & Tomn (C) & Temp (F) & Comments \\
\hline $6 / 22 / 53$ & & 110 & & $8 / 22 / 53$ & reilip pin & 110 & S \\
\hline $6 / 23 / 53$ & & 110 & & $8 / 23 / 53$ & & 110 & \\
\hline $6 / 24 / 53$ & & 110 & & $8 / 24 / 53$ & & 110 & \\
\hline $6 / 25 / 53$ & & 110 & & $8 / 25 / 53$ & & 110 & \\
\hline $6 / 26 / 53$ & & 110 & & $8 / 26 / 53$ & & 110 & \\
\hline $6 / 29 / 53$ & & 100 & & $8 / 27 / 53$ & & 110 & \\
\hline $7 / 1 / 53$ & & 110 & & $8 / 28 / 53$ & & 110 & \\
\hline $7 / 2 / 53$ & & 110 & & $8 / 29 / 53$ & & 910 & \\
\hline $7 / 3 / 53$ & & 110 & & $8 / 30 / 53$ & & 110 & \\
\hline $7 / 4 / 53$ & & 110 & & $8 / 31 / 53$ & & 110 & \\
\hline $7 / 5 / 53$ & & 110 & & $9 / 1 / 53$ & & 110 & \\
\hline $7 / 6 / 53$ & & 110 & & $9 / 2 / 53$ & & 110 & \\
\hline $7 / 7 / 53$ & & 110 & & $9 / 3 / 53$ & & 110 & \\
\hline $7 / 8 / 53$ & & 110 & & 9/4/53 & & 110 & \\
\hline $7 / 9 / 53$ & & 110 & & $9 / 5 / 53$ & & 110 & \\
\hline $7 / 10 / 53$ & & 110 & & $9 / 6 / 53$ & & 110 & \\
\hline $7 / 11 / 53$ & & 110 & & $9 / 7 / 53$ & & 110 & \\
\hline $7 / 13 / 53$ & & 110 & & $9 / 8 / 53$ & & 110 & \\
\hline $7 / 15 / 53$ & & 110 & & $9 / 9 / 53$ & & 110 & \\
\hline $7 / 16 / 53$ & & 110 & & $9 / 10 / 53$ & & 110 & \\
\hline $7 / 17 / 53$ & & 110 & & $9 / 11 / 53$ & & 110 & \\
\hline $7 / 20 / 53$ & & 110 & & $9 / 13 / 53$ & & 110 & \\
\hline $7 / 21 / 53$ & & 110 & & $9 / 14 / 53$ & & 110 & \\
\hline $7 / 22 / 53$ & & 111 & & $9 / 15 / 53$ & & 110 & \\
\hline $7 / 23 / 53$ & & 111 & & $9 / 16 / 53$ & & 109 & \\
\hline $7 / 24 / 53$ & & 110 & & $9 / 17 / 53$ & & 109 & \\
\hline $7 / 25 / 53$ & & 110 & & $9 / 18 / 53$ & & 109 & \\
\hline $7 / 26 / 53$ & & 110 & & $9 / 19 / 53$ & & 110 & \\
\hline $7 / 27 / 53$ & & 112 & & $9 / 20 / 53$ & & 110 & \\
\hline $7 / 28 / 53$ & & 112 & & $9 / 21 / 53$ & & 110 & \\
\hline $7 / 29 / 53$ & & 112 & & $9 / 22 / 53$ & & 110 & \\
\hline $7 / 30 / 53$ & & 112 & & $9 / 23 / 53$ & & 109 & \\
\hline $7 / 31 / 53$ & & 112 & & $9 / 24 / 53$ & & 109 & \\
\hline $8 / 1 / 53$ & $\cdot$ & 112 & & $9 / 25 / 53$ & & 107 & \\
\hline $8 / 2 / 53$ & & 112 & & $9 / 28 / 53$ & & 109 & \\
\hline $8 / 3 / 53$ & & 112 & & $9 / 30 / 53$ & & 109 & \\
\hline $8 / 4 / 53$ & & 112 & & $10 / 1 / 53$ & & 104 & \\
\hline $8 / 5 / 53$ & & 112 & & $10 / 2 / 53$ & & 110 & \\
\hline $8 / 6 / 53$ & & 112 & & $10 / 3 / 53$ & & 110 & \\
\hline $8 / 7 / 53$ & & 112 & & $10 / 4 / 53$ & & 110 & \\
\hline $8 / 8 / 53$ & & 112 & & $10 / 5 / 53$ & & 110 & \\
\hline $8 / 9 / 53$ & & 110 & & $10 / 6 / 53$ & & 110 & \\
\hline $8 / 10 / 53$ & & 112 & & $10 / 7 / 53$ & & 108 & \\
\hline $8 / 11 / 53$ & & 112 & & $10 / 8 / 53$ & & 108 & \\
\hline $8 / 12 / 53$ & & 112 & & $10 / 9 / 53$ & & 108 & \\
\hline $8 / 13 / 53$ & & 112 & & $10 / 10 / 53$ & & 107 & \\
\hline $8 / 14 / 53$ & & 112 & & $10 / 11 / 53$ & & 106 & \\
\hline $8 / 15 / 53$ & & 115 & & $10 / 12 / 53$ & & 106 & \\
\hline $8 / 16 / 53$ & & 110 & & $10 / 14 / 53$ & & 108 & \\
\hline $8 / 17 / 53$ & & 112 & & $10 / 15 / 53$ & & 108 & \\
\hline $8 / 18 / 53$ & & 113 & & $10 / 16 / 53$ & & 108 & \\
\hline $8 / 19 / 53$ & & 110 & & $10 / 17 / 53$ & & 108 & \\
\hline $8 / 20 / 53$ & & 110 & & $10 / 18 / 53$ & & 108 & \\
\hline $8 / 21 / 53$ & & 910 & & $10 / 19 / 53$ & & 108 & \\
\hline
\end{tabular}

Data obtained from HW-83906. 


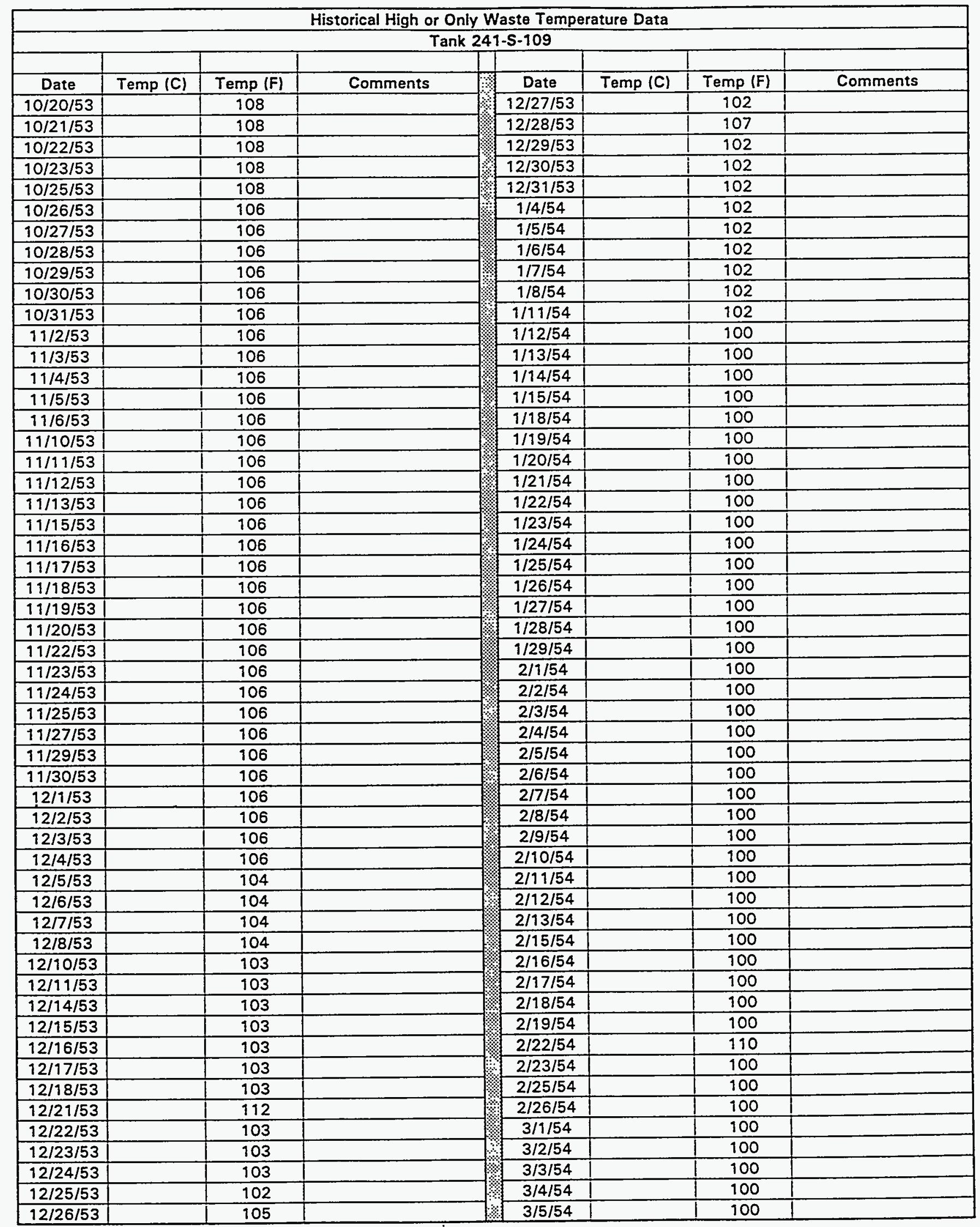

Data obtained from HW-83906. 


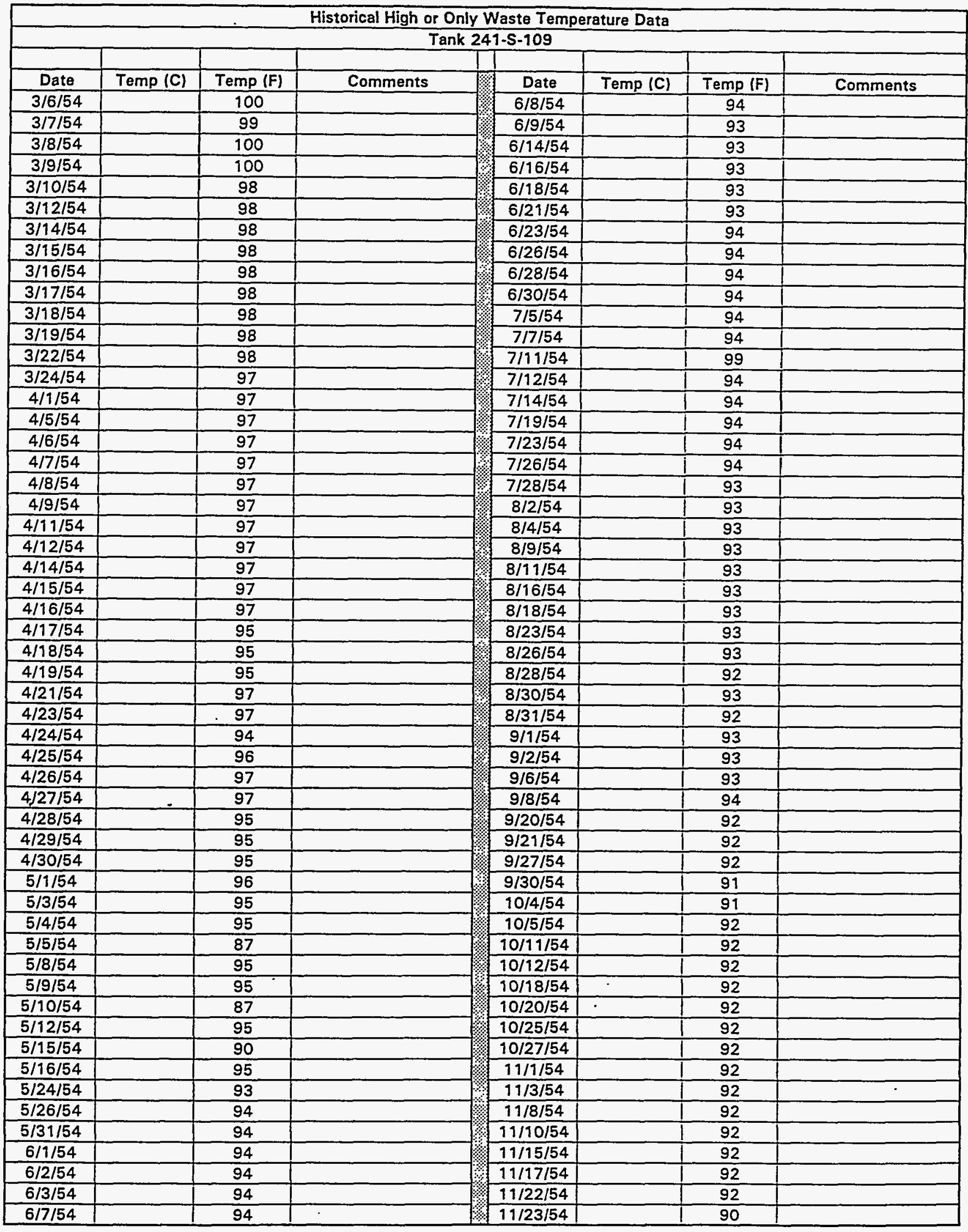

Data obtained from HW-83906. 
WHC-SD-WM-ER-323, Rev. O 1

\begin{tabular}{|c|c|c|c|c|c|c|c|c|}
\hline \multicolumn{9}{|c|}{ Historical High or Only Waste Temperature Data } \\
\hline \multicolumn{9}{|c|}{ Tank 241-S-109 } \\
\hline & & & & & & & & \\
\hline Date & $\operatorname{Temp}(\mathrm{C})$ & Temp (F) & Comments & & Date & Temp (C) & Temp (F) & Comments \\
\hline $11 / 24 / 54$ & & 90 & & & $12 / 15 / 54$ & & 90 & \\
\hline $11 / 26 / 54$ & & 90 & & 9 & $12 / 16 / 54$ & & 90 & \\
\hline $11 / 29 / 54$ & & 90 & & & $12 / 17 / 54$ & & 90 & \\
\hline $11 / 30 / 54$ & & 90 & & 8 & $12 / 20 / 54$ & & 90 & \\
\hline $12 / 1 / 54$ & & 90 & & & $12 / 21 / 54$ & & 90 & \\
\hline $12 / 2 / 54$ & & 90 & & & $12 / 22 / 54$ & & 90 & \\
\hline $12 / 3 / 54$ & & 90 & & $\$$ & $12 / 23 / 54$ & & 90 & \\
\hline $12 / 4 / 54$ & & 90 & & & $12 / 24 / 54$ & & 88 & \\
\hline $12 / 5 / 54$ & & 90 & & & $12 / 25 / 54$ & & 88 & \\
\hline $12 / 6 / 54$ & & 90 & & 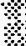 & $12 / 26 / 54$ & & 88 & \\
\hline $12 / 7 / 54$ & & 90 & & & $12 / 27 / 54$ & & 90 & \\
\hline $12 / 8 / 54$ & & 90 & & 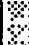 & $12 / 28 / 54$ & & 90 & \\
\hline $12 / 9 / 54$ & & 92 & & & $12 / 29 / 54$ & & 90 & \\
\hline $12 / 10 / 54$ & & 92 & & $\ddot{*}$ & $12 / 30 / 54$ & & 90 & \\
\hline $12 / 12 / 54$ & & 90 & & 4 & $12 / 31 / 54$ & & 90 & \\
\hline $12 / 13 / 54$ & & 90 & & 4 & $1 / 1 / 55$ & & 89 & \\
\hline $12 / 14 / 54$ & & 90 & & 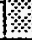 & $1 / 2 / 55$ & & 90 & \\
\hline
\end{tabular}

Data obtained from HW-83906: 


\begin{tabular}{|c|c|c|c|c|c|c|c|}
\hline \multicolumn{8}{|c|}{ Historical Vapor Space Temperature Data } \\
\hline \multicolumn{8}{|c|}{ Tank 241-S-110 } \\
\hline Date & Temp (C) & Temp (F) & Comments & Date & Temp (C) & Temn (F) & Comments \\
\hline $7 / 30 / 56$ & & 144 & & $10 / 22 / 56$ & & 145 & \\
\hline $8 / 2 / 56$ & & 144 & & $10 / 24 / 56$ & & 146 & \\
\hline $8 / 5 / 56$ & & 145 & & $10 / 27 / 56$ & & 146 & \\
\hline $8 / 7 / 56$ & & 145 & & $10 / 29 / 56$ & & 145 & \\
\hline $8 / 9 / 56$ & & 145 & & $10 / 30 / 56$ & & 144 & \\
\hline $8 / 11 / 56$ & & 145 & & $10 / 30 / 56$ & & 144 & \\
\hline $8 / 13 / 56$ & & 145 & & $10 / 31 / 56$ & & 143 & \\
\hline $8 / 15 / 56$ & & 145 & & $11 / 1 / 56$ & & 142 & \\
\hline $8 / 17 / 56$ & & 144 & & $11 / 2 / 56$ & & 142 & \\
\hline $8 / 19 / 56$ & & 143 & & $11 / 3 / 56$ & & 142 & \\
\hline $8 / 21 / 56$ & & 143 & & $11 / 4 / 56$ & & 141 & \\
\hline $8 / 22 / 56$ & & 143 & & $11 / 5 / 56$ & & 140 & \\
\hline $8 / 24 / 56$ & & 143 & & $11 / 7 / 56$ & & 140 & \\
\hline $8 / 26 / 56$ & & 144 & & $11 / 9 / 56$ & & 140 & \\
\hline $8 / 28 / 56$ & & 144 & & $11 / 11 / 56$ & & 141 & \\
\hline $8 / 31 / 56$ & & 144 & & $11 / 14 / 56$ & & 140 & \\
\hline $9 / 2 / 56$ & & 145 & & $11 / 14 / 56$ & & 140 & \\
\hline $9 / 5 / 56$ & & 144 & & $11 / 17 / 56$ & & 140 & \\
\hline $9 / 7 / 56$ & & 145 & & $11 / 19 / 56$ & & 140 & \\
\hline $9 / 8 / 56$ & & 145 & & $11 / 21 / 56$ & & 141 & \\
\hline $9 / 10 / 56$ & & 144 & & $11 / 23 / 56$ & & 140 & \\
\hline $9 / 12 / 56$ & & 144 & & $11 / 26 / 56$ & & 140 & \\
\hline $9 / 13 / 56$ & & 143 & & $11 / 28 / 56$ & & 140 & \\
\hline $9 / 14 / 56$ & & 143 & & $11 / 30 / 56$ & & 140 & \\
\hline $9 / 15 / 56$ & & 142 & & $12 / 3 / 56$ & & 140 & \\
\hline $9 / 16 / 56$ & & 141 & & $12 / 4 / 56$ & & 140 & \\
\hline $9 / 18 / 56$ & & 140 & & $12 / 7 / 56$ & & 141 & \\
\hline $9 / 20 / 56$ & & 140 & & $12 / 9 / 56$ & & 140 & \\
\hline $9 / 23 / 56$ & & 140 & & $12 / 11 / 56$ & & 140 & \\
\hline $9 / 24 / 56$ & & 141 & & $12 / 14 / 56$ & & 140 & \\
\hline $9 / 26 / 56$ & & 141 & & $12 / 16 / 56$ & & 140 & \\
\hline $9 / 27 / 56$ & & 142 & & $12 / 18 / 56$ & & 140 & \\
\hline $9 / 27 / 56$ & & 142 & & $12 / 20 / 56$ & & 140 & \\
\hline $9 / 29 / 56$ & $\therefore$ & 143 & & $12 / 23 / 56$ & & 140 & \\
\hline $9 / 30 / 56$ & & 144 & & $12 / 25 / 56$ & & 140 & \\
\hline $10 / 1 / 56$ & & 145 & & $12 / 26 / 56$ & & 140 & \\
\hline $10 / 2 / 56$ & & 145 & & $12 / 28 / 56$ & & 140 & \\
\hline $10 / 3 / 56$ & & 145 & & $2 / 2 / 57$ & & 140 & \\
\hline $10 / 5 / 56$ & & 144 & & $2 / 22 / 57$ & & 137 & \\
\hline $10 / 5 / 56$ & & 144 & & $3 / 1 / 57$ & & 135 & \\
\hline $10 / 7 / 56$ & & 143 & & $3 / 6 / 57$ & & 135 & \\
\hline $10 / 8 / 56$ & & 143 & & $3 / 30 / 57$ & & 135 & \\
\hline $10 / 9 / 56$ & & 143 & & $4 / 5 / 57$ & & 133 & \\
\hline $10 / 11 / 56$ & & 142 & & $5 / 1 / 57$ & & 119 & \\
\hline $10 / 13 / 56$ & & 142 & & $5 / 30 / 57$ & & 129 & \\
\hline $10 / 15 / 56$ & & 141 & & $6 / 4 / 57$ & & 130 & \\
\hline $10 / 15 / 56$ & & 141 & & $6 / 14 / 57$ & & 131 & \\
\hline $10 / 16 / 56$ & & 141 & & $6 / 29 / 57$ & & 132 & \\
\hline $10 / 17 / 56$ & & 141 & & $7 / 4 / 57$ & & 133 & \\
\hline $10 / 17 / 56$ & & 142 & & $7 / 11 / 57$ & & 132 & \\
\hline $10 / 17 / 56$ & & 143 & & $7 / 22 / 57$ & & 130 & \\
\hline $10 / 19 / 56$ & & 143 & & $8 / 10 / 57$ & & 128 & \\
\hline $10 / 20 / 56$ & & 144 & & $8 / 22 / 57$ & & 127 & \\
\hline $10 / 22 / 56$ & & 144 & - & $9 / 8 / 57$ & & 126 & \\
\hline
\end{tabular}

Data obtained from RHO-CD-1172. 
WHC-SD-WM-ER-323, Rev. 0 1

\begin{tabular}{|c|c|c|c|c|c|c|c|c|}
\hline \multicolumn{9}{|c|}{ Historical Vapor Space Temperature Data } \\
\hline \multicolumn{9}{|c|}{ Tank 241-S-110 } \\
\hline & & & & & & & & \\
\hline Date & Temp (C) & Temp (F) & Comments & 3 & Date & Temp (C) & Temp (F) & Comments \\
\hline $9 / 21 / 57$ & & 125 & & 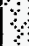 & $11 / 19 / 58$ & & 118 & \\
\hline $10 / 1 / 57$ & & 125 & & 3 & $12 / 10 / 58$ & & 117 & \\
\hline $10 / 21 / 57$ & & 128 & & 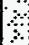 & $1 / 4 / 59$ & & 116 & \\
\hline $10 / 30 / 57$ & & 130 & & & $2 / 1 / 59$ & & 131 & \\
\hline $11 / 5 / 57$ & & 129 & & & $3 / 4 / 59$ & & 113 & \\
\hline $11 / 19 / 57$ & & 129 & & & $3 / 20 / 59$ & & 112 & \\
\hline $12 / 8 / 57$ & & 127 & & & $3 / 28 / 59$ & & 112 & \\
\hline $12 / 28 / 57$ & & 126 & & & $5 / 1 / 59$ & & 109 & \\
\hline $9 / 2 / 58$ & & 117 & & & $12 / 2 / 59$ & & 107 & \\
\hline $10 / 15 / 58$ & & 115 & & 3 & $12 / 15 / 59$ & & 108 & \\
\hline $10 / 28 / 58$ & & 117 & & & $12 / 29 / 59$ & & 110 & \\
\hline
\end{tabular}

Data obtained from RHO-CD-1172. 


\begin{tabular}{|c|c|c|c|c|c|c|c|}
\hline \multicolumn{8}{|c|}{ Historical High or Only Waste Temperature Data } \\
\hline \multicolumn{8}{|c|}{ Tank 241-S-110 } \\
\hline & & & & & & & \\
\hline Date & Temp (C) & Temp (F) & Comments & Date & Temp (C) & Temp (F) & Comments \\
\hline $1 / 14 / 52$ & & 42 & & $8 / 2 / 53$ & & 225 & \\
\hline $1 / 17 / 52$ & & 71 & & $8 / 3 / 53$ & & 224 & \\
\hline $1 / 28 / 52$ & & 95 & & $8 / 4 / 53$ & & 224 & \\
\hline $2 / 6 / 52$ & & 101 & & $8 / 5 / 53$ & & 224 & \\
\hline $2 / 23 / 52$ & & 102 & & $8 / 6 / 53$ & & 224 & \\
\hline $3 / 14 / 52$ & & 111 & & $8 / 7 / 53$ & & 224 & \\
\hline $4 / 2 / 52$ & & 142 & & $8 / 8 / 53$ & & 222 & \\
\hline $4 / 29 / 52$ & & 170 & & $8 / 9 / 53$ & & 222 & \\
\hline $6 / 9 / 52$ & & 191 & & $8 / 10 / 53$ & & 224 & \\
\hline $7 / 18 / 52$ & & 215 & & $8 / 11 / 53$ & & 224 & \\
\hline $8 / 6 / 52$ & & 220 & & $8 / 12 / 53$ & & 224 & \\
\hline $9 / 2 / 52$ & & 220 & & $8 / 13 / 53$ & & 224 & \\
\hline $9 / 27 / 52$ & & 238 & & $8 / 14 / 53$ & & 224 & \\
\hline $10 / 12 / 52$ & & 249 & & $8 / 15 / 53$ & & 220 & \\
\hline $11 / 7 / 52$ & & 252 & & $8 / 16 / 53$ & & 221 & \\
\hline $12 / 3 / 52$ & & 261 & & $8 / 17 / 53$ & & 224 & \\
\hline $1 / 5 / 53$ & & 252 & & $8 / 18 / 53$ & & 224 & \\
\hline $2 / 26 / 53$ & & 239 & & $8 / 19 / 53$ & & 222 & \\
\hline $4 / 9 / 53$ & & 233 & & $8 / 20 / 53$ & & 222 & \\
\hline $5 / 29 / 53$ & & 228 & & $8 / 21 / 53$ & & 222 & \\
\hline $6 / 22 / 53$ & & 228 & & $8 / 22 / 53$ & & 221 & \\
\hline $6 / 23 / 53$ & & 228 & & $8 / 23 / 53$ & & 222 & \\
\hline $6 / 24 / 53$ & & 228 & & $8 / 24 / 53$ & & 222 & \\
\hline $6 / 25 / 53$ & & 228 & & $8 / 25 / 53$ & & 224 & \\
\hline $6 / 26 / 53$ & & 228 & & $8 / 26 / 53$ & & 223 & \\
\hline $6 / 29 / 53$ & & 224 & & $8 / 27 / 53$ & & 223 & \\
\hline $7 / 1 / 53$ & & 224 & & $8 / 28 / 53$ & & 223 & \\
\hline $7 / 2 / 53$ & & 224 & & $8 / 29 / 53$ & & 223 & \\
\hline $7 / 3 / 53$ & & 224 & & $8 / 30 / 53$ & & 225 & \\
\hline $7 / 4 / 53$ & & 224 & & $8 / 31 / 53$ & & 223 & \\
\hline $7 / 5 / 53$ & & 224 & & $9 / 1 / 53$ & & 223 & \\
\hline $7 / 6 / 53$ & & 229 & & $9 / 2 / 53$ & & 223 & \\
\hline $7 / 7 / 53$ & & 234 & & $9 / 3 / 53$ & & 222 & \\
\hline $7 / 8 / 53$ & & 224 & & $9 / 4 / 53$ & & 223 & \\
\hline $7 / 9 / 53$ & & 234 & & $9 / 5 / 53$ & & 222 & \\
\hline $7 / 10 / 53$ & & 224 & & $9 / 6 / 53$ & & 222 & \\
\hline $7 / 11 / 53$ & & 226 & & $9 / 7 / 53$ & & 223 & \\
\hline $7 / 13 / 53$ & & 226 & & $9 / 8 / 53$ & & 224 & \\
\hline $7 / 15 / 53$ & & 226 & & $9 / 9 / 53$ & & 222 & \\
\hline $7 / 16 / 53$ & & 226 & & $9 / 10 / 53$ & & 222 & \\
\hline $7 / 17 / 53$ & & 226 & & $9 / 11 / 53$ & & 222 & \\
\hline $7 / 20 / 53$ & & 226 & & $9 / 13 / 53$ & & 222 & \\
\hline $7 / 21 / 53$ & & 224 & & $9 / 14 / 53$ & & 222 & \\
\hline $7 / 22 / 53$ & & 222 & & $9 / 15 / 53$ & & 222 & \\
\hline $7 / 23 / 53$ & & 220 & & $9 / 16 / 53$ & & 221 & \\
\hline $7 / 24 / 53$ & & 220 & & $9 / 17 / 53$ & & 222 & \\
\hline $7 / 25 / 53$ & & 222 & & $9 / 18 / 53$ & & 222 & \\
\hline $7 / 26 / 53$ & & 222 & & $9 / 19 / 53$ & & 222 & \\
\hline $7 / 27 / 53$ & & 225 & & 9/20/53 & & 222 & \\
\hline $7 / 28 / 53$ & & 225 & & $9 / 21 / 53$ & & 222 & \\
\hline $7 / 29 / 53$ & & 225 & & $9 / 22 / 53$ & & 222 & \\
\hline $7 / 30 / 53$ & & 225 & & $9 / 23 / 53$ & & 222 & \\
\hline $7 / 31 / 53$ & & 225 & & $9 / 24 / 53$ & & 222 & \\
\hline $8 / 1 / 53$ & & 225 & & $9 / 25 / 53$ & & 222 & \\
\hline
\end{tabular}

.. Data obtained from RHO-CD-1172 and HW-83906. 


\begin{tabular}{|c|c|c|c|c|c|c|c|}
\hline \multicolumn{8}{|c|}{ Historical High or Only Waste Temperature Data } \\
\hline \multicolumn{8}{|c|}{ Tank $241-\mathrm{S}-110$} \\
\hline & & & & & & & \\
\hline Date & Temp (C) & Temp (F) & Comments & Date & Temp (C) & Temp (F) & Comments \\
\hline $9 / 28 / 53$ & & 222 & & $12 / 1 / 53$ & & 225 & \\
\hline $9 / 29 / 53$ & & 222 & & $12 / 2 / 53$ & & 220 & \\
\hline $9 / 30 / 53$ & & 224 & & $12 / 3 / 53$ & & 220 & \\
\hline $10 / 1 / 53$ & & 224 & & $12 / 4 / 53$ & & 220 & \\
\hline $10 / 2 / 53$ & & 224 & & $12 / 5 / 53$ & & 220 & \\
\hline $10 / 3 / 53$ & & 224 & & $12 / 6 / 53$ & & 220 & \\
\hline $10 / 4 / 53$ & & 224 & & $12 / 7 / 53$ & & 220 & \\
\hline $10 / 5 / 53$ & & 224 & & $12 / 8 / 53$ & & 220 & \\
\hline $10 / 6 / 53$ & & 224 & & $12 / 10 / 53$ & & 220 & \\
\hline $10 / 7 / 53$ & & 222 & & $12 / 14 / 53$ & & 220 & \\
\hline $10 / 8 / 53$ & & 222 & & $12 / 15 / 53$ & & 220 & \\
\hline $10 / 9 / 53$ & & 222 & & $12 / 16 / 53$ & & 220 & \\
\hline $10 / 10 / 53$ & & 224 & & $12 / 17 / 53$ & & 220 & \\
\hline $10 / 11 / 53$ & & 222 & & $12 / 18 / 53$ & & 220 & \\
\hline $10 / 12 / 53$ & & 222 & & $12 / 21 / 53$ & & 220 & \\
\hline $10 / 14 / 53$ & & 222 & & $12 / 22 / 53$ & & 220 & \\
\hline $10 / 15 / 53$ & & 222 & & $12 / 23 / 53$ & & 220 & \\
\hline $10 / 16 / 53$ & & 222 & & $12 / 24 / 53$ & & 220 & \\
\hline $10 / 17 / 53$ & & 222 & & $12 / 25 / 53$ & & 220 & \\
\hline $10 / 20 / 53$ & & 222 & & $12 / 26 / 53$ & & 220 & \\
\hline $10 / 21 / 53$ & & 222 & & $12 / 27 / 53$ & & 220 & \\
\hline $10 / 22 / 53$ & & 222 & & $12 / 28 / 53$ & & 220 & \\
\hline $10 / 23 / 53$ & & 222 & & $12 / 29 / 53$ & & 220 & \\
\hline $10 / 24 / 53$ & & 222 & & $12 / 30 / 53$ & & 220 & \\
\hline $10 / 25 / 53$ & & 222 & & $12 / 31 / 53$ & & 220 & \\
\hline $10 / 26 / 53$ & & 222 & & $1 / 4 / 54$ & & 220 & \\
\hline $10 / 27 / 53$ & & 222 & & $1 / 5 / 54$ & & 220 & \\
\hline $10 / 28 / 53$ & & 222 & & $1 / 6 / 54$ & & 220 & \\
\hline $10 / 29 / 53$ & & 222 & & $1 / 7 / 54$ & & 220 & \\
\hline $10 / 30 / 53$ & & 222 & & $1 / 8 / 54$ & & 220 & \\
\hline $10 / 31 / 53$ & & 225 & & $1 / 11 / 54$ & & 220 & \\
\hline $11 / 2 / 53$ & & 225 & & $1 / 12 / 54$ & & 220 & \\
\hline $11 / 3 / 53$ & & 225 & & $1 / 13 / 54$ & & 220 & \\
\hline $11 / 4 / 53$ & ? & 225 & & $1 / 14 / 54$ & & 220 & \\
\hline $11 / 5 / 53$ & & 225 & & $1 / 15 / 54$ & & 220 & \\
\hline $11 / 6 / 53$ & & 225 & & $1 / 18 / 54$ & & 220 & \\
\hline $11 / 10 / 53$ & & 225 & & $1 / 19 / 54$ & & 220 & \\
\hline $11 / 111 / 53$ & & 225 & & $1 / 20 / 54$ & & 216 & \\
\hline $11 / 12 / 53$ & & 225 & & $1 / 21 / 54$ & & 216 & \\
\hline $11 / 13 / 53$ & & 225 & & $1 / 22 / 54$ & & 216 & \\
\hline $11 / 15 / 53$ & & 225 & & $1 / 23 / 54$ & & 216 & \\
\hline $11 / 16 / 53$ & & 225 & & $1 / 24 / 54$ & & 219 & \\
\hline $11 / 17 / 53$ & & 225 & & $1 / 25 / 54$ & & 216 & \\
\hline $11 / 18 / 53$ & & 225 & & $1 / 26 / 54$ & & 216 & \\
\hline $11 / 19 / 53$ & & 225 & & $1 / 27 / 54$ & & 218 & \\
\hline $11 / 20 / 53$ & & 225 & & $1 / 28 / 54$ & & 218 & \\
\hline $11 / 22 / 53$ & & 220 & & $1 / 29 / 54$ & & 218 & \\
\hline $11 / 23 / 53$ & & 225 & & $2 / 1 / 54$ & & 218 & \\
\hline $11 / 24 / 53$ & & 225 & & $2 / 2 / 54$ & & 218 & \\
\hline $11 / 25 / 53$ & & 225 & & $2 / 3 / 54$ & & 220 & \\
\hline $11 / 26 / 53$ & & 220 & & $2 / 4 / 54$ & & 220 & \\
\hline $11 / 27 / 53$ & & 221 & & $2 / 5 / 54$ & & 220 & \\
\hline $11 / 29 / 53$ & & 220 & & $2 / 6 / 54$ & & 220 & \\
\hline $11 / 30 / 53$ & & 225 & & $2 / 7 / 54$ & & 220 & \\
\hline
\end{tabular}

Data obtained from RHO-CD-1172 and HW-83906. 


\begin{tabular}{|c|c|c|c|c|c|c|c|}
\hline \multicolumn{8}{|c|}{ Historical High or Only Waste Temperature Data } \\
\hline \multicolumn{8}{|c|}{ Tank 241-S-110 } \\
\hline & & & & & & & \\
\hline Date & Temp (C) & Temp (F) & Comments & Date & $\operatorname{Temp}(\mathrm{C})$ & Temp (F) & Comments \\
\hline $2 / 8 / 54$ & & 220 & & $4 / 28 / 54$ & & 210 & \\
\hline $2 / 9 / 54$ & & 220 & & $4 / 29 / 54$ & & 210 & \\
\hline $2 / 10 / 54$ & & 220 & & $4 / 30 / 54$ & & 210 & \\
\hline $2 / 11 / 54$ & & 220 & & $5 / 1 / 54$ & & 210 & \\
\hline $2 / 12 / 54$ & & 220 & & $5 / 3 / 54$ & & 210 & \\
\hline $2 / 13 / 54$ & & 220 & & $5 / 4 / 54$ & & 210 & \\
\hline $2 / 15 / 54$ & & 220 & & $5 / 5 / 54$ & & 209 & \\
\hline $2 / 16 / 54$ & & 220 & & $5 / 8 / 54$ & & 210 & \\
\hline $2 / 17 / 54$ & & 220 & & $5 / 9 / 54$ & & 210 & \\
\hline $2 / 18 / 54$ & & 221 & & $5 / 10 / 54$ & & 209 & \\
\hline $2 / 19 / 54$ & & 221 & & $5 / 12 / 54$ & & 207 & \\
\hline $2 / 22 / 54$ & & 221 & & $5 / 15 / 54$ & & 210 & \\
\hline $2 / 23 / 54$ & & 221 & & $5 / 16 / 54$ & & 210 & \\
\hline $2 / 25 / 54$ & & 221 & & $5 / 24 / 54$ & & 209 & \\
\hline $2 / 26 / 54$ & & 220 & & $5 / 26 / 54$ & & 206 & \\
\hline $3 / 1 / 54$ & & 220 & & $5 / 31 / 54$ & & 206 & \\
\hline $3 / 2 / 54$ & & 220 & & $6 / 1 / 54$ & & 206 & \\
\hline $3 / 3 / 54$ & & 216 & & $6 / 2 / 54$ & & 207 & \\
\hline $3 / 4 / 54$ & & 216 & & $6 / 3 / 54$ & & 207 & \\
\hline $3 / 5 / 54$ & & 216 & & $6 / 7 / 54$ & & 207 & \\
\hline $3 / 6 / 54$ & & 220 & & $6 / 8 / 54$ & & 207 & \\
\hline $3 / 7 / 54$ & & 218 & & $6 / 9 / 54$ & & 209 & \\
\hline $3 / 8 / 54$ & & 217 & & $6 / 14 / 54$ & & 209 & \\
\hline $3 / 9 / 54$ & & 217 & & $6 / 16 / 54$ & & 209 & \\
\hline $3 / 10 / 54$ & & 219 & & $6 / 18 / 54$ & & 209 & \\
\hline $3 / 12 / 54$ & & 219 & & $6 / 21 / 54$ & & 209 & \\
\hline $3 / 14 / 54$ & & 216 & & $6 / 22 / 54$ & & 206 & \\
\hline $3 / 15 / 54$ & & 219 & & $6 / 23 / 54$ & & 206 & \\
\hline $3 / 16 / 54$ & & 219 & & $6 / 26 / 54$ & & 206 & \\
\hline $3 / 17 / 54$ & & 216 & & $6 / 28 / 54$ & & 206 & \\
\hline $3 / 18 / 54$ & & 217 & & $6 / 30 / 54$ & & 206 & \\
\hline $3 / 19 / 54$ & & 217 & & $7 / 5 / 54$ & & 206 & \\
\hline $3 / 22 / 54$ & & 216 & & $7 / 7 / 54$ & & 204 & \\
\hline $3 / 24 / 54$ & $\cdot$ & 216 & & $7 / 11 / 54$ & & 205 & \\
\hline $4 / 1 / 54$ & & 212 & & $7 / 12 / 54$ & & 204 & \\
\hline $4 / 5 / 54$ & & 212 & & $7 / 14 / 54$ & & 204 & \\
\hline $4 / 6 / 54$ & & 212 & & $7 / 19 / 54$ & & 204 & \\
\hline $4 / 7 / 54$ & & 212 & & $7 / 23 / 54$ & & 203 & \\
\hline $4 / 8 / 54$ & & 212 & & $7 / 26 / 54$ & & 203 & \\
\hline $4 / 9 / 54$ & & 212 & & $7 / 28 / 54$ & & 201 & \\
\hline $4 / 11 / 54$ & & 212 & & $8 / 2 / 54$ & & 201 & \\
\hline $4 / 12 / 54$ & & 212 & & $8 / 4 / 54$ & & 201 & \\
\hline $4 / 14 / 54$ & & 212 & & $8 / 9 / 54$ & & 201 & \\
\hline $4 / 15 / 54$ & & 212 & & $8 / 11 / 54$ & & 200 & \\
\hline $4 / 16 / 54$ & & 212 & & $8 / 16 / 54$ & & 200 & \\
\hline $4 / 17 / 54$ & & 210 & & $8 / 18 / 54$ & & 200 & \\
\hline $4 / 18 / 54$ & & 210 & & $8 / 23 / 54$ & & 200 & \\
\hline $4 / 19 / 54$ & & 210 & & $8 / 26 / 54$ & & 200 & \\
\hline $4 / 21 / 54$ & & 210 & & $8 / 28 / 54$ & & 200 & \\
\hline $4 / 23 / 54$ & & 210 & & $8 / 30 / 54$ & & 200 & \\
\hline $4 / 24 / 54$ & & 210 & & $8 / 31 / 54$ & & 201 & \\
\hline $4 / 25 / 54$ & & 212 & & $9 / 1 / 54$ & & 198 & \\
\hline $4 / 26 / 54$ & & 210 & & $9 / 2 / 54$ & & 200 & \\
\hline $4 / 27 / 54$ & & 210 & & $9 / 6 / 54$ & & 198 & \\
\hline
\end{tabular}

Data obtained from RHO-CD-1172 and HW-83906. 
1

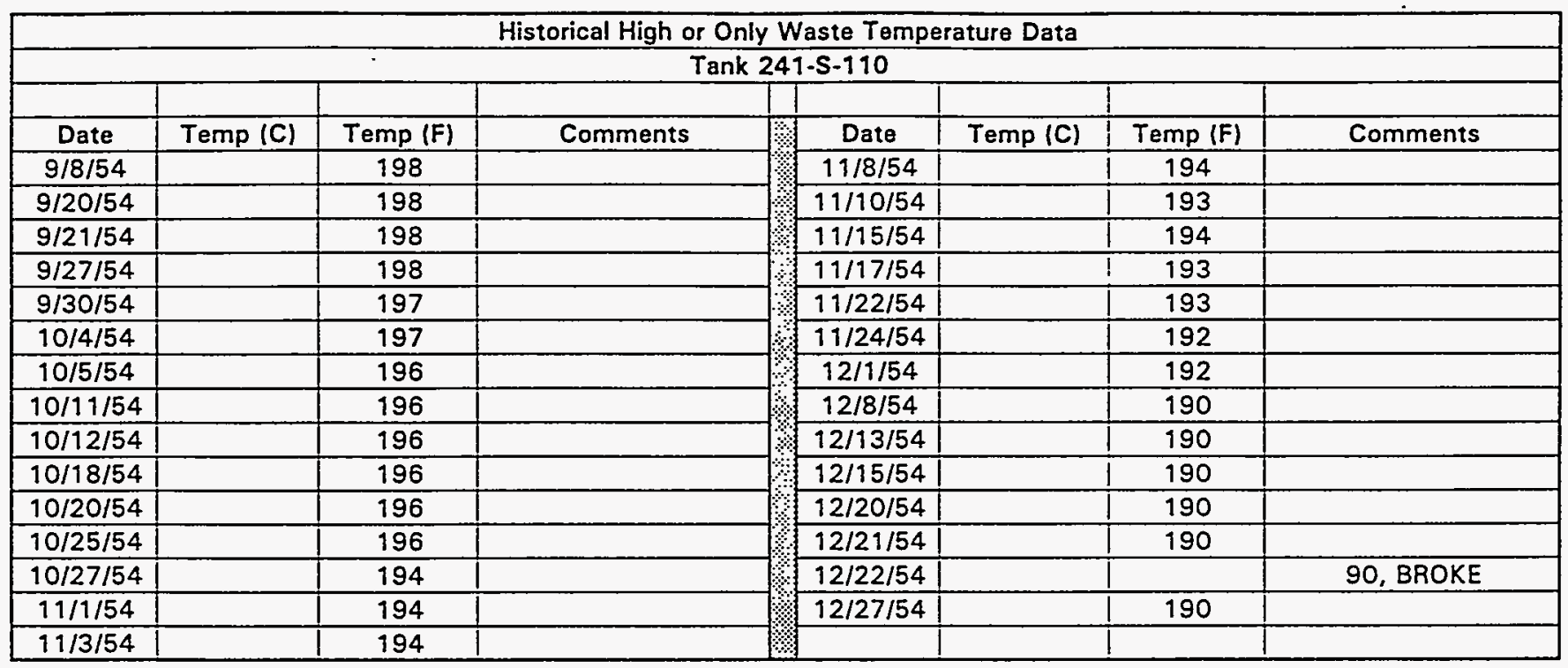




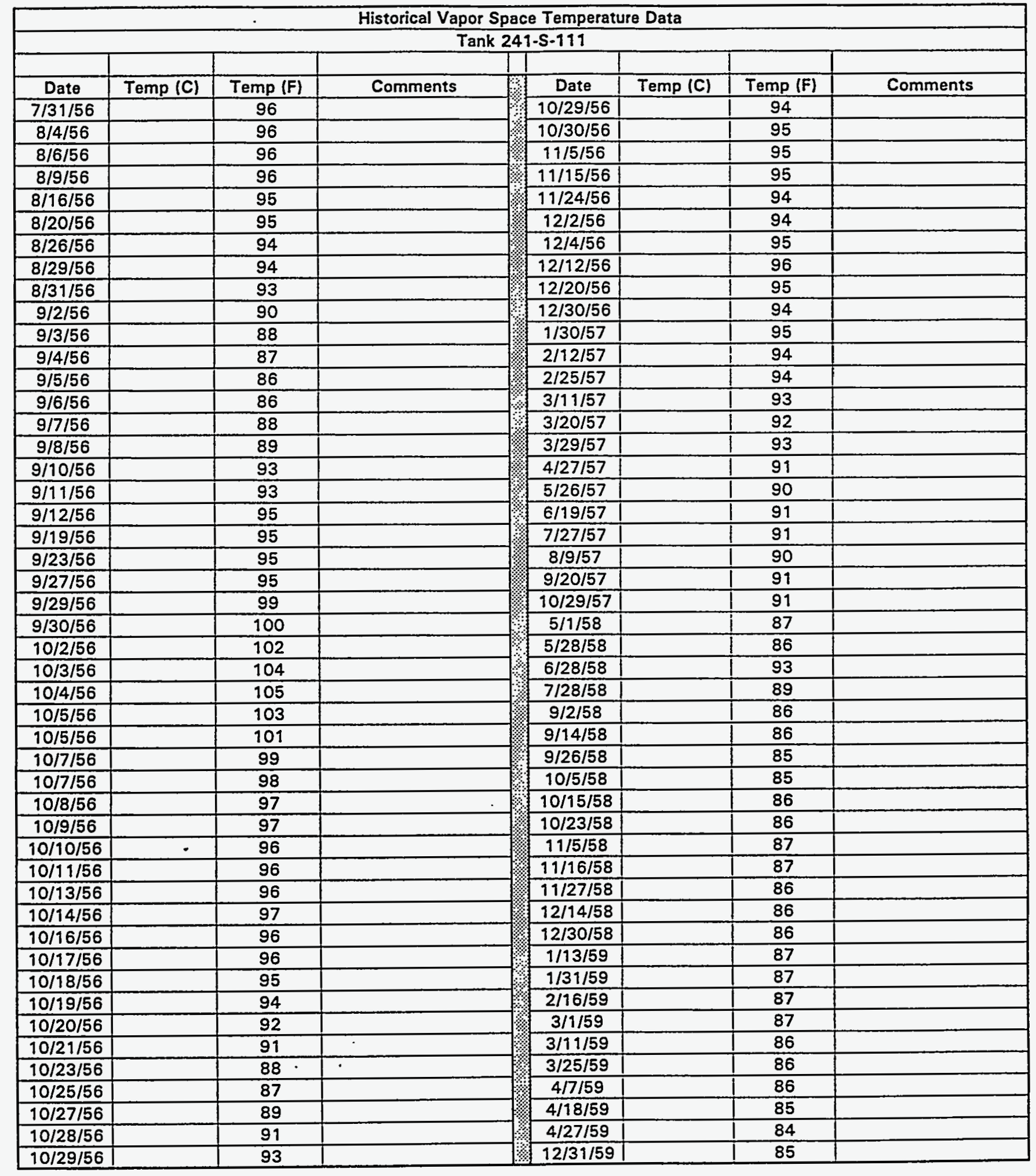

Data obtained from RHO-CD-1172. 


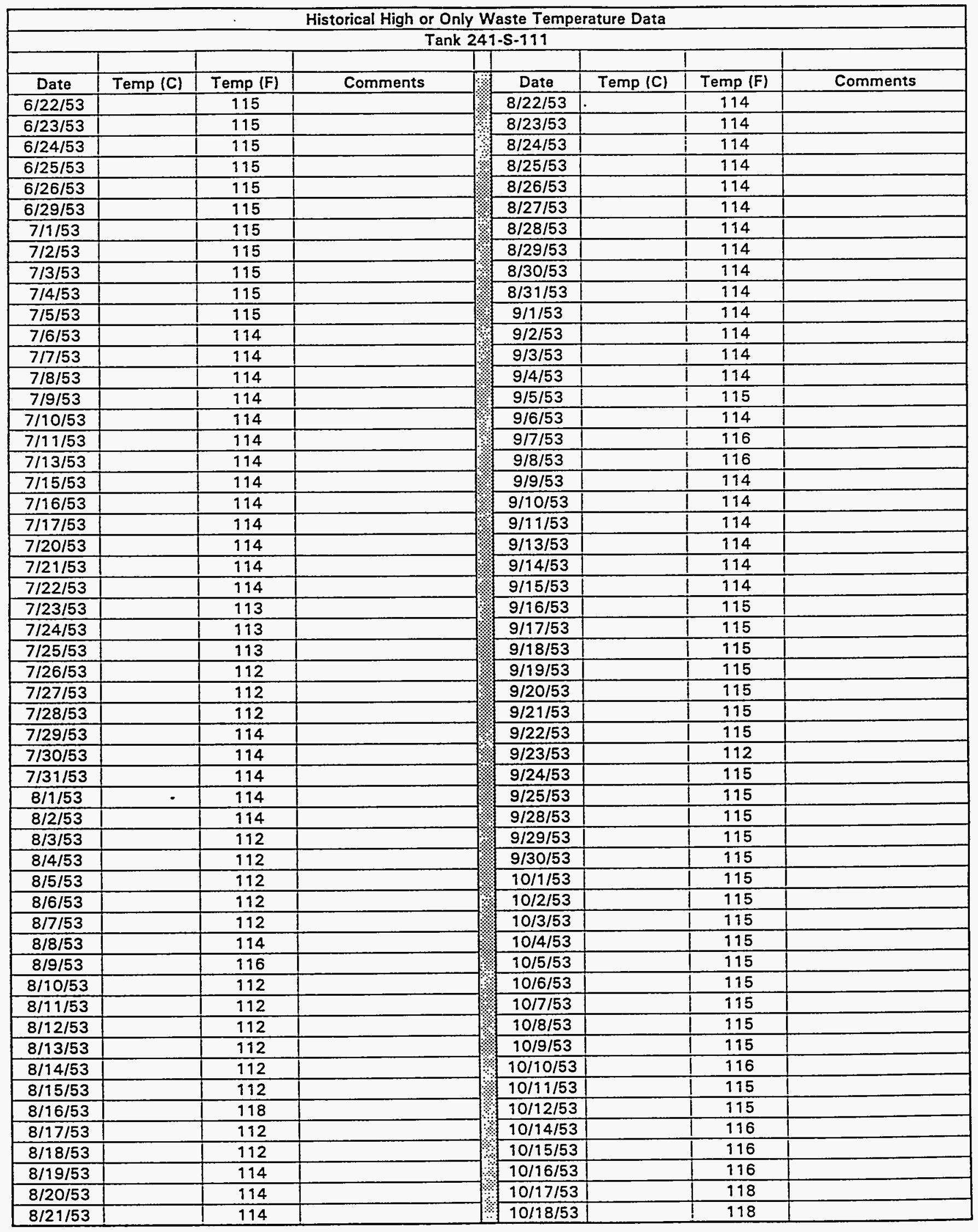

Data obtained from HW-83906. 


\begin{tabular}{|c|c|c|c|c|c|c|c|}
\hline \multicolumn{8}{|c|}{$\begin{array}{c}\text { Historical High or Only Waste Temperature Data } \\
\text { Tank 241-S-111 }\end{array}$} \\
\hline & & & Tank 2 & $1-5-111$ & & & \\
\hline & & & & & & & \\
\hline$\frac{\text { Date }}{10 / 20 / 53}$ & Temp (C) & Temp (F) & Comments & Date & Temp (C) & Temp (F) & Comments \\
\hline $10 / 20 / 53$ & & 118 & & $12 / 26 / 53$ & & 118 & \\
\hline $10 / 21 / 53$ & & 118 & & $12 / 27 / 53$ & & 118 & \\
\hline $10 / 22 / 53$ & & 116 & & $12 / 28 / 53$ & & 118 & \\
\hline $10 / 23 / 53$ & & 117 & & $12 / 29 / 53$ & & 118 & \\
\hline $10 / 24 / 53$ & & 117 & & $12 / 30 / 53$ & & 117 & \\
\hline $10 / 25 / 53$ & & 117 & & $12 / 31 / 53$ & & 117 & \\
\hline $10 / 26 / 53$ & & 116 & & $1 / 4 / 54$ & & 117 & \\
\hline $10 / 27 / 53$ & & 116 & & $1 / 5 / 54$ & & 117 & \\
\hline $10 / 28 / 53$ & & 116 & & $1 / 6 / 54$ & & 117 & \\
\hline $10 / 29 / 53$ & & 116 & & $1 / 7 / 54$ & & 117 & \\
\hline $10 / 30 / 53$ & & 116 & & $1 / 8 / 54$ & & 117 & \\
\hline $10 / 31 / 53$ & & 117 & & $1 / 11 / 54$ & & 117 & \\
\hline $11 / 1 / 53$ & & 116 & & $1 / 12 / 54$ & & 119 & \\
\hline $11 / 2 / 53$ & & 116 & & $1 / 13 / 54$ & & 118 & \\
\hline $11 / 3 / 53$ & & 116 & & $1 / 14 / 54$ & & 118 & \\
\hline $11 / 4 / 53$ & & 116 & & $1 / 15 / 54$ & & 118 & \\
\hline $11 / 5 / 53$ & & 116 & & $1 / 18 / 54$ & & 118 & \\
\hline $11 / 6 / 53$ & & 116 & & $1 / 19 / 54$ & & 118 & \\
\hline $11 / 10 / 53$ & & 116 & & $1 / 20 / 54$ & & 118 & \\
\hline $11 / 11 / 53$ & & 116 & & $1 / 21 / 54$ & & 118 & \\
\hline $11 / 12 / 53$ & & 116 & & $1 / 22 / 54$ & & 118 & \\
\hline $11 / 13 / 53$ & & 116 & & $1 / 23 / 54$ & & 118 & \\
\hline $11 / 15 / 53$ & & 116 & & $1 / 24 / 54$ & & 118 & \\
\hline $11 / 16 / 53$ & & 116 & & $1 / 25 / 54$ & & 118 & \\
\hline $11 / 17 / 53$ & & 116 & & $1 / 26 / 54$ & & 118 & \\
\hline $11 / 18 / 53$ & & 116 & & $1 / 27 / 54$ & & 118 & \\
\hline $11 / 19 / 53$ & & 116 & & $1 / 28 / 54$ & & 118 & \\
\hline $11 / 20 / 53$ & & 916 & & $1 / 29 / 54$ & & 118 & \\
\hline $11 / 22 / 53$ & & 119 & & $2 / 1 / 54$ & & 118 & \\
\hline $11 / 23 / 53$ & & 119 & & $2 / 2 / 54$ & & 118 & \\
\hline $11 / 24 / 53$ & & 119 & & $2 / 3 / 54$ & & 117 & \\
\hline $11 / 25 / 53$ & & 119 & & $2 / 4 / 54$ & & 117 & \\
\hline $11 / 27 / 53$ & & 119 & & $2 / 5 / 54$ & & 117 & \\
\hline $111 / 29 / 53$ & $\div$ & 118 & & $2 / 6 / 54$ & & 118 & \\
\hline $11 / 30 / 53$ & & 119 & & $2 / 7 / 54$ & & 118 & \\
\hline $12 / 1 / 53$ & & 119 & & $2 / 8 / 54$ & & 118 & \\
\hline $12 / 2 / 53$ & & 116 & & $2 / 9 / 54$ & & 118 & \\
\hline $12 / 3 / 53$ & & 116 & & $2 / 10 / 54$ & & 118 & \\
\hline $12 / 4 / 53$ & & 110 & & $2 / 11 / 54$ & & 118 & \\
\hline $12 / 5 / 53$ & & 117 & & $2 / 12 / 54$ & & 118 & \\
\hline $12 / 6 / 53$ & & 118 & & $2 / 13 / 54$ & & 120 & \\
\hline $12 / 7 / 53$ & & 118 & & $2 / 15 / 54$ & & 118 & \\
\hline $12 / 8 / 53$ & & 118 & & $2 / 16 / 54$ & & 118 & \\
\hline $12 / 10 / 53$ & & 118 & & $2 / 17 / 54$ & & 118 & \\
\hline $12 / 11 / 53$ & & 118 & & $2 / 18 / 54$ & & 118 & \\
\hline $12 / 14 / 53$ & & 118 & & $2 / 19 / 54$ & & 118 & \\
\hline $12 / 15 / 53$ & & 118 & & $2 / 22 / 54$ & & 118 & \\
\hline $12 / 16 / 53$ & & 118 & & $2 / 23 / 54$ & & 118 & \\
\hline $12 / 17 / 53$ & & 118 & & $2 / 25 / 54$ & & 118 & \\
\hline $12 / 18 / 53$ & & 118 & & $2 / 26 / 54$ & & 118 & \\
\hline $12 / 21 / 53$ & & 118 & & $3 / 1 / 54$ & & 118 & \\
\hline $12 / 22 / 53$ & & 118 & & $3 / 2 / 54$ & & 118 & \\
\hline $12 / 23 / 53$ & & 118 & & $3 / 3 / 54$ & & 118 & \\
\hline $12 / 25 / 53$ & & 117 & & $3 / 4 / 54$ & & 118 & \\
\hline
\end{tabular}

Data obtained from HW-83906. 


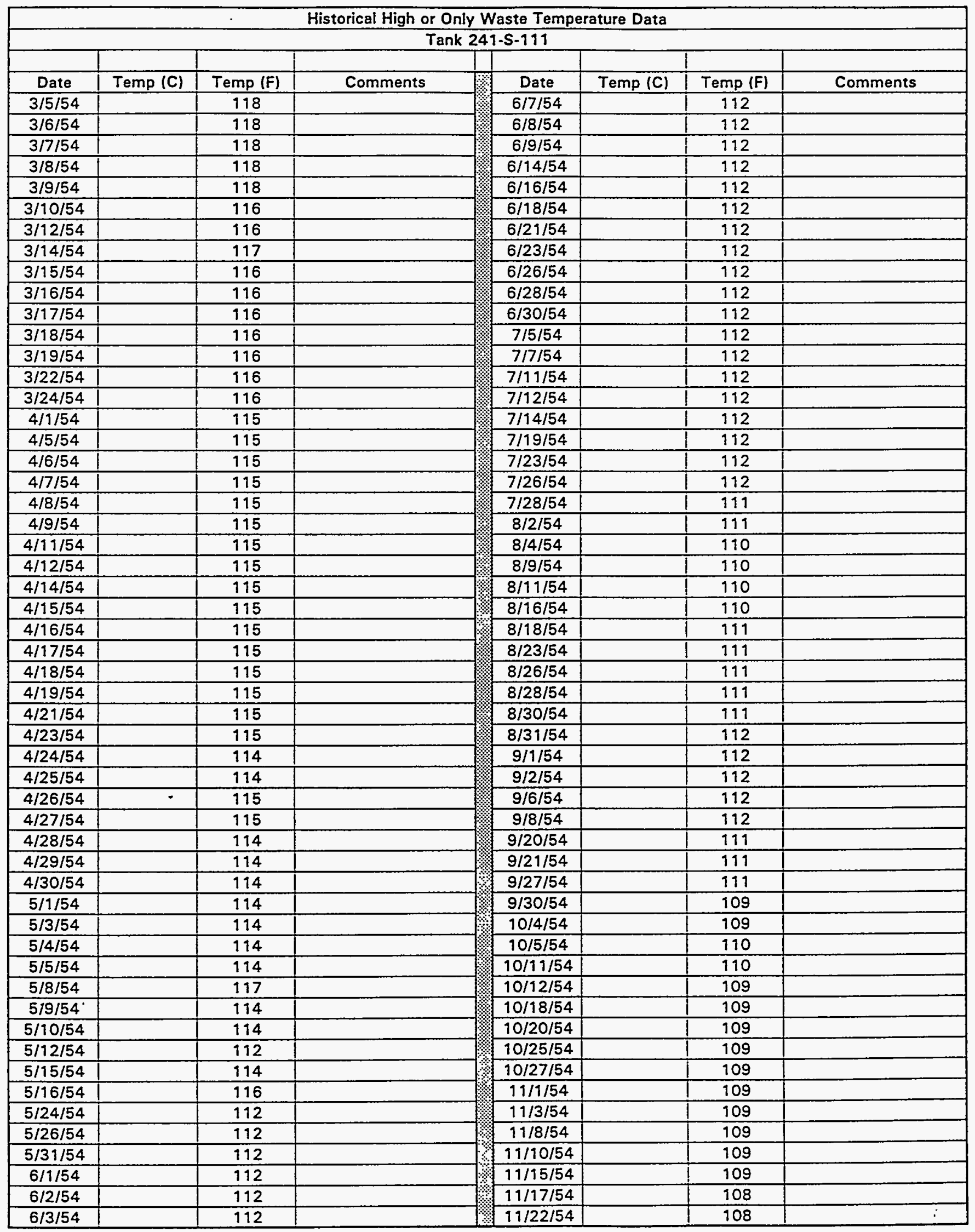

Data obtained from HW-83906. 
WHC-SD-WM-ER-323, Rev. O

\begin{tabular}{|c|c|c|c|c|c|c|c|c|}
\hline \multicolumn{9}{|c|}{ Historical High or Only Waste Temperature Data } \\
\hline \multicolumn{9}{|c|}{ Tank 241-S-111 } \\
\hline Date & Temp (C) & Temp (F) & Comments & & Date & Temp (C) & Temp (F) & Comments \\
\hline $11 / 24 / 54$ & & 108 & & & $12 / 22 / 54$ & & 107 & \\
\hline $12 / 1 / 54$ & & 107 & & & $12 / 27 / 54$ & & 107 & \\
\hline $12 / 8 / 54$ & & 107 & & 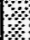 & $12 / 29 / 54$ & & 106 & \\
\hline $12 / 20 / 54$ & & 107 & & 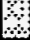 & & & & \\
\hline
\end{tabular}




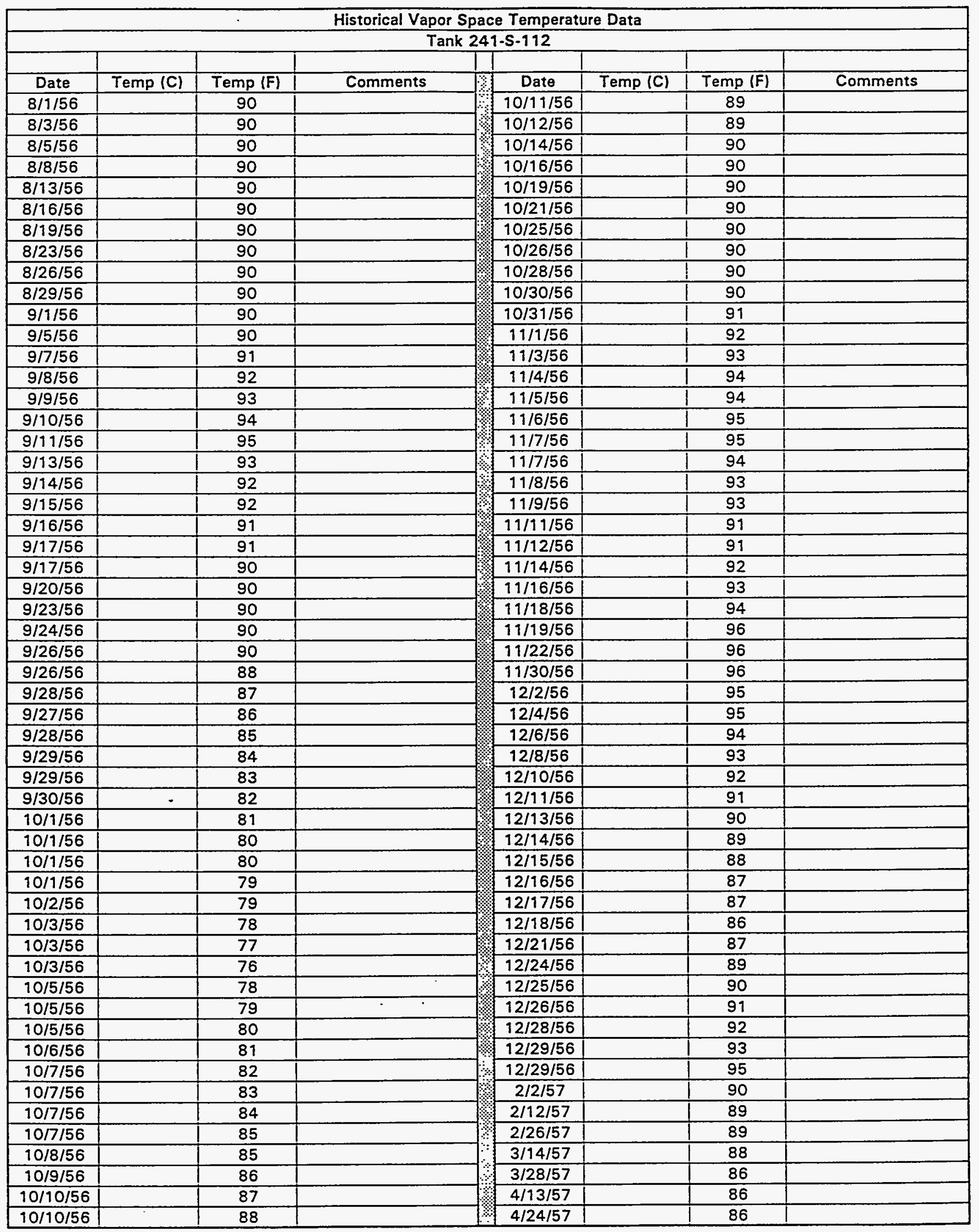

Data obtained from RHO-CD-1172. 
Historical Vapor Space Temperature Data

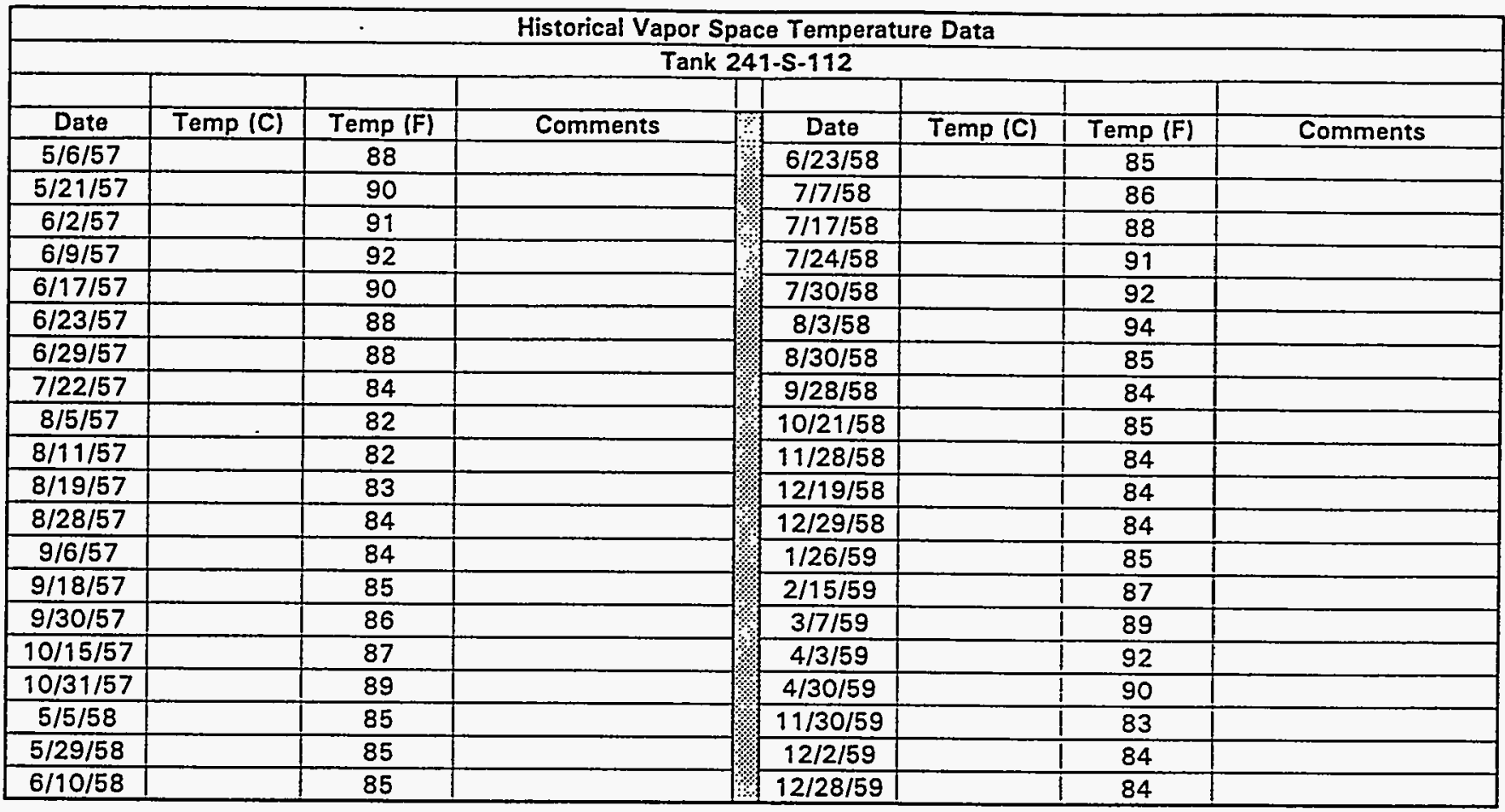

-Data obtained from RHO-CD-1172. 


\begin{tabular}{|c|c|c|c|c|c|c|c|}
\hline \multicolumn{8}{|c|}{ Historical High or Only Waste Temperature Data } \\
\hline \multicolumn{8}{|c|}{ Tank 241-S-112 } \\
\hline & & & & & & & \\
\hline Date & Temp (C) & Temp (F) & Comments & Date & Temp (C) & Temp (F) & Comments \\
\hline $6 / 22 / 53$ & & 100 & & $8 / 20 / 53$ & & 100 & \\
\hline $6 / 23 / 53$ & & 100 & & $8 / 21 / 53$ & & 100 & \\
\hline $6 / 24 / 53$ & & 100 & & $8 / 22 / 53$ & & 100 & \\
\hline $6 / 25 / 53$ & & 100 & & $8 / 23 / 53$ & & 100 & \\
\hline $6 / 26 / 53$ & & 100 & & $8 / 24 / 53$ & & 100 & \\
\hline $6 / 29 / 53$ & & 100 & & $8 / 25 / 53$ & & 101 & \\
\hline $7 / 1 / 53$ & & 100 & & $8 / 26 / 53$ & & 100 & \\
\hline $7 / 2 / 53$ & & 100 & & $8 / 28 / 53$ & & 100 & \\
\hline $7 / 3 / 53$ & & 100 & & $8 / 29 / 53$ & & 100 & \\
\hline $7 / 4 / 53$ & & 100 & & $8 / 30 / 53$ & & 100 & \\
\hline $7 / 5 / 53$ & & 100 & & $8 / 31 / 53$ & & 100 & \\
\hline $7 / 6 / 53$ & & 100 & & $9 / 1 / 53$ & & 100 & \\
\hline $7 / 7 / 53$ & & 100 & & $9 / 2 / 53$ & & 100 & \\
\hline $7 / 8 / 53$ & & 100 & & $9 / 3 / 53$ & & 100 & \\
\hline $7 / 9 / 53$ & & 100 & & $9 / 4 / 53$ & & 100 & \\
\hline $7 / 10 / 53$ & & 100 & & $9 / 5 / 53$ & & 102 & \\
\hline $7 / 11 / 53$ & & 100 & & $9 / 6 / 53$ & & 102 & \\
\hline $7 / 13 / 53$ & & 100 & & $9 / 7 / 53$ & & 102 & \\
\hline $7 / 15 / 53$ & & 100 & & $9 / 8 / 53$ & & 102 & \\
\hline $7 / 16 / 53$ & & 100 & & $9 / 9 / 53$ & & 100 & \\
\hline $7 / 17 / 53$ & & 108 & & $9 / 10 / 53$ & & 100 & \\
\hline $7 / 18 / 53$ & & 100 & & $9 / 11 / 53$ & & 100 & \\
\hline $7 / 19 / 53$ & & 100 & & $9 / 13 / 53$ & & 100 & \\
\hline $7 / 20 / 53$ & & 900 & & $9 / 14 / 53$ & & 100 & \\
\hline $7 / 21 / 53$ & & 100 & & $9 / 15 / 53$ & & 100 & \\
\hline $7 / 22 / 53$ & & 100 & & $9 / 16 / 53$ & & 100 & \\
\hline $7 / 23 / 53$ & & 100 & & $9 / 17 / 53$ & & 100 & \\
\hline $7 / 24 / 53$ & & 100 & & $9 / 18 / 53$ & & 100 & \\
\hline $7 / 25 / 53$ & & 100 & & $9 / 19 / 53$ & & 100 & \\
\hline $7 / 26 / 53$ & & 110 & & $9 / 20 / 53$ & & 100 & \\
\hline $7 / 27 / 53$ & & 100 & & $9 / 21 / 53$ & & 100 & \\
\hline $7 / 28 / 53$ & & 100 & & $9 / 22 / 53$ & & 100 & \\
\hline $7 / 29 / 53$ & & 100 & & $9 / 23 / 53$ & & 102 & \\
\hline $7 / 30 / 53$ & & 100 & & $9 / 24 / 53$ & & 102 & \\
\hline $7 / 31 / 53$ & & 100 & & $9 / 25 / 53$ & & 102 & \\
\hline $8 / 1 / 53$ & & 100 & & $9 / 26 / 53$ & & 102 & \\
\hline $8 / 2 / 53$ & & 100 & & $9 / 27 / 53$ & & 102 & \\
\hline $8 / 3 / 53$ & & 100 & & $9 / 28 / 53$ & & 102 & \\
\hline $8 / 4 / 53$ & & 100 & & $9 / 29 / 53$ & & 102 & \\
\hline $8 / 5 / 53$ & & 100 & & $9 / 30 / 53$ & & 102 & \\
\hline $8 / 6 / 53$ & & 100 & & $10 / 1 / 53$ & & 102 & \\
\hline $8 / 7 / 53$ & & 100 & & $10 / 2 / 53$ & & 102 & \\
\hline $8 / 8 / 53$ & & 100 & & $10 / 3 / 53$ & & 102 & \\
\hline $8 / 9 / 53$ & & 100 & & $10 / 4 / 53$ & & 102 & \\
\hline $8 / 10 / 53$ & & 100 & & $10 / 5 / 53$ & & 102 & \\
\hline $8 / 11 / 53$ & & 100 & & $10 / 6 / 53$ & & 102 & \\
\hline $8 / 12 / 53$ & & 100 & & $10 / 7 / 53$ & & 102 & \\
\hline $8 / 13 / 53$ & & 100 & & $10 / 8 / 53$ & & 102 & \\
\hline $8 / 14 / 53$ & & 100 & & $10 / 9 / 53$ & & 102 & \\
\hline $8 / 15 / 53$ & & 100 & & $10 / 10 / 53$ & & 101 & \\
\hline $8 / 16 / 53$ & & 100 & & $10 / 11 / 53$ & & 100 & \\
\hline $8 / 17 / 53$ & & 100 & & $10 / 12 / 53$ & & 100 & \\
\hline $8 / 18 / 53$ & & 100 & & $10 / 14 / 53$ & & 103 & \\
\hline $8 / 19 / 53$ & & 100 & & $10 / 15 / 53$ & & 103 & \\
\hline
\end{tabular}

Data obtained from HW-83906. 


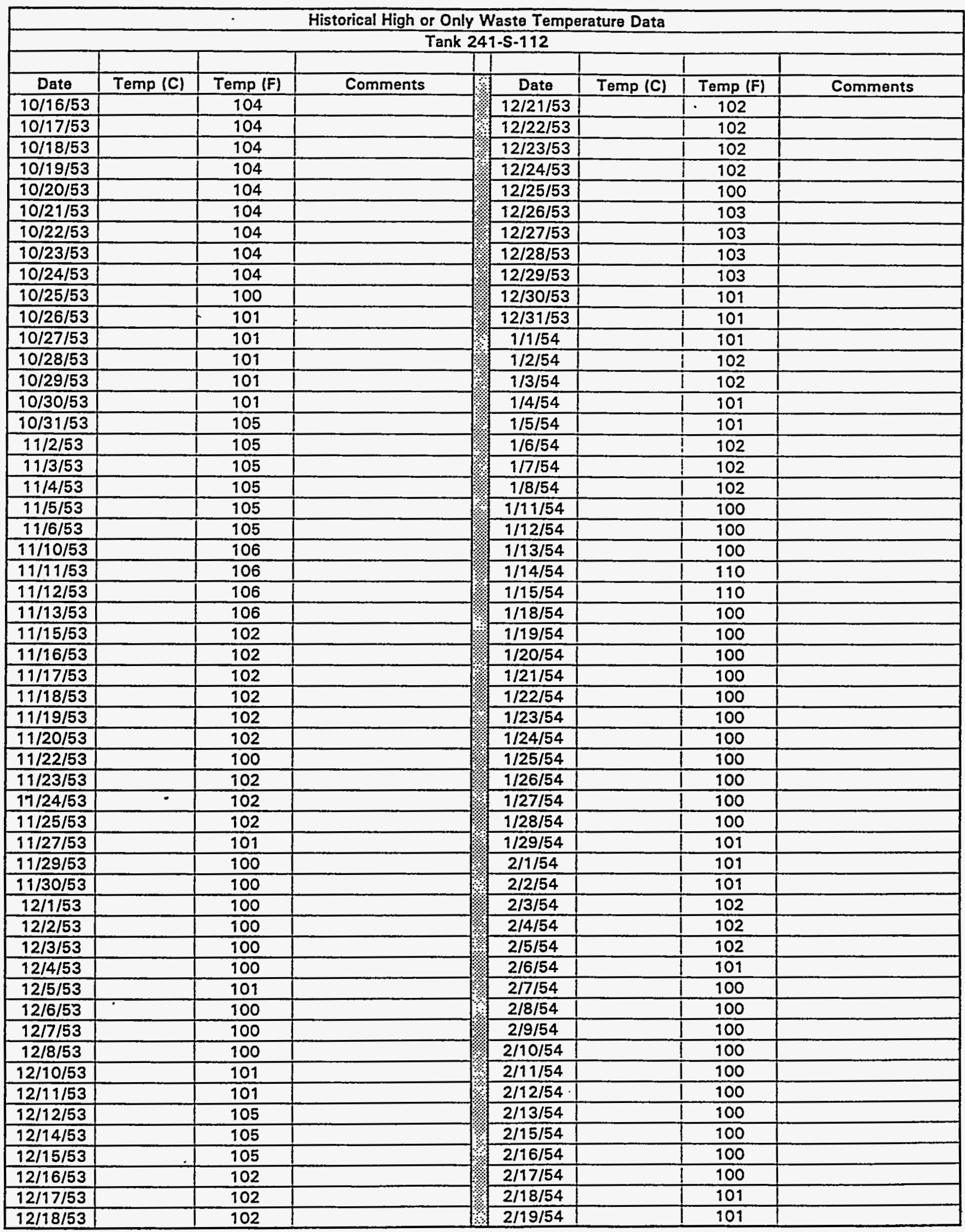

Data obtained from HW-83906. 


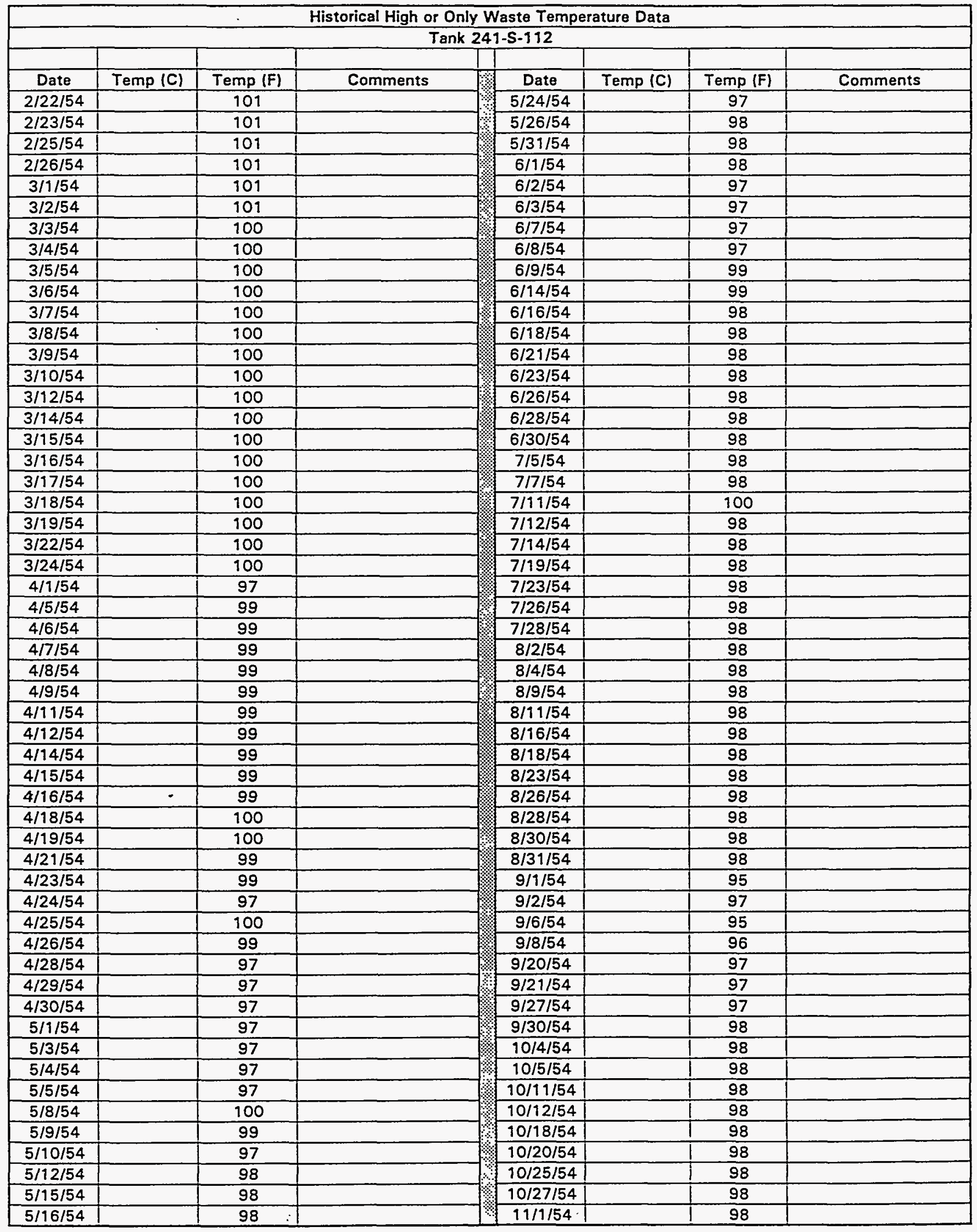

Data obtained from HW-83906. 


\begin{tabular}{|c|c|c|c|c|c|c|c|}
\hline \multicolumn{8}{|c|}{ Historical High or Only Waste Temperature Data } \\
\hline \multicolumn{8}{|c|}{ Tank 241-S-112 } \\
\hline & & & & & & & \\
\hline Date & Temp (C) & Temp (F) & Comments & Date & Temp (C) & Temp (F) & Comments \\
\hline $11 / 3 / 54$ & & 98 & & $12 / 8 / 54$ & & 98 & \\
\hline $11 / 8 / 54$ & & 98 & & $12 / 13 / 54$ & & 98 & \\
\hline $11 / 10 / 54$ & & 96 & & $12 / 15 / 54$ & & 98 & \\
\hline $11 / 15 / 54$ & & 98 & & $12 / 20 / 54$ & & 98 & \\
\hline $11 / 17 / 54$ & & 98 & & $12 / 22 / 54$ & & 94 & \\
\hline $11 / 22 / 54$ & & 98 & & $12 / 27 / 54$ & & 94 & \\
\hline $11 / 24 / 54$ & & 98 & & $12 / 29 / 54$ & & 98 & \\
\hline $12 / 1 / 54$ & & 98 & & & & & \\
\hline
\end{tabular}

Data obtained from HW-83906. 


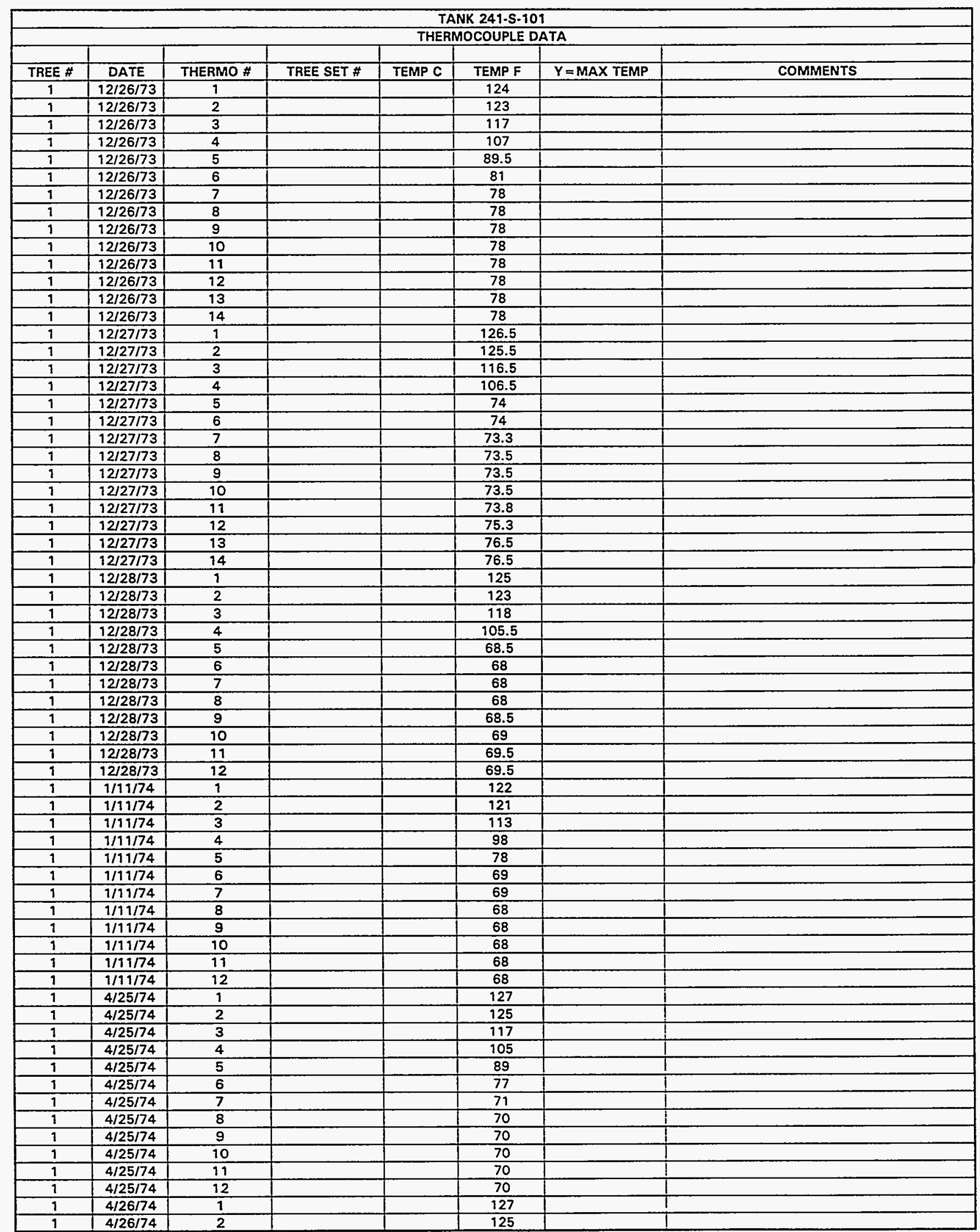

Data obtained from WHC Surveillance Analysis Computer System (SACS), February 24, 1994, Data sheet TK-105-S to TK-101 $S$, and Archive box \#121869. 


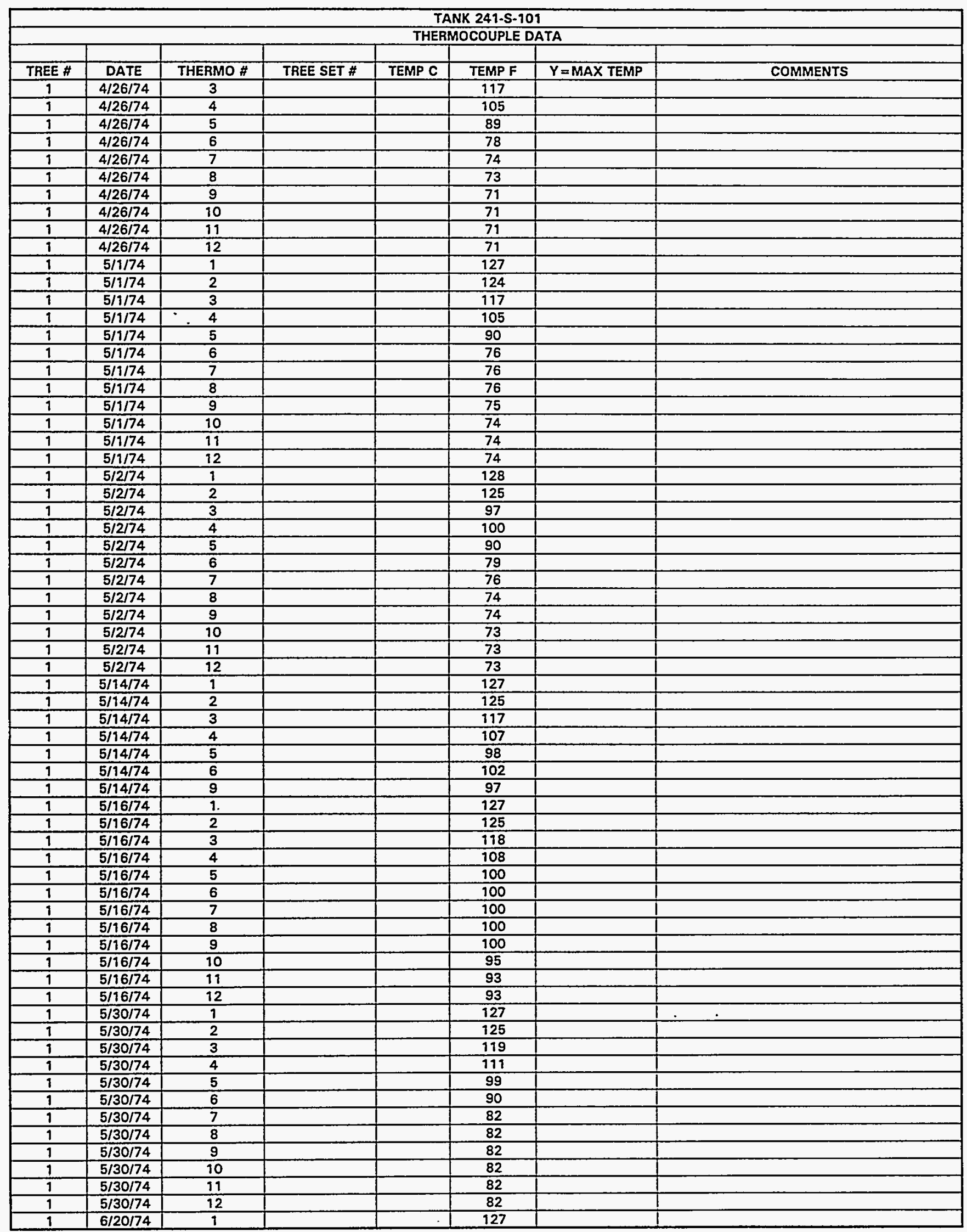

Data obtained from WHC Surveillance Analysis Computer System (SACS), February 24, 1994, Data sheet TK-105-S to TK-101 $S$, and Archive box \#121869. 
WHC-SD-WM-ER-323, Rev. O

\begin{tabular}{|c|c|c|c|c|c|c|c|}
\hline \multicolumn{8}{|c|}{ TANK 241-S-101 } \\
\hline \multicolumn{8}{|c|}{ THERMOCOUPLE DATA } \\
\hline & & & & & & & \\
\hline TAEE \# & DATE & THERMO \# & TREE SET \# & TEMP C & TEMP F & $Y=M A X$ TEMP & COMMENTS \\
\hline 1 & $6 / 20 / 74$ & 2 & & & 126 & & \\
\hline 1 & $6 / 20 / 74$ & 3 & & & 120 & & \\
\hline 1 & $6 / 20 / 74$ & 4 & & & 111 & & \\
\hline 1 & $6 / 20 / 74$ & 5 & & & 96 & & \\
\hline 1 & $6 / 20 / 74$ & 6 & & & 82 & & \\
\hline 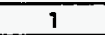 & $6 / 20 / 74$ & 7 & & & 80 & & \\
\hline 1 & $6 / 20 / 74$ & 8 & & & 80 & & \\
\hline 1 & $6 / 20 / 74$ & 9 & & & 81 & & \\
\hline 1 & $6 / 20 / 74$ & 10 & & & 81 & & \\
\hline 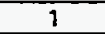 & $6 / 20 / 74$ & 11 & & & 81 & & \\
\hline 9 & $6 / 20 / 74$ & 12 & & & 80 & & \\
\hline 1 & $7 / 12 / 74$ & 1 & & & 128 & & \\
\hline 1 & $7 / 12 / 74$ & 2 & & & 127 & & \\
\hline 1 & $7 / 12 / 74$ & 3 & & & 121 & & \\
\hline 1 & $7 / 12 / 74$ & 4 & & & 112 & & \\
\hline 1 & $7 / 12 / 74$ & 5 & & & 100 & & \\
\hline 9 & $7 / 12 / 74$ & 6 & & & 88 & & \\
\hline 1 & $7 / 12 / 74$ & 7 & & & 83 & & \\
\hline 1 & $7 / 12 / 74$ & 8 & & & 81 & & \\
\hline 1 & $7 / 12 / 74$ & 9 & & & 80 & & \\
\hline 1 & $7 / 12 / 74$ & 10 & & & 80 & & \\
\hline 1 & $7 / 12 / 74$ & 11 & & & 78 & & \\
\hline 1 & $7 / 12 / 74$ & 12 & & & 78 & & \\
\hline 1 & $11 / 26 / 74$ & 1 & & & 133 & & \\
\hline 1 & $11 / 26 / 74$ & 2 & & & 133 & & \\
\hline 1 & $11 / 26 / 74$ & 3 & & & 128 & & \\
\hline$\frac{1}{1}$ & $11 / 26 / 74$ & 4 & & & 119 & & \\
\hline 1 & $11 / 26 / 74$ & 5 & & & 105 & & \\
\hline 1 & $11 / 26 / 74$ & 6 & & & 89 & & \\
\hline$\frac{1}{1}$ & $11 / 26 / 74$ & 7 & & & 80 & & \\
\hline 1 & $11 / 26 / 74$ & 8 & & & 80 & & \\
\hline 1 & $11 / 26 / 74$ & 9 & & & 80 & & \\
\hline 1 & $11 / 26 / 74$ & 10 & & & 78 & & \\
\hline 1 & $11 / 26 / 74$ & 11 & & & 78 & & \\
\hline 1 & $11 / 26 / 74$ & 12 & & & 78 & & \\
\hline 1 & $11 / 27 / 74$ & 1 & & & 133 & & \\
\hline 1 & $11 / 27 / 74$ & 2 & & & 133 & & \\
\hline 1 & $11 / 27 / 74$ & 3 & & & 128 & & \\
\hline 1 & $11 / 27 / 74$ & 4 & & & 119 & & \\
\hline 1 & $11 / 27 / 74$ & 5 & & & 105 & & \\
\hline 1 & $11 / 27 / 74$ & 6 & & & 89 & & \\
\hline 1 & $11 / 27 / 74$ & 7 & & & 80 & & \\
\hline 1 & $11 / 27 / 74$ & 8 & & & 80 & & \\
\hline 1 & $11 / 27 / 74$ & 9 & & & 80 & & \\
\hline 1 & $11 / 27 / 74$ & 10 & & & 78 & & \\
\hline 1 & $11 / 27 / 74$ & 11 & & & 78 & & \\
\hline 1 & $11 / 27 / 74$ & 12 & & & 78 & & \\
\hline 1 & $12 / 2 / 74$ & 1 & & 56.67 & 134.006 & & raw data - assumed degree C \\
\hline 1 & $12 / 2 / 74$ & 2 & & 56.11 & 132.998 & & raw data - assumed degree C \\
\hline 1 & $12 / 2 / 74$ & 3 & & 53.33 & 127.994 & & raw data - assumed degree C \\
\hline 1 & $12 / 2 / 74$ & 4 & & 48.33 & 118.994 & & raw data - assumed degree C \\
\hline 9 & $12 / 2 / 74$ & 5 & & 41.11 & 105.998 & & Traw data - assumed degree C \\
\hline 1 & $12 / 2 / 74$ & 6 & & 32.22 & 89.996 & & raw data - assumed degree $C$ \\
\hline 1 & $12 / 2 / 74$ & 7 & & 27.78 & 82.004 & & raw data - assumed degree C \\
\hline 1 & $12 / 2 / 74$ & 8 & & 26.67 & 80.006 & & raw data - assumed degree C \\
\hline 1 & $12 / 2 / 74$ & 9 & & 25.56 & 78.008 & & lraw data - assumed degree C \\
\hline 1 & $12 / 2 / 74$ & 10 & & 24.44 & 75.992 & & Traw data - assumed degree C \\
\hline 1 & $12 / 2 / 74$ & 11 & & 24.44 & 75.992 & & Iraw data - assumed degree C \\
\hline 1 & $12 / 2 / 74$ & 12 & & 24.44 & 75.992 & & raw data - assumed degree $C$ \\
\hline 1 & $11 / 3 / 75$ & 1 & & & 154 & & \\
\hline 1 & $11 / 3 / 75$ & 2 & & & 155 & & \\
\hline 9 & $11 / 3 / 75$ & 3 & & & 152 & & \\
\hline 1 & $11 / 3 / 75$ & 4 & & & 147 & & \\
\hline 1 & $11 / 3 / 75$ & 5 & & & 138 & & \\
\hline 1 & $11 / 3 / 75$ & 6 & & & 136 & & \\
\hline 1 & $11 / 3 / 75$ & 7 & & & 136 & & \\
\hline
\end{tabular}

Data obtained from WHC Surveillance Analysis Computer System (SACS). February 24, 1994, Data sheet TK-105-S to TK-101 $\mathrm{S}$, and Archive box \#121869. 


\begin{tabular}{|c|c|c|c|c|c|c|c|}
\hline \multicolumn{8}{|c|}{ TANK 241-S-101 } \\
\hline & & & & THER & OCOUPLE & & \\
\hline TREE \# & DATE & THERMO \# & TREE SET \# & TEMP C & TEMP F & $Y=$ MAX TEMP & COMMENTS \\
\hline 1 & $11 / 3 / 75$ & 8 & & & 136 & & \\
\hline 1 & $11 / 3 / 75$ & 9 & & & 136 & & \\
\hline 1 & $11 / 3 / 75$ & 10 & & & 135 & & \\
\hline 1 & $11 / 3 / 75$ & 11 & & & 129 & & \\
\hline 1 & $11 / 3 / 75$ & 12 & & & 110 & & \\
\hline 1 & $2 / 4 / 76$ & 1 & & & 156 & & \\
\hline 1 & $2 / 4 / 76$ & 2 & & & 158 & & \\
\hline 1 & $2 / 4 / 76$ & 3 & & & 156 & & \\
\hline 1 & $2 / 4 / 76$ & 4 & & & 150 & & \\
\hline 1 & $2 / 4 / 76$ & 5 & & & 142 & & \\
\hline 1 & $2 / 4 / 76$ & 6 & & & 132 & & \\
\hline 1 & $2 / 4 / 76$ & 7 & & & 123 & & \\
\hline 1 & $2 / 4 / 76$ & 8 & & & 123 & & \\
\hline 1 & $2 / 4 / 76$ & 9 & & & 122 & & \\
\hline 1 & $2 / 4 / 76$ & 10 & & & 121 & & \\
\hline 1 & $2 / 4 / 76$ & 11 & & & 112 & & \\
\hline 1 & $2 / 4 / 76$ & 12 & & & 91 & & \\
\hline 1 & $3 / 5 / 76$ & 1 & & & 158 & & \\
\hline 1 & $3 / 5 / 76$ & 2 & & & 158 & & \\
\hline 1 & $3 / 5 / 76$ & 3 & & & 157 & & \\
\hline 1 & $3 / 5 / 76$ & 4 & & & 152 & & \\
\hline 1 & $3 / 5 / 76$ & 5 & & & 144 & & \\
\hline 1 & $3 / 5 / 76$ & 6 & & & 132 & & \\
\hline 1 & $3 / 5 / 76$ & 7 & & & 121 & & \\
\hline 1 & $3 / 5 / 76$ & 8 & & & 121 & & \\
\hline 1 & $3 / 5 / 76$ & 9 & & & 120 & & \\
\hline 1 & $3 / 5 / 76$ & 10 & & & 120 & & \\
\hline 1 & $3 / 5 / 76$ & 11 & & & 116 & & \\
\hline 9 & $3 / 5 / 76$ & 12 & & & 93 & & \\
\hline 1 & $5 / 2 / 76$ & 1 & & & 160 & & \\
\hline 9 & $5 / 2 / 76$ & 2 & & & 163 & & \\
\hline 1 & $5 / 2 / 76$ & 3 & & & & & 112 - suspect data (low) \\
\hline 1 & $5 / 2 / 76$ & 4 & & & 156 & & \\
\hline 1 & $5 / 2 / 76$ & 5 & & & 140 & & \\
\hline 1 & $5 / 2 / 76$ & 6 & & & 128 & & \\
\hline 1 & $5 / 2 / 76$ & 7 & & & 124 & & $\div$ \\
\hline 1 & $5 / 2 / 76$ & 8 & & & 123 & & \\
\hline 1 & $5 / 2 / 76$ & 9 & & & 123 & & \\
\hline 1 & $5 / 2 / 76$ & 10 & & & 123 & & \\
\hline 1 & $5 / 2 / 76$ & 19 & & & 123 & & \\
\hline 1 & $5 / 2 / 76$ & 12 & & & 98 & & \\
\hline 1 & $6 / 1 / 76$ & 1 & & & 160 & & \\
\hline 1 & $6 / 1 / 76$ & 2 & & & 162 & & \\
\hline 1 & $6 / 1 / 76$ & 3 & & & 160 & & \\
\hline 1 & $6 / 1 / 76$ & 4 & & & 158 & & \\
\hline 1 & $6 / 1 / 76$ & 5 & & & 148 & & \\
\hline 1 & $6 / 1 / 76$ & 6 & & & 130 & & \\
\hline 1 & $6 / 1 / 76$ & 7 & & & 120 & & \\
\hline 1 & $6 / 1 / 76$ & 8 & & & 120 & & \\
\hline 1 & $6 / 1 / 76$ & 9 & & & 120 & & \\
\hline 1 & $6 / 1 / 76$ & 10 & & & 120 & & \\
\hline 1 & $6 / 1 / 76$ & 11 & & & 120 & & \\
\hline 1 & $6 / 1 / 76$ & 12 & & & 100 & & \\
\hline 1 & $6 / 1 / 76$ & 13 & & & 98 & & \\
\hline 1 & $6 / 1 / 76$ & 14 & & & 96 & & \\
\hline 1 & $7 / 1 / 76$ & 1 & & & 157 & & \\
\hline 1 & $7 / 1 / 76$ & 2 & & & 158 & & \\
\hline 1 & $7 / 1 / 76$ & 3 & & & 158 & & \\
\hline 1 & $7 / 1 / 76$ & 4 & & & 153 & & \\
\hline 1 & $7 / 1 / 76$ & 5 & & & 145 & & \\
\hline 1 & $7 / 1 / 76$ & 6 & & & 130 & & \\
\hline 1 & $7 / 1 / 76$ & 7 & & & 119 & & \\
\hline 1 & $7 / 1 / 76$ & 8 & & & 119 & & \\
\hline 1 & $7 / 1 / 76$ & 9 & & & 119 & & \\
\hline 1 & $7 / 1 / 76$ & 10 & & & 119 & & \\
\hline 1 & $7 / 1 / 76$ & 1.1 & & & 918 & & \\
\hline
\end{tabular}

Data obtained from WHC Surveillance Analysis Computer System (SACS), February 24, 1994, Data sheet TK-105-S to TK-101 S, and Archive box \#121869. 


\begin{tabular}{|c|c|c|c|c|c|c|c|}
\hline \multicolumn{8}{|c|}{ TANK 241-S-101 } \\
\hline \multicolumn{8}{|c|}{ THERMOCOUPLE DATA } \\
\hline TREE \# & DATE & THERMO \# & TREE SET \# & TEMP C & TEMP F & $Y=M A X$ TEMP & COMMENTS \\
\hline 1 & $7 / 1 / 76$ & 12 & & & 102 & & \\
\hline$\frac{1}{1}$ & $8 / 3 / 76$ & 1 & & & 164 & & \\
\hline 1 & $8 / 3 / 76$ & 2 & & & 166 & & \\
\hline 1 & $8 / 3 / 76$ & 3 & & & 164 & & \\
\hline 9 & $8 / 3 / 76$ & 4 & & & 156 & & \\
\hline 1 & $8 / 3 / 76$ & 5 & & & 142 & & \\
\hline 1 & $8 / 3 / 76$ & 6 & & & 136 & & \\
\hline 1 & $8 / 3 / 76$ & 7 & & & 124 & & \\
\hline 1 & $8 / 3 / 76$ & 8 & & & 124 & & \\
\hline 1 & $8 / 3 / 76$ & 9 & & & 124 & & \\
\hline 1 & $8 / 3 / 76$ & 10 & & & 124 & & \\
\hline 9 & $8 / 3 / 76$ & 91 & & & 124 & & \\
\hline 1 & $8 / 3 / 76$ & 12 & & & 108 & & \\
\hline 1 & $9 / 2 / 76$ & 1 & & & 165 & & \\
\hline 1 & $9 / 2 / 76$ & 2 & & & 167 & & \\
\hline 1 & $9 / 2 / 76$ & 3 & & & 165 & & \\
\hline 1 & $9 / 2 / 76$ & 4 & . & & 159 & & \\
\hline 1 & $9 / 2 / 76$ & 5 & & & 150 & & \\
\hline 1 & $9 / 2 / 76$ & 6 & & & 136 & & \\
\hline 1 & $9 / 2 / 76$ & 7 & & & 124 & & \\
\hline 1 & $9 / 2 / 76$ & 8 & & & 124 & & \\
\hline 1 & $9 / 2 / 76$ & 9 & & & 124 & & \\
\hline 1 & $9 / 2 / 76$ & 10 & & & 124 & & \\
\hline 1 & $9 / 2 / 76$ & 11 & & & 124 & & \\
\hline 1 & $9 / 2 / 76$ & 12 & & & 125 & & \\
\hline 1 & $10 / 3 / 76$ & 1 & & & 167 & & \\
\hline 1 & $10 / 3 / 76$ & 2 & & & 169 & & \\
\hline 1 & $10 / 3 / 76$ & 3 & & & 167 & & \\
\hline 1 & $10 / 3 / 76$ & 4 & & & 163 & & \\
\hline 1 & $10 / 3 / 76$ & 5 & & & 153 & & \\
\hline 1 & $10 / 3 / 76$ & 6 & & & 940 & & \\
\hline 1 & $10 / 3 / 76$ & 7 & & & 127 & & \\
\hline 1 & $10 / 3 / 76$ & 8 & & & 127 & & \\
\hline 1 & $10 / 3 / 76$ & 9 & & & 126 & & \\
\hline 1 & $10 / 3 / 76$ & 10 & & & 126 & & \\
\hline 1 & $10 / 3 / 76$ & 11 & & & 126 & & \\
\hline 1 & $10 / 3 / 76$ & 12 & & & 112 & & \\
\hline 1 & $11 / 1 / 76$ & 1 & & & 170 & & \\
\hline 1 & $11 / 1 / 76$ & 2 & & & 172 & & \\
\hline 1 & $11 / 1 / 76$ & 3 & & & 170 & - & \\
\hline 1 & $11 / 1 / 76$ & 4 & & & 160 & & \\
\hline 1 & $11 / 1 / 76$ & 5 & & & 146 & & \\
\hline 1 & $11 / 1 / 76$ & 6 & & & 136 & & \\
\hline 1 & $11 / 1 / 76$ & 7 & & & 130 & & \\
\hline 1 & $11 / 1 / 76$ & 8 & & & 130 & & \\
\hline 1 & $91 / 1 / 76$ & 9 & & & 130 & & \\
\hline 1 & $91 / 1 / 76$ & 10 & & & 130 & & \\
\hline 1 & $11 / 1 / 76$ & 11 & & & 130 & & \\
\hline 1 & $11 / 1 / 76$ & 12 & & & 122 & & \\
\hline 1 & $12 / 4 / 76$ & 1 & & & 168 & & \\
\hline 1 & $12 / 4 / 76$ & 2 & & & 170 & & \\
\hline 1 & $12 / 4 / 76$ & 3 & & & 169 & & \\
\hline 1 & $12 / 4 / 76$ & 4 & & & 161 & & \\
\hline 1 & $12 / 4 / 76$ & 5 & & 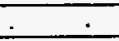 & 153 & & \\
\hline 1 & $12 / 4 / 76$ & 6 & & & 140 & & \\
\hline 1 & $12 / 4 / 76$ & 7 & & & 130 & & \\
\hline 1 & $12 / 4 / 76$ & 8 & & & 139 & & \\
\hline 1 & $12 / 4 / 76$ & 9 & & & 139 & & \\
\hline 1 & $12 / 4 / 76$ & 10 &. & & 139 & & \\
\hline 1 & $12 / 4 / 76$ & 11 & & & 139 & & \\
\hline 1 & $12 / 4 / 76$ & 12 & & & 110 & & \\
\hline 1 & $1 / 1 / 77$ & 1 & & & 169 & & \\
\hline 1 & $1 / 1 / 77$ & 2 & & & 171 & & \\
\hline 1 & $1 / 1 / 77$ & 3 & & & 170 & & \\
\hline 1 & $1 / 1 / 77$ & 4 & & & 166 & & \\
\hline 9 & $1 / 1 / 77$ & 5 & & 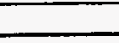 & 156 & & \\
\hline
\end{tabular}

Data obtained from WHC Surveillance Analysis Computer System (SACS), February 24, 1994, Data sheet TK-105-S to TK-101 $S$, and Archive box \#121869. 


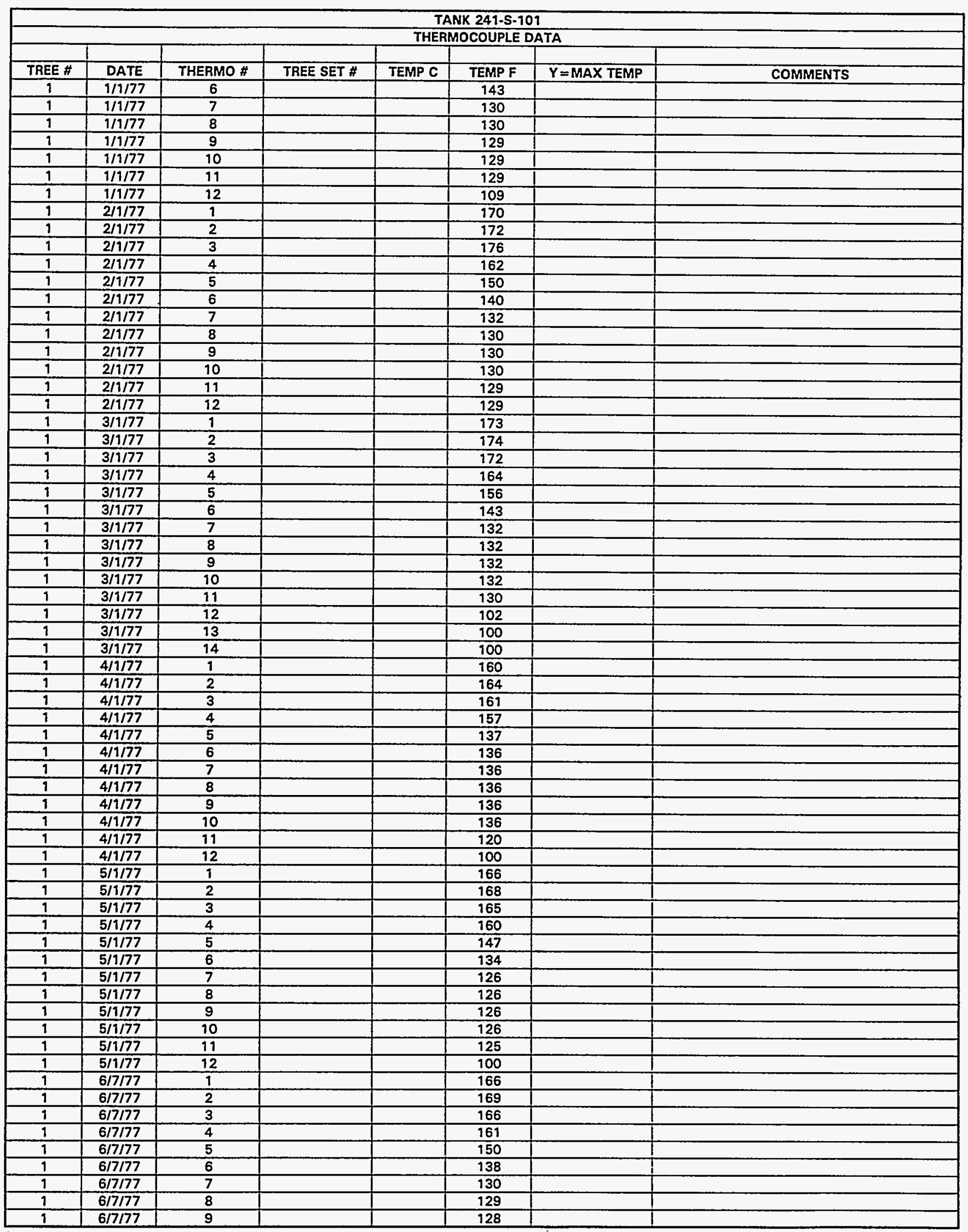

Data obtained from WHC Surveillance Analysis Computer System (SACS), February 24, 1994, Data sheet TK-105-S to TK-101 .. $\quad S$, and Archive box \#121869. 


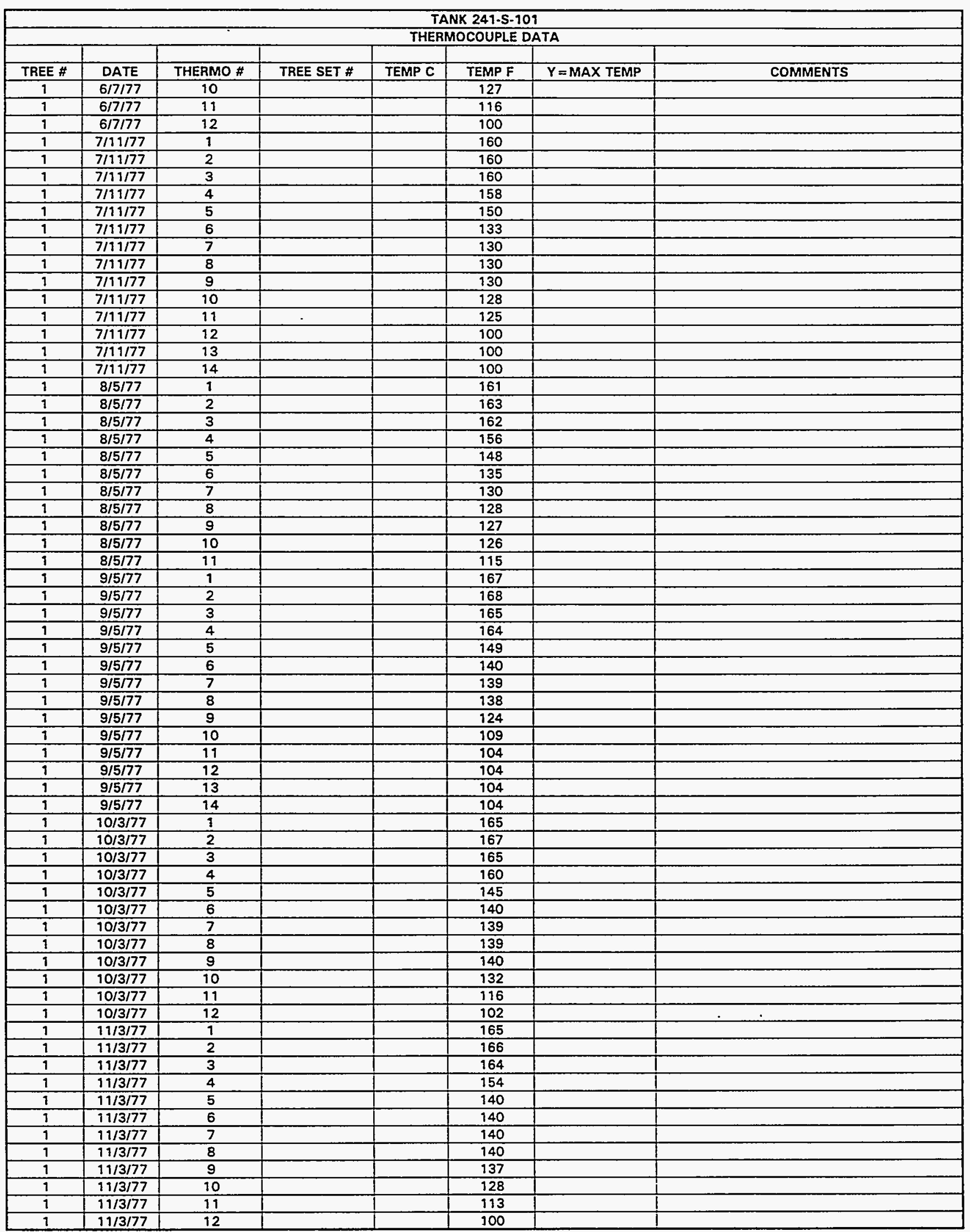

Data obtained from WHC Surveillance Analysis Computer System (SACS), February 24, 1994, Data sheet TK-105-S to TK-101 $S$, and Archive box \#121869. 


\begin{tabular}{|c|c|c|c|c|c|c|c|}
\hline \multicolumn{8}{|c|}{ TANK 241-S-101 } \\
\hline \multicolumn{8}{|c|}{ THERMOCOUPLE DATA } \\
\hline TREE \# & DATE & THERMO \# & TREE SET \# & TEMP C & TEMP F & $\mathrm{Y}=$ MAX TEMP & COMMENTS \\
\hline 1 & $11 / 3 / 77$ & 13 & & & 96 & & \\
\hline$\overline{1}$ & $11 / 3 / 77$ & 14 & & & 96 & & \\
\hline 1 & $12 / 4 / 77$ & 1 & & & 176 & & \\
\hline 9 & $12 / 4 / 77$ & 2 & & & 177 & & \\
\hline 1 & $12 / 4 / 77$ & 3 & & & 173 & & \\
\hline$\frac{1}{1}$ & $12 / 4 / 77$ & 4 & & & 164 & & \\
\hline 1 & $12 / 4 / 77$ & 5 & & & 152 & & \\
\hline 1 & $12 / 4 / 77$ & 6 & & & 152 & & \\
\hline$\frac{1}{1}$ & $12 / 4 / 77$ & 7 & & & 152 & & \\
\hline 1 & $12 / 4 / 77$ & 8 & & & 152 & & \\
\hline 1 & $12 / 4 / 77$ & 9 & & & 148 & & \\
\hline 1 & $12 / 4 / 77$ & 10 & & & 137 & & \\
\hline$\frac{1}{1}$ & $12 / 4 / 77$ & 11 & & & 121 & & \\
\hline 1 & $12 / 4 / 77$ & 12 & & & 104 & & \\
\hline 1 & $12 / 4 / 77$ & 13 & & & 103 & & \\
\hline$\frac{1}{1}$ & $12 / 4 / 77$ & 14 & & & 103 & & \\
\hline 1 & $1 / 8 / 78$ & 1 & & & & & 219 - suspect data (high) \\
\hline 1 & $1 / 8 / 78$ & 2 & & & & & 219 - suspect data (high) \\
\hline 1 & $1 / 8 / 78$ & 3 & & & & & 216 - suspect data (high) \\
\hline 1 & $1 / 8 / 78$ & 4 & & & & & 213 - suspect data (high) \\
\hline 1 & $1 / 8 / 78$ & 5 & & & & & 207 - suspect data (high) \\
\hline 1 & $1 / 8 / 78$ & 6 & & & & & 205 - suspect data (high) \\
\hline 1 & $1 / 8 / 78$ & 7 & & & & & 175 - suspect data (high) \\
\hline 9 & $1 / 8 / 78$ & 8 & & & & & 176 - suspect data (high) \\
\hline 1 & $1 / 8 / 78$ & 9 & & & & & 175 - suspect data (high) \\
\hline 1 & $1 / 8 / 78$ & 10 & & & & & 169 - suspect data (high) \\
\hline$\frac{1}{1}$ & $1 / 8 / 78$ & 11 & & & & & 161 - suspect data (high) \\
\hline 1 & $1 / 8 / 78$ & 12 & & & & & 155 - suspect data (high) \\
\hline 1 & $1 / 8 / 78$ & 13 & & & & & 155 - suspect data (high) \\
\hline 9 & $1 / 8 / 78$ & 14 & & & & & 151 - suspect data (high) \\
\hline 1 & $1 / 11 / 78$ & 1 & & & 168 & & \\
\hline 1 & $1 / 11 / 78$ & 2 & & & 169 & & \\
\hline 1 & $1 / 11 / 78$ & $\frac{2}{3}$ & & & 166 & & \\
\hline 1 & $1 / 11 / 78$ & 4 & & & 157 & & \\
\hline 1 & $1 / 11 / 78$ & 5 & & & 146 & & \\
\hline 1 & $1 / 11 / 78$ & 6 & & & 945 & & \\
\hline 1 & $1 / 11 / 78$ & 7 & & & 145 & & \\
\hline 1 & $1 / 11 / 78$ & 8 & & & 145 & & \\
\hline 1 & $1 / 11 / 78$ & 9 & & & 141 & & \\
\hline 1 & $1 / 11 / 78$ & 10 & & & 129 & & \\
\hline 1 & $1 / 11 / 78$ & 11 & & & 111 & & \\
\hline 1 & $1 / 11 / 78$ & 12 & & & 94 & & \\
\hline 9 & $1 / 11 / 78$ & 13 & & & 92 & & \\
\hline 1 & $1 / 11 / 78$ & 14 & & & 92 & & \\
\hline 1 & $2 / 7 / 78$ & 1 & & & 170 & & \\
\hline 1 & $2 / 7 / 78$ & 2 & & & 179 & & \\
\hline 1 & $2 / 7 / 78$ & 3 & & & 168 & & \\
\hline 1 & $2 / 7 / 78$ & 4 & & & 160 & & \\
\hline 9 & $2 / 7 / 78$ & 5 & & & 148 & & \\
\hline 1 & $2 / 7 / 78$ & 6 & & & 148 & & \\
\hline 1 & $2 / 7 / 78$ & 7 & & & 148 & & \\
\hline 9 & $2 / 7 / 78$ & 8 & & & 148 & & \\
\hline 1 & $2 / 7 / 78$ & 9 & & & 144 & & \\
\hline 1 & $2 / 7 / 78$ & 10 & & & 136 & & \\
\hline 1 & $2 / 7 / 78$ & 11 & & & 112 & & \\
\hline 1 & $2 / 7 / 78$ & 12 & & & 94 & & \\
\hline 1 & $2 / 7 / 78$ & 13 & & & 93 & & \\
\hline 1 & $2 / 7 / 78$ & 14 & & & 93 & & \\
\hline 1 & $3 / 12 / 78$ & 1 & & & 174 & & \\
\hline 1 & $3 / 12 / 78$ & 2 & & & 175 & & \\
\hline 1 & $3 / 12 / 78$ & 3 & & & 172 & & \\
\hline 1 & $3 / 12 / 78$ & 4 & & & 164 & & \\
\hline$\frac{1}{1}$ & $3 / 12 / 78$ & 5 & & & 152 & & \\
\hline$\frac{1}{1}$ & $3 / 12 / 78$ & 6 & & & 151 & & \\
\hline 1 & $3 / 12 / 78$ & 7 & & & 151 & & \\
\hline 1 & $3 / 12 / 78$ & 8 & & & 151 & & \\
\hline
\end{tabular}

Data obtained from WHC Surveillance Analysis Computer System (SACS), February 24, 1994, Data sheet TK-105-S to TK-101 $S$, and Archive box \#121869. 
WHC-SD-WM-ER-323, Rev. 0

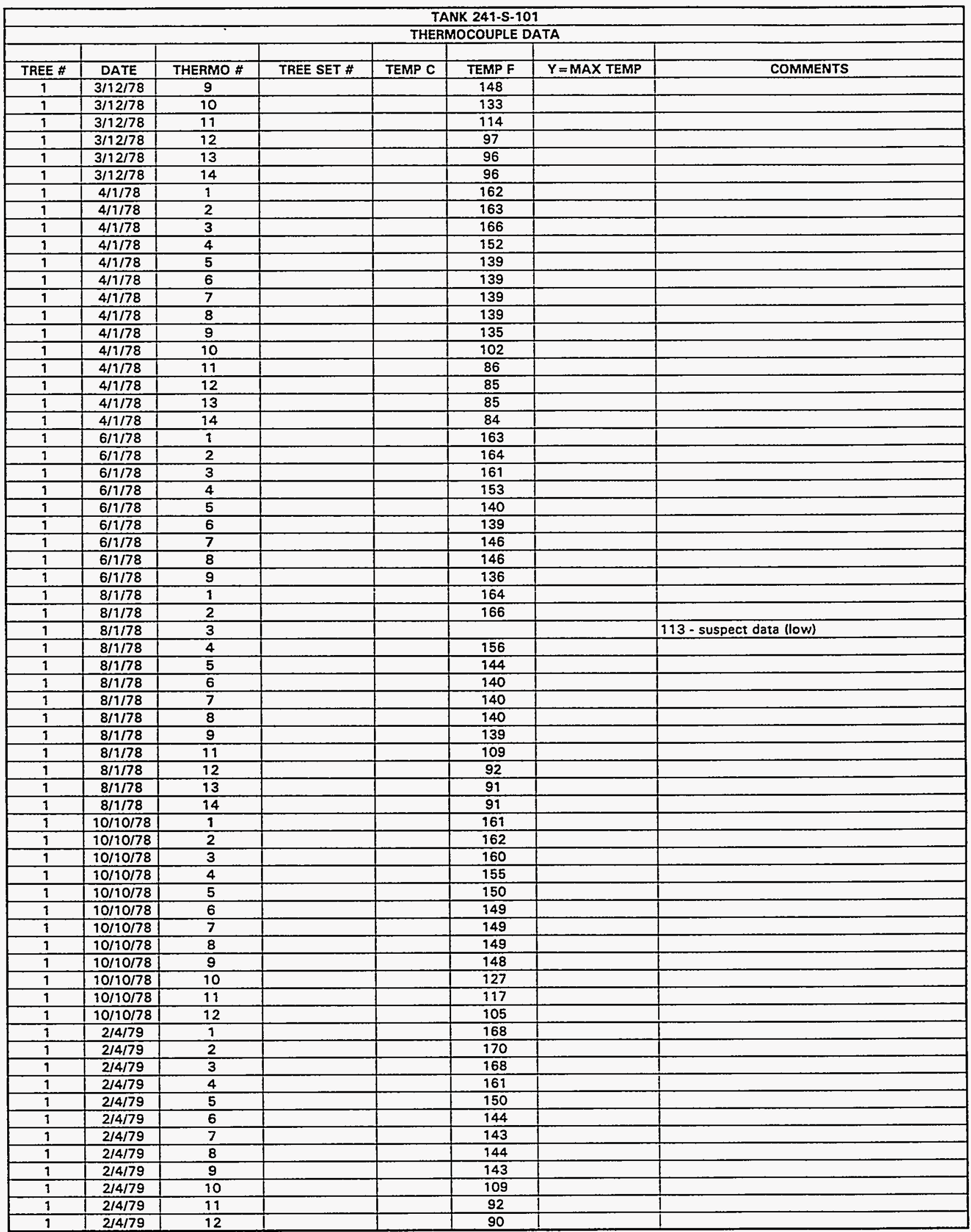

Data obtained from WHC Surveillance Analysis Computer System (SACS), February 24, 1994, Data sheet TK-105-S to TK-101 S, and Archive box \#121869. 


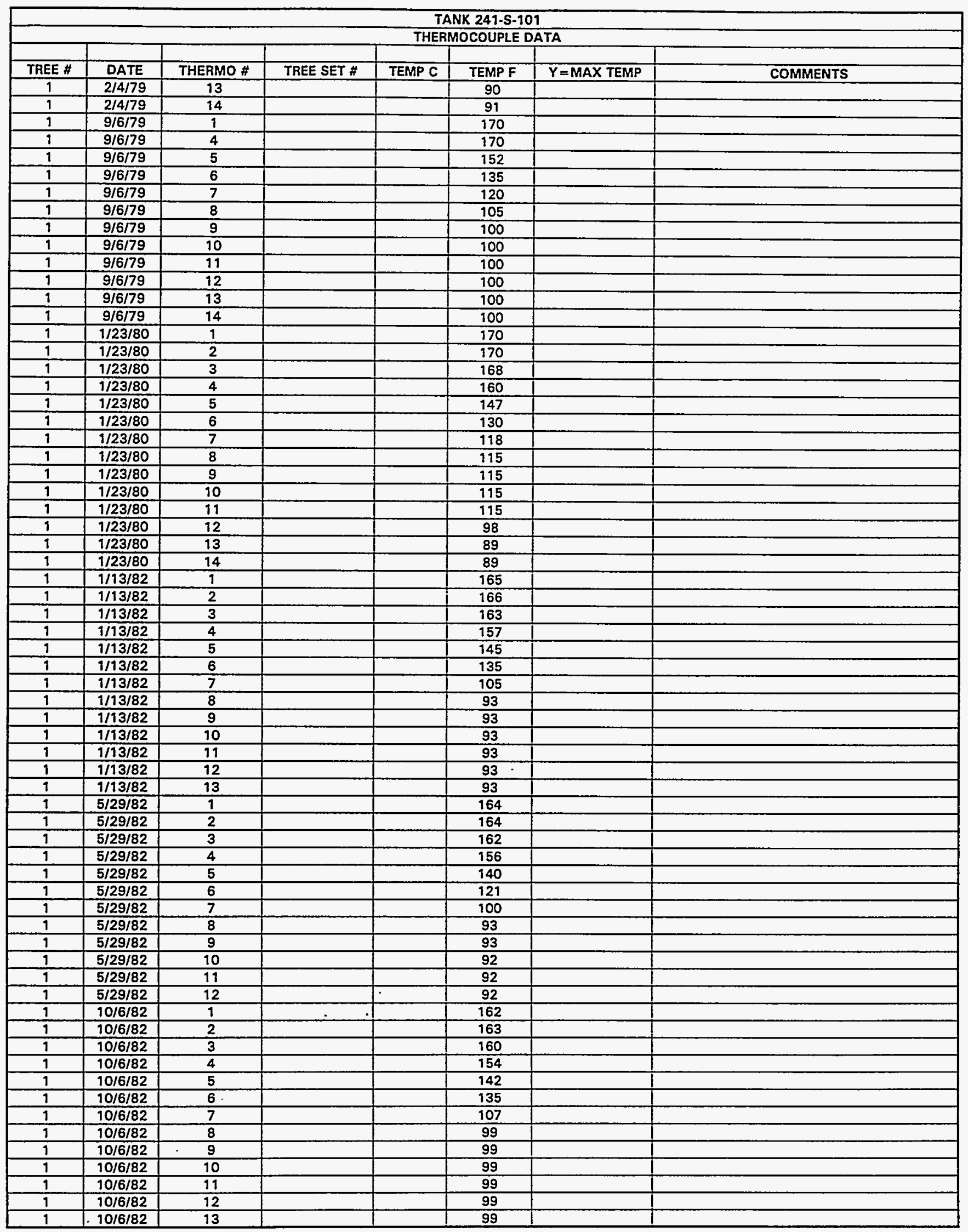

Data obtained from WHC Surveillance Analysis Computer System (SACS), February 24, 1994, Data sheet TK-105-S to TK-101 $S$, and Archive box \#121869. 


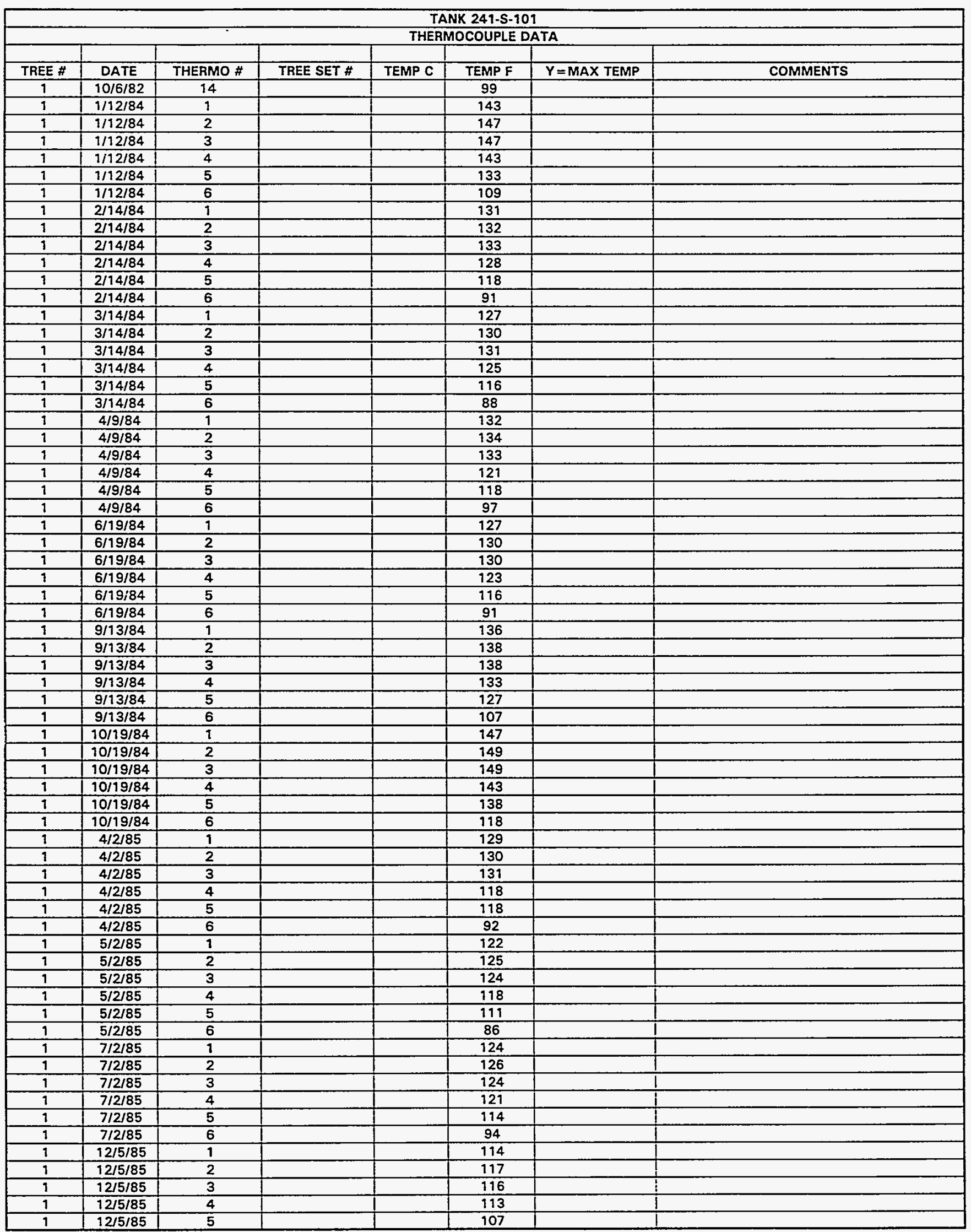

Data obtained from WHC Surveillance Analysis Computer System (SACS), February 24, 1994, Data sheet TK-105-S to TK-101 .. S, and Archive box \#121869. 


\begin{tabular}{|c|c|c|c|c|c|c|c|}
\hline \multicolumn{8}{|c|}{ TANK 241.S-101 } \\
\hline \multicolumn{8}{|c|}{ THERMOCOUPLE DATA } \\
\hline TREE \# & DATE & THERMO \# & TREF SET \# & & & & \\
\hline 1 & $12 / 5 / 85$ & 6 & IMEE SEI H & TEMP C & TEMP F & $Y=$ MAX TEMP & COMMENTS \\
\hline 1 & $1 / 6 / 86$ & $\frac{1}{1}$ & & & 85 & & \\
\hline 1 & $1 / 6 / 86$ & 2 & & & & & 65 - suspect data (low) \\
\hline 1 & $1 / 6 / 86$ & 3 & & & & & 65 - suspect data (low) \\
\hline 1 & $1 / 6 / 86$ & $\frac{3}{4}$ & & & & & 65 - suspect data (low) \\
\hline 1 & $1 / 6 / 86$ & $\frac{4}{5}$ & & & & & 65 - suspect data (low) \\
\hline 1 & $1 / 6 / 86$ & $\frac{5}{6}$ & & & & & 65 - suspect data (low) \\
\hline 1 & $1 / 7 / 86$ & $\frac{0}{1}$ & & & & & 65 - suspect data (low) \\
\hline 1 & $1 / 7 / 86$ & $\frac{1}{2}$ & & & & & 55 - suspect data (low) \\
\hline 1 & $1 / 7 / 86$ & $\frac{2}{3}$ & & & & & 56 - suspect data (low) \\
\hline 1 & $1 / 7 / 86$ & $\frac{5}{4}$ & & & & & 56 - suspect data (low) \\
\hline 1 & $1 / 7 / 86$ & $\frac{4}{5}$ & & & & & 56 - suspect data (low) \\
\hline 1 & $1 / 7 / 86$ & $\frac{5}{6}$ & & & & & 55 - suspect data (low) \\
\hline 9 & $7 / 8 / 86$ & $\frac{6}{1}$ & & & & & 55 - suspect data (low) \\
\hline 1 & $7 / 8 / 86$ & & & & & & 70 - suspect data (low) \\
\hline 1 & $7 / 8 / 86$ & & & & & & 67 - suspect data (low) \\
\hline 1 & $7 / 8 / 86$ & $\frac{3}{4}$ & & & & & 67 - suspect data (low) \\
\hline 1 & $7 / 8 / 86$ & $\frac{4}{5}$ & & & & & 72 - suspect data (low) \\
\hline 9 & $7 / 8 / 86$ & & & & & & 79 - suspect data (low) \\
\hline 1 & $11 / 27 / 86$ & & & & 101.2 & & \\
\hline 1 & $11 / 27 / 86$ & & & & 132.6 & & \\
\hline 1 & $11 / 27 / 86$ & & & & 134.2 & & \\
\hline 1 & $11 / 27 / 86$ & $\frac{3}{4}$ & & & 133.1 & & \\
\hline 1 & $11 / 27 / 86$ & & & & 128.5 & & \\
\hline 1 & $11 / 27 / 86$ & $\frac{5}{6}$ & & & 121.4 & & \\
\hline 1 & $12 / 22 / 86$ & $\frac{6}{1}$ & & & 99.4 & & \\
\hline 1 & $12 / 22 / 86$ & $\frac{1}{2}$ & & & 127.2 & & \\
\hline 1 & $12 / 22 / 86$ & $\frac{2}{3}$ & & & 129.6 & & \\
\hline 1 & $12 / 22 / 86$ & $\frac{3}{4}$ & & & 127.9 & & \\
\hline 1 & $12 / 22 / 86$ & $\frac{4}{5}$ & & & 124.5 & & \\
\hline 1 & $12 / 22 / 86$ & $\frac{5}{6}$ & & & 117.6 & & \\
\hline 1 & $1 / 2 / 87$ & $\frac{6}{1}$ & & & 93.8 & & \\
\hline 1 & $1 / 2 / 87$ & $\frac{1}{2}$ & & & 126.1 & & \\
\hline 1 & $1 / 2 / 87$ & 3 & & & $\frac{128.7}{127.4}$ & & \\
\hline 1 & $1 / 2 / 87$ & 4 & & & 122.9 & & \\
\hline 1 & $1 / 2 / 87$ & 5 & & & 115.8 & & \\
\hline 1 & $1 / 2 / 87$ & 6 & & & 91.8 & & \\
\hline 1 & $2 / 2 / 87$ & 1 & & & 128 & & \\
\hline 1 & $2 / 2 / 87$ & 2 & & & 130 & & \\
\hline 9 & $2 / 2 / 87$ & 3 & & & 128 & & \\
\hline 9 & $2 / 2 / 87$ & 4 & & & 124 & & \\
\hline 1 & $2 / 2 / 87$ & 5 & & & 117 & & \\
\hline 1 & $2 / 2 / 87$ & 6 & & & 91 & & \\
\hline 1 & $3 / 2 / 87$ & 1 & & & 128 & & \\
\hline 1 & $3 / 2 / 87$ & 2 & & & 129.5 & & \\
\hline 1 & $3 / 2 / 87$ & 3 & & & 127 & & \\
\hline 1 & $3 / 2 / 87$ & 4 & & & 123 & & \\
\hline 1 & $3 / 2 / 87$ & 5 & & & 116 & & \\
\hline 1 & $3 / 2 / 87$ & 6 & & & 89 & & \\
\hline 1 & $4 / 6 / 87$ & 1 & & & 128 & & \\
\hline 1 & $4 / 6 / 87$ & 2 & & & 130 & & \\
\hline 1 & $4 / 6 / 87$ & 3 & & & 129 & & \\
\hline 1 & $4 / 6 / 87$ & 4 & & & 125 & & - \\
\hline 1 & $4 / 6 / 87$ & 5 & & & 118 & & \\
\hline 1 & $4 / 6 / 87$ & 6 & & & 94 & & \\
\hline 1 & $5 / 3 / 87$ & 1 & & & 127 & & \\
\hline 1 & $5 / 3 / 87$ & 2 & & & 128 & & \\
\hline 9 & $5 / 3 / 87$ & 3 & & & 126.8 & & \\
\hline 1 & $5 / 3 / 87$ & 4 & & & 122.8 & & \\
\hline 1 & $5 / 3 / 87$ & 5 & & & 115.2 & & \\
\hline 1 & $5 / 3 / 87$ & 6 & & & 90.2 & & \\
\hline 1 & $7 / 6 / 87$ & 1 & & & 126.5 & & \\
\hline 1 & $7 / 6 / 87$ & 2 & & & 127.4 & & \\
\hline 1 & $7 / 6 / 87$ & 3 & & & 126.4 & & \\
\hline 1 & $7 / 6 / 87$ & 4 & & & 122.4 & & \\
\hline 1 & $7 / 6 / 87$ & 5 & & & 115.6 & & \\
\hline
\end{tabular}

Data obtained from WHC Surveillance Analysis Computer System (SACS), February 24, 1994, Data sheet TK-105-S to TK-101 $S$, and Archive box \#121869. 


\begin{tabular}{|c|c|c|c|c|c|c|c|}
\hline \multicolumn{8}{|c|}{ TANK 241-S-101 } \\
\hline \multicolumn{8}{|c|}{ THERMOCOUPLE DATA } \\
\hline TREE \# & DATE & THERMO \# & TREE SET \# & TEMP C & TEMP F & $\mathrm{Y}=$ MAX TEMP & COMMENTS \\
\hline 1 & $7 / 6 / 87$ & 6 & & & 94.4 & & \\
\hline 1 & $8 / 4 / 87$ & 1 & & & 124.9 & & \\
\hline 1 & $8 / 4 / 87$ & 2 & & & 125.3 & & \\
\hline 1 & $8 / 4 / 87$ & 3 & & & 125.1 & & \\
\hline 1 & $8 / 4 / 87$ & 4 & & & 121.6 & & \\
\hline 1 & $8 / 4 / 87$ & 5 & & & 115.6 & & \\
\hline 1 & $8 / 4 / 87$ & 6 & & & 95.1 & & \\
\hline 1 & $10 / 5 / 87$ & 1 & & & 124 & & \\
\hline 1 & $10 / 5 / 87$ & 2 & & & 126 & & \\
\hline 1 & $10 / 5 / 87$ & 3 & & & 126 & & \\
\hline 1 & $10 / 5 / 87$ & 4 & & & 123 & & \\
\hline 1 & $10 / 5 / 87$ & 5 & & & 118 & & \\
\hline 1 & $10 / 5 / 87$ & 6 & & & 100 & & \\
\hline 1 & $11 / 4 / 87$ & 1 & & & 126 & & \\
\hline 1 & $11 / 4 / 87$ & 2 & & & 128 & & \\
\hline 1 & $11 / 4 / 87$ & 3 & & & 127 & & \\
\hline 1 & $11 / 4 / 87$ & 4 & & & 124 & & \\
\hline 9 & $11 / 4 / 87$ & 5 & & & 118 & & \\
\hline 1 & $11 / 4 / 87$ & 6 & & & 98 & & \\
\hline 1 & $12 / 8 / 87$ & 1 & & & 127.4 & & \\
\hline 1 & $12 / 8 / 87$ & 2 & & & 128.8 & & \\
\hline 1 & $12 / 8 / 87$ & 3 & & & 128.8 & & \\
\hline 1 & $12 / 8 / 87$ & 4 & & & 124.7 & & \\
\hline 9 & $12 / 8 / 87$ & 5 & & & 118.1 & & \\
\hline 1 & $12 / 8 / 87$ & 6 & & & 96.4 & & \\
\hline 1 & $1 / 4 / 88$ & 1 & 1 & & 127.7 & $\mathbf{N}$ & \\
\hline 1 & $1 / 4 / 88$ & 2 & 1 & & 129.3 & $\bar{Y}$ & \\
\hline 1 & $1 / 4 / 88$ & 3 & 9 & & 128.2 & $\mathrm{~N}$ & \\
\hline 1 & $1 / 4 / 88$ & 4 & 1 & & 124.7 & $\mathrm{~N}$ & \\
\hline 9 & $9 / 4 / 88$ & 5 & 1 & & 117.8 & $\mathrm{~N}$ & \\
\hline 1 & $1 / 4 / 88$ & 6 & 1 & & 94.3 & $\bar{N}$ & \\
\hline 9 & $2 / 1 / 88$ & 1 & 1 & & & $\bar{N}$ & 91 - suspect data (low) \\
\hline 1 & $2 / 1 / 88$ & 2 & 1 & & 129 & $N$ & \\
\hline 1 & $2 / 1 / 88$ & 3 & 9 & & 124 & $\begin{array}{l} \\
\end{array}$ & \\
\hline 1 & $2 / 9 / 88$ & 4 & 1 & & 121 & $\mathrm{~N}$ & \\
\hline 1 & $2 / 1 / 88$ & 5 & 1 & & 114 & $\bar{N}$ & \\
\hline 1 & $2 / 1 / 88$ & 6 & 1 & & 90 & $\bar{N}$ & \\
\hline 1 & $3 / 8 / 88$ & 1 & 1 & & & $\bar{N}$ & 106 - suspect data (low) \\
\hline 1 & $3 / 8 / 88$ & 3 & 8 & & 128 & $\bar{Y}$ & \\
\hline 1 & $3 / 8 / 88$ & 4 & 1 & & 125 & $\bar{N}$ & \\
\hline 1 & $3 / 8 / 88$ & 5 & 1 & & 117 & $\mathbf{N}$ & \\
\hline 1 & $3 / 8 / 88$ & 6 & 1 & & 94 & $N$ & \\
\hline 1 & $4 / 4 / 88$ & 1 & 1 & & 124 & $\mathbf{N}$ & \\
\hline 1 & $4 / 4 / 88$ & 2 & 1 & & 125 & $\mathrm{Y}$ & \\
\hline 1 & $4 / 4 / 88$ & 3 & 1 & & 124 & $\mathbf{N}$ & \\
\hline 1 & $4 / 4 / 88$ & 4 & 1 & & 120 & $N$ & \\
\hline 1 & $4 / 4 / 88$ & 5 & 1 & & 113 & $\bar{N}$ & \\
\hline 1 & $4 / 4 / 88$ & 6 & 1 & & 90 & $\mathbf{N}$ & \\
\hline 1 & $5 / 2 / 88$ & 1 & 1 & & 133 & $\bar{Y}$ & \\
\hline 1 & $5 / 2 / 88$ & 2 & 1 & & 133 & $\mathbf{N}$ & \\
\hline 1 & $5 / 2 / 88$ & 3 & 1 & & 132 & $\mathbf{N}$ & \\
\hline 1 & $5 / 2 / 88$ & 4 & 1 & & 128 & $\mathbf{N}$ & \\
\hline 1 & $5 / 2 / 88$ & 5 & 1 & & 122 & $\mathbf{N}$ & \\
\hline 1 & $5 / 2 / 88$ & 6 & 9 & & 100 & $\mathrm{~N}$ & \\
\hline 1 & $5 / 4 / 88$ & 1 & 1 & & 125 & $\bar{Y}$ & \\
\hline 1 & $5 / 4 / 88$ & 2 & 1 & & 125 & $\mathrm{~N}$ & \\
\hline 9 & $5 / 4 / 88$ & 3 & 1 & & 125 & $\mathrm{~N}$ & \\
\hline 1 & $5 / 4 / 88$ & 4 & 1 & & 121 & $\mathrm{~N}$ & \\
\hline 1 & $5 / 4 / 88$ & 5 & 1 & & 194 & $N$ & \\
\hline 9 & $5 / 4 / 88$ & 6 & 1 & & 92 & $\bar{N}$ & \\
\hline 1 & $6 / 6 / 88$ & 1 & 1 & & 133 & $Y$ & \\
\hline 1 & $6 / 6 / 88$ & 2 & 9 & & 125 & $\bar{N}$ & \\
\hline 1 & $6 / 6 / 88$ & 3 & 1 & & 128 & $\mathrm{~N}$ & \\
\hline 1 & $6 / 6 / 88$ & 4 & 1 & & 120 & $\bar{N}$ & \\
\hline 1 & $6 / 6 / 88$ & 5 & 7 & & 100 & $N$ & \\
\hline 1 & $6 / 6 / 88$ & 6 & 1 & & 100 & $\mathbf{N}$ & \\
\hline
\end{tabular}




\begin{tabular}{|c|c|c|c|c|c|c|c|}
\hline \multicolumn{8}{|c|}{ TANK 241-S-101 } \\
\hline \multicolumn{8}{|c|}{ THERMOCOUPLE DATA } \\
\hline TREE \# & DATE & THERMO \# & TREF SET \# & & & & \\
\hline 1 & $7 / 4 / 88$ & 1 & $\frac{1 \text { REE SEI H }}{1}$ & TEMP C & TEMPF & $Y=M A X$ TEMP & COMMENTS \\
\hline 1 & $7 / 4 / 88$ & & $\frac{1}{1}$ & & 134 & $\mathbf{N}$ & \\
\hline 1 & $7 / 4 / 88$ & & $\frac{1}{1}$ & & 135 & $Y$ & \\
\hline 1 & $7 / 4 / 88$ & & 1 & & 130 & $\mathbf{N}$ & \\
\hline 1 & $7 / 4 / 88$ & & 1 & & 122 & $\mathbf{N}$ & \\
\hline 1 & $7 / 4 / 88$ & $\frac{5}{6}$ & 1 & & 122 & $\mathbf{N}$ & \\
\hline 9 & $8 / 1 / 88$ & $\frac{6}{9}$ & 1 & & 95 & $\mathrm{~N}$ & \\
\hline 1 & $8 / 1 / 88$ & $\frac{1}{2}$ & 1 & & 125 & $Y$ & \\
\hline 1 & $8 / 1 / 88$ & $\frac{2}{3}$ & $\frac{1}{9}$ & & $\frac{125}{125}$ & $N$ & \\
\hline 1 & $8 / 1 / 88$ & 4 & $\frac{1}{1}$ & & $\frac{125}{122}$ & $\frac{N}{N}$ & \\
\hline 1 & $8 / 1 / 88$ & 6 & 1 & & 99 & $\mathrm{~N}$ & \\
\hline 1 & $9 / 5 / 88$ & 1 & 1 & & 124 & $\mathrm{~N}$ & \\
\hline 1 & $9 / 5 / 88$ & 2 & 1 & & 126 & $\mathbf{Y}$ & \\
\hline 1 & $9 / 5 / 88$ & 3 & 1 & & 126 & $\mathrm{~N}$ & \\
\hline 1 & $9 / 5 / 88$ & 4 & 1 & & 122 & $N$ & \\
\hline 1 & $9 / 5 / 88$ & 6 & 1 & & 97 & $\mathbf{N}$ & \\
\hline 1 & $10 / 3 / 88$ & 2 & 1 & & 125 & $\mathbf{Y}$ & \\
\hline 1 & $10 / 3 / 88$ & 3 & 1 & & 125 & $N$ & \\
\hline 1 & $10 / 3 / 88$ & 4 & 1 & & 122 & $N$ & \\
\hline 1 & $10 / 3 / 88$ & 6 & 1 & & 97 & $\mathrm{~N}$ & \\
\hline 1 & $11 / 7 / 88$ & 1 & 1 & & 169 & $\mathrm{~N}$ & \\
\hline 1 & $11 / 7 / 88$ & 2 & 1 & & 147 & $\mathbf{N}$ & \\
\hline 1 & $11 / 7 / 88$ & 3 & 1 & & 129 & $\mathbf{N}$ & \\
\hline 1 & $91 / 7 / 88$ & 4 & 1 & & 124 & $\mathbf{N}$ & \\
\hline 1 & $11 / 7 / 88$ & 5 & 1 & & & $\bar{Y}$ & 308 - suspect data (high) \\
\hline 1 & $11 / 7 / 88$ & 6 & 1 & & 108 & $\mathbf{N}$ & \\
\hline 1 & $12 / 5 / 88$ & 1 & 1 & & & $\mathbf{N}$ & BAD READINGS \\
\hline 1 & $1 / 2 / 89$ & 1 & 1 & & & $\mathbf{N}$ & $0 / 5$ \\
\hline 1 & $2 / 6 / 89$ & 1 & 1 & & 136.8 & $\bar{Y}$ & \\
\hline 1 & $2 / 6 / 89$ & 2 & 1 & & 128.8 & $\mathbf{N}$ & \\
\hline 1 & $2 / 6 / 89$ & 3 & 1 & & 128.1 & $\mathbf{N}$ & \\
\hline 1 & $2 / 6 / 89$ & 4 & 1 & & 124.8 & $\mathrm{~N}$ & \\
\hline 1 & $2 / 6 / 89$ & 6 & 1 & & 92.4 & $\mathrm{~N}$ & \\
\hline 1 & $3 / 6 / 89$ & 1 & 1 & & 152 & $\mathbf{Y}$ & \\
\hline 1 & $3 / 6 / 89$ & 2 & 1 & & 152 & $\mathbf{N}$ & \\
\hline 1 & $3 / 6 / 89$ & 3 & 1 & & 152 & $\mathbf{N}$ & \\
\hline 1 & $3 / 6 / 89$ & 4 & 1 & & 147 & $\mathbf{N}$ & \\
\hline 1 & $3 / 6 / 89$ & 6 & 1 & & 113 & $\mathbf{N}$ & \\
\hline 1 & $11 / 13 / 91$ & 2 & 1 & & 118.8 & $Y$ & $\begin{array}{c}\text { TC } \# 135 \text { O/S WORK ORDER \#04529 J. } \\
890423320-42\end{array}$ \\
\hline 1 & $11 / 13 / 91$ & 4 & 1 & & 115.2 & $\mathbf{N}$ & \\
\hline 1 & $11 / 13 / 91$ & 6 & 1 & & 90.8 & $\mathbf{N}$ & \\
\hline 1 & $1 / 1 / 92$ & 2 & 1 & & 122.2 & $\bar{Y}$ & J-890523512-20 TC \#135 O/S \\
\hline 1 & $1 / 1 / 92$ & 4 & 1 & & 118.5 & $\mathbf{N}$ & \\
\hline 1 & $1 / 1 / 92$ & 6 & 1 & & 91 & $\mathbf{N}$ & \\
\hline 1 & $11 / 4 / 92$ & 1 & 1 & & & $N$ & $\begin{array}{c}\text { "IS\&DA-92-488 NO RDGS SINCE JANUARY } \\
1992\end{array}$ \\
\hline 1 & $11 / 5 / 92$ & 1 & 1 & & & $\mathbf{N}$ & ENTIRE SYSTEM O/S \\
\hline 1 & $11 / 7 / 92$ & 2 & 1 & & 123 & $Y$ & $\mathrm{~J} 817 \cdot 13-20-019293$ \\
\hline 1 & $11 / 7 / 92$ & 4 & 1 & & 119 & $\mathbf{N}$ & \\
\hline 1 & $11 / 7 / 92$ & 6 & 1 & & 98 & $N$ & \\
\hline 1 & $2 / 13 / 93$ & 1 & 1 & & & $\mathbf{N}$ & OUTOF SERVICE \\
\hline 1 & $7 / 2 / 93$ & 1 & 1 & & & $\mathbf{N}$ & TCs $135 \& 60 / \mathrm{S}$ \\
\hline 1 & $7 / 2 / 93$ & 2 & 1 & & 115 & $\bar{Y}$ & \\
\hline 1 & $7 / 2 / 93$ & 4 & 1 & & 109.9 & $\mathrm{~N}$ & \\
\hline
\end{tabular}

Data obtained from WHC Surveillance Analysis Computer System (SACS), February 24, 1994, Data sheet TK-105-S to TK-101 $\mathrm{S}$, and Archive box \#121869. 
WHC-SD-WM-ER-323, Rev. 0

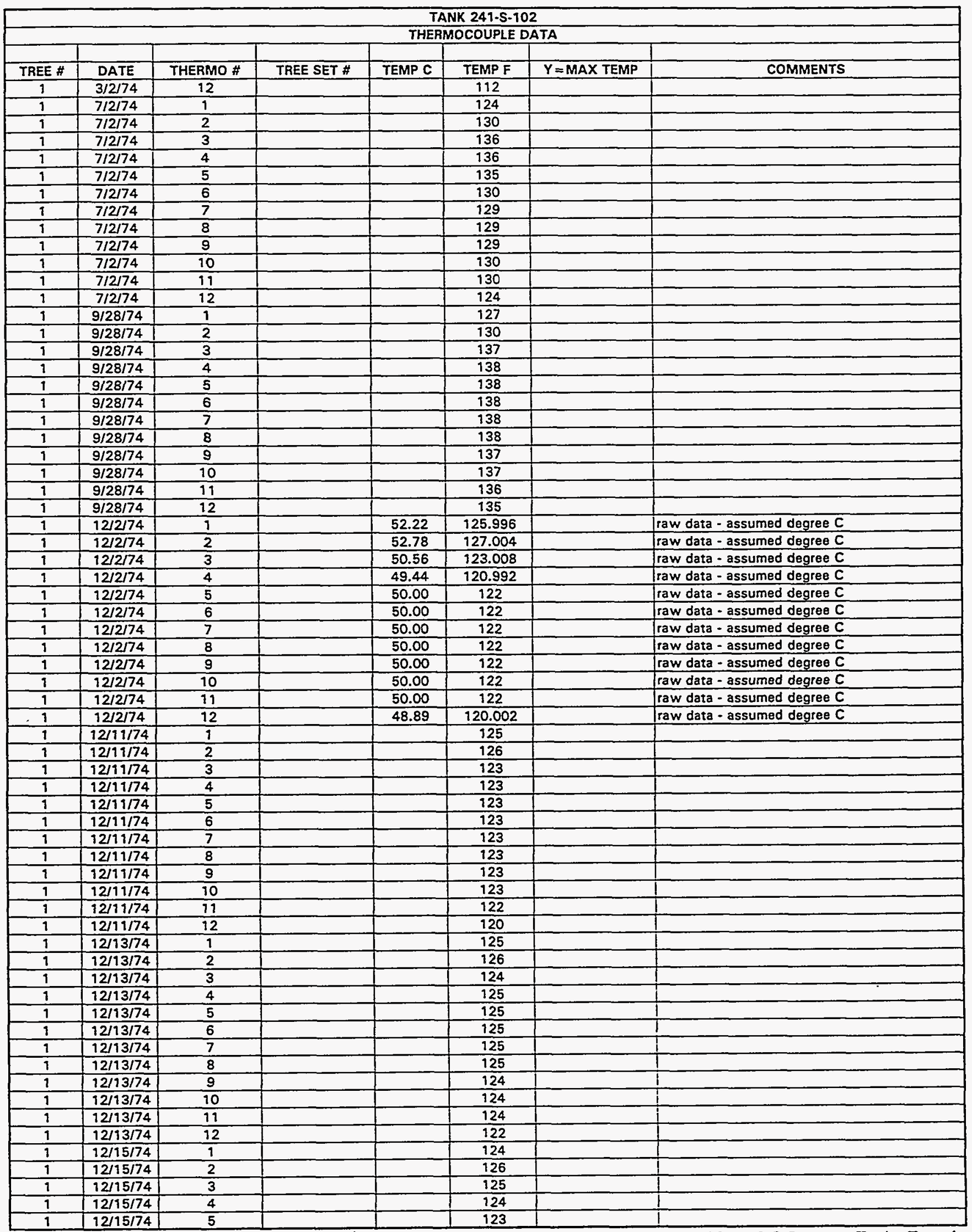

Data obtained from WHC Surveillance Analysis Computer System (SACS), February 24, 1994242-S Bottoms Tanks Transfer Record, Data Sheet TK-105-S to TK-101-S, and Archive box \#121869. 


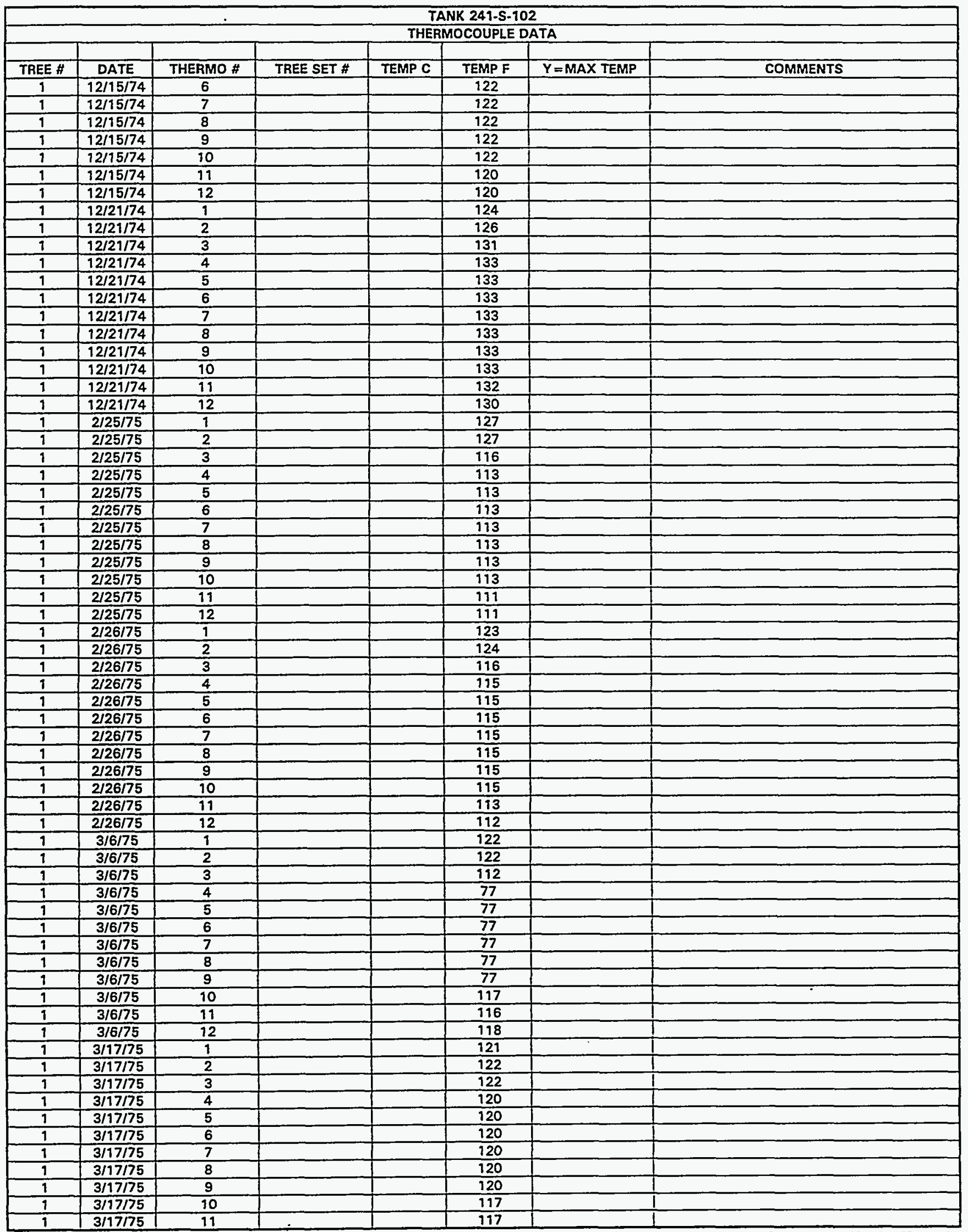

Data obtained from WHC Surveillance Analysis Computer System (SACS), February 24, 1994242-S Bottoms Tanks Transfer -.

Record, Data Sheet TK-105-S to TK-101-S, and Archive box \#121869. 


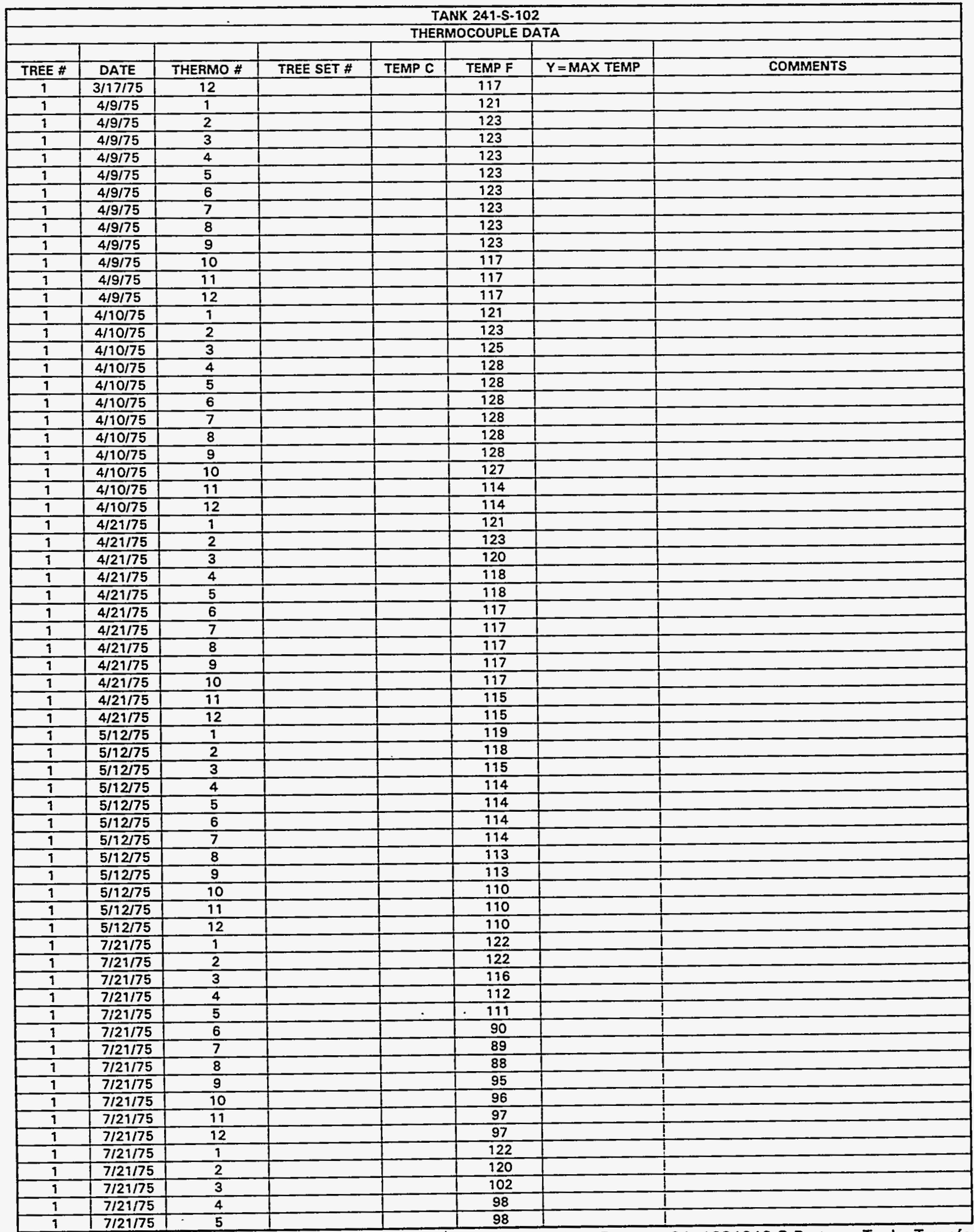

Data obtained from WHC Surveillance Analysis Computer System (SACS), February 24, 1994242-S Bottoms Tanks Transfer Record, Data Sheet TK-105-S to TK-101-S, and Archive box \#121869. 


\begin{tabular}{|c|c|c|c|c|c|c|c|}
\hline \multicolumn{8}{|c|}{ TANK 241-S-102 } \\
\hline \multicolumn{8}{|c|}{ THERMOCOUPLE DATA } \\
\hline TREE \# & DATE & THERMO \# & TREE SET \# & TEMP C & TEMP F & $Y=M A X$ TEMP & COMMENTS \\
\hline 1 & $7 / 21 / 75$ & 6 & & & 98 & & \\
\hline 1 & $7 / 21 / 75$ & 7 & & & 97 & & \\
\hline$T$ & $7 / 21 / 75$ & 8 & & & 97 & & \\
\hline 1 & $7 / 21 / 75$ & 9 & & & 100 & & \\
\hline 1 & $7 / 21 / 75$ & 10 & & & 100 & & \\
\hline 1 & $7 / 21 / 75$ & 11 & & & 100 & & \\
\hline$T$ & $7 / 21 / 75$ & 12 & & & 100 & & \\
\hline 1 & $11 / 3 / 75$ & 1 & & & 127 & & \\
\hline 1 & $11 / 3 / 75$ & 2 & & & 130 & & \\
\hline 1 & $11 / 3 / 75$ & 3 & & & 129 & & \\
\hline$\frac{T}{1}$ & $11 / 3 / 75$ & 4 & & & 126 & & \\
\hline 1 & $11 / 3 / 75$ & 5 & & & 124 & & \\
\hline 1 & $11 / 3 / 75$ & 6 & & & 124 & & \\
\hline$T$ & $11 / 3 / 75$ & 7 & & & 124 & & \\
\hline 1 & $11 / 3 / 75$ & 8 & & & 123 & & \\
\hline 1 & $11 / 3 / 75$ & 9 & & & 923 & & \\
\hline 1 & $11 / 3 / 75$ & 10 & & & 123 & & \\
\hline 1 & $11 / 3 / 75$ & 11 & & & 123 & & \\
\hline 1 & $11 / 3 / 75$ & 12 & & & 123 & & \\
\hline 1 & $2 / 4 / 76$ & 1 & & & 123 & & \\
\hline 1 & $2 / 4 / 76$ & 2 & & & 123 & & \\
\hline 1 & $2 / 4 / 76$ & 3 & & & 121 & & \\
\hline 1 & $2 / 4 / 76$ & 4 & & & 100 & & \\
\hline 1 & $2 / 4 / 76$ & 5 & & & 98 & & \\
\hline 1 & $2 / 4 / 76$ & 6 & & & 98 & & \\
\hline 9 & $2 / 4 / 76$ & 7 & & & 98 & & \\
\hline 1 & $2 / 4 / 76$ & 8 & & & 98 & & \\
\hline 1 & $2 / 4 / 76$ & 9 & & & 98 & & \\
\hline 1 & $2 / 4 / 76$ & 10 & & & 100 & & \\
\hline$\frac{1}{1}$ & $2 / 4 / 76$ & 11 & & & 100 & & \\
\hline 1 & $2 / 4 / 76$ & 12 & & & 101 & & \\
\hline 1 & $3 / 5 / 76$ & 1 & & & 120 & & \\
\hline 1 & $3 / 5 / 76$ & 2 & & & 116 & & \\
\hline 1 & $3 / 5 / 76$ & 3 & & & 108 & & \\
\hline 1 & $3 / 5 / 76$ & 4 & & & 103 & & \\
\hline 1 & $3 / 5 / 76$ & 5 & & & 103 & & \\
\hline 1 & $3 / 5 / 76$ & 6 & & & 100 & & \\
\hline$T$ & $3 / 5 / 76$ & 7 & & & 98 & & \\
\hline 1 & $3 / 5 / 76$ & 8 & & & 99 & & \\
\hline 1 & $3 / 5 / 76$ & 9 & & & 99 & & \\
\hline 1 & $3 / 5 / 76$ & 10 & & & 98 & & \\
\hline$\frac{1}{1}$ & $3 / 5 / 76$ & 91 & & & 98 & & \\
\hline 1 & $3 / 5 / 76$ & 12 & & & 100 & & \\
\hline 1 & $3 / 30 / 76$ & 1 & & & 116 & & \\
\hline 1 & $3 / 30 / 76$ & 2 & & & 113 & & \\
\hline 1 & $3 / 30 / 76$ & 3 & & & 114 & & \\
\hline 1 & $3 / 30 / 76$ & 4 & & & 115 & & \\
\hline 1 & $3 / 30 / 76$ & 5 & & & 115 & & \\
\hline 1 & $3 / 30 / 76$ & 6 & & & 115 & & \\
\hline 1 & $3 / 30 / 76$ & 7 & & & 115 & & \\
\hline$\frac{9}{1}$ & $3 / 30 / 76$ & 8 & & & 115 & & \\
\hline 9 & $3 / 30 / 76$ & 9 & & & 114 & & \\
\hline 1 & $3 / 30 / 76$ & 10 & & & 112 & & \\
\hline 1 & $3 / 30 / 76$ & 11 & & & 112 & & \\
\hline 1 & $3 / 30 / 76$ & 12 & & & 112 & & \\
\hline 1 & $5 / 2 / 76$ & 1 & & & 118 & & \\
\hline 1 & $5 / 2 / 76$ & 2 & & & 119 & & \\
\hline 9 & $5 / 2 / 76$ & 3 & & & 121 & & \\
\hline 1 & $5 / 2 / 76$ & 4 & & & 123 & & \\
\hline 1 & $5 / 2 / 76$ & 5 & & & 123 & & \\
\hline 1 & $5 / 2 / 76$ & 6 & & & 123 & & \\
\hline$\frac{1}{1}$ & $5 / 2 / 76$ & 7 & & & 123 & & \\
\hline 1 & $5 / 2 / 76$ & 8 & & & 121 & & \\
\hline 1 & $5 / 2 / 76$ & 9 & & & 93 & & \\
\hline 9 & $5 / 2 / 76$ & 10 & & & 90 & & \\
\hline 9 & $5 / 2 / 76$ & 11 & & & 90 & & \\
\hline
\end{tabular}

Data obtained from WHC Surveillance Analysis Computer System (SACS), February 24, 1994242-S Bottoms Tanks Transfei Record, Data Sheet TK-105-S to TK-101-S, and Archive box \#121869. 
WHC-SD-WM-ER-323, Rev. 0

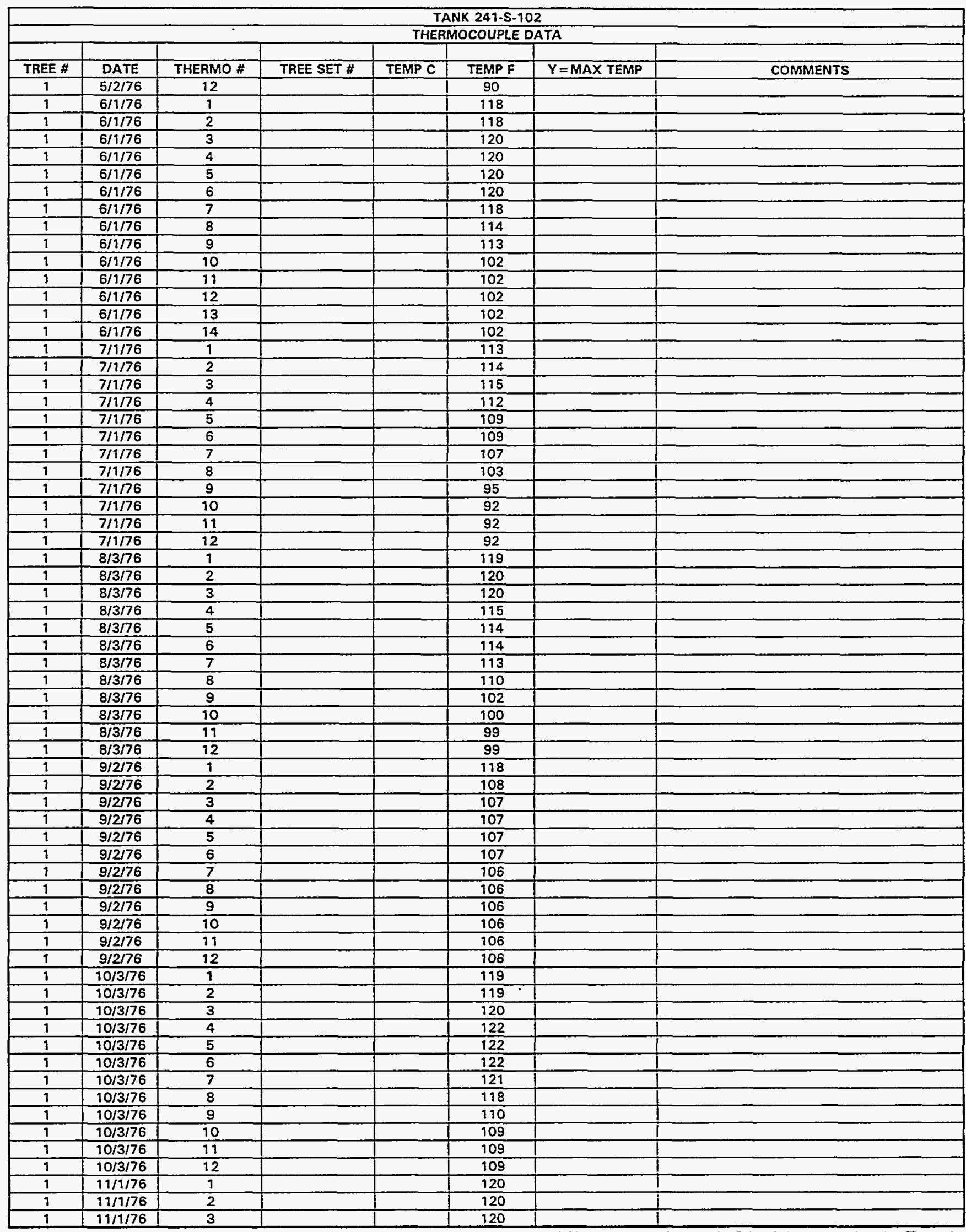

Data obtained from WHC Surveillance Analysis Computer System (SACS), February 24, 1994242-S Bottoms Tanks Transfer Record, Data Sheet TK-105-S to TK-101-S, and Archive box \#121869. 


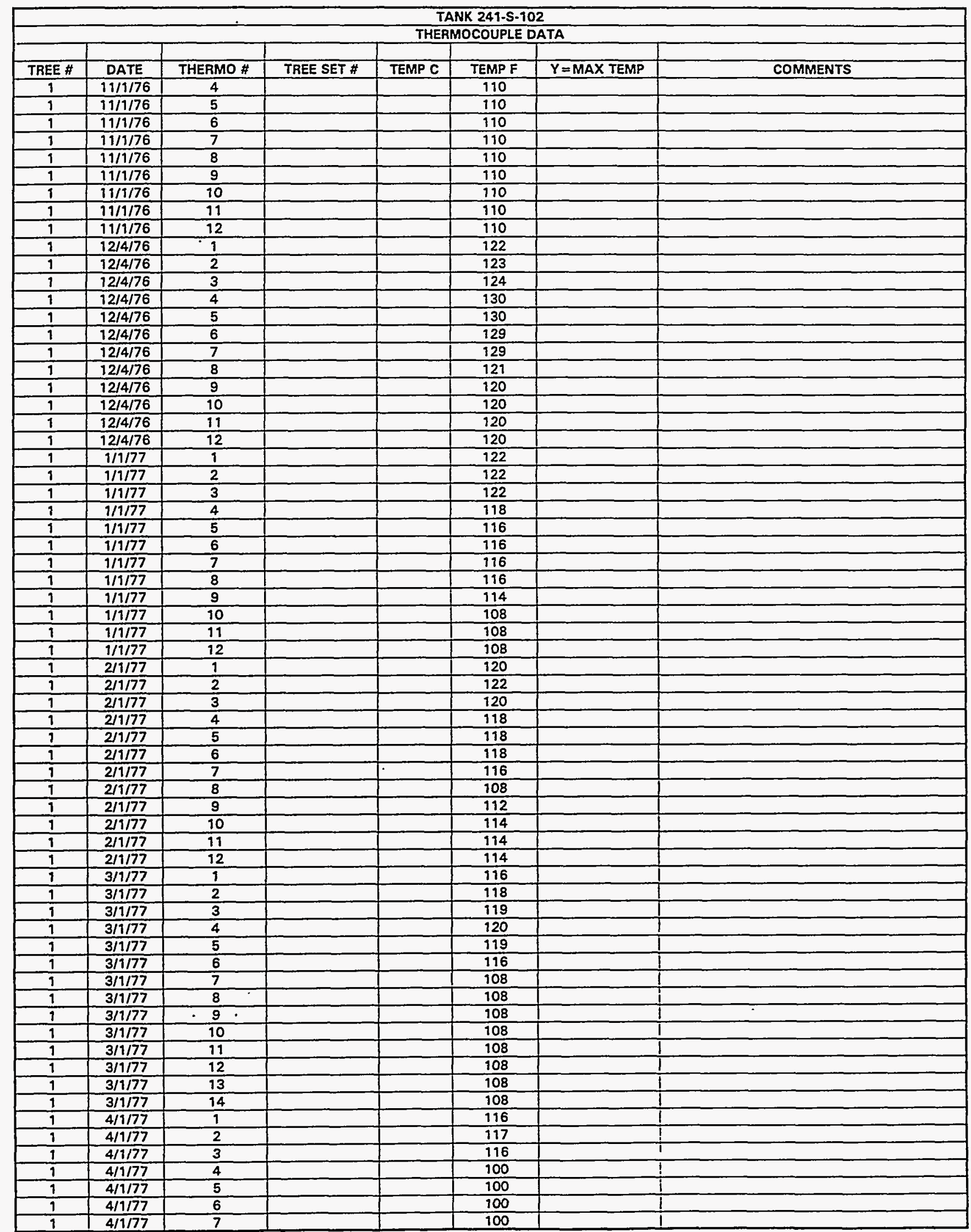

Data obtained from WHC Surveillance Analysis Computer System (SACS), February 24, 1994242-S Bottoms Tanks Transfer Record, Data Sheet TK-105-S to TK-101-S, and Archive box $\# 121869$.

D-193 


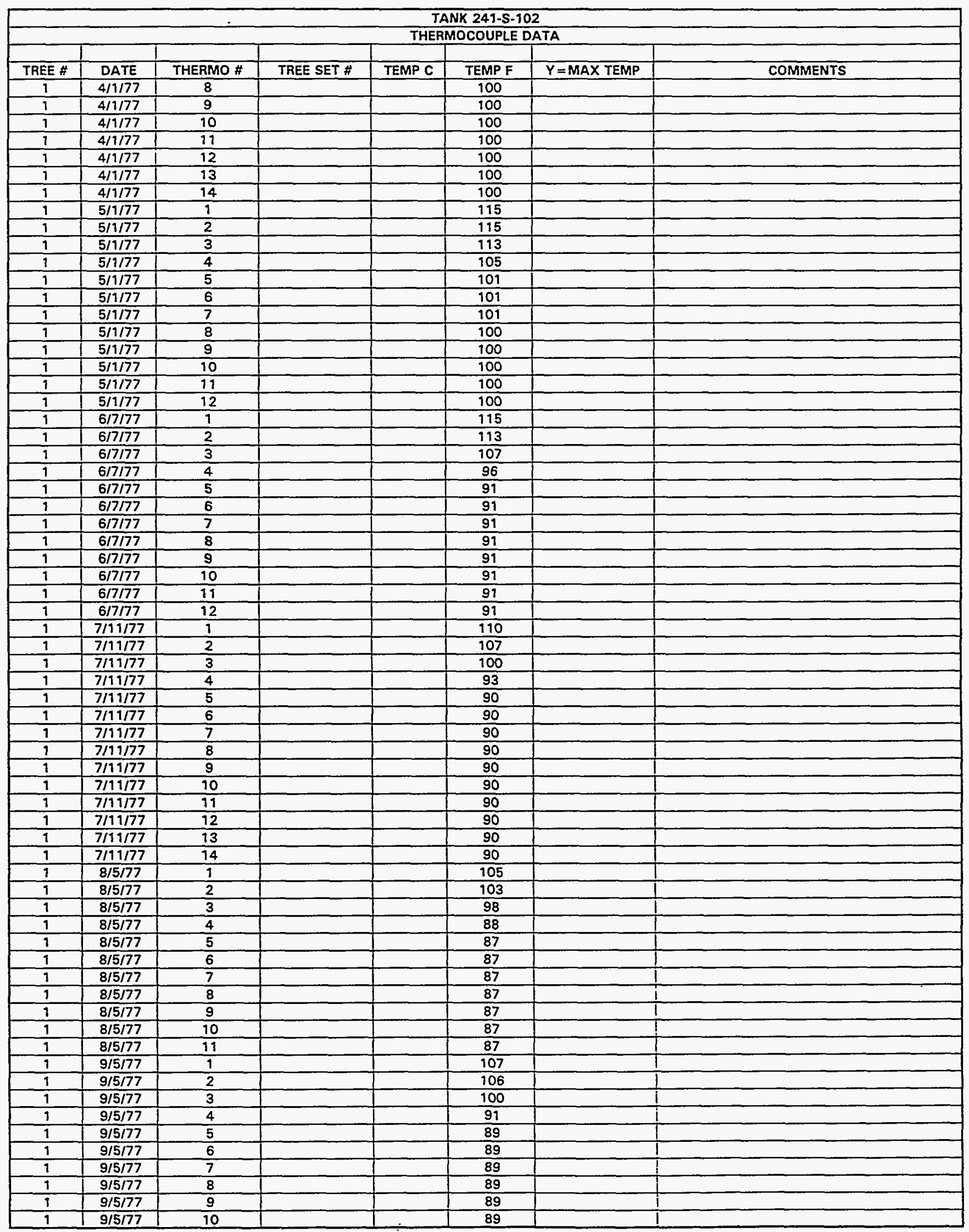

Data obtained from WHC Surveillance Analysis Computer System (SACS), February 24, 1994242-S Bottoms Tanks Transfer Record, Data Sheet TK-105-S to TK-101-S, and Archive box $\# 121869$. 


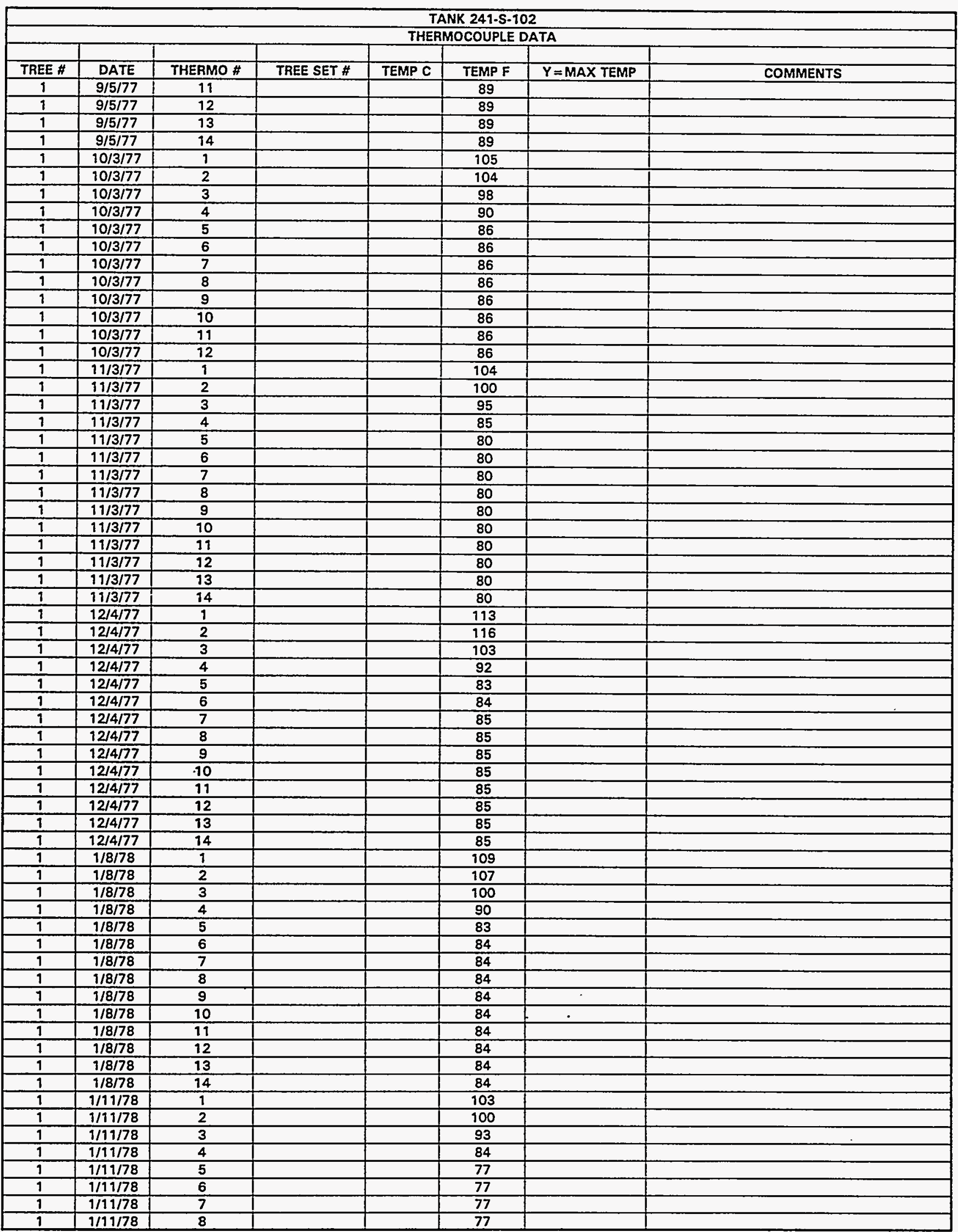

Data obtained from WHC Surveillance Analysis Computer System (SACS), February 24, 1994242-S Bottoms Tanks Transfer Record, Data Sheet TK-105-S to TK-101-S, and Archive box \#121869. 
WHC-SD-WM-ER-323, Rev. 0

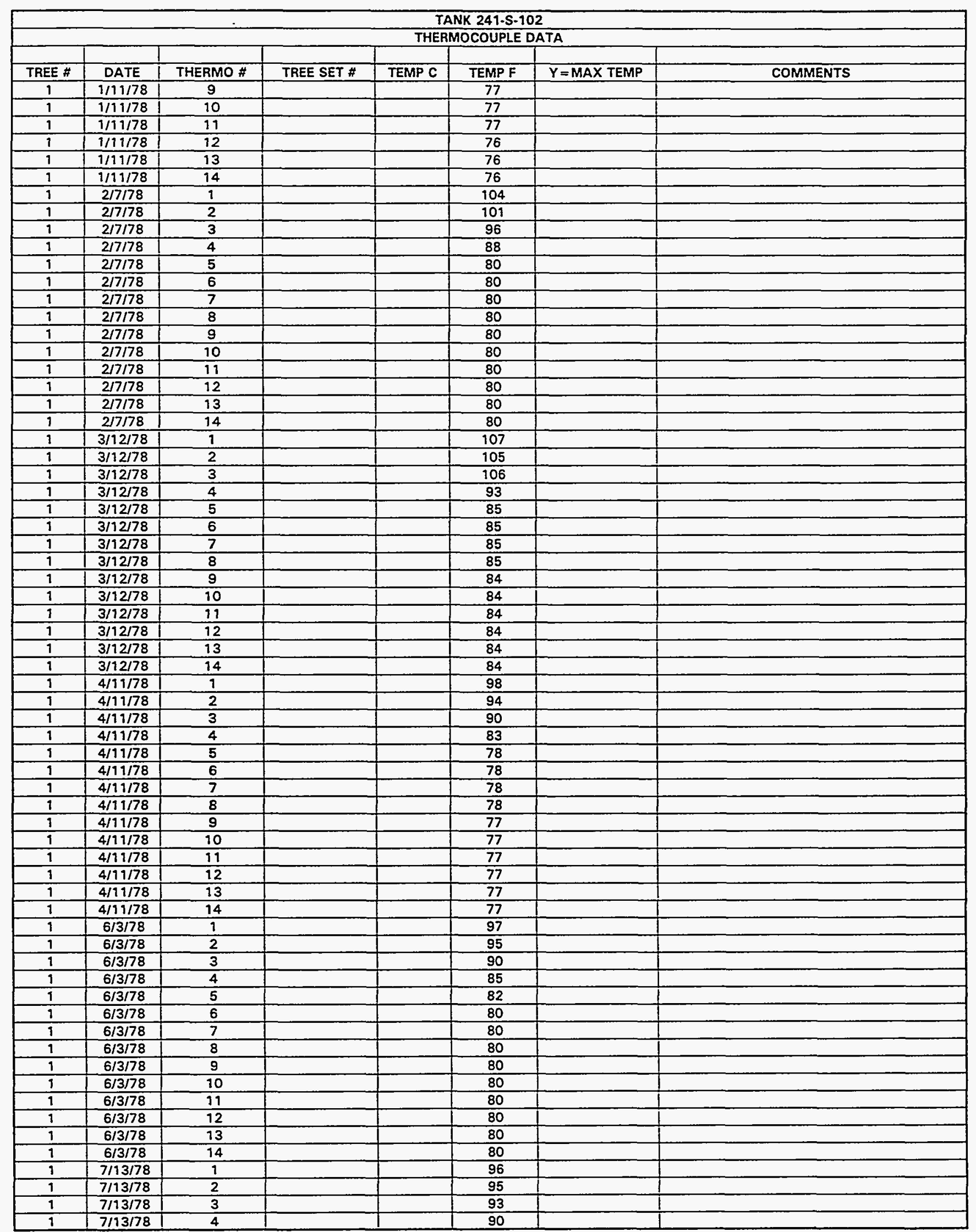

Data obtained from WHC Surveillance Analysis Computer System (SACS), February 24, 1994242-S Bottoms Tanks Transfer Record, Data Sheet TK-105-S to TK-101-S, and Archive box \#121869. 


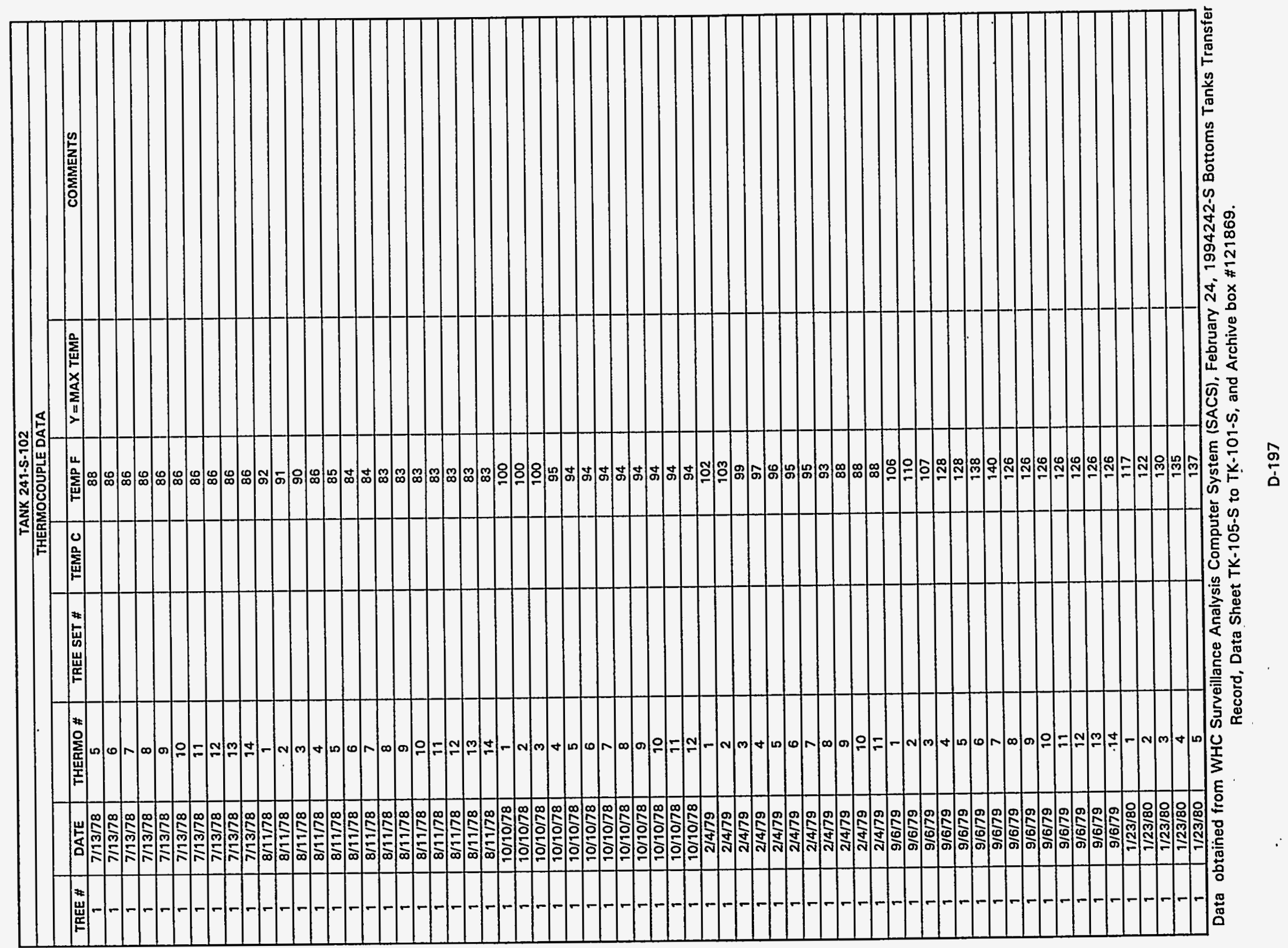


WHC-SD-WM-ER-323, Rev. O

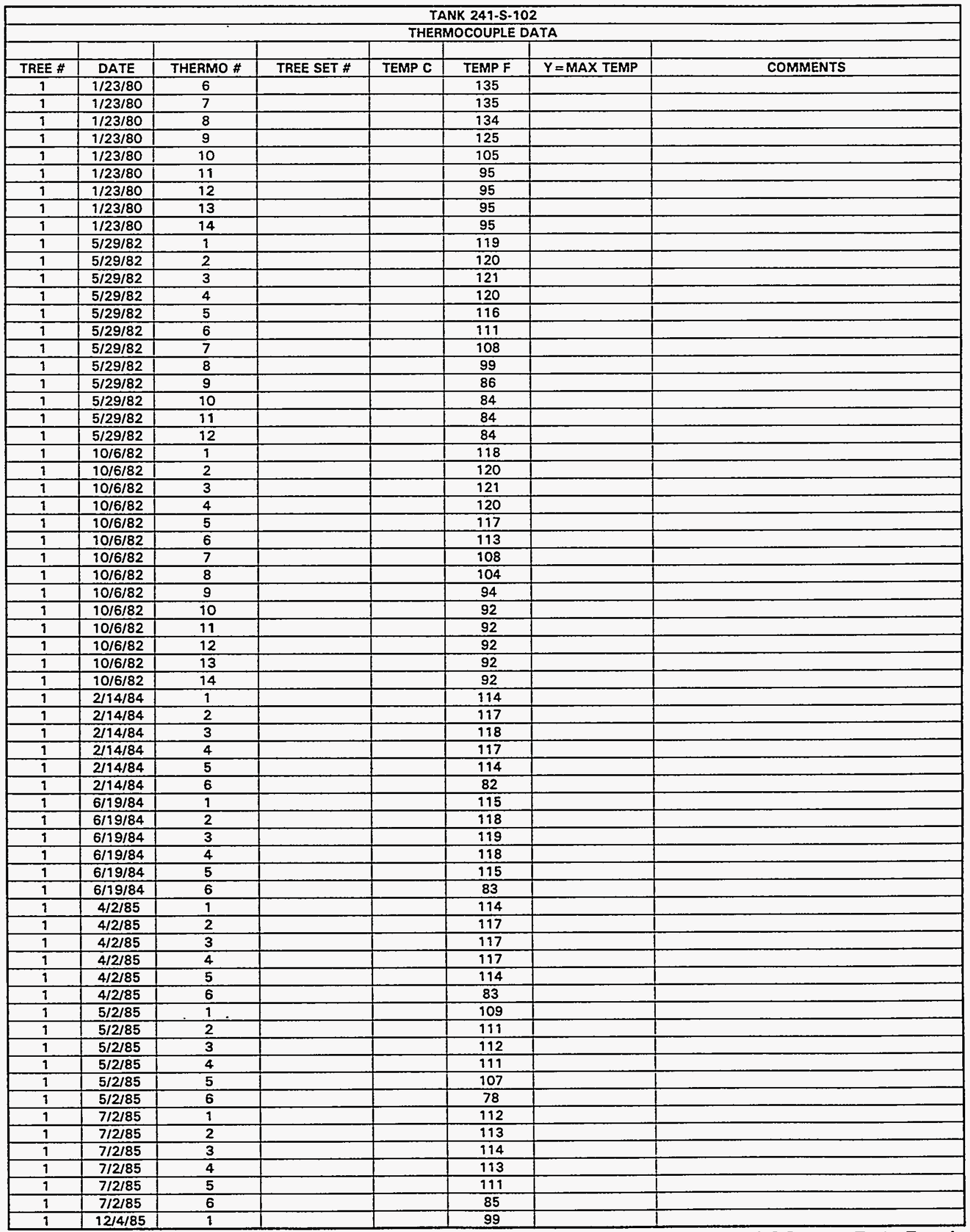

Data obtained from WHC Surveillance Analysis Computer System (SACS), February 24, 1994242-S Bottoms Tanks Transfer Record, Data Sheet TK-105-S to TK-101-S, and Archive box \#121869. 


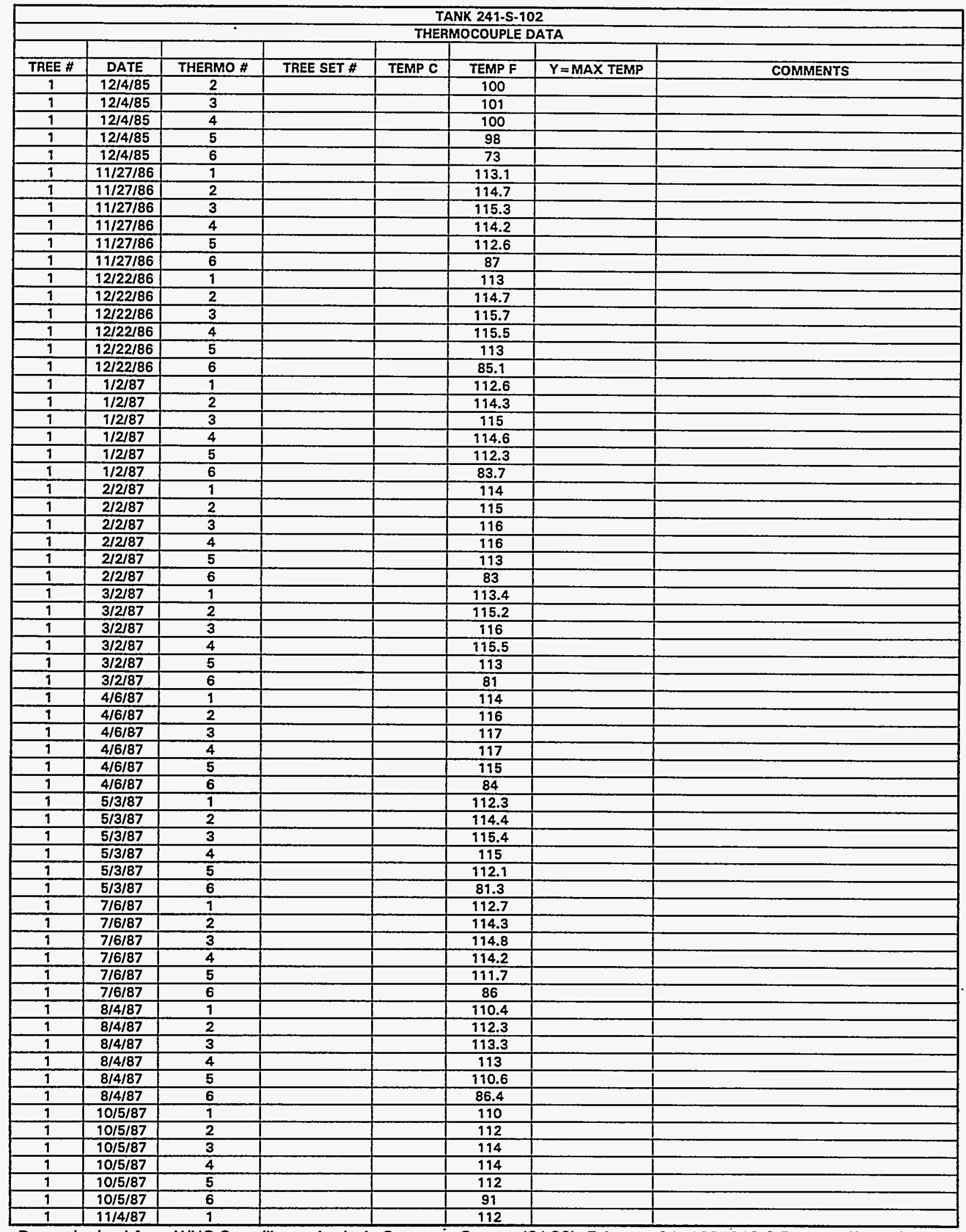

Data obtained from WHC Surveillance Analysis Computer System (SACS), February 24, 1994242-S Bottoms Tanks Transfer .. Record, Data Sheet TK-105-S to TK-101-S, and Archive box \#121869. 
WHC-SD-WM-ER-323, Rev. O

\begin{tabular}{|c|c|c|c|c|c|c|c|}
\hline \multicolumn{8}{|c|}{ TANK 241-S-102 } \\
\hline \multicolumn{8}{|c|}{ THERMOCOUPLE DATA } \\
\hline TREE \# & DATE & THERMO \# & TREE SET \# & TEMP C & TEMP $F$ & $Y=M A X$ TEMP & COMMENTS \\
\hline 1 & $11 / 4 / 87$ & 2 & & & 114 & & \\
\hline 1 & $11 / 4 / 87$ & 3 & & & 115 & & \\
\hline 1 & $11 / 4 / 87$ & 4 & & & 115 & & \\
\hline 1 & $11 / 4 / 87$ & 5 & & & 114 & & \\
\hline 1 & $11 / 4 / 87$ & 6 & & & 90 & & \\
\hline 1 & $12 / 8 / 87$ & 1 & & & 113.2 & & \\
\hline 1 & $12 / 8 / 87$ & 2 & & & 114.9 & & \\
\hline 9 & $12 / 8 / 87$ & 3 & & & 115.8 & & \\
\hline 1 & $12 / 8 / 87$ & 4 & & & 115.7 & & \\
\hline 1 & $12 / 8 / 87$ & 5 & & & 113.8 & & \\
\hline 9 & $12 / 8 / 87$ & 6 & & & 88 & & \\
\hline 1 & $1 / 4 / 88$ & 1 & 1 & & 113.1 & $\mathbf{N}$ & \\
\hline 1 & $1 / 4 / 88$ & 2 & 1 & & 114.9 & $\mathbf{N}$ & \\
\hline$\frac{9}{1}$ & $1 / 4 / 88$ & 3 & $T$ & & 115.7 & $\bar{Y}$ & \\
\hline 1 & $1 / 4 / 88$ & 4 & 1 & & 115.2 & $\mathrm{~N}$ & \\
\hline 1 & $1 / 4 / 88$ & 5 & 1 & & 112.7 & $\mathbf{N}$ & \\
\hline 9 & $1 / 4 / 88$ & 6 & 1 & & 94.3 & $\mathrm{~N}$ & \\
\hline 1 & $2 / 1 / 88$ & 1 & 1 & & 87 & $N$ & \\
\hline 1 & $2 / 1 / 88$ & 2 & 1 & & 90 & $\mathbf{N}$ & \\
\hline 9 & $2 / 1 / 88$ & 3 & 9 & & 94 & $\mathbf{N}$ & \\
\hline 1 & $2 / 1 / 88$ & 4 & 1 & & 98 & $\mathbf{N}$ & \\
\hline 1 & $2 / 1 / 88$ & 5 & 1 & & 99 & $Y$ & \\
\hline 9 & $2 / 1 / 88$ & 6 & 1 & & 99 & $\mathbf{N}$ & \\
\hline 9 & $3 / 8 / 88$ & 1 & 1 & & 112 & $\bar{N}$ & \\
\hline 1 & $3 / 8 / 88$ & 2 & 1 & & 113 & $\mathrm{~N}$ & \\
\hline 1 & $3 / 8 / 88$ & 3 & 9 & & 114 & $\bar{Y}$ & \\
\hline 1 & $3 / 8 / 88$ & 4 & 1 & & 113 & $N$ & \\
\hline 1 & $3 / 8 / 88$ & 5 & 1 & & 112 & $\mathbf{N}$ & \\
\hline$\frac{9}{1}$ & $3 / 8 / 88$ & 6 & 1 & & 84 & $\bar{N}$ & \\
\hline 1 & $4 / 4 / 88$ & 1 & 1 & & 110 & $\mathrm{~N}$ & \\
\hline 1 & $4 / 4 / 88$ & 2 & 1 & & 111 & $\mathbf{N}$ & \\
\hline 9 & $4 / 4 / 88$ & 3 & $\frac{1}{1}$ & & 112 & $\bar{Y}$ & \\
\hline 1 & $4 / 4 / 88$ & 4 & 1 & & 112 & $N$ & \\
\hline 1 & $4 / 4 / 88$ & 5 & 1 & & 110 & $\mathbf{N}$ & \\
\hline 9 & $4 / 4 / 88$ & 6 & 1 & & 82 & $\mathbf{N}$ & \\
\hline 1 & $5 / 2 / 88$ & 1 & 1 & & 121 & $\mathbf{N}$ & \\
\hline 1 & $5 / 2 / 88$ & 2 & 1 & & 122 & $Y$ & \\
\hline 9 & $5 / 2 / 28$ & 3 & 9 & & 122 & $\mathbf{N}$ & \\
\hline 1 & $5 / 2 / 88$ & 4 & 1 & & 122 & $\mathbf{N}$ & \\
\hline 1 & $5 / 2 / 88$ & 5 & 1 & & 119 & $\bar{N}$ & \\
\hline 9 & $5 / 2 / 88$ & 6 & 1 & & 92 & $N$ & \\
\hline 9 & $5 / 4 / 88$ & 1 & 1 & & 110 & $\mathbf{N}$ & \\
\hline 9 & $5 / 4 / 88$ & 2 & 1 & & 112 & $\mathrm{~N}$ & \\
\hline 9 & $5 / 4 / 88$ & 3 & 1 & & 113 & $Y$ & \\
\hline 9 & $5 / 4 / 88$ & 4 & 1 & & 113 & $\mathbf{N}$ & \\
\hline 1 & $5 / 4 / 88$ & 5 & 1 & & 110 & $\mathbf{N}$ & \\
\hline 1 & $5 / 4 / 88$ & 6 & 1 & & 83 & $N$ & \\
\hline 7 & $6 / 6 / 88$ & 9 & 1 & & 120 & $\mathbf{N}$ & \\
\hline$\frac{1}{1}$ & $6 / 6 / 88$ & 2 & 1 & & 122 & $\mathrm{Y}$ & \\
\hline 1 & $6 / 6 / 88$ & 3 & 1 & & 119 & $\mathrm{~N}$ & \\
\hline 1 & $6 / 6 / 88$ & 4 & 1 & & 120 & $\mathbf{N}$ & \\
\hline 1 & $6 / 6 / 88$ & 5 & 1 & & 118 & $\mathrm{~N}$ & \\
\hline 1 & $6 / 6 / 88$ & 6 & 1 & & 90 & $\mathrm{~N}$ & \\
\hline 1 & $7 / 4 / 88$ & 1 & 1 & & 115 & $\mathbf{N}$ & \\
\hline 1 & $7 / 4 / 88$ & 2 & 9 & & 118 & $\bar{N}$ & \\
\hline 1 & $7 / 4 / 88$ & 3 & 9 & & 919 & $\bar{Y}$ & \\
\hline 1 & $7 / 4 / 88$ & 4 & 1 & & 119 & $N$ & \\
\hline 1 & $7 / 4 / 88$ & 5 & 1 & & 116 & $N$ & \\
\hline 1 & $7 / 4 / 88$ & 6 & 1 & & 83 & $\mathrm{~N}$ & \\
\hline 1 & $8 / 1 / 88$ & 1 & 1 & & 109 & $\mathbf{N}$ & \\
\hline 1 & $8 / 1 / 88$ & 2 & 1 & & 111 & $\mathbf{N}$ & \\
\hline 1 & $8 / 1 / 88$ & 3 & 9 & & 112 & $Y$ & \\
\hline 1 & $8 / 1 / 88$ & 4 & 1 & & 192 & $\mathbf{N}$ & \\
\hline 1 & $8 / 1 / 88$ & 5 & 1 & & 110 & $N$ & \\
\hline 1 & $8 / 1 / 88$ & 6 & 1 & & 88 & $\mathbf{N}$ & \\
\hline 1 & $9 / 5 / 88$ & 1 & 1. & & 190 & $\mathbf{N}$ & \\
\hline
\end{tabular}

Data obtained from WHC Surveillance Analysis Computer System (SACS), February 24, 1994242-S Bottoms Tanks Transfer Record, Data Sheet TK-105-S to TK-101-S, and Archive box \#121869. 


\begin{tabular}{|c|c|c|c|c|c|c|c|}
\hline \multicolumn{8}{|c|}{ TANK 241-S-102 } \\
\hline \multicolumn{8}{|c|}{ THERMOCOUPLE DATA } \\
\hline TREE \# & DATE & THERMO \# & TREE SET \# & TEMP C & TEMP F & $Y=$ MAX TEMP & COMMENTS \\
\hline 1 & $9 / 5 / 88$ & 2 & 1 & & 112 & $N$ & \\
\hline 1 & $9 / 5 / 88$ & 3 & 1 & & 113 & $\mathbf{N}$ & \\
\hline 1 & $9 / 5 / 88$ & 4 & 1 & & 113 & $\bar{N}$ & \\
\hline 1 & $9 / 5 / 88$ & 5 & 1 & & 111 & $\bar{N}$ & \\
\hline 1 & $9 / 5 / 88$ & 6 & 1 & & 89 & $\mathbf{N}$ & \\
\hline 1 & $10 / 3 / 88$ & 1 & 1 & & 109 & $\mathrm{~N}$ & \\
\hline 1 & $10 / 3 / 88$ & 2 & 1 & & 111 & $\mathbf{N}$ & \\
\hline 1 & $10 / 3 / 88$ & 3 & 1 & & 112 & $\bar{Y}$ & \\
\hline 1 & $10 / 3 / 88$ & 4 & 1 & & 112 & $\mathrm{~N}$ & \\
\hline 1 & $10 / 3 / 88$ & 5 & 1 & & 110 & $\mathbf{N}$ & \\
\hline 1 & $10 / 3 / 88$ & 6 & 1 & & 89 & $\mathbf{N}$ & \\
\hline 1 & $11 / 7 / 88$ & 1 & 1 & & 109 & $\mathbf{N}$ & \\
\hline 1 & $11 / 7 / 88$ & 2 & 1 & & 111 & $\mathbf{N}$ & \\
\hline 1 & $11 / 7 / 88$ & 3 & 1 & & 113 & $\bar{Y}$ & \\
\hline 1 & $11 / 7 / 88$ & 4 & 1 & & 112 & $N$ & \\
\hline 1 & $1117 / 88$ & 5 & 1 & & 111 & $\mathrm{~N}$ & \\
\hline 1 & $11 / 7 / 88$ & 6 & 1 & & 89 & $\mathbf{N}$ & \\
\hline 1 & $12 / 5 / 88$ & 1 & 1 & & & $\mathbf{N}$ & BAD READINGS \\
\hline 1 & $1 / 2 / 89$ & 1 & 1 & & & $\mathrm{~N}$ & $0 / 5$ \\
\hline 1 & $2 / 6 / 89$ & 1 & 1 & & 112.4 & $\mathbf{N}$ & $\mathrm{J}$ \\
\hline 1 & $2 / 6 / 89$ & 2 & 1 & & 114.5 & $\mathbf{N}$ & \\
\hline 1 & $2 / 6 / 89$ & 3 & 1 & & 115.7 & $\bar{Y}$ & \\
\hline 1 & $2 / 6 / 89$ & 4 & 1 & & 115.5 & $\mathrm{~N}$ & \\
\hline 1 & $2 / 6 / 89$ & 5 & 1 & & 112.8 & $\bar{N}$ & \\
\hline 1 & $2 / 6 / 89$ & 6 & 1 & & 83.7 & $\mathbf{N}$ & \\
\hline 1 & $3 / 8 / 89$ & 1 & 1 & & 111.7 & $\mathbf{N}$ & \\
\hline 1 & $3 / 8 / 89$ & 2 & 9 & & 113.8 & $N$ & \\
\hline 1 & $3 / 8 / 89$ & 3 & 1 & & 114.7 & $Y$ & \\
\hline 1 & $3 / 8 / 89$ & 4 & 1 & & 114.5 & $\bar{N}$ & \\
\hline 1 & $3 / 8 / 89$ & 5 & 1 & & 111.8 & $\mathrm{~N}$ & \\
\hline 1 & $3 / 8 / 89$ & 6 & 1 & & 81.3 & $N$ & \\
\hline 1 & $6 / 5 / 89$ & 1 & 1 & & 110 & $\bar{N}$ & \\
\hline 1 & $6 / 5 / 89$ & 2 & 1 & & 113 & $\mathrm{~N}$ & \\
\hline 1 & $6 / 5 / 89$ & 3 & 1 & & 114 & $\mathbf{Y}$ & \\
\hline 1 & $6 / 5 / 89$ & 4 & 1 & & 114 & $\mathbf{N}$ & \\
\hline 1 & $6 / 5 / 89$ & 5 & 1 & & 112 & $\bar{N}$ & \\
\hline 1 & $6 / 5 / 89$ & 6 & 1 & & 83 & $\mathbf{N}$ & \\
\hline 1 & $7 / 3 / 89$ & 1 & 1 & & 109.9 & $\mathbf{N}$ & \\
\hline 1 & $7 / 3 / 89$ & 2 & 1 & & 112.1 & $\mathbf{N}$ & \\
\hline 1 & $7 / 3 / 89$ & 3 & 1 & & 113.6 & $\mathbf{Y}$ & \\
\hline 1 & $7 / 3 / 89$ & 4 & 1 & & 113.4 & $\mathbf{N}$ & \\
\hline 1 & $7 / 3 / 89$ & 5 & 1 & & 110.7 & $\mathbf{N}$ & \\
\hline 1 & $7 / 3 / 89$ & 6 & 1 & & 84.6 & $\mathbf{N}$ & \\
\hline 1 & $1 / 1 / 90$ & 1 & 1 & & 108.7 & $\mathbf{N}$ & $J$ \\
\hline 1 & $1 / 1 / 90$ & 2 & 1 & & 110.1 & $\mathrm{~N}$ & \\
\hline 1 & $1 / 1 / 90$ & 3 & 1 & & 110.8 & $\mathbf{Y}$ & \\
\hline 1 & $1 / 1 / 90$ & 4 & 1 & & 110.4 & $\mathbf{N}$ & \\
\hline 1 & $1 / 1 / 90$ & 5 & 1 & & 108.3 & $N$ & \\
\hline 1 & $1 / 1 / 90$ & 6 & 1 & & 82.6 & $N$ & \\
\hline 1 & $2 / 5 / 90$ & 1 & 1 & & 107 & $\mathbf{N}$ & $\mathrm{J}$ \\
\hline 1 & $2 / 5 / 90$ & 2 & 1 & & 108 & $\mathbf{N}$ & \\
\hline 1 & $2 / 5 / 90$ & 3 & 1 & & 110 & $\mathbf{Y}$ & \\
\hline 1 & $2 / 5 / 90$ & 4 & 1 & & 110 & $\mathbf{N}$ & \\
\hline 1 & $2 / 5 / 90$ & 5 & 1 & & 107 & $N$ & \\
\hline 1 & $2 / 5 / 90$ & 6 & 1 & & 80 & $\mathbf{N}$ & \\
\hline 1 & $6 / 4 / 90$ & 1 & 1 & & 101 & $\mathbf{N}$ & \\
\hline 1 & $6 / 4 / 90$ & 2 & 1 & & 103 & $\mathbf{N}$ & \\
\hline 1 & $6 / 4 / 90$ & 3 & 1 & & 104 & $\mathbf{Y}$ & \\
\hline 1 & $6 / 4 / 90$ & 4 & 1 & & 104 & $N$ & \\
\hline 1 & $6 / 4 / 90$ & 5 & 9 & & 102 & $N$ & \\
\hline 1 & $6 / 4 / 90$ & 6 & 1 & & 80 & $\mathbf{N}$ & \\
\hline 1 & $10 / 28 / 90$ & 1 & 1 & & 108.6 & $\mathrm{~N}$ & J-2E-OP-02O \\
\hline 1 & $10 / 28 / 90$ & 2 & 9 & & 110.2 & $N$ & \\
\hline 1 & $10 / 28 / 90$ & 3 & 1 & & 111.1 & $\bar{Y}$ & \\
\hline 1 & $10 / 28 / 90$ & 4 & 1 & & 110.8 & $N$ & \\
\hline 1 & $10 / 28 / 90$ & 5 & 1 & & 108.8 & $N$ & \\
\hline
\end{tabular}

Data obtained from WHC Surveillance Analysis Computer System (SACS), February 24, 1994242-S Bottoms Tanks Transfer Record, Data Sheet TK-105-S to TK-101-S, and Archive box \#121869. 
WHC-SD-WM-ER-323, Rev. O

\begin{tabular}{|c|c|c|c|c|c|c|c|}
\hline \multicolumn{8}{|c|}{ TANK 241-S-102 } \\
\hline \multicolumn{8}{|c|}{ THERMOCOUPLE DATA } \\
\hline TREE \# & DATE & THERMO \# & TREE SET \# & TEMP C & TEMP F & $Y=$ MAX TEMP & COMMENTS \\
\hline 1 & $10 / 28 / 90$ & 6 & 1 & & 87.8 & $\mathrm{~N}$ & \\
\hline 1 & $10 / 29 / 90$ & 1 & 1 & & 108.6 & $\bar{Y}$ & J-2E-OP-020 \\
\hline 1 & $10 / 29 / 90$ & 2 & 1 & & 110.2 & $\mathbf{N}$ & \\
\hline$\frac{1}{1}$ & $10 / 29 / 90$ & 3 & 1 & & 111.1 & $\mathbf{N}$ & \\
\hline 1 & $10 / 29 / 90$ & 4 & 1 & & 110.8 & $\mathrm{~N}$ & \\
\hline 1 & $10 / 29 / 90$ & 5 & 1 & & 108.8 & $\mathbf{N}$ & \\
\hline 1 & $10 / 29 / 90$ & 6 & 1 & & 87.8 & $\mathbf{N}$ & \\
\hline 1 & $11 / 4 / 90$ & 1 & 1 & & 110 & $\mathrm{~N}$ & J 2E-OP-020 \\
\hline 1 & $19 / 4 / 90$ & 2 & 1 & & 112 & $\mathrm{~N}$ & \\
\hline 1 & $11 / 4 / 90$ & 3 & 1 & & 113 & $\bar{Y}$ & \\
\hline 1 & $11 / 4 / 90$ & 4 & 1 & & 112 & $N$ & \\
\hline 1 & $11 / 4 / 90$ & 5 & 1 & & 110 & $\mathrm{~N}$ & \\
\hline$\frac{1}{1}$ & $11 / 4 / 90$ & 6 & 1 & & 89 & $\bar{N}$ & \\
\hline 1 & $11 / 12 / 90$ & 1 & 1 & & 108.4 & $\mathbf{N}$ & $2 E-0 P-020$ \\
\hline 1 & $11 / 12 / 90$ & 2 & 1 & & 110.3 & $N$ & \\
\hline$\frac{1}{1}$ & $11 / 12 / 90$ & 3 & $\frac{1}{1}$ & & 111.3 & $\bar{Y}$ & \\
\hline 1 & $11 / 12 / 90$ & 4 & 1 & & 110.9 & $\mathbf{N}$ & \\
\hline 1 & $11 / 12 / 90$ & 5 & 1 & & 108.6 & $\mathrm{~N}$ & \\
\hline 9 & $11 / 12 / 90$ & 6 & 1 & & 86.1 & $\mathbf{N}$ & \\
\hline 1 & $11 / 18 / 90$ & 1 & 1 & & 106.6 & $\mathrm{~N}$ & $2 E-O P-020$ \\
\hline 1 & $11 / 18 / 90$ & 2 & 1 & & 108.3 & $\mathrm{~N}$ & \\
\hline 9 & $11 / 18 / 90$ & 3 & 9 & & 109.1 & $\bar{Y}$ & \\
\hline 1 & $11 / 18 / 90$ & 4 & 1 & & 108.8 & $\bar{N}$ & \\
\hline 1 & $11 / 18 / 90$ & 5 & 1 & & 106.8 & $\bar{N}$ & \\
\hline 1 & $11 / 18 / 90$ & 6 & 1 & & 84.3 & $\mathbf{N}$ & \\
\hline 1 & $11 / 25 / 90$ & 1 & 1 & & 107.6 & $N$ & J.2E-OP-020 \\
\hline 9 & $11 / 25 / 90$ & 2 & 1 & & 109.1 & $\mathbf{N}$ & \\
\hline 1 & $11 / 25 / 90$ & 3 & 1 & & 110 & $\bar{Y}$ & \\
\hline 1 & $11 / 25 / 90$ & 4 & 1 & & 109.8 & $\mathbf{N}$ & \\
\hline 1 & $11 / 25 / 90$ & 5 & 1 & & 107.8 & $\mathbf{N}$ & \\
\hline 1 & $11 / 25 / 90$ & 6 & 9 & & 84.8 & $\bar{N}$ & \\
\hline 1 & $12 / 2 / 90$ & 1 & 1 & & 103.7 & $\mathbf{N}$ & J Used with Tyep E Connector \\
\hline 1 & $12 / 2 / 90$ & 2 & 1 & & 105.4 & $\bar{N}$ & \\
\hline 1 & $12 / 2 / 90$ & 3 & 1 & & 106.3 & $\bar{Y}$ & \\
\hline 1 & $12 / 2 / 90$ & 4 & 1 & & 106 & $\mathbf{N}$ & \\
\hline 1 & $12 / 2 / 90$ & 5 & 1 & & 104 & $\bar{N}$ & \\
\hline 1 & $12 / 2 / 90$ & 6 & 1 & & 81 & $\bar{N}$ & \\
\hline 1 & $12 / 9 / 90$ & 1 & 1 & & 102.9 & $\mathbf{N}$ & HH-99AA-J 890523512.47 \\
\hline 1 & $12 / 9 / 90$ & 2 & 1 & & 104.6 & $\mathbf{N}$ & \\
\hline 1 & $12 / 9 / 90$ & 3 & 9 & & 105.4 & $\bar{Y}$ & \\
\hline 1 & $12 / 9 / 90$ & 4 & 1 & & 105.1 & $\mathbf{N}$ & \\
\hline 1 & $12 / 9 / 90$ & 5 & 1 & & 103.2 & $\mathbf{N}$ & \\
\hline 9 & $12 / 9 / 90$ & 6 & 1 & & 79.7 & $\bar{N}$ & \\
\hline 1 & $12 / 16 / 90$ & 1 & 1 & & 106.7 & $\mathbf{N}$ & 2E-OP-20 \\
\hline 1 & $12 / 16 / 90$ & 2 & 1 & & 108.8 & $\mathbf{N}$ & \\
\hline 1 & $12 / 16 / 90$ & 3 & 1 & & 109.9 & $Y$ & \\
\hline 1 & $12 / 16 / 90$ & 4 & 1 & & 109.5 & $\mathbf{N}$ & \\
\hline 1 & $12 / 16 / 90$ & 5 & 1 & & 106.9 & $\mathbf{N}$ & \\
\hline 1 & $12 / 16 / 90$ & 6 & 1 & & 81.9 & $\mathbf{N}$ & \\
\hline 1 & $12 / 25 / 90$ & 1 & 1 & & 105.1 & $\mathbf{N}$ & $\mathrm{J} 890523512$ \\
\hline 1 & $12 / 25 / 90$ & 2 & 1 & & 106.6 & $\mathbf{N}$ & \\
\hline 1 & $12 / 25 / 90$ & 3 & 1 & & 107.6 & $Y$ & \\
\hline 1 & $12 / 25 / 90$ & 4 & 1 & & 107.3 & $\mathrm{~N}$ & \\
\hline 1 & $12 / 25 / 90$ & 5 & 1 & & 105.2 & $\mathbf{N}$ & \\
\hline 1 & $12 / 25 / 90$ & 6 & 1 & & 80.5 & $\mathbf{N}$ & \\
\hline 1 & $12 / 30 / 90$ & 9 & 1 & & 106 & $\overline{\mathbf{N}}$ & J 890523512-47 \\
\hline 1 & $12 / 30 / 90$ & 2 & 1 & & 107.5 & $\mathbf{N}$ & \\
\hline 1 & $12 / 30 / 90$ & 3 & 1 & & 108 & $\mathbf{Y}$ & \\
\hline$\frac{1}{1}$ & $12 / 30 / 90$ & 4 & 1 & & 108 & $\mathbf{N}$ & \\
\hline 1 & $12 / 30 / 90$ & 5 & 1 & & 106 & $\mathbf{N}$ & \\
\hline 1 & $12 / 30 / 90$ & 6 & $\frac{1}{1}$ & & 80.5 & $\mathbf{N}$ & \\
\hline 1 & $1 / 7 / 91$ & 1 & 1 & & 104.6 & $\mathbf{N}$ & $\mathrm{J}$ \\
\hline 1 & $1 / 7 / 99$ & 2 & 1 & & 106.6 & $\mathbf{N}$ & \\
\hline$\frac{1}{1}$ & $1 / 7 / 99$ & 3 & $\frac{1}{1}$ & & 107.9 & $\bar{Y}$ & \\
\hline 1 & $1 / 7 / 99$ & 4 & 1 & & 107.7 & $\mathbf{N}$ & \\
\hline 1 & $1 / 7 / 91$ & 5 & 1 & & 106.1 & $\mathbf{N}$ & \\
\hline
\end{tabular}

Data obtained from WHC Surveillance Analysis Computer System (SACS), February 24, 1994242-S Bottoms Tanks Transfer Record, Data Sheet TK-105-S to TK-101-S, and Archive box \#121869. 


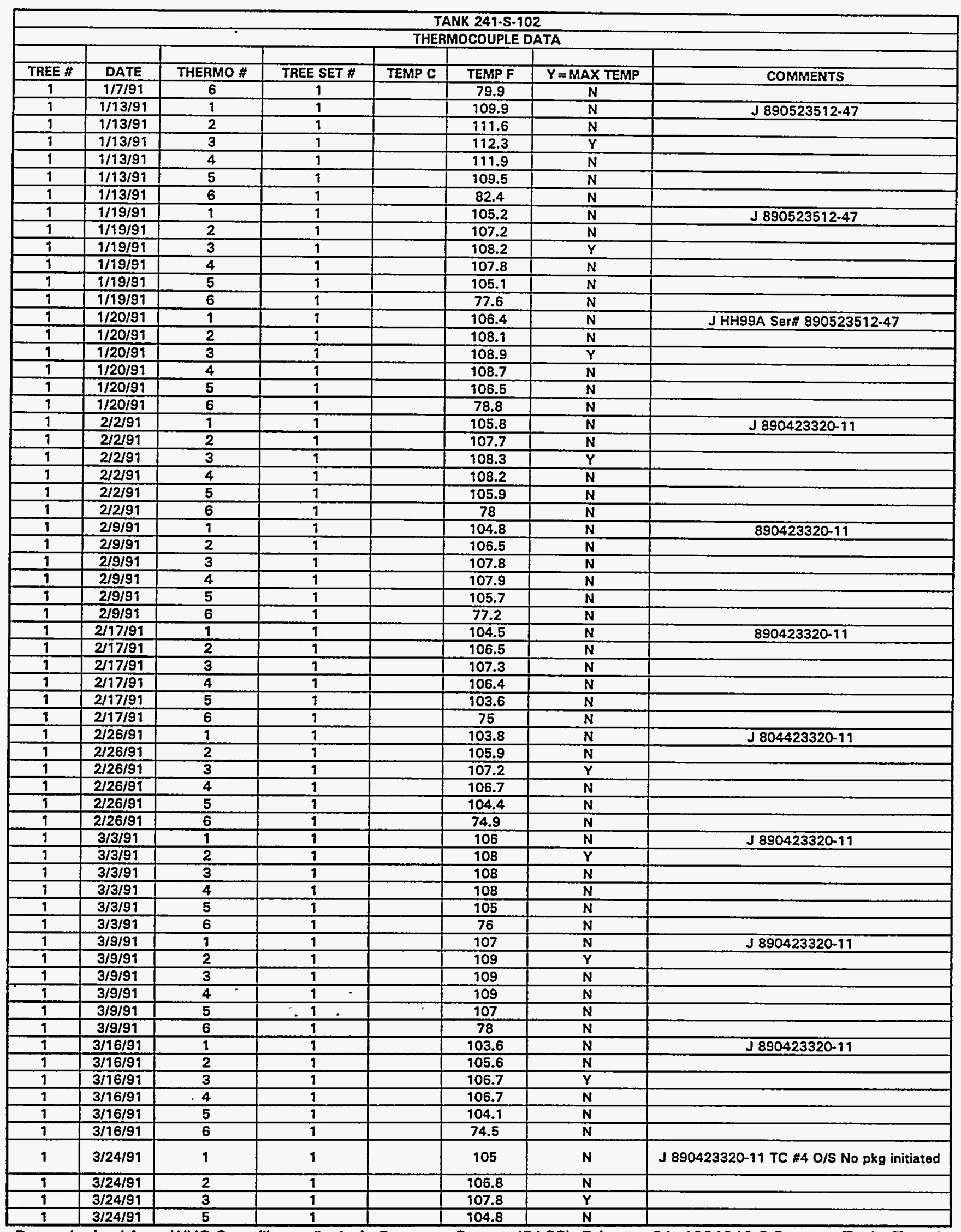

Data obtained from WHC Surveillance Analysis Computer System (SACS), February 24, 1994242-S Bottoms Tanks Transfer Record, Data Sheet TK-105-S to TK-101-S, and Archive box \#121869. 
WHC-SD-WM-ER-323, Rev. O

\begin{tabular}{|c|c|c|c|c|c|c|c|}
\hline \multicolumn{8}{|c|}{ TANK 241.S-102 } \\
\hline \multicolumn{8}{|c|}{ THERMOCOUPLE DATA } \\
\hline TREE \# & DATE & THERMO \# & TREE SET \# & TEMP C & TEMP F & $Y=$ MAX TEMP & COMMENTS \\
\hline 1 & $3 / 24 / 91$ & 6 & 1 & & 75.5 & $N$ & \\
\hline 1 & $3 / 31 / 91$ & 1 & 1 & & 104.3 & $\mathrm{~N}$ & $J 890423320-11$ \\
\hline 1 & $3 / 31 / 91$ & 2 & 1 & & 106.2 & $\mathbf{N}$ & \\
\hline 1 & 3/31/91 & 3 & 1 & & 106.9 & $\mathrm{Y}$ & \\
\hline 1 & $3 / 31 / 91$ & 4 & 1 & & 106.3 & $N$ & \\
\hline 1 & $3 / 31 / 91$ & 5 & 1 & & 103.6 & $\mathrm{~N}$ & \\
\hline 9 & $3 / 31 / 91$ & 6 & 1 & & 74.4 & $\mathbf{N}$ & \\
\hline 1 & $4 / 9 / 91$ & 1 & 1 & & 106.3 & $\bar{N}$ & J.890423320-11 \\
\hline 1 & $4 / 9 / 91$ & 2 & 1 & & 108.3 & $\bar{N}$ & \\
\hline 1 & $4 / 9 / 91$ & 3 & 1 & & 109.4 & $\mathrm{Y}$ & \\
\hline 1 & $4 / 9 / 91$ & 4 & 1 & & 109.4 & $\mathrm{~N}$ & \\
\hline 9 & $4 / 9 / 91$ & 5 & 1 & & 107 & $N$ & \\
\hline 1 & $4 / 9 / 91$ & 6 & 1 & & 77.7 & $\mathrm{~N}$ & \\
\hline$\frac{1}{1}$ & $4 / 14 / 91$ & 1 & 1 & & 107.9 & $\mathrm{~N}$ & J-HH.99-A 89-0423320-11 \\
\hline 9 & $4 / 14 / 91$ & 2 & 1 & & 109.5 & $\mathrm{~N}$ & \\
\hline 1 & $4 / 14 / 91$ & 3 & 1 & & 110 & $\bar{Y}$ & \\
\hline 1 & $4 / 14 / 91$ & 4 & 7 & & 109.6 & $\bar{N}$ & \\
\hline$\dot{1}$ & $4 / 14 / 91$ & 5 & 1 & & 107.1 & $N$ & \\
\hline 1 & $4 / 14 / 91$ & 6 & 1 & & 78.2 & $\mathbf{N}$ & \\
\hline$i$ & $4 / 20 / 91$ & 1 & 1 & & 105.9 & $\mathbf{N}$ & J 890423320-11 \\
\hline 9 & $4 / 20 / 91$ & 2 & 1 & & 108.1 & $\mathrm{~N}$ & \\
\hline 1 & $4 / 20 / 91$ & 3 & 1 & & 109.1 & $\bar{Y}$ & \\
\hline 1 & $4 / 20 / 91$ & 4 & 9 & & 108.9 & $N$ & \\
\hline 1 & $4 / 20 / 91$ & 5 & 1 & & 106.4 & $N$ & \\
\hline 1 & $4 / 20 / 91$ & 6 & 1 & & 77.1 & $N$ & \\
\hline 1 & $4 / 27 / 91$ & 1 & 1 & & 108.2 & $\bar{N}$ & J Keithley 872 \\
\hline 1 & $4 / 27 / 91$ & 2 & 9 & & 110.3 & $\mathrm{~N}$ & \\
\hline 1 & $4 / 27 / 91$ & 3 & 1 & & 111 & $\bar{Y}$ & \\
\hline 1 & $4 / 27 / 91$ & 4 & 1 & & 110.1 & $\bar{N}$ & \\
\hline 1 & $4 / 27 / 91$ & 5 & 9 & & 107.3 & $\mathrm{~N}$ & \\
\hline 9 & $4 / 27 / 91$ & 6 & 1 & & 77.7 & $\bar{N}$ & \\
\hline 1 & $5 / 4 / 91$ & 1 & 1 & & 104.8 & $\mathbf{N}$ & JHH-99A-J \\
\hline 1 & $5 / 4 / 91$ & 2 & 1 & & 107.4 & $\bar{N}$ & \\
\hline 1 & $5 / 4 / 91$ & 3 & 1 & & 108.3 & $\bar{Y}$ & \\
\hline 1 & $5 / 4 / 91$ & 4 & 1 & & 108 & $\mathbf{N}$ & \\
\hline 1 & $5 / 4 / 91$ & 5 & 7 & & 105.5 & $\mathrm{~N}$ & \\
\hline 1 & $5 / 4 / 91$ & 6 & 1 & & 76.7 & $\bar{N}$ & \\
\hline 1 & $5 / 12 / 91$ & 1 & 9 & & 104.9 & $\mathrm{~N}$ & J Omega 199 \\
\hline$i$ & $5 / 12 / 91$ & 2 & 1 & & 106.9 & $\mathrm{~N}$ & \\
\hline 9 & $5 / 12 / 91$ & 3 & 1 & & 107.6 & $\bar{Y}$ & \\
\hline 1 & $5 / 12 / 91$ & 4 & 1 & & 107.1 & $\mathrm{~N}$ & \\
\hline 1 & $5 / 12 / 91$ & 5 & 1 & & 104.6 & $\mathbf{N}$ & \\
\hline 1 & $5 / 12 / 91$ & 6 & 9 & & 76.5 & $\bar{N}$ & \\
\hline 1 & $5 / 19 / 91$ & 1 & 1 & & 106.7 & $\mathrm{~N}$ & J Omega 99-A \\
\hline 1 & $5 / 19 / 91$ & 2 & 1 & & 108.8 & $N$ & \\
\hline 1 & $5 / 19 / 91$ & 3 & 1 & & 109.5 & $Y$ & \\
\hline 1 & $5 / 19 / 91$ & 4 & 1 & & 108.4 & $N$ & \\
\hline 1 & $5 / 19 / 91$ & 5 & 1 & & 105.4 & $\bar{N}$ & \\
\hline 1 & $5 / 19 / 91$ & 6 & 9 & & 77.7 & $\mathbf{N}$ & \\
\hline 9 & $5 / 26 / 99$ & 9 & 1 & & 105.1 & $\bar{N}$ & J 890423320-11 \\
\hline 1 & $5 / 26 / 91$ & 2 & 1 & & 107.6 & $\mathbf{N}$ & \\
\hline 1 & $5 / 26 / 91$ & 3 & 1 & & 108.7 & $\bar{Y}$ & \\
\hline 9 & $5 / 26 / 91$ & 4 & 1 & & 108.5 & $\mathbf{N}$ & \\
\hline 1 & $5 / 26 / 91$ & 5 & 1 & & 105.9 & $\mathrm{~N}$ & \\
\hline 1 & $5 / 26 / 91$ & 6 & 1 & & 77.8 & $N$ & \\
\hline 1 & $6 / 1 / 91$ & 1 & 1 & & 105.2 & $\bar{N}$ & J Omega Model \#199 \\
\hline 1 & $6 / 1 / 91$ & 2 & 1 & & 107.6 & $\mathrm{~N}$ & \\
\hline 1 & $6 / 1 / 91$ & 3 & 1 & & 108.2 & $\bar{Y}$ & \\
\hline 9 & $6 / 1 / 91$ & 4 & 1 & & 106.9 & $\mathbf{N}$ & \\
\hline 1 & $6 / 9 / 91$ & 5 & $\frac{1}{1}$ & & 103.3 & $\mathbf{N}$ & \\
\hline 1 & $6 / 1 / 91$ & 6 & 1 & & 76.2 & $\mathbf{N}$ & \\
\hline 1 & $6 / 9 / 91$ & 1 & 1 & & 105.6 & $\mathbf{N}$ & $J 890423320-11$ \\
\hline 1 & $6 / 9 / 91$ & 2 & $\frac{1}{1}$ & & 107.9 & $\mathbf{N}$ & \\
\hline 1 & $6 / 9 / 91$ & 3 & 1 & & 108.6 & $\bar{Y}$ & \\
\hline 1 & $6 / 9 / 91$ & 4 & 1 & & 107.7 & $\bar{N}$ & \\
\hline 1 & $6 / 9 / 91$ & 5 & 1 & & 104.4 & $\mathbf{N}$ & \\
\hline
\end{tabular}

Data obtained from WHC Surveillance Analysis Computer System (SACS), February 24, 1994242-S Bottoms Tanks Transfer Record, Data Sheet TK-105-S to TK-101-S, and Archive box \#121869. 


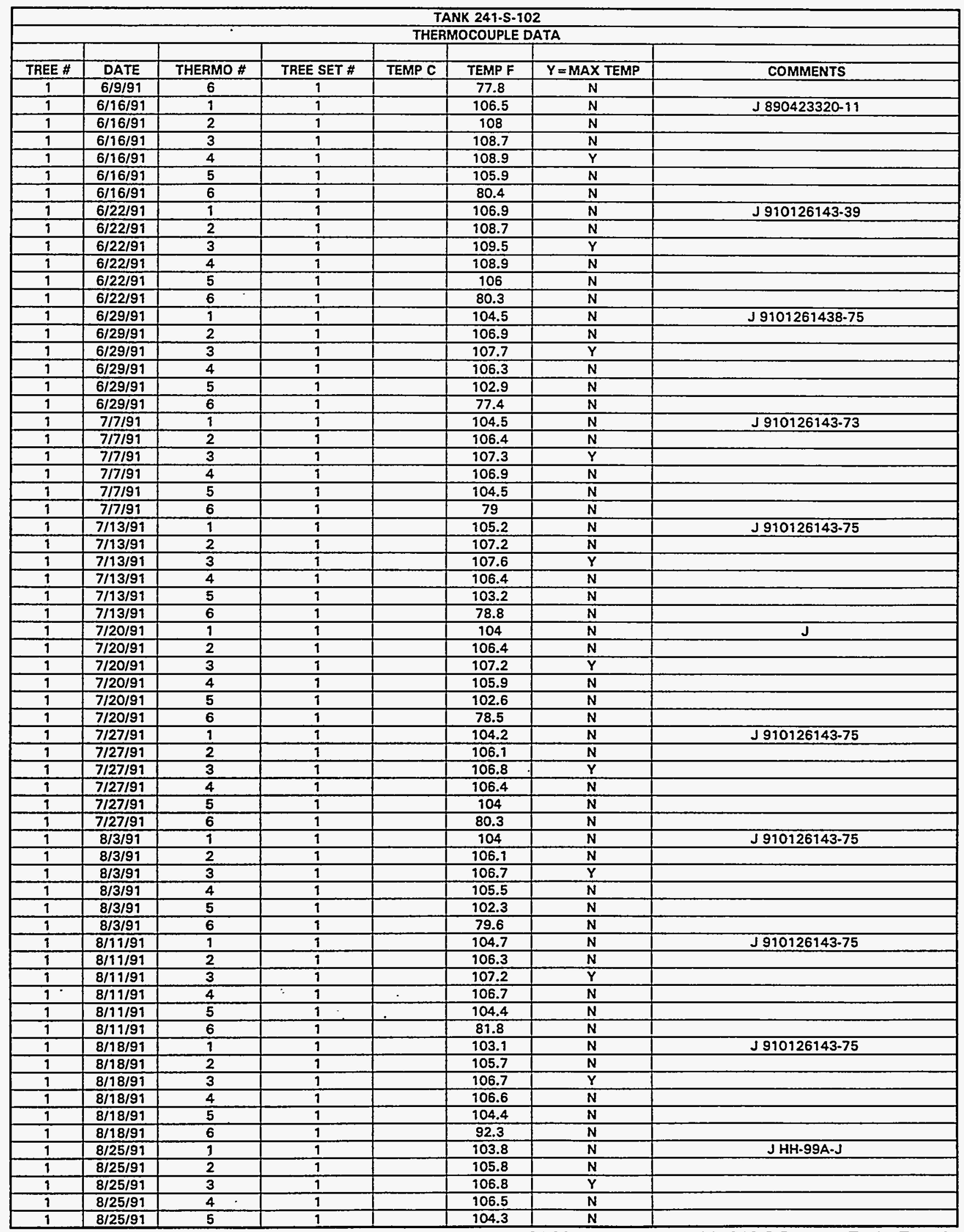


WHC-SD-WM-ER-323, Rev. 0

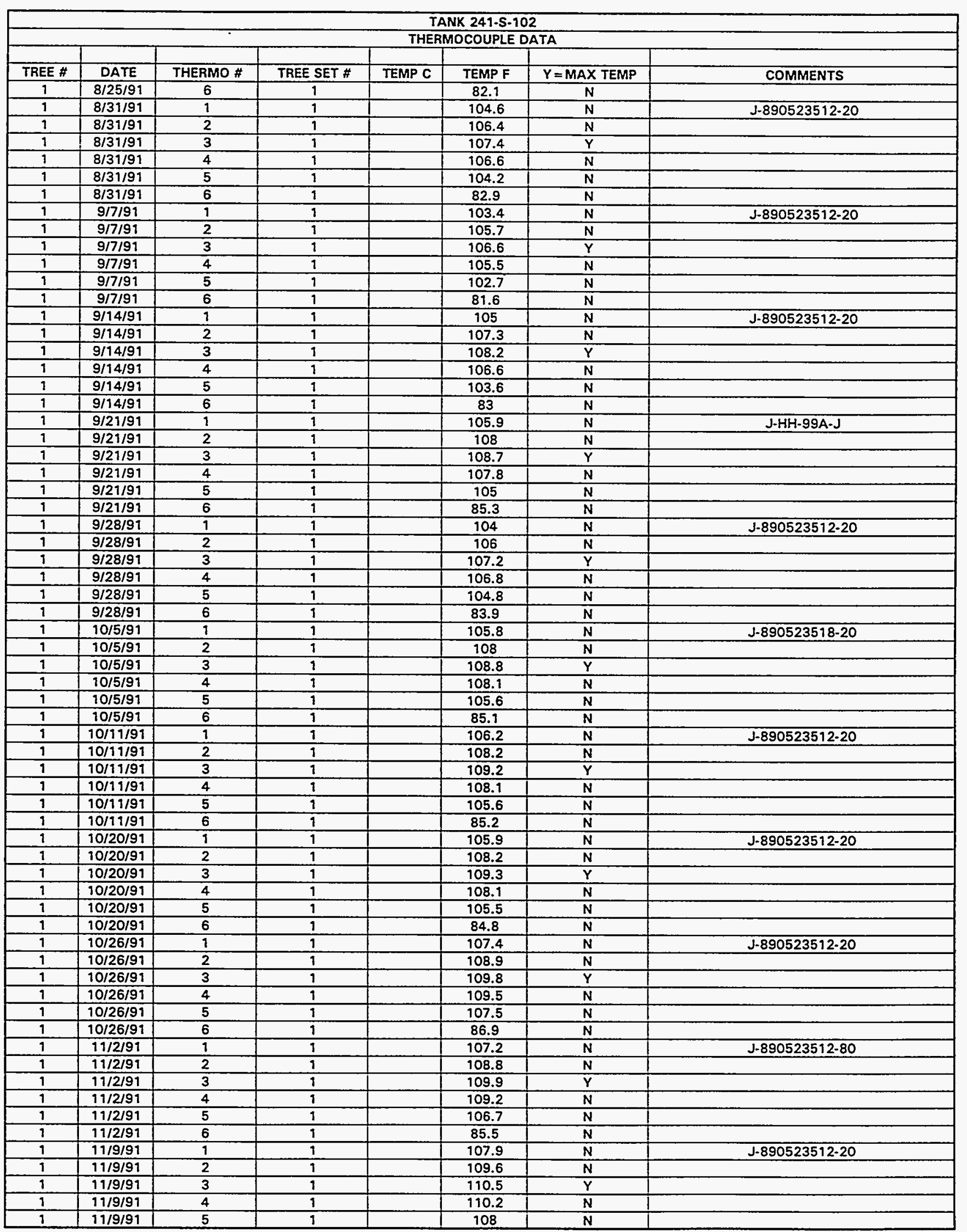

Data obtained from WHC Surveillance Analysis Computer System (SACS), Fébruary 24, 1994242-S Bottoms Tanks Transfer Record, Data Sheet.TK-105-S to TK-101-S, and Archive box \#121869. 


\begin{tabular}{|c|c|c|c|c|c|c|c|}
\hline \multicolumn{8}{|c|}{ TANK 241.S.102 } \\
\hline \multicolumn{8}{|c|}{ THERMOCOUPLE DATA } \\
\hline TREE $\#$ & DATE & THERMO \# & TREE SET \# & TEMP C & TEMP F & $Y=$ MAX TEMP & COMMENTS \\
\hline 1 & $11 / 9 / 91$ & 6 & 1 & & 86.6 & $\mathbf{N}$ & \\
\hline 1 & $11 / 13 / 91$ & 1 & 1 & & 104.1 & $\mathbf{N}$ & $\mathrm{J}-890423320-42$ \\
\hline$T$ & $11 / 13 / 91$ & 2 & 1 & & 105.7 & $\mathbf{N}$ & \\
\hline 1 & $11 / 13 / 91$ & 3 & 1 & & 106.4 & $\overline{\mathrm{Y}}$ & \\
\hline 1 & $11 / 13 / 91$ & 4 & 1 & & 106.1 & $\mathbf{N}$ & \\
\hline 1 & $11 / 13 / 91$ & 5 & 1 & & 104.2 & $\mathbf{N}$ & \\
\hline 1 & $11 / 13 / 91$ & 6 & 1 & & 82.6 & $\mathbf{N}$ & \\
\hline 9 & $11 / 15 / 91$ & 1 & 9 & & 103.7 & $\mathbf{N}$ & $J-817 \cdot 13-55 \cdot 008$ \\
\hline 1 & $11 / 15 / 91$ & 2 & 1 & & 105.3 & $\bar{N}$ & \\
\hline 1 & $11 / 15 / 91$ & 3 & 1 & & 106.2 & $Y$ & \\
\hline 1 & $11 / 15 / 91$ & 4 & 1 & & 105.9 & $\mathbf{N}$ & \\
\hline 9 & $11 / 15 / 91$ & 5 & 1 & & 104 & $\mathbf{N}$ & \\
\hline 1 & $11 / 15 / 91$ & 6 & 1 & & 82.1 & $\mathbf{N}$ & \\
\hline 1 & $11 / 23 / 91$ & 1 & 1 & & 103.4 & $\mathbf{N}$ & $\mathrm{J} \cdot 890423320-42$ \\
\hline 1 & $11 / 23 / 91$ & 2 & 1 & & 105 & $\bar{N}$ & \\
\hline 1 & $11 / 23 / 91$ & 3 & 1 & & 105.8 & $\bar{Y}$ & \\
\hline 1 & $11 / 23 / 91$ & 4 & 1 & & 105.5 & $\mathbf{N}$ & \\
\hline 1 & $11 / 23 / 99$ & 5 & 1 & & 103.6 & $\mathbf{N}$ & \\
\hline 1 & $11 / 23 / 91$ & 6 & 1 & & 81.2 & $\bar{N}$ & \\
\hline 1 & $12 / 1 / 91$ & 1 & 1 & & 103.5 & $N$ & $J-890523512-47$ \\
\hline 1 & $12 / 1 / 91$ & 2 & 1 & & 105.1 & $\mathbf{N}$ & \\
\hline$\frac{1}{1}$ & $12 / 1 / 91$ & 3 & 1 & & 106 & $\bar{N}$ & \\
\hline 1 & $12 / 1 / 91$ & 4 & 1 & & 105.8 & $\bar{Y}$ & \\
\hline 1 & $12 / 1 / 91$ & 5 & 1 & & 103.8 & $\mathbf{N}$ & \\
\hline 1 & $12 / 1 / 91$ & 6 & 1 & & 80.5 & $\mathbf{N}$ & \\
\hline 1 & $12 / 6 / 91$ & 1 & 1 & & 106 & $\mathbf{N}$ & J-910126143-64 \\
\hline 1 & $12 / 6 / 91$ & 2 & 1 & & 108 & $\mathbf{N}$ & \\
\hline 1 & $12 / 6 / 91$ & 3 & 1 & & 109 & $\bar{Y}$ & \\
\hline 1 & $12 / 6 / 91$ & 4 & 1 & & 108 & $\mathbf{N}$ & \\
\hline 1 & $12 / 6 / 91$ & 5 & 1 & & 107 & $\mathbf{N}$ & \\
\hline 1 & $12 / 6 / 91$ & 6 & 1 & & 83 & $\mathbf{N}$ & \\
\hline 1 & $12 / 14 / 91$ & 1 & 1 & & 105.9 & $\mathbf{N}$ & $J-910126143-64$ \\
\hline 1 & $12 / 74 / 99$ & 2 & 1 & & 107.9 & $\mathbf{N}$ & \\
\hline 1 & $12 / 14 / 91$ & 3 & 1 & & 109 & $\overline{\mathbf{Y}}$ & \\
\hline$\frac{1}{1}$ & $12 / 14 / 91$ & 4 & 1 & & 108.5 & $\mathbf{N}$ & \\
\hline 1 & $12 / 14 / 91$ & 5 & 1 & & 106.2 & $\mathbf{N}$ & \\
\hline 1 & $12 / 14 / 91$ & 6 & 1 & & 81.9 & $\mathbf{N}$ & \\
\hline 1 & $12 / 22 / 91$ & 1 & 1 & & 106.8 & $\mathbf{N}$ & $\mathrm{J}-\mathrm{TI}-007$ \\
\hline 1 & $12 / 22 / 91$ & 2 & 1 & & 108.7 & $\mathbf{N}$ & \\
\hline 9 & $12 / 22 / 91$ & 3 & 1 & & 109.6 & $\bar{Y}$ & . \\
\hline 1 & $12 / 22 / 91$ & 4 & 1 & & 109.3 & $\mathbf{N}$ & \\
\hline 1 & $12 / 22 / 91$ & 5 & 1 & & 107.4 & $\bar{N}$ & \\
\hline 1 & $12 / 22 / 91$ & 6 & 1 & & 82.6 & $\mathbf{N}$ & \\
\hline 1 & $12 / 28 / 91$ & 1 & 1 & & 108.7 & $\mathbf{N}$ & $\mathrm{J}-890523512-20$ \\
\hline 1 & $12 / 28 / 91$ & 2 & 1 & & 910.4 & $\mathbf{N}$ & \\
\hline 1 & $12 / 28 / 91$ & 3 & 1 & & 111.3 & $\bar{Y}$ & \\
\hline 1 & $12 / 28 / 91$ & 4 & 1 & & 110.9 & $\mathbf{N}$ & \\
\hline 1 & $12 / 28 / 91$ & 5 & 1 & & 109 & $\mathbf{N}$ & \\
\hline 9 & $12 / 28 / 91$ & 6 & 1 & & 83.5 & $\mathbf{N}$ & \\
\hline 1 & $1 / 6 / 92$ & 1 & 9 & & 105.6 & $\mathbf{N}$ & $J-71-030$ \\
\hline 1 & $1 / 6 / 92$ & 2 & 1 & & 107.3 & $\mathbf{N}$ & \\
\hline 1 & $1 / 6 / 92$ & 3 & 1 & & 108.1 & $\mathbf{Y}$ & \\
\hline 9 & $1 / 6 / 92$ & 4 & 1 & & 107.6 & $N$ & \\
\hline 1 & $1 / 6 / 92$ & 5 & 1 & & 105.8 & $N$ & \\
\hline 1 & $1 / 6 / 92$ & 6 & 9 & & 80 & $\mathbf{N}$ & \\
\hline 9 & $1 / 12 / 92$ & 1 & 1 & & 107.2 & $\mathrm{~N}$ & J 890523512-20 \\
\hline 9 & $7 / 12 / 92$ & 2 & 1 & & 108.2 & $\mathbf{N}$ & \\
\hline 1 & $1 / 12 / 92$ & 3 & 9 & & 109.7 & $\bar{Y}$ & \\
\hline 1 & $1 / 12 / 92$ & 4 & 1 & & 109.5 & $\mathbf{N}$ & \\
\hline 9 & $1 / 12 / 92$ & 5 & 1 & & 107.6 & $\bar{N}$ & \\
\hline 9 & $1 / 12 / 92$ & 6 & $\frac{1}{1}$ & & 81.3 & $\mathbf{N}$ & \\
\hline 1 & $1 / 16 / 92$ & $1 \%$ & 1 & & 105.2 & $N$ & J Omega 199 \\
\hline 1 & $1 / 16 / 92$ & 2 & 1 & & 107.2 & $\mathbf{N}$ & \\
\hline 9 & $1 / 16 / 92$ & 3 & 1 & & 108.1 & $\mathbf{Y}$ & \\
\hline 1 & $1 / 16 / 92$ & 4 & 1 & & 107.9 & $\mathbf{N}$ & \\
\hline 1 & $1 / 16 / 92$ & 5 & 1 & & 105.8 & $\mathrm{~N}$ & \\
\hline
\end{tabular}

Data obtained from WHC Surveillance Analysis Computer System (SACS), February 24, 1994242-S Bottoms Tanks Transfer Record, Data Sheet TK-105-S to TK-101-S, and Archive box \#121869. 
WHC-SD-WM-ER-323, Rev. 0

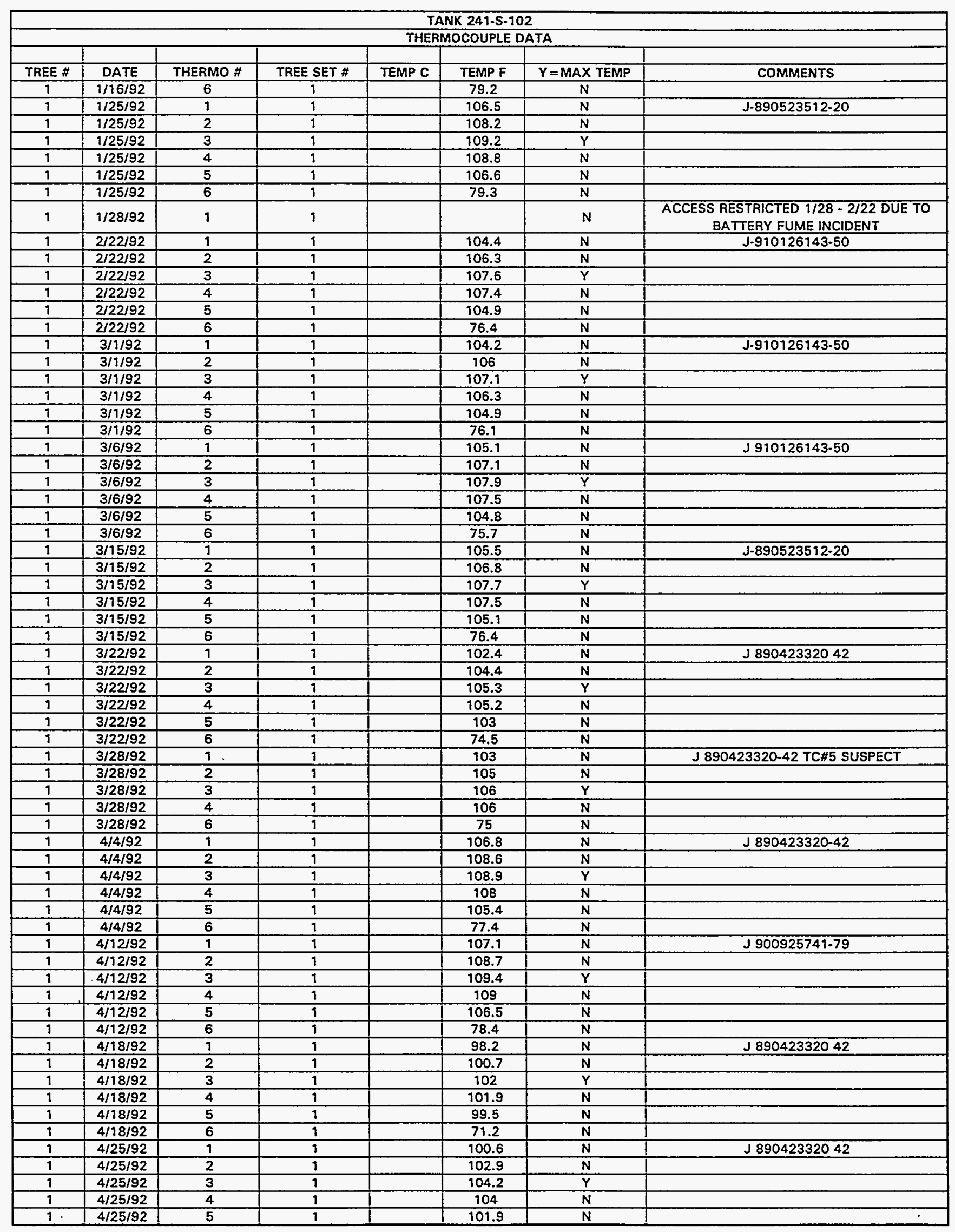

Data obtained from WHC Surveillance Analysis Computer System (SACS), February 24, 1994242-S Bottoms Tanks Transfer Record, Data Sheet TK-105-S to TK-101-S, and Archive box \#121869. 


\begin{tabular}{|c|c|c|c|c|c|c|c|}
\hline \multicolumn{8}{|c|}{ TANK 241-S-102 } \\
\hline \multicolumn{8}{|c|}{ THERMOCOUPLE DATA } \\
\hline TREE \# & DATE & THERMO \# & TREE SET \# & TEMP C & TEMP F & $Y=$ MAX TEMP & COMMENTS \\
\hline 1 & $4 / 25 / 92$ & 6 & 1 & & 73.2 & $\mathrm{~N}$ & \\
\hline 1 & $4 / 30 / 92$ & 1 & 1 & & 106.6 & $\mathrm{~N}$ & $J 900925741.29$ \\
\hline 1 & $4 / 30 / 92$ & 2 & $\frac{1}{1}$ & & 108.6 & $\mathrm{~N}$ & \\
\hline 1 & $4 / 30 / 92$ & 3 & 1 & & 109.2 & $\bar{Y}$ & \\
\hline 1 & $4 / 30 / 92$ & 4 & 1 & & 108.3 & $\mathrm{~N}$ & \\
\hline 1 & $4 / 30 / 92$ & 5 & 1 & & 105.3 & $\mathrm{~N}$ & \\
\hline 1 & $4 / 30 / 92$ & 6 & 1 & & 77.4 & $\bar{N}$ & \\
\hline 1 & $5 / 8 / 92$ & 1 & $\frac{1}{1}$ & & 103.3 & $\mathbf{N}$ & J $890423320-42$ \\
\hline 1 & $5 / 8 / 92$ & 2 & 1 & & 104.9 & $\mathrm{~N}$ & \\
\hline$\frac{T}{1}$ & $5 / 8 / 92$ & 3 & 1 & & 105.6 & $\bar{Y}$ & \\
\hline 9 & $5 / 8 / 92$ & 4 & 1 & & 905 & $\bar{N}$ & \\
\hline 1 & $5 / 8 / 92$ & 5 & 1 & & 102.6 & $\mathrm{~N}$ & \\
\hline 9 & $5 / 8 / 92$ & 6 & 1 & & 75.6 & $N$ & \\
\hline 1 & $5 / 15 / 92$ & 1 & 1 & & 106.9 & $\mathrm{~N}$ & $\mathrm{~J} 90092574179$ \\
\hline 1 & $5 / 15 / 92$ & 2 & 7 & & 108.6 & $\mathbf{N}$ & \\
\hline 9 & $5 / 15 / 92$ & 3 & 1 & & 109.3 & $\mathbf{Y}$ & \\
\hline 1 & $5 / 15 / 92$ & 4 & 1 & & 108.5 & $N$ & \\
\hline$\frac{1}{1}$ & $5 / 15 / 92$ & 5 & 1 & & 105.7 & $\bar{N}$ & \\
\hline 1 & $5 / 15 / 92$ & 6 & 1 & & 78.7 & $\mathbf{N}$ & \\
\hline 1 & $5 / 20 / 92$ & 1 & 1 & & 105.4 & $\mathbf{N}$ & J 89042330-42 \\
\hline 1 & $5 / 20 / 92$ & 2 & 1 & & 107.6 & $\mathbf{N}$ & \\
\hline 1 & $5 / 20 / 92$ & 3 & 1 & & 108.5 & $\bar{Y}$ & \\
\hline 9 & $5 / 20 / 92$ & 4 & 1 & & 108.2 & $\mathbf{N}$ & \\
\hline 1 & $5 / 20 / 92$ & 5 & $\frac{1}{1}$ & & 105.7 & $\bar{N}$ & \\
\hline$\frac{T}{1}$ & $5 / 20 / 92$ & 6 & 1 & & 78.8 & $\bar{N}$ & \\
\hline 1 & $5 / 30 / 92$ & 1 & 1 & & 104.5 & $\mathbf{N}$ & J $900925741-79$ \\
\hline 1 & $5 / 30 / 92$ & 2 & 1 & & 106.8 & $\mathbf{N}$ & \\
\hline$\frac{1}{1}$ & $5 / 30 / 92$ & 3 & 1 & & 107.8 & $\bar{Y}$ & \\
\hline 1 & $5 / 30 / 92$ & 4 & 1 & & 106.7 & $\mathbf{N}$ & \\
\hline 1 & $5 / 30 / 92$ & 5 & 9 & & 103.9 & $\mathbf{N}$ & \\
\hline 1 & $5 / 30 / 92$ & 6 & 1 & & 77.9 & $\mathbf{N}$ & \\
\hline 1 & $6 / 8 / 92$ & $\frac{1}{1}$ & 1 & & 101.6 & $\bar{Y}$ & $J 890423320-42$ \\
\hline 9 & $6 / 8 / 92$ & 2 & 1 & & 904 & $\mathbf{N}$ & \\
\hline 1 & $6 / 8 / 92$ & 3 & 1 & & 104.7 & $\mathbf{N}$ & \\
\hline 1 & $6 / 8 / 92$ & 4 & 1 & & 103.8 & $\mathrm{~N}$ & \\
\hline 1 & $6 / 8 / 92$ & 5 & 1 & & 101.2 & $\mathrm{~N}$ & \\
\hline 1 & $6 / 8 / 92$ & 6 & 1 & & 76.3 & $\mathbf{N}$ & \\
\hline$\frac{1}{1}$ & $6 / 13 / 92$ & $i$ & 1 & & 106.1 & $\mathrm{~N}$ & J $900925741-79$ \\
\hline 1 & $6 / 13 / 92$ & 2 & 1 & & 108.5 & $\bar{N}$ & \\
\hline 1 & $6 / 13 / 92$ & 3 & 1 & & 108.7 & $\mathbf{Y}$ & \\
\hline 1 & $6 / 13 / 92$ & 5 & 1 & & 108.1 & $\mathbf{N}$ & \\
\hline 1 & $6 / 13 / 92$ & 6 & 1 & & 80.5 & $N$ & \\
\hline$\frac{T}{1}$ & $6 / 19 / 92$ & 9 & 9 & & 100.8 & $\mathbf{N}$ & J $890423320-426-92$ \\
\hline 1 & $6 / 19 / 92$ & 2 & 1 & & 103.1 & $\mathbf{N}$ & \\
\hline 1 & $6 / 19 / 92$ & 3 & 1 & & 104 & $\bar{Y}$ & \\
\hline 1 & $6 / 19 / 92$ & 4 & 1 & & 103.9 & $\mathbf{N}$ & \\
\hline 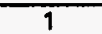 & $6 / 19 / 92$ & 5 & 1 & & 101.8 & $\mathrm{~N}$ & \\
\hline 9 & $6 / 19 / 92$ & 6 & 1 & & 76.7 & $\mathbf{N}$ & \\
\hline 9 & $6 / 29 / 92$ & 9 & 1 & & 102.1 & $\mathbf{N}$ & J TK102-890423320-42 \\
\hline 1 & $6 / 29 / 92$ & 2 & 1 & & 104.3 & $\mathbf{N}$ & \\
\hline 9 & $6 / 29 / 92$ & 3 & 1 & & 104.9 & $\mathbf{Y}$ & \\
\hline$\overline{1}$ & $6 / 29 / 92$ & $\overline{4}$ & 1 & & 103.8 & $\mathbf{N}$ & \\
\hline 1 & $6 / 29 / 92$ & 5 & 1 & & 100.9 & $\mathbf{N}$ & \\
\hline 1 & $6 / 29 / 92$ & 6 & 1 & & 77 & $\mathbf{N}$ & \\
\hline 1 & $7 / 8 / 92$ & 1 & 1 & & 101.2 & $\mathbf{N}$ & $J 890523512-47$ \\
\hline 1 & $7 / 8 / 92$ & 2 & 1 & & 103.5 & $\mathbf{N}$ & \\
\hline 1 & $7 / 8 / 92$ & 3 & $\frac{1}{1}$ & & 104.5 & $\mathbf{Y}$ & \\
\hline 1 & $7 / 8 / 92$ & 4 & 1 & & 103.3 & $\mathbf{N}$ & \\
\hline 1 & $7 / 8 / 92$ & 5 & 1 & & 100.3 & $\mathbf{N}$ & \\
\hline 1 & $7 / 8 / 92$ & 6 & 1 & & 76.9 & $\mathbf{N}$ & \\
\hline 1 & $7 / 17 / 92$ & 1 & 1 & & 101.3 & $\mathbf{N}$ & j $890523512-47$ \\
\hline 1 & $7 / 17 / 92$ & 2 & 1 & & 103.6 & $\mathbf{N}$ & \\
\hline 1 & $7 / 17 / 92$ & 3 & 1 & & 104.4 & $Y$ & \\
\hline 1 & $7 / 17 / 92$ & 4 & 1 & & 102.8 & $\mathbf{N}$ & \\
\hline 1 & $7 / 17 / 92$ & 5 & 1 & & 99.5 & $\mathbf{N}$ & \\
\hline$\frac{1}{1}$ & $7 / 17 / 92$ & 6 & 1 & & 76.6 & $\mathbf{N}$ & \\
\hline
\end{tabular}


WHC-SD-WM-ER-323, Rev. O

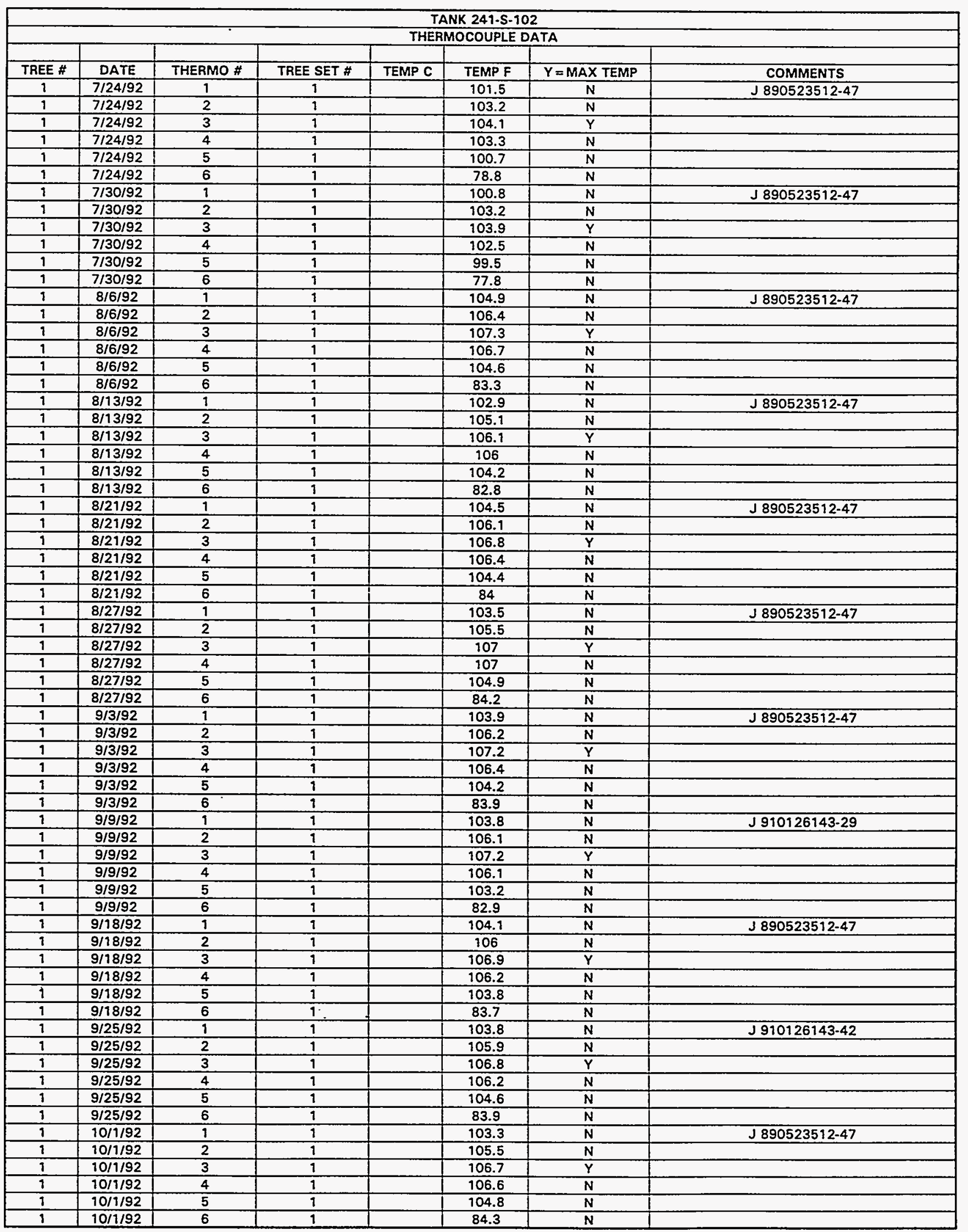

Data obtained from WHC Surveillance Analysis Computer System (SACS), February 24, 1994242-S Bottoms Tanks Transfer Record, Data Sheet TK-105-S to TK-101-S, and Archive box \#121869. 


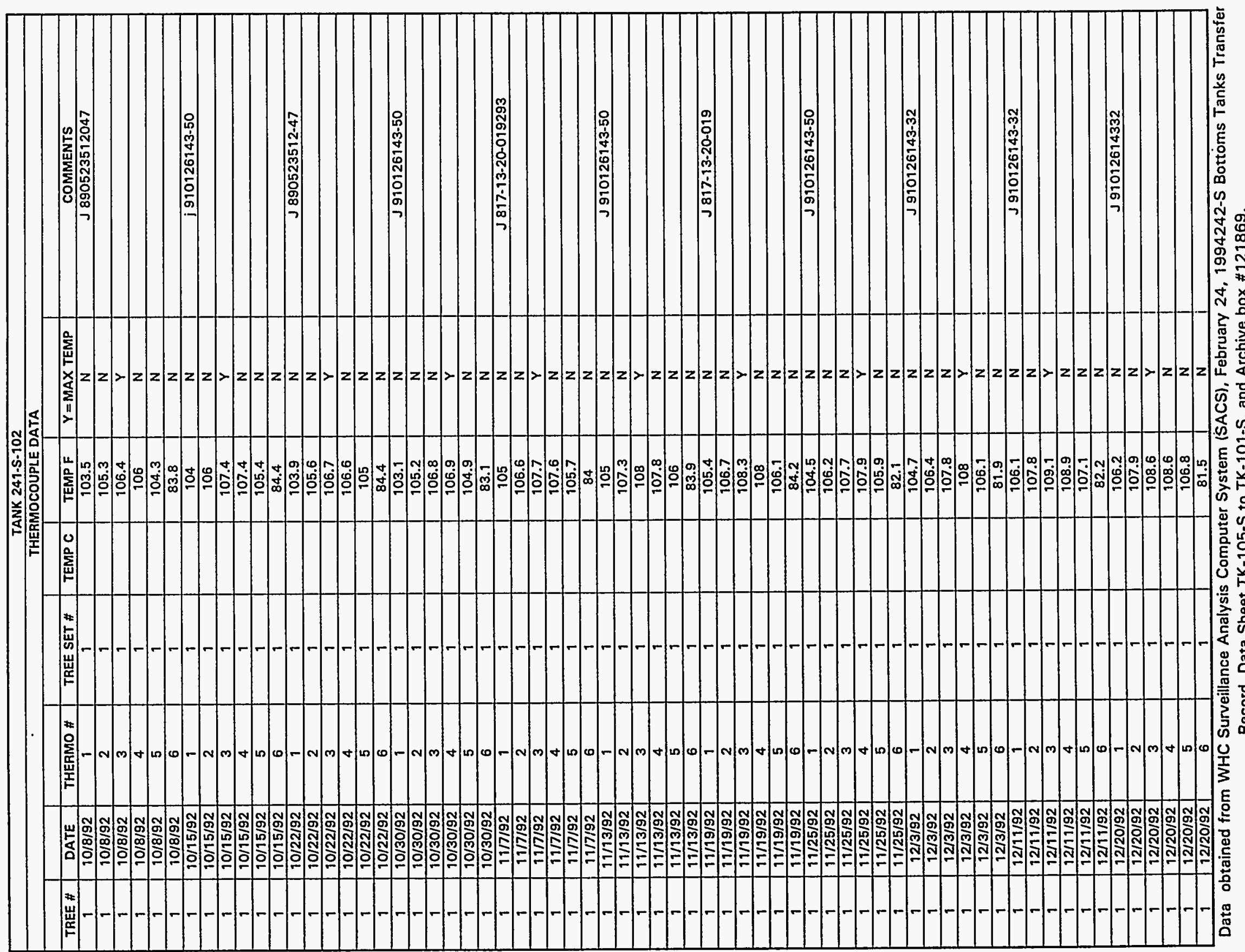




\begin{tabular}{|c|c|c|c|c|c|c|c|}
\hline \multicolumn{8}{|c|}{ TANK 241-S-102 } \\
\hline \multicolumn{8}{|c|}{ THERMOCOUPLE DATA } \\
\hline TREE \# & DATE & THERMO \# & TREE SET \# & TEMP C & TEMP F & $Y=M A X \bar{X}$ TEMP & COMMENTS \\
\hline 1 & $12 / 27 / 92$ & 1 & 1 & & 106.8 & $\mathrm{~N}$ & $J 910126143-32$ \\
\hline 1 & $12 / 27 / 92$ & 2 & 1 & & 108.5 & $\bar{N}$ & \\
\hline 1 & $12 / 27 / 92$ & 3 & 1 & & 109.5 & $\bar{Y}$ & \\
\hline 1 & $12 / 27 / 92$ & 4 & 1 & & 109.4 & $\mathbf{N}$ & \\
\hline 1 & $12 / 27 / 92$ & 5 & 1 & & 107.6 & $\mathrm{~N}$ & \\
\hline 1 & $12 / 27 / 92$ & 6 & 1 & & 81.4 & $\mathbf{N}$ & \\
\hline 1 & $12 / 30 / 92$ & 1 & 1 & & 106.5 & $\mathbf{N}$ & J 91012614332 \\
\hline 1 & $12 / 30 / 92$ & 2 & 1 & & 108 & $\mathrm{~N}$ & \\
\hline 1 & $12 / 30 / 92$ & 3 & 1 & & 109.2 & $\bar{Y}$ & \\
\hline 1 & $12 / 30 / 92$ & 4 & 1 & & 109.1 & $\mathbf{N}$ & \\
\hline 1 & $12 / 30 / 92$ & 5 & 1 & & 107.2 & $N$ & \\
\hline 9 & $12 / 30 / 92$ & 6 & 1 & & 80.7 & $\bar{N}$ & \\
\hline 1 & $1 / 7 / 93$ & 1 & 1 & & 105.6 & $\bar{N}$ & J $910126143-29$ \\
\hline 1 & $1 / 7 / 93$ & 2 & 1 & & 107.4 & $N$ & \\
\hline 1 & $1 / 7 / 93$ & 3 & 1 & & 108.3 & $Y$ & \\
\hline 1 & $1 / 7 / 93$ & 4 & $T$ & & 107.9 & $N$ & \\
\hline 1 & $1 / 7 / 93$ & 5 & 1 & & 105.7 & $\mathbf{N}$ & \\
\hline 1 & $1 / 7 / 93$ & 6 & 1 & & 78.5 & $\mathrm{~N}$ & \\
\hline 1 & $1 / 23 / 93$ & 1 & 1 & & 106.5 & $\bar{N}$ & J 91012614329 \\
\hline 1 & $1 / 23 / 93$ & 2 & 1 & & 107.8 & $\mathbf{N}$ & \\
\hline 1 & $1 / 23 / 93$ & 3 & 1 & & 108.6 & $\bar{N}$ & \\
\hline 1 & $1 / 23 / 93$ & 4 & 1 & & 108.9 & $\bar{Y}$ & \\
\hline 1 & $1 / 23 / 93$ & 5 & 1 & & 107.1 & $\mathrm{~N}$ & \\
\hline 1 & $1 / 23 / 93$ & 6 & 1 & & 78.5 & $\bar{N}$ & \\
\hline 1 & $1 / 31 / 93$ & 1 & 1 & & 104.8 & $\bar{N}$ & J910126143-29 \\
\hline 1 & $1 / 31 / 93$ & 2 & 1 & & 106.9 & $\mathbf{N}$ & \\
\hline 1 & $1 / 31 / 93$ & 3 & 1 & & 107.9 & $\mathrm{~N}$ & \\
\hline 1 & $1 / 31 / 93$ & 4 & 1 & & 108 & $\mathbf{N}$ & \\
\hline 1 & $1 / 31 / 93$ & 5 & 1 & & 105.7 & $N$ & \\
\hline 1 & $1 / 31 / 93$ & 6 & $\frac{1}{1}$ & & 76.6 & $\mathrm{~N}$ & \\
\hline 1 & $2 / 5 / 93$ & 1 & 1 & & 105.6 & $\mathbf{N}$ & J910126143-32 \\
\hline 1 & $2 / 5 / 93$ & 2 & 1 & & 107.7 & $\mathbf{N}$ & \\
\hline 1 & $2 / 5 / 93$ & 3 & 1 & & 108.7 & $\bar{Y}$ & \\
\hline 1 & $2 / 5 / 93$ & 4 & 1 & & 108.4 & $\bar{N}$ & \\
\hline 1 & $2 / 5 / 93$ & 5 & 1 & & 106.1 & $\mathbf{N}$ & \\
\hline 1 & $2 / 5 / 93$ & 6 & 1 & & 76.6 & $\mathrm{~N}$ & \\
\hline 1 & $2 / 13 / 93$ & 1 & 1 & & 104.4 & $\mathrm{~N}$ & $J 910126143.42$ \\
\hline 1 & $2 / 13 / 93$ & 2 & 1 & & 106.2 & $\bar{N}$ & \\
\hline 1 & $2 / 13 / 93$ & 3 & 1 & & 107.4 & $\bar{Y}$ & \\
\hline 1 & $2 / 13 / 93$ & 4 & 1 & & 107.4 & $N$ & \\
\hline 1 & $2 / 13 / 93$ & 5 & 1 & & 104.9 & $\mathbf{N}$ & \\
\hline 1 & $2 / 13 / 93$ & 6 & 1 & & 75.4 & $\bar{N}$ & \\
\hline 1 & $2 / 18 / 93$ & 1 & 1 & & 105.4 & $\mathbf{N}$ & J910126143-29 \\
\hline 1 & $2 / 18 / 93$ & 2 & 1 & & 107.4 & $\mathbf{N}$ & \\
\hline 1 & $2 / 18 / 93$ & 3 & 1 & & 108 & $\mathbf{N}$ & \\
\hline 1 & $2 / 18 / 93$ & 4 & 1 & & 108.3 & $\bar{Y}$ & \\
\hline 1 & $2 / 18 / 93$ & 5 & 1 & & 106 & $\mathrm{~N}$ & \\
\hline 1 & $2 / 18 / 93$ & 6 & 1 & & 76 & $\mathbf{N}$ & \\
\hline 1 & $2 / 25 / 93$ & 1 & 1 & & 105.7 & $\mathbf{N}$ & J 817-13-55-008 \\
\hline 1 & $2 / 25 / 93$ & 2 & 1 & & 108 & $\bar{N}$ & \\
\hline 1 & $2 / 25 / 93$ & 3 & 1 & & 109.2 & $\bar{Y}$ & \\
\hline 1 & $2 / 25 / 93$ & 4 & 1 & & 109.2 & $\mathbf{N}$ & \\
\hline 1 & $2 / 25 / 93$ & 5 & 1 & & 107 & $\bar{N}$ & \\
\hline 1 & $2 / 25 / 93$ & 6 & 1 & & 76.5 & $N$ & \\
\hline 1 & $3 / 4 / 93$ & 1 & 1 & & 104.5 & $\mathbf{N}$ & J 910126143-66 TC\#6 SUSPECT \\
\hline 1 & $3 / 4 / 93$ & 2 & 1 & & 106.6 & $\mathbf{N}$ & \\
\hline 1 & $3 / 4 / 93$ & 3 & 1 & & 107.8 & $Y$ & \\
\hline 1 & $3 / 4 / 93$ & 4 & 1 & & 107.8 & $\mathrm{~N}$ & \\
\hline 1 & $3 / 4 / 93$ & 5 & 1 & & 105.5 & $\mathbf{N}$ & \\
\hline 1 & $3 / 11 / 93$ & 1 & 1 & & 105.1 & $\mathrm{~N}$ & J $910126143-66$ \\
\hline 1 & $3 / 11 / 93$ & 2 & 1 & & 107.2 & $\mathbf{N}$ & \\
\hline 1 & $3 / 11 / 93$ & 3 & 1 & & 108 & $\bar{Y}$ & \\
\hline 1 & $3 / 11 / 93$ & 4 & 1 & & 107.9 & $\mathbf{N}$ & \\
\hline 1 & $3 / 11 / 93$ & 5 & 1 & & 105.4 & $\mathbf{N}$ & \\
\hline 1 & $3 / 11 / 93$ & 6 & 1 & & 75.1 & $\mathbf{N}$ & \\
\hline 1 & $3 / 19 / 93$ & 1 & 1 & & 103.9 & $\bar{N}$ & J 910126143-66 \\
\hline
\end{tabular}




\begin{tabular}{|c|c|c|c|c|c|c|c|}
\hline \multicolumn{8}{|c|}{ TANK 241-S-102 } \\
\hline \multicolumn{8}{|c|}{ THERMOCOUPLE DATA } \\
\hline TREE \# & DATE & THERMO \# & TREE SET \# & TEMP C & TEMP F & $Y=$ MAX TEMP & COMMENTS \\
\hline 1 & $3 / 19 / 93$ & 2 & 1 & & 106 & $\mathbf{N}$ & \\
\hline 1 & $3 / 19 / 93$ & 3 & 1 & & 107.1 & $\bar{Y}$ & \\
\hline 1 & $3 / 19 / 93$ & 4 & 1 & & 107.1 & $\mathbf{N}$ & \\
\hline 1 & $3 / 19 / 93$ & 5 & 1 & & 104.8 & $\mathbf{N}$ & \\
\hline 1 & $3 / 19 / 93$ & 6 & 1 & & 74 & $\mathbf{N}$ & \\
\hline 1 & $3 / 27 / 93$ & 1 & 1 & & 102.3 & $N$ & J91612614360 \\
\hline$\overline{1}$ & $3 / 27 / 93$ & 2 & 1 & & 103.6 & $\mathbf{N}$ & \\
\hline 1 & $3 / 27 / 93$ & 3 & 1 & & 105.1 & $\mathbf{N}$ & \\
\hline 1 & $3 / 27 / 93$ & 4 & 1 & & 105.5 & $\bar{Y}$ & \\
\hline 1 & $3 / 27 / 93$ & 5 & 1 & & 103.3 & $\mathbf{N}$ & \\
\hline 1 & $3 / 27 / 93$ & 6 & 1 & & 72.4 & $\mathrm{~N}$ & \\
\hline 1 & $4 / 1 / 93$ & 1 & 1 & & 101.4 & $\mathbf{N}$ & J 8904-23320-42 \\
\hline 9 & $4 / 1 / 93$ & 2 & 9 & & 104.2 & $\bar{N}$ & \\
\hline 1 & $4 / 1 / 93$ & 3 & 1 & & 105 & $\mathbf{N}$ & \\
\hline$\frac{1}{1}$ & $4 / 1 / 93$ & 4 & 1 & & 104.8 & $\mathbf{N}$ & \\
\hline$\frac{1}{1}$ & $4 / 1 / 93$ & 5 & 1 & & 102.3 & $\mathbf{N}$ & \\
\hline 1 & $4 / 1 / 93$ & 6 & 1 & & 71.8 & $\mathbf{N}$ & \\
\hline$\frac{1}{1}$ & $4 / 8 / 93$ & 1 & 1 & & 103.6 & $\mathbf{N}$ & J 0126143-14 \\
\hline 1 & $4 / 8 / 93$ & 2 & 1 & & 105.5 & $\mathbf{N}$ & \\
\hline T & $4 / 8 / 93$ & 3 & 1 & & 106.1 & $\bar{Y}$ & \\
\hline 1 & $4 / 8 / 93$ & 4 & 1 & & 105.7 & $\mathbf{N}$ & \\
\hline 1 & $4 / 8 / 93$ & 5 & 1 & & 103.4 & $\mathrm{~N}$ & \\
\hline$\frac{1}{1}$ & $4 / 8 / 93$ & 6 & 1 & & 73 & $\mathbf{N}$ & \\
\hline 1 & $4 / 15 / 93$ & 1 & 1 & & 106 & $\mathbf{N}$ & J910126143-66 \\
\hline 1 & $4 / 15 / 93$ & 2 & $\frac{1}{1}$ & & 108 & $\bar{N}$ & \\
\hline 1 & $4 / 15 / 93$ & 3 & 1 & & 109 & $Y$ & \\
\hline 1 & $4 / 15 / 93$ & 4 & 1 & & 108 & $\mathbf{N}$ & \\
\hline 1 & $4 / 15 / 93$ & 5 & 1 & & 106 & $\mathbf{N}$ & \\
\hline 1 & $4 / 15 / 93$ & 6 & 9 & & 75 & $\mathbf{N}$ & \\
\hline 1 & $4 / 21 / 93$ & 1 & 1 & & 103.3 & $\mathbf{N}$ & J 910126143-66 \\
\hline 1 & $4 / 21 / 93$ & 2 & 1 & & 105.3 & $\mathbf{N}$ & \\
\hline 1 & $4 / 21 / 93$ & 3 & 1 & & 106.7 & $\mathbf{N}$ & \\
\hline 1 & $4 / 21 / 93$ & 4 & 1 & & 106.9 & $Y$ & \\
\hline 1 & $4 / 21 / 93$ & 5 & 1 & & 104.5 & $\mathbf{N}$ & \\
\hline$\frac{1}{1}$ & $4 / 21 / 93$ & 6 & 1 & & 74.2 & $\bar{N}$ & \\
\hline 1 & $4 / 23 / 93$ & 1 & 1 & & 105.4 & $\mathbf{N}$ & $\mathrm{J} 910126143-66$ \\
\hline 1 & $4 / 23 / 93$ & 2 & 1 & & 107.1 & $\mathbf{N}$ & \\
\hline 9 & $4 / 23 / 93$ & 3 & 1 & & 107.7 & $\bar{Y}$ & \\
\hline 1 & $4 / 23 / 93$ & 4 & 1 & & 107.3 & $\mathbf{N}$ & \\
\hline 1 & $4 / 23 / 93$ & 5 & 1 & & 104.9 & $\mathbf{N}$ & \\
\hline$\frac{1}{1}$ & $4 / 23 / 93$ & 6 & $\frac{1}{1}$ & & 75 & $\mathbf{N}$ & \\
\hline 1 & $4 / 29 / 93$ & 1 & 1 & & 103 & $\mathbf{N}$ & $J 910126143-66$ \\
\hline 1 & $4 / 29 / 93$ & 2 & 1 & & 106.6 & $\mathbf{N}$ & \\
\hline 1 & $4 / 29 / 93$ & 3 & 1 & & 107.4 & $\bar{Y}$ & \\
\hline 1 & $4 / 29 / 93$ & 4 & 1 & & 106.8 & $\mathbf{N}$ & \\
\hline 1 & $4 / 29 / 93$ & 5 & 1 & & 104.4 & $\mathbf{N}$ & \\
\hline 1 & $4 / 29 / 93$ & 6 & 1 & & 74.7 & $\mathbf{N}$ & \\
\hline 1 & $5 / 6 / 93$ & 1 & 1 & & 104.4 & $\mathbf{N}$ & $J 91012614366$ \\
\hline$\frac{1}{1}$ & $5 / 6 / 93$ & 2 & 1 & & 106.3 & $\mathbf{N}$ & \\
\hline 9 & $5 / 6 / 93$ & 3 & 1 & & 107.1 & $\mathbf{Y}$ & \\
\hline 1 & $5 / 6 / 93$ & 4 & 1 & & 106.5 & $\mathbf{N}$ & \\
\hline 1 & $5 / 6 / 93$ & 5 & 1 & & 103.5 & $\mathbf{N}$ & \\
\hline 1 & $5 / 6 / 93$ & 6 & 7 & & 74.1 & $\mathbf{N}$ & \\
\hline 1 & $5 / 13 / 93$ & 1 & $\frac{T}{1}$ & & 102.7 & $\mathbf{N}$ & J910126143 \\
\hline$\frac{1}{1}$ & $5 / 13 / 93$ & 2 & 1 & & 105 & $\mathbf{N}$ & \\
\hline 1 & $5 / 13 / 93$ & 3 & 1 & & 107.3 & $\underline{Y}$ & \\
\hline 1 & $5 / 13 / 93$ & 4 & 1 & & 106.3 & $\mathbf{N}$ & \\
\hline 1 & $5 / 13 / 93$ & 5 & 1 & & 103.7 & $\mathbf{N}$ & \\
\hline 1 & $5 / 13 / 93$ & 6 & 9 & & 74.2 & $\mathbf{N}$ & \\
\hline 1 & $5 / 20 / 93$ & 9 & 1 & & 104.5 & $N$ & J910126143-66 \\
\hline$\frac{1}{1}$ & $5 / 20 / 93$ & 2 & $\frac{1}{1}$ & & 106.4 & $N$ & \\
\hline 1 & $5 / 20 / 93$ & 3 & 1 & & 107.3 & $Y$ & \\
\hline 1 & $5 / 20 / 93$ & 4 & 1 & & 106.8 & $N$ & \\
\hline 1 & $5 / 20 / 93$ & 5 & 1 & & 104.3 & $\mathbf{N}$ & \\
\hline 1 & $5 / 20 / 93$ & 6 & 1 & & 75.6 & $\mathbf{N}$ & \\
\hline 1 & $5 / 27 / 93$ & 1 & 1 & & 101.6 & $N$ & $J 890423320-42$ \\
\hline
\end{tabular}


WHC-SD-WM-ER-323, Rev. 0

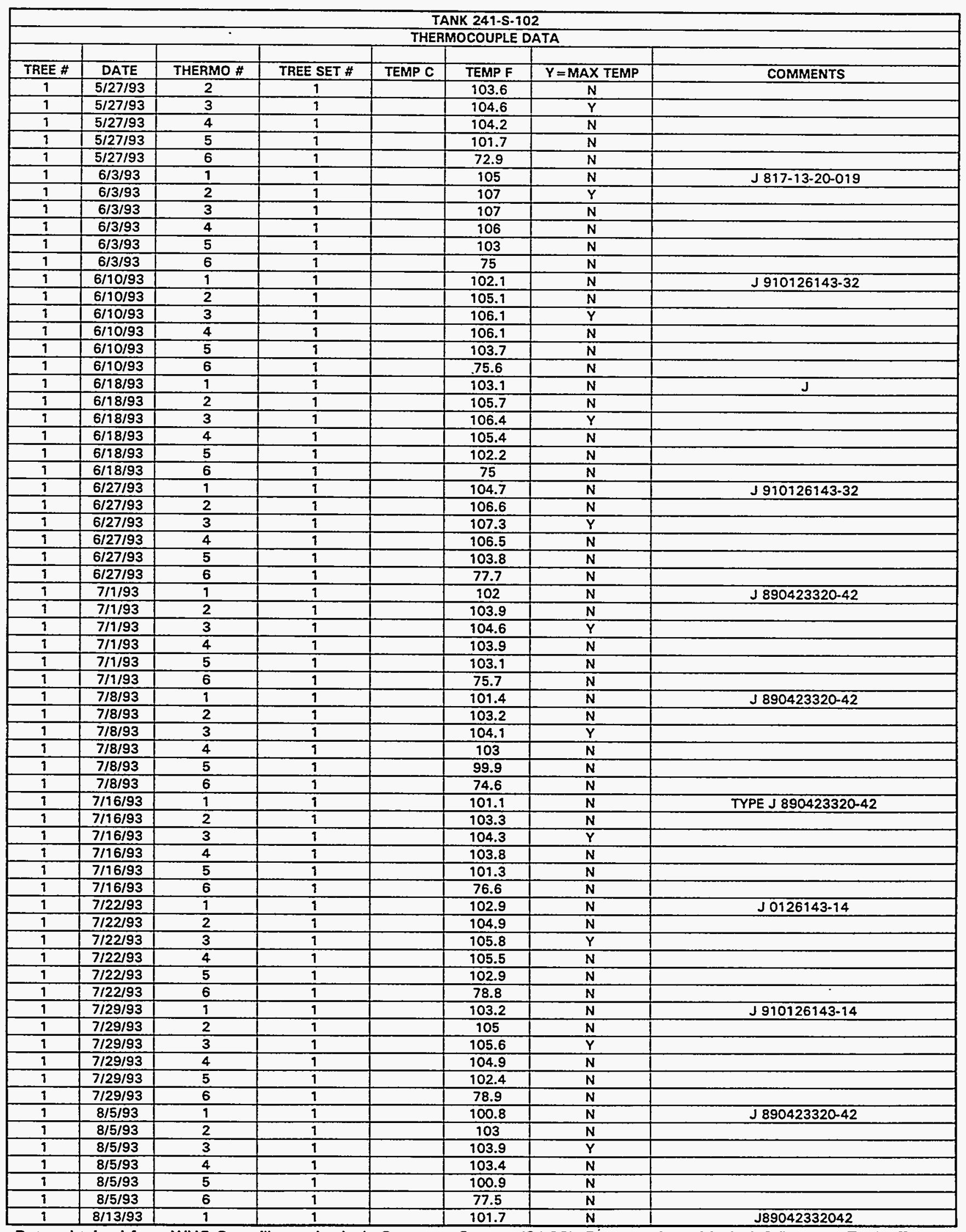

Data obtained from WHC Surveillance Analysis Computer System (SACS), February 24, 1994242-S Bottoms Tanks Transfer Record, Data Sheet TK-1.05-S to TK-101-S, and Archive box \#121869. 
WHC-SD-WM-ER-323, Rev. 0

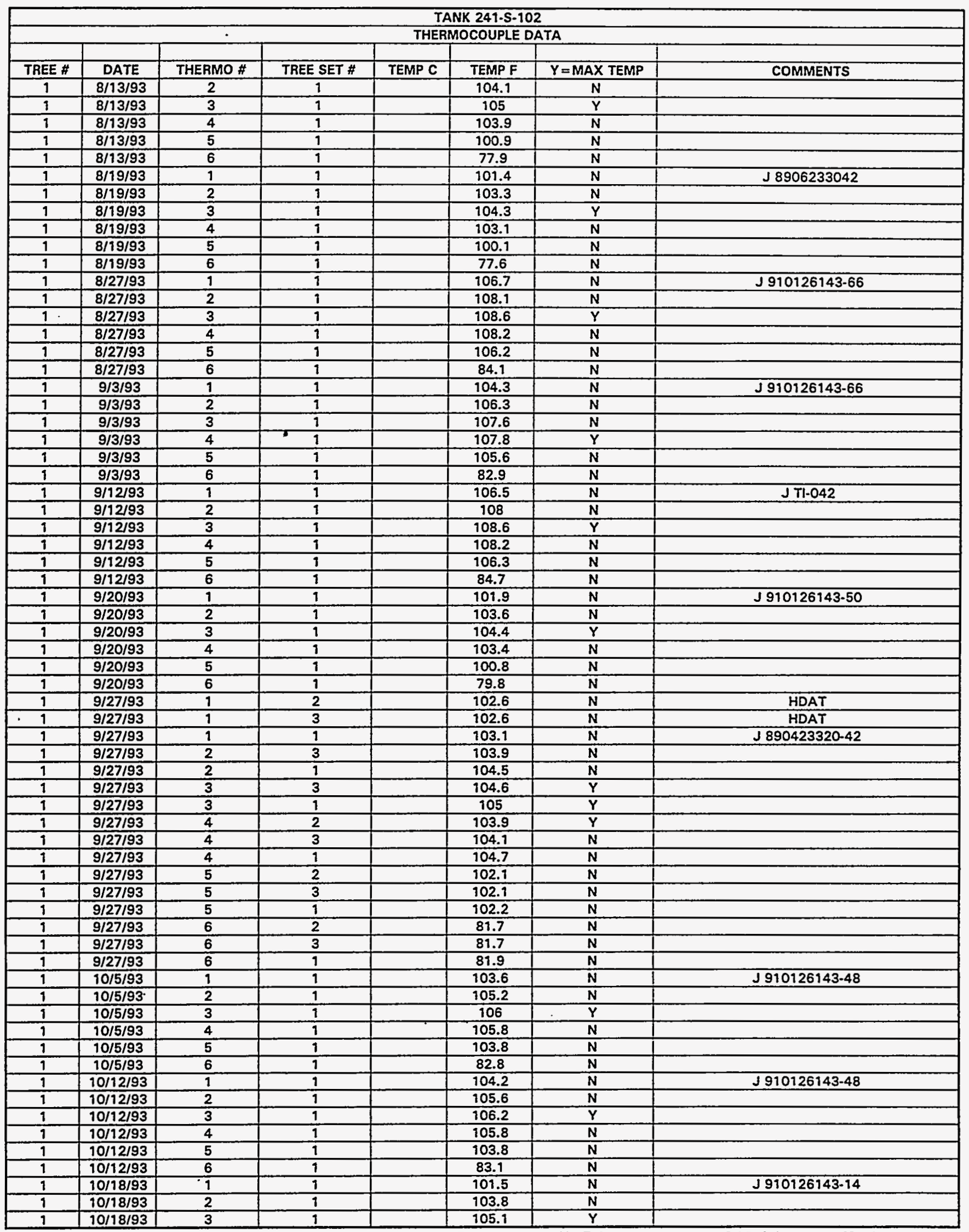

Data obtained from WHC Surveillance Analysis Computer System (SACS), February 24, 1994242-S Bottoms Tanks Transfer Record, Data Sheet TK-105-S to TK-101-S, and Archive box \#121869. 


\begin{tabular}{|c|c|c|c|c|c|c|c|}
\hline \multicolumn{8}{|c|}{ TANK 241.S-102 } \\
\hline \multicolumn{8}{|c|}{ THERMOCOUPLE DATA } \\
\hline TREE \# & DATE & THERMO \# & TREE SET \# & TEMP C & TEMP F & $Y=$ MAX TEMP & COMMENTS \\
\hline 1 & $10 / 18 / 93$ & 4 & 1 & & 104.1 & $\mathbf{N}$ & \\
\hline 1 & $10 / 18 / 93$ & 5 & 1 & & 101.4 & $\mathrm{~N}$ & \\
\hline 1 & $10 / 18 / 93$ & 6 & 1 & & 79.8 & $\mathbf{N}$ & \\
\hline 1 & $10 / 27 / 93$ & 1 & 1 & & 104.4 & $\mathbf{N}$ & J 91012614348 \\
\hline 1 & $10 / 27 / 93$ & 2 & 1 & & 105.8 & $\mathrm{~N}$ & \\
\hline 1 & $10 / 27 / 93$ & 3 & 1 & & 106.4 & $\bar{Y}$ & \\
\hline 1 & $10 / 27 / 93$ & 4 & 1 & & 106.1 & $\mathbf{N}$ & \\
\hline 1 & $10 / 27 / 93$ & 5 & 1 & & 104.3 & $\mathbf{N}$ & \\
\hline 1 & $10 / 27 / 93$ & 6 & 1 & & 83.2 & $\mathbf{N}$ & \\
\hline 1 & $11 / 1 / 93$ & 1 & 1 & & 103.5 & $\mathbf{N}$ & $J 910126143-29$ \\
\hline 1 & $11 / 1 / 93$ & 2 & 1 & & 105.2 & $\bar{N}$ & \\
\hline 1 & $11 / 1 / 93$ & 3 & 1 & & 106 & $Y$ & \\
\hline 1 & $11 / 1 / 93$ & 4 & 1 & & 105.7 & $\mathbf{N}$ & \\
\hline 1 & $11 / 1 / 93$ & 5 & 1 & & 103.8 & $\bar{N}$ & \\
\hline 9 & $11 / 1 / 93$ & 6 & 9 & & 82.3 & $\mathbf{N}$ & \\
\hline 1 & $1118 / 93$ & 1 & 1 & & 105.5 & $\mathbf{N}$ & J $910126143-29$ \\
\hline 1 & $11 / 8 / 93$ & 2 & 1 & & 106.9 & $\bar{N}$ & \\
\hline 9 & $11 / 8 / 93$ & 3 & 1 & & 107.6 & $\bar{Y}$ & \\
\hline 1 & $11 / 8 / 93$ & 4 & 1 & & 107.3 & $\mathbf{N}$ & \\
\hline 1 & $11 / 8 / 93$ & 5 & 1 & & 105.2 & $\mathbf{N}$ & \\
\hline 1 & $11 / 8 / 93$ & 6 & 1 & & 83.5 & $\mathrm{~N}$ & \\
\hline$\frac{1}{1}$ & $11 / 17 / 93$ & 1 & 1 & & 104.6 & $\mathbf{N}$ & $J T 1-041$ \\
\hline 9 & $11 / 17 / 93$ & 2 & 1 & & 106.2 & $\mathbf{N}$ & \\
\hline 9 & $11 / 17 / 93$ & 3 & 1 & & 107.1 & $\bar{Y}$ & \\
\hline 1 & $11 / 17 / 93$ & 4 & 1 & & 106.8 & $N$ & \\
\hline 1 & $11 / 17 / 93$ & 5 & 1 & & 105 & $\mathbf{N}$ & \\
\hline 9 & $11 / 17 / 93$ & 6 & 9 & & 82.2 & $\mathbf{N}$ & \\
\hline 1 & $11 / 23 / 93$ & 1 & 1 & & 104.6 & $N$ & J910126143-29 \\
\hline 9 & $11 / 23 / 93$ & 2 & 1 & & 106.2 & $\mathbf{N}$ & \\
\hline 1 & $11 / 23 / 93$ & 3 & 1 & & 107 & $\bar{Y}$ & \\
\hline 9 & $11 / 23 / 93$ & 4 & 1 & & 106.6 & $\mathbf{N}$ & \\
\hline 1 & $11 / 23 / 93$ & 5 & 1 & & 104.8 & $\mathbf{N}$ & \\
\hline 9 & $11 / 23 / 93$ & 6 & 1 & & 82 & $\mathbf{N}$ & \\
\hline 1 & $11 / 29 / 93$ & 1 & 2 & & 104.8 & $\mathrm{~N}$ & $J-910126143-29$ \\
\hline 1 & $11 / 29 / 93$ & 1 & 1 & & 105.2 & $\mathbf{N}$ & $J 910126143-29$ \\
\hline 1 & $11 / 29 / 93$ & 2 & 2 & & 106.3 & $\mathbf{N}$ & \\
\hline 1 & $11 / 29 / 93$ & 2 & 1 & & 106.8 & $\mathbf{N}$ & \\
\hline 1 & $11 / 29 / 93$ & 3 & 2 & & 107.2 & $Y$ & \\
\hline 1 & $11 / 29 / 93$ & 3 & 1 & & 107.6 & $\mathbf{Y}$ & \\
\hline 7 & $11 / 29 / 93$ & 4 & 2 & & 106.8 & $\mathbf{N}$ & \\
\hline 1 & $11 / 29 / 93$ & 4 & 1 & & 107.4 & $\mathbf{N}$ & \\
\hline 9 & $11 / 29 / 93$ & 5 & 2 & & 105.2 & $\mathrm{~N}$ & \\
\hline 9 & $11 / 29 / 93$ & 5 & 1 & & 105.4 & $\bar{N}$ & \\
\hline 1 & $11 / 29 / 93$ & 6 & 2 & & 89.7 & $\mathbf{N}$ & \\
\hline 1 & $11 / 29 / 93$ & 6 & 1 & & 81.9 & $\mathbf{N}$ & \\
\hline 1 & $12 / 7 / 93$ & 1 & 1 & & 105.1 & $\mathrm{~N}$ & $\mathrm{~J} 910126143-29$ \\
\hline$\frac{1}{1}$ & $12 / 7 / 93$ & 2 & 1 & & 106.4 & $N$ & \\
\hline 1 & $12 / 7 / 93$ & 3 & 1 & & 107.1 & $\mathbf{Y}$ & \\
\hline 9 & $12 / 7 / 93$ & 4 & 1 & & 106.8 & $\mathbf{N}$ & \\
\hline 9 & $12 / 7 / 93$ & 5 & 1 & & 105 & $\mathbf{N}$ & \\
\hline 9 & $12 / 7 / 93$ & 6 & 1 & & 80.8 & $\mathbf{N}$ & \\
\hline 1 & $12 / 14 / 93$ & 9 & 1 & & 104.5 & $\mathbf{N}$ & $\mathrm{J} 910126143-29$ \\
\hline 9 & $12 / 14 / 93$ & 2 & 1 & & 105.9 & $\mathbf{N}$ & \\
\hline$\frac{1}{1}$ & $12 / 14 / 93$ & 3 & 1 & & 106.7 & $\bar{Y}$ & \\
\hline 1 & $12 / 14 / 93$ & 4 & 1 & & 106.4 & $\mathbf{N}$ & \\
\hline 1 & $12 / 14 / 93$ & 5 & 1 & & 104.6 & $\mathbf{N}$ & \\
\hline 1 & $12 / 14 / 93$ & 6 & 9 & & 79.8 & $\mathbf{N}$ & \\
\hline$\frac{1}{1}$ & $12 / 20 / 93$ & 1 & 1 & & 104.7 & $\mathbf{N}$ & $J 910126143-29$ \\
\hline 9 & $12 / 20 / 93$ & $\overline{1}$ & 2 & & 104.7 & $\mathbf{N}$ & $\mathrm{J} 910126143-29$ \\
\hline 1 & $12 / 20 / 93$ & 2 & 1 & & 106.2 & $\mathbf{N}$ & \\
\hline 1 & $12 / 20 / 93$ & 2 & 2 & & 106.3 & $\mathbf{N}$ & \\
\hline 1 & $12 / 20 / 93$ & 3 & 1 & & 107.1 & $\bar{Y}$ & \\
\hline 1 & $12 / 20 / 93$ & 3 & 2 & & 107.2 & $Y$ & \\
\hline 1 & $12 / 20 / 93$ & 4 & 1 & & 106.8 & $\bar{N}$ & \\
\hline 1 & $12 / 20 / 93$ & 4 & 2 & & 106.8 & $\mathbf{N}$ & \\
\hline 1 & $12 / 20 / 93$ & 5 & 1 & & 104.8 & $\mathrm{~N}$ & \\
\hline
\end{tabular}

Data obtained from WHC Surveillance Analysis Computer System (SACS), February 24, 1994242-S Bottoms Tanks Transfer Record, Data Sheet TK-105-S to TK-101-S, and Archive box \#121869. 
WHC-SD-WM-ER-323, Rev. 0

\begin{tabular}{|c|c|c|c|c|c|c|c|}
\hline \multicolumn{8}{|c|}{ TANK 241-S.102 } \\
\hline \multicolumn{8}{|c|}{ THERMOCOUPLE DATA } \\
\hline TREE \# & DATE & THERMO \# & TREE SET \# & TEMP C & TEMP F & $Y=M A X$ TEMP & COMMENTS \\
\hline 1 & $12 / 20 / 93$ & 5 & 2 & & 105.1 & $\mathbf{N}$ & \\
\hline 1 & $12 / 20 / 93$ & 6 & 1 & & 79.5 & $\mathbf{N}$ & \\
\hline 1 & $12 / 20 / 93$ & 6 & 2 & & 79.9 & $\mathbf{N}$ & \\
\hline 1 & $12 / 27 / 93$ & 1 & 1 & & 104.9 & $N$ & J910126143-29 \\
\hline 1 & $12 / 27 / 93$ & 2 & 1 & & 106.4 & $\mathbf{N}$ & \\
\hline 1 & $12 / 27 / 93$ & 3 & 1 & & 107.5 & $Y$ & \\
\hline 1 & $12 / 27 / 93$ & 4 & 1 & & 107.3 & $N$ & \\
\hline 1 & $12 / 27 / 93$ & 5 & 1 & & 105.3 & $N$ & \\
\hline 1 & $12 / 27 / 93$ & 6 & 1 & & 79.5 & $\mathbf{N}$ & \\
\hline & & & & & & & \\
\hline & & & & & & & \\
\hline & & & & & & & \\
\hline & & & & & & & \\
\hline
\end{tabular}

$\therefore$ Data obtained from WHC Surveillance Analysis Computer System (SACS), February 24, 1994242-S Bottoms Tanks Transfer Record, Data Sheet TK-105-S to TK-101-S, and Archive box \#121869. 


\begin{tabular}{|c|c|c|c|c|c|c|c|}
\hline \multicolumn{8}{|c|}{ TANK 241-S-103 } \\
\hline \multicolumn{8}{|c|}{ THERMOCOUPLE DATA } \\
\hline TREE \# & DATE & THERMO \# & TREE SET \# & TEMP C & TEMP F & $Y=M A X T E M P$ & COMMENTS \\
\hline 1 & $12 / 2 / 74$ & 1 & & 41.11 & 105.998 & & raw data-assumed degree C \\
\hline 1 & $12 / 2 / 74$ & 2 & & 37.78 & 100.004 & & raw data - assumed degree C \\
\hline$\frac{1}{1}$ & $12 / 2 / 74$ & 3 & & 36.67 & 98.006 & & raw data - assumed degree $C$ \\
\hline 1 & $12 / 2 / 74$ & 4 & & 36.67 & 98.006 & & raw data - assumed degree $C$ \\
\hline 1 & $12 / 2 / 74$ & 5 & & 36.67 & 98.006 & & raw data - assumed degree $\mathrm{C}$ \\
\hline 1 & $12 / 2 / 74$ & 6 & & 36.11 & 96.998 & & raw data - assumed degree C \\
\hline 1 & $12 / 2 / 74$ & 7 & & 36.11 & 96.998 & & raw data - assumed degree $C$ \\
\hline 9 & $12 / 2 / 74$ & 8 & & 35.56 & 96.008 & & raw data - assumed degree C \\
\hline 9 & $12 / 2 / 74$ & 9 & & 33.89 & 93.002 & & raw data - assumed degree $C$ \\
\hline 1 & $12 / 2 / 74$ & 10 & & 33.89 & 93.002 & & raw data - assumed degree $C$ \\
\hline 1 & $12 / 2 / 74$ & 11 & & 32.78 & 91.004 & & raw data - assumed degree $\mathrm{C}$ \\
\hline 1 & $12 / 2 / 74$ & 12 & & 32.78 & 99.004 & & raw data - assumed degree C \\
\hline 9 & $11 / 3 / 75$ & 1 & & & 114 & & \\
\hline 1 & $11 / 3 / 75$ & 2 & & & 119 & & \\
\hline$T$ & $11 / 3 / 75$ & 3 & & & 120 & & \\
\hline 1 & $11 / 3 / 75$ & 4 & & & 120 & & \\
\hline 1 & $11 / 3 / 75$ & 5 & & & 120 & & \\
\hline 1 & $11 / 3 / 75$ & 6 & & & 120 & & \\
\hline 1 & $11 / 3 / 75$ & 7 & & & 120 & & \\
\hline 1 & $11 / 3 / 75$ & 8 & & & 119 & & \\
\hline 7 & $11 / 3 / 75$ & 9 & & & 114 & & \\
\hline 1 & $11 / 3 / 75$ & 10 & & & 113 & & \\
\hline 1 & $11 / 3 / 75$ & 11 & & & 113 & & \\
\hline 1 & $11 / 3 / 75$ & 12 & & & 113 & & \\
\hline 1 & $2 / 4 / 76$ & 1 & & & 105 & & \\
\hline 1 & $2 / 4 / 76$ & 2 & & & 98 & & \\
\hline 1 & $2 / 4 / 76$ & 3 & & & 96 & & \\
\hline 1 & $2 / 4 / 76$ & 4 & & & 94 & & \\
\hline 1 & $2 / 4 / 76$ & 5 & & & 88 & & \\
\hline 1 & $2 / 4 / 76$ & 6 & & & 84 & & \\
\hline 9 & $2 / 4 / 76$ & 7 & & & 84 & & \\
\hline 1 & $2 / 4 / 76$ & 8 & & & 84 & & \\
\hline 1 & $2 / 4 / 76$ & 9 & & & 84 & & \\
\hline 1 & $2 / 4 / 76$ & 10 & & & 84 & & \\
\hline 1 & $2 / 4 / 76$ & 11 & & & 84 & & \\
\hline 1 & $2 / 4 / 76$ & 12 & & & 84 & & \\
\hline 1 & $3 / 5 / 76$ & 1. & & & 97 & & \\
\hline 1 & $3 / 5 / 76$ & 2 & & & 89 & & \\
\hline 1 & $3 / 5 / 76$ & 3 & & & 84 & & \\
\hline 9 & $3 / 5 / 76$ & 4 & & & 83 & & \\
\hline 1 & $3 / 5 / 76$ & 5 & & & 82 & & \\
\hline 1 & $3 / 5 / 76$ & 6 & & & 82 & & \\
\hline 1 & $3 / 5 / 76$ & 7 & & & 83 & & \\
\hline 9 & $3 / 5 / 76$ & 8 & & & 83 & & \\
\hline 9 & $3 / 5 / 76$ & 9 & & & 82 & & \\
\hline 9 & $3 / 5 / 76$ & 10 & & & 82 & & \\
\hline 1 & $3 / 5 / 76$ & 11 & & & 82 & & \\
\hline 1 & $5 / 2 / 76$ & 1 & & & 97 & & \\
\hline 9 & $5 / 2 / 76$ & 2 & & & 103 & & \\
\hline 1 & $5 / 2 / 76$ & 3 & & & 97 & & \\
\hline 1 & $5 / 2 / 76$ & 4 & & & 96 & & \\
\hline$\frac{1}{1}$ & $5 / 2 / 76$ & 5 & & & 103 & & \\
\hline 9 & $5 / 2 / 76$ & 6 & & & 94 & & \\
\hline 1 & $5 / 2 / 76$ & 7 & & & 90 & & \\
\hline 1 & $5 / 2 / 76$ & 8 & & & 90 & & \\
\hline 1 & $5 / 2 / 76$ & 9 & & & 90 & & \\
\hline 1 & $5 / 2 / 76$ & 10 & & & 90 & & \\
\hline 1 & $5 / 2 / 76$ & 11 & & & 90 & & \\
\hline 1 & $5 / 2 / 76$ & 12 & & & 90 & & \\
\hline 9 & $6 / 9 / 76$ & 1 & & & 98 & & \\
\hline$\frac{1}{1}$ & $6 / 1 / 76$ & 2 & & & 98 & & \\
\hline 1 & $6 / 1 / 76$ & 3 & & & 97 & & \\
\hline 7 & $6 / 1 / 76$ & 4 & & & 94 & & \\
\hline 1 & $6 / 1 / 76$ & 5 & & & 88 & & \\
\hline 7 & $6 / 1 / 76$ & 6 & & & 84 & & \\
\hline 1 & $6 / 1 / 76$ & 7 & & & 84 & & \\
\hline
\end{tabular}

Data obtained from WHC Surveillance Analysis Computer System (SACS), February 24, 1994. 


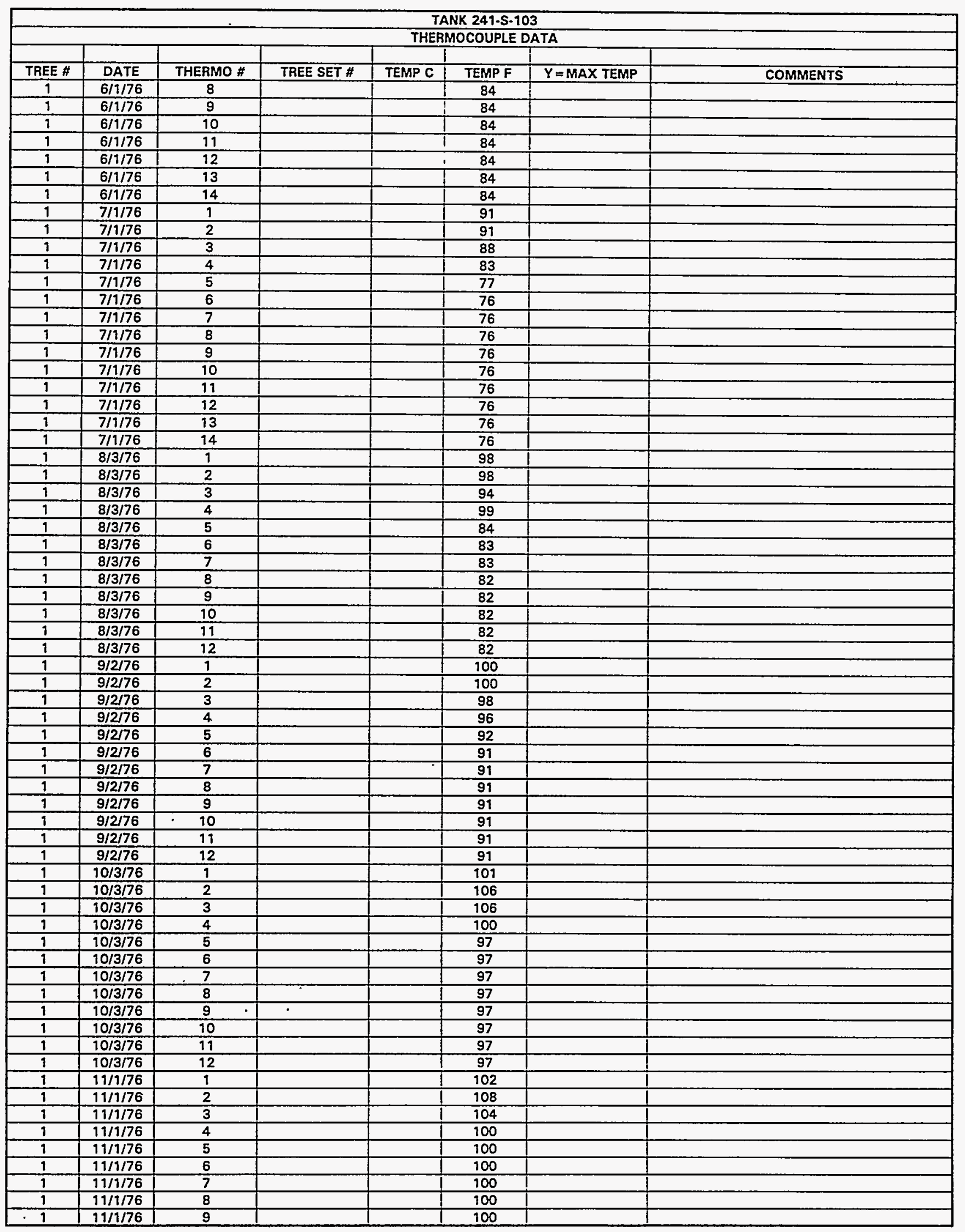

Data obtained from WHC Surveillance Analysis Computer System (SACS), February 24, 1994. 


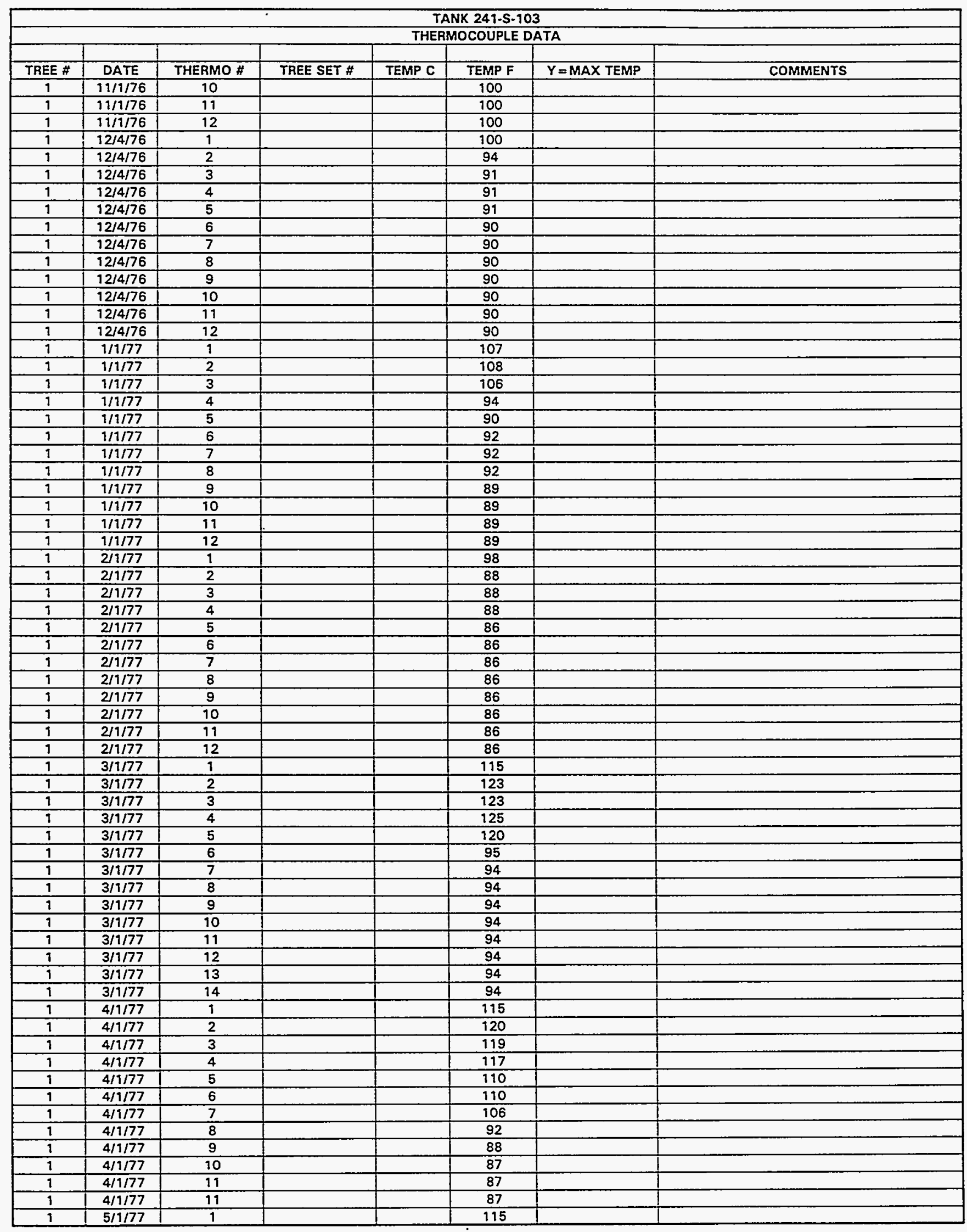

Data obtained from WHC Surveillance Analysis Computer System (SACS), February 24, 1994. 


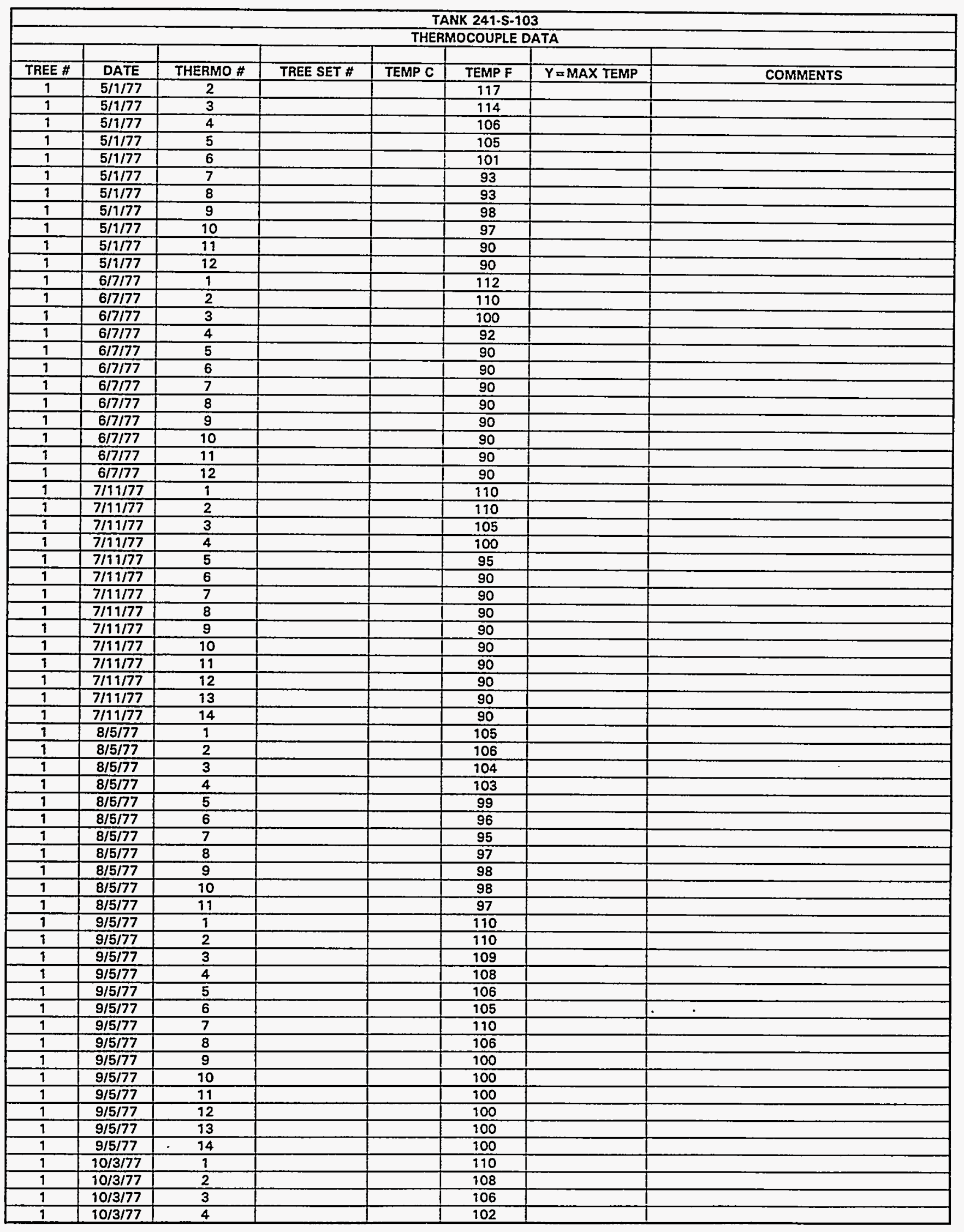

Data obtained from WHC Surveillance Analysis Computer System (SACS), February 24, 1994. 
WHC-SD-WM-ER-323, Rev. 0

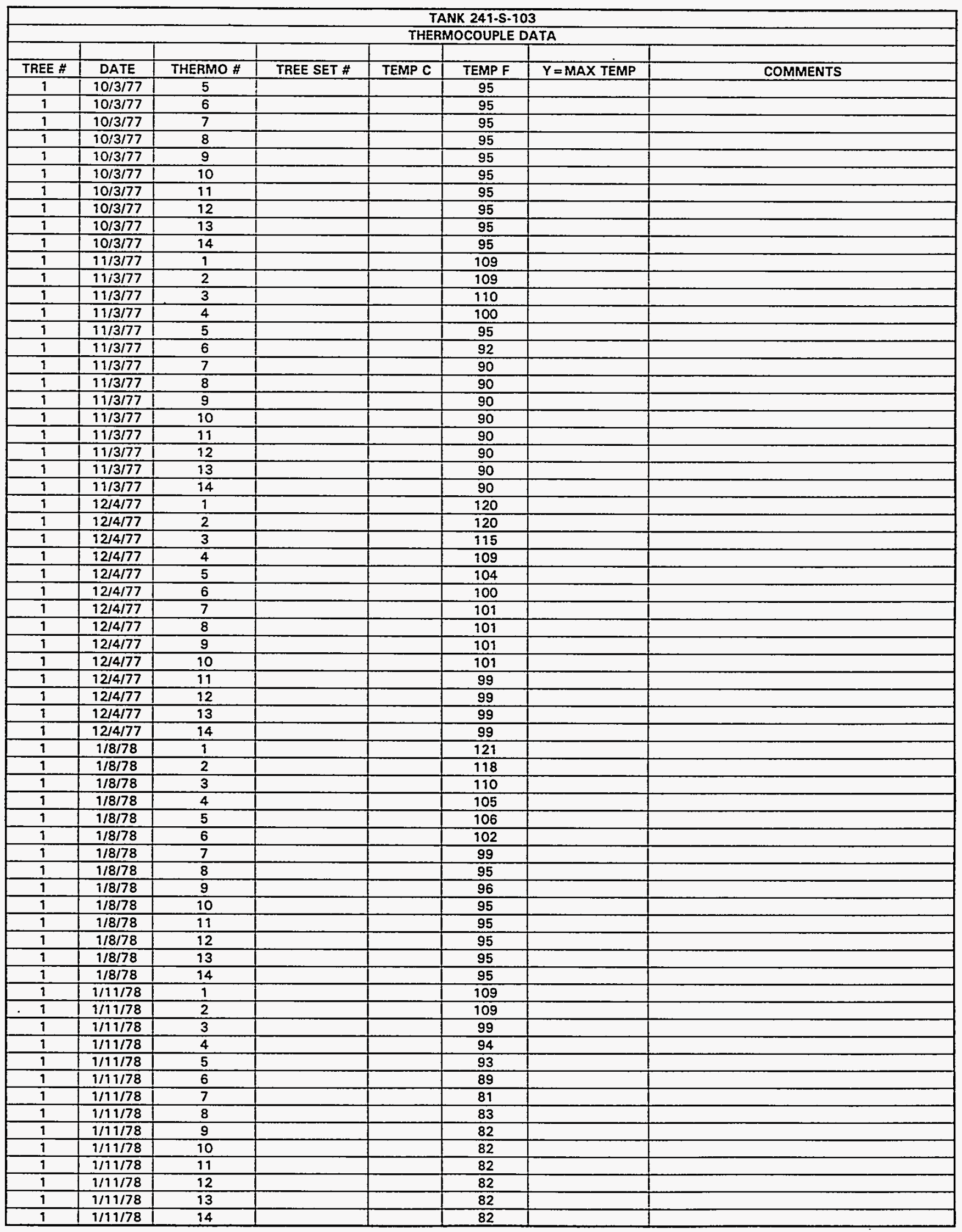

Data obtained from WHC Surveillance Analysis Computer System (SACS), February 24, 1994. 


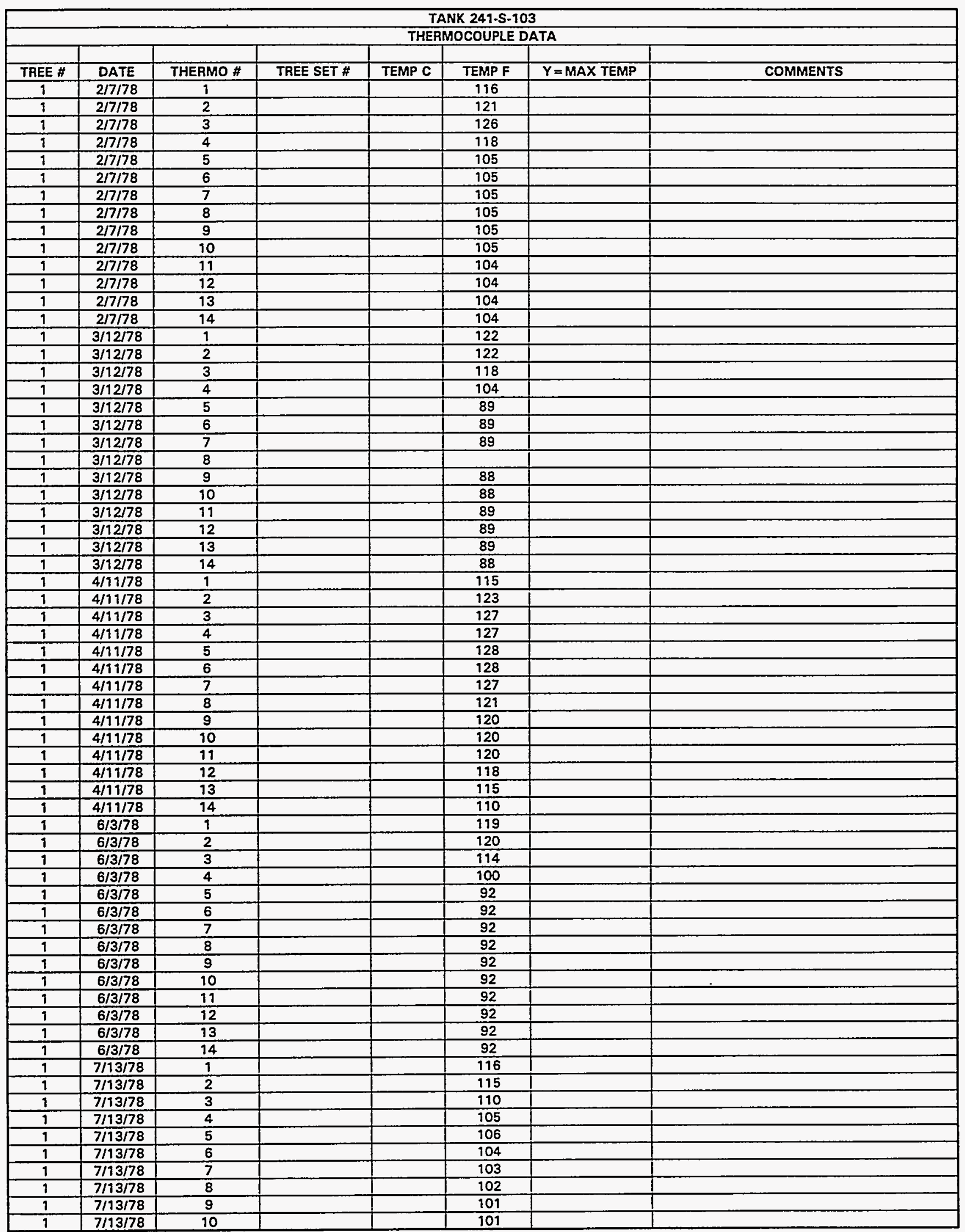

Data obtained from WHC Surveillance Analysis Computer System (SACS), February 24, 1994. 


\begin{tabular}{|c|c|c|c|c|c|c|c|}
\hline \multicolumn{8}{|c|}{ TANK $241.5-103$} \\
\hline & & & & THEI & OCOUPLE & & \\
\hline TREE \# & $\overline{D A T E}$ & THERMO \# & TREE SET \# & TEMP C & TFMP F & & \\
\hline 1 & $7 / 13 / 78$ & 11 & 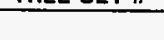 & & 101 & $Y=M A X$ TEMP & COMMENTS \\
\hline 1 & $7 / 13 / 78$ & 12 & & & 101 & & \\
\hline 1 & $7 / 13 / 78$ & 13 & & & 101 & & \\
\hline 9 & $7 / 13 / 78$ & 14 & & & 101 & & \\
\hline 1 & $8 / 11 / 78$ & 1 & & & 110 & & \\
\hline 1 & $8 / 11 / 78$ & 2 & & & 115 & & \\
\hline 1 & $8 / 11 / 78$ & 3 & & & 121 & & \\
\hline 1 & $8 / 11 / 78$ & 4 & & & 123 & & \\
\hline 1 & $8 / 11 / 78$ & 5 & & & 118 & & \\
\hline 1 & $8 / 11 / 78$ & 6 & & & 116 & & \\
\hline$\frac{T}{1}$ & $8 / 11 / 78$ & 7 & & & 105 & & \\
\hline 1 & $8 / 11 / 78$ & 8 & & & 104 & & \\
\hline 1 & $8 / 11 / 78$ & 9 & & & 104 & & \\
\hline 1 & $8 / 11 / 78$ & 10 & & & 104 & & \\
\hline 1 & $8 / 11 / 78$ & 19 & & & 104 & & \\
\hline 1 & $8 / 11 / 78$ & 12 & & & 104 & & \\
\hline 1 & $8 / 71 / 78$ & 13 & & & 104 & & \\
\hline 1 & $8 / 11 / 78$ & 14 & & & 104 & & \\
\hline 1 & $10 / 10 / 78$ & 1 & & & 119 & & \\
\hline 1 & $10 / 10 / 78$ & 2 & & & 125 & & \\
\hline 1 & $10 / 10 / 78$ & 3 & & & 124 & & \\
\hline 1 & $10 / 10 / 78$ & 4 & & & 124 & & \\
\hline 1 & $10 / 10 / 78$ & 5 & & & 124 & & \\
\hline 1 & $10 / 10 / 78$ & 6 & & & 124 & & \\
\hline 1 & $10 / 10 / 78$ & 7 & & & 110 & & \\
\hline 1 & $10 / 10 / 78$ & 8 & & & 105 & & \\
\hline 1 & $10 / 10 / 78$ & 9 & & & 100 & & \\
\hline 1 & $10 / 10 / 78$ & 10 & & & 100 & & \\
\hline 1 & $10 / 10 / 78$ & 11 & & & 100 & & \\
\hline 1 & $10 / 10 / 78$ & 12 & & & 100 & & \\
\hline 1 & $2 / 4 / 79$ & 1 & & & 116 & & \\
\hline 9 & $2 / 4 / 79$ & 2 & & & 112 & & \\
\hline 1 & $2 / 4 / 79$ & 3 & & & 100 & & \\
\hline 1 & $2 / 4 / 79$ & 4 & & & 89 & & \\
\hline 1 & $2 / 4 / 79$ & 5 & & & 87 & & \\
\hline 1 & $2 / 4 / 79$ & 6 & & & 87 & & \\
\hline 1 & $2 / 4 / 79$ & 7 & & & 88 & & \\
\hline 1 & $2 / 4 / 79$ & 8 & & & 83 & & \\
\hline 1 & $2 / 4 / 79$ & 9 & & & 77 & & \\
\hline 1 & $2 / 4 / 79$ & 10 & & & $77^{\circ}$ & & \\
\hline 1 & $2 / 4 / 79$ & 11 & & & 77 & & \\
\hline 1 & $2 / 4 / 79$ & 12 & & & 77 & & \\
\hline 1 & $2 / 4 / 79$ & 13 & & & 77 & & \\
\hline 1 & $2 / 4 / 79$ & 14 & & & 77 & & \\
\hline 1 & $9 / 6 / 79$ & 1 & & & 115 & & \\
\hline 1 & $9 / 6 / 79$ & 2 & & & 120 & & \\
\hline 1 & $9 / 6 / 79$ & 3 & & & 126 & & \\
\hline 1 & $9 / 6 / 79$ & 4 & & & 130 & & \\
\hline 1 & $9 / 6 / 79$ & 5 & & & 130 & & \\
\hline 1 & $9 / 6 / 79$ & 6 & & & 130 & & \\
\hline 1 & $9 / 6 / 79$ & 7 & & & 130 & & \\
\hline 1 & $9 / 6 / 79$ & 8 & & & 130 & & \\
\hline 1 & $9 / 6 / 79$ & 9 & & & 130 & & \\
\hline 1 & $9 / 6 / 79$ & 10 & 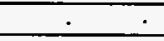 & & 130 & & \\
\hline 1 & $9 / 6 / 79$ & 11 & & & 122 & & \\
\hline 1 & $9 / 6 / 79$ & 12 & & & 122 & & \\
\hline 1 & $9 / 6 / 79$ & 13 & & & 122 & & \\
\hline 1 & $9 / 6 / 79$ & 14 & & & 122 & & \\
\hline 1 & $1 / 23 / 80$ & 1 & & & 125 & & \\
\hline 1 & $1 / 23 / 80$ & 2 & & & 125 & & \\
\hline 9 & $1 / 23 / 80$ & 3 & & & 120 & & \\
\hline 1 & $1 / 23 / 80$ & 4 & & & 115 & & \\
\hline 1 & $1 / 23 / 80$ & 5 & & & 115 & & \\
\hline 1 & $1 / 23 / 80$ & 6 & & & 110 & & \\
\hline 1 & $1 / 23 / 80$ & 7 & & & 190 & & \\
\hline 1 & $1 / 23 / 80$ & 8 & & & 105 & & \\
\hline
\end{tabular}

Data obtained from WHC Surveillance Analysis Computer System (SACS), February 24, 1994. 


\begin{tabular}{|c|c|c|c|c|c|c|c|}
\hline \multicolumn{8}{|c|}{ TANK 241.S-103 } \\
\hline \multicolumn{8}{|c|}{ THERMOCOUPLE DATA } \\
\hline TREE \# & DATE & THERMO \# & TREE SET \# & TEMP C & TEMP F & $Y=$ MAX TEMP & COMMENTS \\
\hline 1 & $1 / 23 / 80$ & 9 & & & 95 & & \\
\hline 1 & $1 / 23 / 80$ & 10 & & & 95 & & \\
\hline 1 & $1 / 23 / 80$ & 11 & & & 95 & & \\
\hline 1 & $1 / 23 / 80$ & 12 & & & 95 & & \\
\hline 1 & $9 / 23 / 80$ & 13 & & & 95 & & \\
\hline 1 & $1 / 23 / 80$ & 14 & & & 95 & & \\
\hline 9 & $1 / 13 / 82$ & 1 & & & 108 & & \\
\hline 1 & $1 / 13 / 82$ & 2 & & & 107 & & \\
\hline 1 & $1 / 13 / 82$ & 3 & & & 100 & & \\
\hline 1 & $1 / 13 / 82$ & 4 & & & 89 & & \\
\hline 1 & $1 / 13 / 82$ & 5 & & & 80 & & \\
\hline 1 & $1 / 13 / 82$ & 6 & & & 78 & & \\
\hline 1 & $1 / 13 / 82$ & 7 & & & 78 & & \\
\hline 1 & $1 / 13 / 82$ & 8 & & & 78 & & \\
\hline 1 & $1 / 13 / 82$ & 9 & & & 78 & & \\
\hline 1 & $1 / 13 / 82$ & 10 & & & 78 & & \\
\hline 1 & $1 / 13 / 82$ & 11 & & & 78 & & \\
\hline 1 & $1 / 13 / 82$ & 12 & & & 78 & & \\
\hline 1 & $1 / 13 / 82$ & 13 & & & 78 & & \\
\hline 1 & $1 / 13 / 82$ & 14 & & & 78 & & \\
\hline 1 & $5 / 29 / 82$ & 1 & & & 101 & & \\
\hline 1 & $5 / 29 / 82$ & 2 & & & 99 & & \\
\hline 1 & $5 / 29 / 82$ & 3 & & & 94 & & \\
\hline 1 & $5 / 29 / 82$ & 4 & & & 96 & & \\
\hline 1 & $5 / 29 / 82$ & 5 & & & 78 & & \\
\hline 1 & $5 / 29 / 82$ & 6 & & & 77 & & \\
\hline 9 & $5 / 29 / 82$ & 7 & & & 77 & & \\
\hline 1 & $5 / 29 / 82$ & 8 & & & 77 & & \\
\hline 1 & $5 / 29 / 82$ & 9 & & & 77 & & \\
\hline 1 & $5 / 29 / 82$ & 10 & & & 77 & & \\
\hline 1 & $5 / 29 / 82$ & 11 & & & 77 & & \\
\hline 9 & $5 / 29 / 82$ & 12 & & & 77 & & \\
\hline 1 & $10 / 6 / 82$ & 1 & & & 101 & & \\
\hline 1 & $10 / 6 / 82$ & 2 & & & 100 & & \\
\hline 1 & $10 / 6 / 82$ & 3 & & & 97 & & \\
\hline 1 & $10 / 6 / 82$ & 4 & & & 91 & & \\
\hline 1 & $10 / 6 / 82$ & 5 & & & 84 & & \\
\hline 1 & $10 / 6 / 82$ & 6 & & & 83 & & \\
\hline 1 & $10 / 6 / 82$ & 7 & & & 83 & & \\
\hline 9 & $10 / 6 / 82$ & 8 & & & 83 & & \\
\hline 9 & $10 / 6 / 82$ & 9 & & & 83 & & \\
\hline 1 & $10 / 6 / 82$ & 10 & & & 83 & & \\
\hline 1 & $10 / 6 / 82$ & 11 & & & 83 & & \\
\hline 1 & $10 / 6 / 82$ & 12 & & & 83 & & \\
\hline 1 & $10 / 6 / 82$ & 13 & & & 83 & & \\
\hline 1 & $10 / 6 / 82$ & 14 & & & 83 & & \\
\hline 1 & $1 / 12 / 84$ & 1 & & & 106 & & \\
\hline 1 & $1 / 12 / 84$ & 2 & & & 104 & & \\
\hline 1 & $1 / 12 / 84$ & 3 & & & 98 & & \\
\hline 1 & $1 / 12 / 84$ & 4 & & & 84 & & \\
\hline 1 & $9 / 12 / 84$ & 5 & & & 83 & & \\
\hline 1 & $1 / 12 / 84$ & 6 & & & 82 & & \\
\hline 1 & $2 / 14 / 84$ & 1 & & & 95 & & \\
\hline 1 & $2 / 74 / 84$ & 2 & & & 92 & & \\
\hline 1 & $2 / 14 / 84$ & 3 & & & 87 & & \\
\hline 1 & $2 / 14 / 84$ & 4 & & & 80 & & \\
\hline 1 & $2 / 14 / 84$ & 5 & & & 71 & & \\
\hline 1 & $2 / 14 / 84$ & 6 & & & 67 & & \\
\hline 1 & $3 / 14 / 84$ & 1 & & & 95 & & \\
\hline 1 & $3 / 14 / 84$ & 2 & & & 92 & & \\
\hline 1 & $3 / 14 / 84$ & 3 & & & 89 & & \\
\hline 1 & $3 / 14 / 84$ & 4 & & & 83 & & \\
\hline 1 & $3 / 14 / 84$ & 5 & & & 74 & & \\
\hline 1 & $3 / 14 / 84$ & 6 & & & 70 & & \\
\hline 1 & $4 / 9 / 84$ & 1 & & & 91 & & \\
\hline 1 & $4 / 9 / 84$ & 2 & & & 94 & & \\
\hline
\end{tabular}

Data obtained from WHC Surveillance Analysis Computer System (SACS), February 24, 1994. 


\begin{tabular}{|c|c|c|c|c|c|c|c|}
\hline \multicolumn{8}{|c|}{ TANK 241.S-103 } \\
\hline \multicolumn{8}{|c|}{ THERMOCOUPLE DATA } \\
\hline TREE \# & DATE & THERMO \# & TREE SET \# & TEMP C & TEMPF & $Y=$ MAX TEMP & COMMFNTS \\
\hline 1 & $4 / 9 / 84$ & 3 & THLL SLT Tr & The & 90 & T- MIAA TERT & COMMENIS \\
\hline 1 & $4 / 9 / 84$ & 4 & & & 83 & & \\
\hline 1 & $4 / 9 / 84$ & 5 & & & 73 & & \\
\hline 1 & $4 / 9 / 84$ & 6 & & & 71 & & \\
\hline 1 & $6 / 19 / 84$ & 1 & & & 66 & & \\
\hline 1 & $6 / 19 / 84$ & 2 & & & 65 & & \\
\hline 1 & $6 / 19 / 84$ & 3 & & & 67 & & \\
\hline 1 & $6 / 19 / 84$ & 4 & & & 60 & & \\
\hline 1 & $6 / 19 / 84$ & 5 & & & 61 & & \\
\hline 1 & $6 / 19 / 84$ & 6 & & & 61 & & \\
\hline 1 & $9 / 13 / 84$ & 1 & & & 100 & & \\
\hline 1 & $9 / 13 / 84$ & 2 & & & 99 & & \\
\hline 1 & $9 / 13 / 84$ & 3 & & & 97 & & \\
\hline 1 & $9 / 13 / 84$ & 4 & & & 92 & & \\
\hline 1 & $9 / 13 / 84$ & 5 & & & 86 & & \\
\hline 1 & $9 / 13 / 84$ & 6 & & & 84 & & \\
\hline 1 & $10 / 19 / 84$ & 1 & & & 115 & & \\
\hline 1 & $10 / 19 / 84$ & 2 & & & 114 & & \\
\hline 1 & $10 / 19 / 84$ & 3 & & & 112 & & \\
\hline 1 & $10 / 19 / 84$ & 4 & & & 108 & & \\
\hline 1 & $10 / 19 / 84$ & 5 & & & 103 & & \\
\hline 1 & $10 / 19 / 84$ & 6 & & & 102 & & \\
\hline 1 & $4 / 2 / 85$ & 1 & & & 94 & & \\
\hline 1 & $4 / 2 / 85$ & 2 & & & 92 & & \\
\hline 1 & $4 / 2 / 85$ & 3 & & & 89 & & \\
\hline 1 & $4 / 2 / 85$ & 4 & & & 83 & & \\
\hline 1 & $4 / 2 / 85$ & 5 & & & 76 & & \\
\hline 1 & $4 / 2 / 85$ & 6 & & & 73 & & \\
\hline 1 & $5 / 2 / 85$ & 1 & & & 88 & & \\
\hline 1 & $5 / 2 / 85$ & 2 & & & 86 & & \\
\hline 1 & $5 / 2 / 85$ & 3 & & & 83 & & \\
\hline 1 & $5 / 2 / 85$ & 4 & & & 79 & & \\
\hline 1 & $5 / 2 / 85$ & 5 & & & 72 & & \\
\hline 1 & $5 / 2 / 85$ & 6 & & & 69 & & \\
\hline 1 & $7 / 2 / 85$ & 1 & & & 90 & & \\
\hline 1 & $7 / 2 / 85$ & 2 & & & 89 & & \\
\hline 1 & $7 / 2 / 85$ & 3 & & & 87 & & \\
\hline 1 & $7 / 2 / 85$ & 4 & & & 83 & & \\
\hline 1 & $7 / 2 / 85$ & 5 & & & 78 & & \\
\hline 1 & $7 / 2 / 85$ & 6 & & & 76 & & \\
\hline 1 & $12 / 4 / 85$ & 1 & & & 74 & & \\
\hline 1 & $12 / 4 / 85$ & 2 & & & 73 & & \\
\hline 1 & $12 / 4 / 85$ & 3 & & & 71 & & \\
\hline 1 & $12 / 4 / 85$ & 4 & & & 67 & & \\
\hline 1 & $12 / 4 / 85$ & 5 & & & 60 & & \\
\hline 1 & $12 / 4 / 85$ & 6 & & & 59 & & \\
\hline 1 & $1 / 6 / 86$ & 1 & & & 85 & & \\
\hline 1 & $1 / 6 / 86$ & 2 & & & 85 & & \\
\hline 1 & $1 / 6 / 86$ & 3 & & & 85 & & \\
\hline 1 & $1 / 6 / 86$ & 4 & & & 84 & & \\
\hline 1 & $1 / 6 / 86$ & 5 & & & 84 & & \\
\hline 1 & $1 / 6 / 86$ & 6 & & & 84 & & \\
\hline 1 & $1 / 7 / 86$ & 1 & & & 72 & & \\
\hline 1 & $1 / 7 / 86$ & 2 & & & 73 & & $\cdot$ \\
\hline 1 & $1 / 7 / 86$ & 3 & & & 73 & & \\
\hline 1 & $1 / 7 / 86$ & 4 & & & 73 & & \\
\hline 1 & $1 / 7 / 86$ & 5 & & & 73 & & \\
\hline 1 & $1 / 7 / 86$ & 6 & & & 72 & & \\
\hline 1 & $11 / 27 / 86$ & 1 & & & 94.2 & & \\
\hline 1 & $11 / 27 / 86$ & 2 & & & 93 & & \\
\hline 1 & $11 / 27 / 86$ & 3 & & & 91.4 & & \\
\hline 1 & $11 / 27 / 86$ & 4 & & & 87.6 & & \\
\hline 1 & $11 / 27 / 86$ & 5 & & & 81.5 & & \\
\hline 1 & $11 / 27 / 86$ & 6 & & & 79.1 & & \\
\hline 1 & $12 / 22 / 86$ & 1 & & & 94.2 & & \\
\hline 1 & $12 / 22 / 86$ & 2 & & & 93.1 & & \\
\hline
\end{tabular}

Data obtained from WHC Surveillance Analysis Computer System (SACS). February 24, 1994. 


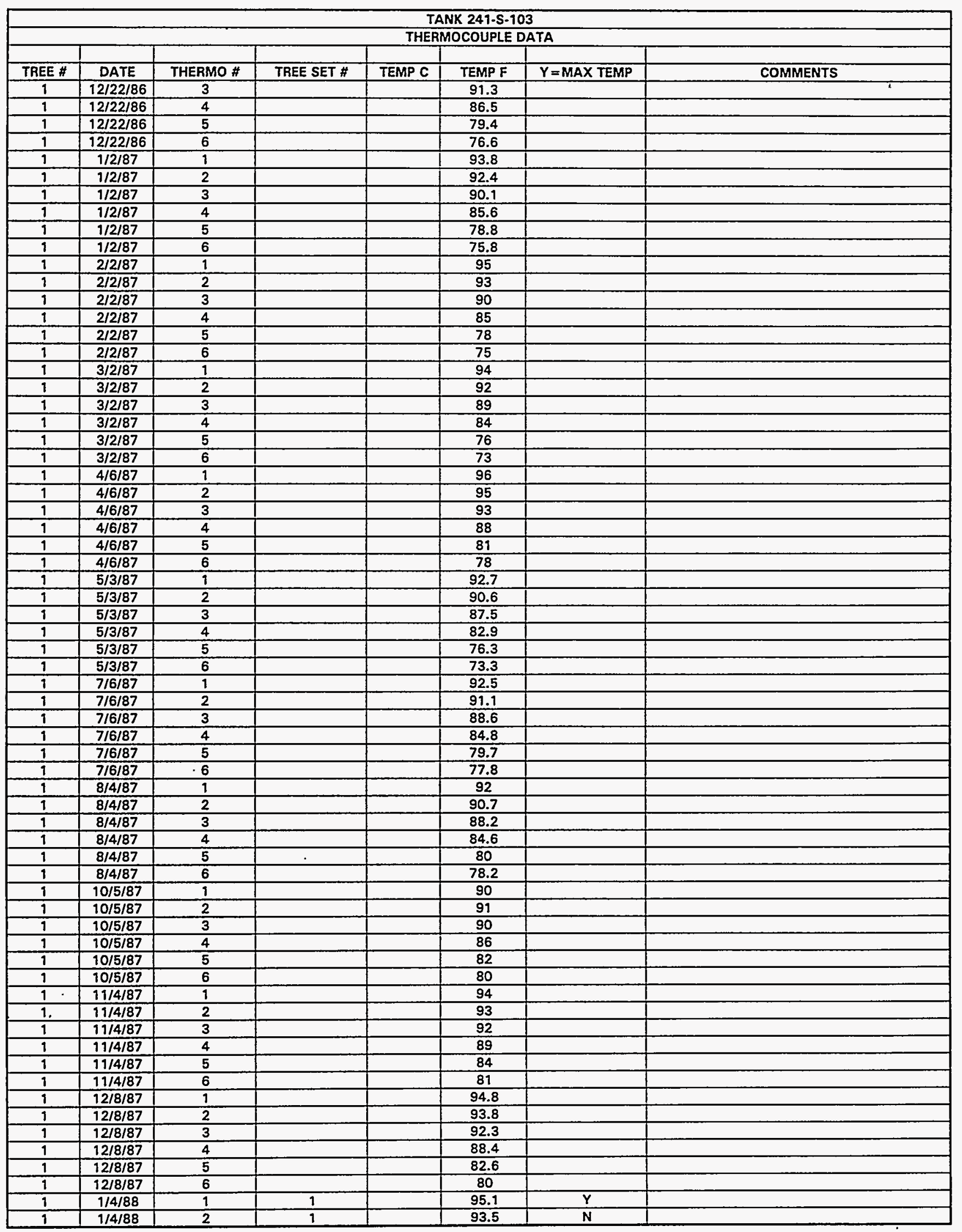

Data obtained from WHC Surveillance Analysis Computer System (SACS), February 24, 1994. 


\begin{tabular}{|c|c|c|c|c|c|c|c|}
\hline \multicolumn{8}{|c|}{ TANK 241-S-103 } \\
\hline & & & & THER & OCOUPLE & TA & \\
\hline TREE \# & DATE & THERMO \# & TREE SET \# & TEMP C & TEMP F & $Y=M A \bar{X}$ TEMP & COMMENTS \\
\hline 1 & $1 / 4 / 88$ & 3 & 1 & & 92.1 & $\mathrm{~N}$ & \\
\hline 1 & $1 / 4 / 88$ & 4 & 1 & & 87.5 & $\mathrm{~N}$ & \\
\hline 1 & $1 / 4 / 88$ & 5 & 1 & & 80.9 & $\mathrm{~N}$ & \\
\hline 1 & $1 / 4 / 88$ & 6 & 1 & & 77.9 & $\bar{N}$ & \\
\hline 1 & $2 / 1 / 88$ & 1 & 1 & & 85 & $Y$ & \\
\hline 1 & $2 / 1 / 88$ & 2 & 1 & & 84 & $\mathrm{~N}$ & \\
\hline 1 & $2 / 1 / 88$ & 3 & 1 & & 84 & $\mathbf{N}$ & \\
\hline 1 & $2 / 1 / 88$ & 4 & 1 & & 84 & $\mathbf{N}$ & \\
\hline 1 & $2 / 1 / 88$ & 5 & 1 & & 83 & $\mathbf{N}$ & \\
\hline 1 & $2 / 1 / 88$ & 6 & 1 & & 82 & $\mathrm{~N}$ & \\
\hline 1 & $3 / 8 / 88$ & 1 & 1 & & 90 & $Y$ & \\
\hline 1 & $3 / 8 / 88$ & 2 & 1 & & 89 & $N$ & \\
\hline 1 & $3 / 8 / 88$ & 3 & 1 & & 87 & $\mathbf{N}$ & \\
\hline 1 & $3 / 8 / 88$ & 4 & 1 & & 82 & $N$ & \\
\hline 1 & $3 / 8 / 88$ & 5 & 1 & & 76 & $\mathrm{~N}$ & \\
\hline 1 & $3 / 8 / 88$ & 6 & 1 & & 75 & $\mathrm{~N}$ & \\
\hline 1 & $4 / 4 / 88$ & 1 & 1 & & 91 & $Y$ & \\
\hline 1 & $4 / 4 / 88$ & 2 & 9 & & 90 & $N$ & \\
\hline 1 & $4 / 4 / 88$ & 3 & 1 & & 88 & $\mathbf{N}$ & \\
\hline 1 & $4 / 4 / 88$ & 4 & 1 & & 83 & $\mathbf{N}$ & \\
\hline 1 & $4 / 4 / 88$ & 5 & 1 & & 76 & $\mathbf{N}$ & \\
\hline 1 & $4 / 4 / 88$ & 6 & 1 & & 73 & $\mathbf{N}$ & \\
\hline 1 & $5 / 2 / 88$ & 1 & 1 & & 105 & $Y$ & \\
\hline 1 & $5 / 2 / 88$ & 2 & 9 & & 102 & $\mathbf{N}$ & \\
\hline 1 & $5 / 2 / 88$ & 3 & 9 & & 99 & $\mathbf{N}$ & \\
\hline 1 & $5 / 2 / 88$ & 4 & 1 & & 95 & $\mathbf{N}$ & \\
\hline 1 & $5 / 2 / 88$ & 5 & 1 & & 89 & $\mathbf{N}$ & \\
\hline 1 & $5 / 2 / 88$ & 6 & 1 & & 86 & $\mathbf{N}$ & \\
\hline 1 & $5 / 4 / 88$ & 1 & 1 & & 92 & $\bar{Y}$ & \\
\hline 1 & $5 / 4 / 88$ & 2 & 1 & & 91 & $\mathrm{~N}$ & \\
\hline 1 & $5 / 4 / 88$ & 3 & 1 & & 89 & $\mathbf{N}$ & \\
\hline 1 & $5 / 4 / 88$ & 4 & 1 & & 85 & $\mathrm{~N}$ & \\
\hline 1 & $5 / 4 / 88$ & 5 & 1 & & 77 & $\mathbf{N}$ & \\
\hline 1 & $5 / 4 / 88$ & 6 & 1 & & 75 & $\mathrm{~N}$ & \\
\hline 1 & $6 / 6 / 88$ & 1 & 1 & & 108 & $\bar{Y}$ & \\
\hline 1 & $6 / 6 / 88$ & 2 & 1 & & 102 & $\mathrm{~N}$ & \\
\hline 1 & $6 / 6 / 88$ & 3 & 1 & & 88 & $\mathbf{N}$ & \\
\hline 1 & $6 / 6 / 88$ & 4 & 1 & & 94 & $\mathbf{N}$ & \\
\hline 1 & $6 / 6 / 88$ & 5 & 1 & & 87 & $\bar{N}$ & \\
\hline 1 & $6 / 6 / 88$ & 6 & 1 & & 82 & $\mathbf{N}$ & \\
\hline 1 & $7 / 4 / 88$ & 1 & 1 & & 72 & $\mathbf{N}$ & \\
\hline 9 & $7 / 4 / 88$ & 2 & 1 & & 71 & $N$ & \\
\hline 1 & $7 / 4 / 88$ & 3 & 1 & & 88 & $N$ & \\
\hline 1 & $7 / 4 / 88$ & 4 & 1 & & 93 & $\bar{Y}$ & \\
\hline 1 & $7 / 4 / 88$ & 5 & 1 & & 89 & $\mathbf{N}$ & \\
\hline 1 & $7 / 4 / 88$ & 6 & 1 & & 89 & $N$ & \\
\hline 1 & $8 / 1 / 88$ & 1 & 1 & & 130 & $Y$ & \\
\hline 1 & $8 / 1 / 88$ & 2 & 1 & & 90 & $\mathrm{~N}$ & \\
\hline 1 & $8 / 1 / 88$ & 3 & 1 & & 88 & $\bar{N}$ & \\
\hline 1 & $8 / 1 / 88$ & 4 & 1 & & 85 & $\mathrm{~N}$ & \\
\hline 1 & $8 / 9 / 88$ & 5 & 1 & & 81 & $\mathrm{~N}$ & \\
\hline 1 & $8 / 1 / 88$ & 6 & 1 & & 85 & $\mathbf{N}$ & \\
\hline 1 & 9/5/88 & 1 & 1 & & 92 & $Y$ & \\
\hline 1 & $9 / 5 / 88$ & 2 & 1 & & 91 & $N$ & \\
\hline 1 & $9 / 5 / 88$ & 3 & 1 & & 90 & $\mathrm{~N}$ & \\
\hline 1 & $9 / 5 / 88$ & 4 & 1 & & 86 & $\mathrm{~N}$ & \\
\hline 1 & $9 / 5 / 88$ & 5 & 1 & & 82 & $\mathrm{~N}$ & \\
\hline 1 & $9 / 5 / 88$ & 6 & 1 & & 80 & $N$ & \\
\hline 1 & $10 / 3 / 88$ & 1 & 1 & & 92 & $\bar{Y}$ & \\
\hline 1 & $10 / 3 / 88$ & 2 & 1 & & 92 & $N$ & \\
\hline 1 & $10 / 3 / 88$ & 3 & 1 & & 90 & $\mathrm{~N}$ & \\
\hline 1 & $10 / 3 / 88$ & 4 & 1 & & 87 & $N$ & \\
\hline 1 & $10 / 3 / 88$ & 5 & 1 & & 82 & $N$ & \\
\hline 1 & $10 / 3 / 88$ & 6 & 1 & & 80 & $N$ & \\
\hline 1 & $11 / 7 / 88$ & 9 & 1 & & 98 & $\bar{Y}$ & \\
\hline 1 & $11 / 7 / 88$ & 2 & 1 & & 94 & $N$ & \\
\hline
\end{tabular}

Data obtained from WHC Surveillance Analysis Computer System (SACS), February 24, 1994. 
WHC-SD-WM-ER-323, Rev. 0

\begin{tabular}{|c|c|c|c|c|c|c|c|}
\hline \multicolumn{8}{|c|}{ TANK 241-S-103 } \\
\hline \multicolumn{8}{|c|}{ THERMOCOUPLE DATA } \\
\hline TREE \# & DATE & THERMO \# & TREE SET \# & TEMP C & TEMP F & $Y=$ MAX TEMP & COMMENTS \\
\hline 1 & $11 / 7 / 88$ & 3 & 1 & & 92 & $\mathbf{N}$ & \\
\hline 1 & $11 / 7 / 88$ & 4 & 1 & & 89 & $\vec{N}$ & \\
\hline 1 & $11 / 7 / 88$ & 5 & 1 & & 85 & $\mathbf{N}$ & \\
\hline 1 & $11 / 7 / 88$ & 6 & 1 & & 82 & $\mathbf{N}$ & \\
\hline 1 & $11 / 7 / 88$ & 7 & 1 & & 80 & $\mathbf{N}$ & \\
\hline 1 & $12 / 5 / 88$ & 1 & 1 & & & $\mathbf{N}$ & BAD READINGS \\
\hline 1 & $1 / 2 / 89$ & 1 & 1 & & & $\mathbf{N}$ & $0 / \mathrm{S}$ \\
\hline 1 & $2 / 6 / 89$ & 1 & 1 & & 96 & $\mathbf{Y}$ & \\
\hline 1 & $2 / 6 / 89$ & 2 & 1 & & 93.5 & $\mathbf{N}$ & \\
\hline 1 & $2 / 6 / 89$ & 3 & 1 & & 91.7 & $\mathbf{N}$ & \\
\hline 1 & $2 / 6 / 89$ & 4 & 1 & & 86.3 & $\mathbf{N}$ & \\
\hline 1 & $2 / 6 / 89$ & 5 & 1 & & 79 & $\mathbf{N}$ & \\
\hline 1 & $2 / 6 / 89$ & 6 & 1 & & 75.9 & $\mathbf{N}$ & \\
\hline 1 & $3 / 6 / 89$ & 1 & 1 & & 116 & $\bar{Y}$ & \\
\hline 1 & $3 / 6 / 89$ & 2 & 1 & & 114 & $\bar{N}$ & \\
\hline 1 & $3 / 6 / 89$ & 3 & 1 & & 112 & $\mathbf{N}$ & \\
\hline 1 & $3 / 6 / 89$ & 4 & 1 & & 106 & $\mathbf{N}$ & \\
\hline 1 & $3 / 6 / 89$ & 5 & 1 & & 97 & $\mathbf{N}$ & \\
\hline 1 & $3 / 6 / 89$ & 6 & 1 & & 92 & $\mathbf{N}$ & \\
\hline 1 & $1 / 4 / 91$ & 1 & 1 & & 89.7 & $\bar{Y}$ & HH-99A-J \#890523512-47 \\
\hline 1 & $1 / 4 / 91$ & 2 & 1 & & 88.5 & $\mathbf{N}$ & \\
\hline 1 & $1 / 4 / 91$ & 3 & 1 & & 87.3 & $\mathbf{N}$ & \\
\hline 1 & $1 / 4 / 91$ & 4 & 1 & & 83 & $\mathbf{N}$ & \\
\hline 1 & $1 / 4 / 91$ & 5 & 1 & & 76.8 & $N$ & \\
\hline 1 & $1 / 4 / 91$ & 6 & 1 & & 73.8 & $N$ & \\
\hline 1 & $11 / 13 / 91$ & 1 & 1 & & 86.7 & $Y$ & $\begin{array}{c}\text { J-890423320-42 TC \#4 O/S WORK ORDER } \\
\text { \#04529 }\end{array}$ \\
\hline 1 & $11 / 13 / 91$ & 2 & 1 & & 86.7 & $\mathbf{N}$ & \\
\hline 1 & $11 / 13 / 91$ & 3 & 1 & & 85.5 & $\mathbf{N}$ & \\
\hline 1 & $11 / 13 / 91$ & 5 & 1 & & 77.8 & $\mathbf{N}$ & \\
\hline 1 & $11 / 13 / 91$ & 6 & 1 & & 75.8 & $\mathbf{N}$ & \\
\hline 1 & $1 / 1 / 92$ & 1 & 1 & & 91.2 & $\mathbf{Y}$ & $\mathrm{J}-890523512-20 \mathrm{TC} \# 4 \mathrm{O} / \mathrm{S}$ \\
\hline 1 & $1 / 1 / 92$ & 2 & 1 & & 89.6 & $\mathbf{N}$ & \\
\hline 1 & $1 / 1 / 92$ & 3 & 1 & & 88 & $\mathbf{N}$ & \\
\hline 1 & $1 / 1 / 92$ & 5 & 1 & & 78.1 & $\mathbf{N}$ & \\
\hline 1 & $1 / 1 / 92$ & 6 & 1 & & 75.2 & $\mathbf{N}$ & \\
\hline 1 & $7 / 3 / 92$ & 6 & 1 & & 77.5 & $Y$ & $\mathrm{~J} 890423320-42$ \\
\hline 1 & $2 / 13 / 93$ & 1 & 1 & & 88.1 & $\mathbf{Y}$ & $J 910126143-42$ \\
\hline 1 & $2 / 13 / 93$ & 2 & 1 & & 86.5 & $N$ & \\
\hline 1 & $2 / 13 / 93$ & 3 & 1 & & 84.6 & $\mathbf{N}$ & \\
\hline 1 & $2 / 13 / 93$ & 5 & 1 & & 72.9 & $N$ & \\
\hline 1 & $2 / 13 / 93$ & 6 & 1 & & 69.5 & $N$ & \\
\hline 1 & $7 / 2 / 93$ & 2 & 1 & & 84.6 & $\mathbf{Y}$ & $J 890423320-42$ \\
\hline 1 & $7 / 2 / 93$ & 3 & 1 & & 82.1 & $N$ & \\
\hline 1 & $7 / 2 / 93$ & 5 & 1 & & 73.7 & $N$ & \\
\hline 1 & $7 / 2 / 93$ & 6 & 1 & & 71.2 & $N$ & \\
\hline
\end{tabular}

Data obtained from WHC Surveillance Analysis Computer System (SACS), February 24, 1994. 
WHC-SD-WM-ER-323, Rev. 0

\begin{tabular}{|c|c|c|c|c|c|c|c|}
\hline \multicolumn{8}{|c|}{ TANK $241 \cdot \mathrm{S}-104$} \\
\hline \multicolumn{8}{|c|}{ THERMOCOUPLE DATA } \\
\hline & & & & & & & \\
\hline TREE \# & DATE & THERMO \# & TREE SET \# & TEMP C & TEMP F & $Y=$ MAX TEMP & COMMENTS \\
\hline 1 & $11 / 3 / 75$ & 1 & & & 56 & & \\
\hline 1 & $11 / 3 / 75$ & 2 & & & 56 & & \\
\hline 1 & $11 / 3 / 75$ & 3 & & & 56 & & \\
\hline 1 & $11 / 3 / 75$ & 4 & & & 56 & & \\
\hline 1 & $11 / 3 / 75$ & 5 & & & 56 & & \\
\hline 1 & $11 / 3 / 75$ & 6 & & & 56 & & \\
\hline 1 & $11 / 3 / 75$ & 7 & & & 102 & & \\
\hline 1 & $11 / 3 / 75$ & 8 & & & 102 & & \\
\hline 1 & $11 / 3 / 75$ & 9 & & & 102 & & \\
\hline 1 & $11 / 3 / 75$ & 10 & & & 56 & & \\
\hline 1 & $11 / 3 / 75$ & 11 & & & 102 & & \\
\hline 1 & $11 / 3 / 75$ & 12 & & & 102 & & \\
\hline 1 & $2 / 4 / 76$ & 1 & & & 74 & & \\
\hline 1 & $2 / 4 / 76$ & 2 & & & 74 & & \\
\hline 1 & $2 / 4 / 76$ & 3 & & & 74 & & \\
\hline 1 & $2 / 4 / 76$ & 4 & & & 74 & & \\
\hline 1 & $2 / 4 / 76$ & 5 & & & 74 & & \\
\hline 1 & $2 / 4 / 76$ & 6 & & & 74 & & \\
\hline 1 & $2 / 4 / 76$ & 7 & & & 75 & & \\
\hline 1 & $2 / 4 / 76$ & 8 & & & 86 & & \\
\hline 1 & $2 / 4 / 76$ & 9 & & & 75 & & \\
\hline 1 & $2 / 4 / 76$ & 10 & & & 86 & & \\
\hline 1 & $2 / 4 / 76$ & 11 & & & 87 & & \\
\hline 1 & $2 / 4 / 76$ & 12 & & & 87 & & \\
\hline 1 & $5 / 2 / 76$ & 1 & & & 78 & & \\
\hline 1 & $5 / 2 / 76$ & 2 & & & 77 & & \\
\hline 1 & $5 / 2 / 76$ & 3 & & & 77 & & \\
\hline 1 & $5 / 2 / 76$ & 4 & & & 78 & & \\
\hline 1 & $5 / 2 / 76$ & 5 & & & 77 & & \\
\hline 1 & $5 / 2 / 76$ & 6 & & & 77 & & \\
\hline 1 & $5 / 2 / 76$ & 7 & & & 77 & & \\
\hline 1 & $5 / 2 / 76$ & 8 & & & 77 & & \\
\hline 1 & $5 / 2 / 76$ & 9 & & & 93 & & \\
\hline 1 & $5 / 2 / 76$ & 10 & & & 78 & & \\
\hline 1 & $5 / 2 / 76$ & 11 & & & 93 & & \\
\hline 1 & $5 / 2 / 76$ & 12 & & & 93 & & \\
\hline 1 & $6 / 1 / 76$ & 1 & & & 78 & & \\
\hline 1 & $6 / 1 / 76$ & 2 & & & 77 & & \\
\hline 1 & $6 / 1 / 76$ & 3 & & & 77 & & \\
\hline 1 & $6 / 1 / 76$ & 4 & & & 78 & & \\
\hline 1 & $6 / 1 / 76$ & 5 & & & 77 & & \\
\hline 1 & $6 / 1 / 76$ & 6 & & & 78 & & \\
\hline 1 & $6 / 1 / 76$ & 7 & & & 77 & & \\
\hline 1 & $6 / 1 / 76$ & 8 & & & 77 & & \\
\hline 1 & $6 / 1 / 76$ & 9 & & & 93 & & \\
\hline 1 & $6 / 1 / 76$ & 10 & & & 78 & & \\
\hline 1 & $6 / 1 / 76$ & 11 & & & 93 & & \\
\hline 1 & $6 / 1 / 76$ & 12 & & & 93 & & \\
\hline 1 & $7 / 1 / 76$ & 1 & & & 84 & & \\
\hline 1 & $7 / 1 / 76$ & 2 & & & 84 & & \\
\hline 1 & $7 / 1 / 76$ & 3 & & & 85 & & \\
\hline 1 & $7 / 1 / 76$ & 4 & & & 88 & & \\
\hline 1 & $7 / 1 / 76$ & 5 & & & 87 & & \\
\hline 1 & $7 / 1 / 76$ & 6 & & & 87 & & \\
\hline 1 & $7 / 1 / 76$ & 7 & & & 87 & & \\
\hline 1 & $7 / 1 / 76$ & 8 & & & 86 & & \\
\hline 1 & $7 / 1 / 76$ & 9 & & & 93 & & \\
\hline 1 & $7 / 1 / 76$ & 10 & & & 92 & & \\
\hline 1 & $7 / 1 / 76$ & 11 & & & 92 & & \\
\hline 1 & $7 / 1 / 76$ & 12 & & & 91 & & \\
\hline 1 & $8 / 3 / 76$ & 1 & & & 84 & & \\
\hline 1 & $8 / 3 / 76$ & 2 & & & 84 & & \\
\hline 1 & $8 / 3 / 76$ & 3 & & & 84 & & \\
\hline 1 & $8 / 3 / 76$ & 4 & & & 84 & & \\
\hline 1 & $8 / 3 / 76$ & 5 & & & 84 & & \\
\hline 1 & $8 / 3 / 76$ & 6 & & & 84 & & \\
\hline
\end{tabular}

Data obtained from WHC Surveillance Analysis Computer System (SACS), February 24, 1994. 
WHC-SD-WM-ER-323, Rev. 0

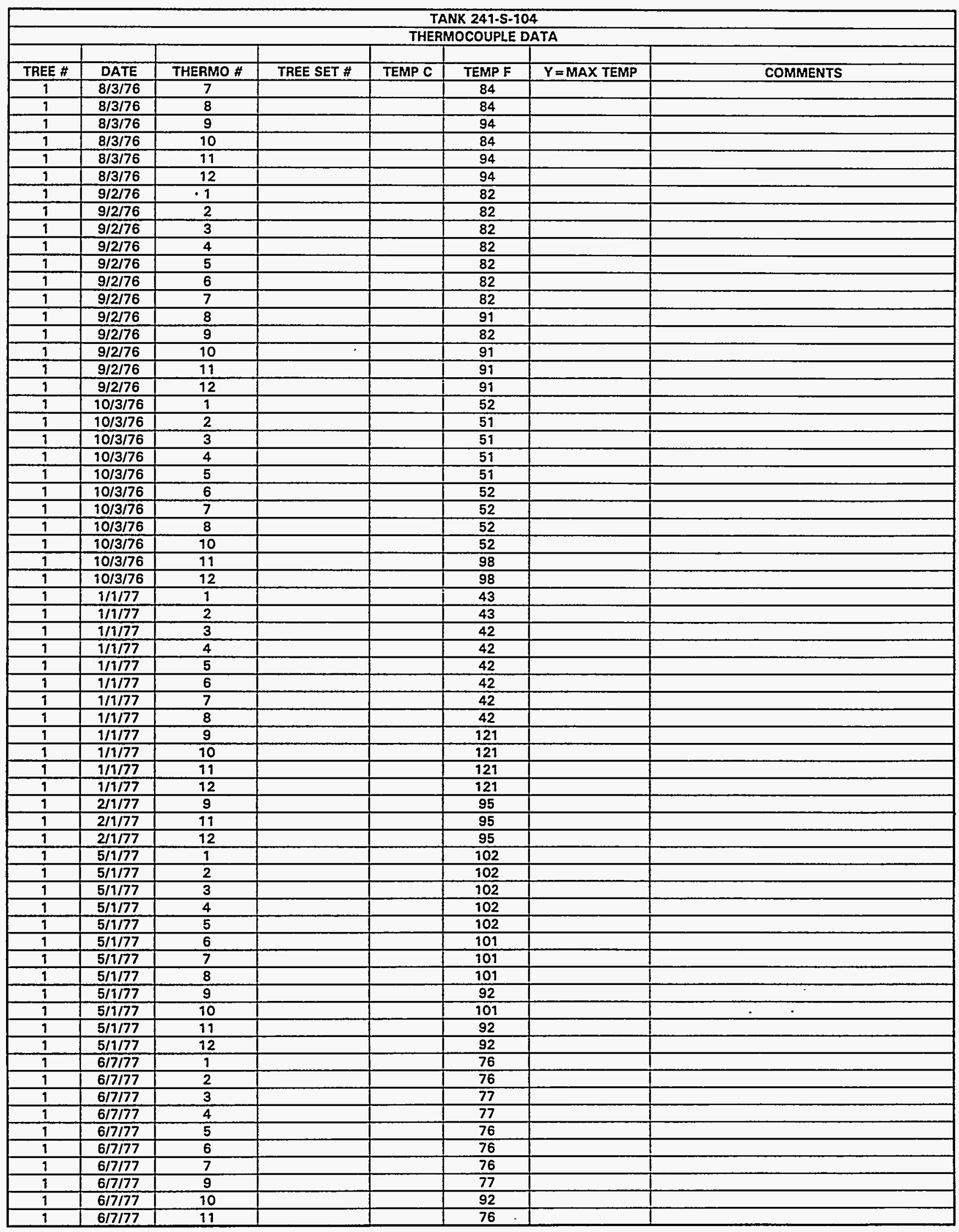

Data obtained from WHC Surveillance Analysis Computer System (SACS), February 24, 1994. 


\begin{tabular}{|c|c|c|c|c|c|c|c|}
\hline \multicolumn{8}{|c|}{ TANK 241-S-104 } \\
\hline \multicolumn{8}{|c|}{ THERMOCOUPLE DATA } \\
\hline & & & & & & & \\
\hline TREE \# & DATE & THERMO \# & TREE SET \# & TEMP C & TEMP F & $\bar{Y}=$ MAX TEMP & COMMENTS \\
\hline 1 & $2 / 7 / 78$ & 1 & & & 40 & & \\
\hline 1 & $2 / 7 / 78$ & 2 & & & 40 & & \\
\hline 1 & $2 / 7 / 78$ & 3 & & & 40 & & \\
\hline 1 & $2 / 7 / 78$ & 4 & & & 41 & & \\
\hline$\frac{9}{1}$ & $2 / 7 / 78$ & 5 & & & 41 & & \\
\hline 1 & $2 / 7 / 78$ & 6 & & & 41 & & \\
\hline 1 & $2 / 7 / 78$ & 7 & & & 41 & & $\cdot$ \\
\hline 1 & $2 / 7 / 78$ & 8 & & & 41 & & \\
\hline 1 & $2 / 7 / 78$ & 9 & & & 88 & & \\
\hline 1 & $2 / 7 / 78$ & 10 & & & 82 & & \\
\hline 1 & $2 / 7 / 78$ & 12 & & & 42 & & \\
\hline$\frac{1}{1}$ & $2 / 7 / 78$ & 13 & & & 43 & & \\
\hline 1 & $2 / 7 / 78$ & 14 & & & 43 & & \\
\hline 1 & $3 / 12 / 78$ & 1 & & & 45 & & \\
\hline 1 & $3 / 12 / 78$ & 2 & & & 45 & & \\
\hline 1 & $3 / 12 / 78$ & 3 & & & 45 & & \\
\hline 1 & $3 / 12 / 78$ & 4 & & & 45 & & \\
\hline 1 & $3 / 12 / 78$ & 5 & & & 45 & & \\
\hline 1 & $3 / 12 / 78$ & 6 & & & 45 & & \\
\hline$\frac{1}{1}$ & $3 / 12 / 78$ & 7 & & & 45 & & \\
\hline 1 & $3 / 12 / 78$ & 8 & & & 45 & & \\
\hline 1 & $3 / 12 / 78$ & 9 & & & 45 & & \\
\hline 1 & $3 / 12 / 78$ & 10 & & & 48 & & \\
\hline 1 & $3 / 12 / 78$ & 13 & & & 50 & & \\
\hline 1 & $3 / 12 / 78$ & 14 & & & 50 & & \\
\hline 1 & $4 / 11 / 78$ & 1 & & & 46 & & \\
\hline$\frac{1}{1}$ & $4 / 11 / 78$ & 2 & & & 47 & & \\
\hline 1 & $4 / 11 / 78$ & 3 & & & 47 & & \\
\hline 1 & $4 / 11 / 78$ & 4 & & & 47 & & \\
\hline 1 & $4 / 11 / 78$ & 5 & & & 47 & & \\
\hline 1 & $4 / 11 / 78$ & 6 & & & 47 & & \\
\hline 1 & $4 / 11 / 78$ & 7 & & & 47 & & \\
\hline 1 & $4 / 11 / 78$ & 8 & & & 47 & & \\
\hline 1 & $4 / 11 / 78$ & 10 & & & 48 & & \\
\hline 1 & $4 / 11 / 78$ & 12 & & & 47 & & \\
\hline 1 & $4 / 11 / 78$ & 14 & & & 48 & & \\
\hline 1 & $6 / 3 / 78$ & 1 & & & 60 & & \\
\hline 1 & $6 / 3 / 78$ & 2 & & & 60 & & \\
\hline 1 & $6 / 3 / 78$ & 3 & & & 60 & & \\
\hline 1 & $6 / 3 / 78$ & 4 & & & 60 & & \\
\hline 1 & $6 / 3 / 78$ & 5 & & & 60 & & \\
\hline 1 & $6 / 3 / 78$ & 6 & & & 60 & & \\
\hline 1 & $6 / 3 / 78$ & 7 & & & 60 & & \\
\hline 1 & $6 / 3 / 78$ & 8 & & & 60 & & \\
\hline 1 & $6 / 3 / 78$ & 9 & & & 92 & & \\
\hline 1 & $6 / 3 / 78$ & 12 & & & 58 & & \\
\hline 1 & $6 / 3 / 78$ & 13 & & & 58 & & \\
\hline 1 & $6 / 3 / 78$ & 14 & & & 58 & & \\
\hline 1 & $7 / 13 / 78$ & 1 & & & 86 & & \\
\hline 1 & $7 / 13 / 78$ & 2 & & & 86 & & \\
\hline 1 & $7 / 13 / 78$ & 3 & & & 86 & & \\
\hline 1 & $7 / 13 / 78$ & 4 & & & 86 & & \\
\hline 1 & $7 / 13178$ & 5 & & & 86 & & \\
\hline 1 & $7 / 13 / 78$ & 6 & & & 86 & & \\
\hline 1 & $7 / 13 / 78$ & 7 & & & 86 & & \\
\hline 1 & $7 / 13 / 78$ & 8 & & & 86 & & \\
\hline 1 & $7 / 13 / 78$ & 9 & & & 90 & & \\
\hline 1 & $7 / 13 / 78$ & 10 & & & 87 & & \\
\hline 1 & $7 / 13 / 78$ & 12 & & & 85 & & \\
\hline 1 & $7 / 13 / 78$ & 13 & & & 87 & & \\
\hline 1 & $7 / 13 / 78$ & 14 & & & 86 & & \\
\hline 1 & $8 / 11 / 78$ & 1 & & & 74 & & \\
\hline 1 & $8 / 11 / 78$ & 2 & & & 74 & & \\
\hline 1 & $8 / 11 / 78$ & 3 & & & 74 & & \\
\hline 1 & $8 / 11 / 78$ & 4 & & & 74 & & \\
\hline 1 & $8 / 111778$ & 5 & & & 75 & & \\
\hline
\end{tabular}

Data obtained from WHC Surveillance Analysis Computer System (SACS), February 24, 1994. 
WHC-SD-WM-ER-323, Rev. 0

\begin{tabular}{|c|c|c|c|c|c|c|c|}
\hline \multicolumn{8}{|c|}{ TANK 241-S-104 } \\
\hline \multicolumn{8}{|c|}{ THERMOCOUPLE DATA } \\
\hline TREE \# & DATE & THERMO \# & TREE SET \# & TEMP C & TEMP F & $Y=$ MAX TEMP & COMMENTS \\
\hline 1 & $8 / 11 / 78$ & 6 & & & 75 & & \\
\hline 1 & $8 / 11 / 78$ & 7 & & & 75 & & \\
\hline 1 & $8 / 11 / 78$ & 8 & & & 75 & & \\
\hline 1 & $8 / 11 / 78$ & 10 & & & 75 & & \\
\hline$\frac{1}{1}$ & $10 / 10 / 78$ & 1 & & & 72 & & \\
\hline 1 & $10 / 10 / 78$ & 2 & & & 72 & & \\
\hline 1 & $10 / 10 / 78$ & 3 & & & 73 & & \\
\hline 1 & $10 / 10 / 78$ & 4 & & & 73 & & \\
\hline 9 & $10 / 10 / 78$ & 5 & & & 72 & & \\
\hline 1 & $10 / 10 / 78$ & 6 & & & 72 & & \\
\hline 9 & $10 / 10 / 78$ & 7 & & & 73 & & \\
\hline 1 & $10 / 10 / 78$ & 8 & & & 73 & & \\
\hline 1 & $10 / 10 / 78$ & 9 & & & 99 & & \\
\hline 1 & $10 / 10 / 78$ & 10 & & & 73 & & \\
\hline 1 & $10 / 10 / 78$ & 12 & & & 99 & & \\
\hline 1 & $2 / 4 / 79$ & 1 & & & 42 & & \\
\hline 1 & $2 / 4 / 79$ & 2 & & & 43 & & \\
\hline 1 & $2 / 4 / 79$ & 3 & & & 43 & & \\
\hline 1 & $2 / 4 / 79$ & 4 & & & 43 & & \\
\hline 1 & $2 / 4 / 79$ & 5 & & & 43 & & \\
\hline 1 & $2 / 4 / 79$ & 6 & & & 43 & & \\
\hline$\frac{1}{1}$ & $2 / 4 / 79$ & 7 & & & 44 & & \\
\hline 1 & $2 / 4 / 79$ & 8 & & & 44 & & \\
\hline 1 & $2 / 4 / 79$ & 9 & & & 101 & & \\
\hline 1 & $2 / 4 / 79$ & 10 & & & 46 & & \\
\hline 1 & $2 / 4 / 79$ & 11 & & & 101 & & \\
\hline 1 & $2 / 4 / 79$ & 12 & & & 44 & & \\
\hline 1 & $2 / 4 / 79$ & 13 & & & 46 & & \\
\hline 1 & $2 / 4 / 79$ & 14 & & & 45 & & \\
\hline 1 & $2 / 4 / 79$ & 15 & & & 46 & & \\
\hline 1 & $2 / 4 / 79$ & 16 & & & 45 & & \\
\hline 1 & $2 / 8 / 79$ & 9 & & & 131 & & \\
\hline 1 & $2 / 8 / 79$ & 2 & & & 130 & & \\
\hline 9 & $2 / 8 / 79$ & 3 & & & 124 & & \\
\hline 9 & $2 / 8 / 79$ & 4 & & & 115 & & \\
\hline 1 & $2 / 8 / 79$ & 5 & & & 101 & & \\
\hline 9 & $2 / 8 / 79$ & 6 & & & 92 & & \\
\hline 1 & $2 / 8 / 79$ & 7 & & & 93 & & \\
\hline 1 & $2 / 8 / 79$ & 8 & & & 93 & & \\
\hline 9 & $2 / 8 / 79$ & 9 & & & 93 & & \\
\hline 9 & $2 / 8 / 79$ & 10 & & & 93 & & \\
\hline 9 & $2 / 8 / 79$ & 11 & & & 92 & & \\
\hline 1 & $2 / 8 / 79$ & 12 & & & 93 & & \\
\hline 1 & $2 / 8 / 79$ & 13 & & & 93 & & \\
\hline 1 & $2 / 8 / 79$ & 14 & & & 93 & & \\
\hline 1 & $2 / 14 / 84$ & 1 & & & 119 & & \\
\hline 9 & $2 / 14 / 84$ & 2 & & & 118 & & \\
\hline 9 & $2 / 14 / 84$ & 3 & & & 116 & & \\
\hline 1 & $2 / 74 / 84$ & 4 & & & 109 & & \\
\hline 1 & $2 / 14 / 84$ & 5 & & & 95 & & \\
\hline 1 & $2 / 14 / 84$ & 6 & & & 87 & & \\
\hline 1 & $2 / 14 / 84$ & 7 & & & 87 & & \\
\hline 1 & $2 / 14 / 84$ & 8 & & & 87 & & \\
\hline 1 & $2 / 14 / 84$ & 9 & & & 87 & & \\
\hline 1 & $2 / 14 / 84$ & 10 & & & 87 & & \\
\hline 1 & $2 / 14 / 84$ & 11 & & & 87 & & \\
\hline 1 & $2 / 14 / 84$ & 12 & & & 87 & & \\
\hline 1 & $2 / 14 / 84$ & 13 & & & 87 & & \\
\hline 1 & $2 / 14 / 84$ & 14 & & & 87 & & \\
\hline 1 & $1 / 4 / 91$ & 1 & 1 & & & $\mathbf{N}$ & $0 / 5$ \\
\hline 1 & $11 / 13 / 91$ & 1 & 1 & & 111.8 & N & J-890423320-42 TC \#1516 O/S W/O\#04529 \\
\hline 1 & $11 / 13 / 91$ & 2 & 1 & & 111 & $\mathbf{N}$ & \\
\hline 1 & $11 / 13 / 91$ & 3 & 1 & & 112.7 & $\bar{Y}$ & \\
\hline$\frac{1}{1}$ & $11 / 13 / 91$ & 4 & 1 & & 109.3 & $\mathbf{N}$ & \\
\hline 1 & $11 / 13 / 91$ & 5 & 1 & & 99.1 & $\mathbf{N}$ & \\
\hline 1 & $11 / 13 / 91$ & 6 & 1 & & 94 & $N$ & \\
\hline
\end{tabular}

-. Data obtained from WHC Surveillance Analysis Computer System (SACS), February 24, 1994. 
WHC-SD-WM-ER-323, Rev. 0

\begin{tabular}{|c|c|c|c|c|c|c|c|}
\hline \multicolumn{8}{|c|}{ TANK 241.S-104 } \\
\hline \multicolumn{8}{|c|}{ THERMOCOUPLE DATA } \\
\hline & & & & & & & \\
\hline TREE \# & DATE & THERMO \# & TREE SET \# & TEMP C & TEMP F & $Y=$ MAX TEMP & COMMENTS \\
\hline 1 & $11 / 13 / 91$ & 7 & 1 & & 93.7 & $\mathbf{N}$ & \\
\hline 1 & $11 / 13 / 91$ & 8 & 1 & & 93.6 & $\mathbf{N}$ & \\
\hline 1 & $11 / 13 / 91$ & 9 & 1 & & 93.6 & $N$ & \\
\hline 1 & $11 / 13 / 91$ & 10 & 1 & & 93.6 & $\mathrm{~N}$ & \\
\hline 1 & $11 / 13 / 91$ & 11 & 1 & 1 & 93.4 & $N$ & \\
\hline 1 & $11 / 13 / 91$ & 12 & 1 & & 93.4 & $\mathbf{N}$ & \\
\hline 1 & $11 / 13 / 91$ & 13 & 1 & & 93.2 & $\mathbf{N}$ & \\
\hline 1 & $11 / 13 / 97$ & 14 & 1 & & 93 & $\mathbf{N}$ & \\
\hline 1 & $1 / 1 / 92$ & 1 & 1 & & 111.1 & $\mathrm{~N}$ & $\mathrm{~J}-890523512-20 \mathrm{TC} \# 15.16 \mathrm{O} / \mathrm{S}$ \\
\hline 1 & $1 / 1 / 92$ & 2 & 1 & & 110.7 & $N$ & \\
\hline 1 & $1 / 1 / 92$ & 3 & 1 & & 112.3 & $\bar{Y}$ & \\
\hline 1 & $1 / 1 / 92$ & 4 & 1 & & 108.3 & $N$ & \\
\hline 1 & $1 / 1 / 92$ & 5 & 1 & & 96.1 & $N$ & \\
\hline 1 & $1 / 1 / 92$ & 6 & 1 & & 90.2 & $\mathrm{~N}$ & \\
\hline 1 & $1 / 1 / 92$ & 7 & 1 & & 89.9 & $N$ & \\
\hline 1 & $1 / 1 / 92$ & 8 & 1 & & 89.8 & $N$ & \\
\hline 1 & $1 / 1 / 92$ & 9 & 1 & & 89.7 & $N$ & \\
\hline 1 & $1 / 1 / 92$ & 10 & 1 & & 89.7 & $N$ & \\
\hline 1 & $1 / 1 / 92$ & 11 & 1 & & 89.6 & $N$ & \\
\hline 1 & $1 / 1 / 92$ & 12 & 1 & & 89.6 & $\mathrm{~N}$ & \\
\hline 1 & $1 / 1 / 92$ & 13 & 1 & & 89.4 & $N$ & \\
\hline 1 & $1 / 1 / 92$ & 14 & 1 & & 89.3 & $\mathrm{~N}$ & \\
\hline 1 & $7 / 3 / 92$ & 1 & 1 & & 103.5 & $\mathbf{N}$ & $\mathrm{J} 890423320-42$ \\
\hline 1 & $7 / 3 / 92$ & 2 & 1 & & 102.7 & $\mathbf{N}$ & \\
\hline 1 & $7 / 3 / 92$ & 3 & 1 & & 104.3 & $\mathbf{Y}$ & \\
\hline 1 & $7 / 3 / 92$ & 4 & 1 & & 100.3 & $\mathbf{N}$ & \\
\hline 1 & $7 / 3 / 92$ & 5 & 1 & & 89.6 & $\mathbf{N}$ & \\
\hline 1 & $7 / 3 / 92$ & 6 & 1 & & 84.7 & $\mathbf{N}$ & \\
\hline 1 & $7 / 3 / 92$ & 7 & 1 & & 84.5 & $\mathbf{N}$ & \\
\hline 1 & $7 / 3 / 92$ & 8 & 1 & & 84.6 & $N$ & \\
\hline 1 & $7 / 3 / 92$ & 9 & 1 & & 84.5 & $N$ & \\
\hline 1 & $7 / 3 / 92$ & 10 & 1 & & 84.6 & $N$ & \\
\hline 1 & $7 / 3 / 92$ & 11 & 1 & & 84.7 & $\mathbf{N}$ & \\
\hline 1 & $7 / 3 / 92$ & 12 & 1 & & 84.7 & $\mathbf{N}$ & \\
\hline 1 & $7 / 3 / 92$ & 13 & 1 & & 84.7 & $\mathbf{N}$ & \\
\hline 1 & $7 / 3 / 92$ & 14 & 1 & & 84.7 & $N$ & \\
\hline 1 & $2 / 13 / 93$ & 1 & 1 & & 107.6 & $Y$ & $\mathrm{~J} 910126143-42$ \\
\hline 1 & $2 / 13 / 93$ & 2 & 1 & & 106.1 & $\mathbf{N}$ & \\
\hline 1 & $2 / 13 / 93$ & 3 & 1 & & 108.4 & $\mathbf{N}$ & \\
\hline 1 & $2 / 13 / 93$ & 4 & 1 & & 103.7 & $\mathbf{N}$ & \\
\hline 1 & $2 / 13 / 93$ & 5 & 1 & & 90.6 & $\mathrm{~N}$ & \\
\hline 1 & $2 / 13 / 93$ & 6 & 1 & & 84.2 & $\mathbf{N}$ & \\
\hline 1 & $2 / 13 / 93$ & 7 & 1 & & 84 & $\mathbf{N}$ & \\
\hline 1 & $2 / 13 / 93$ & 8 & 1 & & 83.9 & $N$ & \\
\hline 1 & $2 / 13 / 93$ & 9 & 1 & & 83.7 & $\mathrm{~N}$ & \\
\hline 1 & $2 / 13 / 93$ & 10 & 1 & & 83.8 & $\mathbf{N}$ & \\
\hline 1 & $2 / 13 / 93$ & 11 & 1 & & 83.3 & $\mathbf{N}$ & \\
\hline 1 & $2 / 13 / 93$ & 12 & 1 & & 83.6 & $\mathbf{N}$ & \\
\hline 1 & $2 / 13 / 93$ & 13 & 1 & & 83.5 & $N$ & \\
\hline 1 & $2 / 13 / 93$ & 14 & 1 & & 83.1 & $\mathrm{~N}$ & \\
\hline 1 & $7 / 2 / 93$ & 1 & 1 & & 102.3 & $N$ & $\mathrm{~J} 890423320-42$ \\
\hline 1 & $7 / 2 / 93$ & 2 & 1 & & 102.1 & $\mathbf{N}$ & \\
\hline 1 & $7 / 2 / 93$ & 3 & 1 & & 103.5 & $Y$ & \\
\hline 1 & $7 / 2 / 93$ & 4 & 1 & & 99.5 & $\mathbf{N}$ & \\
\hline 1 & $7 / 2 / 93$ & 5 & 1 & & 88.1 & $\mathbf{N}$ & \\
\hline 1 & $7 / 2 / 93$ & 6 & 1 & & 83.1 & $\mathbf{N}$ & \\
\hline 1 & $7 / 2 / 93$ & 7 & 1 & & 83.1 & $\mathbf{N}$ & \\
\hline 1 & $7 / 2 / 93$ & 8 & 1 & & 83.3 & $N$ & \\
\hline 1 & $7 / 2 / 93$ & 9 & 1 & & 83.5 & $\mathbf{N}$ & \\
\hline 1 & $7 / 2 / 93$ & 10 & 1 & & 83.6 & $\mathrm{~N}$ & \\
\hline 1 & $7 / 2 / 93$ & 11 & 1 & & 83.6 & $\mathbf{N}$ & \\
\hline 1 & $7 / 2 / 93$ & 12 & 1 & & 83.6 & $N$ & \\
\hline 1 & $7 / 2 / 93$ & 13 & 1 & & 83.6 & $N$ & \\
\hline 1 & $7 / 2 / 93$ & 14 & 1 & & 83.5 & $\mathbf{N}$ & \\
\hline
\end{tabular}

Data obtained from WHC Surveillance Analysis Computer System (SACS), February 24, 1994. 
WHC-SD-WM-ER-323, Rev. 0

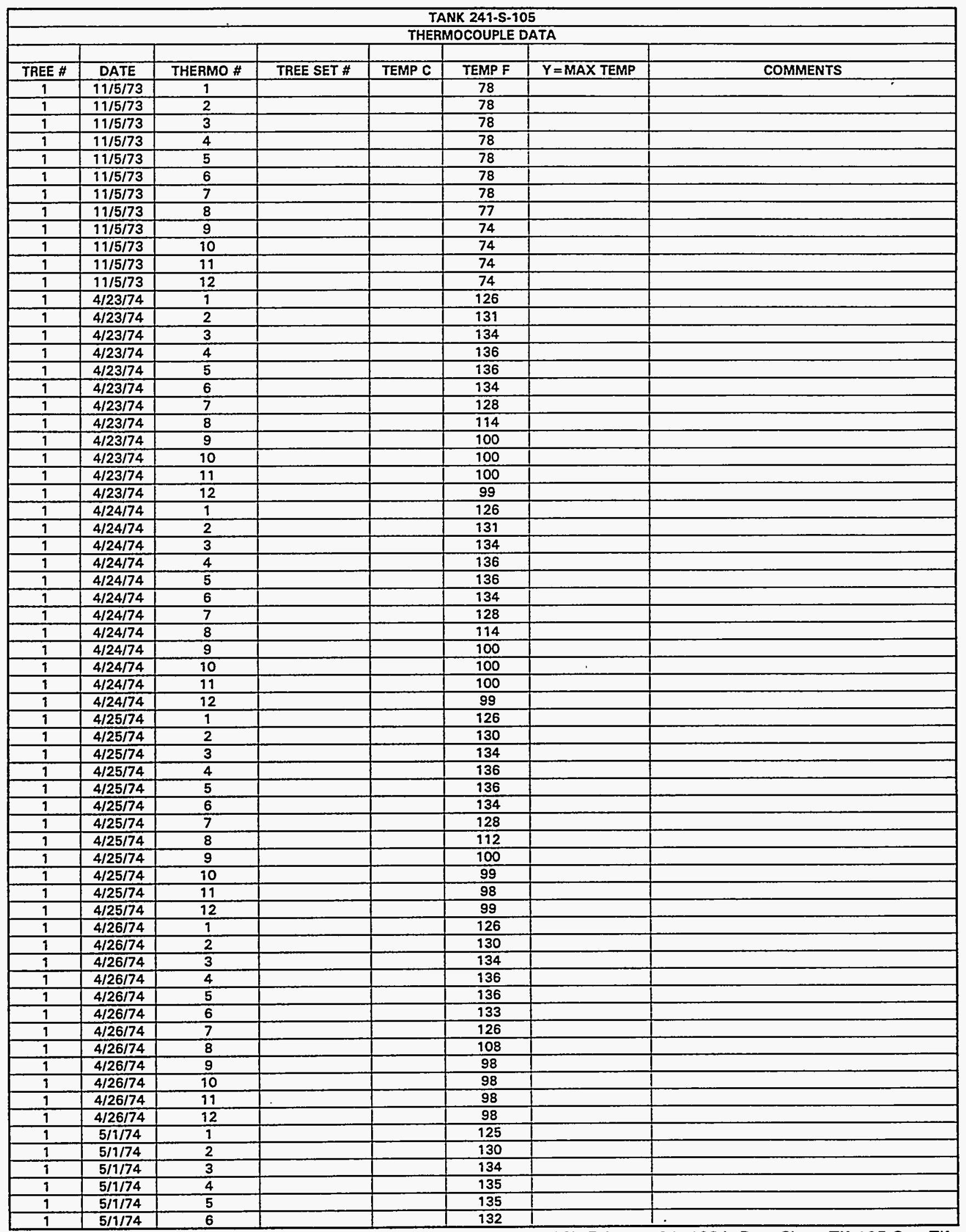

Data obtained from WHC Surveillance Analysis Computer System (SACS), February 24, 1994, Data Sheet TK-105-S to TK$101-S$, and Archive box $\# 121869$. 


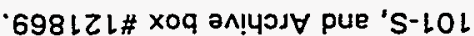

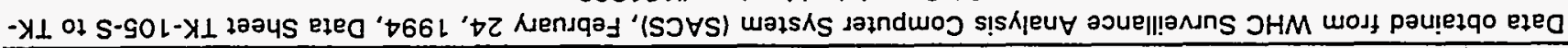

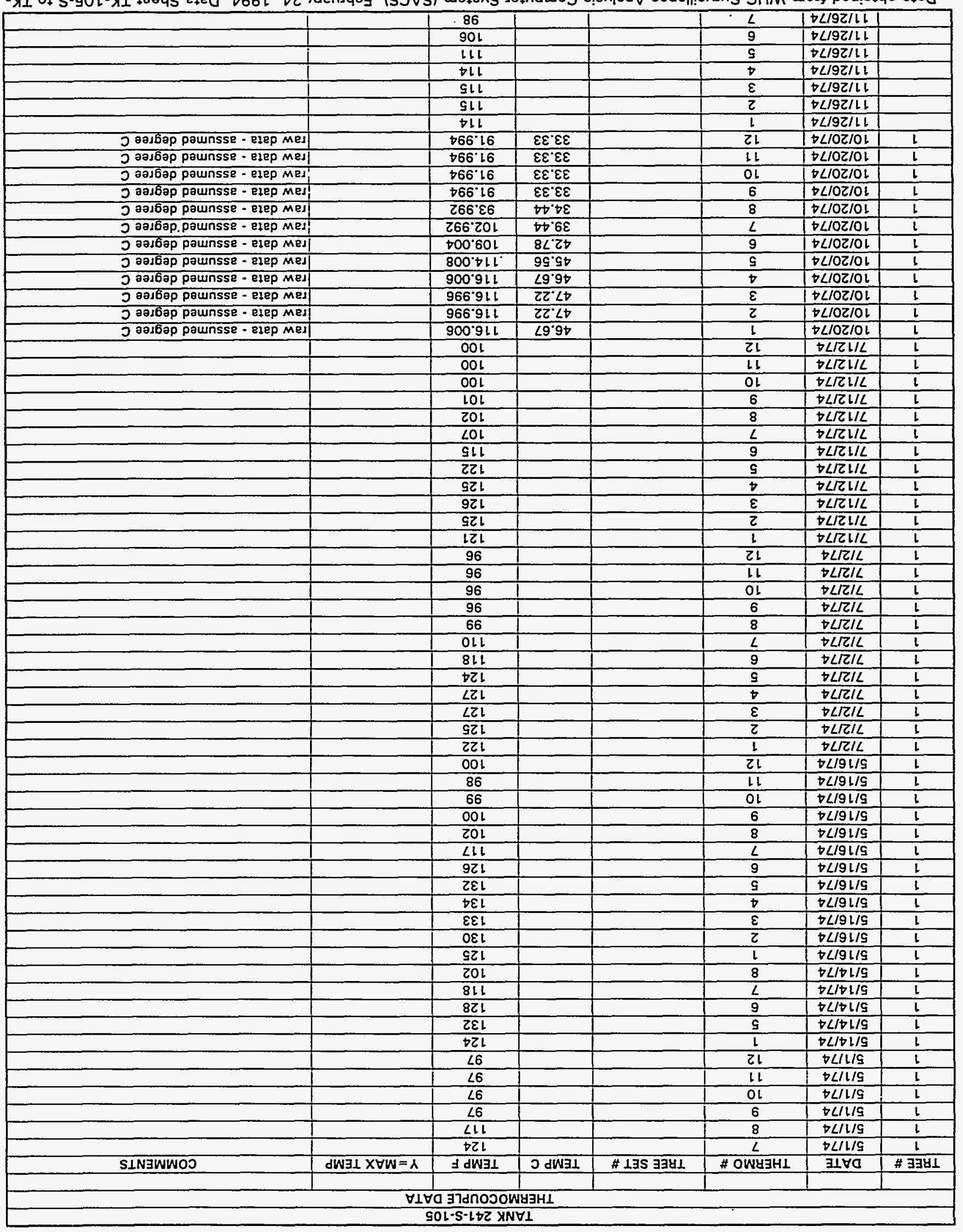




\begin{tabular}{|c|c|c|c|c|c|c|c|}
\hline \multicolumn{8}{|c|}{ TANK 241-S-105 } \\
\hline TREE \# & DATE & THERMO \# & TREE SET \# & TEMP C & TEMP F & $Y=$ MAX TEMP & COMMENTS \\
\hline & $11 / 26 / 74$ & 8 & & & 88 & & \\
\hline & $11 / 26 / 74$ & 9 & & & 87 & & \\
\hline & $11 / 26 / 74$ & 10 & & & 87 & & \\
\hline & $11 / 26 / 74$ & 11 & & & 87 & & \\
\hline & $11 / 26 / 74$ & 12 & & & 87 & & \\
\hline & $11 / 27 / 74$ & 1 & & & 114 & & \\
\hline & $11 / 27 / 74$ & 2 & & & 115 & & \\
\hline & $11 / 27 / 74$ & 3 & & & 115 & & \\
\hline & $11 / 27 / 74$ & 4 & & & 114 & & \\
\hline & $11 / 27 / 74$ & 5 & & & 911 & & \\
\hline & $11 / 27 / 74$ & 6 & & & 106 & & \\
\hline & $11 / 27 / 74$ & 7 & & & 98 & & \\
\hline & $11 / 27 / 74$ & 8 & & & 88 & & \\
\hline & $11 / 27 / 74$ & 9 & & & 87 & & \\
\hline & $11 / 27 / 74$ & 10 & & & 87 & & \\
\hline & $11 / 27 / 74$ & 11 & & & 87 & & \\
\hline & $11 / 27 / 74$ & 12 & & & 87 & & \\
\hline 1 & $12 / 2 / 74$ & 1 & & 45.56 & 114.008 & & raw data - assumed degree $C$ \\
\hline 1 & $12 / 2 / 74$ & 2 & & 46.11 & 114.998 & & raw data - assumed degreo $C$ \\
\hline 1 & $12 / 2 / 74$ & 3 & & 46.11 & 114.998 & & raw data - assumed degree C \\
\hline 1 & $12 / 2 / 74$ & 4 & & 45.56 & 114.008 & & Iraw data - assumed degree C \\
\hline 1 & $12 / 2 / 74$ & 5 & & 43.33 & 109.994 & & raw data - assumed degree C \\
\hline 1 & $12 / 2 / 74$ & 6 & & 40.56 & 105.008 & & raw data - assumed degree C \\
\hline 1 & $12 / 2 / 74$ & 7 & & 35.56 & 96.008 & & raw data - assumed degree C \\
\hline 1 & $12 / 2 / 74$ & 8 & & 30.56 & 87.008 & & raw data - assumed degree C \\
\hline 1 & $12 / 2 / 74$ & 9 & & 30.00 & 86 & & raw data - assumed degree C \\
\hline 1 & $12 / 2 / 74$ & 10 & & 30.00 & 86 & & raw data - assumed degree C \\
\hline 1 & $12 / 2 / 74$ & 11 & & 30.00 & 86 & & raw data - assumed degree C \\
\hline 1 & $12 / 2 / 74$ & 12 & & 30.00 & 86 & & raw data - assumed degree $C$ \\
\hline 1 & $11 / 2 / 75$ & 1 & & & 104 & & \\
\hline 1 & $11 / 2 / 75$ & 2 & & & 104 & & \\
\hline 1 & $11 / 2 / 75$ & 3 & & & 103 & & \\
\hline 1 & $11 / 2 / 75$ & 4 & & & 112 & & \\
\hline 1 & $11 / 2 / 75$ & 5 & & & 100 & & \\
\hline 1 & $11 / 2 / 75$ & 6 & & & 97 & & \\
\hline 1 & $11 / 2 / 75$ & 7 & & & 92 & & \\
\hline 1 & $11 / 2 / 75$ & 8 & & & 88 & & \\
\hline 1 & $11 / 2 / 75$ & 9 & & & 88 & & \\
\hline 1 & $11 / 2 / 75$ & 10 & & & 88 & & \\
\hline 1 & $11 / 2 / 75$ & 11 & & & 88 & & \\
\hline 1 & $11 / 2 / 75$ & 12 & & & 88 & & \\
\hline 1 & $2 / 4 / 76$ & 1 & & & 102 & & \\
\hline 1 & $2 / 4 / 76$ & 2 & & & 102 & & \\
\hline 1 & $2 / 4 / 76$ & 3 & & & 100 & & \\
\hline 1 & $2 / 4 / 76$ & 4 & & & 98 & & \\
\hline 1 & $2 / 4 / 76$ & 5 & & & 96 & & \\
\hline 1 & $2 / 4 / 76$ & 6 & & & 91 & & \\
\hline 1 & $2 / 4 / 76$ & 7 & & & 83 & & \\
\hline 9 & $2 / 4 / 76$ & 8 & & & 76 & & \\
\hline 1 & $2 / 4 / 76$ & 9 & & & 76 & & \\
\hline 1 & $2 / 4 / 76$ & 10 & & & 76 & & \\
\hline 1 & $2 / 4 / 76$ & 11 & & & 76 & & \\
\hline 1 & $2 / 4 / 76$ & 12 & & & 76 & & \\
\hline 1 & $3 / 5 / 76$ & 1 & & & 101 & & \\
\hline 1 & $3 / 5 / 76$ & 2 & & & 101 & & \\
\hline 1 & $3 / 5 / 76$ & 3 & & & 99 & & \\
\hline 1 & $3 / 5 / 76$ & 4 & & & 97 & & \\
\hline 1 & $3 / 5 / 76$ & 5 & & & 94 & & \\
\hline 1 & $3 / 5 / 76$ & 6 & & & 89 & & \\
\hline 1 & $3 / 5 / 76$ & 7 & & & 83 & & \\
\hline 1 & $3 / 5 / 76$ & 8 & & & 77 & & \\
\hline 1 & $3 / 5 / 76$ & 9 & & & 77 & & \\
\hline 1 & $3 / 5 / 76$ & 10 & & & 77 & & \\
\hline 1 & $3 / 5 / 76$ & 11 & & & 77 & & \\
\hline 1 & $3 / 5 / 76$ & 12 & & & 77 & & \\
\hline 9 & $5 / 2 / 76$ & 1 & & & 100 & & \\
\hline
\end{tabular}

Data obtained from WHC Surveillance Analysis Computer System (SACS), February 24, 1994, Data Sheet TK-105-S to TK101-S, and Archive box \#121869. 


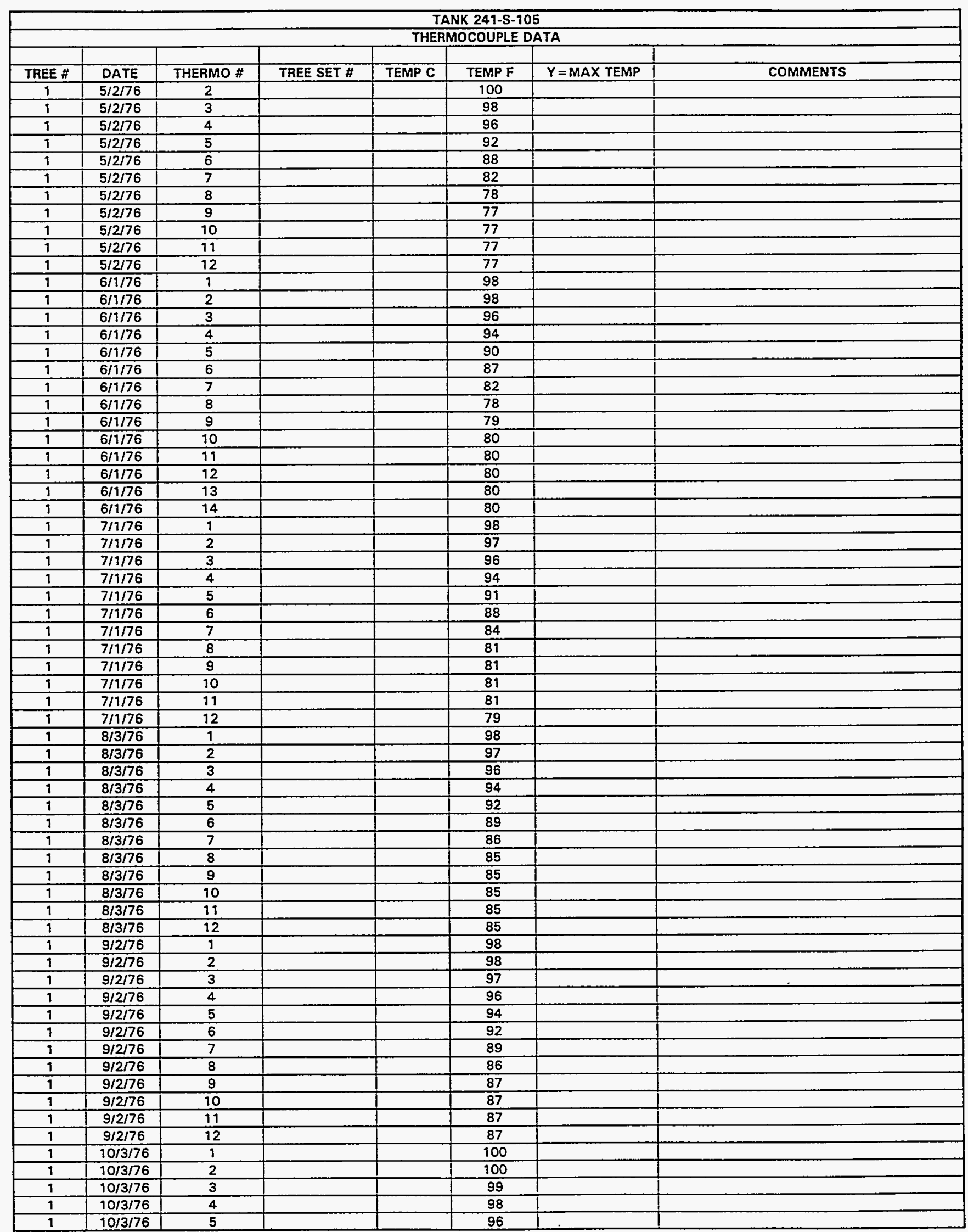

Data obtained from WHC Surveillance Analysis Computer System (SACS). February 24, 1994, Data Sheet TK-105-S to TK-. 101-S, and Archive box \#121869. 


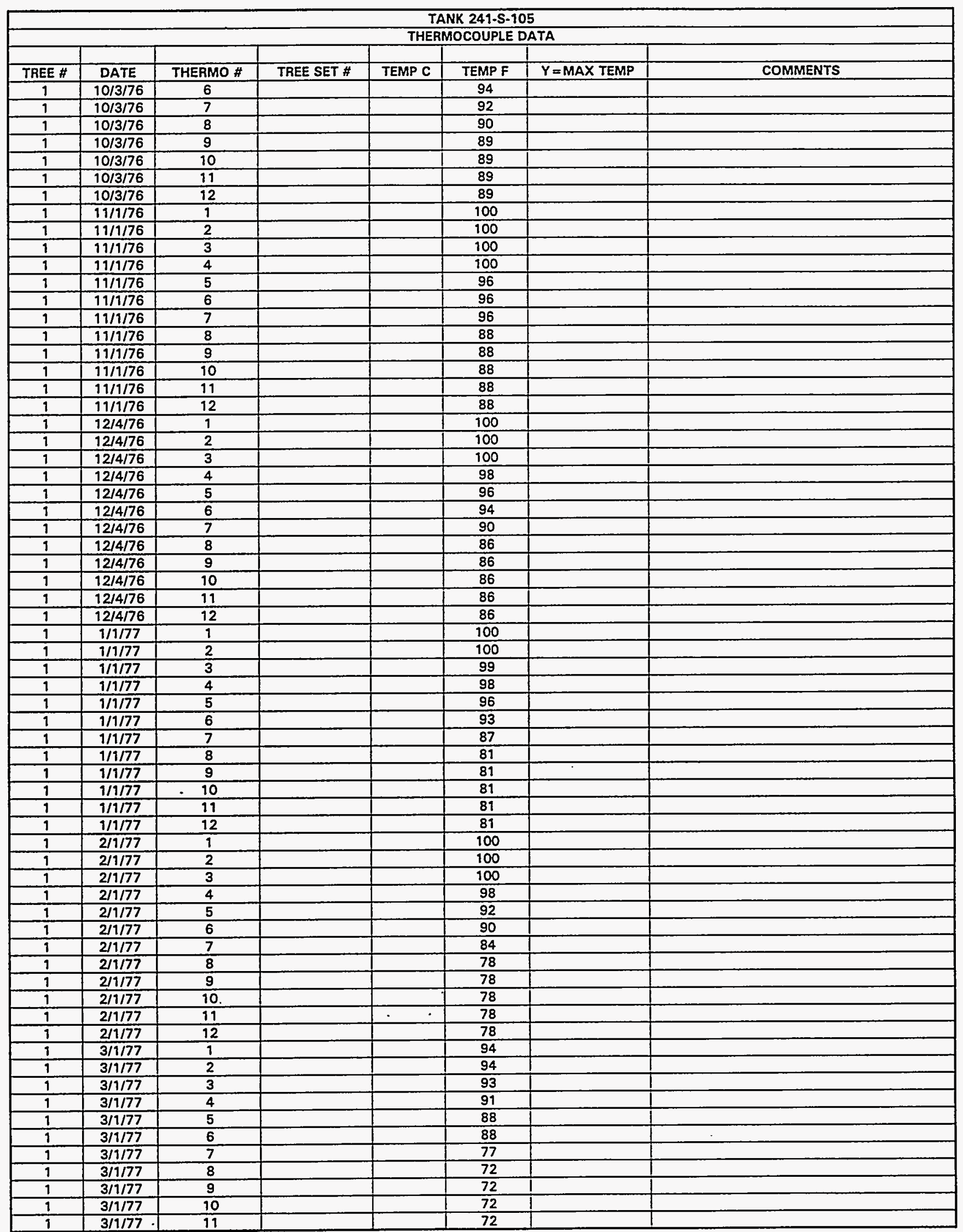

Data obtained from WHC Surveillance Analysis Computer System (SACS), February 24, 1994, Data Sheet TK-105-S to TK101-S, and Archive box $\# 121869$. 


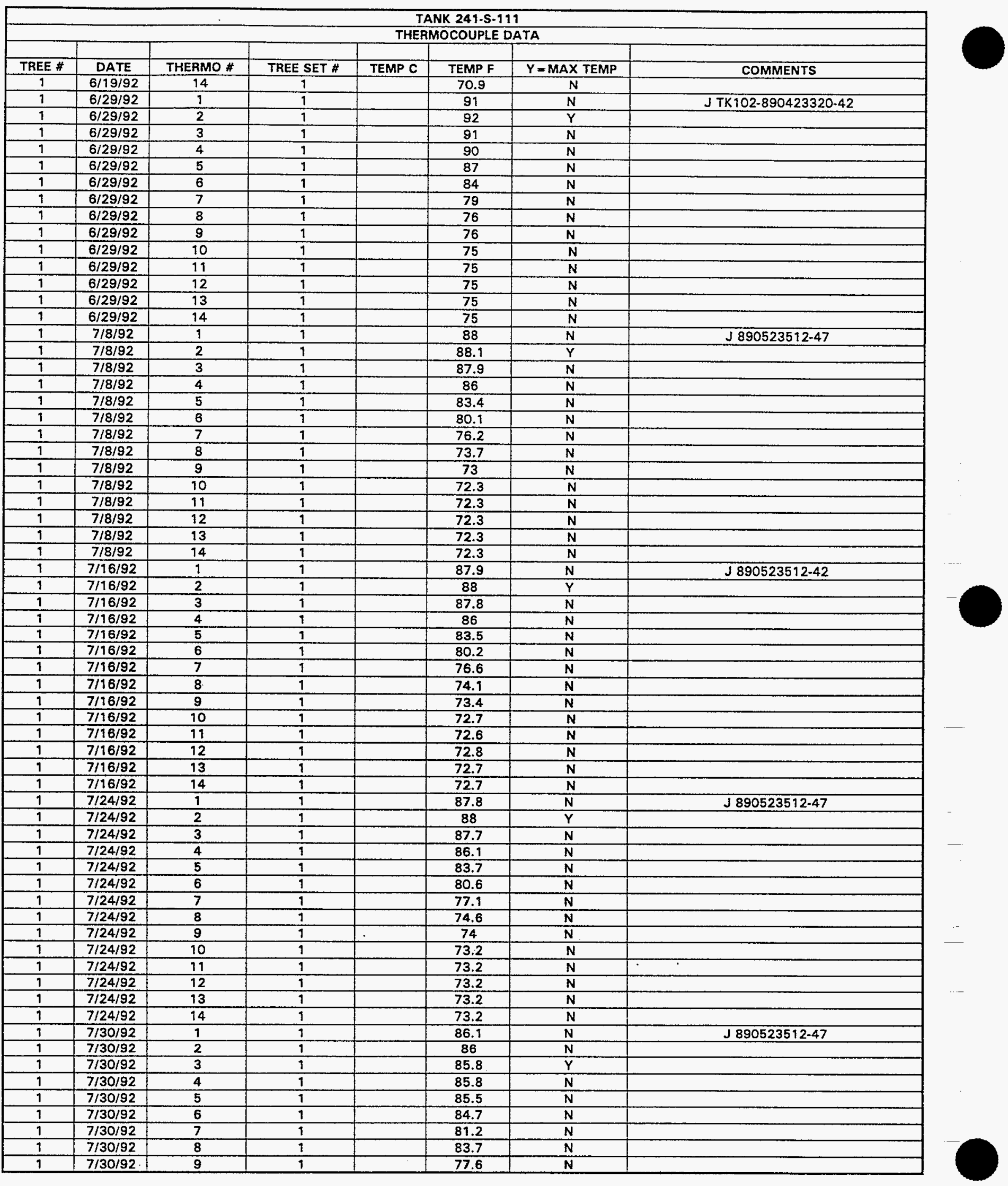

Data obtained from WHC Surveillance Analysis Computer System (SACS), February 24, 1994. 


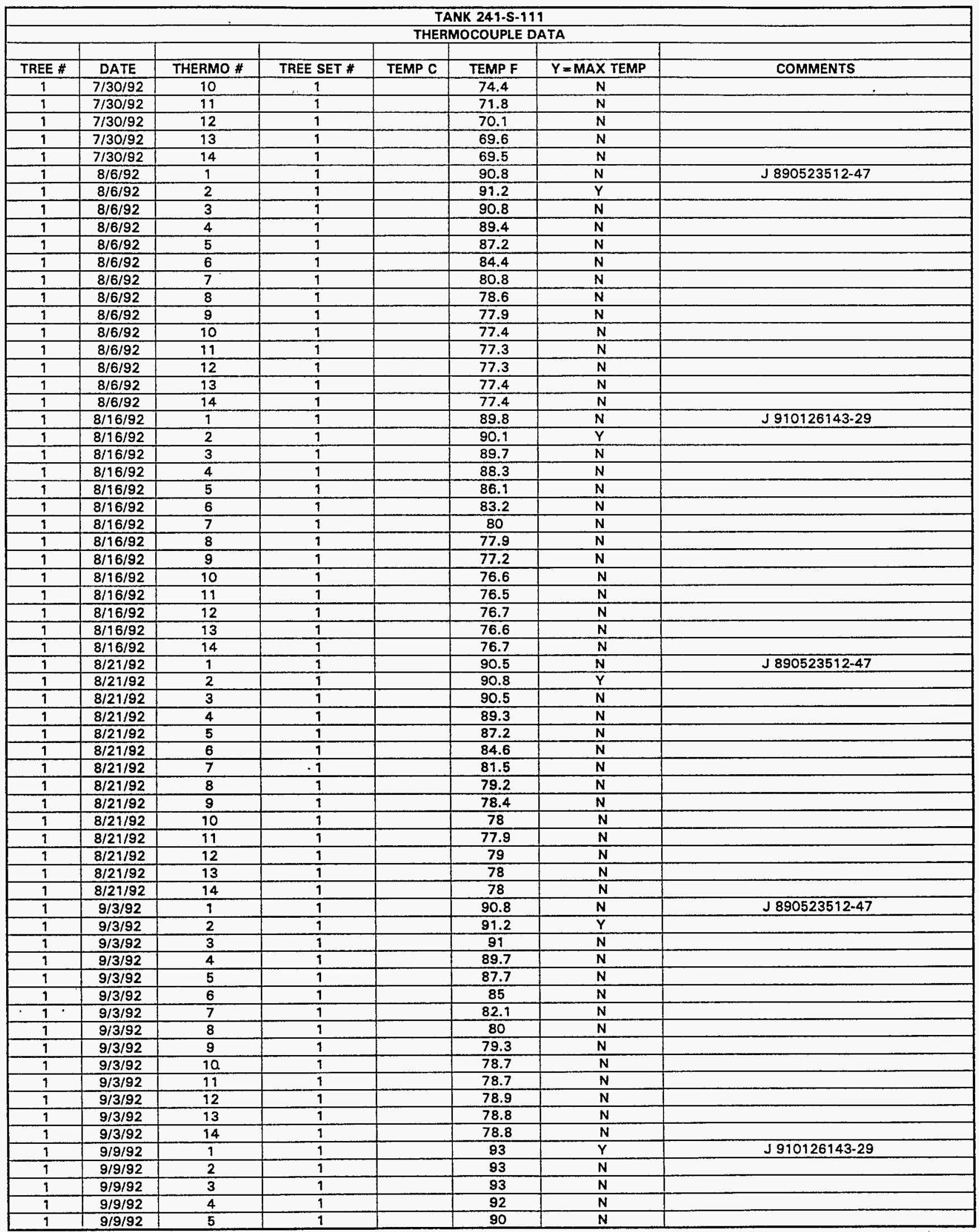

Data obtained from WHC Surveillance Analysis Computer System (SACS), February 24, 1994. 


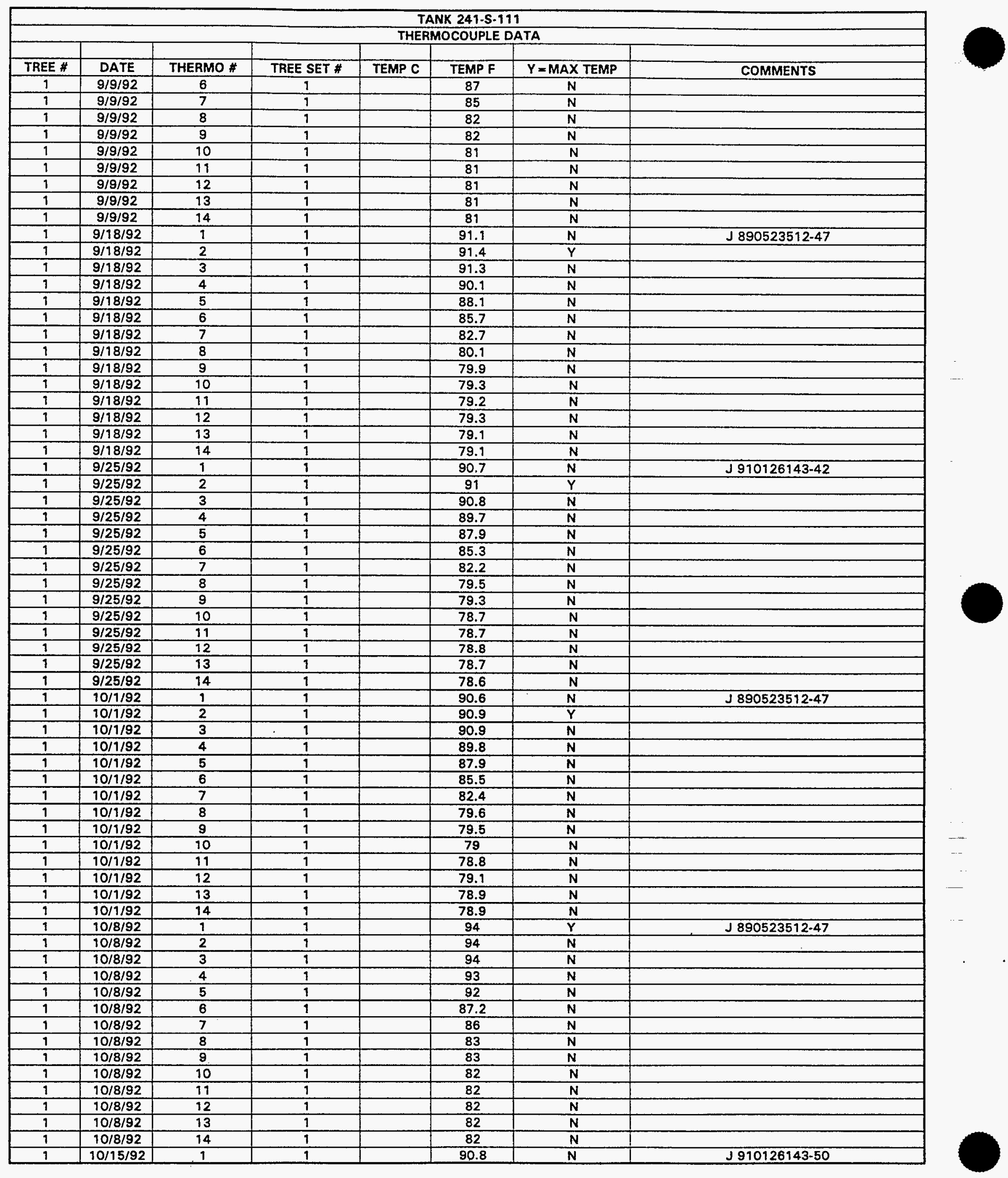

Data obtained from WHC Surveillance Analysis Computer System (SACS), February 24, 1994. 


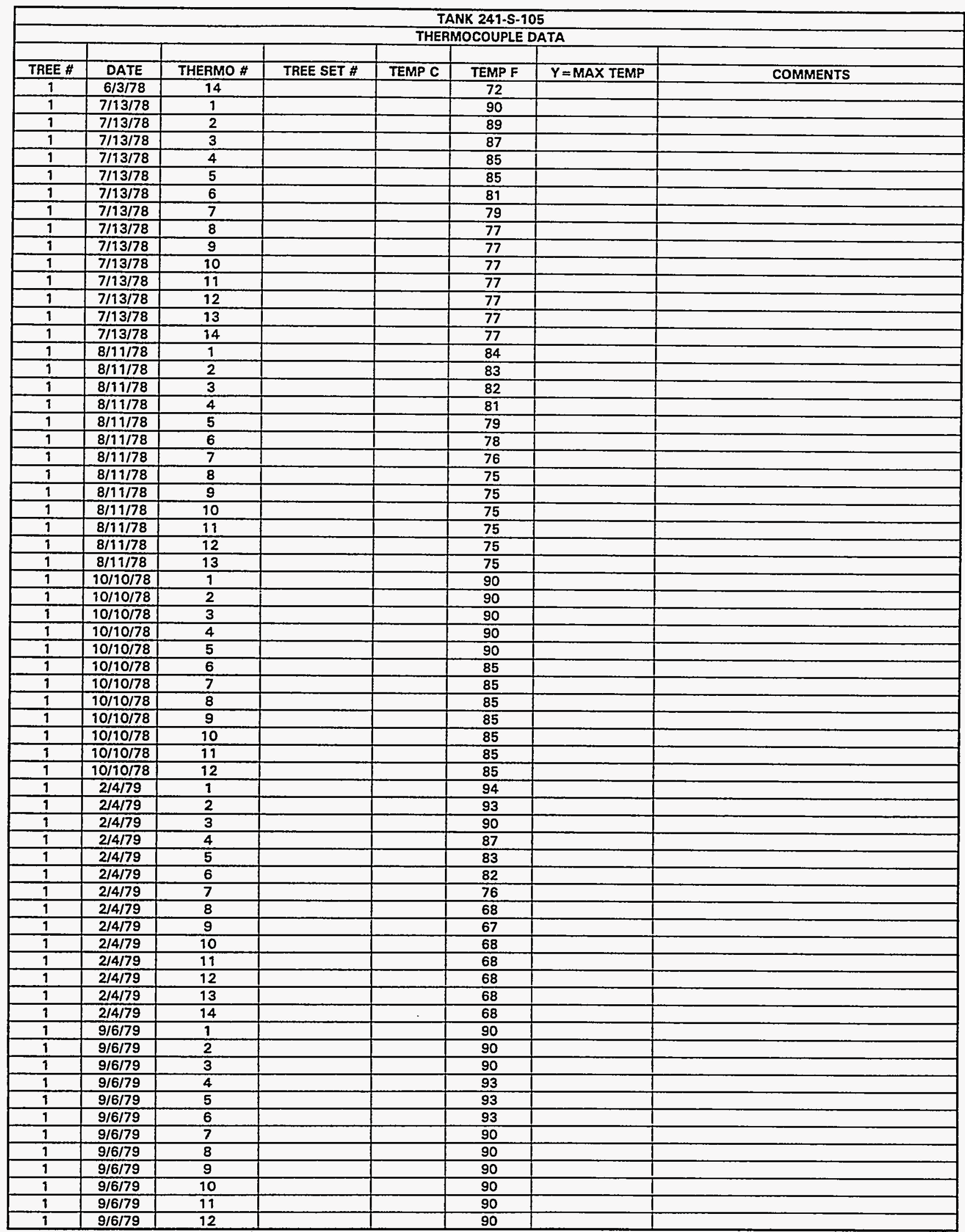

Data obtained from WHC Surveillance Analysis Computer System (SACS), February 24, 1994, Data Sheet TK-105-S to TK-. 101-S, and Archive box \#121869. 


\begin{tabular}{|c|c|c|c|c|c|c|c|}
\hline \multicolumn{8}{|c|}{ TANK 241-S-105 } \\
\hline \multicolumn{8}{|c|}{ THERMOCOUPLE DATA } \\
\hline TREE \# & DATE & THERMO \# & TREE SET \# & TEMP C & TEMP F & $Y=$ MAX TEMP & COMMENTS \\
\hline 1 & $9 / 6 / 79$ & 13 & & & 90 & & \\
\hline 1 & $9 / 6 / 79$ & 94 & & & 90 & & \\
\hline 1 & $1 / 23 / 80$ & 1 & & & 90 & & \\
\hline 1 & $1 / 23 / 80$ & 2 & & & 90 & & \\
\hline 1 & $1 / 23 / 80$ & 3 & & & 90 & & \\
\hline 1 & $1 / 23 / 80$ & 4 & & & 90 & & \\
\hline 1 & $1 / 23 / 80$ & 5 & & & & & 125 - suspect data (high) \\
\hline 1 & $1 / 23 / 80$ & 6 & & & & & 120 - suspect data (high) \\
\hline 1 & $1 / 23 / 80$ & 7 & & & 75 & & \\
\hline 1 & $1 / 23 / 80$ & 8 & & & 75 & & \\
\hline 1 & $1 / 23 / 80$ & 9 & & & 75 & & \\
\hline 1 & $1 / 23 / 80$ & 10 & & & 75 & & \\
\hline 1 & $1 / 23 / 80$ & 11 & & & 75 & & \\
\hline 1 & $1 / 23 / 80$ & 12 & & & 75 & & \\
\hline 1 & $1 / 23 / 80$ & 13 & & & 75 & & \\
\hline 1 & $1 / 23 / 80$ & 14 & & & 75 & & \\
\hline 1 & $2 / 1 / 80$ & 1 & & & 75 & & \\
\hline 1 & $2 / 1 / 80$ & 2 & & & 80 & & \\
\hline 1 & $2 / 1 / 80$ & 3 & & & 80 & & \\
\hline 1 & $2 / 1 / 80$ & 4 & & & 80 & & \\
\hline 1 & $2 / 1 / 80$ & 5 & & & 85 & & \\
\hline 1 & $2 / 1 / 80$ & 6 & & & 75 & & \\
\hline 1 & $2 / 1 / 80$ & 7 & & & & & 120 - suspect data (high) \\
\hline 1 & $2 / 1 / 80$ & 8 & & & 75 & & \\
\hline 1 & $2 / 1 / 80$ & 9 & & & 75 & & \\
\hline 1 & $2 / 1 / 80$ & 10 & & & 75 & & \\
\hline 1 & $2 / 1 / 80$ & 11 & & & 75 & & \\
\hline 1 & $2 / 1 / 80$ & 12 & & & 75 & & \\
\hline 1 & $2 / 1 / 80$ & 13 & & & 75 & & \\
\hline 1 & $2 / 1 / 80$ & 14 & & & 75 & & \\
\hline 1 & $4 / 12 / 82$ & 1 & & & 87 & & \\
\hline 1 & $4 / 12 / 82$ & 2 & & & 86 & & \\
\hline 1 & $4 / 12 / 82$ & 3 & & & 84 & & \\
\hline 1 & $4 / 12 / 82$ & 4 & & & 83 & & \\
\hline 1 & $4 / 12 / 82$ & 5 & & & 75 & & \\
\hline 1 & $4 / 12 / 82$ & 6 & & & 76 & & \\
\hline 1 & $4 / 12 / 82$ & 7 & & & 73 & & \\
\hline 1 & $4 / 12 / 82$ & 8 & & & 72 & & \\
\hline 1 & $4 / 12 / 82$ & 9 & & & 72 & & \\
\hline 1 & $4 / 12 / 82$ & 10 & & & 72 & & \\
\hline 1 & $4 / 12 / 82$ & 11 & & & 72 & & \\
\hline 1 & $4 / 12 / 82$ & 12 & & & 72 & & \\
\hline 1 & $4 / 12 / 82$ & 13 & & & 72 & & \\
\hline 1 & $4 / 12 / 82$ & 14 & & & 72 & & \\
\hline 1 & $4 / 12 / 82$ & 15 & & & 72 & & \\
\hline 1 & $4 / 12 / 82$ & 16 & & & 66 & & \\
\hline 1 & $5 / 29 / 82$ & 1 & & & 84 & & \\
\hline 1 & $5 / 29 / 82$ & 2 & & & 83 & & \\
\hline 1 & $5 / 29 / 82$ & 3 & & & 81 & & \\
\hline 1 & $5 / 29 / 82$ & 4 & & & 78 & & \\
\hline 1 & $5 / 29 / 82$ & 5 & & & 76 & & \\
\hline 1 & $5 / 29 / 82$ & 6 & & & 74 & & \\
\hline 1 & $5 / 29 / 82$ & 7 & & & 73 & & \\
\hline 1 & $5 / 29 / 82$ & 8 & & . & 73 & & \\
\hline 1 & $5 / 29 / 82$ & 9 & & & 72 & & \\
\hline 1 & $5 / 29 / 82$ & 10 & & & 73 & & \\
\hline 1 & $5 / 29 / 82$ & 11 & & & 72 & & \\
\hline 9 & $5 / 29 / 82$ & 12 & & & 72 & & \\
\hline 1 & $10 / 6 / 82$ & 1 & & & 84 & & \\
\hline 1 & $10 / 6 / 82$ & 2 & & & 84 & & \\
\hline 1 & $10 / 6 / 82$ & 3 & & & 83 & & \\
\hline 1 & $10 / 6 / 82$ & 4 & & & 82 & & \\
\hline$\frac{1}{1}$ & $10 / 6 / 82$ & 5 & & & 81 & & \\
\hline 1 & $10 / 6 / 82$ & 6 & & & 81 & & \\
\hline 9 & $10 / 6 / 82$ & 7 & & & 80 & & \\
\hline 1 & $10 / 6 / 82$ & 8 & & & 80 & & \\
\hline
\end{tabular}

Data obtained from WHC Surveillance Analysis Computer System (SACS), February 24, 1994, Data Sheet TK-105-S to TK101-S, and Archive box \#121869. 


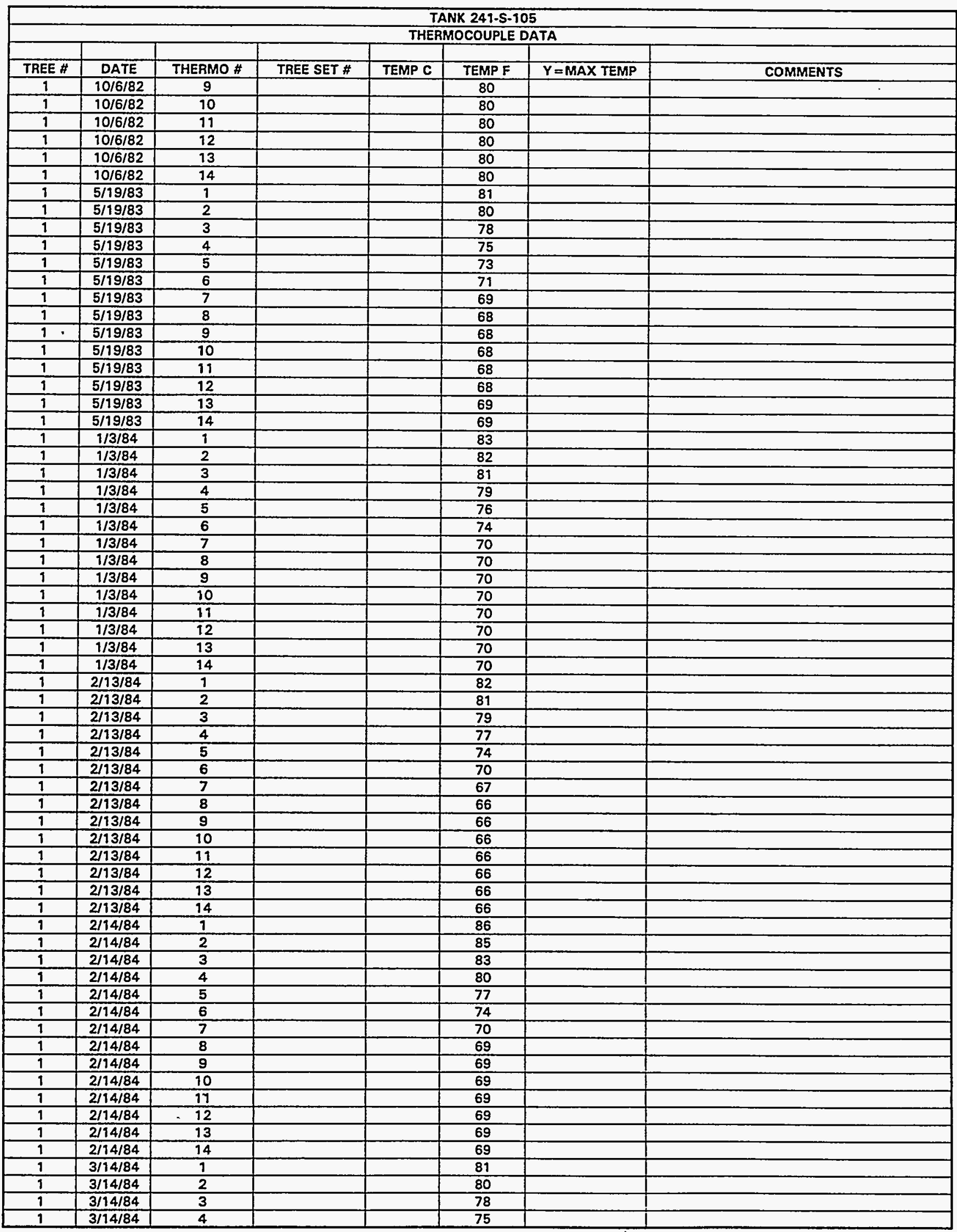

Data obtained from WHC Surveillance Analysis Computer System (SACS), February 24, 1994, Data Sheet TK-105-S to TK101 -S, and Archive box \#121869. 


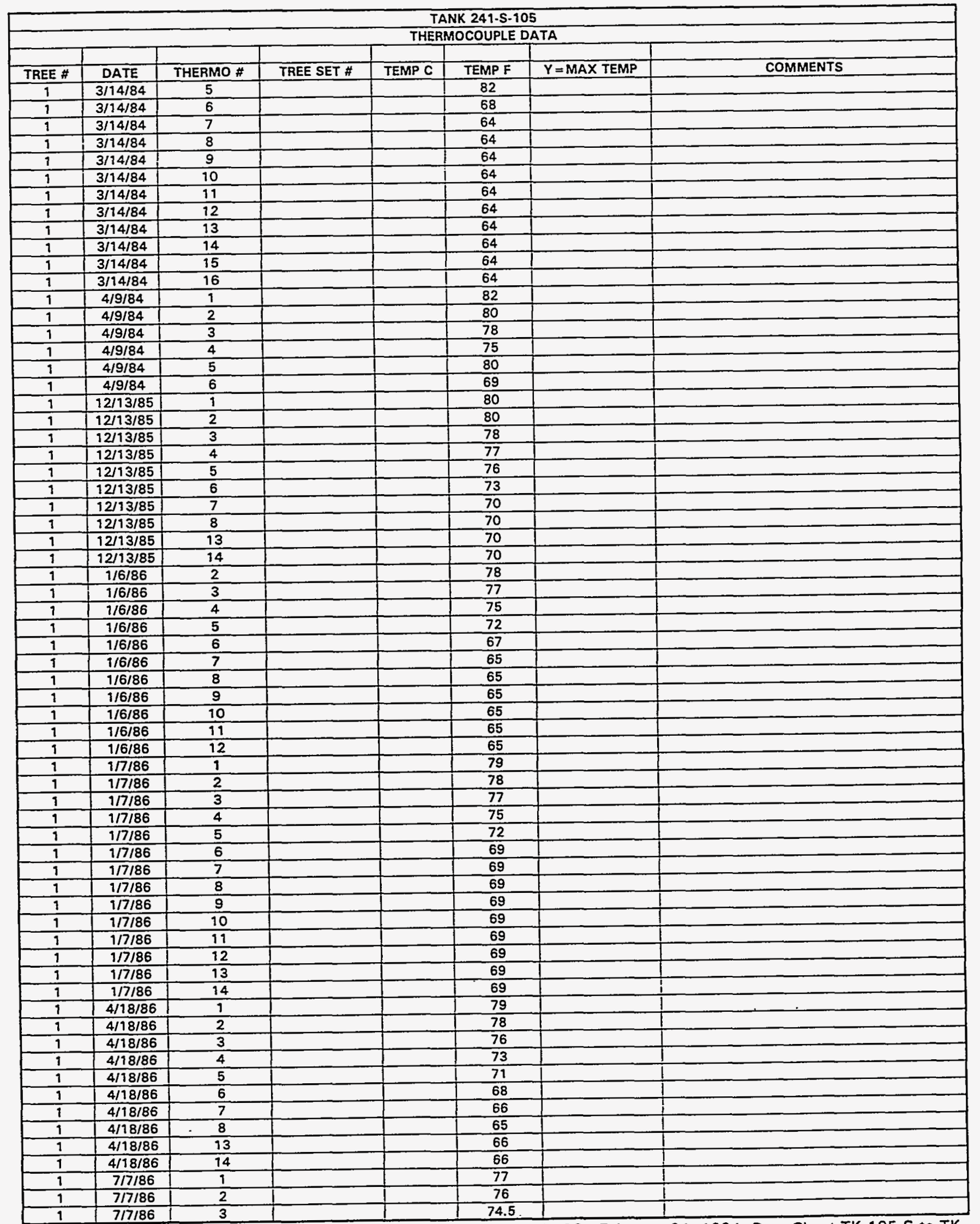

Data obtained from WHC Surveillance Analysis Computer System (SACS), February 24, 1994, Data Sheet TK-105-S to TK$101-S$, and Archive box $\$ 121869$. 


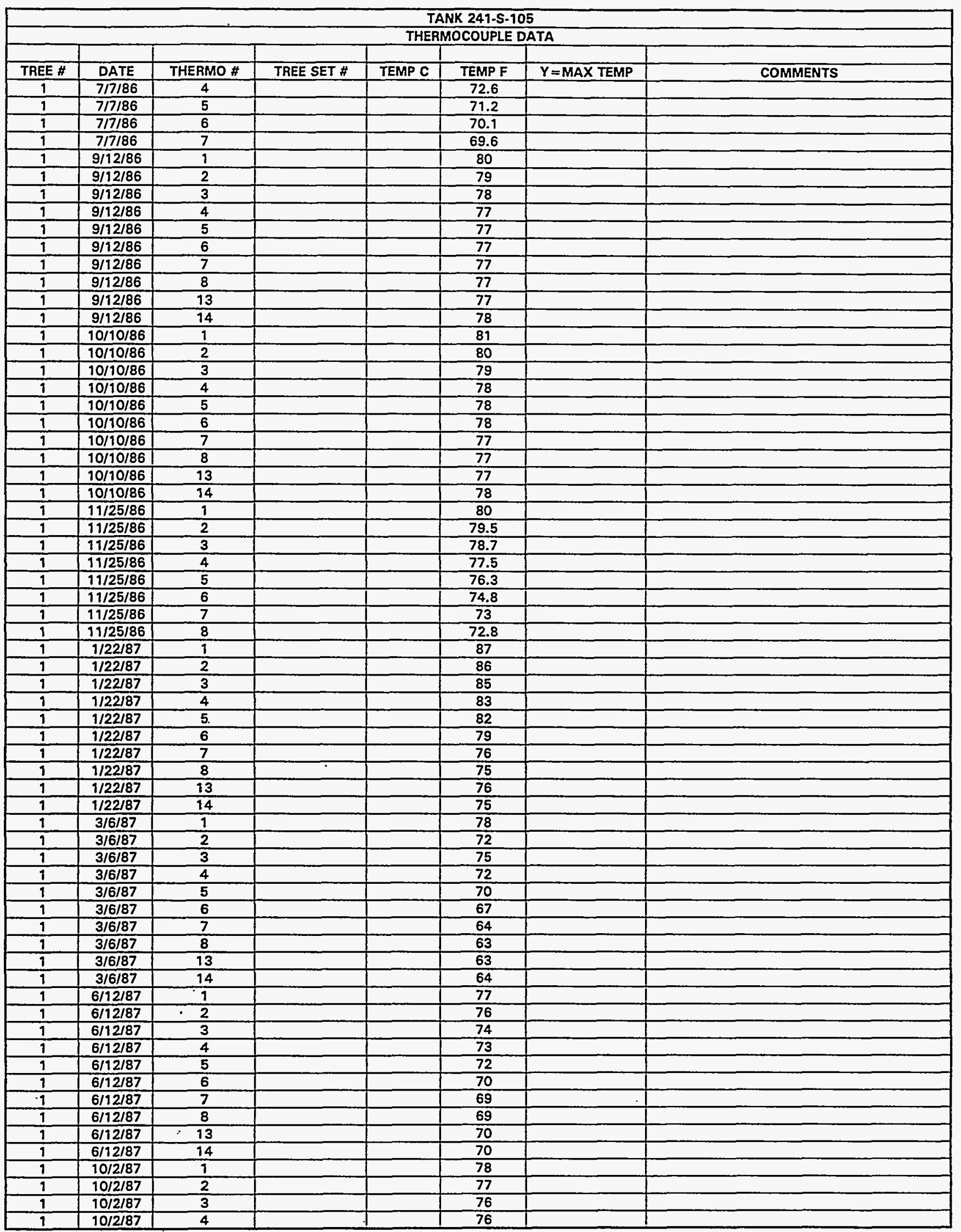

Data obtained from WHC Surveillance Analysis Computer System (SACS), February 24, 1994, Data Sheet TK-105-S to TK101-S, and Archive box \#121869. 


\begin{tabular}{|c|c|c|c|c|c|c|c|}
\hline \multicolumn{8}{|c|}{ TANK 241.S.105 } \\
\hline & & 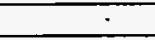 & & THER & COUPLE & & \\
\hline TREE \# & DATE & THERMO \# & TREE SET \# & TEMP C & TEMP $F$ & $\bar{Y}=$ MAX TEMP & COMMENTS \\
\hline 1 & $10 / 2 / 87$ & 5 & & & 76 & & \\
\hline 1 & $10 / 2 / 87$ & 6 & & & 75 & & \\
\hline 1 & $10 / 2 / 87$ & 7 & & & 76 & & \\
\hline 1 & $10 / 2 / 87$ & 8 & & & 76 & & \\
\hline 1 & $10 / 2 / 87$ & 13 & & & 76 & & \\
\hline 1 & $10 / 2 / 87$ & 14 & & & 76 & & \\
\hline 1 & $12 / 18 / 87$ & 1 & & & 86 & & \\
\hline 1 & $12 / 18 / 87$ & 2 & & & 85 & & \\
\hline 1 & $12 / 18 / 87$ & 3 & & & 84 & & \\
\hline 1 & $12 / 18 / 87$ & 4 & & & 83 & & \\
\hline 1 & $12 / 18 / 87$ & 5 & & & 82 & & \\
\hline 1 & $12 / 18 / 87$ & 6 & & & 80 & & \\
\hline 1 & $12 / 18 / 87$ & 7 & & & 78 & & \\
\hline 1 & $12 / 18 / 87$ & 8 & & & 78 & & \\
\hline 1 & $12 / 18 / 87$ & 13 & & & 78 & & \\
\hline 9 & $12 / 18 / 87$ & 14 & & & 78 & & \\
\hline 1 & $2 / 5 / 88$ & 1 & & & 86 & & \\
\hline 9 & $2 / 5 / 88$ & 2 & & & 86 & & \\
\hline 1 & $2 / 5 / 88$ & 3 & & & 85 & & \\
\hline 1 & $2 / 5 / 88$ & 4 & & & 83 & & \\
\hline 9 & $2 / 5 / 88$ & 5 & & & 81 & & \\
\hline 1 & $2 / 5 / 88$ & 6 & & & 78 & & \\
\hline 1 & $2 / 5 / 88$ & 7 & & & 74 & & \\
\hline 9 & $2 / 5 / 88$ & 8 & & & 74 & & \\
\hline 9 & $2 / 5 / 88$ & 13 & & & 74 & & \\
\hline 1 & $2 / 5 / 88$ & 14 & & & 74 & & \\
\hline 9 & $1 / 4 / 91$ & 1 & 9 & & 75.6 & $\mathbf{Y}$ & HH-99A-J \#890523512-47 \\
\hline 1 & $1 / 4 / 91$ & 2 & 1 & & 75.2 & $\mathrm{~N}$ & \\
\hline 1 & $1 / 4 / 91$ & $\overline{3}$ & 9 & & 74.4 & $\mathbf{N}$ & \\
\hline 1 & $1 / 4 / 91$ & 4 & 1 & & 73.1 & $\mathrm{~N}$ & \\
\hline 1 & $1 / 4 / 91$ & 5 & 1 & & 71.6 & $\mathbf{N}$ & \\
\hline 1 & $1 / 4 / 91$ & 6 & $T$ & & 69.3 & $\mathrm{~N}$ & \\
\hline 1 & $1 / 4 / 91$ & 7 & 1 & & 66.7 & $\mathrm{~N}$ & \\
\hline 1 & $9 / 4 / 91$ & 8 & 1 & & 66.6 & $\overline{\mathbf{N}}$ & \\
\hline 1 & $1 / 4 / 91$ & 9 & 1 & & 66.6 & $\mathbf{N}$ & \\
\hline 1 & $1 / 4 / 91$ & 10 & 1 & & 66.6 & $\mathrm{~N}$ & \\
\hline 1 & $1 / 4 / 91$ & 11 & 1 & & 66.6 & $\mathbf{N}$ & \\
\hline 1 & $1 / 4 / 91$ & 12 & 1 & & 66.6 & $N$ & \\
\hline 1 & $1 / 4 / 91$ & 13 & 1 & & 66.7 & $\mathbf{N}$ & \\
\hline 1 & $1 / 4 / 91$ & 14 & 1 & & 66.7 & $\mathbf{N}$ & \\
\hline 1 & $11 / 13 / 91$ & 1 & 1 & & 74.3 & $\bar{Y}$ & J-890423320-42 TC \#14-16 O/S W/O \#04529 \\
\hline 1 & $11 / 13 / 99$ & 2 & 1 & & 73.8 & $\mathrm{~N}$ & \\
\hline 9 & $11 / 13 / 91$ & 3 & 1 & & 73.8 & $\mathbf{N}$ & \\
\hline 1 & $11 / 13 / 91$ & 4 & 1 & & 73.2 & $\mathbf{N}$ & \\
\hline 1 & $11 / 13 / 91$ & 5 & 9 & & 72.8 & $\bar{N}$ & \\
\hline 1 & $11 / 13 / 91$ & 6 & 1 & & 72 & $\bar{N}$ & \\
\hline 1 & $11 / 13 / 99$ & 7 & 1 & & 70.8 & $\mathrm{~N}$ & \\
\hline 1 & $11 / 13 / 91$ & 8 & 1 & & 70.8 & $\mathbf{N}$ & \\
\hline 1 & $11 / 13 / 91$ & 9 & 1 & & 70.8 & $\bar{N}$ & \\
\hline 9 & $11 / 13 / 91$ & 10 & 1 & & 70.8 & $\bar{N}$ & \\
\hline 9 & $11 / 13 / 91$ & 19 & 1 & & 70.7 & $\mathrm{~N}$ & \\
\hline 9 & $11 / 13 / 91$ & 12 & 1 & & 70.8 & $\mathrm{~N}$ & \\
\hline 1 & $11 / 13 / 91$ & 13 & 1 & & 70.9 & $N$ & \\
\hline 1 & $1 / 1 / 92$ & 1 & 1 & & 79.1 & $\mathbf{N}$ & $J-890523512.20$ TC $\# 15160 / S$ \\
\hline 1 & $1 / 1 / 92$ & 2 & 1 & & 78.7 & $\mathbf{N}$ & \\
\hline 1 & $1 / 1 / 92$ & 3 & 1 & & 78 & $\mathrm{~N}$ & \\
\hline 1 & $1 / 1 / 92$ & 4 & 1 & & 76.6 & $\bar{N}$ & \\
\hline 1 & $1 / 1 / 92$ & 5 & 1 & & 75.2 & $\mathrm{~N}$ & \\
\hline 1 & $1 / 1 / 92$ & 6 & 1 & & 73 & $\mathrm{~N}$ & \\
\hline 1 & $1 / 1 / 92$ & 7 & 1 & & 70.6 & $\mathbf{N}$ & \\
\hline 1 & $1 / 1 / 92$ & 8 & 1 & & 70.3 & $\mathbf{N}$ & \\
\hline 1 & $1 / 1 / 92$ & 9 & 1 & & 70.3 & $N$ & \\
\hline 1 & $1 / 1 / 92$ & 10 & 1 & & 70.4 & $\mathbf{N}$ & \\
\hline 1 & $9 / 9 / 92$ & 11 & 1 & & 70.3 & $\mathbf{N}$ & \\
\hline 1 & $1 / 1 / 92$ & 12 & 1 & & 70.3 & $\bar{N}$ & \\
\hline 1 & $1 / 1 / 92$ & 13 & 1 & & 70.4 & $N$ & \\
\hline
\end{tabular}

Data obtained from WHC Surveillance Analysis Computer System (SACS), February 24, 1994, Data Sheet TK-105-S to TK101-S, and Archive box \#121869. 


\begin{tabular}{|c|c|c|c|c|c|c|c|}
\hline \multicolumn{8}{|c|}{ TANK 241-S-105 } \\
\hline & & & & THEF & OCOUPLE & ITA & \\
\hline TREE \# & DATE & THERMO \# & TREE SET \# & TEMP C & TEMP F & $Y=$ MAX TEMP & COMMENTS \\
\hline 1 & $1 / 1 / 92$ & 14 & 1 & & 70.3 & $N$ & \\
\hline 1 & $7 / 3 / 92$ & 1 & 1 & & 77 & $\bar{Y}$ & J 890423320-42 \\
\hline 1 & $7 / 3 / 92$ & 2 & 1 & & 76 & $\mathbf{N}$ & \\
\hline 1 & $7 / 3 / 92$ & 3 & 1 & & 75 & $\mathrm{~N}$ & \\
\hline 1 & $7 / 3 / 92$ & 4 & 1 & & 74 & $\mathrm{~N}$ & \\
\hline 1 & $7 / 3 / 92$ & 5 & 1 & & 73 & $\mathbf{N}$ & \\
\hline 1 & $7 / 3 / 92$ & 6 & 1 & & 72 & $\mathbf{N}$ & \\
\hline 1 & $7 / 3 / 92$ & 7 & 1 & & 72 & $\mathbf{N}$ & \\
\hline 1 & $7 / 3 / 92$ & 8 & 1 & & 71 & $\mathbf{N}$ & \\
\hline 1 & $7 / 3 / 92$ & 9 & 1 & & 72 & $\mathbf{N}$ & \\
\hline 1 & $7 / 3 / 92$ & 10 & 1 & & 72 & $\mathbf{N}$ & \\
\hline 1 & $7 / 3 / 92$ & 11 & 1 & & 72 & $\mathbf{N}$ & \\
\hline 1 & $7 / 3 / 92$ & 12 & 1 & & 72 & $\mathbf{N}$ & \\
\hline 1 & $7 / 3 / 92$ & 13 & 1 & & 72 & $\mathbf{N}$ & \\
\hline 1 & $7 / 3 / 92$ & 14 & 1 & & 72 & $\bar{N}$ & \\
\hline 1 & $1 / 3 / 93$ & 1 & 1 & & 77.2 & $\bar{Y}$ & J $910126143-29$ \\
\hline 1 & $1 / 3 / 93$ & 2 & 1 & & 76.8 & $\mathbf{N}$ & \\
\hline 1 & $1 / 3 / 93$ & 3 & 1 & & 76.1 & $\mathbf{N}$ & \\
\hline 1 & $1 / 3 / 93$ & 4 & 1 & & 74.8 & $\mathbf{N}$ & \\
\hline 1 & $1 / 3 / 93$ & 5 & 1 & & 73.2 & $\mathbf{N}$ & \\
\hline 1 & $1 / 3 / 93$ & 6 & 1 & & 70.7 & $\mathbf{N}$ & \\
\hline 1 & $1 / 3 / 93$ & 7 & 1 & & 68.1 & $\bar{N}$ & \\
\hline 1 & $1 / 3 / 93$ & 8 & 1 & & 67.9 & $\mathbf{N}$ & \\
\hline 1 & $1 / 3 / 93$ & 9 & 1 & & 67.9 & $\mathbf{N}$ & \\
\hline 1 & $1 / 3 / 93$ & 10 & 1 & & 67.9 & $\mathrm{~N}$ & \\
\hline 1 & $1 / 3 / 93$ & 11 & 1 & & 67.8 & $\mathbf{N}$ & \\
\hline 1 & $1 / 3 / 93$ & 12 & 1 & & 67.8 & $N$ & \\
\hline 1 & $1 / 3 / 93$ & 13 & 1 & & 67.8 & $\mathbf{N}$ & \\
\hline 1 & $1 / 3 / 93$ & 14 & 1 & & 67.7 & $\mathbf{N}$ & \\
\hline 1 & $2 / 13 / 93$ & 1 & 1 & & 76.6 & $Y$ & J 910126143.42 \\
\hline 1 & $2 / 13 / 93$ & 2 & 9 & & 76 & $\mathbf{N}$ & \\
\hline 1 & $2 / 13 / 93$ & 3 & 1 & & 74.8 & $\mathrm{~N}$ & \\
\hline 1 & $2 / 13 / 93$ & 4 & $\frac{1}{1}$ & & 72.7 & $\mathbf{N}$ & \\
\hline 1 & $2 / 13 / 93$ & 5 & 1 & & 70.5 & $N$ & \\
\hline 1 & $2 / 13 / 93$ & 6 & 1 & & 67.4 & $\mathbf{N}$ & \\
\hline 1 & $2 / 13 / 93$ & 7 & 1 & & 64.2 & $\mathbf{N}$ & \\
\hline 1 & $2 / 13 / 93$ & 8 & 1 & & 63.9 & $\mathbf{N}$ & \\
\hline 1 & $2 / 13 / 93$ & 9 & 1 & & 63.8 & $\mathbf{N}$ & \\
\hline 1 & $2 / 13 / 93$ & 10 & 1 & & 63.8 & $N$ & \\
\hline 1 & $2 / 13 / 93$ & 11 & 1 & & 63.8 & $N$ & $\cdot$ \\
\hline 1 & $2 / 13 / 93$ & 12 & 1 & & 63.8 & $N$ & \\
\hline 1 & $2 / 13 / 93$ & 13 & 1 & & 63.8 & $N$ & \\
\hline 1 & $2 / 13 / 93$ & 14 & 1 & & 63.7 & $\mathbf{N}$ & \\
\hline 1 & $7 / 2 / 93$ & 1 & 1 & & 73 & $Y$ & J $890423320-42$ \\
\hline 1 & $7 / 2 / 93$ & 2 & 1 & & 72.2 & $\mathbf{N}$ & \\
\hline 1 & $7 / 2 / 93$ & 3 & 1 & & 70.9 & $\mathbf{N}$ & \\
\hline 1 & $7 / 2 / 93$ & 4 & 1 & & 69.1 & $\mathbf{N}$ & \\
\hline 1 & $7 / 2 / 93$ & 5 & 1 & & 67.6 & $\mathrm{~N}$ & \\
\hline 1 & $7 / 2 / 93$ & 6 & 1 & & 66.2 & $\mathbf{N}$ & \\
\hline 1 & $7 / 2 / 93$ & 7 & 1 & & 65.6 & $\mathbf{N}$ & \\
\hline 1 & $7 / 2 / 93$ & 8 & 1 & & 65.5 & $\mathbf{N}$ & \\
\hline 1 & $7 / 2 / 93$ & 9 & 1 & & 65.5 & $\mathbf{N}$ & \\
\hline 1 & $7 / 2 / 93$ & 10 & 1 & & 65.5 & $\mathbf{N}$ & \\
\hline 1 & $7 / 2 / 93$ & 11 & 1 & & 65.6 & $\mathbf{N}$ & \\
\hline 1 & $7 / 2 / 93$ & 12 & 1 & & 65.6 & $\mathbf{N}$ & \\
\hline 1 & $7 / 2 / 93$ & 13 & 1 & & 65.7 & $\mathbf{N}$ & \\
\hline 1 & $7 / 2 / 93$ & 14 & 1 & & 65.8 & $N$ & \\
\hline
\end{tabular}

Data obtained from WHC Surveillance Analysis Computer System (SACS), February 24, 1994, Data Sheet TK-105-S to TK101-S, and Archive box \#121869. 


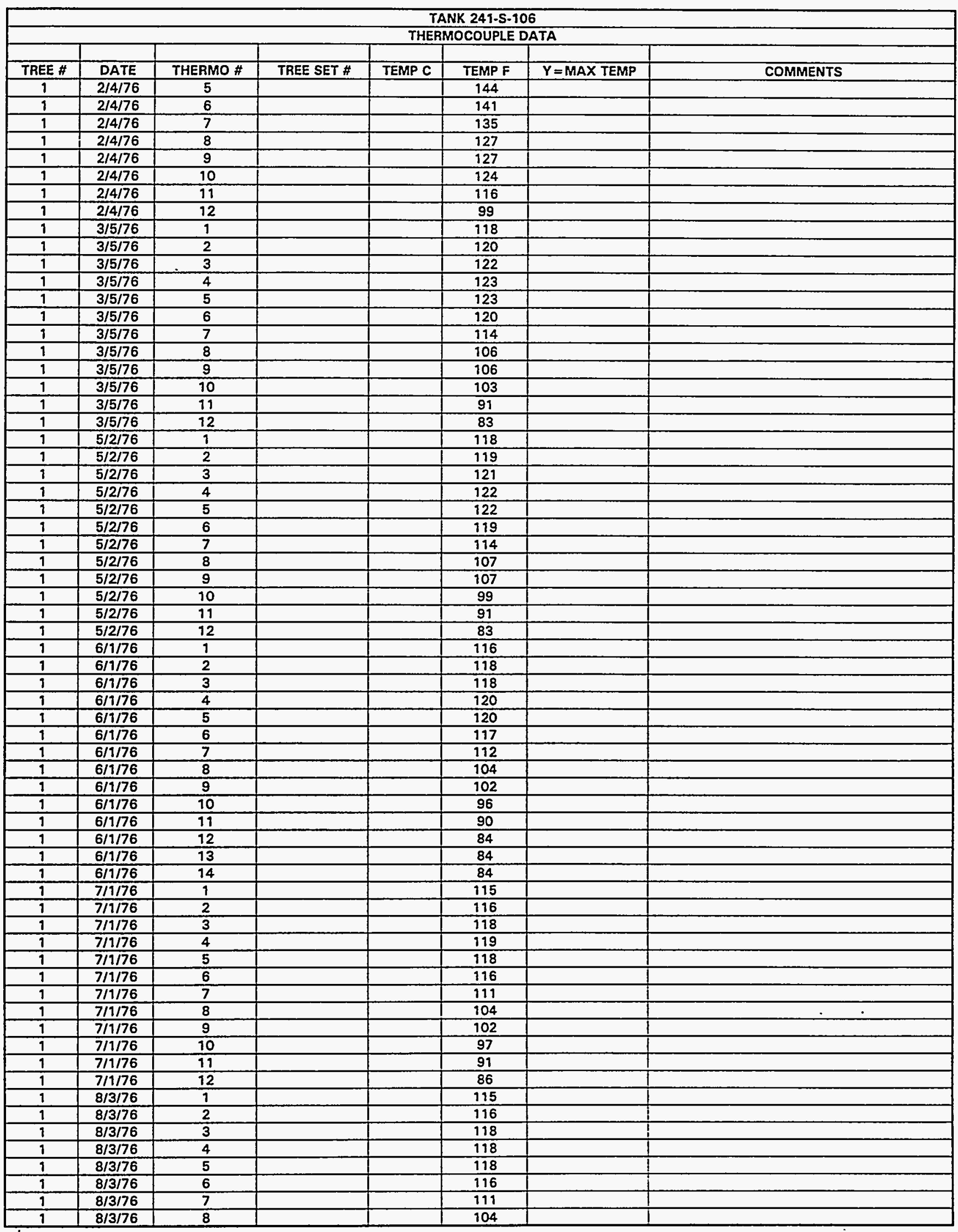

Data obtained from WHC Surveillance Analysis Computer System (SACS), February 24, 1994 and Archive box \#121869. 


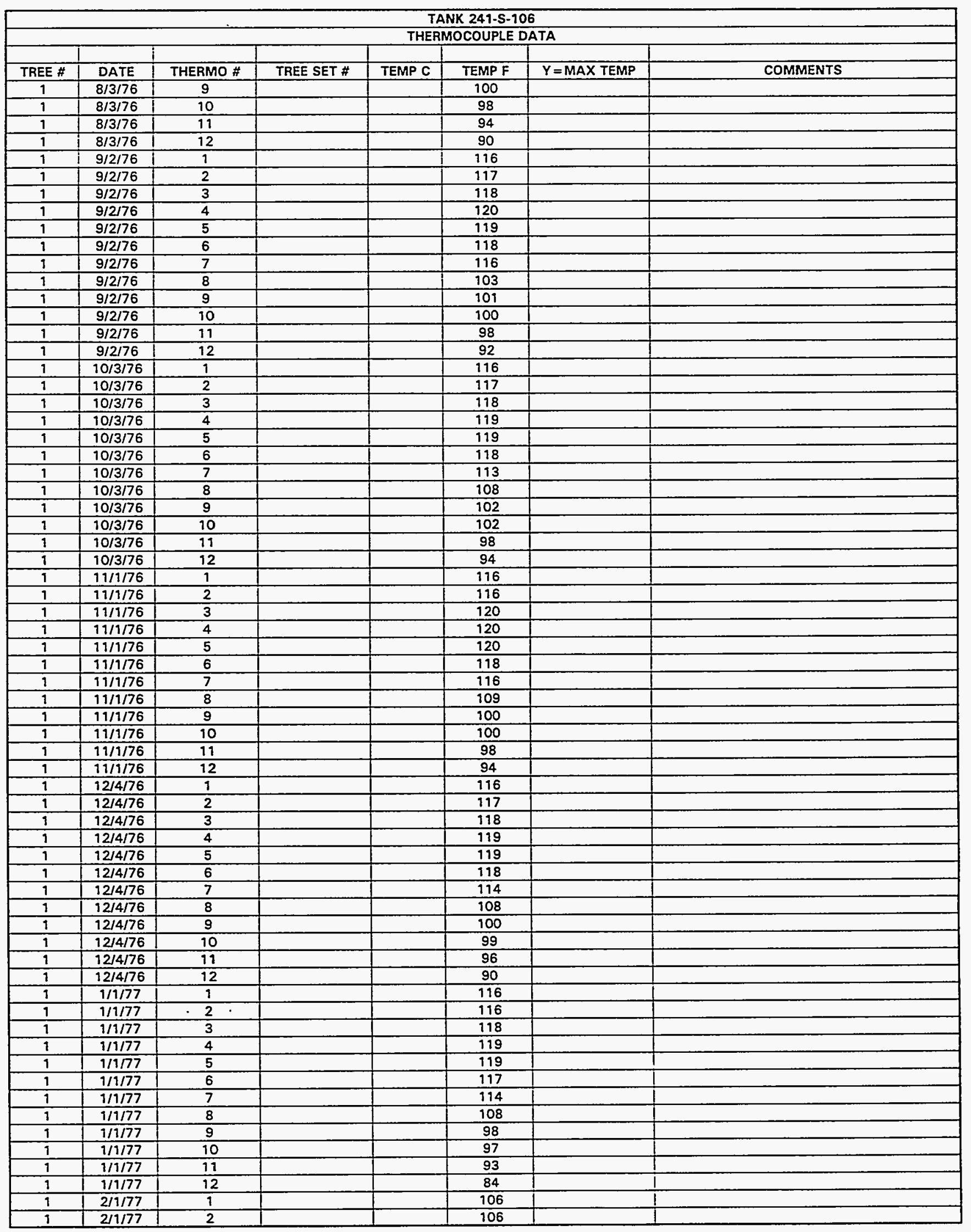

Data obtained from WHC Surveillance Analysis Computer System (SACS), February 24, 1994 and Archive box \#121869. 


\begin{tabular}{|c|c|c|c|c|c|c|c|}
\hline \multicolumn{8}{|c|}{ TANK 241-S-106 } \\
\hline \multicolumn{8}{|c|}{ THERMOCOUPLE DATA } \\
\hline TREE \# & DATE & THERMO \# & TREE SET \# & TEMP C & TEMP F & $Y=$ MAX TEMP & COMMENTS \\
\hline 1 & $2 / 1 / 77$ & 3 & & & 108 & & \\
\hline 1 & $2 / 1 / 77$ & 4 & & & 110 & & \\
\hline$\frac{1}{1}$ & $2 / 1 / 77$ & 5 & & & 110 & & \\
\hline 1 & $2 / 1 / 77$ & 6 & & & 108 & & \\
\hline 1 & $2 / 1 / 77$ & 7 & & & 102 & & \\
\hline 1 & $2 / 1 / 77$ & 8 & & & 104 & & \\
\hline 1 & $2 / 1 / 77$ & 9 & & & 98 & & \\
\hline 1 & $2 / 1 / 77$ & 10 & & & 99 & & \\
\hline 1 & $2 / 1 / 77$ & 11 & & & 90 & & \\
\hline 1 & $2 / 1 / 77$ & 12 & & & 82 & & \\
\hline 1 & $3 / 1 / 77$ & 1 & & & 110 & & \\
\hline 1 & $3 / 1 / 77$ & 2 & & & 110 & & \\
\hline 1 & $3 / 1 / 77$ & 3 & & & 111 & & \\
\hline 1 & $3 / 1 / 77$ & 4 & & & 112 & & \\
\hline 1 & $3 / 1 / 77$ & 5 & & & 112 & & \\
\hline 1 & $3 / 1 / 77$ & 6 & & & 110 & & \\
\hline 1 & $3 / 1 / 77$ & 7 & & & 105 & & \\
\hline 1 & $3 / 1 / 77$ & 8 & & & 99 & & \\
\hline 1 & $3 / 1 / 77$ & 9 & & & 90 & & \\
\hline 1 & $3 / 1 / 77$ & 10 & & & 88 & & \\
\hline 9 & $3 / 1 / 77$ & 11 & & & 84 & & \\
\hline 1 & $3 / 1 / 77$ & 12 & & & 76 & & \\
\hline 1 & $3 / 1 / 77$ & 13 & & & 75 & & \\
\hline 1 & $3 / 1 / 77$ & 14 & & & 75 & & \\
\hline 1 & $4 / 1 / 77$ & 1 & & & 109 & & \\
\hline 1 & $4 / 1 / 77$ & 2 & & & 110 & & \\
\hline 1 & $4 / 1 / 77$ & 3 & & & 111 & & \\
\hline 1 & $4 / 1 / 77$ & 4 & & & 112 & & \\
\hline 1 & $4 / 1 / 77$ & 5 & & & 112 & & \\
\hline 1 & $4 / 1 / 77$ & 6 & & & 110 & & \\
\hline 1 & $4 / 1 / 77$ & 7 & & & 105 & & \\
\hline 1 & $4 / 1 / 77$ & 8 & & & 100 & & \\
\hline 1 & $4 / 1 / 77$ & 9 & & & 89 & & \\
\hline 1 & $4 / 1 / 77$ & 10 & & & 88 & & \\
\hline$\frac{1}{1}$ & $4 / 1 / 77$ & 11 & & & 84 & & \\
\hline 1 & $4 / 1 / 77$ & 12 & & & 75 & & \\
\hline 1 & $5 / 1 / 77$ & 1 & & & 110 & & \\
\hline 1 & $5 / 1 / 77$ & 2 & & & 115 & & \\
\hline 1 & $5 / 1 / 77$ & 3 & & & 116 & & \\
\hline 1 & $5 / 1 / 77$ & 4 & & & 117 & & \\
\hline 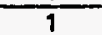 & $5 / 1 / 77$ & 5 & & & 116 & & \\
\hline 1 & $5 / 1 / 77$ & 6 & & & 115 & & \\
\hline 1 & $5 / 1 / 77$ & 7 & & & 105 & & \\
\hline 1 & $5 / 1 / 77$ & 8 & & & 99 & & \\
\hline 1 & $5 / 1 / 77$ & 9 & & & 90 & & \\
\hline 1 & $5 / 1 / 77$ & 10 & & & 89 & & \\
\hline 1 & $5 / 1 / 77$ & 19 & & & 85 & & \\
\hline 1 & $5 / 1 / 77$ & 12 & & & 80 & & \\
\hline 1 & $6 / 7 / 77$ & 1 & & & 107 & & \\
\hline 1 & $6 / 7 / 77$ & 2 & & & 109 & & \\
\hline 1 & $6 / 7 / 77$ & 3 & & & 110 & & \\
\hline 1 & $6 / 7 / 77$ & 4 & & & 110 & & \\
\hline 1 & $6 / 7 / 77$ & 5 & & & 110 & & \\
\hline 1 & $6 / 7 / 77$ & 6 & & & 108 & & \\
\hline 1 & $6 / 7 / 77$ & 7 & & & 104 & & \\
\hline 1 & $6 / 7 / 77$ & 8 & & & 98 & & \\
\hline$\frac{1}{1}$ & $6 / 7 / 77$ & 9 & & & 90 & & \\
\hline 1 & $6 / 7 / 77$ & 10 & & & 90 & & \\
\hline 1 & $6 / 7 / 77$ & 11 & & & 85 & & \\
\hline 1 & $6 / 7 / 77$ & 12 & & & 80 & & \\
\hline 1 & $7 / 11 / 77$ & 1 & & & 105 & & \\
\hline 1 & $7 / 11 / 77$ & 2 & & & 105 & & \\
\hline 1 & $7 / 11 / 77$ & 3 & & & 107 & & \\
\hline$\frac{1}{1}$ & $7 / 11 / 77$ & 4 & & & 107 & & \\
\hline 1 & $7 / 11 / 77$ & 5 & & & 107 & & \\
\hline 1 & $7 / 11 / 77$ & 6 & & . & 105 & & \\
\hline
\end{tabular}

Data obtained from WHC Surveillance Analysis Computer System (SACS), February 24, 1994 and Archive box \#121869. 
WHC-SD-WM-ER-323, Rev. 0

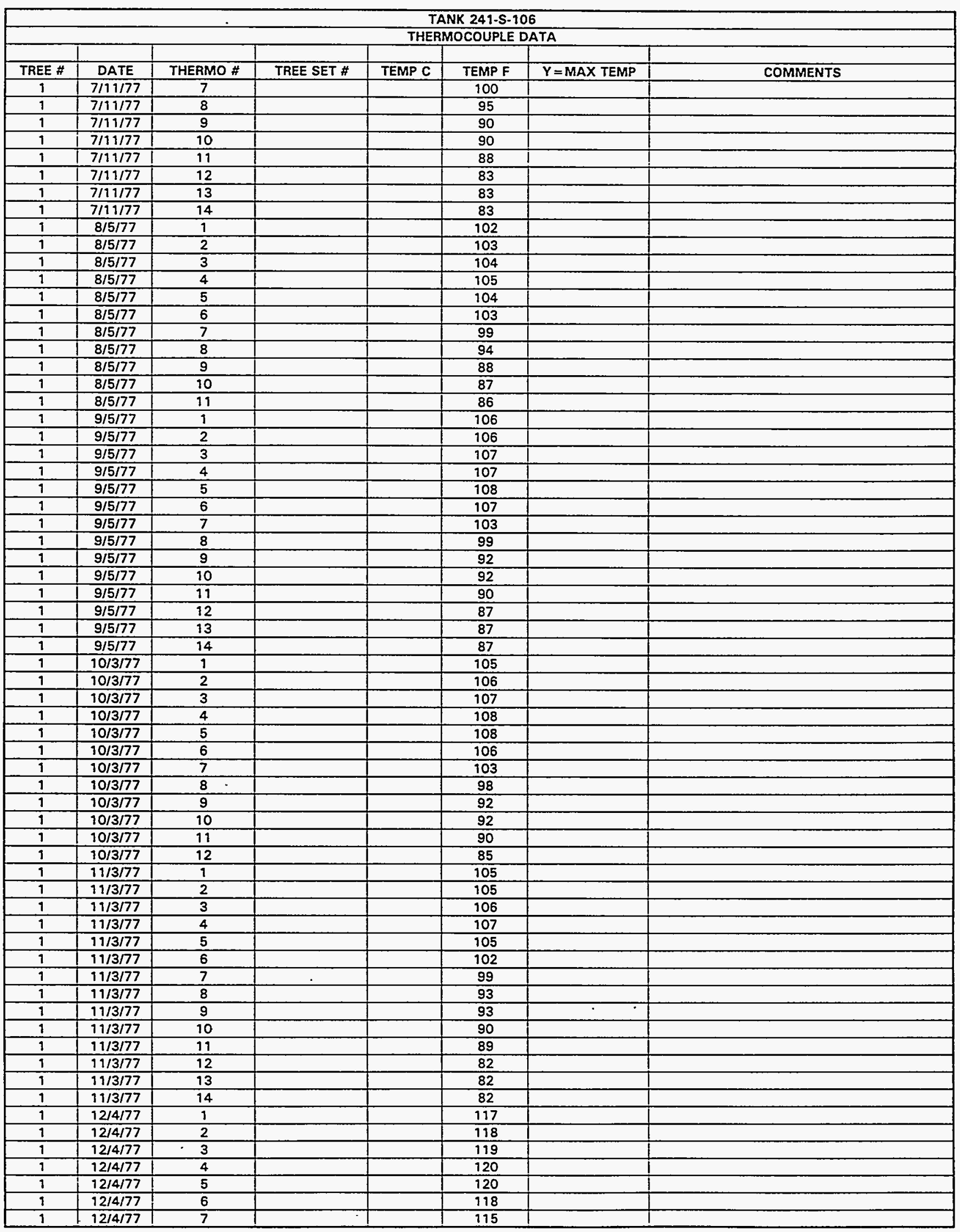

Data obtained from WHC Surveillance Analysis Computer System (SACS), February 24, 1994 and Archive box \#121869. 


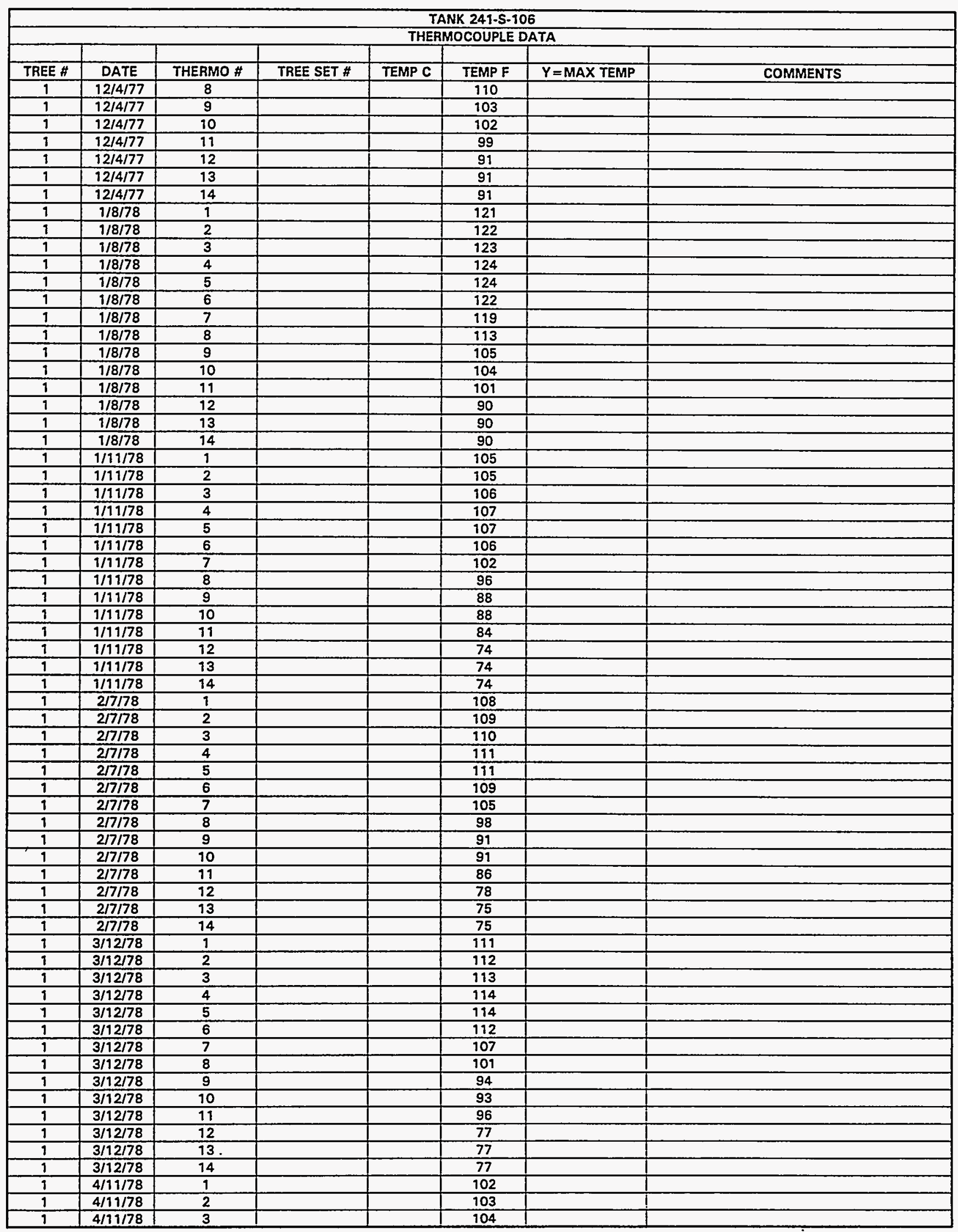

Data obtained from WHC Surveillance Analysis Computer System (SACS), February 24, 1994 and Archive box \#121869. 


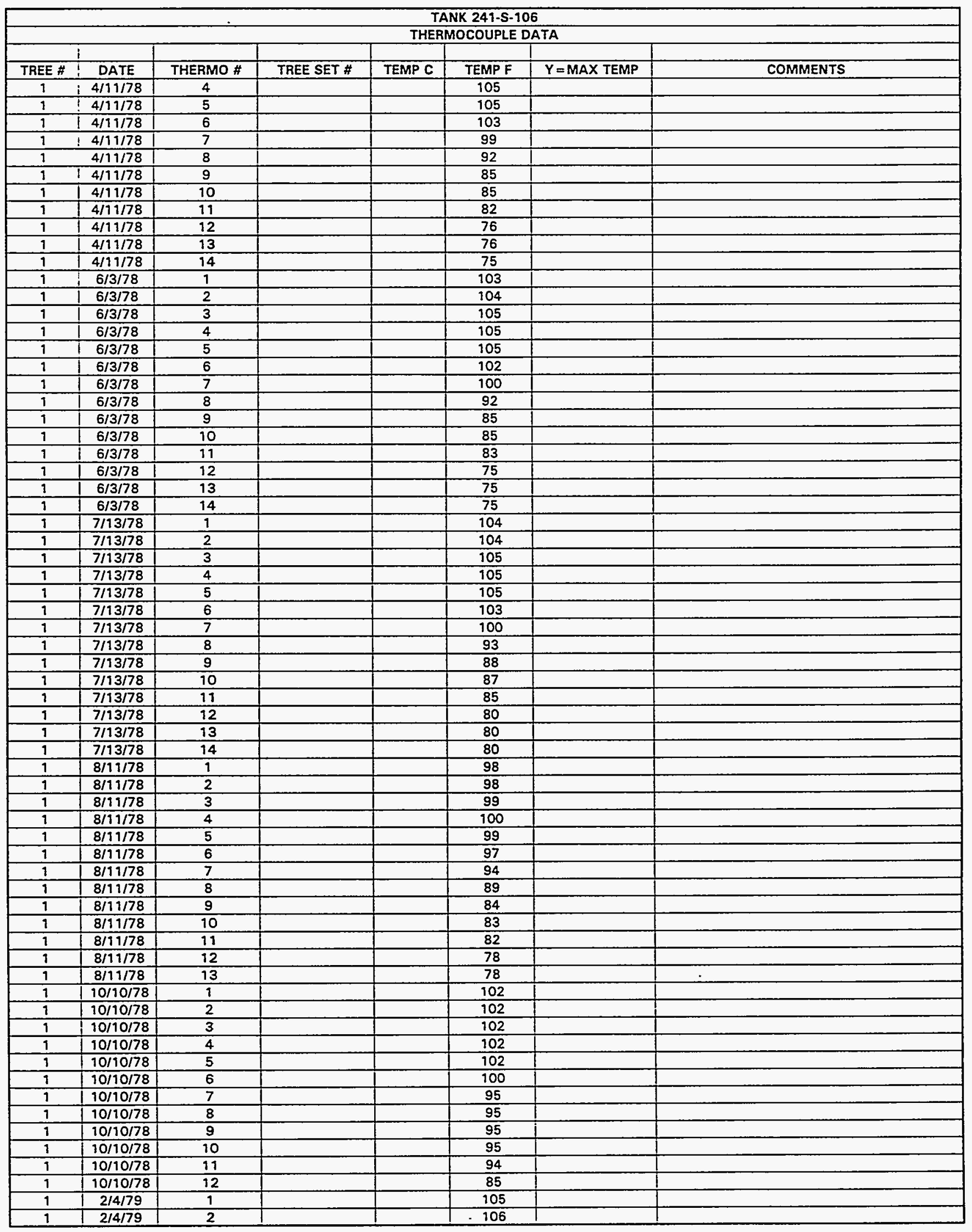

Data obtained from WHC Surveillance Analysis Computer System (SACS), February 24, 1994 and Archive box \#121869. 


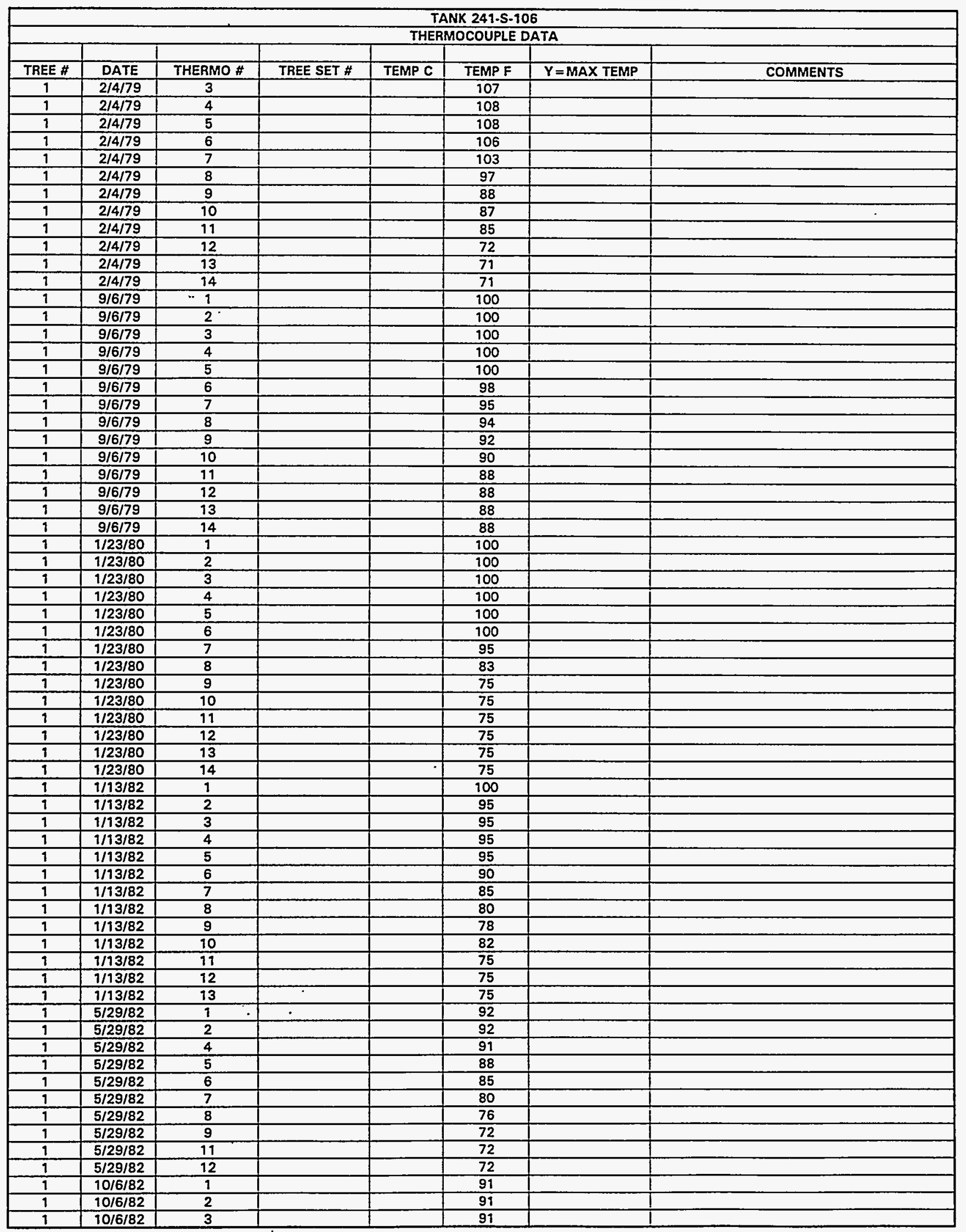

Data obtained from WHC Surveillance Analysis Computer System (SACS), February 24, 1994 and Archive box \#121869. 
WHC-SD-WM-ER-323, Rev. 0

\begin{tabular}{|c|c|c|c|c|c|c|c|}
\hline \multicolumn{8}{|c|}{ TANK 241-S-106 } \\
\hline \multicolumn{8}{|c|}{ THERMOCOUPLE DATA } \\
\hline TREE \# & DATE & THERMO \# & TREE SET \# & TEMP C & TEMP F & $Y=$ MAX TEMP & COMMENTS \\
\hline 1 & $10 / 6 / 82$ & 4 & & & 91 & & \\
\hline 1 & $10 / 6 / 82$ & 5 & & & 89 & & \\
\hline$T$ & $10 / 6 / 82$ & 6 & & & 88 & & \\
\hline 1 & $10 / 6 / 82$ & 7 & & & 85 & & \\
\hline 1 & $10 / 6 / 82$ & 8 & & & 82 & & \\
\hline$\frac{1}{1}$ & $10 / 6 / 82$ & 9 & & & 80 & & \\
\hline 1 & $10 / 6 / 82$ & 10 & & & 80 & & \\
\hline 1 & $10 / 6 / 82$ & 11 & & & 80 & & \\
\hline 9 & $10 / 6 / 82$ & 12 & & & 80 & & \\
\hline 1 & $10 / 6 / 82$ & 13 & & & 80 & & \\
\hline 1 & $10 / 6 / 82$ & 14 & & & 80 & & \\
\hline 1 & $5 / 19 / 83$ & 1 & & & 86 & & \\
\hline 1 & $5 / 19 / 83$ & 2 & & & 86 & & \\
\hline 1 & $5 / 19 / 83$ & 3 & & & 85 & & \\
\hline 1 & $5 / 19 / 83$ & 4 & & & 84 & & \\
\hline 1 & $5 / 19 / 83$ & 5 & & & 82 & & \\
\hline 1 & $5 / 19 / 83$ & 6 & & & 78 & & \\
\hline 1 & $5 / 19 / 83$ & 7 & & & 74 & & \\
\hline 1 & $5 / 19 / 83$ & 8 & & & 69 & & \\
\hline 9 & $5 / 19 / 83$ & 9 & & & 65 & & \\
\hline$\frac{1}{1}$ & $5 / 19 / 83$ & 10 & & & 65 & & \\
\hline 1 & $5 / 19 / 83$ & 11 & & & 65 & & \\
\hline 1 & $5 / 19 / 83$ & 12 & & & 65 & & \\
\hline$\frac{1}{1}$ & $5 / 19 / 83$ & 13 & & & 65 & & \\
\hline 1 & $5 / 19 / 83$ & 14 & & & 65 & & \\
\hline 1 & $1 / 3 / 84$ & 1 & & & 93 & & \\
\hline 1 & $1 / 3 / 84$ & 2 & & & 90 & & \\
\hline 1 & $1 / 3 / 84$ & 3 & & & 90 & & \\
\hline 1 & $1 / 3 / 84$ & 4 & & & 90 & & \\
\hline 1 & $1 / 3 / 84$ & 5 & & & 88 & & \\
\hline 1 & $1 / 3 / 84$ & 6 & & & 85 & & \\
\hline 1 & $1 / 3 / 84$ & 7 & & & 80 & & \\
\hline 1 & $1 / 3 / 84$ & 8 & & & 74 & & \\
\hline 1 & $1 / 3 / 84$ & 9 & & & 69 & & \\
\hline 1 & $1 / 3 / 84$ & 10 & & & 69 & & \\
\hline 1 & $1 / 3 / 84$ & 11 & & & 69 & & \\
\hline 1 & $1 / 3 / 84$ & 12 & & & 69 & & \\
\hline 1 & $1 / 3 / 84$ & 13 & & & 69 & & \\
\hline 1 & $1 / 3 / 84$ & 14 & & & 69 & & \\
\hline 1 & $2 / 13 / 84$ & 1 & & & & & 101 - suspect data (high) \\
\hline 1 & $2 / 13 / 84$ & 2 & & & & & 104 - suspect data (high) \\
\hline 1 & $2 / 13 / 84$ & 3 & & & & & (118- suspect data (high) \\
\hline 1 & $2 / 13 / 84$ & 5 & & & & & 97 - suspect data (high) \\
\hline 1 & $2 / 13 / 84$ & 6 & & & & & 103 - suspect data (high) \\
\hline 1 & $2 / 13 / 84$ & 7 & & & & & 141 - suspect data (high) \\
\hline 1 & $2 / 13 / 84$ & 8 & & & & & 119- suspect data (high) \\
\hline 1 & $2 / 13 / 84$ & 19 & & & & & 102- suspect data (high) \\
\hline 1 & $2 / 14 / 84$ & 1 & & & 92 & & \\
\hline 1 & $2 / 14 / 84$ & 2 & & & 92 & & \\
\hline 1 & $2 / 14 / 84$ & 3 & & & 92 & & \\
\hline 1 & $2 / 14 / 84$ & 4 & & & 91 & & \\
\hline 1 & $2 / 74 / 84$ & 5 & & & 89 & & \\
\hline 1 & $2 / 14 / 84$ & 6 & & & 86 & & \\
\hline 1 & $2 / 14 / 84$ & 7 & & & 81 & & \\
\hline 1 & $2 / 14 / 84$ & 8 & & & 74 & & \\
\hline 1 & $2 / 14 / 84$ & 9 & & & 69 & & \\
\hline 1 & $2 / 14 / 84$ & 10 & & & 69 & & \\
\hline 1 & $2 / 14 / 84$ & 19 & & & 69 & & \\
\hline 1 & $2 / 14 / 84$ & 12 & & & 69 & & I \\
\hline 1 & $2 / 14 / 84$ & 13 & & & 68 & & \\
\hline 1 & $2 / 14 / 84$ & 14 & & & 68 & & \\
\hline 1 & $4 / 19 / 85$ & 1 & & & 86 & & \\
\hline 1 & $4 / 19 / 85$ & 2 & & & 86 & & \\
\hline 1 & $4 / 19 / 85$ & 3 & & & 86 & & \\
\hline 1 & $4 / 19 / 85$ & 4 & & & 85 & & \\
\hline 1 & $4 / 19 / 85$ & 5 & & & 83 & & \\
\hline
\end{tabular}

Data obtained from WHC Surveiliance Analysis Computer System (SACS), February 24, 1994 and Archive box \#121869. 


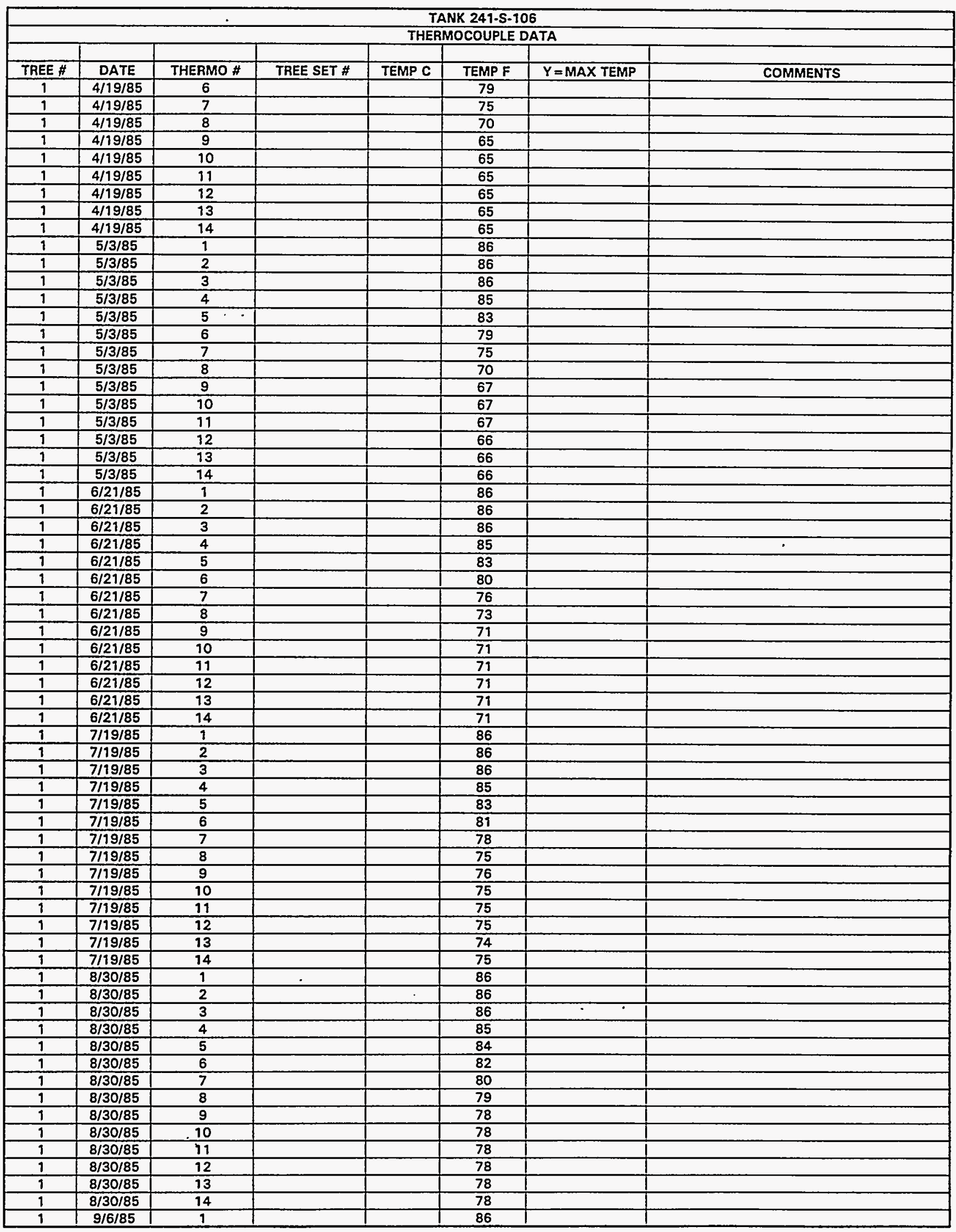

Data obtained from WHC Surveillance Analysis Computer System (SACS), February 24, 1994 and Archive box \#121869. 


\begin{tabular}{|c|c|c|c|c|c|c|c|}
\hline \multicolumn{8}{|c|}{ TANK 241-S-106 } \\
\hline & & & & THER & OCOUPLE & TA & \\
\hline TREE \# & DATE & THERMO \# & TREE SET \# & TEMP C & TEMPF & $Y=M A X$ TFMP & COMMENTS \\
\hline 1 & $9 / 6 / 85$ & 2 & & & 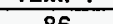 & & \\
\hline 1 & & & & & & & \\
\hline 1 & 910180 & 3 & & & 85 & & \\
\hline 1 & $9 / 6 / 85$ & 4 & & & 85 & & \\
\hline 1 & $9 / 6 / 85$ & 5 & & & 84 & & \\
\hline 1 & $9 / 6 / 85$ & 6 & & & 82 & & \\
\hline 1 & $9 / 6 / 85$ & 7 & & & 80 & & \\
\hline 1 & $9 / 6 / 85$ & 8 & & & 78 & & \\
\hline 1 & $9 / 6 / 85$ & 9 & & & 78 & & \\
\hline 1 & $9 / 6 / 85$ & 10 & & & 78 & & \\
\hline 1 & $9 / 6 / 85$ & 91 & & & 78 & & \\
\hline 1 & $9 / 6 / 85$ & 12 & & & 78 & & \\
\hline 1 & $9 / 6 / 85$ & 13 & & & 78 & & \\
\hline 1 & $9 / 6 / 85$ & 14 & & & 78 & & \\
\hline 1 & $10 / 31 / 85$ & 1 & & & 85 & & \\
\hline 1 & $10 / 31 / 85$ & 2 & & & 85 & & \\
\hline 1 & $10 / 31 / 85$ & 3 & & & 85 & & \\
\hline 1 & $10 / 31 / 85$ & 4 & & & 85 & & \\
\hline 1 & $10 / 31 / 85$ & 5 & & & 84 & & \\
\hline 1 & $10 / 31 / 85$ & 6 & & & 83 & & \\
\hline 1 & $10 / 31 / 85$ & 7 & & & 81 & $\cdot$ & \\
\hline 1 & $10 / 31 / 85$ & 8 & & & 78 & & \\
\hline 1 & $10 / 31 / 85$ & 9 & & & 75 & & \\
\hline 1 & $10 / 31 / 85$ & 10 & & & 75 & & \\
\hline 1 & $10 / 31 / 85$ & 11 & & & 75 & & \\
\hline 1 & $10 / 31 / 85$ & 12 & & & 75 & & \\
\hline 1 & $10 / 31 / 85$ & 13 & & & 75 & & \\
\hline 1 & $10 / 31 / 85$ & 14 & & & 75 & & \\
\hline 1 & $19 / 22 / 85$ & 9 & & & 84 & & \\
\hline 1 & $11 / 22 / 85$ & 2 & & & 84 & & \\
\hline 1 & $11 / 22 / 85$ & 3 & & & 84 & & \\
\hline 1 & $11 / 22 / 85$ & 4 & & & 84 & & \\
\hline 1 & $11 / 22 / 85$ & 5 & & & 83 & & \\
\hline 1 & $11 / 22 / 85$ & 6 & & & 82 & & \\
\hline 1 & $11 / 22 / 85$ & 7 & & & 79 & & \\
\hline 1 & $11 / 22 / 85$ & 8 & & & 75 & & \\
\hline 1 & $11 / 22 / 85$ & 9 & & & 72 & & \\
\hline 1 & $11 / 22 / 85$ & 10 & & & 72 & & \\
\hline 1 & $11 / 22 / 85$ & 11 & & & 72 & & \\
\hline 1 & $11 / 22 / 85$ & 12 & & & 72 & & \\
\hline 9 & $11 / 22 / 85$ & 13 & & & 72 & & \\
\hline 1 & $11 / 22 / 85$ & 14 & & & 72 & & \\
\hline 1 & $12 / 13 / 85$ & 1 & & & 85 & & \\
\hline 1 & $12 / 13 / 85$ & 2 & & & 85 & & \\
\hline 1 & $12 / 13 / 85$ & 3 & & & 85 & & \\
\hline 1 & $12 / 13 / 85$ & 4 & & & 85 & & \\
\hline 1 & $12 / 13 / 85$ & 5 & & & 84 & & \\
\hline 1 & $12 / 13 / 85$ & 6 & & & 82 & & \\
\hline 1 & $12 / 13 / 85$ & 7 & & & 79 & & \\
\hline 1 & $12 / 13 / 85$ & 8 & & & 74 & & \\
\hline 1 & $12 / 13 / 85$ & 9 & & & 70 & & \\
\hline 1 & $12 / 13 / 85$ & 10 & & & 70 & & \\
\hline 9 & $12 / 13 / 85$ & 11 & & & 70 & & \\
\hline 1 & $12 / 13 / 85$ & 12 & & & 70 & & \\
\hline 1 & $12 / 13 / 85$ & 13 & & & 70 & & \\
\hline 1 & $12 / 13 / 85$ & 14 & & & 70 & & \\
\hline 1 & $1 / 17 / 86$ & 1 & & & 85 & & \\
\hline 1 & $1 / 17 / 86$ & 2 & & & 85 & & \\
\hline 1 & $1 / 17 / 86$ & 3 & & & 85 & & \\
\hline 1 & $1 / 17 / 86$ & 4 & & & 85 & & \\
\hline 1 & $1 / 17 / 86$ & 5 & & & 84 & & \\
\hline 1 & $1 / 17 / 86$ & 6 & & & 81 & & \\
\hline 1 & $1 / 17 / 86$ & 7 & & & 78 & & \\
\hline 1 & $1 / 17 / 86$ & 8 & & & 72 & & \\
\hline 1 & $1 / 17 / 86$ & 9 & & & 67 & & \\
\hline 1 & $1 / 17 / 86$ & 10 & & & 67 & & \\
\hline 1 & $1 / 17 / 86$ & 11 & & & 67 & & \\
\hline
\end{tabular}

Data obtained from WHC Surveillance Analysis Computer System (SACS), February 24, 1994 and Archive box \#121869. 
WHC-SD-WM-ER-323, Rev. 0

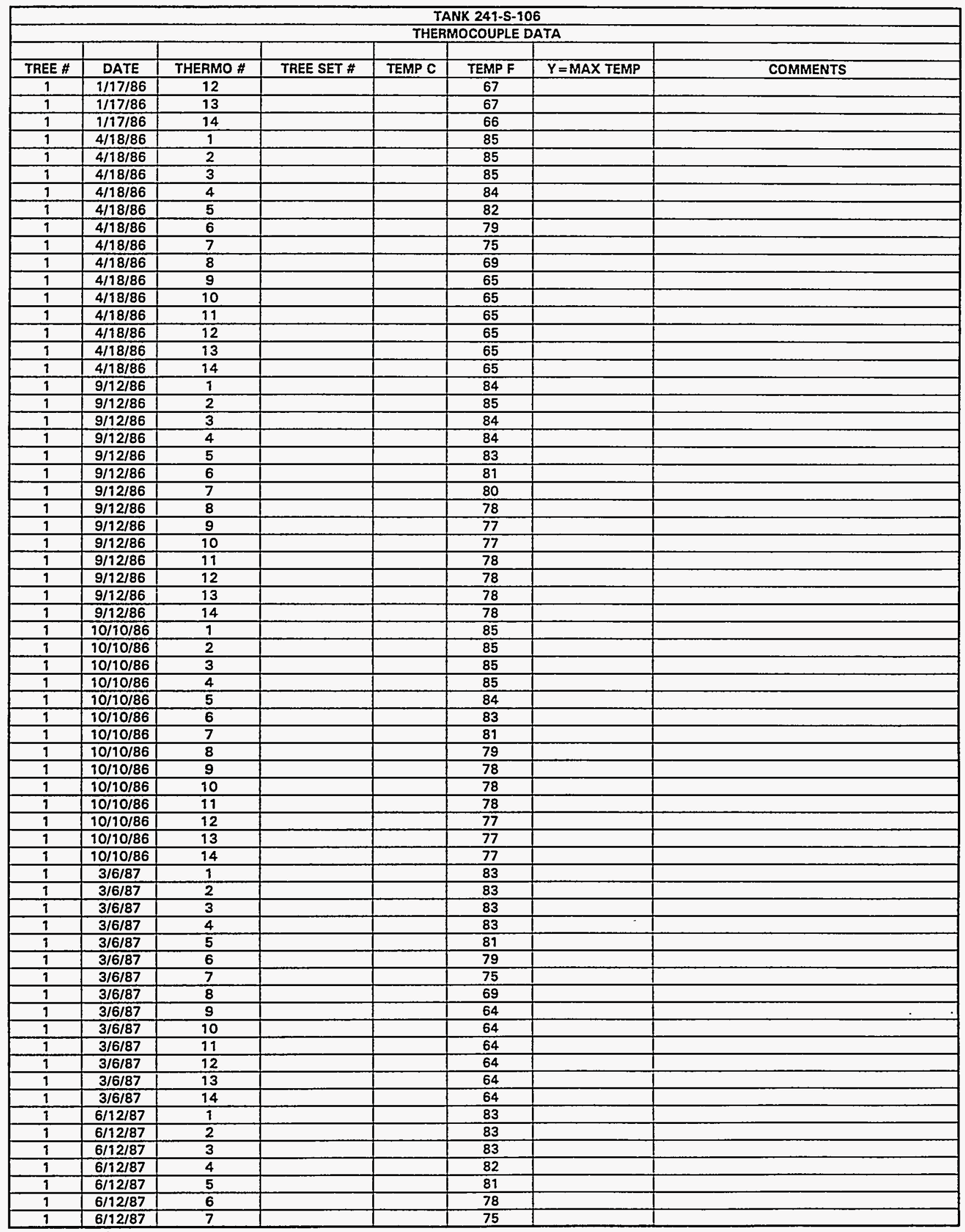

Data obtained from WHC Surveillance Analysis Computer System (SACS), February 24, 1994 and Archive box \#121869. 


\begin{tabular}{|c|c|c|c|c|c|c|c|}
\hline \multicolumn{8}{|c|}{ TANK 241.S-106 } \\
\hline \multicolumn{8}{|c|}{ THERMOCOUPLE DATA } \\
\hline TREF \# & RATF & THEPMO \# & TREF SET \# & TFMP $\mathrm{C}$ & TEMPE & $Y-M A Y$ TEAD & COMMENTS \\
\hline$\frac{1}{1}$ & WAIE & $\frac{1 \text { MEN }}{8}$ & IREE SEI \# & TEMP C & $\frac{1 E M P F}{72}$ & $Y=\operatorname{MAX} \quad T E M P$ & COMMENTS \\
\hline 1 & $6 / 12 / 87$ & 9 & & & 70 & & \\
\hline 1 & $6 / 12 / 87$ & 10 & & & 70 & & \\
\hline 9 & $6 / 12 / 87$ & 11 & & & 70 & & \\
\hline$\frac{1}{1}$ & $6 / 12 / 87$ & 12 & & & 70 & & \\
\hline 1 & $6 / 12 / 87$ & 13 & & & 70 & & \\
\hline 7 & $6 / 12 / 87$ & 14 & & & 70 & & \\
\hline 1 & $10 / 2 / 87$ & 1 & & & 82 & & \\
\hline 1 & $10 / 2 / 87$ & 2 & & & 82 & & \\
\hline 9 & $10 / 2 / 87$ & 3 & & & 82 & & \\
\hline 9 & $10 / 2 / 87$ & 4 & & & 82 & & \\
\hline 1 & $10 / 2 / 87$ & 5 & & & 82 & & \\
\hline$\frac{9}{1}$ & $10 / 2 / 87$ & 6 & & & 81 & & \\
\hline 1 & $10 / 2 / 87$ & 7 & & & 79 & & \\
\hline 1 & $10 / 2 / 87$ & 8 & & & 78 & & \\
\hline 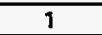 & $10 / 2 / 87$ & 9 & & & 77 & & \\
\hline 1 & $10 / 2 / 87$ & 10 & & & 77 & & \\
\hline 1 & $10 / 2 / 87$ & 11 & & & 77 & & \\
\hline 9 & $10 / 2 / 87$ & 12 & & & 77 & & \\
\hline 1 & $10 / 2 / 87$ & 13 & & & 77 & & \\
\hline 9 & $10 / 2 / 87$ & 14 & & & 77 & & \\
\hline 7 & $12 / 18 / 87$ & 1 & & & 89 & & \\
\hline 1 & $12 / 18 / 87$ & 2 & & & 89 & & \\
\hline 1 & $12 / 18 / 87$ & 3 & & & 90 & & \\
\hline 1 & $12 / 18 / 87$ & 4 & & & 90 & & \\
\hline 1 & $12 / 18 / 87$ & 5 & & & 89 & & \\
\hline 1 & $12 / 18 / 87$ & 6 & & & 88 & & \\
\hline 1 & $12 / 18 / 87$ & 7 & & & 86 & & \\
\hline 1 & $12 / 18 / 87$ & 8 & & & 82 & & \\
\hline 1 & $12 / 18 / 87$ & 9 & & & 78 & & \\
\hline 7 & $12 / 18 / 87$ & 10 & & & 78 & & \\
\hline 1 & $12 / 18 / 87$ & 11 & & & 79 & & \\
\hline 1 & $12 / 18 / 87$ & 12 & & & 79 & & \\
\hline 1 & $12 / 18 / 87$ & 13 & & & 79 & & \\
\hline 9 & $12 / 18 / 87$ & 14 & & & 79 & & \\
\hline 1 & $1 / 22 / 88$ & 1 & & & 91 & & \\
\hline 9 & $1 / 22 / 88$ & 2 & & & 99 & & \\
\hline 1 & $1 / 22 / 88$ & 3 & & & 91 & & \\
\hline 1 & $1 / 22 / 88$ & 4 & & & 99 & & \\
\hline 9 & $1 / 22 / 88$ & 5 & & & 90 & & \\
\hline 1 & $1 / 22 / 88$ & 6 & & & 88 & & \\
\hline 1 & $1 / 22 / 88$ & 7 & & & 85 & & \\
\hline 9 & $1 / 22 / 88$ & 8 & & & 80 & & \\
\hline 1 & $1 / 22 / 88$ & 9 & & & 76 & & \\
\hline 1 & $1 / 22 / 88$ & 10 & & & 76 & & \\
\hline 1 & $1 / 22 / 88$ & 11 & & & 77 & & \\
\hline 1 & $1 / 22 / 88$ & 12 & & & 77 & & \\
\hline 1 & $1 / 22 / 88$ & 13 & & & 77 & & \\
\hline 1 & $1 / 22 / 88$ & 14 & & & 76 & & \\
\hline 1 & $2 / 5 / 88$ & 1 & & & 91 & & \\
\hline 1 & $2 / 5 / 88$ & 2 & & & 91 & & \\
\hline$\frac{1}{1}$ & $2 / 5 / 88$ & 3 & & & 91 & & \\
\hline 1 & $2 / 5 / 88$ & 4 & & & 91 & & \\
\hline 1 & $2 / 5 / 88$ & 5 & $\cdot$ & & 90 & & \\
\hline 1 & $2 / 5 / 88$ & 6 & & & 88 & & \\
\hline 1 & $2 / 5 / 88$ & 7 & & & 84 & & \\
\hline 1 & $2 / 5 / 88$ & 8 & & & 79 & & \\
\hline 1 & $2 / 5 / 88$ & 9 & & & 74 & & \\
\hline 1 & $2 / 5 / 88$ & 10 & & & 74 & & \\
\hline 1 & $2 / 5 / 88$ & 11 & & & 75 & & \\
\hline$T$ & $2 / 5 / 88$ & 12 & & & 75 & & \\
\hline$\frac{1}{1}$ & $2 / 5 / 88$ & 13 & & & 75 & & \\
\hline 1 & $2 / 5 / 88$ & 14 & & & 75 & & 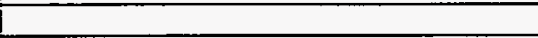 \\
\hline 1 & $1 / 4 / 91$ & 3 & 1 & & 80.2 & $\bar{Y}$ & $\mathrm{HH}-99 \mathrm{~A}-\mathrm{J} \# 890523512-47 \mathrm{TC} \# \mathrm{~s} 1,2,4 \mathrm{O} / \mathrm{S}$ \\
\hline 1 & $1 / 4 / 91$ & 5 & 1 & & 79.5 & $\mathbf{N}$ & \\
\hline 1 & $1 / 4 / 91$ & 6 & 1 & & 78.1 & $\mathbf{N}$ & \\
\hline
\end{tabular}

Data obtained from WHC Surveillance Analysis Computer System (SACS), February 24, 1994 and Archive box \#121869: 
WHC-SD-WM-ER-323, Rev. 0

\begin{tabular}{|c|c|c|c|c|c|c|c|}
\hline \multicolumn{8}{|c|}{ TANK 241-S.106 } \\
\hline \multicolumn{8}{|c|}{ THERMOCOUPLE DATA } \\
\hline TREE \# & DATE & THERMO \# & TREE SET \# & TEMP C & TEMP F & $Y=M A X T E M P$ & COMMENTS \\
\hline 1 & $1 / 4 / 91$ & 7 & 1 & & 75.3 & $\mathbf{N}$ & \\
\hline 1 & $1 / 4 / 91$ & 8 & 1 & & 70.6 & $\mathbf{N}$ & \\
\hline 1 & $1 / 4 / 91$ & 9 & 9 & & 67.2 & $\mathbf{N}$ & \\
\hline 1 & $1 / 4 / 91$ & 10 & 1 & & 67.2 & $\mathbf{N}$ & \\
\hline 1 & $1 / 4 / 91$ & 11 & 1 & & 67.2 & $\bar{N}$ & \\
\hline 1 & $1 / 4 / 91$ & 12 & 1 & & 67.2 & $\mathbf{N}$ & \\
\hline 1 & $1 / 4 / 91$ & 13 & 1 & & 67.3 & $\mathbf{N}$ & \\
\hline 1 & $1 / 4 / 91$ & 14 & 1 & & 67 & $\mathbf{N}$ & \\
\hline 1 & 11/13/91 & 1 & $i$ & & 78.9 & $\mathbf{N}$ & $J-890423320-42$ TC \#14-16 O/S W/O \#04529 \\
\hline 1 & $11 / 13 / 91$ & 2 & 9 & & 78.5 & $\mathbf{N}$ & \\
\hline 1 & $11 / 13 / 91$ & 3 & 1 & & 75.9 & $\mathbf{N}$ & \\
\hline 9 & $11 / 13 / 91$ & 4 & 9 & & 78.7 & $\bar{Y}$ & \\
\hline 1 & $11 / 13 / 91$ & 5 & 9 & & 78.4 & $\mathbf{N}$ & \\
\hline 1 & $11 / 13 / 91$ & 6 & 1 & & 77.7 & $\mathbf{N}$ & \\
\hline 1 & $11 / 13 / 91$ & 7 & 1 & & 75.8 & $\mathbf{N}$ & \\
\hline 1 & $11 / 13 / 91$ & 8 & 1 & & 73.1 & $\mathbf{N}$ & \\
\hline 1 & $11 / 13 / 91$ & 9 & 1 & & 71 & $\mathbf{N}$ & \\
\hline 1 & $11 / 13 / 91$ & 10 & 1 & & 70.9 & $\mathbf{N}$ & \\
\hline 1 & $11 / 13 / 91$ & 11 & 1 & & 70.8 & $\mathbf{N}$ & \\
\hline 1 & $11 / 13 / 91$ & 12 & 1 & & 70.8 & $\mathrm{~N}$ & \\
\hline$T$ & $11 / 13 / 91$ & 13 & 1 & & 70.9 & $\bar{N}$ & \\
\hline 1 & $1 / 1 / 92$ & 1 & 1 & & 82.7 & $\bar{N}$ & $\mathrm{~J} .890523512 .20 \mathrm{TC} \# 1315.16 \mathrm{O} / \mathrm{S}$ \\
\hline 1 & $1 / 1 / 92$ & 2 & 1 & & 83.2 & $\mathbf{N}$ & \\
\hline 1 & $1 / 1 / 92$ & 3 & 1 & & 87 & $\bar{Y}$ & \\
\hline 1 & $1 / 1 / 92$ & 4 & 1 & & 83.3 & $\mathbf{N}$ & \\
\hline 1 & $1 / 1 / 92$ & 5 & 1 & & 82.7 & $\mathbf{N}$ & \\
\hline 1 & $1 / 1 / 92$ & 6 & 1 & & 81.3 & $\mathbf{N}$ & \\
\hline 1 & $1 / 1 / 92$ & 7 & 1 & & 78.4 & $\mathbf{N}$ & \\
\hline 1 & $1 / 1 / 92$ & 8 & 1 & & 74 & $\mathbf{N}$ & \\
\hline 1 & $1 / 1 / 92$ & 9 & 1 & & 70.4 & $\mathbf{N}$ & \\
\hline 1 & $1 / 1 / 92$ & 10 & 1 & & 70.4 & $\mathbf{N}$ & \\
\hline 1 & $1 / 1 / 92$ & 11 & 9 & & 70.3 & $\bar{N}$ & \\
\hline 1 & $1 / 1 / 92$ & 12 & 1 & & 70.3 & $\mathbf{N}$ & \\
\hline 1 & $9 / 1 / 92$ & 74 & 1 & & 70 & $\mathrm{~N}$ & \\
\hline 1 & $7 / 3 / 92$ & 1 & 1 & & 93 & $\mathbf{Y}$ & $\mathrm{J} 890423320-42$ \\
\hline$T$ & $7 / 3 / 92$ & 2 & 1 & & 82 & $\mathbf{N}$ & \\
\hline 1 & $7 / 3 / 92$ & 3 & 1 & & 86 & $\mathbf{N}$ & \\
\hline 1 & $7 / 3 / 92$ & 4 & 1 & & 81 & $\overline{\mathbf{N}}$ & \\
\hline 1 & $7 / 3 / 92$ & 5 & 1 & & 80 & $\mathbf{N}$ & \\
\hline 1 & $7 / 3 / 92$ & 6 & 1 & & 78 & $\mathbf{N}$ & \\
\hline 1 & $7 / 3 / 92$ & 7 & 1 & & 76 & $\mathbf{N}$ & \\
\hline 1 & $7 / 3 / 92$ & 8 & 1 & & 74 & $\mathbf{N}$ & \\
\hline 1 & $7 / 3 / 92$ & 9 & 1 & & 73 & $\mathbf{N}$ & \\
\hline 1 & $7 / 3 / 92$ & 10 & 1 & & 73 & $\mathbf{N}$ & \\
\hline 1 & $7 / 3 / 92$ & 11 & 1 & & 73 & $\mathbf{N}$ & \\
\hline 1 & $7 / 3 / 92$ & 12 & 1 & & 73 & $N$ & \\
\hline 1 & $7 / 3 / 92$ & 13 & 1 & & 73 & $\mathbf{N}$ & \\
\hline 1 & $7 / 3 / 92$ & 14 & 1 & & 73 & $\mathbf{N}$ & \\
\hline 1 & $1 / 3 / 93$ & 1 & 1 & & 80 & $\bar{N}$ & J910126143-29 \\
\hline$T$ & $9 / 3 / 93$ & 2 & 1 & & 81.1 & $\mathbf{N}$ & \\
\hline 1 & $1 / 3 / 93$ & 3 & 1 & & 73 & $\mathbf{N}$ & \\
\hline 1 & $1 / 3 / 93$ & 4 & 1 & & 81.3 & $\bar{Y}$ & \\
\hline 1 & $1 / 3 / 93$ & 5 & 1 & & 80.5 & $\mathbf{N}$ & \\
\hline 1 & $1 / 3 / 93$ & 6 & 1 & & 79 & $\mathbf{N}$ & \\
\hline 1 & $1 / 3 / 93$ & 7 & 1 & & 76.1 & $\mathbf{N}$ & \\
\hline 1 & $1 / 3 / 93$ & 8 & 1 & & 71.3 & $\mathrm{~N}$ & \\
\hline 1 & $1 / 3 / 93$ & 9 & 1 & & 67.6 & $\mathbf{N}$ & \\
\hline$\frac{1}{1}$ & $1 / 3 / 93$ & 10 & 1 & & 67.6 & $\bar{N}$ & \\
\hline 9 & $1 / 3 / 93$ & 11 & 1 & & 67.6 & $\mathbf{N}$ & \\
\hline 1 & $1 / 3 / 93$ & 12 & 1 & & 67.5 & $\mathbf{N}$ & \\
\hline 1 & $1 / 3 / 93$ & 13 & 1 & & 67.5 & $\mathrm{~N}$ & \\
\hline 1 & $1 / 3 / 93$ & 14 & 9 & & 67.2 & $\bar{N}$ & \\
\hline 1 & $2 / 13 / 93$ & 1 & 1 & & 79.6 & $\mathrm{~N}$ & J $910126143-42$ \\
\hline 9 & $2 / 13 / 93$ & 2 & 1 & & 80.8 & $\bar{Y}$ & \\
\hline 1 & $2 / 13 / 93$ & 4 & 1 & & 80.6 & $\mathrm{~N}$ & 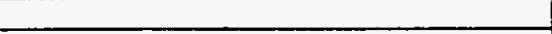 \\
\hline 1 & $2 / 13 / 93$ & 5 & 1 & & 79.5 & $N$ & \\
\hline
\end{tabular}

Data obtained from WHC Surveillance Analysis Computer System (SACS), February 24, 1994 and Archive box.\#121869. 
WHC-SD-WM-ER-323, Rev. O

\begin{tabular}{|c|c|c|c|c|c|c|c|}
\hline \multicolumn{8}{|c|}{ TANK 241-S-106 } \\
\hline \multirow{2}{*}{\multicolumn{8}{|c|}{ THERMOCOUPLE DATA }} \\
\hline & & & & & & & \\
\hline TREE \# & DATE & THERMO \# & TREE SET \# & TEMP C & TEMP F & $Y=$ MAX TEMP & COMMENTS \\
\hline 1 & $2 / 13 / 93$ & 6 & 1 & & 77.3 & $\mathbf{N}$ & \\
\hline 1 & $2 / 13 / 93$ & 7 & 1 & & 73.4 & $\mathbf{N}$ & \\
\hline 1 & $2 / 13 / 93$ & 8 & 1 & & 67.7 & $N$ & \\
\hline 1 & $2 / 13 / 93$ & 9 & 1 & & 63.7 & $\mathbf{N}$ & \\
\hline 1 & $2 / 13 / 93$ & 10 & 1 & & 63.6 & $\mathbf{N}$ & \\
\hline 1 & $2 / 13 / 93$ & 11 & 1 & & 63.5 & $\mathbf{N}$ & \\
\hline 1 & $2 / 13 / 93$ & 12 & 1 & & 63.5 & $\mathbf{N}$ & \\
\hline 1 & $2 / 13 / 93$ & 13 & 1 & & 63.5 & $N$ & \\
\hline 1 & $2 / 13 / 93$ & 14 & 1 & & 63.2 & $\mathbf{N}$ & \\
\hline 1 & $7 / 2 / 93$ & 1 & 1 & & 93.7 & $\mathrm{Y}$ & J890423320-42 \\
\hline 1 & $7 / 2 / 93$ & 2 & 1 & & 77.9 & $\mathbf{N}$ & \\
\hline 1 & $7 / 2 / 93$ & 3 & 1 & & 57.6 & $\mathbf{N}$ & \\
\hline 1 & $7 / 2 / 93$ & 4 & 1 & & 76.5 & $\mathbf{N}$ & \\
\hline 1 & $7 / 2 / 93$ & 5 & 1 & & 74.9 & $N$ & \\
\hline 1 & $7 / 2 / 93$ & 6 & 1 & & 72.8 & $N$ & \\
\hline 1 & $7 / 2 / 93$ & 7 & 1 & & 70.2 & $\mathbf{N}$ & \\
\hline 1 & $7 / 2 / 93$ & 8 & 1 & & 67.3 & $N$ & \\
\hline 1 & $7 / 2 / 93$ & 9 & 1 & & 65.9 & $\mathbf{N}$ & \\
\hline 1 & $7 / 2 / 93$ & 10 & 1 & & 65.9 & $\mathbf{N}$ & \\
\hline 1 & $7 / 2 / 93$ & 11 & 1 & & 65.9 & $\mathbf{N}$ & \\
\hline 1 & $7 / 2 / 93$ & 12 & 1 & & 65.9 & $\mathrm{~N}$ & \\
\hline 1 & $7 / 2 / 93$ & 13 & 1 & & 65.9 & $\mathbf{N}$ & $\div$ \\
\hline 1 & $7 / 2 / 93$ & 14 & 1 & & 65.7 & $\mathrm{~N}$ & \\
\hline
\end{tabular}

Data obtained from WHC Surveillance Analysis Computer System (SACS), February 24, 1994 and Archive box \#121869. 
WHC-SD-WM-ER-323, Rev. 0

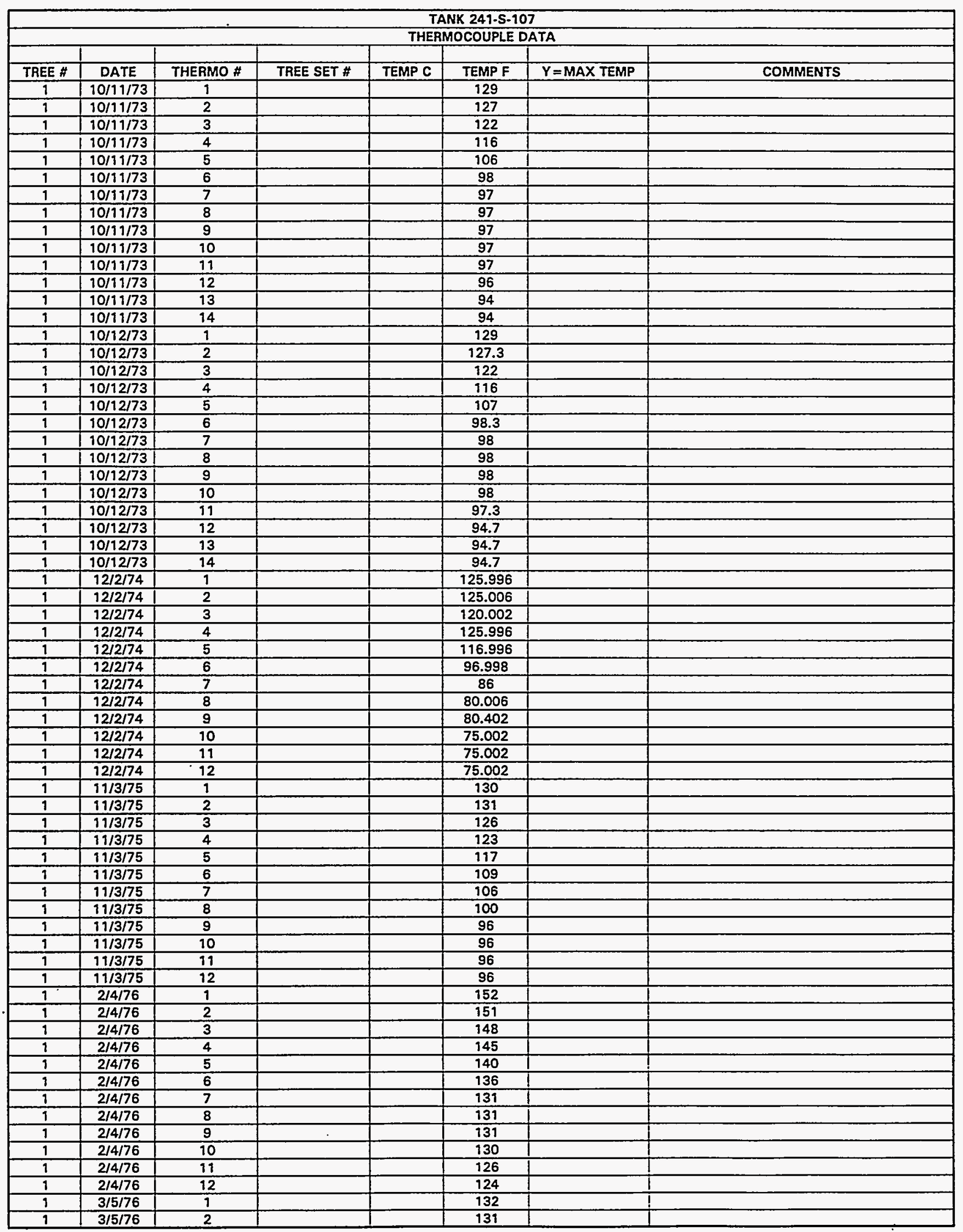

Data obtained from WHC Surveillance Analysis Computer System (SACS), February 24, 1994. 


\begin{tabular}{|c|c|c|c|c|c|c|c|}
\hline \multicolumn{8}{|c|}{ TANK 241-S.107 } \\
\hline \multicolumn{8}{|c|}{ THERMOCOUPLE DATA } \\
\hline TREE \# & DATE & THERMO \# & TREE SET \# & TEMP C & TEMP F & $\mathrm{Y}=\mathrm{MAX}$ TEMP & COMMENTS \\
\hline 1 & $3 / 5 / 76$ & 3 & & & 128 & & \\
\hline 1 & $3 / 5 / 76$ & 4 & & & 126 & & \\
\hline 1 & $3 / 5 / 76$ & 5 & & & 121 & & \\
\hline$\frac{1}{1}$ & $3 / 5 / 76$ & 6 & & & 116 & & \\
\hline 1 & $3 / 5 / 76$ & 7 & & & 110 & & \\
\hline 1 & $3 / 5 / 76$ & 8 & & & 110 & & \\
\hline 1 & $3 / 5 / 76$ & 9 & & & 110 & & \\
\hline 1 & $3 / 5 / 76$ & 10 & & & 109 & & \\
\hline 1 & $3 / 5 / 76$ & 11 & & & 109 & & \\
\hline 1 & $3 / 5 / 76$ & 12 & & & 109 & & \\
\hline 1 & $5 / 2 / 76$ & 1 & & & 135 & & \\
\hline 1 & $5 / 2 / 76$ & 2 & & & 135 & & \\
\hline 1 & $5 / 2 / 76$ & 3 & & & 132 & & \\
\hline 1 & $5 / 2 / 76$ & 4 & & & 129 & & \\
\hline$\frac{1}{1}$ & $5 / 2 / 76$ & 5 & & & 123 & & \\
\hline 1 & $5 / 2 / 76$ & 6 & & & 116 & & \\
\hline 1 & $5 / 2 / 76$ & 7 & & & 109 & & \\
\hline$\frac{1}{1}$ & $5 / 2 / 76$ & 8 & & & 109 & & \\
\hline 1 & $5 / 2 / 76$ & 9 & & & 109 & & \\
\hline 9 & $5 / 2 / 76$ & 10 & & & 109 & & \\
\hline 1 & $5 / 2 / 76$ & 11 & & & 109 & & \\
\hline 1 & $5 / 2 / 76$ & 12 & & & 105 & & \\
\hline 1 & $6 / 1 / 76$ & 1 & & & 134 & & \\
\hline 1 & $6 / 1 / 76$ & 2 & & & 134 & & \\
\hline 1 & $6 / 1 / 76$ & 3 & & & 124 & & \\
\hline 9 & $6 / 1 / 76$ & 4 & & & 120 & & \\
\hline 1 & $6 / 1 / 76$ & 5 & & & 112 & & \\
\hline 9 & $6 / 1 / 76$ & 6 & & & 104 & & \\
\hline 1 & $6 / 1 / 76$ & 7 & & & 104 & & \\
\hline$\frac{1}{1}$ & $6 / 1 / 76$ & 8 & & & 104 & & \\
\hline 1 & $6 / 1 / 76$ & 9 & & & 104 & & \\
\hline 1 & $6 / 1 / 76$ & 10 & & & 104 & & \\
\hline 1 & $6 / 1 / 76$ & 11 & & & 104 & & \\
\hline 1 & $6 / 1 / 76$ & 12 & & & 100 & & \\
\hline 1 & $6 / 1 / 76$ & 13 & & & 100 & & \\
\hline 1 & $6 / 1 / 76$ & 14 & & & 100 & & \\
\hline 1 & $7 / 1 / 76$ & 9 & & & 135 & & \\
\hline 1 & $7 / 1 / 76$ & 2 & & & 135 & & \\
\hline 1 & $7 / 1 / 76$ & 3 & & & 131 & & \\
\hline 9 & $7 / 1 / 76$ & 4 & & & 128 & & \\
\hline 9 & $7 / 1776$ & 5 & & & 121 & & \\
\hline 1 & $7 / 1 / 76$ & 6 & & & 111 & & \\
\hline 1 & $7 / 1 / 76$ & 7 & & & 102 & & \\
\hline 1 & $7 / 1 / 76$ & 8 & & & 102 & & \\
\hline 1 & $7 / 1 / 76$ & 9 & & & 101 & & \\
\hline 1 & $711 / 76$ & 10 & & & 101 & & \\
\hline 1 & $7 / 1 / 76$ & 11 & & & 101 & & \\
\hline 1 & $7 / 1 / 76$ & 12 & & & 100 & & \\
\hline 9 & $8 / 3 / 76$ & 1 & & & 136 & & \\
\hline 1 & $8 / 3 / 76$ & 2 & & & 136 & & \\
\hline 9 & $8 / 3 / 76$ & 3 & & & 132 & & \\
\hline 9 & $8 / 3 / 76$ & 4 & & & 129 & & \\
\hline 1 & $8 / 3 / 76$ & 5 & & & 121 & & \\
\hline 1 & $8 / 3 / 76$ & 6 & & & 191 & & \\
\hline 1 & $8 / 3 / 76$ & 7 & & & 102 & & \\
\hline 1 & $8 / 3 / 76$ & 8 & & & 102 & & \\
\hline 1 & $8 / 3 / 76$ & 9 & & & 102 & & \\
\hline 1 & $8 / 3 / 76$ & 10 & & & 102 & & \\
\hline 1 & $8 / 3 / 76$ & 11 & & & 102 & & \\
\hline 1 & $8 / 3 / 76$ & 12 & & & 102 & & \\
\hline 9 & $9 / 2 / 76$ & 1 & & & 138 & & \\
\hline 1 & $9 / 2 / 76$ & 2 & & & 138 & & \\
\hline 1 & $9 / 2 / 76$ & 3 & & & 134 & & \\
\hline 1 & $9 / 2 / 76$ & 4 & & & 130 & & \\
\hline 1 & $9 / 2 / 76$ & 5 & & & 122 & & \\
\hline 1 & $9 / 2 / 76$ & 6 & & & 112 & & \\
\hline
\end{tabular}

Data obtained from WHC Surveillance Analysis Computer System (SACS), February 24, 1994. 


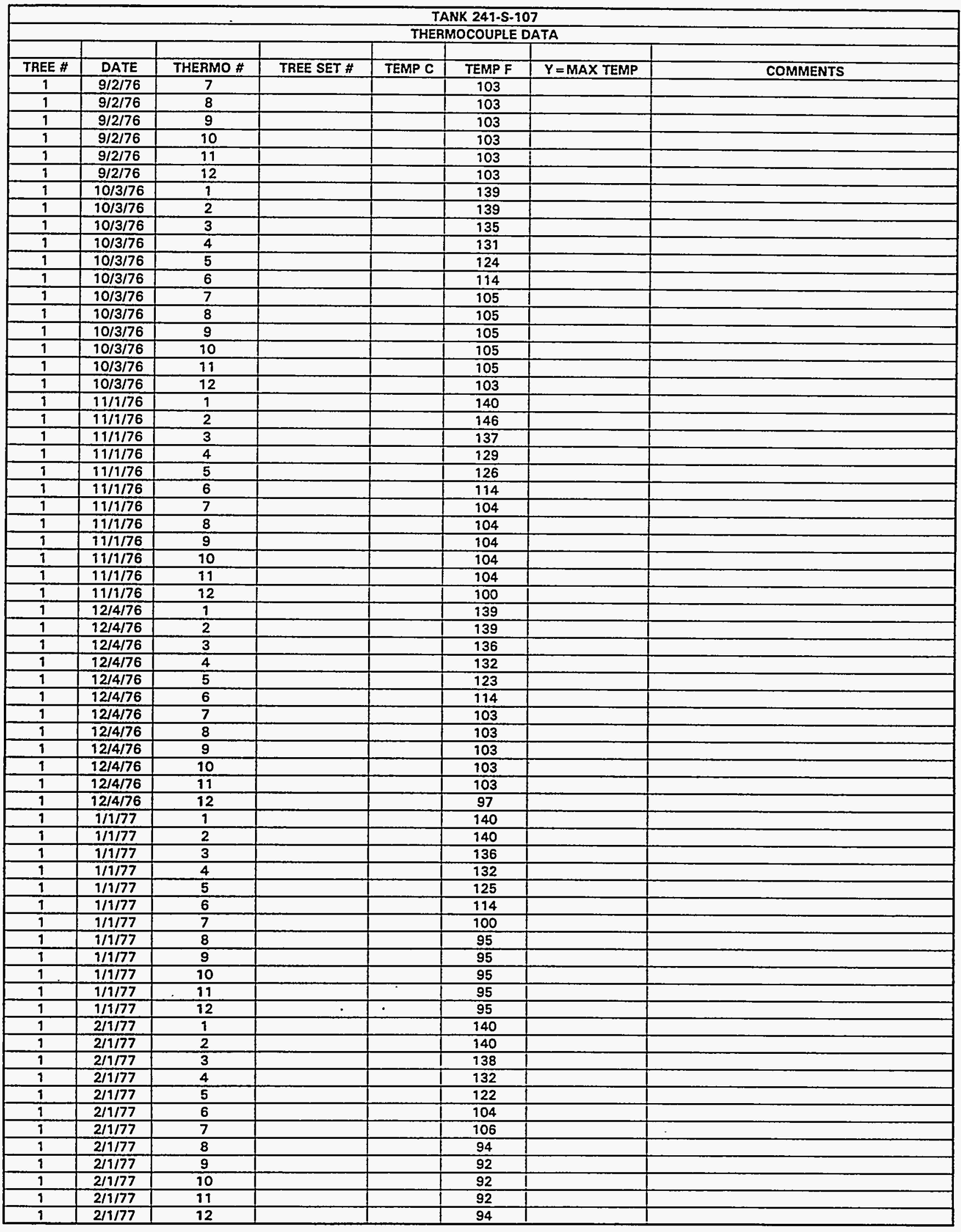

Data obtained from WHC Surveillance Analysis Computer System (SACS), February 24, 1994. 


\begin{tabular}{|c|c|c|c|c|c|c|c|}
\hline \multicolumn{8}{|c|}{ TANK 241-S-107 } \\
\hline \multicolumn{8}{|c|}{ THERMOCOUPLE DATA } \\
\hline & & & & & & & \\
\hline TREE \# & DATE & THERMO \# & TREE SET \# & TEMP C & TEMP F & $Y=$ MAX TEMP & COMMENTS \\
\hline 1 & $3 / 1 / 77$ & 1 & & & 135 & & \\
\hline 1 & $3 / 1 / 77$ & 2 & & & 134 & & \\
\hline 1 & $3 / 1 / 77$ & 3 & & & 130 & & \\
\hline 1 & $3 / 1 / 77$ & 4 & & & 127 & & \\
\hline 1 & $3 / 1 / 77$ & 5 & & & 118 & & \\
\hline 1 & $3 / 1 / 77$ & 6 & & & 102 & & \\
\hline 7 & $3 / 1 / 77$ & 7 & & & 92 & & \\
\hline 1 & $3 / 9 / 77$ & 8 & & & 85 & & \\
\hline 1 & $3 / 1 / 77$ & 9 & & & 84 & & \\
\hline 1 & $3 / 1 / 77$ & 10 & & & 84 & & \\
\hline 1 & $3 / 1 / 77$ & 11 & & & 84 & & \\
\hline 1 & $3 / 1 / 77$ & 12 & & & 84 & & \\
\hline 1 & $3 / 1 / 77$ & 13 & & & 84 & & \\
\hline 1 & $3 / 1 / 77$ & 14 & & & 84 & & \\
\hline 1 & $4 / 1 / 77$ & 1 & & & 135 & & \\
\hline 1 & $4 / 1 / 77$ & 2 & & & 134 & & \\
\hline 1 & $4 / 1 / 77$ & 3 & & & 130 & & \\
\hline 1 & $4 / 1 / 77$ & 4 & & & 125 & & \\
\hline 7 & $4 / 1 / 77$ & 5 & & & 116 & & \\
\hline 1 & $4 / 1 / 77$ & 6 & & & 101 & & \\
\hline 1 & $4 / 1 / 77$ & 7 & & & 87 & & \\
\hline 9 & $4 / 1 / 77$ & 8 & & & 84 & & \\
\hline 1 & $4 / 1 / 77$ & 9 & & & 83 & & \\
\hline 1 & $4 / 1 / 77$ & 10 & & & 83 & & \\
\hline 1 & $4 / 1 / 77$ & 11 & & & 83 & & \\
\hline 1 & $4 / 7 / 77$ & 12 & & & 83 & & \\
\hline 1 & $4 / 1 / 77$ & 13 & & & 83 & & \\
\hline 1 & $4 / 1 / 77$ & 14 & & & 83 & & \\
\hline 1 & $5 / 1 / 77$ & 1 & & & 135 & & \\
\hline 1 & $5 / 1 / 77$ & 2 & & & 134 & & \\
\hline 1 & $5 / 1 / 77$ & 3 & & & 130 & & \\
\hline 1 & $5 / 1 / 77$ & 4 & & & 125 & & \\
\hline 1 & $5 / 1 / 77$ & 5 & & & 115 & & \\
\hline 1 & $5 / 1 / 77$ & 6 & & & 101 & & \\
\hline 1 & $5 / 1 / 77$ & 7 & & & 90 & & \\
\hline 1 & $5 / 1 / 77$ & 8 & & & 85 & & \\
\hline 9 & $5 / 1 / 77$ & 9 & & & 84 & & \\
\hline 1 & $5 / 9 / 77$ & 10 & & & 85 & & \\
\hline 9 & $5 / 1 / 77$ & 11 & & & 84 & & \\
\hline 1 & $5 / 1 / 77$ & 12 & & & 84 & & \\
\hline 1 & $6 / 7 / 77$ & 1 & & & 134 & & \\
\hline 9 & $6 / 7 / 77$ & 2 & & & 134 & & \\
\hline 1 & $6 / 7 / 77$ & 3 & & & 129 & & \\
\hline 1 & $6 / 7 / 77$ & 4 & & & 125 & & \\
\hline$i$ & $6 / 7 / 77$ & 5 & & & 115 & & \\
\hline 9 & $6 / 7 / 77$ & 6 & & & 104 & & \\
\hline 1 & $6 / 7 / 77$ & 7 & & & 92 & & \\
\hline 9 & $6 / 7 / 77$ & 8 & & & 90 & & \\
\hline 9 & $6 / 7 / 77$ & 9 & & & 89 & & \\
\hline 9 & $6 / 7 / 77$ & 10 & & & 89 & & \\
\hline 9 & $6 / 7 / 77$ & 11 & & & 89 & & \\
\hline 1 & $6 / 7 / 77$ & 12 & & & 89 & & \\
\hline 1 & $7 / 11 / 77$ & 1 & & & 133 & & \\
\hline 1 & $7 / 11 / 77$ & 2 & & & 133 & & \\
\hline 1 & $7 / 11 / 77$ & 3 & & & 125 & & \\
\hline 9 & $7 / 11 / 77$ & 4 & & & 125 & & \\
\hline 9 & $7 / 11 / 77$ & 5 & & & 120 & & \\
\hline 9 & $7 / 11 / 77$ & 6 & & & 100 & & \\
\hline 1 & $7 / 19 / 77$ & 7 & & & 90 & & \\
\hline 1 & $7 / 11 / 77$ & 8 & & & 85 & & \\
\hline 1 & $7 / 11 / 77$ & 9 & & & 75 & & \\
\hline 1 & $7 / 19 / 77$ & 10 & & & 75 & & \\
\hline 1 & $7 / 11 / 77$ & 11 & & & 70 & & \\
\hline 1 & $7 / 11 / 77$ & 12 & & & 70 & & \\
\hline 9 & $7 / 19 / 77$ & 13 & & & 70 & & \\
\hline 1 & $7 / 19 / 77$ & 14 & & & 60 & & \\
\hline
\end{tabular}

Data obtained from WHC Surveillance Analysis Computer System (SACS), February 24, 1994. 


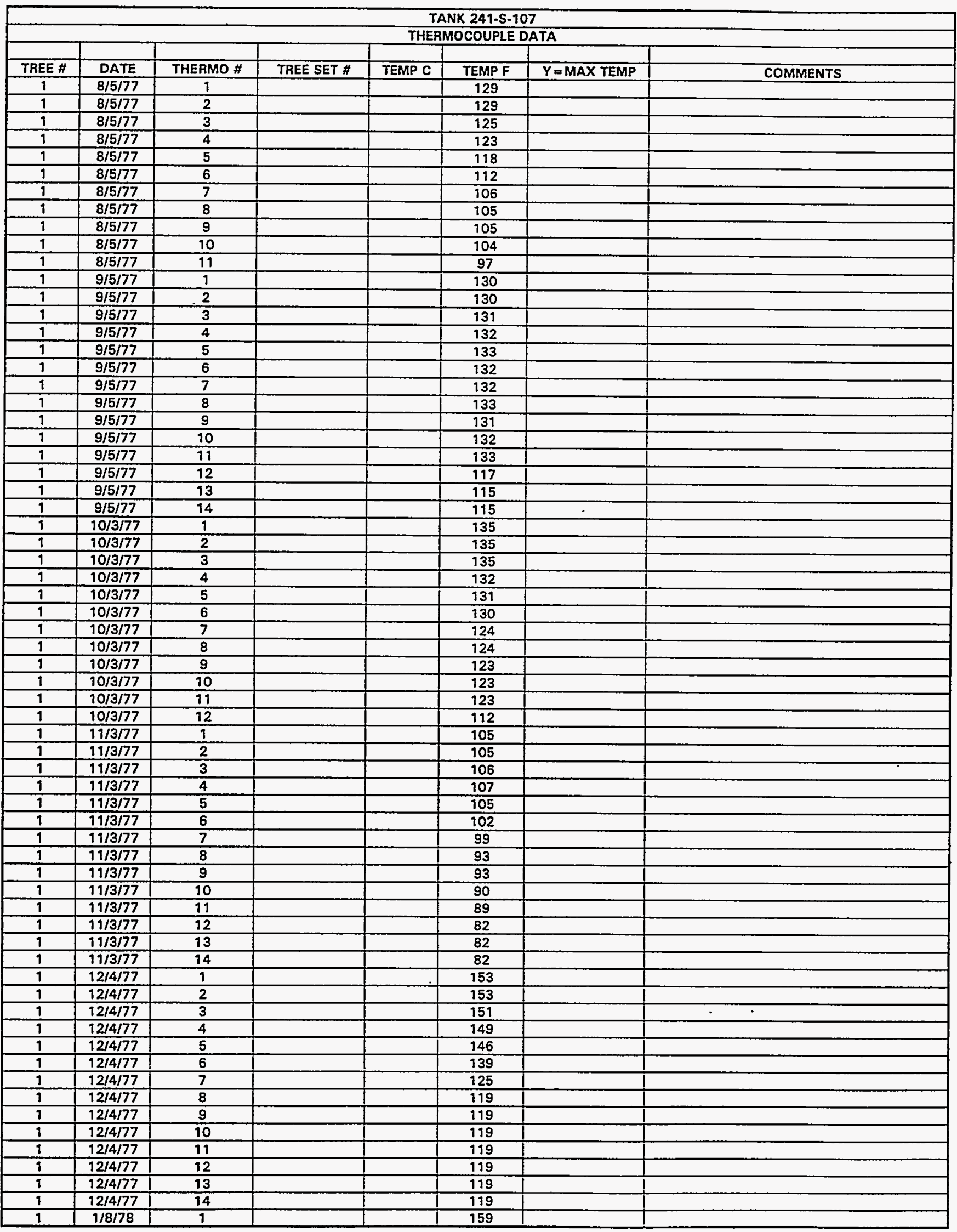

Data obtained from WHC Surveillance Analysis Computer System (SACS). February 24, 1994. 
WHC-SD-WM-ER-323, Rev. 0

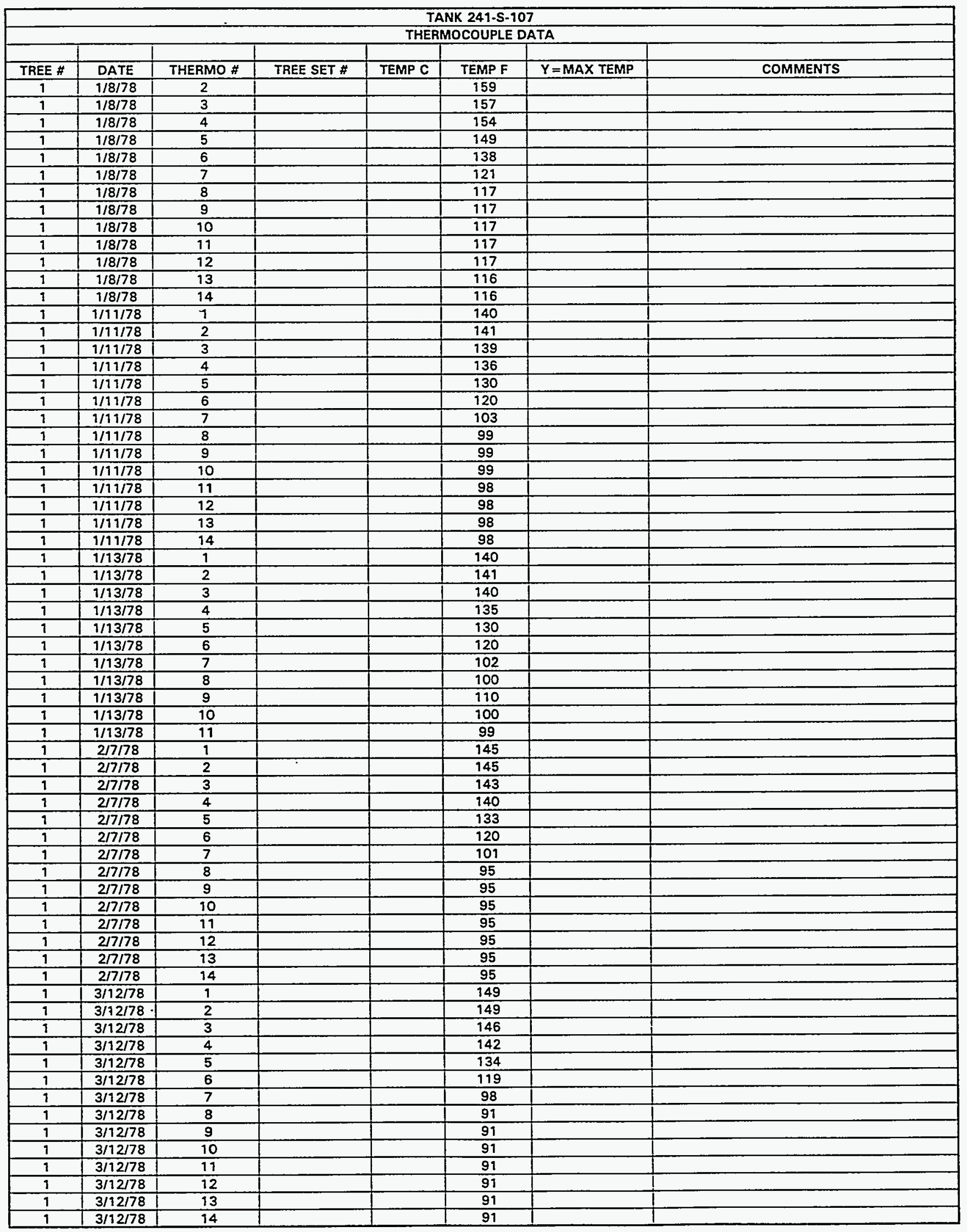

Data obtained from WHC Surveillance Analysis Computer System (SACS), February -24, 1994. 


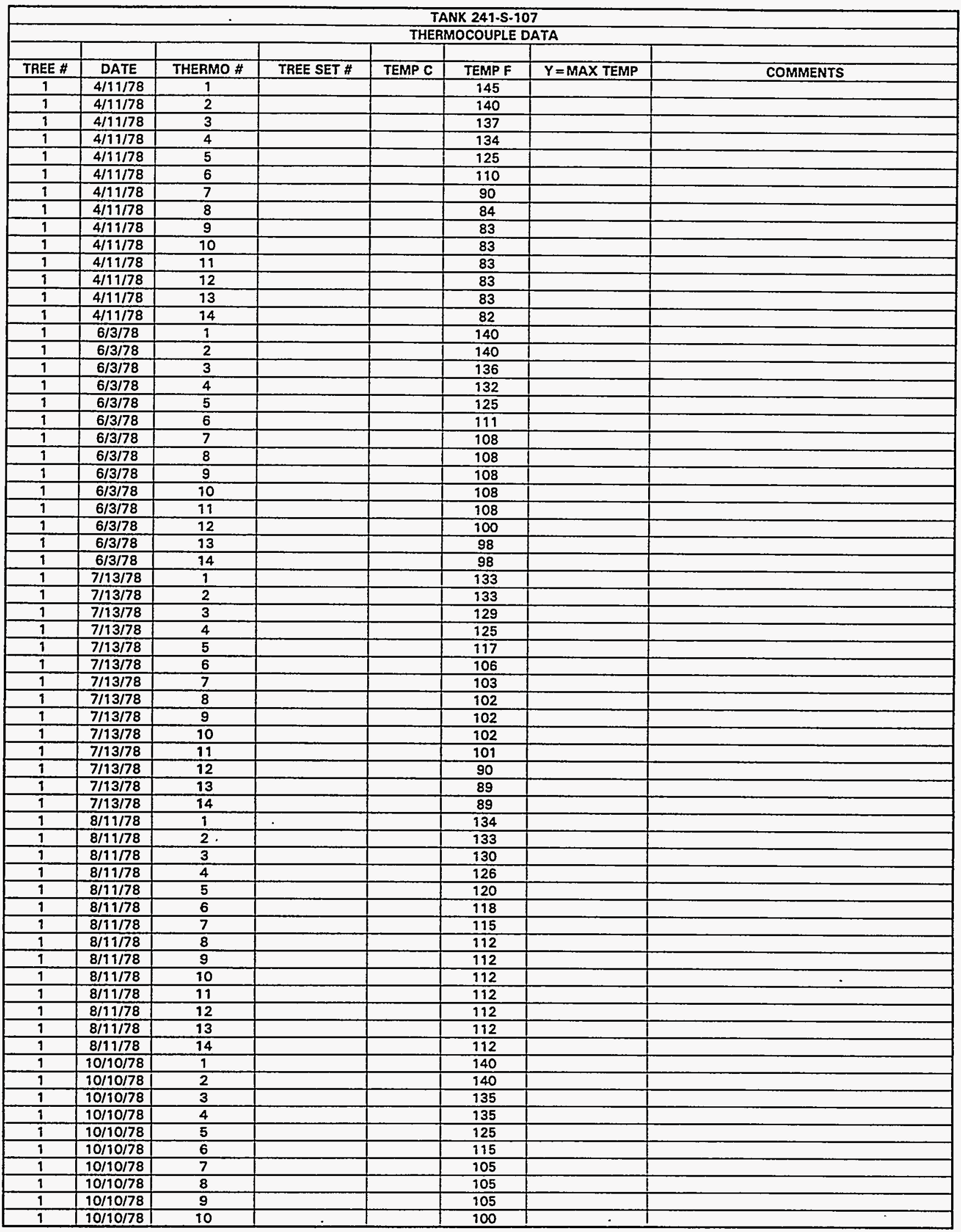

-. Data obtained from WHC Surveillance Analysis Computer System (SACS), February 24, 1994. 


\begin{tabular}{|c|c|c|c|c|c|c|c|}
\hline \multicolumn{8}{|c|}{ TANK 241-S-107 } \\
\hline \multicolumn{8}{|c|}{ THERMOCOUPLE DATA } \\
\hline TREE \# & DATE & THERMO \# & TREE SET & TEMP C & TEMP F & $Y=M A X$ TEMP & COMMENTS \\
\hline 1 & $10 / 10 / 78$ & 11 & & & 100 & & S \\
\hline 1 & $10 / 10 / 78$ & 12 & & & 100 & & \\
\hline 1 & $2 / 4 / 79$ & $\frac{1}{1}$ & & & 141 & & \\
\hline 9 & $2 / 4 / 79$ & 2 & & & 141 & & \\
\hline$\frac{1}{1}$ & $2 / 4 / 79$ & 3 & & & 138 & & \\
\hline 1 & $2 / 4 / 79$ & 4 & & & 134 & & \\
\hline 1 & $2 / 4 / 79$ & 5 & & & 125 & & \\
\hline 1 & $2 / 4 / 79$ & 6 & & & 111 & & \\
\hline 1 & $2 / 4 / 79$ & 7 & & & 104 & & \\
\hline 1 & $2 / 4 / 79$ & 8 & & & 103 & & \\
\hline 1 & $2 / 4 / 79$ & 9 & & & 103 & & \\
\hline 1 & $2 / 4 / 79$ & 10 & & & 103 & & \\
\hline 1 & $2 / 4 / 79$ & 11 & & & 103 & & \\
\hline 1 & $2 / 4 / 79$ & 12 & & & 93 & & \\
\hline 1 & $2 / 4 / 79$ & 13 & & & 93 & & \\
\hline 1 & $2 / 4 / 79$ & 14 & & & 92 & & \\
\hline 1 & $9 / 6 / 79$ & 1 & & & 140 & & \\
\hline 1 & $9 / 6 / 79$ & 2 & & & 140 & & \\
\hline 1 & $9 / 6 / 79$ & 3 & & & 135 & & \\
\hline 1 & $9 / 6 / 79$ & 4 & & & 132 & & \\
\hline 1 & $9 / 6 / 79$ & 5 & & & 125 & & \\
\hline 1 & $9 / 6 / 79$ & 6 & & & 112 & & \\
\hline 1 & $9 / 6 / 79$ & 7 & & & 110 & & \\
\hline 1 & $9 / 6 / 79$ & 8 & & & 110 & & \\
\hline 1 & $9 / 6 / 79$ & 9 & & & 110 & & \\
\hline 1 & $9 / 6 / 79$ & 10 & & & 110 & & \\
\hline 1 & $9 / 6 / 79$ & 11 & & & 105 & & \\
\hline 1 & $9 / 6 / 79$ & 12 & & & 105 & & \\
\hline 1 & $9 / 6 / 79$ & 13 & & & 105 & & \\
\hline 1 & $9 / 6 / 79$ & 14 & & & 105 & & \\
\hline 1 & $1 / 23 / 80$ & 1 & & & 140 & & \\
\hline 1 & $1 / 23 / 80$ & 2 & & & 140 & & \\
\hline 1 & $1 / 23 / 80$ & 3 & & & 135 & & \\
\hline 1 & $1 / 23 / 80$ & 4 & & & 135 & & \\
\hline 1 & $1 / 23 / 80$ & 5 & & & 130 & & \\
\hline 1 & $1 / 23 / 80$ & 6 & & & 125 & & \\
\hline 1 & $1 / 23 / 80$ & 7 & & & 120 & & \\
\hline 1 & $1 / 23 / 80$ & 8 & & & 120 & & \\
\hline 1 & $1 / 23 / 80$ & 9 & & & 118 & & \\
\hline 1 & $1 / 23 / 80$ & 10 & & & 118 & & \\
\hline 9 & $1 / 23 / 80$ & 11 & & & 115 & & \\
\hline 1 & $1 / 23 / 80$ & 12 & & & 110 & & \\
\hline 1 & $1 / 23 / 80$ & 13 & & & 105 & & \\
\hline 1 & $1 / 23 / 80$ & 14 & & & 105 & & \\
\hline 1 & $1 / 13 / 82$ & 1 & & & 123 & & \\
\hline 1 & $1 / 13 / 82$ & 2 & & & 125 & & \\
\hline 1 & $1 / 13 / 82$ & 3 & & & 120 & & \\
\hline 1 & $1 / 13 / 82$ & 4 & & & 118 & & \\
\hline 1 & $1 / 13 / 82$ & 5 & & & 107 & & \\
\hline 1 & $1 / 13 / 82$ & 6 & & & 95 & & \\
\hline 9 & $1 / 13 / 82$ & 7 & & & 88 & & \\
\hline 9 & $1 / 13 / 82$ & 8. & & & 88 & & \\
\hline 1 & $1 / 13 / 82$ & 9 & & & 88 & & \\
\hline 1 & $1 / 13 / 82$ & 10 & & & 89 & & \\
\hline 1 & $1 / 13 / 82$ & 11 & & & 90 & & \\
\hline 1 & $1 / 13 / 82$ & 12 & & & 90 & & \\
\hline 1 & $1 / 13 / 82$ & 13 & & & 90 & & \\
\hline 9 & $5 / 29 / 82$ & 1 & & & 121 & & \\
\hline 1 & $5 / 29 / 82$ & 2 & & & 111 & & \\
\hline 1 & $5 / 29 / 82$ & 3 & & & 117 & & \\
\hline 1 & $5 / 29 / 82$ & 4 & & & 114 & & \\
\hline 1 & $5 / 29 / 82$ & 5 & & & 104 & & \\
\hline 1 & $5 / 29 / 82$ & 6 & & & 93 & & \\
\hline 1 & $5 / 29 / 82$ & 7 & & & 88 & & \\
\hline 1 & $5 / 29 / 82$ & 8 & & & 88 & & \\
\hline 1 & $5 / 29 / 82$ & 9 & & & 88 & & \\
\hline
\end{tabular}

Data obtained from WHC Surveillance Analysis Computer System (SACS), February 24, 1994. 


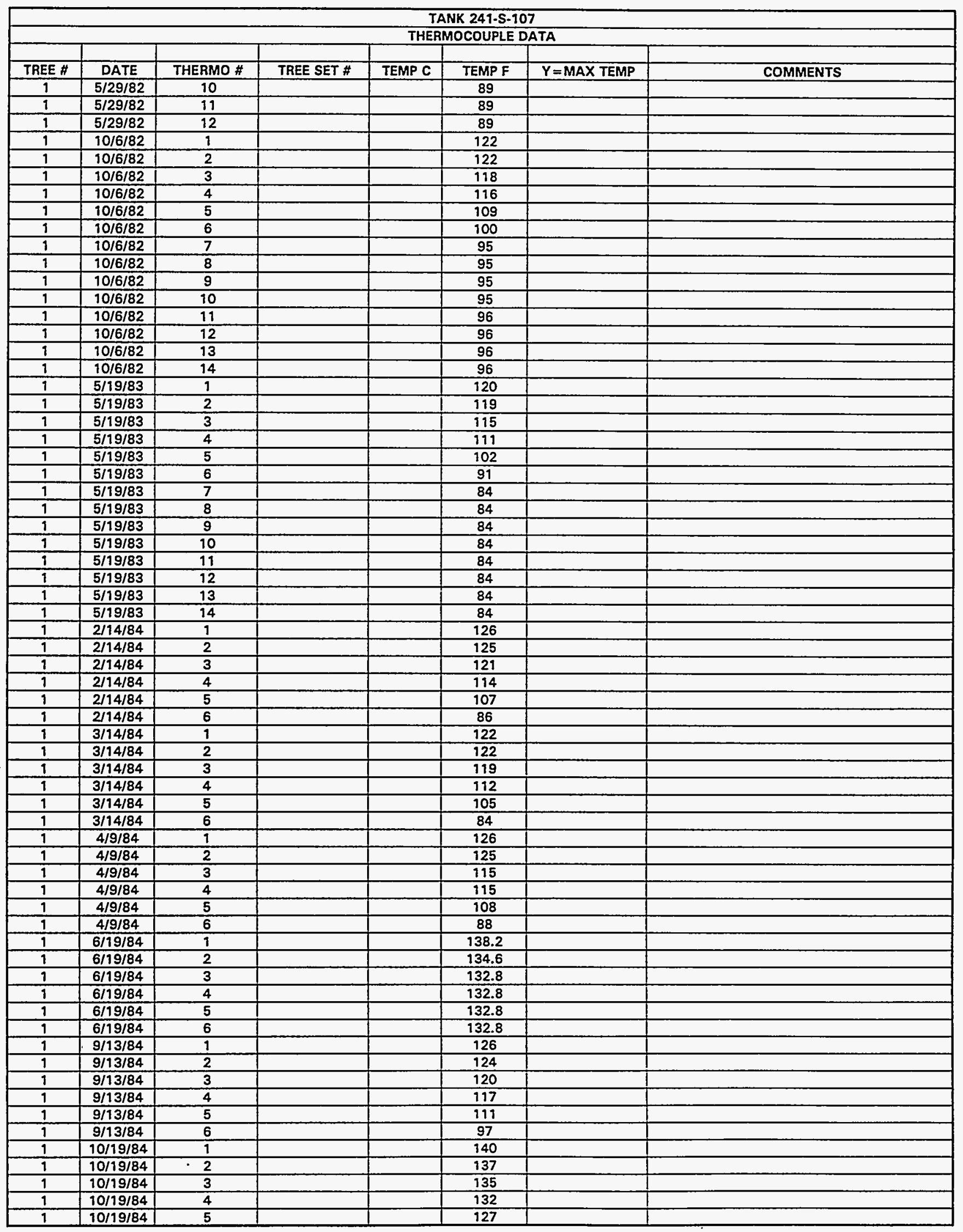

Data obtained from WHC Surveillance Analysis Computer System (SACS), February 24, 1994. 


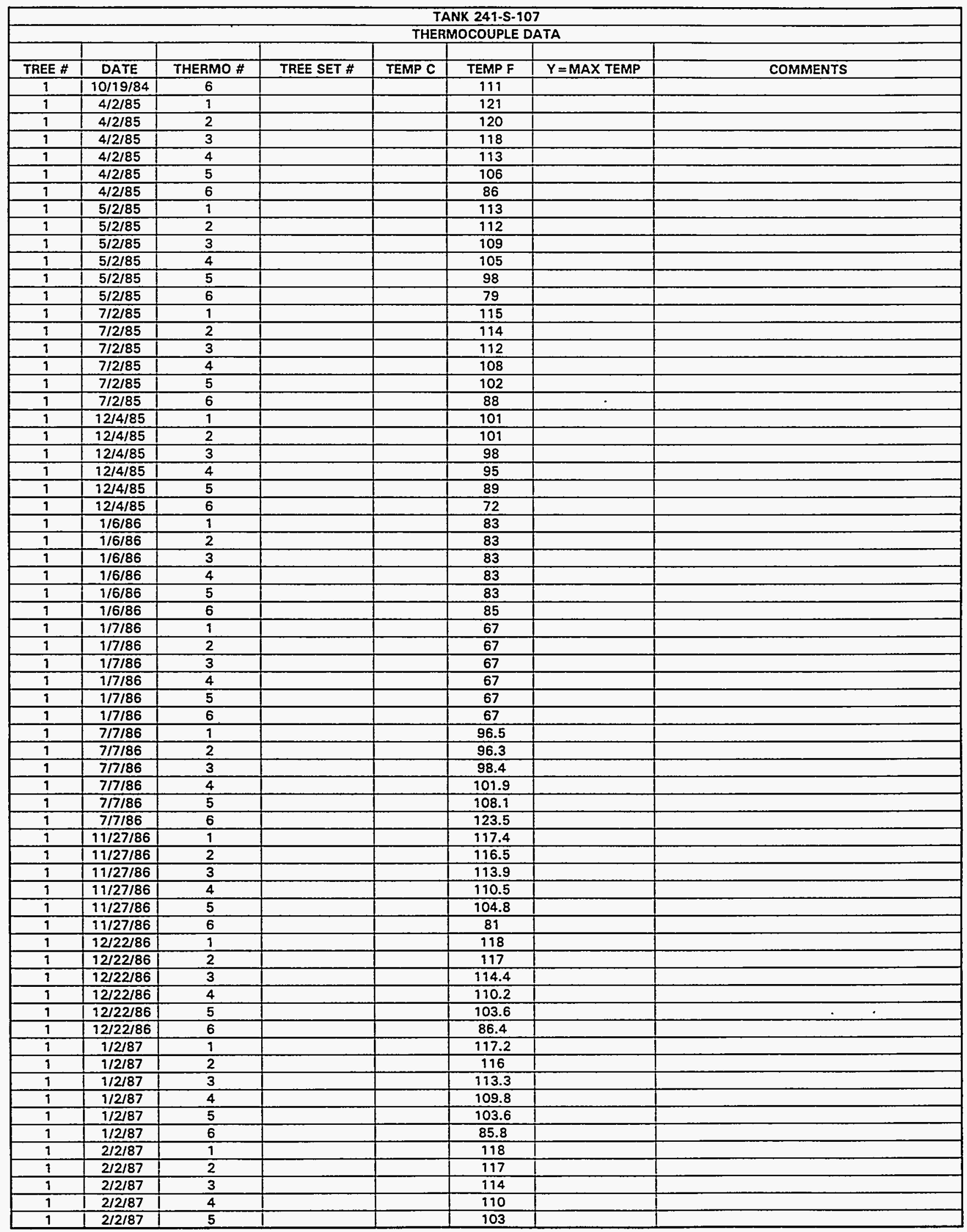

Data obtained from WHC Surveillance Analysis Computer System (SACS), February 24, 1994. 
WHC-SD-WM-ER-323, Rev. 0

\begin{tabular}{|c|c|c|c|c|c|c|c|}
\hline \multicolumn{8}{|c|}{ TANK 241-S-107 } \\
\hline \multicolumn{8}{|c|}{ THERMOCOUPLE DATA } \\
\hline TREE \# & DATE & THERMO \# & TREE SET \# & TEMP C & TEMP F & $Y=M A X$ TEMP & COMMENTC \\
\hline$\frac{1}{1}$ & $2 / 2 / 87$ & $\frac{10}{6}$ & THEE SEI H & IETMF C & 85 & $8=$ MAX IENMI & COMMENIS \\
\hline 1 & $3 / 2 / 87$ & 1 & & & 117 & & \\
\hline 1 & $3 / 2 / 87$ & 2 & & & 116 & & \\
\hline 1 & $3 / 2 / 87$ & 3 & & & 113 & & \\
\hline 1 & $3 / 2 / 87$ & 4 & & & 109 & & \\
\hline 1 & $3 / 2 / 87$ & 5 & & & 102 & & \\
\hline 9 & $3 / 2 / 87$ & 6 & & & 84 & & \\
\hline 1 & $4 / 6 / 87$ & 1 & & & 119 & & \\
\hline 1 & $4 / 6 / 87$ & 2 & & & 918 & & \\
\hline 9 & $4 / 6 / 87$ & 3 & & & 116 & & \\
\hline 1 & $4 / 6 / 87$ & 4 & & & 112 & & \\
\hline 1 & $4 / 6 / 87$ & 5 & & & 905 & & \\
\hline 1 & $4 / 6 / 87$ & 6 & & & 78 & & \\
\hline 1 & $4 / 11 / 87$ & 1 & & & 117 & & \\
\hline 1 & $4 / 11 / 87$ & 2 & & & 116 & & \\
\hline 1 & $4 / 11 / 87$ & 3 & & & 113 & & \\
\hline 1 & $4 / 11 / 87$ & 4 & & & 110 & & \\
\hline 9 & $4 / 11 / 87$ & 5 & & & 104 & & \\
\hline 1 & $4 / 11 / 87$ & 6 & & & 91 & & \\
\hline 1 & $5 / 3 / 87$ & 1 & & & 115.6 & & \\
\hline 1 & $5 / 3 / 87$ & 2 & & & 114.4 & & \\
\hline 1 & $5 / 3 / 87$ & 3 & & & 111.4 & & \\
\hline 1 & $5 / 3 / 87$ & 4 & & & 107.5 & & \\
\hline 1 & $5 / 3 / 87$ & 5 & & & 100.7 & & \\
\hline 1 & $5 / 3 / 87$ & 6 & & & 83.7 & & \\
\hline 1 & $7 / 6 / 87$ & 1 & & & 115.5 & & \\
\hline 1 & $7 / 6 / 87$ & 2 & & & 114.3 & & \\
\hline 1 & $7 / 6 / 87$ & 3 & & & 111.4 & & \\
\hline 1 & $7 / 6 / 87$ & 4 & & & 107.8 & & \\
\hline 1 & $7 / 6 / 87$ & 5 & & & 102 & & \\
\hline 1 & $7 / 6 / 87$ & 6 & & & 88.1 & & \\
\hline 1 & $8 / 4 / 87$ & 1 & & & 114.4 & & \\
\hline 1 & $8 / 4 / 87$ & 2 & & & 113.4 & & \\
\hline 1 & $8 / 4 / 87$ & 3 & & & 110.5 & & \\
\hline 1 & $8 / 4 / 87$ & 4 & & & 106.9 & & \\
\hline 1 & $8 / 4 / 87$ & 5 & & & 101.4 & & \\
\hline 1 & $8 / 4 / 87$ & 6 & & & 88.2 & & \\
\hline 1 & $10 / 5 / 87$ & 7 & & & 114 & & \\
\hline 1 & $10 / 5 / 87$ & 2 & & & 114 & & \\
\hline 1 & $10 / 5 / 87$ & 3 & & & 112 & & \\
\hline 1 & $10 / 5 / 87$ & 4 & & & 109 & & \\
\hline 1 & $10 / 5 / 87$ & 5 & & & 104 & & \\
\hline 1 & $10 / 5 / 87$ & 6 & & & 92 & & \\
\hline 1 & $12 / 8 / 87$ & 1 & & & 117.7 & & \\
\hline 1 & $12 / 8 / 87$ & 2 & & & 116.3 & & \\
\hline 1 & $12 / 8 / 87$ & 3 & & & 113.8 & & \\
\hline 1 & $12 / 8 / 87$ & 4 & & & 110.5 & & \\
\hline 9 & $12 / 8 / 87$ & 5 & & & 104.8 & & \\
\hline 1 & $12 / 8 / 87$ & 6 & & & 89.2 & & \\
\hline 1 & $1 / 4 / 88$ & 1 & 1 & & 117.8 & $\bar{Y}$ & \\
\hline 1 & $9 / 4 / 88$ & 2 & 1 & & 116.6 & $\mathrm{~N}$ & \\
\hline 1 & $1 / 4 / 88$ & 3 & 7 & & 114 & $\bar{N}$ & \\
\hline 1 & $1 / 4 / 88$ & 4 & 1 & & 110.5 & $N$ & \\
\hline 1 & T/4/88. & 5 & 7 & & 104.7 & $\mathrm{~N}$ & \\
\hline 1 & $1 / 4 / 88$ & 6 & 9 & & 87.3 & $\mathrm{~N}$ & \\
\hline 1 & $2 / 1 / 88$ & 9 & 9 & & 113 & $\bar{Y}$ & \\
\hline 1 & $2 / 1 / 88$ & 2 & 1 & & 110 & $N$ & \\
\hline 1 & $2 / 1 / 88$ & 3 & 1 & & 109 & $\mathrm{~N}$ & \\
\hline 1 & $2 / 1 / 88$ & 4 & 7 & & 106 & $\mathbf{N}$ & \\
\hline 1 & $2 / 1 / 88$ & 5 & 1 & & 103 & $\mathrm{~N}$ & \\
\hline$\frac{1}{1}$ & $2 / 1 / 88$ & 6 & 1 & & 83 & $\bar{N}$ & \\
\hline 1 & $3 / 8 / 88$ & 2 & 7 & & 122 & $\bar{Y}$ & \\
\hline 1 & $3 / 8 / 88$ & 4 & $\frac{1}{1}$ & & 113 & $N$ & \\
\hline 1 & $3 / 8 / 88$ & 5 & 1 & & 106 & $N$ & \\
\hline 1 & $3 / 8 / 88$ & 6 & 1 & & 90 & $N$ & \\
\hline 1 & $4 / 4 / 88$ & 9 & 1 & & 196 & $\mathrm{~N}$ & \\
\hline
\end{tabular}

Data obtained from WHC Surveillance Analysis Computer System (SACS), February 24, 1994. 


\begin{tabular}{|c|c|c|c|c|c|c|c|}
\hline \multicolumn{8}{|c|}{ TANK 241-S-107 } \\
\hline & & & & THEF & OCOUPLE & ITA & \\
\hline TREE \# & DATE & THERMO \# & TREE SET \# & TEMP C & TEMPF & $\bar{Y}=$ MAX TEMP & COMMENTS \\
\hline 1 & $4 / 4 / 88$ & 2 & 1 & & 117 & $\mathrm{Y}$ & \\
\hline 1 & $4 / 4 / 88$ & 3 & 1 & & 112 & $\mathbf{N}$ & \\
\hline 1 & $4 / 4 / 88$ & 4 & 1 & & 108 & $\mathbf{N}$ & \\
\hline 1 & $4 / 4 / 88$ & 5 & 1 & & 102 & $\mathbf{N}$ & \\
\hline 1 & $4 / 4 / 88$ & 6 & 1 & & 86 & $\mathrm{~N}$ & \\
\hline 1 & $5 / 2 / 88$ & 1 & 1 & & 123 & $\bar{Y}$ & \\
\hline$\frac{1}{1}$ & $5 / 2 / 88$ & 2 & 1 & & 122 & $\mathbf{N}$ & \\
\hline 9 & $5 / 2 / 88$ & 3 & 1 & & 120 & $\mathbf{N}$ & \\
\hline 1 & $5 / 2 / 88$ & 4 & 1 & & 116 & $\mathbf{N}$ & \\
\hline 1 & $5 / 2 / 88$ & 5 & 1 & & 110 & $\mathbf{N}$ & \\
\hline 1 & $5 / 2 / 88$ & 6 & 1 & & 95 & $\mathbf{N}$ & \\
\hline 1 & $5 / 4 / 88$ & 1 & 1 & & 118 & $Y$ & \\
\hline 1 & $5 / 4 / 88$ & 2 & 1 & & 117 & $\mathbf{N}$ & \\
\hline 1 & $5 / 4 / 88$ & $\overline{3}$ & $T$ & & 115 & $\mathbf{N}$ & \\
\hline 1 & $5 / 4 / 88$ & 4 & 1 & & 111 & $\mathbf{N}$ & \\
\hline 1 & $5 / 4 / 88$ & 5 & 1 & & 105 & $\mathbf{N}$ & \\
\hline 1 & $5 / 4 / 88$ & 6 & 1 & & 89 & $\mathbf{N}$ & \\
\hline 1 & $6 / 6 / 88$ & 1 & 1 & & 120 & $Y$ & \\
\hline 1 & $6 / 6 / 88$ & 2 & 1 & & 118 & $\mathrm{~N}$ & \\
\hline 1 & $6 / 6 / 88$ & 3 & 1 & & 118 & $\bar{N}$ & \\
\hline 1 & $6 / 6 / 88$ & 4 & 1 & & 115 & $\mathbf{N}$ & \\
\hline 1 & $6 / 6 / 88$ & 5 & 1 & & 112 & $\mathbf{N}$ & \\
\hline 1 & $6 / 6 / 88$ & 6 & 1 & & 95 & $N$ & \\
\hline 1 & $7 / 4 / 88$ & 1 & 1 & & 120 & $\bar{Y}$ & \\
\hline 1 & $7 / 4 / 88$ & 2 & 1 & & 119 & $N$ & \\
\hline 1 & $7 / 4 / 88$ & 3 & 1 & & 116 & $N$ & \\
\hline 1 & $7 / 4 / 88$ & 4 & 1 & & 112 & $N$ & \\
\hline 1 & $7 / 4 / 88$ & 5 & 1 & & 106 & $\mathbf{N}$ & \\
\hline 1 & $7 / 4 / 88$ & 6 & 1 & & 86 & $N$ & \\
\hline 1 & $8 / 1 / 88$ & 1 & 1 & & 116 & $Y$ & \\
\hline 1 & $8 / 1 / 88$ & 2 & 1 & & 116 & $\mathrm{~N}$ & \\
\hline 1 & $8 / 1 / 88$ & 3 & 9 & & 113 & $\mathrm{~N}$ & \\
\hline 1 & $8 / 1 / 88$ & 4 & 1 & & 110 & $N$ & \\
\hline 9 & $8 / 1 / 88$ & 5 & 1 & & 106 & $N$ & \\
\hline$\frac{1}{1}$ & $8 / 1 / 88$ & 6 & 1 & & 93 & $\bar{N}$ & \\
\hline 1 & $9 / 5 / 88$ & 1 & 1 & & 114 & $Y$ & \\
\hline 1 & $9 / 5 / 88$ & 2 & 1 & & 113 & $\mathrm{~N}$ & \\
\hline 1 & $9 / 5 / 88$ & 3 & 1 & & 111 & $N$ & \\
\hline 1 & $9 / 5 / 88$ & 4 & 1 & & 108 & $N$ & \\
\hline 1 & $9 / 5 / 88$ & 5 & 1 & & 103 & $N$ & \\
\hline 1 & $9 / 5 / 88$ & 6 & 1 & & 90 & $\mathbf{N}$ & \\
\hline 1 & $10 / 3 / 88$ & 1 & 1 & & 114 & $\bar{Y}$ & \\
\hline 1 & $10 / 3 / 88$ & 2 & 1 & & 114 & $N$ & \\
\hline 1 & $10 / 3 / 88$ & 3 & 7 & & 111 & $\mathrm{~N}$ & \\
\hline 1 & $10 / 3 / 88$ & 4 & 1 & & 108 & $\mathrm{~N}$ & \\
\hline 1 & $10 / 3 / 88$ & 5 & $\frac{T}{1}$ & & 103 & $\mathrm{~N}$ & \\
\hline 1 & $10 / 3 / 88$ & 6 & 1 & & 90 & $\mathrm{~N}$ & \\
\hline 1 & $11 / 7 / 88$ & 1 & 1 & & 114 & $\mathbf{Y}$ & \\
\hline 1 & $11 / 7 / 88$ & 2 & 1 & & 114 & $N$ & \\
\hline 9 & $11 / 7 / 88$ & 3 & 1 & & 111 & $\mathbf{N}$ & \\
\hline 1 & $11 / 7 / 88$ & 4 & 1 & & 109 & $\mathbf{N}$ & \\
\hline 1 & $11 / 7 / 88$ & 5 & 1 & & 104 & $\bar{N}$ & \\
\hline 1 & $11 / 7 / 88$ & 6 & 1 & & 91 & $\mathbf{N}$ & \\
\hline 1 & $12 / 5 / 88$ & 1 & 1 & & & $\mathbf{N}$ & BAD READINGS \\
\hline 1 & $1 / 2 / 89$ & 1 & 1 & & & $N$ & o/s \\
\hline 1 & $2 / 6 / 89$ & 1 & 1 & & 117.5 & $\bar{Y}$ & \\
\hline 1 & $2 / 6 / 89$ & 2 & 1 & & 116.7 & $\bar{N}$ & \\
\hline 1 & $2 / 6 / 89$ & 3 & 1 & & 114.1 & $\mathbf{N}$ & \\
\hline 1 & $2 / 6 / 89$ & 4 & 1 & & 110.1 & $\mathbf{N}$ & \\
\hline 9 & $2 / 6 / 89$ & 5 & 1 & & 103.1 & $\mathrm{~N}$ & \\
\hline 1 & $2 / 6 / 89$ & 6 & 1 & & 86.3 & $\mathbf{N}$ & \\
\hline 1 & $3 / 6 / 89$ & 1 & $\overline{1}$ & & 139 & $\bar{Y}$ & \\
\hline 1 & $3 / 6 / 89$ & 2 & 1 & & 139 & $\mathbf{N}$ & \\
\hline 1 & $3 / 6 / 89$ & 3 & 1 & & 136 & $N$ & \\
\hline 1 & $3 / 6 / 89$ & 4 & 1 & & 132 & $\mathrm{~N}$ & \\
\hline 1 & $3 / 6 / 89$ & 5 & 1 & & 125 & $\mathbf{N}$. & \\
\hline
\end{tabular}

Data obtained from WHC Surveillance Analysis Computer System (SACS), February 24, 1994. 


\begin{tabular}{|c|c|c|c|c|c|c|c|}
\hline \multicolumn{8}{|c|}{ TANK 241-S-107 } \\
\hline \multicolumn{8}{|c|}{ THERMOCOUPLE DATA } \\
\hline TREE \# & DATE & THERMO \# & TREE SET \# & TEMP C & TEMP F & $Y=M A X$ TEMP & COMMENTS \\
\hline 1 & $3 / 6 / 89$ & 6 & 1 & & 105 & $\mathrm{~N}$ & \\
\hline 1 & $1 / 4 / 91$ & 1 & 1 & & 108.9 & $\bar{Y}$ & HH-99A-J \#890523512-47 \\
\hline 1 & $1 / 4 / 91$ & 2 & 1 & & 108.3 & $\mathbf{N}$ & \\
\hline 1 & $1 / 4 / 91$ & 3 & 1 & & 106.2 & $\bar{N}$ & \\
\hline 1 & $1 / 4 / 91$ & 4 & 1 & & 103 & $N$ & \\
\hline 1 & $1 / 4 / 91$ & 5 & 1 & & 97.5 & $N$ & \\
\hline 1 & $1 / 4 / 91$ & 6 & 1 & & 82 & $N$ & \\
\hline 1 & $11 / 13 / 91$ & 1 & 1 & & 108.4 & $\bar{Y}$ & $\mathrm{~J}-890423320-42$ \\
\hline 1 & $11 / 13 / 91$ & 2 & 1 & & 107.3 & $N$ & \\
\hline 1 & $11 / 13 / 91$ & 3 & 1 & & 105.3 & $\mathrm{~N}$ & \\
\hline 1 & $11 / 13 / 91$ & 4 & 1 & & 102.8 & $\mathbf{N}$ & \\
\hline 1 & $11 / 13 / 91$ & 5 & 1 & & 98.9 & $\bar{N}$ & \\
\hline 1 & $11 / 13 / 91$ & 6 & 1 & & 84.3 & $\mathbf{N}$ & \\
\hline 1 & $1 / 1 / 92$ & 1 & 1 & & 113 & $\bar{Y}$ & $J-890523512-20$ \\
\hline 1 & $1 / 1 / 92$ & 2 & 1 & & 111.9 & $\mathbf{N}$ & \\
\hline 1 & $1 / 1 / 92$ & 3 & 1 & & 109.9 & $\bar{N}$ & \\
\hline 1 & $1 / 1 / 92$ & 4 & 1 & & 107.1 & $\mathrm{~N}$ & \\
\hline 1 & $1 / 1 / 92$ & 5 & 1 & & 102.3 & $\mathrm{~N}$ & \\
\hline 1 & $1 / 1 / 92$ & 6 & 1 & & 84.7 & $\mathbf{N}$ & \\
\hline 1 & $7 / 2 / 92$ & 1 & 1 & & & $\mathbf{N}$ & O/S \\
\hline 1 & $11 / 4 / 92$ & 1 & 1 & & & $\mathbf{N}$ & $\begin{array}{l}\text { "US\&DA-92-488 NO RDGS SINCE JANUARY } \\
1992\end{array}$ \\
\hline 1 & $11 / 5 / 92$ & 1 & 1 & & 112 & $\mathbf{Y}$ & $J 817-13 \cdot 20-019$ \\
\hline 1 & $11 / 5 / 92$ & 2 & 1 & & 111 & $\mathbf{N}$ & \\
\hline 1 & $11 / 5 / 92$ & 3 & 1 & & 109 & $N$ & \\
\hline 1 & $11 / 5 / 92$ & 4 & 1 & & 106 & $\bar{N}$ & \\
\hline 9 & $11 / 5 / 92$ & 5 & 1 & & 102 & $\mathbf{N}$ & \\
\hline 1 & $11 / 5 / 92$ & 6 & 1 & & 87 & $\bar{N}$ & \\
\hline 1 & $2 / 13 / 93$ & $\mathrm{~J}$ & 1 & & 106.5 & $\bar{Y}$ & $\mathrm{~J} 910126143-42$ \\
\hline 1 & $2 / 13 / 93$ & 2 & 1 & & 105.3 & $\mathbf{N}$ & \\
\hline 1 & $2 / 13 / 93$ & 3 & 1 & & 102 & $\mathbf{N}$ & \\
\hline 1 & $2 / 13 / 93$ & 4 & 1 & & 96.1 & $\bar{N}$ & \\
\hline 1 & $2 / 13 / 93$ & 5 & 1 & & 78 & $\mathbf{N}$ & \\
\hline 1 & $2 / 13 / 93$ & 6 & 1 & & 78.3 & $\mathrm{~N}$ & \\
\hline 1 & $7 / 2 / 93$ & 1 & 1 & & 106.8 & $\bar{Y}$ & J 890423320-42 \\
\hline 1 & $7 / 2 / 93$ & 2 & 1 & & 104.6 & $\mathbf{N}$ & \\
\hline 1 & $7 / 2 / 93$ & 3 & 1 & & 101.9 & $\mathbf{N}$ & \\
\hline 1 & $7 / 2 / 93$ & 4 & 1 & & 98.7 & $\mathbf{N}$ & \\
\hline 1 & $7 / 2 / 93$ & 5 & 1 & & 93.2 & $\mathbf{N}$ & \\
\hline 1 & $7 / 2 / 93$ & 6 & 1 & & 80.1 & $N$ & \\
\hline
\end{tabular}

Data obtained from WHC Surveillance Analysis Computer System (SACS), February 24, 1994. 


\begin{tabular}{|c|c|c|c|c|c|c|c|}
\hline \multicolumn{8}{|c|}{ TANK 241-S-108 } \\
\hline \multicolumn{8}{|c|}{ THERMOCOUPLE DATA } \\
\hline TREE \# & DATE & THERMO \# & TREE SET \# & TEMP C & TEMP F & $Y=$ MAX TEMP & COMMENTS \\
\hline 1 & $10 / 20 / 74$ & 1 & & 50.56 & 123.01 & & raw data - assumed degree C \\
\hline 1 & $10 / 20 / 74$ & 2 & & 53.33 & 127.99 & & raw data - assumed degree $C$ \\
\hline 1 & $10 / 20 / 74$ & 3 & & 56.67 & 134.006 & & raw data - assumed degree C \\
\hline 1 & $10 / 20 / 74$ & 4 & & 60.00 & 140 & & raw data - assumed degree C \\
\hline 1 & $10 / 20 / 74$ & 5 & & 62.78 & 145.004 & & raw data - assumed degree C \\
\hline 7 & $10 / 20 / 74$ & 6 & & 64.44 & 147.992 & & raw data - assumed degree C \\
\hline 1 & $10 / 20 / 74$ & 7 & & 65.00 & 149 & & raw data - assumed degree C \\
\hline 1 & $10 / 20 / 74$ & 8 & & 65.00 & 149 & & raw data - assumed degree $C$ \\
\hline 9 & $10 / 20 / 74$ & 9 & & 63.33 & 145.994 & & raw data - assumed degree C \\
\hline 1 & $10 / 20 / 74$ & 10 & & 58.33 & 136.994 & & raw data - assumed degree C \\
\hline 1 & $10 / 20 / 74$ & 11 & & 53.89 & 129.002 & & raw data - assumed degree $C$ \\
\hline 1 & $10 / 20 / 74$ & 12 & & 47.22 & 116.996 & & raw data - assumed degree $C$ \\
\hline 1 & $12 / 2 / 74$ & 1 & & 51.67 & 125.006 & & raw data - assumed degree C \\
\hline 1 & $12 / 2 / 74$ & 2 & & 54.44 & 129.992 & & raw data - assumed degree $C$ \\
\hline 1 & $12 / 2 / 74$ & 3 & & 57.78 & 136.004 & & raw data - assumed degree C \\
\hline 1 & $12 / 2 / 74$ & 4 & & 61.11 & 141.998 & & raw data - assumed degree C \\
\hline 1 & $12 / 2 / 74$ & 5 & & 63.33 & 145.994 & & raw data - assumed degree C \\
\hline 1 & $12 / 2 / 74$ & 6 & & 64.44 & 147.992 & & raw data - assumed degree C \\
\hline 1 & $12 / 2 / 74$ & 7 & & 65.00 & 149 & & raw data - assumed degree C \\
\hline 1 & $12 / 2 / 74$ & 8 & & 63.89 & 147.002 & & raw data - assumed degree $C$ \\
\hline 1 & $12 / 2 / 74$ & 9 & & 60.56 & 141.008 & & raw data - assumed degree C \\
\hline 1 & $12 / 2 / 74$ & 10 & & 51.11 & 123.998 & & raw data - assumed degree C \\
\hline 1 & $12 / 2 / 74$ & 11 & & 40.56 & 105.008 & & raw data - assumed degree C \\
\hline 1 & $12 / 2 / 74$ & 12 & & 36.67 & 98.006 & & raw data - assumed degree C \\
\hline 1 & $11 / 3 / 75$ & 1 & & & 129 & & \\
\hline 1 & $11 / 3 / 75$ & 2 & & & 132 & & \\
\hline 1 & $11 / 3 / 75$ & 3 & & & 131 & & \\
\hline 1 & $11 / 3 / 75$ & 4 & & & 139 & & \\
\hline 1 & $11 / 3 / 75$ & 5 & & & 140 & & \\
\hline 1 & $11 / 3 / 75$ & 6 & & & 139 & & \\
\hline 1 & $11 / 3 / 75$ & 7 & & & 136 & & \\
\hline 1 & $11 / 3 / 75$ & 8 & & & 130 & & \\
\hline 1 & $11 / 3 / 75$ & 9 & & & 126 & & \\
\hline 1 & $11 / 3 / 75$ & 10 & & & 120 & & \\
\hline 1 & $11 / 3 / 75$ & 11 & & & 110 & & \\
\hline 1 & $11 / 3 / 75$ & 12 & & & 98 & & \\
\hline 9 & $2 / 4 / 76$ & 1 & & & 149 & & \\
\hline 1 & $2 / 4 / 76$ & 2 & & & 152 & & \\
\hline 1 & $2 / 4 / 76$ & 3 & & & 156 & & \\
\hline 1 & $2 / 4 / 76$ & 4 & & & 159 & & \\
\hline 1 & $2 / 4 / 76$ & 5 & & & 160 & & \\
\hline 1 & $2 / 4 / 76$ & 6 & & & 159 & & \\
\hline 9 & $2 / 4 / 76$ & 7 & & & 156 & & \\
\hline 1 & $2 / 4 / 76$ & 8 & & & 156 & & \\
\hline 1 & $2 / 4 / 76$ & 9 & & & 146 & & \\
\hline 9 & $2 / 4 / 76$ & 10 & & & 139 & & \\
\hline 1 & $2 / 4 / 76$ & 11 & & & 128 & & \\
\hline 1 & $2 / 4 / 76$ & 12 & & & 114 & & \\
\hline 1 & $3 / 5 / 76$ & 1 & & & 126 & & \\
\hline 1 & $3 / 5 / 76$ & 2 & & & 129 & & \\
\hline 1 & $3 / 5 / 76$ & 3 & & & 132 & & \\
\hline 1 & $3 / 5 / 76$ & 4 & & & 135 & & \\
\hline 1 & $3 / 5 / 76$ & 5 & & & 136 & & \\
\hline 1 & $3 / 5 / 76$ & 6 & & & 135 & & \\
\hline 1 & $3 / 5 / 76$ & 7 & & & 131 & & \\
\hline 1 & $3 / 5 / 76$ & 8 & & & 124 & & \\
\hline 1 & $3 / 5 / 76$ & 9 & & & 120 & & \\
\hline 1 & $3 / 5 / 76$ & 10 & & & 114 & & \\
\hline 1 & $3 / 5 / 76$ & 11 & & & 103 & & \\
\hline 1 & $5 / 2 / 76$ & 1 & & & 126 & & \\
\hline 1 & $5 / 2 / 76$ & 2 & & & 130 & & \\
\hline 1 & $5 / 2 / 76$ & 3 & & & 132 & & \\
\hline 1 & $5 / 2 / 76$ & 4 & & & 135 & & \\
\hline 1 & $5 / 2 / 76$ & 5 & & & 135 & & \\
\hline$T$ & $5 / 2 / 76$ & 6 & & & 134 & & I \\
\hline 9 & $5 / 2 / 76$ & 7 & & & 131 & & i \\
\hline
\end{tabular}

Data obtained from WHC Surveillance Analysis Computer System (SACS), February 24, 1994. 


\begin{tabular}{|c|c|c|c|c|c|c|c|c|}
\hline \multicolumn{9}{|c|}{ TANK 241-S-108 } \\
\hline \multicolumn{8}{|c|}{ THERMOCOUPLE DATA } & \\
\hline TREE \# & DATE & THERMO \# & TREE SET \# & TEMP C & TEMP F & $Y=M A X$ TEMP & COMMENTS & \\
\hline 1 & $5 / 2 / 76$ & 8 & & & 126 & & & \\
\hline 1 & $5 / 2 / 76$ & 9 & & & 120 & & & \\
\hline 1 & $5 / 2 / 76$ & 10 & & & 113 & & & \\
\hline 1 & $5 / 2 / 76$ & 11 & & & 103 & & & \\
\hline 1 & $5 / 2 / 76$ & 12 & & & 92 & & & \\
\hline 1 & $6 / 1 / 76$ & 1 & & & 124 & & & \\
\hline 1 & $6 / 1 / 76$ & 2 & & & 126 & & & \\
\hline 1 & $6 / 1 / 76$ & 3 & & & 130 & & & \\
\hline 1 & $6 / 1 / 76$ & 4 & & & 132 & & & \\
\hline 1 & $6 / 7 / 76$ & 5 & & & 134 & & & \\
\hline 1 & $6 / 1 / 76$ & 6 & & & 134 & & & \\
\hline 1 & $6 / 1 / 76$ & 7 & & & 130 & & & \\
\hline 1 & $6 / 1 / 76$ & 8 & & & 126 & & & \\
\hline 1 & $6 / 1 / 76$ & 9 & & & 120 & & & \\
\hline 1 & $6 / 1 / 76$ & 10 & & & 112 & & & \\
\hline 1 & $6 / 1 / 76$ & 11 & & & 103 & & & \\
\hline 1 & $6 / 1 / 76$ & 12 & & & 92 & & & \\
\hline 1 & $6 / 9 / 76$ & 13 & & & 88 & & & \\
\hline 1 & $6 / 1 / 76$ & 14 & & & 88 & & & \\
\hline 1 & $7 / 1 / 76$ & 1 & & & 115 & & & \\
\hline 1 & $7 / 1 / 76$ & 2 & & & 118 & & & \\
\hline 1 & $7 / 1 / 76$ & 3 & & & 121 & & & \\
\hline 1 & $7 / 1 / 76$ & 4 & & & 123 & & & \\
\hline 1 & $7 / 1 / 76$ & 5 & & & 123 & & & \\
\hline 1 & $7 / 1 / 76$ & 6 & & & 122 & & & \\
\hline 1 & $7 / 1 / 76$ & 7 & & & 118 & & & \\
\hline 1 & $7 / 1 / 76$ & 8 & & & 112 & & & \\
\hline 1 & $7 / 1 / 76$ & 9 & & & 111 & & & \\
\hline 1 & $7 / 1 / 76$ & 10 & & & 104 & & & \\
\hline 1 & $7 / 1 / 76$ & 11 & & & 95 & & & \\
\hline 1 & $7 / 1 / 76$ & 12 & & & 86 & & & \\
\hline 1 & $8 / 3 / 76$ & 1 & & & 122 & & & \\
\hline 1 & $8 / 3 / 76$ & 2 & & & 125 & & & \\
\hline 1 & $8 / 3 / 76$ & 3 & & & 130 & & & \\
\hline 1 & $8 / 3 / 76$ & 4 & & & 131 & & & \\
\hline 1 & $8 / 3 / 76$ & 5 & & & 137 & & & \\
\hline 1 & $8 / 3 / 76$ & 6 & & & 130 & & & \\
\hline 1 & $8 / 3 / 76$ & 7 & & & 126 & & $\cdot$ & \\
\hline 1 & $8 / 3 / 76$ & 8 & & & 120 & & & \\
\hline 1 & $8 / 3 / 76$ & 9 & & & 120 & & & \\
\hline 1 & $8 / 3 / 76$ & 10 & & & 124 & & & \\
\hline 1 & $8 / 3 / 76$ & 11 & & & 106 & & & \\
\hline 1 & $8 / 3 / 76$ & 12 & & & 98 & & & \\
\hline 1 & $9 / 3 / 76$ & 1 & & & 124 & & & \\
\hline 1 & $9 / 3 / 76$ & 2 & & & 128 & & & \\
\hline 1 & $9 / 3 / 76$ & 3 & & & 130 & & & \\
\hline 1 & $9 / 3 / 76$ & 4 & & & 132 & & & \\
\hline 1 & $9 / 3 / 76$ & 5 & & & 132 & & & \\
\hline 1 & $9 / 3 / 76$ & 6 & & & 132 & & & \\
\hline 1 & $9 / 3 / 76$ & 7 & & & 128 & & & \\
\hline 1 & $9 / 3 / 76$ & 8 & & & 122 & & & \\
\hline 1 & $9 / 3 / 76$ & 9 & & & 122 & & & \\
\hline 1 & $9 / 3 / 76$ & 10 & & & 116 & & & \\
\hline 1 & $9 / 3 / 76$ & 11 & & & 109 & & $\cdot$ & \\
\hline 1 & $9 / 3 / 76$ & 12 & & & 100 & & & \\
\hline 1 & $10 / 3 / 76$ & 1 & & & 126 & & & \\
\hline 1 & $10 / 3 / 76$ & 2 & & & 129 & & & \\
\hline 1 & $10 / 3 / 76$ & 3 & & & 113 & & & \\
\hline 1 & $10 / 3 / 76$ & 4 & & & 133 & & & \\
\hline 1 & $10 / 3 / 76$ & 5 & & & 134 & & & \\
\hline 1 & $10 / 3 / 76$ & 6 & & & 133 & & & \\
\hline 1 & $10 / 3 / 76$ & 7 & & & 130 & & & \\
\hline 1 & $10 / 3 / 76$ & 8 & & & 124 & & & \\
\hline 1 & $10 / 3 / 76$ & 9 & & & 123 & & & \\
\hline 1 & $10 / 3 / 76$ & 10 & & & 118 & & & \\
\hline 1 & $10 / 3 / 76$ & 11 & & & 191 & $\cdot$ & & \\
\hline
\end{tabular}

Data obtained from WHC Surveillance Analysis Computer System (SACS), February 24, 1994. 
WHC-SD-WM-ER-323, Rev. 0

\begin{tabular}{|c|c|c|c|c|c|c|c|}
\hline \multicolumn{8}{|c|}{ TANK 241-S-108 } \\
\hline \multirow{2}{*}{\multicolumn{8}{|c|}{ THERMOCOUPLE DATA }} \\
\hline & & & & & & & \\
\hline TREE \# & DATE & THERMO \# & TREE SET \# & TEMP C & TEMP F & $Y=$ MAX TEMP & COMMENTS \\
\hline 1 & $10 / 3 / 76$ & 12 & & & 103 & & \\
\hline 1 & $11 / 1 / 76$ & 1 & & & 126 & & \\
\hline 1 & $11 / 1 / 76$ & 2 & & & 130 & & \\
\hline 1 & $11 / 1 / 76$ & 3 & & & 132 & & \\
\hline 1 & $11 / 1 / 76$ & 4 & & & 134 & & \\
\hline 1 & $11 / 1 / 76$ & 5 & & & 134 & & \\
\hline 1 & $11 / 1 / 76$ & 6 & & & 134 & & \\
\hline 1 & $11 / 1 / 76$ & 7 & & & 130 & & \\
\hline 1 & $11 / 1 / 76$ & 8 & & & 124 & & \\
\hline 1 & $11 / 1 / 76$ & 9 & & & 122 & & \\
\hline 1 & $11 / 1 / 76$ & 10 & & & 119 & & \\
\hline 1 & $11 / 1 / 76$ & 11 & & & 112 & & \\
\hline 1 & $11 / 1 / 76$ & 12 & & & 100 & & \\
\hline 1 & $12 / 4 / 76$ & 1 & & & 126 & & \\
\hline 1 & $12 / 4 / 76$ & 2 & & & 128 & & \\
\hline 9 & $12 / 4 / 76$ & 3 & & & 131 & & \\
\hline 1 & $12 / 4 / 76$ & 4 & & & 133 & & \\
\hline 1 & $12 / 4 / 76$ & 5 & & & 134 & & \\
\hline 1 & $12 / 4 / 76$ & 6 & & & 132 & & \\
\hline 1 & $12 / 4 / 76$ & 7 & & & 126 & & \\
\hline 1 & $12 / 4 / 76$ & 8 & & & 118 & & \\
\hline 1 & $12 / 4 / 76$ & 9 & & & 118 & & \\
\hline 1 & $12 / 4 / 76$ & 10 & & & 117 & & \\
\hline 1 & $12 / 4 / 76$ & 11 & & & 110 & & \\
\hline 9 & $12 / 4 / 76$ & 12 & & & 100 & & \\
\hline 1 & $1 / 1 / 77$ & 1 & & & 126 & & \\
\hline 1 & $1 / 1 / 77$ & 2 & & & 128 & & \\
\hline 9 & $1 / 1 / 77$ & 3 & & & 131 & & \\
\hline 1 & $1 / 1 / 77$ & 4 & & & 133 & & \\
\hline 9 & $1 / 1 / 77$ & 5 & & & 133 & & \\
\hline 1 & $1 / 1 / 77$ & 6 & & & 131 & & \\
\hline 1 & $1 / 1 / 77$ & 7 & & & 124 & & \\
\hline 1 & $1 / 1 / 77$ & 8 & & & 114 & & \\
\hline 1 & $1 / 1 / 77$ & 9 & & & 115 & & \\
\hline 1 & $1 / 1 / 77$ & 10 & & & 114 & & \\
\hline 1 & $1 / 1 / 77$ & 11 & & & 108 & & \\
\hline 1 & $1 / 1 / 77$ & 12 & & & 97 & & \\
\hline 1 & $2 / 1 / 77$ & 1 & & & 126 & & \\
\hline 1 & $2 / 1 / 77$ & 2 & & & 128 & & \\
\hline 1 & $2 / 1 / 77$ & 3 & & $\cdot$ & 130 & & \\
\hline 1 & $2 / 1 / 77$ & 4 & & & 132 & & \\
\hline 1 & $2 / 1 / 77$ & 5 & & & 132 & & \\
\hline 1 & $2 / 1 / 77$ & 6 & & & 128 & & \\
\hline 1 & $2 / 1 / 77$ & 7 & & & 120 & & \\
\hline 1 & $2 / 1 / 77$ & 8 & & & 110 & & \\
\hline 1 & $2 / 1 / 77$ & 9 & & & 110 & & \\
\hline 1 & $2 / 1 / 77$ & 10 & & & 110 & & \\
\hline 1 & $2 / 1 / 77$ & 11 & & & 110 & & \\
\hline 1 & $2 / 1 / 77$ & 12 & & & 104 & & \\
\hline 1 & $3 / 1 / 77$ & 1 & & & 120 & & \\
\hline 1 & $3 / 1 / 77$ & 2 & & & 123 & & \\
\hline 1 & $3 / 1 / 77$ & 3 & & & 925 & & \\
\hline 1 & $3 / 1 / 77$ & 4 & & & 127 & & \\
\hline 9 & $3 / 1 / 77$ & 5 & & & 126 & & \\
\hline 1 & $3 / 1 / 77$ & 6 & & & 123 & & \\
\hline 1 & $3 / 1 / 77$ & 7 & & & 115 & & \\
\hline 1 & $3 / 1 / 77$ & 8 & & & 104 & & \\
\hline 1 & $3 / 1 / 77$ & 9 & & & 104 & & \\
\hline 1 & $3 / 1 / 77$ & 10 & & & 104 & & \\
\hline 1 & $3 / 1 / 77$ & 11 & & & 98 & & \\
\hline 1 & $3 / 1 / 77$ & 12 & & & 88 & & \\
\hline 1 & $3 / 1 / 77$ & 13 & & & 85 & & \\
\hline 1 & $3 / 1 / 77$ & 14 & & & 85 & & \\
\hline 1 & $4 / 1 / 77$ & 1 & & & 120 & & \\
\hline 1 & $4 / 1 / 77$ & 2 & & & 123 & & \\
\hline 1 & $4 / 1 / 77$ & 3 & & & 125 & & \\
\hline
\end{tabular}

Data obtained from WHC Surveillance Analysis Computer System (SACS), February 24, 1994. 


\begin{tabular}{|c|c|c|c|c|c|c|c|}
\hline \multicolumn{8}{|c|}{ TANK 241.S-108 } \\
\hline & & & & THER & OCOUPLE & & \\
\hline TREE \# & DATE & THERMO \# & TREE SET \# & TEMP C & TEMP F & $Y=\operatorname{MAX}$ TEMP & COMMENTS \\
\hline 1 & $4 / 1 / 77$ & 4 & & & 126 & & \\
\hline 1 & $4 / 1 / 77$ & 5 & & & 125 & & \\
\hline 1 & $4 / 1 / 77$ & 6 & & & 121 & & \\
\hline 1 & $4 / 1 / 77$ & 7 & & & 114 & & \\
\hline 1 & $4 / 1 / 77$ & 8 & & & 102 & & \\
\hline 1 & $4 / 1 / 77$ & 9 & & & 102 & & \\
\hline 9 & $4 / 1 / 77$ & 10 & & & 101 & & \\
\hline 1 & $4 / 1 / 77$ & 11 & & & 96 & & \\
\hline 1 & $4 / 9 / 77$ & 12 & & & 87 & & \\
\hline 1 & $5 / 1 / 77$ & 1 & & & 120 & & \\
\hline 1 & $5 / 1 / 77$ & 2 & & & 121 & & \\
\hline 1 & $5 / 1 / 77$ & 3 & & & 125 & & \\
\hline 1 & $5 / 1 / 77$ & 4 & & & 125 & & \\
\hline 1 & $5 / 1 / 77$ & 5 & & & 125 & & \\
\hline 1 & $5 / 1 / 77$ & 6 & & & 120 & & \\
\hline 1 & $5 / 1 / 77$ & 7 & & & 114 & & \\
\hline 1 & $5 / 1 / 77$ & 8 & & & & & 164-suspect data (difficult to read on orginal) \\
\hline 1 & $5 / 1 / 77$ & 9 & & & & & 162-suspect data (difficult to read on orginal) \\
\hline 1 & $5 / 1 / 77$ & 10 & & & & & 160-suspect data (difficult to read on orginal) \\
\hline 1 & $5 / 1 / 77$ & 11 & & & 95 & & \\
\hline 1 & $5 / 1 / 77$ & 12 & & & 90 & & \\
\hline 1 & $6 / 7 / 77$ & 1 & & & 119 & & \\
\hline 1 & $6 / 7 / 77$ & 2 & & & 120 & & \\
\hline 1 & $6 / 7 / 77$ & 3 & & & 124 & & \\
\hline 1 & $6 / 7 / 77$ & 4 & & & 124 & & \\
\hline 1 & $6 / 7 / 77$ & 5 & & & 124 & & \\
\hline 1 & $6 / 7 / 77$ & 6 & & & 120 & & \\
\hline 1 & $6 / 7 / 77$ & 7 & & & 117 & & \\
\hline 1 & $6 / 7 / 77$ & 8 & & & 100 & & \\
\hline 1 & $6 / 7 / 77$ & 9 & & & 100 & & \\
\hline 1 & $6 / 7 / 77$ & 10 & & & 100 & & \\
\hline 1 & $6 / 7 / 77$ & 11 & & & 95 & & \\
\hline 1 & $6 / 7 / 77$ & 12 & & & 90 & & \\
\hline 1 & $7 / 11 / 77$ & 1 & & & 118 & & \\
\hline 1 & $7 / 11 / 77$ & 2 & & & 120 & & \\
\hline 1 & $7 / 11 / 77$ & 3 & & & 122 & & \\
\hline 1 & $7 / 11 / 77$ & 4 & & & 122 & & \\
\hline 1 & $7 / 11 / 77$ & 5 & & & 122 & & \\
\hline 1 & $7 / 11 / 77$ & 6 & & & 118 & & \\
\hline 1 & $7 / 11 / 77$ & 7 & & & 110 & & \\
\hline 1 & $7 / 11 / 77$ & 8 & & & 100 & & \\
\hline 1 & $7 / 11 / 77$ & 9 & & & 100 & & \\
\hline 1 & $7 / 11 / 77$ & 10 & & & 100 & & \\
\hline 1 & $7 / 11 / 77$ & 11 & & & 98 & & \\
\hline 1 & $7 / 91 / 77$ & 12 & & & 92 & & \\
\hline 1 & $7 / 11 / 77$ & 13 & & & 90 & & \\
\hline 1 & $7 / 11 / 77$ & 14 & & & 90 & & \\
\hline 1 & $8 / 5 / 77$ & 1 & & & 113 & & \\
\hline 1 & $8 / 5 / 77$ & 2 & & & 115 & & \\
\hline 1 & $8 / 5 / 77$ & 3 & & & 117 & & \\
\hline 1 & $8 / 5 / 77$ & 4 & & & 118 & & \\
\hline 1 & $8 / 5 / 77$ & 5 & & & 118 & & \\
\hline 1 & $8 / 5 / 77$ & 6 & & & 115 & & \\
\hline 1 & $8 / 5 / 77$ & 7 & & & 108 & & \\
\hline 1 & $8 / 5 / 77$ & 8 & & & 97 & & \\
\hline 1 & $8 / 5 / 77$ & 9 & & & 96 & & \\
\hline 1 & $8 / 5 / 77$ & 10 & & & 95 & & \\
\hline 1 & $8 / 5 / 77$ & 11 & & & 94 & & \\
\hline 1 & $9 / 5 / 77$ & 1 & & & 120 & & \\
\hline 1 & $9 / 5 / 77$ & 2 & & & 121 & & \\
\hline 1 & $9 / 5 / 77$ & 3 & & & 122 & & \\
\hline 1 & $9 / 5 / 77$ & 4 & & & 122 & & \\
\hline 1 & $9 / 5 / 77$ & 5 & & & 122 & & \\
\hline
\end{tabular}

Data obtained from WHC Surveillance Analysis Computer System (SACS), February 24, 1994. 


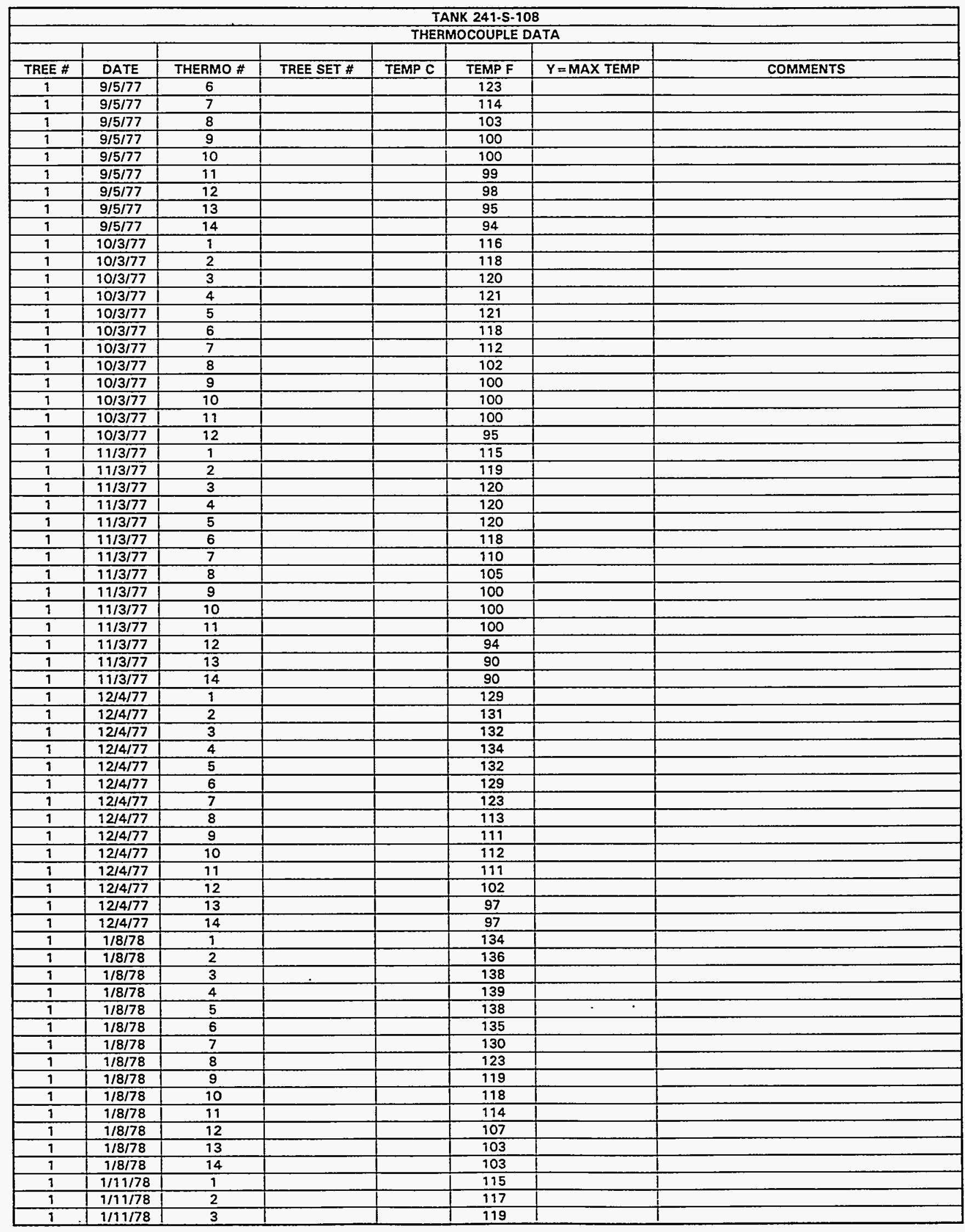

Data obtained from WHC Surveillance Analysis Computer System (SACS), February 24, 1994. 


\begin{tabular}{|c|c|c|c|c|c|c|c|}
\hline \multicolumn{8}{|c|}{ TANK 241-S-108 } \\
\hline & & & & THEF & DCOUPLE & & \\
\hline TREE \# & DATE & THERMO \# & TREE SET \# & TEMP C & TEMP $\bar{F}$ & $Y=M A X$ TEMP & COMMENTS \\
\hline 1 & $1 / 11 / 78$ & 4 & & & 120 & & \\
\hline$\frac{1}{1}$ & $1 / 11 / 78$ & 5 & & & 120 & & \\
\hline 1 & $1 / 11 / 78$ & 6 & & & 117 & & \\
\hline 1 & $1 / 11 / 78$ & 7 & & & 112 & & \\
\hline 1 & $1 / 11 / 78$ & 8 & & & 104 & & \\
\hline 1 & $1 / 11 / 78$ & 9 & & & 101 & & \\
\hline 1 & $1 / 11 / 78$ & 10 & & & 99 & & \\
\hline 1 & $1 / 11 / 78$ & 11 & & & 91 & & \\
\hline 1 & $1 / 11 / 78$ & 12 & & & 88 & & \\
\hline 1 & $1 / 11 / 78$ & 13 & & & 86 & & \\
\hline 1 & $1 / 71 / 78$ & 14 & & & 85 & & \\
\hline 1 & $1 / 13 / 78$ & 1 & & & 115 & & \\
\hline 1 & $1 / 13 / 78$ & 2 & & & 118 & & \\
\hline 1 & $1 / 13 / 78$ & 3 & & & 120 & & \\
\hline 1 & $1 / 13 / 78$ & 4 & & & 120 & & \\
\hline 1 & $1 / 13 / 78$ & 5 & & & 120 & & \\
\hline 1 & $1 / 13 / 78$ & 6 & & & 117 & & \\
\hline 9 & $1 / 13 / 78$ & 7 & & & 113 & & \\
\hline 1 & $1 / 13 / 78$ & 8 & & & 105 & & \\
\hline 1 & $1 / 13 / 78$ & 9 & & & 106 & & \\
\hline 1 & $1 / 13 / 78$ & 10 & & & 100 & & \\
\hline 1 & $1 / 13 / 78$ & 11 & & & 95 & & \\
\hline 1 & $2 / 7 / 78$ & 1 & & & 119 & & \\
\hline 1 & $2 / 7 / 78$ & 2 & & & 121 & & \\
\hline 1 & $2 / 7 / 78$ & 3 & & & 123 & & \\
\hline$T$ & $2 / 7 / 78$ & 4 & & & 124 & & \\
\hline 1 & $2 / 7 / 78$ & 5 & & & 123 & & \\
\hline 1 & $2 / 7 / 78$ & 6 & & & 121 & & \\
\hline 1 & $2 / 7 / 78$ & 7 & & & 116 & & \\
\hline 1 & $2 / 7 / 78$ & 8 & & & 116 & & \\
\hline 1 & $2 / 7 / 78$ & 9 & & & 106 & & \\
\hline 1 & $2 / 7 / 78$ & 10 & & & 103 & & \\
\hline 1 & $2 / 7 / 78$ & 11 & & & 98 & & \\
\hline 1 & $2 / 7 / 78$ & 12 & & & 96 & & \\
\hline 1 & $2 / 7 / 78$ & 13 & & & 86 & & \\
\hline 1 & $2 / 7 / 78$ & 14 & & & 86 & & \\
\hline 9 & $3 / 12 / 78$ & 1 & & & 122 & & \\
\hline 1 & $3 / 12 / 78$ & 2 & & & 124 & & \\
\hline 1 & $3 / 12 / 78$ & 3 & & & 126 & & \\
\hline 1 & $3 / 12 / 78$ & 4 & & & 127 & & \\
\hline 1 & $3 / 12 / 78$ & 5 & & & 127 & & \\
\hline 1 & $3 / 12 / 78$ & 6 & & & 125 & & \\
\hline 1 & $3 / 12 / 78$ & 7 & & & 121 & & \\
\hline 1 & $3 / 12 / 78$ & 8 & & & 115 & & \\
\hline 1 & $3 / 12 / 78$ & 9 & & & 111 & & \\
\hline 1 & $3 / 12 / 78$ & 10 & & & 108 & & \\
\hline 1 & $3 / 12 / 78$ & 11 & & & 101 & & \\
\hline 1 & $3 / 12 / 78$ & 12 & & & 92 & & \\
\hline 1 & $3 / 12 / 78$ & 13 & & & 87 & & \\
\hline 1 & $3 / 12 / 78$ & 14 & & & 87 & & \\
\hline 1 & $6 / 3 / 78$ & 1 & & & 115 & & \\
\hline 1 & $6 / 3 / 78$ & 2 & & & 115 & & \\
\hline 1 & $6 / 3 / 78$ & 3 & & & 117 & & \\
\hline 1 & $6 / 3 / 78$ & 4 & & & 120 & & \\
\hline 1 & $6 / 3 / 78$ & 5 & & & 120 & & \\
\hline$\frac{1}{1}$ & $6 / 3 / 78$ & 6 & & & 118 & & \\
\hline 1 & $6 / 3 / 78$ & 7 & & & 115 & & \\
\hline 1 & $6 / 3 / 78$ & 8 & & & 110 & & \\
\hline 1 & $6 / 3 / 78$ & 9 & & & 105 & & \\
\hline 9 & $6 / 3 / 78$ & 10 & & & 100 & & \\
\hline 9 & $6 / 3 / 78$ & 11 & & & 95 & & \\
\hline 1 & $6 / 3 / 78$ & 12 & & & 86 & & \\
\hline 1 & $6 / 3 / 78$ & 13 & & & 83 & & \\
\hline$\frac{1}{1}$ & $6 / 3 / 78$ & 14 & & & 83 & & \\
\hline 1 & $7 / 13 / 78$ & 1 & & & 115 & & \\
\hline 1 & $7 / 13 / 78$ & 2 & & & 118 & & \\
\hline
\end{tabular}

Data obtained from WHC Surveillance Analysis Computer System (SACS), February 24, 1994. 
WHC-SD-WM-ER-323, Rev. 0

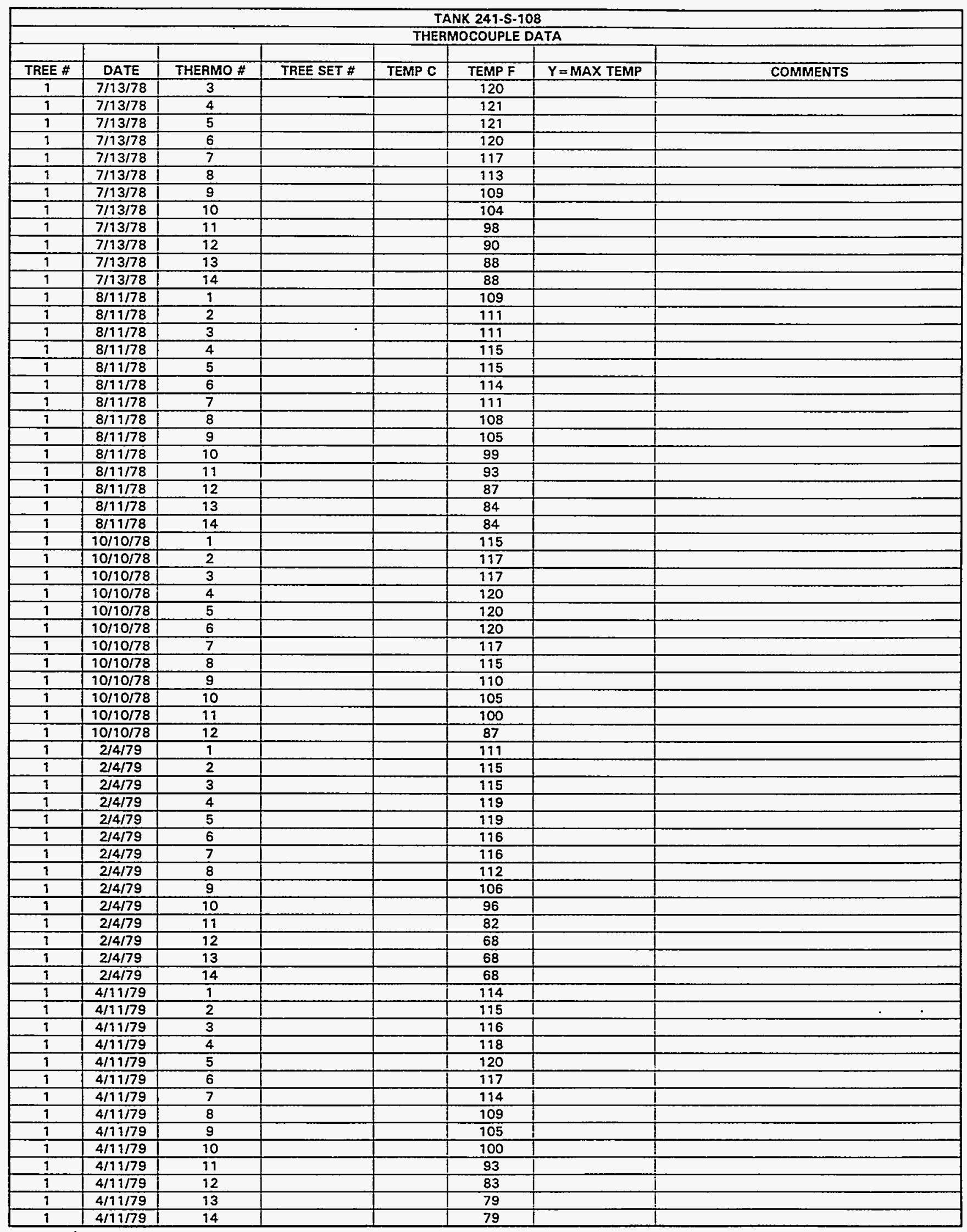

Data obtained from WHC Surveillance Analysis Computer System (SACS), February 24, 1994. 
WHC-SD-WM-ER-323, Rev. O

\begin{tabular}{|c|c|c|c|c|c|c|c|}
\hline \multicolumn{8}{|c|}{ TANK 241-S-108 } \\
\hline \multicolumn{8}{|c|}{ THERMOCOUPLE DATA } \\
\hline TREE \# & DATE & THERMO \# & TREE SET \# & TEMP C & TEMP F & $Y=M A X$ TEMP & COMMENTS \\
\hline 1 & $9 / 6 / 79$ & 1 & & & 110 & & \\
\hline 1 & $9 / 6 / 79$ & 2 & & & 112 & & \\
\hline 1 & $9 / 6 / 79$ & 3 & & & 114 & & \\
\hline 1 & $9 / 6 / 79$ & 4 & & & 915 & & \\
\hline 1 & $9 / 6 / 79$ & 5 & & & 110 & & \\
\hline 9 & $9 / 6 / 79$ & 6 & & & 106 & & \\
\hline 1 & $9 / 6 / 79$ & 7 & & & 105 & & - \\
\hline 1 & $9 / 6 / 79$ & 8 & & & 100 & & \\
\hline 1 & $9 / 6 / 79$ & 9 & & & 94 & & \\
\hline 1 & $9 / 6 / 79$ & 10 & & & 94 & & \\
\hline 1 & $9 / 6 / 79$ & 11 & & & 94 & & \\
\hline 1 & $9 / 6 / 79$ & 12 & & & 94 & & \\
\hline 1 & $9 / 6 / 79$ & 13 & & & 94 & & \\
\hline 9 & $9 / 6 / 79$ & 14 & & & 94 & & \\
\hline 1 & $1 / 23 / 80$ & 1 & & & 110 & & \\
\hline 1 & $1 / 23 / 80$ & 2 & & & & & 80-suspect data-(low) \\
\hline 1 & $1 / 23 / 80$ & 3 & & & 110 & & \\
\hline 1 & $1 / 23 / 80$ & 4 & & & & & 160-suspect data-(high) \\
\hline 1 & $1 / 23 / 80$ & 5 & & & & & 145-suspect data-(high) \\
\hline 1 & $1 / 23 / 80$ & 6 & & & & & 190-suspect data-(high) \\
\hline 9 & $1 / 23 / 80$ & 7 & & & 110 & & \\
\hline 9 & $1 / 23 / 80$ & 9 & & & 95 & & \\
\hline 1 & $1 / 23 / 80$ & 10 & & & 80 & & \\
\hline 1 & $1 / 23 / 80$ & 12 & & & 100 & & \\
\hline 1 & $1 / 23 / 80$ & 13 & & & 90 & & \\
\hline 1 & $1 / 23 / 80$ & 14 & & & 120 & & \\
\hline 1 & $2 / 1 / 80$ & 1 & & & 105 & & \\
\hline 1 & $2 / 1 / 80$ & 2 & & & 110 & & \\
\hline 1 & $2 / 1 / 80$ & 3 & & & 105 & & \\
\hline 1 & $2 / 1 / 80$ & 4 & & & 100 & & \\
\hline 1 & $2 / 1 / 80$ & 5 & & & 115 & & \\
\hline 1 & $2 / 1 / 80$ & 7 & & & 115 & & \\
\hline 1 & $2 / 1 / 80$ & 9 & & & 110 & & \\
\hline 1 & $2 / 1 / 80$ & 10 & & & 80 & & \\
\hline 1 & $2 / 1 / 80$ & 91 & & & 80 & & \\
\hline 1 & $2 / 1 / 80$ & 92 & & & 110 & & \\
\hline 1 & $2 / 1 / 80$ & 13 & & & & & 150-suspect data-(high) \\
\hline 1 & $2 / 1 / 80$ & 14 & & & 125 & & \\
\hline 1 & $1 / 13 / 82$ & 1 & & & 105 & & \\
\hline 1 & $1 / 13 / 82$ & 5 & & & & & 65-suspect data-(low) \\
\hline 1 & $1 / 13 / 82$ & 6 & & & 125 & & \\
\hline 1 & $1 / 13 / 82$ & 7 & & & & & 190-suspect data-(high) \\
\hline 1 & $1 / 13 / 82$ & 8 & & & & & 190-suspect data-(high) \\
\hline 1 & $1 / 13 / 82$ & 9 & & & & & 195-suspect data-(high) \\
\hline 1 & $1 / 13 / 82$ & 10 & & & & & 145-suspect data-(high) \\
\hline 1 & $1 / 13 / 82$ & 11 & & & & & 150-suspect data (high) \\
\hline 1 & $1 / 13 / 82$ & 12 & & & 70 & & \\
\hline 1 & $10 / 6 / 82$ & 1 & & & 103 & & \\
\hline 1 & $10 / 6 / 82$ & 6 & & & 102 & & \\
\hline 1 & $10 / 6 / 82$ & 7 & & & 98 & & \\
\hline 1 & $10 / 6 / 82$ & 8 & & & 93 & & \\
\hline 1 & $5 / 19 / 83$ & $\frac{1}{1}$ & & & 98 & & \\
\hline 1 & $5 / 19 / 83$ & 3 & & & 99 & & \\
\hline 1 & $5 / 19 / 83$ & 4 & & & 98 & & \\
\hline 1 & $5 / 19 / 83$ & 5 & & & 97 & & \\
\hline 1 & $5 / 19 / 83$ & 6 & & & 94 & & \\
\hline 1 & $5 / 19 / 83$ & 7 & & & 88 & & \\
\hline 1 & $5 / 19 / 83$ & 8 & & & 80 & & \\
\hline 1 & $5 / 19 / 83$ & 9 & & & 73 & & \\
\hline 1 & $5 / 19 / 83$ & 10 & & & 70 & & \\
\hline 1 & $5 / 19 / 83$ & 11 & & & 70 & & \\
\hline$\frac{1}{1}$ & $5 / 19 / 83$ & 12 & & & 70 & & \\
\hline 1 & $5 / 19 / 83$ & 13 & & & 70 & & \\
\hline 1 & $5 / 19 / 83$ & 14 & & & 136 & & \\
\hline 1 & $1 / 3 / 84$ & 7 & & & 94 & & \\
\hline 1 & $2 / 14 / 84$ & 9 & & & 104 & & \\
\hline
\end{tabular}

Data obtained from WHC Surveillance Analysis Computer System (SACS), February 24, 1994r. 


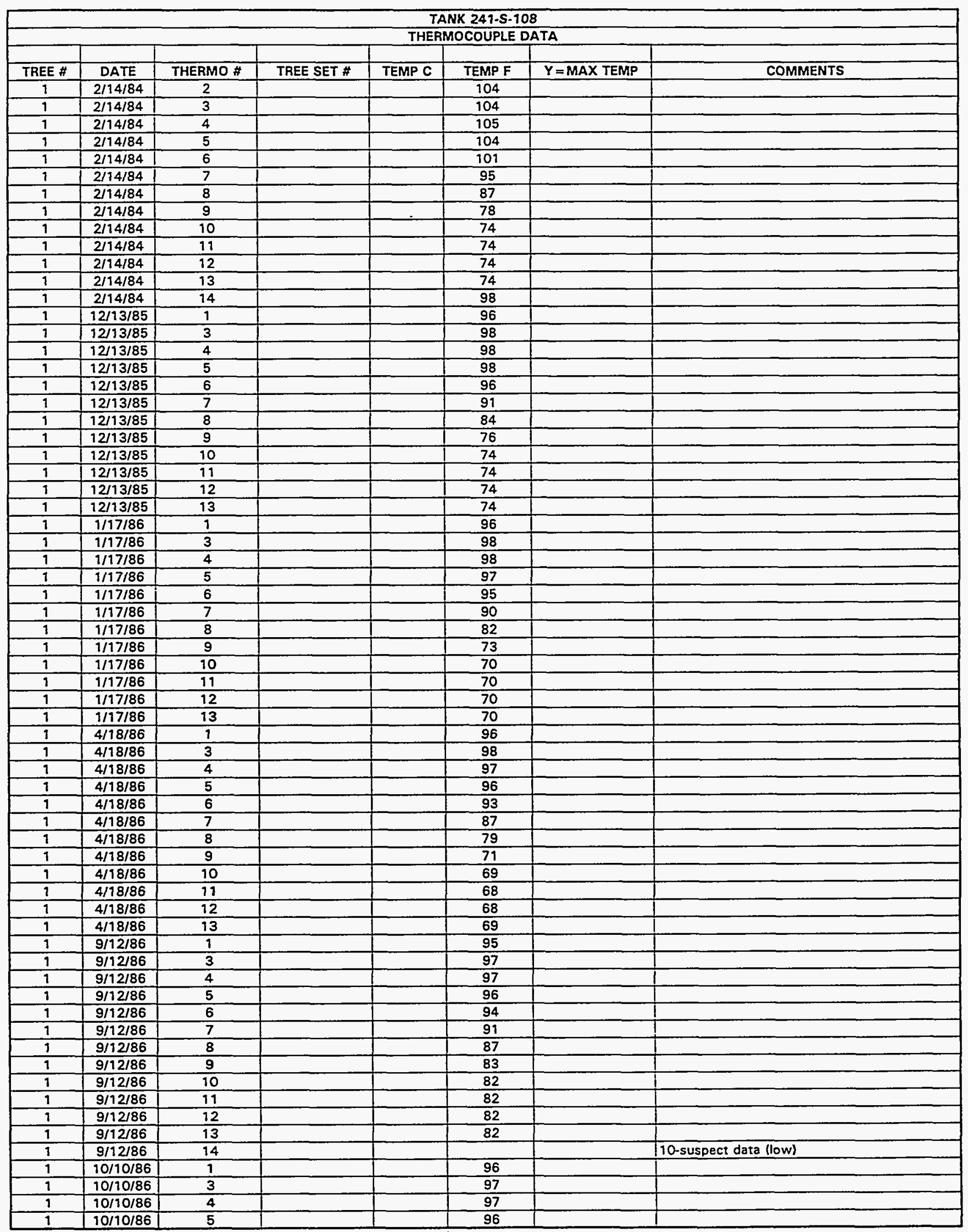

Data obtained from WHC Surveillance Analysis Computer System (SACS), February 24, 1994. 
WHC-SD-WM-ER-323, Rev. 0

\begin{tabular}{|c|c|c|c|c|c|c|c|}
\hline \multicolumn{8}{|c|}{ TANK 241-S-108 } \\
\hline \multicolumn{8}{|c|}{ THERMOCOUPLE DATA } \\
\hline TREE \# & DATE & THERMO \# & TREE SET \# & TEMP C & TEMP F & $Y=$ MAX TEMP & COMMENTS \\
\hline 1 & $10 / 10 / 86$ & 6 & & & 95 & & \\
\hline 1 & $10 / 10 / 86$ & 7 & & & 92 & & \\
\hline 1 & $10 / 10 / 86$ & 8 & & & 88 & & \\
\hline$\frac{1}{1}$ & $10 / 10 / 86$ & 9 & & & 83 & & \\
\hline 1 & $10 / 10 / 86$ & 10 & & & 82 & & \\
\hline 1 & $10 / 10 / 86$ & 11 & & & 82 & & \\
\hline 1 & $10 / 10 / 86$ & 12 & & & 82 & & \\
\hline 1 & $10 / 10 / 86$ & 13 & & & 82 & & \\
\hline 1 & $10 / 10 / 86$ & 14 & & & & & 22-suspect data (low) \\
\hline 1 & $3 / 6 / 87$ & 1 & & & 94 & & \\
\hline 1 & $3 / 6 / 87$ & 3 & & & 93 & & \\
\hline 1 & $3 / 6 / 87$ & 4 & & & 93 & & \\
\hline 1 & $3 / 6 / 87$ & 5 & & & 92 & & \\
\hline 1 & $3 / 6 / 87$ & 6 & & & 89 & & \\
\hline 1 & $3 / 6 / 87$ & 7 & & & 84 & & \\
\hline 1 & $3 / 6 / 87$ & 8 & & & 76 & & \\
\hline 1 & $3 / 6 / 87$ & 9 & & & 67 & & \\
\hline 1 & $3 / 6 / 87$ & 10 & & & 65 & & \\
\hline 1 & $3 / 6 / 87$ & 11 & & & 65 & & \\
\hline 1 & $3 / 6 / 87$ & 12 & & & 64 & & \\
\hline 1 & $3 / 6 / 87$ & 13 & & & 65 & & \\
\hline$T$ & $3 / 6 / 87$ & 74 & & & & & 8-suspect data (low) \\
\hline 1 & $6 / 12 / 87$ & 1 & & & 94 & & \\
\hline 1 & $6 / 12 / 87$ & 3 & & & 92 & & \\
\hline 1 & $6 / 12 / 87$ & 4 & & & 92 & & \\
\hline 1 & $6 / 12 / 87$ & 5 & & & 90 & & \\
\hline$\frac{1}{1}$ & $6 / 12 / 87$ & 6 & & & 88 & & \\
\hline$\frac{1}{1}$ & $6 / 12 / 87$ & 7 & & & 84 & & \\
\hline 1 & $6 / 12 / 87$ & 8 & & & 78 & & \\
\hline 1 & $6 / 12 / 87$ & 9 & & & 73 & & \\
\hline$\frac{1}{1}$ & $6 / 12 / 87$ & 10 & & & 71 & & \\
\hline 1 & $6 / 12 / 87$ & 11 & & & 71 & & \\
\hline 9 & $6 / 12 / 87$ & 72 & & & 71 & & \\
\hline 1 & $6 / 12 / 87$ & 13 & & & 71 & & \\
\hline 1 & $6 / 12 / 87$ & 14 & & & & & 41-suspect data (low) \\
\hline 1 & $10 / 2 / 87$ & 1 & & & 93 & & \\
\hline 1 & $10 / 2 / 87$ & 3 & & & 93 & & \\
\hline 1 & $10 / 2 / 87$ & 4 & & & 93 & & \\
\hline 1 & $10 / 2 / 87$ & 5 & & & 99 & & \\
\hline 1 & $10 / 2 / 87$ & 6 & & & 90 & & \\
\hline 1 & $10 / 2 / 87$ & 7 & & & 88 & & \\
\hline 1 & $10 / 2 / 87$ & 8 & & & 84 & & \\
\hline 1 & $10 / 2 / 87$ & 9 & & & 80 & & \\
\hline 1 & $10 / 2 / 87$ & 10 & & & 78 & & \\
\hline 1 & $10 / 2 / 87$ & 91 & & & 78 & & \\
\hline$\frac{1}{1}$ & $10 / 2 / 87$ & 72 & & & 78 & & \\
\hline 1 & $10 / 2 / 87$ & 13 & & & 78 & & \\
\hline 1 & $10 / 2 / 87$ & 14 & & & & & 32-suspect data (low) \\
\hline 1 & $12 / 18 / 87$ & 1 & & & 100 & & \\
\hline 1 & $12 / 18 / 87$ & 3 & & & 101 & & \\
\hline 1 & $12 / 18 / 87$ & 4 & & & 101 & & \\
\hline 9 & $12 / 18 / 87$ & 5 & & & 101 & & \\
\hline 1 & $12 / 18 / 87$ & 6 & & & 99 & & \\
\hline 1 & $12 / 18 / 87$ & 7 & & & 95 & $\cdot$ & \\
\hline 7 & $12 / 18 / 87$ & 8 & & & 89 & & \\
\hline 1 & $12 / 18 / 87$ & 9 & & & 81 & & \\
\hline 1 & $12 / 18 / 87$ & 10 & & & 79 & & \\
\hline 1 & $12 / 18 / 87$ & 71 & & & 79 & & \\
\hline 1 & $12 / 18 / 87$ & 72 & & & 79 & & \\
\hline 1 & $12 / 18 / 87$ & 13 & & & 79 & & \\
\hline 1 & $1 / 22 / 88$ & 1 & & & 101 & & \\
\hline 1 & $1 / 22 / 88$ & 3 & & & 102 & & \\
\hline 1 & $1 / 22 / 88$ & 4 & & & 102 & & \\
\hline 1 & $1 / 22 / 88$ & 5 & & & 101 & & \\
\hline 1 & $1 / 22 / 88$ & 6 & & & 99 & & \\
\hline 9 & $1 / 22 / 88$ & 7 & & & 94 & & \\
\hline
\end{tabular}

Data obtained from WHC Surveillance Analysis Computer System (SACS), February 24, 1994. 


\begin{tabular}{|c|c|c|c|c|c|c|c|}
\hline \multicolumn{8}{|c|}{ TANK 241-S-108 } \\
\hline \multicolumn{8}{|c|}{ THERMOCOUPLE DATA } \\
\hline & & & & & & & \\
\hline TREE \# & DATE & THERMO \# & TREE SET \# & TEMP C & TEMP F & $Y=$ MAX TEMP & COMMENTS \\
\hline 1 & $1 / 22 / 88$ & 8 & & & 87 & & \\
\hline 1 & $1 / 22 / 88$ & 9 & & & 79 & & \\
\hline 1 & $1 / 22 / 88$ & 10 & & & 76 & & \\
\hline 9 & $1 / 22 / 88$ & 11 & & & 76 & & \\
\hline 1 & $1 / 22 / 88$ & 12 & & & 76 & & \\
\hline 1 & $1 / 22 / 88$ & 13 & & & 76 & & \\
\hline 1 & $2 / 5 / 88$ & 1 & & & 101 & & \\
\hline 1 & $2 / 5 / 88$ & 3 & & & 102 & & \\
\hline 1 & $2 / 5 / 88$ & 4 & & & 101 & & \\
\hline 9 & $2 / 5 / 88$ & 5 & & & 100 & & \\
\hline 1 & $2 / 5 / 88$ & 6 & & & 98 & & \\
\hline 1 & $2 / 5 / 88$ & 7 & & & 93 & & \\
\hline 1 & $2 / 5 / 88$ & 8 & & & 86 & & \\
\hline 1 & $2 / 5 / 88$ & 9 & & & 77 & & \\
\hline 1 & $2 / 5 / 88$ & 10 & & & 75 & & \\
\hline 9 & $2 / 5 / 88$ & 11 & & & 75 & & \\
\hline 1 & $2 / 5 / 88$ & 12 & & & 75 & & \\
\hline 1 & $2 / 5 / 88$ & 13 & & & 75 & & \\
\hline 1 & $2 / 5 / 88$ & 14 & & & & & 5-suspect data (low) \\
\hline 1 & $1 / 4 / 91$ & 1 & 1 & & 87.2 & $\mathbf{N}$ & $\mathrm{HH}-99 \mathrm{~A} . \mathrm{J} \# 890523512.47 \mathrm{TC} \# \mathrm{~S} 241112 \mathrm{O} / \mathrm{S}$ \\
\hline 1 & $1 / 4 / 91$ & 2 & 1 & & & $\mathbf{N}$ & $0 / 5$ \\
\hline 1 & $1 / 4 / 91$ & 3 & 1 & & 88.1 & $\bar{Y}$ & \\
\hline 1 & $1 / 4 / 91$ & 4 & 1 & & & $\mathrm{Y}$ & $0 / 5$ \\
\hline 9 & $1 / 4 / 91$ & 5 & 1 & & 86.8 & $\mathbf{N}$ & \\
\hline 1 & $1 / 4 / 91$ & 6 & 1 & & 85.7 & $\mathbf{N}$ & \\
\hline 1 & $1 / 4 / 91$ & 7 & 1 & & 81.6 & $\bar{N}$ & \\
\hline 1 & $1 / 4 / 91$ & 8 & 1 & & 75.2 & $\mathrm{~N}$ & \\
\hline 1 & $1 / 4 / 91$ & 9 & 1 & & 67.5 & $\mathbf{N}$ & \\
\hline 1 & $1 / 4 / 91$ & 10 & 1 & & 64.8 & $N$ & \\
\hline 1 & $1 / 4 / 91$ & 11 & 1 & & & $\mathrm{~N}$ & $0 / \mathrm{S}$ \\
\hline 1 & $1 / 4 / 91$ & 12 & 1 & & & $N$ & $\mathrm{O} / \mathrm{s}$ \\
\hline 1 & $1 / 4 / 91$ & 13 & 1 & & 65.5 & $\mathbf{N}$ & \\
\hline 1 & $11 / 13 / 91$ & 1 & 1 & & 82.2 & $\mathbf{N}$ & J-890423320-42 TC \#21516 O/S W/O\#04529 \\
\hline 1 & $11 / 13 / 91$ & 3 & 1 & & 86.2 & $\bar{Y}$ & \\
\hline 1 & $11 / 13 / 91$ & 4 & 1 & & 86.2 & $\mathbf{N}$ & \\
\hline 1 & $11 / 13 / 91$ & 5 & 1 & & 86.2 & $\mathbf{N}$ & \\
\hline 1 & $11 / 13 / 91$ & 6 & 1 & & 85.9 & $\mathbf{N}$ & \\
\hline 1 & $11 / 13 / 91$ & 7 & 9 & & 84 & $\mathbf{N}$ & \\
\hline 1 & $11 / 13 / 91$ & 8 & 1 & & 81.9 & $\mathbf{N}$ & \\
\hline 1 & $11 / 13 / 91$ & 9 & $\frac{1}{1}$ & & 78.1 & $\mathbf{N}$ & \\
\hline 1 & $11 / 13 / 91$ & 10 & 1 & & 72.9 & $\mathbf{N}$ & \\
\hline 1 & $11 / 13 / 91$ & 11 & 1 & & 69.8 & $\bar{N}$ & \\
\hline 1 & $11 / 13 / 91$ & 12 & 1 & & 71.5 & $\bar{N}$ & \\
\hline 1 & $11 / 13 / 91$ & 13 & 1 & & 71.4 & $N$ & \\
\hline 1 & $11 / 13 / 91$ & 14 & 1 & & 71.6 & $\mathrm{~N}$ & \\
\hline 1 & $1 / 1 / 92$ & 1 & 9 & & 94 & $\bar{Y}$ & $\mathrm{~J}-890523512.20 \mathrm{TC} \# 2-415.16 \mathrm{O} / \mathrm{S}$ \\
\hline 1 & $1 / 1 / 92$ & 5 & 9 & & 89.7 & $\mathbf{N}$ & \\
\hline 1 & $1 / 1 / 92$ & 6 & 9 & & 87.6 & $\mathbf{N}$ & \\
\hline 1 & $1 / 1 / 92$ & 7 & 1 & & 84.5 & $\mathbf{N}$ & \\
\hline 1 & $1 / 1 / 92$ & 8 & 1 & & 79 & $\mathbf{N}$ & \\
\hline 9 & $1 / 1 / 92$ & 9 & 1 & & 72.3 & $\mathbf{N}$ & \\
\hline 1 & $1 / 1 / 92$ & 10 & 1 & & 69.5 & $\mathrm{~N}$ & \\
\hline 1 & $1 / 1 / 92$ & 11 & 1 & & 70.4 & $\mathbf{N}$ & \\
\hline 1 & $1 / 1 / 92$ & 12 & 1 & & 70.3 & $\bar{N}$ & \\
\hline 1 & $1 / 1 / 92$ & 13 & 1 & & 70.7 & $\mathbf{N}$ & \\
\hline 1 & $1 / 1 / 92$ & 14 & 1 & & 64 & $\mathbf{N}$ & \\
\hline 1 & $7 / 3 / 92$ & 1 & 1 & & 88 & $N$ & $J 890423320-42$ \\
\hline 9 & $7 / 3 / 92$ & 3 & $i$ & & 89 & $\mathrm{~N}$ & \\
\hline 1 & $7 / 3 / 92$ & 4 & 9 & & 91 & $\bar{Y}$ & \\
\hline 1 & $7 / 3 / 92$ & 5 & 1 & & 87 & $\mathbf{N}$ & \\
\hline 1 & $7 / 3 / 92$ & 6 & 9 & & 84 & $\mathrm{~N}$ & \\
\hline 1 & $7 / 3 / 92$ & 7 & 1 & & 81 & $\bar{N}$ & \\
\hline 1 & $7 / 3 / 92$ & 8 & 1 & & 78 & $N$ & \\
\hline 1 & $7 / 3 / 92$ & 9 & 1 & & 75 & $N$ & \\
\hline
\end{tabular}

Data obtained from WHC Surveillance Analysis Computer System (SACS), February 24, 1994. 
WHC-SD-WM-ER-323, Rev. 0

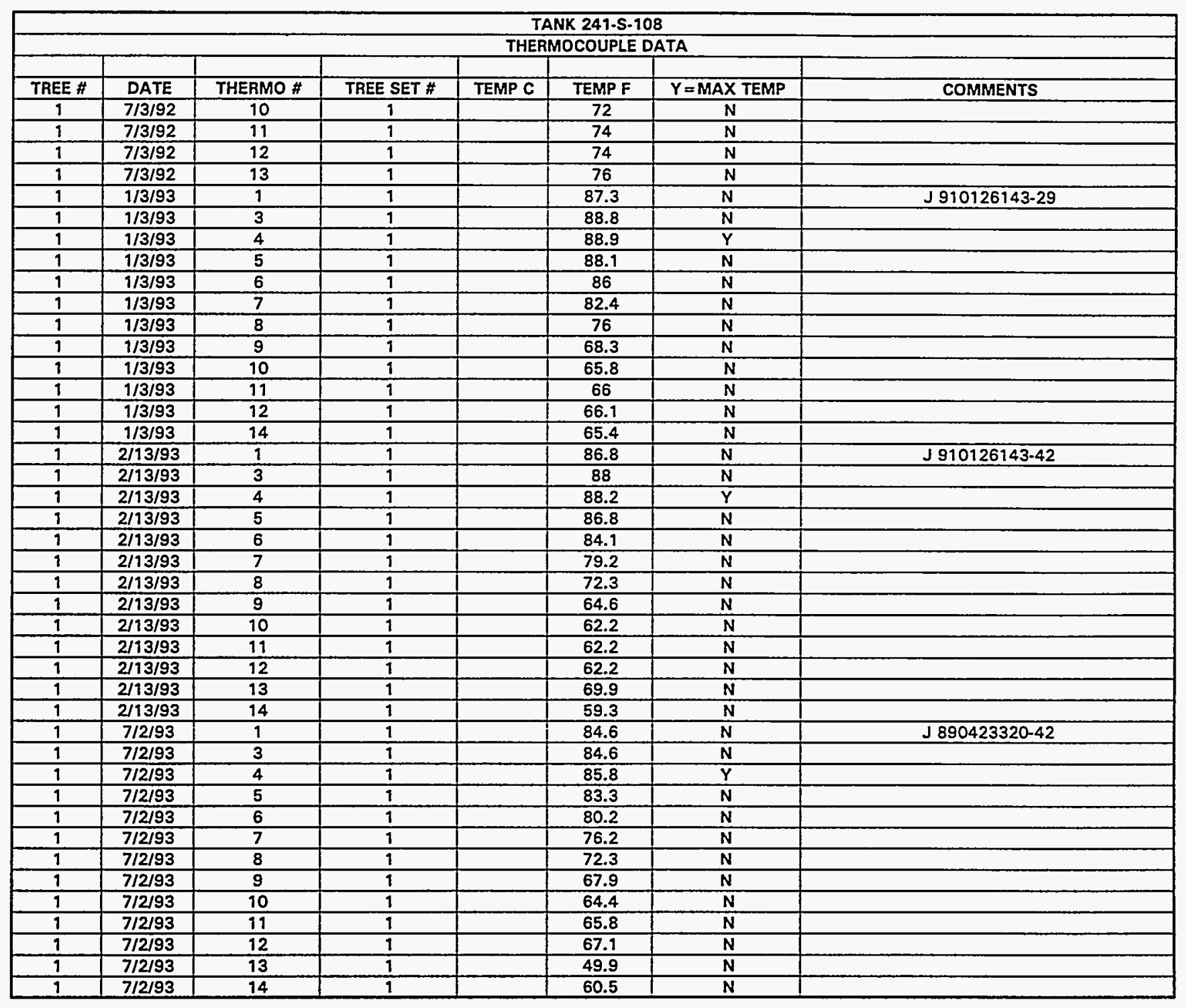

Data obtained from WHC Surveillance Analysis Computer System (SACS), February 24, 1994. 


\begin{tabular}{|c|c|c|c|c|c|c|c|}
\hline \multicolumn{8}{|c|}{ TANK 241.S.109 } \\
\hline \multicolumn{8}{|c|}{ THERMOCOUPLE DATA } \\
\hline & & & & & & & \\
\hline TREE \# & DATE & THERMO \# & TREE SET \# & TEMP C & TEMPF & $Y=$ MAX TEMP & COMMENTS \\
\hline 1 & $2 / 10 / 74$ & 12 & & & 109 & & \\
\hline 1 & $2 / 10 / 74$ & 12 & & & 104 & & \\
\hline 9 & $10 / 20 / 74$ & 1 & & 48.89 & 120.002 & & raw data - assumed degree $\mathrm{C}$ \\
\hline 1 & $10 / 20 / 74$ & 2 & & 52.78 & 127.004 & & raw data - assumed degree C \\
\hline 1 & $10 / 20 / 74$ & 3 & & 57.22 & 134.996 & & raw data - assumed degree C \\
\hline 7 & $10 / 20 / 74$ & 4 & & 61.11 & 141.998 & & raw data - assumed degree C \\
\hline 9 & $10 / 20 / 74$ & 5 & & 63.89 & 147.002 & & raw data - assumed degree C \\
\hline 1 & $10 / 20 / 74$ & 6 & & 65.56 & 150.008 & & raw data - assumed degree C \\
\hline 1 & $10 / 20 / 74$ & 7 & & 65.56 & 150.008 & & raw data - assumed degree C \\
\hline 1 & $10 / 20 / 74$ & 8 & & 64.44 & 147.992 & & raw data - assumed degree C \\
\hline 1 & $10 / 20 / 74$ & 9 & & 61.67 & 143.006 & & raw data-assumed degree $C$ \\
\hline 1 & $10 / 20 / 74$ & 10 & & 58.89 & 138.002 & & raw data - assumed degree C \\
\hline 1 & $10 / 20 / 74$ & 11 & & 53.89 & 129.002 & & raw data - assumed degree $\mathrm{C}$ \\
\hline 1 & $10 / 20 / 74$ & 12 & & 47.22 & 116.996 & & raw data - assumed degree C \\
\hline 1 & $12 / 2 / 74$ & 1 & & 50 & 122 & & raw data - assumed degree C \\
\hline 1 & $12 / 2 / 74$ & 2 & & 53.89 & 129.002 & & raw data - assumed degree C \\
\hline 1 & $92 / 2 / 74$ & 3 & & 58.33 & 136.994 & & raw data - assumed degree $C$ \\
\hline 1 & $12 / 2 / 74$ & 4 & & 62.22 & 143.996 & & raw data - assumed degree $C$ \\
\hline 1 & $12 / 2 / 74$ & 5 & & 65 & 149 & & raw data - assumed degree $C$ \\
\hline 1 & $12 / 2 / 74$ & 6 & & 65.56 & 150.008 & & raw data - assumed degree $C$ \\
\hline 1 & $92 / 2 / 74$ & 7 & & 65.56 & 150.008 & & raw data - assumed degree C \\
\hline 9 & $12 / 2 / 74$ & 8 & & 63.33 & 145.994 & & raw data - assumed degree $C$ \\
\hline 1 & $92 / 2 / 74$ & 9 & & 60 & 140 & & raw data - assumed degree $C$ \\
\hline 1 & $12 / 2 / 74$ & 10 & & 56.67 & 134.006 & & raw data - assumed degree C \\
\hline 1 & $12 / 2 / 74$ & 19 & & 50 & 122 & & raw data - assumed degree C \\
\hline 1 & $12 / 2 / 74$ & 12 & & 42.22 & 107.996 & & raw data - assumed degree $C$ \\
\hline 1 & $11 / 3 / 75$ & 1 & & & 129 & & \\
\hline 1 & $11 / 3 / 75$ & 2 & & & 134 & & \\
\hline 1 & $11 / 3 / 75$ & 3 & & & 139 & & \\
\hline 1 & $11 / 3 / 75$ & 4 & & & 143 & & \\
\hline 1 & $11 / 3 / 75$ & 5 & & & 145 & & \\
\hline 1 & $11 / 3 / 75$ & 6 & & & 145 & & \\
\hline 1 & $11 / 3 / 75$ & 7 & & & 942 & & \\
\hline 1 & $11 / 3 / 75$ & 8 & & & 136 & & \\
\hline 1 & $11 / 3 / 75$ & 9 & & & 131 & & \\
\hline 1 & $11 / 3 / 75$ & 10 & & & 126 & & \\
\hline 1 & $11 / 3 / 75$ & 11 & & & 116 & & \\
\hline 1 & $11 / 3 / 75$ & 12 & & & 103 & & \\
\hline 1 & $1 / 11 / 76$ & 1 & & & 125 & & \\
\hline 1 & $1 / 11 / 76$ & 2 & & & 130 & & \\
\hline 1 & $9 / 19 / 76$ & 3 & & & 135 & & \\
\hline 9 & $9 / 11 / 76$ & 4 & & & 138 & & \\
\hline 1 & $1 / 11 / 76$ & 5 & & & 138 & & \\
\hline 1 & $1 / 11 / 76$ & 6 & & & 138 & & \\
\hline 1 & $1 / 11 / 76$ & 7 & & & 134 & & \\
\hline 1 & $1 / 11 / 76$ & 8 & & & 127 & & \\
\hline 1 & $1 / 11 / 76$ & 9 & & & 121 & & \\
\hline 1 & $1 / 11 / 76$ & 10 & & & 120 & & \\
\hline 9 & $1 / 11 / 76$ & 19 & & & 114 & & \\
\hline 1 & $1 / 11 / 76$ & 12 & & & 102 & & \\
\hline 1 & $3 / 5 / 76$ & 1 & & & 126 & & \\
\hline 1 & $3 / 5 / 76$ & 2 & & & 130 & & \\
\hline 1 & $3 / 5 / 76$ & 3 & & & 135 & & \\
\hline 1 & $3 / 5 / 76$ & 4 & & & 139 & & \\
\hline 7 & $3 / 5 / 76$ & 5 & & & 141 & & \\
\hline 9 & $3 / 5 / 76$ & 6 & & & 140 & & \\
\hline 1 & $3 / 5 / 76$ & 7 & & & 136 & & \\
\hline 1 & $3 / 5 / 76$ & 8 & & & 130 & & \\
\hline 1 & $3 / 5 / 76$ & 9 & & & 125 & & \\
\hline 1 & $3 / 5 / 76$ & 10 & & & 119 & & \\
\hline 1 & $3 / 5 / 76$ & 11 & & & 116 & & \\
\hline 1 & $3 / 5 / 76$ & 12 & & & 102 & & \\
\hline 1 & $5 / 2 / 76$ & 1 & & & 126 & & \\
\hline 1 & $5 / 2 / 76$ & 2 & & & 131 & & \\
\hline 1 & $5 / 2 / 76$ & 3 & & & 136 & & \\
\hline 1 & $5 / 2 / 76$ & 4 & & & 139 & & \\
\hline 1 & $5 / 2 / 76$ & 5 & & & 140 & & I \\
\hline 1 & $5 / 2 / 76$ & 6 & & & 139 & & \\
\hline
\end{tabular}

Data obtained from WHC Surveillance Analysis Computer System (SACS), February 24, 1994 and Archive box_\#121869. 


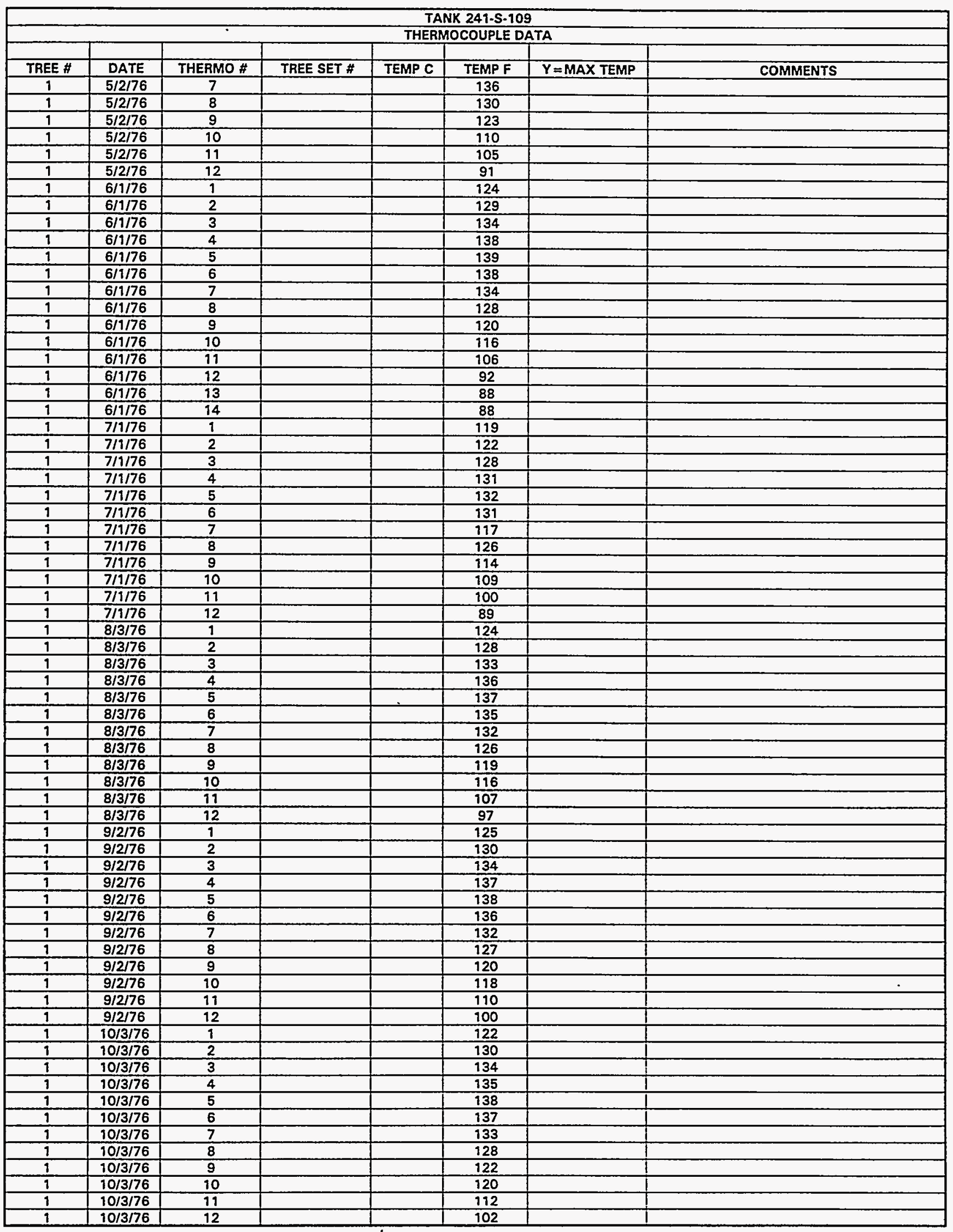

Data obtained from WHC Surveillance Analysis Computer System (SACS), February 24, 1994 and Archive box \#121869. 
WHC-SD-WM-ER-323, Rev. 0

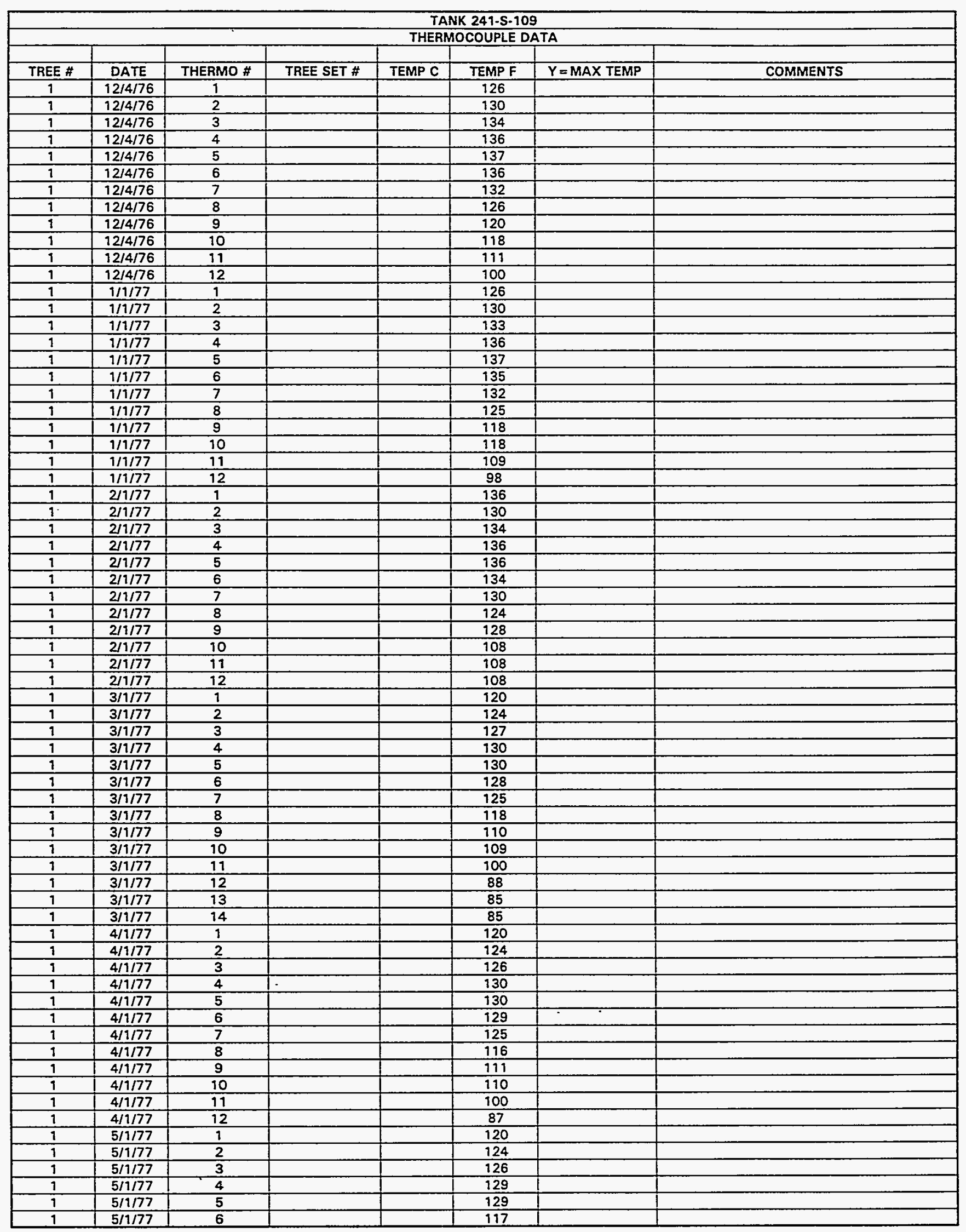

Data obtained from WHC Surveillance Analysis Computer System (SACS), February 24, 1994 and Archive box \#121869. 
WHC-SD-WM-ER-323, Rev. 0

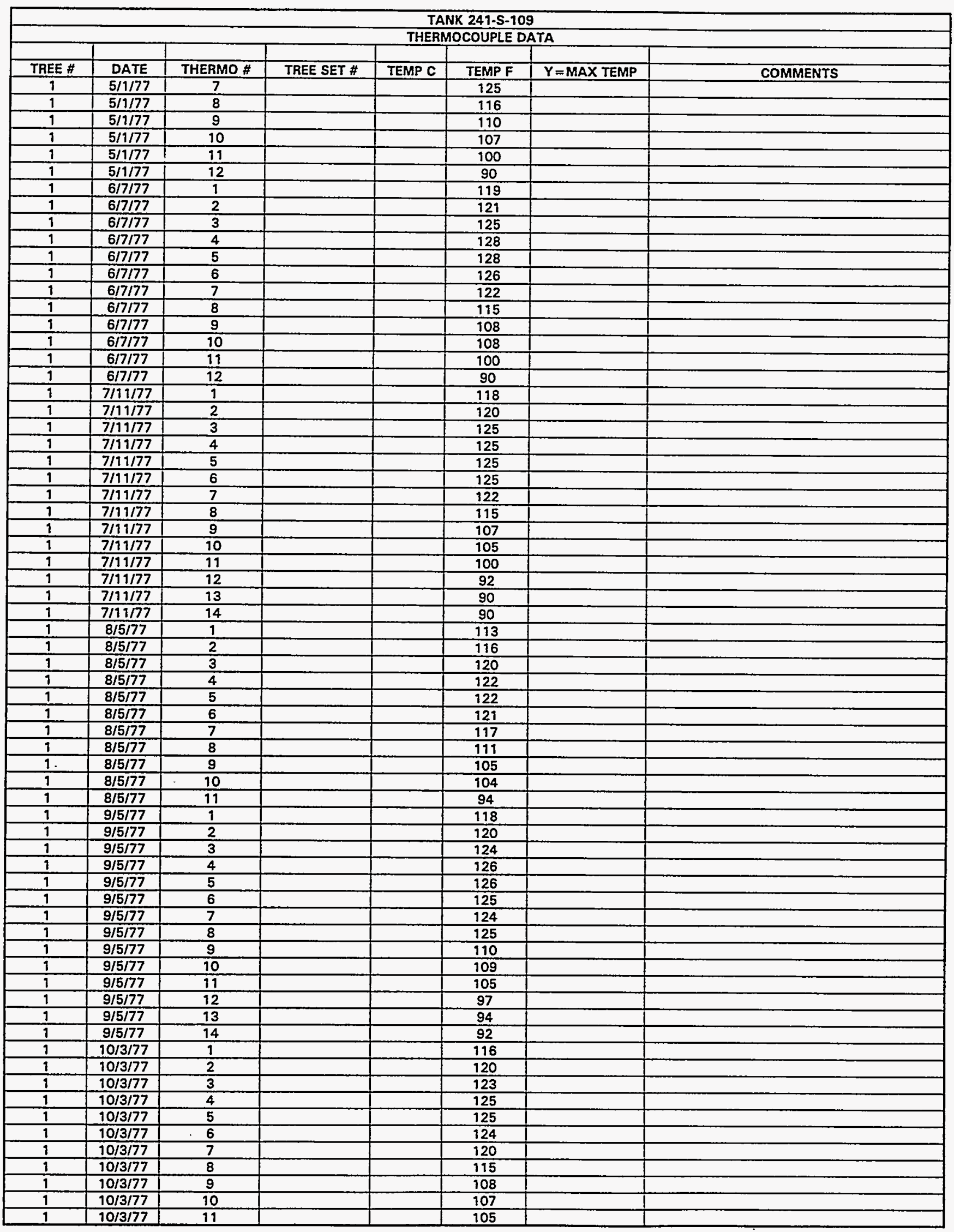

Data obtained from WHC Surveillance Analysis Computer System_(SACS), February 24, 1994 and Archive box \#121869. 


\begin{tabular}{|c|c|c|c|c|c|c|c|}
\hline \multicolumn{8}{|c|}{ TANK 241-S-109 } \\
\hline & & & & THERA & COUPLE D & & \\
\hline TREE \# & DATE & THERMO \# & TREE SET \# & TEMP C & TEMP F & $Y=$ MAX TEMP & COMMENTS \\
\hline 1 & $10 / 3 / 77$ & 12 & & & 95 & & \\
\hline 1 & $11 / 3 / 77$ & 1 & & & 116 & & \\
\hline 1 & $11 / 3 / 77$ & 2 & & & 119 & & \\
\hline 1 & $11 / 3 / 77$ & 3 & & & 123 & & \\
\hline 1 & $19 / 3 / 77$ & 4 & & & 125 & & \\
\hline 1 & $11 / 3 / 77$ & 5 & & & 125 & & \\
\hline 1 & $11 / 3 / 77$ & 6 & & & 124 & & \\
\hline 1 & $11 / 3 / 77$ & 7 & & & 120 & & \\
\hline 1 & $11 / 3 / 77$ & 8 & & & 115 & & \\
\hline 1 & $11 / 3 / 77$ & 9 & & & 109 & & \\
\hline 1 & $11 / 3 / 77$ & 10 & & & 107 & & \\
\hline 1 & $11 / 3 / 77$ & 11 & & & 104 & & \\
\hline 1 & $11 / 3 / 77$ & 12 & & & 94 & & \\
\hline 1 & $11 / 3 / 77$ & 13 & & & 90 & & \\
\hline 1 & $11 / 3 / 77$ & 14 & & & 90 & & \\
\hline 1 & $12 / 4 / 77$ & 1 & & & 125 & & \\
\hline 9 & $12 / 4 / 77$ & 2 & & & 128 & & \\
\hline 1 & $12 / 4 / 77$ & 3 & & & 131 & & \\
\hline 1 & $12 / 4 / 77$ & 4 & & & 133 & & \\
\hline 1 & $12 / 4 / 77$ & 5 & & & 133 & & \\
\hline 1 & $12 / 4 / 77$ & 6 & & & 121 & & \\
\hline 9 & $12 / 4 / 77$ & 7 & & & 117 & & \\
\hline 9 & $12 / 4 / 77$ & 8 & & & 121 & & \\
\hline 1 & $12 / 4 / 77$ & 9 & & & 115 & & \\
\hline 1 & $12 / 4 / 77$ & 10 & & & 114 & & \\
\hline 1 & $12 / 4 / 77$ & 11 & & & 108 & & \\
\hline 1 & $12 / 4 / 77$ & 12 & & & 96 & & \\
\hline 1 & $12 / 4 / 77$ & 13 & & & 91 & & \\
\hline 1 & $12 / 4 / 77$ & 14 & & & 91 & & \\
\hline 1 & $1 / 8 / 78$ & 1 & & & 135 & & \\
\hline 1 & $1 / 8 / 78$ & 2 & & & 137 & & \\
\hline 1 & $1 / 8 / 78$ & 3 & & & 141 & & \\
\hline 1 & $1 / 8 / 78$ & 4 & & & 143 & & \\
\hline 1 & $1 / 8 / 78$ & 6 & & & 141 & & \\
\hline 1 & $1 / 8 / 78$ & 7 & & & 137 & & \\
\hline 1 & $1 / 8 / 78$ & 8 & & & 132 & & \\
\hline 1 & $1 / 8 / 78$ & 9 & & & 125 & & \\
\hline 1 & $1 / 8 / 78$ & 10 & & & 123 & & \\
\hline 1 & $1 / 8 / 78$ & 11 & & & 119 & & \\
\hline 1 & $1 / 8 / 78$ & 12 & & & 107 & & \\
\hline 1 & $1 / 8 / 78$ & 13 & & & 103 & & \\
\hline 1 & $1 / 8 / 78$ & 14 & & & 103 & & \\
\hline 1 & $1 / 11 / 78$ & 1 & & & 115 & & \\
\hline 1 & $1 / 11 / 78$ & 2 & & & 118 & & \\
\hline 1 & $1 / 11 / 78$ & 3 & & & 921 & & \\
\hline 1 & $1 / 11 / 78$ & 4 & & & 124 & & \\
\hline 1 & $1 / 11 / 78$ & 5 & & & 124 & & \\
\hline 1 & $1 / 11 / 78$ & 6 & & & 122 & & \\
\hline 1 & $1 / 11 / 78$ & 7 & & & 118 & & \\
\hline 1 & $1 / 11 / 78$ & 8 & & & 193 & & \\
\hline 9 & $1 / 19 / 78$ & 9 & & & 106 & & \\
\hline 1 & $1 / 11 / 78$ & 10 & & & 104 & & \\
\hline 1 & $1 / 11 / 78$ & 11 & & & 100 & & \\
\hline 1 & $1 / 11 / 78$ & 12 & & & 88 & & \\
\hline 1 & $1 / 11 / 78$ & 13 & & & 85 & & \\
\hline 1 & $1 / 11 / 78$ & 14 & & & 85 & & \\
\hline 1 & $1 / 13 / 78$ & 1 & & & 115 & & \\
\hline 1 & $1 / 13 / 78$ & 2 & & & 120 & & \\
\hline 1 & $1 / 13 / 78$ & 3 & & & 121 & & \\
\hline 1 & $1 / 13 / 78$ & 4 & & & 125 & & \\
\hline 1 & $1 / 13 / 78$ & 5 & & & 125 & & \\
\hline 1 & $1 / 13 / 78$ & 6 & & & 123 & & \\
\hline 1 & $1 / 13 / 78$ & 7 & & & 119 & & \\
\hline 1 & $1 / 13 / 78$ & 8 & & & 114 & & \\
\hline 1 & $1 / 13 / 78$ & 9 & & & 106 & & \\
\hline 9 & $1 / 13 / 78$ & 10 & & & 104 & & \\
\hline 1 & $1 / 13 / 78$ & 11 & & & 100 & & \\
\hline 1 & $2 / 7 / 78$ & 1 & & & 119 & & \\
\hline
\end{tabular}

Data obtained from WHC Surveillance Analysis Computer System (SACS), February 24, 1994 and Archive box \#121869. 


\begin{tabular}{|c|c|c|c|c|c|c|c|}
\hline \multicolumn{8}{|c|}{ TANK 241-S-109 } \\
\hline & & & & THER & COUPLE L & & \\
\hline & & & & & & & \\
\hline TREE \# & DATE & THERMO \# & TREE SET \# & TEMP C & TEMP F & $Y=M A X$ TEMP & COMMENTS \\
\hline 9 & $2 / 7 / 78$ & 2 & & & 122 & & \\
\hline$\frac{1}{1}$ & $2 / 7 / 78$ & 3 & & & 125 & & \\
\hline 1 & $2 / 7 / 78$ & 4 & & & 127 & & \\
\hline 1 & $2 / 7 / 78$ & 5 & & & 127 & & \\
\hline 1 & $2 / 7 / 78$ & 6 & & & 125 & & \\
\hline 1 & $2 / 7 / 78$ & 7 & & & 121 & & \\
\hline$\frac{1}{1}$ & $2 / 7 / 78$ & 8 & & & 116 & & \\
\hline 1 & $2 / 7 / 78$ & 9 & & & 109 & & \\
\hline 1 & $2 / 7 / 78$ & 10 & & & 107 & & \\
\hline 1 & $2 / 7 / 78$ & 11 & & & 103 & & \\
\hline 1 & $2 / 7 / 78$ & 12 & & & 91 & & \\
\hline 1 & $2 / 7 / 78$ & 13 & & & 86 & & \\
\hline 9 & $2 / 7 / 78$ & 14 & & & 86 & & \\
\hline$\frac{1}{1}$ & $3 / 12 / 78$ & 1 & & & 122 & & \\
\hline 9 & $3 / 12 / 78$ & 2 & & & 125 & & \\
\hline 1 & $3 / 12 / 78$ & 3 & & & 128 & & \\
\hline 1 & $3 / 12 / 78$ & 4 & & & 130 & & \\
\hline 1 & $3 / 12 / 78$ & 5 & & & 130 & & \\
\hline 1 & $3 / 12 / 78$ & 6 & & & 128 & & \\
\hline 1 & $3 / 12 / 78$ & 7 & & & 124 & & \\
\hline 1 & $3 / 12 / 78$ & 8 & & & 119 & & \\
\hline 1 & $3 / 12 / 78$ & 9 & & & 112 & & \\
\hline 1 & $3 / 12 / 78$ & 10 & & & 110 & & \\
\hline 1 & $3 / 12 / 78$ & 11 & & & 105 & & \\
\hline 1 & $3 / 12 / 78$ & 12 & & & 91 & & \\
\hline 1 & $3 / 12 / 78$ & 13 & & & 87 & & \\
\hline$\frac{1}{1}$ & $3 / 12 / 78$ & 14 & & & 87 & & \\
\hline$T$ & $4 / 19 / 78$ & 1 & & & 114 & & \\
\hline 1 & $4 / 71 / 78$ & 2 & & & 116 & & \\
\hline$\frac{1}{1}$ & $4 / 71 / 78$ & 3 & & & 120 & & \\
\hline 1 & $4 / 11 / 78$ & 4 & & & 121 & & \\
\hline$\frac{1}{1}$ & $4 / 11 / 78$ & 5 & & & 122 & & \\
\hline 1 & $4 / 11 / 78$ & 6 & & & 120 & & \\
\hline$\frac{1}{1}$ & $4 / 19 / 78$ & 7 & & & 115 & & \\
\hline 1 & $4 / 19 / 78$ & 8 & & & 110 & & \\
\hline 1 & $4 / 19 / 78$ & 9 & & & 103 & & \\
\hline 1 & $4 / 11 / 78$ & 10 & & & 100 & & \\
\hline 1 & $4 / 11 / 78$ & 11 & & & 97 & & \\
\hline 1 & $4 / 11 / 78$ & 12 & & & 83 & & \\
\hline 1 & $4 / 11 / 78$ & 13 & & & 79 & & \\
\hline 1 & $4 / 11 / 78$ & 14 & & & 79 & & \\
\hline 1 & $6 / 3 / 78$ & 1 & & & 115 & & \\
\hline 1 & $6 / 3 / 78$ & 2 & & & 117 & & \\
\hline$\frac{1}{1}$ & $6 / 3 / 78$ & 3 & & & 120 & & \\
\hline$\frac{1}{1}$ & $6 / 3 / 78$ & 4 & & & 122 & & \\
\hline 1 & $6 / 3 / 78$ & 5 & & & 122 & & \\
\hline 1 & $6 / 3 / 78$ & 6 & & & 120 & & \\
\hline 1 & $6 / 3 / 78$ & 7 & & & 915 & & \\
\hline 1 & $6 / 3 / 78$ & 8 & & & 110 & & \\
\hline 1 & $6 / 3 / 78$ & 9 & & & 105 & & \\
\hline$\frac{1}{1}$ & $6 / 3 / 78$ & 10 & & & 100 & & \\
\hline 1 & $6 / 3 / 78$ & 11 & & & 95 & & \\
\hline 1 & $6 / 3 / 78$ & 12 & & & 85 & & \\
\hline 1 & $6 / 3 / 78$ & 13 & & & 85 & & \\
\hline 1 & $.6 / 3 / 78$ & 14 & & & 85 & & \\
\hline 1 & $7 / 13 / 78$ & 1 & & & 115 & & \\
\hline$\frac{1}{1}$ & $7 / 73 / 78$ & 2 & & & 115 & & \\
\hline$\frac{1}{1}$ & $7 / 13 / 78$ & 3 & & & 120 & & \\
\hline$\frac{1}{1}$ & $7 / 13 / 78$ & 4 & & & 123 & & \\
\hline 1 & $7 / 13 / 78$ & 5 & & & 123 & & \\
\hline 1 & $7 / 13 / 78$ & 6 & & & 120 & & \\
\hline 1 & $7 / 13 / 78$ & 7 & & & 917 & & \\
\hline$\frac{T}{1}$ & $7 / 13 / 78$ & 8 & & & 114 & & \\
\hline 1 & $7 / 13 / 78$ & 9 & & & 109 & & \\
\hline 1 & $7 / 13 / 78$ & 10 & & & 105 & & \\
\hline 9 & $7 / 13 / 78$ & 11 & & & 98 & & \\
\hline 1 & $7 / 13 / 78$ & 12 & & & 90 & & \\
\hline 1 & $7 / 13 / 78$ & 13 & & & 87 & & \\
\hline
\end{tabular}

Data obtained from WHC Surveillance Analysis Computer System (SACS), February 24, 1994 and Archive box \#121869. 


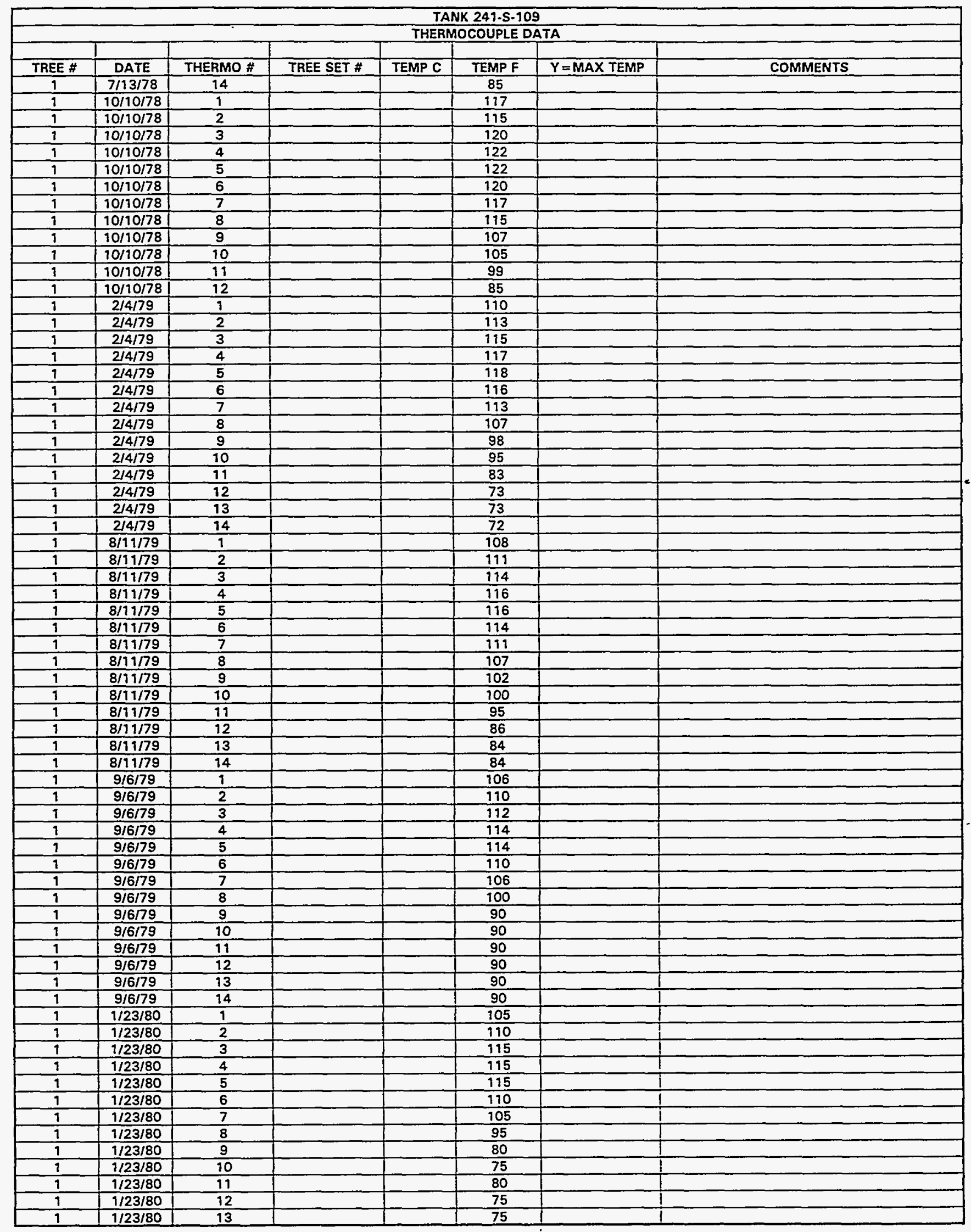

Data obtained from WHC Surveillance Analysis Computer System (SACS), February 24, 1994 and Archive box \#121869. 


\begin{tabular}{|c|c|c|c|c|c|c|c|}
\hline \multicolumn{8}{|c|}{ TANK 241-S-109 } \\
\hline & & - & & THERN & COUPLE D & & \\
\hline TREE \# & DATE & THERMO \# & TREE SET \# & TEMP C & TEMP F & $Y-M A Y$ TFOD & \\
\hline 1 & $1 / 23 / 80$ & 14 & & & 80 & $T=M A X$ IEMP & COMMENTS \\
\hline 1 & $1 / 13 / 82$ & 1 & & & 102 & & \\
\hline 1 & $1 / 13 / 82$ & 2 & & & 120 & & \\
\hline 1 & $1 / 13 / 82$ & 4 & & & 107 & & \\
\hline 1 & $1 / 13 / 82$ & 5 & & & 100 & & \\
\hline 1 & $1 / 13 / 82$ & 6 & & & 97 & & \\
\hline 1 & $1 / 13 / 82$ & 7 & & & 90 & & \\
\hline 1 & $1 / 13 / 82$ & 9 & & & 75 & & \\
\hline 1 & $1 / 13 / 82$ & 10 & & & 75 & & \\
\hline 1 & $1 / 13 / 82$ & 91 & & & 85 & & \\
\hline 1 & $1 / 13 / 82$ & 12 & & & 75 & & \\
\hline 1 & $5 / 29 / 82$ & 1 & & & 102 & & \\
\hline 1 & $5 / 29 / 82$ & 4 & & & 101 & & \\
\hline 1 & $5 / 29 / 82$ & 5 & & & 98 & & \\
\hline 1 & $5 / 29 / 82$ & 6 & & & 94 & & \\
\hline 1 & $5 / 29 / 82$ & 7 & & & 88 & & \\
\hline 1 & $5 / 29 / 82$ & 9 & & & 75 & & \\
\hline 1 & $5 / 29 / 82$ & 10 & & & 74 & & \\
\hline 1 & $5 / 29 / 82$ & 11 & & & & & 129 - suspect data (high) \\
\hline 9 & $5 / 29 / 82$ & 12 & & & 74 & & \\
\hline 1 & $10 / 6 / 82$ & 1 & & & 100 & & \\
\hline 1 & $10 / 6 / 82$ & 4 & & & 101 & & \\
\hline 1 & $10 / 6 / 82$ & 5 & & & 99 & & \\
\hline 1 & $10 / 6 / 82$ & 6 & & & 97 & & \\
\hline 1 & $10 / 6 / 82$ & 7 & & & 93 & & \\
\hline 1 & $10 / 6 / 82$ & 9 & & & 83 & & \\
\hline 1 & $10 / 6 / 82$ & 10 & & & 82 & & \\
\hline 1 & $10 / 6 / 82$ & 12 & & & 82 & & \\
\hline 1 & $10 / 6 / 82$ & 14 & & & 82 & & \\
\hline 1 & $5 / 19 / 83$ & 1 & & & 96 & & \\
\hline 1 & $5 / 19 / 83$ & 2 & & & & & 187 - suspect data (high) \\
\hline 7 & $5 / 19 / 83$ & 3 & & & 33 & & \\
\hline 1 & $5 / 19 / 83$ & 4 & & & 94 & & \\
\hline 1 & $5 / 19 / 83$ & 5 & & & 91 & & \\
\hline 1 & $5 / 19 / 83$ & 6 & & & 88 & & \\
\hline$\frac{1}{1}$ & $5 / 19 / 83$ & 7 & & & 83 & & \\
\hline 1 & $5 / 19 / 83$ & 9 & & & 70 & & \\
\hline$\frac{1}{1}$ & $5 / 19 / 83$ & 10 & & & 70 & & \\
\hline 9 & $5 / 19 / 83$ & 11 & & & & & 132- suspect data (high) \\
\hline 9 & $5 / 19 / 83$ & 12 & & & 70 & & \\
\hline 1 & $5 / 19 / 83$ & 14 & & & 70 & & \\
\hline$\frac{1}{1}$ & $1 / 3 / 84$ & 9 & & & 99 & & \\
\hline$\frac{1}{1}$ & $1 / 3 / 84$ & 4 & & & 99 & & \\
\hline 1 & $1 / 3 / 84$ & 5 & & & 98 & & \\
\hline$\frac{1}{1}$ & $1 / 3 / 84$ & 6 & & & 97 & & \\
\hline$\frac{1}{1}$ & $1 / 3 / 84$ & 7 & & & 95 & & \\
\hline 1 & $1 / 3 / 84$ & 9 & & & 74 & & \\
\hline 1 & $1 / 3 / 84$ & 10 & & & 74 & & \\
\hline 1 & $1 / 3 / 84$ & 11 & & & 74 & & \\
\hline 1 & $1 / 3 / 84$ & 12 & & & 75 & & \\
\hline$\frac{1}{1}$ & $2 / 13 / 84$ & 1 & & & 98 & & \\
\hline 1 & $2 / 13 / 84$ & 4 & & & 98 & & \\
\hline 1 & $2 / 13 / 84$ & 5 & & & 96 & & \\
\hline 1 & $2 / 13 / 84$ & 6 & & & 92 & & \\
\hline 1 & $2 / 13 / 84$ & 7 & & & 87 & & \\
\hline 1 & $2 / 13 / 84$ & 9 & & & 70 & & \\
\hline 1 & $2 / 13 / 84$ & 10 & & & 69 & & \\
\hline 1 & $2 / 13 / 84$ & 12 & & & 69 & & \\
\hline 1 & $2 / 13 / 84$ & 14 & & & 67 & & \\
\hline 1 & $2 / 14 / 84$ & 1 & & & 100 & & \\
\hline 1 & $2 / 14 / 84$ & 4 & & & 101 & & \\
\hline 1 & $2 / 14 / 84$ & 5 & & & 96 & & \\
\hline 1 & $2 / 14 / 84$ & 6 & & & 95 & & \\
\hline 1 & $2 / 14 / 84$ & 7 & & & 90 & & \\
\hline 1 & $2 / 14 / 84$ & 9 & & & 72 & & \\
\hline 1 & $2 / 14 / 84$ & 10 & & & 72 & & \\
\hline 1 & $2 / 14 / 84$ & 12 & & & 72 & & \\
\hline 1 & $2 / 14 / 84$ & 14 & & & 71 & & i \\
\hline
\end{tabular}

Data obtained from WHC Surveillance Analysis Computer System (SACS), February 24, 1994 and Archive box \#121869. 


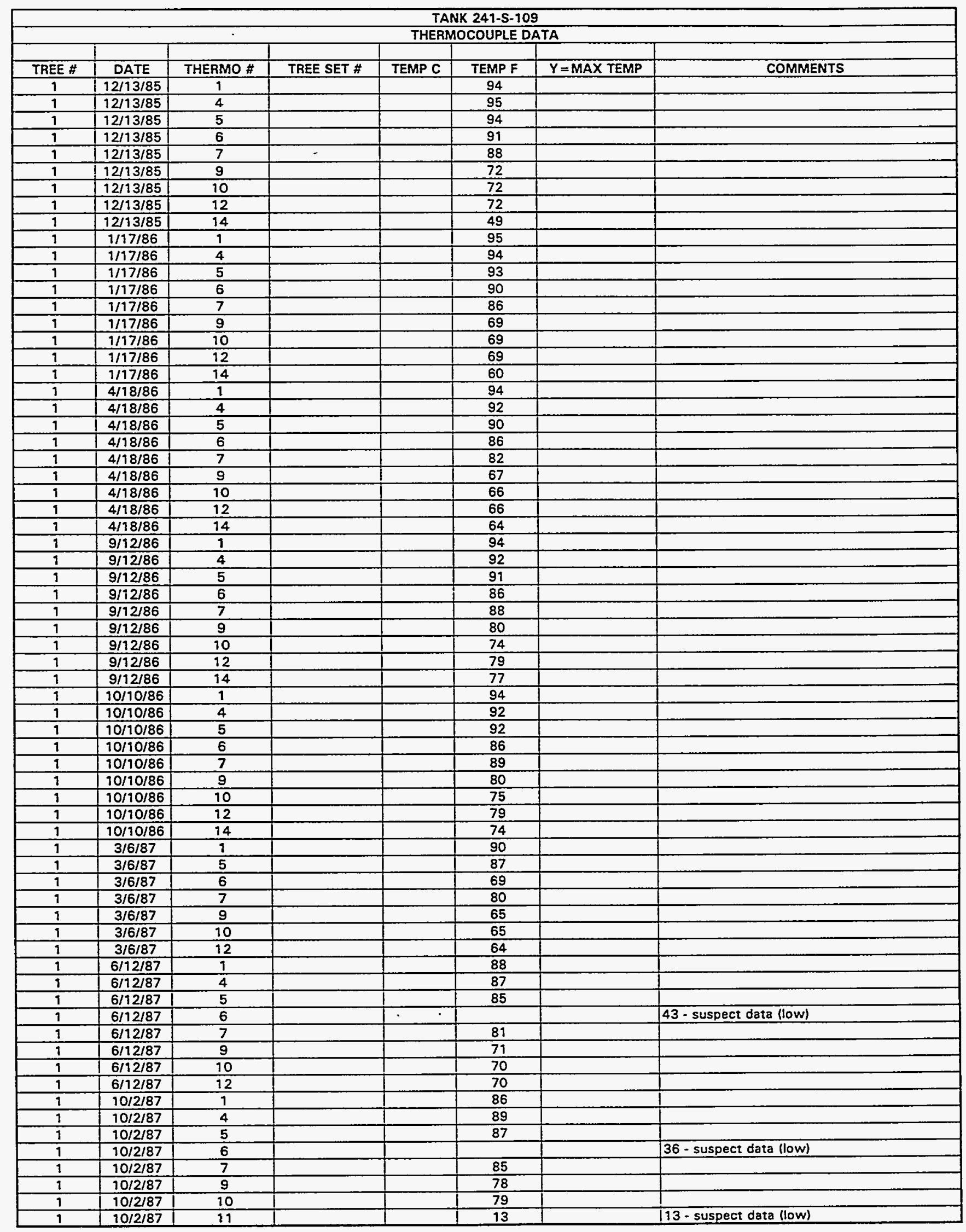

Data obtained from WHC Surveillance Analysis Computer System (SACS), February 24, 1994 and Archive box \#121869. 


\begin{tabular}{|c|c|c|c|c|c|c|c|}
\hline \multicolumn{8}{|c|}{ TANK 241-S-109 } \\
\hline & & & & THERI & COUPLE D & & \\
\hline TREE \# & DATE & THERMO \# & TREE SET \# & TEMP C & TEMP F & $Y=$ MAX TEMP & COMMENTS \\
\hline 1 & $10 / 2 / 87$ & 12 & & & 78 & & \\
\hline 1 & $12 / 18 / 87$ & 1 & & & 96 & & \\
\hline 1 & $12 / 18 / 87$ & 5 & & & 97 & & \\
\hline 1 & $12 / 18 / 87$ & 6 & & & 74 & & \\
\hline 1 & $12 / 18 / 87$ & 7 & & & 92 & & \\
\hline 1 & $12 / 18 / 87$ & 9 & & & 80 & & \\
\hline 1 & $12 / 18 / 87$ & 10 & & & 79 & & \\
\hline 1 & $12 / 18 / 87$ & 11 & & & & & 153 - suspect data (high) \\
\hline 1 & $12 / 18 / 87$ & 12 & & & 79 & & \\
\hline 1 & $1 / 22 / 88$ & 1 & & & & & 170 - suspect data (high) \\
\hline 1 & $1 / 22 / 88$ & 5 & & & 97 & & \\
\hline 1 & $1 / 22 / 88$ & 6 & & & 77 & & \\
\hline 1 & $1 / 22 / 88$ & 7 & & & 91 & & \\
\hline 1 & $1 / 22 / 88$ & 9 & & & 77 & & \\
\hline 1 & $1 / 22 / 88$ & 10 & & & 77 & & \\
\hline 1 & $1 / 22 / 88$ & 11 & & & & & 212 - suspect data (high) \\
\hline 1 & $1 / 22 / 88$ & 12 & & & 77 & & \\
\hline 1 & $2 / 4 / 88$ & 1 & & & & & 172 - suspect data (high) \\
\hline 1 & $2 / 4 / 88$ & 5 & & & 97 & & \\
\hline 1 & $2 / 4 / 88$ & 6 & & & 77 & & \\
\hline 1 & $2 / 4 / 88$ & 7 & & & 90 & & \\
\hline 1 & $2 / 4 / 88$ & 9 & & & 76 & & \\
\hline 1 & $2 / 4 / 88$ & 10 & & & 67 & & \\
\hline 1 & $2 / 4 / 88$ & 11 & & & & & 205 - suspect data (high) \\
\hline 1 & $2 / 4 / 88$ & 12 & & & 75 & & \\
\hline 1 & $1 / 4 / 91$ & 7 & 1 & & 77.9 & & B \\
\hline 1 & $1 / 4 / 91$ & 9 & 1 & & 63.9 & $\mathbf{N}$ & \\
\hline 1 & $1 / 4 / 91$ & 10 & 1 & & 64.7 & $\mathbf{N}$ & \\
\hline 1 & $1 / 4 / 91$ & 12 & 1 & & 65.1 & $\mathbf{N}$ & \\
\hline 1 & $11 / 13 / 91$ & 7 & 1 & & 72.3 & $\mathbf{Y}$ & $\begin{array}{c}\text { TC } \approx 1.68-10 i 3-16 \text { O/S W/O } \# 04529 \mathrm{TC} \\
\# 11 \text { BAD DATA }\end{array}$ \\
\hline 1 & $11 / 13 / 91$ & 12 & 1 & & 64.2 & $\mathbf{N}$ & \\
\hline 1 & $1 / 1 / 92$ & 7 & 1 & & 77.2 & $Y$ & $\mathrm{~J}-890523512-20 \mathrm{TC} \# 1-6813-16 \mathrm{O} / \mathrm{S}$ \\
\hline 1 & $1 / 1 / 92$ & 12 & 1 & & 65.5 & $\mathbf{N}$ & \\
\hline 1 & $7 / 3 / 92$ & 7 & 1 & & 71 & $\bar{N}$ & $J 890423320-42$ \\
\hline 1 & $7 / 3 / 92$ & 11 & 1 & & 75.7 & $Y$ & \\
\hline 1 & $2 / 13 / 93$ & 1 & 1 & & 82.9 & $Y$ & J 910126143-42 TC\#2-68-10 O/S \\
\hline 1 & $2 / 13 / 93$ & 7 & 1 & & 66.3 & $\bar{N}$ & \\
\hline 1 & $2 / 13 / 93$ & 11 & 1 & & 55.3 & $\mathrm{~N}$ & \\
\hline 1 & $7 / 2 / 93$ & 1 & 1 & & & $N$ & TCs $1.60 / \mathrm{S}$ \\
\hline
\end{tabular}

Data obtained from WHC Surveillance Analyșis Computer System (SACS), February 24, 1994 and Archive box \#121869. 


\begin{tabular}{|c|c|c|c|c|c|c|c|}
\hline \multicolumn{8}{|c|}{ TANK 241-S.110 } \\
\hline \multicolumn{8}{|c|}{ THERMOCOUPLE DATA } \\
\hline & & & & & & & \\
\hline TREE \# & DATE & THERMO \# & TREE SET \# & TEMP C & TEMP F & $Y=M A X$ TEMP & $\begin{array}{r}\text { COMMENTS } \\
\end{array}$ \\
\hline & $4 / 23 / 74$ & 1 & & & & & 88 - suspect data (low) \\
\hline & $4 / 23 / 74$ & 2 & & & & & 84 - suspect data (low) \\
\hline & $4 / 23 / 74$ & 3 & & & & & 77 - suspect data (low) \\
\hline & $4 / 23 / 74$ & 4 & & & & & 70 - suspect data (low) \\
\hline & $4 / 23 / 74$ & 5 & & & & & 68 - suspect data (low) \\
\hline & $4 / 23 / 74$ & 6 & & & & & 68 - suspect data (low) \\
\hline & $4 / 23 / 74$ & 7 & & & & & $68 \cdot$ suspect data (low) \\
\hline & $4 / 23 / 74$ & 8 & & & & & 68 - suspect data (low) \\
\hline & $4 / 23 / 74$ & 9 & & & & & 68 - suspect data (low) \\
\hline & $4 / 23 / 74$ & 10 & & & & & 68 - suspect data (low) \\
\hline & $4 / 23 / 74$ & 11 & & & & & 68 - suspect data (low) \\
\hline & $4 / 23 / 74$ & 12 & & & & & 68- suspect data (low) \\
\hline & $4 / 24 / 74$ & 1 & & & & & 87 - suspect data (low) \\
\hline & $4 / 24 / 74$ & 2 & & & & & 84 - suspect data (low) \\
\hline & $4 / 24 / 74$ & 3 & & & & & 76 - suspect data (low) \\
\hline & $4 / 24 / 74$ & 4 & & & & & 74 - suspect data (low) \\
\hline & $4 / 24 / 74$ & 5 & & & & & 70 - suspect data (low) \\
\hline & $4 / 24 / 74$ & 6 & & & & & 70 - suspect data (low) \\
\hline & $4 / 24 / 74$ & 7 & & & & & 70 - suspect data (low) \\
\hline & $4 / 24 / 74$ & 8 & & & & & 69 - suspect data (low) \\
\hline & $4 / 24 / 74$ & 9 & & & & & 68 - suspect data (low) \\
\hline & $4 / 24 / 74$ & 10 & & & & & 68 - suspect data (low) \\
\hline & $4 / 24 / 74$ & 11 & & & & & 68 - suspect data (low) \\
\hline & $4 / 24 / 74$ & 12 & & & & & 68 -suspect data (low) \\
\hline & $5 / 5 / 74$ & 3 & & & 127 & & \\
\hline & $5 / 5 / 74$ & 10 & & & 97 & & \\
\hline 1 & $12 / 2 / 74$ & 1 & & 52.22 & 125.996 & & raw data - assumed degree C \\
\hline 1 & $12 / 2 / 74$ & 2 & & 55.56 & 132.008 & & raw data - assumed degree $C$ \\
\hline 9 & $12 / 2 / 74$ & 3 & & 56.67 & 134.006 & & raw data - assumed degree C \\
\hline 1 & $12 / 2 / 74$ & 4 & & 58.33 & 136.994 & & raw data - assumed degree $C$ \\
\hline 1 & $12 / 2 / 74$ & 5 & & 58.33 & 136.994 & & raw data - assumed degree C \\
\hline 1 & $12 / 2 / 74$ & 6 & & 58.33 & 136.994 & & raw data - assumed degree C \\
\hline 1 & $12 / 2 / 74$ & 7 & & 58.33 & 136.994 & & raw data - assumed degree $C$ \\
\hline 1 & $12 / 2 / 74$ & 8 & & 58.33 & 136.994 & & raw data - assumed degree $\mathrm{C}$ \\
\hline 1 & $12 / 2 / 74$ & 9 & & 57.78 & 136.004 & & raw data - assumed degree $C$ \\
\hline 1 & $12 / 2 / 74$ & 10 & & 55 & 131 & & raw data - assumed degree $C$ \\
\hline 1 & $12 / 2 / 74$ & 11 & & 52.22 & 125.996 & & raw data - assumed degree C \\
\hline 1 & $12 / 2 / 74$ & 12 & & 52.22 & 125.996 & & raw data - assumed degree $C$ \\
\hline 1 & $4 / 13 / 75$ & 1 & & & 138 & & \\
\hline 1 & $4 / 13 / 75$ & 2 & 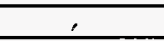 & & 142 & & \\
\hline 1 & $4 / 13 / 75$ & 3 & & & 142 & & \\
\hline 1 & $4 / 13 / 75$ & 4 & & & 141 & & \\
\hline 1 & $4 / 13 / 75$ & 5 & & & 140 & & \\
\hline$\overline{1}$ & $4 / 13 / 75$ & 6 & & & 138 & & \\
\hline 1 & $4 / 13 / 75$ & 7 & & & 136 & & \\
\hline 1 & $4 / 13 / 75$ & 8 & & & 136 & & \\
\hline 1 & $4 / 13 / 75$ & 9 & & & 136 & & \\
\hline 1 & $4 / 13 / 75$ & 10 & & & 136 & & \\
\hline 1 & $4 / 13 / 75$ & 11 & & & 130 & & \\
\hline 1 & $4 / 13 / 75$ & 12 & & & 114 & & \\
\hline 1 & $6 / 3 / 75$ & 1 & & & 142 & & \\
\hline 1 & $6 / 3 / 75$ & 2 & & & 145 & & \\
\hline 1 & $6 / 3 / 75$ & 3 & & & 145 & & \\
\hline 1 & $6 / 3 / 75$ & 4 & & & 145 & & \\
\hline 1 & $6 / 3 / 75$ & 5 & & & 144 & & \\
\hline 1 & $6 / 3 / 75$ & 6 & & & 142 & & \\
\hline 1 & $6 / 3 / 75$ & 7 & & & 141 & & \\
\hline 9 & $6 / 3 / 75$ & 8 & & & 138 & & 1 \\
\hline 1 & $6 / 3 / 75$ & 9 & & & 133 & & \\
\hline 1 & $6 / 3 / 75$ & 10 & & & 130 & & \\
\hline 1 & $6 / 3 / 75$ & 11 & & & 124 & & \\
\hline 1 & $6 / 3 / 75$ & 12 & & & 107 & & \\
\hline 1 & $11 / 3 / 75$ & 1 & & & 153 & & \\
\hline 1 & $11 / 3 / 75$ & 2 & & & 158 & & \\
\hline 1 & $11 / 3 / 75$ & 3 & & & 158 & & \\
\hline 1 & $11 / 3 / 75$ & 4 & & & 154 & & \\
\hline
\end{tabular}

Data obtained from WHC Surveillance Analysis Computer System (SACS), February 24, 1994, Data Sheet TK-105-S to TK$110-5$, and Archive box \#121869. 


\begin{tabular}{|c|c|c|c|c|c|c|c|}
\hline \multicolumn{8}{|c|}{ TANK 241-S.110 } \\
\hline & & & & THEF & OCOUPLE & & \\
\hline TREE \# & DATE & THERMO \# & TREE SET \# & TEMP C & TEMP F & $Y=$ MAX TEMP & COMMENTS \\
\hline 1 & $19 / 3 / 75$ & 5 & & & 150 & & \\
\hline 1 & $11 / 3 / 75$ & 6 & & & 145 & & \\
\hline 1 & $11 / 3 / 75$ & 7 & & & 138 & & \\
\hline 1 & $11 / 3 / 75$ & 8 & & & 136 & & \\
\hline 1 & $11 / 3 / 75$ & 9 & & & 136 & & \\
\hline 1 & $11 / 3 / 75$ & 10 & & & 132 & & \\
\hline 1 & $11 / 3 / 75$ & 11 & & & 115 & & \\
\hline 1 & $11 / 3 / 75$ & 12 & & & 98 & & \\
\hline 1 & $2 / 4 / 76$ & 1 & & & 182 & & \\
\hline 1 & $2 / 4 / 76$ & 2 & & & 185 & & \\
\hline 1 & $2 / 4 / 76$ & 3 & & & 185 & & \\
\hline 1 & $2 / 4 / 76$ & 4 & & & 182 & & \\
\hline 1 & $2 / 4 / 76$ & 5 & & & 178 & & \\
\hline 1 & $2 / 4 / 76$ & 6 & & & 172 & & \\
\hline 1 & $2 / 4 / 76$ & 7 & & & 166 & & \\
\hline 1 & $2 / 4 / 76$ & 8 & & & 162 & & \\
\hline 1 & $2 / 4 / 76$ & 9 & & & 161 & & \\
\hline 1 & $2 / 4 / 76$ & 10 & & & 149 & & \\
\hline 1 & $2 / 4 / 76$ & 11 & & & 130 & & \\
\hline 1 & $2 / 4 / 76$ & 12 & & & 110 & & \\
\hline 1 & $3 / 5 / 76$ & 1 & & & 155 & & \\
\hline 1 & $3 / 5 / 76$ & 2 & & & 158 & & \\
\hline 1 & $3 / 5 / 76$ & 3 & & & 158 & & \\
\hline 1 & $3 / 5 / 76$ & 4 & & & 154 & & \\
\hline 1 & $3 / 5 / 76$ & 5 & & & 149 & & \\
\hline 1 & $3 / 5 / 76$ & 6 & & & 142 & & \\
\hline 9 & $3 / 5 / 76$ & 7 & & & 134 & & \\
\hline 1 & $3 / 5 / 76$ & 8 & & & 134 & & \\
\hline 1 & $3 / 5 / 76$ & 9 & & & 133 & & \\
\hline 1 & $3 / 5 / 76$ & 10 & & & 130 & & \\
\hline 1 & $3 / 5 / 76$ & 11 & & & 106 & & \\
\hline 1 & $3 / 5 / 76$ & 12 & & & 100 & & \\
\hline 1 & $5 / 2 / 76$ & 1 & & & 157 & & \\
\hline 1 & $5 / 2 / 76$ & 2 & & & 160 & & \\
\hline 1 & $5 / 2 / 76$ & 3 & & & 160 & & \\
\hline 1 & $5 / 2 / 76$ & 4 & & & 155 & & \\
\hline 9 & $5 / 2 / 76$ & 5 & & & 149 & & \\
\hline 1 & $5 / 2 / 76$ & 6 & & & 142 & & \\
\hline 1 & $5 / 2 / 76$ & $\frac{7}{7}$ & & & 135 & & \\
\hline 1 & $5 / 2 / 76$ & 8 & & & 134 & & \\
\hline 1 & $5 / 2 / 76$ & 9 & & & 133 & & \\
\hline 1 & $5 / 2 / 76$ & 10 & & & 120 & & \\
\hline 1 & $5 / 2 / 76^{\circ}$ & 19 & & & 103 & & \\
\hline 1 & $5 / 2 / 76$ & 12 & & & 88 & & \\
\hline 1 & $6 / 1 / 76$ & 1 & & & 158 & & \\
\hline 1 & $6 / 1 / 76$ & 2 & & & 160 & & \\
\hline 1 & $6 / 1 / 76$ & 3 & & & 158 & & \\
\hline 1 & $6 / 1 / 76$ & 4 & & & 154 & & \\
\hline 1 & $6 / 1 / 76$ & 5 & & & 148 & & \\
\hline 1 & $6 / 1 / 76$ & 6 & & & 142 & & \\
\hline 1 & $6 / 1 / 76$ & 7 & & & 134 & & \\
\hline 1 & $6 / 1 / 76$ & 8 & & & 132 & & \\
\hline 1 & $6 / 1 / 76$ & 9 & & & 129 & & \\
\hline 1 & $6 / 1 / 76$ & 10 & & & 118 & & \\
\hline 1 & $6 / 1 / 76$ & 11 & & & 104 & & \\
\hline 1 & $6 / 1 / 76$ & 12 & & & 90 & & \\
\hline 1 & $6 / 1 / 76$ & 13 & & & 88 & & \\
\hline 1 & $6 / 1 / 76$ & 14 & & & 88 & & \\
\hline 1 & $7 / 1 / 76$ & 1 & & & 151 & & \\
\hline$\frac{1}{1}$ & $7 / 1 / 76$ & 2 & & & 154 & & \\
\hline 1 & $7 / 1 / 76$ & 3 & & & 153 & & \\
\hline 1 & $7 / 1 / 76$ & 4 & & & 148 & & \\
\hline 1 & $7 / 1 / 76$ & 5 & & & 142 & & \\
\hline 1 & $7 / 1 / 76$ & 6 & & & 135 & & \\
\hline 1 & $7 / 1 / 76$ & 7 & & & 128 & & \\
\hline$\frac{T}{1}$ & $7 / 1 / 76$ & 8 & & & 127 & & \\
\hline
\end{tabular}

Data obtained from WHC Surveillance Analysis Computer System (SACS), February 24, 1994, Data Sheet TK-105-S to TK110-S, and Archive box \#121869. 
WHC-SD-WM-ER-323, Rev. O

\begin{tabular}{|c|c|c|c|c|c|c|c|}
\hline \multicolumn{8}{|c|}{ TANK 241-S-110 } \\
\hline \multicolumn{8}{|c|}{ THERMOCOUPLE DATA } \\
\hline TREE \# & DATE & THERMO \# & TREE SET \# & TEMP C & TEMP F & $Y=M A X$ TEMP & COMMENTS \\
\hline 1 & $7 / 1 / 76$ & 9 & & & 126 & & \\
\hline$\frac{1}{1}$ & $7 / 1 / 76$ & 10 & & & 115 & & \\
\hline 1 & $7 / 1 / 76$ & 11 & & & 100 & & \\
\hline 1 & $7 / 1 / 76$ & 12 & & & 88 & & \\
\hline 1 & $8 / 3 / 76$ & 1 & & & 158 & & \\
\hline 1 & $8 / 3 / 76$ & $\overline{2}$ & & & 160 & & \\
\hline 9 & $8 / 3 / 76$ & 3 & & & 160 & & \\
\hline 1 & $8 / 3 / 76$ & 4 & & & 154 & & \\
\hline 9 & $8 / 3 / 76$ & 5 & & & 148 & & \\
\hline 9 & $8 / 3 / 76$ & 6 & & & 142 & & \\
\hline 1 & $8 / 3 / 76$ & 7 & & & 134 & & \\
\hline 1 & $8 / 3 / 76$ & 8 & & & 133 & & \\
\hline 9 & $8 / 3 / 76$ & 9 & & & 132 & & \\
\hline 1 & $8 / 3 / 76$ & 10 & & & 121 & & $\because$ \\
\hline 1 & $8 / 3 / 76$ & 91 & & & 110 & & \\
\hline 1 & $8 / 3 / 76$ & 12 & & & 98 & & \\
\hline 1 & $9 / 2 / 76$ & 1 & & & 160 & & \\
\hline 1 & $9 / 2 / 76$ & 2 & & & 162 & & \\
\hline 9 & $9 / 2 / 76$ & 3 & & & 160 & & \\
\hline 1 & $9 / 2 / 76$ & 4 & & & 156 & & \\
\hline 1 & $9 / 2 / 76$ & 5 & & & 150 & & \\
\hline 9 & $9 / 2 / 76$ & 6 & & & 142 & & \\
\hline 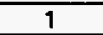 & $9 / 2 / 76$ & 7 & & & 136 & & \\
\hline 1 & $9 / 2 / 76$ & 8 & & & 134 & & \\
\hline 9 & $9 / 2 / 76$ & 9 & & & 133 & & \\
\hline 1 & $9 / 2 / 76$ & 10 & & & 128 & & \\
\hline 1 & $9 / 2 / 76$ & 11 & & & 113 & & \\
\hline 1 & $9 / 2 / 76$ & 12 & & & 102 & & \\
\hline$i$ & $10 / 3 / 76$ & 1 & & & 160 & & \\
\hline 1 & $10 / 3 / 76$ & 2 & & & 164 & & \\
\hline 9 & $10 / 3 / 76$ & 3 & & & 162 & & \\
\hline 1 & $10 / 3 / 76$ & 4 & & & 156 & & \\
\hline 1 & $10 / 3 / 76$ & 5 & & & 150 & & \\
\hline 1 & $10 / 3 / 76$ & 6 & & & 143 & & \\
\hline 1 & $10 / 3 / 76$ & 7 & & & 136 & & \\
\hline 1 & $10 / 3 / 76$ & 8 & & & 136 & & \\
\hline 1 & $10 / 3 / 76$ & 9 & & & 134 & & \\
\hline 1 & $10 / 3 / 76$ & 10 & & & 126 & & \\
\hline 1 & $10 / 3 / 76$ & 11 & & & 115 & & \\
\hline 9 & $10 / 3 / 76$ & 12 & & & 103 & & \\
\hline 1 & $11 / 1 / 76$ & 1 & & & 162 & & \\
\hline 1 & $11 / 1 / 76$ & 2 & & & 165 & & \\
\hline 9 & $11 / 1 / 76$ & 3 & & & 163 & & \\
\hline 1 & $11 / 1 / 76$ & 4 & & & 158 & & \\
\hline 1 & $11 / 1 / 76$ & 5 & & & 152 & & \\
\hline 9 & $11 / 1 / 76$ & 6 & & & 144 & & \\
\hline 1 & $11 / 1 / 76$ & 7 & & & 137 & & \\
\hline 1 & $11 / 1 / 76$ & 8 & & & 137 & & \\
\hline 9 & $1919 / 76$ & 9 & & & 135 & & \\
\hline 1 & $11 / 1 / 76$ & 10 & & & 126 & & \\
\hline 1 & $11 / 1 / 76$ & 11 & & & 114 & & \\
\hline 1 & $11 / 1 / 76$ & 12 & & & 100 & & \\
\hline 9 & $12 / 4 / 76$ & 1 & & & 160 & & \\
\hline 1 & $12 / 4 / 76$ & 2 & & & 164 & & \\
\hline$\frac{1}{1}$ & $12 / 4 / 76$ & 3 & & & 163 & & \\
\hline 1 & $12 / 4 / 76$ & 4 & & & 158 & & \\
\hline 1 & $12 / 4 / 76$ & 5 & & & 152 & & \\
\hline 1 & $12 / 4 / 76$ & 6 & & & 144 & & \\
\hline 1 & $12 / 4 / 76$ & 7 & & & 137 & & \\
\hline 9 & $12 / 4 / 76$ & 8 & & & 137 & & \\
\hline 1 & $12 / 4 / 76$ & 9 & & & 135 & & \\
\hline 1 & $12 / 4 / 76$ & 10 & & & 126 & & \\
\hline 1 & $12 / 4 / 76$ & 11 & & & 112 & & \\
\hline 9 & $12 / 4 / 76$ & 12 & & & 97 & & \\
\hline 1 & $1 / 1 / 77$ & 1 & & & 162 & & \\
\hline 1 & $1 / 1 / 77$ & 2 & & & 165 & & \\
\hline
\end{tabular}

Data obtained from WHC Surveillance Analysis Computer System (SACS), February 24, 1994, Data Sheet TK-105-S to TK$110-S$, and Archive box $\# 121869$. 


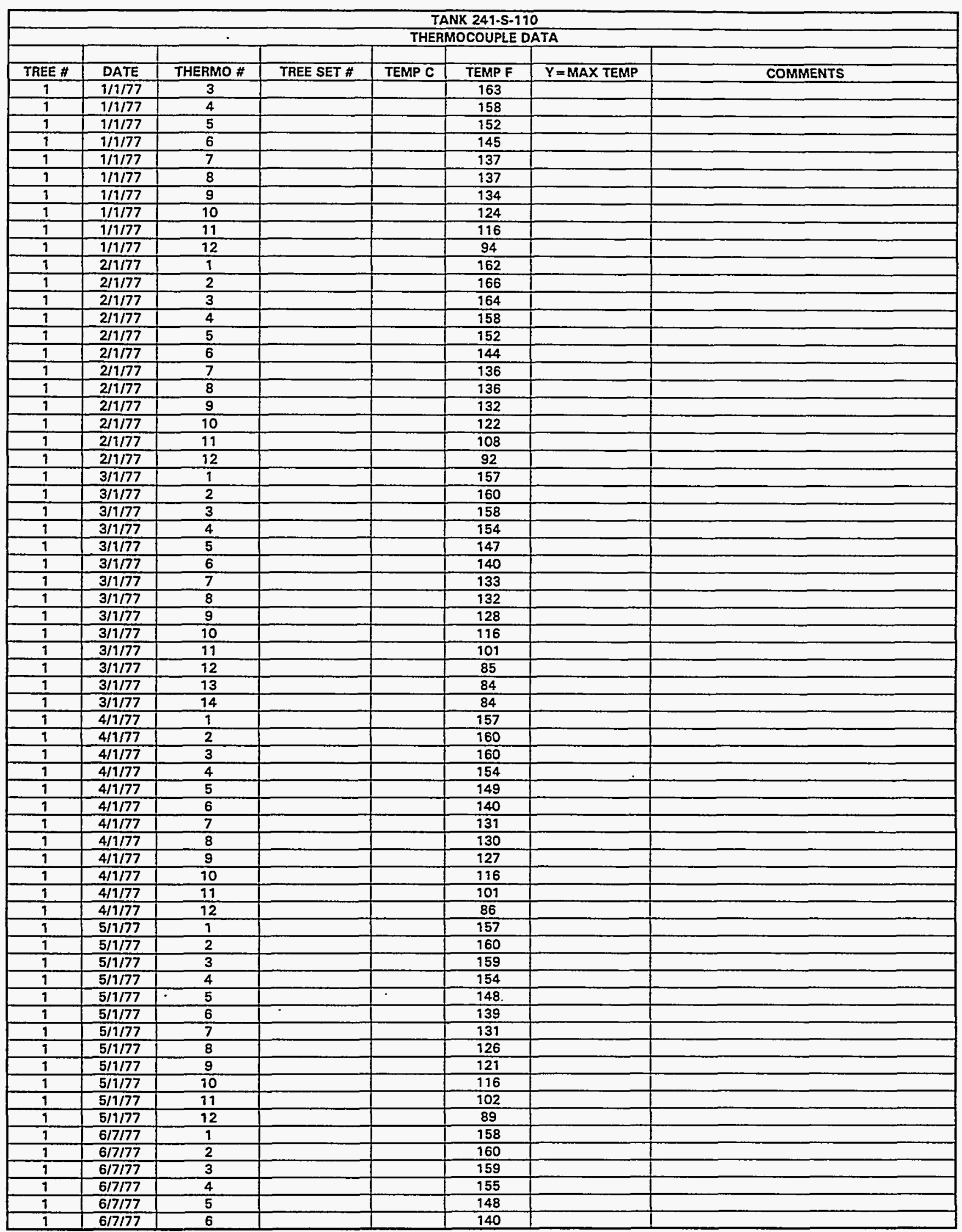

Data obtained from WHC Surveillance Analysis Computer System (SACS), February 24, 1994, Data Sheet TK-105-S to TK110-S, and Archive box \#121869. 


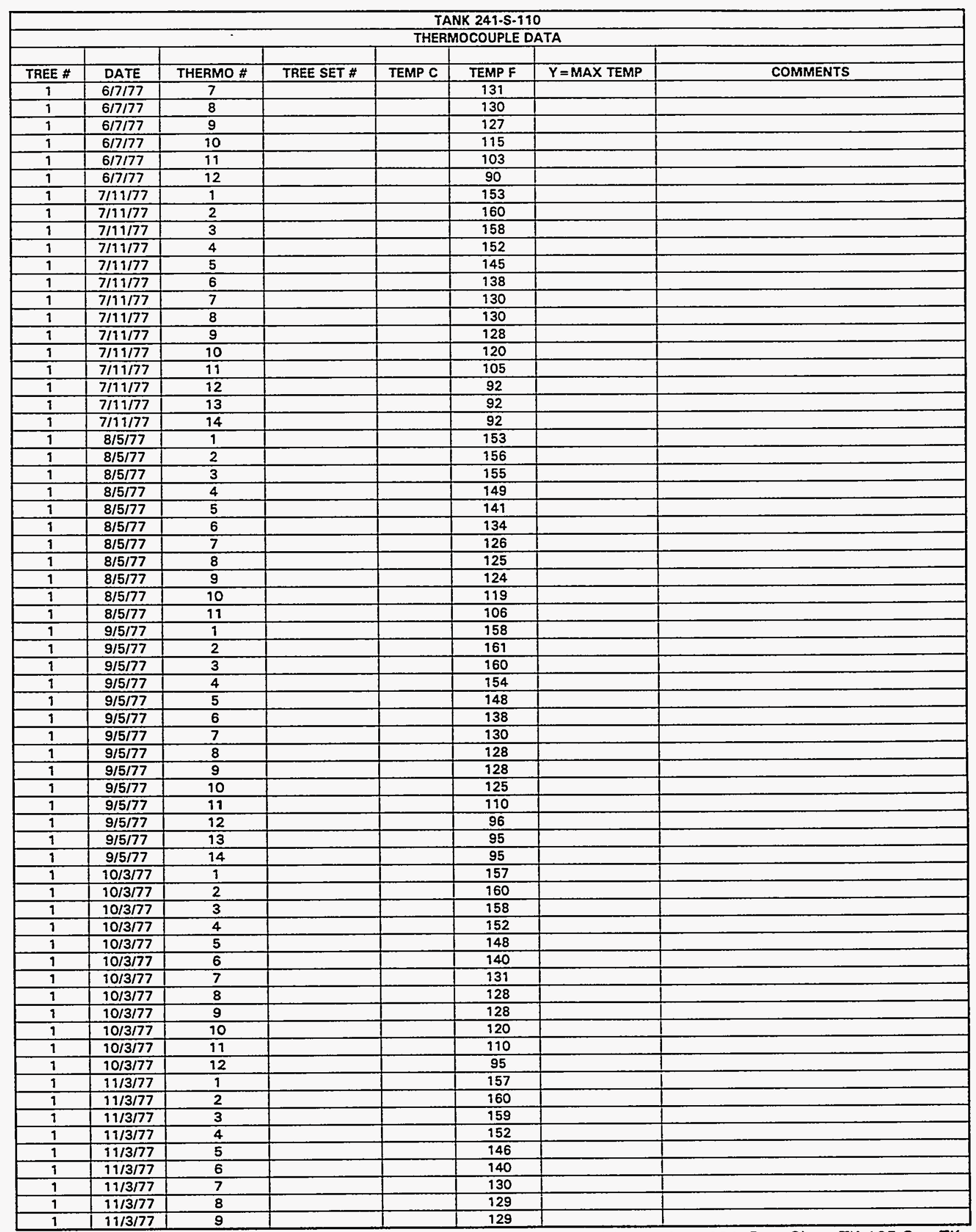

Data obtained from WHC Surveillance Analysis Computer System (SACS), February 24, 1994, Data Sheet TK-105-S to TK110-S, and Archive box \#121869. 
WHC-SD-WM-ER-323, Rev. 0

\begin{tabular}{|c|c|c|c|c|c|c|c|}
\hline \multicolumn{8}{|c|}{ TANK 241-S-110 } \\
\hline & & & & THEF & OCOUPLE & & \\
\hline TREE \# & DATE & THERMO \# & TREE SET \# & TEMP C & TEMP F & $Y=$ MAX TEMP & COMMENTS \\
\hline 1 & $11 / 3 / 77$ & 10 & & & 121 & & \\
\hline 9 & $11 / 3 / 77$ & 11 & & & 106 & & \\
\hline 1 & $11 / 3 / 77$ & 12 & & & 96 & & \\
\hline$\frac{1}{1}$ & $11 / 3 / 77$ & 13 & & & 88 & & \\
\hline 1 & $11 / 3 / 77$ & 14 & & & 88 & & \\
\hline 1 & $12 / 4 / 77$ & 9 & & & 162 & & \\
\hline 9 & $12 / 4 / 77$ & 2 & & & 164 & & \\
\hline 1 & $12 / 4 / 77$ & 3 & & & 162 & & \\
\hline 1 & $12 / 4 / 77$ & 4 & & & 156 & & \\
\hline 1 & $12 / 4 / 77$ & 5 & & & 149 & & \\
\hline 1 & $12 / 4 / 77$ & 6 & & & 142 & & \\
\hline 1 & $12 / 4 / 77$ & 7 & & & 132 & & \\
\hline 1 & $12 / 4 / 77$ & 8 & & & 129 & & \\
\hline 1 & $12 / 4 / 77$ & 9 & & & 128 & & \\
\hline 1 & $12 / 4 / 77$ & 10 & & & 117 & & \\
\hline 1 & $12 / 4 / 77$ & 11 & & & 101 & & \\
\hline$\frac{1}{1}$ & $12 / 4 / 77$ & 12 & & & 81 & & \\
\hline 1 & $12 / 4 / 77$ & 13 & & & 79 & & \\
\hline 1 & $12 / 4 / 77$ & 14 & & & 78 & & \\
\hline$\frac{1}{1}$ & $1 / 8 / 78$ & 1 & & & 177 & & \\
\hline 9 & $1 / 8 / 78$ & 2 & & & 180 & & \\
\hline 9 & $1 / 8 / 78$ & 3 & & & 178 & & \\
\hline 9 & $1 / 8 / 78$ & 4 & & & 173 & & \\
\hline 1 & $1 / 8 / 78$ & 5 & & & 167 & & \\
\hline 9 & $1 / 8 / 78$ & 6 & & & 159 & & \\
\hline 1 & $1 / 8 / 78$ & 7 & & & 150 & & \\
\hline 9 & $1 / 8 / 78$ & 8 & & & 147 & & \\
\hline 1 & $1 / 8 / 78$ & 9 & & & 145 & & \\
\hline 1 & $1 / 8 / 78$ & 10 & & & 134 & & \\
\hline 1 & $1 / 8 / 78$ & 11 & & & 120 & & \\
\hline 1 & $1 / 8 / 78$ & 12 & & & 104 & & \\
\hline 1 & $1 / 8 / 78$ & 13 & & & 102 & & \\
\hline 1 & $1 / 8 / 78$ & 14 & & & 102 & & \\
\hline 1 & $1 / 11 / 78$ & 1 & & & & & 116 - suspect data (low) \\
\hline 1 & $1 / 199 / 78$ & 2 & & & & & 117 - suspect data (low) \\
\hline 1 & $1 / 11 / 78$ & 3 & & & & & 716 - suspect data (low) \\
\hline 1 & $1 / 11 / 78$ & 4 & & & & & 114 - suspect data (low) \\
\hline 1 & $1 / 11 / 78$ & 5 & & & & & 111 - suspect data (low) \\
\hline 1 & $1 / 11 / 78$ & 6 & & & & & 106 - suspect data (low) \\
\hline 1 & $1 / 11 / 78$ & 7 & & & & & 100 - suspect data (low) \\
\hline$i$ & $1 / 11 / 78$ & 8 & & & & & 97 - suspect data (low) \\
\hline 1 & $1 / 11 / 78$ & 9 & & & & & 95 - suspect data (low) \\
\hline 1 & $1 / 11 / 78$ & 10 & & & & & 95 - suspect data (low) \\
\hline 9 & $1 / 11 / 78$ & 11 & & & 95 & & \\
\hline 1 & $1 / 11 / 78$ & 12 & & & 95 & & \\
\hline 1 & $1 / 11 / 78$ & 13 & & & 95 & & \\
\hline 1 & $1 / 11 / 78$ & 14 & & & 95 & & \\
\hline 1 & $1 / 13 / 78$ & 1 & & & 156 & & \\
\hline 1 & $1 / 13 / 78$ & 2 & & & 160 & & \\
\hline 1 & $1 / 13 / 78$ & 3 & & & 158 & & \\
\hline 1 & $1 / 13 / 78$ & 4 & & & 154 & & \\
\hline$\frac{1}{1}$ & $1 / 13 / 78$ & 5 & & & 156 & & \\
\hline 1 & $1 / 13 / 78$ & 6 & & & 140 & & \\
\hline 1 & $1 / 13 / 78$ & 7 & & & 130 & & \\
\hline 1 & $1 / 13 / 78$ & 8 & & & 128 & & \\
\hline 1 & $1 / 13 / 78$ & 9 & & & 115 & & \\
\hline 1 & $1 / 13 / 78$ & 10 & & & 115 & & \\
\hline 1 & $1 / 13 / 78$ & 11 & & & 100 & & \\
\hline 1 & $2 / 7 / 78$ & 1 & & & 161 & & \\
\hline 1 & $2 / 7 / 78$ & 2 & & & 164 & & \\
\hline 1 & $2 / 7 / 78$ & 3 & & & 162 & & \\
\hline 1 & $2 / 7 / 78$ & 4 & & & 156 & & \\
\hline 1 & $2 / 7 / 78$ & 5 & & & 150 & & \\
\hline$\frac{1}{1}$ & $2 / 7 / 78$ & 6 & & & 142 & & \\
\hline 9 & $2 / 7 / 78$ & 7 & & & 133 & & \\
\hline 9 & $2 / 7 / 78$ & 8 & & & 131 & & \\
\hline
\end{tabular}

Data obtained from WHC Surveillance Analysis Computer System (SACS). February 24, 1994, Data Sheet TK-105-S to TK110-S, and Archive box \#121869. 


\begin{tabular}{|c|c|c|c|c|c|c|c|}
\hline \multicolumn{8}{|c|}{ TANK 241-S-110 } \\
\hline \multicolumn{8}{|c|}{ THERMOCOUPLE DATA } \\
\hline & & & & & & & \\
\hline TREE \# & DATE & THERMO \# & TREE SET \# & TEMP C & TEMP F & $Y=$ MAX TEMP & COMMENTS \\
\hline 1 & $2 / 7 / 78$ & 9 & & & 127 & & \\
\hline 1 & $2 / 7 / 78$ & 10 & & & 117 & & \\
\hline 1 & $2 / 7 / 78$ & 11 & & & 102 & & \\
\hline 1 & $2 / 7 / 78$ & 12 & & & 86 & & \\
\hline 1 & $2 / 7 / 78$ & 13 & & & 84 & & \\
\hline 1 & $2 / 7 / 78$ & 14 & & & 84 & & \\
\hline 1 & $3 / 12 / 78$ & 1 & & & 164 & & \\
\hline 1 & $3 / 12 / 78$ & 2 & & & 167 & & \\
\hline 1 & $3 / 12 / 78$ & 3 & & & 166 & & \\
\hline 1 & $3 / 12 / 78$ & 4 & & & 166 & & \\
\hline 1 & $3 / 12 / 78$ & 5 & & & 154 & & \\
\hline 1 & $3 / 12 / 78$ & 6 & & & 946 & & \\
\hline 1 & $3 / 12 / 78$ & 7 & & & 136 & & \\
\hline 1 & $3 / 12 / 78$ & 8 & & & 134 & & \\
\hline 1 & $3 / 12 / 78$ & 9 & & & 130 & & \\
\hline$T$ & $3 / 12 / 78$ & 10 & & & 119 & & \\
\hline 1 & $3 / 12 / 78$ & 11 & & & 104 & & \\
\hline 1 & $3 / 12 / 78$ & 12 & & & 88 & & \\
\hline$\frac{1}{1}$ & $3 / 12 / 78$ & 13 & & & 86 & & \\
\hline 1 & $3 / 12 / 78$ & 14 & & & 86 & & \\
\hline 1 & $4 / 6 / 78$ & 1 & & & 157 & & \\
\hline 1 & $4 / 6 / 78$ & 2 & & & 160 & & \\
\hline 1 & $4 / 6 / 78$ & 3 & & & 158 & & \\
\hline 1 & $4 / 6 / 78$ & 4 & & & 152 & & \\
\hline 1 & $4 / 6 / 78$ & 5 & & & 146 & & \\
\hline 1 & $4 / 6 / 78$ & 6 & & & 138 & & \\
\hline 1 & $4 / 6 / 78$ & 7 & & & 127 & & \\
\hline 1 & $4 / 6 / 78$ & 8 & & & 125 & & \\
\hline 1 & $4 / 6 / 78$ & 9 & & & 121 & & \\
\hline 1 & $4 / 6 / 78$ & 10 & & & 109 & & \\
\hline 1 & $4 / 6 / 78$ & 11 & & & 95 & & \\
\hline 1 & $4 / 6 / 78$ & 12 & & & 80 & & \\
\hline 1 & $4 / 6 / 78$ & 13 & & & 78 & & \\
\hline 1 & $4 / 6 / 78$ & 14 & & & 78 & & \\
\hline 1 & $4 / 11 / 78$ & 1 & & & 157 & & \\
\hline 9 & $4 / 11 / 78$ & 2 & & & 160 & & \\
\hline 1 & $4 / 11 / 78$ & 3 & & & 157 & & \\
\hline 1 & $4 / 11 / 78$ & 4 & & & 152 & & \\
\hline$\frac{1}{1}$ & $4 / 11 / 78$ & 5 & & & 146 & & \\
\hline 1 & $4 / 11 / 78$ & 6 & & & 138 & & \\
\hline 1 & $4 / 11 / 78$ & 7 & & & 127 & & \\
\hline 1 & $4 / 11 / 78$ & 8 & & & 125 & & \\
\hline 1 & $4 / 11 / 78$ & 9 & & & 120 & & \\
\hline 1 & $4 / 11 / 78$ & 10 & & & 119 & & \\
\hline 1 & $4 / 11 / 78$ & 11 & & & 97 & & \\
\hline 1 & $4 / 11 / 78$ & 12 & & & 115 & & \\
\hline 1 & $4 / 11 / 78$ & 13 & & & 80 & & \\
\hline 9 & $4 / 11 / 78$ & 14 & & & 79 & & \\
\hline 1 & $6 / 1 / 78$ & 1 & & & 158 & & \\
\hline 1 & $6 / 1 / 78$ & 2 & & & 161 & & \\
\hline 1 & $6 / 1 / 78$ & 3 & & & 958 & & \\
\hline 1 & $6 / 1 / 78$ & 4 & & & 153 & & \\
\hline 1 & $6 / 1 / 78$ & 5 & & & 146 & & \\
\hline 1 & $6 / 1 / 78$ & 6 & & & 137 & & \\
\hline 1 & $6 / 1 / 78$ & 7 & & & 128 & & \\
\hline 1 & $6 / 1 / 78$ & 8 & & & 122 & & \\
\hline 1 & $6 / 1 / 78$ & 9 & & & 718 & & \\
\hline 1 & $6 / 1 / 78$ & 10 & & & 110 & & \\
\hline 1 & $6 / 1 / 78$ & 11 & & & 98 & & \\
\hline 1 & $6 / 1 / 78$ & 12 & & & 87 & & \\
\hline 9 & $6 / 1 / 78$ & 13 & & & 85 & & \\
\hline 1 & $6 / 1 / 78$ & 14 & & & 85 & & \\
\hline 1 & $6 / 3 / 78$ & 1 & & & 157 & & \\
\hline 9 & $6 / 3 / 78$ & 2 & & & 160 & & \\
\hline 1 & $6 / 3 / 78$ & 3 & & & 158 & & \\
\hline 1 & $6 / 3 / 78$ & 4 & & & 152 & & \\
\hline
\end{tabular}

Data obtained from WHC Surveillance Analysis Computer System (SACS), February 24, 1994, Data Sheet TK-105-S to TK110-S, and Archive box \#121869. 
WHC-SD-WM-ER-323, Rev. 0

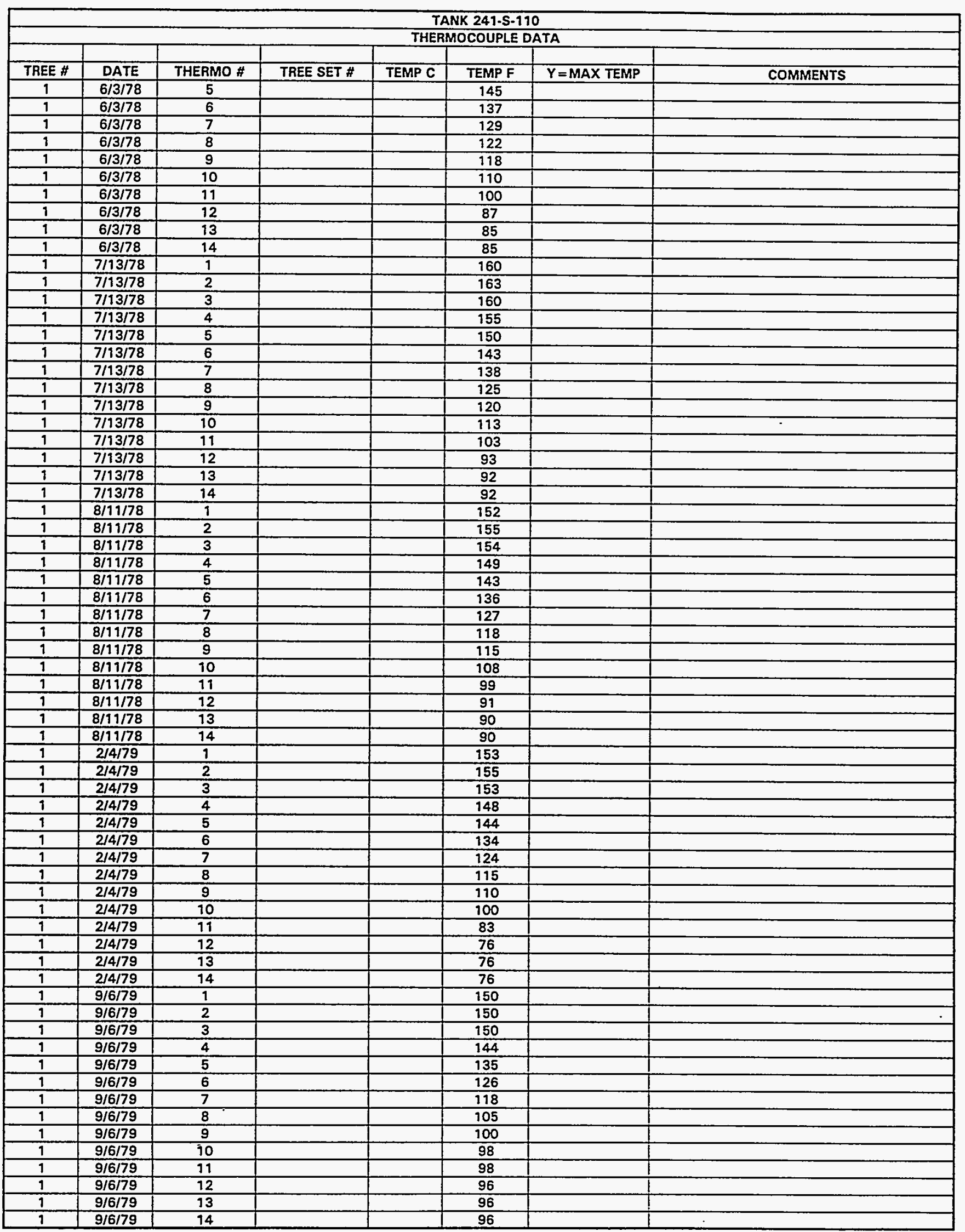

Data obtained from WHC Surveillance Analysis Computer System (SACS), February 24, 1994, Data Sheet TK-105-S to TK$110-\mathrm{S}$, and Archive box \#121869. 


\begin{tabular}{|c|c|c|c|c|c|c|c|}
\hline \multicolumn{8}{|c|}{ TANK 241-S-110 } \\
\hline \multicolumn{8}{|c|}{ THERMOCOUPLE DATA } \\
\hline & & & & & & & \\
\hline TREE \# & DATE & THERMO \# & TREE SET \# & TEMP C & TEMP F & $\mathrm{Y}=\mathrm{MAX}$ TEMP & COMMENTS \\
\hline 1 & $1 / 23 / 80$ & 1 & & & & & 90 - suspect data (low) \\
\hline 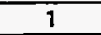 & $1 / 23 / 80$ & 2 & & & & & 90 - suspect data (low) \\
\hline 1 & $1 / 23 / 80$ & 3 & & & & & 90 - suspect data (low) \\
\hline 1 & $1 / 23 / 80$ & 4 & & & & & 90 - suspect data (low) \\
\hline 1 & $1 / 23 / 80$ & 5 & & & & & 90 - suspect data (low) \\
\hline 9 & $1 / 23 / 80$ & 11 & & & & & 145 - suspect data (high) \\
\hline 1 & $1 / 23 / 80$ & 12 & & & & & 745 - suspect data (high) \\
\hline 1 & $1 / 23 / 80$ & 13 & & & & & 145 - suspect data (high) \\
\hline 1 & $9 / 23 / 80$ & 14 & & & & & 145 - suspect data (high) \\
\hline 1 & $2 / 1 / 80$ & 1 & & & 145 & & \\
\hline 1 & $2 / 1 / 80$ & 2 & & & 143 & & \\
\hline 1 & $2 / 1 / 80$ & 3 & & & 143 & & \\
\hline 1 & $2 / 1 / 80$ & 4 & & & 137 & & \\
\hline 1 & $2 / 1 / 80$ & 5 & & & 133 & & \\
\hline 1 & $2 / 1 / 80$ & 6 & & & 125 & & \\
\hline 1 & $2 / 1 / 80$ & 7 & & & 106 & & \\
\hline 1 & $2 / 1 / 80$ & 8 & & & 85 & & \\
\hline 1 & $2 / 1 / 80$ & 9 & & & 85 & & \\
\hline 1 & $2 / 1 / 80$ & 10 & & & 85 & & \\
\hline 1 & $2 / 1 / 80$ & 11 & & & 85 & & \\
\hline 1 & $2 / 1 / 80$ & 12 & & & 85 & & \\
\hline 1 & $2 / 1 / 80$ & 13 & & & 85 & & \\
\hline 1 & $2 / 1 / 80$ & 14 & & & 85 & & \\
\hline 1 & $1 / 13 / 82$ & 9 & & & 140 & & \\
\hline 1 & $1 / 13 / 82$ & 2 & & & & & 85 - suspect data (low) \\
\hline 1 & $1 / 13 / 82$ & 3 & & & 140 & & \\
\hline 1 & $1 / 13 / 82$ & 4 & & & 135 & & \\
\hline 1 & $1 / 13 / 82$ & 5 & & & 127 & & \\
\hline 1 & $1 / 13 / 82$ & 6 & & & 118 & & \\
\hline 1 & $1 / 13 / 82$ & 7 & & & 101 & & \\
\hline 1 & $1 / 13 / 82$ & 8 & & & 85 & & \\
\hline 1 & $1 / 13 / 82$ & 9 & & & 85 & & \\
\hline 1 & $1 / 13 / 82$ & 10 & & & 83 & & \\
\hline 1 & $1 / 13 / 82$ & 13 & & & 83 & & \\
\hline 1 & $1 / 13 / 82$ & 74 & & & 83 & & \\
\hline 1 & $5 / 29 / 82$ & 1 & & & 141 & & \\
\hline 1 & $5 / 29 / 82$ & 3 & & & 137 & & \\
\hline 1 & $5 / 29 / 82$ & 4 & & & 131 & & \\
\hline 1 & $5 / 29 / 82$ & 5 & & & 125 & & \\
\hline 1 & $5 / 29 / 82$ & 6 & & & $\$ 15$ & & \\
\hline 1 & $5 / 29 / 82$ & 7 & & & 100 & & \\
\hline 1 & $5 / 29 / 82$ & 8 & & & 85 & & \\
\hline 9 & $5 / 29 / 82$ & 9 & & & 84 & & \\
\hline 1 & $5 / 29 / 82$ & 10 & & & 84 & & \\
\hline 1 & $5 / 29 / 82$ & 11 & & & 84 & & \\
\hline 1 & $10 / 6 / 82$ & 1 & & & 140 & & \\
\hline 1 & $10 / 6 / 82$ & 2 & & & 140 & & \\
\hline 1 & $10 / 6 / 82$ & 3 & & & 136 & & \\
\hline 1 & $10 / 6 / 82$ & 4 & & & 130 & & \\
\hline 1 & $10 / 6 / 82$ & 5 & & & 125 & & \\
\hline 1 & $10 / 6 / 82$ & 6 & & & 119 & & \\
\hline 1 & $10 / 6 / 82$ & 7 & & & 106 & & \\
\hline 1 & $10 / 6 / 82$ & 8 & & & 93 & & \\
\hline 1 & $10 / 6 / 82$ & 9 & . & & 92 & & \\
\hline 7 & $10 / 6 / 82$ & 10 & & & 92 & & \\
\hline 1 & $10 / 6 / 82$ & 11 & & & 92 & & \\
\hline 1 & $10 / 6 / 82$ & 13 & & & 92 & & \\
\hline 1 & $10 / 6 / 82$ & 14 & & & 92 & & \\
\hline 1 & $5 / 19 / 83$ & 1 & & & 136 & & \\
\hline 1 & $5 / 19 / 83$ & 2 & & & 136 & & \\
\hline 1 & $5 / 19 / 83$ & 3 & & & 132 & & \\
\hline 1 & $5 / 19 / 83$ & 4 & & & 126 & & \\
\hline 1 & $5 / 19 / 83$ & 5 & & & 120 & & \\
\hline 1 & $5 / 19 / 83$ & 6 & & & 111 & & \\
\hline 1 & $5 / 19 / 83$ & 7 & & & 97 & & \\
\hline 1 & $5 / 19 / 83$ & 8 & & & 83 & & \\
\hline
\end{tabular}

Data obtained from WHC Surveillance Analysis Computer System (SACS), February 24, 1994, Data Sheet TK-105-S to TK110-S, and Archive box \#121869. 


\begin{tabular}{|c|c|c|c|c|c|c|c|}
\hline \multicolumn{8}{|c|}{ TANK 241-S-110 } \\
\hline & & & & THEI & DCOUPLE & & \\
\hline TREE \# & DATE & THERMO \# & TREE SET \# & TEMP C & TEMP F & $\bar{Y}=$ MAX TEMP & COMMENTS \\
\hline 1 & $5 / 19 / 83$ & 9 & & & 82 & & \\
\hline 1 & $5 / 19 / 83$ & 10 & & & 81 & & \\
\hline 1 & $5 / 19 / 83$ & 11 & & & 81 & & \\
\hline 1 & $5 / 19 / 83$ & 12 & & & 81 & & \\
\hline 1 & $5 / 19 / 83$ & 13 & & & 81 & & \\
\hline 1 & $5 / 19 / 83$ & 14 & & & 81 & & \\
\hline 1 & $1 / 12 / 84$ & 1 & & & 148 & & \\
\hline 1 & $1 / 12 / 84$ & 2 & & & 142 & & \\
\hline 1 & $1 / 12 / 84$ & 3 & & & 138 & & \\
\hline 1 & $1 / 12 / 84$ & 4 & & & 116 & & \\
\hline 1 & $1 / 12 / 84$ & 5 & & & 102 & & \\
\hline 9 & $9 / 12 / 84$ & 6 & & & 105 & & \\
\hline 1 & $2 / 14 / 84$ & 1 & & & 133 & & \\
\hline 1 & $2 / 14 / 84$ & 2 & & & 132 & & \\
\hline 1 & $2 / 14 / 84$ & 3 & & & 125 & & \\
\hline 1 & $2 / 14 / 84$ & 4 & & & 118 & & \\
\hline 1 & $2 / 14 / 84$ & 5 & & & 116 & & \\
\hline 1 & $2 / 14 / 84$ & 6 & & & 76 & & \\
\hline 1 & $3 / 14 / 84$ & 1 & & & 131 & & \\
\hline 1 & $3 / 14 / 84$ & 2 & & & 131 & & \\
\hline 1 & $3 / 14 / 84$ & 3 & & & 126 & & \\
\hline 1 & $3 / 14 / 84$ & 4 & & & 118 & & \\
\hline 1 & $3 / 14 / 84$ & 5 & & & 115 & & \\
\hline 1 & $3 / 14 / 84$ & 6 & & & 76 & & \\
\hline 1 & $4 / 9 / 84$ & 1 & & & 134 & & - \\
\hline 1 & $4 / 9 / 84$ & 2 & & & 134 & & \\
\hline 1 & $4 / 9 / 84$ & 3 & & & 128 & & \\
\hline 1 & $4 / 9 / 84$ & 4 & & & 121 & & \\
\hline 1 & $4 / 9 / 84$ & 5 & & & 119 & & \\
\hline 1 & $4 / 9 / 84$ & 6 & & & 80 & & \\
\hline 1 & $6 / 19 / 84$ & 1 & & & & & 70 - suspect data (low) \\
\hline 1 & $6 / 19 / 84$ & 2 & & & & & 53 - suspect data (low) \\
\hline 1 & $6 / 19 / 84$ & 3 & & & & & 53 - suspect data (low) \\
\hline 1 & $6 / 19 / 84$ & 4 & & & & & 53 - suspect data (low) \\
\hline 1 & $6 / 19 / 84$ & 5 & & & & & 53 - suspect data (low) \\
\hline 1 & $6 / 19 / 84$ & 6 & & & & & 53 - suspect data (low) \\
\hline 1 & $9 / 13 / 84$ & 1 & & & 129 & & \\
\hline 1 & $9 / 13 / 84$ & 2 & & & 130 & & \\
\hline 1 & $9 / 13 / 84$ & 3 & & & 124 & & \\
\hline 1 & $9 / 13 / 84$ & 4 & & & 119 & & \\
\hline 1 & $9 / 13 / 84$ & 5 & & & 118 & & \\
\hline 1 & $9 / 13 / 84$ & 6 & & & 91 & & \\
\hline 1 & $4 / 2 / 85$ & 1 & & & 131 & & \\
\hline 1 & $4 / 2 / 85$ & 2 & & & 132 & & \\
\hline 1 & $4 / 2 / 85$ & 3 & & & 128 & & \\
\hline 1 & $4 / 2 / 85$ & 4 & & & 119 & & \\
\hline 1 & $4 / 2 / 85$ & 5 & & & 115 & & \\
\hline 1 & $4 / 2 / 85$ & 6 & & & 79 & & \\
\hline 1 & $5 / 2 / 85$ & 1 & & & 123 & & \\
\hline 1 & $5 / 2 / 85$ & 2 & & & 124 & & \\
\hline 1 & $5 / 2 / 85$ & 3 & & & 118 & & \\
\hline 1 & $5 / 2 / 85$ & 4 & & & 109 & & \\
\hline 1 & $5 / 2 / 85$ & 5 & & & 107 & & \\
\hline 1 & $5 / 2 / 85$ & 6 & & & 73 & & \\
\hline 1 & $7 / 2 / 85$ & 1 & & & 125 & & \\
\hline 1 & $7 / 2 / 85$ & 2 & & & 126 & & \\
\hline 1 & $7 / 2 / 85$ & 3 & & & 121 & & \\
\hline 1 & $7 / 2 / 85$ & 4 & & & 113 & & \\
\hline 1 & $7 / 2 / 85$ & 5 & & & 112 & & \\
\hline 1 & $7 / 2 / 85$ & 6 & & & 83 & & \\
\hline 1 & $12 / 4 / 85$ & 1 & & & 114 & & \\
\hline 1 & $12 / 4 / 85$ & 2 & & & 115 & & \\
\hline 1 & $12 / 4 / 85$ & 3 & & & 111 & & \\
\hline 1 & $12 / 4 / 85$ & 4 & & & 102 & & \\
\hline 1 & $12 / 4 / 85$ & 5 & & & 101 & & \\
\hline 1 & $12 / 4 / 85$ & 6 & & & 67 & & \\
\hline
\end{tabular}

Data obtained from WHC Surveillance Analysis Computer System (SACS), February 24, 1994, Data Sheet TK-105-S to TK$110-S$, and Archive box \#121869. 


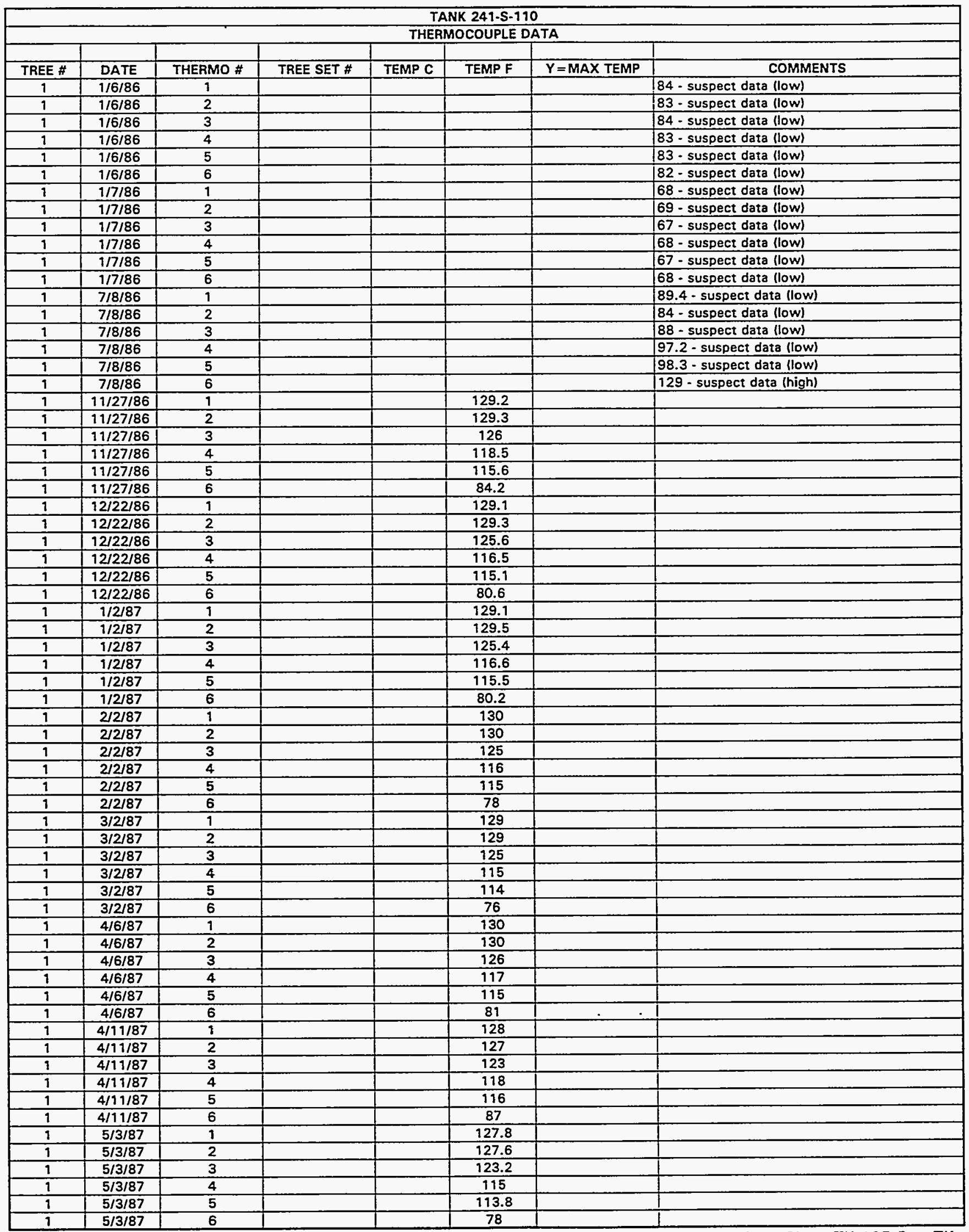

Data obtained from WHC Surveillance Analysis Computer System (SACS), February 24, 1994, Data Sheet TK-105-S to TK110-S, and Archive box \#121869. 


\begin{tabular}{|c|c|c|c|c|c|c|c|}
\hline \multicolumn{8}{|c|}{ TANK 241-S-110 } \\
\hline & & & & THER & COUPLE & TA & \\
\hline TREE \# & DATE & THERMO \# & TREE SET \# & TEMP C & TEMP F & $Y=$ MAX TEMP & COMMENTS \\
\hline 1 & $7 / 6 / 87$ & 1 & & & 128.1 & & \\
\hline 1 & $7 / 6 / 87$ & 2 & & & 127.5 & & \\
\hline 1 & $7 / 6 / 87$ & 3 & & & 122.6 & & \\
\hline 1 & $7 / 6 / 87$ & 4 & & & 115 & & \\
\hline 1 & $7 / 6 / 87$ & 5 & & & 114 & & \\
\hline 1 & $7 / 6 / 87$ & 6 & & & 84.4 & & \\
\hline 1 & $8 / 4 / 87$ & 1 & & & 126.9 & & \\
\hline 1 & $8 / 4 / 87$ & 2 & & & 126.4 & & \\
\hline 9 & $8 / 4 / 87$ & 3 & & & 121.8 & & \\
\hline 1 & $8 / 4 / 87$ & 4 & & & 114.3 & & \\
\hline 9 & $8 / 4 / 87$ & 5 & & & 113 & & \\
\hline 1 & $8 / 4 / 87$ & 6 & & & 85.1 & & \\
\hline 1 & $10 / 5 / 87$ & 1 & & & 126 & & \\
\hline 1 & $10 / 5 / 87$ & 2 & & & 125 & & \\
\hline 1 & $10 / 5 / 87$ & 3 & & & 122 & & \\
\hline 1 & $10 / 5 / 87$ & 4 & & & 116 & & \\
\hline 1 & $10 / 5 / 87$ & 5 & & & 115 & & \\
\hline 1 & $10 / 5 / 87$ & 6 & & & 89 & & \\
\hline 1 & $12 / 8 / 87$ & 1 & & & 136.8 & & \\
\hline 1 & $12 / 8 / 87$ & 2 & & & 135.8 & & \\
\hline 1 & $12 / 8 / 87$ & 3 & & & 130.9 & & \\
\hline 1 & $12 / 8 / 87$ & 4 & & & 126.2 & & \\
\hline 1 & $12 / 8 / 87$ & 5 & & & 124.3 & & \\
\hline 1 & $12 / 8 / 87$ & 6 & & & 91.8 & & \\
\hline 1 & $1 / 4 / 88$ & 1 & 1 & & 128.8 & $Y$ & \\
\hline 1 & $1 / 4 / 88$ & 2 & 1 & & 128 & $\mathbf{N}$ & \\
\hline 1 & $1 / 4 / 88$ & 3 & 1 & & 124 & $\bar{N}$ & \\
\hline 1 & $1 / 4 / 88$ & 4 & 1 & & 198 & $N$ & \\
\hline 1 & $1 / 4 / 88$ & 5 & 1 & & 116.4 & $N$ & \\
\hline 1 & $1 / 4 / 88$ & 6 & 1 & & 81.2 & $\mathrm{~N}$ & \\
\hline 1 & $2 / 1 / 88$ & 1 & 1 & & 124 & $\bar{Y}$ & \\
\hline 1 & $2 / 1 / 88$ & 2 & 1 & & 123 & $\bar{N}$ & \\
\hline 1 & $2 / 1 / 88$ & 3 & 1 & & 120 & $\mathrm{~N}$ & \\
\hline 1 & $2 / 1 / 88$ & 4 & 1 & & 115 & $N$ & \\
\hline 1 & $2 / 1 / 88$ & 5 & 1 & & 112 & $\mathrm{~N}$ & \\
\hline 1 & $2 / 1 / 88$ & 6 & 1 & & 78 & $\bar{N}$ & \\
\hline 1 & $3 / 8 / 88$ & 1 & 1 & & 132 & $\mathrm{Y}$ & \\
\hline 1 & $3 / 8 / 88$ & 2 & 7 & & 131 & $N$ & \\
\hline 1 & $3 / 8 / 88$ & 3 & 1 & & 126 & $\mathbf{N}$ & \\
\hline 1 & $3 / 8 / 88$ & 4 & 1 & & 120 & $N$ & \\
\hline 1 & $3 / 8 / 88$ & 5 & 9 & & 119 & $\bar{N}$ & \\
\hline 1 & $3 / 8 / 88$ & 6 & 1 & & 84 & $\bar{N}$ & \\
\hline 9 & $4 / 4 / 88$ & 1 & 1 & & 126 & $\bar{Y}$ & \\
\hline 1 & $4 / 4 / 88$ & 2 & 1 & & 125 & 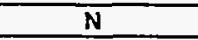 & \\
\hline 9 & $4 / 4 / 88$ & 3 & 1 & & 120 & $N$ & \\
\hline 1 & $4 / 4 / 88$ & 4 & 1 & & 114 & $\bar{N}$ & \\
\hline 1 & $4 / 4 / 88$ & 5 & 9 & & 113 & $\bar{N}$ & \\
\hline 1 & $4 / 4 / 88$ & 6 & 9 & & 79 & $N$ & \\
\hline 1 & $5 / 2 / 88$ & 1 & 1 & & 136 & $\bar{Y}$ & \\
\hline 1 & $5 / 2 / 88$ & 2 & 1 & & 135 & $\bar{N}$ & \\
\hline 1 & $5 / 2 / 88$ & 3 & 1 & & 130 & $\mathbf{N}$ & \\
\hline 1 & $5 / 2 / 88$ & 4 & 1 & & 125 & $\mathrm{~N}$ & \\
\hline 1 & $5 / 2 / 88$ & 5 & 1 & & 123 & $\mathbf{N}$ & \\
\hline 1 & $5 / 2 / 88$ & 6 & 1 & & 91 & $\mathbf{N}$ & \\
\hline 1 & $6 / 6 / 88$ & 1 & 1 & & 135 & $\bar{Y}$ & \\
\hline 1 & $6 / 6 / 88$ & 2 & 1 & & 134 & $\mathbf{N}$ & \\
\hline 1 & $6 / 6 / 88$ & 3 & 1 & & 125 & $\bar{N}$ & \\
\hline 1 & $6 / 6 / 88$ & 4 & 1 & & 120 & $\bar{N}$ & \\
\hline 1 & $6 / 6 / 88$ & 5 & 1 & & 120 & $\mathbf{N}$ & \\
\hline 1 & $6 / 6 / 88$ & 6 & 1 & & 91 & $\mathbf{N}$ & \\
\hline 1 & $7 / 4 / 88$ & 1 & 1 & & 134 & $\bar{Y}$ & \\
\hline 1 & $7 / 4 / 88$ & 2 & 1 & & 133 & $\mathbf{N}$ & \\
\hline 1 & $7 / 4 / 88$ & 3 & 1 & & 127 & $N$ & \\
\hline 1 & $7 / 4188$ & 4 & 1 & & 120 & $\mathbf{N}$ & \\
\hline 1 & $7 / 4 / 88$ & 5 & 1 & & 118 & $\mathbf{N}$ & \\
\hline 1 & $7 / 4 / 88$ & 6 & 1 & & 81 & $\mathbf{N}$ & \\
\hline
\end{tabular}

Data obtained from WHC Surveillance Analysis Computer System (SACS), February 24, 1994, Data Sheet TK-105-S to TK110-S; and Archive box \#121869. 


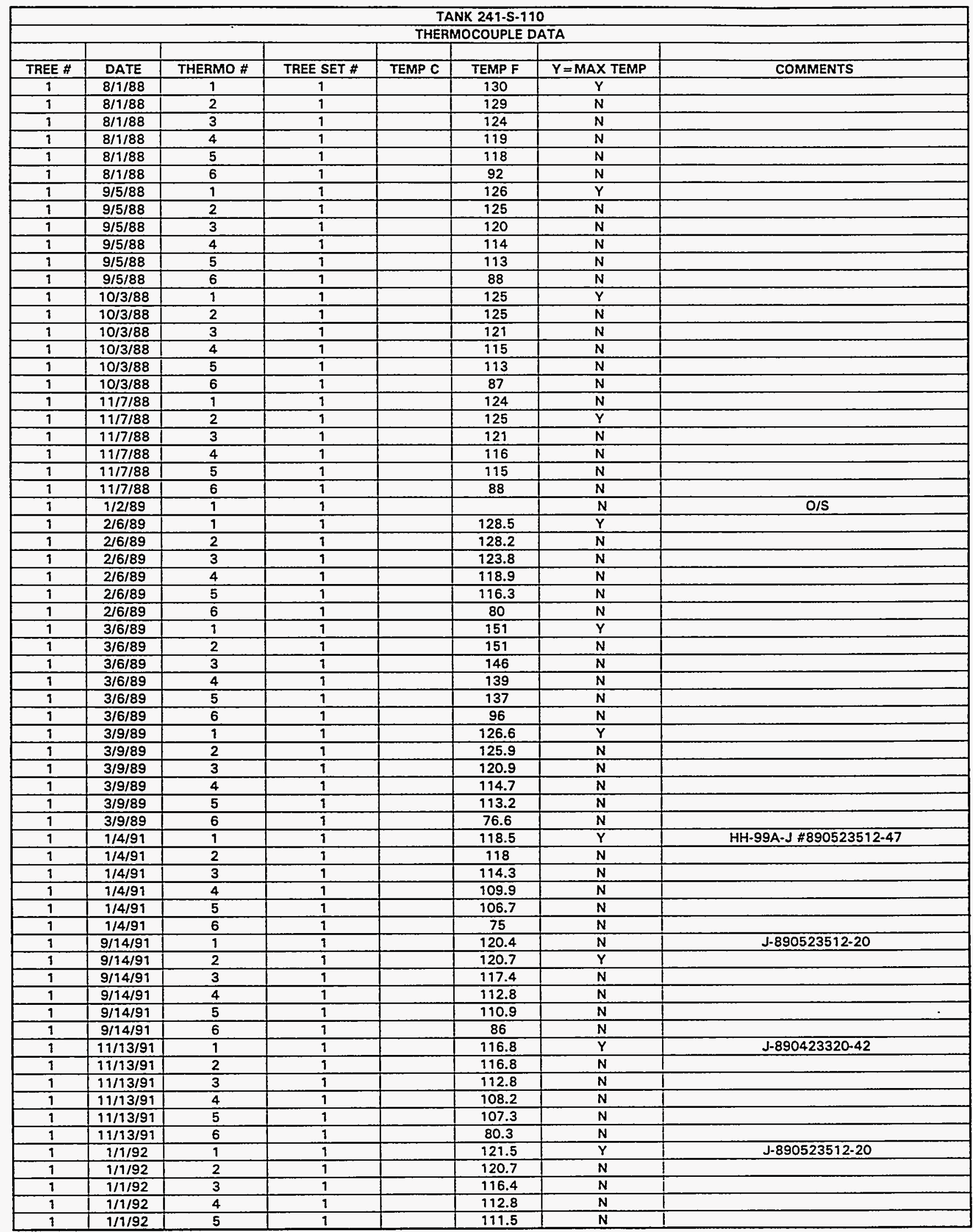

Data obtained from WHC Surveillance Analysis Computer System (SACS), February 24, 1994, Data Sheet TK-105-S to TK$110-S$, and Archive box \#121869. 
WHC-SD-WM-ER-323, Rev. 0

\begin{tabular}{|c|c|c|c|c|c|c|c|}
\hline \multicolumn{8}{|c|}{ TANK 241-S-110 } \\
\hline \multicolumn{8}{|c|}{ THERMOCOUPLE DATA } \\
\hline TREE \# & DATE & THERMO \# & TREE SET \# & TEMP C & TEMP F & $\bar{Y}=$ MAX TEMP & COMMENTS \\
\hline 1 & $1 / 1 / 92$ & 6 & 1 & & 79.1 & $\mathbf{N}$ & \\
\hline 1 & $7 / 3 / 92$ & 1 & 1 & & & $\mathbf{N}$ & OUT OF SERVICE \\
\hline 1 & $9 / 18 / 92$ & 1 & 1 & & 117.7 & N & J 890523512.47 RDGS TAKEN BY MISTAKE \\
\hline 1 & $9 / 18 / 92$ & 2 & 1 & & 118.7 & $\bar{Y}$ & \\
\hline 1 & $9 / 18 / 92$ & 3 & 1 & & 115.2 & $N$ & \\
\hline 1 & $9 / 18 / 92$ & 4 & 1 & & 111.6 & $\mathbf{N}$ & \\
\hline$T$ & $9 / 18 / 92$ & 5 & 7 & & 109.9 & $\mathbf{N}$ & \\
\hline 1 & $9 / 18 / 92$ & 6 & 1 & & 85.9 & $\mathbf{N}$ & \\
\hline 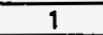 & $11 / 5 / 92$ & 1 & 7 & & 120 & $\bar{Y}$ & J $817-13-20-019$ \\
\hline 1 & $11 / 5 / 92$ & 2 & 1 & & 120 & $\mathbf{N}$ & \\
\hline 1 & $11 / 5 / 92$ & 3 & 9 & & 117 & $\bar{N}$ & \\
\hline 1 & $11 / 5 / 92$ & 4 & 1 & & 113 & $\bar{N}$ & \\
\hline 1 & $11 / 5 / 92$ & 5 & 1 & & 109 & $\mathbf{N}$ & \\
\hline 1 & $11 / 5 / 92$ & 6 & $\frac{1}{1}$ & & 85 & $\mathbf{N}$ & \\
\hline 1 & $2 / 13 / 93$ & 1 & 9 & & 117.4 & $Y$ & $J 910126143-42$ \\
\hline 1 & $2 / 13 / 93$ & 2 & 1 & & 117.2 & $\mathbf{N}$ & \\
\hline 1 & $2 / 13 / 93$ & 3 & 1 & & 113.8 & $\mathbf{N}$ & \\
\hline 1 & $2 / 13 / 93$ & 4 & 1 & & 110 & $\mathbf{N}$ & \\
\hline$T$ & $2 / 13 / 93$ & 5 & 1 & & 105.7 & $\mathbf{N}$ & \\
\hline$T$ & $2 / 13 / 93$ & 6 & $\frac{1}{1}$ & & 72.6 & $\mathbf{N}$ & \\
\hline 1 & $7 / 2 / 93$ & 1 & 1 & & 115.9 & $\bar{Y}$ & $J 890423320-42$ \\
\hline 1 & $7 / 2 / 93$ & 2 & 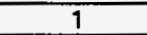 & & 115.7 & $\mathrm{~N}$ & \\
\hline 1 & $7 / 2 / 93$ & 3 & 1 & & 110.8 & $\mathrm{~N}$ & \\
\hline 1 & $7 / 2 / 93$ & 4 & 1 & & 106 & $\mathbf{N}$ & \\
\hline 1 & $7 / 2 / 93$ & 5 & 1 & & 104.7 & $\bar{N}$ & \\
\hline 1 & $7 / 2 / 93$ & 6 & 1 & & 76.2 & $\mathbf{N}$ & \\
\hline
\end{tabular}

Data obtained from WHC Surveillance Analysis Computer System (SACS), February 24, 1994, Data Sheet TK-105-S to TK$110-S$, and Archive box \#121869. 


\begin{tabular}{|c|c|c|c|c|c|c|c|}
\hline \multicolumn{8}{|c|}{ TANK 241-S-111 } \\
\hline & & - & & THEF & OCOUPLE & $\overline{T A}$ & \\
\hline TREE \# & DATE & THERMO \# & TREE SET \# & TEMP C & TEMP F & $Y=$ MAX TEMP & COMMENTS \\
\hline 1 & $12 / 2 / 74$ & 1 & & 53.33 & 127.994 & & raw data - assumed degree C \\
\hline 1 & $12 / 2 / 74$ & 2 & & 55 & 131 & & Iraw data - assumed degree $C$ \\
\hline 1 & $12 / 2 / 74$ & 3 & & 57.22 & 134.996 & & raw data - assumed degree $C$ \\
\hline 1 & $12 / 2 / 74$ & 4 & & 59.44 & 138.992 & & raw data - assumed degree C \\
\hline 1 & $12 / 2 / 74$ & 5 & & 58.89 & 138.002 & & Iraw data-assumed degree $\mathrm{C}$ \\
\hline$T$ & $12 / 2 / 74$ & 6 & & 58.33 & 136.994 & & Iraw data - assumed degree C \\
\hline 1 & $12 / 2 / 74$ & 7 & & 57.78 & 136.004 & & raw data - assumed degree $C$ \\
\hline 1 & $12 / 2 / 74$ & 8 & & 57.78 & 136.004 & & raw data - assumed degree C \\
\hline 1 & $12 / 2 / 74$ & 9 & & 57.78 & 136.004 & & Iraw data - assumed degree $C$ \\
\hline 1 & $12 / 2 / 74$ & 10 & & 56.67 & 134.006 & & raw data - assumed degree C \\
\hline 1 & $12 / 2 / 74$ & 11 & & 55 & 131 & & raw data - assumed degree C \\
\hline 1 & $12 / 2 / 74$ & 12 & & 50.56 & 123.008 & & raw data - assumed degree $C$ \\
\hline 1 & $4 / 15 / 75$ & 1 & & & 131 & & \\
\hline 1 & $4 / 15 / 75$ & 2 & & & 133 & & \\
\hline 1 & $4 / 15 / 75$ & 3 & & & 137 & & \\
\hline 1 & $4 / 15 / 75$ & 4 & & & 142 & & \\
\hline 1 & $4 / 15 / 75$ & 5 & & & 139 & & \\
\hline 1 & $4 / 15 / 75$ & 6 & & & 129 & & \\
\hline 1 & $4 / 15 / 75$ & 7 & & & 131 & & \\
\hline 1 & $4 / 15 / 75$ & 8 & & & 129 & & \\
\hline 1 & $4 / 15 / 75$ & 9 & & & 128 & & \\
\hline 1 & $4 / 15 / 75$ & 10 & & & 127 & & \\
\hline 1 & $4 / 15 / 75$ & 11 & & & 126 & & \\
\hline 1 & $4 / 15 / 75$ & 12 & & & 119 & & \\
\hline 1 & $6 / 3 / 75$ & 1 & & & 131 & & \\
\hline 1 & $6 / 3 / 75$ & 2 & & & 133 & & \\
\hline 1 & $6 / 3 / 75$ & 3 & & & 126 & & \\
\hline 1 & $6 / 3 / 75$ & 4 & & & 140 & & \\
\hline 1 & $6 / 3 / 75$ & 5 & & & 141 & & \\
\hline 1 & $6 / 3 / 75$ & 6 & & & 140 & & \\
\hline 1 & $6 / 3 / 75$ & 7 & & & 137 & & \\
\hline 1 & $6 / 3 / 75$ & 8 & & & 133 & & \\
\hline$\frac{1}{1}$ & $6 / 3 / 75$ & 9 & & & 127 & & \\
\hline 1 & $6 / 3 / 75$ & 10 & & & 127 & & \\
\hline 1 & $6 / 3 / 75$ & 11 & & & 121 & & \\
\hline 1 & $6 / 3 / 75$ & 12 & & & 104 & & \\
\hline 1 & $11 / 3 / 75$ & 1 & & & 125 & & \\
\hline 1 & $11 / 3 / 75$ & 2 & & & 107 & & \\
\hline 1 & $11 / 3 / 75$ & 3 & & & 140 & & \\
\hline 1 & $11 / 3 / 75$ & 4 & & & 145 & & \\
\hline 1 & $11 / 3 / 75$ & 5 & & & 146 & & \\
\hline 1 & $11 / 3 / 75$ & 6 & & & 144 & & \\
\hline 1 & $11 / 3 / 75$ & 7 & & & 139 & & \\
\hline 1 & $11 / 3 / 75$ & 8 & & & 133 & & \\
\hline 1 & $91 / 3 / 75$ & 9 & & & 132 & & \\
\hline 1 & $11 / 3 / 75$ & 10 & & & 130 & & \\
\hline 1 & $11 / 3 / 75$ & 11 & & & 103 & & \\
\hline 9 & $91 / 3 / 75$ & 12 & & & 104 & & \\
\hline 1 & $2 / 4 / 76$ & 1 & & & 160 & & \\
\hline 9 & $2 / 4 / 76$ & 2 & & & 161 & & \\
\hline 9 & $2 / 4 / 76$ & 3 & & & 165 & & \\
\hline 9 & $2 / 4 / 76$ & 4 & & & 169 & & \\
\hline 1 & $2 / 4 / 76$ & 5 & & & 169 & & \\
\hline 1 & $2 / 4 / 76$ & 6 & & & 166 & & \\
\hline 1 & $2 / 4 / 76$ & 7 & & & 160 & & \\
\hline 1 & $2 / 4 / 76$ & 8 & & & 152 & & \\
\hline 9 & $2 / 4 / 76$ & 9 & & & 152 & & \\
\hline 1 & $2 / 4 / 76$ & 10 & & & 152 & & \\
\hline 1 & $2 / 4 / 76$ & 11 & & & 150 & & \\
\hline 1 & $2 / 4 / 76$ & 12 & & & 128 & & \\
\hline 1 & $3 / 5 / 76$ & 1 & & & 132 & & \\
\hline 9 & $3 / 5 / 76$ & 2 & & & 933 & & \\
\hline 1 & $3 / 5 / 76$ & 3 & & & 137 & & \\
\hline 1 & $3 / 5 / 76$ & 4 & & & 146 & & \\
\hline 1 & $3 / 5 / 76$ & 5 & & & 139 & & \\
\hline 1 & $3 / 5 / 76$ & 6 & & & 136 & & \\
\hline
\end{tabular}

Data obtained from WHC Surveillance Analysis Computer System (SACS), February 24, 1994. 
WHC-SD-WM-ER-323, Rev. 0

\begin{tabular}{|c|c|c|c|c|c|c|c|}
\hline \multicolumn{8}{|c|}{ TANK 241-S-111 } \\
\hline & & & & THER & DCOUPLE & TA & \\
\hline TREE \# & DATE & THERMO \# & TREE SET \# & TEMP C & TEMP F & $Y=$ MAX TEMP & COMMENTS \\
\hline 1 & $3 / 5 / 76$ & 7 & & & 136 & & \\
\hline 1 & $3 / 5 / 76$ & 8 & & & 124 & & \\
\hline 1 & $3 / 5 / 76$ & 9 & & & 124 & & \\
\hline 1 & $3 / 5 / 76$ & 10 & & & 118 & & \\
\hline 1 & $3 / 5 / 76$ & 19 & & & 95 & & \\
\hline 1 & $5 / 2 / 76$ & 1 & & & 132 & & \\
\hline 1 & $5 / 2 / 76$ & 2 & & & 133 & & \\
\hline 1 & $5 / 2 / 76$ & $\overline{3}$ & & & 136 & & \\
\hline 1 & $5 / 2 / 76$ & 4 & & & 138 & & \\
\hline 1 & $5 / 2 / 76$ & 5 & & & 134 & & \\
\hline 1 & $5 / 2 / 76$ & 6 & & & 129 & & \\
\hline 1 & $5 / 2 / 76$ & 7 & & & 120 & & \\
\hline 1 & $5 / 2 / 76$ & 8 & & & 121 & & \\
\hline 1 & $5 / 2 / 76$ & 9 & & & 96 & & \\
\hline 1 & $5 / 2 / 76$ & 10 & & & 92 & & \\
\hline 1 & $5 / 2 / 76$ & 11 & & & 92 & & \\
\hline 1 & $5 / 2 / 76$ & 12 & & & 92 & & \\
\hline 1 & $6 / 1 / 76$ & 1 & & & 130 & & \\
\hline 1 & $6 / 1 / 76$ & 2 & & & 132 & & \\
\hline 1 & $6 / 1 / 76$ & 3 & & & 134 & & \\
\hline 1 & $6 / 1 / 76$ & 4 & & & 134 & & \\
\hline 1 & $6 / 1 / 76$ & 5 & & & 132 & & \\
\hline 1 & $6 / 1 / 76$ & 6 & & & 136 & & \\
\hline 1 & $6 / 1 / 76$ & 7 & & & 128 & & \\
\hline 1 & $6 / 1 / 76$ & 8 & & & 126 & & \\
\hline 1 & $6 / 1 / 76$ & 9 & & & 104 & & \\
\hline 1 & $6 / 1 / 76$ & 10 & & & 92 & & \\
\hline 1 & $6 / 1 / 76$ & 11 & & & 92 & & \\
\hline 1 & $6 / 1 / 76$ & 12 & & & 92 & & \\
\hline 1 & $6 / 1 / 76$ & 13 & & & 92 & & \\
\hline 1 & $6 / 1 / 76$ & 14 & & & 92 & & \\
\hline 1 & $7 / 1 / 76$ & 1 & & & 924 & & \\
\hline 1 & $7 / 1 / 76$ & 2 & & & 125 & & \\
\hline 1 & $7 / 1 / 76$ & 3 & & & 127 & & \\
\hline 1 & $7 / 1 / 76$ & 4 & & & 128 & & \\
\hline$T$ & $7 / 1 / 76$ & 5 & & & 126 & & \\
\hline 1 & $7 / 1 / 76$ & 6 & & & 124 & & \\
\hline 1 & $7 / 1176$ & 7 & & & 121 & & \\
\hline 1 & $7 / 1 / 76$ & 8 & & & 119 & & \\
\hline 1 & $7 / 1 / 76$ & 9 & & & 103 & & \\
\hline 1 & $7 / 1 / 76$ & 10 & & & 89 & & \\
\hline 9 & $7 / 1 / 76$ & .11 & & & 88 & & \\
\hline 1 & $7 / 1 / 76$ & 12 & & & 88 & & \\
\hline 1 & $8 / 3 / 76$ & 1 & & & 130 & & \\
\hline 1 & $8 / 3 / 76$ & 2 & & & 131 & & \\
\hline 1 & $8 / 3 / 76$ & 3 & & & 133 & & \\
\hline 1 & $8 / 3 / 76$ & 4 & & & 134 & & \\
\hline 1 & $8 / 3 / 76$ & 5 & & & 133 & & \\
\hline 1 & $8 / 3 / 76$ & 6 & & & 130 & & \\
\hline 1 & $8 / 3 / 76$ & 7 & & & 126 & & \\
\hline 1 & $8 / 3 / 76$ & 8 & & & 124 & & \\
\hline 1 & $8 / 3 / 76$ & 9 & & & 112 & & \\
\hline 1 & $8 / 3 / 76$ & 10 & & & 98 & & \\
\hline 1 & $8 / 3 / 76$ & 11 & & & 98 & & \\
\hline 1 & $8 / 3 / 76$ & 12 & & & 98 & & \\
\hline 1 & $9 / 2 / 76$ & 1 & & & 130 & & \\
\hline 1 & $9 / 2 / 76$ & 2 & & & 132 & & \\
\hline 1 & $9 / 2 / 76$ & 3 & & & 134 & & \\
\hline 1 & $9 / 2 / 76$ & 4 & & & 135 & & \\
\hline 1 & $9 / 2 / 76$ & 5 & & & 134 & & \\
\hline$\frac{1}{1}$ & $9 / 2 / 76$ & 6 & & & 131 & & \\
\hline 1 & $9 / 2 / 76$ & 7 & & & 127 & & \\
\hline 9 & $9 / 2 / 76$ & 8 & & & 124 & & \\
\hline 9 & $9 / 2 / 76$ & 9 & & & 118 & & \\
\hline 1 & $9 / 2 / 76$ & 10 & & & 101 & & \\
\hline 1 & $9 / 2 / 76$ & 11 & & & 100 & & \\
\hline
\end{tabular}

Data obțained from WHC Surveillance Analysis Computer System (SACS), February 24, 1994. 


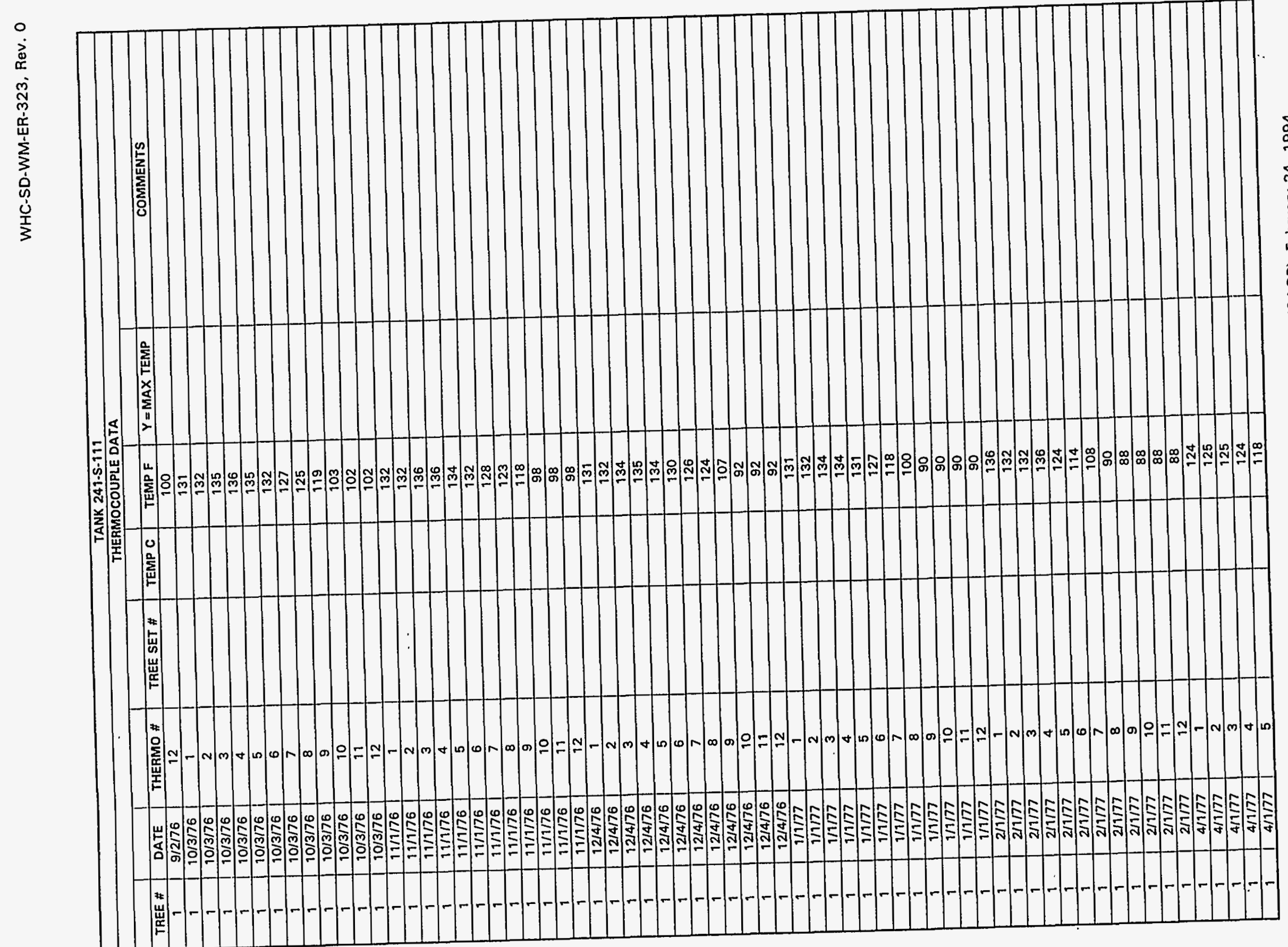




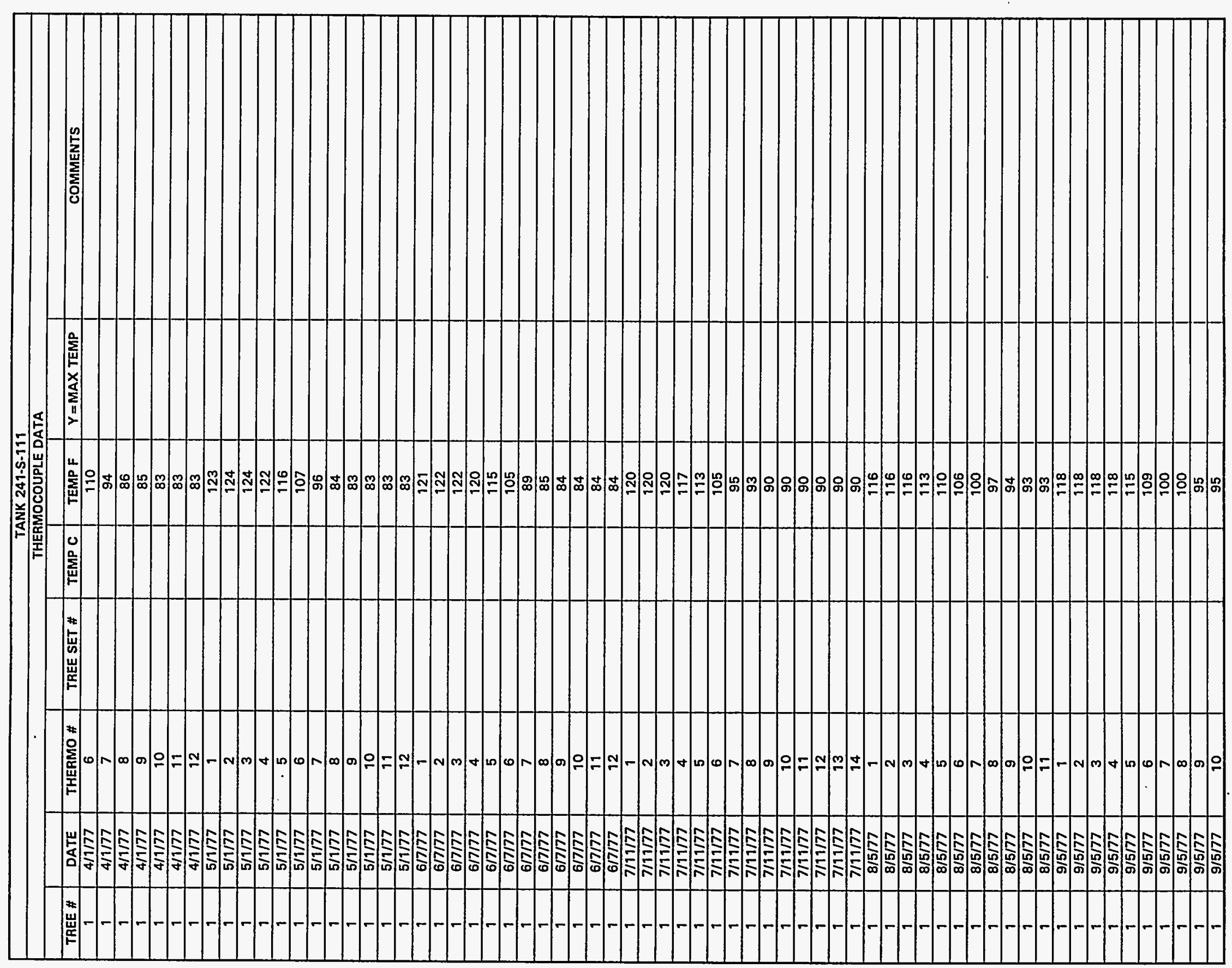

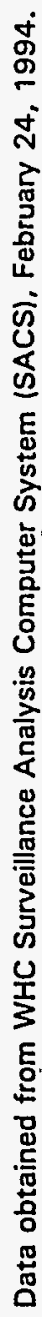


WHC-SD-WM-ER-323, Rev. 0

\begin{tabular}{|c|c|c|c|c|c|c|c|}
\hline \multicolumn{8}{|c|}{ TANK 241-S-111 } \\
\hline & & & & THEF & $\overline{\text { DCOUPLE }}$ & TA & \\
\hline TREE \# & DATE & THERMO \# & TREE SET \# & TEMP C & TEMP F & $Y=$ MAX TEMP & COMMENTS \\
\hline 1 & $9 / 5 / 77$ & 19 & & & 95 & & \\
\hline 1 & $9 / 5 / 77$ & 12 & & & 95 & & \\
\hline 1 & $9 / 5 / 77$ & 13 & & & 95 & & \\
\hline 1 & $9 / 5 / 77$ & 14 & & & 95 & & \\
\hline 1 & $10 / 3 / 77$ & 1 & & & 118 & & \\
\hline 1 & $10 / 3 / 77$ & 2 & & & 119 & & \\
\hline 1 & $10 / 3 / 77$ & 3 & & & 119 & & \\
\hline 1 & $10 / 3 / 77$ & 4 & & & 118 & & \\
\hline 1 & $10 / 3 / 77$ & 5 & & & 115 & & \\
\hline 1 & $10 / 3 / 77$ & 6 & & & 110 & & \\
\hline 1 & $10 / 3 / 77$ & 7 & & & 101 & & \\
\hline 1 & $10 / 3 / 77$ & 8 & & & 97 & & \\
\hline 1 & $10 / 3 / 77$ & 9 & & & 95 & & \\
\hline 1 & $10 / 3 / 77$ & 10 & & & 95 & & \\
\hline 1 & $10 / 3 / 77$ & 11 & & & 95 & & \\
\hline 1 & $10 / 3 / 77$ & 12 & & & 95 & & \\
\hline 1 & $11 / 3 / 77$ & 1 & & & 117 & & \\
\hline 1 & $11 / 3 / 77$ & 2 & & & 117 & & \\
\hline 1 & $11 / 3 / 77$ & 3 & & & 117 & & \\
\hline 1 & $11 / 3 / 77$ & 4 & & & 117 & & \\
\hline 1 & $11 / 3 / 77$ & 5 & & & 114 & & \\
\hline 1 & $11 / 3 / 77$ & 6 & & & 109 & & \\
\hline 1 & $11 / 3 / 77$ & 7 & & & 100 & & \\
\hline 1 & $111 / 3 / 77$ & 8 & & & 95 & & \\
\hline 1 & $911 / 3 / 77$ & 9 & & & 90 & & \\
\hline 1 & $11 / 3 / 77$ & 10 & & & 90 & & \\
\hline 1 & $11 / 3 / 77$ & 11 & & & 90 & & \\
\hline 1 & $919 / 3 / 77$ & 12 & & & 90 & & \\
\hline 1 & $11 / 3 / 77$ & 13 & & & 90 & & \\
\hline 1 & $11 / 3 / 77$ & 14 & & & 90 & & \\
\hline 1 & $12 / 4 / 77$ & 1 & & & 113 & & \\
\hline 1 & $12 / 4 / 77$ & 2 & & & 113 & & \\
\hline 1 & $12 / 4 / 77$ & 3 & & & 113 & & \\
\hline 1 & $12 / 4 / 77$ & 4 & & & 111 & & \\
\hline 1 & $12 / 4 / 77$ & 5 & & & 106 & & \\
\hline 1 & $12 / 4 / 77$ & 6 & & & 98 & & \\
\hline 1 & $12 / 4 / 77$ & 7 & & & 86 & & \\
\hline 1 & $12 / 4 / 77$ & 8 & & & 77 & & \\
\hline 1 & $12 / 4 / 77$ & 9 & & & 73 & & \\
\hline 1 & $12 / 4 / 77$ & 10 & & & 71 & & \\
\hline 1 & $12 / 4 / 77$ & 11 & & & 79 & & \\
\hline 1 & $12 / 4 / 77$ & 12 & & & 68 & & \\
\hline 1 & $12 / 4 / 77$ & 13 & & & 67 & & \\
\hline 1 & $12 / 4 / 77$ & 14 & & & 66 & & \\
\hline 1 & $1 / 8 / 78$ & 1 & & & & & 136 - suspect data (high) \\
\hline 1 & $1 / 8 / 78$ & 2 & & & & & 137 - suspect data (high) \\
\hline 1 & $1 / 8 / 78$ & 3 & & & & & 136 - suspect data (high) \\
\hline 1 & $1 / 8 / 78$ & 4 & & & & & 134 - suspect data (high) \\
\hline 1 & $1 / 8 / 78$ & 5 & & & & & 131 - suspect data (high) \\
\hline 1 & $1 / 8 / 78$ & 6 & & & & & 126 - suspect data (high) \\
\hline 1 & $1 / 8 / 78$ & 7 & & & & & 119 - suspect data (high) \\
\hline 1 & $1 / 8 / 78$ & 8 & & & & & 116 - suspect data (high) \\
\hline 1 & $1 / 8 / 78$ & 9 & & & & & 114 - suspect data (high) \\
\hline 1 & $1 / 8 / 78$ & 10 & & - & & & 114 - suspect data (high) \\
\hline 1 & $1 / 8 / 78$ & 11 & & & & & 114 - suspect data (high) \\
\hline 1 & $1 / 8 / 78$ & 12 & & & & & 114 - suspect data (high) \\
\hline 1 & $1 / 8 / 78$ & 13 & & & & & 114 - suspect data (high) \\
\hline 1 & $1 / 8 / 78$ & 14 & & & & & 114 - suspect data (high) \\
\hline 1 & $1 / 11 / 78$ & 1 & & & 116 & & \\
\hline 1 & $1 / 11 / 78$ & 2 & & & 117 & & \\
\hline 1 & $1 / 11 / 78$ & 3 & & & 116 & & \\
\hline 1 & $1 / 19 / 78$ & 4 & & & 114 & & \\
\hline 1 & $1 / 91 / 78$ & 5 & & & 111 & & \\
\hline 1 & $1 / 11 / 78$ & 6 & & & 106 & & \\
\hline 1 & $1 / 11 / 78$ & 7 & & & 100 & & \\
\hline 1 & $1 / 11 / 78$ & 8 & & & 97 & & \\
\hline
\end{tabular}

Data obtained from WHC Surveillance Analysis Computer System (SACS), February 24, 1994. 


\begin{tabular}{|c|c|c|c|c|c|c|c|}
\hline \multicolumn{8}{|c|}{ TANK 241-S-111 } \\
\hline & & & & THEF & OCOUPLE & TA & \\
\hline TREE \# & DATE & THERMO \# & TREE SET \# & TEMP C & TEMP F & $Y=$ MAX TEMP & COMMENTS \\
\hline 1 & $1 / 11 / 78$ & 9 & & & 95 & & \\
\hline$\frac{1}{1}$ & $1 / 11 / 78$ & 10 & & & 95 & & \\
\hline 1 & $1 / 11 / 78$ & 19 & & & 95 & & \\
\hline 1 & $1 / 11 / 78$ & 12 & & & 95 & & \\
\hline 1 & $1 / 19 / 78$ & 13 & & & 95 & & \\
\hline 1 & $1 / 11 / 78$ & 14 & & & 95 & & \\
\hline 1 & $1 / 13 / 78$ & 1 & & & 117 & & \\
\hline 1 & $1 / 13 / 78$ & 2 & & & 117 & & \\
\hline 1 & $1 / 13 / 78$ & 3 & & & 117 & & \\
\hline 1 & $1 / 13 / 78$ & 4 & & & 115 & & \\
\hline$\frac{1}{1}$ & $1 / 13 / 78$ & .5 & & & 110 & & \\
\hline 1 & $1 / 13 / 78$ & 6 & & & 106 & & \\
\hline 1 & $1 / 13 / 78$ & 7 & & & 101 & & \\
\hline 1 & $1 / 13 / 78$ & 8 & & & 98 & & \\
\hline 1 & $1 / 13 / 78$ & 9 & & & 95 & & \\
\hline 1 & $1 / 13 / 78$ & 10 & & & 95 & & \\
\hline 1 & $1 / 13 / 78$ & 11 & & & 96 & & \\
\hline 1 & $2 / 7 / 78$ & 1 & & & 119 & & \\
\hline 1 & $2 / 7 / 78$ & 2 & & & 120 & & \\
\hline 1 & $2 / 7 / 78$ & 3 & & & 126 & & \\
\hline 1 & $2 / 7 / 78$ & 4 & & & 118 & & \\
\hline$\frac{1}{1}$ & $2 / 7 / 78$ & 5 & & & 114 & & \\
\hline$\frac{1}{1}$ & $2 / 7 / 78$ & 6 & & & 1111 & & \\
\hline 1 & $2 / 7 / 78$ & 7 & & & 103 & & \\
\hline 1 & $2 / 7 / 78$ & 8 & & & 98 & & \\
\hline 1 & $2 / 7 / 78$ & 9 & & & 96 & & \\
\hline 1 & $2 / 7 / 78$ & 10 & & & 96 & & \\
\hline 1 & $2 / 7 / 78$ & 11 & & & 96 & & \\
\hline 1 & $2 / 7 / 78$ & 12 & & & 95 & & \\
\hline 1 & $2 / 7 / 78$ & 13 & & & 95 & & \\
\hline 1 & $2 / 7 / 78$ & 14 & & & 95 & & \\
\hline 9 & $3 / 12 / 78$ & 1 & & & 923 & & \\
\hline 1 & $3 / 12 / 78$ & 2 & & & 113 & & \\
\hline 1 & $3 / 12 / 78$ & 3 & & & 123 & & \\
\hline 7 & $3 / 12 / 78$ & 4 & & & 921 & & \\
\hline 1 & $3 / 12 / 78$ & 5 & & & 118 & & \\
\hline 1 & $3 / 12 / 78$ & 6 & & & 119 & & \\
\hline 1 & $3 / 12 / 78$ & 7 & & & 106 & & \\
\hline 9 & $3 / 12 / 78$ & 8 & & & 98 & & \\
\hline 1 & $3 / 12 / 78$ & 9 & & & 97 & & \\
\hline 1 & $3 / 12 / 78$ & 10 & & & 95 & & \\
\hline 1 & $3 / 12 / 78$ & 11 & & & 95 & & \\
\hline 1 & $3 / 12 / 78$ & 12 & & & 95 & & \\
\hline 9 & $3 / 12 / 78$ & 13 & & & 95 & & \\
\hline 1 & $3 / 12 / 78$ & 14 & & & 95 & & \\
\hline 9 & $4 / 11 / 78$ & 1 & & & 174 & & \\
\hline 1 & $4 / 11 / 78$ & 2 & & & 114 & & \\
\hline 1 & $4 / 11 / 78$ & 3 & & & 114 & & \\
\hline 9 & $4 / 11 / 78$ & 4 & & & 113 & & \\
\hline 1 & $4 / 11 / 78$ & 5 & & & 108 & & \\
\hline 9 & $4 / 11 / 78$ & 6 & & & 103 & & \\
\hline 9 & $4 / 11 / 78$ & 7 & & & 94 & & \\
\hline 9 & $4 / 11 / 78$ & 8 & & & 87 & & \\
\hline 1 & $4 / 11 / 78$ & 9 & & & 85 & & \\
\hline 1 & $4 / 11 / 78$ & 10 & & & 84 & & \\
\hline$\frac{1}{1}$ & $4 / 19 / 78$ & 19 & & & 84 & & \\
\hline 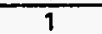 & $4 / 11 / 78$ & 12 & & & 84 & & \\
\hline 1 & $4 / 11 / 78$ & 13 & & & 84 & & \\
\hline$\frac{1}{9}$ & $4 / 19 / 78$ & 14 & & & 84 & & \\
\hline 1 & $6 / 3 / 78$ & .1 & & & 114 & & \\
\hline 1 & $6 / 3 / 78$ & 2 & & & 115 & & \\
\hline 9 & $6 / 3 / 78$ & 3 & & & 115 & & \\
\hline 9 & $6 / 3 / 78$ & 4 & & & 112 & & \\
\hline 1 & $6 / 3 / 78$ & 5 & & & 107 & & \\
\hline 1 & $6 / 3 / 78$ & 6 & & & 100 & & \\
\hline 9 & $6 / 3 / 78$ & 7 & & & 94 & & \\
\hline
\end{tabular}

Data obtained from WHC Surveillance Analysis Computer System (SACS), February 24, 1994. 


\begin{tabular}{|c|c|c|c|c|c|c|c|}
\hline \multicolumn{8}{|c|}{ TANK 241.S.111 } \\
\hline \multicolumn{8}{|c|}{ THERMOCOUPLE DATA } \\
\hline TREE \# & DATE & THERMO \# & TREE SET \# & TEMP C & TEMP F & $Y=M A X$ TEMP & COMMENTS \\
\hline 1 & $6 / 3 / 78$ & 8 & & & 88 & & \\
\hline 1 & $6 / 3 / 78$ & 9 & & & 87 & & \\
\hline 1 & $6 / 3 / 78$ & 10 & & & 87 & & \\
\hline 1 & $6 / 3 / 78$ & 11 & & & 87 & & \\
\hline 1 & $6 / 3 / 78$ & 12 & & & 87 & & \\
\hline 1 & $6 / 3 / 78$ & 13 & & & 87 & & \\
\hline 1 & $6 / 3 / 78$ & 14 & & & 87 & & \\
\hline 1 & $7 / 13 / 78$ & 1 & & & 115 & & \\
\hline 1 & $7 / 13 / 78$ & 2 & & & 115 & & \\
\hline 1 & $7 / 13 / 78$ & 3 & & & 115 & & \\
\hline 1 & $7 / 13 / 78$ & 4 & & & 112 & & \\
\hline 1 & $7 / 13 / 78$ & 5 & & & 108 & & \\
\hline 1 & $7 / 13 / 78$ & 6 & & & 103 & & \\
\hline 1 & $7 / 13 / 78$ & 7 & & & 96 & & \\
\hline 1 & $7 / 13 / 78$ & 8 & & & 91 & & \\
\hline 1 & $7 / 13 / 78$ & 9 & & & 91 & & \\
\hline 9 & $7 / 13 / 78$ & 10 & & & 90 & & \\
\hline 1 & $7 / 13 / 78$ & 91 & & & 90 & & \\
\hline 1 & $7 / 13 / 78$ & 12 & & & 90 & & \\
\hline 1 & $7 / 13 / 78$ & 13 & & & 90 & & \\
\hline 9 & $7 / 13 / 78$ & 94 & & & 90 & & \\
\hline 1 & $8 / 11 / 78$ & 1 & & & 108 & & \\
\hline 1 & $8 / 11 / 78$ & $\overline{2}$ & & & 108 & & \\
\hline 1 & $8 / 11 / 78$ & 3 & & & 108 & & \\
\hline 1 & $8 / 11 / 78$ & 4 & & & 106 & & \\
\hline 1 & $8 / 11 / 78$ & 5 & & & 102 & & \\
\hline 1 & $8 / 19 / 78$ & 6 & & & 98 & & \\
\hline 1 & $8 / 11 / 78$ & 7 & & & 92 & & \\
\hline 1 & $8 / 11 / 78$ & 8 & & & 85 & & \\
\hline 1 & $8 / 11 / 78$ & 9 & & & 85 & & \\
\hline 1 & $8 / 11 / 78$ & 10 & & & 85 & & \\
\hline 1 & $8 / 11 / 78$ & 11 & & & 84 & & \\
\hline 1 & $8 / 11 / 78$ & 12 & & & 84 & & \\
\hline 1 & $8 / 11 / 78$ & 13 & & & 84 & & \\
\hline 1 & $8 / 11 / 78$ & 14 & & & 84 & & \\
\hline 1 & $2 / 4 / 79$ & 1 & & & 106 & & \\
\hline 1 & $2 / 4 / 79$ & 2 & & & 107 & & \\
\hline 1 & $2 / 4 / 79$ & 3 & & & 107 & & \\
\hline 1 & $2 / 4 / 79$ & 4 & & & 104 & & \\
\hline 1 & $2 / 4 / 79$ & 5 & & & 100 & & \\
\hline 1 & $2 / 4 / 79$ & 6 & & & 95 & & \\
\hline 1 & $2 / 4 / 79$ & 7 & & & 86 & & \\
\hline 1 & $2 / 4 / 79$ & 8 & & & 72 & & \\
\hline 1 & $2 / 4 / 79$ & 9 & & & 70 & & \\
\hline 1 & $2 / 4 / 79$ & 10 & & & 68 & & \\
\hline$\frac{1}{1}$ & $2 / 4 / 79$ & 11 & & & 68 & & \\
\hline 1 & $2 / 4 / 79$ & 12 & & & 68 & & \\
\hline 1 & $2 / 4 / 79$ & 13 & & & 68 & & \\
\hline 1 & $2 / 4 / 79$ & 14 & & & 68 & & \\
\hline 9 & $9 / 6 / 79$ & 9 & & & 108 & & \\
\hline 1 & $9 / 6 / 79$ & 2 & & & 108 & & \\
\hline 9 & $9 / 6 / 79$ & 3 & & & 108 & & \\
\hline 9 & $9 / 6 / 79$ & 4 & & & 105 & & \\
\hline 1 & $9 / 6 / 79$ & 5 & & & 100 & & \\
\hline 1 & $9 / 6 / 79$ & 6 & & & 95 & & \\
\hline 1 & $9 / 6 / 79$ & 7 & & & 90 & & \\
\hline 9 & $9 / 6 / 79$ & 8 & & & 85 & & \\
\hline 1 & $9 / 6 / 79$ & 9 & & & 85 & & \\
\hline 1 & $9 / 6 / 79$ & 10 & & & 85 & & \\
\hline 1 & $9 / 6 / 79$ & 91 & & & 85 & & \\
\hline 1 & $9 / 6 / 79$ & 12 & & & 85 & & \\
\hline 1 & $9 / 6 / 79$ & 13 & & & 85 & & \\
\hline 7 & $9 / 6 / 79$ & 14 & & & 85 & & \\
\hline 1 & $1 / 23 / 80$ & 1 & & & 105 & & \\
\hline 1 & $1 / 23 / 80$ & 2 & & & 105 & & \\
\hline 1 & $1 / 23 / 80$ & 3 & & & 105 & & \\
\hline
\end{tabular}

Data obtained from WHC Surveillance Analysis Computer System (SACS), February 24, 1994. 
TANK 241-S-111

THERMOCOUPLE DATA

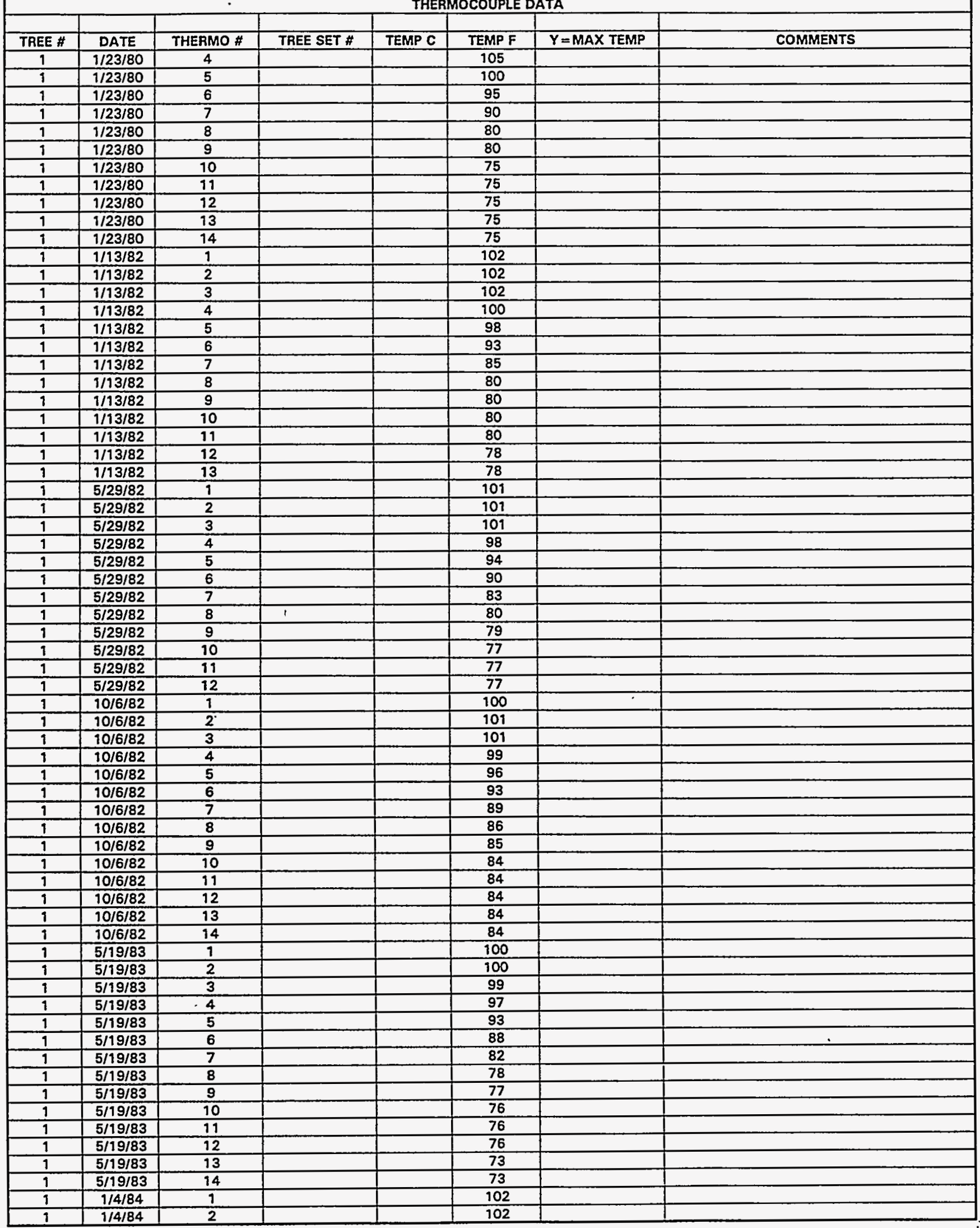

Data obtained from WHC Surveillance Analysis Computer System (SACS), February 24, 1994. 


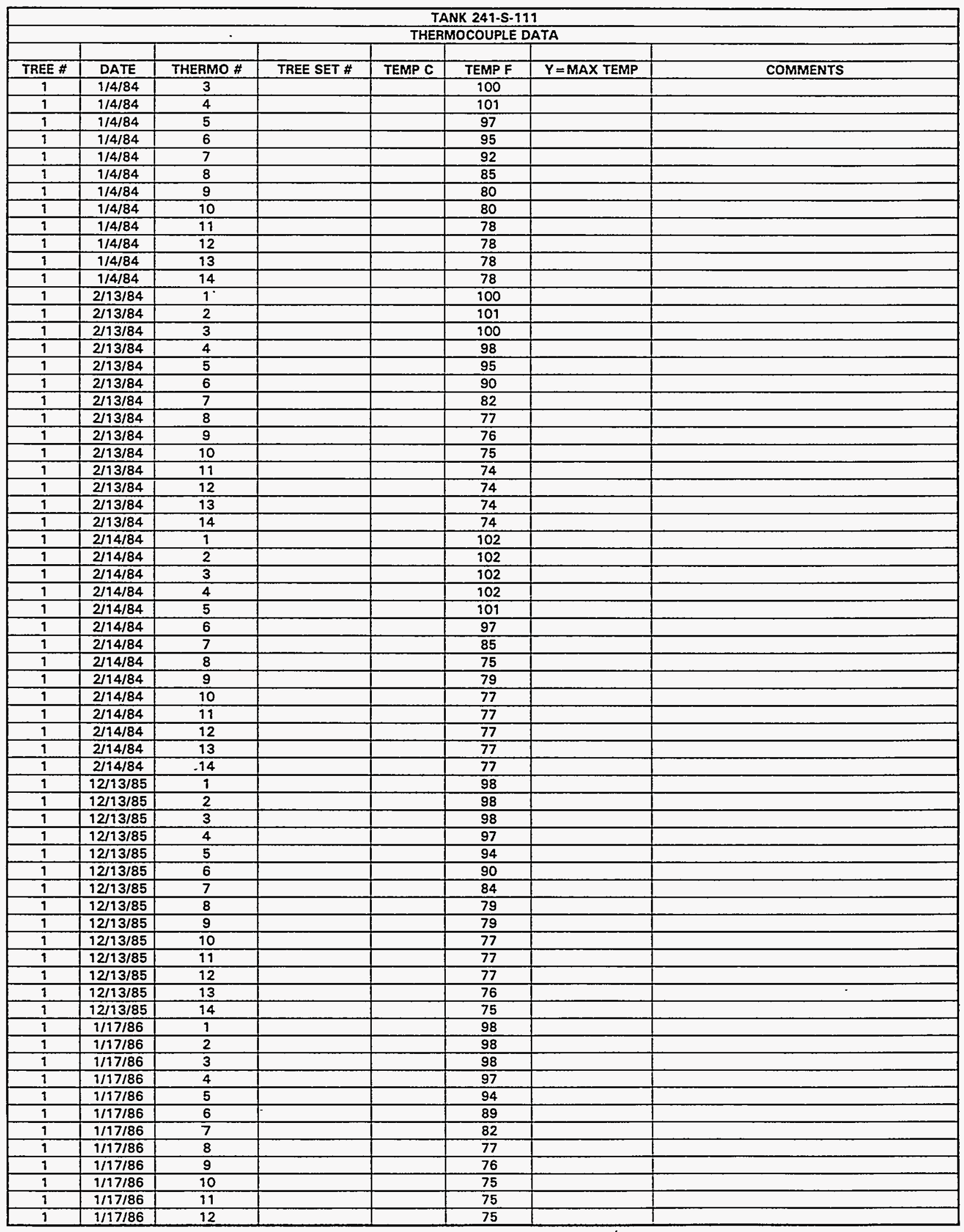

Data obtained from WHC Surveillance Analysis Computer System (SACS), February 24, 1994. 


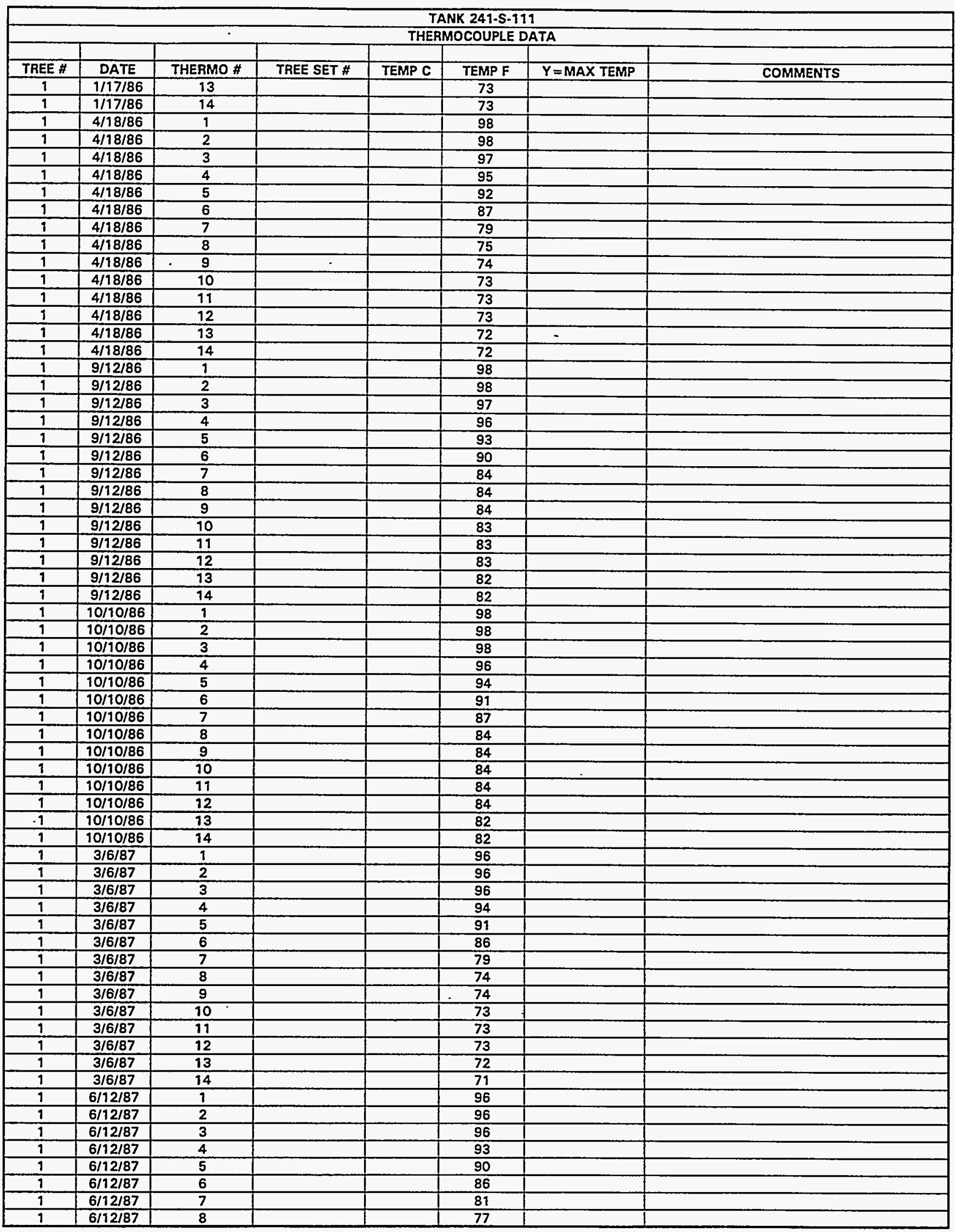

Data obtained from WHC Surveillance Analysis Computer System (SACS), February 24, 1994. 


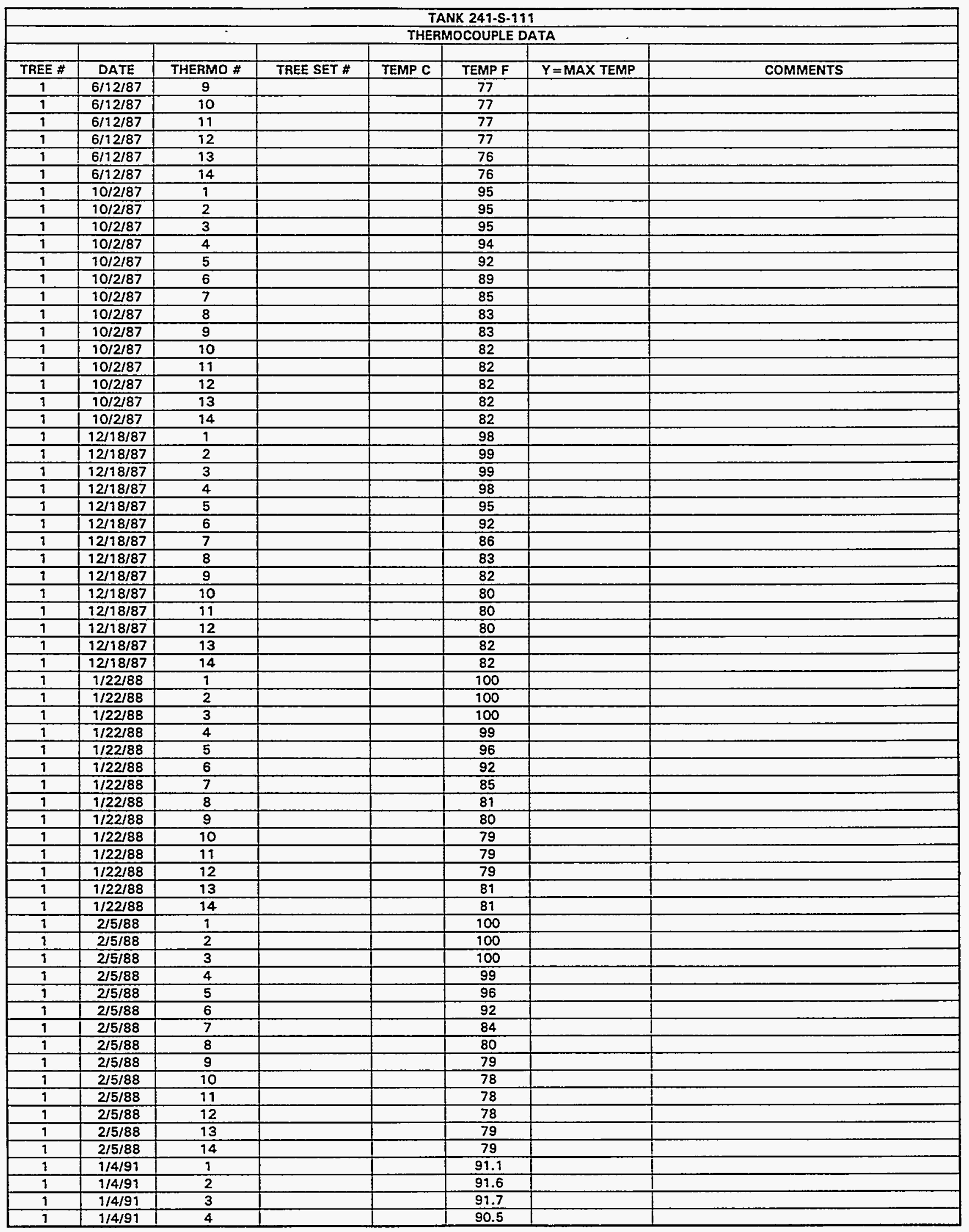

Data obtained from WHC Surveillance_Analysis Computer System (SACS), February 24, 1994. 


\begin{tabular}{|c|c|c|c|c|c|c|c|}
\hline \multicolumn{8}{|c|}{ TANK 241-S-111 } \\
\hline \multicolumn{8}{|c|}{ THERMOCOUPLE DATA } \\
\hline & & & & & & & \\
\hline TREE \# & DATE & THERMO \# & TREE SET \# & TEMP C & TEMP F & $Y=$ MAX TEMP & COMMENTS \\
\hline 1 & $1 / 4 / 91$ & 5 & & & 88.3 & & \\
\hline 1 & $1 / 4 / 91$ & 6 & & & 84.8 & & \\
\hline 1 & $1 / 4 / 91$ & 7 & & & 78.4 & & \\
\hline 9 & $1 / 4 / 91$ & 8 & & & 74.2 & & \\
\hline 1 & $1 / 4 / 91$ & 9 & & & 73.9 & & \\
\hline 1 & $1 / 4 / 91$ & 10 & & & 72.6 & & \\
\hline$\frac{1}{1}$ & $1 / 4 / 91$ & 11 & & & 72.5 & & \\
\hline 1 & $1 / 4 / 91$ & 12 & & & 72.3 & & \\
\hline 1 & $1 / 4 / 91$ & 13 & & & 72.3 & & \\
\hline$\frac{1}{1}$ & $1 / 4 / 91$ & 14 & & & 72.3 & & \\
\hline 1 & $2 / 9 / 91$ & 1 & & & 91.9 & & \\
\hline 1 & $2 / 9 / 91$ & 2 & & & 92.2 & & \\
\hline 1 & $2 / 9 / 91$ & 3 & & & 92.1 & & \\
\hline 1 & $2 / 9 / 91$ & 4 & & & 90.7 & & \\
\hline 1 & $2 / 9 / 91$ & 5 & & & 88 & & \\
\hline 1 & $2 / 9 / 91$ & 6 & & & 83.7 & & \\
\hline 1 & $2 / 9 / 91$ & 7 & & & 76.8 & & \\
\hline$T$ & $2 / 9 / 91$ & 8 & & & 72.2 & & \\
\hline 1 & $2 / 9 / 91$ & 9 & & & 71.5 & & \\
\hline 1 & $2 / 9 / 91$ & 10 & & & 70.2 & & \\
\hline 1 & $2 / 9 / 91$ & 71 & & & 70.1 & & \\
\hline 1 & $2 / 9 / 91$ & 12 & & & 70.1 & & \\
\hline 1 & $2 / 9 / 91$ & 13 & & & 70 & & \\
\hline 1 & $2 / 9 / 91$ & 14 & & & 70 & & \\
\hline 1 & $2 / 17 / 91$ & 1 & & & 91.7 & & \\
\hline 1 & $2 / 17 / 91$ & 2 & & & 92.1 & & \\
\hline 1 & $2 / 17 / 91$ & 3 & & & 92 & & \\
\hline 1 & $2 / 17 / 91$ & 4 & & & 90.6 & & \\
\hline 1 & $2 / 17 / 91$ & 5 & & & 87.8 & & \\
\hline 1 & $2 / 17 / 91$ & 6 & & & 83.4 & & \\
\hline 1 & $2 / 17 / 91$ & 7 & & & 76.7 & & \\
\hline 1 & $2 / 17 / 91$ & 8 & & & 72 & & \\
\hline 1 & $2 / 17 / 91$ & 9 & & & 71.5 & & \\
\hline 1 & $2 / 17 / 91$ & 10 & & & 70.3 & & \\
\hline 1 & $2 / 17 / 91$ & 11 & & & 70 & & \\
\hline 1 & $2 / 17 / 91$ & 12 & & & 70 & & \\
\hline$\frac{1}{1}$ & $2 / 17 / 91$ & 13 & & & 69.9 & & \\
\hline 1 & $2 / 17 / 91$ & 14 & & & 69.9 & & \\
\hline 1 & $2 / 24 / 91$ & 1 & & & 95 & & \\
\hline 1 & $2 / 24 / 91$ & 2 & & & 95 & & $\cdot$ \\
\hline 1 & $2 / 24 / 91$ & 3 & & & 95 & & \\
\hline 1 & $2 / 24 / 91$ & 4 & & & 93 & & \\
\hline 1 & $2 / 24 / 91$ & 5 & & & 91 & & \\
\hline 1 & $2 / 24 / 91$ & 6 & & & 86 & & \\
\hline 1 & $2 / 24 / 91$ & 7 & & & 79 & & \\
\hline 1 & $2 / 24 / 91$ & 8 & & & 75 & & \\
\hline 1 & $2 / 24 / 91$ & 9 & & & 74 & & \\
\hline 1 & $2 / 24 / 91$ & 10 & & & 73 & & \\
\hline 1 & $2 / 24 / 91$ & 11 & & & 73 & & \\
\hline 1 & $2 / 24 / 91$ & 12 & & & 73 & & \\
\hline 9 & $2 / 24 / 91$ & 13 & & & 73 & & \\
\hline 1 & $2 / 24 / 91$ & 14 & & & 73 & & \\
\hline$\frac{T}{1}$ & $3 / 3 / 91$ & 1 & & & 96 & & \\
\hline 9 & $3 / 3 / 91$ & 2 & & & 96 & & \\
\hline 1 & $3 / 3 / 91$ & 3 & & & 96 & & \\
\hline 1 & $3 / 3 / 99$ & 4 & & & 94 & & \\
\hline 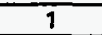 & $3 / 3 / 91$ & 5 & & & 91 & & \\
\hline 1 & $3 / 3 / 99$ & 6 & & & 87 & & \\
\hline 1 & $3 / 3 / 91$ & 7 & & & 80 & & \\
\hline 1 & $3 / 3 / 91$ & 8 & & & 75 & & \\
\hline 1 & $3 / 3 / 91$ & .9 & & & 75 & & \\
\hline 1 & $3 / 3 / 91$ & 10 & & & 74 & & \\
\hline 1 & $3 / 3 / 91$ & 11 & & & 74 & & \\
\hline 1 & $3 / 3 / 91$ & 12 & & & 74 & & \\
\hline 1 & $3 / 3 / 91$ & 13 & & & 74 & & \\
\hline 1 & $3 / 3 / 91$ & 14 & & & 73 & & \\
\hline
\end{tabular}

Data obtained from WHC Surveillance Analysis Computer System (SACS), February 24, 1994. 


\begin{tabular}{|c|c|c|c|c|c|c|c|}
\hline \multicolumn{8}{|c|}{ TANK 241-S-111 } \\
\hline \multicolumn{8}{|c|}{ THERMOCOUPLE DATA } \\
\hline TREE \# & DATE & THERMO \# & TPEE CET H & TFMP & TFMP F & $Y=M A X$ TFMP & COMMENTS \\
\hline 1 & $3 / 9 / 91$ & 1 & onst ver $\pi$ & Lnim & 97 & 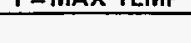 & Comineivis \\
\hline 1 & $3 / 9 / 91$ & 2 & & & 97 & & \\
\hline 1 & $3 / 9 / 91$ & 3 & & & 97 & & \\
\hline 1 & $3 / 9 / 91$ & 4 & & & 95 & & \\
\hline$T$ & $3 / 9 / 91$ & 5 & & & 92 & & \\
\hline 1 & $3 / 9 / 91$ & 6 & & & 88 & & \\
\hline 1 & $3 / 9 / 91$ & 7 & & & 81 & & \\
\hline 1 & $3 / 9 / 91$ & 8 & & & 76 & & \\
\hline 1 & $3 / 9 / 91$ & 9 & & & 75 & & \\
\hline 1 & $3 / 9 / 91$ & 10 & & & 75 & & \\
\hline 1 & $3 / 9 / 99$ & 11 & & & 75 & & \\
\hline 1 & $3 / 9 / 91$ & $\overline{12}$ & & & 75 & & \\
\hline 1 & $3 / 9 / 91$ & 13 & & & 74 & & \\
\hline 1 & $3 / 9 / 91$ & 14 & & & 74 & & \\
\hline 1 & $3 / 16 / 91$ & 1 & & & 95 & & \\
\hline 1 & $3 / 16 / 91$ & 1 & & & 93 & & \\
\hline 1 & $3 / 16 / 91$ & 2 & & & 95 & & \\
\hline 1 & $3 / 16 / 91$ & 2 & & & 91.4 & & \\
\hline 1 & $3 / 16 / 91$ & 3 & & & 95 & & \\
\hline 1 & $3 / 16 / 91$ & 3 & & & 91.4 & & \\
\hline 1 & $3 / 16 / 91$ & 4 & & & 93 & & \\
\hline 1 & $3 / 16 / 91$ & 4 & & & 89.7 & & \\
\hline 9 & $3 / 16 / 91$ & 5 & & & 90 & & \\
\hline 9 & $3 / 16 / 91$ & 5 & & & 86.6 & & \\
\hline 1 & $3 / 16 / 91$ & 6 & & & 85 & & \\
\hline 1 & $3 / 16 / 91$ & 6 & & & 82 & & \\
\hline 1 & $3 / 16 / 91$ & 7 & & & 79 & & \\
\hline 9 & $3 / 16 / 91$ & 7 & & & 75.5 & & \\
\hline 9 & $3 / 16 / 91$ & 8 & & & 75 & & \\
\hline 1 & $3 / 16 / 91$ & 8 & & & 70.9 & & \\
\hline 1 & $3 / 16 / 91$ & 9 & & & 74 & & \\
\hline 9 & $3 / 16 / 91$ & 9 & & & 70.2 & & \\
\hline 1 & $3 / 16 / 91$ & 10 & & & 72 & & \\
\hline 9 & $3 / 16 / 91$ & 10 & & & 68.9 & & \\
\hline 9 & $3 / 16 / 91$ & 19 & & & 72 & & \\
\hline 1 & $3 / 16 / 91$ & 11 & & & 68.7 & & \\
\hline 9 & $3 / 16 / 91$ & 12 & & & 72 & & \\
\hline 1 & $3 / 96 / 91$ & 12 & & & 68.9 & & \\
\hline 1 & $3 / 16 / 91$ & 13 & & & 72 & & \\
\hline 1 & $3 / 16 / 91$ & 13 & & & 68.8 & & \\
\hline 9 & $3 / 16 / 91$ & 14 & & & 72 & & \\
\hline 1 & $3 / 16 / 91$ & 14 & & & 68.7 & & \\
\hline 1 & $3 / 24 / 91$ & 1 & & & 97 & & \\
\hline 1 & $3 / 24 / 91$ & 2 & & & 97 & & \\
\hline 1 & $3 / 24 / 91$ & 3 & & & 96 & & \\
\hline 1 & $3 / 24 / 91$ & 4 & & & 95 & & \\
\hline 1 & $3 / 24 / 91$ & 5 & & & 92 & & \\
\hline 1 & $3 / 24 / 91$ & 6 & & & 88 & & \\
\hline 1 & $3 / 24 / 91$ & 7 & & & 81 & & \\
\hline 1 & $3 / 24 / 91$ & 8 & & & 77 & & \\
\hline 1 & $3 / 24 / 91$ & 9 & & & 76 & & \\
\hline 1 & $3 / 24 / 91$ & 10 & & & 75 & & \\
\hline 9 & $3 / 24 / 91$ & 91 & & & 75 & & \\
\hline 1 & $3 / 24 / 91$ & 12 & & & 74 & & \\
\hline 1 & $3 / 24 / 91$ & 13 & & & 74 & & \\
\hline 1 & $3 / 24 / 91$ & 14 & & & 74 & & \\
\hline 1 & $3 / 31 / 91$ & 1 & & & 97 & & \\
\hline 1 & $3 / 31 / 91$ & 2 & & & 96 & & \\
\hline 1 & $3 / 31 / 91$ & 3 & & & 96 & & \\
\hline 1 & $3 / 31 / 99$ & 4 & & & 94 & & \\
\hline 1 & $3 / 31 / 91$ & 5 & & & 91 & & \\
\hline 1 & $3 / 31 / 91$ & 6 & & & 86 & & \\
\hline 1 & $3 / 31 / 99$ & 7 & & & 82 & & \\
\hline 1 & $3 / 31 / 91$ & 8 & & & 75 & & \\
\hline 1 & $3 / 31 / 91$ & 9 & 1 & & 75 & $\mathrm{~N}$ & \\
\hline 1 & $3 / 31 / 91$ & 10 & 1 & & 73 & $N$ & \\
\hline
\end{tabular}

Data obtained from WHC Surveillance Analysis Computer System (SACS), February 24, 1994. 


\begin{tabular}{|c|c|c|c|c|c|c|c|}
\hline \multicolumn{8}{|c|}{ TANK 241-S-111 } \\
\hline \multicolumn{8}{|c|}{ THERMOCOUPLE DATA } \\
\hline TREE \# & DATE & THERMO \# & TREE SET \# & TEMP C & TEMP F & $Y=$ MAX TEMP & COMMENTS \\
\hline 1 & $3 / 31 / 91$ & 11 & 1 & & 73 & $\mathbf{N}$ & \\
\hline 1 & $3 / 31 / 91$ & 12 & 1 & & 73 & $\bar{N}$ & \\
\hline 1 & $3 / 31 / 91$ & 13 & 1 & & 73 & $\mathbf{N}$ & \\
\hline 1 & $3 / 31 / 91$ & 14 & 1 & & 73 & $\mathbf{N}$ & \\
\hline 1 & $4 / 6 / 91$ & 1 & 1 & & 97 & $\mathbf{Y}$ & OMEGA 199 \\
\hline 1 & $4 / 6 / 91$ & 2 & 1 & & 96 & $\mathbf{N}$ & \\
\hline 1 & $4 / 6 / 91$ & 3 & 1 & & 95 & $\mathbf{N}$ & \\
\hline 1 & $4 / 6 / 91$ & 4 & 1 & & 94 & $N$ & \\
\hline 1 & $4 / 6 / 91$ & 5 & 1 & & 91 & $\mathbf{N}$ & \\
\hline 1 & $4 / 6 / 91$ & 6 & 1 & & 86 & $\mathbf{N}$ & \\
\hline 1 & $4 / 6 / 91$ & 7 & 1 & & 80 & $\mathbf{N}$ & \\
\hline 1 & $4 / 6 / 91$ & 8 & 1 & & 75 & $\mathbf{N}$ & \\
\hline 1 & $4 / 6 / 91$ & 9 & 1 & & 75 & $\mathbf{N}$ & \\
\hline 1 & $4 / 6 / 91$ & 10 & 1 & & 73 & $\mathbf{N}$ & \\
\hline 1 & $4 / 6 / 91$ & 11 & 1 & & 73 & $\mathbf{N}$ & \\
\hline 1 & $4 / 6 / 91$ & 12 & 1 & & 73 & $\mathbf{N}$ & \\
\hline 1 & $4 / 6 / 91$ & 13 & 1 & & 73 & $\mathbf{N}$ & \\
\hline 1 & $4 / 6 / 91$ & 14 & 1 & & 73 & $\mathbf{N}$ & \\
\hline 1 & $4 / 74 / 91$ & 1 & 1 & & 97 & $\mathbf{Y}$ & J-HH-99-A 89-0423320-11 \\
\hline 1 & $4 / 14 / 91$ & 2 & 1 & & 96 & $\mathbf{N}$ & \\
\hline 1 & $4 / 14 / 91$ & 3 & 1 & & 96 & $\mathbf{N}$ & \\
\hline 1 & $4 / 14 / 91$ & 4 & 1 & & 94 & $\mathbf{N}$ & \\
\hline 1 & $4 / 14 / 91$ & 5 & 1 & & 91 & $\mathbf{N}$ & \\
\hline 1 & $4 / 14 / 91$ & 6 & 1 & & 86 & $\mathbf{N}$ & \\
\hline 1 & $4 / 14 / 91$ & 7 & 1 & & 80 & $\mathbf{N}$ & \\
\hline 1 & $4 / 14 / 91$ & 8 & $\frac{9}{1}$ & & 76 & $\mathbf{N}$ & \\
\hline 1 & $4 / 14 / 91$ & 9 & 1 & & 75 & $\mathbf{N}$ & \\
\hline 1 & $4 / 14 / 91$ & 10 & 1 & & 74 & $N$ & \\
\hline 1 & $4 / 14 / 91$ & 11 & 1 & & 74 & $\mathbf{N}$ & \\
\hline 1 & $4 / 14 / 91$ & 12 & 1 & & 73 & $N$ & \\
\hline 1 & $4 / 14 / 91$ & 13 & $\frac{1}{1}$ & & 73 & $\mathbf{N}$ & \\
\hline 1 & $4 / 14 / 91$ & 14 & 1 & & 73 & $\mathrm{~N}$ & \\
\hline 9 & $4 / 20 / 91$ & 1 & 1 & & 95 & $\mathbf{Y}$ & J $8904233202-11$ \\
\hline 1 & $4 / 20 / 91$ & 2 & 1 & & 95 & $\bar{N}$ & \\
\hline 1 & $4 / 20 / 91$ & 3 & 1 & & 94 & $\mathbf{N}$ & \\
\hline 1 & $4 / 20 / 91$ & 4 & 1 & & 92 & $\mathbf{N}$ & \\
\hline 1 & $4 / 20 / 91$ & 5 & 1 & & 89 & $\mathbf{N}$ & \\
\hline 1 & $4 / 20 / 91$ & 6 & 1 & & 85 & $\mathbf{N}$ & \\
\hline 1 & $4 / 20 / 91$ & 7 & 1 & & 79 & $\mathbf{N}$ & \\
\hline 1 & $4 / 20 / 91$ & 8 & 1 & & 74 & $\mathbf{N}$ & \\
\hline 1 & $4 / 20 / 91$ & 9 & 1 & & 74 & $\mathbf{N}$ & \\
\hline 1 & $4 / 20 / 91$ & 10 & $\frac{1}{1}$ & & 72 & $\bar{N}$ & \\
\hline 1 & $4 / 20 / 91$ & 11 & 1 & & 72 & $\mathrm{~N}$ & \\
\hline 1 & $4 / 20 / 91$ & 12 & 1 & & 72 & $N$ & \\
\hline 7 & $4 / 20 / 91$ & 13 & 9 & & 72 & $\bar{N}$ & \\
\hline 1 & $4 / 20 / 91$ & 14 & 1 & & 72 & $N$ & \\
\hline 1 & $4 / 27 / 91$ & 1 & 1 & & 96 & $\bar{Y}$ & $\mathrm{~J}$ \\
\hline 1 & $4 / 27 / 91$ & 2 & 1 & & 96 & $\mathrm{~N}$ & \\
\hline 1 & $4 / 27 / 91$ & 3 & 1 & & 95 & $\mathrm{~N}$ & \\
\hline 1 & $4 / 27 / 91$ & 4 & 1 & & 93 & $\mathbf{N}$ & \\
\hline 1 & $4 / 27 / 91$ & 5 & 1 & & 90 & $\mathbf{N}$ & \\
\hline 9 & $4 / 27 / 91$ & 6 & 9 & & 86 & $\bar{N}$ & \\
\hline 1 & $4 / 27 / 99$ & 7 & 1 & & 80 & $\mathrm{~N}$ & \\
\hline 1 & $4 / 27 / 91$ & 8 & 1 & & 76 & $\mathrm{~N}$ & \\
\hline 1 & $4 / 27 / 91$ & 9 & 1 & & 75 & $\mathbf{N}$ & \\
\hline 1 & $4 / 27 / 91$ & 10 & 1 & & 74 & $N$ & \\
\hline 1 & $4 / 27 / 91$ & 11 & 1 & & 73 & $\mathrm{~N}$ & \\
\hline 1 & $4 / 27 / 91$ & 12 & 1 & & 73 & $\mathbf{N}$ & \\
\hline 1 & $4 / 27 / 91$ & 13 & 1 & & 73 & $\bar{N}$ & \\
\hline 1 & $4 / 27 / 91$ & 14 & 1 & & 73 & $N$ & \\
\hline 1 & $5 / 4 / 91$ & 1 & 1 & & 95 & $Y$ & J HH-99A-J \\
\hline 1 & $5 / 4 / 91$ & 2 & 1 & & 95 & $\mathbf{N}$ & \\
\hline 1 & $5 / 4 / 91$ & 3 & 1 & & 94 & $\mathbf{N}$ & \\
\hline 1 & $5 / 4 / 91$ & 4 & 1 & & 92 & $\mathbf{N}$ & \\
\hline 1 & $5 / 4 / 91$ & 5 & 1 & & 89 & $N$ & \\
\hline 1 & $5 / 4 / 91$ & 6 & 1 & & 85 & $\mathbf{N}$ & \\
\hline
\end{tabular}

Data obtained from WHC Surveillance Analysis Computer System (SACS), February 24, 1994. 


\begin{tabular}{|c|c|c|c|c|c|c|c|}
\hline \multicolumn{8}{|c|}{ TANK 241.S.111 } \\
\hline & & & & THER & DCOUPLE & TA & \\
\hline TREE \# & DATE & THERMO \# & TREE SET \# & TEMP C & TEMP F & $Y=$ MAX TEMP & COMMENTS \\
\hline 1 & $5 / 4 / 91$ & 7 & 1 & & 79 & $N$ & \\
\hline 1 & $5 / 4 / 91$ & 8 & 1 & & 75 & $\mathbf{N}$ & \\
\hline 1 & $5 / 4 / 91$ & 9 & 1 & & 75 & $\mathbf{N}$ & \\
\hline 1 & $5 / 4 / 91$ & 10 & 1 & & 73 & $N$ & \\
\hline 1 & $5 / 4 / 99$ & 11 & 1 & & 73 & $\mathrm{~N}$ & \\
\hline 1 & $5 / 4 / 91$ & 12 & 1 & & 73 & $\mathbf{N}$ & \\
\hline 1 & $5 / 4 / 91$ & 13 & 1 & & 73 & $\bar{N}$ & \\
\hline 1 & $5 / 4 / 91$ & 14 & 1 & & 73 & $\mathbf{N}$ & \\
\hline 1 & $5 / 12 / 91$ & 1 & $T$ & & 95 & $\bar{Y}$ & J $910126143-75$ \\
\hline 1 & $5 / 12 / 91$ & 2 & 1 & & 95 & $\mathbf{N}$ & \\
\hline 1 & $5 / 12 / 91$ & 3 & 1 & & 95 & $\mathbf{N}$ & \\
\hline 9 & $5 / 12 / 91$ & 4 & 1 & & 93 & $\mathbf{N}$ & \\
\hline 1 & $5 / 12 / 91$ & 5 & 1 & & 90 & $\bar{N}$ & \\
\hline 1 & $5 / 12 / 91$ & 6 & 1 & & 85 & $\mathbf{N}$ & \\
\hline 1 & $5 / 12 / 91$ & 7 & 1 & & 80 & $\mathbf{N}$ & \\
\hline 1 & $5 / 12 / 91$ & 8 & 1 & & 76 & $\bar{N}$ & \\
\hline 1 & $5 / 12 / 91$ & 9 & 1 & & 75 & $\mathbf{N}$ & \\
\hline 1 & $5 / 12 / 91$ & 10 & 1 & & 74 & $\mathbf{N}$ & \\
\hline 1 & $5 / 12 / 91$ & 11 & 1 & & 74 & $\mathbf{N}$ & \\
\hline 1 & $5 / 12 / 91$ & 12 & 1 & & 74 & $\mathbf{N}$ & \\
\hline 1 & $5 / 12 / 91$ & 13 & 1 & & 74 & $\mathbf{N}$ & \\
\hline 1 & $5 / 12 / 91$ & 14 & 1 & & 74 & $\mathbf{N}$ & \\
\hline 1 & $5 / 19 / 91$ & 1 & 1 & & 95 & $\mathrm{Y}$ & J Omega 99A \\
\hline 1 & $5 / 19 / 91$ & 2 & 1 & & 95 & $\mathbf{N}$ & \\
\hline 1 & $5 / 19 / 91$ & 3 & 1 & & 94 & $\mathbf{N}$ & \\
\hline 1 & $5 / 19 / 91$ & 4 & 1 & & 93 & $\mathbf{N}$ & \\
\hline$\frac{1}{1}$ & $5 / 19 / 91$ & 5 & 1 & & 89 & $\mathbf{N}$ & \\
\hline 1 & $5 / 19 / 91$ & 6 & 1 & & 85 & $\mathbf{N}$ & \\
\hline 1 & $5 / 19 / 91$ & 7 & 1 & & 80 & $\bar{N}$ & \\
\hline 1 & $5 / 19 / 91$ & 8 & 1 & & 76 & $\mathbf{N}$ & \\
\hline 1 & $5 / 19 / 91$ & 9 & 1 & & 75 & $\bar{N}$ & \\
\hline 1 & $5 / 19 / 91$ & 10 & 9 & & 74 & $\mathbf{N}$ & \\
\hline 1 & $5 / 19 / 91$ & 11 & 1 & & 74 & $\mathbf{N}$ & \\
\hline 1 & $5 / 19 / 91$ & 12 & 1 & & 74 & $\mathbf{N}$ & \\
\hline 1 & $5 / 19 / 91$ & 13 & 1 & & 74 & $\mathbf{N}$ & \\
\hline 1 & $5 / 19 / 91$ & 14 & 1 & & 74 & $\mathrm{~N}$ & \\
\hline 1 & $5 / 24 / 91$ & 1 & 1 & & 95 & $\mathbf{Y}$ & JOmega \#199 \\
\hline 1 & $5 / 24 / 91$ & 2 & 1 & & 95 & $\mathbf{N}$ & \\
\hline 1 & $5 / 24 / 91$ & 3 & 1 & & 94 & $\mathbf{N}$ & \\
\hline 1 & $5 / 24 / 91$ & 4 & 1 & & 92 & $\mathbf{N}$ & \\
\hline 1 & $5 / 24 / 91$ & $5^{-}$ & 1 & & 89 & $\mathbf{N}$ & \\
\hline 1 & $5 / 24 / 91$ & 6 & 1 & & 85 & $\mathbf{N}$ & \\
\hline 1 & $5 / 24 / 91$ & 7 & 1 & & 80 & $\mathbf{N}$ & \\
\hline 1 & $5 / 24 / 91$ & 8 & 1 & & 76 & $\mathbf{N}$ & \\
\hline 1 & $5 / 24 / 91$ & 9 & 1 & & 75 & $\mathbf{N}$ & \\
\hline 1 & $5 / 24 / 91$ & 10 & 1 & & 74 & $\mathbf{N}$ & \\
\hline 1 & $5 / 24 / 91$ & 11 & 1 & & 74 & $\mathbf{N}$ & \\
\hline 1 & $5 / 24 / 99$ & 12 & 1 & & 74 & $\mathbf{N}$ & \\
\hline 1 & $5 / 24 / 91$ & 13 & 9 & & 74 & $\mathbf{N}$ & \\
\hline 1 & $5 / 24 / 91$ & 14 & 1 & & 74 & $\mathbf{N}$ & \\
\hline 1 & $6 / 1 / 91$ & 1 & 1 & & 94 & $\mathbf{Y}$ & J Omega Model \#199 \\
\hline 1 & $6 / 1 / 91$ & 2 & 1 & & 94 & $\bar{N}$ & \\
\hline 1 & $6 / 1 / 91$ & 3 & 1 & & 93 & $\mathbf{N}$ & \\
\hline 1 & $6 / 1 / 91$ & 4 & 1 & & 99 & $\mathbf{N}$ & \\
\hline 1 & $6 / 1 / 91$ & 5 & 1 & & 88 & $\mathbf{N}$ & \\
\hline 1 & $6 / 1 / 91$ & 6 & 1 & & 84 & $N$ & \\
\hline 1 & $6 / 1 / 91$ & 7 & 1 & & 79 & $N$ & \\
\hline 1 & $6 / 1 / 91$ & 8 & 1 & & 75 & $N$ & \\
\hline 1 & $6 / 1 / 91$ & 9 & 1 & & 75 & $N$ & \\
\hline 1 & $6 / 1 / 91$ & 10 & 1 & & 73 & $N$ & \\
\hline 1 & $6 / 1 / 91$ & 19 & 9 & & 74 & $\mathbf{N}$ & \\
\hline 1 & $6 / 1 / 91$ & 12 & 1 & & 74 & $\mathbf{N}$ & \\
\hline 1 & $6 / 1 / 91$ & 13 & 1 & & 74 & $\mathbf{N}$ & \\
\hline 1 & $6 / 1 / 91$ & 14 & 1 & & 74 & $\mathrm{~N}$ & \\
\hline 1 & $6 / 9 / 91$ & 1 & 1 & & 92 & $\mathbf{N}$ & $\mathrm{J} 890423320-11 \mathrm{TCA} 140 / \mathrm{s}$ \\
\hline 1 & $6 / 9 / 91$ & 2 & 1 & & 92.1 & $\mathbf{Y}$ & \\
\hline
\end{tabular}

Data obtained from WHC Surveillance Analysis Computer System (SACS), February 24, 1994. 


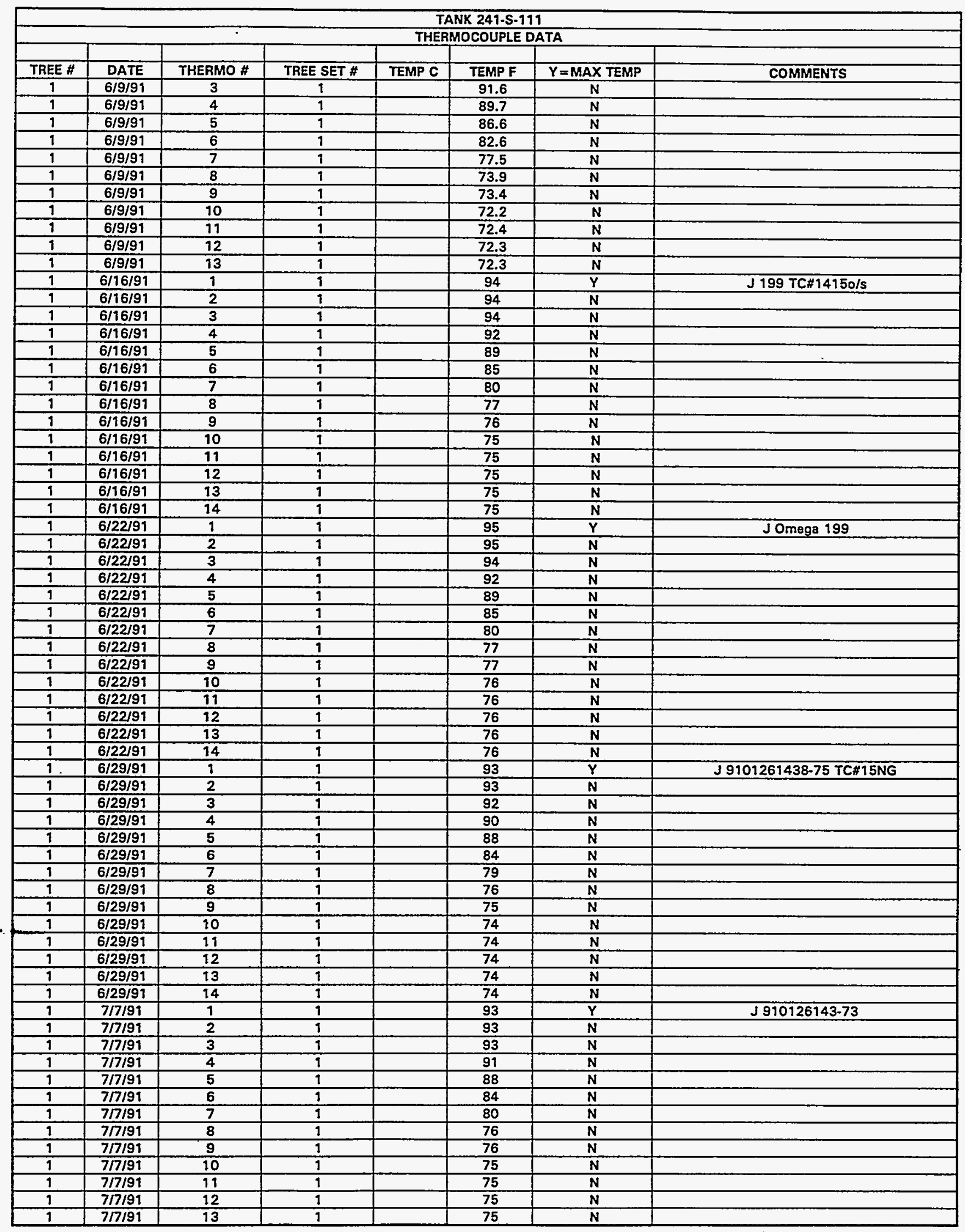

Data obtained from WHC Surveillance Analysis Computer System (SACS), February 24, 1994. 
WHC-SD-WM-ER-323, Rev. 0

\begin{tabular}{|c|c|c|c|c|c|c|c|}
\hline \multicolumn{8}{|c|}{ TANK 241-S-111 } \\
\hline \multicolumn{8}{|c|}{ THERMOCOUPLE DATA } \\
\hline TREE \# & DATE & THERMO \# & TREE SET \# & TEMP C & TEMP F & $Y=$ MAX TEMP & COMMENTS \\
\hline 1 & $7 / 7 / 99$ & 14 & 1 & & 75 & $\mathbf{N}$ & \\
\hline 9 & $7 / 13 / 91$ & 1 & 1 & & 93 & $\bar{Y}$ & Omega Eng199 \\
\hline 1 & $7 / 13 / 91$ & 2 & 1 & & 93 & $\mathbf{N}$ & \\
\hline 1 & $7 / 13 / 91$ & 3 & 1 & & 93 & $\mathrm{~N}$ & \\
\hline 1 & $7 / 13 / 91$ & 4 & 1 & & 91 & $\mathrm{~N}$ & \\
\hline 1 & $7 / 13 / 91$ & 5 & 1 & & 88 & $\mathrm{~N}$ & \\
\hline 9 & $7 / 13 / 91$ & 6 & 9 & & 84 & $\mathbf{N}$ & \\
\hline 1 & $7 / 13 / 91$ & 7 & 9 & & 80 & $\mathbf{N}$ & \\
\hline 1 & $7 / 13 / 91$ & 8 & 1 & & 77 & $\mathbf{N}$ & \\
\hline 1 & $7 / 13 / 91$ & 9 & 9 & & 76 & $\mathrm{~N}$ & \\
\hline 1 & $7 / 13 / 91$ & 10 & 1 & & 76 & $N$ & \\
\hline 1 & $7 / 13 / 91$ & 11 & 1 & & 76 & $\mathbf{N}$ & \\
\hline 1 & $7 / 13 / 91$ & 12 & 9 & & 76 & $N$ & \\
\hline 1 & $7 / 13 / 91$ & 13 & 1 & & 76 & $\bar{N}$ & \\
\hline 1 & $7 / 13 / 91$ & 14 & 1 & & 76 & $\mathrm{~N}$ & \\
\hline 9 & $7 / 20 / 91$ & 1 & 9 & & 94 & $\bar{Y}$ & J \\
\hline 1 & $7 / 20 / 91$ & 2 & 1 & & 93 & $\mathrm{~N}$ & \\
\hline 1 & $7 / 20 / 91$ & 3 & 1 & & 93 & $\bar{N}$ & \\
\hline 1 & $7 / 20 / 91$ & 4 & 9 & & 91 & $\bar{N}$ & \\
\hline 1 & $7 / 20 / 91$ & 5 & 1 & & 88 & $\bar{N}$ & \\
\hline 1 & $7 / 20 / 91$ & 6 & 1 & & 84 & $\mathbf{N}$ & \\
\hline 1 & $7 / 20 / 91$ & 7 & 9 & & 80 & $\bar{N}$ & \\
\hline 1 & $7 / 20 / 91$ & 8 & 1 & & 77 & $N$ & \\
\hline 1 & $7 / 20 / 91$ & 9 & 1 & & 77 & $\mathrm{~N}$ & \\
\hline 1 & $7 / 20 / 91$ & 10 & 1 & & 76 & $\mathrm{~N}$ & \\
\hline 1 & $7 / 20 / 91$ & 11 & 1 & & 76 & $\mathbf{N}$ & \\
\hline 1 & $7 / 20 / 91$ & 12 & 1 & & 76 & $\bar{N}$ & \\
\hline 1 & $7 / 20 / 91$ & 13 & 1 & & 76 & $\mathbf{N}$ & \\
\hline 1 & $7 / 20 / 91$ & 14 & 1 & & 76 & $\mathbf{N}$ & \\
\hline 1 & $7 / 27 / 91$ & 1 & 1 & & 90.5 & $\mathbf{N}$ & J910126143-75 \\
\hline 1 & $7 / 27 / 91$ & 2 & 1 & & 90.8 & $Y$ & \\
\hline 1 & $7 / 27 / 91$ & 3 & 1 & & 90.4 & $\bar{N}$ & \\
\hline 1 & $7 / 27 / 91$ & 4 & 1 & & 88.6 & $\mathbf{N}$ & \\
\hline 1 & $7 / 27 / 91$ & 5 & 1 & & 82.4 & $N$ & \\
\hline 1 & $7 / 27 / 91$ & 6 & 1 & & 78.3 & $N$ & \\
\hline 1 & $7 / 27 / 91$ & 7 & 1 & & 75.5 & $\mathbf{N}$ & \\
\hline 1 & $7 / 27 / 91$ & 8 & 1 & & 74.9 & $\mathbf{N}$ & \\
\hline 1 & $7 / 27 / 91$ & 9 & 1 & & 74.3 & $\bar{N}$ & \\
\hline 1 & $7 / 27 / 91$ & 10 & 9 & & 74.2 & $\mathbf{N}$ & \\
\hline 1 & $7 / 27 / 91$ & 11 & 1 & & 74.3 & $\mathbf{N}$ & \\
\hline 1 & $7 / 27 / 91$ & 12 & 1 & & 74.3 & $\mathbf{N}$ & \\
\hline 1 & $7 / 27 / 91$ & 13 & 1 & & 74.4 & $\mathbf{N}$ & \\
\hline 1 & $8 / 3 / 99$ & 9 & 9 & & 93 & $\bar{Y}$ & J910126143-75 \\
\hline 1 & $8 / 3 / 91$ & 2 & 1 & & 93 & $\mathbf{N}$ & \\
\hline 1 & $8 / 3 / 91$ & 3 & 9 & & 93 & $\mathbf{N}$ & \\
\hline 1 & $8 / 3 / 91$ & 4 & 9 & & 91 & $\bar{N}$ & \\
\hline 1 & $8 / 3 / 91$ & 5 & 1 & & 88 & $\mathbf{N}$ & \\
\hline 1 & $8 / 3 / 91$ & 6 & 1 & & 85 & $\bar{N}$ & \\
\hline 1 & $8 / 3 / 91$ & 7 & 1 & & 81 & $\bar{N}$ & \\
\hline 1 & $8 / 3 / 91$ & 8 & 1 & & 78 & $\mathbf{N}$ & \\
\hline 1 & $8 / 3 / 91$ & 9 & 9 & & 78 & $\mathbf{N}$ & \\
\hline 9 & $8 / 3 / 91$ & 10 & 1 & & 77 & $\mathbf{N}$ & \\
\hline 1 & $8 / 3 / 91$ & 11 & 1 & & 77 & $\mathbf{N}$ & \\
\hline 1 & $8 / 3 / 91$ & 12 & 1 & & 77 & $\mathbf{N}$ & \\
\hline 1 & $8 / 3 / 97$ & 13 & 1 & & 77 & $\mathbf{N}$ & \\
\hline 1 & $8 / 3 / 91$ & 14 & 1 & & 77 & $\mathbf{N}$ & \\
\hline 1 & $8 / 11 / 91$ & 1 & 1 & & 93 & $\bar{Y}$ & J 910126143-75 \\
\hline 1 & $8 / 11 / 91$ & 2 & 1 & & 93 & $\mathbf{N}$ & \\
\hline 1 & $8 / 11 / 91$ & 3 & 1 & & 93 & $\mathbf{N}$ & \\
\hline 1 & $8 / 11 / 91$ & 4 & 1 & & 91 & $N$ & \\
\hline 1 & $8 / 11 / 91$ & 5 & 1 & & 88 & $\mathbf{N}$ & \\
\hline 9 & $8 / 11 / 91$ & 6 & 1 & & 85 & $\mathbf{N}$ & \\
\hline 1 & $8 / 11 / 91$ & 7 & 1 & & 81 & $N$ & \\
\hline 1 & $8 / 11 / 91$ & 8 & 1 & & 79 & $\mathbf{N}$ & \\
\hline 1 & $8 / 11 / 91$ & 9 & 1 & & 78 & $\bar{N}$ & \\
\hline 1 & $8 / 11 / 91$ & 10 & 1 & & 78 & $N$ & \\
\hline
\end{tabular}

Data obtained from WHC Surveillance Analysis Computer System (SACS), February 24, 1994. 


\begin{tabular}{|c|c|c|c|c|c|c|c|}
\hline \multicolumn{8}{|c|}{ TANK 241-S-111 } \\
\hline & & $\therefore$ & & THEF & OCOUPLE & TA & \\
\hline TREE \# & DATE & THERMO \# & TREE SET \# & TEMP C & TEMP F & $Y=$ MAX TEMP & COMMENTS \\
\hline 1 & $8 / 11 / 91$ & 11 & 1 & & 78 & $\mathbf{N}$ & \\
\hline 1 & $8 / 11 / 91$ & 12 & 1 & & 78 & $\mathbf{N}$ & \\
\hline 1 & $8 / 11 / 91$ & 13 & 1 & & 77 & $\mathbf{N}$ & \\
\hline 1 & $8 / 11 / 91$ & 14 & 1 & & 78 & $\mathbf{N}$ & \\
\hline 1 & $8 / 18 / 91$ & 1 & 1 & & 92 & $\mathrm{Y}$ & J910126143-75 \\
\hline 1 & $8 / 18 / 91$ & 2 & 1 & & 92 & $N$ & \\
\hline 1 & $8 / 18 / 91$ & 3 & 1 & & 92 & $\mathrm{~N}$ & \\
\hline 1 & $8 / 18 / 91$ & 4 & 1 & & 90 & $\bar{N}$ & \\
\hline 1 & $8 / 18 / 91$ & 5 & 1 & & 88 & $\mathbf{N}$ & \\
\hline 1 & $8 / 18 / 91$ & 6 & 1 & & 84 & $\mathbf{N}$ & \\
\hline 1 & $8 / 18 / 91$ & 7 & 1 & & 81 & $\mathbf{N}$ & \\
\hline 1 & $8 / 18 / 91$ & 8 & 1 & & 78 & $\mathbf{N}$ & \\
\hline 1 & $8 / 18 / 91$ & 9 & 1 & & 78 & $\mathbf{N}$ & \\
\hline 1 & $8 / 18 / 91$ & 70 & 1 & & 77 & $\bar{N}$ & \\
\hline 1 & $8 / 18 / 91$ & 11 & 1 & & 77 & $N$ & \\
\hline 1 & $8 / 18 / 91$ & 12 & 1 & & 78 & $\mathbf{N}$ & \\
\hline 1 & $8 / 18 / 91$ & 13 & 1 & & 76 & $\mathbf{N}$ & \\
\hline 1 & $8 / 18 / 91$ & 14 & 1 & & 77 & $N$ & \\
\hline 1 & $8 / 25 / 91$ & 1 & 1 & & 91 & $\mathbf{N}$ & JHH-99A-J \\
\hline 1 & $8 / 25 / 91$ & 2 & $T$ & & 93 & $\bar{Y}$ & \\
\hline 1 & $8 / 25 / 91$ & 3 & 1 & & 93 & $\bar{Y}$ & \\
\hline 1 & $8 / 25 / 91$ & 4 & 1 & & 91 & $\mathbf{N}$ & \\
\hline 1 & $8 / 25 / 91$ & 5 & 1 & & 89 & $\mathbf{N}$ & \\
\hline 1 & $8 / 25 / 91$ & 6 & 1 & & 86 & $\mathbf{N}$ & \\
\hline 1 & $8 / 25 / 91$ & 7 & 1 & & 82 & $\mathrm{~N}$ & \\
\hline 1 & $8 / 25 / 91$ & 8 & 1 & & 80 & $\mathbf{N}$ & \\
\hline 1 & $8 / 25 / 91$ & 9 & 1 & & 80 & $\mathbf{N}$ & \\
\hline 1 & $8 / 25 / 91$ & 10 & 1 & & 79 & $\mathbf{N}$ & \\
\hline 1 & $8 / 25 / 91$ & 19 & 1 & & 79 & $\mathbf{N}$ & \\
\hline 1 & $8 / 25 / 91$ & 12 & 1 & & 79 & $\bar{N}$ & \\
\hline 1 & $8 / 25 / 91$ & 13 & 1 & & 78 & $\mathbf{N}$ & \\
\hline 1 & $8 / 25 / 91$ & 14 & 1 & & 79 & $N$ & \\
\hline 1 & $8 / 31 / 91$ & 1 & 1 & & 90.2 & $\mathbf{N}$ & $J .890523512-20$ \\
\hline 1 & $8 / 39 / 91$ & 2 & 1 & & 90.4 & $Y$ & \\
\hline 1 & $8 / 31 / 91$ & 3 & 1 & & 90.1 & $\bar{N}$ & \\
\hline 1 & $8 / 31 / 91$ & 4 & 1 & & 88.6 & $\mathbf{N}$ & \\
\hline 9 & $8 / 31 / 91$ & 5 & 1 & & 86.2 & $\mathbf{N}$ & \\
\hline 1 & $8 / 31 / 91$ & 6 & 1 & & 83.3 & $\mathbf{N}$ & \\
\hline 1 & $8 / 39 / 91$ & 7 & 1 & & 79.9 & $\mathbf{N}$ & \\
\hline$\frac{1}{1}$ & $8 / 31 / 91$ & 8 & $\frac{1}{1}$ & & 77.4 & $\mathbf{N}$ & \\
\hline 1 & $8 / 31 / 91$ & 9 & 1 & & 77.1 & $\mathbf{N}$ & \\
\hline 1 & $8 / 31 / 91$ & 10 & 1 & & 76.7 & $\mathbf{N}$ & \\
\hline 1 & $8 / 39 / 91$ & 11 & 1 & & 76.6 & $\mathbf{N}$ & \\
\hline 1 & $8 / 31 / 91$ & 12 & 1 & & 76.7 & $\mathbf{N}$ & \\
\hline 1 & $8 / 31 / 91$ & 13 & 1 & & 75.6 & $\mathbf{N}$ & \\
\hline 1 & $8 / 31 / 91$ & 14 & 1 & & 76.8 & $\mathbf{N}$ & \\
\hline 1 & $9 / 7 / 91$ & 1 & 1 & & 91.1 & $\mathbf{N}$ & $J-890523512-20$ \\
\hline 1 & $9 / 7 / 91$ & 2 & 1 & & 91.4 & $\bar{Y}$ & \\
\hline 1 & $9 / 7 / 91$ & 3 & 1 & & 91.1 & $\mathbf{N}$ & \\
\hline 1 & $9 / 7 / 91$ & 4 & 1 & & 89.6 & $\mathbf{N}$ & \\
\hline 1 & $9 / 7 / 91$ & 5 & 1 & & 87.4 & $\mathbf{N}$ & \\
\hline 1 & $9 / 7 / 91$ & 6 & 1 & & 84.6 & $\mathbf{N}$ & \\
\hline 9 & $9 / 7 / 91$ & 7 & 1 & & 81.2 & $\bar{N}$ & \\
\hline 1 & $9 / 7 / 91$ & 8 & 1 & & 78.8 & $\mathbf{N}$ & \\
\hline 1 & $9 / 7 / 91$ & 9 & 1 & & 78.5 & $\mathbf{N}$ & \\
\hline 1 & $9 / 7 / 91$ & 10 & 1 & & 78.1 & $\mathbf{N}$ & \\
\hline 1 & $9 / 7 / 91$ & 19 & 1 & & 78.1 & $\mathbf{N}$ & \\
\hline 1 & $9 / 7 / 91$ & 12 & 1 & & 78.1 & $N$ & \\
\hline 1 & $9 / 7 / 91$ & 13 & 1 & & 77 & $N$ & \\
\hline 1 & $9 / 7 / 91$ & 14 & 1 & & 78.1 & $\mathbf{N}$ & \\
\hline 1 & 9/14/91 & 1 & 1 & & 96 & $\mathbf{Y}$ & $J-890523512-20$ \\
\hline 1 & $9 / 14 / 91$ & 2 & 1 & & 95 & $\mathbf{N}$ & \\
\hline 1 & 9/14/91 & 3 & 1 & & 95 & $\mathbf{N}$ & \\
\hline 1 & 9/14/91 & 4 & 1 & & 93 & $\mathbf{N}$ & \\
\hline 1 & $9 / 14 / 91$ & 5 & 9 & & 91 & $N$ & \\
\hline 1 & $9 / 14 / 91$ & 6 & 1 & & 88 & $\mathbf{N}$ & \\
\hline
\end{tabular}

Data obtained from WHC Surveillance Analysis Computer System (SACS), February 24, 1994. 


\begin{tabular}{|c|c|c|c|c|c|c|c|}
\hline \multicolumn{8}{|c|}{ TANK 241-S-111 } \\
\hline \multicolumn{8}{|c|}{ THERMOCOUPLE DATA } \\
\hline TREE \# & DATE & THERMO \# & TREE SET \# & TEMP C & TEMP F & $\mathrm{Y}=\mathrm{MAX}$ TEMP & COMMENTS \\
\hline 1 & $9 / 14 / 91$ & 7 & 1 & & 85 & $\mathrm{~N}$ & \\
\hline 1 & 9/14/91 & 8 & 1 & & 83 & $\mathrm{~N}$ & \\
\hline 1 & $9 / 14 / 91$ & 9 & 1 & & 82 & $\mathrm{~N}$ & \\
\hline 1 & $9 / 14 / 91$ & 10 & 1 & & 82 & $\mathrm{~N}$ & \\
\hline 1 & $9 / 14 / 91$ & 11 & 9 & & 82 & $\mathrm{~N}$ & \\
\hline 1 & $9 / 14 / 91$ & 12 & 1 & & 82 & $\mathrm{~N}$ & \\
\hline 1 & $9 / 14 / 91$ & 13 & 1 & & 82 & $\mathbf{N}$ & \\
\hline 1 & 9/14/91 & 14 & 1 & & 82 & $N$ & \\
\hline 1 & $9 / 21 / 91$ & 1 & 1 & & 92.2 & $N$ & J-HH-99A-J \\
\hline 1 & $9 / 21 / 91$ & 2 & 1 & & 92.5 & $\bar{Y}$ & \\
\hline 1 & $9 / 21 / 91$ & 3 & $\frac{1}{1}$ & & 92.2 & $\mathrm{~N}$ & \\
\hline 1 & $9 / 21 / 91$ & 4 & 9 & & 90.9 & $\mathbf{N}$ & \\
\hline 1 & $9 / 21 / 91$ & 5 & 1 & & 88.7 & $\bar{N}$ & \\
\hline 1 & $9 / 21 / 91$ & 6 & 1 & & 86 & $\mathbf{N}$ & \\
\hline 1 & $9 / 21 / 91$ & 7 & $T$ & & 82.8 & $\mathbf{N}$ & \\
\hline 1 & $9 / 21 / 91$ & 8 & 1 & & 80.3 & $\mathbf{N}$ & \\
\hline 1 & $9 / 21 / 91$ & 9 & 1 & & 80 & $N$ & \\
\hline 1 & $9 / 21 / 91$ & 10 & 9 & & 79.8 & $\mathrm{~N}$ & \\
\hline 1 & $9 / 21 / 91$ & 11 & 1 & & 79.7 & $\mathrm{~N}$ & \\
\hline 1 & $9 / 21 / 91$ & 12 & 9 & & 79.8 & $\mathbf{N}$ & \\
\hline 1 & $9 / 21 / 91$ & 13 & 9 & & 79.7 & $\mathbf{N}$ & \\
\hline 1 & $9 / 21 / 91$ & 14 & 1 & & 79.7 & $N$ & \\
\hline 1 & $9 / 28 / 91$ & 1 & 9 & & 93 & $\bar{Y}$ & $J-890523512-20$ \\
\hline 1 & $9 / 28 / 91$ & 2 & 9 & & 93 & $\bar{N}$ & \\
\hline 1 & $9 / 28 / 91$ & 3 & 1 & & 92 & $N$ & \\
\hline 9 & $9 / 28 / 91$ & 4 & 1 & & 91 & $\bar{N}$ & \\
\hline 9 & $9 / 28 / 91$ & 5 & 1 & & 89 & $N$ & \\
\hline 1 & $9 / 28 / 91$ & 6 & 1 & & 86 & $\mathbf{N}$ & \\
\hline 9 & $9 / 28 / 91$ & 7 & 1 & & 83 & $\mathbf{N}$ & \\
\hline 1 & $9 / 28 / 99$ & 8 & 9 & & 81 & $\bar{N}$ & \\
\hline 1 & $9 / 28 / 91$ & 9 & 1 & & 81 & $\mathbf{N}$ & \\
\hline 1 & $9 / 28 / 91$ & 10 & 1 & & 80 & $N$ & \\
\hline 1 & $9 / 28 / 91$ & 17 & 9 & & 80 & $\bar{N}$ & \\
\hline 1 & $9 / 28 / 91$ & 12 & 1 & & 80 & $N$ & \\
\hline 1 & $9 / 28 / 91$ & 13 & 1 & & 80 & $\mathbf{N}$ & \\
\hline 1 & $9 / 28 / 91$ & 14 & 7 & & 80 & $\mathrm{~N}$ & \\
\hline$\frac{1}{1}$ & $10 / 5 / 91$ & 1 & 9 & & 94 & $\mathbf{N}$ & $\mathrm{J}-890523518-20$ \\
\hline 1 & $10 / 5 / 91$ & 2 & 9 & & 95 & $Y$ & \\
\hline 1 & $10 / 5 / 91$ & 3 & 1 & & 94 & $\mathbf{N}$ & \\
\hline 9 & $10 / 5 / 91$ & 4 & 9 & & 93 & $\mathbf{N}$ & \\
\hline 1 & $10 / 5 / 91$ & 5 & 1 & & 91 & $\bar{N}$ & \\
\hline 1 & $10 / 5 / 91$ & 6 & 1 & & 88 & $\mathbf{N}$ & \\
\hline 9 & $10 / 5 / 91$ & 7 & 9 & & 85 & $\mathbf{N}$ & \\
\hline 1 & $10 / 5 / 91$ & 8 & 1 & & 83 & $\mathbf{N}$ & \\
\hline 1 & $10 / 5 / 91$ & 9 & 1 & & 82 & $\mathrm{~N}$ & \\
\hline 9 & $10 / 5 / 91$ & 10 & 1 & & 82 & $\overline{\mathbf{N}}$ & \\
\hline 1 & $10 / 5 / 91$ & 11 & 1 & & 82 & $\mathbf{N}$ & \\
\hline 1 & $10 / 5 / 91$ & 12 & 1 & & 82 & $\mathbf{N}$ & \\
\hline 1 & $10 / 5 / 91$ & 13 & 7 & & 82 & $\bar{N}$ & \\
\hline 1 & $10 / 5 / 91$ & 14 & 1 & & 82 & $N$ & \\
\hline 9 & $10 / 11 / 91$ & 1 & 1 & & 95 & $\bar{Y}$ & $J-890523512-20$ \\
\hline 1 & $10 / 11 / 91$ & 2 & 1 & & 95 & $\mathbf{N}$ & \\
\hline 1 & $10 / 11 / 91$ & 3 & $\frac{1}{1}$ & & 95 & $\mathbf{N}$ & \\
\hline 1 & $10 / 11 / 91$ & 4 & 1 & & 94 & $\overline{\mathbf{N}}$ & \\
\hline 1 & $10 / 11 / 91$ & 5 & 1 & & 92 & $\mathbf{N}$ & \\
\hline 1 & $10 / 19 / 91$ & 6 & 1 & & 89 & $\bar{N}$ & \\
\hline 1 & $10 / 19 / 91$ & 7 & 9 & & 86 & $\bar{N}$ & \\
\hline 1 & $10 / 11 / 91$ & 8 & 1 & & 83 & $\mathbf{N}$ & \\
\hline 1 & $10 / 11 / 91$ & 9 & 1 & & 83 & $\bar{N}$ & \\
\hline 1 & $10 / 11 / 91$ & 10 & 1 & & 82 & $\mathbf{N}$ & \\
\hline 9 & $10 / 11 / 91$ & 11 & 1 & & 82 & $\mathbf{N}$ & \\
\hline 1 & $10 / 11 / 91$ & 12 & 1 & & 83 & $\mathbf{N}$ & \\
\hline 1 & $10 / 11 / 91$ & 13 & 1 & & 82 & $N$ & \\
\hline 1 & $10 / 11 / 91$ & 14 & 1 & & 82 & $\bar{N}$ & \\
\hline 1 & $10 / 20 / 91$ & 1 & 1 & & 93.1 & $\mathbf{N}$ & $\mathrm{J}-890523512-20$ \\
\hline 1 & $10 / 20 / 91$ & 2 & 1 & & 93.5 & $\mathrm{Y}$ & \\
\hline
\end{tabular}

Data obtained from WHC Surveillance Analysis Computer System (SACS), February 24, 1994. 
WHC-SD-WM-ER-323, Rev. 0

\begin{tabular}{|c|c|c|c|c|c|c|c|}
\hline \multicolumn{8}{|c|}{ TANK 241-S-111 } \\
\hline & & & & THER & COUPLE & & \\
\hline TREE \# & DATE & THERMO \# & TREE SET \# & TEMP C & TEMP F & $Y=$ MAX TEMP & COMMENTS \\
\hline 1 & $10 / 20 / 91$ & 3 & 1 & & 93.4 & $N$ & \\
\hline 1 & $10 / 20 / 91$ & 4 & 1 & & 92.2 & $\mathrm{~N}$ & \\
\hline 1 & $10 / 20 / 91$ & 5 & 1 & & 90.2 & $\mathbf{N}$ & \\
\hline 1 & $10 / 20 / 91$ & 6 & 1 & & 87.7 & $\mathrm{~N}$ & \\
\hline 1 & $10 / 20 / 91$ & 7 & 1 & & 84.2 & $\mathbf{N}$ & \\
\hline 1 & $10 / 20 / 91$ & 8 & 1 & & 81.4 & $\mathbf{N}$ & \\
\hline 1 & $10 / 20 / 91$ & 9 & 1 & & 81.2 & $\mathbf{N}$ & \\
\hline 1 & $10 / 20 / 91$ & 10 & 1 & & 80.6 & $\mathbf{N}$ & \\
\hline 1 & $10 / 20 / 91$ & 11 & 1 & & 80.5 & $\mathbf{N}$ & \\
\hline 1 & $10 / 20 / 91$ & 12 & 1 & & 80.7 & $\mathbf{N}$ & \\
\hline 1 & $10 / 20 / 91$ & 13 & 1 & & 80.6 & $\mathbf{N}$ & \\
\hline 1 & $10 / 20 / 91$ & 14 & 1 & & 80.5 & $\mathbf{N}$ & \\
\hline 1 & $10 / 26 / 91$ & 1 & 1 & & 95 & $Y$ & $\mathrm{~J}-890523512.20$ \\
\hline 1 & $10 / 26 / 91$ & 2 & 1 & & 95 & $N$ & \\
\hline 1 & $10 / 26 / 91$ & 3 & 1 & & 95 & $\mathbf{N}$ & \\
\hline 1 & $10 / 26 / 91$ & 4 & 1 & & 94 & $\mathbf{N}$ & \\
\hline 1 & $10 / 26 / 91$ & 5 & 1 & & 92 & $\mathrm{~N}$ & \\
\hline 1 & $10 / 26 / 91$ & 6 & 1 & & 89 & $\mathrm{~N}$ & \\
\hline 1 & $10 / 26 / 91$ & 7 & 1 & & 86 & $N$ & \\
\hline 9 & $10 / 26 / 91$ & 8 & 1 & & 83 & $N$ & \\
\hline 1 & $10 / 26 / 91$ & 9 & 1 & & 83 & $\mathbf{N}$ & \\
\hline 1 & $10 / 26 / 91$ & 10 & 1 & & 82 & $\mathbf{N}$ & \\
\hline 1 & $10 / 26 / 91$ & 11 & 1 & & 82 & $\mathbf{N}$ & \\
\hline 1 & $10 / 26 / 91$ & 12 & 1 & & 82 & $\mathbf{N}$ & \\
\hline 1 & $10 / 26 / 91$ & 13 & 1 & & 82 & $\mathbf{N}$ & \\
\hline 1 & $10 / 26 / 91$ & 14 & 1 & & 82 & $N$ & \\
\hline 1 & $11 / 2 / 91$ & 1 & 1 & & 94.8 & $Y$ & $\mathrm{~J}-890523512-80$ \\
\hline 1 & $11 / 2 / 91$ & 2 & 1 & & 94.9 & $\mathbf{N}$ & \\
\hline 1 & $11 / 2 / 91$ & 3 & 1 & & 94.8 & $\mathrm{~N}$ & \\
\hline 1 & $11 / 2 / 91$ & 4 & 1 & & 93.7 & $\mathrm{~N}$ & \\
\hline 1 & $11 / 2 / 91$ & 5 & 1 & & 91.8 & $N$ & \\
\hline 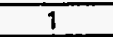 & $11 / 2 / 91$ & 6 & 1 & & 89.1 & $\mathbf{N}$ & \\
\hline 1 & $11 / 2 / 91$ & 7 & 1 & & 85.2 & $\mathbf{N}$ & \\
\hline 1 & $11 / 2 / 91$ & 8 & 1 & & 82.2 & $\mathrm{~N}$ & \\
\hline 1 & $11 / 2 / 91$ & 9 & 1 & & 81.9 & $\mathbf{N}$ & \\
\hline 1 & $11 / 2 / 91$ & 10 & 1 & & 81.1 & $N$ & \\
\hline 1 & $11 / 2 / 91$ & 11 & 1 & & 81.1 & $\mathbf{N}$ & \\
\hline 1 & $11 / 2 / 91$ & 12 & 1 & & 81.1 & $\mathbf{N}$ & \\
\hline 1 & $11 / 2 / 91$ & 13 & 1 & & 81.1 & $\bar{N}$ & \\
\hline 1 & $11 / 2 / 91$ & 14 & 1 & & 81.1 & $\mathbf{N}$ & \\
\hline 1 & $11 / 9 / 91$ & 1 & 1 & & 95 & $\mathbf{N}$ & $J-890523512-20$ \\
\hline 1 & $11 / 9 / 91$ & 2 & 1 & & 96 & $\bar{Y}$ & \\
\hline 1 & $11 / 9 / 91$ & 3 & 1 & & 95 & $\mathbf{N}$ & \\
\hline 1 & $11 / 9 / 91$ & 4 & 1 & & 95 & $\mathbf{N}$ & \\
\hline 1 & $111 / 9 / 91$ & 5 & 1 & & 93 & $\mathbf{N}$ & \\
\hline 1 & $11 / 9 / 91$ & 6 & 1 & & 90 & $\mathbf{N}$ & \\
\hline 1 & $11 / 9 / 91$ & 7 & 1 & & 86 & $\mathbf{N}$ & \\
\hline 1 & $11 / 9 / 91$ & 8 & 1 & & 83 & $\mathbf{N}$ & \\
\hline 1 & $11 / 9 / 91$ & 9 & 1 & & 83 & $\mathbf{N}$ & \\
\hline 1 & $11 / 9 / 91$ & 10 & 1 & & 82 & $N$ & \\
\hline 9 & $11 / 9 / 91$ & 11 & 1 & & 82 & $\mathbf{N}$ & \\
\hline 1 & $11 / 9 / 91$ & 12 & 1 & & 82 & $\mathbf{N}$ & \\
\hline 1 & $11 / 9 / 91$ & 13 & 1 & & 82 & $\mathbf{N}$ & \\
\hline 1 & $11 / 9 / 91$ & 14 & 1 & & 82 & $\mathbf{N}$ & \\
\hline 1 & $11 / 13 / 91$ & 1 & 1 & & 89.4 & $N$ & J.890423320-42 TC \#1516 O/S W/O \#04529 \\
\hline 1 & $11 / 13 / 91$ & 2 & 1 & & 89.8 & $\bar{Y}$ & \\
\hline 1 & $11 / 13 / 91$ & 3 & 1 & & 89.8 & $N$ & \\
\hline 1 & $11 / 13 / 91$ & 4 & 1 & & 88.8 & $\mathbf{N}$ & \\
\hline 1 & $11 / 13 / 91$ & 5 & 1 & & 87.1 & $\mathbf{N}$ & \\
\hline 1 & $11 / 13 / 91$ & 6 & 1 & & 84.6 & $\mathbf{N}$ & \\
\hline 1 & $11 / 13 / 91$ & 7 & 1 & & 80.1 & $\mathbf{N}$ & \\
\hline 1 & $11 / 13 / 91$ & 8 & 1 & & 77.1 & $\mathbf{N}$ & \\
\hline 1 & $11 / 13 / 91$ & 9 & 1 & & 76.8 & $\mathbf{N}$ & \\
\hline 1 & $11 / 13 / 91$ & 10 & 1 & & 75.7 & $\mathbf{N}$ & \\
\hline 1 & $917 / 13 / 91$ & 11 & 1 & & 75.7 & $\mathbf{N}$ & \\
\hline
\end{tabular}

Data obtained from WHC Surveillance Analysis Computer System (SACS). February 24, 1994. 
WHC-SD-WM-ER-323, Rev. O

\begin{tabular}{|c|c|c|c|c|c|c|c|}
\hline \multicolumn{8}{|c|}{ TANK 241-S-111 } \\
\hline \multicolumn{8}{|c|}{ THERMOCOUPLE DATA } \\
\hline TREE \# & DATE & THERMO \# & TREE SET \# & TEMP C & TEMPF & $Y=\operatorname{MAX}$ TEMP & COMMENTS \\
\hline 1 & $11 / 13 / 91$ & 12 & 1 & & 75.5 & $N$ & \\
\hline 1 & $11 / 13 / 91$ & 13 & 1 & & 75.6 & $\mathrm{~N}$ & \\
\hline 1 & $11 / 13 / 91$ & 14 & 1 & & 75.6 & $\mathbf{N}$ & \\
\hline 1 & $11 / 15 / 91$ & 1 & 1 & & 90.4 & $\mathrm{~N}$ & J.817-13-55-008 \\
\hline 1 & $11 / 15 / 91$ & 2 & 1 & & 90.7 & $Y$ & \\
\hline 9 & $11 / 15 / 91$ & 3 & 1 & & 90.6 & $\mathbf{N}$ & \\
\hline 1 & $11 / 15 / 91$ & 4 & 1 & & 89.6 & $\mathbf{N}$ & \\
\hline 1 & $11 / 15 / 91$ & 5 & 1 & & 87.9 & $\mathrm{~N}$ & \\
\hline 9 & $11 / 15 / 91$ & 6 & 9 & & 85.2 & $\mathbf{N}$ & \\
\hline 1 & $11 / 15 / 91$ & 7 & 1 & & 80.7 & $\mathrm{~N}$ & \\
\hline 1 & $11 / 15 / 91$ & 8 & 1 & & 77.6 & $\mathbf{N}$ & \\
\hline 1 & $11 / 15 / 91$ & 9 & 1 & & 77.4 & $\mathrm{~N}$ & \\
\hline 1 & $11 / 15 / 91$ & 10 & 1 & & 76.4 & $\mathrm{~N}$ & \\
\hline 1 & $11 / 15 / 91$ & 11 & 1 & & 76.4 & $\mathrm{~N}$ & \\
\hline 1 & $11 / 15 / 91$ & 12 & 1 & & 76.3 & $N$ & \\
\hline 1 & $11 / 15 / 91$ & 13 & 1 & & 76.3 & $\mathbf{N}$ & \\
\hline 1 & $11 / 15 / 91$ & 14 & 1 & & 76.2 & $\mathrm{~N}$ & \\
\hline 1 & $11 / 23 / 91$ & 1 & 1 & & 89.7 & $\mathbf{N}$ & $\mathrm{J}-890423320-42$ \\
\hline 1 & $11 / 23 / 91$ & 2 & 1 & & 90.1 & $Y$ & \\
\hline 1 & $11 / 23 / 91$ & 3 & 1 & & 90 & $N$ & \\
\hline 1 & $11 / 23 / 91$ & 4 & 1 & & 89.1 & $\mathbf{N}$ & \\
\hline 1 & $11 / 23 / 91$ & 5 & 1 & & 87.3 & $N$ & \\
\hline 1 & $11 / 23 / 91$ & 6 & 1 & & 84.5 & $\mathbf{N}$ & \\
\hline 1 & $11 / 23 / 91$ & 7 & 1 & & 79.8 & $\mathrm{~N}$ & \\
\hline 1 & $11 / 23 / 91$ & 8 & 1 & & 76.3 & $\mathrm{~N}$ & \\
\hline 1 & $11 / 23 / 91$ & 9 & 1 & & 76.1 & $\mathbf{N}$ & \\
\hline 1 & $11 / 23 / 91$ & 10 & 1 & & 75.2 & $\mathbf{N}$ & \\
\hline 1 & $11 / 23 / 91$ & 11 & 1 & & 75.2 & $N$ & \\
\hline$\frac{1}{1}$ & $11 / 23 / 91$ & 12 & 1 & & 75.1 & $N$ & \\
\hline 1 & $11 / 23 / 91$ & 13 & 1 & & 75.1 & $\mathrm{~N}$ & \\
\hline 1 & $11 / 23 / 91$ & 14 & 1 & & 75 & $\mathbf{N}$ & \\
\hline 1 & $12 / 1 / 91$ & 1 & 1 & & 96 & $\mathrm{Y}$ & $J-890523512.47$ \\
\hline 1 & $12 / 1 / 91$ & 2 & 1 & & 96 & $\mathbf{N}$ & \\
\hline 1 & $12 / 1 / 91$ & 3 & 1 & & 96 & $\mathbf{N}$ & \\
\hline$\frac{1}{1}$ & $12 / 1 / 91$ & 4 & 1 & & 95 & $\mathrm{~N}$ & \\
\hline 1 & $12 / 1 / 91$ & 5 & 1 & & 93 & $\mathbf{N}$ & \\
\hline 1 & $12 / 1 / 91$ & 6 & 1 & & 90 & $\mathbf{N}$ & \\
\hline 9 & $12 / 1 / 91$ & 7 & 1 & & 85 & $\mathbf{N}$ & \\
\hline 1 & $12 / 1 / 91$ & 8 & 1 & & 81 & $\mathbf{N}$ & \\
\hline 1 & $12 / 1 / 91$ & 9 & 1 & & 81 & $\mathbf{N}$ & \\
\hline 1 & $12 / 1 / 91$ & 10 & 1 & & 80 & $\mathbf{N}$ & \\
\hline 1 & $12 / 1 / 91$ & 11 & 1 & & 80 & $\mathbf{N}$ & \\
\hline 1 & $12 / 1 / 91$ & 12 & 1 & & 80 & $\mathbf{N}$ & \\
\hline 1 & $12 / 1 / 91$ & 13 & 1 & & 80 & $\mathbf{N}$ & \\
\hline 1 & $12 / 1 / 91$ & 14 & 1 & & 80 & $\mathbf{N}$ & \\
\hline 1 & $12 / 8 / 91$ & 1 & 1 & & 95 & $\mathbf{N}$ & $J$ \\
\hline 1 & $12 / 8 / 91$ & 2 & 1 & & 96 & $\bar{Y}$ & \\
\hline 1 & $12 / 8 / 91$ & 3 & 1 & & 96 & $\mathbf{N}$ & \\
\hline 1 & $12 / 8 / 91$ & 4 & 1 & & 95 & $\mathbf{N}$ & \\
\hline 1 & $12 / 8 / 91$ & 5 & 1 & & 93 & $N$ & \\
\hline 1 & $12 / 8 / 91$ & 6 & 1 & & 90 & $\mathbf{N}$ & \\
\hline 1 & $12 / 8 / 91$ & 7 & $\frac{1}{1}$ & & 85 & $\mathbf{N}$ & \\
\hline 1 & $12 / 8 / 91$ & 8 & 1 & & 81 & $\mathbf{N}$ & \\
\hline 1 & $12 / 8 / 91$ & 9 & 1 & & 89 & $\mathrm{~N}$ & \\
\hline 1 & $12 / 8 / 91$ & 10 & $\frac{T}{1}$ & & 79 & $\mathbf{N}$ & \\
\hline 1 & $12 / 8 / 91$ & 11 & 1 & & 79 & $N$ & \\
\hline 1 & $12 / 8 / 91$ & 12 & 1 & & 79 & $\mathrm{~N}$ & \\
\hline 1 & $12 / 8 / 91$ & 13 & 1 & & 79 & $\mathbf{N}$ & \\
\hline 1 & $12 / 8 / 91$ & 14 & 1 & & 79 & $\mathbf{N}$ & \\
\hline 1 & $12 / 14 / 91$ & 1 & 1 & & 97 & $Y$ & $J-910126143-64$ \\
\hline 1 & $12 / 14 / 91$ & 2 & 1 & & 97 & $\mathbf{N}$ & \\
\hline 1 & $12 / 14 / 91$ & 3 & 1 & & 97 & $\mathbf{N}$ & \\
\hline 1 & $12 / 14 / 91$ & 4 & 1 & & 96 & $\mathrm{~N}$ & \\
\hline 1 & $12 / 14 / 91$ & 5 & 1 & & 93 & $\mathbf{N}$ & \\
\hline 1 & $12 / 14 / 91$ & 6 & 1 & & 90 & $\mathrm{~N}$ & \\
\hline 1 & $12 / 14 / 91$ & 7 & 1 & & 85 & $\mathrm{~N}$ & \\
\hline
\end{tabular}

Data obtained from WHC Surveillance Analysis Computer System (SACS). February 24, 1994. 


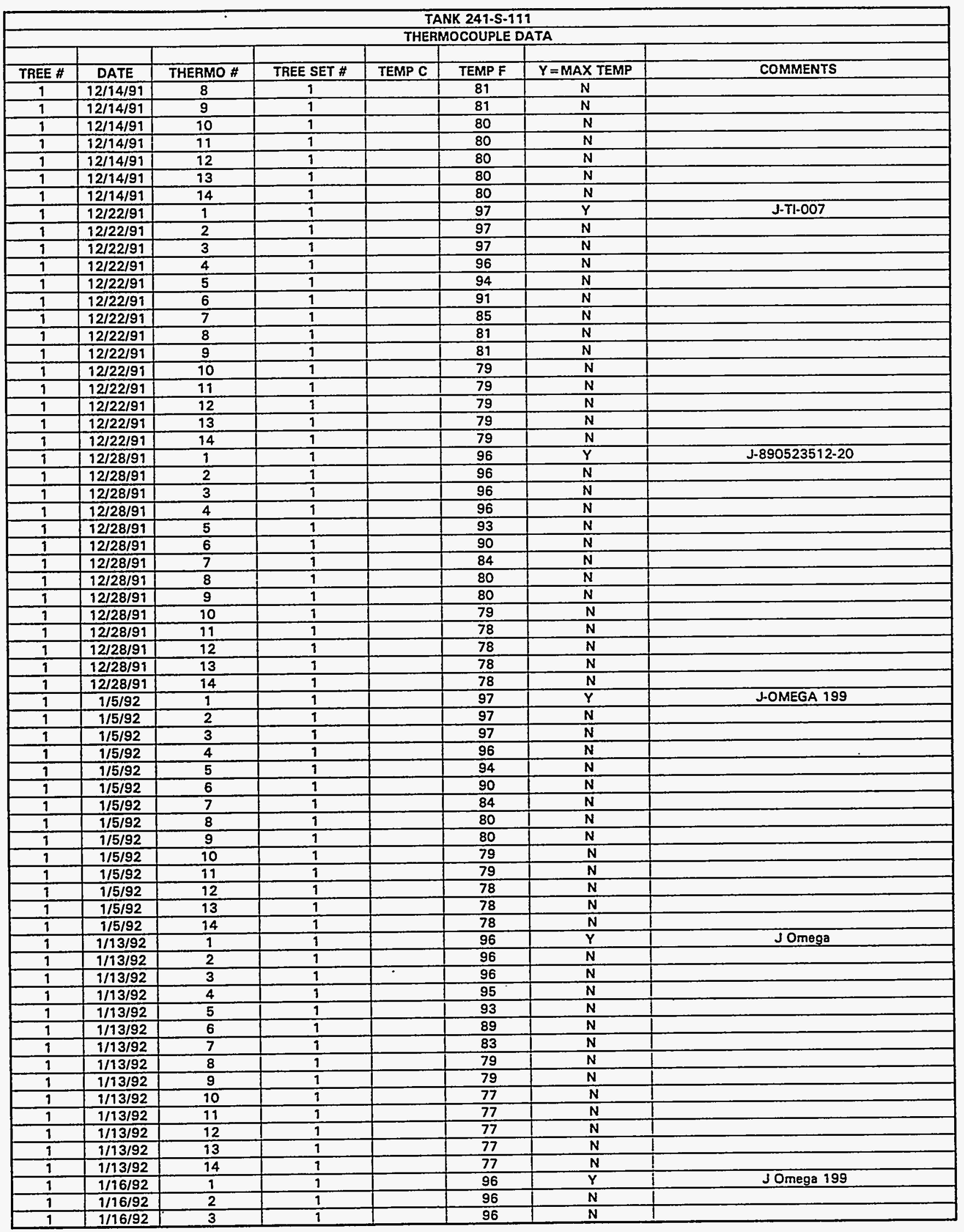

Data obtained from WHC Surveillance Analysis Computer System (SACS), February 24, 1994. 
WHC-SD-WM-ER-323, Rev. 0

\begin{tabular}{|c|c|c|c|c|c|c|c|}
\hline \multicolumn{8}{|c|}{ TANK 241-S.111 } \\
\hline \multicolumn{8}{|c|}{ THERMOCOUPLE DATA } \\
\hline TREE \# & DATE & THERMO \# & TREE SET \# & TEMP C & TEMP F & $Y=M A X$ TEMP & COMMENTS \\
\hline 1 & $1 / 16 / 92$ & 4 & 1 & & 95 & $N$ & coministo \\
\hline 1 & $1 / 16 / 92$ & 5 & 1 & & 93 & $\mathbf{N}$ & \\
\hline 1 & $1 / 16 / 92$ & 6 & 1 & & 89 & $\mathbf{N}$ & \\
\hline 1 & $1 / 16 / 92$ & 7 & 1 & & 83 & $\mathrm{~N}$ & \\
\hline 1 & $1 / 16 / 92$ & 8 & 1 & & 79 & $\mathrm{~N}$ & \\
\hline 1 & $1 / 16 / 92$ & 9 & 1 & & 78 & $\mathbf{N}$ & \\
\hline 1 & $1 / 16 / 92$ & 10 & 1 & & 77 & $\mathbf{N}$ & \\
\hline 1 & $7 / 16 / 92$ & 11 & 1 & & 77 & $\mathbf{N}$ & \\
\hline 1 & $9 / 16 / 92$ & 12 & 1 & & 77 & $\mathbf{N}$ & \\
\hline 1 & $1 / 16 / 92$ & 13 & 1 & & 77 & $\mathbf{N}$ & \\
\hline 1 & $1 / 16 / 92$ & 14 & 1 & & 77 & $N$ & \\
\hline 1 & $1 / 25 / 92$ & 1 & 1 & & 96 & $\bar{Y}$ & \\
\hline 1 & $1 / 25 / 92$ & 2 & 9 & & 96 & $\mathbf{N}$ & \\
\hline 1 & $1 / 25 / 92$ & 3 & 1 & & 96 & $\mathbf{N}$ & \\
\hline 1 & $1 / 25 / 92$ & 4 & 1 & & 95 & $\mathrm{~N}$ & \\
\hline 1 & $1 / 25 / 92$ & 5 & 9 & & 92 & $N$ & \\
\hline 1 & $1 / 25 / 92$ & 6 & 1 & & 88 & $\mathbf{N}$ & \\
\hline 1 & $1 / 25 / 92$ & 7 & 1 & & 82 & $N$ & \\
\hline 1 & $1 / 25 / 92$ & 8 & 9 & & 78 & $\mathbf{N}$ & \\
\hline 1 & $9 / 25 / 92$ & 9 & 1 & & 77 & $\mathbf{N}$ & \\
\hline 1 & $1 / 25 / 92$ & 10 & 1 & & 76 & $\mathbf{N}$ & \\
\hline 1 & $1 / 25 / 92$ & 17 & 1 & & 76 & $\mathbf{N}$ & \\
\hline 1 & $7 / 25 / 92$ & 12 & 1 & & 76 & N & \\
\hline 1 & $1 / 25 / 92$ & 13 & 1 & & 76 & $\mathbf{N}$ & \\
\hline 1 & $1 / 25 / 92$ & 14 & 1 & & 76 & $\mathbf{N}$ & \\
\hline 1 & $1 / 28 / 92$ & 1 & 1 & & & $\mathbf{N}$ & $\begin{array}{l}\text { ACCESS RESTRICTED } 1 / 28-2 / 22 \text { DUE TO } \\
\text { BATTERY FUME INCIDENT }\end{array}$ \\
\hline 1 & $2 / 22 / 92$ & 1 & 1 & & & $\bar{N}$ & OUT OF SERVICE \\
\hline 1 & $3 / 1 / 92$ & 1 & 1 & & 97 & $\bar{Y}$ & \\
\hline 1 & $3 / 1 / 92$ & 2 & 1 & & 97 & $\mathrm{~N}$ & \\
\hline 1 & $3 / 1 / 92$ & 3 & 1 & & 97 & $\mathbf{N}$ & \\
\hline 1 & $3 / 1 / 92$ & 4 & $T$ & & 95 & $\bar{N}$ & \\
\hline 1 & $3 / 1 / 92$ & 5 & 1 & & 93 & $\mathbf{N}$ & \\
\hline 1 & $3 / 1 / 92$ & 6 & 1 & & 89 & $\mathbf{N}$ & \\
\hline 1 & $3 / 1 / 92$ & 7 & 1 & & 82 & $\mathbf{N}$ & \\
\hline 1 & $3 / 1 / 92$ & 8 & 9 & & 78 & $\mathbf{N}$ & \\
\hline 1 & $3 / 1 / 92$ & 9 & 1 & & 77 & $\mathbf{N}$ & \\
\hline 1 & $3 / 9 / 92$ & 10 & 9 & & 75 & $\mathbf{N}$ & \\
\hline 1 & $3 / 1 / 92$ & 11 & 1 & & 75 & $\bar{N}$ & \\
\hline 1 & $3 / 9 / 92$ & 12 & 1 & & 75 & $\mathbf{N}$ & \\
\hline 1 & $3 / 1 / 92$ & 13 & 1 & & 75 & $N$ & \\
\hline 9 & $3 / 1 / 92$ & 94 & 1 & & 75 & $\mathrm{~N}$ & \\
\hline 1 & $3 / 6 / 92$ & 1 & 1 & & 95 & $\bar{Y}$ & $J 910126143-50$ \\
\hline 1 & $3 / 6 / 92$ & 2 & 1 & & 95 & $\mathrm{~N}$ & \\
\hline 1 & $3 / 6 / 92$ & 3 & 1 & & 95 & N & \\
\hline 1 & $3 / 6 / 92$ & 4 & 1 & & 93 & $\mathbf{N}$ & \\
\hline 1 & $3 / 6 / 92$ & 5 & $\frac{1}{1}$ & & 99 & $\mathbf{N}$ & \\
\hline 1 & $3 / 6 / 92$ & 6 & 1 & & 86 & $\mathbf{N}$ & \\
\hline 1 & $3 / 6 / 92$ & 7 & 1 & & 80 & $\mathbf{N}$ & \\
\hline 1 & $3 / 6 / 92$ & 8 & 1 & & 76 & $\mathbf{N}$ & \\
\hline 1 & $3 / 6 / 92$ & 9 & 1 & & 75 & $\mathbf{N}$ & \\
\hline 1 & $3 / 6 / 92$ & 10 & 1 & & 74 & $\mathbf{N}$ & \\
\hline 1 & $3 / 6 / 92$ & 11 & 1 & & 73 & $\mathbf{N}$ & \\
\hline 1 & $3 / 6 / 92$ & 12 & 1 & & 73 & $\mathbf{N}$ & \\
\hline 1 & $3 / 6 / 92$ & 13 & 1 & & 73 & $\mathbf{N}$ & \\
\hline 1 & $3 / 6 / 92$ & 14 & 1 & & 73 & $\mathbf{N}$ & \\
\hline 1 & $3 / 15 / 92$ & 1 & 1 & & 93 & $N$ & $J-890523512-20$ \\
\hline$\frac{1}{1}$ & $3 / 15 / 92$ & 2 & $\frac{1}{1}$ & & 94 & $\bar{Y}$ & \\
\hline 1 & $3 / 15 / 92$ & 3 & 1 & & 93 & $\mathbf{N}$ & \\
\hline 1 & $3 / 15 / 92$ & 4 & 1 & & 92 & $\mathbf{N}$ & \\
\hline 1 & $3 / 15 / 92$ & 5 & 1 & & 89 & $\mathbf{N}$ & \\
\hline 1 & $3 / 15 / 92$ & 6 & 1 & & 85 & $\mathbf{N}$ & \\
\hline 1 & $3 / 15 / 92$ & 7 & 1 & & 79 & $N$ & \\
\hline 1 & $3 / 15 / 92$ & 8 & 1 & & 74 & $\mathbf{N}$ & \\
\hline 1 & $3 / 15 / 92$ & 9 & $\frac{1}{1}$ & & 74 & $\mathrm{~N}$ & \\
\hline 1 & $3 / 15 / 92$ & 10 & 1 & & 72 & $\mathbf{N}$ & \\
\hline
\end{tabular}

Data obtained from WHC Surveillance Analysis Computer System (SÄCS), February 24, 1994. 
WHC-SD-WM-ER-323, Rev. 0

\begin{tabular}{|c|c|c|c|c|c|c|c|}
\hline \multicolumn{8}{|c|}{ TANK 247-S-111 } \\
\hline \multicolumn{8}{|c|}{ THERMOCOUPLE DATA } \\
\hline TREE \# & DATE & THERMO \# & TREE SET \# & TEMP C & TEMPF & $\bar{Y}=\overline{M A X}$ TEMP & COMMENTS \\
\hline 1 & $3 / 15 / 92$ & 11 & 1 & & 72 & $\mathrm{~N}$ & \\
\hline$\frac{1}{1}$ & $3 / 15 / 92$ & 12 & 1 & & 72 & $\mathrm{~N}$ & \\
\hline 1 & $3 / 15 / 92$ & 13 & 1 & & 72 & $\mathbf{N}$ & \\
\hline$\frac{1}{1}$ & $3 / 15 / 92$ & 14 & 1 & & 72 & $N$ & \\
\hline 1 & $3 / 22 / 92$ & 9 & 1 & & 94 & $\mathbf{N}$ & J $890423320 \mathrm{TC} \# 15-16 \mathrm{o} / \mathrm{S}$ \\
\hline 1 & $3 / 22 / 92$ & 2 & 1 & & 95 & $\bar{Y}$ & \\
\hline 1 & $3 / 22 / 92$ & 3 & 1 & & 94 & $\mathrm{~N}$ & \\
\hline 9 & $3 / 22 / 92$ & 4 & 1 & & 92 & $\bar{N}$ & \\
\hline 1 & $3 / 22 / 92$ & 5 & 1 & & 89 & $\mathbf{N}$ & \\
\hline 1 & $3 / 22 / 92$ & 6 & 1 & & 85 & $\bar{N}$ & \\
\hline 1 & $3 / 22 / 92$ & 7 & 1 & & 79 & $\mathrm{~N}$ & \\
\hline 1 & $3 / 22 / 92$ & 8 & 1 & & 75 & $\mathrm{~N}$ & \\
\hline 1 & $3 / 22 / 92$ & 9 & 1 & & 74 & $\mathrm{~N}$ & \\
\hline$\frac{1}{1}$ & $3 / 22 / 92$ & 10 & 1 & & 73 & $N$ & \\
\hline 1 & $3 / 22 / 92$ & 11 & 1 & & 73 & $\bar{N}$ & \\
\hline 9 & $3 / 22 / 92$ & 12 & 1 & & 73 & $\mathbf{N}$ & \\
\hline$\frac{1}{1}$ & $3 / 22 / 92$ & 14 & 1 & & 72 & $\mathbf{N}$ & \\
\hline 1 & $3 / 29 / 92$ & 1 & 1 & & 95 & $\mathbf{N}$ & J 199 OMEGA \\
\hline 1 & $3 / 29 / 92$ & 2 & 1 & & 96 & $\bar{Y}$ & \\
\hline 1 & $3 / 29 / 92$ & 3 & 1 & & 95 & $\mathrm{~N}$ & \\
\hline 1 & $3 / 29 / 92$ & 4 & $\frac{1}{1}$ & & 94 & $\mathbf{N}$ & \\
\hline 9 & $3 / 29 / 92$ & 5 & 1 & & 91 & $\mathrm{~N}$ & \\
\hline 1 & $3 / 29 / 92$ & 6 & 1 & & 86 & $\bar{N}$ & \\
\hline 1 & $3 / 29 / 92$ & 7 & 1 & & 81 & $N$ & \\
\hline 1 & $3 / 29 / 92$ & 8 & 1 & & 77 & $\bar{N}$ & \\
\hline 9 & $3 / 29 / 92$ & 9 & $\frac{1}{1}$ & & 76 & $\mathbf{N}$ & \\
\hline 1 & $3 / 29 / 92$ & 10 & 9 & & 74 & $N$ & \\
\hline$\frac{1}{1}$ & $3 / 29 / 92$ & 11 & 1 & & 74 & $\mathrm{~N}$ & \\
\hline 1 & $3 / 29 / 92$ & 12 & 1 & & 74 & $N$ & \\
\hline 9 & $3 / 29 / 92$ & 13 & 1 & & 74 & $N$ & \\
\hline 9 & $3 / 29 / 92$ & 14 & 1 & & 74 & $\mathbf{N}$ & \\
\hline 1 & $4 / 4 / 92$ & 1 & 1 & & 93 & $\mathbf{N}$ & $J 890423320-42$ \\
\hline 1 & $4 / 4 / 92$ & 2 & 9 & & 93.4 & $\bar{Y}$ & \\
\hline 1 & $4 / 4 / 92$ & 3 & 1 & & 93.1 & $\mathbf{N}$ & \\
\hline 1 & $4 / 4 / 92$ & 4 & 1 & & 91.4 & $\mathbf{N}$ & \\
\hline 9 & $4 / 4 / 92$ & 5 & 1 & & 88.3 & $\mathrm{~N}$ & \\
\hline 1 & $4 / 4 / 92$ & 6 & 1 & & 84.1 & $N$ & \\
\hline 9 & $4 / 4 / 92$ & 7 & 1 & & 78 & $\mathrm{~N}$ & \\
\hline 1 & $4 / 4 / 92$ & 8 & 1 & & 74 & $N$ & \\
\hline 1 & $4 / 4 / 92$ & 9 & 9 & & 73 & $N$ & \\
\hline$T$ & $4 / 4 / 92$ & 10 & 1 & & 71.6 & $\mathbf{N}$ & \\
\hline 1 & $4 / 4 / 92$ & 11 & 9 & & 71.5 & $\mathbf{N}$ & \\
\hline 9 & $4 / 4 / 92$ & 12 & 1 & & 71.6 & $\mathbf{N}$ & \\
\hline 1 & $4 / 4 / 92$ & 13 & 1 & & 71.4 & $\overline{\mathbf{N}}$ & \\
\hline 1 & $4 / 4 / 92$ & 14 & 9 & & 71.4 & $\mathbf{N}$ & \\
\hline 9 & $4 / 12 / 92$ & 1 & 7 & & 93.6 & $\mathbf{N}$ & J 900925741.79 \\
\hline 9 & $4 / 12 / 92$ & 2 & $\frac{1}{1}$ & & 94 & $\bar{Y}$ & \\
\hline 1 & $4 / 12 / 92$ & 3 & 1 & & 93.6 & $\mathbf{N}$ & \\
\hline 1 & $4 / 12 / 92$ & 4 & 1 & & 92 & $\mathbf{N}$ & \\
\hline 1 & $4 / 12 / 92$ & 5 & 1 & & 88.9 & $\mathbf{N}$ & \\
\hline 1 & $4 / 12 / 92$ & 6 & 1 & & 84.9 & $\mathbf{N}$ & \\
\hline 1 & $4 / 12 / 92$ & 7 & 1 & & 78.7 & $\mathbf{N}$ & $\cdot$ \\
\hline 7 & $4 / 12 / 92$ & 8 & 7 & & 74.8 & $\bar{N}$ & \\
\hline 1 & $4 / 12 / 92$ & 9 & 1 & & 74 & $\mathbf{N}$ & \\
\hline 9 & $4 / 12 / 92$ & 10 & 1 & & 72.4 & $\mathbf{N}$ & \\
\hline 1 & $4 / 12 / 92$ & 11 & 1 & & 72.3 & $\mathbf{N}$ & \\
\hline 1 & $4 / 12 / 92$ & 12 & $\frac{1}{1}$ & & 72.2 & $\mathbf{N}$ & \\
\hline 1 & $4 / 12 / 92$ & 13 & 1 & & 72.2 & $\mathbf{N}$ & \\
\hline 1 & $4 / 12 / 92$ & 14 & 1 & & 72.1 & $\bar{N}$ & \\
\hline 9 & $4 / 18 / 92$ & 1 & $\frac{1}{1}$ & & 86.7 & $\bar{Y}$ & $J 89042332042$ \\
\hline 1 & $4 / 18 / 92$ & 2 & $\frac{1}{1}$ & & 87.1 & $\mathbf{N}$ & \\
\hline 1 & $4 / 18 / 92$ & 3 & 1 & & 86.2 & $N$ & \\
\hline 1 & $4 / 18 / 92$ & 4 & 1 & & 84.9 & $\mathbf{N}$ & \\
\hline 1 & $4 / 18 / 92$ & 5 & 9 & & 82 & $\mathbf{N}$ & \\
\hline 1 & $4 / 18 / 92$ & 6 & 1 & & 78 & $\mathrm{~N}$ & \\
\hline 1 & $4 / 18 / 92$ & 7 & 1 & & 72.2 & $\mathbf{N}$ & \\
\hline
\end{tabular}

Data obtained from WHC Surveillance Analysis Computer System (SACS), February 24, 1994. 


\begin{tabular}{|c|c|c|c|c|c|c|c|}
\hline \multicolumn{8}{|c|}{ TANK 241-S-111 } \\
\hline \multicolumn{8}{|c|}{ THEAMOCOUPLE DATA } \\
\hline TREE \# & DATE & THERMO \# & TREE SET \# & TEMP C & TEMP F & $Y=M A X$ TEMP & COMMENTS \\
\hline 1 & $4 / 18 / 92$ & 8 & 1 & & 68.4 & $N$ & \\
\hline 1 & $4 / 18 / 92$ & 9 & 1 & & 67.5 & $\mathbf{N}$ & \\
\hline 1 & $4 / 18 / 92$ & 10 & 1 & & 66.2 & $\mathbf{N}$ & \\
\hline 1 & $4 / 18 / 92$ & 11 & 1 & & 65.9 & $\mathbf{N}$ & \\
\hline 1 & $4 / 18 / 92$ & 12 & 1 & & 65.5 & $\mathbf{N}$ & \\
\hline 1 & $4 / 18 / 92$ & 13 & 1 & & 65.4 & $\mathbf{N}$ & \\
\hline 1 & $4 / 18 / 92$ & 14 & 1 & & 65.2 & $\mathbf{N}$ & \\
\hline 1 & $4 / 25 / 92$ & 1 & 1 & & 94 & $\bar{Y}$ & $\mathrm{~J} 89042332042$ \\
\hline 1 & $4 / 25 / 92$ & 2 & 1 & & 94 & $\mathbf{N}$ & \\
\hline 1 & $4 / 25 / 92$ & 3 & 1 & & 94 & $\mathbf{N}$ & \\
\hline 1 & $4 / 25 / 92$ & 4 & 1 & & 92 & $\mathbf{N}$ & \\
\hline 1 & $4 / 25 / 92$ & 5 & 1 & & 89 & $\mathbf{N}$ & \\
\hline 1 & $4 / 25 / 92$ & 6 & 1 & & 85 & $\mathbf{N}$ & \\
\hline 1 & $4 / 25 / 92$ & 7 & 1 & & 80 & $\mathbf{N}$ & \\
\hline 9 & $4 / 25 / 92$ & 8 & 1 & & 76 & $\overline{\mathbf{N}}$ & \\
\hline 1 & $4 / 25 / 92$ & 9 & 1 & & 75 & $\mathbf{N}$ & \\
\hline 1 & $4 / 25 / 92$ & 10 & 1 & & 73 & $\mathbf{N}$ & \\
\hline 1 & $4 / 25 / 92$ & 11 & 1 & & 73 & $\mathbf{N}$ & \\
\hline 1 & $4 / 25 / 92$ & 12 & 1 & & 73 & $\mathbf{N}$ & \\
\hline 1 & $4 / 25 / 92$ & 13 & 1 & & 73 & $\mathbf{N}$ & \\
\hline 1 & $4 / 25 / 92$ & 14 & 9 & & 73 & $\mathbf{N}$ & \\
\hline 1 & $4 / 30 / 92$ & 1 & 1 & & 93 & $N$ & J 900925741.79 \\
\hline 1 & $4 / 30 / 92$ & 2 & 1 & & 93.3 & $\bar{Y}$ & \\
\hline 1 & $4 / 30 / 92$ & 3 & 1 & & 92.9 & $\mathbf{N}$ & \\
\hline 1 & $4 / 30 / 92$ & 4 & 1 & & 91.2 & $\mathbf{N}$ & \\
\hline 1 & $4 / 30 / 92$ & 5 & 1 & & 88.1 & $\mathbf{N}$ & \\
\hline 1 & $4 / 30 / 92$ & 6 & 1 & & 84.1 & $\mathbf{N}$ & \\
\hline 1 & $4 / 30 / 92$ & 7 & 1 & & 78.3 & $\mathbf{N}$ & \\
\hline 1 & $4 / 30 / 92$ & 8 & 1 & & 74.7 & $\mathrm{~N}$ & \\
\hline 1 & $4 / 30 / 92$ & 9 & 1 & & 73.7 & $\mathbf{N}$ & \\
\hline 1 & $4 / 30 / 92$ & 10 & 1 & & 72.2 & $N$ & \\
\hline 1 & $4 / 30 / 92$ & 11 & 1 & & 72.2 & $N$ & \\
\hline 1 & $4 / 30 / 92$ & 12 & 1 & & 72.2 & $\mathbf{N}$ & \\
\hline 9 & $4 / 30 / 92$ & 13 & 1 & & 72.1 & $\mathbf{N}$ & \\
\hline 1 & $4 / 30 / 92$ & 14 & 1 & & 72 & $\mathbf{N}$ & \\
\hline 1 & $5 / 8 / 92$ & 1 & 1 & & 89.6 & $\mathbf{N}$ & $\mathrm{J} 890423320-42$ \\
\hline 1 & $5 / 8 / 92$ & 2 & 1 & & 89.8 & $\mathrm{Y}$ & \\
\hline 1 & $5 / 8 / 92$ & 3 & 1 & & 89.5 & $\mathbf{N}$ & \\
\hline 1 & $5 / 8 / 92$ & 4 & 1 & & 87.8 & $\mathbf{N}$ & \\
\hline 1 & $5 / 8 / 92$ & 5 & 1 & & 84.9 & $\mathbf{N}$ & \\
\hline 1 & $5 / 8 / 92$ & 6 & 1 & & 81 & $\bar{N}$ & \\
\hline 1 & $5 / 8 / 92$ & 7 & 1 & & 75.4 & $\mathbf{N}$ & \\
\hline 1 & $5 / 8 / 92$ & 8 & 1 & & 71.8 & $\mathbf{N}$ & \\
\hline 1 & $5 / 8 / 92$ & 9 & 1 & & 70.9 & $\mathbf{N}$ & \\
\hline 1 & $5 / 8 / 92$ & 10 & 1 & & 69.7 & $\mathbf{N}$ & \\
\hline 1 & $5 / 8 / 92$ & 11 & 1 & & 69.5 & $\bar{N}$ & \\
\hline 1 & $5 / 8 / 92$ & 12 & 1 & & 69.5 & $\mathrm{~N}$ & \\
\hline 1 & $5 / 8 / 92$ & 13 & 1 & & 69.4 & $\mathbf{N}$ & \\
\hline 1 & $5 / 8 / 92$ & 14 & 1 & & 69.3 & $\mathbf{N}$ & \\
\hline 1 & $5 / 16 / 92$ & 1 & 1 & & 89.7 & $\mathrm{~N}$ & $1890423320-42$ \\
\hline 1 & $5 / 16 / 92$ & 2 & 1 & & 89.9 & $\mathbf{Y}$ & \\
\hline 1 & $5 / 16 / 92$ & 3 & 1 & & 89.5 & $\mathbf{N}$ & \\
\hline 1 & $5 / 16 / 92$ & 4 & 1 & & 87.8 & $\mathbf{N}$ & \\
\hline 1 & $5 / 16 / 92$ & 5 & 1 & & 84.9 & $\mathbf{N}$ & \\
\hline 1 & $5 / 16 / 92$ & 6 & 1 & & 80.9 & $\mathbf{N}$ & \\
\hline 1 & $5 / 16 / 92$ & 7 & 1 & & 75.6 & $\mathbf{N}$ & \\
\hline 1 & $5 / 16 / 92$ & 8 & 1 & & 72.2 & $\mathbf{N}$ & \\
\hline 1 & $5 / 16 / 92$ & 9 & 1 & & 71.3 & $\mathbf{N}$ & \\
\hline 1 & $5 / 16 / 92$ & 10 & 1 & & 70.1 & $\mathrm{~N}$ & \\
\hline 1 & $5 / 16 / 92$ & 11 & 1 & & 70 & $\mathbf{N}$ & \\
\hline 1 & $5 / 16 / 92$ & 12 & 1 & & 70.1 & $N$ & \\
\hline 1 & $5 / 16 / 92$ & 13 & 1 & & 70.1 & $\mathbf{N}$ & \\
\hline 1 & $5 / 16 / 92$ & 14 & 1 & & 70 & $\mathbf{N}$ & \\
\hline 1 & $5 / 20 / 92$ & 1 & 1 & & 94 & $\mathbf{Y}$ & $\mathrm{J} 89042330-42$ \\
\hline 1 & $5 / 20 / 92$ & 2 & $\frac{1}{1}$ & & 94 & $\mathbf{N}$ & \\
\hline 1 & $5 / 20 / 92$ & 3 & 1 & & 94 & $\mathbf{N}$ & \\
\hline
\end{tabular}

Data obtained from WHC Surveillance Analysis Computer System (SACS), February 24, 1994. 
WHC-SD-WM-ER-323, Rev. 0

\begin{tabular}{|c|c|c|c|c|c|c|c|}
\hline \multicolumn{8}{|c|}{ TANK 241-S-111 } \\
\hline & & & & THEF & $\overline{\text { OCOUPLE }}$ & ITA & \\
\hline TREE \# & DATE & THERMO \# & TREE SET \# & TEMP $\bar{C}$ & TEMP F & $Y=$ MAX TEMP & COMMENTS \\
\hline 9 & $5 / 20 / 92$ & 4 & 1 & & 92 & $\mathrm{~N}$ & \\
\hline 1 & $5 / 20 / 92$ & 5 & 1 & & 89 & $\mathbf{N}$ & \\
\hline 1 & $5 / 20 / 92$ & 6 & 1 & & 85 & $\bar{N}$ & \\
\hline 1 & $5 / 20 / 92$ & 7 & 1 & & 80 & $\mathrm{~N}$ & \\
\hline$\frac{1}{1}$ & $5 / 20 / 92$ & 8 & 1 & & 76 & $\mathbf{N}$ & \\
\hline 1 & $5 / 20 / 92$ & 9 & 1 & & 75 & $\mathrm{~N}$ & \\
\hline 1 & $5 / 20 / 92$ & 10 & 1 & & 74 & $\bar{N}$ & \\
\hline 1 & $5 / 20 / 92$ & 11 & 1 & & 74 & $N$ & \\
\hline 1 & $5 / 20 / 92$ & 12 & 1 & & 74 & $\mathrm{~N}$ & \\
\hline 1 & $5 / 20 / 92$ & 13 & 1 & & 74 & $\mathrm{~N}$ & \\
\hline 1 & $5 / 20 / 92$ & 14 & 1 & & 74 & $\mathbf{N}$ & \\
\hline 1 & $5 / 30 / 92$ & 1 & 1 & & 91.5 & $\mathbf{N}$ & J $900925741-79$ \\
\hline 1 & $5 / 30 / 92$ & 2 & 1 & & 91.7 & $\bar{Y}$ & \\
\hline 1 & $5 / 30 / 92$ & 3 & 1 & & 91.2 & 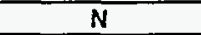 & \\
\hline 1 & $5 / 30 / 92$ & 4 & 1 & & 89.4 & $\mathbf{N}$ & \\
\hline 1 & $5 / 30 / 92$ & 5 & 1 & & 86.4 & $N$ & \\
\hline 1 & $5 / 30 / 92$ & 6 & 1 & & 82.5 & $\mathbf{N}$ & \\
\hline 1 & $5 / 30 / 92$ & 7 & 1 & & 77.6 & $\mathbf{N}$ & \\
\hline 1 & $5 / 30 / 92$ & 8 & 1 & & 74.3 & $\bar{N}$ & \\
\hline 1 & $5 / 30 / 92$ & 9 & 1 & & 73.5 & $\mathrm{~N}$ & \\
\hline 1 & $5 / 30 / 92$ & 10 & 1 & & 72.4 & $\bar{N}$ & \\
\hline 1 & $5 / 30 / 92$ & 11 & 1 & & 72.3 & $\bar{N}$ & \\
\hline 1 & $5 / 30 / 92$ & 12 & 1 & & 72.5 & $\mathbf{N}$ & \\
\hline 1 & $5 / 30 / 92$ & 13 & 1 & & 72.3 & $\mathbf{N}$ & \\
\hline 1 & $5 / 30 / 92$ & 94 & 1 & & 72.3 & $\mathrm{~N}$ & \\
\hline 1 & $6 / 8 / 92$ & 1 & 1 & & 88.2 & $N$ & $\mathrm{~J} 890423320-42$ \\
\hline 1 & $6 / 8 / 92$ & 2 & 1 & & 88.5 & $\bar{Y}$ & \\
\hline 1 & $6 / 8 / 92$ & 3 & 1 & & 88 & $\bar{N}$ & \\
\hline 1 & $6 / 8 / 92$ & 4 & 1 & & 86.3 & $\mathbf{N}$ & \\
\hline 1 & $6 / 8 / 92$ & 5 & 1 & & 83.5 & $\bar{N}$ & \\
\hline 1 & $6 / 8 / 92$ & 6 & 1 & & 79.7 & $\mathbf{N}$ & \\
\hline 9 & $6 / 8 / 92$ & 7 & 1 & & 75 & $\bar{N}$ & \\
\hline 1 & $6 / 8 / 92$ & 8 & 1 & & 72 & $\mathbf{N}$ & \\
\hline 1 & $6 / 8 / 92$ & 9 & 1 & & 70.9 & $\mathrm{~N}$ & \\
\hline 1 & $6 / 8 / 92$ & 10 & 1 & & 70.1 & $N$ & \\
\hline 1 & $6 / 8 / 92$ & 11 & 1 & & 70 & $N$ & \\
\hline 1 & $6 / 8 / 92$ & 12 & 1 & & 70.2 & $\mathbf{N}$ & \\
\hline 1 & $6 / 8 / 92$ & 13 & 1 & & 70.1 & $\bar{N}$ & \\
\hline 1 & $6 / 8 / 92$ & 14 & 1 & & 70.2 & $N$ & \\
\hline 1 & $6 / 13 / 92$ & 1 & 1 & & 92.7 & $N$ & $J 900925741-79$ \\
\hline 9 & $6 / 13 / 92$ & 2 & 1 & & 92.9 & $\bar{Y}$ & \\
\hline 1 & $6 / 73 / 92$ & 3 & 1 & & 92.5 & $\bar{N}$ & \\
\hline 1 & $6 / 13 / 92$ & 4 & 1 & & 90.7 & $\mathbf{N}$ & \\
\hline 1 & $6 / 73 / 92$ & 5 & 1 & & 87.9 & $\mathbf{N}$ & \\
\hline 1 & $6 / 13 / 92$ & 5 & 1 & & 84.3 & $\bar{N}$ & \\
\hline 1 & $6 / 13 / 92$ & 7 & 1 & & 79.6 & $\bar{N}$ & \\
\hline 1 & $6 / 73 / 92$ & 8 & 1 & & 76.5 & $\mathbf{N}$ & \\
\hline 1 & $6 / 13 / 92$ & 9 & 1 & & 75.7 & $N$ & \\
\hline 1 & $6 / 13 / 92$ & 10 & 1 & & 74.8 & $N$ & \\
\hline 1 & $6 / 13 / 92$ & 11 & 1 & & 74.8 & $\bar{N}$ & \\
\hline 1 & $6 / 13 / 92$ & 92 & 1 & & 74.8 & $\mathbf{N}$ & \\
\hline 1 & $6 / 13 / 92$ & 13 & 1 & & 74.8 & $\mathbf{N}$ & \\
\hline 1 & $6 / 13 / 92$ & 14 & 1 & & 74.7 & $\mathbf{N}$ & \\
\hline 1 & $6 / 19 / 92$ & 1 & 1 & & 87.6 & $N$ & J $890423320-426-92$ \\
\hline 1 & $6 / 19 / 92$ & 2 & 1 & & 88 & $\bar{Y}$ & \\
\hline 1 & $6 / 19 / 92$ & 3 & 1 & & 87.6 & $N$ & \\
\hline 1 & $6 / 19 / 92$ & 4 & 1 & & 85.9 & $\mathbf{N}$ & \\
\hline 1 & $6 / 19 / 92$ & 5 & 1 & & 83.2 & $\bar{N}$ & \\
\hline 1 & $6 / 19 / 92$ & 6. & 1 & & 79.6 & $\mathbf{N}$ & \\
\hline 9 & $6 / 19 / 92$ & 7 & 9 & & 75.2 & $\mathrm{~N}$ & \\
\hline 1 & $6 / 19 / 92$ & 8 & 1 & & 72.2 & $\mathbf{N}$ & \\
\hline 1 & $6 / 19 / 92$ & 9 & 1 & & 71.7 & $\mathbf{N}$ & \\
\hline 1 & $6 / 19 / 92$ & 10 & 1 & & 70.8 & $\mathbf{N}$ & \\
\hline 9 & $6 / 19 / 92$ & 11 & 1 & & 70.7 & $\mathbf{N}$ & \\
\hline 1 & $6 / 19 / 92$ & 12 & 9 & & 70.9 & $\mathbf{N}$ & \\
\hline 1 & $6 / 19 / 92$ & 13 & 1 & & 70.8 & $\mathbf{N}$ & \\
\hline
\end{tabular}

Data obtained from WHC Surveillance Analysis Computer System (SACS), February 24, 1994. 
WHC-SD-WM-ER-323, Rev. 0

\begin{tabular}{|c|c|c|c|c|c|c|c|}
\hline \multicolumn{8}{|c|}{ TANK 241.S-111 } \\
\hline \multicolumn{8}{|c|}{ THERMOCOUPLE DATA } \\
\hline TREE \# & DATE & THERMO \# & TREE SET \# & TEMP C & TEMP F & $Y=M A X$ TEMP & COMMENTS \\
\hline 1 & $6 / 19 / 92$ & 14 & 1 & ston & 70.9 & $\mathrm{~N}$ & comincois \\
\hline 1 & $6 / 29 / 92$ & 1 & 1 & & 91 & $\mathbf{N}$ & J TK102-890423320-42 \\
\hline 1 & $6 / 29 / 92$ & 2 & 1 & & 92 & $\bar{Y}$ & \\
\hline 1 & $6 / 29 / 92$ & 3 & 1 & & 91 & $\mathbf{N}$ & \\
\hline 1 & $6 / 29 / 92$ & 4 & 1 & & 90 & $\mathbf{N}$ & \\
\hline 1 & $6 / 29 / 92$ & 5 & $T$ & & 87 & $\mathbf{N}$ & \\
\hline 1 & $6 / 29 / 92$ & 6 & 1 & & 84 & $\mathbf{N}$ & \\
\hline 1 & $6 / 29 / 92$ & 7 & 1 & & 79 & $\mathbf{N}$ & \\
\hline 1 & $6 / 29 / 92$ & 8 & 1 & & 76 & $\mathbf{N}$ & \\
\hline 1 & $6 / 29 / 92$ & 9 & 1 & & 76 & $\mathbf{N}$ & \\
\hline 1 & $6 / 29 / 92$ & 10 & 1 & & 75 & $\mathbf{N}$ & \\
\hline 1 & $6 / 29 / 92$ & 19 & 1 & & 75 & $\mathbf{N}$ & \\
\hline 1 & $6 / 29 / 92$ & 12 & 1 & & 75 & $\mathbf{N}$ & \\
\hline 1 & $6 / 29 / 92$ & 13 & 1 & & 75 & $\bar{N}$ & \\
\hline 9 & $6 / 29 / 92$ & 14 & 1 & & 75 & $\mathrm{~N}$ & \\
\hline 1 & $7 / 8 / 92$ & 1 & 1 & & 88 & $N$ & $J 890523512-47$ \\
\hline 1 & $7 / 8 / 92$ & 2 & 1 & & 88.1 & $Y$ & \\
\hline 1 & $7 / 8 / 92$ & 3 & 1 & & 87.9 & $\mathrm{~N}$ & \\
\hline 1 & $7 / 8 / 92$ & 4 & 1 & & 86 & $\mathbf{N}$ & \\
\hline 1 & $7 / 8 / 92$ & 5 & 9 & & 83.4 & $N$ & \\
\hline 1 & $7 / 8 / 92$ & 6 & 1 & & 80.1 & $\mathrm{~N}$ & \\
\hline 1 & $7 / 8 / 92$ & 7 & 1 & & 76.2 & $\mathbf{N}$ & \\
\hline 1 & $7 / 8 / 92$ & 8 & 1 & & 73.7 & $N$ & \\
\hline 1 & $7 / 8 / 92$ & 9 & 1 & & 73 & $\mathbf{N}$ & \\
\hline 1 & $7 / 8 / 92$ & 10 & 1 & & 72.3 & $\mathbf{N}$ & \\
\hline 1 & $7 / 8 / 92$ & 11 & 1 & & 72.3 & $\mathrm{~N}$ & \\
\hline 1 & $7 / 8 / 92$ & 12 & 1 & & 72.3 & $N$ & \\
\hline 9 & $7 / 8 / 92$ & 13 & 1 & & 72.3 & $\mathbf{N}$ & \\
\hline 1 & $7 / 8 / 92$ & 14 & 1 & & 72.3 & $\mathbf{N}$ & \\
\hline 1 & $7 / 16 / 92$ & 1 & 1 & & 87.9 & $\mathbf{N}$ & J 890523512-42 \\
\hline 1 & $7 / 16 / 92$ & 2 & 1 & & 88 & $\bar{Y}$ & \\
\hline 1 & $7 / 16 / 92$ & 3 & 1 & & 87.8 & $\mathrm{~N}$ & \\
\hline 1 & $7 / 16 / 92$ & 4 & 1 & & 86 & $\mathrm{~N}$ & \\
\hline$\frac{1}{1}$ & $7 / 16 / 92$ & 5 & 1 & & 83.5 & $\mathbf{N}$ & \\
\hline 1 & $7 / 16 / 92$ & 6 & 1 & & 80.2 & $\bar{N}$ & \\
\hline 1 & $7 / 16 / 92$ & 7 & 1 & & 76.6 & $\bar{N}$ & \\
\hline 1 & $7 / 16 / 92$ & 8 & 1 & & 74.1 & $\mathbf{N}$ & \\
\hline 1 & $7 / 16 / 92$ & 9 & 1 & & 73.4 & $N$ & \\
\hline 1 & $7 / 16 / 92$ & 10 & 1 & & 72.7 & $N$ & \\
\hline 1 & $7 / 16 / 92$ & 11 & 1 & & 72.6 & $\mathrm{~N}$ & \\
\hline 1 & $7 / 16 / 92$ & 12 & 1 & & 72.8 & $\mathbf{N}$ & \\
\hline$\frac{1}{1}$ & $7 / 16 / 92$ & 13 & 1 & & 72.7 & $N$ & \\
\hline 1 & $7 / 16 / 92$ & 14 & 1 & & 72.7 & $\mathrm{~N}$ & \\
\hline 1 & $7 / 24 / 92$ & 1 & 1 & & 87.8 & $\mathbf{N}$ & $J 890523512.47$ \\
\hline 1 & $7 / 24 / 92$ & 2 & 1 & & 88 & $\bar{Y}$ & \\
\hline 1 & $7 / 24 / 92$ & 3 & 1 & & 87.7 & $\bar{N}$ & \\
\hline 1 & $7 / 24 / 92$ & 4 & 1 & & 86.1 & $N$ & \\
\hline 1 & $7 / 24 / 92$ & 5 & 1 & & 83.7 & $\mathrm{~N}$ & \\
\hline 1 & $7 / 24 / 92$ & 6 & 1 & & 80.6 & $\mathrm{~N}$ & \\
\hline 9 & $7 / 24 / 92$ & 7 & 1 & & 77.1 & $\mathbf{N}$ & \\
\hline 1 & $7 / 24 / 92$ & 8 & 1 & & 74.6 & $\mathbf{N}$ & \\
\hline 1 & $7 / 24 / 92$ & 9 & 9 & - & 74 & $\mathrm{~N}$ & \\
\hline$\frac{1}{1}$ & $7 / 24 / 92$ & 10 & 1 & & 73.2 & $\mathbf{N}$ & \\
\hline 1 & $7 / 24 / 92$ & 11 & 1 & & 73.2 & $N$ & $\cdot$ \\
\hline 1 & $7 / 24 / 92$ & 12 & 1 & & 73.2 & $\mathrm{~N}$ & \\
\hline 1 & $7 / 24 / 92$ & 13 & 1 & & 73.2 & $\mathbf{N}$ & \\
\hline 1 & $7 / 24 / 92$ & 14 & 1 & & 73.2 & $N$ & \\
\hline 1 & $7 / 30 / 92$ & 1 & 1 & & 86.1 & $\mathbf{N}$ & J 890523512.47 \\
\hline 9 & $7 / 30 / 92$ & 2 & 1 & & 86 & $\mathbf{N}$ & \\
\hline 1 & $7 / 30 / 92$ & 3 & 1 & & 85.8 & $Y$ & \\
\hline 1 & $7 / 30 / 92$ & 4 & 1 & & 85.8 & $\bar{N}$ & \\
\hline 9 & $7 / 30 / 92$ & 5 & 1 & & 85.5 & $\mathbf{N}$ & \\
\hline 9 & $7 / 30 / 92$ & 6 & 1 & & 84.7 & $\mathrm{~N}$ & \\
\hline 1 & $7 / 30 / 92$ & 7 & 1 & & 81.2 & $\mathbf{N}$ & \\
\hline 1 & $7 / 30 / 92$ & 8 & 1 & & 83.7 & $\mathbf{N}$ & \\
\hline 1 & $7 / 30 / 92$ & 9 & 1 & & 77.6 & $\mathbf{N}$ & \\
\hline
\end{tabular}

Data obtained from WHC Surveillance Analysis Computer System (SACS), February 24, 1994. 


\begin{tabular}{|c|c|c|c|c|c|c|c|}
\hline \multicolumn{8}{|c|}{ TANK 241-S-111 } \\
\hline \multicolumn{8}{|c|}{ THERMOCOUPLE DATA } \\
\hline TREE \# & DATE & THERMO \# & TREE SET \# & TEMP C & TEMP F & $Y=$ MAX TEMP & COMMENTS \\
\hline 1 & $7 / 30 / 92$ & 10 & 1 & & 74.4 & $\mathrm{~N}$ & \\
\hline 1 & $7 / 30 / 92$ & 11 & 9 & & 71.8 & $\mathrm{~N}$ & \\
\hline 1 & $7 / 30 / 92$ & 12 & 1 & & 70.1 & $\bar{N}$ & \\
\hline 1 & $7 / 30 / 92$ & 13 & 1 & & 69.6 & $\mathrm{~N}$ & \\
\hline 9 & $7 / 30 / 92$ & 14 & $\frac{1}{1}$ & & 69.5 & $\mathbf{N}$ & \\
\hline 1 & $8 / 6 / 92$ & 1 & $\frac{1}{1}$ & & 90.8 & $\bar{N}$ & $J 890523512-47$ \\
\hline 1 & $8 / 6 / 92$ & 2 & 1 & & 91.2 & $\bar{Y}$ & \\
\hline 1 & $8 / 6 / 92$ & $\overline{3}$ & 1 & & 90.8 & $\bar{N}$ & \\
\hline 9 & $8 / 6 / 92$ & 4 & 1 & & 89.4 & $N$ & \\
\hline 1 & $8 / 6 / 92$ & 5 & 9 & & 87.2 & $\bar{N}$ & \\
\hline 1 & $8 / 6 / 92$ & 6 & 1 & & 84.4 & $\bar{N}$ & \\
\hline 1 & $8 / 6 / 92$ & 7 & 1 & & 80.8 & $\bar{N}$ & \\
\hline 1 & $8 / 6 / 92$ & 8 & 1 & & 78.6 & $\mathbf{N}$ & \\
\hline 1 & $8 / 6 / 92$ & 9 & 1 & & 77.9 & $N$ & \\
\hline 1 & $8 / 6 / 92$ & 10 & $T$ & & 77.4 & $\bar{N}$ & \\
\hline 1 & $8 / 6 / 92$ & 11 & 1 & & 77.3 & $N$ & \\
\hline 1 & $8 / 6 / 92$ & 12 & $\frac{1}{1}$ & & 77.3 & $\bar{N}$ & \\
\hline 1 & $8 / 6 / 92$ & 13 & 1 & & 77.4 & $\bar{N}$ & \\
\hline 1 & $8 / 6 / 92$ & 14 & 1 & & 77.4 & $\bar{N}$ & \\
\hline 9 & $8 / 16 / 92$ & 1 & 1 & & 89.8 & $\bar{N}$ & J910126143-29 \\
\hline 1 & $8 / 16 / 92$ & 2 & 1 & & 90.1 & $\bar{Y}$ & \\
\hline 1 & $8 / 16 / 92$ & 3 & 1 & & 89.7 & $\mathbf{N}$ & \\
\hline 1 & $8 / 16 / 92$ & 4 & 1 & & 88.3 & $\bar{N}$ & \\
\hline 1 & $8 / 16 / 92$ & 5 & 1 & & 86.1 & $\bar{N}$ & \\
\hline 1 & $8 / 16 / 92$ & 6 & 1 & & 83.2 & $\bar{N}$ & \\
\hline 1 & $8 / 16 / 92$ & 7 & 1 & & 80 & $\bar{N}$ & \\
\hline 1 & $8 / 16 / 92$ & 8 & 1 & & 77.9 & $\mathbf{N}$ & \\
\hline 9 & $8 / 16 / 92$ & 9 & 1 & & 77.2 & $\mathbf{N}$ & \\
\hline 1 & $8 / 16 / 92$ & 10 & 1 & & 76.6 & $\mathbf{N}$ & \\
\hline 1 & $8 / 16 / 92$ & 11 & $\frac{1}{1}$ & & 76.5 & $\bar{N}$ & \\
\hline 1 & $8 / 16 / 92$ & 12 & 1 & & 76.7 & $\mathbf{N}$ & \\
\hline 1 & $8 / 16 / 92$ & 13 & 1 & & 76.6 & $\mathbf{N}$ & \\
\hline 1 & $8 / 16 / 92$ & 14 & 1 & & 76.7 & $\mathbf{N}$ & \\
\hline 1 & $8 / 21 / 92$ & 1 & 1 & & 90.5 & $\mathbf{N}$ & $J 890523512-47$ \\
\hline 1 & $8 / 21 / 92$ & 2 & 1 & & 90.8 & $\bar{Y}$ & \\
\hline 1 & $8 / 21 / 92$ & 3 & 1 & & 90.5 & $\mathbf{N}$ & \\
\hline$\frac{1}{1}$ & $8 / 21 / 92$ & 4 & $T$ & & 89.3 & $\bar{N}$ & \\
\hline 1 & $8 / 21 / 92$ & 5 & 1 & & 87.2 & $\mathbf{N}$ & \\
\hline 1 & $8 / 21 / 92$ & 6 & 1 & & 84.6 & $\mathbf{N}$ & \\
\hline 1 & $8 / 21 / 92$ & 7 & .1 & & 81.5 & $\bar{N}$ & \\
\hline$\frac{1}{1}$ & $8 / 21 / 92$ & 8 & 1 & & 79.2 & $\mathbf{N}$ & \\
\hline 1 & $8 / 21 / 92$ & 9 & $\frac{1}{1}$ & & 78.4 & $\mathrm{~N}$ & \\
\hline 1 & $8 / 21 / 92$ & 10 & 1 & & 78 & $N$ & \\
\hline 1 & $8 / 21 / 92$ & 11 & 1 & & 77.9 & $\mathbf{N}$ & \\
\hline 1 & $8 / 21 / 92$ & 12 & 1 & & 79 & $\mathbf{N}$ & \\
\hline 1 & $8 / 21 / 92$ & 13 & 1 & & 78 & $\mathbf{N}$ & \\
\hline 1 & $8 / 27 / 92$ & 14 & $\overline{1}$ & & 78 & $\mathbf{N}$ & \\
\hline 1 & $9 / 3 / 92$ & 1 & 1 & & 90.8 & $N$ & $J 890523512.47$ \\
\hline 1 & $9 / 3 / 92$ & 2 & 1 & & 91.2 & $\bar{Y}$ & \\
\hline 1 & $9 / 3 / 92$ & 3 & 1 & & 91 & $\mathbf{N}$ & \\
\hline 1 & $9 / 3 / 92$ & 4 & 1 & & 89.7 & $\mathbf{N}$ & \\
\hline$\frac{1}{1}$ & $9 / 3 / 92$ & 5 & 1 & & 87.7 & $\mathbf{N}$ & \\
\hline 1 & $9 / 3 / 92$ & 6 & 1 & & 85 & $\mathbf{N}$ & \\
\hline 1 & $9 / 3 / 92$ & 7 & $\frac{1}{1}$ & & 82.1 & $\bar{N}$ & \\
\hline 1 & $9 / 3 / 92$ & 8 & 1 & & 80 & $\mathbf{N}$ & \\
\hline 1 & $9 / 3 / 92$ & 9 & 1 & & 79.3 & $\mathbf{N}$ & \\
\hline 1 & $9 / 3 / 92$ & 10 & $\overline{1}$ & & 78.7 & $\mathbf{N}$ & \\
\hline$-\frac{1}{1}$ & $9 / 3 / 92$ & 11 & 1 & & 78.7 & $\mathbf{N}$ & \\
\hline 1 & $9 / 3 / 92$ & 12 & 1 & & 78.9 & $\mathbf{N}$ & \\
\hline 1 & $9 / 3 / 92$ & 13 & 1 & & 78.8 & $\mathbf{N}$ & \\
\hline 1 & $9 / 3 / 92$ & 34 & 1 & & 78.8 & $N$ & \\
\hline 1 & $9 / 9 / 92$ & 1 & 1 & & 93 & $Y$ & J $910126143-29$ \\
\hline 1 & $9 / 9 / 92$ & 2 & 1 & & 93 & $\bar{N}$ & \\
\hline 1 & $9 / 9 / 92$ & 3 & $\frac{1}{1}$ & & 93 & $\mathbf{N}$ & \\
\hline 1 & $9 / 9 / 92$ & 4 & $\frac{1}{1}$ & & 92 & $N$ & \\
\hline 1 & $9 / 9 / 92$ & 5 & 1 & & 90 & $\bar{N}$ & \\
\hline
\end{tabular}

Data obtained from WHC Surveillance Analysis Computer System (SACS), February 24, 1994. 


\begin{tabular}{|c|c|c|c|c|c|c|c|}
\hline \multicolumn{8}{|c|}{ TANK 241-S-111 } \\
\hline \multicolumn{8}{|c|}{ THERMOCOUPLE DATA } \\
\hline TREE \# & DATE & THERMO \# & TREE SET \# & TEMP C & TEMP F & $Y=M A X$ TEMP & COMMENTS \\
\hline 1 & 9/9/92 & 6 & 1 & & 87 & $N$ & \\
\hline 1 & $9 / 9 / 92$ & 7 & 1 & & 85 & $\mathbf{N}$ & \\
\hline 1 & $9 / 9 / 92$ & 8 & 1 & & 82 & $N$ & \\
\hline 1 & 9/9/92 & 9 & 1 & & 82 & $\bar{N}$ & \\
\hline 1 & $9 / 9 / 92$ & 10 & 1 & & 81 & $N$ & \\
\hline 1 & $9 / 9 / 92$ & 11 & $\overline{1}$ & & 81 & $N$ & \\
\hline 1 & 9/9/92 & 12 & 9 & & 81 & $\mathbf{N}$ & \\
\hline 1 & $9 / 9 / 92$ & 13 & 1 & & 81 & $N$ & \\
\hline 1 & $9 / 9 / 92$ & 14 & $T$ & & 81 & $\mathbf{N}$ & \\
\hline 1 & $9 / 18 / 92$ & 1 & 1 & & 91.1 & $\mathbf{N}$ & $J 890523512-47$ \\
\hline 1 & $9 / 18 / 92$ & 2 & 1 & & 91.4 & $Y$ & \\
\hline 1 & $9 / 18 / 92$ & 3 & 1 & & 91.3 & $\bar{N}$ & \\
\hline 1 & $9 / 18 / 92$ & 4 & 1 & & 90.1 & $\bar{N}$ & \\
\hline 1 & $9 / 18 / 92$ & 5 & 1 & & 88.1 & $\mathbf{N}$ & \\
\hline 1 & $9 / 18 / 92$ & 6 & 1 & & 85.7 & $\mathbf{N}$ & \\
\hline 1 & $9 / 18 / 92$ & 7 & 1 & & 82.7 & $\bar{N}$ & \\
\hline 1 & $9 / 18 / 92$ & 8 & 1 & & 80.1 & $N$ & \\
\hline 1 & $9 / 18 / 92$ & 9 & 1 & & 79.9 & $\mathrm{~N}$ & \\
\hline 1 & $9 / 18 / 92$ & 10 & 1 & & 79.3 & $\bar{N}$ & \\
\hline 1 & $9 / 18 / 92$ & 11 & 1 & & 79.2 & $\mathbf{N}$ & \\
\hline 1 & $9 / 18 / 92$ & 12 & 1 & & 79.3 & $N$ & \\
\hline 1 & $9 / 18 / 92$ & 13 & 1 & & 79.1 & $N$ & \\
\hline 1 & $9 / 18 / 92$ & 14 & 1 & & 79.1 & $N$ & \\
\hline 1 & $9 / 25 / 92$ & 1 & 1 & & 90.7 & $\bar{N}$ & J910126143-42 \\
\hline 1 & $9 / 25 / 92$ & 2 & 9 & & 91 & $\bar{Y}$ & \\
\hline 1 & $9 / 25 / 92$ & 3 & 1 & & 90.8 & $\mathbf{N}$ & \\
\hline 1 & $9 / 25 / 92$ & 4 & 1 & & 89.7 & $\mathrm{~N}$ & \\
\hline 1 & $9 / 25 / 92$ & 5 & 1 & & 87.9 & $\mathbf{N}$ & \\
\hline 1 & $9 / 25 / 92$ & 6 & 1 & & 85.3 & $\mathrm{~N}$ & \\
\hline 1 & $9 / 25 / 92$ & 7 & 1 & & 82.2 & $\mathbf{N}$ & \\
\hline 9 & $9 / 25 / 92$ & 8 & 1 & & 79.5 & $\mathbf{N}$ & \\
\hline 1 & $9 / 25 / 92$ & 9 & 1 & & 79.3 & $N$ & \\
\hline 1 & $9 / 25 / 92$ & 10 & 1 & & 78.7 & $\mathrm{~N}$ & \\
\hline 1 & $9 / 25 / 92$ & 11 & 1 & & 78.7 & $\bar{N}$ & \\
\hline 1 & $9 / 25 / 92$ & 12 & 1 & & 78.8 & $N$ & \\
\hline 1 & $9 / 25 / 92$ & 13 & 1 & & 78.7 & $\mathbf{N}$ & \\
\hline 9 & $9 / 25 / 92$ & 14 & 1 & & 78.6 & $\overline{\mathbf{N}}$ & \\
\hline 1 & $10 / 1 / 92$ & 1 & 1 & & 90.6 & $\mathbf{N}$ & $\mathrm{J} 890523512-47$ \\
\hline 1 & $10 / 1 / 92$ & 2 & 1 & & 90.9 & $Y$ & \\
\hline 9 & $10 / 1 / 92$ & 3 & 1 & & 90.9 & $\mathbf{N}$ & \\
\hline 1 & $10 / 1 / 92$ & 4 & 1 & & 89.8 & $\mathbf{N}$ & \\
\hline 1 & $10 / 1 / 92$ & 5 & 1 & & 87.9 & $\mathbf{N}$ & \\
\hline 9 & $10 / 1 / 92$ & 6 & $\frac{1}{1}$ & & 85.5 & $\overline{\mathbf{N}}$ & \\
\hline 1 & $10 / 1 / 92$ & 7 & 1 & & 82.4 & $\mathbf{N}$ & \\
\hline 1 & $10 / 1 / 92$ & 8 & 1 & & 79.6 & $\bar{N}$ & \\
\hline 1 & $10 / 1 / 92$ & 9 & 1 & & 79.5 & $\mathbf{N}$ & \\
\hline 1 & $10 / 1 / 92$ & 10 & 1 & & 79 & $N$ & \\
\hline 1 & $10 / 1 / 92$ & 11 & 1 & & 78.8 & $\mathbf{N}$ & \\
\hline 9 & $10 / 1 / 92$ & 12 & 1 & & 79.1 & $\mathbf{N}$ & \\
\hline 1 & $10 / 1 / 92$ & 13 & 1 & & 78.9 & $\mathbf{N}$ & \\
\hline 1 & $10 / 1 / 92$ & 14 & 1 & & 78.9 & $\bar{N}$ & \\
\hline 9 & $10 / 8 / 92$ & 1 & 1 & & 94 & $\bar{Y}$ & J 890523512.47 \\
\hline 1 & $10 / 8 / 92$ & 2 & 1 & & 94 & $\mathbf{N}$ & \\
\hline 9 & $10 / 8 / 92$ & 3 & 9 & & 94 & $\mathbf{N}$ & \\
\hline 1 & $10 / 8 / 92$ & 4 & 9 & & 93 & $\mathrm{~N}$ & \\
\hline 1 & $10 / 8 / 92$ & 5 & 1 & & 92 & $\mathbf{N}$ & \\
\hline 1 & $10 / 8 / 92$ & 6 & 1 & & 87.2 & $\mathbf{N}$ & \\
\hline 1 & $10 / 8 / 92$ & 7 & 1 & & 86 & $\mathbf{N}$ & \\
\hline 1 & $10 / 8 / 92$ & 8 & 1 & & 83 & $N$ & \\
\hline 1 & $10 / 8 / 92$ & 9 & 1 & & 83 & $N$ & \\
\hline 1 & $10 / 8 / 92$ & 10 & 1 & & 82 & $\mathbf{N}$ & \\
\hline 1 & $10 / 8 / 92$ & 11 & 1 & & 82 & $\mathbf{N}$ & \\
\hline 1 & $10 / 8 / 92$ & 12 & 1 & & 82 & $\mathbf{N}$ & \\
\hline 1 & $10 / 8 / 92$ & 13 & 1 & & 82 & $\mathrm{~N}$ & \\
\hline 1 & $10 / 8 / 92$ & 14 & 7 & & 82 & $\mathbf{N}$ & \\
\hline 1 & $10 / 15 / 92$ & 1 & 1 & & 90.8 & $N$ & J $910126143-50$ \\
\hline
\end{tabular}

Data obtained from WHC Surveillance Analysis Computer System (SACS), February 24, 1994. 


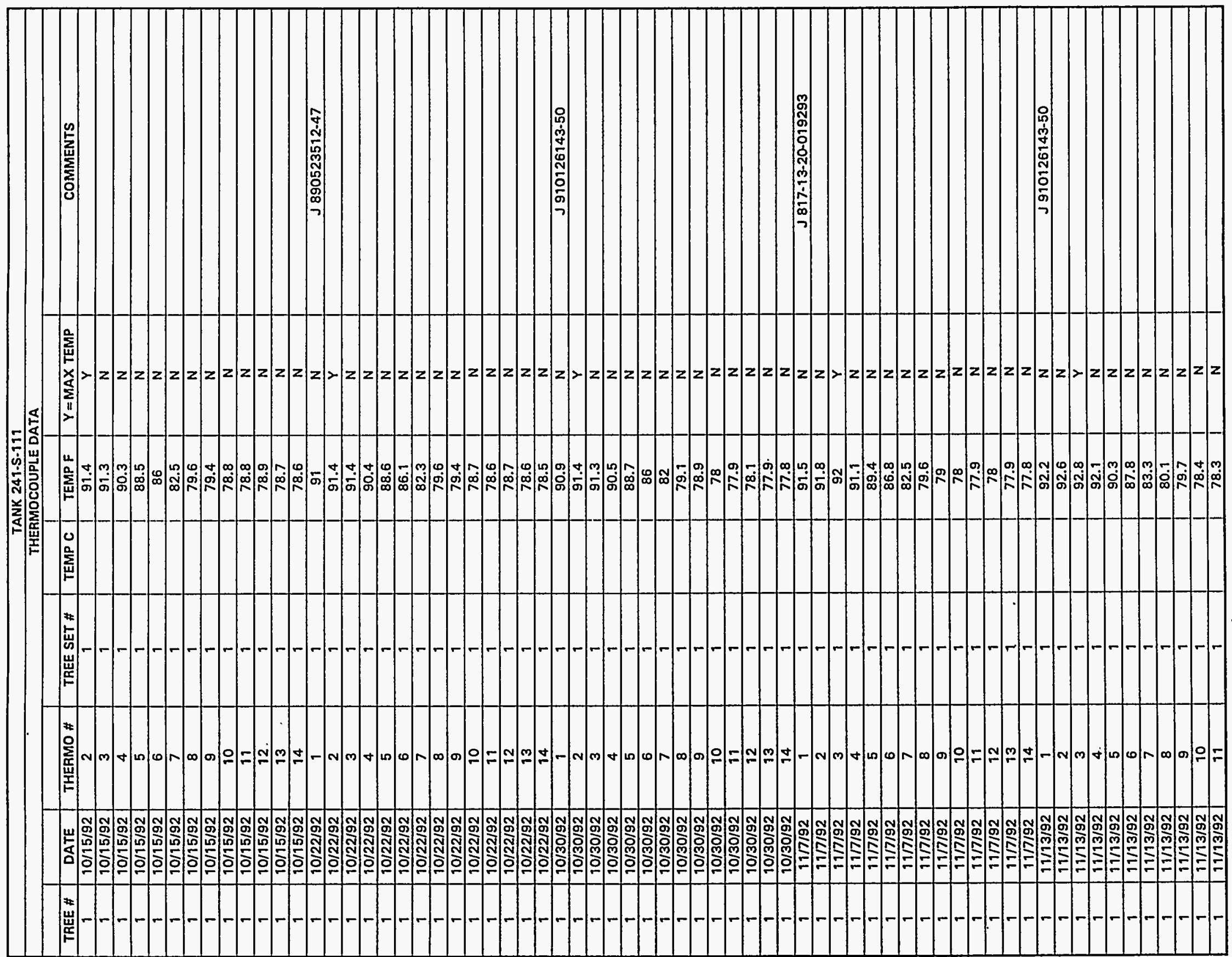

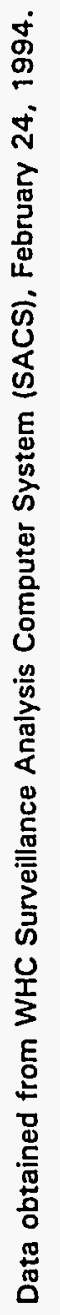




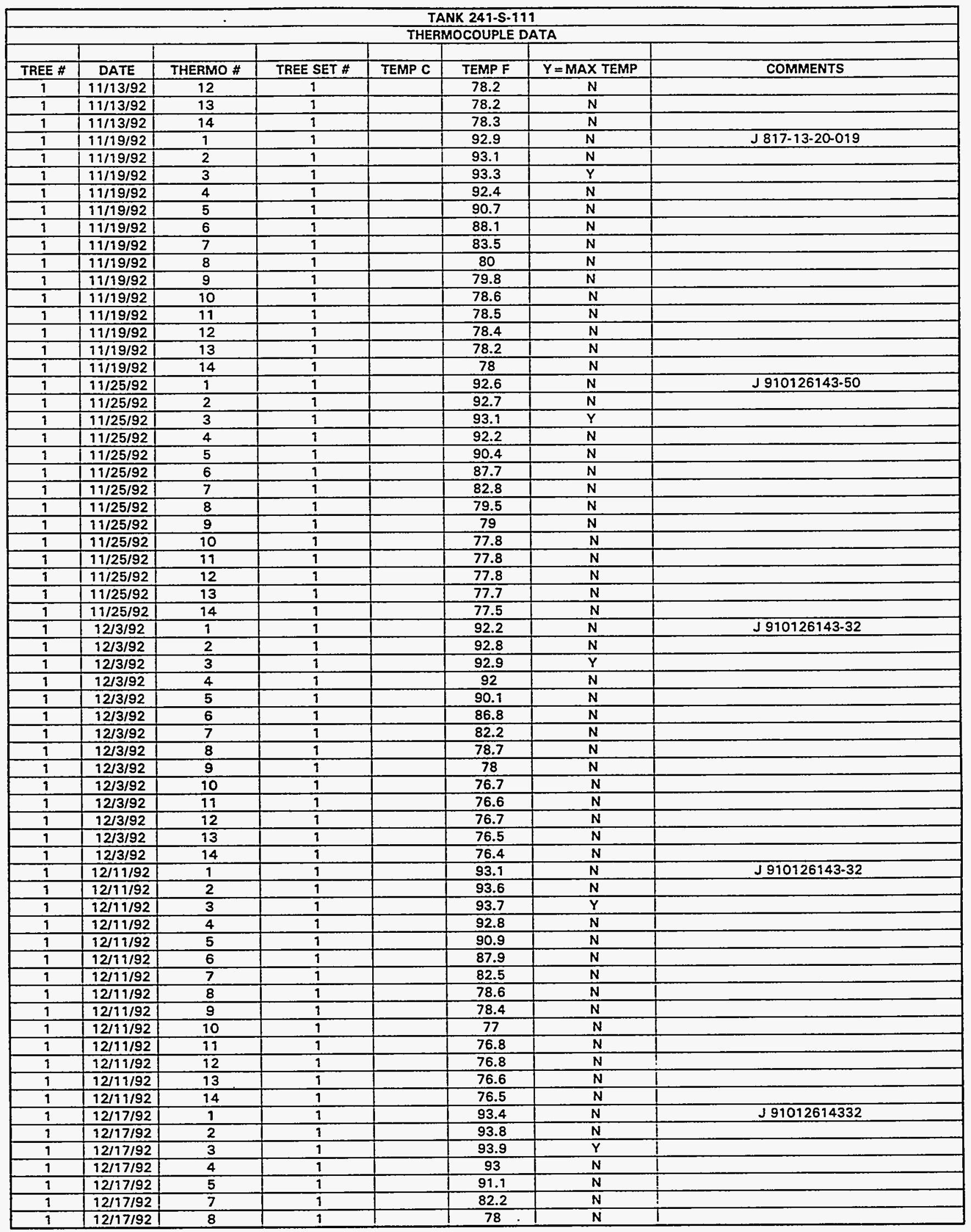

Data obtained from-WHC Surveillance Analysis Computer System (SACS), February 24, 1994. 
WHC-SD-WM-ER-323, Rev. 0

\begin{tabular}{|c|c|c|c|c|c|c|c|}
\hline \multicolumn{8}{|c|}{ TANK 241-S-111 } \\
\hline \multicolumn{8}{|c|}{ THERMOCOUPLE DATA } \\
\hline & & & & & & & \\
\hline TREE \# & DATE & THERMO \# & TREE SET \# & TEMP C & TEMP F & $Y=$ MAX TEMP & COMMENTS \\
\hline 1 & $12 / 17 / 92$ & 9 & 1 & & 77.9 & $\mathrm{~N}$ & \\
\hline 1 & $12 / 17 / 92$ & 10 & 1 & & 76.6 & $\bar{N}$ & \\
\hline 1 & $12 / 17 / 92$ & 11 & 9 & & 76.5 & $\mathbf{N}$ & \\
\hline 1 & $12 / 17 / 92$ & 12 & 1 & & 76.6 & $\mathbf{N}$ & \\
\hline 1 & $12 / 17 / 92$ & 13 & 9 & & 76.4 & $\mathbf{N}$ & \\
\hline$T$ & $12 / 17 / 92$ & 14 & $\frac{1}{1}$ & & 76.2 & $\mathbf{N}$ & \\
\hline$\frac{1}{1}$ & $12 / 28 / 92$ & 1 & $T$ & & 96 & $\mathrm{~N}$ & J $910126143-32$ \\
\hline 1 & $12 / 28 / 92$ & 2 & 1 & & 96.3 & $\mathbf{N}$ & \\
\hline$\frac{1}{1}$ & $12 / 28 / 92$ & 3 & 9 & & 96.4 & $\bar{Y}$ & \\
\hline 1 & $12 / 28 / 92$ & 4 & 1 & & 95.6 & $\mathbf{N}$ & \\
\hline 1 & $12 / 28 / 92$ & 5 & 1 & & 93.5 & $\mathrm{~N}$ & \\
\hline 1 & $12 / 28 / 92$ & 6 & 1 & & 90 & $\mathbf{N}$ & \\
\hline 1 & $12 / 28 / 92$ & 7 & 7 & & 83.8 & $\bar{N}$ & \\
\hline 9 & $12 / 28 / 92$ & 8 & 1 & & 79.6 & $\mathbf{N}$ & \\
\hline 1 & $12 / 28 / 92$ & 9 & 1 & & 79.4 & $\mathrm{~N}$ & \\
\hline 1 & $12 / 28 / 92$ & 10 & 1 & & 78 & $\mathbf{N}$ & \\
\hline 1 & $12 / 28 / 92$ & 11 & 1 & & 77.9 & $\mathbf{N}$ & \\
\hline 1 & $12 / 28 / 92$ & 12 & 9 & & 77.9 & $\mathrm{~N}$ & \\
\hline$\frac{1}{1}$ & $12 / 28 / 92$ & 13 & 1 & & 77.7 & $\mathbf{N}$ & \\
\hline 1 & $12 / 28 / 92$ & 14 & $\overline{1}$ & & 77.6 & $\bar{N}$ & \\
\hline 9 & $12 / 30 / 92$ & 1 & 1 & & 93.8 & $\bar{N}$ & J 91012614332 \\
\hline 1 & $12 / 30 / 92$ & 2 & 1 & & 94.2 & $\mathrm{~N}$ & \\
\hline 1 & $12 / 30 / 92$ & 3 & 1 & & 94.3 & $\bar{N}$ & \\
\hline 1 & $12 / 30 / 92$ & 4 & 1 & & 94.5 & $\bar{Y}$ & \\
\hline 9 & $12 / 30 / 92$ & 5 & $\frac{1}{1}$ & & 91.5 & $\mathbf{N}$ & \\
\hline 1 & $12 / 30 / 92$ & 6 & $\frac{1}{1}$ & & 88 & $\mathbf{N}$ & \\
\hline 1 & $12 / 30 / 92$ & 7 & 1 & & 81.6 & $\mathbf{N}$ & \\
\hline 1 & $12 / 30 / 92$ & 8 & 1 & & 77.4 & $\mathrm{~N}$ & \\
\hline 1 & $12 / 30 / 92$ & 9 & 1 & & 77.1 & $\bar{N}$ & \\
\hline 1 & $12 / 30 / 92$ & 10 & 1 & & 75.8 & $\mathbf{N}$ & \\
\hline 1 & $12 / 30 / 92$ & 11 & 1 & & 75.8 & $\mathrm{~N}$ & \\
\hline 1 & $12 / 30 / 92$ & 12 & $\frac{1}{1}$ & & 75.6 & $N$ & \\
\hline$\frac{1}{1}$ & $12 / 30 / 92$ & 13 & 1 & & 75.5 & $\mathbf{N}$ & \\
\hline 1 & $12 / 30 / 92$ & 14 & 1 & & 75.4 & $\bar{N}$ & \\
\hline 1 & $1 / 7 / 93$ & 1 & 1 & & 93.5 & $\mathbf{N}$ & J $910126143-29$ \\
\hline$\frac{1}{1}$ & $1 / 7 / 93$ & 2 & 1 & & 93.8 & $Y$ & \\
\hline 9 & $1 / 7 / 93$ & 3 & 1 & & 93.8 & $N$ & \\
\hline 1 & $1 / 7 / 93$ & 4 & $\frac{1}{1}$ & & 92.8 & $\mathbf{N}$ & \\
\hline 1 & $1 / 7 / 93$ & 5 & 1 & & 90.7 & $\mathbf{N}$ & \\
\hline 9 & $1 / 7 / 93$ & 7 & 1 & & 80.3 & $\mathbf{N}$ & \\
\hline 1 & $1 / 7 / 93$ & 8 & 1 & & 76.1 & $N$ & \\
\hline 1 & $1 / 7 / 93$ & 9 & 1 & & 75.6 & $\mathbf{N}$ & \\
\hline$\frac{1}{1}$ & $1 / 7 / 93$ & 10 & 1 & & 74.4 & $\bar{N}$ & \\
\hline 1 & $1 / 7 / 93$ & 11 & 1 & & 74.3 & $\mathbf{N}$ & \\
\hline 1 & $1 / 7 / 93$ & 12 & 1 & & 74.3 & $\mathbf{N}$ & \\
\hline 1 & $1 / 7 / 93$ & 13 & 1 & & 74.1 & $\bar{N}$ & \\
\hline 1 & $1 / 7 / 93$ & 14 & 1 & & 73.9 & $N$ & \\
\hline 1 & $1 / 16 / 93$ & 1 & 1 & & 94.2 & $\mathbf{N}$ & J910126143-32 \\
\hline 1 & $1 / 16 / 93$ & 2 & 1 & & 94.6 & $\mathbf{N}$ & \\
\hline 7 & $1 / 16 / 93$ & 3 & 1 & & 94.7 & $\mathbf{Y}$ & \\
\hline 1 & $1 / 16 / 93$ & 4 & 1 & & 93.7 & $\mathbf{N}$ & \\
\hline 1 & $1 / 16 / 93$ & 5 & 1 & & 91.4 & $\mathbf{N}$ & \\
\hline$\frac{1}{1}$ & $1 / 16 / 93$ & 6 & 1 & & 87.6 & $\mathbf{N}$ & \\
\hline 1 & $1 / 16 / 93$ & 7 & 9 & & 80.8 & $\mathbf{N}$ & . \\
\hline 1 & $1 / 16 / 93$ & 8 & 1 & & 76.5 & $\overline{\mathbf{N}}$ & \\
\hline 1 & $1 / 16 / 93$ & 9 & 1 & & 75.8 & $\mathbf{N}$ & \\
\hline 1 & $1 / 16 / 93$ & 10 & 1 & & 74.4 & $\mathbf{N}$ & \\
\hline 1 & $1 / 16 / 93$ & 11 & 1 & & 74.3 & $\mathbf{N}$ & \\
\hline 1 & $1 / 16 / 93$ & 12 & 1 & & 74.2 & $\mathbf{N}$ & \\
\hline 1 & $1 / 16 / 93$ & 13 & 1 & & 74.1 & $\mathbf{N}$ & \\
\hline 1 & $1 / 16 / 93$ & 14 & 1 & & 74 & $\mathbf{N}$ & \\
\hline 1 & $1 / 23 / 93$ & 1 & 1 & & 93.3 & $\mathbf{N}$ & J 91012614329 \\
\hline 1 & $1 / 23 / 93$ & 2 & 1 & & 93.3 & $\mathbf{N}$ & \\
\hline 1 & $1 / 23 / 93$ & 3 & 1 & & 93.7 & $\mathbf{N}$ & \\
\hline 1 & $1 / 23 / 93$ & 4 & 1 & & 92.8 & $\mathbf{N}$ & \\
\hline 1 & $1 / 23 / 93$ & 5 & 1 & & 90.4 & $\mathbf{N}$ & \\
\hline
\end{tabular}

Data obtained from WHC Surveillance Analysis Computer System (SACS), February 24, 1994. 


\begin{tabular}{|c|c|c|c|c|c|c|c|}
\hline \multicolumn{8}{|c|}{ TANK 241-S-111 } \\
\hline \multicolumn{8}{|c|}{ THERMOCOUPLE DATA } \\
\hline & & & & & & & \\
\hline TREE \# & DATE & THERMO \# & TREE SET \# & TEMP C & TEMP F & $Y=M A X$ TEMP & COMMENTS \\
\hline 1 & $1 / 23 / 93$ & 6 & 1 & & 96.4 & $Y$ & \\
\hline 1 & $9 / 23 / 93$ & 7 & 1 & & 79.6 & N & \\
\hline 1 & $1 / 23 / 93$ & 8 & 1 & & 75.2 & $\mathrm{~N}$ & \\
\hline 1 & $1 / 23 / 93$ & 9 & 9 & & 74.4 & $\mathbf{N}$ & \\
\hline 1 & $1 / 23 / 93$ & 10 & 1 & & 72.7 & $\mathrm{~N}$ & \\
\hline 1 & $1 / 23 / 93$ & 11 & 1 & & 72.6 & $\mathrm{~N}$ & \\
\hline 1 & $1 / 23 / 93$ & 12 & 1 & & 72.5 & $\mathbf{N}$ & \\
\hline 1 & $1 / 23 / 93$ & 13 & 1 & & 72.5 & $\mathbf{N}$ & \\
\hline 1 & $1 / 23 / 93$ & 14 & 1 & & 72.3 & $\ddot{N}$ & \\
\hline 1 & $1 / 29 / 93$ & 9 & 9 & & 92.7 & $\mathbf{N}$ & $J 910126143.29$ \\
\hline 1 & $1 / 29 / 93$ & 2 & 1 & & 93 & $\mathbf{N}$ & \\
\hline 1 & $1 / 29 / 93$ & 3 & 1 & & 93.1 & $\bar{Y}$ & \\
\hline 1 & 1/29/93 & 4 & 1 & & 91.9 & $\mathbf{N}$ & \\
\hline 1 & $1 / 29 / 93$ & 5 & 1 & & 89.5 & $\ddot{N}$ & \\
\hline 9 & $1 / 29 / 93$ & 6 & 1 & & 85.6 & $\mathbf{N}$ & \\
\hline 9 & $1 / 29 / 93$ & 7 & 1 & & 78.6 & $\mathbf{N}$ & \\
\hline 1 & $1 / 29 / 93$ & 8 & 1 & & 74.3 & $\mathrm{~N}$ & \\
\hline 1 & $1 / 29 / 93$ & 9 & 1 & & 73.4 & $\bar{N}$ & \\
\hline 1 & $1 / 29 / 93$ & 10 & 1 & & 79.7 & $N$ & \\
\hline 1 & $1 / 29 / 93$ & 11 & 9 & & 71.7 & $N$ & \\
\hline 1 & $9 / 29 / 93$ & 12 & $\frac{T}{1}$ & & 71.5 & $\mathrm{~N}$ & \\
\hline 1 & $1 / 29 / 93$ & 13 & 1 & & 71.5 & $\bar{N}$ & \\
\hline 1 & $1 / 29 / 93$ & 14 & 1 & & 71.4 & $\mathrm{~N}$ & \\
\hline 1 & $2 / 5 / 93$ & 1 & 1 & & 92.9 & $\bar{N}$ & J910126143-32 \\
\hline 1 & $2 / 5 / 93$ & 2 & 1 & & 93.4 & $\bar{Y}$ & \\
\hline 1 & $2 / 5 / 93$ & 3 & 1 & & 93.4 & $\mathrm{~N}$ & \\
\hline 1 & $2 / 5 / 93$ & 4 & 1 & & 92.2 & $\bar{N}$ & \\
\hline 1 & $2 / 5 / 93$ & 5 & 1 & & 89.6 & $\mathbf{N}$ & \\
\hline 9 & $2 / 5 / 93$ & 7 & 1 & & 78.6 & $\mathrm{~N}$ & \\
\hline 1 & $2 / 5 / 93$ & 8 & 1 & & 74.3 & $\mathrm{~N}$ & \\
\hline 1 & $2 / 5 / 93$ & 9 & 1 & & 73.4 & $\ddot{N}$ & \\
\hline 1 & $2 / 5 / 93$ & 10 & 1 & & 71.5 & $N$ & \\
\hline 9 & $2 / 5 / 93$ & 11 & 1 & & 71.5 & $\mathrm{~N}$ & \\
\hline 1 & $2 / 5 / 93$ & 12 & 1 & & 71.4 & $\mathbf{N}$ & \\
\hline 1 & $2 / 5 / 93$ & 13 & 1 & & 71.4 & $\mathbf{N}$ & \\
\hline 1 & $2 / 5 / 93$ & 14 & 9 & & 71.3 & $\mathbf{N}$ & \\
\hline 1 & $2 / 13 / 93$ & 1 & 1 & & 91.6 & $\mathbf{N}$ & J 91026143-42 \\
\hline 1 & $2 / 13 / 93$ & 2 & 1 & & 92 & $\bar{Y}$ & \\
\hline 1 & $2 / 13 / 93$ & 3 & 1 & & 91.9 & $\mathrm{~N}$ & \\
\hline 1 & $2 / 13 / 93$ & 4 & 1 & & 90.5 & $\mathbf{N}$ & \\
\hline 1 & $2 / 13 / 93$ & 5 & 1 & & 87.9 & $\mathbf{N}$ & \\
\hline 1 & $2 / 13 / 93$ & 7 & 1 & & 76.8 & $\mathrm{~N}$ & \\
\hline 1 & $2 / 13 / 93$ & 8 & 1 & & 72.6 & $\bar{N}$ & \\
\hline 1 & $2 / 13 / 93$ & 9 & 1 & & 71.5 & $\bar{N}$ & \\
\hline 1 & $2 / 13 / 93$ & 10 & 1 & & 69.6 & $N$ & \\
\hline 1 & $2 / 13 / 93$ & 11 & 1 & & 69.5 & $\mathrm{~N}$ & \\
\hline 1 & $2 / 13 / 93$ & 12 & 1 & & 69.4 & $N$ & \\
\hline$\frac{1}{1}$ & $2 / 13 / 93$ & 13 & 1 & & 69.4 & $\bar{N}$ & \\
\hline 1 & $2 / 13 / 93$ & 14 & 1 & & 69.2 & $\mathrm{~N}$ & \\
\hline 1 & $2 / 18 / 93$ & 1 & 1 & & 92.5 & $N$ & $J 910126143.29$ \\
\hline 1 & $2 / 18 / 93$ & 2 & 1 & & 92.6 & $\mathrm{~N}$ & \\
\hline 9 & $2 / 18 / 93$ & 3 & 1 & & 92.8 & $\bar{Y}$ & \\
\hline 1 & $2 / 18 / 93$ & 4 & 1 & & 91.7 & $\bar{N}$ & \\
\hline 1. & $2 / 18 / 93$ & 5 & 1 & & 89.1 & $\bar{N}$ & \\
\hline 1 & $2 / 18 / 93$ & 6 & 9 & & 84.8 & $\mathbf{N}$ & \\
\hline 1 & $2 / 18 / 93$ & 7 & 1 & & 77.9 & $\mathrm{~N}$ & \\
\hline 1 & $2 / 18 / 93$ & 8 & 1 & & 73.4 & $\mathrm{~N}$ & \\
\hline 1 & $2 / 18 / 93$ & 9 & 1 & & 72.3 & $N$ & \\
\hline 1 & $2 / 18 / 93$ & 10 & 1 & & 70.4 & $N$ & \\
\hline 1 & $2 / 18 / 93$ & 11 & 1 & & 70.2 & $\mathbf{N}$ & \\
\hline 1 & $2 / 18 / 93$ & 12 & 1 & & 70.1 & $\mathbf{N}$ & \\
\hline 1 & $2 / 18 / 93$ & 13 & 1 & & 70 & $\mathbf{N}$ & \\
\hline 1 & $2 / 18 / 93$ & 14 & 1 & & 69.9 & $N$ & \\
\hline 1 & $2 / 25 / 93$ & 1 & 1 & & 93.4 & $N$ & J $817-13.55-008$ \\
\hline 1 & $2 / 25 / 93$ & 2 & 9 & & 93.8 & $\bar{Y}$ & \\
\hline 1 & $2 / 25 / 93$ & 3 & 1 & & 93.7 & $\mathbf{N}$ & \\
\hline
\end{tabular}

Data obtained from WHC Surveillance Analysis Computer System (SACS), February 24, 1994. 


\begin{tabular}{|c|c|c|c|c|c|c|c|}
\hline \multicolumn{8}{|c|}{ TANK 241-S-111 } \\
\hline \multicolumn{8}{|c|}{ THERMOCOUPLE DATA } \\
\hline TREE \# & DATE & THERMO \# & TREE SET \# & & & & \\
\hline 1 & $2 / 25 / 93$ & 4 & 1 & Deint 6 & $\frac{\text { IEMPr }}{92.3}$ & $Y=$ MAX TEMP & COMMENTS \\
\hline 1 & $2 / 25 / 93$ & 5 & 1 & & 89.5 & $\frac{N}{N}$ & \\
\hline 1 & $2 / 25 / 93$ & 6 & 1 & & 85.3 & $N$ & \\
\hline 1 & $2 / 25 / 93$ & 7 & 1 & & 78.1 & $N$ & \\
\hline 1 & $2 / 25 / 93$ & 8 & 1 & & 73.3 & $N$ & \\
\hline 1 & $2 / 25 / 93$ & 9 & 9 & & 73 & $\bar{N}$ & \\
\hline 1 & $2 / 25 / 93$ & 10 & $\frac{1}{1}$ & & 71.4 & $\mathbf{N}$ & \\
\hline 1 & $2 / 25 / 93$ & 11 & 1 & & 71.4 & $N$ & \\
\hline 1 & $2 / 25 / 93$ & 12 & 1 & & 71.2 & $\mathbf{N}$ & \\
\hline 1 & $2 / 25 / 93$ & 13 & 1 & & 71.1 & $\mathbf{N}$ & \\
\hline 1 & $2 / 25 / 93$ & 14 & 1 & & 71 & $\mathbf{N}$ & \\
\hline 1 & $3 / 4 / 93$ & 1 & 1 & & 92.5 & $\mathbf{N}$ & J 910126143.66 \\
\hline 1 & $3 / 4 / 93$ & 2 & 1 & & 92.9 & $Y$ & \\
\hline 9 & $3 / 4 / 93$ & 3 & 1 & & 92.8 & $\mathbf{N}$ & \\
\hline 1 & $3 / 4 / 93$ & 4 & 1 & & 91.4 & $\mathbf{N}$ & \\
\hline 1 & $3 / 4 / 93$ & 5 & 1 & & 88.5 & $\mathbf{N}$ & \\
\hline 1 & $3 / 4 / 93$ & 6 & 1 & & 84.3 & $\mathbf{N}$ & \\
\hline 1 & $3 / 4 / 93$ & 7 & 1 & & 77 & $\mathbf{N}$ & \\
\hline 1 & $3 / 4 / 93$ & 8 & 1 & & 72.1 & $\mathbf{N}$ & \\
\hline 1 & $3 / 4 / 93$ & 9 & 1 & & 71.3 & $\mathbf{N}$ & \\
\hline 1 & $3 / 4 / 93$ & 10 & 1 & & 70 & $\mathbf{N}$ & \\
\hline 1 & $3 / 4 / 93$ & 11 & 1 & & 70 & $\mathbf{N}$ & \\
\hline 1 & $3 / 4 / 93$ & 12 & 1 & & 69.9 & $\mathbf{N}$ & \\
\hline 1 & $3 / 4 / 93$ & 13 & 1 & & 69.8 & $\mathbf{N}$ & \\
\hline 1 & $3 / 4 / 93$ & 14 & 1 & & 69.8 & $\mathbf{N}$ & \\
\hline 1 & $3 / 11 / 93$ & 1 & 1 & & 92 & $\mathbf{N}$ & J 910126143.66 \\
\hline 1 & $3 / 11 / 93$ & 2 & 1 & & 92.5 & $Y$ & \\
\hline 9 & $3 / 11 / 93$ & 3 & 1 & & 92.3 & $\bar{N}$ & \\
\hline 1 & $3 / 11 / 93$ & 4 & 1 & & 90.9 & $N$ & \\
\hline 1 & $3 / 11 / 93$ & 5 & 1 & & 88 & $\mathbf{N}$ & \\
\hline 1 & $3 / 11 / 93$ & 6 & 1 & & 83.6 & $\mathbf{N}$ & \\
\hline 1 & $3 / 11 / 93$ & 7 & 1 & & 76.4 & $\mathbf{N}$ & \\
\hline 1 & $3 / 11 / 93$ & 8 & 1 & & 71.6 & $\mathbf{N}$ & \\
\hline 1 & $3 / 11 / 93$ & 9 & 1 & & 70.8 & $\mathbf{N}$ & \\
\hline 1 & $3 / 11 / 93$ & 10 & 1 & & 69.3 & $\mathbf{N}$ & \\
\hline 1 & $3 / 11 / 93$ & 11 & 1 & & 69.2 & $\mathbf{N}$ & \\
\hline 1 & $3 / 11 / 93$ & 12 & 1 & & 69.1 & $\mathbf{N}$ & \\
\hline 1 & $3 / 11 / 93$ & 13 & 1 & & 69 & $\mathbf{N}$ & \\
\hline 1 & $3 / 11 / 93$ & 14 & 1 & & 68.9 & $\mathrm{~N}$ & \\
\hline 1 & $3 / 19 / 93$ & 1 & 1 & & 93 & $\mathbf{Y}$ & J910126143-66 \\
\hline 1 & $3 / 19 / 93$ & 2 & 1 & & 93 & $\mathbf{N}$ & \\
\hline 1 & $3 / 19 / 93$ & 3 & 1 & & 93 & $\mathbf{N}$ & \\
\hline 1 & $3 / 19 / 93$ & 4 & 1 & & 91 & $\mathbf{N}$ & \\
\hline 1 & $3 / 19 / 93$ & 5 & 1 & & 88 & $\mathbf{N}$ & \\
\hline$\frac{1}{1}$ & $3 / 19 / 93$ & 6 & 1 & & 84 & $\bar{N}$ & \\
\hline 1 & $3 / 19 / 93$ & 7 & 1 & & 77 & $\mathbf{N}$ & \\
\hline 1 & $3 / 19 / 93$ & 8 & 1 & & 72 & $\mathbf{N}$ & \\
\hline 1 & $3 / 19 / 93$ & 9 & 1 & & 71 & $\mathbf{N}$ & \\
\hline 1 & $3 / 19 / 93$ & 10 & 1 & & 70 & $\mathbf{N}$ & \\
\hline 1 & $3 / 19 / 93$ & 11 & 1 & & 70 & $\mathbf{N}$ & \\
\hline 1 & $3 / 19 / 93$ & 13 & 1 & & 70 & $\mathbf{N}$ & \\
\hline 1 & $3 / 19 / 93$ & 14 & 1 & & 70 & $\mathbf{N}$ & \\
\hline 1 & $3 / 27 / 93$ & 1 & 1 & & 90 & $Y$ & J 91612614360 \\
\hline 1 & $3 / 27 / 93$ & 2 & 1 & 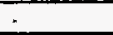 & 89.1 & $\mathbf{N}$ & \\
\hline 1 & $3 / 27 / 93$ & 3 & 1 & & 89.7 & $\mathbf{N}$ & \\
\hline 1 & $3 / 27 / 93$ & 4 & 1 & & 88 & $\mathbf{N}$ & \\
\hline 1 & $3 / 27 / 93$ & 5 & 1 & & 85.5 & $\mathbf{N}$ & \\
\hline 1 & $3 / 27 / 93$ & 6 & 1 & & 80.6 & $\mathbf{N}$ & \\
\hline 1 & $3 / 27 / 93$ & 7 & 1 & & 73.4 & $\mathbf{N}$ & \\
\hline 1 & $3 / 27 / 93$ & 8 & 1 & & 68.9 & $\mathbf{N}$ & \\
\hline 1 & $3 / 27 / 93$ & 9 & 1 & & 68.3 & $\mathrm{~N}$ & \\
\hline 1 & $3 / 27 / 93$ & 10 & 1 & & 67.2 & $\mathbf{N}$ & \\
\hline 1 & $3 / 27 / 93$ & 11 & 1 & & 66.9 & $\mathbf{N}$ & \\
\hline 1 & $3 / 27 / 93$ & 12 & 1 & & 66.9 & $\mathbf{N}$ & \\
\hline 1 & $3 / 27 / 93$ & 13 & 1 & & 66.9 & $\mathbf{N}$ & \\
\hline 1 & $3 / 27 / 93$ & 14 & 1 & & 66.9 & $\mathbf{N}$ & \\
\hline
\end{tabular}

Data obtained from WHC Surveillance Analysis Computer System (SACS), February 24, 1994. 


\begin{tabular}{|c|c|c|c|c|c|c|c|}
\hline \multicolumn{8}{|c|}{ TANK 241.S-111 } \\
\hline & & & & THER & OCOUPLE & TA & \\
\hline TREE \# & DATE & THERMO \# & TREE SET \# & TEMP C & TEMP F & $Y=M A X$ TEMP & COMMENTS \\
\hline 1 & $4 / 1 / 93$ & 1 & 1 & & 89.5 & $\frac{N}{N}$ & J 8904-23320-42 \\
\hline 1 & $4 / 1 / 93$ & 2 & 1 & & 89.7 & Y & \\
\hline 1 & $4 / 1 / 93$ & 3 & 1 & & 89.5 & $\mathbf{N}$ & \\
\hline 1 & $4 / 1 / 93$ & 4 & 1 & & 87.9 & $\bar{N}$ & \\
\hline 1 & $4 / 1 / 93$ & 5 & 1 & & 87.8 & $N$ & \\
\hline 1 & $4 / 1 / 93$ & 6 & 9 & & 80.3 & $\mathrm{~N}$ & \\
\hline 1 & $4 / 1 / 93$ & 7 & 1 & & 73.3 & $\mathrm{~N}$ & \\
\hline 9 & $4 / 1 / 93$ & 8 & 1 & & 68.5 & $\mathbf{N}$ & \\
\hline 1 & $4 / 1 / 93$ & 9 & 1 & & 67.9 & $\mathrm{~N}$ & \\
\hline 1 & $4 / 1 / 93$ & 10 & 1 & & 66.4 & $\mathbf{N}$ & \\
\hline 1 & $4 / 1 / 93$ & 11 & 1 & & 66.3 & $\mathrm{~N}$ & \\
\hline 1 & $4 / 1 / 93$ & 12 & 1 & & 66.2 & $\mathbf{N}$ & \\
\hline 1 & $4 / 1 / 93$ & 13 & 1 & & 66.2 & $N$ & \\
\hline 1 & $4 / 1 / 93$ & 14 & 1 & & 66.2 & $\mathbf{N}$ & \\
\hline 1 & $4 / 8 / 93$ & 9 & 1 & & 90.6 & $\mathbf{N}$ & $\mathrm{J} 0126143-14$ \\
\hline 1 & $4 / 8 / 93$ & 2 & 1 & & 90.9 & $\bar{Y}$ & \\
\hline 1 & $4 / 8 / 93$ & 3 & 1 & & 90.6 & $\mathbf{N}$ & \\
\hline 1 & $4 / 8 / 93$ & 4 & 1 & & 88.9 & $\bar{N}$ & \\
\hline 1 & $4 / 8 / 93$ & 5 & 1 & & 85.9 & $\bar{N}$ & \\
\hline 1 & $4 / 8 / 93$ & 6 & 1 & & 81.4 & $\mathrm{~N}$ & \\
\hline 1 & $4 / 8 / 93$ & 7 & 7 & & 74.5 & $\mathbf{N}$ & \\
\hline 1 & $4 / 8 / 93$ & 8 & 1 & & 69.9 & $\mathbf{N}$ & \\
\hline 1 & $4 / 8 / 93$ & 9 & 1 & & 69.2 & $\mathbf{N}$ & \\
\hline 1 & $4 / 8 / 93$ & 10 & 9 & & 67.7 & $\mathbf{N}$ & \\
\hline 1 & $4 / 8 / 93$ & 11 & 1 & & 67.6 & $\mathrm{~N}$ & \\
\hline 1 & $4 / 8 / 93$ & 12 & 1 & & 67.6 & $N$ & \\
\hline 1 & $4 / 8 / 93$ & 13 & 1 & & 67.5 & $\mathrm{~N}$ & \\
\hline 1 & $4 / 8 / 93$ & 14 & 1 & & 67.5 & $\mathrm{~N}$ & \\
\hline 1 & $4 / 15 / 93$ & 1 & 1 & & 93 & $Y$ & J910126143-66 \\
\hline 1 & $4 / 15 / 93$ & 2 & 9 & & 93 & $\mathbf{N}$ & \\
\hline 1 & $4 / 15 / 93$ & 3 & 1 & & 93 & $\mathrm{~N}$ & \\
\hline 1 & $4 / 15 / 93$ & 4 & 1 & & 91 & $N$ & \\
\hline 1 & $4 / 15 / 93$ & 5 & 1 & & 88 & $\bar{N}$ & \\
\hline 1 & $4 / 15 / 93$ & 7 & 1 & & 76 & $\mathrm{~N}$ & \\
\hline 1 & $4 / 15 / 93$ & 8 & 1 & & 72 & $\mathrm{~N}$ & \\
\hline 1 & $4 / 15 / 93$ & 9 & 1 & & 71 & $\mathbf{N}$ & \\
\hline 1 & $4 / 15 / 93$ & 10 & 1 & & 70 & $\mathbf{N}$ & \\
\hline 1 & $4 / 15 / 93$ & 11 & 1 & & 69 & $N$ & \\
\hline 1 & $4 / 15 / 93$ & 12 & 1 & & 69 & $N$ & \\
\hline 1 & $4 / 15 / 93$ & 13 & 1 & & 69 & $\mathbf{N}$ & \\
\hline 1 & $4 / 15 / 93$ & 14 & 1 & & 69 & $N$ & \\
\hline 1 & $4 / 21 / 93$ & 1 & 1 & & 91.2 & $N$ & J910126143-66 \\
\hline 1 & $4 / 21 / 93$ & 2 & 1 & & 91.5 & $Y$ & \\
\hline 1 & $4 / 21 / 93$ & 3 & 1 & & 91 & $\mathbf{N}$ & \\
\hline 1 & $4 / 21 / 93$ & 4 & 1 & & 89.2 & $N$ & \\
\hline 1 & $4 / 21 / 93$ & 5 & 1 & & 86 & $\bar{N}$ & \\
\hline 1 & $4 / 21 / 93$ & 6 & 1 & & 81.6 & $\mathbf{N}$ & \\
\hline 1 & $4 / 21 / 93$ & 7 & 1 & & 74.9 & $N$ & \\
\hline 1 & $4 / 21 / 93$ & 8 & 1 & & 70.6 & $\bar{N}$ & \\
\hline 1 & $4 / 21 / 93$ & 9 & 1 & & 69.8 & $N$ & \\
\hline 9 & $4 / 21 / 93$ & 10 & 1 & & 68.4 & $N$ & \\
\hline 1 & $4 / 21 / 93$ & 11 & 1 & & 68.3 & $\mathbf{N}$ & \\
\hline 1 & $4 / 21 / 93$ & 12 & 1 & & 68.3 & $\mathbf{N}$ & \\
\hline 1 & $4 / 21 / 93$ & 13 & 1 & & 68.3 & $\bar{N}$ & \\
\hline 1 & $4 / 21 / 93$ & 14 & 1 & & 68.2 & $\mathbf{N}$ & \\
\hline 1 & $4 / 23 / 93$ & 1 & 1 & & 92 & $N$ & J910126143-66 \\
\hline 1 & $4 / 23 / 93$ & 2 & 1 & & 92.3 & $Y$ & \\
\hline 1 & $4 / 23 / 93$ & 3 & 1 & & 91.9 & $N$ & \\
\hline 1 & $4 / 23 / 93$ & 4 & 1 & & 90.1 & $N$ & \\
\hline 1 & $4 / 23 / 93$ & 5 & 1 & & 87 & $N$ & \\
\hline 1 & $4 / 23 / 93$ & 6 & 1 & & 82.5 & $N$ & \\
\hline 1 & $4 / 23 / 93$ & 7 & 1 & & 75.8 & $N$ & \\
\hline 1 & $4 / 23 / 93$ & 8 & 1 & & 71.6 & $N$ & \\
\hline 1 & $4 / 23 / 93$ & 9 & 1 & & 70.7 & $N$ & \\
\hline 1 & $4 / 23 / 93$ & 10 & 1 & & 69.2 & $\mathbf{N}$ & \\
\hline 1 & $4 / 23 / 93$ & 11 & 1 & & 69.2 & $\mathbf{N}$ & \\
\hline
\end{tabular}

Data obtained from WHC Surveillance Analysis Computer System (SACS), February 24, 1994. 
WHC-SD-WM-ER-323, Rev. 0

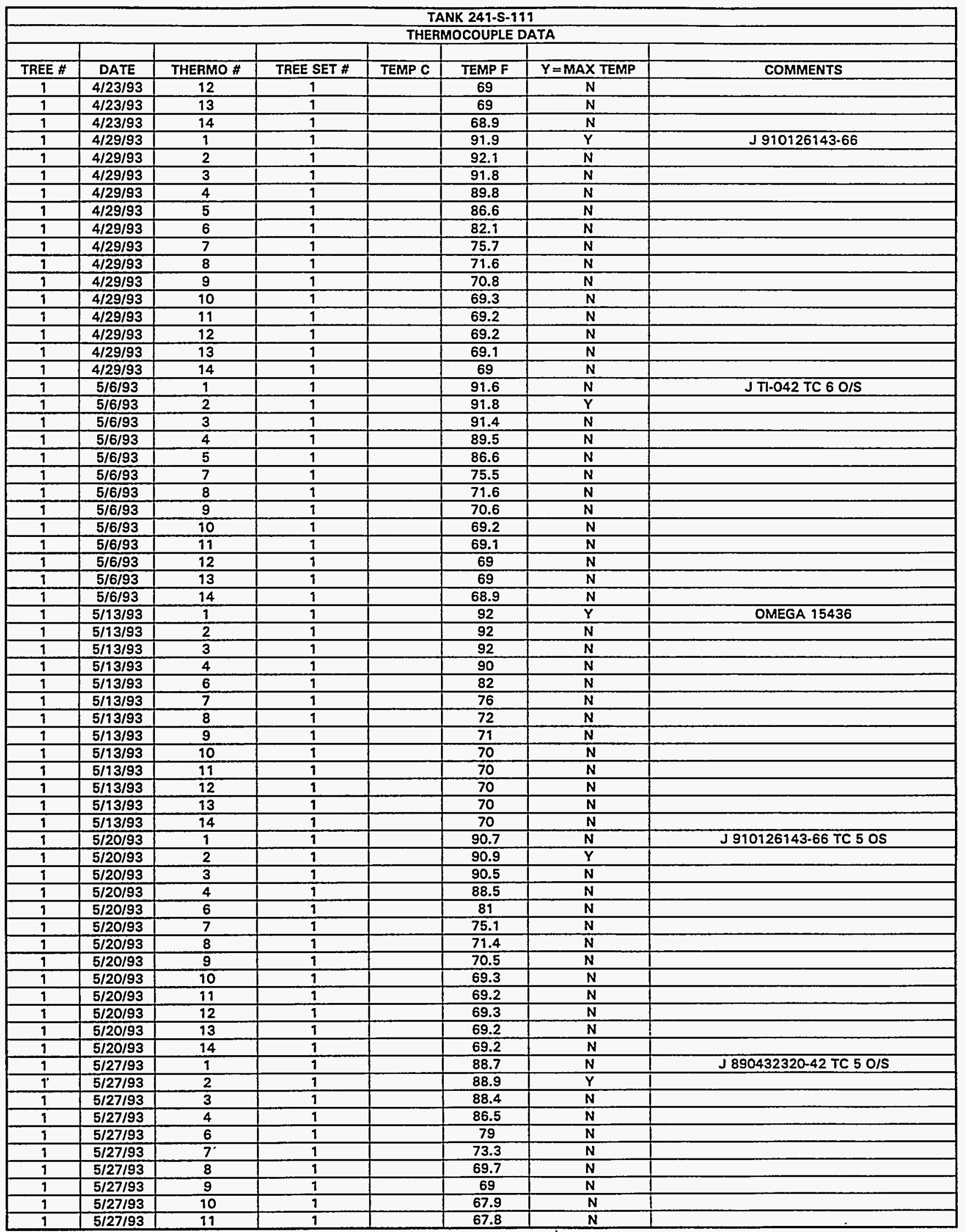

Data obtained from WHC Surveillance Analysis Computer System (SACS), February 24, 1994. 


\begin{tabular}{|c|c|c|c|c|c|c|c|}
\hline \multicolumn{8}{|c|}{ TANK 241-S-111 } \\
\hline \multicolumn{8}{|c|}{ THERMOCOUPLE DATA } \\
\hline TREE \# & DATE & THERMO \# & TREE SET \# & TEMP C & TEMP F & $Y=\operatorname{MAX} T E M P$ & COMMENTS \\
\hline 9 & $5 / 27 / 93$ & 12 & 1 & & 67.9 & $\mathbf{N}$ & \\
\hline 1 & $5 / 27 / 93$ & 13 & 1 & & 67.8 & $\mathbf{N}$ & \\
\hline 1 & $5 / 27 / 93$ & 14 & 1 & & 67.8 & $\mathbf{N}$ & \\
\hline 1 & $6 / 3 / 93$ & 1 & 1 & & 91 & $\bar{Y}$ & J $817 \cdot 13-20-019$ \\
\hline 1 & $6 / 3 / 93$ & 2 & 1 & & 99 & $\mathbf{N}$ & \\
\hline 1 & $6 / 3 / 93$ & 3 & 1 & & 91 & $\mathbf{N}$ & \\
\hline 1 & $6 / 3 / 93$ & 4 & 1 & & 89 & $\mathrm{~N}$ & \\
\hline 1 & $6 / 3 / 93$ & 5 & 1 & & 86 & $\mathbf{N}$ & \\
\hline 1 & $6 / 3 / 93$ & 6 & 1 & & 78 & $\mathbf{N}$ & \\
\hline 1 & $6 / 3 / 93$ & 7 & 1 & & 76 & $\mathbf{N}$ & \\
\hline 1 & $6 / 3 / 93$ & 8 & 1 & & 73 & $\mathbf{N}$ & \\
\hline 1 & $6 / 3 / 93$ & 9 & 1 & & 72 & $N$ & \\
\hline 1 & $6 / 3 / 93$ & 10 & 1 & & 71 & $\mathbf{N}$ & \\
\hline 1 & $6 / 3 / 93$ & 11 & 1 & & 71 & $\mathbf{N}$ & \\
\hline 1 & $6 / 3 / 93$ & 12 & 1 & & 71 & $\mathbf{N}$ & \\
\hline 1 & $6 / 3 / 93$ & 13 & 1 & & 71 & $\mathbf{N}$ & \\
\hline 1 & $6 / 3 / 93$ & 14 & 1 & & 71 & $\mathbf{N}$ & \\
\hline 1 & $6 / 10 / 93$ & 1 & 1 & & 90.8 & $\mathbf{N}$ & J $910126143-32$ \\
\hline 1 & $6 / 10 / 93$ & 2 & 1 & & 91 & $\bar{Y}$ & \\
\hline 9 & $6 / 10 / 93$ & 3 & 1 & & 90.5 & $\mathbf{N}$ & \\
\hline 1 & $6 / 10 / 93$ & 4 & 1 & & 85.8 & $N$ & \\
\hline 1 & $6 / 10 / 93$ & 5 & 1 & & 63.6 & $\bar{N}$ & \\
\hline 9 & $6 / 10 / 93$ & 6 & 1 & & 81.1 & $N$ & \\
\hline 1 & $6 / 10 / 93$ & 7 & 1 & & 75.8 & $\mathbf{N}$ & \\
\hline 1 & $6 / 10 / 93$ & 8 & 1 & & 72.4 & $\mathbf{N}$ & \\
\hline 1 & $6 / 10 / 93$ & 9 & 1 & & 71.5 & $\mathrm{~N}$ & \\
\hline 1 & $6 / 10 / 93$ & 10 & 1 & & 70.6 & $\mathbf{N}$ & \\
\hline 1 & $6 / 10 / 93$ & 11 & 1 & & 70.5 & $\mathbf{N}$ & \\
\hline 9 & $6 / 10 / 93$ & 12 & 1 & & 70.6 & $\mathbf{N}$ & \\
\hline 1 & $6 / 10 / 93$ & 13 & 1 & & 70.5 & $\mathbf{N}$ & \\
\hline 1 & $6 / 10 / 93$ & 14 & 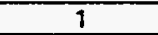 & & 70.5 & $\bar{N}$ & \\
\hline 1 & $6 / 18 / 93$ & 1 & 1 & & 90.1 & $\mathbf{N}$ & $\mathrm{J}$ \\
\hline 1 & $6 / 18 / 93$ & 2 & 1 & & 90.3 & $\mathrm{~N}$ & \\
\hline 1 & $6 / 18 / 93$ & 3 & 1 & & 89.8 & $\mathbf{N}$ & \\
\hline 1 & $6 / 18 / 93$ & 4 & 1 & & 87.8 & $N$ & \\
\hline 1 & $6 / 18 / 93$ & 5 & 1 & & 84.6 & $N$ & \\
\hline 1 & $6 / 18 / 93$ & 6 & $\frac{1}{1}$ & & 80.5 & $\bar{N}$ & \\
\hline 1 & $6 / 18 / 93$ & 7 & 1 & & 75.6 & $N$ & \\
\hline 1 & $6 / 18 / 93$ & 8 & 1 & & 72.1 & $\mathbf{N}$ & \\
\hline 1 & $6 / 18 / 93$ & 9 & 1 & & 72.5 & $\mathbf{N}$ & \\
\hline 1 & $6 / 18 / 93$ & 10 & 1 & & 70.6 & $\mathbf{N}$ & \\
\hline 1 & $6 / 18 / 93$ & 11 & 1 & & 70.5 & $\mathbf{N}$ & \\
\hline 1 & $6 / 18 / 93$ & 12 & 1 & & 70.7 & $\mathbf{N}$ & \\
\hline 1 & $6 / 18 / 93$ & 13 & 1 & & 70.6 & $N$ & \\
\hline 1 & $6 / 18 / 93$ & 14 & 1 & & 70.6 & $\mathrm{~N}$ & \\
\hline 1 & $6 / 27 / 93$ & 1 & 1 & & 91.2 & $\mathbf{N}$ & J910126143-32 TC $50 / 5$ \\
\hline 1 & $6 / 27 / 93$ & 2 & 1 & & 91.4 & $\bar{Y}$ & \\
\hline 1 & $6 / 27 / 93$ & 3 & 1 & & 90.8 & $\mathbf{N}$ & \\
\hline 1 & $6 / 27 / 93$ & 4 & $\frac{1}{1}$ & & 88.8 & $\mathbf{N}$ & \\
\hline 1 & $6 / 27 / 93$ & 6 & 1 & & 82 & $\mathbf{N}$ & \\
\hline 1 & $6 / 27 / 93$ & 7 & 1 & & 77.1 & $\mathbf{N}$ & \\
\hline 1 & $6 / 27 / 93$ & 8 & 1 & & 73.8 & $\mathbf{N}$ & \\
\hline 1 & $6 / 27 / 93$ & 9 & 1 & & 73.1 & $\mathbf{N}$ & \\
\hline 1 & $6 / 27 / 93$ & 10 & 1 & & 72.3 & $\mathrm{~N}$ & - \\
\hline 1 & $6 / 27 / 93$ & 11 & 9 & & 72.2 & $\mathbf{N}$ & \\
\hline 1 & $6 / 27 / 93$ & 12 & 1 & & 72.3 & $\mathrm{~N}$ & \\
\hline 1 & $6 / 27 / 93$ & 13 & 1 & & 72.3 & $\mathrm{~N}$ & \\
\hline 9 & $6 / 27 / 93$ & 14 & 1 & & 72.3 & $N$ & \\
\hline 1 & $7 / 1 / 93$ & 1 & 1 & & 88.7 & $\mathbf{N}$ & ¡890423320-42 TC $515160 / \mathrm{S}$ \\
\hline 1 & $7 / 1 / 93$ & 2 & 1 & & 88.9 & $\bar{Y}$ & \\
\hline 1 & $7 / 1 / 93$ & 3 & 1 & & 88.4 & $\mathbf{N}$ & \\
\hline 1 & $7 / 1 / 93$ & 4 & 9 & & 86.6 & $\mathrm{~N}$ & \\
\hline 1 & $7 / 1 / 93$ & 6 & 9 & & 80 & $N$ & \\
\hline 1 & $7 / 1 / 93$ & 7 & 3 & & 75.3 & $\mathbf{N}$ & \\
\hline 9 & $7 / 1 / 93$ & 8 & 1 & & 72.2 & $\mathbf{N}$ & \\
\hline 1 & $7 / 1 / 93$ & 9 & 9 & & 71.6 & $N$ & \\
\hline
\end{tabular}

Data obtained from WHC Surveillance Analysis Computer System (SACS), February 24, 1994. 


\begin{tabular}{|c|c|c|c|c|c|c|c|}
\hline \multicolumn{8}{|c|}{ TANK 241-S-111 } \\
\hline & & & & THEF & JCOUPLE & TA & \\
\hline TREE \# & DATE & THERMO \# & TREE SET \# & TEMP C & TEMP F & $Y=$ MAX TEMP & COMMENTS \\
\hline 1 & $7 / 1 / 93$ & 10 & 1 & & 70.7 & $\mathbf{N}$ & \\
\hline 1 & $7 / 1 / 93$ & 11 & 1 & & 70.6 & $\mathbf{N}$ & \\
\hline 1 & $7 / 1 / 93$ & 12 & 1 & & 70.7 & $\mathbf{N}$ & \\
\hline 1 & $7 / 1 / 93$ & 13 & 1 & & 70.7 & $\bar{N}$ & \\
\hline 1 & $7 / 1 / 93$ & 14 & 1 & & 70.7 & $\mathbf{N}$ & \\
\hline 1 & $7 / 8 / 93$ & 1 & 1 & & 88.5 & $\mathbf{N}$ & J $890423320-42$ TC 56 O/S \\
\hline 1 & $7 / 8 / 93$ & 2 & 1 & & 88.7 & $\bar{Y}$ & \\
\hline 9 & $7 / 8 / 93$ & 3 & 1 & & 88.2 & $\bar{N}$ & \\
\hline 1 & $7 / 8 / 93$ & 4 & 1 & & 86.3 & $\mathbf{N}$ & \\
\hline 1 & $7 / 8 / 93$ & 7 & 1 & & 75.3 & $\mathbf{N}$ & \\
\hline 1 & $7 / 8 / 93$ & 8 & 1 & & 72.3 & $\mathbf{N}$ & \\
\hline 1 & $7 / 8 / 93$ & 9 & 1 & & 71.7 & $\mathbf{N}$ & \\
\hline 1 & $7 / 8 / 93$ & 10 & 1 & & 70.9 & $\mathbf{N}$ & \\
\hline 1 & $7 / 8 / 93$ & 11 & $\frac{1}{1}$ & & 70.9 & $\mathbf{N}$ & \\
\hline 1 & $7 / 8 / 93$ & 12 & 1 & & 71.1 & $\mathbf{N}$ & \\
\hline 1 & $7 / 8 / 93$ & 13 & 1 & & 71.1 & $\mathbf{N}$ & \\
\hline 1 & $7 / 8 / 93$ & 74 & 1 & & 71.1 & $\mathbf{N}$ & \\
\hline 1 & $7 / 16 / 93$ & 1 & 1 & & 88.4 & $\mathbf{N}$ & TYPE J 890423320-42 \\
\hline 1 & $7 / 16 / 93$ & 2 & 1 & & 88.6 & $\overline{\mathbf{Y}}$ & \\
\hline 1 & $7 / 16 / 93$ & 3 & 1 & & 88.1 & $\mathbf{N}$ & \\
\hline 1 & $7 / 16 / 93$ & 4 & 1 & & 86.2 & $\mathbf{N}$ & \\
\hline 1 & $7 / 16 / 93$ & 5 & 1 & & 83.5 & $\mathbf{N}$ & \\
\hline 1 & $7 / 16 / 93$ & 6 & 1 & & 80 & $\mathbf{N}$ & \\
\hline 1 & $7 / 16 / 93$ & 7 & 1 & & 75.7 & $\mathbf{N}$ & \\
\hline 1 & $7 / 16 / 93$ & 8 & 1 & & 72.7 & $\mathbf{N}$ & \\
\hline 1 & $7 / 16 / 93$ & 9 & 1 & & 72.1 & $\mathbf{N}$ & \\
\hline 1 & $7 / 16 / 93$ & 10 & 1 & & 71.5 & $\mathbf{N}$ & \\
\hline 1 & $7 / 16 / 93$ & 11 & 9 & & 71.5 & $\mathbf{N}$ & \\
\hline 1 & $7 / 16 / 93$ & 12 & 1 & & 71.6 & $\mathbf{N}$ & \\
\hline 1 & $7 / 16 / 93$ & 13 & 1 & & 71.5 & $\mathbf{N}$ & \\
\hline 1 & $7 / 16 / 93$ & 14 & 1 & & 71.6 & $\mathbf{N}$ & \\
\hline 1 & $7 / 22 / 93$ & 1 & 1 & & 89.4 & $\mathbf{N}$ & J 0126143-14 TC-56 O/S \\
\hline 1 & $7 / 22 / 93$ & 2 & 1 & & 89.6 & $\bar{Y}$ & \\
\hline 1 & $7 / 22 / 93$ & 3 & 1 & & 89.1 & $\mathbf{N}$ & \\
\hline 1 & $7 / 22 / 93$ & 4 & 1 & & 87.4 & $\mathbf{N}$ & \\
\hline 1 & $7 / 22 / 93$ & 7 & 1 & & 77.1 & $\mathbf{N}$ & \\
\hline 1 & $7 / 22 / 93$ & 8 & 1 & & 74.3 & $\mathbf{N}$ & \\
\hline 1 & $7 / 22 / 93$ & 9 & 1 & & 73.4 & $\mathbf{N}$ & \\
\hline 1 & $7 / 22 / 93$ & 10 & 1 & & 72.8 & $\mathbf{N}$ & \\
\hline 1 & $7 / 22 / 93$ & 11 & 1 & & 72.8 & $\mathbf{N}$ & \\
\hline 1 & $7 / 22 / 93$ & 12 & 1 & & 72.9 & $\mathrm{~N}$ & \\
\hline 1 & $7 / 22 / 93$ & 13 & 1 & & 72.9 & $\mathbf{N}$ & \\
\hline 1 & $7 / 22 / 93$ & 74 & 1 & & 73 & $\mathbf{N}$ & \\
\hline 1 & $7 / 29 / 93$ & 1 & 1 & & 89.8 & $\mathbf{N}$ & J 910126143.14 TCH $12 \& 16$ O/S \\
\hline 1 & $7 / 29 / 93$ & 2 & 1 & & 90 & $\mathbf{Y}$ & \\
\hline 1 & $7 / 29 / 93$ & 3 & 1 & & 89.6 & $\mathbf{N}$ & \\
\hline 1 & $7 / 29 / 93$ & 4 & 1 & & 87.9 & $\mathbf{N}$ & \\
\hline 1 & $7 / 29 / 93$ & 7 & 1 & & 78 & $\mathbf{N}$ & \\
\hline 1 & $7 / 29 / 93$ & 8 & 1 & & 75.3 & $\mathbf{N}$ & \\
\hline 1 & $7 / 29 / 93$ & 9 & 1 & & 74.4 & $\mathbf{N}$ & \\
\hline 1 & $7 / 29 / 93$ & 10 & 1 & & 73.8 & $\mathbf{N}$ & \\
\hline 1 & $7 / 29 / 93$ & 11 & 1 & & 73.8 & $\mathbf{N}$ & \\
\hline 1 & $7 / 29 / 93$ & 12 & 1 & & 73.8 & $\mathbf{N}$ & \\
\hline .1 & $7 / 29 / 93$ & 13 & 1 & & 73.8 & $\mathbf{N}$ & \\
\hline 1 & $7 / 29 / 93$ & 14 & 1 & & 73.8 & $N$ & \\
\hline 1 & $8 / 5 / 93$ & 1 & 1 & & 88.1 & $\mathbf{N}$ & J $890423320-42$ \\
\hline 1 & $8 / 5 / 93$ & 2 & 1 & & 88.4 & $\bar{Y}$ & \\
\hline 1 & $8 / 5 / 93$ & 3 & 1 & & 88 & $\mathbf{N}$ & \\
\hline 1 & $8 / 5 / 93$ & 4 & 1 & & 86.3 & $\mathbf{N}$ & \\
\hline 1 & $8 / 5 / 93$ & 5 & 1 & & 83.7 & $\mathbf{N}$ & \\
\hline 1 & $8 / 5 / 93$ & 6 & 1. & & 80.4 & $\mathbf{N}$ & \\
\hline 1 & $8 / 5 / 93$ & 7 & 1 & & 76.8 & $\mathbf{N}$ & \\
\hline 1 & $8 / 5 / 93$ & 8 & 1 & & 74 & $\mathbf{N}$ & \\
\hline$\frac{1}{1}$ & $8 / 5 / 93$ & 9 & 9 & & 73.2 & $\mathbf{N}$ & \\
\hline 1 & $8 / 5 / 93$ & 10 & 1 & & 72.6 & $\mathbf{N}$ & \\
\hline 1 & $8 / 5 / 93$ & 11 & 1 & & 72.5 & $\mathbf{N}$ & \\
\hline
\end{tabular}

Data obtained from WHC Surveillance Analysis Computer System (SACS), February 24, 1994. 


\begin{tabular}{|c|c|c|c|c|c|c|c|}
\hline \multicolumn{8}{|c|}{ TANK 241-S.111 } \\
\hline & & & & THEF & OCOUPLE & & \\
\hline TREE \# & DATE & THERMO \# & TREE SET \# & TEMP C & TEMP F & $Y=$ MAX TEMP & COMMENTS \\
\hline 1 & $8 / 5 / 93$ & 12 & 1 & & 72.7 & $\mathrm{~N}$ & \\
\hline 1 & $8 / 5 / 93$ & 13 & 1 & & 72.6 & $\mathbf{N}$ & \\
\hline 1 & $8 / 5 / 93$ & 14 & 1 & & 72.7 & $N$ & \\
\hline 1 & $8 / 19 / 93$ & 1 & 1 & & 91.2 & $\mathrm{~N}$ & J910126143-66 \\
\hline 1 & $8 / 19 / 93$ & 2 & 1 & & 91.5 & $Y$ & \\
\hline 1 & $8 / 19 / 93$ & 3 & 1 & & 99 & $\mathbf{N}$ & \\
\hline 1 & $8 / 19 / 93$ & 4 & 1 & & 89.3 & $\mathbf{N}$ & \\
\hline 1 & $8 / 19 / 93$ & 5 & 1 & & 86.7 & $\mathrm{~N}$ & \\
\hline 1 & $8 / 19 / 93$ & 6 & 1 & & 83.7 & $\mathrm{~N}$ & \\
\hline 1 & $8 / 19 / 93$ & 7 & 1 & & 80.2 & $\mathbf{N}$ & \\
\hline 1 & $8 / 19 / 93$ & 8 & 1 & & 77.7 & $\mathrm{~N}$ & \\
\hline 1 & $8 / 19 / 93$ & 9 & 1 & & 76.6 & $\mathbf{N}$ & \\
\hline 1 & $8 / 19 / 93$ & 10 & 1 & & 76 & $\mathbf{N}$ & \\
\hline 1 & $8 / 19 / 93$ & 11 & 1 & & 75.9 & $\mathbf{N}$ & \\
\hline 1 & $8 / 19 / 93$ & 12 & 1 & & 76.2 & $\mathbf{N}$ & \\
\hline 1 & $8 / 19 / 93$ & 13 & 1 & & 76.1 & $\mathbf{N}$ & \\
\hline 1 & $8 / 19 / 93$ & 14 & 1 & & 76.2 & $\mathbf{N}$ & \\
\hline 1 & $8 / 27 / 93$ & 1 & 1 & & 92.3 & $\mathbf{N}$ & J $910126143-66$ TC $5 \& 6$ O/S \\
\hline 1 & $8 / 27 / 93$ & 2 & 1 & & 92.5 & $\bar{Y}$ & \\
\hline 1 & $8 / 27 / 93$ & 3 & 1 & & 92.1 & $N$ & \\
\hline 1 & $8 / 27 / 93$ & 4 & 1 & & 90.5 & $\bar{N}$ & \\
\hline 1 & $8 / 27 / 93$ & 7 & 1 & & 82 & $\mathbf{N}$ & \\
\hline 1 & $8 / 27 / 93$ & 8 & 1 & & 79.4 & $\mathrm{~N}$ & \\
\hline 1 & $8 / 27 / 93$ & 9 & 1 & & 78.4 & $\mathbf{N}$ & \\
\hline 1 & $8 / 27 / 93$ & 10 & 1 & & 77.9 & $\mathbf{N}$ & \\
\hline 1 & $8 / 27 / 93$ & 11 & 1 & & 77.9 & $\mathrm{~N}$ & \\
\hline 1 & $8 / 27 / 93$ & 12 & 1 & & 77.8 & $\mathrm{~N}$ & \\
\hline 1 & $8 / 27 / 93$ & 13 & 1 & & 77.9 & $\mathbf{N}$ & \\
\hline 1 & $8 / 27 / 93$ & 14 & 1 & & 77.9 & $\mathbf{N}$ & \\
\hline 1 & $9 / 3 / 93$ & 1 & 1 & & 91.2 & $\mathbf{N}$ & $J 910126143-66 \mathrm{TC} 515160 / \mathrm{s}$ \\
\hline 1 & $9 / 3 / 93$ & 2 & 1 & & 91.6 & $Y$ & \\
\hline 1 & $9 / 3 / 93$ & 3 & 1 & & 91.2 & $\mathrm{~N}$ & \\
\hline 1 & $9 / 3 / 93$ & 4 & 1 & & 89.6 & $\mathrm{~N}$ & \\
\hline 1 & $9 / 3 / 93$ & 6 & 1 & & 84.6 & $\mathrm{~N}$ & \\
\hline 1 & $9 / 3 / 93$ & 7 & 1 & & 81.5 & $\mathrm{~N}$ & \\
\hline 1 & $9 / 3 / 93$ & 8 & 1 & & 78.8 & $N$ & \\
\hline 1 & $9 / 3 / 93$ & 9 & 1 & & 77.8 & $\mathrm{~N}$ & \\
\hline 1 & $9 / 3 / 93$ & 10 & 1 & & 77.3 & $N$ & \\
\hline 1 & 9/3/93 & 11 & 1 & & 77.2 & $N$ & \\
\hline 1 & $9 / 3 / 93$ & 12 & $i$ & & 77.4 & $\mathbf{N}$ & \\
\hline 1 & $9 / 3 / 93$ & 13 & 1 & & 77.3 & $N$ & \\
\hline 9 & $9 / 3 / 93$ & 14 & 1 & & 77.6 & $\mathbf{N}$ & \\
\hline 1 & $9 / 12 / 93$ & 1 & 1 & & 92.7 & $N$ & 3 TI-042 TC $515160 / \mathrm{s}$ \\
\hline 1 & 9/12/93 & 2 & 1 & & 93 & $Y$ & \\
\hline 1 & $9 / 12 / 93$ & 3 & 1 & & 92.7 & $\mathrm{~N}$ & \\
\hline 1 & $9 / 12 / 93$ & 4 & 1 & & 91.4 & $\mathrm{~N}$ & \\
\hline 1 & $9 / 12 / 93$ & 6 & 1 & & 86.6 & $\mathbf{N}$ & \\
\hline 1 & $9 / 12 / 93$ & 7 & 1 & & 83.4 & $N$ & \\
\hline 1 & $9 / 12 / 93$ & 8 & 1 & & 80.8 & $\mathbf{N}$ & \\
\hline 1 & $9 / 12 / 93$ & 9 & 1 & & 79.5 & $N$ & \\
\hline 1 & $9 / 12 / 93$ & 10 & 1 & & 79.1 & $\mathbf{N}$ & \\
\hline 1 & $9 / 12 / 93$ & 11 & 1 & & 79 & $\mathbf{N}$ & \\
\hline 1 & $9 / 12 / 93$ & 12 & 1 & & 79 & $\mathbf{N}$ & \\
\hline 1 & $9 / 12 / 93$ & 13 & 1 & & 79 & $\mathrm{~N}$ & \\
\hline 1 & $9 / 12 / 93$ & 14 & 1 & & 79 & $\mathbf{N}$ & \\
\hline 1 & $9 / 20 / 93$ & 1 & 1 & & 89.1 & $\mathbf{N}$ & J $910126143-50$ \\
\hline 9 & $9 / 20 / 93$ & 2 & 1 & & 89.3 & $Y$ & \\
\hline 1 & $9 / 20 / 93$ & 3 & 1 & & 89.1 & $\mathrm{~N}$ & \\
\hline 1 & $9 / 20 / 93$ & 4 & 1 & & 87.8 & $N$ & \\
\hline 1 & $9 / 20 / 93$ & 5 & 1 & & 85.7 & $\mathbf{N}$ & \\
\hline 1 & $9 / 20 / 93$ & 6 & 1 & & 83.3 & $\mathbf{N}$ & \\
\hline 1 & $9 / 20 / 93$ & 7 & 1 & & 80.6 & $\mathbf{N}$ & \\
\hline 1 & $9 / 20 / 93$ & 8 & 1 & & 78 & $\mathbf{N}$ & \\
\hline 1 & 9/20/93 & 9 & 1 & & 76.9 & $\mathbf{N}$ & \\
\hline 1 & 9/20/93 & 10 & 1 & & 76.4 & $\mathbf{N}$ & \\
\hline 1 & $9 / 20 / 93$ & 11 & 1 & & 76.3 & $\mathbf{N}$ & \\
\hline
\end{tabular}

Data obtained from WHC Surveillance Analysis Computer System (SACS), February 24, 1994. 
WHC-SD-WM-ER-323, Rev. 0

\begin{tabular}{|c|c|c|c|c|c|c|c|}
\hline \multicolumn{8}{|c|}{ TANK 241-S-111 } \\
\hline \multicolumn{8}{|c|}{ THERMOCOUPLE DATA } \\
\hline TREE \# & DATE & THERMO \# & TREE SET \# & TEMP C & TEMP F & $Y=M A X$ TEMP & \\
\hline 1 & $9 / 20 / 93$ & 12 & 1 & Thin 0 & 76.3 & $\mathrm{~N}$ & comolome \\
\hline 1 & $9 / 20 / 93$ & 13 & 1 & & 76.2 & $\mathrm{~N}$ & \\
\hline 1 & $9 / 20 / 93$ & 14 & 1 & & 76.1 & $N$ & \\
\hline 1 & $9 / 27 / 93$ & 1 & 2 & & 89.3 & $N$ & HDAT TC 56 O/S \\
\hline 1 & $9 / 27 / 93$ & 1 & 9 & & 89.5 & $\mathbf{N}$ & J 890423320-42 TC $110 / 5$ \\
\hline 1 & $9 / 27 / 93$ & 2 & 2 & & 89.5 & $\bar{Y}$ & \\
\hline 1 & $9 / 27 / 93$ & 2 & 1 & & 89.6 & $\bar{Y}$ & \\
\hline 1 & $9 / 27 / 93$ & 3 & 2 & & 89.2 & $\mathbf{N}$ & \\
\hline 1 & $9 / 27 / 93$ & 3 & 1 & & 89.3 & $\mathbf{N}$ & \\
\hline$\frac{1}{1}$ & $9 / 27 / 93$ & 4 & 2 & & 88 & $\mathbf{N}$ & \\
\hline$\frac{1}{1}$ & $9 / 27 / 93$ & 4 & 1 & & 88.2 & $\mathrm{~N}$ & \\
\hline 1 & $9 / 27 / 93$ & 5 & 1 & & 86.3 & $\mathbf{N}$ & \\
\hline 1 & $9 / 27 / 93$ & 6 & 1 & & 84.2 & $\mathrm{~N}$ & \\
\hline 9 & $9 / 27 / 93$ & 7 & 2 & & 80.7 & $\mathbf{N}$ & \\
\hline 1 & $9 / 27 / 93$ & 7 & 1 & & 81.1 & $\mathbf{N}$ & \\
\hline 1 & $9 / 27 / 93$ & 8 & 2 & & 77.9 & $\mathbf{N}$ & \\
\hline 1 & $9 / 27 / 93$ & 8 & 1 & & 78.1 & $\bar{N}$ & \\
\hline$\frac{1}{1}$ & $9 / 27 / 93$ & 9 & 2 & & 77 & $\mathbf{N}$ & \\
\hline 1 & $9 / 27 / 93$ & 9 & 1 & & 77.4 & $\mathrm{~N}$ & \\
\hline 1 & $9 / 27 / 93$ & 10 & 2 & & 76.5 & $\mathbf{N}$ & \\
\hline 1 & $9 / 27 / 93$ & 10 & 1 & & 76.8 & $\mathbf{N}$ & \\
\hline 9 & $9 / 27 / 93$ & 11 & 2 & & 76.3 & $\mathbf{N}$ & \\
\hline 1 & $9 / 27 / 93$ & 12 & 2 & & 76.4 & $N$ & \\
\hline 1 & $9 / 27 / 93$ & 12 & 1 & & 76.7 & $\mathbf{N}$ & \\
\hline 9 & $9 / 27 / 93$ & 13 & 2 & & 76.4 & $\mathrm{~N}$ & \\
\hline 1 & $9 / 27 / 93$ & 13 & 1 & & 76.7 & $\bar{N}$ & \\
\hline 1 & $9 / 27 / 93$ & 14 & 2 & & 76.4 & $\mathbf{N}$ & \\
\hline 9 & $9 / 27 / 93$ & 14 & 1 & & 76.7 & $N$ & \\
\hline 1 & $10 / 5 / 93$ & 9 & 1 & & 90.2 & $\mathbf{N}$ & $\mathrm{J} 910126143-48$ TC $56111516 \mathrm{O} / \mathrm{S}$ \\
\hline$\frac{1}{1}$ & $10 / 5 / 93$ & 2 & 1 & & 90.4 & $\bar{Y}$ & \\
\hline 1 & $10 / 5 / 93$ & 3 & 1 & & 90.2 & $\mathbf{N}$ & \\
\hline 1 & $10 / 5 / 93$ & 4 & 1 & & 88.9 & $\mathrm{~N}$ & \\
\hline$\frac{1}{1}$ & $10 / 5 / 93$ & 7 & 1 & & 81.7 & $\mathbf{N}$ & \\
\hline$\frac{1}{1}$ & $10 / 5 / 93$ & 8 & 1 & & 78.5 & $\mathrm{~N}$ & \\
\hline 1 & $10 / 5 / 93$ & 9 & 1 & & 77.8 & $\mathbf{N}$ & \\
\hline$T$ & $10 / 5 / 93$ & 10 & 1 & & 77.3 & $\bar{N}$ & \\
\hline 1 & $10 / 5 / 93$ & 12 & 1 & & 77.2 & $\mathbf{N}$ & \\
\hline 1 & $10 / 5 / 93$ & 13 & 1 & & 77.2 & $\mathbf{N}$ & \\
\hline 1 & $10 / 5 / 93$ & 14 & 1 & & 77.2 & $\mathbf{N}$ & \\
\hline 1 & $10 / 12 / 93$ & 1 & 1 & & 90.8 & $\mathbf{N}$ & $J 910126143-48$ \\
\hline 9 & $10 / 12 / 93$ & 2 & 1 & & 91.1 & $\bar{Y}$ & \\
\hline 1 & $10 / 12 / 93$ & 3 & 1 & & 90.8 & $N$ & \\
\hline 1 & $10 / 12 / 93$ & 4 & 1 & & 89.7 & $\bar{N}$ & \\
\hline 1 & $10 / 12 / 93$ & 5 & 1 & & 87.9 & $\mathbf{N}$ & \\
\hline 1 & $10 / 12 / 93$ & 6 & 1 & & 85.7 & $\mathbf{N}$ & \\
\hline 1 & $10 / 12 / 93$ & 7 & 1 & & 82.6 & $\mathbf{N}$ & \\
\hline 1 & $10 / 12 / 93$ & 8 & 1 & & 79.2 & $\mathbf{N}$ & \\
\hline 1 & $10 / 12 / 93$ & 9 & 1 & & 78.5 & $\mathbf{N}$ & \\
\hline 1 & $10 / 12 / 93$ & 10 & 1 & & 78 & $\mathbf{N}$ & \\
\hline 1 & $10 / 12 / 93$ & 11 & 1 & & 77.9 & $\mathbf{N}$ & \\
\hline 1 & $10 / 12 / 93$ & 12 & 1 & & 77.9 & $N$ & \\
\hline$\frac{1}{1}$ & $10 / 72 / 93$ & 93 & 1. & & 77.9 & $\mathrm{~N}$ & \\
\hline 1 & $10 / 12 / 93$ & 14 & .1 & & 77.9 & $N$ & \\
\hline 1 & $10 / 18 / 93$ & 1 & 1 & & 89.8 & $\mathbf{N}$ & $J 910126143-14$ \\
\hline 1 & $10 / 18 / 93$ & 2 & 1 & & 90.1 & $\mathbf{Y}$ & \\
\hline 1 & $10 / 18 / 93$ & 3 & 1 & & 90 & N & \\
\hline 1 & $10 / 18 / 93$ & 4 & 1 & & 88.9 & $\mathbf{N}$ & \\
\hline 1 & $10 / 18 / 93$ & 5 & 1 & & 87.1 & $\mathbf{N}$ & \\
\hline 1 & $10 / 18 / 93$ & 6 & 1 & & 84.8 & $\mathbf{N}$ & \\
\hline 1 & $10 / 18 / 93$ & 7 & 1 & & 81.9 & $\mathbf{N}$ & \\
\hline 1 & $10 / 18 / 93$ & 8 & 1 & & 78.4 & $\mathbf{N}$ & \\
\hline 1 & $10 / 18 / 93$ & 9 & 1 & & 77.9 & $\bar{N}$ & \\
\hline 1 & $10 / 18 / 93$ & 10 & 1 & & 77.3 & $\mathbf{N}$ & \\
\hline 1 & $10 / 18 / 93$ & 11 & 9 & & 77.3 & $N$ & \\
\hline 1 & $10 / 18 / 93$ & 12 & 1 & & 77.4 & $\mathbf{N}$ & \\
\hline 1 & $10 / 18 / 93$ & 13 & 1 & & 77.2 & $\mathbf{N}$ & \\
\hline
\end{tabular}

Data obtained from WHC Surveillance Analysis Computer System (SACS), February 24, 1994. 


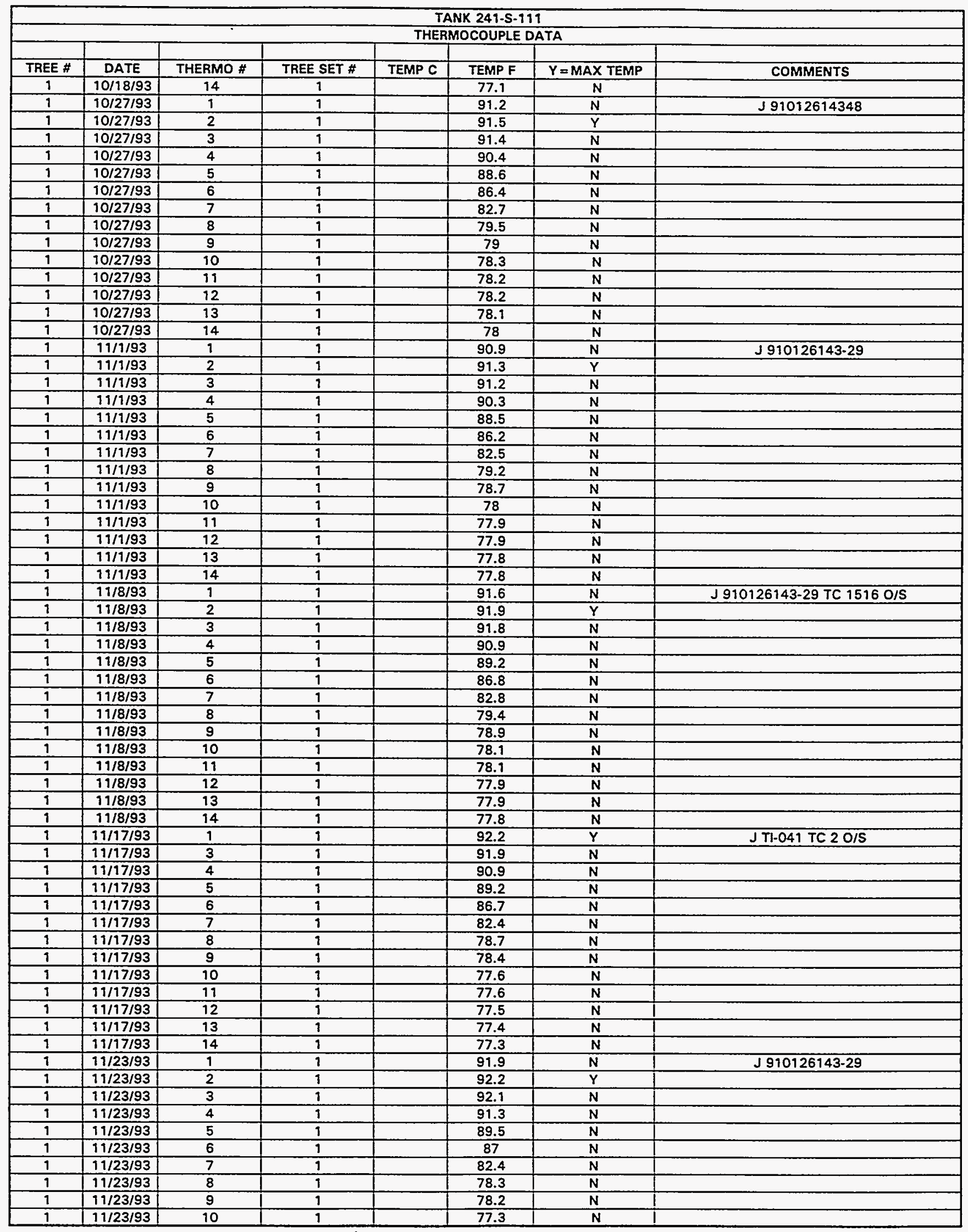

Data obtained from WHC Surveillance Analysis Computer System (SACS), February 24, 1994. 
WHC-SD-WM-ER-323, Rev. 0

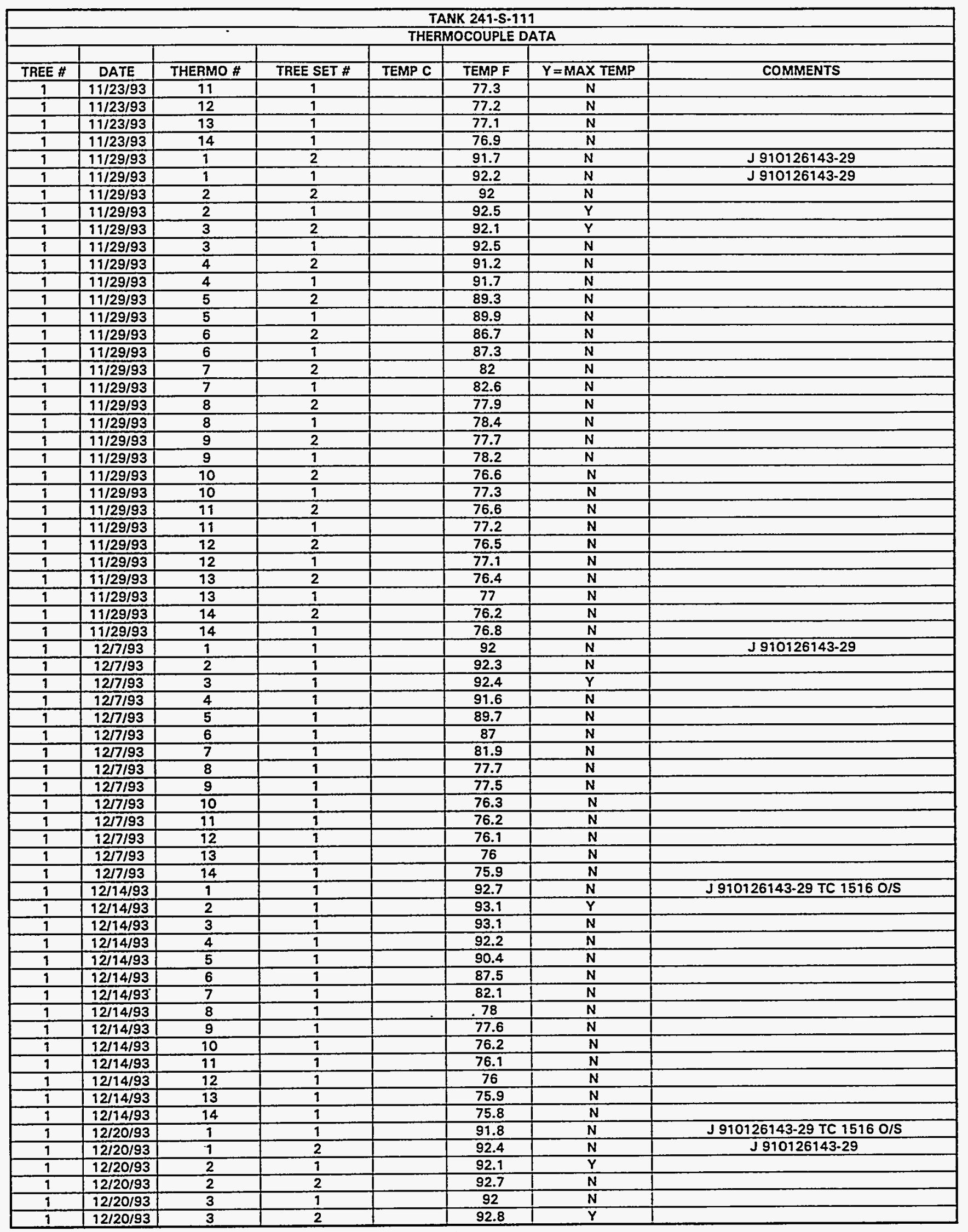

Data obtained from WHC Surveillance Analysis Computer System (SACS), February 24, 1994. 


\begin{tabular}{|c|c|c|c|c|c|c|c|}
\hline \multicolumn{8}{|c|}{ TANK 241-S-111 } \\
\hline \multicolumn{8}{|c|}{ THERMOCOUPLE DATA } \\
\hline TREE \# & DATE & THERMO \# & TREE SET \# & TEMP C & TEMP F & $Y=M A X$ TEMP & COMMENTS \\
\hline 1 & $12 / 20 / 93$ & 4 & 1 & & 91.2 & $\mathrm{~N}$ & \\
\hline 1 & $12 / 20 / 93$ & 4 & 2 & & 91.9 & $N$ & \\
\hline 1 & $12 / 20 / 93$ & 5 & 1 & & 89.2 & $N$ & \\
\hline 1 & $12 / 20 / 93$ & 5 & 2 & & 89.9 & $N$ & \\
\hline 1 & $12 / 20 / 93$ & 6 & 1 & & 86.1 & $\mathbf{N}$ & \\
\hline 1 & $12 / 20 / 93$ & 6 & 2 & & 86.8 & $\mathbf{N}$ & \\
\hline 1 & $12 / 20 / 93$ & 7 & 1 & & 80.5 & $\bar{N}$ & \\
\hline 1 & $12 / 20 / 93$ & 7 & 2 & & 81.4 & $\mathbf{N}$ & \\
\hline 1 & $12 / 20 / 93$ & 8 & 1 & & 76.4 & $\mathrm{~N}$ & \\
\hline 1 & $12 / 20 / 93$ & 8 & 2 & & 77.3 & $N$ & \\
\hline 1 & $12 / 20 / 93$ & 9 & 9 & & 76.2 & $N$ & \\
\hline 1 & $12 / 20 / 93$ & 9 & 2 & & 76.9 & $N$ & \\
\hline 1 & $12 / 20 / 93$ & 10 & 1 & & 74.8 & $\mathbf{N}$ & \\
\hline 1 & $12 / 20 / 93$ & 10 & 2 & & 75.5 & $\mathbf{N}$ & \\
\hline 1 & $12 / 20 / 93$ & 11 & 1 & & 74.7 & $N$ & \\
\hline 1 & $12 / 20 / 93$ & 11 & 2 & & 75.4 & $\mathbf{N}$ & \\
\hline 1 & $12 / 20 / 93$ & 12 & 1 & & 74.6 & $\mathbf{N}$ & \\
\hline 1 & $12 / 20 / 93$ & 12 & 2 & & 75.3 & $N$ & \\
\hline 1 & $12 / 20 / 93$ & 13 & 1 & & 74.5 & $N$ & \\
\hline 1 & $12 / 20 / 93$ & 13 & 2 & & 75.2 & $N$ & \\
\hline 1 & $12 / 20 / 93$ & 14 & 1 & & 74.4 & $\mathbf{N}$ & \\
\hline 1 & $12 / 20 / 93$ & 14 & 2 & & 75.1 & $N$ & \\
\hline 1 & $12 / 27 / 93$ & 1 & 1 & & 91.9 & $N$ & J $910126143-29$ TC 1516 o/S \\
\hline 1 & $12 / 27 / 93$ & 2 & 1 & & 92.2 & $\mathbf{N}$ & \\
\hline 1 & $12 / 27 / 93$ & 3 & 1 & & 92.3 & $\bar{Y}$ & \\
\hline 1 & $12 / 27 / 93$ & 4 & 1 & & 91.4 & $\mathbf{N}$ & \\
\hline 1 & $12 / 27 / 93$ & 5 & 1 & & 89.4 & $N$ & \\
\hline 1 & $12 / 27 / 93$ & 6 & 1 & & 86.2 & $\bar{N}$ & \\
\hline 1 & $12 / 27 / 93$ & 7 & 1 & & 80.2 & $N$ & \\
\hline 1 & $12 / 27 / 93$ & 8 & 1 & & 76 & $\mathrm{~N}$ & \\
\hline 1 & $12 / 27 / 93$ & 9 & 1 & & 75.7 & $N$ & \\
\hline 1 & $12 / 27 / 93$ & 10 & 1 & & 74.5 & $N$ & \\
\hline 1 & $12 / 27 / 93$ & 11 & 1 & & 74.5 & $N$ & \\
\hline 1 & $12 / 27 / 93$ & 12 & 9 & & 74.3 & $\mathbf{N}$ & \\
\hline 1 & $12 / 27 / 93$ & 13 & 1 & & 74.3 & $\mathbf{N}$ & \\
\hline 1 & $12 / 27 / 93$ & 14 & 1 & & 74.1 & $\mathbf{N}$ & \\
\hline
\end{tabular}

Data obtained from WHC Surveillance Analysis Computer System (SACS), February 24, 1994. 


\begin{tabular}{|c|c|c|c|c|c|c|c|}
\hline \multicolumn{8}{|c|}{ TANK 241-S-112 } \\
\hline \multicolumn{8}{|c|}{ THERMOCOUPLE DATA } \\
\hline TREE \# & DATE & THERMO \# & TREE SET \# & TEMP C & TEMP F & $Y=$ MAX TEMP & COMMENTS \\
\hline 1 & $10 / 20 / 74$ & 1 & & 47.78 & 118.004 & & raw data - assumed degree $\mathrm{C}$ \\
\hline 1 & $10 / 20 / 74$ & 2 & & 50.56 & 123.008 & & raw data - assumed degree $C$ \\
\hline 1 & $10 / 20 / 74$ & 4 & & 57.78 & 136.004 & & raw data - assumed degree $C$ \\
\hline 1 & $10 / 20 / 74$ & 5 & & 61.67 & 143.006 & & raw data - assumed degree $C$ \\
\hline 1 & $10 / 20 / 74$ & 6 & & 63.33 & 145.994 & & raw data - assumed degree C \\
\hline 1 & $10 / 20 / 74$ & 7 & & 63.89 & 147.002 & & raw data - assumed degree $\mathrm{C}$ \\
\hline 1 & $10 / 20 / 74$ & 8 & & 63.33 & 145.994 & & raw data - assumed degree $C$ \\
\hline 1 & $10 / 20 / 74$ & 9 & & 62.78 & 145.004 & & raw data - assumed degree C \\
\hline 1 & $10 / 20 / 74$ & 10 & & 61.67 & 143.006 & & raw data - assumed degree $C$ \\
\hline 1 & $10 / 20 / 74$ & 11 & & 56.67 & 134.006 & & raw data - assumed degree $C$ \\
\hline 1 & $10 / 20 / 74$ & 12 & & 46.67 & 116.006 & & raw data - assumed degree C \\
\hline 1 & $12 / 2 / 74$ & 9 & & 48.89 & 120.002 & & raw data - assumed degree $C$ \\
\hline 1 & $12 / 2 / 74$ & 2 & & 52.22 & 125.996 & & raw data - assumed degree C \\
\hline 1 & $12 / 2 / 74$ & 3 & & 55.56 & 132.008 & & raw data - assumed degree C \\
\hline 1 & $12 / 2 / 74$ & 4 & & 58.89 & 138.002 & & raw data - assumed degree C \\
\hline 1 & $12 / 2 / 74$ & 5 & & 62.22 & 143.996 & & raw data - assumed degree $C$ \\
\hline 1 & $12 / 2 / 74$ & 6 & & 63.89 & 147.002 & & raw data - assumed degree C \\
\hline 1 & $12 / 2 / 74$ & 7 & & 64.44 & 147.992 & & raw data - assumed degree $C$ \\
\hline 1 & $12 / 2 / 74$ & 8 & & 63.33 & 145.994 & & raw data - assumed degree $C$ \\
\hline 1 & $12 / 2 / 74$ & 9 & & 63.33 & 145.994 & & raw data - assumed degree $C$ \\
\hline 1 & $12 / 2 / 74$ & 10 & & 60 & 140 & & raw data - assumed degree C \\
\hline 1 & $12 / 2 / 74$ & 91 & & 52.78 & 127.004 & & raw data - assumed degree C \\
\hline 1 & $12 / 2 / 74$ & 12 & & 43.89 & 111.002 & & raw data - assumed degree C \\
\hline 1 & $6 / 3 / 75$ & 1 & & & 120 & & \\
\hline 1 & $6 / 3 / 75$ & 2 & & & 125 & & \\
\hline 1 & $6 / 3 / 75$ & 4 & & & 136 & & \\
\hline 1 & $6 / 3 / 75$ & 5 & & & 940 & & \\
\hline 1 & $6 / 3 / 75$ & 6 & & & 141 & & \\
\hline 1 & $6 / 3 / 75$ & 7 & & & 139 & & \\
\hline 1 & $6 / 3 / 75$ & 8 & & & 135 & & \\
\hline 1 & $6 / 3 / 75$ & 9 & & & 135 & & \\
\hline 9 & $6 / 3 / 75$ & 10 & & & 133 & & \\
\hline 1 & $6 / 3 / 75$ & 11 & & & 113 & & \\
\hline 1 & $6 / 3 / 75$ & 12 & & & 98 & & \\
\hline 1 & $11 / 3 / 75$ & 1 & & & 124 & & \\
\hline 1 & $11 / 3 / 75$ & 2 & & & 125 & & \\
\hline 1 & $11 / 3 / 75$ & 4 & & & 138 & & \\
\hline 1 & $11 / 3 / 75$ & 5 & & & 140 & & \\
\hline 1 & $11 / 3 / 75$ & 6 & & & 140 & & \\
\hline 1 & $11 / 3 / 75$ & 8 & & & 132 & & \\
\hline 9 & $11 / 3 / 75$ & 9 & & & 126 & & \\
\hline 9 & $11 / 3 / 75$ & 10 & & & 120 & & \\
\hline 1 & $11 / 3 / 75$ & 11 & & & 110 & & \\
\hline 1 & $11 / 3 / 75$ & 12 & & & 100 & & \\
\hline 1 & $2 / 4 / 76$ & 1 & & & & & 155 - suspect data (high) \\
\hline 7 & $2 / 4 / 76$ & 2 & & & & & 163 - suspect data (high) \\
\hline 1 & $2 / 4 / 76$ & 4 & & & & & 166 - suspect data (high) \\
\hline 1 & $2 / 4 / 76$ & 5 & & & & & 166 - suspect data (high) \\
\hline 1 & $2 / 4 / 76$ & 6 & & & & & 161 - suspect data (high) \\
\hline 1 & $2 / 4 / 76$ & 8 & & & & & 155 - suspect data (high) \\
\hline 1 & $2 / 4 / 76$ & 9 & & & & & 146 - suspect data (high) \\
\hline 1 & $2 / 4 / 76$ & 10 & & & & & 131 - suspect date (high) \\
\hline$\frac{1}{1}$ & $2 / 4 / 76$ & 11 & & & & & 119 - suspect data (high) \\
\hline$T$ & $2 / 4 / 76$ & 12 & & & & & 119 - suspect data (high) \\
\hline 1 & $3 / 5 / 76$ & 1 & & & 122 & & \\
\hline 1 & $3 / 5 / 76$ & 2 & & & 126 & & \\
\hline 9 & $3 / 5 / 76$ & 4 & & & 134 & & \\
\hline 1 & $3 / 5 / 76$ & 5 & & & 136 & & \\
\hline 1 & $3 / 5 / 76$ & 6 & & & 137 & & \\
\hline 1 & $3 / 5 / 76$ & 8 & & & 131 & & \\
\hline 1 & $3 / 5 \Pi 6$ & 9 & & & 125 & & \\
\hline 1 & $3 / 5 / 76$ & 10 & & & 115 & & \\
\hline 1 & $3 / 5 / 76$ & 11 & & & 101 & & \\
\hline 1 & $3 / 5 / 76$ & 12 & & & 91 & & \\
\hline 1 & $5 / 2 / 76$ & 1 & & & 121 & & \\
\hline
\end{tabular}

Data obtained from WHC Surveillance Analysis Computer System (SACS), February 24, 1994. 
WHC-SD-WM-ER-323, Rev. 0

TANK 241-S-112

THERMOCOUPLE DATA

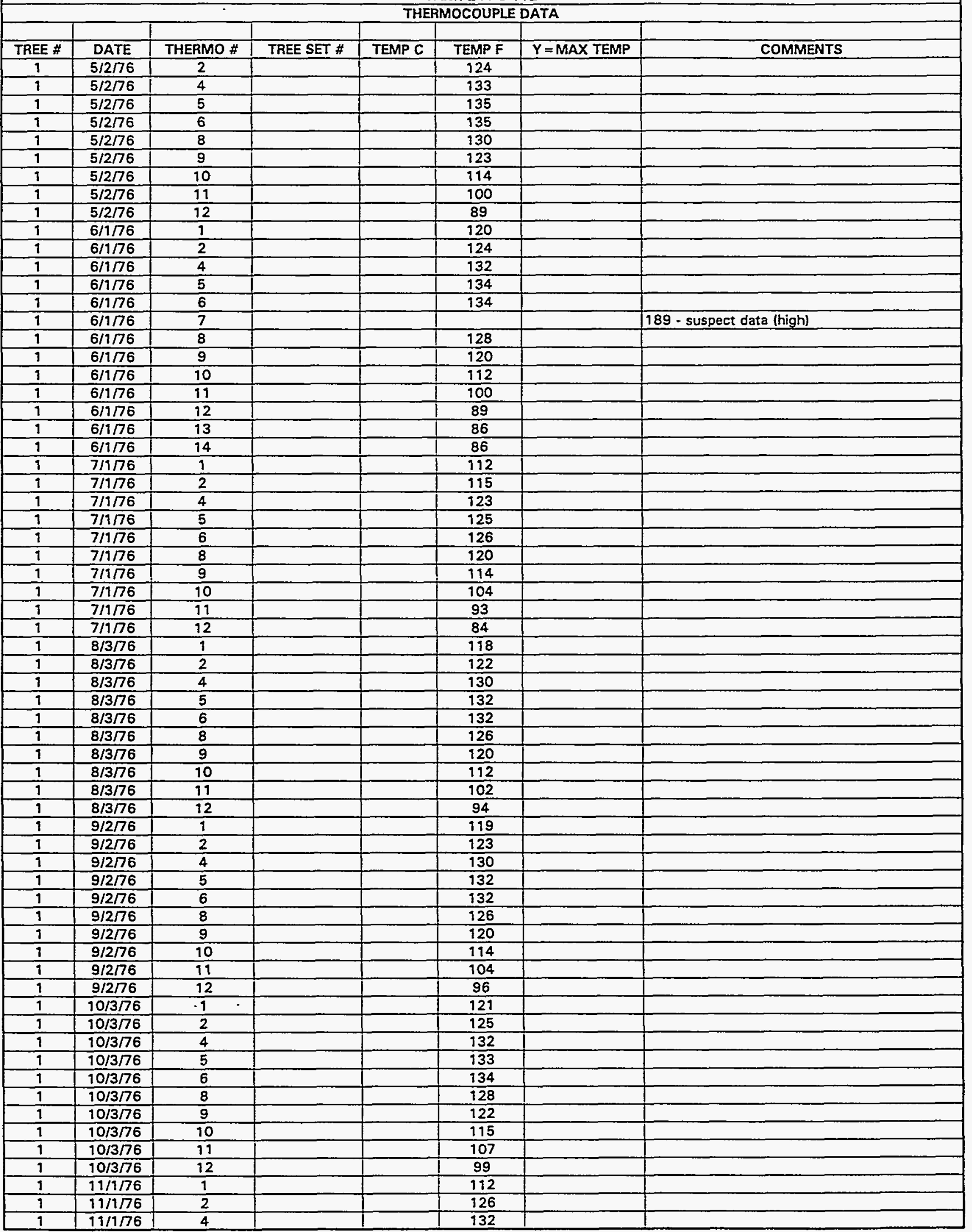

Data obtained from WHC Surveillance Analysis Computer System (SACS), February 24, 1994. . 


\begin{tabular}{|c|c|c|c|c|c|c|c|}
\hline \multicolumn{8}{|c|}{ TANK 241-S-112 } \\
\hline \multicolumn{8}{|c|}{ THERMOCOUPLE DATA } \\
\hline TAEE \# & DATE & THERMO \# & TREE SET \# & TEMP C & TEMP F & $Y=$ MAX TEMP & COMMENTS \\
\hline 1 & $11 / 1 / 76$ & 5 & & & 134 & & C vinosive \\
\hline 1 & $11 / 1 / 76$ & 6 & & & 134 & & \\
\hline 1 & $11 / 1 / 76$ & 7 & & & & & 200 - suspect data (high) \\
\hline 1 & $11 / 1 / 76$ & 8 & & & 128 & & \\
\hline 1 & $11 / 1 / 76$ & 9 & & & 122 & & \\
\hline 1 & $11 / 1 / 76$ & 10 & & & 118 & & \\
\hline 1 & $11 / 1 / 76$ & 11 & & & 106 & & \\
\hline 1 & $19 / 1 / 76$ & 12 & & & 98 & & \\
\hline 1 & $12 / 4 / 76$ & 9 & & & 122 & & \\
\hline 1 & $12 / 4 / 76$ & 2 & & & 125 & & \\
\hline 1 & $12 / 4 / 76$ & 4 & & & 122 & & \\
\hline 1 & $12 / 4 / 76$ & 5 & & & 123 & & \\
\hline 1 & $12 / 4 / 76$ & 6 & & & 123 & & \\
\hline 1 & $12 / 4 / 76$ & 8 & & & 128 & & \\
\hline 1 & $12 / 4 / 76$ & 9 & & & 123 & & \\
\hline 1 & $12 / 4 / 76$ & 10 & & & 116 & & \\
\hline 1 & $12 / 4 / 76$ & 11 & & & 106 & & \\
\hline 1 & $12 / 4 / 76$ & 12 & & & 96 & & \\
\hline 1 & $1 / 1 / 77$ & 1 & & & 122 & & \\
\hline 1 & $1 / 1 / 77$ & 2 & & & 124 & & \\
\hline 1 & $1 / 1 / 77$ & 4 & & & 131 & & \\
\hline 1 & $1 / 1 / 77$ & 5 & & & 133 & & \\
\hline 1 & $1 / 1 / 77$ & 6 & & & 133 & & \\
\hline 1 & $1 / 1 / 77$ & 7 & & & & & 200 - suspect data (high) \\
\hline 9 & $1 / 1 / 77$ & 8 & & & 128 & & \\
\hline 1 & $1 / 1 / 77$ & 9 & & & 121 & & \\
\hline 1 & $1 / 1 / 77$ & 10 & & & 115 & & \\
\hline 1 & $1 / 1 / 77$ & 11 & & & 104 & & \\
\hline 1 & $1 / 1 / 77$ & 12 & & & 94 & & \\
\hline 1 & $2 / 1 / 77$ & 1 & & & 120 & & \\
\hline 1 & $2 / 1 / 77$ & 2 & & & 124 & & \\
\hline 1 & $2 / 1 / 77$ & 4 & & & 132 & & \\
\hline 1 & $2 / 1 / 77$ & 5 & & & 132 & & \\
\hline 9 & $2 / 1 / 77$ & 6 & & & 134 & & \\
\hline 1 & $2 / 1 / 77$ & 7 & & & 132 & & \\
\hline 9 & $2 / 1 / 77$ & 9 & & & 126 & & \\
\hline 1 & $2 / 1 / 77$ & 10 & & & 120 & & \\
\hline 1 & $2 / 1 / 77$ & 11 & & & 112 & & \\
\hline 1 & $2 / 1 \pi 7$ & 12 & & & 112 & & \\
\hline 9 & $4 / 1 / 77$ & 1 & & & 115 & & \\
\hline 1 & $4 / 1 / 77$ & 2 & & & 117 & & \\
\hline 1 & $4 / 1 / 77$ & 4 & & & 125 & & \\
\hline 9 & $4 / 1 / 77$ & 5 & & & 126 & & \\
\hline 1 & $4 / 1 / 77$ & 6 & & & 126 & & \\
\hline 1 & $4 / 1 / 77$ & 8 & & & 120 & & \\
\hline 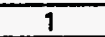 & $4 / 1 / 77$ & 9 & & & 116 & & \\
\hline 9 & $4 / 1 / 77$ & 10 & & & 106 & & \\
\hline 9 & $4 / 1 / 77$ & 11 & & & 95 & & \\
\hline 1 & $4 / 1 / 77$ & 12 & & & 83 & & \\
\hline 1 & $5 / 9 / 77$ & 1 & & & 115 & & \\
\hline 1 & $5 / 1 / 77$ & 2 & & & 117 & & \\
\hline 1 & $5 / 1 / 77$ & 4 & & & 125 & & \\
\hline 1 & $5 / 1 / 77$ & 5 & & & 126 & & \\
\hline 1 & $5 / 1 / 77$ & 6 & & & 127 & & \\
\hline$\frac{T}{1}$ & $5 / 1777$ & 8 & & & 113 & & \\
\hline 1 & $5 / 1 / 77$ & 9 & & & 115 & & \\
\hline 1 & $5 / 1 / 77$ & 10 & & & 105 & & \\
\hline 1 & $5 / 1 / 77$ & 11 & & & 95 & & \\
\hline$T$ & $5 / 1 / 77$ & 12 & & & 85 & & \\
\hline 1 & $6 / 7 / 77$ & 1 & & & 113 & & \\
\hline 1 & $6 / 7 / 77$ & 2 & & & 116 & & \\
\hline 1 & $6 / 7 / 77$ & 4 & & & 124 & & \\
\hline 1 & $6 / 7 / 77$ & 5 & & & 125 & & \\
\hline 1 & $6 / 7 / 77$ & 6 & & & 125 & & \\
\hline 1 & $6 / 7 / 77$ & 8 & & $:$ & 117 & & \\
\hline
\end{tabular}

Data obtained from WHC Surveillance Analysis Computer System (SACS). February 24, 1994. 
WHC-SD-WM-ER-323, Rev. 0

\begin{tabular}{|c|c|c|c|c|c|c|c|}
\hline \multicolumn{8}{|c|}{ TANK 241-S-112 } \\
\hline \multicolumn{8}{|c|}{ THERMOCOUPLE DATA } \\
\hline & & & & & & & \\
\hline TREE \# & DATE & THERMO \# & TREE SET \# & TEMP C & TEMP F & $\mathrm{Y}=\mathrm{MAX}$ TEMP & COMMENTS \\
\hline 1 & $6 / 7 / 77$ & 9 & & & 113 & & \\
\hline 1 & 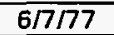 & 10 & & & 105 & & \\
\hline 1 & $6 / 7 / 77$ & 11 & & & 96 & & \\
\hline 1 & $6 / 7 / 77$ & 12 & & & 85 & & \\
\hline 1 & $7 / 11 / 77$ & 9 & & & 110 & & \\
\hline 1 & $7 / 11 / 77$ & 2 & & & 115 & & \\
\hline 1 & $7 / 11 / 77$ & 3 & & & 116 & & \\
\hline$\frac{1}{1}$ & $7 / 11 / 77$ & $\overline{4}$ & & & 120 & & \\
\hline 9 & $7 / 11 / 77$ & 5 & & & 122 & & \\
\hline 1 & $7 / 11 / 77$ & 6 & & & 122 & & \\
\hline 9 & $7 / 19 / 77$ & 8 & & & 115 & & \\
\hline 1 & $7 / 11 / 77$ & 9 & & & 113 & & \\
\hline 1 & $7 / 11 / 77$ & 10 & & & 105 & & \\
\hline 9 & $7 / 11 / 77$ & 11 & & & 100 & & \\
\hline 9 & $7 / 11 / 77$ & 12 & & & 88 & & \\
\hline 1 & $7 / 11 / 77$ & 14 & & & 85 & & \\
\hline 1 & $8 / 5 / 77$ & 1 & & & 108 & & \\
\hline 1 & $8 / 5 / 77$ & 2 & & & 112 & & \\
\hline 1 & $8 / 5 / 77$ & 4 & & & 115 & & \\
\hline 1 & $8 / 5 / 77$ & 5 & & & 118 & & \\
\hline 1 & $8 / 5 / 77$ & 6 & & & 119 & & \\
\hline 1 & $8 / 5 / 77$ & 8 & & & 113 & & \\
\hline 1 & $8 / 5 / 77$ & 9 & & & 110 & & \\
\hline 1 & $8 / 5 / 77$ & 10 & & & 103 & & \\
\hline 1 & $8 / 5 / 77$ & 11 & & & 96 & & \\
\hline 1 & $9 / 5 / 77$ & 1 & & & 111 & & \\
\hline 9 & $9 / 5 / 77$ & 2 & & & 115 & & \\
\hline 1 & $9 / 5 / 77$ & 4 & & & 121 & & \\
\hline 7 & $9 / 5 / 77$ & 5 & & & 122 & & \\
\hline 1 & $9 / 5 / 77$ & 6 & & & 123 & & \\
\hline 1 & $9 / 5 / 77$ & 8 & & & 116 & & \\
\hline 1 & $9 / 5 / 77$ & 9 & & & 114 & & \\
\hline 1 & $9 / 5 / 77$ & 10 & & & 107 & & \\
\hline 1 & $9 / 5 / 77$ & 11 & & & 100 & & \\
\hline 9 & $9 / 5 / 77$ & 12 & & & 96 & & \\
\hline 1 & $9 / 5 / 77$ & 14 & & & 89 & & \\
\hline 1 & $10 / 3 / 77$ & 1 & & & 111 & & \\
\hline 1 & $10 / 3 / 77$ & 2 & & & 115 & & \\
\hline 1 & $10 / 3 / 77$ & 4 & & & 120 & & \\
\hline 1 & $10 / 3 / 77$ & 5 & & & 122 & & \\
\hline 9 & $10 / 3 / 77$ & 6 & & & 122 & & \\
\hline 1 & $10 / 3 / 77$ & 8 & & & 118 & & \\
\hline 1 & $10 / 3 / 77$ & 9 & & & 115 & & \\
\hline 1 & $10 / 3 / 77$ & 10 & & & 108 & & \\
\hline 1 & $10 / 3 / 77$ & 19 & & & 100 & & \\
\hline 1 & $10 / 3 / 77$ & 12 & & & 92 & & \\
\hline 1 & $11 / 3 / 77$ & 1 & & & 112 & & \\
\hline 1 & $11 / 3 / 77$ & 2 & & & 115 & & \\
\hline 1 & $11 / 3 / 77$ & 4 & & & 120 & & \\
\hline 1 & $11 / 3 / 77$ & 5 & & & 122 & & \\
\hline 1 & $11 / 3 / 77$ & 6 & & & 122 & & \\
\hline 1 & $11 / 3 / 77$ & 8 & & & 117 & & \\
\hline 1 & $11 / 3 / 77$ & 9 & & & 113 & $\dot{\square}$ & \\
\hline 1 & $11 / 3 / 77$ & 10 & & & 112 & & \\
\hline$\frac{1}{1}$ & $11 / 3 / 77$ & 19 & & & 100 & & \\
\hline 1 & $11 / 3 / 77$ & 12 & & & 88 & & \\
\hline 1 & $11 / 3 / 77$ & 14 & & & 85 & & \\
\hline 9 & $1 / 8 / 78$ & 1 & & & & & 132 - suspect data (high) \\
\hline 1 & $9 / 8 / 78$ & 2 & & & & & 134 - suspect data (high) \\
\hline 1 & $1 / 8 / 78$ & 4 & & & & & 139 - suspect data (high) \\
\hline 1 & $1 / 8 / 78$ & 5 & & & & & 141 - suspect data (high) \\
\hline 1 & $1 / 8 / 78$ & 6 & & & & & 141 - suspect data (high) \\
\hline 1 & $1 / 8 / 78$ & 9 & & & & & 132 - suspect data (high) \\
\hline 1 & $1 / 8 / 78$ & 10 & & & & & 124 - suspect data (high) \\
\hline 1 & $1 / 8 / 78$ & 11 & & & & & 114 - suspect data (high) \\
\hline
\end{tabular}

Data obtained from WHC Surveillance Analysis Computer System (SACS), February 24, 1994. 


\begin{tabular}{|c|c|c|c|c|c|c|c|}
\hline \multicolumn{8}{|c|}{ TANK 241-S-112 } \\
\hline \multicolumn{8}{|c|}{ THERMOCOUPLE DATA } \\
\hline & & & & & & & \\
\hline TREE \# & DATE & THERMO \# & TREE SET \# & TEMP C & TEMP F & $Y=$ MAX TEMP & $\begin{array}{r}\text { COMMENTS } \\
\end{array}$ \\
\hline 1 & $1 / 8 / 78$ & 12 & & & & & $101 \cdot$ suspect data (high) \\
\hline 1 & $1 / 8 / 78$ & 14 & & & & & 97 - suspect data (high) \\
\hline 1 & $1 / 11 / 78$ & 1 & & & 111 & & \\
\hline 1 & $1 / 11 / 78$ & 2 & & & 114 & & \\
\hline 1 & $1 / 11 / 78$ & 4 & & & 117 & & \\
\hline 1 & $1 / 11 / 78$ & 5 & & & 120 & & \\
\hline 1 & $1 / 11 / 78$ & 6 & & & 120 & & \\
\hline 1 & $1 / 11 / 78$ & 9 & & & 112 & & \\
\hline 1 & $1 / 11 / 78$ & 10 & & & 105 & & \\
\hline 1 & $1 / 11 / 78$ & 11 & & & 96 & & \\
\hline 1 & $1 / 11 / 78$ & 12 & & & 83 & & \\
\hline 1 & $1 / 11 / 78$ & 14 & & & 78 & & \\
\hline 1 & $1 / 13 / 78$ & 1 & & & 119 & & \\
\hline 1 & $1 / 13 / 78$ & 2 & & & 115 & & \\
\hline 1 & $1 / 13 / 78$ & 4 & & & 120 & & \\
\hline 1 & $1 / 13 / 78$ & 5 & & & 121 & & \\
\hline 1 & $1 / 13 / 78$ & 6 & & & 121 & & \\
\hline 1 & $1 / 13 / 78$ & 9 & & & 113 & & \\
\hline 1 & $1 / 13 / 78$ & 10 & & & 105 & & \\
\hline 1 & $1 / 13 / 78$ & 11 & & & 95 & & \\
\hline 1 & $1 / 13 / 78$ & 12 & & & 95 & & \\
\hline 1 & $1 / 13 / 78$ & 13 & & & 95 & & \\
\hline 1 & $1 / 13 / 78$ & 14 & & & 95 & & \\
\hline 1 & $2 / 7 / 78$ & 1 & & & 114 & & \\
\hline 1 & $2 / 7 / 78$ & 2 & & & 114 & & \\
\hline 1 & $2 \Pi 7 \Pi 8$ & 4 & & & 122 & & \\
\hline 1 & $2 / 7 / 78$ & 5 & & & 124 & & \\
\hline 1 & $2 / 7 / 78$ & 6 & & & 124 & & \\
\hline 1 & $2 / 7 / 78$ & 9 & & & 194 & & \\
\hline 1 & $2 / 7 / 78$ & 10 & & & 107 & & \\
\hline 1 & 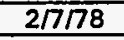 & 12 & & & 83 & & \\
\hline 1 & $2 / 7 / 78$ & 14 & & & 79 & & \\
\hline 1 & $3 / 12 / 78$ & 1 & & & 118 & & \\
\hline 1 & $3 / 12 / 78$ & 2 & & & 121 & & \\
\hline 1 & $3 / 12 / 78$ & 4 & & & 125 & & \\
\hline 1 & $3 / 12 / 78$ & 5 & & & 128 & & \\
\hline 1 & $3 / 12 / 78$ & 6 & & & 128 & & \\
\hline 1 & $3 / 12 / 78$ & 9 & & & 118 & & \\
\hline 1 & $3 / 12 / 78$ & 10 & & & 110 & & \\
\hline 1 & $3 / 12 / 78$ & 12 & & & 85 & & \\
\hline 1 & $3 / 12 / 78$ & 14 & & & 81 & & \\
\hline 1 & $4 / 11 / 78$ & 1 & & & 110 & & \\
\hline 1 & $4 / 11 / 78$ & 2 & & & 113 & & \\
\hline 1 & $4 / 11 / 78$ & 4 & & & 118 & & \\
\hline 1 & $4 / 11 / 78$ & 5 & $\cdot$ & & 120 & & \\
\hline 1 & $4 / 11 / 78$ & 6 & & & 120 & & \\
\hline 1 & $4 / 11 / 78$ & 9 & & & 107 & & \\
\hline 1 & $4 / 11 / 78$ & 10 & & & 100 & & \\
\hline 1 & $4 / 11 / 78$ & 12 & & & 77 & & \\
\hline 1 & $4 / 11 / 78$ & 14 & & & 75 & & \\
\hline 1 & $6 / 3 / 78$ & 1 & & & 110 & & \\
\hline 1 & $6 / 3 / 78$ & 2 & & & 113 & & \\
\hline 1 & $6 / 3 / 78$ & 4 & & & 118 & & \\
\hline 1 & $6 / 3 / 78$ & 5 & & & 120 & & \\
\hline 1 & $6 / 3 / 78$ & 6 & & & 120 & & \\
\hline 9 & $6 / 3 / 78$ & 9 & & & 108 & & \\
\hline 1 & $6 / 3 / 78$ & 10 & & & 100 & & \\
\hline 1 & $6 / 3 / 78$ & 12 & & & 81 & & \\
\hline 1 & $6 / 3 / 78$ & 14 & & & 77 & & \\
\hline 1 & $7 / 13 / 78$ & 1 & & & 110 & & \\
\hline 1 & $7 / 13 / 78$ & 2 & & & 113 & & \\
\hline 1 & $7 / 13 / 78$ & 4 & & & 118 & & \\
\hline 1 & $7 / 13 / 78$ & 5 & & & 120 & & \\
\hline 1 & $7 / 13 / 78$ & 6 & & & 120 & & \\
\hline 1 & $7 / 13 / 78$ & 9 & & & 108 & & $\therefore$ \\
\hline
\end{tabular}

Data obtained from WHC Surveillance Analysis Computer System (SACS), February 24, 1994. 
WHC-SD-WM-ER-323, Rev. 0

\begin{tabular}{|c|c|c|c|c|c|c|c|}
\hline \multicolumn{8}{|c|}{ TANK 241-S-112 } \\
\hline \multicolumn{8}{|c|}{ THERMOCOUPLE DATA } \\
\hline TREE \# & DATE & THERMO \# & TREE SET \# & TEMP C & TEMPF & $Y=M A X$ TEMP & COMMENTS \\
\hline 1 & $7 / 13 / 78$ & 10 & & & 101 & & \\
\hline 1 & $8 / 11 / 78$ & 1 & & & 904 & & \\
\hline 1 & $8 / 11 / 78$ & 2 & & & 107 & & \\
\hline 1 & $8 / 11 / 78$ & 4 & & & 912 & & \\
\hline 1 & $8 / 11 / 78$ & 5 & & & 113 & & \\
\hline 1 & $8 / 11 / 78$ & 6 & & & 113 & & \\
\hline 1 & $8 / 11 / 78$ & 9 & & & 101 & & \\
\hline 1 & $8 / 11 / 78$ & 10 & & & 97 & & \\
\hline 1 & $2 / 4 / 79$ & 1 & & & 902 & & \\
\hline 1 & $2 / 4 / 79$ & 2 & & & 104 & & \\
\hline 1 & $2 / 4 / 79$ & 4 & & & 110 & & \\
\hline 1 & $2 / 4 / 79$ & 5 & & & 110 & & \\
\hline 1 & $2 / 4 / 79$ & 6 & & & 100 & & \\
\hline 1 & $2 / 4 / 79$ & 9 & & & 98 & & \\
\hline 1 & $2 / 4 / 79$ & 10 & & & 87 & & \\
\hline 1 & $2 / 4 \Pi 79$ & 14 & & & 63 & & \\
\hline 1 & $9 / 6 / 79$ & 1 & & & 105 & & \\
\hline 1 & $9 / 6 / 79$ & 2 & & & 106 & & \\
\hline 1 & $9 / 6 / 79$ & 4 & & & 110 & & \\
\hline 1 & $9 / 6 / 79$ & 5 & & & 110 & & \\
\hline 1 & $9 / 6 / 79$ & 6 & & & 110 & & \\
\hline 1 & $9 / 6 / 79$ & 9 & & & 95 & & \\
\hline 1 & $9 / 6 / 79$ & 10 & & & 90 & & \\
\hline 1 & $9 / 6 / 79$ & 14 & & & 90 & & \\
\hline 1 & $1 / 23 / 80$ & 1 & & & 105 & & \\
\hline 1 & $1 / 23 / 80$ & 2 & & & 110 & & \\
\hline 1 & $1 / 23 / 80$ & 4 & & & 195 & & \\
\hline 1 & $1 / 23 / 80$ & 5 & & & 115 & & \\
\hline 1 & $1 / 23 / 80$ & 6 & & & 115 & & \\
\hline 1 & $1 / 23 / 80$ & 9 & & & 85 & & \\
\hline 1 & $1 / 23 / 80$ & 10 & & & 80 & & \\
\hline 1 & $1 / 23 / 80$ & 14 & & & 75 & & \\
\hline 1 & $10 / 6 / 81$ & 1 & & & 93 & & \\
\hline 1 & $10 / 6 / 81$ & 2 & & & 94 & & \\
\hline 1 & $10 / 6 / 81$ & 4 & & & 95 & & \\
\hline 1 & $10 / 6 / 81$ & 6 & & & 95 & & \\
\hline 1 & $10 / 6 / 81$ & 9 & & & 81 & & \\
\hline 1 & $10 / 6 / 81$ & 10 & & & 80 & & \\
\hline 1 & $10 / 6 / 81$ & 14 & & & 45 & & \\
\hline 1 & $1 / 13 / 82$ & 1 & & & 95 & & \\
\hline 1 & $1 / 13 / 82$ & 2 & & & 97 & & \\
\hline 1 & $1 / 13 / 82$ & 3 & & & & & 185 - suspect data (high) \\
\hline 1 & $1 / 13 / 82$ & 4 & & & 97 & & \\
\hline 1 & $1 / 13 / 82$ & 6 & & & 85 & & \\
\hline 1 & $1 / 13 / 82$ & 7 & & & 75 & & \\
\hline 1 & $1 / 13 / 82$ & 9 & & & 75 & & \\
\hline 1 & $1 / 13 / 82$ & 10 & & & 70 & & \\
\hline 1 & $5 / 29 / 82$ & 1 & & & 94 & & \\
\hline 1 & $5 / 29 / 82$ & 2 & & & 95 & & \\
\hline 1 & $5 / 29 / 82$ & 4 & & & 95 & & \\
\hline 1 & $5 / 29 / 82$ & 6 & & & 90 & & \\
\hline 1 & $5 / 29 / 82$ & 9 & & & 74 & & \\
\hline 1 & $5 / 29 / 82$ & 10 & & & 71 & & \\
\hline 1 & $5 / 19 / 83$ & 1 & & & 89 & & \\
\hline 1 & $5 / 19 / 83$ & 2 & & & 90 & & \\
\hline 1 & $5 / 19 / 83$ & 4 & & & 90 & & \\
\hline 1 & $5 / 19 / 83$ & 6 & & & 85 & & \\
\hline 1 & $5 / 19 / 83$ & 9 & & & 48 & & \\
\hline 1 & $5 / 19 / 83$ & 10 & & & 65 & & \\
\hline 1 & $5 / 19 / 83$ & 13 & & & & & 142 - suspect data (high) \\
\hline 1 & $5 / 19 / 83$ & 14 & & & & & 34 - suspect data (low) \\
\hline 1 & $1 / 3 / 84$ & 1 & & & 94 & & \\
\hline 1 & $1 / 3 / 84$ & 4 & & & 95 & & \\
\hline 1 & $1 / 3 / 84$ & 6 & & & & & 163 - suspect data (high) \\
\hline 1 & $1 / 3 / 84$ & 10 & & & 75 & & \\
\hline
\end{tabular}

Data obtained from WHC Surveillance Analysis Computer System (SACS), February 24, 1994. 
WHC-SD-WM-ER-323, Rev. 0

\begin{tabular}{|c|c|c|c|c|c|c|c|}
\hline \multicolumn{8}{|c|}{ TANK 241.S-112 } \\
\hline \multicolumn{8}{|c|}{ THERMOCOUPLE DATA } \\
\hline TREE \# & DATE & THERMO \# & TREE SET \# & TEMP C & TEMPF & $Y=$ MAX TEMP & COMMENTS \\
\hline 1 & $2 / 13 / 84$ & 3 & & & 94 & & 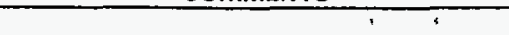 \\
\hline 1 & $2 / 13 / B 4$ & 10 & & & 69 & & \\
\hline 1 & $2 / 13 / 84$ & 13 & & & 45 & & \\
\hline 1 & $2 / 13 / 84$ & 14 & & & 80 & & \\
\hline 1 & $2 / 14 / 84$ & 1 & & & 90 & & \\
\hline 1 & $2 / 14 / 84$ & 5 & & & 92 & & \\
\hline 1 & $2 / 14 / 84$ & 10 & & & 68 & & \\
\hline 1 & $2 / 14 / 84$ & 14 & & & 70 & & \\
\hline 1 & $12 / 18 / 87$ & 1 & & & 92 & & \\
\hline 1 & $12 / 18 / 87$ & 10 & & & 76 & & \\
\hline 1 & $1 / 22 / 88$ & 1 & & & 93 & & \\
\hline 1 & $1 / 22 / 88$ & 10 & & & 73 & & \\
\hline 1 & $5 / 2 / 88$ & 1 & & & 93 & & \\
\hline 1 & $5 / 2 / 88$ & 10 & & & 72 & & \\
\hline 1 & $2 / 24 / 91$ & 1 & 1 & & & $\mathbf{N}$ & J O/S Letter written to OPS \\
\hline$\frac{1}{1}$ & $3 / 3 / 91$ & $\frac{1}{1}$ & 1 & & & $N$ & No data received \\
\hline 1 & $3 / 23 / 91$ & 4 & 1 & & 86.5 & $\bar{Y}$ & \\
\hline 1 & $3 / 23 / 91$ & 6 & 1 & & 84.2 & $\mathbf{N}$ & \\
\hline 1 & $3 / 23 / 91$ & 7 & 1 & & 81.8 & $\mathbf{N}$ & \\
\hline 1 & $3 / 23 / 91$ & 8 & 1 & & 75.1 & $\mathbf{N}$ & \\
\hline 1 & $3 / 23 / 91$ & 9 & 1 & & 66.9 & $\mathrm{~N}$ & \\
\hline 1 & $3 / 23 / 91$ & 10 & 1 & & 77.5 & $\bar{N}$ & \\
\hline 1 & $3 / 23 / 91$ & 11 & 1 & & 77.5 & $\mathbf{N}$ & \\
\hline 1 & $3 / 23 / 91$ & 12 & 1 & & 64.4 & $\mathbf{N}$ & \\
\hline 1 & $3 / 31 / 91$ & 1 & 1 & & & $\mathbf{N}$ & $\begin{array}{l}\text { No readings -requested retest or remedial } \\
\text { action }\end{array}$ \\
\hline 1 & $4 / 1 / 91$ & 4 & 1 & & 88.5 & $\bar{Y}$ & \\
\hline 1 & $4 / 1 / 91$ & 6 & 1 & & 86.4 & $\mathbf{N}$ & \\
\hline 1 & $4 / 1 / 91$ & 7 & 1 & & 83.4 & $\mathbf{N}$ & \\
\hline 1 & $4 / 1 / 91$ & 8 & 1 & & 76.4 & $\mathbf{N}$ & \\
\hline 1 & $4 / 1 / 91$ & 9 & 1 & & 68.1 & $\mathbf{N}$ & \\
\hline 1 & $4 / 1 / 91$ & 10 & 1 & & 65.7 & $\mathrm{~N}$ & \\
\hline 1 & $4 / 1 / 91$ & 11 & 1 & & 66.4 & $\mathbf{N}$ & \\
\hline 1 & $4 / 1 / 91$ & 12 & 1 & & 65.5 & $\mathbf{N}$ & \\
\hline 1 & $4 / 11 / 91$ & 4 & 1 & & 83.9 & $\bar{Y}$ & \\
\hline 1 & $4 / 11 / 91$ & 6 & 1 & & 81.2 & $\bar{N}$ & \\
\hline 1 & $4 / 11 / 91$ & 7 & 1 & & 79.1 & $\mathbf{N}$ & \\
\hline 9 & $4 / 11 / 91$ & 8 & 1 & & 72.9 & $N$ & \\
\hline 1 & $4 / 11 / 91$ & 9 & 1 & $\cdot$ & 64.9 & $\mathbf{N}$ & \\
\hline 1 & $4 / 11 / 91$ & 10 & 9 & & 62.7 & $\mathbf{N}$ & \\
\hline 1 & $4 / 11 / 91$ & 91 & 1 & & 62.5 & $\mathrm{~N}$ & \\
\hline 1 & $4 / 11 / 91$ & $\cdot 12$ & 1 & & 72.1 & $\mathbf{N}$ & \\
\hline 1 & $4 / 18 / 91$ & 4 & 1 & & 92 & $\bar{Y}$ & \\
\hline 1 & $4 / 18 / 91$ & 6 & 1 & & 82.4 & $\mathbf{N}$ & \\
\hline 1 & $4 / 18 / 91$ & 7 & 1 & & 79.9 & $\mathbf{N}$ & \\
\hline 1 & $4 / 18 / 91$ & 8 & 1 & & 73.5 & $\mathbf{N}$ & \\
\hline 1 & $4 / 18 / 91$ & 9 & 1 & & 66.1 & $\mathbf{N}$ & \\
\hline 1 & $4 / 18 / 91$ & 10 & 1 & & 63.7 & N & \\
\hline 9 & $4 / 18 / 91$ & 11 & 9 & & 64.1 & $\mathbf{N}$ & \\
\hline 1 & $4 / 18 / 91$ & 12 & 1 & & 64.4 & $\mathbf{N}$ & \\
\hline 1 & $4 / 26 / 91$ & 3 & 1 & & 60.4 & $\overline{\mathbf{N}}$ & J Omni-Cal-8A-110 \\
\hline 1 & $4 / 26 / 91$ & 4 & 1 & & 84 & $\mathbf{Y}$ & \\
\hline 1 & $4 / 26 / 91$ & 6 & 1 & & 82.5 & $\mathbf{N}$ & \\
\hline 1 & $4 / 26 / 91$ & 7 & 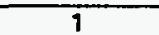 & & 78.6 & $\bar{N}$ & \\
\hline 1 & $4 / 26 / 91$ & 8 & 1 & & 73.3 & $N$ & \\
\hline 1 & $4 / 26 / 91$ & 9 & 1 & & 65.9 & $\mathbf{N}$ & \\
\hline 1 & $4 / 26 / 91$ & 10 & 1 & & 63.8 & $N$ & \\
\hline 1 & $4 / 26 / 91$ & 11 & 1 & & 64.2 & $N$ & \\
\hline 1 & $4 / 26 / 91$ & 12 & $\frac{1}{1}$ & & 64.4 & $N$ & \\
\hline 1 & $5 / 2 / 91$ & 3 & 1 & & 63.5 & $\mathbf{N}$ & J $128904240-5$ Rechecked \\
\hline 1 & $5 / 2 / 91$ & 4 & 9 & & 86.4 & $\mathrm{Y}$ & \\
\hline 1 & $5 / 2 / 91$ & 6 & 1 & & 84.1 & $\mathbf{N}$ & \\
\hline 1 & $5 / 2 / 91$ & 7 & $\frac{1}{1}$ & & 81.9 & $N$ & \\
\hline 1 & $5 / 2 / 91$ & 8 & 1 & & 76.1 & $\mathrm{~N}$ & \\
\hline
\end{tabular}

Data obtained from WHC Surveillance Analysis Computer System (SACS), February 24, 1994. 
WHC-SD-WM-ER-323, Rev. 0

\begin{tabular}{|c|c|c|c|c|c|c|c|}
\hline \multicolumn{8}{|c|}{ TANK 241.S.112 } \\
\hline & & & & THE & IOCOUPLE & ATA & \\
\hline TREE \# & DATE & THERMO \# & TREE SET \# & TEMP C & TEMP F & $Y=$ MAX TEMP & COMMENTS \\
\hline 9 & $5 / 2 / 99$ & 9 & 1 & & 68.4 & $N$ & COMMEN IS \\
\hline 1 & $5 / 2 / 91$ & 10 & 1 & & 66 & $\mathrm{~N}$ & \\
\hline 1 & $5 / 2 / 91$ & 11 & 1 & & 65.6 & $N$ & \\
\hline 1 & $5 / 2 / 91$ & 12 & 1 & & 65.9 & $\mathbf{N}$ & \\
\hline 1 & $5 / 9 / 91$ & 4 & 1 & & 83.5 & $Y$ & \\
\hline 1 & $5 / 9 / 91$ & 6 & 1 & & 81.2 & $\mathrm{~N}$ & \\
\hline 1 & $5 / 9 / 91$ & 7 & 1 & & 79.1 & $\mathbf{N}$ & \\
\hline 1 & $5 / 9 / 91$ & 8 & 1 & & 73.2 & $\mathrm{~N}$ & \\
\hline 1 & $5 / 9 / 91$ & 9 & 1 & & 66.4 & $N$ & \\
\hline 1 & $5 / 9 / 91$ & 10 & 1 & & 64.9 & $\mathrm{~N}$ & \\
\hline 1 & $5 / 9 / 91$ & 11 & 1 & & 64.7 & $N$ & \\
\hline 1 & $5 / 9 / 91$ & 12 & 1 & & 65 & $N$ & \\
\hline 1 & $5 / 16 / 91$ & 3 & 1 & & 65.3 & $\mathbf{N}$ & J 128-9042405 TC \#5 Bad data \\
\hline 1 & $5 / 16 / 91$ & 4 & 1 & & 85.1 & $Y$ & \\
\hline 1 & $5 / 16 / 91$ & 6 & 1 & & 83.2 & $\mathrm{~N}$ & \\
\hline 1 & $5 / 16 / 91$ & 7 & 1 & & 80.6 & $\mathbf{N}$ & \\
\hline 1 & $5 / 16 / 91$ & 8 & 1 & & 74.8 & $\mathbf{N}$ & \\
\hline 1 & $5 / 16 / 91$ & 9 & 1 & & 68 & $N$ & \\
\hline 1 & $5 / 16 / 91$ & 10 & 1 & & 66 & $\mathbf{N}$ & \\
\hline 1 & $5 / 16 / 91$ & 11 & 1 & & 66 & $N$ & \\
\hline 1 & $5 / 16 / 91$ & 12 & 1 & & 66 & $N$ & \\
\hline 1 & $5 / 23 / 91$ & 4 & 1 & & 83.5 & $\bar{Y}$ & \\
\hline 1 & $5 / 23 / 91$ & 6 & 1 & & 80.9 & $\bar{N}$ & \\
\hline 1 & $5 / 23 / 91$ & 7 & 1 & & 78.5 & $\mathrm{~N}$ & \\
\hline 1 & $5 / 23 / 91$ & 8 & 1 & & 73.4 & $\mathbf{N}$ & \\
\hline 1 & $5 / 23 / 91$ & 9 & 1 & & 66.8 & $N$ & \\
\hline 1 & $5 / 23 / 91$ & 10 & 1 & & 64.5 & $\mathrm{~N}$ & \\
\hline 1 & $5 / 23 / 91$ & 11 & 1 & & 64.6 & $N$ & \\
\hline 1 & $5 / 23 / 91$ & 12 & 1 & & 64.5 & $\bar{N}$ & \\
\hline 1 & $5 / 30 / 91$ & 3 & 1 & & 63.2 & $N$ & J SN-128904240-5 \\
\hline 1 & $5 / 30 / 91$ & 4 & 1 & & 83.7 & $Y$ & \\
\hline 1 & $5 / 30 / 91$ & 6 & 1 & & 81.7 & $N$ & \\
\hline 1 & $5 / 30 / 91$ & 7 & 1 & & 79 & $\mathbf{N}$ & \\
\hline 1 & $5 / 30 / 91$ & 8 & 1 & & 73.6 & $\mathrm{~N}$ & \\
\hline 1 & $5 / 30 / 91$ & 9 & 1 & & 67.4 & $\mathbf{N}$ & \\
\hline 1 & $5 / 30 / 91$ & 10 & 1 & & 65.5 & $N$ & \\
\hline 1 & $5 / 30 / 91$ & 11 & 1 & & 65.5 & $N$ & \\
\hline 1 & $5 / 30 / 91$ & 12 & 1 & & 65.5 & $\mathrm{~N}$ & \\
\hline 1 & $6 / 6 / 91$ & 4 & 1 & & 83.3 & $\bar{Y}$ & \\
\hline 1 & $6 / 6 / 91$ & 6 & 1 & & 81.3 & $N$ & \\
\hline 1 & $6 / 6 / 91$ & 7 & 1 & & 79.5 & $N$ & \\
\hline 1 & $6 / 6 / 91$ & 8 & 1 & & 74.1 & $N$ & \\
\hline 1 & $6 / 6 / 91$ & 9 & 1 & & 68 & $\bar{N}$ & \\
\hline 1 & $6 / 6 / 91$ & 10 & 1 & & 66.3 & $N$ & \\
\hline 1 & $6 / 6 / 91$ & 11 & 1 & & 74.1 & $\mathrm{~N}$ & \\
\hline 1 & $6 / 6 / 91$ & 12 & 1 & & 66.3 & $\bar{N}$ & \\
\hline 1 & $6 / 9 / 91$ & 4 & 1 & & 81.4 & $\bar{Y}$ & \\
\hline 1 & $6 / 9 / 91$ & 7 & 1 & & 77.3 & $\mathbf{N}$ & \\
\hline 1 & $6 / 9 / 91$ & 8 & 1 & & 73.6 & $\mathbf{N}$ & \\
\hline 1 & $6 / 9 / 91$ & 9 & 9 & & 67 & $\mathbf{N}$ & \\
\hline 1 & $6 / 9 / 91$ & 10 & 1 & & 64.6 & $\mathbf{N}$ & \\
\hline 1 & $6 / 9 / 91$ & 11 & 1 & & 64.2 & $\mathbf{N}$ & \\
\hline 1 & $6 / 9 / 91$ & 12 & 1 & & 66.7 & $N$ & \\
\hline 1 & $6 / 20 / 91$ & 3 & 1 & & 68.8 & $\mathbf{N}$ & J Omega Omni-Cal TCs \#125 Bad Data \\
\hline 1 & $6 / 20 / 91$ & 4 & 1 & & 91 & $\mathbf{Y}$ & \\
\hline 1 & $6 / 20 / 91$ & 6 & 1 & & 88.4 & $\mathbf{N}$ & \\
\hline 1 & $6 / 20 / 91$ & 7 & 1 & & 88.3 & $\mathbf{N}$ & \\
\hline 1 & $6 / 20 / 91$ & 8 & 1 & & 79.9 & $\mathbf{N}$ & \\
\hline 1 & $6 / 20 / 91$ & 9 & 1 & & 74.1 & $\mathbf{N}$ & \\
\hline 1 & $6 / 20 / 91$ & 10 & 1 & & 73.1 & $\mathbf{N}$ & \\
\hline 1 & $6 / 20 / 91$ & 11 & 1 & & 73.4 & $\mathbf{N}$ & \\
\hline 1 & $6 / 20 / 91$ & 12 & 1 & & 74 & $\mathbf{N}$ & \\
\hline 1 & $6 / 27 / 91$ & 3 & 1 & & 63.2 & $\mathbf{N}$ & J 128-9042405 TC\#125 Bad data \\
\hline 1 & $6 / 27 / 91$ & 4 & 1 & & 83.1 & $Y$ & \\
\hline 1 & $6 / 27 / 91$ & 6 & 1 & & 81.1 & $\mathbf{N}$ & \\
\hline
\end{tabular}

Data obtained from WHC Surveillance Analysis Computer System (SACS), February 24, 1994. 


\begin{tabular}{|c|c|c|c|c|c|c|c|}
\hline \multicolumn{8}{|c|}{ TANK 241-S.112 } \\
\hline \multicolumn{8}{|c|}{ THERMOCOUPLE DATA } \\
\hline & & & & & & & \\
\hline TREE \# & DATE & THERMO \# & TREE SET \# & TEMP C & TEMP F & $Y=$ MAX TEMP & COMMENTS \\
\hline 1 & $6 / 27 / 91$ & 7 & 1 & & 78.8 & $\mathrm{~N}$ & 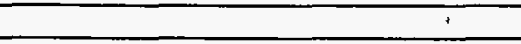 \\
\hline 1 & $6 / 27 / 91$ & 8 & 1 & & 73.9 & $\mathbf{N}$ & \\
\hline 1 & $6 / 27 / 91$ & 9 & 1 & & 68.4 & $\mathrm{~N}$ & \\
\hline 1 & $6 / 27 / 91$ & 10 & 1 & & 67 & $\mathbf{N}$ & \\
\hline 1 & $6 / 27 / 91$ & 11 & 1 & & 67 & $\bar{N}$ & \\
\hline 1 & $6 / 27 / 91$ & 12 & 1 & & 66.9 & $\mathbf{N}$ & \\
\hline 1 & $7 / 3 / 91$ & 3 & 1 & & 63.5 & $\mathrm{~N}$ & J $128.9042212 \mathrm{TC} \# 125 \mathrm{Bad}$ data \\
\hline 1 & $7 / 3 / 91$ & 4 & 1 & & 87 & $Y$ & \\
\hline 1 & $7 / 3 / 91$ & 6 & 1 & & 81.1 & $\mathbf{N}$ & \\
\hline 1 & $7 / 3 / 91$ & 7 & $\overline{1}$ & & 78.9 & $\mathbf{N}$ & \\
\hline 1 & $7 / 3 / 91$ & 8 & 1 & & 74.4 & $N$ & \\
\hline 1 & $7 / 3 / 91$ & 9 & 1 & & 73.9 & $\mathbf{N}$ & \\
\hline 1 & $7 / 3 / 91$ & 10 & 9 & & 71.3 & $\mathbf{N}$ & \\
\hline 1 & $7 / 3 / 91$ & 11 & 1 & & 71.5 & $\mathbf{N}$ & \\
\hline 1 & $7 / 3 / 91$ & 12 & 1 & & 67.8 & $\mathbf{N}$ & \\
\hline 1 & $7 / 3 / 91$ & 14 & 1 & & 53.5 & $\mathbf{N}$ & \\
\hline 1 & $7 / 7 / 91$ & 3 & 1 & & 66 & $N$ & $J 910126143-73$ \\
\hline 1 & $7 / 7 / 91$ & 4 & 1 & & 83 & $\bar{Y}$ & \\
\hline 1 & $7 / 7 / 91$ & 7 & 1 & & 79 & $\mathbf{N}$ & \\
\hline 1 & $7 / 7 / 91$ & 8 & 9 & & 76 & $\mathbf{N}$ & \\
\hline 1 & $7 / 7 / 91$ & 9 & 1 & & $\overline{71}$ & $\bar{N}$ & \\
\hline 1 & $7 / 7 / 91$ & 10 & 1 & & 70 & $\mathrm{~N}$ & \\
\hline 1 & $7 / 7 / 91$ & 11 & 1 & & 68 & $\mathbf{N}$ & \\
\hline 1 & $7 / 7 / 91$ & 12 & 1 & & 71 & $\mathbf{N}$ & \\
\hline 1 & $7 / 11 / 91$ & 3 & 1 & & 65.5 & $\mathrm{~N}$ & J 128-904 2212 TC\#12LO TC\#6Bad data \\
\hline 1 & $7 / 11 / 91$ & 4 & 1 & & 84.2 & $\bar{Y}$ & \\
\hline 1 & $7 / 16 / 91$ & 3 & 1 & & 65.9 & $\mathbf{N}$ & J 817-13-55-008 TC\#513Bad data $12 \mathrm{Lo}$ \\
\hline 1 & $7 / 16 / 91$ & 4 & 1 & & 85.5 & $Y$ & \\
\hline 1 & $7 / 16 / 91$ & 6 & 1 & & 83.5 & $\mathbf{N}$ & \\
\hline 1 & $7 / 16 / 91$ & 7 & $\frac{1}{1}$ & & 85 & $\mathbf{N}$ & \\
\hline 1 & $7 / 16 / 91$ & 8 & 1 & & 77.4 & $N$ & \\
\hline 1 & $7 / 16 / 91$ & 9 & 1 & & 72.5 & $\mathbf{N}$ & \\
\hline 1 & $7 / 16 / 91$ & 10 & 1 & & 71.3 & $\mathbf{N}$ & \\
\hline 1 & $7 / 16 / 91$ & 11 & $\overline{1}$ & & 71.4 & $\mathbf{N}$ & \\
\hline 1 & $7 / 16 / 91$ & 12 & 1 & & 71.4 & $\mathbf{N}$ & \\
\hline 1 & $7 / 16 / 91$ & 14 & 1 & & 57.4 & $\mathbf{N}$ & \\
\hline 1 & $7 / 25 / 91$ & 3 & 1 & & 63 & $\mathbf{N}$ & $T C \# 125130 / \mathrm{s}$ \\
\hline 1 & $7 / 25 / 91$ & 4 & 9 & & 83.1 & $\bar{Y}$ & \\
\hline 1 & $7 / 25 / 91$ & 6 & 1 & & 81.7 & $\mathbf{N}$ & $\cdot$ \\
\hline 1 & $7 / 25 / 91$ & 7 & 1 & & 79.9 & $\mathbf{N}$ & \\
\hline 1 & $7 / 25 / 91$ & 8 & 9 & & 76 & $\mathbf{N}$ & \\
\hline 1 & $7 / 25 / 91$ & 9 & 9 & & 79.7 & $\bar{N}$ & \\
\hline$T$ & $7 / 25 / 91$ & 10 & 1 & & 70.7 & $\mathbf{N}$ & \\
\hline 1 & $7 / 25 / 91$ & 19 & 1 & & 70.8 & $\mathrm{~N}$ & \\
\hline 1 & $7 / 25 / 91$ & 12 & 1 & & 70.9 & $\mathbf{N}$ & \\
\hline 1 & $7 / 25 / 91$ & 14 & 9 & & 57.3 & $N$ & \\
\hline 1 & $8 / 8 / 91$ & 3 & 1 & & 61.4 & $N$ & J 128.9042405 TC\#5 Bad Data \\
\hline 1 & $8 / 8 / 91$ & 4 & 1 & & 82.5 & $\mathbf{N}$ & \\
\hline 1 & $8 / 8 / 91$ & 6 & $\frac{1}{1}$ & & 81.5 & $\bar{N}$ & \\
\hline 1 & $8 / 8 / 91$ & 7 & 1 & & 91.9 & $\bar{Y}$ & \\
\hline 1 & $8 / 8 / 91$ & 8 & 1 & & 76.7 & $\bar{N}$ & \\
\hline 1 & $8 / 8 / 91$ & 9 & 1 & & 72.8 & $N$ & \\
\hline 1 & $8 / 8 / 91$ & 10 & 1 & & 72 & $\bar{N}$ & $\cdot$ \\
\hline 1 & $8 / 8 / 91$ & 11 & 1 & & 72.1 & $N$ & \\
\hline 1 & $8 / 8 / 91$ & 12 & 1 & & 72.5 & $N$ & \\
\hline 1 & $8 / 8 / 91$ & 14 & 1 & & 59.6 & $N$ & \\
\hline 1 & $8 / 15 / 91$ & 3 & 1 & & 61.9 & $\mathrm{~N}$ & $\mathrm{~J} 128904240-\mathrm{S}$ \\
\hline 1 & $8 / 15 / 91$ & 6 & 1 & & 82.3 & $\mathbf{N}$ & \\
\hline 1 & $8 / 15 / 91$ & 7 & 1 & & 81 & $\mathbf{N}$ & \\
\hline 9 & $8 / 15 / 91$ & 8 & 1 & & 86 & $\bar{Y}$ & \\
\hline 1 & $8 / 15 / 91$ & 9 & 1 & & 74.2 & $\mathbf{N}$ & \\
\hline 1 & $8 / 15 / 91$ & 10 & 1 & & 73.5 & $\mathbf{N}$ & \\
\hline 1 & $8 / 15 / 91$ & 11 & 1 & & 73.3 & $\mathbf{N}$ & \\
\hline 1 & $8 / 15 / 91$ & 12 & 1 & & 73.5 & $\mathbf{N}$ & \\
\hline 1 & $8 / 15191$ & 14 & 1 & & 60.4 & $\mathbf{N}$ & \\
\hline
\end{tabular}

Data obtained from WHC Surveillance Analysis Computer System (SACS), February 24, 1994. 


\begin{tabular}{|c|c|c|c|c|c|c|c|}
\hline \multicolumn{8}{|c|}{ TANK 241-S-112 } \\
\hline \multicolumn{8}{|c|}{ THERMOCOUPLE DATA } \\
\hline TREE \# & DATE & THERMO \# & TREE SET \# & TEMP C & TEMP F & $Y=$ MAX TEMP & COMMENTS \\
\hline 1 & $8 / 18 / 91$ & 4 & 1 & & 82 & $\mathrm{~N}$ & $J 910126143-75$ \\
\hline 1 & $8 / 18 / 91$ & 7 & 1 & & 90 & $\bar{Y}$ & \\
\hline 1 & $8 / 18 / 91$ & 8 & 1 & & 78 & $\bar{N}$ & \\
\hline 1 & $8 / 18 / 91$ & 9 & 1 & & 74 & $\mathbf{N}$ & \\
\hline 1 & $8 / 18 / 91$ & 10 & 1 & & 74 & $\bar{N}$ & \\
\hline 1 & $8 / 18 / 91$ & 11 & 1 & & 72 & $\mathbf{N}$ & \\
\hline 1 & $8 / 18 / 91$ & 12 & 1 & & 76 & $\mathbf{N}$ & \\
\hline 1 & $8 / 22 / 91$ & 1 & 1 & & & $\bar{N}$ & J omega 128.9042405 LOW \\
\hline 1 & $8 / 22 / 91$ & 2 & 1 & & & $\mathbf{N}$ & LOW \\
\hline 1 & $8 / 22 / 91$ & 3 & 1 & & 60.9 & $N$ & J omega 128-9042405 \\
\hline 9 & $8 / 22 / 91$ & 4 & 1 & & 82.8 & $\bar{Y}$ & \\
\hline 1 & $8 / 22 / 91$ & 6 & 1 & & 81.6 & $\bar{N}$ & \\
\hline 9 & $8 / 22 / 91$ & 7 & 1 & & 80.4 & $\mathbf{N}$ & \\
\hline 1 & $8 / 25 / 91$ & 1 & 1 & & & $\mathbf{N}$ & J HH.99A-J O/S \\
\hline 1 & $8 / 25 / 91$ & 2 & 9 & & & $\bar{N}$ & $0 / 5$ \\
\hline 1 & $8 / 25 / 91$ & 3 & 1 & & 64 & $\mathbf{N}$ & \\
\hline 1 & $8 / 25 / 91$ & 4 & 1 & & 83 & $\bar{Y}$ & \\
\hline 1 & $8 / 25 / 91$ & 5 & 1 & & & $N$ & $0 / S$ \\
\hline 1 & $8 / 25 / 91$ & 6 & 1 & & & $\mathbf{N}$ & $0 / 5$ \\
\hline 1 & $8 / 25 / 91$ & 7 & 1 & & 82 & $\mathbf{N}$ & \\
\hline$\frac{1}{1}$ & $8 / 25 / 91$ & 8 & 1 & & 80 & $\mathbf{N}$ & \\
\hline 1 & $8 / 25 / 91$ & 9 & 1 & & 76 & N & \\
\hline 1 & $8 / 25 / 91$ & 10 & 1 & & 76 & $\mathbf{N}$ & \\
\hline 1 & $8 / 25 / 91$ & 11 & 1 & & 74 & $\mathbf{N}$ & \\
\hline$\frac{1}{1}$ & $8 / 25 / 91$ & 12 & 9 & & 78 & $\mathbf{N}$ & \\
\hline 1 & $8 / 25 / 91$ & 13 & 1 & & & $\mathrm{~N}$ & $0 / 5$ \\
\hline$\frac{1}{1}$ & $8 / 25 / 91$ & 14 & 1 & & & $\mathrm{~N}$ & $0 / 5$ \\
\hline 1 & $8 / 29 / 91$ & 3 & 1 & & 63.5 & $\mathbf{N}$ & J-Omega 128.9042405 \\
\hline 1 & $8 / 29 / 91$ & 4 & 1 & & 85.3 & $\bar{Y}$ & \\
\hline 1 & $8 / 29 / 91$ & 6 & 1 & & 84.1 & $\mathbf{N}$ & \\
\hline 1 & $8 / 29 / 91$ & 7 & 1 & & 83.1 & $\mathbf{N}$ & \\
\hline 1 & $8 / 29 / 91$ & 8 & 1 & & 80.1 & $\mathbf{N}$ & \\
\hline 1 & $8 / 29 / 91$ & 9 & 1 & & 76.8 & $\mathbf{N}$ & \\
\hline 1 & $8 / 29 / 91$ & 10 & 1 & & 75.9 & $N$ & \\
\hline 1 & $8 / 29 / 91$ & 11 & 1 & & 75.9 & $\mathbf{N}$ & \\
\hline 1 & $8 / 29 / 91$ & 12 & 1 & & 75.8 & $\mathbf{N}$ & \\
\hline 1 & $8 / 29 / 91$ & 14 & $\frac{1}{1}$ & & 62.4 & $\mathbf{N}$ & \\
\hline 1 & $9 / 5 / 91$ & 4 & 1 & & 82.9 & $Y$ & \\
\hline 1 & $9 / 5 / 91$ & 6 & 1 & & 82.1 & $\mathbf{N}$ & \\
\hline 1 & $9 / 5 / 91$ & 7 & 1 & & 81.3 & $\mathbf{N}$ & \\
\hline$i$ & $9 / 5 / 91$ & 8 & 1 & & 78.9 & $\mathbf{N}$ & \\
\hline 9 & $9 / 5 / 91$ & 9 & 1 & & 75 & $\mathbf{N}$ & \\
\hline 1 & $9 / 5 / 91$ & 10 & 1 & & 74 & $\mathbf{N}$ & \\
\hline 1 & $9 / 5 / 91$ & 11 & 1 & & 74 & $\mathbf{N}$ & \\
\hline 1 & $9 / 5 / 91$ & 12 & 1 & & 73.8 & $\mathbf{N}$ & \\
\hline 1 & $9 / 12 / 91$ & 4 & 1 & & 83.7 & $N$ & \\
\hline 1 & $9 / 12 / 91$ & 6 & 1 & & 83.5 & $\bar{Y}$ & \\
\hline 1 & $9 / 12 / 91$ & 7 & 1 & & 82.6 & $\mathbf{N}$ & \\
\hline 1 & $9 / 12 / 91$ & 8 & 1 & & 79.9 & $N$ & \\
\hline 1 & $9 / 12 / 91$ & 9 & 1 & & 76.8 & $\mathbf{N}$ & \\
\hline 1 & $9 / 12 / 91$ & 10 & 1 & & 76 & $\mathbf{N}$ & \\
\hline 1 & $9 / 12 / 91$ & 19 & 9 & & 76.4 & $N$ & \\
\hline 1 & $9 / 12 / 91$ & 12 & 9 & & 76.6 & $N$ & \\
\hline 9 & $9 / 12 / 91$ & 14 & 1 & & 63.4 & $N$ & \\
\hline 1 & $9 / 19 / 91$ & 4 & 1 & & 89.9 & $\bar{Y}$ & \\
\hline 1 & $9 / 19 / 91$ & 6 & 9 & & 83.5 & $\bar{N}$ & \\
\hline 1 & $9 / 19 / 91$ & 7 & 9 & & 82.8 & $\mathbf{N}$ & \\
\hline 1 & $9 / 19 / 91$ & 8 & 1 & & 80.3 & $\mathrm{~N}$ & \\
\hline 1 & $9 / 19 / 91$ & 9 & 1 & & 77.2 & $N$ & \\
\hline 1 & $9 / 19 / 91$ & 10 & 1 & & 76.6 & $N$ & \\
\hline 1 & $9 / 19 / 91$ & 11 & 1 & & 76 & $N$ & \\
\hline 1 & $9 / 19 / 91$ & 12 & 1 & & 76.1 & $N$ & \\
\hline 1 & $9 / 19 / 91$ & 14 & 1 & & 62.1 & $\mathrm{~N}$ & \\
\hline 1 & $9 / 26 / 91$ & 4 & 1 & & 85 & $\bar{Y}$ & \\
\hline 1 & $9 / 26 / 91$ & 6 & 1 & & 84.1 & $\mathbf{N}$ & \\
\hline
\end{tabular}

Data obtained from WHC Surveillance Analysis Computer System (SACS), February 24, 1994. 


\begin{tabular}{|c|c|c|c|c|c|c|c|}
\hline \multicolumn{8}{|c|}{ TANK 241-S-112 } \\
\hline \multicolumn{8}{|c|}{ THERMOCOUPLE DATA } \\
\hline & & & & & & & \\
\hline TREE \# & DATE & THERMO \# & TREE SET \# & TEMP C & TEMP F & $Y=\operatorname{MAX}$ TEMP & COMMENTS \\
\hline 1 & $9 / 26 / 91$ & 7 & 1 & & 83.6 & $N$ & \\
\hline 1 & $9 / 26 / 91$ & 8 & 1 & & 81 & $N$ & \\
\hline 1 & $9 / 26 / 91$ & 9 & 1 & & 79.1 & $\mathrm{~N}$ & \\
\hline 1 & $9 / 26 / 91$ & 10 & 1 & & 76.9 & $\mathrm{~N}$ & \\
\hline 1 & $9 / 26 / 91$ & 11 & 1 & & 77.1 & $\mathrm{~N}$ & \\
\hline 1 & $9 / 26 / 91$ & 12 & 1 & & 77.4 & $\mathbf{N}$ & \\
\hline$T$ & $9 / 26 / 91$ & 14 & 9 & & 62.8 & $\mathbf{N}$ & \\
\hline 1 & $9 / 28 / 91$ & 1 & 1 & & & $\mathbf{N}$ & No Reading \\
\hline 1 & $10 / 7 / 91$ & 4 & 1 & & 84 & $Y$ & \\
\hline 1 & $10 / 7 / 91$ & 6 & 1 & & 83.6 & $\mathbf{N}$ & \\
\hline 1 & $10 / 7 / 91$ & 7 & 1 & & 83 & $\mathbf{N}$ & \\
\hline 1 & $10 / 7 / 91$ & 8 & 1 & & 80.5 & $\mathbf{N}$ & \\
\hline 1 & $10 / 7 / 91$ & 9 & 1 & & 77.1 & $\mathbf{N}$ & \\
\hline 1 & $10 / 7 / 91$ & 10 & 1 & & 76.1 & $N$ & \\
\hline 1 & $10 / 7 / 91$ & 11 & 1 & & 80.5 & $N$ & \\
\hline 1 & $10 / 7 / 99$ & 12 & 1 & & 76.6 & $N$ & \\
\hline 1 & $10 / 7 / 91$ & 14 & 1 & & 62.6 & $\mathbf{N}$ & \\
\hline 1 & $10 / 10 / 91$ & 3 & 1 & & 59.9 & $\mathbf{N}$ & $\mathrm{J}-817-13-35-008$ \\
\hline 1 & $10 / 10 / 91$ & 4 & 1 & & 82.9 & $\mathbf{N}$ & \\
\hline 1 & $10 / 10 / 91$ & 6 & 1 & & 82 & $\bar{Y}$ & \\
\hline 1 & $10 / 10 / 91$ & 7 & 1 & & 81.6 & $\mathbf{N}$ & \\
\hline 1 & $10 / 10 / 91$ & 8 & 1 & & 79.2 & $\mathbf{N}$ & \\
\hline 1 & $10 / 10 / 91$ & 9 & 1 & & 75.9 & $\mathbf{N}$ & \\
\hline 1 & $10 / 10 / 91$ & 10 & 1 & & 78.9 & $N$ & \\
\hline 1 & $10 / 10 / 91$ & 11 & 1 & & 79 & $\mathbf{N}$ & \\
\hline 1 & $10 / 10 / 91$ & 92 & 1 & & 79.2 & $N$ & \\
\hline 1 & $10 / 10 / 91$ & 14 & 1 & & 65 & $\mathbf{N}$ & \\
\hline 1 & $10 / 17 / 91$ & 4 & 1 & & 85.5 & $\mathbf{Y}$ & \\
\hline 1 & $10 / 17 / 91$ & 6 & 1 & & 84.9 & $\mathrm{~N}$ & \\
\hline 1 & $10 / 17 / 91$ & 7 & 1 & & 84.5 & $N$ & \\
\hline 1 & $10 / 17 / 91$ & 8 & 1 & & 82 & $\mathbf{N}$ & \\
\hline 1 & $10 / 17 / 91$ & 9 & 1 & & 78.2 & $N$ & \\
\hline 1 & $10 / 17 / 91$ & 10 & 1 & & 77 & $N$ & \\
\hline 1 & $10 / 17 / 91$ & 11 & 1 & & 77.6 & $\mathrm{~N}$ & \\
\hline 1 & $10 / 17 / 91$ & 12 & 1 & & 77.9 & $\mathbf{N}$ & \\
\hline 1 & $10 / 17 / 91$ & 14 & 1 & & 65.5 & $\mathbf{N}$ & \\
\hline 1 & $10 / 26 / 91$ & 3 & 1 & & 63.6 & $\mathbf{N}$ & J.817-13-55-008 TC \#5 Bad Data \\
\hline 1 & $10 / 26 / 91$ & 4 & 1 & & 85.9 & $\mathbf{Y}$ & \\
\hline 1 & $10 / 26 / 91$ & 6 & 1 & & 85.1 & $\mathbf{N}$ & \\
\hline 1 & $10 / 26 / 91$ & 7 & 1 & & 84.6 & $\mathbf{N}$ & \\
\hline 1 & $10 / 26 / 91$ & 8 & 1 & & 82 & $N$ & \\
\hline 1 & $10 / 26 / 91$ & 9 & 1 & & 78.1 & $N$ & \\
\hline 1 & $10 / 26 / 91$ & 10 & 1 & & 77.4 & $\mathrm{~N}$ & \\
\hline 1 & $10 / 26 / 91$ & 11 & 1 & & 76.9 & $\mathbf{N}$ & \\
\hline 1 & $10 / 26 / 91$ & 12 & 1 & & 77.1 & $\mathbf{N}$ & \\
\hline 1 & $10 / 26 / 91$ & 14 & 1 & & 63.2 & $N$ & \\
\hline 1 & $10 / 31 / 91$ & 3 & 1 & & 65 & $N$ & TC \#5 Bad Data TC \#713 Open \\
\hline 1 & $10 / 31 / 91$ & 4 & 1 & & 87.7 & $\mathrm{~N}$ & \\
\hline 1 & $10 / 31 / 91$ & 6 & 1 & & 87.8 & $\mathbf{Y}$ & \\
\hline 1 & $10 / 31 / 91$ & 8 & 1 & & 84.3 & $\mathbf{N}$ & \\
\hline 1 & $10 / 31 / 91$ & 9 & 9 & & 80.2 & $\mathbf{N}$ & \\
\hline 1 & $10 / 31 / 91$ & 10 & 1 & & 79.1 & $\mathbf{N}$ & \\
\hline 1 & $10 / 31 / 91$ & 11 & 1 & $\cdot$ & 79.4 & $\mathbf{N}$ & \\
\hline 1 & $10 / 31 / 91$ & 12 & 1 & & 79.2 & $\mathbf{N}$ & \\
\hline 1 & $10 / 31 / 91$ & 14 & 1 & & 66 & $\mathbf{N}$ & \\
\hline 1 & $11 / 9 / 91$ & 4 & 1 & & 87.6 & $Y$ & \\
\hline 1 & $11 / 9 / 91$ & 6 & 1 & & 87.5 & $N$ & \\
\hline 1 & $11 / 9 / 91$ & 7 & 1 & & 86.1 & $\mathbf{N}$ & \\
\hline 1 & $11 / 9 / 91$ & 8 & 1 & & 82.8 & $\mathbf{N}$ & \\
\hline 1 & $11 / 9 / 91$ & 9 & 1 & & 77.8 & $\mathbf{N}$ & \\
\hline 1 & $11 / 9 / 91$ & 10 & 1 & & 76.5 & $\mathbf{N}$ & \\
\hline 1 & $11 / 9 / 91$ & 11 & 1 & & 76.2 & $\mathbf{N}$ & \\
\hline 1 & $11 / 9 / 91$ & 12 & 1 & & 76.5 & $\mathbf{N}$ & \\
\hline 1 & $11 / 9 / 91$ & 14 & 1 & & 53.6 & $N$ & \\
\hline
\end{tabular}

Data obtained from. WHC Surveillance Analysis Computer System (SACS), February 24, 1994. 
TANK 241-S-112

THERMOCOUPLE DATA

\begin{tabular}{|c|c|c|c|c|c|c|c|}
\hline \multicolumn{8}{|c|}{ THERMOCOUPLE DATA } \\
\hline & & & & & & & \\
\hline TREE \# & DATE & THERMO \# & TREE SET \# & TEMP C & TEMP F & $Y=$ MAX TEMP & COMMENTS \\
\hline 1 & $11 / 13 / 91$ & 4 & 1 & & 80.8 & -1 & $\begin{array}{c}\text { TC } \# 1.35-613-160 / S \text { W/O \#04529 J- } \\
890423320.42 \\
\end{array}$ \\
\hline 1 & $11 / 13 / 91$ & 7 & 9 & & 80.3 & $\mathbf{N}$ & \\
\hline 1 & $11 / 13 / 91$ & 8 & 1 & & 77.6 & $\mathbf{N}$ & \\
\hline 1 & $11 / 13 / 91$ & 9 & 1 & & 72.2 & $\mathbf{N}$ & \\
\hline 1 & $11 / 13 / 91$ & 10 & 1 & & 70.4 & $\mathbf{N}$ & \\
\hline 1 & $11 / 13 / 91$ & 11 & 1 & & 70 & $\mathbf{N}$ & \\
\hline 1 & $11 / 13 / 91$ & 12 & 1 & & 79.8 & $\mathbf{N}$ & \\
\hline 1 & $11 / 15 / 91$ & 4 & 2 & & 81.7 & $\bar{Y}$ & \\
\hline 1 & $11 / 15 / 91$ & 4 & 1 & & 88 & $\mathbf{N}$ & \\
\hline 1 & $11 / 15 / 91$ & 6 & 1 & & 88.5 & $\bar{Y}$ & \\
\hline 1 & $11 / 15 / 91$ & 7 & 2 & & 81.1 & $\mathbf{N}$ & \\
\hline 1 & $11 / 15 / 91$ & .7 & 1 & & 86.8 & $\mathbf{N}$ & \\
\hline 1 & $11 / 15 / 91$ & 8 & 2 & & 78.1 & $\mathbf{N}$ & \\
\hline 1 & $11 / 15 / 91$ & 8 & 1 & & 83.1 & $\mathbf{N}$ & \\
\hline 1 & $11 / 15 / 91$ & 9 & 2 & & 72.6 & $\mathbf{N}$ & \\
\hline 1 & $11 / 15 / 91$ & 9 & 1 & & 78 & $\mathbf{N}$ & \\
\hline 1 & $11 / 75 / 91$ & 10 & 2 & & 70.7 & $\mathrm{~N}$ & \\
\hline 1 & $11 / 15 / 91$ & 10 & 1 & & 76.5 & $\mathrm{~N}$ & \\
\hline 1 & $11 / 15 / 91$ & 11 & 2 & & 70.4 & $N$ & \\
\hline 1 & $11 / 15 / 91$ & 11 & 1 & & 76.5 & $N$ & \\
\hline 1 & $11 / 15 / 91$ & 12 & 2 & & 72 & $\mathrm{~N}$ & \\
\hline 1 & $11 / 15 / 91$ & 12 & 1 & & 76.3 & $N$ & \\
\hline 1 & $11 / 21 / 91$ & 4 & 1 & & 89.4 & $\bar{Y}$ & \\
\hline 1 & $11 / 21 / 91$ & 6 & 1 & & 88.7 & $N$ & \\
\hline 1 & $11 / 21 / 91$ & 7 & 1 & & 87.4 & $N$ & \\
\hline 1 & $11 / 21 / 91$ & 8 & 1 & & 83.6 & $\mathbf{N}$ & \\
\hline 1 & $11 / 21 / 91$ & 9 & 1 & & 78 & $N$ & \\
\hline 1 & $11 / 21 / 91$ & 10 & 1 & & 76.4 & $N$ & \\
\hline 1 & $11 / 21 / 91$ & 11 & 1 & & 76.3 & $N$ & \\
\hline 1 & $11 / 21 / 91$ & 12 & 1 & & 76.5 & $\mathbf{N}$ & \\
\hline 1 & $11 / 21 / 91$ & 14 & 1 & & 52.7 & $\mathbf{N}$ & \\
\hline 1 & $11 / 27 / 91$ & 4 & 1 & & 91.8 & $Y$ & \\
\hline 1 & $11 / 27 / 91$ & 6 & 1 & & 90.2 & $N$ & \\
\hline 1 & $11 / 27 / 91$ & 7 & 1 & & 89.9 & $\mathbf{N}$ & \\
\hline 1 & $11 / 27 / 91$ & 8 & 1 & & 85.5 & $\mathbf{N}$ & \\
\hline 1 & $11 / 27 / 91$ & 9 & 1 & & 79.6 & $\mathbf{N}$ & \\
\hline 1 & $11 / 27 / 91$ & 10 & 1 & & 78.1 & $\mathbf{N}$ & \\
\hline 1 & $11 / 27 / 91$ & 11 & 1 & & 78 & $\mathbf{N}$ & \\
\hline 1 & $11 / 27 / 91$ & 12 & 1 & & 77.9 & $\mathbf{N}$ & \\
\hline 1 & $11 / 27 / 91$ & 14 & 1 & & 60.1 & $\mathbf{N}$ & \\
\hline 1 & $12 / 5 / 91$ & 4 & 1 & & 87.6 & $Y$ & \\
\hline 1 & $12 / 5 / 91$ & 6 & 1 & & 87.6 & $\mathbf{N}$ & \\
\hline 1 & $12 / 5 / 91$ & 7 & 1 & & 86 & $\mathbf{N}$ & \\
\hline 1 & $12 / 5 / 91$ & 8 & 1 & & 81.5 & $N$ & \\
\hline 1 & $12 / 5 / 91$ & 9 & 1 & & 75.4 & $\mathbf{N}$ & \\
\hline 1 & $12 / 5 / 91$ & 10 & 1 & & 73.6 & $\mathbf{N}$ & \\
\hline 1 & $12 / 5 / 91$ & 11 & 1 & & 73.6 & $\mathbf{N}$ & \\
\hline 1 & $12 / 5 / 91$ & 12 & 1 & & 73.5 & $N$ & \\
\hline 1 & $12 / 5 / 91$ & 14 & 1 & & 58 & $N$ & \\
\hline 1 & $12 / 1,3 / 91$ & 4 & 1 & & 83.9 & $N$ & \\
\hline 1 & $12 / 13 / 91$ & 6 & 1 & & 84.5 & $\bar{Y}$ & \\
\hline 1 & $12 / 13 / 91$ & 7 & 1 & & 82.7 & $N$ & \\
\hline 1 & $12 / 13 / 91$ & 8 & 1 & & 78.6 & $\mathrm{~N}$ & \\
\hline 1 & $12 / 13 / 91$ & 9 & 1 & & 72.5 & $\mathrm{~N}$ & \\
\hline 1 & $12 / 13 / 91$ & 10 & 1 & & 70.1 & $N$ & \\
\hline 1 & $12 / 13 / 91$ & 11 & 1 & & 70.2 & $N$ & \\
\hline 1 & $12 / 13 / 91$ & 12 & 1 & & 70.8 & $N$ & \\
\hline 1 & $12 / 13 / 91$ & 14 & 1 & & 53.9 & $N$ & \\
\hline 1 & $12 / 19 / 91$ & 4 & 1 & & 89.4 & $Y$ & \\
\hline 1 & $12 / 19 / 91$ & 6 & 1 & & 88.9 & $N$ & \\
\hline 1 & $12 / 19 / 91$ & 7 & 1 & & 86.7 & $N$ & \\
\hline 1 & $12 / 19 / 91$ & 8 & 1 & & 81.9 & $\mathbf{N}$ & \\
\hline 1 & $12 / 19 / 91$ & 9 & 1 & & 74.9 & $\mathrm{~N}$ & \\
\hline 1 & $12 / 19 / 91$ & 10 & 1 & & 73 & $\mathbf{N}$ & \\
\hline
\end{tabular}

Data obtained from WHC Surveillance Analysis Computer System (SACS), February 24, 1994. 


\begin{tabular}{|c|c|c|c|c|c|c|c|}
\hline \multicolumn{8}{|c|}{ TANK 241-S-112 } \\
\hline \multicolumn{8}{|c|}{ THERMOCOUPLE DATA } \\
\hline & & & & & & & \\
\hline TREE \# & DATE & THERMO \# & TREE SET \# & TEMP C & TEMP F & $Y=\operatorname{MAX}$ TEMP & COMMENTS \\
\hline 1 & $12 / 19 / 91$ & 11 & 1 & & 76.5 & $N$ & \\
\hline 1 & $12 / 19 / 91$ & 12 & 1 & & 76.5 & $N$ & \\
\hline 1 & $12 / 19 / 91$ & 14 & 1 & & 58 & $\mathbf{N}$ & \\
\hline 1 & $12 / 26 / 91$ & 4 & 1 & & 89.2 & $\mathrm{~N}$ & \\
\hline 1 & $12 / 26 / 91$ & 6 & 1 & & 89.3 & $Y$ & \\
\hline 1 & $12 / 26 / 91$ & 7 & 1 & & 87.2 & $\mathbf{N}$ & \\
\hline 1 & $12 / 26 / 91$ & 8 & 1 & & 81.8 & $\mathbf{N}$ & \\
\hline 1 & $12 / 26 / 91$ & 9 & 1 & & 74.4 & $\mathbf{N}$ & \\
\hline 1 & $12 / 26 / 91$ & 10 & 1 & & 72.5 & $\bar{N}$ & \\
\hline 1 & $12 / 26 / 91$ & 11 & 1 & & 72.8 & $N$ & \\
\hline 1 & $12 / 26 / 91$ & 12 & 1 & & 73 & $N$ & \\
\hline 1 & $12 / 26 / 91$ & 14 & 1 & & 57.7 & $N$ & \\
\hline 1 & $1 / 2 / 92$ & 4 & 1 & & 89 & $\bar{Y}$ & J $8171355008 \mathrm{TC} \# 1-350 / \mathrm{S}$ \\
\hline 1 & $1 / 2 / 92$ & 6 & 1 & & 85 & $\mathrm{~N}$ & \\
\hline 1 & $1 / 2 / 92$ & 7 & 1 & & 83 & $N$ & \\
\hline 1 & $1 / 2 / 92$ & 8 & 1 & & 77 & $\mathbf{N}$ & \\
\hline 1 & $1 / 9 / 92$ & 4 & 1 & & 88.9 & $Y$ & \\
\hline 1 & $1 / 9 / 92$ & 6 & 1 & & 88 & $\mathbf{N}$ & \\
\hline 1 & $1 / 9 / 92$ & 7 & 1 & & 85.6 & $\mathrm{~N}$ & \\
\hline 1 & $1 / 9 / 92$ & 8 & 1 & & 79.9 & $\mathrm{~N}$ & \\
\hline 1 & $1 / 9 / 92$ & 9 & 1 & & 72.2 & $\mathbf{N}$ & \\
\hline 1 & $1 / 9 / 92$ & 10 & 1 & & 70 & $N$ & \\
\hline 1 & $1 / 9 / 92$ & 11 & 1 & & 70.3 & $N$ & \\
\hline 1 & $1 / 9 / 92$ & 12 & 1 & & 70 & $N$ & \\
\hline 1 & $1 / 9 / 92$ & 14 & 1 & & 57.5 & $N$ & \\
\hline 11 & $1 / 9 / 92$ & 1 & 4 & & 57.5 & $\mathbf{N}$ & \\
\hline 11 & $1 / 9 / 92$ & 1 & 0 & & 70 & $\mathrm{~N}$ & \\
\hline 11 & $1 / 9 / 92$ & 1 & 2 & & 70 & $\mathbf{N}$ & \\
\hline 11 & $1 / 9 / 92$ & 1 & 9 & & 70.3 & $\mathbf{N}$ & \\
\hline 11 & $1 / 9 / 92$ & 4 & 1 & & 88.9 & $Y$ & $J-817-13-55-008$ \\
\hline 11 & $1 / 9 / 92$ & 5 & 1 & & & $N$ & Out of service \\
\hline 11 & $1 / 9 / 92$ & 6 & 1 & & 88 & $\mathbf{N}$ & \\
\hline 19 & $1 / 9 / 92$ & 7 & 1 & & 85.6 & $\mathbf{N}$ & \\
\hline 19 & $1 / 9 / 92$ & 8 & 1 & & 79.9 & $\mathbf{N}$ & \\
\hline 11 & $1 / 9 / 92$ & 9 & 1 & & 72.2 & $\mathbf{N}$ & \\
\hline 1 & $1 / 16 / 92$ & 1 & 1 & & & $\mathbf{N}$ & $\begin{array}{c}\text { No Data on } 1 / 16 / 92 \text { data sheet. Called shift } \\
\text { office } 1 / 21 / 92 \text {. }\end{array}$ \\
\hline 1 & $1 / 17 / 92$ & 4 & 1 & & 87.7 & $\mathbf{Y}$ & \\
\hline 1 & $1 / 17 / 92$ & 6 & 1 & & 87 & $\mathbf{N}$ & \\
\hline 1 & $1 / 17 / 92$ & 7 & 1 & & 84.5 & $\mathbf{N}$ & \\
\hline 1 & $1 / 17 / 92$ & 8 & 1 & & 78.5 & $\mathbf{N}$ & \\
\hline 1 & $1 / 17 / 92$ & 9 & 1 & & 70.6 & $\mathbf{N}$ & \\
\hline 1 & $1 / 17 / 92$ & 10 & 1 & & 68.4 & $\mathbf{N}$ & \\
\hline 1 & $1 / 17 / 92$ & 11 & 1 & & 68.2 & $\mathbf{N}$ & \\
\hline 1 & $1 / 17 / 92$ & 12 & 1 & & 68.2 & $\mathbf{N}$ & \\
\hline 1 & $1 / 17 / 92$ & 14 & 1 & & 52.5 & $\mathbf{N}$ & \\
\hline 1 & $1 / 23 / 92$ & 4 & 1 & & 86.1 & $Y$ & \\
\hline 1 & $1 / 23 / 92$ & 6 & 1 & & 85.4 & $N$ & \\
\hline 1 & $1 / 23 / 92$ & 7 & 1 & & 82.9 & $\mathbf{N}$ & - \\
\hline 1 & $1 / 23 / 92$ & 8 & 1 & & 76.7 & $\mathbf{N}$ & \\
\hline 1 & $1 / 23 / 92$ & 9 & 1 & & 68.6 & $\mathrm{~N}$ & \\
\hline 1 & $1 / 23 / 92$ & 10 & 1 & & 66.1 & $N$ & \\
\hline 1 & $1 / 23 / 92$ & 11 & 1 & & 66.1 & $N$ & \\
\hline 1 & $1 / 23 / 92$ & 12 & 1 & & 66.4 & $\mathbf{N}$ & \\
\hline 1 & $1 / 23 / 92$ & 14 & 1 & & 50.4 & $N$ & \\
\hline 1 & $1 / 28 / 92$ & 1 & 1 & & & $N$ & $\begin{array}{c}\text { ACCESS RESTRICTED } 1 / 28-2 / 22 \text { DUE TO } \\
\text { BATTERY FUME INCIDENT }\end{array}$ \\
\hline 1 & $3 / 8 / 92$ & 1 & 1 & & & $\mathbf{N}$ & $0 / 5 J 890423320.42$ \\
\hline 1 & $3 / 9 / 92$ & 4 & 1 & & 84.3 & $Y$ & \\
\hline 1 & $3 / 9 / 92$ & 7 & 1 & & 81.1 & $\mathbf{N}$ & \\
\hline 1 & $3 / 9 / 92$ & 8 & 1 & & 75 & $\mathbf{N}$ & \\
\hline 1 & $3 / 9 / 92$ & 9 & 1 & & 65.5 & $\mathbf{N}$ & \\
\hline 1 & $3 / 9 / 92$ & 10 & 1 & & 63.2 & $\mathrm{~N}$ & \\
\hline
\end{tabular}

-Data obtained from WHC Surveillance Analysis Computer System (SACS), February 24, 1994. 


\begin{tabular}{|c|c|c|c|c|c|c|c|}
\hline & & $\cdot$ & & & IK 241-S & & \\
\hline & & & & THE & OCOUPL & ATA & \\
\hline TREE \# & DATE & THERMO \# & TREE SET \# & TEMP C & TEMP F & $\bar{Y}=$ MAX TEMP & COMMENTS \\
\hline 1 & $3 / 9 / 92$ & 11 & 1 & & 63.3 & $\mathrm{~N}$ & \\
\hline 1 & $3 / 9 / 92$ & 12 & 1 & & 64.5 & $\bar{N}$ & \\
\hline 1 & $3 / 12 / 92$ & 4 & 1 & & 76 & $Y$ & \\
\hline 1 & $3 / 12 / 92$ & 6 & 1 & & 75 & $\mathrm{~N}$ & \\
\hline 1 & $3 / 12 / 92$ & 7 & 1 & & $7 \overline{3}$ & $N$ & \\
\hline 1 & $3 / 12 / 92$ & 8 & 1 & & 67 & $N$ & \\
\hline 1 & $3 / 12 / 92$ & 9 & 1 & & 58 & $N$ & \\
\hline 1 & $3 / 12 / 92$ & 10 & 1 & & 56 & $\bar{N}$ & \\
\hline 9 & $3 / 12 / 92$ & 11 & 1 & & 57 & $\mathrm{~N}$ & \\
\hline 1 & $3 / 12 / 92$ & 12 & 1 & & 58 & $N$ & \\
\hline 1 & $3 / 39 / 92$ & 4 & 1 & & 87 & $N$ & \\
\hline 1 & $3 / 19 / 92$ & 6 & 1 & & 83 & $\bar{N}$ & \\
\hline 1 & $3 / 19 / 92$ & 7 & 1 & & 82 & $N$ & \\
\hline 1 & $3 / 19 / 92$ & 8 & 1 & & 76 & $\mathrm{~N}$ & \\
\hline 1 & $3 / 19 / 92$ & 9 & 1 & & 68 & $N$ & \\
\hline 1 & $3 / 19 / 92$ & 10 & 1 & & 66 & $\mathrm{~N}$ & \\
\hline 9 & $3 / 19 / 92$ & 11 & 1 & & 66 & $\mathbf{N}$ & \\
\hline 1 & $3 / 19 / 92$ & 12 & 1 & & 68 & $N$ & \\
\hline 1 & $3 / 19 / 92$ & 14 & 1 & & 50 & $N$ & \\
\hline 1 & $3 / 26 / 92$ & 6 & 1 & & 92 & $\bar{Y}$ & \\
\hline 1 & $3 / 26 / 92$ & 7 & 1 & & 90 & $\mathbf{N}$ & \\
\hline 1 & $3 / 26 / 92$ & 8 & 1 & & 84 & $\mathrm{~N}$ & \\
\hline 1 & $3 / 26 / 92$ & 9 & 1 & & 76 & $\bar{N}$ & \\
\hline 1 & $3 / 26 / 92$ & 10 & 1 & & 74 & $N$ & \\
\hline 1 & $3 / 26 / 92$ & 11 & 1 & & 74 & $\mathbf{N}$ & \\
\hline 1 & $3 / 26 / 92$ & 12 & 1 & & 74 & $N$ & \\
\hline 1 & $3 / 26 / 92$ & 14 & 1 & & 56 & $\mathrm{~N}$ & \\
\hline 1 & $3 / 27 / 92$ & 4 & $\overline{1}$ & & 91 & $\bar{Y}$ & \\
\hline 9 & $4 / 4 / 92$ & 4 & 1 & & 81.9 & $Y$ & $\mathrm{~J} 890423320-42$ \\
\hline 1 & $4 / 4 / 92$ & 7 & 1 & & 78.1 & $\mathrm{~N}$ & \\
\hline 1 & $4 / 4 / 92$ & 8 & 1 & & 73.5 & $N$ & \\
\hline 1 & $4 / 4 / 92$ & 9 & 1 & & 65 & $\mathrm{~N}$ & \\
\hline 1 & $4 / 4 / 92$ & 10 & 1 & & 62.8 & $\mathrm{~N}$ & \\
\hline 1 & $4 / 4 / 92$ & 11 & 1 & & 62.5 & $\bar{N}$ & \\
\hline 1 & $4 / 4 / 92$ & 12 & $\overline{1}$ & & 64 & $\bar{N}$ & \\
\hline 1 & $4 / 9 / 92$ & 4 & 1 & & 88.5 & $\bar{Y}$ & \\
\hline 1 & $4 / 9 / 92$ & 6 & 1 & & 82.1 & $\mathbf{N}$ & \\
\hline$\frac{1}{1}$ & $4 / 9 / 92$ & 7 & 1 & & 80.6 & $\bar{N}$ & \\
\hline 9 & $4 / 9 / 92$ & 8 & 1 & & 75.4 & $\mathrm{~N}$ & \\
\hline 1 & $4 / 9 / 92$ & 9 & 1 & & 68.1 & $N$ & \\
\hline 1 & $4 / 9 / 92$ & 10 & 1 & & 66 & $N$ & \\
\hline$\frac{1}{1}$ & $4 / 9 / 92$ & 11 & 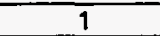 & & 66 & $\bar{N}$ & \\
\hline 1 & $4 / 9 / 92$ & 12 & 1 & & 65.9 & $N$ & \\
\hline 1 & $4 / 16 / 92$ & 4 & 1 & & 84 & $Y$ & \\
\hline 1 & $4 / 16 / 92$ & 6 & 1 & & 81 & $\bar{N}$ & \\
\hline 1 & $4 / 16 / 92$ & 7 & 1 & & 79 & $\bar{N}$ & \\
\hline 1 & $4 / 16 / 92$ & 8 & 1 & & 74 & $\mathbf{N}$ & \\
\hline 1 & $4 / 16 / 92$ & 9 & 1 & & 67 & $N$ & \\
\hline 1 & $4 / 16 / 92$ & 10 & T & & 65 & $\mathbf{N}$ & \\
\hline 1 & $4 / 16 / 92$ & 11 & 1 & & 65 & $N$ & \\
\hline 1 & $4 / 16 / 92$ & 12 & 1 & & 65 & $\mathbf{N}$ & \\
\hline 1 & $4 / 23 / 92$ & 4 & $\frac{1}{1}$ & & 77 & $Y$. & J 8171355010 \\
\hline 1 & $4 / 23 / 92$ & 6 & 1 & & 74 & $\mathrm{~N}$ & \\
\hline 1 & $4 / 23 / 92$ & 7 & 9 & & 72 & $N$ & \\
\hline 1 & $4 / 23 / 92$ & 8 & 1 & & 66 & $\mathbf{N}$ & \\
\hline 7 & $4 / 23 / 92$ & 9 & 9 & & 60 & $\mathbf{N}$ & \\
\hline 1 & $4 / 23 / 92$ & 10 & 1 & & 59 & $\mathbf{N}$ & \\
\hline 1 & $4 / 23 / 92$ & 11 & 1 & & 61 & $\mathbf{N}$ & \\
\hline 1 & $4 / 23 / 92$ & 12 & 1 & & 63 & $\mathbf{N}$ & \\
\hline 1 & $4 / 30 / 92$ & 4 & 1 & & 88 & $Y$ & \\
\hline 1 & $4 / 30 / 92$ & 6 & 1 & & 87 & $\mathrm{~N}$ & \\
\hline 9 & $4 / 30 / 92$ & 7 & 9 & & 85 & $\mathrm{~N}$ & \\
\hline 1 & $4 / 30 / 92$ & 8 & 1 & & 73 & $\mathbf{N}$ & \\
\hline 1 & $4 / 30 / 92$ & 9 & 1 & & 73 & $\mathrm{~N}$ & \\
\hline 1 & $4 / 30 / 92$ & 10 & 1 & & 72 & $\mathbf{N}$ & \\
\hline
\end{tabular}

Data obtained from WHC Surveillance Analysis Computer System (SACS), February 24, 1994. 


\begin{tabular}{|c|c|c|c|c|c|c|c|}
\hline \multicolumn{8}{|c|}{ TANK 241-S-112 } \\
\hline \multicolumn{8}{|c|}{ THERMOCOUPLE DATA } \\
\hline TREE \# & DATE & THERMO \# & TREE SET \# & TEMP C & TEMP F & $Y=$ MAX TEMP & COMMENTS \\
\hline 1 & $4 / 30 / 92$ & 11 & 1 & & 72 & $N$ & \\
\hline 1 & $4 / 30 / 92$ & 12 & 1 & & 75 & $\mathbf{N}$ & \\
\hline 1 & $4 / 30 / 92$ & 14 & 1 & & 54 & $N$ & \\
\hline 1 & $5 / 7 / 92$ & 4 & 1 & & 81.9 & $Y$ & J 8171355008 TC $\# 35$ BAD DATA \\
\hline 1 & $5 / 7 / 92$ & 6 & 1 & & 80.3 & $\mathbf{N}$ & \\
\hline 1 & $5 / 7 / 92$ & 7 & 1 & & 77.8 & $\mathbf{N}$ & \\
\hline 1 & $5 / 7 / 92$ & 8 & 1 & & 72 & $\mathbf{N}$ & \\
\hline 1 & $5 / 7 / 92$ & 9 & 1 & & 65.2 & $\mathbf{N}$ & \\
\hline 1 & $5 / 7 / 92$ & 10 & 1 & & 63.5 & $\mathbf{N}$ & \\
\hline 1 & $5 / 7 / 92$ & 11 & 1 & & 63.5 & $\mathbf{N}$ & \\
\hline 1 & $5 / 7 / 92$ & 12 & 1 & & 64.2 & $N$ & \\
\hline 1 & $5 / 15 / 92$ & 4 & 1 & & 83 & $\bar{N}$ & \\
\hline 1 & $5 / 15 / 92$ & 6 & 1 & & 80 & $Y$ & \\
\hline 1 & $5 / 15 / 92$ & 7 & 1 & & 78 & $\mathbf{N}$ & \\
\hline 1 & $5 / 15 / 92$ & 8 & 1 & & 72 & $\mathbf{N}$ & \\
\hline 1 & $5 / 15 / 92$ & 9 & 1 & & 66 & $N$ & \\
\hline 1 & $5 / 15 / 92$ & 10 & 1 & & 64 & $N$ & \\
\hline 1 & $5 / 15 / 92$ & 11 & 1 & & 64 & $\mathbf{N}$ & \\
\hline 1 & $5 / 15 / 92$ & 12 & 1 & & 63 & $\bar{N}$ & \\
\hline 1 & $5 / 26 / 92$ & 4 & 1 & & 77.5 & $Y$ & \\
\hline 1 & $5 / 26 / 92$ & 7 & 1 & & 76 & $\mathrm{~N}$ & \\
\hline 1 & $5 / 26 / 92$ & 8 & 1 & & 71.5 & $\mathbf{N}$ & \\
\hline 1 & $5 / 26 / 92$ & 9 & 1 & & 64.2 & $N$ & \\
\hline 1 & $5 / 26 / 92$ & 10 & 1 & & 62.2 & $\mathbf{N}$ & \\
\hline 1 & $5 / 26 / 92$ & 11 & 1 & & 61.7 & $\mathbf{N}$ & \\
\hline 1 & $5 / 26 / 92$ & 12 & 1 & & 63.8 & $\mathbf{N}$ & \\
\hline 1 & $5 / 28 / 92$ & 4 & 1 & & 71 & $\mathbf{N}$ & \\
\hline 1 & $5 / 28 / 92$ & 6 & 1 & & 80 & $\mathbf{N}$ & \\
\hline 1 & $5 / 28 / 92$ & 7 & 1 & & 82 & $Y$ & \\
\hline 1 & $5 / 28 / 92$ & 8 & 1 & & 75 & $\mathbf{N}$ & \\
\hline 1 & $5 / 28 / 92$ & 9 & 1 & & 67 & $\mathbf{N}$ & \\
\hline 1 & $5 / 28 / 92$ & 10 & 1 & & 69 & $\mathbf{N}$ & \\
\hline 1 & $5 / 28 / 92$ & 11 & 1 & & 69 & $\mathbf{N}$ & \\
\hline 1 & $5 / 28 / 92$ & 12 & 1 & & 69 & $\mathbf{N}$ & \\
\hline 1 & $6 / 4 / 92$ & 4 & 1 & & 72 & $\mathbf{Y}$ & J 8171320019 TC\#14 BAD DATA \\
\hline 1 & $6 / 4 / 92$ & 6 & 1 & & 72 & $\mathbf{N}$ & \\
\hline 1 & $6 / 4 / 92$ & 7 & 1 & & 71 & $\mathbf{N}$ & \\
\hline 1 & $6 / 4 / 92$ & 8 & 1 & & 66 & $\mathbf{N}$ & \\
\hline 1 & $6 / 4 / 92$ & 9 & 1 & & 61 & $\mathbf{N}$ & \\
\hline 1 & $6 / 4 / 92$ & 10 & 1 & & 59 & $\mathbf{N}$ & \\
\hline 1 & $6 / 4 / 92$ & 11 & 1 & & 61 & $N$ & \\
\hline 1 & $6 / 4 / 92$ & 12 & 1 & & 61 & $\mathbf{N}$ & \\
\hline 1 & $6 / 11 / 92$ & 1 & 1 & & 78 & $\mathbf{N}$ & J NO-817-13-20-019 TC $\# 67814$ \\
\hline 1 & $6 / 11 / 92$ & 2 & 1 & & 80 & $\mathbf{Y}$ & \\
\hline 1 & $6 / 11 / 92$ & 3 & 1 & & 79 & $\mathbf{N}$ & \\
\hline 1 & $6 / 11 / 92$ & 4 & 1 & & 78 & $\mathbf{N}$ & \\
\hline 1 & $6 / 11 / 92$ & 5 & 1 & & 78 & $\mathbf{N}$ & \\
\hline 1 & $6 / 11 / 92$ & 9 & 1 & & 65 & $\mathbf{N}$ & \\
\hline 1 & $6 / 11 / 92$ & 10 & 1 & & 64 & $\mathbf{N}$ & \\
\hline 1 & $6 / 11 / 92$ & 11 & 1 & & 65 & $\mathbf{N}$ & \\
\hline 1 & $6 / 11 / 92$ & 12 & 1 & & 65 & $\mathbf{N}$ & \\
\hline 1 & $6 / 11 / 92$ & 13 & 1 & & 66 & $\mathbf{N}$ & \\
\hline 1 & $6 / 19 / 92$ & 1 & 1 & & 78 & $Y$ & J 817-13-20-019 TC\# 14 BAD \\
\hline 1 & $6 / 19 / 92$ & 2 & 1 & & 75 & $\mathbf{N}$ & \\
\hline 1 & $6 / 19 / 92$ & 3 & 1 & & 75 & $\mathbf{N}$ & \\
\hline 1 & $6 / 19 / 92$ & 4 & 1 & & 75 & $\mathbf{N}$ & \\
\hline 1 & $6 / 19 / 92$ & 5 & 1 & & 74 & $\mathbf{N}$ & \\
\hline 1 & $6 / 19 / 92$ & 6 & 1 & & 73 & $\mathbf{N}$ & \\
\hline 1 & $6 / 19 / 92$ & 7 & 1 & & 72 & $\mathbf{N}$ & \\
\hline 1 & $6 / 19 / 92$ & 8 & 1 & & 69 & $\mathbf{N}$ & \\
\hline 1 & $6 / 19 / 92$ & 9 & 1 & & 64 & $\mathbf{N}$ & \\
\hline 1 & $6 / 19 / 92$ & 10 & 1 & & 63 & $\mathbf{N}$ & \\
\hline 1 & $6 / 19 / 92$ & 11 & 1 & & 63 & $\mathbf{N}$ & \\
\hline 1 & $6 / 19 / 92$ & 12 & 1 & & 64 & $\mathbf{N}$ & . \\
\hline 1 & $6 / 19 / 92$ & 13 & 1 & & 65 & $\mathbf{N}$ & \\
\hline
\end{tabular}

Data obtained from WHC Surveillance Analysis Computer System (SACS), February 24, 1994. 
WHC-SD-WM-ER-323, Rev. 0

\begin{tabular}{|c|c|c|c|c|c|c|c|}
\hline \multicolumn{8}{|c|}{ TANK 241-S-112 } \\
\hline \multicolumn{8}{|c|}{ THERMOCOUPLE DATA } \\
\hline TREE \# & DATE & THERMO \# & TREE SET \# & TEMP $\mathrm{C}$ & TEMP F & $Y=$ MAX TEMP & COMMENTS \\
\hline 1 & $6 / 19 / 92$ & 14 & 1 & & 47 & $\mathrm{~N}$ & \\
\hline 9 & $7 / 9 / 92$ & 1 & 1 & & 78 & $\mathbf{N}$ & JC19950 \\
\hline 1 & $7 / 1 / 92$ & 2 & 1 & & 79 & $Y$ & \\
\hline 1 & $7 / 1 / 92$ & 3 & 1 & & 79 & $\mathrm{~N}$ & \\
\hline 1 & $7 / 1 / 92$ & 4 & 1 & & 79 & $\mathbf{N}$ & \\
\hline 1 & $7 / 1 / 92$ & 5 & 1 & & 78 & $\mathbf{N}$ & \\
\hline 1 & $7 / 1 / 92$ & 6 & 1 & & 78 & $\mathrm{~N}$ & \\
\hline 1 & $7 / 1 / 92$ & 7 & 1 & & 76 & $\mathbf{N}$ & \\
\hline 1 & $7 / 1 / 92$ & 8 & 1 & & 72 & $\mathbf{N}$ & \\
\hline 1 & $7 / 1 / 92$ & 9 & 1 & & 68 & $\mathbf{N}$ & \\
\hline 1 & $7 / 1 / 92$ & 10 & 1 & & 68 & $N$ & \\
\hline 1 & $7 / 1 / 92$ & 11 & 1 & & 67 & $\mathbf{N}$ & \\
\hline 1 & $7 / 1 / 92$ & 12 & 1 & & 67 & $\mathrm{~N}$ & \\
\hline 1 & $7 / 1 / 92$ & 13 & 1 & & 67 & $\mathrm{~N}$ & \\
\hline 1 & $7 / 13 / 92$ & 1 & 1 & & 78 & $\mathrm{~N}$ & J 817-13-20-019 \\
\hline 1 & $7 / 13 / 92$ & 2 & 1 & & 79 & $\mathbf{N}$ & \\
\hline 1 & $7 / 13 / 92$ & 3 & 1 & & 79 & $Y$ & \\
\hline 1 & $7 / 13 / 92$ & 4 & 9 & & 79 & $\mathrm{~N}$ & \\
\hline 1 & $7 / 13 / 92$ & 5 & 1 & & 78 & $\mathrm{~N}$ & \\
\hline 1 & $7 / 13 / 92$ & 6 & 1 & & 78 & $\mathrm{~N}$ & \\
\hline 1 & $7 / 13 / 92$ & 7 & 1 & & 76 & $\mathrm{~N}$ & \\
\hline 1 & $7 / 13 / 92$ & 8 & 1 & & 72 & $N$ & \\
\hline 1 & $7 / 13 / 92$ & 9 & 1 & & 68 & $N$ & \\
\hline 1 & $7 / 13 / 92$ & 10 & 1 & & 68 & $N$ & \\
\hline 1 & $7 / 13 / 92$ & 11 & 1 & & 67 & $\mathrm{~N}$ & \\
\hline 1 & $7 / 13 / 92$ & 12 & 1 & & 67 & $\mathrm{~N}$ & \\
\hline 1 & $7 / 13 / 92$ & 13 & 1 & & 67 & $\mathrm{~N}$ & \\
\hline 1 & $7 / 13 / 92$ & 14 & 1 & & 68 & $\mathrm{~N}$ & \\
\hline 1 & $7 / 16 / 92$ & 1 & 1 & & 79 & $\mathrm{~N}$ & J $817-13-20-019$ \\
\hline 1 & $7 / 16 / 92$ & 2 & 1 & & 80 & $Y$ & \\
\hline 1 & $7 / 16 / 92$ & 3 & 1 & & 80 & $\mathrm{~N}$ & \\
\hline 1 & $7 / 16 / 92$ & 4 & 1 & & 80 & $\mathrm{~N}$ & \\
\hline 1 & $7 / 16 / 92$ & 5 & 1 & & 80 & $N$ & \\
\hline 1 & $7 / 16 / 92$ & 6 & 1 & & 79 & $\mathrm{~N}$ & \\
\hline 1 & $7 / 16 / 92$ & 7 & 1 & & 77 & $\mathrm{~N}$ & \\
\hline 1 & $7 / 16 / 92$ & 8 & 1 & & 74 & $N$ & \\
\hline 1 & $7 / 16 / 92$ & 9 & 1 & & 70 & $N$ & \\
\hline 1 & $7 / 16 / 92$ & 10 & 1 & & 69 & $N$ & \\
\hline 1 & $7 / 16 / 92$ & 11 & 1 & & 69 & $N$ & \\
\hline 1 & $7 / 16 / 92$ & 12 & 1 & & 69 & $N$ & \\
\hline 1 & $7 / 16 / 92$ & 13 & 1 & & 69 & $N$ & \\
\hline 1 & $7 / 16 / 92$ & 14 & 1 & & 70 & $\mathrm{~N}$ & \\
\hline 1 & $7 / 23 / 92$ & 1 & 1 & & 81 & $Y$ & \\
\hline 1 & $7 / 23 / 92$ & 2 & 1 & & 82 & $\mathbf{N}$ & \\
\hline 1 & $7 / 23 / 92$ & 3 & 1 & & 83 & $\mathrm{~N}$ & \\
\hline 1 & $7 / 23 / 92$ & 4 & 1 & & 83 & $Y$ & \\
\hline 1 & $7 / 23 / 92$ & 5 & 1 & & 82 & $\mathrm{~N}$ & \\
\hline 1 & $7 / 23 / 92$ & 6 & 1 & & 82 & $N$ & \\
\hline 1 & $7 / 23 / 92$ & 7 & 1 & & 81 & $\mathbf{N}$ & \\
\hline 1 & $7 / 23 / 92$ & 8 & 1 & & 78 & $\mathrm{~N}$ & \\
\hline 1 & $7 / 23 / 92$ & 9 & 1 & & 74 & $N$ & \\
\hline 1 & $7 / 23 / 92$ & 10 & 1 & & 73 & $\mathbf{N}$ & \\
\hline 1 & $7 / 23 / 92$ & 11 & 1 & & 73 & $\mathbf{N}$ & \\
\hline 1 & $7 / 23 / 92$ & 12 & 1 & & 73 & $N$ & \\
\hline 1 & $7 / 23 / 92$ & 13 & 1 & & 73 & $N$ & \\
\hline 1 & $7 / 23 / 92$ & 14 & 1 & & 54 & $N$ & \\
\hline 1 & $7 / 30 / 92$ & 1 & 1 & & 81 & $Y$ & J 813-13-55-008 \\
\hline 1 & $7 / 30 / 92$ & 2 & 1 & & 80.1 & $N$ & \\
\hline 1 & $7 / 30 / 92$ & 3 & 1 & & 80 & $N$ & \\
\hline 1 & $7 / 30 / 92$ & 4 & 1 & & 80.3 & $N$ & \\
\hline 1 & $7 / 30 / 92$ & 5 & 1 & & 81 & $\mathrm{~N}$ & \\
\hline 1 & $7 / 30 / 92$ & 6 & 1 & & 80.3 & $\mathrm{~N}$ & \\
\hline 1 & $7 / 30 / 92$ & 7 & 1 & & 80 & $\mathbf{N}$ & \\
\hline 1 & $7 / 30 / 92$ & 8 & 1 & & 77.4 & $\mathbf{N}$ & \\
\hline 1 & $7 / 30 / 92$ & 9 & 1 & & 75 & $N$ & \\
\hline
\end{tabular}

Data obtained from WHC Surveillance Analysis Computer System (SACS), February 24, 1994. 


\begin{tabular}{|c|c|c|c|c|c|c|c|}
\hline \multirow{2}{*}{\multicolumn{8}{|c|}{ TANK 241-S-112 }} \\
\hline \multicolumn{7}{|c|}{ THERMOCOUPLE DATA } & \\
\hline TREE \# & DATE & THERMO \# & TREE SET \# & TEMP C & TEMPF & $Y=$ MAX TEMP & COMMENTS \\
\hline 1 & $7 / 30 / 92$ & 10 & 1 & & 73.1 & $\mathrm{~N}$ & \\
\hline 1 & $7 / 30 / 92$ & 11 & $i$ & & 73.2 & $\mathbf{N}$ & \\
\hline 1 & $7 / 30 / 92$ & 12 & 1 & & 70.5 & $\mathbf{N}$ & \\
\hline 1 & $7 / 30 / 92$ & 13 & 1 & & 71.4 & $\bar{N}$ & \\
\hline 1 & $7 / 30 / 92$ & 94 & 1 & & 72 & $\bar{N}$ & \\
\hline 1 & $8 / 7 / 92$ & 4 & 1 & & 83.9 & $\bar{Y}$ & \\
\hline 9 & $8 / 7 / 92$ & 6 & 1 & & 83 & $N$ & \\
\hline 1 & $8 / 7 / 92$ & 7 & 1 & & 81.5 & $\bar{N}$ & \\
\hline 1 & $8 / 7 / 92$ & 8 & 9 & & 78.4 & $N$ & \\
\hline 1 & $8 / 7 / 92$ & 9 & 1 & & 75.1 & $N$ & \\
\hline 1 & $8 / 7 / 92$ & 10 & 1 & & 74.2 & $N$ & \\
\hline 1 & $8 / 7 / 92$ & 11 & 1 & & 74.1 & $N$ & \\
\hline 1 & $8 / 7 / 92$ & 12 & 1 & & 74.1 & $\mathbf{N}$ & \\
\hline 1 & $8 / 7 / 92$ & 14 & 1 & & 54.6 & $\mathbf{N}$ & \\
\hline 1 & $8 / 13 / 92$ & 1 & 1 & & 80.4 & $\mathbf{N}$ & J $817-13-55-008$ \\
\hline 1 & $8 / 13 / 92$ & 2 & 1 & & 81.5 & $\mathrm{~N}$ & \\
\hline 1 & $8 / 13 / 92$ & 3 & 1 & & 81.9 & $\mathrm{~N}$ & \\
\hline 1 & $8 / 13 / 92$ & 4 & 1 & & 82.4 & $\mathrm{Y}$ & \\
\hline 1 & $8 / 13 / 92$ & 5 & 1 & & 81.5 & $N$ & \\
\hline 1 & $8 / 13 / 92$ & 6 & 1 & & 82 & $N$ & \\
\hline 1 & $8 / 13 / 92$ & 7 & 1 & & 80.5 & $\bar{N}$ & \\
\hline 1 & $8 / 13 / 92$ & 8 & 1 & & 77.6 & $\mathrm{~N}$ & \\
\hline 1 & $8 / 13 / 92$ & 9 & 1 & & 74.5 & $\mathbf{N}$ & \\
\hline 1 & $8 / 13 / 92$ & 10 & 1 & & 73.5 & $\mathbf{N}$ & \\
\hline$\frac{T}{1}$ & $8 / 13 / 92$ & 11 & 1 & & 77.8 & $\mathbf{N}$ & \\
\hline 1 & $8 / 13 / 92$ & 12 & 1 & & 73.7 & $\mathbf{N}$ & \\
\hline 1 & $8 / 13 / 92$ & 13 & 1 & & 74.1 & $\mathbf{N}$ & \\
\hline 1 & $8 / 13 / 92$ & 74 & 1 & & 74.3 & $N$ & \\
\hline 1 & $8 / 20 / 92$ & 1 & 1 & & 77.5 & $\mathbf{N}$ & $J 817.13 .55 .008$ \\
\hline 1 & $8 / 20 / 92$ & 2 & 1 & & 80.7 & $\mathbf{N}$ & \\
\hline 1 & $8 / 20 / 92$ & 3 & 1 & & 80.9 & $\mathbf{N}$ & \\
\hline 1 & $8 / 20 / 92$ & 4 & 1 & & 85.6 & $\mathrm{Y}$ & \\
\hline 1 & $8 / 20 / 92$ & 5 & 1 & & 81 & $N$ & \\
\hline 1 & $8 / 20 / 92$ & 6 & 1 & & 80.6 & $N$ & \\
\hline 1 & $8 / 20 / 92$ & 7 & 1 & & 79.4 & $N$ & \\
\hline 1 & $8 / 20 / 92$ & 8 & 1 & & 76.6 & $N$ & \\
\hline 1 & $8 / 20 / 92$ & 9 & 1 & & 77.9 & $\mathbf{N}$ & \\
\hline 9 & $8 / 20 / 92$ & 10 & 1 & & 72.8 & $\mathbf{N}$ & \\
\hline 1 & $8 / 20 / 92$ & 11 & 1 & & 72.8 & $\bar{N}$ & \\
\hline 1 & $8 / 20 / 92$ & 12 & 1 & & 79.5 & $\mathbf{N}$ & \\
\hline 1 & $8 / 20 / 92$ & 13 & 1 & & 73.7 & $\mathbf{N}$ & \\
\hline 1 & $8 / 20 / 92$ & 14 & 1 & & 73.9 & $N$ & \\
\hline 1 & $9 / 3 / 92$ & 1 & 1 & & 81.9 & $\mathbf{N}$ & $J 890523512.47$ \\
\hline 1 & $9 / 3 / 92$ & 2 & 1 & & 82.4 & $Y$ & \\
\hline$\overline{1}$ & $9 / 3 / 92$ & 3 & 1 & & 82.1 & $\mathbf{N}$ & \\
\hline 1 & $9 / 3 / 92$ & 4 & 1 & & 81.9 & $\mathbf{N}$ & \\
\hline 1 & $9 / 3 / 92$ & 5 & 1 & & 81.5 & $N$ & \\
\hline 1 & $9 / 3 / 92$ & 6 & 1 & & 81 & $\bar{N}$ & \\
\hline 1 & $9 / 3 / 92$ & 7 & 1 & & 80.5 & $\mathbf{N}$ & \\
\hline 1 & $9 / 3 / 92$ & 8 & 1 & & 78 & $\mathbf{N}$ & \\
\hline 1 & $9 / 3 / 92$ & 10 & 1 & & 74.3 & $\mathbf{N}$ & \\
\hline 1 & $9 / 3 / 92$ & 11 & 1 & & 74.8 & $\mathbf{N}$ & \\
\hline 1 & $9 / 3 / 92$ & 12 & 1 & & 75.1 & $\mathbf{N}$ & \\
\hline 1 & $9 / 3 / 92$ & 13 & 1 & & 75.6 & $\mathbf{N}$ & \\
\hline 1 & $9 / 3 / 92$ & 14 & $\frac{1}{1}$ & & 75.9 & $\mathbf{N}$ & \\
\hline 1 & 9/9/92 & 1 & 1 & & 79 & $\mathbf{N}$ & J910126143-29 \\
\hline 1 & 9/9/92 & 2 & 1 & & 80 & $\mathbf{N}$ & \\
\hline 1 & $9 / 9 / 92$ & 3 & 1 & & 81 & $\mathbf{Y}$ & \\
\hline 1 & $9 / 9 / 92$ & 4 & 1 & & 81 & $N$ & \\
\hline 1 & $9 / 9 / 92$ & 5 & 1 & & 80 & $N$ & \\
\hline 9 & 9/9/92 & 6 & 1 & & 80 & $\mathbf{N}$ & \\
\hline 1 & 9/9/92 & 7 & 1 & & 79 & $\bar{N}$ & \\
\hline 1 & $9 / 9 / 92$ & 8 & $\frac{1}{1}$ & & 72 & $\mathrm{~N}$ & \\
\hline 1 & $9 / 9 / 92$ & 9 & 1 & & 74 & $\bar{N}$ & \\
\hline 1 & 9/9/92 & 10 & 1 & & 73 & $\mathrm{~N}$ & \\
\hline
\end{tabular}

Data obtained from WHC Surveillance Analysis Computer System (SACS), February 24, 1994. 


\begin{tabular}{|c|c|c|c|c|c|c|c|}
\hline \multicolumn{8}{|c|}{ TANK 241.S.112 } \\
\hline \multicolumn{8}{|c|}{ THERMOCOUPLE DATA } \\
\hline TREE \# & DATE & THERMO \# & TREE SET \# & TEMP C & TEMP F & $Y=$ MAX TEMP & COMMENTS \\
\hline 1 & $9 / 9 / 92$ & 11 & 1 & & 73 & $\mathrm{~N}$ & \\
\hline 1 & 9/9/92 & 12 & 1 & & 74 & $\mathbf{N}$ & \\
\hline 1 & $9 / 9 / 92$ & 13 & 1 & & 73 & $\mathbf{N}$ & \\
\hline 1 & 9/9/92 & 14 & 1 & & 73 & $\mathbf{N}$ & \\
\hline 9 & $9 / 15 / 92$ & 1 & 1 & & 84 & $\bar{N}$ & \\
\hline 1 & $9 / 15 / 92$ & 2 & 9 & & 85 & $Y$ & \\
\hline 1 & $9 / 15 / 92$ & 3 & 1 & & 85 & $\mathbf{N}$ & \\
\hline 1 & $9 / 15 / 92$ & 4 & 1 & & 85 & $\mathbf{N}$ & \\
\hline 1 & $9 / 15 / 92$ & 5 & 1 & & 85 & $\mathbf{N}$ & \\
\hline$\frac{1}{1}$ & $9 / 15 / 92$ & 6 & 1 & & 85 & $\mathbf{N}$ & \\
\hline 1 & $9 / 15 / 92$ & 7 & 1 & & 84 & $\mathbf{N}$ & \\
\hline 9 & $9 / 15 / 92$ & 8 & 1 & & 81 & $\mathbf{N}$ & \\
\hline 1 & $9 / 15 / 92$ & 9 & 9 & & 78 & $\bar{N}$ & \\
\hline 1 & $9 / 15 / 92$ & 10 & 1 & & 77 & $\mathrm{~N}$ & \\
\hline 1 & $9 / 15 / 92$ & 11 & 1 & & 77 & $\mathbf{N}$ & \\
\hline 9 & $9 / 15 / 92$ & 12 & 1 & & 77 & $\bar{N}$ & \\
\hline 1 & $9 / 15 / 92$ & 13 & 1 & & 77 & $N$ & \\
\hline 1 & $9 / 15 / 92$ & 14 & 1 & & 77 & $\bar{N}$ & \\
\hline 1 & $10 / 1 / 92$ & 1 & 1 & & 81 & $N$ & J 890523512-47 \\
\hline 1 & $10 / 1 / 92$ & 2 & 1 & & 80 & $N$ & \\
\hline 1 & $10 / 1 / 92$ & 3 & 9 & & 81 & $\mathrm{~N}$ & \\
\hline 9 & $10 / 1 / 92$ & 4 & 1 & & 81 & $\bar{Y}$ & \\
\hline 9 & $10 / 1 / 92$ & 5 & 1 & & 81 & $N$ & \\
\hline 1 & $10 / 1 / 92$ & 6 & 1 & & 81 & $\mathrm{~N}$ & \\
\hline 1 & $10 / 1 / 92$ & 7 & 1 & & 80 & $\mathrm{~N}$ & \\
\hline 1 & $10 / 1 / 92$ & 8 & 1 & & 77 & $N$ & \\
\hline 1 & $10 / 1 / 92$ & 9 & 1 & & 74 & $N$ & \\
\hline 9 & $10 / 1 / 92$ & 10 & 9 & & 73 & $\mathrm{~N}$ & \\
\hline 1 & $10 / 1 / 92$ & 11 & 1 & & 73 & $\mathbf{N}$ & \\
\hline 1 & $10 / 1 / 92$ & 12 & 1 & & 73 & $\bar{N}$ & \\
\hline 1 & $10 / 1 / 92$ & 13 & 1 & & 73 & $N$ & \\
\hline$\frac{1}{1}$ & $10 / 1 / 92$ & 14 & 1 & & 73 & $N$ & \\
\hline 1 & $10 / 8 / 92$ & 1 & 1 & & 83 & $Y$ & \\
\hline 1 & $10 / 8 / 92$ & 2 & 1 & & 82 & $\mathbf{N}$ & \\
\hline 1 & $10 / 8 / 92$ & 3 & 1 & & 83 & $N$ & \\
\hline 1 & $10 / 8 / 92$ & 4 & 1 & & 82 & $N$ & \\
\hline 1 & $10 / 8 / 92$ & 5 & 1 & & 83 & $\mathbf{N}$ & \\
\hline 1 & $10 / 8 / 92$ & 6 & 9 & & 83 & $\mathrm{~N}$ & \\
\hline 1 & $10 / 8 / 92$ & 7 & 9 & & 82 & $\bar{N}$ & \\
\hline 1 & $10 / 8 / 92$ & 8 & 1 & & 80 & $\mathbf{N}$ & \\
\hline 9 & $10 / 8 / 92$ & 9 & $\frac{1}{1}$ & & 80 & $\mathrm{~N}$ & \\
\hline 9 & $10 / 8 / 92$ & 10 & 9 & & 76 & $\bar{N}$ & \\
\hline 1 & $10 / 8 / 92$ & 11 & 1 & & 76 & $N$ & \\
\hline 1 & $10 / 8 / 92$ & 12 & 9 & & 75 & $\bar{N}$ & \\
\hline 1 & $10 / 8 / 92$ & 13 & 1 & & 75 & $N$ & \\
\hline 1 & $10 / 8 / 92$ & 14 & 1 & & 74 & $N$ & RETESTED \\
\hline 9 & $10 / 15 / 92$ & 9 & $\frac{1}{1}$ & & 81 & $N$ & $J 817-13-20-019$ \\
\hline 1 & $10 / 15 / 92$ & 2 & 9 & & 82 & $\mathrm{~N}$ & \\
\hline 9 & $10 / 15 / 92$ & 3 & 1 & & 83 & $N$ & \\
\hline 1 & $10 / 15 / 92$ & 4 & 1 & & 84 & $\mathrm{Y}$ & \\
\hline 9 & $10 / 15 / 92$ & 5 & 1 & & 84 & $\mathbf{N}$ & \\
\hline 1 & $10 / 15 / 92$ & 6 & 1 & & 83 & $N$ & \\
\hline 1 & $10 / 15 / 92$ & 7 & 9 & & 83 & $N$ & \\
\hline 1 & $10 / 15 / 92$ & 8 & 1 & & 80 & $\mathbf{N}$ & \\
\hline 1 & $10 / 15 / 92$ & 9 & 1 & & 76 & $\mathbf{N}$ & \\
\hline 1 & $10 / 15 / 92$ & 10 & 9 & & 75 & $\mathrm{~N}$ & \\
\hline 1 & $10 / 15 / 92$ & 11 & 9 & & 74 & $\mathrm{~N}$ & \\
\hline 1 & $10 / 15 / 92$ & 12 & 1 & & 74 & $\mathbf{N}$ & \\
\hline 9 & $10 / 15 / 92$ & 13 & 1 & & 75 & $\bar{N}$ & \\
\hline 9 & $10 / 15 / 92$ & 14 & 1 & & 74 & $\mathrm{~N}$ & \\
\hline 1 & $10 / 22 / 92$ & 1 & 1 & & 85 & $\mathbf{N}$ & J $817-13-20-019$ \\
\hline 1 & $10 / 22 / 92$ & 2 & 9 & & 86 & $\mathbf{N}$ & \\
\hline 1 & $10 / 22 / 92$ & 3 & 1 & & 86 & $N$ & \\
\hline 9 & $10 / 22 / 92$ & 4 & 1 &. & 87 & $\bar{Y}$ & \\
\hline$\frac{9}{1}$ & $10 / 22 / 92$ & 5 & 1 & & 86 & $\mathbf{N}$ & \\
\hline
\end{tabular}

Data obtained from WHC Surveillance Analysis Computer System (SACS), February 24, 1994. 


\begin{tabular}{|c|c|c|c|c|c|c|c|}
\hline \multicolumn{8}{|c|}{ TANK 241-S-112 } \\
\hline \multicolumn{8}{|c|}{ THERMOCOUPLE DATA } \\
\hline TREE \# & DATE & THERMO \# & TREE SET \# & TEMP C & TEMP F & $Y=M A X$ TEMP & COMMENTS \\
\hline 1 & $10 / 22 / 92$ & 6 & 1 & & 86 & $\mathrm{~N}$ & \\
\hline 1 & $10 / 22 / 92$ & 7 & 1 & & 85 & $N$ & \\
\hline 1 & $10 / 22 / 92$ & 8 & 1 & & 82 & $\mathrm{~N}$ & \\
\hline 1 & $10 / 22 / 92$ & 9 & 1 & & 78 & $\mathrm{~N}$ & \\
\hline 1 & $10 / 22 / 92$ & 10 & 1 & & 77 & $\bar{N}$ & \\
\hline 1 & $10 / 22 / 92$ & 11 & 1 & & 77 & $\mathbf{N}$ & \\
\hline 1 & $10 / 22 / 92$ & 12 & 1 & & 77 & $N$ & \\
\hline 1 & $10 / 22 / 92$ & 13 & 1 & & 77 & $\mathbf{N}$ & \\
\hline 1 & $10 / 22 / 92$ & 14 & 1 & & 76 & $N$ & \\
\hline 1 & $10 / 29 / 92$ & 1 & 1 & & 84 & $N$ & J 819-20.13-019 \\
\hline 1 & $10 / 29 / 92$ & 2 & 1 & & 86 & $\mathbf{N}$ & \\
\hline 1 & $10 / 29 / 92$ & 3 & 1 & & 86 & $\mathbf{N}$ & \\
\hline 1 & $10 / 29 / 92$ & 4 & 1 & & 85 & $\mathbf{N}$ & \\
\hline 1 & $10 / 29 / 92$ & 5 & 1 & & 86 & $\mathbf{N}$ & \\
\hline 1 & $10 / 29 / 92$ & 6 & 1 & & 84 & $\mathbf{N}$ & \\
\hline 1 & $10 / 29 / 92$ & 7 & 1 & & 84 & $\mathbf{N}$ & \\
\hline 1 & $10 / 29 / 92$ & 8 & 1 & & 85 & $\mathrm{~N}$ & \\
\hline 1 & $10 / 29 / 92$ & 9 & 1 & & 84 & $N$ & \\
\hline 1 & $10 / 29 / 92$ & 10 & 1 & & 86 & $N$ & \\
\hline 1 & $10 / 29 / 92$ & 11 & 1 & & 87 & $\bar{N}$ & \\
\hline 1 & $10 / 29 / 92$ & 13 & 1 & & 77 & $\mathbf{N}$ & \\
\hline 1 & $10 / 29 / 92$ & 14 & 1 & & 86 & $N$ & \\
\hline 1 & $11 / 5 / 92$ & 1 & 1 & & 85 & $\mathbf{N}$ & J 817.13-20-019 \\
\hline 1 & $11 / 5 / 92$ & 2 & 1 & & 86 & $\mathbf{N}$ & \\
\hline 1 & $11 / 5 / 92$ & 3 & 1 & & 85 & $\mathbf{N}$ & \\
\hline 1 & $11 / 5 / 92$ & 4 & 1 & & 86 & $\mathbf{N}$ & \\
\hline 1 & $11 / 5 / 92$ & 5 & 1 & & 86 & $\overline{\mathbf{N}}$ & \\
\hline 1 & $11 / 5 / 92$ & 6 & 1 & & 89 & $\bar{Y}$ & \\
\hline 1 & $11 / 5 / 92$ & 7 & 1 & & 84 & $\mathbf{N}$ & \\
\hline 1 & $11 / 5 / 92$ & 8 & 1 & & 81 & $N$ & \\
\hline 9 & $11 / 5 / 92$ & 9 & 1 & & 76 & $\mathrm{~N}$ & \\
\hline 1 & $11 / 5 / 92$ & 10 & 1 & & 75 & $\bar{N}$ & \\
\hline 1 & $11 / 5 / 92$ & 11 & 1 & & 75 & $\mathbf{N}$ & \\
\hline 1 & $11 / 5 / 92$ & 12 & 1 & & 75 & $\mathbf{N}$ & \\
\hline 1 & $11 / 5 / 92$ & 13 & 1 & & 75 & $\mathbf{N}$ & \\
\hline 1 & $11 / 5 / 92$ & 14 & 1 & & 75 & $\mathbf{N}$ & \\
\hline 1 & $11 / 7 / 92$ & 1 & 1 & & 85 & $\mathbf{N}$ & $\mathrm{J} 817-13-20-019293$ \\
\hline 1 & $11 / 7 / 92$ & 2 & 1 & & 86 & $\mathbf{N}$ & \\
\hline 1 & $11 / 7 / 92$ & 4 & 1 & & 88 & $\mathbf{Y}$ & \\
\hline 1 & $11 / 7 / 92$ & 6 & 1 & & 87 & $\mathrm{~N}$ & \\
\hline 1 & $11 / 7 / 92$ & 7 & $\frac{1}{1}$ & & 85 & $\mathbf{N}$ & \\
\hline 1 & $11 / 7 / 92$ & 8 & 1 & & 82 & $\mathbf{N}$ & \\
\hline 1 & $11 / 7 / 92$ & 9 & 1 & & 77 & $\mathbf{N}$ & \\
\hline 1 & $11 / 7 / 92$ & 10 & 1 & & 76 & $N$ & \\
\hline 1 & $11 / 7 / 92$ & 11 & 1 & & 76 & $N$ & \\
\hline 1 & $11 / 7 / 92$ & 12 & 1 & & 76 & $N$ & \\
\hline 1 & $11 / 12 / 92$ & 1 & 9 & & 88 & $\bar{N}$ & $817-13-20-019$ \\
\hline 1 & $11 / 12 / 92$ & 2 & 1 & & 88 & $\mathrm{~N}$ & \\
\hline 1 & $11 / 12 / 92$ & 3 & 1 & & 89 & $\mathbf{Y}$ & \\
\hline 1 & $11 / 12 / 92$ & 4 & 1 & & 88 & $\mathbf{N}$ & \\
\hline 1 & $11 / 12 / 92$ & 5 & $\frac{1}{1}$ & & 89 & $\mathbf{N}$ & \\
\hline 1 & $11 / 12 / 92$ & 6 & 1 & & 88 & $\mathbf{N}$ & \\
\hline 1 & $11 / 12 / 92$ & 7 & 1 & & 85 & $\mathbf{N}$ & \\
\hline 1 & $11 / 12 / 92$ & 8 & 1 & & 83 & $\mathbf{N}$ & \\
\hline 1 & $11 / 12 / 92$ & 9 & 1 & & 79 & $\mathbf{N}$ & \\
\hline$\frac{1}{1}$ & $11 / 12 / 92$ & 10 & $\frac{1}{1}$ & & 79 & $\mathbf{N}$ & \\
\hline 1 & $11 / 12 / 92$ & 11 & 1 & & 78 & $\mathbf{N}$ & \\
\hline 1 & $11 / 12 / 92$ & 12 & 1 & & 78 & $\mathbf{N}$ & \\
\hline 1 & $11 / 12 / 92$ & 13 & 1 & & 78 & $\mathbf{N}$ & \\
\hline 1 & $11 / 12 / 92$ & 14 & 1 & & 78 & $\mathbf{N}$ & \\
\hline 1 & $11 / 24 / 92$ & 1 & 1 & & 83 & $\mathbf{N}$ & J $817-13 \cdot 20-019$ \\
\hline 1 & $11 / 24 / 92$ & 2 & 1 & & 84 & $\mathbf{N}$ & \\
\hline 1 & $11 / 24 / 92$ & 3 & 1 & & 85 & $Y$ & \\
\hline 1 & $11 / 24 / 92$ & 4 & 1 & & 85 & $\mathbf{N}$ & \\
\hline 1 & $11 / 24 / 92$ & 6 & 1 & & 85 & $\mathbf{N}$ & \\
\hline
\end{tabular}

Data obtained from WHC Surveillance Analysis Computer System (SACS), February 24, 1994. 
WHC-SD-WM-ER-323, Rev. O

\begin{tabular}{|c|c|c|c|c|c|c|c|}
\hline \multicolumn{8}{|c|}{ TANK 241-S-112 } \\
\hline \multicolumn{8}{|c|}{ THERMOCOUPLE DATA } \\
\hline TREE \# & DATE & THERMO \# & TREE SET \# & TEMP C & TEMP F & $\mathbf{Y}=$ MAX TEMP & COMMENTS \\
\hline 1 & $11 / 24 / 92$ & 7 & 1 & & 84 & $\mathrm{~N}$ & \\
\hline 1 & $11 / 24 / 92$ & 8 & 1 & & 80 & $\mathbf{N}$ & \\
\hline 1 & $11 / 24 / 92$ & 9 & 1 & & 74 & $\mathrm{~N}$ & \\
\hline 1 & $11 / 24 / 92$ & 10 & 1 & & 72 & $\mathrm{~N}$ & \\
\hline 1 & $11 / 24 / 92$ & 11 & 1 & & 73 & $\mathbf{N}$ & \\
\hline 1 & $11 / 24 / 92$ & 12 & 1 & & 73 & $\mathrm{~N}$ & \\
\hline 1 & $11 / 24 / 92$ & 13 & 1 & & 73 & $\mathbf{N}$ & \\
\hline 1 & $11 / 24 / 92$ & 14 & 1 & & 72 & $\mathrm{~N}$ & \\
\hline 1 & $12 / 3 / 92$ & 1 & 1 & & 85 & $\mathbf{N}$ & $817-13-20-019$ \\
\hline 1 & $12 / 3 / 92$ & 2 & $\frac{1}{1}$ & & 86 & $\mathbf{N}$ & \\
\hline 1 & $12 / 3 / 92$ & 3 & 1 & & 87 & $\bar{Y}$ & \\
\hline 1 & $12 / 3 / 92$ & 4 & 1 & & 87 & $\mathbf{Y}$ & \\
\hline 1 & $12 / 3 / 92$ & 5 & 1 & & 86 & $\mathrm{~N}$ & \\
\hline 1 & $12 / 3 / 92$ & 6 & 1 & & 87 & $\mathbf{N}$ & \\
\hline 1 & $12 / 3 / 92$ & 7 & 1 & & 85 & $\bar{N}$ & \\
\hline 1 & $12 / 3 / 92$ & 8 & 1 & & 80 & $\mathrm{~N}$ & \\
\hline 1 & $12 / 3 / 92$ & 9 & 1 & & 74 & $\mathbf{N}$ & \\
\hline 1 & $12 / 3 / 92$ & 10 & 1 & & 72 & $\mathrm{~N}$ & \\
\hline 1 & $12 / 3 / 92$ & 11 & 1 & & 72 & $\mathbf{N}$ & \\
\hline 1 & $12 / 3 / 92$ & 12 & 1 & & 72 & $\mathrm{~N}$ & \\
\hline 1 & $12 / 3 / 92$ & 13 & 1 & & 72 & $\mathrm{~N}$ & \\
\hline 1 & $12 / 3 / 92$ & 14 & 1 & & 71 & $N$ & \\
\hline 1 & $12 / 10 / 92$ & 1 & 1 & & 89 & $\mathbf{N}$ & $817-13-20-019$ \\
\hline 9 & $12 / 10 / 92$ & 2 & 1 & & 90 & $Y$ & \\
\hline 1 & $12 / 10 / 92$ & 3 & 1 & & 90 & $\bar{N}$ & \\
\hline 1 & $12 / 10 / 92$ & 4 & 1 & & 90 & $N$ & \\
\hline 1 & $12 / 10 / 92$ & 5 & 1 & & 89 & $\mathrm{~N}$ & \\
\hline 1 & $12 / 10 / 92$ & 6 & 1 & & 89 & $\mathbf{N}$ & \\
\hline 1 & $12 / 10 / 92$ & 7 & 1 & & 88 & $\mathbf{N}$ & \\
\hline 1 & $12 / 10 / 92$ & 8 & 1 & & 83 & $\mathrm{~N}$ & \\
\hline 1 & $12 / 10 / 92$ & 9 & 1 & & 76 & $\mathrm{~N}$ & \\
\hline 1 & $12 / 10 / 92$ & 10 & 1 & & 78 & $\mathbf{N}$ & \\
\hline 1 & $12 / 10 / 92$ & 11 & 1 & & 75 & $\mathrm{~N}$ & \\
\hline 1 & $12 / 10 / 92$ & 12 & 1 & & 75 & $\bar{N}$ & \\
\hline 1 & $12 / 10 / 92$ & 13 & 1 & & 74 & $\mathrm{~N}$ & \\
\hline 1 & $12 / 10 / 92$ & 14 & 9 & & 73 & $\mathbf{N}$ & \\
\hline 1 & $12 / 14 / 92$ & 1 & 1 & & & $\mathbf{N}$ & $\begin{array}{c}\text { DISCREP\#S\&DA-92-503 NO RDGS SINCE } \\
11 / 24 / 92\end{array}$ \\
\hline 1 & $12 / 17 / 92$ & 9 & 1 & & 85 & $N$ & $817-13-20-019$ \\
\hline 1 & $12 / 17 / 92$ & 2 & 1 & & 86 & $\mathbf{N}$ & \\
\hline 1 & $12 / 17 / 92$ & 3 & 1 & & 87 & $\mathbf{Y}$ & \\
\hline 1 & $12 / 17 / 92$ & 4 & 1 & & 87 & $\mathbf{N}$ & \\
\hline 1 & $12 / 17 / 92$ & 5 & 1 & & 86 & $\mathbf{N}$ & \\
\hline 1 & $12 / 17 / 92$ & 6 & 1 & & 87 & $\mathrm{~N}$ & \\
\hline 1 & $12 / 17 / 92$ & 7 & 1 & & 87 & $\mathbf{N}$ & \\
\hline 1 & $12 / 17 / 92$ & 8 & 1 & & 81 & $\mathbf{N}$ & \\
\hline 9 & $12 / 17 / 92$ & 9 & 9 & & 75 & $\bar{N}$ & \\
\hline 9 & $12 / 17 / 92$ & 10 & 1 & & 73 & $N$ & \\
\hline 9 & $12 / 17 / 92$ & 11 & 1 & & 73 & $\mathrm{~N}$ & \\
\hline 1 & $12 / 17 / 92$ & 12 & 1 & & 73 & $N$ & \\
\hline 9 & $12 / 17 / 92$ & 13 & 1 & & 73 & $\mathbf{N}$ & \\
\hline 1 & $12 / 17 / 92$ & 14 & 9 & & 71 & $\mathbf{N}$ & \\
\hline 1 & $12 / 22 / 92$ & 1 & 9 & & 86 & $\mathbf{N}$ & $J 817-13-20-019$ \\
\hline 1 & $12 / 22 / 92$ & 2 & 1 & & 87 & $\mathbf{N}$ & \\
\hline 1 & $12 / 22 / 92$ & 3 & 1 & & 88 & $\mathbf{Y}$ & \\
\hline 1 & $12 / 22 / 92$ & 4 & 1 & & 88 & $\bar{N}$ & \\
\hline 1 & $12 / 22 / 92$ & 5 & 1 & & 86 & $\mathrm{~N}$ & \\
\hline 1 & $12 / 22 / 92$ & 6 & 1 & & 85 & $N$ & \\
\hline 1 & $12 / 22 / 92$ & 7 & 1 & & 84 & $\mathrm{~N}$ & \\
\hline 1 & $12 / 22 / 92$ & 8 & $\frac{1}{1}$ & & 80 & $\mathrm{~N}$ & \\
\hline 1 & $12 / 22 / 92$ & 9 & 1 & & 73 & $\mathbf{N}$ & \\
\hline 1 & $12 / 22 / 92$ & 10 & 1 & & 79 & $N$ & \\
\hline 1 & $12 / 22 / 92$ & 11 & 1 & & 70 & $N$ & \\
\hline 1 & $12 / 22 / 92$ & 12 & $\frac{1}{1}$ & & 70 & $\mathbf{N}$ & \\
\hline
\end{tabular}

Data obtained from WHC Surveillance Analysis Computer System (SACS). February 24, 1994. 


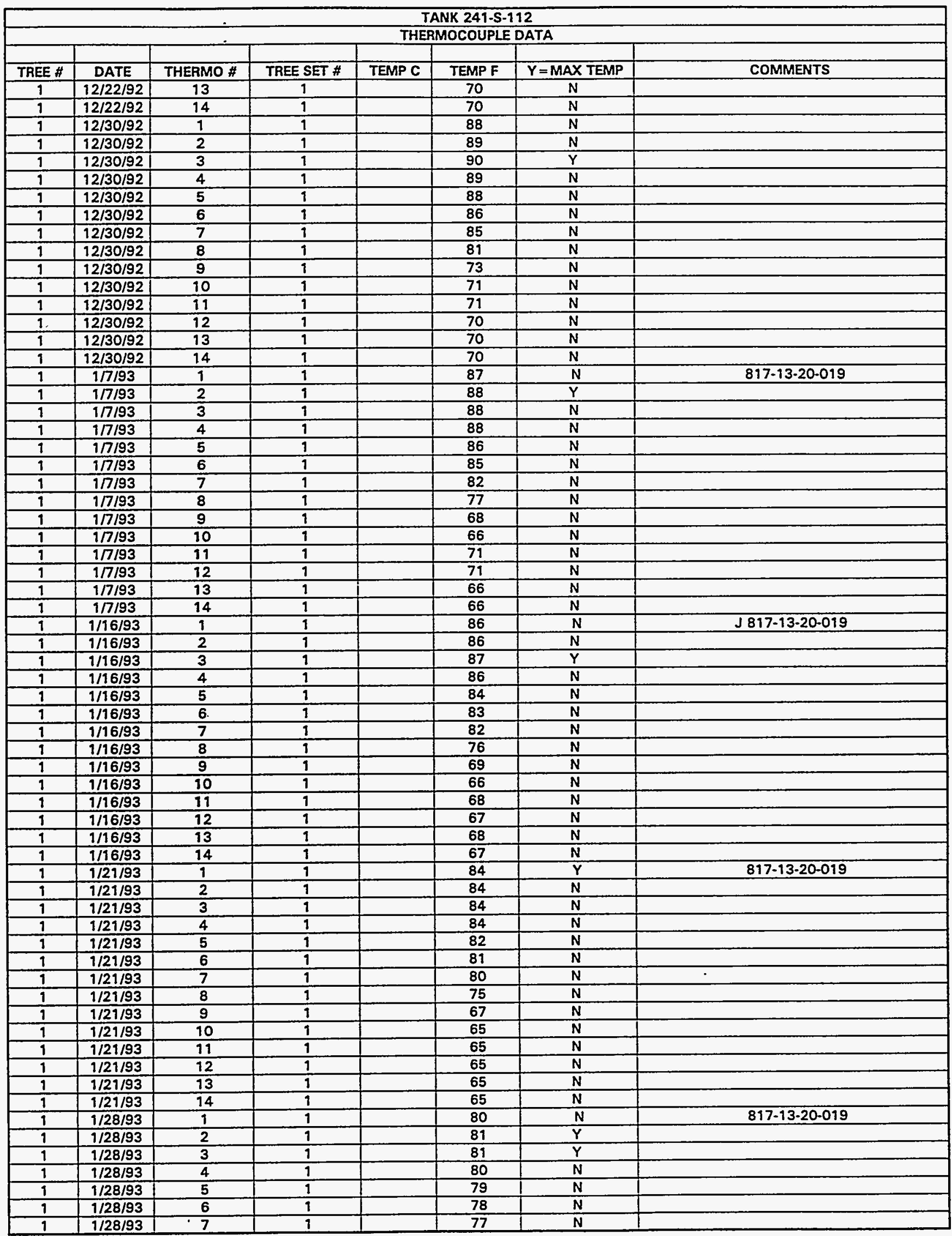

Data obtained from WHC Surveillance Analysis Computer System (SACS), February 24, 1994. 


\begin{tabular}{|c|c|c|c|c|c|c|c|}
\hline \multicolumn{8}{|c|}{ TANK 241.S-112 } \\
\hline \multicolumn{8}{|c|}{ THERMOCOUPLE DATA } \\
\hline & & & & & & & \\
\hline TREE \# & DATE & THERMO \# & TREE SET \# & TEMP C & TEMP F & $Y=M A X$ TEMP & COMMENTS \\
\hline 1 & $1 / 28 / 93$ & 8 & 1 & & 72 & $N$ & \\
\hline 1 & $1 / 28 / 93$ & 9 & 1 & & 65 & $N$ & \\
\hline 1 & $1 / 28 / 93$ & 10 & 1 & & 64 & $\mathrm{~N}$ & \\
\hline 1 & $1 / 28 / 93$ & 91 & 1 & & 64 & $N$ & \\
\hline 1 & $1 / 28 / 93$ & $\overline{12}$ & 1 & & 63 & $N$ & \\
\hline 1 & $1 / 28 / 93$ & 13 & 1 & & 63 & $\mathrm{~N}$ & \\
\hline 1 & $1 / 28 / 93$ & 14 & 1 & & 63 & $\mathbf{N}$ & \\
\hline 1 & $2 / 3 / 93$ & 1 & 1 & & 84 & $\mathrm{~N}$ & $817-13-20-019$ \\
\hline 1 & $2 / 3 / 93$ & 2 & 1 & & 86 & $Y$ & \\
\hline 1 & $2 / 3 / 93$ & 3 & 1 & & 86 & $\mathrm{~N}$ & \\
\hline 1 & $2 / 3 / 93$ & 4 & 1 & & 85 & $\bar{N}$ & \\
\hline 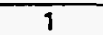 & $2 / 3 / 93$ & 5 & 1 & & 84 & $N$ & \\
\hline 1 & $2 / 3 / 93$ & 6 & 1 & & 84 & $\mathrm{~N}$ & \\
\hline 1 & $2 / 3 / 93$ & 7 & 1 & & 82 & $\mathrm{~N}$ & \\
\hline 1 & $2 / 3 / 93$ & 8 & 9 & & 76 & $\bar{N}$ & \\
\hline 1 & $2 / 3 / 93$ & 9 & 1 & & 68 & $\mathrm{~N}$ & \\
\hline 1 & $2 / 3 / 93$ & 10 & 1 & & 65 & $\bar{N}$ & \\
\hline 1 & $2 / 3 / 93$ & 11 & 1 & & 64 & $\overline{\mathrm{N}}$ & \\
\hline 1 & $2 / 3 / 93$ & 12 & 1 & & 64 & $\bar{N}$ & \\
\hline 1 & $2 / 3 / 93$ & 13 & 1 & & 64 & $N$ & \\
\hline 1 & $2 / 3 / 93$ & 14 & 1 & & 64 & $N$ & \\
\hline 1 & $2 / 25 / 93$ & 1 & 1 & & 85.9 & $\bar{Y}$ & $\sqrt{817-13-55-008}$ \\
\hline 1 & $2 / 25 / 93$ & 2 & 1 & & 85.6 & $\bar{N}$ & \\
\hline 1 & $2 / 25 / 93$ & 3 & 1 & & 86.1 & $\mathrm{~N}$ & \\
\hline 1 & $2 / 25 / 93$ & 4 & $\frac{1}{1}$ & & 85.6 & $N$ & \\
\hline 1 & $2 / 25 / 93$ & 5 & 1 & & 83.5 & $N$ & \\
\hline 1 & $2 / 25 / 93$ & 6 & 1 & & 83 & $\bar{N}$ & \\
\hline 1 & $2 / 25 / 93$ & 7 & 1 & & 81 & $\bar{N}$ & \\
\hline 1 & $2 / 25 / 93$ & 8 & 1 & & 74.5 & $N$ & \\
\hline 1 & $2 / 25 / 93$ & 9 & 1 & & 66 & $N$ & \\
\hline 1 & $2 / 25 / 93$ & 10 & 1 & & 63.4 & $\mathrm{~N}$ & \\
\hline 1 & $2 / 25 / 93$ & 11 & 1 & & 63.3 & $\mathrm{~N}$ & \\
\hline 1 & $2 / 25 / 93$ & 12 & 9 & & 63.7 & $\bar{N}$ & \\
\hline 1 & $2 / 25 / 93$ & 13 & 1 & & 63.6 & $\mathrm{~N}$ & \\
\hline 1 & $2 / 25 / 93$ & 14 & 1 & & 63.6 & $\bar{N}$ & \\
\hline 1 & $3 / 4 / 93$ & 1 & 1 & & 82 & $N$ & OMEGA $817-13-20-019$ \\
\hline 1 & $3 / 4 / 93$ & 2 & 1 & & 85 & $\bar{Y}$ & \\
\hline 9 & $3 / 4 / 93$ & 3 & 1 & & 85 & $\bar{N}$ & \\
\hline 1 & $3 / 4 / 93$ & 4 & 1 & & 83 & $N$ & \\
\hline 1 & $3 / 4 / 93$ & 5 & 1 & & 77 & $N$ & \\
\hline 1 & $3 / 4 / 93$ & 6 & 1 & & 83 & $\bar{N}$ & \\
\hline 1 & $3 / 4 / 93$ & 7 & 1 & & 89 & $\mathrm{~N}$ & \\
\hline 1 & $3 / 4 / 93$ & 8 & 1 & & 74 & $N$ & \\
\hline 1 & $3 / 4 / 93$ & 9 & 1 & & 65 & $\mathrm{~N}$ & \\
\hline 1 & $3 / 4 / 93$ & 10 & 1 & & 62 & $N$ & \\
\hline 1 & $3 / 4 / 93$ & 17 & 1 & & 63 & $\mathrm{~N}$ & \\
\hline 1 & $3 / 4 / 93$ & 12 & $\frac{1}{1}$ & & 63 & $\mathrm{~N}$ & \\
\hline 1 & $3 / 4 / 93$ & 93 & 1 & & 66 & $\bar{N}$ & \\
\hline 1 & $3 / 4 / 93$ & 14 & 1 & & 61 & $\bar{N}$ & \\
\hline 1 & $3 / 11 / 93$ & 1 & 1 & & 84 & $\bar{N}$ & J $817-13-20-019$ \\
\hline 1 & $3 / 11 / 93$ & 2 & 1 & & 85 & $Y$ & \\
\hline 1 & $3 / 11 / 93$ & 3 & 1 & & 85 & $\mathrm{~N}$ & \\
\hline 1 & $3 / 11 / 93$ & 4 & 1 & & 85 & $N$ & \\
\hline 1 & $3 / 11 / 93$ & 5 & 1 & & 84 & $\mathrm{~N}$ & \\
\hline 1 & $3 / 11 / 93$ & 6 & 1 & & 84 & $N$ & \\
\hline 1 & $3 / 11 / 93$ & 7 & 1 & & 89 & $\bar{N}$ & \\
\hline 1 & $3 / 11 / 93$ & 8 & 1 & & 74 & $\mathrm{~N}$ & \\
\hline 9 & $3 / 11 / 93$ & 9 & 1 & & 65 & $\mathrm{~N}$ & \\
\hline 1 & $3 / 11 / 93$ & 10 & 1 & & 62 & $\bar{N}$ & \\
\hline 1 & $3 / 11 / 93$ & 11 & 1 & & 63 & $\mathrm{~N}$ & \\
\hline 1 & $3 / 11 / 93$ & 12 & 1 & & 62 & $\bar{N}$ & \\
\hline 1 & $3 / 11 / 93$ & 13 & $\overline{1}$ & & 62 & $\mathbf{N}$ & \\
\hline 1 & $3 / 11 / 93$ & 14 & 1 & & 62 & $\mathbf{N}$ & \\
\hline$\frac{1}{1}$ & $3 / 18 / 93$ & 1 & 1 & & 83 & $N$ & $817-13-20-019$ \\
\hline 1 & $3 / 18 / 93$ & 2 & 1 & & 83 & $\mathbf{N}$ & \\
\hline
\end{tabular}

Data obtained from WHC Surveillance Analysis Computer System (SACS), February 24, 1994. 


\begin{tabular}{|c|c|c|c|c|c|c|c|}
\hline \multicolumn{8}{|c|}{ TANK 241-S-112 } \\
\hline \multicolumn{8}{|c|}{ THERMOCOUPLE DATA } \\
\hline & & THFRMO \# & TAEE SET \# & TEMP C & TEMP F & $\bar{Y}=$ MAX TEMP & COMMENTS \\
\hline$\frac{\text { TREE \# }}{1}$ & $\frac{\text { DATE }}{3 / 18 / 93}$ & $\frac{\text { THERMO \# }}{3}$ & $\frac{1 \text { IHEE SEI H }}{1}$ & acini co & 84 & $Y$ & \\
\hline 1 & $3 / 18 / 93$ & 4 & 1 & & 83 & $\mathrm{~N}$ & \\
\hline 1 & $3 / 18 / 93$ & 5 & 1 & & 82 & $\mathbf{N}$ & \\
\hline 1 & $3 / 18 / 93$ & 6 & 1 & & 81 & $\mathbf{N}$ & \\
\hline$\overline{1}$ & $3 / 18 / 93$ & 7 & 1 & & 77 & $N$ & \\
\hline 1 & $3 / 18 / 93$ & 8 & 1 & & 70 & $N$ & \\
\hline 1 & $3 / 18 / 93$ & 9 & 1 & & 61 & $\mathrm{~N}$ & \\
\hline 1 & $3 / 18 / 93$ & 10 & 1 & & 58 & $\mathbf{N}$ & \\
\hline 1 & $3 / 18 / 93$ & 11 & 1 & & 60 & $\mathbf{N}$ & \\
\hline 9 & $3 / 18 / 93$ & 12 & 1 & & 59 & $\mathbf{N}$ & \\
\hline 1 & $3 / 18 / 93$ & 13 & 1 & & 60 & $\bar{N}$ & \\
\hline 1 & $3 / 18 / 93$ & 14 & 1 & & 61 & $N$ & \\
\hline 1 & $3 / 25 / 93$ & 1 & 1 & & 86 & $Y$ & $\mathrm{~J} 817-13-20-019$ \\
\hline 1 & $3 / 25 / 93$ & 2 & 1 & & 86 & $\mathrm{~N}$ & \\
\hline 1 & $3 / 25 / 93$ & 3 & 1 & & 86 & $N$ & \\
\hline 1 & $3 / 25 / 93$ & 4 & 1 & & 86 & $N$ & \\
\hline 1 & $3 / 25 / 93$ & 5 & 1 & & 84 & $N$ & \\
\hline 1 & $3 / 25 / 93$ & 6 & 1 & & 84 & $N$ & \\
\hline 1 & $3 / 25 / 93$ & 7 & 1 & & 78 & $\mathrm{~N}$ & \\
\hline 1 & $3 / 25 / 93$ & 8 & 1 & & 72 & $N$ & \\
\hline 1 & $3 / 25 / 93$ & 9 & 1 & & 63 & $\mathrm{~N}$ & \\
\hline 1 & $3 / 25 / 93$ & 10 & 1 & & 60 & $N$ & \\
\hline 1 & $3 / 25 / 93$ & 11 & 1 & & 60 & $N$ & \\
\hline 1 & $3 / 25 / 93$ & 12 & 1 & & 54 & $\mathrm{~N}$ & \\
\hline 1 & $3 / 25 / 93$ & 13 & 1 & & 55 & $\mathbf{N}$ & \\
\hline 1 & $3 / 25 / 93$ & 14 & 1 & & 60 & $\mathbf{N}$ & \\
\hline 1 & $4 / 1 / 93$ & 1 & 1 & & 85 & $N$ & TYPE J \\
\hline 1 & $4 / 1 / 93$ & 2 & 1 & & 85 & $\mathrm{~N}$ & \\
\hline 1 & $4 / 9 / 93$ & 3 & 1 & & 86 & $Y$ & \\
\hline 1 & $4 / 1 / 93$ & 4 & 1 & & 85 & $N$ & \\
\hline 1 & $4 / 1 / 93$ & 5 & 1 & & 83 & $N$ & \\
\hline 1 & $4 / 1 / 93$ & 6 & 1 & & 82 & $\mathbf{N}$ & \\
\hline 1 & $4 / 1 / 93$ & 7 & 1 & & 79 & $\mathrm{~N}$ & \\
\hline 1 & $4 / 1 / 93$ & 8 & 1 & & 73 & $N$ & \\
\hline 1 & $4 / 1 / 93$ & 9 & 9 & & 64 & $\mathbf{N}$ & \\
\hline 1 & $4 / 1 / 93$ & 10 & 1 & & 62 & $N$ & \\
\hline 1 & $4 / 1 / 93$ & 11 & 1 & & 62 & $N$ & \\
\hline 1 & $4 / 1 / 93$ & 12 & 9 & & 62 & $N$ & \\
\hline 1 & $4 / 1 / 93$ & 13 & 1 & & 61 & $\mathbf{N}$ & \\
\hline 1 & $4 / 1 / 93$ & 14 & 1 & & 61 & $N$ & \\
\hline 1 & $4 / 8 / 93$ & 1 & 1 & & 84 & $\mathbf{N}$ & $J 817-13-20-019$ \\
\hline 1 & $4 / 8 / 93$ & 2 & 1 & & 84 & $\mathbf{N}$ & \\
\hline 1 & $4 / 8 / 93$ & 3 & 1 & & 85 & $\bar{Y}$ & \\
\hline 1 & $4 / 8 / 93$ & 4 & 1 & & 84 & $\bar{N}$ & 1 \\
\hline 1 & $4 / 8 / 93$ & 5 & 1 & & 83 & $\mathrm{~N}$ & \\
\hline 1 & $4 / 8 / 93$ & 6 & 1 & & 82 & $\mathbf{N}$ & \\
\hline 1 & $4 / 8 / 93$ & 7 & 1 & & 79 & $\mathbf{N}$ & \\
\hline 1 & $4 / 8 / 93$ & 8 & 1 & & 72 & $\mathbf{N}$ & \\
\hline 1 & $4 / 8 / 93$ & 9 & 1 & & 64 & $N$ & \\
\hline 1 & $4 / 8 / 93$ & 10 & 1 & & 62 & $\mathbf{N}$ & \\
\hline 1 & $4 / 8 / 93$ & 11 & 1 & & 61 & $\mathbf{N}$ & \\
\hline 1 & $4 / 8 / 93$ & 12 & 1 & & 61 & $N$ & \\
\hline 1 & $4 / 8 / 93$ & 13 & $i$ & & 61 & $\mathbf{N}$ & \\
\hline 1 & $4 / 8 / 93$ & 14 & 1 & & 61 & $N$ & \\
\hline 1 & $4 / 15 / 93$ & 1 & 1 & & 83 & $\mathbf{N}$ & $\mathrm{J} 910126143-66$ \\
\hline 1 & $4 / 15 / 93$ & 2 & 1 & & 84 & $Y$ & \\
\hline 1 & $4 / 15 / 93$ & 4 & 9 & & 84 & $\mathbf{N}$ & \\
\hline 1 & $4 / 15 / 93$ & 6 & 1 & & 81 & $\mathbf{N}$ & \\
\hline 1 & $4 / 15 / 93$ & 7 & 1 & & 78 & $N$ & \\
\hline 1 & $4 / 15 / 93$ & 8 & 1 & & 71 & $\mathbf{N}$ & \\
\hline 1 & $4 / 15 / 93$ & 9 & 1 & & 64 & $\mathbf{N}$ & \\
\hline 1 & $4 / 15 / 93$ & 10 & 1 & & 61 & $\mathbf{N}$ & \\
\hline 1 & $4 / 15 / 93$ & 11 & 1 & & 61 & $\mathbf{N}$ & \\
\hline 1 & $4 / 15 / 93$ & 12 & 1 & & 61 & $\mathrm{~N}$ & \\
\hline 1 & $4 / 15 / 93$ & 14 & 1 & & 61 & $\therefore N$ & \\
\hline
\end{tabular}

Data obtained from WHC Surveillance Analysis Computer System (SACS), February 24, 1994. 


\begin{tabular}{|c|c|c|c|c|c|c|c|}
\hline \multicolumn{8}{|c|}{ TANK 241-S-112 } \\
\hline \multicolumn{8}{|c|}{ THERMOCOUPLE DATA } \\
\hline TREE \# & DATE & THERMO \# & TREE SET \# & TEMP $\mathrm{C}$ & TEMP F & $Y=M A X$ TEMP & COMMENTS \\
\hline 1 & $4 / 22 / 93$ & 1 & 1 & & 78 & $\mathrm{Y}$ & TYPE J $817-13-20.019$ \\
\hline 1 & $4 / 22 / 93$ & 2 & 1 & & 78 & $N$ & \\
\hline 9 & $4 / 22 / 93$ & 3 & 1 & & 78 & $\mathbf{N}$ & \\
\hline 9 & $4 / 22 / 93$ & 4 & 1 & & 78 & $N$ & \\
\hline 1 & $4 / 22 / 93$ & 5 & 1 & & 76 & $\mathrm{~N}$ & \\
\hline 1 & $4 / 22 / 93$ & 6 & 1 & & 75 & $\bar{N}$ & \\
\hline 1 & $4 / 22 / 93$ & 7 & 1 & & 72 & $N$ & \\
\hline 1 & $4 / 22 / 93$ & 8 & 1 & & 65 & $\mathbf{N}$ & \\
\hline 1 & $4 / 22 / 93$ & 9 & 1 & & 56 & $N$ & \\
\hline 9 & $4 / 22 / 93$ & 10 & 1 & & 54 & $\bar{N}$ & \\
\hline 1 & $4 / 22 / 93$ & 11 & 1 & & 54 & $\mathbf{N}$ & \\
\hline 1 & $4 / 22 / 93$ & 12 & 1 & & 54 & $\mathrm{~N}$ & \\
\hline 1 & $4 / 22 / 93$ & 13 & $i$ & & 54 & $N$ & \\
\hline 1 & $4 / 22 / 93$ & 14 & $\overline{1}$ & & 54 & $\mathrm{~N}$ & \\
\hline 1 & $4 / 29 / 93$ & 1 & 1 & & 81 & $Y$ & $\mathrm{~J} 8971320019$ \\
\hline 1 & $4 / 29 / 93$ & 2 & 1 & & 82 & $\mathbf{N}$ & \\
\hline 1 & $4 / 29 / 93$ & 3 & 1 & & 83 & $\bar{Y}$ & \\
\hline 1 & $4 / 29 / 93$ & 4 & 1 & & 82 & $\mathrm{~N}$ & \\
\hline 1 & $4 / 29 / 93$ & 5 & 1 & & 81 & $N$ & \\
\hline 1 & $4 / 29 / 93$ & 6 & 1 & & 79 & $\mathrm{~N}$ & \\
\hline 1 & $4 / 29 / 93$ & 7 & 1 & & 77 & $N$ & \\
\hline 1 & $4 / 29 / 93$ & 8 & 1 & & 70 & $\mathbf{N}$ & \\
\hline 1 & $4 / 29 / 93$ & 9 & 1 & & 63 & $\mathbf{N}$ & \\
\hline 1 & $4 / 29 / 93$ & 10 & 1 & & 60 & $\mathrm{~N}$ & \\
\hline 9 & $4 / 29 / 93$ & 19 & 1 & & 60 & $\mathbf{N}$ & \\
\hline 1 & $4 / 29 / 93$ & 12 & 1 & & 60 & $\mathrm{~N}$ & \\
\hline 1 & $4 / 29 / 93$ & 13 & $\frac{1}{1}$ & & 60 & $\mathbf{N}$ & \\
\hline 1 & $4 / 29 / 93$ & 14 & 1 & & 61 & $N$ & \\
\hline 1 & $5 / 6 / 93$ & 1 & 1 & & 82 & $\mathrm{~N}$ & \\
\hline 1 & $5 / 6 / 93$ & 2 & 1 & & 83 & $\mathbf{N}$ & \\
\hline 1 & $5 / 6 / 93$ & 3 & 1 & & 84 & $Y$ & \\
\hline 1 & $5 / 6 / 93$ & 4 & 1 & & 83 & $N$ & \\
\hline 9 & $5 / 6 / 93$ & 5 & 9 & & 82 & $\mathbf{N}$ & \\
\hline 1 & $5 / 6 / 93$ & 6 & 1 & & 81 & $\mathrm{~N}$ & \\
\hline 1 & $5 / 6 / 93$ & 7 & 1 & & 77 & $\mathrm{~N}$ & \\
\hline 1 & $5 / 6 / 93$ & 8 & 1 & & 71 & $\mathbf{N}$ & \\
\hline 1 & $5 / 6 / 93$ & 9 & 1 & & 64 & $\mathbf{N}$ & \\
\hline 1 & $5 / 6 / 93$ & 10 & 1 & & 62 & $\mathbf{N}$ & \\
\hline 1 & $5 / 6 / 93$ & 11 & 1 & & 62 & $N$ & \\
\hline 1 & $5 / 6 / 93$ & 12 & 1 & & 61 & $\mathbf{N}$ & \\
\hline 1 & $5 / 6 / 93$ & 13 & 1 & & 69 & $\mathbf{N}$ & \\
\hline 1 & $5 / 6 / 93$ & 14 & 1 & & 61 & $\mathrm{~N}$ & \\
\hline 1 & $5 / 13 / 93$ & 1 & 1 & & 81 & $\mathrm{~N}$ & J 817-13-20-019 \\
\hline 1 & $5 / 13 / 93$ & 2 & 1 & & 82 & $Y$ & \\
\hline$\frac{1}{1}$ & $5 / 13 / 93$ & 3 & 1 & & 82 & $N$ & \\
\hline 1 & $5 / 13 / 93$ & 4 & 1 & & 81 & $\mathbf{N}$ & \\
\hline 1 & $5 / 13 / 93$ & 5 & 1 & & 80 & $\mathbf{N}$ & \\
\hline 1 & $5 / 13 / 93$ & 6 & 1 & & 79 & $\mathrm{~N}$ & \\
\hline 1 & $5 / 13 / 93$ & 7 & 1 & & 76 & $N$ & \\
\hline 1 & $5 / 13 / 93$ & 8 & 1 & & 70 & $\mathrm{~N}$ & \\
\hline 1 & $5 / 13 / 93$ & 9 & 1 & & 59 & $N$ & \\
\hline 1 & $5 / 13 / 93$ & 10 & 1 & & 57 & $\bar{N}$ & \\
\hline 1 & $5 / 13 / 93$ & 11 & 1 & & 57 & $N$ & \\
\hline 1 & $5 / 13 / 93$ & 12 & 1 & & 57 & $\mathrm{~N}$ & \\
\hline 1 & $5 / 13 / 93$ & 13 & 1 & & 57 & $N$ & \\
\hline 1 & $5 / 13 / 93$ & 14 & 1 & & 58 & $N$ & \\
\hline 1 & $5 / 20 / 93$ & 1 & 1 & & 81 & $N$ & J910126143-66 \\
\hline 1 & $5 / 20 / 93$ & 2 & 1 & & 82 & $Y$ & \\
\hline 1 & $5 / 20 / 93$ & 3 & 1 & & 82 & $N$ & \\
\hline 1 & $5 / 20 / 93$ & 4 & 1 & & 80 & $\mathrm{~N}$ & \\
\hline 1 & $5 / 20 / 93$ & 5 & 1 & & 80 & $N$ & \\
\hline 1 & $5 / 20 / 93$ & 6 & 1 & & 77 & $N$ & \\
\hline 7 & $5 / 20 / 93$ & 7 & 1 & & 74 & $\mathbf{N}$ & \\
\hline 1 & $5 / 20 / 93$ & 8 & 1 & & 68 & $N$ & \\
\hline 1 & $5 / 20 / 93$ & 9 & 1 & & 62 & $\mathrm{~N}$ & \\
\hline
\end{tabular}

Data obtained from WHC Surveillance Analysis Computer System (SACS), February 24, 1994. 


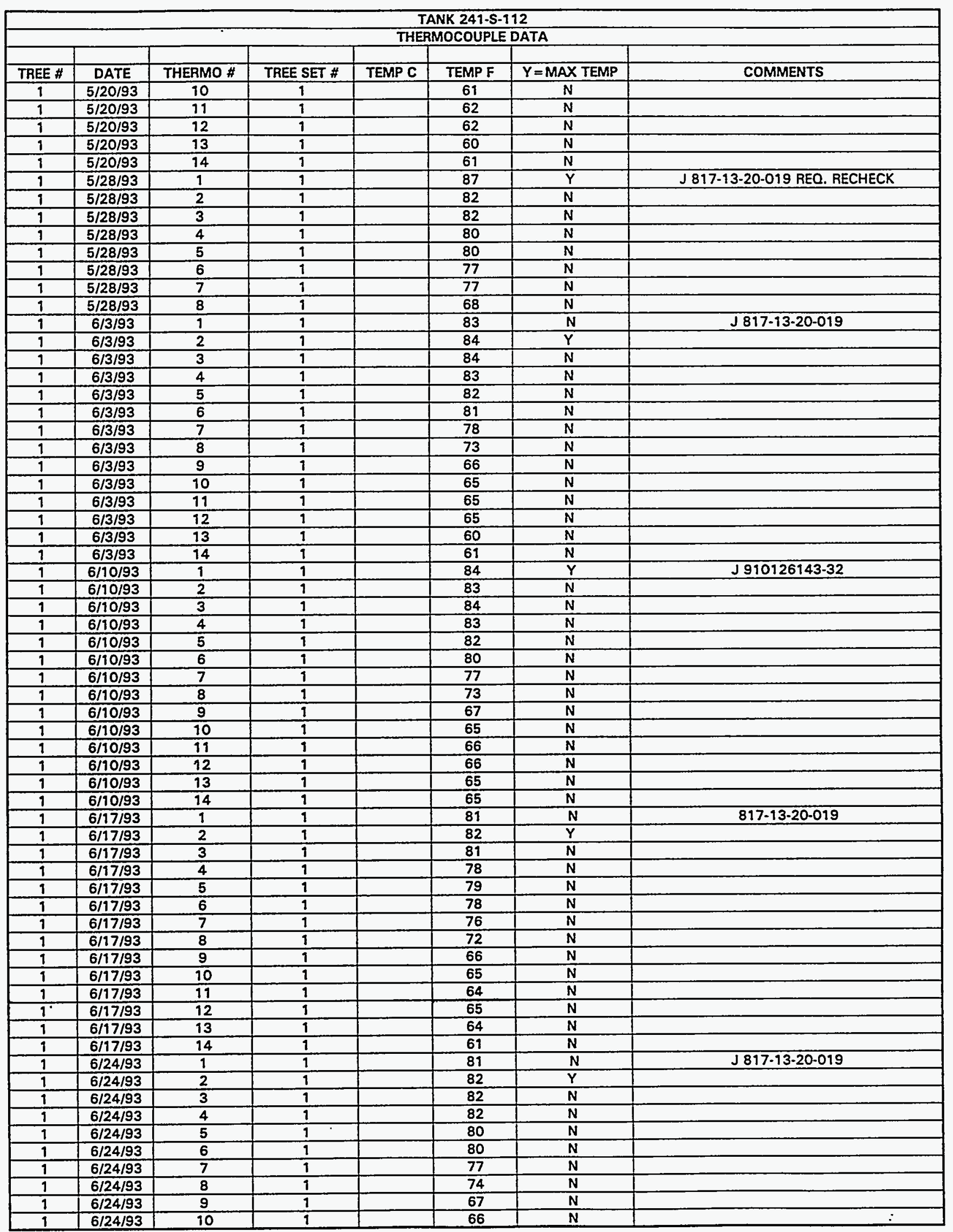

Data obtained from WHC Surveillance Analysis Computer System (SACS), February 24, 1994. 


\begin{tabular}{|c|c|c|c|c|c|c|c|}
\hline \multicolumn{8}{|c|}{ TANK 241-S-112 } \\
\hline \multicolumn{8}{|c|}{ THERMOCOUPLE DATA } \\
\hline TREE \# & DATE & THERMO \# & TREE SET \# & TEMP C & TEMP F & $Y=$ MAX TEMP & COMMENTS \\
\hline 1 & $6 / 24 / 93$ & 11 & 9 & & 66 & $N$ & \\
\hline 9 & $6 / 24 / 93$ & 12 & 1 & & 66 & $\mathrm{~N}$ & \\
\hline 1 & $6 / 24 / 93$ & 13 & 1 & & 65 & $\bar{N}$ & \\
\hline 1 & $6 / 24 / 93$ & 14 & 1 & & 64 & $\mathrm{~N}$ & \\
\hline 1 & $7 / 8 / 93$ & 4 & 1 & & 76.6 & $\bar{Y}$ & \\
\hline 1 & $7 / 8 / 93$ & 7 & 1 & & 74.3 & $\mathrm{~N}$ & \\
\hline 1 & $7 / 8 / 93$ & 8 & 1 & & 72 & $\ddot{N}$ & \\
\hline 1 & $7 / 8 / 93$ & 10 & 1 & & 62.1 & $\mathrm{~N}$ & \\
\hline 1 & $7 / 8 / 93$ & 11 & 1 & & 62.8 & $\mathbf{N}$ & \\
\hline 1 & $7 / 8 / 93$ & 12 & 1 & & 64.9 & $\mathbf{N}$ & \\
\hline 1 & $7 / 16 / 93$ & 1 & 1 & & 73 & $\mathbf{N}$ & J 817-13-55-001 \\
\hline 9 & $7 / 16 / 93$ & 2 & 9 & & 72 & $\mathbf{N}$ & \\
\hline 1 & $7 / 16 / 93$ & 3 & 1 & & 72 & $\bar{N}$ & \\
\hline 9 & $7 / 16 / 93$ & 4 & 1 & & 72 & $\mathbf{N}$ & \\
\hline 1 & $7 / 16 / 93$ & 5 & 1 & & 73 & N & \\
\hline 1 & $7 / 16 / 93$ & 6 & 1 & & 75 & $\mathbf{N}$ & \\
\hline 1 & $7 / 16 / 93$ & 7 & 1 & & 77 & $\mathbf{N}$ & \\
\hline 1 & $7 / 16 / 93$ & 8 & 1 & & 81 & $\mathbf{N}$ & \\
\hline$\frac{1}{1}$ & $7 / 16 / 93$ & 9 & 1 & & 86 & $\bar{N}$ & \\
\hline 1 & $7 / 16 / 93$ & 10 & 1 & & 88 & $Y$ & \\
\hline 1 & $7 / 16 / 93$ & 11 & 1 & & 88 & $\mathbf{N}$ & \\
\hline 1 & $7 / 16 / 93$ & 13 & 1 & & 88 & $\mathbf{N}$ & \\
\hline 1 & $7 / 16 / 93$ & 14 & 1 & & 88 & $\mathbf{N}$ & \\
\hline 1 & $7 / 22 / 93$ & 1 & 1 & & 82.4 & $N$ & J 817.013 .55 .009 \\
\hline 1 & $7 / 22 / 93$ & 2 & 1 & & 83 & $N$ & \\
\hline 1 & $7 / 22 / 93$ & 3 & 1 & & 83.3 & $\bar{Y}$ & \\
\hline 1 & $7 / 22 / 93$ & 4 & 1 & & 83 & $\mathrm{~N}$ & \\
\hline 1 & $7 / 22 / 93$ & 5 & 1 & & 76.9 & $\mathbf{N}$ & \\
\hline 1 & $7 / 22 / 93$ & 6 & 1 & & 79.5 & $\bar{N}$ & \\
\hline 1 & $7 / 22 / 93$ & 7 & 1 & & 79.1 & $\mathrm{~N}$ & \\
\hline 1 & $7 / 22 / 93$ & 8 & 1 & & 75.1 & $\mathbf{N}$ & \\
\hline 1 & $7 / 22 / 93$ & 9 & 1 & & 70.5 & $\mathbf{N}$ & \\
\hline 1 & $7 / 22 / 93$ & 10 & 1 & & 69.4 & $N$ & \\
\hline 1 & $7 / 22 / 93$ & 11 & 1 & & 63.5 & $\mathbf{N}$ & \\
\hline 1 & $7 / 22 / 93$ & 12 & 1 & & 69.5 & $\mathrm{~N}$ & \\
\hline$\frac{1}{1}$ & $7 / 22 / 93$ & 13 & 1 & & 65 & $\mathbf{N}$ & \\
\hline 1 & $7 / 22 / 93$ & 14 & 1 & & 71.6 & $\mathbf{N}$ & \\
\hline 1 & $7 / 29 / 93$ & 1 & 1 & & & $\mathbf{N}$ & J910126143-14 TC\# 1, 2,5,6, \& 13-16 \\
\hline 1 & $8 / 5 / 93$ & 1 & 1 & & 80 & $\mathbf{N}$ & $J 890423320-42$ \\
\hline 1 & $8 / 5 / 93$ & 2 & 1 & & 80 & $N$ & \\
\hline 1 & $8 / 5 / 93$ & 3 & 1 & & 89 & $\bar{Y}$ & \\
\hline 1 & $8 / 5 / 93$ & 4 & 1 & & 81 & $\dot{N}$ & \\
\hline 1 & $8 / 5 / 93$ & 5 & 1 & & 80 & $\mathbf{N}$ & \\
\hline 1 & $8 / 5 / 93$ & 6 & 1 & & 79 & $\bar{N}$ & \\
\hline 1 & $8 / 5 / 93$ & 7 & 1 & & 78 & $\mathbf{N}$ & \\
\hline 1 & $8 / 5 / 93$ & 8 & 1 & & 74 & $\mathbf{N}$ & \\
\hline 1 & $8 / 5 / 93$ & 9 & 1 & & 70 & $\mathrm{~N}$ & \\
\hline 1 & $8 / 5 / 93$ & 10 & 1 & & 69 & $\bar{N}$ & \\
\hline 1 & $8 / 5 / 93$ & 11 & 1 & & 69 & $N$ & \\
\hline 1 & $8 / 5 / 93$ & 12 & 1 & & 69 & $\bar{N}$ & \\
\hline$\overline{1}$ & $8 / 5 / 93$ & 13 & 1 & & 69 & $\mathbf{N}$ & . \\
\hline 1 & $8 / 5 / 93$ & 14 & 1 & & 69 & $\mathbf{N}$ & \\
\hline 1 & $8 / 19 / 93$ & 3 & 1 & & 67.7 & $\mathrm{~N}$ & J910126143-66 TC 1,25,6,13814 O/S \\
\hline 1 & $8 / 19 / 93$ & 4 & 1 & & 79.2 & $\mathbf{N}$ & \\
\hline 1 & $8 / 19 / 93$ & 7 & 1 & & 79.4 & $Y$ & \\
\hline 1 & $8 / 19 / 93$ & 8 & 1 & & 76.9 & $\overline{\mathbf{N}}$ & \\
\hline 1 & $8 / 19 / 93$ & 9 & 1 & & 72.1 & $\mathbf{N}$ & \\
\hline 1 & $8 / 19 / 93$ & 10 & 1 & & 69 & $\mathbf{N}$ & \\
\hline 1 & $8 / 19 / 93$ & -11 & 1 & & 69.1 & $\mathbf{N}$ & \\
\hline 1 & $8 / 19 / 93$ & 12 & 1 & & 71.5 & $\mathrm{~N}$ & \\
\hline 1 & $8 / 27 / 93$ & 1 & 1 & & 79.6 & $\mathbf{N}$ & J $817-13.55 .009$ \\
\hline 9 & $8 / 27 / 93$ & 2 & 1 & & 80 & $\mathbf{N}$ & \\
\hline 1 & $8 / 27 / 93$ & 3 & 1 & & 80.5 & $\bar{N}$ & \\
\hline 1 & $8 / 27 / 93$ & 4 & 1 & & 83.6 & $Y$ & \\
\hline 1 & $8 / 27 / 93$ & 5 & 1 & & 82.9 & $N$ & \\
\hline
\end{tabular}

Data obtained from WHC Surveillance Analysis Computer System (SACS), February 24, 1994. 


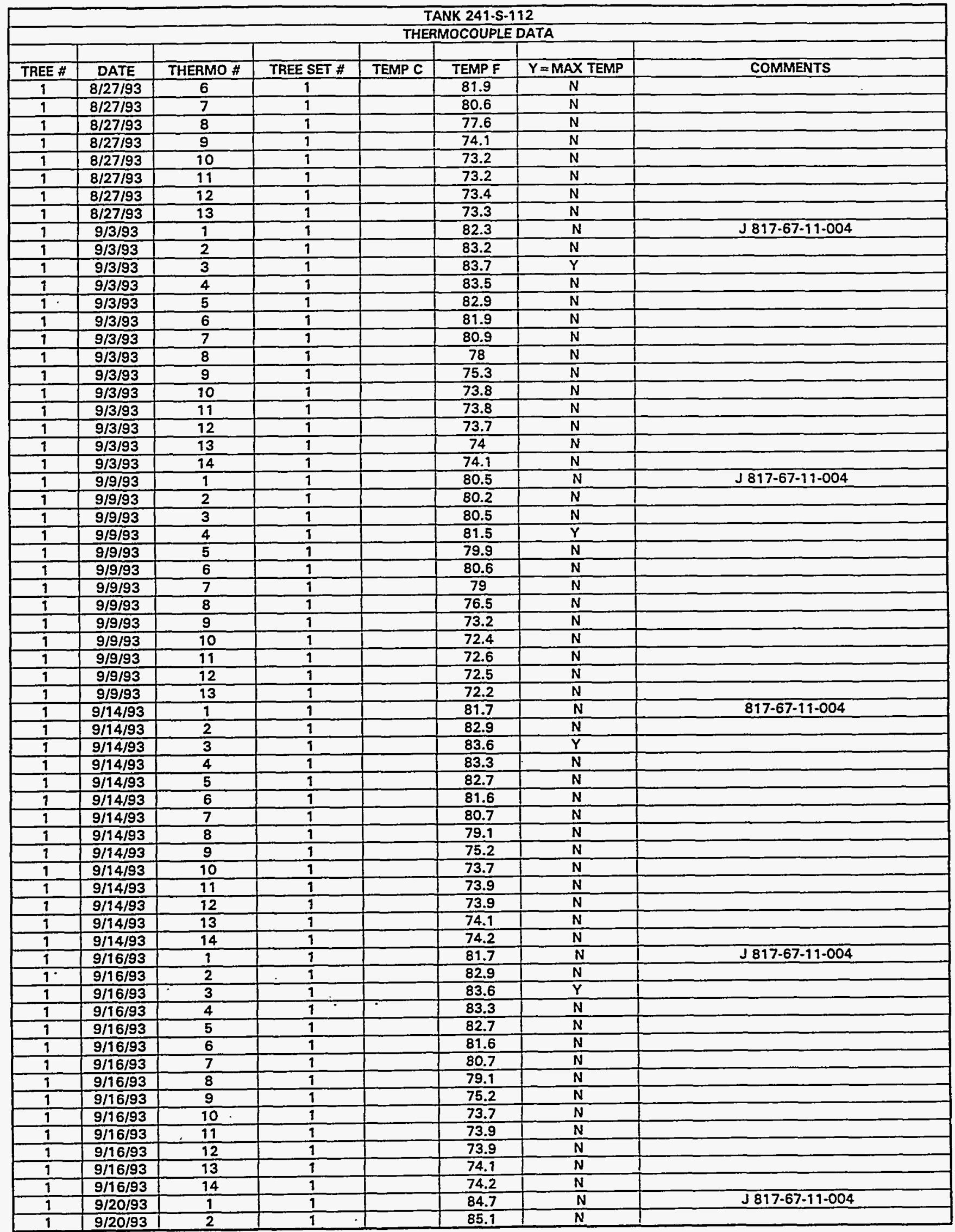

Data obtained from WHC Surveillance Analysis Computer System (SACS). February 24, 1994. 


\begin{tabular}{|c|c|c|c|c|c|c|c|}
\hline \multicolumn{8}{|c|}{ TANK 241-S-112 } \\
\hline \multicolumn{8}{|c|}{ THERMOCOUPLE DATA } \\
\hline TREE \# & DATE & THERMO \# & TREE SET \# & TEMP C & TEMP F & $Y=M A X$ TEMP & COMMENTS \\
\hline 1 & $9 / 20 / 93$ & 3 & 1 & & 85.4 & $\mathrm{Y}$ & \\
\hline 1 & $9 / 20 / 93$ & 4 & 9 & & 85.2 & $\mathbf{N}$ & \\
\hline 1 & $9 / 20 / 93$ & 5 & 1 & & 84.2 & $\mathrm{~N}$ & \\
\hline 1 & $9 / 20 / 93$ & 6 & 1 & & 83.1 & $\mathrm{~N}$ & \\
\hline 1 & $9 / 20 / 93$ & 7 & 1 & & 82.6 & $\mathbf{N}$ & \\
\hline 1 & $9 / 20 / 93$ & 8 & 1 & & 79.5 & $\mathbf{N}$ & \\
\hline 1 & $9 / 27 / 93$ & 1 & 1 & & 82.4 & $\mathbf{N}$ & $817-13-20-019$ \\
\hline 1 & $9 / 27 / 93$ & 2 & 9 & & 81.6 & $\mathbf{N}$ & \\
\hline$\frac{1}{1}$ & $9 / 27 / 93$ & 3 & 1 & & 81.7 & $\mathbf{N}$ & \\
\hline 1 & $9 / 27 / 93$ & 4 & 1 & & 81.8 & $\mathbf{N}$ & \\
\hline 1 & $9 / 27 / 93$ & 5 & 1 & & 81.1 & $N$ & \\
\hline 1 & $9 / 27 / 93$ & 6 & 1 & & 83 & $\bar{Y}$ & \\
\hline 1 & $9 / 27 / 93$ & 7 & 1 & & 80.2 & $\mathbf{N}$ & \\
\hline 1 & $9 / 27 / 93$ & 8 & 1 & & 78.1 & $\mathbf{N}$ & \\
\hline 1 & $10 / 4 / 93$ & 1 & 1 & & 83 & $N$ & $817-67-11-004$ \\
\hline 1 & $10 / 4 / 93$ & 2 & 1 & & 82.9 & $N$ & \\
\hline 1 & $10 / 4 / 93$ & 3 & 1 & & 83.4 & $\mathrm{~N}$ & \\
\hline 1 & $10 / 4 / 93$ & 4 & 1 & & 83.6 & $Y$ & \\
\hline 1 & $10 / 4 / 93$ & 5 & 1 & & 79.1 & $N$ & \\
\hline 1 & $10 / 4 / 93$ & 6 & 1 & & 75.7 & $\mathrm{~N}$ & \\
\hline 1 & $10 / 4 / 93$ & 7 & 1 & & 74.9 & $N$ & \\
\hline 1 & $10 / 4 / 93$ & 8 & 1 & & 74.8 & $N$ & 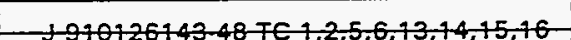 \\
\hline 1 & $10 / 5 / 93$ & 3 & 1 & & 66.3 & $\mathrm{~N}$ & $0 / 2$ \\
\hline 1 & $10 / 5 / 93$ & 4 & 9 & & 79.2 & $\mathrm{~N}$ & \\
\hline 1 & $10 / 5 / 93$ & 7 & 1 & & 79.7 & $Y$ & \\
\hline 1 & $10 / 5 / 93$ & 8 & 1 & & 78.1 & $\dot{N}$ & \\
\hline 9 & $10 / 5 / 93$ & 9 & 1 & & 73.7 & $\mathbf{N}$ & \\
\hline 1 & $10 / 5 / 93$ & 10 & 1 & & 71.4 & $\mathbf{N}$ & \\
\hline 1 & $10 / 5 / 93$ & 11 & 1 & & 71.2 & N & \\
\hline 1 & $10 / 5 / 93$ & 12 & 1 & & 72.7 & $N$ & \\
\hline 1 & $10 / 11 / 93$ & 1 & 1 & & 82.9 & $\mathbf{N}$ & $817-67-11-004$ \\
\hline 1 & $10 / 11 / 93$ & 2 & 1 & & 83.4 & $\mathbf{N}$ & \\
\hline 1 & $10 / 11 / 93$ & 3 & 1 & & 83.6 & $\mathbf{N}$ & \\
\hline 1 & $10 / 11 / 93$ & 4 & 1 & & 83.7 & $\mathbf{Y}$ & \\
\hline 1 & $10 / 11 / 93$ & 5 & 1 & & 80 & $\mathrm{~N}$ & \\
\hline 1 & $10 / 11 / 93$ & 6 & 1 & & 76.2 & $\mathbf{N}$ & \\
\hline 1 & $10 / 11 / 93$ & 7 & 1 & & 75.2 & $\mathbf{N}$ & \\
\hline 1 & $10 / 11 / 93$ & 8 & 1 & & 75.1 & $\mathbf{N}$ & \\
\hline 1 & $10 / 12 / 93$ & 1 & 1 & & & $N$ & $\begin{array}{c}\text { J } 910126143-48 \text { TC } 1,2,5,6,13,14 \text { O/S BAD } \\
\text { DATA }\end{array}$ \\
\hline 1 & $10 / 18 / 93$ & 1 & 1 & & 83.2 & $\mathbf{Y}$ & $817-67-11-004$ \\
\hline 1 & $10 / 18 / 93$ & 2 & 1 & & 82.9 & $N$ & \\
\hline 1 & $10 / 18 / 93$ & 3 & 1 & & 83.1 & $\mathrm{~N}$ & \\
\hline 1 & $10 / 18 / 93$ & 4 & 1 & & 83 & $\mathrm{~N}$ & \\
\hline 1 & $10 / 18 / 93$ & 5 & 1 & & 79.1 & $\overline{\mathbf{N}}$ & \\
\hline 1 & $10 / 18 / 93$ & 6 & 1 & & 75.2 & $N$ & \\
\hline 1 & $10 / 18 / 93$ & 7 & 1 & & 74.9 & $\mathbf{N}$ & \\
\hline 1 & $10 / 18 / 93$ & 8 & 1 & & 74.5 & $\mathbf{N}$ & \\
\hline 1 & $10 / 25 / 93$ & 1 & 1 & & 83.2 & $\bar{Y}$ & $817-67-11-004$ \\
\hline 1 & $10 / 25 / 93$ & 2 & 1 & & 81.9 & $\mathbf{N}$ & \\
\hline 1 & $10 / 25 / 93$ & 3 & 1 & & 82.7 & $N$ & \\
\hline 1 & $10 / 25 / 93$ & 4 & 1 & & 82.9 & $N$ & \\
\hline 1 & $10 / 25 / 93$ & 5 & 1 & & 79.3 & $\mathrm{~N}$ & \\
\hline 1 & $10 / 25 / 93$ & 6 & 1 & & 76 & $\bar{N}$ & \\
\hline 1 & $10 / 25 / 93$ & 7 & 1 & & 75.1 & $\mathrm{~N}$ & \\
\hline 1 & $10 / 25 / 93$ & 8 & 1 & & 74.7 & $\mathbf{N}$ & \\
\hline 1 & $11 / 1 / 93$ & 9 & 1 & & 53.8 & $\mathbf{N}$ & 53.8 - suspect data (low), J $910126143-29$ \\
\hline 9 & $11 / 1 / 93$ & 2 & 9 & & & $\mathrm{~N}$ & 52.7 - suspect data (low) \\
\hline 1 & $11 / 1 / 93$ & 3 & 1 & & & $\mathbf{N}$ & 52.9 - suspect data (low) \\
\hline 1 & $11 / 1 / 93$ & 4 & 1 & & & $N$ & 51.6 - suspect data (low) \\
\hline 1 & $11 / 1 / 93$ & 5 & 1 & & & $\mathbf{N}$ & 52.2 - suspect data (fow) \\
\hline 1 & $11 / 1 / 93$ & 6 & 9 & & & $\mathbf{N}$ & 53.1 - suspect data (low) \\
\hline 1 & $11 / 1 / 93$ & 7 & 1 & & & $\mathbf{N}$ & 53.6 - suspect data (low) \\
\hline 1 & $11 / 1 / 93$ & 8 & 1 & & & $\bar{N}$ & 56 - suspect data (low) \\
\hline
\end{tabular}

Data obtained from WHC. Surveillance Analysis Computer System (SACS), February 24, 1994. 


\begin{tabular}{|c|c|c|c|c|c|c|c|}
\hline \multicolumn{8}{|c|}{ TANK 241-S-112 } \\
\hline \multicolumn{8}{|c|}{ THERMOCOUPLE DATA } \\
\hline TREE \# & DATE & THERMO \# & TREE SET \# & TEMP C & TEMPF & $Y=$ MAX TEMP & COMMENTS \\
\hline 1 & $11 / 1 / 93$ & 9 & 1 & & & $\mathrm{~N}$ & 60.2 - suspect data (low) \\
\hline 1 & $11 / 1 / 93$ & 11 & 1 & & & $\mathbf{N}$ & 60.8 - suspect data (low) \\
\hline 1 & $11 / 1 / 93$ & 12 & 1 & & & $\mathbf{N}$ & 60.9 - suspect data (low) \\
\hline 1 & $11 / 1 / 93$ & 13 & 1 & & & $\mathbf{N}$ & 60.9 - suspect data (low) \\
\hline 1 & $11 / 1 / 93$ & 14 & 1 & & & $\mathbf{N}$ & 61 - suspect data (low) \\
\hline 1 & $11 / 8 / 93$ & 1 & 2 & & 80 & $\mathrm{~N}$ & 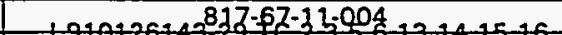 \\
\hline 1 & $11 / 8 / 93$ & 1 & 1 & & & $\mathbf{N}$ & $59+0,26+43,29+1,2,3,5,6,13,74,75,76$ \\
\hline 1 & $11 / 8 / 93$ & $\overline{2}$ & 2 & & 80.6 & $\mathbf{N}$ & \\
\hline 1 & $11 / 8 / 93$ & 3 & 1 & & 68.7 & $\mathrm{~N}$ & \\
\hline 1 & $11 / 8 / 93$ & 3 & 2 & & 81.2 & $\mathbf{N}$ & \\
\hline 1 & $11 / 8 / 93$ & 4 & 2 & & 81.4 & $\bar{Y}$ & \\
\hline 1 & $11 / 8 / 93$ & 4 & 1 & & 82.3 & $\bar{Y}$ & \\
\hline 1 . & $11 / 8 / 93$ & 5 & 2 & & 80.9 & $\mathbf{N}$ & \\
\hline 1 & $11 / 8 / 93$ & 6 & 2 & & 79.8 & $\mathbf{N}$ & \\
\hline 1 & $11 / 8 / 93$ & 7 & 2 & & 78.8 & $\mathbf{N}$ & \\
\hline 1 & $11 / 8 / 93$ & 7 & 1 & & 81.7 & $\mathbf{N}$ & \\
\hline 1 & $11 / 8 / 93$ & 8 & 2 & & 75.9 & $\mathbf{N}$ & \\
\hline 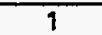 & $11 / 8 / 93$ & 8 & 1 & & 79.6 & $\mathbf{N}$ & \\
\hline 1 & $11 / 8 / 93$ & 9 & 2 & & 71.9 & $\mathbf{N}$ & \\
\hline 1 & $11 / 8 / 93$ & 9 & 1 & & 74.5 & $\mathbf{N}$ & \\
\hline 1 & $11 / 8 / 93$ & 10 & 2 & & 70.7 & $\mathbf{N}$ & \\
\hline 1 & $19 / 8 / 93$ & 10 & 1 & & 72.3 & $\mathbf{N}$ & \\
\hline 1 & $11 / 8 / 93$ & 11 & 2 & & 70.9 & $\mathbf{N}$ & \\
\hline 1 & $11 / 8 / 93$ & 11 & 1 & & 72.2 & $\mathbf{N}$ & \\
\hline 1 & $11 / 8 / 93$ & 12 & 2 & & 71 & $\mathbf{N}$ & \\
\hline 1 & $11 / 8 / 93$ & 12 & 1 & & 72.9 & $\mathrm{~N}$ & \\
\hline 1 & $11 / 8 / 93$ & 13 & 2 & & 70.8 & $\mathbf{N}$ & \\
\hline 9 & $11 / 8 / 93$ & 14 & 2 & & 70.6 & $\bar{N}$ & \\
\hline$T$ & $11 / 15 / 93$ & 1 & 1 & & 86.5 & $\bar{Y}$ & $817-67 \cdot 11.004$ \\
\hline 1 & $11 / 15 / 93$ & 2 & 1 & & 84.6 & $N$ & \\
\hline 1 & $11 / 15 / 93$ & 3 & 9 & & 85.3 & $\mathbf{N}$ & \\
\hline 1 & $11 / 15 / 93$ & 4 & 1 & & 85.4 & $\mathbf{N}$ & \\
\hline 1 & $11 / 15 / 93$ & 5 & 1 & & 84.9 & $\mathbf{N}$ & \\
\hline 1 & $11 / 15 / 93$ & 6 & 1 & & 84.1 & $\mathbf{N}$ & \\
\hline 1 & $11 / 15 / 93$ & 7 & 1 & & 80.1 & $\mathbf{N}$ & \\
\hline 1 & $11 / 15 / 93$ & 8 & 1 & & 75.3 & $\mathbf{N}$ & \\
\hline 1 & $11 / 15 / 93$ & 9 & 1 & & 73.8 & $\mathbf{N}$ & \\
\hline$\frac{T}{1}$ & $11 / 15 / 93$ & 10 & 1 & & 73.8 & $\mathbf{N}$ & \\
\hline 1 & $11 / 15 / 93$ & 11 & 1 & & 73.8 & $\mathbf{N}$ & \\
\hline 1 & $11 / 15 / 93$ & 12 & 1 & & 73.7 & $\mathbf{N}$ & \\
\hline 1 & $11 / 15 / 93$ & 93 & 1 & & 73.8 & $\mathbf{N}$ & \\
\hline 9 & $11 / 15 / 93$ & 14 & 9 & & 73.8 & $\mathbf{N}$ & \\
\hline 1 & $11 / 17 / 93$ & 3 & 1 & & 69.4 & $\mathbf{N}$ & $\mathrm{J}$ TI.041 TC $1256 \mathrm{O} / \mathrm{S}$ \\
\hline 1 & $11 / 17 / 93$ & 4 & 1 & & 82.7 & $\mathbf{Y}$ & \\
\hline 1 & $11 / 17 / 93$ & 7 & 1 & & 81.8 & $\mathbf{N}$ & \\
\hline 1 & $11 / 17 / 93$ & 8 & 1 & & 79.3 & $\mathbf{N}$ & \\
\hline 9 & $11 / 17 / 93$ & 9 & 1 & & 73.8 & $\mathbf{N}$ & \\
\hline 1 & $11 / 17 / 93$ & 10 & 1 & & 71.9 & $\mathbf{N}$ & \\
\hline 1 & $11 / 17 / 93$ & 11 & 1 & & 71.6 & $N$ & \\
\hline 1 & $11 / 17 / 93$ & 12 & 1 & & 72 & $\bar{N}$ & \\
\hline 1 & $11 / 23 / 93$ & 3 & 1 & & 69.6 & $\mathbf{N}$ & J910126143-29 TC 1256 O/S \\
\hline$\frac{1}{1}$ & $11 / 23 / 93$ & 4 & 1 & & 83 & $\mathbf{Y}$ & \\
\hline 1 & $11 / 23 / 93$ & 7 & 1 & & 82 & $\mathbf{N}$ & \\
\hline 1 & $11 / 23 / 93$ & 8 & 1 & & 79.2 & $\mathbf{N}$ & \\
\hline$\frac{1}{1}$ & $11 / 23 / 93$ & 9 & 1 & & 73.4 & $\mathbf{N}$ & \\
\hline 1 & $11 / 23 / 93$ & 10 & 1 & & 71.5 & $N$ & \\
\hline 1 & $11 / 23 / 93$ & 11 & 1 & & 71.1 & $\mathbf{N}$ & \\
\hline 1 & $19 / 23 / 93$ & 12 & 1 & & 71.6 & $\mathbf{N}$ & \\
\hline 1 & $11 / 29 / 93$ & 1 & 1 & & 87 & $\mathbf{Y}$ & $\mathrm{J}-910126143-29$ \\
\hline 9 & $11 / 29 / 93$ & 1 & 2 & & 87 & $Y$ & \\
\hline 9 & $11 / 29 / 93$ & 2 & 1 & & 85 & $\bar{N}$ & \\
\hline 1 & $11 / 29 / 93$ & 2 & 2 & & 85 & $\mathbf{N}$ & \\
\hline 1 & $11 / 29 / 93$ & 3 & 1 & & 84.8 & $\mathbf{N}$ & \\
\hline$\frac{T}{1}$ & $11 / 29 / 93$ & 3 & 2 & & 84.8 & $\mathbf{N}$ & \\
\hline 1 & $11 / 29 / 93$ & 4 & $\frac{T}{1}$ & & 84.7 & $N$ & \\
\hline
\end{tabular}

Data obtained from WHC Surveillance Analysis Computer System (SACS), February 24, 1994. 


\begin{tabular}{|c|c|c|c|c|c|c|c|}
\hline \multicolumn{8}{|c|}{ TANK 241.S-112 } \\
\hline \multicolumn{8}{|c|}{ THERMOCOUPLE DATA } \\
\hline TREE \# & DATE & THERMO \# & TREE SET \# & TFMP & TEMP F & $Y=$ MAX TEMP & \\
\hline 1 & $11 / 29 / 93$ & 4 & 2 & I Livit 1 & 84.7 & $\mathrm{~N}$ & COMIMENATS \\
\hline 1 & $11 / 29 / 93$ & 5 & 9 & & 84.7 & $\mathbf{N}$ & \\
\hline 1 & $11 / 29 / 93$ & 5 & 2 & & 84.7 & $\mathrm{~N}$ & \\
\hline 1 & $11 / 29 / 93$ & 6 & 1 & & 84.6 & $N$ & \\
\hline 1 & $11 / 29 / 93$ & 6 & 2 & & 84.7 & $\mathrm{~N}$ & \\
\hline 1 & $11 / 29 / 93$ & 7 & 1 & & 80 & $\mathrm{~N}$ & \\
\hline 1 & $11 / 29 / 93$ & 7 & 2 & & 80.1 & $N$ & \\
\hline 9 & $11 / 29 / 93$ & 8 & 1 & & 74.9 & $N$ & \\
\hline 1 & $11 / 29 / 93$ & 8 & 2 & & 74.9 & $N$ & \\
\hline 1 & $12 / 7 / 93$ & 3 & 1 & & 70.3 & $\mathbf{N}$ & J 910126143.29 TC $9,2,5,6,13,140 / 5$ \\
\hline 1 & $12 / 7 / 93$ & 4 & 1 & & 83.4 & $Y$ & \\
\hline 1 & $12 / 7 / 93$ & 7 & 1 & & 82.3 & $\mathrm{~N}$ & \\
\hline 1 & $12 / 7 / 93$ & 8 & 1 & & 78.7 & $N$ & \\
\hline 1 & $12 / 7 / 93$ & 9 & 1 & & 72 & $\mathbf{N}$ & \\
\hline 1 & $12 / 7 / 93$ & 10 & 1 & & 69.6 & $\mathrm{~N}$ & \\
\hline 1 & $12 / 7 / 93$ & 11 & 1 & & 69.5 & $\mathbf{N}$ & \\
\hline 1 & $12 / 7 / 93$ & 12 & 1 & & 69.8 & $N$ & \\
\hline 1 & $12 / 14 / 93$ & 3 & 1 & & 71.5 & $\mathbf{N}$ & $\begin{array}{c}\text { J910126143-29 TC } 1,2,5,6,13,14,15,16 \\
\text { O/S }\end{array}$ \\
\hline 1 & $12 / 14 / 93$ & 4 & 1 & & 83.9 & $\bar{Y}$ & \\
\hline 1 & $12 / 14 / 93$ & 7 & 1 & & 82.5 & $\mathrm{~N}$ & \\
\hline 1 & $12 / 14 / 93$ & 8 & 1 & & 78.8 & $N$ & \\
\hline 1 & $12 / 14 / 93$ & 9 & 1 & & 71.8 & N & \\
\hline 1 & $12 / 14 / 93$ & 10 & 1 & & 69.4 & $N$ & \\
\hline 1 & $12 / 14 / 93$ & 11 & 1 & & 69.1 & $\mathbf{N}$ & \\
\hline 1 & $12 / 14 / 93$ & 12 & 1 & & 69.4 & $\mathbf{N}$ & \\
\hline 1 & $12 / 20 / 93$ & 1 & 1 & & 86.5 & $\mathbf{N}$ & 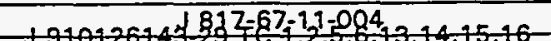 \\
\hline 1 & $12 / 20 / 93$ & 1 & 2 & & & $\mathbf{N}$ & $J 910+26+43-29+6,4,2,5,6,13,14,75,76-$ \\
\hline 1 & $12 / 20 / 93$ & 1 & 3 & & & $N$ & $\mathrm{~J} 910126143-29$, $2,5,6,13,14 \mathrm{O} / \mathrm{S}$ \\
\hline 1 & $12 / 20 / 93$ & 2 & 1 & & 86.8 & $\mathbf{N}$ & \\
\hline 1 & $12 / 20 / 93$ & 3 & 3 & & 70.5 & $\mathbf{N}$ & \\
\hline 1 & $12 / 20 / 93$ & 3 & 2 & & 70.6 & $N$ & \\
\hline 1 & $12 / 20 / 93$ & 3 & 1 & & 87.5 & $Y$ & \\
\hline 1 & $12 / 20 / 93$ & 4 & 2 & & 83.1 & $Y$ & \\
\hline 1 & $12 / 20 / 93$ & 4 & 3 & & 83.8 & $\bar{Y}$ & \\
\hline 1 & $12 / 20 / 93$ & 4 & 1 & & 87.5 & $\mathbf{N}$ & \\
\hline 1 & $12 / 20 / 93$ & 5 & 1 & & 87.1 & $\mathbf{N}$ & \\
\hline 1 & $12 / 20 / 93$ & 6 & 1 & & 86.2 & $N$ & \\
\hline 1 & $12 / 20 / 93$ & 7 & 2 & & 81.6 & $N$ & \\
\hline 1 & $12 / 20 / 93$ & 7 & 3 & & 82.2 & $\mathbf{N}$ & \\
\hline 1 & $12 / 20 / 93$ & 7 & 1 & & 84.5 & $N$ & \\
\hline 1 & $12 / 20 / 93$ & 8 & 2 & & 77.5 & $\mathbf{N}$ & \\
\hline 1 & $12 / 20 / 93$ & 8 & 3 & & 78.3 & $\mathbf{N}$ & \\
\hline 1 & $12 / 20 / 93$ & 8 & 1 & & 80.2 & $N$ & \\
\hline 1 & $12 / 20 / 93$ & 9 & 2 & & 70.2 & $\mathrm{~N}$ & \\
\hline 1 & $12 / 20 / 93$ & 9 & 3 & & 71.1 & $\mathrm{~N}$ & \\
\hline 1 & $12 / 20 / 93$ & 10 & 2 & & 67.7 & $\mathbf{N}$ & \\
\hline 1 & $12 / 20 / 93$ & 10 & 3 & & 68.4 & $\mathrm{~N}$ & \\
\hline 1 & $12 / 20 / 93$ & 11 & 2 & & 67.5 & $\mathbf{N}$ & \\
\hline 1 & $12 / 20 / 93$ & 11 & 3 & & 68.2 & $\mathbf{N}$ & \\
\hline 1 & $12 / 20 / 93$ & 12 & 2 & & 67.8 & $\mathbf{N}$ & \\
\hline 1 & $12 / 20 / 93$ & 12 & 3 & & 68.5 & $\mathbf{N}$ & \\
\hline 1 & $12 / 27 / 93$ & 1 & 1 & & 86.3 & $\mathbf{N}$ & INST TECH \\
\hline 1 & $12 / 27 / 93$ & 2 & 1 & & 87.3 & $\bar{N}$ & \\
\hline 1 & $12 / 27 / 93$ & 3 & 1 & & 88.1 & $Y$ & \\
\hline 1 & $12 / 27 / 93$ & 4 & 1 & & 88.1 & $N$ & \\
\hline 1 & $12 / 27 / 93$ & 5 & 1 & & 87.9 & $\mathbf{N}$ & \\
\hline 1 & $12 / 27 / 93$ & 6 & 1 & & 86.9 & $N$ & \\
\hline 1 & $12 / 27 / 93$ & 7 & 1 & & 85.1 & $\mathbf{N}$ & \\
\hline 1 & $12 / 27 / 93$ & 8 & 1 & & 80.5 & $N$ & \\
\hline
\end{tabular}

Data obtained from WHC Surveillance Analysis Computer System (SACS), February 24, 1994. 
WHC-SD-WM-ER-323, Rev. 0
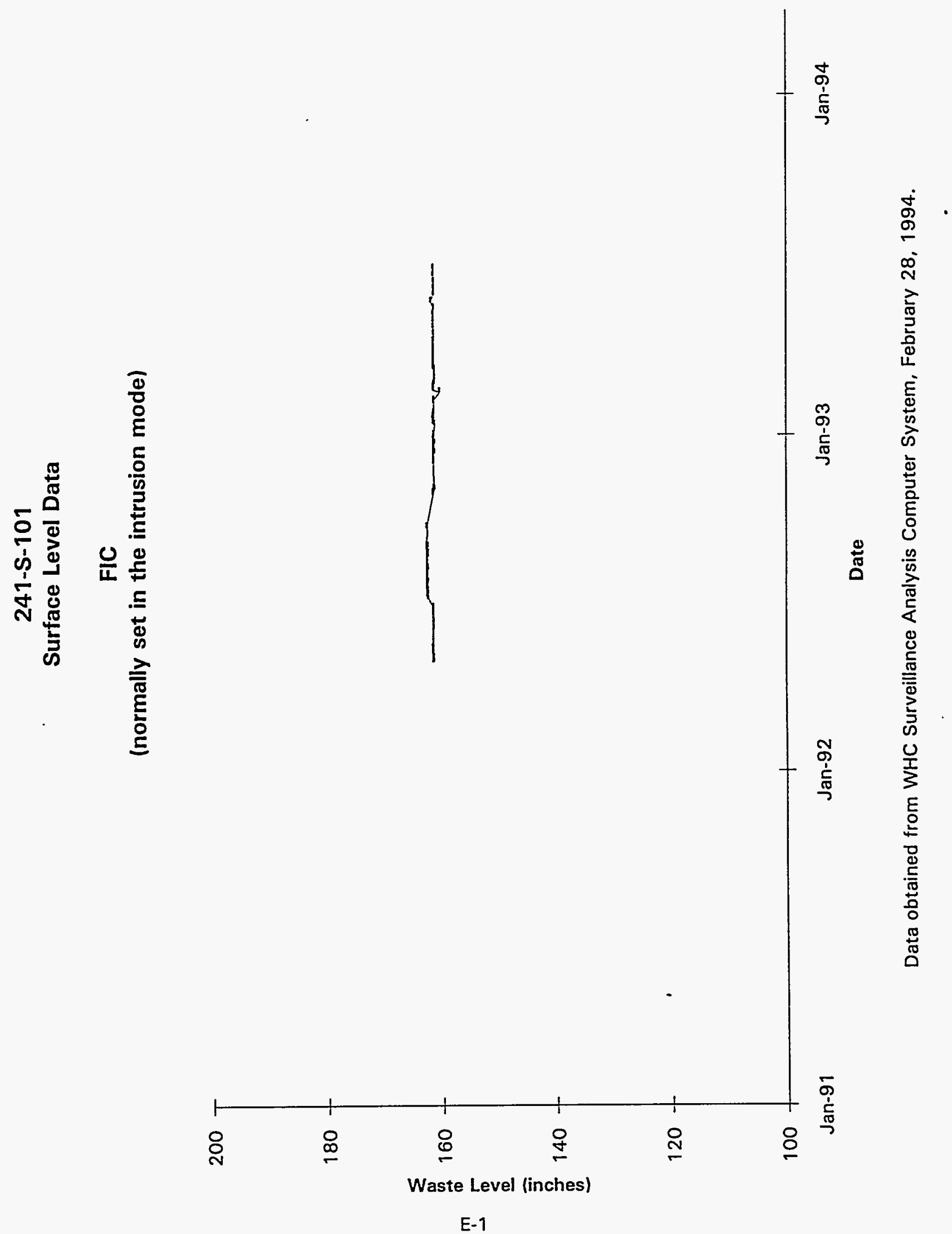
241-S-102

Surface Level Data

FIC

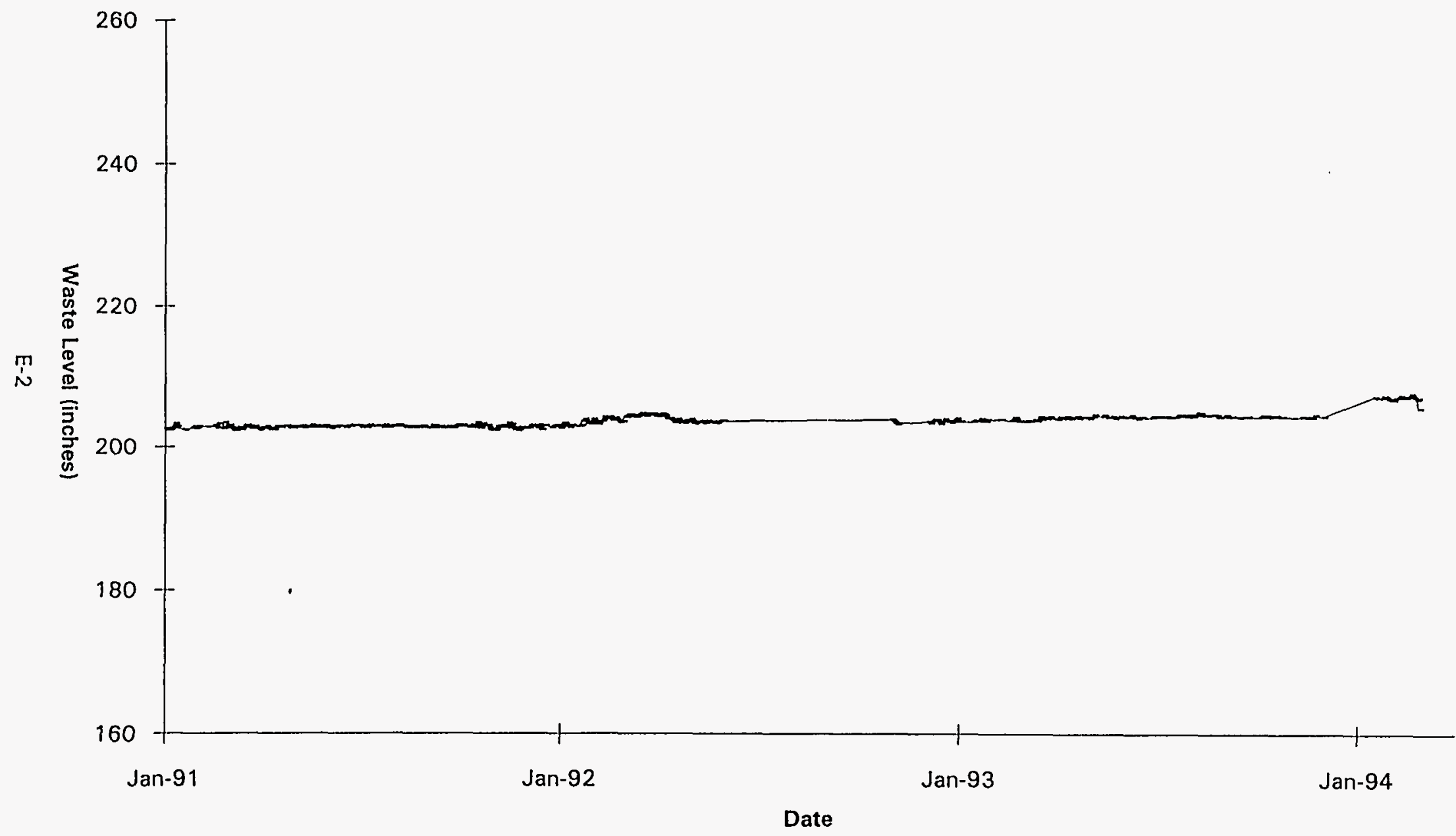

Data obtained from WHC Surveillance Analysis Computer System, February 28, 1994. 
WHC-SD-WM-ER-323, Rev. 0

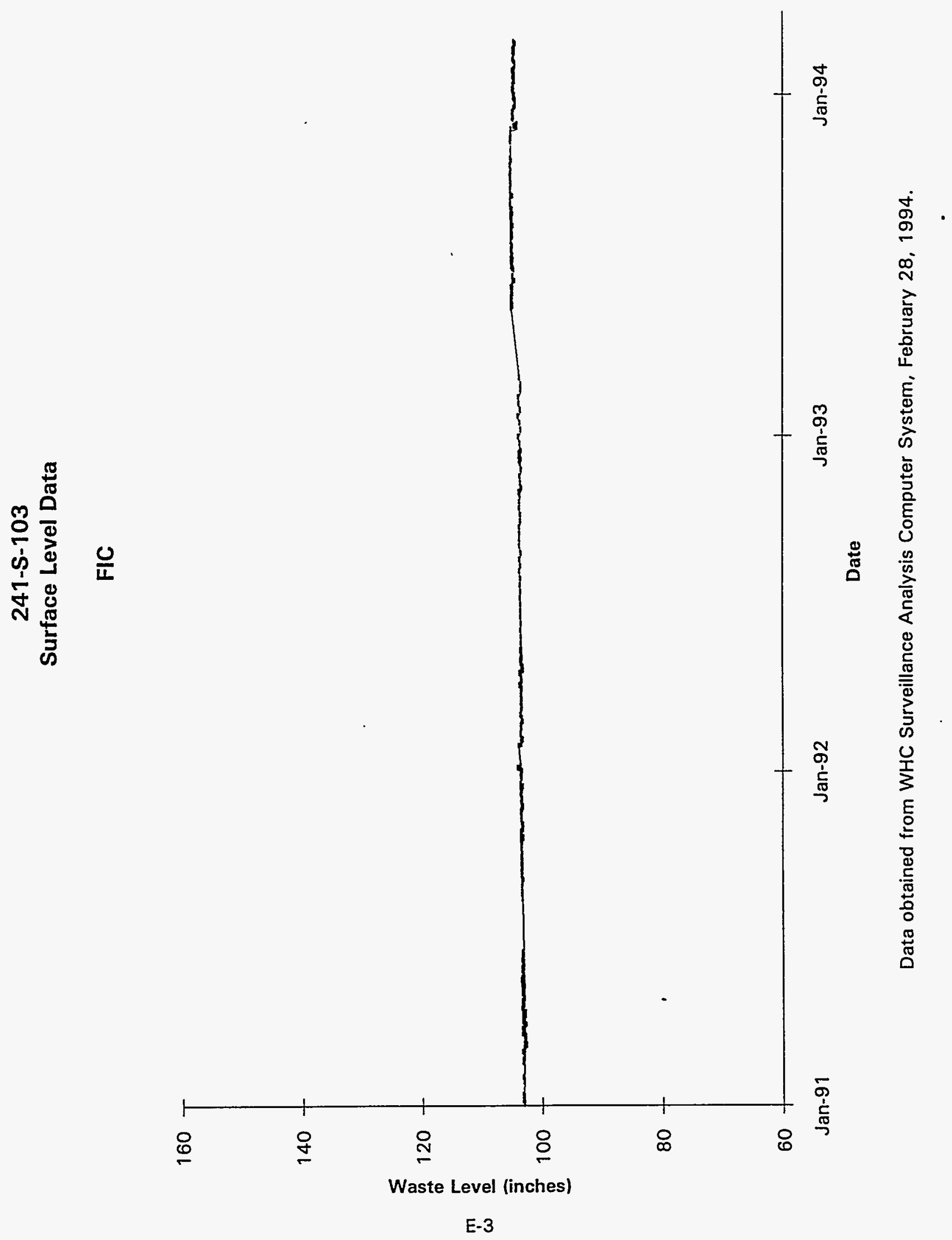


WHC-SD-WM-ER-323, Rev. 0

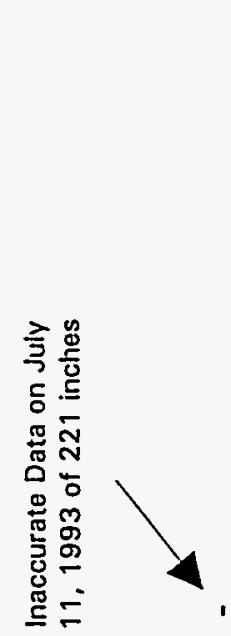

吉兽

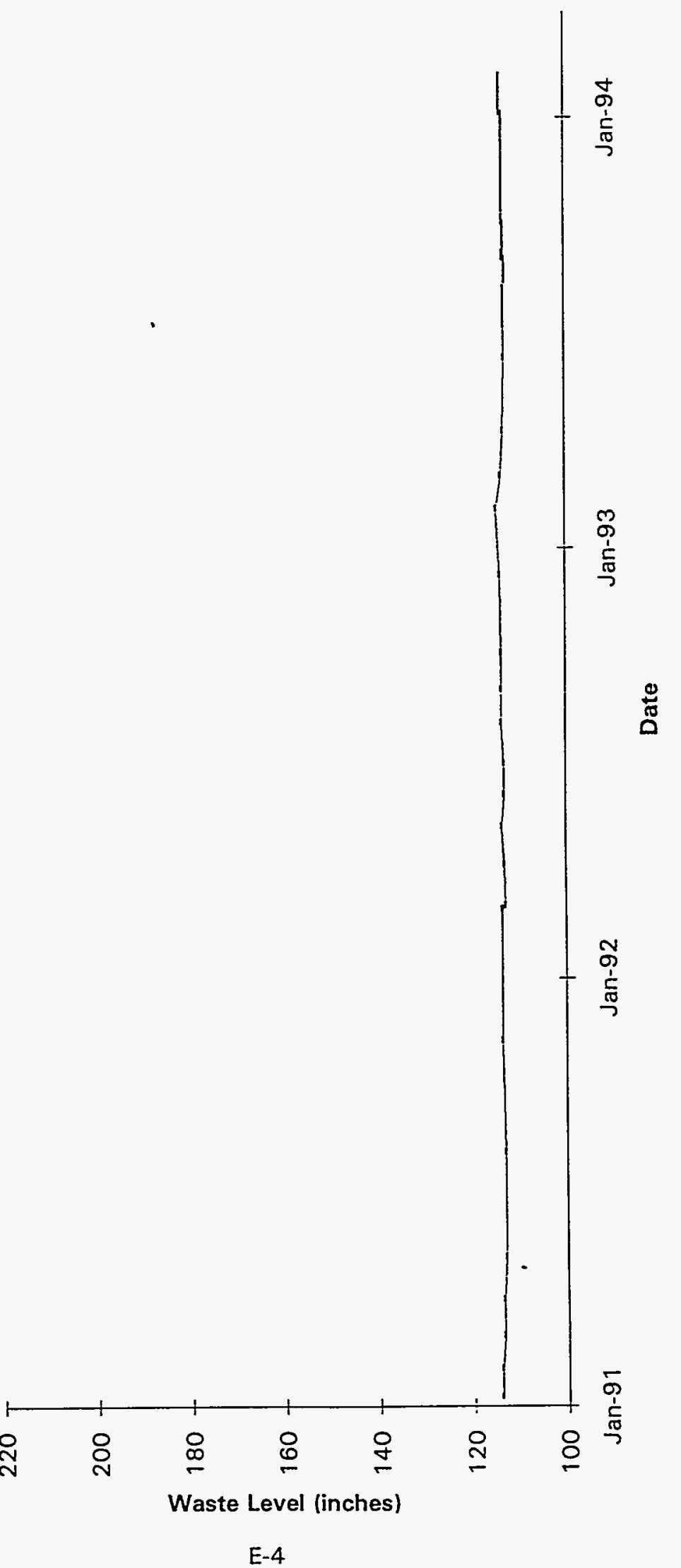


WHC-SD-WM-ER-323, Rev. 0

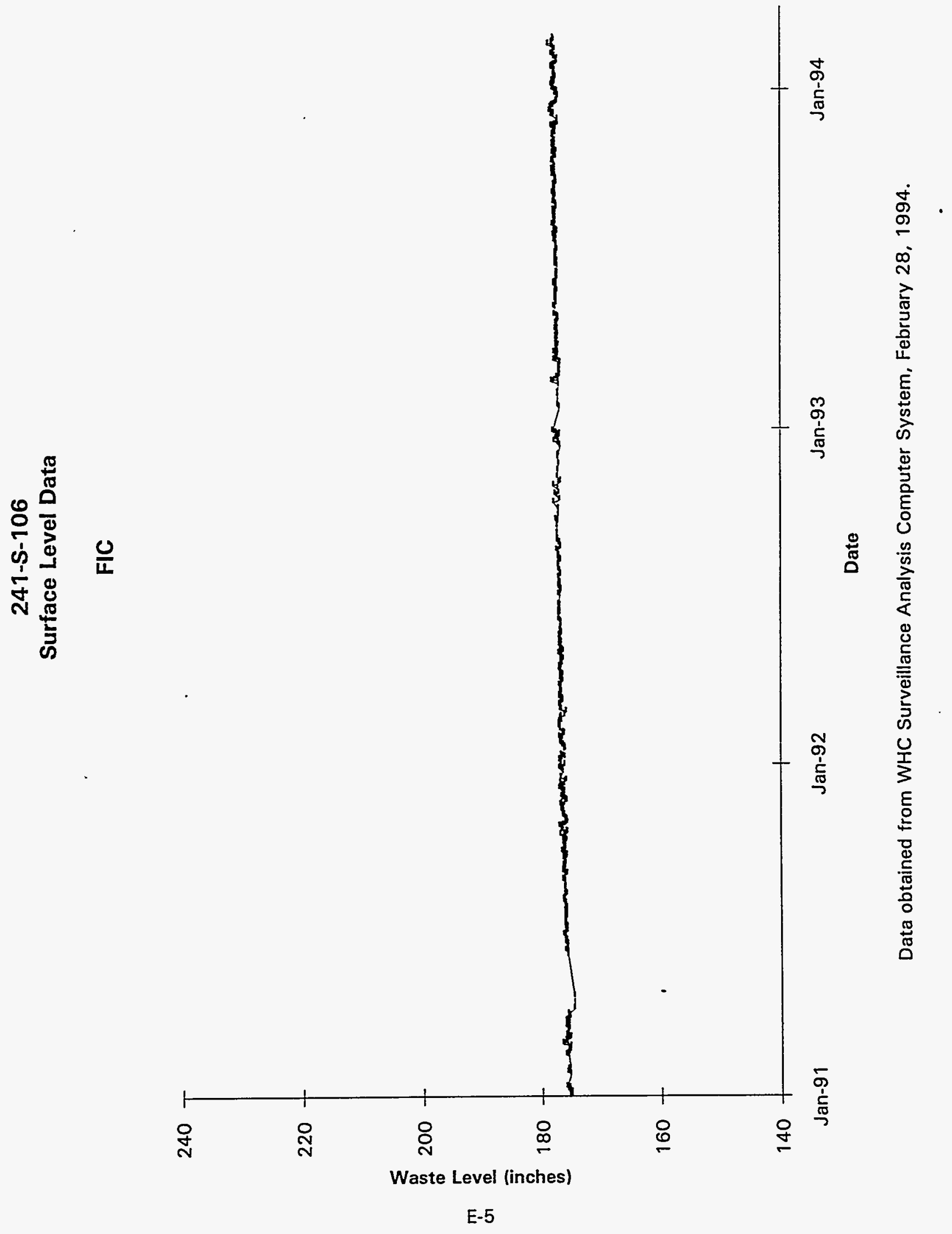


WHC-SD-WM-ER-323, Rev. 0

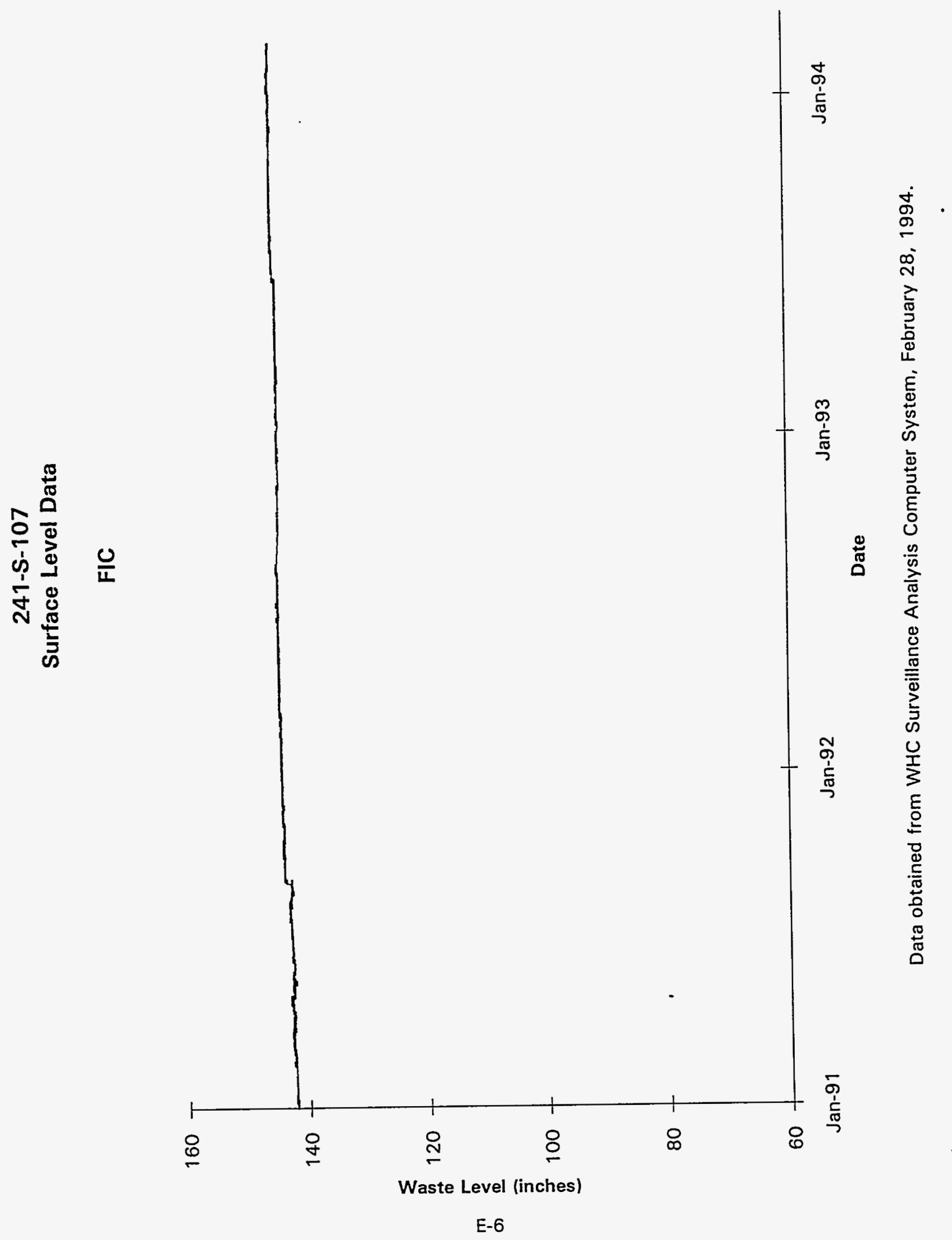




\section{1-S-108 \\ Surface Level Data \\ Manual Tape \\ (this tank has an FIC set in the intrusion mode)}

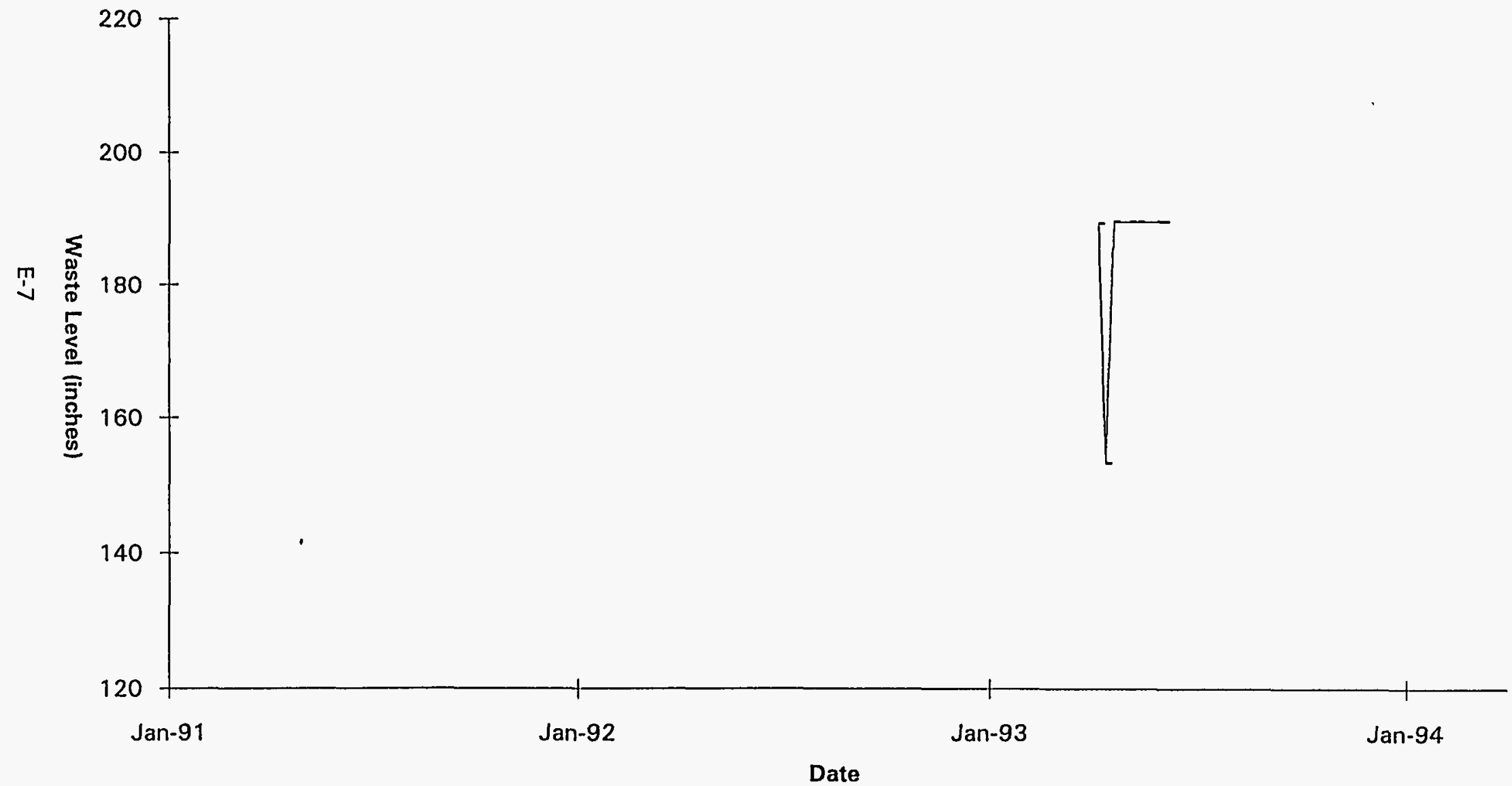

Data obtained from WHC Surveillance Analysis Computer System, February 28, 1994. 
241-S-109

\section{Surface Level Data}

FIC

(normally set in the intrusion mode)

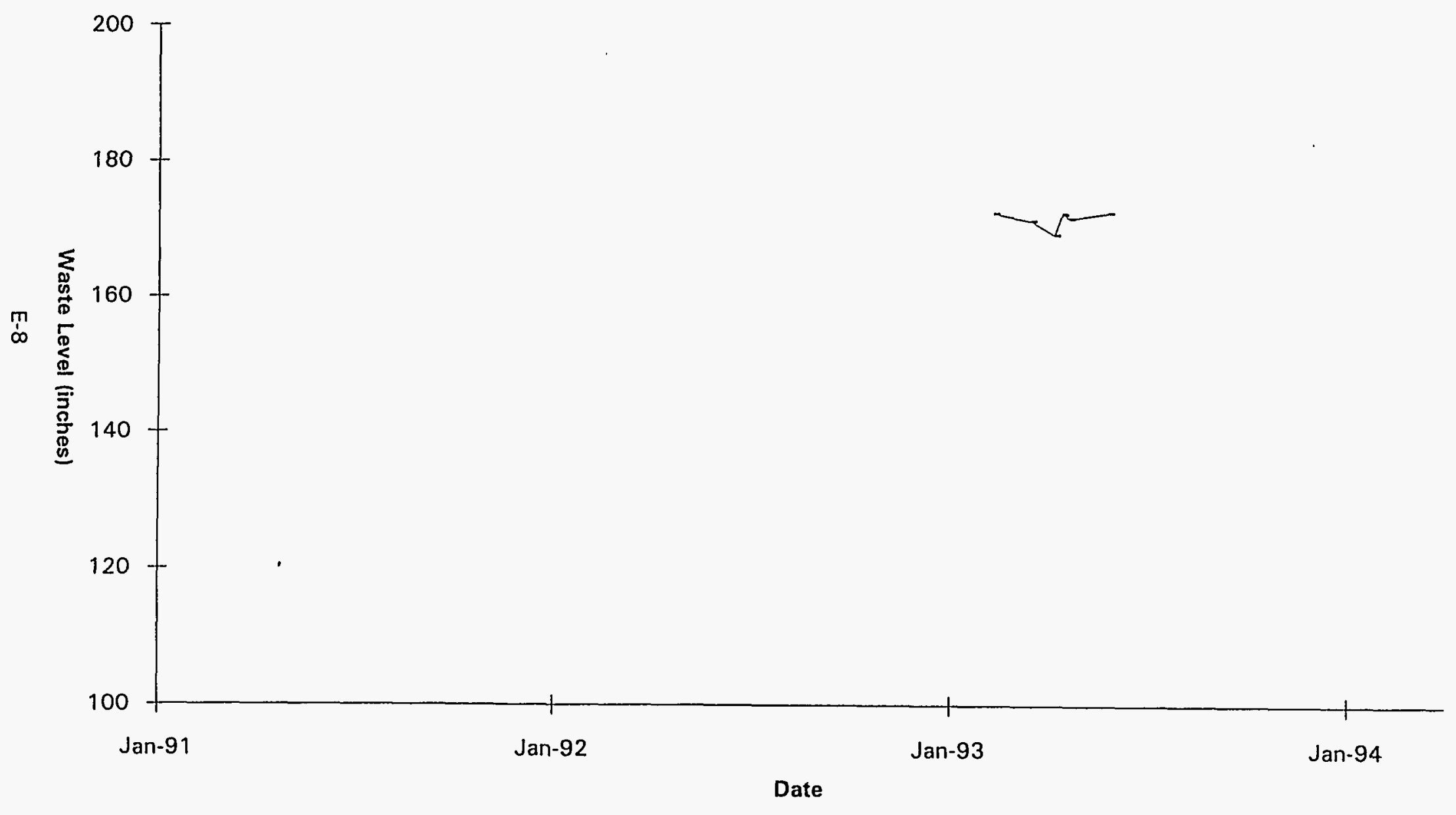

Data obtained from WHC Surveillance Analysis Computer System, February 28, 1994. 

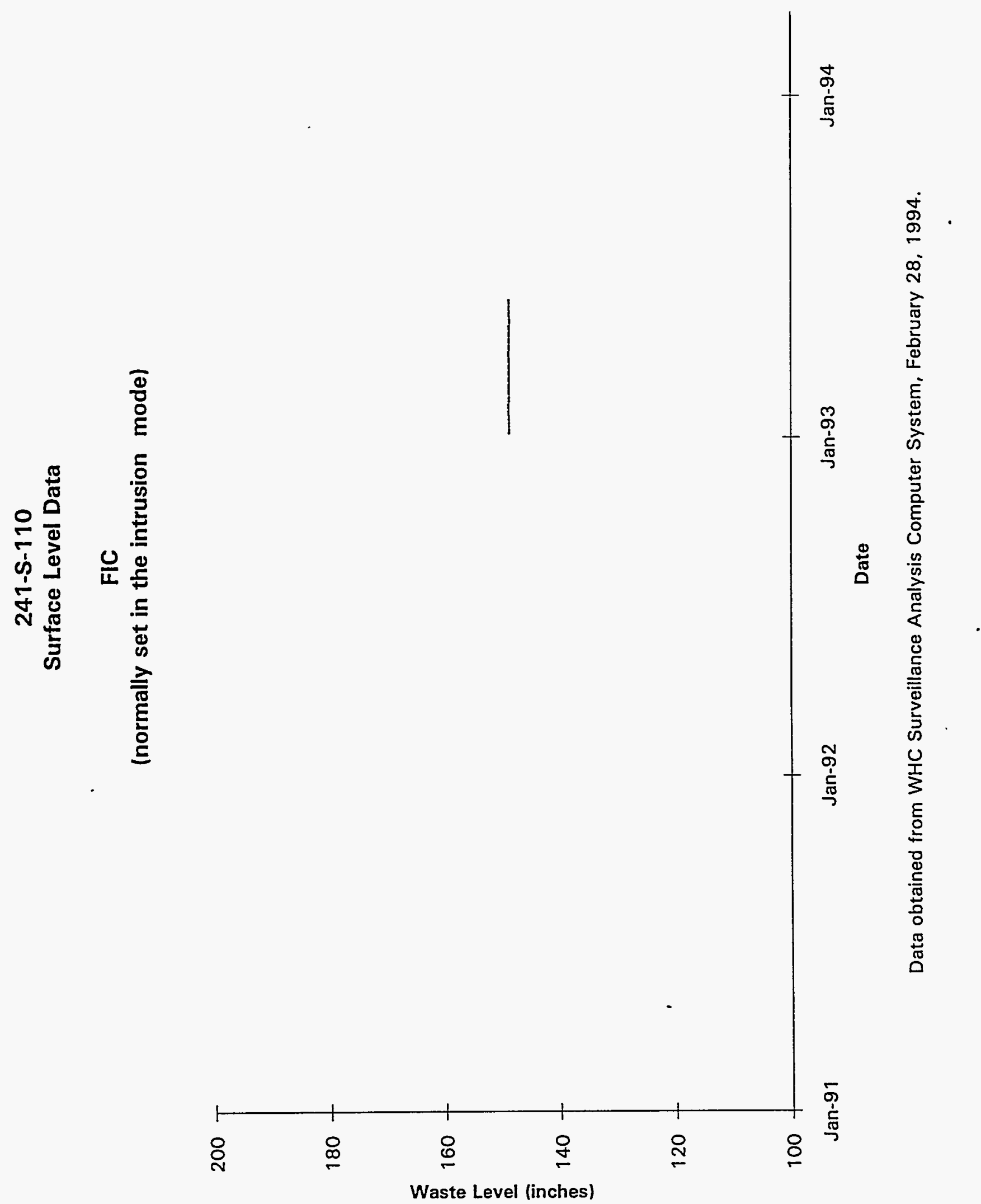

E-9 
WHC-SD-WM-ER-323, Rev. 0

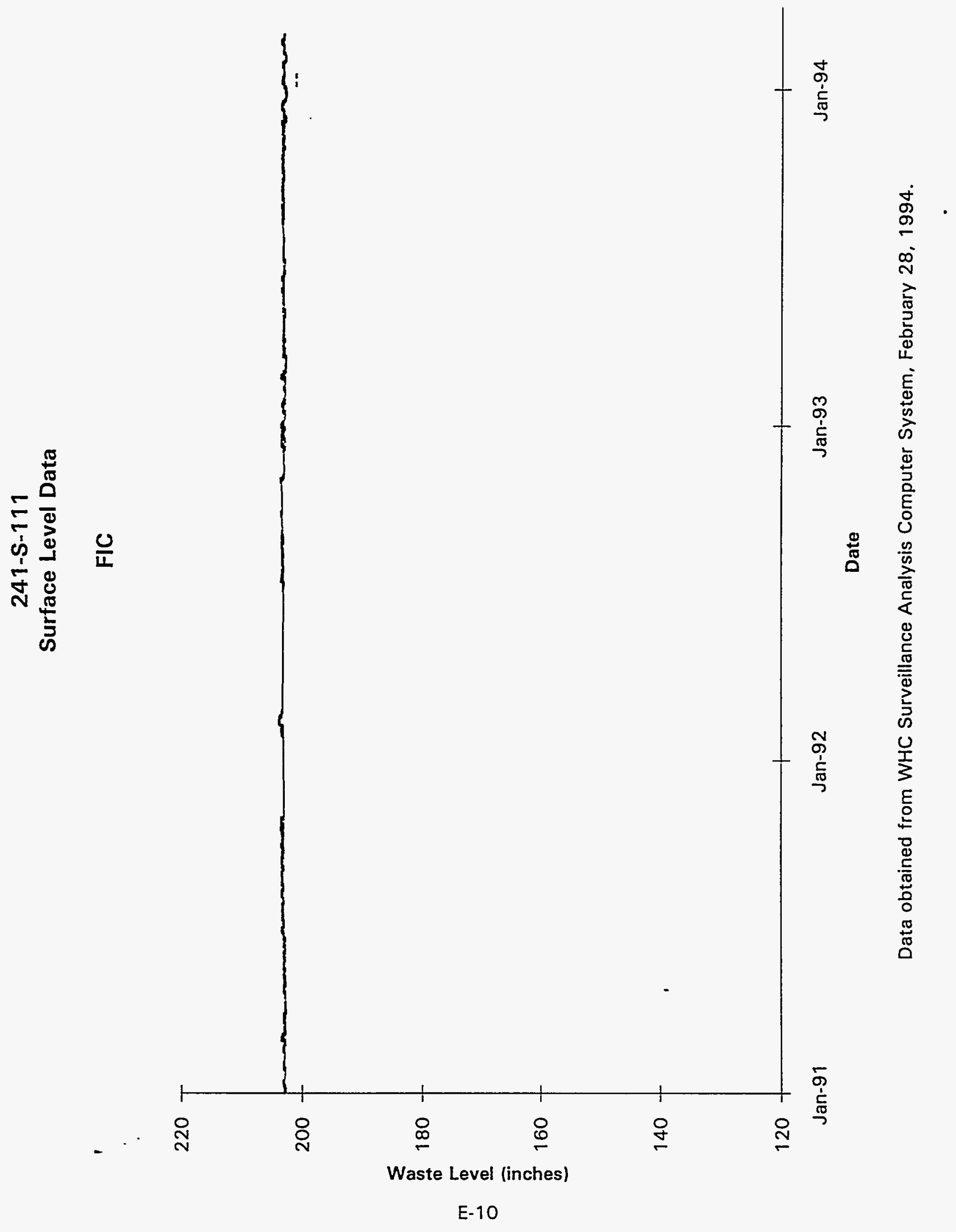


241-S-112

\section{Surface Level Data}

Inormally set in the

(normally set in the intrusion mode)

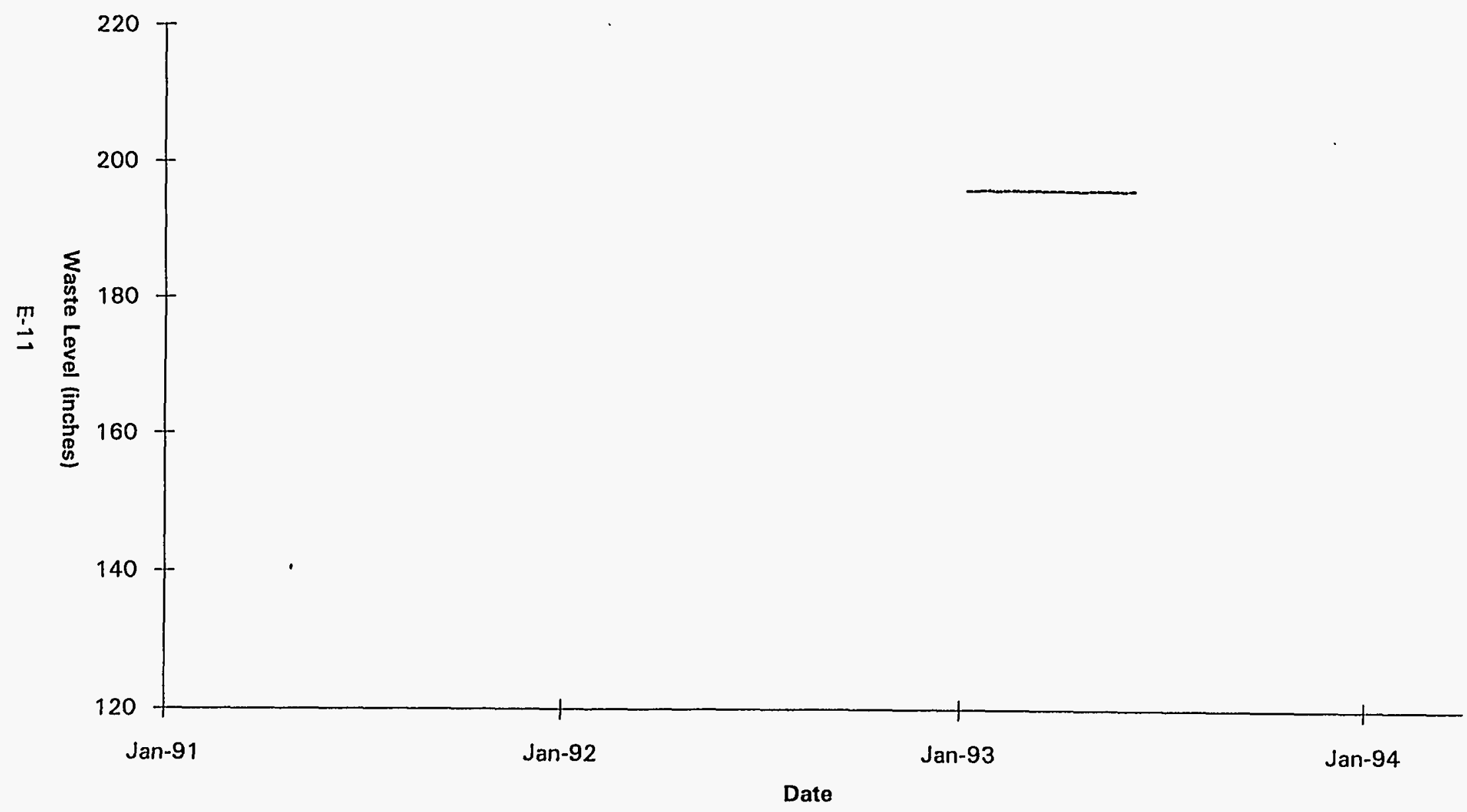

Data obtained from WHC Surveillance Analysis Computer System, February 28, 1994. 


\begin{tabular}{|c|c|c|c|c|c|}
\hline \multicolumn{6}{|c|}{ Surface Level Data } \\
\hline \multicolumn{6}{|c|}{ FIC } \\
\hline Year & Month & Day & Hour & Minute & Surface Level \\
\hline 92 & 4 & 30 & 7 & 3 & 161.3 \\
\hline 92 & 5 & 1 & 7 & 3 & 161.2 \\
\hline 92 & 5 & 2 & 7 & 3 & 161.3 \\
\hline 92 & 5 & 3 & 7 & 3 & 161.2 \\
\hline 92 & 5 & 4 & 7 & 3 & 161.2 \\
\hline 92 & 5 & 5 & 7 & 4 & 161.3 \\
\hline 92 & 5 & 6 & 7 & 3 & 161.3 \\
\hline 92 & 5 & 7 & 7 & 4 & 161.3 \\
\hline 92 & 5 & 8 & 7 & 3 & 161.3 \\
\hline 92 & 5 & 9 & 7 & 4 & 161.3 \\
\hline 92 & 5 & 10 & 7 & 4 & 161.4 \\
\hline 92 & 5 & 11 & 7 & 3 & 161.4 \\
\hline 92 & 5 & 12 & 7 & 3 & 161.4 \\
\hline 92 & 5 & 13 & 7 & 3 & 161.3 \\
\hline 92 & 5 & 14 & 7 & 3 & 161.4 \\
\hline 92 & 5 & 15 & 7 & 3 & 161.4 \\
\hline 92 & 5 & 16 & 7 & 3 & 161.3 \\
\hline 92 & 5 & 17 & 7 & 2 & 161.3 \\
\hline 92 & 5 & 18 & 7 & 3 & 161.3 \\
\hline 92 & 5 & 19 & 7 & 3 & 161.3 \\
\hline 92 & 5 & 20 & 7 & 3 & 161.3 \\
\hline 92 & 5 & 21 & 7 & 3 & 161.3 \\
\hline 92 & 5 & 22 & 7 & 2 & 161.3 \\
\hline 92 & 5 & 23 & 7 & 3 & 161.3 \\
\hline 92 & 5 & 24 & 7 & 3 & 161.3 \\
\hline 92 & 5 & 25 & 7 & 3 & 161.3 \\
\hline 92 & 5 & 27 & 7 & 3 & 161.3 \\
\hline 92 & 5 & 28 & 7 & 2 & 161.3 \\
\hline 92 & 5 & 29 & 7 & 2 & 161.3 \\
\hline 92 & 5 & 30 & 7 & 3 & 161.3 \\
\hline 92 & 5 & 31 & 7 & 3 & 161.3 \\
\hline 92 & 6 & 1 & 7 & 3 & 161.3 \\
\hline 92 & 6 & 2 & 7 & 3 & 161.3 \\
\hline 92 & 6 & 3 & 7 & 3 & 161.3 \\
\hline 92 & 6 & 4 & 7 & 3 & 161.3 \\
\hline 92 & 6 & 5 & 7 & 2 & 161.2 \\
\hline 92 & 6 & 6 & 7 & 3 & 161.3 \\
\hline 92 & 6 & 7 & 7 & 3 & 161.3 \\
\hline 92 & 6 & 8 & 7 & 3 & 161.3 \\
\hline 92 & 6 & 9 & 7 & 3 & 161.3 \\
\hline 92 & 6 & 10 & 7 & 3 & 161.3 \\
\hline 92 & 6 & 11 & 7 & 3 & 161.3 \\
\hline 92 & 6 & 12 & 7 & 5 & 161.2 \\
\hline 92 & 6 & 13 & 7 & 5 & 161.3 \\
\hline 92 & 6 & 15 & 7 & 3 & 161.3 \\
\hline 92 & 6 & 16 & 7 & 3 & 161.3 \\
\hline
\end{tabular}

Data obtained from WHC Surveillance Analysis Computer System (SACS), February 28, 1994. E-12 


\begin{tabular}{|c|c|c|c|c|c|}
\hline Year & Month & Day & Hour & Minute & Surface Level \\
\hline 92 & 6 & 17 & 7 & 3 & 161.3 \\
\hline 92 & 6 & 18 & 7 & 3 & 161.3 \\
\hline 92 & 6 & 19 & 7 & 2 & 161.3 \\
\hline 92 & 6 & 20 & 7 & 2 & 161.3 \\
\hline 92 & 6 & 21 & 7 & 3 & 161.3 \\
\hline 92 & 6 & 22 & 7 & 3 & 161.3 \\
\hline 92 & 6 & 23 & 7 & 3 & 161.3 \\
\hline 92 & 6 & 24 & 7 & 3 & 161.3 \\
\hline 92 & 6 & 25 & 7 & 3 & 161.3 \\
\hline 92 & 6 & 26 & 7 & 2 & 161.3 \\
\hline 92 & 6 & 27 & 7 & 3 & 161.3 \\
\hline 92 & 6 & 28 & 6 & 6 & 161.3 \\
\hline 92 & 7 & 8 & 7 & 6 & 162.2 \\
\hline 92 & 7 & 9 & 7 & 6 & 162.2 \\
\hline 92 & 7 & 10 & 7 & 6 & 162.2 \\
\hline 92 & 7 & 11 & 7 & 6 & 162.3 \\
\hline 92 & 7 & 12 & 7 & 6 & 162.3 \\
\hline 92 & 7 & 13 & 7 & 6 & 162.3 \\
\hline 92 & 7 & 14 & 7 & 6 & 162.2 \\
\hline 92 & 7 & 15 & 7 & 6 & 162.2 \\
\hline 92 & 7 & 16 & 7 & 6 & 162.2 \\
\hline 92 & 7 & 17 & 7 & 6 & 162.3 \\
\hline 92 & 7 & 18 & 7 & 6 & 162.3 \\
\hline 92 & 7 & 19 & 7 & 6 & 162.3 \\
\hline 92 & 7 & 20 & 7 & 6 & 162.3 \\
\hline 92 & 7 & 21 & 7 & 5 & 162.3 \\
\hline 92 & 7 & 22 & 7 & 6 & 162.3 \\
\hline 92 & 7 & 24 & 7 & 5 & 162.3 \\
\hline 92 & 7 & 25 & 7 & 6 & 162.2 \\
\hline .92 & 7 & 26 & 7 & 6 & 162.3 \\
\hline 92 & 7 & 27 & 7 & 5 & 162.3 \\
\hline 92 & 7 & 28 & 7 & 6 & 162.3 \\
\hline 92 & 7 & 29 & 7 & 6 & 162.3 \\
\hline 92 & 7 & 30 & 7 & 6 & 162.3 \\
\hline 92 & 7 & 31 & 7 & 5 & 162.3 \\
\hline 92 & 8 & 1 & 7 & 6 & 162.3 \\
\hline 92 & 8 & 2 & 6 & 6 & 162.3 \\
\hline 92 & 8 & 3 & 7 & 6 & 162.3 \\
\hline 92 & 8 & 4 & 7 & 6 & 162.3 \\
\hline 92 & 8 & 5 & 7 & 5 & 162.3 \\
\hline 92 & 8 & 6 & 7 & 5 & 162.3 \\
\hline 92 & 8 & 7 & 7 & 5 & 162.3 \\
\hline 92 & 8 & 8 & 7 & 6 & 162.3 \\
\hline 92 & 8 & 9 & 7 & 6 & 162.2 \\
\hline 92 & 8 & 10 & 7 & 5 & 162.2 \\
\hline 92 & 8 & 11 & 7 & 6 & 162.3 \\
\hline 92 & 8 & 12 & 7 & 6 & 162.3 \\
\hline 92 & 8 & 13 & 7 & 6 & 162.3 \\
\hline
\end{tabular}

Data obtained from WHC Surveillance Analysis Computer System (SACS), February 28, 1994. 


\begin{tabular}{|c|c|c|c|c|c|}
\hline Year & Month & Day & Hour & Minute & Surface Level \\
\hline 92 & 8 & 14 & 7 & 6 & 162.3 \\
\hline 92 & 8 & 15 & 7 & 6 & 162.3 \\
\hline 92 & 8 & 16 & 7 & 6 & 162.3 \\
\hline 92 & 8 & 17 & 7 & 6 & 162.3 \\
\hline 92 & 8 & 18 & 7 & 6 & 162.3 \\
\hline 92 & 8 & 19 & 7 & 6 & 162.3 \\
\hline 92 & 8 & 20 & 7 & 6 & 162.3 \\
\hline 92 & 8 & 21 & 7 & 5 & 162.3 \\
\hline 92 & 8 & 22 & 7 & 4 & 162.3 \\
\hline 92 & 8 & 23 & 7 & 4 & 162.2 \\
\hline 92 & 8 & 24 & 7 & 4 & 162.2 \\
\hline 92 & 8 & 25 & 7 & 4 & 162.2 \\
\hline 92 & 8 & 26 & 7 & 3 & 162.2 \\
\hline 92 & 8 & 27 & 7 & 4 & 162.3 \\
\hline 92 & 8 & 28 & 7 & 2 & 162.3 \\
\hline 92 & 8 & 29 & 7 & 2 & 162.3 \\
\hline 92 & 8 & 30 & 7 & 2 & 162.3 \\
\hline 92 & 8 & 31 & 7 & 2 & 162.4 \\
\hline 92 & 9 & 1 & 7 & 2 & 162.3 \\
\hline 92 & 9 & 2 & 7 & 2 & 162.2 \\
\hline 92 & 9 & 3 & 7 & 4 & 162.4 \\
\hline 92 & 9 & 22 & 7 & 3 & 162.3 \\
\hline 92 & 9 & 23 & 7 & 3 & 162.4 \\
\hline 92 & 10 & 29 & 7 & 3 & 161.3 \\
\hline 92 & 10 & 30 & 7 & 3 & 161.3 \\
\hline 92 & 10 & 31 & 7 & 3 & 161.2 \\
\hline 92 & 11 & 1 & 7 & 3 & 161.1 \\
\hline 92 & 11 & 2 & 7 & 3 & 161.1 \\
\hline 92 & 11 & 3 & 7 & 3 & 161 \\
\hline 92 & 11 & 4 & 7 & 3 & 161.1 \\
\hline 92 & 12 & 2 & 6 & 3 & 161.2 \\
\hline 92 & 12 & 3 & 6 & 3 & 161.1 \\
\hline 92 & 12 & 4 & 7 & 3 & 161.1 \\
\hline 92 & 12 & 6 & 7 & 2 & 161.2 \\
\hline 92 & 12 & 7 & 7 & 3 & 161.2 \\
\hline 92 & 12 & 8 & 7 & 3 & 161.2 \\
\hline 92 & 12 & 9 & 7 & 3 & 161.2 \\
\hline 92 & 12 & 10 & 7 & 3 & 161.2 \\
\hline 92 & 12 & 11 & 7 & 3 & 161.2 \\
\hline 92 & 12 & 13 & 7 & 4 & 161 \\
\hline 92 & 12 & 14 & 7 & 3 & 161.1 \\
\hline 92 & 12 & 15 & 7 & 3 & 161.1 \\
\hline 92 & 12 & 16 & 7 & 4 & 161.1 \\
\hline 92 & 12 & 17 & 7 & 3 & 161.2 \\
\hline 92 & 12 & 18 & 7 & 3 & 161.1 \\
\hline 92 & 12 & 19 & 7 & 3 & 161.1 \\
\hline 92 & 12 & 20 & 7 & 4 & 161.2 \\
\hline 92 & 12 & 21 & 7 & 4 & 161.1 \\
\hline
\end{tabular}

Data obtained from WHC Surveillance Analysis Computer System (SACS), February 28, 1994. 


\begin{tabular}{|c|c|c|c|c|c|}
\hline Year & Month & Day & Hour & Minute & Surface Level \\
\hline 92 & 12 & 23 & 7 & 4 & 161 \\
\hline 92 & 12 & 24 & 7 & 3 & 161.2 \\
\hline 92 & 12 & 25 & 7 & 3 & 161.2 \\
\hline 92 & 12 & 26 & 7 & 3 & 161.2 \\
\hline 92 & 12 & 27 & 7 & 3 & 161.2 \\
\hline 92 & 12 & 28 & 7 & 3 & 161.2 \\
\hline 92 & 12 & 30 & 7 & 3 & 161.1 \\
\hline 92 & 12 & 31 & 7 & 3 & 161.1 \\
\hline 93 & 1 & 1 & 7 & 4 & 161.1 \\
\hline 93 & 1 & 12 & 7 & 6 & 161 \\
\hline 93 & 1 & 13 & 7 & 6 & 161.1 \\
\hline 93 & 1 & 14 & 7 & 3 & 161.2 \\
\hline 93 & 1 & 15 & 7 & 3 & 161.1 \\
\hline 93 & 1 & 17 & 7 & 2 & 161.1 \\
\hline 93 & 1 & 18 & 7 & 2 & 161.1 \\
\hline 93 & 1 & 19 & 7 & 2 & 161.2 \\
\hline 93 & 1 & 20 & 7 & 2 & 161.3 \\
\hline 93 & 1 & 21 & 7 & 2 & 161.1 \\
\hline 93 & 1 & 22 & 7 & 4 & 161.2 \\
\hline 93 & 1 & 23 & 7 & 3 & 161.1 \\
\hline 93 & 1 & 24 & 7 & 3 & 161.1 \\
\hline 93 & 1 & 25 & 6 & 3 & 161.1 \\
\hline 93 & 2 & 5 & 6 & 4 & 161.1 \\
\hline 93 & 2 & 6 & 2 & 3 & 161.1 \\
\hline 93 & 2 & 7 & 7 & 3 & 161.1 \\
\hline 93 & 2 & 16 & 6 & 3 & 160.1 \\
\hline 93 & 2 & 18 & 7 & 3 & 161.1 \\
\hline 93 & 2 & 19 & 7 & 2 & 161.2 \\
\hline 93 & 2 & 20 & 7 & 3 & 161.2 \\
\hline 93 & 2 & 21 & 7 & 4 & 161.2 \\
\hline 93 & 2 & 22 & 7 & 4 & 161.1 \\
\hline 93 & 2 & 23 & 7 & 3 & 161.1 \\
\hline 93 & 2 & 24 & 7 & 4 & 161.1 \\
\hline 93 & 2 & 25 & 7 & 3 & 161 \\
\hline 93 & 2 & 26 & 7 & 3 & 161 \\
\hline 93 & 2 & 27 & 7 & 4 & 161 \\
\hline 93 & 2 & 28 & 7 & 3 & 161 \\
\hline 93 & 3 & 1 & 7 & 3 & 161 \\
\hline 93 & 3 & 2 & 7 & 4 & 161 \\
\hline 93 & 3 & 3 & 7 & 3 & 161 \\
\hline 93 & 3 & 4 & 7 & 4 & 161 \\
\hline 93 & 3 & 5 & 7 & 4 & 161 \\
\hline 93 & 3 & 6 & 7 & 3 & 161 \\
\hline 93 & 3 & 9 & 7 & 4 & 161 \\
\hline 93 & 3 & 10 & 7 & 5 & 161 \\
\hline 93 & 3 & 11 & 7 & 4 & 161 \\
\hline 93 & 3 & 12 & 7 & 4 & 161 \\
\hline 93 & 3 & 13 & 7 & 3 & 161.1 \\
\hline
\end{tabular}

Data obtained from WHC Surveillance Analysis Computer System (SACS), February 28, 1994. E-15 


\begin{tabular}{|c|c|c|c|c|c|}
\hline Year & Month & Day & Hour & Minute & Surface Level \\
\hline 93 & 3 & 14 & 7 & 4 & 161.2 \\
\hline 93 & 3 & 15 & 7 & 3 & 161.2 \\
\hline 93 & 3 & 16 & 7 & 4 & 161.1 \\
\hline 93 & 3 & 17 & 7 & 3 & 161.1 \\
\hline 93 & 3 & 18 & 7 & 4 & 161.2 \\
\hline 93 & 3 & 19 & 7 & 3 & 161.1 \\
\hline 93 & 3 & 20 & 7 & 4 & 161.1 \\
\hline 93 & 3 & 21 & 7 & 4 & 161.1 \\
\hline 93 & 3 & 22 & 7 & 3 & 161.1 \\
\hline 93 & 3 & 23 & 7 & 3 & 161.2 \\
\hline 93 & 3 & 24 & 7 & 4 & 161.1 \\
\hline 93 & 3 & 25 & 7 & 3 & 161.1 \\
\hline 93 & 3 & 26 & 7 & 3 & 161.2 \\
\hline 93 & 3 & 27 & 7 & 3 & 161.2 \\
\hline 93 & 3 & 28 & 7 & 3 & 161.1 \\
\hline 93 & 3 & 29 & 6 & 3 & 161.1 \\
\hline 93 & 3 & 30 & 7 & 3 & 161.1 \\
\hline 93 & 3 & 31 & 7 & 3 & 161.1 \\
\hline 93 & 4 & 1 & 7 & 3 & 161.2 \\
\hline 93 & 4 & 2 & 7 & 6 & 161.2 \\
\hline 93 & 4 & 3 & 7 & 6 & 161.2 \\
\hline 93 & 4 & 4 & 7 & 5 & 161.1 \\
\hline 93 & 4 & 5 & 7 & 6 & 161.2 \\
\hline 93 & 4 & 10 & 7 & 5 & 161.2 \\
\hline 93 & 4 & 11 & 7 & 6 & 161.2 \\
\hline 93 & 4 & 12 & 7 & 6 & 161.1 \\
\hline 93 & 4 & 12 & 10 & 3 & 161.1 \\
\hline 93 & 4 & 19 & 10 & 3 & 161.1 \\
\hline 93 & 4 & 26 & 10 & 3 & 161.2 \\
\hline 93 & 5 & 3 & 10 & 5 & 161.2 \\
\hline 93 & 5 & 10 & 10 & 5 & 161.2 \\
\hline 93 & 5 & 17 & 10 & 5 & 161.1 \\
\hline 93 & 5 & 24 & 10 & 5 & 161.6 \\
\hline 93 & 6 & 1 & 10 & 6 & 161.1 \\
\hline 93 & 6 & 7 & 10 & 6 & 161.1 \\
\hline 93 & 6 & 14 & 10 & 6 & 161.15 \\
\hline 93 & 6 & 21 & 10 & 6 & 161.2 \\
\hline 93 & 6 & 28 & 10 & 6 & 161.2 \\
\hline & & & & & \\
\hline & & & & & \\
\hline & & & & & \\
\hline & & & & & \\
\hline & & & & & \\
\hline & & & & & \\
\hline & & & & & \\
\hline & & & & & \\
\hline & & & & & \\
\hline & & & & & \\
\hline
\end{tabular}

Data obtained from WHC Surveillance Analysis Computer System (SACS), February 28, 1994. 


\begin{tabular}{|c|c|c|c|c|c|}
\hline \multicolumn{6}{|c|}{ Surface Level Data } \\
\hline \multicolumn{6}{|c|}{$\mathrm{FIC}$} \\
\hline Year & Month & Day & Hour & Minute & Surface Level \\
\hline 91 & 1 & 1 & 7 & 3 & 202.5 \\
\hline 91 & 1 & 2 & 7 & 2 & 202.3 \\
\hline 91 & 1 & 3 & 7 & 3 & 202.3 \\
\hline 91 & 1 & 4 & 7 & 2 & 202.5 \\
\hline 91 & 1 & 5 & 7 & 2 & 202.5 \\
\hline 91 & 1 & 6 & 7 & 2 & 202.4 \\
\hline 91 & 1 & 7 & 7 & 2 & 202.6 \\
\hline 91 & 1 & 8 & 7 & 3 & 202.6 \\
\hline 91 & 1 & 9 & 7 & 2 & 202.7 \\
\hline 91 & 1 & 10 & 7 & 2 & 203.2 \\
\hline 91 & 1 & 11 & 7 & 3 & 202.6 \\
\hline 91 & 1 & 12 & 7 & 2 & 202.8 \\
\hline 91 & 1 & 19 & 7 & 3 & 202.4 \\
\hline 91 & 1 & 26 & 7 & 3 & 202.6 \\
\hline 91 & 1 & 27 & 7 & 3 & 202.7 \\
\hline 91 & 1 & 28 & 7 & 10 & 202.8 \\
\hline 91 & 1 & 29 & 7 & 2 & 202.5 \\
\hline 91 & 1 & 30 & 7 & 10 & 202.7 \\
\hline 91 & 1 & 31 & 7 & 2 & 202.7 \\
\hline 91 & 2 & 1 & 7 & 2 & 202.8 \\
\hline 91 & 2 & 15 & 7 & 3 & 202.8 \\
\hline 91 & 2 & 16 & 7 & 2 & 203 \\
\hline 91 & 2 & 17 & 7 & 2 & 202.8 \\
\hline 91 & 2 & 18 & 6 & 2 & 202.6 \\
\hline 91 & 2 & 19 & 7 & 2 & 203.2 \\
\hline 91 & 2 & 20 & 7 & 3 & 202.6 \\
\hline 91 & 2 & 21 & 7 & 2 & 202.6 \\
\hline 91 & 2 & 22 & 7 & 2 & 202.7 \\
\hline 91 & 2 & 23 & 7 & 2 & 202.6 \\
\hline 91 & 2 & 24 & 7 & 3 & 202.5 \\
\hline 91 & 2 & 25 & 7 & 3 & 203.4 \\
\hline 91 & 2 & 26 & 6 & 2 & 202.6 \\
\hline 91 & 2 & 27 & 7 & 2 & 202.9 \\
\hline 91 & 2 & 28 & 7 & 2 & 203 \\
\hline 91 & 3 & 1 & 7 & 2 & 202.8 \\
\hline 91 & 3 & 2 & 7 & 2 & 203 \\
\hline 91 & 3 & 3 & 4 & 3 & 202.6 \\
\hline 91 & 3 & 4 & 7 & 3 & 202.7 \\
\hline 91 & 3 & 5 & 7 & 3 & 202.4 \\
\hline 91 & 3 & 6 & 7 & 2 & 202.3 \\
\hline 91 & 3 & 7 & 7 & 2 & 202.2 \\
\hline 91 & 3 & 8 & 7 & 2 & 202.2 \\
\hline 91 & 3 & 9 & 7 & 2 & 202.5 \\
\hline 91 & 3 & 10 & 7 & 2 & 202.6 \\
\hline 91 & 3 & 11 & 7 & 2 & 202.7 \\
\hline 91 & 3 & 12 & 7 & 3 & 202.6 \\
\hline
\end{tabular}

Data obtained from WHC Surveillance Analysis Computer System (SACS), February 28, 1994. E-17 


\begin{tabular}{|c|c|c|c|c|c|}
\hline Year & Month & Day & Hour & Minute & Surface Level \\
\hline 91 & 3 & 13 & 7 & 3 & 202.4 \\
\hline 91 & 3 & 14 & 7 & 3 & 202.6 \\
\hline 91 & 3 & 15 & 7 & 3 & 202.7 \\
\hline 91 & 3 & 16 & 7 & 3 & 202.9 \\
\hline 91 & 3 & 17 & 7 & 2 & 203 \\
\hline 91 & 3 & 18 & 7 & 3 & 203 \\
\hline 91 & 3 & 19 & 7 & 3 & 202.8 \\
\hline 91 & 3 & 20 & 7 & 2 & 202.9 \\
\hline 91 & 3 & 21 & 7 & 3 & 202.6 \\
\hline 91 & 3 & 22 & 7 & 2 & 202.5 \\
\hline 91 & 3 & 23 & 7 & 3 & 202.5 \\
\hline 91 & 3 & 24 & 7 & 3 & 202.7 \\
\hline 91 & 3 & 25 & 7 & 3 & 202.8 \\
\hline 91 & 3 & 26 & 7 & 3 & 202.7 \\
\hline 91 & 3 & 27 & 7 & 3 & 202.6 \\
\hline 91 & 3 & 28 & 7 & 3 & 202.5 \\
\hline 91 & 3 & 29 & 7 & 3 & 202.3 \\
\hline 91 & 3 & 30 & 7 & 3 & 202.4 \\
\hline 91 & 3 & 31 & 7 & 3 & 202.6 \\
\hline 91 & 4 & 1 & 7 & 3 & 202.7 \\
\hline 91 & 4 & 2 & 7 & 3 & 202.6 \\
\hline 91 & 4 & 3 & 7 & 3 & 202.5 \\
\hline 91 & 4 & 4 & 7 & 3 & 202.7 \\
\hline 91 & 4 & 5 & 7 & 3 & 202.8 \\
\hline 91 & 4 & 6 & 7 & 3 & 202.6 \\
\hline 91 & 4 & 7 & 7 & 3 & 202.6 \\
\hline 91 & 4 & 8 & 7 & 4 & 202.4 \\
\hline 91 & 4 & 9 & 7 & 3 & 202.6 \\
\hline 91 & 4 & 10 & 7 & 3 & 202.6 \\
\hline 91 & 4 & 11 & 7 & 3 & 202.4 \\
\hline 91 & 4 & 12 & 7 & 3 & 202.4 \\
\hline 91 & 4 & 13 & 7 & 4 & 202.6 \\
\hline 91 & 4 & 14 & 7 & 3 & 202.8 \\
\hline 91 & 4 & 15 & 7 & 3 & 202.8 \\
\hline 91 & 4 & 16 & 7 & 10 & 202.8 \\
\hline 91 & 4 & 17 & 7 & 3 & 202.7 \\
\hline 91 & 4 & 18 & 7 & 6 & 202.7 \\
\hline 91 & 4 & 19 & 7 & 5 & 202.7 \\
\hline 91 & 4 & 20 & 7 & 6 & 202.8 \\
\hline 91 & 4 & 21 & 7 & 5 & 202.8 \\
\hline 91 & 4 & 22 & 7 & 6 & 202.8 \\
\hline 91 & 4 & 23 & 7 & 6 & 202.9 \\
\hline 91 & 4 & 24 & 7 & 5 & 202.9 \\
\hline 91 & 4 & 25 & 7 & 5 & 202.8 \\
\hline 91 & 4 & 26 & 3 & 5 & 202.7 \\
\hline 91 & 4 & 27 & 7 & 5 & 202.6 \\
\hline 91 & 4 & 28 & 7 & 6 & 202.6 \\
\hline 91 & 4 & 29 & 7 & 5 & 202.6 \\
\hline
\end{tabular}

Data obtained from WHC Surveillance Analysis Computer System (SACS), February 28, 1994. $\mathrm{E}-18$ 


\begin{tabular}{|c|c|c|c|c|c|}
\hline Year & Month & Day & Hour & Minute & Surface Level \\
\hline 91 & 4 & 30 & 7 & 6 & 202.6 \\
\hline 91 & 5 & 1 & 6 & 6 & 202.8 \\
\hline 91 & 5 & 2 & 7 & 5 & 202.8 \\
\hline 91 & 5 & 3 & 7 & 6 & 202.8 \\
\hline 91 & 5 & 4 & 7 & 5 & 202.7 \\
\hline 91 & 5 & 5 & 7 & 5 & 202.7 \\
\hline 91 & 5 & 6 & 7 & 5 & 202.7 \\
\hline 91 & 5 & 7 & 7 & 5 & 202.8 \\
\hline 91 & 5 & 8 & 7 & 5 & 202.9 \\
\hline 91 & 5 & 9 & 7 & 5 & 202.7 \\
\hline 91 & 5 & 10 & 7 & 5 & 202.6 \\
\hline 91 & 5 & 11 & 7 & 5 & 202.7 \\
\hline 91 & 5 & 12 & 7 & 5 & 202.8 \\
\hline 91 & 5 & 13 & 7 & 5 & 202.8 \\
\hline 91 & 5 & 14 & 7 & 5 & 202.8 \\
\hline 91 & 5 & 15 & 7 & 5 & 202.7 \\
\hline 91 & 5 & 16 & 7 & 6 & 202.8 \\
\hline 91 & 5 & 17 & 7 & 6 & 203 \\
\hline 91 & 5 & 18 & 7 & 6 & 202.9 \\
\hline 91 & 5 & 19 & 7 & 5 & 202.9 \\
\hline 91 & 5 & 20 & 7 & 6 & 202.7 \\
\hline 91 & 5 & 21 & 7 & 6 & 202.9 \\
\hline 91 & 5 & 23 & 7 & 5 & 202.7 \\
\hline 91 & 5 & 24 & 7 & 5 & 202.8 \\
\hline 91 & 5 & 25 & 7 & 5 & 202.7 \\
\hline 91 & 5 & 26 & 7 & 5 & 202.7 \\
\hline 91 & 5 & 27 & 7 & 5 & 202.7 \\
\hline 91 & 5 & 28 & 7 & 5 & 202.7 \\
\hline 91 & 5 & 29 & 7 & 5 & 202.9 \\
\hline 91 & 5 & 30 & 7 & 5 & 202.9 \\
\hline 91 & 5 & 31 & 7 & 5 & 202.6 \\
\hline 91 & 6 & 1 & 7 & 5 & 202.6 \\
\hline 91 & 6 & 2 & 7 & 5 & 202.8 \\
\hline 91 & 6 & 3 & 7 & 5 & 202.7 \\
\hline 91 & 6 & 4 & 7 & 5 & 202.5 \\
\hline 91 & 6 & 5 & 7 & 5 & 202.7 \\
\hline 91 & 6 & 6 & 7 & 6 & 202.7 \\
\hline 91 & 6 & 7 & 7 & 5 & 202.8 \\
\hline 91 & 6 & 8 & 7 & 5 & 202.8 \\
\hline 91 & 6 & 9 & 7 & 5 & 202.7 \\
\hline 91 & 6 & 10 & 7 & 6 & 202.8 \\
\hline 91 & 6 & 11 & 7 & 5 & 202.9 \\
\hline 91 & 6 & 12 & 7 & 4 & 202.8 \\
\hline 91 & 6 & 13 & 7 & 5 & 202.8 \\
\hline 91 & 6 & 14 & 7 & 5 & 202.8 \\
\hline 91 & 6 & 15 & 7 & 5 & 202.8 \\
\hline 91 & 6 & 16 & 7 & 5 & 202.8 \\
\hline 91 & 6 & 17 & 7 & 5 & 202.7 \\
\hline
\end{tabular}

Data obtained from WHC Surveillance Analysis Computer System (SACS), February 28, 1994. 


\begin{tabular}{|c|c|c|c|c|c|}
\hline Year & Month & Day & Hour & Minute & Surface Level \\
\hline 91 & 6 & 18 & 7 & 5 & 202.7 \\
\hline 91 & 6 & 20 & 7 & 5 & 202.9 \\
\hline 91 & 6 & 21 & 7 & 6 & 202.8 \\
\hline 91 & 6 & 22 & 7 & 5 & 202.8 \\
\hline 91 & 6 & 23 & 7 & 5 & 202.9 \\
\hline 91 & 6 & 24 & 7 & 5 & 203 \\
\hline 91 & 6 & 25 & 7 & 6 & 203 \\
\hline 91 & 6 & 26 & 7 & 3 & 203 \\
\hline 91 & 6 & 27 & 7 & 2 & 203 \\
\hline 91 & 6 & 28 & 7 & 3 & 203 \\
\hline 91 & 6 & 29 & 7 & 2 & 203 \\
\hline 91 & 7 & 2 & 7 & 6 & 202.7 \\
\hline 91 & 7 & 3 & 7 & 6 & 202.9 \\
\hline 91 & 7 & 4 & 7 & 10 & 202.8 \\
\hline 91 & 7 & 5 & 7 & 6 & 202.9 \\
\hline 91 & 7 & 6 & 7 & 6 & 202.8 \\
\hline 91 & 7 & 7 & 7 & 10 & 202.7 \\
\hline 91 & 7 & 8 & 7 & 6 & 202.8 \\
\hline 91 & 7 & 9 & 7 & 6 & 203 \\
\hline 91 & 7 & 10 & 6 & 6 & 202.9 \\
\hline 91 & 7 & 11 & 7 & 6 & 202.8 \\
\hline 91 & 7 & 12 & 7 & 6 & 202.8 \\
\hline 91 & 7 & 13 & 7 & 6 & 202.9 \\
\hline 91 & 7 & 14 & 7 & 6 & 202.8 \\
\hline 91 & 7 & 15 & 7 & 6 & 202.9 \\
\hline 91 & 7 & 16 & 7 & 6 & 202.8 \\
\hline 91 & 7 & 17 & 7 & 5 & 202.8 \\
\hline 91 & 7 & 18 & 7 & 5 & 202.8 \\
\hline 91 & 7 & 19 & 7 & 5 & 202.9 \\
\hline 91 & 7 & 21 & 7 & 5 & 202.8 \\
\hline 91 & 7 & 22 & 7 & 5 & 202.7 \\
\hline 91 & 7 & 23 & 7 & 5 & 202.8 \\
\hline 91 & 7 & 24 & 7 & 5 & 203 \\
\hline 91 & 7 & 26 & 7 & 2 & 202.8 \\
\hline 91 & 7 & 27 & 7 & 2 & 202.7 \\
\hline 91 & 7 & 28 & 7 & 2 & 202.7 \\
\hline 91 & 7 & 29 & 7 & 2 & 202.8 \\
\hline 91 & 7 & 30 & 7 & 2 & 202.8 \\
\hline 91 & 7 & 31 & 7 & 2 & 202.9 \\
\hline 91 & 8 & 1 & 7 & 2 & 202.9 \\
\hline 91 & 8 & 2 & 7 & 2 & 202.8 \\
\hline 91 & 8 & 3 & 7 & 2 & 202.9 \\
\hline 91 & 8 & 4 & 7 & 2 & 203 \\
\hline 91 & 8 & 5 & 7 & 2 & 203 \\
\hline 91 & 8 & 6 & 7 & 2 & 203 \\
\hline 91 & 8 & 7 & 7 & 6 & 202.9 \\
\hline 91 & 8 & 8 & 7 & 6 & 202.8 \\
\hline 91 & 8 & 9 & 7 & 4 & 202.9 \\
\hline
\end{tabular}

Data obtained from WHC Surveillance Analysis Computer System (SACS), February 28, 1994. 


\begin{tabular}{|c|c|c|c|c|c|}
\hline Year & Month & Day & Hour & Minute & Surface Level \\
\hline 91 & 8 & 10 & 7 & 4 & 202.8 \\
\hline 91 & 8 & 11 & 7 & 4 & 202.7 \\
\hline 91 & 8 & 12 & 7 & 4 & 202.7 \\
\hline 91 & 8 & 13 & 7 & 4 & 202.7 \\
\hline 91 & 8 & 14 & 7 & 4 & 202.7 \\
\hline 91 & 8 & 15 & 7 & 3 & 202.8 \\
\hline 91 & 8 & 17 & 7 & 2 & 202.7 \\
\hline 91 & 8 & 18 & 7 & 3 & 202.8 \\
\hline 91 & 8 & 19 & 7 & 2 & 202.8 \\
\hline 91 & 8 & 20 & 7 & 4 & 202.7 \\
\hline 91 & 8 & 21 & 7 & 3 & 202.7 \\
\hline 91 & 8 & 22 & 7 & 3 & 202.7 \\
\hline 91 & 8 & 23 & 7 & 2 & 202.8 \\
\hline 91 & 8 & 24 & 7 & 2 & 202.8 \\
\hline 91 & 8 & 25 & 7 & 2 & 202.7 \\
\hline 91 & 8 & 26 & 7 & 3 & 202.7 \\
\hline 91 & 8 & 27 & 7 & 3 & 202.9 \\
\hline 91 & 8 & 28 & 7 & 3 & 202.8 \\
\hline 91 & 8 & 29 & 7 & 2 & 202.7 \\
\hline 91 & 8 & 30 & 7 & 10 & 202.7 \\
\hline 91 & 8 & 31 & 7 & 2 & 202.7 \\
\hline 91 & 9 & 1 & 7 & 2 & 202.7 \\
\hline 91 & 9 & 2 & 7 & 2 & 202.6 \\
\hline 91 & 9 & 3 & 7 & 2 & 202.6 \\
\hline 91 & 9 & 4 & 7 & 3 & 202.6 \\
\hline 91 & 9 & 5 & 7 & 2 & 203 \\
\hline 91 & 9 & 6 & 7 & 2 & 203 \\
\hline 91 & 9 & 7 & 7 & 2 & 203 \\
\hline 91 & 9 & 8 & 7 & 2 & 202.7 \\
\hline 91 & 9 & 9 & 7 & 3 & 202.6 \\
\hline 91 & 9 & 10 & 7 & 2 & 202.8 \\
\hline 91 & 9 & 11 & 7 & 3 & 202.8 \\
\hline 91 & 9 & 12 & 7 & 2 & 202.8 \\
\hline 91 & 9 & 13 & 7 & 2 & 202.8 \\
\hline 91 & 9 & 14 & 7 & 3 & 202.7 \\
\hline 91 & 9 & 15 & 7 & 2 & 202.6 \\
\hline 91 & 9 & 16 & 7 & 3 & 202.7 \\
\hline 91 & 9 & 17 & 7 & 3 & 202.7 \\
\hline 91 & 9 & 18 & 7 & 3 & 202.7 \\
\hline 91 & 9 & 19 & 7 & 3 & 202.7 \\
\hline 91 & 9 & 20 & 7 & 2 & 202.8 \\
\hline 91 & 9 & 21 & 7 & 2 & 202.7 \\
\hline 91 & 9 & 22 & 7 & 3 & 202.6 \\
\hline 91 & 9 & 23 & 7 & 3 & 202.6 \\
\hline 91 & 9 & 24 & 7 & 2 & 202.6 \\
\hline 91 & 9 & 25 & 7 & 3 & 202.7 \\
\hline 91 & 9 & 26 & 7 & 3 & 202.8 \\
\hline 91 & 9 & 27 & 7 & 2 & 202.9 \\
\hline
\end{tabular}

Data obtained from WHC Surveillance Analysis Computer System (SACS), February 28, 1994. 


\begin{tabular}{|c|c|c|c|c|c|}
\hline Year & Month & Day & Hour & Minute & Surface Level \\
\hline 91 & 9 & 28 & 7 & 2 & 203 \\
\hline 91 & 9 & 29 & 7 & 3 & 202.9 \\
\hline 91 & 9 & 30 & 7 & 3 & 202.8 \\
\hline 91 & 10 & 1 & 7 & 2 & 203 \\
\hline 91 & 10 & 2 & 7 & 2 & 203 \\
\hline 91 & 10 & 3 & 7 & 2 & 203 \\
\hline 91 & 10 & 4 & 7 & 3 & 202.8 \\
\hline 91 & 10 & 6 & 6 & 2 & 202.8 \\
\hline 91 & 10 & 7 & 6 & 2 & 202.8 \\
\hline 91 & 10 & 8 & 7 & 2 & 202.7 \\
\hline 91 & 10 & 9 & 7 & 2 & 202.7 \\
\hline 91 & 10 & 10 & 7 & 2 & 202.7 \\
\hline 91 & 10 & 11 & 7 & 2 & 202.9 \\
\hline 91 & 10 & 12 & 7 & 2 & 203 \\
\hline 91 & 10 & 13 & 7 & 3 & 202.7 \\
\hline 91 & 10 & 14 & 7 & 2 & 202.8 \\
\hline 91 & 10 & 15 & 7 & 2 & 203 \\
\hline 91 & 10 & 16 & 7 & 3 & 203.4 \\
\hline 91 & 10 & 17 & 7 & 5 & 202.8 \\
\hline 91 & 10 & 18 & 7 & 6 & 202.6 \\
\hline 91 & 10 & 19 & 7 & 6 & 202.6 \\
\hline 91 & 10 & 20 & 7 & 2 & 202.8 \\
\hline 91 & 10 & 21 & 7 & 2 & 203.2 \\
\hline 91 & 10 & 22 & 7 & 3 & 203 \\
\hline 91 & 10 & 23 & 7 & 2 & 202.8 \\
\hline 91 & 10 & 24 & 7 & 2 & 202.9 \\
\hline 91 & 10 & 25 & 7 & 3 & 202.9 \\
\hline 91 & 10 & 26 & 7 & 3 & 203 \\
\hline 91 & 10 & 27 & 7 & 2 & 202.7 \\
\hline 91 & 10 & 28 & 7 & 2 & 202.4 \\
\hline 91 & 10 & 29 & 7 & 3 & 202.5 \\
\hline 91 & 10 & 30 & 7 & 3 & 202.3 \\
\hline 91 & 10 & 31 & 7 & 3 & 202.5 \\
\hline 91 & 11 & 1 & 7 & 2 & 202.5 \\
\hline 91 & 11 & 2 & 7 & 3 & 202.3 \\
\hline 91 & 11 & 3 & 7 & 3 & 202.4 \\
\hline 91 & 11 & 4 & 7 & 2 & 202.8 \\
\hline 91 & 11 & 5 & 7 & 3 & 202.8 \\
\hline 91 & 11 & 6 & 7 & 3 & 202.9 \\
\hline 91 & 11 & 7 & 7 & 2 & 202.7 \\
\hline 91 & 11 & 8 & 7 & 3 & 202.9 \\
\hline 91 & 11 & 9 & 7 & 2 & 203 \\
\hline 91 & 11 & 10 & 7 & 3 & 202.7 \\
\hline 91 & 11 & 11 & 7 & 10 & 202.8 \\
\hline 91 & 11 & 12 & 7 & 3 & 203 \\
\hline 91 & 11 & 13 & 7 & 3 & 203 \\
\hline 91 & 11 & 14 & 7 & 3 & 203.3 \\
\hline 91 & 11 & 15 & 7 & 3 & 202.6 \\
\hline
\end{tabular}

Data obtained from WHC Surveillance Analysis Computer System (SACS), February 28, 1994. 


\begin{tabular}{|c|c|c|c|c|c|}
\hline Year & Month & Day & Hour & Minute & Surface Level \\
\hline 91 & 11 & 16 & 7 & 3 & 202.9 \\
\hline 91 & 11 & 17 & 7 & 2 & 203.1 \\
\hline 91 & 11 & 18 & 7 & 3 & 202.7 \\
\hline 91 & 11 & 19 & 7 & 3 & 202.4 \\
\hline 91 & 11 & 20 & 7 & 3 & 202.6 \\
\hline 91 & 11 & 21 & 7 & 2 & 202.4 \\
\hline 91 & 11 & 22 & 7 & 3 & 202.2 \\
\hline 91 & 11 & 23 & 7 & 2 & 202.3 \\
\hline 91 & 11 & 24 & 7 & 3 & 202.4 \\
\hline 91 & 11 & 25 & 7 & 3 & 202.7 \\
\hline 91 & 11 & 27 & 7 & 3 & 202.7 \\
\hline 91 & 11 & 28 & 7 & 3 & 202.6 \\
\hline 91 & 11 & 29 & 7 & 3 & 202.6 \\
\hline 91 & 11 & 30 & 7 & 3 & 202.5 \\
\hline 91 & 12 & 1 & 7 & 3 & 202.6 \\
\hline 91 & 12 & 2 & 7 & 3 & 202.7 \\
\hline 91 & 12 & 3 & 7 & 3 & 202.6 \\
\hline 91 & 12 & 4 & 7 & 3 & 202.9 \\
\hline 91 & 12 & 5 & 7 & 3 & 202.9 \\
\hline 91 & 12 & 6 & 7 & 4 & 203 \\
\hline 91 & 12 & 7 & 7 & 3 & 202.9 \\
\hline 91 & 12 & 8 & 7 & 3 & 202.8 \\
\hline 91 & 12 & 9 & 7 & 3 & 202.8 \\
\hline 91 & 12 & 10 & 7 & 3 & 202.6 \\
\hline 91 & 12 & 11 & 7 & 2 & 202.9 \\
\hline 91 & 12 & 12 & 7 & 3 & 203 \\
\hline 91 & 12 & 13 & 6 & 6 & 202.5 \\
\hline 91 & 12 & 14 & 7 & 2 & 202.4 \\
\hline 91 & 12 & 15 & 7 & 2 & 202.4 \\
\hline 91 & 12 & 18 & 7 & 3 & 202.9 \\
\hline 91 & 12 & 24 & 7 & 3 & 202.7 \\
\hline 91 & 12 & 25 & 7 & 3 & 202.8 \\
\hline 91 & 12 & 26 & 7 & 3 & 202.8 \\
\hline 91 & 12 & 27 & 7 & 3 & 202.9 \\
\hline 91 & 12 & 28 & 7 & 2 & 203 \\
\hline 91 & 12 & 29 & 7 & 3 & 202.9 \\
\hline 91 & 12 & 30 & 7 & 3 & 202.9 \\
\hline 91 & 12 & 31 & 7 & 3 & 202.7 \\
\hline 92 & 1 & 1 & 7 & 3 & 202.6 \\
\hline 92 & 1 & 2 & 7 & 2 & 202.9 \\
\hline 92 & 1 & 3 & 7 & 4 & 203.1 \\
\hline 92 & 1 & 4 & 7 & 3 & 203.3 \\
\hline 92 & 1 & 5 & 7 & 3 & 203.2 \\
\hline 92 & 1 & 6 & 7 & 3 & 203 \\
\hline 92 & 1 & 7 & 7 & 4 & 202.8 \\
\hline 92 & 1 & 9 & 7 & 3 & 202.7 \\
\hline 92 & 1 & 10 & 7 & 4 & 202.9 \\
\hline 92 & 1 & 11 & 7 & 4 & 203 \\
\hline
\end{tabular}

Data obtained from WHC Surveillance Analysis Computer System (SACS), February 28, 1994.

E-23 


\begin{tabular}{|c|c|c|c|c|c|}
\hline Year & Month & Day & Hour & Minute & Surface Level \\
\hline 92 & 1 & 17 & 7 & 4 & 202.8 \\
\hline 92 & 1 & 18 & 7 & 4 & 202.8 \\
\hline 92 & 1 & 19 & 7 & 3 & 202.9 \\
\hline 92 & 1 & 20 & 7 & 3 & 203 \\
\hline 92 & 1 & 21 & 7 & 3 & 203.6 \\
\hline 92 & 1 & 22 & 7 & 6 & 203.5 \\
\hline 92 & 1 & 23 & 7 & 3 & 203.8 \\
\hline 92 & 1 & 24 & 7 & 2 & 203.5 \\
\hline 92 & 1 & 25 & 7 & 3 & 203.5 \\
\hline 92 & 1 & 26 & 7 & 10 & 203.2 \\
\hline 92 & 1 & 27 & 7 & 2 & 203.3 \\
\hline 92 & 1 & 28 & 7 & 3 & 203.4 \\
\hline 92 & 1 & 29 & 7 & 3 & 203.1 \\
\hline 92 & 1 & 30 & 7 & 3 & 203.4 \\
\hline 92 & 1 & 31 & 7 & 3 & 203.8 \\
\hline 92 & 2 & 1 & 7 & 2 & 203.5 \\
\hline 92 & 2 & 2 & 7 & 3 & 203.5 \\
\hline 92 & 2 & 3 & 7 & 3 & 203.2 \\
\hline 92 & 2 & 4 & 7 & 3 & 203.1 \\
\hline 92 & 2 & 5 & 7 & 2 & 203.2 \\
\hline 92 & 2 & 8 & 7 & 4 & 203.5 \\
\hline 92 & 2 & 9 & 7 & 3 & 203.6 \\
\hline 92 & 2 & 10 & 7 & 4 & 204.1 \\
\hline 92 & 2 & 11 & 7 & 3 & 203.9 \\
\hline 92 & 2 & 12 & 7 & 3 & 204 \\
\hline 92 & 2 & 13 & 7 & 3 & 204 \\
\hline 92 & 2 & 14 & 7 & 3 & 204 \\
\hline 92 & 2 & 15 & 7 & 3 & 204.1 \\
\hline 92 & 2 & 16 & 7 & 3 & 204 \\
\hline 92 & 2 & 17 & 7 & 3 & 203.7 \\
\hline 92 & 2 & 18 & 7 & 3 & 203.9 \\
\hline 92 & 2 & 19 & 7 & 3 & 204 \\
\hline 92 & 2 & 20 & 7 & 3 & 204 \\
\hline 92 & 2 & 21 & 7 & 3 & 203.8 \\
\hline 92 & 2 & 22 & 7 & 3 & 203.7 \\
\hline 92 & 2 & 23 & 7 & 3 & 203.5 \\
\hline 92 & 2 & 24 & 7 & 3 & 203.4 \\
\hline 92 & 2 & 27 & 0 & 3 & 203.5 \\
\hline 92 & 2 & 29 & 7 & 3 & 204.1 \\
\hline 92 & 3 & 1 & 7 & 3 & 204.3 \\
\hline 92 & 3 & 2 & 7 & 3 & 204.1 \\
\hline 92 & 3 & 3 & 7 & 4 & 204.4 \\
\hline 92 & 3 & 4 & 7 & 4 & 204.3 \\
\hline 92 & 3 & 5 & 7 & 3 & 204.4 \\
\hline 92 & 3 & 6 & 7 & 3 & 204.4 \\
\hline 92 & 3 & 7 & 7 & 2 & 204.4 \\
\hline 92 & 3 & 8 & 7 & 2 & 204.2 \\
\hline 92 & 3 & 9 & 7 & 2 & 204.1 \\
\hline
\end{tabular}

Data obtained from WHC Surveillance Analysis Computer System (SACS), February 28, 1994. 


\begin{tabular}{|c|c|c|c|c|c|}
\hline Year & Month & Day & Hour & Minute & Surface Level \\
\hline 92 & 3 & 10 & 6 & 3 & 204.1 \\
\hline 92 & 3 & 11 & 7 & 3 & 204.2 \\
\hline 92 & 3 & 12 & 7 & 3 & 204.3 \\
\hline 92 & 3 & 13 & 7 & 3 & 204.4 \\
\hline 92 & 3 & 14 & 7 & 3 & 204.5 \\
\hline 92 & 3 & 15 & 7 & 6 & 204.7 \\
\hline 92 & 3 & 16 & 7 & 3 & 204.7 \\
\hline 92 & 3 & 17 & 7 & 3 & 204.6 \\
\hline 92 & 3 & 18 & 7 & 3 & 204.4 \\
\hline 92 & 3 & 19 & 7 & 3 & 204.3 \\
\hline 92 & 3 & 20 & 7 & 2 & 204.3 \\
\hline 92 & 3 & 21 & 7 & 3 & 204.5 \\
\hline 92 & 3 & 22 & 7 & 2 & 204.6 \\
\hline 92 & 3 & 23 & 7 & 2 & 204.6 \\
\hline 92 & 3 & 24 & 7 & 2 & 204.4 \\
\hline 92 & 3 & 25 & 7 & 3 & 204.4 \\
\hline 92 & 3 & 26 & 7 & 3 & 204.6 \\
\hline 92 & 3 & 27 & 7 & 2 & 204.6 \\
\hline 92 & 3 & 28 & 7 & 3 & 204.3 \\
\hline 92 & 3 & 29 & 7 & 3 & 204.3 \\
\hline 92 & 3 & 30 & 7 & 3 & 204.4 \\
\hline 92 & 3 & 31 & 7 & 3 & 204.2 \\
\hline 92 & 4 & 1 & 7 & 3 & 204.3 \\
\hline 92 & 4 & 2 & 7 & 3 & 204.5 \\
\hline 92 & 4 & 3 & 7 & 3 & 204.6 \\
\hline 92 & 4 & 4 & 7 & 3 & 204.5 \\
\hline 92 & 4 & 5 & 7 & 3 & 204.4 \\
\hline 92 & 4 & 6 & 7 & 3 & 204.3 \\
\hline 92 & 4 & 7 & 7 & 3 & 204 \\
\hline 92 & 4 & 8 & 7 & 10 & 203.9 \\
\hline 92 & 4 & 9 & 7 & 3 & 204 \\
\hline 92 & 4 & 10 & 7 & 3 & 204 \\
\hline 92 & 4 & 11 & 7 & 3 & 203.8 \\
\hline 92 & 4 & 12 & 7 & 4 & 203.7 \\
\hline 92 & 4 & 13 & 7 & 3 & 203.8 \\
\hline 92 & 4 & 14 & 7 & 3 & 203.4 \\
\hline 92 & 4 & 15 & 7 & 2 & 203.7 \\
\hline 92 & 4 & 16 & 7 & 3 & 203.8 \\
\hline 92 & 4 & 17 & 7 & 3 & 203.8 \\
\hline 92 & 4 & 18 & 7 & 2 & 203.4 \\
\hline 92 & 4 & 19 & 7 & 2 & 203.3 \\
\hline 92 & 4 & 20 & 7 & 3 & 203.5 \\
\hline 92 & 4 & 21 & 7 & 3 & 203.6 \\
\hline 92 & 4 & 22 & 7 & 2 & 203.6 \\
\hline 92 & 4 & 23 & 7 & 3 & 203.3 \\
\hline 92 & 4 & 24 & 7 & 3 & 203.4 \\
\hline 92 & 4 & 25 & 7 & 4 & 203.5 \\
\hline 92 & 4 & 26 & 7 & 3 & 203.7 \\
\hline
\end{tabular}

Data obtained from WHC Surveillance Analysis Computer System (SACS), February 28, 1994. 


\begin{tabular}{|c|c|c|c|c|c|}
\hline Year & Month & Day & Hour & Minute & Surface Level \\
\hline 92 & 4 & 27 & 7 & 4 & 203.5 \\
\hline 92 & 4 & 28 & 7 & 3 & 203.5 \\
\hline 92 & 4 & 29 & 7 & 3 & 203.8 \\
\hline 92 & 4 & 30 & 7 & 3 & 203.7 \\
\hline 92 & 5 & 1 & 7 & 3 & 203.3 \\
\hline 92 & 5 & 2 & 7 & 3 & 203.2 \\
\hline 92 & 5 & 3 & 7 & 3 & 203.3 \\
\hline 92 & 5 & 4 & 7 & 3 & 203.4 \\
\hline 92 & 5 & 5 & 7 & 4 & 203.5 \\
\hline 92 & 5 & 6 & 7 & 3 & 203.5 \\
\hline 92 & 5 & 7 & 7 & 4 & 203.5 \\
\hline 92 & 5 & 8 & 7 & 3 & 203.5 \\
\hline 92 & 5 & 9 & 7 & 4 & 203.4 \\
\hline 92 & 5 & 10 & 7 & 4 & 203.6 \\
\hline 92 & 5 & 11 & 7 & 3 & 203.4 \\
\hline 92 & 5 & 12 & 7 & 3 & 203.3 \\
\hline 92 & 5 & 13 & 7 & 3 & 203.4 \\
\hline 92 & 5 & 14 & 7 & 3 & 203.5 \\
\hline 92 & 5 & 15 & 7 & 3 & 203.6 \\
\hline 92 & 5 & 16 & 7 & .3 & 203.4 \\
\hline 92 & 5 & 17 & 7 & 2 & 203.5 \\
\hline 92 & 5 & 18 & 7 & 3 & 203.5 \\
\hline 92 & 5 & 19 & 7 & 3 & 203.5 \\
\hline 92 & 5 & 20 & 7 & 3 & 203.5 \\
\hline 92 & 5 & 21 & 7 & 3 & 203.3 \\
\hline 92 & 5 & 22 & 7 & 2 & 203.3 \\
\hline 92 & 5 & 23 & 7 & 3 & 203.4 \\
\hline 92 & 5 & 24 & 7 & 3 & 203.4 \\
\hline 92 & 5 & 25 & 7 & 3 & 203.5 \\
\hline 92 & 5 & 27 & 7 & 3 & 203.6 \\
\hline 92 & 5 & 28 & 7 & 2 & 203.6 \\
\hline 92 & 5 & 29 & 7 & 2 & 203.6 \\
\hline 92 & 10 & 29 & 7 & 3 & 203.8 \\
\hline 92 & 10 & 30 & 7 & 3 & 203.7 \\
\hline 92 & 10 & 31 & 7 & 3 & 203.5 \\
\hline 92 & 11 & 1 & 7 & 3 & 203.4 \\
\hline 92 & 11 & 2 & 7 & 3 & 203.3 \\
\hline 92 & 11 & 3 & 7 & 3 & 203.1 \\
\hline 92 & 11 & 4 & 7 & 3 & 203.3 \\
\hline 92 & 12 & 2 & 6 & 3 & 203.5 \\
\hline 92 & 12 & 3 & 6 & 3 & 203.4 \\
\hline 92 & 12 & 4 & 7 & 3 & 203.3 \\
\hline 92 & 12 & 6 & 7 & 2 & 203.6 \\
\hline 92 & 12 & 7 & 7 & 3 & 203.7 \\
\hline 92 & 12 & 8 & 7 & 3 & 203.8 \\
\hline 92 & 12 & 9 & 7 & 3 & 203.6 \\
\hline 92 & 12 & 10 & 7 & 3 & 203.8 \\
\hline 92 & 12 & 11 & 7 & 3 & 203.6 \\
\hline
\end{tabular}

Data obtained from WHC Surveillance Analysis Computer System (SACS), February 28, 1994. 


\begin{tabular}{|c|c|c|c|c|c|}
\hline Year & Month & Day & Hour & Minute & Surface Level \\
\hline 92 & 12 & 13 & 7 & 4 & 203.2 \\
\hline 92 & 12 & 14 & 7 & 3 & 203.5 \\
\hline 92 & 12 & 15 & 7 & 3 & 203.5 \\
\hline 92 & 12 & 16 & 7 & 4 & 203.5 \\
\hline 92 & 12 & 17 & 7 & 3 & 203.9 \\
\hline 92 & 12 & 18 & 7 & 3 & 203.6 \\
\hline 92 & 12 & 19 & 7 & 3 & 203.5 \\
\hline 92 & 12 & 20 & 7 & 4 & 203.7 \\
\hline 92 & 12 & 21 & 7 & 4 & 203.7 \\
\hline 92 & 12 & 23 & 7 & 4 & 203.4 \\
\hline 92 & 12 & 24 & 7 & 3 & 203.5 \\
\hline 92 & 12 & 25 & 7 & 3 & 203.5 \\
\hline 92 & 12 & 26 & 7 & 3 & 203.7 \\
\hline 92 & 12 & 27 & 7 & 3 & 203.8 \\
\hline 92 & 12 & 28 & 7 & 3 & 204 \\
\hline 92 & 12 & 30 & 7 & 3 & 203.6 \\
\hline 92 & 12 & 31 & 7 & 3 & 203.5 \\
\hline 93 & 1 & 1 & 7 & 4 & 203.6 \\
\hline 93 & 1 & 12 & 7 & 6 & 203.5 \\
\hline 93 & 1 & 13 & 7 & 6 & 203.6 \\
\hline 93 & 1 & 14 & 7 & 3 & 203.7 \\
\hline 93 & 1 & 15 & 7 & 3 & 203.6 \\
\hline 93 & 1 & 17 & 7 & 2 & 203.6 \\
\hline 93 & 1 & 18 & 7 & 2 & 203.7 \\
\hline 93 & 1 & 19 & 7 & 2 & 203.8 \\
\hline 93 & 1 & 20 & 7 & 2 & 204 \\
\hline 93 & 1 & 21 & 7 & 2 & 203.7 \\
\hline 93 & 1 & 22 & 7 & 4 & 203.9 \\
\hline 93 & 1 & 23 & 7 & 3 & 203.6 \\
\hline 93 & 1 & 24 & 6 & 3 & 203.6 \\
\hline 93 & 1 & 25 & 6 & 3 & 203.8 \\
\hline 93 & 2 & 5 & 6 & 4 & 203.8 \\
\hline 93 & 2 & 6 & 2 & 3 & 203.8 \\
\hline 93 & 2 & 7 & 7 & 3 & 203.8 \\
\hline 93 & 2 & 16 & 6 & 3 & 203.7 \\
\hline 93 & 2 & 18 & 7 & 3 & 203.8 \\
\hline 93 & 2 & 19 & 7 & 2 & 204.1 \\
\hline 93 & 2 & 20 & 7 & 3 & 204.1 \\
\hline 93 & 2 & 21 & 7 & 4 & 203.9 \\
\hline 93 & 2 & 22 & 7 & 4 & 203.6 \\
\hline 93 & 2 & 23 & 7 & 3 & 203.7 \\
\hline 93 & 2 & 24 & 7 & 4 & 203.7 \\
\hline 93 & 2 & 25 & 7 & 3 & 203.6 \\
\hline 93 & 2 & 26 & 7 & 3 & 203.5 \\
\hline 93 & 2 & 27 & 7 & 4 & 203.5 \\
\hline 93 & 2 & 28 & 7 & 3 & 203.5 \\
\hline 93 & 3 & 1 & 7 & 3 & 203.6 \\
\hline 93 & 3 & 2 & 7 & 4 & 203.5 \\
\hline
\end{tabular}

Data obtained from WHC Surveillance Analysis Computer System (SACS), February 28, 1994. 


\begin{tabular}{|c|c|c|c|c|c|}
\hline Year & Month & Day & Hour & Minute & Surface Level \\
\hline 93 & 3 & 3 & 7 & 3 & 203.7 \\
\hline 93 & 3 & 4 & 7 & 4 & 203.6 \\
\hline 93 & 3 & 5 & 7 & 4 & 203.6 \\
\hline 93 & 3 & 6 & 7 & 3 & 203.6 \\
\hline 93 & 3 & 9 & 7 & 4 & 203.7 \\
\hline 93 & 3 & 10 & 7 & 5 & 203.8 \\
\hline 93 & 3 & 11 & 7 & 4 & 203.6 \\
\hline 93 & 3 & 12 & 7 & 4 & 203.6 \\
\hline 93 & 3 & 13 & 7 & 3 & 203.8 \\
\hline 93 & 3 & 14 & 7 & 4 & 204.1 \\
\hline 93 & 3 & 15 & 7 & 3 & 204.2 \\
\hline 93 & 3 & 16 & 7 & 4 & 203.8 \\
\hline 93 & 3 & 17 & 7 & 3 & 204 \\
\hline 93 & 3 & 18 & 7 & 4 & 204.1 \\
\hline 93 & 3 & 19 & 7 & 3 & 203.9 \\
\hline 93 & 3 & 20 & 7 & 4 & 204 \\
\hline 93 & 3 & 21 & 7 & 4 & 203.9 \\
\hline 93 & 3 & 22 & 7 & 3 & 204 \\
\hline 93 & 3 & 23 & 7 & 3 & 204.2 \\
\hline 93 & 3 & 24 & 7 & 4 & 204.1 \\
\hline 93 & 3 & 25 & 7 & 3 & 204 \\
\hline 93 & 3 & 26 & 7 & 3 & 204 \\
\hline 93 & 3 & 27 & 7 & 3 & 204 \\
\hline 93 & 3 & 28 & 7 & 3 & 204 \\
\hline 93 & 3 & 29 & 6 & 3 & 204 \\
\hline 93 & 3 & 30 & 7 & 3 & 204 \\
\hline 93 & 3 & 31 & 7 & 3 & 203.9 \\
\hline 93 & 4 & 1 & 7 & 3 & 204.2 \\
\hline 93 & 4 & 2 & 7 & 6 & 204.1 \\
\hline 93 & 4 & 3 & 7 & 6 & 204.1 \\
\hline 93 & 4 & 4 & 7 & 5 & 204.2 \\
\hline 93 & 4 & 5 & 7 & 6 & 204.2 \\
\hline 93 & 4 & 6 & 7 & 6 & 204.1 \\
\hline 93 & 4 & 7 & 7 & 6 & 204 \\
\hline 93 & 4 & 8 & 7 & 6 & 204.3 \\
\hline 93 & 4 & 9 & 7 & 6 & 204.2 \\
\hline 93 & 4 & 10 & 7 & 5 & 204.2 \\
\hline 93 & 4 & 11 & 7 & 6 & 204.1 \\
\hline 93 & 4 & 12 & 6 & 6 & 204 \\
\hline 93 & 4 & 14 & 7 & 6 & 204.1 \\
\hline 93 & 4 & 16 & 7 & 6 & 204.1 \\
\hline 93 & 4 & 17 & 7 & 5 & 204.3 \\
\hline 93 & 4 & 18 & 7 & 6 & 204.2 \\
\hline 93 & 4 & 19 & 7 & 6 & 204 \\
\hline 93 & 4 & 23 & 7 & 6 & 204.2 \\
\hline 93 & 4 & 24 & 2 & 6 & 204.2 \\
\hline 93 & 4 & 25 & 7 & 6 & 204.1 \\
\hline 93 & 4 & 26 & 7 & 6 & 204.1 \\
\hline
\end{tabular}

Data obtained from WHC Surveillance Analysis Computer System (SACS), February 28, 1994. 


\begin{tabular}{|c|c|c|c|c|c|}
\hline Year & Month & Day & Hour & Minute & Surface Level \\
\hline 93 & 4 & 29 & 6 & 2 & 204 \\
\hline 93 & 4 & 30 & 7 & 6 & 204.2 \\
\hline 93 & 5 & 1 & 7 & 5 & 204.2 \\
\hline 93 & 5 & 2 & 7 & 6 & 204.2 \\
\hline 93 & 5 & 3 & 7 & 6 & 204.5 \\
\hline 93 & 5 & 11 & 7 & 6 & 204.3 \\
\hline 93 & 5 & 12 & 2 & 6 & 204.4 \\
\hline 93 & 5 & 15 & 7 & 6 & 204.1 \\
\hline 93 & 5 & 16 & 7 & 5 & 204.1 \\
\hline 93 & 5 & 17 & 7 & 3 & 204.1 \\
\hline 93 & 5 & 18 & 7 & 3 & 204.2 \\
\hline 93 & 5 & 19 & 7 & 3 & 204.2 \\
\hline 93 & 5 & 20 & 7 & 3 & 204.3 \\
\hline 93 & 5 & 21 & 7 & 2 & 204.2 \\
\hline 93 & 5 & 22 & 7 & 3 & 204 \\
\hline 93 & 5 & 23 & 7 & 4 & 204.1 \\
\hline 93 & 5 & 24 & 7 & 3 & 204.1 \\
\hline 93 & 5 & 25 & 7 & 3 & 204.2 \\
\hline 93 & 5 & 26 & 7 & 3 & 204.2 \\
\hline 93 & 5 & 27 & 7 & 3 & 204.3 \\
\hline 93 & 5 & 28 & 7 & 3 & 204.2 \\
\hline 93 & 5 & 29 & 7 & 5 & 204.1 \\
\hline 93 & 5 & 30 & 7 & 5 & 204.1 \\
\hline 93 & 5 & 31 & 7 & 6 & 204.3 \\
\hline 93 & 6 & 1 & 7 & 6 & 204.2 \\
\hline 93 & 6 & 2 & 7 & 6 & 204.2 \\
\hline 93 & 6 & 3 & 7 & 6 & 204.2 \\
\hline 93 & 6 & 4 & 7 & 6 & 204.3 \\
\hline 93 & 6 & 5 & 7 & 6 & 204.3 \\
\hline 93 & 6 & 6 & 7 & 5 & 204.4 \\
\hline 93 & 6 & 7 & 7 & 5 & 204.2 \\
\hline 93 & 6 & 8 & 7 & 5 & 204.1 \\
\hline 93 & 6 & 9 & 7 & 5 & 204.2 \\
\hline 93 & 6 & 10 & 7 & 4 & 204.1 \\
\hline 93 & 6 & 11 & 7 & 5 & 204.2 \\
\hline 93 & 6 & 12 & 7 & 5 & 204 \\
\hline 93 & 6 & 13 & 7 & 4 & 204.1 \\
\hline 93 & 6 & 14 & 7 & 5 & 204.2 \\
\hline 93 & 6 & 15 & 7 & 5 & 204.2 \\
\hline 93 & 6 & 16 & 7 & 5 & 204.1 \\
\hline 93 & 6 & 25 & 7 & 3 & 204.1 \\
\hline 93 & 6 & 26 & 7 & 3 & 204.3 \\
\hline 93 & 6 & 27 & 7 & 4 & 204.3 \\
\hline 93 & 6 & 28 & 7 & 3 & 204.2 \\
\hline 93 & 6 & 29 & 7 & 4 & 204.2 \\
\hline 93 & 6 & 30 & 6 & 3 & 204.2 \\
\hline 93 & 7 & 1 & 6 & 6 & 204.2 \\
\hline 93 & 7 & 2 & 7 & 6 & 204.3 \\
\hline
\end{tabular}

Data obtained from WHC Surveillance Analysis Computer System (SACS), February 28, 1994. 


\begin{tabular}{|c|c|c|c|c|c|}
\hline Year & Month & Day & Hour & Minute & Surface Level \\
\hline 93 & 7 & 3 & 7 & 3 & 204.3 \\
\hline 93 & 7 & 4 & 7 & 4 & 204.2 \\
\hline 93 & 7 & 5 & 7 & 3 & 204.2 \\
\hline 93 & 7 & 6 & 7 & 3 & 204.2 \\
\hline 93 & 7 & 7 & 7 & 4 & 204.2 \\
\hline 93 & 7 & 8 & 7 & 4 & 204.2 \\
\hline 93 & 7 & 9 & 7 & 5 & 204.3 \\
\hline 93 & 7 & 10 & 7 & 3 & 204.1 \\
\hline 93 & 7 & 11 & 7 & 3 & 204.1 \\
\hline 93 & 7 & 12 & 7 & 2 & 204.1 \\
\hline 93 & 7 & 13 & 7 & 3 & 204.1 \\
\hline 93 & 7 & 14 & 7 & 6 & 204.2 \\
\hline 93 & 7 & 15 & 7 & 6 & 204.2 \\
\hline 93 & 7 & 16 & 7 & 6 & 204.2 \\
\hline 93 & 7 & 17 & 7 & 7 & 204.2 \\
\hline 93 & 7 & 18 & 7 & 6 & 204.3 \\
\hline 93 & 7 & 19 & 7 & 3 & 204.4 \\
\hline 93 & 7 & 20 & 7 & 3 & 204.5 \\
\hline 93 & 7 & 21 & 7 & 3 & 204.4 \\
\hline 93 & 7 & 22 & 7 & 3 & 204.5 \\
\hline 93 & 7 & 23 & 6 & 2 & 204.4 \\
\hline 93 & 7 & 24 & 7 & 3 & 204.3 \\
\hline 93 & 7 & 25 & 7 & 4 & 204.3 \\
\hline 93 & 7 & 26 & 7 & 3 & 204.3 \\
\hline 93 & 7 & 27 & 4 & 3 & 204.4 \\
\hline 93 & 7 & 28 & 7 & 4 & 204.5 \\
\hline 93 & 7 & 29 & 7 & 3 & 204.3 \\
\hline 93 & 7 & 30 & 7 & 3 & 204.2 \\
\hline 93 & 7 & 31 & 7 & 3 & 204.5 \\
\hline 93 & 8 & 1 & 7 & 3 & 204.3 \\
\hline 93 & 8 & 2 & 7 & 6 & 204.3 \\
\hline 93 & 8 & 3 & 7 & 3 & 204.4 \\
\hline 93 & 8 & 4 & 7 & 3 & 204.6 \\
\hline 93 & 8 & 5 & 7 & 4 & 204.5 \\
\hline 93 & 8 & 6 & 15 & 4 & 204.8 \\
\hline 93 & 8 & 8 & 7 & 4 & 204.8 \\
\hline 93 & 8 & 9 & 6 & 4 & 204.6 \\
\hline 93 & 8 & 10 & 7 & 4 & 204.5 \\
\hline 93 & 8 & 11 & 6 & 3 & 204.4 \\
\hline 93 & 8 & 12 & 7 & 4 & 204.4 \\
\hline 93 & 8 & 13 & 7 & 4 & 204.4 \\
\hline 93 & 8 & 14 & 7 & 3 & 204.4 \\
\hline 93 & 8 & 15 & 7 & 4 & 204.5 \\
\hline 93 & 8 & 16 & 4 & 4 & 204.5 \\
\hline 93 & 8 & 20 & 7 & 4 & 204.6 \\
\hline 93 & 8 & 21 & 7 & 4 & 204.5 \\
\hline 93 & 8 & 22 & 7 & 4 & 204.5 \\
\hline 93 & 8 & 23 & 7 & 4 & 204.5 \\
\hline
\end{tabular}

Data obtained from WHC Surveillance Analysis Computer System (SACS), February 28, 1994. 


\begin{tabular}{|c|c|c|c|c|c|}
\hline Year & Month & Day & Hour & Minute & Surface Level \\
\hline 93 & 8 & 24 & 7 & 4 & 204.3 \\
\hline 93 & 8 & 25 & 7 & 4 & 204.2 \\
\hline 93 & 8 & 26 & 7 & 4 & 204.2 \\
\hline 93 & 8 & 27 & 7 & 5 & 204.3 \\
\hline 93 & 8 & 28 & 6 & 3 & 204.3 \\
\hline 93 & 8 & 29 & 7 & 3 & 204.3 \\
\hline 93 & 8 & 30 & 7 & 4 & 204.3 \\
\hline 93 & 8 & 31 & 7 & 4 & 204.4 \\
\hline 93 & 9 & 1 & 7 & 4 & 204.5 \\
\hline 93 & 9 & 2 & 7 & 4 & 204.3 \\
\hline 93 & 9 & 3 & 7 & 4 & 204.2 \\
\hline 93 & 9 & 4 & 7 & 4 & 204.3 \\
\hline 93 & 9 & 5 & 7 & 4 & 204.3 \\
\hline 93 & 9 & 6 & 7 & 4 & 204.3 \\
\hline 93 & 9 & 12 & 7 & 4 & 204.2 \\
\hline 93 & 9 & 13 & 7 & 4 & 204.1 \\
\hline 93 & 9 & 16 & 7 & 4 & 204.2 \\
\hline 93 & 9 & 18 & 7 & 4 & 204.3 \\
\hline 93 & 9 & 20 & 7 & 4 & 204.3 \\
\hline 93 & 9 & 21 & 7 & 4 & 204.2 \\
\hline 93 & 10 & 2 & 7 & 5 & 204.2 \\
\hline 93 & 10 & 3 & 7 & 6 & 204.3 \\
\hline 93 & 10 & 4 & 7 & 6 & 204.4 \\
\hline 93 & 10 & 5 & 6 & 6 & 204.4 \\
\hline 93 & 10 & 6 & 6 & 6 & 204.4 \\
\hline 93 & 10 & 8 & 6 & 4 & 204.2 \\
\hline 93 & 10 & 9 & 7 & 3 & 204.3 \\
\hline 93 & 10 & 10 & 7 & 10 & 204.2 \\
\hline 93 & 10 & 11 & 7 & 3 & 204.4 \\
\hline 93 & 10 & 12 & 7 & 4 & 204.3 \\
\hline .93 & 10 & 13 & 7 & 3 & 204.3 \\
\hline 93 & 10 & 14 & 7 & 4 & 204.4 \\
\hline 93 & 10 & 15 & 7 & 4 & 204.4 \\
\hline 93 & 10 & 16 & 6 & 3 & 204.4 \\
\hline 93 & 10 & 17 & 7 & 3 & 204.4 \\
\hline 93 & 10 & 18 & 7 & 4 & 204.3 \\
\hline 93 & 10 & 19 & 3 & 4 & 204.3 \\
\hline 93 & 10 & 20 & 7 & 3 & 204.2 \\
\hline 93 & 10 & 21 & 7 & 4 & 204.3 \\
\hline 93 & 10 & 22 & 6 & 4 & 204.3 \\
\hline 93 & 11 & 9 & 6 & 4 & 204.2 \\
\hline 93 & 11 & 10 & 7 & 4 & 204.3 \\
\hline 93 & 11 & 11 & 6 & 3 & 204.3 \\
\hline 93 & 11 & 12 & 6 & 4 & 204.3 \\
\hline 93 & 11 & 13 & 0 & 4 & 204.3 \\
\hline 93 & 11 & 14 & 2 & 4 & 204.1 \\
\hline 93 & 11 & 15 & 5 & 4 & 204.3 \\
\hline 93 & 11 & 18 & 1 & 3 & 204.3 \\
\hline
\end{tabular}

Data obtained from WHC Surveillance Analysis Computer System (SACS), February 28, 1994. E-31 


\begin{tabular}{|c|c|c|c|c|c|}
\hline Year & Month & Day & Hour & Minute & Surface Level \\
\hline 93 & 11 & 19 & 15 & 4 & 204.1 \\
\hline 93 & 11 & 21 & 7 & 4 & 204.5 \\
\hline 93 & 11 & 29 & 4 & 4 & 204.3 \\
\hline 94 & 1 & 15 & 7 & 10 & 207.1 \\
\hline 94 & 1 & 16 & 7 & 7 & 207 \\
\hline 94 & 1 & 17 & 7 & 7 & 207 \\
\hline 94 & 1 & 18 & 7 & 7 & 207 \\
\hline 94 & 1 & 19 & 7 & 7 & 207 \\
\hline 94 & 1 & 20 & 7 & 7 & 207 \\
\hline 94 & 1 & 21 & 7 & 7 & 207 \\
\hline 94 & 1 & 22 & 7 & 8 & 207 \\
\hline 94 & 1 & 23 & 7 & 7 & 207.2 \\
\hline 94 & 1 & 24 & 7 & 8 & 207.2 \\
\hline 94 & 1 & 25 & 7 & 7 & 207.1 \\
\hline 94 & 1 & 26 & 7 & 6 & 207 \\
\hline 94 & 1 & 27 & 7 & 10 & 206.9 \\
\hline 94 & 1 & 28 & 7 & 7 & 206.8 \\
\hline 94 & 1 & 29 & 7 & 7 & 206.8 \\
\hline 94 & 1 & 30 & 7 & 6 & 206.7 \\
\hline 94 & 1 & 31 & 7 & 7 & 206.7 \\
\hline 94 & 2 & 1 & 7 & 6 & 206.7 \\
\hline 94 & 2 & 2 & 7 & 7 & 206.9 \\
\hline 94 & 2 & 3 & 7 & 7 & 207 \\
\hline 94 & 2 & 4 & 7 & 7 & 207 \\
\hline 94 & 2 & 5 & 7 & 7 & 207 \\
\hline 94 & 2 & 6 & 7 & 7 & 207.1 \\
\hline 94 & 2 & 7 & 7 & 6 & 207.3 \\
\hline 94 & 2 & 8 & 7 & 7 & 207 \\
\hline 94 & 2 & 9 & 7 & 4 & 207.2 \\
\hline 94 & 2 & 10 & 7 & 4 & 207.1 \\
\hline 94 & 2 & 11 & 7 & 6 & 207.1 \\
\hline 94 & 2 & 12 & 7 & 3 & 207 \\
\hline 94 & 2 & 13 & 7 & 4 & 207.2 \\
\hline 94 & 2 & 14 & 7 & 4 & 207.1 \\
\hline 94 & 2 & 15 & 7 & 4 & 207.2 \\
\hline 94 & 2 & 16 & 7 & 3 & 207.3 \\
\hline 94 & 2 & 17 & 7 & 4 & 207.5 \\
\hline 94 & 2 & 18 & 7 & 6 & 207.3 \\
\hline 94 & 2 & 19 & 7 & 6 & 207.1 \\
\hline 94 & 2 & 20 & 7 & 7 & 207 \\
\hline 94 & 2 & 21 & 7 & 6 & 207 \\
\hline 94 & 2 & 22 & 7 & 5 & 206.8 \\
\hline 94 & 2 & 23 & 7 & 6 & 207 \\
\hline 94 & 2 & 24 & 7 & 6 & 205.5 \\
\hline & & & & & \\
\hline & & & & & \\
\hline & & & & & \\
\hline
\end{tabular}

Data obtained from WHC Surveillance Analysis Computer System (SACS), February 28, 1994. 


\begin{tabular}{|c|c|c|c|c|c|}
\hline \multicolumn{6}{|c|}{ Surface Level Data } \\
\hline \multicolumn{6}{|c|}{ FIC } \\
\hline Year & Month & Day & Hour & Minute & Surface Level \\
\hline 91 & 1 & 1 & 7 & 3 & 103 \\
\hline 91 & 1 & 2 & 7 & 2 & 103 \\
\hline 91 & 1 & 3 & 7 & 3 & 102.9 \\
\hline 91 & 1 & 4 & 7 & 2 & 103 \\
\hline 91 & 1 & 5 & 7 & 2 & 103.1 \\
\hline 91 & 1 & 6 & 7 & 2 & 103 \\
\hline 91 & 1 & 7 & 7 & 2 & 103 \\
\hline 91 & 1 & 8 & 7 & 3 & 103 \\
\hline 91 & 1 & 9 & 7 & 2 & 103.1 \\
\hline 91 & 1 & 10 & 7 & 2 & 103.1 \\
\hline 91 & 1 & 11 & 7 & 3 & 103 \\
\hline 91 & 1 & 12 & 7 & 2 & 103.1 \\
\hline 91 & 1 & 26 & 7 & 3 & 103 \\
\hline 91 & 1 & 27 & 7 & 3 & 103 \\
\hline 91 & 1 & 28 & 7 & 10 & 103 \\
\hline 91 & 1 & 29 & 7 & 2 & 103 \\
\hline 91 & 1 & 30 & 7 & 10 & 103 \\
\hline 91 & 1 & 31 & 7 & 2 & 103.1 \\
\hline 91 & 2 & 1 & 7 & 2 & 103 \\
\hline 91 & 2 & 15 & 7 & 3 & 103 \\
\hline 91 & 2 & 16 & 7 & 2 & 103.1 \\
\hline 91 & 2 & 17 & 7 & 2 & 103.1 \\
\hline 91 & 2 & 18 & 7 & 3 & 103.1 \\
\hline 91 & 2 & 19 & 7 & 2 & 103.1 \\
\hline 91 & 2 & 20 & 7 & 3 & 102.9 \\
\hline 91 & 2 & 21 & 7 & 2 & 102.9 \\
\hline 91 & 2 & 22 & 7 & 2 & 103 \\
\hline 91 & 2 & 23 & 7 & 2 & 103 \\
\hline 91 & 2 & 24 & 7 & 3 & 102.9 \\
\hline 91 & 2 & 25 & 7 & 3 & 102.9 \\
\hline 91 & 2 & 26 & 7 & 3 & 103 \\
\hline 91 & 2 & 27 & 7 & 2 & 103.1 \\
\hline 91 & 2 & 28 & 7 & 2 & 103.2 \\
\hline 91 & 3 & 2 & 7 & 2 & 102.8 \\
\hline 91 & 3 & 3 & 7 & 3 & 102.9 \\
\hline 91 & 3 & 4 & 7 & 3 & 103 \\
\hline 91 & 3 & 5 & 7 & 3 & 102.8 \\
\hline 91 & 3 & 6 & 7 & 2 & 102.6 \\
\hline 91 & 3 & 7 & 7 & 2 & 102.5 \\
\hline 91 & 3 & 8 & 7 & 2 & 102.5 \\
\hline 91 & 3 & 9 & 7 & 2 & 102.9 \\
\hline 91 & 3 & 10 & 7 & 2 & 102.8 \\
\hline 91 & 3 & 11 & 7 & 2 & 102.7 \\
\hline 91 & 3 & 12 & 7 & 3 & 102.7 \\
\hline 91 & 3 & 13 & 7 & 3 & 102.7 \\
\hline 91 & 3 & 14 & 7 & 3 & 102.8 \\
\hline
\end{tabular}

Data obtained from WHC Surveillance Analysis Computer System (SACS), February 28, 1994. E-33 


\begin{tabular}{|c|c|c|c|c|c|}
\hline Year & Month & Day & Hour & Minute & Surface Level \\
\hline 91 & 3 & 15 & 7 & 3 & 102.7 \\
\hline 91 & 3 & 16 & 7 & 3 & 102.9 \\
\hline 91 & 3 & 17 & 7 & 2 & 102.7 \\
\hline 91 & 3 & 18 & 7 & 3 & 102.8 \\
\hline 91 & 3 & 19 & 7 & 3 & 103.2 \\
\hline 91 & 3 & 20 & 7 & 2 & 103 \\
\hline 91 & 3 & 21 & 7 & 3 & 102.9 \\
\hline 91 & 3 & 22 & 7 & 2 & 103 \\
\hline 91 & 3 & 23 & 7 & 3 & 102.8 \\
\hline 91 & 3 & 24 & 7 & 3 & 102.9 \\
\hline 91 & 3 & 25 & 7 & 3 & 103.2 \\
\hline 91 & 3 & 26 & 7 & 3 & 103 \\
\hline 91 & 3 & 27 & 7 & 3 & 102.8 \\
\hline 91 & 3 & 28 & 7 & 3 & 102.8 \\
\hline 91 & 3 & 29 & 7 & 3 & 102.7 \\
\hline 91 & 3 & 30 & 7 & 3 & 102.6 \\
\hline 91 & 3 & 31 & 7 & 3 & 103 \\
\hline 91 & 4 & 1 & 7 & 3 & 103.1 \\
\hline 91 & 4 & 2 & 7 & 3 & 102.8 \\
\hline 91 & 4 & 3 & 7 & 3 & 102.9 \\
\hline 91 & 4 & 4 & 7 & 3 & 103.1 \\
\hline 91 & 4 & 5 & 7 & 3 & 103.1 \\
\hline 91 & 4 & 6 & 7 & 3 & 102.8 \\
\hline 91 & 4 & 7 & 7 & 3 & 103 \\
\hline 91 & 4 & 8 & 7 & 4 & 102.7 \\
\hline 91 & 4 & 9 & 7 & 3 & 103.1 \\
\hline 91 & 4 & 10 & 7 & 3 & 102.8 \\
\hline 91 & 4 & 11 & 7 & 3 & 102.7 \\
\hline 91 & 4 & 12 & 7 & 3 & 102.7 \\
\hline 91 & 4 & 13 & 7 & 4 & 103 \\
\hline 91 & 4 & 14 & 7 & 3 & 103.1 \\
\hline 91 & 4 & 15 & 7 & 3 & 103.1 \\
\hline 91 & 4 & 16 & 7 & 10 & 103.3 \\
\hline 91 & 4 & 17 & 7 & 3 & 103.1 \\
\hline 91 & 4 & 18 & 7 & 6 & 103.1 \\
\hline 91 & 4 & 19 & 7 & 5 & 103 \\
\hline 91 & 4 & 20 & 7 & 6 & 102.9 \\
\hline 91 & 4 & 21 & 7 & 5 & 103.1 \\
\hline 91 & 4 & 22 & 7 & 6 & 103.1 \\
\hline 91 & 4 & 23 & 7 & 6 & 103.2 \\
\hline 91 & 4 & 24 & 7 & 5 & 103.2 \\
\hline 91 & 4 & 25 & 7 & 5 & 103.1 \\
\hline 91 & 4 & 26 & 3 & 5 & 102.9 \\
\hline 91 & 4 & 27 & 7 & 5 & 102.8 \\
\hline 91 & 4 & 28 & 7 & 6 & 102.8 \\
\hline 91 & 4 & 29 & 7 & 5 & 103 \\
\hline 91 & 4 & 30 & 7 & 6 & 103 \\
\hline 91 & 5 & 1 & 6 & 6 & 103 \\
\hline
\end{tabular}

Data obtained from WHC Surveillance Analysis Computer System (SACS), February 28, 1994. 


\begin{tabular}{|c|c|c|c|c|c|}
\hline Year & Month & Day & Hour & Minute & Surface Level \\
\hline 91 & 5 & 2 & 7 & 5 & 103.2 \\
\hline 91 & 5 & 3 & 7 & 6 & 103 \\
\hline 91 & 5 & 4 & 7 & 5 & 102.9 \\
\hline 91 & 5 & 5 & 7 & 5 & 103 \\
\hline 91 & 5 & 6 & 7 & 5 & 103 \\
\hline 91 & 5 & 7 & 7 & 5 & 103.2 \\
\hline 91 & 5 & 8 & 7 & 5 & 103 \\
\hline 91 & 5 & 9 & 7 & 5 & 103.1 \\
\hline 91 & 5 & 10 & 7 & 5 & 102.8 \\
\hline 91 & 5 & 11 & 7 & 5 & 103.2 \\
\hline 91 & 5 & 12 & 7 & 5 & 103.3 \\
\hline 91 & 5 & 13 & 7 & 5 & 103.1 \\
\hline 91 & 5 & 14 & 7 & 5 & 103 \\
\hline 91 & 5 & 15 & 7 & 5 & 103.1 \\
\hline 91 & 5 & 16 & 7 & 6 & 102.9 \\
\hline 91 & 5 & 17 & 7 & 6 & 103.4 \\
\hline 91 & 5 & 18 & 7 & 6 & 103.1 \\
\hline 91 & 5 & 19 & 7 & 5 & 103.3 \\
\hline 91 & 5 & 20 & 7 & 6 & 102.9 \\
\hline 91 & 5 & 21 & 7 & 6 & 103.1 \\
\hline 91 & 5 & 22 & 7 & 5 & 103 \\
\hline 91 & 5 & 23 & 7 & 5 & 103.1 \\
\hline 91 & 5 & 24 & 7 & 5 & 103 \\
\hline 91 & 5 & 25 & 7 & 5 & 103 \\
\hline 91 & 5 & 26 & 7 & 5 & 103 \\
\hline 91 & 5 & 27 & 7 & 5 & 103.2 \\
\hline 91 & 5 & 28 & 7 & 5 & 103 \\
\hline 91 & 5 & 29 & 7 & 5 & 103.2 \\
\hline 91 & 5 & 30 & 7 & 5 & 103.2 \\
\hline 91 & 5 & 31 & 7 & 5 & 102.9 \\
\hline 91 & 6 & 1 & 7 & 5 & 103 \\
\hline 91 & 6 & 2 & 7 & 5 & 103.1 \\
\hline 91 & 6 & 3 & 7 & 5 & 103.1 \\
\hline 91 & 6 & 4 & 7 & 5 & 102.9 \\
\hline 91 & 6 & 5 & 7 & 5 & 102.9 \\
\hline 91 & 6 & 6 & 7 & 6 & 102.9 \\
\hline$\overline{91}$ & 6 & 7 & 7 & 5 & 103.1 \\
\hline 91 & 6 & 8 & 7 & 5 & 103.2 \\
\hline 91 & 6 & 9 & 7 & 5 & 103.1 \\
\hline 91 & 6 & 10 & 7 & 6 & 103.1 \\
\hline 91 & 6 & 11 & 7 & 5 & 103.1 \\
\hline 91 & 6 & 12 & 7 & 4 & 103 \\
\hline 91 & 6 & 13 & 7 & 5 & 103 \\
\hline 91 & 6 & 14 & 7 & 5 & 103 \\
\hline 91 & 6 & 15 & 7 & 5 & 103.1 \\
\hline 91 & 6 & 16 & 7 & 5 & 103.1 \\
\hline 91 & 6 & 17 & 7 & 5 & 103 \\
\hline 91 & 8 & 13 & 7 & 4 & 103.1 \\
\hline
\end{tabular}

Data obtained from WHC Surveillance Analysis Computer System (SACS), February 28, 1994. E-35 


\begin{tabular}{|c|c|c|c|c|c|}
\hline Year & Month & Day & Hour & Minute & Surface Level \\
\hline 91 & 8 & 14 & 7 & 4 & 103.1 \\
\hline 91 & 8 & 15 & 7 & 3 & 103.1 \\
\hline 91 & 8 & 17 & 7 & 2 & 103.1 \\
\hline 91 & 8 & 18 & 7 & 3 & 103.2 \\
\hline 91 & 8 & 19 & 7 & 2 & 103.2 \\
\hline 91 & 8 & 20 & 7 & 4 & 103.1 \\
\hline 91 & 8 & 21 & 7 & 3 & 103.2 \\
\hline 91 & 8 & 22 & 7 & 3 & 103.2 \\
\hline 91 & 8 & 23 & 7 & 2 & 103.2 \\
\hline 91 & 8 & 24 & 7 & 2 & 103.2 \\
\hline 91 & 8 & 25 & 7 & 2 & 103.2 \\
\hline 91 & 8 & 26 & 7 & 3 & 103.2 \\
\hline 91 & 8 & 27 & 7 & 3 & 103.2 \\
\hline 91 & 8 & 28 & 7 & 3 & 103.2 \\
\hline 91 & 8 & 29 & 7 & 2 & 103.1 \\
\hline 91 & 8 & 30 & 7 & 10 & 103.1 \\
\hline 91 & 8 & 31 & 7 & 2 & 103.2 \\
\hline 91 & 9 & 1 & 7 & 2 & 103.1 \\
\hline 91 & 9 & 2 & 7 & 2 & 103.1 \\
\hline 91 & 9 & 3 & 7 & 2 & 103 \\
\hline 91 & 9 & 4 & 7 & 3 & 103.1 \\
\hline 91 & 9 & 5 & 7 & 2 & 103.2 \\
\hline 91 & 9 & 6 & 7 & 2 & 103.2 \\
\hline 91 & 9 & 7 & 7 & 2 & 103.3 \\
\hline 91 & 9 & 8 & 7 & 2 & 103.2 \\
\hline 91 & 9 & 9 & 7 & 3 & 103.1 \\
\hline 91 & 9 & 10 & 7 & 2 & 103.3 \\
\hline 91 & 9 & 11 & 7 & 3 & 103.2 \\
\hline 91 & 9 & 12 & 7 & 2 & 103.2 \\
\hline 91 & 9 & 13 & 7 & 2 & 103.3 \\
\hline 91 & 9 & 14 & 7 & 3 & 103.2 \\
\hline 91 & 9 & 15 & 7 & 2 & 103.1 \\
\hline 91 & 9 & 16 & 7 & 3 & 103.2 \\
\hline 91 & 9 & 17 & 7 & 3 & 103.1 \\
\hline 91 & 9 & 18 & 7 & 3 & 103.2 \\
\hline 91 & 9 & 19 & 7 & 3 & 103.2 \\
\hline 91 & 9 & 20 & 7 & 2 & 103.3 \\
\hline 91 & 9 & 21 & 7 & 2 & 103.2 \\
\hline 91 & 9 & 22 & 7 & 3 & 103.1 \\
\hline 91 & 9 & 23 & 7 & 3 & 103.1 \\
\hline 91 & 9 & 24 & 7 & 2 & 103.1 \\
\hline 91 & 9 & 25 & 7 & 3 & 103.2 \\
\hline 91 & 9 & 26 & 7 & 3 & 103.3 \\
\hline 91 & 9 & 27 & 7 & 2 & 103.3 \\
\hline 91 & 9 & 28 & 7 & 2 & 103.3 \\
\hline 91 & 9 & 29 & 7 & 3 & 103.2 \\
\hline 91 & 9 & 30 & 7 & 3 & 103.1 \\
\hline 91 & 10 & 1 & 7 & 2 & 103.2 \\
\hline
\end{tabular}

Data obtained from WHC Surveillance Analysis Computer System (SACS), February 28, 1994. 


\begin{tabular}{|c|c|c|c|c|c|}
\hline Year & Month & Day & Hour & Minute & Surface Level \\
\hline 91 & 10 & 2 & 7 & 2 & 103.2 \\
\hline 91 & 10 & 3 & 7 & 2 & 103.2 \\
\hline 91 & 10 & 4 & 7 & 3 & 103.2 \\
\hline 91 & 10 & 6 & 6 & 2 & 103.2 \\
\hline 91 & 10 & 7 & 6 & 2 & 103.3 \\
\hline 91 & 10 & 8 & 7 & 2 & 103.2 \\
\hline 91 & 10 & 9 & 7 & 2 & 103.2 \\
\hline 91 & 10 & 10 & 7 & 2 & 103.2 \\
\hline 91 & 10 & 11 & 7 & 2 & 103.3 \\
\hline 91 & 10 & 12 & 7 & 2 & 103.3 \\
\hline 91 & 10 & 13 & 7 & 3 & 103.2 \\
\hline 91 & 10 & 14 & 7 & 2 & 103.1 \\
\hline 91 & 10 & 15 & 7 & 2 & 103.3 \\
\hline 91 & 10 & 16 & 7 & 3 & 103.4 \\
\hline 91 & 10 & 17 & 7 & 5 & 103.1 \\
\hline 91 & 10 & 18 & 7 & 6 & 102.9 \\
\hline 91 & 10 & 19 & 7 & 6 & 102.9 \\
\hline 91 & 10 & 20 & 7 & 2 & 103 \\
\hline 91 & 10 & 21 & 7 & 2 & 103.4 \\
\hline 91 & 10 & 22 & 7 & 3 & 103.2 \\
\hline 91 & 10 & 23 & 7 & 2 & 103.2 \\
\hline 91 & 10 & 24 & 7 & 2 & 103.2 \\
\hline 91 & 10 & 25 & 7 & 3 & 103.3 \\
\hline 91 & 10 & 26 & 7 & 3 & 103.4 \\
\hline 91 & 10 & 27 & 7 & 2 & 103.2 \\
\hline 91 & 10 & 28 & 7 & 2 & 103 \\
\hline 91 & 10 & 29 & 7 & 3 & 103.1 \\
\hline 91 & 10 & 30 & 7 & 3 & 103 \\
\hline 91 & 10 & 31 & 7 & 3 & 103.1 \\
\hline 91 & 11 & 1 & 7 & 2 & 103 \\
\hline 91 & 11 & 2 & 7 & 3 & 103 \\
\hline 91 & 11 & 3 & 7 & 3 & 103 \\
\hline 91 & 11 & 4 & 7 & 2 & 103.1 \\
\hline 91 & 11 & 5 & 7 & 3 & 103.2 \\
\hline 91 & 11 & 6 & 7 & 3 & 103.2 \\
\hline 91 & 11 & 7 & 7 & 2 & 103.2 \\
\hline 91 & 11 & 8 & 7 & 3 & 103.3 \\
\hline 91 & 11 & 9 & 7 & 2 & 103.2 \\
\hline 91 & 11 & 10 & 7 & 3 & 103.2 \\
\hline 91 & 11 & 11 & 7 & 10 & 103.3 \\
\hline 91 & 11 & 12 & 7 & 3 & 103.3 \\
\hline 91 & 11 & 13 & 7 & 3 & 103.3 \\
\hline 91 & 11 & 14 & 7 & 3 & 103.3 \\
\hline 91 & 11 & 15 & 7 & 3 & 103.2 \\
\hline 91 & 11 & 16 & 7 & 3 & 103.4 \\
\hline 91 & 11 & 17 & 7 & 2 & 103.4 \\
\hline 91 & 11 & 18 & 7 & 3 & 103.3 \\
\hline 91 & 11 & 19 & 7 & 3 & 103.1 \\
\hline
\end{tabular}

Data obtained from WHC Surveillance Analysis Computer System (SACS), February 28, 1994. E-37 


\begin{tabular}{|c|c|c|c|c|c|}
\hline Year & Month & Day & Hour & Minute & Surface Level \\
\hline 91 & 11 & 20 & 7 & 3 & 103.2 \\
\hline 91 & 11 & 21 & 7 & 2 & 103 \\
\hline 91 & 11 & 22 & 7 & 3 & 103 \\
\hline 91 & 11 & 23 & 7 & 2 & 103 \\
\hline 91 & 11 & 24 & 7 & 3 & 103.2 \\
\hline 91 & 11 & 25 & 7 & 3 & 103.2 \\
\hline 91 & 11 & 27 & 7 & 3 & 103.3 \\
\hline 91 & 11 & 28 & 7 & 3 & 103.1 \\
\hline 91 & 11 & 29 & 7 & 3 & 103.1 \\
\hline 91 & 11 & 30 & 7 & 3 & 103.1 \\
\hline 91 & 12 & 1 & 7 & 3 & 103.1 \\
\hline 91 & 12 & 2 & 7 & 3 & 103.2 \\
\hline 91 & 12 & 3 & 7 & 3 & 103.2 \\
\hline 91 & 12 & 4 & 7 & 3 & 103.3 \\
\hline 91 & 12 & 5 & 7 & 3 & 103.3 \\
\hline 91 & 12 & 6 & 7 & 4 & 103.4 \\
\hline 91 & 12 & 7 & 7 & 3 & 103.3 \\
\hline 91 & 12 & 8 & 7 & 3 & 103.2 \\
\hline 91 & 12 & 9 & 7 & 3 & 103.2 \\
\hline 91 & 12 & 10 & 7 & 3 & 103.1 \\
\hline 91 & 12 & 11 & 7 & 2 & 103.3 \\
\hline 91 & 12 & 12 & 7 & 3 & 103.4 \\
\hline 91 & 12 & 13 & 6 & 6 & 103.1 \\
\hline 91 & 12 & 14 & 7 & 2 & 103 \\
\hline 91 & 12 & 15 & 7 & 2 & 103 \\
\hline 91 & 12 & 18 & 7 & 3 & 103.4 \\
\hline 91 & 12 & 24 & 7 & 3 & 103.2 \\
\hline 91 & 12 & 25 & 7 & 3 & 103.3 \\
\hline 91 & 12 & 26 & 7 & 3 & 103.2 \\
\hline 91 & 12 & 27 & 7 & 3 & 103.3 \\
\hline 91 & 12 & 28 & 7 & 2 & 103.4 \\
\hline 91 & 12 & 29 & 7 & 3 & 103.3 \\
\hline 91 & 12 & 30 & 7 & 3 & 103.3 \\
\hline 91 & 12 & 31 & 7 & 3 & 103.1 \\
\hline 92 & 1 & 1 & 7 & 3 & 103.2 \\
\hline 92 & 1 & 2 & 7 & 2 & 103.4 \\
\hline 92 & 1 & 3 & 7 & 4 & 103.7 \\
\hline 92 & 1 & 4 & 7 & 3 & 103.8 \\
\hline 92 & 1 & 5 & 7 & 3 & 103.4 \\
\hline 92 & 1 & 6 & 7 & 3 & 103.4 \\
\hline 92 & 1 & 28 & 7 & 3 & 103.7 \\
\hline 92 & 1 & 29 & 7 & 3 & 103.2 \\
\hline 92 & 1 & 30 & 7 & 3 & 103.3 \\
\hline 92 & 1 & 31 & 7 & 3 & 103.3 \\
\hline 92 & 2 & 1 & 7 & 2 & 103.3 \\
\hline 92 & 2 & 2 & 7 & 3 & 103.1 \\
\hline 92 & 2 & 3 & 7 & 3 & 103 \\
\hline 92 & 2 & 4 & 7 & 3 & 103 \\
\hline
\end{tabular}

Data obtained from WHC Surveillance Analysis Computer System (SACS), February 28, 1994. 


\begin{tabular}{|c|c|c|c|c|c|}
\hline Year & Month & Day & Hour & Minute & Surface Level \\
\hline 92 & 2 & 5 & 7 & 2 & 103.2 \\
\hline 92 & 2 & 8 & 7 & 4 & 103.3 \\
\hline 92 & 2 & 9 & 7 & 3 & 103.3 \\
\hline 92 & 2 & 10 & 7 & 4 & 103.4 \\
\hline 92 & 2 & 11 & 7 & 3 & 103.3 \\
\hline 92 & 2 & 12 & 7 & 3 & 103.3 \\
\hline 92 & 2 & 13 & 7 & 3 & 103.3 \\
\hline 92 & 2 & 14 & 7 & 3 & 103.3 \\
\hline 92 & 2 & 15 & 7 & 3 & 103.3 \\
\hline 92 & 2 & 16 & 7 & 3 & 103.2 \\
\hline 92 & 2 & 17 & 7 & 3 & 103.2 \\
\hline 92 & 2 & 18 & 7 & 3 & 103.2 \\
\hline 92 & 2 & 19 & 7 & 3 & 103.3 \\
\hline 92 & 2 & 20 & 7 & 3 & 103.2 \\
\hline 92 & 2 & 21 & 7 & 3 & 103.2 \\
\hline 92 & 2 & 22 & 7 & 3 & 103.1 \\
\hline 92 & 2 & 23 & 7 & 3 & 103 \\
\hline 92 & 2 & 24 & 7 & 3 & 103.1 \\
\hline 92 & 2 & 27 & 0 & 3 & 103.1 \\
\hline 92 & 2 & 29 & 7 & 3 & 103.3 \\
\hline 92 & 3 & 1 & 7 & 3 & 103.4 \\
\hline 92 & 3 & 2 & 7 & 3 & 103.3 \\
\hline 92 & 3 & 3 & 7 & 4 & 103.3 \\
\hline 92 & 3 & 4 & 7 & 4 & 103.3 \\
\hline 92 & 3 & 5 & 7 & 3 & 103.3 \\
\hline 92 & 3 & 6 & 7 & 3 & 103.3 \\
\hline 92 & 3 & 7 & 7 & 2 & 103.2 \\
\hline 92 & 3 & 8 & 7 & 2 & 103.2 \\
\hline 92 & 3 & 9 & 7 & 2 & 103.1 \\
\hline 92 & 3 & 10 & 6 & 3 & 103.1 \\
\hline 92 & 3 & 11 & 7 & 3 & 103.1 \\
\hline 92 & 3 & 12 & 7 & 3 & 103.2 \\
\hline 92 & 3 & 13 & 7 & 3 & 103.2 \\
\hline 92 & 3 & 14 & 7 & 3 & 103.3 \\
\hline 92 & 3 & 15 & 7 & 6 & 103.3 \\
\hline 92 & 3 & 16 & 7 & 3 & 103.3 \\
\hline 92 & 3 & 17 & 7 & 3 & 103.2 \\
\hline 92 & 3 & 18 & 7 & 3 & 103.1 \\
\hline 92 & 3 & 19 & 7 & 3 & 103.1 \\
\hline 92 & 3 & 20 & 7 & 2 & 103.2 \\
\hline 92 & 3 & 21 & 7 & 3 & 103.2 \\
\hline 92 & 3 & 22 & 7 & 2 & 103.3 \\
\hline 92 & 3 & 23 & 7 & 2 & 103.3 \\
\hline 92 & 3 & 24 & 7 & 2 & 103.2 \\
\hline 92 & 3 & 25 & 7 & 3 & 103.2 \\
\hline 92 & 3 & 26 & 7 & 3 & 103.3 \\
\hline 92 & 3 & 27 & 7 & 2 & 103.3 \\
\hline 92 & 3 & 28 & 7 & 3 & 103.2 \\
\hline
\end{tabular}

Data obtained from WHC Surveillance Analysis Computer System (SACS), February 28, 1994. E-39 


\begin{tabular}{|c|c|c|c|c|c|}
\hline Year & Month & Day & Hour & Minute & Surface Level \\
\hline 92 & 3 & 29 & 7 & 3 & 103.1 \\
\hline 92 & 3 & 30 & 7 & 3 & 103.2 \\
\hline 92 & 3 & 31 & 7 & 3 & 103.1 \\
\hline 92 & 4 & 1 & 7 & 3 & 103.3 \\
\hline 92 & 4 & 2 & 7 & 3 & 103.5 \\
\hline 92 & 4 & 3 & 7 & 3 & 103.5 \\
\hline 92 & 4 & 4 & 7 & 3 & 103.2 \\
\hline 92 & 4 & 5 & 7 & 3 & 103.3 \\
\hline 92 & 4 & 6 & 7 & 3 & 103.2 \\
\hline 92 & 4 & 7 & 7 & 3 & 103.1 \\
\hline 92 & 4 & 8 & 7 & 10 & 103.2 \\
\hline 92 & 4 & 9 & 7 & 3 & 103.3 \\
\hline 92 & 4 & 10 & 7 & 3 & 103.3 \\
\hline 92 & 4 & 11 & 7 & 3 & 103.2 \\
\hline 92 & 4 & 12 & 7 & 4 & 103.3 \\
\hline 92 & 4 & 13 & 7 & 3 & 103.3 \\
\hline 92 & 4 & 14 & 7 & 3 & 103.2 \\
\hline 92 & 4 & 15 & 7 & 2 & 103.5 \\
\hline 92 & 4 & 16 & 7 & 3 & 103.5 \\
\hline 92 & 4 & 17 & 7 & 3 & 103.2 \\
\hline 92 & 4 & 18 & 7 & 2 & 103 \\
\hline 92 & 4 & 19 & 7 & 2 & 102.9 \\
\hline 92 & 4 & 20 & 7 & 3 & 103.2 \\
\hline 92 & 4 & 21 & 7 & 3 & 103.2 \\
\hline 92 & 4 & 22 & 7 & 2 & 103.2 \\
\hline 92 & 4 & 23 & 7 & 3 & 103 \\
\hline 92 & 4 & 24 & 7 & 3 & 103.1 \\
\hline 92 & 4 & 25 & 7 & 4 & 103.2 \\
\hline 92 & 4 & 26 & 7 & 3 & 103.3 \\
\hline 92 & 4 & 27 & 7 & 4 & 103.2 \\
\hline 92 & 4 & 28 & 7 & 3 & 103.2 \\
\hline 92 & 4 & 29 & 7 & 3 & 103.4 \\
\hline 92 & 4 & 30 & 7 & 3 & 103.4 \\
\hline 92 & 5 & 1 & 7 & 3 & 103.1 \\
\hline 92 & 5 & 2 & 7 & 3 & 103.1 \\
\hline 92 & 5 & 3 & 7 & 3 & 103.1 \\
\hline 92 & 5 & 4 & 7 & 3 & 103.2 \\
\hline 92 & 5 & 4 & 10 & 3 & 103.2 \\
\hline 92 & 5 & 5 & 7 & 4 & 103.3 \\
\hline 92 & 5 & 6 & 7 & 3 & 103.3 \\
\hline 92 & 5 & 7 & 7 & 4 & 103.3 \\
\hline 92 & 5 & 8 & 7 & 3 & 103.3 \\
\hline 92 & 5 & 9 & 7 & 4 & 103.2 \\
\hline 92 & 5 & 10 & 7 & 4 & 103.4 \\
\hline 92 & 5 & 11 & 7 & 3 & 103.2 \\
\hline 92 & 5 & 11 & 10 & 3 & 103.4 \\
\hline 92 & 5 & 18 & 10 & 3 & 103.3 \\
\hline 92 & 5 & 25 & 10 & 3 & 103.3 \\
\hline
\end{tabular}

Data obtained from WHC Surveillance Analysis Computer System (SACS), February 28, 1994. 


\begin{tabular}{|c|c|c|c|c|c|}
\hline Year & Month & Day & Hour & Minute & Surface Level \\
\hline 92 & 6 & 1 & 10 & 6 & 103.4 \\
\hline 92 & 6 & 8 & 10 & 6 & 103.25 \\
\hline 92 & 6 & 15 & 10 & 6 & 103.4 \\
\hline 92 & 6 & 22 & 10 & 6 & 103.4 \\
\hline 92 & 6 & 29 & 10 & 6 & 103.4 \\
\hline 92 & 7 & 6 & 10 & 6 & 103.43 \\
\hline 92 & 7 & 13 & 10 & 6 & 103.4 \\
\hline 92 & 7 & 20 & 10 & 6 & 103.45 \\
\hline 92 & 7 & 27 & 10 & 6 & 103.4 \\
\hline 92 & 8 & 4 & 10 & 6 & 103.5 \\
\hline 92 & 8 & 10 & 10 & 6 & 103.35 \\
\hline 92 & 8 & 17 & 10 & 6 & 103.5 \\
\hline 92 & 8 & 24 & 10 & 6 & 103.3 \\
\hline 92 & 9 & 1 & 10 & 2 & 103.5 \\
\hline 92 & 9 & 8 & 10 & 2 & 103.55 \\
\hline 92 & 9 & 14 & 10 & 2 & 103.55 \\
\hline 92 & 9 & 21 & 10 & 2 & 103.5 \\
\hline 92 & 9 & 28 & 10 & 2 & 103.4 \\
\hline 92 & 10 & 5 & 10 & 3 & 103.4 \\
\hline 92 & 10 & 12 & 10 & 3 & 103.55 \\
\hline 92 & 10 & 19 & 10 & 3 & 103.5 \\
\hline 92 & 10 & 26 & 10 & 3 & 103.45 \\
\hline 92 & 10 & 29 & 7 & 3 & 103.6 \\
\hline 92 & 10 & 30 & 7 & 3 & 103.6 \\
\hline 92 & 10 & 31 & 7 & 3 & 103.4 \\
\hline 92 & 11 & 1 & 7 & 3 & 103.3 \\
\hline 92 & 11 & 2 & 7 & 3 & 103.3 \\
\hline 92 & 11 & 3 & 7 & 3 & 103.1 \\
\hline 92 & 11 & 4 & 7 & 3 & 103.3 \\
\hline 92 & 11 & 9 & 10 & 3 & 103.4 \\
\hline 92 & 11 & 16 & 10 & 3 & 103.45 \\
\hline 92 & 11 & 23 & 10 & 3 & 103.2 \\
\hline 92 & 11 & 30 & 10 & 3 & 103.4 \\
\hline 92 & 12 & 2 & 6 & 3 & 103.5 \\
\hline 92 & 12 & 3 & 6 & 3 & 103.3 \\
\hline 92 & 12 & 4 & 7 & 3 & 103.2 \\
\hline 92 & 12 & 6 & 7 & 2 & 103.4 \\
\hline 92 & 12 & 7 & 7 & 3 & 103.6 \\
\hline 92 & 12 & 8 & 7 & 3 & 103.6 \\
\hline 92 & .12 & 9 & 7 & 3 & 103.4 \\
\hline 92 & 12 & 10 & 7 & 3 & 103.6 \\
\hline 92 & 12 & 11 & 7 & 3 & 103.5 \\
\hline 92 & 12 & 13 & 7 & 4 & 103.2 \\
\hline 92 & 12 & 14 & 7 & 3 & 103.4 \\
\hline 92 & 12 & 21 & 10 & 3 & 103.45 \\
\hline 92 & 12 & 28 & 10 & 3 & 103.65 \\
\hline 93 & 1 & 5 & 10 & 3 & 103.3 \\
\hline 93 & 1 & 12 & 10 & 3 & 103.45 \\
\hline
\end{tabular}

Data obtained from WHC Surveillance Analysis Computer System (SACS), February 28, 1994. 


\begin{tabular}{|c|c|c|c|c|c|}
\hline Year & Month & Day & Hour & Minute & Surface Level \\
\hline 93 & 1 & 19 & 10 & 3 & 103.75 \\
\hline 93 & 1 & 25 & 10 & 3 & 103.4 \\
\hline 93 & 1 & 26 & 10 & 3 & 103.4 \\
\hline 93 & 2 & 1 & 10 & 3 & 103.6 \\
\hline 93 & 2 & 8 & 10 & 3 & 103.65 \\
\hline 93 & 2 & 16 & 10 & 3 & 103.3 \\
\hline 93 & 2 & 22 & 10 & 3 & 103.25 \\
\hline 93 & 3 & 1 & 10 & 3 & 103.55 \\
\hline 93 & 5 & 14 & 10 & 6 & 104.8 \\
\hline 93 & 5 & 15 & 10 & 6 & 104.9 \\
\hline 93 & 5 & 16 & 11 & 6 & 104.6 \\
\hline 93 & 5 & 17 & 11 & 6 & 104.8 \\
\hline 93 & 5 & 18 & 7 & 3 & 104.6 \\
\hline 93 & 5 & 20 & 7 & 3 & 104.9 \\
\hline 93 & 5 & 21 & 5 & 2 & 104.7 \\
\hline 93 & 5 & 22 & 7 & 3 & 104.8 \\
\hline 93 & 5 & 23 & 7 & 4 & 104.8 \\
\hline 93 & 5 & 24 & 7 & 3 & 104.8 \\
\hline 93 & 5 & 25 & 7 & 3 & 104.9 \\
\hline 93 & 5 & 26 & 7 & 3 & 104.7 \\
\hline 93 & 5 & 27 & 7 & 3 & 104.9 \\
\hline 93 & 5 & 28 & 7 & 3 & 104.8 \\
\hline 93 & 5 & 29 & 7 & 5 & 104.7 \\
\hline 93 & 5 & 30 & 7 & 5 & 104.6 \\
\hline 93 & 5 & 31 & 7 & 6 & 104.7 \\
\hline 93 & 6 & 1 & 7 & 6 & 104.8 \\
\hline 93 & 6 & 2 & 7 & 6 & 104.6 \\
\hline 93 & 6 & 3 & 7 & 6 & 104.8 \\
\hline 93 & 6 & 4 & 7 & 6 & 104.7 \\
\hline 93 & 6 & 5 & 7 & 6 & 104.7 \\
\hline 93 & 6 & 6 & 7 & 5 & 104.9 \\
\hline 93 & 6 & 7 & 7 & 5 & 104.6 \\
\hline 93 & 6 & 8 & 7 & 5 & 104.7 \\
\hline 93 & 6 & 9 & 7 & 5 & 104.6 \\
\hline 93 & 6 & 10 & 7 & 4 & 104.6 \\
\hline 93 & 6 & 11 & 7 & 5 & 104.6 \\
\hline 93 & 6 & 12 & 7 & 5 & 104.6 \\
\hline 93 & 6 & 13 & 7 & 4 & 104.3 \\
\hline 93 & 6 & 14 & 7 & 5 & 104.6 \\
\hline 93 & 6 & 15 & 7 & 5 & 104.6 \\
\hline 93 & 6 & 16 & 7 & 5 & 104.6 \\
\hline 93 & 6 & 17 & 7 & 5 & 104.6 \\
\hline 93 & 6 & 18 & 7 & 6 & 104.6 \\
\hline 93 & 6 & 25 & 7 & 3 & 104.4 \\
\hline 93 & 6 & 26 & 7 & 3 & 104.7 \\
\hline 93 & 6 & 27 & 7 & 4 & 104.5 \\
\hline 93 & 6 & 28 & 7 & 3 & 104.8 \\
\hline 93 & 6 & 29 & 7 & 4 & 104.8 \\
\hline
\end{tabular}

Data obtained from WHC Surveillance Analysis Computer System (SACS), February 28, 1994. 


\begin{tabular}{|c|c|c|c|c|c|}
\hline Year & Month & Day & Hour & Minute & Surface Level \\
\hline 93 & 6 & 30 & 6 & 3 & 104.7 \\
\hline 93 & 7 & 1 & 7 & 6 & 104.6 \\
\hline 93 & 7 & 2 & 7 & 6 & 104.8 \\
\hline 93 & 7 & 3 & 7 & 3 & 104.9 \\
\hline 93 & 7 & 4 & 7 & 4 & 104.9 \\
\hline 93 & 7 & 5 & 7 & 3 & 104.5 \\
\hline 93 & 7 & 6 & 7 & 3 & 104.6 \\
\hline 93 & 7 & 7 & 7 & 4 & 104.9 \\
\hline 93 & 7 & 8 & 7 & 4 & 104.7 \\
\hline 93 & 7 & 9 & 7 & 5 & 104.8 \\
\hline 93 & 7 & 10 & 7 & 3 & 104.8 \\
\hline 93 & 7 & 11 & 7 & 3 & 104.6 \\
\hline 93 & 7 & 12 & 7 & 2 & 104.8 \\
\hline 93 & 7 & 13 & 7 & 3 & 104.8 \\
\hline 93 & 7 & 14 & 7 & 6 & 104.7 \\
\hline 93 & 7 & 15 & 7 & 6 & 104.8 \\
\hline 93 & 7 & 16 & 7 & 6 & 104.9 \\
\hline 93 & 7 & 17 & 7 & 7 & 104.9 \\
\hline 93 & 7 & 18 & 7 & 6 & 104.7 \\
\hline 93 & 7 & 19 & 7 & 3 & 104.8 \\
\hline 93 & 7 & 20 & 7 & 3 & 104.7 \\
\hline 93 & 7 & 21 & 7 & 3 & 104.7 \\
\hline 93 & 7 & 22 & 7 & 3 & 104.8 \\
\hline 93 & 7 & 23 & 7 & 3 & 104.8 \\
\hline 93 & 7 & 24 & 7 & 3 & 104.8 \\
\hline 93 & 7 & 25 & 7 & 4 & 104.9 \\
\hline 93 & 7 & 26 & 7 & 3 & 104.8 \\
\hline 93 & 7 & 27 & 7 & 3 & 104.9 \\
\hline 93 & 7 & 28 & 7 & 4 & 104.9 \\
\hline 93 & 7 & 29 & 7 & 3 & 104.8 \\
\hline 93 & 7 & 30 & 7 & 3 & 104.7 \\
\hline 93 & 7 & 31 & 7 & 3 & 104.6 \\
\hline 93 & 8 & 1 & 7 & 3 & 104.7 \\
\hline 93 & 8 & 2 & 7 & 6 & 104.6 \\
\hline 93 & 8 & 3 & 7 & 3 & 104.9 \\
\hline 93 & 8 & 4 & 7 & 3 & 104.9 \\
\hline 93 & 8 & 5 & 7 & 4 & 104.7 \\
\hline 93 & 8 & 6 & 7 & 4 & 104.9 \\
\hline 93 & 8 & 8 & 7 & 4 & 104.9 \\
\hline 93 & 8 & 9 & 7 & 4 & 104.8 \\
\hline 93 & 8 & 10 & 7 & 4 & 104.7 \\
\hline 93 & 8 & 11 & 7 & 4 & 104.5 \\
\hline .93 & 8 & 12 & 7 & 4 & 104.7 \\
\hline 93 & 8 & 13 & 7 & 4 & 104.7 \\
\hline 93 & 8 & 14 & 7 & 3 & 104.9 \\
\hline 93 & 8 & 15 & 7 & 4 & 104.7 \\
\hline 93 & 8 & 16 & 7 & 4 & 104.9 \\
\hline 93 & 8 & 17 & 7 & 4 & 104.8 \\
\hline
\end{tabular}

Data obtained from WHC Surveillance Analysis Computer System (SACS), February 28, 1994. 


\begin{tabular}{|c|c|c|c|c|c|}
\hline Year & Month & Day & Hour & Minute & Surface Level \\
\hline 93 & 8 & 18 & 7 & 3 & 104.8 \\
\hline 93 & 8 & 19 & 7 & 4 & 104.7 \\
\hline 93 & 8 & 20 & 7 & 4 & 105 \\
\hline 93 & 8 & 21 & 7 & 4 & 104.9 \\
\hline 93 & 8 & 22 & 7 & 4 & 104.9 \\
\hline 93 & 8 & 23 & 7 & 4 & 105 \\
\hline 93 & 8 & 24 & 7 & 4 & 104.6 \\
\hline 93 & 8 & 25 & 7 & 4 & 104.8 \\
\hline 93 & 8 & 26 & 7 & 4 & 104.8 \\
\hline 93 & 8 & 27 & 7 & 5 & 104.9 \\
\hline 93 & 8 & 28 & 7 & 4 & 104.7 \\
\hline 93 & 8 & 29 & 7 & 3 & 104.7 \\
\hline 93 & 8 & 30 & 7 & 4 & 104.9 \\
\hline 93 & 8 & 31 & 7 & 4 & 105 \\
\hline 93 & 9 & 1 & 7 & 4 & 105 \\
\hline 93 & 9 & 2 & 7 & 4 & 105 \\
\hline 93 & 9 & 3 & 7 & 4 & 105 \\
\hline 93 & 9 & 4 & 7 & 4 & 105 \\
\hline 93 & 9 & 5 & 7 & 4 & 105 \\
\hline 93 & 9 & 6 & 7 & 4 & 105 \\
\hline 93 & 9 & 7 & 7 & 4 & 105 \\
\hline 93 & 9 & 8 & 7 & 4 & 105 \\
\hline 93 & 9 & 9 & 7 & 4 & 105 \\
\hline 93 & 9 & 10 & 7 & 4 & 105 \\
\hline 93 & 9 & 11 & 7 & 4 & 105 \\
\hline 93 & 9 & 12 & 7 & 4 & 104.6 \\
\hline 93 & 9 & 13 & 7 & 4 & 104.8 \\
\hline 93 & 9 & 14 & 7 & 4 & 105 \\
\hline 93 & 9 & 15 & 7 & 4 & 105 \\
\hline 93 & 9 & 16 & 7 & 4 & 105 \\
\hline 93 & 9 & 17 & 7 & 4 & 105 \\
\hline 93 & 9 & 18 & 7 & 4 & 105 \\
\hline 93 & 9 & 19 & 7 & 4 & 105 \\
\hline 93 & 9 & 20 & 7 & 4 & 105 \\
\hline 93 & 9 & 21 & 7 & 4 & 105 \\
\hline 93 & 9 & 22 & 7 & 4 & 104.9 \\
\hline 93 & 9 & 23 & 7 & 4 & 104.9 \\
\hline 93 & 9 & 24 & 7 & 4 & 105 \\
\hline 93 & 9 & 25 & 7 & 4 & 105 \\
\hline 93 & 9 & 26 & 7 & 4 & 104.9 \\
\hline 93 & 9 & 27 & 7 & 4 & 105 \\
\hline 93 & 9 & 28 & 7 & 4 & 105 \\
\hline 93 & 9 & 29 & 7 & 4 & 105 \\
\hline 93 & 9 & 30 & 7 & 3 & 105 \\
\hline 93 & 10 & 1 & 7 & 6 & 105 \\
\hline 93 & 10 & 2 & 7 & 5 & 105 \\
\hline 93 & 10 & 3 & 7 & 6 & 105 \\
\hline 93 & 10 & 4 & 7 & 6 & 105 \\
\hline
\end{tabular}

Data obtained from WHC Surveillance Analysis Computer System (SACS), February 28, 1994. 


\begin{tabular}{|c|c|c|c|c|c|}
\hline Year & Month & Day & Hour & Minute & Surface Level \\
\hline 93 & 10 & 5 & 7 & 6 & 105.1 \\
\hline 93 & 10 & 6 & 7 & 6 & 105 \\
\hline 93 & 10 & 7 & 7 & 5 & 105 \\
\hline 93 & 10 & 8 & 7 & 4 & 104.9 \\
\hline 93 & 10 & 9 & 7 & 3 & 104.9 \\
\hline 93 & 10 & 10 & 7 & 10 & 105 \\
\hline 93 & 10 & 11 & 7 & 3 & 105 \\
\hline 93 & 10 & 12 & 7 & 4 & 105 \\
\hline 93 & 10 & 13 & 7 & 3 & 105 \\
\hline 93 & 10 & 14 & 7 & 4 & 105 \\
\hline 93 & 10 & 15 & 7 & 4 & 105.1 \\
\hline 93 & 10 & 16 & 7 & 4 & 105 \\
\hline 93 & 10 & 17 & 7 & 3 & 105 \\
\hline 93 & 10 & 18 & 7 & 4 & 105 \\
\hline 93 & 10 & 19 & 7 & 4 & 105 \\
\hline 93 & 10 & 20 & 7 & 3 & 104.8 \\
\hline 93 & 10 & 21 & 7 & 4 & 105 \\
\hline 93 & 10 & 22 & 7 & 4 & 105 \\
\hline 93 & 11 & 9 & 7 & 3 & 105 \\
\hline 93 & 11 & 10 & 7 & 4 & 105 \\
\hline 93 & 11 & 11 & 7 & 4 & 105.1 \\
\hline 93 & 11 & 12 & 7 & 4 & 104.9 \\
\hline 93 & 11 & 13 & 7 & 4 & 105 \\
\hline 93 & 11 & 14 & 7 & 3 & 104.9 \\
\hline 93 & 11 & 15 & 7 & 4 & 105 \\
\hline 93 & 11 & 16 & 7 & 3 & 105 \\
\hline 93 & 11 & 17 & 7 & 4 & 105 \\
\hline 93 & 11 & 18 & 7 & 4 & 105 \\
\hline 93 & 11 & 22 & 15 & 4 & 104.9 \\
\hline 93 & 11 & 24 & 7 & 4 & 103.9 \\
\hline 93 & 11 & 25 & 7 & 4 & 104.4 \\
\hline 93 & 11 & 26 & 7 & 4 & 104.3 \\
\hline 93 & 11 & 27 & 7 & 4 & 103.9 \\
\hline 93 & 11 & 28 & 7 & 3 & 103.9 \\
\hline 93 & 12 & 3 & 15 & 4 & 104.5 \\
\hline 93 & 12 & 4 & 7 & 5 & 104.7 \\
\hline 93 & 12 & 5 & 7 & 4 & 104.4 \\
\hline 93 & 12 & 6 & 7 & 5 & 104.5 \\
\hline 93 & 12 & 7 & 7 & 4 & 104.7 \\
\hline 93 & 12 & 8 & 7 & 6 & 104.7 \\
\hline 93 & 12 & 9 & 7 & 4 & 104.5 \\
\hline 93 & 12 & 10 & 7 & 10 & 104.7 \\
\hline 93 & 12 & 11 & 7 & 6 & 104.7 \\
\hline 93 & 12 & 12 & 7 & 6 & 104.5 \\
\hline 93 & 12 & 13 & 7 & 6 & 104.4 \\
\hline 93 & 12 & 14 & 7 & 6 & 104.4 \\
\hline 93 & 12 & 15 & 7 & 6 & 104.4 \\
\hline 93 & 12 & 16 & 7 & 6 & 104.2 \\
\hline
\end{tabular}

Data obtained from WHC Surveillance Analysis Computer System (SACS), February 28, 1994. E-45 


\begin{tabular}{|c|c|c|c|c|c|}
\hline Year & Month & Day & Hour & Minute & Surface Level \\
\hline 93 & 12 & 17 & 7 & 5 & 104.2 \\
\hline 93 & 12 & 18 & 7 & 6 & 104.1 \\
\hline 93 & 12 & 19 & 7 & 6 & 104.2 \\
\hline 93 & 12 & 20 & 7 & 6 & 104.3 \\
\hline 93 & 12 & 21 & 7 & 6 & 104.2 \\
\hline 93 & 12 & 22 & 7 & 5 & 104.3 \\
\hline 93 & 12 & 23 & 7 & .10 & 104.1 \\
\hline 93 & 12 & 24 & 7 & 6 & 104.2 \\
\hline 93 & 12 & 25 & 7 & 6 & 104.4 \\
\hline 93 & 12 & 26 & 7 & 6 & 104.5 \\
\hline 93 & 12 & 27 & 7 & 6 & 104.5 \\
\hline 93 & 12 & 28 & 6 & 6 & 104.4 \\
\hline 93 & 12 & 29 & 7 & 6 & 104.3 \\
\hline 93 & 12 & 30 & 0 & 5 & 104.4 \\
\hline 93 & 12 & 31 & 7 & 6 & 104.5 \\
\hline 94 & 1 & 1 & 7 & 6 & 104.6 \\
\hline 94 & 1 & 2 & 7 & 6 & 104.4 \\
\hline 94 & 1 & 3 & 7 & 7 & 104.6 \\
\hline 94 & 1 & 4 & 7 & 7 & 104.7 \\
\hline 94 & 1 & 5 & 7 & 6 & 104.6 \\
\hline 94 & 1 & 6 & 7 & 6 & 104.4 \\
\hline 94 & 1 & 7 & 7 & 7 & 104.5 \\
\hline 94 & 1 & 8 & 7 & 6 & 104.6 \\
\hline 94 & 1 & 9 & 7 & 6 & 104.6 \\
\hline 94 & 1 & 10 & 7 & 7 & 104.5 \\
\hline 94 & 1 & 11 & 7 & 7 & 104.4 \\
\hline 94 & 1 & 12 & 7 & 6 & 104.3 \\
\hline 94 & 1 & 13 & 7 & 10 & 104.4 \\
\hline 94 & 1 & 14 & 7 & 6 & 104.4 \\
\hline 94 & 1 & 15 & 7 & 10 & 104.4 \\
\hline 94 & 1 & 16 & 7 & 7 & 104.4 \\
\hline 94 & 1 & 17 & 7 & 7 & 104.6 \\
\hline 94 & 1 & 18 & 7 & 7 & 104.3 \\
\hline 94 & 1 & 19 & 7 & 7 & 104.5 \\
\hline 94 & 1 & 20 & 7 & 7 & 104.6 \\
\hline$\overline{94}$ & 1 & 21 & 7 & 7 & 104.3 \\
\hline 94 & 1 & 22 & 7 & 8 & 104.6 \\
\hline 94 & 1 & 23 & 7 & 7 & 104.6 \\
\hline 94 & 1 & 24 & 7 & 8 & 104.7 \\
\hline 94 & 1 & 25 & 7 & 7 & 104.5 \\
\hline 94 & 1 & 26 & 7 & 6 & 104.4 \\
\hline 94 & 1 & 27 & 7 & 10 & 104.3 \\
\hline 94 & 1 & 28 & 7 & 7 & 104.3 \\
\hline 94 & 1 & 29 & 7 & 7 & 104.2 \\
\hline 94 & 1 & 30 & 7 & 6 & 104.2 \\
\hline 94 & 1 & 31 & 7 & 7 & 104.3 \\
\hline 94 & 2 & 1 & 7 & 6 & 104.2 \\
\hline 94 & 2 & 2 & 7 & 7 & 104.3 \\
\hline
\end{tabular}

Data obtained from WHC Surveillance Analysis Computer System (SACS), February 28, 1994. 


\begin{tabular}{|c|c|c|c|c|c|}
\hline Year & Month & Day & Hour & Minute & Surface Level \\
\hline 94 & 2 & 3 & 7 & 7 & 104.4 \\
\hline 94 & 2 & 4 & 7 & 7 & 104.4 \\
\hline 94 & 2 & 5 & 7 & 7 & 104.6 \\
\hline 94 & 2 & 6 & 7 & 7 & 104.4 \\
\hline 94 & 2 & 7 & 7 & 6 & 104.5 \\
\hline 94 & 2 & 8 & 7 & 7 & 104.4 \\
\hline 94 & 2 & 9 & 7 & 4 & 104.4 \\
\hline 94 & 2 & 10 & 7 & 4 & 104.4 \\
\hline 94 & 2 & 11 & 7 & 6 & 104.5 \\
\hline 94 & 2 & 12 & 7 & 3 & 104.3 \\
\hline 94 & 2 & 13 & 7 & 4 & 104.6 \\
\hline 94 & 2 & 14 & 7 & 4 & 104.3 \\
\hline 94 & 2 & 15 & 7 & 4 & 104.6 \\
\hline 94 & 2 & 16 & 7 & 3 & 104.6 \\
\hline 94 & 2 & 17 & 7 & 4 & 104.5 \\
\hline 94 & 2 & 18 & 7 & 6 & 104.4 \\
\hline 94 & 2 & 19 & 7 & 6 & 104.3 \\
\hline 94 & 2 & 20 & 7 & 7 & 104.3 \\
\hline 94 & 2 & 21 & 7 & 6 & 104.2 \\
\hline 94 & 2 & 22 & 7 & 5 & 104.1 \\
\hline 94 & 2 & 23 & 7 & 6 & 104.3 \\
\hline 94 & 2 & 24 & 7 & 6 & 104.4 \\
\hline & & & & & \\
\hline & & & & & \\
\hline & & & & & \\
\hline & & & & & \\
\hline & & & & & \\
\hline & & & & & \\
\hline & & & & & \\
\hline & & & & & \\
\hline & & & & & \\
\hline & & & & & \\
\hline & & & & & \\
\hline & & & & & \\
\hline & & & & & \\
\hline & & & & & \\
\hline & & & & & \\
\hline & & & & & \\
\hline & & & & & \\
\hline & & & & & \\
\hline & & & & $\therefore$ & \\
\hline & & & & & \\
\hline & & & & & \\
\hline & & & & & \\
\hline & & & & & \\
\hline & & & & & \\
\hline & & & & & \\
\hline & & & & & \\
\hline
\end{tabular}

Data obtained from WHC Surveillance Analysis Computer System (SACS), February 28, 1994. E-47 


\begin{tabular}{|c|c|c|c|c|c|}
\hline \multicolumn{6}{|c|}{ Surface Level Data } \\
\hline \multicolumn{6}{|c|}{ Manual Tape } \\
\hline Year & Month & Day & Hour & Minute & Surface Level \\
\hline 91 & 1 & 8 & 13 & 28 & 114 \\
\hline 91 & 2 & 1 & 14 & 17 & 114 \\
\hline 91 & 3 & 1 & 14 & 46 & 113.5 \\
\hline 91 & 4 & 1 & 15 & 0 & 113.5 \\
\hline 91 & 5 & 7 & 14 & 42 & 113 \\
\hline 91 & 8 & 5 & 18 & 12 & 113 \\
\hline 91 & 11 & 8 & 7 & 49 & 113.5 \\
\hline 92 & 2 & 28 & 15 & 51 & 113.5 \\
\hline 92 & 3 & 2 & 15 & 47 & 112.75 \\
\hline 92 & 4 & 6 & 17 & 30 & 113 \\
\hline 92 & 5 & 8 & 20 & 26 & 113.5 \\
\hline 92 & 6 & 4 & 17 & 45 & 113 \\
\hline 92 & 7 & 1 & 15 & 19 & 113 \\
\hline 92 & 8 & 4 & 21 & 41 & 113.5 \\
\hline 92 & 9 & 1 & 15 & 46 & 113.5 \\
\hline 92 & 10 & 2 & 15 & 34 & 113.5 \\
\hline 92 & 12 & 7 & 22 & 7 & 113.75 \\
\hline 93 & 2 & 1 & 15 & 22 & 114.5 \\
\hline 93 & 3 & 1 & 15 & 18 & 113.5 \\
\hline 93 & 4 & 8 & 3 & 11 & ${ }^{*} 221$ \\
\hline 93 & 4 & 8 & 4 & 18 & 113 \\
\hline 93 & 6 & 9 & 9 & 35 & 112.75 \\
\hline 93 & 7 & 7 & 19 & 29 & 112.75 \\
\hline 93 & 7 & 11 & 7 & 4 & 112.75 \\
\hline 93 & 7 & 12 & 7 & 4 & 112.75 \\
\hline 93 & 7 & 13 & 7 & 4 & 112.75 \\
\hline 93 & 7 & 14 & 7 & 4 & 112.75 \\
\hline 93 & 7 & 15. & 7 & 4 & 112.75 \\
\hline 93 & 7 & 16 & 7 & 4 & 112.75 \\
\hline 93 & 7 & 17 & 7 & 4 & 112.75 \\
\hline 93 & 7 & 18 & 7 & 4 & 112.75 \\
\hline 93 & 7 & 19 & 7 & 4 & 112.75 \\
\hline 93 & 7 & 20 & 7 & 4 & 112.75 \\
\hline 93 & 7 & 21 & 7 & 4 & 112.75 \\
\hline 93 & 7 & 22 & 7 & 4 & 112.75 \\
\hline 93 & 7 & 23 & 7 & 4 & 112.75 \\
\hline 93 & 7 & 24 & 7 & 4 & 112.75 \\
\hline 93 & 7 & 25 & 7 & 4 & 112.75 \\
\hline 93 & 7 & 26 & 7 & 4 & 112.75 \\
\hline 93 & 7 & 27 & 7 & 4 & 112.75 \\
\hline 93 & 7 & 28 & 7 & 4 & 112.75 \\
\hline 93 & 7 & 29 & 7 & 4 & 112.75 \\
\hline 93 & 7 & 30 & 7 & 4 & 112.75 \\
\hline 93 & 7 & 31 & 7 & 4 & 112.75 \\
\hline 93 & 8 & 1 & 7 & 4 & 112.75 \\
\hline 93 & 8 & 2 & 7 & 4 & 112.75 \\
\hline
\end{tabular}

Data obtained from WHC Surveillance Analysis Computer System (SACS), February 28, 1994. 


\begin{tabular}{|c|c|c|c|c|c|}
\hline Year & Month & Day & Hour & Minute & Surface Level \\
\hline 93 & 8 & 3 & 7 & 4 & 112.75 \\
\hline 93 & 8 & 4 & 7 & 4 & 112.75 \\
\hline 93 & 8 & 5 & 7 & 4 & 112.75 \\
\hline 93 & 8 & 6 & 7 & 4 & 112.75 \\
\hline 93 & 8 & 7 & 7 & 4 & 112.75 \\
\hline 93 & 8 & 14 & 9 & 41 & 112.5 \\
\hline 93 & 8 & 17 & 7 & 4 & 112.5 \\
\hline 93 & 8 & 18 & 7 & 4 & 112.5 \\
\hline 93 & 8 & 19 & 7 & 4 & 112.5 \\
\hline 93 & 8 & 20 & 7 & 4 & 112.5 \\
\hline 93 & 8 & 21 & 7 & 4 & 112.5 \\
\hline 93 & 8 & 22 & 7 & 4 & 112.5 \\
\hline 93 & 8 & 23 & 7 & 4 & 112.5 \\
\hline 93 & 8 & 24 & 7 & 4 & 112.5 \\
\hline 93 & 8 & 25 & 7 & 4 & 112.5 \\
\hline 93 & 8 & 26 & 7 & 4 & 112.5 \\
\hline 93 & 8 & 27 & 7 & 4 & 112.5 \\
\hline 93 & 8 & 28 & 7 & 4 & 112.5 \\
\hline 93 & 8 & 29 & 7 & 4 & 112.5 \\
\hline 93 & 8 & 30 & 7 & 4 & 112.5 \\
\hline 93 & 8 & 31 & 7 & 4 & 112.5 \\
\hline 93 & 9 & 1 & 7 & 4 & 112.5 \\
\hline 93 & 9 & 2 & 7 & 4 & 113 \\
\hline 93 & 9 & 3 & 7 & 4 & 113 \\
\hline 93 & 9 & 4 & 7 & 4 & 113 \\
\hline 93 & 9 & 5 & 7 & 4 & 113 \\
\hline 93 & 9 & 6 & 7 & 4 & 113 \\
\hline 93 & 9 & 7 & 7 & 4 & 112.75 \\
\hline 93 & 9 & 8 & 7 & 4 & 112.75 \\
\hline 93 & 9 & 9 & 7 & 4 & 112.75 \\
\hline 93 & 9 & 10 & 7 & 4 & 112.75 \\
\hline 93 & 9 & 11 & 7 & 4 & 112.75 \\
\hline 93 & 9 & 12 & 7 & 4 & 112.75 \\
\hline 93 & 9 & 13 & 7 & 4 & 112.75 \\
\hline 93 & 9 & 14 & 7 & 4 & 112.75 \\
\hline 93 & 9 & 15 & 7 & 4 & 112.75 \\
\hline 93 & 9 & 16 & 7 & 4 & 112.75 \\
\hline 93 & 9 & 17 & 7 & 4 & 112.75 \\
\hline 93 & 9 & 18 & 7 & 4 & 112.75 \\
\hline 93 & 9 & 19 & 7 & 4 & 112.75 \\
\hline 93 & 9 & 20 & 7 & 4 & 112.75 \\
\hline 93 & 9 & 21 & 7 & 4 & 112.75 \\
\hline 93 & 9 & 22 & 7 & 4 & 112.75 \\
\hline 93 & 9 & 23 & 7 & 4 & 112.75 \\
\hline 93 & 9 & 24 & 7 & 4 & 112.75 \\
\hline 93 & 9 & 25 & 7 & 4 & 112.75 \\
\hline 93 & 9 & 26 & 7 & 4 & 112.75 \\
\hline 93 & 9 & 27 & 7 & 4 & 112.75 \\
\hline
\end{tabular}

Data obtained from WHC Surveillance Analysis Computer System (SACS), February 28, 1994. 


\begin{tabular}{|c|c|c|c|c|c|}
\hline Year & Month & Day & Hour & Minute & Surface Level \\
\hline 93 & 9 & 28 & 7 & 4 & 112.75 \\
\hline 93 & 9 & 29 & 7 & 4 & 112.75 \\
\hline 93 & 9 & 30 & 7 & 4 & 112.75 \\
\hline 93 & 10 & 1 & 7 & 4 & 112.75 \\
\hline 93 & 10 & 2 & 20 & 58 & 113 \\
\hline 93 & 10 & 3 & 7 & 4 & 113 \\
\hline 93 & 10 & 4 & 7 & 4 & 113 \\
\hline 93 & 10 & 5 & 7 & 4 & 113 \\
\hline 93 & 10 & 6 & 7 & 4 & 113 \\
\hline 93 & 10 & 13 & 7 & 4 & 113 \\
\hline 93 & 10 & 14 & 7 & 4 & 113 \\
\hline 93 & 10 & 15 & 7 & 4 & 113 \\
\hline 93 & 10 & 16 & 7 & 4 & 113 \\
\hline 93 & 10 & 17 & 7 & 4 & 113 \\
\hline 93 & 10 & 18 & 7 & 4 & 113 \\
\hline 93 & 10 & 19 & 7 & 4 & 113 \\
\hline 93 & 10 & 20 & 7 & 4 & 113 \\
\hline 93 & 10 & 21 & 7 & 4 & 113 \\
\hline 93 & 10 & 22 & 7 & 4 & 113 \\
\hline 93 & 10 & 23 & 7 & 4 & 113 \\
\hline 93 & 10 & 24 & 7 & 4 & 113 \\
\hline 93 & 10 & 25 & 7 & 4 & 113 \\
\hline 93 & 10 & 26 & 7 & 4 & 113 \\
\hline 93 & 10 & 27 & 7 & 4 & 113 \\
\hline 93 & 10 & 28 & 7 & 4 & 113 \\
\hline 93 & 10 & 29 & 7 & 4 & 113 \\
\hline 93 & 10 & 30 & 7 & 4 & 113 \\
\hline 93 & 10 & 31 & 7 & 4 & 113 \\
\hline 93 & 11 & 1 & 7 & 4 & 113 \\
\hline 93 & 11 & 2 & 7 & 4 & 113 \\
\hline 93 & 11 & 3 & 7 & 4 & 113 \\
\hline 93 & 11 & 4 & 7 & 4 & 113 \\
\hline 93 & 11 & 5 & 7 & 4 & 113 \\
\hline 93 & 11 & 6 & 7 & 4 & 113 \\
\hline 93 & 11 & 7 & 7 & 4 & 113 \\
\hline 93 & 11 & 8 & 7 & 4 & 113 \\
\hline 93 & 11 & 9 & 7 & 4 & 113 \\
\hline 93 & 11 & 10 & 7 & 4 & 113 \\
\hline 93 & 11 & 11 & 7 & 4 & 113 \\
\hline 93 & 11 & 12 & 7 & 4 & 113 \\
\hline 93 & 11 & 13 & 7 & 4 & 113 \\
\hline 93 & 11 & 14 & 7 & 4 & 113 \\
\hline 93 & 11 & 15 & 7 & 4 & 113 \\
\hline 93 & 11 & 16 & 7 & 4 & 113 \\
\hline 93 & 11 & 17 & 7 & 4 & 113 \\
\hline 93 & 11 & 18 & 7 & 4 & 113 \\
\hline 93 & 11 & 19 & 7 & 4 & 113 \\
\hline 93 & 11 & 20 & 7 & 4 & 113 \\
\hline
\end{tabular}

Data obtained from WHC Surveillance Analysis Computer System (SACS), February 28, 1994. 


\begin{tabular}{|c|c|c|c|c|c|}
\hline Year & Month & Day & Hour & Minute & Surface Level \\
\hline 93 & 11 & 21 & 7 & 4 & 113 \\
\hline 93 & 11 & 22 & 7 & 4 & 113 \\
\hline 93 & 11 & 23 & 7 & 4 & 113 \\
\hline 93 & 11 & 24 & 7 & 4 & 113 \\
\hline 93 & 11 & 25 & 7 & 4 & 113 \\
\hline 93 & 11 & 26 & 7 & 4 & 113 \\
\hline 93 & 11 & 27 & 7 & 4 & 113 \\
\hline 93 & 11 & 28 & 7 & 4 & 113 \\
\hline 93 & 11 & 29 & 7 & 4 & 113 \\
\hline 93 & 11 & 30 & 7 & 4 & 113 \\
\hline 93 & 12 & 1 & 7 & 4 & 113 \\
\hline 93 & 12 & 2 & 7 & 4 & 113 \\
\hline 93 & 12 & 3 & 7 & 4 & 113 \\
\hline 93 & 12 & 4 & 7 & 4 & 113 \\
\hline 93 & 12 & 5 & 7 & 4 & 113 \\
\hline 93 & 12 & 6 & 7 & 4 & 113 \\
\hline 93 & 12 & 7 & 7 & 4 & 113 \\
\hline 93 & 12 & 8 & 7 & 4 & 113 \\
\hline 93 & 12 & 13 & 7 & 4 & 113 \\
\hline 93 & 12 & 14 & 7 & 4 & 113 \\
\hline 93 & 12 & 15 & 7 & 4 & 113 \\
\hline 93 & 12 & 16 & 7 & 4 & 113 \\
\hline 93 & 12 & 17 & 7 & 4 & 113 \\
\hline 93 & 12 & 18 & 7 & 4 & 113 \\
\hline 93 & 12 & 19 & 7 & 4 & 113 \\
\hline 93 & 12 & 20 & 7 & 4 & 113 \\
\hline 93 & 12 & 21 & 7 & 4 & 113 \\
\hline 93 & 12 & 22 & 7 & 4 & 113 \\
\hline 93 & 12 & 23 & 7 & 4 & 113 \\
\hline 93 & 12 & 24 & 7 & 4 & 113 \\
\hline .93 & 12 & 25 & 7 & 4 & 113 \\
\hline 93 & 12 & 26 & 7 & 4 & 113 \\
\hline 93 & 12 & 27 & 7 & 4 & 113 \\
\hline 93 & 12 & 28 & 7 & 4 & 113 \\
\hline 93 & 12 & 29 & 7 & 4 & 113 \\
\hline 93 & 12 & 30 & 7 & 4 & 113 \\
\hline 93 & 12 & 31 & 7 & 4 & 113 \\
\hline 94 & 1 & 1 & 7 & 4 & 113 \\
\hline 94 & 1 & 2 & 7 & 4 & 113 \\
\hline 94 & 1 & 3 & 15 & 26 & 113.5 \\
\hline 94 & 1 & 4 & 7 & 4 & 113.5 \\
\hline 94 & 1 & 5 & 7 & 4 & 113.5 \\
\hline 94 & 1 & 6 & 7 & 4 & 113.5 \\
\hline 94 & 1 & 7 & 7 & 4 & 113.5 \\
\hline 94 & 1 & 8 & 7 & 4 & 113.5 \\
\hline 94 & 1 & 9 & 7 & 4 & 113.5 \\
\hline 94 & 1 & 10 & 7 & 4 & 113.5 \\
\hline 94 & 1 & 11 & 7 & 4 & 113.5 \\
\hline
\end{tabular}

Data obtained from WHC Surveillance Analysis Computer System (SACS), February 28, 1994. 


\begin{tabular}{|c|c|c|c|c|c|}
\hline Year & Month & Day & Hour & Minute & Surface Level \\
\hline 94 & 1 & 12 & 7 & 4 & 113.5 \\
\hline 94 & 1 & 13 & 7 & 4 & 113.5 \\
\hline 94 & 1 & 14 & 7 & 4 & 113.5 \\
\hline 94 & 1 & 15 & 7 & 4 & 113.5 \\
\hline 94 & 1 & 16 & 7 & 4 & 113.5 \\
\hline 94 & 1 & 17 & 7 & 4 & 113.5 \\
\hline 94 & 1 & 18 & 7 & 4 & 113.5 \\
\hline 94 & 1 & 19 & 7 & 4 & 113.5 \\
\hline 94 & 1 & 20 & 7 & 4 & 113.5 \\
\hline 94 & 1 & 21 & 7 & 4 & 113.5 \\
\hline 94 & 1 & 22 & 7 & 4 & 113.5 \\
\hline 94 & $\frac{1}{1}$ & 23 & 7 & 4 & 113.5 \\
\hline 94 & 1 & 24 & 7 & 4 & 113.5 \\
\hline 94 & 1 & 25 & 7 & 4 & 113.5 \\
\hline 94 & 1 & 26 & 7 & 4 & 113.5 \\
\hline 94 & 1 & 27 & 7 & 4 & 113.5 \\
\hline 94 & 1 & 28 & 7 & 4 & 113.5 \\
\hline 94 & 1 & 29 & 7 & 4 & 113.5 \\
\hline 94 & 1 & 30 & 7 & 4 & 113.5 \\
\hline 94 & 1 & 31 & 7 & 4 & 113.5 \\
\hline 94 & 2 & 1 & 7 & 4 & 113.5 \\
\hline 94 & 2 & 2 & 7 & 4 & 113.5 \\
\hline 94 & 2 & 3 & 7 & 4 & 113.5 \\
\hline & & & & & \\
\hline & & & & & \\
\hline & & & & & \\
\hline & & & & & \\
\hline & & & & & \\
\hline & & & & & \\
\hline & & & & & \\
\hline & & & & & \\
\hline & & & & & \\
\hline & & & & & \\
\hline & & & & & \\
\hline & & & & & \\
\hline & & & & & \\
\hline & & & & & \\
\hline & & & & & \\
\hline & & & & & \\
\hline & & & & & \\
\hline & & & & & \\
\hline & & & & & \\
\hline & & & & & \\
\hline & & & & & \\
\hline & & & & & \\
\hline & & & & & \\
\hline & & & & & \\
\hline & & & & & \\
\hline
\end{tabular}

Data obtained from WHC Surveillance Analysis Computer System (SACS), February 28, 1994. 


\begin{tabular}{|c|c|c|c|c|c|}
\hline \multicolumn{6}{|c|}{ Surface Level Data } \\
\hline \multicolumn{6}{|c|}{$\mathrm{FIC}$} \\
\hline Year & Month & Day & Hour & Minute & Surface Level \\
\hline 91 & 1 & 1 & 7 & 3 & 175.3 \\
\hline 91 & 1 & 2 & 7 & 2 & 175 \\
\hline 91 & 1 & 3 & 7 & 3 & 175 \\
\hline 91 & 1 & 4 & 7 & 2 & 175.2 \\
\hline 91 & 1 & 5 & 7 & 2 & 175.2 \\
\hline 91 & 1 & 6 & 7 & 2 & 175 \\
\hline 91 & 1 & 7 & 7 & 2 & 175.4 \\
\hline 91 & 1 & 8 & 7 & 3 & 175.3 \\
\hline 91 & 1 & 9 & 7 & 2 & 175.6 \\
\hline 91 & 1 & 10 & 7 & 2 & 175.5 \\
\hline 91 & 1 & 11 & 7 & 3 & 175.4 \\
\hline 91 & 1 & 12 & 7 & 2 & 175.8 \\
\hline 91 & 1 & 26 & 7 & 3 & 175.2 \\
\hline 91 & 1 & 27 & 7 & 3 & 175.4 \\
\hline 91 & 1 & 28 & 7 & 10 & 175.7 \\
\hline 91 & 1 & 29 & 7 & 2 & 175.3 \\
\hline 91 & 1 & 30 & 7 & 10 & 175.5 \\
\hline 91 & 1 & 31 & 7 & 2 & 175.5 \\
\hline 91 & 2 & 1 & 7 & 2 & 175.3 \\
\hline 91 & 2 & 15 & 7 & 3 & 175.5 \\
\hline 91 & 2 & 16 & 7 & 2 & 175.9 \\
\hline 91 & 2 & 17 & 7 & 2 & 175.7 \\
\hline 91 & 2 & 18 & 7 & 3 & 175.4 \\
\hline 91 & 2 & 19 & 7 & 2 & 175.5 \\
\hline 91 & 2 & 20 & 7 & 3 & 175.4 \\
\hline 91 & 2 & 21 & 7 & 2 & 175.2 \\
\hline 91 & 2 & 22 & 7 & 2 & 175.5 \\
\hline 91 & 2 & .23 & 7 & 2 & 175.3 \\
\hline 91 & 2 & 24 & 7 & 3 & 175.1 \\
\hline 91 & 2 & 25 & 7 & 3 & 175.1 \\
\hline 91 & 2 & 26 & 7 & 3 & 175.2 \\
\hline 91 & 2 & 27 & 7 & 2 & 175.7 \\
\hline 91 & 2 & 28 & 7 & 2 & 176.3 \\
\hline 91 & 3 & 1 & 7 & 2 & 176.4 \\
\hline 91 & 3 & 2 & 7 & 2 & 176 \\
\hline 91 & 3 & 3 & 7 & 3 & 175.8 \\
\hline 91 & 3 & 4 & 7 & 3 & 176 \\
\hline 91 & 3 & 5 & 7 & 3 & 175.7 \\
\hline 91 & 3 & 6 & 7 & 2 & 175.4 \\
\hline 91 & 3 & 7 & 7 & 2 & 175.2 \\
\hline 91 & 3 & 8 & 7 & 2 & 175.1 \\
\hline 91 & 3 & 9 & 7 & 2 & 175.6 \\
\hline 91 & 3 & 10 & 7 & 2 & 175.8 \\
\hline 91 & 3 & 11 & 7 & 2 & 175.6 \\
\hline 91 & 3 & 12 & 7 & 3 & 175.6 \\
\hline 91 & 3 & 13 & 7 & 3 & 175.5 \\
\hline
\end{tabular}

Data obtained from WHC Surveillance Analysis Computer System (SACS), February 28, 1994. E-53 


\begin{tabular}{|c|c|c|c|c|c|}
\hline Year & Month & Day & Hour & Minute & Surface Level \\
\hline 91 & 3 & 14 & 7 & 3 & 175.5 \\
\hline 91 & 3 & 15 & 7 & 3 & 175.5 \\
\hline 91 & 3 & 16 & 7 & 3 & 175.6 \\
\hline 91 & 3 & 17 & 7 & 2 & 175.4 \\
\hline 91 & 3 & 18 & 7 & 3 & 175.5 \\
\hline 91 & 3 & 19 & 7 & 3 & 175.8 \\
\hline 91 & 3 & 20 & 7 & 2 & 175.9 \\
\hline 91 & 3 & 21 & 7 & 3 & 175.8 \\
\hline 91 & 3 & 22 & 7 & 2 & 175.6 \\
\hline 91 & 3 & 23 & 7 & 3 & 175.4 \\
\hline 91 & 3 & 24 & 7 & 3 & 175.7 \\
\hline 91 & 3 & 25 & 7 & 3 & 175.9 \\
\hline 91 & 3 & 26 & 7 & 3 & 175.8 \\
\hline 91 & 3 & 27 & 7 & 3 & 175.6 \\
\hline 91 & 3 & 28 & 7 & 3 & 175.4 \\
\hline 91 & 3 & 29 & 7 & 3 & 175.2 \\
\hline 91 & 3 & 30 & 7 & 3 & 175.2 \\
\hline 91 & 3 & 31 & 7 & 3 & 175.3 \\
\hline 91 & 4 & 1 & 7 & 3 & 175.5 \\
\hline 91 & 4 & 2 & 4 & 3 & 175.5 \\
\hline 91 & 4 & 8 & 7 & 4 & 174.5 \\
\hline 91 & 4 & 22 & 7 & 6 & 174.5 \\
\hline 91 & 6 & 6 & 7 & 6 & 175.5 \\
\hline 91 & 6 & 7 & 7 & 5 & 175.5 \\
\hline 91 & 6 & 8 & 7 & 5 & 175.5 \\
\hline 91 & 6 & 9 & 7 & 5 & 175.6 \\
\hline 91 & 6 & 10 & 7 & 6 & 175.5 \\
\hline 91 & 6 & 11 & 7 & 5 & 175.9 \\
\hline 91 & 6 & 12 & 7 & 4 & 175.7 \\
\hline 91 & 6 & 13 & 7 & 5 & 175.7 \\
\hline 91 & 6 & 14 & 7 & 5 & 175.6 \\
\hline 91 & 6 & 15 & 7 & 5 & 175.7 \\
\hline 91 & 6 & 16 & 7 & 5 & 175.7 \\
\hline 91 & 6 & 17 & 7 & 5 & 175.5 \\
\hline 91 & 6 & 18 & 7 & 5 & 175.5 \\
\hline 91 & 6 & 19 & 7 & 5 & 175.8 \\
\hline 91 & 6 & 20 & 7 & 5 & 175.9 \\
\hline 91 & 6 & 21 & 7 & 6 & 175.7 \\
\hline 91 & 6 & 22 & 7 & 5 & 175.7 \\
\hline 91 & 6 & 23 & 7 & 5 & 175.8 \\
\hline 91 & 6 & 24 & 7 & 5 & 175.9 \\
\hline 91 & 6 & 25 & 7 & 6 & 176 \\
\hline 91 & 6 & 26 & 7 & 3 & 176 \\
\hline 91 & 6 & 27 & 7 & 2 & 176 \\
\hline 91 & 6 & 28 & 7 & 3 & 176.2 \\
\hline 91 & 6 & 29 & 7 & 2 & 176 \\
\hline 91 & 7 & 2 & 7 & 6 & 175.6 \\
\hline 91 & 7 & 3 & 7 & 6 & 175.6 \\
\hline
\end{tabular}

Data obtained from WHC Surveillance Analysis Computer System (SACS), February 28, 1994. 


\begin{tabular}{|c|c|c|c|c|c|}
\hline Year & Month & Day & Hour & Minute & Surface Level \\
\hline 91 & 7 & 4 & 7 & 10 & 175.7 \\
\hline 91 & 7 & 5 & 7 & 6 & 175.9 \\
\hline 91 & 7 & 6 & 7 & 6 & 175.9 \\
\hline 91 & 7 & 7 & 7 & 10 & 175.6 \\
\hline 91 & 7 & 8 & 7 & 6 & 175.8 \\
\hline 91 & 7 & 9 & 7 & 6 & 176 \\
\hline 91 & 7 & 10 & 6 & 6 & 176 \\
\hline 91 & 7 & 11 & 7 & 6 & 175.8 \\
\hline 91 & 7 & 12 & 7 & 6 & 175.8 \\
\hline 91 & 7 & 13 & 7 & 6 & 176 \\
\hline 91 & 7 & 14 & 7 & 6 & 175.8 \\
\hline 91 & 7 & 15 & 7 & 6 & 176 \\
\hline 91 & 7 & 16 & 7 & 6 & 175.9 \\
\hline 91 & 7 & 17 & 7 & 5 & 175.8 \\
\hline 91 & 7 & 18 & 7 & 5 & 175.7 \\
\hline 91 & 7 & 19 & 7 & 5 & 175.9 \\
\hline 91 & 7 & 20 & 7 & 5 & 176 \\
\hline 91 & 7 & 21 & 7 & 5 & 175.9 \\
\hline 91 & 7 & 22 & 7 & 5 & 175.7 \\
\hline 91 & 7 & 23 & 7 & 5 & 175.8 \\
\hline 91 & 7 & 24 & 7 & 5 & 176 \\
\hline 91 & 7 & 25 & 7 & 1 & 176 \\
\hline 91 & 7 & 26 & 7 & 2 & 175.8 \\
\hline 91 & 7 & 27 & 7 & 2 & 175.7 \\
\hline 91 & 7 & 28 & 7 & 2 & 175.7 \\
\hline 91 & 7 & 29 & 7 & 2 & 175.8 \\
\hline 91 & 7 & 30 & 7 & 2 & 175.8 \\
\hline 91 & 7 & 31 & 7 & 2 & 175.9 \\
\hline 91 & 8 & 1 & 7 & 2 & 176 \\
\hline 91 & 8 & 2 & 7 & 2 & 175.8 \\
\hline 91 & 8 & 3 & 7 & 2 & 176 \\
\hline 91 & 8 & 4 & 7 & 2 & 176.1 \\
\hline 91 & 8 & 5 & 7 & 2 & 176.2 \\
\hline 91 & 8 & 6 & 7 & 2 & 176.2 \\
\hline 91 & 8 & 7 & 7 & 6 & 176.1 \\
\hline 91 & 8 & 8 & 7 & 6 & 176 \\
\hline 91 & 8 & 9 & 7 & 4 & 176 \\
\hline 91 & 8 & 10 & 7 & 4 & 176 \\
\hline 91 & 8 & 11 & 7 & 4 & 175.8 \\
\hline 91 & 8 & 12 & 7 & 4 & 175.7 \\
\hline 91 & 8 & 13 & 7 & 4 & 175.8 \\
\hline 91 & 8 & 14 & 7 & 4 & 175.9 \\
\hline 91 & 8 & 15 & 7 & 3 & 176 \\
\hline 91 & 8 & 17 & 7 & 2 & 175.9 \\
\hline 91 & 8 & 18 & 7 & 3 & 176 \\
\hline 91 & 8 & 19 & 7 & 2 & 176 \\
\hline 91 & 8 & 20 & 7 & 4 & 176 \\
\hline 91 & 8 & 21 & 7 & 3 & 176 \\
\hline
\end{tabular}

Data obtained from WHC Surveillance Analysis Computer System (SACS), February 28, 1994. E-55 


\begin{tabular}{|c|c|c|c|c|c|}
\hline Year & Month & Day & Hour & Minute & Surface Level \\
\hline 91 & 8 & 22 & 7 & 3 & 175.9 \\
\hline 91 & 8 & 23 & 7 & 2 & 176 \\
\hline 91 & 8 & 24 & 7 & 2 & 176 \\
\hline 91 & 8 & 25 & 7 & 2 & 176 \\
\hline 91 & 8 & 26 & 7 & 3 & 175.9 \\
\hline 91 & 8 & 27 & 7 & 3 & 176.3 \\
\hline 91 & 8 & 28 & 7 & 3 & 176.3 \\
\hline 91 & 8 & 29 & 7 & 2 & 176 \\
\hline 91 & 8 & 30 & 7 & 10 & 176 \\
\hline 91 & 8 & 31 & 7 & 2 & 176 \\
\hline 91 & 9 & 1 & 7 & 2 & 176 \\
\hline 91 & 9 & 2 & 7 & 2 & 175.8 \\
\hline 91 & 9 & 3 & 7 & 2 & 175.6 \\
\hline 91 & 9 & 4 & 7 & 3 & 175.7 \\
\hline 91 & 9 & 5 & 7 & 2 & 176 \\
\hline 91 & 9 & 6 & 7 & 2 & 176.3 \\
\hline 91 & 9 & 7 & 7 & 2 & 176.3 \\
\hline 91 & 9 & 8 & 7 & 2 & 176 \\
\hline 91 & 9 & 9 & 7 & 3 & 175.9 \\
\hline 91 & 9 & 10 & 7 & 2 & 176 \\
\hline 91 & 9 & 11 & 7 & 3 & 176 \\
\hline 91 & 9 & 12 & 7 & 2 & 176 \\
\hline 91 & 9 & 13 & 7 & 2 & 176.2 \\
\hline 91 & 9 & 14 & 7 & 3 & 176 \\
\hline 91 & 9 & 15 & 7 & 2 & 175.8 \\
\hline 91 & 9 & 16 & 7 & 3 & 175.8 \\
\hline 91 & 9 & 17 & 7 & 3 & 175.8 \\
\hline 91 & 9 & 18 & 7 & 3 & 175.8 \\
\hline 91 & 9 & 19 & 7 & 3 & 176 \\
\hline 91 & 9 & 20 & 7 & 2 & 176.2 \\
\hline 91 & 9 & 21 & 7 & 2 & 176 \\
\hline 91 & 9 & 22 & 7 & 3 & 175.7 \\
\hline 91 & 9 & 23 & 7 & 3 & 175.7 \\
\hline 91 & 9 & 24 & 7 & 2 & 175.6 \\
\hline 91 & 9 & 25 & 7 & 3 & $175.9^{\circ}$ \\
\hline 91 & 9 & 26 & 7 & 3 & 176.2 \\
\hline 91 & 9 & 27 & 7 & 2 & 176.3 \\
\hline 91 & 9 & 28 & 7 & 2 & 176.4 \\
\hline 91 & 9 & 29 & 7 & 3 & 176.1 \\
\hline 91 & 9 & 30 & 7 & 3 & 175.8 \\
\hline 91 & 10 & 1 & 7 & 2 & 176.1 \\
\hline 91 & 10 & 2 & 7 & 2 & 176 \\
\hline 91 & 10 & 3 & 7 & 2 & 176.1 \\
\hline 91 & 10 & 4 & 7 & 3 & 175.8 \\
\hline 91 & 10 & 6 & 6 & 2 & 176.1 \\
\hline 91 & 10 & 7 & 6 & 2 & 176.2 \\
\hline 91 & 10 & 8 & 7 & 2 & 176 \\
\hline 91 & 10 & 9 & 7 & 2 & 175.8 \\
\hline
\end{tabular}

Data obtained from WHC Surveillance Analysis Computer System (SACS), February 28, 1994. 


\begin{tabular}{|c|c|c|c|c|c|}
\hline Year & Month & Day & Hour & Minute & Surface Level \\
\hline 91 & 10 & 10 & 7 & 2 & 175.7 \\
\hline 91 & 10 & 11 & 7 & 2 & 176 \\
\hline 91 & 10 & 12 & 7 & 2 & 176.3 \\
\hline 91 & 10 & 13 & 7 & 3 & 176 \\
\hline 91 & 10 & 14 & 7 & 2 & 175.8 \\
\hline 91 & 10 & 15 & 7 & 2 & 176.1 \\
\hline 91 & 10 & 16 & 7 & 3 & 176.7 \\
\hline 91 & 10 & 17 & 7 & 5 & 176 \\
\hline 91 & 10 & 18 & 7 & 6 & 175.6 \\
\hline 91 & 10 & 19 & 7 & 6 & 175.5 \\
\hline 91 & 10 & 20 & 7 & 2 & 175.7 \\
\hline 91 & 10 & 21 & 7 & 2 & 176.5 \\
\hline 91 & 10 & 22 & 7 & 3 & 176.4 \\
\hline 91 & 10 & 23 & 7 & 2 & 176.2 \\
\hline 91 & 10 & 24 & 7 & 2 & 176.2 \\
\hline 91 & 10 & 25 & 7 & 3 & 176.5 \\
\hline 91 & 10 & 26 & 7 & 3 & 176.8 \\
\hline 91 & 10 & 27 & 7 & 2 & 176.3 \\
\hline 91 & 10 & 28 & 7 & 2 & 175.8 \\
\hline 91 & 10 & 29 & 7 & 3 & 175.9 \\
\hline 91 & 10 & 30 & 7 & 3 & 175.5 \\
\hline 91 & 10 & 31 & 7 & 3 & 175.7 \\
\hline 91 & 11 & 1 & 7 & 2 & 175.7 \\
\hline 91 & 11 & 2 & 7 & 3 & 175.6 \\
\hline 91 & 11 & 3 & 7 & 3 & 175.7 \\
\hline 91 & 11 & 4 & 7 & 2 & 175.9 \\
\hline 91 & 11 & 5 & 7 & 3 & 176 \\
\hline 91 & 11 & 6 & 7 & 3 & 176.2 \\
\hline 91 & 11 & 7 & 7 & 2 & 176 \\
\hline 91 & 11 & 8 & 7 & 3 & 176.3 \\
\hline 91 & 11 & 9 & 7 & 2 & 176.2 \\
\hline 91 & 11 & 10 & 7 & 3 & 176.1 \\
\hline 91 & 11 & 11 & 7 & 10 & 176.1 \\
\hline 91 & 11 & 12 & 7 & 3 & 176.4 \\
\hline 91 & 11 & 13 & 7 & 3 & 176.5 \\
\hline 91 & 11 & 14 & 7 & 3 & 176.5 \\
\hline 91 & 11 & 15 & 7 & 3 & 176.1 \\
\hline 91 & 11 & 16 & 7 & 3 & 176.3 \\
\hline 91 & 11 & 18 & 7 & 3 & 176.7 \\
\hline 91 & 11 & 19 & 7 & 3 & 176 \\
\hline 91 & 11 & 20 & 7 & 3 & 176.4 \\
\hline 91 & 11 & 21 & 7 & 2 & 176 \\
\hline 91 & 11 & 22 & 7 & 3 & 175.6 \\
\hline 91 & 11 & 23 & 7 & 2 & 175.6 \\
\hline 91 & 11 & 24 & 7 & 3 & 175.8 \\
\hline 91 & 11 & 25 & 7 & 3 & 176.3 \\
\hline 91 & 11 & 27 & 7 & 3 & 176.5 \\
\hline 91 & 11 & 28 & 7 & 3 & 176 \\
\hline
\end{tabular}

Data obtained from WHC Surveillance Analysis Computer System (SACS), February 28, 1994. 


\begin{tabular}{|c|c|c|c|c|c|}
\hline Year & Month & Day & Hour & Minute & Surface Level \\
\hline 91 & 11 & 29 & 7 & 3 & 176.1 \\
\hline 91 & 11 & 30 & 7 & 3 & 176 \\
\hline 91 & 12 & 1 & 7 & 3 & 176 \\
\hline 91 & 12 & 2 & 7 & 3 & 176.3 \\
\hline 91 & 12 & 3 & 7 & 3 & 176 \\
\hline 91 & 12 & 4 & 7 & 3 & 176.5 \\
\hline 91 & 12 & 5 & 7 & 3 & 176.5 \\
\hline 91 & 12 & 6 & 7 & 4 & 176.8 \\
\hline 91 & 12 & 7 & 7 & 3 & 176.7 \\
\hline 91 & 12 & 8 & 7 & 3 & 176.3 \\
\hline 91 & 12 & 9 & 7 & 3 & 176.3 \\
\hline 91 & 12 & 10 & 7 & 3 & 176 \\
\hline 91 & 12 & 11 & 7 & 2 & 176.6 \\
\hline 91 & 12 & 12 & 7 & 3 & 176.7 \\
\hline 91 & 12 & 13 & 6 & 6 & 176 \\
\hline 91 & 12 & 14 & 7 & 2 & 175.8 \\
\hline 91 & 12 & 15 & 7 & 2 & 175.7 \\
\hline 91 & 12 & 18 & 7 & 3 & 176.6 \\
\hline 91 & 12 & 24 & 7 & 3 & 176.2 \\
\hline 91 & 12 & 25 & 7 & 3 & 176.3 \\
\hline 91 & 12 & 26 & 7 & 3 & 176.4 \\
\hline 91 & 12 & 27 & 7 & 3 & 176.5 \\
\hline 91 & $\overline{12}$ & 28 & 7 & 2 & 176.8 \\
\hline 91 & 12 & 29 & 7 & 3 & 176.7 \\
\hline 91 & 12 & 30 & 7 & 3 & 176.6 \\
\hline 91 & 12 & 31 & 7 & 3 & 176 \\
\hline 92 & 1 & 1 & 7 & 3 & 176 \\
\hline 92 & 1 & 2 & 7 & 2 & 176.5 \\
\hline 92 & 1 & 3 & 7 & 4 & 176.6 \\
\hline 92 & 1 & 5 & 7 & 3 & 176.8 \\
\hline 92 & 1 & 6 & 7 & 3 & 176.8 \\
\hline 92 & 1 & 7 & 7 & 4 & 176.4 \\
\hline 92 & 1 & 9 & 7 & 3 & 175.9 \\
\hline 92 & 1 & 10 & 7 & 4 & 176.2 \\
\hline 92 & 1 & 11 & 7 & 4 & 176.5 \\
\hline 92 & 1 & 17 & 7 & 4 & 176 \\
\hline 92 & 1 & 18 & 7 & 4 & 175.8 \\
\hline 92 & 1 & 19 & 7 & 3 & 175.9 \\
\hline 92 & 1 & 20 & 7 & 3 & 176 \\
\hline 92 & 1 & 21 & 7 & 3 & 176.3 \\
\hline 92 & 1 & 22 & 7 & 6 & 176.1 \\
\hline 92 & 1 & 23 & 7 & 3 & 176.4 \\
\hline 92 & 1 & 24 & 7 & 2 & 176.6 \\
\hline 92 & 1 & 25 & 7 & 3 & 176.7 \\
\hline 92 & 1 & 26 & 7 & 10 & 176.4 \\
\hline 92 & 1 & 27 & 7 & 2 & 176.5 \\
\hline 92 & 1 & 28 & 7 & 3 & 176.7 \\
\hline 92 & 1 & 29 & 7 & 3 & 176.3 \\
\hline
\end{tabular}

Data obtained from WHC Surveillance Analysis Computer System (SACS), February 28, 1994. 


\begin{tabular}{|c|c|c|c|c|c|}
\hline Year & Month & Day & Hour & Minute & Surface Level \\
\hline 92 & 1 & 30 & 7 & 3 & 176.4 \\
\hline 92 & 1 & 31 & 7 & 3 & 176.8 \\
\hline 92 & 2 & 2 & 7 & 3 & 176.3 \\
\hline 92 & 2 & 3 & 7 & 3 & 176 \\
\hline 92 & 2 & 4 & 7 & 3 & 176 \\
\hline 92 & 2 & 5 & 7 & 2 & 176.2 \\
\hline 92 & 2 & 8 & 7 & 4 & 176.7 \\
\hline 92 & 2 & 9 & 7 & 3 & 176.7 \\
\hline 92 & 2 & 11 & 7 & 3 & 176.9 \\
\hline 92 & 2 & 12 & 7 & 3 & 176.7 \\
\hline 92 & 2 & 14 & 7 & 3 & 176.9 \\
\hline 92 & 2 & 16 & 7 & 3 & 176.8 \\
\hline 92 & 2 & 17 & 7 & 3 & 176.4 \\
\hline 92 & 2 & 18 & 7 & 3 & 176.5 \\
\hline 92 & 2 & 19 & 7 & 3 & 176.7 \\
\hline 92 & 2 & 20 & 7 & 3 & 176.7 \\
\hline 92 & 2 & 21 & 7 & 3 & 176.6 \\
\hline 92 & 2 & 22 & 7 & 3 & 176.5 \\
\hline 92 & 2 & 23 & 7 & 3 & 176 \\
\hline 92 & 2 & 24 & 7 & 3 & 176 \\
\hline 92 & 2 & 27 & 0 & 3 & 175.7 \\
\hline 92 & 2 & 29 & 7 & 3 & 176.4 \\
\hline 92 & 3 & 1 & 7 & 3 & 176.7 \\
\hline 92 & 3 & 2 & 7 & 3 & 176.5 \\
\hline 92 & 3 & 3 & 7 & 4 & 176.7 \\
\hline 92 & 3 & 4 & 7 & 4 & 176.6 \\
\hline 92 & 3 & 5 & 7 & 3 & 176.8 \\
\hline 92 & 3 & 6 & 7 & 3 & 176.8 \\
\hline 92 & 3 & 7 & 7 & 2 & 176.6 \\
\hline 92 & 3 & 8 & 7 & 2 & 176.5 \\
\hline 92 & 3 & 9 & 7 & 2 & 176.3 \\
\hline 92 & 3 & 10 & 6 & 3 & 176.2 \\
\hline 92 & 3 & 11 & 7 & 3 & 176.3 \\
\hline 92 & 3 & 12 & 7 & 3 & 176.3 \\
\hline 92 & 3 & 13 & 7 & 3 & 176.5 \\
\hline 92 & 3 & 14 & 7 & 3 & 176.8 \\
\hline 92 & 3 & 15 & 7 & 6 & 176.8 \\
\hline 92 & 3 & 17 & 7 & 3 & 176.7 \\
\hline 92 & 3 & 18 & 7 & 3 & 176.5 \\
\hline 92 & 3 & 19 & 7 & 3 & 176.5 \\
\hline 92 & 3 & 20 & 7 & 2 & 176.4 \\
\hline 92 & 3 & 21 & 7 & 3 & 176.6 \\
\hline 92 & 3 & 22 & 7 & 2 & 176.6 \\
\hline 92 & 3 & 23 & 7 & 2 & 176.7 \\
\hline 92 & 3 & 24 & 7 & 2 & 176.5 \\
\hline 92 & 3 & 25 & 7 & 3 & 176.5 \\
\hline 92 & 3 & 26 & 7 & 3 & 176.6 \\
\hline 92 & 3 & 27 & 7 & 2 & 176.8 \\
\hline
\end{tabular}

Data obtained from WHC Surveillance Analysis Computer System (SACS), February 28, 1994. 


\begin{tabular}{|c|c|c|c|c|c|}
\hline Year & Month & Day & Hour & Minute & Surface Level \\
\hline 92 & 3 & 28 & 7 & 3 & 176.5 \\
\hline 92 & 3 & 29 & 7 & 3 & 176.4 \\
\hline 92 & 3 & 30 & 7 & 3 & 176.4 \\
\hline 92 & 3 & 31 & 7 & 3 & 176.3 \\
\hline 92 & 4 & 1 & 7 & 3 & 176.2 \\
\hline 92 & 4 & 2 & 7 & 3 & 176.5 \\
\hline 92 & 4 & 3 & 7 & 3 & 176.7 \\
\hline 92 & 4 & 4 & 7 & 3 & 176.7 \\
\hline 92 & 4 & 5 & 7 & 3 & 176.6 \\
\hline 92 & 4 & 6 & 7 & 3 & 176.4 \\
\hline 92 & 4 & 7 & 7 & 3 & 176.2 \\
\hline 92 & 4 & 8 & 7 & 10 & 176.3 \\
\hline 92 & 4 & 9 & 7 & 3 & 176.6 \\
\hline 92 & 4 & 10 & 7 & 3 & 176.7 \\
\hline 92 & 4 & 11 & 7 & 3 & 176.6 \\
\hline 92 & 4 & 12 & 7 & 4 & 176.7 \\
\hline 92 & 4 & 13 & 7 & 3 & 176.8 \\
\hline 92 & 4 & 14 & 7 & 3 & 176.5 \\
\hline 92 & 4 & 15 & 7 & 2 & 176.6 \\
\hline 92 & 4 & 16 & 7 & 3 & 176.8 \\
\hline 92 & 4 & 18 & 7 & 2 & 176.6 \\
\hline 92 & 4 & 19 & 7 & 2 & 176.3 \\
\hline 92 & 4 & 20 & 7 & 3 & 176.4 \\
\hline 92 & 4 & 21 & 7 & 3 & 176.5 \\
\hline 92 & 4 & 22 & 7 & 2 & 176.6 \\
\hline 92 & 4 & 23 & 7 & 3 & 176.3 \\
\hline 92 & 4 & 24 & 7 & 3 & 176.1 \\
\hline 92 & 4 & 25 & 7 & 4 & 176.3 \\
\hline 92 & 4 & 26 & 7 & 3 & 176.6 \\
\hline 92 & 4 & 27 & 7 & 4 & 176.6 \\
\hline 92 & 4 & 28 & 7 & 3 & 176.4 \\
\hline 92 & 4 & 29 & 7 & 3 & 176.7 \\
\hline 92 & 4 & 30 & 7 & 3 & 176.9 \\
\hline 92 & 5 & 1 & 7 & 3 & 176.4 \\
\hline 92 & 5 & 2 & 7 & 3 & 176.2 \\
\hline 92 & 5 & 3 & 7 & 3 & 176.1 \\
\hline 92 & 5 & 4 & 7 & 3 & 176.3 \\
\hline 92 & 5 & 5 & 7 & 4 & 176.6 \\
\hline 92 & 5 & 6 & 7 & 3 & 176.7 \\
\hline 92 & 5 & 7 & 7 & 4 & 176.7 \\
\hline 92 & 5 & 8 & 7 & 3 & 176.6 \\
\hline 92 & 5 & 9 & 7 & 4 & 176.5 \\
\hline 92 & 5 & 10 & 7 & 4 & 176.8 \\
\hline 92 & 5 & 11 & 7 & 3 & 176.6 \\
\hline 92 & 5 & 12 & 7 & 3 & 176.4 \\
\hline 92 & 5 & 13 & 7 & 3 & 176.4 \\
\hline 92 & 5 & 14 & 7 & 3 & 176.6 \\
\hline 92 & 5 & 15 & 7 & 3 & 176.8 \\
\hline
\end{tabular}

Data obtained from WHC Surveillance Analysis Computer System (SACS), February 28, 1994. E-60 


\begin{tabular}{|c|c|c|c|c|c|}
\hline Year & Month & Day & Hour & Minute & Surface Level \\
\hline 92 & 5 & 16 & 7 & 3 & 176.6 \\
\hline 92 & 5 & 17 & 7 & 2 & 176.6 \\
\hline 92 & 5 & 18 & 7 & 3 & 176.8 \\
\hline 92 & 5 & 19 & 7 & 3 & 176.8 \\
\hline 92 & 5 & 20 & 7 & 3 & 176.8 \\
\hline 92 & 5 & 21 & 7 & 3 & 176.5 \\
\hline 92 & 5 & 22 & 7 & 2 & 176.4 \\
\hline 92 & 5 & 23 & 7 & 3 & 176.4 \\
\hline 92 & 5 & 24 & 6 & 2 & 176.5 \\
\hline 92 & 5 & 25 & 7 & 3 & 176.7 \\
\hline 92 & 5 & 27 & 7 & 3 & 176.6 \\
\hline 92 & 5 & 28 & 7 & 2 & 176.6 \\
\hline 92 & 5 & 29 & 7 & 2 & 176.6 \\
\hline 92 & 5 & 30 & 7 & 3 & 176.6 \\
\hline 92 & 5 & 31 & 7 & 3 & 176.5 \\
\hline 92 & 6 & 1 & 7 & 3 & 176.5 \\
\hline 92 & 6 & 2 & 7 & 3 & 176.6 \\
\hline 92 & 6 & 3 & 7 & 3 & 176.5 \\
\hline 92 & 6 & 4 & 7 & 3 & 176.6 \\
\hline 92 & 6 & 5 & 7 & 2 & 176.7 \\
\hline 92 & 6 & 6 & 7 & 3 & 176.9 \\
\hline 92 & 6 & 8 & 7 & 3 & 176.8 \\
\hline 92 & 6 & 10 & 7 & 3 & 176.8 \\
\hline 92 & 6 & 11 & 7 & 3 & 176.9 \\
\hline 92 & 6 & 12 & 7 & 5 & 176.9 \\
\hline 92 & 6 & 13 & 7 & 5 & 176.7 \\
\hline 92 & 6 & 15 & 7 & 3 & 176.8 \\
\hline 92 & 6 & 16 & 7 & 3 & 176.9 \\
\hline 92 & 6 & 17 & 7 & 3 & 176.7 \\
\hline 92 & 6 & 18 & 7 & 3 & 176.7 \\
\hline 92 & 6 & 19 & 7 & 2 & 176.7 \\
\hline 92 & 6 & 20 & 7 & 2 & 176.8 \\
\hline 92 & 6 & 21 & 7 & 3 & 176.7 \\
\hline 92 & 6 & 22 & 7 & 3 & 176.7 \\
\hline 92 & 6 & 23 & 7 & 3 & 176.9 \\
\hline 92 & 7 & 3 & 7 & 6 & 176.7 \\
\hline 92 & 7 & 4 & 7 & 6 & 176.9 \\
\hline 92 & 7 & 5 & 7 & 6 & 176.7 \\
\hline 92 & 7 & 6 & 7 & 6 & 176.6 \\
\hline 92 & 7 & 7 & 7 & 6 & 176.5 \\
\hline 92 & 7 & 8 & 7 & 6 & 176.5 \\
\hline 92 & 7 & 9 & 7 & 6 & 176.4 \\
\hline 92 & 7 & 10 & 7 & 6 & 176.5 \\
\hline 92 & 7 & 11 & 7 & 6 & 176.8 \\
\hline 92 & 7 & 12 & 7 & 6 & 176.8 \\
\hline 92 & 7 & 13 & 7 & 6 & 176.9 \\
\hline 92 & 7 & 14 & 7 & 6 & 176.8 \\
\hline 92 & 7 & 15 & 7 & 6 & 176.6 \\
\hline
\end{tabular}

Data obtained from WHC Surveillance Analysis Computer System (SACS), February 28, 1994. E-61 


\begin{tabular}{|c|c|c|c|c|c|}
\hline Year & Month & Day & Hour & Minute & Surface Level \\
\hline 92 & 7 & 16 & 7 & 6 & 176.6 \\
\hline 92 & 7 & 17 & 7 & 6 & 176.7 \\
\hline 92 & 7 & 18 & 7 & 6 & 176.8 \\
\hline 92 & 7 & 19 & 7 & 6 & 176.8 \\
\hline 92 & 7 & 20 & 7 & 6 & 176.8 \\
\hline 92 & 7 & 21 & 7 & 5 & 176.8 \\
\hline 92 & 7 & 22 & 7 & 6 & 176.9 \\
\hline 92 & 7 & 24 & 7 & 5 & 176.6 \\
\hline 92 & 7 & 25 & 7 & 6 & 176.5 \\
\hline 92 & 7 & 26 & 7 & 6 & 176.6 \\
\hline 92 & 7 & 27 & 7 & 5 & 176.7 \\
\hline 92 & 7 & 28 & 7 & 6 & 176.7 \\
\hline 92 & 7 & 29 & 7 & 6 & 176.7 \\
\hline 92 & 7 & 30 & 7 & 6 & 176.8 \\
\hline 92 & 7 & 31 & 7 & 5 & 176.9 \\
\hline 92 & 8 & 2 & 6 & 6 & 176.8 \\
\hline 92 & 8 & 3 & 7 & 6 & 176.9 \\
\hline 92 & 8 & 4 & 7 & 6 & 177 \\
\hline 92 & 8 & 5 & 7 & 5 & 176.9 \\
\hline 92 & 8 & 6 & 7 & 5 & 176.9 \\
\hline 92 & 8 & 8 & 7 & 6 & 176.8 \\
\hline 92 & 8 & 9 & 7 & 6 & 176.6 \\
\hline 92 & 8 & 10 & 7 & 5 & 176.5 \\
\hline 92 & 8 & 11 & 7 & 6 & 176.6 \\
\hline 92 & 8 & 12 & 7 & 6 & 176.8 \\
\hline 92 & 8 & 13 & 7 & 6 & 177 \\
\hline 92 & 8 & 14 & 7 & 6 & 176.9 \\
\hline 92 & 8 & 15 & 7 & 6 & 176.8 \\
\hline 92 & 8 & 16 & 7 & 6 & 176.9 \\
\hline 92 & 8 & 17 & 7 & 6 & 177 \\
\hline 92 & 8 & 18 & 7 & 6 & 177 \\
\hline 92 & 8 & 19 & 7 & 6 & 177 \\
\hline 92 & 8 & 20 & 7 & 6 & 177 \\
\hline 92 & 8 & 21 & 7 & 5 & 177.2 \\
\hline 92 & 8 & 23 & 7 & 4 & 176.7 \\
\hline 92 & 8 & 24 & 7 & 4 & 176.5 \\
\hline 92 & 8 & 25 & 7 & 4 & 176.5 \\
\hline 92 & 8 & 26 & 7 & 3 & 176.6 \\
\hline 92 & 8 & 27 & 7 & 4 & 176.7 \\
\hline 92 & 8 & 28 & 7 & 2 & 176.8 \\
\hline 92 & 8 & 29 & 7 & 2 & 176.7 \\
\hline 92 & 8 & 30 & 7 & 2 & 176.9 \\
\hline 92 & 8 & 31 & 7 & 2 & 177.1 \\
\hline 92 & 9 & 1 & 7 & 2 & 177 \\
\hline 92 & 9 & 2 & 7 & 2 & 177 \\
\hline 92 & 9 & 3 & 7 & 4 & 177.1 \\
\hline 92 & 9 & 8 & 7 & 4 & 177.1 \\
\hline 92 & 9 & 16 & 7 & 4 & 177.1 \\
\hline
\end{tabular}

Data obtained from WHC Surveillance Analysis Computer System (SACS), February 28, 1994. 


\begin{tabular}{|c|c|c|c|c|c|}
\hline Year & Month & Day & Hour & Minute & Surface Level \\
\hline 92 & 9 & 21 & 7 & 4 & 177 \\
\hline 92 & 9 & 22 & 7 & 3 & 177.2 \\
\hline 92 & 9 & 23 & 7 & 3 & 177.4 \\
\hline 92 & 9 & 28 & 10 & 2 & 176.9 \\
\hline 92 & 10 & 5 & 10 & 3 & 176.9 \\
\hline 92 & 10 & 12 & 10 & 3 & 177.65 \\
\hline$\overline{92}$ & 10 & 15 & 10 & 3 & 176.65 \\
\hline 92 & 10 & 19 & 10 & 3 & 177.1 \\
\hline 92 & 10 & 21 & 10 & 3 & 177.45 \\
\hline 92 & 10 & 22 & 10 & 3 & 176.85 \\
\hline 92 & 10 & 26 & 10 & 3 & 177 \\
\hline 92 & 10 & 30 & 10 & 3 & 177.7 \\
\hline 92 & 11 & 2 & 7 & 3 & 176.8 \\
\hline 92 & 11 & 3 & 7 & 3 & 176.5 \\
\hline 92 & 11 & 4 & 4 & 3 & 176.5 \\
\hline 92 & 11 & 9 & 4 & 3 & 177.05 \\
\hline 92 & 11 & 16 & 4 & 3 & 177 \\
\hline 92 & 11 & 23 & 4 & 3 & 176.8 \\
\hline 92 & 11 & 30 & 4 & 3 & 176.95 \\
\hline 92 & 12 & 2 & 6 & 3 & 177 \\
\hline 92 & 12 & 3 & 6 & 3 & 176.8 \\
\hline 92 & 12 & 12 & 12 & 4 & 176.6 \\
\hline 92 & 12 & 13 & 7 & 4 & 176.6 \\
\hline 92 & 12 & 14 & 7 & 3 & 176.8 \\
\hline 92 & 12 & 15 & 7 & 3 & 177 \\
\hline 92 & 12 & 16 & 7 & 4 & 176.8 \\
\hline 92 & 12 & 17 & 7 & 3 & 177.6 \\
\hline 92 & 12 & 18 & 7 & 3 & 177 \\
\hline 92 & 12 & 19 & 7 & 3 & 176.8 \\
\hline 92 & 12 & 20 & 7 & 4 & 177.4 \\
\hline 92 & 12 & 21 & 7 & 4 & 177.2 \\
\hline 92 & 12 & 23 & 7 & 4 & 176.7 \\
\hline 92 & 12 & 24 & 7 & 3 & 176.8 \\
\hline 92 & 12 & 25 & 7 & 3 & 176.7 \\
\hline 92 & 12 & 26 & 7 & 3 & 177.2 \\
\hline 92 & 12 & 27 & 7 & 3 & 177.5 \\
\hline 92 & 12 & 28 & 7 & 3 & 177.8 \\
\hline 93 & 1 & 23 & 7 & 3 & 176.7 \\
\hline 93 & 1 & 24 & 7 & 3 & 176.8 \\
\hline 93 & 1 & 25 & 6 & 3 & 177 \\
\hline 93 & 2 & 5 & 6 & 4 & 177 \\
\hline 93 & 2 & 6 & 2 & 3 & 177 \\
\hline 93 & 2 & 7 & 7 & 3 & 177 \\
\hline 93 & 2 & 16 & 6 & 3 & 176.8 \\
\hline 93 & 2 & 18 & 7 & 3 & 176.8 \\
\hline 93 & 2 & 19 & 7 & 2 & 177.6 \\
\hline 93 & 2 & 20 & 7 & 3 & 178 \\
\hline 93 & 2 & 21 & 7 & 4 & 177.5 \\
\hline
\end{tabular}

Data obtained from WHC Surveillance Analysis Computer System (SACS), February 28, 1994. E-63 


\begin{tabular}{|c|c|c|c|c|c|}
\hline Year & Month & Day & Hour & Minute & Surface Level \\
\hline 93 & 2 & 22 & 7 & 4 & 177 \\
\hline 93 & 2 & 23 & 7 & 3 & 176.9 \\
\hline 93 & 2 & 24 & 7 & 4 & 177.1 \\
\hline 93 & 2 & 25 & 7 & 3 & 176.8 \\
\hline 93 & 2 & 26 & 7 & 3 & 176.7 \\
\hline 93 & 2 & 27 & 7 & 4 & 176.6 \\
\hline 93 & 2 & 28 & 7 & 3 & 176.6 \\
\hline 93 & 3 & 1 & 7 & 3 & 176.7 \\
\hline 93 & 3 & 2 & 7 & 4 & 176.7 \\
\hline 93 & 3 & 3 & 7 & 3 & 176.8 \\
\hline 93 & 3 & 4 & 7 & 4 & 176.7 \\
\hline 93 & 3 & 5 & 7 & 4 & 176.7 \\
\hline 93 & 3 & 6 & 7 & 3 & 176.6 \\
\hline 93 & 3 & 9 & 7 & 4 & 176.8 \\
\hline 93 & 3 & 10 & 7 & 5 & 176.7 \\
\hline 93 & 3 & 11 & 7 & 4 & 176.6 \\
\hline 93 & 3 & 12 & 7 & 4 & 176.5 \\
\hline 93 & 3 & 13 & 7 & 3 & 176.7 \\
\hline 93 & 3 & 14 & 7 & 4 & 177.2 \\
\hline 93 & 3 & 15 & 7 & 3 & 177.6 \\
\hline 93 & 3 & 16 & 7 & 4 & 177 \\
\hline 93 & 3 & 17 & 7 & 3 & 177.1 \\
\hline 93 & 3 & 18 & 7 & 4 & 177.4 \\
\hline 93 & 3 & 19 & 7 & 3 & 177 \\
\hline 93 & 3 & 20 & 7 & 4 & 177 \\
\hline 93 & 3 & 21 & 7 & 4 & 176.9 \\
\hline 93 & 3 & 22 & 7 & 3 & 177.2 \\
\hline 93 & 3 & 23 & 7 & 3 & 177.6 \\
\hline 93 & 3 & 24 & 7 & 4 & 177.2 \\
\hline 93 & 3 & 25 & 7 & 3 & 177 \\
\hline 93 & 3 & 26 & 7 & 3 & 177.1 \\
\hline 93 & 3 & 27 & 7 & 3 & 177.3 \\
\hline 93 & 3 & 29 & 6 & 3 & 177.1 \\
\hline 93 & 3 & 30 & 7 & 3 & 177 \\
\hline 93 & 3 & 31 & 7 & 3 & 176.8 \\
\hline 93 & 4 & 1 & 7 & 3 & 177.3 \\
\hline 93 & 4 & 2 & 7 & 6 & 177.1 \\
\hline 93 & 4 & 3 & 7 & 6 & 177.2 \\
\hline 93 & 4 & 4 & 7 & 5 & 177.1 \\
\hline 93 & 4 & 5 & 7 & 6 & 177.2 \\
\hline 93 & 4 & 6 & 7 & 6 & 177.2 \\
\hline 93 & 4 & 7 & 7 & 6 & 177 \\
\hline 93 & 4 & 8 & 7 & 6 & 177.3 \\
\hline 93 & 4 & 9 & 7 & 6 & 177.3 \\
\hline 93 & 4 & 10 & 7 & 5 & 177.3 \\
\hline 93 & 4 & 11 & 7 & 6 & 177.1 \\
\hline 93 & 4 & 12 & 7 & 6 & 176.9 \\
\hline 93 & 4 & 13 & 7 & 6 & 177.1 \\
\hline
\end{tabular}

Data obtained from WHC Surveillance Analysis Computer System (SACS), February 28, 1994. 


\begin{tabular}{|c|c|c|c|c|c|}
\hline Year & Month & Day & Hour & Minute & Surface Level \\
\hline 93 & 4 & 14 & 7 & 6 & 177.1 \\
\hline 93 & 4 & 15 & 7 & 3 & 177.1 \\
\hline 93 & 4 & 16 & 7 & 6 & 177 \\
\hline 93 & 4 & 17 & 7 & 5 & 177.3 \\
\hline 93 & 4 & 18 & 7 & 6 & 177.4 \\
\hline 93 & 4 & 19 & 7 & 6 & 177 \\
\hline 93 & 4 & 22 & 7 & 6 & 177.2 \\
\hline 93 & 4 & 23 & 7 & 6 & 177.1 \\
\hline 93 & 4 & 24 & 7 & 5 & 177.3 \\
\hline 93 & 4 & 25 & 7 & 6 & 177 \\
\hline 93 & 4 & 26 & 7 & 6 & 177.1 \\
\hline 93 & 4 & 27 & 7 & 6 & 176.9 \\
\hline 93 & 4 & 28 & 7 & 3 & 176.8 \\
\hline 93 & 4 & 29 & 7 & 3 & 176.8 \\
\hline 93 & 4 & 30 & 7 & 6 & 176.9 \\
\hline 93 & 5 & 1 & 7 & 5 & 176.9 \\
\hline 93 & 5 & 2 & 7 & 6 & 177.1 \\
\hline 93 & 5 & 3 & 7 & 6 & 177.5 \\
\hline 93 & 5 & 11 & 7 & 6 & 177.4 \\
\hline 93 & 5 & 12 & 2 & 6 & 177.6 \\
\hline 93 & 5 & 15 & 7 & 6 & 177.1 \\
\hline 93 & 5 & 16 & 7 & 5 & 177.3 \\
\hline 93 & 5 & 17 & 7 & 3 & 177.2 \\
\hline 93 & 5 & 18 & 7 & 3 & 177.3 \\
\hline 93 & 5 & 19 & 7 & 3 & 177.4 \\
\hline 93 & 5 & 20 & 7 & 3 & 177.4 \\
\hline 93 & 5 & 21 & 7 & 2 & 177.4 \\
\hline 93 & 5 & 22 & 7 & 3 & 177.1 \\
\hline 93 & 5 & 23 & 7 & 4 & 177.1 \\
\hline 93 & 5 & 24 & 7 & 3 & 177.1 \\
\hline 93 & 5 & 25 & 7 & 3 & 177.2 \\
\hline 93 & 5 & 26 & 7 & 3 & 177.3 \\
\hline 93 & 5 & 27 & 7 & 3 & 177.4 \\
\hline 93 & 5 & 28 & 7 & 3 & 177.4 \\
\hline 93 & 5 & 29 & 7 & 5 & 177 \\
\hline 93 & 5 & 30 & 7 & 5 & 177.1 \\
\hline 93 & 5 & 31 & 7 & 6 & 177.4 \\
\hline 93 & 6 & 1 & 7 & 6 & 177.4 \\
\hline 93 & 6 & 2 & 7 & 6 & 177.1 \\
\hline 93 & 6 & 3 & 7 & 6 & 177.2 \\
\hline 93 & 6 & 4 & 7 & 6 & 177.4 \\
\hline 93 & 6 & 5 & 7 & 6 & 177.6 \\
\hline 93 & 6 & 6 & 7 & 5 & 177.6 \\
\hline 93 & 6 & 7 & 7 & 5 & 177.5 \\
\hline 93 & 6 & 8 & 7 & 5 & 177.1 \\
\hline 93 & 6 & 9 & 7 & 5 & 177.2 \\
\hline 93 & 6 & 10 & 7 & 4 & 177.1 \\
\hline 93 & 6 & 11 & 7 & 5 & 177.2 \\
\hline
\end{tabular}

Data obtained from WHC Surveillance Analysis Computer System (SACS), February 28, 1994. 


\begin{tabular}{|c|c|c|c|c|c|}
\hline Year & Month & Day & Hour & Minute & Surface Level \\
\hline 93 & 6 & 12 & 7 & 5 & 177 \\
\hline 93 & 6 & 13 & 7 & 4 & 177 \\
\hline 93 & 6 & 14 & 7 & 5 & 177.1 \\
\hline 93 & 6 & 15 & 7 & 5 & 177.2 \\
\hline 93 & 6 & 16 & 7 & 5 & 177.1 \\
\hline 93 & 6 & 17 & 7 & 5 & 177 \\
\hline 93 & 6 & 18 & 7 & 6 & 177.1 \\
\hline 93 & 6 & 25 & 7 & 3 & 177 \\
\hline 93 & 6 & 26 & 7 & 3 & 177.3 \\
\hline 93 & 6 & 27 & 7 & 4 & 177.4 \\
\hline 93 & 6 & 28 & 7 & 3 & 177.4 \\
\hline 93 & 6 & 29 & 7 & 4 & 177.2 \\
\hline 93 & 6 & 30 & 6 & 3 & 177 \\
\hline 93 & 7 & 1 & 7 & 6 & 177.2 \\
\hline 93 & 7 & 2 & 7 & 6 & 177.4 \\
\hline 93 & 7 & 3 & 7 & 3 & 177.4 \\
\hline 93 & 7 & 4 & 7 & 4 & 177.4 \\
\hline 93 & 7 & 5 & 7 & 3 & 177.3 \\
\hline 93 & 7 & 6 & 7 & 3 & 177.1 \\
\hline 93 & 7 & 7 & 7 & 4 & 177.2 \\
\hline 93 & 7 & 8 & 7 & 4 & 177.3 \\
\hline 93 & 7 & 9 & 7 & 5 & 177.4 \\
\hline 93 & 7 & 10 & 7 & 3 & 177.2 \\
\hline 93 & 7 & 11 & 7 & 3 & 177.2 \\
\hline 93 & 7 & 12 & 7 & 2 & 177 \\
\hline 93 & 7 & 13 & 7 & 3 & 177.1 \\
\hline 93 & 7 & 14 & 7 & 6 & 177.2 \\
\hline 93 & 7 & 16 & 7 & 6 & 177.2 \\
\hline 93 & 7 & 17 & 7 & 7 & 177.2 \\
\hline 93 & 7 & 18 & 7 & 6 & 177.1 \\
\hline 93 & 7 & 19 & 7 & 3 & 177.2 \\
\hline 93 & 7 & 20 & 7 & 3 & 177.3 \\
\hline 93 & 7 & 21 & 7 & 3 & 177.3 \\
\hline 93 & 7 & 22 & 7 & 3 & 177.5 \\
\hline 93 & 7 & 23 & 7 & 3 & 177.5 \\
\hline 93 & 7 & 24 & 7 & 3 & 177.3 \\
\hline 93 & 7 & 25 & 7 & 4 & 177.1 \\
\hline 93 & 7 & 26 & 7 & 3 & 177.2 \\
\hline 93 & 7 & 27 & 7 & 3 & 177.3 \\
\hline 93 & 7 & 28 & 7 & 4 & 177.7 \\
\hline 93 & 7 & 29 & 7 & 3 & 177.5 \\
\hline 93 & 7 & 30 & 7 & 3 & 177.1 \\
\hline 93 & 7 & 31 & 7 & 3 & 177 \\
\hline 93 & 8 & 1 & 7 & 3 & 177 \\
\hline 93 & 8 & 2 & 7 & 6 & 177.1 \\
\hline 93 & 8 & 3 & 7 & 3 & 177.3 \\
\hline 93 & 8 & 4 & 7 & 3 & 177.5 \\
\hline 93 & 8 & 5 & 7 & 4 & 177.4 \\
\hline
\end{tabular}

Data obtained from WHC Surveillance Analysis Computer System (SACS), February 28, 1994. 


\begin{tabular}{|c|c|c|c|c|c|}
\hline Year & Month & Day & Hour & Minute & Surface Level \\
\hline 93 & 8 & 6 & 7 & 4 & 177.5 \\
\hline 93 & 8 & 8 & 7 & 4 & 177.8 \\
\hline 93 & 8 & 9 & 7 & 4 & 177.5 \\
\hline 93 & 8 & 10 & 7 & 4 & 177.3 \\
\hline 93 & 8 & 11 & 7 & 4 & 177.3 \\
\hline 93 & 8 & 12 & 7 & 4 & 177.3 \\
\hline 93 & 8 & 13 & 7 & 4 & 177.5 \\
\hline 93 & 8 & 14 & 7 & 3 & 177.5 \\
\hline 93 & 8 & 15 & 7 & 4 & 177.6 \\
\hline 93 & 8 & 16 & 7 & 4 & 177.6 \\
\hline 93 & 8 & 17 & 7 & 4 & 177.3 \\
\hline 93 & 8 & 18 & 7 & 3 & 177.3 \\
\hline 93 & 8 & 19 & 7 & 4 & 177.5 \\
\hline 93 & 8 & 20 & 7 & 4 & 177.7 \\
\hline 93 & 8 & 21 & 7 & 4 & 177.5 \\
\hline 93 & 8 & 22 & 7 & 4 & 177.4 \\
\hline 93 & 8 & 23 & 7 & 4 & 177.7 \\
\hline 93 & 8 & 24 & 7 & 4 & 177.3 \\
\hline 93 & 8 & 25 & 7 & 4 & 177.2 \\
\hline 93 & 8 & 26 & 7 & 4 & 177.1 \\
\hline 93 & 8 & 27 & 7 & 5 & 177.1 \\
\hline 93 & 8 & 28 & 7 & 4 & 177.3 \\
\hline 93 & 8 & 29 & 7 & 3 & 177.2 \\
\hline 93 & 8 & 30 & 7 & 4 & 177.2 \\
\hline 93 & 8 & 31 & 7 & 4 & 177.4 \\
\hline 93 & 9 & 1 & 7 & 4 & 177.7 \\
\hline 93 & 9 & 2 & 7 & 4 & 177.5 \\
\hline 93 & 9 & 3 & 7 & 4 & 177.4 \\
\hline 93 & 9 & 4 & 7 & 4 & 177.5 \\
\hline 93 & 9 & 5 & 7 & 4 & 177.6 \\
\hline .93 & 9 & 6 & 7 & 4 & 177.6 \\
\hline 93 & 9 & 7 & 7 & 4 & 177.4 \\
\hline 93 & 9 & 8 & 7 & 4 & 177.3 \\
\hline 93 & 9 & 9 & 7 & 4 & 177.5 \\
\hline 93 & 9 & 10 & 7 & 4 & 177.7 \\
\hline 93 & 9 & 11 & 7 & 4 & 177.8 \\
\hline 93 & 9 & 12 & 7 & 4 & 177.6 \\
\hline 93 & 9 & 13 & 7 & 4 & 177.4 \\
\hline 93 & 9 & 14 & 7 & 4 & 177.6 \\
\hline 93 & 9 & 15 & 7 & 4 & 177.6 \\
\hline 93 & 9 & 16 & 7 & 4 & 177.4 \\
\hline 93 & 9 & 17 & 7 & 4 & 177.5 \\
\hline 93 & 9 & 18 & 7 & 4 & 177.5 \\
\hline 93 & 9 & 19 & 7 & 4 & 177.6 \\
\hline 93 & 9 & 20 & 7 & 4 & 177.6 \\
\hline 93 & 9 & 21 & 7 & 4 & 177.3 \\
\hline 93 & 9 & 22 & 7 & 4 & 177.1 \\
\hline 93 & 9 & 23 & 7 & 4 & 177.3 \\
\hline
\end{tabular}

Data obtained from WHC Surveillance Analysis Computer System (SACS), February 28, 1994. 


\begin{tabular}{|c|c|c|c|c|c|}
\hline Year & Month & Day & Hour & Minute & Surface Level \\
\hline 93 & 9 & 24 & 7 & 4 & 177.3 \\
\hline 93 & 9 & 25 & 7 & 4 & 177.4 \\
\hline 93 & 9 & 26 & 7 & 4 & 177.2 \\
\hline 93 & 9 & 27 & 7 & 4 & 177.3 \\
\hline 93 & 9 & 28 & 7 & 4 & 177.3 \\
\hline 93 & 9 & 29 & 7 & 4 & 177.4 \\
\hline 93 & 9 & 30 & 7 & 3 & 177.8 \\
\hline 93 & 10 & 1 & 7 & 6 & 177.6 \\
\hline 93 & 10 & 2 & 7 & 5 & 177.4 \\
\hline 93 & 10 & 3 & 7 & 6 & 177.4 \\
\hline 93 & 10 & 4 & 7 & 6 & 177.6 \\
\hline 93 & 10 & 5 & 7 & 6 & 177.9 \\
\hline 93 & 10 & 6 & 7 & 6 & 177.9 \\
\hline 93 & 10 & 7 & 7 & 5 & 177.6 \\
\hline 93 & 10 & 8 & 7 & 4 & 177.4 \\
\hline 93 & 10 & 9 & 7 & 3 & 177.4 \\
\hline 93 & 10 & 10 & 7 & 10 & 177.5 \\
\hline 93 & 10 & 11 & 7 & 3 & 177.6 \\
\hline 93 & 10 & 12 & 7 & 4 & 177.5 \\
\hline 93 & 10 & 13 & 7 & 3 & 177.6 \\
\hline 93 & 10 & 14 & 7 & 4 & 177.7 \\
\hline 93 & 10 & 15 & 7 & 4 & 177.9 \\
\hline 93 & 10 & 16 & 7 & 4 & 177.8 \\
\hline 93 & 10 & 17 & 7 & 3 & 177.7 \\
\hline 93 & 10 & 18 & 7 & 4 & 177.6 \\
\hline 93 & 10 & 19 & 7 & 4 & 177.5 \\
\hline 93 & 10 & 20 & 7 & 3 & 177.2 \\
\hline 93 & 10 & 21 & 7 & 4 & 177.4 \\
\hline 93 & 10 & 22 & 7 & 4 & 177.5 \\
\hline 93 & 10 & 23 & 7 & 4 & 177.6 \\
\hline 93 & 10 & 24 & 7 & 4 & 177.8 \\
\hline 93 & 10 & 25 & 7 & 4 & 177.4 \\
\hline 93 & 10 & 26 & 7 & 4 & 177.1 \\
\hline 93 & 10 & 27 & 7 & 4 & 177.4 \\
\hline 93 & 10 & 28 & 7 & 4 & 177.7 \\
\hline 93 & 10 & 29 & 7 & 4 & 177.6 \\
\hline 93 & 10 & 30 & 7 & 4 & 177.5 \\
\hline 93 & 10 & 31 & 7 & 4 & 177.8 \\
\hline 93 & 11 & 1 & 7 & 3 & 177.6 \\
\hline 93 & 11 & 2 & 7 & 3 & 177.4 \\
\hline 93 & 11 & 3 & 7 & 3 & 177.8 \\
\hline 93 & 11 & 4 & 7 & 3 & 177.7 \\
\hline 93 & 11 & 5 & 7 & 3 & 177.4 \\
\hline 93 & 11 & 6 & 7 & 3 & 177.35 \\
\hline 93 & 11 & 7 & 7 & 3 & 177.6 \\
\hline 93 & 11 & 8 & 7 & 3 & 177.6 \\
\hline 93 & 11 & 9 & 7 & 3 & 177.6 \\
\hline 93 & 11 & 10 & 7 & 4 & 177.6 \\
\hline
\end{tabular}

Data obtained from WHC Surveillance Analysis Computer System (SACS), February 28, 1994. 


\begin{tabular}{|c|c|c|c|c|c|}
\hline Year & Month & Day & Hour & Minute & Surface Level \\
\hline 93 & 11 & 11 & 7 & 4 & 177.8 \\
\hline 93 & 11 & 12 & 7 & 4 & 177.8 \\
\hline 93 & 11 & 13 & 7 & 4 & 177.8 \\
\hline 93 & 11 & 14 & 7 & 3 & 177.3 \\
\hline 93 & 11 & 15 & 7 & 4 & 177.6 \\
\hline 93 & 11 & 16 & 7 & 3 & 177.7 \\
\hline 93 & 11 & 17 & 7 & 4 & 177.7 \\
\hline 93 & 11 & 18 & 7 & 4 & 177.6 \\
\hline 93 & 11 & 19 & 7 & 4 & 177.2 \\
\hline 93 & 11 & 19 & 7 & 4 & 177.2 \\
\hline 93 & 11 & 20 & 7 & 4 & 177.4 \\
\hline 93 & 11 & 20 & 7 & 4 & 177.3 \\
\hline 93 & 11 & 21 & 7 & 4 & 177.9 \\
\hline 93 & 11 & 21 & 7 & 4 & 177.7 \\
\hline 93 & 11 & 22 & 7 & 4 & 178 \\
\hline 93 & 11 & 22 & 7 & 4 & 177.9 \\
\hline 93 & 11 & 23 & 7 & 4 & 177.4 \\
\hline 93 & 11 & 23 & 7 & 4 & 177.5 \\
\hline 93 & 11 & 24 & 7 & 4 & 177 \\
\hline 93 & 11 & 24 & 7 & 4 & 177.1 \\
\hline 93 & 11 & 25 & 7 & 4 & 176.9 \\
\hline 93 & 11 & 25 & 7 & 4 & 177 \\
\hline 93 & 11 & 26 & 7 & 4 & 176.9 \\
\hline 93 & 11 & 26 & 7 & 4 & 176.9 \\
\hline 93 & 11 & 27 & 7 & 4 & 177 \\
\hline 93 & 11 & 29 & 7 & 4 & 177 \\
\hline 93 & 12 & 3 & 15 & 4 & 177.6 \\
\hline 93 & 12 & 4 & 7 & 5 & 178 \\
\hline 93 & 12 & 5 & 7 & 4 & 177.5 \\
\hline 93 & 12 & 6 & 7 & 5 & 177.6 \\
\hline 93 & 12 & 7 & 7 & 4 & 177.9 \\
\hline 93 & 12 & 8 & 7 & 6 & 178.3 \\
\hline 93 & 12 & 9 & 7 & 4 & 178.1 \\
\hline 93 & 12 & 10 & 7 & 10 & 178 \\
\hline 93 & 12 & 11 & 7 & 6 & 178.1 \\
\hline 93 & 12 & 12 & 7 & 6 & 178.1 \\
\hline 93 & 12 & 13 & 7 & 6 & 177.6 \\
\hline 93 & 12 & 14 & 7 & 6 & 177.9 \\
\hline 93 & 12 & 15 & 7 & 6 & 177.6 \\
\hline 93 & 12 & 16 & 7 & 6 & 177.3 \\
\hline 93 & 12 & 17 & 7 & 5 & 177.2 \\
\hline 93 & 12 & 18 & 7 & 6 & 177 \\
\hline 93 & 12 & 19 & 7 & 6 & 177.1 \\
\hline 93 & 12 & 20 & 7 & 6 & 177 \\
\hline 93 & 12 & 21 & 7 & 6 & 177 \\
\hline 93 & 12 & 22 & 7 & 5 & 177 \\
\hline 93 & 12 & 23 & 7 & 10 & 176.9 \\
\hline 93 & 12 & 24 & 7 & 6 & 176.8 \\
\hline
\end{tabular}

Data obtained from WHC Surveillance Analysis Computer System (SACS), February 28, 1994. 


\begin{tabular}{|c|c|c|c|c|c|}
\hline Year & Month & Day & Hour & Minute & Surface Level \\
\hline 93 & 12 & 25 & 7 & 6 & 176.9 \\
\hline 93 & 12 & 26 & 7 & 6 & 177.3 \\
\hline 93 & 12 & 27 & 7 & 6 & 177.4 \\
\hline 93 & 12 & 28 & 6 & 6 & 177.2 \\
\hline 93 & 12 & 29 & 7 & 6 & 177 \\
\hline 93 & 12 & 30 & 0 & 5 & 177.1 \\
\hline 93 & 12 & 31 & 7 & 6 & 177.6 \\
\hline 94 & 1 & 1 & 7 & 6 & 177.8 \\
\hline 94 & 1 & 2 & 7 & 6 & 177.5 \\
\hline 94 & 1 & 3 & 7 & 7 & 177.5 \\
\hline 94 & 1 & 4 & 7 & 7 & 177.8 \\
\hline 94 & 1 & 5 & 7 & 6 & 178 \\
\hline 94 & 1 & 6 & 7 & 6 & 177.6 \\
\hline 94 & 1 & 7 & 7 & 7 & 177.4 \\
\hline 94 & 1 & 8 & 7 & 6 & 177.7 \\
\hline 94 & 1 & 9 & 7 & 6 & 177.8 \\
\hline 94 & 1 & 10 & 7 & 7 & 177.6 \\
\hline 94 & 1 & 11 & 7 & 7 & 177.6 \\
\hline 94 & 1 & 12 & 7 & 6 & 177.3 \\
\hline 94 & 1 & 13 & 7 & 10 & 177.2 \\
\hline 94 & 1 & 14 & 7 & 6 & 177.4 \\
\hline 94 & 1 & 15 & 7 & 10 & 177.6 \\
\hline 94 & 1 & 16 & 7 & 7 & 177.4 \\
\hline 94 & 1 & 17 & 7 & 7 & 177.4 \\
\hline 94 & 1 & 18 & 7 & 7 & 177.5 \\
\hline 94 & 1 & 19 & 7 & 7 & 177.6 \\
\hline 94 & 1 & 20 & 7 & 7 & 177.4 \\
\hline 94 & 1 & 21 & 7 & 7 & 177.4 \\
\hline 94 & 1 & 22 & 7 & 8 & 177.5 \\
\hline 94 & 1 & 23 & 7 & 7 & 177.9 \\
\hline 94 & 1 & 24 & 7 & 8 & 178 \\
\hline 94 & 1 & 25 & 7 & 7 & 177.9 \\
\hline 94 & 1 & 26 & 7 & 6 & 177.7 \\
\hline 94 & 1 & 27 & 7 & 10 & 177.5 \\
\hline 94 & 1 & 28 & 7 & 7 & 177.2 \\
\hline 94 & 1 & 29 & 7 & 7 & 177.1 \\
\hline 94 & 1 & 30 & 7 & 6 & 177 \\
\hline 94 & 1 & 31 & 7 & 7 & 177 \\
\hline 94 & 2 & 1 & 7 & 6 & 177 \\
\hline 94 & 2 & 2 & 7 & 7 & 177 \\
\hline 94 & 2 & 3 & 7 & 7 & 177.5 \\
\hline 94 & 2 & 4 & 7 & 7 & 177.4 \\
\hline 94 & 2 & 5 & 7 & 7 & 177.4 \\
\hline 94 & 2 & 6 & 7 & 7 & 177.7 \\
\hline 94 & 2 & 7 & 7 & 6 & 178 \\
\hline 94 & 2 & 8 & 7 & 7 & 177.7 \\
\hline 94 & 2 & 9 & 7 & 4 & 177.7 \\
\hline 94 & 2 & 10 & 7 & 4 & 177.7 \\
\hline
\end{tabular}

Data obtained from WHC Surveillance Analysis Computer System (SACS), February 28, 1994. E-70 


\begin{tabular}{|c|c|c|c|c|c|}
\hline Year & Month & Day & Hour & Minute & Surface Level \\
\hline 94 & 2 & 11 & 7 & 6 & 177.6 \\
\hline 94 & 2 & 12 & 7 & 3 & 177.4 \\
\hline 94 & 2 & 13 & 7 & 4 & 177.7 \\
\hline 94 & 2 & 14 & 7 & 4 & 177.7 \\
\hline 94 & 2 & 15 & 7 & 4 & 177.8 \\
\hline 94 & 2 & 16 & 7 & 3 & 178 \\
\hline 94 & 2 & 17 & 7 & 4 & 178.5 \\
\hline 94 & 2 & 18 & 7 & 6 & 178.4 \\
\hline 94 & 2 & 19 & 7 & 6 & 178 \\
\hline 94 & 2 & 20 & 7 & 7 & 177.8 \\
\hline 94 & 2 & 21 & 7 & 6 & 177.7 \\
\hline 94 & 2 & 22 & 7 & 5 & 177.5 \\
\hline 94 & 2 & 23 & 7 & 6 & 177.7 \\
\hline 94 & 2 & 24 & 7 & 6 & 177.7 \\
\hline & & & & & \\
\hline & & & & & \\
\hline & & & & & \\
\hline & & & & & \\
\hline & & & & & \\
\hline & & & & & \\
\hline & & & & & \\
\hline & & & & & \\
\hline & & & & & \\
\hline & & & & & \\
\hline & & & & & \\
\hline & & & & & \\
\hline & & & & & \\
\hline & & & & & \\
\hline & & & & & \\
\hline . & & & & & \\
\hline & & & & & \\
\hline & & & & & \\
\hline & & & & & \\
\hline & & & & & \\
\hline & & & & & \\
\hline & & & & & \\
\hline & & & & & \\
\hline & & & & & \\
\hline & & & & & \\
\hline & & & & & \\
\hline & & & & & \\
\hline & & & & & \\
\hline & & & & & \\
\hline & & & & & \\
\hline & & & & & \\
\hline & & & & & \\
\hline & & & & & \\
\hline
\end{tabular}

Data obtained from WHC Surveillance Analysis Computer System (SACS), February 28, 1994. E-71 


\begin{tabular}{|c|c|c|c|c|c|}
\hline \multicolumn{6}{|c|}{ Surface Level Data } \\
\hline \multicolumn{6}{|c|}{ FIC } \\
\hline Year & Month & Day & Hour & Minute & Surface Level \\
\hline 91 & 1 & 1 & 7 & 3 & 142.1 \\
\hline 91 & 1 & 2 & 7 & 2 & 142.1 \\
\hline 91 & 1 & 3 & 7 & 3 & 142.1 \\
\hline 91 & 1 & 4 & 7 & 2 & 142.1 \\
\hline 91 & 1 & 5 & 7 & 2 & 142.1 \\
\hline 91 & 1 & 6 & 7 & 2 & 142.1 \\
\hline 91 & 1 & 7 & 7 & 2 & 142.2 \\
\hline 91 & 1 & 8 & 7 & 3 & 142.2 \\
\hline 91 & 1 & 9 & 7 & 2 & 142.2 \\
\hline 91 & 1 & 10 & 7 & 2 & 142.2 \\
\hline 91 & 1 & 11 & 7 & 3 & 142.2 \\
\hline 91 & 1 & 12 & 7 & 2 & 142.2 \\
\hline 91 & 1 & 26 & 7 & 3 & 142.2 \\
\hline 91 & 1 & 27 & 7 & 3 & 142.2 \\
\hline 91 & 1 & 28 & 7 & 10 & 142.2 \\
\hline 91 & 1 & 29 & 7 & 2 & 142.2 \\
\hline 91 & 1 & 30 & 7 & 10 & 142.2 \\
\hline 91 & 1 & 31 & 7 & 2 & 142.2 \\
\hline 91 & 2 & 1 & 7 & 2 & 142.2 \\
\hline 91 & 2 & 15 & 7 & 3 & 142.3 \\
\hline 91 & 2 & 16 & 7 & 2 & 142.4 \\
\hline 91 & 2 & 17 & 7 & 2 & 142.5 \\
\hline 91 & 2 & 18 & 7 & 3 & 142.3 \\
\hline 91 & 2 & 19 & 7 & 2 & 142.4 \\
\hline 91 & 2 & 20 & 7 & 3 & 142.4 \\
\hline 91 & 2 & 21 & 7 & 2 & 142.3 \\
\hline 91 & 2 & 22 & 7 & 2 & 142.4 \\
\hline 91 & 2 & 23 & 7 & 2 & 142.3 \\
\hline 91 & 2 & 24 & 7 & 3 & 142.3 \\
\hline 91 & 2 & 25 & 7 & 3 & 142.3 \\
\hline 91 & 2 & 26 & 7 & 3 & 142.4 \\
\hline 91 & 2 & 27 & 7 & 2 & 142.4 \\
\hline 91 & 2 & 28 & 7 & 2 & 142.4 \\
\hline 91 & 3 & 1 & 7 & 2 & 142.5 \\
\hline 91 & 3 & 2 & 7 & 2 & 142.5 \\
\hline 91 & 3 & 3 & 7 & 3 & 142.4 \\
\hline 91 & 3 & 4 & 7 & 3 & 142.5 \\
\hline 91 & 3 & 5 & 7 & 3 & 142.5 \\
\hline 91 & 3 & 6 & 7 & 2 & 142.6 \\
\hline 91 & 3 & 7 & 7 & 2 & 142.5 \\
\hline 91 & 3 & 8 & 7 & 2 & 142.5 \\
\hline 91 & 3 & 9 & 7 & 2 & 142.6 \\
\hline 91 & 3 & 10 & 7 & 2 & 142.7 \\
\hline 91 & 3 & 11 & 7 & 2 & 142.7 \\
\hline 91 & 3 & 12 & 7 & 3 & 142.6 \\
\hline 91 & 3 & 13 & 7 & 3 & 142.6 \\
\hline
\end{tabular}

Data obtained from WHC Surveillance Analysis Computer System (SACS), February 28, 1994. 


\begin{tabular}{|c|c|c|c|c|c|}
\hline Year & Month & Day & Hour & Minute & Surface Level \\
\hline 91 & 3 & 14 & 7 & 3 & 142.5 \\
\hline 91 & 3 & 15 & 7 & 3 & 142.5 \\
\hline 91 & 3 & 16 & 7 & 3 & 142.6 \\
\hline 91 & 3 & 17 & 7 & 2 & 142.6 \\
\hline 91 & 3 & 18 & 7 & 3 & 142.7 \\
\hline 91 & 3 & 19 & 7 & 3 & 142.7 \\
\hline 91 & 3 & 20 & 7 & 2 & 142.8 \\
\hline 91 & 3 & 21 & 7 & 3 & 142.8 \\
\hline 91 & 3 & 22 & 7 & 2 & 142.5 \\
\hline 91 & 3 & 23 & 7 & 3 & 142.5 \\
\hline 91 & 3 & 24 & 7 & 3 & 142.6 \\
\hline 91 & 3 & 25 & 7 & 3 & 142.7 \\
\hline 91 & 3 & 26 & 7 & 3 & 142.3 \\
\hline 91 & 3 & 27 & 7 & 3 & 142.5 \\
\hline 91 & 3 & 28 & 7 & 3 & 142.5 \\
\hline 91 & 3 & 29 & 7 & 3 & 142.6 \\
\hline 91 & 3 & 30 & 7 & 3 & 142.6 \\
\hline 91 & 3 & 31 & 7 & 3 & 142.6 \\
\hline 91 & 4 & 1 & 7 & 3 & 142.4 \\
\hline 91 & 4 & 2 & 7 & 3 & 142.4 \\
\hline 91 & 4 & 3 & 7 & 3 & 142.4 \\
\hline 91 & 4 & 4 & 7 & 3 & 142.5 \\
\hline 91 & 4 & 5 & 7 & 3 & 142.6 \\
\hline 91 & 4 & 6 & 7 & 3 & 142.6 \\
\hline 91 & 4 & 7 & 7 & 3 & 142.6 \\
\hline 91 & 4 & 8 & 7 & 4 & 142.3 \\
\hline 91 & 4 & 9 & 7 & 3 & 142.4 \\
\hline 91 & 4 & 10 & 7 & 3 & 142.4 \\
\hline 91 & 4 & 11 & 7 & 3 & 142.4 \\
\hline 91 & 4 & 12 & 7 & 3 & 142.4 \\
\hline 91 & 4 & 13 & 7 & 4 & 142.4 \\
\hline 91 & 4 & 14 & 7 & 3 & 142.5 \\
\hline 91 & 4 & 15 & 7 & 3 & 142.6 \\
\hline 91 & 4 & 16 & 7 & 10 & 142.6 \\
\hline 91 & 4 & 17 & 7 & 3 & 142.6 \\
\hline 91 & 4 & 18 & 7 & 6 & 142.7 \\
\hline 91 & 4 & 19 & 7 & 5 & 142.7 \\
\hline 91 & 4 & 20 & 7 & 6 & 142.6 \\
\hline 91 & 4 & 21 & 7 & 5 & 142.6 \\
\hline 91 & 4 & 22 & 7 & 6 & 142.6 \\
\hline 91 & 4 & 23 & 7 & 6 & 142.8 \\
\hline 91 & 4 & 24 & 7 & 5 & 142.9 \\
\hline 91 & 4 & 25 & 7 & 5 & 142.9 \\
\hline 91 & 4 & 26 & 3 & 5 & 142.8 \\
\hline 91 & 4 & 27 & 7 & 5 & 142.7 \\
\hline 91 & 4 & 28 & 7 & 6 & 142.8 \\
\hline 91 & 4 & 29 & 7 & 5 & 142.8 \\
\hline 91 & 4 & 30 & 7 & 6 & 142.9 \\
\hline
\end{tabular}

Data obtained from WHC Surveillance Analysis Computer System (SACS), February 28, 1994. E-73 


\begin{tabular}{|c|c|c|c|c|c|}
\hline Year & Month & Day & Hour & Minute & Surface Level \\
\hline 91 & 5 & 1 & 6 & 6 & 142.5 \\
\hline 91 & 5 & 2 & 7 & 5 & 142.4 \\
\hline 91 & 5 & 3 & 7 & 6 & 142.4 \\
\hline 91 & 5 & 4 & 7 & 5 & 142.4 \\
\hline 91 & 5 & 5 & 7 & 5 & 142.4 \\
\hline 91 & 5 & 6 & 7 & 5 & 142.4 \\
\hline 91 & 5 & 7 & 7 & 5 & 142.4 \\
\hline 91 & 5 & 8 & 7 & 5 & 142.5 \\
\hline 91 & 5 & 9 & 7 & 5 & 142.5 \\
\hline 91 & 5 & 10 & 7 & 5 & 142.6 \\
\hline 91 & 5 & 11 & 7 & 5 & 142.6 \\
\hline 91 & 5 & 12 & 7 & 5 & 142.4 \\
\hline 91 & 5 & 13 & 7 & 5 & 142.5 \\
\hline 91 & 5 & 14 & 7 & 5 & 142.5 \\
\hline 91 & 5 & 15 & 7 & 5 & 142.4 \\
\hline 91 & 5 & 16 & 7 & 6 & 142.1 \\
\hline 91 & 5 & 17 & 7 & 6 & 142.3 \\
\hline 91 & 5 & 18 & 7 & 6 & 142.3 \\
\hline 91 & 5 & 19 & 7 & 5 & 142.4 \\
\hline 91 & 5 & 20 & 7 & 6 & 142.5 \\
\hline 91 & 5 & 21 & 7 & 6 & 142.5 \\
\hline 91 & 5 & 22 & 7 & 5 & 142.6 \\
\hline 91 & 5 & 23 & 7 & 5 & 142.6 \\
\hline 91 & 5 & 24 & 7 & 5 & 142.5 \\
\hline 91 & 5 & 25 & 7 & 5 & 142.5 \\
\hline 91 & 5 & 26 & 7 & 5 & 142.5 \\
\hline 91 & 5 & 27 & 7 & 5 & 142.4 \\
\hline 91 & 5 & 28 & 7 & 5 & 142.4 \\
\hline 91 & 5 & 29 & 7 & 5 & 142.4 \\
\hline 91 & 5 & 30 & 7 & 5 & 142.4 \\
\hline 91 & 5 & 31 & 7 & 5 & 142.4 \\
\hline 91 & 6 & 1 & 7 & 5 & 142.4 \\
\hline 91 & 6 & 2 & 7 & 5 & 142.3 \\
\hline 91 & 6 & 3 & 7 & 5 & 142.4 \\
\hline 91 & 6 & 4 & 7 & 5 & 142.3 \\
\hline 91 & 6 & 5 & 7 & 5 & 142.4 \\
\hline 91 & 6 & 6 & 7 & 6 & 142.4 \\
\hline 91 & 6 & 7 & 7 & 5 & 142.4 \\
\hline 91 & 6 & 8 & 7 & 5 & 142.4 \\
\hline 91 & 6 & 9 & 7 & 5 & 142.4 \\
\hline 91 & 6 & 10 & 7 & 6 & 142.6 \\
\hline 91 & 6 & 11 & 7 & 5 & 142.7 \\
\hline 91 & 6 & 12 & 7 & 4 & 142.7 \\
\hline 91 & 6 & 13 & 7 & 5 & 142.5 \\
\hline 91 & 6 & 14 & 7 & 5 & 142.5 \\
\hline 91 & 6 & 15 & 7 & 5 & 142.5 \\
\hline 91 & 6 & 16 & 7 & 5 & 142.5 \\
\hline 91 & 6 & 17 & 7 & 5 & 142.5 \\
\hline
\end{tabular}

Data obtained from WHC Surveillance Analysis Computer System (SACS), February 28, 1994. 


\begin{tabular}{|c|c|c|c|c|c|}
\hline Year & Month & Day & Hour & Minute & Surface Level \\
\hline 91 & 6 & 18 & 7 & 5 & 142.5 \\
\hline 91 & 6 & 19 & 7 & 5 & 142.5 \\
\hline 91 & 6 & 20 & 7 & 5 & 142.5 \\
\hline 91 & 6 & 21 & 7 & 6 & 142.5 \\
\hline 91 & 6 & 22 & 7 & 5 & 142.5 \\
\hline 91 & 6 & 23 & 7 & 5 & 142.6 \\
\hline 91 & 6 & 24 & 7 & 5 & 142.6 \\
\hline 91 & 6 & 25 & 7 & 6 & 142.6 \\
\hline 91 & 6 & 26 & 7 & 3 & 142.6 \\
\hline 91 & 6 & 27 & 7 & 2 & 142.6 \\
\hline 91 & 6 & 28 & 7 & 3 & 142.6 \\
\hline 91 & 6 & 29 & 7 & 2 & 142.6 \\
\hline 91 & 7 & 2 & 7 & 6 & 142.6 \\
\hline 91 & 7 & 3 & 7 & 6 & 142.6 \\
\hline 91 & 7 & 4 & 7 & 10 & 142.6 \\
\hline 91 & 7 & 5 & 7 & 6 & 142.7 \\
\hline 91 & 7 & 6 & 7 & 6 & 142.8 \\
\hline 91 & 7 & 7 & 7 & 10 & 142.7 \\
\hline 91 & 7 & 8 & 7 & 6 & 142.7 \\
\hline 91 & 7 & 9 & 7 & 6 & 142.8 \\
\hline 91 & 7 & 10 & 6 & 6 & 142.8 \\
\hline 91 & 7 & 11 & 7 & 6 & 142.7 \\
\hline 91 & 7 & 12 & 7 & 6 & 142.7 \\
\hline 91 & 7 & 13 & 7 & 6 & 142.8 \\
\hline 91 & 7 & 14 & 7 & 6 & 142.8 \\
\hline 91 & 7 & 15 & 7 & 6 & 142.8 \\
\hline 91 & 7 & 16 & 7 & 6 & 142.8 \\
\hline 91 & 7 & 17 & 7 & 5 & 142.8 \\
\hline 91 & 7 & 18 & 7 & 5 & 142.8 \\
\hline 91 & 7 & 19 & 7 & 5 & 142.8 \\
\hline 91 & 7 & 20 & 7 & 5 & 142.8 \\
\hline 91 & 7 & 21 & 7 & 5 & 142.8 \\
\hline 91 & 7 & 22 & 7 & 5 & 142.8 \\
\hline 91 & 7 & 23 & 7 & 5 & 142.8 \\
\hline 91 & 7 & 24 & 7 & 5 & 143 \\
\hline 91 & 7 & 25 & 7 & 1 & 142.9 \\
\hline 91 & 7 & 26 & 7 & 2 & 142.9 \\
\hline 91 & 7 & 27 & 7 & 2 & 142.9 \\
\hline 91 & 7 & 28 & 7 & 2 & 142.9 \\
\hline 91 & 7 & 29 & 7 & 2 & 142.9 \\
\hline 91 & 7 & 30 & 7 & 2 & 142.9 \\
\hline 91 & 7 & 31 & 7 & 2 & 142.9 \\
\hline 91 & 8 & 1 & 7 & 2 & 142.9 \\
\hline 91 & 8 & 2 & 7 & 2 & 143 \\
\hline 91 & 8 & 3 & 7 & 2 & 143 \\
\hline 91 & 8 & 4 & 7 & 2 & 143 \\
\hline 91 & 8 & 5 & 7 & 2 & 143 \\
\hline 91 & 8 & 6 & 7 & 2 & 143 \\
\hline
\end{tabular}

Data obtained from WHC Surveillance Analysis Computer System (SACS), February 28, 1994. E-75 


\begin{tabular}{|c|c|c|c|c|c|}
\hline Year & Month & Day & Hour & Minute & Surface Level \\
\hline 91 & 8 & 7 & 7 & 6 & 143 \\
\hline 91 & 8 & 8 & 7 & 6 & 142.7 \\
\hline 91 & 8 & 9 & 7 & 4 & 142.9 \\
\hline 91 & 8 & 10 & 7 & 4 & 143 \\
\hline 91 & 8 & 11 & 7 & 4 & 142.9 \\
\hline 91 & 8 & 12 & 7 & 4 & 142.9 \\
\hline 91 & 8 & 13 & 7 & 4 & 142.8 \\
\hline 91 & 8 & 14 & 7 & 4 & 142.8 \\
\hline 91 & 8 & 15 & 7 & 3 & 142.8 \\
\hline 91 & 8 & 17 & 7 & 2 & 142.7 \\
\hline 91 & 8 & 18 & 7 & 3 & 142.7 \\
\hline 91 & 8 & 19 & 7 & 2 & 142.7 \\
\hline 91 & 8 & 20 & 7 & 4 & 142.7 \\
\hline 91 & 8 & 21 & 7 & 3 & 142.7 \\
\hline 91 & 8 & 22 & 7 & 3 & 142.4 \\
\hline 91 & 8 & 23 & 7 & 2 & 142.5 \\
\hline 91 & 8 & 24 & 7 & 2 & 142.5 \\
\hline 91 & 8 & 25 & 7 & 2 & 142.5 \\
\hline 91 & 8 & 26 & 7 & 3 & 142.5 \\
\hline 91 & 8 & 27 & 7 & 3 & 142.6 \\
\hline 91 & 8 & 28 & 7 & 3 & 142.6 \\
\hline 91 & 8 & 29 & 7 & 2 & 142.6 \\
\hline 91 & 8 & 30 & 7 & 10 & 142.6 \\
\hline 91 & 8 & 31 & 7 & 2 & 142.7 \\
\hline 91 & 9 & 1 & 7 & 2 & 142.6 \\
\hline 91 & 9 & 2 & 7 & 2 & 142.6 \\
\hline 91 & 9 & 3 & 7 & 2 & 142.6 \\
\hline 91 & 9 & 4 & 7 & 3 & 143.5 \\
\hline 91 & 9 & 5 & 7 & 2 & 143.7 \\
\hline 91 & 9 & 6 & 7 & 2 & 143.7 \\
\hline 91 & 9 & 7 & 7 & 2 & 143.8 \\
\hline 91 & 9 & 8 & 7 & 2 & 143.8 \\
\hline 91 & 9 & 9 & 7 & 3 & 143.8 \\
\hline 91 & 9 & 10 & 7 & 2 & 143.8 \\
\hline 91 & 9 & 11 & 7 & 3 & 143.8 \\
\hline 91 & 9 & 12 & 7 & 2 & 143.8 \\
\hline 91 & 9 & 13 & 7 & 2 & 143.8 \\
\hline 91 & 9 & 14 & 7 & 3 & 143.8 \\
\hline 91 & 9 & 15 & 7 & 2 & 143.8 \\
\hline 91 & 9 & 16 & 7 & 3 & 143.8 \\
\hline 91 & 9 & 17 & 7 & 3 & 143.8 \\
\hline 91 & 9 & 18 & 7 & 3 & 143.8 \\
\hline 91 & 9 & 19 & 7 & 3 & 143.8 \\
\hline 91 & 9 & 20 & 7 & 2 & 143.8 \\
\hline 91 & 9 & 21 & 7 & 2 & 143.8 \\
\hline 91 & 9 & 22 & 7 & 3 & 143.8 \\
\hline 91 & 9 & 23 & 7 & 3 & 143.8 \\
\hline 91 & 9 & 24 & 7 & 2 & 143.8 \\
\hline
\end{tabular}

Data obtained from WHC Surveillance Analysis Computer System (SACS), February 28, 1994. 


\begin{tabular}{|c|c|c|c|c|c|}
\hline Year & Month & Day & Hour & Minute & Surface Level \\
\hline 91 & 9 & 25 & 7 & 3 & 143.8 \\
\hline 91 & 9 & 26 & 7 & 3 & 143.8 \\
\hline 91 & 9 & 27 & 7 & 2 & 143.8 \\
\hline 91 & 9 & 28 & 7 & 2 & 143.8 \\
\hline 91 & 9 & 29 & 7 & 3 & 143.8 \\
\hline 91 & 9 & 30 & 7 & 3 & 143.7 \\
\hline 91 & 10 & 1 & 7 & 2 & 143.9 \\
\hline 91 & 10 & 2 & 7 & 2 & 143.9 \\
\hline 91 & 10 & 3 & 7 & 2 & 143.9 \\
\hline 91 & 10 & 4 & 7 & 3 & 143.8 \\
\hline 91 & 10 & 6 & 6 & 2 & 143.9 \\
\hline 91 & 10 & 7 & 6 & 2 & 143.9 \\
\hline 91 & 10 & 8 & 7 & 2 & 143.9 \\
\hline 91 & 10 & 9 & 7 & 2 & 143.8 \\
\hline 91 & 10 & 10 & 7 & 2 & 143.8 \\
\hline 91 & 10 & 11 & 7 & 2 & 143.9 \\
\hline 91 & 10 & 12 & 7 & 2 & 143.9 \\
\hline 91 & 10 & 13 & 7 & 3 & 143.9 \\
\hline 91 & 10 & 14 & 7 & 2 & 143.8 \\
\hline 91 & 10 & 15 & 7 & 2 & 143.9 \\
\hline 91 & 10 & 16 & 7 & 3 & 143.9 \\
\hline 91 & 10 & 17 & 7 & 5 & 143.7 \\
\hline 91 & 10 & 18 & 7 & 6 & 143.7 \\
\hline 91 & 10 & 19 & 7 & 6 & 143.7 \\
\hline 91 & 10 & 20 & 7 & 2 & 143.7 \\
\hline 91 & 10 & 21 & 7 & 2 & 143.8 \\
\hline 91 & 10 & 23 & 7 & 2 & 143.8 \\
\hline 91 & 10 & 24 & 7 & 2 & 143.8 \\
\hline 91 & 10 & 25 & 7 & 3 & 143.8 \\
\hline 91. & 10 & 26 & 7 & 3 & 143.8 \\
\hline 91 & 10 & 27 & 7 & 2 & 143.8 \\
\hline 91 & 10 & 28 & 7 & 2 & 143.7 \\
\hline 91 & 10 & 29 & 7 & 3 & 143.7 \\
\hline 91 & 10 & 30 & 7 & 3 & 143.7 \\
\hline 91 & 10 & 31 & 7 & 3 & 143.7 \\
\hline 91 & 11 & 1 & 7 & 2 & 143.7 \\
\hline 91 & 11 & 2 & 7 & 3 & 143.7 \\
\hline 91 & 11 & 3 & 7 & 3 & 143.8 \\
\hline 91 & 11 & 4 & 7 & 2 & 143.8 \\
\hline 91 & 11 & 5 & 7 & 3 & 143.9 \\
\hline 91 & 11 & 6 & 7 & 3 & 143.9 \\
\hline 91 & 11 & 7 & 7 & 2 & 143.9 \\
\hline 91 & 11 & 8 & 7 & 3 & 143.9 \\
\hline 91 & 11 & 9 & 7 & 2 & 143.9 \\
\hline 91 & 11 & 10 & 7 & 3 & 143.9 \\
\hline 91 & 11 & 11 & 7 & 10 & 143.9 \\
\hline 91 & 11 & 12 & 7 & 3 & 143.9 \\
\hline 91 & 11 & 13 & 7 & 3 & 143.9 \\
\hline
\end{tabular}

Data obtained from WHC Surveillance Analysis Computer System (SACS), February 28, 1994. 


\begin{tabular}{|c|c|c|c|c|c|}
\hline Year & Month & Day & Hour & Minute & Surface Level \\
\hline 91 & 11 & 14 & 7 & 3 & 143.9 \\
\hline 91 & 11 & 15 & 7 & 3 & 143.9 \\
\hline 91 & 11 & 16 & 7 & 3 & 143.9 \\
\hline 91 & 11 & 17 & 7 & 2 & 144 \\
\hline 91 & 11 & 18 & 7 & 3 & 143.9 \\
\hline 91 & 11 & 19 & 7 & 3 & 143.9 \\
\hline 91 & 11 & 20 & 7 & 3 & 143.9 \\
\hline 91 & 11 & 21 & 7 & 2 & 143.9 \\
\hline 91 & 11 & 22 & 7 & 3 & 143.8 \\
\hline 91 & 11 & 23 & 7 & 2 & 143.8 \\
\hline 91 & 11 & 24 & 7 & 3 & 143.9 \\
\hline 91 & 11 & 25 & 7 & 3 & 143.9 \\
\hline 91 & 11 & 27 & 7 & 3 & 144 \\
\hline 91 & 11 & 28 & 7 & 3 & 143.9 \\
\hline 91 & 11 & 29 & 7 & 3 & 144 \\
\hline 91 & 11 & 30 & 7 & 3 & 143.9 \\
\hline 91 & 12 & 1 & 7 & 3 & 144 \\
\hline 91 & 12 & 2 & 7 & 3 & 144 \\
\hline 91 & 12 & 3 & 7 & 3 & 144 \\
\hline 91 & 12 & 4 & 7 & 3 & 144 \\
\hline 91 & 12 & 5 & 7 & 3 & 144 \\
\hline 91 & 12 & 6 & 7 & 4 & 144 \\
\hline 91 & 12 & 7 & 7 & 3 & 144 \\
\hline 91 & 12 & 8 & 7 & 3 & 144 \\
\hline 91 & 12 & 9 & 7 & 3 & 144 \\
\hline 91 & 12 & 10 & 7 & 3 & 144 \\
\hline 91 & 12 & 11 & 7 & 2 & 144 \\
\hline 91 & 12 & 12 & 7 & 3 & 144 \\
\hline 91 & 12 & 13 & 6 & 6 & 144 \\
\hline 91 & 12 & 14 & 7 & 2 & 143.9 \\
\hline 91 & 12 & 15 & 7 & 2 & 144 \\
\hline 91 & 12 & 18 & 7 & 3 & 144 \\
\hline 91 & 12 & 24 & 7 & 3 & 144 \\
\hline 91 & 12 & 25 & 7 & 3 & 144.1 \\
\hline 91 & 12 & 26 & 7 & 3 & 144.1 \\
\hline 91 & 12 & 27 & 7 & 3 & 144.1 \\
\hline 91 & 12 & 28 & 7 & 2 & 144.1 \\
\hline 91 & 12 & 29 & 7 & 3 & 144 \\
\hline 91 & 12 & 30 & 7 & 3 & 144 \\
\hline 91 & 12 & 31 & 7 & 3 & 144 \\
\hline 92 & 1 & 1 & 7 & 3 & 144 \\
\hline 92 & 1 & 2 & 7 & 2 & 144.1 \\
\hline 92 & 1 & 3 & 7 & 4 & 144.1 \\
\hline 92 & 1 & 4 & 7 & 3 & 144.1 \\
\hline 92 & 1 & 5 & 7 & 3 & 144.1 \\
\hline 92 & 1 & 6 & 7 & 3 & 144.1 \\
\hline 92 & 1 & 7 & 7 & 4 & 144.1 \\
\hline 92 & 1 & 9 & 7 & 3 & 144 \\
\hline
\end{tabular}

Data obtained from WHC Surveillance Analysis Computer System (SACS), February 28, 1994. 


\begin{tabular}{|c|c|c|c|c|c|}
\hline Year & Month & Day & Hour & Minute & Surface Level \\
\hline 92 & 1 & 10 & 7 & 4 & 144.1 \\
\hline 92 & 1 & 11 & 7 & 4 & 144.1 \\
\hline 92 & 1 & 17 & 7 & 4 & 144.1 \\
\hline 92 & 1 & 18 & 7 & 4 & 144 \\
\hline 92 & 1 & 19 & 7 & 3 & 144.1 \\
\hline 92 & 1 & 20 & 7 & 3 & 144.1 \\
\hline 92 & 1 & 21 & 7 & 3 & 144.1 \\
\hline 92 & 1 & 22 & 7 & 6 & 144.1 \\
\hline 92 & 1 & 23 & 7 & 3 & 144.1 \\
\hline 92 & 1 & 24 & 7 & 2 & 144.1 \\
\hline 92 & 1 & 25 & 7 & 3 & 144.1 \\
\hline 92 & 1 & 26 & 7 & 10 & 144.1 \\
\hline 92 & 1 & 27 & 7 & 2 & 144.1 \\
\hline 92 & 1 & 28 & 7 & 3 & 144.1 \\
\hline 92 & 1 & 29 & 7 & 3 & 144.1 \\
\hline 92 & 1 & 30 & 7 & 3 & 144.1 \\
\hline 92 & 1 & 31 & 7 & 3 & 144.1 \\
\hline 92 & 2 & 1 & 7 & 2 & 144.1 \\
\hline 92 & 2 & 2 & 7 & 3 & 144.1 \\
\hline 92 & 2 & 3 & 7 & 3 & 144 \\
\hline 92 & 2 & 4 & 7 & 3 & 144 \\
\hline 92 & 2 & 5 & 7 & 2 & 144.1 \\
\hline 92 & 2 & 8 & 7 & 4 & 144.1 \\
\hline 92 & 2 & 9 & 7 & 3 & 144.1 \\
\hline 92 & 2 & 10 & 7 & 4 & 144.2 \\
\hline 92 & 2 & 11 & 7 & 3 & 144.1 \\
\hline 92 & 2 & 12 & 7 & 3 & 144.1 \\
\hline 92 & 2 & 13 & 7 & 3 & 144.1 \\
\hline 92 & 2 & 14 & 7 & 3 & 144.1 \\
\hline 92 & 2 & 15 & 7 & 3 & 144.1 \\
\hline 92 & 2 & 16 & 7 & 3 & 144.1 \\
\hline 92 & 2 & 17 & 7 & 3 & 144.1 \\
\hline 92 & 2 & 18 & 7 & 3 & 144.1 \\
\hline 92 & 2 & 19 & 7 & 3 & 144.1 \\
\hline 92 & 2 & 20 & 7 & 3 & 144.1 \\
\hline 92 & 2 & 21 & 7 & 3 & 144.1 \\
\hline 92 & 2 & 22 & 6 & 3 & 144.2 \\
\hline 92 & 2 & 23 & 7 & 3 & 144.1 \\
\hline 92 & 2 & 24 & 7 & 3 & 144.1 \\
\hline 92 & 2 & 27 & 0 & 3 & 144 \\
\hline 92 & 2 & 29 & 7 & 3 & 144.1 \\
\hline 92 & 3 & 1 & 7 & 3 & 144.2 \\
\hline 92 & 3 & 2 & 7 & 3 & 144.1 \\
\hline 92 & 3 & 3 & 7 & 4 & 144.3 \\
\hline 92 & 3 & 4 & 7 & 4 & 144.3 \\
\hline 92 & 3 & 5 & 7 & 3 & 144.3 \\
\hline 92 & 3 & 6 & 7 & 3 & 144.3 \\
\hline 92 & 3 & 7 & 7 & 2 & 144.3 \\
\hline
\end{tabular}

Data obtained from WHC Surveillance Analysis Computer System (SACS), February 28, 1994. 


\begin{tabular}{|c|c|c|c|c|c|}
\hline Year & Month & Day & Hour & Minute & Surface Level \\
\hline 92 & 3 & 8 & 7 & 2 & 144.3 \\
\hline 92 & 3 & 9 & 7 & 2 & 144.3 \\
\hline 92 & 3 & 10 & 6 & 3 & 144.3 \\
\hline 92 & 3 & 11 & 7 & 3 & 144.3 \\
\hline 92 & 3 & 12 & 7 & 3 & 144.3 \\
\hline 92 & 3 & 13 & 7 & 3 & 144.3 \\
\hline 92 & 3 & 14 & 7 & 3 & 144.3 \\
\hline 92 & 3 & 15 & 7 & 6 & 144.3 \\
\hline 92 & 3 & 16 & 7 & 3 & 144.3 \\
\hline 92 & 3 & 17 & 7 & 3 & 144.3 \\
\hline 92 & 3 & 18 & 7 & 3 & 144.3 \\
\hline 92 & 3 & 19 & 7 & 3 & 144.2 \\
\hline 92 & 3 & 20 & 7 & 2 & 144.3 \\
\hline 92 & 3 & 21 & 7 & 3 & 144.3 \\
\hline 92 & 3 & 22 & 7 & 2 & 144.3 \\
\hline 92 & 3 & 23 & 6 & 3 & 144.3 \\
\hline 92 & 3 & 24 & 7 & 2 & 144.3 \\
\hline 92 & 3 & 25 & 7 & 3 & 144.3 \\
\hline 92 & 3 & 26 & 7 & 3 & 144.3 \\
\hline 92 & 3 & 27 & 7 & 2 & 144.3 \\
\hline 92 & 3 & 28 & 7 & 3 & 144.3 \\
\hline 92 & 3 & 29 & 7 & 3 & 144.2 \\
\hline 92 & 3 & 30 & 7 & 3 & 144.3 \\
\hline 92 & 3 & 31 & 6 & 3 & 144.2 \\
\hline 92 & 4 & 1 & 7 & 3 & 144.2 \\
\hline 92 & 4 & 2 & 7 & 3 & 144.3 \\
\hline 92 & 4 & 3 & 7 & 3 & 144.3 \\
\hline 92 & 4 & 4 & 7 & 3 & 144.3 \\
\hline 92 & 4 & 5 & 7 & 3 & 144.3 \\
\hline 92 & 4 & 6 & 7 & 3 & 144.2 \\
\hline 92 & 4 & 7 & 7 & 3 & 144.2 \\
\hline 92 & 4 & 8 & 7 & 10 & 144.2 \\
\hline 92 & 4 & 9 & 7 & 3 & 144.3 \\
\hline 92 & 4 & 10 & 7 & 3 & 144.3 \\
\hline 92 & 4 & 11 & 7 & 3 & 144.3 \\
\hline 92 & 4 & 12 & 7 & 4 & 144.3 \\
\hline 92 & 4 & 13 & 7 & 3 & 144.3 \\
\hline 92 & 4 & 14 & 4 & 3 & 144.3 \\
\hline 92 & 4 & 15 & 6 & 3 & 144.3 \\
\hline 92 & 4 & 16 & 7 & 3 & 144.3 \\
\hline 92 & 4 & 17 & 7 & 3 & 144.3 \\
\hline 92 & 4 & 18 & 7 & 2 & 144.3 \\
\hline 92 & 4 & 19 & 7 & 2 & 144.3 \\
\hline 92 & 4 & 20 & 7 & 3 & 144.3 \\
\hline 92 & 4 & 21 & 7 & 3 & 144.3 \\
\hline 92 & 4 & 22 & 7 & 2 & 144.3 \\
\hline 92 & 4 & 23 & 7 & 3 & 144.3 \\
\hline 92 & 4 & 24 & 7 & 3 & 144.3 \\
\hline
\end{tabular}

Data obtained from WHC Surveillance Analysis Computer System (SACS), February 28, 1994. 


\begin{tabular}{|c|c|c|c|c|c|}
\hline Year & Month & Day & Hour & Minute & Surface Level \\
\hline 92 & 4 & 25 & 7 & 4 & 144.3 \\
\hline 92 & 4 & 26 & 7 & 3 & 144.3 \\
\hline 92 & 4 & 27 & 7 & 4 & 144.2 \\
\hline 92 & 4 & 28 & 7 & 3 & 144.3 \\
\hline 92 & 4 & 29 & 7 & 3 & 144.4 \\
\hline 92 & 4 & 30 & 7 & 3 & 144.4 \\
\hline 92 & 5 & 1 & 7 & 3 & 144.2 \\
\hline 92 & 5 & 2 & 7 & 3 & 144.3 \\
\hline 92 & 5 & 3 & 7 & 3 & 144.3 \\
\hline 92 & 5 & 4 & 7 & 3 & 144.3 \\
\hline 92 & 5 & 5 & 7 & 4 & 144.3 \\
\hline 92 & 5 & 6 & 7 & 3 & 144.4 \\
\hline 92 & 5 & 7 & 7 & 4 & 144.3 \\
\hline 92 & 5 & 8 & 7 & 3 & 144.4 \\
\hline 92 & 5 & 9 & 4 & 4 & 144.4 \\
\hline 92 & 5 & 10 & 7 & 4 & 144.4 \\
\hline 92 & 5 & 11 & 4 & 4 & 144.4 \\
\hline 92 & 5 & 12 & 7 & 3 & 144.2 \\
\hline 92 & 5 & 13 & 7 & 3 & 144.4 \\
\hline 92 & 5 & 14 & 7 & 3 & 144.3 \\
\hline 92 & 5 & 15 & 7 & 3 & 144.4 \\
\hline 92 & 5 & 16 & 6 & 3 & 144.4 \\
\hline 92 & 5 & 17 & 6 & 3 & 144.4 \\
\hline 92 & 5 & 18 & 7 & 3 & 144.4 \\
\hline 92 & 5 & 19 & 7 & 3 & 144.4 \\
\hline 92 & 5 & 20 & 7 & 3 & 144.4 \\
\hline 92 & 5 & 21 & 7 & 3 & 144.3 \\
\hline 92 & 5 & 22 & 7 & 2 & 144.3 \\
\hline 92 & 5 & 23 & 7 & 3 & 144.3 \\
\hline 92 & 5 & 24 & 7 & 3 & 144.3 \\
\hline 92 & 5 & 25 & 7 & 3 & 144.4 \\
\hline 92 & 5 & 27 & 7 & 3 & 144.4 \\
\hline 92 & 5 & 28 & 7 & 2 & 144.4 \\
\hline 92 & 5 & 29 & 7 & 2 & 144.4 \\
\hline 92 & 5 & 30 & 7 & 3 & 144.4 \\
\hline 92 & 5 & 31 & 6 & 3 & 144.4 \\
\hline 92 & 6 & 1 & 7 & 3 & 144.4 \\
\hline 92 & 6 & 2 & 7 & 3 & 144.4 \\
\hline 92 & 6 & 3 & 7 & 3 & 144.4 \\
\hline 92 & 6 & 4 & 7 & 3 & 144.4 \\
\hline 92 & 6 & 5 & 7 & 2 & 144.4 \\
\hline 92 & 6 & 6 & 7 & 3 & 144.4 \\
\hline 92 & 6 & 7 & 7 & 3 & 144.4 \\
\hline 92 & 6 & 8 & 7 & 3 & 144.4 \\
\hline 92 & 6 & 9 & 7 & 3 & 144.4 \\
\hline 92 & 6 & 10 & 7 & 3 & 144.4 \\
\hline 92 & 6 & 11 & 7 & 3 & 144.4 \\
\hline 92 & 6 & 12 & 7 & 5 & 144.4 \\
\hline
\end{tabular}

Data obtained from WHC Surveillance Analysis Computer System (SACS), February 28, 1994. E-81 


\begin{tabular}{|c|c|c|c|c|c|}
\hline Year & Month & Day & Hour & Minute & Surface Level \\
\hline 92 & 6 & 13 & 6 & 5 & 144.5 \\
\hline 92 & 6 & 15 & 7 & 3 & 144.3 \\
\hline 92 & 6 & 16 & 7 & 3 & 144.3 \\
\hline 92 & 6 & 17 & 7 & 3 & 144.3 \\
\hline 92 & 6 & 18 & 7 & 3 & 144.3 \\
\hline 92 & 6 & 19 & 7 & 2 & 144.4 \\
\hline 92 & 6 & 20 & 7 & 2 & 144.4 \\
\hline 92 & 6 & 21 & 7 & 3 & 144.4 \\
\hline 92 & 6 & 22 & 7 & 3 & 144.4 \\
\hline 92 & 6 & 23 & 7 & 3 & 144.4 \\
\hline 92 & 6 & 24 & 6 & 3 & 144.4 \\
\hline 92 & 6 & 25 & 7 & 3 & 144.4 \\
\hline 92 & 6 & 26 & 6 & 3 & 144.4 \\
\hline 92 & 6 & 27 & 7 & 3 & 144.4 \\
\hline 92 & 6 & 28 & 5 & 6 & 144.4 \\
\hline 92 & 6 & 29 & 5 & 6 & 144.6 \\
\hline 92 & 6 & 30 & 7 & 6 & 144.4 \\
\hline 92 & 7 & 3 & 7 & 6 & 144.4 \\
\hline 92 & 7 & 4 & 7 & 6 & 144.4 \\
\hline 92 & 7 & 5 & 7 & 6 & 144.4 \\
\hline 92 & 7 & 6 & 6 & 6 & 144.4 \\
\hline 92 & 7 & 7 & 7 & 6 & 144.4 \\
\hline 92 & 7 & 8 & 7 & 6 & 144.4 \\
\hline 92 & 7 & 9 & 7 & 6 & 144.4 \\
\hline 92 & 7 & 10 & 7 & 6 & 144.4 \\
\hline 92 & 7 & 11 & 7 & 6 & 144.4 \\
\hline 92 & 7 & 12 & 7 & 6 & 144.4 \\
\hline 92 & 7 & 13 & 7 & 6 & 144.4 \\
\hline 92 & 7 & 14 & 5 & 6 & 144.4 \\
\hline 92 & 7 & 15 & 7 & 6 & 144.4 \\
\hline 92 & 7 & 16 & 7 & 6 & 144.4 \\
\hline 92 & 7 & 17 & 7 & 6 & 144.4 \\
\hline 92 & 7 & 18 & 7 & 6 & 144.4 \\
\hline 92 & 7 & 19 & 7 & 6 & 144.4 \\
\hline 92 & 7 & 20 & 7 & 6 & 144.4 \\
\hline 92 & 7 & 21 & 7 & 5 & 144.4 \\
\hline 92 & 7 & 22 & 7 & 6 & 144.4 \\
\hline 92 & 7 & 24 & 7 & 5 & 144.4 \\
\hline 92 & 7 & 25 & 7 & 6 & 144.4 \\
\hline 92 & 7 & 26 & 7 & 6 & 144.4 \\
\hline 92 & 7 & 27 & 6 & 5 & 144.4 \\
\hline 92 & 7 & 28 & 6 & 6 & 144.4 \\
\hline 92 & 7 & 29 & 7 & 6 & 144.4 \\
\hline 92 & 7 & 30 & 7 & 6 & 144.4 \\
\hline 92 & 7 & 31 & 7 & 5 & 144.4 \\
\hline 92 & 8 & 1 & 7 & 6 & 144.4 \\
\hline 92 & 8 & 2 & 6 & 6 & 144.5 \\
\hline 92 & 8 & 3 & 7 & 6 & 144.5 \\
\hline
\end{tabular}

Data obtained from WHC Surveillance Analysis Computer System (SACS), February 28, 1994. E-82 


\begin{tabular}{|c|c|c|c|c|c|}
\hline Year & Month & Day & Hour & Minute & Surface Level \\
\hline 92 & 8 & 4 & 7 & 6 & 144.5 \\
\hline 92 & 8 & 5 & 6 & 6 & 144.6 \\
\hline 92 & 8 & 6 & 6 & 6 & 144.6 \\
\hline 92 & 8 & 7 & 7 & 5 & 144.6 \\
\hline 92 & 8 & 8 & 7 & 6 & 144.5 \\
\hline 92 & 8 & 9 & 7 & 6 & 144.5 \\
\hline 92 & 8 & 10 & 7 & 5 & 144.5 \\
\hline 92 & 9 & 19 & 10 & 3 & 144.2 \\
\hline 92 & 9 & 21 & 10 & 3 & 144.2 \\
\hline 92 & 9 & 22 & 7 & 3 & 144.2 \\
\hline 92 & 9 & 23 & 7 & 3 & 144.2 \\
\hline 92 & 9 & 24 & 7 & 3 & 144.2 \\
\hline 92 & 9 & 25 & 7 & 3 & 144.2 \\
\hline 92 & 9 & 28 & 10 & 3 & 144.2 \\
\hline 92 & 10 & 5 & 10 & 3 & 144.2 \\
\hline 92 & 10 & 29 & 7 & 3 & 144.2 \\
\hline 92 & 10 & 30 & 7 & 3 & 144.2 \\
\hline 92 & 10 & 31 & 7 & 3 & 144.2 \\
\hline 92 & 11 & 1 & 7 & 3 & 144.2 \\
\hline 92 & 11 & 2 & 7 & 3 & 144.2 \\
\hline 92 & 11 & 3 & 7 & 3 & 144.1 \\
\hline 92 & 11 & 4 & 7 & 3 & 144.1 \\
\hline 92 & 12 & 2 & 6 & 3 & 144.2 \\
\hline 92 & 12 & 3 & 6 & 3 & 144.2 \\
\hline 92 & 12 & 4 & 7 & 3 & 144.1 \\
\hline 92 & 12 & 6 & 7 & 2 & 144.2 \\
\hline 92 & 12 & 7 & 7 & 3 & 144.2 \\
\hline 92 & 12 & 8 & 7 & 3 & 144.2 \\
\hline 92 & 12 & 9 & 7 & 3 & 144.2 \\
\hline 92 & 12 & 10 & 7 & 3 & 144.2 \\
\hline 92 & 12 & 11 & 7 & 3 & 144.2 \\
\hline 92 & 12 & 13 & 7 & 4 & 144.1 \\
\hline 92 & 12 & 14 & 7 & 3 & 144.2 \\
\hline 92 & 12 & 15 & 7 & 3 & 144.2 \\
\hline 92 & 12 & 16 & 7 & 4 & 144.2 \\
\hline 92 & 12 & 17 & 7 & 3 & 144.2 \\
\hline 92 & 12 & 18 & 7 & 3 & 144.2 \\
\hline 92 & 12 & 19 & 7 & 3 & 144.2 \\
\hline 92 & 12 & 20 & 7 & 4 & 144.2 \\
\hline 92 & 12 & 21 & 7 & 4 & 144.2 \\
\hline 92 & 12 & 23 & 7 & 4 & 144.2 \\
\hline 92 & 12 & 24 & 7 & 3 & 144.2 \\
\hline 92 & 12 & 25 & 7 & 3 & 144.2 \\
\hline 92 & 12 & 26 & 7 & 3 & 144.3 \\
\hline 92 & 12 & 27 & 7 & 3 & 144.3 \\
\hline 92 & 12 & 28 & 7 & 3 & 144.3 \\
\hline 92 & 12 & 30 & 7 & 3 & 144.2 \\
\hline 92 & 12 & 31 & 7 & 3 & 144.2 \\
\hline
\end{tabular}

Data obtained from WHC Surveillance Analysis Computer System (SACS), February 28, 1994. 


\begin{tabular}{|c|c|c|c|c|c|}
\hline Year & Month & Day & Hour & Minute & Surface Level \\
\hline 93 & 1 & 1 & 7 & 4 & 144.2 \\
\hline 93 & 1 & 12 & 7 & 6 & 144.1 \\
\hline 93 & 1 & 13 & 7 & 6 & 144.2 \\
\hline 93 & 1 & 14 & 7 & 3 & 144.2 \\
\hline 93 & 1 & 15 & 7 & 3 & 144.2 \\
\hline 93 & 1 & 17 & 7 & 2 & 144.2 \\
\hline 93 & 1 & 18 & 7 & 2 & 144.2 \\
\hline 93 & 1 & 19 & 7 & 2 & 144.2 \\
\hline 93 & 1 & 20 & 7 & 2 & 144.3 \\
\hline 93 & 1 & 21 & 7 & 2 & 144.2 \\
\hline 93 & 1 & 22 & 7 & 4 & 144.2 \\
\hline 93 & 1 & 23 & 7 & 3 & 144.2 \\
\hline 93 & 1 & 24 & 7 & 3 & 144.2 \\
\hline 93 & 1 & 25 & 6 & 3 & 144.2 \\
\hline 93 & 2 & 5 & 6 & 4 & 144.2 \\
\hline 93 & 2 & 6 & 2 & 3 & 144.2 \\
\hline 93 & 2 & 7 & 7 & 3 & 144.2 \\
\hline 93 & 2 & 16 & 6 & 3 & 144.2 \\
\hline 93 & 2 & 18 & 7 & 3 & 144.2 \\
\hline 93 & 2 & 19 & 7 & 2 & 144.3 \\
\hline 93 & 2 & 20 & 7 & 3 & 144.3 \\
\hline 93 & 2 & 21 & 7 & 4 & 144.2 \\
\hline 93 & 2 & 22 & 7 & 4 & 144.2 \\
\hline 93 & 2 & 23 & 7 & 3 & 144.2 \\
\hline 93 & 2 & 24 & 7 & 4 & 144.2 \\
\hline 93 & 2 & 25 & 7 & 3 & 144.2 \\
\hline 93 & 2 & 26 & 7 & 3 & 144.1 \\
\hline 93 & 2 & 27 & 7 & 4 & 144.1 \\
\hline 93 & 2 & 28 & 7 & 3 & 144.1 \\
\hline 93 & 3 & 1 & 7. & 3 & 144.1 \\
\hline 93 & 3 & 2 & 7 & 4 & 144.1 \\
\hline 93 & 3 & 3 & 7 & 3 & 144.2 \\
\hline 93 & 3 & 4 & 7 & 4 & 144.1 \\
\hline 93 & 3 & 5 & 7 & 4 & 144.1 \\
\hline 93 & 3 & 6 & 7 & 3 & 144.1 \\
\hline 93 & 3 & 9 & 7 & 4 & 144.2 \\
\hline 93 & 3 & 10 & 7 & 5 & 144.2 \\
\hline 93 & 3 & 11 & 7 & 4 & 144.2 \\
\hline 93 & 3 & 12 & 7 & 4 & 144.1 \\
\hline 93 & 3 & 13 & 7 & 3 & 144.2 \\
\hline 93 & 3 & 14 & 7 & 4 & 144.2 \\
\hline 93 & 3 & 15 & 7 & 3 & 144.2 \\
\hline 93 & 3 & 16 & 7 & 4 & 144.2 \\
\hline 93 & 3 & 17 & 7 & 3 & 144.2 \\
\hline 93 & 3 & 18 & 7 & 4 & 144.2 \\
\hline 93 & 3 & 19 & 7 & 3 & 144.2 \\
\hline 93 & 3 & 20 & 7 & 4 & 144.2 \\
\hline 93 & 3 & 21 & 7 & 4 & 144.2 \\
\hline
\end{tabular}

Data obtained from WHC Surveillance Analysis Computer System (SACS), February 28, 1994. 


\begin{tabular}{|c|c|c|c|c|c|}
\hline Year & Month & Day & Hour & Minute & Surface Level \\
\hline 93 & 3 & 22 & 7 & 3 & 144.2 \\
\hline 93 & 3 & 23 & 7 & 3 & 144.3 \\
\hline 93 & 3 & 24 & 7 & 4 & 144.2 \\
\hline 93 & 3 & 25 & 7 & 3 & 144.2 \\
\hline 93 & 3 & 26 & 7 & 3 & 144.2 \\
\hline 93 & 3 & 27 & 7 & 3 & 144.2 \\
\hline 93 & 3 & 28 & 7 & 3 & 144.2 \\
\hline 93 & 3 & 29 & 6 & 3 & 144.2 \\
\hline 93 & 3 & 30 & 7 & 3 & 144.2 \\
\hline 93 & 3 & 31 & 7 & 3 & 144.2 \\
\hline 93 & $\overline{4}$ & 1 & 7 & 3 & 144.2 \\
\hline 93 & 4 & 2 & 7 & 6 & 144.2 \\
\hline 93 & 4 & 3 & 7 & 6 & 144.2 \\
\hline 93 & 4 & 4 & 7 & 5 & 144.3 \\
\hline 93 & 4 & 5 & 7 & 6 & 144.3 \\
\hline 93 & 4 & 6 & 7 & 6 & 144.3 \\
\hline 93 & 4 & 7 & 7 & 6 & 144.2 \\
\hline 93 & 4 & 8 & 7 & 6 & 144.3 \\
\hline 93 & 4 & 9 & 7 & 6 & 144.3 \\
\hline 93 & 4 & 10 & 7 & 5 & 144.3 \\
\hline 93 & $\overline{4}$ & 11 & 7 & 6 & 144.3 \\
\hline 93 & 4 & 12 & 7 & 6 & 144.2 \\
\hline 93 & 4 & 13 & 7 & 6 & 144.3 \\
\hline 93 & 4 & 14 & 7 & 6 & 144.2 \\
\hline 93 & 4 & 15 & 7 & 3 & 144.3 \\
\hline 93 & 4 & 16 & 7 & 6 & 144.3 \\
\hline 93 & 4 & 17 & 7 & 5 & 144.3 \\
\hline 93 & 4 & 18 & 7 & 6 & 144.3 \\
\hline 93 & 4 & 19 & 7 & 6 & 144.2 \\
\hline 93 & 4 & 22 & 7 & 6 & 144.3 \\
\hline 93 & 4 & 23 & 7 & 6 & 144.3 \\
\hline 93 & 4 & 24 & 7 & 5 & 144.3 \\
\hline 93 & 4 & 25 & 7 & 6 & 144.3 \\
\hline 93 & 4 & 26 & 7 & 6 & 144.3 \\
\hline 93 & 4 & 27 & 7 & 6 & 144.2 \\
\hline 93 & 4 & 28 & 7 & 3 & 144.2 \\
\hline 93 & 4 & 29 & 7 & 3 & 144.2 \\
\hline 93 & 4 & 30 & 7 & 6 & 144.2 \\
\hline 93 & 5 & 1 & 7 & 5 & 144.2 \\
\hline 93 & 5 & 2 & 7 & 6 & 144.3 \\
\hline 93 & 5 & 3 & 7 & 6 & 144.3 \\
\hline 93 & 5 & 11 & 7 & 6 & 144.3 \\
\hline 93 & 5 & 12 & 2 & 6 & 144.3 \\
\hline 93 & 5 & 15 & 7 & 6 & 144.3 \\
\hline 93 & 5 & 16 & 7 & 5 & 144.3 \\
\hline 93 & 5 & 17 & 7 & 3 & 144.2 \\
\hline 93 & 5 & 18 & 7 & 3 & 144.2 \\
\hline 93 & 5 & 19 & 7 & 3 & 144.3 \\
\hline
\end{tabular}

Data obtained from WHC Surveillance Analysis Computer System (SACS), February 28, 1994. 


\begin{tabular}{|c|c|c|c|c|c|}
\hline Year & Month & Day & Hour & Minute & Surface Level \\
\hline 93 & 5 & 20 & 7 & 3 & 144.2 \\
\hline 93 & 5 & 21 & 7 & 2 & 144.2 \\
\hline 93 & 5 & 22 & 7 & 3 & 144.2 \\
\hline 93 & 5 & 23 & 7 & 4 & 144.2 \\
\hline 93 & 5 & 24 & 7 & 3 & 144.2 \\
\hline 93 & 5 & 25 & 7 & 3 & 144.2 \\
\hline 93 & 5 & 26 & 7 & 3 & 144.3 \\
\hline 93 & 5 & 27 & 7 & 3 & 144.3 \\
\hline 93 & 5 & 28 & 7 & 3 & 144.2 \\
\hline 93 & 5 & 29 & 7 & 5 & 144.2 \\
\hline 93 & 5 & 30 & 7 & 5 & 144.2 \\
\hline 93 & 5 & 31 & 7 & 6 & 144.3 \\
\hline 93 & 6 & 1 & 7 & 6 & 144.3 \\
\hline 93 & 6 & 2 & 7 & 6 & 144.3 \\
\hline 93 & 6 & 3 & 7 & 6 & 144.3 \\
\hline 93 & 6 & 4 & 7 & 6 & 144.2 \\
\hline 93 & 6 & 5 & 7 & 6 & 144.3 \\
\hline 93 & 6 & 6 & 7 & 5 & 144.3 \\
\hline 93 & 6 & 7 & 7 & 5 & 144.3 \\
\hline 93 & 6 & 8 & 7 & 5 & 144.2 \\
\hline 93 & 6 & 9 & 7 & 5 & 144.3 \\
\hline 93 & 6 & 10 & 7 & 4 & 144.2 \\
\hline 93 & 6 & 11 & 7 & 5 & 144.3 \\
\hline 93 & 6 & 12 & 7 & 5 & 144.2 \\
\hline 93 & 6 & 13 & 7 & 4 & 144.2 \\
\hline 93 & 6 & 14 & 7 & 5 & 144.3 \\
\hline 93 & 6 & 15 & 7 & 5 & 144.3 \\
\hline 93 & 6 & 16 & 7 & 5 & 144.6 \\
\hline 93 & 6 & 25 & 7 & 3 & 144.7 \\
\hline 93 & 6 & 26 & 7 & 3 & 144.8 \\
\hline 93 & 6 & 27 & 7 & 4 & 144.8 \\
\hline 93 & 6 & 28 & 7 & 3 & 144.8 \\
\hline 93 & 6 & 29 & 7 & 4 & 144.8 \\
\hline 93 & 6 & 30 & 6 & 3 & 144.8 \\
\hline 93 & 7 & 1 & 7 & 6 & 144.8 \\
\hline 93 & 7 & 2 & 7 & 6 & 144.8 \\
\hline 93 & 7 & 3 & 7 & 3 & 144.8 \\
\hline 93 & 7 & 4 & 7 & 4 & 144.8 \\
\hline 93 & 7 & 5 & 7 & 3 & 144.8 \\
\hline 93 & 7 & 6 & 7 & 3 & 144.8 \\
\hline 93 & 7 & 7 & 7 & 4 & 144.8 \\
\hline 93 & 7 & 8 & 7 & 4 & 144.8 \\
\hline 93 & 7 & 9 & 7 & 5 & 144.8 \\
\hline 93 & 7 & 10 & 7 & 3 & 144.8 \\
\hline 93 & 7 & 11 & 7 & 3 & 144.8 \\
\hline 93 & 7 & 12 & 7 & 2 & 144.8 \\
\hline 93 & 7 & 13 & 7 & 3 & 144.8 \\
\hline 93 & 7 & 14 & 7 & 6 & 144.8 \\
\hline
\end{tabular}

Data obtained from WHC Surveillance Analysis Computer System (SACS), February 28, 1994. 


\begin{tabular}{|c|c|c|c|c|c|}
\hline Year & Month & Day & Hour & Minute & Surface Level \\
\hline 93 & 7 & 15 & 7 & 6 & 144.8 \\
\hline 93 & 7 & 16 & 7 & 6 & 144.8 \\
\hline 93 & 7 & 17 & 7 & 7 & 144.8 \\
\hline 93 & 7 & 18 & 7 & 6 & 144.9 \\
\hline 93 & 7 & 19 & 7 & 3 & 144.9 \\
\hline 93 & 7 & 20 & 7 & 3 & 144.9 \\
\hline 93 & 7 & 21 & 7 & 3 & 144.9 \\
\hline$\overline{93}$ & 7 & 22 & 7 & 3 & 144.9 \\
\hline 93 & 7 & 23 & 7 & 3 & 144.9 \\
\hline 93 & 7 & 24 & 7 & 3 & 144.9 \\
\hline 93 & 7 & 25 & 7 & 4 & 144.9 \\
\hline 93 & 7 & 26 & 7 & 3 & 144.9 \\
\hline 93 & 7 & 27 & 7 & 3 & 144.9 \\
\hline 93 & 7 & 28 & 7 & 4 & 144.9 \\
\hline 93 & 7 & 29 & 7 & 3 & 144.9 \\
\hline 93 & 7 & 30 & 7 & 3 & 144.9 \\
\hline 93 & 7 & 31 & 7 & 3 & 144.8 \\
\hline 93 & 8 & 1 & 7 & 3 & 144.8 \\
\hline 93 & 8 & 2 & 7 & 6 & 144.9 \\
\hline 93 & 8 & 3 & 7 & 3 & 144.9 \\
\hline 93 & 8 & 4 & 7 & 3 & 144.9 \\
\hline 93 & 8 & 5 & 7 & 4 & 144.9 \\
\hline 93 & 8 & 6 & 7 & 4 & 144.9 \\
\hline 93 & 8 & 8 & 7 & 4 & 144.9 \\
\hline 93 & 8 & 9 & 7 & 4 & 144.9 \\
\hline 93 & 8 & 10 & 7 & 4 & 144.9 \\
\hline 93 & 8 & 11 & 7 & 4 & 144.9 \\
\hline 93 & 8 & 12 & 7 & 4 & 144.9 \\
\hline 93 & 8 & 13 & 7 & 4 & 144.9 \\
\hline 93 & 8 & 14 & 7 & 3 & 144.9 \\
\hline 93 & 8 & 15 & 7 & 4 & 144.9 \\
\hline 93 & 8 & 16 & 7 & 4 & 144.9 \\
\hline 93 & 8 & 17 & 7 & 4 & 144.9 \\
\hline 93 & 8 & 18 & 7 & 3 & 144.9 \\
\hline 93 & 8 & 19 & 7 & 4 & 145 \\
\hline 93 & 8 & 20 & 7 & 4 & 145 \\
\hline 93 & 8 & 21 & 7 & 4 & 145 \\
\hline 93 & 8 & 22 & 7 & 4 & 145 \\
\hline 93 & 8 & 23 & 7 & 4 & 145 \\
\hline 93 & 8 & 24 & 7 & 4 & 144.9 \\
\hline 93 & 8 & 25 & 7 & 4 & 144.9 \\
\hline 93 & 8 & 26 & 7 & 4 & 144.9 \\
\hline 93 & 8 & 27 & 7 & 5 & 144.9 \\
\hline 93 & 8 & 28 & 7 & 4 & 145 \\
\hline 93 & 8 & 29 & 7 & 3 & 144.9 \\
\hline 93 & 8 & 30 & 7 & 4 & 144.9 \\
\hline 93 & 8 & 31 & 7 & 4 & 145 \\
\hline 93 & 9 & 1 & 7 & 4 & 145 \\
\hline
\end{tabular}

Data obtained from WHC Surveillance Analysis Computer System (SACS), February 28, 1994. 


\begin{tabular}{|c|c|c|c|c|c|}
\hline Year & Month & Day & Hour & Minute & Surface Level \\
\hline 93 & 9 & 2 & 7 & 4 & 145 \\
\hline 93 & 9 & 3 & 7 & 4 & 145 \\
\hline 93 & 9 & 4 & 7 & 4 & 145 \\
\hline 93 & 9 & 5 & 7 & 4 & 145 \\
\hline 93 & 9 & 6 & 7 & 4 & 145 \\
\hline 93 & 9 & 7 & 7 & 4 & 145 \\
\hline 93 & 9 & 8 & 7 & 4 & 145 \\
\hline 93 & 9 & 9 & 7 & 4 & 145 \\
\hline 93 & 9 & 10 & 7 & 4 & 145 \\
\hline 93 & 9 & 11 & 7 & 4 & 145 \\
\hline 93 & 9 & 12 & 7 & 4 & 145 \\
\hline 93 & 9 & 13 & 7 & 4 & 145 \\
\hline 93 & 9 & 14 & 7 & 4 & 145 \\
\hline 93 & 9 & 15 & 7 & 4 & 145 \\
\hline 93 & 9 & 16 & 7 & 4 & 145 \\
\hline 93 & 9 & 17 & 7 & 4 & 145 \\
\hline 93 & 9 & 18 & 7 & 4 & 145 \\
\hline 93 & 9 & 19 & 7 & 4 & 145 \\
\hline 93 & 9 & 20 & 7 & 4 & 145 \\
\hline 93 & 9 & 21 & 7 & 4 & 145 \\
\hline 93 & 9 & 22 & 7 & 4 & 144.9 \\
\hline 93 & 9 & 23 & 7 & 4 & 145 \\
\hline 93 & 9 & 24 & 7 & 4 & 145 \\
\hline 93 & 9 & 25 & 7 & 4 & 145 \\
\hline 93 & 9 & 26 & 7 & 4 & 144.9 \\
\hline 93 & 9 & 27 & 7 & 4 & 145 \\
\hline 93 & 9 & 28 & 7 & 4 & 145 \\
\hline 93 & 9 & 29 & 7 & 4 & 145 \\
\hline 93 & 9 & 30 & 7 & 3 & 145 \\
\hline 93 & 10 & 1 & 7 & 6 & 145 \\
\hline 93 & 10 & 2 & 7 & 5 & 145 \\
\hline 93 & 10 & 3 & 7 & 6 & 145 \\
\hline 93 & 10 & 4 & 7 & 6 & 145 \\
\hline 93 & 10 & 5 & 7 & 6 & 145 \\
\hline 93 & 10 & 6 & 7 & 6 & 145 \\
\hline 93 & 10 & 7 & 7 & 5 & 145 \\
\hline 93 & 10 & 8 & 7 & 4 & 145 \\
\hline 93 & 10 & 9 & 7 & 3 & 145 \\
\hline 93 & 10 & 10 & 7 & 10 & 145 \\
\hline 93 & 10 & 11 & 7 & 3 & 145 \\
\hline 93 & 10 & 12 & 7 & 4 & 145 \\
\hline 93 & 10 & 13 & 7 & 3 & 145 \\
\hline 93 & 10 & 14 & 7 & 4 & 145 \\
\hline 93 & 10 & 15 & 7 & 4 & 145 \\
\hline 93 & 10 & 16 & 7 & 4 & 145 \\
\hline 93 & 10 & 17 & 7 & 3 & 145 \\
\hline 93 & 10 & 18 & 7 & 4 & 145 \\
\hline 93 & 10 & 19 & 7 & 4 & 145 \\
\hline
\end{tabular}

Data obtained from WHC Surveillance Analysis Computer System (SACS), February 28, 1994. E-88 


\begin{tabular}{|c|c|c|c|c|c|}
\hline Year & Month & Day & Hour & Minute & Surface Level \\
\hline 93 & 10 & 20 & 7 & 3 & 144.9 \\
\hline 93 & 10 & 21 & 7 & 4 & 145 \\
\hline 93 & 10 & 22 & 7 & 4 & 145 \\
\hline 93 & 11 & 9 & 7 & 3 & 145 \\
\hline 93 & 11 & 10 & 7 & 4 & 145 \\
\hline 93 & 11 & 11 & 7 & 4 & 145 \\
\hline 93 & 11 & 12 & 7 & 4 & 145 \\
\hline 93 & 11 & 13 & 7 & 4 & 145 \\
\hline 93 & 11 & 14 & 7 & 3 & 144.9 \\
\hline 93 & 11 & 15 & 7 & 4 & 145 \\
\hline 93 & 11 & 16 & 7 & 3 & 145 \\
\hline 93 & 11 & 17 & 7 & 4 & 145 \\
\hline 93 & 11 & 18 & 7 & 4 & 144.9 \\
\hline 93 & 11 & 19 & 7 & 4 & 144.9 \\
\hline 93 & 11 & 20 & 7 & 4 & 144.9 \\
\hline 93 & 11 & 21 & 7 & 4 & 145 \\
\hline 93 & 11 & 22 & 7 & 4 & 145 \\
\hline 93 & 11 & 23 & 7 & 4 & 144.9 \\
\hline 93 & 11 & 24 & 7 & 4 & 144.8 \\
\hline 93 & 11 & 25 & 7 & 4 & 144.8 \\
\hline 93 & 11 & 26 & 7 & 4 & 144.8 \\
\hline 93 & 11 & 27 & 7 & 4 & 144.9 \\
\hline 93 & 11 & 28 & 7 & 3 & 144.9 \\
\hline 93 & 11 & 29 & 7 & 4 & 145 \\
\hline 93 & 11 & 30 & 7 & 3 & 145 \\
\hline 93 & 12 & 3 & 15 & 4 & 145 \\
\hline 93 & 12 & 4 & 7 & 5 & 145 \\
\hline 93 & 12 & 5 & 7 & 4 & 144.9 \\
\hline 93 & 12 & 6 & 7 & 5 & 145 \\
\hline 93 & 12 & 7 & 7 & 4 & 145 \\
\hline 93 & 12 & 8 & 7 & 6 & 145 \\
\hline 93 & 12 & 9 & 7 & 4 & 145 \\
\hline 93 & 12 & 10 & 7 & 10 & 145 \\
\hline 93 & 12 & 11 & 7 & 6 & 145 \\
\hline 93 & 12 & 12 & 7 & 6 & 145 \\
\hline 93 & 12 & 13 & 7 & 6 & 145 \\
\hline 93 & 12 & 14 & 7 & 6 & 145.1 \\
\hline 93 & 12 & 15 & 7 & 6 & 145 \\
\hline 93 & 12 & 16 & 7 & 6 & 145 \\
\hline 93 & 12 & 17 & 7 & 5 & 144.9 \\
\hline 93 & 12 & 18 & 7 & 6 & 144.9 \\
\hline 93 & 12 & 19 & 7 & 6 & 144.9 \\
\hline 93 & 12 & 20 & 7 & 6 & 144.9 \\
\hline 93 & 12 & 21 & 7 & 6 & 144.9 \\
\hline 93 & 12 & 22 & 7 & 5 & 144.9 \\
\hline 93 & 12 & 23 & 7 & 10 & 144.9 \\
\hline 93 & 12 & 24 & 7 & 6 & 144.9 \\
\hline 93 & 12 & 25 & 7 & 6 & 144.9 \\
\hline
\end{tabular}

Data obtained from WHC Surveillance Analysis Computer System (SACS), February 28, 1994. 


\begin{tabular}{|c|c|c|c|c|c|}
\hline Year & Month & Day & Hour & Minute & Surface Level \\
\hline 93 & 12 & 26 & 7 & 6 & 145 \\
\hline 93 & 12 & 27 & 7 & 6 & 145 \\
\hline 93 & 12 & 28 & 6 & 6 & 144.9 \\
\hline 93 & 12 & 29 & 7 & 6 & 144.9 \\
\hline 93 & 12 & 30 & 0 & 5 & 144.9 \\
\hline 93 & 12 & 31 & 7 & 6 & 145 \\
\hline 94 & 1 & 1 & 7 & 6 & 145 \\
\hline 94 & 1 & 2 & 7 & 6 & 145 \\
\hline 94 & 1 & 3 & 7 & 7 & 145 \\
\hline 94 & 1 & 4 & 7 & 7 & 145.1 \\
\hline 94 & 1 & 5 & 7 & 6 & 145.2 \\
\hline 94 & 1 & 6 & 7 & 6 & 145.1 \\
\hline 94 & 1 & 7 & 7 & 7 & 145.1 \\
\hline 94 & 1 & 8 & 7 & 6 & 145.1 \\
\hline 94 & 1 & 9 & 7 & 6 & 145.2 \\
\hline 94 & 1 & 10 & 7 & 7 & 145.1 \\
\hline 94 & 1 & 11 & 7 & 7 & 145.1 \\
\hline 94 & 1 & .12 & 7 & 6 & 145.1 \\
\hline 94 & 1 & 13 & 7 & 10 & 145.1 \\
\hline 94 & 1 & 14 & 7 & 6 & 145.1 \\
\hline 94 & 1 & 15 & 7 & 10 & 145.1 \\
\hline 94 & 1 & 16 & 7 & 7 & 145.1 \\
\hline 94 & 1 & 17 & 7 & 7 & 145.1 \\
\hline 94 & 1 & 18 & 7 & 7 & 145.1 \\
\hline 94 & 1 & 19 & 7 & 7 & 145.1 \\
\hline 94 & 1 & 20 & 7 & 7 & 145.1 \\
\hline 94 & 1 & 21 & 7 & 7 & 145.1 \\
\hline 94 & 1 & 22 & 7 & 8 & 145.1 \\
\hline 94 & 1 & 23 & 7 & 7 & 145.2 \\
\hline 94 & 1 & 24 & 7 & 8 & 145.1 \\
\hline 94 & 1 & 25 & 7 & 7 & 145.1 \\
\hline 94 & 1 & 26 & 7 & 6 & 145.1 \\
\hline 94 & 1 & 27 & 7 & 10 & 145.1 \\
\hline 94 & 1 & 28 & 7 & 7 & 145.1 \\
\hline 94 & 1 & 29 & 7 & 7 & 145 \\
\hline 94 & 1 & 30 & 7 & 6 & 144.9 \\
\hline 94 & 1 & 31 & 7 & 7 & 144.9 \\
\hline 94 & 2 & 1 & 7 & 6 & 144.9 \\
\hline 94 & 2 & 2 & 7 & 7 & 145 \\
\hline 94 & 2 & 3 & 7 & 7 & 145 \\
\hline 94 & 2 & 4 & 7 & 7 & 145 \\
\hline 94 & 2 & 5 & 7 & 7 & 145 \\
\hline 94 & 2 & 6 & 7 & 7 & 145 \\
\hline 94 & 2 & 7 & 7 & 6 & 145 \\
\hline 94 & 2 & 8 & 7 & 7 & 145 \\
\hline 94 & 2 & 9 & 7 & 4 & 145 \\
\hline 94 & 2 & 10 & 7 & 4 & 145 \\
\hline 94 & 2 & 11 & 7 & 6 & 145 \\
\hline
\end{tabular}

Data obtained from WHC Surveillance Analysis Computer System (SACS), February 28, 1994. 


\begin{tabular}{|c|c|c|c|c|c|}
\hline Year & Month & Day & Hour & Minute & Surface Level \\
\hline 94 & 2 & 12 & 7 & 3 & 145 \\
\hline 94 & 2 & 13 & 7 & 4 & 145 \\
\hline 94 & 2 & 14 & 7 & 4 & 145 \\
\hline 94 & 2 & 15 & 7 & 4 & 145 \\
\hline 94 & 2 & 16 & 7 & 3 & 145 \\
\hline 94 & 2 & 17 & 7 & 4 & 145.1 \\
\hline 94 & 2 & 18 & 7 & 6 & 145 \\
\hline 94 & 2 & 19 & 7 & 6 & 145 \\
\hline 94 & 2 & 20 & 7 & 7 & 145 \\
\hline 94 & 2 & 21 & 7 & 6 & 145 \\
\hline 94 & 2 & 22 & 7 & 5 & 144.9 \\
\hline 94 & 2 & 23 & 7 & 6 & 145 \\
\hline 94 & 2 & 24 & 7 & 6 & 144.9 \\
\hline & & & & & \\
\hline & & & & & \\
\hline & & & & & \\
\hline & & & & & \\
\hline & & & & & \\
\hline & & & & & \\
\hline & & & & & \\
\hline & & & & & \\
\hline & & & & & \\
\hline & & & & & \\
\hline & & & & & \\
\hline & & & & & \\
\hline & & & & & \\
\hline & & & & & \\
\hline & & & & & \\
\hline & & & & & \\
\hline & & & & & \\
\hline & & & & & \\
\hline & & & & & \\
\hline & & & & & \\
\hline & & & & & \\
\hline & & & & & \\
\hline & & & & & \\
\hline & & & & & \\
\hline & & & & & \\
\hline & & & & & \\
\hline & & & & & \\
\hline & & & & & \\
\hline & & & & & \\
\hline
\end{tabular}

Data obtained from WHC Surveillance Analysis Computer System (SACS), February 28, 1994. E-91 


\begin{tabular}{|c|c|c|c|c|c|}
\hline \multicolumn{6}{|c|}{ Surface Level Data } \\
\hline \multicolumn{6}{|c|}{ Manual Tape } \\
\hline Year & Month & Day & Hour & Minute & Surface Level \\
\hline 93 & 4 & 5 & 10 & 3 & 189.25 \\
\hline 93 & 4 & 12 & 10 & 3 & 153.25 \\
\hline 93 & 4 & 19 & 10 & 3 & 189.5 \\
\hline 93 & 5 & 3 & 10 & 5 & 189.5 \\
\hline 93 & 5 & 10 & 10 & 5 & 189.5 \\
\hline 93 & 5 & 27 & 10 & 5 & 189.5 \\
\hline 93 & 6 & 1 & 10 & 6 & 189.5 \\
\hline & & & & & \\
\hline & & & & & \\
\hline & & & & & \\
\hline & & & & & \\
\hline & & & & & \\
\hline & & & & & \\
\hline & & & & & \\
\hline & & & & & \\
\hline & & & & & \\
\hline & & & & & \\
\hline & & & & & \\
\hline & & & & & \\
\hline & & & & & \\
\hline & & & & & \\
\hline & & & & & \\
\hline & & & & & \\
\hline & & & & & \\
\hline & & & & & \\
\hline & & & & & \\
\hline & & & & & \\
\hline & & & & & \\
\hline & & & & & \\
\hline & & & & & \\
\hline & & & & & \\
\hline & & & & & \\
\hline & & & & & \\
\hline & & & & & \\
\hline & & & & & \\
\hline & & & & & \\
\hline & & & & & \\
\hline & & & & & \\
\hline & & & & & \\
\hline & & & & & \\
\hline & & & & & \\
\hline
\end{tabular}

Data obtained from WHC Surveillance Analysis Computer System (SACS), February 28, 1994. E-92 


\begin{tabular}{|c|c|c|c|c|c|}
\hline \multicolumn{6}{|c|}{ Surface Level Data } \\
\hline \multicolumn{6}{|c|}{ FIC } \\
\hline Year & Month & Day & Hour & Minute & Surface Level \\
\hline 93 & 2 & 8 & 10 & 4 & 172.5 \\
\hline 93 & 3 & 15 & 10 & 3 & 171.4 \\
\hline 93 & 4 & 5 & 10 & 3 & 169.3 \\
\hline 93 & 4 & 12 & 10 & 3 & 172.4 \\
\hline 93 & 4 & 19 & 10 & 3 & 171.8 \\
\hline 93 & 5 & 24 & 10 & 5 & 172.6 \\
\hline & & & & & \\
\hline & & & & & \\
\hline & & & & & \\
\hline & & & & & \\
\hline & & & & & \\
\hline & & & & & \\
\hline & & & & & \\
\hline & & & & & \\
\hline & & & & & \\
\hline & & & & & \\
\hline & & & & & \\
\hline & & & & & \\
\hline & & & & & \\
\hline & & & & & \\
\hline & & & & & \\
\hline & & & & & \\
\hline & & & & & \\
\hline & & & & & \\
\hline & & & & & \\
\hline & & & & & \\
\hline & & & & & \\
\hline & & & & & \\
\hline & & & & & \\
\hline & & & & & \\
\hline & & & & & \\
\hline & & & & & \\
\hline & & & & & \\
\hline & & & & & \\
\hline & & & & & \\
\hline & & & & & \\
\hline & & & & & \\
\hline & & & & & \\
\hline & & & & & \\
\hline & & & & & \\
\hline & & & & & \\
\hline & & & & & \\
\hline & & & & & \\
\hline & & & & & \\
\hline & & & & & \\
\hline & & & & & \\
\hline
\end{tabular}

Data obtained from WHC Surveillance Analysis Computer System (SACS), February 28, 1994. E-93 


\begin{tabular}{|c|c|c|c|c|c|}
\hline \multicolumn{6}{|c|}{ - $\quad$ Surface Level Data } \\
\hline \multicolumn{6}{|c|}{ FIC } \\
\hline Year & Month & Day & Hour & Minute & Surface Level \\
\hline 91 & 1 & 1 & 7 & 3 & 202.9 \\
\hline 91 & 1 & 2 & 7 & 2 & 202.8 \\
\hline 91 & 1 & 3 & 7 & 3 & 202.7 \\
\hline 91 & 1 & 4 & 7 & 2 & 202.8 \\
\hline 91 & 1 & 5 & 7 & 2 & 202.8 \\
\hline 91 & 1 & 6 & 7 & 2 & 202.8 \\
\hline 91 & 1 & 7 & 7 & 2 & 202.9 \\
\hline 91 & 1 & 8 & 7 & 3 & 202.9 \\
\hline 91 & 1 & 9 & 7 & 2 & 203 \\
\hline 91 & 1 & 10 & 7 & 2 & 203 \\
\hline 91 & 1 & 11 & 7 & 3 & 203 \\
\hline 91 & 1 & 12 & 7 & 2 & 203.1 \\
\hline 91 & 1 & 26 & 7 & 3 & 202.8 \\
\hline 91 & 1 & 27 & 7 & 3 & 202.9 \\
\hline 91 & 1 & 28 & 7 & 10 & 203 \\
\hline 91 & 1 & 29 & 7 & 2 & 202.9 \\
\hline 91 & 1 & 30 & 7 & 10 & 203 \\
\hline 91 & 1 & 31 & 7 & 2 & 203 \\
\hline 91 & 2 & 1 & 7 & 2 & 203 \\
\hline 91 & 2 & 15 & 7 & 3 & 202.9 \\
\hline 91 & 2 & 16 & 7 & 2 & 203.1 \\
\hline 91 & 2 & 17 & 7 & 2 & 203.1 \\
\hline 91 & 2 & 18 & 7 & 3 & 203 \\
\hline 91 & 2 & 19 & 7 & 2 & 203 \\
\hline 91 & 2 & 21 & 7 & 2 & 202.9 \\
\hline 91 & 2 & 23 & 7 & 2 & 202.8 \\
\hline 91 & 2 & 24 & 7 & 3 & 202.8 \\
\hline 91 & 2 & 25 & 7 & 3 & 202.8 \\
\hline 91 & 2 & 26 & 7 & 3 & 202.8 \\
\hline 91 & 2 & 27 & 7 & 2 & 203 \\
\hline 91 & 2 & 28 & 7 & 2 & 203.3 \\
\hline 91 & 3 & 1 & 7 & 2 & 203.4 \\
\hline 91 & 3 & 2 & 7 & 2 & 203.2 \\
\hline 91 & 3 & 3 & 7 & 3 & 203.1 \\
\hline 91 & 3 & 4 & 7 & 3 & 203.2 \\
\hline 91 & 3 & 5 & 7 & 3 & 203.1 \\
\hline 91 & 3 & 6 & 7 & 2 & 202.9 \\
\hline 91 & 3 & 7 & 7 & 2 & 202.8 \\
\hline 91 & 3 & 8 & 7 & 2 & 202.7 \\
\hline 91 & 3 & 9 & 7 & 2 & 202.8 \\
\hline 91 & 3 & 10 & 7 & 2 & 202.9 \\
\hline 91 & 3 & 11 & 7 & 2 & 202.8 \\
\hline 91 & 3 & 12 & 7 & 3 & 202.9 \\
\hline 91 & 3 & 13 & 7 & 3 & 202.8 \\
\hline 91 & 3 & 14 & 7 & 3 & 202.8 \\
\hline 91 & 3 & 15 & 7 & 3 & 202.8 \\
\hline
\end{tabular}

Data obtained from WHC Surveillance Analysis Computer System (SACS), February 28, 1994. 


\begin{tabular}{|c|c|c|c|c|c|}
\hline Year & Month & Day & Hour & Minute & Surface Level \\
\hline 91 & 3 & 16 & 7 & 3 & 202.8 \\
\hline 91 & 3 & 17 & 7 & 2 & 202.8 \\
\hline 91 & 3 & 18 & 7 & 3 & 202.8 \\
\hline 91 & 3 & 19 & 7 & 3 & 202.9 \\
\hline 91 & 3 & 20 & 7 & 1 & 203 \\
\hline 91 & 3 & 21 & 7 & 3 & 203 \\
\hline 91 & 3 & 22 & 7 & 2 & 202.9 \\
\hline 91 & 3 & 23 & 7 & 3 & 202.8 \\
\hline 91 & 3 & 24 & 7 & 3 & 202.9 \\
\hline 91 & 3 & 26 & 7 & 3 & 203 \\
\hline 91 & 3 & 27 & 7 & 3 & 202.9 \\
\hline 91 & 3 & 28 & 7 & 3 & 202.8 \\
\hline 91 & 3 & 29 & 7 & 3 & 202.7 \\
\hline 91 & 3 & 30 & 7 & 3 & 202.6 \\
\hline 91 & 3 & 31 & 7 & 3 & 202.7 \\
\hline 91 & 4 & 1 & 7 & 3 & 202.8 \\
\hline 91 & 4 & 2 & 7 & 3 & 202.8 \\
\hline 91 & 4 & 3 & 7 & 3 & 202.7 \\
\hline 91 & 4 & 4 & 7 & 3 & 202.7 \\
\hline 91 & 4 & 5 & 7 & 3 & 202.8 \\
\hline 91 & 4 & 6 & 7 & 3 & 202.8 \\
\hline 91 & 4 & 7 & 7 & 3 & 202.8 \\
\hline 91 & 4 & 8 & 7 & 4 & 202.7 \\
\hline 91 & 4 & 16 & 7 & 10 & 202.8 \\
\hline 91 & 4 & 17 & 7 & 3 & 202.8 \\
\hline 91 & 4 & 18 & 7 & 6 & 202.8 \\
\hline 91 & 4 & 19 & 7 & 5 & 202.8 \\
\hline 91 & 4 & 20 & 7 & 6 & 202.8 \\
\hline 91 & 4 & 21 & 7 & 5 & 202.9 \\
\hline 91 & 4 & 22 & 7 & 6 & 202.9 \\
\hline 91 & 4 & 23 & 7 & 6 & 203 \\
\hline 91 & 4 & 24 & 7 & 5 & 203 \\
\hline 91 & 4 & 25 & 7 & 5 & 203 \\
\hline 91 & 4 & 26 & 3 & 5 & 203 \\
\hline 91 & 4 & 27 & 7 & 5 & 202.9 \\
\hline 91 & 4 & 28 & 7 & 6 & 202.8 \\
\hline 91 & 4 & 29 & 7 & 5 & 202.7 \\
\hline 91 & 4 & 30 & 7 & 6 & 202.7 \\
\hline 91 & 5 & 1 & 6 & 6 & 202.8 \\
\hline 91 & 5 & 2 & 7 & 5 & 202.9 \\
\hline 91 & 5 & 3 & 7 & 6 & 202.9 \\
\hline 91 & 5 & 4 & 7 & 5 & 202.8 \\
\hline 91 & 5 & 5 & 7 & 5 & 202.8 \\
\hline 91 & 5 & 6 & 7 & 5 & 202.8 \\
\hline 91 & 5 & 7 & 7 & 5 & 202.8 \\
\hline 91 & 5 & 8 & 7 & 5 & 203 \\
\hline 91 & 5 & 9 & 7 & 5 & 202.9 \\
\hline 91 & 5 & 10 & 7 & 5 & 202.9 \\
\hline
\end{tabular}

Data obtained from WHC Surveillance Analysis Computer System (SACS), February 28, 1994. 


\begin{tabular}{|c|c|c|c|c|c|}
\hline Year & Month & Day & Hour & Minute & Surface Level \\
\hline 91 & 5 & 11 & 7 & 5 & 202.9 \\
\hline 91 & 5 & 12 & 7 & 5 & 202.9 \\
\hline 91 & 5 & 13 & 7 & 5 & 202.9 \\
\hline 91 & 5 & 14 & 7 & 5 & 202.9 \\
\hline 91 & 5 & 15 & 7 & 5 & 202.8 \\
\hline 91 & 5 & 16 & 7 & 6 & 202.9 \\
\hline 91 & 5 & 17 & 7 & 6 & 203 \\
\hline 91 & 5 & 18 & 7 & 6 & 203 \\
\hline 91 & 5 & 19 & 7 & 5 & 203 \\
\hline 91 & 5 & 20 & 7 & 6 & 202.9 \\
\hline 91 & 5 & 21 & 7 & 6 & 203 \\
\hline 91 & 5 & 22 & 7 & 5 & 202.9 \\
\hline 91 & 5 & 23 & 7 & 5 & 202.9 \\
\hline 91 & 5 & 24 & 7 & 5 & 202.9 \\
\hline 91 & 5 & 25 & 7 & 5 & 202.9 \\
\hline 91 & 5 & 26 & 7 & 5 & 202.8 \\
\hline 91 & 5 & 27 & 7 & 5 & 202.8 \\
\hline 91 & 5 & 28 & 7 & 5 & 202.9 \\
\hline 91 & 5 & 29 & 7 & 5 & 203 \\
\hline 91 & 5 & 30 & 7 & 5 & 203 \\
\hline 91 & 5 & 31 & 7 & 5 & 203 \\
\hline 91 & 6 & 1 & 7 & 5 & 202.9 \\
\hline 91 & 6 & 2 & 7 & 5 & 203 \\
\hline 91 & 6 & 3 & 7 & 5 & 202.9 \\
\hline 91 & 6 & 4 & 7 & 5 & 202.8 \\
\hline 91 & 6 & 5 & 7 & 5 & 202.8 \\
\hline 91 & 6 & 6 & 0 & 10 & 202.8 \\
\hline 91 & 6 & 10 & 7 & 6 & 202.8 \\
\hline 91 & 6 & 11 & 7 & 5 & 203 \\
\hline 91 & 6 & 12 & 7 & 4 & 202.9 \\
\hline 91 & 6 & 13 & 7 & 5 & 202.9 \\
\hline 91 & 6 & 14 & 7 & 5 & 202.9 \\
\hline 91 & 6 & 15 & 7 & 5 & 202.9 \\
\hline 91 & 6 & 17 & 7 & 5 & 202.8 \\
\hline 91 & 6 & 18 & 7 & 5 & 202.8 \\
\hline 91 & 6 & 19 & 7 & 5 & 203 \\
\hline 91 & 6 & 20 & 2 & 6 & 203 \\
\hline 91 & 6 & 22 & 7 & 5 & 203 \\
\hline 91 & 6 & 23 & 7 & 5 & 203 \\
\hline 91 & 6 & 24 & 7 & 5 & 203.1 \\
\hline 91 & 6 & 25 & 7 & 6 & 203.1 \\
\hline 91 & 6 & 26 & 7 & 3 & 203.2 \\
\hline 91 & 6 & 27 & 7 & 2 & 203.2 \\
\hline 91 & 6 & 28 & 7 & 3 & 203.3 \\
\hline 91 & 6 & 29 & 7 & 2 & 203.2 \\
\hline 91 & 7 & 2 & 7 & 6 & 203 \\
\hline 91 & 7 & 3 & 7 & 6 & 203 \\
\hline 91 & 7 & 4 & 7 & 10 & 203 \\
\hline
\end{tabular}

Data obtained from WHC Surveillance Analysis Computer System (SACS), February 28, 1994. 


\begin{tabular}{|c|c|c|c|c|c|}
\hline Year & Month & Day & Hour & Minute & Surface Level \\
\hline 91 & 7 & 5 & 7 & 6 & 203.1 \\
\hline 91 & 7 & 6 & 7 & 6 & 203.1 \\
\hline 91 & 7 & 7 & 7 & 10 & 203 \\
\hline 91 & 7 & 8 & 7 & 6 & 203.1 \\
\hline 91 & 7 & 9 & 7 & 6 & 203.1 \\
\hline 91 & 7 & 10 & 6 & 6 & 203.2 \\
\hline 91 & 7 & 11 & 7 & 6 & 203.1 \\
\hline 91 & 7 & 12 & 7 & 6 & 203.1 \\
\hline 91 & 7 & 13 & 7 & 6 & 203.1 \\
\hline 91 & 7 & 14 & 7 & 6 & 203.1 \\
\hline 91 & 7 & 15 & 7 & 6 & 203.2 \\
\hline 91 & 7 & 16 & 7 & 6 & 203.1 \\
\hline 91 & 7 & 17 & 7 & 5 & 203.1 \\
\hline 91 & 7 & 18 & 7 & 5 & 203.1 \\
\hline 91 & 7 & 19 & 7 & 5 & 203.1 \\
\hline 91 & 7 & 20 & 7 & 5 & 203.2 \\
\hline 91 & 7 & 21 & 7 & 5 & 203.2 \\
\hline 91 & 7 & 22 & 7 & 5 & 203.1 \\
\hline 91 & 7 & 23 & 7 & 5 & 203.1 \\
\hline 91 & 7 & 24 & 7 & 5 & 203.2 \\
\hline 91 & 7 & 25 & 7 & 1 & 203.2 \\
\hline 91 & 7 & 26 & 7 & 2 & 203.1 \\
\hline 91 & 7 & 27 & 7 & 2 & 203.1 \\
\hline 91 & 7 & 28 & 7 & 2 & 203 \\
\hline 91 & 7 & 29 & 7 & 2 & 203.1 \\
\hline 91 & 7 & 30 & 7 & 2 & 203.1 \\
\hline 91 & 7 & 31 & 7 & 2 & 203.1 \\
\hline 91 & 8 & 4 & 7 & 2 & 203.2 \\
\hline 91 & 8 & 5 & 7 & 2 & 203.3 \\
\hline 91 & 8 & 6 & 7 & 2 & 203.3 \\
\hline 91 & 8 & 7 & 7 & 6 & 203.3 \\
\hline 91 & 8 & 8 & 7 & 6 & 203.3 \\
\hline 91 & 8 & 9 & 7 & 4 & 203.2 \\
\hline 91 & 8 & 10 & 7 & 4 & 203.2 \\
\hline 91 & 8 & 11 & 7 & 4 & 203.1 \\
\hline 91 & 8 & 12 & 7 & 4 & 203.1 \\
\hline 91 & 8 & 13 & 7 & 4 & 203.1 \\
\hline 91 & 8 & 14 & 7 & 4 & 203.1 \\
\hline 91 & 8 & 15 & 7 & 3 & 203.1 \\
\hline 91 & 8 & 17 & 7 & 2 & 203.3 \\
\hline 91 & 8 & 18 & 7 & 3 & 203.2 \\
\hline 91 & 8 & 19 & 7 & 2 & 203.2 \\
\hline 91 & 8 & 20 & 7 & 4 & 203.2 \\
\hline 91 & 8 & 21 & 7 & 3 & 203.2 \\
\hline 91 & 8 & 22 & 7 & 3 & 203.2 \\
\hline 91 & 8 & 23 & 7 & 2 & 203.2 \\
\hline 91 & 8 & 24 & 7 & 2 & 203.2 \\
\hline 91 & 8 & 25 & 7 & 2 & 203.2 \\
\hline
\end{tabular}

Data obtained from WHC Surveillance Analysis Computer System (SACS), February 28, 1994. 


\begin{tabular}{|c|c|c|c|c|c|}
\hline Year & Month & Day & Hour & Minute & Surface Level \\
\hline 91 & 8 & 26 & 7 & 3 & 203.2 \\
\hline 91 & 8 & 27 & 7 & 3 & 203.3 \\
\hline 91 & 8 & 28 & 7 & 3 & 203.3 \\
\hline 91 & 8 & 29 & 7 & 2 & 203.2 \\
\hline 91 & 8 & 30 & 7 & 10 & 203.2 \\
\hline 91 & 8 & 31 & 7 & 2 & 203.2 \\
\hline 91 & 9 & 1 & 7 & 2 & 203.2 \\
\hline 91 & 9 & 2 & 7 & 2 & 203.1 \\
\hline 91 & 9 & 3 & 7 & 2 & 203 \\
\hline 91 & 9 & 4 & 7 & 3 & 203 \\
\hline 91 & 9 & 5 & 7 & 2 & 203.2 \\
\hline 91 & 9 & 6 & 7 & 2 & 203.3 \\
\hline 91 & 9 & 7 & 7 & 2 & 203.3 \\
\hline 91 & 9 & 8 & 7 & 2 & 203.2 \\
\hline 91 & 9 & 9 & 7 & 3 & 203.2 \\
\hline 91 & 9 & 10 & 7 & 2 & 203.2 \\
\hline 91 & 9 & 11 & 7 & 3 & 203.2 \\
\hline 91 & 9 & 12 & 7 & 2 & 203.2 \\
\hline 91 & 9 & 13 & 7 & 2 & 203.3 \\
\hline 91 & 9 & 14 & 7 & 3 & 203.3 \\
\hline 91 & 9 & 15 & 7 & 2 & 203.2 \\
\hline 91 & 9 & 16 & 7 & 3 & 203.1 \\
\hline 91 & 9 & 17 & 7 & 3 & 203.1 \\
\hline 91 & 9 & 18 & 7 & 3 & 203.1 \\
\hline 91 & 9 & 19 & 7 & 3 & 203.2 \\
\hline 91 & 9 & 20 & 7 & 2 & 203.2 \\
\hline 91 & 9 & 21 & 7 & 2 & 203.2 \\
\hline 91 & 9 & 22 & 7 & 3 & 203 \\
\hline 91 & 9 & 23 & 7 & 3 & 203 \\
\hline 91 & 9 & 24 & 7 & 2 & 203 \\
\hline 91 & 9 & 25 & 7 & 3 & 203.1 \\
\hline 91 & 9 & 26 & 7 & 3 & 203.2 \\
\hline 91 & 9 & 27 & 7 & 2 & 203.3 \\
\hline 91 & 9 & 28 & 7 & 2 & 203.4 \\
\hline 91 & 9 & 29 & 7 & 3 & 203.2 \\
\hline 91 & 9 & 30 & 7 & 3 & 203.1 \\
\hline 91 & 10 & 1 & 7 & 2 & 203.2 \\
\hline 91 & 10 & 2 & 7 & 2 & 203.1 \\
\hline 91 & 10 & 3 & 7 & 2 & 203.2 \\
\hline 91 & 10 & 4 & 7 & 3 & 203.1 \\
\hline 91 & 10 & 6 & 6 & 2 & 203.2 \\
\hline 91 & 10 & 7 & 6 & 2 & 203.2 \\
\hline 91 & 10 & 8 & 7 & 2 & 203.1 \\
\hline 91 & 10 & 9 & 7 & 2 & 203.1 \\
\hline 91 & 10 & 10 & 7 & 2 & 203 \\
\hline 91 & 10 & 11 & 7 & 2 & 203.1 \\
\hline 91 & 10 & 12 & 7 & 2 & 203.3 \\
\hline 91 & 10 & 13 & 7 & 3 & 203.1 \\
\hline
\end{tabular}

Data obtained from WHC Surveillance Analysis Computer System (SACS), February 28, 1994. 


\begin{tabular}{|c|c|c|c|c|c|}
\hline Year & Month & Day & Hour & Minute & Surface Level \\
\hline 91 & 10 & 14 & 7 & 2 & 203.1 \\
\hline 91 & 10 & 15 & 7 & 2 & 203.2 \\
\hline 91 & 10 & 16 & 7 & 3 & 203.5 \\
\hline 91 & 10 & 17 & 7 & 5 & 203.2 \\
\hline 91 & 10 & 18 & 7 & 6 & 203 \\
\hline 91 & 10 & 20 & 7 & 2 & 203 \\
\hline 91 & 10 & 21 & 7 & 2 & 203.4 \\
\hline 91 & 10 & 22 & 7 & 3 & 203.4 \\
\hline 91 & 10 & 23 & 7 & 2 & 203.3 \\
\hline 91 & 10 & 24 & 6 & 3 & 203.3 \\
\hline 91 & 10 & 25 & 7 & 3 & 203.4 \\
\hline 91 & 10 & 27 & 4 & 3 & 203.4 \\
\hline 91 & 10 & 28 & 10 & 2 & 203 \\
\hline 92 & 1 & 26 & 7 & 10 & 203.1 \\
\hline 92 & 1 & 27 & 7 & 2 & 203.1 \\
\hline 92 & 1 & 27 & 10 & 2 & 203.1 \\
\hline 92 & 1 & 28 & 7 & 3 & 203.3 \\
\hline 92 & 1 & 29 & 7 & 3 & 203.1 \\
\hline 92 & 1 & 30 & 7 & 3 & 203.1 \\
\hline 92 & 1 & 31 & 7 & 3 & 203.3 \\
\hline 92 & 2 & 1 & 7 & 2 & 203.4 \\
\hline 92 & 2 & 2 & 7 & 3 & 203.3 \\
\hline 92 & 2 & 3 & 7 & 3 & 203.1 \\
\hline 92 & 2 & 3 & 10 & 2 & 203.1 \\
\hline 92 & 2 & 4 & 7 & 3 & 203.1 \\
\hline 92 & 2 & 5 & 7 & 2 & 203.2 \\
\hline 92 & 2 & 8 & 7 & 4 & 203.6 \\
\hline 92 & 2 & 9 & 7 & 3 & 203.7 \\
\hline 92 & 2 & 10 & 7 & 4 & 203.8 \\
\hline 92 & 2 & 10 & 10 & 2 & 203.8 \\
\hline 92 & 2 & 11 & 7 & 3 & 203.7 \\
\hline 92 & 2 & 12 & 7 & 3 & 203.6 \\
\hline 92 & 2 & 13 & 7 & 3 & 203.7 \\
\hline 92 & 2 & 14 & 7 & 3 & 203.6 \\
\hline 92 & 2 & 15 & 7 & 3 & 203.6 \\
\hline 92 & 2 & 16 & 7 & 3 & 203.4 \\
\hline 92 & 2 & 17 & 7 & 3 & 203.3 \\
\hline 92 & 2 & 17 & 10 & 2 & 203.3 \\
\hline 92 & 2 & 18 & 7 & 3 & 203.3 \\
\hline 92 & 2 & 19 & 7 & 3 & 203.3 \\
\hline 92 & 2 & 21 & 7 & 3 & 203.2 \\
\hline 92 & 7 & 3 & 7 & 6 & 203.1 \\
\hline 92 & 7 & 4 & 7 & 6 & 203.1 \\
\hline 92 & 7 & 5 & 7 & 6 & 203.1 \\
\hline 92 & 7 & 6 & 7 & 6 & 203 \\
\hline 92 & 7 & 6 & 10 & 2 & 203 \\
\hline 92 & 7 & 7 & 7 & 6 & 203 \\
\hline 92 & 7 & 8 & 7 & 6 & 203 \\
\hline
\end{tabular}

Data obtained from WHC Surveillance Analysis Computer System (SACS), February 28, 1994. 


\begin{tabular}{|c|c|c|c|c|c|}
\hline Year & Month & Day & Hour & Minute & Surface Level \\
\hline 92 & 7 & 9 & 7 & 6 & 202.9 \\
\hline 92 & 7 & 10 & 7 & 6 & 203 \\
\hline 92 & 7 & 11 & 7 & 6 & 203.1 \\
\hline 92 & 7 & 12 & 7 & 6 & 203.1 \\
\hline 92 & 7 & 13 & 7 & 6 & 203.4 \\
\hline 92 & 7 & 13 & 10 & 2 & 203.4 \\
\hline 92 & 7 & 14 & 7 & 6 & 203.3 \\
\hline 92 & 7 & 15 & 7 & 6 & 203.2 \\
\hline 92 & 7 & 16 & 7 & 6 & 203.2 \\
\hline 92 & 7 & 17 & 7 & 6 & 203.3 \\
\hline 92 & 7 & 18 & 7 & 6 & 203.1 \\
\hline 92 & 7 & 19 & 7 & 6 & 203.1 \\
\hline 92 & 7 & 20 & 7 & 6 & 203.1 \\
\hline 92 & 7 & 20 & 10 & 2 & 203.1 \\
\hline 92 & 7 & 21 & 7 & 5 & 203.1 \\
\hline 92 & 7 & 22 & 7 & 6 & 203.2 \\
\hline 92 & 7 & 24 & 7 & 5 & 203 \\
\hline 92 & 7 & 25 & 7 & 6 & 203 \\
\hline 92 & 7 & 26 & 7 & 6 & 203 \\
\hline 92 & 7 & 27 & 7 & 5 & 203.1 \\
\hline 92 & 7 & 27 & 10 & 2 & 203.1 \\
\hline 92 & 7 & 28 & 7 & 6 & 203.1 \\
\hline 92 & 7 & 29 & 7 & 6 & 203.1 \\
\hline 92 & 7 & 30 & 7 & 6 & 203.1 \\
\hline 92 & 7 & 31 & 7 & 5 & 203.2 \\
\hline 92 & 8 & 1 & 7 & 6 & 203.2 \\
\hline 92 & 8 & 2 & 6 & 6 & 203.1 \\
\hline 92 & 8 & 3 & 7 & 6 & 203.2 \\
\hline 92 & 8 & 3 & 10 & 2 & 203.2 \\
\hline 92 & 8 & 4 & 7 & 6 & 203.2 \\
\hline 92 & 8 & 5 & 7 & 5 & 203.2 \\
\hline 92 & 8 & 6 & 7 & 5 & 203.2 \\
\hline 92 & 8 & 7 & 7 & 5 & 203.2 \\
\hline 92 & 8 & 8 & 7 & 6 & 203.1 \\
\hline 92 & 8 & 9 & 7 & 6 & 203 \\
\hline 92 & 8 & 10 & 7 & 5 & 203.1 \\
\hline 92 & 8 & 10 & 10 & 2 & 203.1 \\
\hline 92 & 8 & 11 & 7 & 6 & 203.1 \\
\hline 92 & 8 & 12 & 7 & 6 & 203.2 \\
\hline 92 & 8 & 13 & 7 & 6 & 203.2 \\
\hline 92 & 8 & 14 & 7 & 6 & 203.2 \\
\hline 92 & 8 & 15 & 7 & 6 & 203.1 \\
\hline 92 & 8 & 16 & 7 & 6 & 203.2 \\
\hline 92 & 8 & 17 & 7 & 6 & 203.2 \\
\hline 92 & 8 & 17 & 10 & 2 & 203.2 \\
\hline 92 & 8 & 18 & 7 & 6 & 203.2 \\
\hline 92 & 8 & 19 & 7 & 6 & 203.3 \\
\hline 92 & 8 & 20 & 7 & 6 & 203.2 \\
\hline
\end{tabular}

Data obtained from WHC Surveillance Analysis Computer System (SACS), February 28, 1994. 


\begin{tabular}{|c|c|c|c|c|c|}
\hline Year & Month & Day & Hour & Minute & Surface Level \\
\hline 92 & 8 & 21 & 7 & 5 & 203.3 \\
\hline 92 & 8 & 22 & 7 & 4 & 203.3 \\
\hline 92 & 8 & 23 & 7 & 4 & 203.1 \\
\hline 92 & 8 & 24 & 7 & 4 & 203 \\
\hline 92 & 8 & 24 & 10 & 2 & 203.2 \\
\hline 92 & 8 & 25 & 7 & 4 & 203 \\
\hline 92 & 8 & 26 & 7 & 3 & 203 \\
\hline 92 & 8 & 27 & 7 & 4 & 203 \\
\hline 92 & 8 & 28 & 7 & 2 & 203.1 \\
\hline 92 & 8 & 29 & 7 & 2 & 203.3 \\
\hline 92 & 8 & 30 & 7 & 2 & 203.1 \\
\hline 92 & 8 & 31 & 7 & 2 & 203.2 \\
\hline 92 & 9 & 1 & 7 & 2 & 203.2 \\
\hline 92 & 9 & 2 & 7 & 2 & 203.2 \\
\hline 92 & 9 & 3 & 7 & 4 & 203.2 \\
\hline 92 & 9 & 22 & 7 & 3 & 203.3 \\
\hline 92 & 9 & 23 & 7 & 3 & 203.4 \\
\hline 92 & 9 & 24 & 7 & 3 & 203.4 \\
\hline 92 & 9 & 25 & 7 & 3 & 203.2 \\
\hline 92 & 10 & 29 & 7 & 3 & 203.4 \\
\hline 92 & 10 & 30 & 7 & 3 & 203.5 \\
\hline 92 & 10 & 31 & 7 & 3 & 203.3 \\
\hline 92 & 11 & 1 & 7 & 3 & 203.1 \\
\hline 92 & 11 & 2 & 7 & 3 & 203.1 \\
\hline 92 & 11 & 3 & 7 & 3 & 202.9 \\
\hline 92 & 11 & 4 & 7 & 3 & 202.9 \\
\hline 92 & 12 & 2 & 6 & 3 & 203 \\
\hline 92 & 12 & 3 & 6 & 3 & 202.9 \\
\hline 92 & 12 & 4 & 7 & 3 & 202.8 \\
\hline 92 & 12 & 6 & 7 & 2 & 203 \\
\hline 92 & 12 & 7 & 7 & 3 & 203.2 \\
\hline 92 & 12 & 8 & 7 & 3 & 203.3 \\
\hline 92 & 12 & 9 & 7 & 3 & 203.3 \\
\hline 92 & 12 & 10 & 7 & 3 & 203.3 \\
\hline 92 & 12 & 11 & 7 & 3 & 203.3 \\
\hline 92 & 12 & 13 & 7 & 4 & 202.8 \\
\hline 92 & 12 & 15 & 7 & 3 & 203 \\
\hline 92 & 12 & 16 & 7 & 4 & 202.9 \\
\hline 92 & 12 & 17 & 7 & 3 & 203.3 \\
\hline 92 & 12 & 18 & 7 & 3 & 203.1 \\
\hline 92 & 12 & 19 & 7 & 3 & 203 \\
\hline 92 & 12 & 20 & 7 & 4 & 203.2 \\
\hline 92 & 12 & 21 & 7 & 4 & 203.2 \\
\hline 92 & 12 & 23 & 7 & 4 & 202.9 \\
\hline 92 & 12 & 24 & 7 & 3 & 202.9 \\
\hline 92 & 12 & 25 & 7 & 3 & 202.8 \\
\hline 92 & 12 & 26 & 7 & 3 & 203.1 \\
\hline 92 & 12 & 27 & 7 & 3 & 203.2 \\
\hline
\end{tabular}

Data obtained from WHC Surveillance Analysis Computer System (SACS), February 28, 1994. 


\begin{tabular}{|c|c|c|c|c|c|}
\hline Year & Month & Day & Hour & Minute & Surface Level \\
\hline 92 & 12 & 28 & 7 & 3 & 203.4 \\
\hline 92 & 12 & 30 & 7 & 3 & 203.2 \\
\hline 92 & 12 & 31 & 7 & 3 & 203 \\
\hline 93 & 1 & 1 & 7 & 4 & 203 \\
\hline 93 & 1 & 12 & 7 & 6 & 202.8 \\
\hline 93 & 1 & 13 & 7 & 6 & 203 \\
\hline 93 & 1 & 14 & 7 & 3 & 203.1 \\
\hline 93 & 1 & 15 & 7 & 3 & 203 \\
\hline 93 & 1 & 17 & 7 & 2 & 203 \\
\hline 93 & 1 & 18 & 7 & 2 & 203 \\
\hline 93 & 1 & 19 & 7 & 2 & 203 \\
\hline 93 & 1 & 20 & 7 & 2 & 203.2 \\
\hline 93 & 1 & 21 & 7 & 2 & 203.1 \\
\hline 93 & 1 & 22 & 7 & 4 & 203.1 \\
\hline 93 & 1 & 23 & 7 & 3 & 202.8 \\
\hline 93 & 1 & 24 & 7 & 3 & 202.8 \\
\hline 93 & 1 & 25 & 6 & 3 & 202.9 \\
\hline 93 & 2 & 5 & 6 & 4 & 202.8 \\
\hline 93 & 2 & 6 & 2 & 3 & 202.8 \\
\hline 93 & 2 & 7 & 7 & 3 & 202.9 \\
\hline 93 & 2 & 16 & 6 & 3 & 202.8 \\
\hline 93 & 2 & 18 & 7 & 3 & 202.8 \\
\hline 93 & 2 & 19 & 7 & 2 & 203.2 \\
\hline 93 & 2 & 20 & 7 & 3 & 203.4 \\
\hline 93 & 2 & 21 & 7 & 4 & 203.2 \\
\hline 93 & 2 & 22 & 7 & 4 & 203 \\
\hline 93 & 2 & 23 & 7 & 3 & 202.9 \\
\hline 93 & 2 & 24 & 7 & 4 & 203 \\
\hline 93 & 2 & 25 & 7 & 3 & 202.8 \\
\hline 93 & 2 & 26 & 7 & 3 & 202.7 \\
\hline 93 & 2 & 27 & 7 & 4 & 202.6 \\
\hline 93 & 2 & 28 & 7 & 3 & 202.6 \\
\hline 93 & 3 & 1 & 7 & 3 & 202.6 \\
\hline 93 & 3 & 2 & 7 & 4 & 202.6 \\
\hline 93 & 3 & 3 & 7 & 3 & 202.7 \\
\hline 93 & 3 & 4 & 7 & 4 & 202.6 \\
\hline 93 & 3 & 5 & 7 & 4 & 202.6 \\
\hline 93 & 3 & 6 & 7 & 3 & 202.6 \\
\hline 93 & 3 & 9 & 7 & 4 & 202.6 \\
\hline 93 & 3 & 10 & 7 & 5 & 202.6 \\
\hline 93 & 3 & 11 & 7 & 4 & 202.6 \\
\hline 93 & 3 & 12 & 7 & 4 & 202.5 \\
\hline 93 & 3 & 13 & 7 & 3 & 202.6 \\
\hline 93 & 3 & 14 & 7 & 4 & 202.8 \\
\hline 93 & 3 & 15 & 7 & 3 & 203.1 \\
\hline 93 & 3 & 16 & 7 & 4 & 202.9 \\
\hline 93 & 3 & 17 & 7 & 3 & 202.9 \\
\hline 93 & 3 & 18 & 7 & 4 & 203 \\
\hline
\end{tabular}

Data obtained from WHC Surveillance Analysis Computer System (SACS), February 28, 1994. 


\begin{tabular}{|c|c|c|c|c|c|}
\hline Year & Month & Day & Hour & Minute & Surface Level \\
\hline 93 & 3 & 19 & 7 & 3 & 202.8 \\
\hline 93 & 3 & 20 & 7 & 4 & 202.9 \\
\hline 93 & 3 & 21 & 7 & 4 & 202.8 \\
\hline 93 & 3 & 22 & 7 & 3 & 202.9 \\
\hline 93 & 3 & 23 & 7 & 3 & 203.1 \\
\hline 93 & 3 & 24 & 7 & 4 & 203 \\
\hline 93 & 3 & 25 & 7 & 3 & 202.9 \\
\hline 93 & 3 & 26 & 7 & 3 & 203 \\
\hline 93 & 3 & 27 & 7 & 3 & 203 \\
\hline 93 & 3 & 28 & 7 & 3 & 203 \\
\hline$\overline{93}$ & 3 & 29 & 6 & 3 & 203 \\
\hline 93 & 3 & 30 & 7 & 3 & 202.9 \\
\hline 93 & 3 & 31 & 7 & 3 & 202.8 \\
\hline 93 & 4 & 1 & 7 & 3 & 203.1 \\
\hline 93 & 4 & 2 & 7 & 6 & 203 \\
\hline 93 & 4 & 3 & 7 & 6 & 203 \\
\hline 93 & 4 & 4 & 7 & 5 & 203 \\
\hline 93 & 4 & 5 & 7 & 6 & 203 \\
\hline 93 & 4 & 6 & 7 & 6 & 203 \\
\hline 93 & 4 & 7 & 7 & 6 & 202.9 \\
\hline 93 & 4 & 8 & 7 & 6 & 203 \\
\hline 93 & 4 & 9 & 7 & 6 & 203 \\
\hline 93 & 4 & 10 & 7 & 5 & 203 \\
\hline 93 & 4 & 11 & 7 & 6 & 203 \\
\hline 93 & 4 & 12 & 7 & 6 & 202.9 \\
\hline 93 & 4 & 13 & 7 & 6 & 202.9 \\
\hline 93 & 4 & 14 & 7 & 6 & 202.9 \\
\hline 93 & 4 & 15 & 7 & 3 & 203 \\
\hline 93 & 4 & 16 & 7 & 6 & 203 \\
\hline 93 & 4 & 17 & 7 & 5 & 203 \\
\hline 93 & 4 & 18 & 7 & 6 & 203 \\
\hline 93 & 4 & 19 & 7 & 6 & 202.9 \\
\hline 93 & 4 & 22 & 7 & 6 & 202.9 \\
\hline 93 & 4 & 23 & 7 & 6 & 203 \\
\hline 93 & 4 & 24 & 7 & 5 & 203 \\
\hline 93 & 4 & 25 & 7 & 6 & 203 \\
\hline 93 & 4 & 27 & 7 & 6 & 202.8 \\
\hline 93 & 4 & 28 & 7 & 3 & 202.8 \\
\hline 93 & 4 & 29 & 7 & 3 & 202.8 \\
\hline 93 & 4 & 30 & 7 & 6 & 202.8 \\
\hline 93 & 5 & 1 & 7 & 5 & 202.8 \\
\hline 93 & 5 & 2 & 7 & 6 & 202.8 \\
\hline 93 & 5 & 3 & 7 & 6 & 203.1 \\
\hline 93 & 5 & 11 & 7 & 6 & 203 \\
\hline 93 & 5 & 12 & 2 & 6 & 203.1 \\
\hline 93 & 5 & 15 & 7 & 6 & 203 \\
\hline 93 & 5 & 16 & 7 & 5 & 203 \\
\hline 93 & 5 & 17 & 7 & 3 & 203 \\
\hline
\end{tabular}

Data obtained from WHC Surveillance Analysis Computer System (SACS), February 28, 1994. 


\begin{tabular}{|c|c|c|c|c|c|}
\hline Year & Month & Day & Hour & Minute & Surface Level \\
\hline 93 & 5 & 18 & 7 & 3 & 203 \\
\hline 93 & 5 & 19 & 7 & 3 & 203 \\
\hline 93 & 5 & 20 & 7 & 3 & 203.1 \\
\hline 93 & 5 & 21 & 7 & 2 & 203 \\
\hline 93 & 5 & 22 & 7 & 3 & 203 \\
\hline 93 & 5 & 23 & 7 & 4 & 203.1 \\
\hline 93 & 5 & 24 & 7 & 3 & 203.2 \\
\hline 93 & 5 & 25 & 7 & 3 & 203.3 \\
\hline 93 & 5 & 26 & 7 & 3 & 203 \\
\hline 93 & 5 & 27 & 7 & 3 & 203.1 \\
\hline 93 & 5 & 28 & 7 & 3 & 203.1 \\
\hline 93 & 5 & 29 & 7 & 5 & 203 \\
\hline 93 & 5 & 31 & 7 & 6 & 203.1 \\
\hline 93 & 6 & 1 & 7 & 6 & 203.1 \\
\hline 93 & 6 & 2 & 7 & 6 & 203.1 \\
\hline 93 & 6 & 3 & 7 & 6 & 203 \\
\hline 93 & 6 & 4 & 7 & 6 & 203.1 \\
\hline 93 & 6 & 5 & 7 & 6 & 203.1 \\
\hline 93 & 6 & 6 & 7 & 5 & 203.3 \\
\hline 93 & 6 & 7 & 7 & 5 & 203.2 \\
\hline 93 & 6 & 8 & 7 & 5 & 203 \\
\hline 93 & 6 & 9 & 7 & 5 & 203 \\
\hline 93 & 6 & 10 & 7 & 4 & 202.9 \\
\hline 93 & 6 & 11 & 7 & 5 & 203 \\
\hline 93 & 6 & 13 & 7 & 4 & 202.8 \\
\hline 93 & 6 & 14 & 7 & 5 & 202.9 \\
\hline 93 & 6 & 16 & 7 & 5 & 202.9 \\
\hline 93 & 6 & 17 & 7 & 5 & 202.9 \\
\hline 93 & 6 & 18 & 7 & 6 & 202.9 \\
\hline 93 & 6 & 25 & 7 & 3 & 202.8 \\
\hline 93 & 6 & 26 & 7 & 3 & 203 \\
\hline 93 & 6 & 27 & 7 & 4 & 203 \\
\hline 93 & 6 & 28 & 7 & 3 & 203.1 \\
\hline 93 & 6 & 29 & 7 & 4 & 203 \\
\hline 93 & 6 & 30 & 6 & 3 & 203 \\
\hline 93 & 7 & 1 & 7 & 6 & 203 \\
\hline 93 & 7 & 2 & 7 & 6 & 203.1 \\
\hline 93 & 7 & 3 & 7 & 3 & 203.1 \\
\hline 93 & 7 & 4 & 7 & 4 & 203.1 \\
\hline 93 & 7 & 5 & 7 & 3 & 203 \\
\hline 93 & 7 & 6 & 7 & 3 & 203 \\
\hline 93 & 7 & 7 & 7 & 4 & 203 \\
\hline 93 & 7 & 8 & 7 & 4 & 203 \\
\hline 93 & 7 & 9 & 7 & 5 & 203.1 \\
\hline 93 & 7 & 10 & 7 & 3 & 203 \\
\hline 93 & 7 & 11 & 7 & 3 & 203 \\
\hline 93 & 7 & 12 & 7 & 2 & 203 \\
\hline 93 & 7 & 13 & 7 & 3 & 203 \\
\hline
\end{tabular}

Data obtained from WHC Surveillance Analysis Computer System (SACS), February 28, 1994. 


\begin{tabular}{|c|c|c|c|c|c|}
\hline Year & Month & Day & Hour & Minute & Surface Level \\
\hline 93 & 7 & 14 & 7 & 6 & 203 \\
\hline 93 & 7 & 15 & 7 & 6 & 203 \\
\hline 93 & 7 & 16 & 7 & 6 & 203 \\
\hline 93 & 7 & 17 & 7 & 7 & 203 \\
\hline 93 & 7 & 18 & 16 & 6 & 202.9 \\
\hline 93 & 7 & 19 & 7 & 3 & 203 \\
\hline 93 & 8 & 6 & 15 & 4 & 203.2 \\
\hline 93 & 8 & 6 & 16 & 4 & 203.1 \\
\hline 93 & 8 & 7 & 16 & 4 & 203.25 \\
\hline 93 & 8 & 8 & 7 & 4 & 203.2 \\
\hline 93 & 8 & 9 & 7 & 4 & 203.1 \\
\hline 93 & 8 & 10 & 7 & 4 & 203.1 \\
\hline 93 & 8 & 11 & 7 & 4 & 203 \\
\hline 93 & 8 & 12 & 7 & 4 & 203 \\
\hline 93 & 8 & 13 & 7 & 4 & 203.1 \\
\hline 93 & 8 & 14 & 7 & 3 & 203.2 \\
\hline 93 & 8 & 15 & 7 & 4 & 203.2 \\
\hline 93 & 8 & 16 & 7 & 4 & 203.2 \\
\hline 93 & 8 & 17 & 7 & 4 & 203.1 \\
\hline 93 & 8 & 18 & 7 & 3 & 203 \\
\hline 93 & 8 & 19 & 7 & 4 & 203.1 \\
\hline 93 & 8 & 20 & 7 & 4 & 203.2 \\
\hline 93 & 8 & 21 & 7 & 4 & 203.2 \\
\hline 93 & 8 & 22 & 7 & 4 & 203.1 \\
\hline 93 & 8 & 23 & 7 & 4 & 203.3 \\
\hline 93 & 8 & 24 & 7 & 4 & 203.1 \\
\hline 93 & 8 & 25 & 7 & 4 & 203 \\
\hline 93 & 8 & 26 & 7 & 4 & 203 \\
\hline 93 & 8 & 27 & 7 & 5 & 203 \\
\hline 93 & 8 & 28 & 7 & 4 & 203 \\
\hline 93 & 8 & 29 & 7 & 3 & 203 \\
\hline 93 & 8 & 30 & 7 & 4 & 203 \\
\hline 93 & 8 & 31 & 7 & 4 & 203.1 \\
\hline 93 & 9 & 1 & 7 & 4 & 203.2 \\
\hline 93 & 9 & 2 & 7 & 4 & 203.1 \\
\hline 93 & 9 & 2 & 16 & 4 & 203.1 \\
\hline 93 & 9 & 3 & 7 & 4 & 203.1 \\
\hline 93 & 9 & 3 & 16 & 4 & 203.1 \\
\hline 93 & 9 & 4 & 7 & 4 & 203.1 \\
\hline 93 & 9 & 4 & 16 & 4 & 203.1 \\
\hline 93 & 9 & 5 & 7 & 4 & 203.2 \\
\hline 93 & 9 & 5 & 16 & 4 & 203.2 \\
\hline 93 & 9 & 6 & 7 & 4 & 203.2 \\
\hline 93 & 9 & 6 & 16 & 4 & 203.2 \\
\hline 93 & 9 & 7 & 7 & 4 & 203.1 \\
\hline 93 & 9 & 7 & 16 & 4 & 203.15 \\
\hline 93 & 9 & 8 & 7 & 4 & 203.1 \\
\hline 93 & 9 & 8 & 16 & 4 & 203.1 \\
\hline
\end{tabular}

Data obtained from WHC Surveillance Analysis Computer System (SACS), February 28, 1994. 


\begin{tabular}{|c|c|c|c|c|c|}
\hline Year & Month & Day & Hour & Minute & Surface Level \\
\hline 93 & 9 & 9 & 7 & 4 & 203.2 \\
\hline 93 & 9 & 9 & 16 & 4 & 203.2 \\
\hline 93 & 9 & 10 & 7 & 4 & 203.3 \\
\hline 93 & 9 & 10 & 16 & 4 & 203.2 \\
\hline 93 & 9 & 11 & 7 & 4 & 203.3 \\
\hline 93 & 9 & 11 & 16 & 4 & 203.3 \\
\hline 93 & 9 & 12 & 7 & 4 & 203.2 \\
\hline 93 & 9 & 12 & 16 & 4 & 203.3 \\
\hline 93 & 9 & 13 & 7 & 4 & 203.1 \\
\hline 93 & 9 & 13 & 16 & 4 & 203.2 \\
\hline 93 & 9 & 14 & 7 & 4 & 203.2 \\
\hline 93 & 9 & 14 & 16 & 4 & 203.2 \\
\hline 93 & 9 & 15 & 7 & 4 & 203.2 \\
\hline 93 & 9 & 15 & 16 & 4 & 203.2 \\
\hline 93 & 9 & 16 & 7 & 4 & 203.1 \\
\hline 93 & 9 & 16 & 16 & 4 & 203.2 \\
\hline 93 & 9 & 17 & 7 & 4 & 203.1 \\
\hline 93 & 9 & 17 & 16 & 4 & 203.2 \\
\hline 93 & 9 & 18 & 7 & 4 & 203.2 \\
\hline 93 & 9 & 18 & 16 & 4 & 203.2 \\
\hline 93 & 9 & 19 & 7 & 4 & 203.2 \\
\hline 93 & 9 & 19 & 16 & 4 & 203.2 \\
\hline 93 & 9 & 20 & 7 & 4 & 203.2 \\
\hline 93 & 9 & 20 & 16 & 4 & 203.2 \\
\hline 93 & 9 & 21 & 7 & 4 & 203.1 \\
\hline 93 & 9 & 21 & 16 & 4 & 203.1 \\
\hline 93 & 9 & 22 & 7 & 4 & 203 \\
\hline 93 & 9 & 22 & 16 & 4 & 203 \\
\hline 93 & 9 & 23 & 7 & 4 & 203 \\
\hline 93 & 9 & 23 & 16 & 4 & 203 \\
\hline 93 & 9 & 24 & 7 & 4 & 203 \\
\hline 93 & 9 & 24 & 16 & 4 & 203.1 \\
\hline 93 & 9 & 25 & 7 & 4 & 203.1 \\
\hline 93 & 9 & 25 & 16 & 4 & 203.1 \\
\hline 93 & 9 & 26 & 7 & 4 & 203 \\
\hline 93 & 9 & 26 & 16 & 4 & 203 \\
\hline 93 & 9 & 27 & 7 & 4 & 203 \\
\hline 93 & 9 & 27 & 16 & 4 & 203 \\
\hline 93 & 9 & 28 & 7 & 4 & 203 \\
\hline 93 & 9 & 28 & 16 & 4 & 203.1 \\
\hline 93 & 9 & 29 & 7 & 4 & 203.1 \\
\hline 93 & 9 & 29 & 16 & 4 & 203 \\
\hline 93 & 9 & 30 & 7 & 3 & 203.2 \\
\hline 93 & 10 & 1 & 7 & 6 & 203.2 \\
\hline 93 & 10 & 2 & 7 & 5 & 203.1 \\
\hline 93 & 10 & 3 & 7 & 6 & 203.1 \\
\hline 93 & 10 & 4 & 7 & 6 & 203.2 \\
\hline 93 & 10 & 5 & 7 & 6 & 203.3 \\
\hline
\end{tabular}

Data obtained from WHC Surveillance Analysis Computer System (SACS), February 28, 1994. 


\begin{tabular}{|c|c|c|c|c|c|}
\hline Year & Month & Day & Hour & Minute & Surface Level \\
\hline 93 & 10 & 6 & 7 & 6 & 203.3 \\
\hline 93 & 10 & 7 & 7 & 5 & 203.2 \\
\hline 93 & 10 & 8 & 7 & 4 & 203.1 \\
\hline 93 & 10 & 9 & 7 & 3 & 203.1 \\
\hline 93 & 10 & 10 & 7 & 10 & 203.1 \\
\hline 93 & 10 & 11 & 7 & 3 & 203.2 \\
\hline 93 & 10 & 12 & 7 & 4 & 203.1 \\
\hline 93 & 10 & 13 & 7 & 3 & 203.1 \\
\hline 93 & 10 & 14 & 7 & 4 & 203.2 \\
\hline 93 & 10 & 15 & 7 & 4 & 203.3 \\
\hline 93 & 10 & 16 & 7 & 4 & 203.3 \\
\hline 93 & 10 & 17 & 7 & 3 & 203.2 \\
\hline 93 & 10 & 18 & 7 & 4 & 203.1 \\
\hline 93 & 10 & 19 & 7 & 4 & 203.1 \\
\hline 93 & 10 & 20 & 7 & 3 & 203 \\
\hline 93 & 10 & 21 & 7 & 4 & 203 \\
\hline 93 & 10 & 22 & 7 & 4 & 203.1 \\
\hline 93 & 10 & 23 & 16 & 4 & 203.15 \\
\hline 93 & 10 & 24 & 16 & 4 & 203.2 \\
\hline 93 & 10 & 25 & 16 & 4 & 203 \\
\hline 93 & 10 & 26 & 16 & 4 & 202.85 \\
\hline 93 & 10 & 27 & 16 & 4 & 203 \\
\hline 93 & 10 & 28 & 16 & 4 & 203.1 \\
\hline 93 & 10 & 29 & 16 & 4 & 203.1 \\
\hline 93 & 10 & 30 & 16 & 4 & 203.05 \\
\hline 93 & 10 & 31 & 16 & 4 & 203.2 \\
\hline 93 & 11 & 1 & 16 & 3 & 203.1 \\
\hline 93 & 11 & 2 & 16 & 3 & 203 \\
\hline 93 & 11 & 3 & 16 & 3 & 203.2 \\
\hline 93 & 11 & 4 & 16 & 3 & 203.1 \\
\hline 93 & 11 & 5 & 16 & 3 & 203 \\
\hline 93 & 11 & 6 & 16 & 3 & 202.95 \\
\hline 93 & 11 & 8 & 16 & 3 & 203.1 \\
\hline 93 & 11 & 9 & 7 & 3 & 203.1 \\
\hline 93 & 11 & 10 & 7 & 4 & 203.1 \\
\hline 93 & 11 & 11 & 7 & 4 & 203.2 \\
\hline 93 & 11 & 12 & 7 & 4 & 203.2 \\
\hline 93 & 11 & 13 & 7 & 4 & 203.2 \\
\hline 93 & 11 & 14 & 7 & 3 & 203 \\
\hline 93 & 11 & 15 & 7 & 4 & 203.1 \\
\hline 93 & 11 & 16 & 7 & 3 & 203.1 \\
\hline 93 & 11 & 17 & 7 & 4 & 203.2 \\
\hline 93 & 11 & 17 & 16 & 4 & 203.2 \\
\hline 93 & 11 & 18 & 7 & 4 & 203.1 \\
\hline 93 & 11 & 19 & 7 & 4 & 202.9 \\
\hline 93 & 11 & 19 & 16 & 4 & 202.9 \\
\hline 93 & 11 & 20 & 7 & 4 & 203 \\
\hline 93 & 11 & 20 & 16 & 4 & 203 \\
\hline
\end{tabular}

Data obtained from WHC Surveillance Analysis Computer System (SACS), February 28, 1994. 


\begin{tabular}{|c|c|c|c|c|c|}
\hline Year & Month & Day & Hour & Minute & Surface Level \\
\hline 93 & 11 & 21 & 7 & 4 & 203.3 \\
\hline 93 & 11 & 21 & 16 & 4 & 203.2 \\
\hline 93 & 11 & 22 & 7 & 4 & 203.3 \\
\hline 93 & 11 & 22 & 16 & 4 & 203.4 \\
\hline 93 & 11 & 23 & 7 & 4 & 203 \\
\hline 93 & 11 & 23 & 16 & 4 & 203.1 \\
\hline 93 & 11 & 24 & 7 & 4 & 202.7 \\
\hline 93 & 11 & 24 & 16 & 4 & 202.8 \\
\hline 93 & 11 & 25 & 7 & 4 & 202.6 \\
\hline 93 & 11 & 25 & 16 & 4 & 202.7 \\
\hline 93 & 11 & 26 & 7 & 4 & 202.6 \\
\hline 93 & 11 & 26 & 16 & 4 & 202.6 \\
\hline 93 & 11 & 27 & 7 & 4 & 202.7 \\
\hline 93 & 11 & 28 & 7 & 3 & 202.8 \\
\hline 93 & 11 & 29 & 7 & 4 & 203.1 \\
\hline 93 & 11 & 30 & 7 & 3 & 203.1 \\
\hline 93 & 12 & 1 & 16 & 4 & 203.1 \\
\hline 93 & 12 & 2 & 16 & 4 & 202.9 \\
\hline 93 & 12 & 3 & 16 & 4 & 202.9 \\
\hline 93 & 12 & 5 & 7 & 4 & 203 \\
\hline 93 & 12 & 6 & 7 & 5 & 203.1 \\
\hline 93 & 12 & 7 & 7 & 4 & 203.2 \\
\hline 93 & 12 & 8 & 7 & 6 & 203.4 \\
\hline 93 & 12 & 9 & 7 & 4 & 203.3 \\
\hline 93 & 12 & 10 & 7 & 10 & 203.3 \\
\hline 93 & 12 & 11 & 7 & 6 & 203.4 \\
\hline 93 & 12 & 12 & 7 & 6 & 203.3 \\
\hline 93 & 12 & 13 & 7 & 6 & 203.1 \\
\hline 93 & 12 & 14 & 7 & 6 & 203.2 \\
\hline 93 & 12 & 15 & 7 & 6 & 203 \\
\hline 93 & 12 & 16 & 7 & 6 & 202.9 \\
\hline 93 & 12 & 17 & 7 & 5 & 202.8 \\
\hline 93 & 12 & 18 & 7 & 6 & 202.7 \\
\hline 93 & 12 & 19 & 7 & 6 & 202.7 \\
\hline 93 & 12 & 20 & 7 & 6 & 202.7 \\
\hline 93 & 12 & 21 & 7 & 6 & 202.6 \\
\hline 93 & 12 & 22 & 7 & 5 & 202.6 \\
\hline 93 & 12 & 23 & 7 & 10 & 202.5 \\
\hline 93 & 12 & 24 & 7 & 6 & 202.5 \\
\hline 93 & 12 & 25 & 7 & 6 & 202.6 \\
\hline 93 & 12 & 26 & 7 & 6 & 202.8 \\
\hline 93 & 12 & 27 & 7 & 6 & 202.8 \\
\hline 93 & 12 & 28 & 6 & 6 & 202.7 \\
\hline 93 & 12 & 29 & 7 & 6 & 202.6 \\
\hline 93 & 12 & 30 & 0 & 5 & 202.7 \\
\hline 93 & 12 & 31 & 16 & 6 & 202.9 \\
\hline 94 & 1 & 1 & 7 & 6 & 203 \\
\hline 94 & 1 & 2 & 7 & 6 & 202.9 \\
\hline
\end{tabular}

Data obtained from WHC Surveillance Analysis Computer System (SACS), February 28, 1994. 


\begin{tabular}{|c|c|c|c|c|c|}
\hline Year & Month & Day & Hour & Minute & Surface Level \\
\hline 94 & 1 & 3 & 7 & 7 & $* 200.9$ \\
\hline 94 & 1 & 4 & 7 & 7 & 203.1 \\
\hline 94 & 1 & 5 & 7 & 6 & 203.3 \\
\hline 94 & 1 & 6 & 7 & 6 & 203.1 \\
\hline 94 & 1 & 7 & 7 & 7 & 203 \\
\hline 94 & 1 & 8 & 7 & 6 & 203.1 \\
\hline 94 & 1 & 9 & 7 & 6 & 203.2 \\
\hline 94 & 1 & 10 & 7 & 7 & 203.1 \\
\hline 94 & 1 & 11 & 7 & 7 & 203.1 \\
\hline 94 & 1 & 12 & 7 & 6 & *200.9 \\
\hline 94 & 1 & 13 & 7 & 10 & 202.9 \\
\hline 94 & 1 & 14 & 7 & 6 & 202.9 \\
\hline 94 & 1 & 15 & 7 & 10 & 203 \\
\hline 94 & 1 & 16 & 7 & 7 & 203 \\
\hline 94 & 1 & 17 & 7 & 7 & 203 \\
\hline 94 & 1 & 18 & 7 & 7 & 203 \\
\hline 94 & 1 & 19 & 7 & 7 & 203 \\
\hline 94 & 1 & 20 & 6 & 7 & 203 \\
\hline 94 & 1 & 21 & 7 & 7 & 203 \\
\hline 94 & 1 & 22 & 7 & 8 & 203 \\
\hline 94 & 1 & 23 & 7 & 7 & 203.2 \\
\hline 94 & 1 & 24 & 7 & 8 & 203.2 \\
\hline 94 & 1 & 25 & 7 & 7 & 203.2 \\
\hline 94 & 1 & 26 & 7 & 6 & 203.1 \\
\hline 94 & 1 & 27 & 7 & 10 & 202.9 \\
\hline 94 & 1 & 28 & 7 & 7 & 202.8 \\
\hline 94 & 1 & 29 & 7 & 7 & 202.7 \\
\hline 94 & 1 & 30 & 7 & 6 & 202.6 \\
\hline 94 & 1 & 31 & 7 & 7 & 202.6 \\
\hline 94 & 2 & 1 & 7 & 6 & 202.6 \\
\hline 94 & 2 & 2 & 7 & 7 & 202.7 \\
\hline 94 & 2 & 3 & 7 & 7 & 202.8 \\
\hline 94 & 2 & 4 & 7 & 7 & 202.8 \\
\hline 94 & 2 & 5 & 7 & 7 & 202.8 \\
\hline 94 & 2 & 6 & 7 & 7 & 203 \\
\hline 94 & 2 & 7 & 7 & 6 & 203.1 \\
\hline 94 & 2 & 8 & 7 & 7 & 203 \\
\hline 94 & 2 & 9 & 7 & 4 & 203 \\
\hline 94 & 2 & 10 & 7 & 4 & 203 \\
\hline 94 & 2 & 11 & 7 & 6 & 203 \\
\hline 94 & 2 & 12 & 7 & 3 & 202.9 \\
\hline 94 & 2 & 13 & 7 & 4 & 203 \\
\hline 94 & 2 & 14 & 7 & 4 & 203 \\
\hline 94 & 2 & 15 & 7 & 4 & 203.1 \\
\hline 94 & 2 & 16 & 7 & 3 & 203.2 \\
\hline 94 & 2 & 17 & 7 & 4 & 203.4 \\
\hline 94 & 2 & 18 & 7 & 6 & 203.4 \\
\hline 94 & 2 & 19 & 7 & 6 & 203.2 \\
\hline
\end{tabular}

Data obtained from WHC Surveillance Analysis Computer System (SACS), February 28, 1994. 


\begin{tabular}{|c|c|c|c|c|c|}
\hline Year & Month & Day & Hour & Minute & Surface Level \\
\hline 94 & 2 & 20 & 7 & 7 & 203.1 \\
\hline 94 & 2 & 21 & 7 & 6 & 203 \\
\hline 94 & 2 & 22 & 7 & 5 & 202.9 \\
\hline 94 & 2 & 23 & 7 & 6 & 203 \\
\hline 94 & 2 & 24 & 7 & 6 & 202.9 \\
\hline & & & & & \\
\hline & & & & & \\
\hline & & & & & \\
\hline & & & & & \\
\hline & & & & & \\
\hline & & & & & \\
\hline & & & & & \\
\hline & & & & & \\
\hline & & & & & \\
\hline & & & & & \\
\hline & & & & & \\
\hline & & & & & \\
\hline & & & & & \\
\hline & & & & & \\
\hline & & & & & \\
\hline & & & & & \\
\hline & & & & & \\
\hline & & & & & \\
\hline & & & & & \\
\hline & & & & & \\
\hline & & & & & \\
\hline & & & & & \\
\hline & & & & & \\
\hline & & & & & \\
\hline & & & & & \\
\hline & & & & & \\
\hline & & & & & \\
\hline & & & & & \\
\hline & & & & & \\
\hline & & & & & \\
\hline & & & & & \\
\hline & & & & & \\
\hline & & & & & \\
\hline & & & & & \\
\hline & & & & & \\
\hline & & & & & \\
\hline
\end{tabular}

Data obtained from WHC Surveillance Analysis Computer System (SACS), February 28, 1994. 


\begin{tabular}{|c|c|c|c|c|c|}
\hline \multicolumn{6}{|c|}{ Surface Level Data } \\
\hline \multicolumn{6}{|c|}{ FIC } \\
\hline Year & Month & Day & Hour & Minute & Surface Level \\
\hline 93 & 1 & 5 & 10 & 4 & 195.8 \\
\hline 93 & 1 & 12 & 10 & 4 & 195.8 \\
\hline 93 & 1 & 18 & 10 & 4 & 195.85 \\
\hline 93 & 1 & 25 & 10 & 4 & 195.85 \\
\hline 93 & 2 & 1 & 10 & 4 & 195.8 \\
\hline 93 & 2 & 8 & 10 & 4 & 195.9 \\
\hline 93 & 2 & 16 & 10 & 4 & 195.85 \\
\hline 93 & 2 & 22 & 10 & 4 & 195.8 \\
\hline 93 & 3 & 1 & 10 & 3 & 195.8 \\
\hline 93 & 3 & 8 & 10 & 3 & 195.9 \\
\hline 93 & 3 & 15 & 10 & 3 & 195.8 \\
\hline 93 & 3 & 22 & 10 & 3 & 195.8 \\
\hline 93 & 3 & 29 & 10 & 3 & 195.8 \\
\hline 93 & 4 & 5 & 10 & 3 & 195.7 \\
\hline 93 & 4 & 12 & 10 & 3 & 195.7 \\
\hline 93 & 4 & 19 & 10 & 3 & 195.7 \\
\hline 93 & 4 & 26 & 10 & 3 & 195.8 \\
\hline 93 & 5 & 3 & 10 & 5 & 195.8 \\
\hline 93 & 5 & 10 & 10 & 5 & 195.8 \\
\hline 93 & 5 & 17 & 10 & 5 & 195.7 \\
\hline 93 & 5 & 24 & 10 & 5 & 195.7 \\
\hline 93 & 6 & 1 & 10 & 6 & 195.8 \\
\hline & & & & & \\
\hline & & & & & \\
\hline & & & & & \\
\hline & & & & & \\
\hline & & & & & \\
\hline & & & & & \\
\hline & & & & & \\
\hline & & & & & \\
\hline & & & & & \\
\hline & & & & & \\
\hline & & & & & \\
\hline & & & & & \\
\hline & & & & & \\
\hline & & & & & \\
\hline & & & & & \\
\hline & & & & & \\
\hline & & & & & \\
\hline & & & & & \\
\hline & & & & & \\
\hline & & & & & \\
\hline & & & & & \\
\hline & & & & & \\
\hline & & & & & \\
\hline
\end{tabular}

Data obtained from WHC Surveillance Analysis Computer System (SACS), February 28, 1994. 
WHC-SD-WM-ER-323, Rev. 0

$\widehat{N}$
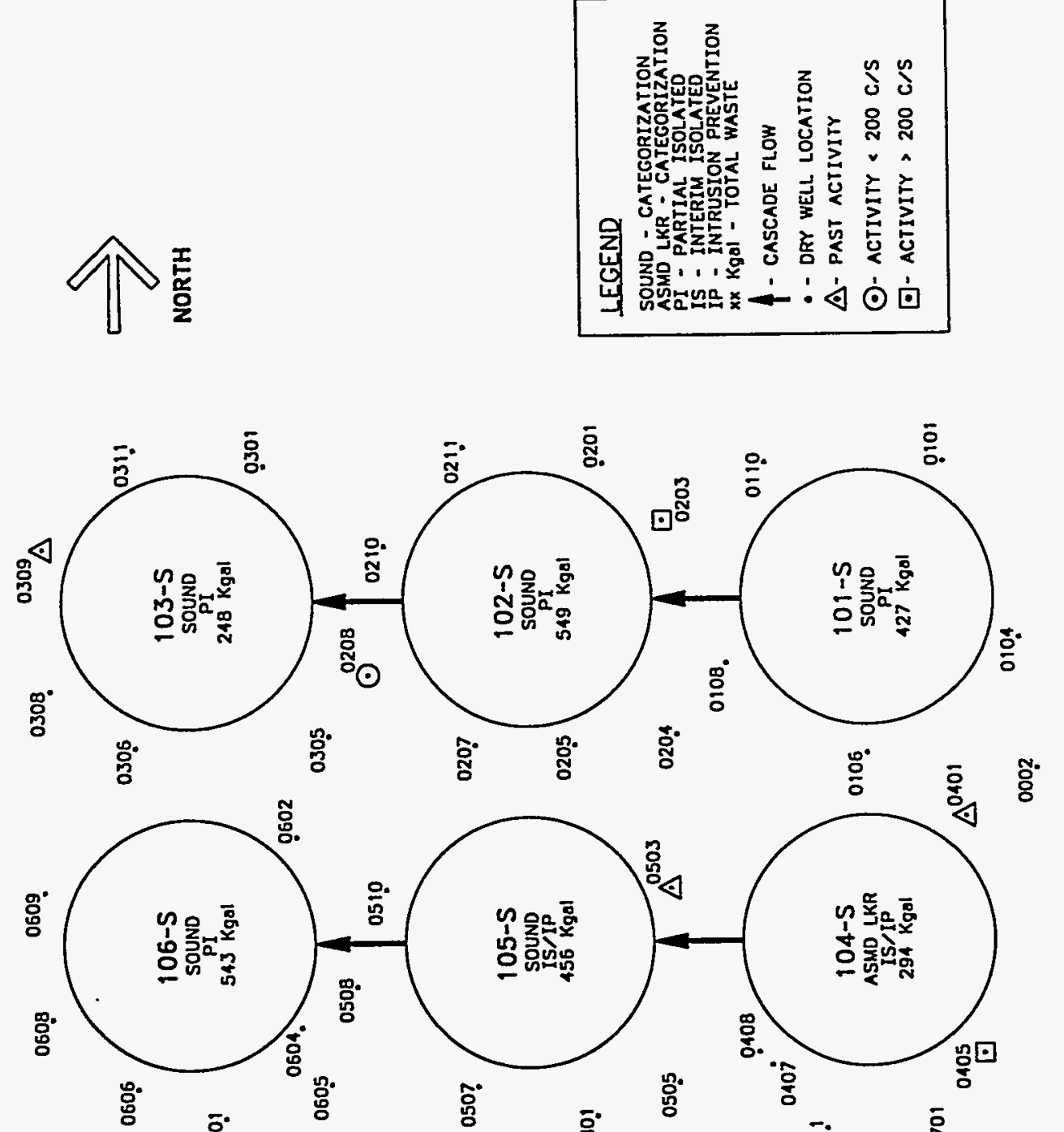

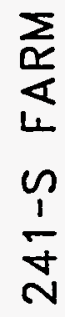
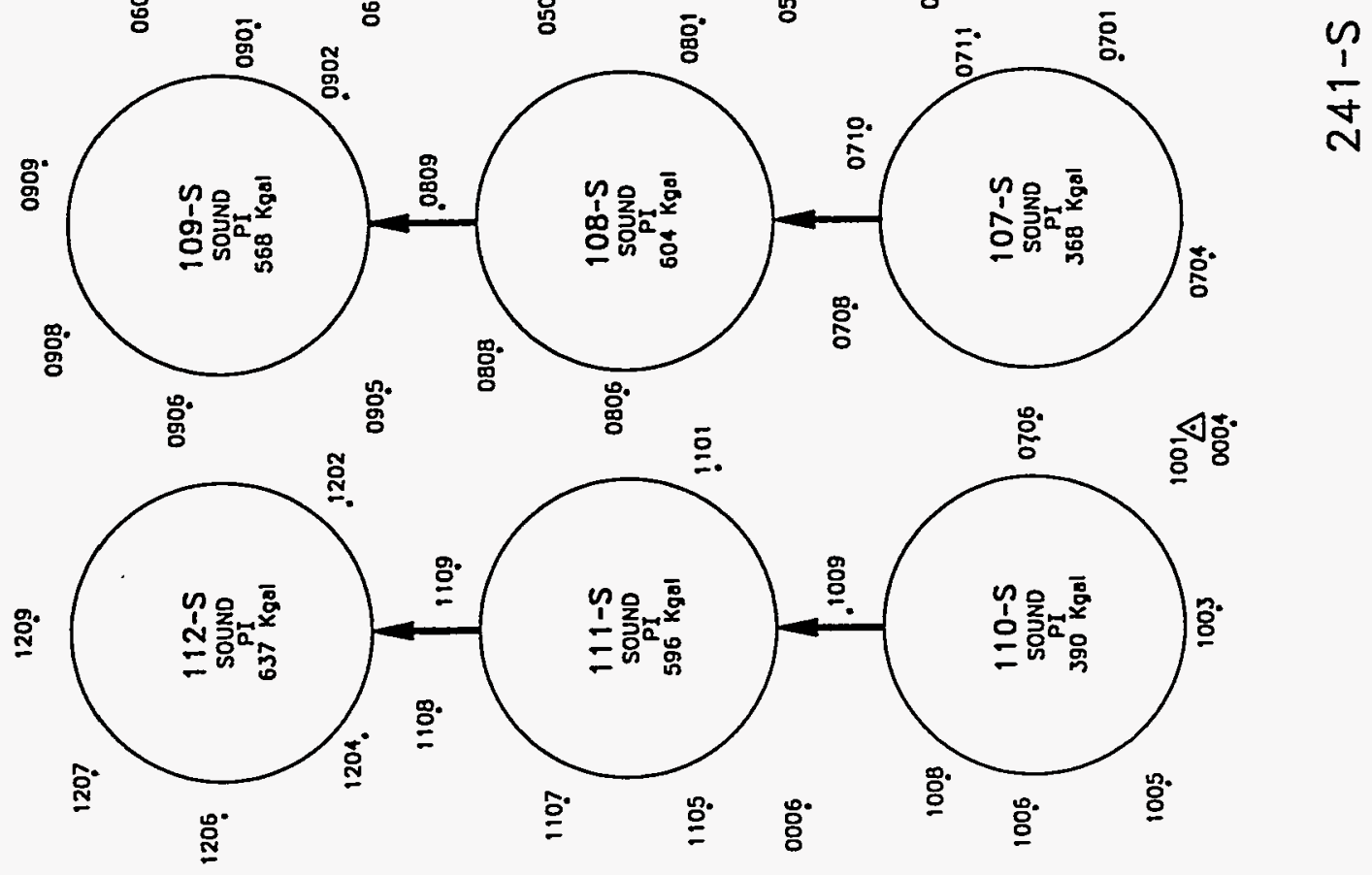

$F-1$ 
241-S-102 Drywell Activity

Well \#40-02-03 Well Depth 100'

GM \# 1 Probe

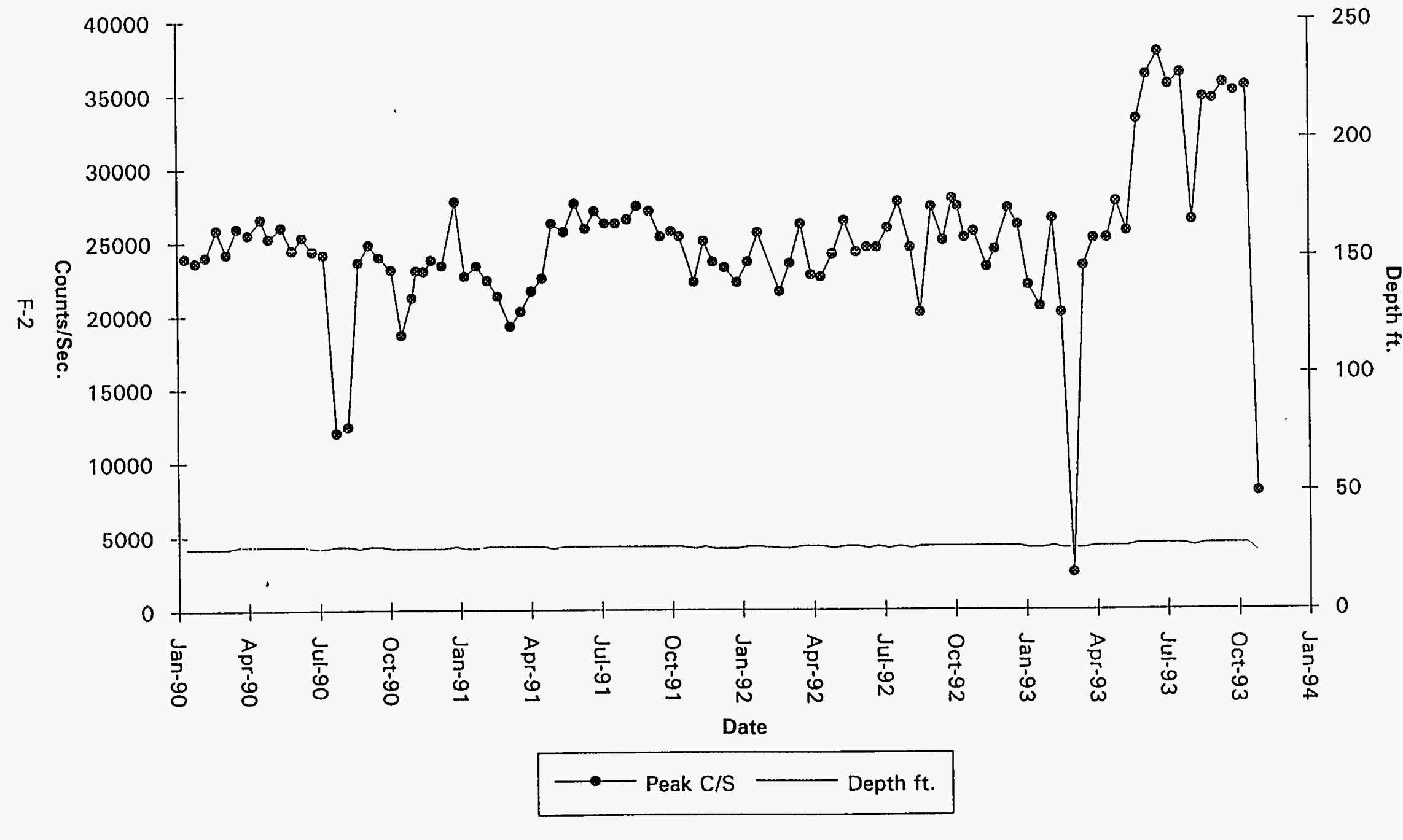


241-S-102 Drywell Activity

Well \#40-02-03 Well Depth 100'

GM \# 2 Probe

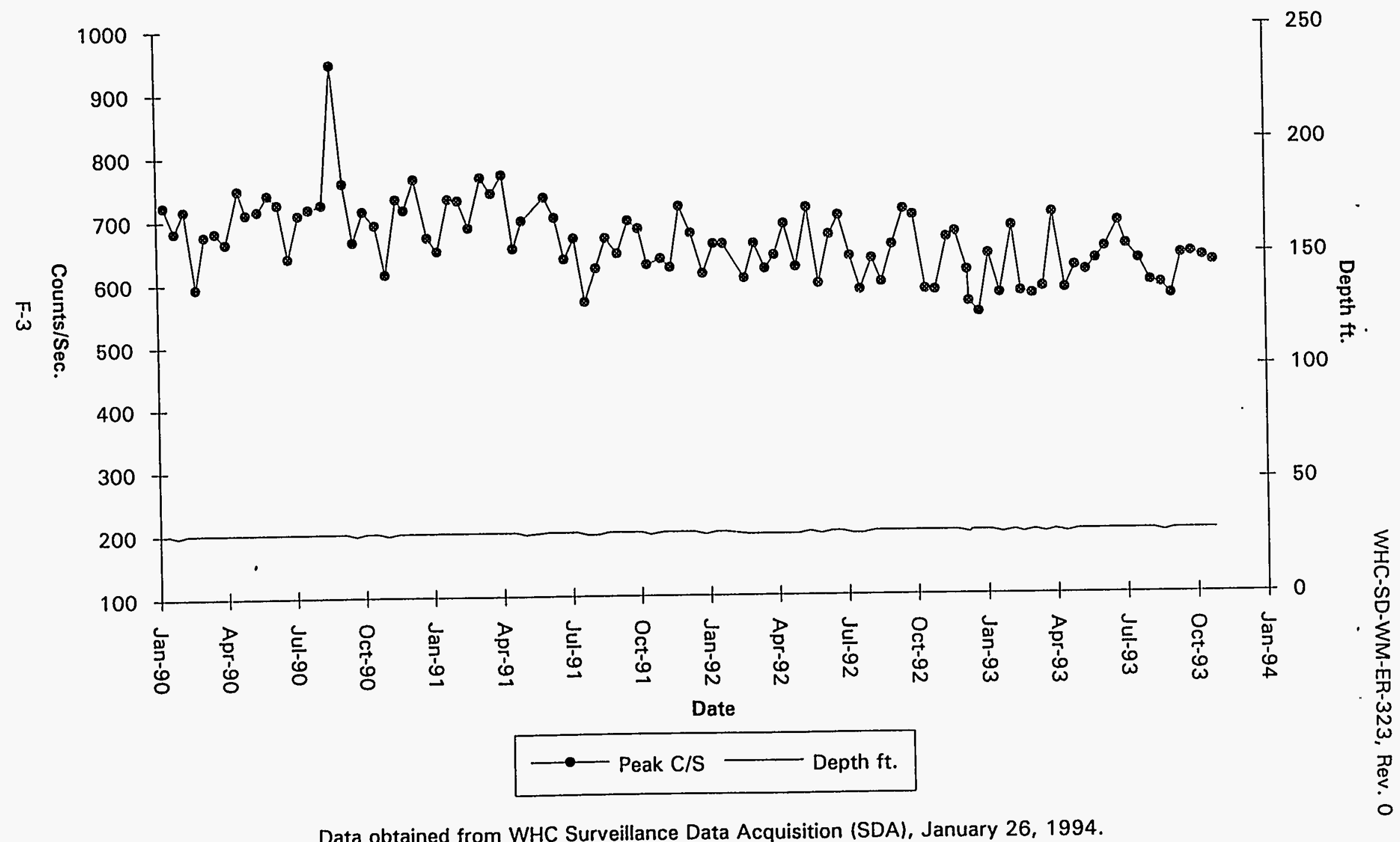




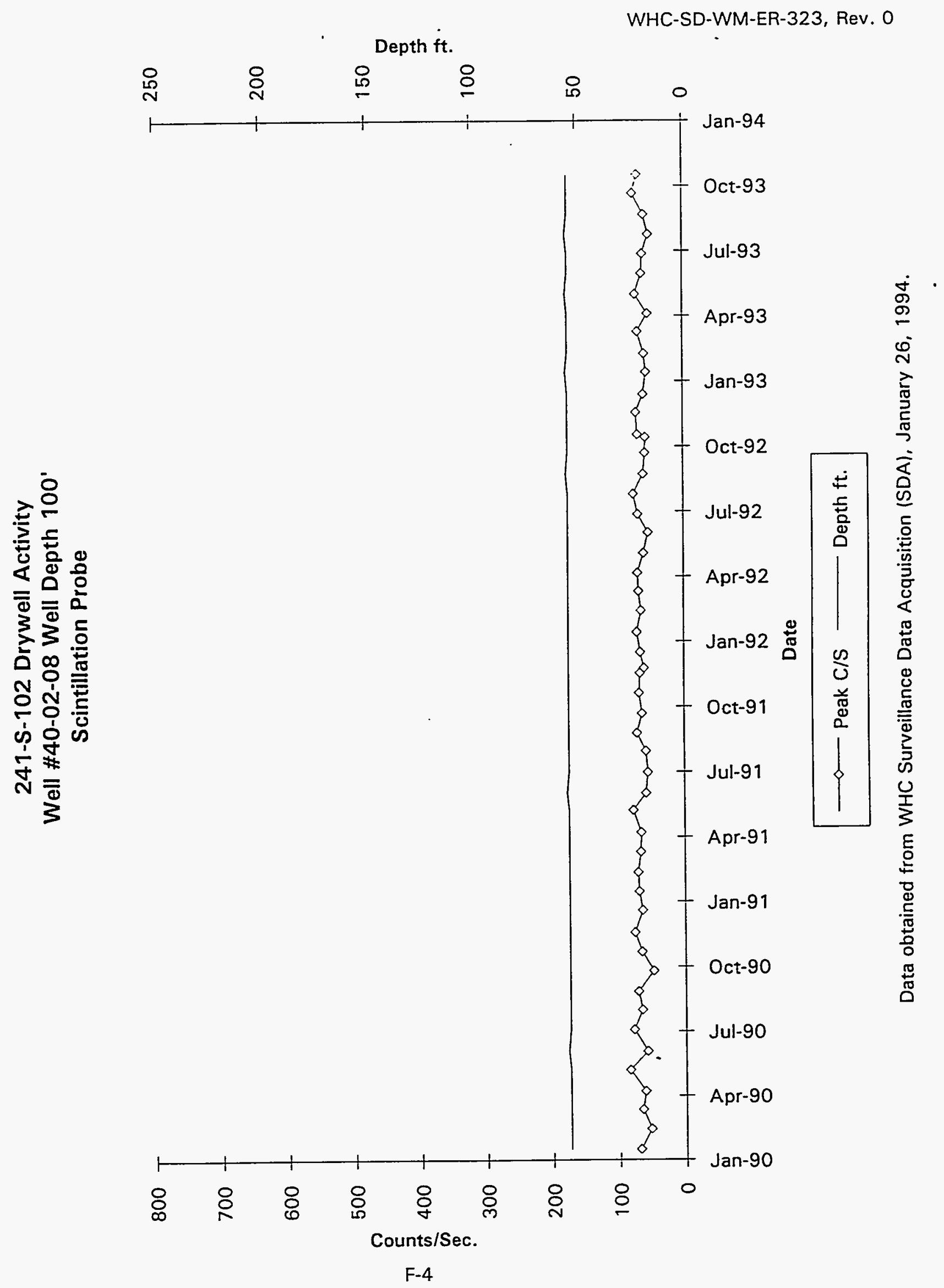




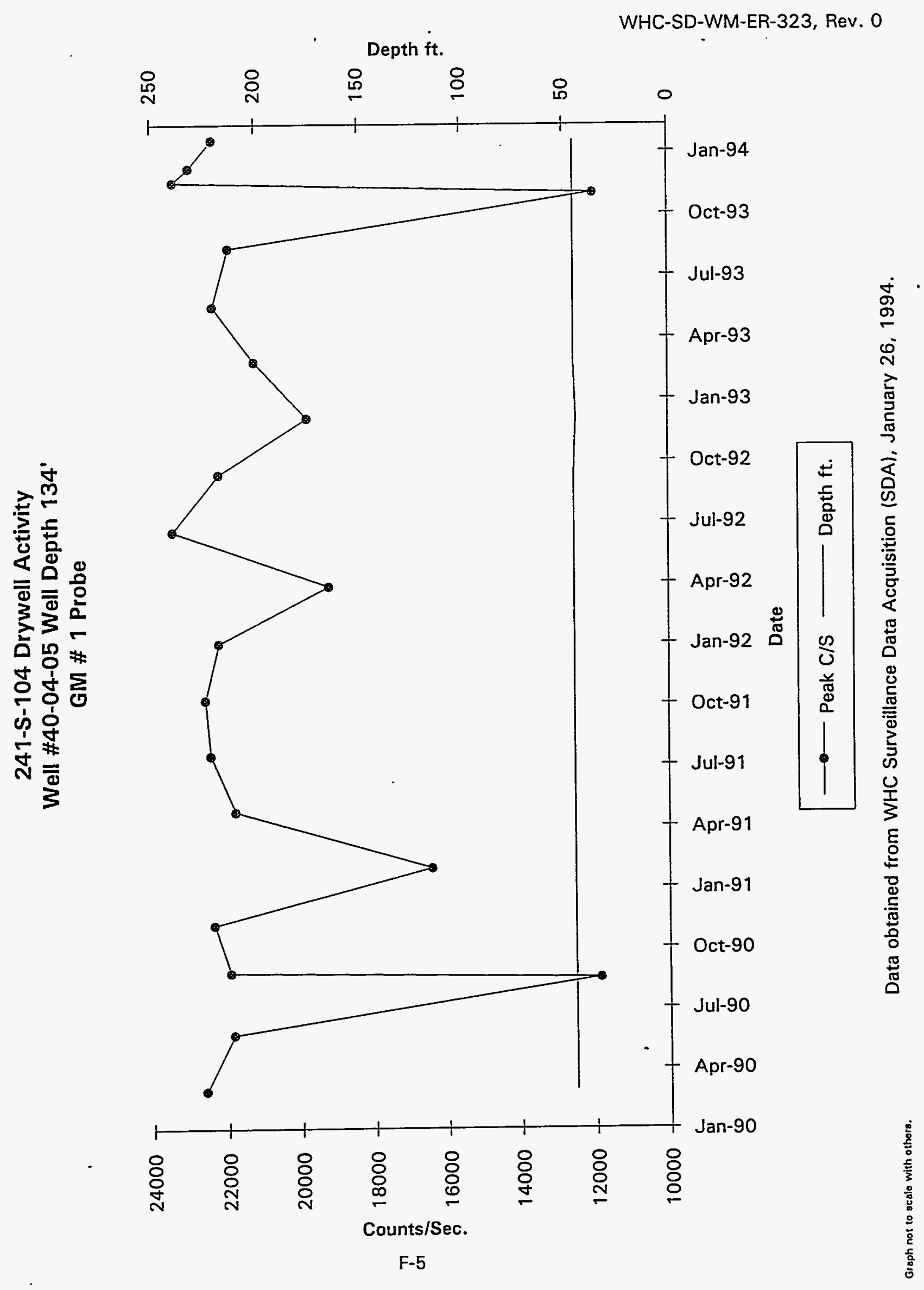




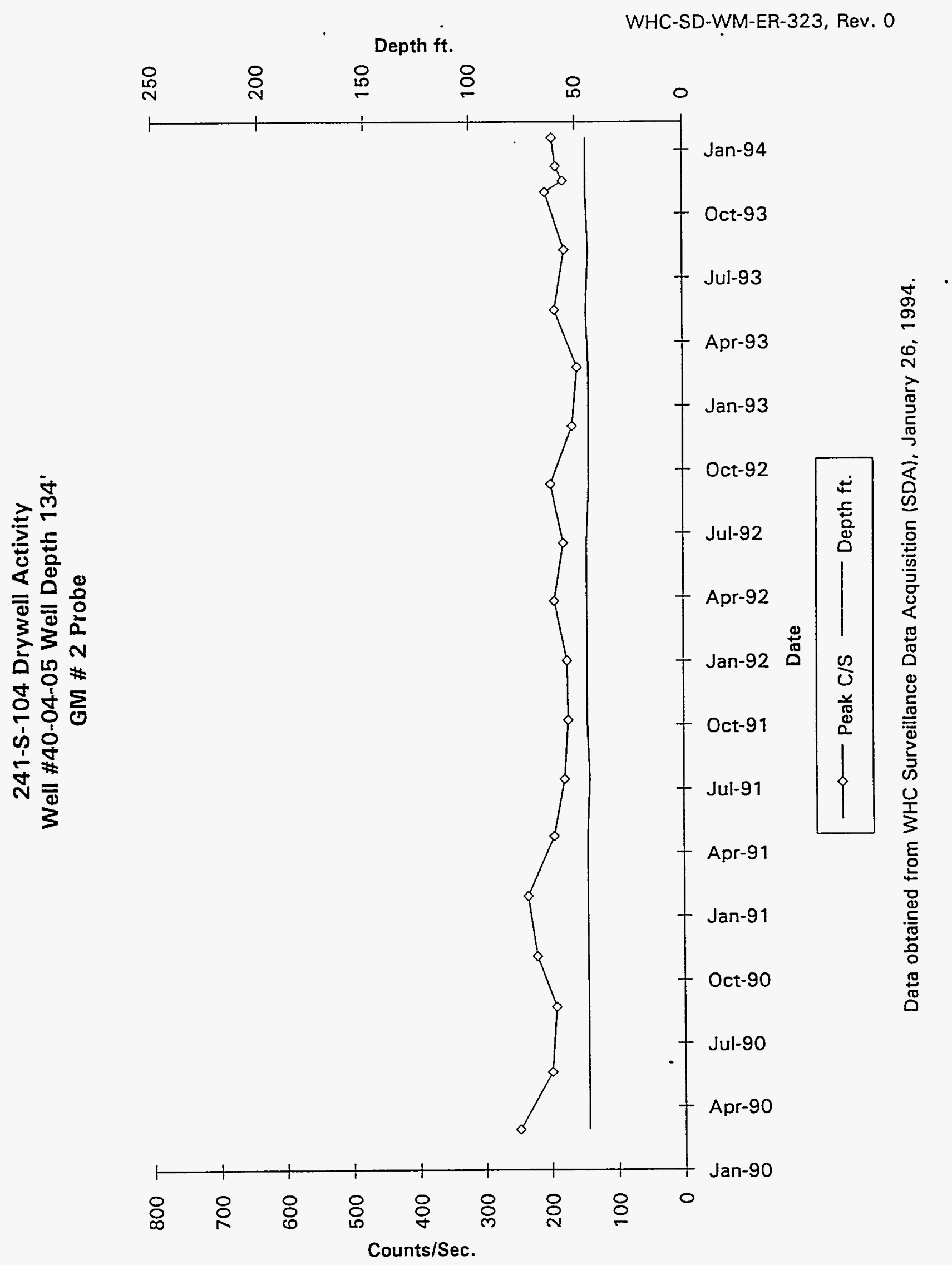

F-6 


\begin{tabular}{|c|c|c|c|c|c|c|}
\hline \multicolumn{6}{|c|}{ 241-S-102 DRYWELL ACTIVITY } & \multirow[t]{2}{*}{ • } \\
\hline \multicolumn{6}{|c|}{ WELL \#40-02-03 WELL DEPTH 100' } & \\
\hline \multicolumn{7}{|c|}{$\mathrm{GM} \# 1$ PROBE } \\
\hline Date & Peak C/S & Depth ft. & & Date & Peak C/S & Depth $\mathrm{ft}$. \\
\hline $1 / 11 / 90$ & 23907 & 26 & & $6 / 26 / 91$ & 27037 & 27 \\
\hline $1 / 25 / 90$ & 23615 & 26 & & $7 / 9 / 91$ & 26204 & 27 \\
\hline $2 / 7 / 90$ & 23991 & 26 & & $7 / 23 / 91$ & 26235 & 27 \\
\hline $2 / 21 / 90$ & 25818 & 26 & & $8 / 7 / 91$ & 26484 & 27 \\
\hline $3 / 6 / 90$ & 24203 & 26 & & $8 / 20 / 91$ & 27396 & 27 \\
\hline $3 / 20 / 90$ & 25938 & 27 & & $9 / 5 / 91$ & 27046 & 27 \\
\hline $4 / 3 / 90$ & 25470 & 27 & & $9 / 19 / 91$ & 25327 & 27 \\
\hline $4 / 20 / 90$ & 26581 & 27 & & $10 / 3 / 91$ & 25661 & 27 \\
\hline $4 / 30 / 90$ & 25226 & 27 & & $10 / 14 / 91$ & 25322 & 27 \\
\hline $5 / 16 / 90$ & 26010 & 27 & & $11 / 1 / 91$ & 22208 & 26 \\
\hline $5 / 30 / 90$ & 24440 & 27 & & $11 / 14 / 91$ & 25006 & 27 \\
\hline $6 / 12 / 90$ & 25321 & 27 & & $11 / 26 / 91$ & 23563 & 26 \\
\hline $6 / 25 / 90$ & 24388 & 26 & & $12 / 11 / 91$ & 23179 & 26 \\
\hline $7 / 9 / 90$ & 24135 & 26 & & $* 12 / 27 / 91$ & 23349 & 26 \\
\hline$* 7 / 23 / 90$ & 6822 & 28 & & $12 / 27 / 91$ & 22183 & 26 \\
\hline${ }^{*} 7 / 24 / 90$ & 6413 & 30 & & $* 1 / 9 / 92$ & 23568 & 26 \\
\hline $7 / 25 / 90$ & 11998 & 27 & & $1 / 10 / 92$ & 23559 & 27 \\
\hline $8 / 9 / 90$ & 12411 & 27 & & $1 / 23 / 92$ & 25554 & 27 \\
\hline $8 / 21 / 90$ & 12193 & 27 & & $2 / 20 / 92$ & 21571 & 26 \\
\hline $8 / 23 / 90$ & 23611 & 26 & & $3 / 4 / 92$ & 23461 & 26 \\
\hline $9 / 6 / 90$ & 24789 & 27 & & $3 / 18 / 92$ & 26129 & 27 \\
\hline $9 / 19 / 90$ & 23949 & 27 & & $3 / 31 / 92$ & 22689 & 27 \\
\hline * $10 / 3 / 90$ & 18312 & 26 & & $4 / 13 / 92$ & 22518 & 27 \\
\hline $10 / 5 / 90$ & 23090 & 26 & & $4 / 28 / 92$ & 24080 & 26 \\
\hline$* 10 / 18 / 90$ & 17908 & 26 & & $5 / 13 / 92$ & 26342 & 27 \\
\hline $10 / 18 / 90$ & 18655 & 26 & & $5 / 28 / 92$ & 24249 & 26 \\
\hline $11 / 1 / 90$ & 21174 & 26 & & $5 / 28 / 92$ & 24251 & 27 \\
\hline $11 / 6 / 90$ & 23028 & 26 & & $6 / 11 / 92$ & 24552 & 26 \\
\hline $11 / 16 / 90$ & 22989 & 26 & & $6 / 24 / 92$ & 24543 & 27 \\
\hline $11 / 26 / 90$ & 23756 & 26 & & $7 / 8 / 92$ & 25872 & 26 \\
\hline $12 / 10 / 90$ & 23375 & 26 & & $7 / 21 / 92$ & 27634 & 27 \\
\hline $12 / 27 / 90$ & 27700 & 27 & & $8 / 6 / 92$ & 24542 & 26 \\
\hline $1 / 9 / 91$ & 22646 & 26 & & $8 / 18 / 92$ & 20132 & 27 \\
\hline $1 / 24 / 91$ & 23316 & 26 & & $9 / 2 / 92$ & 27302 & 27 \\
\hline *2/6/91 & 2356 & 31 & & $9 / 17 / 92$ & 25026 & 27 \\
\hline $2 / 7 / 91$ & 22345 & 27 & : & $9 / 29 / 92$ & 27851 & 27 \\
\hline $2 / 20 / 91$ & 21276 & 27 & & $* 10 / 5 / 92$ & 23034 & 26 \\
\hline $3 / 8 / 91$ & 19220 & 27 & & $10 / 6 / 92$ & 27333 & 27 \\
\hline $3 / 22 / 91$ & 20245 & 27 & & $10 / 15 / 92$ & 25210 & 27 \\
\hline $4 / 5 / 91$ & 21638 & 27 & & $10 / 27 / 92$ & 25624 & 27 \\
\hline $4 / 19 / 91$ & 22493 & 27 & & $11 / 12 / 92$ & 23228 & 27 \\
\hline $5 / 1 / 91$ & 26230 & 26 & & $11 / 23 / 92$ & 24413 & 27 \\
\hline $5 / 17 / 91$ & 25673 & 27 & & $* 12 / 8 / 92$ & 19407 & 26 \\
\hline $5 / 31 / 91$ & 27558 & 27 & & $12 / 10 / 92$ & 27176 & 27 \\
\hline $6 / 14 / 91$ & 25872 & 27 & मे. & $* 12 / 23 / 92$ & 26232 & 27 \\
\hline
\end{tabular}

Data obtained from WHC Surveillance Data Acquisition (SDA), January 26, 1994. 
WHC-SD-WM-ER-323, Rev. 0

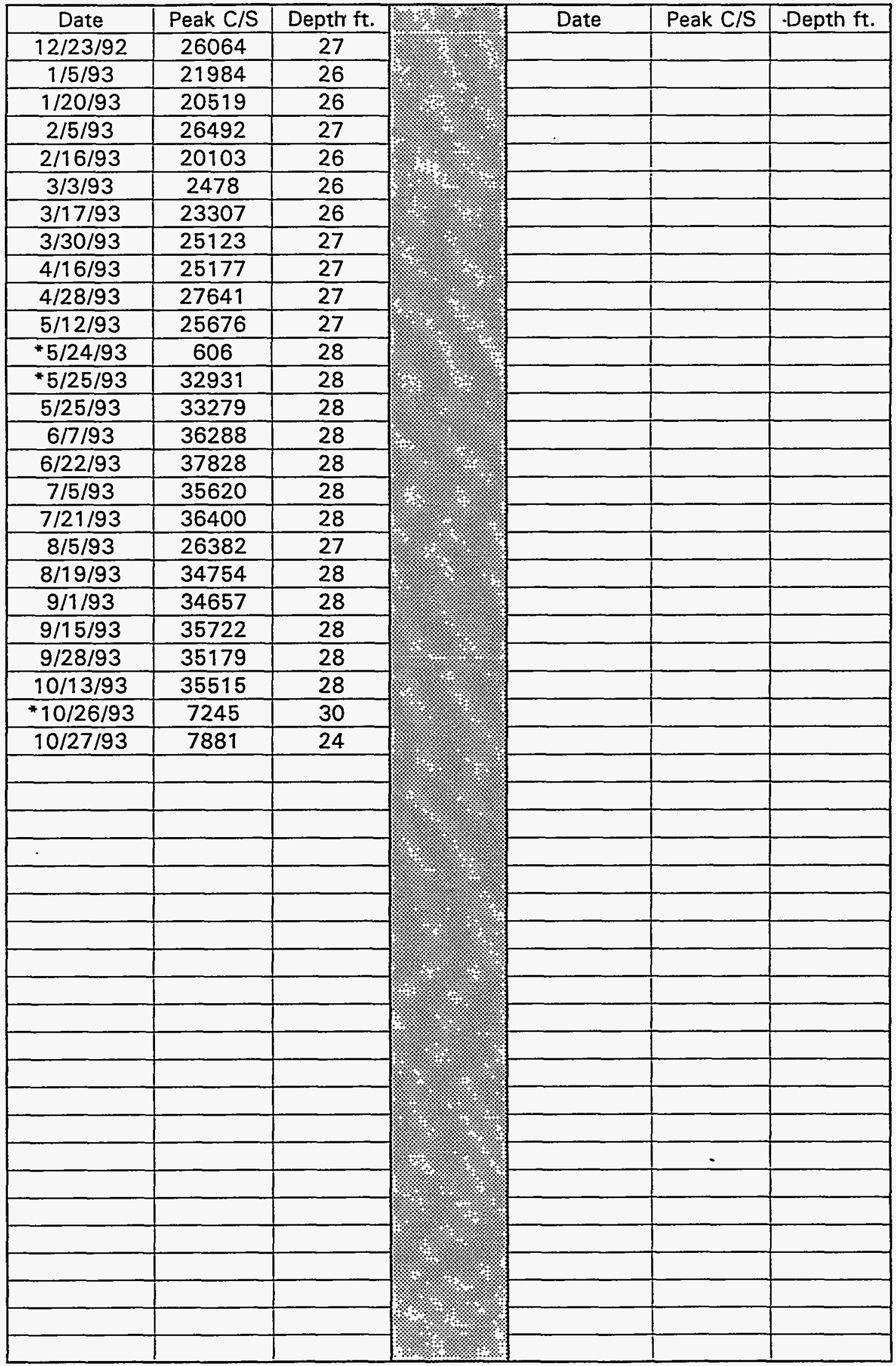

Data obtained from WHC Surveillance Data Acquisition (SDA), January 26, 1994. 


\begin{tabular}{|c|c|c|c|c|c|c|}
\hline \multicolumn{7}{|c|}{ 241-S-102 DRYWELL ACTIVITY } \\
\hline \multicolumn{7}{|c|}{ WELL \#40-02-03 WELL DEPTH 100' } \\
\hline \multicolumn{7}{|c|}{ GM \# 2 PROBE } \\
\hline Date & Peak C/S & Depth ft. & & Date & Peak C/S & Depth ft. \\
\hline $1 / 11 / 90$ & 723 & 28 & & $9 / 19 / 91$ & 695 & 28 \\
\hline $1 / 25 / 90$ & 682 & 27 & & $10 / 3 / 91$ & 683 & 28 \\
\hline $2 / 7 / 90$ & 716 & 28 & & $10 / 14 / 91$ & 626 & 27 \\
\hline $2 / 21 / 90$ & 593 & 28 & & $11 / 1 / 91$ & 635 & 28 \\
\hline $3 / 6 / 90$ & 676 & 28 & & $11 / 14 / 91$ & 621 & 28 \\
\hline $3 / 20 / 90$ & 681 & 28 & & $11 / 26 / 91$ & 717 & 28 \\
\hline * 4/3/90 & 709 & 28 & & $12 / 11 / 91$ & 675 & 28 \\
\hline $4 / 3 / 90$ & 664 & 28 & & $* 12 / 27 / 91$ & 686 & 28 \\
\hline $4 / 20 / 90$ & 748 & 28 & & $12 / 27 / 91$ & 611 & 27 \\
\hline $4 / 30 / 90$ & 710 & 28 & & $1 / 19 / 92$ & 659 & 28 \\
\hline $5 / 16 / 90$ & 715 & 28 & & $1 / 10 / 92$ & 658 & 28 \\
\hline $5 / 30 / 90$ & 740 & 28 & & $1 / 23 / 92$ & 658 & 28 \\
\hline $6 / 12 / 90^{\circ}$ & 725 & 28 & & $2 / 20 / 92$ & 603 & 27 \\
\hline $6 / 25 / 90$ & 640 & 28 & & $3 / 4 / 92$ & 658 & 27 \\
\hline $7 / 9 / 90$ & 708 & 28 & & $3 / 18 / 92$ & 618 & 27 \\
\hline $7 / 23 / 90$ & 717 & 28 & & $3 / 31 / 92$ & 639 & 27 \\
\hline $8 / 9 / 90$ & 724 & 28 & & $4 / 13 / 92$ & 688 & 27 \\
\hline $8 / 21 / 90$ & 0 & 95 & & $4 / 28 / 92$ & 620 & 27 \\
\hline $8 / 23 / 90$ & 945 & 28 & & $5 / 13 / 92$ & 713 & 28 \\
\hline $9 / 6 / 90$ & 758 & 28 & & $5 / 28 / 92$ & 594 & 27 \\
\hline $9 / 19 / 90$ & 665 & 27 & & $6 / 11 / 92$ & 671 & 28 \\
\hline $10 / 3 / 90$ & 713 & 28 & & $6 / 24 / 92$ & 701 & 28 \\
\hline $10 / 19 / 90$ & 691 & 28 & & $7 / 8 / 92$ & 637 & 27 \\
\hline $11 / 1 / 90$ & 613 & 27 & & $7 / 21 / 92$ & 584 & 27 \\
\hline $11 / 16 / 90$ & 732 & 28 & & $8 / 6 / 92$ & 633 & 28 \\
\hline $11 / 26 / 90$ & 715 & 28 & & $8 / 18 / 92$ & 595 & 28 \\
\hline $12 / 10 / 90$ & 763 & 28 & & $9 / 2 / 92$ & 655 & 28 \\
\hline $12 / 27 / 90$ & 671 & 28 & & $9 / 17 / 92$ & 710 & 28 \\
\hline $1 / 9 / 91$ & 649 & 28 & & $9 / 29 / 92$ & 701 & 28 \\
\hline $1 / 24 / 91$ & 731 & 28 & & $10 / 15 / 92$ & 584 & 28 \\
\hline $2 / 6 / 91$ & 729 & 28 & & $10 / 27 / 92$ & 582 & 28 \\
\hline $2 / 20 / 91$ & 686 & 28 & & $11 / 12 / 92$ & 666 & 28 \\
\hline $3 / 8 / 91$ & 765 & 28 & & $11 / 23 / 92$ & 674 & 28 \\
\hline $3 / 22 / 91$ & 740 & 28 & & $12 / 8 / 92$ & 613 & 27 \\
\hline $4 / 5 / 91$ & 769 & 28 & & $12 / 10 / 92$ & 563 & 28 \\
\hline $4 / 19 / 91$ & 652 & 28 & & $12 / 23 / 92$ & 546 & 28 \\
\hline $5 / 1 / 91$ & 696 & 27 & & $1 / 5 / 93$ & 639 & 28 \\
\hline $5 / 31 / 91$ & 733 & 28 & & $1 / 20 / 93$ & 577 & 27 \\
\hline $6 / 14 / 91$ & 701 & 28 & & $2 / 5 / 93$ & 683 & 28 \\
\hline $6 / 26 / 91$ & 635 & 28 & 6 & $2 / 16 / 93$ & 579 & 27 \\
\hline $7 / 9 / 91$ & 668 & 28 & & $3 / 3 / 93$ & 575 & 28 \\
\hline $7 / 23 / 91$ & 567 & 27 & & $3 / 17 / 93$ & 586 & 27 \\
\hline $8 / 7 / 91$ & 620 & 27 & & $3 / 30 / 93$ & 703 & 28 \\
\hline $8 / 20 / 91$ & 668 & 28 & & $4 / 15 / 93$ & 584 & 27 \\
\hline $9 / 5 / 91$ & 644 & 28 & 3.14 .18$. & $4 / 28 / 93$ & 619 & 28 \\
\hline
\end{tabular}

Data obtained from WHC Surveillance Data Acquisition (SDA), January 26, 1994. 
WHC-SD-WM-ER-323, Rev. 0

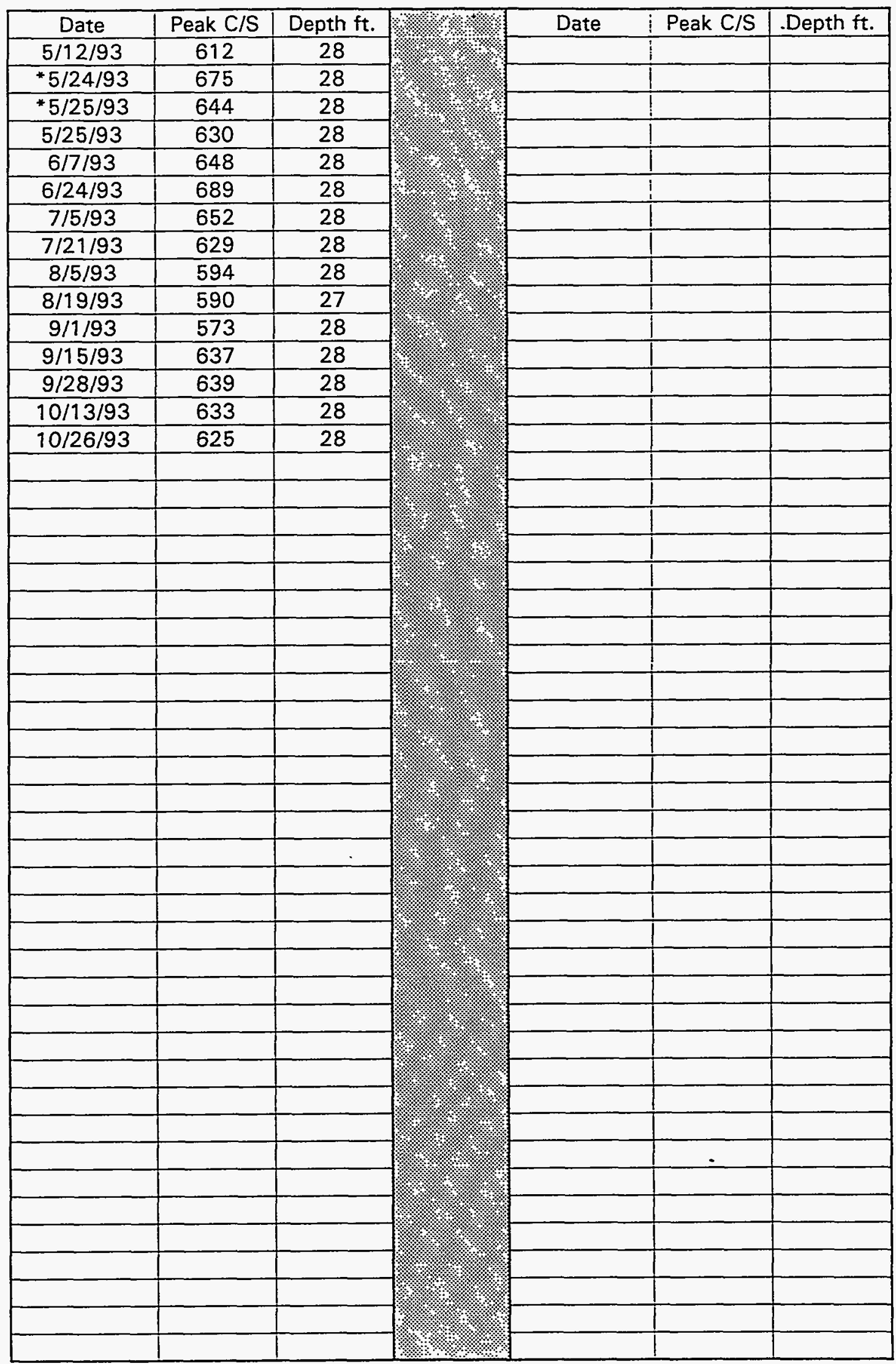

Data obtained from WHC Surveillance Data Acquisition (SDA), January 26, 1994. 


\begin{tabular}{|c|c|c|c|c|c|c|}
\hline \multicolumn{7}{|c|}{ 241-S-102 DRYWELL ACTIVITY } \\
\hline \multicolumn{7}{|c|}{ WELL \#40-02-08 WELL DEPTH 100' } \\
\hline \multicolumn{7}{|c|}{ SCINTILLATION PROBE } \\
\hline Date & Peak C/S & Depth ft. & & Date & Peak C/S & Depth $\mathrm{ft}$. \\
\hline $1 / 16 / 90$ & 69 & 54 & & $4 / 6 / 93$ & 52 & 54 \\
\hline $2 / 14 / 90$ & 53 & 54 & & $5 / 3 / 93$ & 71 & 55 \\
\hline $3 / 14 / 90$ & 65 & 54 & & $* 5 / 31 / 93$ & 338 & 85 \\
\hline $4 / 9 / 90$ & 61 & 54 & & $6 / 1 / 93$ & 61 & 54 \\
\hline $5 / 9 / 90$ & 84 & 54 & & $6 / 29 / 93$ & 60 & 54 \\
\hline $6 / 5 / 90$ & 58 & 55 & & $7 / 26 / 93$ & 51 & 55 \\
\hline $7 / 5 / 90$ & 78 & 54 & & $8 / 23 / 93$ & 58 & 54 \\
\hline $8 / 2 / 90$ & 65 & 54 & & $9 / 22 / 93$ & 74 & 54 \\
\hline $8 / 28 / 90$ & 71 & 54 & & $10 / 18 / 93$ & 67 & 54 \\
\hline $9 / 26 / 90$ & 48 & 54 & & & & \\
\hline $10 / 23 / 90$ & 65 & 54 & & & & \\
\hline $11 / 19 / 90$ & 76 & 54 & & & & \\
\hline $12 / 20 / 90$ & 64 & 54 & & & & \\
\hline $1 / 16 / 91$ & 69 & 54 & & & & \\
\hline $2 / 12 / 91$ & 70 & 54 & & & & \\
\hline $3 / 13 / 91$ & 66 & 54 & & & & \\
\hline $4 / 9 / 91$ & 65 & 54 & & & & \\
\hline $5 / 10 / 91$ & 77 & 54 & & & & \\
\hline $6 / 3 / 91$ & 58 & 55 & & & & \\
\hline $7 / 2 / 91$ & 55 & 54 & & & & \\
\hline $8 / 1 / 91$ & 58 & 54 & & & & \\
\hline $8 / 26 / 91$ & 71 & 54 & & & & \\
\hline $9 / 23 / 91$ & 63 & 54 & & & & \\
\hline $10 / 22 / 91$ & 67 & 54 & & & & \\
\hline $11 / 19 / 91$ & 66 & 54 & & & & \\
\hline $11 / 26 / 91$ & 60 & 54 & & & & \\
\hline $12 / 18 / 91$ & 65 & 54 & & & & \\
\hline $1 / 15 / 92$ & 70 & 54 & & & & \\
\hline $2 / 14 / 92$ & 64 & 54 & & & & \\
\hline $3 / 12 / 92$ & 67 & 54 & & & & \\
\hline $4 / 7 / 92$ & 69 & 54 & & & & \\
\hline$* 5 / 4 / 92$ & 62 & 54 & & & & \\
\hline $5 / 5 / 92$ & 60 & 54 & & & & \\
\hline $6 / 3 / 92$ & 53 & 54 & & & & \\
\hline $6 / 29 / 92$ & 68 & 54 & & & & \\
\hline $7 / 27 / 92$ & 75 & 54 & & & & \\
\hline $8 / 24 / 92$ & 60 & 55 & & & & \\
\hline $9 / 23 / 92$ & 57 & 54 & & & $\therefore$ & \\
\hline $10 / 15 / 92$ & 56 & 54 & & & & \\
\hline $10 / 19 / 92$ & 68 & 54 & & & & \\
\hline $11 / 19 / 92$ & 70 & 54 & & & & \\
\hline $12 / 14 / 92$ & 59 & 54 & & & & \\
\hline $1 / 14 / 93$ & 55 & 55 & & & & \\
\hline $2 / 9 / 93$ & 58 & 54 & 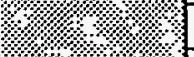 & & & \\
\hline $3 / 12 / 93$ & 67 & 54 & 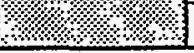 & & & \\
\hline
\end{tabular}

Data obtained from WHC Surveillance Data Acquisition (SDA), January 26, 1994. 
WHC-SD-WM-ER-323, Rev. 0

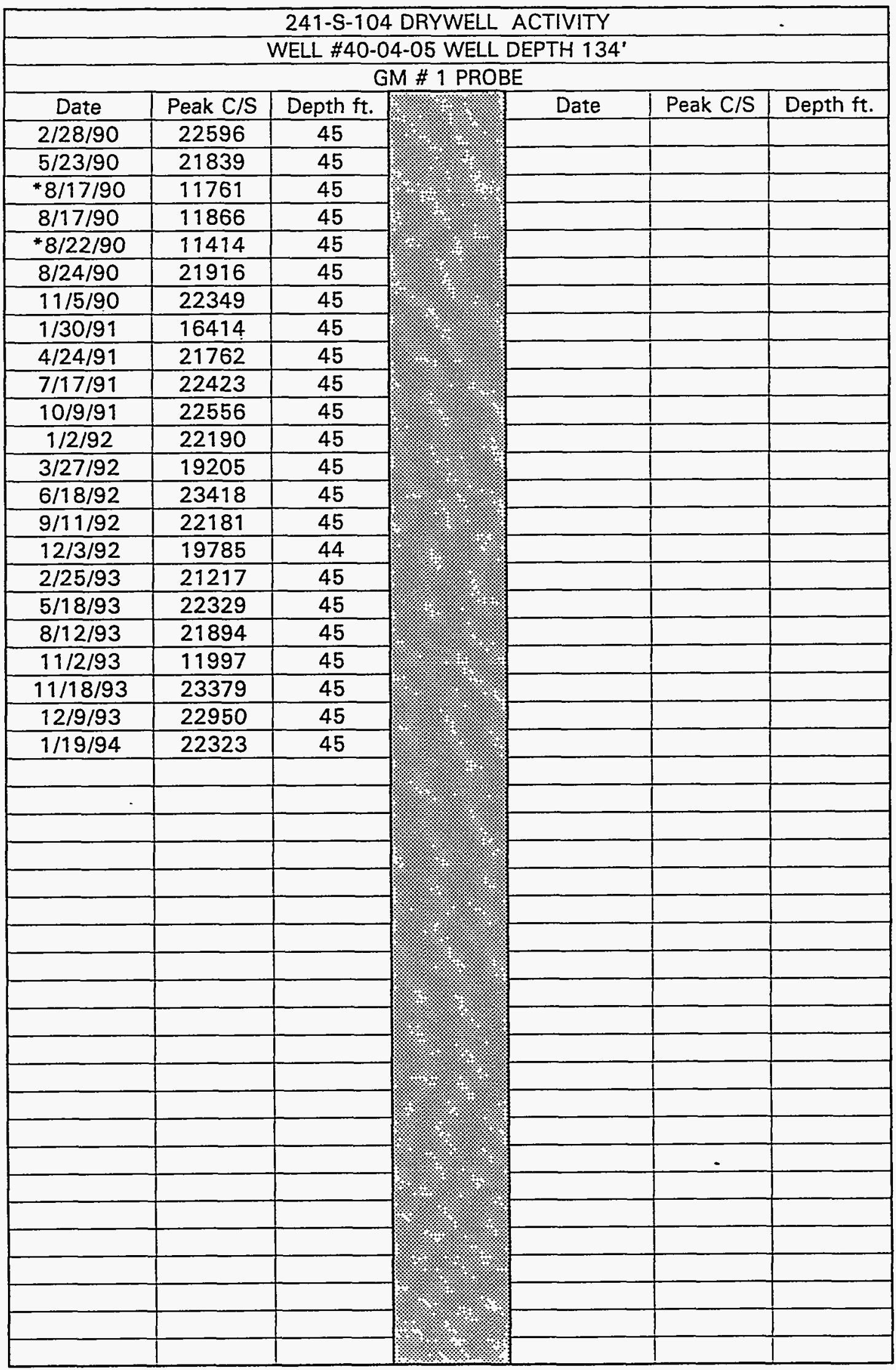

Data obtained from WHC Surveillance Data Acquisition (SDA), January 26, 1994. 


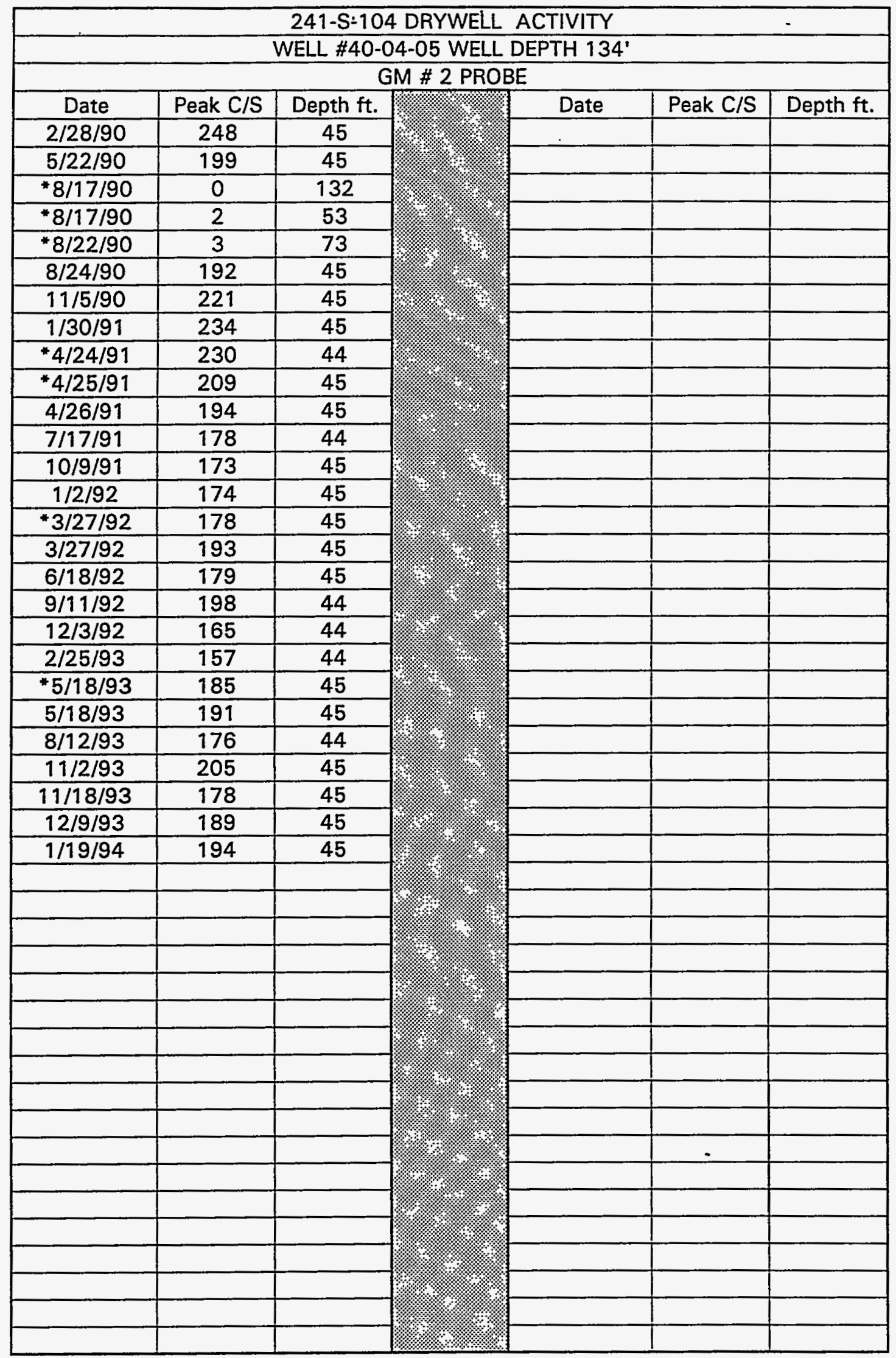

Data obtained from WHC Surveillance Data Acquisition (SDA), January 26, 1994. 
WHC-SD-WM-ER-323，Rev. 0

THIS PAGE INTENTIONALLY LEFT BLANK

G-1 
WHC-SD-WM-ER-323, REV. 0

\begin{tabular}{|c|c|c|}
\hline NO. & DIA. & DESCRIPTION AND COMMENTS \\
\hline 1 & $4 "$ & (CONN NOZZLE) PUMP PIT DRAIN \\
\hline 2 & $4 "$ & FLANGE WITH SPOOL PIECE/LOW, BM \\
\hline 3 & $4 "$ & FIC INTRUSION MODE \\
\hline 4 & $4 "$ & FI_ANGE \\
\hline 5 & $12^{\prime \prime}$ & SALTWELI SCREEN AND PUMP \\
\hline 6 & $12 "$ & PLUG/OBSV PORT \\
\hline 7 & $12 "$ & FLANGE \\
\hline 8 & $12^{\prime \prime}$ & PLUG \\
\hline 11 & $4 "$ & SMP \\
\hline 13 & $42^{\prime \prime}$ & (SLURRY DISTRIBUTION) \\
\hline 14 & $4^{\prime \prime}$ & TEMPERATURE PROBE, MANUAL B-221 \\
\hline 16 & $4 "$ & BM, BREATHER FILTER, G-I HOUSING \\
\hline
\end{tabular}




\section{$241-S-101$}

3.790,000 Liters

[758,000 Gallons]

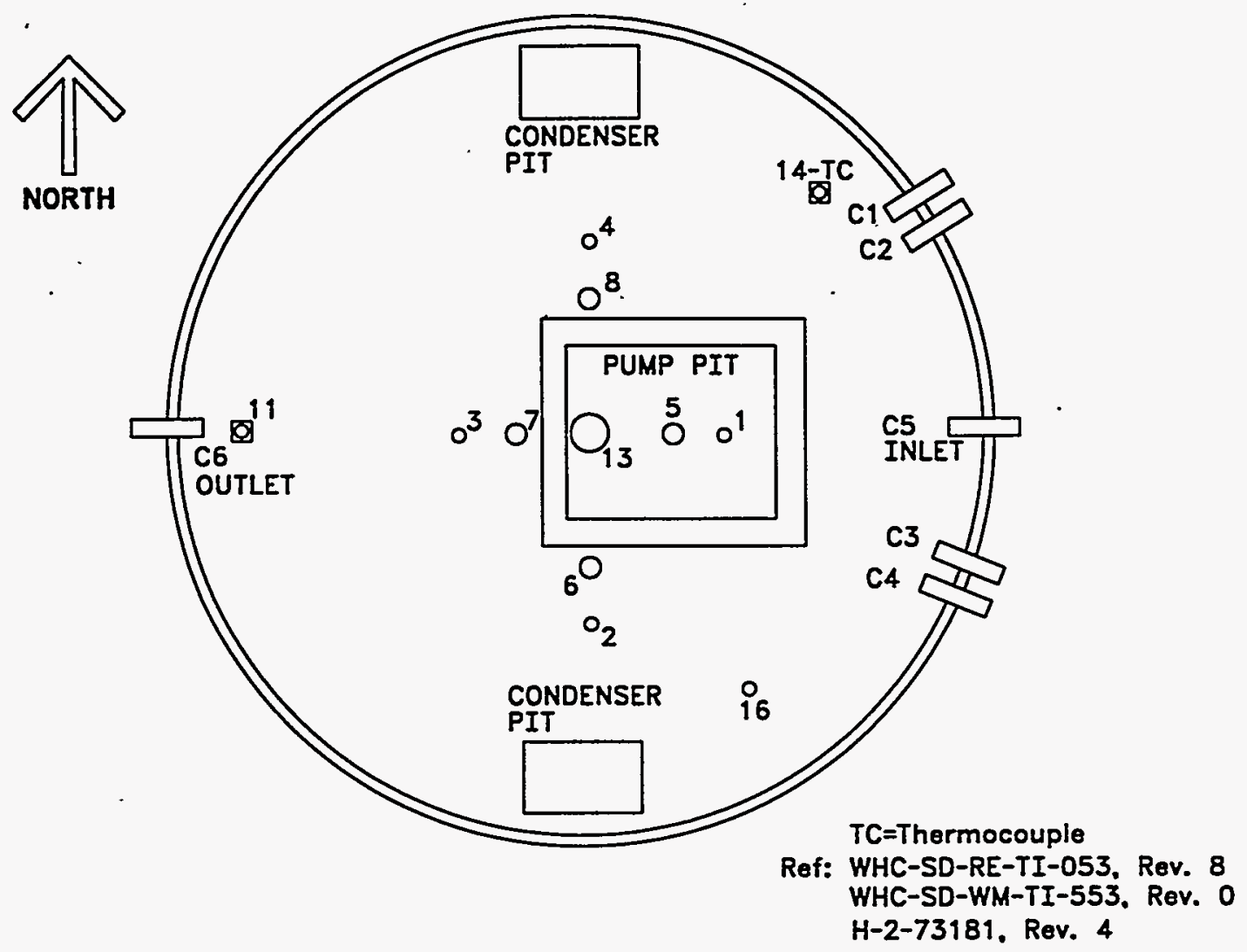

\section{TANK RISER LOCATION}

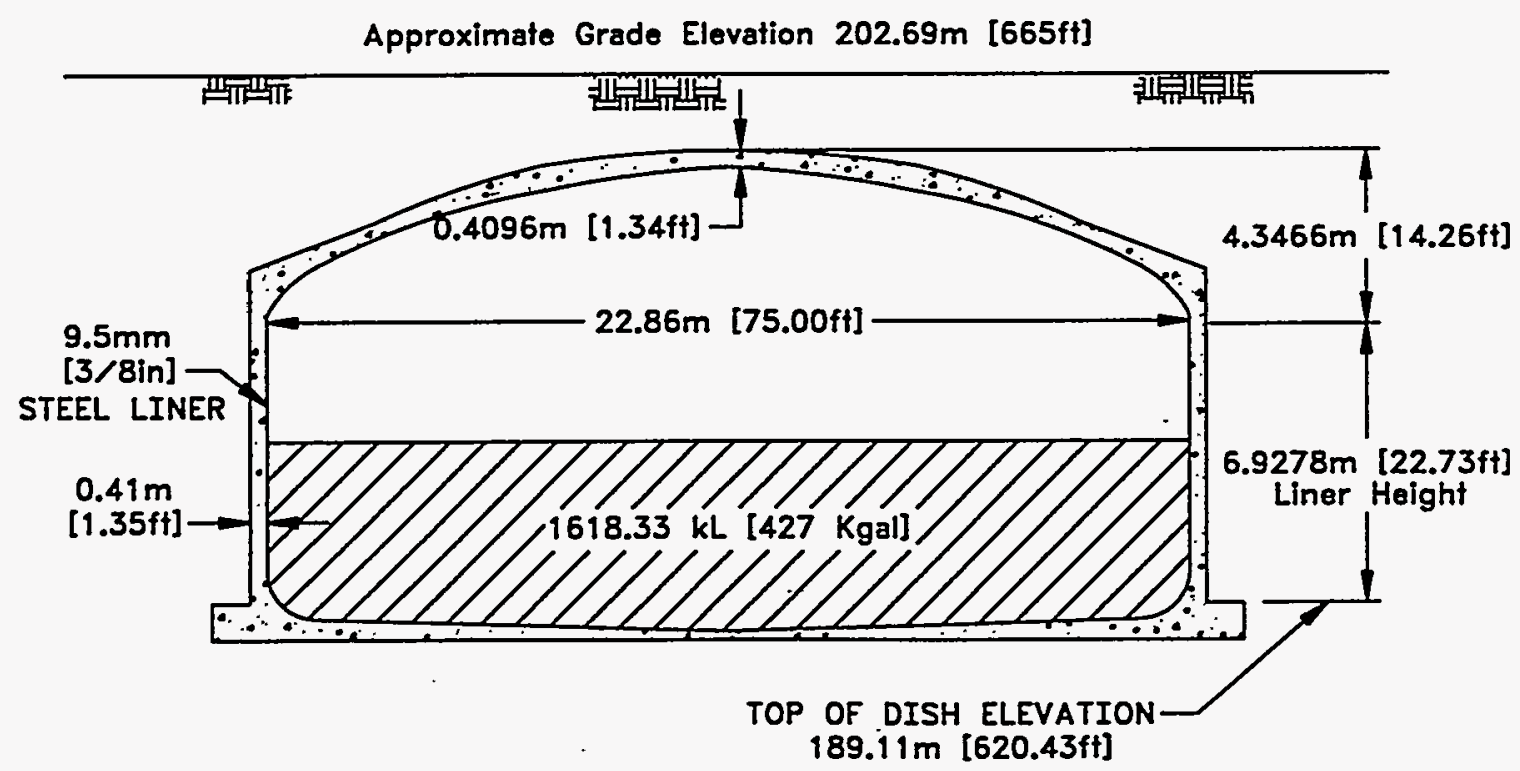

Ref: H-2-1783, Rev. 3

Ref: H-2-46293. Rev, 3 


\begin{tabular}{|c||c||l||}
\hline \multicolumn{3}{|c||}{ 241-S-102 } \\
\hline \hline NO. & DIA. & \multicolumn{1}{|c||}{ DESCRIPTION AND COMMENTS } \\
\hline \hline 1 & $4^{\prime \prime}$ & BREATHER FILTER, G-1 HOUSING \\
\hline 2 & $4^{\prime \prime}$ & FIC, BM INTRUSION MODE \\
\hline 3 & $4^{\prime \prime}$ & TEMPERATURE PROBE, MANUAL B-221 \\
\hline 4 & $4^{\prime \prime}$ & PIT DRAIN WC \\
\hline 5 & $12^{\prime \prime}$ & FLANGE/B-436 LOW, BM \\
\hline 6 & $12^{\prime \prime}$ & FLANGE \\
\hline 7 & $12^{\prime \prime}$ & FIANGE/B-222 OBSV PORT \\
\hline 8 & $12^{\prime \prime}$ & PUMP MOUNT WC \\
\hline 11 & $4^{\prime \prime}$ & SMP \\
\hline 13 & $42^{\prime \prime}$ & SALTWELI SCREEN AND PUMP \\
\hline 14 & $4^{\prime \prime}$ & SMP \\
\hline 16 & $4^{\prime \prime}$ & SMP, BM \\
\hline
\end{tabular}




\section{1-S-102}

3,790,000 Liters

[758,000 Gallons]

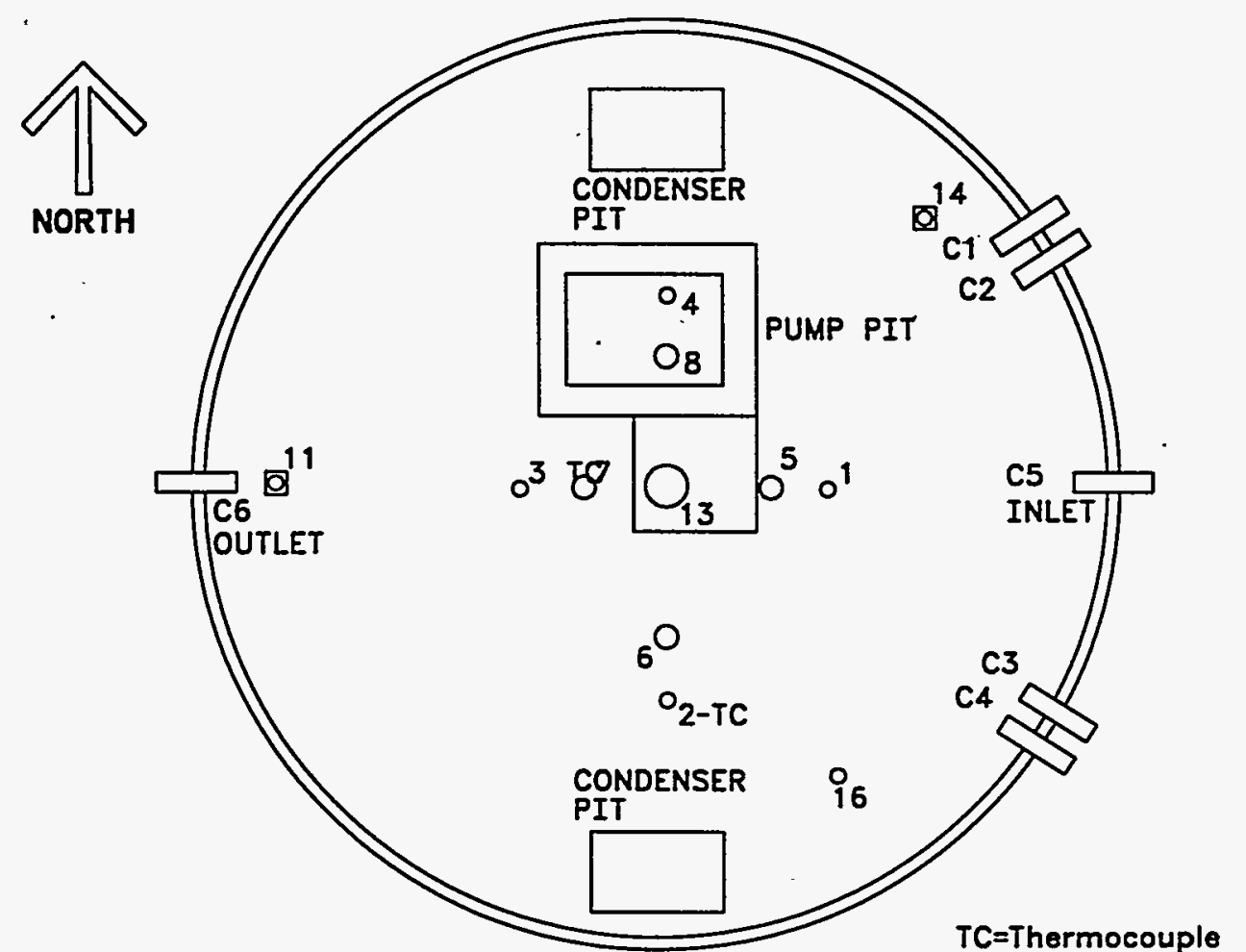

Ref: WHC-SD-RE-TI-053, Rev. 8 WHC-SD-WM-TI-553, Rev. 0 $\mathrm{H}-2-73$ 182. Rev. 4

\section{TANK RISER LOCATION}

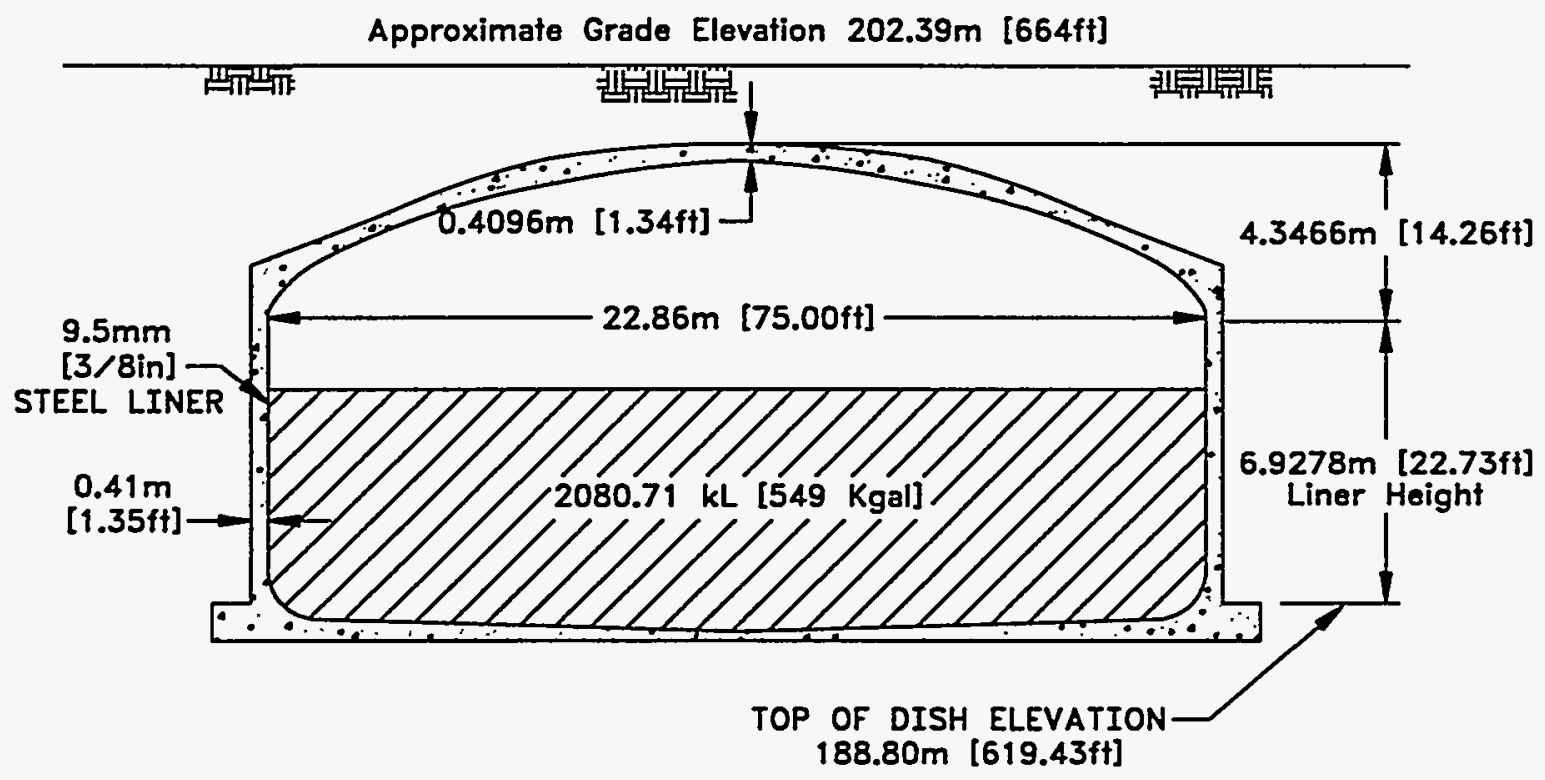

Ref: H-2-1783, Rev. 3 


\begin{tabular}{|c|c|c|}
\hline \multicolumn{3}{|r|}{ 241-S-103 } \\
\hline NO. & DIA. & DESCRIPTION AND COMMENTS \\
\hline 1 & $4 "$ & (CONN NOZZLE) PUMP RIT DRAIN \\
\hline 2 & $4 "$ & FLANGE/B-436 LOW，BM \\
\hline 3 & $4 "$ & FIC INTRUSION MODE \\
\hline 4 & 4" & TEMPERATURE PROBE, MANUAI B-221 \\
\hline 5 & $12 "$ & SALTWELL SCREEN AND PUMP \\
\hline 6 & $12 "$ & CONCRETE PLUG-LB \\
\hline$\overline{7}$ & $12 "$ & FLANGE \\
\hline 8 & $12 "$ & OBSV PORT \\
\hline II & $4 "$ & SMP \\
\hline 13 & $42 "$ & SLURRY DISTRIBUTOR \\
\hline 14 & $4 "$ & BREATHER FILTER, G-I HOUSING \\
\hline 16 & 41 & SMP, BM \\
\hline
\end{tabular}




\section{$241-S-103$ \\ $3,790,000$ Liters \\ [758,000 Gallons]}

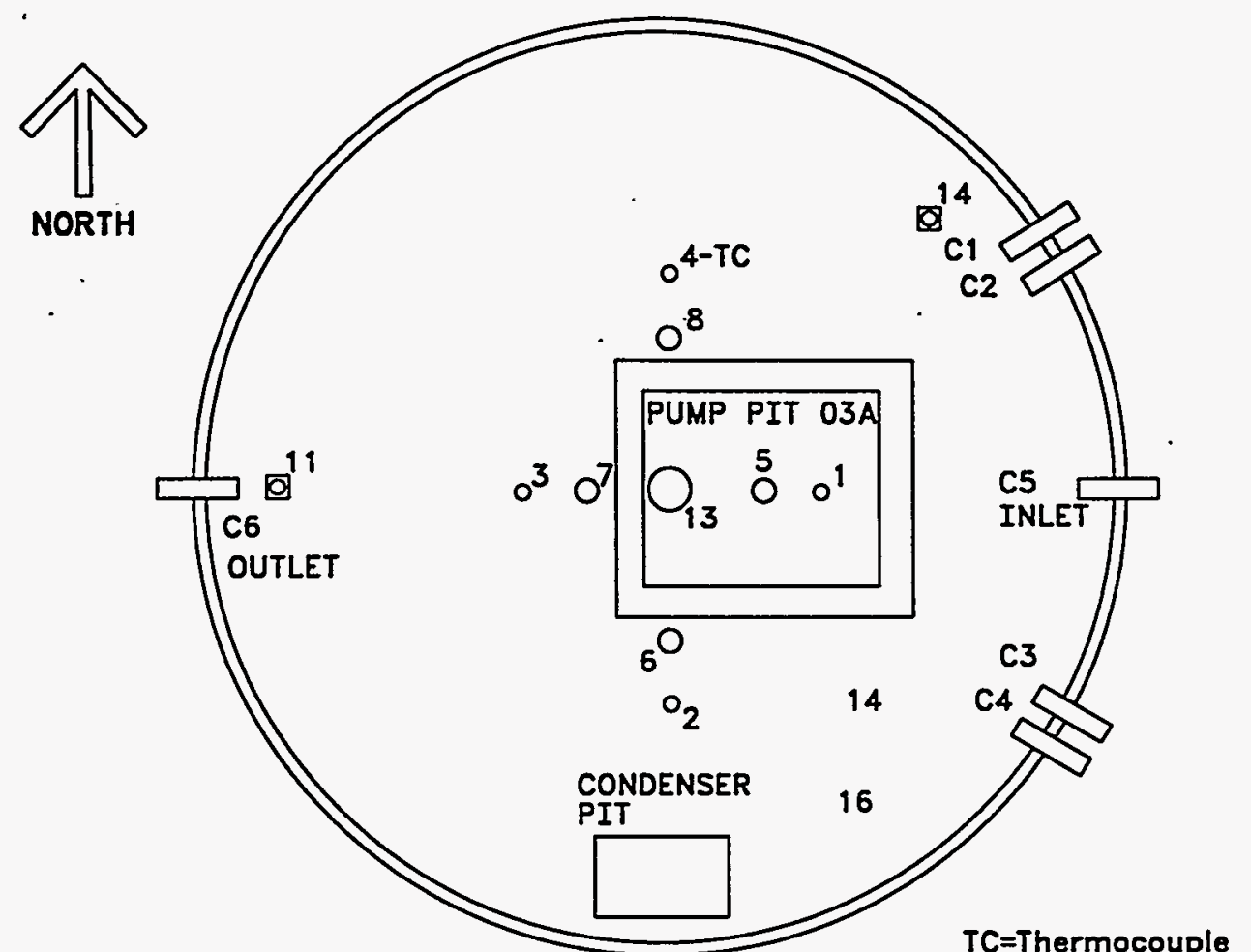

Ref: WHC-SD-RE-TI-053, Rev. 8 WHC-SD-WM-TI-553, Rev. 0 $\mathrm{H}-2-73183$, Rev. 4

\section{TANK RISER LOCATION}

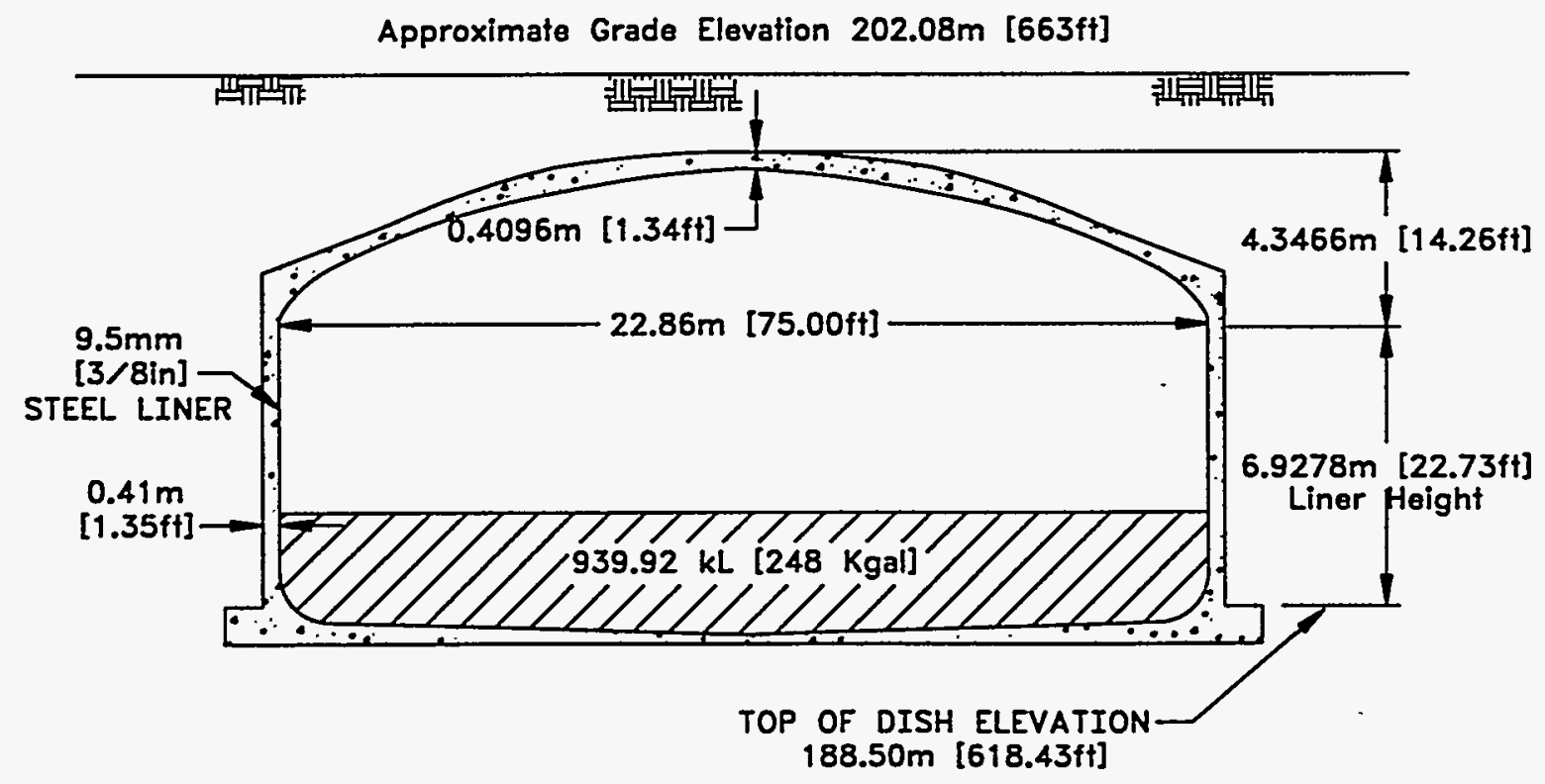

Ref: $\mathrm{H}-2-1783$, Rev. 3 
WHC-SD-WM-ER-323, REV. 0

\begin{tabular}{||c||c||l||}
\hline \multicolumn{2}{|c||}{ 241-S-104 } \\
\hline \hline NO. & DIA. & \multicolumn{1}{|c||}{ DESCRIPTION AND COMMENTS } \\
\hline \hline 1 & $4 "$ & FLANGE \\
\hline 2 & $4 "$ & FLAANGE, BM \\
\hline 3 & $4 "$ & BREATHER FILTER \\
\hline 4 & $4 "$ & TEMPERATURE PROBE, INOPERATIVE \\
\hline 5 & $12^{\prime \prime}$ & IIQUID LEVEL REEL, BM \\
\hline 6 & $12^{\prime \prime}$ & SALTWELI SCREEN AND PUMP \\
\hline 7 & $12^{\prime \prime}$ & |BSV PORT, BM \\
\hline 8 & $12^{\prime \prime}$ & FLANGE WITH LEAD \\
\hline 13 & $42^{\prime \prime}$ & BG \\
\hline
\end{tabular}




\section{1-S-104 \\ 3,790,000 Liters \\ [758,000 Gallons]}

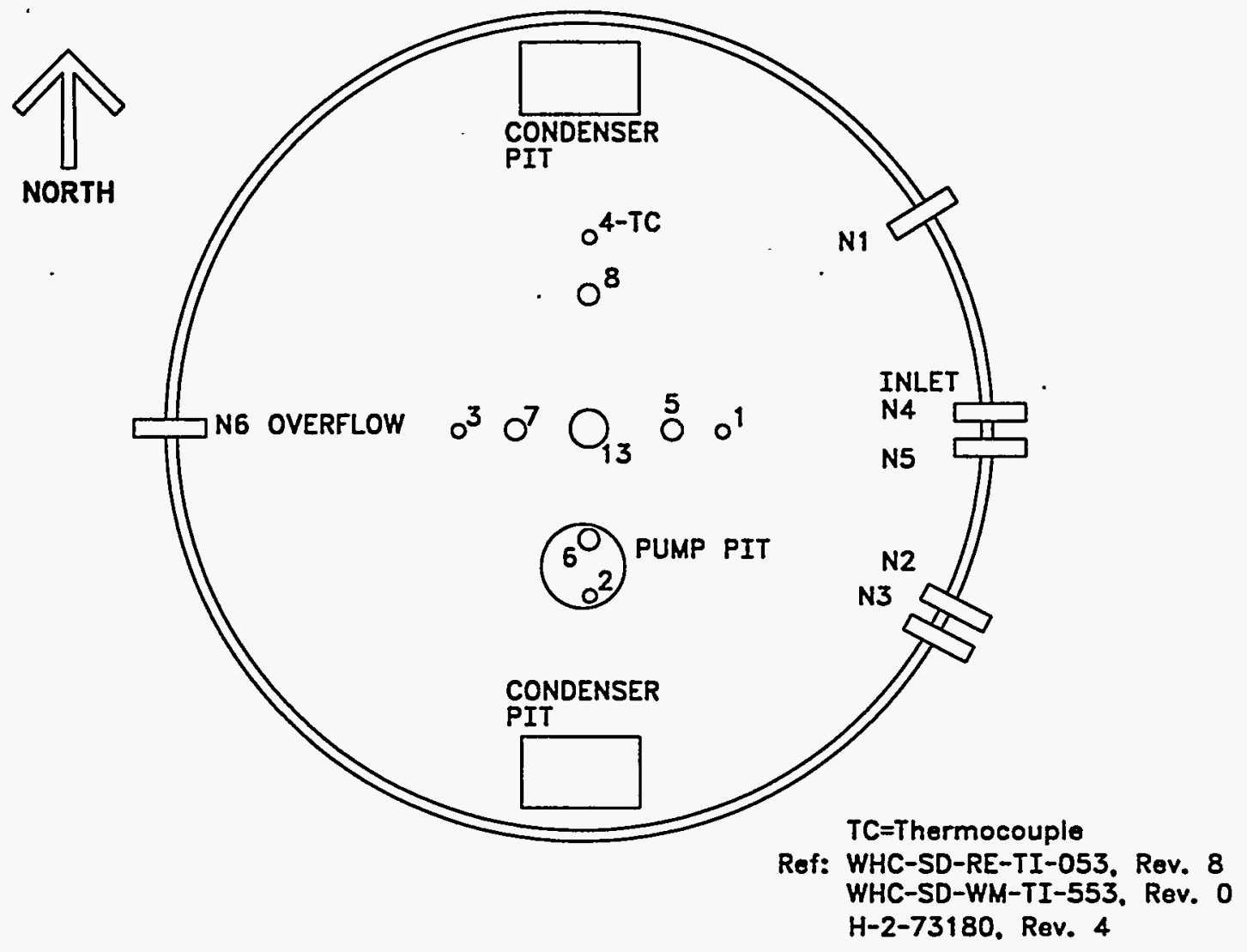

TANK RISER LOCATION

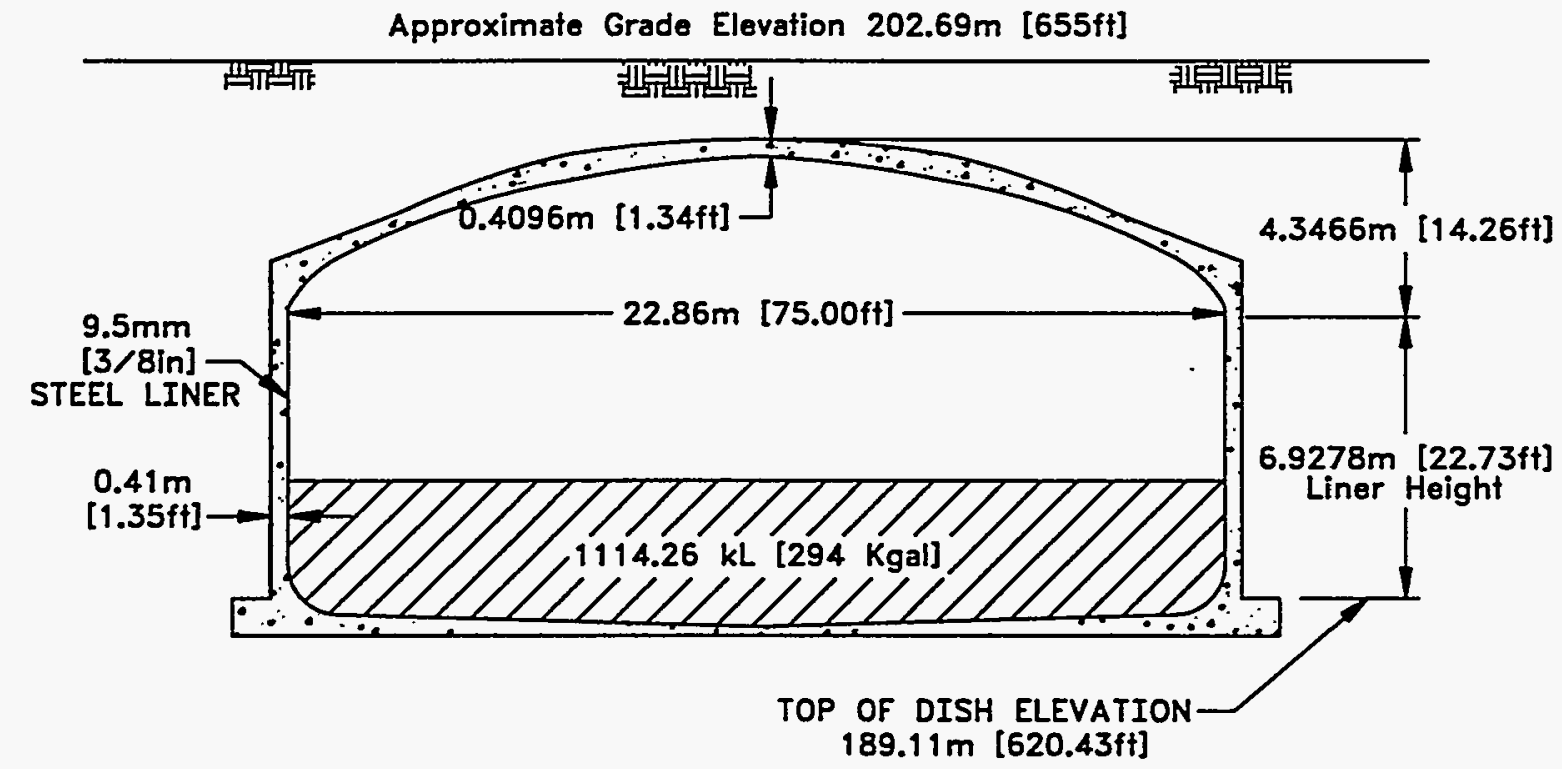

Ref: H-2-1783, Rev. 3 


\begin{tabular}{|c||c||l||}
\hline \multicolumn{3}{|c||}{ 241-S-105 } \\
\hline \hline NO. & DIA. & \multicolumn{1}{|c||}{ DESCRIPTION AND COMMENTS } \\
\hline \hline 1 & $4 "$ & (CONN NOZZLE) PUMP PIT DRAIN \\
\hline 2 & $4^{\prime \prime}$ & FLANGE, BM \\
\hline 3 & $4^{\prime \prime}$ & FIC INTRUSION MODE \\
\hline 4 & $4^{\prime \prime}$ & TEMPERATURE PROBE, TO CASS \\
\hline 5 & $12^{\prime \prime}$ & SALTWELL SCREEN AND PUMP \\
\hline 6 & $12^{\prime \prime}$ & FLANGE WITH LIFTING BALE \\
\hline 7 & $12^{\prime \prime}$ & FLANGE \\
\hline 8 & $12^{\prime \prime}$ & FLANGE/B-222 OBSV PORT \\
\hline 11 & $4^{\prime \prime}$ & SMP \\
\hline 13 & $42^{\prime \prime}$ & SLURRY DISTRIBUTOR \\
\hline 14 & $4^{\prime \prime}$ & SMP/B-436 LOW \\
\hline 16 & $4^{\prime \prime}$ & BM, BREATHER FILTER, G-1 HOUSING \\
\hline
\end{tabular}




\section{1-S-105}

3,790,000 Liters

[758,000 Gallons]

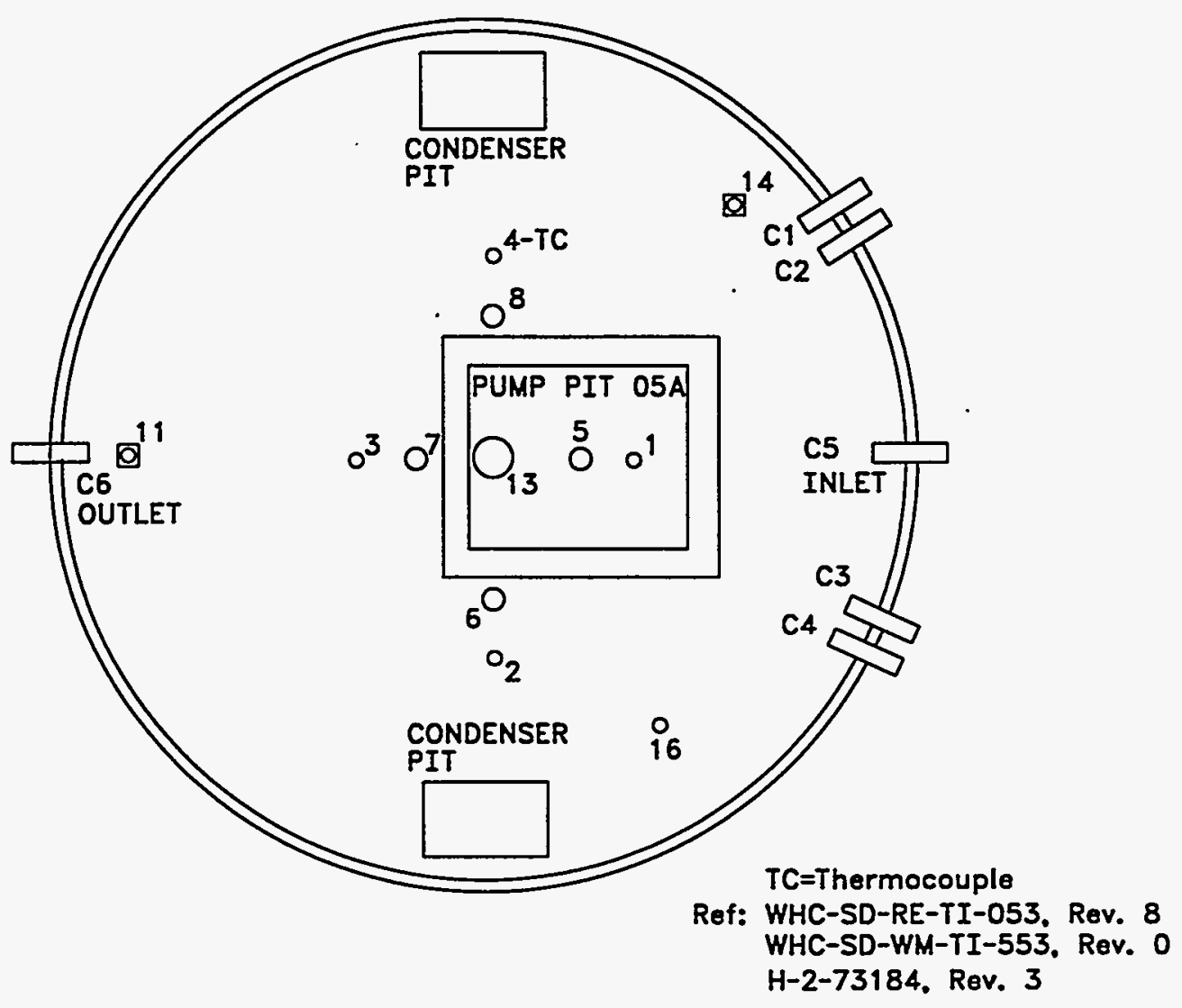

TANK RISER LOCATION

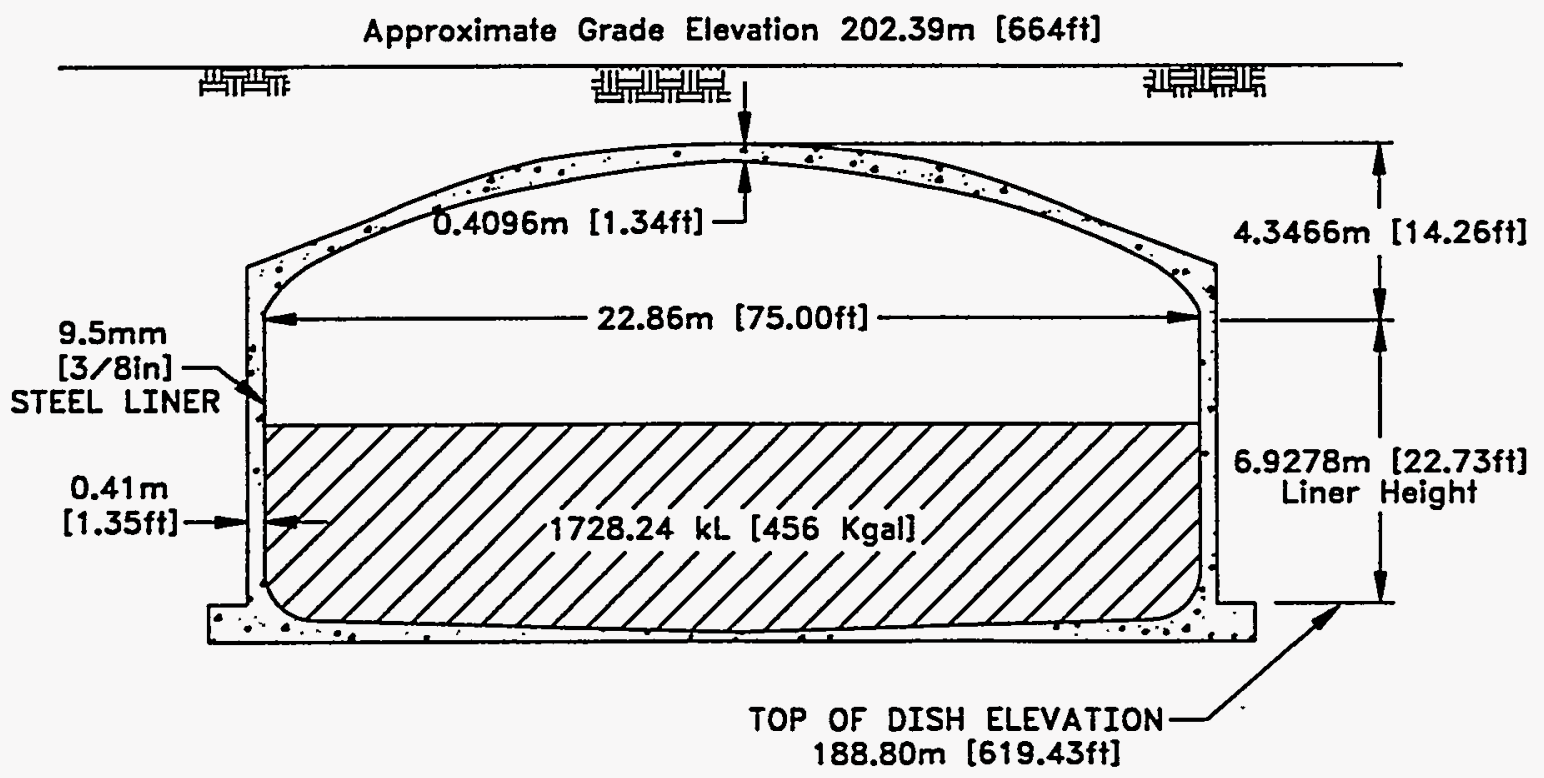

Ref: H-2-1783, Rev. 3

Ref: $\mathrm{H}-2-46293$, Rev. 3 
WHC-SD-WM-ER-323, REV. 0

\begin{tabular}{|c||c||l||}
\hline \multicolumn{3}{|c||}{ 241-S-106 } \\
\hline \hline NO. & DIA. & \multicolumn{1}{|||}{ DESCRIPTION AND COMMENTS } \\
\hline \hline 1 & $4 "$ & (CONN NOZZLE) PUMP PIT DRAIN \\
\hline 2 & $4 "$ & TEMPERATURE PROBE, TO CASS, BM \\
\hline 3 & $4 "$ & FIC - INTRUSION MODE \\
\hline 4 & $4 "$ & FLANGE/B-436 LOW \\
\hline 5 & $12 "$ & SALTWELL SCREEN AND PUMP \\
\hline 6 & $12 "$ & FLANGE - INLET NOZZLE IN RISER, FOAMED \\
\hline 7 & $12^{\prime \prime}$ & FLANGE \\
\hline 8 & $12^{\prime \prime}$ & OBSV PORT \\
\hline 11 & $4 "$ & FLANGE/SMP . \\
\hline 13 & $42^{\prime \prime}$ & SLURRY DISTRIBUTOR \\
\hline 14 & $4 "$ & FLANGE/SMP \\
\hline 16 & $4 "$ & BM, BREATHER FILTER, G-1 HOUS ING \\
\hline
\end{tabular}




\section{$241-S-106$ \\ $3,790,000$ Liters \\ [758,000 Gallons]}

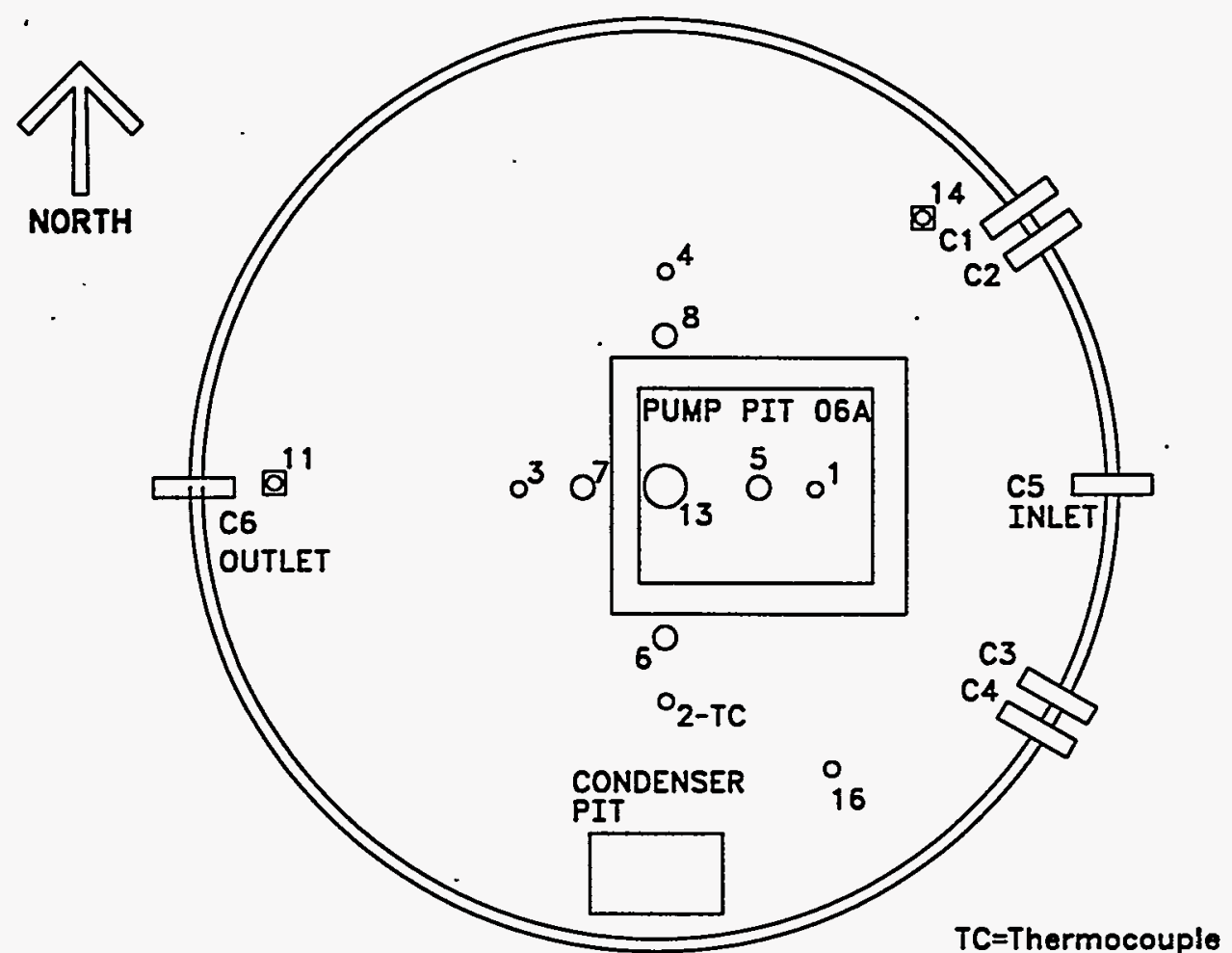

Ref: WHC-SD-RE-TI-053, Rev. 8 WHC-SD-WM-TI-553, Rev. 0 H-2-73185, Rev. 4

\section{TANK RISER LOCATION} Approximate Grade Elevation $202.08 \mathrm{~m}$ [663ft]

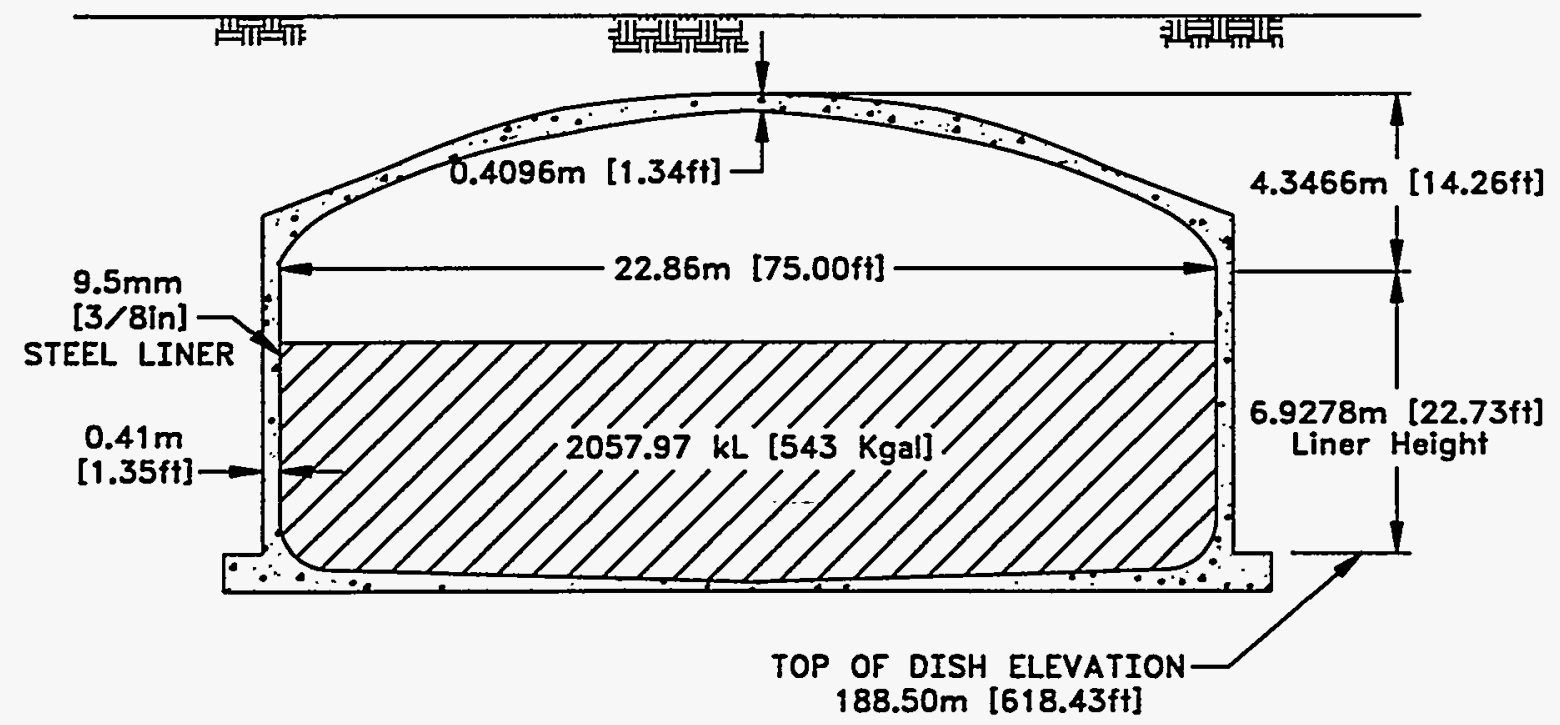

Ref: H-2-1783, Rev. 3 


\begin{tabular}{||c||c||l||}
\hline \multicolumn{3}{|c||}{ 241-S-107 } \\
\hline \hline NO. & DIA. & \multicolumn{1}{|||}{ DESCRIPTION AND COMMENTS } \\
\hline \hline 1 & $4 "$ & (CONN NOZZLE) PUMP PIT DRAIN \\
\hline 2 & $4 "$ & FLANGE WITH SPOOL PIECE, BM \\
\hline 3 & $4 "$ & FIC - INTRUSION MODE \\
\hline 4 & $4 "$ & TEMPERATURE PROBE, MANUAL B-221 \\
\hline 5 & $12 "$ & SALTWELL SCREEN AND PUMP \\
\hline 6 & $12^{\prime \prime}$ & FLANGE/B-222 OBSV PORT \\
\hline 7 & $12^{\prime \prime}$ & FLANGE \\
\hline 8 & $12^{\prime \prime}$ & BG \\
\hline 11 & $4 "$ & FLANGE/SMP \\
\hline 13 & $42^{\prime \prime}$ & (SLURRY DISTRIBUTOR) \\
\hline 14 & $4 "$ & FLANGE/SMP \\
\hline 16 & $4 "$ & BM, BREATHER FILTER, G-1 HOUSING \\
\hline
\end{tabular}




\section{$241-S-107$}

3,790,000 Liters

[758,000 Gallons]

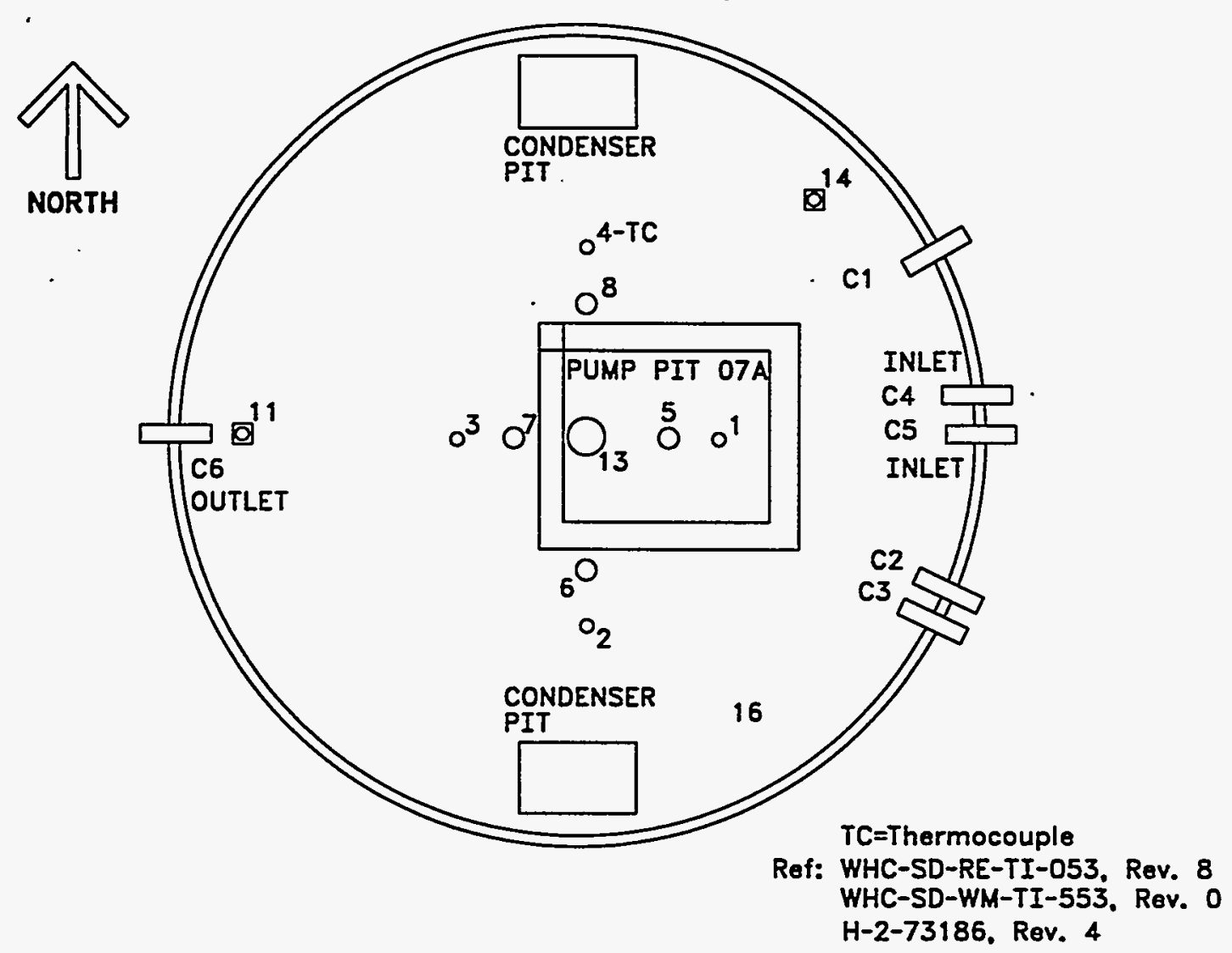

TANK RISER LOCATION

Approximate Grade Elevation 202.69m [665ft]

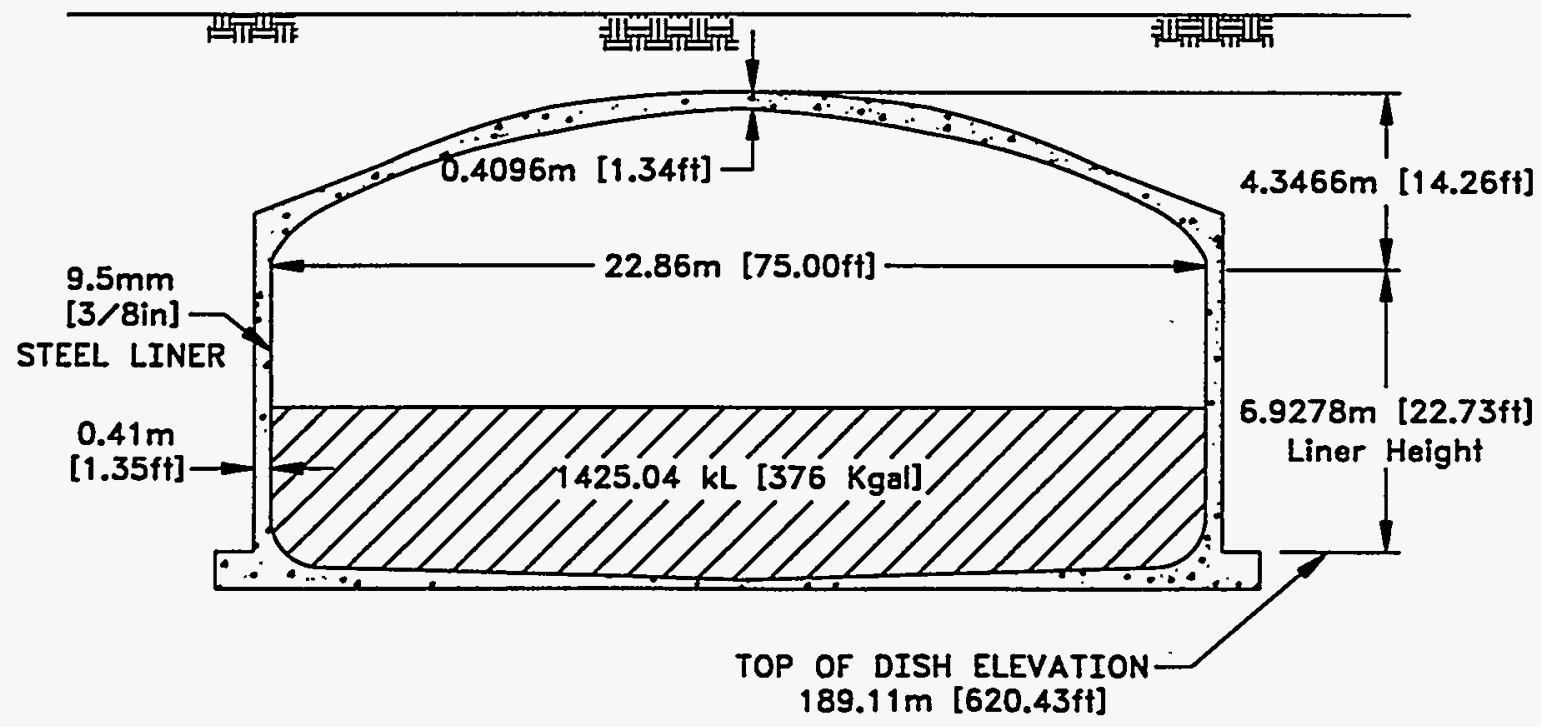

Ref: H-2-1783, Rev. 3

G-15

Ref: H-2-46293, Rev. 3 
WHC-SD-WM-ER-323, REV. 0

\begin{tabular}{|c|c|c|}
\hline NO. & DIA. & DESCRIPTION AND COMMENTS \\
\hline 1 & $4^{\prime \prime}$ & (CONN NOZZLE) PUMP PIT DRAIN \\
\hline 2 & $4 "$ & IIQUID LEVEL REEL, BM \\
\hline 3 & $4 "$ & FIC \\
\hline 4 & $4 n$ & TEMPERATURE PROBE, TO CASS \\
\hline 5 & $12 "$ & SALTWELL SCREEN AND PUMP \\
\hline 6 & $12^{\prime \prime}$ & FLANGE \\
\hline 7 & $12 "$ & FLANGE WITH LIFTING BALE/B-222 OBSV PORT \\
\hline 8 & $12 "$ & LEAD ENCASEMENT \\
\hline 11 & $4 "$ & SMP \\
\hline 13 & $42^{\prime \prime}$ & (SLURRY DISTRIBUTOR) \\
\hline 14 & $4 "$ & BREATHER FILTER， G-I HOUSING \\
\hline 16 & $4 "$ & LOW, BM \\
\hline
\end{tabular}




\section{$241-S-108$}

3,970,000 Liters

[758,000 Gallons]

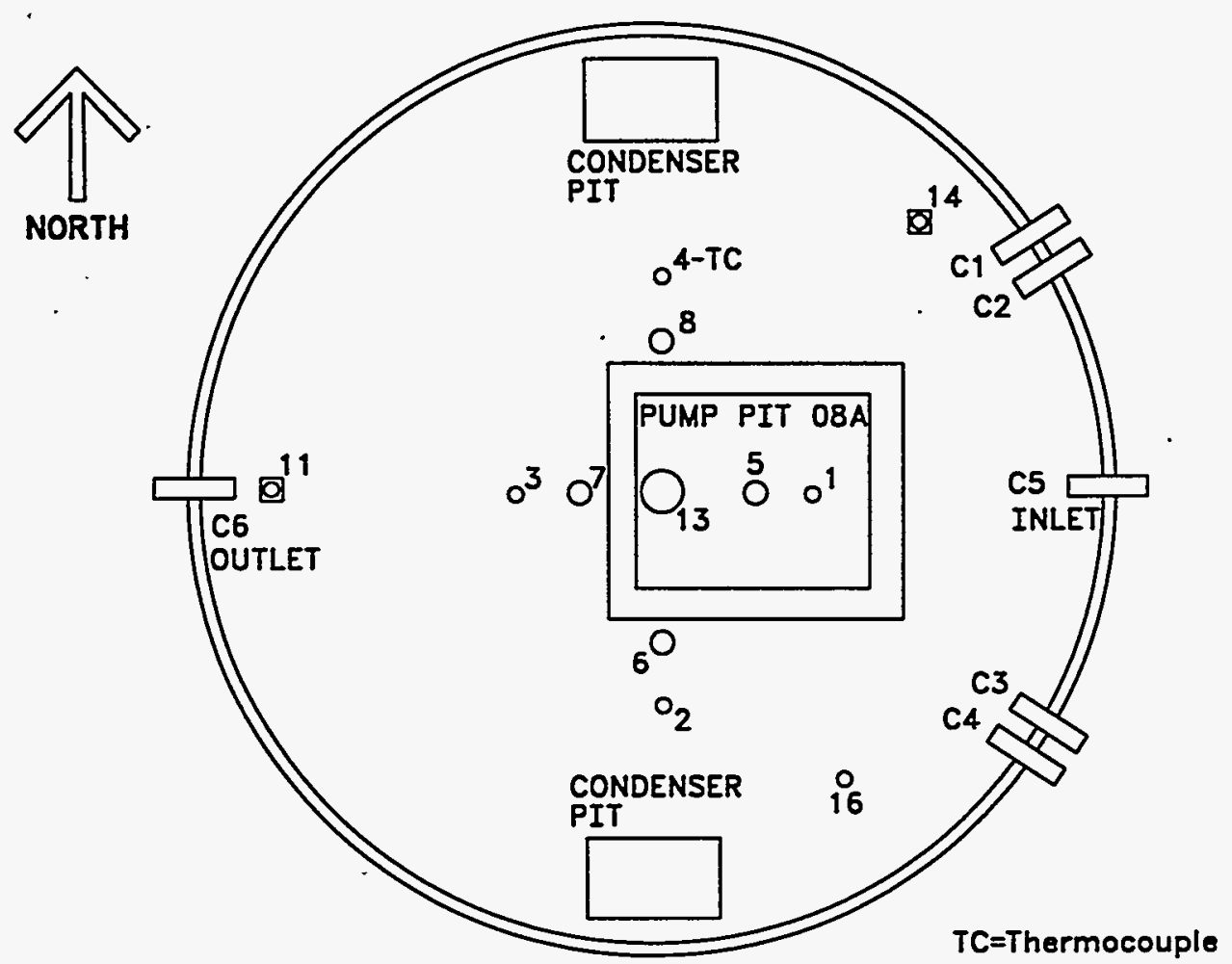

Ref: WHC-SD-RE-TI-053, Rev. 8 WHC-SD-WM-TI-553, Rev. 0 H-2-73187. Rev. 4

\section{TANK RISER LOCATION}

Approximate Grade Elevation 202.39m [664ft]

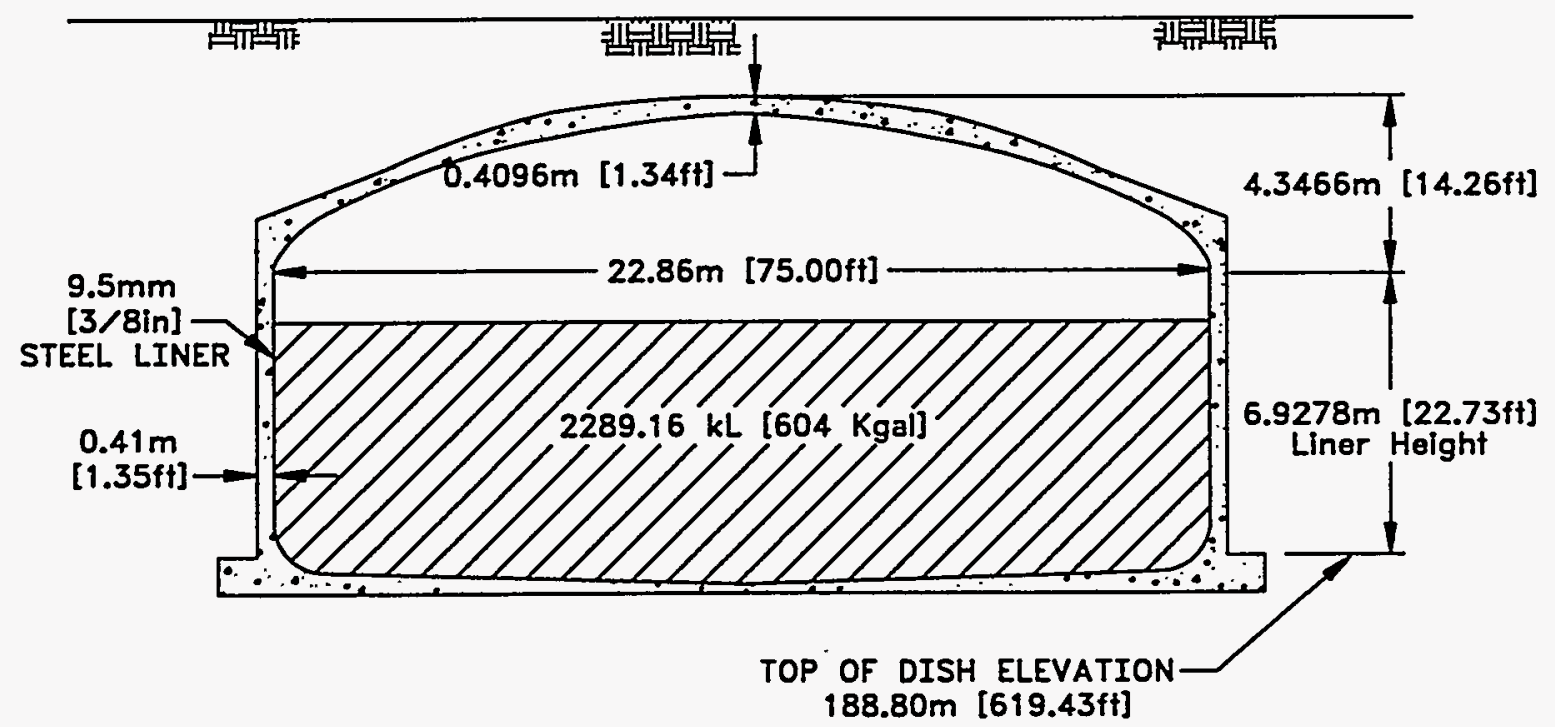

Ref: H-2-1783, Rev. 3 
WHC-SD-WM-ER-323, REV. 0

\begin{tabular}{||c||c||l||}
\hline \multicolumn{2}{|c||}{ 241-S-109 } \\
\hline \hline NO. & DIA. & \multicolumn{1}{|c||}{ DESCRIPTION AND COMMENTS } \\
\hline \hline 1 & $4 "$ & (CONN NOZZLE) PUMP PIT DRAIN \\
\hline 2 & $4 "$ & BREATHER FILTER, G-1 HOUSING \\
\hline 3 & $4 "$ & FIC \\
\hline 4 & $4 "$ & TEMPERATURE PROBE, TO CASS \\
\hline 5 & $12^{\prime \prime}$ & SALTWELI SCREEN AND PUMP \\
\hline 6 & $12^{\prime \prime}$ & OBSV PORT \\
\hline 7 & $12^{\prime \prime}$ & FLANGE \\
\hline 8 & $12^{\prime \prime}$ & LOW/B-436 LOW \\
\hline 11 & $4 "$ & FLANGE/SMP \\
\hline 13 & $42^{\prime \prime}$ & SLURRY DISTRIBUTOR \\
\hline 14 & $4 "$ & FLANGE/SMP \\
\hline 16 & $4 "$ & FLANGE/SMP, BM \\
\hline
\end{tabular}




\section{1-S-109 \\ 3,970,000 Liters \\ [758,000 Gallons]}

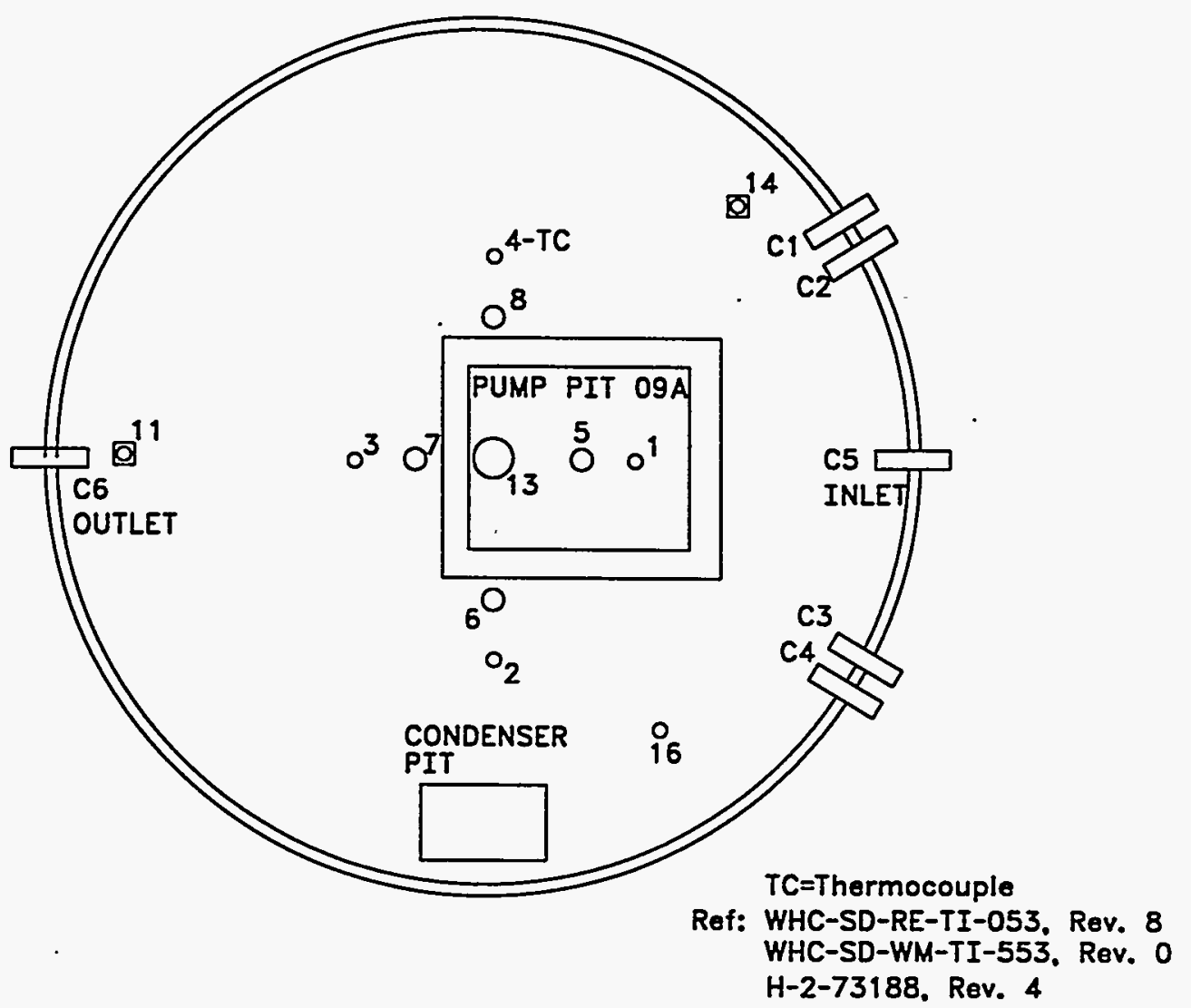

TANK RISER LOCATION

Approximate Grade Elevation 202.08m [663ft]

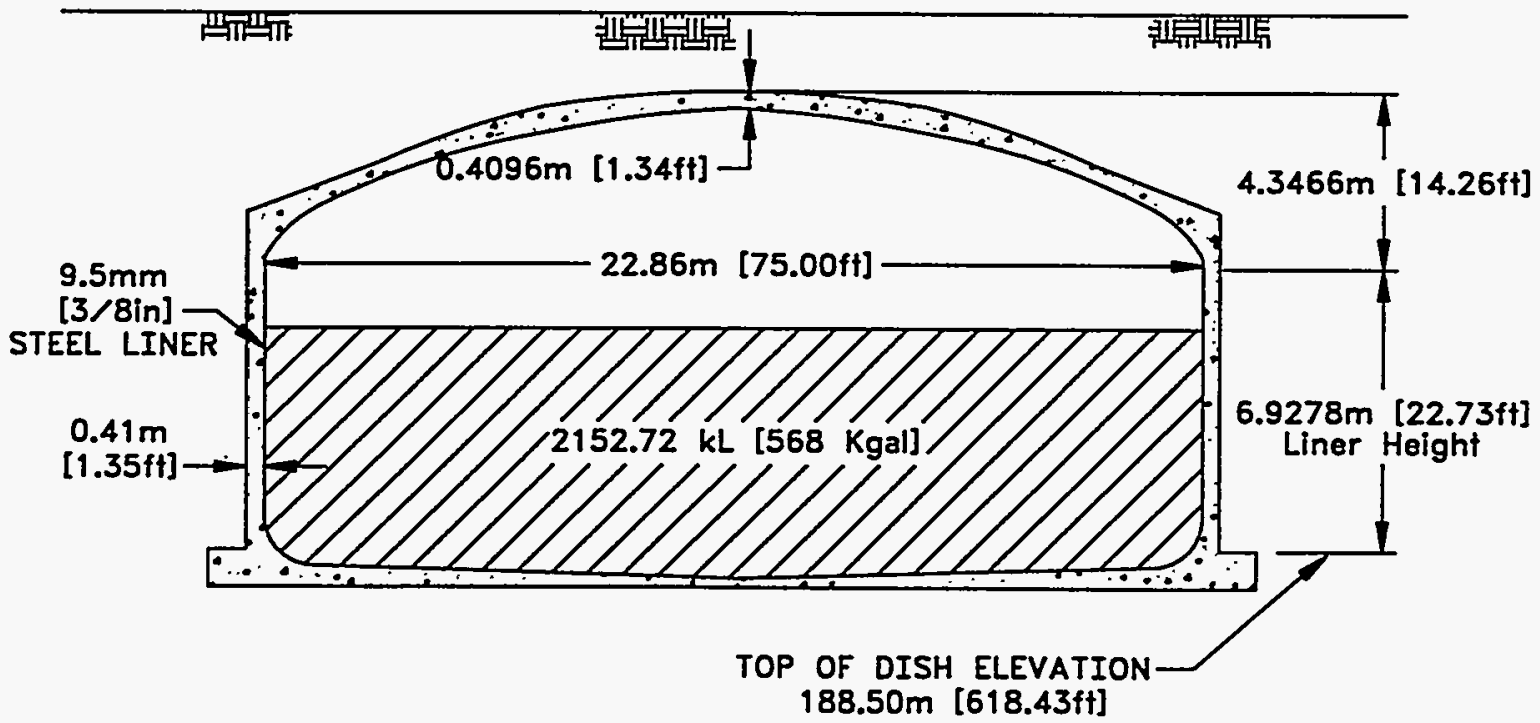

Ref: H-2-1783, Rev. 3

G-19

Ref: H-2-46293. Rev. 3 
WHC-SD-WM-ER-323, REV. 0

\begin{tabular}{||c||c||l||}
\hline \multicolumn{3}{|c||}{ 241-S-110 } \\
\hline \hline NO. & DIA. & \multicolumn{1}{||}{ DESCRIPTION AND COMMENTS } \\
\hline \hline 1 & $4^{\prime \prime}$ & (CONN NOZZLE) PUMP PIT DRAIN \\
\hline 2 & $4^{\prime \prime}$ & FLANGE/B-436 LOW, BM \\
\hline 3 & $4 "$ & FIC \\
\hline 4 & $4 "$ & TEMPERATURE PROBE, MANUAL B-221 \\
\hline 5 & $12^{\prime \prime}$ & SALTWELL SCREEN AND PUMP \\
\hline 6 & $12^{\prime \prime}$ & FLANGE \\
\hline 7 & $12^{\prime \prime}$ & OBSV PORT \\
\hline 8 & $12^{\prime \prime}$ & FLANGE W/2" CAPS \\
\hline 11 & $4^{\prime \prime}$ & SMP \\
\hline 13 & $42^{\prime \prime}$ & SLURRY DISTRIBUTOR \\
\hline 14 & $4 "$ & SMP \\
\hline 16 & $4 "$ & BM, BREATHER FILTER, G-1 HOUSING \\
\hline
\end{tabular}




\section{1-S-110}

$3,970,000$ Lifers

[758,000 Gallons]

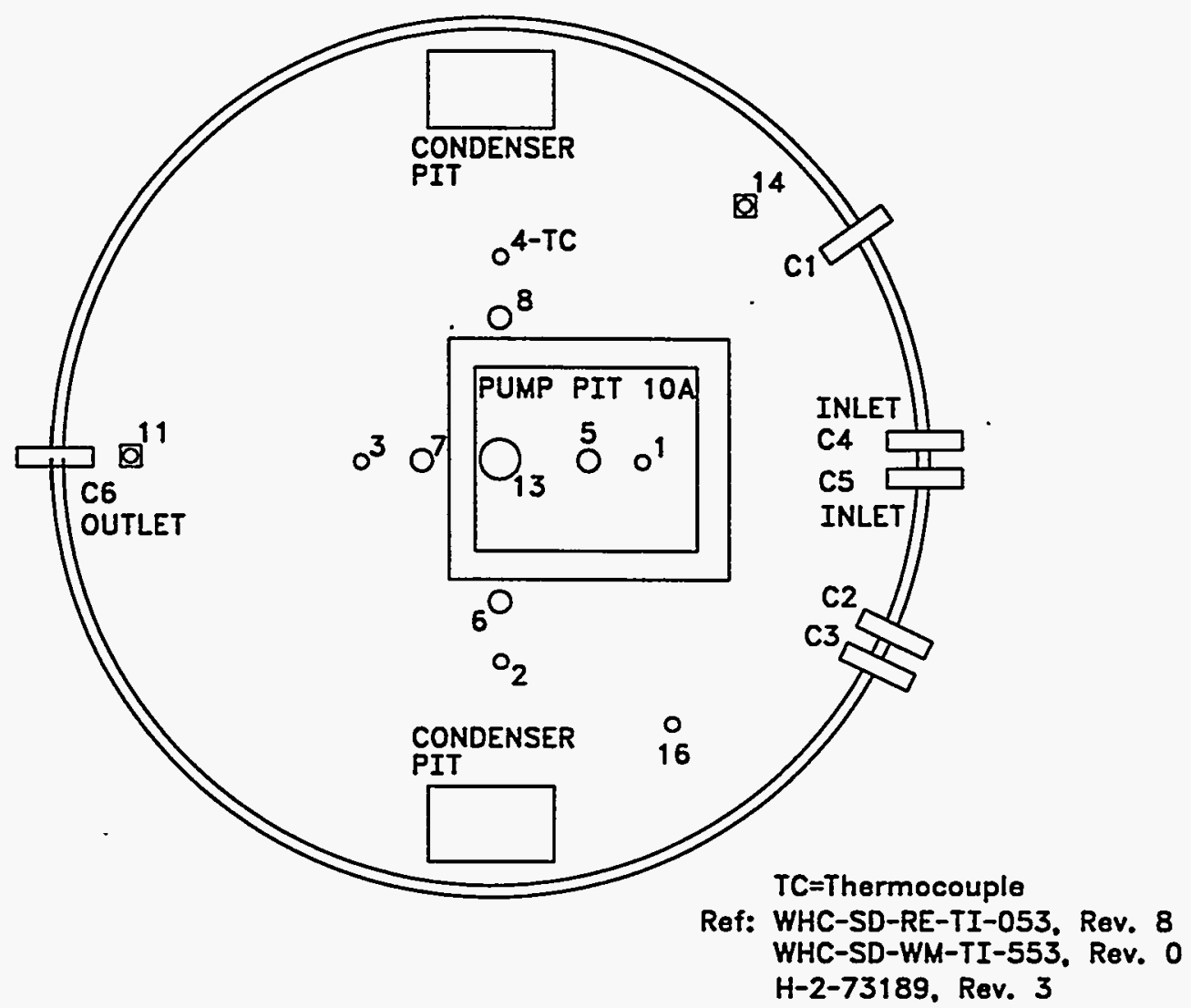

\section{TANK RISER LOCATION}

Approximate Grade Elevation 202.69m [665ft]

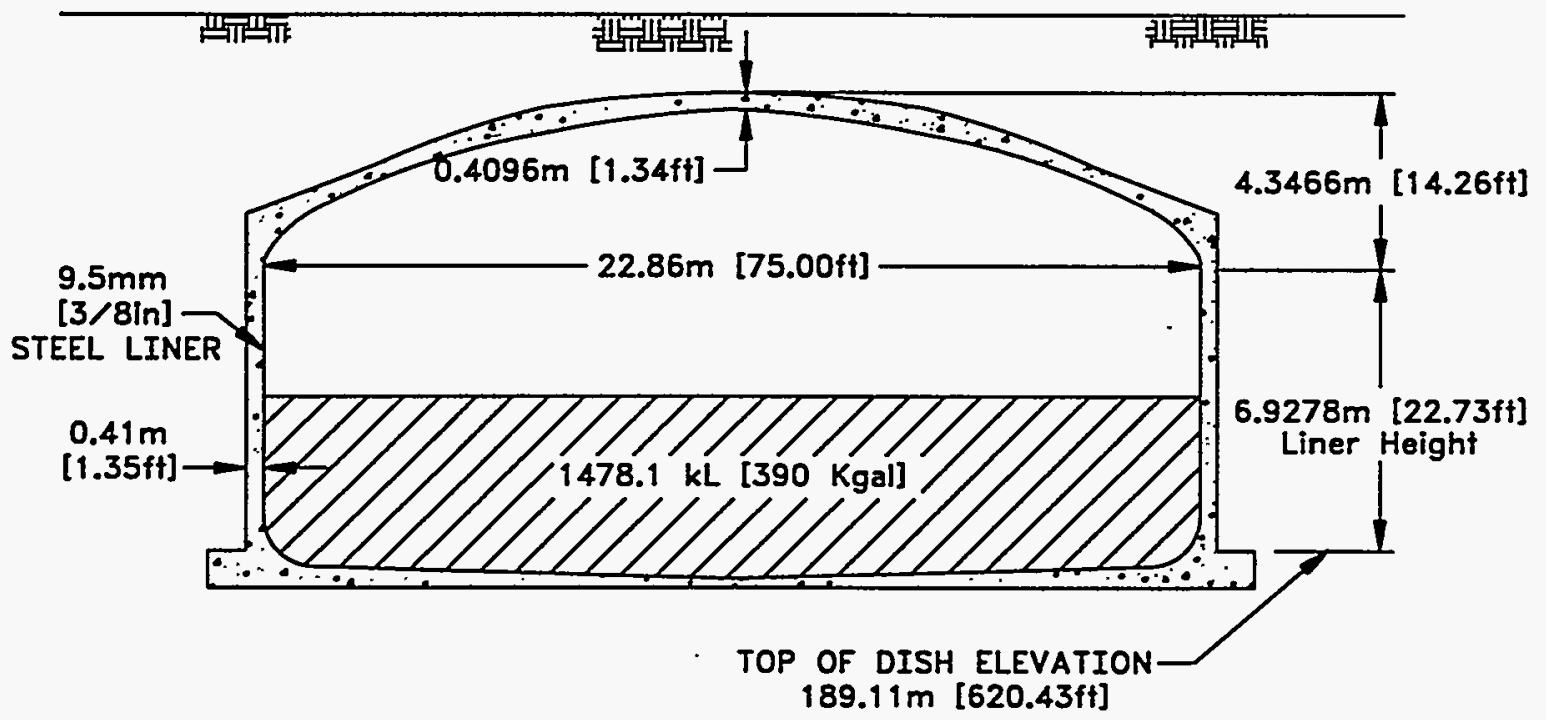

Ref; H-2-1783, Rev. 3

Ref: H-2-46293, Rev. 3 
WHC-SD-WM-ER-323, REV. 0

\begin{tabular}{||c||c||l||}
\hline \multicolumn{1}{|c||}{} \\
\hline \hline NO. & DIA. & \multicolumn{1}{|c||}{ DESCRIPTION AND COMMENTS } \\
\hline \hline 1 & $4 "$ & (CONN NOZZLE) PUMP PIT DRAIN \\
\hline 2 & $4 "$ & FIC, BM \\
\hline 3 & $4 "$ & FIC \\
\hline 4 & $4 "$ & TEMPERATURE PROBE, TO CASS \\
\hline 5 & $12^{\prime \prime}$ & SALTWELI SCREEN AND PUMP \\
\hline 6 & $12 "$ & FLANGE \\
\hline 7 & $12 "$ & FLANGE WITH TUBING \\
\hline 8 & $12^{\prime \prime}$ & FLANGE WITH LIFTING BALE/B-222 OBSV PORT \\
\hline 11 & $4^{\prime \prime}$ & FLAANGE WITH TUBING \\
\hline 13 & $42^{\prime \prime}$ & SLURRY DISTRIBUTOR \\
\hline 14 & $4^{\prime \prime}$ & BREATHER FILTER, G-I HOUSING \\
\hline 16 & $4 "$ & SMP/B-436 LOW, BM \\
\hline
\end{tabular}




\section{$241-S-111$}

3,970,000 Liters [758,000 Gallons]

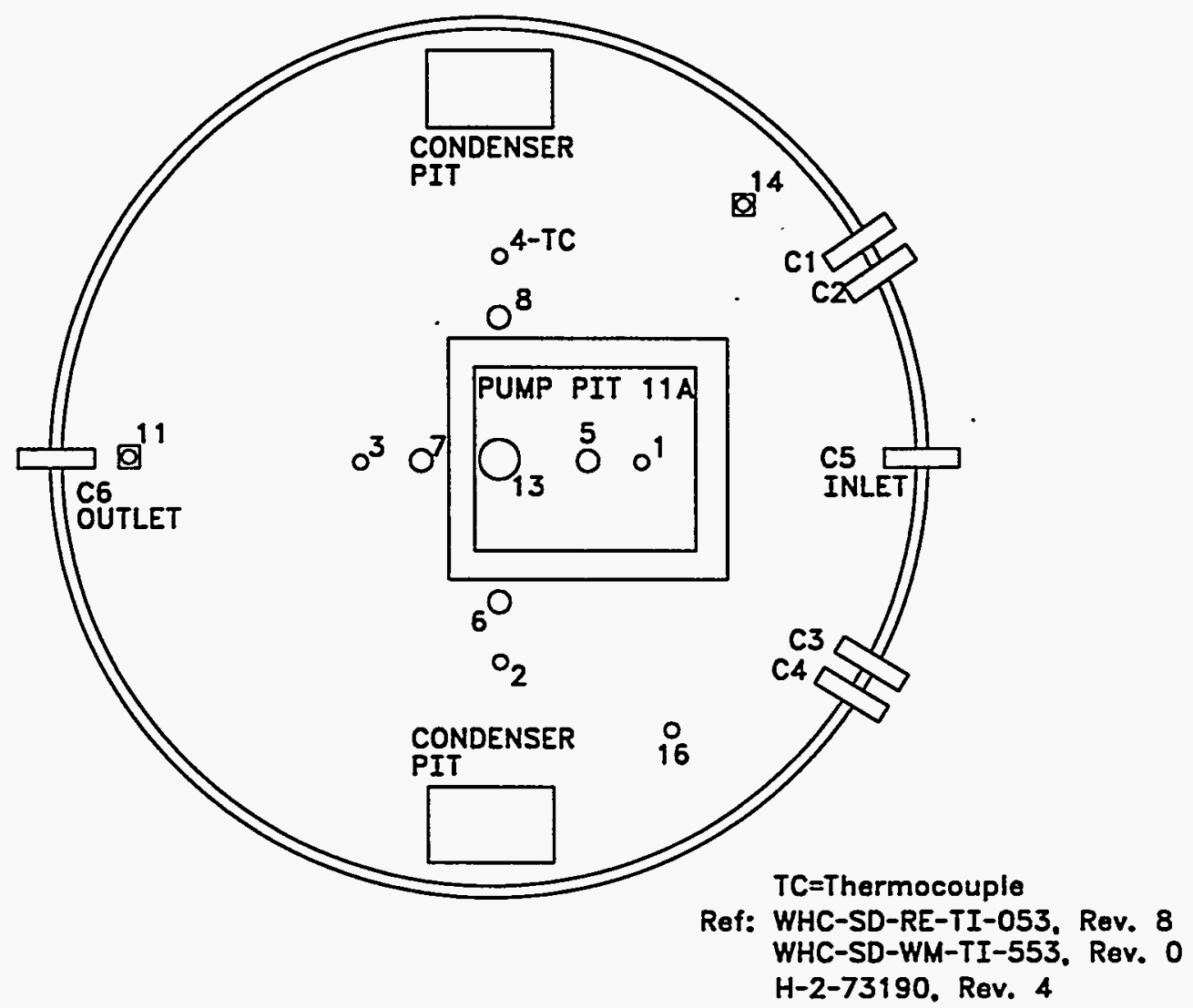

TANK RISER LOCATION

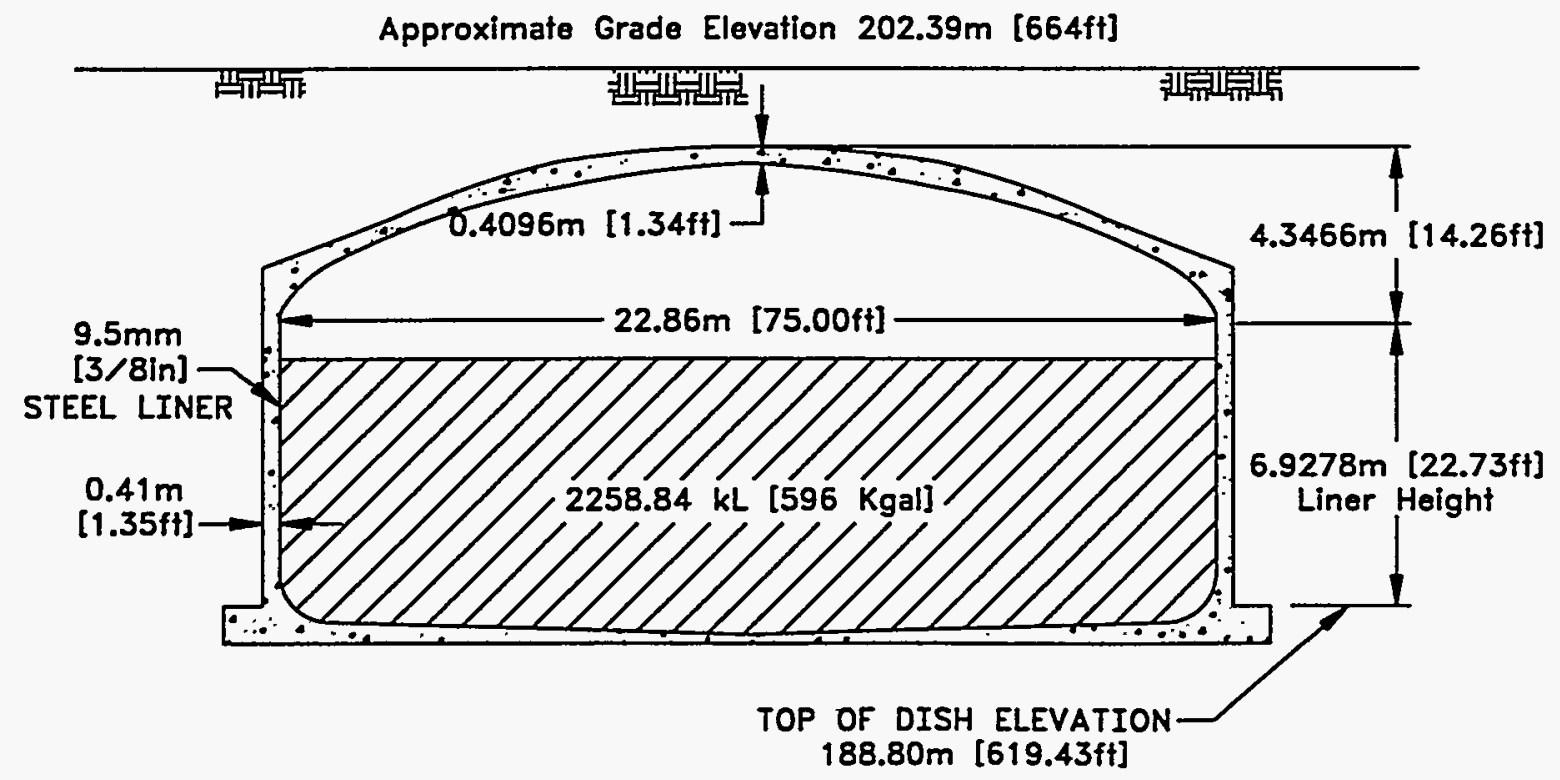

Ref: H-2-1783, Rev. 3 


\begin{tabular}{||c|c||l||}
\hline \multicolumn{2}{|c||}{ 241-S-112 } \\
\hline \hline NO. & DIA. & \multicolumn{1}{|c||}{ DESCRIPTION AND COMMENTS } \\
\hline 1 & $4^{\prime \prime}$ & (CONN NOZZLE) PUMP PIT DRAIN \\
\hline 2 & $4 "$ & FLANGE, BM \\
\hline 3 & $4 "$ & FIC \\
\hline 4 & $4^{\prime \prime}$ & TEMPERATURE PROBE, TO CASS \\
\hline 5 & $12^{\prime \prime}$ & SALTWELI SCREEN AND PUMP \\
\hline 6 & $12^{\prime \prime}$ & OBSV PORT \\
\hline 7 & $12^{\prime \prime}$ & FLANGE \\
\hline 8 & $12^{\prime \prime}$ & B-436 LOW \\
\hline 11 & $4^{\prime \prime}$ & FLANGE/SMP \\
\hline 13 & $42^{\prime \prime}$ & SLURRY DISTRIBUTOR \\
\hline 14 & $4 "$ & BREATHER FILTER, G-1 HOUSING \\
\hline 16 & $4 "$ & FLANGE/SMP, BM \\
\hline
\end{tabular}




\section{$241-S-112$}

3,970,000 Liters

[758,000 Gallons]

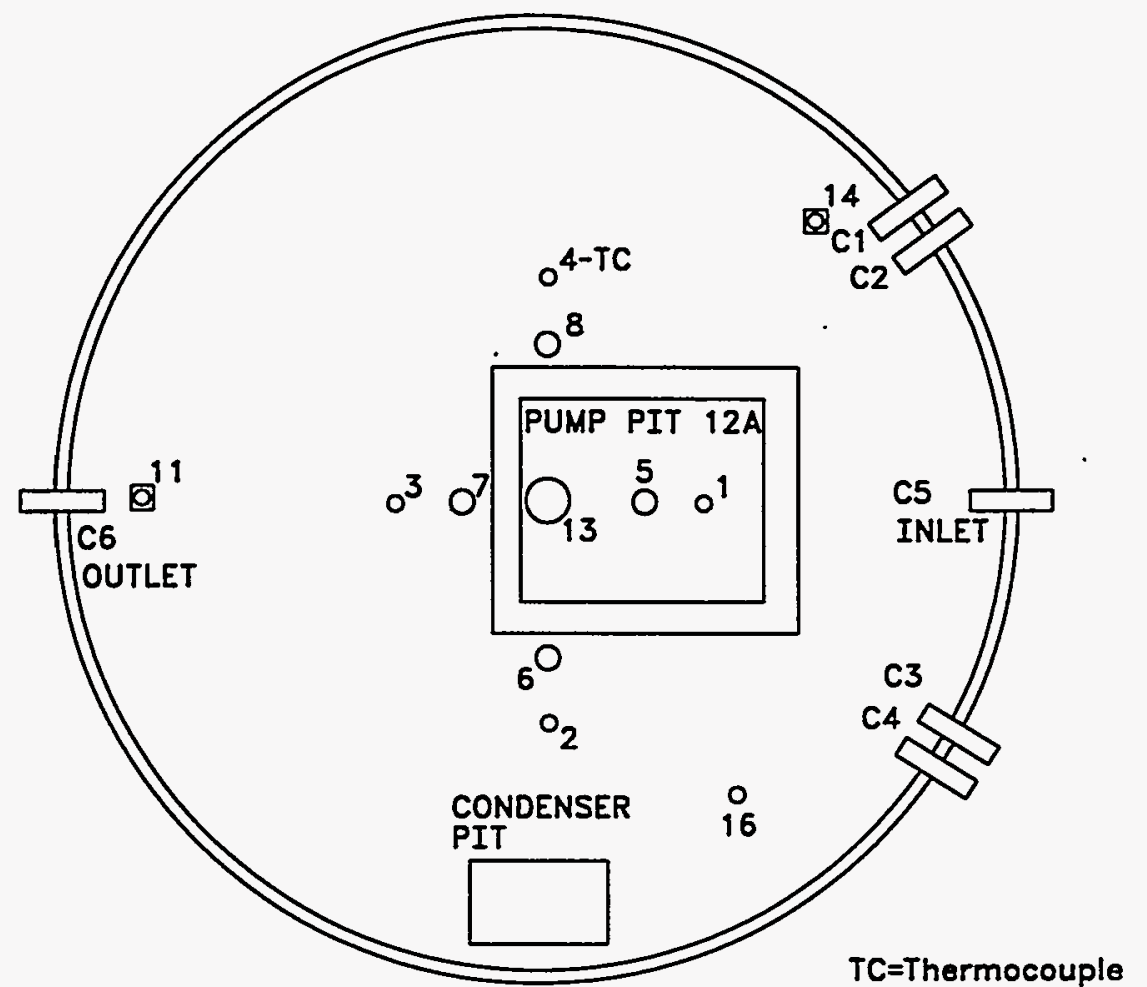

Ref: WHC-SD-RE-TI-053, Rev. 8 WHC-SD-WM-TI-553, Rev. 0

H-2-73191. Rev. 4

\section{TANK RISER LOCATION}

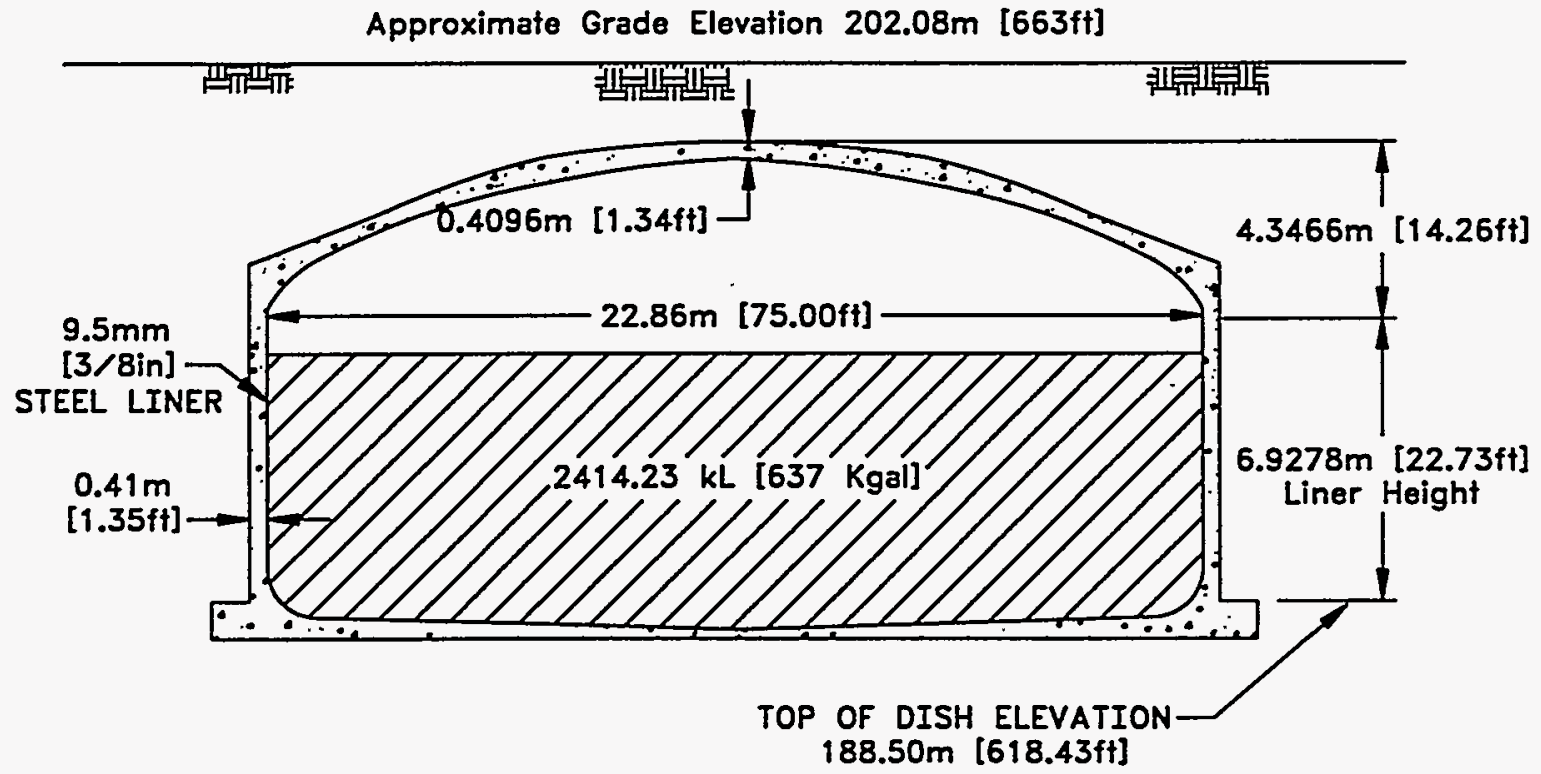

Ref: $\mathrm{H}-2-1783$, Rev. 3 
'त्रue? s!

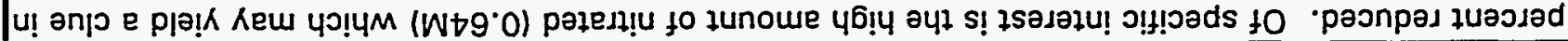

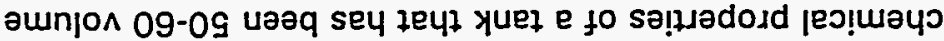

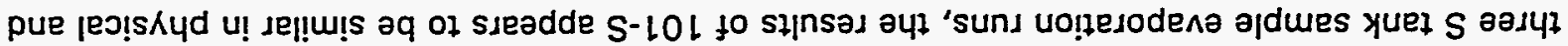

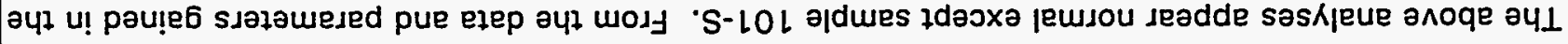

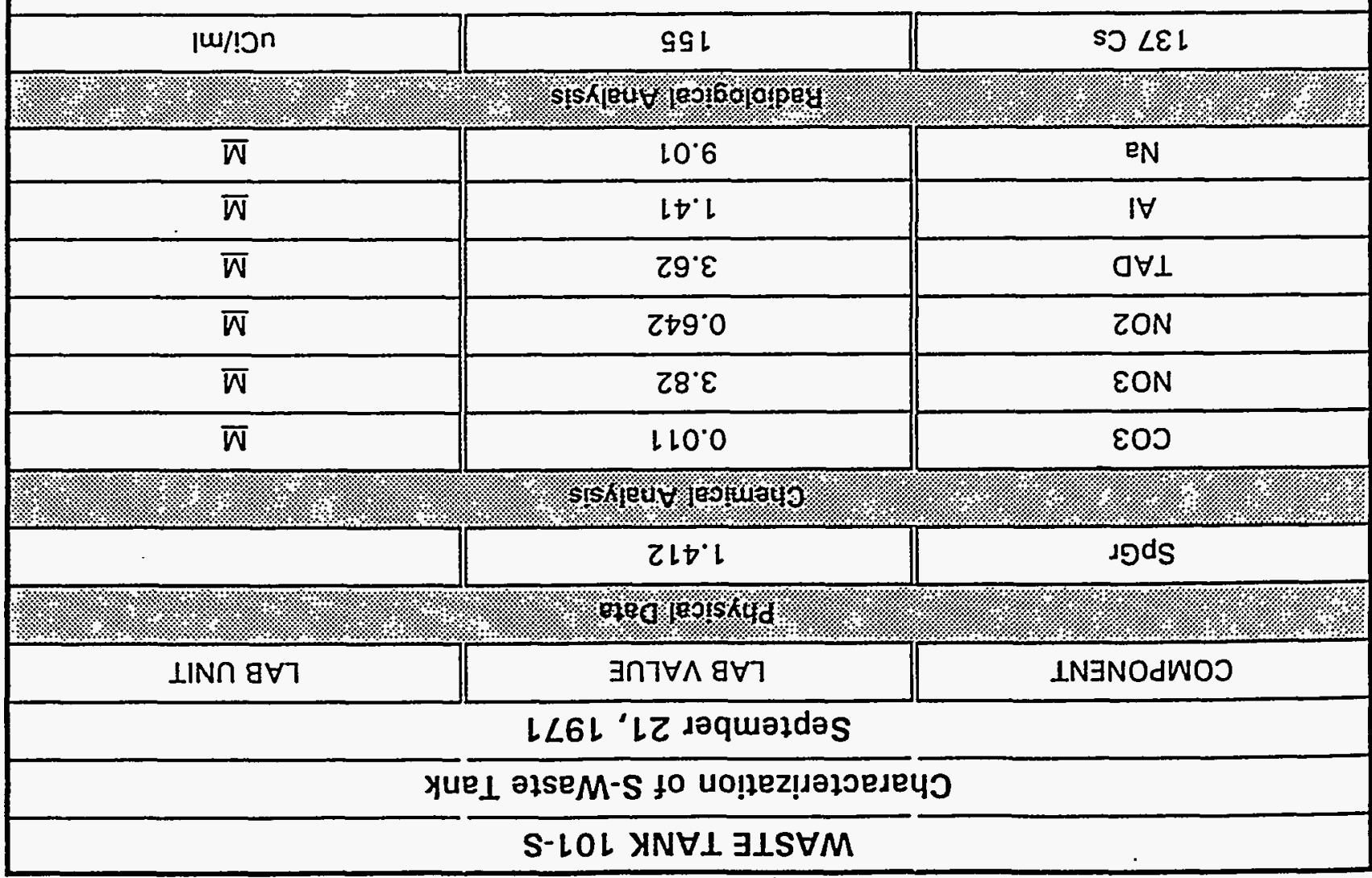




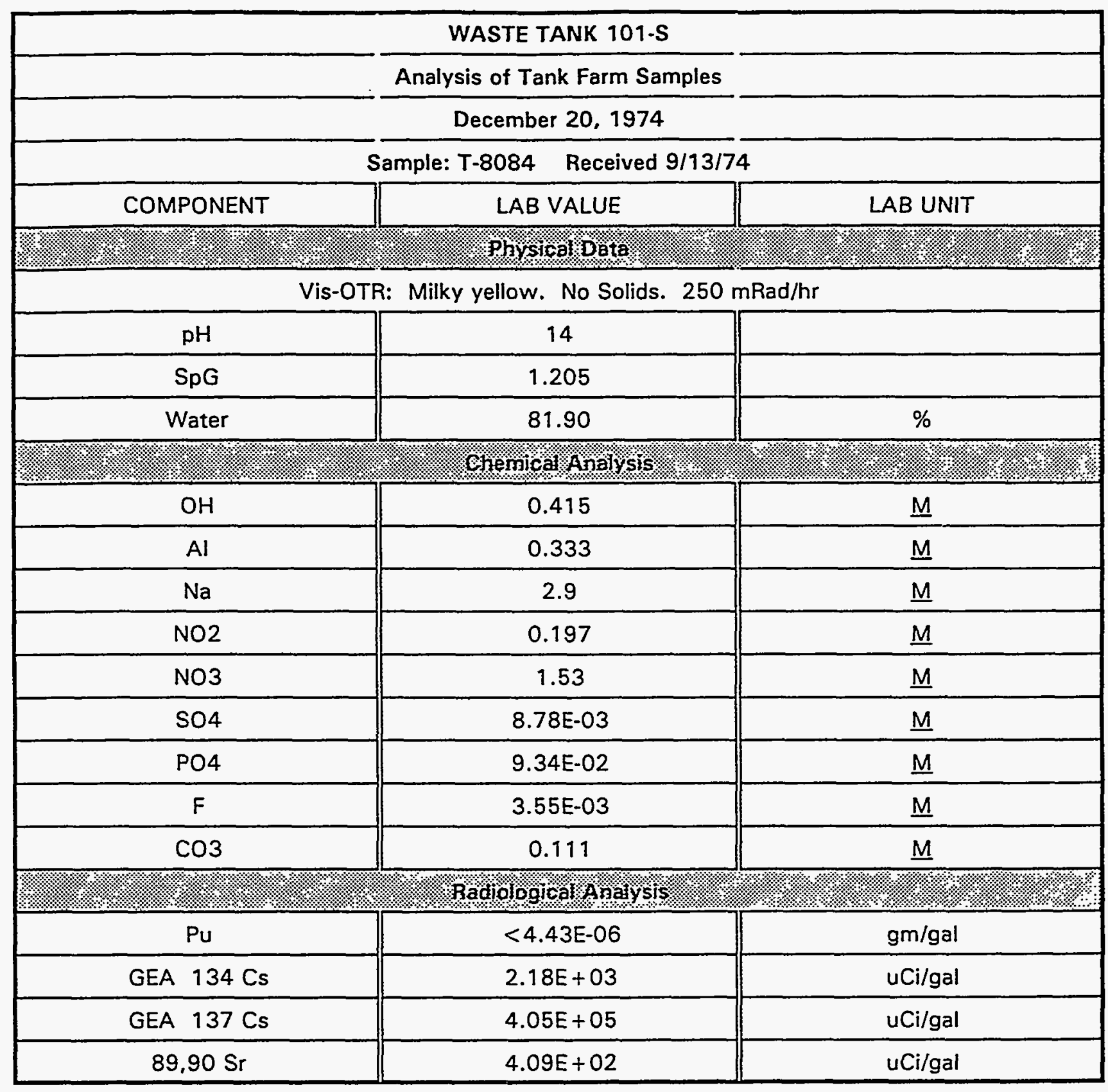




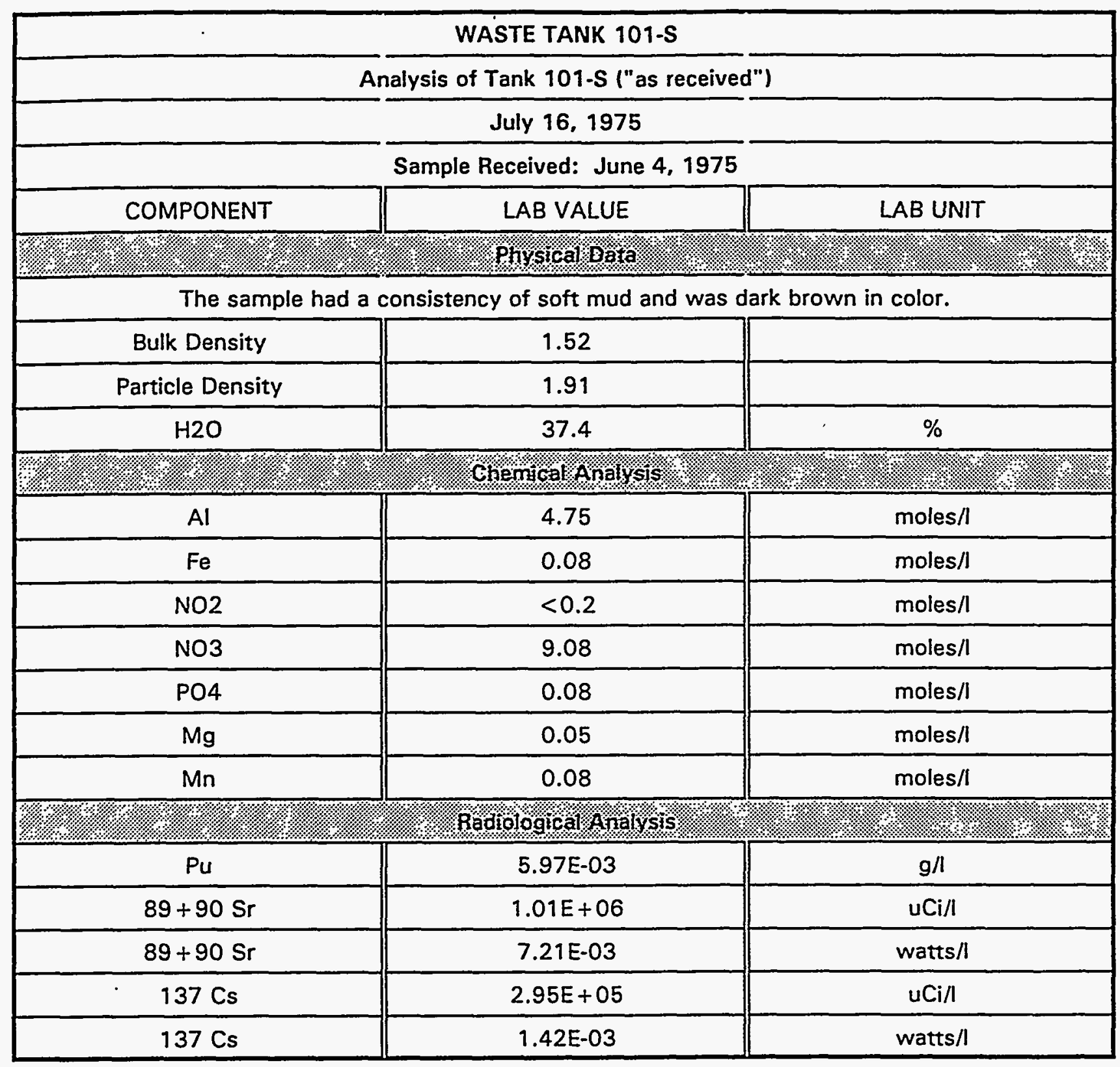




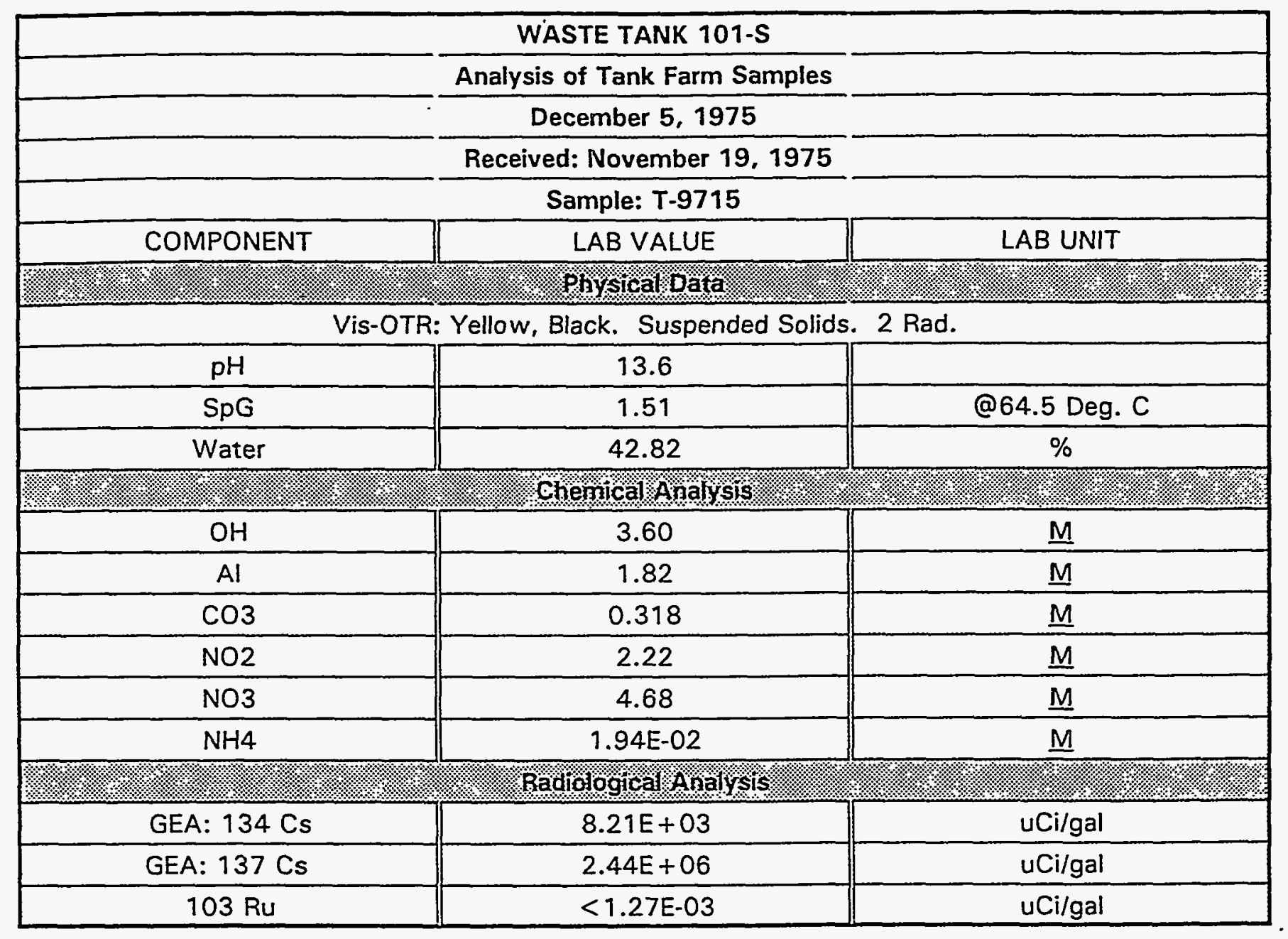




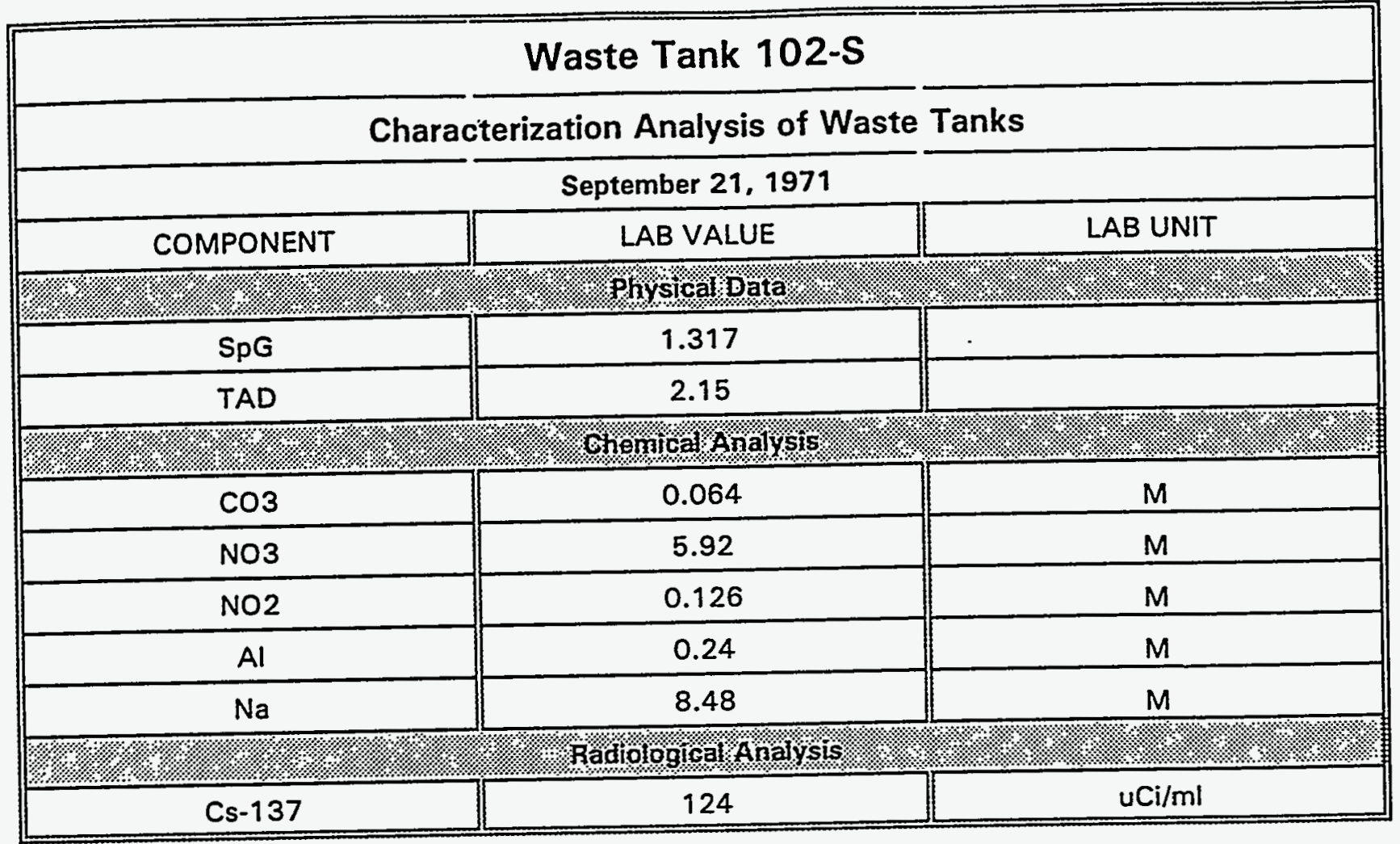




\begin{tabular}{|c|c|c|}
\hline \multicolumn{3}{|c|}{ Waste Tank 102-S } \\
\hline \multicolumn{3}{|c|}{ Analysis and Identification of Solids } \\
\hline \multicolumn{3}{|c|}{ November 27,1973} \\
\hline \multicolumn{3}{|c|}{ Sample Date: November 5,1973 } \\
\hline COMPONENT & LAB VALUE & LAB UNIT \\
\hline$\because \|$ & Hy sical Data & 1. \\
\hline $\mathrm{SpG}$ & 1.2297 & \\
\hline \multicolumn{3}{|c|}{ 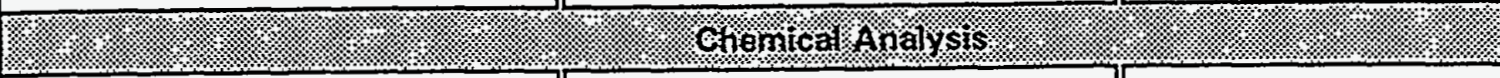 } \\
\hline $\mathrm{Na}$ & 5.09 & $M$ \\
\hline $\mathrm{Al}$ & 0.23 & $M$ \\
\hline NO2 & 0.118 & $M$ \\
\hline NO3 & 4.25 & $M$. \\
\hline $\mathrm{OH}$ & 1.22 & $M$ \\
\hline $\mathrm{PO4}$ & NA & $M$ \\
\hline $\mathrm{CO} 3$ & 0.019 & M \\
\hline SO4 & 0.00988 & $M$ \\
\hline $\mathrm{F}$ & 0.00154 & $\mathrm{M}$ \\
\hline
\end{tabular}




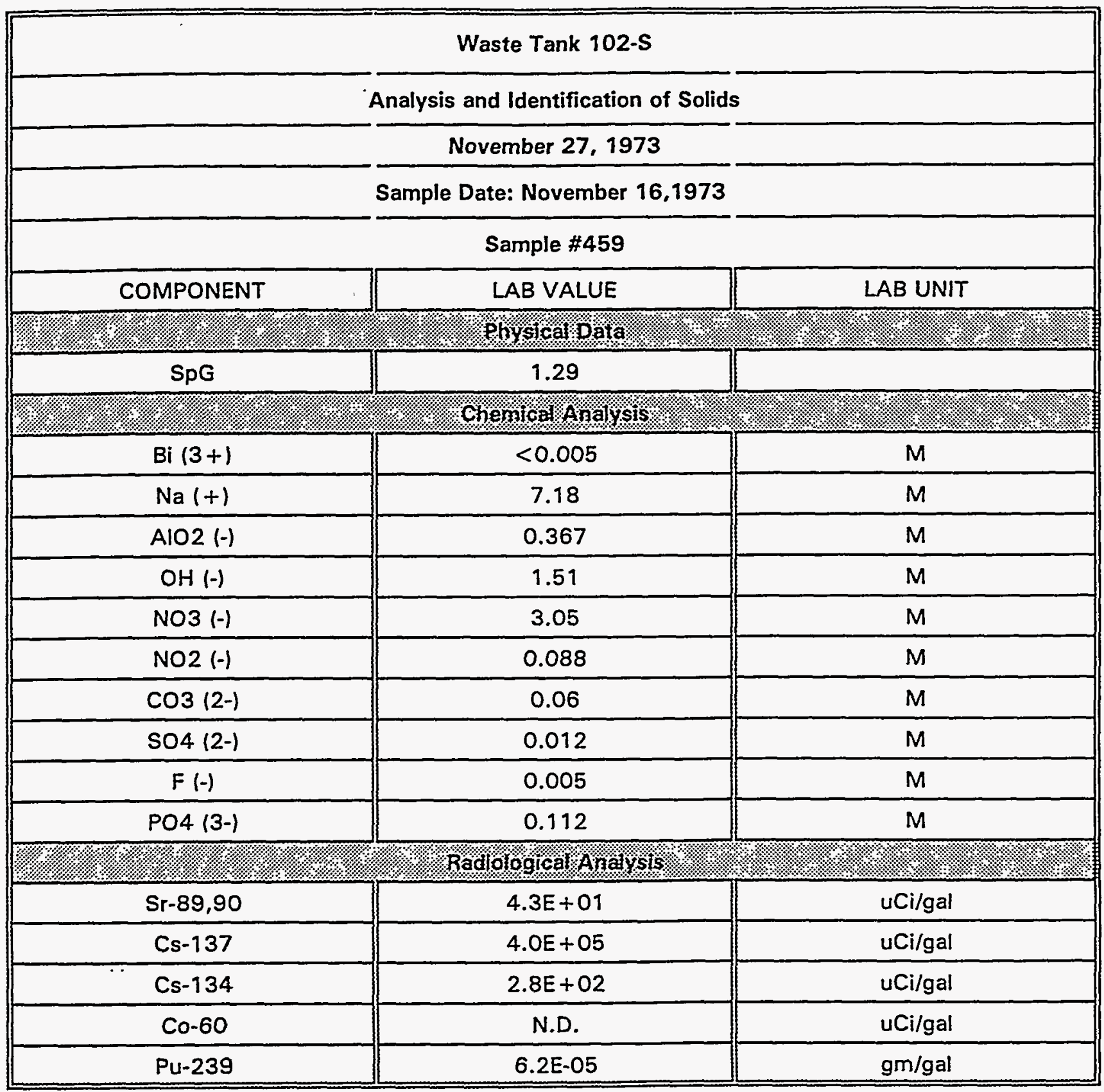




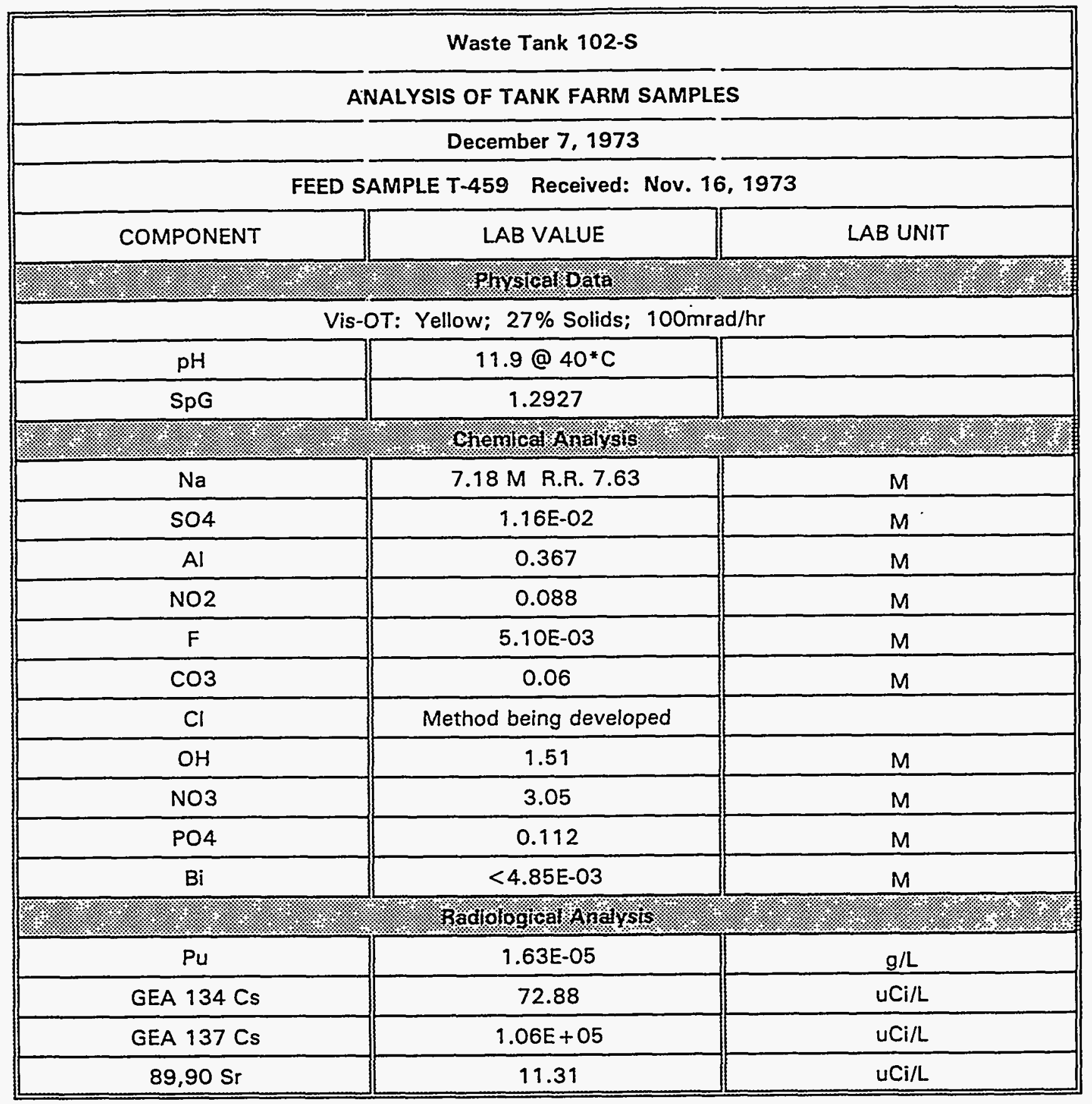




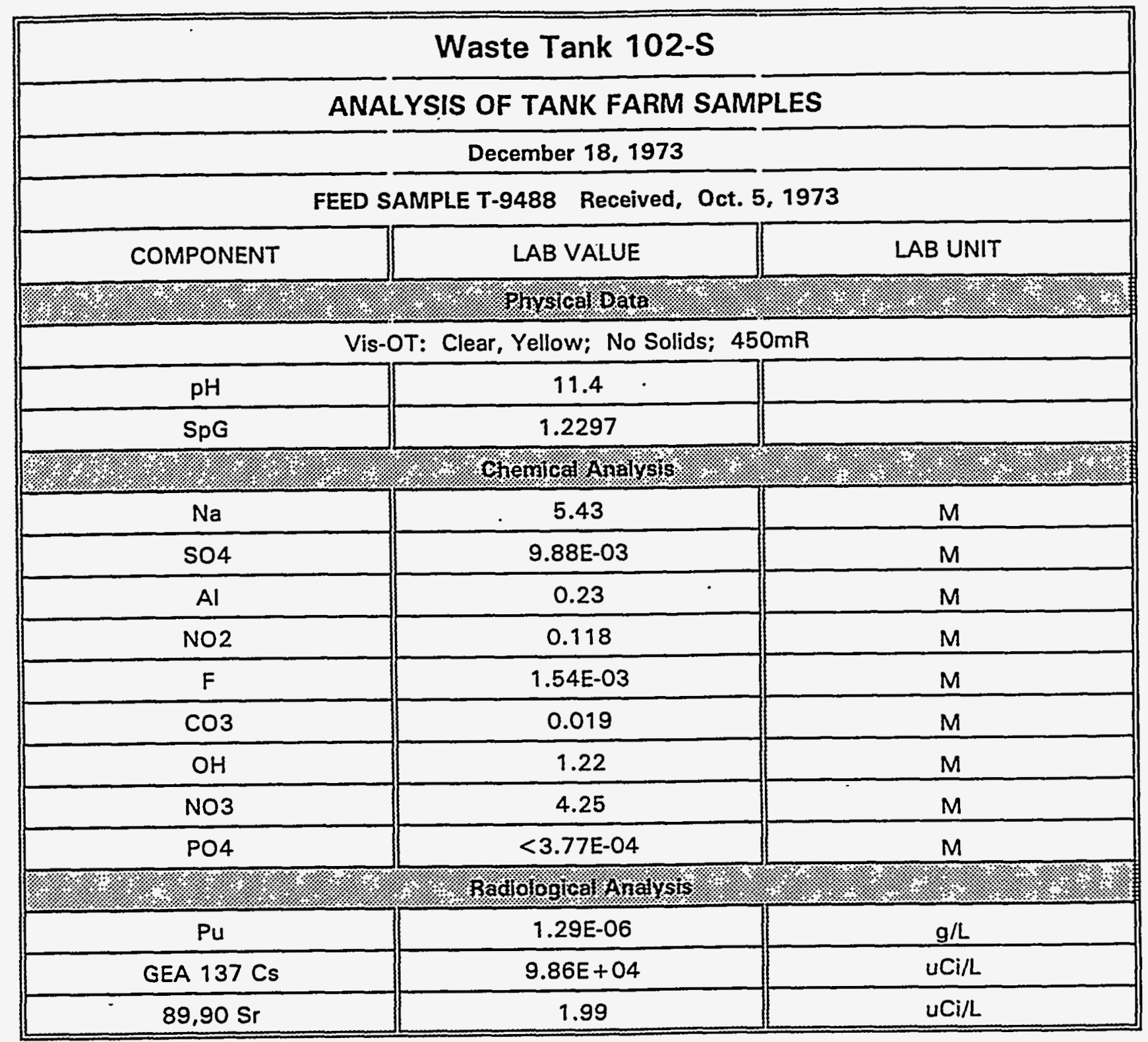




\begin{tabular}{|c|c|c|}
\hline \multicolumn{3}{|c|}{ Waste Tank 102-S } \\
\hline \multicolumn{3}{|c|}{ ANALYSIS OF TANK FARM SAMPLES } \\
\hline \multicolumn{3}{|c|}{ January 25,1974} \\
\hline \multicolumn{3}{|c|}{ SAMPLE T-1746 } \\
\hline COMPONENT & LAB VALUE & LAB UNIT \\
\hline ?.:य & brsical pat: & $2 \geqslant$ \\
\hline \multicolumn{3}{|c|}{ Vis-OTR: Clear, Yellow; $1000 \mathrm{mRad} / \mathrm{hr}$} \\
\hline \multicolumn{3}{|c|}{ Appearance - $90 \%$ Solids at Room Temperature } \\
\hline $\mathrm{pH}$ & 10.5 & \\
\hline $\operatorname{SpG}$ & 1.4081 & \\
\hline Water & 59.39 & $\%$ \\
\hline ?:4: & floglcal/Ana & 1.8 \\
\hline GEA $137 \mathrm{Cs}$ & $1.98 E+05$ & uCi/L \\
\hline
\end{tabular}


WHC-SD-WM-ER-323, Rev. 0

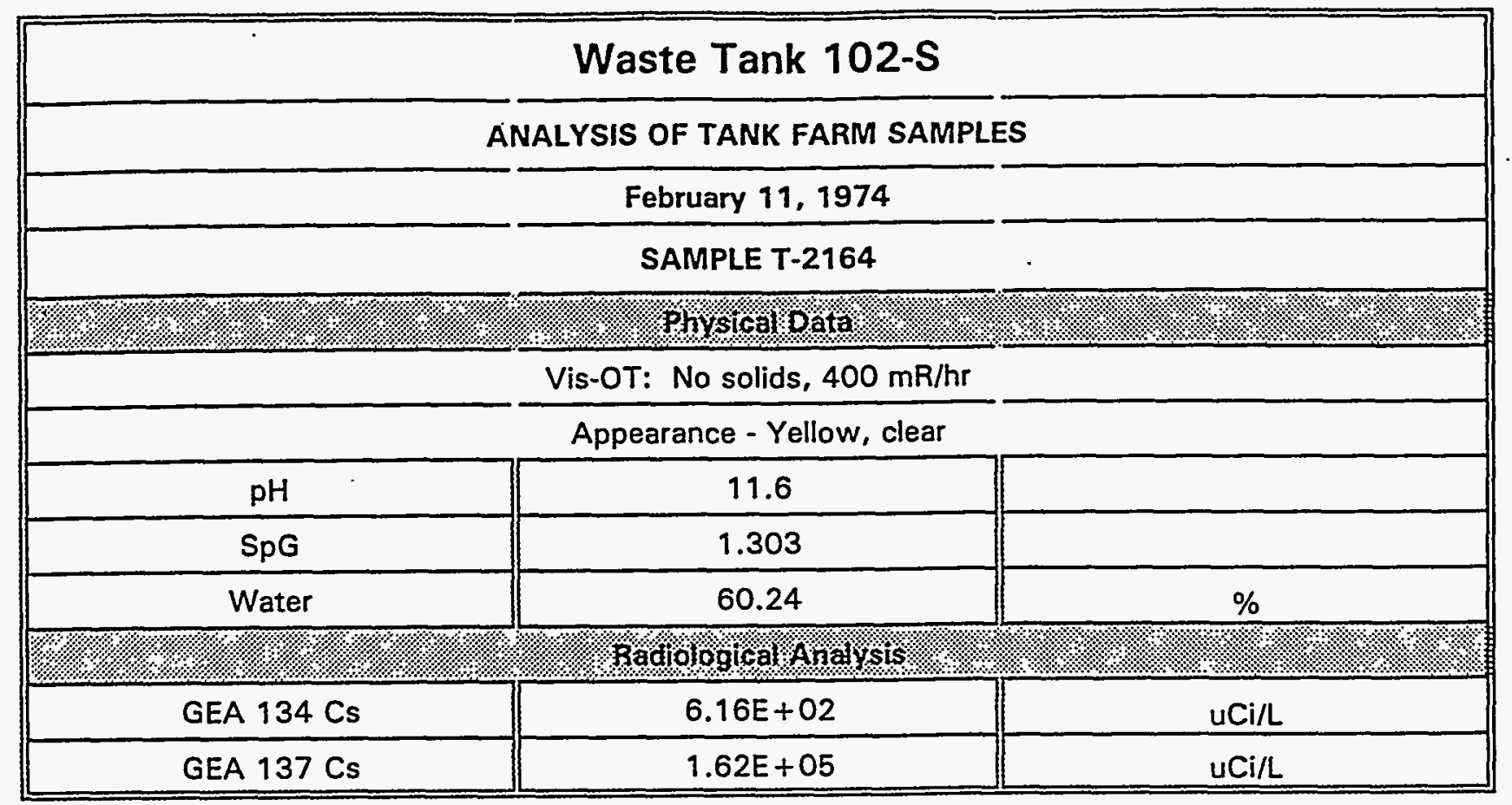


WHC-SD-WM-ER-323, Rev. 0

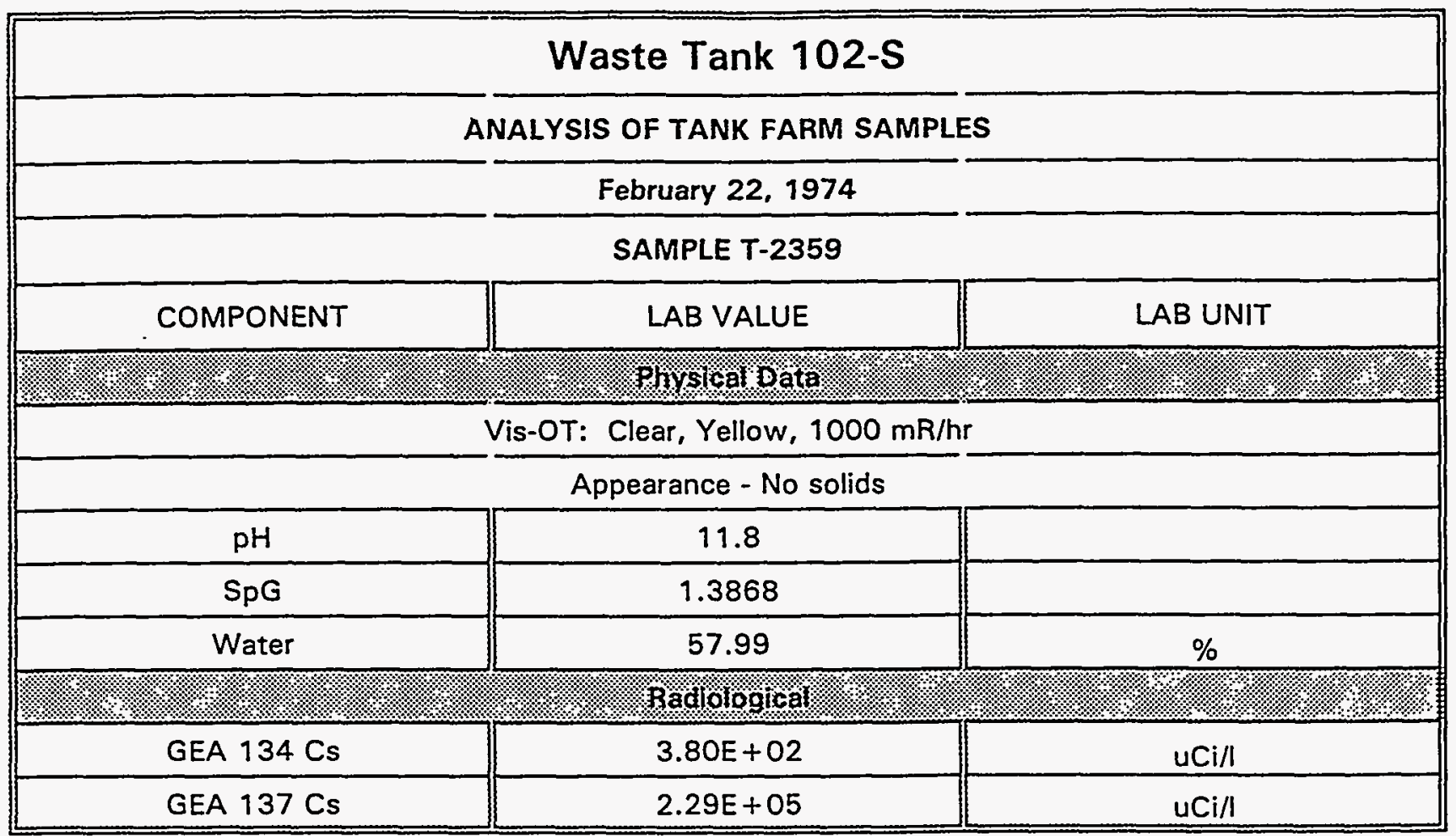


WHC-SD-WM-ER-323, Rev. O

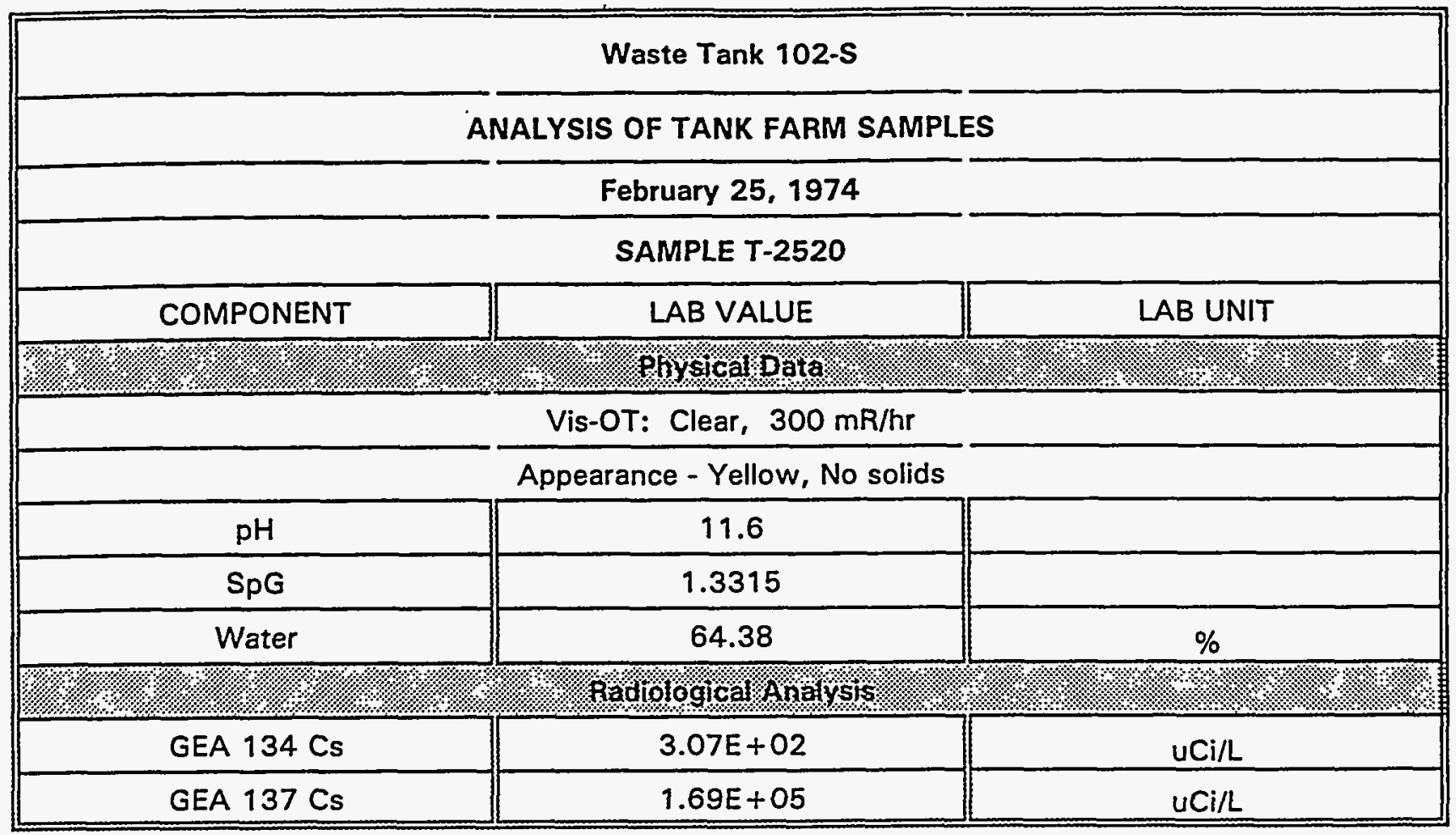


WHC-SD-WM-ER-323, Rev. O

\begin{tabular}{|c|c|c|}
\hline \multicolumn{3}{|c|}{ Waste Tank 102-S } \\
\hline \multicolumn{3}{|c|}{ ANALYSIS OF TANK FARM SAMPLES } \\
\hline \multicolumn{3}{|c|}{ March 7, 1974} \\
\hline \multicolumn{3}{|c|}{ SAMPLE T-2759 } \\
\hline COMPONENT & LAB VALUE & LAB UNIT \\
\hline স. & Prysicalloata & \\
\hline \multicolumn{3}{|c|}{ Vis-OT: $200 \mathrm{mR} / \mathrm{hr}$} \\
\hline \multicolumn{3}{|c|}{ Appearance - Yellow, $85 \%$ solids at room temperature } \\
\hline $\mathrm{pH}$ & 10.9 & \\
\hline SpG & 1.310 at $49^{*} \mathrm{C}$ & \\
\hline Water & 62.77 & $\%$ \\
\hline \multicolumn{3}{|c|}{ 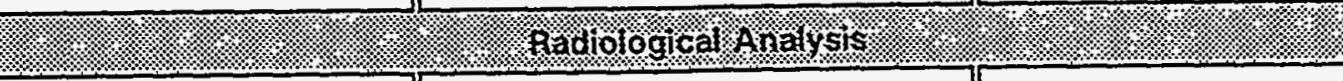 } \\
\hline GEA $134 \mathrm{Cs}$ & $9.01 E+02$ & uCi/gal \\
\hline GEA $137 \mathrm{Cs}$ & $4.81 E+05$ & uCi/gal \\
\hline
\end{tabular}


WHC-SD-WM-ER-323, Rev. 0

\begin{tabular}{|c|c|c|}
\hline \multicolumn{3}{|c|}{ Waste Tank 102-S } \\
\hline \multicolumn{3}{|c|}{ ANALYSIS OF TANK FARM SAMPLES } \\
\hline \multicolumn{3}{|c|}{ March 25, 1974} \\
\hline \multicolumn{3}{|c|}{ SAMPLE T-3005 } \\
\hline COMPONENT & LAB VALUE & LAB UNIT \\
\hline $4.1 \%, 1.1 \%$ & GYsicallopata & ঋ. \\
\hline \multicolumn{3}{|c|}{ Vis-OTR: Clear, Yellow, $100 \mathrm{mR} / \mathrm{hr}$} \\
\hline $\mathrm{pH}$ & 12.1 & \\
\hline SpG & 1.204 & \\
\hline Water & 76.99 & $\%$ \\
\hline \multicolumn{3}{|c|}{ 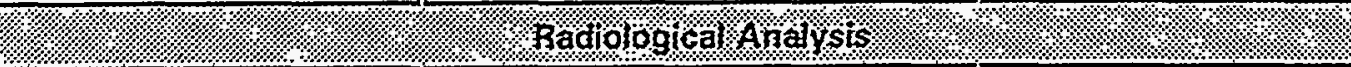 } \\
\hline GEA $134 \mathrm{Cs}$ & $1.31 E+03$ & uCi/gal \\
\hline GEA $137 \mathrm{Cs}$ & $4.62 E+05$ & uCi/gal \\
\hline GEA 106 RURh & $2.78 E+05$ & uCi/gal \\
\hline
\end{tabular}


WHC-SD-WM-ER-323, Rev. 0

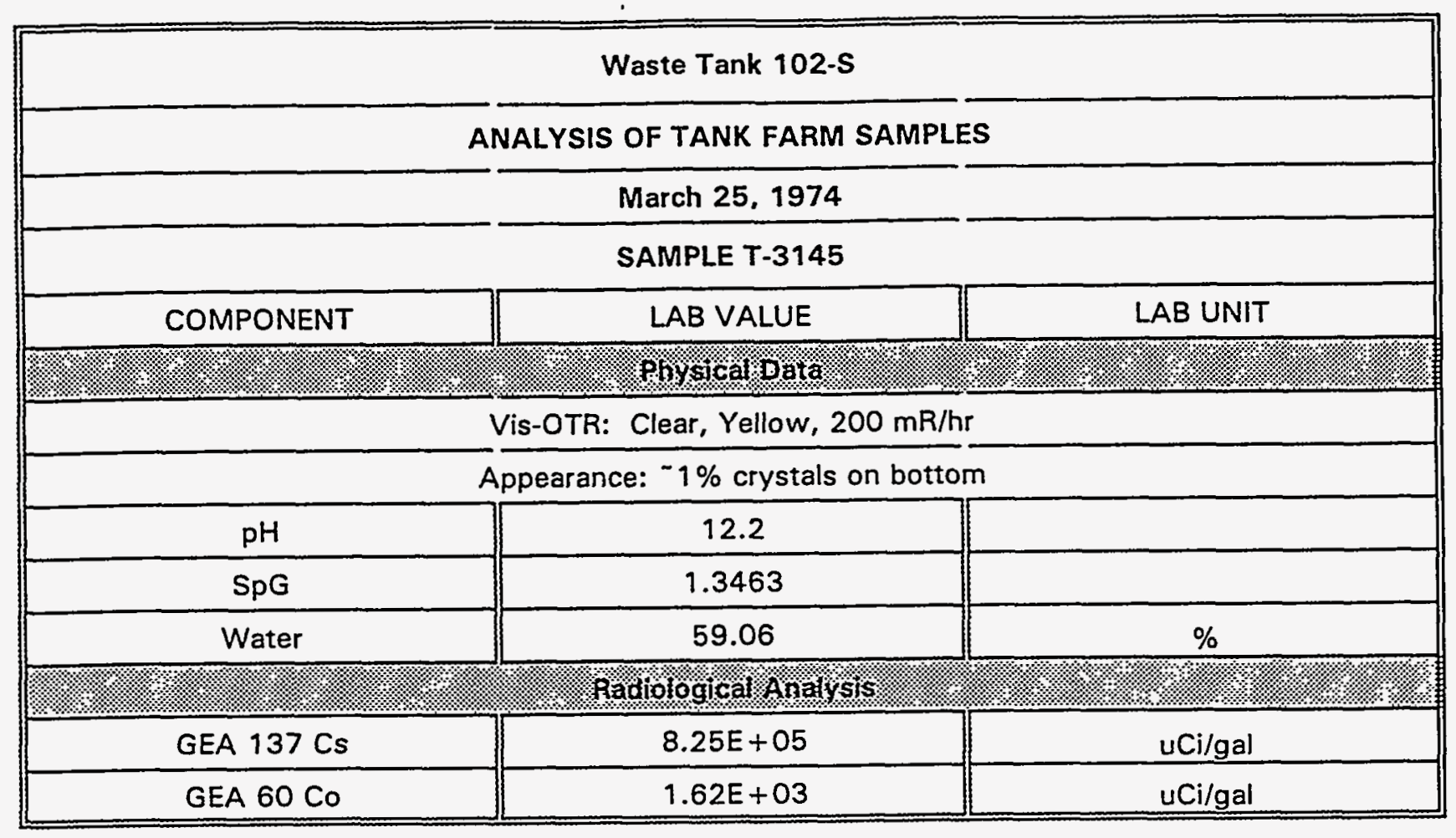


WHC-SD-WM-ER-323, Rev. 0

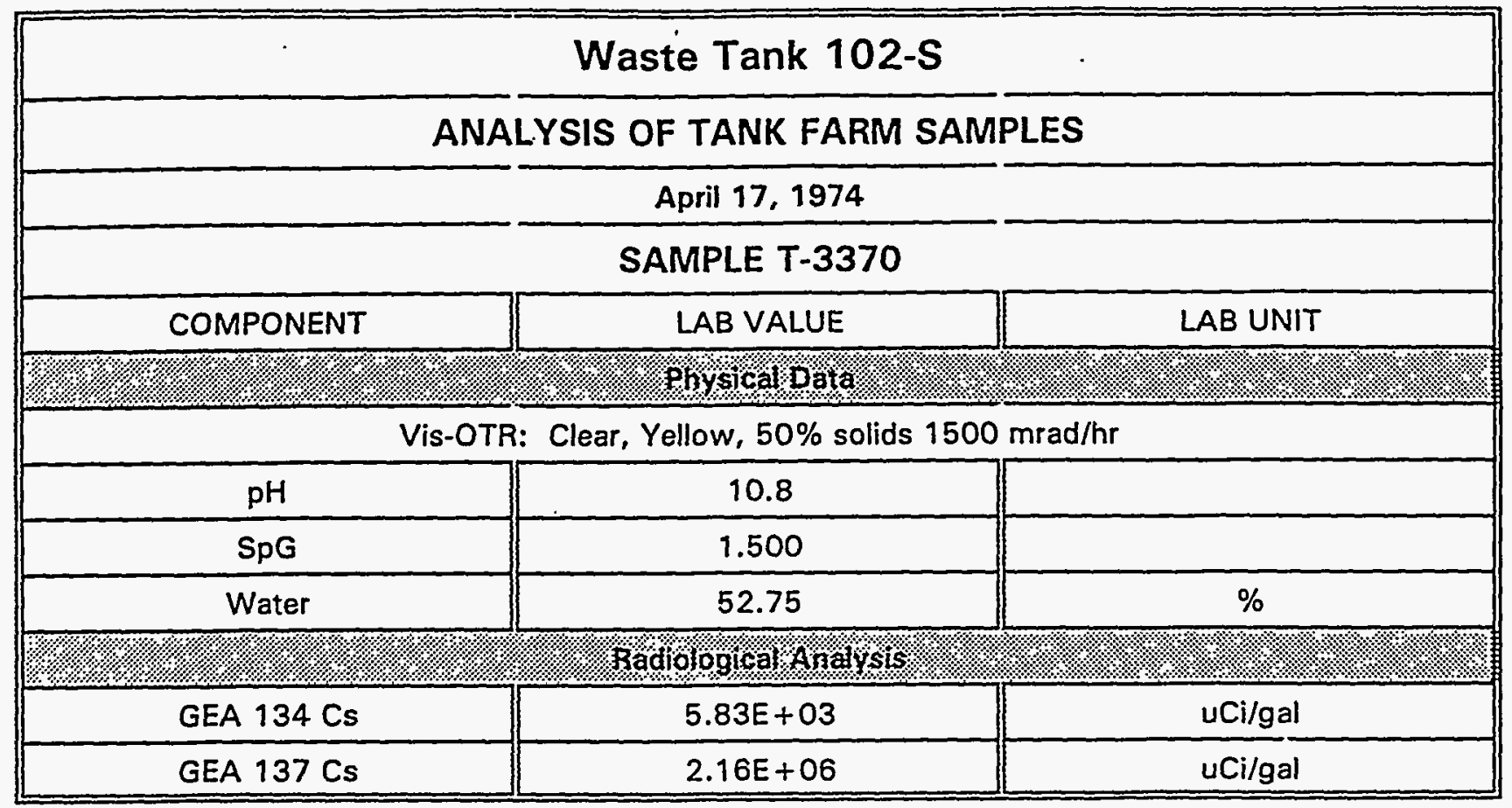




\begin{tabular}{|c|c|c|}
\hline \multicolumn{3}{|c|}{ Waste Tank 102-S } \\
\hline \multicolumn{3}{|c|}{ ANALYSIS OF TANK FARM SAMPLES } \\
\hline \multicolumn{3}{|c|}{ May 13,1974} \\
\hline \multicolumn{3}{|c|}{ FEED SAMPLE T-3601 } \\
\hline COMPONENT & LAB VALUE & LAB UNIT \\
\hline 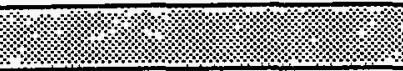 & 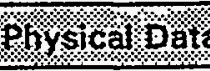 & 198 \\
\hline \multicolumn{3}{|c|}{ Vis-OTR: Yellow; $50 \%$ Solids; $500 \mathrm{mrad} / \mathrm{hr}$} \\
\hline $\mathrm{pH}$ & 12.4 & \\
\hline SpG & 1.2839 & \\
\hline Water & 53.48 & $\%$ \\
\hline \multicolumn{3}{|c|}{ Chenical Analysis: } \\
\hline $\mathrm{OH}$ & 4.52 & M \\
\hline Al & 0.62 & $M$ \\
\hline NO2 & 0.69 & $M$ \\
\hline $\mathrm{Na}$ & 6.66 & M \\
\hline NO3 & 2.51 & $M$ \\
\hline $\mathrm{SO} 4$ & $5.32 \mathrm{E}-02$ & $M$ \\
\hline $\mathrm{PO} 4$ & 3.87E-02 & M \\
\hline $\mathrm{F}$ & $1.11 \mathrm{E}-02$ & M \\
\hline $\mathrm{co3}$ & 0.259 & $M$ \\
\hline $\mathrm{NH} 4$ & $1.92 \mathrm{E}-02$ & M \\
\hline \multicolumn{3}{|c|}{ 7. } \\
\hline $\mathrm{Pu}$ & $1.43 \mathrm{E}-05$ & $\mathrm{~g} / \mathrm{L}$ \\
\hline GEA $134 \mathrm{Cs}$ & $3.66 \mathrm{E}+03$ & $\mathrm{uCi} / \mathrm{L}$ \\
\hline GEA $137 \mathrm{Cs}$ & $9.58 \mathrm{E}+05$ & $u C i / L$ \\
\hline
\end{tabular}




\begin{tabular}{|c|c|c|}
\hline \multicolumn{3}{|c|}{ Waste Tank 102-S } \\
\hline \multicolumn{3}{|c|}{ ANALYSIS OF TANK FARM SAMPLES } \\
\hline \multicolumn{3}{|c|}{ May 22, 1974} \\
\hline \multicolumn{3}{|c|}{ SAMPLE T-4372 } \\
\hline COMPONENT & LAB VALUE & LAB UNIT \\
\hline 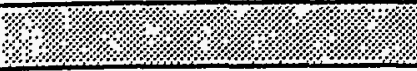 & \% Physical pata & \\
\hline \multicolumn{3}{|c|}{ Vis-OTR: Dark Amber; No solids at room temp; $700 \mathrm{mrad} / \mathrm{hr}$} \\
\hline $\mathrm{pH}$ & 11.7 & . \\
\hline SpG & 1.400 & \\
\hline Water & 53.48 & $\%$ \\
\hline $11 \% 18$. & Radiologloal anat & 2. \\
\hline GEA $134 \mathrm{Cs}$ & $4.31 E+03$ & uCi/gal \\
\hline GEA $137 \mathrm{Cs}$ & $1.18 E+06$ & uCi/gal \\
\hline GEA 106 RuRh & $4.01 E+05$ & uCi/gal \\
\hline
\end{tabular}


WHC-SD-WM-ER-323, Rev. 0

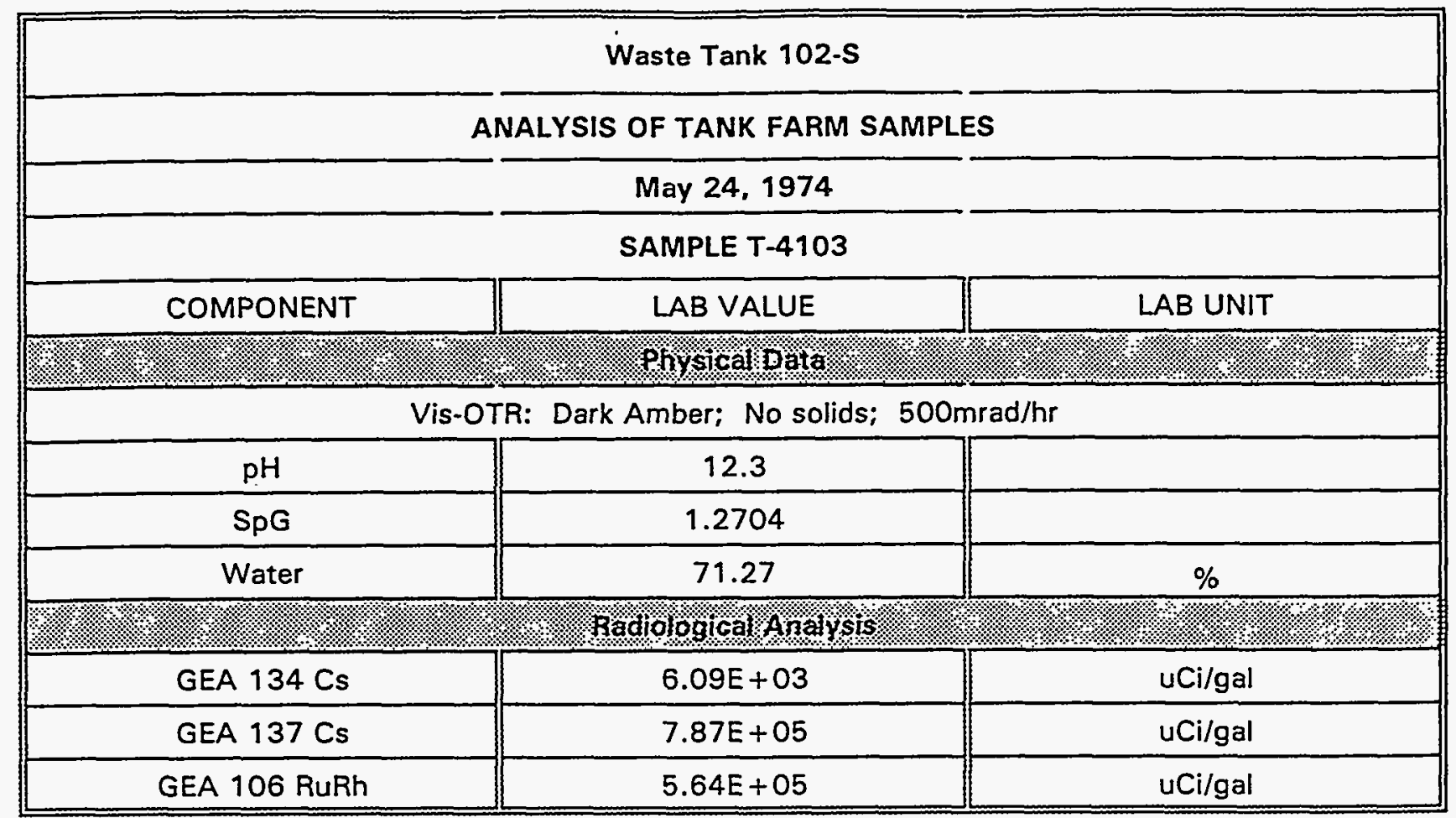




\begin{tabular}{|c|c|c|}
\hline \multicolumn{3}{|c|}{ Waste Tank 102-S } \\
\hline \multicolumn{3}{|c|}{ ANALYSIS OF TANK FARM SAMPLES } \\
\hline \multicolumn{3}{|c|}{ June 7, 1974} \\
\hline \multicolumn{3}{|c|}{ SAMPLE T-4652 } \\
\hline COMPONENT & LAB VALUE & LAB UNIT \\
\hline ২\% \% পু & physicals att & 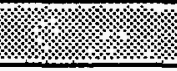 \\
\hline \multicolumn{3}{|c|}{ Vis-OTR: Dark Amber; No solids; $280 \mathrm{mrad} / \mathrm{hr}$} \\
\hline $\mathrm{pH}$ & 11.8 & \\
\hline SpG & 1.4564 & \\
\hline Water & 55.29 & $\%$ \\
\hline \multicolumn{3}{|c|}{ 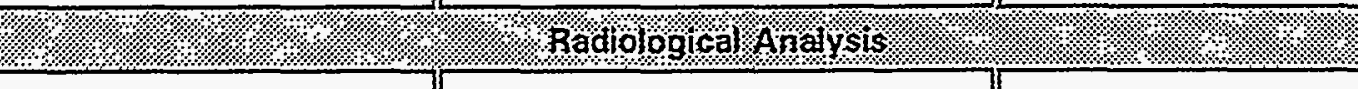 } \\
\hline GEA 134 Cs & $4.35 E+03$ & uCi/gal \\
\hline GEA 137 Cs & $1.18 E+06$ & uCi/gal \\
\hline GEA 106 RuRh & $2.86 \mathrm{E}+05$ & $\mathrm{uCi} / \mathrm{gal}$ \\
\hline
\end{tabular}




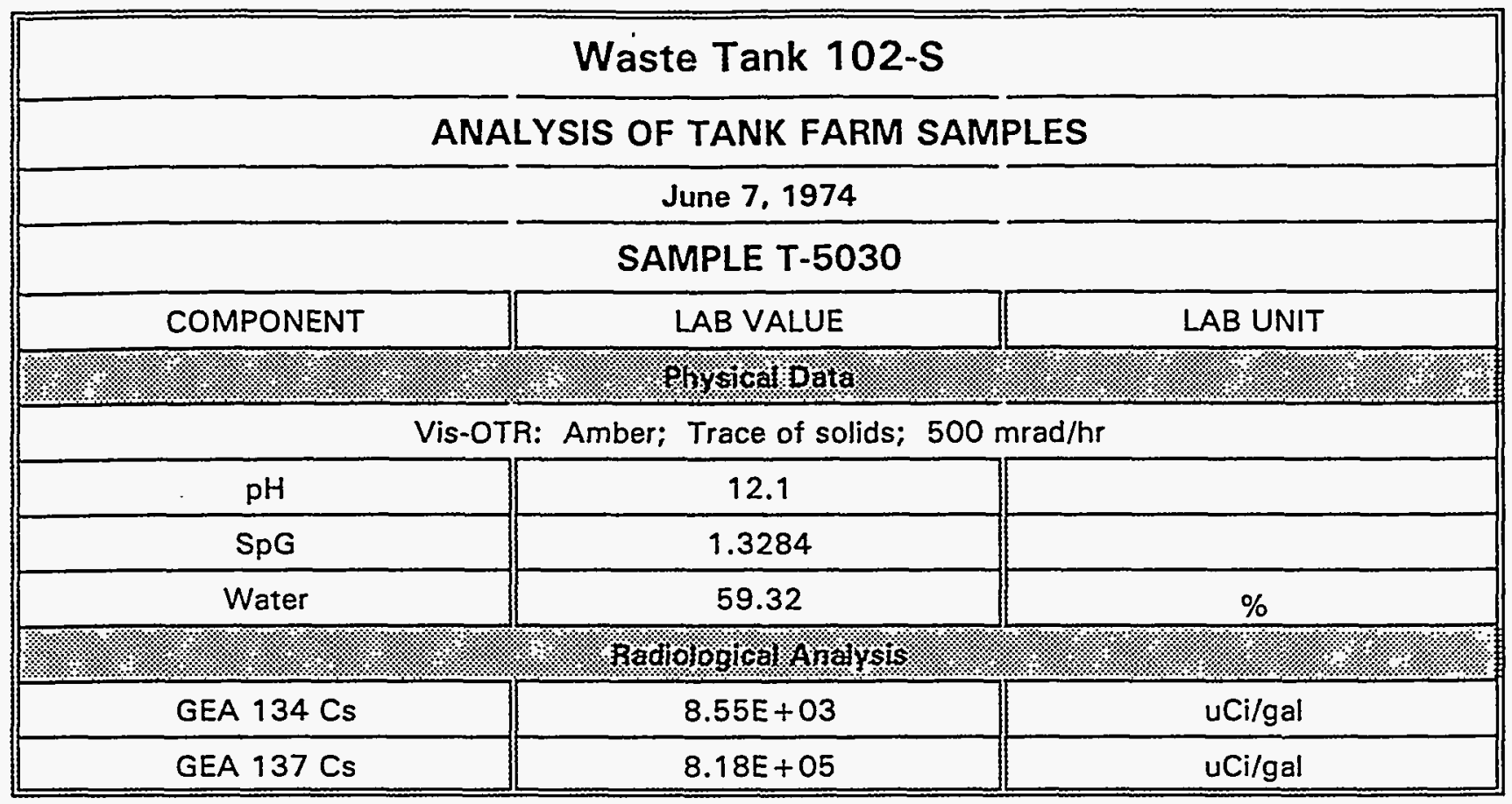




\begin{tabular}{|c|c|c|}
\hline \multicolumn{3}{|c|}{ Waste Tank 102-S } \\
\hline \multicolumn{3}{|c|}{ Analysis of Tank Farm Samples } \\
\hline \multicolumn{3}{|c|}{ June 17,1974} \\
\hline \multicolumn{3}{|c|}{ Feed Sample T-3770 } \\
\hline COMPONENT & LAB VALUE & LAB UNIT \\
\hline 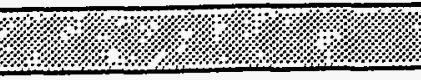 & Physical Datz & 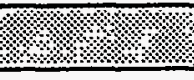 \\
\hline \multicolumn{3}{|c|}{ Vis-OTR: Yellow; $<1 \%$ Solids; $375 \mathrm{mrad} / \mathrm{hr}$} \\
\hline $\mathrm{pH}$ & 12.6 & \\
\hline $\mathrm{SpG}$ & 1.315 & \\
\hline Water & 55.20 & $\%$ \\
\hline \multicolumn{3}{|c|}{ 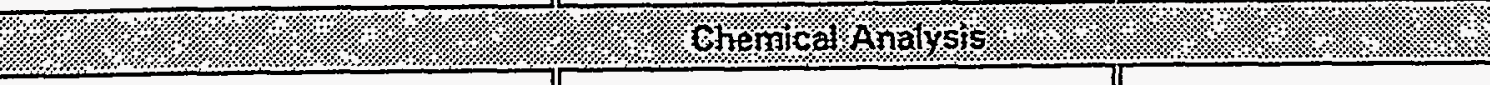 } \\
\hline $\mathrm{OH}$ & 2.78 & $\underline{M}$ \\
\hline $\mathrm{Al}$ & 0.671 & $\underline{M}$ \\
\hline $\mathrm{Na}$ & 6.92 & $\underline{M}$ \\
\hline NO2 & 0.958 & $\underline{M}$ \\
\hline NO3 & 2.56 & $\underline{M}$ \\
\hline SO4 & $4.20 \mathrm{E}-02$ & $\underline{M}$ \\
\hline $\mathrm{PO} 4$ & 1.89E-02 & $\underline{M}$ \\
\hline $\mathrm{F}$ & $5.36 \mathrm{E}-03$ & $\underline{\mathrm{M}}$ \\
\hline $\mathrm{CO} 3$ & 0.176 & $\mathrm{M}$ \\
\hline \multicolumn{3}{|c|}{ (1) } \\
\hline $\mathrm{Pu}$ & $6.92 E-06$ & $\mathrm{~g} / \mathrm{L}$ \\
\hline GEA $134 \mathrm{Cs}$ & $7.68 \mathrm{E}+02$ & uCi/gal \\
\hline GEA $137 \mathrm{Cs}$ & $5.45 \mathrm{E}+05$ & $\mathrm{uCi} / \mathrm{gal}$ \\
\hline GEA $60 \mathrm{CO}$ & $4.66 \mathrm{E}+02$ & uCi/gal \\
\hline GEA 106 RuRh & $3.85 \mathrm{E}+04$ & $\mathrm{uCi} / \mathrm{gal}$ \\
\hline
\end{tabular}




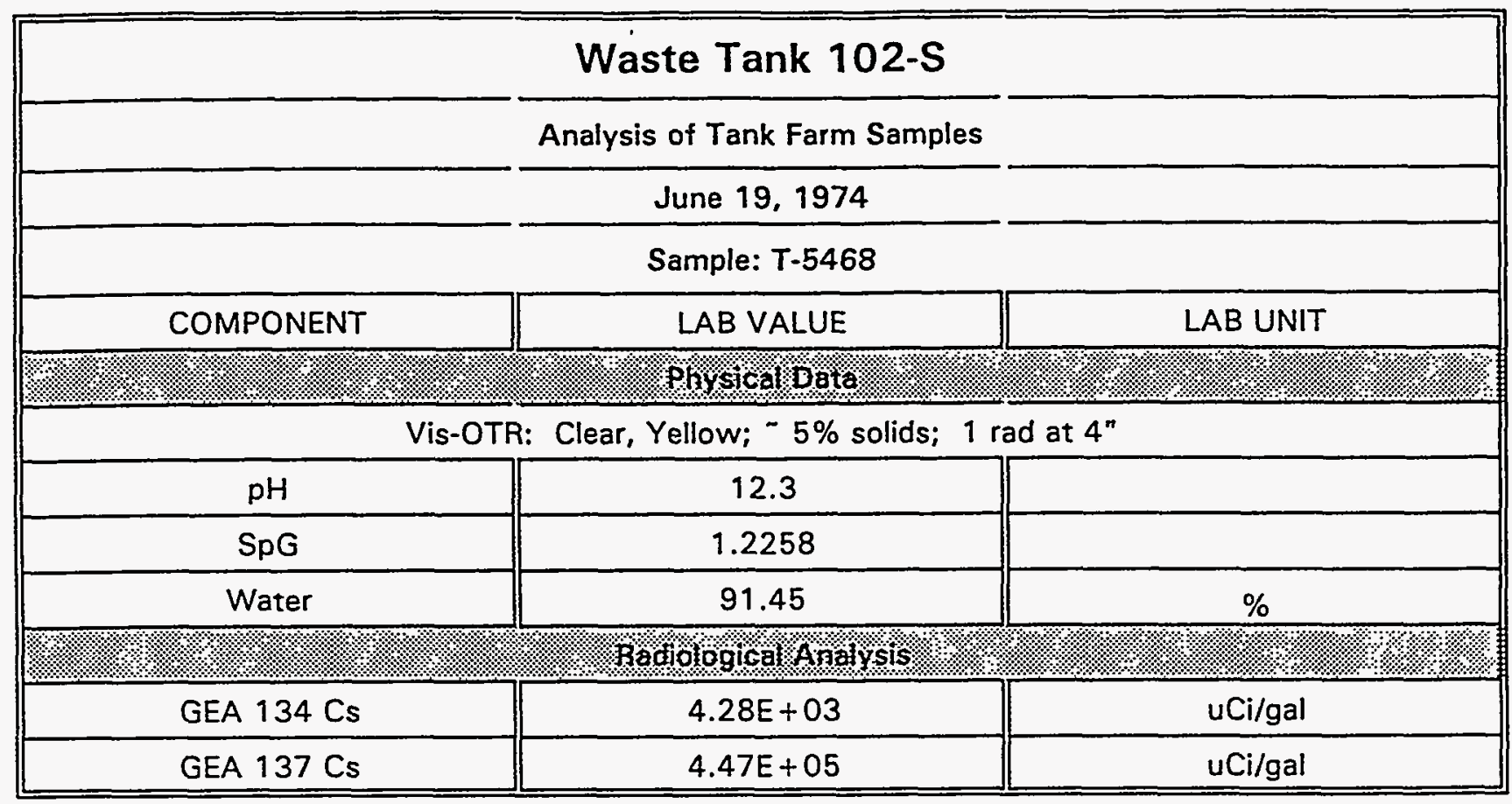




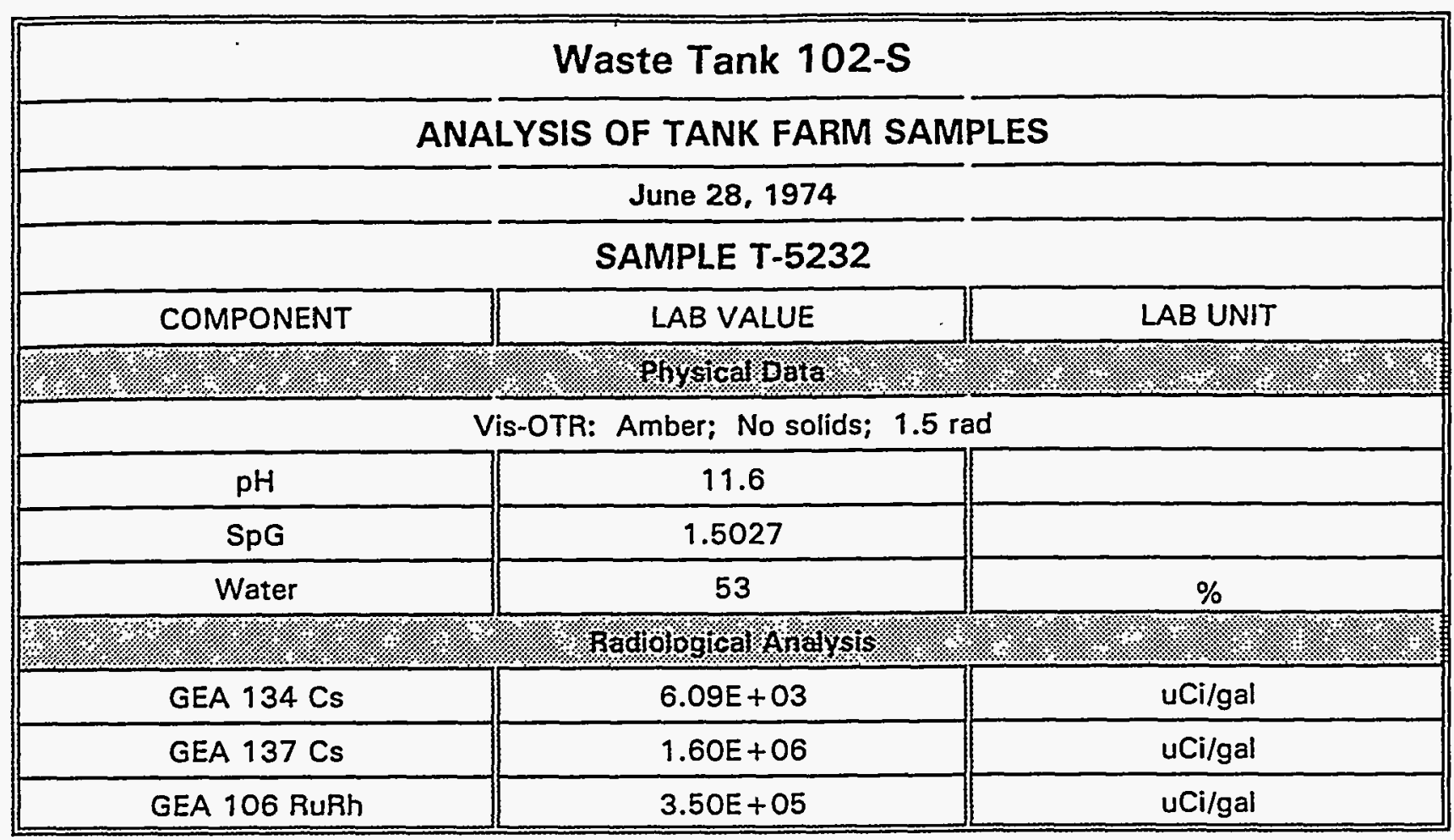




\begin{tabular}{|c|c|c|}
\hline \multicolumn{3}{|c|}{ Waste Tank 102-S } \\
\hline \multicolumn{3}{|c|}{ ANALYSIS OF TANK FARM SAMPLES } \\
\hline \multicolumn{3}{|c|}{ July 2, 1974} \\
\hline \multicolumn{3}{|c|}{ SAMPLE T-5775 } \\
\hline COMPONENT & LAB VALUE & LAB UNIT \\
\hline & physicalopata & \\
\hline \multicolumn{3}{|c|}{ Vis-OTR: Dark brown; $<5 \%$ solids; $1500 \mathrm{mrad} / \mathrm{hr}$} \\
\hline $\mathrm{pH}$ & 11.9 & \\
\hline SpG & 1.0450 & \\
\hline Water & 65.83 & $\%$ \\
\hline ২. & 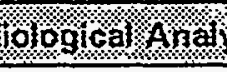 & \\
\hline GEA $134 \mathrm{Cs}$ & $2.02 E+03$ & uCi/gal \\
\hline GEA $137 \mathrm{Cs}$ & $2.18 E+05$ & uCi/gal \\
\hline GEA 106 RURh & $4.24 E+05$ & uCi/gal \\
\hline
\end{tabular}


WHC-SD-WM-ER-323, Rev. 0

\begin{tabular}{|c|c|c|}
\hline \multicolumn{3}{|c|}{ Waste Tank 102-S } \\
\hline \multicolumn{3}{|c|}{ ANALYSIS OF TANK FARM SAMPLES } \\
\hline \multicolumn{3}{|c|}{ July 3, 1974} \\
\hline \multicolumn{3}{|c|}{ SAMPLE T-5458 } \\
\hline COMPONENT & LAB VALUE & LAB UNIT \\
\hline 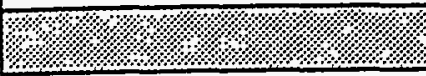 & YYysicardatio & (1., \\
\hline \multicolumn{3}{|c|}{ Vis-OTR: Green; $<5 \%$ solids; 1 rad } \\
\hline $\mathrm{pH}$ & 11.0 & \\
\hline SpG & 1.4923 & \\
\hline Water & 48 & $\%$ \\
\hline \multicolumn{3}{|l|}{ 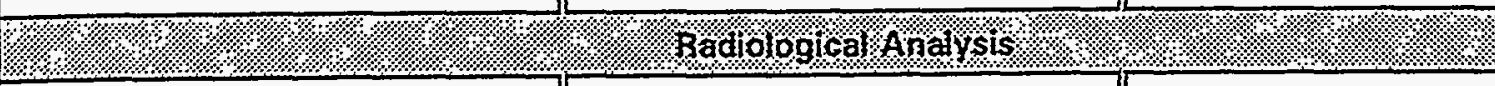 } \\
\hline GEA $134 \mathrm{Cs}$ & $6.28 \mathrm{E}+04$ & uCi/gal \\
\hline GEA $137 \mathrm{Cs}$ & $1.67 E+06$ & uCi/gal \\
\hline GEA 106 RuRh & $3.07 E+05$ & uCi/gal \\
\hline
\end{tabular}




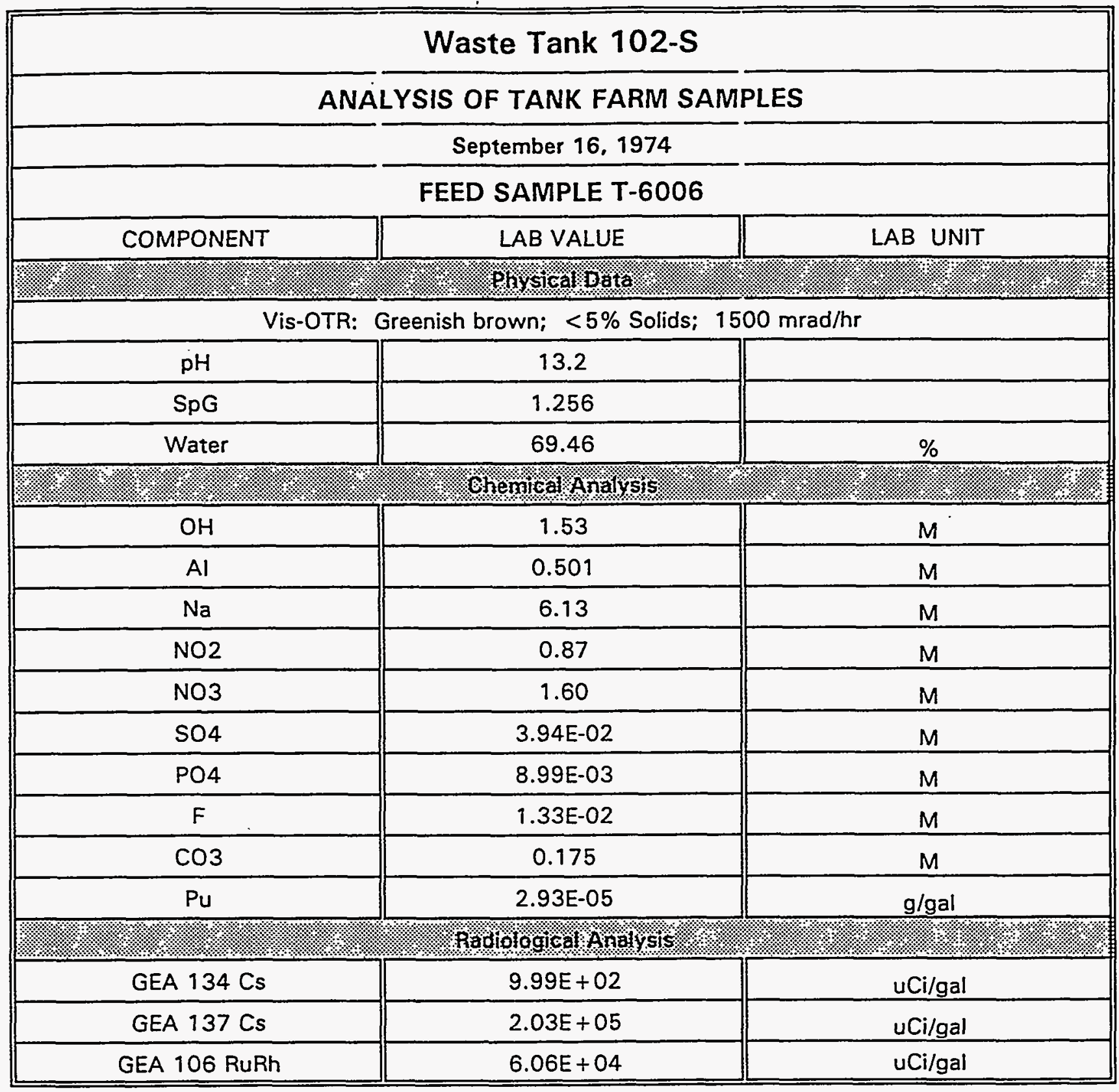




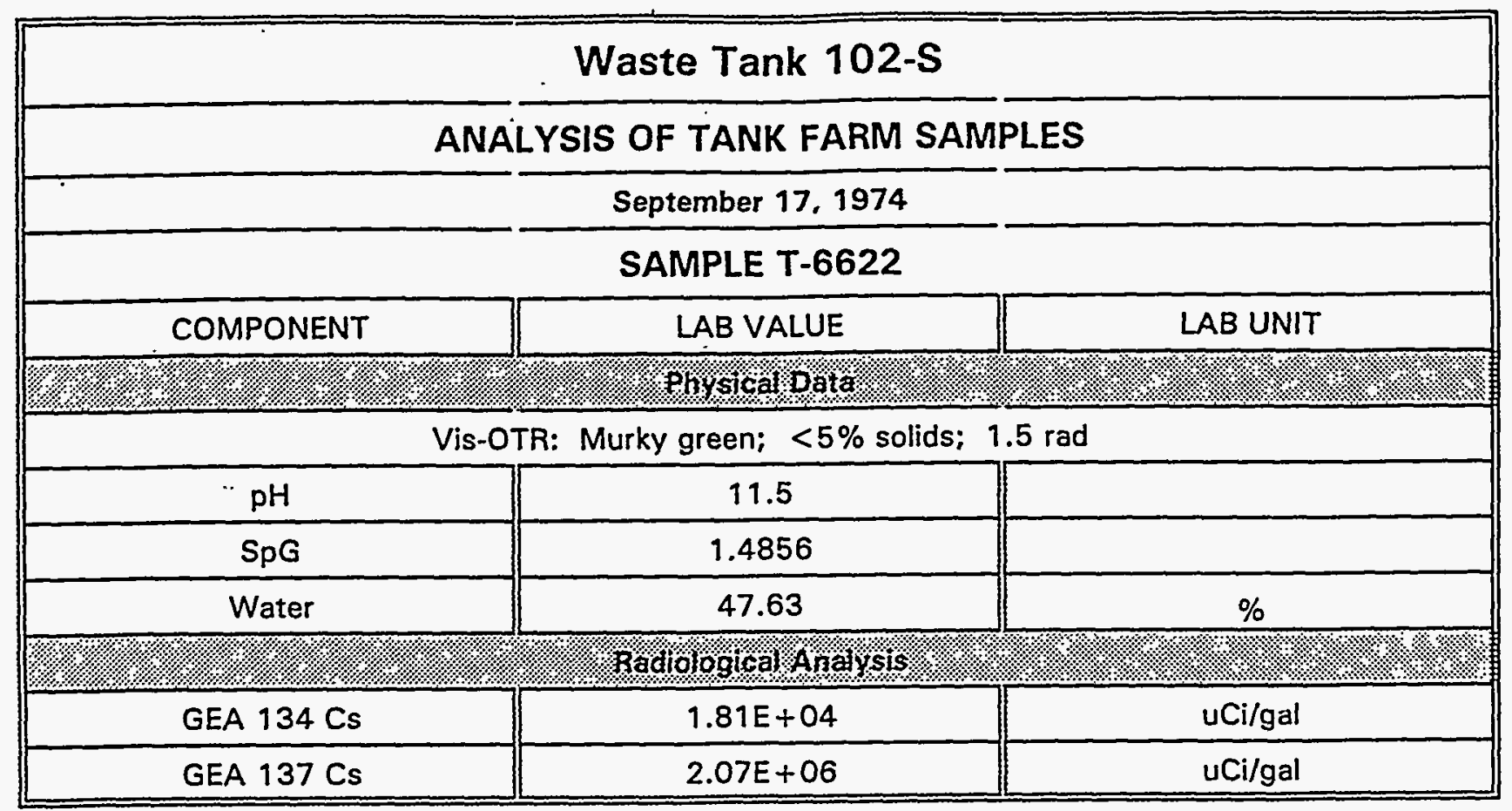




\begin{tabular}{|c|c|c|}
\hline \multicolumn{3}{|c|}{ Waste Tank 102-S } \\
\hline \multicolumn{3}{|c|}{ ANALYSIS OF TANK FARM SAMPLES } \\
\hline \multicolumn{3}{|c|}{ October 14,1974} \\
\hline \multicolumn{3}{|c|}{ SAMIPLE T-6214 } \\
\hline COMPONENT & LAB VALUE & LAB UNIT \\
\hline & Hystal pata & \\
\hline \multicolumn{3}{|c|}{ Vis-OTR: Brown; $<5 \%$ solids; 3 rad } \\
\hline $\mathrm{pH} \quad-$ & 11.5 & \\
\hline SpG & 1.4005 & \\
\hline Water & 92.89 & $\%$ \\
\hline & fogical ana & 1. \\
\hline GEA 134 Cs & $1.12 E+04$ & uCi/gal \\
\hline GEA 137 Cs & $1.54 \mathrm{E}+06$ & uCi/gal \\
\hline
\end{tabular}




\begin{tabular}{|c|c|c|}
\hline \multicolumn{3}{|c|}{ Waste Tank 102-S } \\
\hline \multicolumn{3}{|c|}{ ANALYSIS OF TANK FARM SAMPLES } \\
\hline \multicolumn{3}{|c|}{ October 14,1974} \\
\hline \multicolumn{3}{|c|}{ SAMPLE T-6416 } \\
\hline COMPONENT & LAB VALUE & LAB UNIT \\
\hline 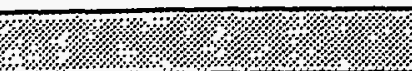 & hysicalpata & ?. \\
\hline \multicolumn{3}{|c|}{ Vis-OTR: Amber, brown, cloudy; $<5 \%$ solids; $2500 \mathrm{mRad} / \mathrm{hr}$} \\
\hline $\mathrm{pH}$ & 11.6 & \\
\hline SpG & 1.3780 & \\
\hline Water & 49.05 & $\%$ \\
\hline \multicolumn{3}{|c|}{ 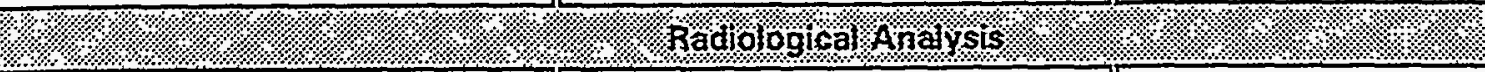 } \\
\hline GEA: 134 Cs & $5.45 E+03$ & uCi/gal \\
\hline GEA: $137 \mathrm{Cs}$ & $1.36 E+06$ & uCi/gal \\
\hline GEA: 106 RuRh & $4.05 E+05$ & uCi/gal \\
\hline
\end{tabular}




\begin{tabular}{|c|c|c|}
\hline \multicolumn{3}{|c|}{ Waste Tank 102-S } \\
\hline \multicolumn{3}{|c|}{ ANALYSIS OF TANK FARM SAMPLES } \\
\hline \multicolumn{3}{|c|}{ October 14,1974} \\
\hline \multicolumn{3}{|c|}{ SAMPLE T-7300 } \\
\hline COMPONENT & LAB VALUE & LAB UNIT \\
\hline ras: & physicainota & \\
\hline \multicolumn{3}{|c|}{ Vis-OTR: Yellow, cloudy; Unknown amount of solids; 1 Rad } \\
\hline $\mathrm{pH}$ & 11.7 & \\
\hline SpG & 1.3360 & \\
\hline Water & 60.46 & $\%$ \\
\hline \multicolumn{3}{|c|}{ Radiological Anarysis. } \\
\hline GEA $134 \mathrm{Cs}$ & $7.34 \mathrm{E}+03$ & uCi/gal \\
\hline GEA $137 \mathrm{Cs}$ & $1.11 E+06$ & uCi/gal \\
\hline GEA $125 \mathrm{Sb}$ & $3.52 E+03$ & $u C i / g a l$ \\
\hline
\end{tabular}




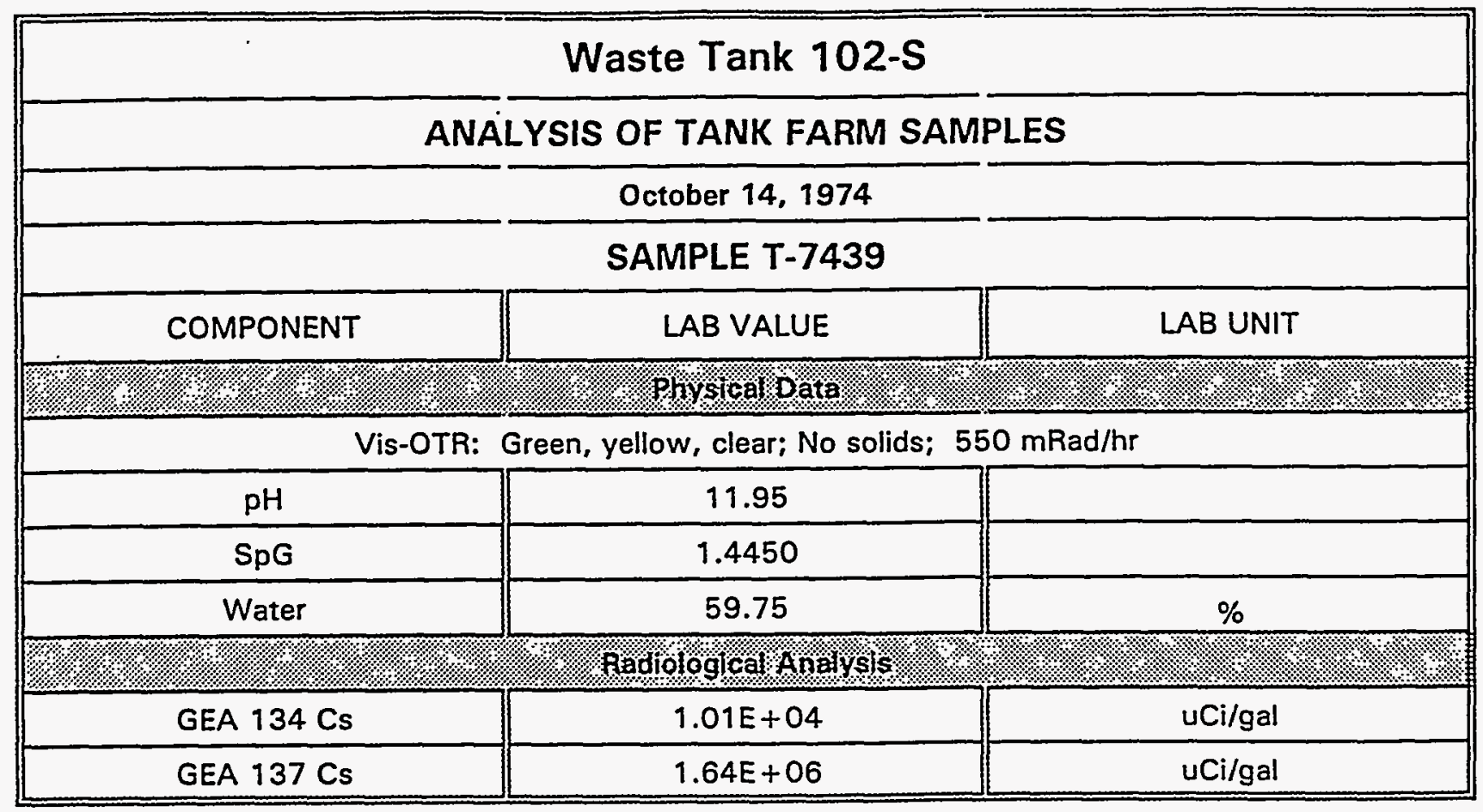




\begin{tabular}{|c|c|c|}
\hline \multicolumn{3}{|c|}{ Waste Tank 102-S } \\
\hline \multicolumn{3}{|c|}{ ANALYSIS OF TANK FARM SAMPLES } \\
\hline \multicolumn{3}{|c|}{ October 21, 1974} \\
\hline \multicolumn{3}{|c|}{ SAMPLE T-6761 } \\
\hline COMPONENT & LAB VALUE & LAB UNIT \\
\hline 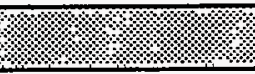 & Plysicatonata & 9.18 .9 \\
\hline \multicolumn{3}{|c|}{ Vis-OTR: Green brown; $<5 \%$ Solids; $300 \mathrm{mRad} / \mathrm{hr}$} \\
\hline $\mathrm{pH}$ & $>12.8$ & \\
\hline $\operatorname{SpG}$ & 1.4669 & \\
\hline Water & 49.82 & $\%$ \\
\hline \multicolumn{3}{|c|}{ chernical Arizlysis: } \\
\hline $\mathrm{OH}$ & 3.65 & M \\
\hline $\mathrm{Al}$ & $1.34 E+00$ & M \\
\hline $\mathrm{Na}$ & 12.51 & $M$ \\
\hline NO2 & 1.89 & M \\
\hline NO3 & $3.29 E+00$ & M \\
\hline $\mathrm{SO} 4$ & Sample slurped & \\
\hline PO4 & 3.34E-02 & $M$ \\
\hline $\mathrm{F}$ & $4.10 E-03$ & $M$ \\
\hline $\mathrm{CO} 3$ & $1.03 \mathrm{E}-02$ & $M$ \\
\hline \multicolumn{3}{|c|}{ Radiologica $A$ alas,sis } \\
\hline $\mathrm{Pu}$ & Sample slurped & \\
\hline GEA $134 \mathrm{Cs}$ & $1.80 E+04$ & $u C i / g a l$ \\
\hline GEA $137 \mathrm{Cs}$ & $2.05 E+06$ & uCi/gal \\
\hline
\end{tabular}


WHC-SD-WM-ER-323, Rev. 0

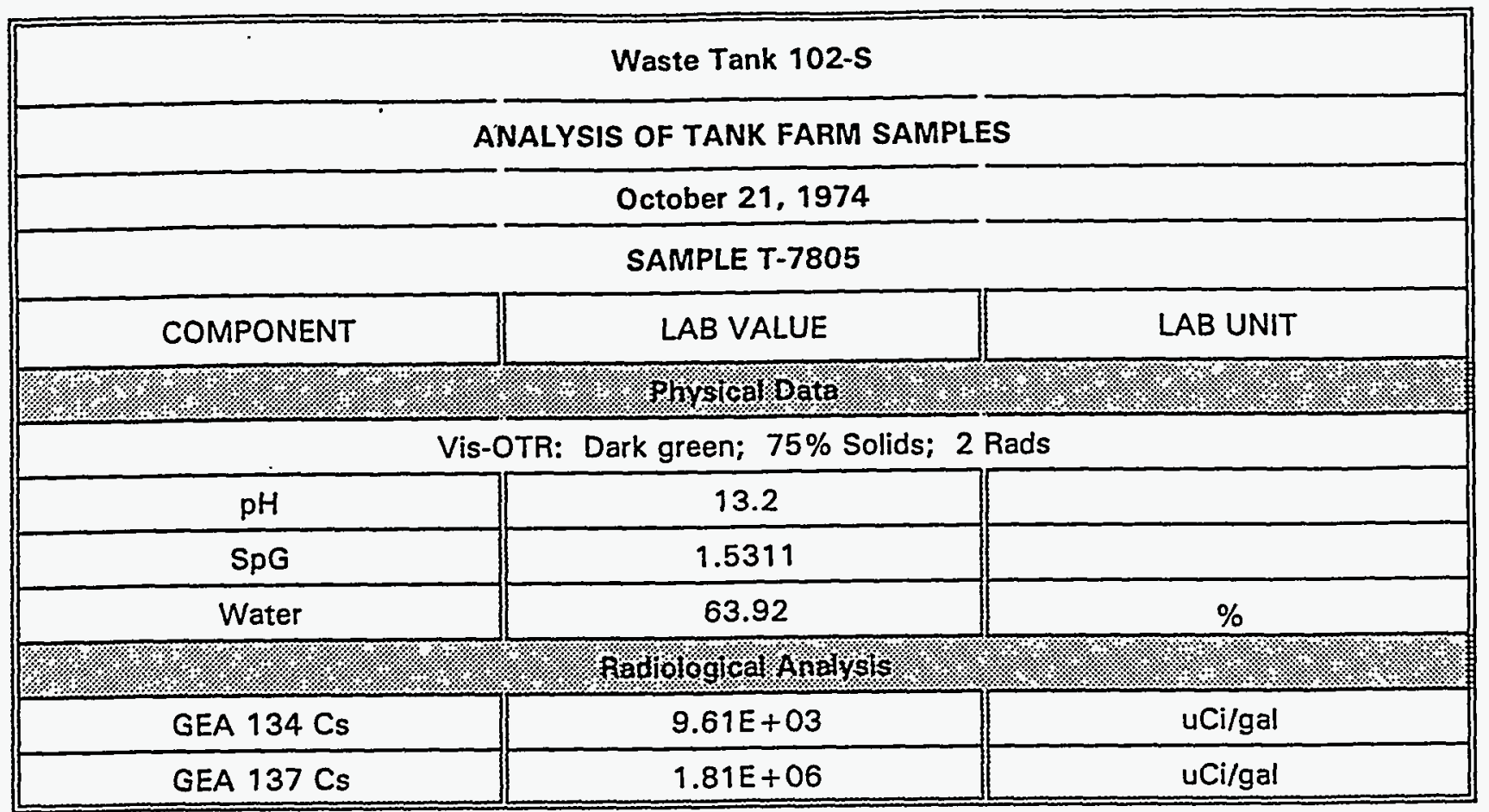




\begin{tabular}{|c|c|c|}
\hline \multicolumn{3}{|c|}{ Waste Tank 102-S } \\
\hline \multicolumn{3}{|c|}{ ANALYSIS OF TANK FARM SAMPLES } \\
\hline \multicolumn{3}{|c|}{ October 21, 1974} \\
\hline \multicolumn{3}{|c|}{ SAMPLE T-8077 } \\
\hline COMPONENT & LAB VALUE & LAB UNIT \\
\hline ("., & Hysicatouata & (2.: \\
\hline \multicolumn{3}{|c|}{ Vis-OTR: Murky green; - $25 \%$ Solids; 1.4 Rads } \\
\hline $\mathrm{pH}$ & 14 & \\
\hline SpG & 1.5357 & \\
\hline Water & 47.48 & $\%$ \\
\hline \multicolumn{3}{|c|}{ Badologicar Anarsis } \\
\hline GEA $134 \mathrm{Cs}$ & $8.93 E+03$ & $\mathrm{uCi} / \mathrm{gal}$ \\
\hline GEA $137 \mathrm{Cs}$ & $2.15 E+06$ & uCi/gal \\
\hline
\end{tabular}




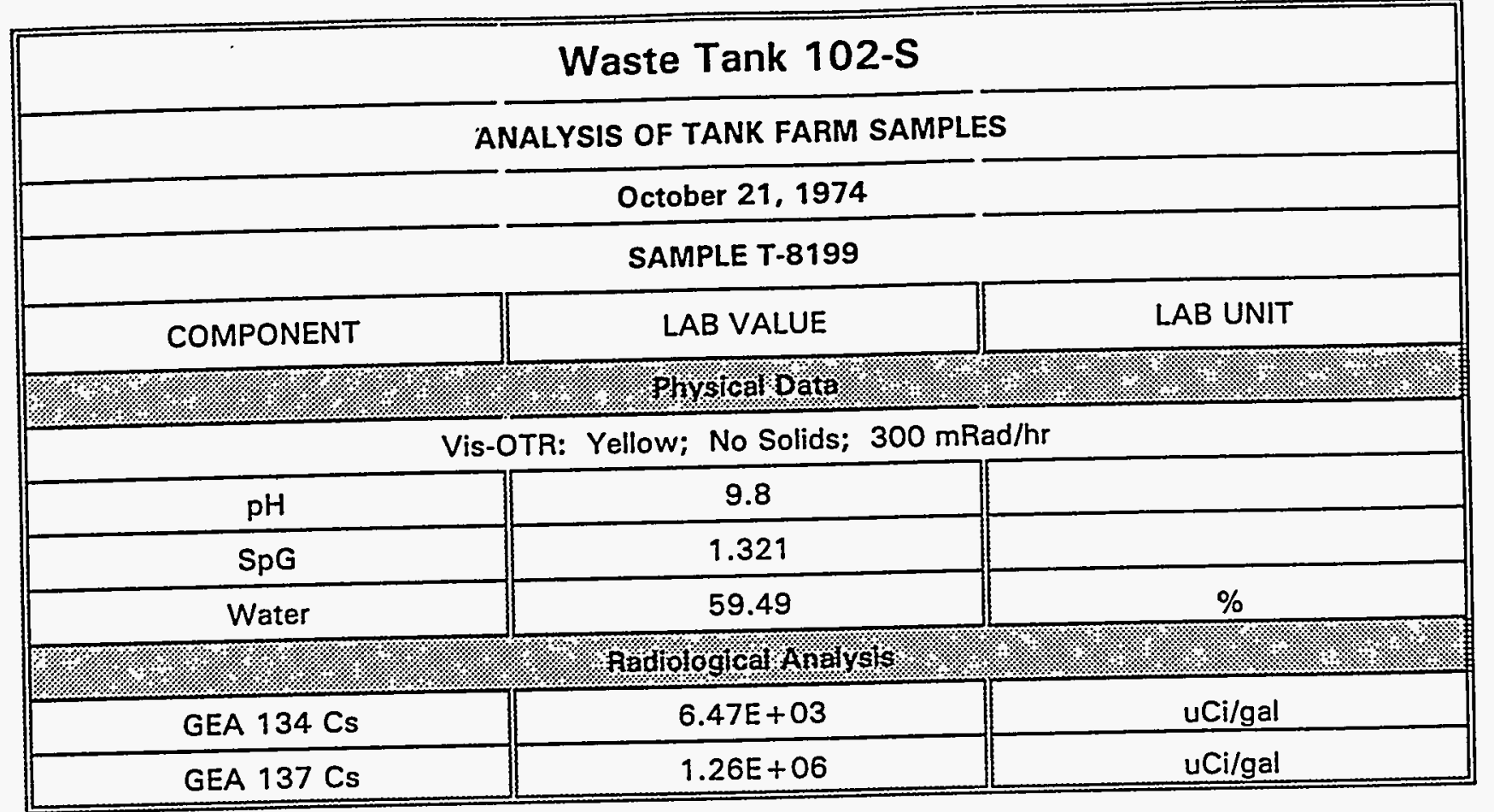




\begin{tabular}{|c|c|c|}
\hline \multicolumn{3}{|c|}{ Waste Tank 102-S } \\
\hline \multicolumn{3}{|c|}{ ANALYSIS OF TANK FARM SAMPLES } \\
\hline \multicolumn{3}{|c|}{ October 23,1974} \\
\hline \multicolumn{3}{|c|}{ SAMPLE T-9007 } \\
\hline COMPONENT & LAB VALUE & LAB UNIT \\
\hline 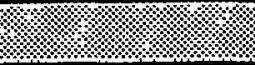 & Physicalopata & \%: \\
\hline \multicolumn{3}{|c|}{ Vis-OTR: Cloudy green; $5 \%$ Solids; $1.3 \mathrm{Rad} / \mathrm{hr}$} \\
\hline $\mathrm{pH}$ & 14.0 & \\
\hline SpG & 1.4866 & \\
\hline Water & 54.56 & $\%$ \\
\hline \multicolumn{3}{|c|}{ 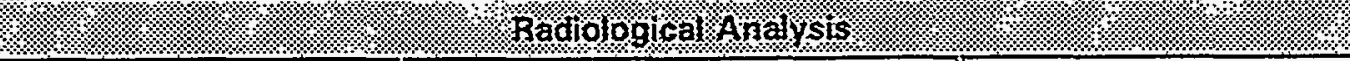 } \\
\hline GEA $134 \mathrm{Cs}$ & $4.35 E+03$ & uCi/gal \\
\hline GEA 137 Cs & $7.68 E+05$ & uCi/gal \\
\hline GEA $125 \mathrm{Sb}$ & $2.04 E+03$ & $u C i / g a l$ \\
\hline
\end{tabular}




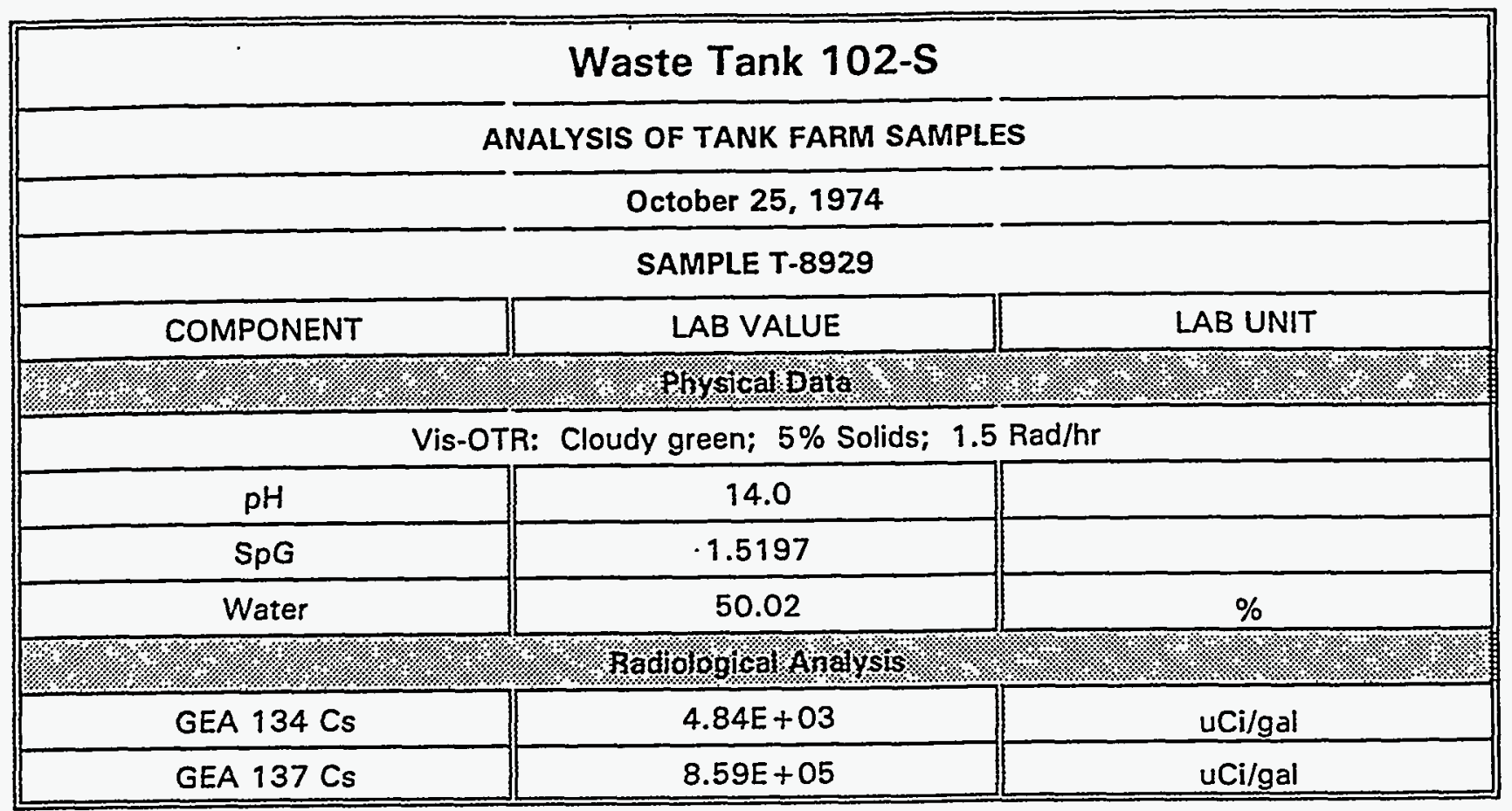




\begin{tabular}{|c|c|c|}
\hline \multicolumn{3}{|c|}{ Waste Tank 102-S } \\
\hline \multicolumn{3}{|c|}{ ANALYSIS OF TANK FARM SAMPLES } \\
\hline \multicolumn{3}{|c|}{ October 29,1974} \\
\hline \multicolumn{3}{|c|}{ FEED SAMPLE T-8779 } \\
\hline COMPONENT & LAB VALUE & LAB UNIT \\
\hline 2 & Privsical Data & $\because$ \\
\hline \multicolumn{3}{|c|}{ Vis-OTR: Cloudy yellow; $25 \%$ solids; $0.70 \mathrm{Rad} / \mathrm{hr}$} \\
\hline $\mathrm{pH}$ & 10.1 & \\
\hline $\mathrm{SpG}$ & 1.3691 & \\
\hline Water & 44.50 & $\%$ \\
\hline \multicolumn{3}{|c|}{ य》च } \\
\hline GEA $134 \mathrm{Cs}$ & $8.86 E+03$ & $\mathrm{uCi} / \mathrm{gal}$ \\
\hline GEA $137 \mathrm{Cs}$ & $1.67 E+06$ & $\mathrm{uCi} / \mathrm{gal}$ \\
\hline
\end{tabular}


WHC-SD-WM-ER-323, Rev. 0

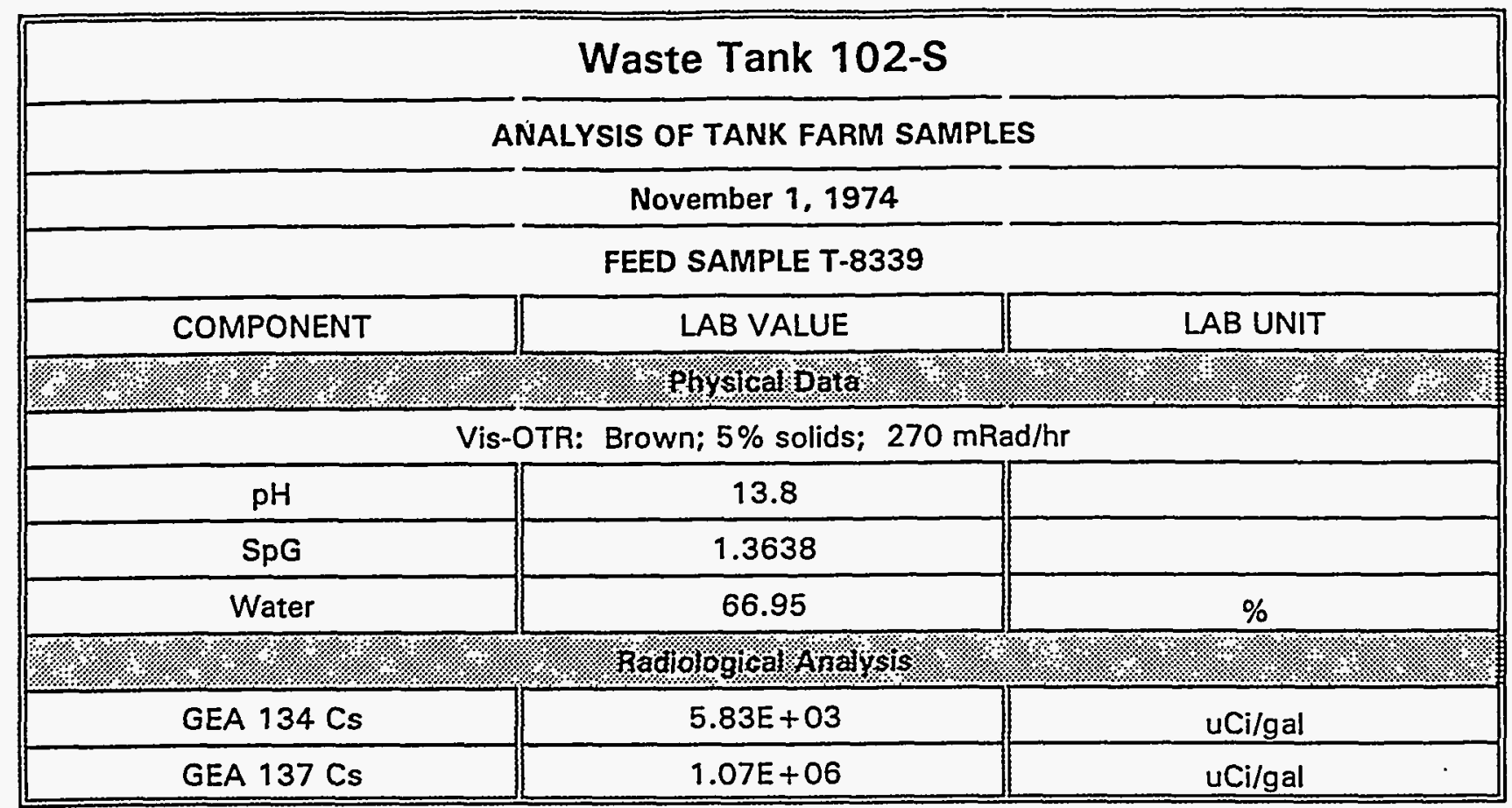




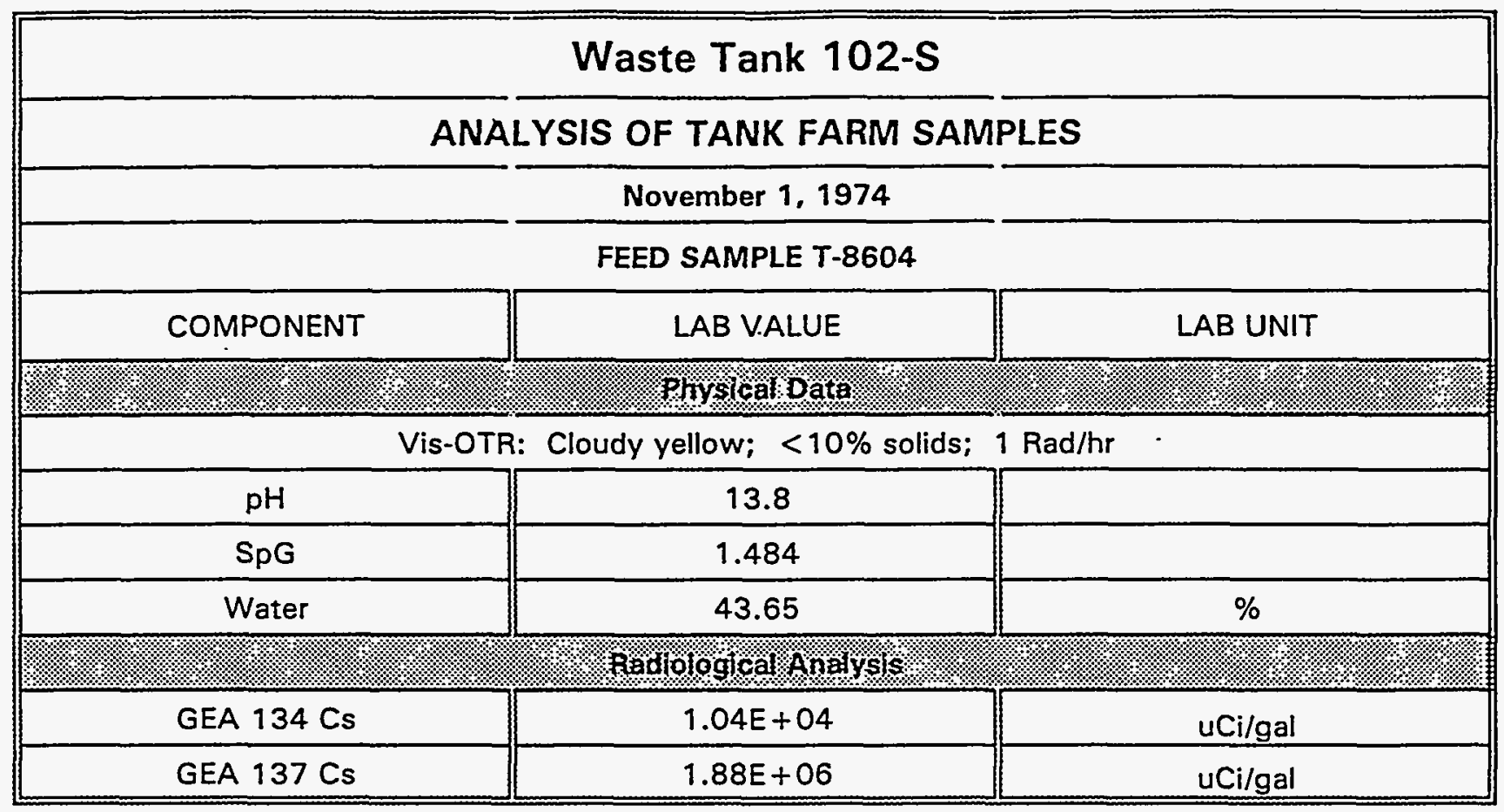


WHC-SD-WM-ER-323, Rev. 0

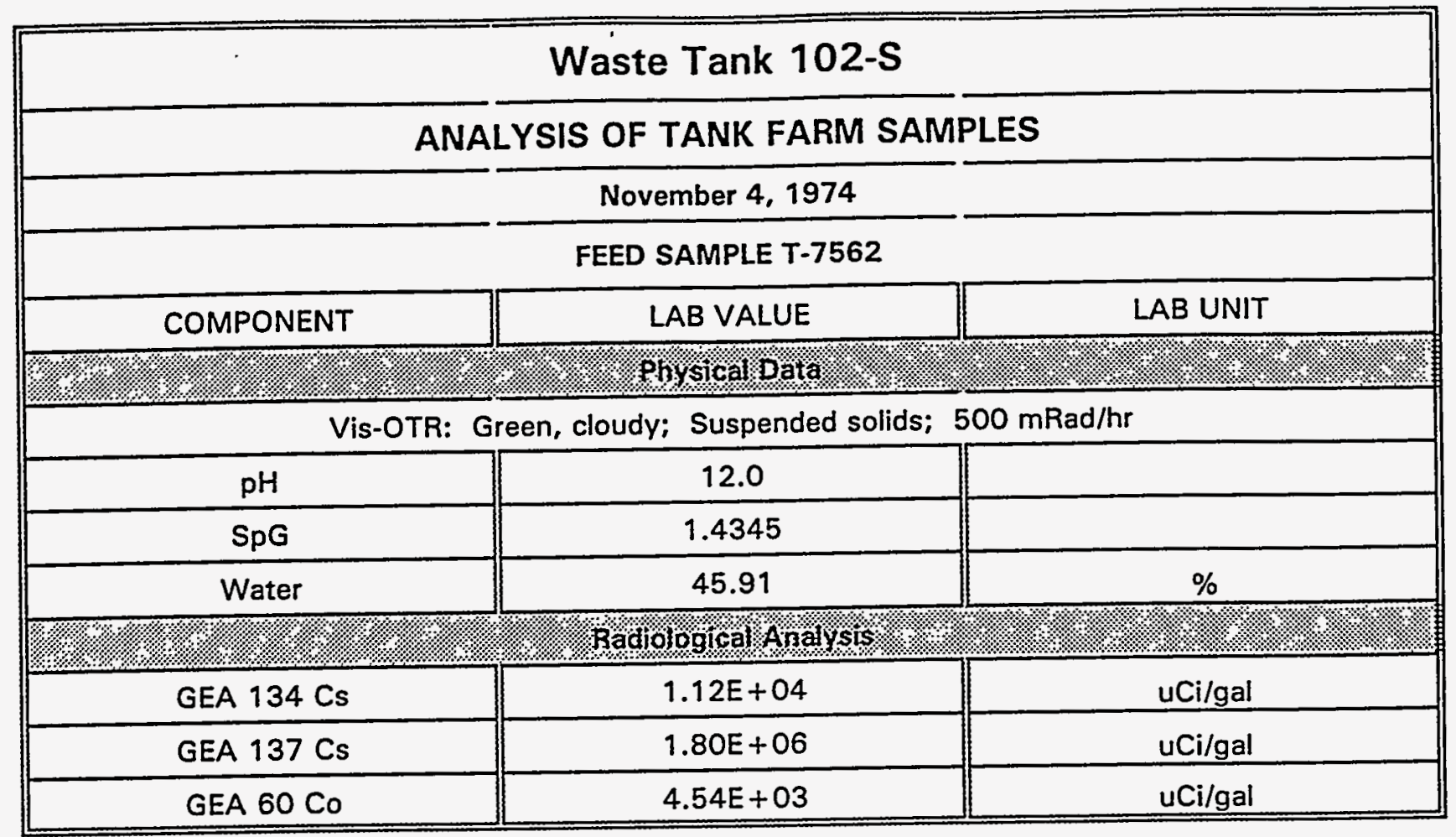




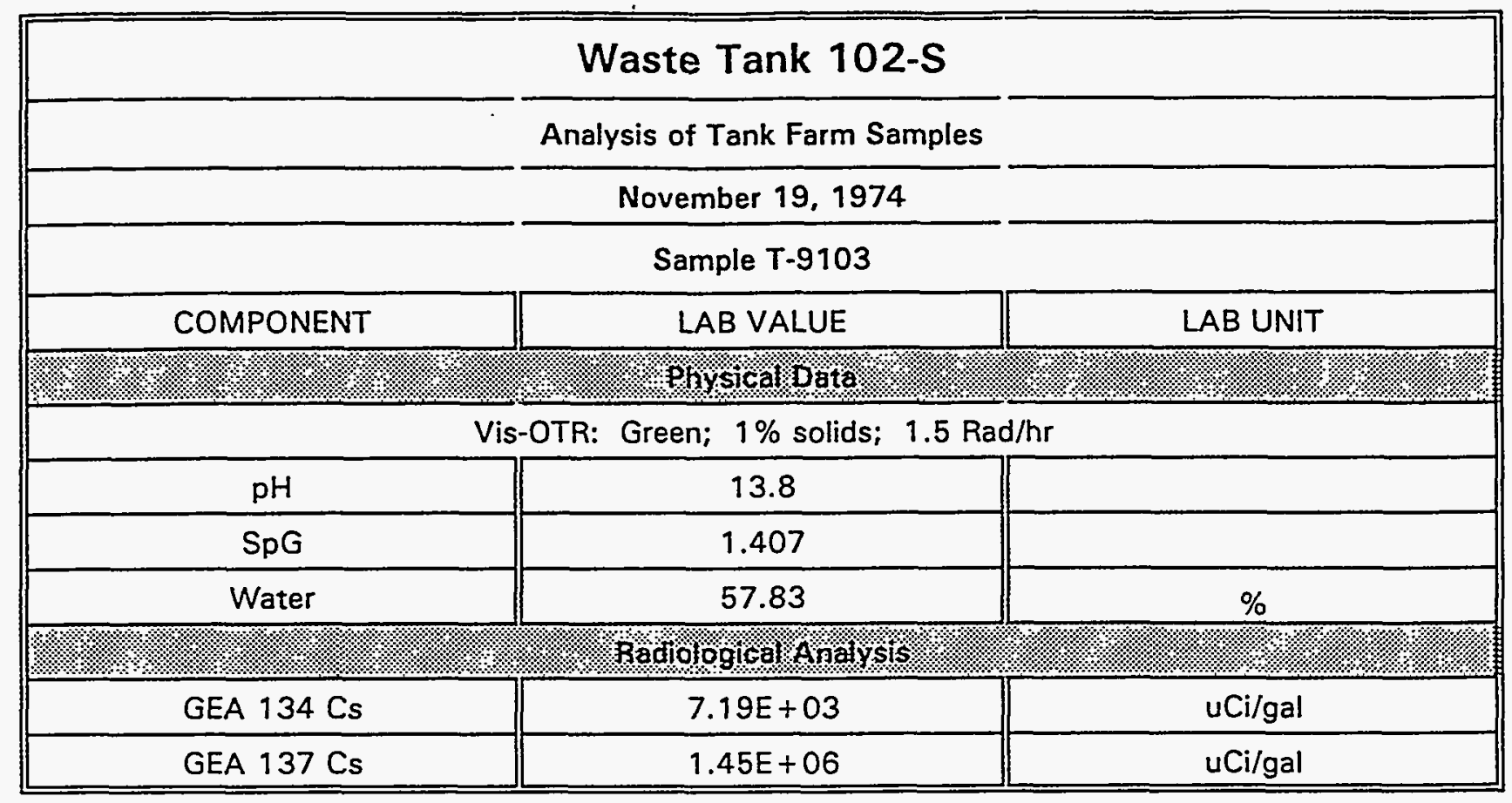




\begin{tabular}{|c|c|c|}
\hline \multicolumn{3}{|c|}{ Waste Tank 102-S } \\
\hline \multicolumn{3}{|c|}{ ANALYSIS OF TANK FARM SAMPLES } \\
\hline \multicolumn{3}{|c|}{ November 19,1974} \\
\hline \multicolumn{3}{|c|}{ SAMPLE T-9601 } \\
\hline COMPONENT & LAB VALUE & LAB UNIT \\
\hline \multirow{2}{*}{\multicolumn{3}{|c|}{ 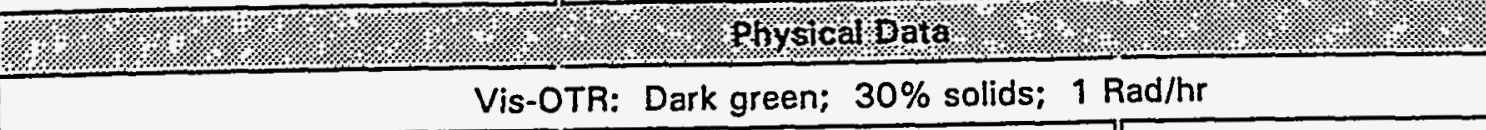 }} \\
\hline & & \\
\hline \multicolumn{3}{|c|}{$\begin{array}{cc}\mathrm{pH} & 13.1 \\
\end{array}$} \\
\hline \multicolumn{3}{|c|}{1.4554} \\
\hline Water & 54.89 & $\%$ \\
\hline \multicolumn{3}{|c|}{ (1) } \\
\hline GEA $134 \mathrm{Cs}$ & $7.04 E+03$ & uCi/gal \\
\hline GEA $137 \mathrm{Cs}$ & $1.54 E+06$ & uCi/gal \\
\hline GEA $60 \mathrm{Co}$ & $1.16 E+03$ & uCi/gal \\
\hline
\end{tabular}




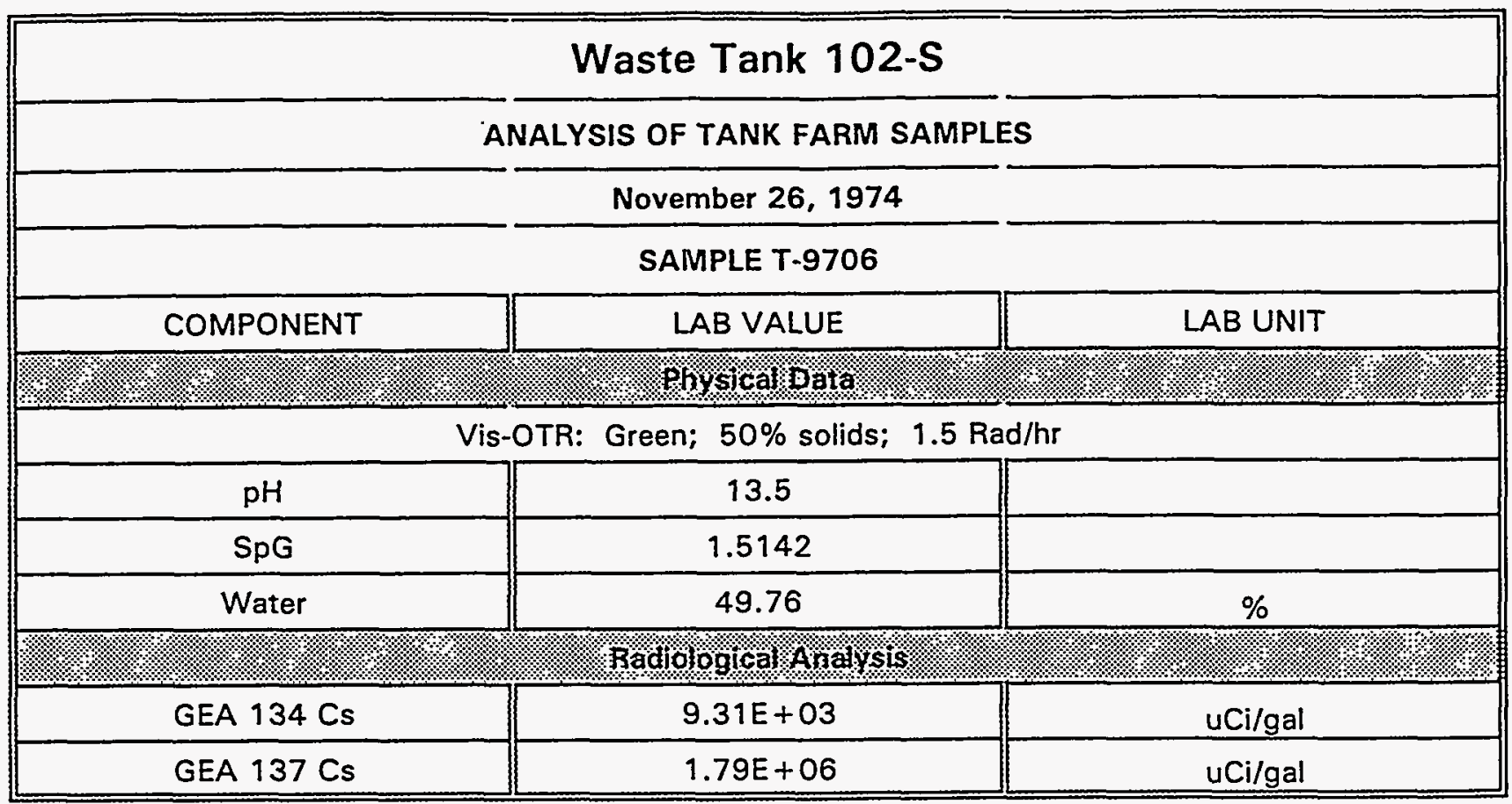


WHC-SD-WM-ER-323, Rev. O

\begin{tabular}{|c|c|c|}
\hline \multicolumn{3}{|c|}{ Waste Tank 102-S } \\
\hline \multicolumn{3}{|c|}{ AṄALYSIS OF TANK FARM SAMPLES } \\
\hline \multicolumn{3}{|c|}{ December 4, 1974} \\
\hline \multicolumn{3}{|c|}{ SAMPLE T-8416 } \\
\hline COMPONENT & LAB VALUE & LAB UNIT \\
\hline \multicolumn{3}{|c|}{ 1981 } \\
\hline \multicolumn{3}{|c|}{ Vis-OTR: Green; $<10 \%$ solids; 1 Rad $/ \mathrm{hr}$} \\
\hline $\mathrm{pH}$ & 13.3 & \\
\hline SpG & 1.4327 & \\
\hline Water & 57.98 & $\%$ \\
\hline \multicolumn{3}{|l|}{18.18 .18} \\
\hline GEA $134 \mathrm{Cs}$ & $3.45 E+04$ & uCi/gal \\
\hline GEA $137 \mathrm{Cs}$ & $5.83 E+06$ & $\mathrm{uCi} / \mathrm{gal}$ \\
\hline
\end{tabular}




\begin{tabular}{|c|c|c|}
\hline \multicolumn{3}{|c|}{ Waste Tank 102-S } \\
\hline \multicolumn{3}{|c|}{ ANALYSIS OF TANK FARM SAMPLES } \\
\hline \multicolumn{3}{|c|}{ December 26, 1974} \\
\hline \multicolumn{3}{|c|}{ SAMPLE T-423 Received, Dec. 6, 1974} \\
\hline COMPONENT & LAB VALUE & LAB UNIT \\
\hline I, & / & 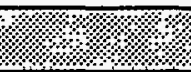 \\
\hline \multicolumn{3}{|c|}{ Vis-OTR: Green; $50 \%$ crystal solids; $1.5 \mathrm{Rad} / \mathrm{hr}$} \\
\hline $\mathrm{pH}$ & 14.0 & \\
\hline SpG & 1.4272 & \\
\hline Water & 58.15 & $\%$ \\
\hline (4, & Chemica Analys & \\
\hline $\mathrm{Al}$ & 1.14 & $M$ \\
\hline \multicolumn{3}{|c|}{ 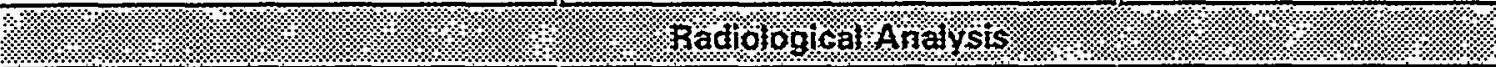 } \\
\hline GEA $134 \mathrm{Cs}$ & $6.09 E+03$ & uCi/gal \\
\hline GEA 137 Cs & $1.40 E+06$ & uCi/gal \\
\hline
\end{tabular}




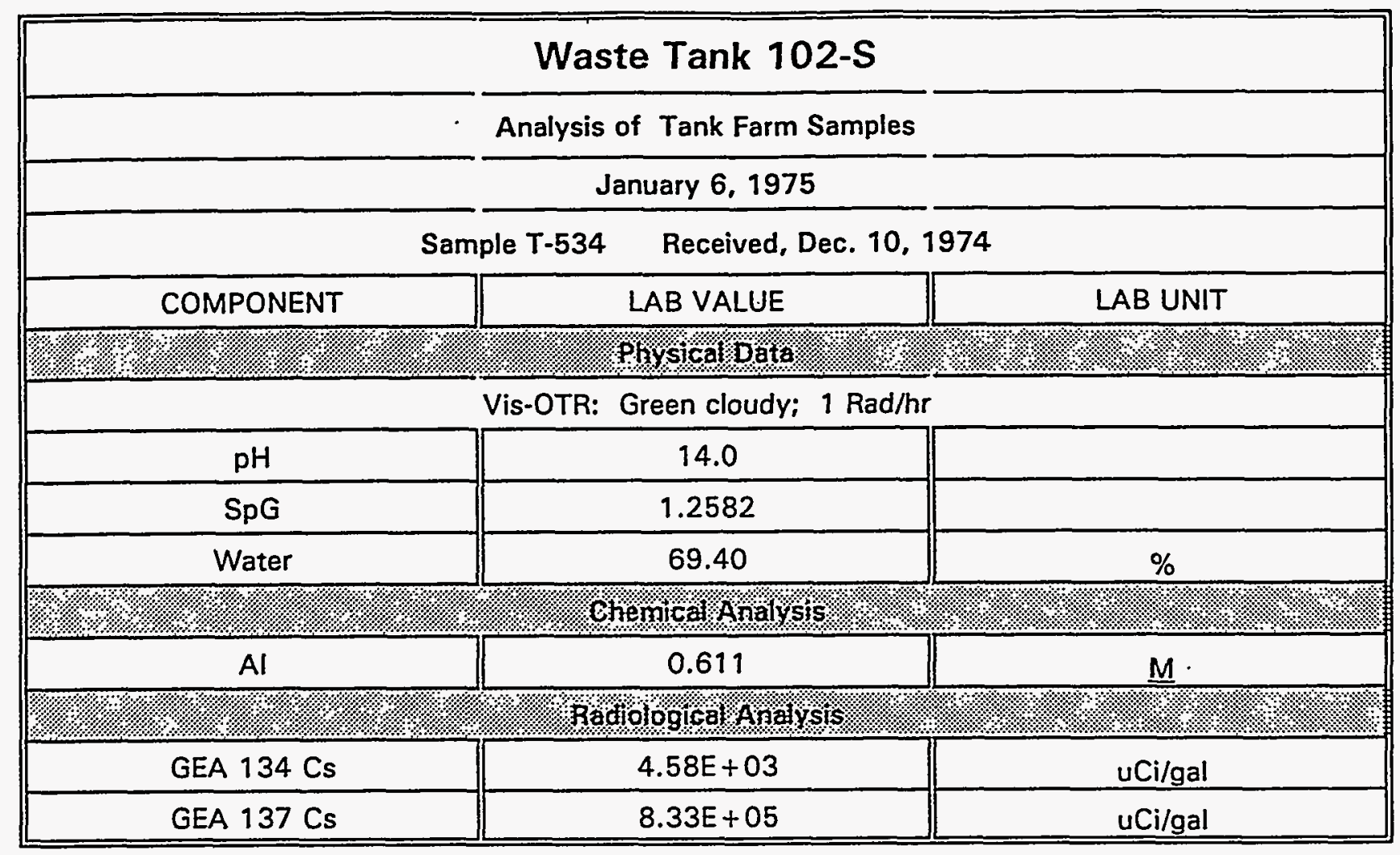


WHC-SD-WM-ER-323, Rev. O

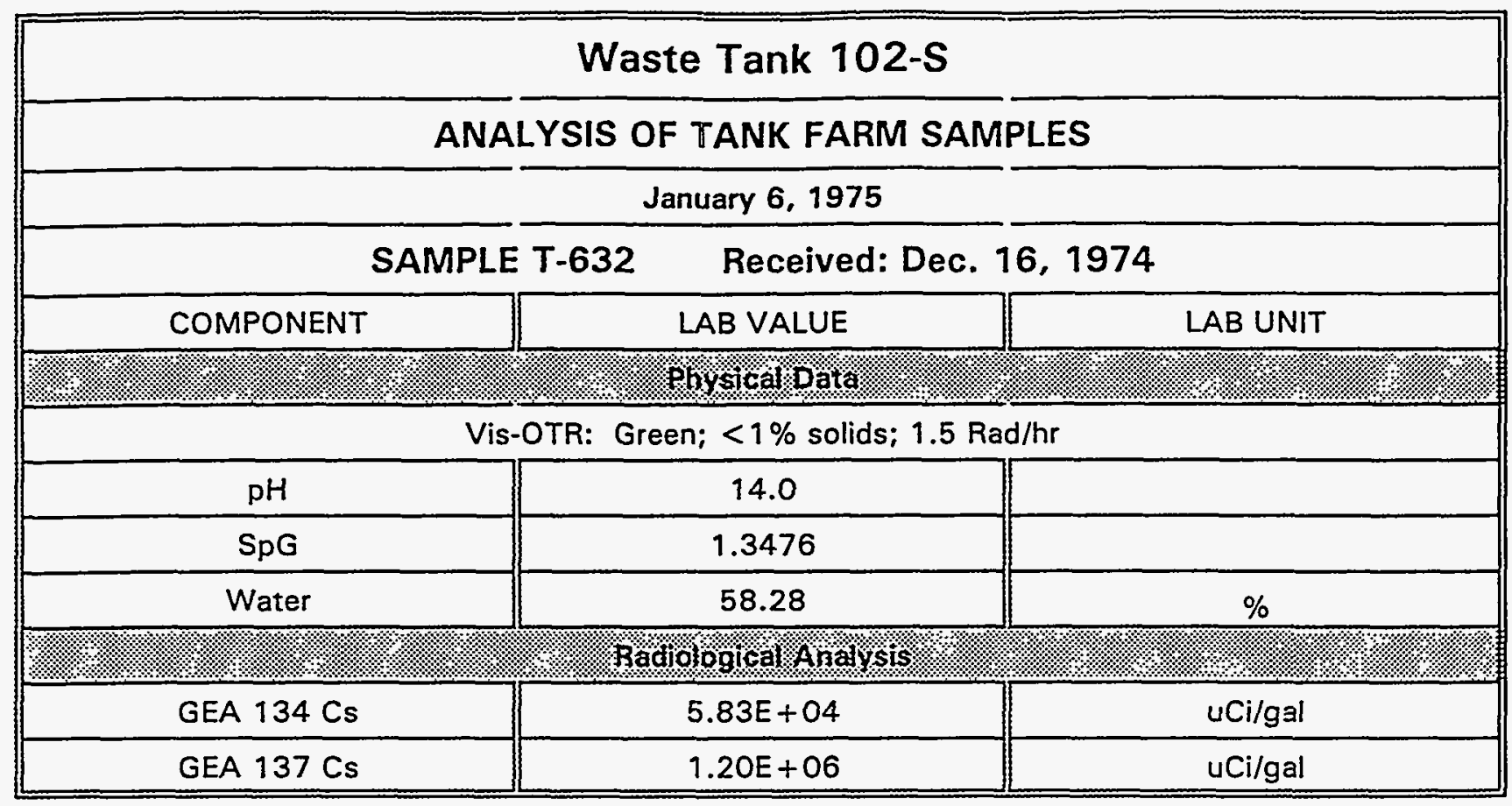




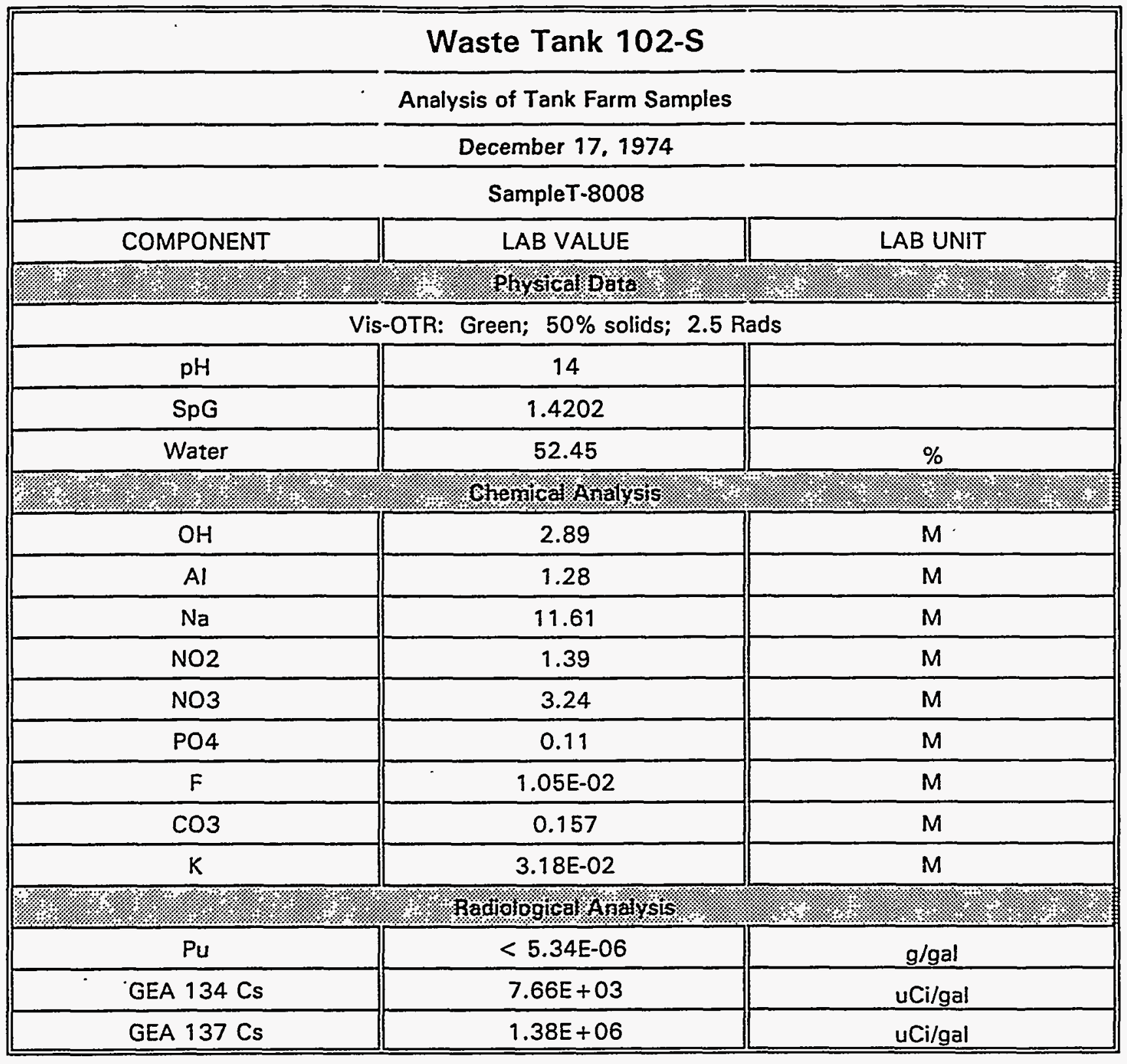




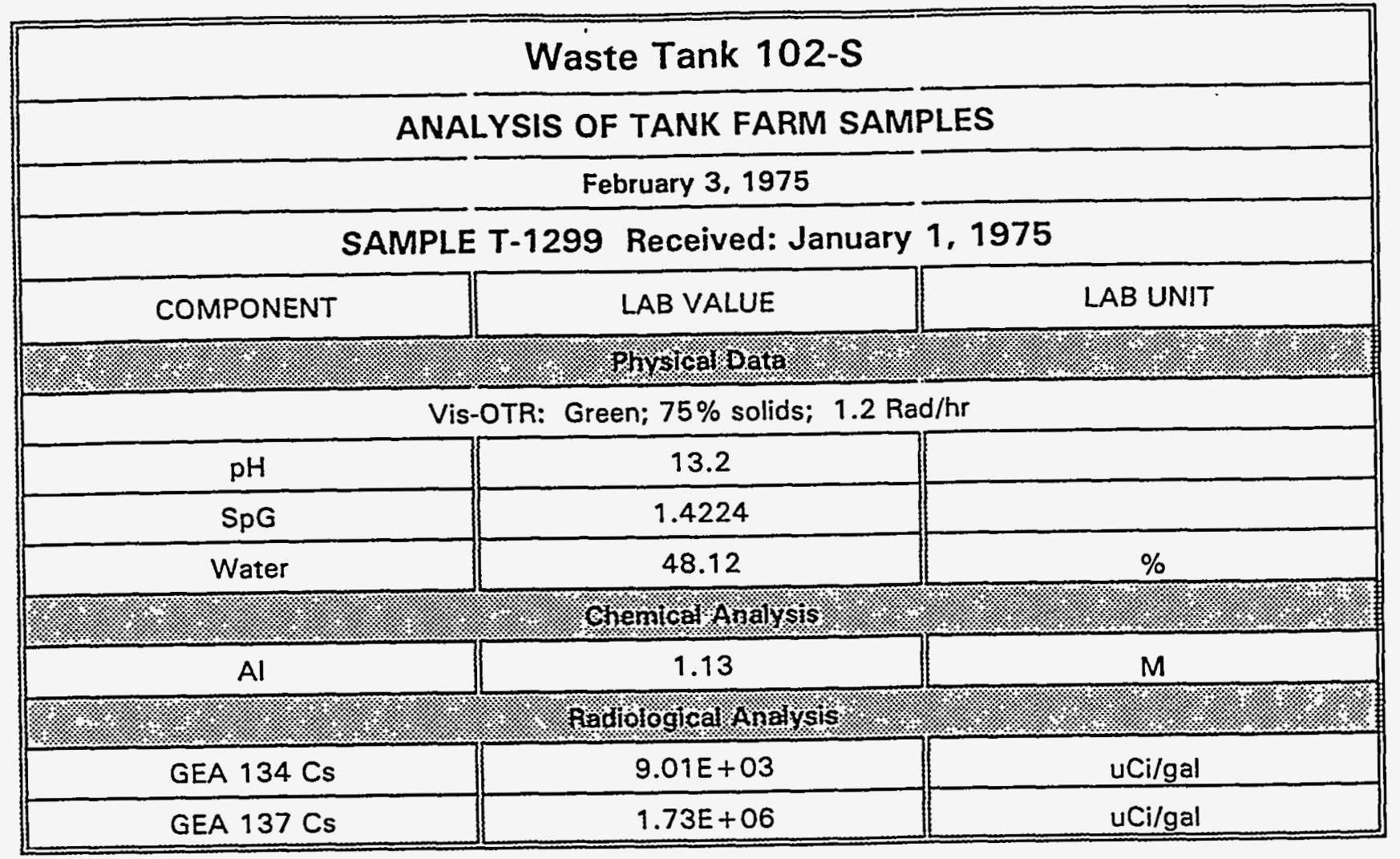




\begin{tabular}{|c|c|c|}
\hline \multicolumn{3}{|c|}{ Waste Tank 102-S } \\
\hline \multicolumn{3}{|c|}{ ANALYSIS OF TANK FARM SAMPLES } \\
\hline \multicolumn{3}{|c|}{ October 20, 1975} \\
\hline \multicolumn{3}{|c|}{ Received: January 2, 1975} \\
\hline COMPONENT & LAB VALUE & LAB UNIT \\
\hline (1) & Prysicoloate & (1., \\
\hline \multicolumn{3}{|c|}{ Vis-OTR: Green, cloudy; No solids; $1 \mathrm{rad} / \mathrm{hr}$} \\
\hline $\mathrm{pH}$ & 14.0 & \\
\hline SPG & 1.580 & \\
\hline Water & 51.68 & $\%$ \\
\hline \multicolumn{3}{|c|}{ 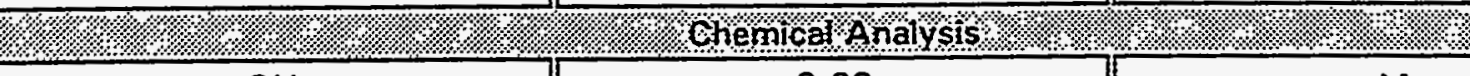 } \\
\hline $\mathrm{OH}$ & 2.63 & $\mathrm{M}$ \\
\hline Al & 1.13 & M \\
\hline $\mathrm{Na}$ & 9.93 & M \\
\hline NO2 & 1.87 & $\mathrm{M}$ \\
\hline NO3 & 3.64 & M \\
\hline SO4 & cancelled & \\
\hline PO4 & $5.68 \mathrm{E}-02$ & $M$ \\
\hline $\mathrm{F}$ & $1.34 \mathrm{E}-02$ & M \\
\hline $\mathrm{CO} 3$ & 0.257 & M \\
\hline \multicolumn{3}{|c|}{ 1.8. } \\
\hline $\mathrm{Pu}$ & $2.40 \mathrm{E}-05$ & g/gal \\
\hline GEA $134 \mathrm{Cs}$ & $1.53 E+04$ & uCi/gal \\
\hline GEA 137 Cs & $2.69 \mathrm{E}+06$ & uCi/gal \\
\hline
\end{tabular}




\begin{tabular}{|c|c|c|}
\hline \multicolumn{3}{|c|}{ Waste Tank 102-S } \\
\hline \multicolumn{3}{|c|}{ ANALYSIS OF TANK FARM SAMPLES } \\
\hline \multicolumn{3}{|c|}{ January 6, 1975} \\
\hline \multicolumn{3}{|c|}{ SAMPLE T-841 } \\
\hline COMPONENT & LAB VALUE & LAB UNIT \\
\hline 1. & efiysicaliopata & 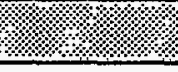 \\
\hline \multicolumn{3}{|c|}{ Vis-OTR: Yellow; No solids } \\
\hline $\mathrm{pH}$ & 14.0 & \\
\hline SpG & 1.4346 & \\
\hline Water & 51.45 & $\%$ \\
\hline \multicolumn{3}{|c|}{ 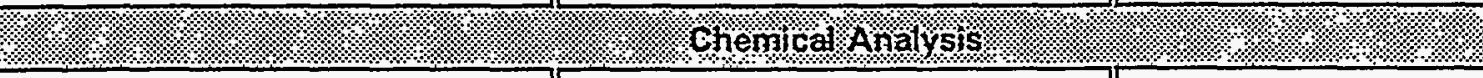 } \\
\hline \multirow{2}{*}{\multicolumn{3}{|c|}{ 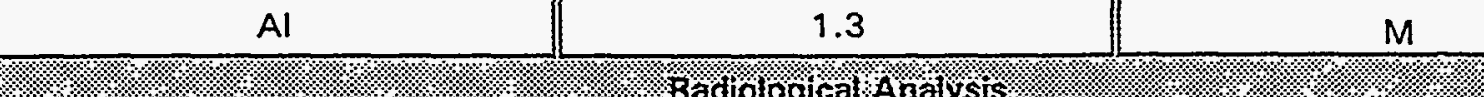 }} \\
\hline (4) & Radiologicalo Ana & \\
\hline GEA 134 Cs & $2.13 E+04$ & uCi/gal \\
\hline GEA $137 \mathrm{Cs}$ & $3.75 E+05$ & uCi/gal \\
\hline GEA 60 Co & $1.56 E+04$ & $\mathrm{uCi} / \mathrm{gal}$ \\
\hline GEA $125 \mathrm{Sb}$ & $1.52 E+05$ & uCi/gal \\
\hline
\end{tabular}




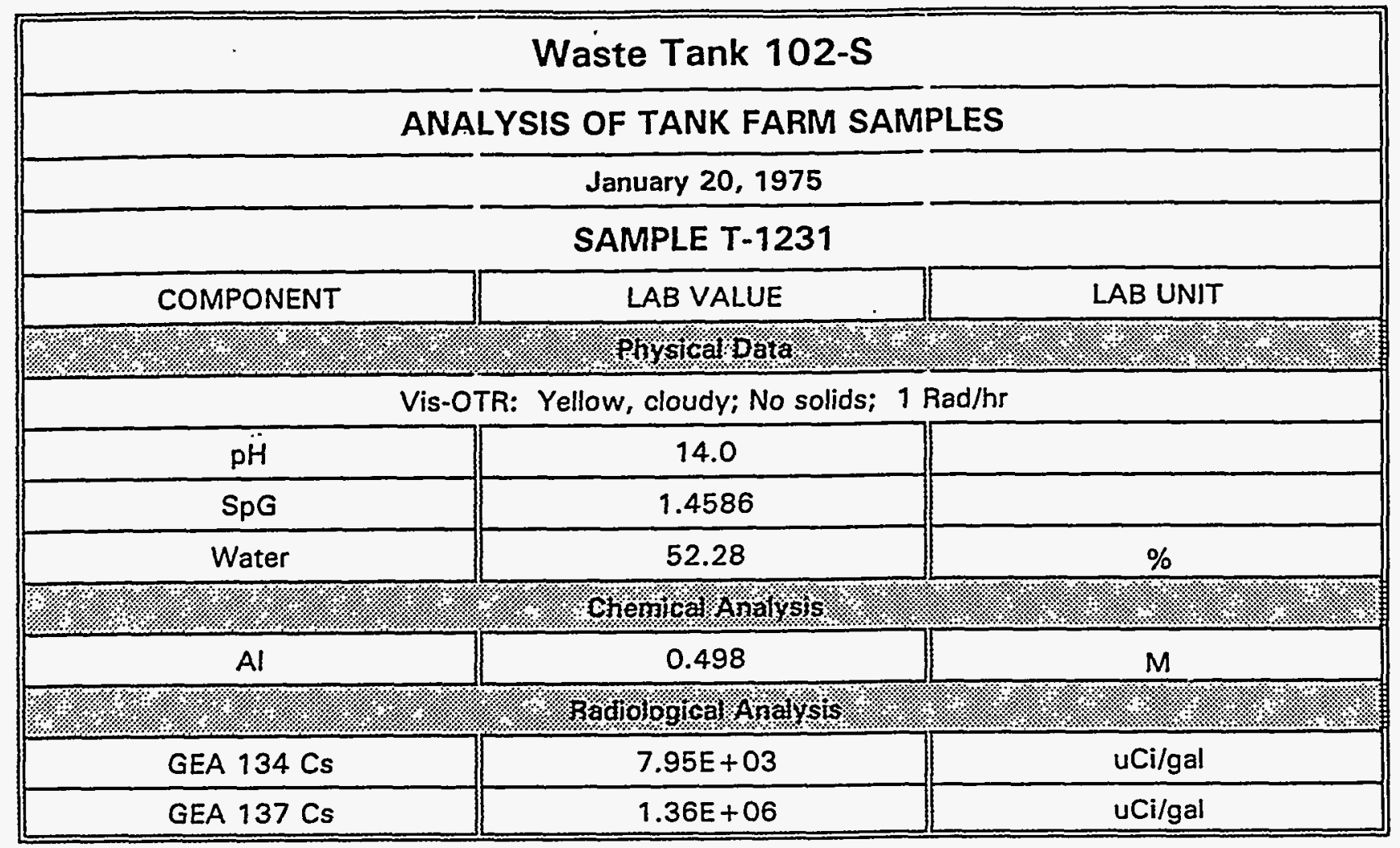


WHC-SD-WM-ER-323, Rev. 0

\begin{tabular}{|c|c|c|}
\hline \multicolumn{3}{|c|}{ Waste Tank 102-S } \\
\hline \multicolumn{3}{|c|}{ ANALYSIS OF TANK FARM SAMPLES } \\
\hline \multicolumn{3}{|c|}{ January 22, 1975} \\
\hline \multicolumn{3}{|c|}{ SAMPLE T-933 } \\
\hline COMPONENT & LAB VALUE & LAB UNIT \\
\hline 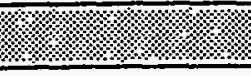 & hyisical oate & 4.1 .1 .1 .2 .1$. \\
\hline \multicolumn{3}{|c|}{ Vis-OTR: Green; No solids; $1.3 \mathrm{Rad} / \mathrm{hr}$} \\
\hline $\mathrm{pH}$ & 14.0 & \\
\hline SpG & 1.6515 & \\
\hline Water & 51.10 & $\%$ \\
\hline \multicolumn{3}{|c|}{ Chericar Analysis, } \\
\hline Al & 1.29 & $M$ \\
\hline \multicolumn{3}{|c|}{ Radiologica's snalysis } \\
\hline GEA $134 \mathrm{Cs}$ & $8.02 E+03$ & uCi/gal \\
\hline GEA $137 \mathrm{Cs}$ & $1.60 E+06$ & uCi/gal \\
\hline
\end{tabular}




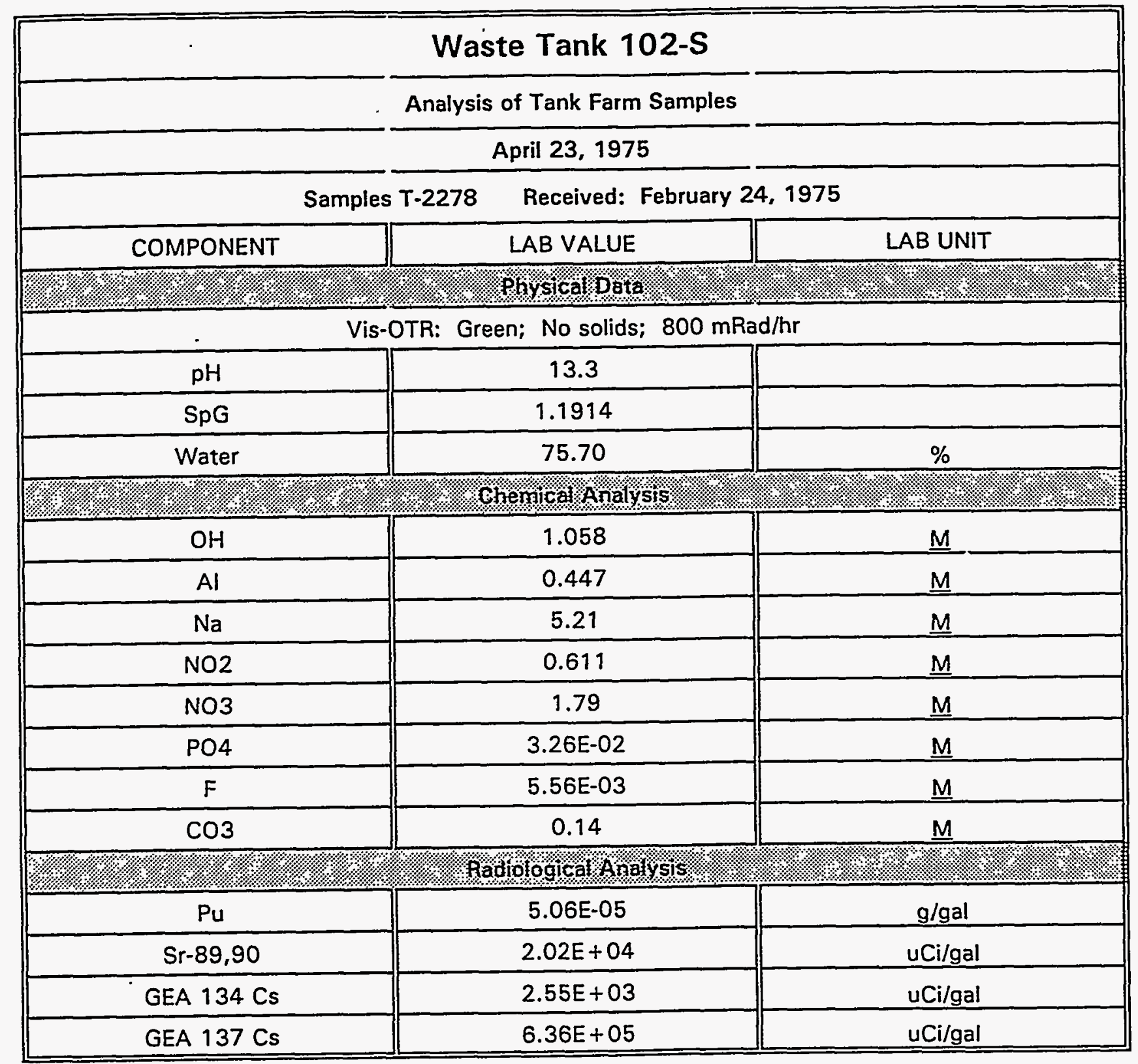




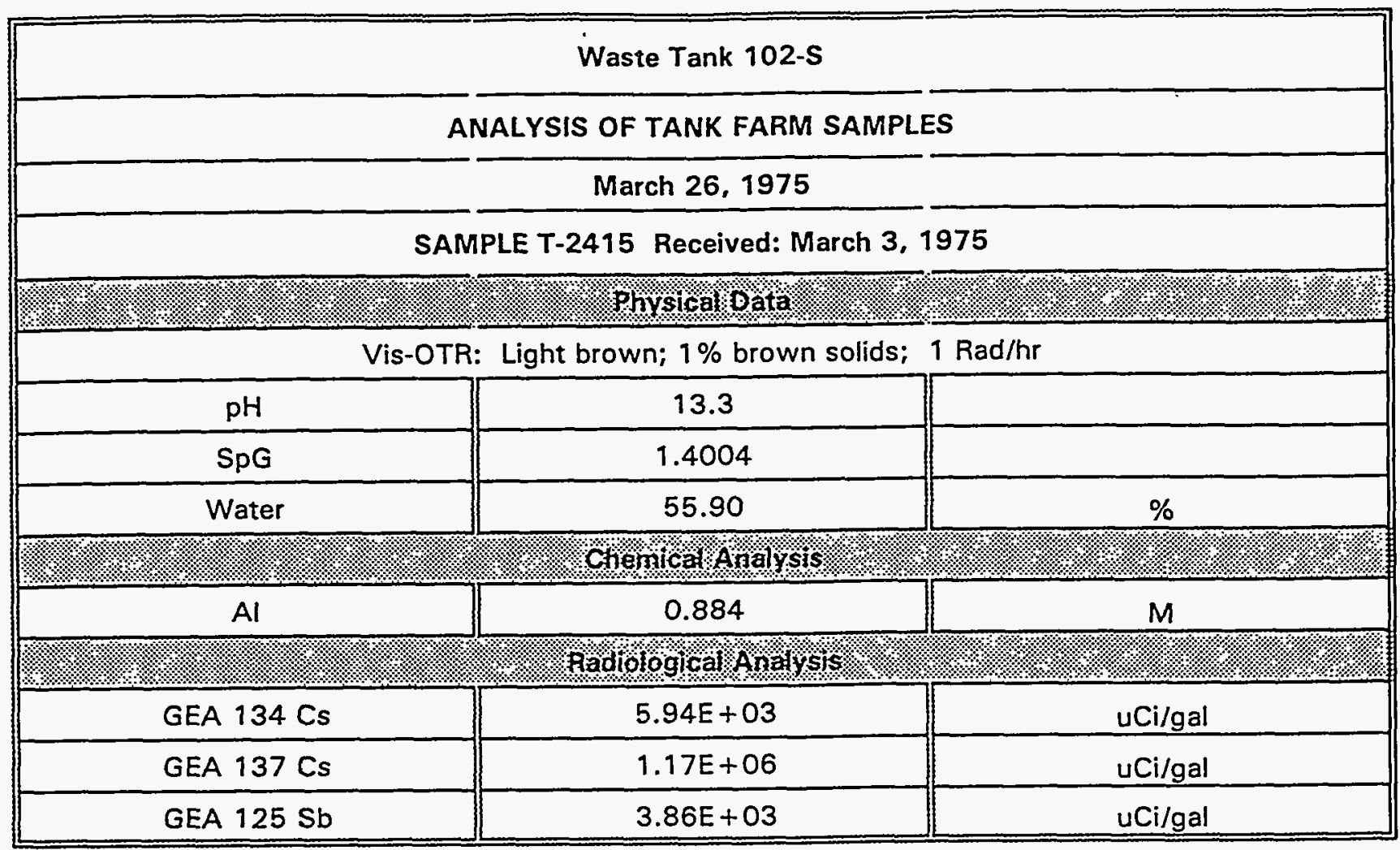




\begin{tabular}{|c|c|c|}
\hline \multicolumn{3}{|c|}{ Waste Tank 102-S } \\
\hline \multicolumn{3}{|c|}{ ANALYSIS OF TANK FARM SAMPLES } \\
\hline \multicolumn{3}{|c|}{ April 11, 1975} \\
\hline \multicolumn{3}{|c|}{ SAMPLE T-2617 Received: March 10, 1975} \\
\hline COMPONENT & LAB VALUE & LAB UNIT \\
\hline 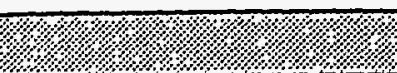 & Physicalpata) & 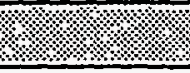 \\
\hline \multicolumn{3}{|c|}{ Vis-OTR: Black; 2 Rad/hr } \\
\hline $\mathrm{pH}$ & 13.5 & \\
\hline SpG & $1.32 @ 60 * \mathrm{C}$ & \\
\hline Water & 54.52 & $\%$ \\
\hline \multicolumn{3}{|l|}{ 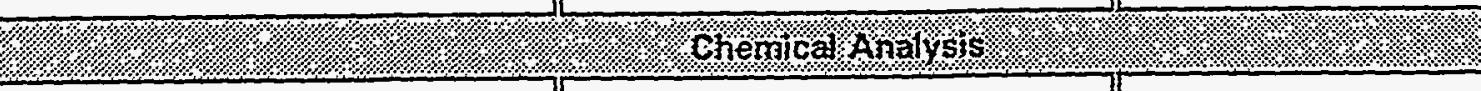 } \\
\hline Al & 0.589 & $M$ \\
\hline \multicolumn{3}{|c|}{ l. } \\
\hline GEA $134 \mathrm{Cs}$ & $6.06 E+03$ & uCi/gal \\
\hline GEA 137 Cs & $1.38 E+06$ & uCi/gal \\
\hline
\end{tabular}


WHC-SD-WM-ER-323, Rev. 0

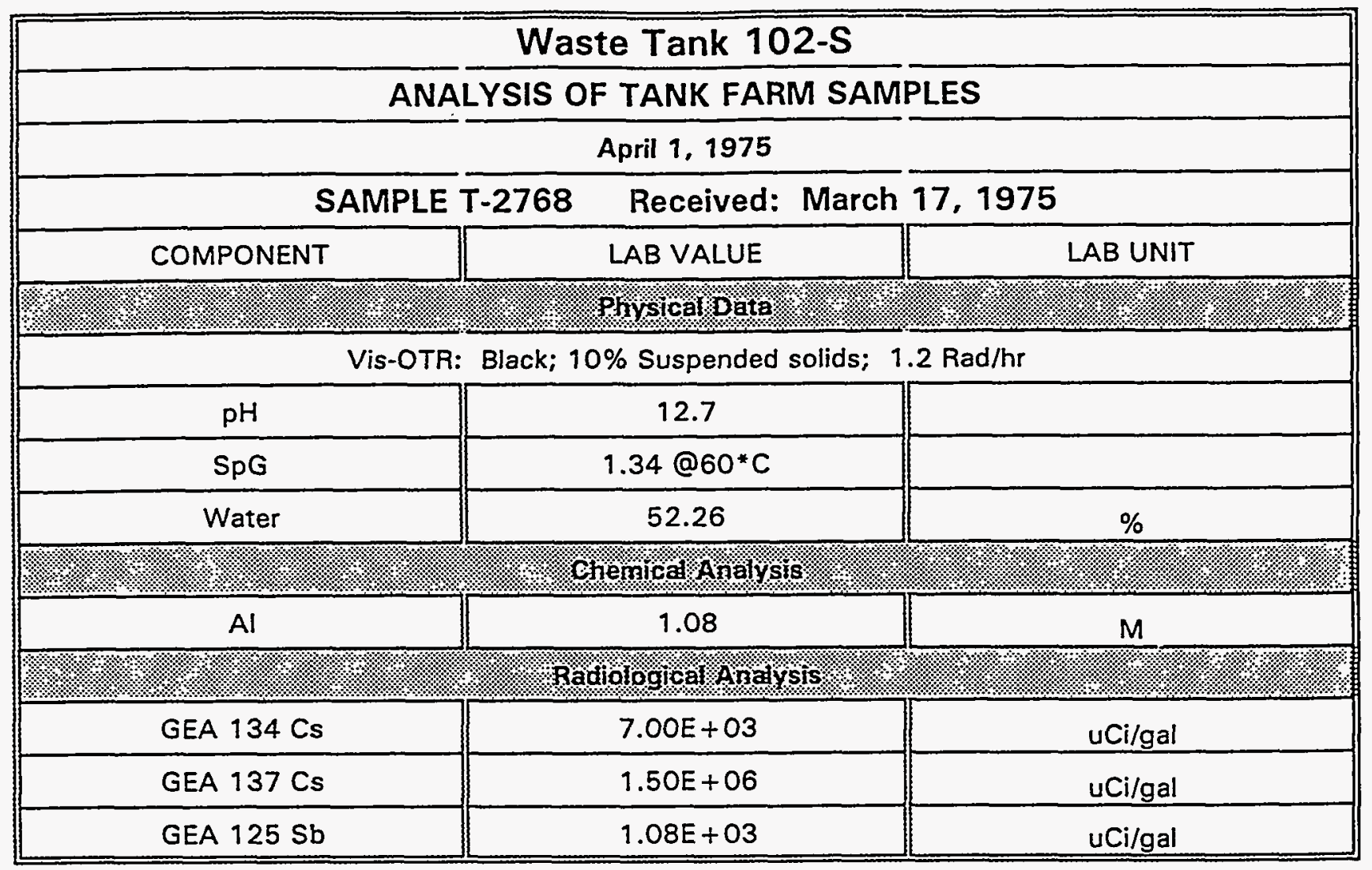




\begin{tabular}{|c|c|c|}
\hline \multicolumn{3}{|c|}{ Waste Tank 102-S } \\
\hline \multicolumn{3}{|c|}{ ANALYSIS OF TANK FARM SAMPLES } \\
\hline \multicolumn{3}{|c|}{ April 23, 1975} \\
\hline SAMPLE T-3109 & \multicolumn{2}{|c|}{ Received: March 28, 1975} \\
\hline COMPONENT & LAB VALUE & LAB UNIT \\
\hline \multicolumn{3}{|c|}{ 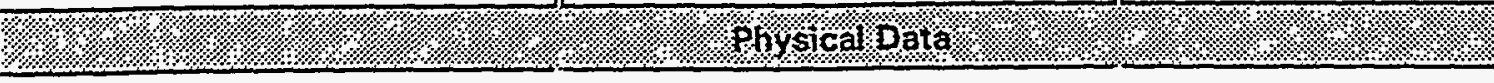 } \\
\hline \multicolumn{3}{|c|}{ Vis-OTR: Brown, cloudy; $600 \mathrm{mrad} / \mathrm{hr}$ on contact } \\
\hline $\mathrm{pH}$ & 12.8 & \\
\hline SpG & $1.36 @ 60 * \mathrm{C}$ & \\
\hline Water & 50.71 & $\%$ \\
\hline \multicolumn{3}{|c|}{ 1. } \\
\hline $\mathrm{Al}$ & 0.978 & M \\
\hline \multicolumn{3}{|c|}{ 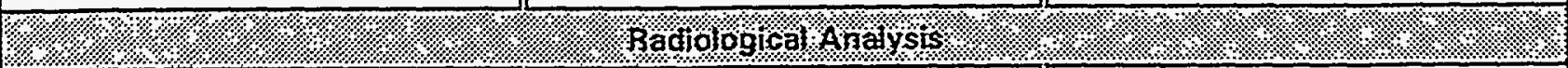 } \\
\hline GEA $134 \mathrm{Cs}$ & $7.50 E+03$ & uCi/gal \\
\hline GEA $137 \mathrm{Cs}$ & $1.77 E+06$ & uCi/gal \\
\hline GEA $125 \mathrm{Sb}$ & $3.54 E+03$ & $\mathrm{uCi} / \mathrm{gal}$ \\
\hline
\end{tabular}




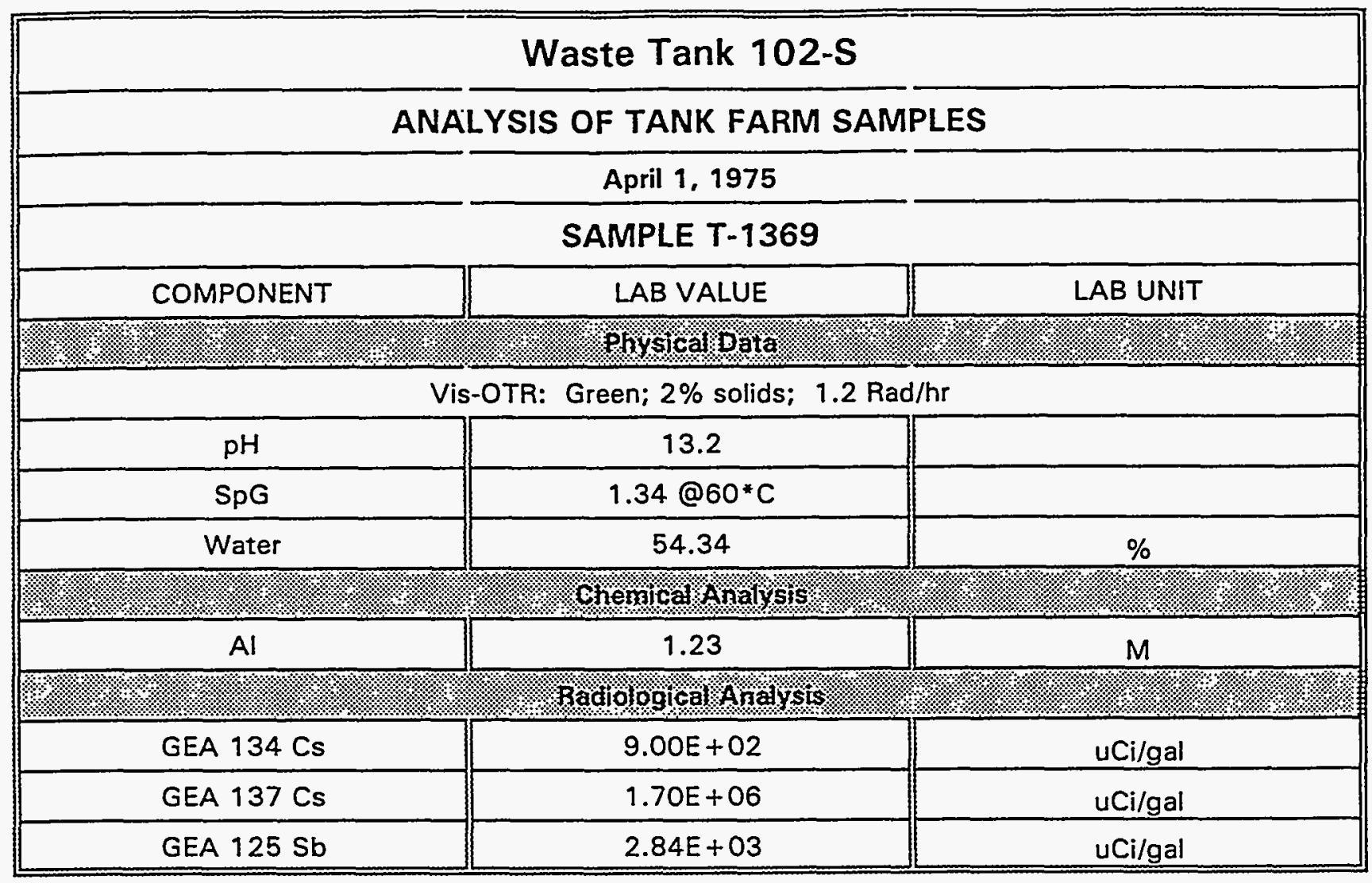




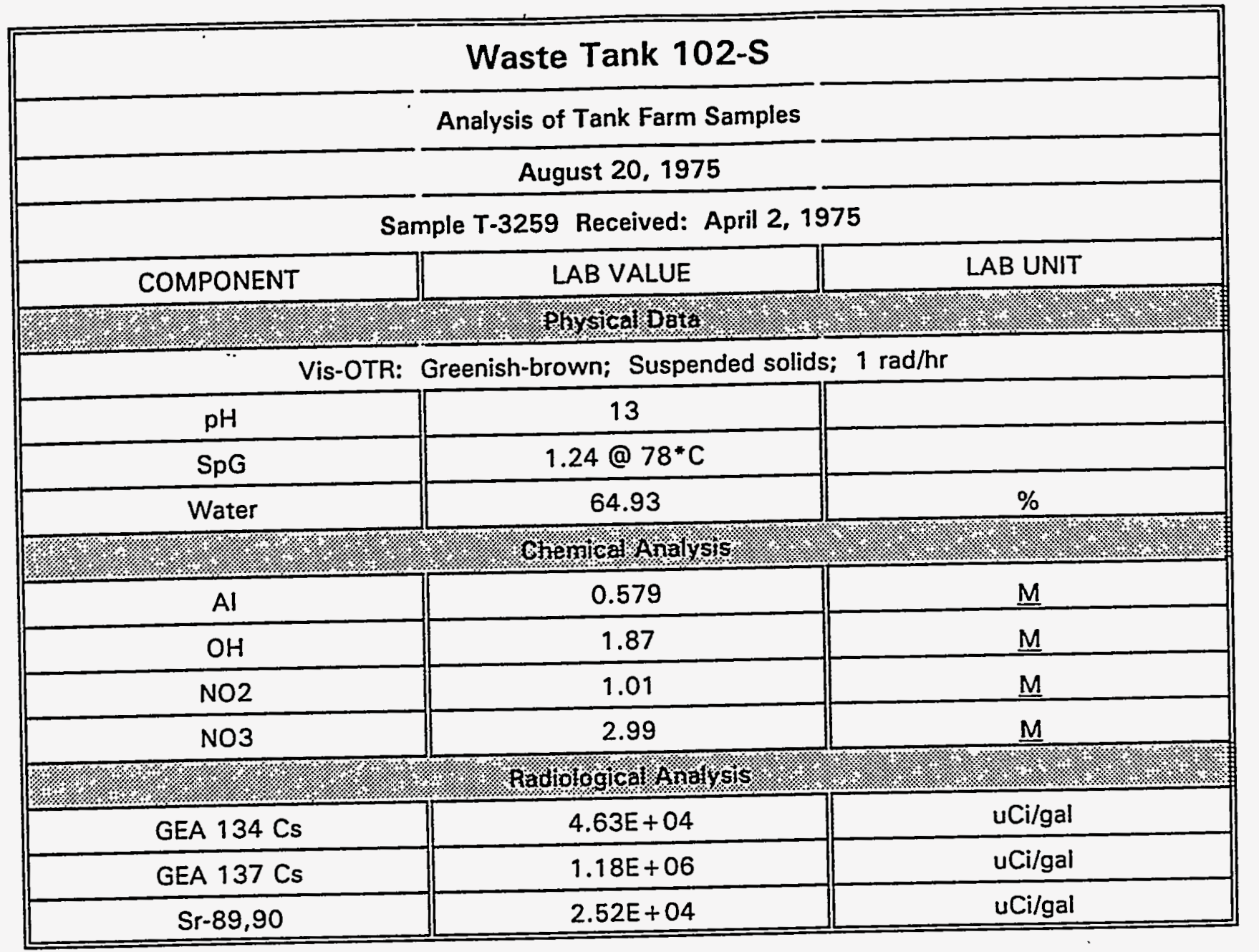


WHC-SD-WM-ER-323, Rev. O

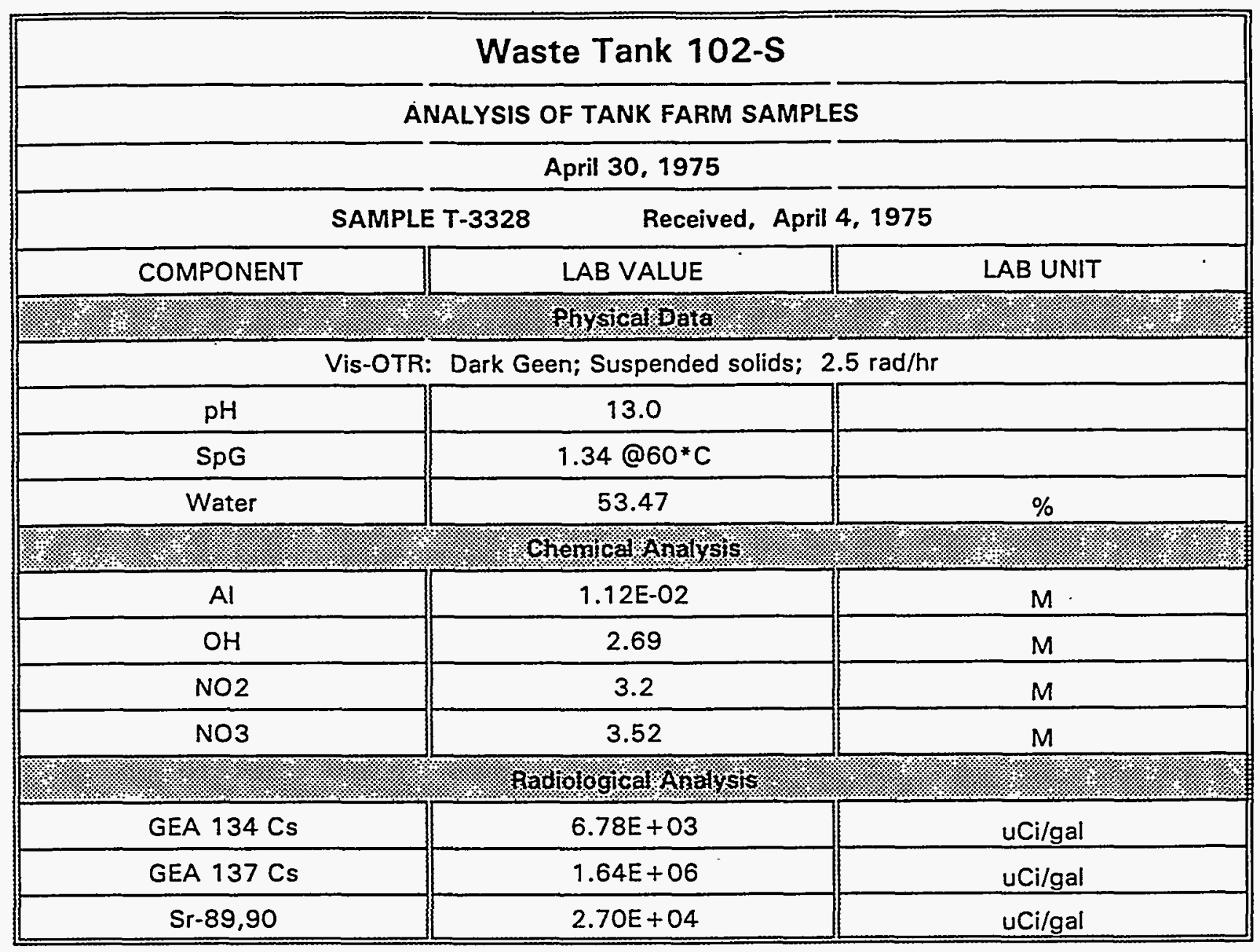




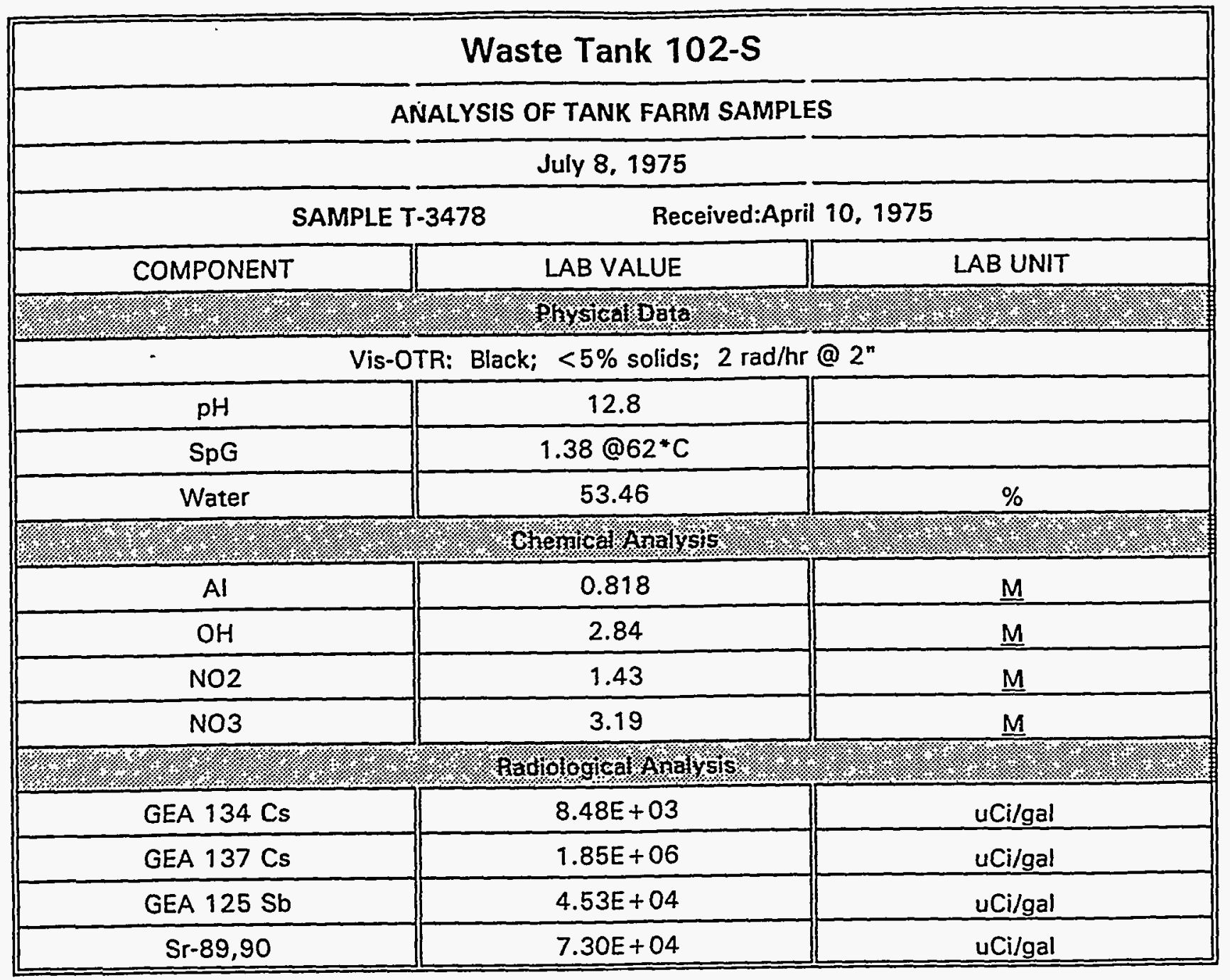




\begin{tabular}{|c|c|c|}
\hline \multicolumn{3}{|c|}{ Waste Tank 102-S } \\
\hline \multicolumn{3}{|c|}{ Analysis of $102-\mathrm{S}$ Top Layer } \\
\hline \multicolumn{3}{|c|}{ April 17, 1975} \\
\hline COMPONENT & LAB VALUE & LAB UNIT \\
\hline 4. & Ply sical patz: & 1.1. \\
\hline \multicolumn{3}{|c|}{ Appearance: The top layer was made up of coarse black crystals. } \\
\hline Bulk Density & 1.3 & $\mathrm{~g} / \mathrm{cc}$ \\
\hline Particle Density & 1.74 & $\mathrm{~g} / \mathrm{cc}$ \\
\hline \multicolumn{3}{|c|}{ Chemical Analysis } \\
\hline $\mathrm{H} 2 \mathrm{O}$ & 45.00 & $\%$ \\
\hline $\mathrm{NaNO} 2$ & $<0.1$ & $\%$ \\
\hline NaNO3 & 20.80 & $\%$ \\
\hline $\mathrm{Na} 3 \mathrm{PO} 4$ & 29.90 & $\%$ \\
\hline $\mathrm{SiO} 2$ & 1.70 & $\%$ \\
\hline $\mathrm{Fe} 2 \mathrm{O} 3$ & 0.20 & $\%$ \\
\hline $\mathrm{A} 1203$ & 5.50 & $\%$ \\
\hline TOTAL & 102.90 & $\%$ \\
\hline প. & Radiological Analysis & \%1\%. \\
\hline $\mathrm{Pu}$ & $5.20 \mathrm{E}-07$ & $\mathrm{~g} / \mathrm{g}$ \\
\hline $\mathrm{Pu}$ & $4.00 \mathrm{E}-04$ & $g / 1$ \\
\hline $\mathrm{Sr}-89,90$ & 19.20 & $\mathrm{uCi} / \mathrm{g}$ \\
\hline $\mathrm{Sr}-89,90$ & $1.50 E+04$ & uCi/L \\
\hline Cs-137 & 17.30 & $u C i / g$ \\
\hline Cs-137 & $1.30 E+04$ & $\mathrm{uCi} / \mathrm{L}$ \\
\hline
\end{tabular}




\begin{tabular}{|c|c|c|}
\hline \multicolumn{3}{|c|}{ Waste Tank 102-S } \\
\hline \multicolumn{3}{|c|}{ Analysis of $102-\mathrm{S}$ Bottom Layer } \\
\hline \multicolumn{3}{|c|}{ April 17,1975} \\
\hline COMPONENT & LAB VALUE & LAB UNIT \\
\hline \multicolumn{3}{|c|}{ (1) } \\
\hline Bulk Density & 1.5 & $\mathrm{~g} / \mathrm{cc}$ \\
\hline Particle Density & 2.09 & $\mathrm{~g} / \mathrm{cc}$ \\
\hline \multicolumn{3}{|c|}{ 1\% } \\
\hline $\mathrm{H} 2 \mathrm{O}$ & 10.00 & $\%$ \\
\hline $\mathrm{NaNO} 2$ & 0.10 & $\%$ \\
\hline $\mathrm{NaNO} 3$ & (Note 1) & $\%$ \\
\hline $\mathrm{Na} 3 \mathrm{PO} 4$ & 1.00 & $\%$ \\
\hline $\mathrm{SiO} 2$ & 0.70 & $\%$ \\
\hline $\mathrm{Fe} 2 \mathrm{O} 3$ & 1.40 & $\%$ \\
\hline $\mathrm{Al} 203$ & 87.00 & $\%$ \\
\hline TOTAL & 100.20 & $\%$ \\
\hline \multicolumn{3}{|c|}{ 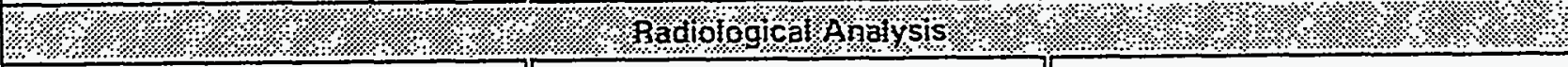 } \\
\hline $\mathrm{Pu}$ & 7.50E-07 & $\mathrm{g} / \mathrm{g}$ \\
\hline $\mathrm{Pu}$ & 4.90E-04 & $g / 1$ \\
\hline Sr-89,90 & 0.50 & $u \mathrm{Ci} / \mathrm{g}$ \\
\hline Sr-89,90 & $3.30 E+02$ & uCi/l \\
\hline Cs-137 & 6.60 & $\mathrm{uCi} / \mathrm{g}$ \\
\hline Cs-137 & $4.30 E+03$ & uCi/L \\
\hline \multicolumn{3}{|c|}{ The nitrate analysis of the sample was impossibly high ( 520 percent } \\
\hline \multicolumn{3}{|c|}{ NaNO3 1. It is assumed that the sample was contaminated during } \\
\hline \multicolumn{3}{|c|}{ preparation. However, based on the material balance calculation, we } \\
\hline feel that th & e content of the salt is essentially $z$ & \\
\hline
\end{tabular}




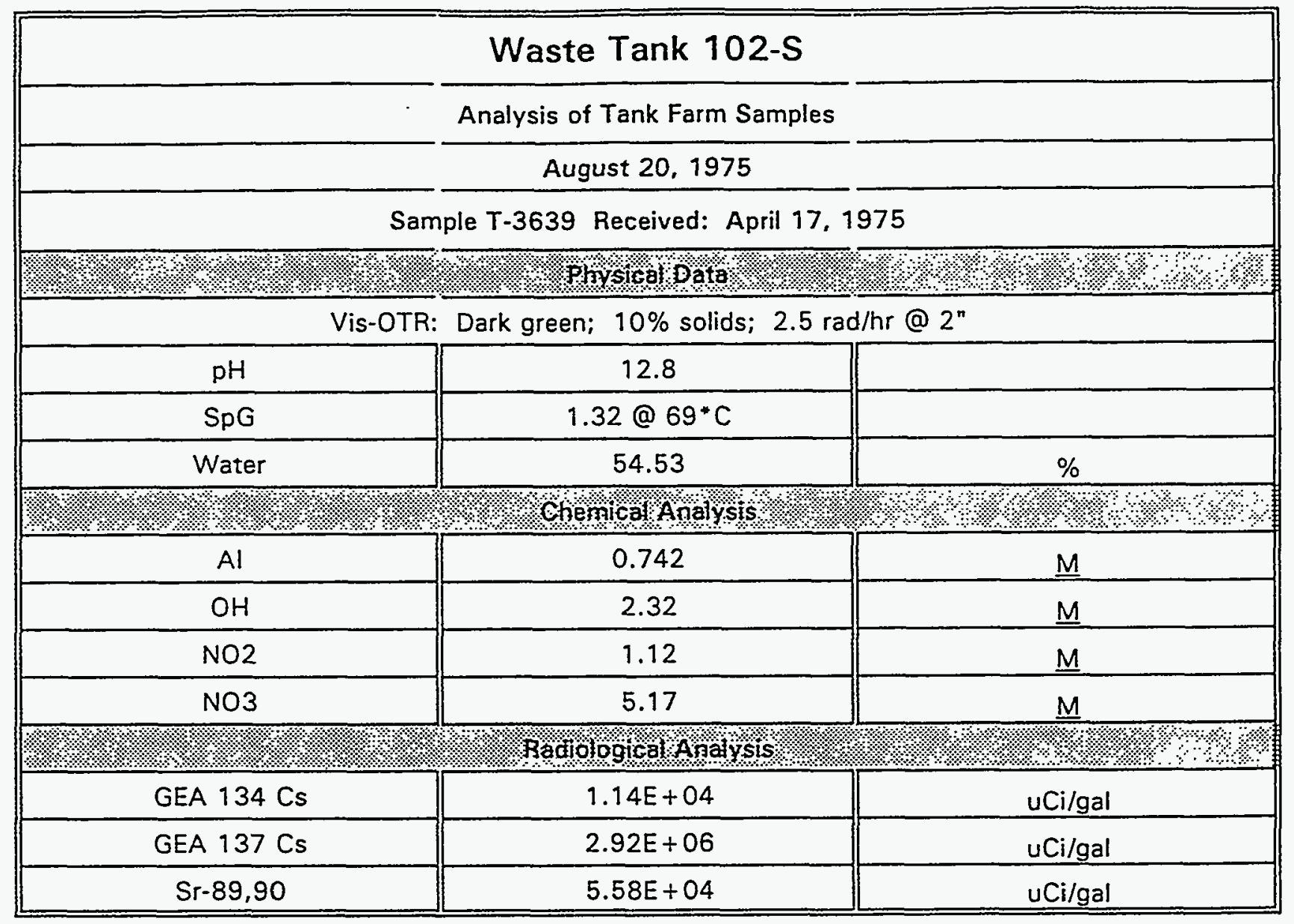




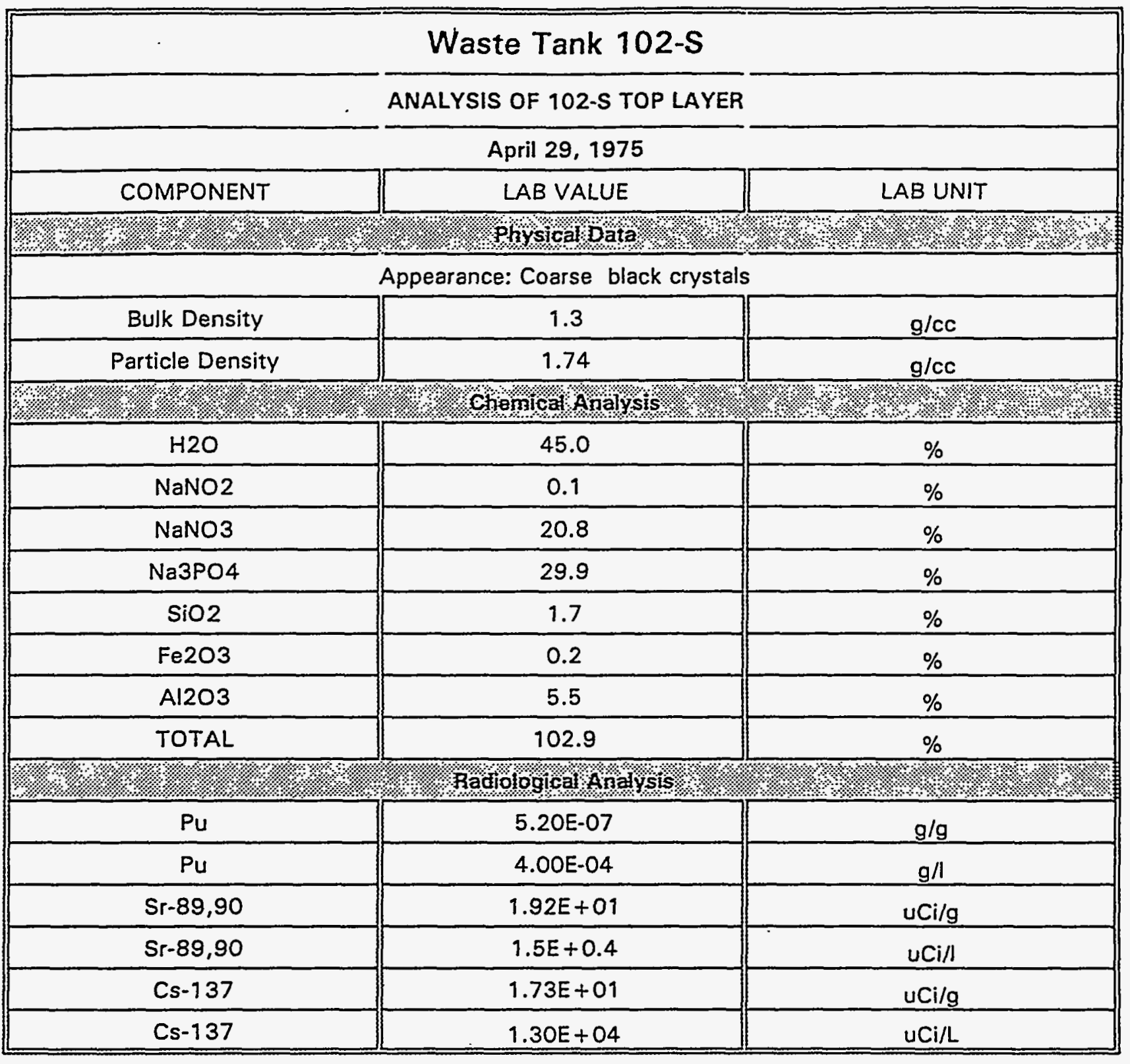




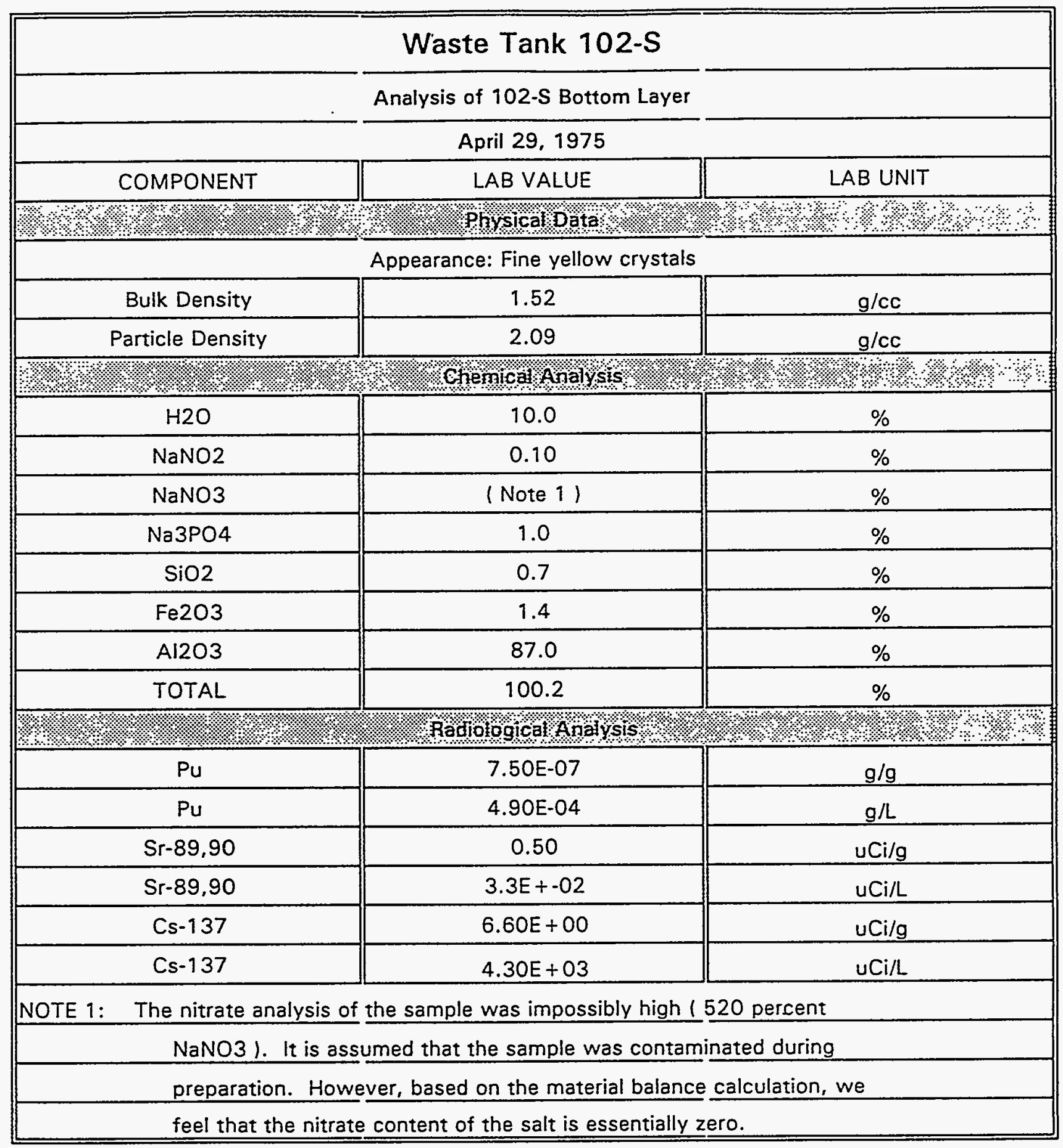




\begin{tabular}{|c|c|c|}
\hline \multicolumn{3}{|c|}{ Waste Tank 102-S } \\
\hline \multicolumn{3}{|c|}{ ANALYSIS OF TANK FARM SAMPLES } \\
\hline \multicolumn{3}{|c|}{ May 13,1975} \\
\hline \multicolumn{3}{|c|}{ Received, April 29, 1975} \\
\hline COMPONENT & LAB VALUE & LAB UNIT \\
\hline \multicolumn{3}{|c|}{ \%४थ } \\
\hline \multicolumn{3}{|c|}{$\begin{array}{ll} & \text { Vis-OTR: Clear, geen; No solids; } 600 \mathrm{mrad} / \mathrm{hr} \\
\end{array}$} \\
\hline $\mathrm{pH}$ & 13.0 & \\
\hline $\mathrm{SpG}$ & 1.2538 & \\
\hline Water & 70.04 & $\%$ \\
\hline \multicolumn{3}{|c|}{ 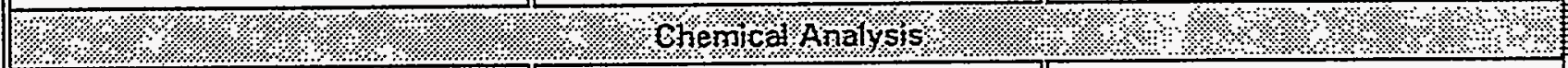 } \\
\hline $\mathrm{Al}$ & 0.448 & $\underline{M}$ \\
\hline $\mathrm{OH}$ & 1.16 & $\underline{M}$ \\
\hline NO2 & 0.818 & $\underline{M}$ \\
\hline $\mathrm{NO} 3$ & 1.61 & M \\
\hline \multicolumn{3}{|c|}{ 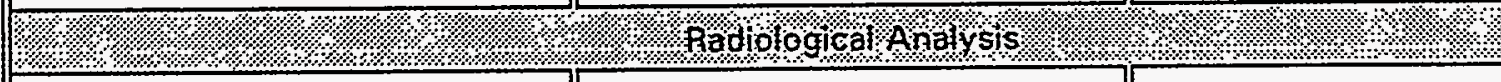 } \\
\hline GEA $134 \mathrm{Cs}$ & $4.84 E+03$ & $u \mathrm{Ci}_{\mathrm{i} / \mathrm{gal}}$ \\
\hline GEA $137 \mathrm{Cs}$ & $9.24 \mathrm{E}+05$ & uCi/gal \\
\hline $\mathrm{Sr}-89,90$ & $2.71 E+04$ & $u \mathrm{Ci} / \mathrm{gal}$ \\
\hline
\end{tabular}




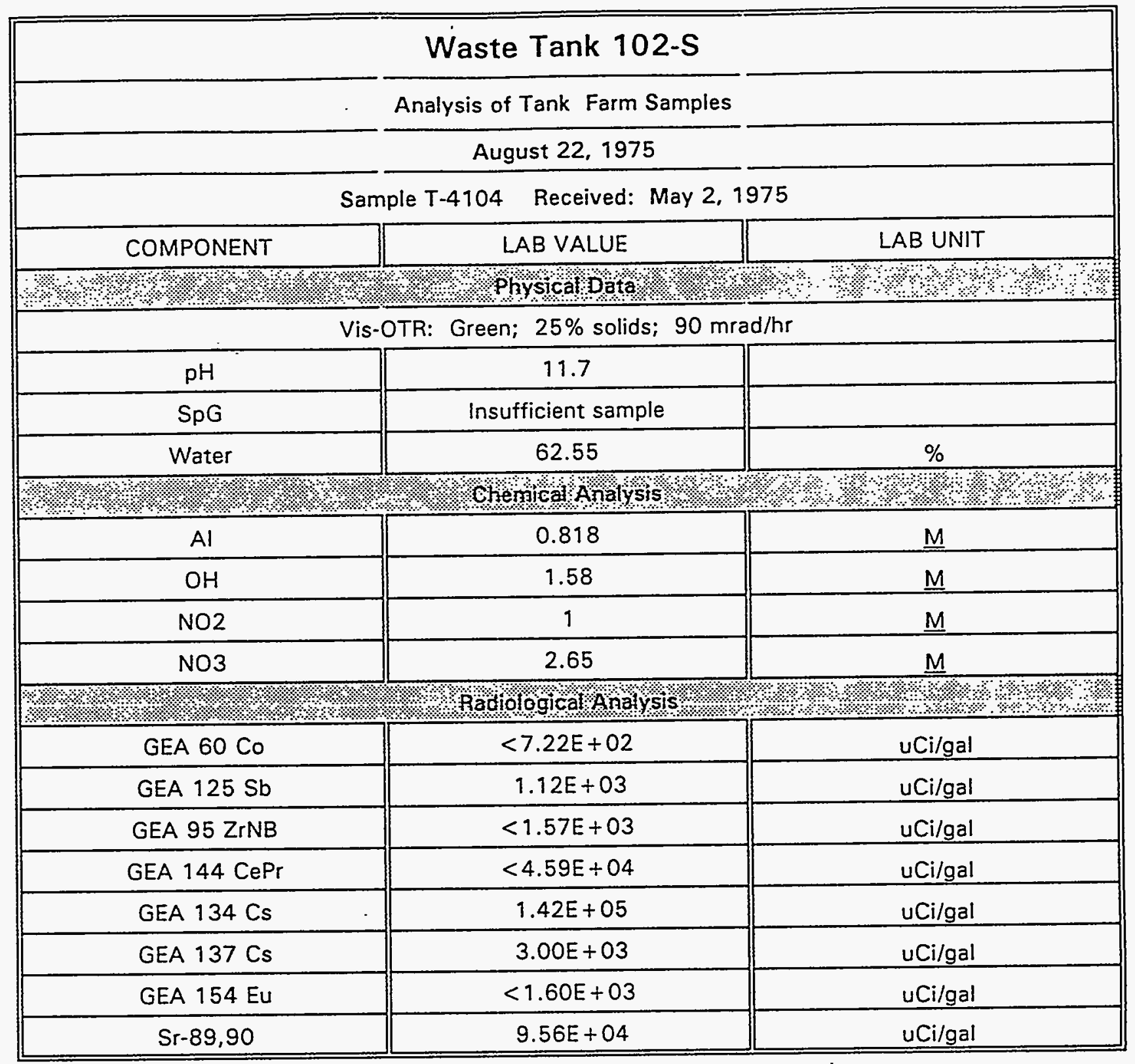




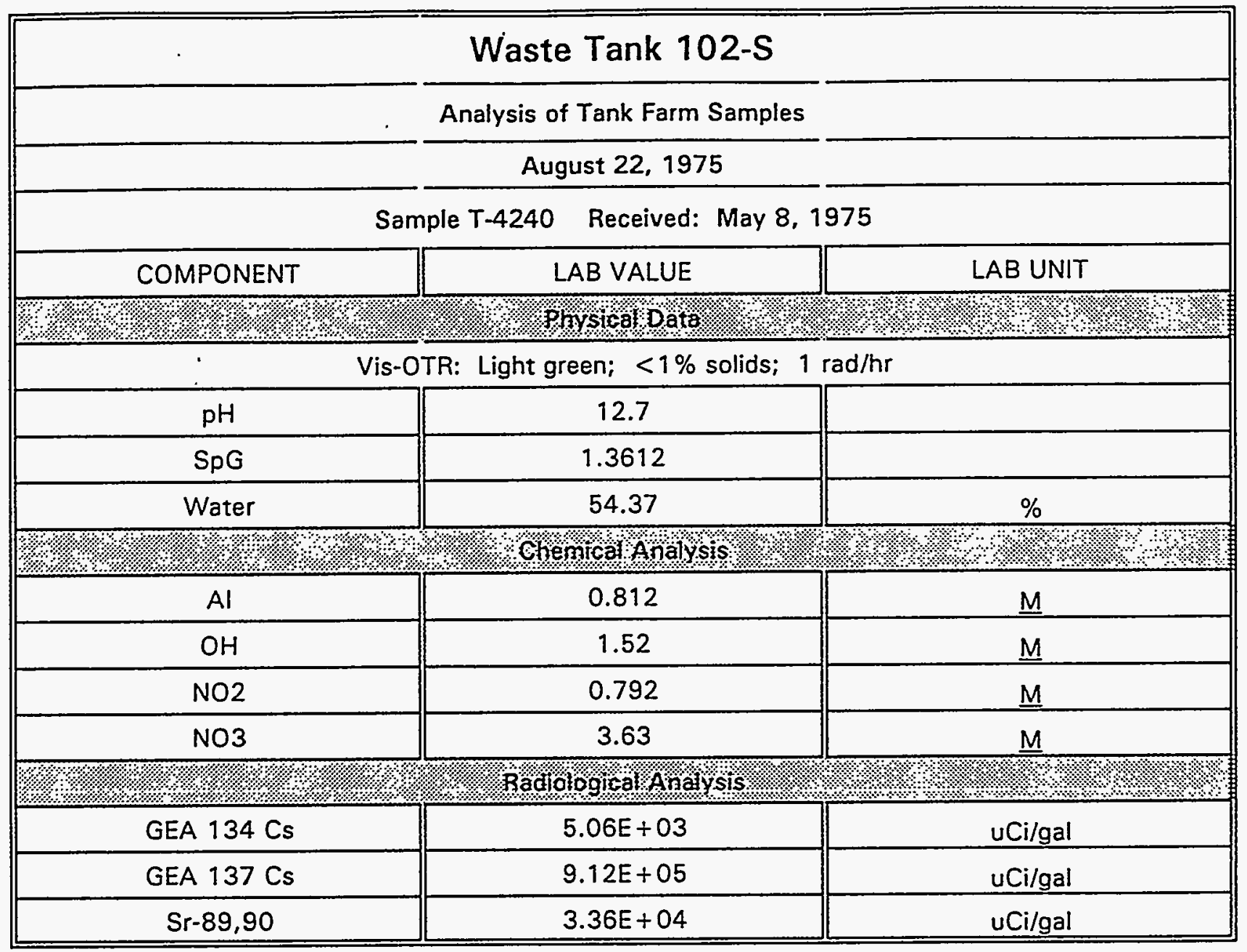




\begin{tabular}{|c|c|c|}
\hline \multicolumn{3}{|c|}{ Waste Tank 102-S } \\
\hline \multicolumn{3}{|c|}{ ANALYSIS OF TANK FARM SAMPLES } \\
\hline \multicolumn{3}{|c|}{ July 8,1975} \\
\hline \multicolumn{3}{|c|}{ Received, May 13, 1975} \\
\hline COMPONENT & LAB VALUE & LAB UNIT \\
\hline $1.12 .12 .1 \%$ & Physical Datt & ४. \\
\hline \multicolumn{3}{|c|}{ Vis-OTR: Green, clear; No solids; $200 \mathrm{mrad} / \mathrm{hr}$} \\
\hline SpG & 1.369 & \\
\hline Water & 52.76 & $\%$ \\
\hline \multicolumn{3}{|c|}{ ४४४४ ४ } \\
\hline Al & 0.747 & $\underline{M}$ \\
\hline $\mathrm{OH}$ & 1.66 & $\underline{\mathrm{M}}$ \\
\hline NO2 & 0.929 & $\underline{M}$ \\
\hline NO3 & 3.74 & $\underline{M}$ \\
\hline $\mathrm{CO} 3$ & 0.316 & M \\
\hline \multicolumn{3}{|c|}{ 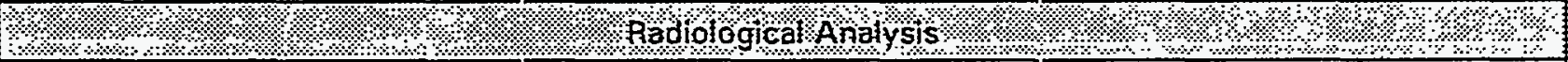 } \\
\hline GEA $134 \mathrm{Cs}$ & $5.07 E+03$ & uCi/gal \\
\hline GEA $137 \mathrm{Cs}$ & $9.63 E+05$ & $u C i / g a l$ \\
\hline GEA $125 \mathrm{Sb}$ & $1.13 E+03$ & uCi/gal \\
\hline
\end{tabular}




\begin{tabular}{|c|c|c|}
\hline \multicolumn{3}{|c|}{ Waste Tank 102-S } \\
\hline \multicolumn{3}{|c|}{ ANALYSIS OF ALKALINE WASTE SOLUTIONS } \\
\hline \multicolumn{3}{|c|}{ May 20, 1975} \\
\hline COMPONENT & LAB VALUE & LAB UNIT \\
\hline $1 \% 2.1 \%$ & \% Physicalgata & : \\
\hline Density & 1.3 & $\mathrm{~g} / \mathrm{ml}$ at $75^{*} \mathrm{C}$ \\
\hline \multicolumn{3}{|c|}{ \%1\% } \\
\hline $\mathrm{H} 2 \mathrm{O}$ & 41.6 & $\%$ \\
\hline $\mathrm{NaAlO2}$ & 0.97 & $\underline{M}$ \\
\hline $\mathrm{NaAlO} 2$ & 6.0 & $\%$ \\
\hline NaNO2 & 1.09 & $\underline{M}$ \\
\hline $\mathrm{NaNO} 2$ & 5.6 & $\%$ \\
\hline $\mathrm{NaNO} 3$ & 3.78 & $\underline{M}$ \\
\hline $\mathrm{NaNO} 3$ & 24.1 & $\%$ \\
\hline $\mathrm{NaOH}$ & 2.02 & $\underline{M}$ \\
\hline $\mathrm{NaOH}$ & 6.1 & $\%$ \\
\hline $\mathrm{Na} 2 \mathrm{CO} 3$ & 0.2 & $\underline{M}$ \\
\hline $\mathrm{Na} 2 \mathrm{CO} 3$ & 1.6 & $\%$ \\
\hline $\mathrm{Na} 3 \mathrm{PO} 4$ & 0.06 & $\underline{\mathrm{M}}$ \\
\hline $\mathrm{Na} 3 \mathrm{PO} 4$ & 0.7 & $\%$ \\
\hline $\mathrm{Na} 2 \mathrm{SiO} 3$ & 0.003 & $\underline{M}$ \\
\hline $\mathrm{Na} 2 \mathrm{SiO} 3$ & $<0.1$ & $\%$ \\
\hline $\mathrm{NaF}$ & 0.006 & $\%$ \\
\hline $\mathrm{NaF}$ & $<0.1$ & $\%$ \\
\hline TOTAL & 85.9 & $\%$ \\
\hline \multicolumn{3}{|c|}{$\%: 1 \%$ \% } \\
\hline Cs-137 & $3.61 \mathrm{E}+05$ & $\mathrm{uCi} / \mathrm{L}$ \\
\hline Cs-134 & $1.05 E+03$ & uCi/L \\
\hline $\mathrm{Sb}-125$ & $2.97 \mathrm{E}+03$ & uCi/L \\
\hline Pu-239,240 & $3.27 E+00$ & uCi/L \\
\hline Pu-238 & 0.19 & uCi/L \\
\hline $\mathrm{Np}-237$ & 0.05 & uCi/L \\
\hline Am-241 & 9.10 & $\mathrm{uCi} / \mathrm{L}$ \\
\hline
\end{tabular}




\begin{tabular}{|c|c|}
\hline \multicolumn{2}{|c|}{ Waste Tank 102-S } \\
\hline \multicolumn{2}{|c|}{ FILTERABLE ACTIVITY IN ALKALINE WASTE SOLUTIONS } \\
\hline \multicolumn{2}{|c|}{ UCi/L UNFILTERED SOLUTION } \\
\hline \multicolumn{2}{|c|}{ May 20, 1975} \\
\hline 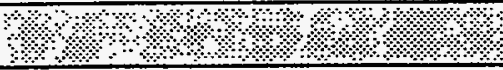 & ब४ \\
\hline$A m-241$ & $2.11 E+03$ \\
\hline $\mathrm{Ce}-144$ & $2.07 E+04$ \\
\hline Eu-154 & $6.60 E \div 03$ \\
\hline Eu-155 & $2.23 E \div 04$ \\
\hline Cs-134 & $5.24 E \div 02$ \\
\hline Cs-137 & $1.34 \mathrm{E} \div 04$ \\
\hline $\mathrm{Co}-57$ & $\cdots$ \\
\hline Co-60 & $1.78 \mathrm{E} \div 03$ \\
\hline $\mathrm{Rh}-106$ & $8.45 \mathrm{E} \div 03$ \\
\hline $\mathrm{Zr}-95$ & $3.09 E+02$ \\
\hline Sb-125 & $2.61 E+03$ \\
\hline
\end{tabular}




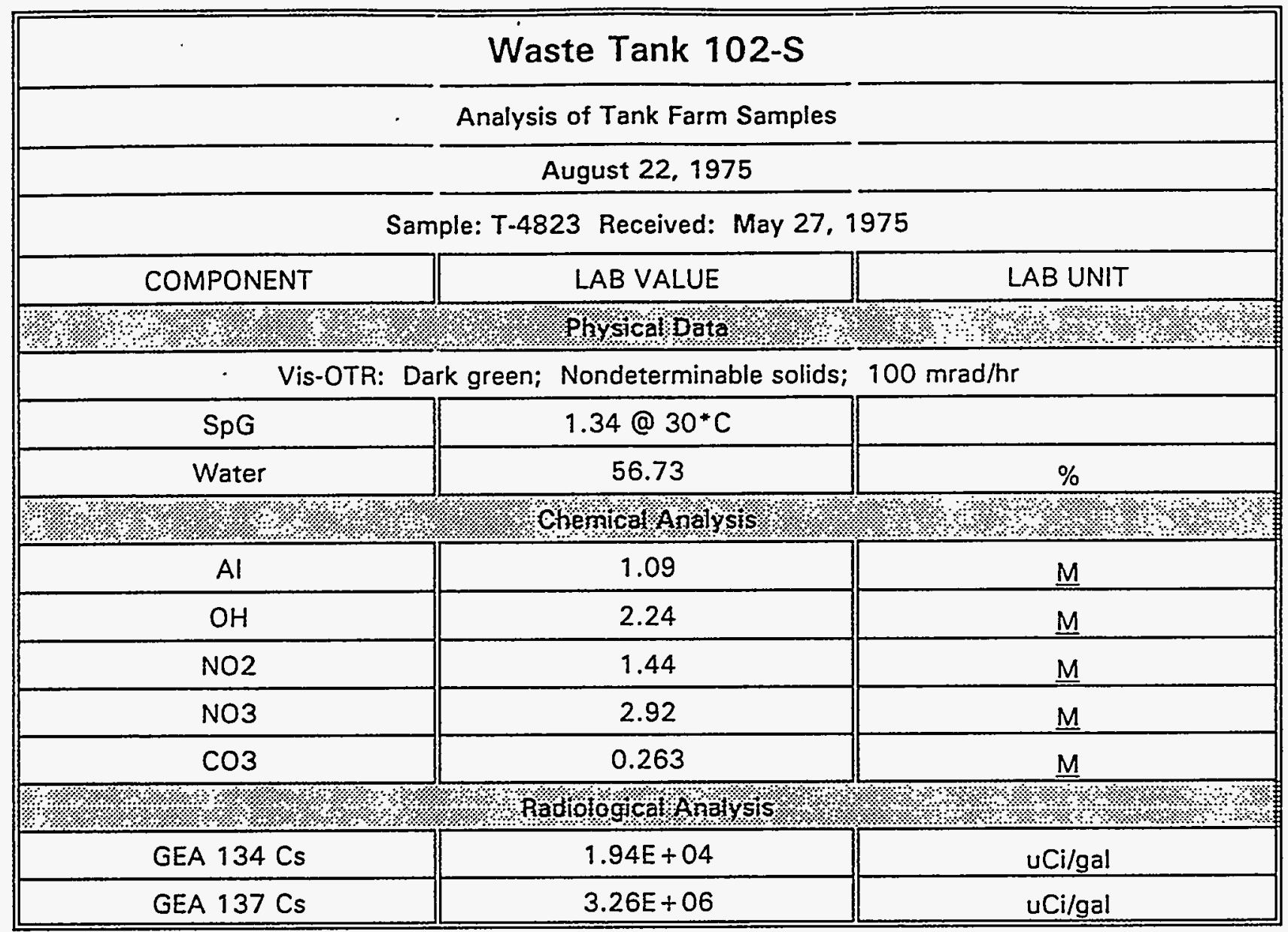




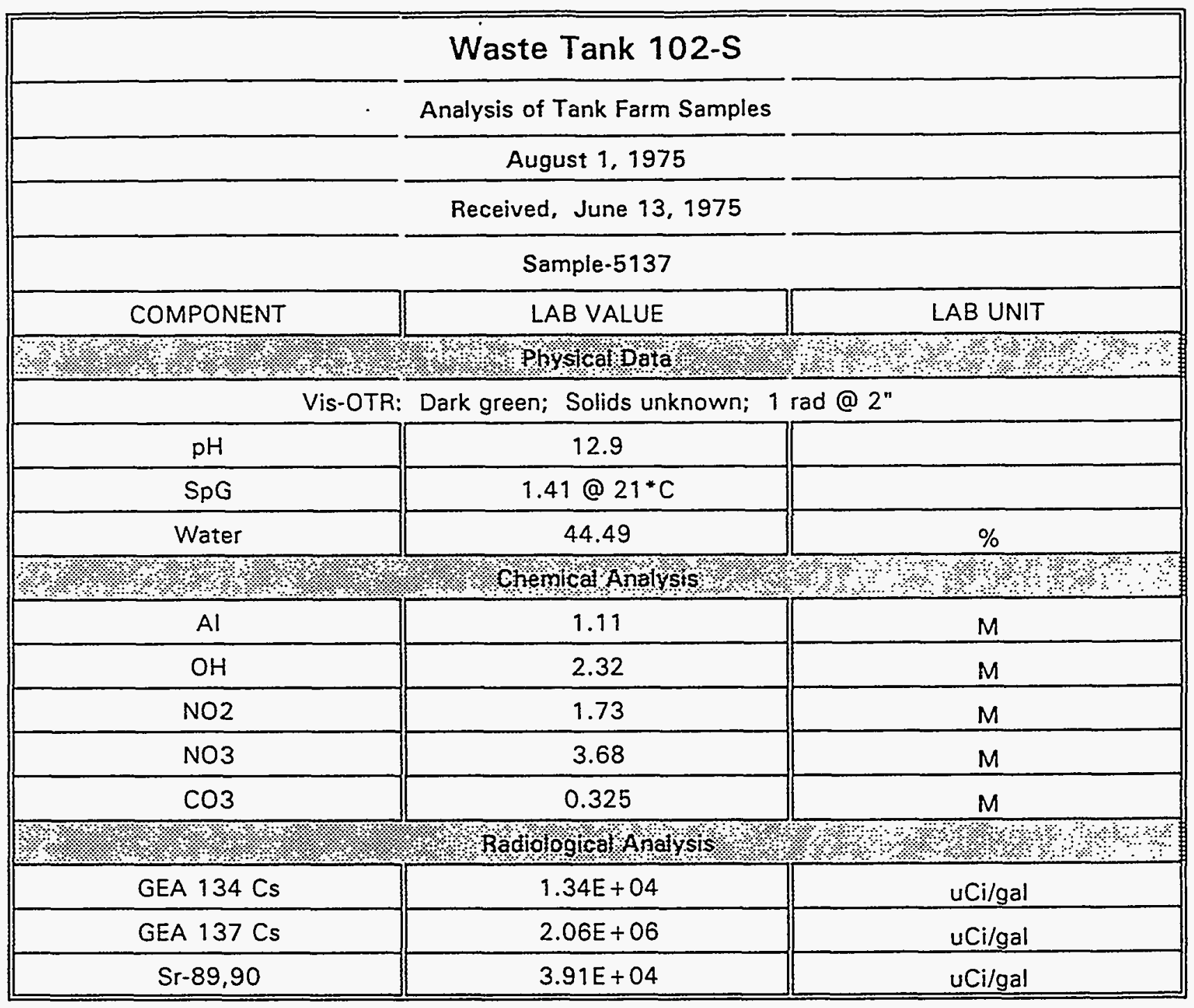




\begin{tabular}{|c|c|c|}
\hline \multicolumn{3}{|c|}{ Waste Tank 102-S } \\
\hline \multicolumn{3}{|c|}{ ANALYSIS OF TANK FARM SAMPLES } \\
\hline \multicolumn{3}{|c|}{ July 28, 1975} \\
\hline \multicolumn{3}{|c|}{ Received, June 20, 1975} \\
\hline \multicolumn{3}{|c|}{ Sample:T-5514 } \\
\hline COMPONENT & LAB VALUE & LAB UNIT \\
\hline 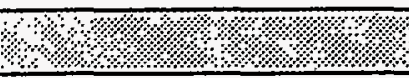 & (1.2. Physicaloata & (... \\
\hline \multicolumn{3}{|c|}{ Vis-OTR: Greenish black; Solids undetermined; $1.5 \mathrm{rad} / \mathrm{hr}$} \\
\hline SpG & 1.5202 & \\
\hline Water & 47.00 & $\%$ \\
\hline 18.8. & \multicolumn{2}{|c|}{ 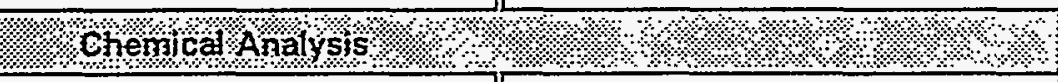 } \\
\hline Al & $0.553-$ Rerun 0.812 & $\mathrm{M}$ \\
\hline $\mathrm{OH}$ & 4.29 & $M$ \\
\hline NO2 & 2.38 & $M$ \\
\hline NO3 & 2.63 & M \\
\hline $\mathrm{CO} 3$ & 0.223 & $\mathrm{M}$ \\
\hline \multicolumn{3}{|c|}{ 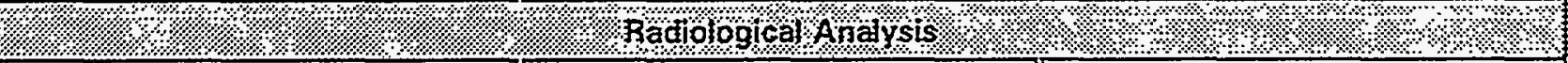 } \\
\hline GEA 134 Cs & $1.44 \mathrm{E}+04$ & uCi/gal \\
\hline GEA $137 \mathrm{Cs}$ & $2.88 E+06$ & uCi/gal \\
\hline Sr-89,90 & $1.20 E+05$ & uCi/gal \\
\hline
\end{tabular}




\begin{tabular}{|c|c|c|}
\hline \multicolumn{3}{|c|}{ Waste Tank 102-S } \\
\hline \multicolumn{3}{|c|}{ Analysis of Tank Farm Samples } \\
\hline \multicolumn{3}{|c|}{ July 31,1975} \\
\hline \multicolumn{3}{|c|}{ Received, July 2, 1975} \\
\hline \multicolumn{3}{|c|}{ Sample T-5792 } \\
\hline COMPONENT & LAB VALUE & LAB UNIT \\
\hline \multicolumn{3}{|c|}{ 1०४\% } \\
\hline \multicolumn{3}{|c|}{ Vis-OTR: Dark brown; 25\% solids; $50 \mathrm{mrad} / \mathrm{hr} @ 4^{\mathrm{n}}$} \\
\hline $\mathrm{pH}$ & 13.5 & \\
\hline SpG & Insufficient sample & \\
\hline Water & 60.31 & $\%$ \\
\hline \multicolumn{3}{|c|}{ 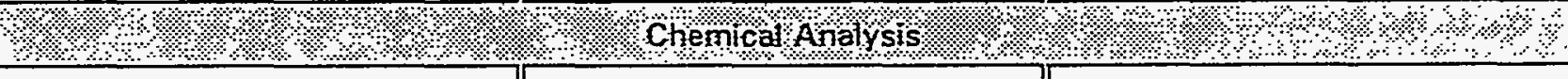 } \\
\hline $\mathrm{Al}$ & 1.00 & M \\
\hline $\mathrm{OH}$ & 1.74 & M \\
\hline NO2 & 1.15 & M \\
\hline NO3 & 2.92 & M \\
\hline \multicolumn{3}{|c|}{ 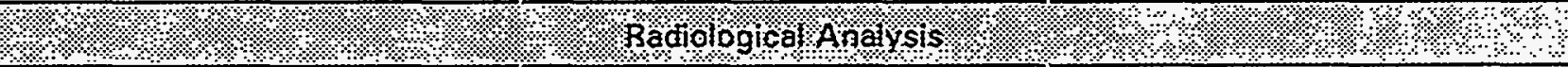 } \\
\hline GEA $134 \mathrm{Cs}$ & $6.61 \mathrm{E}+03$ & uCi/gal \\
\hline GEA $137 \mathrm{Cs}$ & $1.14 E+06$ & uCi/gal \\
\hline $\mathrm{Sr}-89,90$ & $4.02 E+04$ & $\mathrm{uCi} / \mathrm{gal}$ \\
\hline
\end{tabular}




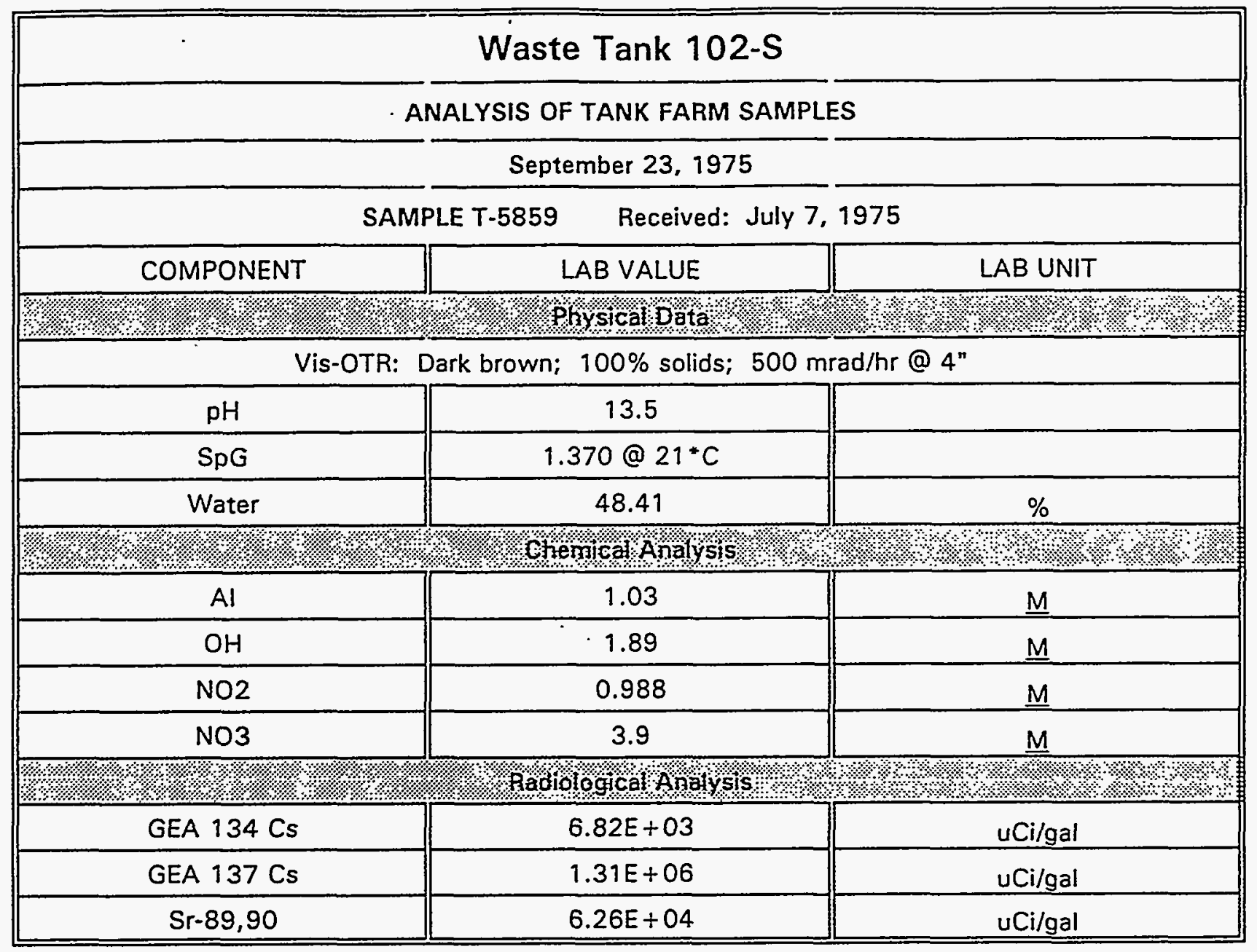




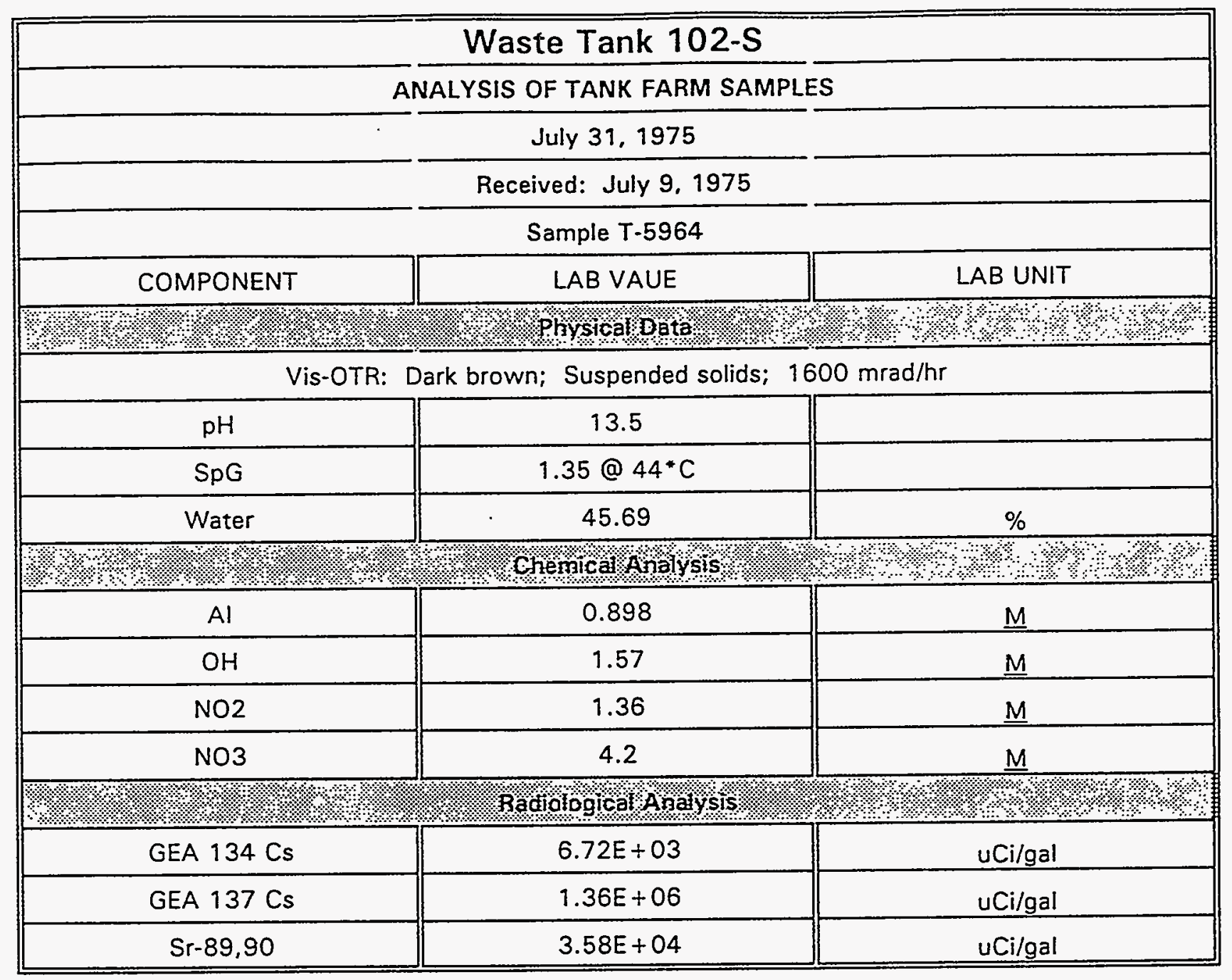




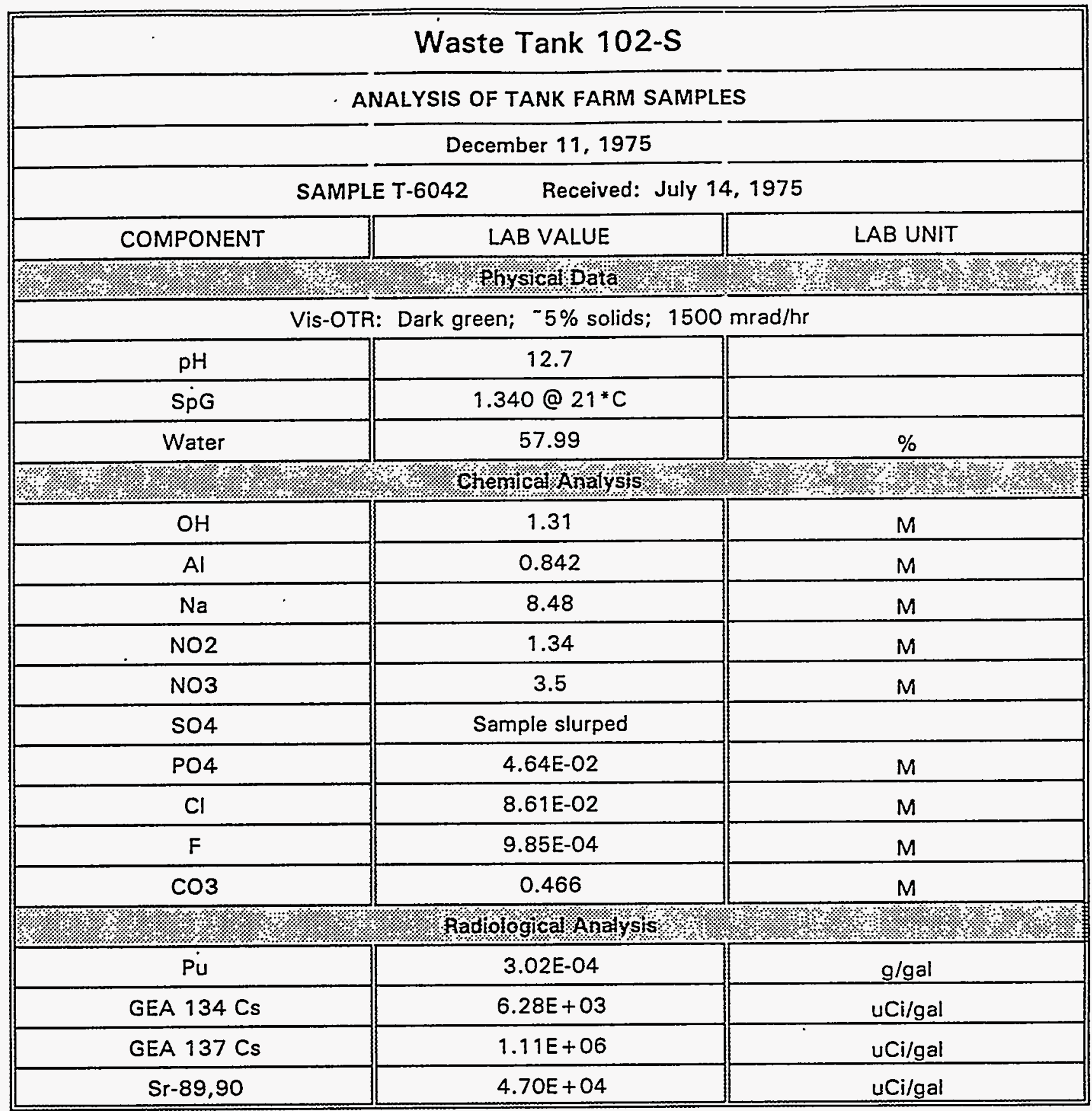




\begin{tabular}{|c|c|c|}
\hline \multicolumn{3}{|c|}{ Waste Tank 102-S } \\
\hline \multicolumn{3}{|c|}{ Analysis of Tank Farm Samples } \\
\hline \multicolumn{3}{|c|}{ July 31,1975} \\
\hline \multicolumn{3}{|c|}{ Received, July 18,1975} \\
\hline \multicolumn{3}{|c|}{ Sample T-6226 } \\
\hline COMPONENT & LAB VALUE & LAB UNIT \\
\hline 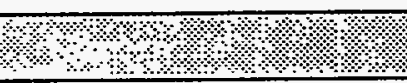 & 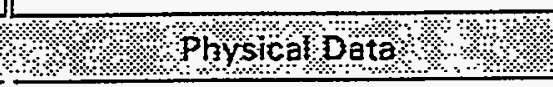 & 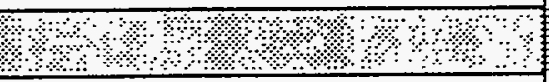 \\
\hline \multicolumn{3}{|c|}{ Vis-OTR: Dark green; $-25 \%$ solids; $500 \mathrm{mrad} / \mathrm{hr}$} \\
\hline $\mathrm{pH}$ & 13.4 & \\
\hline$S p G$ & $1.31 @ 21^{*} \mathrm{C}$ & \\
\hline Water & 41.14 & $\%$ \\
\hline \%? & Chemical Anatysis:? & 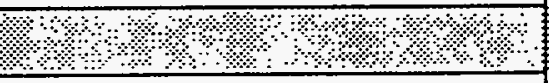 \\
\hline $\mathrm{Al}$ & 0.837 & $\underline{M}$ \\
\hline $\mathrm{OH}$ & 1.72 & $\underline{M}$ \\
\hline NO2 & 1.4 & $\underline{M}$ \\
\hline $\mathrm{NO} 3$ & 3.1 & $\underline{M}$ \\
\hline 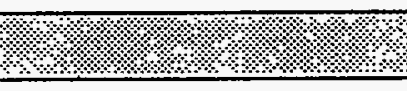 & 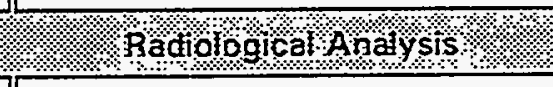 & (.) \\
\hline GEA $134 \mathrm{Cs}$ & $6.96 \mathrm{E}+03$ & $\mathrm{uCi} / \mathrm{gal}$ \\
\hline GEA $137 \mathrm{Cs}$ & $1.41 E+06$ & $u C i / g a l$ \\
\hline $\mathrm{Sr}-89,90$ & $4.24 E \div 04$ & uCi/gal \\
\hline
\end{tabular}




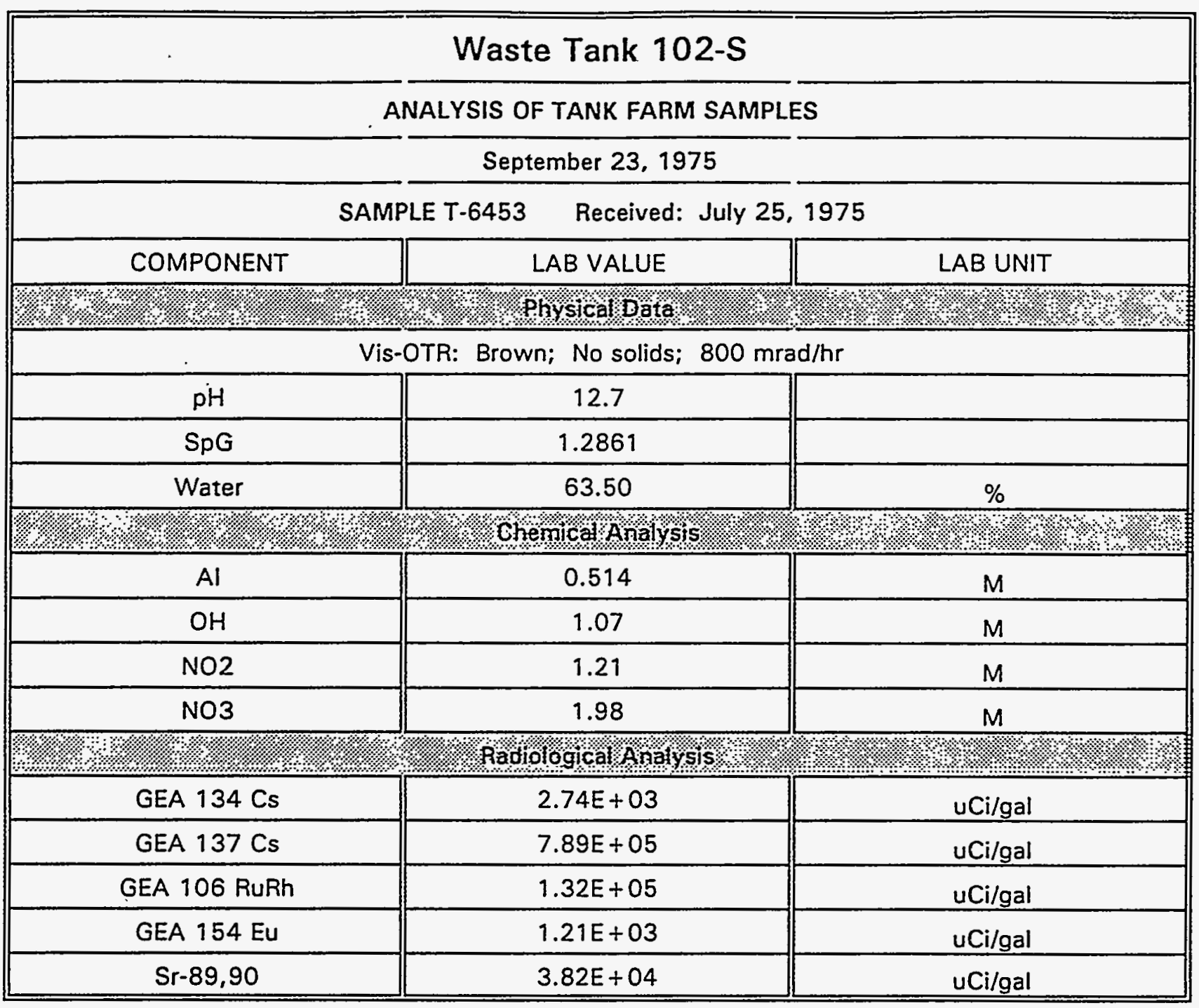




\begin{tabular}{|c|c|c|}
\hline \multicolumn{3}{|c|}{ Waste Tank 102-S } \\
\hline \multicolumn{3}{|c|}{ ANALYSIS OF TANK FARM SAMPLES } \\
\hline \multicolumn{3}{|c|}{ November 13, 1975} \\
\hline \multicolumn{3}{|c|}{ Received: August $5,1975^{\circ}$} \\
\hline COMPONENT & LAB VALUE & LAB UNIT \\
\hline 1. & Phy sicalobata & $1 .+2$ \\
\hline \multicolumn{3}{|c|}{ Vis-OTR: Dark brown; $80 \%$ solids; $800 \mathrm{mrad} / \mathrm{hr}$} \\
\hline $\mathrm{pH}$ & 13.3 & \\
\hline SpG & $1.320 @ 54^{*} \mathrm{C}$ & \\
\hline Water & 54.07 & $\%$ \\
\hline \multicolumn{3}{|l|}{ 1.. } \\
\hline $\mathrm{OH}$ & 1.98 & M \\
\hline $\mathrm{Al}$ & 0.826 & $M$ \\
\hline NO2 & 1.52 & M \\
\hline NO3 & 3.3 & M \\
\hline \multicolumn{3}{|c|}{ (1. } \\
\hline GEA 134 Cs & $2.85 E+03$ & $\mathrm{uCi} / \mathrm{gal}$ \\
\hline GEA $137 \mathrm{Cs}$ & $7.04 E+05$ & uCi/gal \\
\hline Sr-89,90 & $8.27 E+04$ & $\mathrm{uCi} / \mathrm{gal}$ \\
\hline
\end{tabular}




\begin{tabular}{|c|c|c|}
\hline \multicolumn{3}{|c|}{ Waste Tank 102-S } \\
\hline \multicolumn{3}{|c|}{ ANALYSIS OF TANK FARM SAMPLES } \\
\hline \multicolumn{3}{|c|}{ September 19,1975} \\
\hline \multicolumn{3}{|c|}{ SAMPLE T-7092 Received: August 15, 1975} \\
\hline COMPONENT & LAB VALUE & LAB UNIT \\
\hline $1 \% \%: \%$ & Prysicaloat & $1 \%$ \\
\hline \multicolumn{3}{|c|}{ Vis-OTR: Dark brown; $1 \%$ solids; $3 \mathrm{rad} / \mathrm{hr}$} \\
\hline $\mathrm{pH}$ & 12.9 & \\
\hline $\mathrm{SpG}$ & 1.513 & \\
\hline Water & 53.16 & $\%$ \\
\hline \multicolumn{3}{|c|}{ \%। } \\
\hline $\mathrm{Al}$ & 1.91 & $\underline{M}$ \\
\hline $\mathrm{OH}$ & 2.98 & $\underline{\mathrm{M}}$ \\
\hline NO2 & 1.9 & $\underline{M}$ \\
\hline $\mathrm{NO} 3$ & 4.29 & $\underline{M}$ \\
\hline \multicolumn{3}{|c|}{ \%"\% } \\
\hline GEA $134 \mathrm{Cs}$ & $1.29 E+04$ & uCi/gal \\
\hline GEA $137 \mathrm{Cs}$ & $2.17 E+06$ & uCi/gal \\
\hline $\mathrm{Sr}-89,90$ & $5.05 \mathrm{E}+04$ & uCi/gal \\
\hline
\end{tabular}




\begin{tabular}{|c|c|c|}
\hline \multicolumn{3}{|c|}{ Waste Tank 102-S } \\
\hline \multicolumn{3}{|c|}{ ANALYSIS OF TANK FARM SAMPLES } \\
\hline \multicolumn{3}{|c|}{ September 22, 1975} \\
\hline \multicolumn{3}{|c|}{ Received: August 19, 1975} \\
\hline COMPONENT & LAB VALUE & LAB UNIT \\
\hline \multicolumn{3}{|c|}{ ४०\% } \\
\hline \multicolumn{3}{|c|}{ Vis-OTR: Dark brown; No solids; $700 \mathrm{mrad} / \mathrm{hr}$} \\
\hline $\mathrm{pH}$ & 12.5 & \\
\hline SpG & 1.3662 & \\
\hline Water & 69.40 & $\%$ \\
\hline \multicolumn{3}{|c|}{ 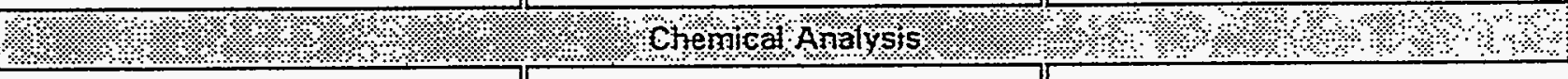 } \\
\hline $\mathrm{Al}$ & 0.754 & $\underline{M}$ \\
\hline $\mathrm{OH}$ & 1.78 & $\underline{M}$ \\
\hline NO2 & 1.96 & $\underline{\mathrm{M}}$ \\
\hline NO3 & 3.33 & $\underline{M}$ \\
\hline \multicolumn{3}{|c|}{ (n) } \\
\hline GEA $134 \mathrm{Cs}$ & $8.63 E+03$ & uCi/gal \\
\hline GEA $137 \mathrm{Cs}$ & $1.50 E+06$ & uCi/gal \\
\hline Sr-89,90 & $4.73 E+04$ & uCi/gal \\
\hline
\end{tabular}




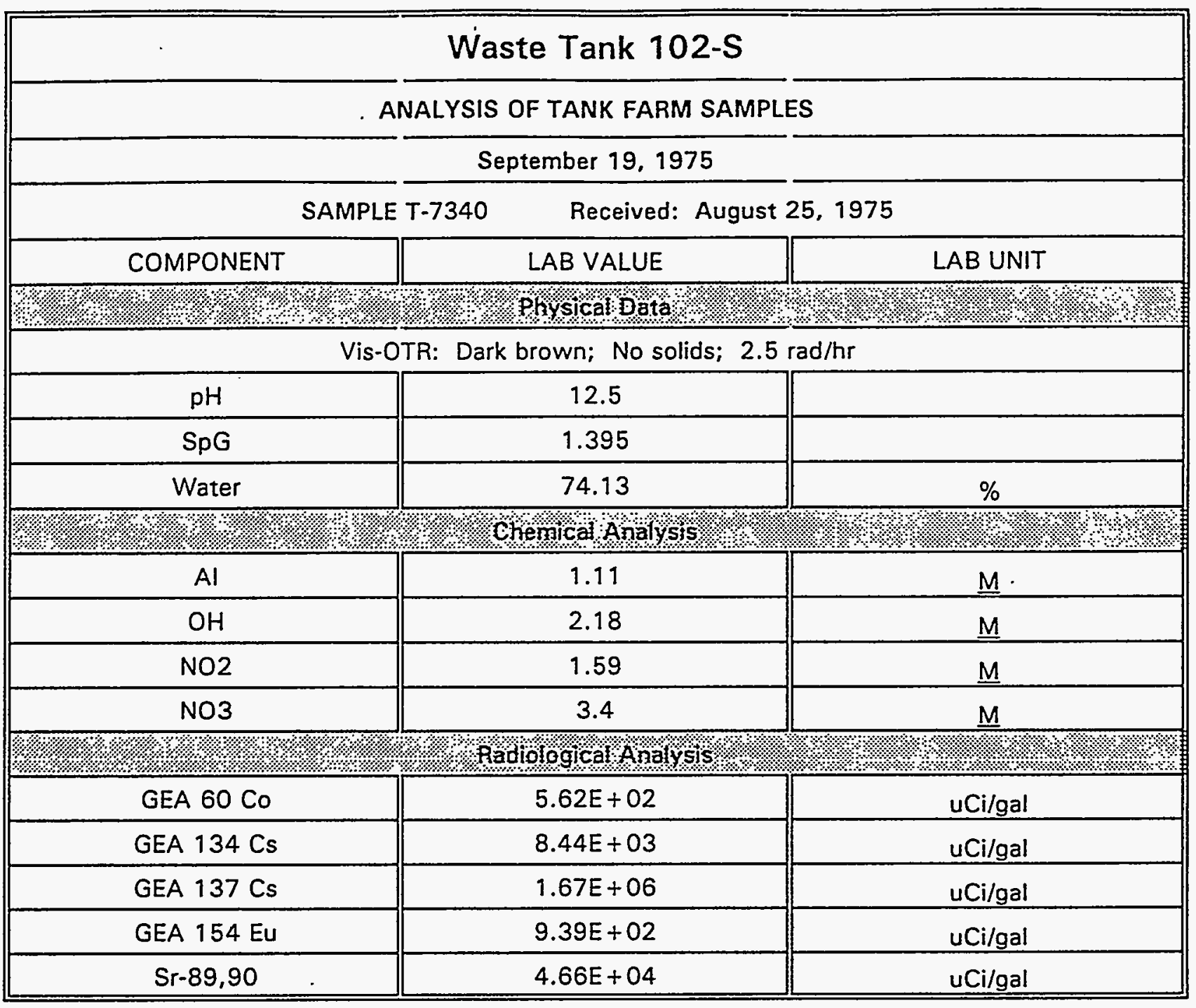




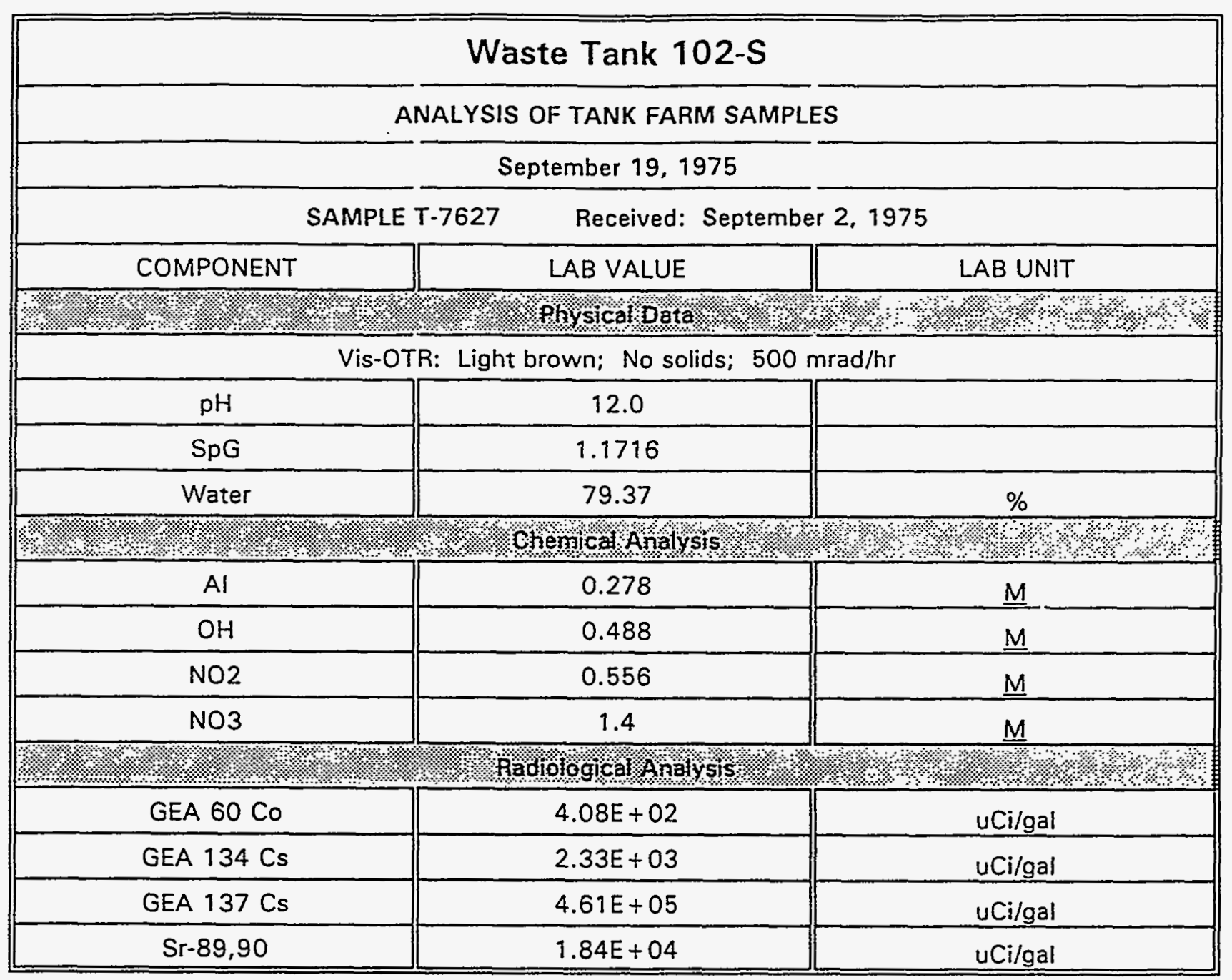




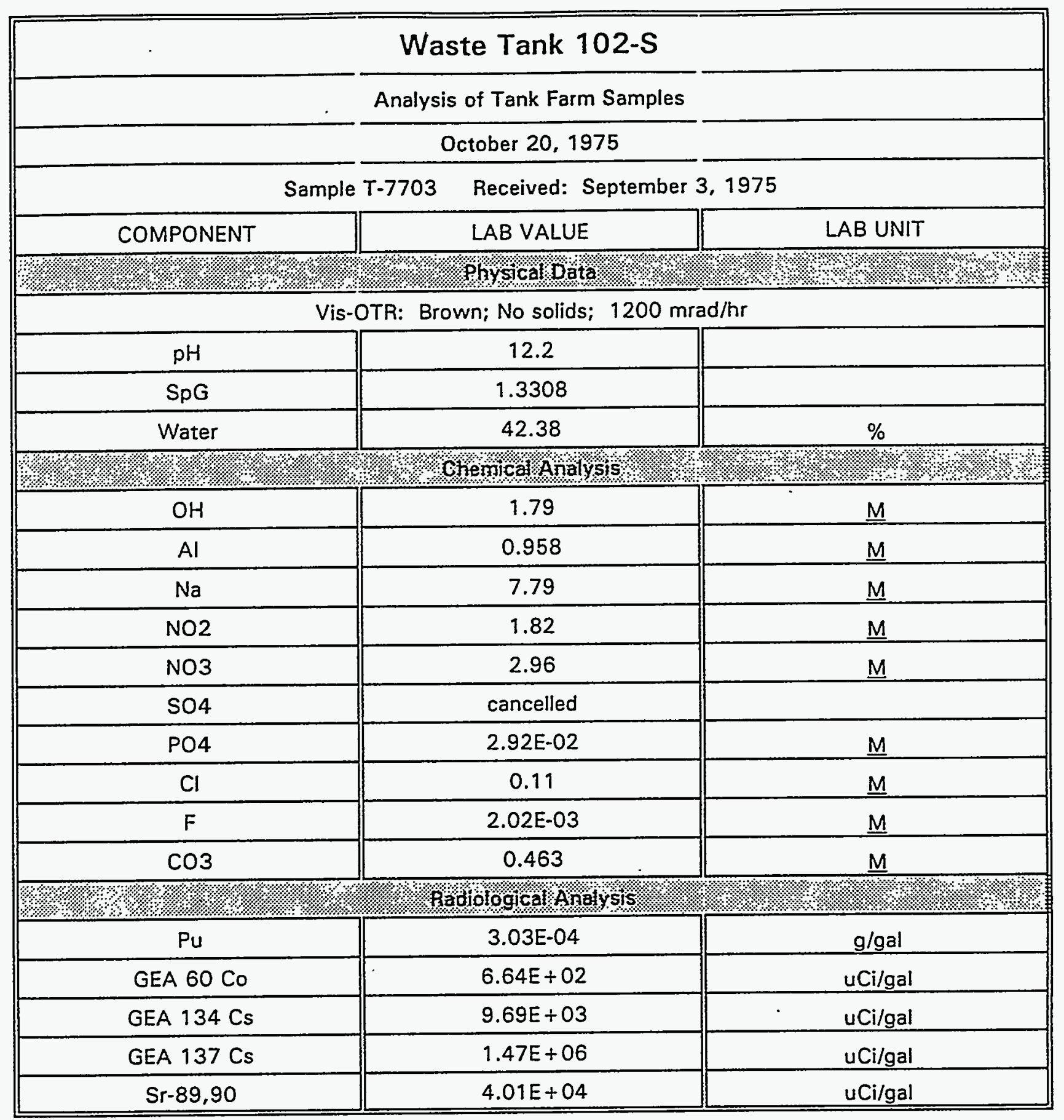




\begin{tabular}{|c|c|c|}
\hline \multicolumn{3}{|c|}{ Waste Tank 102-S } \\
\hline \multicolumn{3}{|c|}{ ANALYSIS OF TANK FARM SAMPLES } \\
\hline \multicolumn{3}{|c|}{ March 16, 1976} \\
\hline \multicolumn{3}{|c|}{ Received: September 8, 1975} \\
\hline COMPONENT & LAB VALUE & LAB UNIT \\
\hline $4: 19$ & Physicalpata & 2.1 .2$. \\
\hline \multicolumn{3}{|c|}{ Vis-OTR: Green; $-1 \%$ solids; $1.5 \mathrm{mRads} / \mathrm{hr}$} \\
\hline $\mathrm{pH}$ & 13.2 & \\
\hline $\mathrm{SpG}$ & 1.2748 & \\
\hline Water & 68.08 & $\%$ \\
\hline \multicolumn{3}{|c|}{ ४ } \\
\hline $\mathrm{OH}$ & 1.21 & M \\
\hline Al & 0.565 & $M$ \\
\hline $\mathrm{Na}$ & 5.21 & M \\
\hline NO2 & 1.27 & M \\
\hline NO3 & 2.76 & M \\
\hline $\mathrm{PO} 4$ & $4.85 \mathrm{E}-02$ & $M$ \\
\hline $\mathrm{Cl}$ & $8.63 E-02$ & M \\
\hline $\mathrm{F}$ & $3.54 \mathrm{E}-03$ & $M$ \\
\hline $\mathrm{CO} 3$ & 0.0138 & M \\
\hline \multicolumn{3}{|c|}{ 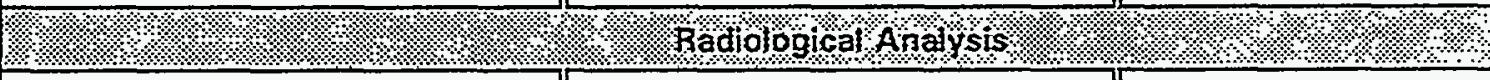 } \\
\hline $\mathrm{Pu}$ & $1.51 \mathrm{E}-04$ & $\mathrm{~g} / \mathrm{gal}$ \\
\hline GEA 60 Co & $1.55 \mathrm{E}+03$ & $u$ Ci/gal \\
\hline GEA $125 \mathrm{Sb}$ & $3.56 E+03$ & $\mathrm{uCi} / \mathrm{gal}$ \\
\hline GEA $134 \mathrm{Cs}$ & $5.84 E+03$ & $u \mathrm{Ci} / \mathrm{gal}$ \\
\hline GEA $137 \mathrm{Cs}$ & $1.08 E+06$ & uCi/gal \\
\hline Sr-89,90 & $2.44 E+04$ & $\mathrm{uCi} / \mathrm{gal}$ \\
\hline
\end{tabular}




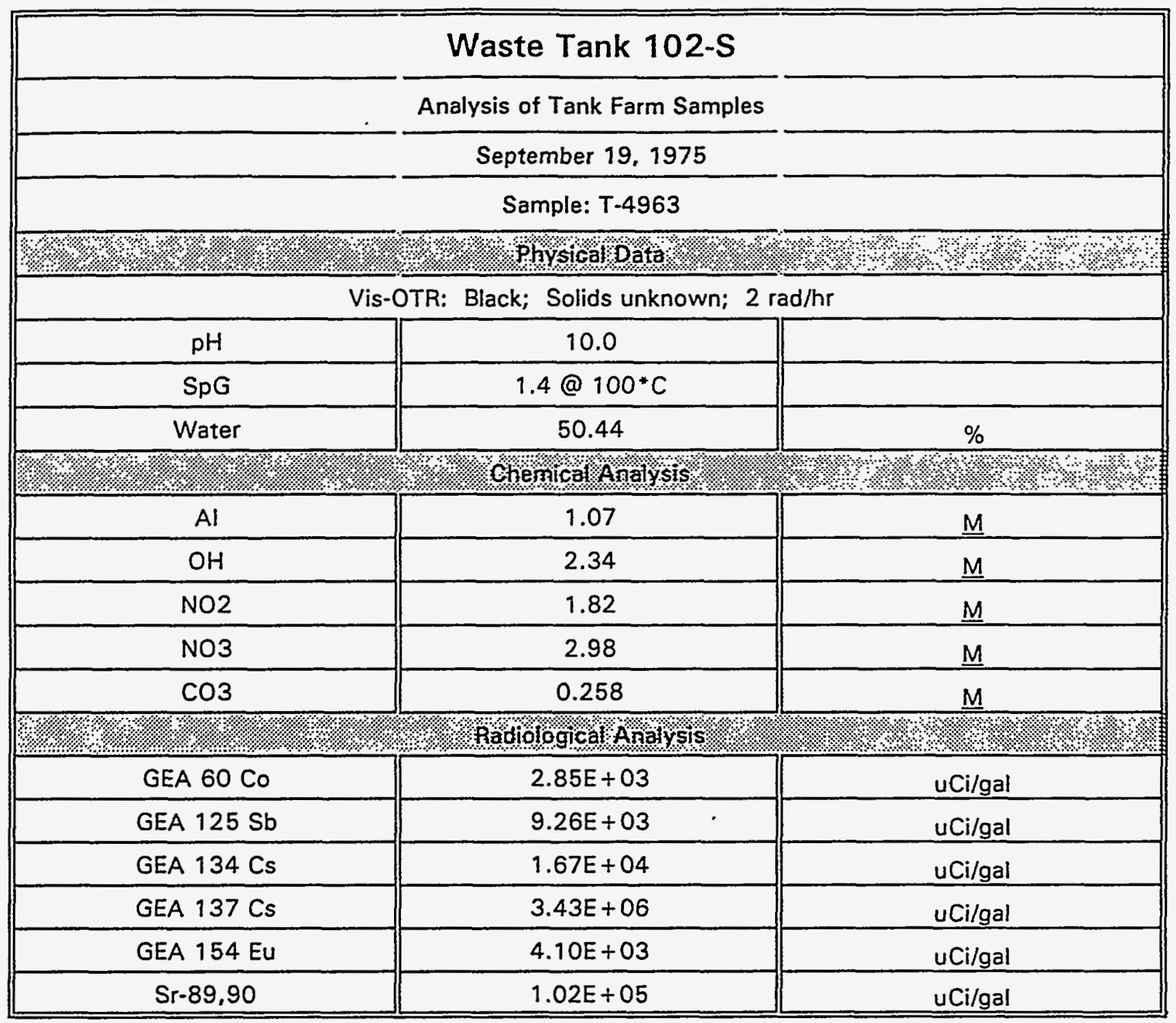




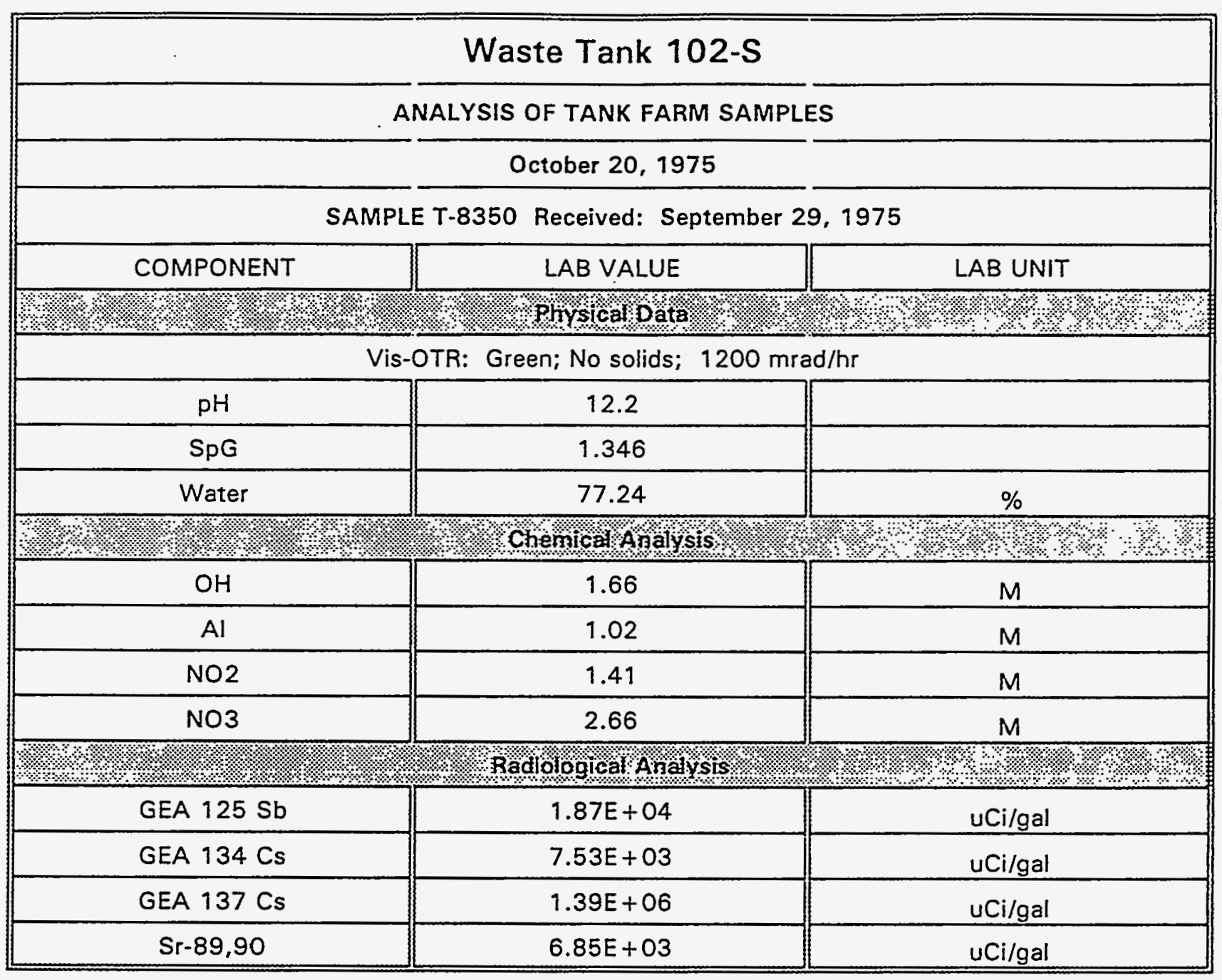


WHC-SD-WM-ER-323, Rev. 0

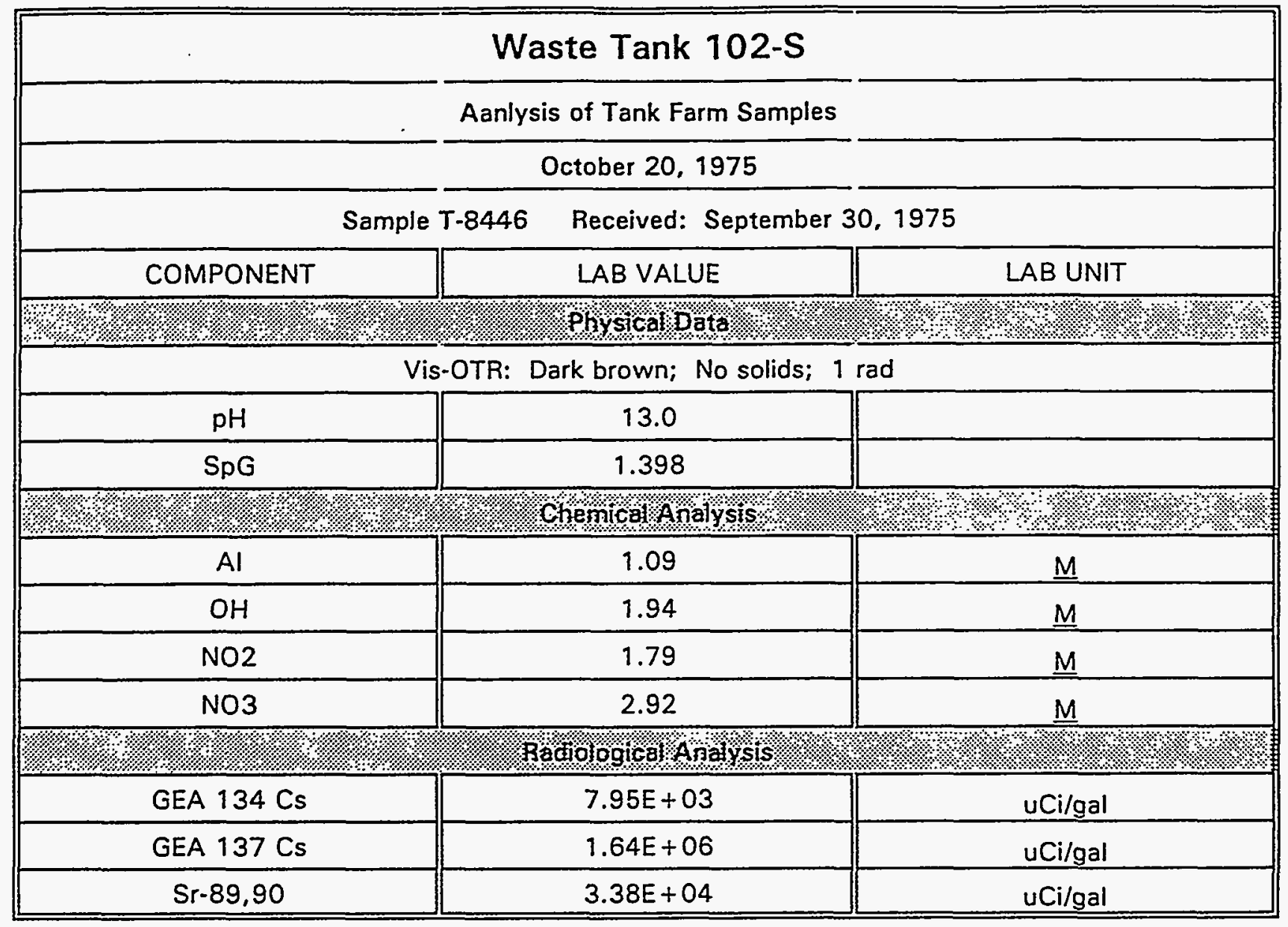




\begin{tabular}{|c|c|c|}
\hline \multicolumn{3}{|c|}{ Waste Tank 102-S } \\
\hline \multicolumn{3}{|c|}{ ANALYSIS OF TANK FARM SAMPLES } \\
\hline \multicolumn{3}{|c|}{ December 3, 1975} \\
\hline \multicolumn{3}{|c|}{ SAMPLE T-8634 Received: October 7, 1975} \\
\hline COMPONENT & LAB VALUE & LAB UNIT \\
\hline 19.17 .19 & Physical bata & ४४.? \\
\hline \multicolumn{3}{|c|}{ Vis-OTR: Green; $10 \%$ solids; $1500 \mathrm{mrad} / \mathrm{hr}$} \\
\hline $\mathrm{pH}$ & 12.7 & \\
\hline $\mathrm{SpG}$ & $1.190 @ 59 * \mathrm{C}$ & \\
\hline Water & 76.82 & $\%$ \\
\hline \multicolumn{3}{|c|}{ 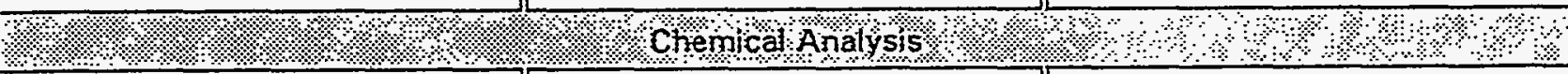 } \\
\hline $\mathrm{OH}$ & 0.97 & M \\
\hline Al & 0.444 & $\underline{M}$ \\
\hline $\mathrm{Na}$ & 7.6 & $\underline{M}$ \\
\hline No2 & 0.596 & $\underline{M}$ \\
\hline NO3 & 1.99 & $\underline{M}$ \\
\hline $\mathrm{SO} 4$ & Cancelled & \\
\hline $\mathrm{PO4}$ & $9.08 \mathrm{E}-02$ & $\underline{M}$ \\
\hline $\mathrm{Cl}$ & $5.04 \mathrm{E}-02$ & $\underline{M}$ \\
\hline $\mathrm{F}$ & $9.72 \mathrm{E}-05$ & $\underline{M}$ \\
\hline $\mathrm{CO} 3$ & 0.115 & M \\
\hline \multicolumn{3}{|c|}{ 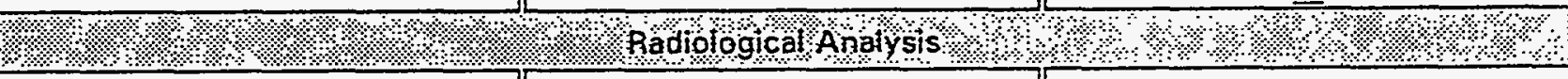 } \\
\hline $\mathrm{Pu}$ & $2.42 \mathrm{E}-04$ & $\mathrm{~g} / \mathrm{gal}$ \\
\hline GEA $134 \mathrm{Cs}$ & $2.70 E+03$ & uCi/gal \\
\hline GEA $137 \mathrm{Cs}$ & $7.06 E+05$ & $\mathrm{uCi} / \mathrm{gal}$ \\
\hline Sr-89,90 & $1.35 E+04$ & $\mathrm{uCi} / \mathrm{gal}$ \\
\hline
\end{tabular}




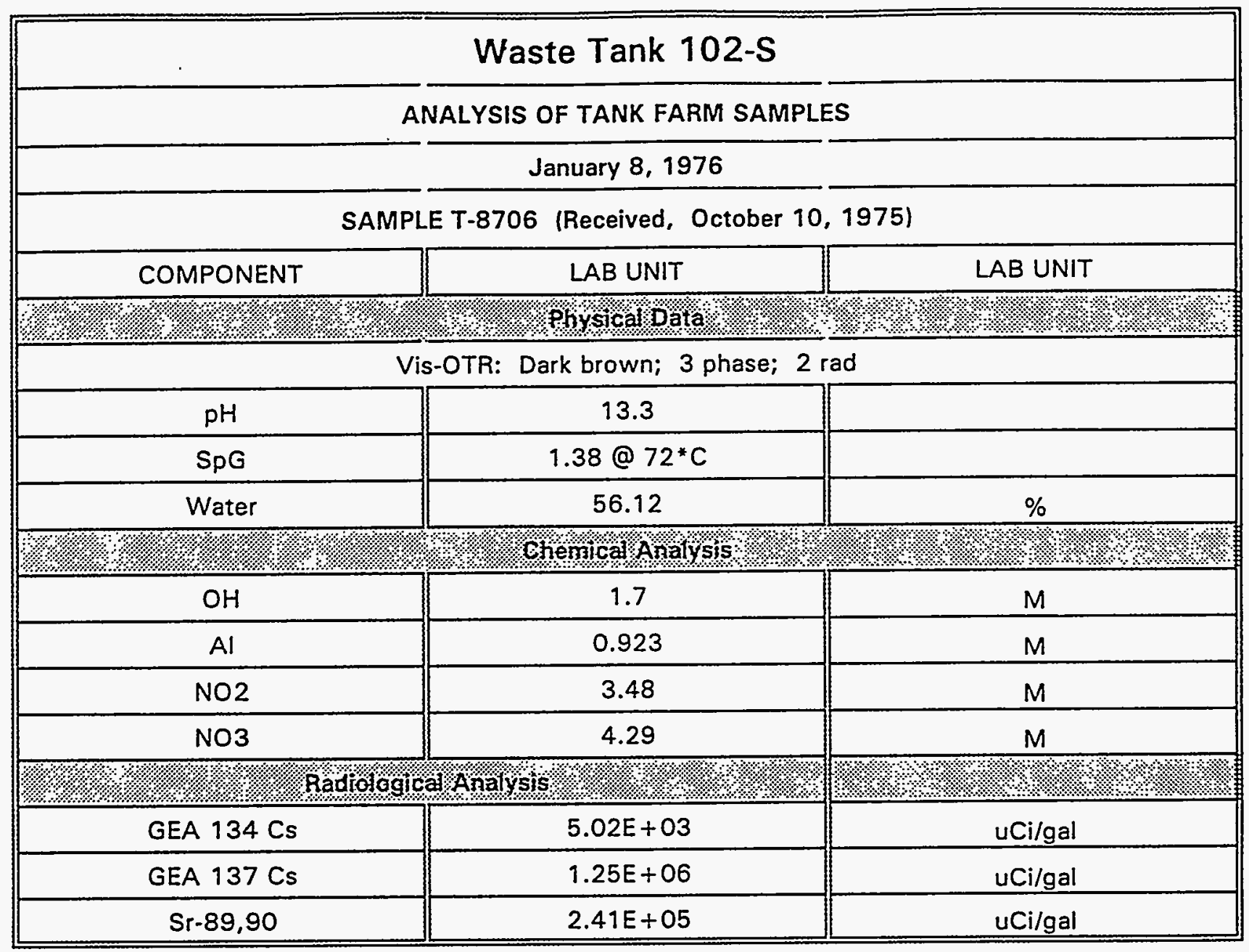




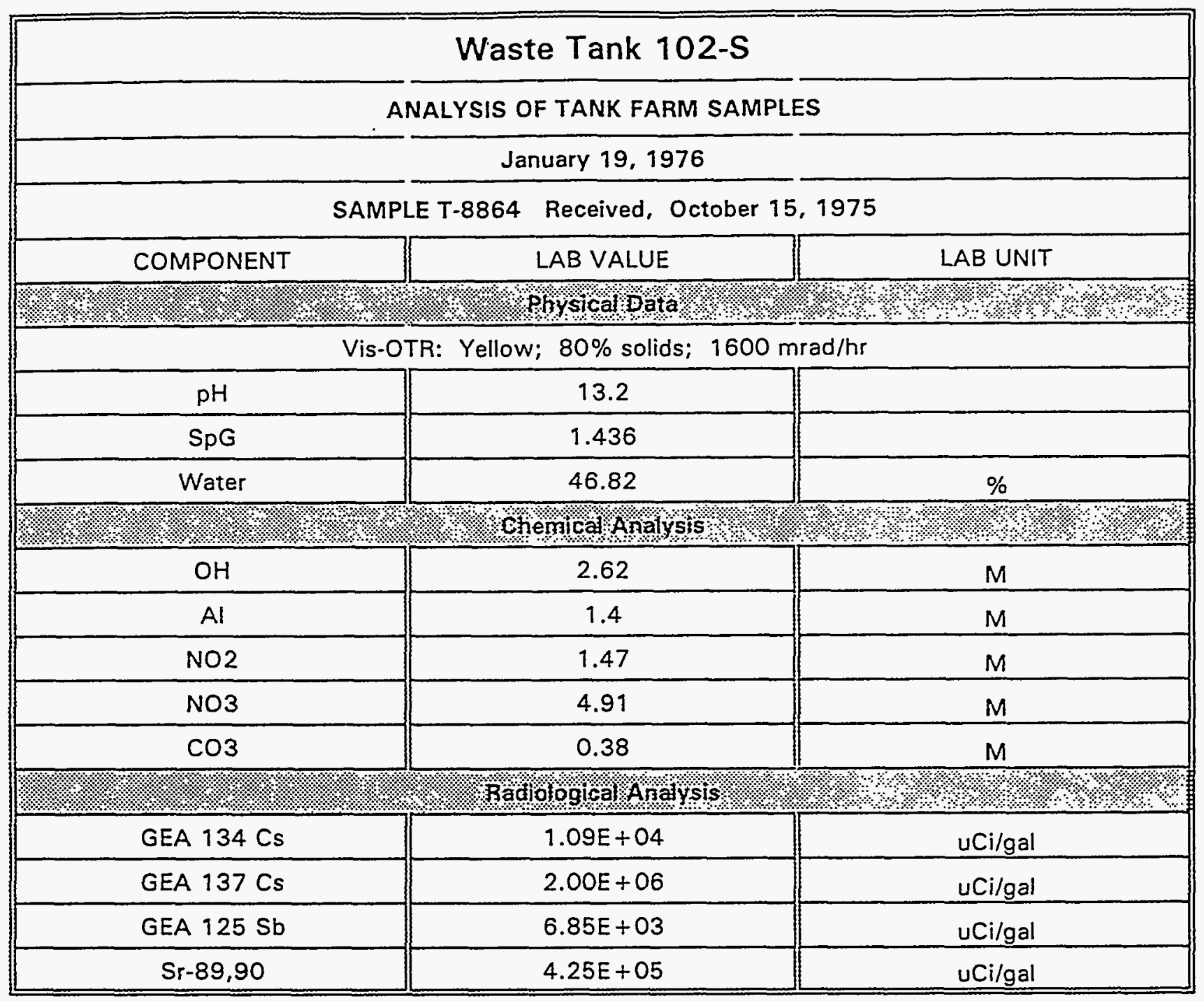




\begin{tabular}{|c|c|c|}
\hline \multicolumn{3}{|c|}{ Waste Tank 102-S } \\
\hline \multicolumn{3}{|c|}{ ANALYSIS OF TANK FARM SAMPLES } \\
\hline \multicolumn{3}{|c|}{ November 6, 1975} \\
\hline \multicolumn{3}{|c|}{ SAMPLE T-9049 Received: October 21, 1975} \\
\hline COMPONENT & LAB VALUE & LAB UNIT \\
\hline 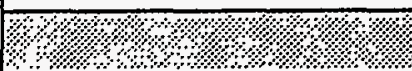 & Prysicalobia & (.). \\
\hline \multicolumn{3}{|c|}{ Vis-OTR: Brown; $>75 \%$ solids; $1800 \mathrm{mrad} / \mathrm{hr}$} \\
\hline $\mathrm{pH}$ & 13.7 & \\
\hline$S p G$ & $1.480 @ 61 * \mathrm{C}$ & \\
\hline Water & 65.00 & $\%$ \\
\hline \multicolumn{3}{|c|}{ \% $\%$ \% } \\
\hline $\mathrm{OH}$ & 3.02 & $M$ \\
\hline $\mathrm{Al}$ & 1.64 & M \\
\hline No2 & 2.58 & M \\
\hline NO3 & 4.4 & $M$ \\
\hline \multicolumn{3}{|c|}{ l. } \\
\hline GEA $134 \mathrm{Cs}$ & $1.36 E+04$ & uCi/gal \\
\hline GEA $137 \mathrm{Cs}$ & $3.19 E+06$ & uCi/gal \\
\hline Sr-89,90 & $5.56 E+04$ & uCi/gal \\
\hline
\end{tabular}




\begin{tabular}{|c|c|c|}
\hline \multicolumn{3}{|c|}{ Waste Tank 102-S } \\
\hline \multicolumn{3}{|c|}{ ANALYSIS OF TANK FARM SAMPLES } \\
\hline \multicolumn{3}{|c|}{ February 12,1976} \\
\hline \multicolumn{3}{|c|}{ SAMPLE T-9394 Received: November 3, 1975} \\
\hline COMPONENT & LAB VALUE & LAB UNIT \\
\hline 1: & Physical Data & $.1 .4 \%$ \\
\hline \multicolumn{3}{|c|}{ Vis-OTR: Dark brown; No solids; $700 \mathrm{mrad} / \mathrm{hr}$} \\
\hline $\mathrm{pH}$ & 12.8 & \\
\hline SpG & 1.326 & \\
\hline Water & 58.62 & $\%$ \\
\hline \multicolumn{3}{|c|}{ \% Chenical Analysis:? } \\
\hline $\mathrm{OH}$ & 1.57 & $M$ \\
\hline $\mathrm{Al}$ & 0.823 & M \\
\hline $\mathrm{Na}$ & 7.5 & M \\
\hline NO2 & 1.77 & $M$ \\
\hline No3 & 2.92 & M \\
\hline $\mathrm{PO} 4$ & $1.77 \mathrm{E}-02$ & $M$ \\
\hline $\mathrm{Cl}$ & $9.04 \mathrm{E}-02$ & $M$ \\
\hline $\mathrm{F}$ & 4.19E-05 & M \\
\hline $\mathrm{CO} 3$ & 0.468 & $M$ \\
\hline \multicolumn{3}{|c|}{ ( } \\
\hline Pu & $1.17 \mathrm{E}-04$ & g/gal \\
\hline GEA $134 \mathrm{Cs}$ & $5.62 E+03$ & $u \mathrm{Ci} / \mathrm{gal}$ \\
\hline GEA $137 \mathrm{Cs}$ & $1.44 \mathrm{E}+06$ & uCi/gal \\
\hline Sr-89,90 & $3.53 E+04$ & $\mathrm{uCi} / \mathrm{gal}$ \\
\hline
\end{tabular}




\begin{tabular}{|c|c|c|}
\hline \multicolumn{3}{|c|}{ Waste Tank 102-S } \\
\hline \multicolumn{3}{|c|}{ ANALYSIS OF TANK FARM SAMPLES } \\
\hline \multicolumn{3}{|c|}{ November 21, 1975} \\
\hline \multicolumn{3}{|c|}{ Received: November 5, 1975} \\
\hline COMPONENT & LAB VALUE & LAB UNIT \\
\hline 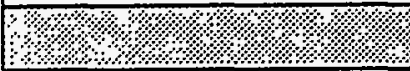 & \% 1. physicalonata & \\
\hline \multicolumn{3}{|c|}{ Vis-OTR: Dark brown; Suspended solids; $1500 \mathrm{mrad} / \mathrm{hr}$} \\
\hline $\mathrm{pH}$ & 13.5 & \\
\hline SpG & $1.410 @ 52 * \mathrm{C}$ & \\
\hline Water & 54.80 & $\%$ \\
\hline \multicolumn{3}{|c|}{ 1/.1.1.1. } \\
\hline $\mathrm{OH}$ & 2 & M \\
\hline Al & 1.06 & M \\
\hline NO2 & 5.14 & M \\
\hline NO3 & 6.44 & M \\
\hline \multicolumn{3}{|c|}{ 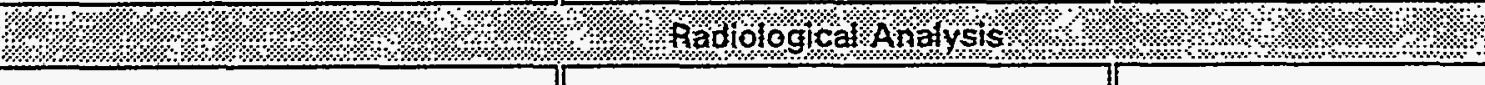 } \\
\hline GEA $134 \mathrm{Cs}$ & $6.40 E+03$ & uCi/gal \\
\hline GEA $137 \mathrm{Cs}$ & $1.82 E+06$ & uCi/gal \\
\hline St-89,90 & $3.64 E+04$ & uCi/gal \\
\hline
\end{tabular}




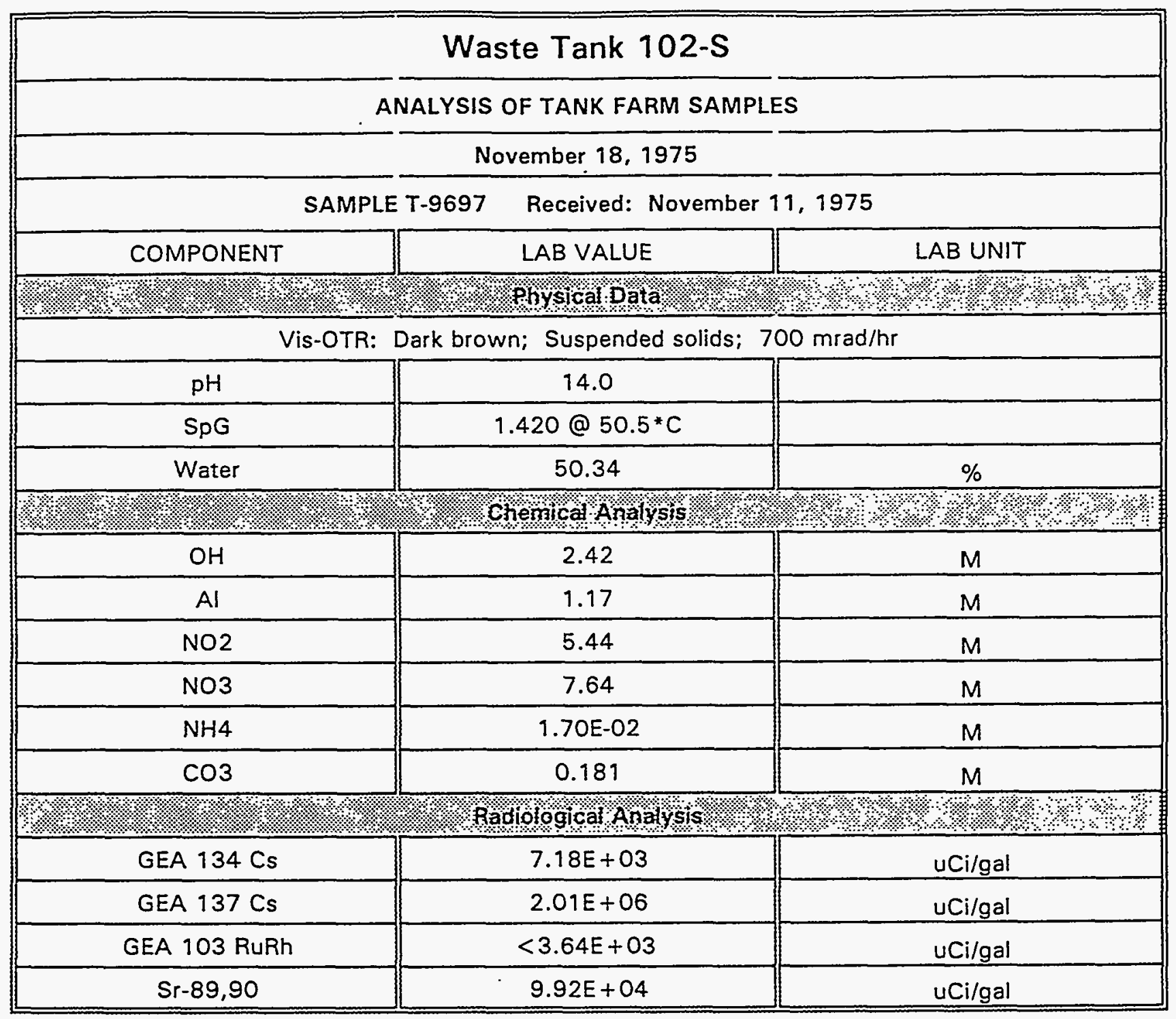




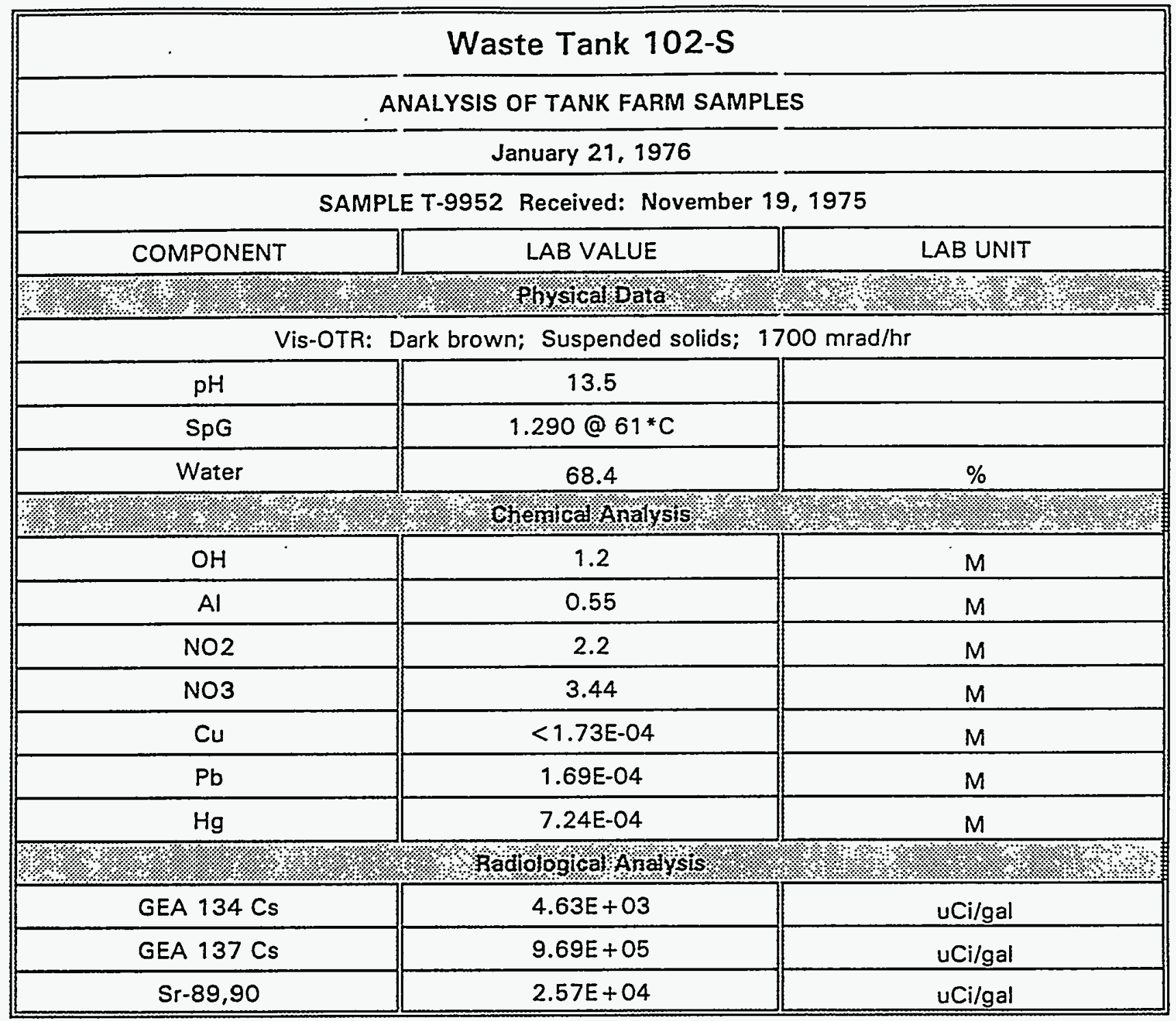




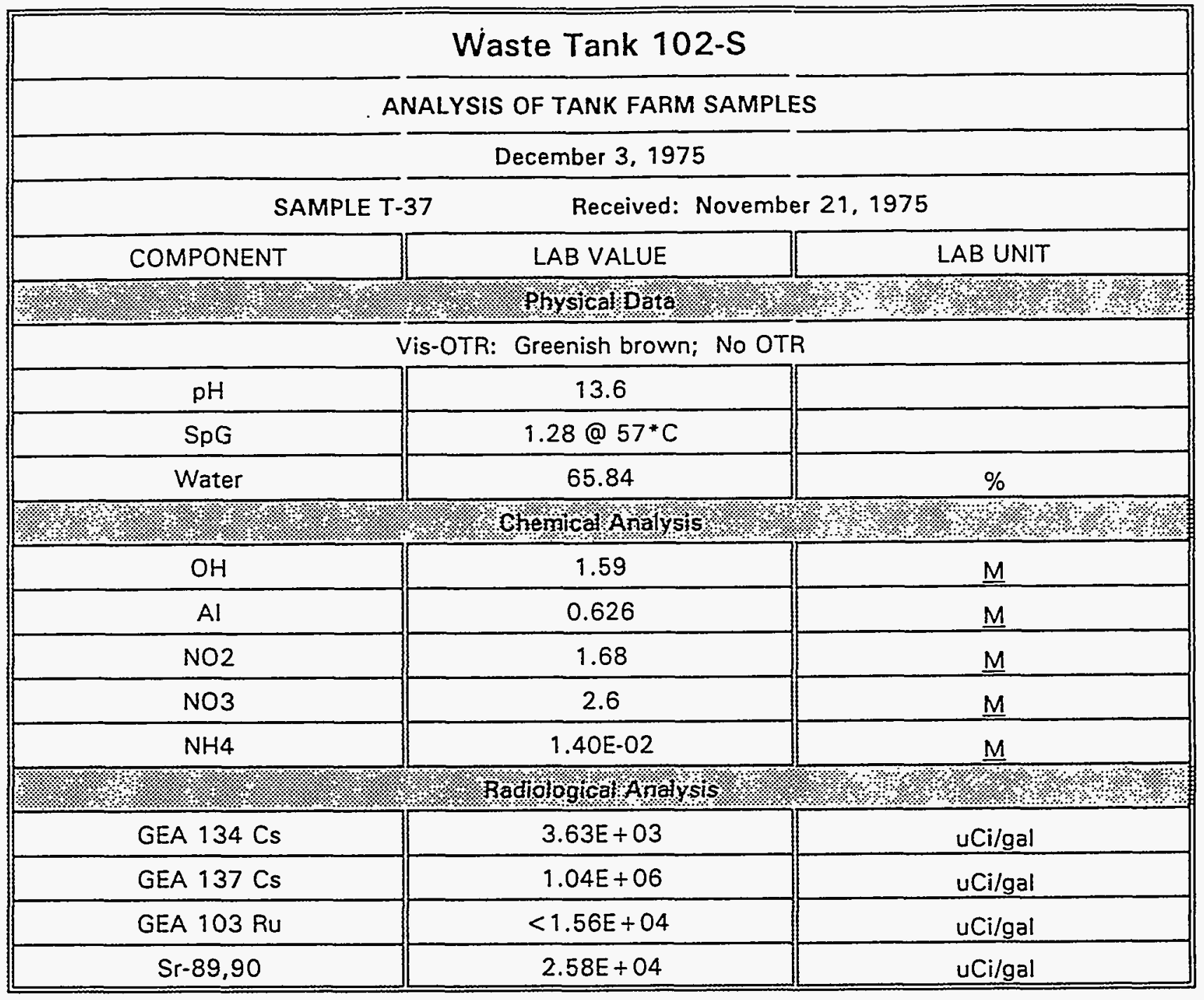




\begin{tabular}{|c|c|}
\hline \multicolumn{2}{|c|}{ Waste Tank 102-S } \\
\hline \multicolumn{2}{|c|}{ ANALYSIS OF TANK FARM SAMPLES } \\
\hline \multicolumn{2}{|c|}{ December 3, 1975} \\
\hline SAMPLE T-119 & Received: November 24, 1975 \\
\hline COMPONENT & 3 UNIT \\
\hline \multicolumn{2}{|c|}{ (1) } \\
\hline \multicolumn{2}{|c|}{ Vis-OTR: Dark brown, cruddy; $2300 \mathrm{mrad} / \mathrm{hr}$} \\
\hline $\mathrm{pH}$ & \\
\hline SpG & \\
\hline Water & $\%$ \\
\hline \multicolumn{2}{|c|}{ 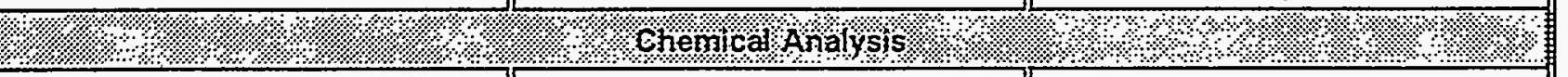 } \\
\hline $\mathrm{OH}$ & M \\
\hline Al & M \\
\hline No2 & M \\
\hline NO3 & M \\
\hline $\mathrm{NH} 4$ & M \\
\hline \multicolumn{2}{|c|}{ 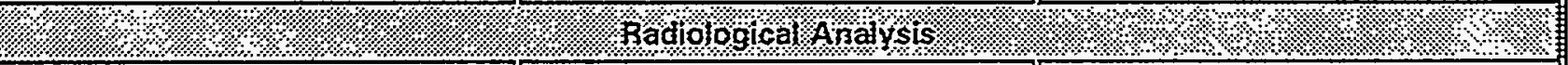 } \\
\hline GEA $134 \mathrm{Cs}$ & i/gal \\
\hline GEA $137 \mathrm{Cs}$ & Ci/gal \\
\hline GEA 103 Ru & Ci/gal \\
\hline Sr-89,90 & $\mathrm{uCi} / \mathrm{gal}$ \\
\hline
\end{tabular}




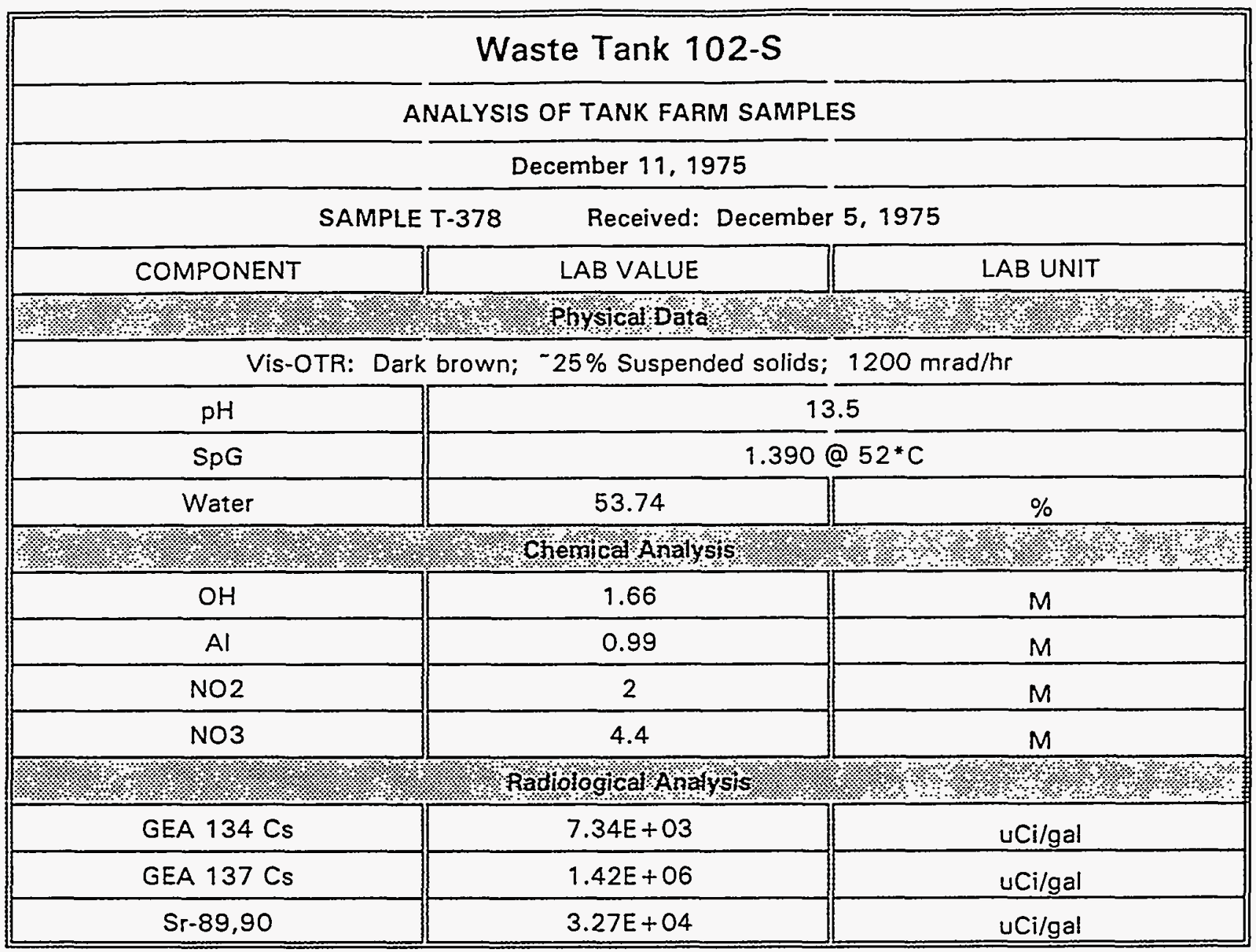

1. 


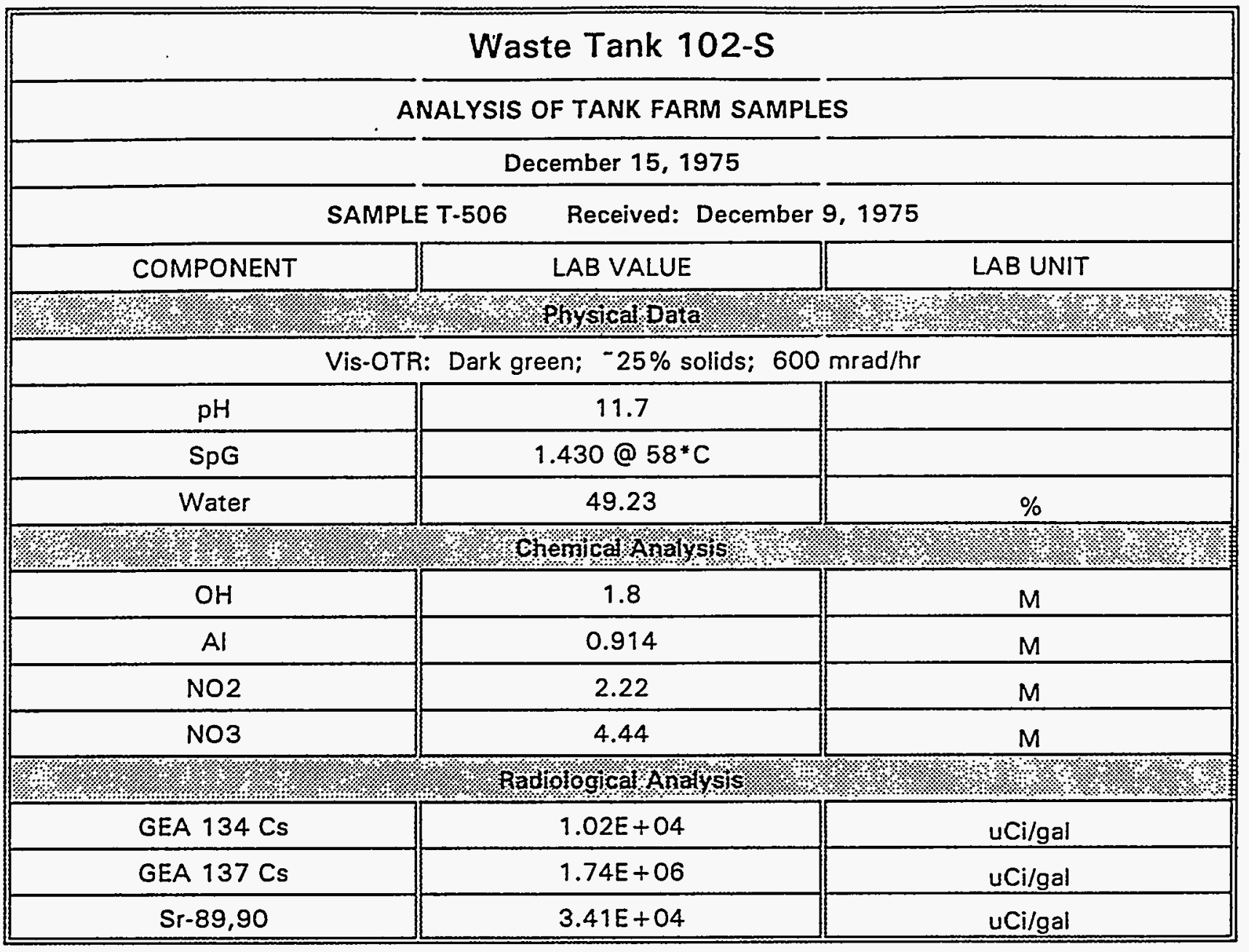




\begin{tabular}{|c|c|c|}
\hline \multicolumn{3}{|c|}{ Waste Tank 102-S } \\
\hline \multicolumn{3}{|c|}{ ANALYSIS OF TANK FARM SAMPLES } \\
\hline \multicolumn{3}{|c|}{ March 16, 1976} \\
\hline \multicolumn{3}{|c|}{ Received: December 28, 1975} \\
\hline COMPONENT & LAB VALUE & LAB UNIT \\
\hline $1.17 \%: 1 \%$ & Physica o ota & ?., \\
\hline \multicolumn{3}{|c|}{ Vis-OTR: Dark brown; Suspended solids; $1.5 \mathrm{Rad} / \mathrm{hr}$} \\
\hline $\mathrm{pH}$ & 13.0 & \\
\hline $\mathrm{SpG}$ & $1.4 @ 55^{*} \mathrm{C}$ & \\
\hline Water & 50.12 & $\%$ \\
\hline \multicolumn{3}{|c|}{ 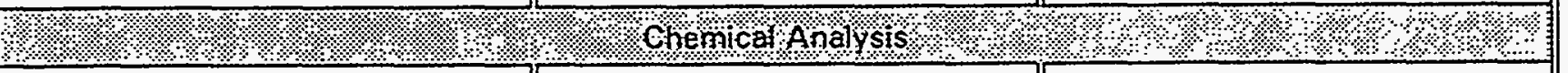 } \\
\hline $\mathrm{OH}$ & 2.22 & M \\
\hline Al & 1.54 & M \\
\hline $\mathrm{Na}$ & 11.34 & M \\
\hline $\mathrm{Cu}$ & $8.18 E-05$ & M \\
\hline $\mathrm{Pb}$ & $3.02 \mathrm{E}-04$ & M \\
\hline $\mathrm{Hg}$ & $2.46 \mathrm{E}-03$ & M \\
\hline NO2 & 2.06 & M \\
\hline NO3 & 4.88 & M \\
\hline $\mathrm{PO} 4$ & $6.78 \mathrm{E}-02$ & M \\
\hline $\mathrm{SO} 4$ & $9.30 \mathrm{E}-02$ & M \\
\hline $\mathrm{Cl}$ & 0.157 & M \\
\hline $\mathrm{F}$ & $1.50 \mathrm{E}-03$ & M \\
\hline $\mathrm{CO} 3$ & 0.43 & $M$ \\
\hline \multicolumn{3}{|c|}{ ४४१ } \\
\hline Pu & 4.84E-06 & g/gal \\
\hline GEA $134 \mathrm{Cs}$ & $9.22 E+03$ & uCi/gal \\
\hline GEA $137 \mathrm{Cs}$ & $2.16 E+06$ & $u \mathrm{Ci} / \mathrm{gal}$ \\
\hline $\mathrm{Sr}-89,90$ & $3.49 E+04$ & uCi/gal \\
\hline
\end{tabular}




\begin{tabular}{|c|c|c|}
\hline \multicolumn{3}{|c|}{ Waste Tank 102-S } \\
\hline \multicolumn{3}{|c|}{ ANALYSIS OF TANK FARM SAMPLES } \\
\hline \multicolumn{3}{|c|}{ January 19, 1976} \\
\hline \multicolumn{3}{|c|}{ SAMPLE T-1193 Received, January 5, 1976} \\
\hline 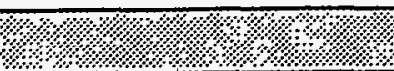 & Physilar oata & $2.1 .2 .1 . \% . \%$. \\
\hline \multicolumn{3}{|c|}{ Vis-OTR: Dark brown; Suspended solids; $1800 \mathrm{mrad} / \mathrm{hr}$} \\
\hline $\mathrm{pH}$ & 13.7 & \\
\hline $\mathrm{SpG}$ & $1.40 @ 67 * \mathrm{C}$ & \\
\hline Water & 50.32 & $\%$ \\
\hline \multicolumn{3}{|c|}{ 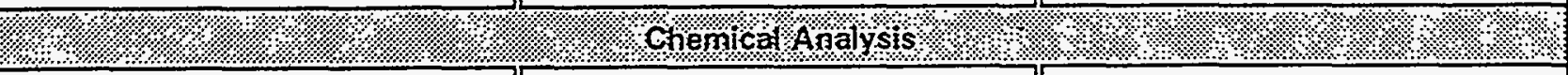 } \\
\hline $\mathrm{OH}$ & 2.06 & M \\
\hline Al & 1.21 & M \\
\hline NO2 & 2.26 & $M$ \\
\hline NO3 & 4.94 & $\mathrm{M}$ \\
\hline \multicolumn{3}{|c|}{ 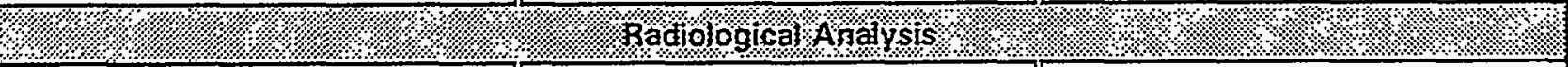 } \\
\hline GEA $134 \mathrm{Cs}$ & $8.80 E+03$ & uCi/gal \\
\hline GEA $137 \mathrm{Cs}$ & $1.91 E+06$ & uCi/gal \\
\hline $\mathrm{Sr}-89,90$ & $2.99 E+04$ & $\mathrm{uCi} / \mathrm{gal}$ \\
\hline
\end{tabular}




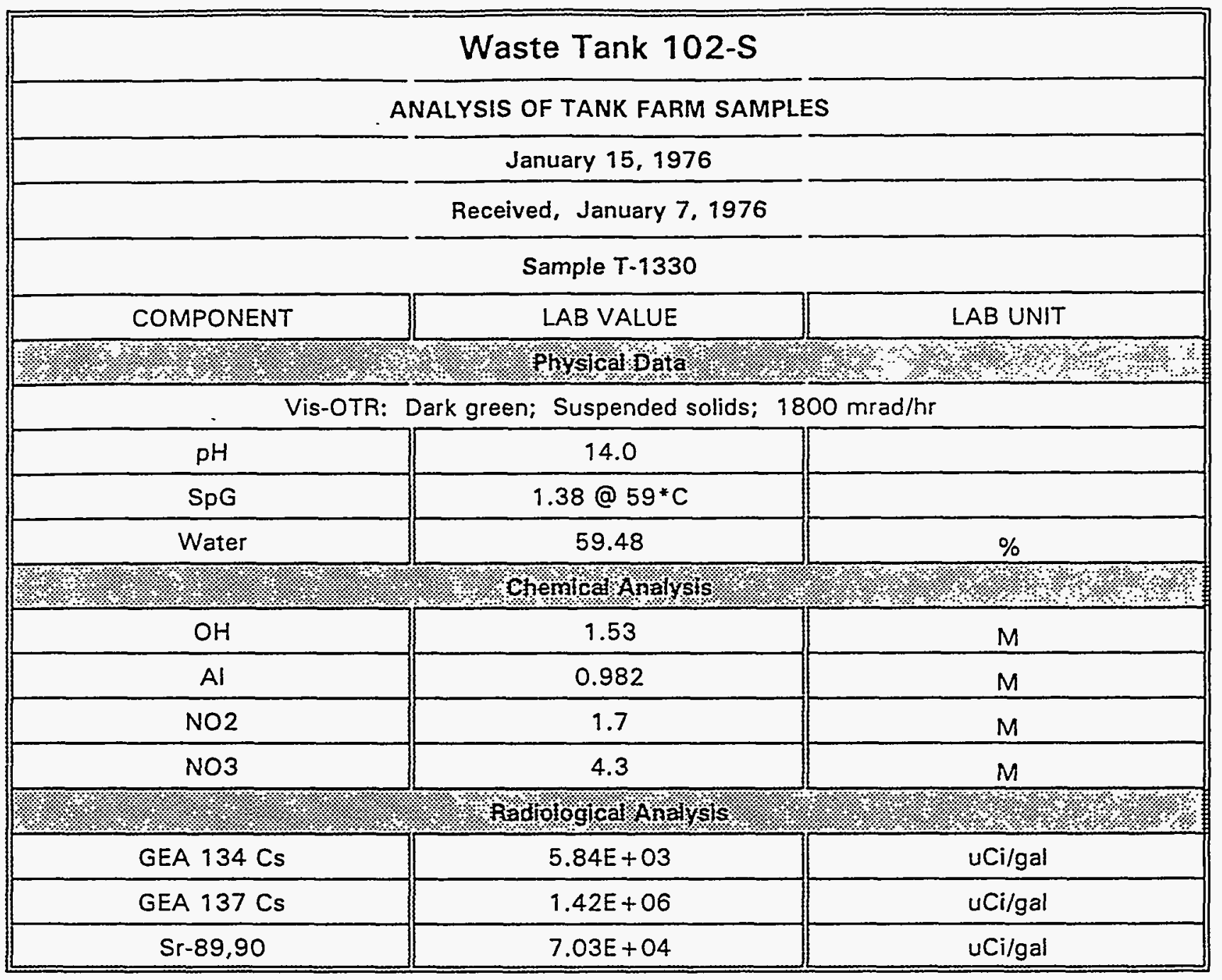




\begin{tabular}{|c|c|c|}
\hline \multicolumn{3}{|c|}{ Waste Tank 102-S } \\
\hline \multicolumn{3}{|c|}{ ANALYSIS OF TANK FARM SAMPLES } \\
\hline \multicolumn{3}{|c|}{ February 13,1976} \\
\hline \multicolumn{3}{|c|}{ Received: January 20, 1976} \\
\hline COMPONENT & LAB VALUE & LAB UNIT \\
\hline \multicolumn{3}{|c|}{ 1\%\%". } \\
\hline \multicolumn{3}{|c|}{ Vis-OTR: Dark green; Suspended solids; 2 rad } \\
\hline $\mathrm{pH}$ & 13.0 & \\
\hline $\mathrm{SpG}$ & $1.39 @ 60^{\circ} \mathrm{C}$ & \\
\hline Water & 54.86 & $\%$ \\
\hline \multicolumn{3}{|c|}{ 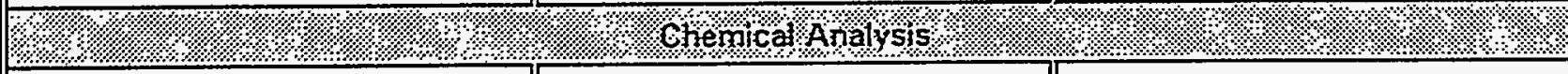 } \\
\hline $\mathrm{OH}$ & 1.33 & $M$ \\
\hline $\mathrm{Al}$ & 0.978 & M \\
\hline $\mathrm{Na}$ & 8.1 & $M$ \\
\hline NO2 & 1.75 & M \\
\hline NO3 & 3.9 & $M$ \\
\hline $\mathrm{PO4}$ & $1.04 \mathrm{E}-01$ & $M$ \\
\hline $\mathrm{Cl}$ & 0.154 & $M$ \\
\hline$F$ & $2.42 \mathrm{E}-03$ & $M$ \\
\hline $\mathrm{CO}$ & 0.442 & $M$ \\
\hline $\mathrm{Cu}$ & $<9.6 \mathrm{E}-05$ & $M$ \\
\hline $\mathrm{Hg}$ & $2.22 \mathrm{E}-03$ & $M$ \\
\hline $\mathrm{Pb}$ & $2.32 \mathrm{E}-04$ & $M$ \\
\hline \multicolumn{3}{|c|}{ 1.\% } \\
\hline $\mathrm{Pu}$ & $5.22 \mathrm{E}-04$ & $\mathrm{gm} / \mathrm{gal}$ \\
\hline GEA $134 \mathrm{Cs}$ & $6.32 E+03$ & uCi/gal \\
\hline GEA $137 \mathrm{Cs}$ & $1.56 E+06$ & uCi/gal \\
\hline Sr-89,90 & $2.32 E+04$ & uCi/gal \\
\hline
\end{tabular}




\begin{tabular}{|c|c|c|}
\hline \multicolumn{3}{|c|}{ Waste Tank 102-S } \\
\hline \multicolumn{3}{|c|}{ ANALYSIS OF TANK FARM SAMPLES } \\
\hline \multicolumn{3}{|c|}{ April 27, 1976} \\
\hline \multicolumn{3}{|c|}{ SAMPLE T-2395 (Received, February 17, 1976) } \\
\hline $4.1 \%$ & Prystcat orata & ४४४४४४ ४ै। ४ै। \\
\hline \multicolumn{3}{|c|}{ Vis-OTR: Dark brown; No solids; $1500 \mathrm{mrad} / \mathrm{hr}$} \\
\hline $\mathrm{pH}$ & 13.2 & \\
\hline $\mathrm{SpG}$ & 1.250 & \\
\hline Water & 66.58 & $\%$ \\
\hline \multicolumn{3}{|c|}{ ?.4: } \\
\hline $\mathrm{OH}$ & 0.773 & $M$ \\
\hline Al & 0.453 & $M$ \\
\hline $\mathrm{Na}$ & 5.03 & M \\
\hline $\mathrm{Cu}$ & $1.51 \mathrm{E}-04$ & M \\
\hline $\mathrm{Pb}$ & 5.17E-04 & $M$ \\
\hline $\mathrm{Hg}$ & 8.04E-04 & $M$ \\
\hline $\mathrm{NO} 2$ & 0.531 & $M$ \\
\hline NO3 & 2.42 & $M$ \\
\hline $\mathrm{PO4}$ & $4.70 \mathrm{E}-02$ & $M$ \\
\hline 504 & Unable to run & \\
\hline $\mathrm{Cl}$ & $6.02 \mathrm{E}-02$ & M \\
\hline$F$ & $9.38 \mathrm{E}-03$ & $M$ \\
\hline $\mathrm{CO} 3$ & 0.238 & $M$ \\
\hline \multicolumn{3}{|c|}{ 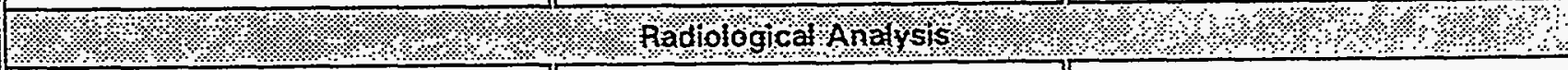 } \\
\hline $\mathrm{Pu}$ & $3.33 E-03$ & $\mathrm{~g} / \mathrm{gal}$ \\
\hline GEA 60 Co & $2.36 \mathrm{E}+03$ & $u C i / g a l$ \\
\hline GEA $144 \mathrm{Ce}$ & $1.86 E+04$ & uCi/gal \\
\hline GEA $155 \mathrm{Eu}$ & $1.46 \mathrm{E}+04$ & uCi/gal \\
\hline GEA $134 \mathrm{Cs}$ & $2.77 E+03$ & uCi/gal \\
\hline GEA $137 \mathrm{Cs}$ & $8.19 E+05$ & uCi/gal \\
\hline Sr-89,90 & $1.97 E+05$ & $u \mathrm{Ci} / \mathrm{gal}$ \\
\hline
\end{tabular}




\begin{tabular}{|c|c|c|c|}
\hline \multicolumn{4}{|c|}{ Waste Tank 102-S } \\
\hline \multicolumn{4}{|c|}{ CUSTOMER WASTE ANALYSIS } \\
\hline \multicolumn{4}{|c|}{ April 5, 1979} \\
\hline COMPONENT & LAB VALUE & LAB VALUE (RERUN) & LAB UNIT \\
\hline $1.1 .1 .1 \%$ & 1\%.:- & $6 a+5 \%$ \% & +... \\
\hline \multicolumn{4}{|c|}{ Notes: Light green color, ${ }^{-}-10 \%$ settled solids, solids dissolved at $60^{*} \mathrm{C}$} \\
\hline Density & 1.257 & & \\
\hline TOC & 10.60 & 13 & $\mathrm{gm} / \mathrm{L}$ \\
\hline Water & 61.74 & & $\%$ \\
\hline \multicolumn{4}{|c|}{ Chemecal Analysis. } \\
\hline NO3 (-) & 2.04 & 1.65 & M \\
\hline NO2 (-) & 1.55 & & $M$ \\
\hline SO4 (2-) & 0.27 & & M \\
\hline PO4 (3-) & 0.12 & & $M$ \\
\hline $\mathrm{Al}(3+1$ & IS** & 0.51 & M \\
\hline $\mathrm{OH}(-)$ & IS & 0.49 & $M$ \\
\hline $\cos (2-)$ & 0.62 & 0.56 & M \\
\hline \multicolumn{4}{|c|}{ 1/1\%, Radiologicaly Analysis } \\
\hline Cs-137 & $2.63 E+05$ & & $\mathrm{uCi} / \mathrm{l}$ \\
\hline Cs-134 & $4.07 E+02$ & & $\mathrm{uCi} / \mathrm{l}$ \\
\hline Co. 60 & $4.17 E+02$ & & $u \mathrm{Ci} / \mathrm{I}$ \\
\hline \multicolumn{4}{|l|}{ - Total Organic Carbon } \\
\hline * Insufficient Sample & & & \\
\hline
\end{tabular}




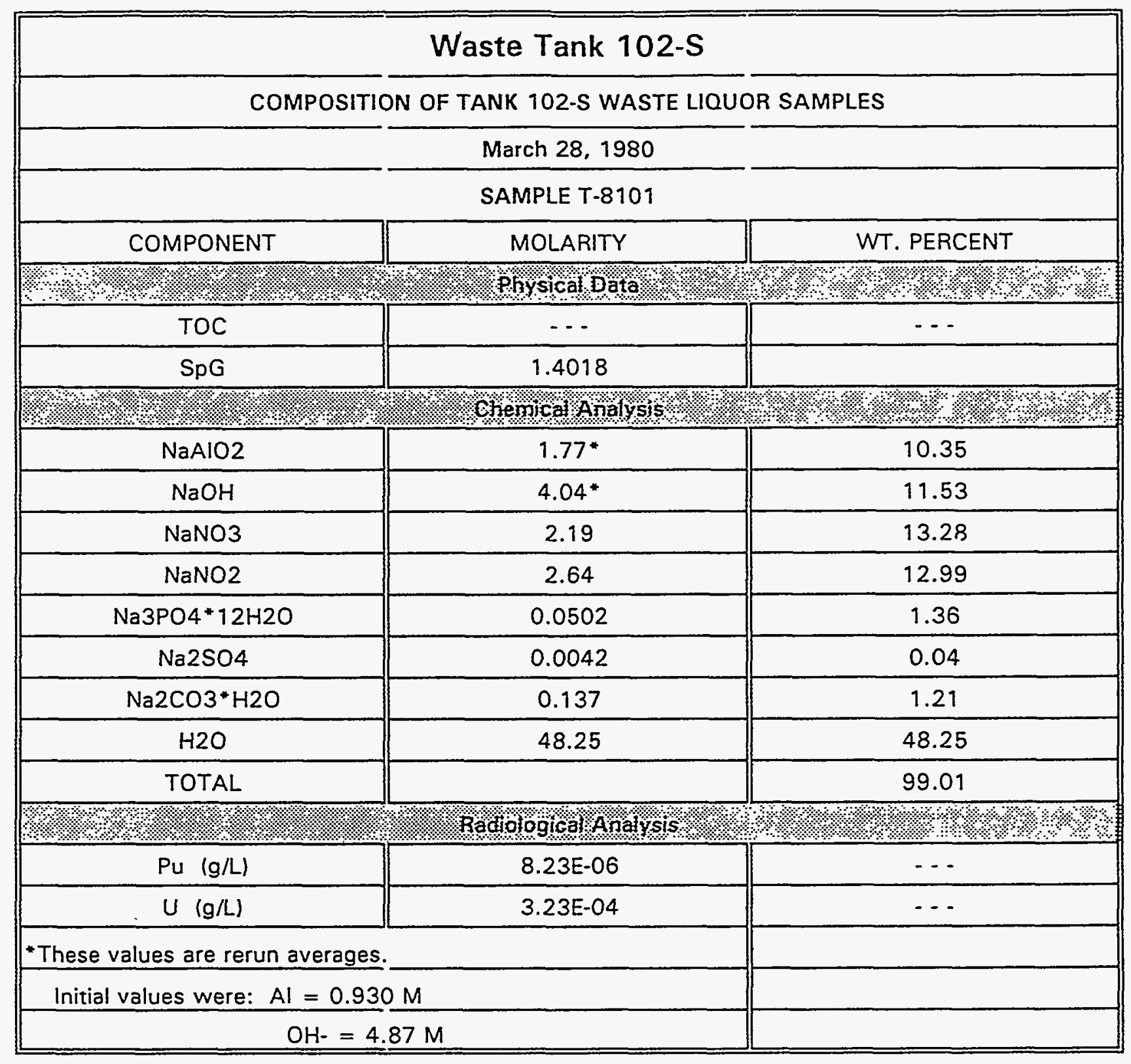




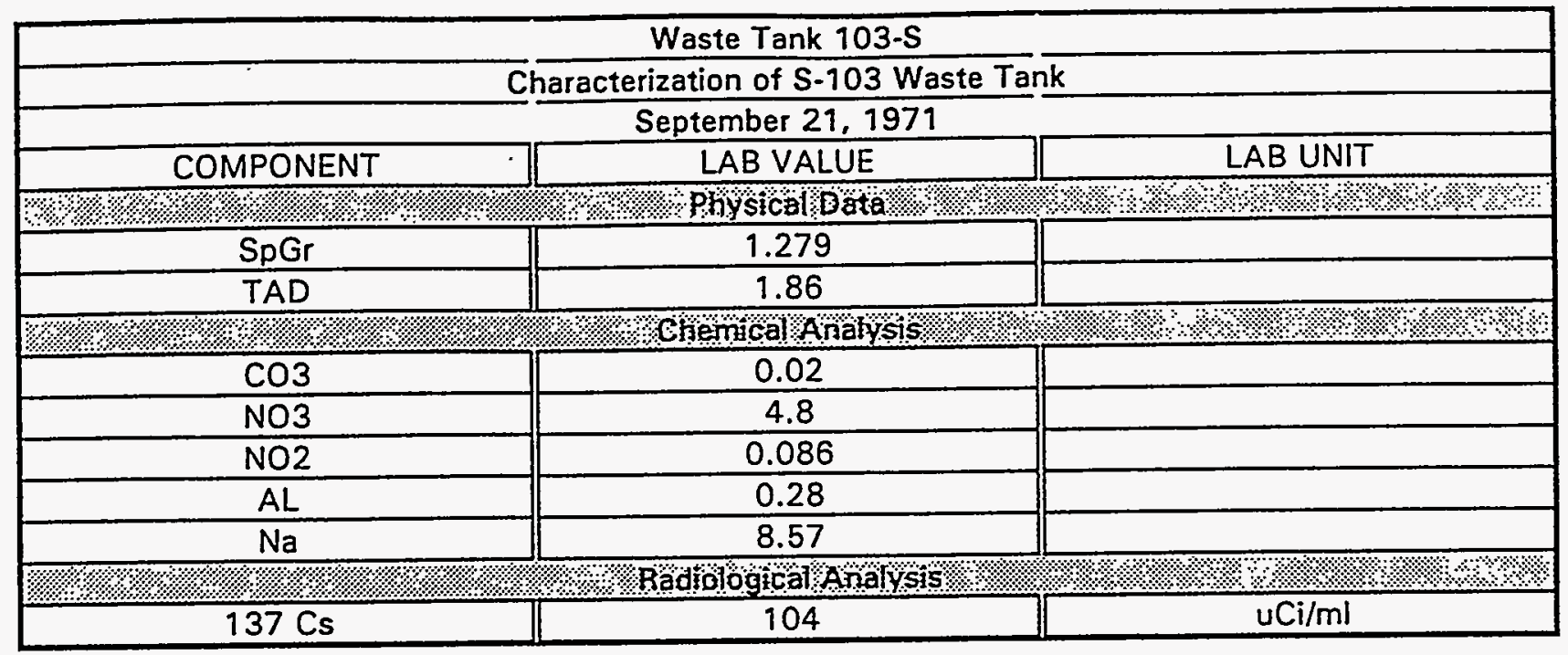




\begin{tabular}{|c|c|c|}
\hline \multicolumn{3}{|c|}{ Waste Tank S-103 } \\
\hline \multicolumn{3}{|c|}{ 242-S Feed Sample } \\
\hline \multicolumn{3}{|c|}{ December 7,1973 } \\
\hline \multicolumn{3}{|c|}{ Sample: T-9490 Received: October 5,1973} \\
\hline COMPONENT & LAB VALUE & LAB UNIT \\
\hline 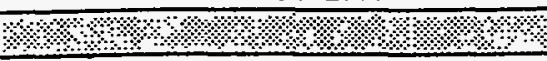 & ,. Physicaloata & ४. \\
\hline \multicolumn{3}{|c|}{ Vis-OTR: Clear, yellow, $600 \mathrm{mR}$} \\
\hline $\mathrm{pH}$ & 11 & \\
\hline SpG & 1.3517 & \\
\hline 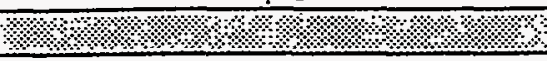 & . Ghemicall Arafysis $/ 4$ & 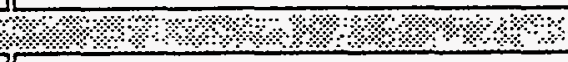 \\
\hline $\mathrm{OH}$ & 1.96 & $\underline{M}$ \\
\hline $\mathrm{Al}$ & 1.22 & $\underline{M}$ \\
\hline $\mathrm{Na}$ & 8.68 & $\underline{M}$ \\
\hline NO2 & 0.852 & $\underline{\underline{M}}$ \\
\hline NO3 & 4.36 & $\underline{M}$ \\
\hline $\mathrm{SO4}$ & $8.78 \mathrm{E}-03$ & $\underline{\underline{M}}$ \\
\hline $\mathrm{PO4}$ & $<3.77 \mathrm{E}-04$ & $\underline{M}$ \\
\hline $\mathrm{F}$ & $2.07 E-03$ & $\underline{M}$ \\
\hline $\mathrm{CO} 3$ & 0.042 & $\underline{M}$ \\
\hline 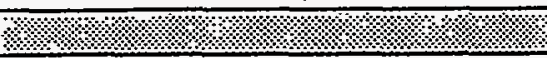 & 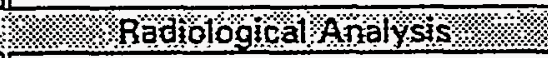 & 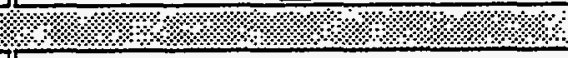 \\
\hline $\mathrm{Pu}$ & $4.93 \mathrm{E}-07$ & \begin{tabular}{|c|}
$g / l$ \\
\end{tabular} \\
\hline GEA: $137 \mathrm{Cs}$ & $1.61 \mathrm{E}+05$ & $\mathrm{uCi} / \mathrm{l}$ \\
\hline $89,90 \mathrm{Sr}$ & 11.24 & $\mathrm{uCi} / \mathrm{l}$ \\
\hline
\end{tabular}




\begin{tabular}{|c|c|c|}
\hline \multicolumn{3}{|c|}{ Waste Tank S-103 } \\
\hline & 242 S Feed Samples & \\
\hline \multicolumn{3}{|c|}{ January 21,1974} \\
\hline \multicolumn{3}{|c|}{ Sample: T-736 } \\
\hline COMPONENT & LAB VALUE & LAB UNIT \\
\hline ४४\% \% \% & Physfcallobata & (1. \\
\hline Vis-OTR & Clear, ye & $200 \mathrm{mRad} / \mathrm{hr}$. \\
\hline $\mathrm{pH}$ & 11.8 & \\
\hline $\mathrm{SpG}$ & 1.0742 & \\
\hline Water & 86.59 & $\%$ \\
\hline 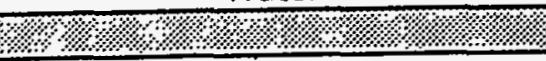 & Chemacarlaraly & \% \\
\hline $\mathrm{OH}$ & 0.523 & $\underline{\underline{M}}$ \\
\hline $\mathrm{Al}$ & 0.122 & $\underline{M}$ \\
\hline $\mathrm{Na}$ & 1.89 & $\underline{\underline{M}}$ \\
\hline NO2 & 0.067 & $\underline{\underline{M}}$ \\
\hline NO3 & 1.31 & $\underline{\bar{M}}$ \\
\hline SO4 & $6.12 \mathrm{E}-03$ & $\underline{M}$ \\
\hline PO4 & $<3.77 \mathrm{E}-04$ & $\underline{\underline{M}}$ \\
\hline$F$ & $5.26 E-04$ & $\underline{M}$ \\
\hline $\mathrm{CO} 3$ & 0.011 & $\underline{M}$ \\
\hline \% \% \%\%: & 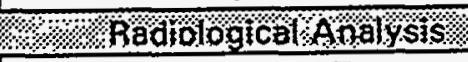 & \%. \\
\hline $\mathrm{Pu}$ & $9.51 \mathrm{E}-07$ & $g / 1$ \\
\hline GEA: $137 \mathrm{Cs}$ & $8.42 \mathrm{E}+04$ & $\mathrm{uCi} / \mathrm{l}$ \\
\hline $89,90 \mathrm{Sr}$ & Not Requested & \\
\hline
\end{tabular}


WHC-SD-WM-ER-323, Rev. O

\begin{tabular}{|c|c|c|}
\hline \multicolumn{3}{|c|}{ Waste Tank S-103 } \\
\hline & Analysis of Tank Farm Sample & \\
\hline \multicolumn{3}{|c|}{ December 6, 1974} \\
\hline \multicolumn{3}{|c|}{ Sample: T-8009 } \\
\hline COMPONENT & LAB VALUE & LAB UNIT \\
\hline 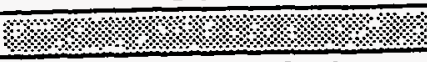 & Pptysicalobata & $4 \% \%, \%$ \\
\hline Vis-OTR & \multicolumn{2}{|c|}{ Clear, yellow, no solids. $1000 \mathrm{mRad} / \mathrm{hr}$. } \\
\hline $\mathrm{pH}$ & $<14.5$ & \\
\hline SpG & 1.235 & \\
\hline Water & 77.55 & $\%$ \\
\hline (m. & 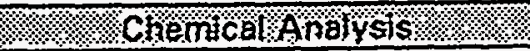 & 1. \\
\hline $\mathrm{OH}$ & 1.18 & $\underline{\underline{M}}$ \\
\hline $\mathrm{Al}$ & 0.471 & $\underline{\underline{M}}$ \\
\hline $\mathrm{Na}$ & 3.84 & $\underline{M}$ \\
\hline $\mathrm{NO2}$ & 0.579 & $\underline{M}$ \\
\hline NO3 & 1.43 & $\underline{M}$ \\
\hline SO4 & $1.21 \mathrm{E}-02$ & $\underline{M}$ \\
\hline $\mathrm{PO4}$ & $3.44 \mathrm{E}-02$ & $\underline{M}$ \\
\hline $\mathrm{F}$ & $4.27 E-03$ & $M$ \\
\hline $\mathrm{CO} 3$ & $6.73 \mathrm{E}-02$ & $\underline{M}$ \\
\hline 7/: & 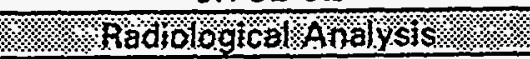 & 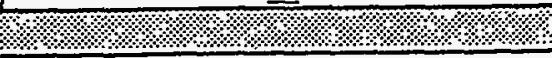 \\
\hline $\mathrm{Pu}$ & $<6.21 \mathrm{E}-06$ & $\mathrm{gm} / \mathrm{gal}$ \\
\hline GEA $134 \mathrm{Cs}$ & $2.88 E+03$ & uCi/gal \\
\hline GEA $137 \mathrm{Cs}$ & $6.74 \mathrm{E}+05$ & uCi/gal \\
\hline GEA 106 RuRh & $1.21 E+05$ & uCi/gal \\
\hline $89,90 \mathrm{Sr}$ & $9.08 E+02$ & uCi/gal \\
\hline
\end{tabular}




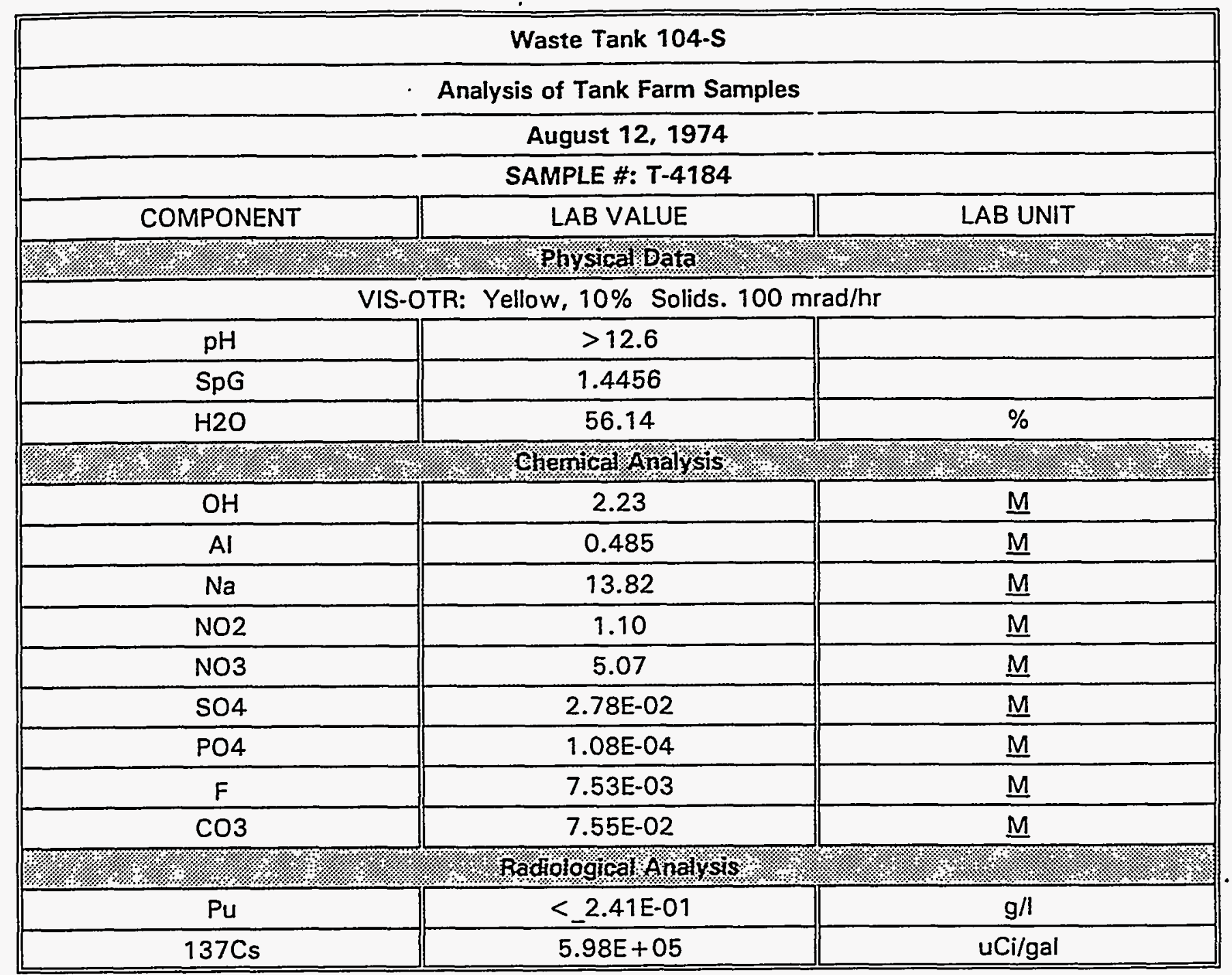




\begin{tabular}{|c|c|c|}
\hline \multicolumn{3}{|c|}{ Waste Tank 104-S } \\
\hline & Analysis of Tank S-104 Sludge & \\
\hline \multicolumn{3}{|c|}{ February 24,1976} \\
\hline \multicolumn{3}{|c|}{ Received: January 9, 1976} \\
\hline COMPONENT & LAB VALUE & LAB UNIT \\
\hline \multicolumn{3}{|c|}{ Prysicalobata } \\
\hline \multicolumn{3}{|c|}{$\begin{array}{c}\text { The consistency of the sample was like a stiff dough or putty with a tendency to be sticky, being } \\
\text { grayish in color }\end{array}$} \\
\hline Bulk Density & 1.21 & $\mathrm{~g} / \mathrm{cc}$ \\
\hline Particle Density & 2.45 & $\mathrm{~g} / \mathrm{cc}$ \\
\hline $\mathrm{H} 2 \mathrm{O}$ & 30.3 & $\%$ \\
\hline \% & Chemicat Analysis & $\%$ \\
\hline $\mathrm{Al}$ & 3.2 & moles/L \\
\hline$\overline{\mathrm{Fe}}$ & 0.06 & moles/L \\
\hline $\mathrm{Si}$ & 0.3 & moles/L \\
\hline $\mathrm{NO3}$ & 13.2 & moles/L \\
\hline $\mathrm{Mg}$ & 0.01 & moles/L \\
\hline $\mathrm{Mn}$ & 0.02 & moles /L \\
\hline $\mathrm{PO4}$ & $<0.01$ & moles/L \\
\hline $\mathrm{Ca}$ & 0.09 & moles/L \\
\hline $\mathrm{Ba}$ & $<0.09$ & moles/L \\
\hline (1., & Radiofogical Analysis & 6.4 .8 .8 \\
\hline $\mathrm{Pu}$ & $4.46 \mathrm{E}-03$ & $g / 1$ \\
\hline $89+90 \mathrm{Sr}$ & 0.38 & $\mathrm{Ci} / \mathrm{L}$ \\
\hline $89+90 \mathrm{Sr}$ & $2.55 \mathrm{E}-03$ & watts/L \\
\hline $137 \mathrm{Cs}$ & 0.11 & $\mathrm{Ci} / \mathrm{L}$ \\
\hline $137 \mathrm{Cs}$ & $5.23 E-04$ & watts/L \\
\hline
\end{tabular}


WHC-SD-WM-ER-323, Rev. 0

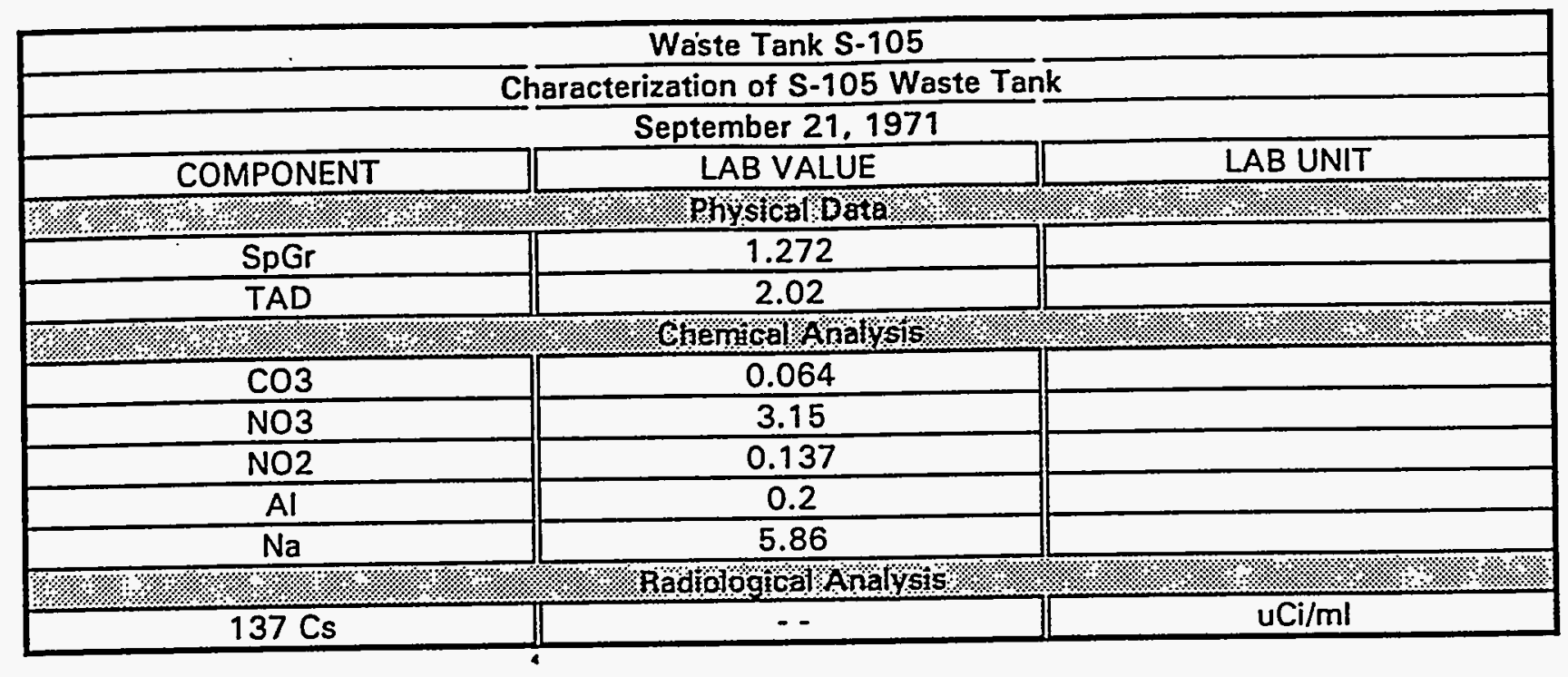


WHC-SD-WM-ER-323, Rev. O

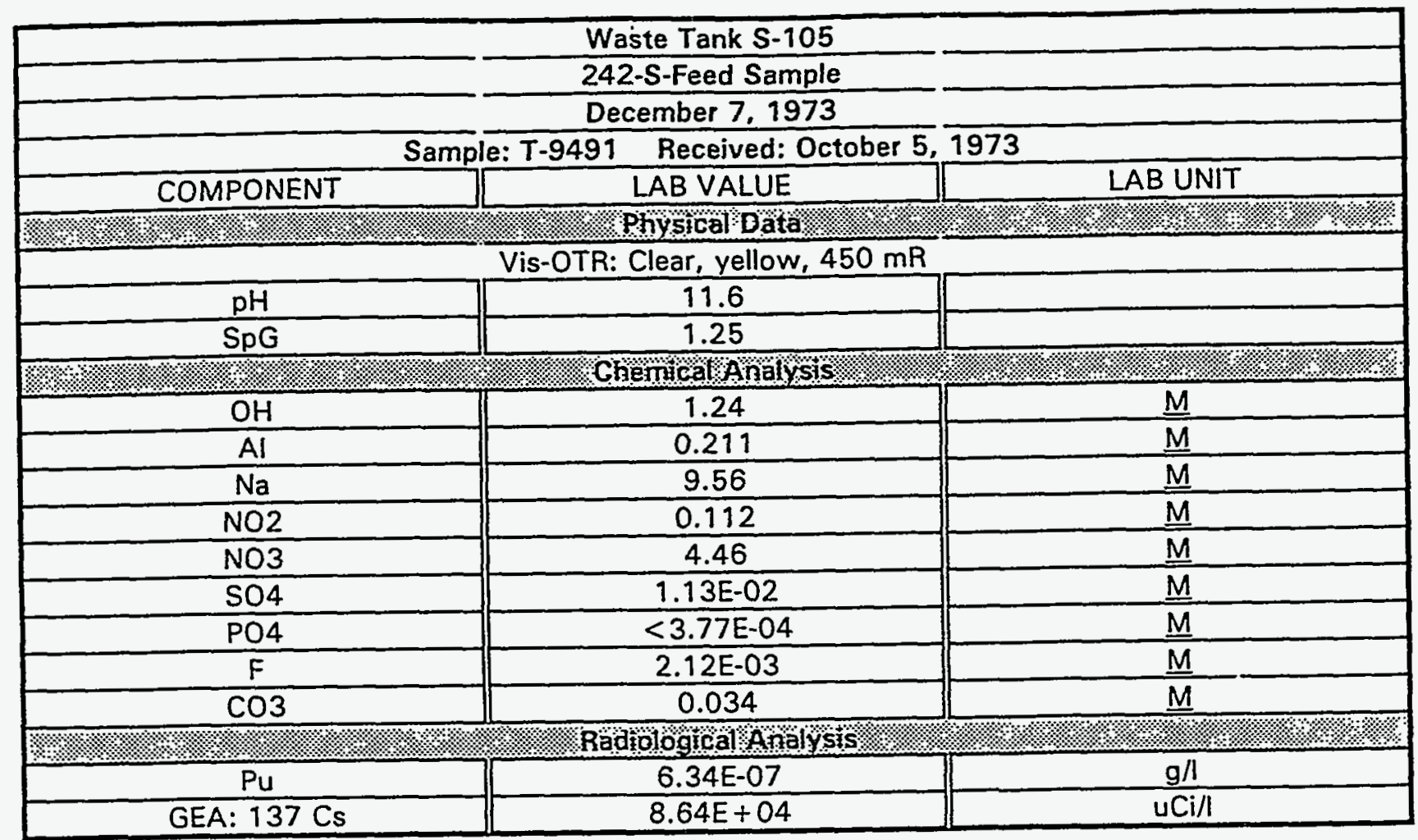




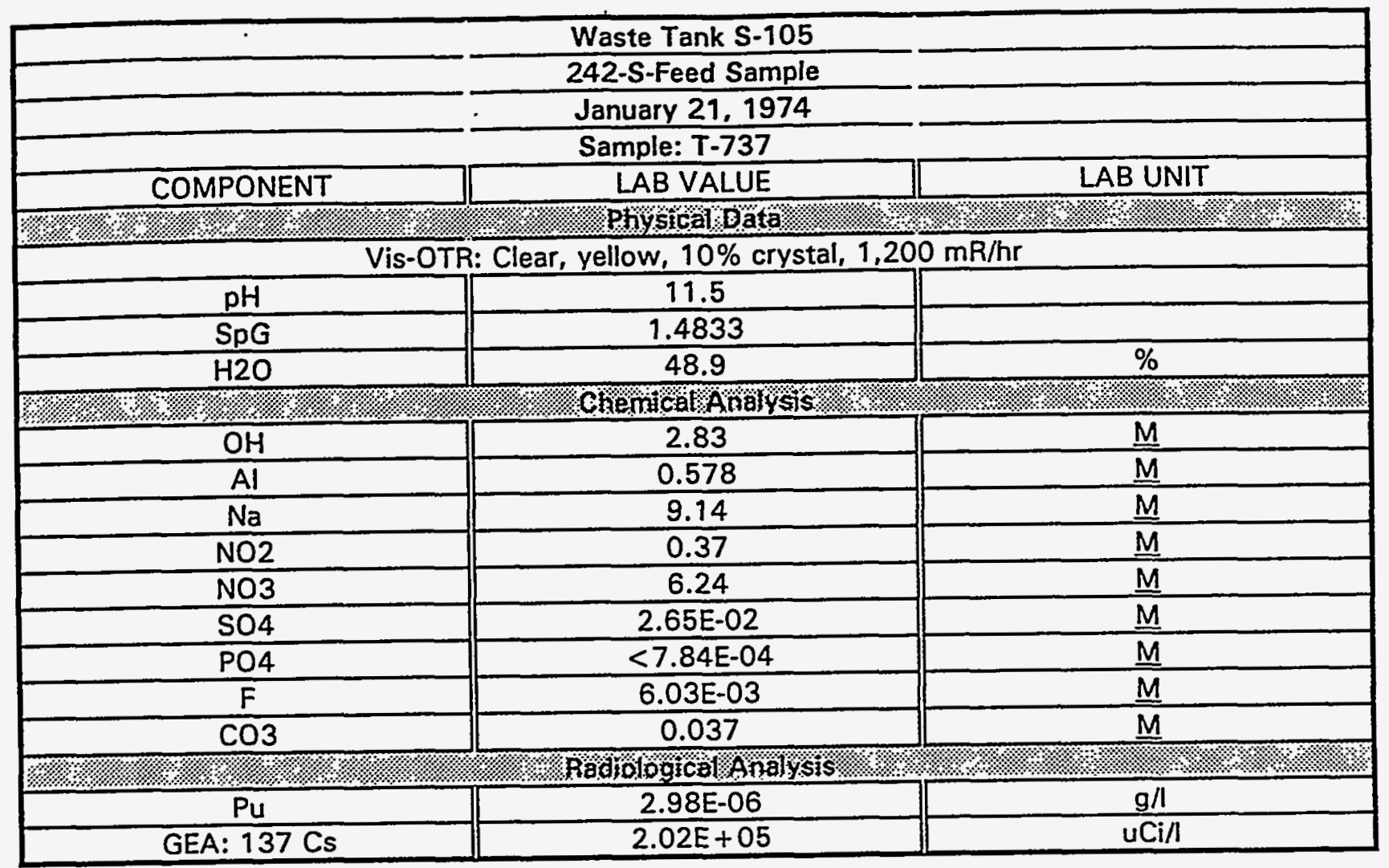


WHC-SD-WM-ER-323, Rev. 0

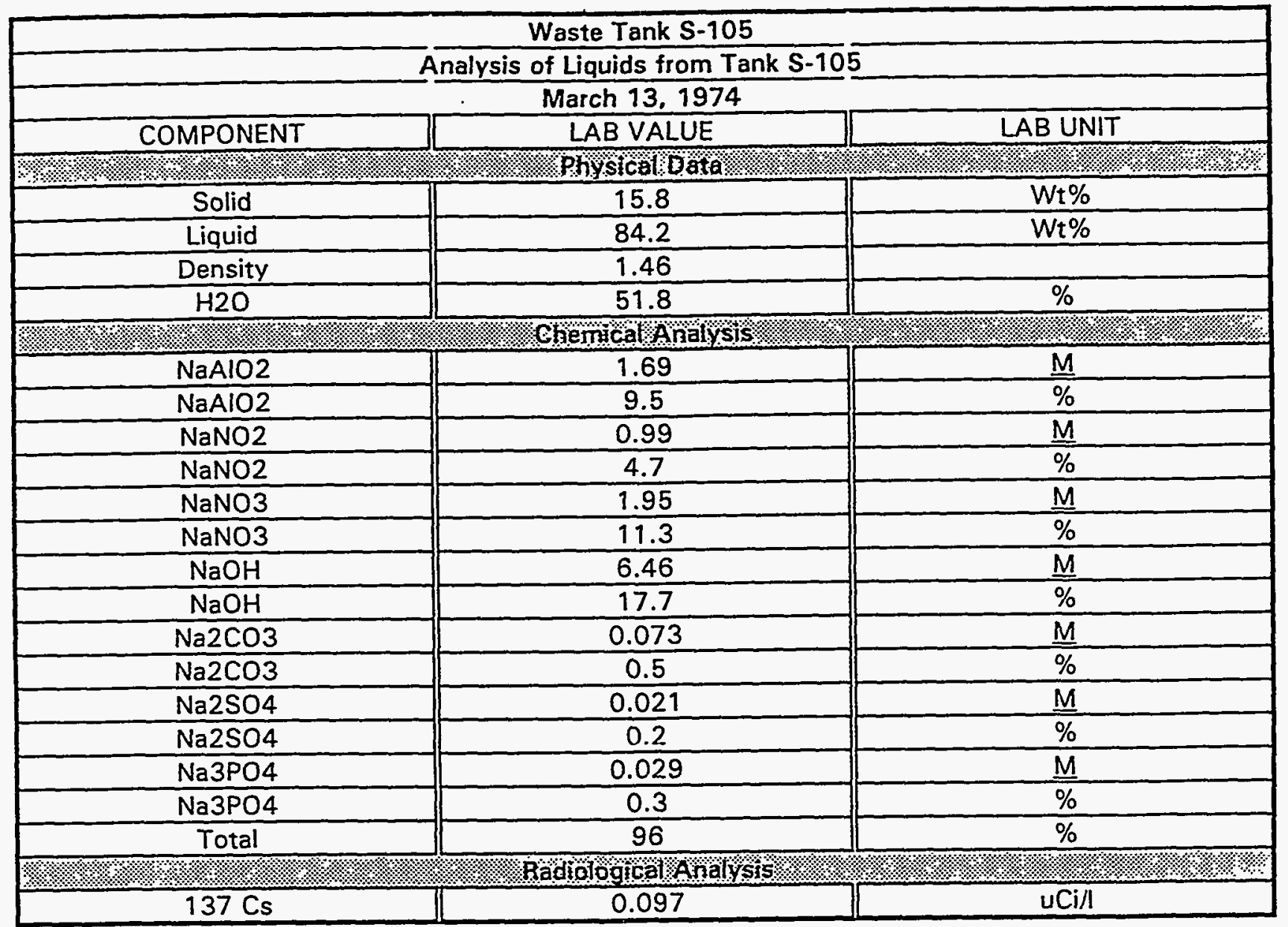




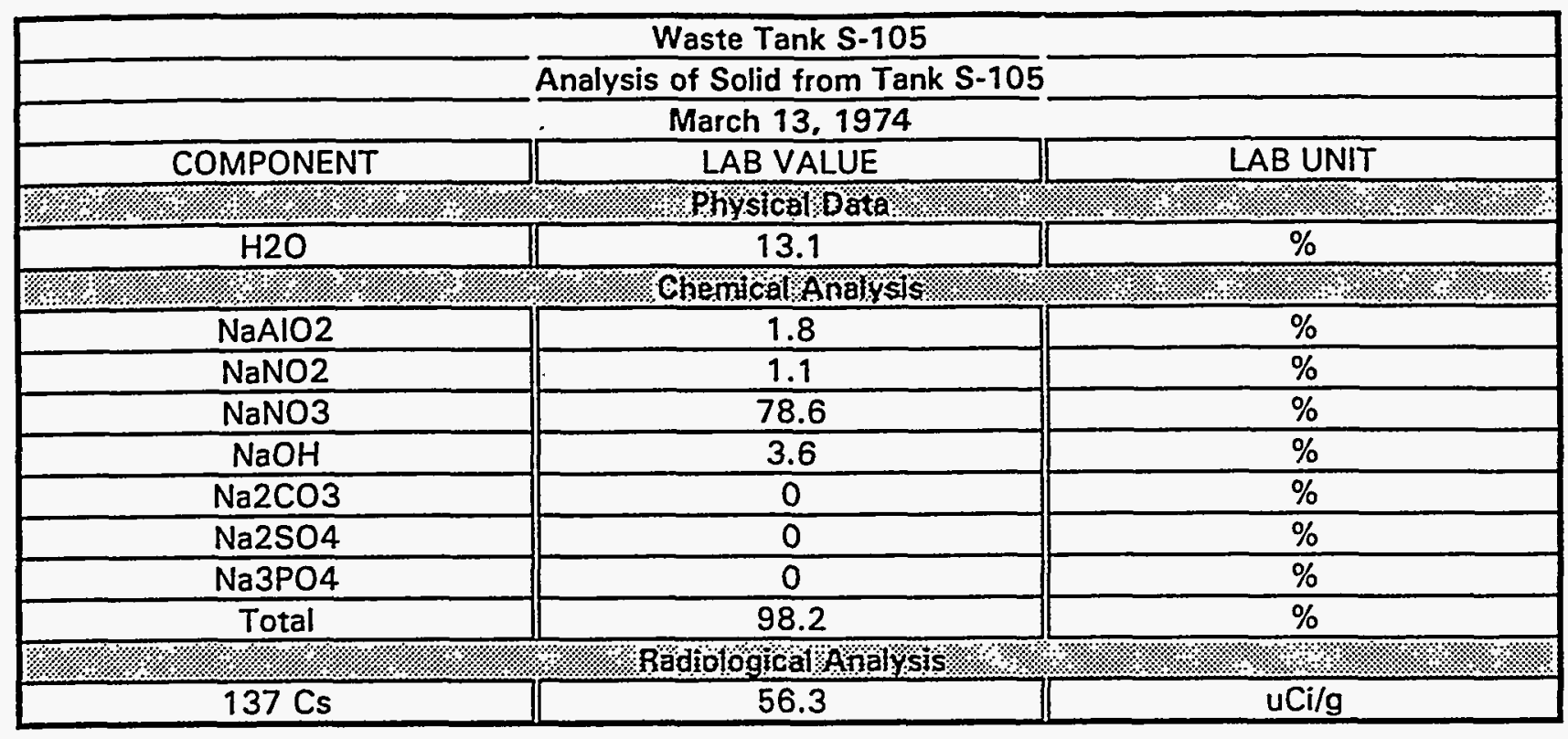




\begin{tabular}{|c|c|c|}
\hline \multicolumn{3}{|c|}{ Waste Tank S-105 } \\
\hline & Salt Cake Sample & \\
\hline & August 21, 1974 & \\
\hline COMPONENT & LAB VALUE & LAB UNIT \\
\hline স) & (ে. Physicaldatata & (4) \\
\hline Vis-OTR & Coarse yellow crystals, & very dry in appearance. \\
\hline As Received Density & 1.26 & \\
\hline Wet Density & 1.78 & \\
\hline NPH Density & 2.52 & \\
\hline 1) & Ghemacal Analysis & 1.8 .8 \\
\hline$\frac{\mathrm{H} 2 \mathrm{O}}{2}$ & 3.4 & $\%$ \\
\hline $\mathrm{NaAlO} 2$ & $1.34 \mathrm{E}-03$ & $\underline{M}$ \\
\hline $\mathrm{NaAlO} 2$ & 0.11 & $\%$ \\
\hline NaNO2 & $3.73 \mathrm{E}-03$ & $\underline{M}$ \\
\hline $\mathrm{NaNO} 2$ & 0.26 & $\%$ \\
\hline $\mathrm{NaOH}$ & $1.26 \mathrm{E}-02$ & $\underline{M}$ \\
\hline $\mathrm{NaOH}$ & 0.5 & $\%$ \\
\hline $\mathrm{Na} 2 \mathrm{CO} 3$ & $2.36 \mathrm{E}-02$ & $\underline{M}$ \\
\hline $\mathrm{Na} 2 \mathrm{CO} 3$ & 2.54 & $\%$ \\
\hline $\mathrm{NaNO} 3$ & 1.16 & $\underline{M}$ \\
\hline NaNO3 & 100.3 & $\ddot{\%}$ \\
\hline Total & 107.1 & $\%$ \\
\hline (6. & 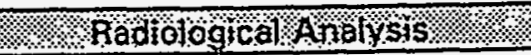 & \% \\
\hline $137 \mathrm{Cs}$ & $8.76 \mathrm{E}+02$ & $\mathrm{uCi} / \mathrm{I}$ \\
\hline $137 \mathrm{Cs}$ & 8.9 & $\mathrm{uCi} / \mathrm{g}$ \\
\hline $125 \mathrm{Sb}$ & 2.2 & $\mathrm{uCi} / \mathrm{l}$ \\
\hline $125 \mathrm{Sb}$ & 0.02 & $\mathrm{uCi} / \mathrm{g}$ \\
\hline
\end{tabular}




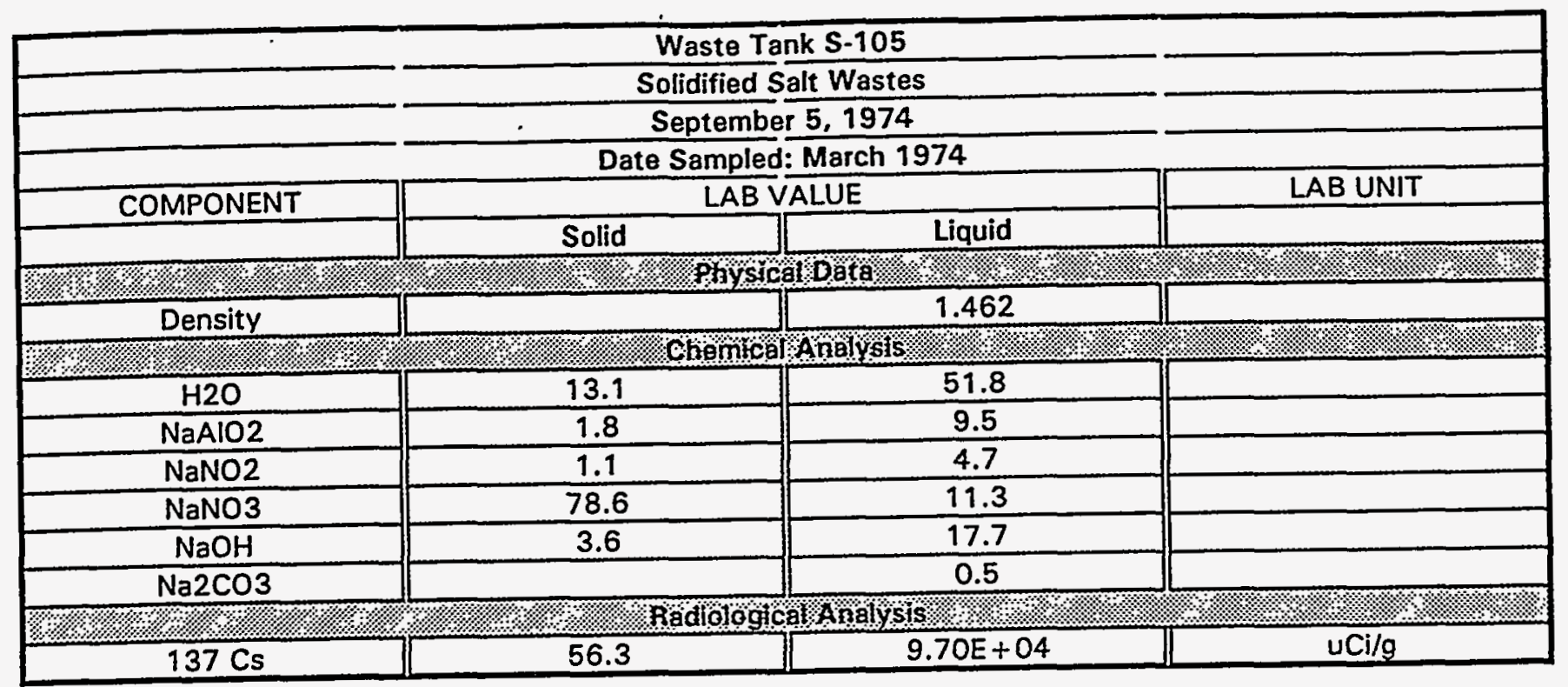


WHC-SD-WM-ER-323, Rev. O

\begin{tabular}{|c|c|c|}
\hline \multicolumn{3}{|c|}{ Waste Tank S-105 } \\
\hline \multicolumn{3}{|c|}{ Solidified Salt Wastes } \\
\hline \multicolumn{3}{|c|}{ September 5,1974} \\
\hline \multicolumn{3}{|c|}{ Date Sample:July 26, 1974} \\
\hline COMPONENT & LAB VALUE & LAB UNIT \\
\hline (6) & PSitystcal Date & 7) \\
\hline Bulb Density & 1.261 & \\
\hline Particle Density & 2.521 & \\
\hline (4) & Ghentcal Analysis. & \% \\
\hline $\mathrm{H} 2 \mathrm{O}$ & 3.4 & $\%$ \\
\hline $\mathrm{NaAlO2}$ & 0.1 & $\%$ \\
\hline $\mathrm{NaNO} 2$ & 0.3 & $\%$ \\
\hline NaNO3 & 100.3 & $\%$ \\
\hline $\mathrm{NaOH}$ & 0.5 & $\%$ \\
\hline $\mathrm{Na} 2 \mathrm{CO} 3$ & 2.5 & $\%$ \\
\hline \% & Baddological Analysis & 18.1 .2 .8 .8 .8 \\
\hline $137 \mathrm{Cs}$ & 8.9 & $\mathrm{uCi} / \mathrm{g}$ \\
\hline
\end{tabular}


WHC-SD-WM-ER-323, Rev. 0

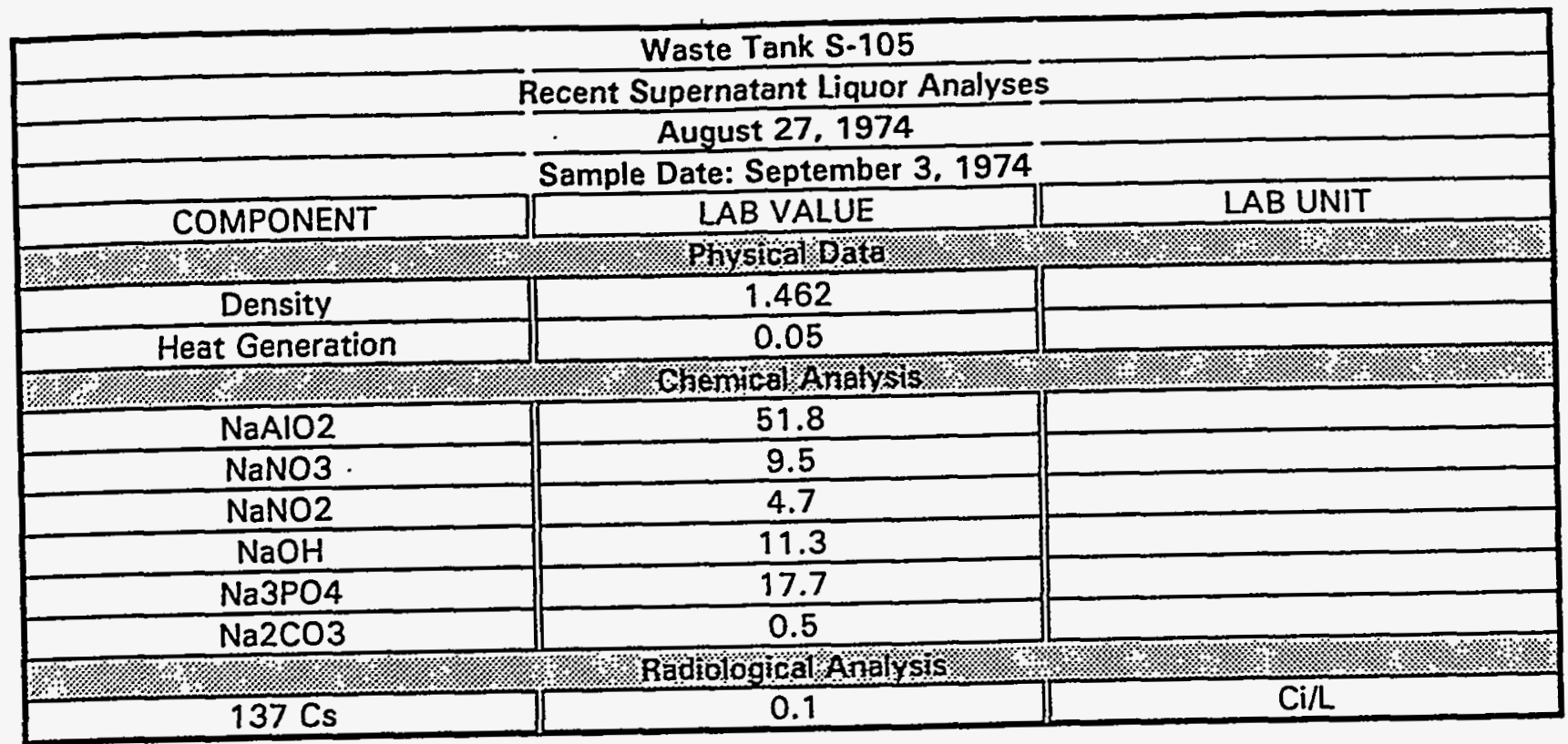




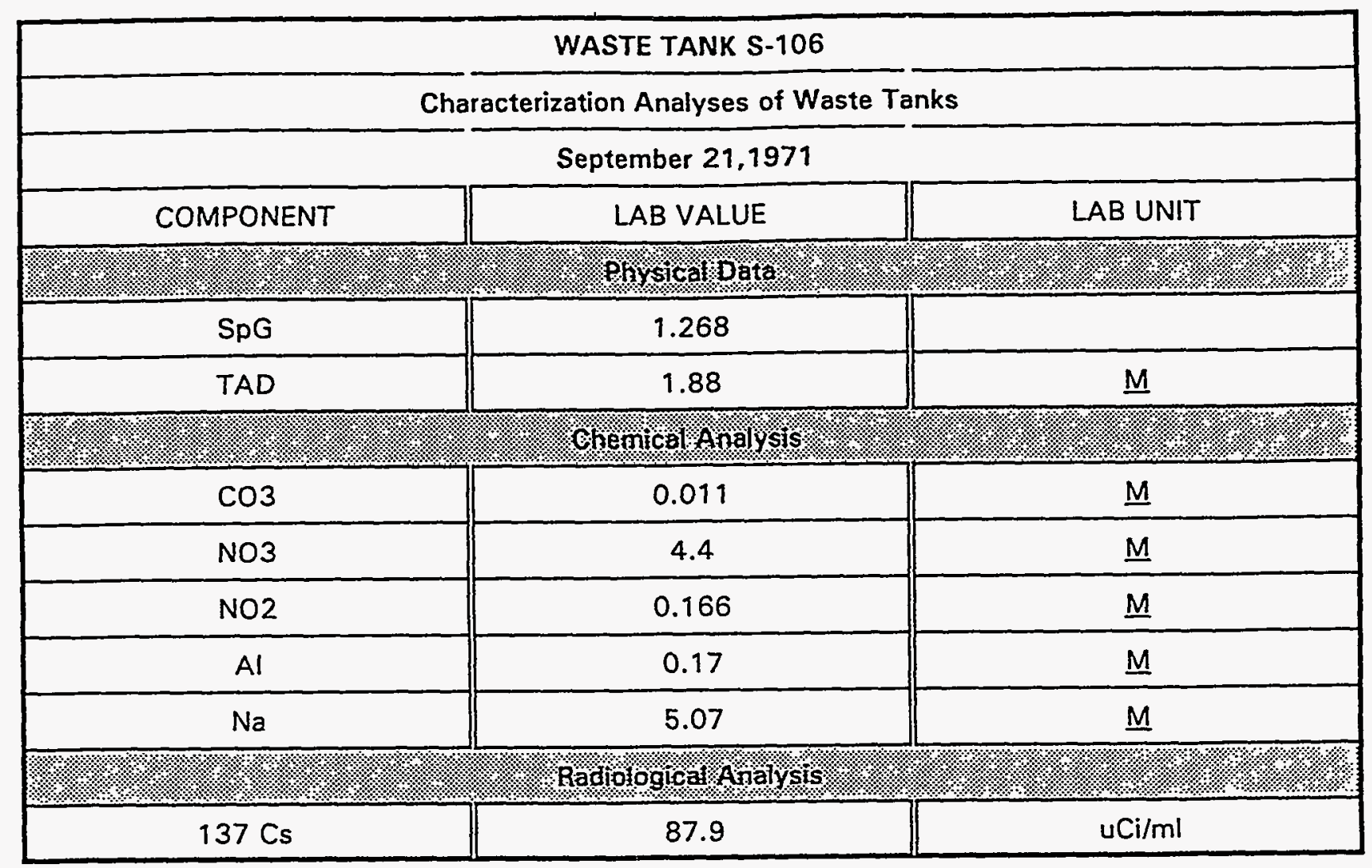




\begin{tabular}{|c|c|c|}
\hline \multicolumn{3}{|c|}{ WASTE TANK S-106 } \\
\hline & Analysis of 106-S Salt & \\
\hline \multicolumn{3}{|c|}{ September 21,1971} \\
\hline COMPONENT & LAB VALUE & LAB UNIT \\
\hline 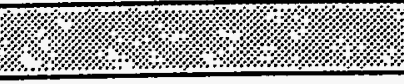 & $1 \%$ Physicalpota. & \%. \\
\hline Bulk Density & 1.27 & g/cc \\
\hline $\mathrm{H} 2 \mathrm{O}$ & 4.2 & $\%$ \\
\hline \%) & Chenricelaralysis. & (2.:- \\
\hline $\mathrm{NaAlO} 2$ & 0.6 & $\%$ \\
\hline $\mathrm{NaNO} 2$ & $<0.1$ & $\%$ \\
\hline $\mathrm{NaNO} 3$ & 90.1 & $\%$ \\
\hline $\mathrm{NaOH}$ & 1 & $\%$ \\
\hline $\mathrm{Na} 2 \mathrm{CO} 3$ & 4.1 & $\%$ \\
\hline $\mathrm{Na} 3 \mathrm{PO} 4$ & $\cdots$ & $\%$ \\
\hline TOTAL & 100 & $\%$ \\
\hline $1 \% 1 \%$ & Raglouglcal Anulysis. & 4 (4.) \\
\hline $137 \mathrm{Cs}$ & 17.2 & $u \mathrm{Ci} / \mathrm{g}$ \\
\hline $89+90 \mathrm{Sr}$ & $\cdots$ & $\mathrm{uCi} / \mathrm{g}$ \\
\hline
\end{tabular}


WHC-SD-WM-ER-323, Rev. 0

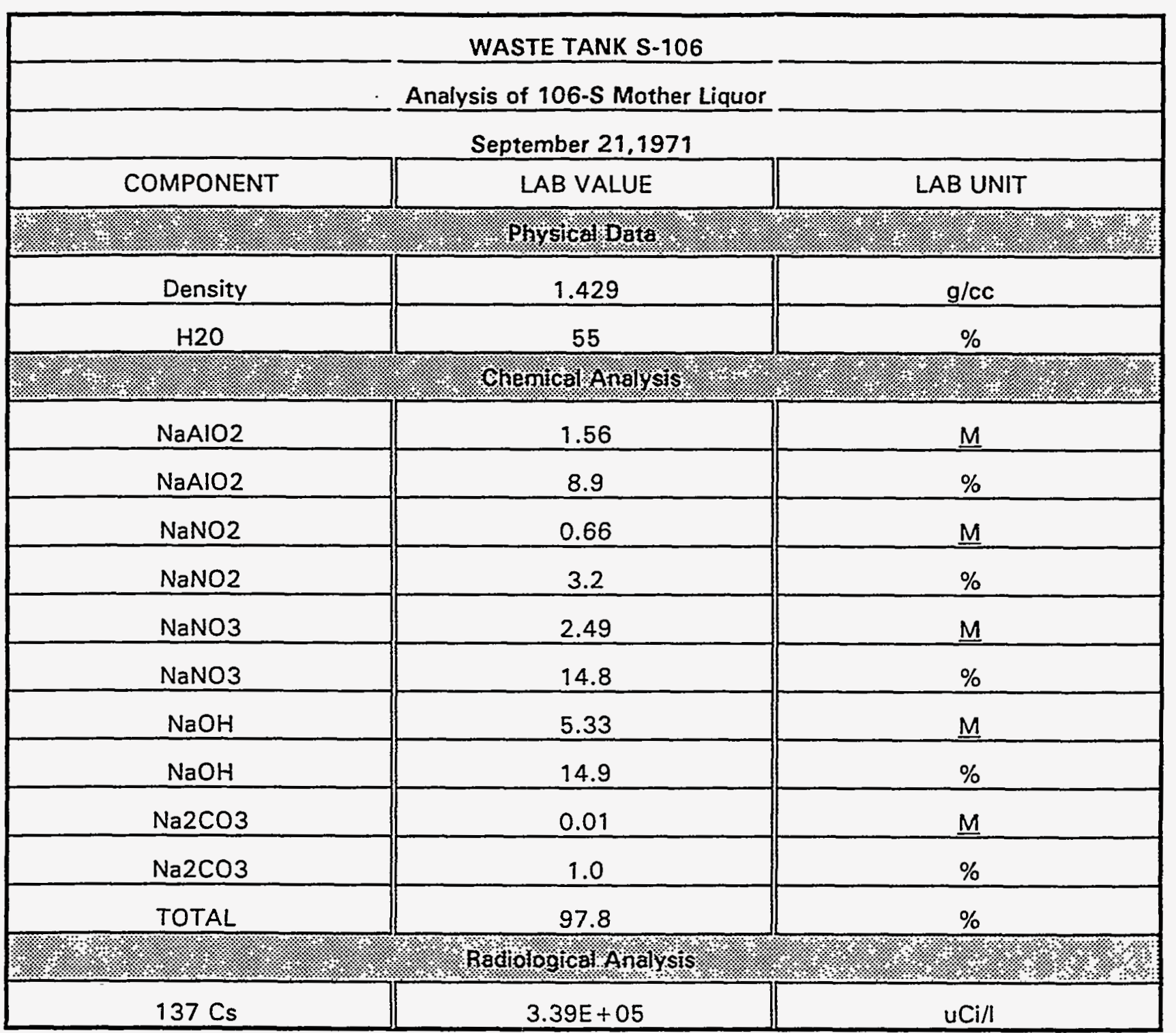




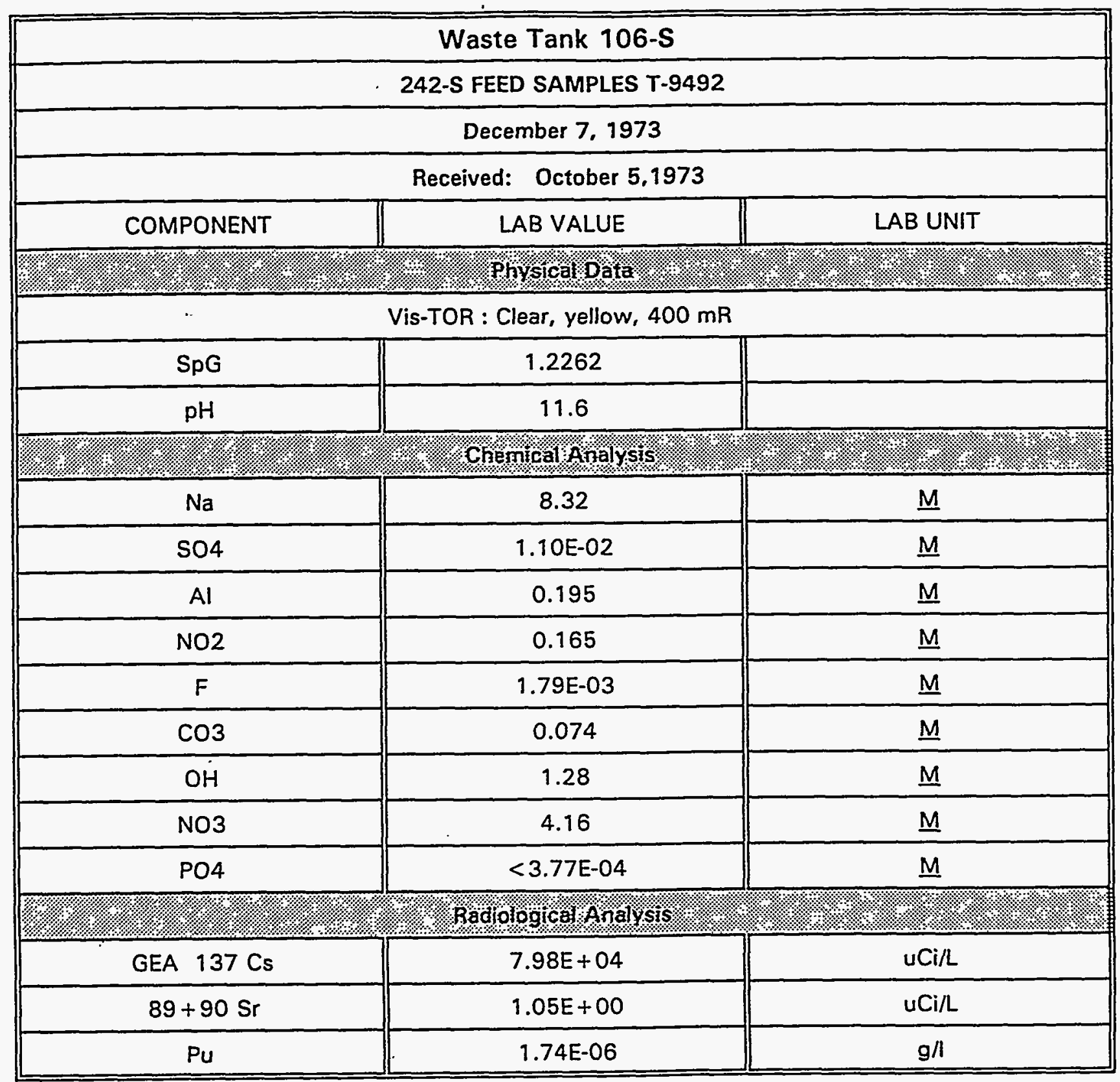


WHC-SD-WM-ER-323, Rev. O

\begin{tabular}{|c|c|c|}
\hline \multicolumn{3}{|c|}{ Waste Tank 106-S } \\
\hline \multicolumn{3}{|c|}{ 242-S Feed Samples } \\
\hline \multicolumn{3}{|c|}{ January 21, 1974} \\
\hline \multicolumn{3}{|c|}{ Sample: T-738 } \\
\hline COMPONENT & LAB VALUE & LAB UNIT \\
\hline 1. & Physical pata & 4.1. \\
\hline \multicolumn{3}{|c|}{ Vis-OTR : Clear, yellow, 10\% Solids, 500 mRAD/Hr.@2" } \\
\hline $\mathrm{pH}$ & 10.7 & \\
\hline SpG & 1.49125 & \\
\hline $\mathrm{H} 2 \mathrm{O}$ & 49.6 & $\%$ \\
\hline \multicolumn{3}{|c|}{ Gheminal Analysis } \\
\hline $\mathrm{Na}$ & 10.94 & $\underline{M}$ \\
\hline SO4 & $1.51 \mathrm{E}-02$ & $\underline{M}$ \\
\hline Al & 0.758 & $\underline{M}$ \\
\hline NO2 & 0.296 & $\underline{M}$ \\
\hline $\mathrm{F}$ & $2.55 \mathrm{E}-03$ & $\underline{M}$ \\
\hline $\mathrm{CO} 3$ & 0.037 & $\underline{M}$ \\
\hline $\mathrm{OH}$ & 2.86 & $\underline{M}$ \\
\hline NO3 & 5.66 & $\underline{M}$ \\
\hline PO4 & $<7.87$ E-04 & $\underline{M}$ \\
\hline $\mathrm{Pu}$ & $4.28 \mathrm{E}-06$ & $g / l$ \\
\hline (1.1.: & ologicat Analy & ४ै. \\
\hline GEA $137 \mathrm{Cs}$ & $2.17 E+05$ & $u C i / L$ \\
\hline $89+90 \mathrm{Sr}$ & Vot Requested & $u C i / L$ \\
\hline
\end{tabular}




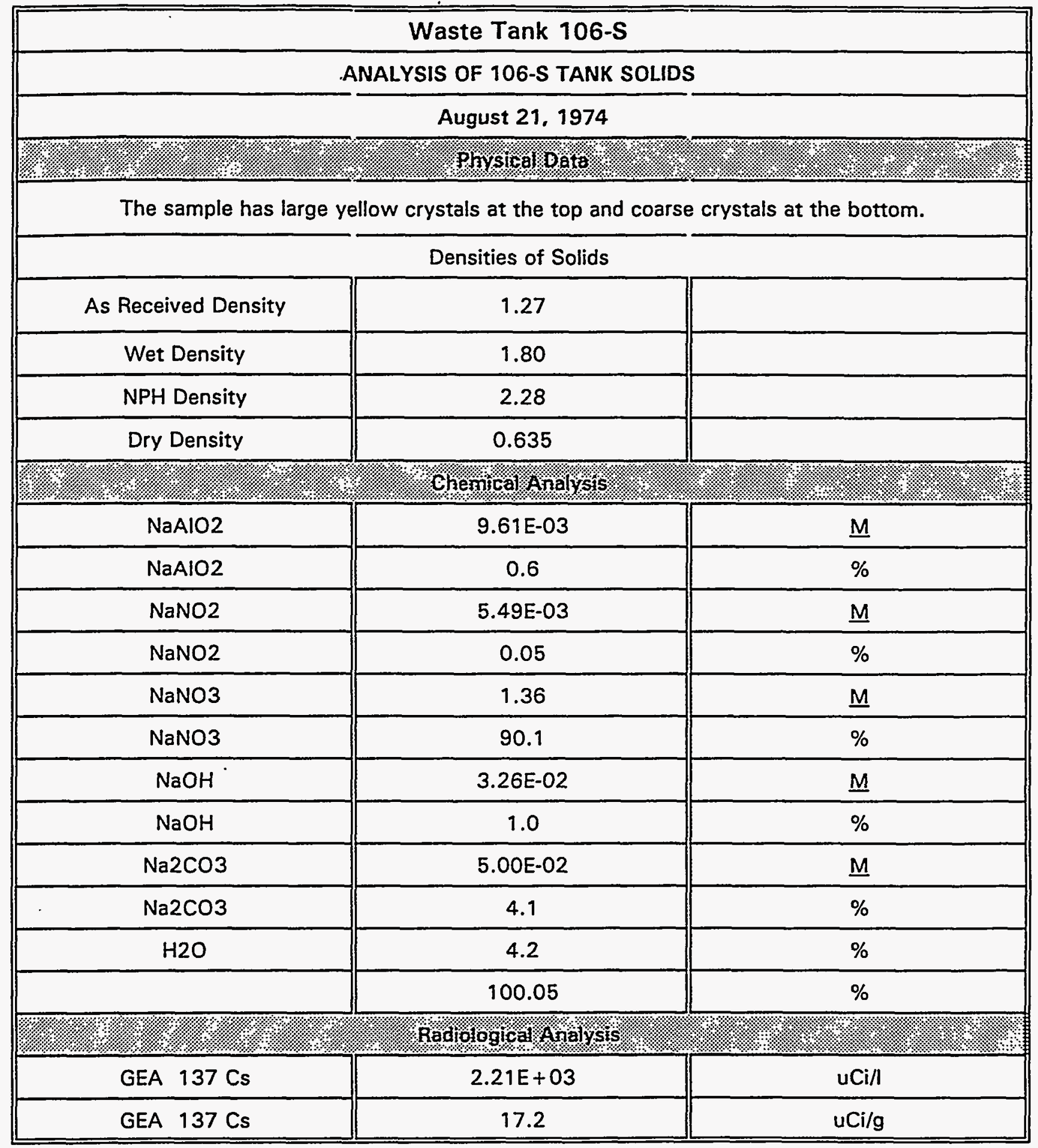


WHC-SD-WM-ER-323, Rev. 0

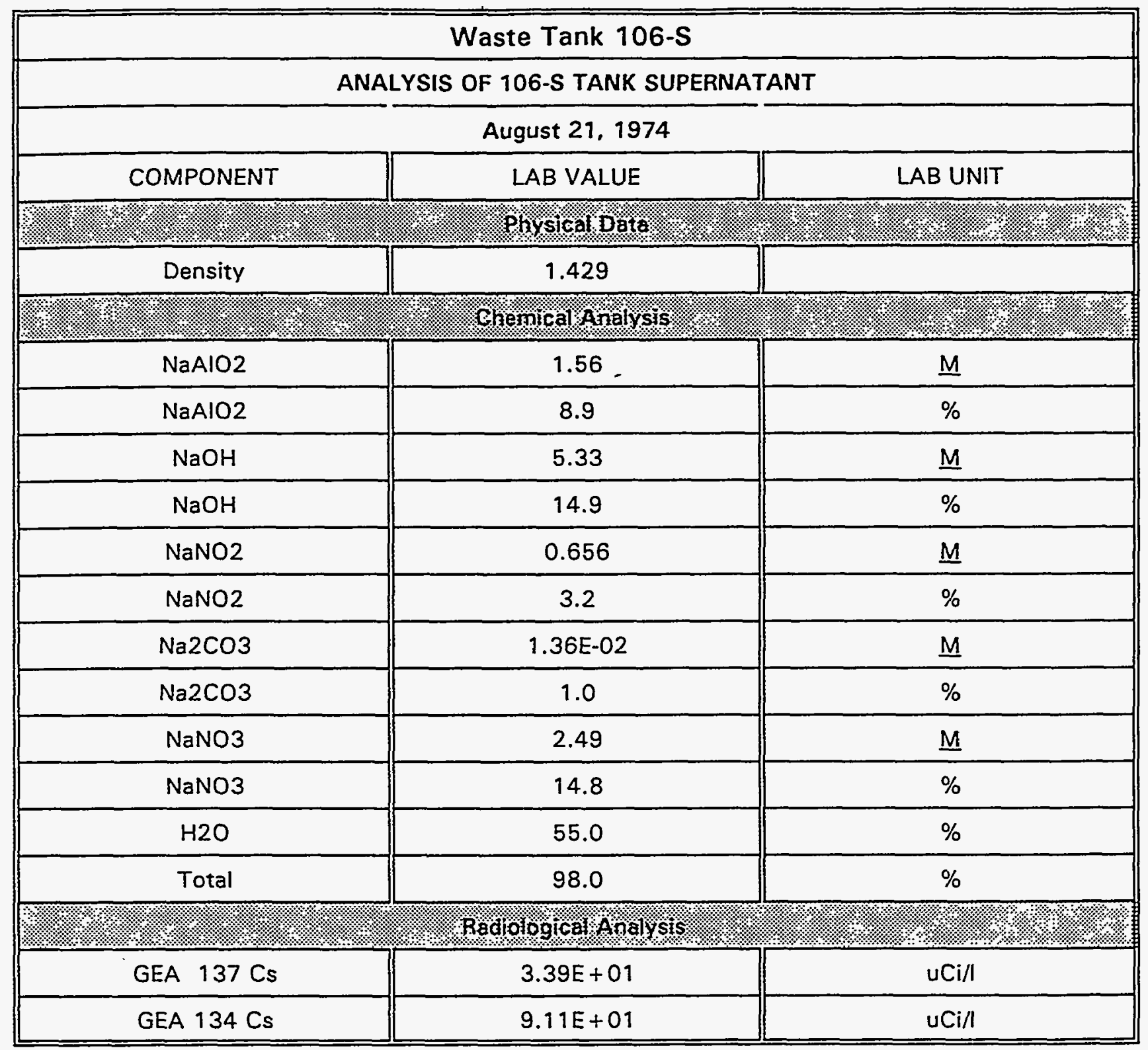


WHC-SD-WM-ER-323, Rev. 0

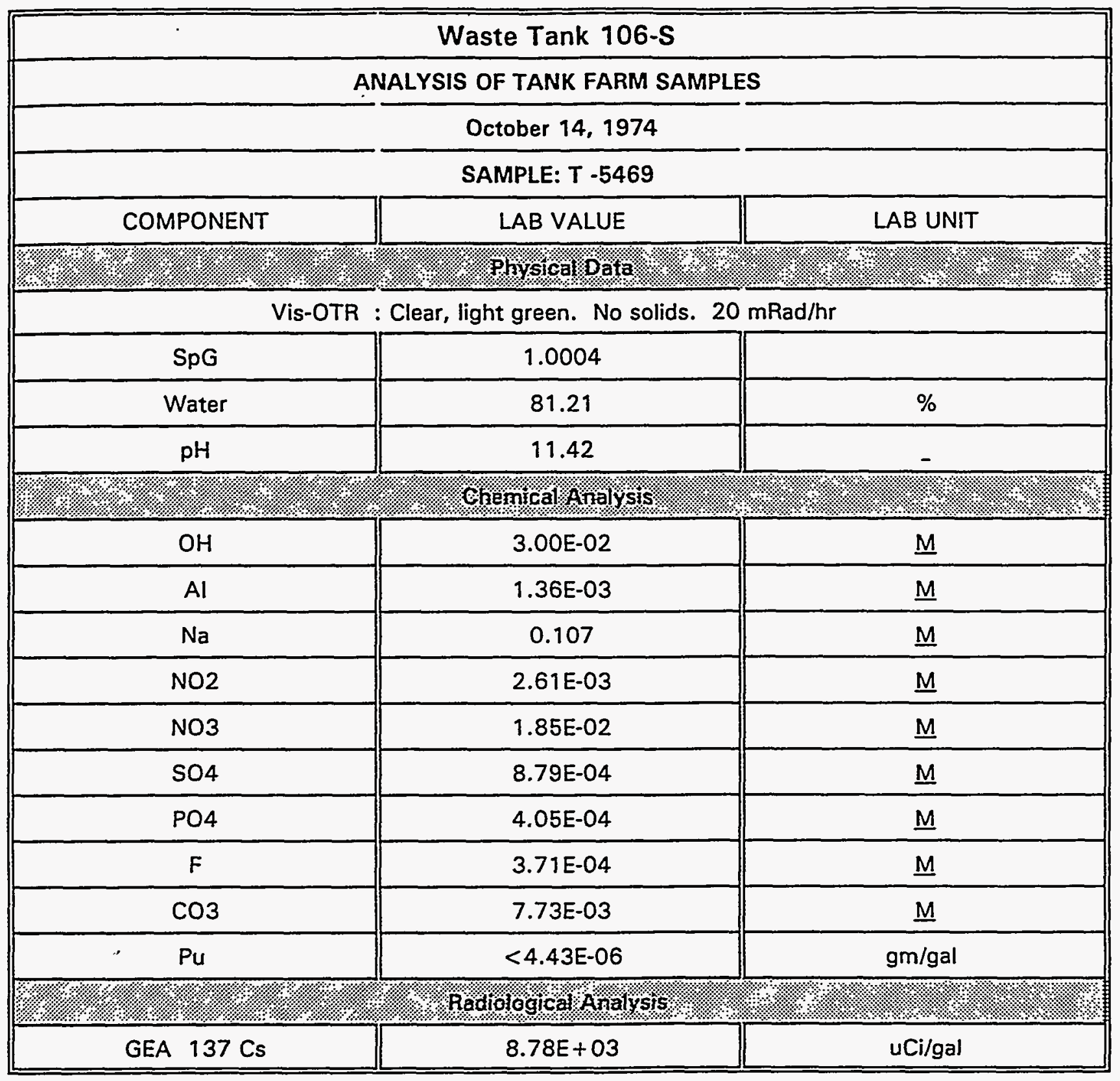




\begin{tabular}{|c|c|c|}
\hline \multicolumn{3}{|c|}{ Waste Tank 106-S } \\
\hline \multicolumn{3}{|c|}{ ANALYSIS OF TANK FARM SAMPLES } \\
\hline \multicolumn{3}{|c|}{ December 16, 1974} \\
\hline \multicolumn{3}{|c|}{ SAMPLE: T-8035 } \\
\hline COMPONENT & LAB VALUE & LAB UNIT \\
\hline \multicolumn{3}{|c|}{ 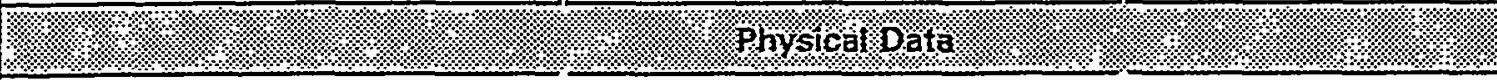 } \\
\hline \multicolumn{3}{|c|}{ Vis-ORT : Clear yellow, $50 \%$ solids. 2 Rads. } \\
\hline $\mathrm{pH}$ & 14 & - \\
\hline SpG & 1.5414 & \\
\hline Water & 46.62 & $\%$ \\
\hline \multicolumn{3}{|c|}{ m: } \\
\hline $\mathrm{OH}$ & 5.20 & $\underline{M}$ \\
\hline Al & 1.45 & $\underline{\mathrm{M}}$ \\
\hline $\mathrm{Na}$ & 14.20 & $\underline{M}$ \\
\hline NO2 & 1.02 & $\underline{M}$ \\
\hline NO3 & 2.90 & $\underline{M}$ \\
\hline $\mathrm{SO} 4$ & \multicolumn{2}{|c|}{ Cancelled by R. L. Walser } \\
\hline PO4 & $5.10 \mathrm{E}-02$ & $\underline{M}$ \\
\hline F & $7.49 \mathrm{E}-04$ & $\underline{M}$ \\
\hline $\mathrm{CO}$ & 0.145 & _ \\
\hline K & 2.77E-02 & $\underline{M}$ \\
\hline $\mathrm{Pu}$ & $1.90 E-05$ & $\mathrm{~g} / \mathrm{gal}$ \\
\hline \multicolumn{3}{|c|}{ (1) } \\
\hline GEA $137 \mathrm{Cs}$ & $1.44 E+06$ & uCi/gal \\
\hline GEA $134 \mathrm{Cs}$ & $3.94 E+02$ & uCi/gal \\
\hline $89,90 \mathrm{Sr}$ & 78.80 & uCi/gal \\
\hline
\end{tabular}


WHC-SD-WM-ER-323, Rev. 0

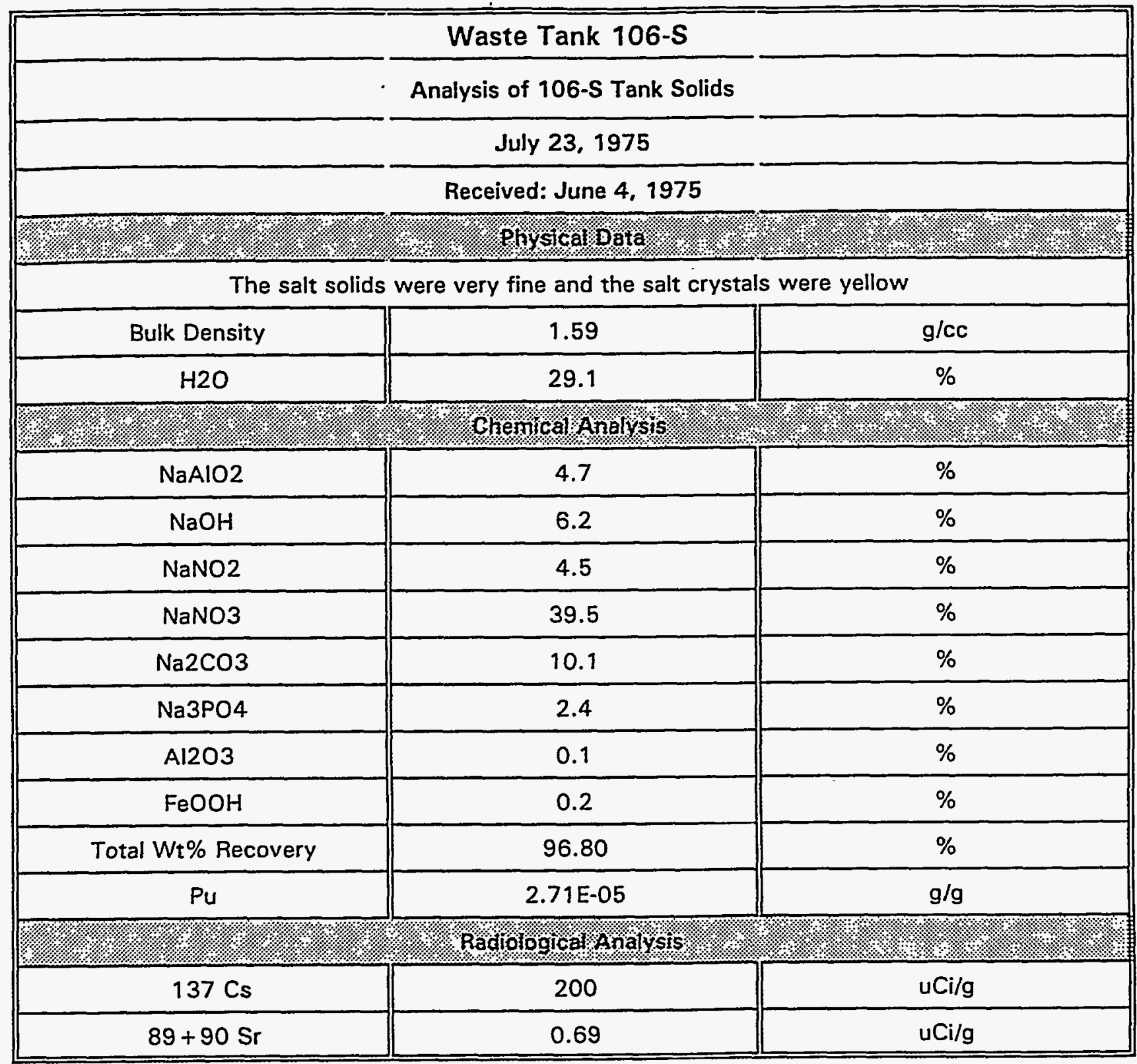


WHC-SD-WM-ER-323, Rev. 0

\begin{tabular}{|c|c|c|}
\hline \multicolumn{3}{|c|}{ Waste Tank 106-S } \\
\hline \multicolumn{3}{|c|}{ Analysis of 106-S Supernatant liquid } \\
\hline \multicolumn{3}{|c|}{ July 23,1975} \\
\hline \multicolumn{3}{|c|}{ Received: June 4, 1975} \\
\hline COMPONENT & LAB VALUE & LAB UNIT \\
\hline & Hysicaitoats & ?. \\
\hline Density & 1.45 & \\
\hline $\mathrm{H} 2 \mathrm{O}$ & 50.0 & $\%$ \\
\hline \multicolumn{3}{|c|}{ Chemical Analy sis? } \\
\hline $\mathrm{NaNO} 2$ & 1.78 & $M$ \\
\hline $\mathrm{NaNO} 2$ & 8.5 & $\%$ \\
\hline $\mathrm{NaAlO} 2$ & 1.85 & $M$ \\
\hline $\mathrm{NaAlO} 2$ & 10.5 & $\%$ \\
\hline NaNO3 & 2.42 & $M$ \\
\hline $\mathrm{NaNO} 3$ & 14.2 & $\%$ \\
\hline $\mathrm{NaOH}$ & 4.44 & $M$ \\
\hline $\mathrm{NaOH}$ & 12.2 & $\%$ \\
\hline $\mathrm{Na} 2 \mathrm{CO} 3$ & 0.27 & $M$ \\
\hline $\mathrm{Na} 2 \mathrm{CO} 3$ & 1.9 & $\%$ \\
\hline $\mathrm{Na} 3 \mathrm{PO}_{4}$ & 0.028 & M \\
\hline $\mathrm{Na} 3 \mathrm{PO} 4$ & 0.3 & $\%$ \\
\hline Total Wt\% Recovery & 98.3 & $\%$ \\
\hline $\mathrm{Pu}$ & $1.17 E-06$ & $g / 1$ \\
\hline & foglical Anol & \\
\hline $137 \mathrm{Cs}$ & $5.29 E+05$ & UCi/L \\
\hline $89+90 \mathrm{Sr}$ & $1.22 E+03$ & uCi/L \\
\hline $134 \mathrm{Cs}$ & $2.02 E+03$ & $\mathrm{uCi} / \mathrm{L}$ \\
\hline
\end{tabular}


WHC-SD-WM-ER-323, Rev. 0

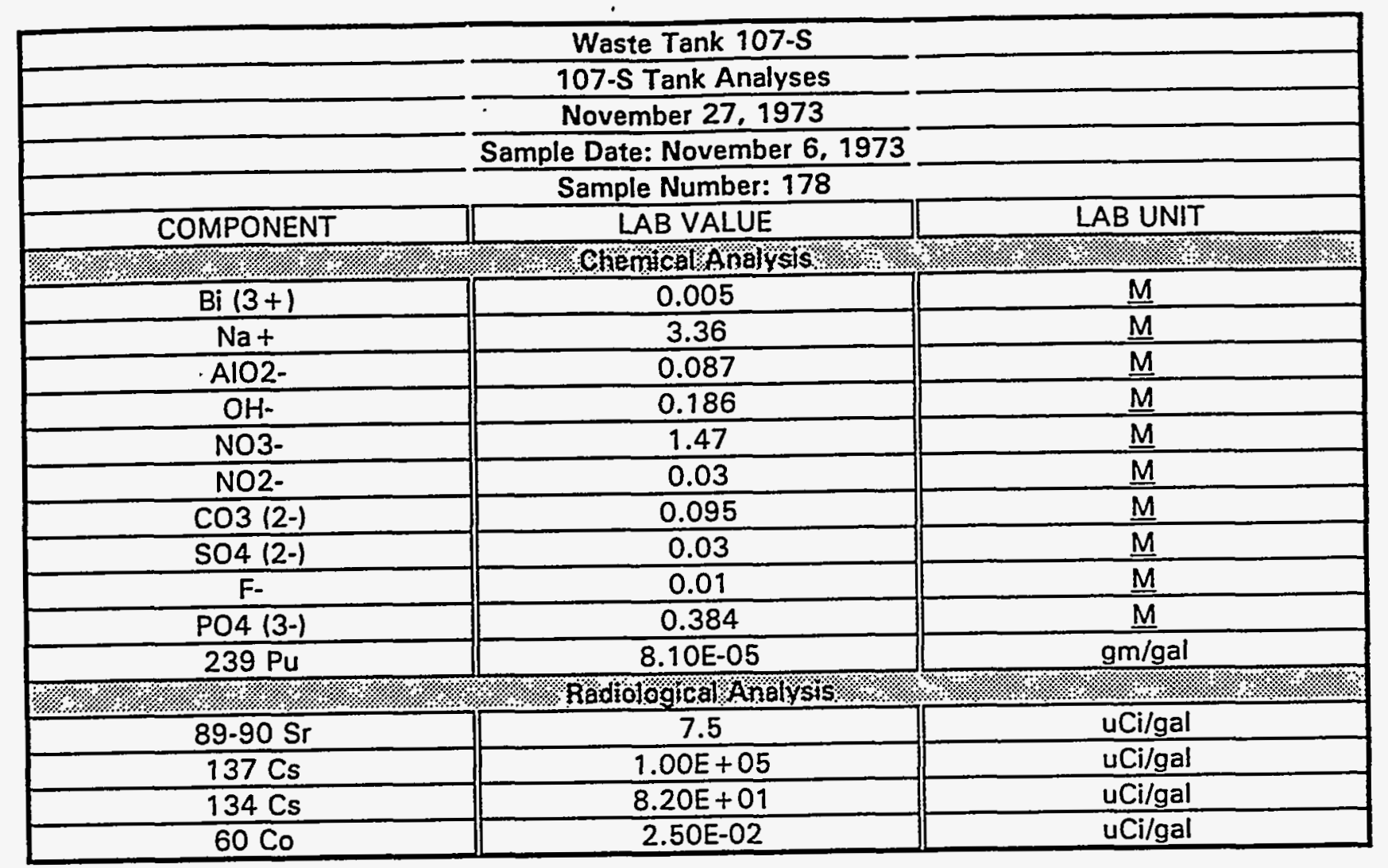


WHC-SD-WM-ER-323, Rev. O

\begin{tabular}{|c|c|c|}
\hline \multicolumn{3}{|c|}{ Waste Tank 107-S } \\
\hline & 107-S Tank Analyses & \\
\hline \multicolumn{3}{|c|}{$\begin{array}{l}\text { 107-S Tank Analyses } \\
\text { November 27, } 1973 \\
\end{array}$} \\
\hline & Sample Date: November 17,1973 & \\
\hline \multicolumn{3}{|c|}{ Sample Number: 476} \\
\hline COMPONENT & LAB VALUE & LAB UNIT \\
\hline Y. & 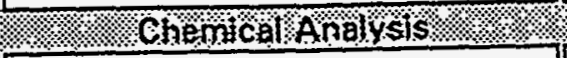 & 4, \\
\hline $\mathrm{Bi}(3+)$ & $<0.005$ & $\underline{M}$ \\
\hline $\mathrm{Na}+$ & 3.39 & $\underline{M}$ \\
\hline AlO2- & 0.091 & $\underline{\underline{M}}$ \\
\hline $\mathrm{OH}-$ & 0.292 & $\underline{M}$ \\
\hline NO3- & 1.73 & $\underline{M}$ \\
\hline NO2- & 0.024 & $\underline{M}$ \\
\hline $\cos (2-)$ & 0.099 & $\underline{M}$ \\
\hline SO4 (2-1 & 0.022 & $M$ \\
\hline $\mathrm{F}-$ & 0.01 & $\underline{M}$ \\
\hline $\mathrm{PO4}(3)$ & 0.53 & $\underline{M}$ \\
\hline $239 \mathrm{Pu}$ & $1.07 \mathrm{E}-06$ & $\mathrm{gm} / \mathrm{gal}$ \\
\hline 19.6. & 1. & (4) \\
\hline $89-90 \mathrm{Sr}$ & $4.40 E+01$ & uCi/gal \\
\hline $137 \mathrm{Cs}$ & $8.60 E+04$ & uCi/gal \\
\hline $134 \mathrm{Cs}$ & $1.50 E+02$ & uCi/gal \\
\hline $60 \mathrm{Co}$ & $1.90 E+02$ & uCi/gal \\
\hline
\end{tabular}


WHC-SD-WM-ER-323, Rev. O

\begin{tabular}{|c|c|c|}
\hline \multicolumn{3}{|c|}{ Waste Tank S-107 } \\
\hline \multicolumn{3}{|c|}{$242-S$-Feed Samples (Supernate)T-178 } \\
\hline \multicolumn{3}{|c|}{ December 7,1973 } \\
\hline \multicolumn{3}{|c|}{ Date Sample: November 6, 1973} \\
\hline COMPONENT & \begin{tabular}{|c|} 
LAB VALUE \\
\end{tabular} & LAB UNIT \\
\hline \%া & (1) Physacallata & 1. \\
\hline \multicolumn{3}{|c|}{ Vis-OTR: Yellow, $50 \%$ crystals, $180 \mathrm{mR} / \mathrm{hr}$} \\
\hline $\mathrm{pH}$ & \begin{tabular}{|c|}
12.2 \\
\end{tabular} & \\
\hline SpG & 1.1071 & \\
\hline \% & Chamical Andysis & \%. \\
\hline $\mathrm{Na}$ & 2.55 & $\underline{M}$ \\
\hline SO4 & $2.96 \mathrm{E}-02$ & $\underline{M}$ \\
\hline $\mathrm{Al}$ & 0.105 & $\underline{\underline{M}}$ \\
\hline NO2 & $2.70 \mathrm{E}-02$ & $\underline{\mathrm{M}}$ \\
\hline $\mathrm{Fe}$ & $2.26 \mathrm{E}-04$ & $\underline{M}$ \\
\hline $\mathrm{CO3}$ & $9.40 \mathrm{E}-02$ & $\underline{\underline{M}}$ \\
\hline $\mathrm{OH}$ & 0.186 & $\underline{M}$ \\
\hline NO3 & 1.72 & $\underline{M}$ \\
\hline $\mathrm{Pu}$ & $1.43 \mathrm{E}-05$ & $\mathrm{~g} / \mathrm{h}$ \\
\hline : & Radiological Aralysis: & 1. \\
\hline GEA: 137 & $3.11 E+04$ & $\mathrm{uCi} / \mathrm{l}$ \\
\hline GEA: $134 \mathrm{Cs}$ & 31.86 & $\mathrm{uCi} / \mathrm{I}$ \\
\hline GEA: $60 \mathrm{Co}$ & 37.97 & $\mathrm{uCi} / \mathrm{l}$ \\
\hline $89+90 \mathrm{Sr}$ & 6.156 & $\mathrm{uCi} / \mathrm{l}$ \\
\hline
\end{tabular}


WHC-SD-WM-ER-323, Rev. O

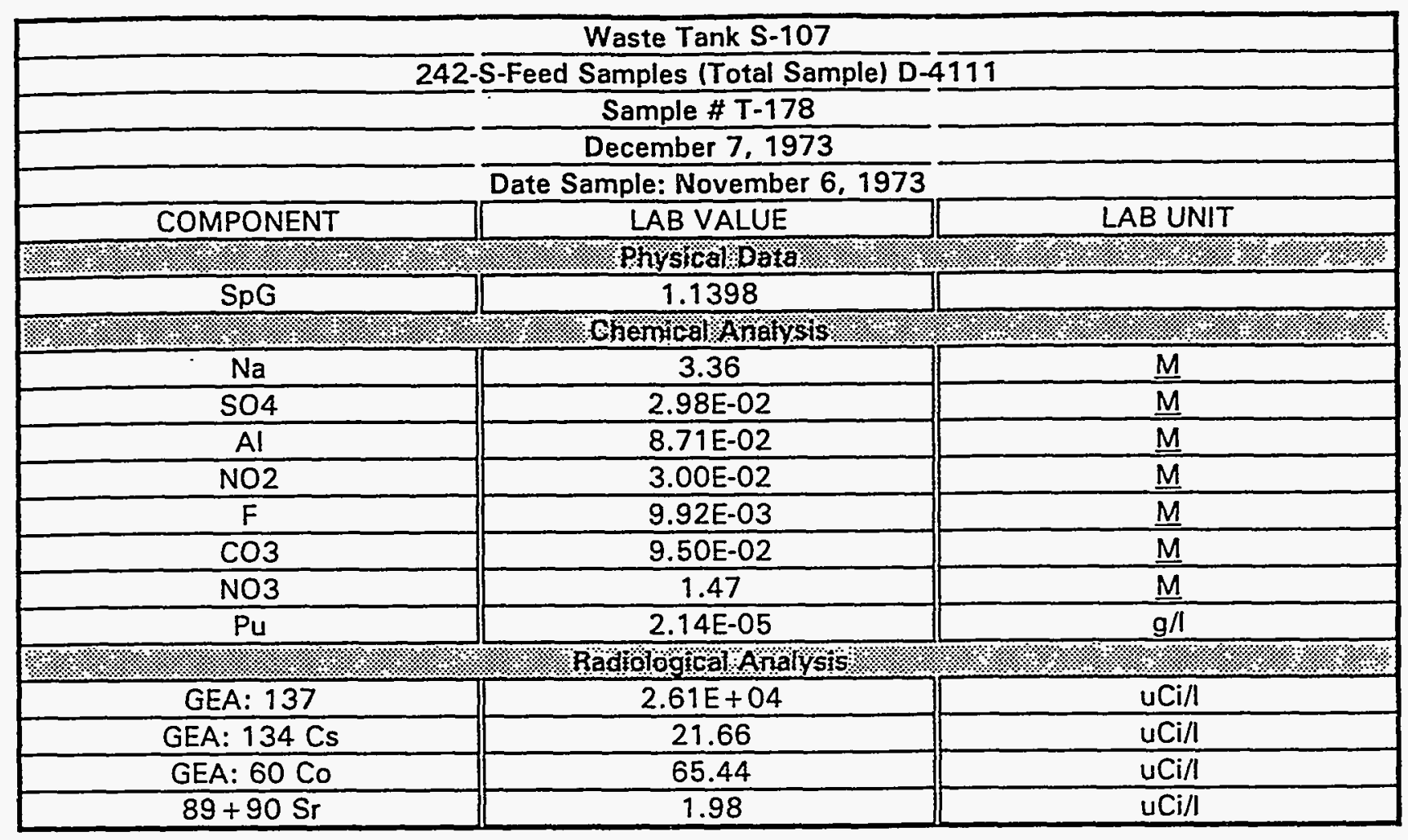




\begin{tabular}{|c|c|c|}
\hline \multicolumn{3}{|c|}{ Waste Tank 107-S } \\
\hline & 242-S Feed Sample T-476 & \\
\hline \multicolumn{3}{|c|}{ December 7, 1973} \\
\hline \multicolumn{3}{|c|}{ Received: November 16,1973} \\
\hline COMPONENT & LAB VALUE & LAB UNIT \\
\hline \% & 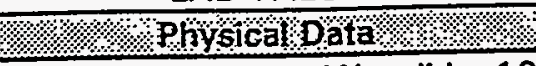 & (1.7. \\
\hline \multicolumn{3}{|c|}{ Vis-OTR: Yellow crystals, $75 \%$ solids, $100 \mathrm{mR} / \mathrm{hr}$} \\
\hline $\mathrm{pH}$ & $11.9 @ 40^{*} \mathrm{C}$ & \\
\hline SpG & 1.1592 & \\
\hline 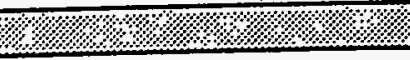 & / & (1). \\
\hline $\mathrm{Na}$ & 3.39 & $\underline{M}$ \\
\hline SO4 & $2.18 \mathrm{E}-02$ & $\underline{M}$ \\
\hline $\mathrm{Al}$ & $9.07 \mathrm{E}-02$ & $\underline{\underline{M}}$ \\
\hline $\mathrm{NO} 2$ & 0.024 & $\underline{M}$ \\
\hline$F$ & $1.03 \mathrm{E}-02$ & $\underline{M}$ \\
\hline $\mathrm{CO3}$ & 0.099 R.R. 0.090 & $\underline{M}$ \\
\hline $\mathrm{Cl}$ & Method being developed & $\underline{M}$ \\
\hline $\mathrm{OH}$ & 0.292 & $\underline{M}$ \\
\hline NO3 & 1.73 & $\underline{\mathrm{M}}$ \\
\hline $\mathrm{PO4}$ & 0.53 & $\underline{M}$ \\
\hline $\mathrm{Bi}$ & $<4.98 \mathrm{E}-03$ & $\underline{\underline{M}}$ \\
\hline $\mathrm{Pu}$ & $2.82 \mathrm{E}-05$ & $\mathrm{~g} / \mathrm{l}$ \\
\hline \%४\% & /Radiologicalshalysis: & \% \% \\
\hline GEA: $134 \mathrm{Cs}$ & 40.58 & $u \mathrm{Ci} / \mathrm{I}$ \\
\hline GEA: $137 \mathrm{Cs}$ & $2.28 \mathrm{E}+04$ & $\mathrm{uCi} / \mathrm{l}$ \\
\hline GEA: 60 Co & 50.05 & $\mathrm{uCi} / \mathrm{I}$ \\
\hline
\end{tabular}




\begin{tabular}{|c|c|c|}
\hline & Waste Tank S-107 & \\
\hline \multicolumn{3}{|c|}{ Analysis of Tank Farm Samples } \\
\hline \multicolumn{3}{|c|}{ December 17,1974} \\
\hline \multicolumn{3}{|c|}{ Sample: T-8078 } \\
\hline COMPONENT & LAB VALUE & LAB UNIT \\
\hline (1.6. & (1) Physicalobata & 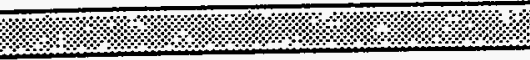 \\
\hline \multicolumn{3}{|c|}{ Vis-OTR: Yellow, No solids, $150 \mathrm{mRad} / \mathrm{hr}$} \\
\hline $\mathrm{pH}$ & 14 & \\
\hline SpG & 1.201 & \\
\hline $\mathrm{H} 2 \mathrm{O}$ & 76.65 & $\%$ \\
\hline ২) & 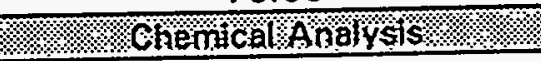 & (1./. \\
\hline $\mathrm{OH}$ & 101 & $\underline{\mathrm{M}}$ \\
\hline $\mathrm{Al}$ & 0.297 & $\underline{M}$ \\
\hline $\mathrm{Na}$ & 3.72 & $\underline{M}$ \\
\hline NO2 & 0.323 & $\underline{M}$ \\
\hline $\mathrm{NO3}$ & 1.82 & $\underline{M}$ \\
\hline SO4 & $3.14 \mathrm{E}-02$ & $\underline{M}$ \\
\hline PO4 & 0.106 & $\underline{\bar{M}}$ \\
\hline $\mathrm{F}$ & $8.36 \mathrm{E}-03$ & $\underline{\bar{M}}$ \\
\hline $\mathrm{co3}$ & 0.168 & $\underline{M}$ \\
\hline $\mathrm{Pu}$ & $1.78 \mathrm{E}-05$ & $\mathrm{~g} / \mathrm{gal}$ \\
\hline 16/2/, & 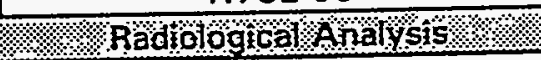 & 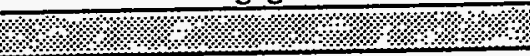 \\
\hline GEA: $134 \mathrm{Cs}$ & $3.35 E+03$ & uCi/gal \\
\hline GEA: $137 \mathrm{Cs}$ & $4.81 \mathrm{E}+05$ & uCi/gal \\
\hline GEA: $125 \mathrm{Sb}$ & $1.34 \mathrm{E}+02$ & $\mathrm{uCi} / \mathrm{gal}$ \\
\hline GEA: $60 \mathrm{Co}$ & 54.54 & $u \mathrm{Ci} / \mathrm{gal}$ \\
\hline $89,90 \mathrm{Sr}$ & $6.253 E+03$ & uCi/gal \\
\hline
\end{tabular}


WHC-SD-WM-ER-323, Rev. 0

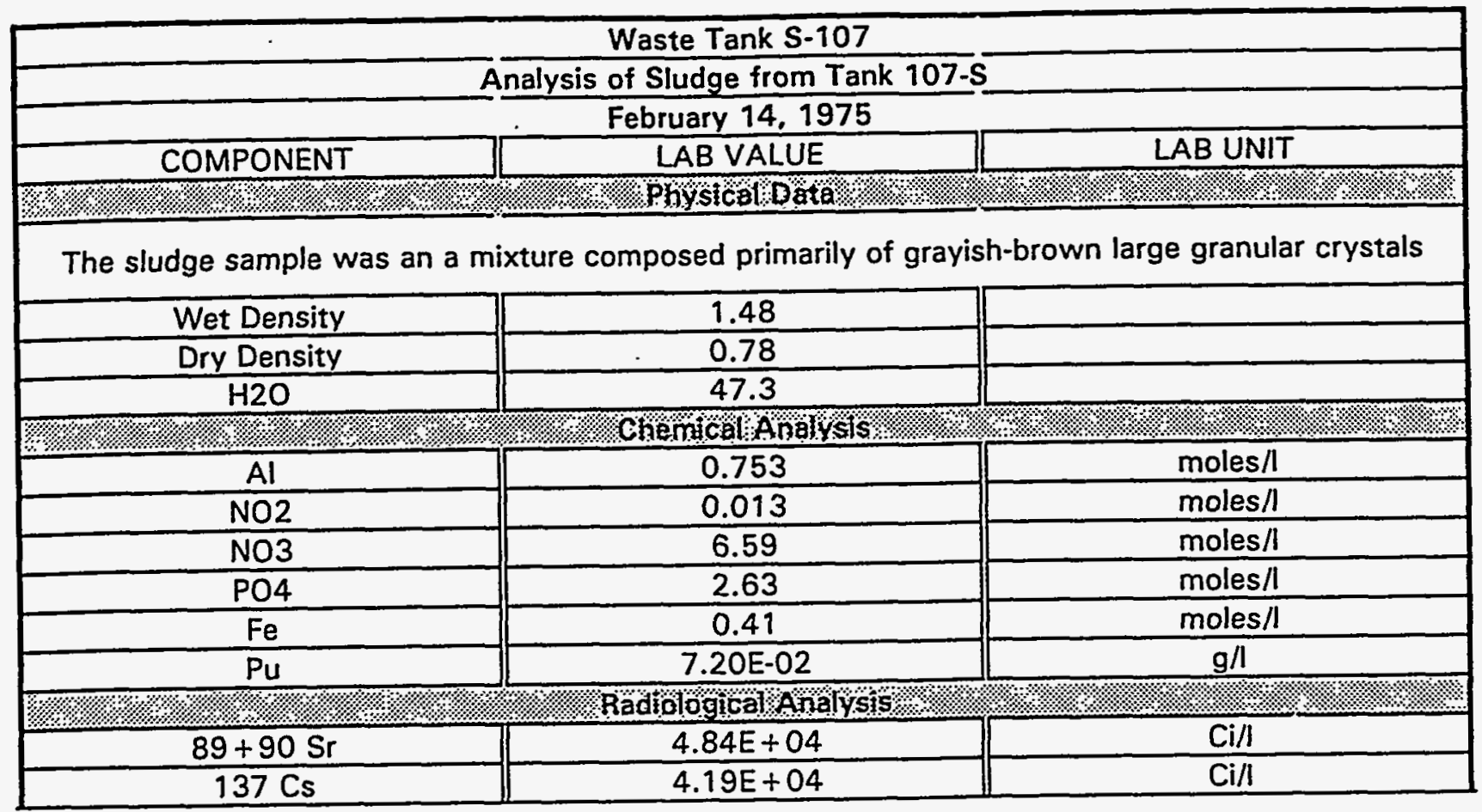


WHC-SD-WM-ER-323, Rev. 0

\begin{tabular}{|c|c|c|c|}
\hline \multicolumn{4}{|c|}{ Waste Tank S-107 } \\
\hline \multicolumn{4}{|c|}{ 107.S Analyses } \\
\hline \multicolumn{4}{|c|}{ July 10,1978} \\
\hline & Feed & Concentrate & \\
\hline COMPONENT & \multicolumn{2}{|c|}{ LAB VALUE } & LAB UNIT \\
\hline ২. & \multicolumn{2}{|c|}{ Chimical Anialysis } & \% \\
\hline $\mathrm{Al}$ & 1.59 & 2.74 & $\mathrm{M}$ \\
\hline $\mathrm{OH}$ & 1.52 & 2.6 & $M$ \\
\hline NO2 & 2.58 & 2.35 & $M$ \\
\hline $\mathrm{NO3}$ & 3.36 & 2.92 & $M$ \\
\hline $\mathrm{CO} 3$ & 0.19 & 0.18 & $M$ \\
\hline TOC & & 22 & $g / 1$ \\
\hline সে & (7) R Ra & YSis $/$. & ४ \\
\hline $89+90 \mathrm{Sr}$ & $8.19 \mathrm{E}+03$ & $7.15 \mathrm{E}+03$ & $\mathrm{uCi} / \mathrm{I}$ \\
\hline * $35 \%$ Reduction & & & \\
\hline
\end{tabular}




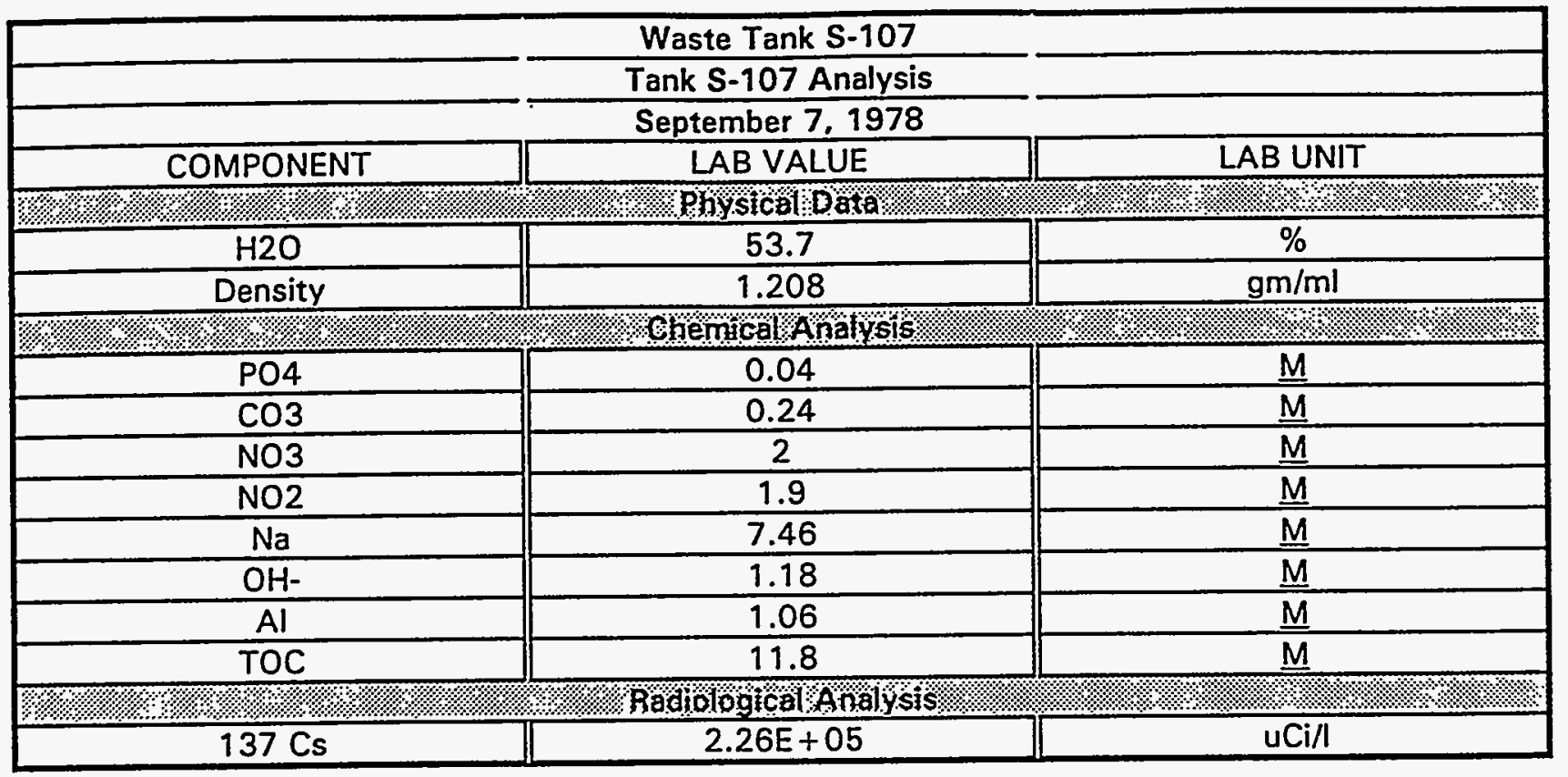




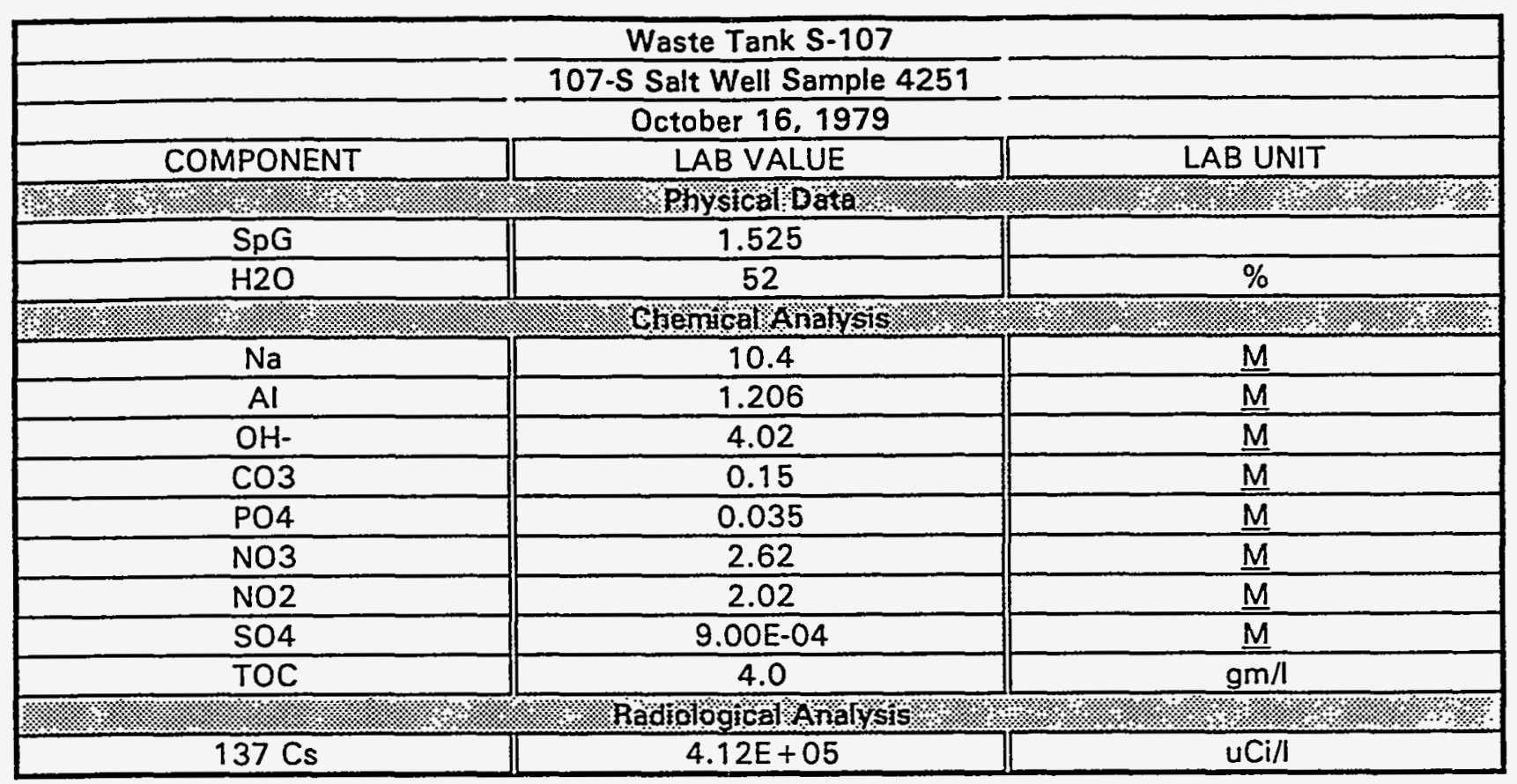




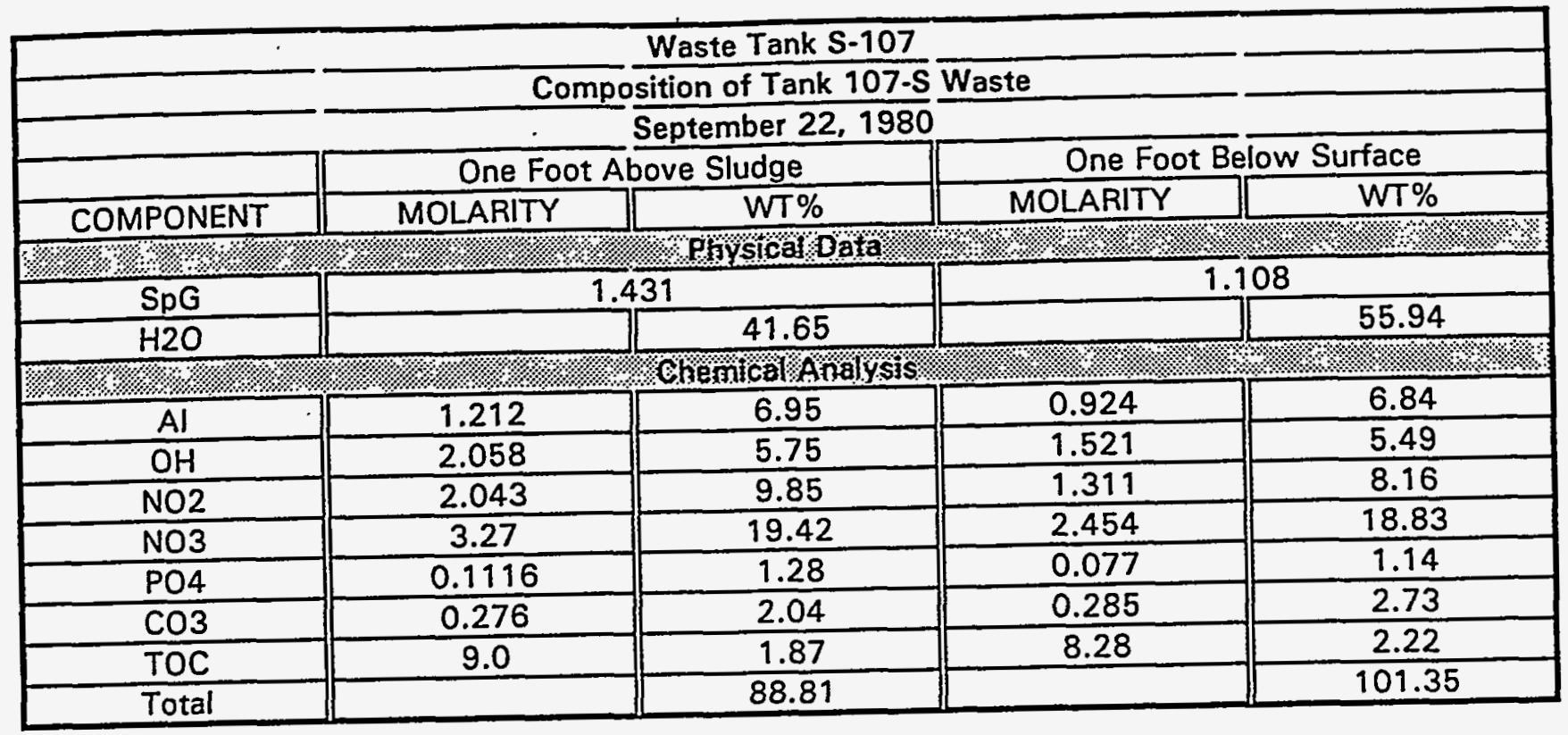




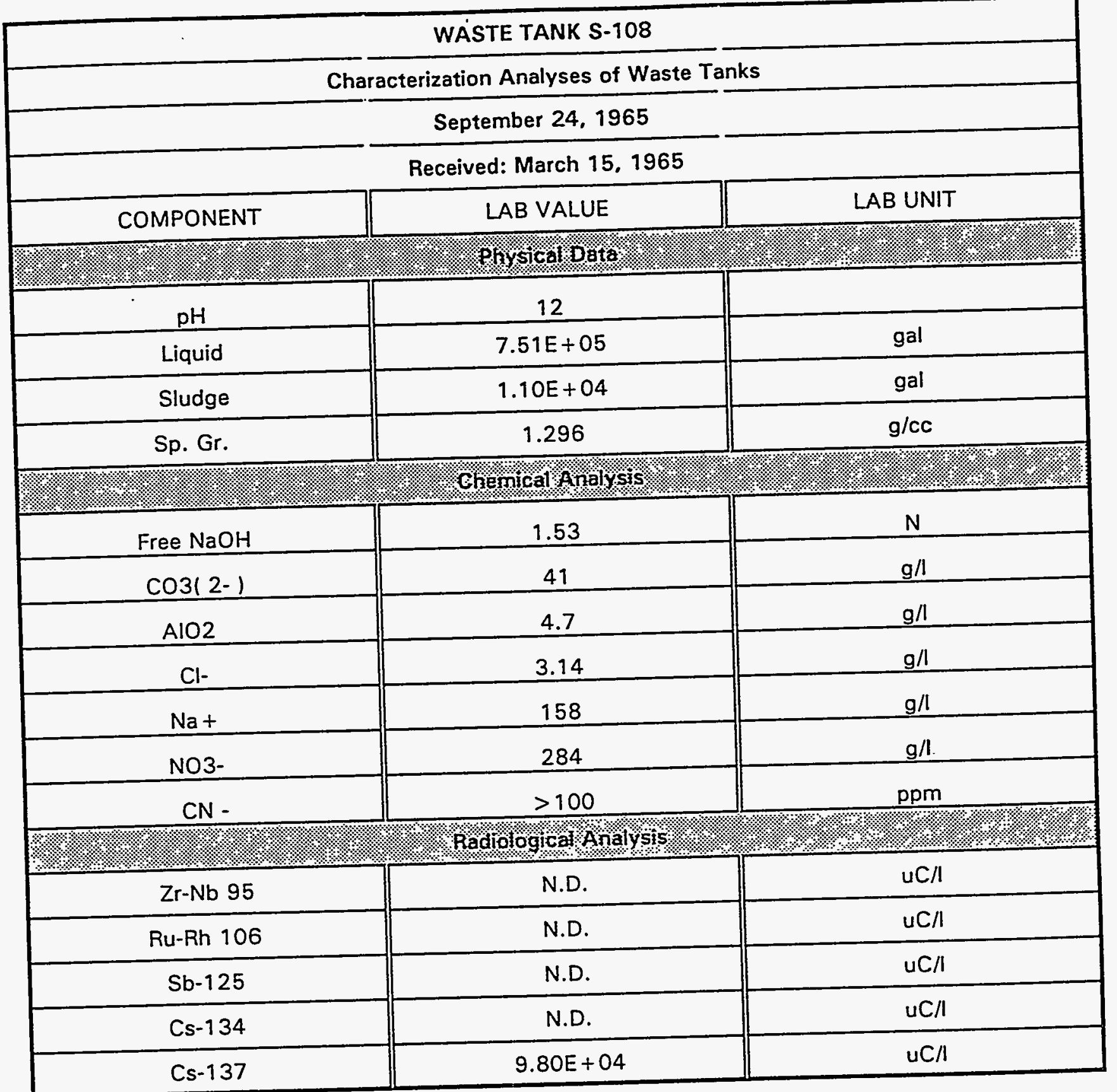


WHC-SD-WM-ER-323, Rev. 0

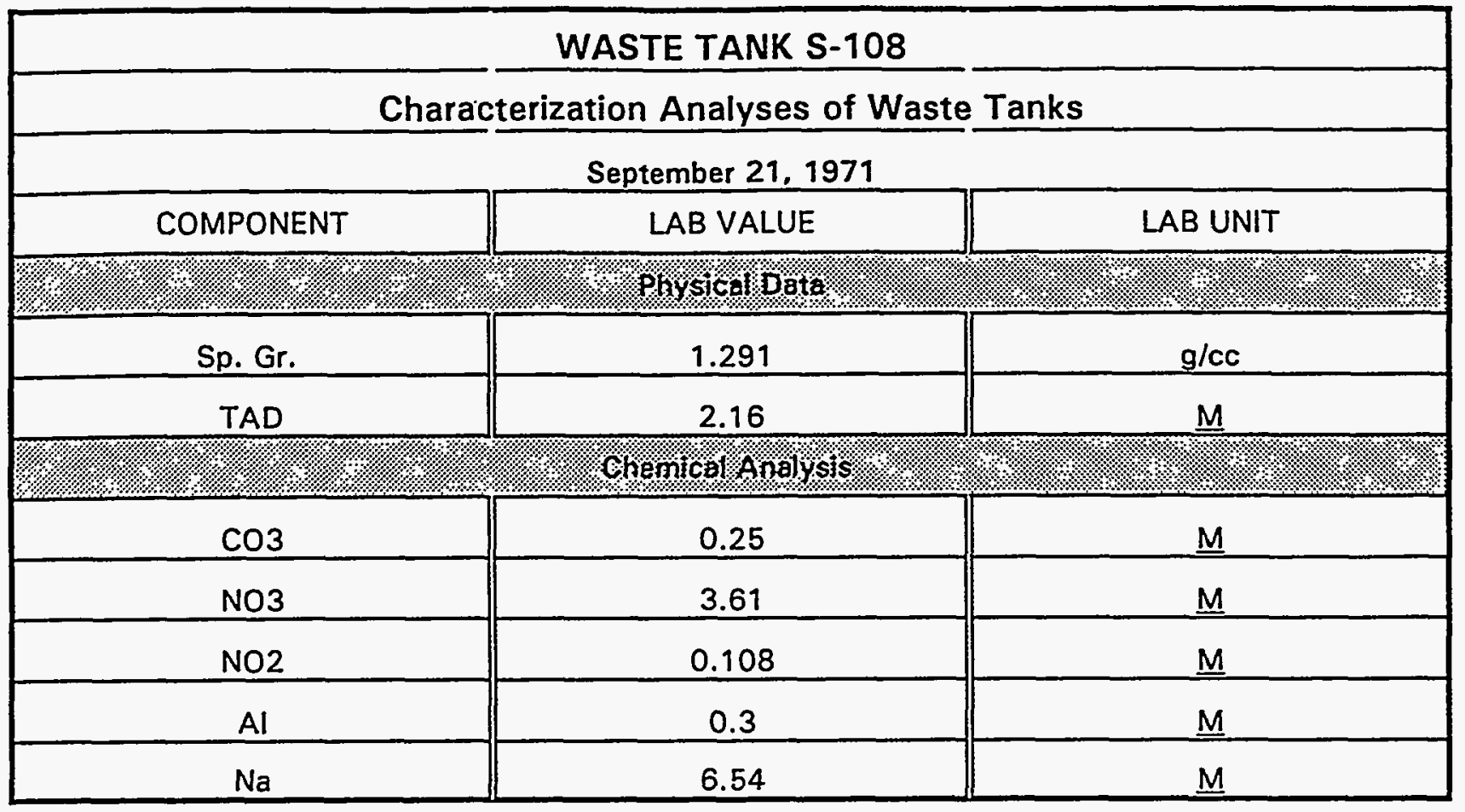


WHC-SD-WM-ER-323, Rev. 0

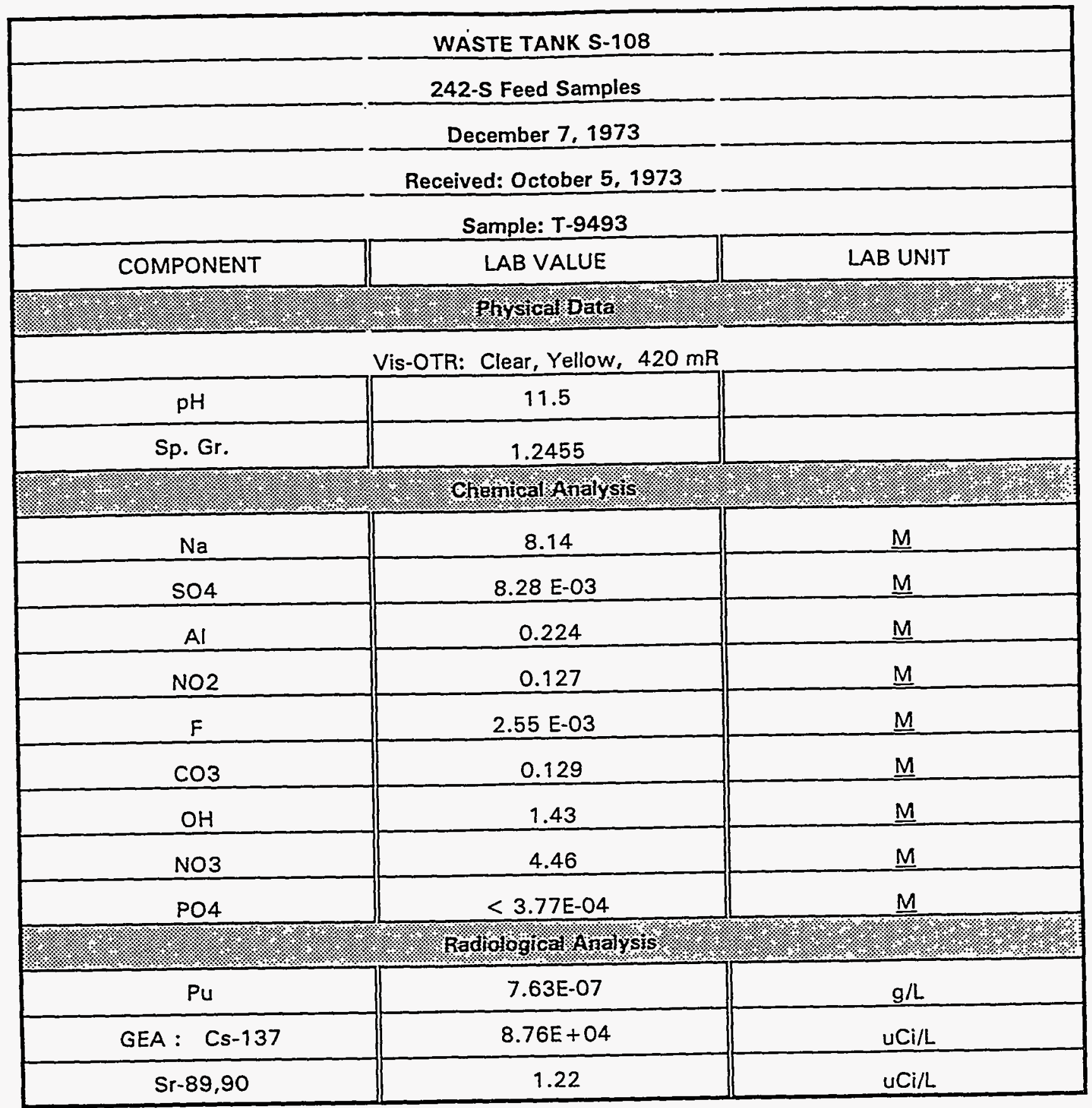




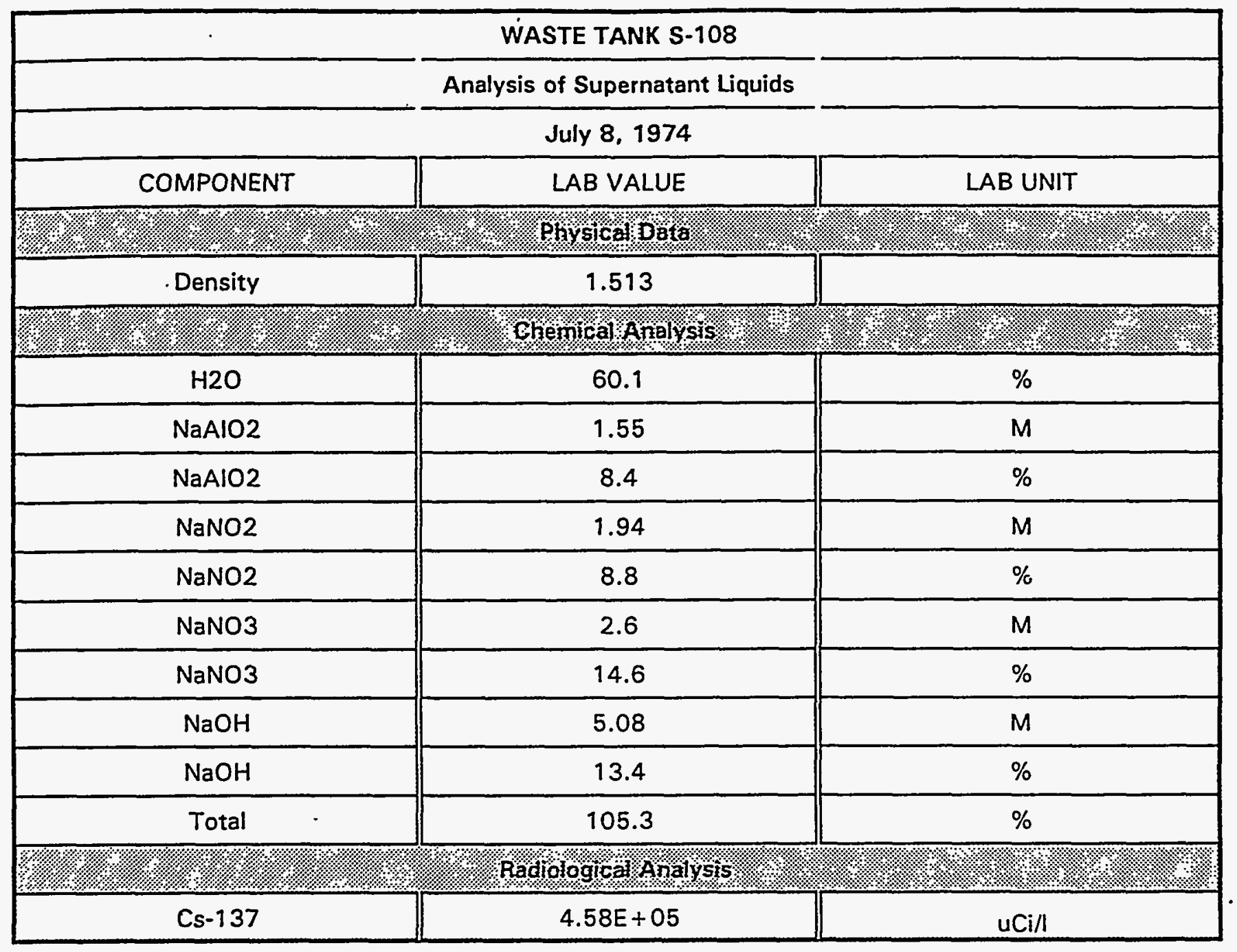




\begin{tabular}{|c|c|c|c|}
\hline & \multicolumn{2}{|c|}{ WASTE TANK S-108 } & \\
\hline & \multicolumn{2}{|c|}{ Analysis of Solid Phase } & \\
\hline & \multicolumn{2}{|c|}{ July 8, 1974} & \\
\hline \multicolumn{4}{|c|}{ Sample taken: June 25, 1974} \\
\hline COMPONENT & LAB VALUE (TOP) & LAB VALUE (BOTTOM) & LAB UNIT \\
\hline \multicolumn{4}{|c|}{ 1.1: } \\
\hline Damp Density & 1.495 & 1.393 & \\
\hline Dry Density & 1.136 & 1.222 & \\
\hline $\mathrm{H} 2 \mathrm{O}$ & 24.0 & 11.5 & $\%$ \\
\hline \multicolumn{4}{|c|}{ 1., } \\
\hline $\mathrm{NaAlO} 2$ & 5.4 & 1.8 & $\%$ \\
\hline $\mathrm{NaNO} 2$ & 4.6 & 1.9 & $\%$ \\
\hline NaNO3 & & 68.1 & $\%$ \\
\hline $\mathrm{NaOH}$ & 6.3 & 3.6 & $\%$ \\
\hline $\mathrm{NaCO} 3$ & & 6.5 & $\%$ \\
\hline $\mathrm{Fe} 2 \mathrm{O} 3$ & 2.0 & & $\%$ \\
\hline $\mathrm{A} 1203$ & $<0.3$ & & $\%$ \\
\hline \multicolumn{4}{|c|}{ (1., } \\
\hline Pu-239 & $1.87 \mathrm{E}-08$ & $1.60 \mathrm{E}-08$ & $\mathrm{~g} / \mathrm{g}$ \\
\hline Sr-89,90 & $3.15 \mathrm{E}-04$ & 0.684 & $u \mathrm{Ci} / \mathrm{g}$ \\
\hline Cs-137 & $1.74 \mathrm{E}-02$ & 4.4 & $\mathrm{uCi} / \mathrm{g}$ \\
\hline Cs-134 & & 0.4 & $\mathrm{uCi} / \mathrm{g}$ \\
\hline Sb-125 & 0.46 & & $\mathrm{uCi} / \mathrm{g}$ \\
\hline Ru-106, Rh-106 & $3.95 E+03$ & & $u \mathrm{Ci} / \mathrm{g}$ \\
\hline
\end{tabular}


WHC-SD-WM-ER-323, Rev. 0

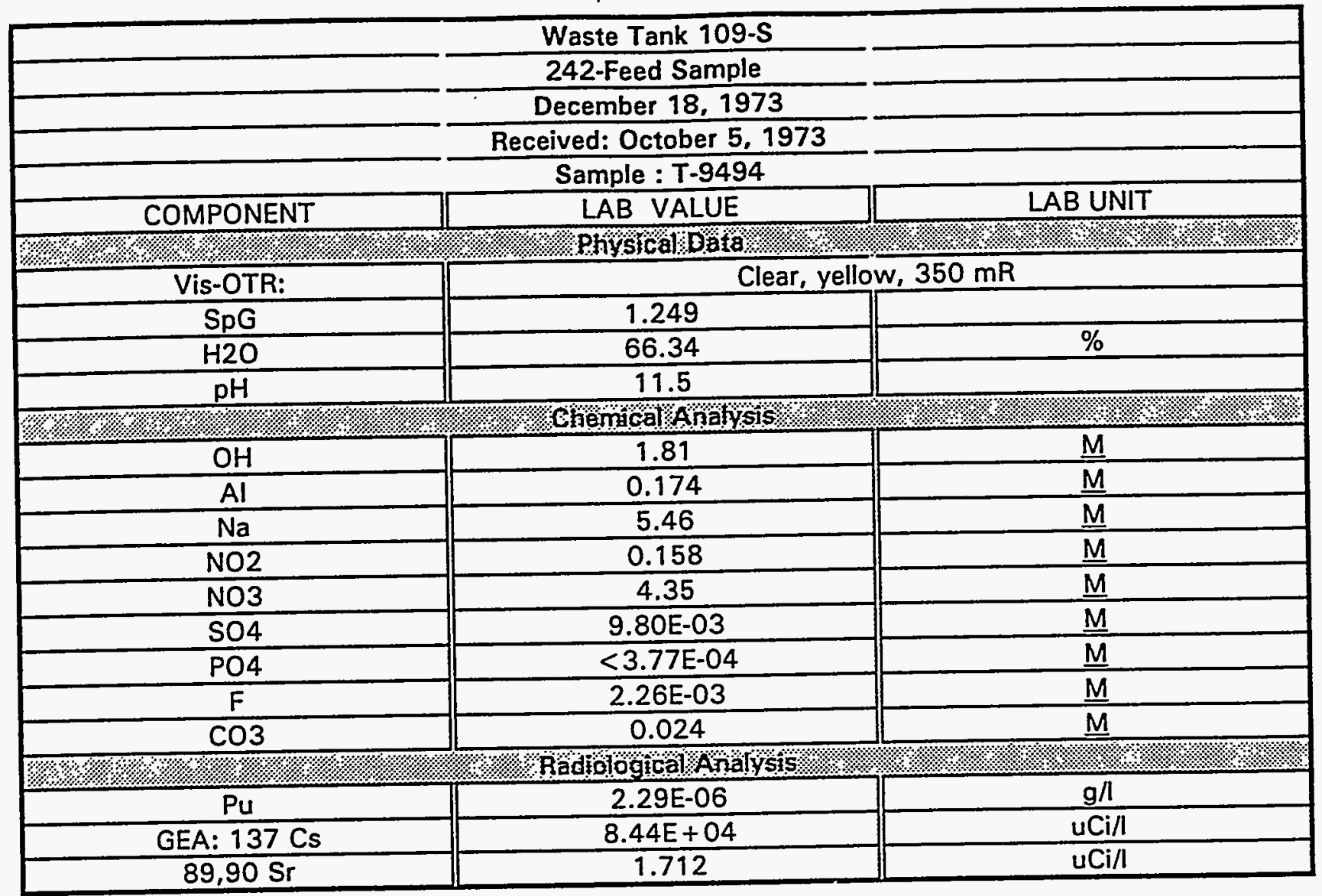


WHC-SD-WM-ER-323, Rev. 0

\begin{tabular}{|c|c|c|}
\hline \multicolumn{3}{|c|}{ Waste Tank S-109 } \\
\hline \multirow{2}{*}{\multicolumn{3}{|c|}{$\begin{array}{c}\text { Analyses of Salt Samples } \\
\text { September } 3,1974\end{array}$}} \\
\hline & & \\
\hline \multicolumn{3}{|c|}{ Received: August 19, 1974} \\
\hline COMPONENT & LAB VALUE & LAB UNIT \\
\hline ) & Phystcal Data & (4) \\
\hline Bulk Density & 1.1 & g/cc \\
\hline $\mathrm{H} 2 \mathrm{O}$ & 9.2 & $\%$ \\
\hline সে। & Ghentcal Ahatysis & (4: \\
\hline $\mathrm{NaAlO} 2$ & 1.1 & $\%$ \\
\hline $\mathrm{NaNO} 2$ & $<0.1$ & $\%$ \\
\hline NaNO3 & 86.1 & $\%$ \\
\hline $\mathrm{NaOH}$ & 2.3 & $\%$ \\
\hline $\mathrm{Na} 2 \mathrm{CO} 3$ & 2.7 & $\%$ \\
\hline Total & 101.4 & $\%$ \\
\hline 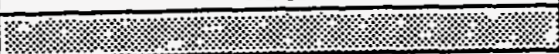 & Radiological Analysis & (I) \\
\hline Cs 137 & 56 & uCi/L \\
\hline $89+90 \mathrm{Sr}$ & 2.08 & $u C \mathrm{i} / \mathrm{L}$ \\
\hline
\end{tabular}




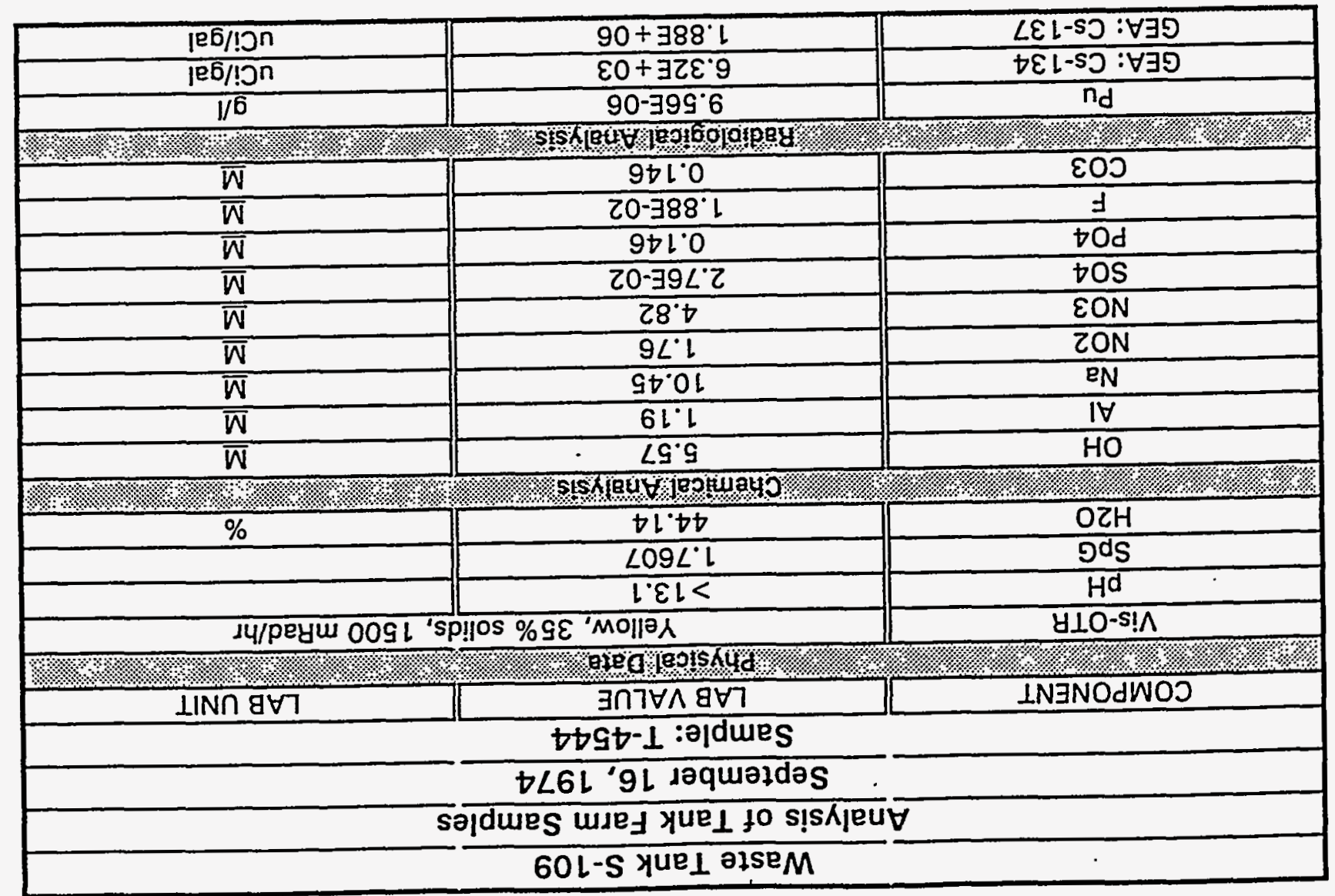


WHC-SD-WM-ER-323, Rev. 0

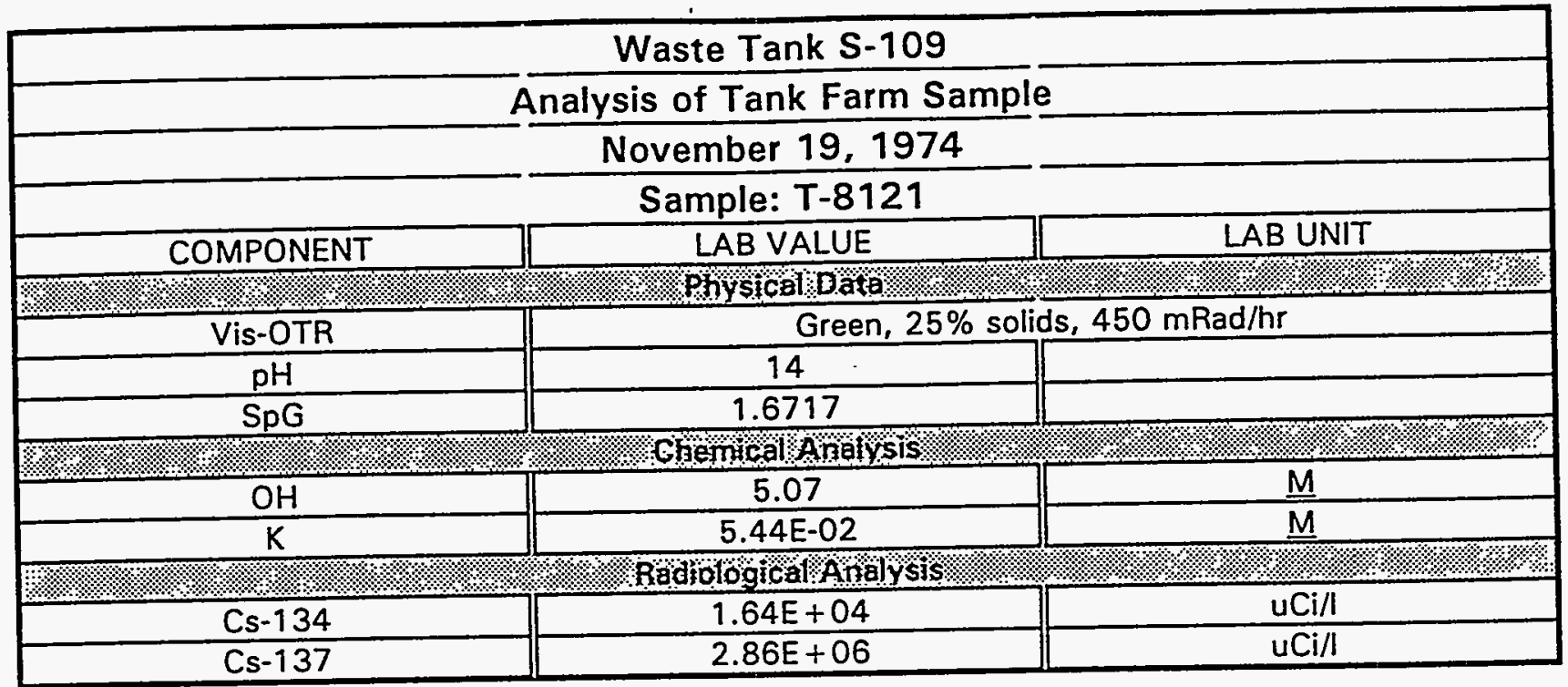




\begin{tabular}{|c|c|c|}
\hline & Waste Tank S-109 & \\
\hline & Analysis of Tank S-109 & \\
\hline & December 8, 1976 & \\
\hline & Received: October 29,1976 & \\
\hline COMPONENT & LAB VALUE & LAB UNIT \\
\hline \%थ & 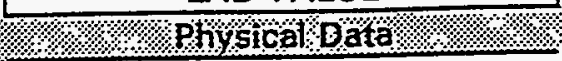 & 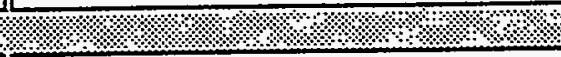 \\
\hline & Yellowish, coarse granular crystals & \\
\hline Bulk Density & 1.27 & $\mathrm{~g} / \mathrm{cc}$ \\
\hline Particle Density & 2.38 & $\mathrm{~g} / \mathrm{cc}$ \\
\hline $\mathrm{H} 2 \mathrm{O}$ & 10.6 & $\%$ \\
\hline (4) & Chemicalanalysss & 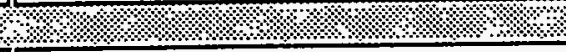 \\
\hline $\mathrm{AlO} 2$ & 1.3 & $\%$ \\
\hline $\mathrm{CO} 3$ & 4.2 & $\%$ \\
\hline $\mathrm{Fe}$ & 0.3 & $\%$ \\
\hline $\mathrm{OH}$ & 0.3 & $\%$ \\
\hline NO2 & 1.4 & $\%$ \\
\hline NO3 & 53.5 & $\%$ \\
\hline $\mathrm{Mn}$ & 0.003 & $\%$ \\
\hline $\mathrm{Mg}$ & 0.02 & $\%$ \\
\hline $\mathrm{Ca}$ & 0.08 & $\%$ \\
\hline $\mathrm{Cr}$ & 0.1 & $\%$ \\
\hline $\mathrm{Si}$ & 0.1 & $\%$ \\
\hline $\mathrm{Na}$ & 35 & $\%$ \\
\hline $\mathrm{PO} 4$ & 0.01 & $\%$ \\
\hline 504 & \begin{tabular}{|c|}
0.9 \\
\end{tabular} & $\%$ \\
\hline \% & Radfologicallanalysts & 4 \\
\hline $\mathrm{Pu}$ & 0.654 & $\frac{u g / g}{4}$ \\
\hline Cs-137 & 71.8 & $\mathrm{uCi} / \mathrm{g}$ \\
\hline $89,90 \mathrm{Sr}$ & 14.6 & $\mathrm{uCi} / \mathrm{g}$ \\
\hline
\end{tabular}




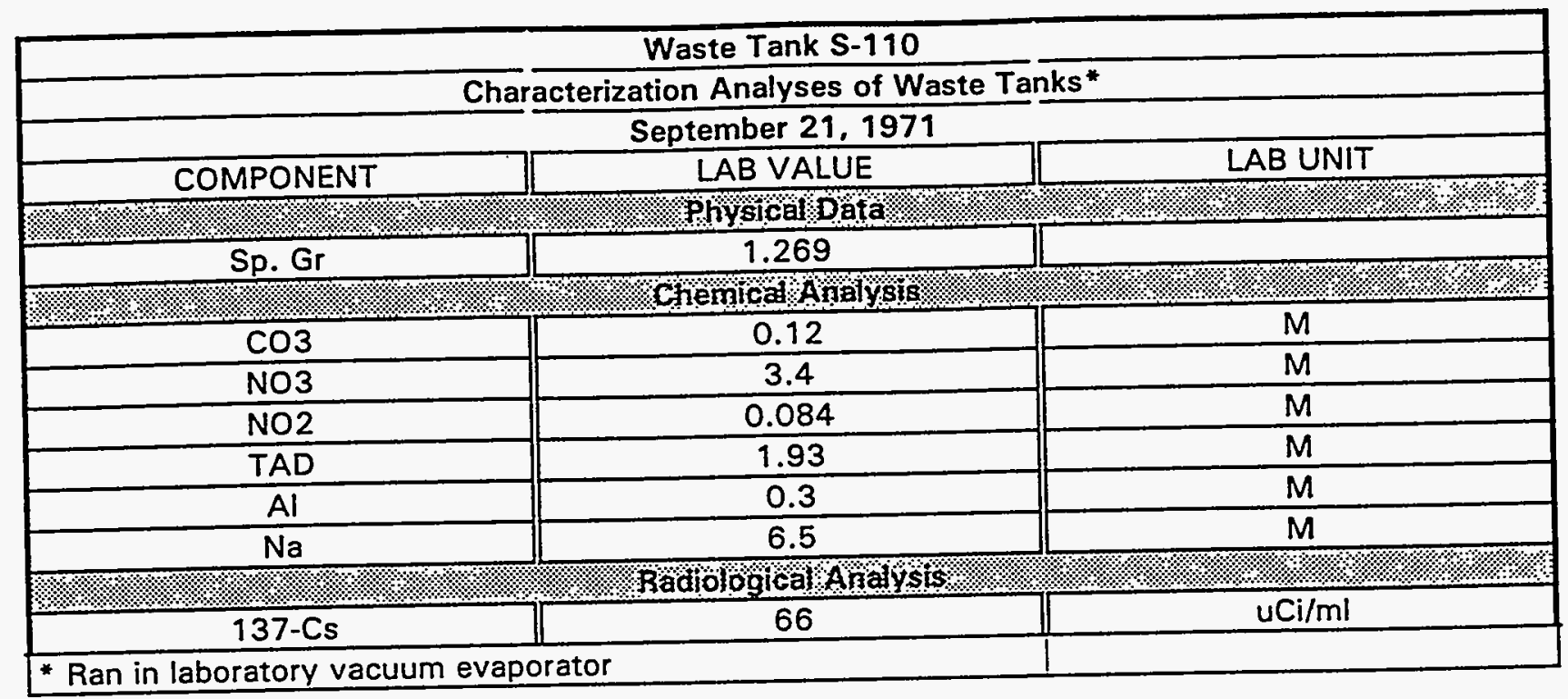


Waste Tank S-110

Analysis of Tank S-110 Sludge Sample

September 25, 1974

Received: August 9, 1974

COMPONENT

Physicabiata

The top layer was dark brown color and represented about 10 volume percent of the total sample

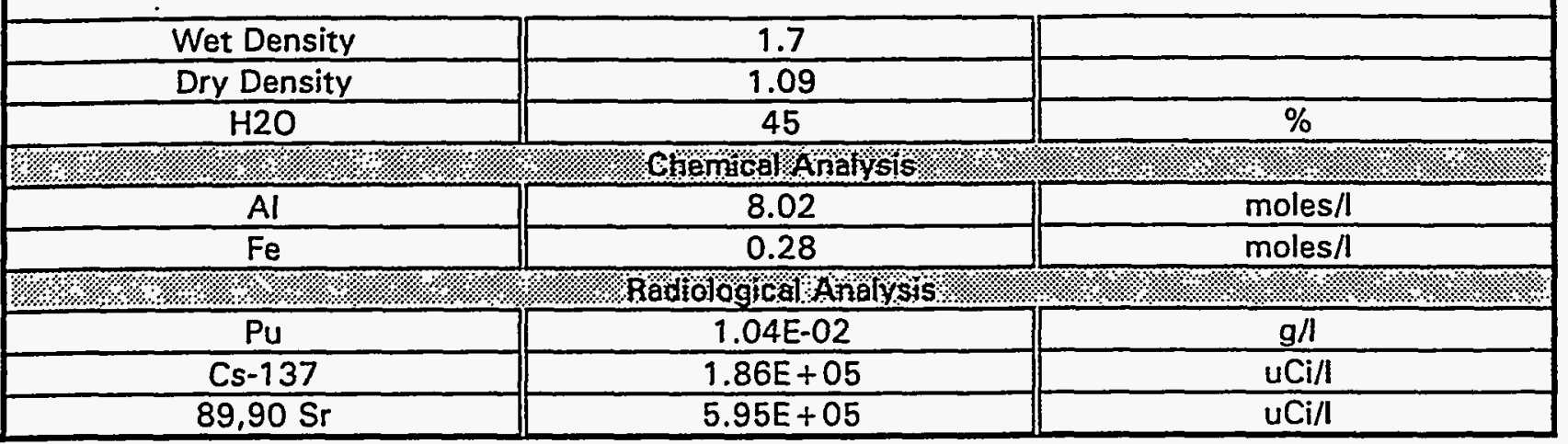




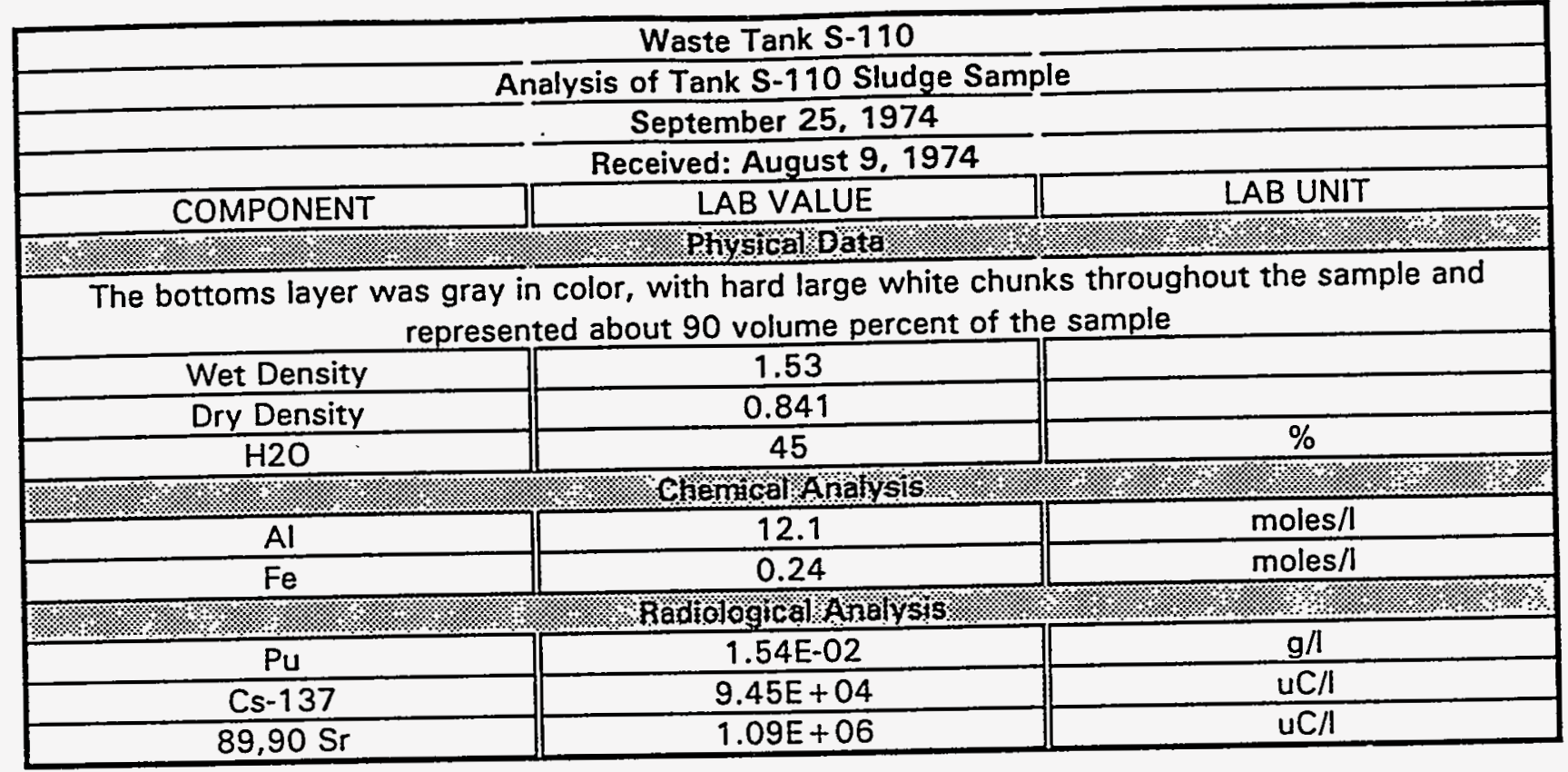




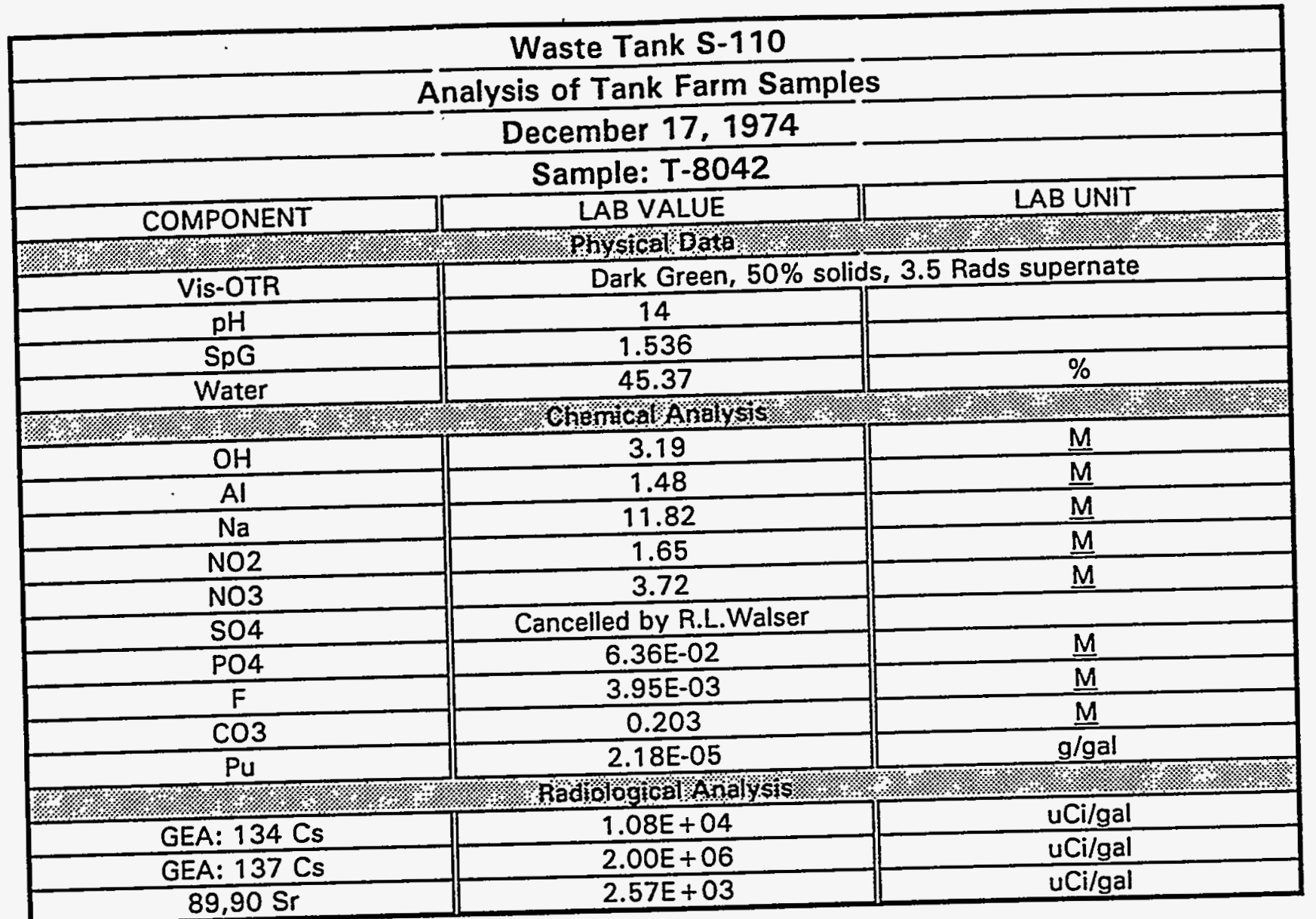


WHC-SD-WM-ER-323, Rev. 0

\begin{tabular}{|c|c|c|}
\hline \multicolumn{3}{|c|}{ Waste Tank 110-S } \\
\hline \multicolumn{3}{|c|}{ Analysis of Tank S-110 Sludge Sample } \\
\hline \multicolumn{3}{|c|}{ January 7,1975} \\
\hline \multicolumn{3}{|c|}{ Received: October 22,1974} \\
\hline COMPONENT & LAB VALUE & LAB UNIT \\
\hline \multicolumn{3}{|c|}{ Radiological A nalysis: } \\
\hline $\mathrm{Pu}$ & $6.29 \mathrm{E}-04$ & $g / 1$ \\
\hline Cs-137 & $8.10 E+05$ & $u C i / L$ \\
\hline Cs-134 & $4.57 E+03$ & $\mathrm{uCi} / \mathrm{I}$ \\
\hline $89,90 \mathrm{Sr}$ & $5.43 E+05$ & $u \mathrm{Ci} / \mathrm{l}$ \\
\hline
\end{tabular}




\begin{tabular}{|c|c|c|c|}
\hline \multicolumn{4}{|c|}{ Waste Tank S-110 } \\
\hline \multicolumn{4}{|c|}{ Analysis of Salt from Tank 110-S } \\
\hline \multicolumn{4}{|c|}{ June 20,1975} \\
\hline \multicolumn{4}{|c|}{ Received: April 23, 1975} \\
\hline COMPONENT & LAB VALUE (SALT) & $\begin{array}{l}\text { LAB VALUE } \\
\text { (SALT FUSION) }\end{array}$ & LAB UNIT \\
\hline \multicolumn{4}{|c|}{ Pphysualobara } \\
\hline \multicolumn{4}{|c|}{ The salt was yellow } \\
\hline Bulk Density & 1.9 & & \\
\hline Parcticle Density & 1.73 & & \\
\hline \multicolumn{4}{|c|}{ (1) Chemical Analysis } \\
\hline $\mathrm{H} 2 \mathrm{O}$ & & 19.3 & Wt\% \\
\hline $\mathrm{NaOH}$ & 0.115 & 3.2 & $\mathrm{Wt} \%$ \\
\hline $\mathrm{NaNO2}$ & $6.39 \mathrm{E}-02$ & 3.1 & $W_{t} \%$ \\
\hline NaNO3 & 0.805 & 57.8 & $W_{t} \%$ \\
\hline $\mathrm{NaAlO} 2$ & 4.27E-02 & 2.5 & $W_{t} \%$ \\
\hline $\mathrm{Na2CO3}$ & $6.82 \mathrm{E}-02$ & 2.9 & $\mathrm{Wt}_{\mathrm{t}} \%$ \\
\hline $\mathrm{Na2PO4}$ & $2.76 \mathrm{E}-02$ & $<3.2$ & $W_{t} \%$ \\
\hline $\mathrm{Fe}$ & 1.44E-03 & 0.2 & $\mathrm{Wt} \%$ \\
\hline $\mathrm{Al}$ & $3.08 \mathrm{E}-02$ & 1.8 & $W t \%$ \\
\hline $\mathrm{Mn}$ & $6.80 \mathrm{E}-04$ & 0.05 & $W_{t} \%$ \\
\hline $\mathrm{Si}$ & $2.00 \mathrm{E}-03$ & 0.15 & $W_{t} \%$ \\
\hline $\mathrm{Na}$ & $2.51 \mathrm{E}-02$ & 1.6 & Wt\% \\
\hline $\mathrm{Cr}$ & $6.58 \mathrm{E}-04$ & 0.1 & $W_{t} \%$ \\
\hline Total Wt\% Recovery & & 96 & $W_{t} \%$ \\
\hline \%" " & (1. & Alyis: & 1. \\
\hline Pu & $9.11 E-09$ & & $\mathrm{~g} / \mathrm{g}$ \\
\hline $\mathrm{Cs}-137$ & 108.8 & & $\mathrm{uCi} / \mathrm{g}$ \\
\hline $89,90 \mathrm{Sr}$ & 81.1 & & $\mathrm{uCi} / \mathrm{g}$ \\
\hline
\end{tabular}




\begin{tabular}{|c|c|c|}
\hline \multicolumn{3}{|c|}{ Waste Tank 111-S } \\
\hline \multicolumn{3}{|c|}{ Characterization Analyses of Waste Tanks } \\
\hline \multicolumn{3}{|c|}{ September 21,1971} \\
\hline COMPONENT & LAB VALUE & LAB UNIT \\
\hline$\sqrt{4}$ & Phystealobara & 1. \\
\hline SpG & \multicolumn{2}{|c|}{1.219} \\
\hline TAD & 1.92 & $\underline{M}$ \\
\hline \multicolumn{3}{|c|}{ Chinmicat Analysis } \\
\hline $\operatorname{co3}$ & 0.025 & $\underline{\mathrm{M}}$ \\
\hline NO3 & 3.40 & $\underline{\mathrm{M}}$ \\
\hline NO2 & 0.30 & $\underline{\mathrm{M}}$ \\
\hline Al & 0.49 & $\underline{M}$ \\
\hline $\mathrm{Na}$ & 8.40 & $\underline{M}$ \\
\hline ঝ/. & Reaciologledr/ & \\
\hline $137 \mathrm{Cs}$ & 51.6 & $\mathrm{uCi} / \mathrm{ml}$ \\
\hline
\end{tabular}




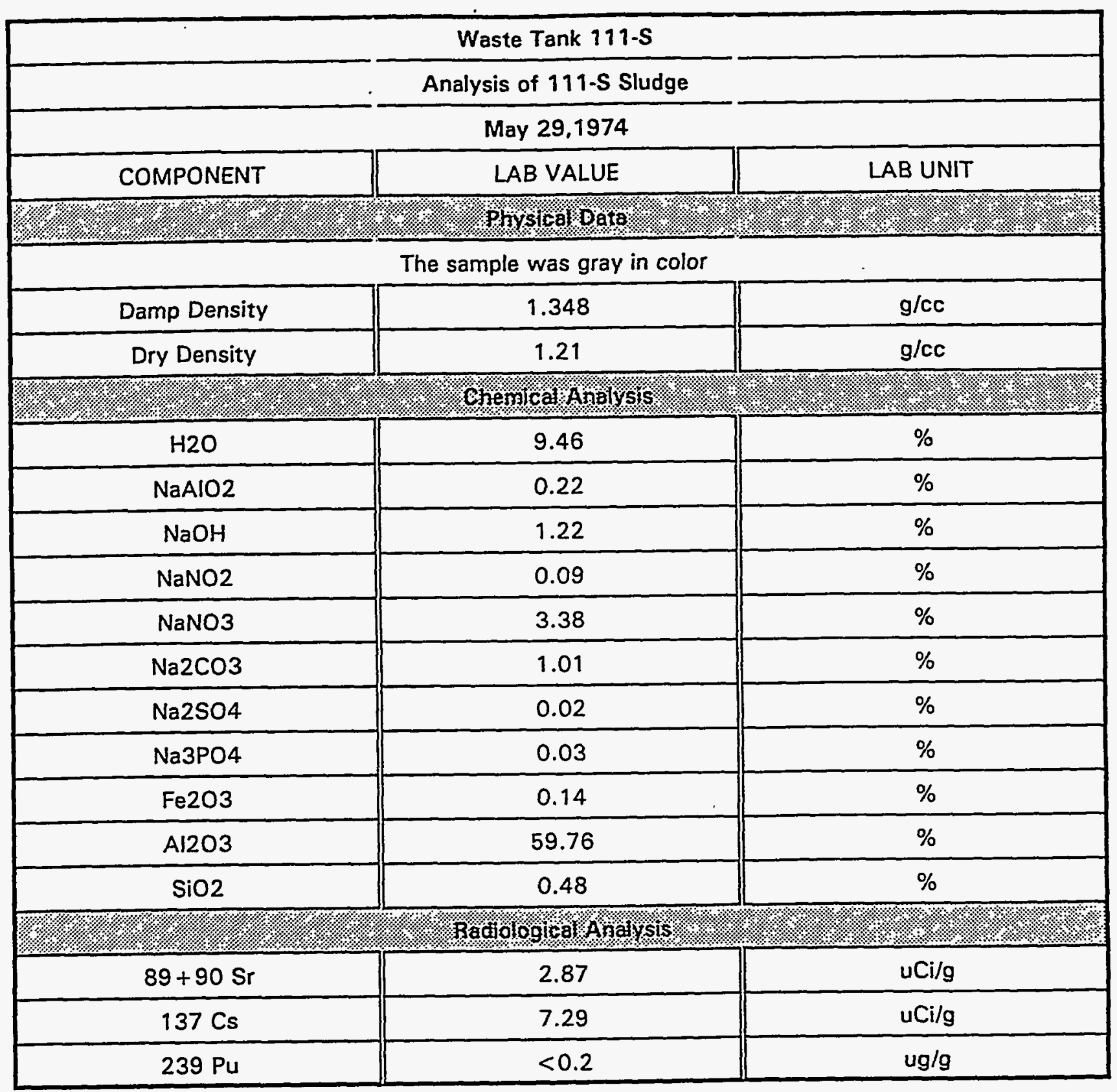




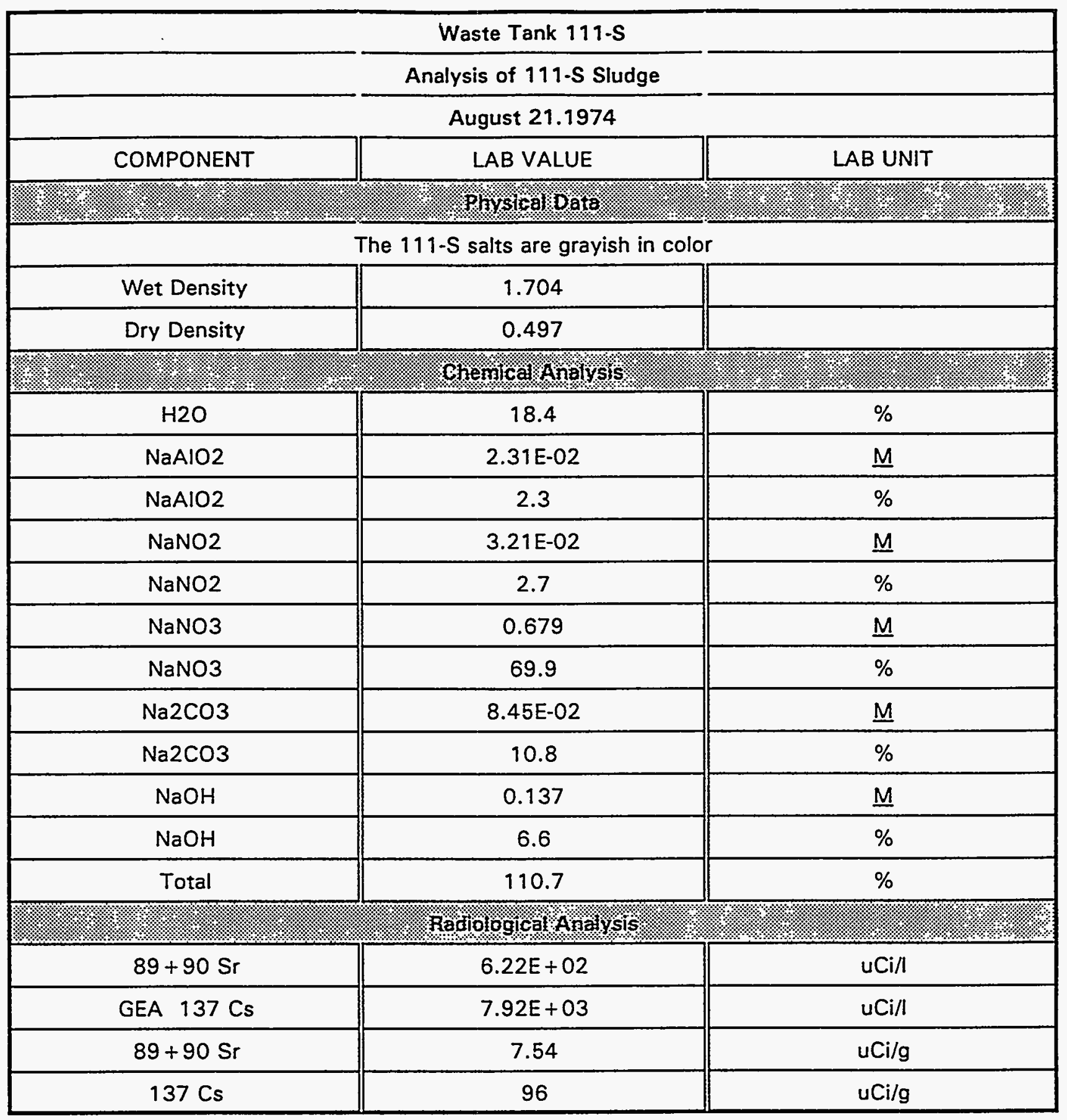




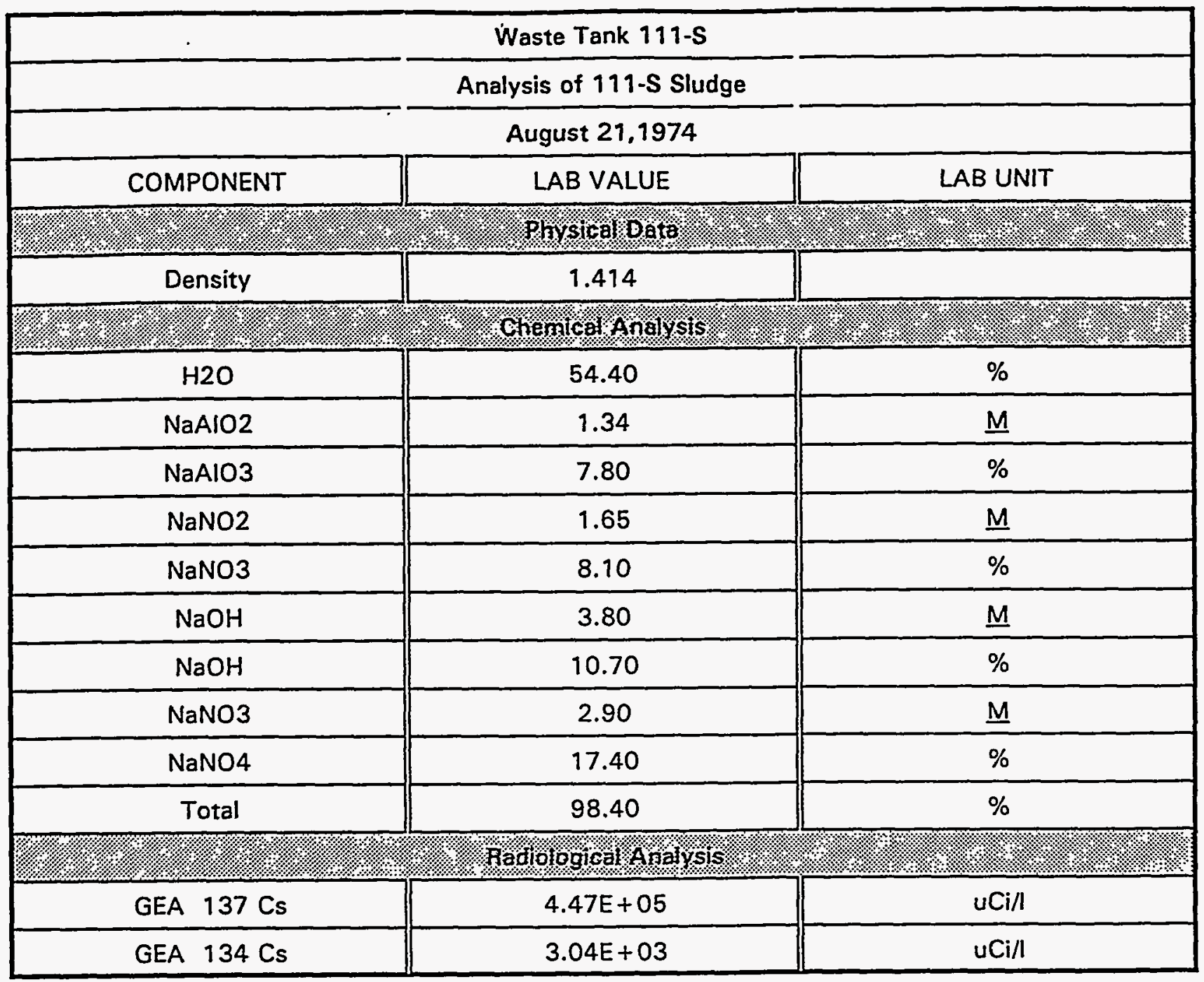




\begin{tabular}{|c|c|c|}
\hline \multicolumn{3}{|c|}{ Waste Tank 111-S } \\
\hline \multicolumn{3}{|c|}{ Analysis of Tank Farm Samples } \\
\hline \multicolumn{3}{|c|}{ December 16, 1974} \\
\hline \multicolumn{3}{|c|}{ Sample: T-8141 } \\
\hline COMPONENT & LAB VALUE & LAB UNIT \\
\hline 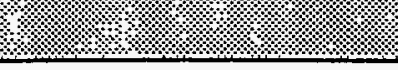 & 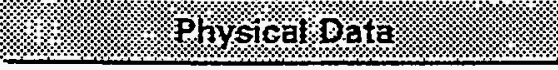 & \\
\hline \multicolumn{3}{|c|}{ Vis-OTR: Yellowish green. $100 \%$ solids. $500 \mathrm{mRad} / \mathrm{hr}$} \\
\hline $\mathrm{pH}$ & $>13.4$ & \\
\hline $\mathrm{SpG}$ & 1.7953 & \\
\hline Water & 34.29 & $\%$ \\
\hline \multicolumn{3}{|c|}{ Ghemicat } \\
\hline $\mathrm{OH}$ & 5.16 & M \\
\hline Al & 1.34 & $M$ \\
\hline $\mathrm{Na}$ & 23.84 & $M$ \\
\hline NO2 & 2.761 & $M$ \\
\hline NO3 & 4.91 & $M$ \\
\hline SO4 & \multicolumn{2}{|c|}{ Cancelled by R. L. Walser } \\
\hline PO4 & $4.52 E-02$ & $M$ \\
\hline$F$ & $5.10 E-03$ & M \\
\hline $\mathrm{CO} 3$ & 0.293 & $M$ \\
\hline$\sqrt[3]{1.4 .1}$ & \multicolumn{2}{|l|}{ Radiologloal Anaysis } \\
\hline $\mathrm{Pu}$ & $3.44 \mathrm{E}-05$ & $\mathrm{gm} / \mathrm{gal}$ \\
\hline GEA $134 \mathrm{Cs}$ & $1.13 E+04$ & uCi/gal \\
\hline GEA $137 \mathrm{Cs}$ & $2.93 E+06$ & uCi/gal \\
\hline GEA $60 \mathrm{Co}$ & $7.12 E+02$ & uCi/gal \\
\hline $89,90 \mathrm{Sr}$ & $2.33 E+06$ & uCi/gal \\
\hline
\end{tabular}




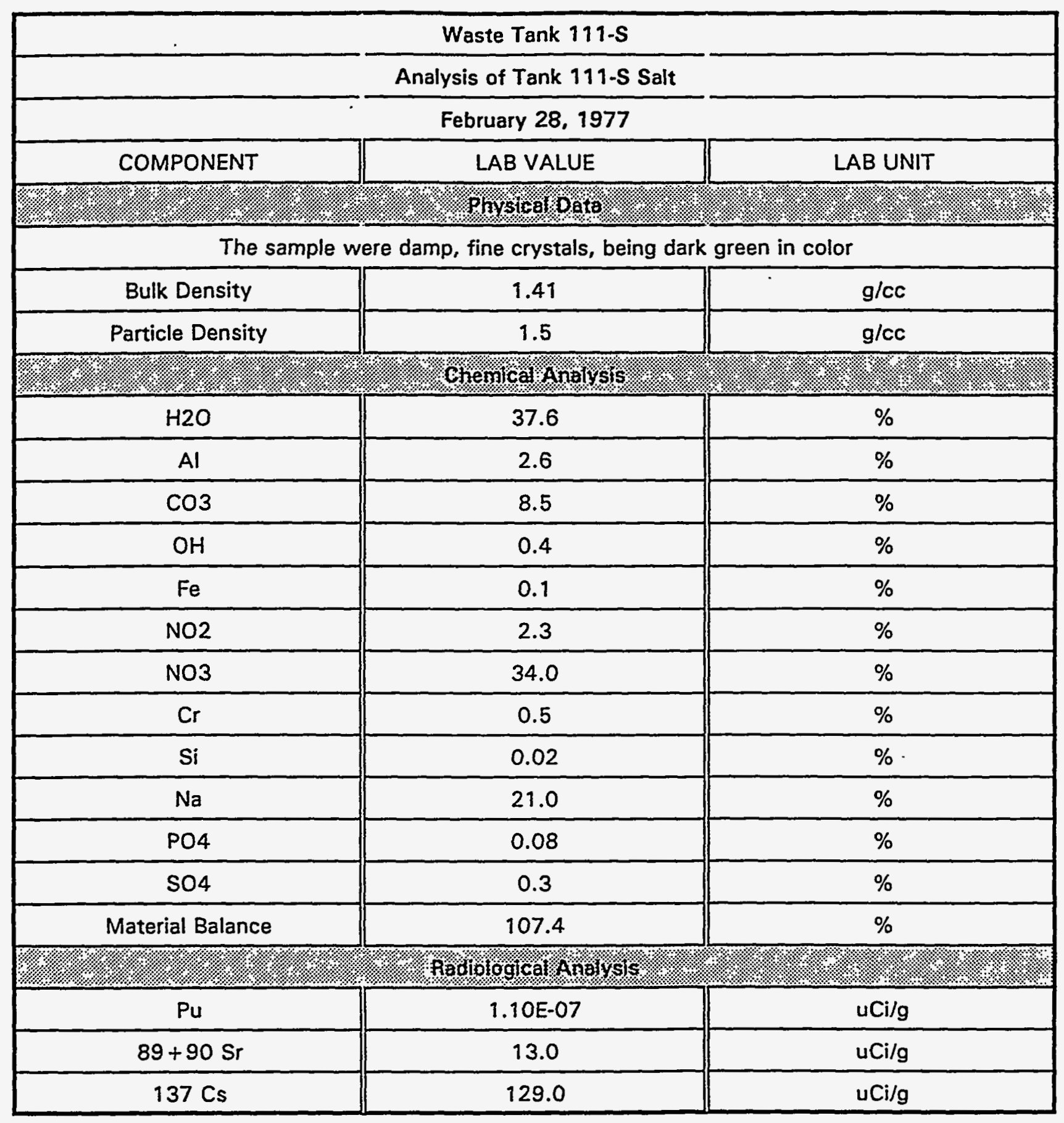




\begin{tabular}{|c|c|c|c|}
\hline \multicolumn{4}{|c|}{ - Waste Tank 111-S } \\
\hline \multicolumn{4}{|c|}{ Chemical and Physical Analysis (content \& percent) for Tonk 111.5} \\
\hline \multicolumn{4}{|c|}{ August 25, 1978} \\
\hline \multicolumn{4}{|c|}{ Sample \#1001-C } \\
\hline & WATER SOLUBLE & ACID (KOH FUSION) & \\
\hline COMPONENT & LAB VALUE & LAB VALUE & LAB UNIT \\
\hline \multicolumn{4}{|c|}{ 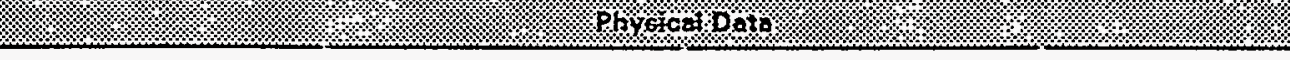 } \\
\hline \multicolumn{4}{|c|}{ The salt were yellowish green in color, with variable crystal sizes. } \\
\hline Bulk Density & 1.27 & & \\
\hline Particle Density & 1.34 & & \\
\hline Percent Water & 10.7 & & \\
\hline \multicolumn{4}{|c|}{ 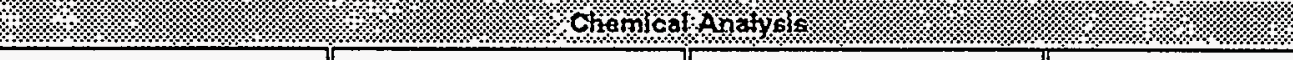 } \\
\hline Al & 0.6 & 0.06 & $\%$ \\
\hline $\mathrm{Cl}$ & 0.1 & & $\%$ \\
\hline cd & $<0.0002$ & 0.003 & $\%$ \\
\hline $\mathrm{Fe}$ & & 0.02 & $\%$ \\
\hline$F$ & 0.02 & & $\%$ \\
\hline $\cos$ & 5.0 & & $\%$ \\
\hline $\mathrm{OH}$ & 1.0 & & $\%$ \\
\hline $\mathrm{Hg}$ & 0.004 & & $\%$ \\
\hline $\mathrm{Na}$ & 26.2 & & $\%$ \\
\hline $\mathrm{NO} 2$ & 0.9 & & $\%$ \\
\hline $\mathrm{NO} 3$ & 51.4 & $<0.3$ & $\%$ \\
\hline PO4 & 0.3 & 0.20 & $\%$ \\
\hline SO4 & 1.5 & $<0.5$ & $\%$ \\
\hline CrO4 & 0.5 & & $\%$ \\
\hline $\mathrm{SiO} 2$ & 0.03 & 1.3 & $\%$ \\
\hline Total Organic Carbon & 1.29 & & $g /$ \\
\hline Material Balance & 99.8 & & $\%$ \\
\hline \multicolumn{4}{|c|}{ W } \\
\hline$U$ & & 2.47E-05 & $g / 9$ \\
\hline $239 \mathrm{Pu}$ & $3.60 E-10$ & $6.42 \mathrm{E}-08$ & $g / g$ \\
\hline $241 \mathrm{Am}$ & $1.02 \mathrm{E}-10$ & & $g / g$ \\
\hline $89+90 \mathrm{Sr}$ & 1.03 & 94.3 & $\mathrm{uCi} / \mathrm{g}$ \\
\hline $137 \mathrm{Cs}$ & 72.1 & 0.3 & $u C i / g$ \\
\hline $134 \mathrm{Cs}$ & 0.1 & & $u C i / g$ \\
\hline $106 \mathrm{Ru}$ & & 0.608 & $u \mathrm{Ci} / \mathrm{g}$ \\
\hline $60 \mathrm{Co}$ & & 0.056 & uCi/g \\
\hline $125 \mathrm{Sb}$ & & 0.007 & $\mathrm{uCi} / \mathrm{g}$ \\
\hline $154 \mathrm{Eu}$ & & 0.150 & $\mathrm{uCi} / \mathrm{g}$ \\
\hline $755 \mathrm{Eu}$ & & 0.307 & $u C i / g$ \\
\hline
\end{tabular}




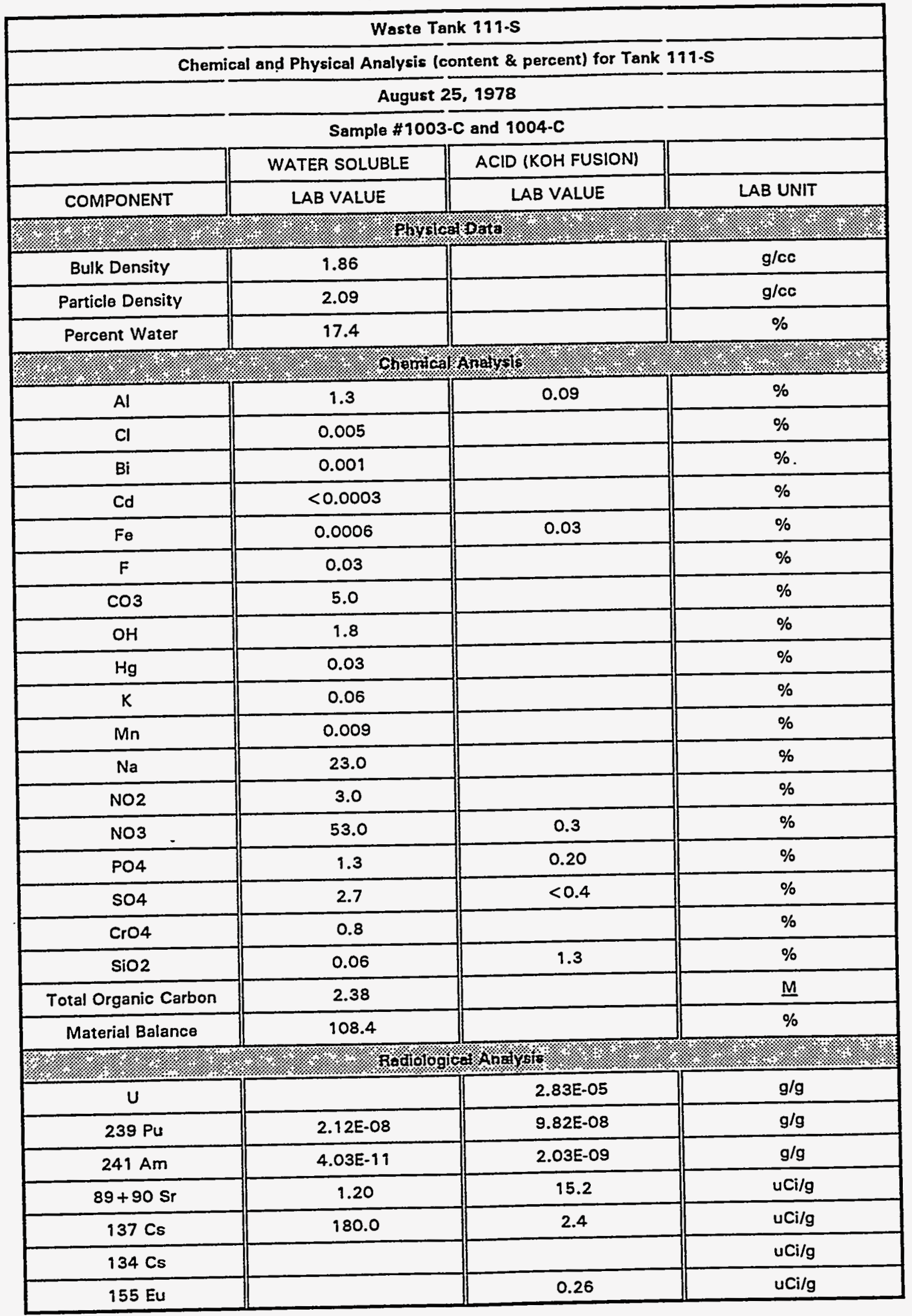




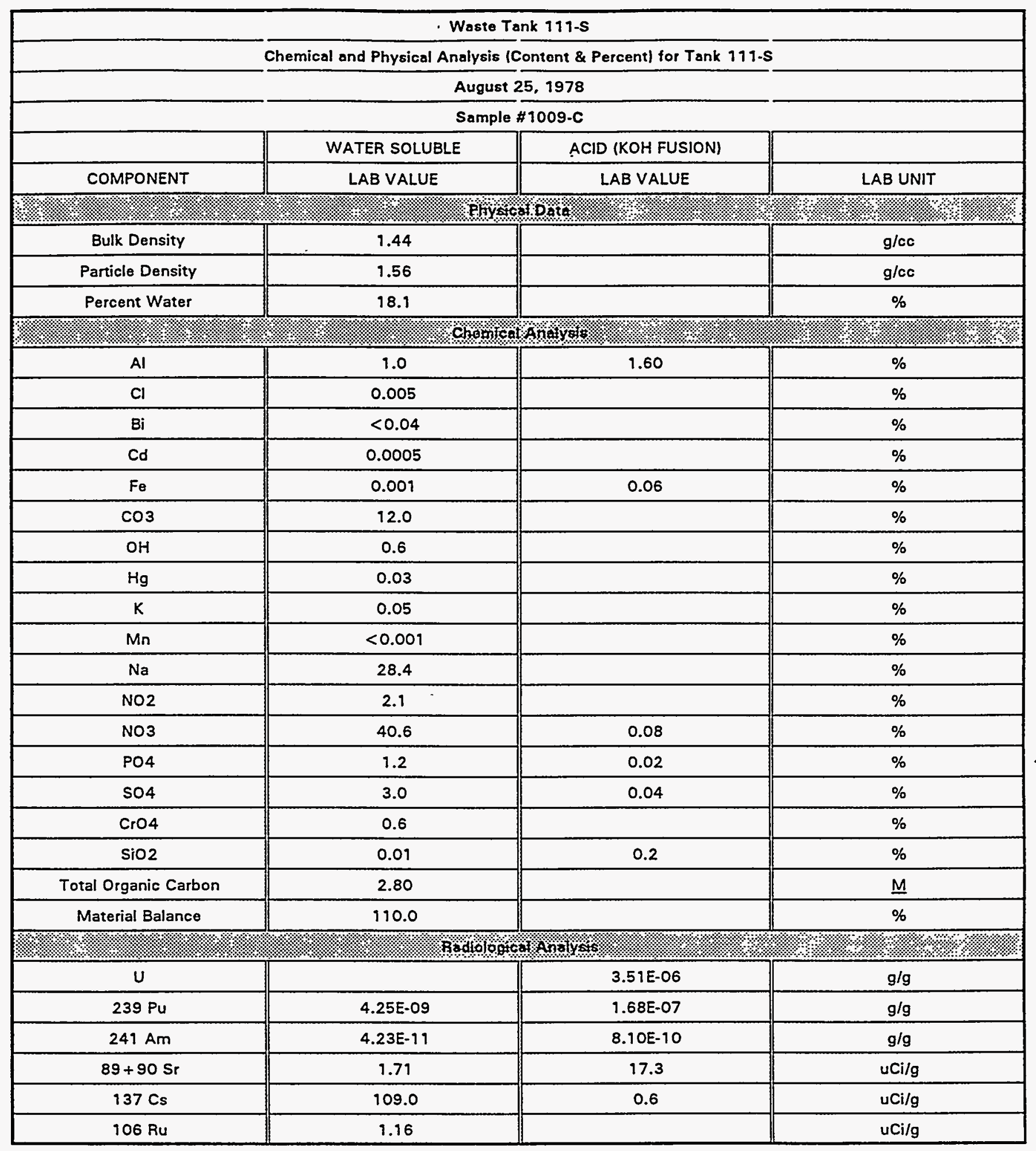




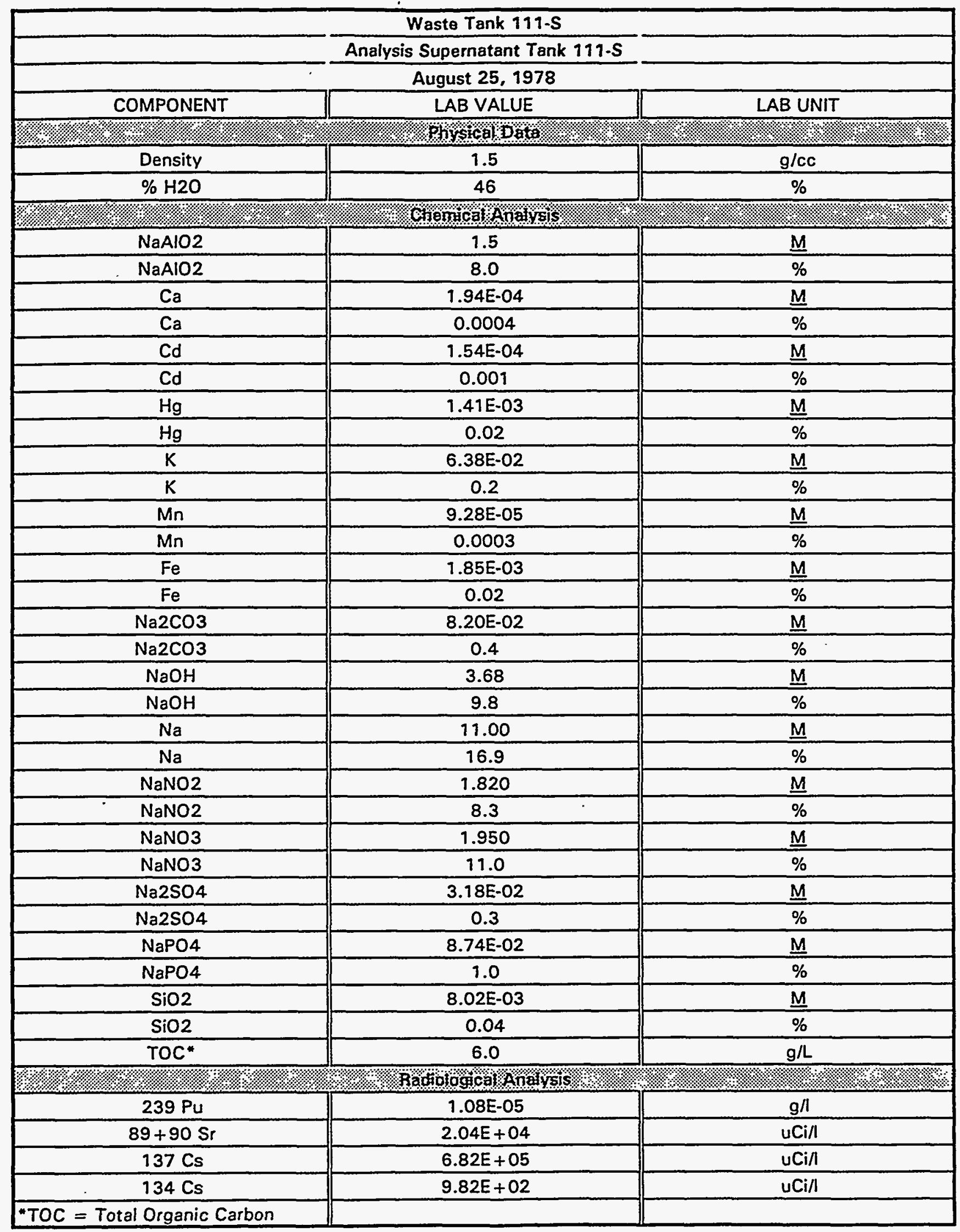




\begin{tabular}{|c|c|c|}
\hline \multicolumn{3}{|c|}{ Waste Tank 111-S } \\
\hline & Analysis Supernatant Tank 111-S & \\
\hline \multicolumn{3}{|c|}{ August 25, 1978} \\
\hline COMPONENT & LAB VALUE & LAB UNIT \\
\hline 1: & Pfysicalobuta & \%" \\
\hline Density & 1.46 & $\mathrm{~g} / \mathrm{cc}$ \\
\hline$\% \mathrm{H} 2 \mathrm{O}$ & 44.6 & $\%$ \\
\hline 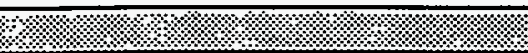 & Cherical Analysis: & II: \\
\hline $\mathrm{NaAlO} 2$ & 1.88 & $M$ \\
\hline $\mathrm{NaAlO} 2$ & 10.60 & $\%$ \\
\hline $\mathrm{Ca}$ & $3.70 \mathrm{E}-04$ & $\bar{M}$ \\
\hline $\mathrm{Ca}$ & 0.001 & $\%$ \\
\hline $\mathrm{Cd}$ & $1.62 \mathrm{E}-04$ & $\bar{M}$ \\
\hline $\mathrm{Cd}$ & 0.001 & $\%$ \\
\hline $\mathrm{Hg}$ & $1.53 \mathrm{E}-03$ & $\bar{M}$ \\
\hline $\mathrm{Hg}$ & 0.02 & $\%$ \\
\hline $\mathrm{K}$ & $4.21 \mathrm{E}-02$ & $\bar{M}$ \\
\hline $\mathrm{K}$ & 0.1 & $\%$ \\
\hline $\mathrm{Mn}$ & $1.13 \mathrm{E}-04$ & $M$ \\
\hline $\mathrm{Mn}$ & 0.0004 & $\%$ \\
\hline $\mathrm{Fe}$ & $<6.0 \mathrm{E}-04$ & $M$ \\
\hline $\mathrm{Fe}$ & $<0.004$ & $\%$ \\
\hline $\mathrm{Na} 2 \mathrm{CO} 3$ & 0.21 & $M$ \\
\hline $\mathrm{Na} 2 \mathrm{CO} 3$ & 1.5 & $\%$ \\
\hline $\mathrm{NaOH}$ & 3.77 & $\bar{M}$ \\
\hline $\mathrm{NaOH}$ & 10.33 & $\%$ \\
\hline $\mathrm{Na}$ & 9.6 & $M$ \\
\hline $\mathrm{Na}$ & 15.11 & $\%$ \\
\hline $\mathrm{NaNO} 2$ & 1.74 & $M$ \\
\hline $\mathrm{NaNO} 2$ & 8.20 & $\%$ \\
\hline $\mathrm{NaNO3}$ & 0.75 & $\bar{M}$ \\
\hline NaNO3 & 4.40 & $\%$ \\
\hline $\mathrm{Na3PO4}$ & $3.46 \mathrm{E}-02$ & $\bar{M}$ \\
\hline $\mathrm{Na3PO4}$ & 0.4 & $\%$ \\
\hline $\mathrm{Na} 2 \mathrm{SO}_{4}$ & 0.011 & $\bar{M}$ \\
\hline $\mathrm{Na2SO} 4$ & 0.1 & $\%$ \\
\hline $\mathrm{SiO} 2$ & $4.58 \mathrm{E}-03$ & $M$ \\
\hline $\mathrm{SiO2}$ & 0.02 & $\%$ \\
\hline $\mathrm{Pb}$ & $3.44 \mathrm{E}-04$ & $M$ \\
\hline $\mathrm{Pb}$ & 0.005 & $\%$ \\
\hline TOC* & 6.2 & $g / 1$ \\
\hline W W: & Radiological Aralysis & $1 \%$ \\
\hline $\mathrm{Pu}$ & $8.95 \mathrm{E}-06$ & $g / 1$ \\
\hline $89+90 \mathrm{Sr}$ & $2.51 E+03$ & $\mathrm{uCi} / \mathrm{I}$ \\
\hline $137 \mathrm{Cs}$ & $4.68 \mathrm{E}+05$ & $u \mathrm{Ci} / \mathrm{I}$ \\
\hline TOC $=$ Total Organic Carbon & & \\
\hline
\end{tabular}




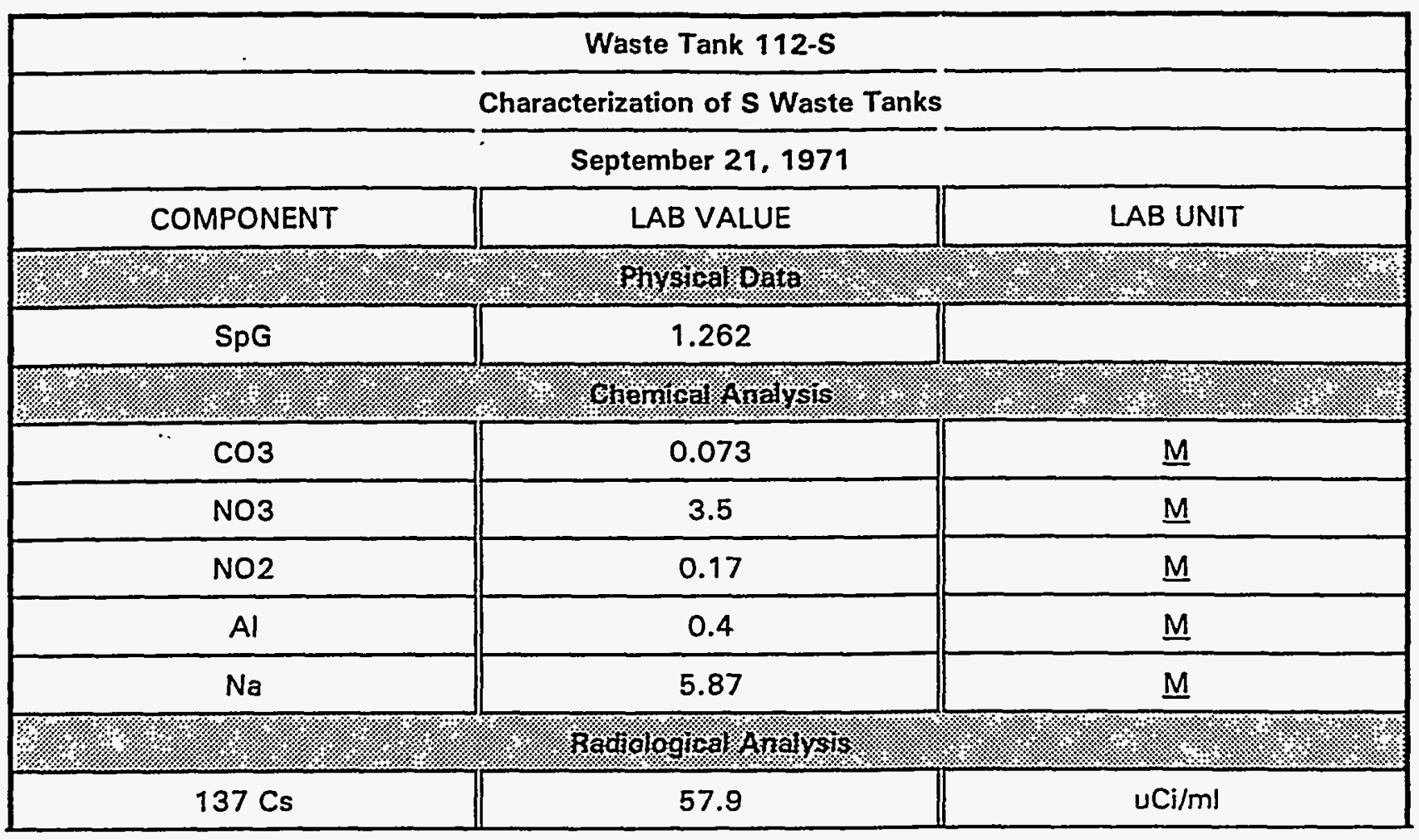




\begin{tabular}{|c|c|c|}
\hline \multicolumn{3}{|c|}{ Waste Tank 112-S } \\
\hline \multicolumn{3}{|c|}{ 242-S Feed Sample } \\
\hline \multicolumn{3}{|c|}{ January 7,1974} \\
\hline \multicolumn{3}{|c|}{ Sample: $T-181$} \\
\hline COMPONENT & LAB VALUE & LAB UNIT \\
\hline \multicolumn{3}{|c|}{ Prysicul Dara } \\
\hline \multicolumn{3}{|c|}{ Vis-OTR Clear, yellow, no solids $200 \mathrm{mR}$} \\
\hline $\mathrm{pH}$ & 12.2 & \\
\hline $\mathrm{SpG}$ & 1.1739 & \\
\hline Water & 66.74 & $\%$ \\
\hline \multicolumn{3}{|c|}{ 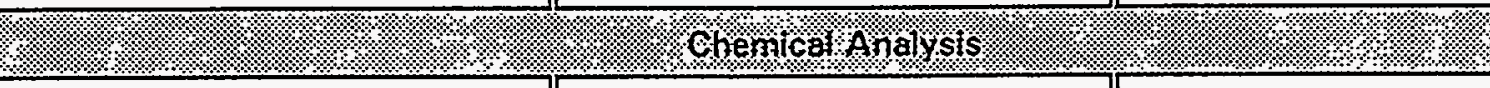 } \\
\hline $\mathrm{Na}$ & 4.95 & $\underline{M}$ \\
\hline $\mathrm{SO} 4$ & $1.60 \mathrm{E}-02$ & $\underline{M}$ \\
\hline Al & 0.348 & $\underline{M}$ \\
\hline NO2 & 0.118 & $\underline{M}$ \\
\hline $\mathrm{Fe}$ & $<9.65 E-05$ & $\underline{M}$ \\
\hline $\mathrm{co3}$ & 0.014 & $\underline{M}$ \\
\hline $\mathrm{OH}$ & 1.26 & $\underline{M}$ \\
\hline NO3 & 2.83 & $\underline{M}$ \\
\hline PO4 & $<3.77 E-04$ & $\underline{M}$ \\
\hline $\mathrm{F}$ & $2.95 \mathrm{E}-03$ & $\underline{M}$ \\
\hline $\mathrm{Pu}$ & $2.46 \mathrm{E}-06$ & $g / 1$ \\
\hline \multicolumn{3}{|c|}{ ( } \\
\hline GEA $137 \mathrm{Cs}$ & $5.83 E+04$ & $\mathrm{uCi} / \mathrm{ml}$ \\
\hline $90 \mathrm{Sr}$ & 16.15 & $\mathrm{uCi} / \mathrm{l}$ \\
\hline
\end{tabular}




\begin{tabular}{|c|c|c|c|}
\hline \multicolumn{4}{|c|}{ Waste Tank 112-S } \\
\hline \multicolumn{4}{|c|}{ Analysis of $112-5$ Supernatant Liquids } \\
\hline \multicolumn{4}{|c|}{ July 8, 1974} \\
\hline \multicolumn{4}{|c|}{ Received: May 10-11, 1974} \\
\hline COMPONENT & LAB VALUE & LAB VALUE & LAB UNIT \\
\hline 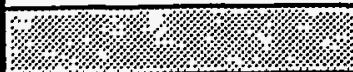 & \% & & ? \\
\hline Density & 1.501 & & \\
\hline $\mathrm{H} 2 \mathrm{O}$. & & 58.3 & $\%$ \\
\hline ২.\% \%. & $1 \%, 8,18 \mathrm{ch}$ & is: & 8. \\
\hline $\mathrm{NaAlO} 2$ & $1.19 \mathrm{M}$ & 6.5 & $\%$ \\
\hline $\mathrm{NaNO} 2$ & $2.02 \mathrm{M}$ & 9.3 & $\%$ \\
\hline NaNO3 & $2.5 M^{*}$ & 14.2 & $\%$ \\
\hline $\mathrm{NaOH}$ & $5.72 \mathrm{M}$ & 15.2 & $\%$ \\
\hline Total & & 103.5 & $\%$ \\
\hline 1. & $4 \% 18.18$ Rad & 4 sis. & \%, \\
\hline $137 \mathrm{Cs}$ & & & $\mathrm{uCi} / \mathrm{l}$ \\
\hline
\end{tabular}




\begin{tabular}{|c|c|c|}
\hline \multicolumn{3}{|c|}{ Waste Tank 112-S } \\
\hline \multicolumn{3}{|c|}{ Analysis of Tank Farm Samples } \\
\hline \multicolumn{3}{|c|}{ December 9, 1974} \\
\hline \multicolumn{3}{|c|}{ Sample T-8122 } \\
\hline COMPONENT & LAB VALUE & LAB UNIT \\
\hline 3... & PPY STGPDare & 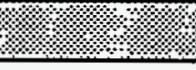 \\
\hline \multicolumn{3}{|c|}{ Vis-OTR: Yellowish green, $50 \%$ solids. $110 \mathrm{mRad} / \mathrm{hr}$} \\
\hline $\mathrm{pH}$ & 13.7 & \\
\hline $\mathrm{SpG}$ & 1.5909 & \\
\hline \multicolumn{3}{|c|}{ Ghenical Analysis } \\
\hline $\mathrm{OH}$ & 4.54 & $\underline{\underline{M}}$ \\
\hline $\mathrm{CO} 3$ & 0.293 & $\underline{M}$ \\
\hline \multicolumn{3}{|c|}{ Radiologlcal analysis } \\
\hline GEA $134 \mathrm{Cs}$ & $8.29 E+03$ & uCi/gal \\
\hline GEA $137 \mathrm{Cs}$ & $1.75 E+06$ & uCi/gal \\
\hline
\end{tabular}




\begin{tabular}{|c|c|c|}
\hline \multicolumn{3}{|c|}{ Waste Tank 112-S } \\
\hline \multicolumn{3}{|c|}{ Analysis of Salt from 112-S } \\
\hline \multicolumn{3}{|c|}{ December 10, 1974} \\
\hline COMPONENT & LAB VALUE & LAB UNIT \\
\hline 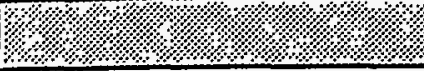 & 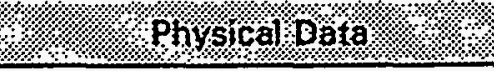 & $\sqrt{3}$ \\
\hline Bulk Density & 1.304 & $g / c c$ \\
\hline Particle Density & 1.918 & $g / c c$ \\
\hline 1. & 18 Gremical 4 Aratysis & 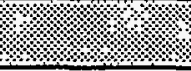 \\
\hline $\mathrm{H} 2 \mathrm{O}$ & 15.8 & $\%$ \\
\hline $\mathrm{NaAlO} 2$ & 2.5 & $\%$ \\
\hline $\mathrm{A} 1203$ & 0.1 & $\%$ \\
\hline $\mathrm{NaNO} 2$ & 3.1 & $\%$ \\
\hline NaNO3 & 58.7 & $\%$ \\
\hline $\mathrm{NaOH}$ & 4.4 & $\%$ \\
\hline $\mathrm{Na} 2 \mathrm{CO} 3$ & 14.6 & $\%$ \\
\hline $\mathrm{Na} 3 \mathrm{PO} 4$ & 0.8 & $\%$ \\
\hline Total & 100 & $\%$ \\
\hline $4: 191.1 \%$ & Radiological Analysis: & \\
\hline $137 \mathrm{Cs}$ & 10.1 & $\mathrm{uCi} / \mathrm{g}$ \\
\hline $8990 \mathrm{Sr}$ & 0.67 & $\mathrm{uCi} / \mathrm{g}$ \\
\hline $239 \mathrm{Pu}$ & $<1.00 \mathrm{E}-08$ & $g / g$ \\
\hline
\end{tabular}


THIS PAGE LEFT INTENTIONALLY BLANK 
Atlantic Richfield Hanford Company

$00: 25 y$

Date: Septembe: 21, 1971

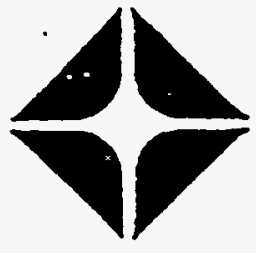

TC:

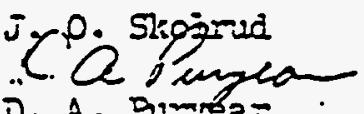

$5 / 0 \% 1$

suce: D. A. Euryez

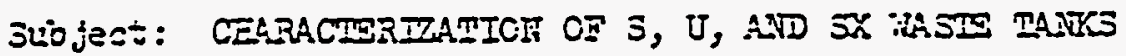

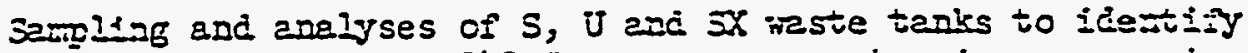
iee. To inte three coe leter samples frcm the 5 Jan tanks iare beel processed tirough the labcretory recuum eveporator. In

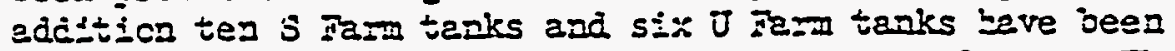
sampled and suoritied for cineracterzation aselyses. The gesults are shom in tie follaring tajle.

\section{Conzacterization Araiyses cf ileste Baniks}

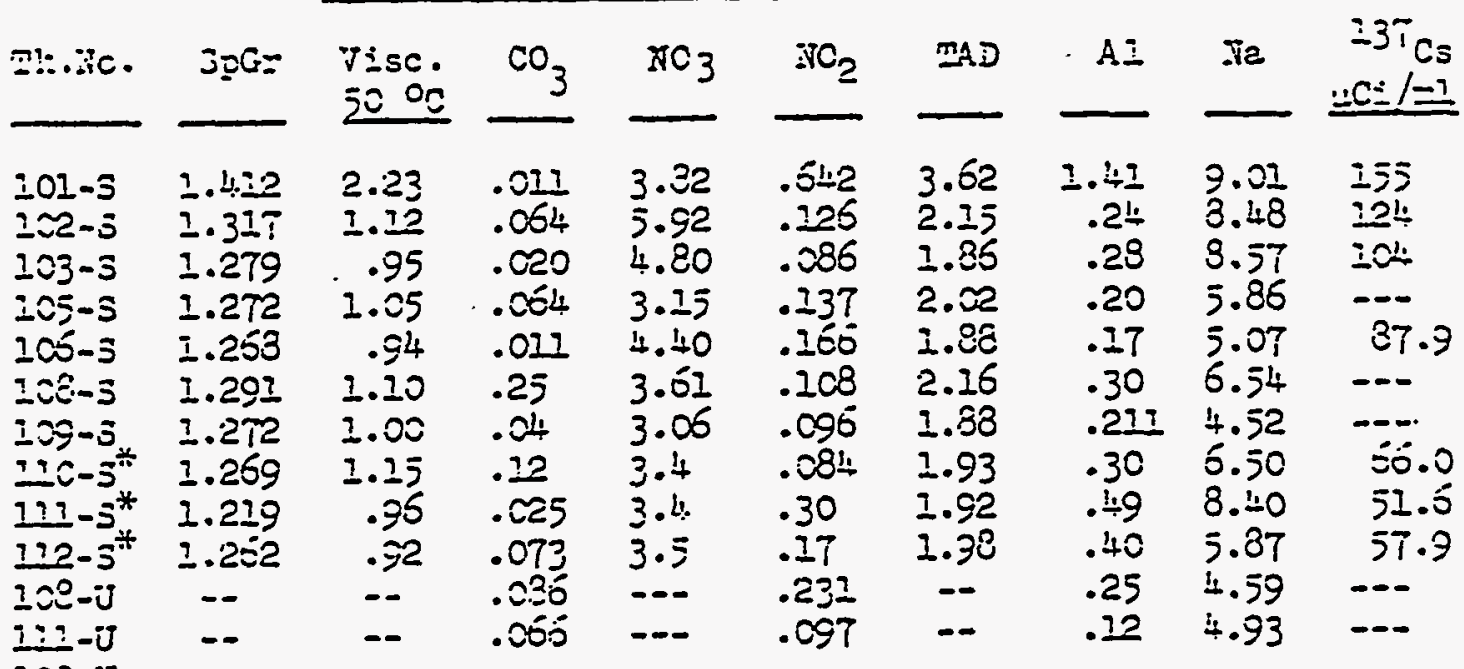

$i \approx 5-i$

$103-i$

$105-U_{1}$

$106-i$

* 3an in lejocratory vacurum evejoretce.

The above areiyses appear notpel except sewole l01-s.

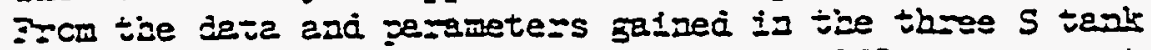
semple evepcraticn was, tie results of lol-s apger=s to

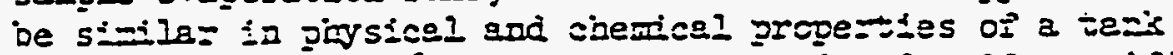

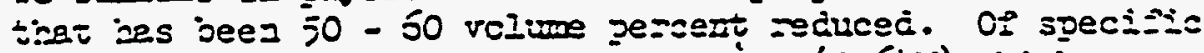

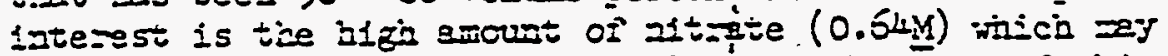

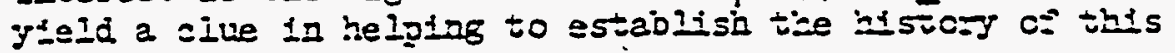
tanis. 
J. 0. Sici-ua

इอ5?

Sezterbe: 21, 1972

If there are afj questicns pleese cell we ca $2-7478$.

DLP:ah

se: GI 3crsicein

is 3ucikingian

ME Cacoibel

DJ Iarled

İie

I.3

Eocess Ains (5) 
Date: December 20, 1974

To:

R. L. Walser

5101.2

From: R. E. wheeler FPlPfictic

SUbject: AMALYSIS OF TARK FARM SAYPLES

SAH:PLE: T-8C84 10?-5 Peceived $9 / 73 / 74$

Vis-OTR: Hitky yellow. No solids. $250 \mathrm{mRad} / \mathrm{hr}$

PH: $\quad 14$

SFG: $\quad 1.205$

OH: $\quad 0.415 \mathrm{H}$

Al: $\quad 0.333 \mathrm{H}$

:iz: $\quad 2.00$ ․․

$: 10_{2}: \quad 0.197$ M

:i०3: $\quad 1.53 \underline{\mu}$

Py: $\quad<4.4 \overline{3} \times 10^{-5} \mathrm{gm} / \mathrm{gal}$

SO $_{4}: \quad 8.78 \times 10^{-3} \mathrm{H}$

$\mathrm{PO}_{4}: \quad 9.34 \times 10^{-2} \underline{\mathrm{M}}$

$\because: \quad 3.55 \times 10^{-3} \underline{\underline{H}}$

Cग: $\quad 0.1111 \mathrm{M}$

EDA: $\quad l \equiv{ }^{L} C S-2.18 \times 10_{5}^{3}: C i j g a l$

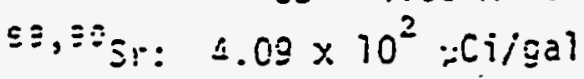

Wa

ב:

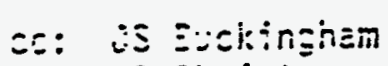

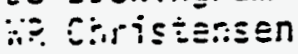

$\because \because . r_{1}=5=e \tau$

$\because \because$ :ee $i \in r$

$\therefore \therefore$ ㅅn. 


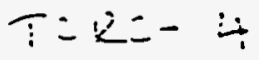

Date: July 16,1975

To: W. R. Christensen

From: J.E. Horton ffoctor

SUbject: ANALYSIS AND CHARACTERIZATION

OF SIUDGE FROM TANK IOI-S

Chemical Technology Laboratory (CTI) received a sample of sludge from Tank lol-s on June 4, 1975. The sample had a consistency of soft mud and was dark brown in color.

The calculated heat generation rate for the l01-s sludge, based on $89+9{ }^{\circ} \mathrm{Sr}$ and $137 \mathrm{Cs}$ content, was $8.63 \times 10^{-3}$ watts/liter sludge.

Sludge analyses were made by fusing $1.78 \mathrm{milli-}$ liters of "as received" sludge with KOH, dissolving the melt with concentrated HCl, and diluting to a volume of 350 milliliters with water. Analyses are shown in Table $I$.

Please call if you have any questions regarding this work.

JEH:ms

Att.

$\begin{array}{ll}\text { cC: } & \text { H Babad } \\ \text { JS Buckingham } \\ \text { RG Geier } \\ \text { DC Iini } \\ \text { NC Rodewald } \\ \text { RE Var der cook } \\ \text { WJ Var Slyke }\end{array}$

RI Walser

JC Womack

Process Aids (7)

CTI-123

File (2)

IB 
WHC-SD-WM-ER-323, Rev. 0

5101.3

TABLEI

ANALYSIS OF TANK lO1-S ("as receivea")

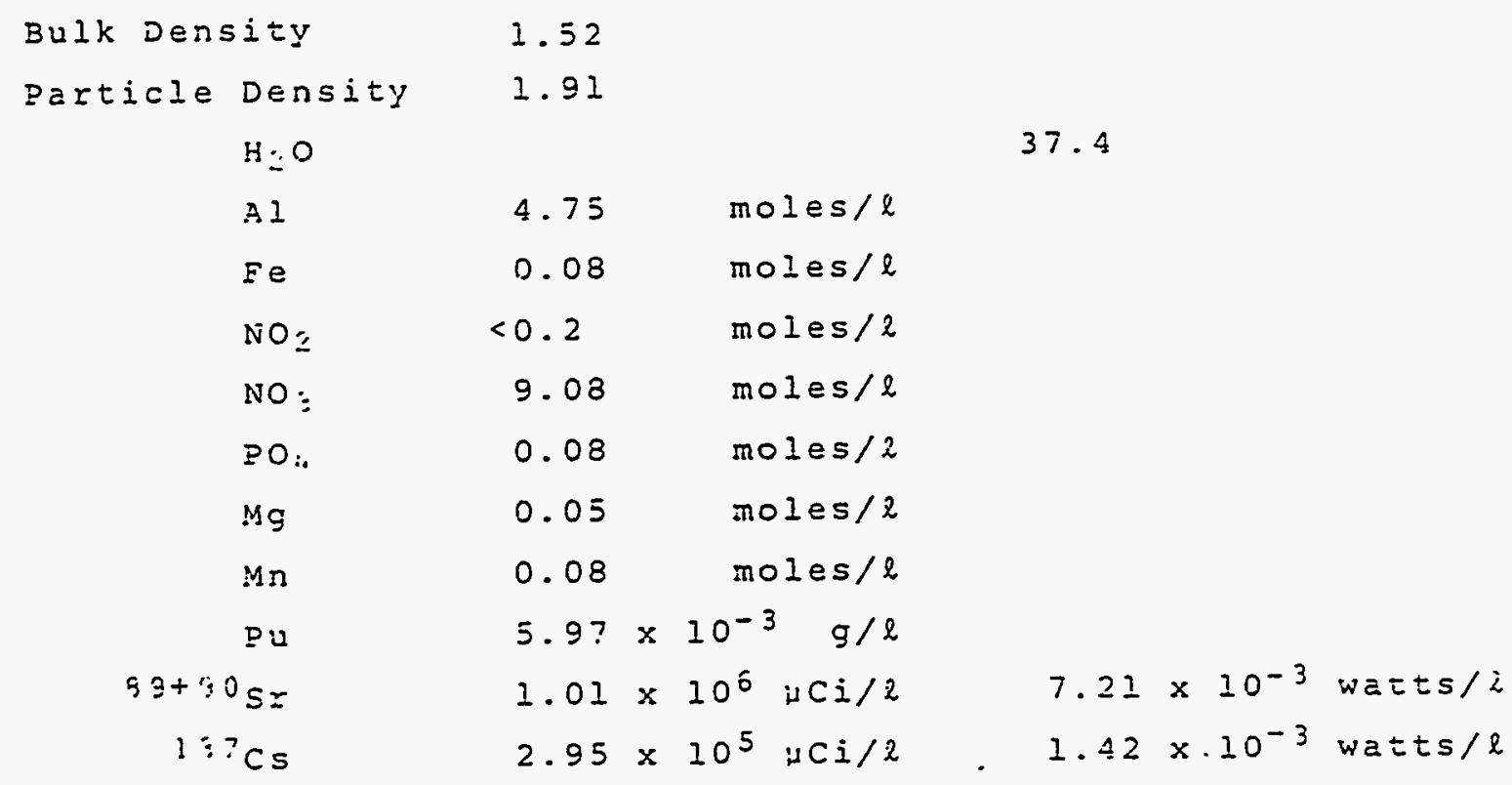


Date: Decenber 5, 1975

To: R. L. Walser

From: R. E. Wheeler, 2-2449 Re. rplezen

$\begin{array}{ll}\text { Subject: } & \text { ANALYSIS OF TANK FARM SAMPLES } \\ & \text { Sample: T-9715 Tank: } 101-S \text { S }\end{array}$ Received: Novenber 19, 10j5

Vis-OTR: Yellow, black Suspended solids 2 rad

$\mathrm{pH}: \quad 13.6$

SPG: $\quad 1.51 @ 64.5^{\circ} \mathrm{C}$

OH: $\quad 3.60 \underline{M}$

A1: $\quad 1.82$ M

$\mathrm{CO}_{3}: \quad 0.318 \mathrm{M}$

$\mathrm{NO}_{2}: \quad 2.22 \mathrm{M}$

$\mathrm{NO}_{3}: \quad 4.68 \mathrm{M}$

GEA: $\quad{ }_{134} \mathrm{Cs}-8.21 \times 10_{6}^{3} \mathrm{pCi} / \mathrm{gal}$

${ }^{137} \mathrm{Cs}-2.44 \times 10^{6} \mathrm{HCi} / \mathrm{gal}$

${ }^{103} \mathrm{Ru}-<1.27 \times 10^{3} \mu \mathrm{Ci} / \mathrm{gal}$

$\mathrm{NH}_{4}: \quad \quad 1.94 \times 10^{-2} \underline{\mathrm{M}}$

Water: $\quad 42.82 \%$

REW: eyw

Cc: JS Buckingham

WR Christensen

1. RM Ginestet

KE Nelson

JC Womack 


\section{$\because \div \therefore-5$}

Atsentic Richfield Hantord Company
WHC-SD-WM-ER-323, Rev. 0

$00=25$

Dа亡е: $\quad$ sepiemice: 21,1971

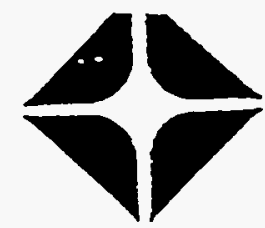

Ic:

İส:

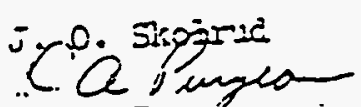

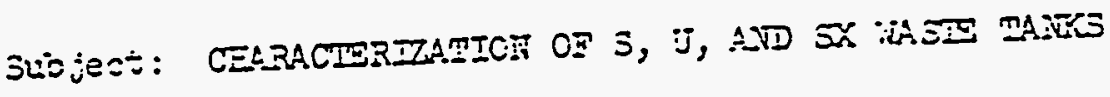

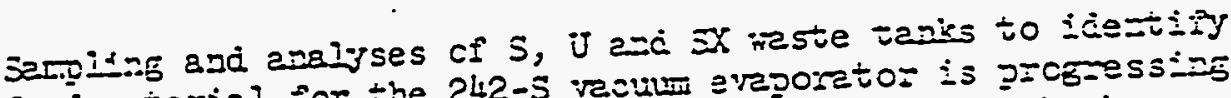

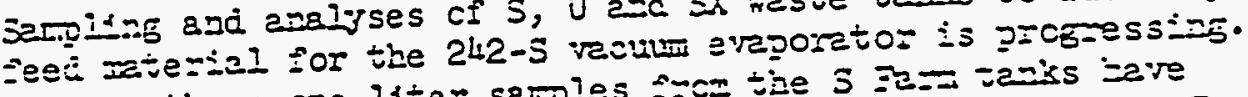

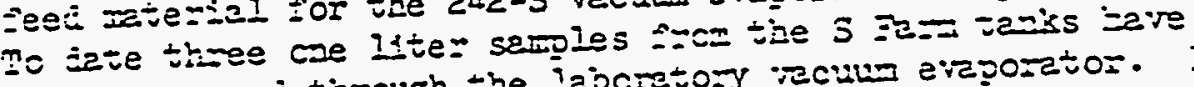

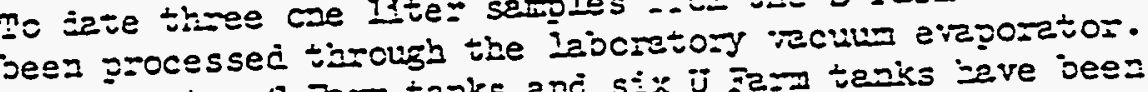

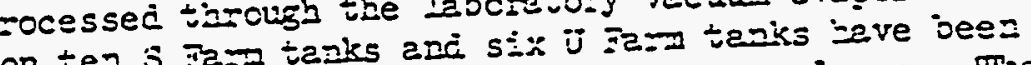

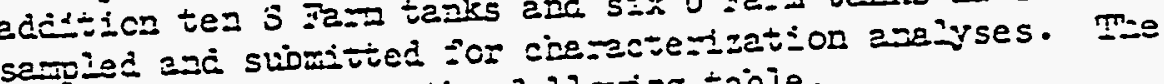

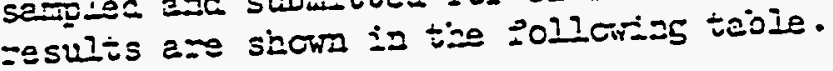

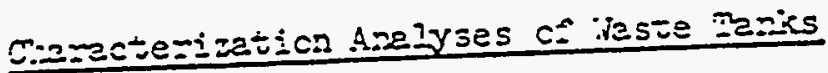

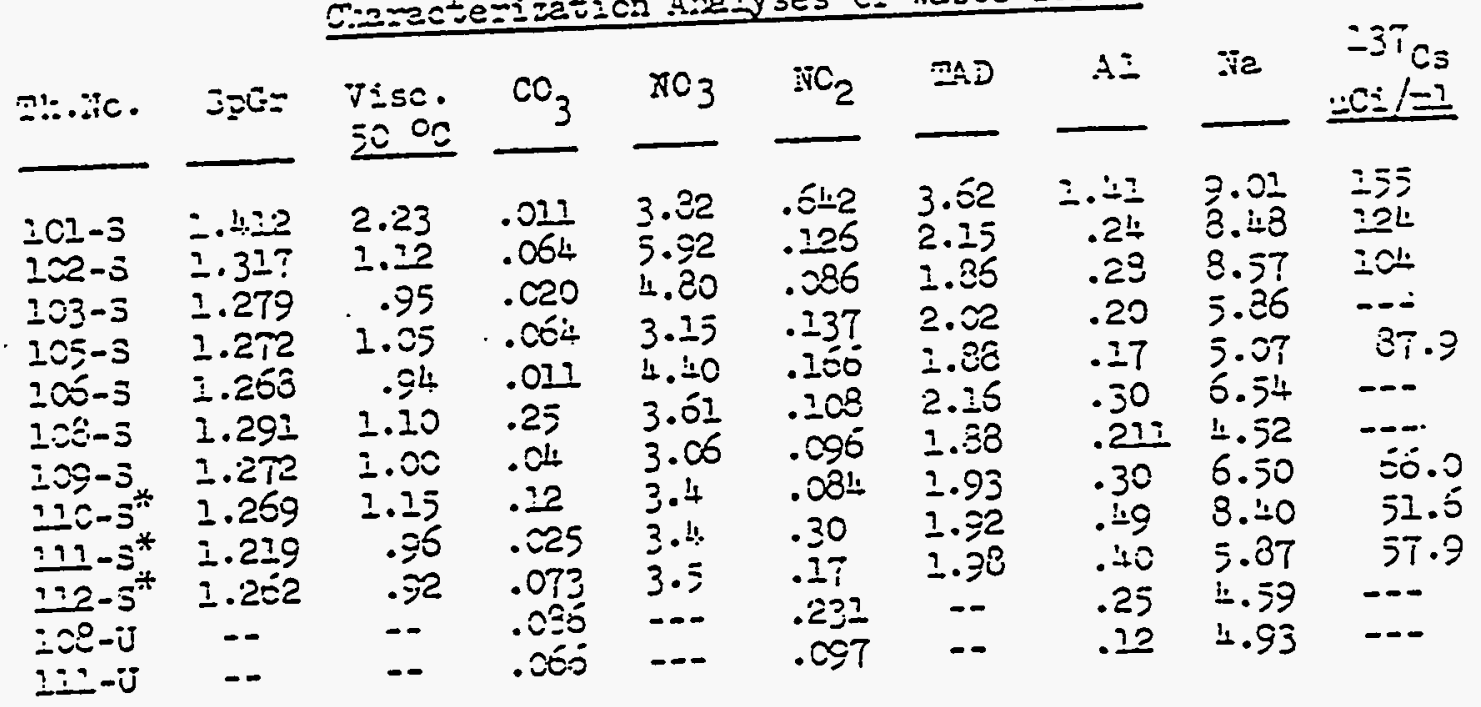

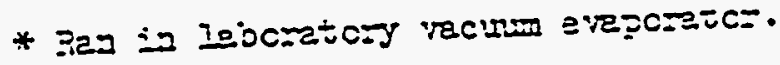

The abcre aneiyses apper- nchel excezt samis I0I-s.

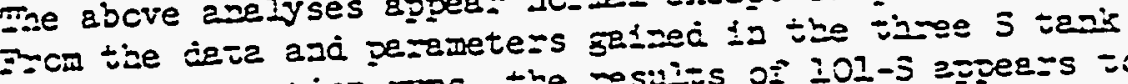

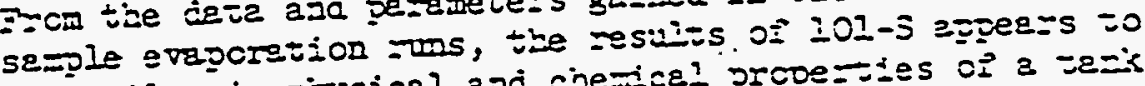

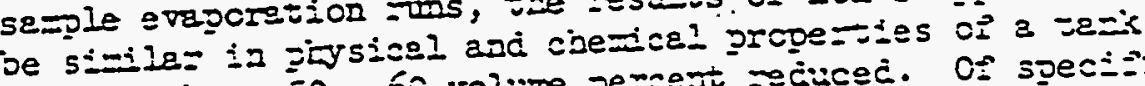

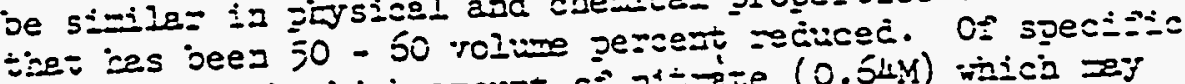

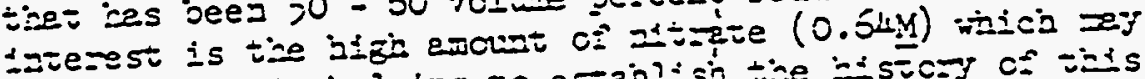

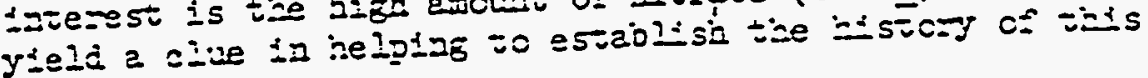
vank. 
J. 0. Sici-sic

इ5e 2

Seztexice= 2I, I9T

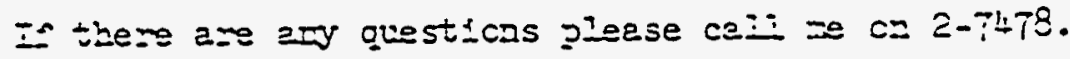

D洋: 形

sะ: GI 3crsisein

is 3uclinginem

证 Cambei

DJ Ie-lei

Fivis

I. 3

Zrceess Aids (5) 
Atlantic Richfield Hanforc Company

$$
5-107
$$

Date: $\quad$ November 27,1973

Io:

G. I. Borsheim

From:

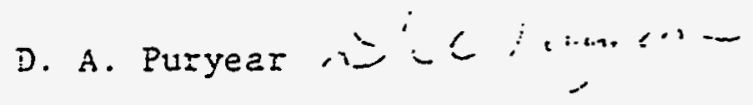

Subject: ANALYSIS AND IDENTIFICATION OF SOIIDS IN TK 107 AND 102-S.

Reference: D. A. Puryear, Phosphate Solidification of caustic process Solution Waste, ARH-SA-120 May 1972.

Samples from Tank 107 and $102-S$ were referred co CIL for identification of solids which plugged a jumper line irom Tank 107-S to 102-S. The temperature of the tanks are $90{ }^{\circ} \mathrm{F}$ and the samples were licuid when taken. However, the sample from TK $107-5$ was about 50 percent crystalline when received by $C T L$.

Analysis of the original sample from IX 107-s showed a phosphace molarity of 0.384 and a water content of 81 weight percent. From the reference above on phosphate solidification of waste, experience was gained that shows prosphates are only partially soluable in caustic solution because they Form a solid solution of the formulas $7\left(\mathrm{Na}_{3} \mathrm{PO}_{4} \cdot 12 \mathrm{H}_{2} \mathrm{O}\right) \mathrm{NaOH}$ or $5\left(\mathrm{Na}_{3} \mathrm{PO}_{4} \cdot 12 \mathrm{H}_{2} \mathrm{O}\right) \mathrm{NaOH}$ at a sodium to phosphace mole ratio of 3.12 and 3.21 respectiveiy. Tank $107-5$ has by analysis an available sodium to phosphate mole ratio of 4.36 . The high percentage of water actributes to tie crystals being in solution at.90 $=\mathrm{F}$. Tests on solids formation as a function of cooling showed that crystallization began to occur at $89.6^{\circ} \mathrm{F}$ and was near maximum solidification at $82.4 \mathrm{~F}$.

Table I below shows the chemical analyses of sank 107 and $102-5$. The first column is the original analysis oi the analycical laboratory as designated in ARH-1601. The second column shows the analysis of the same sample aiter it was melced and dilured $1: 1$ with water. Ali answers have been calculared to the original sample. The third column is a resample of rank 107 taken on $11 / 16 / 73$. Sample preparation of the resample in the laboratory was made as a $1: 1$ dilution after melting the sample. 
G. L. Borshein

Page 2

November $27,-1973$

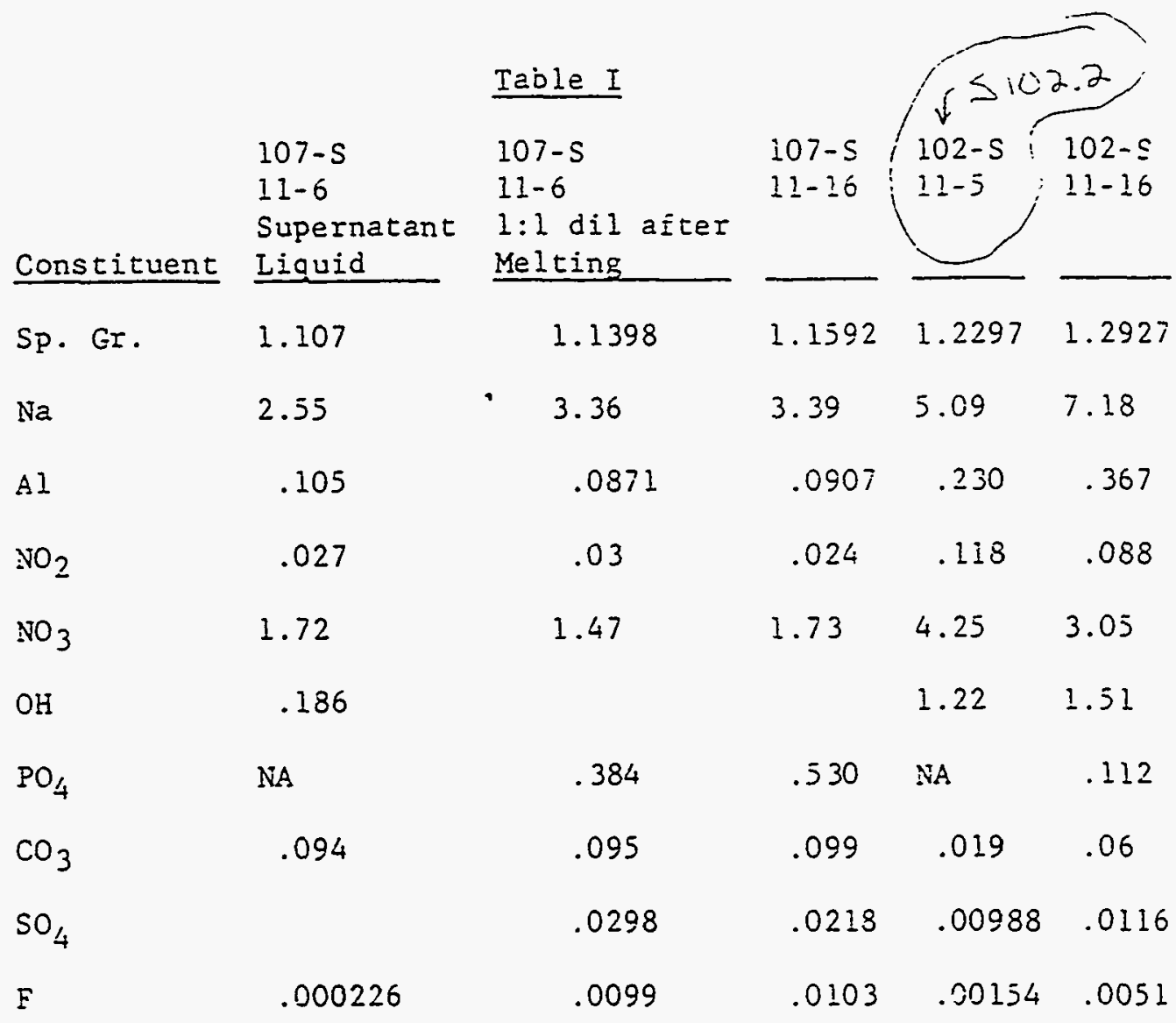

Column four is the chemical composition of tank 102 prior to 242-S start-up and is really not related to the plugged line whereas column five is a sample of tank 102 aiter it received some transfer from tank 107 prior to the jumper line plug up.

All the results shown in Table 1 appear to be realistic. The important discrepancies can be explained. Eor example the specific gravity of column 1 appears light as does the scdium result. The lower specific gravity and sodiun zesult is the result of analyzing the supernatant liquid. 3y meliting the samp-es and obtaining a homogeneous solution the total of the sample is accounted for.

A plot of solids formation as a function of cemperature is shown in Figure 1 for tank $107-5,102-s$ and $a 2: 1$ blend of 107-S wich 102-S. Solidification begins at $32^{\circ}, 18^{\circ}$, and $30^{\circ} \mathrm{C}$ respectively for the three solutions.

DAP: jf

cc: JS Buckingham

MH Campbell

WR Christensen

HH Hopkins

RI Walser 
SAMPLE ANALYSES

IANKS 107-S AND 102-S

iank

Date Sampled

Samole Number

soecific Gravity

DTA

?adioisotoces

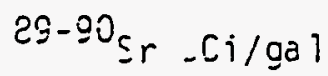

i37 cs :ci/gal

$\left.{ }^{134} \mathrm{Cs}=\mathrm{Ci} / \mathrm{ga}\right]$

${ }^{E 0}$ Co $\quad \mathrm{Ci} / \mathrm{gal}$

$239_{\mathrm{pu}} \mathrm{gm} / \mathrm{gal}$

Cnemical
7.5

$1.0 \times 10^{5}$

$8.2 \times 10^{\prime}$

$2.5 \times 10^{2}$

$8.1 \times 10^{-5}$
$107-5$

$11 / 17 / 73$

475

1.16

lio Exotherm

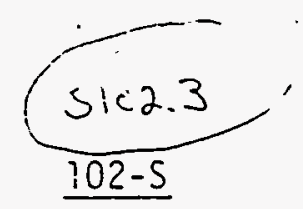

$11 / 16 / 73$ 450

1.29

No Exotherm
$3 i^{2++} \quad$ II

Haे

$\mathrm{AlO}_{2}^{-}$诖

$\mathrm{SH}^{-2} \quad \mathrm{H}$

$\mathrm{NO}_{3}{ }^{-} \quad M$

$\mathrm{NO}_{2}{ }^{-}$in

$\mathrm{CO}_{2}=\quad M$

$\mathrm{SO}_{4}=\quad M$

$F-y$

cl $\quad M$

$\mathrm{PO}_{4} \equiv \quad \overline{\mathrm{M}}$
.0 .005

3.36

0.287

0.186

1.47

0.030

0.095

0.030

0.010

0.384

FREEZING POINT DATA
$4.4 \times 10^{\prime}$

$8.6 \times 10^{4}$

$1.5 \times 10^{2}$

$1.9 \times 10^{2}$

$10.7 \times 10^{-5}$
4. $3 \times 10^{1}$

$4.0 \times 10^{5}$

$2.8 \times 10^{2}$

iv.D.

$5.2 \times 10^{-5}$
.0 .005

3.39

0.091

0.292

1.73

0.024

0.099

0.022

0.010

0.53
.0 .005

7.18

0.367

1.51

3.05

0.088

0.06

0.012

0.005

0.112
102-S

${ }^{\circ} \mathrm{C} v / 0$ solids 18 Solids appear 15 : $100^{2}=$
$25 \quad 15$

$24 \quad 17.5$

$22 \quad 23$

$20 ! 25$

$73 \quad 57$

$15: \div 100$ 
TCR -5

Atlantic Richfield Hanford Company

WHC-SD-WM-ER-323, Rev. 0
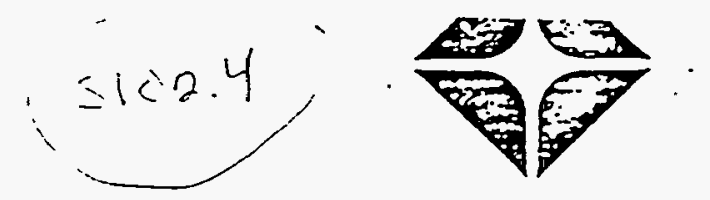

Date: December 7, 1973

To: R. L. Waiser

From: H. H. Sant $\geqslant=0,7, \frac{1}{\pi}, T$

Subject: 242-S FEED SAMPLE T-459 (11/16/73) 102-S

\begin{tabular}{|c|c|c|}
\hline Vis-OT & Yellow, $27 \%$ solids, 100 & $\mathrm{mR} /$ hour \\
\hline $\mathrm{pH}$ & $11.9 \odot 40^{\circ} \mathrm{C}$ & \\
\hline SpG & 1.2927 & \\
\hline DTA & No Exotherm & \\
\hline Pu & $1.63 \times 10^{-5} \mathrm{~g} / 1$ & \\
\hline $\mathrm{Na}$ & $7.18 \mathrm{M}$ R.R.7.63 M & \\
\hline $\mathrm{SO}_{4}$ & $1.16 \times 10^{-2} \underline{M}$ & \\
\hline A1 & $0.367 \mathrm{M}$ & \\
\hline $\mathrm{NO}_{2}$ & $0.088 \mathrm{M}$ & \\
\hline$F$ & $5.10 \times 10^{-3} \underline{M}$ & - \\
\hline $\mathrm{CO}_{3}$ & $0.060 \underline{M}$ & $\vdots$ \\
\hline $\mathrm{Cr}$ & Method being developed & ' \\
\hline $\mathrm{OH}$ & $1.51 \underline{M}$ & $\therefore$ \\
\hline GEA & $\begin{array}{l}134 \mathrm{Cs}-72.88 \mu \mathrm{Ci} / 1 \\
{ }_{137} \mathrm{CS}_{5}-1.06 \times 10^{5} \mathrm{Ci} / 1\end{array}$ & \\
\hline $\mathrm{NO}_{3}$ & $3.05 \mathrm{M}$ & \\
\hline \multicolumn{2}{|c|}{$\begin{array}{l}30 \% \text { Reduction } 33.8 \% \\
50 \% \text { Reduction } 53.8 \%\end{array}$} & $\begin{array}{l}\approx \text { Solids - 1.79\% } \\
\approx \text { Solids - } 93.9 \% \\
\%\end{array}$ \\
\hline \multicolumn{3}{|c|}{$8990 \mathrm{Sr}-11.31 \mathrm{\mu Ci} / \mathrm{T}$} \\
\hline $\mathrm{PO}_{4}$ & $0.112 \mathrm{M}$ & $\cdot$ \\
\hline$B i$ & $<4.85 \times 10^{-3} \underline{M}$ & \\
\hline
\end{tabular}

WHS: bp

CC: WR Christensen

GW Reddick, Jr. WH Sant 
Analyses of 107-S and 102-S samples show that the 107-S material was vary close to its freezing point even while in TK-107-S. Specific gravity determinations indicate the 107-5 material was less coricentrated than the 242-S feed in 102-S and this is confirmed by the chemical analyses which show lesser concentrations of all major cations and anions with the exception of phosphate ion. The high phosphate ion in 107-5 presumabiy originated in $N$ Reactor flushes where phosphoric acid is one of the major decontaminating agents. The freezing point data for the two to one blend of 107-S to $102-j$ (the approximate volume ratio below the 242-5 feed recirculation line in the "problem" riser) and the individual solution freezing point curves indicate that there was no reaction between the solutions thet promoted solids formation.

High phosphate ion concentrations in caustic solutions are known to produce high freezing point trisodium orthophosphate hydrates - indeed, this is ine basic chemical reaction for the phosphoric acid solidification prototype. The 107-5 solution approached the stoichiometric rasio of phosphate ion to available sodium ion planned for the solidification ilowsheet ( $1 \pm n 3.2$ ) and only the high percentage of water (approximately 21 weight percent v.S. approximately 50 weight percent for the solidification process) prevented 107-S from solidifying. The 107-5 tank bulk temperature (ca. $97^{\circ} \mathrm{F}$ ) was nigh enough to prevent freezing, although the solution wes essentially saturated, as was the heat traced line from 107-5 to 102-5. However, when the solution

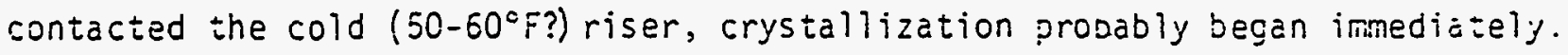


WHC-SD-WM-ER-323, ReV. O $\leq 102.5$

Date: Decemider 18, 1973

To: R. L. Hâtser

From: H. H. Sant $\theta=$ Hith

Subject: 242-S FEED SAMPLES T-0.488 (10/5/73) i02-S

Vis-OTR Clear, yeilow, no solids, $450 \mathrm{mR}$

$\mathrm{pH}$

11.4

SPG

1.2297

DTA

No Exotherm

$\mathrm{Pu}$

$1.29 \times 10^{-5} \mathrm{~g} / 1$

$1220 \%=1.0410^{-9}$

$\mathrm{Na}$

5.43 픈

$\mathrm{SO}_{4}$

$9.38 \times 10^{-3}$

A1

0.230 is

$\mathrm{NO}_{2}$

$0.118 \mathrm{in}$

$\mathrm{F}$

$1.54 \times 10^{-3}$ in

$\mathrm{CO}_{3}$

0.019 is

$\mathrm{Cl}$

$\mathrm{OH}$

1.22 is

GEA $\quad{ }^{1.37} \mathrm{CS}-9.86 \times 10^{4} \% \mathrm{Ci} / 7$

$\mathrm{NO}_{3}$

4.25 프

$30 \%$ Reduction - 31.25\%

$\%$ Solids - $0.909 \%$

$50 \%$ Reduction - 50.0\%

$\%$ Solids - $11.25 \%$

$39{ }^{90} \mathrm{Sr} ; 1.99 \mathrm{HCi} / \mathrm{l}$

$\mathrm{PO}_{4}$

$<3.77 \times 10^{-4}$ is

:SHS:bp

CC: UR Christensen

G'd Reddick,.Jr.

INH Sent. : 
Date: January 25,1974

To: R. L. Walser

From: W. H. Sant Py?

Subject: ANALYSIS OF TAIN FARM SAMPLES

SAMPLE: T 1746 102-S

\begin{tabular}{|c|c|c|}
\hline VIS-OTR & \multicolumn{2}{|c|}{ Clear, yellow $\quad 1,000 \mathrm{mRad} / \mathrm{hr}$} \\
\hline Appearance & \multicolumn{2}{|c|}{$90 \%$ solids at room temperature } \\
\hline $\mathrm{pH}$ & \multicolumn{2}{|c|}{10.5} \\
\hline$D$ & \multicolumn{2}{|l|}{1.4081} \\
\hline$E A$ & \multicolumn{2}{|c|}{${ }^{137} \mathrm{Cs}-1.98 \times 10^{5} \mu \mathrm{Ci} / 1$} \\
\hline$\%$ Water & \multicolumn{2}{|c|}{59.39} \\
\hline \multicolumn{2}{|c|}{$30 \%$ Reduction - 27.5} & $\%$ Solids -5.17 \\
\hline \multicolumn{2}{|c|}{$50 \%$ Reduction - 51.25} & $\%$ Solids $-88.46^{x}$ \\
\hline \multirow[t]{4}{*}{ Cooling Curv } & $25^{\circ} \mathrm{C}$ & No solids for 45 minutes \\
\hline & $20^{\circ} \mathrm{C}$ & $<10 \%$ solids for 45 minutes \\
\hline & $15^{\circ} \mathrm{C}$ & $\sim 30 \%$ solids for 45 minutes \\
\hline & $10^{\circ} \mathrm{C}$ & $\leadsto 90 \%$ solids for 60 minutes \\
\hline
\end{tabular}

WHS: bp

cc: WR Christensen

GW Reddick, Jr.

WH Sant 

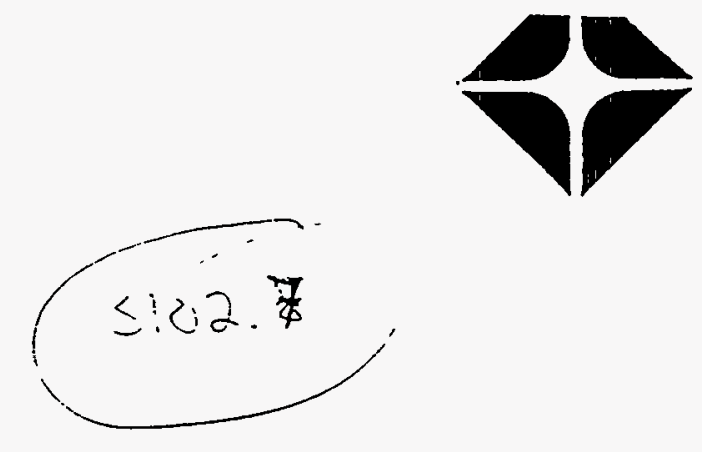

Date: $\quad$ February 11, 1974

To: R. L. Walser

From: W. H. Sant थ2:-

Subject: ANALYSIS OF TANK FARM SAMPLES

SAMPLE: $T 2164$ 102-S

VIS-OT No solids, $400 \mathrm{mR} / \mathrm{hr}$.

Appearance Yellow, clear

$\mathrm{pH} \quad 11.6$

Spg $\quad 1.3030$

GEA $\quad{ }^{134} \mathrm{Cs}_{5}-6.16 \times 10^{2} \mathrm{iCi} / 1$

${ }^{137} \mathrm{Cs}-1.62 \times 10^{5} \mathrm{iCi} / 1$

$\%$ Water $\quad 60.24 \%$

$30 \%$ Reduction - 26.25

$50 \%$ Reduction -51.25

\% Solids - 2.71

: Solids -70.51

Cooling Curve - Room temp. No Solids

$10^{\circ} \mathrm{C} \quad$ No Solids

$5^{\circ} \mathrm{C} \quad$ No Solids

WHS: bp

cc: WR Christensen

GW Reddick, Jr.

WH Sant 

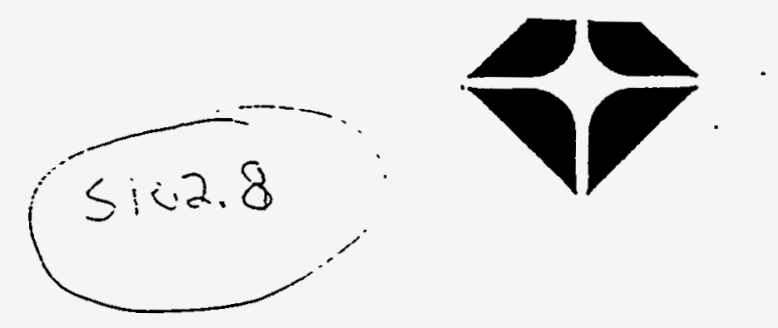

Date: February 22, 1974

To:

R. L. Waiser

From:

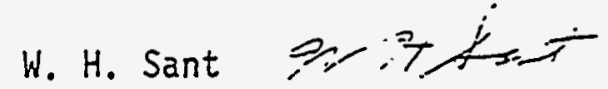

Subject: ANALYSIS OF TANK FARM SAMPLES

Sample: T-2359 102-S

VIS-OT: Clear, Yellow $1000 \mathrm{mr} / \mathrm{hr}$.

Appearance: No solids

$\mathrm{pH}: \quad 11.8$

SPG: $\quad 1.3868$

GEA: $\quad \begin{aligned} & 134 \mathrm{Cs}-3.8 \times \times 10^{2} \mu \mathrm{Ci} / 7 \\ & \quad 137_{\mathrm{Cs}}-2.29 \times 10^{5} \mathrm{HC} / 1\end{aligned}$

$\%$ Water: 57.99

Cooling Curve: No solids at room temperature. $10^{\circ} \mathrm{C}$ for $45 \mathrm{~min}$. no solids. $5^{\circ} \mathrm{C}$ for $60 \mathrm{~min}$. no solids.

30\% Reduction: 27.5

50\% Reduction: 50

$\%$ solids - 19.83

solids -93.75

WHS: jd

cc: WR Christensen

GW Reddick Jr.

WH Sant 
Date: FeEruary 25, 1974

To: R. L. Halser

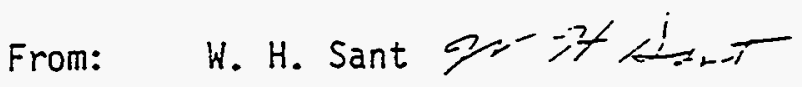

Subject: ANALYSIS OF TANK FARM SAMPLES

Sample: T 2520 102-S

VIS-OT: Clear $\quad 300 \mathrm{mrad} / \mathrm{hr}$.

Appear: Yellow No solids

SPG: $\quad 1.3315$

$30 \%$ Reduction - 27.5 \% Solids -3.96

$50 \%$ Reduction - $51.25 \quad \%$ Solids -75.64

\% Water: 64.38

Cooling curve: No solids at room temperature. $10^{\circ} \mathrm{C}$ for 45 min. no solids. $5^{\circ} \mathrm{C}$ for $60 \mathrm{~min}$. no solids.

$\mathrm{pH}: \quad 11.6$

GEA: $\quad \begin{aligned} & 134 \mathrm{CS}-3.07 \times 10_{5}^{2} \mu \mathrm{Ci} / 1 \\ & \quad 137 \mathrm{CS}-1.69 \times 10^{5} \mathrm{\mu i} / 1\end{aligned}$

WHS:jd

CC: WR Christensen

GW Reddick Jr.

WH Sant 

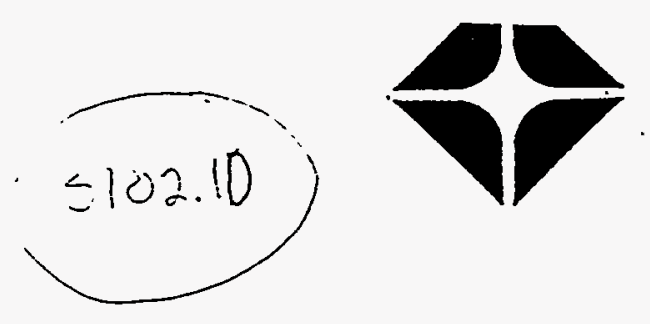

Date: $\quad$ March 7, 1974

To: R. L. Walser

From:

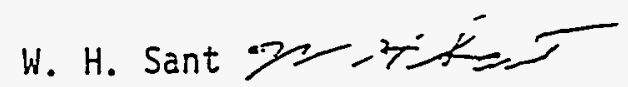

Subject: ANALYSIS OF TANK FARM SAMPLES

Sample: $\quad$ T2759 102-S

Vis-OT: $\quad 200 \mathrm{mrads} / \mathrm{hr}$.

Appearance: Yellow $\quad 85 \%$ solids at room temperature

pH: $\quad 10.9$

Sp̣: $\quad \quad \quad 1.310$ at $49^{\circ} \mathrm{C}$

GEA:

$$
\begin{aligned}
& \left.{ }_{134} C_{5}-9.01 \times 10^{2} \mathrm{HCi} / g a\right] \\
& \left.{ }_{137} C_{5}-4.81 \times 10^{5} \mathrm{HCi} / \mathrm{ga}\right]
\end{aligned}
$$

Water:

$62.77 \%$

$30 \%$ Reduction - 27.5

$50 \%$ Reduction - 50.0

Solids - $2.76 \%$

Solids $-7 i .25 \%$

Cooling Curve: $85 \%$ solids at room temperature $50^{\circ} \mathrm{C}$ for $45 \mathrm{~min}$. Trace of solids $45^{\circ} \mathrm{C}$ for $60 \mathrm{~min}$. $2 \%$ solids $40^{\circ} \mathrm{C}$ for $45 \mathrm{~min}$. $5 \%$ solids $35^{\circ} \mathrm{C}$ for $i 5 \mathrm{~min}$. $240 \%$ solids $30^{\circ} \mathrm{C}$ for $45 \mathrm{~min}$. $100 \%$ solids

WHS: jd

CC: WR Christensen GW Reddick. Jr. WH Sant 


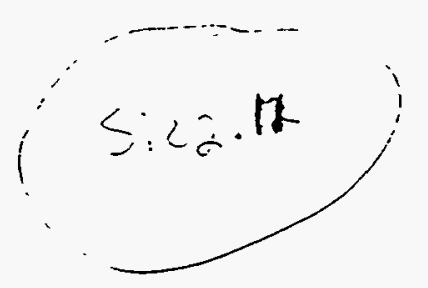

Date: $\quad$ March 25, 1974

To: R. L. Walser

From: R. E. Wheeler Teritheith

Subject: ANALYSIS OF TANK FARM SAMPLES

SAMPLE: T-3005 TANK 102-S

VIS-OTR: Clear, Yellow $100 \mathrm{mrad} / \mathrm{hr}$.

$\mathrm{pH}: \quad 12.1$

SpG: $\quad 1.204$

GEA:

$$
\begin{aligned}
& { }_{137}^{134} \mathrm{Cs}-1.31 \times 10_{5}^{3} \mathrm{HCi} / \mathrm{gal} \\
& { }^{137} \mathrm{Cs}-4.62 \times 10^{5} \mathrm{j} \mathrm{Ci} / \mathrm{gal} \\
& \left.{ }^{106} \mathrm{RuRh}-2.78 \times 10^{5} \mathrm{\mu} \mathrm{Ci} / \mathrm{ga}\right]
\end{aligned}
$$

Water: $\quad 76.99 \%$

Cooling Curve: $27 \%$ sol.ids at room temperature. $30^{\circ} \mathrm{C} \mathrm{Clear,} \mathrm{no} \mathrm{solids} \mathrm{for} 45 \mathrm{~min}$. $20^{\circ} \mathrm{C}$ Clear, no solids for $45 \mathrm{~min}$. $15^{\circ} \mathrm{C}$ Clear, no solids for $45 \mathrm{~min}$. $10^{\circ} \mathrm{C} \mathrm{Clear,} \mathrm{no} \mathrm{solids} \mathrm{for} 45 \mathrm{~min}$. $5^{\circ} \mathrm{C}$ Clear, no solids for $45 \mathrm{~min}$.

$30 \%$ Reduction - 27.5

Solids - $1.38 \%$

$50 \%$ Reduction -51.25

Solids - $23.59 \%$

REW:jd

cc: LG Johnson (2)

RE Wheeler 
Date: $\quad$ March 25, 1974

To: R. L. Walser

From: R. E. Wheeler repoptee

Subject: $\quad$ ANALYSIS OF TANK FARM SAMPLES

SAMPLE: T-3145 TANK 102-S

VIS-0TR: Yellow, clear $200 \mathrm{mrad} / \mathrm{hr}$.

Appear: $\quad 27 \%$ crystals on bottom

$\mathrm{pH}: \quad \quad 72.2$

SPG: $\quad 1.3463$

$30 \%$ Reduction - $26.25 \quad$ Solids $-2.88 \%$

$50 \%$ Reduction - $51.25 \quad$ Solids - 91.02\%

Water: $\quad 59.06 \%$.

Cooling Curve: $25^{\circ} \mathrm{C}$ - No solids.

$20^{\circ} \mathrm{C}$ for $45 \mathrm{~min}$. Trace of solids.

- $15^{\circ} \mathrm{C}$ for $45 \mathrm{~min} .20 \%$ solids. $10^{\circ} \mathrm{C}$ for $45 \mathrm{~min} .60 \%$ solids. $5^{\circ} \mathrm{C}$ for $60 \mathrm{~min}$. $100 \%$ solids.

GEA:

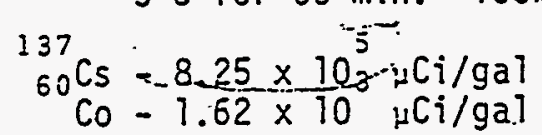

$R E W: j d$

CC: LG Johnson (2)

RE Wheeler 
TCRC- 15

Date: Apri1 17, 1974

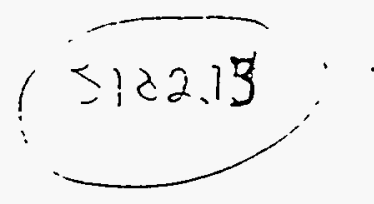

To: - R. L. Halser

From: W. E. WheëTer :

Subject: AIALYYSIS OF TAIK FARM SAMPLES

SAMPLE: T-337D 102-S

VIS-OTR: Clear, Yellow, 50\% solids. 1,500 $\mathrm{mrad} / \mathrm{hr}$

DH: $\quad 10.8$

OTA: Ho Exotherm

GEA: $\quad \begin{array}{ll}134 \\ \end{array} \quad 137 \mathrm{Cs}-5.83 \times 10^{3} \mathrm{uCF} / \mathrm{gal}$

SNG: $\quad 1.500$

Water - 52.75\%

Cooling Curve: . 100\% solids when cooling curve yas started at room temperature: $35^{\circ} \mathrm{C}$ for $45 \mathrm{~min}$. Ho solids.

$30^{\circ} \mathrm{C}$ for $45 \mathrm{~min}$. Ho solids.

$25^{\circ} \mathrm{C}$ for $60 \mathrm{~min} .60^{\circ}$ solids.

$20^{\circ} \mathrm{C}$ for $10 \mathrm{~min}$. 1002 solids.

REW:jd cc: LG Johnsen (2) 
Date: May 13, 1974

$$
1 \leq 102.14
$$

To: $\quad$ R. L. Waiser

From: R. E. Wheeler :Prphlictic

Subject: ANALYSIS OF TANK FARM SAMPLES

SAMPLE T-3601 102-S

Vis-0TR: Yellow, 50\% solids. $500 \mathrm{mrad} / \mathrm{hr}$

$\mathrm{pH}: \quad 12.4$

SPG: $\quad 1.2839$

$\mathrm{OH}: \quad 4.52 \underline{\mathrm{M}}$

A1: $\quad 0.620 \underline{M}$

$\mathrm{NO}_{2}: \quad 0.690 \underline{\mathrm{M}}$

$\mathrm{Na}: \quad 6.66 \underline{M}$

$\mathrm{NO}_{3}: \quad 2.57 \mathrm{M}$

Pu: $\quad 1.43 \times 10^{-5} \mathrm{~g} / 1$

DTA: No Exotherm

$\mathrm{SO}_{4}: \quad 5.32 \times 10^{-2} \underline{\mathrm{M}}$

$\mathrm{PO}_{4}: \quad 3.87 \times 10^{-2} \underline{\mathrm{M}}$

F: $\quad 1.11 \times 10^{-2} \underline{\mathrm{M}}$

$\mathrm{CO}_{3}: \quad 0.259 \underline{M}$

GEA: $\quad{ }^{134} C_{S}-3.66 \times 10^{3} \mu \mathrm{Ci} / \mathrm{gal}$

$30 \%$ Reduction - 26.25 Solids - $2.03 \%$

$50 \%$ Reduction $-51.25 \quad$ Solids - $19.74 \%$

Water: $\quad 58.31 \%$

$\mathrm{NH}_{4}: \quad 1.92 \times 10^{-2} \underline{\mathrm{M}}$

Cooling curve: $50 \%$ Solids at room temperature. $25^{\circ} \mathrm{C}$ for $45 \mathrm{~min}$. Trace of Solids. $20^{\circ} \mathrm{C}$ for $45 \mathrm{~min} .5 \%$ solids. $15^{\circ} \mathrm{C}$ for 45 min. $15 \%$ solides. $10^{\circ} \mathrm{C}$ for $45 \mathrm{~min} .20^{\circ}$ solids.

REN:jd $5^{\circ} \mathrm{C}$ for 45 min. $50 \%$ solids.

CC: LG Johnson (2) 
Date: May 22, 1974

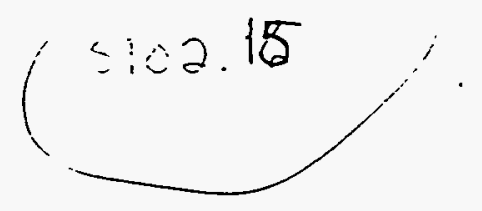

To: R. L. Walser

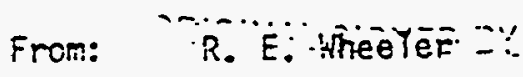

SUbject: AMARLYSIS DF TAIIK FARY SAMPLES

SAMPLE: $T-4372$ TO2-S

Vis-OTR: Dark Amber. :io sollds at room temp. '7nn mrad/hr

pH: $\quad 11.7$

SoG: $\quad 1.400$

GEA: $\quad \begin{aligned} & 134 \mathrm{CS}-4.31 \times 10^{3} \mu \mathrm{CH} / \mathrm{gal} \\ & 137 \mathrm{CS}-1.18 \times 10^{6} \mu \mathrm{CJ} / \mathrm{gal}\end{aligned}$

106 RuRh $-4.01 \times 10^{5}{ }_{\mu C i} / g a l$

Water: $\quad 53.43^{\circ}$

$30 \%$ peduetion - 31.25

$50 \%$ Peduction - 51.25

Solids -12.73

Solids - 20.74

Cooling Eurve: 25: C for 45 min. ito 5011 ds.

$20 \% \mathrm{C}$ for $45 \mathrm{~min}$. lo solids.

15: C for $60 \mathrm{~min}$. Trace of Solids.

lof $C$ for $45 \mathrm{~min}$. 502 solids.

5) C for $45 \mathrm{~min}$. $90 \%$ solids.

REW:jd

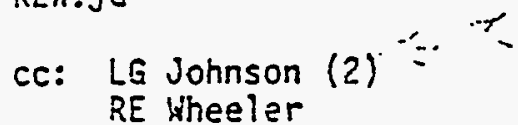


Late: May 24,1974

To: R. L. Waiser

From: R. E. Wheeler

Subject: AHALYSIS OF TAHK FARH SAMPLES

SAMPLE: T-4IN3 1M2-S

Vis-0TR: Dark amber. No solids. $500 \mathrm{mrad} / \mathrm{hr}$

pH: $\quad 12.3$

SpG: $\quad 1.2704$

GEA: $\quad \begin{aligned} & 134 \mathrm{Cs}-6.09 \times 10^{3} \mathrm{yCi} / \mathrm{gaT} \\ & \quad 137 \mathrm{Cs}-7.87 \times 10^{3} \mathrm{yCt} / \mathrm{gal}\end{aligned}$

${ }^{106}$ RuRh $-5.64 \times 10^{2}$ yCi/gal

Nater: 71.27\%

$30 \%$ Reduction -28.75

50\% Reduction $=57.25$

Solids - O.525:

Solicis - 13.97\%

Cooling Curve: $25^{\circ} \mathrm{C}$ for $45^{\circ} \mathrm{min}$. :io solids. $20^{\circ} \mathrm{C}$ for $45 \mathrm{~min}$. ilo solids. $15^{\circ} \mathrm{C}$ for $45 \mathrm{~min}$. to solids. $10^{\circ} \mathrm{C}$ for $60 \mathrm{~min}$. Ho solits. $5^{\circ} \mathrm{C}$ for $45 \mathrm{~min}$. ito solics.
REW:jd
cc: LG Johnson (2)
P.E Wheeler 
Atlantic Richfield Hanford Company

Date: June 7, 1974

To:

R. L. Walser

From:

Subject: $\leq 1 \leq 2.17$
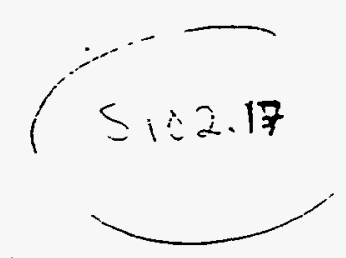

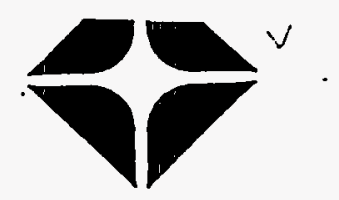

Vis-OTR: Dark Amber. No solids. $280 \mathrm{mrad} / \mathrm{hr}$

$\mathrm{pH}: \quad 11.8$

SpG: $\quad 1.4564$

GEA:

$$
\begin{aligned}
& 134 \mathrm{Cs}-4.35 \times 10^{3} \mu \mathrm{Ci} / \mathrm{gal} \\
& 137 \mathrm{Cs}-1.18 \times 10^{5} \mathrm{Hi} / \mathrm{gal} \\
& \left.106 \mathrm{RuRh}-2.86 \times 10^{5} \mu \mathrm{Ci} / \mathrm{ga}\right]
\end{aligned}
$$

Water: $\quad 55.29 \%$
$30 \%$ Reduction - 30
$50 \%$ Reduction -51.25
Solids - 22.14\%
Solids - 68.97\%

Cooling Curve: $35^{\circ} \mathrm{C}$ for $45 \mathrm{~min}$. No solids.

$30^{\circ} \mathrm{C}$ for $45 \mathrm{~min}$. No solids.

$25^{\circ} \mathrm{C}$ for $60 \mathrm{~min}$. About $2 \%$ salts.

$20^{\circ} \mathrm{C}$ for $45 \mathrm{~min}$ : About $2 \%$ salts.

$15^{\circ} \mathrm{C}$ for $45 \mathrm{~min}$. About $2 \%$ salts.

$10^{\circ} \mathrm{C}$ for $45 \mathrm{~min}$. About $2 \%$ salts.

$5^{\circ} \mathrm{C}$ for $45 \mathrm{~min}$. About $2 \%$ salts.

REW:jd

cC: LG Johnson (2)

RE Wheeler 
Date: June 7, 1974

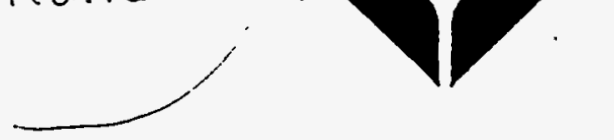

To: R. L. Walser

From: R. E. Wheeler ip pirhecl:

Subject: ANALYSIS OF TAilK FARM SAMPLES

SAMPLE; T-5030 102-5

Vis-OTR: Amber. Trace of solids. $500 \mathrm{mrad} / \mathrm{hr}$.

$\mathrm{pH}: \quad 12.1$

SPG: $\quad 1.3284$

GEA: $\quad \begin{aligned} & { }^{134} \mathrm{Cs}-8.55 \times 10^{3} \mu \mathrm{Ci} / \mathrm{gaT} \\ & \left.\quad{ }_{137} \mathrm{Cs}-8.18 \times 10^{5} \mathrm{\mu Ci} / \mathrm{ga}\right]\end{aligned}$

Water: $\quad 59.32 \%$

$30 \%$ Reduction - $28.75 \quad$ Solids $-1.75 \%$

$50 \%$ Reduction - $50.0 \quad$ Solids - $18.25 \%$

Cooling Curve: $25^{\circ} \mathrm{C}$ for $45 \mathrm{~min}$. No solids. $20^{\circ} \mathrm{C}$ for $45 \mathrm{~min}$. No solids. $15^{\circ} \mathrm{C}$ for $45 \mathrm{~min}$. No solids. $10^{\circ} \mathrm{C}$ for $45 \mathrm{~min}$. No solids. $5^{\circ} \mathrm{C}$ for $45 \mathrm{~min}$. No solids.

$<1 \%$ solids setting over night.

REW:jd

CC: LG Johnson (2)

RE Wheeler 


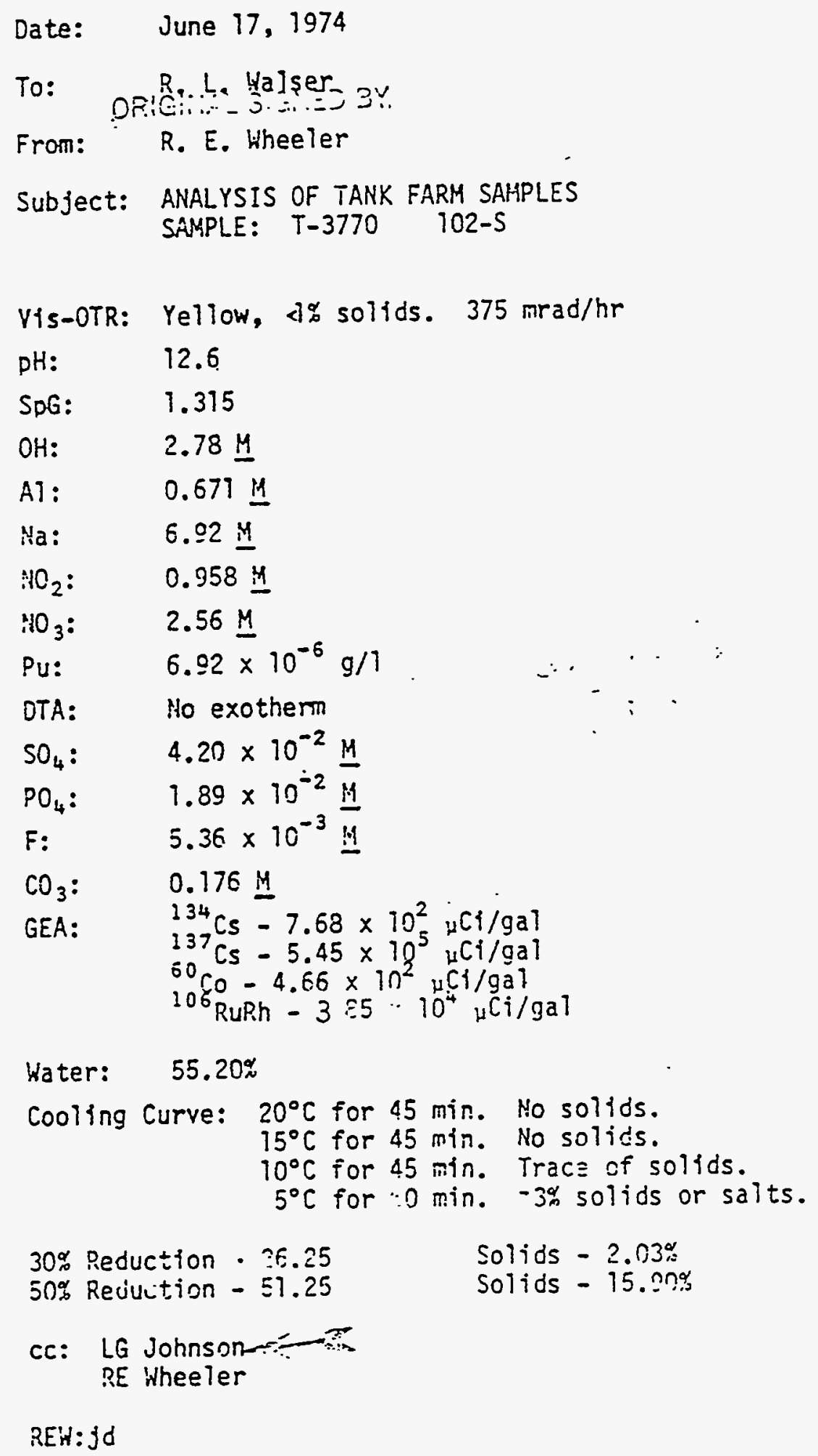

REW:jd 
$T C R C-25$

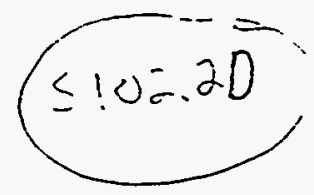

Date: June 19, 1974

To: R. L. Walser

From: R. E. Wheeter

Subject: ARALYSIS OF TAIIK FAFM SAMPLES

SAAPLE: T-54E3 102-5

Vis-0TR: Clear, yellow. -5\% solids. 1 rad at $4^{\prime \prime}$

pH: $\quad 12.3$

SPG: $\quad 1.2258$

GEA: $\quad \begin{aligned} & 134 \mathrm{CS}-4.28 \times 10^{3} \mu \mathrm{Ci} / \mathrm{gal} \\ & 137 \mathrm{CS}-4.47 \times 10^{5} \mathrm{HCi} / \mathrm{gal}\end{aligned}$

Water: $\quad 91.45 \%$

$3 \%$ Reduction - 27.5

$50 \%$ Reduction - 50

Solids $-0.172 \%$

Solids $-1 \%$

Cooling Curve: $20^{\circ} \mathrm{C}$ for $45 \mathrm{~min}$. Ho soltis. $15^{\circ} \mathrm{C}$ for 45 min. No solids. $10^{\circ} \mathrm{C}$ for $45 \mathrm{~min}$. ilo solids. $5^{\circ} \mathrm{C}$ for $50 \mathrm{~min}$. Ho solids.

REW:jd

cc: LG Johnson (2)

RE Wheeler 


$$
\leq: 2=. j
$$

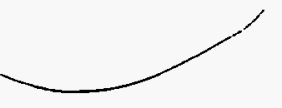

Date: June 28,1974

To: R. L. Watser

From: R. E. Wheeler if Pfhieg

Subject: ANALYSIS OF TAHK FARM SAMPLES

SAMPLE: T- 5232 102-S

Vis-OTR: Amber. No solids. $1.5 \mathrm{rad}$

$\mathrm{pH}: \quad 11.6$

SPG: $\quad 1.5027$

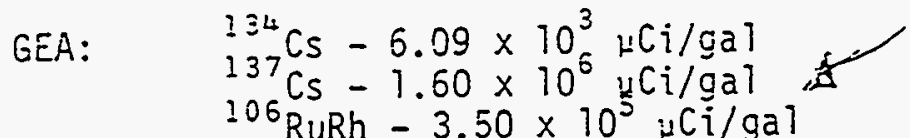

Water: $53 \%$

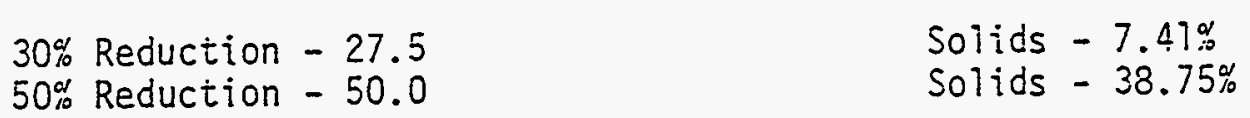

$50 \%$ Reduction - 50.0

Solids - 7.41\%

Solids - $38.75 \%$

Cooling Curve: $35^{\circ} \mathrm{C}$ for 60 min. No solids. $30^{\circ} \mathrm{C}$ for $45 \mathrm{~min}$. No solids. $25^{\circ} \mathrm{C}$ for $45 \mathrm{~min}$. No solids. $20^{\circ} \mathrm{C}$ for $45 \mathrm{~min}$. About $10 \%$ solids. $15^{\circ} \mathrm{C}$ for $45 \mathrm{~min}$. About $90 \%$ solids. $10^{\circ} \mathrm{C}$ for $60 \mathrm{~min}$. About $90 \%$ solids. $5^{\circ} \mathrm{C}$ for $60 \mathrm{~min}$. About $90 \%$ solids.

REW:jd

cc: LG Johnson (2) 


$$
5102.22
$$

Date: Juiy 2, 1974

To: R. L. Walser

From: R. E. Wheeler tripthewe

Subject: ANALYSIS OF TANK FARM SAMPLES

SAMPLE: 5775 102-S

Vis-0TR: Dark brown, <5\% solids. $1500 \mathrm{mrad} / \mathrm{hr}$

$\mathrm{pH}: \quad 11.9$

SPG: $\quad 1.045^{?}$ ?

GEA: $\quad \begin{array}{ll}134 \mathrm{Cs}-2.02 \times 10^{3} \mu \mathrm{Hi} / \mathrm{gaT} \\ \quad & \left.137 \mathrm{Cs}-2.18 \times 10^{5} \mathrm{Hi} / \mathrm{ga}\right]\end{array}$ 106 RuRh $-4.24 \times 10^{5} ; \mathrm{Ci} / \mathrm{gal}$

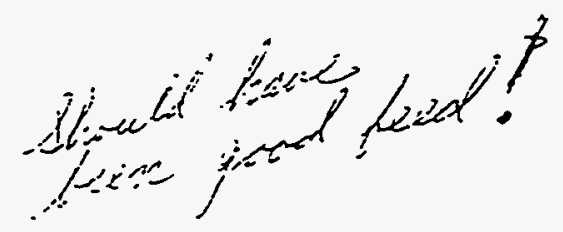

Water: $\quad 65.83 \%$

$30 \%$ Reduction: 27.5

50\% Reduction: 50.0

Solids: $1.55 \%$

Solids: $14.0 \%$

Cooling Curve: $25^{\circ} \mathrm{C}$ for $45 \mathrm{~min}$. No solids. $20^{\circ} \mathrm{C}$ for $45 \mathrm{~min}$. No solids. $15^{\circ} \mathrm{C}$ for $60 \mathrm{~min}$. No solids. $10^{\circ} \mathrm{C}$ for $45 \mathrm{~min}$. No solids.

$5^{\circ} \mathrm{C}$ for $45 \mathrm{~min}$. No solids.

REW:jd 
TCRC -23

$$
5102.25
$$

Date: July 3, 1974

To: ...... Halser

From: $\quad \ddot{R}$. E. Wheeler

Subject: AMALYSIS OF TANK FARM SAMPLES

SAMPLE: T-5458 102-S

VIS-OTR: Green. \& 5\% solids. $1 \mathrm{rad}$

$\mathrm{pH}: \quad 11.0$

SPG: $\quad 1.4923$

GEA: $\quad{ }^{134} \mathrm{Cs}-6.23 \times 10^{4} \mu \mathrm{Ci} / \mathrm{gaT}$

$106 \mathrm{CS}-1.67 \times 10^{6} \mathrm{HCi} / \mathrm{gal}$

RuRh - $3.07 \times 10^{5} \ldots \mathrm{C} / \mathrm{gal}$

Water: 48:

30\% Peduction - 25.25 Solids - $4.24 \%$

$50 \%$ reduction -50.0 Solids - $92.5 \%$

Cooling Curve: $55^{\circ} \mathrm{C}$ for $45 \mathrm{~min}$. $17^{\circ}$ solids. $50^{\circ} \mathrm{C}$ for $45 \mathrm{~min}$. $132 \mathrm{~s} \cdot 11 \mathrm{ds}$. $45^{\circ} \mathrm{C}$ for 45 min. $16 \%$ solids. . $40^{\circ} \mathrm{C}$ for $60 \mathrm{~min}$. 17\% solids. $35^{\circ} \mathrm{C}$ for 60 min. $18 \%$ solics. $30^{\circ} \mathrm{C}$ for 45 nin. ho solids. $25^{\circ} \mathrm{C}$ for $45 \mathrm{~min}$. $37 \%$ solids. $20^{\circ} \mathrm{C}$ for 60 min. $322^{\circ}$ solids. $15^{\circ} \mathrm{C}$ for on min. 33. solits. $10^{\circ} \mathrm{C}$ for $45 \mathrm{~min}$. $38^{\circ}$ solitis. $5^{\circ} \mathrm{C}$ for 60 min. $49 \%$ solids.

PEN: $j d$

cs: LG Johnson (2).

P.E Wheeler 


\section{Sica. 2 每}

Date: $\quad$ September 16, 1974

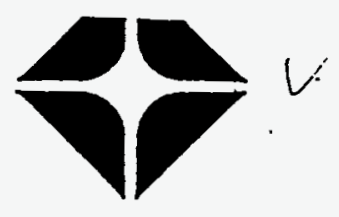

To: R. L. Waiser

From: R. E. Wheeler fersfleetic

Subject: ANALYSIS OF TANK FARM SAI?PLES

SAMPLE: T-6006 102-S.

Vis-0TR: Greenish brown. <-5\% solids, $1500 \mathrm{mrad} / \mathrm{hr}$

$\mathrm{pH}: \quad 13.2$

SPG: $\quad 1.256$

$\mathrm{OH}: \quad 1.53 \mathrm{M}$

A1: $\quad 0.501 \underline{M}$

$\mathrm{Na}: \quad 6.13 \mathrm{M}$

$\mathrm{NO}_{2}: \quad 0.870 \underline{\mathrm{M}}$

$\mathrm{NO}_{3}: \quad 1.60 \mathrm{M}$

Pu: $\quad 2.93 \times 10^{-5} \mathrm{gm} / \mathrm{gal}$

DTA: No Exotherm

$\mathrm{SO}_{4}: \quad 3.94 \times 10^{-2} \mathrm{M}$

$\mathrm{PO}_{4}: \quad 8.99 \times 10^{-3} \frac{\mathrm{M}}{\mathrm{M}}$

$\mathrm{F}: \quad \quad 1.33 \times 10^{-2} \underline{\mathrm{M}}$

$\mathrm{CO}_{3}: \quad 0.175$ M

GEA: $\quad i \equiv 4 \mathrm{Cs}-9.99 \times 10^{2} \mu \mathrm{Ci} / \mathrm{gat}$

$137 \mathrm{Cs}-2.03 \times 10^{5} \mathrm{HCi} / \mathrm{gal}$

$106 \mathrm{RuRh}-6.06 \times 10^{4} \mathrm{\mu Ci} / \mathrm{gal}$

Water: $69.46 \%$

Cooling curve: $25^{\circ} \mathrm{C}$ for $45 \mathrm{~min}$. No solids.

$20^{\circ} \mathrm{C}$ for $45 \mathrm{~min}$. tho solids. .

$15^{\circ} \mathrm{C}$ for $60 \mathrm{~min}$. No solias.

$10^{\circ} \mathrm{C}$ for $45 \mathrm{~min}$. No solids.

$5^{\circ} \mathrm{C}$ for $45 \mathrm{~min}$. No solids.

REW:jd

CC: JS Buckingham

WR Christensen

RE Wheeler 


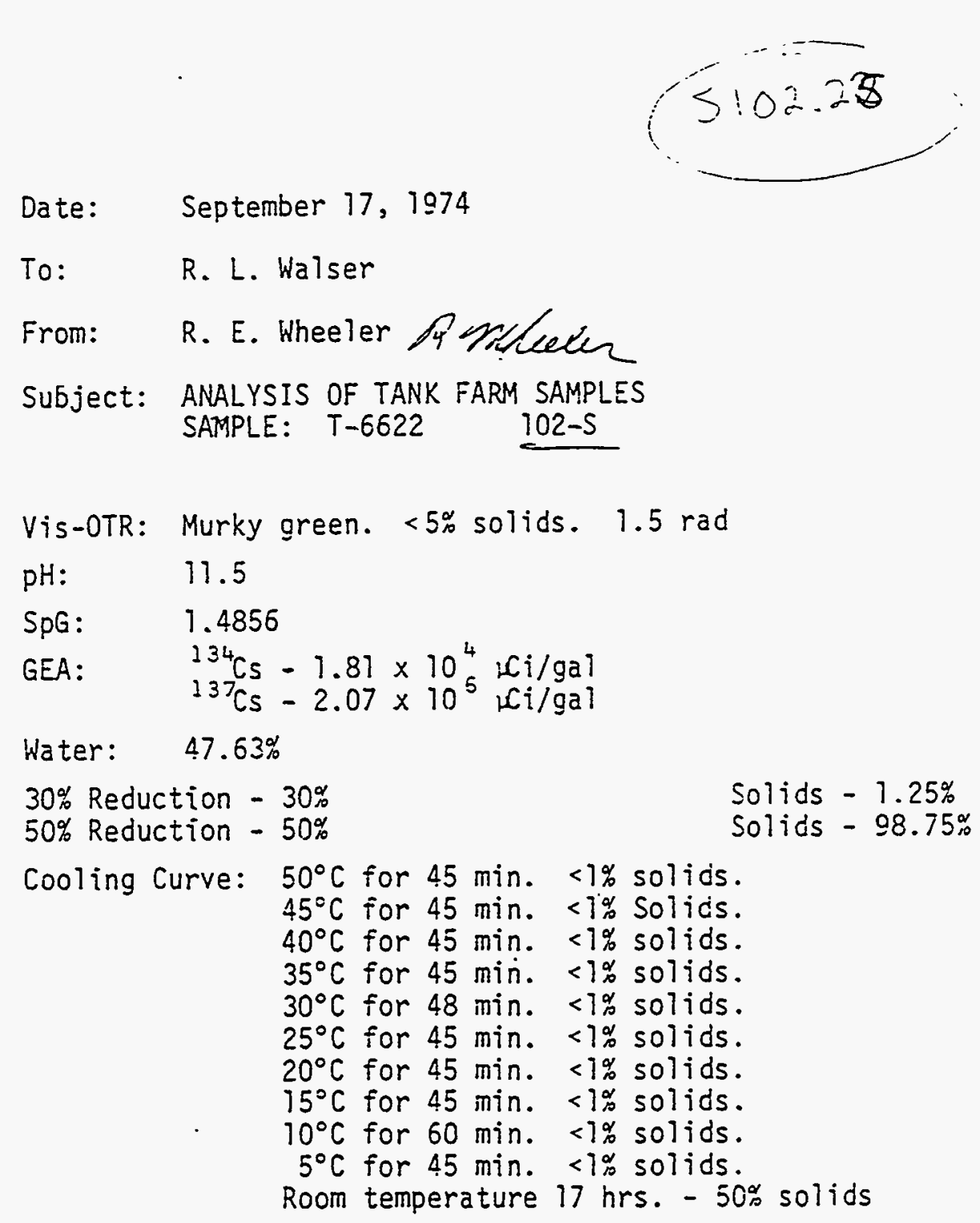

Date: September 17, 1974

To: R. L. Walser

From: R. E. Wheeler pyefeeter

Subject: ANALYSIS OF TANK FARM SAMPLES

SAMPLE: T-6622 102-S

Vis-OTR: Murky green. $<5 \%$ solids. 1.5 rad

$\mathrm{pH}: \quad 11.5$

SpG: $\quad 1.4856$

GEA: $\quad \begin{aligned} & 13{ }^{4} \mathrm{Cs}-1.81 \times 10^{4} \mathrm{Hi} / \mathrm{gaT} \\ & \quad{ }^{37} \mathrm{Cs}-2.07 \times 10^{5} \mathrm{Li} / \mathrm{gal}\end{aligned}$

Water: $\quad 47.63 \%$

$30 \%$ Reduction - 30\% Solids - $1.25 \%$

$50 \%$ Reduction - 50\%

Solids - $98.75 \%$

Cooling Curve: $50^{\circ} \mathrm{C}$ for $45 \mathrm{~min}$. $<1 \%$ solids. $45^{\circ} \mathrm{C}$ for $45 \mathrm{~min}$. < $\%$ Solids. $40^{\circ} \mathrm{C}$ for $45 \mathrm{~min}$. $<7 \%$ solids. $35^{\circ} \mathrm{C}$ for $45 \mathrm{~min}$. <l\% solids. $30^{\circ} \mathrm{C}$ for $48 \mathrm{~min}$. $<7 \%$ solids. $25^{\circ} \mathrm{C}$ for $45 \mathrm{~min}$. <7\% solids. $20^{\circ} \mathrm{C}$ for $45 \mathrm{~min}$. <1\% solids. $15^{\circ} \mathrm{C}$ for $45 \mathrm{~min}$. $<1 \%$ solids. $10^{\circ} \mathrm{C}$ for $60 \mathrm{~min}$. $<7 \%$ solids. $5^{\circ} \mathrm{C}$ for $45 \mathrm{~min}$. <i\% solids. Room temperature 17 hrs. - 50\% solids

REW:jd

cc: JS Buckingham

LG Johnson

WR Christensen 

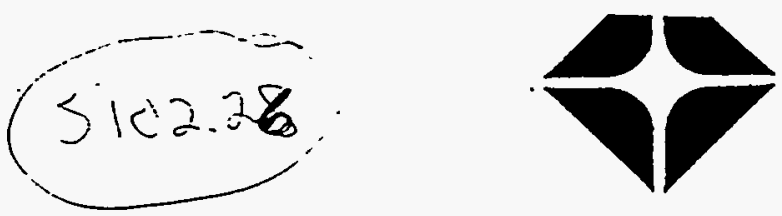

Date: $\quad$ October 14, 1974

To: R. L. Walser

From: R. E. Wheeler $R$ Pthlecter

Subject: ANALYSIS OF TANK FARM SAMPLES

SAMPLE: T-6214 102-S

Vis-OTR: Brown. < 5\% solids. 3 Rad.

$\mathrm{pH}: \quad 11.5$

SPG: $\quad 1.4005$

GEA: $\quad 134 \mathrm{CS}-1.12 \times 10_{5}^{4} \mathrm{\mu Ci} / \mathrm{gal}$$$
137 \mathrm{Cs}-1.54 \times 10^{5} \mathrm{jCi} / \mathrm{gal}
$$

Water: $\quad 92.89 \%$

Cooling Curve: $35^{\circ} \mathrm{C}$ for $60 \mathrm{~min}$. No solids. $30^{\circ} \mathrm{C}$ for $45 \mathrm{~min}$. No solids.

$25^{\circ} \mathrm{C}$ for $45 \mathrm{~min}$. $1 \%$ solids. $20^{\circ} \mathrm{C}$ for $45 \mathrm{~min}$ < $1 \%$ solids. $15^{\circ} \mathrm{C}$ for $45 \mathrm{~min}$. < $7 \%$ solids. $10^{\circ} \mathrm{C}$ for $45 \mathrm{~min}$ : < $1 \%$ solids. $5^{\circ} \mathrm{C}$ for $45 \mathrm{~min}$. $<1 \%$ solids.

REW:jd

cc: JS Buckingham

WR Christensen

RE Wheeler

JC Womack 


\section{$\therefore \leq i c .27$}

Date: $\quad$ October 14,1974

To: R. L. Walser

From: R. E. Wheeler R Plliele

Subject: ANALYSIS OF TANK FARM SAMPLES

SAMPLE: T-6416 102-S

Vis-OTR: Amber, brown, cloudy. <5\% solids. $2500 \mathrm{mRad} / \mathrm{hr}$.

$\mathrm{pH}: \quad 11.6$

SPG: $\quad \quad \quad \quad .378$

GEA: $\quad \begin{array}{ll}134 \\ & 137 \mathrm{CS}-5.45 \times 10^{3} \mathrm{HCi} / \mathrm{gal}\end{array}$

$\left.106 \mathrm{Cs}-1.36 \times 10^{\circ} \mathrm{HCi} / \mathrm{ga}\right]$

RuRh $\left.-4.05 \times 10^{5} ; \mathrm{Ci} / \mathrm{ga}\right]$

Wàter: $49.05 \%$

Cooling Curve: $45^{\circ} \mathrm{C}$ for $45 \mathrm{~min}$. No solids.

$40^{\circ} \mathrm{C}$ for $75 \mathrm{~min}$. No solids.

$35^{\circ} \mathrm{C}$ for $45 \mathrm{~min}$. No solids.

$30^{\circ} \mathrm{C}$ for $45 \mathrm{~min} .<1 \%$ solids.

$25^{\circ} \mathrm{C}$ for $45 \mathrm{~min}$. < $1 \%$ solids.

$20^{\circ} \mathrm{C}$ for $45 \mathrm{~min}$. $5 \%$ solids.

$15^{\circ} \mathrm{C}$ for $45 \mathrm{~min}$. $5.5 \%$

$10^{\circ} \mathrm{C}$ for $45 \mathrm{~min}$. $6.0 \%$

$5^{\circ} \mathrm{C}$ for $60 \mathrm{~min}$. $6.5 \%$

REW: jd

CC: JS Buckingham

WR Christensen

RE Wheeler

JC Womack 
Date: $\quad$ October 14,1974

To: R. L. Walser

From: R. E. Wheeter Apteleles

Subject: ANALYSIS OF TANK FARM SAMPLES

SAMPLE: T-7300 102-S

Vis-OTR: Yellow, cloudy. Unknown amount of solids. 1 Rad.

$\mathrm{pH}: \quad 11.7$

SPG: $\quad 1.336$

GEA: $\quad 134 \mathrm{Cs}-7.34 \times 10^{3} \mu \mathrm{Ci} / \mathrm{gal}$

$137 \mathrm{Cs}-1.11 \times 10^{5} \mathrm{iCi} / \mathrm{gal}$

$125 \mathrm{Sb}-3.52 \times 10^{3} \mathrm{HCi} / \mathrm{gal}$

Water: $\quad 60.46 \%$

Cooling Curve: $25^{\circ} \mathrm{C}$ for $30 \mathrm{~min}$. No solids. $20^{\circ} \mathrm{C}$ for $60 \mathrm{~min}$. No solids. $15^{\circ} \mathrm{C}$ for $45 \mathrm{~min}$. No solids. $10^{\circ} \mathrm{C}$ for $45 \mathrm{~min}$. No solids. $5^{\circ} \mathrm{C}$ for $90 \mathrm{~min}$. No solids.

REW: jd

cc: jS Buckingham WR Christensen JC Womack 

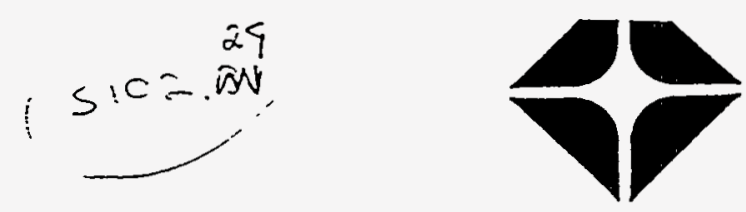

Date: $\quad$ October 14, 1974

To: $\quad$ R. L. Walser

From: R. E. Wheeler Rrthath

$\begin{aligned} \text { Subject: } & \text { ANALYSIS OF TANK FARM SAMPLES } \\ & \text { SAMPLE: T-74.39 102-S }=\frac{1}{4}\end{aligned}$

Vis-0TR: Green, yellow, clear. No solids. $550 \mathrm{mRad} / \mathrm{hr}$

pH: $\quad 11.95$

SpG: $\quad 1.445$

GEA: $\quad \begin{aligned} & 134 \\ & \quad\end{aligned} \quad 137 \mathrm{CS}-1.01 \times 10^{4} \mu \mathrm{Ci} / \mathrm{gal}$

Water: $59.75 \%$

Cooling Curve: $25^{\circ} \mathrm{C}$ for $45 \mathrm{~min}$. No solids.

$20^{\circ} \mathrm{C}$ for 45 min. No solids.

$15^{\circ} \mathrm{C}$ for $40 \mathrm{~min}$. No solids.

$10^{\circ} \mathrm{C}$ for $45 \mathrm{~min}$. No solids.

$5^{\circ} \mathrm{C}$ for $45 \mathrm{~min}$. No solids.

REH:jd

CC: JS Buckingham

WR Christensen

JC Womack 
Date: $\quad$ October 21,1974

To: R. L. Walser

From: R. E. Wheeler Retithele

Subject: ANALYSIS OF TANK FARM SAMPLES

SAMPLE: T-6761 102-S

Vis-OTR: Green brown. $<5 \%$ solids. $300 \mathrm{mRar} / \mathrm{hr}$

$\mathrm{pH}: \quad>12.8$

SpG: $\quad 1.4669$

$\mathrm{OH}: \quad 3.65 \underline{M}$

A1: $\quad 1.34 \underline{M}$

$\mathrm{Na}: \quad 12.51$

$\mathrm{NO}_{2}: \quad 1.89 \underline{\mathrm{M}}$

$\mathrm{NO}_{3}: \quad 3.29 \mathrm{M}$

Pu: $\quad$ Sample sluroed.

DTA: No exotherm

$\mathrm{SO}_{4}: \quad$ Sample siurped.

$\mathrm{PO}_{4}: \quad 3.34 \times 10^{-2} \mathrm{M}$

$\mathrm{F}: \quad 4.10 \times i 0^{-3} \overline{\mathrm{M}}$

$\mathrm{CO}_{3}: \quad 1.03 \times 10^{-2} \underline{\mathrm{M}}$

GEA: $\left.\quad{ }^{134} \mathrm{Cs}-1.80 \times 10^{4} \mathrm{HCi} / \mathrm{ga}\right]$

${ }^{137} \mathrm{Cs}-2.05 \times 10^{6} \mu \mathrm{Ci} / \mathrm{gal}$

Water: $49.82 \%$

Cooling Curve: $45^{\circ} \mathrm{C}$ for $45 \mathrm{~min}$. < $1 \%$ solids.

$40^{\circ} \mathrm{C}$ for $60 \mathrm{~min}$. $<7 \%$ solids.

$35^{\circ} \mathrm{C}$ for 45 min. $2 \%$ solids.

$30^{\circ} \mathrm{C}$ for $45 \mathrm{~min} .5 \%$ solids.

$25^{\circ} \mathrm{C}$ for 18 hours. Sample jelled.

$20^{\circ} \mathrm{C}$ for $60 \mathrm{~min}$. Samble jelled.

$15^{\circ} \mathrm{C}$ for $45 \mathrm{~min}$. Sample jelled.

$10^{\circ} \mathrm{C}$ for $45 \mathrm{~min}$. Samble jelled.

$5^{\circ} \mathrm{C}$ for 60 min. Sample jelled.

Set over weekend $85 \%$ solids.

REW:jd

CC: JS Buckingham

WR Christensen

RE Wheeler

JC Womack 


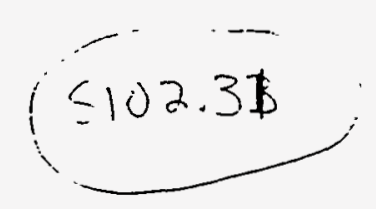 \\ Date: October 21, 1974 \\ To: R. L. Walser \\ From: R. E. Wheeler Reffflitien \\ Subject: ANALYSIS OF TANK FARM SAMPLES \\ SAMPLE: T-7805 102-S \\ Vis-0TR: Dark Green. 75\% solids. 2 Rads. \\ $\mathrm{pH}: \quad 13.2$ \\ SDG: $\quad 1.5311$ \\ GEA: $\quad \begin{aligned} & \left.134 \mathrm{CS}-9.61 \times 10^{3} \mathrm{\mu Ci} / \mathrm{ga}\right] \\ & \quad 137 \mathrm{CS}-1.81 \times 10^{5} \mathrm{\mu i} / \mathrm{gal}\end{aligned}$ \\ Water: $63.92 \%$ \\ Cooling Curve: $40^{\circ} \mathrm{C}$ for 60 min. Cloudy \\ $35^{\circ} \mathrm{C}$ for $90 \mathrm{~min}$. Suspended solids.
$30^{\circ} \mathrm{C}$ for $45 \mathrm{~min}$. Suspended solids.
$25^{\circ} \mathrm{C}$ for $45 \mathrm{~min}$. Suspended solids.
$20^{\circ} \mathrm{C}$ for $45 \mathrm{~min}$. Suspended solids.
$15^{\circ} \mathrm{C}$ for $45 \mathrm{~min}$. Suspended solids.
$10^{\circ} \mathrm{C}$ for $45 \mathrm{~min}$. Suspended solids.
$5^{\circ} \mathrm{C}$ for $65 \mathrm{~min}$. Suspended solids. \\ REW:jd \\ cC: JS Buckingham \\ WR Christensen \\ RE Wheeler \\ JC Womack
}




$$
\operatorname{sic} 2.32
$$

Date: October 21, 1974

To:

R. L. Walser

From:

R. E. Wheeler R.E.theche

Subject: ANALYSIS OF TANK FARM SAMPLES

SAMPLE: T-8077 1ח2-S

Vis-OTR: Murky green. $-25 \%$ solids. 1.4 Rads.

$\mathrm{pH}$ :

14

SPG:

1.5357

GEA:

$$
\begin{aligned}
& \left.134 \mathrm{Cs}-8.93 \times 10^{3} \mu \mathrm{Ci} / \mathrm{ga}\right] \\
& \left.137 \mathrm{Cs}-2.15 \times 10^{6} \mu \mathrm{Ci} / \mathrm{ga}\right]
\end{aligned}
$$

Water:

$$
47.48 \%
$$

Cooling Curve:

$$
\begin{aligned}
& 40^{\circ} \mathrm{C} \text { for } 45 \mathrm{~min} .<1 \% \text { solids. } \\
& 35^{\circ} \mathrm{C} \text { for } 130 \mathrm{~min} \text {. }<1 \% \text { solids. } \\
& 30^{\circ} \mathrm{C} \text { for } 60 \text { min. } 3 \% \text { solids. } \\
& 25^{\circ} \mathrm{C} \text { for } 45 \mathrm{~min} . \quad 3 \% \text { solids. } \\
& \text { Set over night - room temp. } 3 \% \text { solids } \\
& 20^{\circ} \mathrm{C} \text { for } 75 \mathrm{~min} . \quad 3 \% \text { solids. } \\
& 15^{\circ} \mathrm{C} \text { for } 45 \mathrm{~min} . \quad 3 \% \text { solids. } \\
& 10^{\circ} \mathrm{C} \text { for } 45 \mathrm{~min} . \quad 3 \% \text { solids. } \\
& 5^{\circ} \mathrm{C} \text { for } 45 \mathrm{~min} \text {. } \quad 3 \% \text { solids. }
\end{aligned}
$$

REW: jd
cc: JS Buckingham
WR Christensen
RE Wheeler
JC Womack 
Date: $\quad$ October 21, 1974
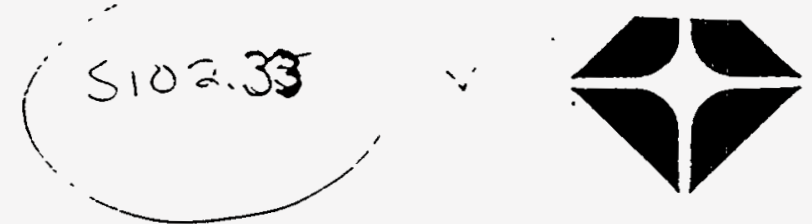

To: R. L. Walser

From: R. E. Wheeter R Extifual

Subject: ANALYSIS OF TANK FARM SAMPLES

SAMPLE: T-8199 102-S

Vis-0TR: Yellow. No solids. $300 \mathrm{mRad} / \mathrm{hr}$

pH: $\quad 9.8$

SPG: $\quad 1.321$

GEA: $\quad \begin{aligned} & 134 \mathrm{CS}-6.47 \times 1 \gamma^{3} \text { u } \mathrm{Ci} / \mathrm{gal} \\ & \quad 137 \mathrm{Cs}-1.26 \times 10^{\circ} \mathrm{Ci} / \mathrm{gal}\end{aligned}$

Water: $\quad 59.40 \%$

Cooling Curve: $40^{\circ} \mathrm{C}$ for $45 \mathrm{~min}$. No solids. $35^{\circ} \mathrm{C}$ for $45 \mathrm{~min}$. $110 \mathrm{solids.}$ $30^{\circ} \mathrm{C}$ for $75 \mathrm{~min}$. No solids. $25^{\circ} \mathrm{C}$ for $45 \mathrm{~min}$. No solids. $20^{\circ} \mathrm{C}$ for $45 \mathrm{~min}$. No solids. $75^{\circ} \mathrm{C}$. for $45 \mathrm{~min}$. No solids.

$10^{\circ} \mathrm{C}$ for $16 \mathrm{hrs} ., 15 \mathrm{~min}$. Susnended Solids. $5^{\circ} \mathrm{C}$ for $45 \mathrm{~min}$. Suspended Solids.

REW:jd

cc: JS Buckingham WR Christensen

JC Womack

RE Wheeler 
Date: October 23, 1974

$510=.3 \%$

To: R. L. Walser

From: R. E. Wheeler ip. kythici

Subject: AIIALYSIS OF TANK FARM SAMPLES

SAMPLE: T-9007 102-S

Vis-0TR: Cloudy green. $5 \%$ solids. $1.3 \mathrm{Rad} / \mathrm{hr}$.

$\mathrm{pH}: \quad \quad 14.0$

SpG: $\quad 1.4866$

GEA: $\quad{ }^{134} \mathrm{CS}_{5}-4.35 \times 10^{3} \mathrm{\mu Ci} / \mathrm{ga}$

$\left.137 \mathrm{Cs}-7.68 \times 10^{5} \mu \mathrm{Ci} / \mathrm{ga}\right]$

$125 \mathrm{Sb}-2.04 \times 10^{3} \mathrm{HCi} / \mathrm{gal}$

Water: $54.56 \%$

Cooling Curve: $35^{\circ} \mathrm{C}$ for $45 \mathrm{~min}$. No solids.

$3 n^{\circ} \mathrm{C}$ for $60 \mathrm{~min}$. No solids.

$25^{\circ} \mathrm{C}$ for $45 \mathrm{~min}$. No solids.

$20^{\circ} \mathrm{C}$ for $45 \mathrm{~min}$. No solids.

$15^{\circ} \mathrm{C}$ for $45 \mathrm{~min}$. Suspended solids.

$10^{\circ} \mathrm{C}$ for $45 \mathrm{~min}$. Suspended solids.

$5^{\circ} \mathrm{C}$ for $45 \mathrm{~min}$. Suspended solids.

REW:jd

CC: JS Buckingham

WR Christensen

RE Wheeler

JC Womack 
Date: Octoier 25, 1974

To: $\quad$ R. I. Walser

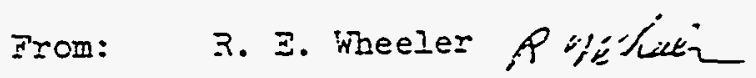

SUDject: ANAIYSIS OF TANK PARM SAMEIES

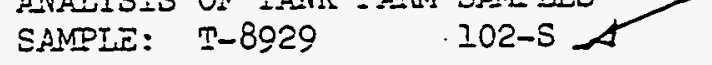

Vis-OTR: Cloudy green. 5\% solids. 1.5 sad/:

Diت: $\quad$ it. 0

SDG : $\quad 2.5197$

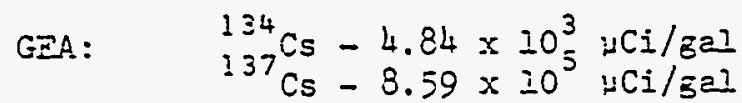

Water: $\quad 50.02 \%$

Cooling Curve: $40^{\circ} \mathrm{C}=0 \mathrm{cr} 60 \mathrm{~min}$. No solicis.

$35^{\circ} \mathrm{C}$ for 45 min. No solids.

$30^{\circ} \mathrm{C}$ =or $60 \mathrm{~min}$. No solics.

$25^{\circ} \mathrm{C}$ for 45 min: No solics.

$20^{\circ} \mathrm{C}$ =or $45 \mathrm{~min}$. No solicis.

$15^{\circ} \mathrm{C}$ icr 45 min. No solics.

$10^{\circ} \mathrm{C}$ :or $45 \mathrm{~min}$. No solids.

$5^{\circ} \mathrm{C}$ for $45 \mathrm{~min}$. Suspenced sclicis.

SEN : jđ

cc: js Zuciringham

WR Christensen

IE wheeler

JC Womack 


\subsection{6}

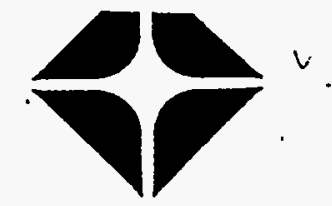

Date: October 29,1974

To:

3. I. Walser

Irom:

R. \pm . Wheeler iprylleth

Sūject:

ANAIYSIS OF TANK FARM SAMPLES

SAMPIE: T-8779

202-S

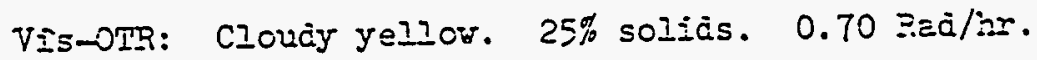

EE:

10.2

SDG:

1.3692

E포:

$$
\begin{aligned}
& 134 \mathrm{Cs}-8.86 \times 10^{3} \mu \mathrm{Hi} / \mathrm{gal} \\
& 137 \mathrm{Cs}-1.67 \times 10^{5} \mathrm{pCi} / \mathrm{EaI}
\end{aligned}
$$

Water: $\quad 44.50 \%$

Cccing Curve: $35^{\circ} \mathrm{C}$ for 60 min. Crear, no soiics. $30^{\circ} \mathrm{C}$ for $45 \mathrm{~min}$. No solids. $25^{\circ} \mathrm{C}$ 아 45 min. No solids. $20^{\circ} \mathrm{C}$ for 45 min. No solics. $15^{\circ} \mathrm{C}=0 \mathrm{r} 45 \mathrm{~min}$. iNo solicis. $20^{\circ} \mathrm{C}$ for $45 \mathrm{~min}$. $5 \%$ solics. $5^{\circ} \mathrm{C}$ ior $65 \mathrm{~min}$. $5 \%$ solicis.

RีW: $\jmath \dot{\alpha}$
cc: js 3uckingiam
WR Curistensen
32 ineeler
JC Nomecir 
Date: November 1, $197^{14}$

To: R. I. Walser

Irom: R. E. wineeler Fhticule

Sudject: ANALYSIS OF TANK IARM SAMPIES

SAMPTE: T-3339 $\underline{202-S}$

Vis-OTR: Brown. 5\% solics. 270 nRac/hr

Dस: $\quad 13.8$

SDG: $\quad 1.3638$

GEA: $\quad \begin{aligned} & 134 \mathrm{Cs}-5.83 \times 10^{3} \mu \mathrm{Hi} / \mathrm{gal} \\ & \quad 137 \mathrm{Cs}-1.07 \times 10^{5} \mathrm{HC} / \mathrm{gal}\end{aligned}$

त̃ater: 66.95\%

Cooing Curve: $10^{\circ} \mathrm{C}$ for $90 \mathrm{~min}$. To solics. $35^{\circ} \mathrm{C}$ ér $45 \min$. No solius. $30^{\circ} \mathrm{C}$ for 45 min. No solicis. $25^{\circ} \mathrm{C}$ for 45 min. No solies. $20^{\circ} \mathrm{C}$ for $45 \mathrm{~min}$. No solicis. $15^{\circ} \mathrm{C}$ for 45 min: No solics. $20^{\circ} \mathrm{C}=$ for $45 \mathrm{~min}$. No solics. $5^{\circ} \mathrm{C}$ for $45 \mathrm{~min}$. to solics.

3EW : jd

cc: is Buckingiam
WR Christensen
TE Theeler
IC Womaci. 
Date: November I, 1974
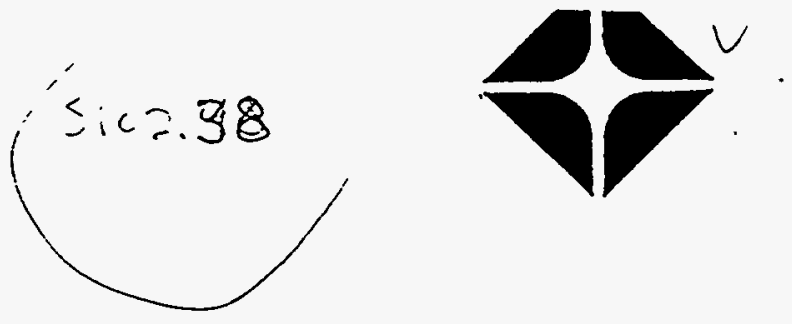

To: 3. I. Walser

From: R. $\mathrm{I}$. Wheeler Qphtulen

Subject: ANAIYSIS OF TANK FARM SAMPIES

SAMPIE: T-6604 102-S it

Vis-OTR: Cloudy yellow. < 10\% solids. I Red/hr

pir: $\quad 13.8$

SpG : $\quad 1.484$

GEA: $\quad 134 \mathrm{Cs}-1.04 \times 10^{4} \mu \mathrm{Ci} / \mathrm{z} 21$

Water: $\quad 43.65 \%$

Cocling Curve: $40^{\circ} \mathrm{C}$ for 45 min. No solicis.

$35^{\circ} \mathrm{C}$ for $45 \mathrm{~min}$. No solics.

$30^{\circ} \mathrm{C}$ for $75 \mathrm{~min}$. No solics.

$25^{\circ} \mathrm{C}$ for $45 \mathrm{~min}$. ilo solics.

$20^{\circ} \mathrm{C}$ for $45 \mathrm{~min}$. No solics.

$15^{\circ} \mathrm{C}$ for $45 \mathrm{~min}$. No soliss.

$10^{\circ} \mathrm{C}$ for 16 irs., 15 min. Suspencied solids.

$5^{\circ} \mathrm{C}$ for 45 min. Suspencied soiics.

నEW: jd

cc: JS 3uckingiam

WR Christensen

SE wheeler

jC Womack 


$$
\begin{aligned}
& \text { Atlante Richneld Hanfor } \\
& (510=.99) \\
& \text { Date: Noveriber 4, } 1974 \\
& \text { To: } \quad \text { S. I. Nalser } \\
& \text { From: R. E. Wheeler R mifuetion } \\
& \text { SUDject: ANAIYSIS OF MAIK FARM SAMPLES } \\
& \text { SAMPTE: T-7562 102-S -- } \\
& \text { Vis-OTR: Green, cloucy. Suspenced solids. } \$ C 0 \mathrm{Mrad} / \mathrm{hr} \\
& \text { DII: } \quad 12.0 \\
& \text { SDG: } \quad 2.4345
\end{aligned}
$$

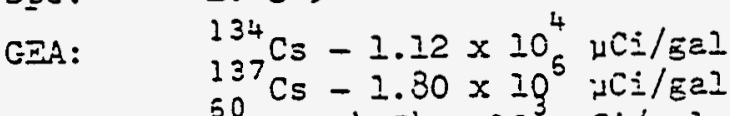

$$
\begin{aligned}
& \text { Co }-4.54 \times 10^{3}, \mathrm{C}: / \mathrm{E} 2 \mathrm{I}
\end{aligned}
$$

REW : jd 
Date: November 19, 1974
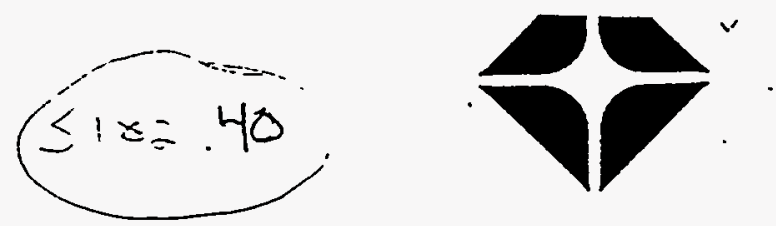

To:

3. I. Walser

From:

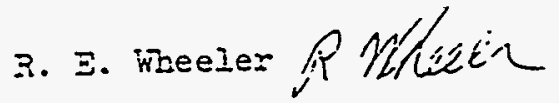

Süject: ANAIYSIS OF TAIKK FARM SAMPIES

SAMPIE: T-9103 102-S :-

Vis-OTR: Green. $1 \%$ solicis. $1.5 \mathrm{Red} / \mathrm{hr}$

표:

13.8

SDG:

1.407

$G \underline{B A}$ :

$134 \mathrm{Cs}-7.19 \times 10^{3} \mathrm{Hi} \mathrm{Ci} / \mathrm{saI}$
$137 \mathrm{Cs}-1.45 \times=0^{\circ} \mathrm{HC} / \mathrm{sal}$

Water: $\quad 57.83 \%$

cooling Curve:

$$
\begin{aligned}
& 25^{\circ} \mathrm{C} \text { for } 45 \text { min. No solics. } \\
& 20^{\circ} \mathrm{C} \text { for } 45 \text { min. No soliss. } \\
& 15^{\circ} \mathrm{C} \text { for } 45 \text { min. No solics. } \\
& 20^{\circ} \mathrm{C} \text { for } 45 \mathrm{~min} \text {. No solias. } \\
& 5^{\circ} \mathrm{C} \text { for } 45 \text { min. No solics. }
\end{aligned}
$$

SER $: j a$

cc: JS Juciringham

NR Christensen

RM Ginestet

PI ineelex

JC Womacis 


\section{$5102.4 B$}

Date: Novemicer 29, 2974

To: R. I. W̉alser

Irom: R. E. Wheeler frofiecin

Subject: AILIYSIS OF TANR FARM SAMTIES

SAMPIE: T-9601 . 102-S -

VismoTR: Dark green. $30 \%$ solicis. 1 Rad/hr

ㅍ: $\quad 13.1$

SDG: $\quad 2.4554$

GEA: $\quad 134 \mathrm{Cs}-7.04 \times 10^{3} \mu \mathrm{Ci} / \mathrm{gaI}$

${ }^{60} \mathrm{Cs}-1.54 \times 1.16 \times 10^{3} \mathrm{\mu Ci} / \mathrm{sel}$

Weter: $\quad 54.89 \%$

Cooling curve: $40^{\circ} \mathrm{C}$ for $45 \mathrm{~min}$. (cloucy) I: solices.

$35^{\circ} \mathrm{C}$ for 45 min. $2 \%$ solias.

$30^{\circ} \mathrm{C}$ for $45 \mathrm{~min} .2 \%$ solids.

$25^{\circ} \mathrm{C}$ for $60 \mathrm{~min}$. $1 \%$ solids.

$20^{\circ} \mathrm{C}$ For 45 min. I\% solias suspenced.

$15^{\circ} \mathrm{C}$ ?or $45 \mathrm{~min}$. $1 \%$ solids suspended.

$10^{\circ} \mathrm{C}$ for $45 \mathrm{~min} .2 \%$ solicis suspenced.

$5^{\circ} \mathrm{C}$ for 45 min. $3 \%$ solicas suspencied.

STEस: $j \bar{c}$

cc: JS Juckingham
WR CDristensen
RM Ginestet
$R E$ Nheeler
JC Homacir




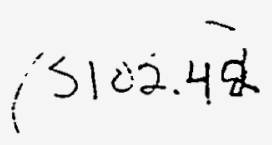

Date: November 26,1974

To: R. L. Waiser

From: R. E. Wheeler $f$ Mhet

Subject: ANALYSIS OF TANK FARM SAMPLES

SAMPLE: T-9706 102-S ..

Vis-OTR: Green. 50\% solids. $1.5 \mathrm{Rad} / \mathrm{hr}$

PH: $\quad 13.5$

SpG: $\quad 1.5142$

GEA : $\quad \begin{aligned} & 134 \mathrm{Cs}-9.31 \times 10^{3} \mu \mathrm{Ci} / \mathrm{gal} \\ & \left.\quad 137 \mathrm{CS}-1.79 \times 10^{5} \mathrm{\mu Ci} / \mathrm{ga}\right]\end{aligned}$

Water: $\quad 49.76 \%$

Cooling Curve: $40^{\circ} \mathrm{C}$ for $45 \mathrm{~min}$. No solids. $35^{\circ} \mathrm{C}$ for $45 \mathrm{~min}$. No solids. $30^{\circ} \mathrm{C}$ for 45 min. No solids. $25^{\circ} \mathrm{C}$ for $45 \mathrm{~min}$. No solids. $20^{\circ} \mathrm{C}$ for $45 \mathrm{~min}$. Suspended solids. $15^{\circ} \mathrm{C}$ for 60 min. Suspended solids. $10^{\circ} \mathrm{C}$ for $45 \mathrm{~min}$. Suspended solids. $5^{\circ} \mathrm{C}$ for $45 \mathrm{~min}$. Suspended solids.

REW: jd
CC: JS Buckingham
WR Christensen
RE Wheeler
JC Womack 


$$
: 5122.45
$$

Date: $\quad$ December 4, 1974

To: R. L. Walser

From: R. E. Wheeler of ikdeith

SUbject: ANALYSIS OF TANK FARM SAMPLES

SAMPLE: T-8476 102-S

Vis-OTR: Green. < $10 \%$ solids. $1 \mathrm{Rad} / \mathrm{hr}$

$\mathrm{pH}: \quad 13.3$

SPG: $\quad 1.4327$

GEA: $\quad \begin{array}{ll}134 \\ \end{array} \quad 137 \mathrm{Cs}-3.45 \times 10^{4} \mathrm{HCi} / \mathrm{gal}$

Nater: $\quad 57.98 \%$

Cooling Curve: $40^{\circ} \mathrm{C}$ for $90 \mathrm{~min}$. No solids. $35^{\circ} \mathrm{C}$ for $75 \mathrm{~min}$. No solids. $30^{\circ} \mathrm{C}$ for $60 \mathrm{~min}$. No solids. $25^{\circ} \mathrm{C}$ for $45 \mathrm{~min}$. No solids. $20^{\circ} \mathrm{C}$ for $17 \mathrm{hrs}$. No solids. $15^{\circ} \mathrm{C}$ for $45 \mathrm{~min}$. No solids. $10^{\circ} \mathrm{C}$ for $45 \mathrm{~min}$. No solids. $5^{\circ} \mathrm{C}$ for $45 \mathrm{~min}$. No solids.

REW:jd
CC: JS Buckingbam
WR Christenser
RM Ginestet
RE Wheeler
JC Womack 
$T E R E-49$

Atlantic Richfield Hanford Company

WHC-SD.WM-ER-323, Rev. 0

Date: $\quad$ December 26,1974

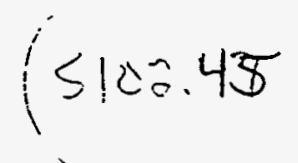

To: R. L. Walser

From: R. E. Wheeler indec

Subject: ANALYSIS OF TANK FARM SAMPLES

SAMPLE: T-423 102-5 Rec. 12/6/74

$<$

Yis-0TR: Green. $50 \%$ crystal solids. $1.5 \mathrm{Rad} / \mathrm{hr}$

$\mathrm{pH}: \quad 14.0$

SpG: $\quad 1.4272$

GEA: $\quad{ }_{137}^{134} \mathrm{Cs}-6.09 \times 10_{5}^{3} \mathrm{vCi} / \mathrm{gal}$

${ }_{137} \mathrm{Cs}-1.40 \times 10^{5} \mathrm{\mu Ci} / \mathrm{gal}$

Al: $\quad 1.14 \underline{M}$

Water: $58.75^{\circ}$

Cooling Curve: $40^{\circ} \mathrm{C}$ for $45 \mathrm{~min}$. Cloudy (no solids)

$35^{\circ} \mathrm{C}$ for $60 \mathrm{~min} .2 \%$ solids.

$30^{\circ} \mathrm{C}$ for $45 \mathrm{~min} .2 \%$ solids.

$25^{\circ} \mathrm{C}$ for $45 \mathrm{~min} .2 \%$ solids.

$20^{\circ} \mathrm{C}$ for 45 min. $2 \%$ solids.

$15^{\circ} \mathrm{C}$ for $45 \mathrm{~min}$. $2 \%$ solids.

$10^{\circ} \mathrm{C}$ for $45 \mathrm{~min}$. $2 \%$ solids.

$5^{\circ} \mathrm{C}$ for $45 \mathrm{~min} .2 \%$ solids.

REH: jd

CC: JS Buckingham

WR Christensen

RM Ginestet

JC Womack

RE Wheeler 
Date: January 6, 1975
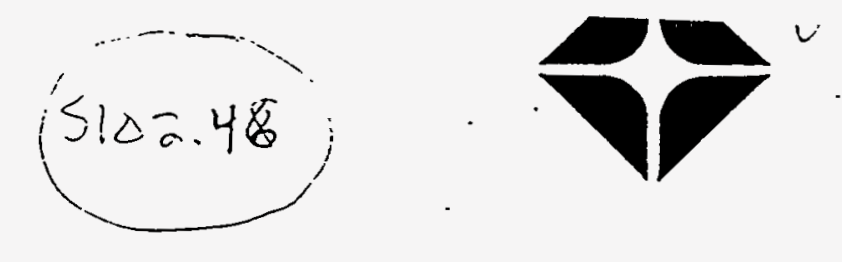

To: R. L. Walser

From: R. E. Wheeler P.Ei-Phlule

SUEject: ANALYSIS OF TANK FARM SAMPLES

SAMPLE: T-534 102-5 Received on 12/10/74

Vis-0TR: Green cloudy, I Rad/hr

$\mathrm{pH}: \quad 14$

SpG: $\quad 1.2582$

Al: $\quad 0.61 T M$

GEA: $\quad \begin{aligned} & 134 \mathrm{CS}-4.58 \times 10^{3} \mathrm{HCi} / \mathrm{gal} \\ & 137 \mathrm{Cs}-8.33 \times 10^{5} \mathrm{iCi} / \mathrm{gal}\end{aligned}$

Water: $69.40 \%$

Cooling Curve: $25^{\circ} \mathrm{C}$ for $45 \mathrm{~min}$. No solids.

$20^{\circ} \mathrm{C}$ for $45 \mathrm{~min}$. No solids.

$15^{\circ} \mathrm{C}$ for $45 \mathrm{~min}$. No solids.

$10^{\circ} \mathrm{C}$ for $45 \mathrm{~min}$. No solids.

$5^{\circ} \mathrm{C}$ for $45 \mathrm{~min}$. No solids.

REW: jd

cc: JS Buckingham

WR CEristensen

RM Ginestet

JC Nomack

RE Theeler 
Date: January 6,1975

$$
\text { (5):2.47 }
$$

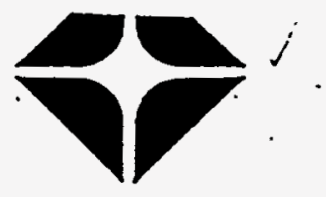

To: R. L. Walser

From: R. E. Wheeler Repthealen

Subject: ANALYSIS OF TANK FARM SAMPLES

SAMPLE: T-632 102-S Received 12/16/74

8

Yis-0TR: Green. <T\% solids. $1.5 \mathrm{Rad} / \mathrm{hr}$

$\mathrm{pH}$ :

14

SpG: $\quad 1.3476$

GEA: $\quad \begin{aligned} & 13{ }^{4} \mathrm{Cs}-5.83 \times 10^{4} \mu \mathrm{Ci} / \mathrm{gal} \\ & \left.\quad{ }^{137} \mathrm{Cs}-1.20 \times 10^{6} \mathrm{Hi} / \mathrm{ga}\right]\end{aligned}$

Water: $58.28 \%$

Cooling Curve: $25^{\circ} \mathrm{C}$ for $45^{\circ} \mathrm{min}$. No solids. $20^{\circ} \mathrm{C}$ for $45 \mathrm{~min}$. No solids. $15^{\circ} \mathrm{C}$ for $75 \mathrm{~min}$. No solids. $10^{\circ} \mathrm{C}$ for $45 \mathrm{~min}$. No solids. $5^{\circ} \mathrm{C}$ for $45 \mathrm{~min}$. No solids.

$R E N: j d$

cc: JS Buckingham

WR Christensen

RM Ginestet

RE WẼeeler

JC Nomack 
TCRC- 47

Atlantic Richfield Hanford Company

$$
5122.44
$$

Date: December 17, 1974

To: R. L. Walser

From: R. E. Wheeler forfllieiti

Subject: ANALYSIS OF TANK FARM SAMPLES

SAMPLE: T-8008 102-S --

Vis-0TR: Green. $50 \%$ solids. 2.5 Rads

$\mathrm{pH}: \quad 14$

SPG: $\quad 1.4202$

OB: $\quad 2.89 \mathrm{M}$

A1: $\quad 1.28 \mathrm{M}$

$\mathrm{Na}: \quad 11.61 \mathrm{M}$

$\mathrm{NO}_{2}: \quad 1.39 \underline{\mathrm{M}}$

$\mathrm{NO}_{3}: \quad 3.24 \underline{\mathrm{M}}$

Pu: $\quad<5.34 \times 10^{-5} \mathrm{gm} / \mathrm{ga} 7$

$\mathrm{SO}_{4}$ : Cancelled by R. L. Waiser

$\mathrm{PO}_{4}: \quad 0.170 \mathrm{M}$

F: $\quad 1.05 \times 10^{-2} \underline{M}$

$\mathrm{CO}_{3}: \quad 0.157 \mathrm{M}$

GEA: $\quad{ }^{134} \mathrm{CS}^{-7.66 \times 10^{3} \mathrm{Hi} / \mathrm{gal}}$

Water: $52.45 \%$

K: $\quad 3.18 \times 10^{-2} \underline{M}$

Cooling Curve: $40^{\circ} \mathrm{C}$ for $45 \mathrm{~min}$. No solids.

$35^{\circ} \mathrm{C}$ for $60 \mathrm{~min}$. No solids.

$30^{\circ} \mathrm{C}$ for $45 \mathrm{~min}$. No solids.

$25^{\circ} \mathrm{C}$ for $45 \mathrm{~min}$. $1 \%$ solids.

$20^{\circ} \mathrm{C}$ for $45 \mathrm{~min}$. Suspended Solids.

$20^{\circ} \mathrm{C}$ for $17 \mathrm{hrs}$. Suspended Solids.

$15^{\circ} \mathrm{C}$ for $60 \mathrm{~min}$. Suspended Solids.

$10^{\circ} \mathrm{C}$ for $45 \mathrm{~min}$. Suspended Solids.

$5^{\circ} \mathrm{C}$ for $45 \mathrm{~min}$. Suspended Solids.

$R E W: j d$

CC: JS Buckingham

WR Christensen

RM Ginestet

LG Johnson

RE Wheeler

JC Womack 


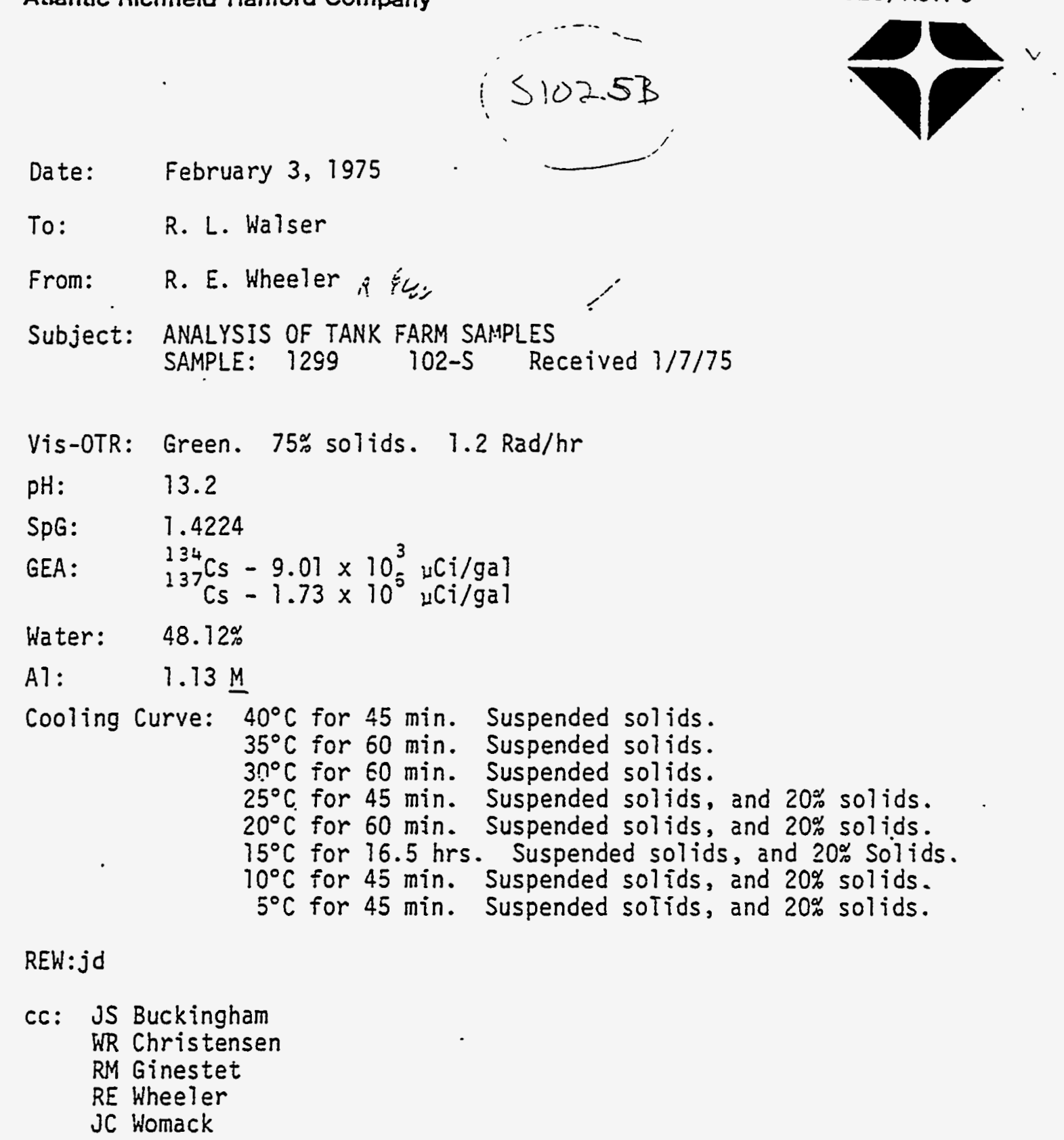

REW:jd

Cc: JS Buckingham

WR Christensen

RM Ginestet

RE Wheeler

JC Womack 
T: $\div$ : $\div$ -

Atlantic Richfleld Hanford Company

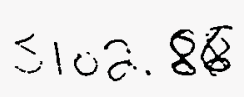

Date: October 20, 1975

To: R. L. Walser

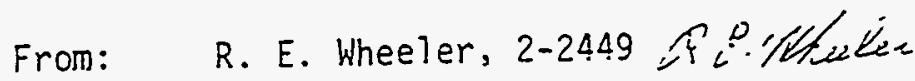

Subject: ANALYSIS OF TANK FARM SAMPLES

Sample: T-1091 Tank: 102-S

Received: January 2, 1975 ?

Vis-OTR: Green, cloudy No solids $1 \mathrm{rad} / \mathrm{hr}$

$\mathrm{pH}: \quad \quad 14.0$

SpG: $\quad 1.580$

$\mathrm{OH}: \quad 2.63 \underline{M}$

A1: $\quad 1.13$ 쓰

$\mathrm{Na}: \quad 9.93 \mathrm{M}$

$\mathrm{NO}_{2}: \quad 1.87 \underline{\mathrm{M}}$

$\mathrm{NO}_{3}: \quad 3.64 \mathrm{M}$

Pu: $\quad 2.40 \times 10^{-5} \mathrm{~g} / \mathrm{gal}$

D.T.A.: No exotherso below $200^{\circ} \mathrm{C}$

$\mathrm{SO}_{4}: \quad$ Cancelled

$\mathrm{PO}_{4}: \quad 5.68 \times 10^{-2} \mathrm{M}$

F: $\quad 1.34 \times 10^{-2} \underline{M}$

$\mathrm{CO}_{3}: \quad 0.257 \underline{M}$

GEA: $\quad \begin{array}{r}i 34 \\ { }^{4} C_{S}-1.53 \times 10^{4} j \mathrm{ji} / \mathrm{gal}\end{array}$

Water: $\quad 51.68 \%$

Cooling Curve: $40^{\circ} \mathrm{C}$ for $60 \mathrm{~min}$. . No soliuss. $30^{\circ} \mathrm{C}$ for $60 \mathrm{~min}$. No solids. $25^{\circ} \mathrm{C}$ for $45 \mathrm{~min}$. No solids. $20^{\circ} \mathrm{C}$ for $45 \mathrm{~min}$. No solids. 
R. L. Walser

Page 2

October 20, 1975

Cooling Curve: $15^{\circ} \mathrm{C}$ for $45 \mathrm{~min}$. Suspended solids. $10^{\circ} \mathrm{C}$ for $45 \mathrm{~min}$. Suspended solids. $5^{\circ} \mathrm{C}$ for $45 \mathrm{~min}$. Suspended solids.

REW:eyw

cc: JS Buckingham

WR Christensen

RM Ginestet

RE Wheeler

JC Womack 


\subsection{8}

Date: January 6, 1975

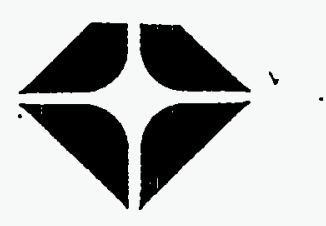

To:

R. L. Walser

From:

R. E. Wheeler ix End hulli

SUbject: ANALYSIS OF TANK FARM SAMPLES

SAMPLE: T-847 102-S

Yis-OTR: Yellow, No solids.

$\mathrm{pH}: \quad 14$

SPG: $\quad 1.4346$

A): $\quad 1.30 \underline{11}$

GEA: $\begin{aligned} & 134 \mathrm{CS}-2.13 \times 10^{4} \mathrm{HCi} / \mathrm{gal} \\ & 137 \mathrm{CS}-3.75 \times 10^{5} \mathrm{WCi} / \mathrm{gal} \\ & 60 \mathrm{CS}-1.56 \times 10^{\circ} \mathrm{HCi} / \mathrm{gal} \\ & 12 \pm \mathrm{Sb}-1.52 \times 10^{5} \mathrm{HCi} / \mathrm{gal}\end{aligned}$

Nater: $57.45 \%$

Cooling Curve: $25^{\circ} \mathrm{C}$ for $60 \mathrm{~min}$. No solids.

$20^{\circ} \mathrm{C}$ for 45 min. No solids.

$15^{\circ} \mathrm{C}$ for $60 \mathrm{~min}$. No solids.

$10^{\circ} \mathrm{C}$ for $45 \mathrm{~min}$. No solids.

$5^{\circ} \mathrm{C}$ for $60 \mathrm{~min}$. No solids.

REN:jd

CC: JS Buckingbam

WR Christensen

RM Ginestet

RE Wheeler

jC Womack 
Date: January 20, 1975
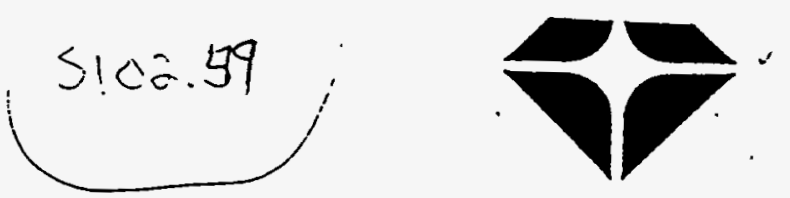

To:

R. L. Walser

From:

R. E. Wheeler, PEthetieter

Subject: ANALYSIS OF TANK FARM SAMPLES

SAMPLE: T-1231 102-S \&

Vis-0TR: Yellow. clovidy. (No solids) $1 \mathrm{Rad} / \mathrm{hr}$

$\mathrm{pH}$ :

14.0

SPG:

7.4586

GEA:

$$
\begin{aligned}
& 134 \\
& 137 \mathrm{Cs}-7.95 \times 10^{3} \mathrm{HCi} / \mathrm{gal}
\end{aligned}
$$

A1:

$$
0.498 M
$$

Water: $\quad 52.28 \%$

Cooling Curve: $40^{\circ} \mathrm{C}$ for $45 \mathrm{~min}$. No solids. $35^{\circ} \mathrm{C}$ for $45 \mathrm{~min}$. No solids. $30^{\circ} \mathrm{C}$ for $60 \mathrm{~min}$. No solids. $25^{\circ} \mathrm{C}$ for $60 \mathrm{~min}$. No solids. $20^{\circ} \mathrm{C}$ for $45 \mathrm{~min}$. No solids. - $15^{\circ} \mathrm{C}$ for $45 \mathrm{~min}$. No solids. $10^{\circ} \mathrm{C}$ for $45 \mathrm{~min}$. No sollids. $5^{\circ} \mathrm{C}$ for $60 \mathrm{~min}$. No solids.

REW:jd

$$
\begin{aligned}
& \text { cc: JS Buckinghan } \\
& \text { WR Christensen } \\
& \text { RM Ginestet } \\
& \text { RE Wheeler } \\
& \text { JC Womack }
\end{aligned}
$$


Atlantic Richfield Hanford Company

Date: January 22, 1975
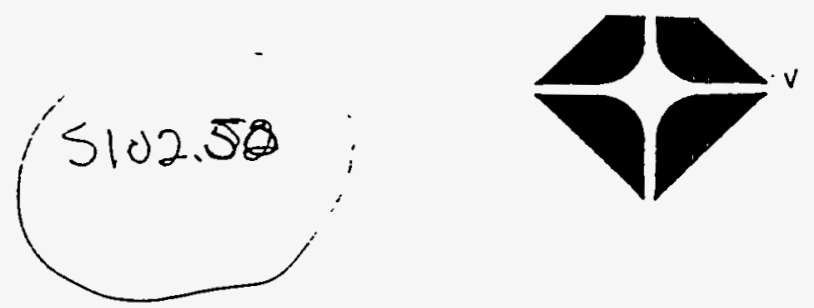

To:

R. L. Walser

From:

R. E. Wheeler

Subject:

ANALYSIS OF TANK FARM SAMPLES

SAMPLE: T-933

Vis-0TR: Green. No solids. 1.3 Rad/hr Received 12/30/74

$\mathrm{pH}: \quad 14.0$

SPG: $\quad 1.6515$

GEA: $\quad \begin{aligned} & \left.134 \mathrm{Cs}-8.02 \times 10^{3} \mu \mathrm{Hi} / \mathrm{ga}\right] \\ & { }_{137} \mathrm{Cs}=1.60 \times 10^{5} \mathrm{\mu Ci} / \mathrm{gal}\end{aligned}$

Water: $\quad 51.10 \%$

A1: $\quad 1.29 \mathrm{M}$

Cooling Curve: $25^{\circ} \mathrm{C}$ for $60 \mathrm{~min}$. No solids.

$20^{\circ} \mathrm{C}$ for $45 \mathrm{~min}$. Suspended solids.

$75^{\circ} \mathrm{C}$ for $60 \mathrm{~min}$. Suspended solids.

$.10^{\circ} \mathrm{C}$ for $45 \cdot \mathrm{min}$. Suspended solids.

$5^{\circ} \mathrm{C}$ for $60 \mathrm{~min}$. Suspended solids.

$R E R: j d$

CC: JS Buckingham

WR Christensen

RP Ginestet

RE Wheeler

JC Nomack 


\section{$T=. \check{-}-\dot{0}:$}

Atlantic Richfield Hanford Company

WHC-SD-WM-ER-323, Rev. 0

Date: April 23, 1975

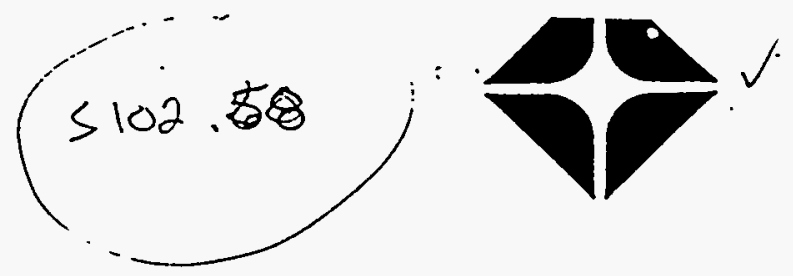

To: R. L. Walser

From: R. E. Wheeler,$\because \therefore$

Subject: ANALYSIS OF TANK FARM SAMPLES

Sample: T-2278 102-S

Received February 24, 1975

Vis-0TR: Green No solids $800 \mathrm{mrad} / \mathrm{hr}$

$\mathrm{pH}: \quad 13.3$

SPG: $\quad 1.1914$

$\mathrm{OH}: \quad 1.058 \underline{\mathrm{M}}$

Pu: $\quad 5.06 \times 10^{-5} \mathrm{~g} / \mathrm{gal}$

${ }^{89},{ }^{90} \mathrm{Sr}: 2.02 \times 10^{4} \mu \mathrm{Ci} / \mathrm{gal}$

D.T.A.: No Exotherm below $200^{\circ} \mathrm{C}$

$\mathrm{Na}: \quad 5.21 \mathrm{M}$

A1: $\quad 0.447 \underline{M}$

$\mathrm{NO}_{2}: \quad 0.611 \underline{\mathrm{M}}$

$\mathrm{NO}_{3}: \quad 1.79 \underline{\mathrm{M}}$

$\mathrm{PO}_{4}: \quad 3.26 \times 10^{-2} \mathrm{M}$

F: $\quad 5.56 \times 10^{-3} \underline{M}$

$\mathrm{CO}_{3}: \quad 0.140 \mathrm{M}$

GEA: $\quad{ }^{134} \mathrm{Cs}-2.55 \times 10^{3} \mathrm{\mu Ci} / \mathrm{gal}$ ${ }^{137} \mathrm{Cs}-6.36 \times 10^{5} \mu \mathrm{Ci} / \mathrm{gal}$

Water: $\quad 75.70 \%$

Cooling Curve: $25^{\circ} \mathrm{C}$ for $60 \mathrm{~min}$. No solids $20^{\circ} \mathrm{C}$ for $60 \mathrm{~min}$. No solids $15^{\circ} \mathrm{C}$ for $60 \mathrm{~min}$. No solids $10^{\circ} \mathrm{C}$ for $60 \mathrm{~min}$. No solids $5^{\circ} \mathrm{C}$ for $60 \mathrm{~min}$. No solids

REN:Vmk

CC: JS Buckingham

WR Christensen

RM Ginestet

JC Womack

RE Wheeler

LB 
TCRC- 55

\section{Atlantic Richfield Hanford Company}

Date: $\quad$ March 26, 1975

$$
\therefore 5102.52
$$

To:

R. G. Geier

R. L. Walser

From:

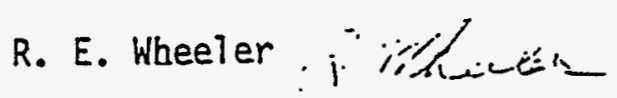

Subject:

ANALYSIS OF TANK FARM SAMPLE

SAMPLE: T-2415 102-S

RECEIVED MARCH 3, 1975

Vis-OTR Light Brown $1 \%$ Brown Solids $1 \mathrm{Rad} / \mathrm{hr}$

SpG:

1.4004

Ph: $\quad 13.3$

Al: $\quad 0.884$.

GEA: $\quad 134 \mathrm{CS}-5.94 \times 10^{3} \mu \mathrm{Ci} / \mathrm{gal}$

$137 \mathrm{CS}-1.17 \times 10^{6} \mathrm{\mu Ci} / \mathrm{ga}$

$125 \mathrm{Sb}-3.86 \times 103 \mu \mathrm{Ci} / \mathrm{gal}$

Water: $55.90 \%$.

Cooling Curve: $25^{\circ} \mathrm{C}$ for $45 \mathrm{~min}$. No solids

$20^{\circ} \mathrm{C}$ for 45 min. No solids

$15^{\circ} \mathrm{C}$ for $45 \mathrm{~min}$. No solids

$10^{\circ} \mathrm{C}$ for $45 \mathrm{~min}$. No solids

$5^{\circ} \mathrm{C}$ for $45 \mathrm{~min}$. No solids

REW: vmk

cc: JS Buckingham

WR Christensen

RM Ginestet

JC Womack

RE Wheeler 


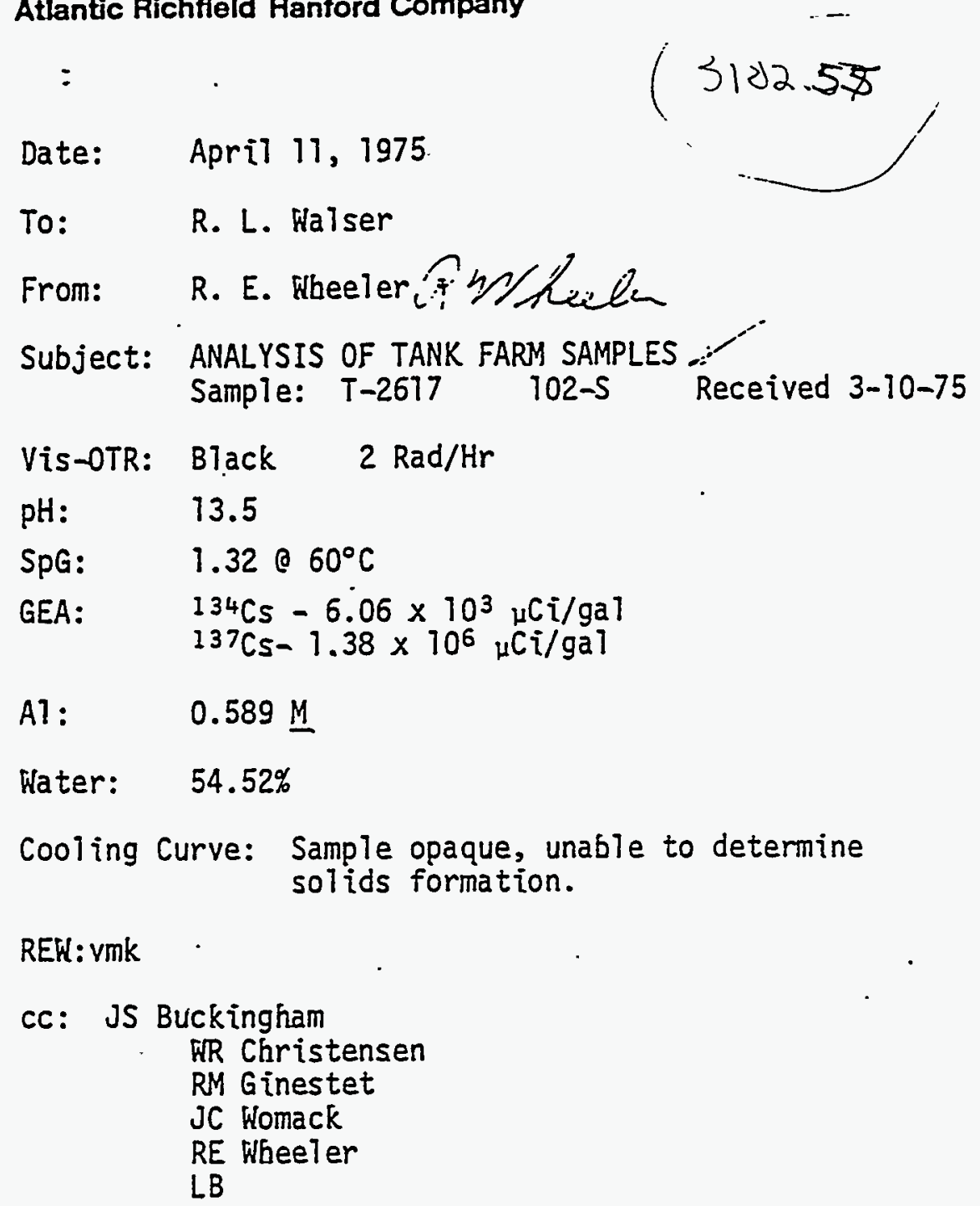

REK: Vmk

cc: JS Buckingham

FR Christensen

RM Ginestet

JC Womack

RE Wheeter

LB 
Date: $\quad$ April 1, 1975
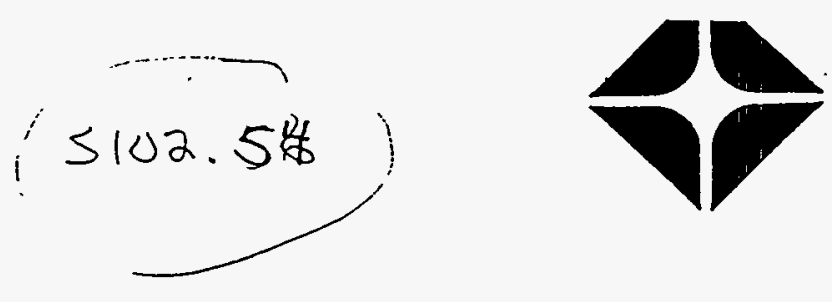

To:

R. L. Walser

From:

R. E. Wheeler $R$ c mlucle

Subject: ANALYSIS OF TANK FARM SAMPLE.

SAMPLE: T-2768 102-S Rंeceived 3-17-75

Vis-OTR: Black. $10 \%$ Suspended Solids. $1.2 \mathrm{Rad} / \mathrm{hr}$

$\mathrm{Ph}$ :

12.7

SPG:

1.34 @ $60^{\circ} \mathrm{C}$

AT:

$1.08 \mathrm{M}$

GEA :

$$
\begin{aligned}
& \left.{ }^{13{ }^{4}} \mathrm{Cs}-7.0 \times 10^{3} \mu \mathrm{Ci} / \mathrm{ga}\right] \\
& \left.{ }^{137} \mathrm{Cs}-1.50 \times 10^{6} \mu \mathrm{Ci} / \mathrm{ga}\right] \\
& \left.{ }^{60} \mathrm{Co}-1.08 \times 10^{3} \mu \mathrm{Ci} / \mathrm{ga}\right]
\end{aligned}
$$

Water:

$52.26 \%$

Cooling

Curve: $\quad 40^{\circ} \mathrm{C} \quad 45 \mathrm{Min}$. Suspended Solids

$35^{\circ} \mathrm{C} \quad 45 \mathrm{Min}$. Suspended Solids

$30^{\circ} \mathrm{C} \quad 45 \mathrm{Min}$. Suspended Solids

$25^{\circ} \mathrm{C} \quad 90 \mathrm{Min}$. Suspended Solids

$20^{\circ} \mathrm{C} \quad 45 \mathrm{Min}$. Suspended Solids

$15^{\circ} \mathrm{C} \quad 45 \mathrm{Min}$. Suspended Solids

$10^{\circ} \mathrm{C} \quad 45 \mathrm{Min}$. Suspended Solids

$5^{\circ} \mathrm{C} \quad 45 \mathrm{Min}$. Suspended Solids

REW: vmk

CC: JS Buckingham

WR Christensen

RM Ginestet

JC Womack

RE Wheeler

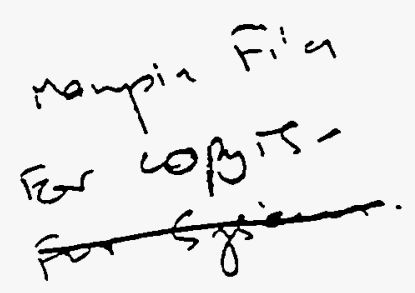




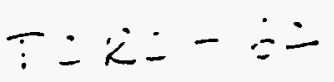

\section{Atlantic Richfield Hanford Company}

WHC-SD-WM-ER-323, Rev. 0

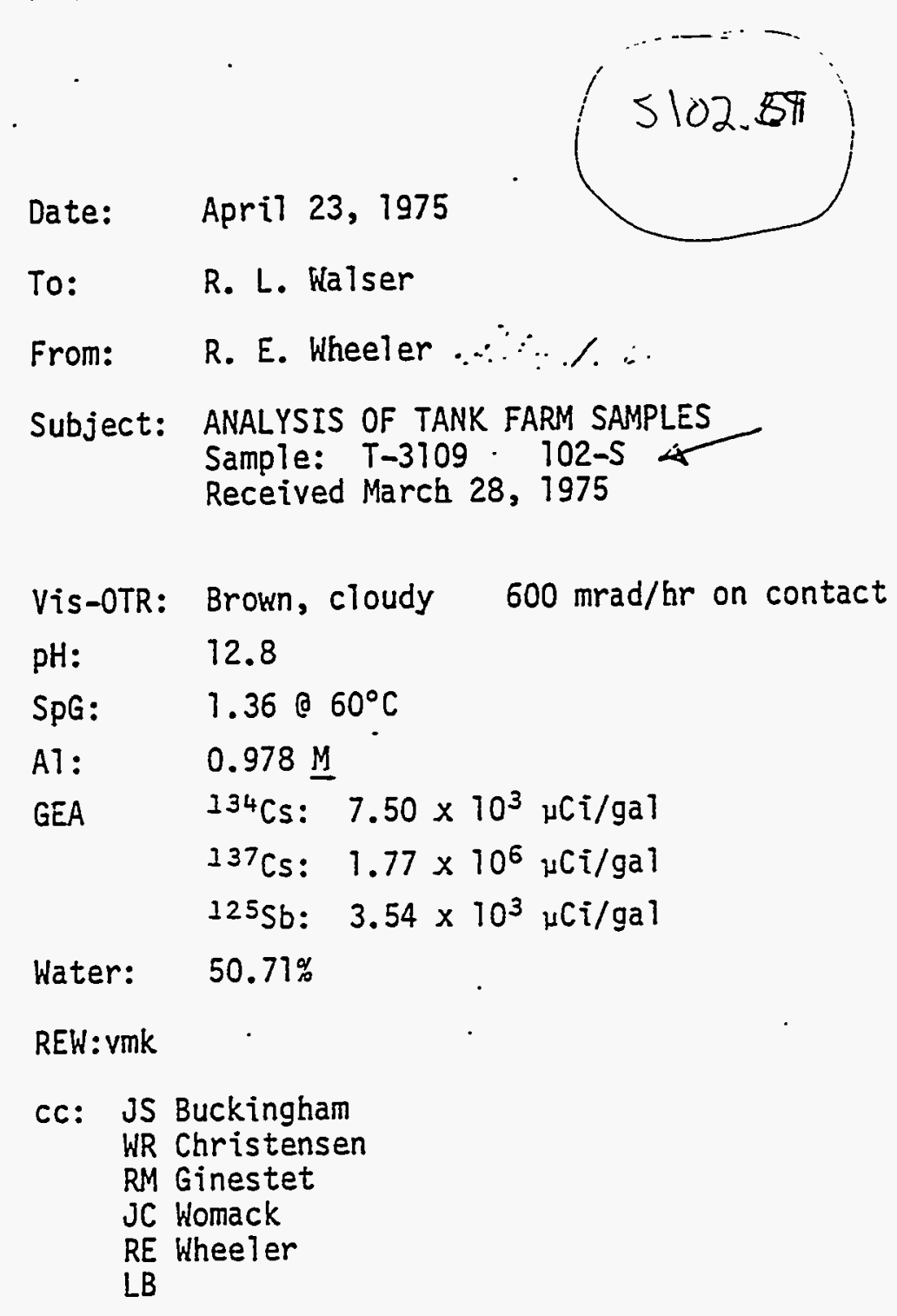


Date: $\quad$ April 1, 1975

To:

R. L. Walser

From:

R. E. Wheeler $R$ gindece

Subject:

ANALYSIS OF TANK FARM SAMPLES

SAMPLE: T-1369 102-S $\quad:$

Vis-OTR: Green $2 \%$ solids $1.2 \mathrm{Rad} / \mathrm{hr}$

Ph:

13.2

SPG:

$1.34 \odot 60^{\circ} \mathrm{C}$

A1:

$1.23 \mathrm{M}$

GEA :

$$
\begin{aligned}
& { }^{134} \mathrm{Cs}-9.0 \times 10^{2} \mu \mathrm{Ci} / \mathrm{gal} \\
& 137 \mathrm{Cs}-1.70 \times 10^{6} \mu \mathrm{Ci} / \mathrm{gal} \\
& 125 \mathrm{Sb}-2.84 \times 10^{3} \mathrm{Ci} / \mathrm{gal}
\end{aligned}
$$

Water:

$54.34 \%$

Cooling Curve: $40^{\circ} \mathrm{C}$ for $45 \mathrm{Min}$. No Solids

$35^{\circ} \mathrm{C}$ for $60 \mathrm{Min}$. No Solids

$30^{\circ} \mathrm{C}$ for $60 \mathrm{Min}$. No Solids

$25^{\circ} \mathrm{C}$ for $45 \mathrm{Min}$. No Solids

$20^{\circ} \mathrm{C}$ for $60 \mathrm{Min}$. No Solids

$15^{\circ} \mathrm{C}$ for $16 \mathrm{l} / 2 \mathrm{hrs}$. Suspended solids

$10^{\circ} \mathrm{C}$ for $45 \mathrm{Min}$. Suspended solids

$5^{\circ} \mathrm{C}$ for $45 \mathrm{Min}$. Suspended solids

REW: vmk
CC: JS Buckingham
WR Christensen
RM Ginestet
JC Womack
RE Wheeler 


\section{- : :- - Á - Atlantic Richfield Hanford Company}

Date: $\quad$ August 20, 1975

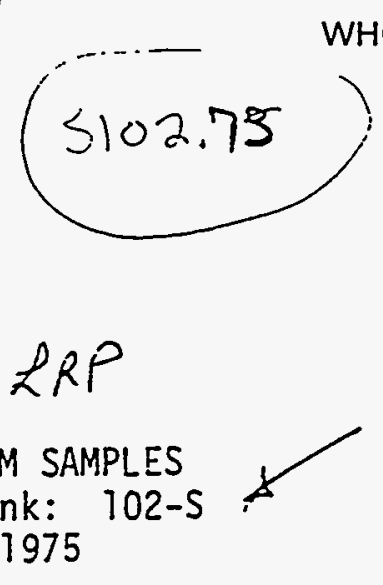

Vis-OTR: Greenish-brown Suspended solids $1 \mathrm{rad} / \mathrm{hr}$

Ph: $\quad 13.0$

$\mathrm{OH}: \quad \quad \quad .87 \underline{M}$

SpG: $\quad 1.24 \div 78^{\circ} \mathrm{C}$

$\mathrm{NO}_{2}: \quad 1.01 \mathrm{M}$

$\mathrm{NO}_{3}: \quad 2.99 \underline{\mathrm{M}}$

AT: $\quad 0.579 \underline{\text { 殅 }}$

GEA: $\quad{ }^{134} \mathrm{Cs}-4.63 \times 10^{4} \mu \mathrm{Ci} / \mathrm{gal}$

${ }^{13.7} \mathrm{Cs}-1.18 \times 10^{5} \mathrm{\mu Ci} / \mathrm{gal}$

$\left.{ }^{89,}{ }^{90} \mathrm{Sr}: 2.52 \times 10^{4} \mu \mathrm{Cj} / \mathrm{ga}\right]$

Water: $\quad 64.93 \%$

LRP : eyw

cc: JS Buckingham

WR Christensen

RM Ginestet

JC Womack

RE Wheeler 
Date: $\quad$ April 30, 1975

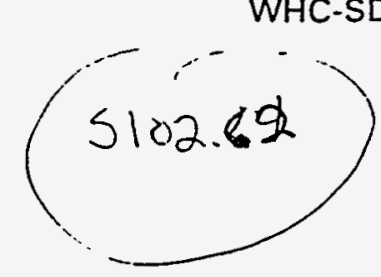

To: R. L. Walser

From: R. E. Wheeler Frypheclin

Subject: ANALYSIS OF TANK FARM SAMPLES

Sample: T-3328 102-S

Received April 4, 1975

Vis-0TR: Dark Green Suspended solids $2.5 \mathrm{rad} / \mathrm{hr}$

PH: $\quad 13.0$

SpG: $\quad 1.34$ @ $60^{\circ} \mathrm{C}$

AT: $\quad 1.12 \times 10^{-2} \mathrm{M}$

$\mathrm{OH}: \quad 2.69 \mathrm{M}$

$\mathrm{NO}_{2}: \quad 3.20 \mathrm{in}$

$\mathrm{NO}_{3}: \quad 3.52 \mathrm{M}$

GEA: $\left.\quad{ }^{134} \mathrm{Cs}-6.78 \times 10^{3} \mathrm{uCi} / \mathrm{ga}\right]$

${ }^{137} \mathrm{Cs}-1.64 \times 10^{6} \mathrm{uCi} / \mathrm{ga} 1$

$39.90 \mathrm{Sr}: \quad 2.70 \times 10^{4} \mathrm{uCi} / \mathrm{gal}$

Water: $\quad 53.47 \%$

REW: eW

CC: US Buckingham

WR Christensen

RM Ginestet

JC Womack

RE Wheeler

LB 


\subsection{8}

Date: July 8, 1975

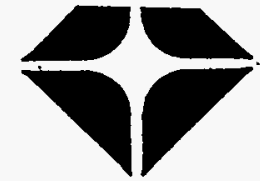

To: R. L. Walser

From: R. E. Wheeter Feryteden

Subject: ANALYSIS OF TANK FARM SAMPLES

Sample: T-3478 Tank: 102-S

Received April 10, 1975

Vis-OTR: Black $<5 \%$ solids $2 \mathrm{rad} / \mathrm{hr}$. $2^{\prime \prime}$

SPG: $\quad 1.38 \oplus 62^{\circ} \mathrm{C}$

Ph: $\quad 12.8$

$\mathrm{OH}: \quad 2.84 \underline{\mathrm{M}}$

$\mathrm{A} 7: \quad 0.818 \underline{\mathrm{M}}$

GEA: $\quad{ }_{137}^{134} \mathrm{Cs}-8.48 \times 10_{6}^{3} \mathrm{\mu Ci} / \mathrm{gat}$

$125 \mathrm{Cs}-1.85 \times 10_{4} \mu \mathrm{Cj} / \mathrm{gal}$

$\mathrm{Sb}-4.53 \times 10^{\mu \mathrm{Ci} / \mathrm{gal}}$

Water: $\quad 53.46 \%$

$89,90 \mathrm{Sr}: 7.30 \times 10^{4} \mu \mathrm{Ci} / \mathrm{gal}$

$\mathrm{NO}_{2}: \quad 1.43 \underline{\mathrm{M}}$

$\mathrm{NO}_{3}: \quad 3.19 \underline{\mathrm{M}}$

REW: eyw

cc: JS Buckingham

WR Christensen

RM Ginestet

JC Womack

RE Wheeler 


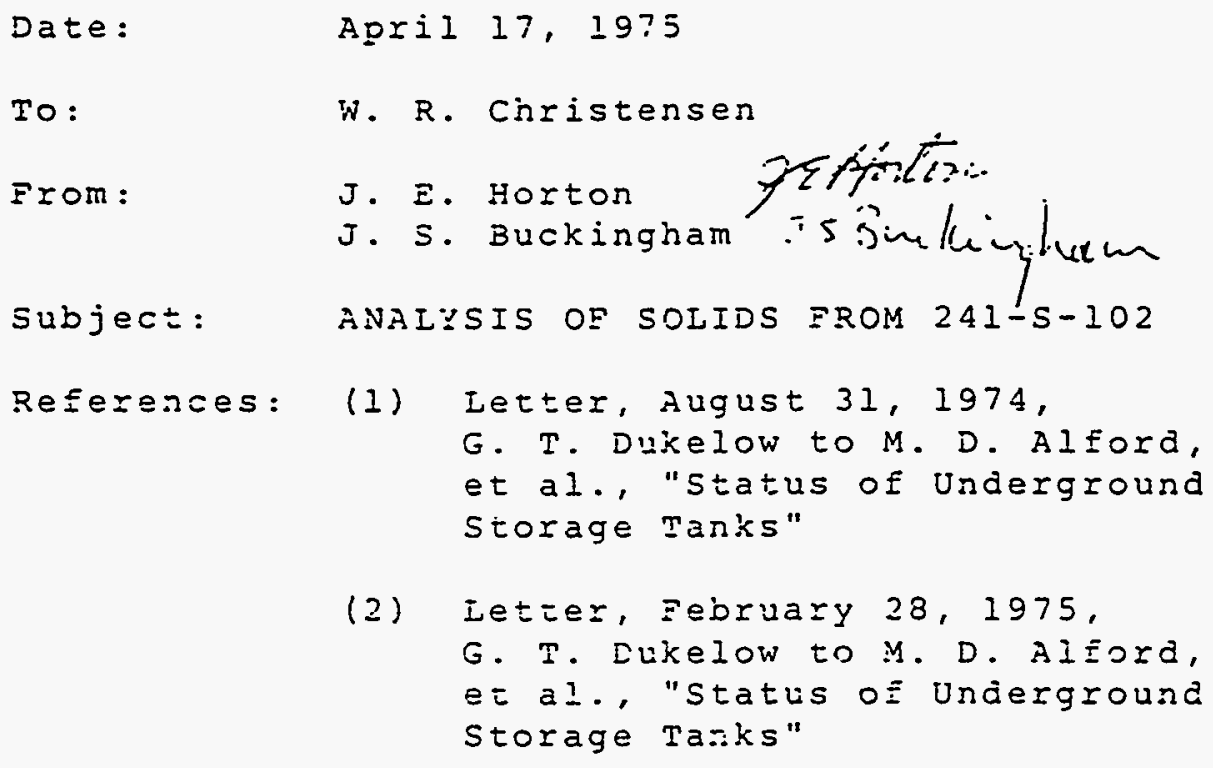

Tank 24l-s-102 is used as a feed tank for the 242-s Evaporator-Crystaliizer. Data in the reference letcers show that the level of solids in Tank 24I-S-lo2 is increasing. A sample oI the soiids was taken in an attempt to determine whether they were salts precipitating irom supersaturaced recycle solutions or something else.

The solids sample was composed of two layers, about 50 percent each. The tup layer was made up of coarse black crystals. The top layer dissolved in water. Analyses showed it to be a mixture of sodium nitrate and sodium phospnate. Only a small portion of the lower (heavier) layer was solible in wazer. Analyses showed it to be primarily an aluminum compound. The aluminum compound is presumed to be $\mathrm{Al}_{2} \mathrm{O}_{3}$. Details of the laboratory work are discussed beiow.

Weighed portions of tine two layers were leacied with water. The water insolubie Eraction was then leached inth corcentrated HCl. The two portions were combined, diluted to $50 \mathrm{milliliters,} \mathrm{and}$ anaiyzed. Any solids not soluble in water and HCl were Eused with koH, the melt dissolved with HCI, 
W. R. Christensen

Page 2

April 17, 1975

and dizuted to a known volume. The analyses of the two layers are shown below, reported as weight percent. Metaliic ions are reported as the oxide. Nitrate, nitrite, and phosphate are reported as tine sodium salt. Plutonium, strontium, and cesium analyses are shown as grams or microcuries per gram of damp "as received" solid and grams or microcuries per liter of "as received" solid.

Please call us if you have any questions regardir.g this work.

JEH : JSB : MS

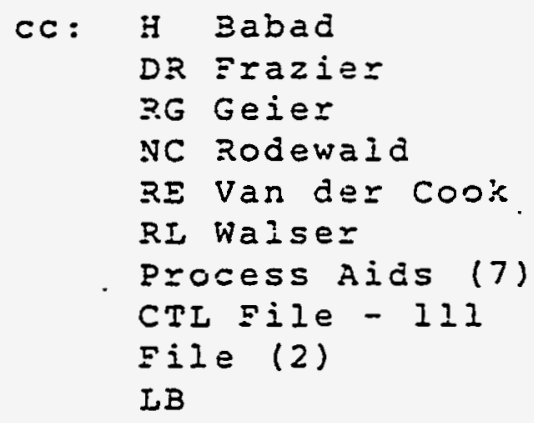


WHC-SD-WM-ER-323, Rev. 0

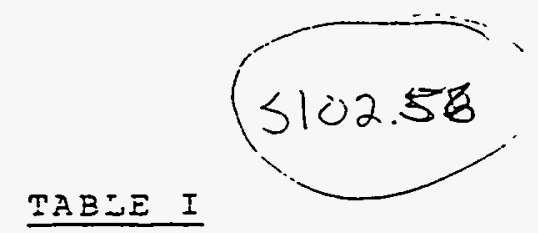

ANALYSIS OF 1O2-S TOP LAYER

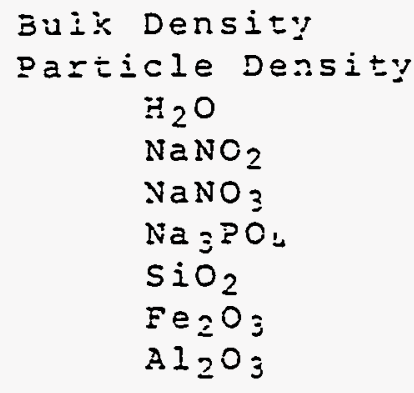

TOTAL

3.02
$39+90 \leq x$
$13:-5 x$
$1.30 \mathrm{~g} / \mathrm{cc}$

$1.74 \mathrm{~g} / \mathrm{CC}$

$45.0 \%$

$<0.1 \%$

$20.8 z$

29.98

1.73

0. 28

$5.5 \frac{8}{1}$

$102.9 \frac{8}{3}$

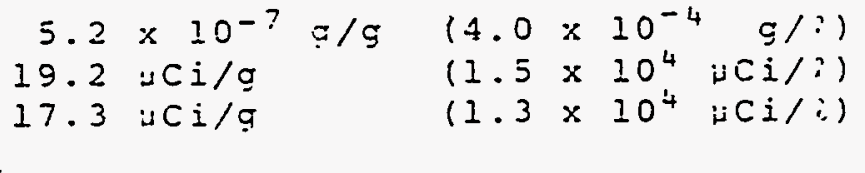




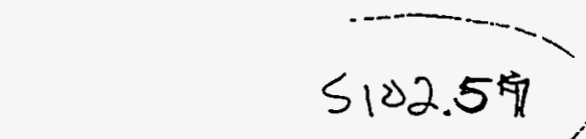

TABLE II

ANALYSIS OE 102-S BOTTOM LAYER

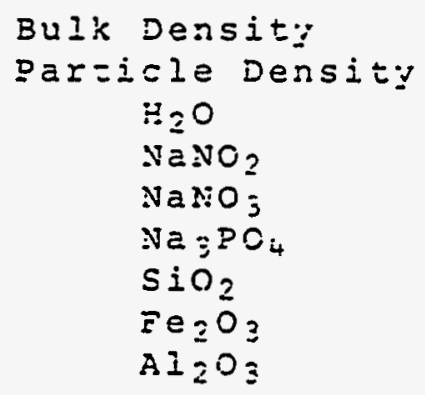

$$
\begin{aligned}
& 2.52 \quad 9 i=0 \\
& 2.09 \quad 9 /=0 \\
& 10.0 \% \\
& 0.1 \% \\
& (.0501) \\
& 1.03 \\
& 0.78 \\
& 1.48 \\
& 87.0 \% \\
& 100.25
\end{aligned}
$$

$$
\begin{aligned}
& 7.5 \times 10^{-7} \mathrm{~g} / \mathrm{g}\left(4.9 \times 10^{-4} \mathrm{~g} / 2\right) \\
& 0.5 \times \mathrm{Ci} / \mathrm{g} \quad\left(3.3 \times 10^{2} \times \mathrm{Ci} / \mathrm{R}\right) \\
& 5.6: 1 \mathrm{C} i / \mathrm{g} \quad\left(4.3 \times 10^{3} \mathrm{\mu Ci} / 2\right)
\end{aligned}
$$

NOrE I: The nitrate analysis o: the sample was impossibly high (520 percent $\left.\mathrm{NaNO}_{3}\right)$. It is assumed that the sample was contaminated during preparation. However, based on the material jalance calculation, we feel that the nitrate content of the salt is essentially zero. 
Date: August 20, 1975

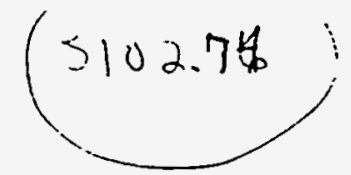

To: R. L. Walser

From: L. R. Perez, 2-2449 LRP

Subject: ANALYSIS OF TANK FARM SAMPLES

Sample: T-3639 Tank: 102-S

Received: April 17, 1975

Vis-OTR: Dark green $\quad 10 \%$ solids $2.5 \mathrm{rad} / \mathrm{hr}$ Q 2"

Ph: $\quad 12.8$

SPG: $\quad 1.32 @ 69^{\circ} \mathrm{C}$

Al: $\quad 0.742 \underline{M}$

$\mathrm{OH}: \quad 2.32 \mathrm{M} \approx$

$\mathrm{NO}_{2}: \quad \cdot 1.12 \mathrm{M}$

$\mathrm{NO}_{3}: \quad 5.17 \underline{\mathrm{M}}$

GEA: $\quad{ }^{34} \mathrm{Cs}-1.14 \times 10^{4} \mathrm{jCj} / \mathrm{gaI}$

$\left.{ }^{137} \mathrm{Cs}-2.92 \times 10^{6} \mu \mathrm{Ci} / \mathrm{ga}\right]$

$\left.{ }^{89,90} \mathrm{Sr}: 5.58 \times 10^{4} \mathrm{\mu Ci} / \mathrm{ga}\right]$

Water: $\quad 54.53 \%$

LRP : eyw

CC: JS Buckingham

WR Christensen

RM Ginestet

JC Womack

RE Wheeler 
WHC-SD-WM-ER-323, Rev. 0

4 Babad

Js Buckingham

WR Christeasen

RG Geier

DG Harlow

JE HOrton

RC Roal

JC Womack

CTL OEfice

iile

circulate:

CTI Exempt

Eile:

CTL-122

CTI-111
Ţo:

D. C. IIDi

E=om: J. E. Horton

$$
\begin{array}{r}
\text { WASTE SOLUTION SOLIDIEICATIOA } \\
\text { BY VOLUME PEDUCIION } \\
\text { [ACHIEVDAENT RESORT EOR PERIOD } \\
\text { ENDING APRIL 29, I9TS] }
\end{array}
$$

Budget Numbe=:

Contributors:

$$
\Xi-52204
$$

J. E. Hั:

J. S. Buckinghan.

\section{ABSTRACT}

mank 242-5-102 is used as a feed tank Eo= the 242-s Evaporator-Czystallizez. A sample OI IOZ-S solids was analyzed to desezmiag if the presipicating salts =esul:ed Exom supezseturated resycle soluzions.

The solicis sample ras composes ojequal quantities 0 E two layers. The top layer dissolved in water. Analyses showed í to be a IIxture oE sodium nit:z=e and sodiug piosphate. Only a small poz=ion oz tie lowe= (heavier) layer was water soluble. Analyses showed it to be primarily an aluminum compound, Presumably $\mathrm{Al}_{2} \mathrm{O}_{3}$. 
WHC-SD-WM-ER-323, Rev. 0

- DISCUSSION

Tanx Earm Assistance Summày

The top layez Exom lo2-s tanx was made up of coarse black crystals and the bottom layer was fine yellow crystals. weighed portions of the two layezs were leached with watez. The water insoluble Exaction was then leached with concentrated acl. The two portions were combined, diluted to 50 milliliters and analyzed. Solids not soluble in water and ïl were Eused with kor, the melt aissolved with riCl, and ailuted so a known volume. The analyses oi the two layers are shown below, zeported as weigit percens. Metallic ions are zepoz=el

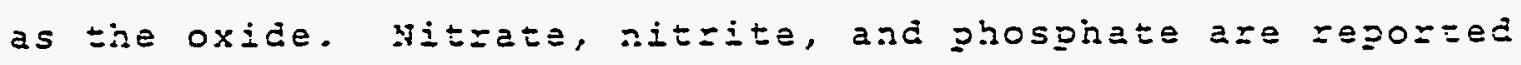
as tie sociun salt. ?lutonium, stzontilin, and sesium asalyses are shown as grams or ricrocuries per gram of camp "as received" solids and grans or microcuries per liter oj "as zeceived" solids. Analyses oE tie laboratory work aze showa ia TabIes I and II. 
WHC-SD-WM-ER-323, Rev. 0

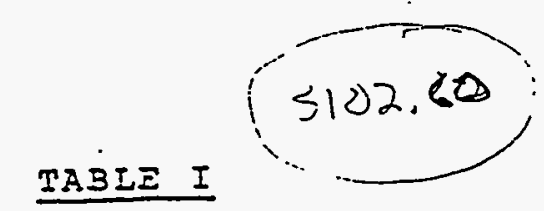

ANAIYSIS OE IOZ-S TOZ IAYZR

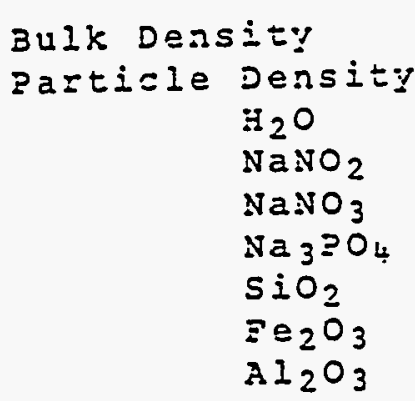

TOTAL

$$
\begin{array}{r}
89+9050 \\
137 \text { C }
\end{array}
$$
$1.30 \mathrm{~g} / \mathrm{co}$
I. $74 \mathrm{~g} / \mathrm{C}=$
$45.0 \%$
0.17
$20.3 \%$
29.93
$1.7 \%$
$0.2 \frac{1}{2}$
$5.5 \%$

102.93

$\begin{array}{rrrr}5.2 \times 10^{-7} g / 9 & \left(4.0 \times 10^{-4}\right. & g / 2) \\ 19.2 & \mu c i / 9 & \left(1.5 \times 10^{4} j(j / 2)\right. \\ 17: 3 & \mu c i / 9 & \left(1.3 \times 10^{4} j(1 / 2)\right.\end{array}$




\section{TA}

\section{ANALYSIS OE :O2-S BOTTOM IAYE?}

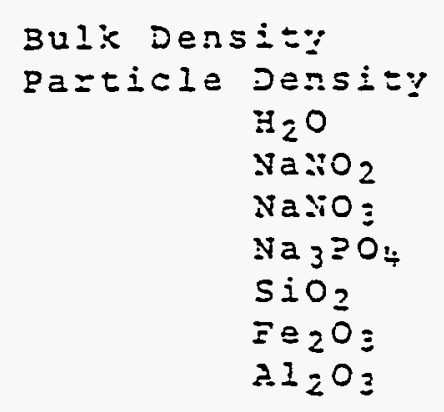

TOTAI

$$
\begin{array}{r}
p u \\
89+905= \\
13705
\end{array}
$$

$\begin{array}{r}1.52 \mathrm{~g} / 00 \\ 2.09 \mathrm{~g} / \mathrm{co} \\ 10.07 \\ 0.13 \\ (1005 \pm 1) \\ 1.03 \\ 0.75 \\ 1.43 \\ 37.03 \\ \hline\end{array}$

$100.2 \overline{3}$

$7.5 \times 10^{-7} \mathrm{~g} / \mathrm{s}$

$0.5 \quad 2 \in i / g$

$6.5 \quad j \in i / g$

HOTE: The nitrate analysis cE tie sample was impossibly high $(520$ gezcen: iavoz). It is assumed tha tine sample was son:azinz=ed duzing preparation. Nowever, based on the neterial jalance calculacion, we Eeel that the niczase content of she salu is essentially zero. 
Date: $\quad$ May 13, 1975

$$
5102.63
$$

To:

R. L. Walser

From:

R. E. Wheeler

Subject:

ANALYSIS OF TANK FARM SAMPLES
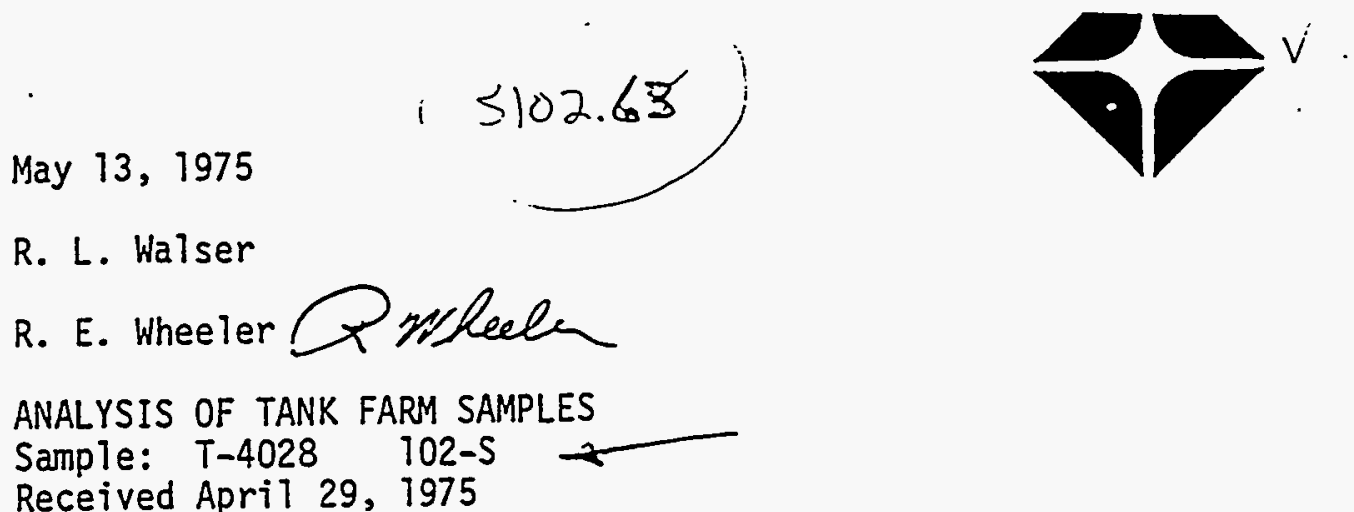

Vis-0TR: Clear, green, no solids $600 \mathrm{mrad} / \mathrm{hr}$

$\mathrm{pH}: \quad 13.0$

SpG: $\quad 1.2538$

$\mathrm{OH}: \quad 1.16 \mathrm{M}$

A1: $\quad 0.448 \underline{M}$

$\mathrm{NO}_{2}: \quad 0.818 \underline{\mathrm{M}}$

$\mathrm{NO}_{3}: \quad 1.61 \underline{\mathrm{M}}$

GEA: $\quad{ }^{1.34} \mathrm{CS}-4.84 \times 10^{3} \mu \mathrm{Ci} / \mathrm{gal}$

${ }^{137} \mathrm{Cs}-9.24 \times 10^{5} \mathrm{\mu Ci} / \mathrm{gal}$

Water: $70.04 \%$

${ }^{89,90} \mathrm{Sr}: 2.71 \times 10^{4} \mu \mathrm{Ci} / \mathrm{gal}$

REW:eyw

CC: JS Buckingham

WR Christensen

RM Ginestet

JC Womack

RE Wheeler 
Date: August 22, 1975

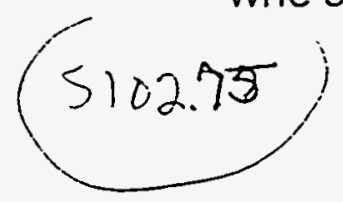

To: R. L. Walser

From: L. R. Perez, 2-2449 XPf

Subject: ANALYSIS OF TANK FARM SAMPLES

Sample: T-4104 Tank: 102-S

Received: May 2, 1975

Vis-0TR: Green $25 \%$ solids $90 \mathrm{mrad} / \mathrm{hr}$

Ph: $\quad 11.70$

SpG: Insufficient sample

$\mathrm{OH}: \quad 1.58 \underline{\mathrm{M}}$

A1: $\quad 0.818 \underline{M}$

$\mathrm{NO}_{2}: \quad \mathrm{T} .0 \mathrm{M}$

$\mathrm{NO}_{3}: \quad 2.65 \underline{M}$

${ }^{89,90}$ Sr: $9.56 \times 10^{4} \mu \mathrm{Ci} / \mathrm{gat}$

GEA: $\quad{ }_{125}^{60} \mathrm{Co}-<7.22 \times 10_{3}^{2} \mu \mathrm{Ci} / \mathrm{gal}$ ${ }_{95} \mathrm{Sb}-1.12 \times 10_{3} \mu \mathrm{Ci} / \mathrm{gal}$ $144 \mathrm{ZrNB}-<1.57 \times 10_{4} \mathrm{\mu Ci} / \mathrm{gaT}$ $\left.134 \mathrm{CePr}-<4.59 \times 10_{3} \mu \mathrm{Ci} / \mathrm{ga}\right]$ $137 \mathrm{Cs}-1.42 \times 10_{5} \mu \mathrm{Ci} / \mathrm{gal}$ $\left.154 \mathrm{Cs}-3.0 \times 10_{3} \mu \mathrm{Cj} / \mathrm{ga}\right]$

Water: $\quad 62.55 \%$

LRP : eyw

CC: JS Buckingham

WR Christensen

RM Ginestet

JC Womack

RE Wheeler 


\section{Atlantic Richfield Ha}

WHC-SD-WM-ER-323, Rev. 0

Date: $\quad$ August 22, 1975

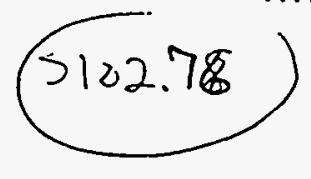

To: R. L. Walser

From: L. R. Perez, 2-2449 बే̂R

Subject: ANALYSIS OF TANK FARM SAMPES Sample: T-4240 Tank: 102-S

Received: May 8, 1975

Vis-OTR: Light green $<1 \%$ Solids $1 \mathrm{rad} / \mathrm{hr}$

$\mathrm{Ph}: \quad 12.7$

SpG: $\quad 1.3612$

$\mathrm{OH}: \quad 1.52 \underline{M}$

Al: $\quad 0.812 \underline{M}$

$\mathrm{NO}_{2}: \quad 0.792 \underline{\mathrm{M}}$

$\mathrm{NO}_{3}: \quad 3.63 \underline{\mathrm{M}}$

${ }^{89}, 90 \mathrm{Sr}: \quad 3.36 \times 10^{4} \mu \mathrm{Cj} / \mathrm{gal}$

GEA $\quad{ }^{134} \mathrm{Cs}-5.06 \times 10^{3} \mathrm{\mu Ci} / \mathrm{gal}$

$\left.{ }^{137} \mathrm{Cs}-9.12 \times 10^{5} \mu \mathrm{Cj} / \mathrm{ga}\right]$

Water: $\quad 54.37 \%$

Cooling Curve: $30^{\circ} \mathrm{C}$ for $45 \mathrm{~min}$. No solids

$25^{\circ} \mathrm{C}$ for $45 \mathrm{~min}$. No solids

$20^{\circ} \mathrm{C}$ for $45 \mathrm{~min}$. No solids

$15^{\circ} \mathrm{C}$ for $45 \mathrm{~min}$. No solids

$10^{\circ} \mathrm{C}$ for $45 \mathrm{~min}$. No solids

$5^{\circ} \mathrm{C}$ for $45 \mathrm{~min}$. No solids

LRP : eyw

CC: JS Buckingham

WR Christensen

RM Ginestet

JC Womack

RE Wheeler 


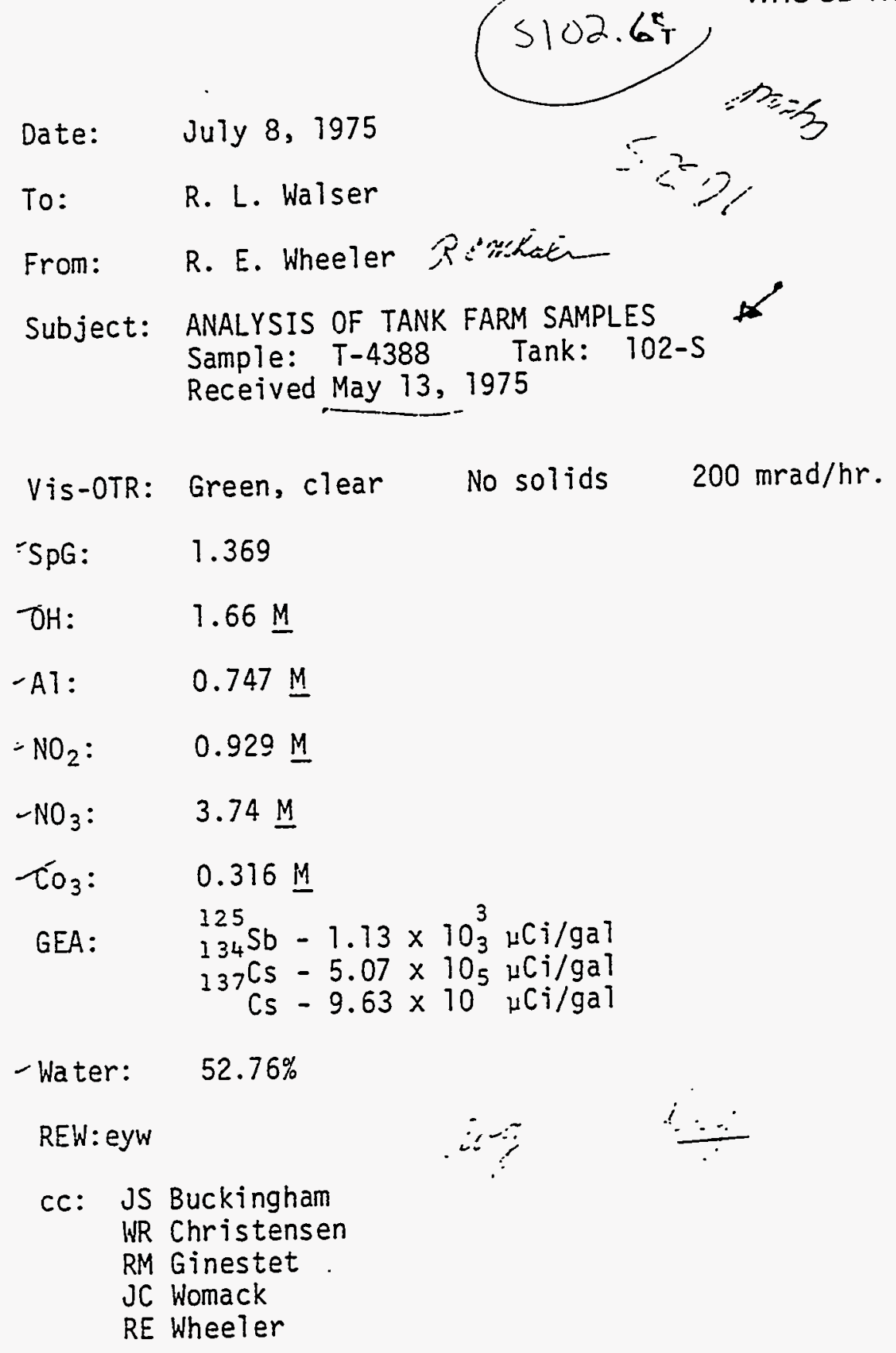
CC: JS Buckingham
WR Christensen
RM Ginestet
JC Womack
RE Wheeler


H. 2. $M a t=$

?age 2

say 20,1975

?30C?00R3

solids had pzectpitated from ali of the samplos by the time they had arzired in ths Iabozato=y. The samples ve=e heated to zedissolve the p=ecipitated solids before aliquots wezo taker. All of the precipitated solids zedissolved between 50 and $35^{\circ} C$ except for the solids la the sample. from log-3x which contained about 20 settled rolume pe=cent insolubla solids. The 109-3X sample vas. SIuz=ied and alfoluots ve=0 taker of the siufyy. The $109-3 x$ sluzil was treated. in the same jamal as the cleal solutions flom tise othei sampies.

Except E0: the sample E=0m I09-3x, when the solids had zedissolred, 50 zillilitess of tie solution weze imediately diluzed with 50 aill1lites of wates. A second aligroit was taken $a=$ bie saze tempezalu=s for densily and pe=seat ฯะะュ.

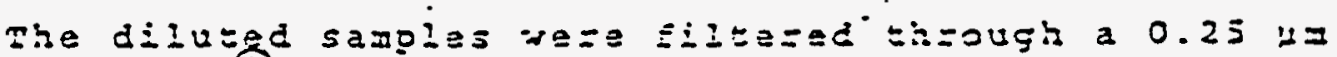

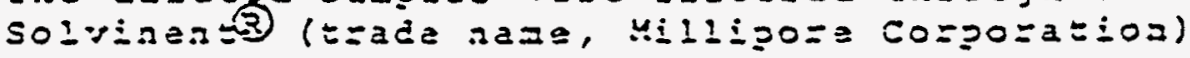

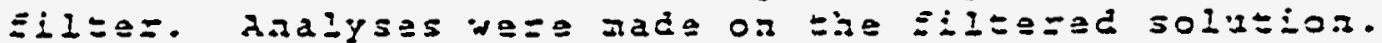

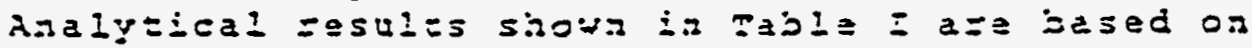
tha yadiluzed beaned solution.

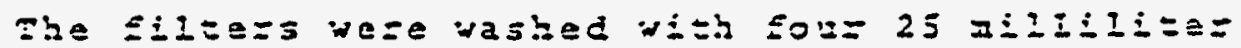

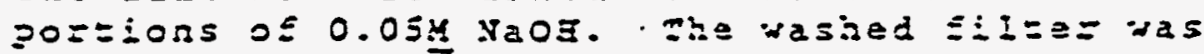

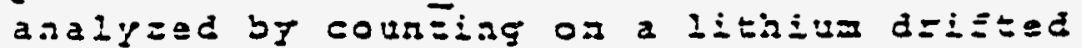

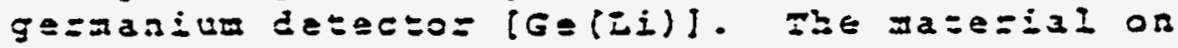

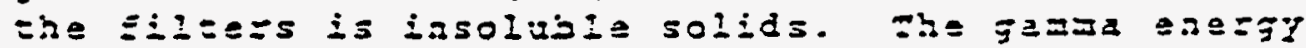

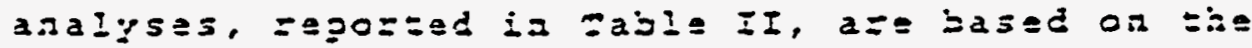
a

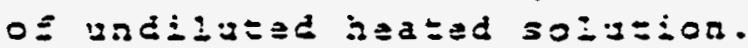


i. 2. $4 \dot{e}=z$

Page 3

MaY 20, 1975

DISCUSSIOX

The 4PC. foz soluble actioides of fatezest iz a contzolled jone are shown in Tabie II. The $=0$ ase undoubtediy lasoluble plutonium solids on the filtezs, but wlti the othez actirity present on the filter, the detectloa lizit for 239 as rould be ia tha $10^{7} \mathrm{hl} / \mathrm{h}$ ange. i. I. Winters is goiag to look at analytical technigues for analyzing tho. insoluble material.

It is interesting to note that amezicium is associated only with $S$ and $\mathrm{rx}$ samples. Hasto E=0I 234-5 buildiag ame=icium zecorazi plocess is processed lazough the 242-T zipapozatoz which accounts for the ilgh amezicium aralyses ia the TX samples.

othe= than $137 \mathrm{Cs}$, yost os the gamma activity ia the waste is associated with insoluble solids. Cesiun Sound on tho Eiltez may be due to small aluminosilicats solids such as cancliniles whici s=aj cesiug.

The sample s=0m log-3x containad so many iasoluble solids $i=$ was $70=$ possisle =0 coun

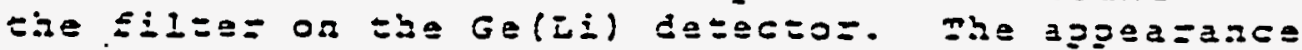

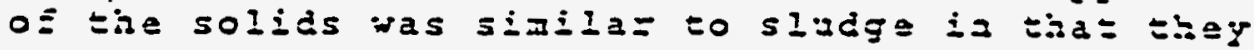

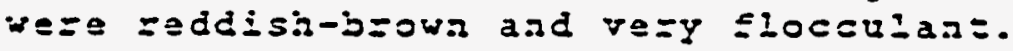

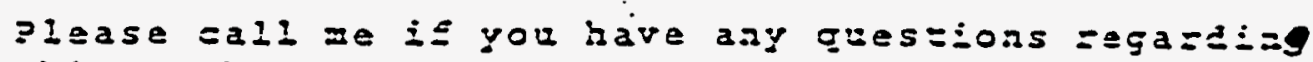
$\therefore i=$ No $=k$.

55: $: 25$

$A==$. (3)

\begin{tabular}{|c|c|c|}
\hline & \multirow{6}{*}{ 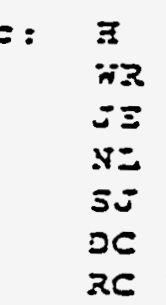 } & 3ajad \\
\hline & & $C .2= \pm 3=9.25$ \\
\hline & & $\exists a=\infty \in I \geq a=$ \\
\hline & & $\exists a=\geq s$ \\
\hline & & $\begin{array}{l}\text { J0h.150.2 } \\
\text { Ii.2: }\end{array}$ \\
\hline & & \\
\hline
\end{tabular}

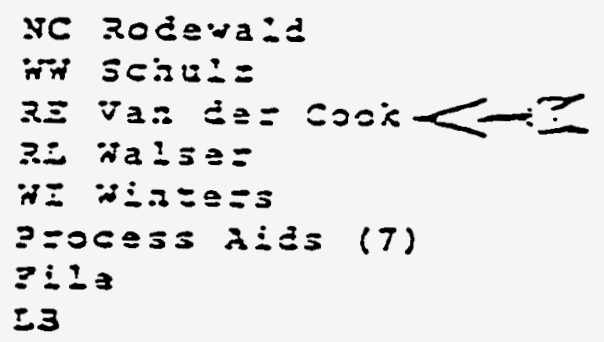




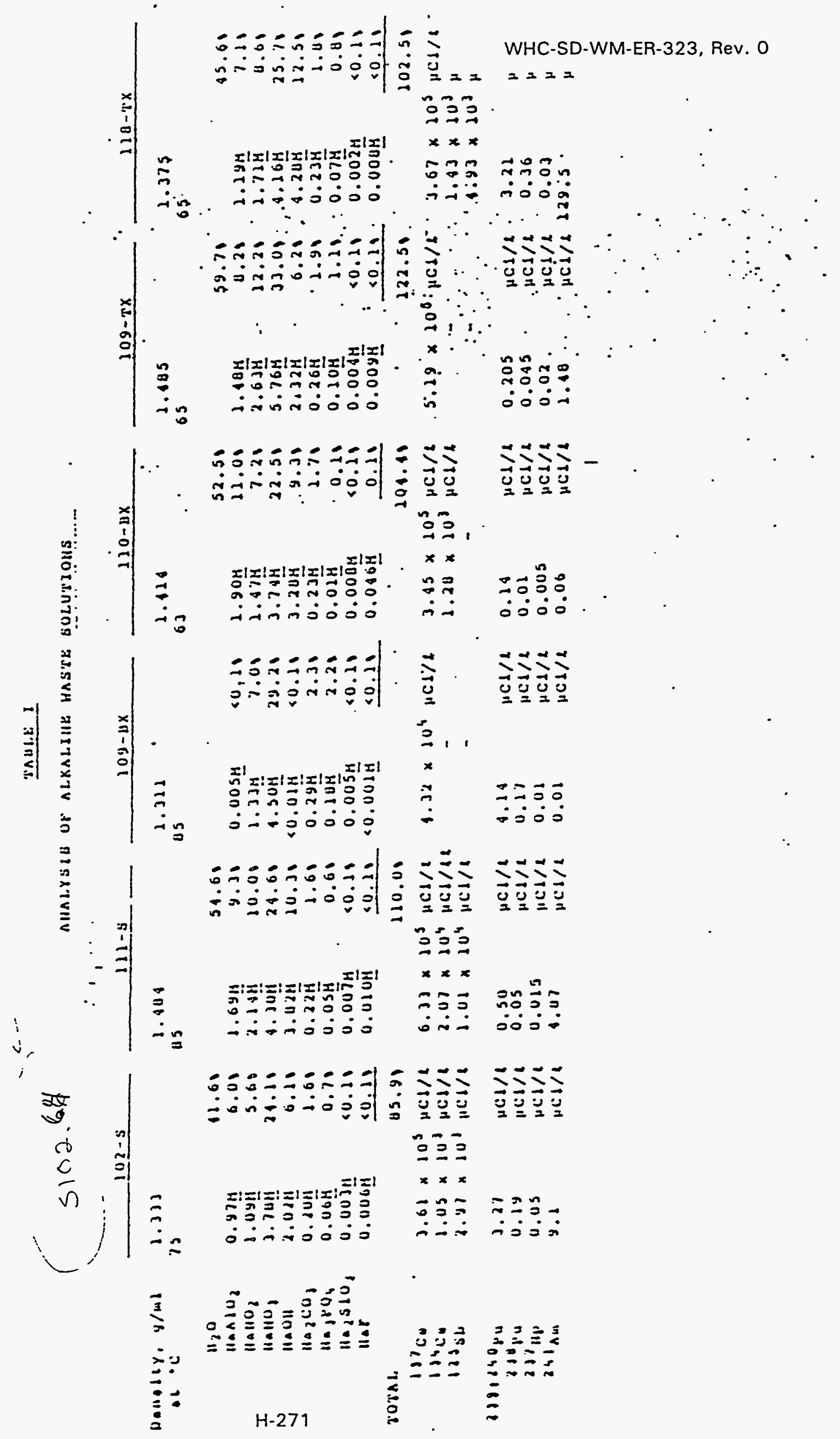




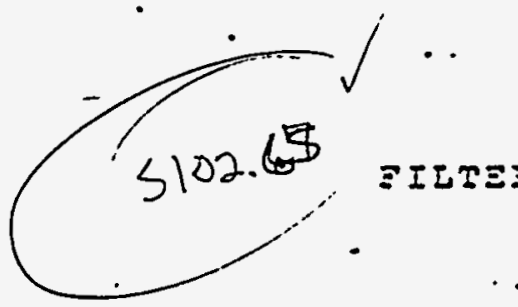

2A3L3 II

\section{2-5}

$241 \mathrm{Am}$

$144 \mathrm{Ce}$

$154 \equiv 0$

$155 \equiv 4$

$134 \mathrm{Cs}$

$137 \mathrm{Cs}$

$57 \mathrm{Co}$

$23 \mathrm{Co}$

10 0 रh

$35 \mathrm{z}=$

$+25 \mathrm{si}$
$2.11 \times 10^{3}$

$2.07 \times 10^{4}$

$6.50 \times 10^{3}$

$2.23 \times 10^{4}$

$5.24 \times 10^{2}$

$1.34 \times 10^{5}$

$1.73 \times 10^{3}$

$8.45 \times 10^{3}$

$3.09 \times 10^{2}$

$2.51 \times 10^{3}$

$.111-5$
$4.92 \times 10^{3}$
$5.51 \times 10^{4}$
$1.21 \times 10^{4}$
$3.99 \times 10^{4}$
$9.93 \times 10^{2}$
$8.95 \times 10^{4}$
$7.40 \times 10^{3}$
$5.57 \times 10^{5}$
$5.63 \times 10^{3}$
$1.37 \times 10^{4}$

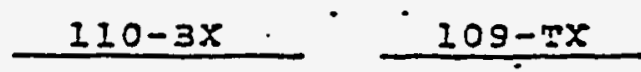

$-\quad 5.61 \times \overline{103}$

$-\quad 8.05 \times 10^{3}$

- $1.41 \times 10^{3}$

$3.01 \times 10^{2}$

32.4

$1.23 \times 10^{3}$ 39.3

$2.25 \times 10^{3}$

$3.23 \times 10^{2}$

- $7: .3$

$-$
$4.52 \times 10^{3}$

$113-2 x$

$8.55 \times 10^{7}$

$3.02 \times 10^{\circ}$

$4.56 \times 10^{2}$

$1.49 \times 10^{3}$

79.5

$1.24 \times 10^{4}$

4. 10 $\times 10^{2}$

$3.25 \times 10^{3}$

$\div .2$

$\times 20^{3}$
$1.73 \times 10^{2}$

22.4

\section{IABIZ IEI}

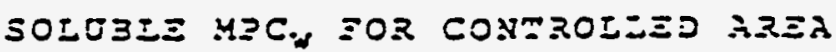

$10: 12$

\begin{tabular}{|c|c|}
\hline $23^{3} \geq 3$ & 0.1 \\
\hline $240 \geq 7$ & 0.1 \\
\hline $233 \geq 2$ & 0.1 \\
\hline 237 פַה 2 & 0.09 \\
\hline $2+1$ & 0.1 \\
\hline
\end{tabular}


Atlantic Richfield Hanford Company

WHC-SD-WM-ER-323, Rev. 0

Date: $\quad$ August 22,1975

$$
5102.79
$$

To: R. L. Walser

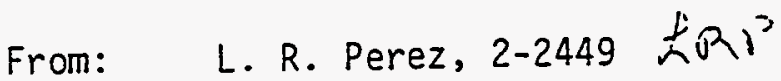

Subject: ANALYSIS OF TANK FARM SAMPLES

Sample: T-4823 Tank: 102-S

Received: May 27, 1975

Vis-OTR: Dark green iNondeterminable solids $100 \mathrm{mrad} / \mathrm{hr}$

SpG: $\quad 1.34 \quad 30^{\circ} \mathrm{C}$

$\mathrm{OH}: \quad 2.24 \mathrm{M}$

A1: $\quad 1.09 \underline{M}$

$\mathrm{NO}_{2}: \quad 1.44 \underline{\mathrm{M}}$

$\mathrm{NO}_{3}: \quad 2.92 \underline{\mathrm{M}}$

$\mathrm{CO}_{3}: \quad 0.263 \mathrm{M}$

GEA: $\left.\quad{ }_{137} \mathrm{Cs}-1.94 \times 10^{4} \mathrm{\mu Ci} / \mathrm{ga}\right]$

${ }^{137} \mathrm{Cs}-3.26 \times 10^{5} \mathrm{HCi} / \mathrm{gal}$

Water: $\quad 56.73 \%$

LRP : eyw

CC: JS Buckingham

WR Christensen

RM Ginestet

RE Wheeler

JC Womack 
Date: August 1, 1975

To: $\quad$ R. L. Walser

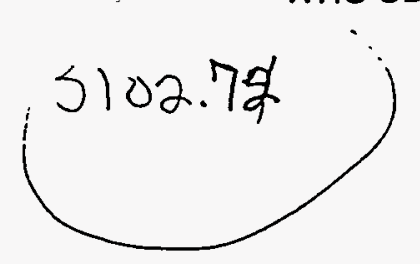

From: R. E. Wheeler FPldeken

Subject: ANALYSIS OF TANK FARM SAMPLES

Sample: T-5137 Tank: 102-S

Received: June 13, 1975

Vis-OTR: Dark green Solids unknown 1 rad a 2"

$\mathrm{Ph}: \quad 12.9$

SpG: $\quad 1.41^{\circ} \circ 21^{\circ} \mathrm{C}$

$\mathrm{OH}: \quad 2.32 \underline{\mathrm{M}}$

Al: $\quad 1.11 \underline{\mathrm{M}}$

$\mathrm{NO}_{2}: \quad 1.73 \underline{M}$

$\mathrm{HO}_{3}: \quad 3.68 \mathrm{M}$

${ }^{89}, 90^{\circ} \mathrm{Sr}: 3.97 \times 10^{4} \mu \mathrm{Cj} / \mathrm{gal}$

$\mathrm{CO}_{3}: \quad 0.325 \mathrm{M}$

GEA: $\quad{ }^{134} \mathrm{CS}-1.34 \times 10^{4} \mu \mathrm{Ci} / \mathrm{ga}$

$\left.{ }^{137} \mathrm{Cs}-2.06 \times 10^{6} \mathrm{HCi} / \mathrm{ga}\right]$

Water: $\quad 44.49 \%$

REW: eyw

CC: JS Buckingham

WR Christensen

RM Ginestet

JC Womack

RE Wheeler 


\subsection{8}

Date: July 28,1975

To: R. L. Walser

From: R. E. Wheeler P P P fleide

Subject: ANALYSIS OF TARK FARM SAMPLES

Sample: T-5514 Tank: 102-S

Received: June 20, 1975

\section{Supernatant}

Vis-0TR: Greenish-black Solids undetermined $1.5 \mathrm{rad} / \mathrm{hr}$

SpG: $\quad 1.5202$

$\mathrm{OH}: \quad 4.29 \mathrm{M}$

A]: $\quad 0.553 \underline{M}-$ Rerun: $0.812 \underline{M}$

$\mathrm{NO}_{2}: \quad 2.38 \underline{\mathrm{M}}$

$\mathrm{NO}_{3}: \quad 2.63$

$\mathrm{CO}_{3}: \quad 0.223 \underline{\mathrm{M}}$

GEA: $\quad 134 \mathrm{CS}-1.44 \times 10_{6}^{4} \mu \mathrm{Ci} / \mathrm{gal}$

$\mathrm{Cs}-2.88 \times 10 \mu \mathrm{Ci} / \mathrm{gal}$

${ }^{89},{ }^{90} \mathrm{Sr}: 1.20 \times 10^{5} \mu \mathrm{Ci} / \mathrm{gal}$

Water: $\quad 47.0 \%$

REW: eyw

Cc: JS Buckingham

WR Christensen

RM Ginestet

JC Womack

RE Wheeler 
Date: July 31,1975

$$
5102.7 B
$$

To: R. L. Walser

From: R. E. Wheeter Ge.Mrlew

Subject: ANALYSIS OF TANK FARM SAMPLES

Sample: T-5792 Tank: 102-S

Received: JuTy 2, 1975

Vis-OTR: Dark brown 25\% Solids $50 \mathrm{mrad} / \mathrm{hr}$ ( $4 "$

$\mathrm{Ph}: \quad 13.50$

SPG: Insufficient sample

$\mathrm{OH}: \quad \quad 1.74 \mathrm{M}$

Al: $\quad 1.0 \underline{M}$

$\mathrm{NO}_{2}: \quad 1.15 \underline{\mathrm{M}}$

$\mathrm{NO}_{3}: \quad 2.92 \mathrm{M}$

${ }^{89},{ }^{90} \mathrm{Sr}: 4.02 \times 10^{4} \mathrm{\mu Ci} / \mathrm{gal}$

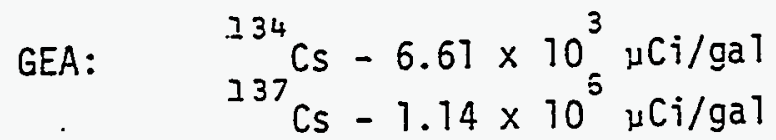

Water: $\quad 60.31 \%$

REW: eyw

cC: JS Buckingham

WR Christensen

RM Ginestet

JC Womack

RE Wheeler 
Date: $\quad$ September 23, 1975

5102.83

To: R. L. Walser

From: R. E. Wheeler, 2-2449 if trhatin

Subject: ANALYSIS OF TANK FARM SAMPLES

Sample: T-5859 Tank: 102-S

Received: July 7, 1975

Vis-0TR: Dark brown $\quad 100 \%$ Solids $\quad 500 \mathrm{mrad} / \mathrm{hr}$ @ 4"

$\mathrm{pH}: \quad 13.50$

SpG: $\quad 1.370 \circ 21^{\circ} \mathrm{C}$

$\mathrm{OH}: \quad 1.89 \underline{\mathrm{M}}$

A1: $\quad 1.03 \underline{M}$

$\mathrm{NO}_{2}: \quad 0.988 \underline{\mathrm{M}}$

$\mathrm{NO}_{3}: \quad 3.90 \underline{\mathrm{M}}$

${ }^{33},{ }^{90} \mathrm{Sr}: 6.26 \times 10^{4} \mathrm{\mu Ci} / \mathrm{gal}$

GEA: $\quad \begin{array}{r}134 \mathrm{Cs}-6.82 \times 10^{3} \mu \mathrm{Ci} / \mathrm{gal} \\ \left.\quad{ }^{137} \mathrm{Cs}-1.31 \times 10^{6} \mu \mathrm{Ci} / \mathrm{ga}\right]\end{array}$

Water: $\quad 48.41 \%$

REW: eyw

CC: JS Buckingham

WR Christensen

RM Ginestet

RE Wheeler

JC Womack 


\title{
$\because: \therefore--\because \quad$ Atlantic Richfield Hanford Company
}

\author{
Date: JuTy 31,1975

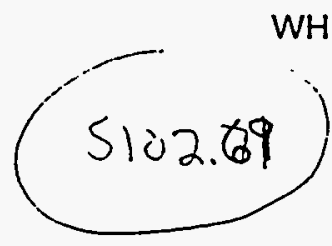 \\ To: R. L. Walser \\ From: R. E. Wheeler Feyshiclize \\ Subject: ANALYSIS OF TANK FARM SAMPLES \\ Sample: T-5964 Tank: 102-S \\ Received: July 9, 1975 \\ Vis-0TR: Dark brown Suspended solids $1600 \mathrm{mrad} / \mathrm{hr}$ \\ Ph: $\quad 13.5$ \\ SPG: $\quad 1.35 \odot 44^{\circ} \mathrm{C}$ \\ $\mathrm{OH}: \quad 1.57 \underline{\mathrm{M}}$ \\ $\mathrm{AT}: \quad 0.898 \mathrm{M}$ \\ $\mathrm{NO}_{2}: \quad 1.36 \underline{\mathrm{M}}$ \\ $\mathrm{NO}_{3}: \quad 4.20 \underline{\mathrm{M}}$ \\ GEA: $\quad 134 \mathrm{Cs}-6.72 \times 10_{6}^{3} \mathrm{HC \textrm {Ci } / \mathrm { ga } ]}$ \\ CS $-1.36 \times 10 \mu \mathrm{Hi} / \mathrm{gal}$ \\ ${ }^{89}, 90 \mathrm{Sr}: 3.58 \times 10^{4}, \mathrm{Ci} / \mathrm{ga}$ \\ Water: $\quad 45.69 \%$ \\ REN: eyw \\ CC: JS Buckingham \\ WR Christensen \\ RM Ginestet \\ JC Womack \\ RE Wheeler
}

WHC-SD-WM-ER-323, Rev. 0 


\section{$\bar{i}=2 L-\because 3$}

\section{Atlantic Richfleld Hanford Company}

Date: . December 11, 1975

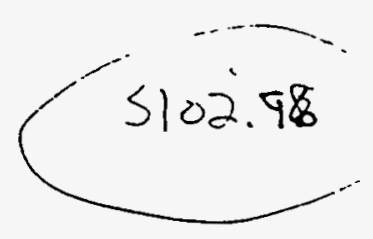

WHC-SD-WM-ER-323, Rev. 0

To: R. L. Walser

From: R. E. Wheeler, 2-2449 . il'j, í. '.

Subject: ANALYSIS OF TANK FARM SAMPLES

Sample: T-6042 Tank: 102-S -

Received: July 14, 1975 .

Vis-OTR: Dark green $\sim 5 \%$ Solids $1500 \mathrm{mrad} / \mathrm{hr}$

$\mathrm{pH}: \quad 12.7$

SpG: $\quad 1.340 \quad 27^{\circ} \mathrm{C}$

$\mathrm{OH}: \quad 1.31 \mathrm{M}$

AT: $\quad 0.842 \underline{M}$

$\mathrm{Na}: \quad 8.48 \mathrm{M}$

$\mathrm{NO}_{2}: \quad 1.34 . \underline{M}$

$\mathrm{NO}_{3}: \quad 3.50 \mathrm{M}$

Pu: $\quad 3.02 \times 10^{-4} \mathrm{~g} / \mathrm{gal}$

D.T.A.: No exotherm below $200^{\circ} \mathrm{C}$

$\mathrm{SO}_{4}: \quad$ Sample slurped

$\mathrm{PO}_{4}: \quad 4.64 \times 10^{-2} \mathrm{M}$

F: $\quad 9.85 \times 10^{-4} \underline{M}$

$\mathrm{CO}_{3}: \quad 0.466 \underline{M}$

GEA: $\quad{ }_{137}^{134} \mathrm{Cs}-6.28 \times 10^{3} \mu \mathrm{Ci} / \mathrm{ga} 1$

${ }^{39}, 90 \mathrm{Sr}: 4.70 \times 10^{4} \mathrm{j \textrm {Ci } / \mathrm { gal }}$

Water: $57.99 \%$

Cl: $\quad 8.67 \times 10^{-2} \underline{M}$

Cooling Curve: $30^{\circ} \mathrm{C}$ for $155 \mathrm{~min}$ < $1 \%$ Solids. $25^{\circ} \mathrm{C}$ for $49 \mathrm{~min}$ < $1 \%$ Solids.
$20^{\circ} \mathrm{C}$ for $45 \mathrm{~min}$ < $7 \%$ Solids. 


\section{Atlantic Richfield Hanford Company}

B. L. Halser

Page 2

December 11, 1975

Cooling Curve: $15^{\circ} \mathrm{C}$ for $60 \mathrm{~min}$. $<1 \%$ Solids. $10^{\circ} \mathrm{C}$ for $45 \mathrm{~min} .<1 \%$ solids.

$5^{\circ} \mathrm{C}$ for $45 \mathrm{~min} .<1 \%$ Solids.

REW: eyw

cc: JS Buckingham

WUR Christensen

RM Ginestet

KE Nelson

JC Womack 


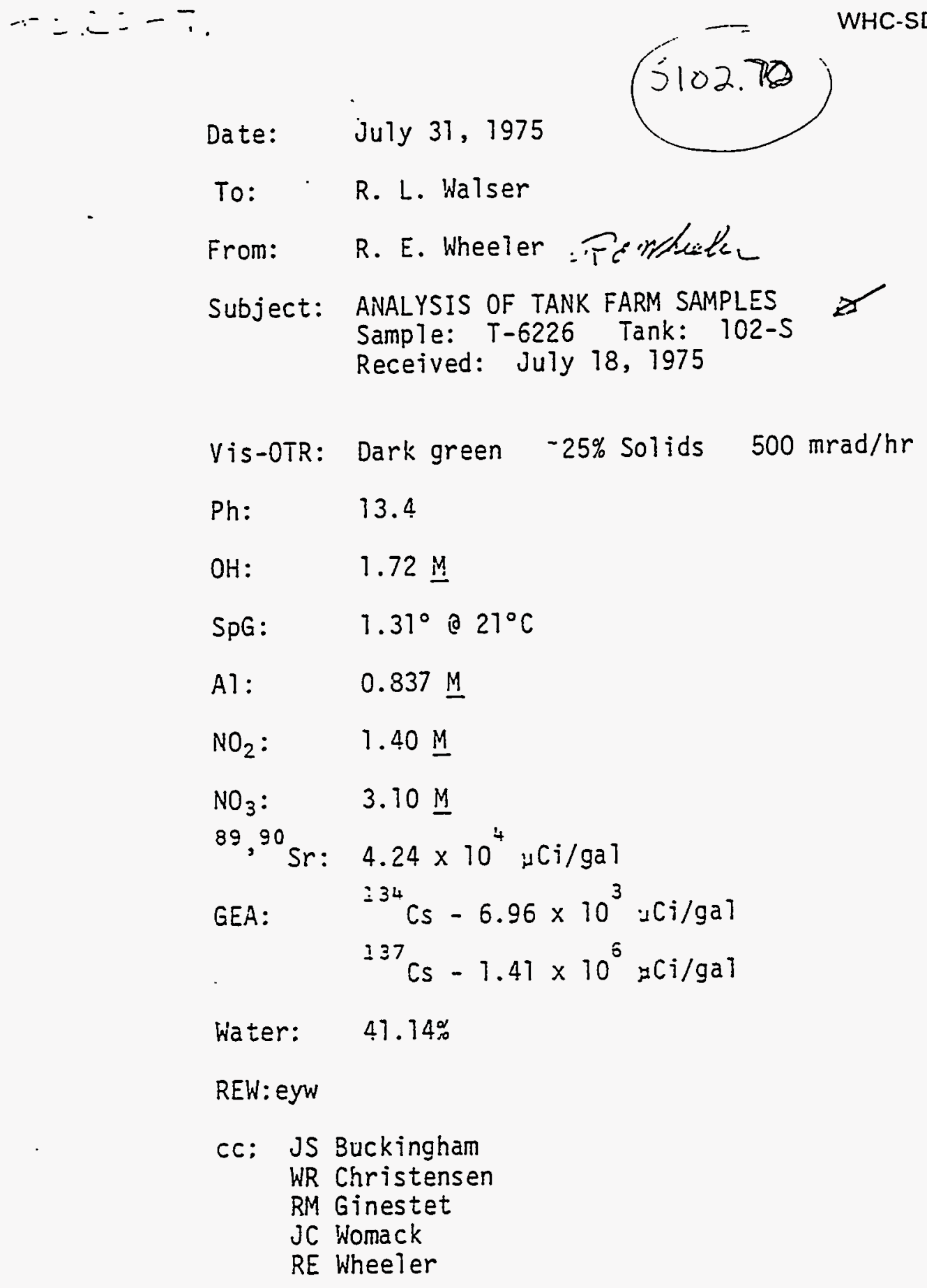


Date: September 23, 1975

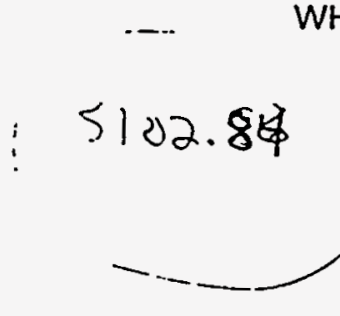

To: R. L. Walser

From: R. E. Wheeler, 2-2449 Gopsleezh

SUbject: ANALYSIS OF TANK FARM SAMPLES

Sample: T-6453 Tank: 102-S ¿

Received: Ju7y 25, 1975

Vis-0TR: Brown No solids $800 \mathrm{mrad} / \mathrm{hr}$

$\mathrm{pH}: \quad 12.7$

SpG: $\quad 1.286]$

$\mathrm{OH}: \quad \quad 1.07 \mathrm{M}$

Al: $\quad 0.574 \underline{M}$

$\mathrm{NO}_{2}: \quad 7.21 \underline{\mathrm{M}}$

$\mathrm{NO}_{3}: \quad 1.98$ i1

$89,90 \mathrm{Sr}: \quad 3.82 \times 10^{4}{ }_{\mu} \mathrm{Ci} / \mathrm{gaT}$

GEA: $\quad{ }_{137}^{134} \mathrm{Cs}-2.74 \times 10^{3} \mu \mathrm{Ci} / \mathrm{gal}$

$\left.{ }^{137} \mathrm{Cs}-7.89 \times 10^{5} \mathrm{HCi} / \mathrm{ga}\right]$

${ }^{306} \mathrm{RuRh}=1.32 \times 10^{5} \mathrm{uCi} / \mathrm{ga}$

${ }^{154} \mathrm{Eu}-1.21 \times 10^{3}{ }_{\mu \mathrm{Ci} / \mathrm{ga}}$

Water: $\quad 63.5 \%$

REW: eyw

cC: JS Buckingham

WR Christensen

RM Ginestet

RE Wheeler

JC Womack 
Date: $\quad$ November 13, 1975

To: R. L. Walser

From: R. E. Wheeler, 2-2449

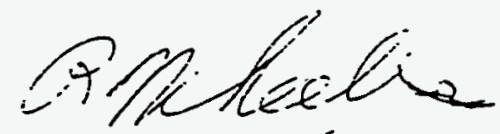

Subject: ANALYSIS OF TANK FARM SAMPLES

Sample: T-6806 Tank: 102-S

Received: August 5, 1975

Vis-0TR: Dark brown $\quad 80 \%$ Solids $800 \mathrm{mrad} / \mathrm{hr}$

$\mathrm{pH}: \quad \quad 13.3$

SPG: $\quad 1.320$ \& $54^{\circ} \mathrm{C}$

OH: $\quad \quad \quad 1.98 M$

Al: $\quad 0.826 \underline{M}$

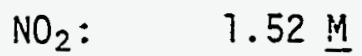

$\mathrm{NO}_{3}: \quad 3.30 \underline{\mathrm{H}}$

${ }^{89}, 90 \mathrm{Sr}: 8.27 \times 10^{4} \mu \mathrm{Cj} / \mathrm{gal}$

GEA: $\left.\quad{ }_{137}^{134} \mathrm{CS}-2.85 \times 10_{5}^{3} \mathrm{pCj} / \mathrm{ga}\right]$

${ }^{237} \mathrm{Cs}-7.04 \times 10^{5} \mathrm{\mu Ci} / \mathrm{gal}$

Water: $\quad 54.07 \%$

REW: eyw

cc: JS Buckingham

WR Christensen

RM Ginestet

RE Wheeler

JC Womack 
Date: September 19, 1975

To: R. L. Walser

From: R. E. Wheeler, 2-2449 R inthece

Subject: ANALYSIS OF TANK FARM SAMPLES

Sample: T-7092 Tank: 102-S
Received: August 15,1975

Vis-OTR: Dark brown $1 \%$ Solids $3 \mathrm{rad} / \mathrm{hr}$

$\mathrm{pH}: \quad 12.90$

SpG: $\quad 1.5130$

$\mathrm{OH}: \quad 2.98 \underline{\mathrm{M}}$

A1: $\quad 1.91 \underline{\mathrm{M}}$

$\mathrm{NO}_{2}: \quad 1.90 \underline{\mathrm{M}}$

$\mathrm{NO}_{3}: \quad 4.29 \mathrm{M}$

89, $\left.{ }^{90} \mathrm{Sf:} \quad 5.05 \times 10^{4}{ }_{\mu} \mathrm{Ci} / \mathrm{ga}\right]$

GEA: $\quad \begin{aligned} & \left.{ }^{134} \mathrm{CS}=1.29 \times 10^{4}{ }_{\mu} \mathrm{Ci} / \mathrm{ga}\right] \\ & \left.{ }^{137} \mathrm{Cs}=2.17 \times 10^{6} \mathrm{\mu Ci} / \mathrm{ga}\right]\end{aligned}$

Water: $\quad 53.16 \%$

REW:eyw

cc: JS Buckingham

WR Christensen

RM Ginestet

RE Wheeler

JC Womack 
Date: $\quad$ September 22, 1975

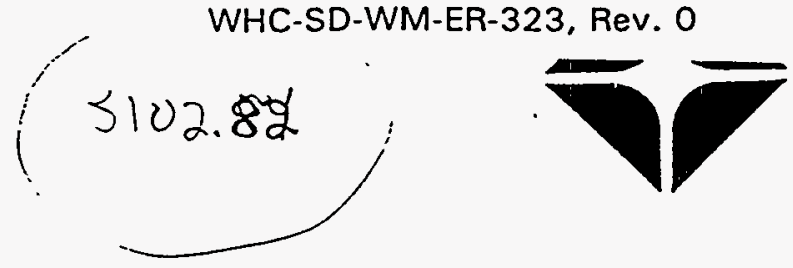

To: R. L. Walser

From: R. E. Wheeler, 2-2499 ritrectu

Subject: ANALYSIS OF TANK FARM SAMPLES

Sample: T-7218 Tank: 102-S $\not 2$

Received: August 19, 1975

Vis-OTR: Dark brown No solids $700 \mathrm{mrad} / \mathrm{hr}$

$\mathrm{pH}: \quad 12.5$

SpG: $\quad 1.3662$

$\mathrm{OH}: \quad 1.78 \underline{\mathrm{M}}$

Al: $\quad 0.754 \underline{M}$

$\mathrm{NO}_{2}: \quad 1.96 \underline{M}$

$\mathrm{NO}_{3}: \quad 3.33 \underline{\mathrm{M}}$

$\left.89,90 \mathrm{Sr}: 4.73 \times 10^{4} \mathrm{\mu [i} / \mathrm{ga}\right]$

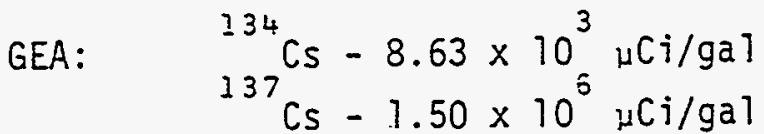

Water: $\quad 69.40 \%$

REW: eyw

cc: JS Buckingham

WR Christensen

RM Ginestet

RE Wheeler

JC Womack 


\subsection{3}

Date: September 19, 1975

To: R. L. Walser

From: R. E. Wheeler, 2-2449

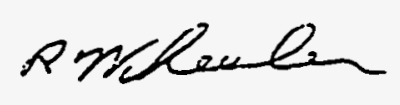

Subject: 'ANALYSIS OF TANK FARM SAMPLES

Sample: T-7340 Tank:
Received: August 25,1975

Vis-OTR: Dark brown No solids $2.5 \mathrm{rad} / \mathrm{hr}$

$\mathrm{pH}: \quad \mathbf{1 2 . 5}$

SPG: $\quad 1.395$

$\mathrm{OH}: \quad 2.18 \mathrm{M}$

Al: $\quad 1.71 \mathrm{M}$

$\mathrm{NO}_{2}: \quad 1.59 \mathrm{M}$

$\mathrm{NO}_{3}: \quad 3.40 \underline{\mathrm{M}}$

${ }^{89},{ }^{90} \mathrm{Sr}: 4.66 \times 10^{4} \mu \mathrm{Ci} / \mathrm{gal}$

GEA: $\quad{ }_{137}^{134} \mathrm{CS}-8.44 \times 10^{3} \mathrm{HCi} / \mathrm{ga}$

$\left.{ }^{137} \mathrm{Cs}-1.67 \times 10^{6} \mathrm{HCi} / \mathrm{ga}\right]$

${ }^{60} \mathrm{Co}-5.62 \times 10^{2} \mathrm{uCi} / \mathrm{gal}$

$\left.{ }^{154} \mathrm{Eu}-9.39 \times 10^{2} \mu \mathrm{Ci} / \mathrm{ga}\right]$

Water: $\quad 74.13 \%$

REW: eyw

CC: JS Buckingham

WR Christensen

RM Ginestet

RE Wheeler

JC Womack 
Date: September 19, 1975

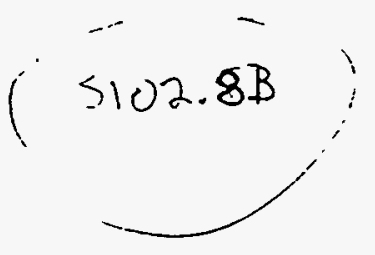

To: R. L. Walser

From: R. E. Wheeler, 2-2449 pwhab

Subject: ANALYSIS OF TANK FARM SAMPLES

Sample: T-7627 Tank: 102-S $\not 2$

Received: September 2, 1975

Vis-OTR: Light brown No solids $500 \mathrm{mrad} / \mathrm{hr}$

$\mathrm{pH}: \quad \quad \quad 12.0$

SpG: $\quad 1.1716$

$\mathrm{OH}: \quad 0.488 \underline{M}$

Al: $\quad 0.278 \underline{M}$

$\mathrm{NO}_{2}: \quad 0.556 \underline{\mathrm{M}}$

$\mathrm{NO}_{3}: \quad 1.40 \mathrm{M}$

$89,90 \mathrm{Sr}: \quad 1.84 \times 10^{4} \mu \mathrm{Ci} / \mathrm{gal}$

GEA: $\left.\quad{ }_{137}^{134} \mathrm{Cs}-2.33 \times 10_{5}^{3} \mathrm{\mu Ci} / \mathrm{ga}\right]$

${ }^{137} \mathrm{Cs}-4.61 \times 10^{5} \mathrm{\mu Ci} / \mathrm{ga} 1$
${ }^{60} \mathrm{Co}-4.08 \times 10^{2} \mathrm{\mu Ci} / \mathrm{ga} 7$

Water: $\quad 79.37 \%$

REW: eyw

CC: JS Buckingham

WR Christensen

RM Ginestet

RE Wheeler

JC Womack 
Date: $\quad$ October 20,1975

$$
15107.87
$$

To: R. L. Walser

From: R. E. Wheeler, 2-2449 Prphater

Subject: ANALYSIS OF TANK FARM SAMPLES

Sample: T-7703 Tank: 102-S

Received: September 3, 1975

Vis-0TR: Brown No solids $1200 \mathrm{mrad} / \mathrm{hr}$

$\mathrm{pH}: \quad 12.2$

SPG: $\quad 1.3308$

$\mathrm{OH}: \quad 1.79 \underline{\mathrm{M}}$

A1: $\quad 0.958$

$\mathrm{Na}: \quad 7.79 \underline{M}$

$\mathrm{NO}_{2}: \quad 1.82 \underline{\mathrm{M}}$

$\mathrm{NO}_{3}: \quad 2.96 \underline{\mathrm{M}}$

Pu: $\quad 3.03 \times 10^{-4} \mathrm{~g} / \mathrm{gal}$

D.T.A.: No exotherm below $200^{\circ} \mathrm{C}$ - exotherm $235^{\circ} \mathrm{C}$

\begin{tabular}{|c|c|}
\hline $\mathrm{SO}_{4}:$ & Cancelled \\
\hline $\mathrm{PO}_{4}:$ & $2.92 \times 10^{-2} \mathrm{M}$ \\
\hline Cl: & 0.110 \\
\hline$F:$ & $2.02 \times 10^{-3} \underline{M}$ \\
\hline $\mathrm{CO}_{3}:$ & $0.463 \underline{M}$ \\
\hline GEA: & $\begin{array}{l}{ }_{20}^{60} C_{0}-6.64 \times 10^{2} \mu \mathrm{Ci} / \mathrm{gal} \\
{ }_{135}{ }^{\mathrm{Cs}}-9.69 \times 10^{3} \mu \mathrm{Ci} / \mathrm{gal} \\
{ }_{\mathrm{Cs}}-1.47 \times 10^{\circ} \mu \mathrm{Ci} / \mathrm{gal}\end{array}$ \\
\hline${ }^{89}, 905 r:$ & $4.01 \times 10^{4}, \mathrm{NCi} / \mathrm{gal}$ \\
\hline Water: & $42.38 \%$ \\
\hline
\end{tabular}


R. L. Walser

Page 2

October 20, 1975

Cooling Curve: $35^{\circ} \mathrm{C}$ for $45 \mathrm{~min}$. No solids. $30^{\circ} \mathrm{C}$ for $45 \mathrm{~min}$. No solids. $25^{\circ} \mathrm{C}$ for $45 \mathrm{~min}$. No solids. $20^{\circ} \mathrm{C}$ for $45 \mathrm{~min}$. No solids. $15^{\circ} \mathrm{C}$ for $45 \mathrm{~min}$. No solids. $10^{\circ} \mathrm{C}$ for $45 \mathrm{~min}$. No solids. $5^{\circ} \mathrm{C}$ for $45 \mathrm{~min}$. No solids.

REW: eyw

CC: JS Buckingham

WR Christensen

RM Ginestet

RE Wheeler

JC Womack 
Oate: $3-16-9$

To: P.L. W.ALSER

From: F.E. IPILicis-

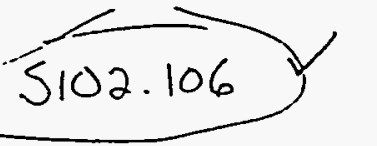

Subject: AAIALYSIS OF TA:HK FARM SAMPIES

SAMPLE NO. $\frac{T 2 ; 45}{4-19}$ TANK:

VIS-OTR: EREEN, $\quad$ I

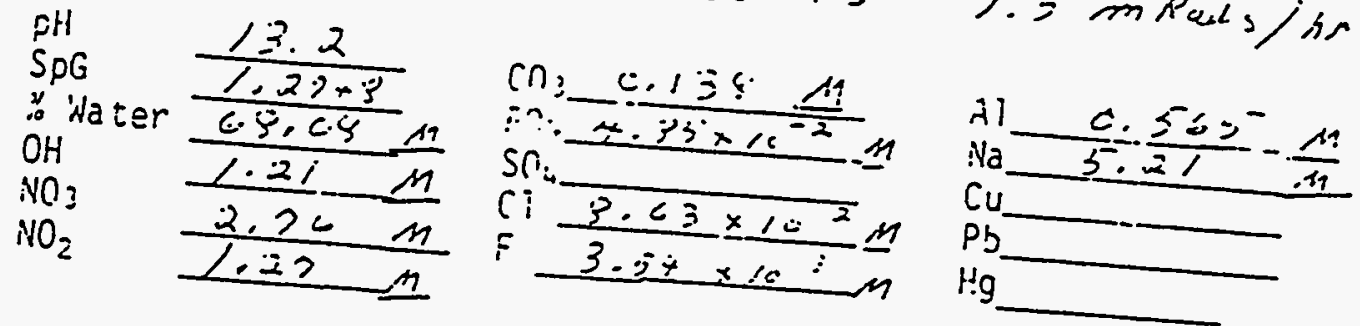

$j={ }^{-}$mPaus $/$hr

Pu $1.51 \times 10^{-4} \quad \dot{y}$ igal

GEA :

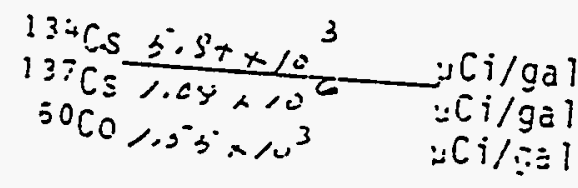

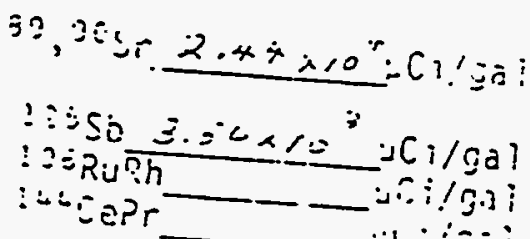

Cooling Curve: 35: Fo: 35 mirutes

$30^{\circ}$ o. 3.5 minutes

$25^{\circ}$ io: $3 \equiv$ ninutes

$20^{\circ}$ For 35 minuzes

$15^{\circ}$ for 3 minutes $^{\circ}$

$10^{\circ}$ ic: 35 minutes

$5^{3}$ for 35 minutes

DiA: $w_{0}=x_{0}$ Therm beime $200^{\circ} \mathrm{C}$ - $6:$ :

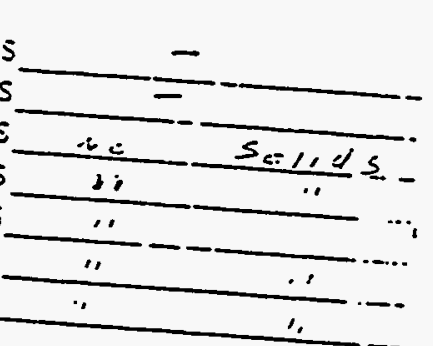


Date: $\quad$ September 19, 1975
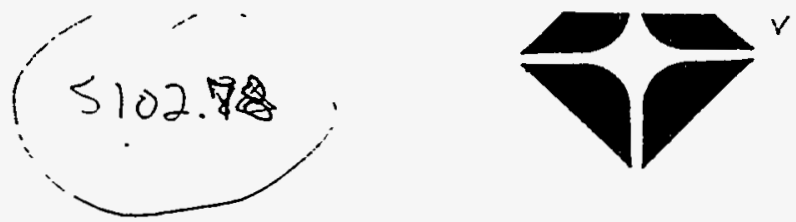

To: R. L. Waiser

From: R. E. Wheeler, 2-2449, Preferein.

Subject: ANALYSIS OF TANK FARM SAMPLES

\section{Sample: $T-4963$}

Tank: $102-5$

Received: June 2, 1975

Vis-OTR: Black Solids unknown $2 \mathrm{rad} / \mathrm{hr}$

$\mathrm{pH}: \quad \quad 10.0$

SpG: $\quad 1.4 \div 100^{\circ} \mathrm{C}$

$\mathrm{OH}: \quad 2.34 \mathrm{M}$

A): $\quad 1.07 \underline{M}$

$\mathrm{NO}_{2}: \quad 1.82 \underline{\mathrm{M}}$

$\mathrm{NO}_{3}: \quad 2.98 \underline{\mathrm{M}}$

89,90. $\mathrm{Sr}: 1.02 \times 10^{5} \mathrm{\mu Ci}_{\mathrm{gat}} \alpha$

$\mathrm{CO}_{3}: \quad 0.258 \underline{\mathrm{M}}$

GEA: $\quad{ }^{60} \mathrm{Co}-2.85 \times 10^{3} \mu \mathrm{Ci} / \mathrm{gal}$

$$
\begin{aligned}
& { }^{125} \mathrm{Sb}-9.26 \times 10^{3} \mu \mathrm{Ci} / \mathrm{gal} \\
& { }_{134} \mathrm{Cs}-1.67 \times 10^{4} \mu \mathrm{Ci} / \mathrm{gal} \\
& 13{ }^{13} \mathrm{Cs}-3.43 \times 10^{5} \mu \mathrm{Ci} / \mathrm{gal} \\
& { }_{154} \mathrm{Eu}-4.10 \times 10^{3} \mu \mathrm{Ci} / \mathrm{gal}
\end{aligned}
$$

Water: $\quad 50.44 \%$

REW: eyw

cc: JS Buckingham

WR Christensen

RM Ginestet

RE Wheeler

JC Womack 
Date: October 20, 1975

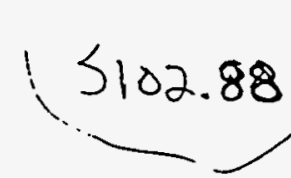

To: R. L. Walser

From: R. E. Wheeler, 2-2449 Rrychulin

Subject: ANALYSIS OF TANK FARM SAMPLES

Sample: T-8350 Tank: 102-S

Received: September 29, 1975

Vis-0TR: Green No solids $1200 \mathrm{mrad} / \mathrm{hr}$

$\mathrm{pH}: \quad 12.2$

SPG: $\quad 1.346$

OH: $\quad 1.66$ in

A7: $\quad 1.02 \underline{M}$

$\mathrm{NO}_{2}: \quad 1.41 \mathrm{M}$

$\mathrm{NO}_{3}: \quad 2.66 \mathrm{M}$

D.T.A.: No exotherm below $200^{\circ} \mathrm{C}$, exotherm a $240^{\circ} \mathrm{C}$

GEA: $\quad{ }_{134}^{125} \mathrm{Sb}-1.87 \times 10_{3}^{4} \mu \mathrm{Ci} / \mathrm{gaT}$

$$
\begin{aligned}
& 134 \\
& { }_{137}{ }^{3}-7.53 \times 10^{3} \\
& \left.C s-1.39 \times 10^{6} \mathrm{Ci} / \mathrm{ga}\right]
\end{aligned}
$$

${ }^{89,90} \mathrm{Sr}: \quad 6.85 \times 10^{3} \mathrm{\mu Ci} / \mathrm{ga}$

Water: $\quad 77.24 \%$

REW: eyw

CC: JS Buckingham

WR Christensen

RM Ginestet

RE Wheeler

JC Womack 


\subsection{5}

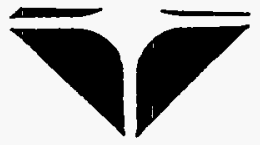

Dated: October 20, 1975

To: R. L. Walser

From: R. E. Wheeler, 2-2449 finthick

Subject: ANALYSIS OF TANK FARM SAMPLES

Sample: T-8446 Tank: 102-S

Received: September 30, 1975

Vis-OTR: Dark brown No solids $1 \mathrm{rad}$

pH: $\quad 13.0$

$S p G: \quad 1.398$

OH: $\quad 1.04$ M

A7: $\quad 1.085 \underline{M}$

$\mathrm{NO}_{2}: \quad 1.79 \mathrm{M}$

$\mathrm{NO}_{3}: \quad 2.92 \mathrm{i}$

GEA: $\quad \begin{aligned} & 134 \\ & \quad{ }^{4} C_{S}-7.95 \times 10^{3} \mu \mathrm{Ci} / g a 1 \\ & { }^{6} C_{5}-1.64 \times 10^{6} \mu \mathrm{Ci} / g a 1\end{aligned}$

$\left.{ }^{39},{ }^{90} \mathrm{sr}: 3.38 \times 10^{4} \mu \mathrm{Ci} / \mathrm{ga}\right]$

REW: gW

CC: JS Buckingham

WR Christensen

RM Ginestet

RE Wheeler

JC Womack 


$$
\text { - } \because=--\because 5
$$

Atlantic Richfield Hanford Company

Date: December 3, 1975
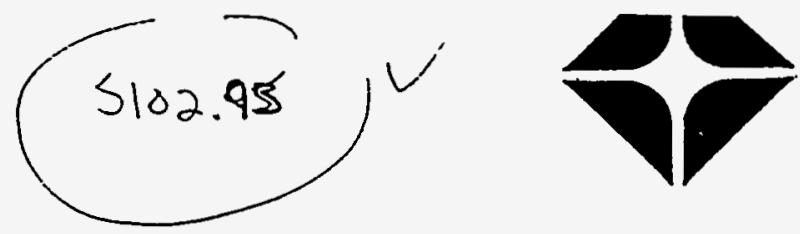

To: R. L. Walser

From: R. E. Wheeler, 2-2449 R Thexen

Subject: ANALYSIS OF TANK FARM SAMPLES

Sample: T-8634 Tank: 102-S

Received: October 7, 1975

Vis-0TR: Green $10 \%$ Solids $1500 \mathrm{mrad} / \mathrm{hr}$

$\mathrm{pH}: \quad 12.7$

SPG: $\quad 1.190 \bigcirc 59^{\circ} \mathrm{C}$

$\mathrm{OH}: \quad 0.970 \underline{\mathrm{M}}$

A1: $\quad 0.444 \underline{M}$

$\mathrm{Na}: \quad 7.60 \underline{\mathrm{M}}$

$\mathrm{NO}_{2}: \quad 0.596 \underline{\mathrm{M}}$

$\mathrm{NO}_{3}: \quad 1.99 \underline{\mathrm{M}}$

Pu: $\left.\quad 2.42 \times 10^{-4} \mathrm{gm} / \mathrm{ga}\right]$

D.T.A.: Non-exotherm

$\mathrm{SO}_{4}: \quad$ Cancelled

$\mathrm{PO}_{4}: \quad 9.08 \times 10^{-2} \underline{\mathrm{M}}$

C1: $\quad 5.04 \times 10^{-2} \mathrm{M}$

F: $\quad 9.72 \times 10^{-5} \underline{M}$

$\mathrm{CO}_{3}: \quad 0.115 \underline{M}$

GEA: $\left.\quad{ }_{137}^{134} \mathrm{Cs}-2.70 \times 10^{3} \mathrm{HCi} / \mathrm{ga}\right]$

${ }^{137} \mathrm{Cs}-7.06 \times 10^{5}{ }_{\mu \mathrm{Ci} / \mathrm{ga} i}$

${ }^{89,90} \mathrm{Sr}: \quad 1.35 \times 10^{4}{ }_{\mu} \mathrm{Ci} / \mathrm{ga} 7$

Water: $\quad 76.82 \%$ 
R. L. Walser

Page 2

December 3, 1975

Cooling Curve: $35^{\circ} \mathrm{C}$ for $35 \mathrm{~min}$. No solids. $30^{\circ} \mathrm{C}$ for $35 \mathrm{~min}$. No solids. $25^{\circ} \mathrm{C}$ for $35 \mathrm{~min}$. No solids. $20^{\circ} \mathrm{C}$ for $35 \mathrm{~min}$. No solids. $15^{\circ} \mathrm{C}$ for $35 \mathrm{~min}$. No solids. $10^{\circ} \mathrm{C}$ for $35 \mathrm{~min}$. No solids. $5^{\circ} \mathrm{C}$ for $35 \mathrm{~min}$. No solids.

REW: eyw

CC: JS Buckingham

WR Christensen

RM Ginestet

KE Neison

JC Womack 
Date: January 8, 1976

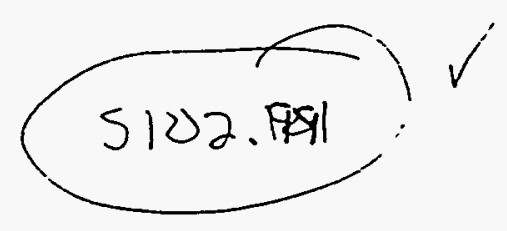

To: R. L. Walser

From: R. E. Wheeter, 2-2449, Preflicliz

$\begin{aligned} \text { Subject: } & \text { ANALYSIS OF TANK FARM SAMPLES } \\ & \text { Sample: T-8706 Tank: InZ-S - }\end{aligned}$

Received: Dctober 10, 1975

Vis-0TR: Dark brown 3 prase 2 rad

$\mathrm{pH}: \quad 13.3$

SpG: $\quad 1.38 \cong 72^{\circ} \mathrm{C}$

$\mathrm{OH}: \quad 1.70 \underline{\mathrm{M}}$

A1: $\quad 0.923 \underline{M}$

$\mathrm{NO}_{2}: \quad 3.48 \underline{\mathrm{M}}$

$\mathrm{NO}_{3}: \quad 4.29 \mathrm{M}$

$\left.{ }^{89,90} \mathrm{Sr}: 2.41 \times 10^{5} \mu \mathrm{Ci} / \mathrm{ga}\right]$

GEA: $\quad{ }_{137}^{134} \mathrm{Cs}-5.02 \times 10^{3} \mathrm{pCi} / \mathrm{gaT}$

Water: $\quad 56.12 \%$

REW: eyw

CC: JS Buckingham

WR Christensen

RM Ginestet

KE Melson

JC Womack 
Date: January 19,1976
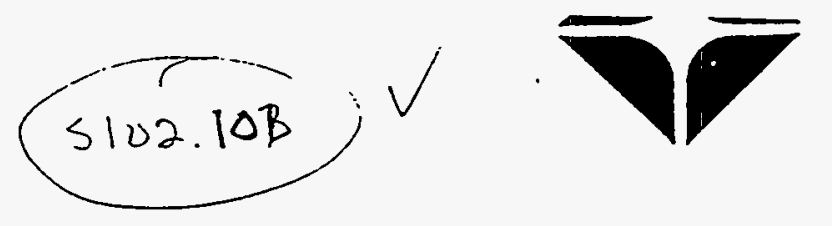

To:

R. L. Walser

From:

R. E. Wheeler, 2-2449

-PPr:ketic

Subject: ANALYSIS OF TAIIK FARIM SAIPPLES

Sample: T-8364

Tank:

Received: Dctober 15, 1975

Vis-OTR: Yellow $30 \%$ solids $1600 \mathrm{mrad} / \mathrm{hr}$

$\mathrm{pH}: \quad 13.2$

SpG: $\quad 1.436$

$\mathrm{OH}: \quad 2.62$ ‥1

Al: $\quad 1.40 \mathrm{M}$

$\mathrm{iO}_{2}: \quad 1.47$

$\mathrm{NO}_{3}: \quad 4.91 \mathrm{M}$

${ }^{89},{ }^{30} \mathrm{Sr}: 4.25 \times 10^{5} \mu \mathrm{Ci} / \mathrm{gal}$

GEA: $\quad{ }_{137}^{134} \mathrm{CS}-1.09 \times 10_{6}^{4} \mu \mathrm{Ci} / g a 1$

${ }_{137}{ }^{37} \mathrm{Cs}-2.0 \times 10^{6}{ }^{3 \mathrm{Ci} / \mathrm{ga} 7}$
$\left.{ }_{\mathrm{Sb}}-6.35 \times 10^{3} \mathrm{HCi} / \mathrm{ga}\right]$

$\mathrm{CO}_{3}: \quad 0.380$ i

Water: $\quad 46.82 \%$

REW: eyw

CC: JS Buckingham

UR Christensen

PM Ginestet

KE Nelson

JC Womack 
Date: November 6, 1975

To: R. L. Walser
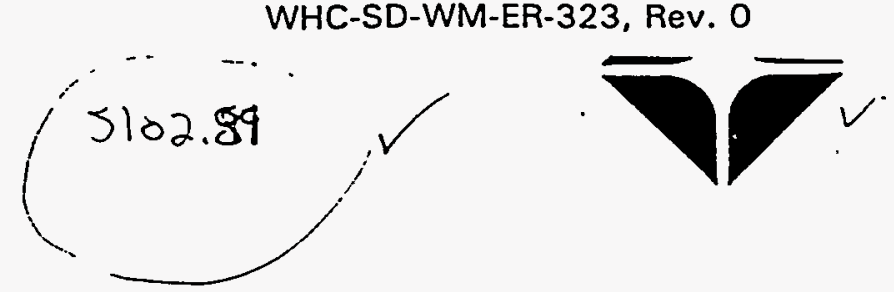

From: R. E. Wheeler, 2-2449 fifflullu

Subject: ANALYSIS OF TANK FARM SAMPLES

Sample: T-90,49

Tank:

Received: October 21, 1975

Vis-OTR: Brown

$>75 \%$ Solids $\quad 1800 \mathrm{mrad} / \mathrm{hr}$

pH:

13.7

SpG:

$1.480 @ 67 \% \mathrm{C}$

OH:

$3.02 \mathrm{M}$

AT:

$1.64 \mathrm{M}$

$\mathrm{NO}_{2}$

$2.58 \mathrm{M}$

$\mathrm{NO}_{3}$

$4.40 \underline{M}$

89,90 Sr:

$\left.5.56 \times 10^{4} \mathrm{jCi} / \mathrm{ga}\right]$

GEA:

${ }_{134} \mathrm{Cs}-1.36 \times 10^{4} \mu \mathrm{Ci} / \mathrm{ga} 1$
$\left.137 \mathrm{Cs}-3.19 \times 10^{6} \mu \mathrm{Ci} / \mathrm{ga}\right]$

Water: $65 \%$

REW: eyw

CC: JS Buckingham

WR Christensen

RM Ginestet

RE Wheeler

JC Womack 


$$
\because: ニ ニ-\because S
$$

Atlantic Richfield Hanford Company

$$
\begin{aligned}
& 5102.104 \\
& \text { Date: } \quad \text { February 12, } 1976 \\
& \text { To: R. L. Walser } \\
& \text { From: } \quad \text { ‥ E. theeler, 2-2449 Prplleele } \\
& \text { Súject: ANALYSIS OF TAIK FARII SAMPLES } \\
& \text { Sample: T-9394 Tank: 102-S } \not- \\
& \text { Received: Hovember 3, } 1976 \\
& \text { Vis-OTR: Dark brown Ho solids } 700 \mathrm{mrad} / \mathrm{hr} \\
& \mathrm{pH}: \quad 12.8 \\
& \text { Sp̣G: } \quad 1.32 E \\
& \mathrm{OH}: \quad 1.57 \text { if }
\end{aligned}
$$

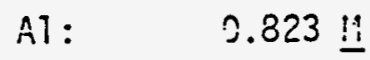

$$
\begin{aligned}
& \text { :a: } \quad 7.5 n \text { í } \\
& \mathrm{HO}_{2}: \quad 1.77: \underline{\mathrm{Z}} \\
& \mathrm{iNO}_{3}: \quad 2.92 \underline{1} \\
& \text { Pu: } \quad 1.17 \times 10^{-4} \mathrm{~g} / \mathrm{gat} \\
& \text { D.T.A.: io exotherm belo: } 200^{\circ} \mathrm{C} \text {, exotherm } 250^{\circ} \mathrm{C} \\
& \mathrm{PO}_{4}: \quad 1.77 \times 10^{-2} \\
& \text { CL: } \quad 3.04 \times 10^{-2} \text { i1 } \\
& \text { F: } \quad 4.19 \times 10^{-5} \underline{14}
\end{aligned}
$$

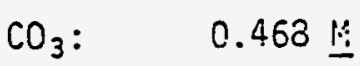

$$
\begin{aligned}
& \text { GEA: } \quad \begin{array}{l}
{ }_{137}^{134} C_{S}-5.62 \times 10^{3} \mu \mathrm{Hi} / \mathrm{gal} \\
\left.{ }^{C S}-1.44 \times 10^{6} \mathrm{HCi} / \mathrm{ga}\right]
\end{array} \\
& 39,{ }^{90} \mathrm{Sr}: 3.52 \times 10^{4} \mathrm{uCi} / \mathrm{gal} \\
& \text { ilater: } 52.62 .5
\end{aligned}
$$


R. L. Walser

Page 2

February 12, 1976

Cooling Curve: $35^{\circ} \mathrm{C}$ for $35 \mathrm{~min}$. ito solids. $30^{\circ} \mathrm{C}$ for $35 \mathrm{~min}$. ilo solids. $25^{\circ} \mathrm{C}$ for 35 min. ilo solicis. $20^{\circ} \mathrm{C}$ for 35 min. ilo solids. $15^{\circ} \mathrm{C}$ for $35 \mathrm{~min}$. 1 lo solids.

$10^{\circ} \mathrm{C}$ for $35 \mathrm{~min}$. llo solids.

$5^{\circ} \mathrm{C}$ for $35 \mathrm{~min}$. tho solids.

\author{
P.E.! : eyw \\ CC: JS Buckingham \\ UR Christensen \\ RI! Ginestet \\ KE Neison \\ JC Homack
}


Date: $\quad$ November 21, 1975

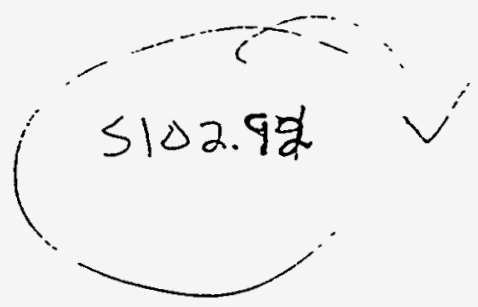

To: R. L. Walser

From: R. E. Wheeler, 2-2449. PPflleclic

Subject: ANALYSIS OF TANK FARM SAMPLES

Sample: T-9515 Tank: 102-S

Received: November 5, 1975

Vis-OTR: Dark Brown Suspended Solids $1500 \mathrm{mrad} / \mathrm{hr}$

pH: $\quad 13.5$

SPG: $\quad 1.410 @ 52^{\circ} \mathrm{C}$

$\mathrm{OH}: \quad 2.00 \mathrm{M}$

A7: $\quad \quad \quad 1.06 \geqq$

$\mathrm{NO}_{2}: \quad 5.14 \mathrm{M}$

$\mathrm{NO}_{3}: \quad 6.44 \mathrm{M}$

${ }^{89}, 90 \mathrm{Sr}: 3.64 \times 10^{4} \mu \mathrm{Ci} / \mathrm{gal}$

GEA: $\quad \begin{aligned} & { }^{134} \mathrm{Cs}-6.40 \times 10^{3} \mathrm{yCi} / \mathrm{ga} 1 \\ & \left.\quad{ }^{37} \mathrm{Cs}-1.32 \times 10^{5} \mathrm{HCi} / \mathrm{ga}\right]\end{aligned}$

Water: $\quad 54.8 \%$

REW: eyw

CC: JS Buckingham

WR Christensen

RM Ginestet

RE Wheeler

JC Womack 
Date: November 18,1975
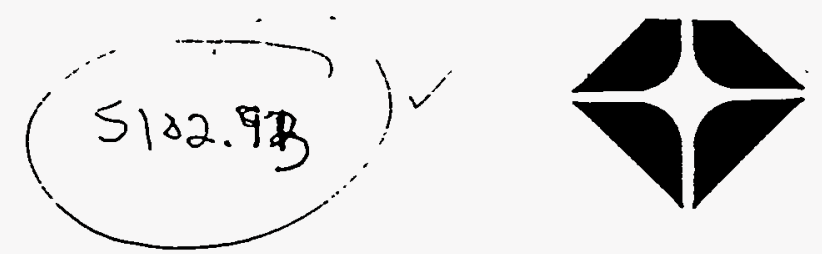

To: R. L. Walser

From: R. E. Wheeler, 2-2449 R'nhtace

Subject: ANALYSIS OF TANK FARM SAMPLES

Sample: T-9697 Tank: 102-S $\searrow$

Received: November 11, 1975

Vis-OTR: Dark brown Suspended solids $700 \mathrm{mrad} / \mathrm{hr}$

$\mathrm{pH}: \quad \quad 14.0$

SpG: $\quad 1.420 \odot 50.5^{\circ} \mathrm{C}$

$\mathrm{OH}: \quad 2.42 \underline{\mathrm{M}}$

$\mathrm{A} 1: \quad 1.17 \mathrm{M}$

$\mathrm{NO}_{2}: \quad 5.44 \underline{\mathrm{M}}$

$\mathrm{NO}_{3}: \quad 7.64 \underline{\mathrm{M}}$

$89,90 \mathrm{Sr}: 9.92 \times 10^{4} \mathrm{pCi} / \mathrm{gal}$

GEA: $\quad{ }_{137}^{134} \mathrm{Cs}-7.18 \times 10^{3} \mathrm{pCi} / \mathrm{gal}$

$\left.{ }_{103}^{137} \mathrm{Cs}-2.01 \times 10^{6} \mathrm{pCi} / \mathrm{ga}\right]$

Water: $\quad 50.34 \%$

$\mathrm{NH}_{4}: \quad 1.70 \times 10^{-2} \underline{\mathrm{M}}$

$\mathrm{CO}_{3}: \quad 0.181 \underline{\mathrm{M}}$

REW: eyw

cc: JS Buckingham

WR Christensen

RM Ginestet

RE Wheeler

JC Womack 


\subsection{5}

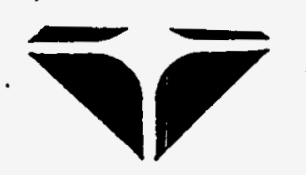

Date: January 21,1976

To: R. L. Walser

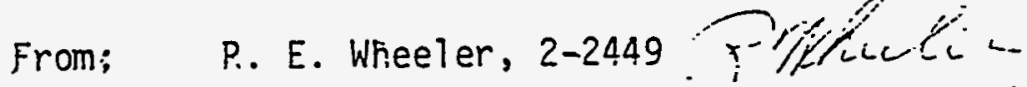

Subject: $\triangle N A L Y S I S$ OF TANK FARM SAMPLES

Sample: T-9.952 Tank: 102-S

D.eceived: November 10, 1975

Vis-nTR: Dark brown Suspended solids $1700 \mathrm{mrad} / \mathrm{hr}$

$\mathrm{pH}: \quad \quad 73.5$

SPG: $\quad 1.290$ A $E 1^{\circ} \mathrm{C}$

$\mathrm{OH}: \quad 1.20 \cdot 1$

A]: $\quad 0.550 \mathrm{M}$

$\mathrm{NO}_{2}: \quad 2.20 \mathrm{M}$

$\mathrm{NO}_{3}: \quad 3.44 . \underline{M}$

${ }^{89}, 90 \mathrm{Sr}: 2.57 \times 10^{4} \mathrm{pCi} / \mathrm{gal}$

GEA: $\quad \begin{array}{r}134 \mathrm{Cs}-4.63 \times 10^{3} \mathrm{\mu Ci} / \mathrm{gal} \\ \quad{ }^{137} \mathrm{Cs}-0.69 \times 10^{5} \mathrm{uCj} / \mathrm{gal}\end{array}$

Water: $\quad 68.40 \%$

Cu: $\quad<1.73 \times 10^{-4} \underline{M}$

PD: $\quad 1.69 \times 10^{-4} \stackrel{M}{-}$

$\mathrm{Hg}: \quad \quad \quad 3.24 \times 10^{-4} \stackrel{\mathrm{M}}{-}$

REW: eyw

cc: JS Buckingham

WR Christensen

PM! Ginestet

KE Nelson

JC Womack. 
Date: December 3, 1975

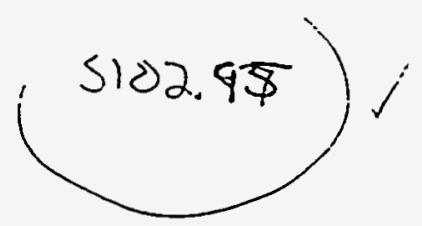

To: R. L. Walser

From: R. E. Wheeler, 2-2449 Trphezi-

Subject: ANALYSIS OF TANK FARM SAMPLES

Sample: T-37 Tank: 102-S

Received: November 21, 1975

Vis-OTR: Greenish brown No OTR

$\mathrm{pH}: \quad 13.6$

SpG: $\quad 1.28 \div 57^{\circ} \mathrm{C}$

OH: $\quad 1.59 \underline{M}$

A1: $\quad 0.626 \underline{M}$

$\mathrm{NO}_{2}: \quad 1.68 \underline{\mathrm{M}}$

$\mathrm{NO}_{3}: \quad 2.60 \mathrm{M}$

${ }^{89}, 90 \mathrm{Sr}: 2.58 \times 10^{4} \mathrm{pCi} / \mathrm{gal}$

GEA: $\quad{ }_{137}^{134} \mathrm{Cs}-3.63 \times 10^{3} \mathrm{j} \mathrm{Ci} / \mathrm{ga}$

$$
\begin{array}{r}
\left.{ }_{137} \mathrm{Cs}-1.04 \times 10^{6} \mathrm{pCi} / \mathrm{ga}\right] \\
203 \mathrm{Ru}-<1.56 \times 10^{4} \mathrm{pCi} / \mathrm{ga} 7
\end{array}
$$

Water: $\quad 65.84 \%$

$\mathrm{NH}_{4}: \quad 1.40 \times 10^{-2}$ is

REW: eyw

CC: JS Buckingham

WR Christensen

RM Ginestet

KE Nelson

JC Womack 
Date: December 3, 1975

\section{is102.9\%}

To: R. L. Walser

From: R. E. Wheeler, 2-2449 fryitizter

Subject: ANALYSIS OF TANK FARM SAMPLES

Sample: T-119 Tank: $102-5$
Received: Novemter 24, 1975

Vis-OTR: Dark brown, cruddy $2300 \mathrm{mrad} / \mathrm{hr}$

pH: $\quad 11.4$

SpG: $\quad 1.44 \oplus 26^{\circ} \mathrm{C}$

$\mathrm{OH}: \quad 2.20 \mathrm{M}$

A1: $\quad 1.13$ M

$\mathrm{NO}_{2}: \quad 2.72 \underline{\mathrm{M}}$

$\mathrm{NO}_{3}: \quad 5.10 \mathrm{M}$

${ }^{39},{ }^{90} \mathrm{Sr}: 4.20 \times 10^{4} \mathrm{jCi} / \mathrm{gal}$

GEA: $\left.\quad{ }_{137}^{134} \mathrm{CS}-7.64 \times 10_{6}^{3} \mathrm{HCi} / \mathrm{ga}\right]$ ${ }_{137} \mathrm{Cs}-1.85 \times 10^{6} \mu \mathrm{Ci} / \mathrm{ga} 1$
$\left.{ }_{103} \mathrm{Ru}-<2.77 \times 10^{4} \mathrm{HCi} / \mathrm{ga}\right]$

Water: $\quad 48.22 \%$

$\mathrm{NH}_{4}: \quad 4.46 \times 10^{-2} \underline{\mathrm{M}}$

REW: eyw

Cc: JS Buckingham

WR Christensen

RM Ginestet

KE Nelson

JC Womack 

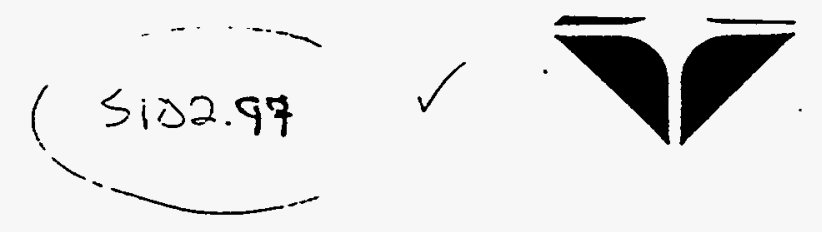

Date: December 11, 1975

To: R. L. Walser

From: R. E. Wheeler, 2-2449 ff fisturie

Subject: ANALYSIS OF TA:IK FARM SAMPLES

Sample: T-378 Tank: 102-S

Received: December 5, 1975

Vis-0TR: Dark brown -25\% Suspended Solids $1200 \mathrm{mrad} / \mathrm{hr}$

$\mathrm{pH}: \quad 13.5$

SpG: $\quad 1.390 \bigcirc 52^{\circ} \mathrm{C}$

$\mathrm{OH}: \quad 1.66 \underline{\mathrm{M}}$

Al: $\quad 0.990 \underline{M}$

$\mathrm{NO}_{2}: \quad 2.0 \mathrm{M}$

$\mathrm{NO}_{3}: \quad 4.40 \underline{\mathrm{M}}$

${ }^{89}, 90 \mathrm{Sr}: 3.27 \times 10^{4} \mathrm{jCi} / \mathrm{gal}$

GEA: $\quad{ }_{137}^{134} C_{S}-7.34 \times 10^{3} \mu \mathrm{Ci} / \mathrm{gal}$

Water: $\quad 53.74 \%$

REW: eyw

cc: JS Buckingham

WR Christensen

RM Ginestet

KE Nelson

JC Womack. 


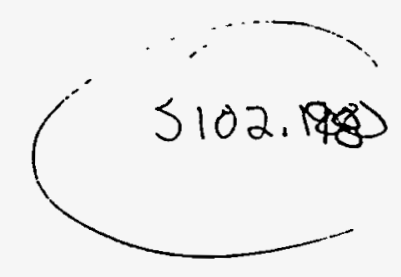

Date: December 15, 1975

To: R. L. Walser

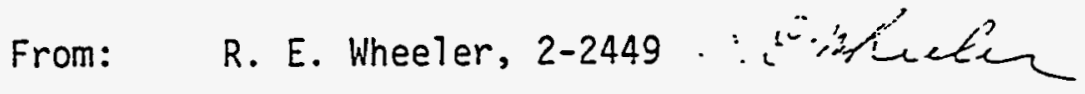

Subject: ANALYSIS OF TANK FARM SAMPLES

Sample: T-506 Tank: 102-S

Received: December 9, 1975

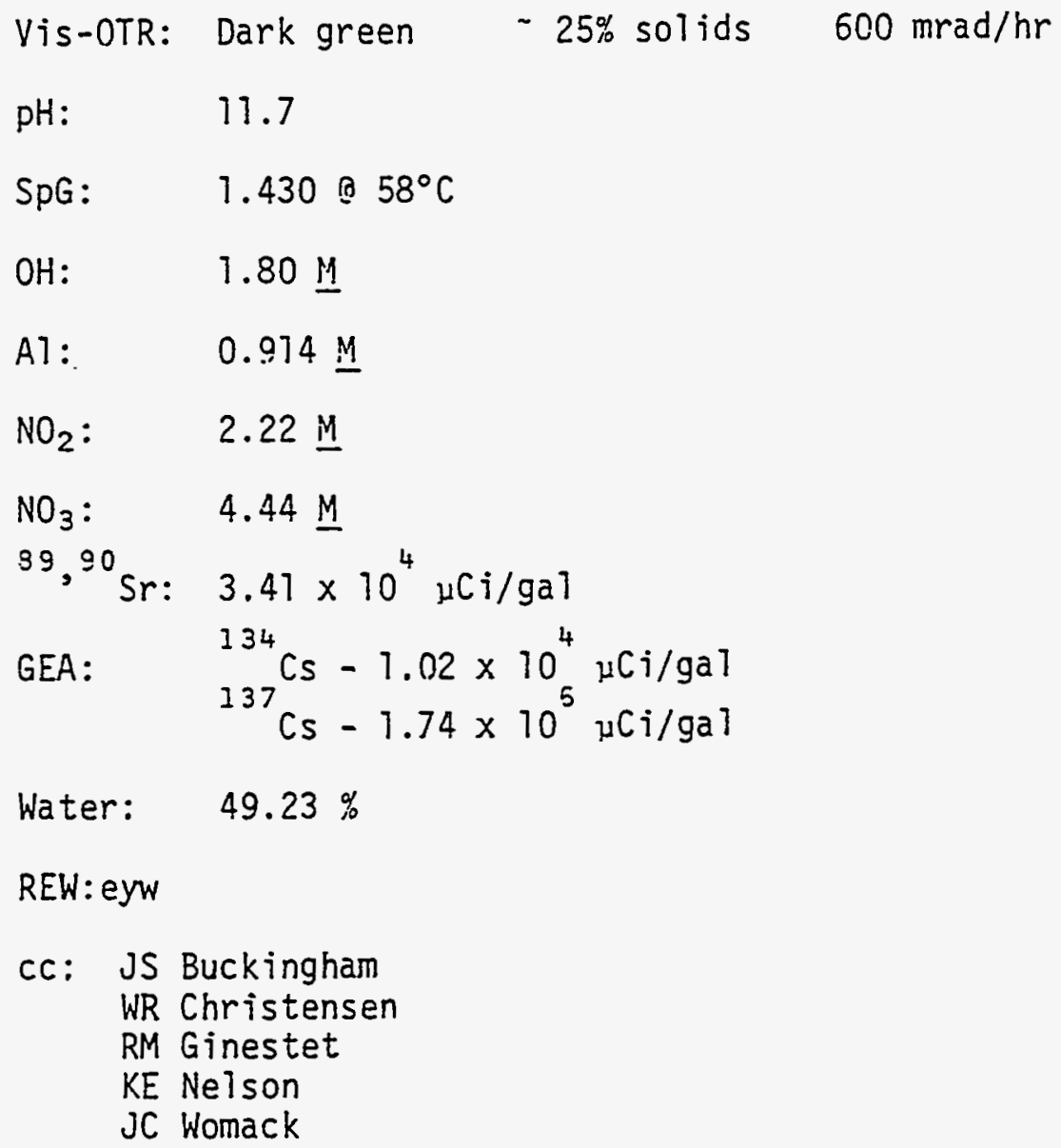


Da te: $3-16->c$

TO: P. L. WALSER

From: Pde ithelen
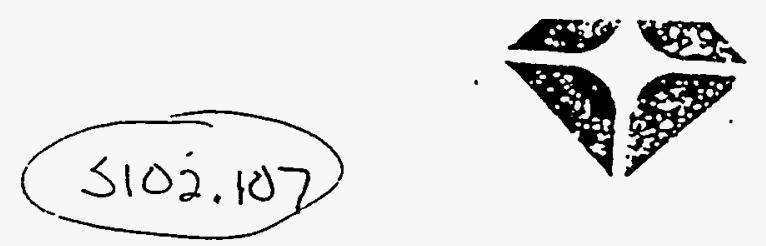

SUbject: ANALYSIS OF TANK FARM SAMPLES

SAMPLE NO. $I / 0 / 8$ RECEIVED: $\frac{1 / 20 / 8}{12-28-.25}$ TANK:

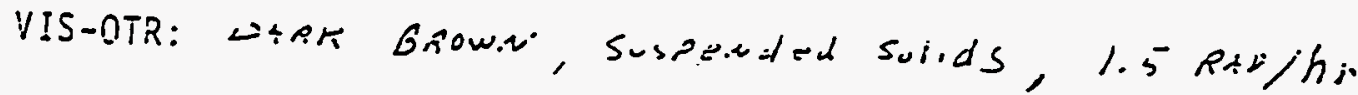

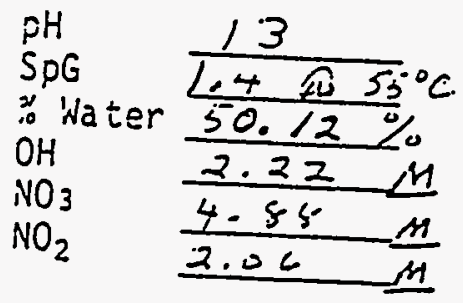

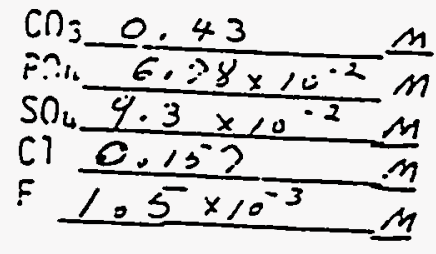
Al $\frac{1.5^{-} 4}{1.34} \frac{\mathrm{m}}{\mathrm{m}}$
$\mathrm{Cu} \frac{8.18 \times 10^{-5}}{\mathrm{~m}}$
$\mathrm{~Pb} \frac{3.02 \times 10^{-4}}{\frac{\mathrm{m}}{2.76 \times 10^{-3}} \mathrm{~m}}$ PU $\frac{4.8 \% \times 10^{-6}}{\mathrm{~g} / \mathrm{ga} I}$ Fi/gal $\quad 1: 5 \mathrm{Sb}$ iCi/gal issRu $\mathrm{WCi} \mathrm{G} / \mathrm{ga}] \quad: 4 \mathrm{H}$ CePr $-\mathrm{Ci} / \mathrm{gal}$ Cijja i ici;gal

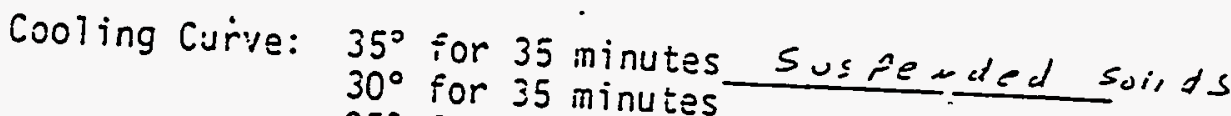
$25^{\circ}$ for 35 minutes

$20^{\circ}$ ior 35 minutes

$15^{\circ}$ for 35 minutes

$10^{\circ}$ for 35 minutes $5^{2}$ for 35 minutes

DTA: N: Exotheims below zo0 ${ }^{\circ} \mathrm{C}$, Excineiph at $250^{\circ} \mathrm{C}$ 

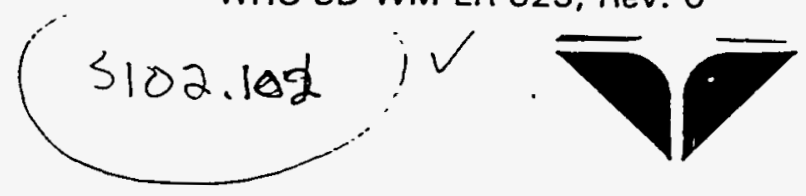

Date: January 19, 1976

To: R. L. Halser

From: R. E. Wheeler, 2-2449 $\Rightarrow$ PPfflectic

Subject: ANALYSIS OF TANK FARM SAMPLES

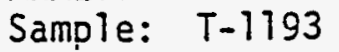

Received: January 5, 1976

Vis-OTR: Dark brown Suspended solids $1800 \mathrm{mrad} / \mathrm{hr}$

$\mathrm{pH}: \quad 13.7$

Spg: $\quad 1.40 @ 67^{\circ} \mathrm{C}$

$\mathrm{OH}: \quad 2.06 \stackrel{1 ! 9}{-}$

AT: $\quad 1.21 \dddot{1}$

$\mathrm{HO}_{2}: \quad 2.26 \mathrm{H}$

$\mathrm{NO}_{3}: \quad 4.94 \mathrm{H}$

${ }^{89}, 90 \mathrm{Sr}: 2.99 \times 10^{4} \mu \mathrm{Cj} / \mathrm{gal}$

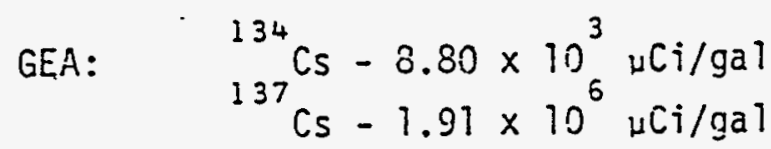

Water: $\quad 50.32 \%$

REW : eyw

cc: JS Suckingham

WR Christensen

PM Ginestet

$K E$ ivelson

JC Womack 
Date: January 15, 1976

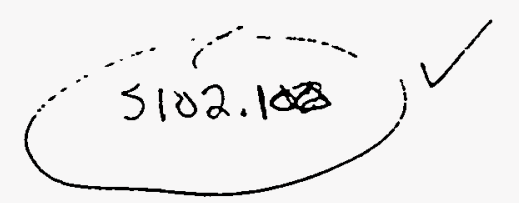

To: R. L. Walser

From:

R. E. Wheeler, 2-2449

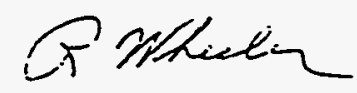

Subject: ANALYSIS OF TANK FARM SAMPLES
Sample: $T-1330$
Tank:
Received: January 7,1976
Vis-OTR: Dark green Suspended solids $1800 \mathrm{mrad} / \mathrm{hr}$
$\mathrm{pH}: \quad 14.0$
SpG: $\quad 1.38$ @ $59^{\circ} \mathrm{C}$
$\mathrm{OH}: \quad \quad \quad 1.53 \underline{\mathrm{M}}$
A.1: $\quad 0.982 M$
$\mathrm{NO}_{2}: \quad 1.70 \underline{\mathrm{M}}$
$\mathrm{NO}_{3}: \quad 4.30 \mathrm{M}$
${ }^{39}, 90 \mathrm{Sr}: 7.03 \times 10^{4} \mathrm{HCi} / \mathrm{ga} 7$

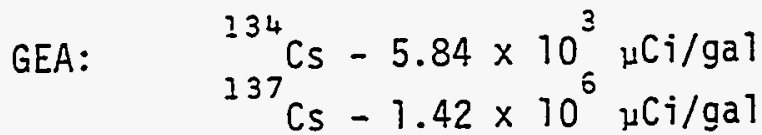
Water: $\quad 59.48 \%$
REW: eyw
CC: JS Buckingham
WR Christensen
RM Ginestet
KE Nelson
JC Womack


Atlantic Richfield Hanford Company

\begin{tabular}{|c|c|}
\hline Date: & February 13, 1976 \\
\hline To: & R. L. Halser \\
\hline From: & ?. E. Wheeier, 2-2449 F P/luele \\
\hline Subject: & $\begin{array}{l}\text { AMIALYSIS OF TAIIK FARA SAI:PLES } \\
\text { Sample: T-lEZ3 Tank: 102-S } \\
\text { Received: January 20, 1976 }\end{array}$ \\
\hline Vis-OTR: & Suspended solids \\
\hline pH: & 13.0 \\
\hline SPG: & $1.39: 50^{\circ} \mathrm{C}$ \\
\hline OH: & 1.33 \\
\hline A.7: & 0.973 \\
\hline ila: & 3.10 \\
\hline $\mathrm{NO}_{2}:$ & $1.75 \mathrm{H}$ \\
\hline $\mathrm{iO}_{3}:$ & $3.90 \mathrm{M}$ \\
\hline Pu: & $5.22 \times 10^{-4} \mathrm{gm} / \mathrm{gat}$ \\
\hline D.T.A.: & No exotherm below $200^{\circ} \mathrm{C}$, exotherm $245^{\circ} \mathrm{C}$ \\
\hline $\mathrm{PO}_{4}:$ & $0.104: 19$ \\
\hline $\mathrm{Cl}:$ & $0.154:$ \\
\hline$F:$ & $2.42 \times 10^{-3} \mathrm{ii}$ \\
\hline $\mathrm{CO}_{3}:$ & $0.442 \stackrel{\because}{-}=$ \\
\hline Cu: & $<9.6 \times 10^{-3}$ \\
\hline $\mathrm{Hg}_{g}:$ & $2.22 \times 10^{-3}$ \\
\hline Pb: & $2.32 \times 10^{-4} \vdots !$ \\
\hline EEA: & $\begin{array}{l}\left.134 \mathrm{Cs}-6.32 \times 10^{3} \mathrm{uCi} / \mathrm{ga}\right] \\
\left.137{ }_{\mathrm{Cs}}-1.56 \times 10^{5} \mathrm{uCi} / \mathrm{ga}\right]\end{array}$ \\
\hline${ }^{89}, 90 \mathrm{Sr}:$ & $2.32 \times 12^{4} \mathrm{HCi} / \mathrm{gal}$ \\
\hline
\end{tabular}


Atlantic Richfield Hanford Company

R. L. Walser

Page 2

February 13, 1976

Hater: $54.86_{\%}^{\% !}$

Cooling Curve: Suspended solids

RE!U: eyn

cc: JS Buckingham

U:R Christensen

跳 Ginestet

KE !!elson

JC Homack 
$\because-\check{c}-i$.

Date: $\quad x-27-76$

To: E, L. WALSER
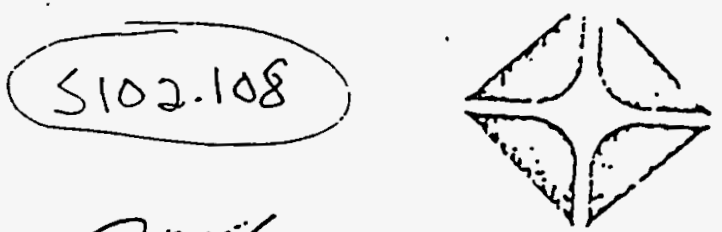

From: Supervisor, Anaiytical Services

Subject: ANALYSES OF TANK FARM SAMPLE

Sample No:T2375Tank: 102-5 Received: $2-17-76$

VIS-OTR: DHRK उRED

No solits 1500 mind $/ H_{L}$

$\mathrm{PH} 13.2 \mathrm{CO} 0.235$ Al $0.453 \mathrm{M}$

SpG $1.250 \quad \mathrm{PO}_{4} 4.20 \times 10^{-\frac{2}{4}}$ i. 5

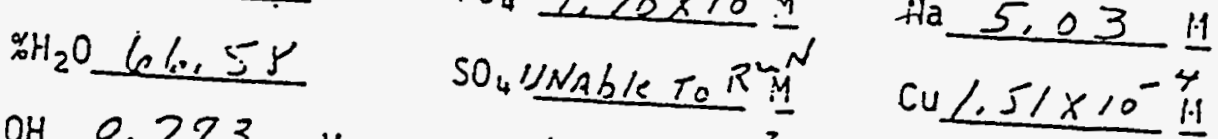

OH 0.793 i1 $\quad$ Cl $6.02 \times 10^{-2}$ in $\mathrm{Pb} 5.17 \times 10^{-14}$

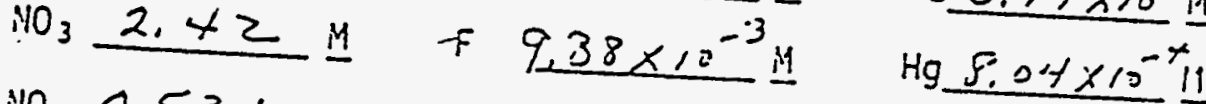

$\mathrm{NO}_{2} 0.53 /$ is

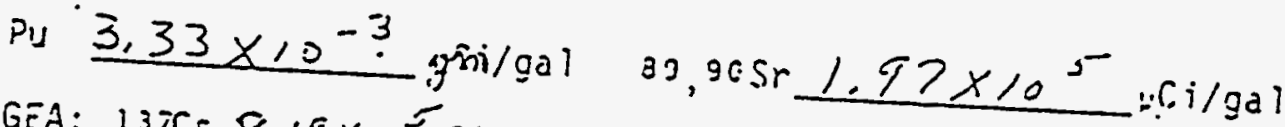

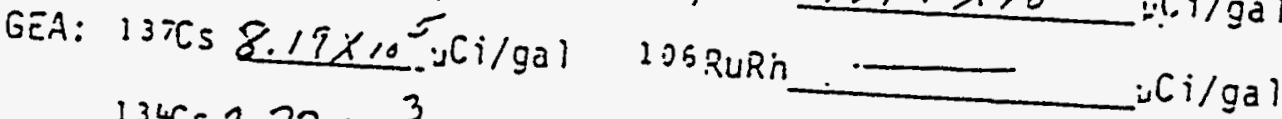

$1.34 \mathrm{Cs} 2.7 .7 \times 10^{3}{ }^{6} \mathrm{Ci} / \mathrm{gal} \quad 154 \mathrm{Eu}$

${ }^{50} \mathrm{Co} 2.36 \times 10^{3} \mathrm{uCi} / \mathrm{gal}$ is $\mathrm{Eu} 1.46 \times 10^{4} \mathrm{Ci} \mathrm{Ci} / \mathrm{gal}$

${ }^{144} \mathrm{Ce}, 1.86 \times 10^{4}, \mathrm{Ci} / \mathrm{gal}$

$144 \mathrm{Pr}=\mathrm{iCi} / \mathrm{gal}$

ici/gal

COOLING CURYE: $35^{\circ}$ for

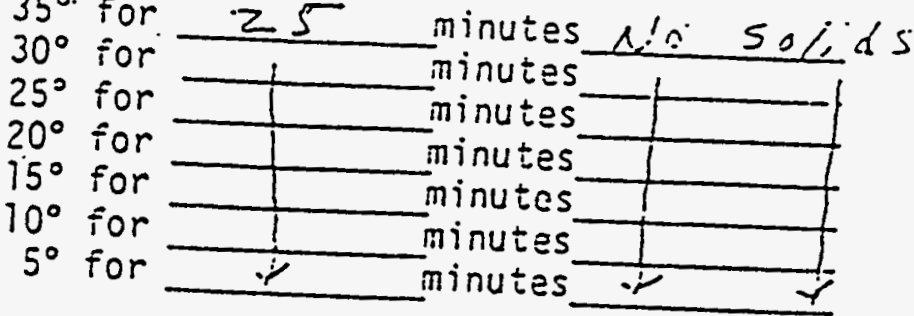

DTA: No Exotherm be low $200^{\circ} \mathrm{C}$ Exotharon e $233^{\circ} \mathrm{C}+315^{\circ} \mathrm{C}$ 
$T \in R E-11 z$

\section{Internal Letter}
Date. Aprit 5, 1979.

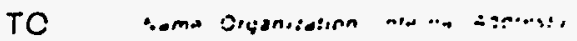
- K. G. Carothers
- Process Engineering
. 2750-E, 200 East

$$
\begin{aligned}
& \therefore \text {. 65120-79-062 J } \\
& \text { G. E. Brown } \\
& \text { - Chemical Sciences Group } \\
& \text { 222-5, } 200 \text { West } \\
& \text {. 2-1571 }
\end{aligned}
$$

Suject. . Tank 102-S Customer Waste Analyses

This letter reports results from Analytical Laboratory on a

\begin{tabular}{|c|c|c|c|}
\hline Analysis & \multicolumn{2}{|c|}{ Results } & Rerun Result \\
\hline $\mathrm{NO}_{3}^{-}$ & $2.04 \mathrm{M}$ & & $1.65 \underline{M}$ \\
\hline $\mathrm{NO}_{2}^{-}$ & $1.55 \mathrm{M}$ & & \\
\hline $\mathrm{SO}_{4}{ }^{2-}$ & $.27 \mathrm{M}$ & & \\
\hline $\mathrm{PO}_{4}{ }^{3-}$ & $.12 \mathrm{M}$ & & \\
\hline $\mathrm{Al}^{+3}$ & $I S^{* *}$ & & $.51 \underline{M}$ \\
\hline $\mathrm{OH}^{-}$ & is & & $.49 \underline{M}$ \\
\hline $\mathrm{CO}_{3}^{2-}$ & $.62 M$ & & $.56 \underline{M}$ \\
\hline TOC* & $10.6 \mathrm{gm} / 1$ & & $13.0 \mathrm{gm} / 1$ \\
\hline Density & 1.257 & & \\
\hline$\% \mathrm{H}_{2} \mathrm{O}$ & 61.74 & & \\
\hline $\mathrm{Cs}^{137}$ & $2.63 \times 10^{5}$ & $\mu \mathrm{Ci} / 1$ & \\
\hline $\mathrm{Cs}^{134}$ & $4.07 \times 10^{2}$ & ${ }_{\mu \mathrm{Ci} / \mathrm{T}}$ & \\
\hline $\cos 0$ & $4.17 \times 10^{2}$ & $\mu \mathrm{Ci} / \mathrm{T}$ & \\
\hline
\end{tabular}
customer waste sample from Tank 102-S, received on January 31. The sample was a light green solution with about 10 percent settled solids. The solids all dissolved when the sample was heated to $60^{\circ} \mathrm{C}$. The table below lists the resuits.

Please contact me if you need further information.

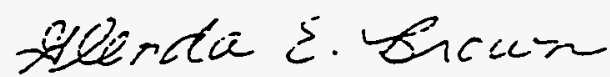

G. E. Brown, Chemist Waste Chemistry Unit

GEB : jkr

Information: D. R. Jorgenson

F. M. Jungfleisch $Y_{m} \not$

T. A. Lane

R. A. Zinsli

File: KF55E

* Total Organic Carbon

** Insufficient Sample 


\section{TCRC-11?.}

WHC-SD-WM-ER-323, Rev. O

\section{Iniernal latior}

2 2.e: March 28,1980

H. J. Eding

Systers Engineering

$27505, A-227$
Rocksyell International

tul
M. T. Jarisk.y

Seperations Process Deveiopment ini 140-037, 200 West

$\because=-$. Hot Boildown of Tank 102-S Liquer

In response to a request, a hot boildown oi iank 102-5 liavor was done. The waste was a product of the second partial reutraization (pi) campaign. The main purpose of this boildown was to see if double shell slurry (OSS) couid be made from lOz-S liciver.

The boiling temperat:ire and pressure data were gathered as a function of waste volume reduction (WVP.) and are shown in Table I. Visccsity cata ivere not obtained due to the presence of solids in the origina? sample as weil as in the product. The "hot" cone viscometer is not useable for mixtures.

The iollowing observaticrs were noted durinc the boildown. The scripie. when placed in the boildown flask, separated into four phases:
a) jelled crust
b) je?ied liquid pinase
c) İyer of suspencied solids
d) sett? 20 solids

The sample foamed at 40 torr and the boiline point. The foan remaired tinroughout the boildown. The sample tehaved as uncomblexed waste.

The product afier $40 \%$ Hiv was in three priases:
a) solid crust
b) viscous middle phase

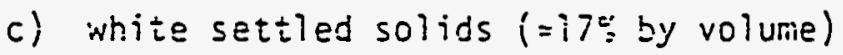

To obiain a uniform mixture for analysis, the sample was reheated (at 30 torr) to dissolve the solids. Sone solids dissolved, aliquots of the liquor transferred to centrifuge cones, and centrifuged. After centriiugation, the aliquots were 100: solids, wita no cel phase present. A portion was submitted to Analytical Laboratories for anaiyses. A second portion was warmed to $80^{\circ} \mathrm{C}$ to dissolve solids, allowed to sit to setile insoluble solids by crayisy, and the lipuid phase decanted and sutmitted for analysis. The low riacer content of the supernatant liquer, as snclwn in $i_{i b l e} i j$, is unreal, and riakes calculations of weight jercent in "sry" 
H. J. Eding

Page 2

March 28, 1980

solids impossible. This, in turn, will not allow any conciusions concerning DSS to be reached. The analyses of the received sample are shown in Table if. The analyses of the product solids arid supernatant are shown in iable II!.

Future boildours will be conducted such thict ine product solids and supernatant will be separated at temperature. Tris will alleviate the problems encountered here.

Please call it you have any questions regarding this work.

M. T. Jansky

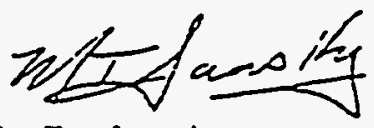

Advanced Chemist

MTJ:nâj

Information:

R. B. Eendixsen.

D. E. Bcwers

L. C. Brown

J. S. Buckingham

G. T. Dukelow

D. L. Herting

T. A. Lane

R. D. Prosser

T.. 3. Veneziano

Process Aids (8)

File Code: KF5JE 
H. J. Eơing

Page 3

Harch 28, 1980

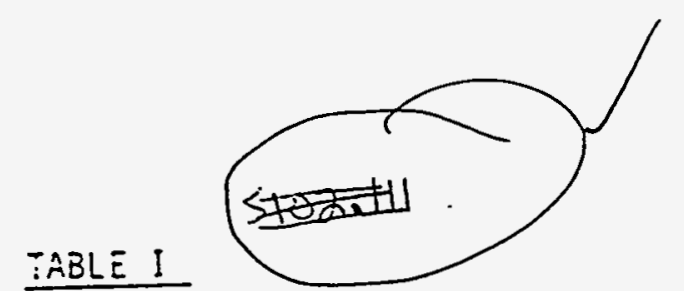

Temperature and Pressure at Boijinn versus biv ior Tank 102-s

Percent WVR

0

10

20

30

40

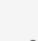

\section{Temperzture ('C;=)}

$51.6 / 125.9$

$50.4 / 137.2$

$64.6 / 749.2$

$54.6 /: 30.2$

$62.2 / 141.0$

ES.2/354.6

$53.2 ; 136.8$

$66.0 / 151.0$

$73.0 / 163.4$

$64.6 / 115.2$

$71.8 / 161.4$

$77.8 / 772.0$

$75.3 / 168.4$

$60.0 / 177.0$

$88.2 / 190.3$
Pressure (Torr)

40

60

30

40

60

3.)

4.)

50

80

40

60

80

40

60

30 
H. J. Eding

Page 4

Merch 28, 1980

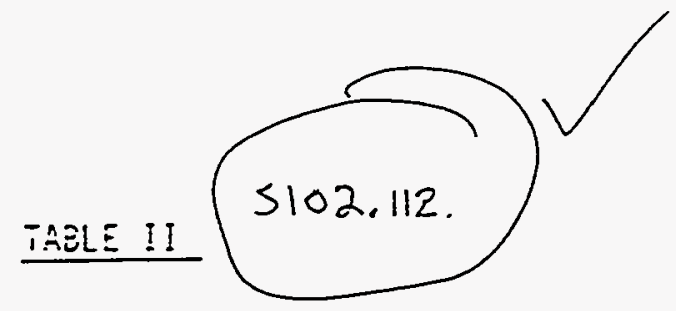

Composition of Tínk i0z-s Híste Liouor Semp?es. Semole =i-9i0l

Component

$\mathrm{MEATO}$

$\mathrm{NAOH}$

$\mathrm{NANO}_{3}$

$\mathrm{NaHO}_{2}$

ila $\mathrm{PO}_{4} . i 2 \mathrm{in}_{2} \mathrm{O}$

$\mathrm{Na}_{2} \mathrm{SO}_{4}$

$\mathrm{Ha}_{2} \mathrm{CO}_{3} \cdot \mathrm{H}_{2} \mathrm{O}$.

TOC

Pu $(g ; 1)$

if $(\Xi / 1)$

$\mathrm{H}_{2} \mathrm{O}$

Tota

SPE.

* These values are rerun everāẹes.

Initial valves were: $A$ l $=0.930 t$ A

$$
0 \mathrm{H}^{-}=4.87 \bar{M}
$$

Molarity

$1.77^{*}$

$4.34^{x}$

2.19

2.64

0.0502

0.0012

0.137

$3.23 \times 10^{-6}$

$3.23 \times 10^{-4}$

43.25

48.25

90.01

10.35

11.53

13.28

12.59

1.35

0.04

1.21

--

$\ldots$ ht. Fercent 
H. J. Eding

Page 5

Hurch 28, 1900

TASLE I.II

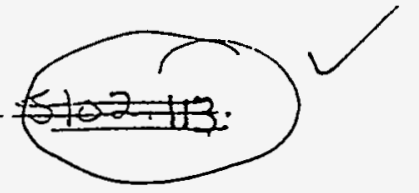

Eomposition of Boildcwn Products from Tank in2-s

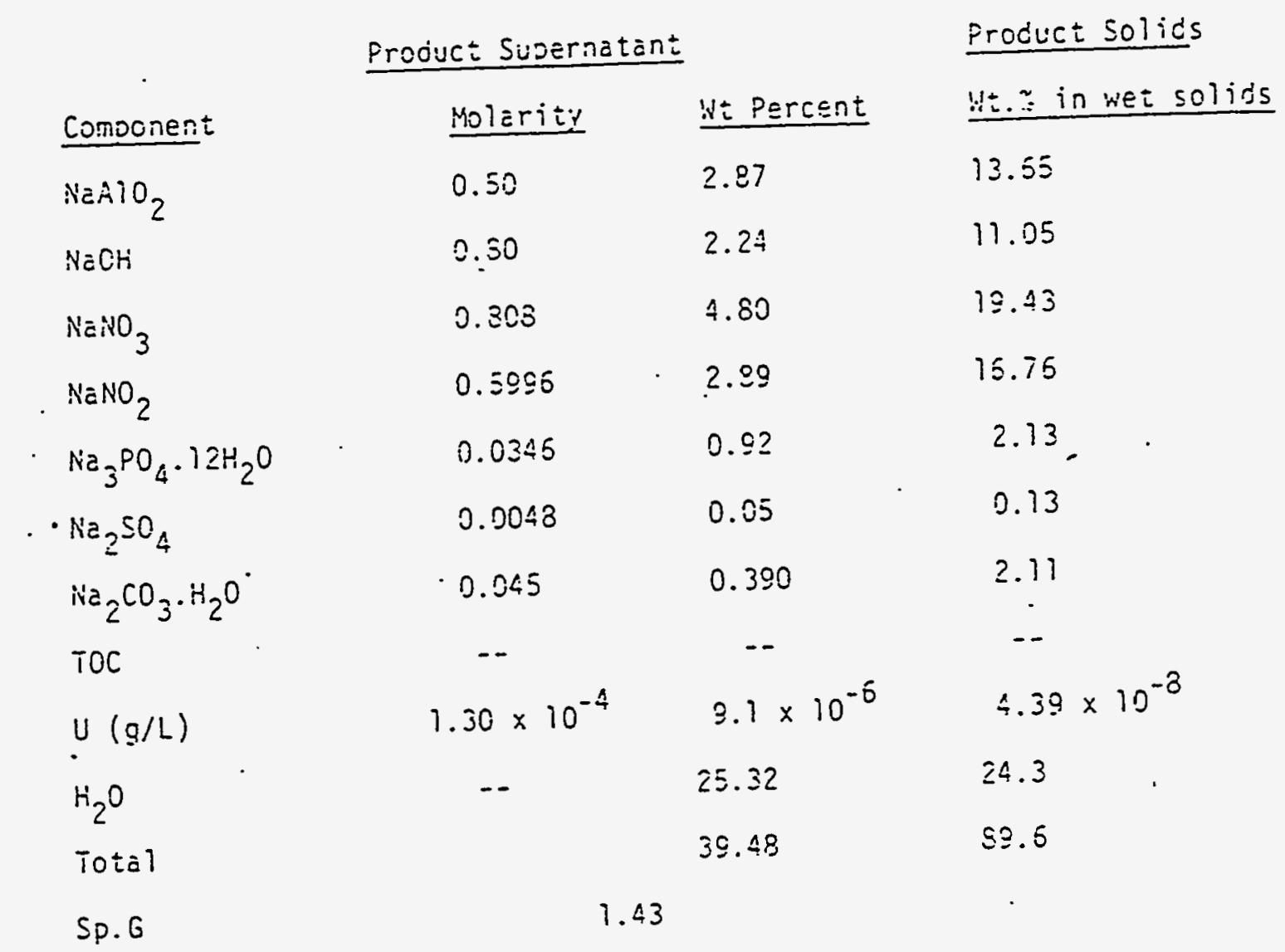


TCRC-1

Atlantic Richfield Hanford Company

Deie: $\quad$ Septembe $=21$, I9T1

$5103 \cdot 4$

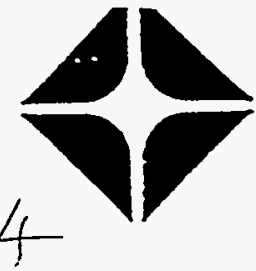

Tc:

In=c5:

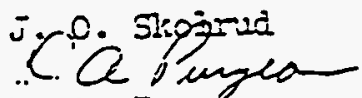

D. A. Eurgar

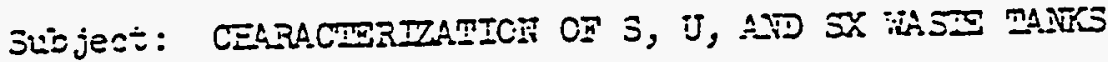

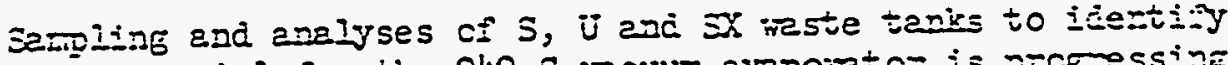
Eee: rateriel for the 242-s veluum erepoidtcr is pros-essing. To jate three che liter semples fro the 3 Jiz janks iave beea processed through the labcretory reculd eveportor. In

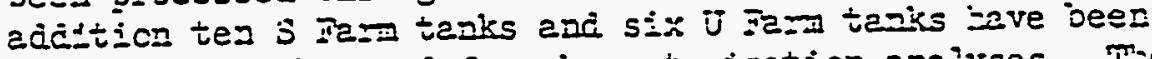

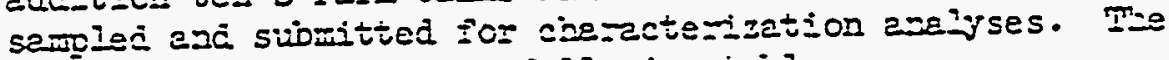
zesults are shewn in tine following tajle.

C:zzactezizaticn Anziyses co ilaste Tanks

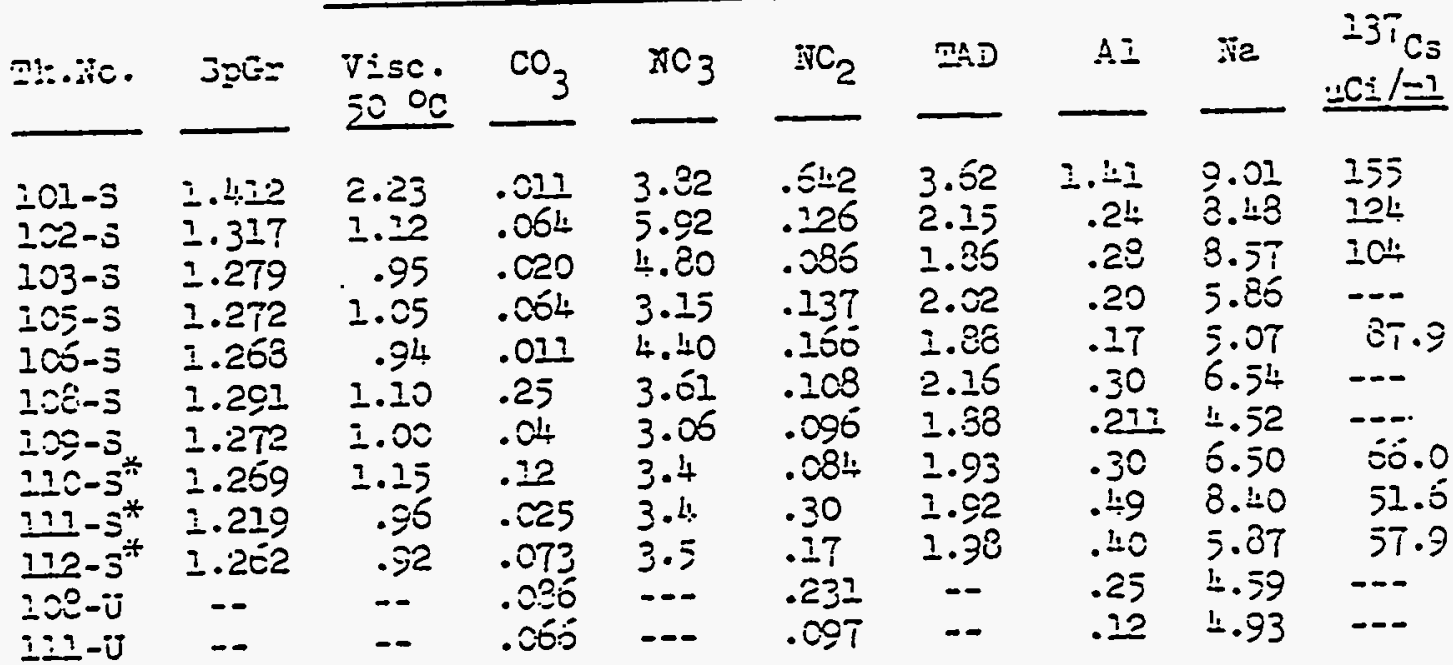

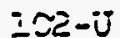

IC:

I $c 5-\mathrm{U}_{1}$

IÓ-i

* Saz in lajocatory racuum evegoretcr.

The above aneiyses eppear nomel except sazile iOI-5. Fron tive date and peremeters jained in the inree $S$ teni sazple evepcreticn zuns, the resulis 0 i lOI-s appen=s to

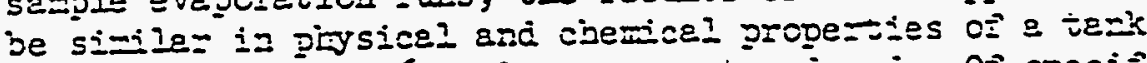

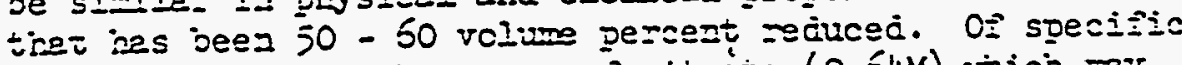

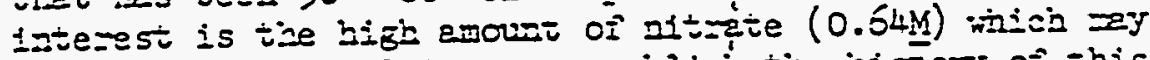
yield a ciue in helping to escablisi tie listogs os this tank. 
J. $0.51: 0 i-25$

इase 2

Seziverise: $21,19 T 1$

If there are aby questions pieese ceii in ca $2-T^{4}+T^{8}$.

DIP:Ah

ce: GI 3crstein

is 3uckinginan

ive Cambeli

DJ Iarliza

Fì

I3

Process Aids (5) 
Date: December 7, 1973

To: R. L. Walser

From: W. H. Sant :ry Yilet

Subject: 242-S FEED SAMPLES T-9490 (10/05/73) 103-S

Vis-0tR Clear, yellow, $600 \mathrm{mR}$

$\mathrm{pH} \quad 11.0$

SpG $\quad 1.3517$

DTA No Exotherm

Pu $\quad 4.93 \times 10^{-7} \mathrm{~g} / 1$

$\mathrm{Na} \quad 8.68 \underline{\mathrm{M}}$

$\mathrm{SO}_{4} \quad 8.78 \times 10^{-3} \underline{\mathrm{M}}$

A1 $\quad 1.22 \mathrm{M}$

$\mathrm{NO}_{2} \quad 0.852$ iM

F $\quad 2.07 \times 10^{-3} \mathrm{M}$

$\mathrm{CO}_{3} \quad 0.042 \underline{\mathrm{M}}$

$\mathrm{Cl}$

$\mathrm{OH} \quad 1.96 \mathrm{M}$

GEA $\quad{ }^{137} \mathrm{CS}-1.61 \times 10^{5} \mu \mathrm{Ci} / \mathrm{T}$

$\mathrm{NO}_{3} \quad 4.36 \underline{M}$

$30 \%$ reduction $-28.8 \%$

$50 \%$ reduction $-52.5 \%$

$8990 \mathrm{Sr} 11.24 \mathrm{jCi} / \mathrm{l}$

$\%$ Solids $-75.0 \%$

$\mathrm{PO}_{4} \quad=3.77 \times 10^{-4} \mathrm{M}$

WHS: bp

cc: WR Christensen

GW Reddick, Jr.

-WH Sant 
Date: January 21, 1974

To: R. L. Walser

From:

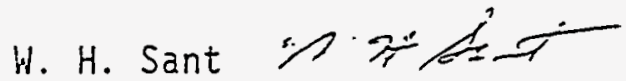

Subject: 242-S FEED SAMPLES NUMBER T- 736 SAMPLE POINT 103-S

Vis-0TR Cleàr, yellow, 0\% solids, 200 mRAD/hr.

$\mathrm{pH}$

11.8

$S P G$

1.0742

DTA

No Exotherm

Pu

$9.51 \times 10^{-7} \mathrm{~g} / 1$

Ha

1.89 嵌

$\mathrm{SO}_{4}$

$6.12 \times 10^{-3} \mathrm{M}$

A)

$0.122 \mathrm{M}$

$\mathrm{NO}_{2}$

$0.067 M$

$\mathrm{F}$

$5.26 \times 10^{-4}$ i

$\mathrm{CO}_{3}$

$0.017 \mathrm{M}$

$\mathrm{Cl}$

$\mathrm{OH}$

0.523 毝

GEA

$$
\begin{aligned}
& { }^{137} \mathrm{CS}-8.42 \times 10^{4} \mathrm{HCi} / 7 \\
& { }^{134} \mathrm{Cs}
\end{aligned}
$$

$\mathrm{NO}_{3}$

3.37 in

$30 \%$ Reduction - 27.5

$\%$ Solids - 0.690

$50 \%$ Reduction - 50

$\%$ Solids - 1.25

$89-90 \mathrm{Sr} \quad$ Not Requested

$\mathrm{PO}_{4} \quad 3.77 \times 10^{-4} \mathrm{M}$

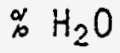

86.59

Cooling Curve $-15^{\circ} \mathrm{C}$ ivo solids

WHS: bp

CC: WR Christensen

GW Reddick, jr.

Hit Sant 
Date: December 6, 1974

To: R. L. Hialser

From: R. E. Whesler of reflicter

SUbject: AHALYYSIS OF TAMK FARM SAILPLES

S.JPLE: T-8009 103-S

Vis-DiR: Ci $\equiv$ ar, yellow, no solics. InCO med $/ \mathrm{hr}$

pH: < $\quad 14.5$

SPE: $\quad 1.235$

A1: $\quad 0.471 \underline{M}$

: : : $\quad 3.84 \underline{M}$

$: 10_{2}: \quad 0.579 \mathrm{M}$

$1: 0_{3}: \quad \quad \quad 1.43 \mathrm{H}$

Pu: $\quad<6.21 \times 10^{-5} \mathrm{gm} / \mathrm{gal}$

$\mathrm{SO}_{4}: \quad 1.21 \times 10^{-2} \mathrm{H}$

$\mathrm{FO}_{4}: \quad 3.44 \times 10^{-2} \frac{\mathrm{H}}{\mathrm{H}}$

F: $\quad 4.27 \times 10^{-3} \frac{1 .}{1 .}$

$\mathrm{CO}_{3}: \quad 6.73 \times 10^{-2}$

GEA: $\quad \because \vdots C_{5}-2.88 \times 10_{s}^{3} \because[i / g a l$

$:=\left[\right.$ Cs $-6.74 \times 10^{5}$ icijoal

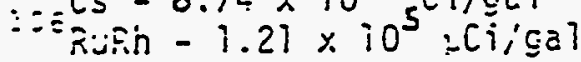

$W \equiv \pm=r: \quad 77.55 \%$

89,90 sr: $9.08 \times 10^{2}: \because i j i g z l$

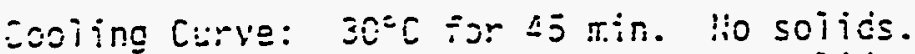
$25^{c} C$ for 1 z min. ilo solitis. ZCEC for eo rin. 10 soilids.

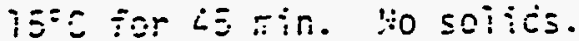
ince for ta rin. lio solics.

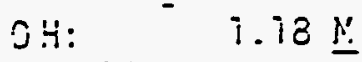

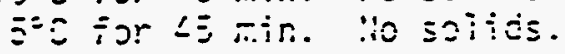

PE: in: jc

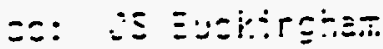

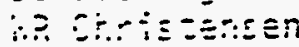

$\because \because \because \because . \equiv \Sigma \Sigma \equiv Z$

$p \equiv: \because: z \equiv i \in r$

ic :is...eck 


$$
\text { S-:Cᄀ.1 }
$$

Date: $\quad$ August 12,1974

To: R. L. Waiser

From: R. E. Wheeler is mikici.

Subject: ANALYSIS OF TANK FARM SAMPLES

SAMPLE: T-4184 104-S

Vis-0TR: Yellow, $10 \%$ solids. $100 \mathrm{mrad} / \mathrm{hr}$

$\mathrm{pH}$

$>12.6$

SPG: $\quad T .4456$

OH: $\quad 2.23 \mathrm{M}$

A1: $\quad 0.485 \underline{M}$

Na: $\quad 13.82$

$\mathrm{NO}_{2}: \quad 1.10 \mathrm{M}$

$\mathrm{NO}_{3}: \quad 5.07 \mathrm{M}$

Pu: $\quad \leq 2.41 \times 10^{-} \mathrm{g} / 7$

DTA: No Exotherm

$\mathrm{SO}_{4}: \quad 2.78 \times 10^{-2} \mathrm{M}$

$\mathrm{PO}_{4}: \quad 1.08 \times 10^{-4} \mathrm{M}$

$\mathrm{F}: \quad 7.53 \times 10^{-3} \mathrm{M}$

$\mathrm{CO}_{3}: \quad 7.55 \times 10^{-2} \bar{M}$

GEA: $\quad{ }^{137} \mathrm{CS}-5.98 \times 10^{5} \mathrm{HCi} / \mathrm{gaT}$

Water: $56.14 \%$

Cooling Curve: $25^{\circ} \mathrm{C}$ for $45 \mathrm{~min}$. No solids. $20^{\circ} \mathrm{C}$ for $45 \mathrm{~min}$. No solids. $15^{\circ} \mathrm{C}$ for $45 \mathrm{~min}$. No solids. $10^{\circ} \mathrm{C}$ for $60 \mathrm{~min}$. Trace of solids. $5^{\circ} \mathrm{C}$ for $45 \mathrm{~min}$. $\sim 7 \%$ solids.

$30 \%$ Reduction - 28.75

$50 \%$ Reduction - 50.0

Solids $-47.37 \%$

Solids - $98.75 \%$

REW:jd

cc: JS Buckingham

LG Johnson

RE Wheeler 


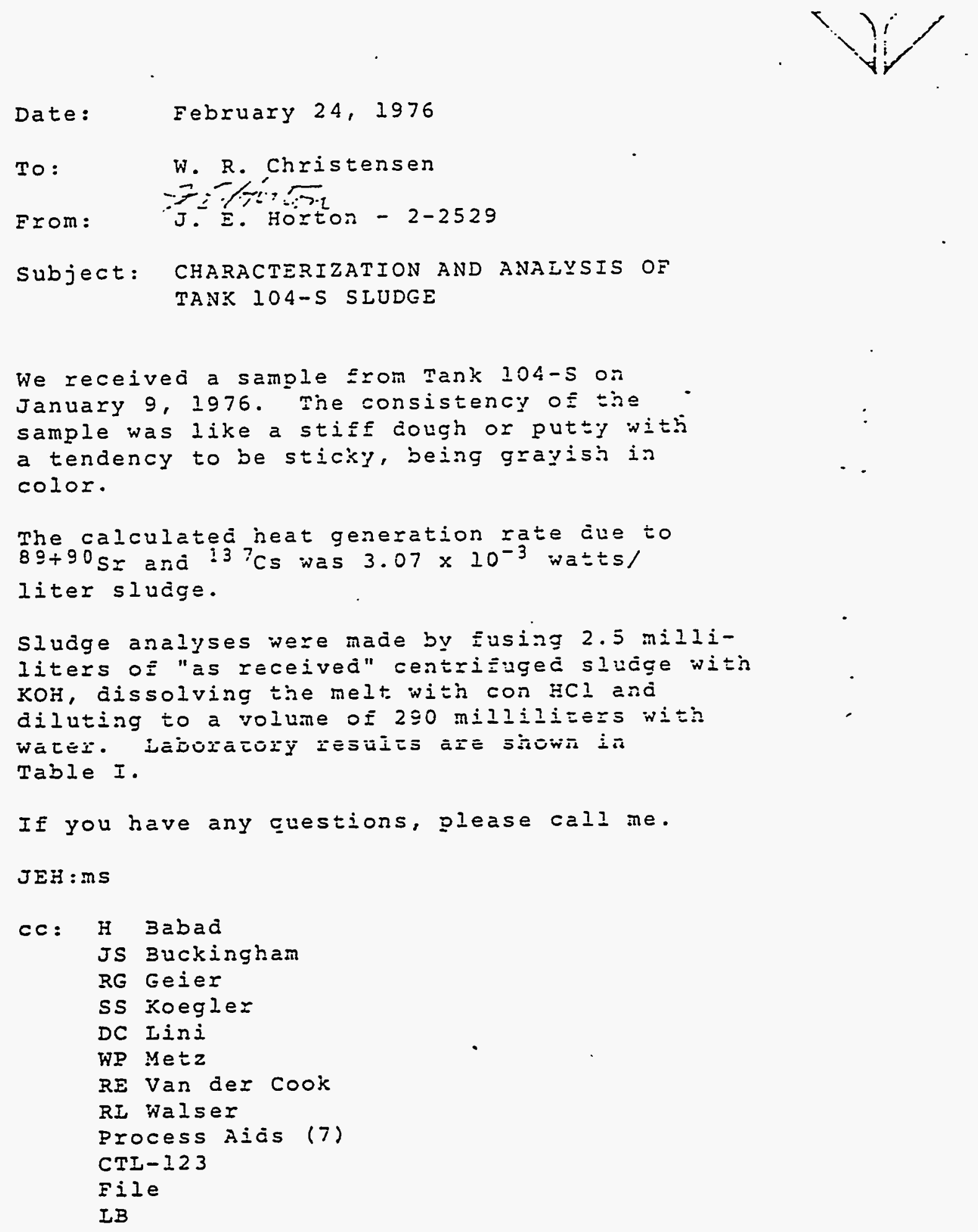




\section{TABLE I}

\section{ANAIYSIS OF IO4-S TANK SIUDGE}

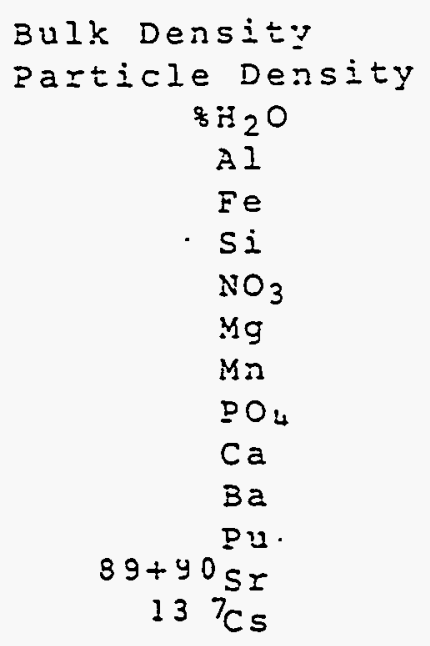

$1.21 \mathrm{~g} / \mathrm{CC}$
$2.45 \mathrm{~g} / \mathrm{Cc}$

30.3

0.06

0.3

$13: 2$

0.01

0.02

$<0.01$

0.09

$<0.09$

4.46

0.38

0.11
3.2 moles/2 moles/2

moles/2

moles $/ 2$

moles/2

moles/2

moles $/ 2$

moles/2

moles/2

$x 10^{-3} \mathrm{~g} / 2$

$c i / 2$

$\mathrm{Ci} / 2$
$2.55 \times 20^{-3}$ wat

$5.23 \times 10^{-4}$ watts/2 
Atlantic Richfield Hanford Company

Date: Septemice= 21, 1971

Tc:

Iscx:

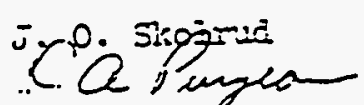

D. A. Zursea:
WHC-SD-WM-ER-323, Rev. 0

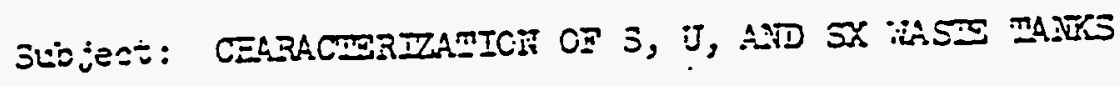

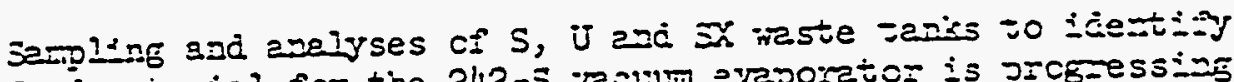

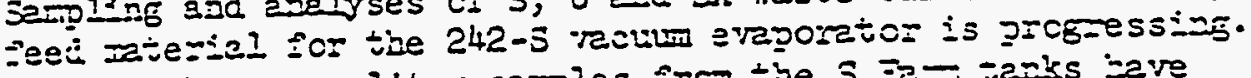

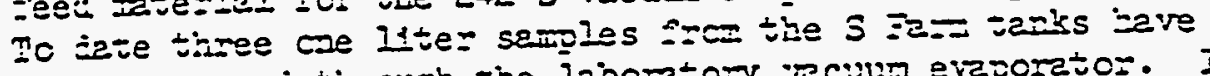
jeen processed tirough the labcretory reculn evaporator. I

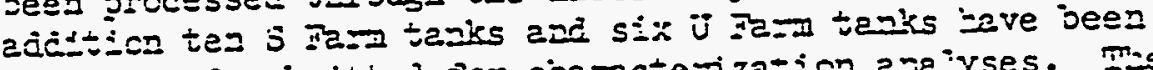

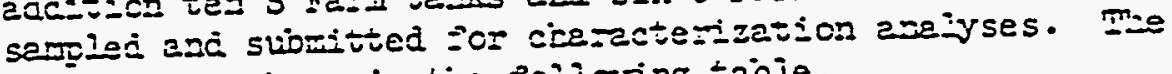
lesults are skown in tie icilcorias taile.

T:azcotorizaticn Azziyses co ivste Taniks

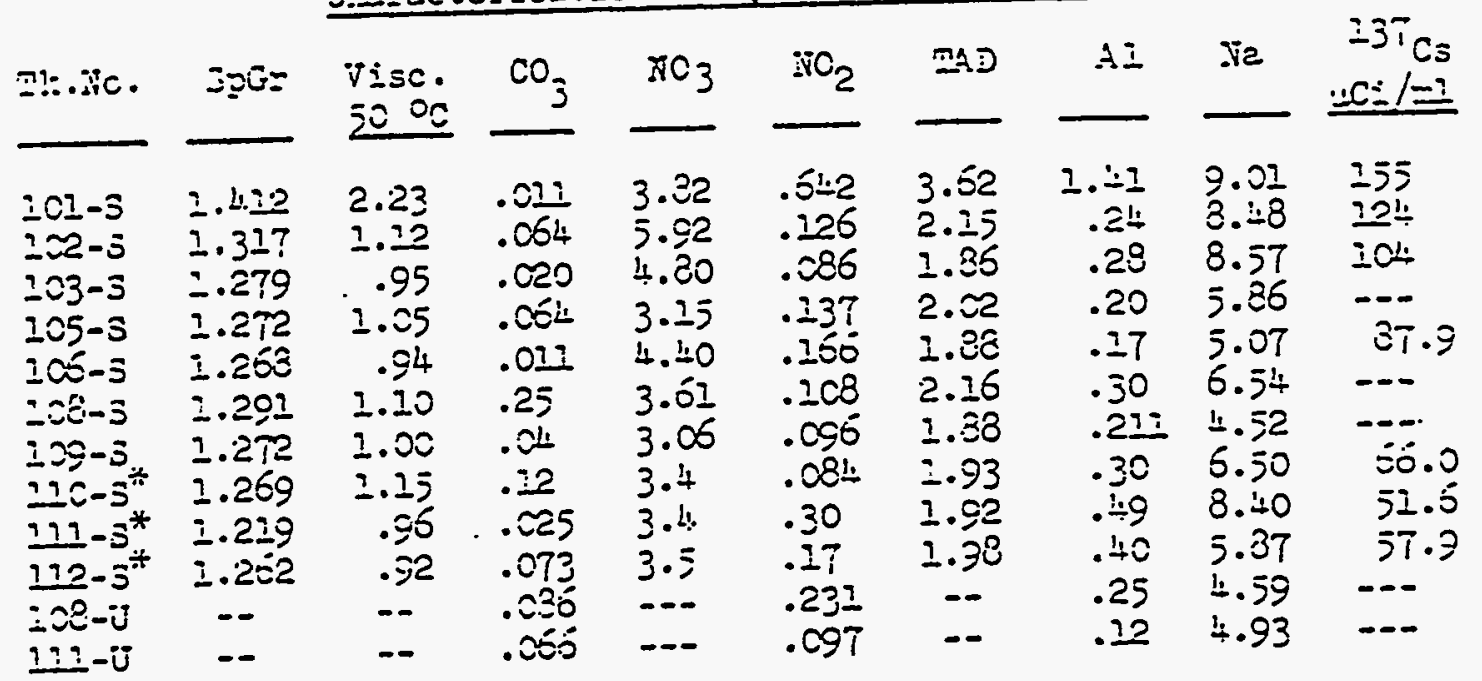

* Jen in lejocztory racum eveporetcr.

The eiove anelyses eppes: zorpl except sappie l0l-s. Irom tive jata and peremeters jeined fin the three $S$ tank semile srepcraticn zins, the Iesults of. I01-s epper=s to

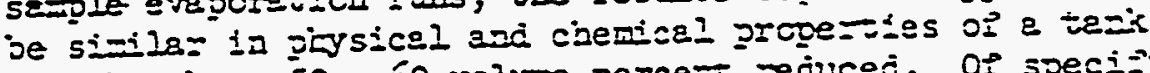
ties ins jeel j0 - 50 rolune pereest rouced. Of specizil

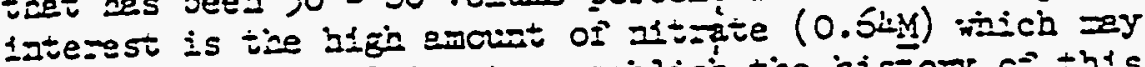

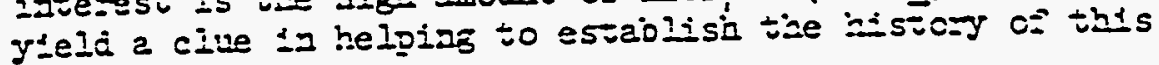
tanis. 


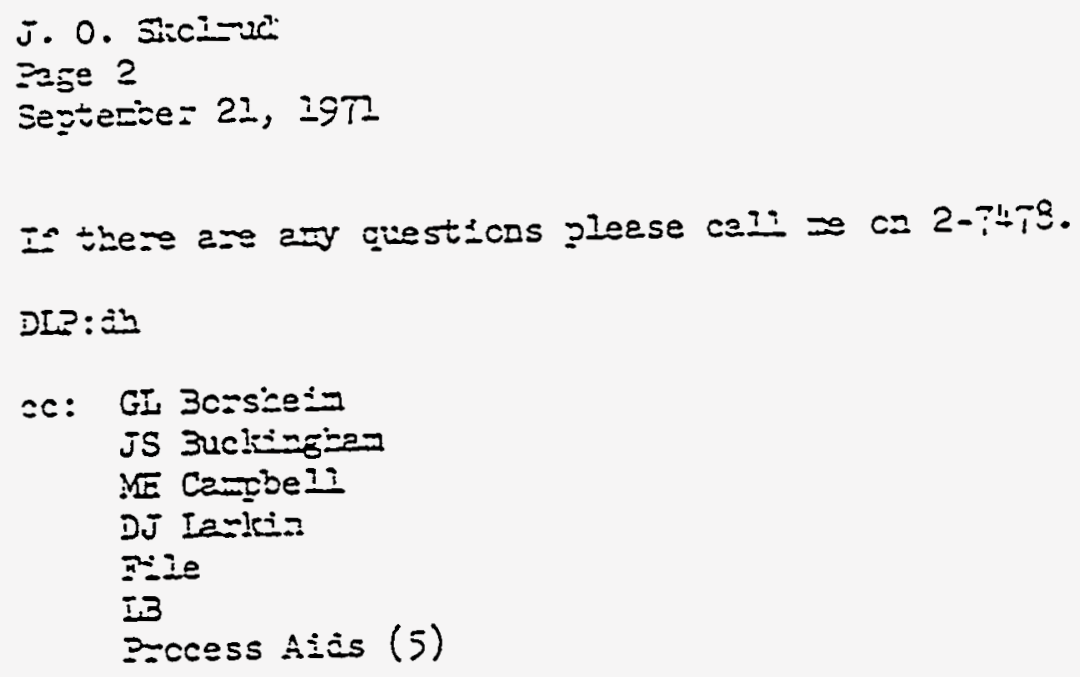


Date: December 7, 1973

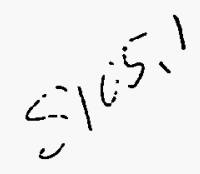

To: R. L. Walser

From: W. H. Sant $\because 7$,

Subject: 242-S FEED SAMPLES T-9491 (10/05/73) 105-S

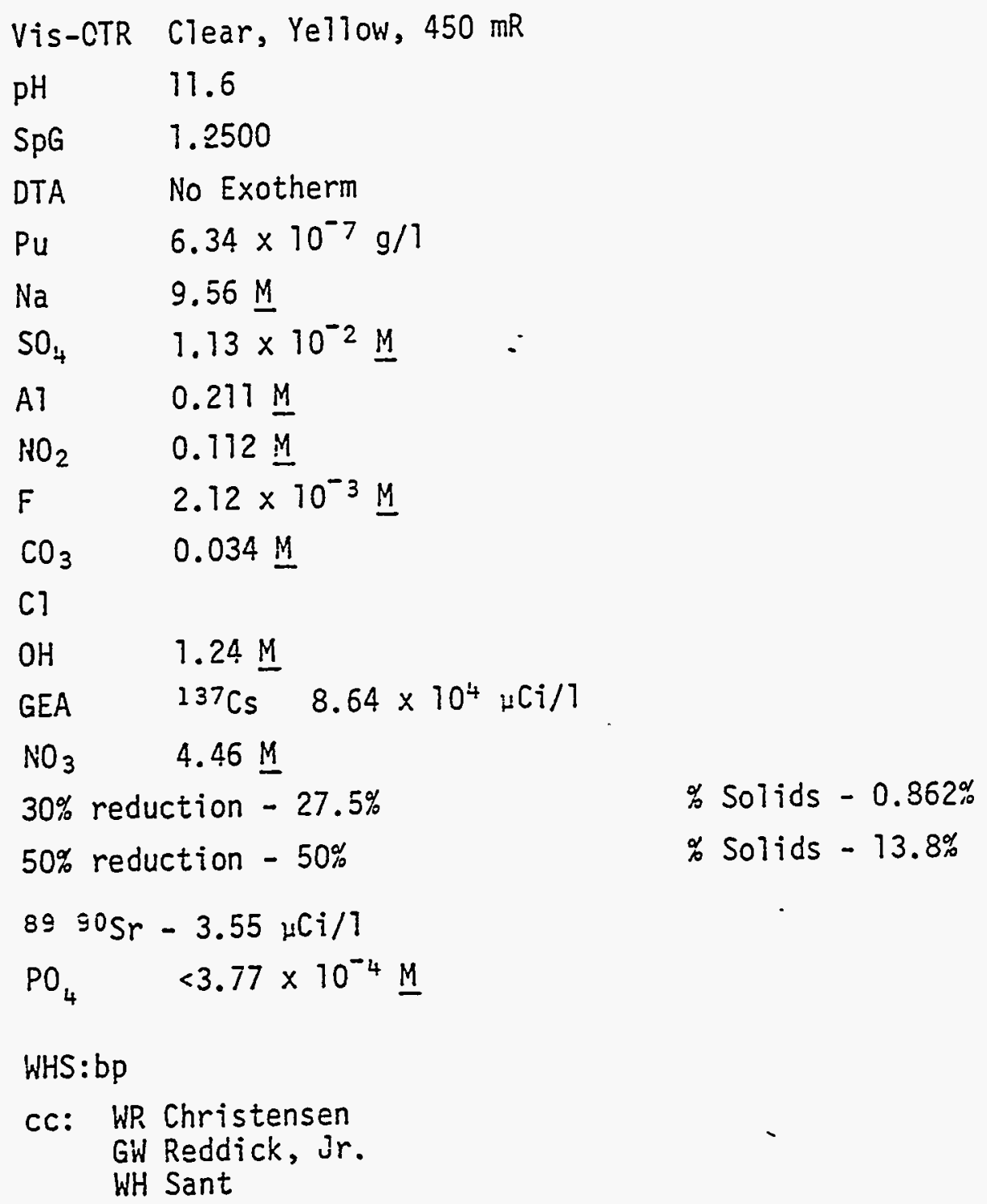


TCR.C $=4$ Atlantic Richtisld ianford Company

WHC-SD-WM-ER-323, Rev. 0

Date: January 21, 1974

To: R. L. Waiser

From: W. H. Sant $9: 2 \%$ Hiz

Subject: 242-S FEED SAMPLES NUMBER T-737 SAMPLE POINT I05-S
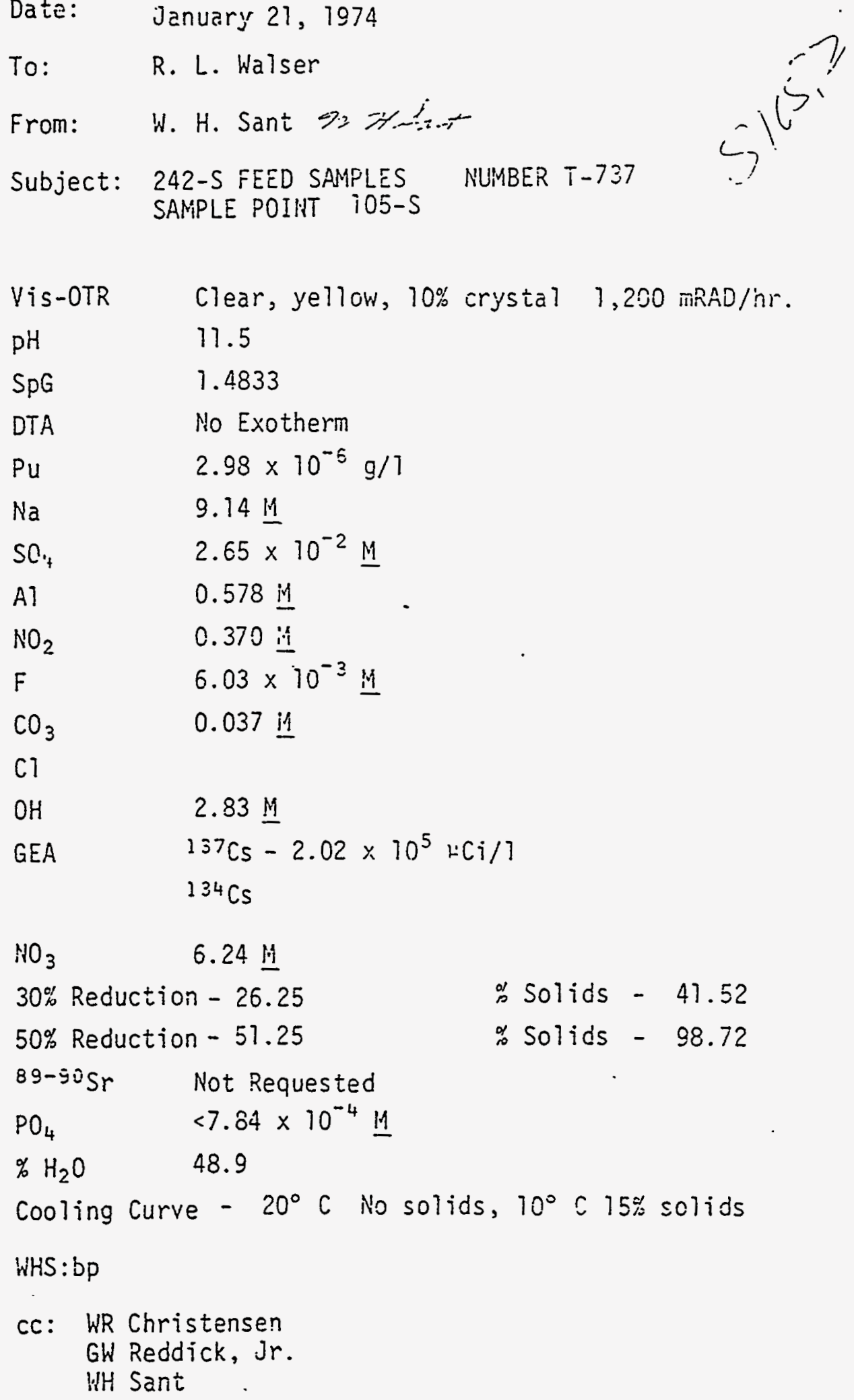
Date: March 13, 1974

To: M. H. Campbell

From: J. S. Buckingham JSBuckinghaud

Subject: ANALYSES OF SAMPLES FROM 242-S SLURRY RECEIVING TANKS

The first phase in the program to reduce the volume of residual, non-processable waste liquors was to analyze samples from three 242-5 slurry receiving tanks. The three tanks sampled were 105-S, 106-5 and 108-5.

The "as received" samples contained 14 to 16 weight. percent solids at room temperature. The volume percent solids was around 10 to 12 percent. Analys is of the solid phase from the 105-S sample indicated that it was essentially damp sodium nitrate. Analyses of the mother liquors showed that the water content ranged from 52 to 55 weight percent and the caustic content ranged from 15 to 18 weight percent. The concentration of other components in the waste are discussed in detail below. The curie percent cesium in the solid and mother liquor from $105-5$ was 14 and 85 percent respectively.

Solids were separated from mother liquors in the "as received" samples by vacuum filtering through $M$ porosity glass frits. Interstitial liquid was removed by pulling water saturated air through the solids for about ten minutes after visible liquidihad been removed. The liquid phases were analyzed and the results are shown in Tabie $I$. The weight percent of sotids and liquids in the "as received" samples are also shown in Table I.

The solids separated from the Tank 105-S sample were analyzed. The results are shown in Table II.

The distribution of ${ }^{137} \mathrm{Cs}$ between the riquid and solid phases from the 105-s sample was calculated based on analyses in Tables I and II. The results are shown below.

Solid $56.3 \mu \mathrm{Ci} / \mathrm{g} \times 0.158=8.90 \mu \mathrm{Ci}=14$ percent

Liquid $64.1 \mu \mathrm{Ci} / \mathrm{g} \times 0.842=53.97 \mu \mathrm{Ci}=86$ percent

Please call me if you have any questions regarding this work.

JSB:jf

Att.

$\begin{array}{lll}\text { cc: } & \text { W/Att. WP Metz } & \text { AE Smith } \\ \text { CH Delegard } & \text { DA Puryear } & \text { Process Aids } \\ \text { RD Fox } & \text { RC Roal } & \text { Extra (5) } \\ \text { INL Harms } & \text { WC Schmidt } & \text { LB } \\ \text { OP MCKnight } & \text { HP Shaw } & \text { File }\end{array}$


Table I

ANALYSIS OF LIQUIDS FROM TANKS 105-S, 106-S, AND 108-S

\begin{tabular}{|c|c|c|c|c|c|c|c|}
\hline & \multicolumn{2}{|c|}{$105-5$} & \multicolumn{2}{|c|}{$106-5$} & \multicolumn{2}{|c|}{ 108-S } & \\
\hline Wt.\& solid & \multicolumn{2}{|c|}{15.8} & \multicolumn{2}{|c|}{13.6} & \multicolumn{2}{|c|}{15.9} & \\
\hline Wt.\% liquid & \multicolumn{2}{|c|}{84.2} & \multicolumn{2}{|c|}{86.4} & \multicolumn{2}{|c|}{84.1} & \\
\hline \multicolumn{8}{|l|}{ Liquid Analysis } \\
\hline density & 1.462 & & 1.441 & & 1.429 & & \\
\hline $\mathrm{H}_{2} \mathrm{O}$ & & $51.8 \%$ & & $55.4 \%$ & & $55.2 \%$ & \\
\hline $\mathrm{NaAlO}_{2}$ & $1.69 \mathrm{M}$ & $9.5 \%$ & $1.26 \mathrm{M}$ & $7.3 \%$ & $1.04 \mathrm{M}$ & $6.0 \%$ & 㝴 \\
\hline $\mathrm{NaNO}_{2}$ & $0.99 M$ & $4.7 \%$ & $0.76 \underline{M}$ & $3.6 \%$ & $1.14 \mathrm{M}$ & $5.5 \%$ & , \\
\hline $\mathrm{VaNO}_{3}$ & i.95i & $11.3 \%$ & $2.32 \mathrm{M}$ & $13.7 \%$ & $2.30 \mathrm{M}$ & $13.7 \%$ & $\therefore=$ \\
\hline $\mathrm{NaOH}$ & $6 . .46 \mathrm{M}$ & $17.7 \%$ & $5.74 M$ & $14.3 \%$ & $5.16 \mathrm{M}$ & $14.5 \%$ & \\
\hline $\mathrm{Na}_{2} \mathrm{CO}_{3}$ & $0.073 M$ & $0.5 \%$ & $0.065 \mathrm{M}$ & $0.5 \%$ & $0.095 \mathrm{M}$ & $0.7 \%$ & \\
\hline $\mathrm{Na}_{2} \mathrm{SO}_{4}$ & $0.021 \mathrm{M}$ & $0.2 \%$ & $0.028 \mathrm{M}$ & $0.3 \%$ & $0.049 \mathrm{M}$ & $0.5 \%$ & \\
\hline$\therefore \mathrm{PO}_{4}$ & 0.029 证 & $0.3 \%$ & $0.017 \mathrm{M}$ & $0.1 \%$ & $0.016 \mathrm{M}$ & $0.2 ! !$ & \\
\hline Total & & $95.0 \%$ & & $95.2 \%$ & & $96.3 \%$ & \\
\hline${ }^{137} \mathrm{Cs}, \mathrm{Ci} / 1$ & 0.097 & & 0.356 & & 0.344 & & \\
\hline
\end{tabular}

Table II

ANALYSIS OF SOLID FROM TANK 105-S SAMPLE

\begin{tabular}{|c|c|}
\hline $\mathrm{H}_{2} \mathrm{O}$ & $13.1 \%$ \\
\hline $\mathrm{NaAlO}_{2}$ & $1.8 \%$ \\
\hline $\mathrm{NanO}_{2}$ & $1.1 \%$ \\
\hline $\mathrm{NaNO}_{3}$ & $78.6 \%$ \\
\hline $\mathrm{NaOH}$ & $3.6 \%$ \\
\hline $\mathrm{Na}_{2} \mathrm{CO}_{3}$ & $0.0 \%$ \\
\hline $\mathrm{Na}_{2} \mathrm{SO}_{4}$ & $0.0 \%$ \\
\hline $\mathrm{Na}_{3} \mathrm{PO}_{4}$ & $0.0 \%$ \\
\hline Total & $98.2 \%$ \\
\hline $137 c s_{5}$ & $56.3 \mathrm{wCi} / \mathrm{g}$ \\
\hline
\end{tabular}

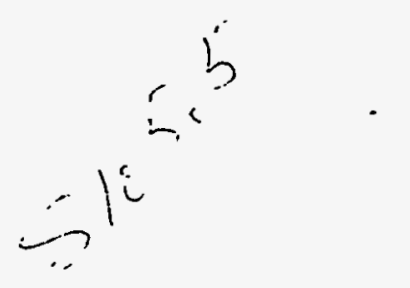


WHC-SD-WM-ER-323, Rev. 0

J. $\Xi$. $\because 0 x=0:$

Augus= 21, 1974

$$
\text { Aาhievemes: seวor: }
$$

\section{อUMMARIIS}

- Salt cake samples Erom tanks 105-s, loE-s, aid Ill-s.

o Theral consuctivities = 
DRAET

WHC-SD-WM-ER-323, ReV. O

J. $\Xi$. HO $= \pm 0$ 次

3u․25: 21, 1974

TANK FARY ASSISTAXCE

Samples $0 \equiv$ tie $5:$ : cakes in tanxs i0j-s, 106-s, and lli-s were taken. Ihese tanks are sluz=y receiving tanks or the 242-s evaporator. Solids iz tanks I05-s, I06-S, and III-S are prinazily sodiun hitrate. Details of laboratory work are aiscussed below. Aaliysis of tia solias and notier ijquors are sinow below.

mie IO5-s salt sample is composac of coanse yeIIow crystals. The yellow czystais are very dry iz appearazca. A weighed amount of 105-s solids was dissolved in $\dddot{z}_{2} 0$ a.a race to a volune of $25 \mathrm{mI}$. Anaiysis are showa in Taile

\section{TASIEI}

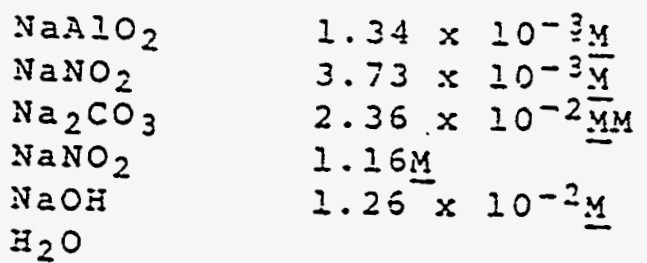

$0.11 \frac{2}{3}$

$0.25 \frac{3}{3}$

$2.54 \%$

100.38

$0.5 \%$

$\frac{3.43}{107.28}$<smiles>CCCCCCCCCC</smiles>

GES

$137 \mathrm{Cs}$

$125 \leq 3$

$$
\begin{aligned}
& 8.76 \times 10^{2} \sim \mathrm{C} i / 8 \\
& 2.20 \times \mathrm{C}=12
\end{aligned}
$$

3. 9 :Ci/g

$0.02 \mathrm{i} C \vdots / g$

$\begin{array}{ll}\text { As Received Density } & 1.25 \\ \text { Wet Density } & 2.79 \\ \text { NPS Density } & 2.52\end{array}$


DRAFT

WHC-SD-WM-ER-323, Rev, 0

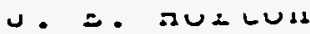

Augus $=2 i, 1974$

HYGROSCOPIDITY

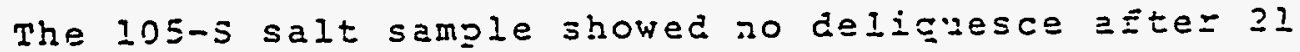

days of testing. Eercent gain after 22 Eays was 11.1

percent. This test was made in air saturated ilitio water vapor at $23-25^{\circ} \mathrm{C}$.

Thermal conductivity measurements $0 \equiv$ los-s salts Nere made. Table II reflects tie data.

\section{TABIE II}

THERMAL CONDUCTIVITIES OE IOJ-S SOIIDS

(DATA BY D. G. BOUSE)

As Received

Held Overnight Dried overaighe Overnigit

over Weekend

\begin{tabular}{|c|c|c|c|}
\hline 21.8 & ${ }^{\circ} \mathrm{C}$ & 0.251 & $3=U /: \leq / \equiv t^{2} / /^{\circ} \Xi$ \\
\hline 52.1 & ${ }^{\circ} \mathrm{C}$ & 0.247 & " \\
\hline 80.0 & ${ }^{\circ} \mathrm{C}$ & 0.235 & $"$ \\
\hline 114.9 & ${ }^{\circ} \mathrm{C}$ & 0.216 & " \\
\hline 156.0 & ${ }^{\circ} \mathrm{C}$ & 0.205 & $"$ \\
\hline
\end{tabular}

$21.8^{\circ} \mathrm{C}$

0.251

0.235

0.216

$156.0^{\circ} \mathrm{C} \quad 0.205$ 


\section{TABIE III}

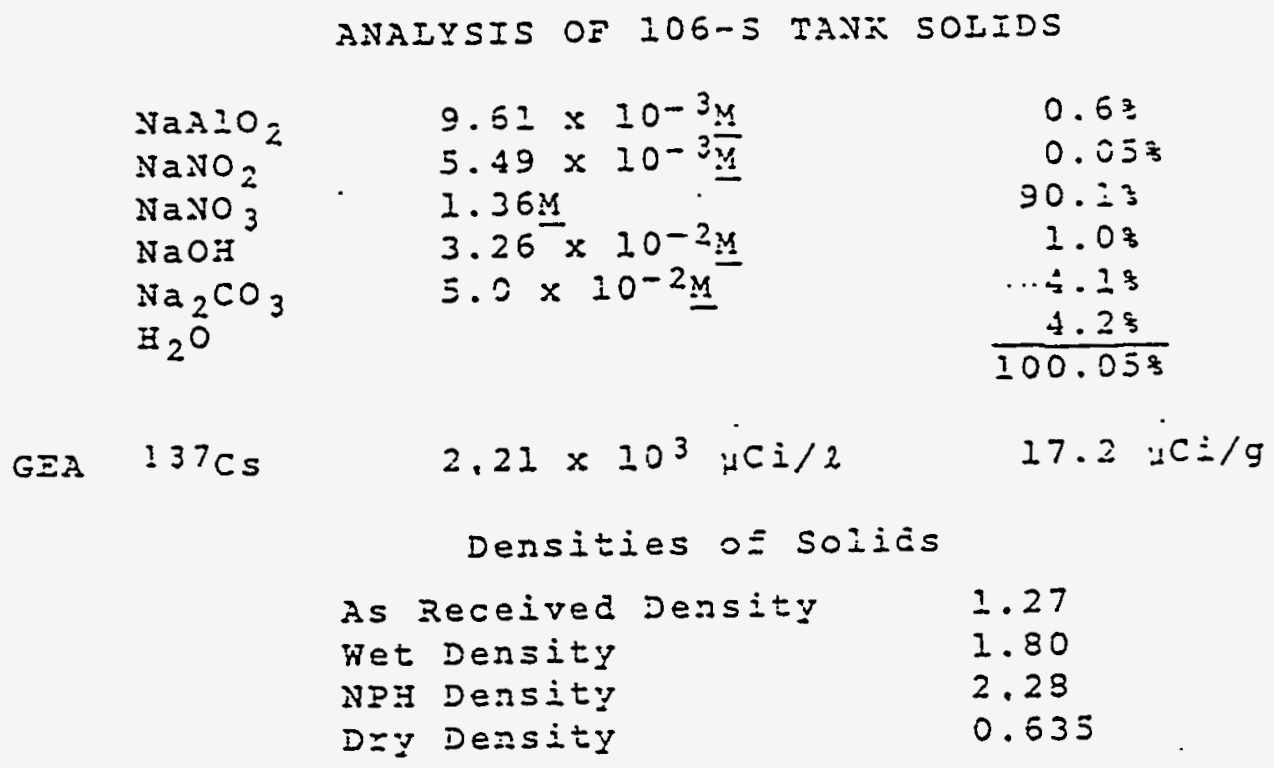

TABIE IV

AMAIYSIS OE 10E-S SUPERNATANM

\begin{tabular}{|c|c|c|}
\hline $\mathrm{NaAlO}_{2}$ & $1.36 \mathrm{M}$ & 3.98 \\
\hline $\mathrm{NaOH}$ & $5.33 \mathrm{M}$ & 24.93 \\
\hline $\mathrm{NaNO}_{2}$ & $0.056 \mathrm{M}$ & 3.2 方 \\
\hline $\begin{array}{l}\mathrm{Na}_{2} \mathrm{CO}_{3} \\
\mathrm{NaNO}\end{array}$ & $\begin{array}{l}1.36 \times 10^{-2} .1 \\
2.49 \mathrm{M}\end{array}$ & $\begin{array}{r}1.03 \\
14.83 \\
=3\end{array}$ \\
\hline$z_{2} \mathrm{O}$ & & $\frac{55.03}{9.9 .08}$ \\
\hline
\end{tabular}

Density $\quad 1.429$

-. GEก
13705
$3.39 \times 10 \quad \mathrm{C} i / 2$
$134 \mathrm{Cs}$
$9.1 I \times 10 \mathrm{HCi} / 2$ 
EYgYOSCO0icities of $106-5$ Salts

The 106-s salt sample. shows no deliquescence ajEer

It days of testiag. Percent gain in 14 days oz testing was 11.4 percent. This test was made in air saturated with water vapor at $23-25^{\circ} \mathrm{C}$.

phe ll1-s salts are a gayish in colol azd very sticky. Laboratory work was cone on boti tise superaatant and solia. A portion was aissolved in water and sent to labozatory Eoz analysis.

\section{TABIE V}

ANAIYSIS OE III-S SAITS

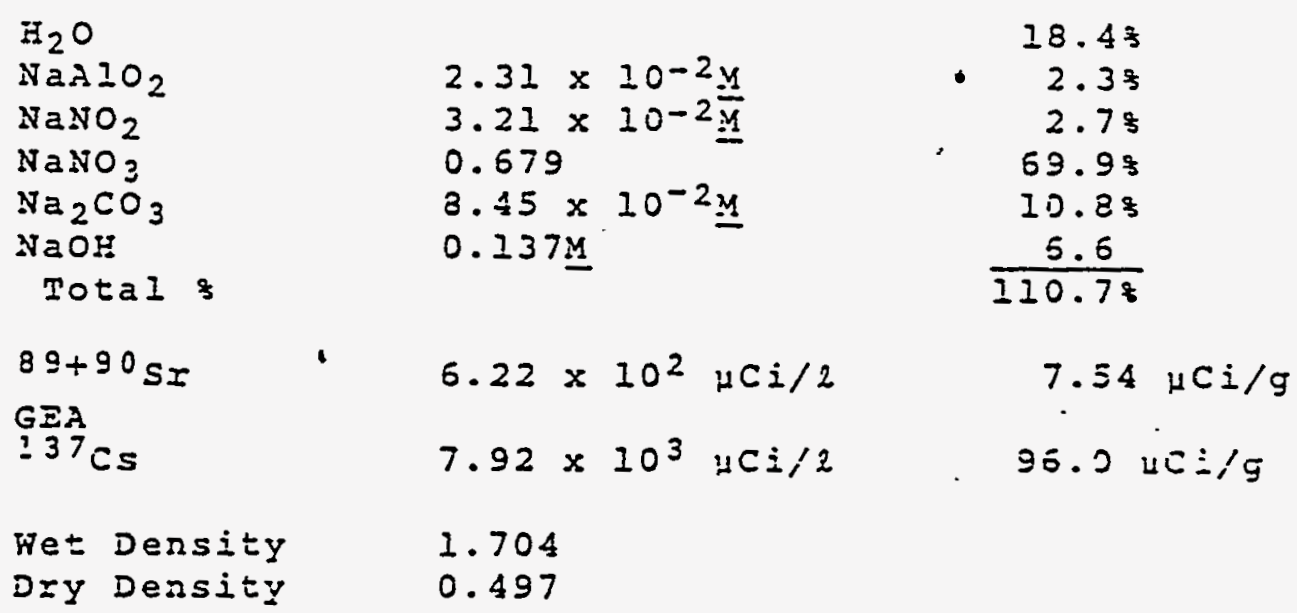



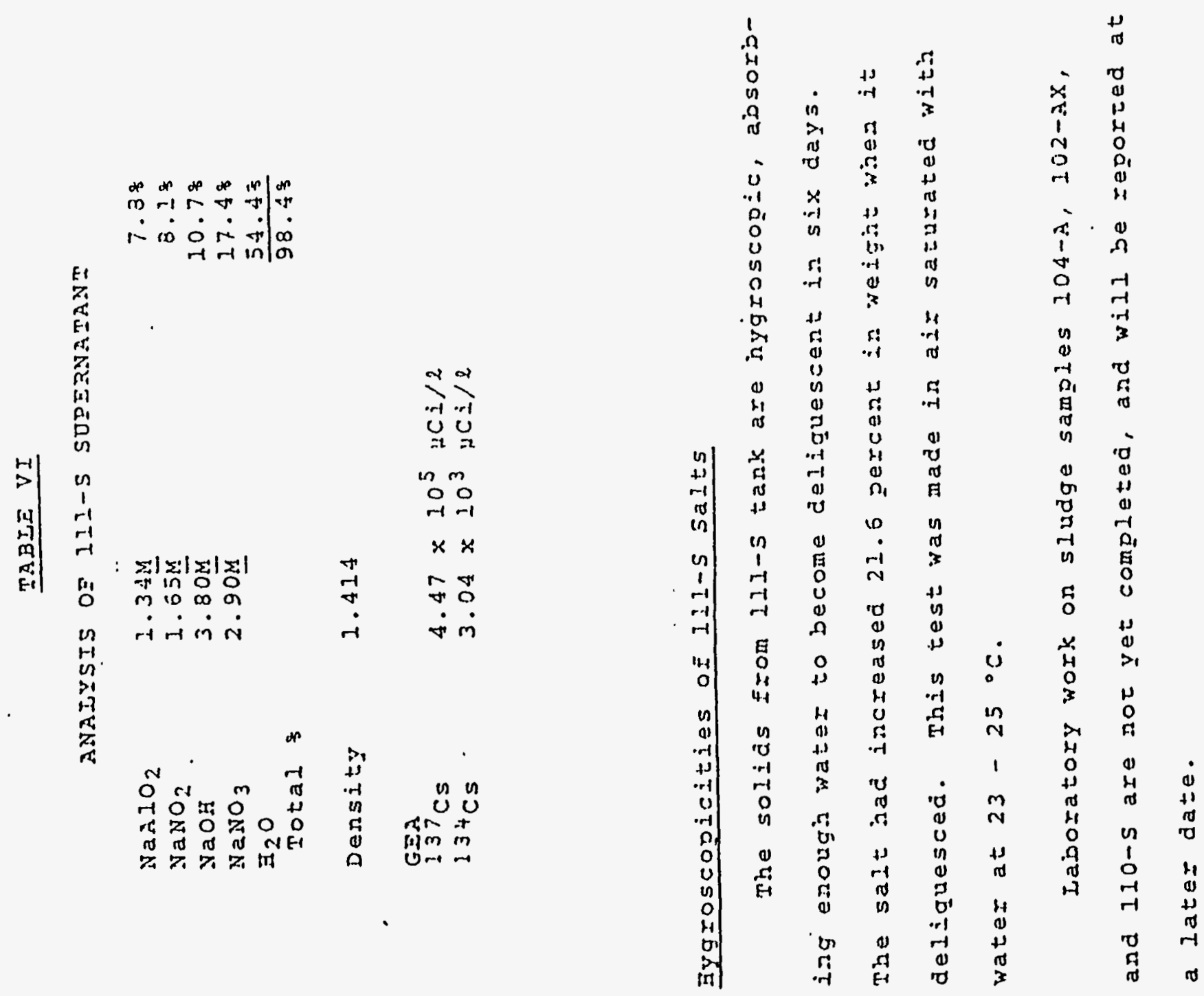
TCRC- 11

Atlantic Richfield Hanford Company
WHC-SD-WM-ER-323, Rev. 0

$$
105-5
$$

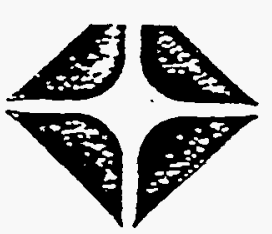

Date: September 5, 1974

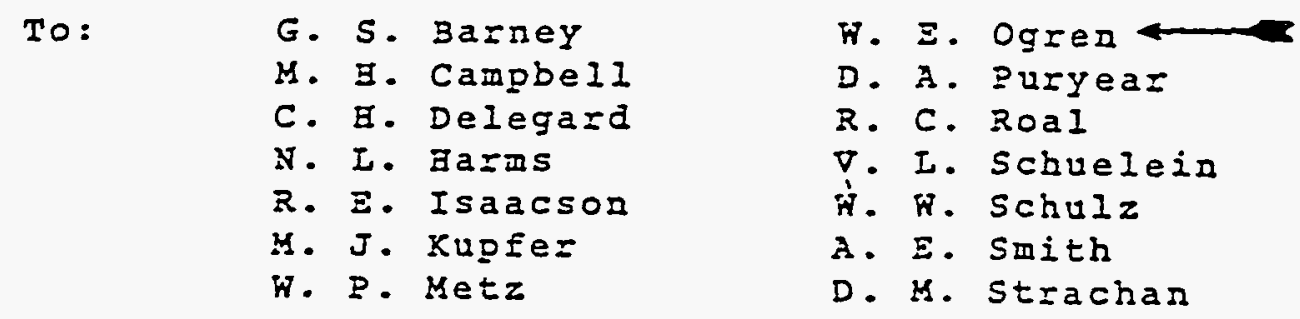

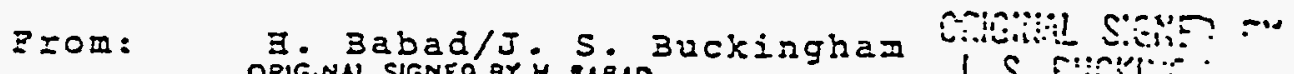

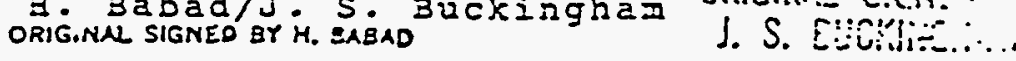

SUbject: ANAIYSES OF SOIIDIFIED SALT KASTES AND ASSOCIATED MOTHER IIQUORS

A number of samples of solidified salt wastes have been analyzed and reported in monthly zeports and technical letters. We thought it would be convenient and useful to accumulate these data on a simple form. Data for salt samples produced by the 242-s evaporator a=e included here along with notes discussing the analytical methods used.

The notes will be issued only once--the table will be updated weekly for current samples. Analyses of older samples will be added as time permits.

You should be cautioned that the data presented in this informal report may at times be of a preliminary nature. Please use discretion in using these data.

月 $3:$ J $33:$ e

Att. 
Za.nk No.

Date sampled

source of.

material

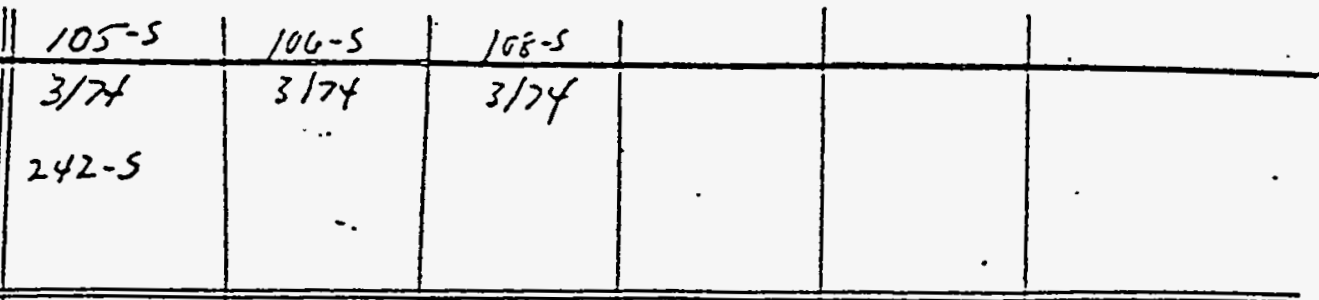

solid

3u1x Dexsity

Paftical Density

$\exists_{2} 0,{ }^{2}$

$\mathrm{NanlO}, 3$

$\mathrm{NaNO}_{2}, 3$

$\mathrm{NaNO}_{3}, 3$

$\mathrm{NaOE},{ }^{3}$

$\mathrm{Na}_{2} \mathrm{CO}_{3},+3$

$\mathrm{Na}_{2} \mathrm{SO}_{4}, 3$

$\mathrm{Na}_{3} \mathrm{PO}_{4},{ }^{3}$

$137 \mathrm{Cs}, 4 \mathrm{Ci} / \mathrm{g}^{3}$

$90^{2} \mathrm{x}, \mu \mathrm{CL} / \mathrm{g}^{3}$

$239 \mathrm{pu}, \mathrm{g} / \mathrm{g}^{3}$

\begin{tabular}{|l|l|l|l|}
- & & & \\
\end{tabular}

Bardiess, $\mathrm{Xg} / \mathrm{cm}^{2}{ }^{4}$

Thermal Conductivity

$50 \cdot \mathrm{C}$

$100 \cdot c$ damp

$100^{\circ} \mathrm{C}$ dry

Eeat Generation

Calculated H watts/g

Calorimeter

\begin{tabular}{|c|c|c|c|}
\hline Ilguid & $84.267 \%$ & $86.40+7$ & $84.1 \mathrm{wn.g}$ \\
\hline Permeab111ty & & & \\
\hline $\begin{array}{l}\text { Bygroscopicitydo } \\
\text { Deliquescenced }\end{array}$ & & & \\
\hline $\begin{array}{l}\text { iquid } \\
\text { Deag1ty, g/ce } \\
\mathrm{B}_{2} \mathrm{O}, \mathrm{P}\end{array}$ & $\begin{array}{l}1.42 \\
51.8\end{array}$ & $\begin{array}{l}1.441 \\
55.4\end{array}$ & $\begin{array}{l}1.429 \\
55.2\end{array}$ \\
\hline $\begin{array}{l}\mathrm{NaAlO}_{2}, \\
\mathrm{NaNO}_{2},\end{array}$ & $\begin{array}{l}9.5 \\
47 \\
\end{array}$ & $\begin{array}{l}7.3 \\
3.6 \\
\end{array}$ & $\begin{array}{l}6.0 \\
5.5\end{array}$ \\
\hline $\begin{array}{l}\mathrm{HaNO}_{3}, \\
\mathrm{NaOB}_{1}\end{array}$ & $\begin{array}{l}1 / 3 \\
177\end{array}$ & $\begin{array}{l}13.7 \\
14.2\end{array}$ & $\begin{array}{l}13.7 \\
14.5\end{array}$ \\
\hline $\begin{array}{l}\operatorname{ma}_{2} \mathrm{CO}_{3}, \\
237 \mathrm{Cs}, H \mathrm{C}_{1 / 2}\end{array}$ & $\begin{array}{l}0.5 \\
9.7 \times 0^{4}\end{array}$ & 0.5 & $\begin{array}{l}0.7 \\
3.54 \times 10^{5}\end{array}$ \\
\hline $9055,401 / 2$ & & & $\begin{array}{r}1 \\
H-341\end{array}$ \\
\hline
\end{tabular}




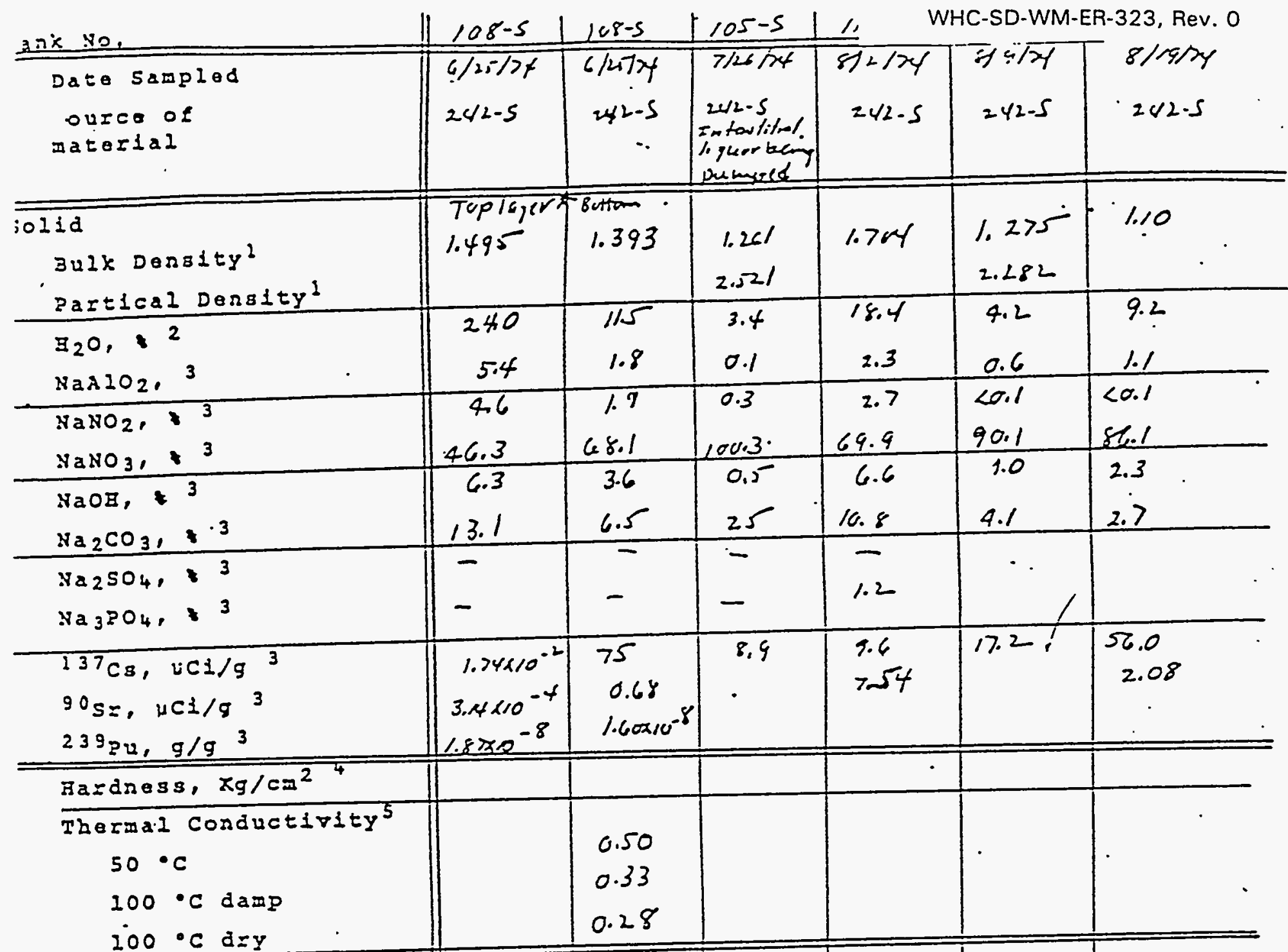

Heat Goneration
Calculated $\mu$ watts/g
Calorimeter

$=$ Iiquid
$=$ Permeability
Hygroscopicity

İqu1d

Dozs1ty, G/CC

$\mathrm{B}_{2} \mathrm{O}$,

$\mathrm{NaA1O}$,

$\mathrm{NaNO}_{2}$,

$\mathrm{KaNO}_{3}$,

$\frac{\mathrm{NaOB}_{1}}{\mathrm{Na}_{2} \mathrm{CO}_{3},}$

$137 \mathrm{Cs}, \mu \mathrm{Cl} / \mathrm{L}$

$905=, \mu C 1 / 2$

$239 \mathrm{gu}, g / 2$

0.36

1

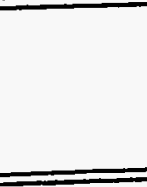


HOTES

Samples of solidified salt waste will, in geatral, be received as a damp salt. All tests and geasuremente will be made or the sample as received. Jowever, if free (Dourable or drainable) Iiquid is observed on the sample, the solid and liguid phases will be separated by vacuum filtration using an "H" porosity fritted glass fungel. Air will be pulled through the crystals until no more liquid is observed axipping into the Elask, then for ten minutes longer. A pad of roistered cheese loth placed over the top of the Eilter will prevent the solid ghase Exom alying out.

in those samples that are extremely slow filtering, phase separation may be made usiag a Millipore(Q) pressure filter equipped with a plungez. phase sepazations should be inade at 25 to 28 psig. 
WHC-SD-WM-ER-323, Rev. 0

1. Bulk density is determined by weighing one to three grams in a graduated 25 mI centrifuge cone. xda exactiy 3 mI r-parafeis hyarocarbon (NPH) to wash crystals into the cone. Centrifuge in a cinical centrifuge at high speed for 15 minutes to pack solid in cone. Note polume of solid and polume of solid DIUS NPH.

$$
\begin{aligned}
& \text { BuIx density }=\frac{\text { wt. of solid }}{\text { volume of solid }} \\
& \text { Paxticle density }=\frac{\text { wt. of solid }}{\text { (Yolume of solid NaB - }}
\end{aligned}
$$

2. Percent water is determined by drying a weighed portion of the sample in a vacuum over at $100^{\circ} \mathrm{C} / 252 \mathrm{Im}$ Ig, absolute, to constant weight.

3. Chemical and radiochemical analyses will be made by dissolving about a five gran weighed portion of the solid phase in 20 milliliters of hot water, cooling and dilutiag to 25 millilitezs. If greater than one percent of the salt is insoluble in water, =emove the water soluble fraction and aissolve the water insolubles with $6 \mathrm{~N}$ HCl. Any remaining acid insoluble fxaction will be fuzed with xor.

The water soluble fraction will be analyaed for alumian by atomic absorption. Aluminum in the water soluble fraction is assumed to be as $\mathrm{NaAlO}_{2}$. SOdiun 
attzlte id the water soluble fraction is determined by a colorometric procedure. Sodium nitrate is measured using a nttrate specific fon electrode. sodium hydroxide is detezmined by titzating tho sample with standard acia to the zirst $D E$ break wich occurs around $2 \pi$ 10.5. Sulfate is determined by reduction to sulfide and measuripg the concentration with a sulfide specific ion electrode. Phosplate is determined spectrophotometzicaliy. Carbonates aze determined by acidifying the solution celeasing $\mathrm{CO}_{2}$ witich is absorbed on Ascarite?.

4. razdness is determined with a pocket penetroneter Model Ci-700 made by soiltest, Inc., Chicago, Illinois. As much as possible hardness siould be made on an uadisturbed sample.

5. Thermal coñductivity tests will be made at $50^{\circ} \mathrm{C}$ and at $100^{\circ} \mathrm{C}$. A second measurement at $100^{\circ} \mathrm{C}$ will be rade after tine sample has been allowed to dry. Thermal conductivity is reported in $3 T u / h z / E t^{2} /{ }^{\circ}$.

6. Heat generation can be calculated from the fission froduct content of the sample and also directiy by measuring it in the microcalorimeter.

7. Percent liquid is determined by pressing a pozion of tha "as received" sample in a Hillipore pressure 
fliter at $25-28 \mathrm{psig}$. If the sample is a slurry, the percezt liquid of the wet cake will be calculated on the vacuum arained sample uniess otherwige noted.

8. A method jor permeability of salt cake has not been. developed as yet. It is being cereloped.

9. On those samples that have "free Ilquid" removed by vacuum araining or by pressure filtering, the separated liguid will be analyzed. Analytical nethods are the same as with the water soluble solid fraction. Results will be reported in weight percent rather than nolaxity.

10. Hygroscopicity is reported as the weight pezcent increase of a salt placed in a cabinet with relative. humidity of 70 to 80 percent and temperature of 22 to $25^{\circ} \mathrm{C}$ for 21 days. If during that period of tine Eree "flowablen liquid is observed on the sample, the sample is consiared to have deliguesced. 
Eate: August 27, 1974
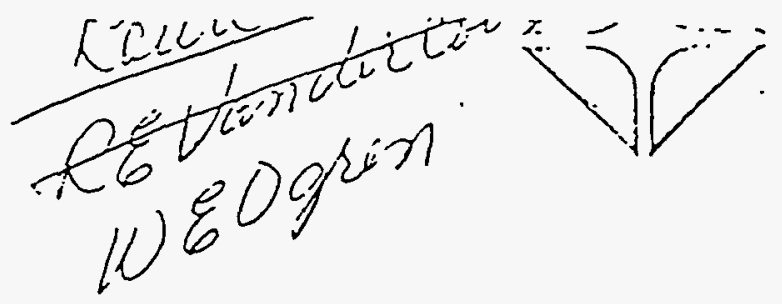

To:

R. I. Ua]ser,

Froin:

WRCOiristinter

SUDjERt: SLUEGE SAMPLEIG STATUS

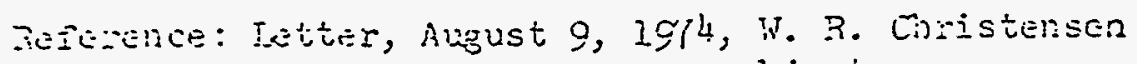

to R. T. Falser, same subject

Sludie sampling was temporarily suspenced to alzow CTI latoratory porsonel to complete processirg a $\vec{i}$ existing asmpies and to clean out the not cell prior to leceiving acoitional slucge seriples. jot cell work will resuie on

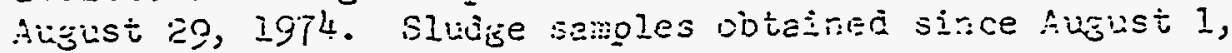
l974 are listed in Table $I$.

Calibretion or the calorivetry apiaratus is expected to be completed in the near îture and heat fencration rates issed on caiorimetric data are expected to be available guring the reek of septemicer 2, igit.

Although no proyress has been mece to iate, the ievalop-" irent of a leburatory procisure to provice "Eelting point" ipta sinould be vieprously pursued since mel ting ciariatueistics are a critical consicicration in the safe stonae or cuaporator salt ca'ee. It is recojinized that the jalt calie probety coes not have a discrete meling point, however the tempereture at inich free licuid inst fors is of prinary interest in teras oi salt cake stjracue.

Thermal conouctivity c̀ata for tank $241-S-105$ salt cake are listed in Table II. The low (0.251 biu/nr. It 0 o $\left./ \mathrm{ft}^{t}\right)$ thermal conouctivity observed at arabient temperature is significant since this sample represents sait cake drained by salt well pumping. These results confirn that the large reduction of thermal concuctivity ocserved on previous semples vas primarily due to evaporation of moisture ratiner than the temperature rise alone, and that a significant reduction in salt cake thermal conductivity will occur when the liquid cover and interstitial licuors are reroved from the salt cake. 
R. T\% Blaser

Page 2

Aleist 27, 1974

Adoitional equipment has been set up to allo: ther...l condictivity analyses of soil samples to be periorned concurrently with sludge and salt cake scmies. Analyses are to be periormed on eleven composite serples representing typical 24l-sX ferm soils. Initial runs at aricient temperathre heve begun.

Ievelopacht of vapor puessure velulis temperature cata for 2li2-s salt care is propessing satisfactorily as are the

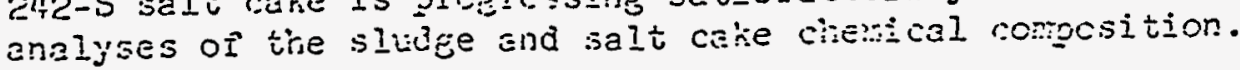

Those letters, issued since August 0 , Igft, that relite to ine slude cieracterization profrem, are listso below.

ihisuti 15, ig74, W. R. Coristensen to J. A. Seal, "Tank

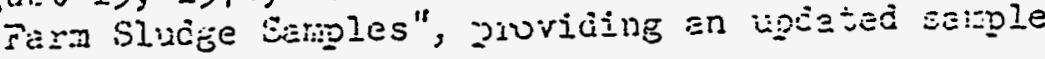
prioricy iist.

Sugust 16, I97h, N. I. Taras to R. I. :Elser, "Isboratory Glucge Cneracteriation", stating that no sairoles will be loaked into hot cell until existing semples are taken care of and hot cell is cleared. out, and reguesing inoroved procecurel assurence tiat the sainle casis is properly ceconteninated.

$\mathrm{BNC}: j$

Aㄴt. (2)

cc: Wh Christensen

CD Compton, $A \bar{E} C-R L$

DG Nariow

NL Farios

GC Oberg

RC Roalcken

Ris Snithers

CP Sutter

JA Teal

JC Worack

LB

.. 
WHC-SD-WM-ER-323, Rev. 0

\author{
TAA BIE I \\ STudre Ssmple Slumirary \\ (since $8-1-7^{4}$ )
}

\begin{tabular}{|c|c|c|c|}
\hline$T \leq n K$ & Sate Obtained & Iate inguesteo & Connents \\
\hline $106-5$ & $2-1-74$ & $7-23-74$ & Good sazple \\
\hline $102-A X$ & $8-7-74$ & $10-4-73$ & rood sampie \\
\hline$I I C-S$ & $8-9-74$ & $6-4-74$ & Good simple \\
\hline $203-5 X$ & $8-i:-74$ & $3-22-74$ & $\begin{array}{l}\because 0 \text { solics - } \\
\text { iill reguire } \\
\text { iesangle }\end{array}$ \\
\hline I0P-S & $8-20-74$ & $8-15-74$ & $\begin{array}{l}\text { Cood sicrole - } \\
\text { iull core }\end{array}$ \\
\hline $101-S X$ & $8-23-74$ & $3-22-74$ & Still in casis \\
\hline
\end{tabular}




\section{TABI.E II}

leat Ceneration/Thernal Conductivity Data

Tinik

$2 C 5-5$

$\operatorname{ing}-2 x(200)$

$102-5 \times\left(200^{2}+0 \mathrm{n}\right)$

$10 !-2 x(T 02)$

$10-5 X(30 \div \operatorname{tom})$$$
\text { icB-s (-op) }
$$

ICB-S (Sottom)

$107-5$

.18 .5

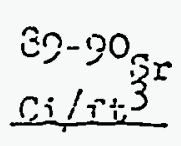

.110

$3 . \overline{3} \mathrm{Cs}_{\mathrm{S}}$

ci/isi

$2.73 \times 10^{-7}$

${ }_{38.8} \vdots \vdots$

$22.6=$

$3.5 \times 10^{-4}$

71.6

3.65

12.8

6.29

3.0

1.72

1.11

Sieat

Dtu/ins

Themal conducilvity

Talue Tengerature

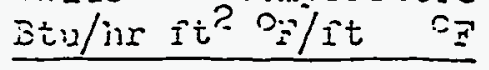

.00593

$--$

.678

$.0: 3$

$.6 \div 5$

.338

.313

$1.01 \times 10^{-5} \cdot \quad 5.60 \times 10^{-4}$

.024

1.52

.052

.547

.279

. $โ$ ?

27

$3: 6$

\section{$\therefore .$.}

$.2: 52$

$.4 \pm 3$

$.5 \% 0$

.103

.ธิร

77

8.6

$\therefore 75$

沪4

110-U

26.2

1.62

0.317

105-S

-

30.9
0.317

2.7
.635

.005

$$
\begin{array}{ll} 
& .251 \\
. & .235 \\
.216 \\
.205
\end{array}
$$

.76
110-S 


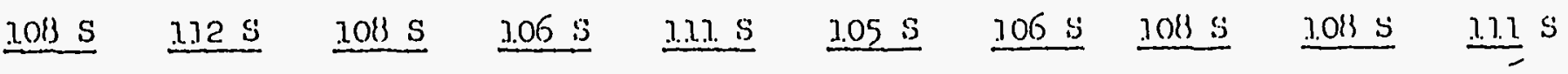

Sumple Date

\section{$5 / 20 / 7 h_{1} \quad 5 / 21 / 7 h \quad 6 / 30 / 7 h$}

Dale Analyses Reported

$7 / 8 / 74$

Nilajyses, Welght Percent

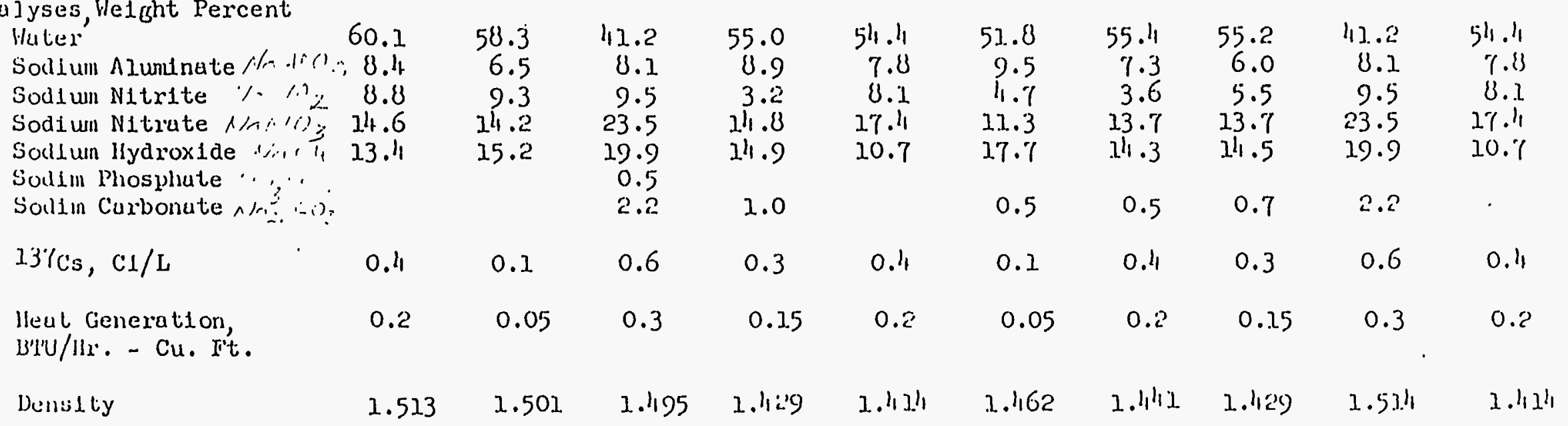

$\underset{\dot{\omega}}{\mathcal{G}}$

$\stackrel{\omega}{\rightarrow}$

\section{Sumple Date}

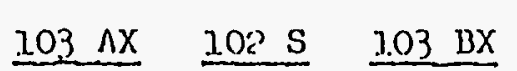

$8 / 9 / 7 h \quad \ldots \quad 3 / 3 / 75 \quad 3 / 27 / 75$

Dale Analyses Reported

9/5/74 10/24/\%3. 3/26/75 3/2\%/75

Ninujyses, Helght Percent

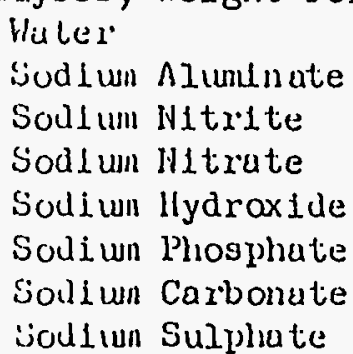

$237 \mathrm{Cs}, \mathrm{Cl} / \mathrm{L}$

lleal Generation, $\left\|^{\prime \prime \cdots} \cdot 1\right\|_{1} .-\mathrm{Cu}$, lic.

$\begin{array}{rr}55.0 & 81.2 \\ 8.9 & 0.2 \\ 3.2 & 8.1 \\ 14.8 & 1.0 \\ 14.9 & 2.7 \\ & 0.2 \\ 1.0 & 3.6 \\ & 2.1\end{array}$

0.3

2.2

$2 . ?$

0.6

$0.15 \quad 1.0$

0.6

0.3 
WHC-SD-WM-ER-323, Rev. 0

ת

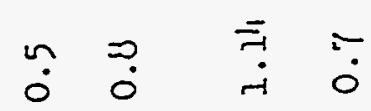

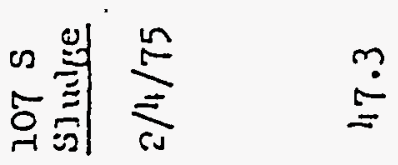

$\Rightarrow \underset{1}{0}$

$\begin{array}{lll}\overrightarrow{5} & \stackrel{0}{\vec{i}} & 1\end{array}$

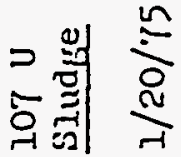

:

$\ddot{\infty}$

$\ddot{0} \quad \stackrel{\vec{H}}{\dot{H}} \quad 1$

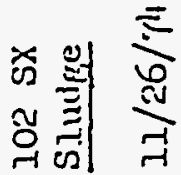

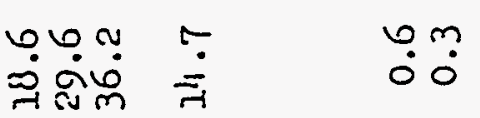

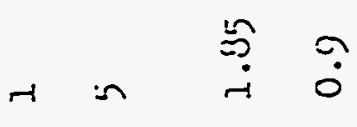

造羿

i

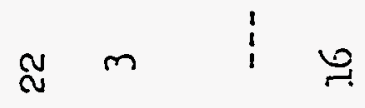

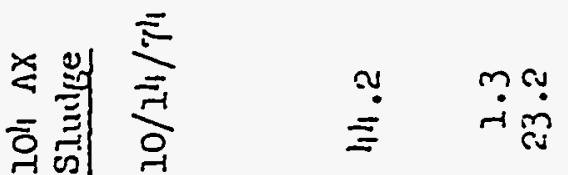

$m \approx \stackrel{\tilde{b}}{i} \approx$

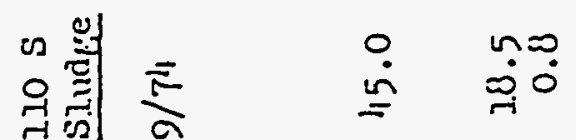

$\rightarrow \quad \dot{0} \quad \stackrel{n}{i} \quad \div$

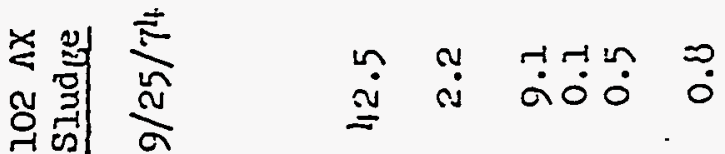

$a \rightarrow \stackrel{i n}{i} 6$

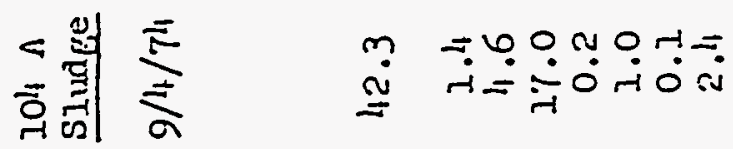

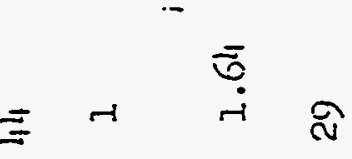

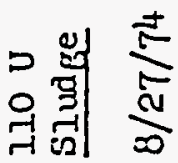

$\stackrel{m}{\equiv}+m$

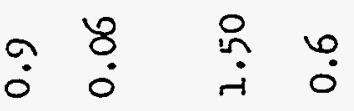

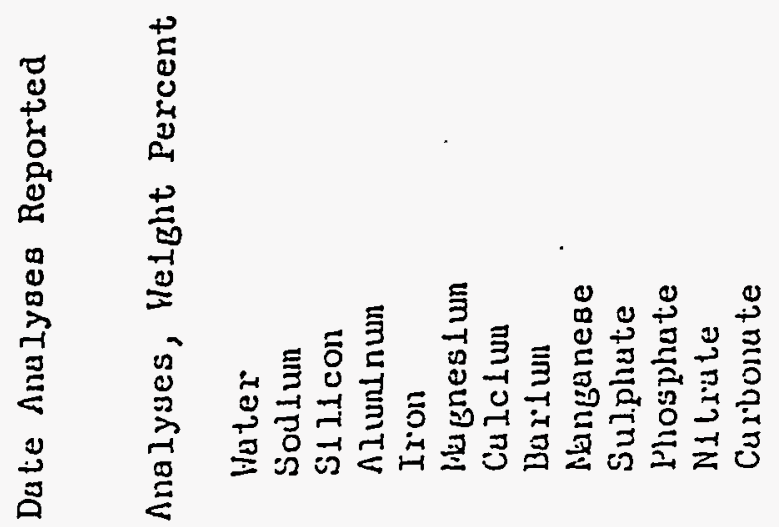

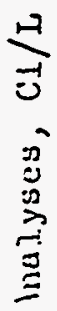

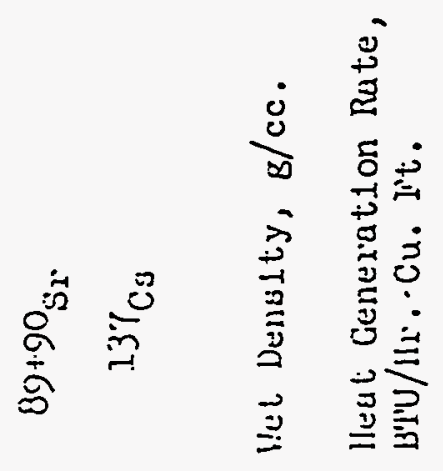


TCRC-2

Atiantic Richfield Hanford Company

WHC-SD-WM-ER-323, Rev. 0

$\checkmark \ll \omega \cdot 1$

$0\{:=5-9$

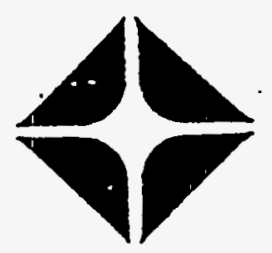

Date: Septembe: 21, 1971

TC:

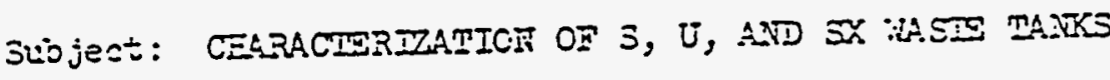

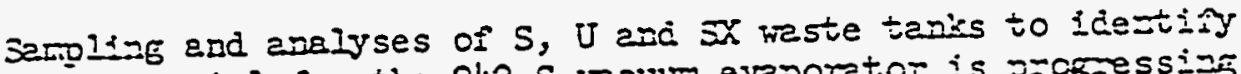

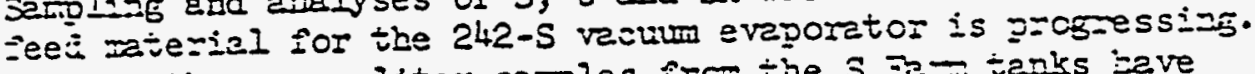

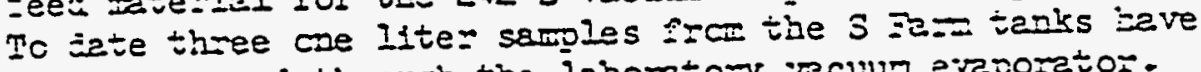
been processed tinrough the labcretory rechum eveporato:. If adciticn tel $S$ Farm tanks and six 0 Fent tanks heve been seruled and subritied for ciezacterization arelyses. Tie zesults are show in tine pollowias tejle.

Cozzecterizaticn Anzlyses co iveste Zanks

\begin{tabular}{|c|c|c|c|c|c|c|c|c|c|}
\hline בI:.sic. & JpG= & $\begin{array}{l}\text { Visc. } \\
\text { so oc }\end{array}$ & $\mathrm{CO}_{3}$ & $\mathrm{XO}_{3}$ & $\mathrm{NC}_{2}$ & MAD & $A I$ & iva & $\begin{array}{l}23 \mathrm{CS}^{2} \\
\mathrm{uC}=1 \mathrm{~m} \\
\end{array}$ \\
\hline $101-3$ & 1.4 .22 & 2.23 & .017 & 3.32 & $.6 \pm 2$ & 3.52 & $1 . \div 1$ & $\frac{0.01}{8.48}$ & $\begin{array}{l}135 \\
124\end{array}$ \\
\hline $1 \approx 2-3$ & 7.317 & 1.12 & .064 & $\begin{array}{l}5.92 \\
4.80\end{array}$ & .126 & $\begin{array}{l}2.15 \\
1.35\end{array}$ & $\begin{array}{l}.24 \\
.28\end{array}$ & 8.57 & IOL \\
\hline $\begin{array}{l}103-3 \\
i 05-3\end{array}$ & $\begin{array}{l}1.279 \\
1.270\end{array}$ & $\begin{array}{r}.95 \\
1.05\end{array}$ & $\begin{array}{l}.020 \\
.0644\end{array}$ & 3.15 & .237 & $2 . \pi 2$ & .20 & 5.86 & $\ldots$ \\
\hline $\begin{array}{l}105-3 \\
106-5\end{array}$ & $\begin{array}{l}1.272 \\
1.263\end{array}$ & $\begin{array}{r}1.07 \\
.94\end{array}$ & .011 & 4.150 & $.160^{\circ}$ & 1.38 & .17 & 5.07 & $37 \cdot 9$ \\
\hline บละ-5 & 1.291 & 1.10 & .25 & 3.61 & .108 & 2.16 & . 30 & $6.5^{1}+$ & $\cdots$ \\
\hline iڤ9-5 & $1.2 T 2$ & 1.00 & .04 & 3.06 & $.096^{\circ}$ & 1.38 & $.27:$ & $\frac{4}{6} \cdot 52$ & 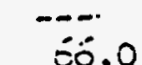 \\
\hline $110-3^{*}$ & 1.269 & 1.15 & $\cdot \frac{12}{\sim 5}$ & 3.4 & $\begin{array}{l}.084 \\
30\end{array}$ & $\begin{array}{l}1.93 \\
1.92\end{array}$ & $\begin{array}{l}.30 \\
.499\end{array}$ & $\begin{array}{l}6.70 \\
8.110\end{array}$ & 51.5 \\
\hline$\frac{111}{112}-5^{*}$ & $\begin{array}{l}1.210 \\
1.2002\end{array}$ & $\begin{array}{l}.95 \\
.92\end{array}$ & $\begin{array}{l}. \approx 25 \\
.073\end{array}$ & $\begin{array}{l}3.4 \\
3.5\end{array}$ & .30 & 1.38 & .40 & 5.37 & $57 \cdot 9$ \\
\hline$\frac{172}{200}$ & $1 \cdot C C<$ & •プ & .036 & $\ldots$ & .231 & -- & .25 & 1.59 & $\cdots$ \\
\hline U-U & - & - & .065 & & .097 & - & .12 & 1.93 & $\cdots$ \\
\hline
\end{tabular}

* Raz in lejooratory vacurr evepcretcr.

The ebove anelyses appear notpel except sepole 101-s.

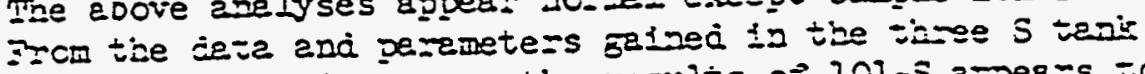
se=ple evepcriticn Jus, the resulis of lol-s epper=s to

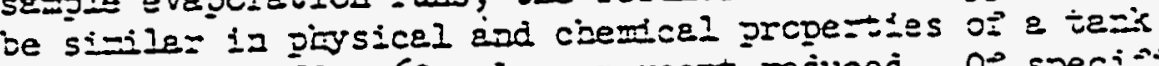

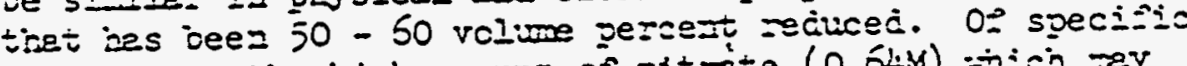

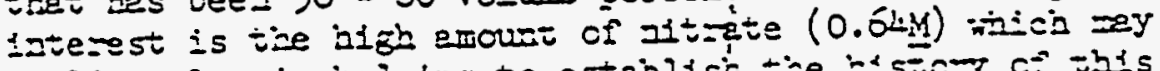

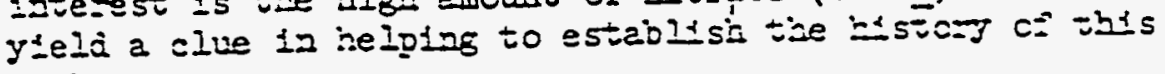
tank. 
3

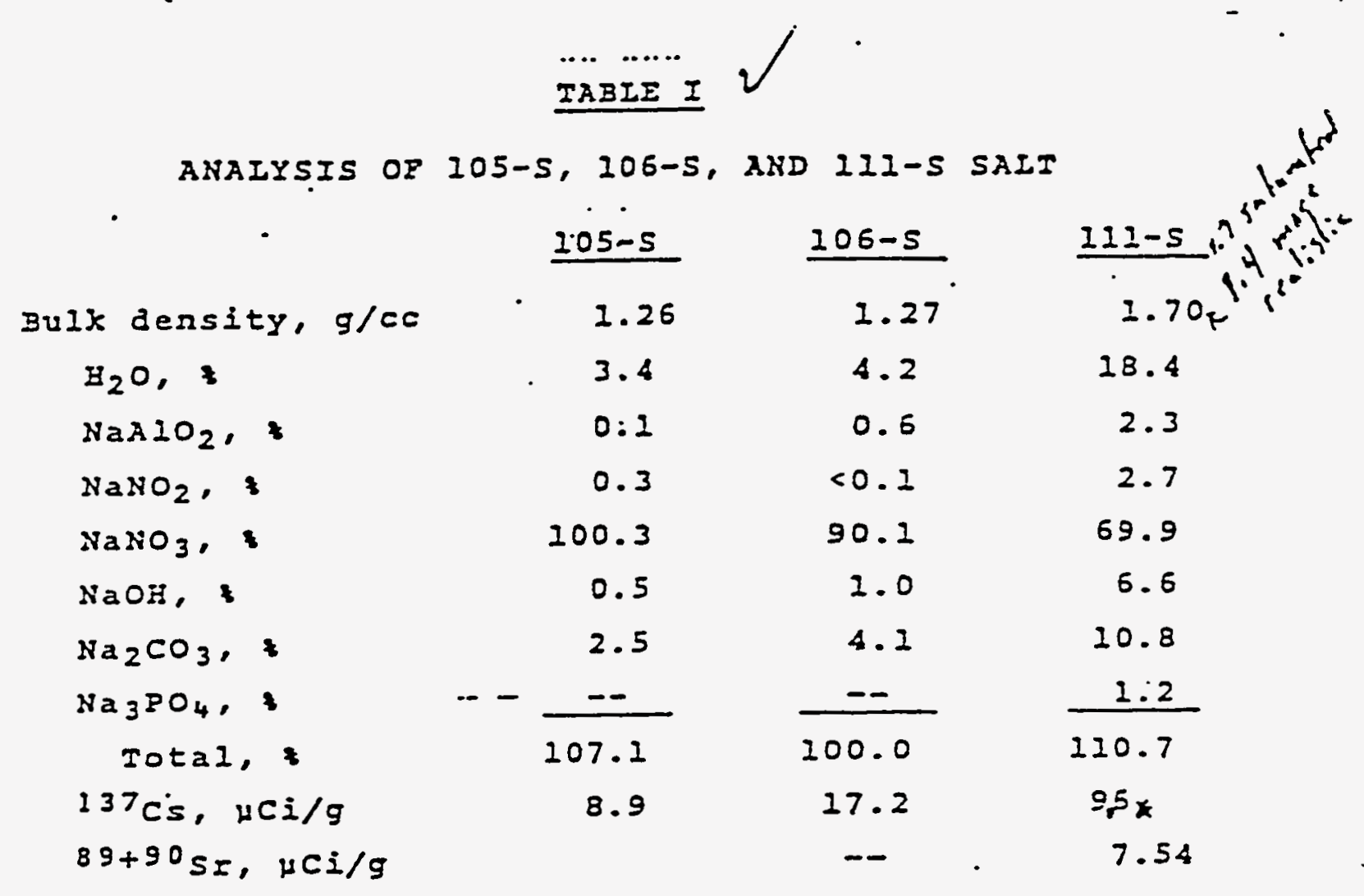


TABIE II

ANAIYSES OF 106-S AED III-S MOTHER IIQUOR

\begin{tabular}{|c|c|c|c|c|}
\hline \multirow[b]{2}{*}{ Density } & \multicolumn{2}{|r|}{$106-5$} & \multicolumn{2}{|c|}{$111-5$} \\
\hline & 1.429 & $g / c=$ & $1.414 \mathrm{~g} / 00$ & \\
\hline $\mathrm{B}_{2} \mathrm{O}$ & & 55.0 & . & 54.43 \\
\hline $\mathrm{NaALO}_{2}$ & $1.56 \underline{\underline{\mu}}$ & 8.94 & $1.34 \mathrm{M}$ & 7.81 \\
\hline $\mathrm{XaSO}_{2}$ & $0.66 \underline{M}$ & 3.23 & $1.65 \underline{H}$ & 8.11 \\
\hline $\mathrm{NaNO}_{3}$ & $2.49 \underline{\underline{4}}$ & 24.81 & $2.90 \underline{1}$ & 27.41 \\
\hline NaOB & $5.33 \mu$ & $14.9 t$ & $3.80 \underline{\underline{\mu}}$ & 10.71 \\
\hline $\mathrm{Na}_{2} \mathrm{CO}_{3}$ & $0.01 \underline{M}$ & 1.08 & & \\
\hline Total & & $-\cdots 97.81$ & & 98.41 \\
\hline $137 \mathrm{Cs}$ & $3.39 \times$ & $10^{5} \mathrm{HCI} / \mathrm{l}$ & $4.47 \times 10^{5}$ & $u C i / 2$ \\
\hline
\end{tabular}


Date: $\quad$ December 7, 1973

To: R. L. Walser

From: W. H. Sant 2 -

Subject: 242-S FEED SAMPLES T-9492 (10/5/73) 106-S

Vis-TOR Clear, yellow, $400 \mathrm{mR}$

$\mathrm{pH} \quad 11.6$

SpG $\quad 1.2262$

DTA No Exotherm

$\mathrm{Pu} \quad 1.74 \times 10^{-6} \mathrm{~g} / 1$

$\mathrm{Na} \quad 8.32 \underline{\mathrm{M}}$

$\mathrm{SO}_{4} \quad 1.10 \times 10^{-2} \mathrm{M}$.

A7 $\quad 0.195 \underline{M}$

$\mathrm{NO}_{2} \quad 0.165 \underline{\mathrm{M}}$

$\mathrm{F} \quad 1.79 \times 10^{-3} \underline{\mathrm{M}}$

$\mathrm{CO}_{3} \quad 0.074 \mathrm{M}$

$\mathrm{Cl}$

$\begin{array}{ll}\mathrm{OH} & 1.28 \underline{\mathrm{M}} \\ \mathrm{GEA} & { }^{37} \mathrm{Cs}-7.98 \times 10^{4} \mu \mathrm{Ci} / 1 \\ \mathrm{NO}_{3} & 4.16 \underline{\mathrm{M}}\end{array}$

$30 \%$ reduction -28.8

$50 \%$ reduction - 53.8

$\%$ Solids $-0.526 \%$

$8990 \mathrm{Sr} \quad 1.05 \mu \mathrm{Ci} / \mathrm{I}$

$\mathrm{PO}_{4}$

$<3.77 \times 10^{-4} \mathrm{M}$

$\%$ Solids - $63.51 \%$

WHS:bp

cc: WR Christensen

GW Reddick, Jr.

WH Sant

$$
\begin{aligned}
& \% \mathrm{H}_{2} \mathrm{O} \quad 65.54 \\
& \text { coding curve } \\
& \\
& \text { No solid }=Q 5^{\circ} \mathrm{C} \text { for } 25 \mathrm{mia}
\end{aligned}
$$


Date: January 21, 1974

To: R. L. Walser

From: W. H. Sant רo if Aj-

Subject: 242-S FEED SAMPLES NUMBER T- 738 SAMPLE POINT 106-S

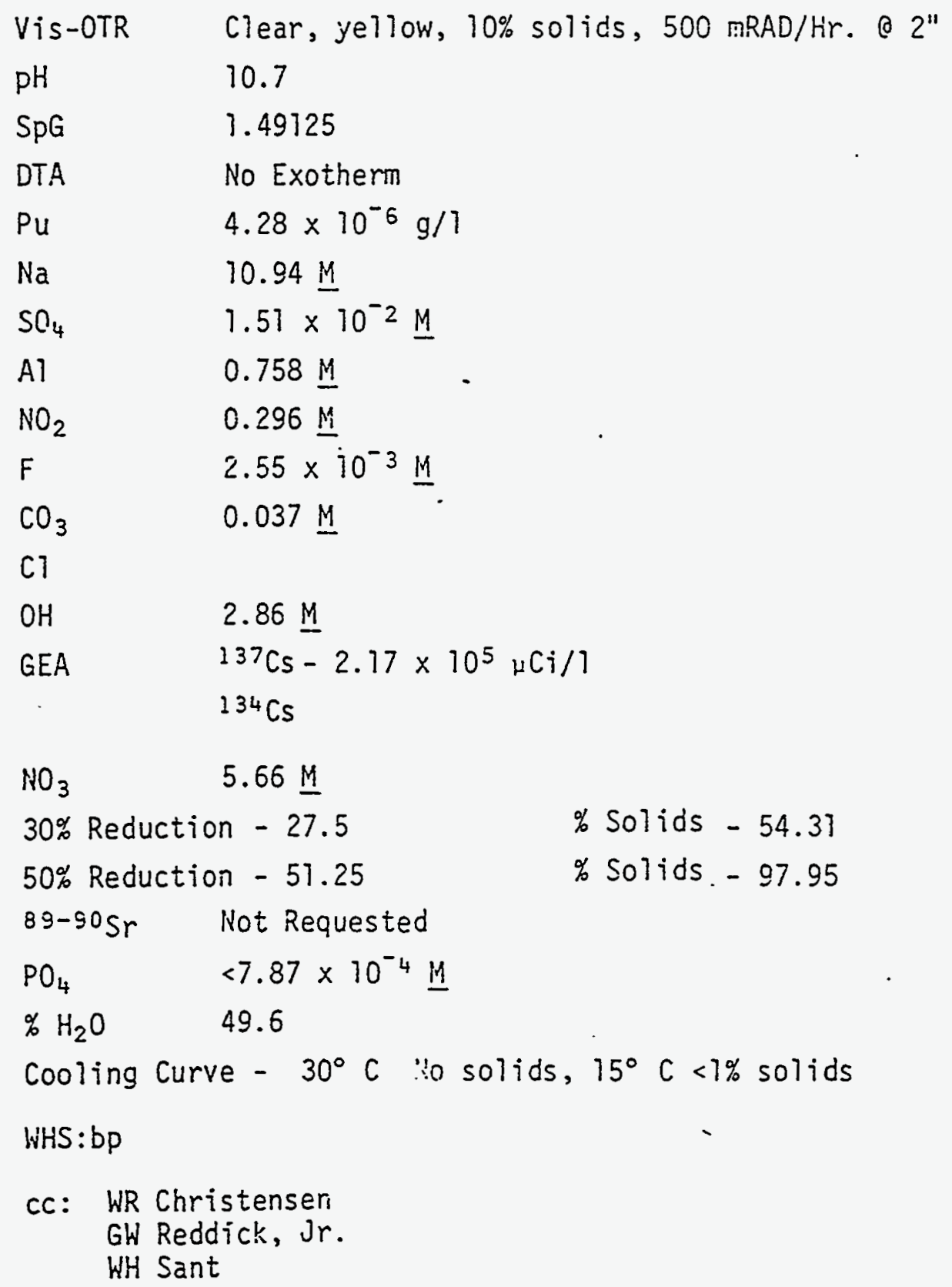




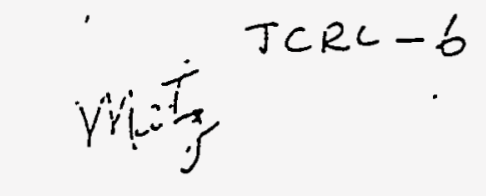

WHC-SD-WM-ER-323, Rev. 0

DRA $2 m$

J. इ. jorton

August 21, 1974

$$
\text { Achierement รeport }
$$

\section{SUMMARIIS}

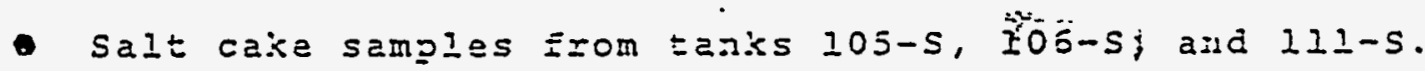

- Theraal. conductivities $= \pm$ l05-s sazt. 
DRAET

J. Z. $\quad 30=50 \Omega$

August 21, 2974

TANX FARM ASSISTAACE

Samples oz the si:- cakes in tanks 105-5, 106-5,

and III-s were taken. Ihese tanks aze siurzy receiving tanks Eor the 242-s evapozator. Solics in tanks 105-s, l06-s, and l11-s are primarily sodiun nitrate. Details of laboratory work are discussed below. Analysis of the solids and mother liquors are shown below.

The 105-s salt sample is composed of coazse yellow crystals. The yellow crystals are very ary in appeazance. A weigied amount of lo5-s solids was aissolved in $z_{2} 0$ and made to a volume of 25 ml. Analysis are snown in Table =.

\section{T33IEI}

$\begin{array}{llr}\mathrm{NaAlO}_{2} & 1.34 \times 10^{-3} \mathrm{M} & 0.118 \\ \mathrm{NaNO}_{2} & 3.73 \times 10^{-3} \frac{\mathrm{M}}{\mathrm{M}} & 0.25 \xi \\ \mathrm{Na}_{2} \mathrm{CO}_{3} & 2.36 \times 10^{-2} \mathrm{MM} & 2.543 \\ \mathrm{NaNO}_{2} & 1.16 \mathrm{M} & 100.3 \xi \\ \mathrm{NaOH} & 2.26 \times 10^{-2} \mathrm{M} & 0.5 \% \\ \mathrm{~B}_{2} \mathrm{O} & & \frac{3.4 \xi}{107.18}\end{array}$

GI:

$137 \mathrm{Cs}$

12535

$8.75 \times 10^{2}+C i / 2$
$2.20 \div C \equiv / 2$

3. $9: 1 C: / 9$

$0.02 \mathrm{j} C i / g$

$\begin{array}{ll}\text { As Received Density } & 1.25 \\ \text { Wet Density } & 2.79 \\ \text { WH Density } & 2.52\end{array}$


DRAFT

J. इ. Horton.

August 2I,. 1974

HYGROSCOPICITY

The 105-s salt sample showed no deliçusce afte= 21 days of testing. Pezcent gain after 21 days was 11.1 percent. This test was made in air saturated iith vater vapor at $23-25^{\circ} \mathrm{C}$.

Thermal conductivity measurements of lo5-s salts were made. Table II reflects the data.

\section{TABIE II}

THERMAI CONDUCTIVITIES OF 105-S SOIIDS

(DATA BY D. G. BOUSE)

\begin{tabular}{|c|c|c|c|c|}
\hline As Received & 21.8 & ${ }^{\circ} \mathrm{C}$ & 0.252 & $3 \geq 0 /: x / E t^{2} /{ }^{\circ} z$ \\
\hline Held overnight & 52.1 & ${ }^{\circ} \mathrm{C}$ & 0.247 & " \\
\hline Dried overaight & 80.0 & ${ }^{\circ} \mathrm{C}$ & 0.235 & $"$ \\
\hline overnignt & 114.9 & ${ }^{\circ} \mathrm{C}$ & 0.216 & $"$ \\
\hline Over weekend & 156.0 & ${ }^{\circ} \mathrm{C}$ & 0.205 & $"$ \\
\hline
\end{tabular}

The 106-s salt smple has large yellow crystals at the top and coanse crystals at the bottom. A rasgtacd amount of l06-s solids was dissolved in $\mathrm{g} 0$ and made to a volume of $25 \mathrm{ml}$. Labo: 5 toy analysis $0 \equiv$ l06-s scidis are shown on the followis- page. 


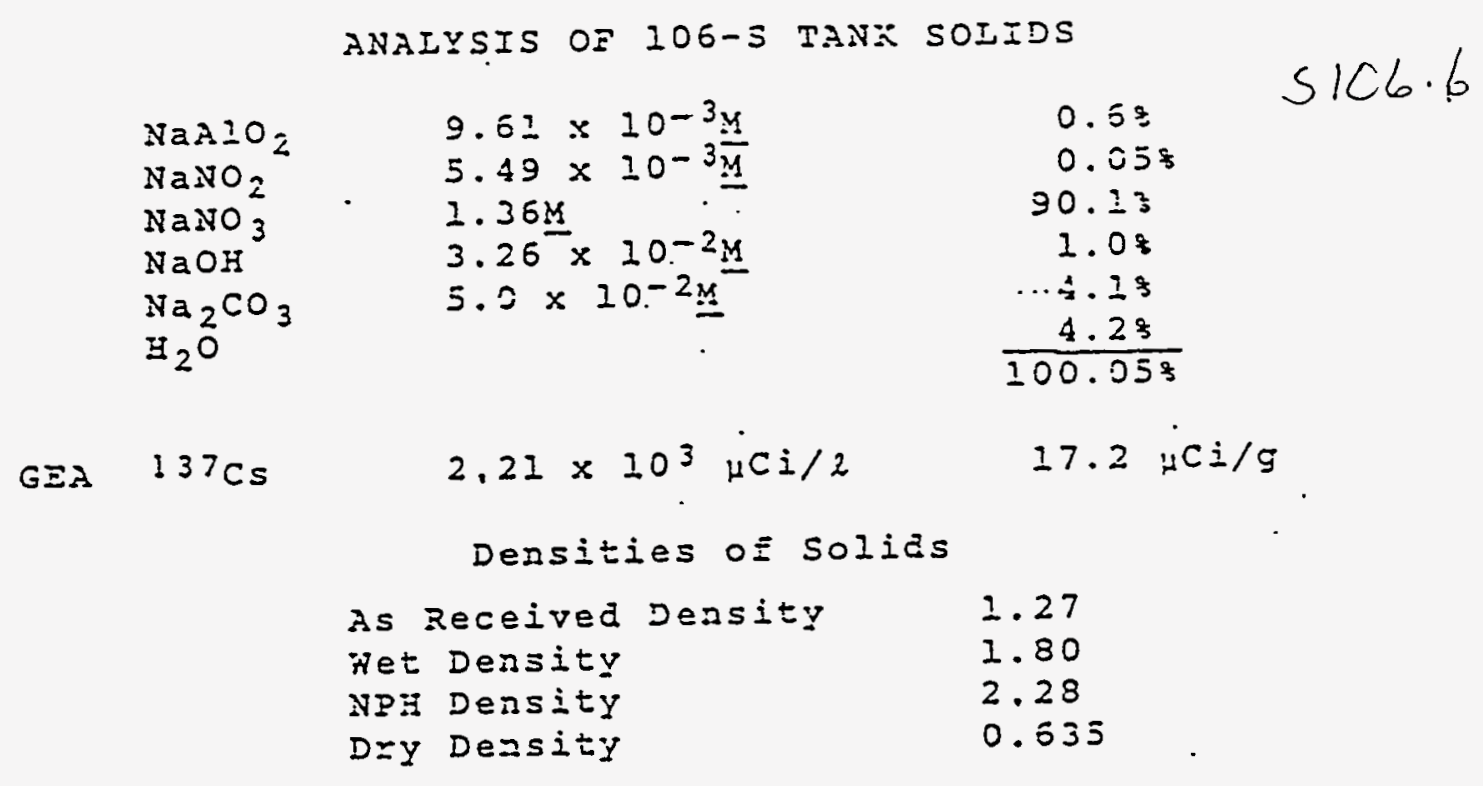

TXIIE IV

ANAEYSIS OF IOG-S SEPERNAMATM

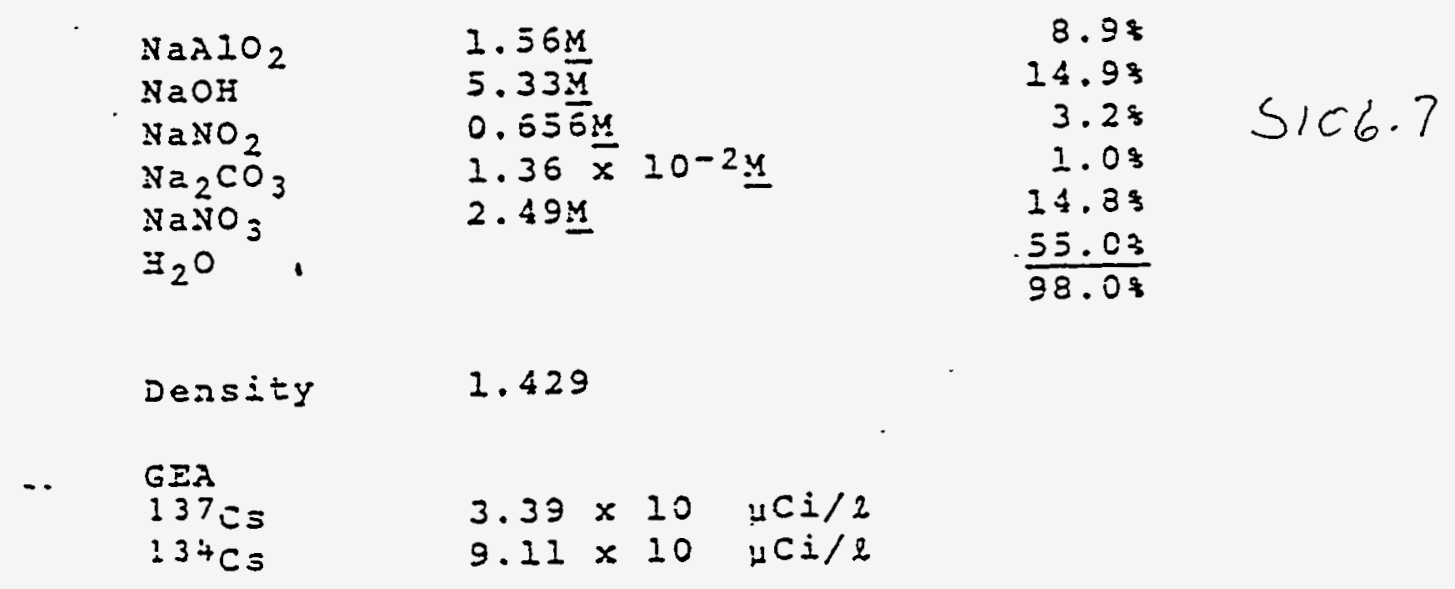


DRAPT

Hygroscodicities of l06-S SalEs

The 106-s salt sample. shows no deliquescence azter 14 days o⿰ testiag. Pexcent gain in 14 days 0 E testing was 11.4 percent. This test was made in air saturated with water vapor at $23-25^{\circ} \mathrm{C}$.

The III-S salts are a grayish in color and very sticky. Iaboratory work was done on boti the supezatant and solia. A portion was aissolved in water and sent to laboratory for analysis.

\section{TABIE V}

ANAIYSIS OE III-S SAITS

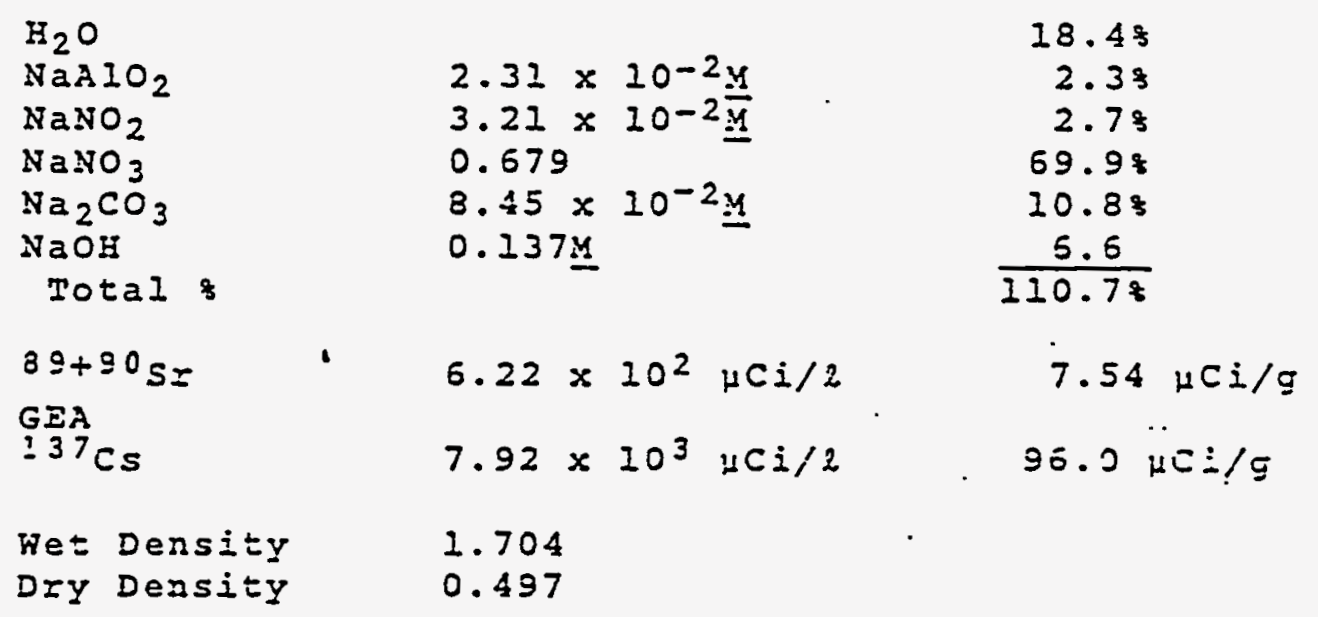




\section{TABIE VI}

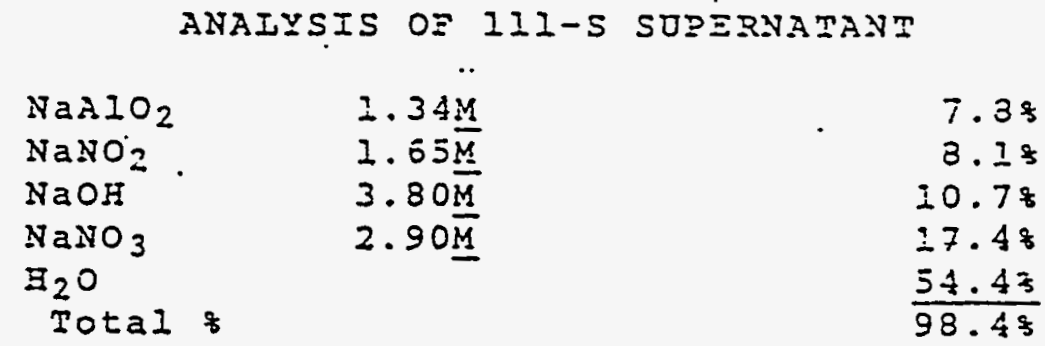

Density $\quad 1.414$

GEA

$137 \mathrm{Cs} \quad 4.47 \times 10^{5} \mathrm{HCi} / 2$

$134 \mathrm{Cs} \quad 3.04 \times 10^{3} \mathrm{HCi} / 2$

\section{Eygroscopicities of III-s salts}

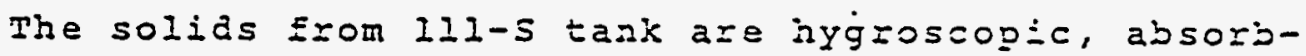
ing enough water to become deIiquescent ia six days. The salt hal increased 21.6 percent in weight when it deliguesced. This test was made in ail saturated with water at $23-25^{\circ} \mathrm{C}$.

Iaboratory work on sludge samples I04-A, I02-AX, and 110-s are nor yet completed, and will be reporied at a latez date. 
Date: October 14, 1974

To: R. L. Walser

From: R. E. Wheeter anthaler

Subject: ANALYSIS OF TANK FARM SAMPLES

SAMPLE: T-5469 106-S

Vis-0TR: Clear, light green. No solids. $20 \mathrm{mRad} / \mathrm{hr}$

pH: $\quad 11.42$

SpG: $\quad 1.0004$

$\mathrm{OH}: \quad 3.0 \times 10^{-2} \mathrm{M}$

A1: $\quad 1.36 \times 10^{-3} \underline{M}$

$\mathrm{Na}: \quad 0.107 \underline{\mathrm{M}}$

$\mathrm{NO}_{2}: \quad 2.61 \times 10^{-3} \mathrm{M}$

$\mathrm{NO}_{3}: \quad 1.85 \times 10^{-2} \overline{\mathrm{M}}$

Pu: $\left.\quad<4.43 \times 10^{-\overline{6}} \mathrm{gm} / \mathrm{ga}\right]$

DTA: No Exotherm

$\mathrm{SO}_{4}: \quad 8.79 \times 10^{-4} \mathrm{M}$

$\mathrm{PO}_{4}: \quad 4.05 \times 10^{-4} \frac{\mathrm{M}}{\mathrm{M}}$

F: $\quad 3.71 \times 10^{-4} \frac{\mathrm{M}}{\mathrm{M}}$

$\mathrm{CO}_{3}: \quad 7.73 \times 10^{-3} \underline{\mathrm{M}}$

GEA: $\quad{ }^{137} C_{S}-8.78 \times 10^{3} \mu \mathrm{Ci} / \mathrm{ga}$

Water: $\quad 81.27 \%$

Cooling Curve: $20^{\circ} \mathrm{C}$ for $45 \mathrm{~min}$. No solids.

$75^{\circ} \mathrm{C}$ for $45 \mathrm{~min}$. No solids.

$10^{\circ} \mathrm{C}$ for $45 \mathrm{~min}$. No solids.

$5^{\circ} \mathrm{C}$ for $45 \mathrm{~min}$. No solids.

REW:jd

cc: JS Buckingham

WR Christensen

RE Wheeler

JC Womack 
Date: Decenter 16, 1974

$$
5106.9
$$

To: R: L. Halser

From: R. E. Wheeier R Englielin

SUDject: RURLYSIS OF TANK FARM SAMPLES

SAI:PLE: T-8035 I06-S

Vis-0iR: Clear yellow, 50: solids. 2 Rads.

PH: $\quad 14$

SPG: $\quad 1.5414$

DH: $\quad 5.20 \underline{11}$

Al: $\quad 1.45 \underline{M}$

$\mathrm{Na}: \quad 14.20 \underline{\mathrm{H}}$

$\mathrm{HO}_{2}: \quad \quad \quad 1.02 \underline{\mathrm{H}}$

$\mathrm{NO}_{3}: \quad 2.90 \underline{\mathrm{H}}$

Pu: $\quad 1.90 \times 10^{-5} \mathrm{~g} / \mathrm{gal}$

$\mathrm{SO}_{4}$ : Cancelled by R. L. Halser

$\mathrm{PO}_{4}: \quad 5.10 \times 10^{-2} \underline{\mathrm{M}}$

F: $\quad 7.49 \times 10^{-4} \underline{M}$

$\mathrm{CO}_{3}: \quad 0.145$

EEA: $\quad: 3{ }^{2} \mathrm{Cs}-3.94 \times 10^{2} \because \mathrm{Ci} / \mathrm{gal}$

$E \equiv, E_{5 r}: \quad 78.80 \psi[\mathrm{Ci} / \mathrm{gal}$

lister: LE. CE.

K: $\quad 2.77 \times 10^{-2} \underline{\underline{H}}$

Cooling Curve: $40^{\circ} \mathrm{C}$ for $90 \mathrm{~min}$. lis solics.

$35^{\circ} \mathrm{C}$ ior $75 \mathrm{~min}$. lio solics.

$30^{\circ} \mathrm{C}$ for ED min. lio solids.

$25^{\circ} \mathrm{C}$ zor 45 rin. iio solics.

$20=C$ for 17 hrs. 3\% solics sus zzerded.

$15=C$ for 45 min. $3 \%$ solics susjenset.

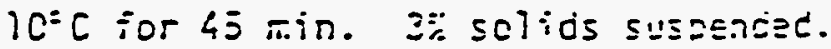

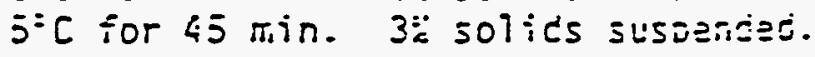

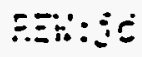

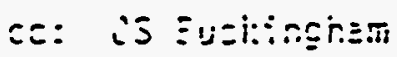

$\therefore$ Ctritisis:ssen

F.? Eir.?2t:

LS icinson

r.: $:$ 'hesier

ic $\because j x \equiv c k$ 
Date: July 23,1975

To: W. R. Christensen

From: J. E. Horton Gyffrition

SUbject: ANAIYSIS OF SAIT AND LIQUID SAMPIE EROM 106-S TANK

A sample was received on June 4, 1975 from Tank 106-s. Sample 106-s contained a liquid phase and a solid phase. Separation was made by centrifuging at high speed for 20 minutes. The salt solids were very fine with a tendency to float even after a 20 minute centrifuging. The salt crystals were yellow in color.

The analyses indicate that the 106-s solid is essentially a mixture of sodium nitrate and sodium carbonate. Details of the analyses are discussed in Tajles I and II.

Analyses were made by weighing 2.03265 grams and dissolving it in water to a volume of 50 milliliters. The remaining insoluble solids were dissolved in con. HCl and diluted to a volume of 10 milliliters with water. About 95 percent of the solids were water solubie.

Please call me if you have any questions. $J \Xi H: m s$ $c c$

GK Allen
H Babad
JS BuCEingnam
RJ Cain
DC Lini
NC Rodewald

RJ Thompson

RE Van der cook

RL WaIsez

JC Nomack

Process Aids (7) CTI- 223 File. 


\section{TABLE I}

\section{ANALYSIS OF $106-5$ TAIN SOLIDS}

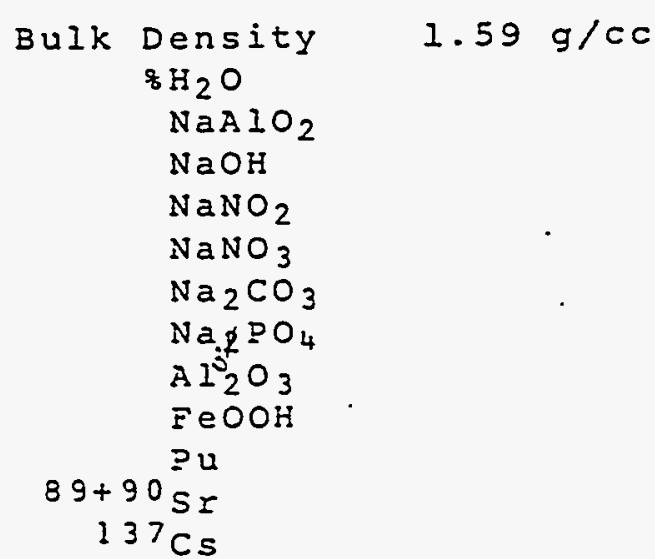

Total Wt: Recovery
$5106 \cdot 10 \mathrm{~s}$

\section{TABIE II}

ANALYSIS OF IO6-S SUPERNATANT IIQUID

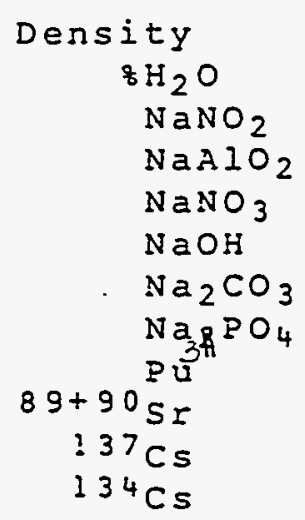

Total wto Recovery

\subsection{5}

$1.78 \mathrm{M}$

$1.85 \bar{M}$

$2.42 \overline{\mathrm{M}}$

$4.44 \bar{M}$

$0.27 \overline{O M}$

$0.028 \overline{\frac{M}{x}}$

$1.17 \times 10^{-6} \mathrm{~g} / 2$

$1.22 \times 10^{3} \mu \mathrm{Ci} / 2$

$5.29 \times 10^{5} \mu \mathrm{Ci} / 2$

$2.02 \times 10^{3} . \mu \mathrm{C} i / 2$

$$
\begin{array}{r}
50.08 \\
8.58 \\
10.58 \\
14.28 \\
12.28 \\
1.98 \\
0.38
\end{array}
$$

98.38
5106.11

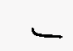




$$
s-107
$$

Date: $\quad$ November 22, 1973

To:

G. L. Borsheim

From:

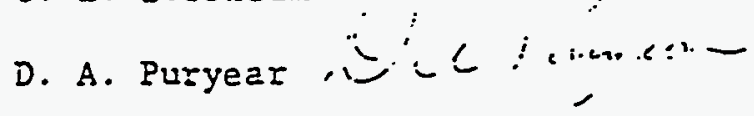

Subject:

ANAIYSIS AND IDENTIFICATION OF SOLIDS

IN TK IOA AND 102-S

Reference: D. A. Puryear, Phosphate Solidification of Caustic Process Solution Waste, ARH-SA-120 May 1972.

Samples from Tank 107 and 102-s were referred to CTL for identification of solids which plugged a jumper line from Tank 107-S to 102-S. The temperature of the tanks are $90^{\circ} \mathrm{F}$ and the samples were liçuid when taken. However, the sample from IK $107-5$ was about 50 percent crystalline when received by CIL.

Analysis of the original sample from TK 107-S showed a phosphace molarity of 0.384 and a water content of 81 weight percent. From the reference above on phosphate solidification of waste, experience was gained that shows phosphates are only partialiy soluable in caustic solution because they form a solid solution of the formulas $7\left(\mathrm{Na}_{3} \mathrm{PO}_{4} \cdot 12 \mathrm{H}_{2} \mathrm{O}\right) \mathrm{NaOH}$ or $5\left(\mathrm{Na}_{3} \mathrm{PO}_{4} \cdot 12 \mathrm{H}_{2} \mathrm{O}\right) \mathrm{NaOH}$ at a sodium to phosphace mole ratio OI 3.12 and 3.21 respectively. Tank $107-5$ has by analysis an available sodium to phosphace mole ratio of 4.36 . The high percentage of water attributes to the crystals being in solution at $90 \mathrm{~F}$. Tests on solids formation as a function of cooling showed that crystallization began to occur at $89.6^{\circ} \bar{F}$ and was near maximum solidification at $82.4 \cdot$.

Table I below shows the chemical analyses of cank 107 and 102-S. The irst column is the original analysis of the analycical laboracory as designated in ARH-1601. The second column shows the analysis of the same sample arter it was melied and diluted $1: 1$ with water. All answers have been calculared to the original sample. The third column is a resample of cank 107 caken on 11/16/73. Sample preparation of the resample in the laboratory was made as a $1: 1$ dilucion aiter relting the sample. 
G. I. Borsheim

Page 2

November 27,1973

Table I

\begin{tabular}{|c|c|c|c|c|c|}
\hline Constituent & $\begin{array}{l}107-5 \\
11-6 \\
\text { Supernatant } \\
\text { Liquid } \\
\end{array}$ & $\begin{array}{l}107-5 \\
11-6 \\
1: 1 \text { dil after } \\
\text { Melcing } \\
\end{array}$ & $\begin{array}{l}107-5 \\
11-16\end{array}$ & $\begin{array}{l}102-5 \\
11-5\end{array}$ & $\begin{array}{l}102-5 \\
11-16\end{array}$ \\
\hline Sp. Gr. & 1.107 & 1.1398 & 1.1592 & 1.2297 & 1.2927 \\
\hline $\mathrm{Na}$ & 2.55 & . $\quad 3.36$ & 3.39 & 5.09 & 7.18 \\
\hline Al & .105 & .0871 & .0907 & .230 & .367 \\
\hline $\mathrm{NO}_{2}$ & .027 & .03 & .024 & .118 & .088 \\
\hline $\mathrm{NO}_{3}$ & 1.72 & 1.47 & 1.73 & 4.25 & 3.05 \\
\hline $\mathrm{OH}$ & .186 & & & 1.22 & 1.51 \\
\hline $\mathrm{PO}_{4}$ & $N A$ & .384 & .530 & $: \mathrm{NA}$ & .112 \\
\hline $\mathrm{CO}_{3}$ & .094 & .095 & .099 & .019 & .06 \\
\hline $\mathrm{SO}_{4}$ & & .0298 & .0218 & .00988 & .0116 \\
\hline F & .000226 & .0099 & .0103 & .00154 & .0051 \\
\hline
\end{tabular}

Column four is the chemical composition of cank 102 prior to 242-S start-up and is really not related to the plugged line whereas column iive is a sample of cank 102 after it received some transier from tank 107 prior to the jumper line plug up.

All the results shown in Table 1 appear to be realistic. The important discrepancies can be explained. For example the specific gravity of column 1 appears light as does the sodium result. The lower specific gravicy and sodium result is the result of analyzing the supernatant liquid. By melting the samp-es and vocaining a homogeneous solution the total of the sample is accounced for.

A plor of solids formation as a function of temperatura is shown in Figure 1 for rank 107-S, $102-S$ and a $2: 1$ blend of 107-s with 102-S. Solidification begins at $32^{\circ}$, $18^{\circ}$, and $30^{\circ} \mathrm{C}$ respectively for the three solutions.

DAP:jf
cc: JS Buckingham
MH Campbe 11
WR Christensen
HH Hopkins
RI Walse: 


\section{SAMPLE ANALYSES}

TANKS 107-S AND 102-S

Tank

Date Sampled

Sample Number

Specific Gravity

DTA

Radioisotopes

$29-90_{s} r=\mathrm{Ci} / \mathrm{gal}$

137 Cs $\quad$-Ci/gal

${ }^{134} \mathrm{Cs} \quad$ ¿Ci/gal

${ }^{50}$ Co $\quad$ Ci/gal

239 $P_{u} \quad g m / g a l$

Chemicai
7.5

$1.0 \times 10^{5}$

$8.2 \times 10^{1}$

$2.5 \times 10^{2}$

$8.1 \times 10^{-5}$

\section{3}

$107-5$

$11 / 17 / 73$

476

1.16

No Exotherm
$102-5$

$11 / 16 / 73$

459

1.29

No Exotherm
$\mathrm{Bi}^{+++}$

$\mathrm{Na}^{\circ} \quad \frac{\mathrm{M}}{\mathrm{M}}$

$\mathrm{A}^{10^{-}} \quad \mathrm{M}$

$\mathrm{OH}^{-2} \quad \mathrm{M}$

$\mathrm{NO}_{3}^{-} \quad \bar{M}$

$\mathrm{NO}_{2}^{-} \quad \underline{M}$

$\mathrm{CO}_{2}=\quad$ M

$\mathrm{SO}_{4}=$. M

$F-\quad M$

CI $-\quad$ id

$\mathrm{PO}_{4} \equiv \quad \underline{M}$
$-0.005$

3.36

0.087

0.186

1.47

0.030

0.095

0.030

0.010

0.384
$4.4 \times 10^{1}$

$8.6 \times 10^{4}$

$1.5 \times 10^{2}$

$1.9 \times 10^{2}$

$10.7 \times 10^{-5}$
$4.3 \times 10^{1}$

$4.0 \times 10^{5}$

$2.8 \times 10^{2}$

N.D.

$5.2 \times 10^{-5}$
.0 .005

3.39

0.091

0.292

1.73

0.024

0.099

0.022

0.010

0.53
.0 .005

7.18

0.367

1.51

3.05

0.088

0.06

0.012

0.005

0.112

FREEZING POINT DATA

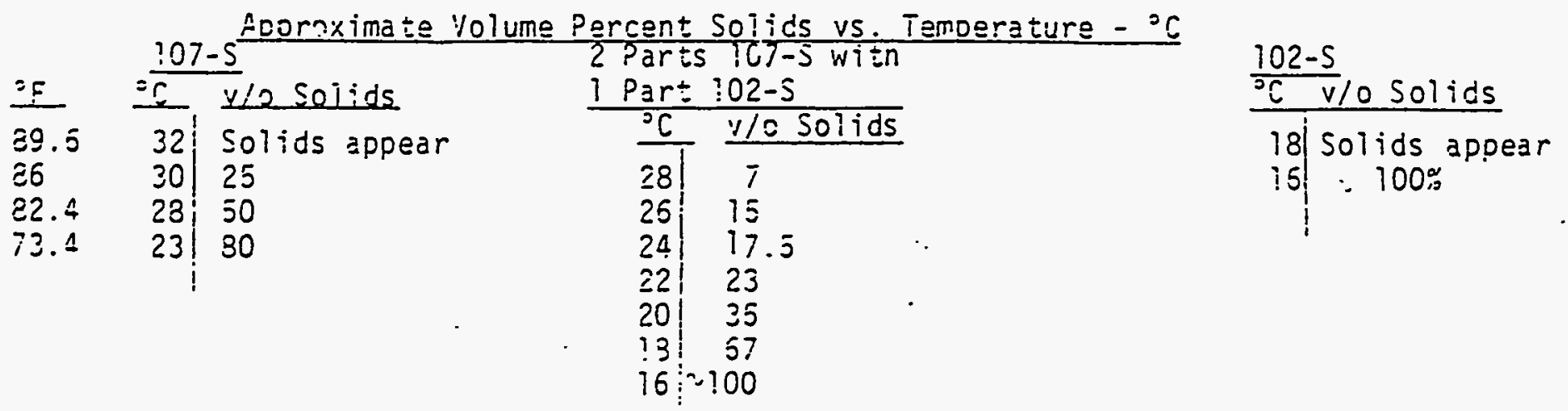


Analyses of 107-5 and 102-S samples show that the 107-5 material was very close to its freezing point even while in TK-107-S. Specific gravity determinations indicate the 107-S material was less concentrated than the 242-S feed in 102-S and this is confirmed by the chemical analyses wnich show lesser concentrations of all major cations and anions with the exception of phosphate ion. The high phosphate ion in 107-5 presumably originated in $N$ Reactor flushes where phosphoric acid is one of the major decontaminating agents. The freezing point data for the two to one blend of 107-5 to 102-5 (the approximate volume ratio below the $242-5$ feed recirculation 1 ine in the "problem" riser) and the individual solution freezing point curves indicate that there was no reaction between the solutions that promoted solids formation.

High phosphate ion concentrations in caustic solutions are known to produce high freezing point trisodium orthophosphate hydrates - indeed, this is the basic chemical reaction for the phosphoric acid solidification prototype. The 107-S solution approached the stoichiometric ratio of phosphate ion to available sodium ion planned for the solidification flowsheet ( 1 to 3.2 ) and only the high percentage of water (approximately 81 weight percent v.s. approximately 50 weight percent for the solidification process) prevented 107-s from solidifying. The 107-s tank bulk temperature (ca. 97 F) was high enough to prevent freezing, although the solution was essentially saturated, as was the heat traced line from 107-5 to 102-S. However, when the solution contactad the cold (50-50\%?) riser, crystallization probably began immediately. 
Date: $\quad$ December 7, 1973

To:

R. L. Watser

From: W. H. Sant orit?-

Subject: 242-S FEED SAMPLES T-178 (11/6/73) 107-S

EUARORATCR

5107.4

Supernate

Vis-0T Yellow, $50 \%$ crystals, $180 \mathrm{mR} / \mathrm{hr}$

$\mathrm{pH} \quad 12.2$

SpG $\quad 1.1071$

1.1398

DTA No Exotherm

Pu $\quad 1.43 \times 10^{-5} \mathrm{~g} / 1$

$\mathrm{Na} \quad 2.55 \mathrm{M}$

$\mathrm{SO}_{4} \quad 2.96 \times 10^{-2} \mathrm{M}$

A1 0.105 if

$\mathrm{NO}_{2} 2.7 \times 10^{-2} \mathrm{M}$

$\mathrm{Fe} \quad 2.26 \times 10^{-4} \mathrm{M}$

$F$

$\mathrm{CO}_{3}$

CI

$\mathrm{OH}$

GEA

$$
9.4 \times 10^{-2} \mathrm{M}
$$

$9.92 \times 10^{-3} \underline{M}$

$9.5 \times 10^{-2} \mathrm{M}$

$2.14 \times 10^{-5} \mathrm{~g} / 7$

$3.36 M$

$2.98 \times 10^{-2} \mathrm{M}$

$8.71 \times 10^{-2} \mathrm{M}$

$3.0 \times 10^{-2} \mathrm{M}$

$2.61 \times 10^{4} \mu \mathrm{Ci} / 1$

$21.66 \mu \mathrm{Ci} / 1$

$65.44 \mu \mathrm{Ci} / 7$

$\mathrm{NO}_{3} \quad 1.72 \underline{\mathrm{M}}$

Boildown:

26.2 reduction, Solids $0.76 \%$

$52.5 \%$ reduction, Solids $3.42 \%$

$89{ }^{90} \mathrm{Sr} 6.156 \mu \mathrm{Ci} / 7$

$\mathrm{PO}_{4}$

$\mathrm{Bi}$

WHS:bp

cc: WR Christensen

GW Reddick, Jr.

WH Sant
1.47 M

$28.8 \%$ reduction, Solids $65.6 \%$

$57.2 \%$ reduction, Solids $98.7 \%$

$1.98 \mu \mathrm{Ci} / 1$

0.384 M

$<4.79 \times 10^{-3} \mathrm{M}$ 
Date: $\quad$ December 7,1973

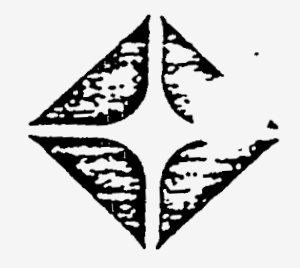

To: R. L. Walser

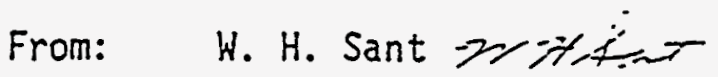

Subject: 242-S FEED SAMPLE T476 (11/16/73) 107-S

$\therefore \quad$ Vis-OTR yellow crystals 75 percent solids $100 \mathrm{mR} / \mathrm{hr}$

$\mathrm{pH} \quad 11.9040^{\circ} \mathrm{C}$

Spg $\quad 1.1592$

DTA No Exotherm

Pu $\quad 2.82 \times 10^{-5} \mathrm{~g} / 1$

$\mathrm{Na} \quad 3.39 \mathrm{M}$

$\mathrm{SO}_{4} \quad 2.18 \times 10^{-2} \mathrm{M}$

A1 $\quad 9.07 \times 10^{-2} \mathrm{H}$

$\mathrm{NO}_{2} \quad 0.024 \mathrm{M}$

F $\quad 1.03 \times 10^{-2} \mathrm{M}$

$\mathrm{CO}_{3} \quad 0.099 \mathrm{M}$ R.R. $-0.090 \mathrm{M}$

Cl Method being developed

OH 0.292 i

GEA $\quad 134 \mathrm{CS}-40.58 \mathrm{HCi} / \mathrm{T}$

$137 \mathrm{Cs}_{5}-2.28 \times 10^{4}, \mathrm{Ci} / \mathrm{T}$

$50 \mathrm{Co}-50.05 ; \mathrm{Ci} / \mathrm{T}$

$\mathrm{NO}_{3} \quad 1.73 \mathrm{~A}$

$30 \%$ Reduction - 27.5\%

$\%$ Solids - 0.862\%

50\% Reduction - 50\%

$\%$ Solids - $53.8 \%$

29 $90 \mathrm{Sr}-11.74 \mathrm{Li} / \mathrm{T}$

$\mathrm{PO}_{4} \quad 0.530 \mathrm{M}$

$\mathrm{Bi} \quad<4.98 \times 10^{-3} \mathrm{M}$

WHS: b?

CC: 'AR Christensen

GW Reddick, Jr. WH Sent 
Date: December 17, 1974

To: R. L. Watser

From: R. E. Wheeler Pisflicelle

SUbject: ANALYSIS OF TANK FARM SAMPLES

SAMPLE: T-8078 107-S

Yis-OTR: Yellow. No solids. $150 \mathrm{mRad} / \mathrm{hr}$

$\mathrm{pH}: \quad 14$

SPG: $\quad 1.201$

OH: $\quad 1.01 \mathrm{M}$

A7: $\quad 0.297 \underline{M}$

$\mathrm{Na}: \quad 3.72 \mathrm{M}$

$\mathrm{NO}_{2}: \quad 0.323 \underline{\mathrm{M}}$

$\mathrm{NO}_{3}: \quad 1.82 \mathrm{M}$

Pu: $\quad 1.78 \times 10^{-5} \mathrm{gm} / \mathrm{gal}$

$\mathrm{SO}_{4}: \quad 3.14 \times 10^{-2} \underline{\mathrm{M}}$

$\mathrm{PO}_{4}: \quad 0.106 \underline{\mathrm{M}}$

$\mathrm{F}: \quad 8.36 \times 10^{-3} \mathrm{M}$

$\mathrm{CO}_{3}: \quad 0.168 \mathrm{M}$

GEA: $\quad 134 \mathrm{Cs}-3.35 \times 10_{5}^{3} \mathrm{\mu Ci} / \mathrm{gal}$ $137 \mathrm{Cs}-4.81 \times 10^{5} \mathrm{\mu Ci} / \mathrm{gal}$ $125 \mathrm{Sb}-1.34 \times 10^{2} \mu \mathrm{Ci} / \mathrm{gal}$ ${ }^{60} \mathrm{Co}-54.54 \mu \mathrm{Ci} / \mathrm{ga} 1$

${ }^{89,90} \mathrm{Sr}: \quad 6.253 \times 10^{3} \mathrm{\mu Ci} / \mathrm{gal}$

Water: $\quad 76.65 \%$

REW:jd

CC: JS Buckingham

WR Christensen

RM Ginestet

RE Wheeler

JC Womack 
Date: $\quad$ Esbuary 14,1975

To: $\quad$ W. R. Christensen

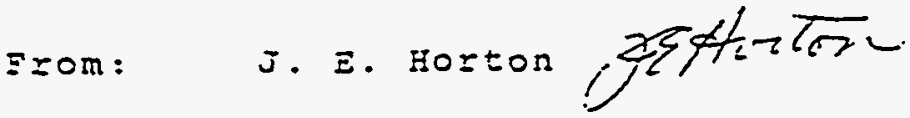

SUDject: ANALYSIS OF SIUDGE FROM TANK 107-S

We received a sample oI l07-s tank sludge on

July 1, 1974. The sludge sample was a mixture composed primarily os grayish-brown large

granular crystals.

The calculated heat genezation rate For the 107-s sludge, based on $3 \equiv+30$ SI and li Cs content was $5.30 \times 10^{-4}$ watts/1iter.

sludge analyses were nade by jusiag 1.05 milliliters of damp "as zeceived" slucge wi=h KoH, dissolving the melt in concentrated $\because C I$, and diluting to 200 milliliters with water. Analyses shown in Table I are reported in liters of damp "as received" sludge.

The mal conductivities $0 \equiv 107-5$ sludge, as reported by D. G. Bolise, are shown in Tajle II.

Please call if you have questions regaring tiis work.

$\Xi \Xi Z: \mathrm{ms}$

Aะニ。

cc: I Babad

is 3uckinginam

_RG Geier

NI Harms

NC Rocewald

RI Nalser

zrocess Aids (7)

CTI-123

Eile

L 3 


\section{TABIEI}

ANAIYSIS OE 107-S TANK

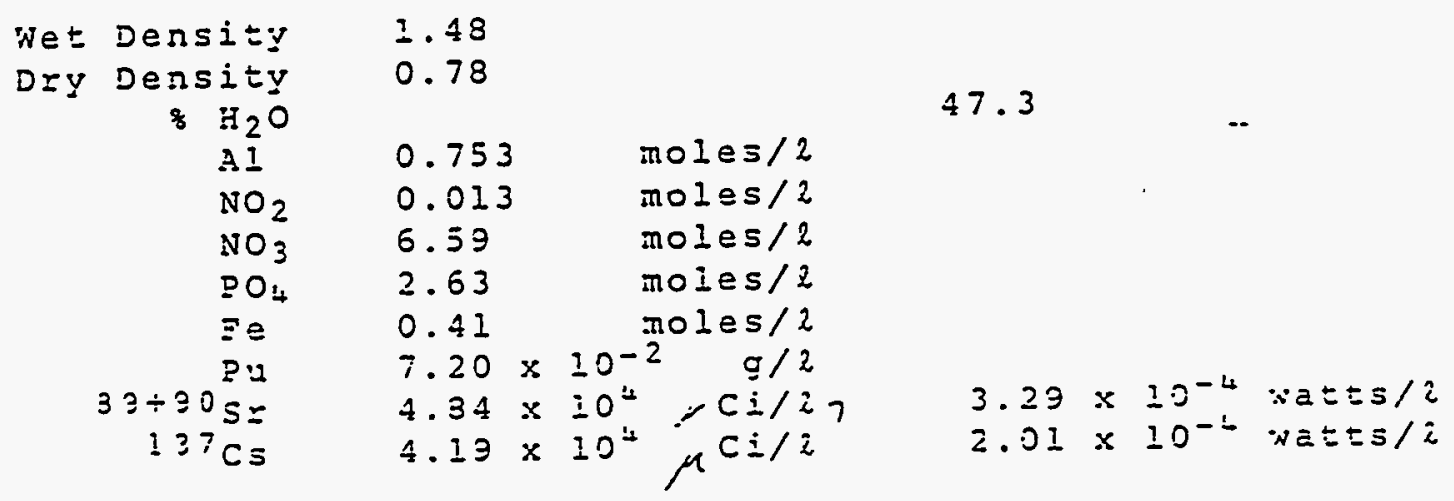

TASIE II

THERMAL CONDUCTIVITIES OE IOT-S SIUDGE

(Data by D. G. 3ouse)

\begin{tabular}{|c|c|c|}
\hline & $\begin{array}{c}T=m ? \\
0 / \mathrm{C}\end{array}$ & $\begin{array}{c}T C \\
N / \pi\end{array}$ \\
\hline As Received & 24.9 & 0.775 \\
\hline 40 Bours at & 101.0 & 0.934 \\
\hline 22 :̈ours at & 135.0 & 0.317 \\
\hline Overaight a= & 201.0 & 0.144 \\
\hline cyer weekend a & 24.3 & 0.151 \\
\hline
\end{tabular}


$T=: 2-\vdots i$

Internal Letter

Dale. . July 10, 1978

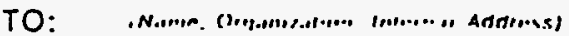

J. 0. Honeyman

- Process Enaineering

. 2750-E Bldg., 200 East
WHC-SD-WM-ER-323, Rev. 0

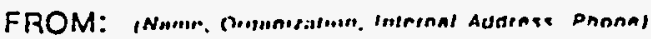

- T. A. Lane

- Chemical Sciences Group

. 222-S Bldạ., 200 Vlest

. 2-1571

Sudecl: - Crosscountry Transfer of 103/107-S Material

Ref: Letter, June 21, 1978, T. A. Lane to J. 0. Honeyman, same subject

In the reference letter we suggested concentrating the 103/107-5 material 60 percent. However, laboratory simulations using synthetic waste (Table I) indicated that 35 percent volume reduction at 40 torr could be achieved without nucleation. A dilution of this material could be made and not cause fibbsite $\left(\mathrm{N}_{2} \mathrm{O}_{3} \cdot 3 \mathrm{H}_{2} \mathrm{O}\right)$ precipitation. See Table II for the boildown data.

The concentrated synthetic was diluted $10-70$ volume percent. The 10 percent dilution formed $\mathrm{NaNO}_{3}$ crystals at $50^{\circ} \mathrm{C}\left(122^{\circ} \mathrm{F}\right)$. Hone of the other dilutions formed solids at $22^{\circ} \mathrm{C}$ a fiter four days. Aluminum and hydroxide analyses were made on each dilution (Table III) and the results plotted on the phase dianram, finure. 1. As you can see the results are a random scatter instead of the expected straight line. I feel this is caused by the analytical method used.

Because of variations in the 103/107-s analyses it was decided to do a "hot" boildown on actual 107-5 material. A new samnle was taken and analyzed. Analytical results are in Table IV. The boildown was made at 40 torr. The temperature of the actual material was $1^{\circ} \mathrm{C}$ higher than the synthetic at each percent reduction. Nucleation occurred at aporoximately 30 percent reduction. The slurry was removed at 35 percent reduction and dilutions of 10,20 , and 50 percent were made. Analysis indicates the actual reduction was 42 percent based on aluminum. See Table III. These results are plotted on the phase dianram, fiaure 2. The 10 percent dilution solidified at $50^{\circ} \mathrm{C}$. No analys is of the solid was made. However, they redissolved in additional water, therefore, I believe they were $\mathrm{NaNh}_{3}$. The 20 percent dilution had about 10 percent. solids at $22^{\circ} \mathrm{C}\left(72^{\mathrm{F}}\right)$. These solids also dissolved in additiona? water. The 50 percent dilution apneared to thicken slightly but formed no. solids. Additional dilution of this dortion to 75 percent at $22^{\circ} \mathrm{C}$ caused solids to form. The solids volume was about five percent of the total diluted volume. The solids analysis has not been completed yet, but I suspect the solid is gibosite.

Analysis of the condensate showed it had $0.065 \mathrm{~d} / \%$ of total ornanic carbon. The condensate strontium analysis was $7.93 \times 10^{-2} \mu \mathrm{Ci} / 2$. 
J. O. Honeyman

Page 2

JuTy 10, 1978

I recommend this material be reduced 35 volume percent at 40 torr prior to dilution and transfer to East Area. I also recommend the transfer and dilution be simultaneous. The possibility of nibbsite formation after dilution can not be ruled out if the solution is allowed to stand.

If you have any question about this work please give me a call.

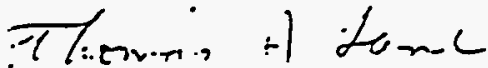

T. A. Lane

Chemist

Waste Chemistry Unit

TAL: rm

Attachments

Information:

G. E. Brown

J. S. Buckingham

R. J. Cain

K. F. Carothers

f. T. Dukelow

F. M. Jungfleisch Fm/

D. L. Merrick

G. Olsen

M. J. Smith

Process Aids (7) 
WHC-SD-WM-ER-323, Rev. 0

\section{TABLE III}

\begin{tabular}{|c|c|c|}
\hline $\begin{array}{l}\text { Dilution } \\
\text { iginal Feed }\end{array}$ & $\begin{array}{l}\mathrm{AT} \\
1.54 \mathrm{M}\end{array}$ & $\begin{array}{l}\mathrm{OH} \\
1.39 \mathrm{M}\end{array}$ \\
\hline 10 & 2.01 & 2.13 \\
\hline 20 & 1.48 & 2.18 \\
\hline 30 & 1.56 & 1.86 \\
\hline 40 & 1.01 & 1.83 \\
\hline 50 & 1.24 & 1.91 \\
\hline 60 & 0.979 & 0.917 \\
\hline 70 & 0.05 & 0,08 \\
\hline
\end{tabular}

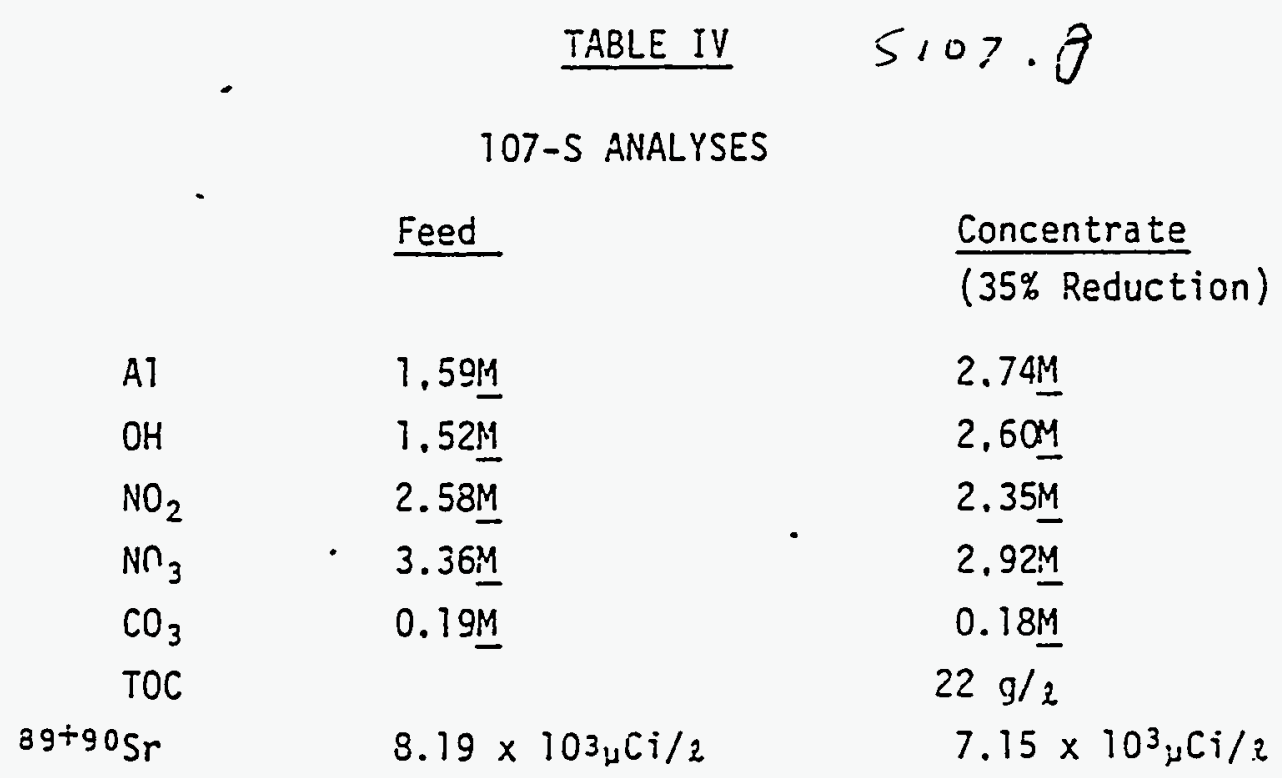




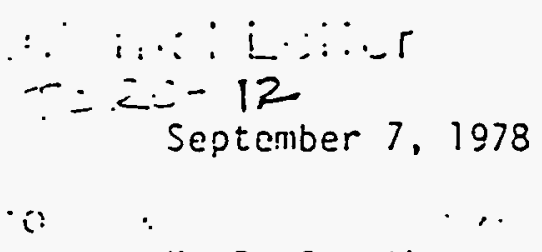

- K. G. Carothers

- Process Engineering

. 2750-E, 200 East Area

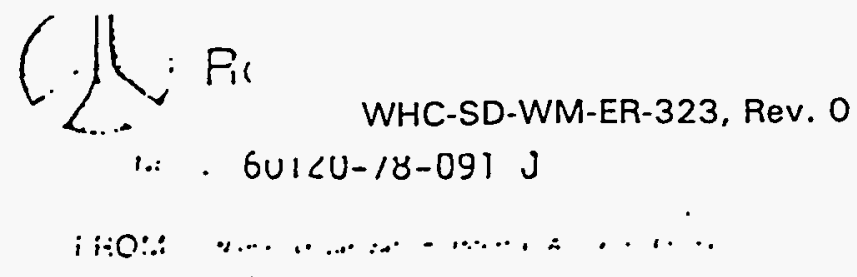

- G. E. Brown

- Chemical Sciences Group

- 222-5, 200 West Area

. $2-1571$

$\therefore$. Analysis of 107-S Cross-Site Transfer Material

During the recent cross-site transfer, line pluggage occurred. A sample of the cross-site feed material, taken from Tank 107-5, was gelatinous at room temperature $\left(22-25^{\circ} \mathrm{C}\right)$. The material did not separate into obvious solid and liquid phases. Because of the gelatinous nature of the material, we decided that viscosity vs. temperature curves would be more meaningful than the usual cooling curves of percent solids vs. temperature.

The laboratory data show that a dilution of 60 percent $(4 \mathrm{ml}$ sample + $6 \mathrm{ml} \mathrm{H}_{2} \mathrm{O}$ ) is necessary before the material rema ins completely liquid at $2^{\circ} \mathrm{C}$. The 40 percent dilution $\left(5 \mathrm{ml}\right.$ sample $+4 \mathrm{ml} \mathrm{H}_{2} \mathrm{O}$ ) is liquid at $15^{\circ} \mathrm{C}$ (nominal ground temperature), but is solid at $10^{\circ} \mathrm{C}$. The undiluted sainple solidified between 30 and $35^{\circ} \mathrm{C}$. Details of the laboratory work, including the chemical analysis, are discussed below.

Dip sample 3143, taken from the 150-inch level of Tank 107-S, is very viscous at room temperature. The material resembles cloudy, thickened gelatin and no obvious crystalline solids can be scen. At $45^{\circ} \mathrm{C}$, the sample is a sligitly cloudy liquid with the consistency of water. Table 1 lists the chemical analys is of the material. The analys is is similar to others for Jank 107-5, with nothing unusual that would explain the gelatinous character.

Ordinarily, a cooling curve of percent solids vs. temperature would give the necessary inionation. The cooling curve is useless in this case, since the material gradually thickens without settling. Because of this, the sample and three dilutions were characterized with viscosity vs. temperature ineasurements. Table 2 sumarizes this data. A Brookifield plate and cone microviscometer with a controlled temperature bath was used for all viscosity measurements. As indicated in Table 2, the original sample thickened betwcen 35 and $29^{\circ} \mathrm{C}$. The 20 percent dilution $\left(3 \mathrm{ml}\right.$ sample $\left.+2 \mathrm{ml} \mathrm{H}_{2} \mathrm{O}\right)$ solidified betiveen 20 and $15^{\circ} \mathrm{C}$; and the 40 percent dilution, between 15 and $10^{\circ} \mathrm{C}$. The 60 percent dilution remained liquid at $2^{\circ} \mathrm{C}$. 


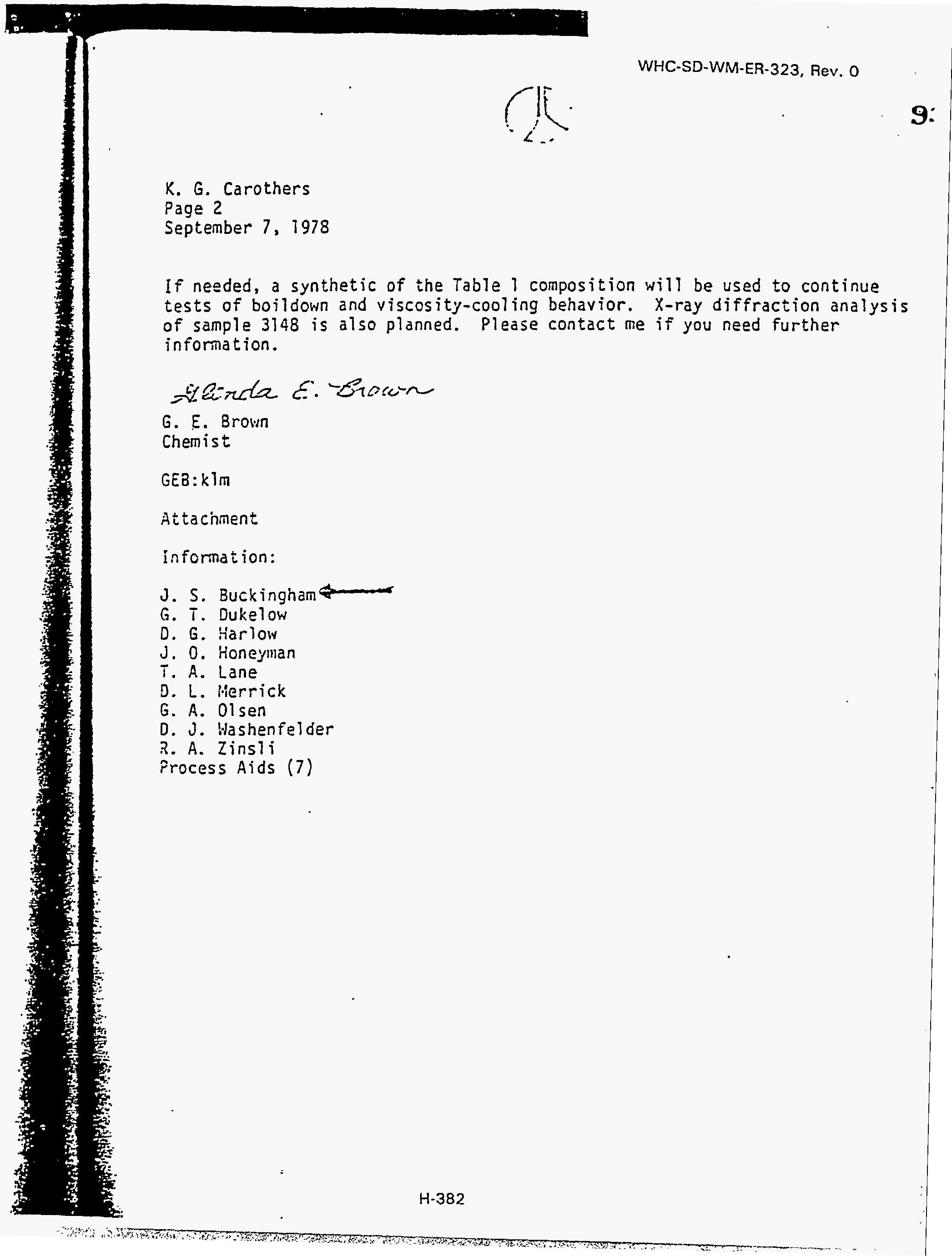




$$
5107.9
$$

TABLE I

TAMK 107-S AHALYSIS

\begin{tabular}{|c|c|c|c|c|}
\hline $\mathrm{PO}_{4}$ & $.04 \mathrm{H}$ & $A 7$ & 1.06 & 梠 \\
\hline $\mathrm{CO}_{3}$ & $.24 \underline{M}$ & TOC & 11.8 & $g m / l$ \\
\hline $\mathrm{NO}_{3}$ & $2.00 \mathrm{M}$ & ${ }^{137} \mathrm{Cs}$ & $2.26 \times 10^{5}$ & $\mathrm{iCi} / \mathrm{T}$ \\
\hline $\mathrm{NO}_{2}$ & 1.90 证 & $\approx \mathrm{H}_{2} \mathrm{O}$ & $53.7 \%$ & \\
\hline $\mathrm{Na}$ & $7.46 \underline{M}$ & Density & 1.208 & $g m / m l$ \\
\hline $\mathrm{OH}^{-}$ & $1.18 \mathrm{M}$ & 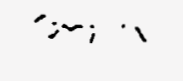 & & \\
\hline
\end{tabular}

$$
\begin{aligned}
& 1: 20 \text { j: } \\
& \text { id, o. } 0.5 \\
& \text {... } \\
& i_{1}, \% \text { यi is }
\end{aligned}
$$

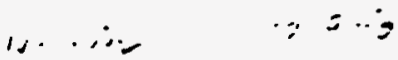

$$
\begin{aligned}
& \text { if is ? ?\% } \\
& \text { l..... } \\
& (x, \ldots) 1
\end{aligned}
$$




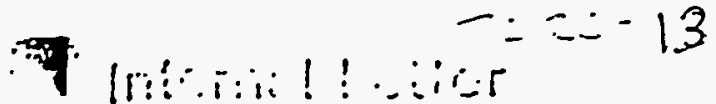

October 16, 1978

G. A. OTsen

Process Ennineering

.2750-E, 200 East

$\left\{b_{i}\right.$

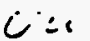

Rock.

WHC-SD-WM-ER-323, Rev. 0

186

rinOl::

$60120-78-106 \mathrm{~J}$

Ret $3 ?$

- G. E. Brown

- Chemical Sciences Grouo

- 222-5, 200 !lest

- 2-1571

s:s,ca .107-S Salt Hell Sample 4251

Ref: Letter, September 7, 1978; G. E. Brown to K. F. Carothers, "Analysis of 107-S Cross-site Transfer Material."

The waste material from Tank 107-S that caused the recent cross-site transfer line pluggage has been moved to Tank 102-SY. Concern over interactions between the heel of 107-5 waste and salt well wastes beinn pumped into Tank 107-S prompted several experinients. The work requested of Chemical Sciences froup included complete clicmical analysis, dilution, and viscosity-cooling curves. This letter reports the results of those experiments.

Sample 4251 , a cloudy, areen liquid from the salt well pumping, was received by Chemical Scicnces Group. A dilution was analyzed by Analytical Laboratory. Those results are listed in Table 1.

Sample 4251 was heated to $55^{\circ} \mathrm{C}$, and then an aliquot was mixed with an equal volume of sainle 3748 (the cross-site transfer material mentioned in the reference letter) also heated to $55^{\circ} \mathrm{C}$. Ho solids formed while the mixture was hot. At room temperature $\left(22^{\circ} \mathrm{C}\right)$, the mixture had solidified into a gelatinous mass closely resembling samole 3148 . The Brookifield plate and cone viscometer was used for a viscosity v.s. cooling curve of the mixture of samples 3148 and 4251 . Table 2 summarizes the viscosity data. The crystallization point was between 29.5 and $24.5^{\circ} \mathrm{C}$, and no larqe increase in viscosity was noted until this point had been reached.

Please contact me if you need further information.

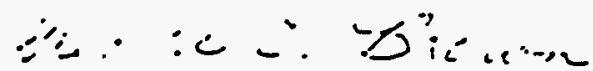

5. E. Brown

Chemist

GEB:bn

Att.

Information:

J. S. Buckingham

K. G. Carothers

G. T. Dukelow

F. 11. Jungfieisch $=\pi 5$

D. J. liashenfeider

R. A. Zinsli 
TABLE I

$$
\text { Analys is of Sample } 4251
$$

$\begin{array}{llll}\mathrm{Na} & 10.4 \mathrm{M} & \mathrm{NO}_{3} & 2.62 \mathrm{M} \\ \mathrm{AT} & 1.206 \mathrm{M} & \mathrm{NO}_{2} & 2.02 \mathrm{M} \\ \mathrm{OH}^{-} & 4.02 \mathrm{M} & \mathrm{SO}_{4} & 9 \times 10^{-4} \mathrm{M} \\ \mathrm{CO}_{3} & 0.15 \mathrm{M} & \mathrm{SPG} & 1.525 \\ \mathrm{PO}_{4} & 0.035 \mathrm{M} & { }^{\circ} \mathrm{H}_{2} \mathrm{O} & 52 \% \\ \mathrm{TOC} & 4.0 \mathrm{gm} / 2 & \mathrm{Cs}^{137} & 4.12 \times 10^{5} \mathrm{HCi} / 2\end{array}$

TABLE 2

Viscosity ys. Temperature Data

Tempera ture
$\left({ }^{\circ} \mathrm{C}\right)$

40.5

35

29.5

24.5
Shear Rate

$\left(\operatorname{Sec}^{-1}\right)$

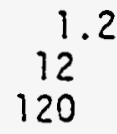

12.2

120
1.2

12

120

1.2

12
Viscosity

(centipoise)

11.1

5.6

6.6

12.8

8.3

8.3

13.0

19.5

10.4

$>1916$

$>191$

$>19$ 


\section{Inetrial Letier}

Date: - September 22, 1980

TO: IName, Organization. Interensl hadress)

- D. E. Bowers

- Plant Engineering

- 2750E/A100/200 East

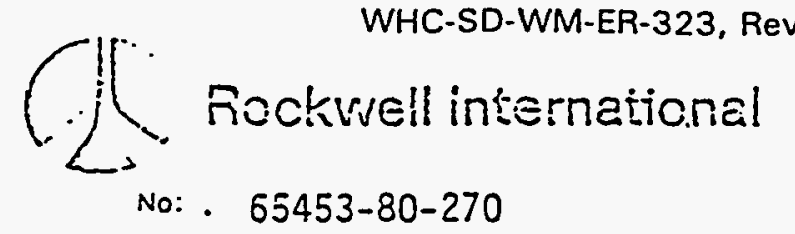

FROM: IName, Organization, Internal ideress. Phonel

- M. T. Jansky

- Separations Process Development Uni-

- 222-5/M0-037/200 West

- 3-1571

Sudiect: Viscosity/Cooling Curves for Tank 1075 Waste

Viscosity versus temperature data was obtained for waste from Tank 1075.

The waste was 242-s evaporator product scheduled for cross-site transfer.

Two samples were taken; one from one foot above the sludge layer in the

tank and the other from one foot below the suriace of the waste. Both samples contained solids. The samples were analyzed, and the results are shown in Table I. Viscosity versus temperature data for the waste samples are shown in Table II and Table III. Laboratory details are discussed below.

Aliquots of each sample were submitted to Analytical Laboratories. The data are shown in Table I. Various dilutions were made on al iquots of the waste sample and placed in a Brookiield LVT cup and cone microviscometer. Viscosity versus temperature data were taken, and are shown in Tables II and III.

Please call if you have any questions or comments regarding this work.

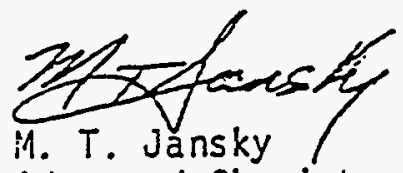

Advanced Chemist

MTJ/naj

Attachments

Distribution:

L. C. Brown

j. S. Buckingham

H. J. Eding

L. A. Gale

D. L. Herting

T. A. Lane

D. A. Reynolds

M. C. Teats

T. B. Veneziano

File Code: KF5zE

Process Aids: (8)

Let terbook 
$S \quad$ WHC-SD-WM-ER-323, Rev. 0

\section{TABLE I}

Composition of Tank 1075 Waste

Component

AT

$\mathrm{OH}$

$\mathrm{NO}_{2}$

$\mathrm{NO}_{3}$

$\mathrm{PO}_{4}$

$\mathrm{CO}_{3}$

TOC

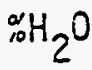

$S p G$

TOTAL

\section{Sample}

One Foot Above Sludge

Molarity

Wt.:

1.212

6.95

2.058

5.75

2.043

9.85

3.27

19.42

1.28

2.04

1.87

41.55

1.431

88.81
One Foot Below Surface Molarity

0.924

6.84

1.521

5.49

1.311

8.16

2.454

18.83

0.077

1.14

.285

2.73

8.28

2.22

55.94

1.108

101.35 


\section{TABLE II}

\section{Viscosity Versus Temperature of Tank 1075 Sample}

$$
\text { One Foot Above Sludge }
$$

\section{Temperature $\left({ }^{\circ} \mathrm{C}\right)$}

50

40

30

25

17

12

6.5

10

5
Viscosity (Centipoise) at RPM $\underline{60} \quad \underline{30} \quad \underline{12} \quad \underline{6} \quad \underline{3}$

$\begin{array}{lllll}14 & 17 & 28 & 42 & 70\end{array}$

$\begin{array}{lllll}17 & 20 & 30 & 40 & 65\end{array}$

$>19$

27

$>19>38$

67

2

1

2

$7 \quad 10$

13

15

21

12

12

15

18

awater: waste liquor

*not measured

** too low to measure

Dilution

$0 / 100$

$50 / 40$

$40 / 60$ 
WHC-SD-WM-ER-323, Rev. 0

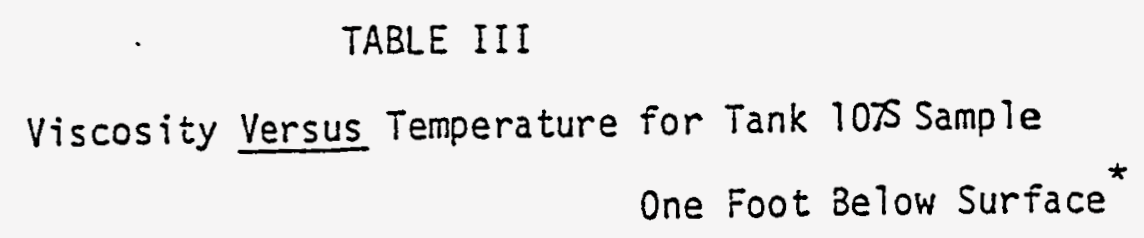

Temperature $\left({ }^{\circ} \mathrm{C}\right)$

51

40

30

20

10

4
Viscosity (centipoise) at RPM

$60 \quad \underline{30} \quad \underline{12} \quad \underline{6} \quad \underline{3}$

4554 *

$\begin{array}{lllll}7 & 7 & 6 & 9\end{array}$

$\begin{array}{lllll}8 & 9 & 8 & 8\end{array}$

$\begin{array}{lllll}11 & 11 & 11 & 12 & 15\end{array}$

$\begin{array}{lllll}14 & 14 & 16 & 18 & 18\end{array}$

$\begin{array}{lllll}18 & 18 & 19 & 19 & 19\end{array}$

*ilo dilution

**Too low to measure 
$\because A \quad T C R C-1$

$\cdot$

WHC-SD-WM-ER-323, Rev. 0

Seqtember 24, 1965

To: S. J. Beard

Irom:-

พ. I. Goderey Wy

242-T EVAPORATOR FEED

The results of analyses of the prospective 242-T Evaporator feed suroply are ettached. Some taniss, notady 118-IX, have ieceived adationgl waste since that time and the and.lyses shown are no longer valia, although they should still be indicative of tine corposition.

Cyenice hes been detected in only four tenirs and they heve been elfminated from consicieztion as feedstocis.

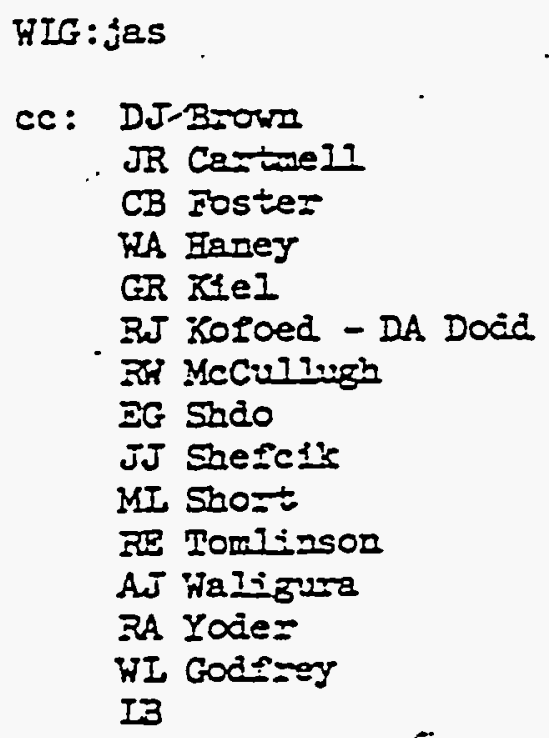




\section{TANK 108-S STATUS}

Iiquid, GeI.

Sludge, Gel.

SD. GI. $(g / c c)$

$751 \times 10^{3}$

$11 \times 10^{3}$

1.296

Chemical Analyses

\begin{tabular}{|c|c|}
\hline Free NaOI & $1.53 \mathrm{~N}$ \\
\hline $\mathrm{CO}_{3}=(\mathrm{g} / \mathrm{I})$ & 41.0 \\
\hline $\mathrm{AlO}_{2}(\mathrm{~B} / \mathrm{I})$ & $4.70^{\circ}$ \\
\hline \multicolumn{2}{|l|}{$F^{-}(B / I)$} \\
\hline $\mathrm{CI}^{-}(\mathrm{g} / \mathrm{I})$ & 3.14. \\
\hline $\mathrm{Ne}^{+}(\mathrm{B} / \mathrm{I})$ & 158 \\
\hline $\mathrm{NO}_{3}^{-}(\mathrm{g} / \mathrm{l})$ & 284 \\
\hline DE & 12.0 \\
\hline $\mathrm{CH}^{-}$ & $>i C O$ Dpm \\
\hline \multicolumn{2}{|l|}{ - Gamme Scen } \\
\hline $\mathrm{Zr}-\mathrm{No} 95(\mathrm{uc} / \mathrm{l})$ & N.D. \\
\hline$R u-R i^{206}(u c / 1)$ & N.D. \\
\hline $50-125(u c / 1)$ & N.D. \\
\hline $\mathrm{Cs}-134(\mathrm{uc} / 1)$ & N.D. \\
\hline $\mathrm{Cs}-137(\mathrm{uc} / \mathrm{I})$ & $9.8 \times 10^{4}$ \\
\hline
\end{tabular}

\section{Boil-Down Studies}

$\begin{array}{lccccc}\text { Percent Boil-Down } & 0 & 45 & 50 & 60 & 70 \\ \text { Boiling Point }\left({ }^{\circ} \mathrm{C}\right) & 100 & 118 & 125 & 128 & 130 \\ & & & & & \\ \begin{array}{l}\text { Percent Solids - } \\ \text { at boiling point }\end{array} & \mathrm{CIr} & 2 & 10 & 50 & 90 \\ \text { at room temperature } & \mathrm{CIr} & 15 & 50 & 90 & 100\end{array}$


Date: September 21, 1971

TC:

Fac:

$$
\text { J.'. Sligirad }
$$

SLio jest: CEGRACTERTZATICI OF $s, U$, AND SK :LASTE TANKS

3amoling and analyses of $S$, U and $5 X$ reste tanks to lientiify Fees raterial for the 242-S vecuum evaporator is progressing. To jate three che lite: semples frcr the 3 Fan taniss have been processed through the iabcretory reculm eveporetor: In edditicn ten $S$ Farm tanks and sio J Farm tanks have been sempled and subritied for cheracterizetion ansiyses. The zesults are shown in tive following taile.

Cinzacterization Anelyses ci ivste manks

\begin{tabular}{|c|c|c|c|c|c|c|c|c|c|}
\hline T!. .Xic. & $30 G=$ & $\begin{array}{l}\text { Visc. } \\
=00 \text { OC }\end{array}$ & $\mathrm{CO}_{3}$ & $\mathrm{XO}_{3}$ & $\mathrm{NO}_{2}$ & TAD & $A I$ & Na & $\begin{array}{r}13 T_{\mathrm{Cs}} \\
. \mathrm{Ci} /=\mathrm{I}\end{array}$ \\
\hline 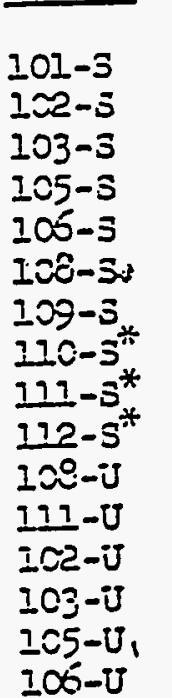 & $\begin{array}{c}1.412 \\
1.317 \\
1.279 \\
1.272 \\
1.263 \\
1.291 \\
1.272 \\
1.269 \\
1.219 \\
1.202 \\
-- \\
-.\end{array}$ & $\begin{array}{r}2.23 \\
1.12 \\
.95 \\
1.05 \\
.94 \\
1.10 \\
1.00 \\
1.15 \\
.95 \\
.92 \\
-.- \\
-.\end{array}$ & $\begin{array}{l}.017 \\
.064 \\
.020 \\
.054 \\
.017 \\
.25 \\
.04 \\
.12 \\
.025 \\
.073 \\
.036 \\
.055\end{array}$ & $\begin{array}{l}3.32 \\
5.92 \\
4.80 \\
3.15 \\
4.10 \\
3.61 \\
3.06 \\
3.4 \\
3.4 \\
3.5 \\
\ldots . . \\
\ldots-.\end{array}$ & $\begin{array}{l}.0112 \\
.126 \\
.086 \\
.137 \\
.160 \\
.108 \\
.096 \\
.084 \\
.30 \\
.17 \\
.231 \\
.097\end{array}$ & $\begin{array}{l}3.52 \\
2.175 \\
1.35 \\
2.02 \\
1.88 \\
2.16 \\
1.88 \\
1.93 \\
1.92 \\
1.38 \\
\ldots \\
\ldots\end{array}$ & $\begin{array}{l}1.41 \\
.21 \\
.28 \\
.20 \\
.17 \\
.30 \\
.211 \\
.30 \\
.19 \\
.40 \\
.25 \\
.12\end{array}$ & $\begin{array}{l}9.01 \\
8.48 \\
8.57 \\
5.86 \\
5.07 \\
6.54 \\
4.52 \\
6.50 \\
8.40 \\
5.37 \\
4.59 \\
4.93\end{array}$ & $\begin{array}{l}155 \\
121 \% \\
104 \\
-57.9 \\
37.9 \\
-0 . \\
-06.0 \\
51.5 \\
57.9 \\
-0- \\
-\cdots\end{array}$ \\
\hline
\end{tabular}

* Ren is leioratory vecurm eveporatcr.

The abcre analyses appear zormel except sawpis l0l-s. From the dete and pezameters gained in the three $S$ tenk sample eveporaticn zus, the resuits of 101-s appears to be si-rlaz in pinsicel and chemical propetiles $0 \vec{i}$ a iejk

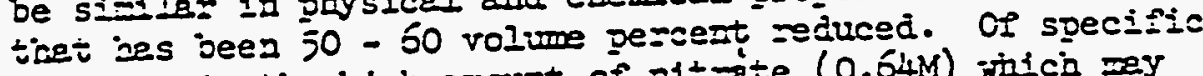

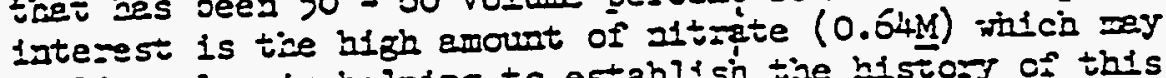
yteld a lue in helping to establisi tie history of this tank. 
J. 0. Sticl-uad

Peूe 2

sepiember 21, 1971

If there are any questicns pleese call we ca 2-7478.

DIP: th

cc: GL Borskein

IS Buckingien

Ma cambelI

DJ Iarletis

File

IB

?ccess Aics (5) 
TCRC-4 Atlantic Richfield Hanford Company

WHC-SD-WM-ER-323, Rev. 0

Date: December 7, 1973

To: R. L. Watser

From: W. H. Sant groffert

Subject: 242-S FEED SAMPLES T-9493 (10/5/73) 108-S

Vis-OTR Clear, yellow, $420 \mathrm{mR}$

$\mathrm{pH} \quad 11.5$

SpG $\quad 7.2455$

DTA No Exotherm

$\mathrm{Pu} \quad 7.63 \times 10^{-7} \mathrm{~g} / 1$

$\mathrm{Na} \quad 8.14 \mathrm{M}$

$\mathrm{SO}_{4} \cdot \quad 8.28 \times 10^{-3} \underline{M}$

Al $0.224 \underline{M}$

$\mathrm{NO}_{2} \quad 0.127 \underline{M}$

F $\quad 2.55 \times 10^{-3} \mathrm{M}$

$\mathrm{CO}_{3} \quad 0.129 \underline{\mathrm{M}}$.

$\mathrm{Cl}$

$\mathrm{OH} \quad 1.43 \mathrm{M}$

GEA $\quad{ }^{137} \mathrm{Cs}-8.76 \times 10^{4} \mathrm{iCi} / \mathrm{l}$

$\mathrm{NO}_{3} \quad 4.46 \mathrm{M}$

$30 \%$ Reduction - $27.5 \%$

$50 \%$ Reduction - $51.25 \%$

$\%$ Solids $-0.690 \%$

$\%$ Solids $-52.56 \%$

$8990 \mathrm{Sr}-1.22 \mu \mathrm{Ci} / 1$

$\mathrm{PO}_{4} \quad<3.77 \times 10^{-4} \mathrm{M}$

$\% \mathrm{H}_{2} \mathrm{O} \quad 63.56$ for $45 \mathrm{~min}$ wo solids

WHS: bp

CC: WR Christensen

GW Reddick, Jr.

WH Sant 


$$
\begin{aligned}
& \text { TCRC- } 6 \\
& \text { |ríx. } \\
& 112-5 \\
& \text { On } \\
& \text { ANALYSIS OP SUPERNATANT LIQUIDS TRON } \\
& \text { 242-S SLURRY RECEIVING TANXS }
\end{aligned}
$$

There vas some concern that the waste belng evaporated in the 242-s evaporator-crystallizer may be approaohing terminal concentration. samples of supernatant 11quid from 242-S sluryy recólolng tanks $108-8$ and $22 Z-5$ were samplod May 20 and $I I$ Lor analyses.

Because the samples solldilled on standing, Process Control Laboratorles could not run tbo analysis. Chemlcal Techaology Laboratory was able to analyze the samples and the results Indicate that the waste 18 not yet at terminal concentratioz. The results of the analyses are shown below.

The solidifled samples ware hested to botween 80 and $90^{\circ} \mathrm{C}$ in a water bath. A magnetlo otly bar was placed in the sample to help in red1s80lving the sample. For chemlcal analysea, a 5 mill111ter allquot of the hot slurty sample was lmmedlately diluted with 5 milililiters of wataz and ohemial analyaer zun on the dilution. Dangltiog and percent waters were rus on separate aliguots of tho hot glurry.

Please call we 1s you have eng questlons regarding Ehla rork.

ISB:52

AtE.

Co: V/Att.

* Babad

DG Barlow

NI BaTA

WP Metz

RC Roal

RI Halsez

Proceas Alda 
TABLEI

ARALYSIS OP 108-S AND 212-S SCPERHATANT IIQUIDS

\begin{tabular}{|c|c|c|c|c|}
\hline \multirow[b]{2}{*}{ Density } & \multicolumn{2}{|c|}{$108-5$} & \multicolumn{2}{|c|}{$112-5$} \\
\hline & 2.513 & " & 2.502 & \\
\hline $\mathrm{H}_{2} \mathrm{O}$ & $\cdot$ & 60.14 & & 58.38 \\
\hline $\operatorname{NaA} 10_{22}$ & $1.55 \mathrm{M}$ & 8.48 & $2.19 M$ & $6.5 \%$ \\
\hline $\mathrm{NaNO}_{2}$ & $1.94 \mathrm{II}$ & 8.8. & $2.02 \mathrm{M}$ & $9.3 *$ \\
\hline $\mathrm{NaNO}_{3}$ & $2.60 \mathrm{H}$ & 24.64 & $2.5 \underline{M}$ & 14.28 \\
\hline SaOI & $5.08 \%$ & 13.48 & $5.72 \underline{M}$ & 25.28 \\
\hline Tota & & 105.38 & & $103.5 \%$ \\
\hline
\end{tabular}

$237 \mathrm{Cg} \quad 4.58 \times 10^{5} \mu \mathrm{Cl} / 7.2 .14 \times 20^{5} \mu \mathrm{Cl} / 2$

*he analyzed nitrate value wa unexplafnably hlgh

by a lactor of 2 , so the result shown here is an

- estimated palue rather than an analyzed value. 
$T C R C-7$

WHC-SD-WM-ER-323, Rev. 0

DRAFT:

J. S. Buckingham

JuIy 8,1978

Achievement Report.

TANK FARM ASSISTANCE

Analysis of pilot Evaporator Samoles

$\therefore \quad \because$ a series of runs was made in the pilot vacuum

evaporator-crystallizer in which the temperature of tho.

concentrate was allowed to reach various levels. The

higher temperatures indicated a greater degree of evaporation of the waste solution. The physical and chemical properties of the feed and product materials are discussed in detail in ARH-ID-I0I, "Chemical and Physical Characteristics of Salt Caxes and Associated Mother Iiquors Produced by the Pilot Vacuum Evaporator-Crystallizez as a Function of Operating Temperature," June 1974 by J. 5. Buckiagham. The data are sumarizied here.

Solids produced at $49^{\circ} \mathrm{C}$ operating temperature were primazily sodium nitzate. The solids were slightiy bygroscopic but not deliquescent. Thomother liquor. associated with the solids was 10.86 M NaOB (28.4 percent).

Solids producad at $60^{\circ} \mathrm{C}$ operating temperature were hygroscopic, absorbing enough water to become deliguescent in 19 days. The composition of the solid phase was 23.8 percent $\mathrm{NaAlO}_{2}, 16.8$ pezcent NaNO, 38.4 pezcent $\mathrm{NaNO}_{3}$ and 20.3 percent $\mathrm{NaOH}$ (water free basis). The 
WHC-SD-WM-ER-323, Rev. 0

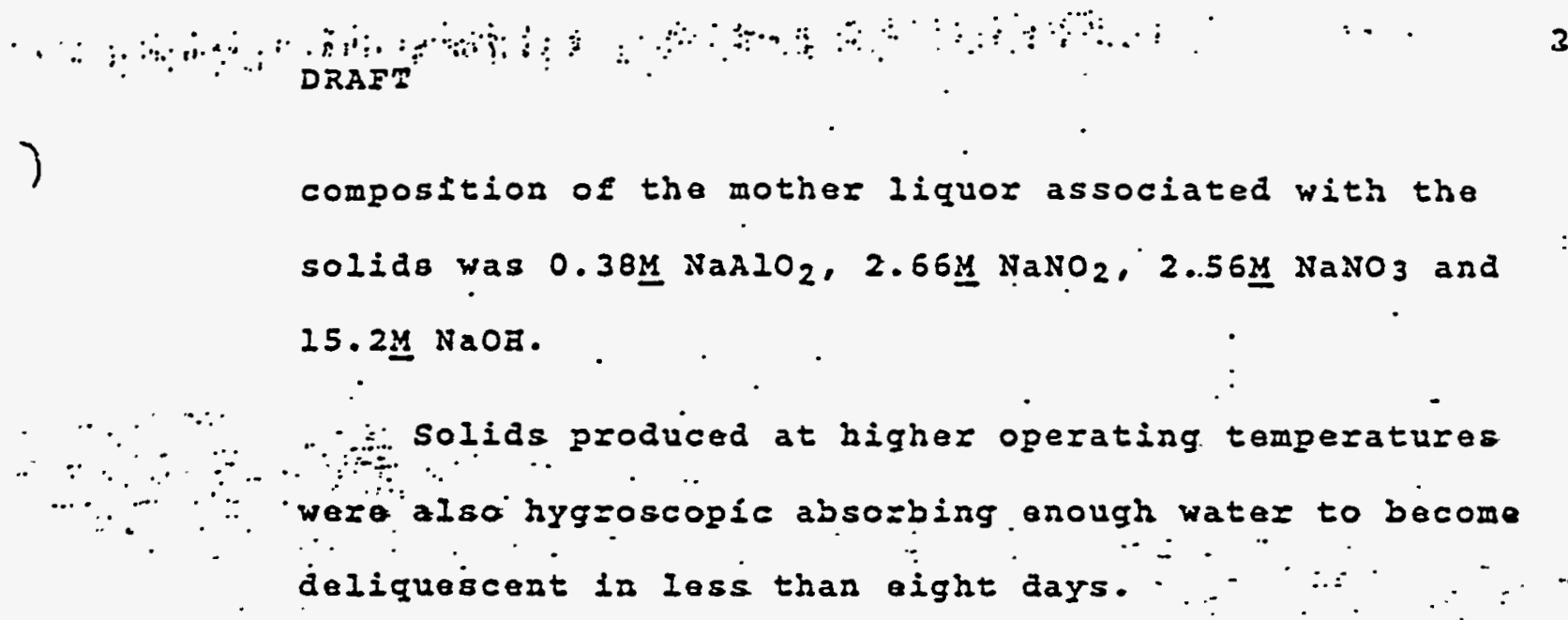


WHC-SD-WM-ER-323, Rev. 0
:

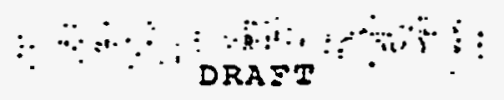

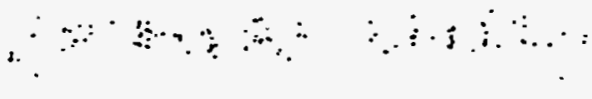
J. S. Buckingham
July 8,1974

Definition of Terminal Iiguia

Terminal waste liquor is now defined as mother liquor associated with a solld phase that. is not $\therefore \because \cdots \quad \because \because \therefore \quad \therefore$.

deliquescent: that is, a solid phase that will not. absorb enough moisture from the atmosphere to become Iiguid. Based on data from ARE-ID-1OI the composition of terminal Iiquor is estimated to be 40 percent $\pi_{2} 0$, $0.5 \underline{\underline{K}} \mathrm{NaAIO}_{2}, 2.5 \underline{\mathrm{M}} \mathrm{NaNO}_{2}, 2.5 \underline{\mathrm{M}} \mathrm{NaNO}_{3}$, and $22 \underline{\mathrm{M}} \mathrm{NaOZ}$. 


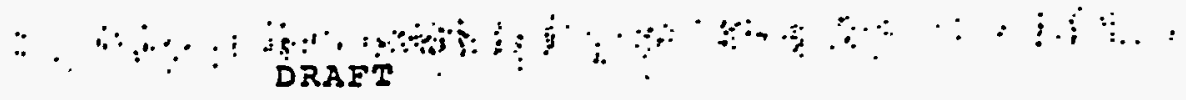

Analysis of Supernatant Liquid (Mother Liquor) Zrom Tanks 108-s and 112-s

There has been some concern that-the waste being evaporated in the 242-s evaporator may be approaching terminal concentration. Samples of supernatant liquid

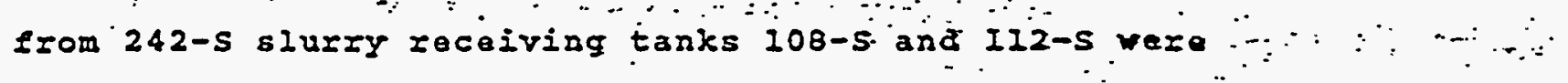
sampled May 10 and 11 for analysis. The results aro shown below.

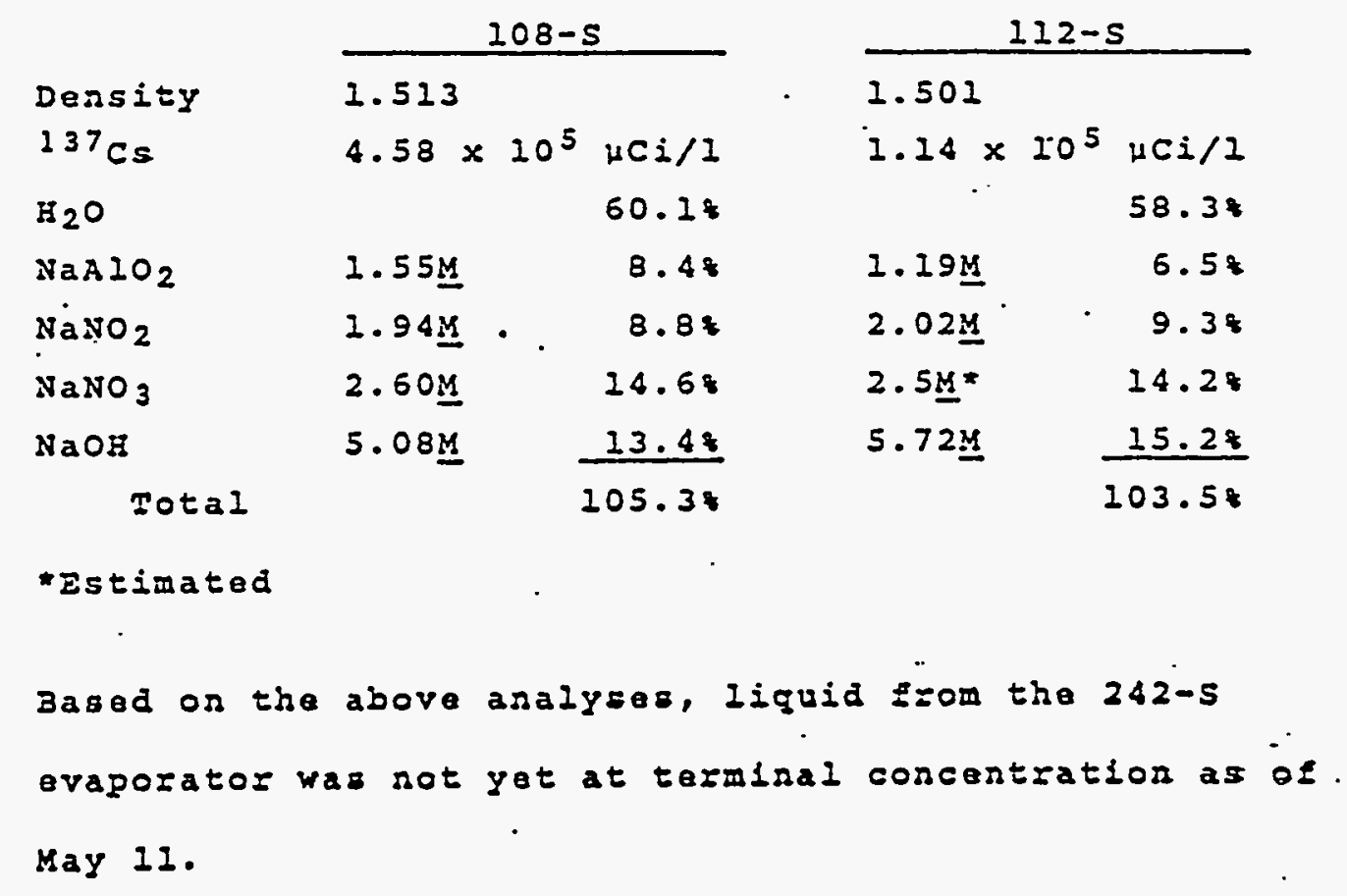


Analysis of Solid phase Erom mx $108-5$

A sample was taken of the solids in Tank 108-s

Iune 25, 1974. The sample contained two layers of salid. The bottom Iayer was composed of rather coarse yeliow cróstals while the upper Iayer appeared to be a soft sludge. Analyses ara not yet complete, but those Einished to date aze shown below.

\section{TK I08-S SOIid}

\begin{tabular}{|c|c|c|c|c|c|}
\hline \multirow[b]{2}{*}{ Damp deasity } & \multicolumn{3}{|c|}{ ToD } & \multicolumn{2}{|l|}{ Bottom } \\
\hline & 1.495 & & & 1.393 & $\cdot$ \\
\hline DIY dansity & 1.236 & & & 1.222 & \\
\hline $\mathrm{Z}_{2} \mathrm{O}$ & 24.08 & & & 11.58 & \\
\hline $\mathrm{NaAIO}_{2}$ & 5.48 & & & $1.8 \%$ & \\
\hline $\mathrm{NaNO}_{2}$ & 4.68 & & & 1.98 & . \\
\hline $\mathrm{NaNO}_{3}$ & & . & & $68.1 *$ & \\
\hline $\mathrm{NaOZ}$ & 6.38 & & & $3.6 z$ & \\
\hline $\mathrm{Na}_{2} \mathrm{CO}_{3}$ & & & & $6.5 t$ & \\
\hline $\mathrm{Ze}_{2} \mathrm{O}_{3}$ & 2.08 & & & & \\
\hline $\mathrm{Al}_{2} \mathrm{O}_{3}$ & $<0.38$ & & & & \\
\hline $239 \geq u$ & $1.87 \times$ & $10^{-8}$ & $g / g$ & $1.60 \times 10^{-8}$ & $g / 9$ \\
\hline $89+905=$. & $3.15 \times$ & $10^{-4}$ & $\mu C i / g$ & $0.584 \mathrm{HCI} / \mathrm{g}$ & - \\
\hline${ }^{137} \mathrm{Cs}$ & $1.74 \times$ & $10^{-2}$ & $\mu C_{i} / g$ & $\$ 4.4 \mathrm{uCi} / \mathrm{g}$ & \\
\hline $134 \mathrm{Cs}$ & & & & $0.40 \mathrm{HCi} / \mathrm{g}$ & \\
\hline $125 \mathrm{sb}$ & $0.46 \mathrm{~L}$ & $C i / g$ & & $\cdot$ & \\
\hline $10 \sigma_{R \mu} 10 \sigma_{R i}$ & $3.95 \times$ & $10^{3}$ & $\mathrm{Ci} / \mathrm{g}$ & & \\
\hline
\end{tabular}

Thermal conductivity of the lower solid is being run. A sample of superattant liquid from 108-S tank was taxen June 30 , but has not been asalyzed. 
TCRC- 3

WHC-SD-WM-ER-323, Rev. 0

$\left.\Leftrightarrow i 0^{C}\right\}, 1$

Date: December 18, 1973

To: R. L. Waiser

From: W. H. Sant $\because->+$ thand

Subject: 242-S FEED SAMPLES T-9494 (10/5/73) 109-S

Vis-OTR Clear, yellow, $350 \mathrm{mR}$

$\mathrm{pH} \quad 11.5$

SpG. $\quad 1.2490$

DTA No Exotherm

Pu $\quad 2.29 \times 10^{-6} \mathrm{~g} / 1$.

$\mathrm{Na} \quad 5.46 \underline{\mathrm{M}}$

$\mathrm{SO}_{4} \quad 9.80 \times 10^{-3} \mathrm{M}$

A7 0.174 in

$\mathrm{NO}_{2} \quad 0.158$ in

F $\quad 2.26 \times 10^{-3} \underline{M}$

$\mathrm{CO}_{3} \quad 0.024$.

$\mathrm{Cl}$

$\mathrm{OH} \quad 1.81 \underline{\mathrm{M}}$

GEA $\quad{ }^{137} \mathrm{Cs}-8.44 \times 10^{4} \mu \mathrm{Ci} / \mathrm{]}$

$\mathrm{NO}_{3} \quad 4.35 \mathrm{M}$

$30 \%$ Reduction - $28.8 \% \quad \%$ Solids $-0.526 \%$

$50 \%$ Reduction - $52.5 \%$

$\%$ Solids $-67.7 \%$

$8990 \mathrm{Sr} \quad 1.712 \mu \mathrm{Ci} / 1$

$\mathrm{PO}_{4} \quad<3.77 \times 10^{-4} \mathrm{M}$

WHS: bp

cc: WR Christensen GW Reddick, Jr. WH Sant

$5^{\circ} \mathrm{C} 45 \min$ mo solid

\% water 66.34

$\%$

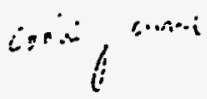



JS Buckingham
DA Dodd
RG Geier
DC Harlow
iE Horton
DC Lini
RL Walser
RA Hatrous $=$
WI Winters
CIL office file
Circulate CTL Exempt

File

CTL 503
To: D. C. Lini

From: J. E. Horton

\section{ENGINEERING ASSISTANCE WASTE CONCENTRATION}

Budget Number:

K.W217
Contributors:

J. E. Horton

E. L. Richards

ABSTRACT

Work has been completed on Tanks ipg-5and 103-5X.

Analysis indicated Tanks 109-S and 103-5X salts were a mix ture of sodium nitrate, associated with intersititial mother liquor. These are the first samples received from Tanks 103-SX and 109-S after receiving salt slurry from 242-5 evaporator process.

\section{PROGRESS DURING REPORTING PERIOD}

Samples were received in September and Octoder from Tanks 109-5 and 103-5X. Analyses were made by weighing a known amount of solids from each tank and diluting them with watar and acid. Laboratory resuits are reported in Tables I and II.

PLANS FOR NEXT REPORTING PERIOD

Report and evaluate any Tank Farm samples recaived.

CONCLUSIONS

He have completed or have in progress, all sampies received from the Tank Farms.

\section{REFERENCES}

1. Letter, December 8, 1976, J. E. Horton to 'H. R. Christiensen, "Analysis of Salts from Tank 109-S"

2. Letter, December 3, i976, J. E. Horton to W. R. Christiansen, "Analysis of Tank i03-SX salts" 


\section{TABLE I}

\section{ANALYSİS OF TANK 103-SX - SALTS}

$\mathrm{Bulk}$ density
Particle density
$\mathrm{H}_{2} \mathrm{O}$
$\mathrm{ALO}_{2}$
$\mathrm{NO}_{2}$
$\mathrm{NO}_{3}$
$\mathrm{CO}_{3}$
$\mathrm{OH}$
$\mathrm{Na}$
$\mathrm{Fe}$
$\mathrm{PO}$
$\mathrm{SO}_{4}$
$\mathrm{Ca}$
$\mathrm{Cr}$
$\mathrm{Mg}$
$\mathrm{Mn}$
$\mathrm{Si}$
$\mathrm{Ni}$
$\mathrm{Pu}$
$89+905 r$
$137 \mathrm{Cs}$

$0.84 \mathrm{~g} / \mathrm{cc}$

$2.24 \mathrm{~g} / \mathrm{CC}$

$15.0 \%$

$1.7 \%$

2. $7 \%$

$51.7 \%$

$6.3 \%$

< 0.009\%

$27.8 \%$

$0.2 \%$

$0.3 \%$

$0.5 \%$

$0.7 \%$

$0.002 \%$

$0.02 \%$

$0.04 \%$

$0.3 \%$

$0.004 \%$

$0.446 \mathrm{ug} / \mathrm{g}$

$38.0 \mathrm{uci} / \mathrm{g}$

$95.0 \mathrm{uci} / \mathrm{g}$ 


\section{TABLE II}

ANALYSIS OF TANK 109-S - SALTS

Bulk Density
Particle density
$\mathrm{H}_{2} \mathrm{O}$
$\mathrm{AL}$
$\mathrm{CO}_{3}$
$\mathrm{Fe}$
$\mathrm{OH}$
$\mathrm{NO}_{2}$
$\mathrm{NO}_{3}$
$\mathrm{MN}$
$\mathrm{Mg}$
$\mathrm{Ca}$
$\mathrm{Cr}$
$\mathrm{Si}$
$\mathrm{Na}$
$\mathrm{PO} 4$
$\mathrm{SO}_{4}$
$\mathrm{Pu}$
$89 \div 90 \mathrm{Or}$
$137 \mathrm{Cs}$

$1.27 \mathrm{~g} / \mathrm{cc}$

$2.38 \mathrm{~g} / \mathrm{cc}$

$10.6 \%$

$1.3 \%$

$4.2 \%$

$0.3 \%$

$0.3 \%$

$1.4 \%$

$53.5 \%$

$0.003 \%$

$0.02 \%$

$0.08 \%$

$0.7 \%$

$0.1 \%$

$35.0 \%$

$0.07 \%$

$0.9 \%$

$0.654 \mathrm{ug} / \mathrm{g}$

$14.6 \mathrm{uci} / \mathrm{g}$

$71.8 \mathrm{uci} / \mathrm{g}$ 
Atlantic Richfield Hanford Company

WHC-SD-WM-ER-323, Rev. 0
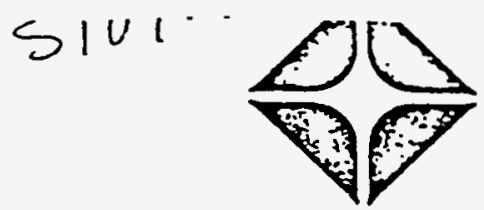

Date: $\quad$ September 16, 1974

To: R. L. Walser

From: R. E. Wheeler prytulen

Subject: ANALYSIS OF TANK FARM SAMPLES . SAMPLE: T-4544 109-S

Vis-0TR: Yellow, 35\% solids, $1500 \mathrm{mrad} / \mathrm{hr}$

pH: $\quad>13.1$

SPG: $\quad 1.7607$

$\mathrm{OH}: \quad 5.57 \mathrm{M}$

A7: $\quad 7.19 \mathrm{M}$

Na: $\quad 10.45 \underline{M}$

$\mathrm{NO}_{2}: \quad 7.76 \mathrm{M}$

$\mathrm{NO}_{3}: \quad 4.82 \mathrm{M}$

Pu: $\quad 9.56 \times 10^{-6} \mathrm{~g} / 1$

DTA: No Exotherm below $200^{\circ} \mathrm{C}$

$\mathrm{SO}_{4}: \quad 2.76 \times 10^{-2} \mathrm{M}$

$\mathrm{PO}_{4}: \quad 0.146 \underline{\mathrm{M}}$

$\mathrm{F}: \quad 1.88 \times 10^{-2} \mathrm{M}$

$\mathrm{CO}_{3}: \quad 0.146 \underline{\mathrm{M}}$

GEA: $\left.\quad{ }^{134} C_{S}-6.32 \times 10^{3} \mu \mathrm{Ci} / \mathrm{ga}\right]$

Hater: $44.14 \%$

Cooling Curve: About 35\% solids at room temperature, About 5\% solids after heating to $100^{\circ} \mathrm{C}$ for 60 minutes.

$\begin{array}{ll}45^{\circ} \mathrm{C} \text { for } 60 \mathrm{~min} . & 2 \% \text { solids } \\ 40^{\circ} \mathrm{C} \text { for } 45 \mathrm{~min} . & 2 \% \text { solids } \\ 35^{\circ} \mathrm{C} \text { for } 45 \mathrm{~min} . & 2 \% \text { solids } \\ 30^{\circ} \mathrm{C} \text { for } 45 \mathrm{~min} . & 3 \% \text { solids. } \\ 25^{\circ} \mathrm{C} \text { for } 60 \mathrm{~min} . & 3 \% \text { solids. } \\ 20^{\circ} \mathrm{C} \text { for } 45 \mathrm{~min} . & 3 \% \text { solids. } \\ 15^{\circ} \mathrm{C} \text { for } 45 \mathrm{~min} . & 4 \% \text { solids. } \\ 10^{\circ} \mathrm{C} \text { for } 45 \mathrm{~min} . & 4 \% \text { solids. } \\ 5^{\circ} \mathrm{C} \text { for } 45 \mathrm{~min} . & 4 \% \text { solids. }\end{array}$

REW: jo

cc: JS Buckingham WR Christensen

RE Wheeler

WP Metz 
Atlantic Richfield Hanford Company

\section{$T C R C-/ 2$}

Date: November 19,1974

To: R. I. Wialser

From:

3. E. wheeler $P$ prptevi

Sudject: ANAIYSIS GF TANK FARM SAMPIES

SAMPLE: T-8I2I I09-S

Vis-OTR: Green. 25\% solids $450 \mathrm{mRad} / \mathrm{hr}$

요:

14.0

SDG :

1.6717

$\mathrm{OH}$ :

$5.07 \mathrm{M}$

DIA: No exctherms below $200^{\circ} \mathrm{C}$

GaA: $\quad \begin{aligned} & 134 \\ & \quad 137 \mathrm{Cs}-1.54 \times 10^{4} \mu \mathrm{Ci} / 521\end{aligned}$

X: $\quad 5.44 \times 10^{-2} \mathrm{M}$

$B \overline{4} \bar{\alpha}: \dot{u} \dot{c}$

co: is Buckingham

WR Christensen

FM Ginestet

Ba ineeier

JC Nomacx
WHC-SD-WM-ER-323, Rev. 0

$\sum_{0} 9.4$ 


$$
\text { TCRL-14 }
$$

Date: $\quad$ December 8, 1976

To:<smiles>CCCC</smiles>

From:

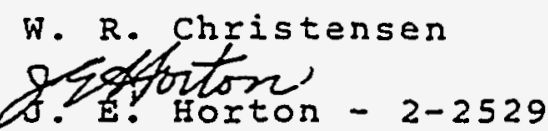

SUbject: ANAIYSIS OF SAITS FROM TANK IO9-S

A sample was received from Tank 109-s on october 29, 1976. Solids from Tank 109-s were coarse granular crystals, yellowish in color, being about 85 to 90 percent water soluble. This is the first sample taken from Tank 109-s after receiving salt siurry waste from 242-s evaporator process.

Analyses indicated $109-5$ salts to be sodium nitrate associated with interstitial mother liquor. Analyses were made by weighing 4.7666 grams of solids, dissolving in water and diluting to a known volume. The fraction not soluble in water was dissolved in concentrated HCI, and diluted with water to a known volume. The results of the laboratory work are reported in Table I (attached).

Please call me if you have any questions regarding this work.

$J E H: j f$

Att.

cc: w/att.

IS Buckingham

RG Geier

SS Koegler

DC Iini

JE Mirabella

WE Ogren

RL Walser

Process Aids (7)

File

$I_{B}$ 
WHC-SD-WM-ER-323, Rev. 0 $\rightarrow 1 U 7 . J$

TABIE I

\section{ANALYSIS OF TANK 109-S SALTS}

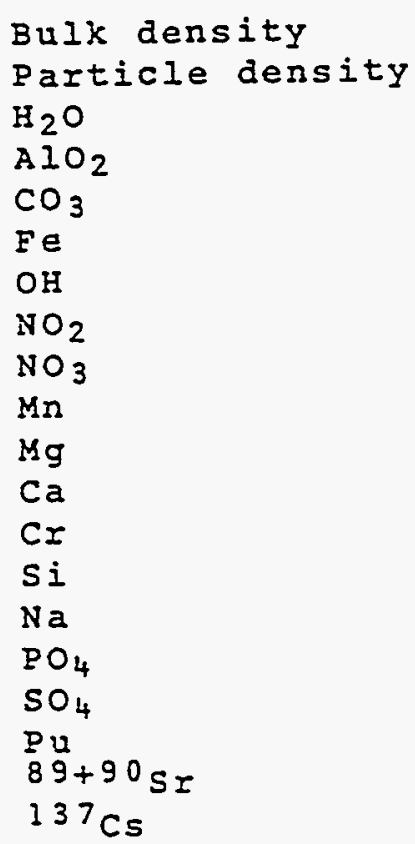

$$
\begin{array}{ll}
1.27 & g / c c \\
2.38 & g / c c
\end{array}
$$$$
10.68
$$$$
1.38
$$$$
4.28
$$$$
0.38
$$$$
0.38
$$$$
1.48
$$$$
53.5 \%
$$$$
0.0038
$$$$
0.028
$$$$
0.08 \%
$$$$
0.1 \%
$$$$
0.18
$$$$
35.08
$$$$
0.01 \%
$$$$
0.98
$$$$
0.654 \mu \mathrm{g} / \mathrm{g}
$$$$
14.6 \mu \mathrm{Ci} / \mathrm{g}
$$

$71.8 \mu \mathrm{Ci} / \mathrm{g}$ 
Atlantic Richfiẹid Hanford Company

Date: Septembe: 21 , I9TI

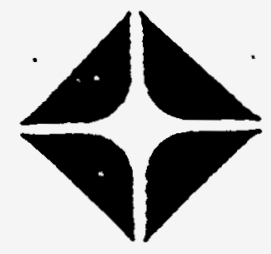

Tc:

Fick: $\quad$ D. A. Pumbar

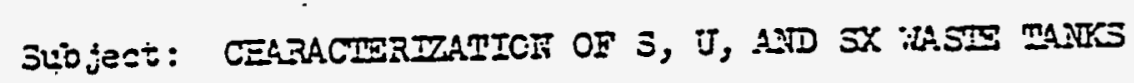

Jampleng and analyses of $S$, IJ and $\$ X$ weste tanks to ilextiny feeü rate:ial for the 242-s vacum evaporator is prcg-essing. Tc inte tiree che liter samples from the 3 Jand tanks have been prccessed tin rough the labcratory reculm evaporator. In additicn tea 3 Farm tarks and 5 i* 0 Fand tanks jave been samiled and subritied for cineracterization anelyses. The zesults are shown in tine follorias taile.

\section{C:aractezization Araivses ci ieste Taniks}

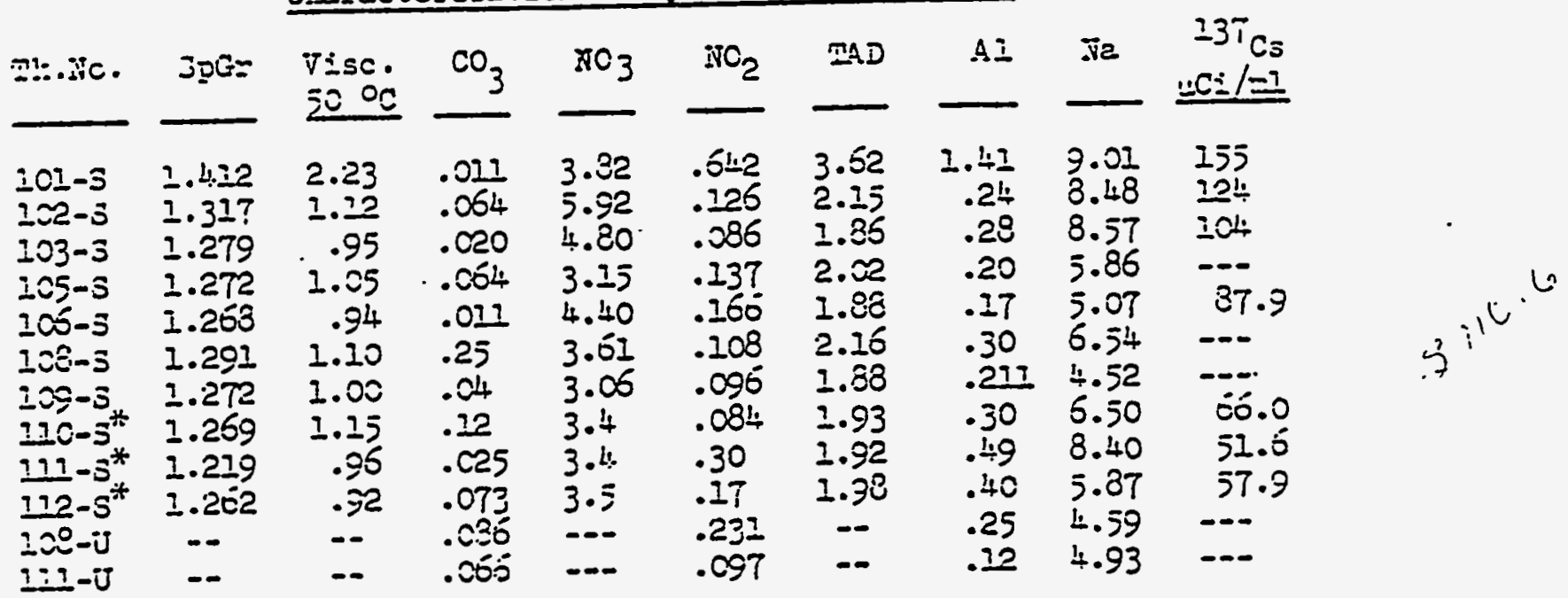

* San in lejoczetcry vecuran eveporatcr.

The above aceiyses appear zormel except sanplo 101-5. Ercll tie jeth and perrmeters galied in the three $S$ tank semple eveporetion zws, the zesults of $101-5$ appeers to be si-iie: 1 in jibysical add chemical prope:ties $0 \vec{i}$ a iank

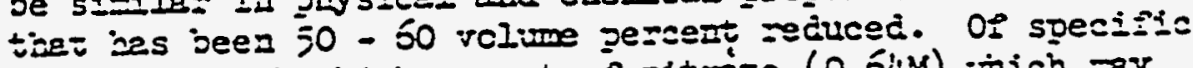

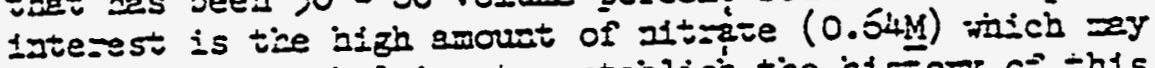

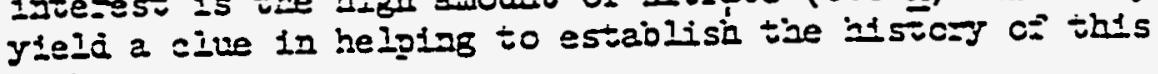
tank. 
J. 0. जicl-uc

गese 2

Seฏterke: 21, 1971

If there are any questicns pleese call ne ca 2-74it.

DLP:an

se: GI 30rstein

JS 3ucivingher

ME Cambeli

DJ Iarkin

File

i3

Process Ains (5) 
Date: September 25, 1974

To: พ. R. Christersen

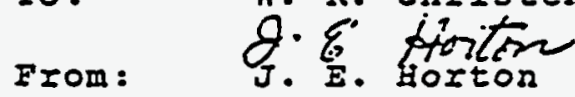

Subject: ANAIYSIS OF SIUDGE SAMPLE FROM TANK 120-S

We received a sample of siudge from Tank II0-s on August 9, 1974. The 110-s sludge sample was composed of two layers. The top layer being a soft dark brown color, and represented about 10 volume percent of the total sample. The bottom layer was gray in color, with hard large white chunks throughout the sample. The bottom layer represented about 90 volume percent of the sample.

Sludge analyses were made by fusing 2.0 milliliters of the top layer of sludge with $\mathrm{KOH}$, dissolving the melt in concentrated $\mathrm{FCl}$, and diluting with $\mathrm{H}_{2} \mathrm{O}$ to a volume of 270 milliliters. Three milliliters of the bottom layer were fused with $\mathrm{KOH}$, and the melt was dissolved in concentrated HCl and brought up to a volume of 210 milliliters. The analysis in the tables below are reported in liters of damp, as-received, sludge.

please call me if you have any questions.

JEH: $j \pm$

Att. (1)

$c c: w / a t t$.

I Babad

IS Buckingham

DG Harlow

NL Harms

RI Walser

Process Aids 
WHC-SD-WM-ER-323, Rev. O

TANK FARM ASSISTANCE

Analysis of Tank IIO-s siudge Sample

Sludge sample 110-s was composed of two layers, the

top layer representing about 10 volume percent of the

total sample, the bottom layer represented about 90 volume

percent of the sample received. Tank 110-s is a possible

receiving tank for waste from the 242-5 Evaporator.

Analysis reported in the tables below are reported in

liters of damp, as received, sludge.

\section{TABLE I}

\section{TOP LAYER}

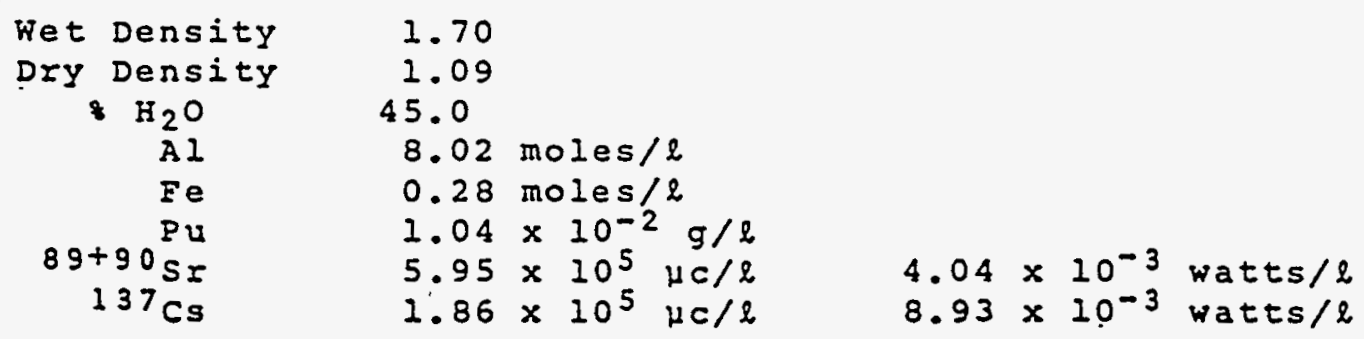

TABLEII

\section{BOTTOM LAYER}

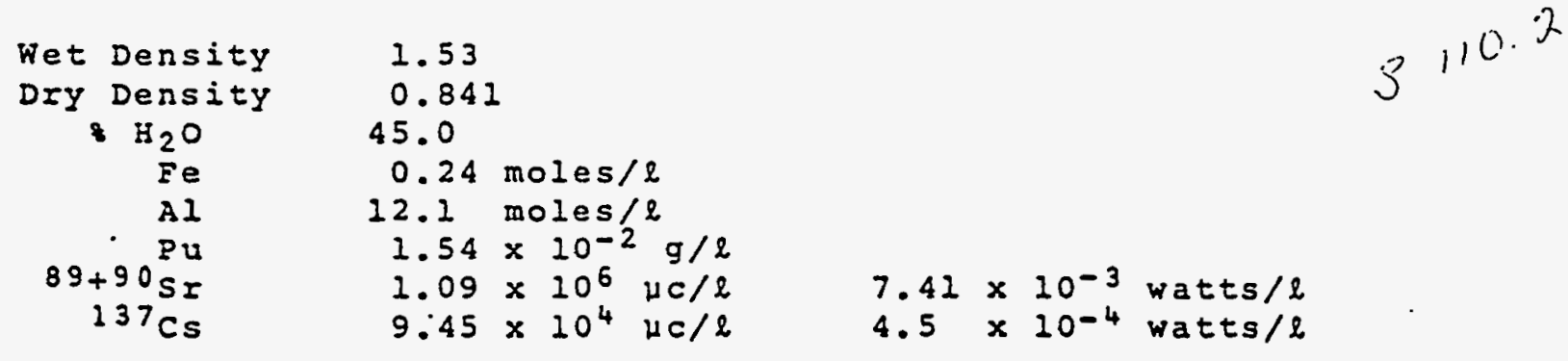


Date:

To:

From:

\author{
December 17, 1974
}

R. L. Walser

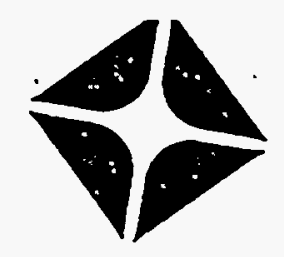
R. E. Wheeler
Subject: AMALYSIS OF TANK FARM SAME LC SAMPLE: T-8042 FARM SAMPLES

- pH: $\quad 14$ Dark Green. 50\% solids. 3.5 Rads supernate.

$\mathrm{OH}:$
$\mathrm{AT}:$
$\mathrm{Na}:$
$-\mathrm{NO}_{2}:$
$-\mathrm{NO}_{3}:$

Pu:

$\mathrm{SO}_{4}: \quad 2.78 \times 10^{-5} \mathrm{~g} / \mathrm{ga} 7$

$\mathrm{PO}_{4}:$

$F:$

$\begin{array}{ll}\mathrm{CO}_{3}: & 3.95 \times 10 \\ \text { GEA: } & 0.203 \mathrm{M} \\ 134 \mathrm{Cs}\end{array}$

17: $\quad 3.79 \mathrm{M}$

7.536
$3.79 \mathrm{M}$
$.48 \mathrm{M}$

$\quad 71.82 \mathrm{M}$

$\mathrm{O}_{3}: \quad \quad 7.65 \mathrm{M}$

$\quad 3.72 \mathrm{M}$

$89,{ }^{\circ 0} \mathrm{Sr}: \quad{ }^{37 \mathrm{Cs}}-2.08 \times 10^{4} \mathrm{HCi} / \mathrm{gal}$

Water: $\quad 45.568 \times 10^{3} \mathrm{\mu Ci} / \mathrm{gal}$

Cooling Curve:

REW:jd

$$
\begin{aligned}
& 40^{\circ} \mathrm{C} \text { for } 45 \mathrm{~min} \text {. No solids. } \\
& 35^{\circ} \mathrm{C} \text { for } 60 \mathrm{~min} \text {. No solids. } \\
& 30^{\circ} \mathrm{C} \text { for } 45 \mathrm{~min} \text {. Nor }
\end{aligned}
$$

$25^{\circ} \mathrm{C}$ for $45 \mathrm{~min}$. No solids.

$20^{\circ} \mathrm{C}$ for $45 \mathrm{~min}$. No solids.

$75^{\circ} \mathrm{C}$ for $45 \mathrm{~min}$. No solids.

$10^{\circ} \mathrm{C}$ for $45 \mathrm{~min}$. Suspended Solids.

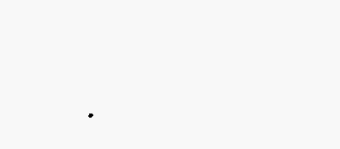




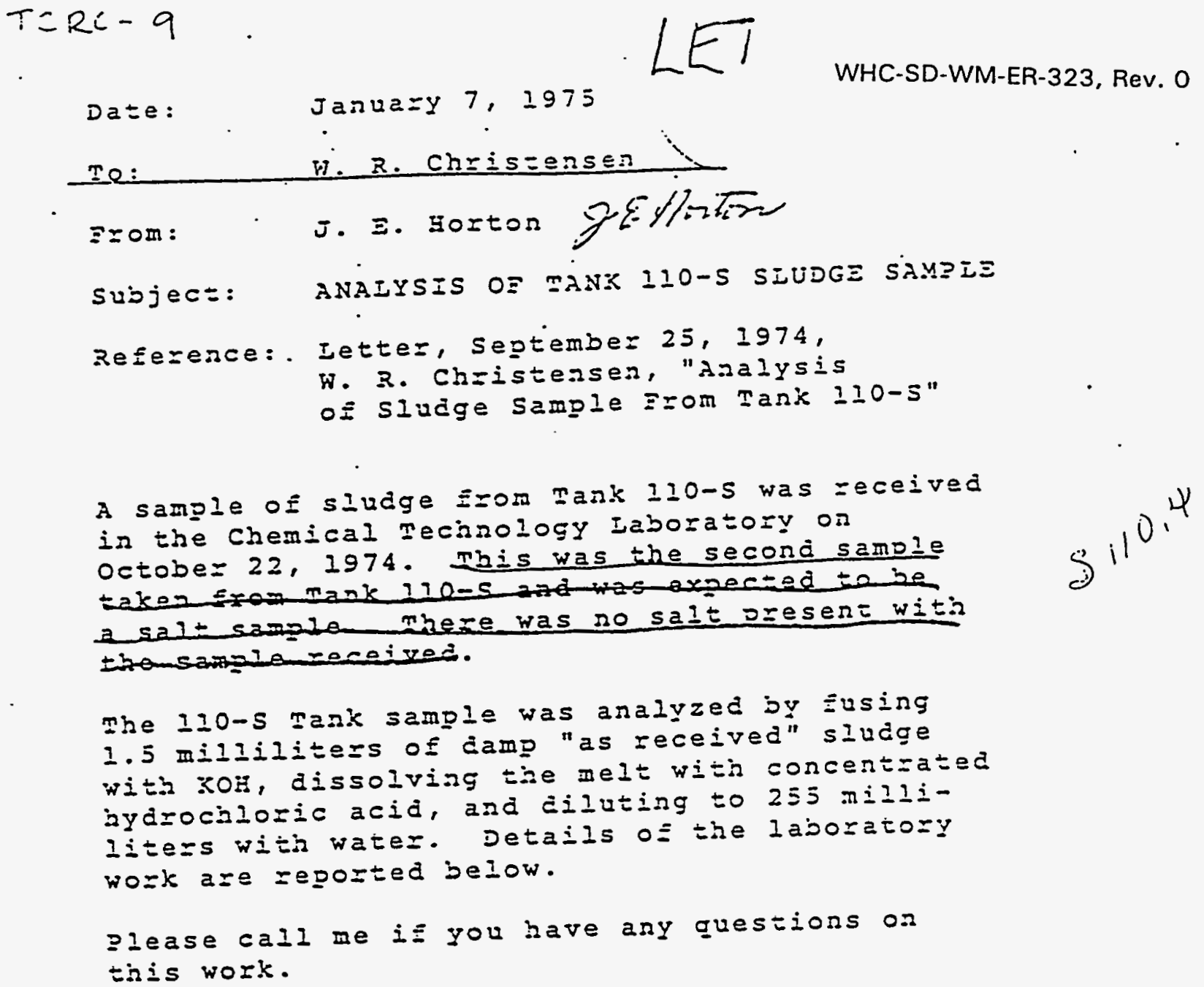

A sample of sludge E=or mank lio-s was =eceived in the chemical pecinology Iaboratory on october 22, 1974 . This was the second sampie

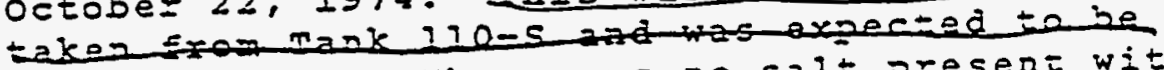
a salt sagle nhese was no salt oresert witi thomsaña -aceived.

The lio-s Tank sample was analyzed by Eusing 1.5 milliliters of camp "as received" slucge with KoH, dissolving the nelt with concent=aちed bydrociloric acid, and eiluting to z5j milliliters with water. Details oj the laboretory wozk are zeported below.

please call me if you have any questions on tinis work.

\section{ANALYSES OE IIO-S SIITDGE}

$$
\begin{array}{r}
2 u \\
89+905= \\
137 \mathrm{Cs} \\
134 \mathrm{Cs}
\end{array}
$$

$6.29 \times 10^{-4} \mathrm{~g} / 2$
$5.43 \times 10^{5} \mu \mathrm{C} / 2$
$8.10 \times 10^{5} \mu \mathrm{C} / 2$
$4.57 \times 10^{3} \mu \mathrm{L} / 2$

$3.69 \times 10^{-4}$ watts/2
$3.89 \times 10^{-3}$ wat $\left.5 / 2\right\}$

$.41 \frac{3 \pi / 4 r}{r^{3}}$

Јコม :

$$
\begin{aligned}
& c=\text { I } 3 a b a d \\
& \text { Js 3uckingham } \\
& \text { DG } \mathrm{Ba}=10 \mathrm{w} \\
& \text { NI } 3 a=n s \\
& R \equiv \text { Van deI Cook } \\
& \text { RI พaIse: } \\
& \text { P=ocess inds (7) } \\
& \text { CII Bile - III } \\
& \text { crd }=122 \\
& \text { I3 }
\end{aligned}
$$


Date :

To:

From:

subject:

References:
June 20,1975

W. R. Christensen

J. E. Horton fromis:-

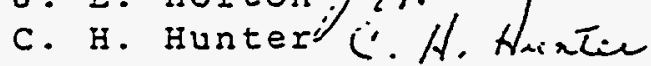

(1) Letter, September 25, 1974, J. E. Horton to i. R. Christensen, "Analysis of sluage from Tank 110-s"

(2) Letter, January 7, 1975,

J. E. Hozton to i. R. Christensen, "Analysis of Tank llo-s sludge sample"

Chemical Technology Laboratory (CII) receivec a sample from Tank Ilo-s on April 23, 1975. This is the third sample taken from the 110-s Tank. Analyses of the first two samples, which were sludge, are discussed in the two reference letters.

The current sample had a small amount oz salt on top of the sludge. This amounted to about tiree to four percent of the total sample received. The salt was yellow in color.

The analyses indicate that the salt in 110-s is essentially sodium nitrate dampened with entrained mother liguor. Details of the analyses are discussed on the following page. 
W. R. Christensen

Page 2

June 20,1975

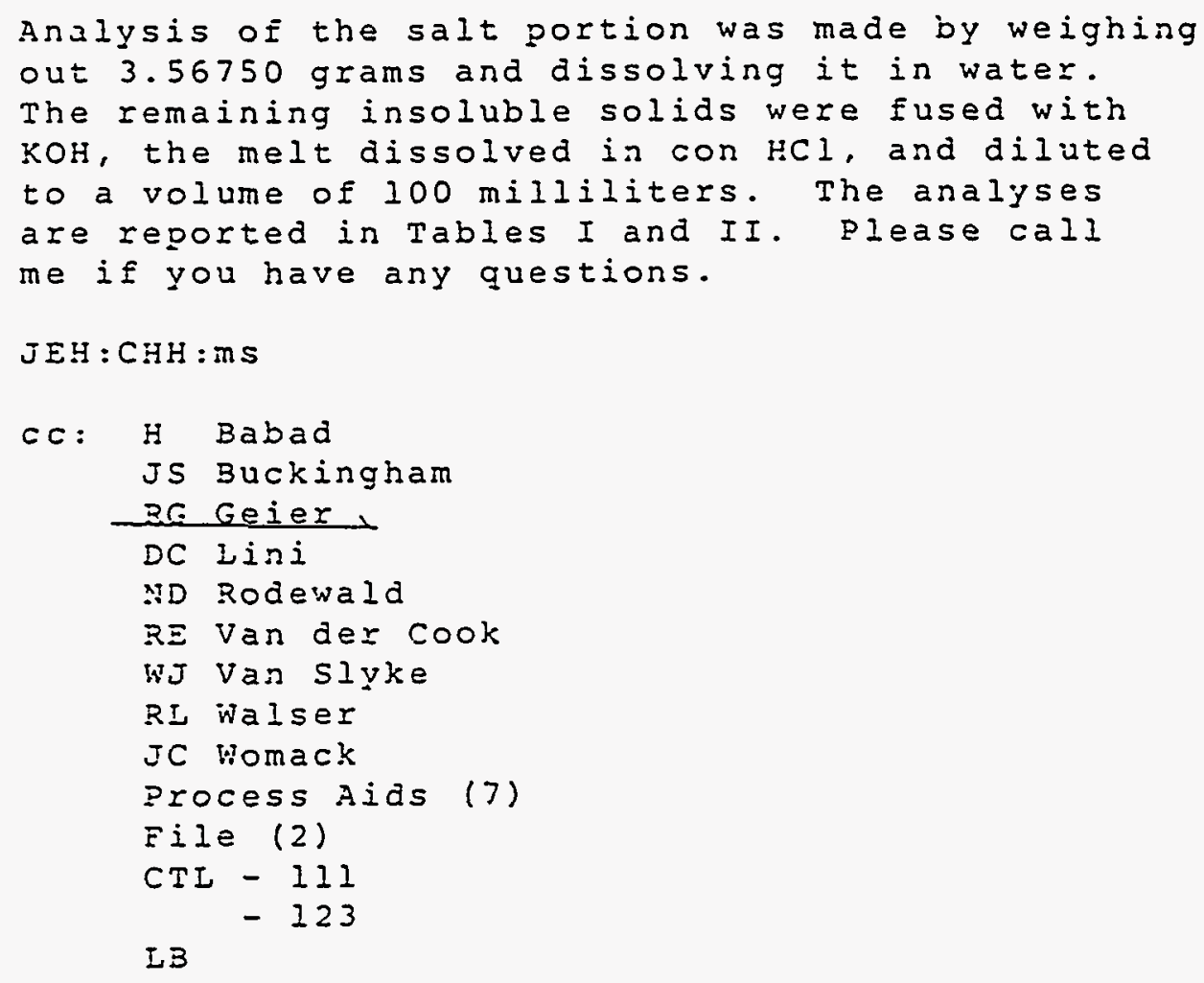


TABIE I

ANALYSIS OF SALT FROM TANK ILO-S

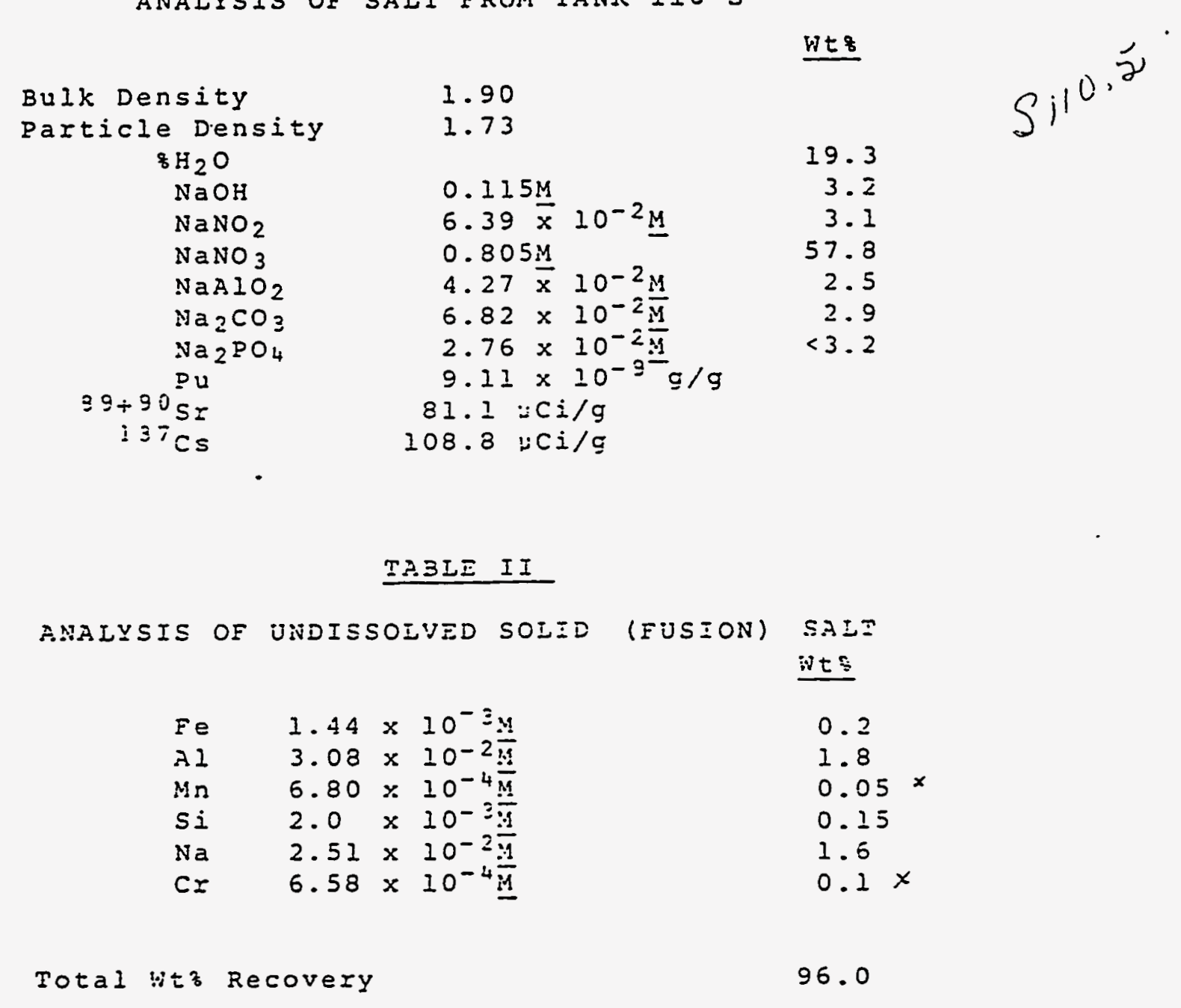


Dete: September 21,1971

Tc:

F⿻ct:

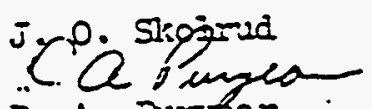

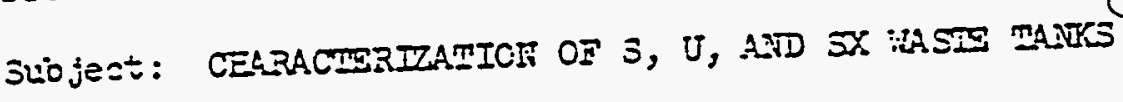

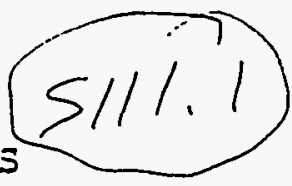

sanoling and analyses of $S$, i and $\mathrm{XX}$ weste tanks to lientify Eee: raterial for the 242-s resulu eveporator is progessins. Tc jate tiree cae lite: samples frcm the 3 Fan tanks bave jeel processed through the lebcretory reculum evaportor. In adcition ten is Fam tanks and sio J Fard tarks iave been semiled and subritted for cinerecterzation anelyses. Tio gesults are shcw in tie follorias taile.

c:aracterizaticn Arajyses cs ileste Tarks

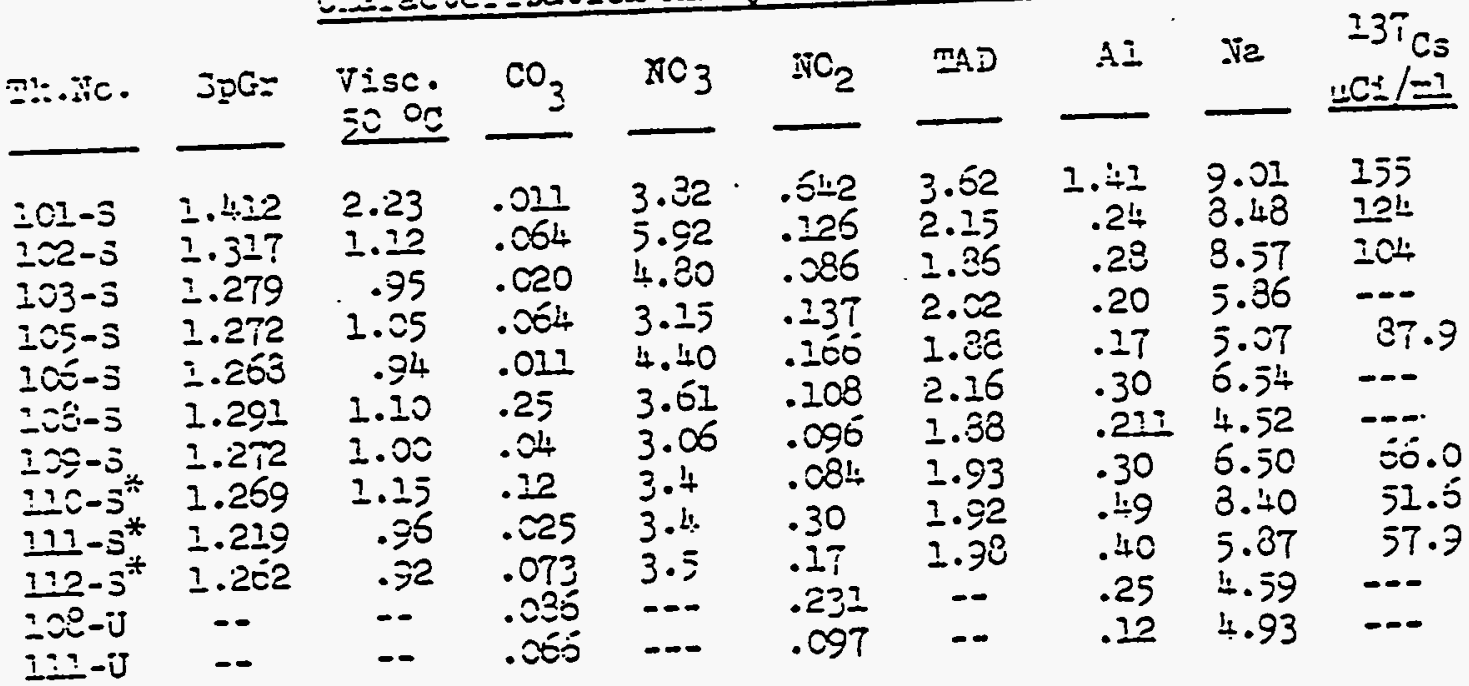

$i \approx 5-i$

$103-4$

ICS-i丁,

iळi-j

* Res in lejoratory vacuun eveporatcr.

The sbove arelyses appear jormel except semplo lol-s. IZCD ije deta and perameters jained ty the three $S$ tajk semple evepcraticn zins, the results of 101-s anpeess to

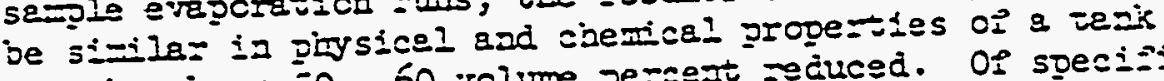

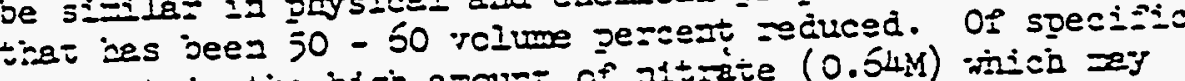

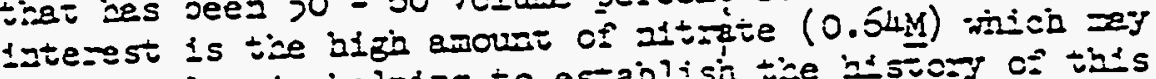

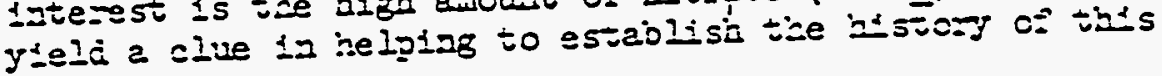
zask. 
J. 0. जicl-xa'

iese 2

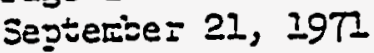

If there are aby questicns pleese ceII $=$ ca 2-7478.

DLF: in

ce: GL 30rsteim

is 3uckinghem

iv CambelI

DJ Iarieta

Fisle

I3

Pocess Ais (5) 
$T C R C-28$

Atlantic Richfield Hanford Company

WHC-SD-WM-ER-323, Rev. 0

Date:

May 29,1974

To:

From:

W. R. Christensen

subject:

J. S. Buckingham

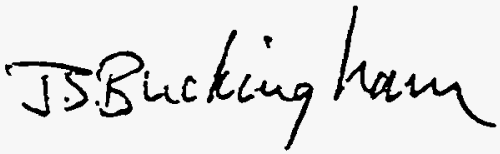

ANAIYSIS OF SIUDGE SAMPLE FROM TANK III-S

Reference: Letter, March 25, 1974, W. R. Christensen to M. H. Campbell, "S and SX Farm Sludge Samples"

A sample of sludge from tank 111-s has been analyzed in the laboratory. The sample was gray in color more nearly resembling an aluminum compound rather than the dark brown color usually associated with iron compounds. Chemical analyses showed that the sample was greater than 60 percent $\mathrm{Al}_{2} \mathrm{O}_{3}$ confirming the suspected high aluminum content.

I suspect that as Tank Ill-s aged, a layer of hydrated aluminum oxide precipitated and formed on top of the iron hydroxide sludge layer. When the sludge sample was taken, the hard aluminum oxide Iilled the sludge sample tube preventing the softer irco hydroxide sludge from entering the tube. The results of the analyses, discussed in detail below, are representative of the sample but in all probability do not represent what is in the entire sludge layer. The low fission product content and heat generation rate of the sample also indicates that the sample is not representative of the entire sludge layer.

Damp or wet density of the sludge was measured at $1.348 \mathrm{~g} / \mathrm{CC}$. Dry, particle density (NPH density) was $1.21 \mathrm{~g} / \mathrm{cc}$. The analysis of the sludge is shown in mable I.

Thermal conductivity and aifferential thermal analyses of the sludge have not yet been made.

About 76 percent of the weight of the sluage can be accounted for by the analyses assuming the compounds listed in Table I aze in the sludge. The ratio of anions to cations in the water soluble fraction was 1.22 incicating that the analyses are reasonable. I suspect that if any analysis is low, it is aluminum. 
W. R. Christensen

Page 2

May 29, 1974

I recommend that another attempt be made to get a more representative sample of the sludge layer in this tank before it be considered as a receiver tank for salt cake.

$\mathrm{JSB}: \mathrm{kh}$

Enc.

CC: MH Campbell
DG Harlow
NI Harms
JE Horton
WP Metz
RC Roal
NC Rodewald
WW Schulz
RE VandeI Cook
RI Walser
JC Womack
Process Aids - 7
File
LB




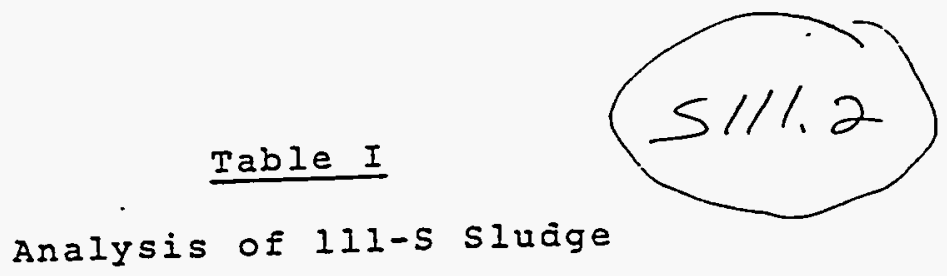

Damp density
Dry density
Analysis
$\mathrm{H}_{2} \mathrm{O}$
$\mathrm{NaAlO}$
$\mathrm{NaOH}_{2}$
$\mathrm{NaNO}_{2}$
$\mathrm{NaNO}_{3}$
$\mathrm{Na}_{2} \mathrm{CO}_{3}$
$\mathrm{Na}_{2} \mathrm{SO}_{4}$
$\mathrm{Na}_{3} \mathrm{PO}_{4}$
$\mathrm{Fe}_{2} \mathrm{O}_{3}$
$\mathrm{Al}_{2} \mathrm{O}_{3}$
$\mathrm{SiO}_{2} \mathrm{O}_{9}$
$89+9 \mathrm{Sr}$
$137 \mathrm{Cs}$
239 Pu
$\mathrm{Heat}$ generation

$1.348 \mathrm{~g} / \mathrm{cc}$

$1.21 \mathrm{~g} / \mathrm{Cc}$

$9.46 \%$

$0.22 \%$

$1.22 \%$

0.098

$3.38 \%$

$1.01 \%$

$0.02 \%$

$0.03 \%$

0.148

$59.76 \%$

$0.48 \%$

$2.87 \mu \mathrm{Ci} / \mathrm{g}$

$7.29 \mu \mathrm{Ci} / \mathrm{g}$

$<0.2 \mu \mathrm{g} / \mathrm{g}$

$12 \mu w a t t s / g$ 
Yifin

DลA.

(1)

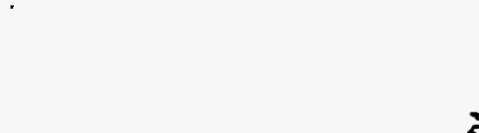

\section{EUMMARIES}

- Salt cake samples Exom tanks 105-s; 10б-s, and 111-s.

- Thermal. concuctivizies $=\sum$ lo5-s salt.

\section{Achievemen= ละอุo:}

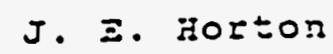 \\ August 21,1974
}

WHC-SD-WM-ER-323, Rev. 0 Theraal conductivies oa $105-5$ salt.

1 
DRAFT

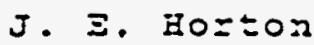

August 21, 1974

TANK FARY ASSISTANCE

Samples of tine sa:- eakes in tanks i0s-s, 106-s, and lil-s were taken. Inese tanks are sluryy receiving tanks for the 242-s evaporator. Solids in tanks 105-s, 106-S, and 11I-s are primarily sodium nitrato. Details of laboratory work are discussed below. Analysis of the solids and motier liguors are sinown below.

The los-s salt sample is composed of coazse yeilow crystals. The yellow czystals a ve very dyy in appeazance. A weighed amount of l05-3 solids was dissolved in ${ }_{2} 0$ and rade to a volume of $25 \mathrm{mI}$. Analysis are shown in mable I.

\section{TA3IZI}

$\begin{array}{llr}\mathrm{NaAlO}_{2} & 1.34 \times 10^{-3} & 0.113 \\ \mathrm{NaNO}_{2} & 3.73 \times 10^{-3} & 0.263 \\ \mathrm{Na}_{2} \mathrm{CO}_{3} & 2.36 \times 10^{-2} \frac{\mathrm{MM}}{4} & 2.543 \\ \mathrm{NaNO}_{2} & 1.15 \mathrm{M} & 100.33 \\ \mathrm{NaOH} & 1.26 \times 10^{-2} \mathrm{M} & 0.53 \\ \mathrm{~N}_{2} \mathrm{O} & & \frac{3.43}{107.13}\end{array}$

GEA

$125 \mathrm{Cs}$

12535
$3.76 \times 10^{2}$ uCi/2.

$2.20 \div 6: / 2$
$3.3: 1 C: / 3$

$0.02 \mathrm{jCi} / \mathrm{g}$
As Received Density
Wet Density
NPA Density
1.25
1.78
2.52 
DRAFT

J. E. Horton

August $21, .2974$

HYGROSCOPIこITY

The I05-5 salt sanple showed so deliguesce after 21 days of testing. Percent gain after 21 days was 11.1 percent. This test was made in aiz saturated iiti water vapor at $23-25^{\circ} \mathrm{C}$.

Thermal conductivity measurements os lo5-s salts were made. Table II reflects the data.

\section{TABIE II}

MYZRMAL CONDUCTIVITIES OP IOS-S SOIIDS

(DAIF 3Y D. G. 3005E)

As Received

Held Ovezagit Dried ovezight Ove $=$ igit Over Heekend $21.3^{\circ} \mathrm{C}$

$52.1^{\circ} \mathrm{C}$ $80.0^{\circ} \mathrm{C}$ $114.9 \circ \mathrm{C}$ $156.0{ }^{\circ} \mathrm{C}$ c. $25 I 3 T U / 4 I / E t^{2} / 0 I$

0.247

0.235

0.216

0.205
"1

18

"

"s

The 106-s salt sample has large yellow cyystals at

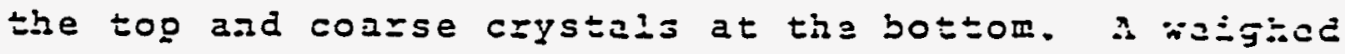
amount $0 \leq 106-5$ solids uas dissolved ia 10 and maze to a volume OE $25 \mathrm{II}$. Iabo: are shown on the followis- page. 


\section{TABIE III}

ANAIYSIS OF 106-S TANA SOLIDS

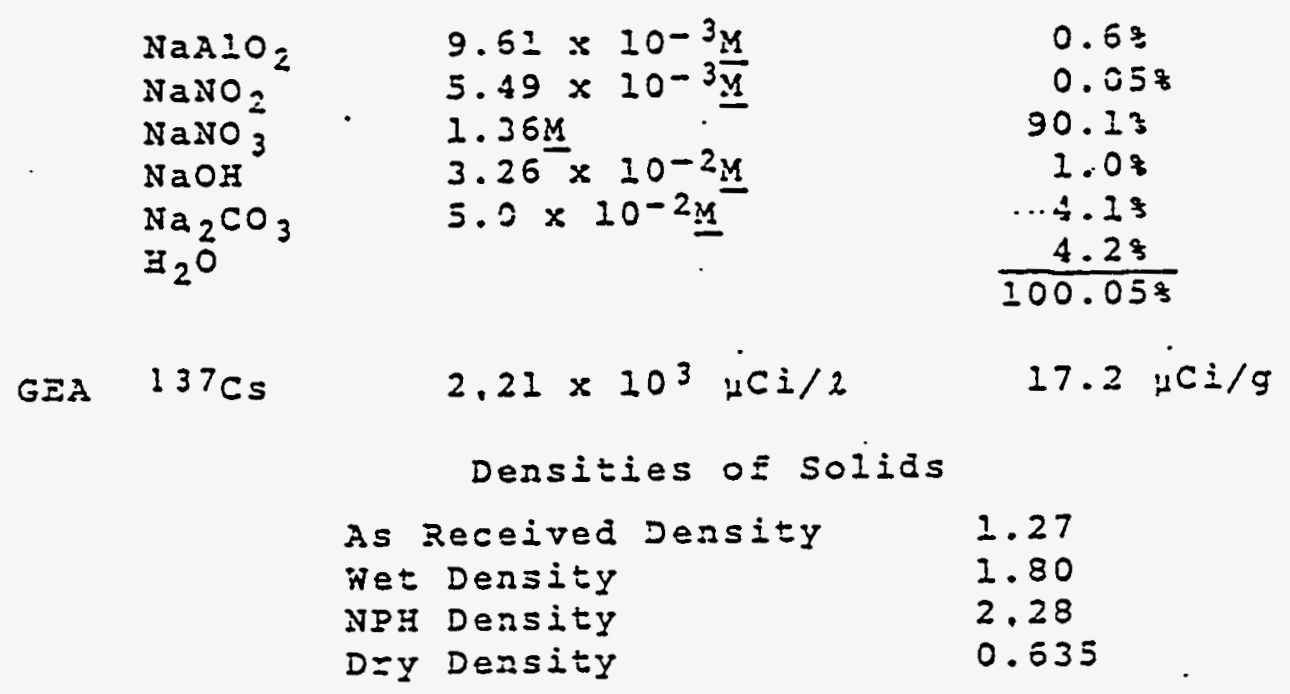

TABIE IV

AHAEYSIS OF IOG-S SUPERNATAMT

\begin{tabular}{|c|c|c|}
\hline $\mathrm{NaAlO} 2$ & $1.56 \mathrm{M}$ & 8.98 \\
\hline $\mathrm{NaOH}$ & $5.33 \bar{M}$ & 14.93 \\
\hline $\mathrm{NaNO}_{2}$ & $0 . \overline{5 \bar{\sigma}} \underline{M}$ & $3.2 \frac{5}{3}$ \\
\hline $\begin{array}{l}\mathrm{Na}_{2} \mathrm{CO}_{3} \\
\mathrm{NaNO}_{3}\end{array}$ & $\begin{array}{l}1.36 \bar{x} 10^{-2} \underline{Y} \\
2.49 \mathrm{M}\end{array}$ & $\begin{array}{r}1.03 \\
14.83\end{array}$ \\
\hline$\Xi_{2} \mathrm{O}$ & & $\frac{55.03}{98.03}$ \\
\hline
\end{tabular}

Density $\quad 2.429$

-. $G E A$
$137=5$
$3.39 \times 10 \quad \mathrm{Ci} / 2$

$: 3+\mathrm{Cs}$

$9.11 \times 10 \mu \mathrm{Ci} / 2$ 
DRAFT

Hygroscodicities oE 106-s saits

The 106-5 salt sample. shows no deliquescence ajter

I4 days of testiag. Percent gain in 14 days of testing was 11.4 percent. This test was made in air saturated

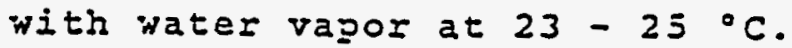

The III-s saits a=e a grayish in color and very sticky. Iaboratory work was done on both the superantant and solia. A portion was dissonved in water and sent to laboratory for analysis.

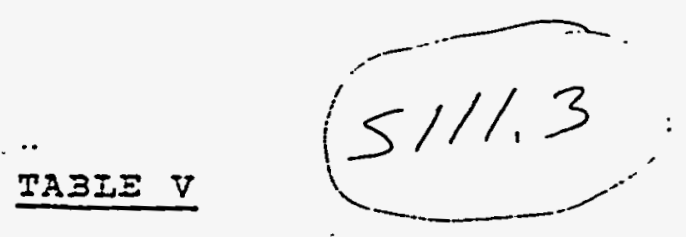

ANAIYSIS OE III-S SAITS

$\mathrm{I}_{2} \mathrm{O}$

$\mathrm{NaAlO}$

$\mathrm{NaNO}_{2}$

$\mathrm{NaNO}_{3}$

$\mathrm{Na}_{2} \mathrm{CO}_{3}$

NaOH

Total

$99+905 x$

GEA

$\div 37 \mathrm{Cs}$

Wet Density

Dry Dezsity
$2.31 \times 10^{-2} \mathrm{y}$

$3.21 \times 10^{-2} \bar{M}$

0.679

$3.45 \times 10^{-2} \mathrm{M}$

0.13719

$6.22 \times 10^{2} \mathrm{HC} / 2$

$7.92 \times 10^{3}, \mathrm{Ci} / 2$

1.704

0.497
18.45

2.35

2.78

69.98

10.83

$\frac{5.5}{110.78}$

$7.54 \mu \mathrm{Ci} / \mathrm{g}$

$90.2 \mu: \Sigma, g$ 
WHC-SD-WM-ER-323, Rev. 0 DRAET

\section{TABIE VI}

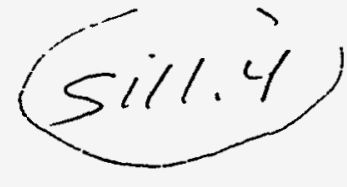

ANAIYSIS OF III-S SUPERNATAXT

$\begin{array}{llr}\mathrm{NaAlO}_{2} & 1.34 \mathrm{M} & 7.33 \\ \mathrm{NaNO}_{2} & 1.55 \mathrm{M} & 3.13 \\ \mathrm{NaOH}^{2} & 3.80 \overline{\mathrm{M}} & 20.73 \\ \mathrm{NaNO}_{3} & 2.90 \mathrm{M} & 17.43 \\ \mathrm{~B}_{2} \mathrm{O} & & \frac{54.43}{98.43} \\ \text { TotaI } & & \end{array}$

Density $\quad 2.414$

GEA

$137 \mathrm{Cs}$

$134 \mathrm{Cs}$

$4.47 \times 10^{5} \mu \mathrm{Ci} / 2$

$3.04 \times 10^{3} \mu \mathrm{Ci} / 2$

\section{Eygroscopicities or Iil-s salts}

The solics Exom III-s tank are iygroscopic, absorbing enougi water to become deliquescent in six days.

The salt sac increased 21.6 percent in weight when it deliquesced. This test was made in aif saturated with water at $23-25^{\circ} \mathrm{C}$.

Laboratory work on sluage samples 104-A, 102-AX, and 110-s are not yet completed, and will be reporzed at a later date. 


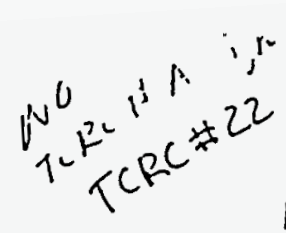

Date: December 16, 1974

To:

R. L. Watser

From:

R. E. Wheeler Pprikietin

Subject: ANALYSIS OF TANK FARM SAMPLES

SAMPLE: T-8747 $171-S$

'Vis-0TR: Yellowish green. $100 \%$ solids. $500 \mathrm{mRad} / \mathrm{hr}$

' $\mathrm{pH}$ :

$>13.4$

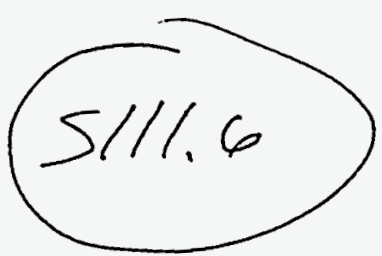

'SpG:

1.7953

- $\mathrm{OH}$ :

$5.16 M$

, A7 :

$1.34 \mathrm{M}$

- Na:

$23.84 M$

- $\mathrm{NO}_{2}$ :

$2.767 M$

- $\mathrm{NO}_{3}$ :

$4.91 M$

- Pu:

$\mathrm{SO}_{4}$ :

$3.44 \times 10^{-5} \mathrm{gm} / \mathrm{gal}$

- $\mathrm{PO}_{4}$ :

Cancelled by R. L. Walser

- $F$ :

- $\mathrm{CO}_{3}$ :

$4.52 \times 10^{-2} \mathrm{M}$

GEA:

$5.10 \times 10^{-3} \frac{\mathrm{M}}{\mathrm{M}}$

$0.293 \mathrm{M}$

$134 \mathrm{Cs}-1.13 \times 10^{4} \mathrm{HCi} / \mathrm{gal}$
${ }_{60} \mathrm{Cs}-2.93 \times 10^{6} \mathrm{Ci} / \mathrm{gal}$

${ }^{39},{ }^{90} \mathrm{Sr}$

- Water:

$\left.2.33 \times 10^{6} \mathrm{\mu Ci} / \mathrm{ga}\right]$

$\mu \mathrm{Ci} / \mathrm{ga}]$

Cooling Curve:

REW:jd

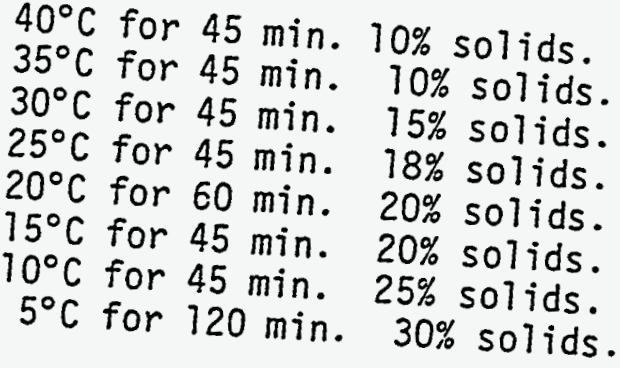

Cc: JS Buckingham

WR Christensen

RM Ginestet

RE Wheeler

JC Womack 
Date: February 28, 1977

To: N. R. Christensen

From: J. E. Horton-2-2529 Byforten

Subject: ANALYSIS OE TANK I11-S SALTS

We received a sample Erom Tank 111-s on

Desember 27, 1976. The salts as received were damp, fine crystals, being dark green in color. Analysis indicated tine salts from Tank 11l-s are essentially sodium nitrate with lesser amounts of $\mathrm{CO}_{3}, \mathrm{Al}, \mathrm{OH}, \mathrm{e}=\mathrm{C}$.

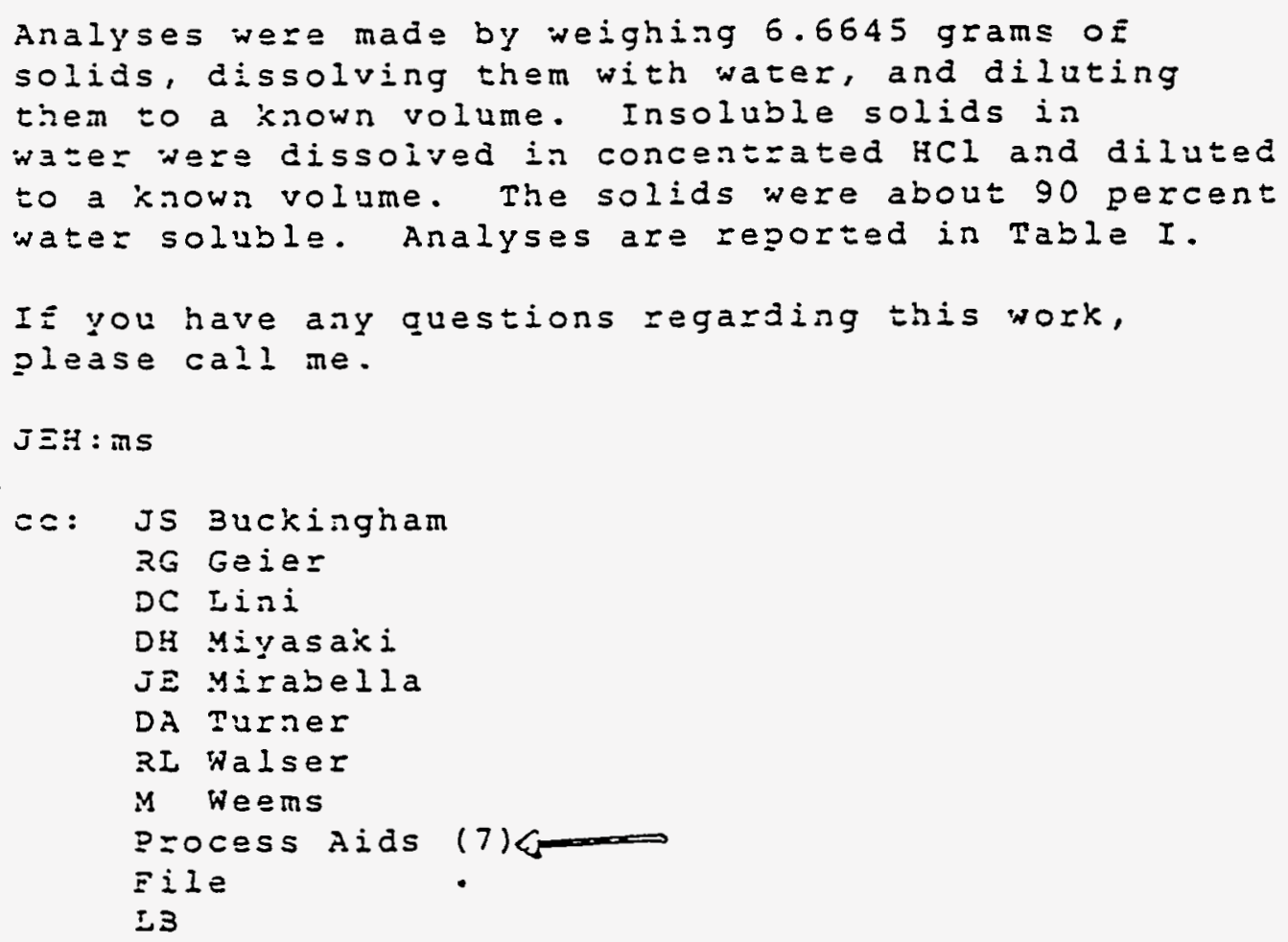




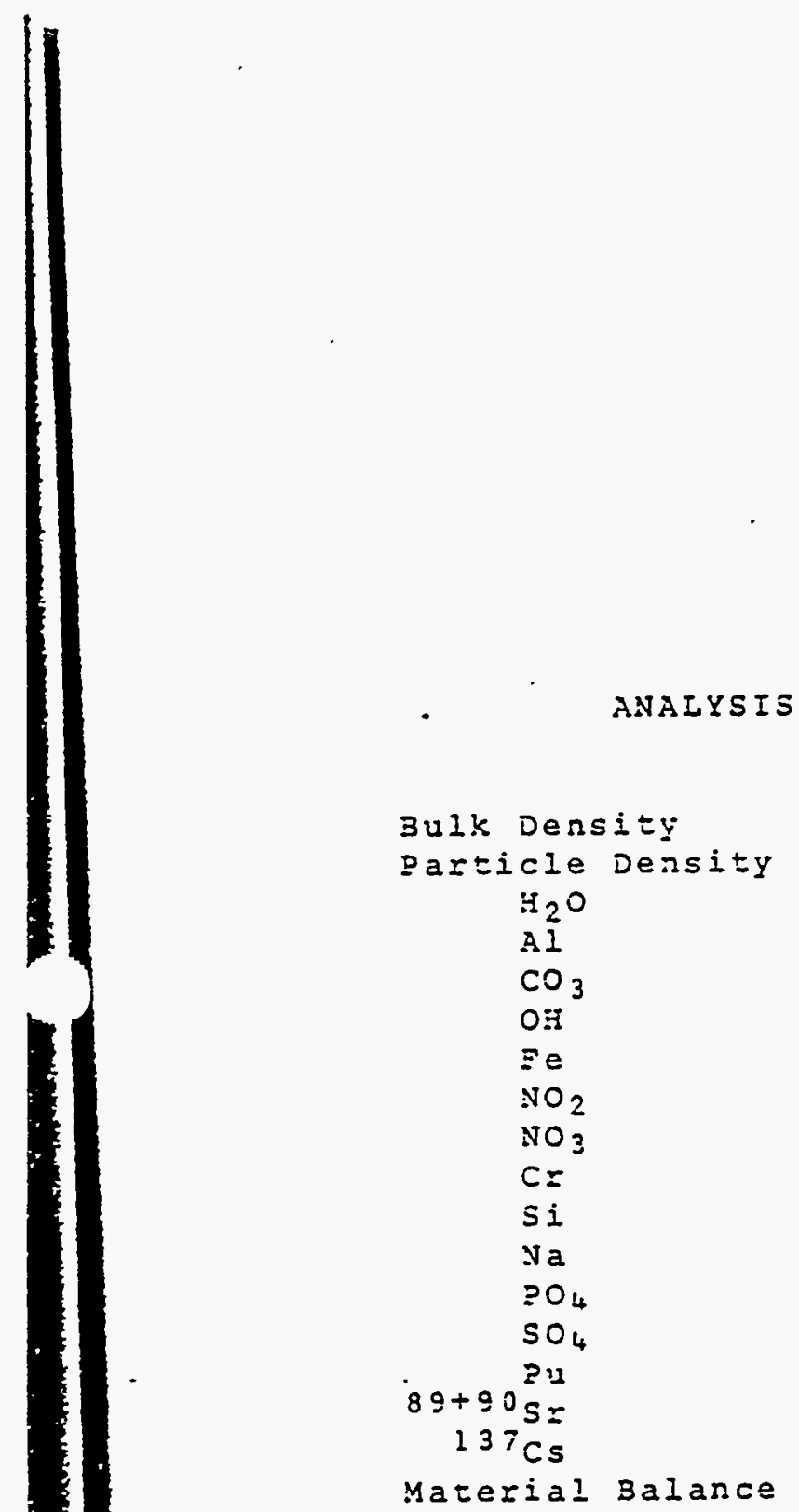

TABLEI

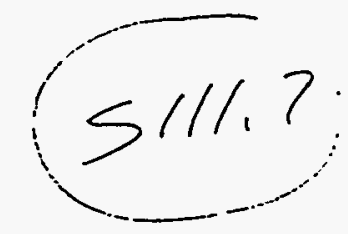

OE TANK 111-S SAIT

Bulk Density

Macerial Balance

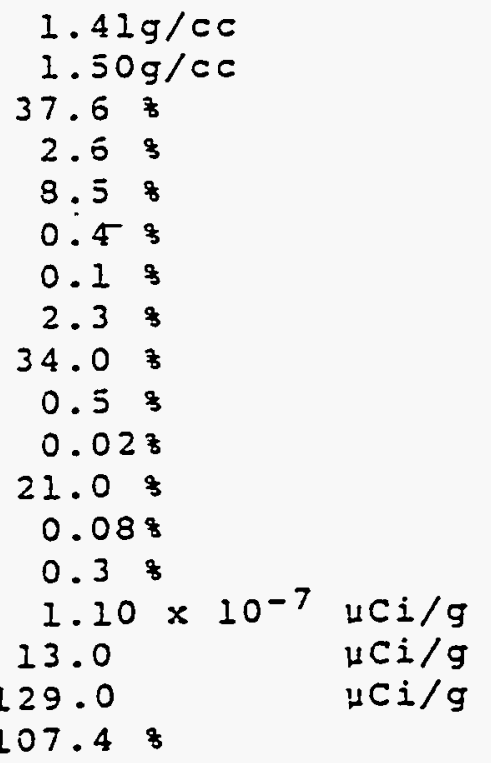


Iniernal Letter

TCRC-2H $5 a / 4$

$\cdots \cdots \cdot$ August 25, 1979

io

- G. K. Allen

Process Enoineerina

- 2750-E, 200 East Area $\therefore$ Rock

$501:$

WHC-SD-WM-ER-323, Rev. 0

J. E. Horton

Chemical Sciences firoup

$222-5,200$ West Area

$2-2529$

: Mw1 . Chemical and Physical Analysis of Core Senments from Tank $111-S$

Ref: Letter, February 23,1977, J. E. Horton to 1. R. Christensen, "Analysis of Tank 117-5 Salts."

Chemical Sciences Group received eight core seaments from Tank 111 -S between February 2,1373 , and June 27,1970 . Senments $i 302-c$, 1003-C, and 1006-C were empty, segments $1001-\mathrm{C}$ contained $43.2 \mathrm{CH}, 130 \mathrm{C}-325.4 \mathrm{CM}, 1004-\mathrm{C} 25.4 \mathrm{CM}$, i007-C 7.6 CH and 1009-C 43.2 C:4 of sait cáke.

Total salt cake retrieved from iank 111-S was i44.3 CM. Core 1009-C aiso contained 37 milliliters of supernatant liquid. Core sentent 1007-C contained drilling sediment and was not a representativa sanple. It was not analyzed. Core segments 1003-C and 1004-r, were blended for chemicai analysis. The salts as received were yellowisn green in color, with variable crystal sizes. The analytical dato shous that they differ in sodiur and sodiun nitrote. The

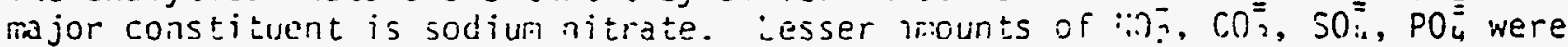
seen. I believe the variation in bulk densities is due to differences in percent water. Al core secanents vere approximately percent water soluble. There were no unususi or unique difierences seen in appearance of core segment received.

The hich radiation reading of $i-5$ rads forced us to use the hot cell for breakdown on all core segments from iank $111-5$.

inaiytical data reported in this letter were ottained by the analytical methods described in document $A R H-C D-571$. Chemical and physical data are reported in Tables I through $V$.

In December 1976, a surface sampie vias taken froin this tank (Reference). Analytical data obtained at that time are in good agreement with the data reported here.

If you have any questions please call me.

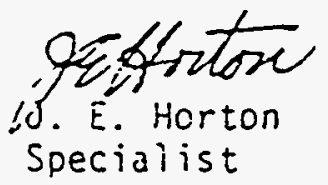

JEH:kim

information:

j. S. Buckinginam

r. id. Frines

F. i. Jungfleisch

i. U. Schulz

D. A. Strachan

File (7)

ittacnments 


\section{Components}

A1

Cl

$\mathrm{Bi}$

Cd

$\mathrm{Fe}$

$F$

$\mathrm{CO}_{3}$

$\mathrm{CH}$

$\mathrm{Hg}$

$x$

$M n$

Na

$\mathrm{NO}_{2}$

$\mathrm{HO}_{3}$

$\mathrm{PO}_{4}$

$\mathrm{SO}_{4}$

$\mathrm{SiO}_{2}$

Totál Organic Carbon U

$239 p_{4}$

$24.2 \mathrm{Am}$

$89+90 S r$

$137 \mathrm{CS}$

$134 \mathrm{CS}$

$106 \mathrm{RU}$

$\therefore O C O$

i25Sb

$154 E U$

$155 E u$

Material 3alance
Water Soiuble
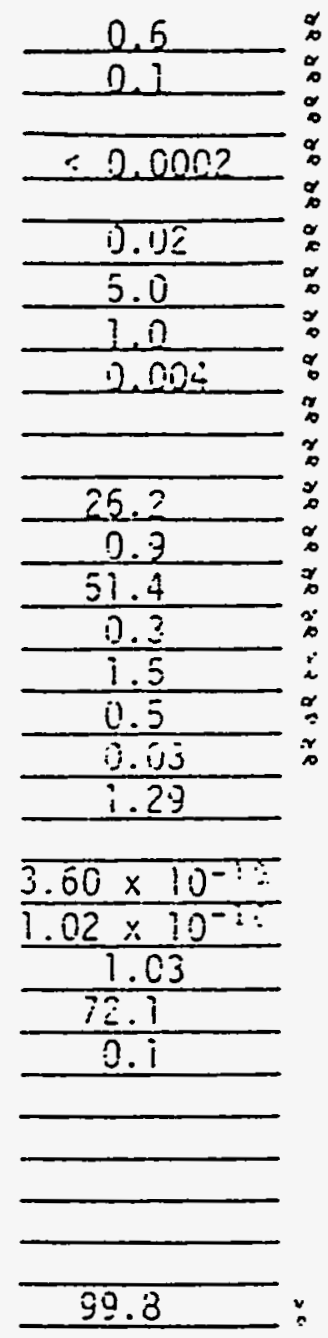

Acid (KOH Fusion)

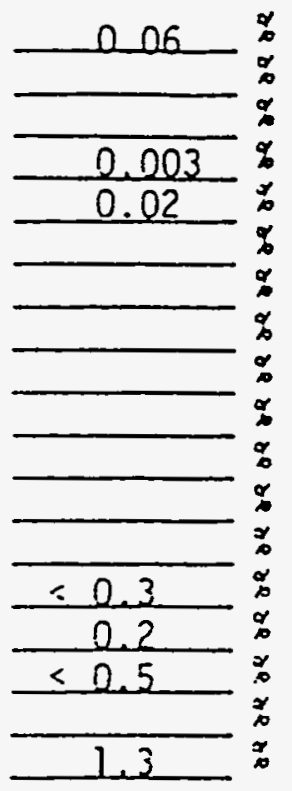

$9 / 1$

$g / 9$

$\mathrm{g} / 9$

:Ci $\mathrm{i} / \mathrm{g}$

$\mu \mathrm{Ci} / \mathrm{G}$

uCi/g
$9 / 9$

$g / 9$

$9 / 9$

$\mathrm{NC} i / g$

$\mathrm{NCi} / \mathrm{g}$

$u \dot{C} i / g$

$山 \mathrm{Ci} / \mathrm{g}$

$\mathrm{LC} i / \mathrm{g}$

$\mu \mathrm{Ci} / \mathrm{g}$

$\mu \mathrm{Ci} / \mathrm{g}$

\section{Physical Properties}

Bulk Density

Particle Density

Percent Hater

DTA

Calculated Heat Content

Sample Size (Core Length)

Hardness

Vapor Pressure

Drainable Liquid

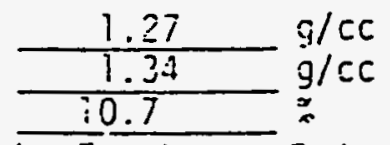

lio Exctierris-Endotinerm at $103^{\circ} \mathrm{C}$ and $285^{\circ} \mathrm{C}$

$5.74 \times 10^{-4}$

$\frac{13.2}{26.0} \mathrm{~kg} / \mathrm{Cm}^{2}$.

$3.3+\min$ at $24.5{ }^{\circ} \mathrm{C}$

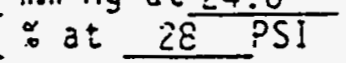


TAELE 13

PARTICLE SIZE OISTRIEUTION"

SAMPLE IOCI-C HI-S TANK

\begin{tabular}{|c|c|c|c|}
\hline $\begin{array}{l}\text { Particle } \\
\text { Size } \mu \mathrm{m} \\
\end{array}$ & $\begin{array}{l}\text { Average } \\
\text { Dia. im }\end{array}$ & $\begin{array}{l}\text { Height } \\
\text { Percent }\end{array}$ & $\begin{array}{l}\text { Cumulative } \\
\text { Wt. Percent }\end{array}$ \\
\hline $\begin{array}{c}5-10 \\
10-15 \\
15-20 \\
20-25 \\
25-30 \\
30-35 \\
35-40 \\
40-45 \\
45-50 \\
50-55 \\
55-60 \\
60-55 \\
65-70 \\
70-75 \\
75-80 \\
80-90 \\
100-110 \\
110-120 \\
120-130 \\
130-140 \\
140-150 \\
>150\end{array}$ & $\begin{array}{l}8.25 \\
12.98 \\
17.85 \\
22.8 \\
27.7 \\
32.7 \\
37.7 \\
42.7 \\
47.6 \\
52.5 \\
57.6 \\
62.5 \\
67.5 \\
72.5 \\
77.5 \\
35.3 \\
105.2 \\
115.2 \\
125.2 \\
135.2 \\
145.2\end{array}$ & $\begin{array}{r}3.2 \\
0.3 \\
1.2 \\
10.5 \\
3.2 \\
9.5 \\
3.5 \\
3.7 \\
1.5 \\
1.2 \\
0.3 \\
12.6 \\
0.4 \\
3.5 \\
0.5 \\
1.1 \\
0.3 \\
1.0 \\
0.5 \\
2 \\
3 \\
33.7\end{array}$ & $\begin{array}{r}99.9 \\
98.9 \\
97.7 \\
87.2 \\
84.0 \\
74.5 \\
70.9 \\
62.2 \\
60.7 \\
59.5 \\
59.2 \\
46.6 \\
46.2 \\
37.7 \\
37.2 \\
36.1 \\
35.3 \\
34.3 \\
\end{array}$ \\
\hline
\end{tabular}

tParticles size 5 to 150 um were run on the HIAC Counter and > 150 um were mechanically sieved. 


\section{Components}

A)

Cl

Bi

Cd

$\mathrm{Fe}$

$F$

$\mathrm{CO}_{3}$

$\mathrm{Hg}$

$K$

$\mathrm{Na}$

$\mathrm{NO}_{2}$

$\mathrm{HO}_{3}$

$\mathrm{PO}_{4}$

SO.

$\mathrm{CrO}_{4}$

$\mathrm{SiO}_{2}$

Total Organic Carbon

$u$

\section{$253 P u$}

$241 \mathrm{Am}$

$89+905 r$

$137 \mathrm{Cs}$

13 CS

$106 \mathrm{RU}$

-OCO

$125 \mathrm{Sb}$

$154 \mathrm{EU}$

ISSEU

Material Balance

Physical Properties

Bulk Density

Particle Density

Percent Water

DTA

Calculaied heat Content

Sample Size (Core Length)

Hardness

Vapor Pressure

Drainable Liquid
Water Soluble

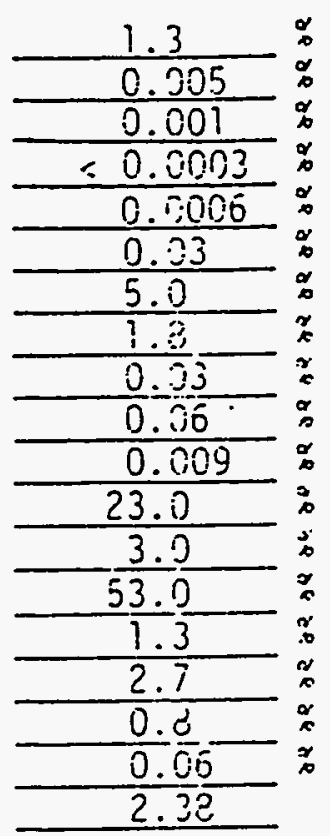

i!

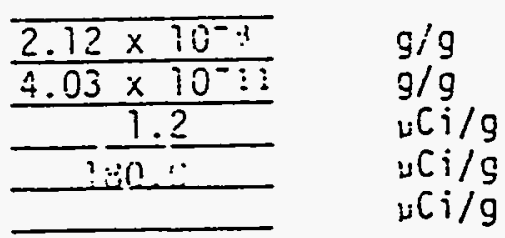

108.4
Acid ( $\mathrm{KOH}$ Fusion)

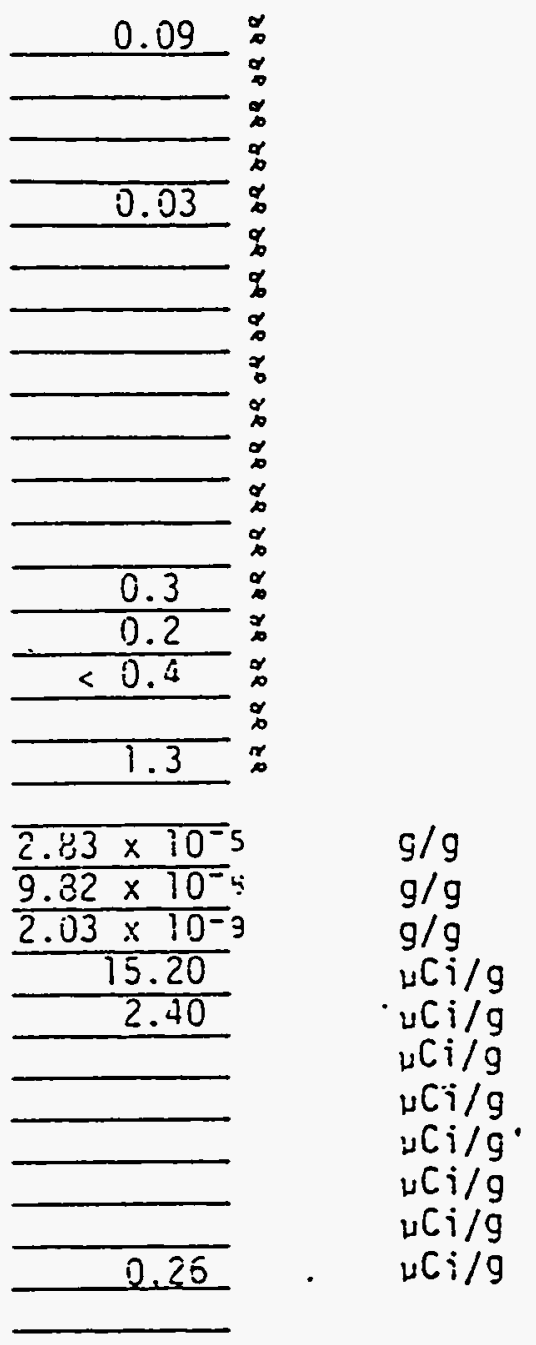


TABLE IIB

PARTICLE SIZE DISTRIBUTION ${ }^{+}$

SAMPLE 1003-1004 li1-5 Tank

$$
\begin{aligned}
& \text { Particle } \\
& \text { Size pm }
\end{aligned}
$$

Average

Diz. um

rieight

Percent

\subsection{5}

12.98

17.85

22.8

27.7

32.7

37.7

42.7

47.5

52.6

57.6

62.6

57.5

72.5

77.6

35.3

105.2

115.2

125.2

135.2

145.2
Cumulative

Wt. Percent

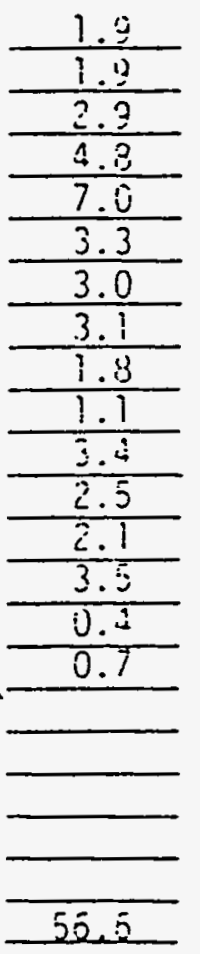

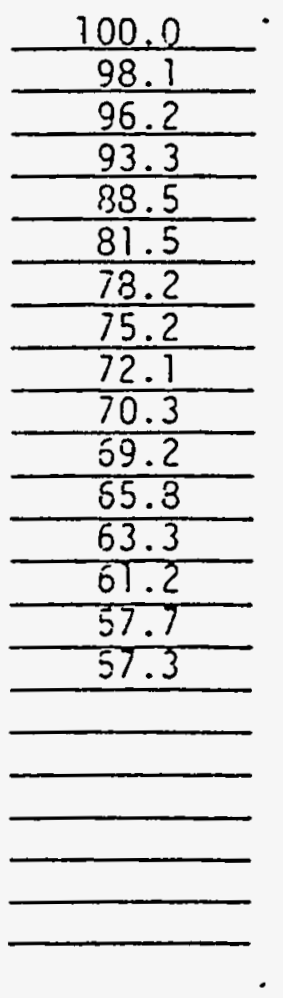

+Particles size 5 to 150 um were run on the HIAC Counter and > $150 \mu \mathrm{m}$ were mechanically sieved. 
CHEMICAL AND PHYSICAL ANALYSIS ${ }^{+}$(COUTENT

FOR TANK $111-5$ SAMPLE = 1029-6
WHC-SD-WM-ER-323, Rev. 0

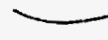

Components

A1

Cl

$B i$

$\mathrm{Cd}$

Fe

$\mathrm{F}$

$\mathrm{CO}_{3}$

$\mathrm{Hg}$

$\mathrm{K}$

in

$\mathrm{Na}$

$\mathrm{NO}_{2}$

$\mathrm{NO}_{3}$

$\mathrm{PO}_{4}$

$\mathrm{SO}_{4}$

$\mathrm{SiO}_{4}$

Total Organic Carbon U

$233 P_{4}$

$241 \mathrm{Am}$

$89+905 r$

$137 \mathrm{Cs}$

$13+C S$

$.105 R h$

BOCO

$1255 \mathrm{~b}$

$154 E U$

ISSEU

Material Balance

Physical Properties

Bulk Density

Particle Density

Percent Water

DiA

Calculated Heat Content

Sample Size (Core Length)

Hardness

Vapor Pressure

Drainable Liquid
Hater Solubie

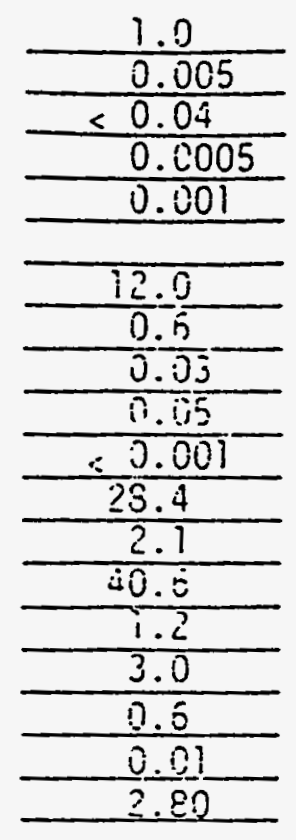

$\frac{\frac{2.25 \times 15-7}{4.23 \times 10=1}}{\frac{1.79}{100.0}}$

11

$9 / 9$

$\mathrm{c} / \mathrm{g}$

. $6 i / 3$

$: \mathrm{Ci} / \mathrm{g}$

:Ci/g

$\mathrm{C} \mathrm{C} / \mathrm{g}$
Acid (KOH Fusion)

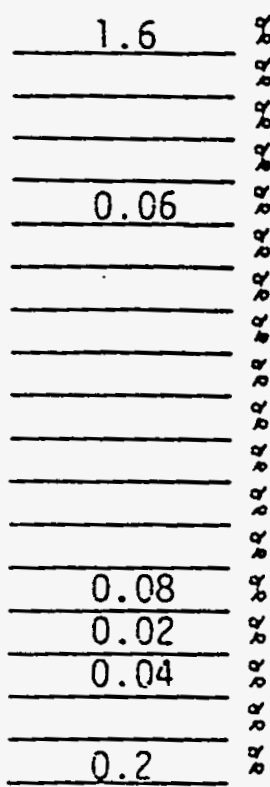

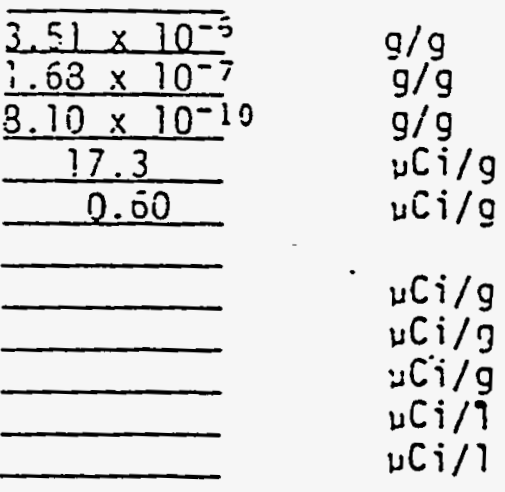

Tio Exothermis-irctherns at $105^{\circ} \mathrm{C}$ and $280^{\circ} \mathrm{C}$

$3.49 \times 10^{-4}$ inatts/liter/content of $89+90 \mathrm{Sr}$ and $137 \mathrm{Cs}$ 43.19 icm or $\mathrm{m}$ $\ddot{\mathrm{cm}}:$ or

6.3 in itg at $\frac{26.3}{\text { PSI }}{ }^{\circ} \mathrm{C}$ 


\section{TABLE IIIC}

PAPTICLE SIZE DISTRIBUTIOH'

SAMMPLE IOEQ-C III-S TANK

\begin{tabular}{|c|c|c|}
\hline $\begin{array}{l}\text { Particle } \\
\text { Size } \mu \mathrm{m} \\
\end{array}$ & $\begin{array}{l}\text { Average } \\
\text { Dia. } \mu^{m}\end{array}$ & $\begin{array}{l}\text { hieight } \\
\text { Percent } \\
\end{array}$ \\
\hline $\begin{array}{r}5-10 \\
10-15 \\
15-20 \\
20-25 \\
25-30 \\
30-35 \\
35-40 \\
40-75 \\
45-50 \\
50-55 \\
55-60 \\
60-65 \\
65-70 \\
70-75 \\
75-80 \\
80-90 \\
100-110 \\
110-120 \\
120-130 \\
130-140 \\
140-150 \\
>150\end{array}$ & $\begin{array}{l}8.25 \\
12.98 \\
17.85 \\
22.8 \\
27.7 \\
32.7 \\
37.7 \\
42.7 \\
47.6 \\
52.6 \\
57.6 \\
62.5 \\
67.5 \\
72.6 \\
77.6 \\
85.3 \\
105.2 \\
115.2 \\
125.2 \\
135.2 \\
145.2\end{array}$ & $\begin{array}{r}9.3 \\
14.3 \\
3.3 \\
3.5 \\
3.4 \\
1.5 \\
1.1 \\
1.3 \\
0.4 \\
1.2 \\
0.4 \\
0.2 \\
0.3 \\
0.1 \\
0.7 \\
\\
\end{array}$ \\
\hline
\end{tabular}

Cumulative Wt. Percent

100.0

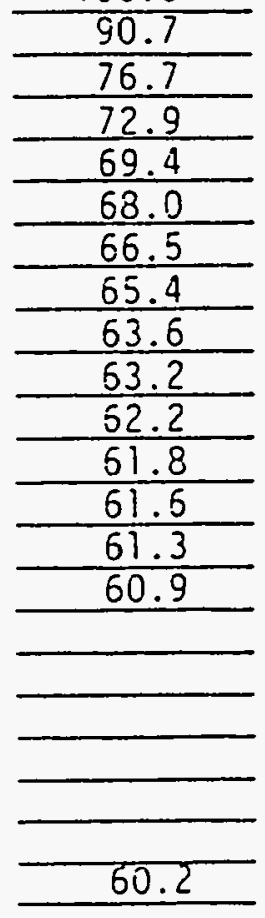

tParticies size 5 to $150 \mathrm{\mu m}$ were run on the $\mathrm{HIAC}$ Coonter and > $150 \mu \mathrm{m}$ were mechanically sieved. 
TABLE IV

\section{ANALYSIS SUPERNATANT TANK $111-5$}

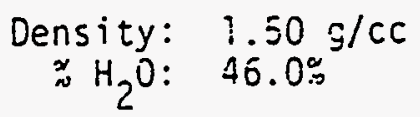

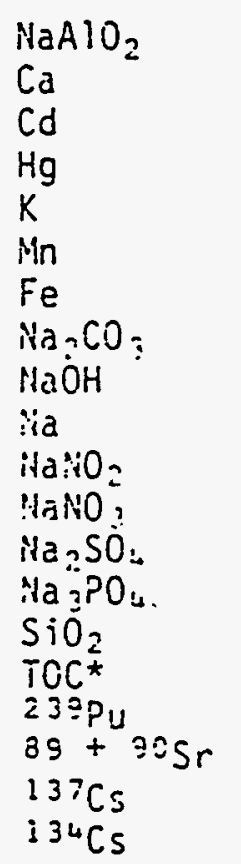

*TOC = Total Organic Carbon
6.10 $9 / 10909$

$2.04 \times 10^{1 / 2} \mathrm{iCi} i$

$5.82 \times 10^{5} \mathrm{ici} / \mathrm{C}$
$982 \times 10^{2} \mathrm{iCi/1}$

$1.94^{-14}$ is

$1.54^{-i}$ i.

$6.38^{-2} . \overline{1}$

$9.28^{-5}$

$3.35^{-3}$

$3.2 \mathrm{C}^{-2}=\frac{1.1}{3.03}$

$1.32 \quad \overline{19}$

1.95

$3.13^{-2}$

$3.74^{-2}$ if

$3.02^{-3}$.
$1.4 i^{-3} \bar{M}$

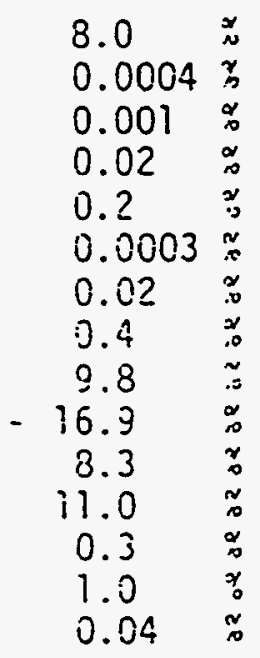

$54.4 \%$

0
0
0
0
0
0
0
0
1
0
0
0
0
0
0
0
0
0
0
0
0
0
0
0
0
$\approx$
0

ร4.4 
Density: $1.46 \mathrm{~g} / \mathrm{cc}$

$\underset{\sim}{\sim} \mathrm{H}_{2} \mathrm{O}: 44.6$

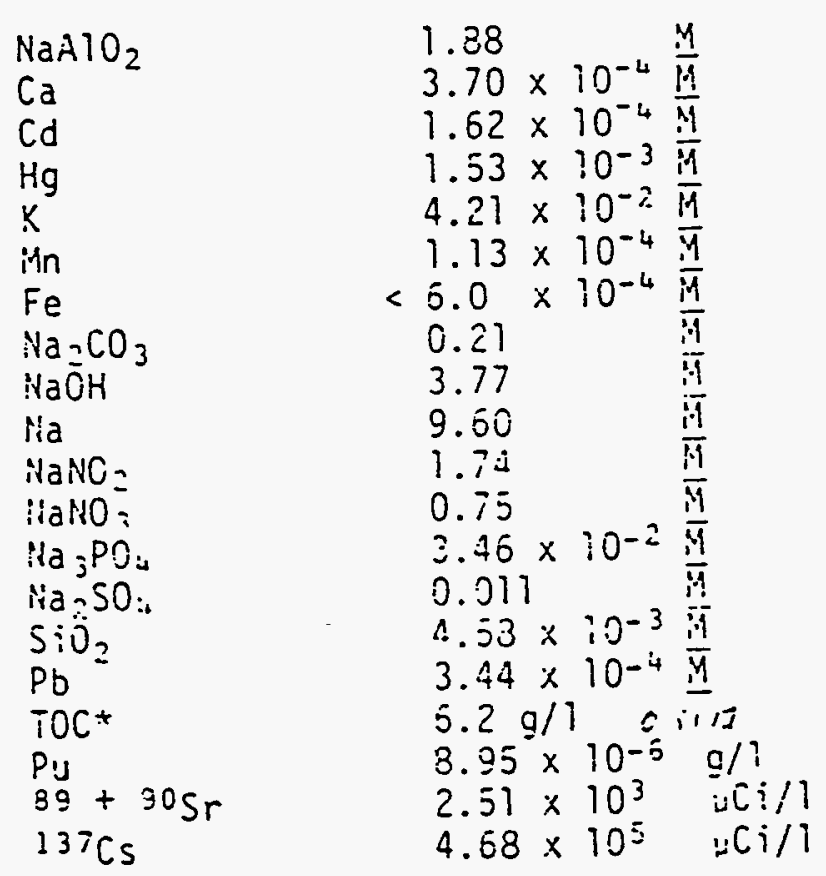

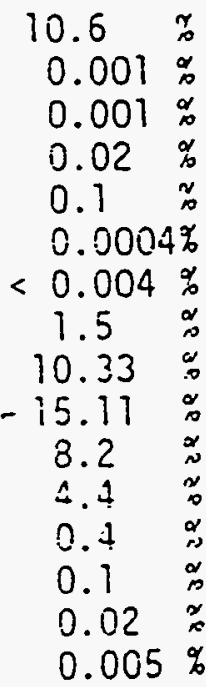

*TOC = Total Organic Carbon 
$\therefore \quad$ TCRC-2

WHC-SD-WM-ER-323, Rev. 0

\section{Atlantic Richfield Hantord Company}

$008 x+7$

Date: Septemive: 21; 1971

Tc:

Inct:

$$
\text { YCo. slipigrud }
$$

D. A. Ruryea:

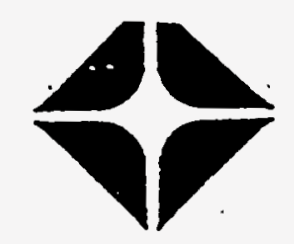

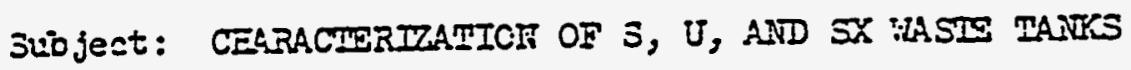

Serroling and arelyses of $S, U$ and $S X$ waste tanks to idextify

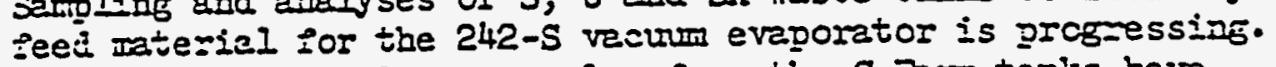
To tate three che liter samoles frcm the 3 Fant tans bave been processed tinrough the labcretory veculum evaporator: in additicn ten $S$ Farm tanks and six $\bar{J}$ Jarm tarks have been serroled ana subritited for cberacterization arslyses. The gesurts axe showa in tie pollowing taile.

Crarecterization Areiyses CF ieste Tanks

\begin{tabular}{|c|c|c|c|c|c|c|c|c|c|}
\hline T!..4C. & $J D G=$ & $\begin{array}{l}\text { Vise. } \\
\text { jo oc }\end{array}$ & $\mathrm{CO}_{3}$ & $\mathrm{xO}_{3}$ & $\mathrm{NO}_{2}$ & TAD & AI & Na & $\begin{array}{r}23 T_{C 5} \\
.0 C I /=1\end{array}$ \\
\hline 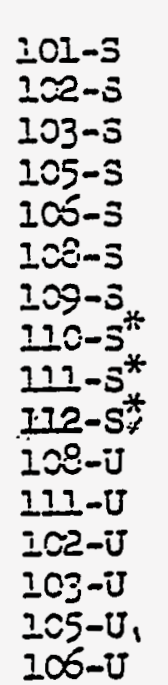 & $\begin{array}{l}1.1 .12 \\
1.317 \\
1.279 \\
1.272 \\
1.263 \\
1.291 \\
1.272 \\
1.269 \\
1.219 \\
1.202 \\
-. \\
-.\end{array}$ & $\begin{array}{l}2.23 \\
1.12 \\
. .95 \\
1.05 \\
.94 \\
1.10 \\
1.00 \\
1.15 \\
.95 \\
.92 \\
-.- \\
--.\end{array}$ & $\begin{array}{l}.011 \\
.064 \\
.020 \\
.064 \\
.017 \\
.25 \\
.041 \\
.022 \\
.025 \\
.073 \\
.035 \\
.065\end{array}$ & $\begin{array}{l}3.32 \\
5.92 \\
4.80 \\
3.15 \\
4.40 \\
3.61 \\
3.06 \\
3.4 \\
3.4 \\
3.5 \\
\cdots-. \\
\cdots-\end{array}$ & $\begin{array}{l}.0112 \\
.126 \\
.086 \\
.137 \\
.150 \\
.108 \\
.096 \\
.084 \\
.30 \\
.17 \\
.231 \\
.097\end{array}$ & $\begin{array}{l}3.62 \\
2.15 \\
1.86 \\
2 . \dddot{10} \\
1.80 \\
2.16 \\
1.88 \\
1.93 \\
1.92 \\
1.38 \\
-- \\
--\end{array}$ & $\begin{array}{l}1.47 \\
.24 \\
.28 \\
.20 \\
.17 \\
.30 \\
.217 \\
.30 \\
.19 \\
.40 \\
.25 \\
.12\end{array}$ & $\begin{array}{l}9.01 \\
8.48 \\
8.57 \\
5.86 \\
5.07 \\
6.54 \\
4.52 \\
6.50 \\
8.40 \\
5.37 \\
4.59 \\
4.93\end{array}$ & \begin{tabular}{l}
155 \\
1204 \\
104 \\
$-\ldots-$ \\
37.9 \\
\hdashline.- \\
\hdashline 60.0 \\
51.5 \\
57.9 \\
--- \\
---
\end{tabular} \\
\hline
\end{tabular}

* Ser in lejoratory vecurum eveporatcr.

The above anelyses appear normal except samplo 101-5. From the deta and perameters gained in the three $S$ tenk semple evepcretion zuns, the results of $101-5$ appes=s to be si-ila in pirysicel and chemical prope:ties oi a tenk thet ins beez 50 - 50 vclume pereent zeduced. Of specisic

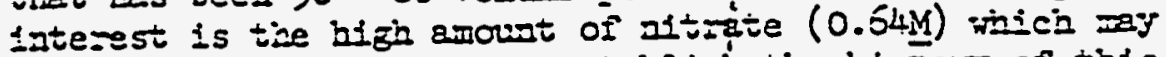
y'eld a clue in helping to establush tie intsong ci this tank. 
TCRC-1

WHC-SD-WM-ER-323, ReV. O

Dxto:

U. $30.27 \pi$

Ero:

S. 0. Tasos

$2 \leq \div \leq 2$

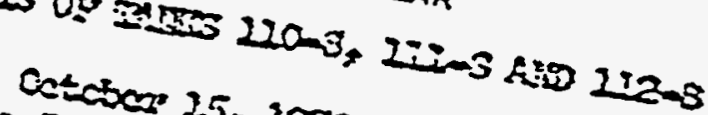

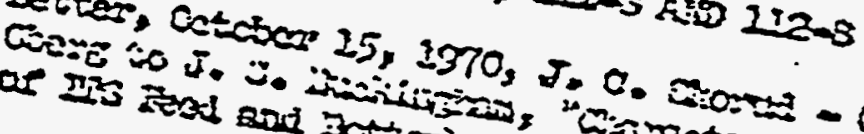

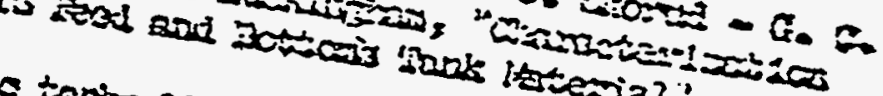

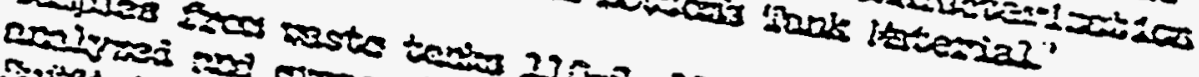

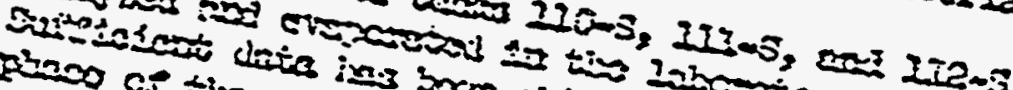

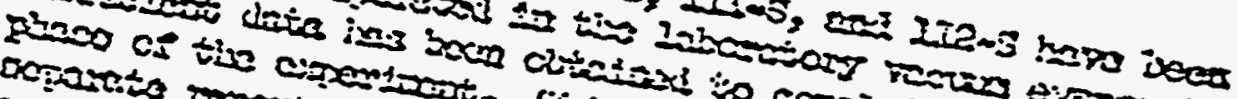

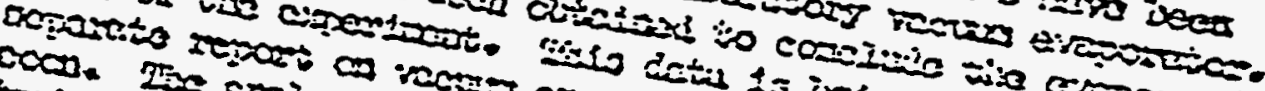

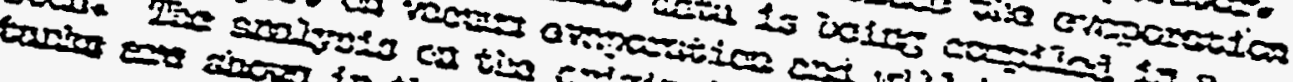

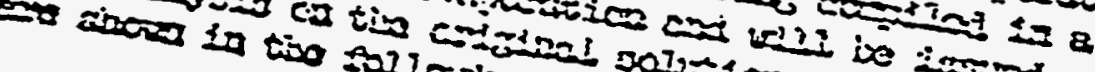
292

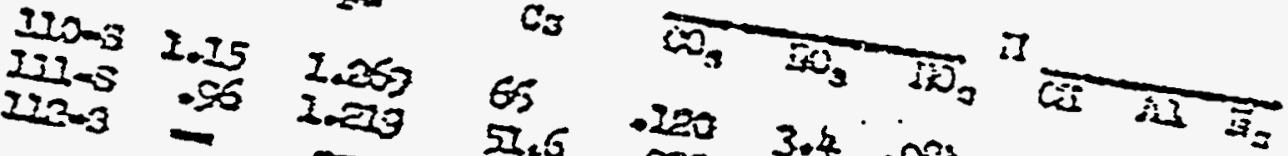

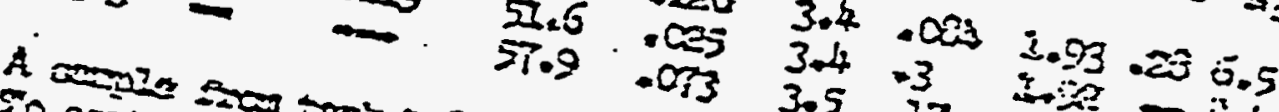

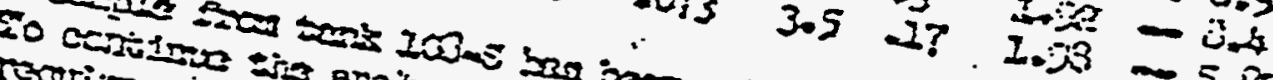

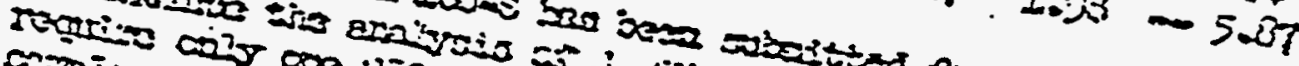

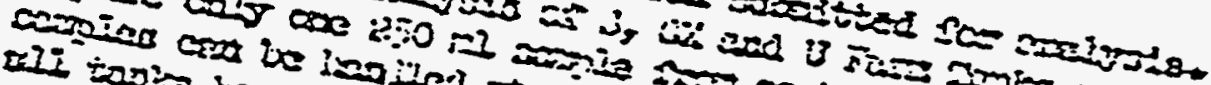

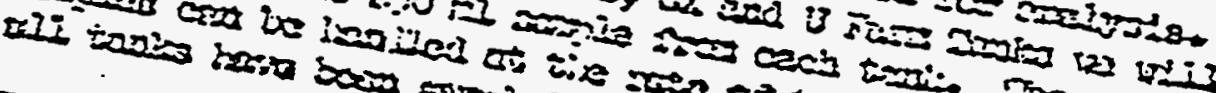

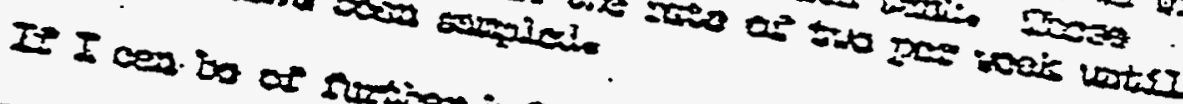

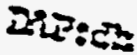

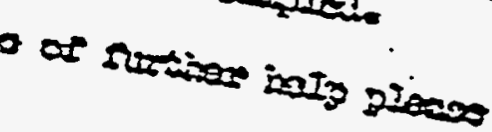

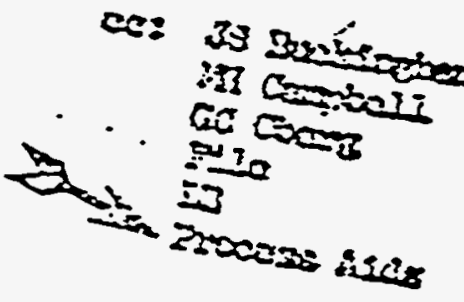

$\cos 202-\pi+\pi$ 
J. 0. Sicizuc

गु5e 2

Septembe: 21, 1971

If there are any questions pleese cell we cn 2-7478.

DIP:dh

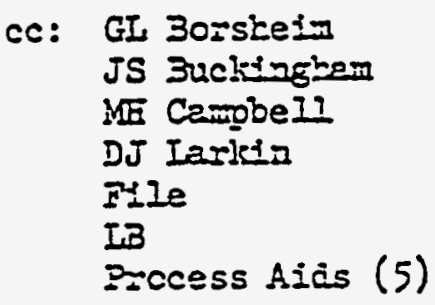


TCRC-3

A it...

WHC-SD-WM-ER-323, Rev. 0

$(5112.2)$

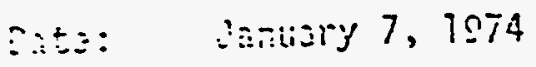

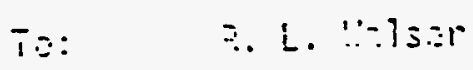

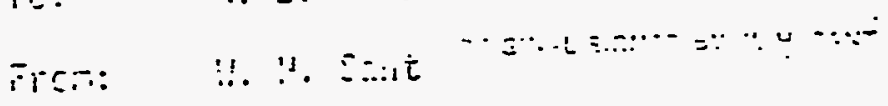

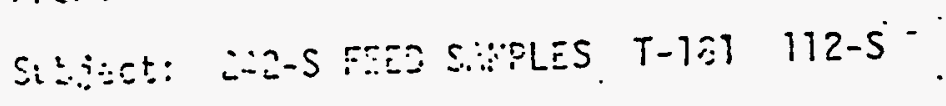

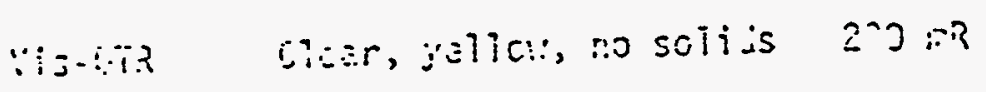

ri: 12.2

s.5 $1.7 \%=0$

活

Pu $\quad 2.95 \times 10^{-5} \mathrm{~g} / 1$

$\mathrm{N3} \quad 4.95 \%$

$5 ?_{1} \quad 1.63 \times 10^{-2}: 1$.

$\because ?$

$0.2: 3 \because$

$\therefore=\quad 0.115$

$\because$

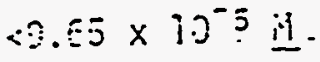

$c=0.014 \because$

$\therefore 1$

Cti $\quad 1.20: 3$

UEA $\quad 137 \mathrm{Cs}-5.83 \times 13 \%[\mathrm{i} i \mathrm{i}$

Ojolvater $\quad 06.74$

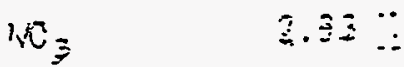

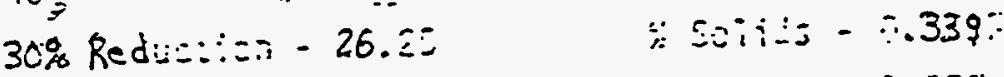

$50 \%$ Red::Z: : $2 \pi-50$

$\therefore 50 j i \leq 5-1.20: 0$

$\therefore 905 \mathrm{r} \quad 16.15 \mathrm{kSi/7}$

$\mathrm{PO}_{4}-\quad<3.77 \times 10^{-4}: 3$

Cooling curke-5. C Pro Solids

:..

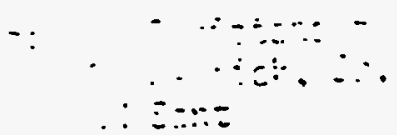

$H-445$ 


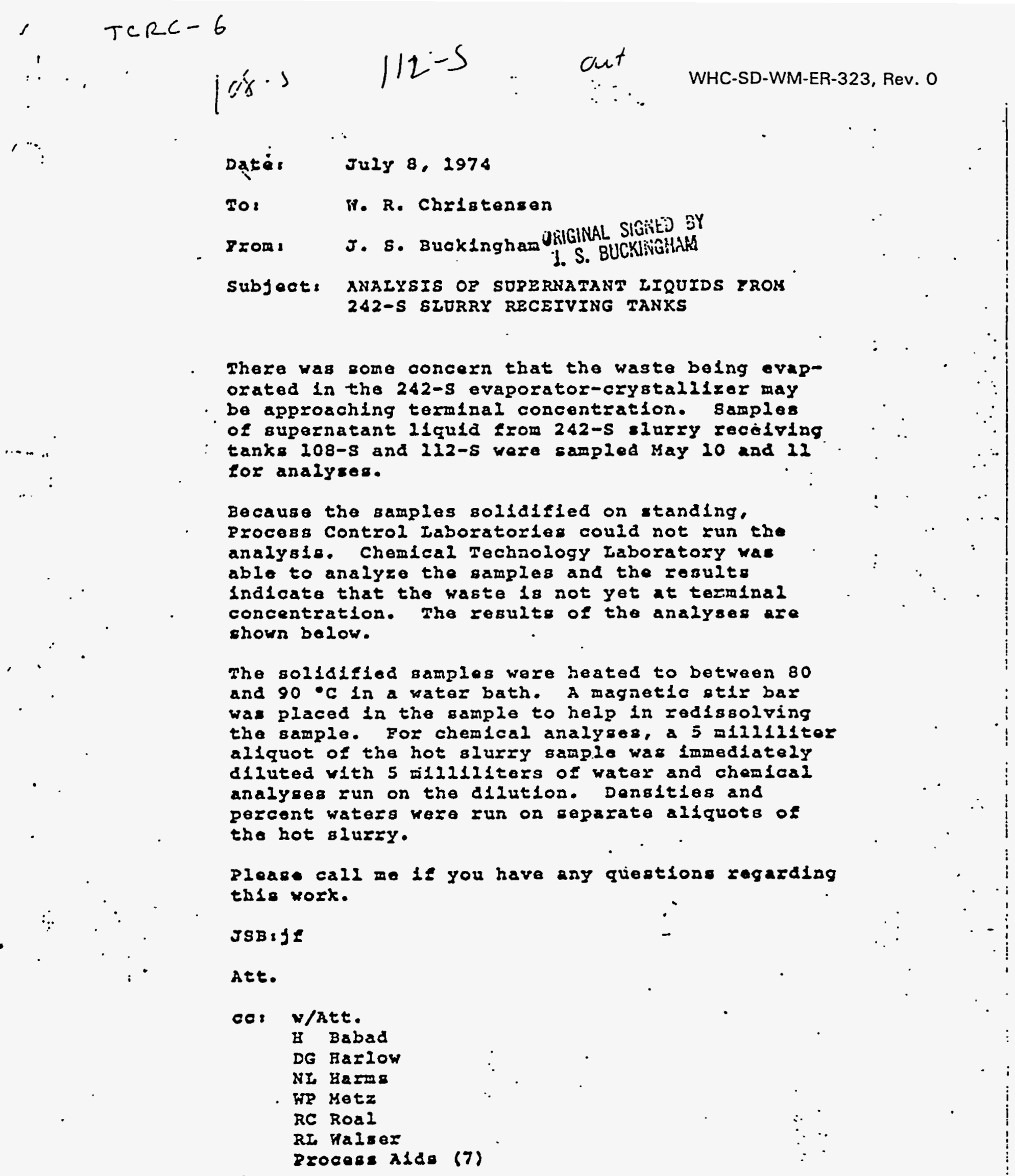

There was some concern that the waste belng evaporated in the 242-s evaporator-crystalilyer may be approaching terminal concentration. samples of Bupernatant 11quid from 242-S slurry recolving tanks $108-s$ and $212-5$ were sampled May 10 and 12 for analyzes.

Because the samples solidifled on standing. process Control Laboratorles could not run the analys10. Chemical Technology Iaboratory ras able to analyzo the samples and the resulto Indicate that the waste 18 not yet at terminal concentration. The results of the analyses are chowr balow.

The solidlfled samples were heated to between 80 and $90^{\circ} \mathrm{C}$ in a sater bath. A magnetio otir bar was placed in the sample to help in red1s80lving the sample. For chemlcal analyges, a 5 mill111ter allquot of the hot slurry gample was immediately diluted with 5 milililters of water and chemleal analyses Ius on tho dilution. Densities and percent waters were rus on separate allquots of tho hot 8 luzry.

Please call me li you have any gliedtons regarding JSBIJ

A ť.

RI. Halser

2500eas $\lambda 180(7)$ 


\section{TABLEI}

ANALYSIS OB IO8-S AND 122-S SERERIATANT IIQUIDS

\begin{tabular}{|c|c|c|c|c|c|}
\hline \multirow{3}{*}{$\begin{array}{l}\text { Dens } 1 t y \\
\mathrm{H}_{2} \mathrm{O}\end{array}$} & \multicolumn{2}{|l|}{$108-5$} & \multicolumn{3}{|c|}{$212-5$} \\
\hline & 1.523 & & 1.501 & & \\
\hline & & 60.11 & & & 58.31 \\
\hline $\mathrm{NaA} 1 \mathrm{O}_{22}$ & $2.55 \mathrm{M}$ & 8.48 & $2.19 \mathrm{M}$ & & 6.58 \\
\hline $\operatorname{NaNO}_{2}$ & $1.94 \mathrm{II}$ & 8.88 & $2.02 \mathrm{M}$ & & 9.31 \\
\hline $\mathrm{NaNO}_{3}$ & $2.60 \%$ & $24.6 \%$ & $2.51 \%$ & & 14.21 \\
\hline NaOE & $5.08 \underline{x}$ & $13.4 t$ & $5.72 \underline{M}$ & & 25.28 \\
\hline Total & & 105.38 & & & 103.51 \\
\hline $237 \mathrm{Cs}$ & $4.58 \times 10^{5}$ & $5 \mu \mathrm{Cl} / 2$ & $1.14 \times$ & $10^{5}$ & $\mu C 1 / 1$ \\
\hline
\end{tabular}


<smiles>[O]C1CCCC1</smiles>

Date: $\quad$ December 9, 1974

To: R. L. Walser

From: R. E. Wheeler Arrdueter

Subject: ANALYSIS OF TANK FARM SAMPLES

SAMPLE: T-8122 112-S

Vis-0TR: Yellowish green, $50 \%$ solids. $110 \mathrm{mRad} / \mathrm{hr}$

$\mathrm{pH}: \quad 13.7$

SpG: $\quad 1.5909$

$\mathrm{OH}: \cdot \quad 4.54 \underline{\mathrm{M}}$

DTA: No exotherm.

$\mathrm{CO}_{3}: \quad 0.293$ M

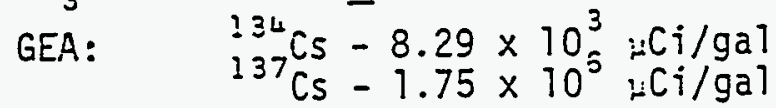

REN: jd

cc: JS Buckingham

NR Christensen

RM Ginesi2:

RE Wheeler

JC Komack 


$-\cdots$ H Babad ( $)$
GS Barney
JS Buckinghamb
CH Delegard
JS Garfield
JE Horton
MJ Rupfer
WP Metz
WE Ogren
DA Puryear
RC Roal
VI Schuelein
WW Schulz
DM Strachan
WJ Van SIyke
CTI Office
File
$\because:$ :

Circulate:

CTI Exempt

File:

CTI- 114
CTI- 122
CTI-503

TCRC-9

WHC-SD-WM-ER-323, Rev. 0

To: N. L. Harms From: J. S. Buckingham

$$
\begin{gathered}
\text { IIQUID WASTE CHAPACTERIZATION } \\
\text { AND SOLIDIFICATION STUDIES } \\
\text { [ACHIEVEMENT REPORT FOR DERIOD ENDING } \\
\text { DECEMBER IO, 1974] }
\end{gathered}
$$

Budget Number:

$$
\begin{aligned}
& E-52204 \\
& E-5420 I
\end{aligned}
$$

Contributors:

$$
\begin{aligned}
& \text { I. I. Curfman } \\
& \text { C. H. Hunter } \\
& \text { J. S. Buckingham }
\end{aligned}
$$

\section{ABSTRACT}

A sampie of salt from 242-s slurry receiving tank, 112-s, was composed primarily of a mixture of sodium nitrate and sodium carbonate. The sodium hydroxide content of the damp salt was high enough to cause the salt to deliquesce in about 13 days when placed in an atmosphere of 70 percent relative humidity at $23^{\circ} \mathrm{C}$. The $13^{7} \mathrm{Cs}$ content of the sait was $10 \mu \mathrm{c} / \mathrm{g}$. The $89+90 \mathrm{~s}=$ content was $0.67 \mathrm{uc} / \mathrm{g}$. The 239 pu content was $<1 \times 10^{-8} \mathrm{~g} / \mathrm{g}$

Solids found pluggiag tie 242-s evaporator's rebciler steam condensate trap were not completa? identified, but are suspected of being valve packing. No ghemicals or cleanizg agents were found that would compietely isssolve the solid. 
A sample of solids from Tank 101-sx, the concentrate receiver for the now shut down Redox concentrator, was found to be primarily sodium nitrate. The $137 \mathrm{Cs}$ and $89+90^{\mathrm{Sr}}$ content was 24.8 and $0.23 \mu \mathrm{c} / 2$, respectively. The 239 pu content was $<8 \times 10^{-9}$.

zelon in the 242-s process condensate ion exchanger had degraded in November. Fifty-three volume percent of the material passed through a 70 mesh screen. The residual fission product content of the eluted zeolon was 0.16 and $0.38 \mu \mathrm{c} / \mathrm{g}^{89+90} \mathrm{Sr}$ and $137 \mathrm{Cs}$, respectively.

\section{NEEDS AND OBJECIIVES}

Iiquid waste solutions are converted to salt cakes by evaporation. Evaporation is accomplisied in a vacuum evaporator-crystallizer operated at approximately 41 torr. The waste is concentrated to 20 to 40 volume percent settled solids slurry, then transferred to underground storage tanks where solids can settle and aditional solids form through cooling. Supernatant liquid is pumped from the tank, blended with fresh, dilute waste, and recycled to tine evaporator. When the tank is filled with solids (salts), interstitial liquor is zemoved by means oł a sal= weII.

Though specifications have not been set for salt cakes, it is felt that among other things the cake should contain no arainabie liquor: that is, liquid that could arain into 
the soil should the integrity of the underground tank be breached. No drainable liquid also means that the salts should not pick up enough moisture from the atmosphere to deliquesce.

DISCUSSION

Analysis of salt from 242-s Evaporator

A sample of salt from $242-s$ slurry receiving Tank $112-5$ was analyzed. The results are shown in Table I.

\section{TABLE I}

ANAIYSIS OF SAIT FROM TANK $112-\mathrm{S}$

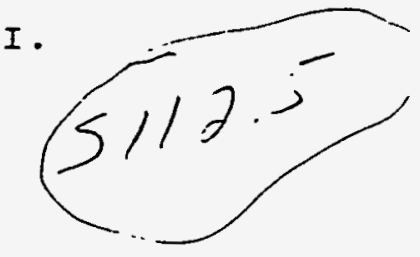

$g / c=$

3ulk density, $g / 0 c$ $\mathrm{H}_{2} \mathrm{O}$. $\mathrm{NaAlO}_{2}$, 学 $\mathrm{Al}_{2} \mathrm{O}_{3}, \quad$ \& $\mathrm{NaNO}_{2}, \quad$ \& $\mathrm{NaNO}_{3}$, \& $\mathrm{NaOH}, \quad$ क $\mathrm{Na}_{2} \mathrm{CO}_{3}$, \% $\mathrm{Na}_{3} \mathrm{PO}_{4}$, \% TOTAI $137 \mathrm{Cs}, \mu \mathrm{C} / \mathrm{g}$
$8990 \mathrm{Sr}, \mu \mathrm{C} / \mathrm{g}$
$239 \mathrm{Pu}, \quad \mathrm{g} / \mathrm{g}$
1. 304

1.918

i5. 8

2.5

0.1

3.1

58.7

4.4

14.6

0.8

100.0

10.1

0.67

$<1 \times 10^{-8}$

The salt deliquesced in about 13 days on gaining about 10 weight percent water when placed in en atmosphere of 70 percent relative bumidity at $23^{\circ} \mathrm{C}$. 


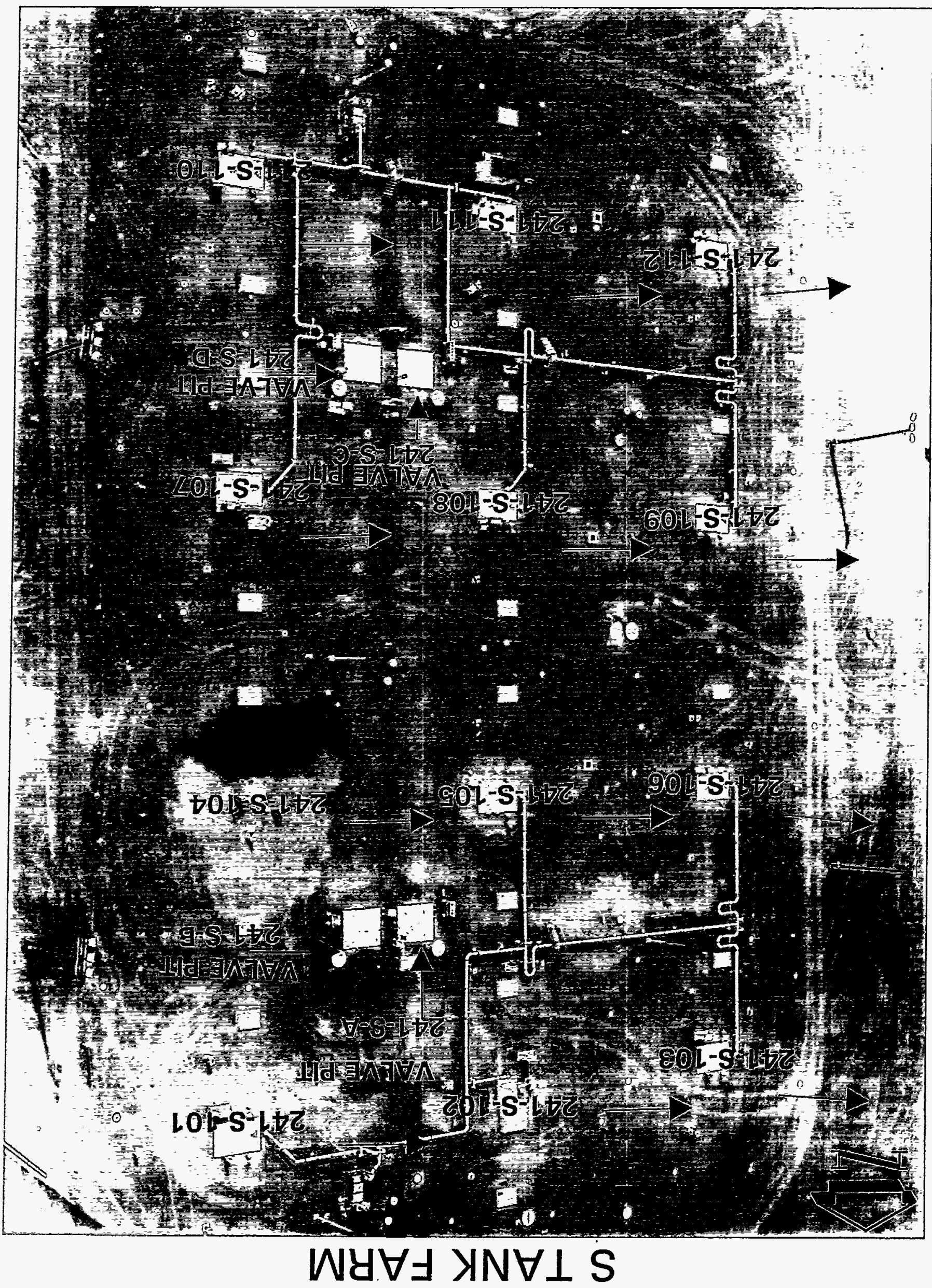




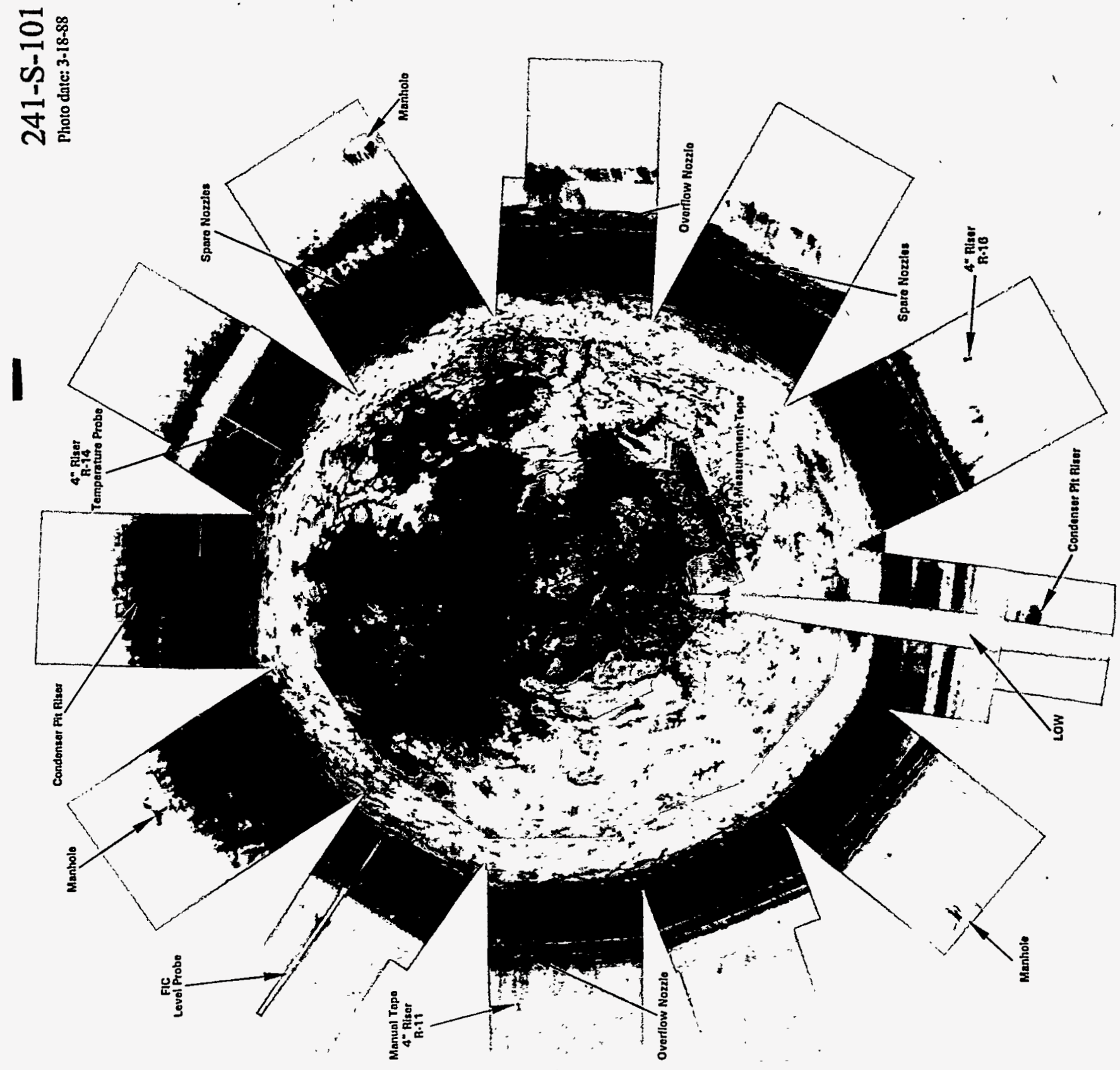


WHC-SD-WM-ER-323， Rev . 0

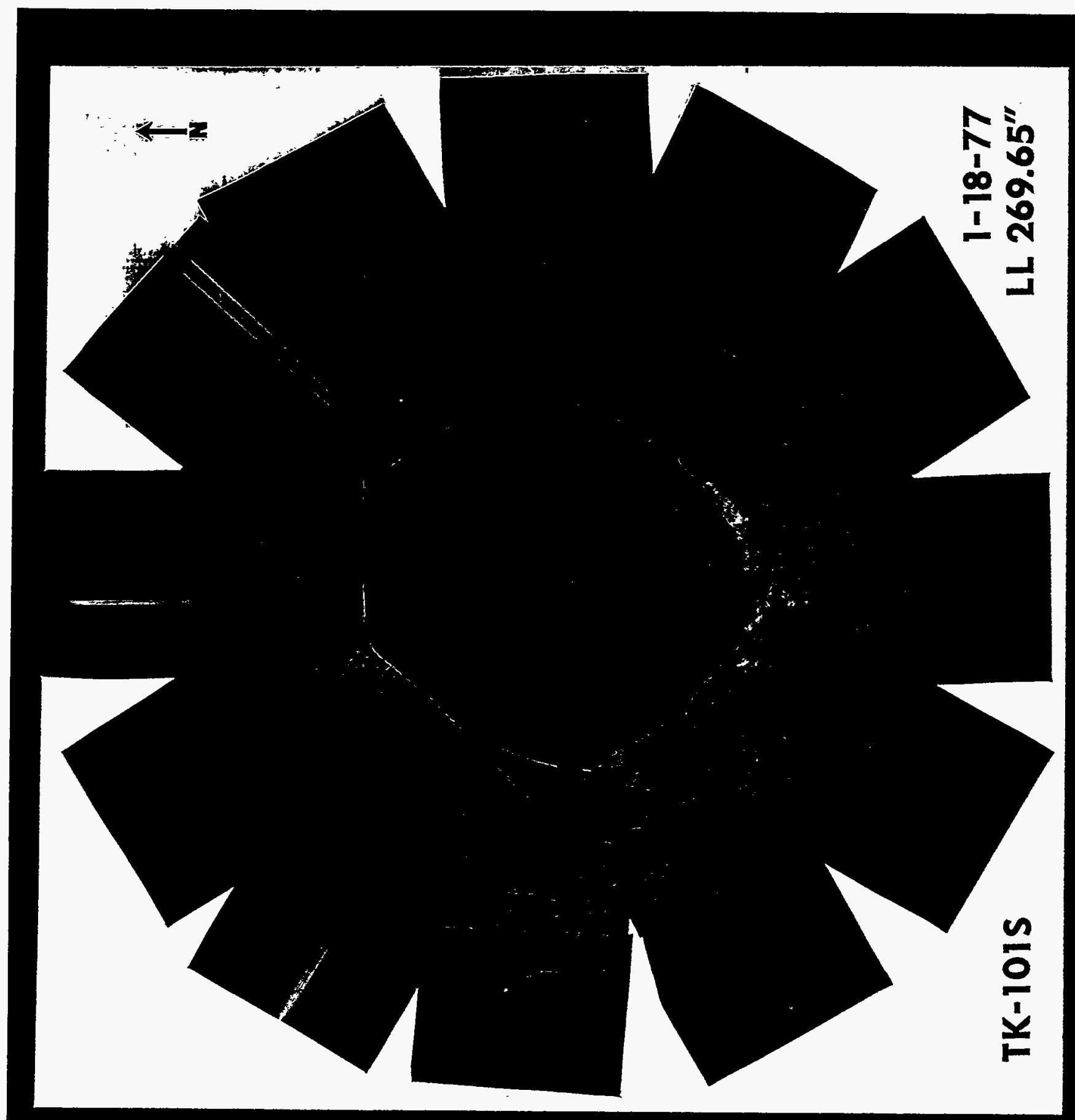

$I-3$ 


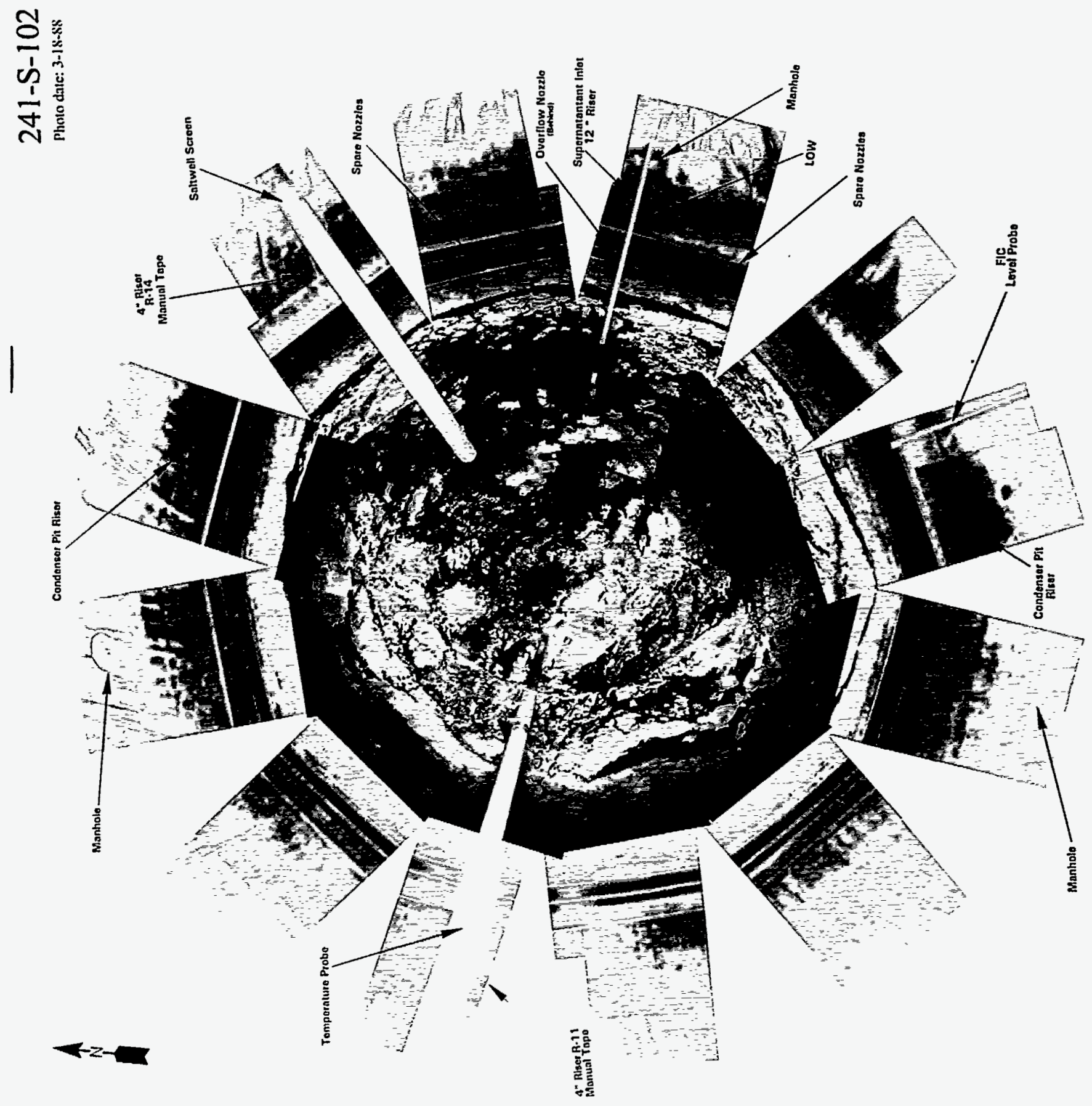




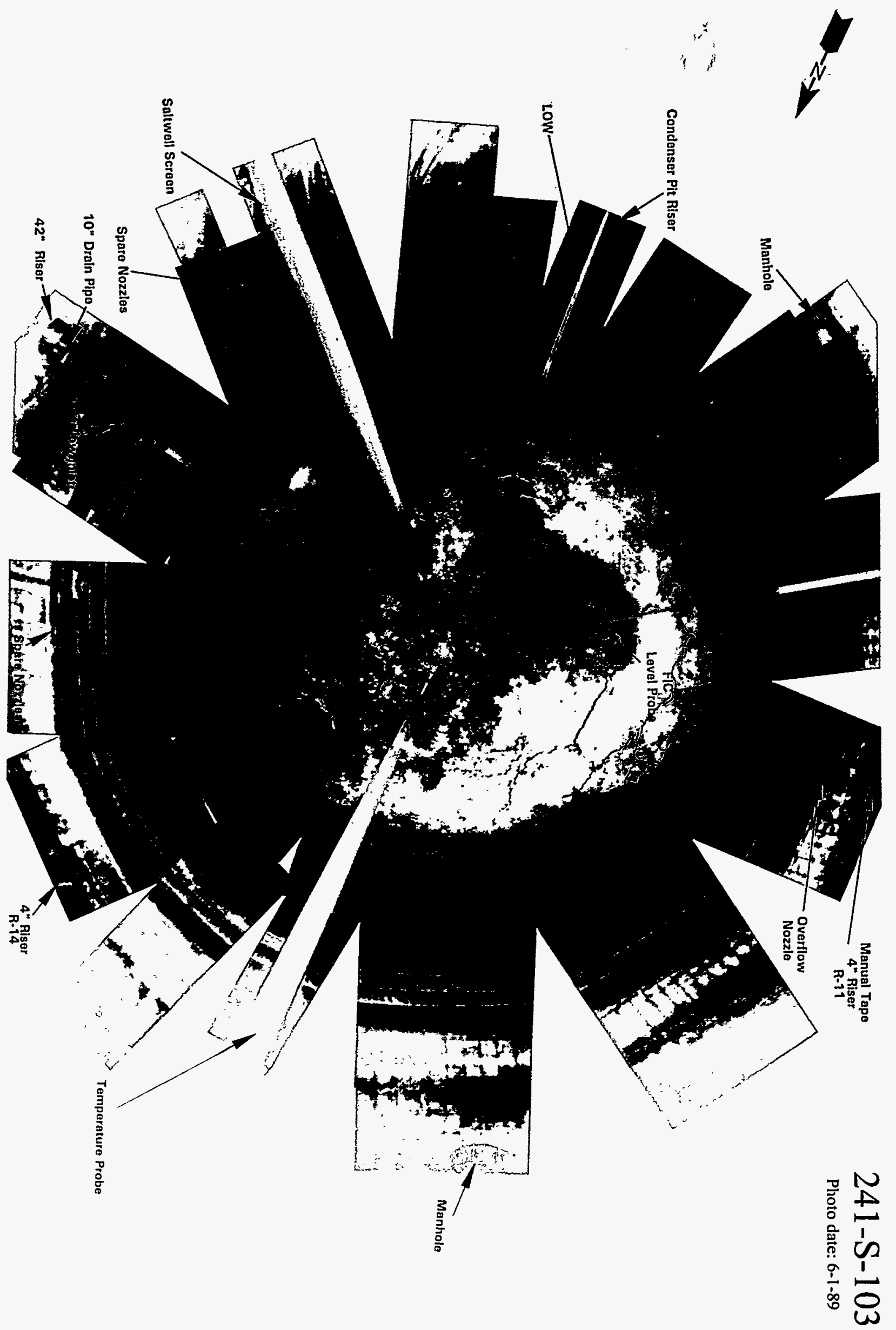


WHC-SD-WM-ER-323, Rev. 0

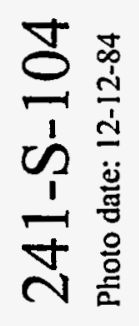

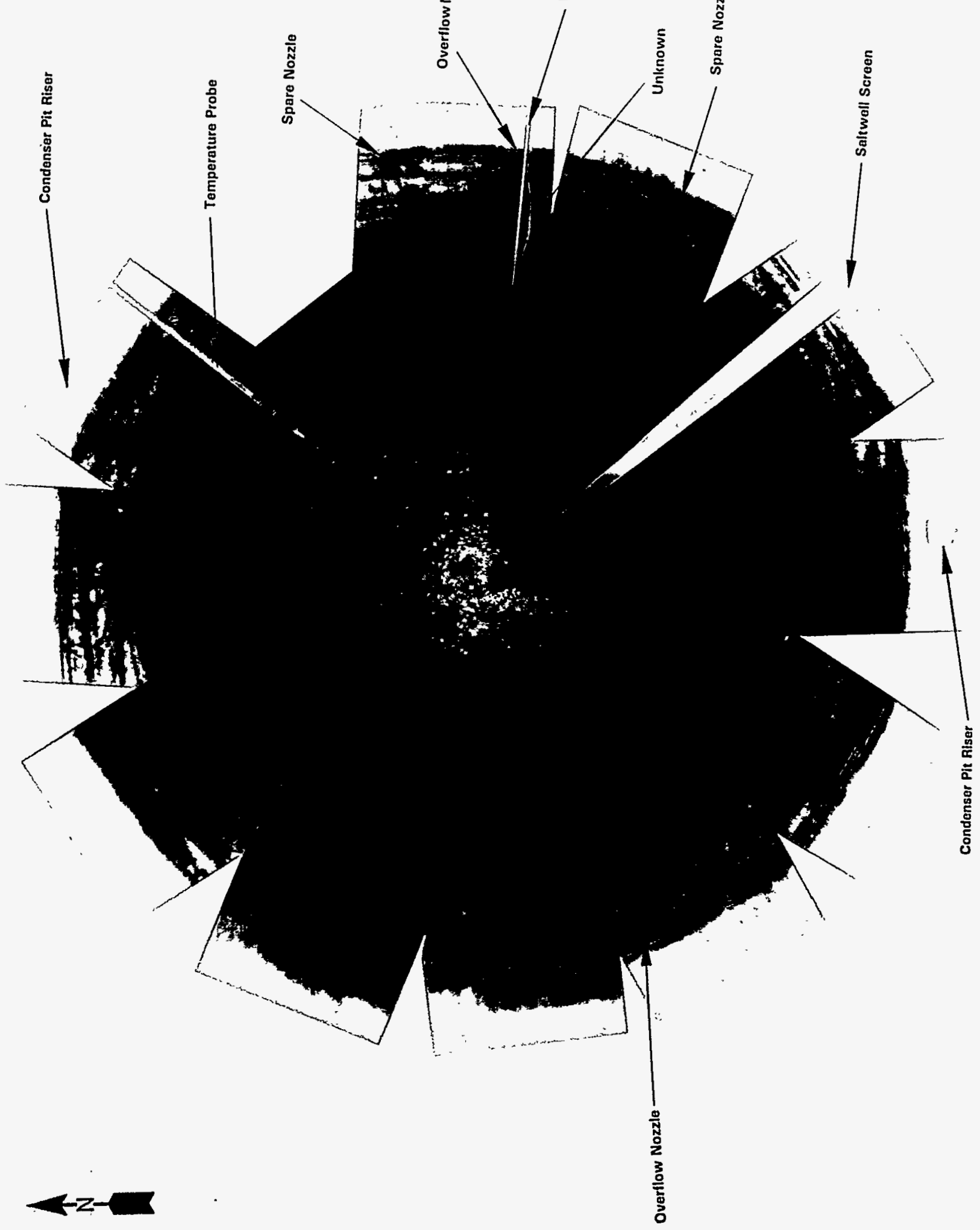

$I-6$ 
WHC-SD-WM-ER-323， Rev. 0

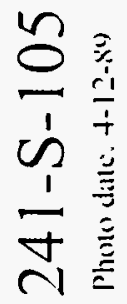
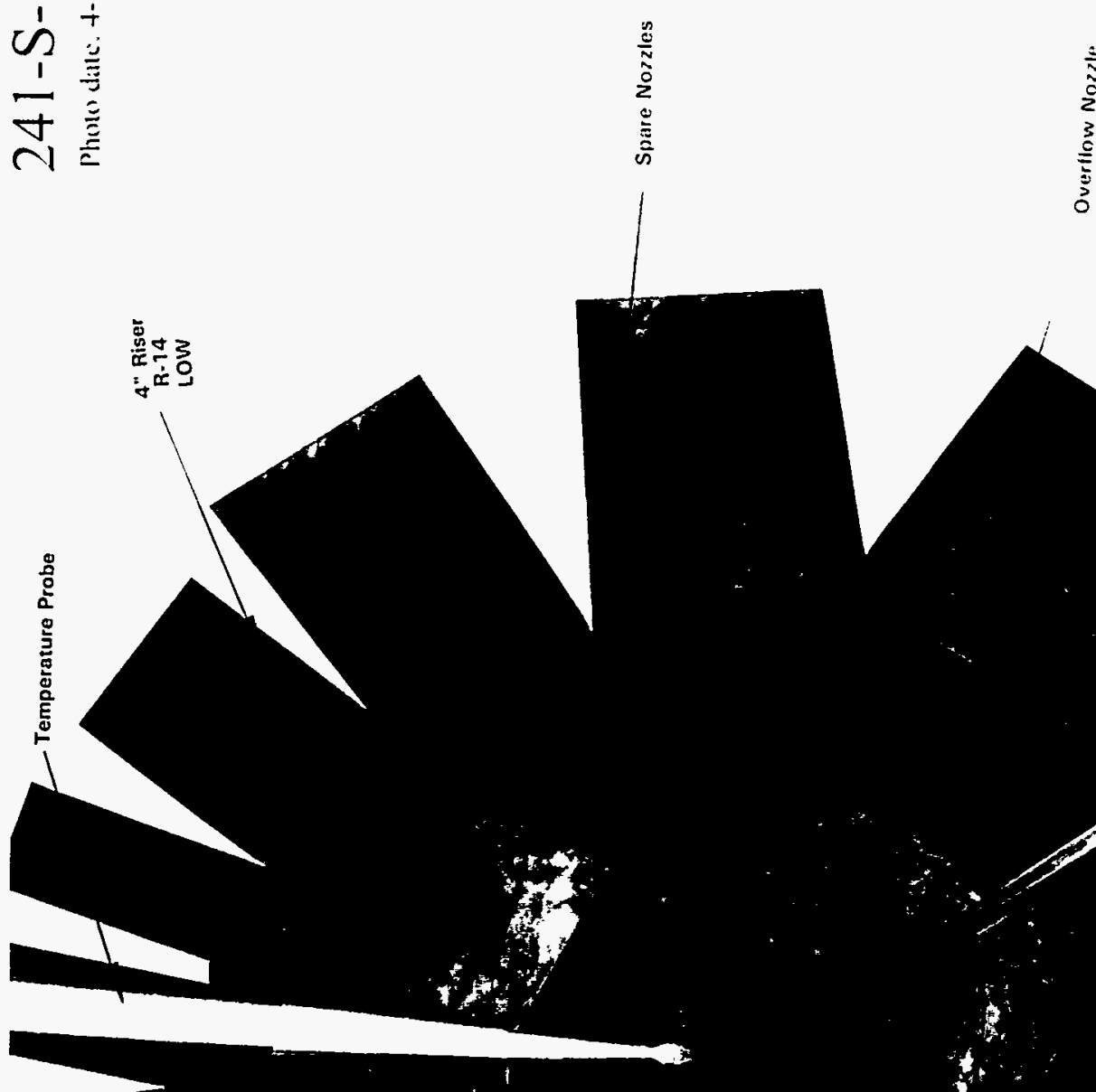

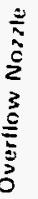
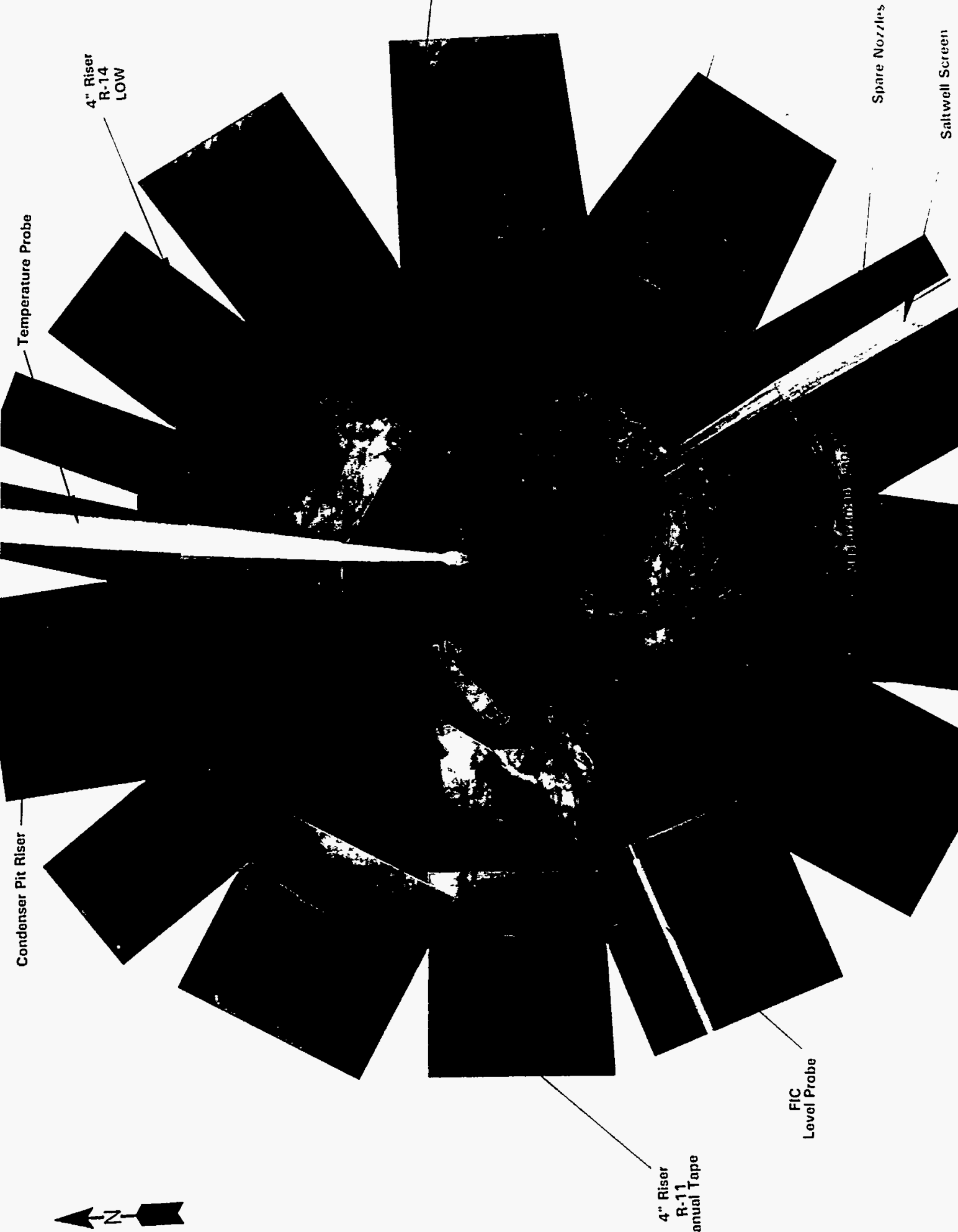

$[x$

$\therefore$

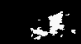
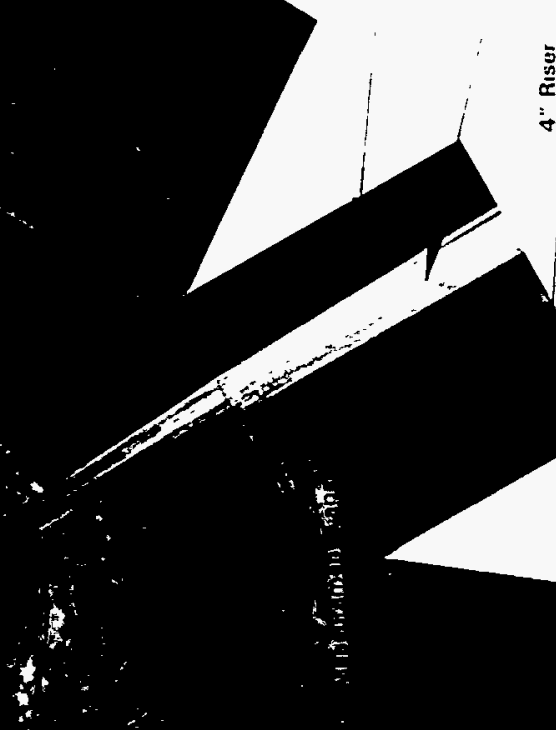

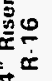
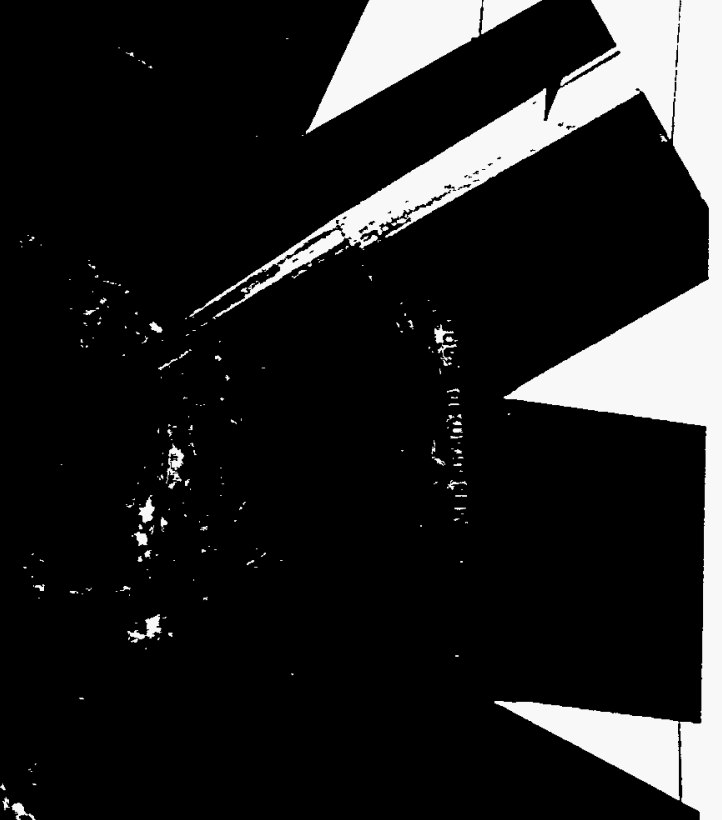
WHC-SD-WM-ER-323， Rev. 0

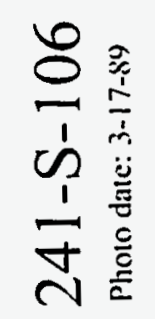

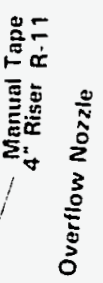

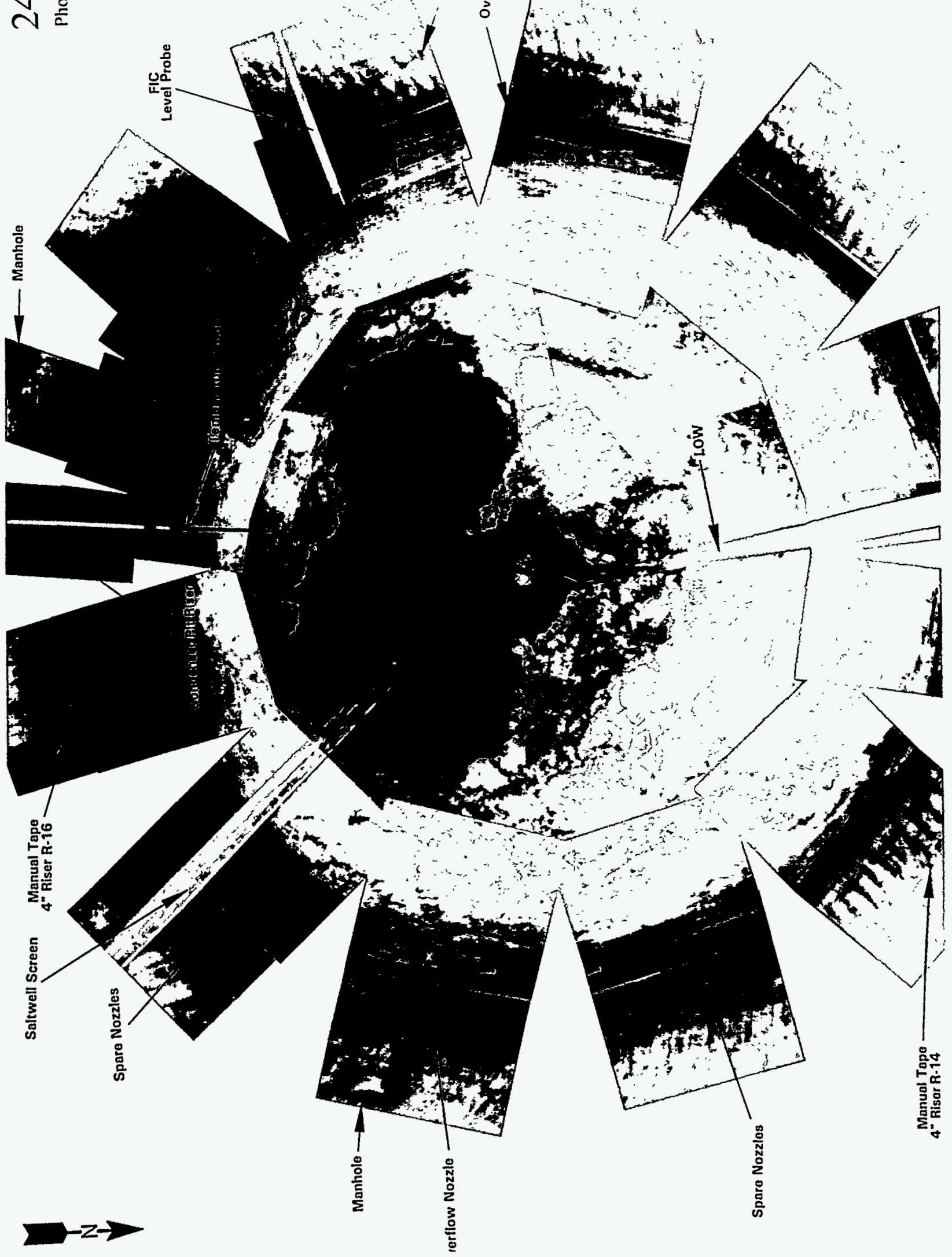


WHC-SD-WM-ER-323， Rev. 0

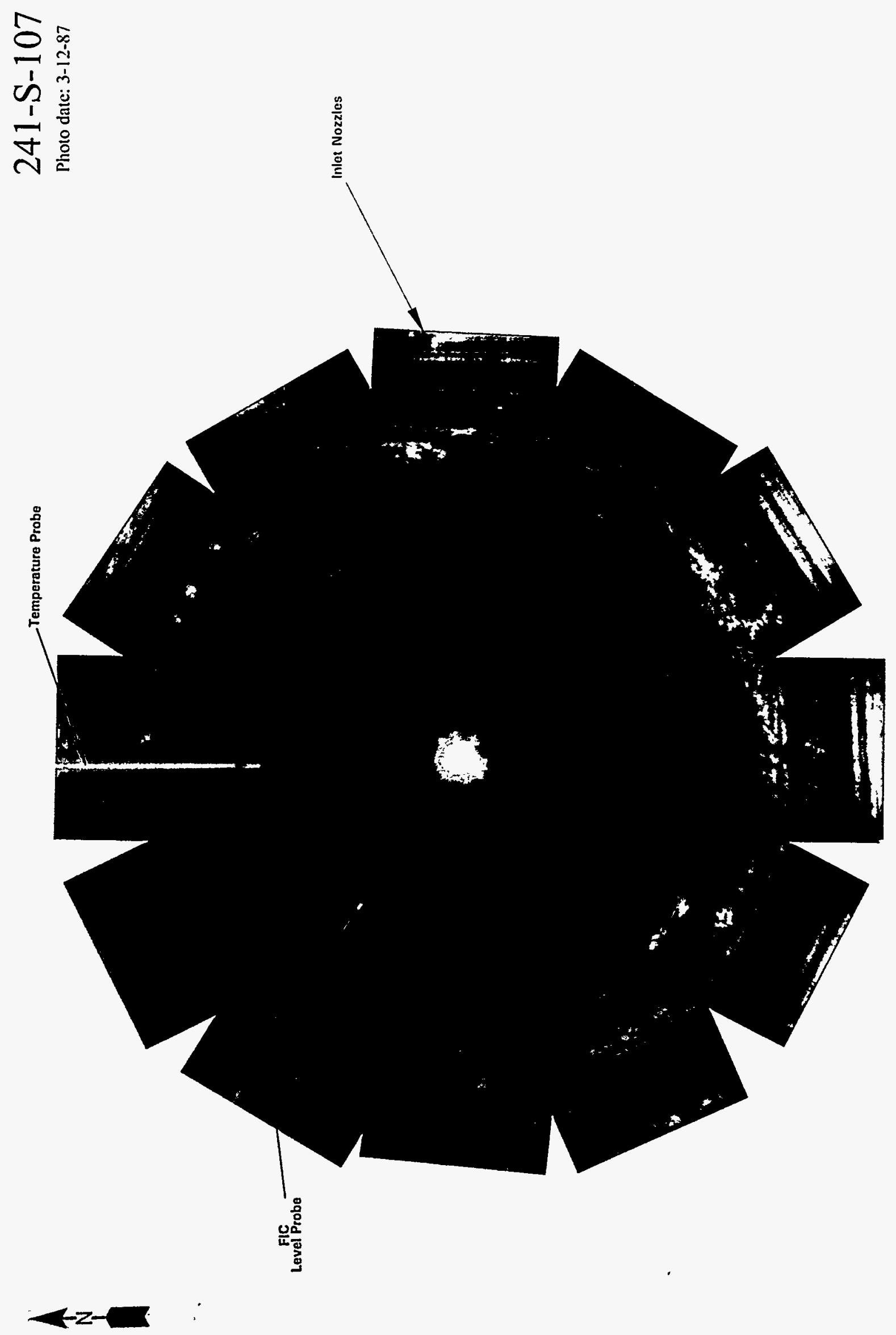

$I-9$ 
WHC-SD-WM-ER-323，Rev. 0

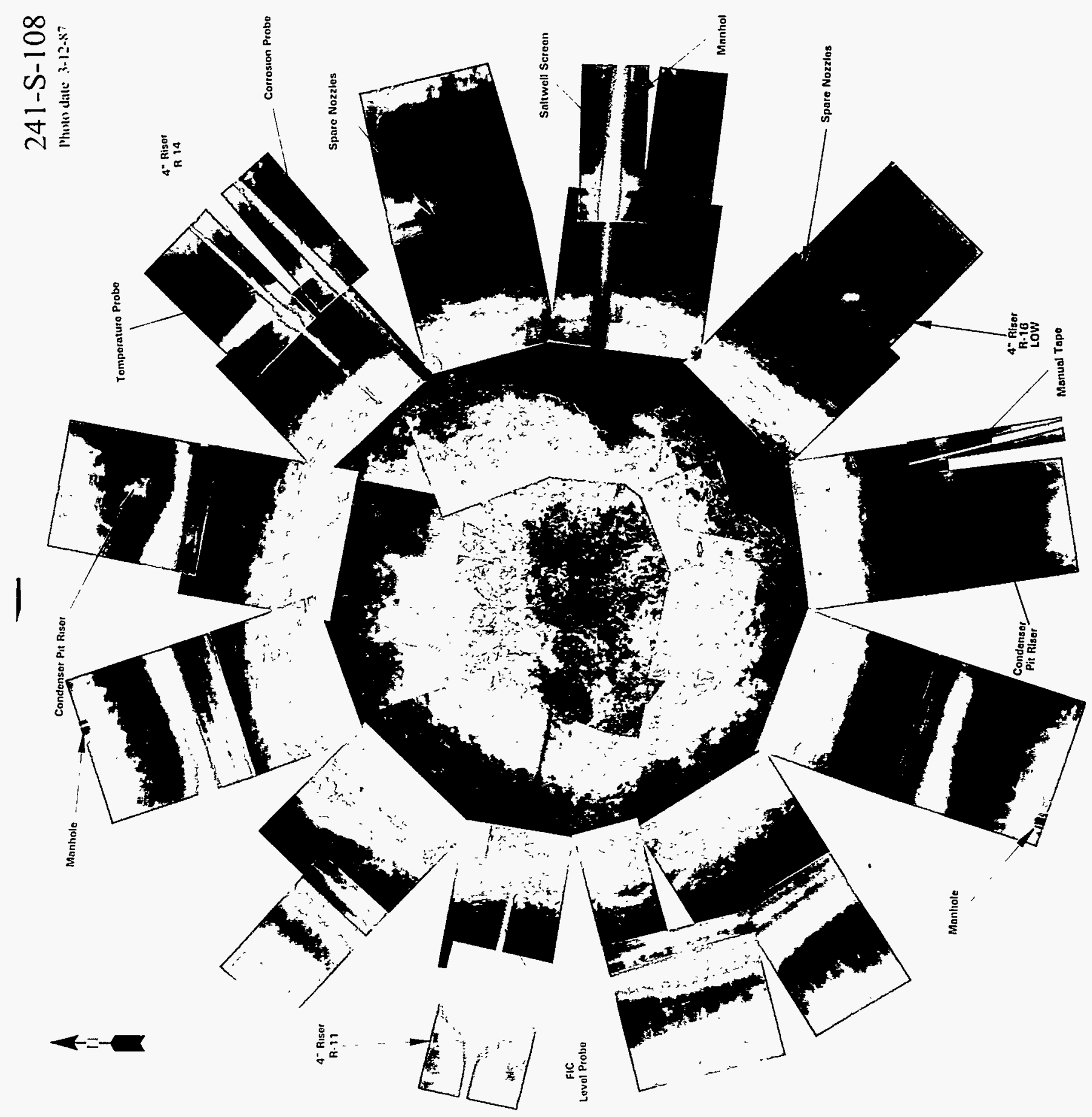




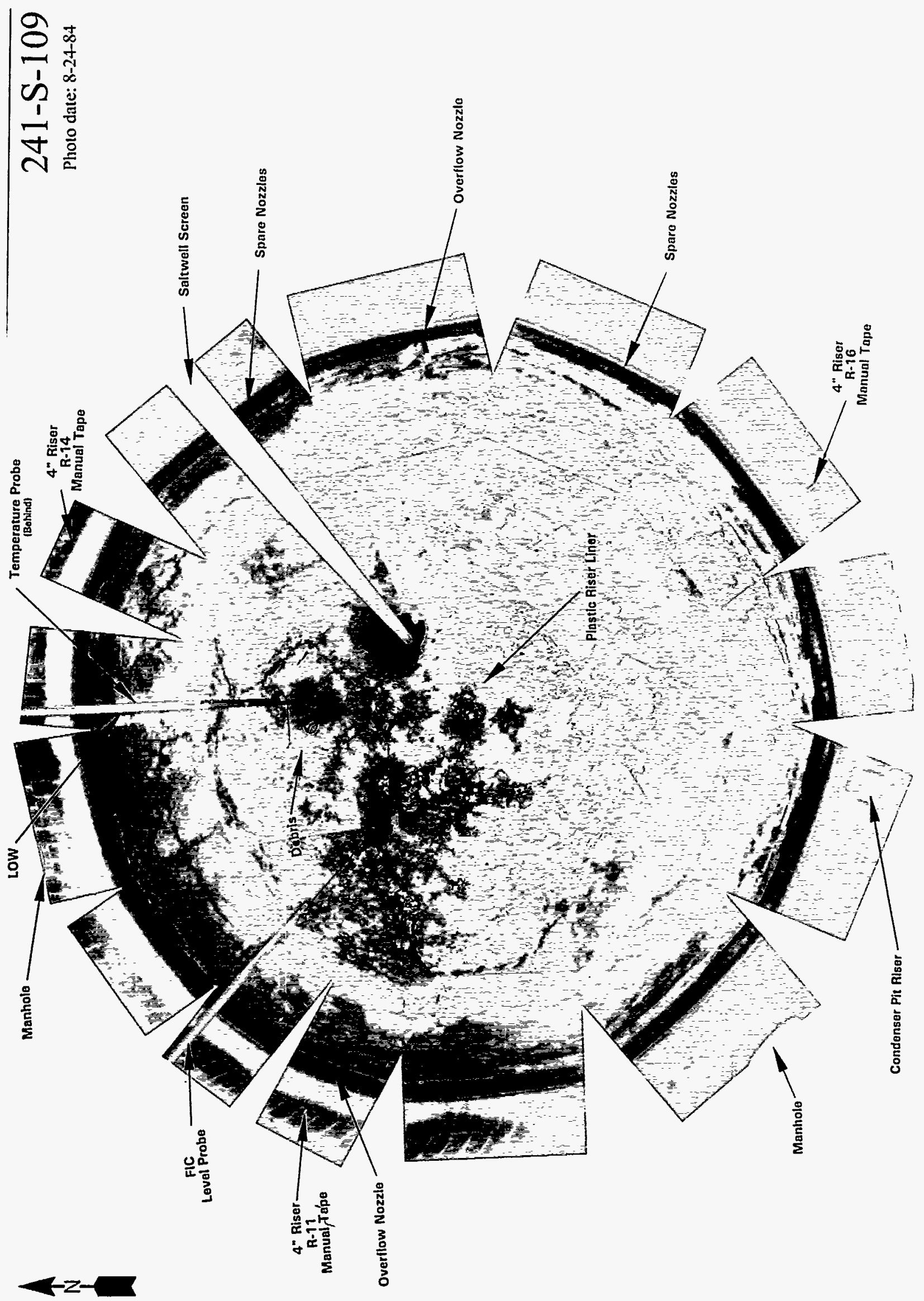




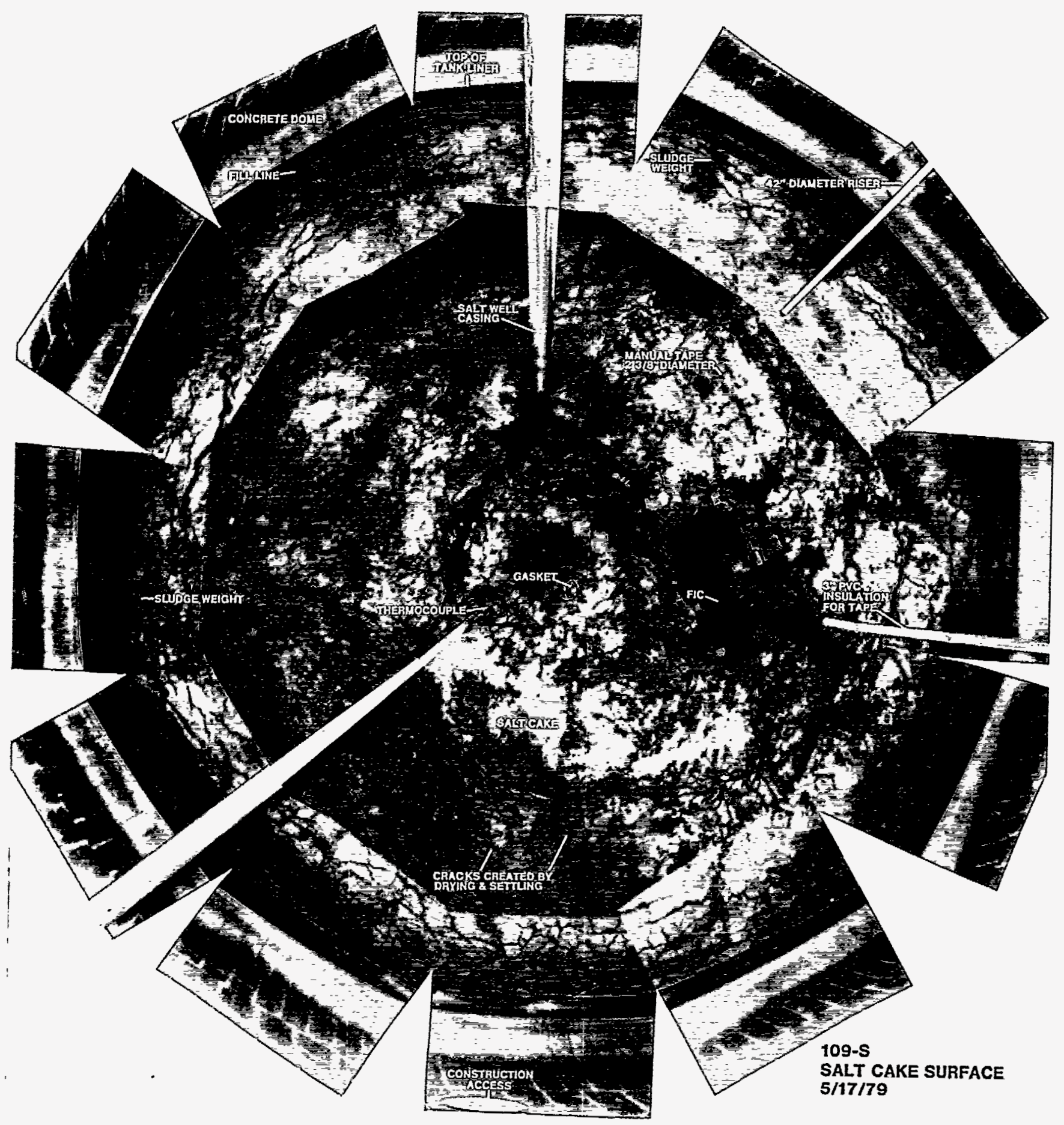




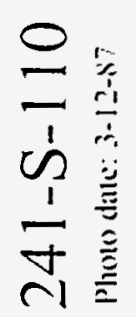

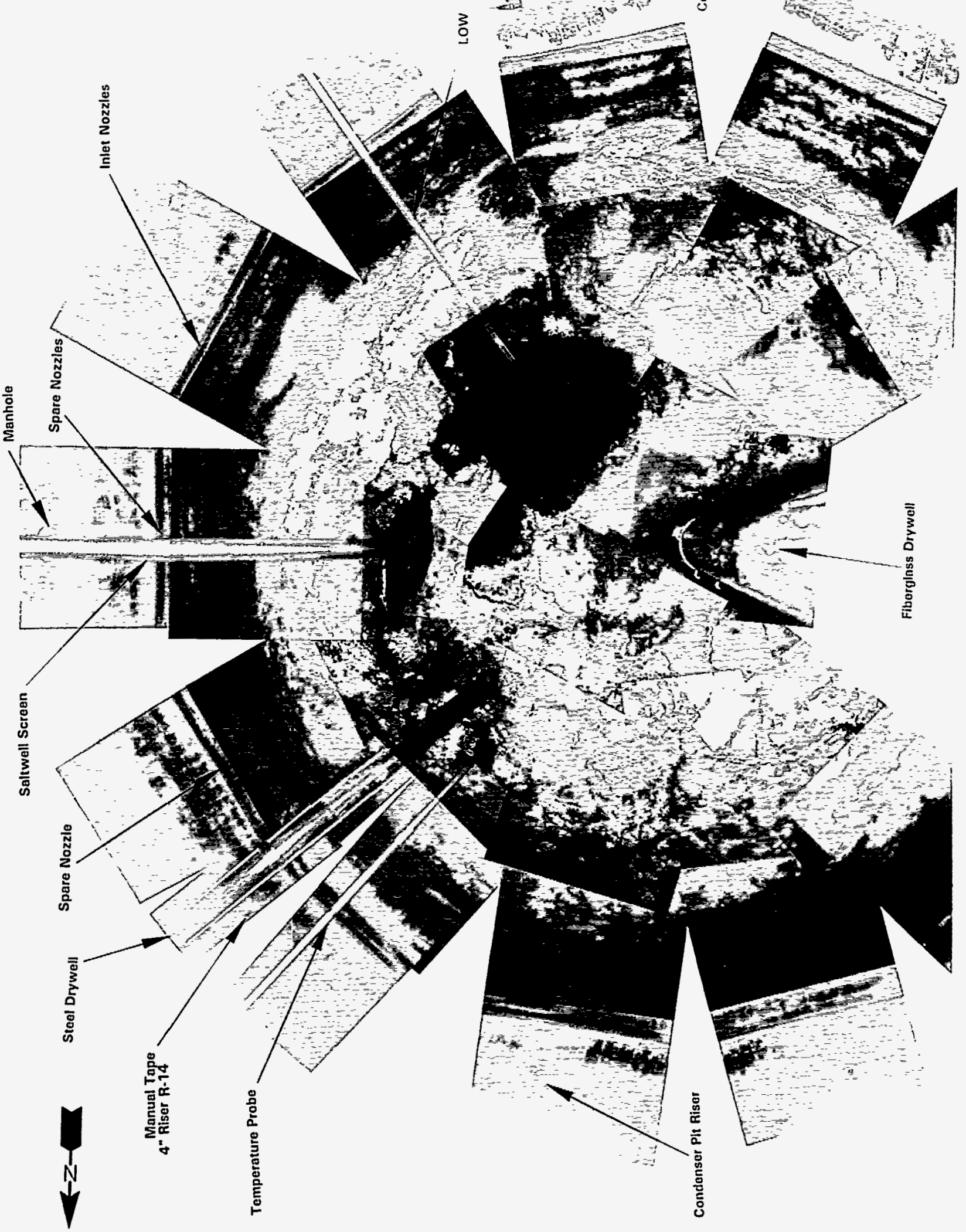




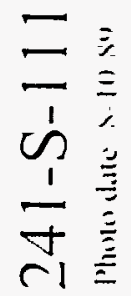
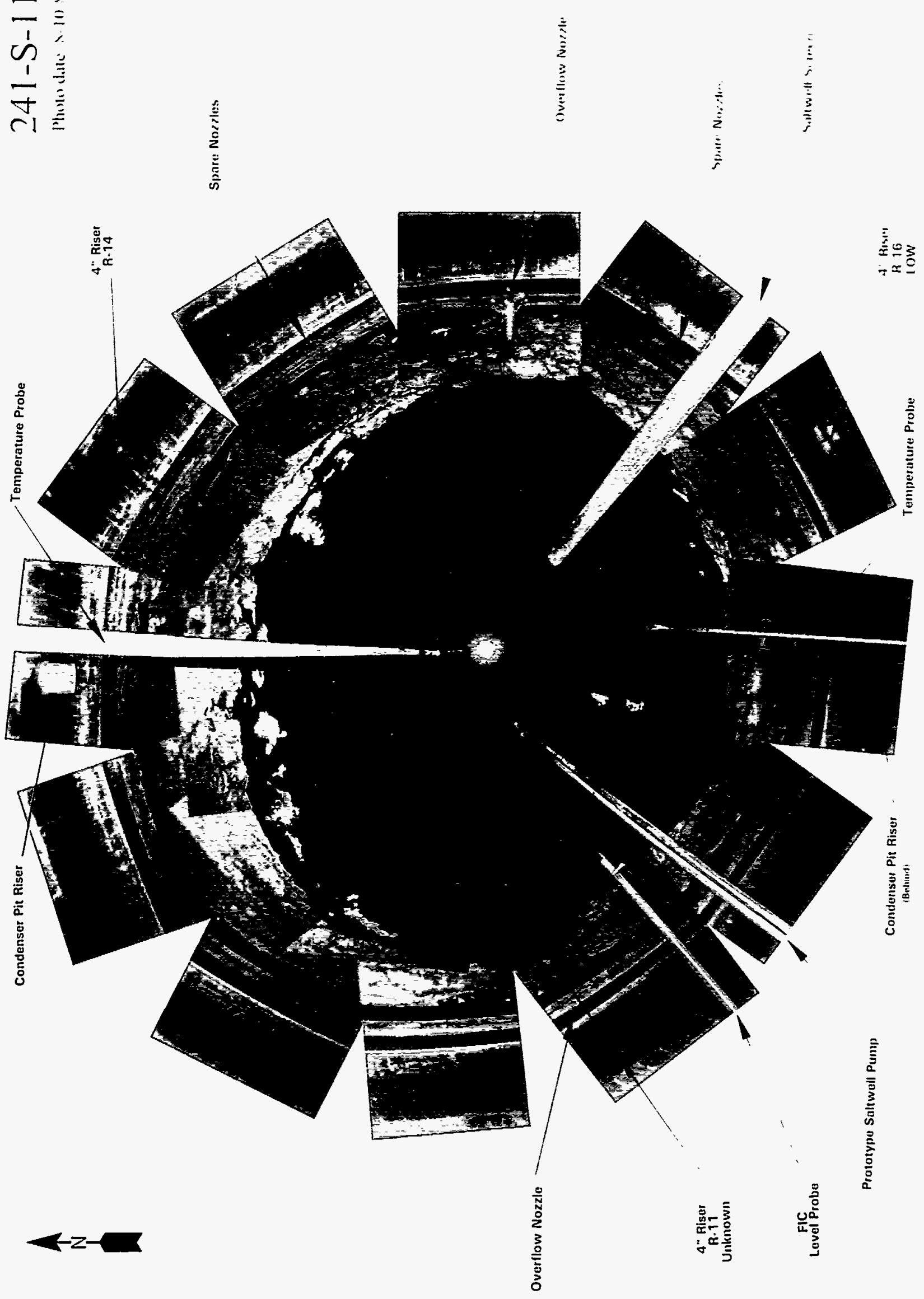
WHC-SD-WM-ER-323， Rev. 0

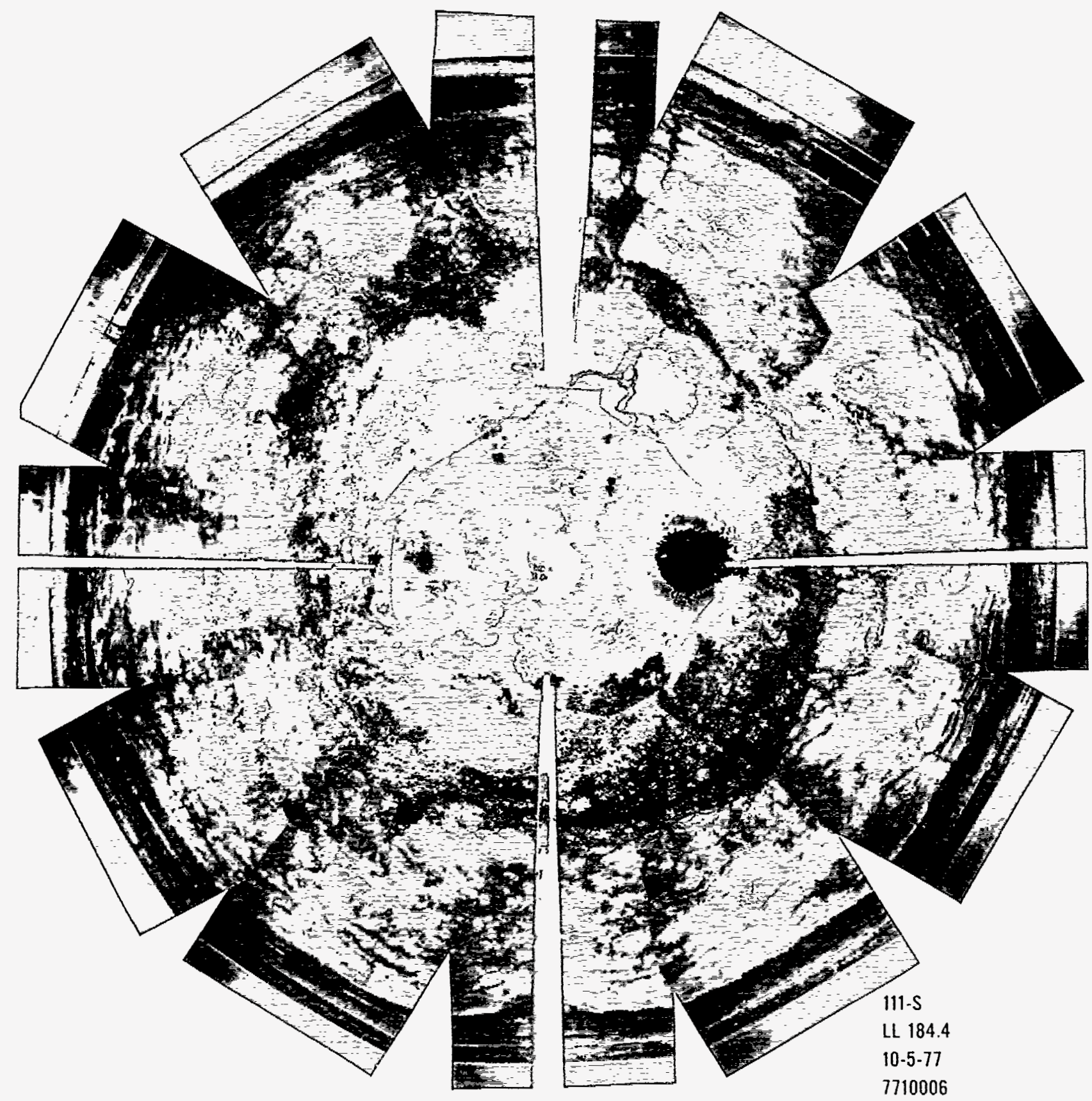


WHC-SD-WM-ER-323， Rev . 0

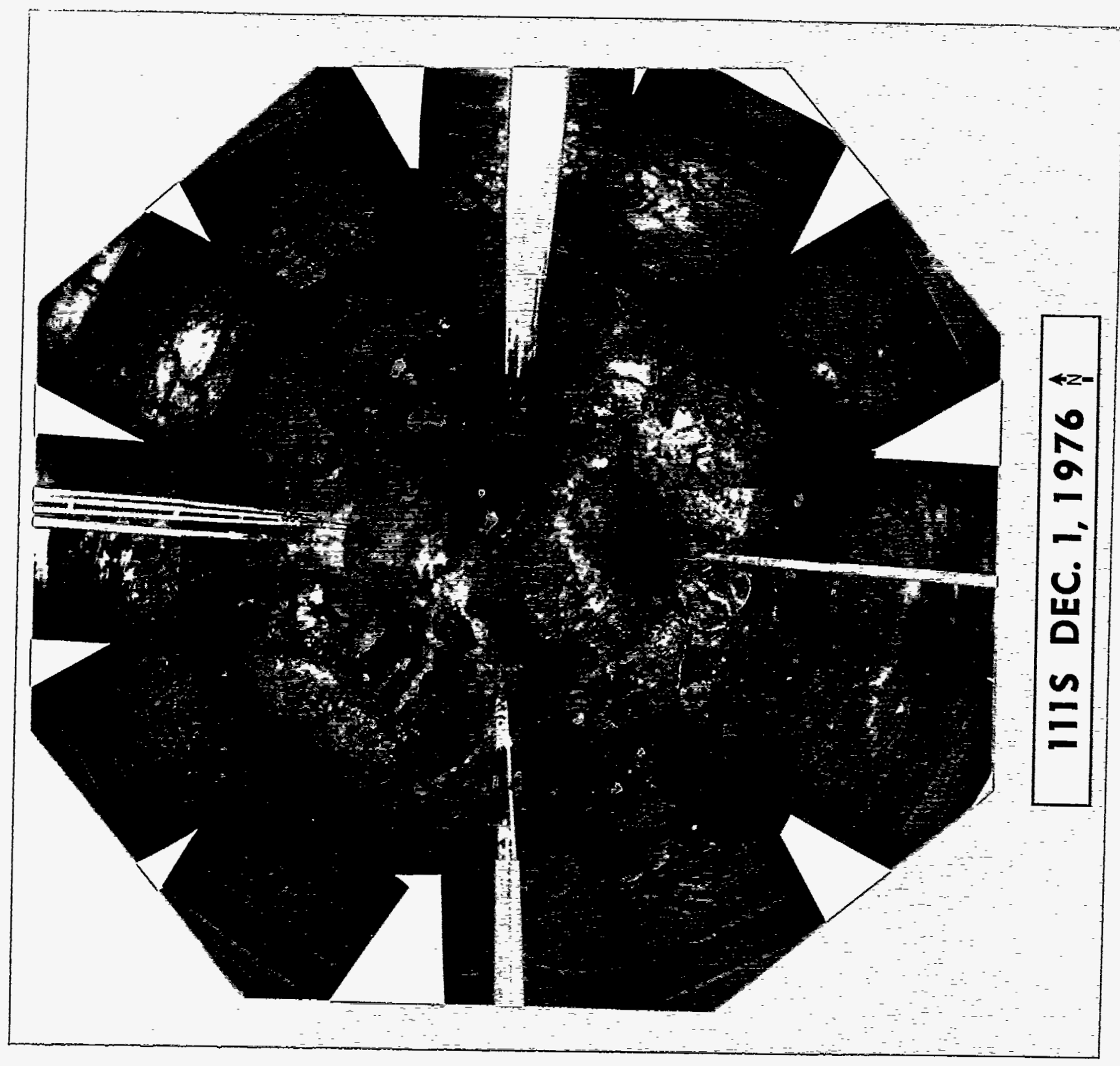




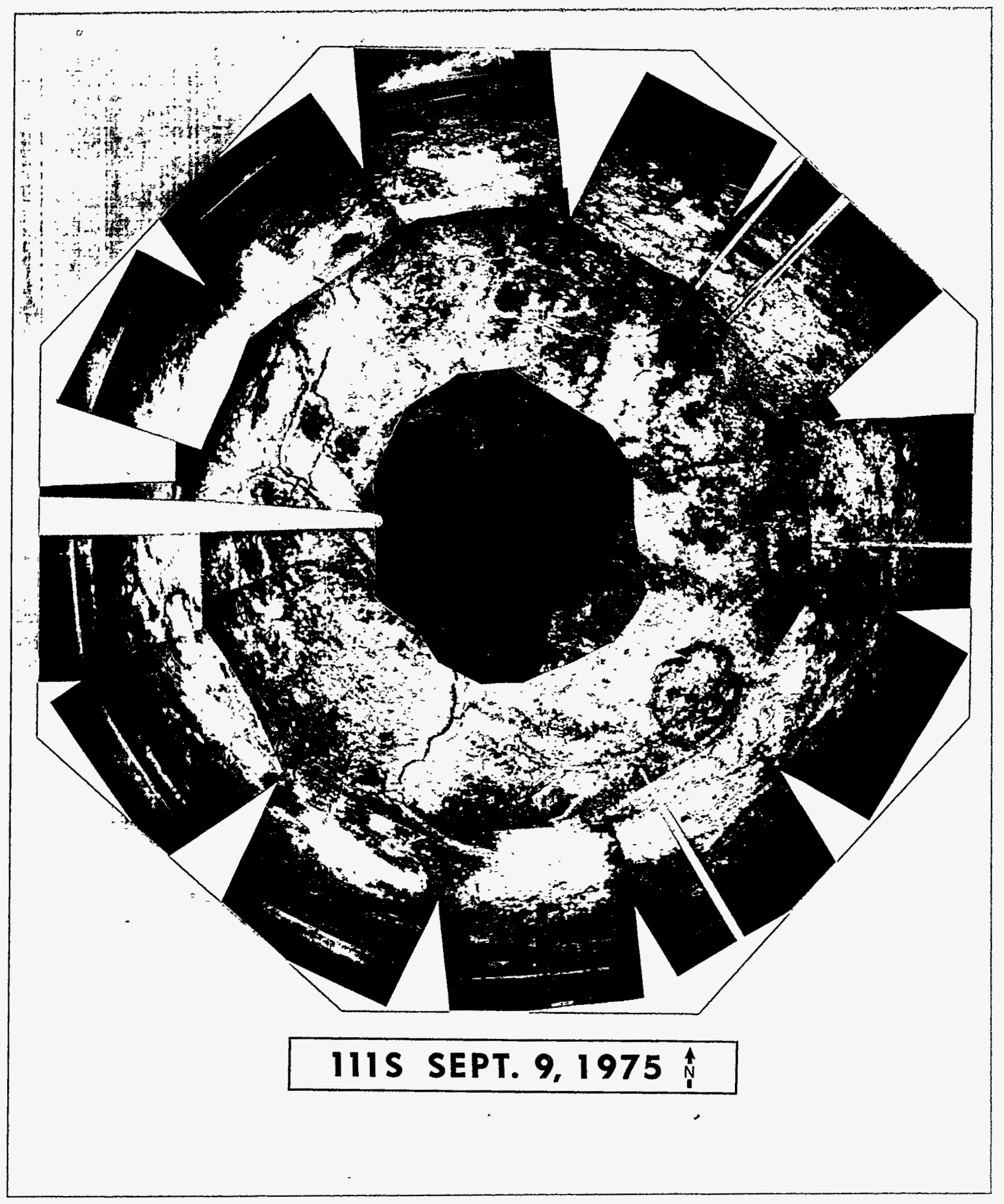




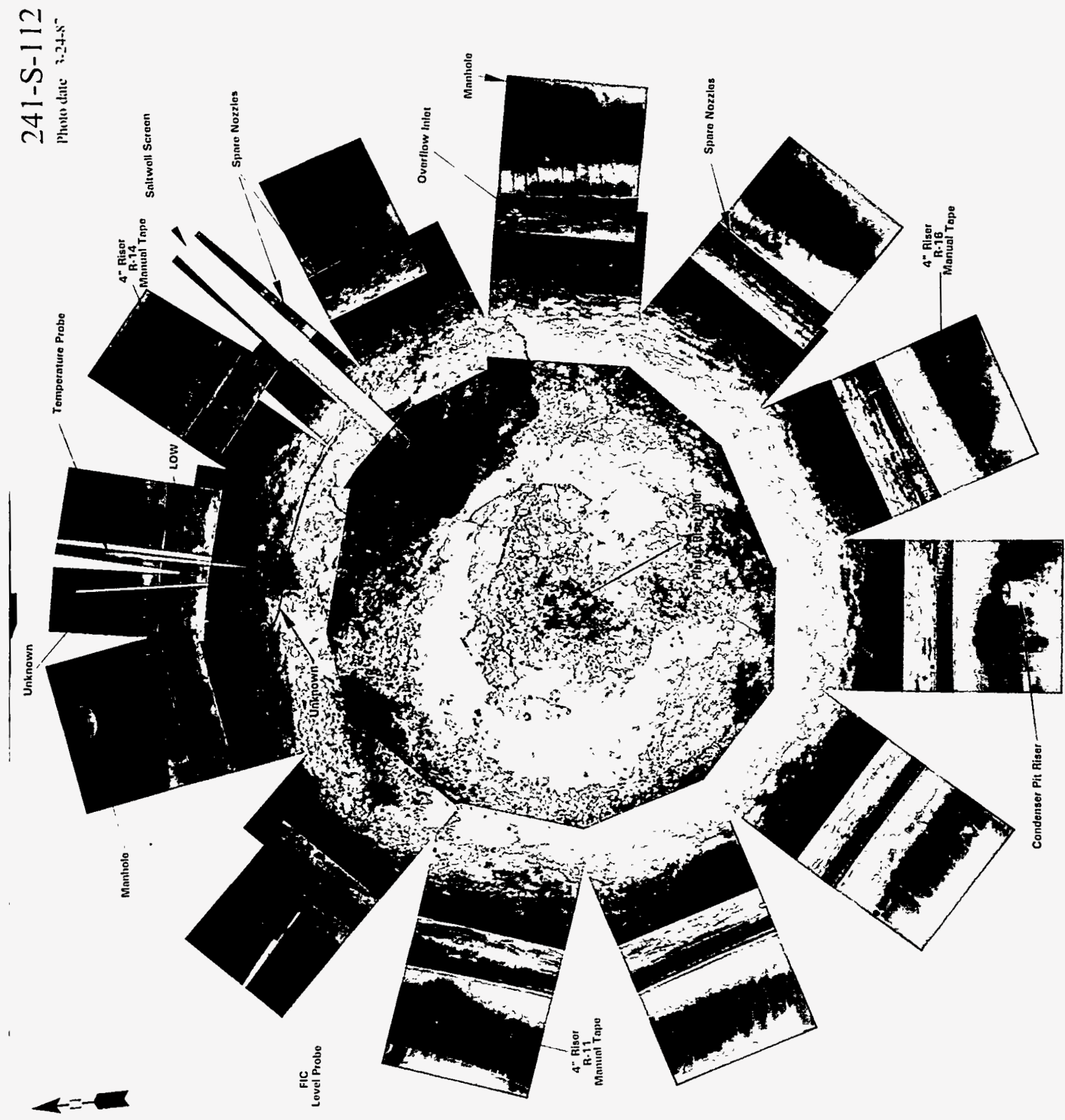


WHC-SD-WM-ER-323, Rev. 0

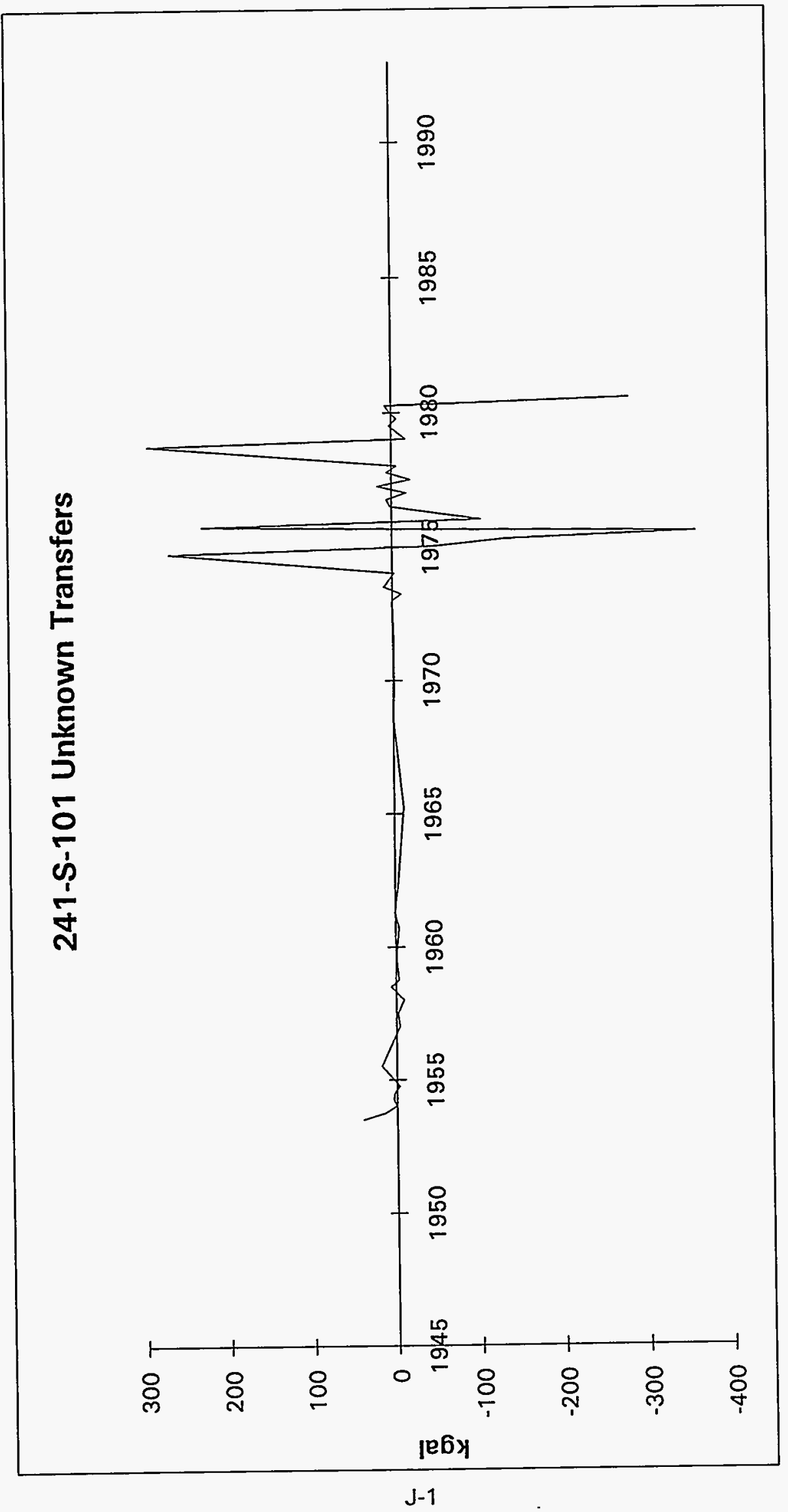

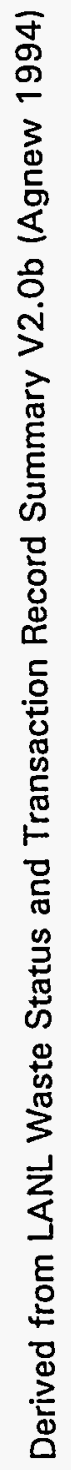


WHC-SD-WM-ER-323, Rev. 0

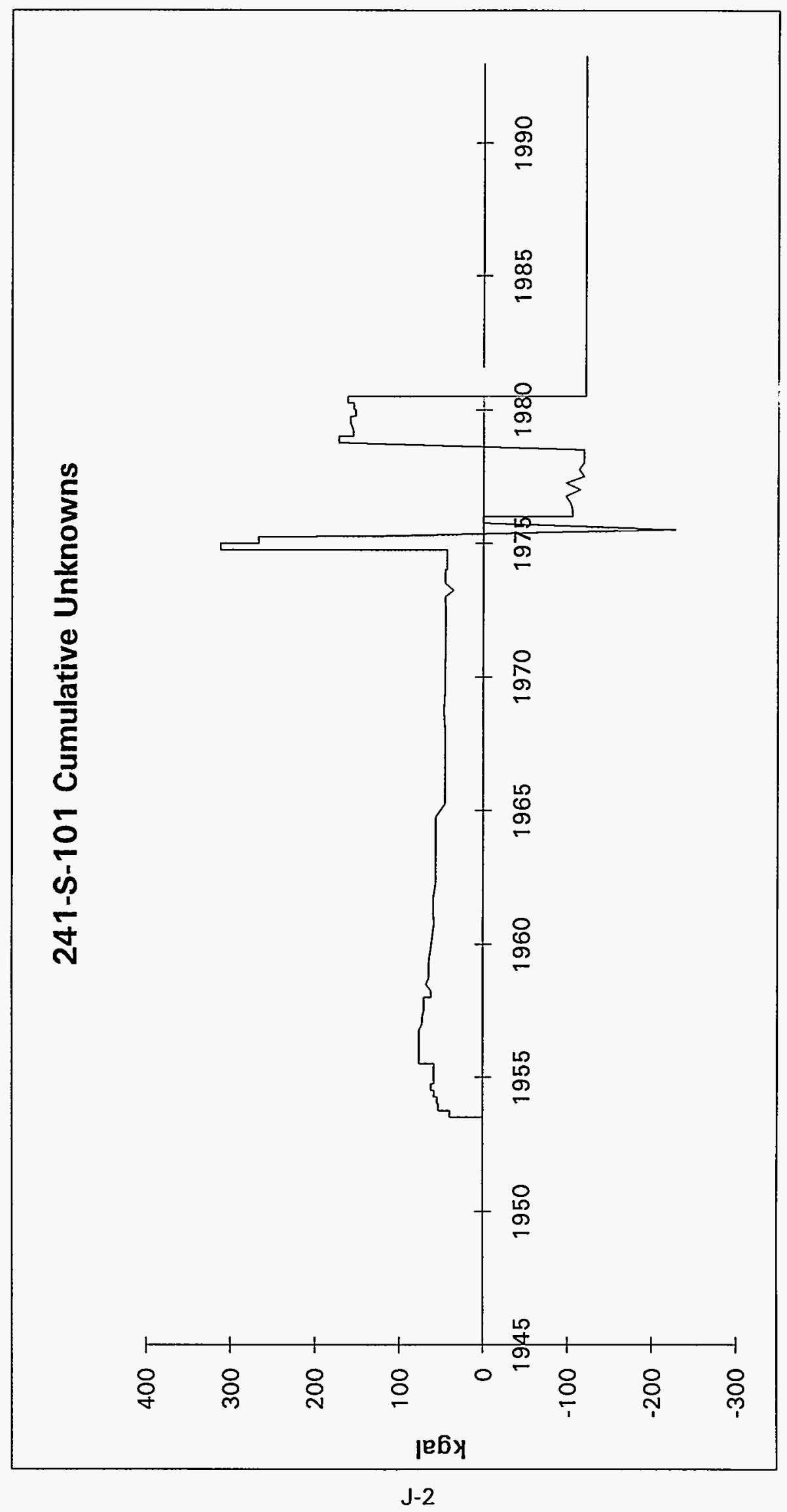

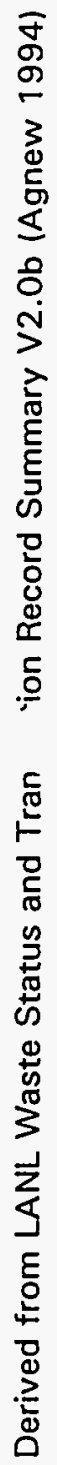




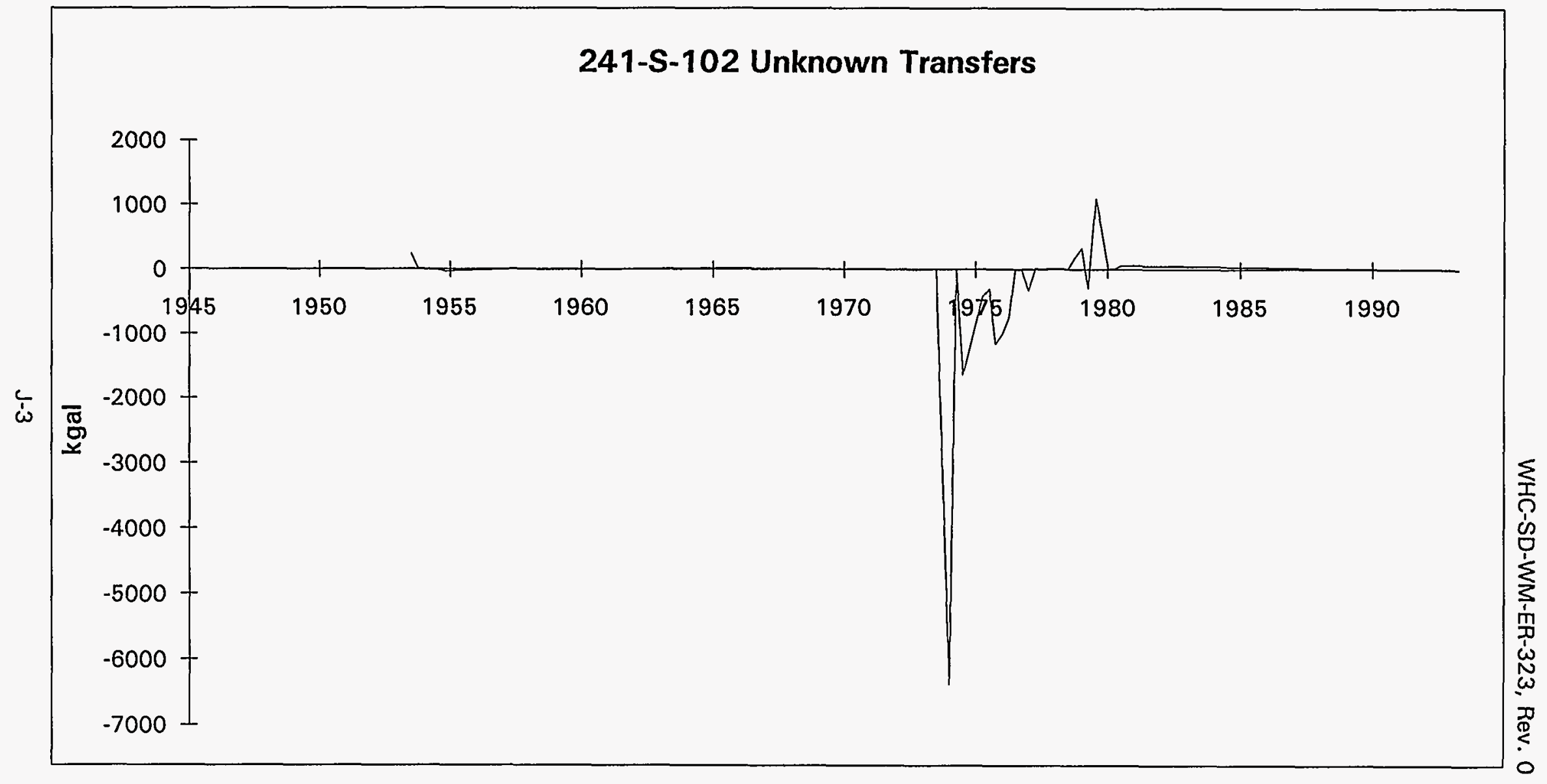




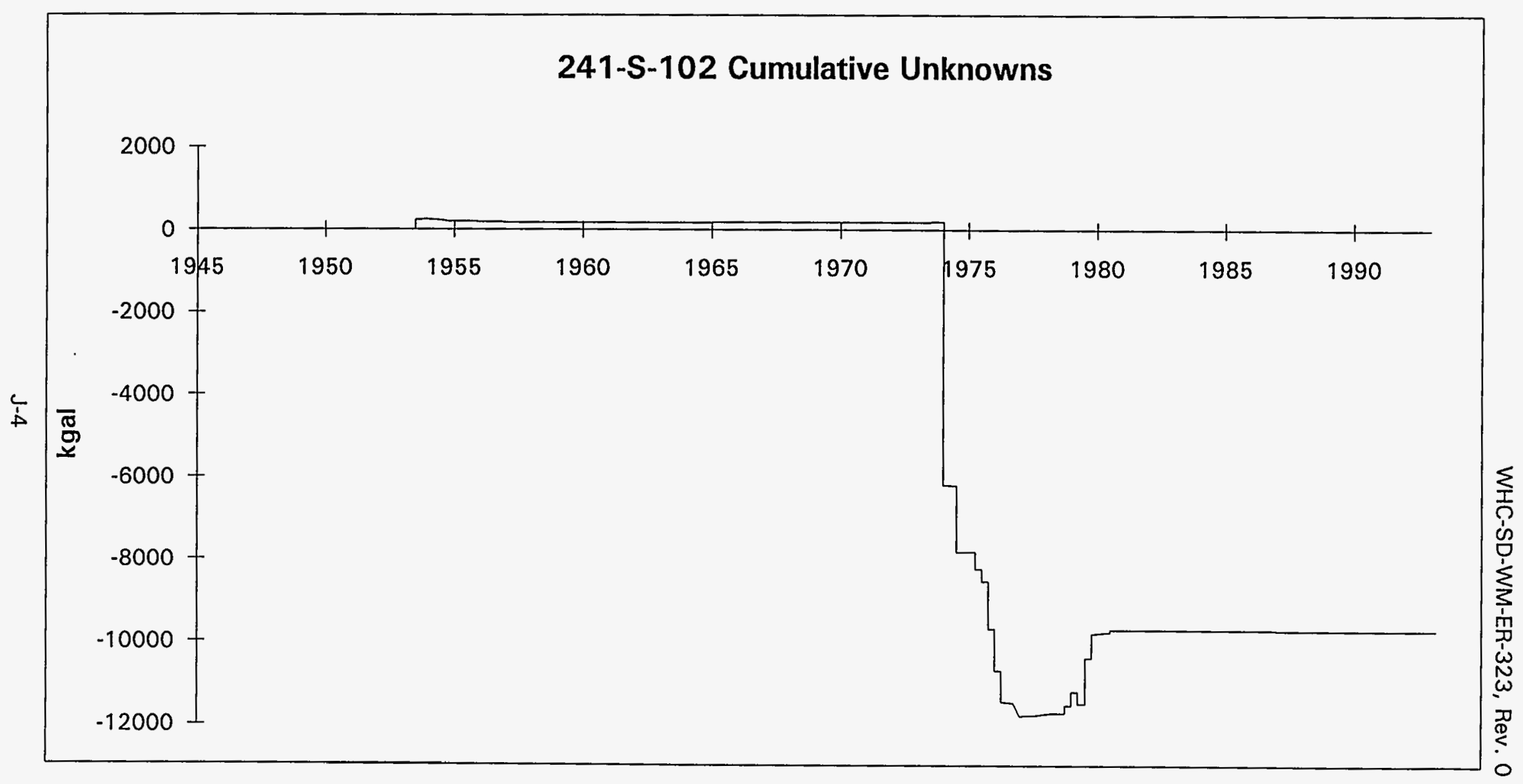

Derived from LANL Waste Status and Transartion Record Summary V2.0b (Agnew 1994) 
WHC-SD-WM-ER-323, Rev. 0

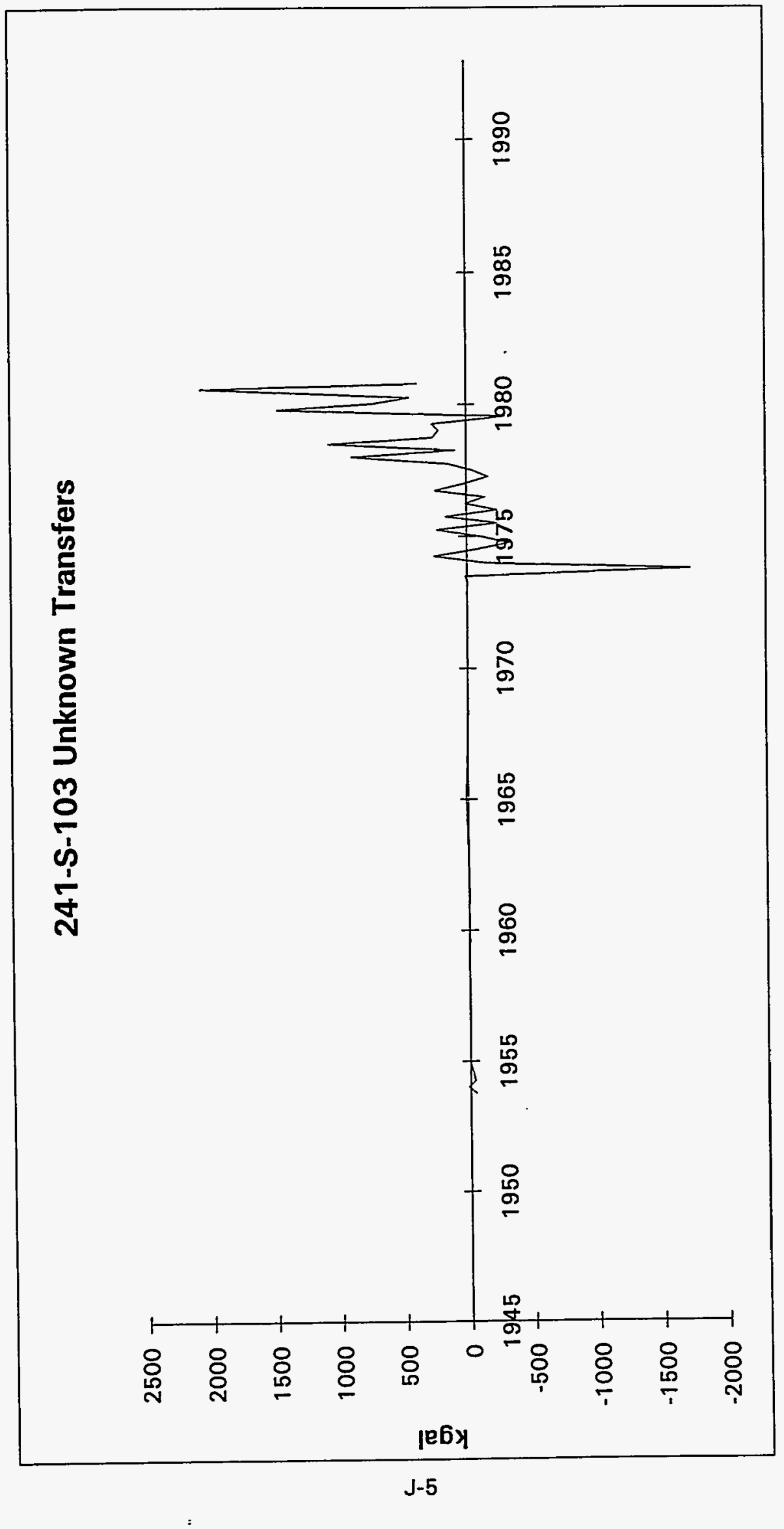

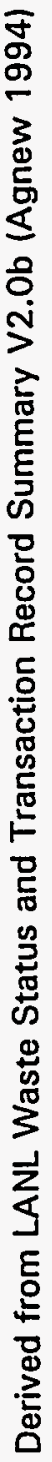




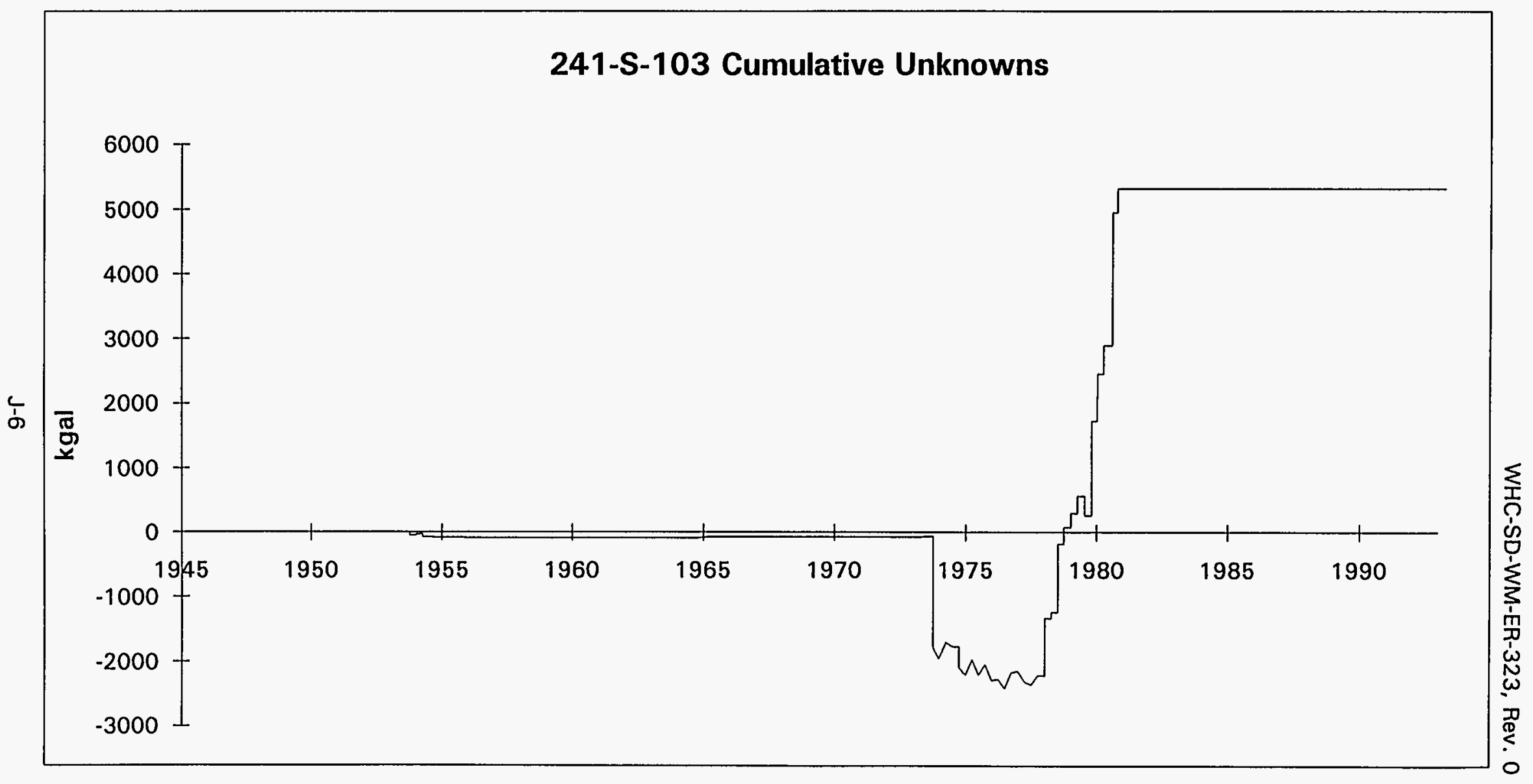

Derived from LANL Waste Status and Transaction Record Summary V2.0b (Agnew 1994) 
WHC-SD-WM-ER-323, Rev. 0

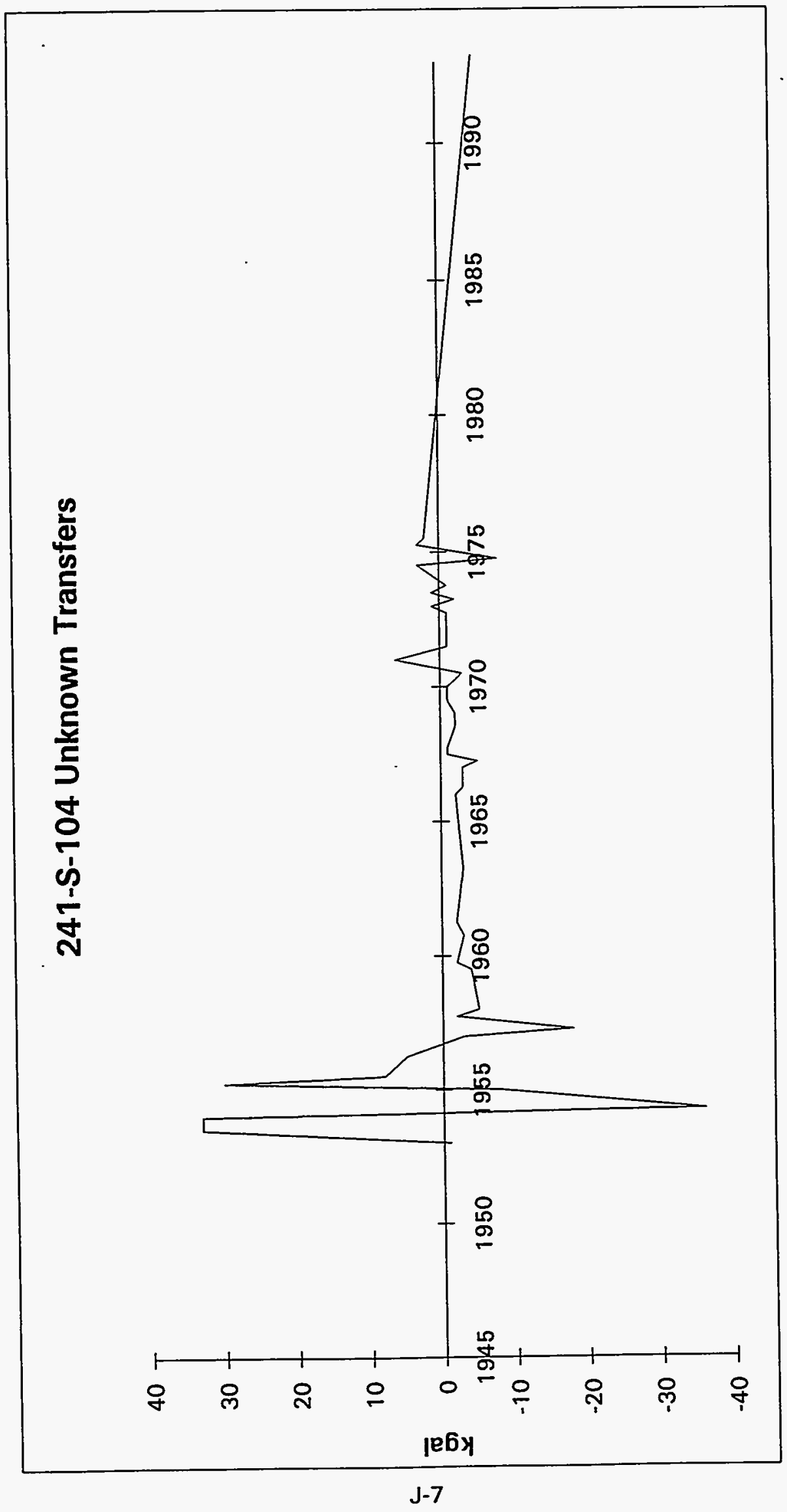

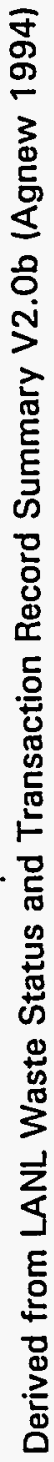


WHC-SD-WM-ER-323, Rev. 0

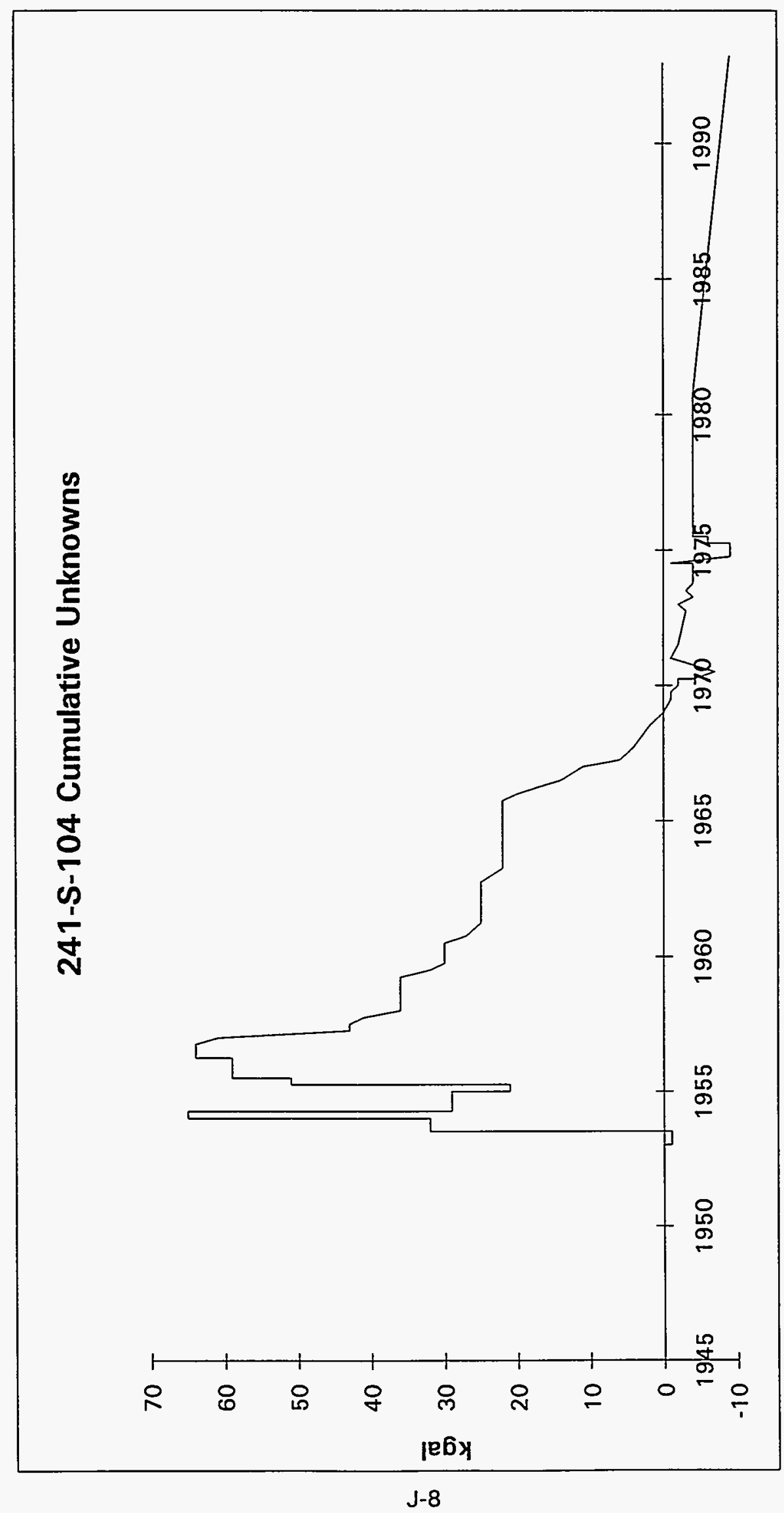

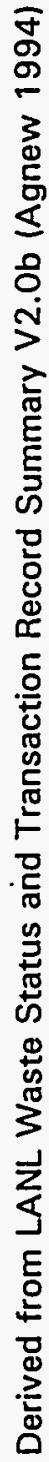


WHC-SD-WM-ER-323, Rev. 0

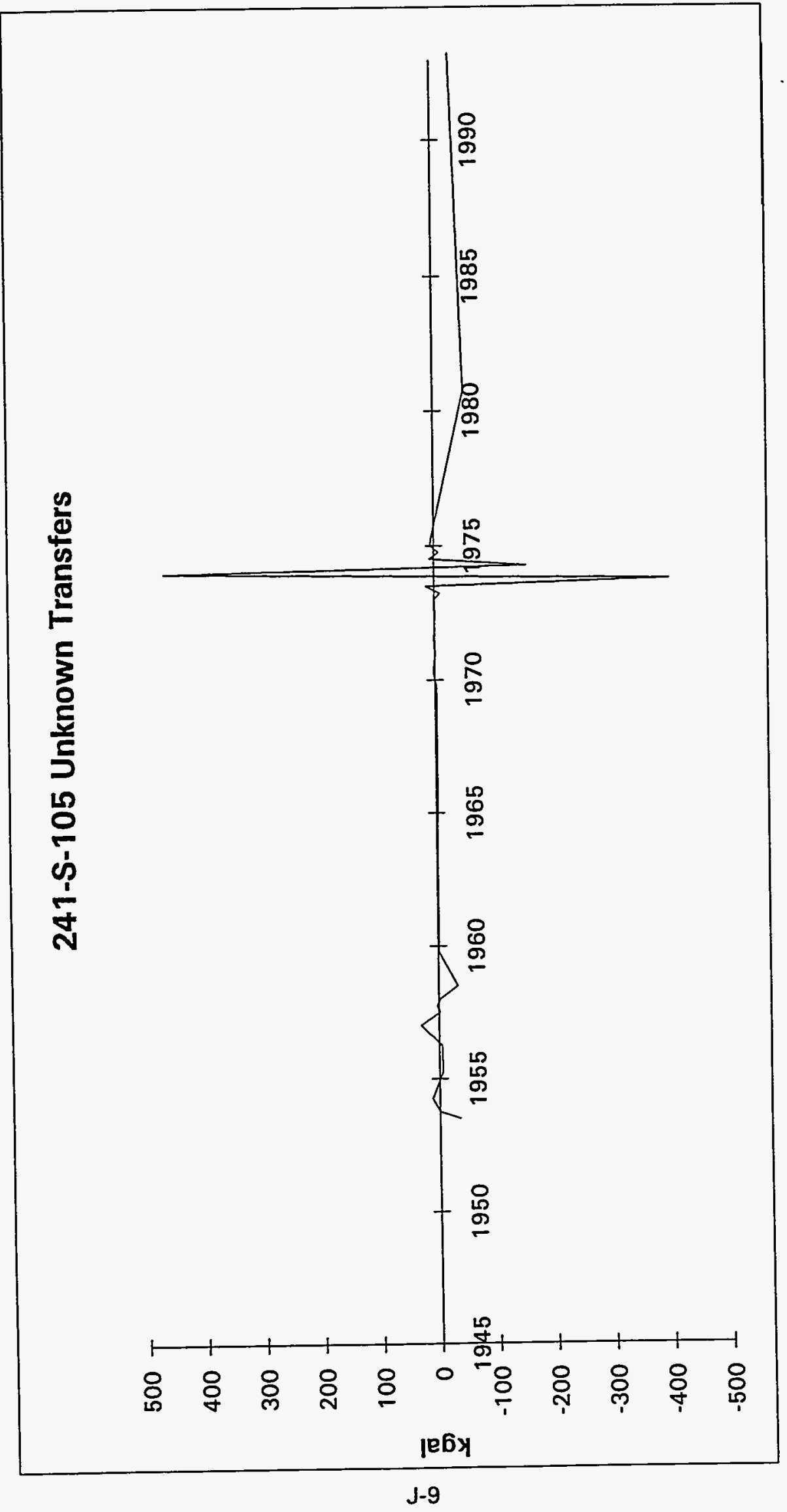

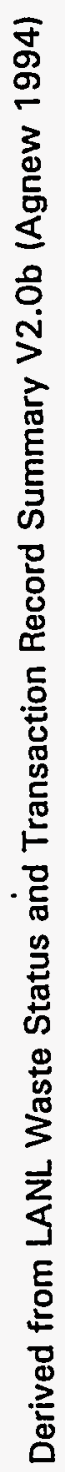


WHC-SD-WM-ER-323, Rev. 0

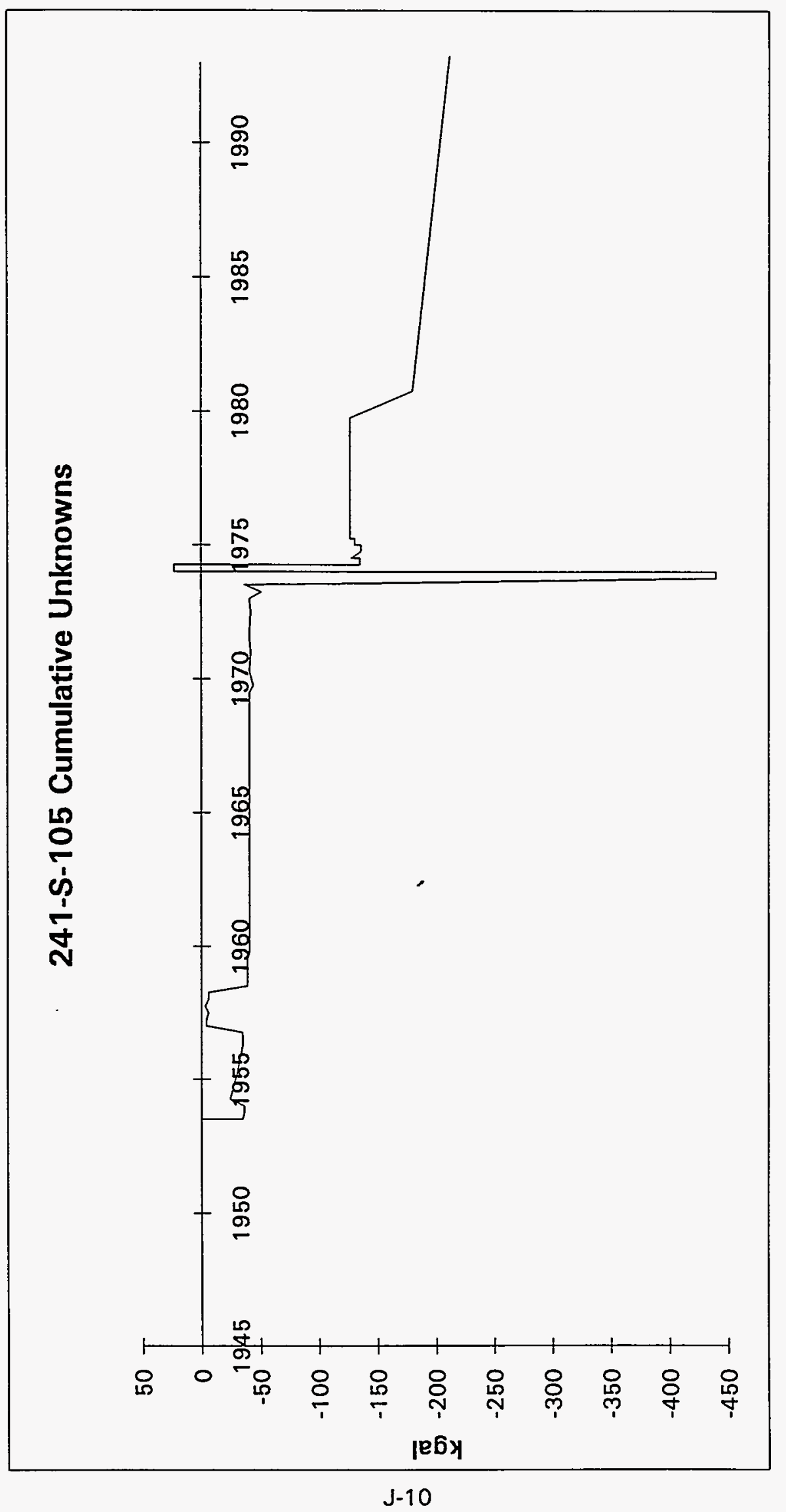

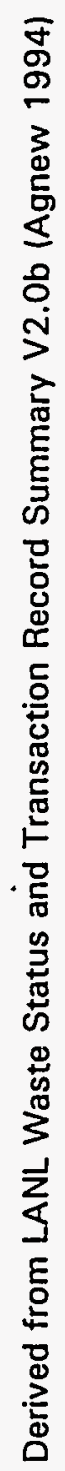


WHC-SD-WM-ER-323, Rev. 0

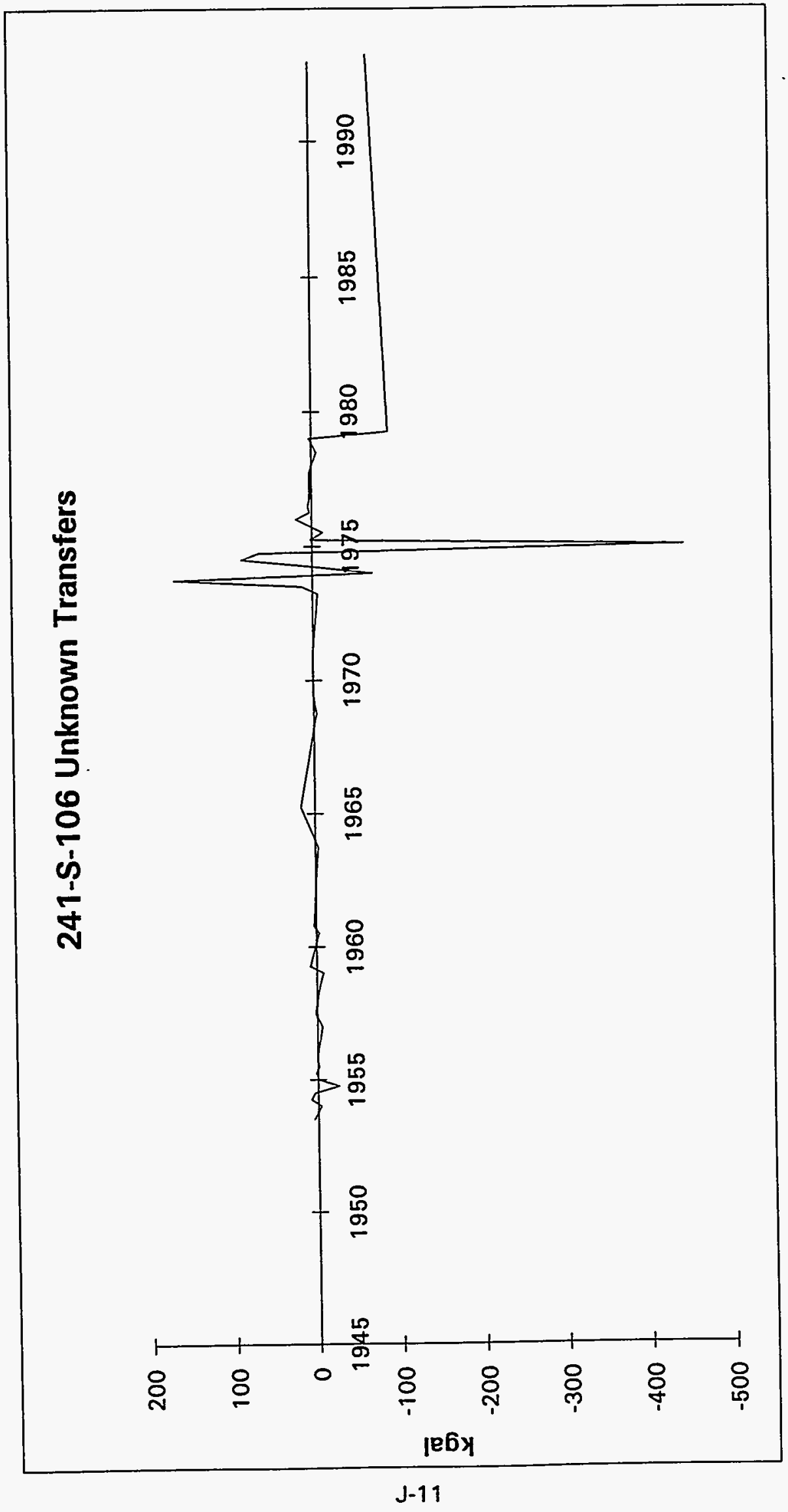

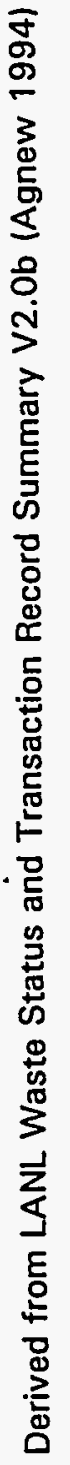


WHC-SD-WM-ER-323, Rev. 0

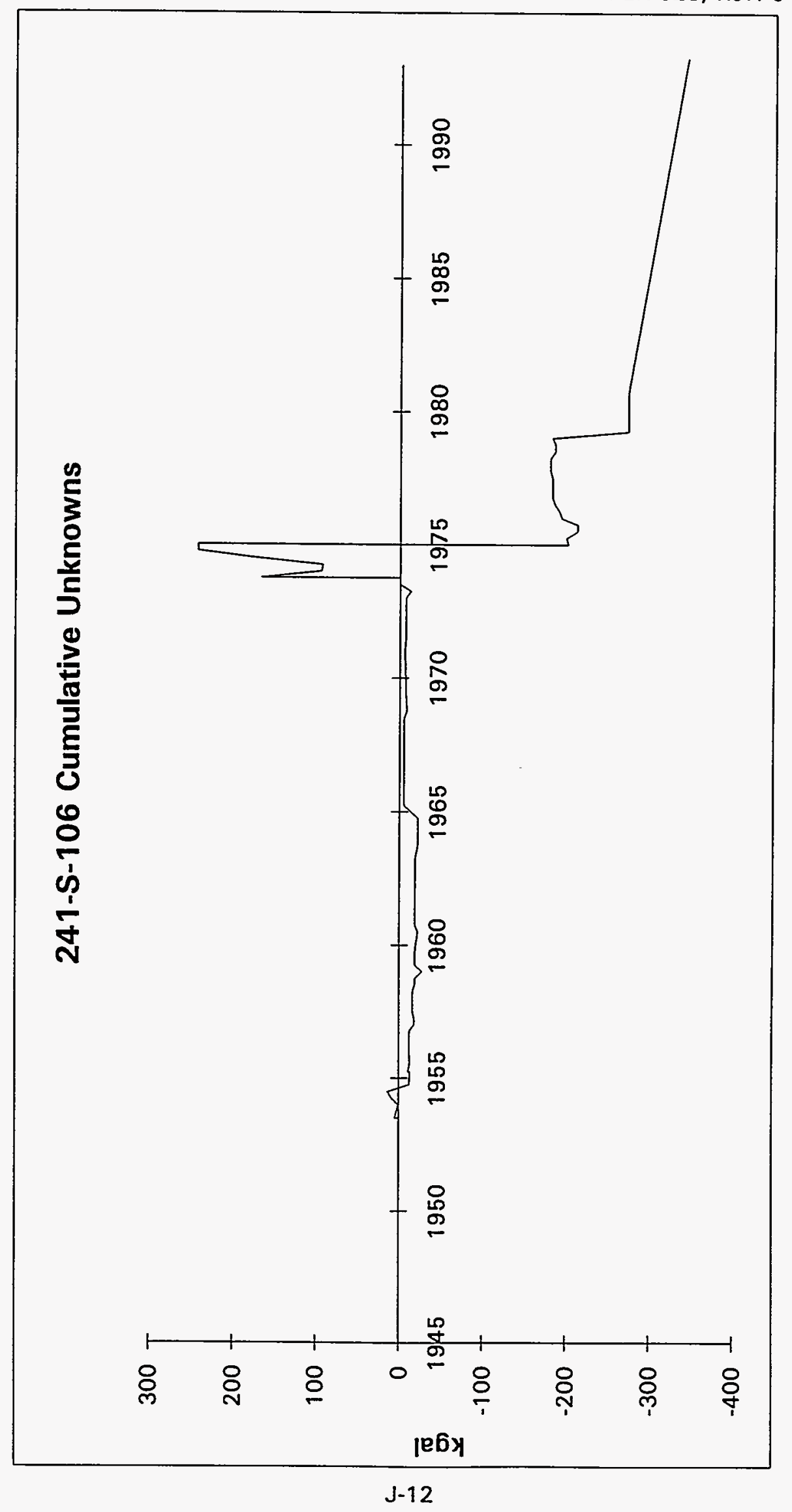

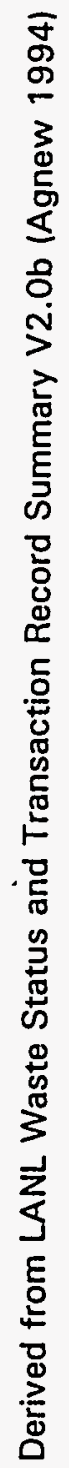


WHC-SD-WM-ER-323, Rev. 0

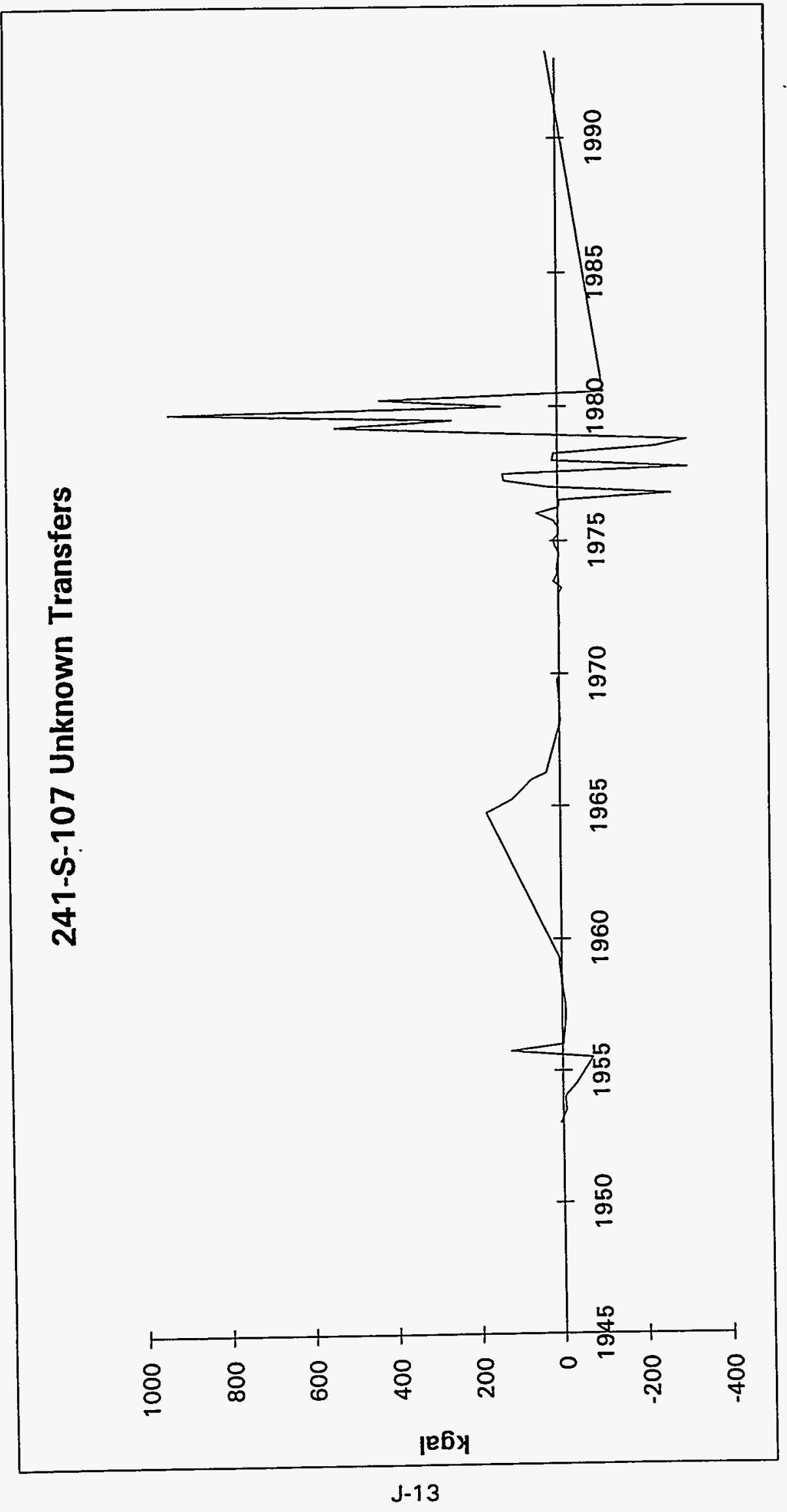

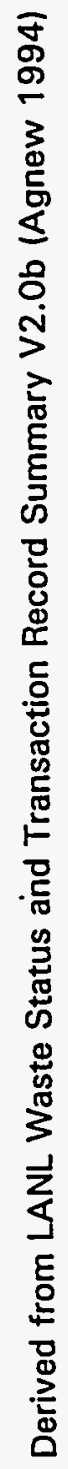




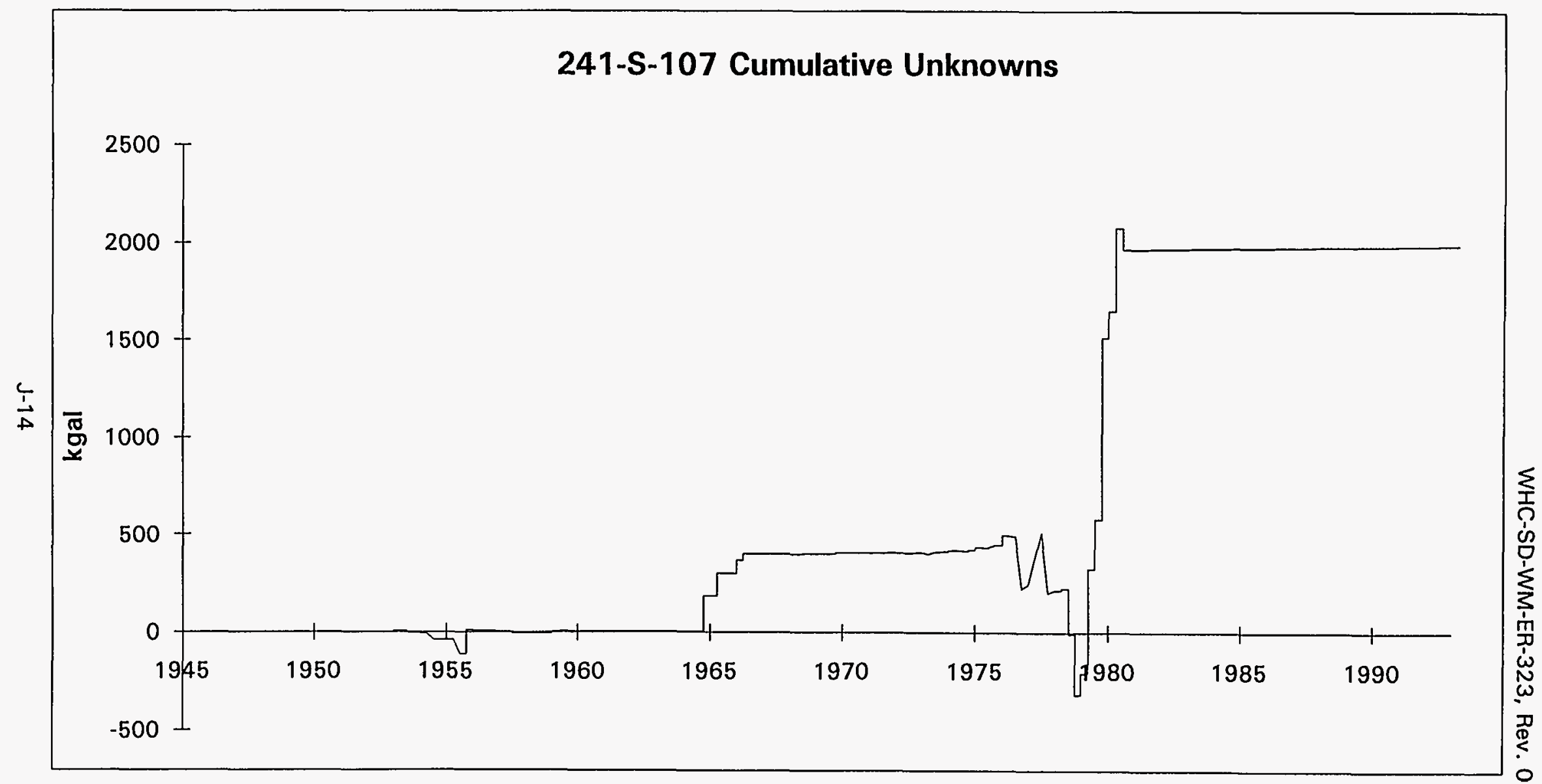

Derived from LANL Waste Status and Transaction Record Summary V2.0b (Agnew 1994) 
WHC-SD-WM-ER-323, Rev. 0

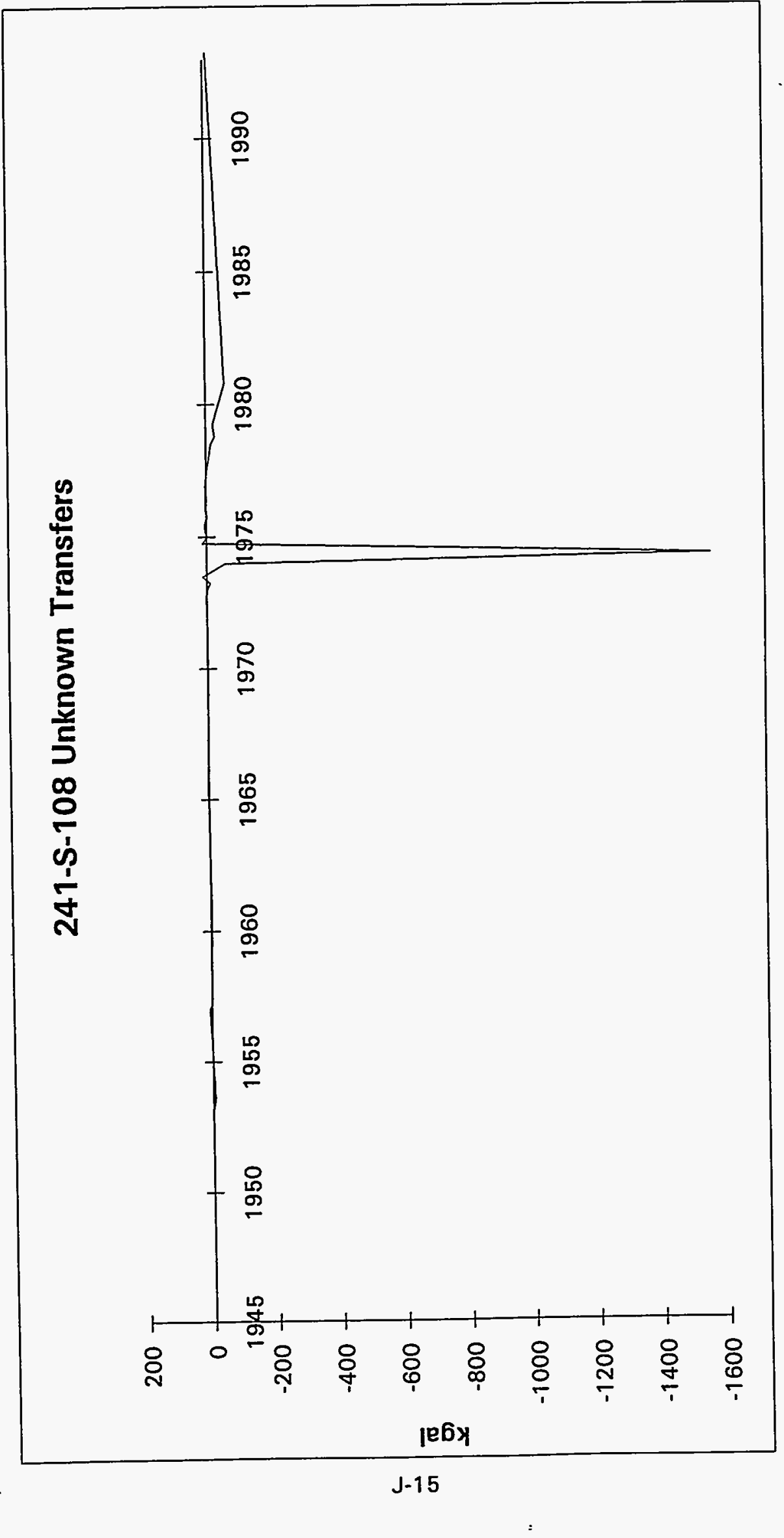

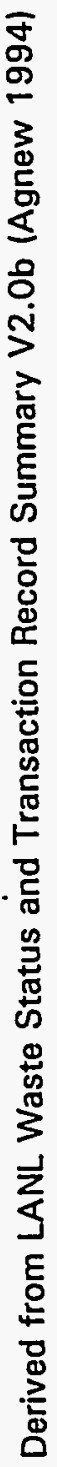


WHC-SD-WM-ER-323, Rev. 0

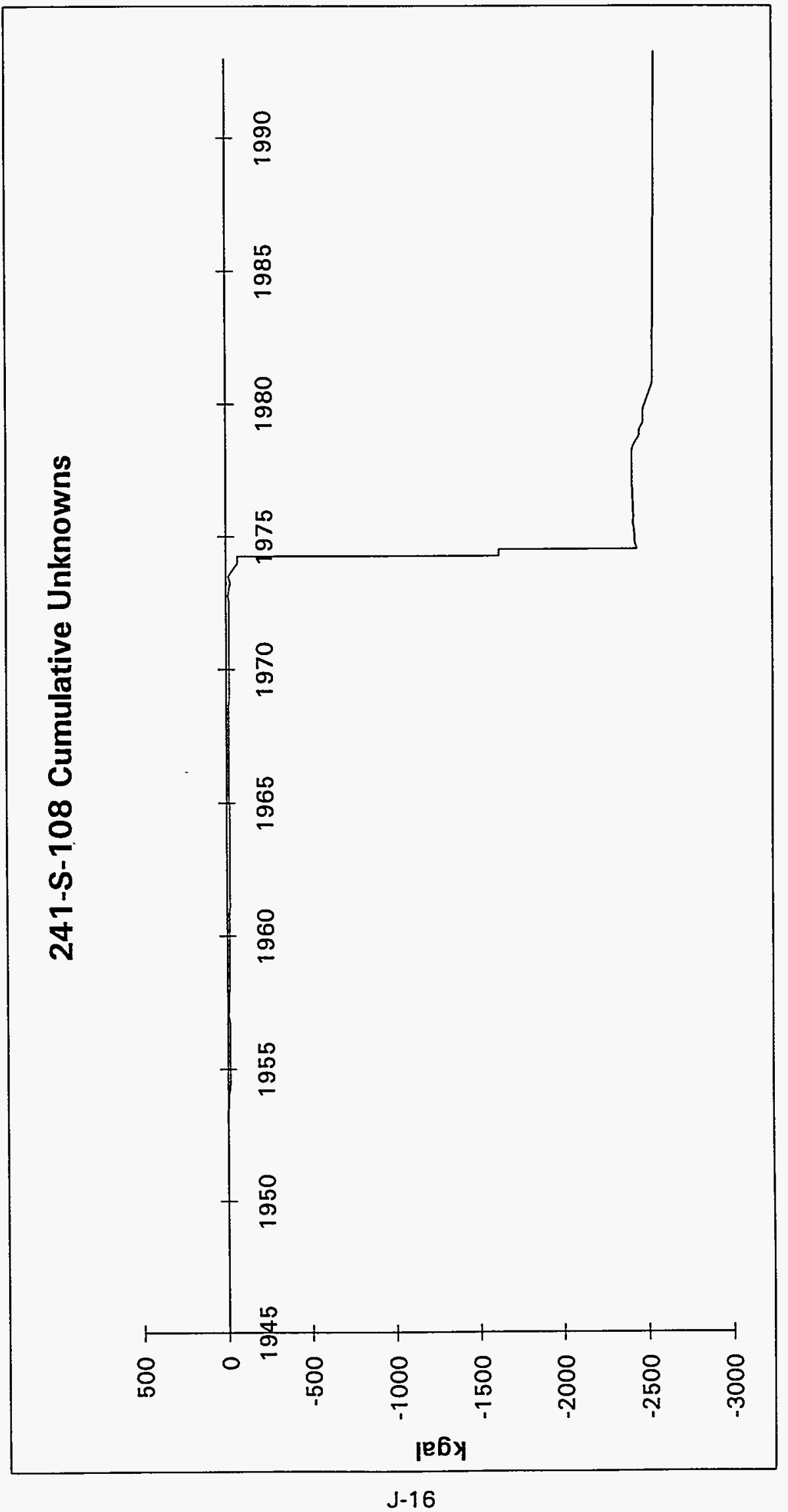

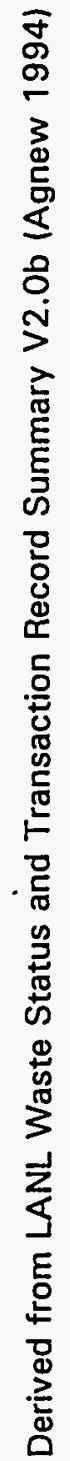


WHC-SD-WM-ER-323, Rev. 0

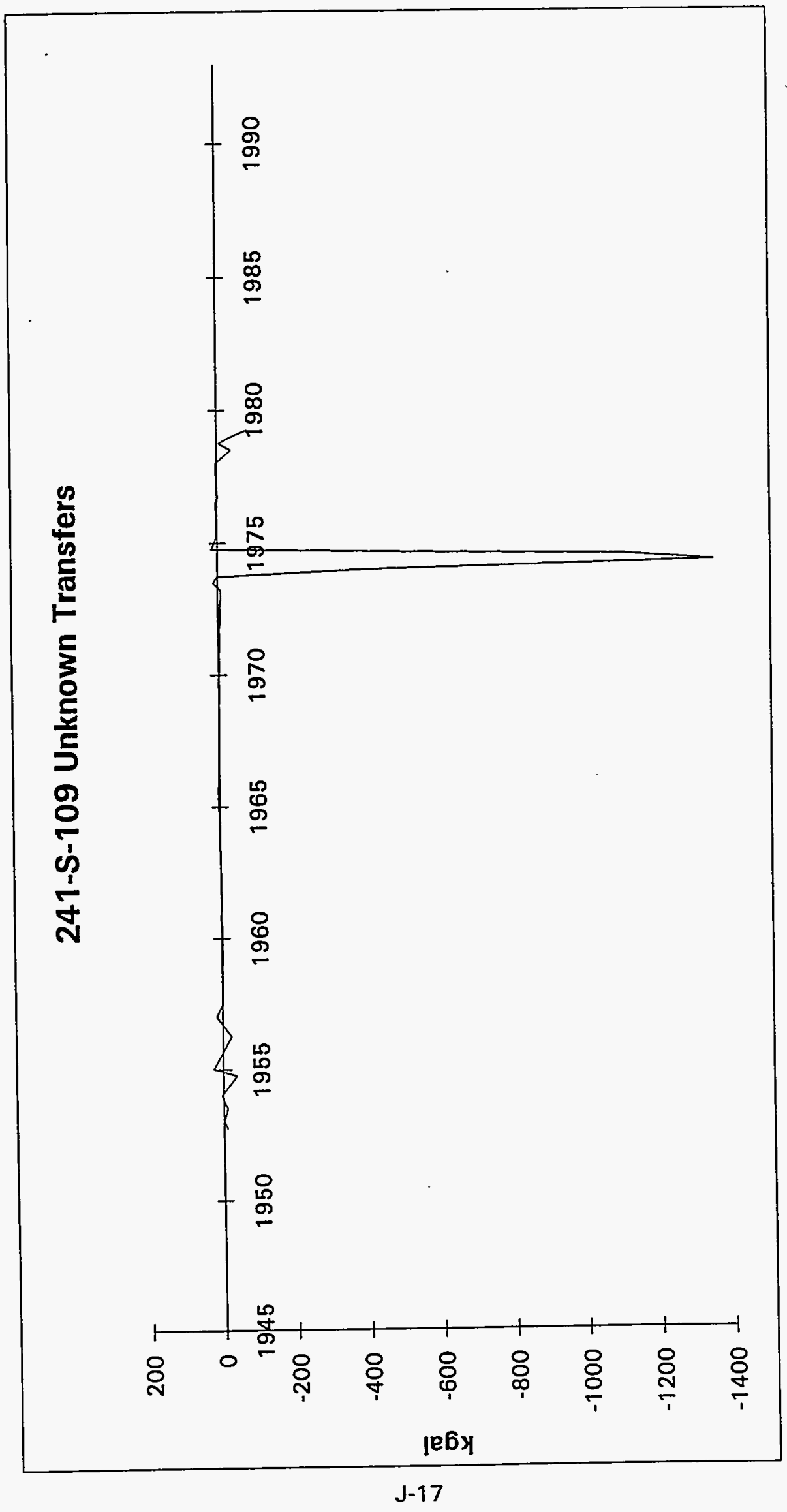

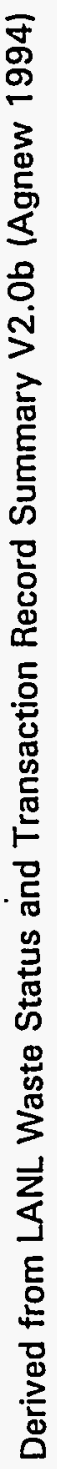




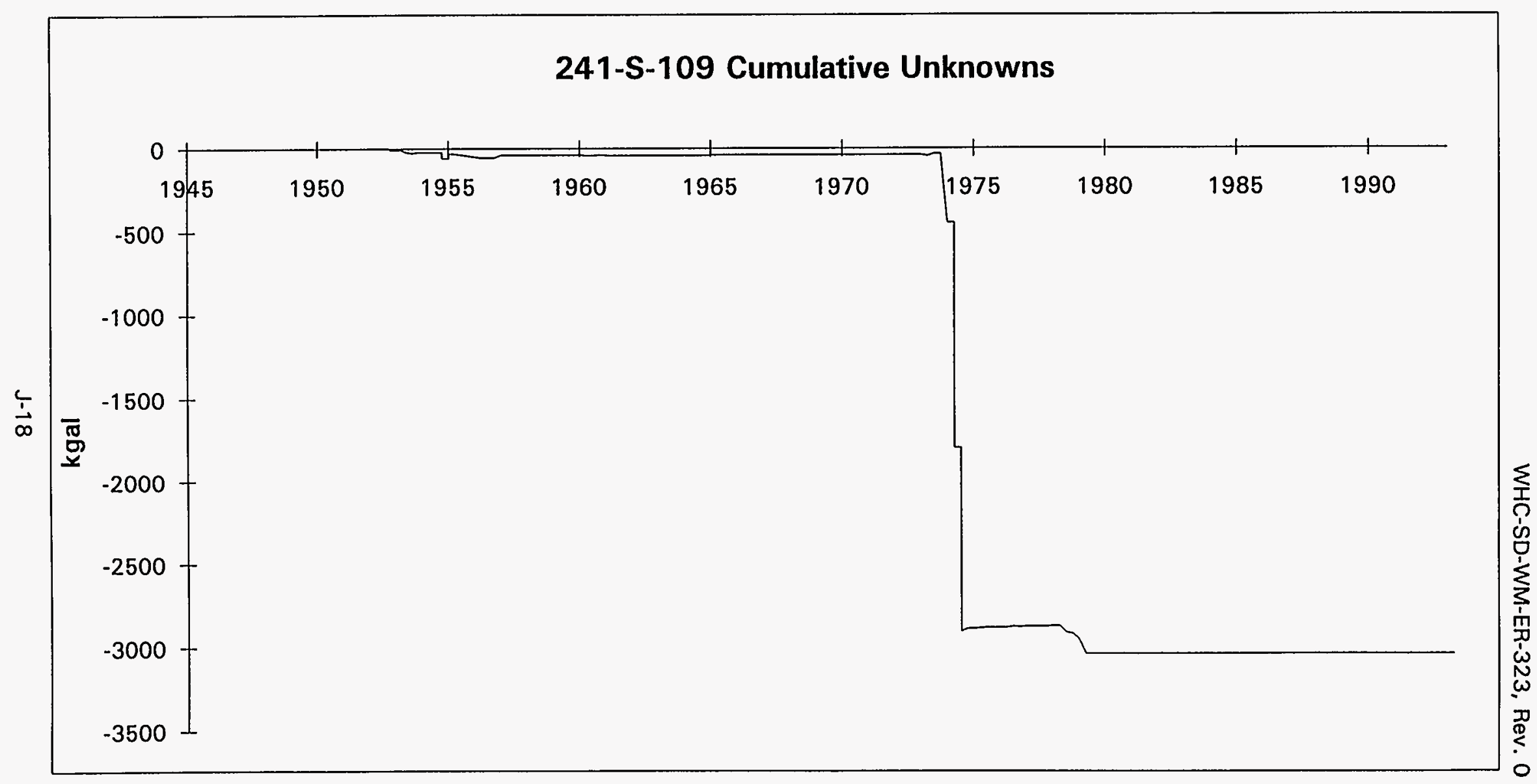

Derived from LANL Waste Status and Transaction Record Summary V2.0b (Agnew 1994) 


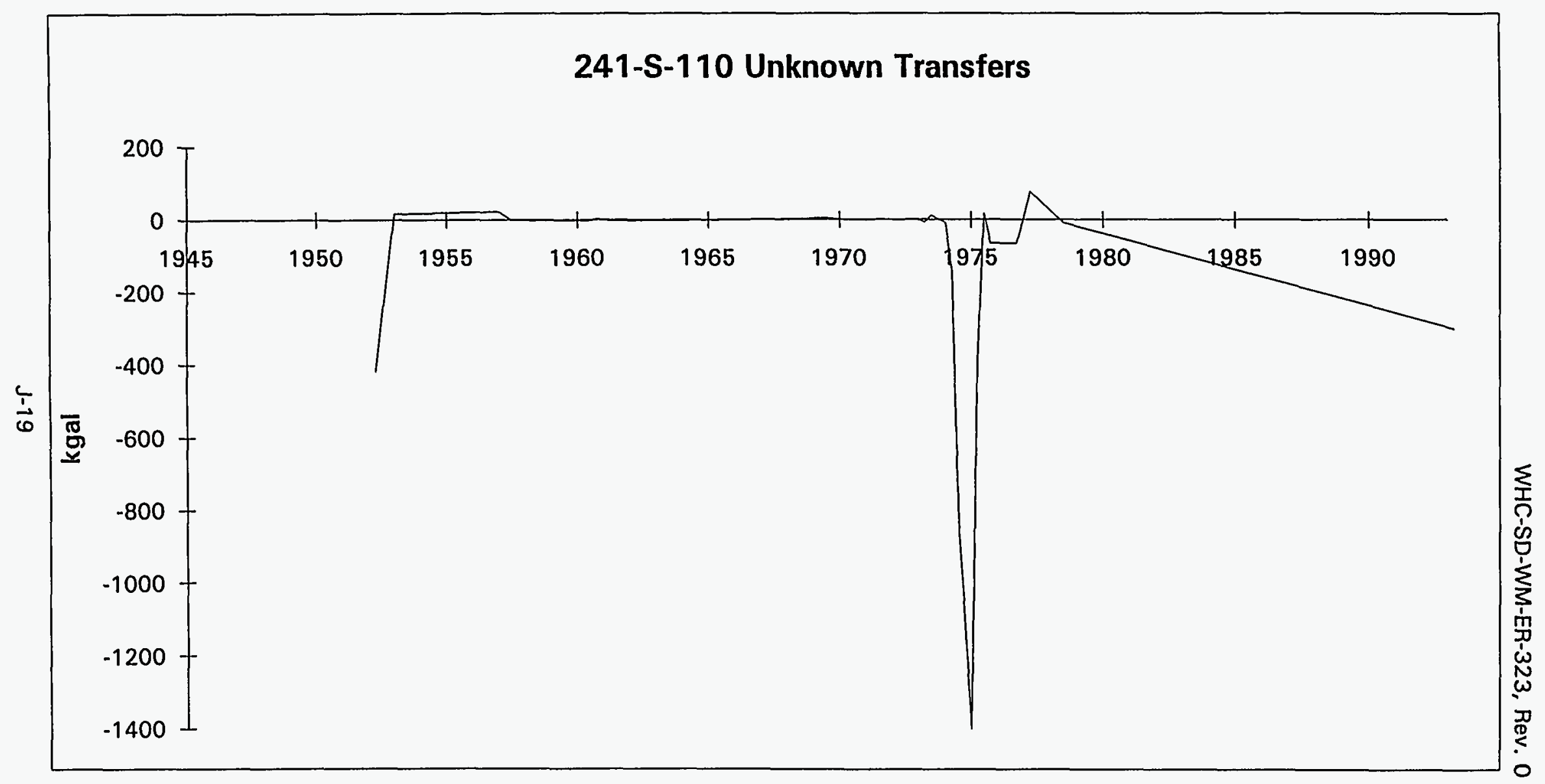

Derived from LANL Waste Status and Transaction Record Summary V2.0b (Agnew 1994) 
WHC-SD-WM-ER-323, Rev. 0

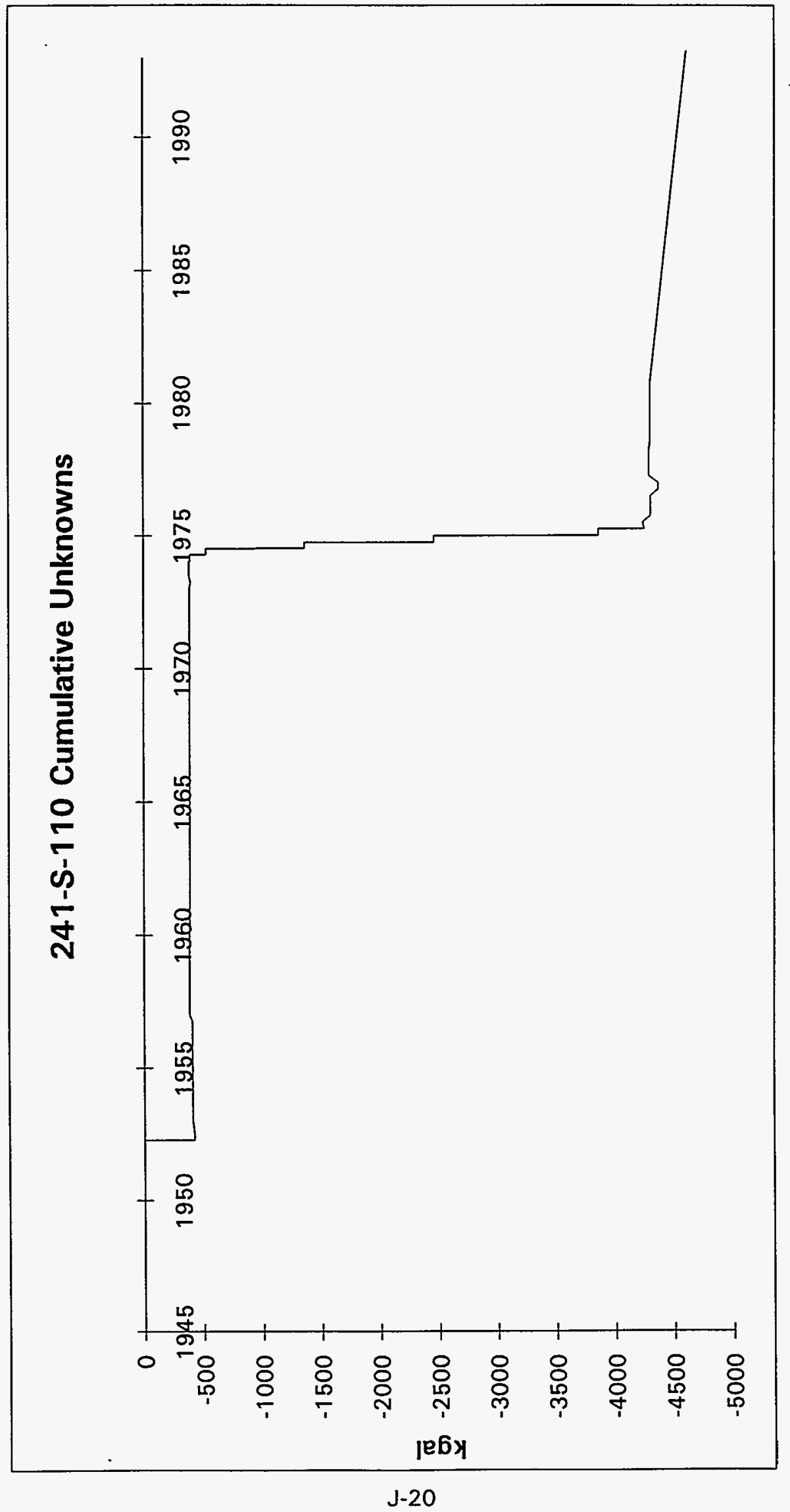

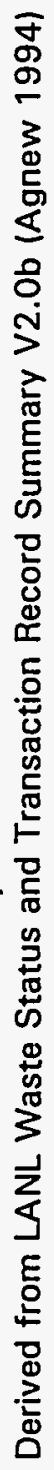


WHC-SD-WM-ER-323, Rev. 0

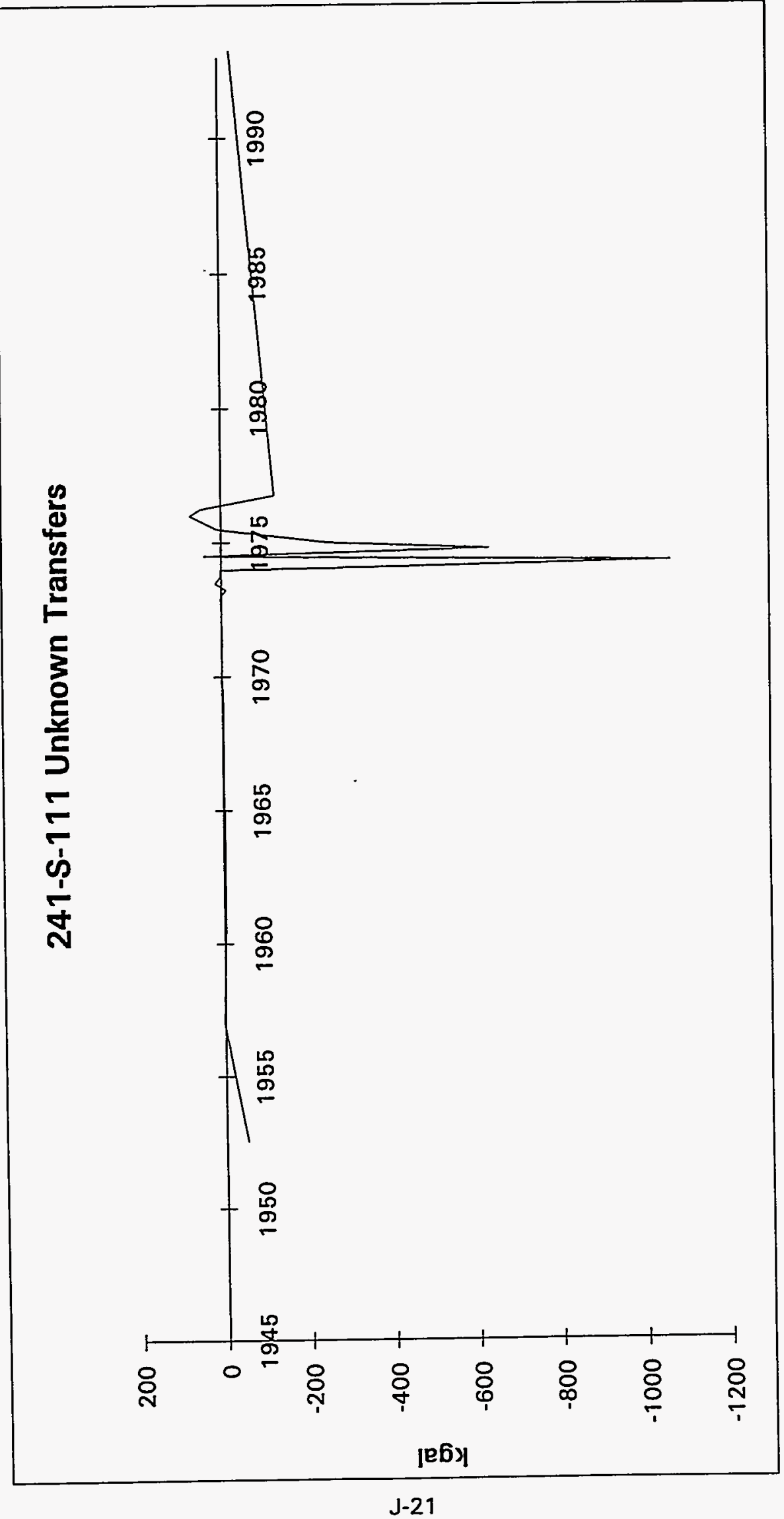

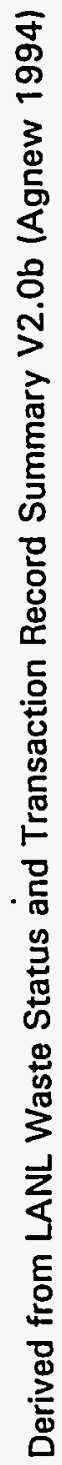


WHC-SD-WM-ER-323, Rev. 0

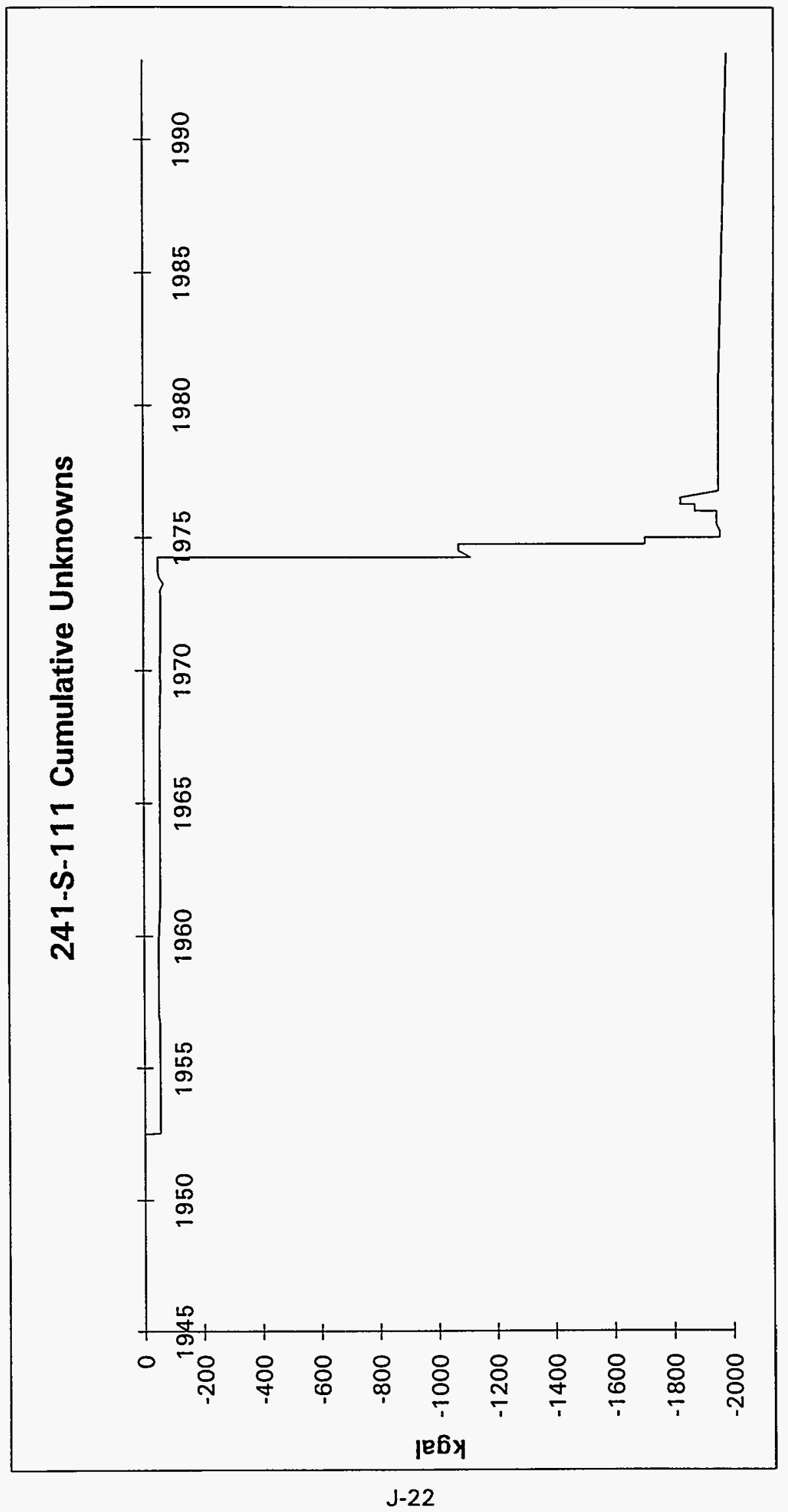

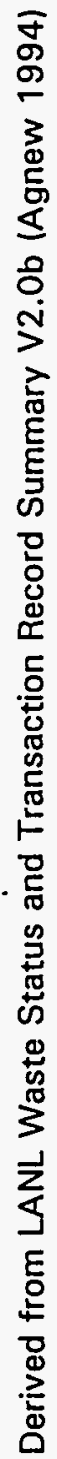




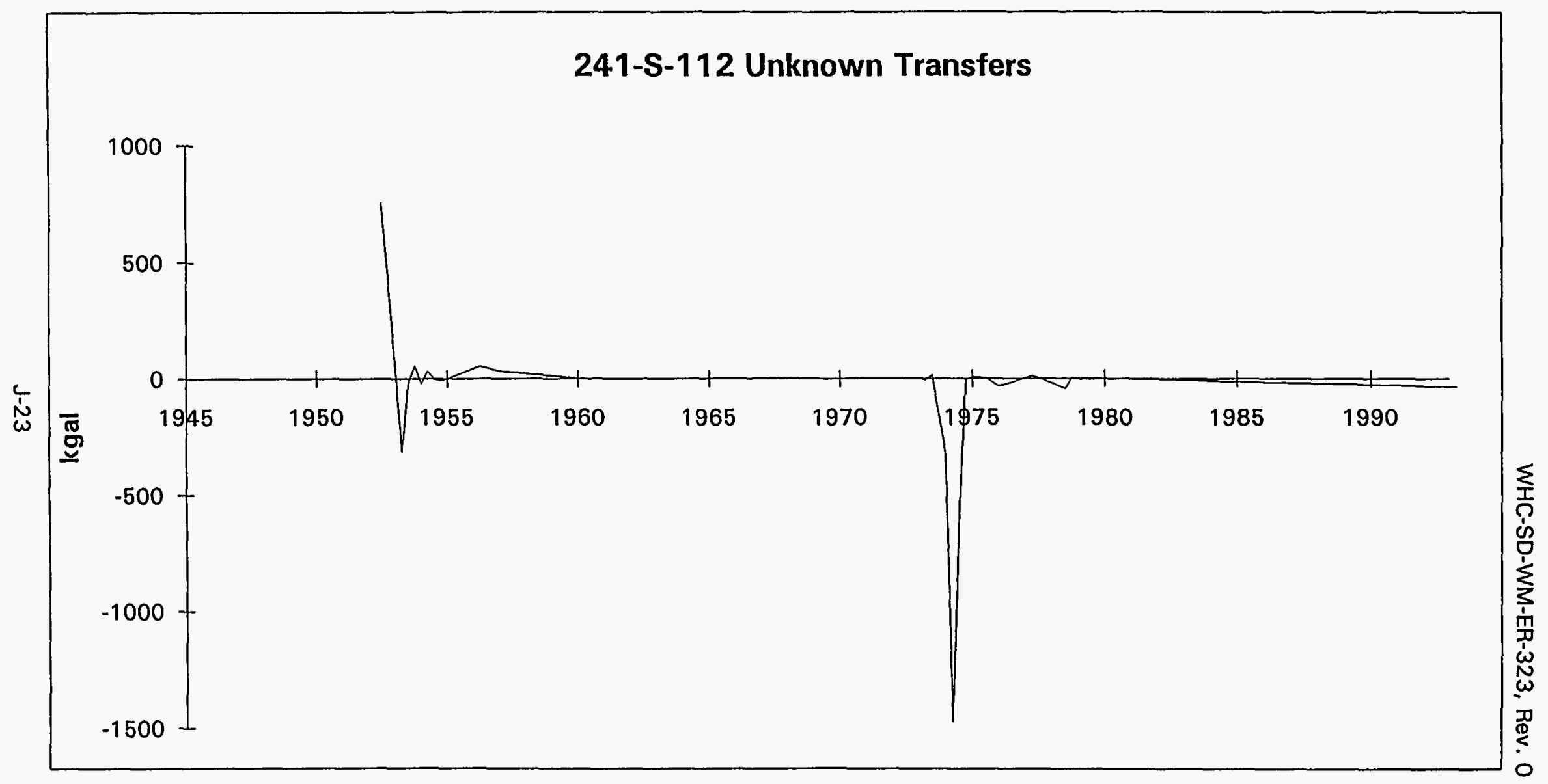

Derived from LANL Waste Status and Transaction Record Summary V2.0b (Agnew 1994) 
WHC-SD-WM-ER-323, Rev. 0

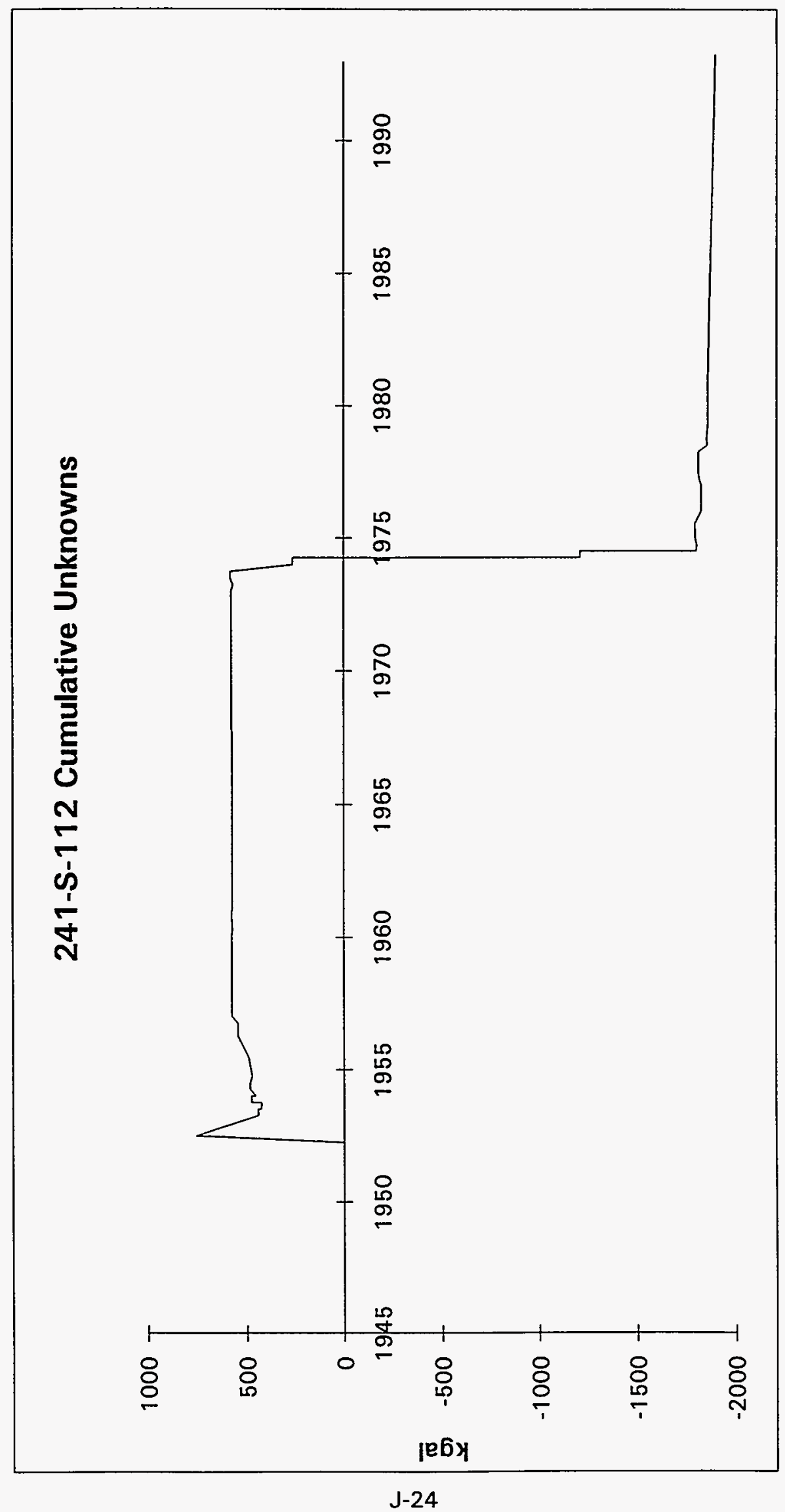

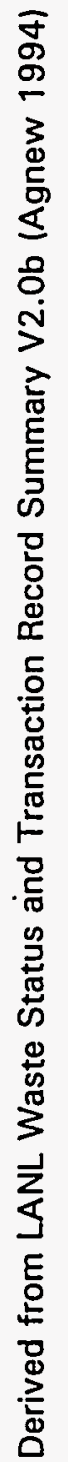




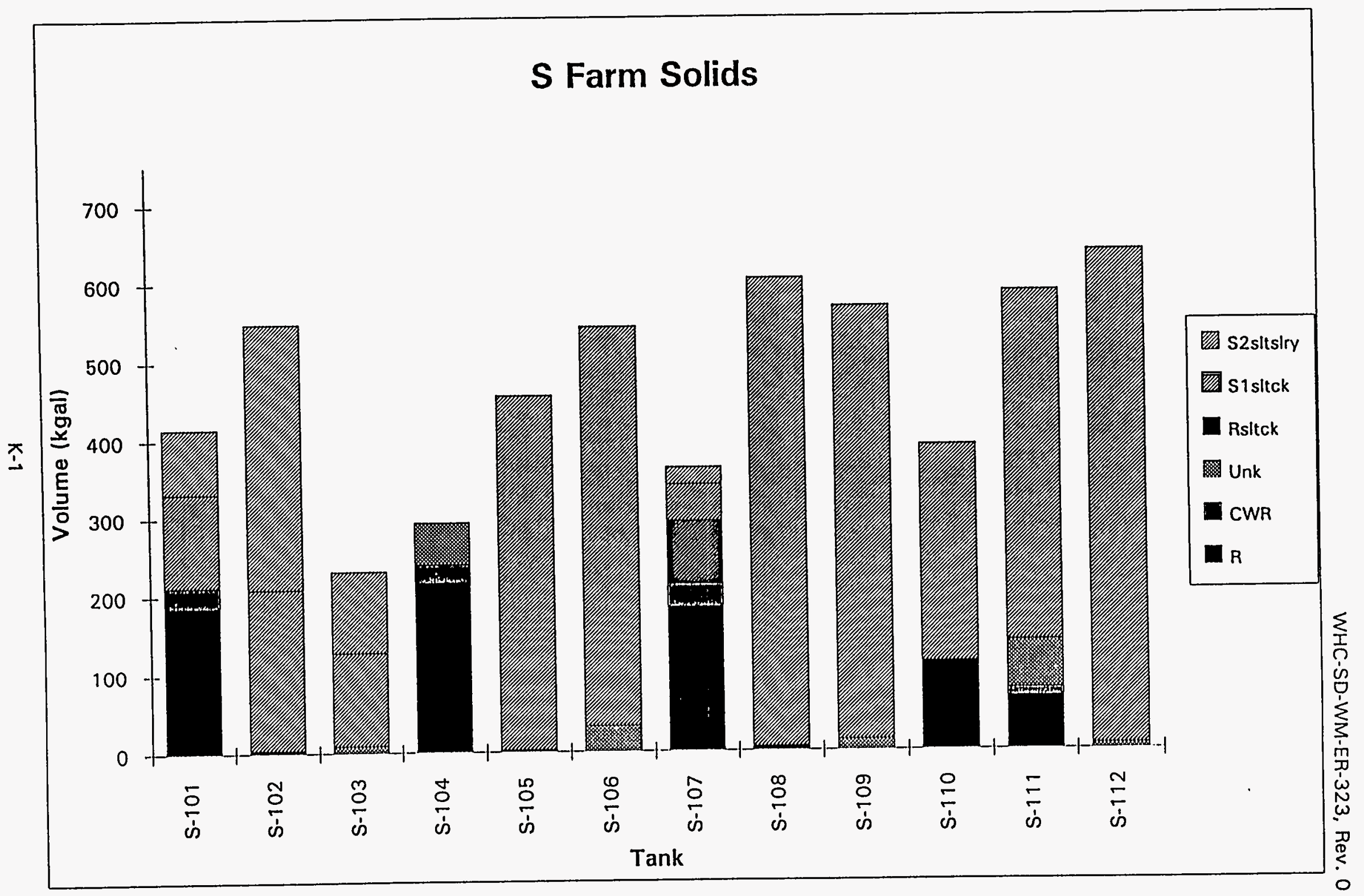

Derived from LANL Waste Status and Transaction Record Summary V2.0b (Agnew 1994) 


\begin{tabular}{|c|c|c|c|c|c|c|c|}
\hline Tank & Year & Qtr & $\begin{array}{l}\text { Meas. } \\
\text { Solids }\end{array}$ & $\begin{array}{c}\text { Solids } \\
\text { Change }\end{array}$ & $\begin{array}{c}\text { Predicted } \\
\text { Layer }\end{array}$ & $\begin{array}{l}\text { Layer } \\
\text { Type }\end{array}$ & $\begin{array}{l}\text { Waste } \\
\text { Volume } \\
\text { (K Gal) }\end{array}$ \\
\hline \multirow[t]{11}{*}{$S-101$} & 1954 & 1 & & 171 & 171 & $R$ & 1924 \\
\hline & 1954 & 1 & & 2 & 2 & CWRI & 21 \\
\hline & 1954 & 1 & & 2 & 2 & $R$ & 23 \\
\hline & 1954 & 2 & & 11 & 11 & CWRI & 131 \\
\hline & 1954 & 2 & & 12 & 12 & $R$ & 135 \\
\hline & 1955 & 3 & & 16 & 13 & CWRI & 197 \\
\hline & 1969 & 4 & 211 & -3 & & & \\
\hline & 1974 & 1 & 244 & & & & \\
\hline & 1975 & 2 & 332 & 121 & 121 & S1Sltck & \\
\hline & 1980 & 4 & 415 & 83 & 83 & S2SItsiry & \\
\hline & 1993 & 2 & 415 & & & & \\
\hline \multirow{10}{*}{$S-102$} & 1960 & & 5 & & & & \\
\hline & 1970 & 4 & 4 & 4 & & $R$ & \\
\hline & 1973 & 4 & 51 & & & & \\
\hline & 1974 & 3 & 103 & & & & \\
\hline & 1974 & 4 & 145 & & & & \\
\hline & 1975 & 4 & 200 & & & & \\
\hline & 1977 & 1 & 208 & 204 & 204 & S1SItck & \\
\hline & 1979 & 4 & 510 & & & & \\
\hline & 1980 & 3 & 555 & 347 & & & \\
\hline & 1993 & 2 & 549 & -6 & 341 & S2Sitsiry & \\
\hline \multirow{9}{*}{$S-103$} & 1954 & 2 & 0 & & & & \\
\hline & 1966 & 4 & 5 & & & & \\
\hline & 1969 & 4 & 9 & 9 & 9 & Unk & \\
\hline & 1978 & 2 & 120 & & & & \\
\hline & 1978 & 3 & 167 & & & & \\
\hline & 1978 & 4 & 128 & 119 & 119 & S1Sltck & \\
\hline & 1979 & 4 & 153 & & & & \\
\hline & 1980 & 4 & 231 & & 103 & S2SItsiry & \\
\hline & 1993 & 2 & 231 & 103 & & & \\
\hline & & & & & & & \\
\hline & & & & & & & \\
\hline & & & & & & & \\
\hline & & & & & & & \\
\hline & & & & & & & \\
\hline
\end{tabular}

Derived from LANL Waste Status and Transaction Record Summary V2.Ob (Agnew 1994) 
WHC-SD-WM-ER-323, Rev. 0

\begin{tabular}{|c|c|c|c|c|c|c|c|}
\hline Tank & Year & Qtr & $\begin{array}{l}\text { Meas. } \\
\text { Solids }\end{array}$ & $\begin{array}{l}\text { Solids } \\
\text { Change }\end{array}$ & $\begin{array}{c}\text { Predicted } \\
\text { Layer }\end{array}$ & $\begin{array}{l}\text { Layer } \\
\text { Type }\end{array}$ & $\begin{array}{l}\text { Waste } \\
\text { Volume } \\
\text { (K Gal) }\end{array}$ \\
\hline \multirow[t]{20}{*}{ S-104 } & 1953 & 1 & o & & & & \\
\hline & 1953 & 1 & & 2 & 2 & CWRI & 22 \\
\hline & 1953 & 1 & & 19 & 19 & $R$ & 212 \\
\hline & 1953 & 1 & & 3 & 3 & CWRI & 39 \\
\hline & 1953 & 1 & & 42 & 42 & $R$ & 469 \\
\hline & 1953 & 2 & & 3 & 3 & CWRI & 35 \\
\hline & 1953 & 2 & & 38 & 38 & $R$ & 423 \\
\hline & 1953 & 2 & & 3 & 3 & CWRI & 34 \\
\hline & 1953 & 2 & & 36 & 36 & $R$ & 409 \\
\hline & 1953 & 2 & & 2 & 2 & CWRI & 23 \\
\hline & 1953 & 2 & & 25 & 25 & $\mathrm{R}$ & 277 \\
\hline & 1953 & 3 & & 3 & 3 & CWRI & 40 \\
\hline & 1953 & 3 & & 23 & 23 & $\mathrm{R}$ & 258 \\
\hline & 1954 & 2 & & 15 & 15 & $\mathrm{R}$ & 163 \\
\hline & 1954 & 2 & & & 1 & CWRI & 8 \\
\hline & 1954 & 2 & & 17 & 17 & $R$ & 186 \\
\hline & 1955 & 3 & & 7 & 7 & CWRI & 83 \\
\hline & 1969 & 4 & 241 & 2 & 2 & UNK & \\
\hline & 1975 & 1 & 299 & 58 & & & \\
\hline & 1993 & 2 & 293 & -6 & 52 & UNK & \\
\hline \multirow{7}{*}{ S-105 } & & & & & & & \\
\hline & 1953 & 2 & 이 & & & & \\
\hline & 1969 & 4 & 2 & 2 & 2 & $R$ & \\
\hline & 1973 & 4 & 249 & & & & \\
\hline & 1974 & 1 & 549 & 547| & & & \\
\hline & 1980 & 4 & 488 & & & & \\
\hline & 1993 & 2 & 456 & -93 & 454 & S1Sltck & \\
\hline & & & & & & & \\
\hline & & & & & & & \\
\hline & & & & & & & \\
\hline & & & & & & & \\
\hline & & & & & & & \\
\hline & & & & & & & \\
\hline & & & & & & & \\
\hline & & & & & & & \\
\hline & & & & & & & \\
\hline & & & & & & & \\
\hline
\end{tabular}


WHC-SD-WM-ER-323, Rev. 0

\begin{tabular}{|c|c|c|c|c|c|c|c|}
\hline Tank & Year & Qtr & $\begin{array}{l}\text { Meas. } \\
\text { Solids }\end{array}$ & $\begin{array}{c}\text { Solids } \\
\text { Change } \\
\end{array}$ & $\begin{array}{l}\text { Predicted } \\
\text { Layer }\end{array}$ & $\begin{array}{l}\text { Layer } \\
\text { Type }\end{array}$ & $\begin{array}{l}\text { Waste } \\
\text { Volume } \\
\text { (K Gal) }\end{array}$ \\
\hline \multirow[t]{11}{*}{$S-106$} & 1953 & 2 & 0 & & & & \\
\hline & 1965 & 2 & 13 & 13 & 13 & UNK & \\
\hline & 1969 & 4 & 32 & 19 & 19 & UNK & \\
\hline & 1973 & 4 & 384 & & & & \\
\hline & 1974 & 1 & 436 & & & & \\
\hline & 1974 & 4 & 557 & & & & \\
\hline & 1975 & 1 & 607 & & & & \\
\hline & 1975 & 3 & 673 & 641 & & & \\
\hline & 1977 & 2 & 582 & & & & \\
\hline & 1980 & 4 & 612 & & & & \\
\hline & 1993 & 2 & 543 & -130 & 511 & S1sltck & \\
\hline \multirow[t]{18}{*}{ S-107 } & 1953 & 1 & 이 & 185 & 182 & $R$ & 2080 \\
\hline & 1953 & 1 & & -3 & & & \\
\hline & 1953 & 2 & & & & & \\
\hline & 1960 & 4 & & 89 & 12 & CWRI & 1108 \\
\hline & 1962 & 4 & & 19 & & CWRII & 433 \\
\hline & 1963 & 4 & & 10 & & CWRIII & 230 \\
\hline & 1964 & 4 & & 16 & & CWRIV & 361 \\
\hline & & & & -122 & & & \\
\hline & 1965 & 2 & 194 & & & & \\
\hline & 1967 & 1 & & 18 & 18 & CWRV & 763 \\
\hline & 1969 & 4 & 285 & & & & \\
\hline & 1970 & 2 & 293 & 81 & 81 & Rsltck & 923 \\
\hline & 1971 & 3 & & & & & \\
\hline & 1974 & 2 & 343 & 50 & & & \\
\hline & 1974 & 3 & 282 & & & & \\
\hline & 1974 & 4 & 343 & & & & \\
\hline & 1980 & 3 & 340 & -3 & 47 & S1 sltck & \\
\hline & 1993 & 2 & 362 & 22 & 22 & S2sitsiry & \\
\hline & & & & & & & \\
\hline & & & & & & & \\
\hline & & & & & & & \\
\hline & & & & & & & \\
\hline & & & & & & & \\
\hline & & & & & & & \\
\hline
\end{tabular}

Derived from LANL Waste Status and Transaction Record Summary V2.0b (Agnew 1994) 


\begin{tabular}{|c|c|c|c|c|c|c|c|}
\hline Tank & Year & Qtr & $\begin{array}{l}\text { Meas. } \\
\text { Solids }\end{array}$ & $\begin{array}{c}\text { Solids } \\
\text { Change } \\
\end{array}$ & $\begin{array}{c}\text { Predicted } \\
\text { Layer }\end{array}$ & $\begin{array}{l}\text { Layer } \\
\text { Type }\end{array}$ & $\begin{array}{l}\text { Waste } \\
\text { Volume } \\
\text { (K Gal) }\end{array}$ \\
\hline \multirow[t]{10}{*}{$S-108$} & 1952 & 4 & 0 & & & & \\
\hline & 1965 & 2 & 4 & & & & \\
\hline & 1969 & 4 & 5 & 5 & 5 & $\mathrm{R}$ & \\
\hline & 1974 & 1 & 373 & & & & \\
\hline & 1974 & 2 & 585 & & & & \\
\hline & 1974 & 3 & 702 & & & & \\
\hline & 1974 & 4 & 706 & & & & \\
\hline & 1977 & 1 & 670 & & & & \\
\hline & 1979 & 4 & 612 & & & & \\
\hline & 1993 & 2 & 604 & 599 & 599 & S1Sltck & \\
\hline & & & & & & & \\
\hline \multirow[t]{9}{*}{$S-109$} & 1953 & 2 & 0 & & & & \\
\hline & 1965 & 2 & 7 & & & & \\
\hline & 1971 & 3 & 13 & 13 & 13 & IUNK & \\
\hline & 1974 & 1 & 263 & & & & \\
\hline & 1974 & 2 & 488 & & & & \\
\hline & 1974 & 3 & 653 & & & & \\
\hline & 1977 & 1 & 568 & 555 & 555 & S1sltck & \\
\hline & 1993 & 2 & 568 & & & & \\
\hline & & & & & & & \\
\hline \multirow[t]{15}{*}{$S-110$} & 1952 & 3 & & 176 & & $R$ & 1983 \\
\hline & 1953 & 3 & & 12 & & CWR & 152 \\
\hline & 1954 & 1 & o & & & & \\
\hline & 1954 & 1 & & 3 & & $R$ & 39 \\
\hline & & & & & & & \\
\hline & 1965 & 2 & 106 & & & & \\
\hline & 1969 & 4 & 113 & -78 & 113 & R & \\
\hline & 1974 & 1 & 131 & & & & \\
\hline & 1974 & 4 & 255 & & & & \\
\hline & 1975 & 1 & 414 & & & & \\
\hline & 1975 & 3 & 755 & 642 & & & \\
\hline & 1975 & 4 & 689 & & & & \\
\hline & 1977 & 1 & 475 & -280 & & & \\
\hline & 1979 & 1 & 692 & 217 & & & \\
\hline & 1993 & 2 & 390 & -302 & 277 & S1sltck & \\
\hline & & & & & & & \\
\hline & & & & & & & \\
\hline
\end{tabular}

Derived from LANL Waste Status and Transaction Record Summary V2.0b (Agnew 1994) 
WHC-SD-WM-ER-323, Rev. 0

\begin{tabular}{|c|c|c|c|c|c|c|c|}
\hline Tank & Year & Qtr & $\begin{array}{l}\text { Meas. } \\
\text { Solids }\end{array}$ & $\begin{array}{c}\text { Solids } \\
\text { Change }\end{array}$ & $\begin{array}{l}\text { Predicted } \\
\text { Layer }\end{array}$ & $\begin{array}{l}\text { Layer } \\
\text { Type }\end{array}$ & $\begin{array}{l}\text { Waste } \\
\text { Volume } \\
\text { (K Gal) }\end{array}$ \\
\hline \multirow[t]{10}{*}{ S-111 } & 1952 & 3 & 0 & & & & \\
\hline & 1965 & 2 & 144 & & 63 & $R$ & \\
\hline & 1969 & 4 & 139 & 139 & 12 & CWRI & \\
\hline & 1974 & 2 & 334 & & 3 & $\mathrm{R}$ & \\
\hline & 1974 & 3 & 502 & & 61 & UNK & \\
\hline & 1974 & 4 & 563 & & & & \\
\hline & 1975 & 1 & 596 & & & & \\
\hline & 1975 & 3 & 750 & & & & \\
\hline & 1976 & 4 & 623 & & & & \\
\hline & 1993 & 2 & 586 & 447 & 447 & S1sltck & \\
\hline \multirow{12}{*}{$S-112$} & 1957 & 1 & 0 & & & & \\
\hline & 1965 & 2 & 4 & & 1 & UNK & \\
\hline & 1971 & 3 & 6 & 6 & 5 & UNK & \\
\hline & 1974 & 1 & 367 & & & & \\
\hline & 1974 & 2 & 598 & & & & \\
\hline & 1974 & 3 & 686 & & & & \\
\hline & 1974 & 4 & 673 & & & & \\
\hline & 1975 & 1 & 714 & & & & \\
\hline & 1976 & 2 & 706 & & & & \\
\hline & 1977 & 1 & 673 & & & & \\
\hline & 1980 & 2 & 672 & & & & \\
\hline & 1993 & 2 & 637 & 631 & 631 & S1sltck & \\
\hline
\end{tabular}

